K/ETO - 162

MARTIN MARUETTA

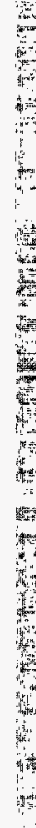

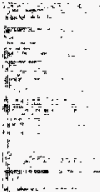

ign.

thend

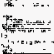

$=$

tis

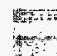

int

it

a.

.

,

in.

in

政=

the

to

tht

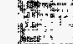

t.

焉

This document has been approved for releas top puplic by:

\section{Flow Boiling Test of GDP Replacement Coolants}

\author{
Compiled by
}

S. H. Park

Enrichment Technical Support Department

\author{
Uranium Enrichment Organization \\ Lockheed Martin Utility Services, Inc.
}




\section{DISCLAIMER}

This report was prepared as an account of work sponsored by an agency of the United States Government. Neither the United States Government nor any agency thereot, nor any of their empioyees, makes any warranty, express or implied, or assumes any legal liability or responsibility for the accuracy, completeness, or usefulness of any information, apparatus, product, or process disclosed, or represents that its use would not infringe privately owned rights. Reference herein to any specific commercial product, process or service by trade name. trademark. manufacturer, or otherwise, does not necessarily constitute or imply its endorsement, recommendation, or favoring by the United States Government or any agency thereof. The views and opinions of authors expressed herein do not necessarily state or reflect those of the United States Govemment or any agency thereof. 


\section{DISCLAIMER}

Portions of this document may be illegible in electronic image products. Images are produced from the best available original document. 


\section{Flow Boiling Test of GDP Replacement Coolants}

Compiled by

S. H. Park

Enrichment Technical Support Department

Prepared by

LOCKHEED MARTIN UTILITY SERVICES, INC.

Oak Ridge, Tennessee 37830

for the

UNITED STATES ENRICHMENT CORPORATION

under Contract No. USECHQ-93-C-0001 


\section{INTRODUCTION}

This report contains the works done by the Cudo Technologies, Ltd. in Lexington, Kentucky under the contract \#11K-NR559. The tests were a part of the CFC Replacement Program to identify and test alternate coolants to replace CFC-114 being used in the uranium enrichment processes at both Paducah, Kentucky and Portsmouth, Ohio. Two coolants identified as possible replacement coolants were $\mathrm{C}_{4} \mathrm{~F}_{10}$ and $\mathrm{C}_{4} \mathrm{~F}_{8}$ and tests were conducted to determine boiling heat transfer coefficients and pressure drop through a 10 foot test section which simulates an average length of the coolers used in the enrichment process. The study consisted of constructing a flow boiling test loop and conducting flow boiling tests with $\mathrm{CFC}-114, \mathrm{C}_{4} \mathrm{~F}_{10}, \mathrm{C}_{4} \mathrm{~F}_{8}$ and mixture of CFC -114 and $\mathrm{C}_{4} \mathrm{~F}_{10}$ at three different weight ratios. This report consists of the final reports from Cudo Technologies, Ltd. and is divided into four sections.
Section 1
Design of test apparatus and test criteria
Section 2
Results of tests with CFC-114, C4F10, C4F8
Section 3
Results of test with mixtures of CFC-114 and C4F10
Section 4
Test data

\section{SUMMARY}

The coolants tested, $\mathrm{C}_{4} \mathrm{~F}_{10}$ and $\mathrm{C}_{4} \mathrm{~F}_{8}$, were selected based on their compatibility with the uranium hexafluoride process gas and how well the boiling temperature and vapor pressure matched that of CFC-114. However, the heat of vaporization of both coolants is lower than that of CFC-114 requiring larger coolant mass flow than CFC-114 to remove the same amount of heat. The vapor pressure of these coolants is higher than $\mathrm{CFC}-114$ within the cascade operational range, and each coolant can be used as a replacement coolant with some limitation at 3,300 hp operation. The results of the CFC-114/ $\mathrm{C}_{4} \mathrm{~F}_{10}$ mixture tests show boiling heat transfer coefficient degraded to a minimum value with about $25 \% \mathrm{C}_{4} \mathrm{~F}_{10}$ weight mixture in $\mathrm{CFC}-114$ and the degree of degradation is about $20 \%$ from that of CFC-114 boiling heat transfer coefficient. 


\section{Section 1}

Design, Construction, and Testing of an Experiment to Measure the Flow Boiling Heat Transfer and Pressure Drop in a Vertical Tube 


\section{Flow and Pool Boiling Heat Transfer Testing of GDP Replacement Coolants}

Enrichment Technical Support Department

Uranium Enrichment Organization Martin Marietta Utility Services, Inc. 


\section{Flow and Pool Boiling Heat Transfer Testing of GDP Replacement Coolants}

Enrichment Technical Support

May 1995

Prepared by the

MARTIN MARIETTA UTILITY SERVICES, INC.

Oak Ridge, Tennessee 37830

for the

UNITED STATES ENRICHMENT CORPORATION

under contract No. USECHQ-93-C-0001 
Design, Construction and Testing of an Experiment to Measure the Flow Boiling Heat Transfer and Pressure Drop in a vertical Tube

Cudo Technologies, Ltd.

P.O. Box 185, Lexington, KY 40584

Contract \# $11 \mathrm{~K}-\mathrm{NR} 559$

Report \# MMES-CTL-NR559-1

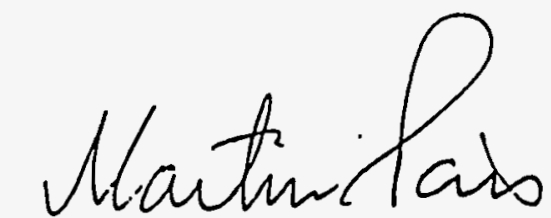

Martin R. Pais, PhD

Principal Investigator

ming \&. Choung

Ming Jr Chang, PhD

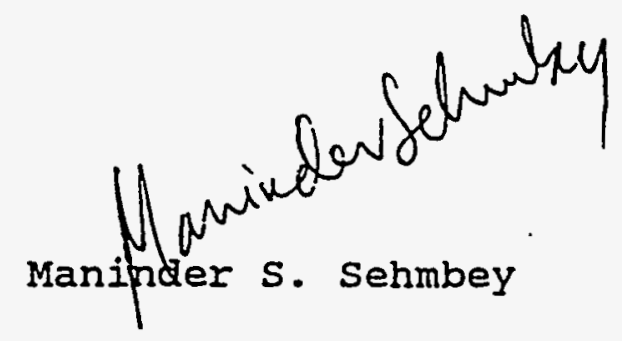


Design, Construction and Testing of an Experiment to Measure the Flow Boiling Heat Transfer and Pressure Drop in a Vertical Tube

by

Cudo Technologies, Itd.

P.O. Box 185, Lexington, KY 40584

Contract \# 11K-NR559

Report \# MMES-CTL-NR559-1

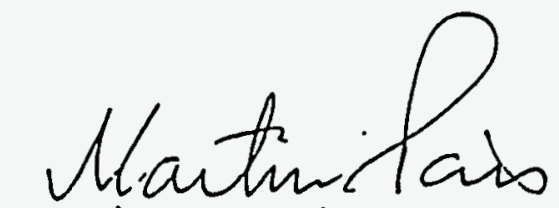

Martin R. Pais, PhD

Principal Investigator

ring \&. Cliang

Ming Jr Chang, PhD

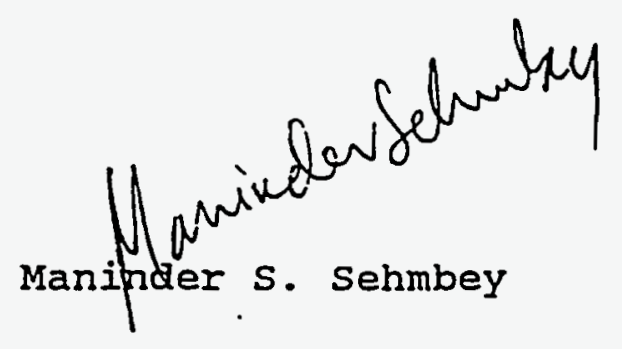




\section{TABLE OF CONTENTS}

Page

NOMENCLATURE

ABSTRACT

INTRODUCTION

4

TECHNICAL OBJECTIVES

TECHNICAL APPROACH

Theory

Effect of Roughness

Correlations

DESIGN OF FLOW BOILING EXPERIMENTAL SETUP 9

Experimental Arrangement 9

1) Storage Tank 10

2) Pumps 10

3) Flow straightener 10

4) Instrumented Vertical Tube 11

5) Heaters, Power Supply, and Power Measurement Systems 11

6) Temperature Measurement and Control systems 12

7) Pressure Measurement System 13

8) Flow Visualization Section 14

9) Vapor-liquid Separator 14

10) Condenser 14

11) Flow Meters 14

12) Valves 15

13) Level Gauges 15

14) Chiller system 16

Roughness Measurement 16

$\begin{array}{ll}\text { SYSTEM OPERATION } & 17\end{array}$

cleaning and charging the system 17

Calibration $\quad 18$

Differential and Absolute Pressure Transducers 18

Turbine Flow Meters $\quad 18$

Heat Input $\quad$ - 19

Heat Loss $r$

Temperature $\quad 20$

Experimental Procedure $\quad 21$

CONCLUSIONS $\quad 23$

ACKNOWLEDGEMENTS $\quad 24$

REFERENCES $\quad 24$ 
Nomenclature

C Specific heat

D Diameter

$G$ Mass flux

h Heat-transfer coefficient

$i$ Latent heat of vaporization

I current

k Thermal conductivity

I Length of tube

m Mass flow rate

n Constant

P Pressure

Pr Prandtl number

$q$ Heat flux

$Q$ Heat input

$\mathrm{R}$ Roughness

Re Reynolds number

$S$ Correction factor

T Temperature

$\checkmark$ Voltage

$x$ Quality of vapor

$z$ Location in vertical direction

Superscripts

$a-i$ Constants

A Area

B Constant

" Heat flux indicator

subscripts

a,q Roughness descriptive

c Convective

co Coolant

f Liquid/fluid

fg of vaporization

g Gas/vapor

m mean

nb Nucleate boiling

$p$ Constant pressure

sat Saturated

sub Sub-cooled

w Wall

Greek

$\mu$ Kinematic viscosity

$\Delta \quad$ Difference

$\rho$ Density

$\sigma$ Surface tension 
This study involves the design, construction and calibration of a flow boiling heat transfer measurement system to study flow boiling from the internal surface of a vertical cylindrical tube. The system is designed to operate over a wide saturation temperature range of $50^{\circ} \mathrm{F}$ to $250^{\circ} \mathrm{F}$, pressure range of 14 to 350 psig, a heat flux range of 0 to $60,000 \mathrm{btu} / \mathrm{hr} . \mathrm{ft}^{2}$ and a mass flux of 0 to $1.4 \mathrm{e} 6 \mathrm{lbm} / \mathrm{hr}$. $\mathrm{ft}^{2}$. Optical access for flow visualization is incorporated into the design. The test section is a 10 foot long, 0.78 " ID instrumented copper tube divided into 10 zones in the longitudinal direction. Facilities to measure temperature, pressure and flow rate within each zone and at various locations in the system are provided. 


\section{INTRODUCTION}

In several proposed cooling applications, the use of fully fluorinated carbon compounds is being seriously considered. This is because the common refrigerants in use today (chlorofluorocarbons, CFCs) have been linked to the depletion of ozone in the stratosphere. The current world plan ironed out by the Montreal Protocol calls for a total ban of all CFCs by the year 2000. The USA has unilaterally called for a ban on all CFCs by 1995. This demands that replacement refrigerant fluids be sought and tested which will not only satisfy the tougher environmental standards but also, more importantly, provide satisfactory cooling capacities within the current existing installations.

Two fluids have been selected as promising replacements for CFC-114 in the uranium enrichment facilities. They are perfluorobutane $C_{4} F_{10}$ and perfluorocyclobutane $C_{4} F_{8}$. These fluids:

(9) are very inert,

( ) have thermophysical properties that are comparable to the currently used refrigerants, and

* can be manufactured with currently available technology.

If the two coolants stated above can be used in the heat exchangers directly as replacements, then:

( ) a new cooling system will not have to be designed and built.

- large savings in new installation costs will be realized.

( ) time will be saved in switch-over to the new coolant.

The proposed effort has a direct relationship with the cooling of uranium-enrichment-plant heat exchanger systems. It is also of interest in the cooling of high-power electronic chips for terrestrial applications and in the refrigeration industry.

Despite the significant advantages of using current installations, the direct use of such replacement fluorocarbons may have some drawbacks that must be checked. The heat transfer capability of the fluid is dependent on a number of parameters [1-4]. Depending on the operating temperatures, pressures and heat loads envisaged, conditions within the heat exchanger system can be reached which can be deleterious to the process.

To date, an extensive Iiterature search fails to reveal any information on the heat transfer characteristics of perfluorobutane and perfluorocyclobutane. Experimentally correlated constitutive equations for boiling and two-phase phenomena appropriate to these particular fluids do not currently exist. Some flow boiling studies have been found [5-11] which have enabled us to estimate the heat transfer rate for the fluids in question. However, these estimates must be certified. Knowledge of the conditions and heat transfer rates is required for the safe design and operation of 
systems cooled by pressurized or boiling liquid coolants. The critical heat flux (CHF) also is a major parameter in the design of units cooled by forced convective boiling for transient and accidental analysis.

Most refrigerants of the fluoro/chloro-carbon series have excellent wetting properties, enhancing evaporation and condensation. However, higher wetting also increases the superheat temperatures for the onset of boiling, these temperature overshoots can sometimes be as high as $55^{\circ} \mathrm{F}$ [1]. The contact angle plays an important part in the nucleation process [12-14]. Specifically, the dynamic contact angle is a strong superheat controlling factor. Therefore, depending on the wetting characteristics of these liquids under various conditions imposed by the thermal and fluid fields and the surface roughness [15-19], situations can arise which are detrimental to the operation.

\section{TECHNICAL OBJECTIVES}

The overall objective of this proposed study is to develop a method of measuring the heat-transfer coefficient and pressure drop on the internal surface of a cylindrical pipe under conditions of uniform heat flux in flow boiling in a vertical orientation with respect to gravity.

The overall objective of the total research effort is to:

A. Design and build an experimental setup to:

1. Measure the boiling heat-transfer coefficient on the internal surface of a tube under conditions of:

a) flow and free convective boiling,

b) different angles of inclination with respect to gravity,

c) uniform heat flux, and

d) different surface roughness conditions.

2. Measure the two-phase flow pressure drop.

3. Measure the critical heat flux.

4. Measure the surface temperature distribution.

5. Measure the flow temperature distribution.

6. Measure and define the surface roughness conditions.

7. Maintain the desired saturation conditions.

8. Provide a means for flow visualization.

B. Compare the results with the currently existing R-114 data from the Oak Ridge Gaseous Diffusion Plant (ORGDP) [7].

c. Compare existing or determine a new correlation for:

1. the heat flux,

2. the CHF, and

3. the pressure drop. 
The specific objective of this study is to:

A: Design a well instrumented experimental setup to:

1. Measure the boiling heat-transfer coefficient on the internal surface of a $0.785^{\prime \prime}$ I.D., 1.75" O.D., 120" long copper tube.

2. Measure the two-phase flow pressure drop at five locations.

3. Measure the critical heat flux under conditions of:

a) flow boiling,

b) vertical orientation, i.e., the axis of the tube will be held in the direction of gravity,

c) uniform heat flux within the ten 11-1/2" heat zones,

d) predefined surface roughness on a copper wall.

4. Provide the following capabilities:
a) fluid boiling temperature of $100{ }^{\circ} \mathrm{F}$ to $250{ }^{\circ} \mathrm{F}$

b) fluid mass flux of $0.1 \mathrm{e} 6$ to $1.1 \mathrm{e} 6 \mathrm{lbm} / \mathrm{hr} . \mathrm{ft}^{2}$

c) test section exit quality of 0 to 1

d) heat flux maximum of 3,000 to $60,000 \mathrm{Btu} / \mathrm{hr}$. $\mathrm{ft}^{2}$ per zone

f) tube saturated length to diameter ratio $L / D=30$ to 150

5. Overall system conditions to be measured include:

a) surface temperature distribution

b) fluid temperature distribution

c) pressure at 6 equally spaced locations along the 120 " test section with one location at the entrance and one at the exit. An absolute pressure is measured at the test section entrance and the remaining five pressures are determined differentially with respect to the inlet reading.

d) heat flux into each region

e) provide a means for exit flow visualization

B: Run tests using:

1. CFC-114 to confirm and check for repeatability with the (ORGDP) data [7].

2. $C_{4} F_{8}$

3. $C_{4} F_{10}$

4. non-azeotropic mixtures of the above three liquids

\section{TECHNICAI APPROACH}

\section{Theory}

Consider a vertical tube heated uniformly over its length. A sub-cooled liquid enters at the base of the tube at a flow rate such that on exiting the tube at the top all the liquid has changed phase to vapor. Figures 1 [1] and 2 illustrate the different regimes of heat transfer encountered over the length of the tube.

Region A: Single phase heat transfer to the Iiquid phase. Here the wall temperature is lower than that required for nucleation $[1,3,4]$ and the liquid being sub-cooled is heated up to the saturation temperature. The heat-transfer coefficient is relatively constant, changing only slightly due to the influence of 
temperature on the liquid thermophysical properties. Both the liquid and wall temperatures increase linearly.

Region B: Sub-cooled nucleate boiling. As the flow moves downstream the wall temperature rises above saturation temperature, i.e., the degree of superheat, $\Delta \mathrm{T}_{\text {sat }}\left(=\mathrm{T}_{w}-\mathrm{T}_{\text {sat }}\right)$ is positive. When some critical value of temperature is reached within the sublayer that is well above that of the saturation temperature $[1,3,4]$ ebullition commences. $\Delta T_{\text {sat, }}$ the temperature difference between the wall and the saturation temperature depends on the interaction of the surface tension at the liquid-vapor-solid interface with roughness $[15,16,20]$. The conditions at the wall support nucleation although the main liquid flow field is below saturation, i.e., $\Delta \mathrm{T}_{\text {sub }}\left(=\mathrm{T}_{\text {sat }}-\mathrm{T}_{\mathrm{f}}\right)$ is positive $[21,22]$. Sub-cooled boiling is characterized by the formation of vapor at the heating surface in the form of vapor bubbles at preferred nucleation points. The bubbles condense in the cold liquid away from the heated surface with the result that no net vapor is produced. Thermocapillary, turbulent, and convective heat transport dominate the flow of heat from the condensing bubble and wall surfaces $[21,23]$. At the onset of nucleation, bubbles form on the surface which maintain the wall temperature constant. However, the fluid being sub-cooled increases in temperature Iinearly up to Region $c$. The heat flux being constant, the heat-transfer coefficient increases linearly with length.

Region C: Saturated nucleate boiling. This region begins when the average bulk liquid temperature is equal to the saturation temperature. The primary mode of heat transfer is within the layer adjacent to the wall and is by conduction and convection. The intermittent generation of bubbles on the surface increases the heat transfer rate via two mechanisms. The latent heat of vaporization in the bubbles carries heat away from the wall. As the individual bubbles leave the wall, they rupture the laminar sublayer, thus reducing the resistance to heat flow by introducing added turbulence at the wall surface. This stage is appropriately termed nucleate boiling. This region is defined from the point at which the liquid reaches the saturation temperature, $i . e .$, the liquid mixed-mean-enthalpy equals that of the saturated liquid. As the liquid flows downstream vapor bubbles become a non-condensible part of the flow field. The heat transfer is characterized by the liquid-vapor quality,

$$
x(z)=\text { mass flow of vapor/total mass flow }
$$

Figure 2 shows the change in the quality ' $x$ ' as the flow moves down the tube.

Region D: Slug flow. The bubbles become larger and more numerous coalescing to form one large bubble that can sometimes be almost as large as the diameter of the pipe. The liquid flow is contained in liquid 'slugs' which separates successive gas bubbles. 
In regions $C \& D$, the $\Delta T_{\text {sat }}$ and hence, the heat-transfer coefficient remain constant.

Region E: Annular flow. The bubble bursts and a thin liquid film forms on the pipe wall with a continuous central vapor core. Heat is transferred by conduction and convection through the liquid film, the effective thermal conductivity of the film being high enough to allow direct evaporation from the liquid-core vapor interface. No nucleation occurs within the film.

Region F: Annular flow with entrainment. Owing to the increase in the vapor velocity, large amplitude waves are whipped up on the surface of the liquid film that continuously break up ejecting droplets into the vapor core flow field. Hence, this is truly a two-phase convective region. Because of the reducing thickness of the liquid film, the heat-transfer coefficient begins to increase.

Region G: Drop Flow. Here the liquid film on the tube wall is completely depleted. The wall temperature shows a significant rise. The heat-transfer coefficient is suddenly reduced from a very high value in the two-phase forced convective region to a value near to that expected for heat transfer by forced convection to a dry saturated vapor. In this liquid deficient region the vapor velocity increases and the heat-transfer coefficient rises correspondingly.

Region H: Dry saturated vapor. The liquid exiting the tube is of $x=1$ quality. This is a single-phase vapor region. The heat-transfer coefficient levels out to that corresponding to the convective heat transfer to a single-phase vapor flow [24]. The regions $G$ and $H$ are associated with the condition of dryout that essentially limits the amount of evaporation that can be supported by a tube with a particular heat flux.

The above processes occur when the heat flux at the walls is kept relatively low. However, if the heat flux is gradually increased, the regions move closer together, the regions $G$ \& $H$ becoming extended.. Finally, a point is reached when a sudden deterioration in the cooling process takes place. The above occurs before the two-phase forced convective region is initiated and while bubble nucleation is still taking place. This condition is called the critical heat flux (CHF) and is associated with a rapid rise in surface temperature from a temperature close to the saturation value. It represents a situation in which there is a more or less a sudden decrease in the value of the heat-transfer coefficient of one or two orders of magnitude as compared with the values obtained in the nucleate boiling and two-phase forced convective regions. This decrease is a consequence of the fact that the heat transfer surface is no longer completely wetted by the liquid phase. Vapor now completely or partially blankets the surface creating an insulating layer between the wall and the liquid (in the center of the tube). Hence, the heat transfer is no longer directly to a 
highly conducting liquid but through a poorly conducting gas/vapor to a liquid.

\section{Effect of Roughness}

Roughness has been shown to have a controlling effect on nucleation under pool boiling conditions [15]. The effect of surface roughness has been studied by Weatherhead [22] and Chirkin [25] who noted that the critical heat flux is lowered for polished surfaces and ascribed the decrease to the lack of nucleation sites. contrary to the above, De Bartoli [26] and Aladyev [27] report no influence of roughness on the CHF for surfaces in the 0.7 to $4 \mu \mathrm{m}$ roughness range.

\section{Correlations}

The Heat-Transfer Coefficient: References $[1,2,5-10,28]$ represent a few examples of the numerous investigations into the theory of boiling. While theoretical investigations have led to a better understanding of the basic physics underlying boiling, the correlations used to model boiling heat transfer [2,5-10] stem in large part from the appropriate parameterization of experimental observations.

The theory of nucleation and the solid-liquid-vapor relationship of boiling is addressed in references $[1,3,4,28-33]$. These authors attempt to explain how the surface temperature for nucleation relates to the composition of the solid surface.

The thermodynamic conditions under which boiling, nucleate or film, take place are well documented for a number of common liquids [34]. However, scarcely any data is available on the effect of the fluid's flow field on boiling. Several empirical theories $[1,4]$ have been developed which can predict the thermal properties of the boiling flow field; however, they have been determined for specific cases, and general application would be questionable.

For fluid mechanics problems involving numerical modeling of two-phase flow, several authors have addressed the problem of optimum equations needed for predicting the flow field. Concerning this question, three particularly illustrative sources are Chawla [33], Ishii [35] and Bergles [36]. These articles contain a theoretical discussion as well as some specific constitutive equations.

\section{DESIGN OF FLOF BOIIING EXPERIMENTAL SETUP}

\section{Experimental Arrangement}

Figures 3 and 4 illustrate the flow boiling system capable of operating at pressures from 0 to $350 \mathrm{psig}$ and temperatures ranging from $50^{\circ} \mathrm{F}$ to $300{ }^{\circ} \mathrm{F}$. The system consists of fourteen major components, namely: 
1. Storage tank

2. Pumps

3. Flow straightener

4. Instrumented vertical tube

5. Heaters, power supply and power measurement systems

6. Temperature measurement and control systems

7. Pressure measurement system

8. Flow visualization zone

9. Vapor-liquid separator

10. Condenser

11. Flow meters

12. Valves

13. Level gages

14. Chiller system

In the following sections each of the individual components will be described in detail.

\section{Storage Tank}

The storage tank was designed to provide sufficient head for the pumps to operate without cavitation. The total volume of the storage tank is approximately 15.6 gallons. This volume was sufficient to allow the tank to be at least a third full at all times during the test. The tank design is shown in figure 5. The tank is provided with a heat exchanger which can be used to subcool the Iiquid. This heat exchanger is a 30 foot coil of $1 / 2$ " copper tubing connected to the chiller. The tank is also provided with a $5 \mathrm{~kW}$ heater wrapped around the outer wall at the bottom. The combination of the heater and sub-cooler is used to control the temperature of the coolant inside the tank. The whole tank is insulated with a thick layer of fiberglass insulation to reduce the heat loss to the environment.

\section{Pumps}

The two pumps used in the test loop are identical magnetic clutch canister gear pumps ( 9 teeth in rotor, 7 teeth in idler). The variable speed pump has a variable speed motor with an electronic speed controller. The speed of the pump can be varied semi-continuously between 0 and 1750 RPM. The fixed speed pump operates at $1750 \mathrm{RPM}$. The performance curve for both the pumps is shown in figure 6 .

\section{Flow straightener}

The flow straightener shown in figures 7 to 10 is used to provide a uniform velocity profile to the flow and eliminate the eddies and turbulence caused by the bends and valves between the pump and the straightener. The flow, on entering from the bottom of the straightener, passes through an aluminum honeycomb that straightens the flow and then through a reducer which accelerates the flow into the test section entrance. The mating flanges between the flow straightener and the test section ensure that the flow is undisturbed by the joint between the two. 
4. Instrumented vertical tube

The test section is primarily a $0.785^{\prime \prime}$ ID \& $1.75^{\prime \prime}$ OD copper tube about 10 feet in length. At each end of the test section is a welded copper flange for mating with the other parts of the setup. Figure 11 shows the overall dimensions of the test section. The test section is divided into ten zones, each 11.5" long and separated from the adjacent zones by a 0.5 " wide groove shown in figure 12. Figures 13,14 , and 15 show the details of the thermocouple wells and pressure taps in each zone, and the corresponding cross-section views are shown in figures 16,17 and 18. Zone 1 (the first zone at the bottom) and zone 10 (the last zone at the top) are welded to copper flanges, figure 19, which in conjunction with the carbon steel slip-on flanges, figure 20, are used to connect the test section to the flow straightener (for zone 1 ) and the flow visualizer (for zone 10). Figure 20 shows the end zones with the welded flanges and the slip-on flanges. As can be seen from the previous figure, due to the addition and welding of the end flanges, the end zones turn out to be slightly longer compared to the other zones (11.8" long as compared to 11.5").

\section{Heaters, power supply, and power measurement systems}

Each zone of the test section is wrapped with heaters capable of providing over $60,000 \mathrm{Btu} / \mathrm{hr} . \mathrm{ft}^{2}$ to the outer surface of the tube. The heater arrangement, as shown in figure 21, consists of two nichrome wires $(80 \% \mathrm{Ni} / 20 \% \mathrm{Cr}$, AWG 20) each $30 \mathrm{ft}$ long connected in parallel and wound helically around each zone. The nichrome wires were insulated with a sleeve of fiberglass capable of withstanding temperatures up to $900^{\circ} \mathrm{F}$. Initially it was felt that wrapping the insulated nichrome wires tightly around the zones would provide enough heat transfer area for the heater to remain below this temperature. However, from initial tests it was ascertained that some form of heat spreader would be needed to obtain the highest heat flux at reasonably low heater temperatures. For this purpose a high temperature, high expansion magnesia (Mgo, $\mathrm{k}=40$ Btu.in/hr. $\mathrm{ft}^{2}{ }^{\circ} \mathrm{F}$ ) cement was coated around the zones, the insulated heater wires were then wrapped around the coated zone and then again covered with two thick layers ( $\approx 1.5 \mathrm{~mm})$ of the cement: This arrangement provided a good insulation between the zone and the heater wire and also increased the heat transfer from the heater wires.

The heater arrangement and the test section was thermally insulated from the environment by three layers of high temperature Fiberfrax insulation $\left(k=0.65 \mathrm{Btu}\right.$. in $/ \mathrm{hr} . \mathrm{ft}^{2}{ }^{\circ} \mathrm{F}$ e $\left.500{ }^{\circ} \mathrm{F}\right)$ in $3 / 8^{\prime \prime}$ diameter rope form and an aluminum foil radiation heat shield.

To reduce electrical and induction noise on the various transducers the direction of current flow was reversed in every zone to counteract induction as much as possible. All supply wiring was run through grounded metal conduits. 
Depending on the level of heat flux desired, a control signal from the potentiometer is applied to the phase-angle SCR power supply, see figure 22. The SCR power supply regulates the power to the heaters by varying the phase angle on each $A C$ cycle proportional to the control signal. This method of applying power provides a continuous uniform heat flux with fine control. The temperature controller, as shown in figure 22 , monitors the temperature of the outer heater surface. In the event of an overshoot in the temperature of the surface, an alarm disconnects the power to the heaters via relays. The power to the heaters along with the temperatures are continuously indicated by the panel meters and recorded by the data acquisition system. All process parameters are displayed and stored on the PC.

By measuring the voltage across and the current flowing through the heater (the power transducer does this), the total heat input into the heater is given by,

$$
Q=V \times I \text { - Losses Watts }
$$

The losses include,

Losses $=$ Conduction losses to end plate + ambient

The losses to the ambient were calibrated as described in Heat Loss.

6. Temperature measurement and control systems

Wall temperatures are measured by five thermocouples imbedded into threaded holes in the wall of each zone of the tube (see figures 13 to 18 ). Each thermocouple was set up to be coaxial with a screw which was threaded into the hole, see figure 23 . The bottom of the hole was filled with a highly conductive omegatherm paste for improving thermal contact. In addition, one thermocouple was positioned so as to be within the flow field to measure the instream flow temperature.

All the thermocouples were of type $E$ stainless steel sheathed (1/16" OD) with ungrounded junctions. The thermocouple extension wires were teflon insulated, twisted-pair cable ( 8 pairs to a cable) made of thermocouple grade wire. Twisted-pair cables were selected to reduce noise in the signal. The specifications on thermocouples are given below.

Type: $\mathbf{E}$

Sheath: Stainless steel, 1/16" OD 3.5 to 4.5 " long

Limits of Error: $3^{\circ} \mathrm{F}$ or $0.75 \%$ (whichever is greater)

Manufacturer: Omega Engineering, CT.

Using the temperature controller in conjunction with another thermocouple affixed to the outer heater wall, the temperatures of the heater wires are monitored. In the event of a sudden 
temperature rise (such as at $\mathrm{CHF}$ ), the controller alarm will disconnect the power to the heater, see figure 22. The power control to the heaters is set up to provide the capability of either maintaining a constant temperature or a constant heat flux on each zone.

Each thermocouple was calibrated using a standard platinum thermistor over the temperature range $100{ }^{\circ} \mathrm{F}$ to $350^{\circ} \mathrm{F}$ in approximately $25^{\circ} \mathrm{F}$ temperature intervals. The thermocouples were calibrated with the same wire, connecting harness and data acquisition cards in place as used in the actual experiment. Hence, any uncertainty due to electrical contacts is removed. The uncertainty in the temperature measurement was reduced to $\pm 1{ }^{\circ} \mathrm{F}$.

\section{Pressure measurement system}

As shown in figures 3, 11-18, the ten zones are identical as far as the location of thermocouples is concerned. However, the pressure taps are located in zones $1,3,5,7,9 \& \cdot 10$ such that the distance between two consecutive taps is $2^{\prime}$ except for the tap in zone 10 which is at a distance of $22.5^{\prime \prime}$ from that in zone 8 .

Seven pressure transducers were positioned along the length of the tube. One at the entrance of the tube and one at the exit that gave the absolute pressures. Additionally, five transducers were placed equidistantly within the test section to provide the differential pressure relative to the inlet pressure. The differential pressure transducers provide the pressure drop information along the length of the tube.

Two types of pressure transducers were used in the experiment. The first were gage pressure transducers connected to the first and the last pressure port in the test section. These were the model \#PX906-500GV manufactured by omega with the following specifications:

Range: 0-500 psig

Accuracy: $0.4 \%$

Zero balance: $3 \%$

Output: $20 \mathrm{mV}$ full scale

Excitation: $10 \mathrm{~V}$ dc

Sensitivity: $2 \mathrm{mV} / \mathrm{V}$

The second type of pressure transducer was used for measuring the pressure drop along the length of the test section. For this purpose 5 differential pressure transducers, model \# DP-30-0003-111, manufactured by Celesco Transducer Products, CA were selected. Following are the specifications for these transducers:

Range: $0-3$ psid

Accuracy: $0.5 \%$ F.S.

Repeatability: $0.1 \%$ F.S. 
Each of the differential pressure transducers were calibrated periodically (after change of each liquid) over a range of 0 to 5 psi using a dead weight tester. The absolute pressure transducer was calibrated by omega.

\section{Flow visualization section}

The flow visualization zone follows zone 10 at the exit or top of the test section, see figures 24 to 26 . The flow visualizer is a pyrex glass tube with an internal diameter of $0.787 " \pm 0.005 "$. The pyrex tube is held between two flanges, see figures 25 and 26 . The lower flange mates to the test section exit flange and ensures a smooth transition from the test section to the view port. The o-rings around the glass tube provide an axial seal for the fluid. The pyrex tube is rated at 350 psig.

\section{Vapor-liquid separator}

The separator is essentially a cyclone separator, in which the liquid-vapor mixture is coarsely separated using gravitational and centrifugal forces. The liquid-vapor mixture enters a large chamber. where the liquid settles to the bottom due to gravity. The vapor with fine entrained liquid droplets then moves toward the elevated vapor exit passing through swirler-vanes which induce a circumferential velocity on the flow. This circumferential motion produces the centrifugal force that throws the heavier droplets outward and allows the vapor to move toward the center where the vapor exit is located. Figure 27 shows the separator design in detail. The separator is also wrapped with a heater and insulated with fiberglass insulation to minimize heat loss and prevent condensation in the separator.

\section{Condenser}

The condenser was designed as a shell and tube type with a capacity of $150,000 \mathrm{Btu} / \mathrm{hr}$ at a condensate temperature of $98.6^{\circ} \mathrm{F}$, antifreeze inlet temperature of $25^{\circ} \mathrm{F}$, and antifreeze flow rate of $25 \mathrm{gpm}$. The capacity increases as the saturation temperature of the coolant rises. The capacity of the condenser is limited only by the temperature and flow rate of the antifreeze. The details of the condenser are shown in figures 28 to 33 . The details of the tube bundle are as follows:

Tube bundle

No. of tubes: 7

Length of each tube: 16 feet

No. of passes: 2

Tube specification: 1/2" nominal, type $\mathrm{K}$ copper tube

\section{Flow meters}

The flow meter locations are shown in figure 3. The three flow meters used in the test loop are identical. The flow meters are model \# FTB-102 turbine meters sold by omega with the following specifications: 
Flow range: $0.75-7.5 \mathrm{gpm}$

Temperature range: -450 to $450{ }^{\circ} \mathrm{F}$

output: pulse output with a minimum amplitude of $30 \mathrm{mv}$

Accuracy: $0.5 \%$ of reading

Repeatability: $0.1 \%$ of reading

End connections: 1/2" NPT male

Max. pressure drop: 5 psid

The turbine flow meters were installed with a straight length of at least 8" upstream and 3" downstream as per the specifications of the manufacturer. The flow meter assembly had an internal diameter of 0.54" which resulted in a substantial pressure drop. Also, to prevent cavitation on the impeller vanes, sub-cooled conditions had to be maintained at the inlet.

The flow meter used to measure the coolant mixture flow rate was a model \# FTB4607 from Omega. The accuracy of this flow meter is $1.5 \%$ of the reading and its operating range is from 0.22 to $20 \mathrm{gpm}$.

The flow meters at the exit of the separator and condenser did not operate in a continuous fashion but rather in an intermittent way. This is because the turbine flow meters used require a threshold impulse to start. This required the liquid level to build up in the separator and condenser lines before the meter would register a reading. Once the turbine started, the liquid would run through in a short period and the process would repeat itself. Therefore, determination of the exit quality in a continuous fashion was not possible.

For flows below $1 \mathrm{gpm}$ three rotameters were installed in parallel with the turbine meters with valves to isolate either one if necessary. The rotameters were Omega series FL-1502A with the following specifications:

Flow range: $0.145-1.45 \mathrm{gpm}$

Temperature range: 40 to $200^{\circ} \mathrm{F}$

Pressure: 300 psig maximum

Output: Visual fused scale on rotameter glass tube

Accuracy: $1 \%$ of full scale

Repeatability: $0.5 \%$ of full scale

End connections: 1/2" NPT male

Max. pressure drop: 14" of water

\section{Valves}

Valves used in the setup were manual ball valves of various sizes depending on the piping. This facilitated isolation of various regions of the flow loop for maintenance and checking purposes.

\section{Level gauges}

clear nylon tube level gages were constructed using compression fittings connected to the top and bottom of the storage tank, 
separator, and condenser to determine the liquid level in the components.

\section{Chiller system}

The chiller system is used for supplying antifreeze to the condenser and the storage tank. As shown in figure 4 the system consists of a 7 ton refrigeration unit, a 1000 gallon tank (with evaporator coils connected to the refrigeration unit) and a 750 gallon tank for additional antifreeze storage. The refrigeration unit removes the heat from the antifreeze mixture in the larger tank via the evaporator. The chilled antifreeze solution is pumped to the condenser and storage tank heat exchangers. The cold antifreeze mixture picks up the heat in these two heat exchangers and is returned to the smaller tank. The smaller tank is also connected to the larger tank. The two tanks contain plastic bottles filled with water which act as heat sinks. The system was generally maintained below $30^{\circ} \mathrm{F}$ before starting the test, hence the water bottles were in frozen condition. During the test, the hot antifreeze mixture returning from the condenser and the storage tank melts the ice in the bottles and is restored to near $30{ }^{\circ} \mathrm{F}$.

During the initial cool-down of the antifreeze solution, the variable speed gear pump is turned on, the valve connecting the pumps to the smaller tank is kept open, and the valve leading to the test loop is left closed. This allows the antifreeze to circulate between the two chiller tanks and aids in the freezing of the water bottles in both of the tanks. The chiller system was successfully used for continuous tests lasting over 120 hours at a time.

\section{Roughness measurement}

The 10-foot copper tube was supplied by Martin Marietta Utility Services, Inc. (MMUS). The inner surface was successively cleaned using 400 grit sand paper, acetone, and methanol. Also supplied was a part of the test section used by Jallouk [7] in his experiments. The roughness profiles of Jallouk's [7] test section and the present one were measured using a surtronic 3P profilometer (made by Rank Taylor-Hobson); figures 34 and 35 show sample roughness profiles and table 1 shows the values of commonly used roughness parameter $R_{a}$ and $R_{q} \cdot R_{a}$ is defined as the arithmetic mean of the departure of the roughness profile from the mean line.

$$
R_{\mathrm{a}}=\frac{1}{L} \int_{0}^{L}|y(x)| d x
$$

$R_{\text {is }}$ isfined as the root-mean-square average of the departure of the roughness profile from the mean line.

$$
R_{q}=\sqrt{\frac{1}{L} \int_{0}^{L} y(x)^{2} d x}
$$


Here $I$ is the scan length, $y$ the roughness profile in the direction parallel to the tube axis.

As shown in table 1, three scans were carried out for each tube. The third scan for each tube is presented in figures 34 and 35 . The results show a marked difference in the roughness of the two test section tubes; moreover, the results of the old test section, see figure 35, show a large variance. This is due to the fact that the old test section had a thick layer of oxide like deposits on the inside that made it impossible to determine its original roughness; any attempt to remove the oxide layer would inevitably result in a change in the original roughness of the surface. Therefore it is not possible to compare the roughness of the present test section with the previous one.

Since the purpose of this study is to simulate flow boiling conditions encountered in actual practice, it would be more appropriate to compare the roughness of the test section with that of a commercially available copper tube. This was carried out by measuring the internal roughness of a piece of $1 / 2$ " outer diameter copper tubing commonly used in the refrigeration industry; the results are presented in figure 36 and table 1 . It can be seen that the roughness values of the new test section and the commercial tube are comparable. Thus the test section can be assumed to be an appropriate representation of the real situation.

Table 1 Roughness Values

\begin{tabular}{|c|c|c|c|}
\hline Material & & $\mathrm{R}_{\mathrm{a}} \mu$ in & $R_{q} \mu$ in \\
\hline $\begin{array}{c}\text { New test section } \\
1^{\text {st }} \text { scan } \\
2^{\text {nd }} \text { scan } \\
3^{\text {rd }} \operatorname{scan}\end{array}$ & (figure 34) & $\begin{array}{l}12.0 \\
13.1 \\
15.7\end{array}$ & $\begin{array}{l}15.1 \\
17.7 \\
20.4\end{array}$ \\
\hline $\begin{array}{c}\text { Jallouk [7] } \\
1^{\text {st }} \text { scan } \\
2^{\text {nd }} \text { scan } \\
3^{\text {rd }} \text { scan }\end{array}$ & (figure 35) & $\begin{array}{l}29.9 \\
59.3 \\
52.9\end{array}$ & $\begin{array}{l}37.7 \\
85.2 \\
76.1\end{array}$ \\
\hline $\begin{array}{l}\text { Commercial 1/2" } \\
1^{\text {st }} \operatorname{scan} \\
2^{\text {nd }} \operatorname{scan} \\
3^{\text {rd }} \operatorname{scan}\end{array}$ & copper tubing & $\begin{array}{c}\text { (figure 36) } \\
10.4 \\
14.7 \\
15.6\end{array}$ & $\begin{array}{l}15.2 \\
21.5 \\
21.8\end{array}$ \\
\hline
\end{tabular}

\section{System operation}

cleaning and charging the system

Initially, after it was assembled, the system was pressure tested with water to $350 \mathrm{psig}$. No leaks were detected. The water was drained and a solution of degreaser/rust-inhibitor was pumped 
through the system for a number of hours. The system was then drained and flushed repeatedly with water and finally drained again. Next, the system was heated and compressed air was passed through for a period of four days to remove as much moisture as possible. Then, a new desiccant filter was installed in-line and the system was evacuated and filled with about ten gallons of CFC-114. Any leaks detected with a halogen monitor were fixed. The CFC-114 was circulated through the system for two hours. The CFC-114 was emptied, a new filter was installed and the system was evacuated again prior to filling with a fresh supply of CFC-114.

\section{Calibration}

In the design of this experiment the number of devices to be measured included over eighty thermocouples, seven pressure transducers, six flow meters, and ten power transducers. To expedite on-site data analysis a PC-based data acquisition was used to measure, perform the necessary calculations, display system conditions and store the data. To reduce the uncertainty in the overall measurement, the devices were calibrated using standards in conjunction with the data acquisition.

\section{Differential and absolute pressure transducers}

The pressure transducer calibration control panel (PDCCP), see figure 37 offers an easily accessible port to conveniently calibrate the differential (DP) and absolute (AP) pressure transducers. This panel comprises of a set of two- and three-way valves that control the flow through the transducers and facilitate the access of any one or all of the transducers to the pressure dead weight tester. Hence, the transducers can be calibrated insitu at any time.

\section{Turbine flow meters}

The turbine flow meter calibration was provided by the manufacturer. This calibration exists in the form of a linear equation that is given in table 2. The voltage reading from the flow meter is substituted into the equation to be converted into the flow rate.

Table 2: Flow meter calibration values

\begin{tabular}{cl} 
Flow Meter & \multicolumn{1}{c}{$A$} \\
1 & 16.721 \\
2 & 26.93 \\
3 & 35.926
\end{tabular}

The equation for the flow rate is:

$$
F=\frac{A \times V}{1000}
$$


where, $v$ is the voltage reading in millivolts (hence, the term 1000) from the flow meter and $F$ is the flow rate in gpm.

Heat input

The heat input, $Q_{\text {in }}$, was measured by a power transducer, see figure 22, and calibrated using a Yokogawa 3-phase power meter. The input range is 0 to 4000 watts with an output at the data acquisition of 0 to 4.5 volts. The input power, in watts, was measured with the power meter while the output was measured with the data acquisition unit. Therefore, for a given reading of power in watts that is supplied to the heater, there was a corresponding value in volts at the data acquisition unit. The two values were correlated using a linear regression scheme. This curve presented an equation with which to calculate the heat flux at any voltage. The following table 3 provides the two parameters for each zone of the tube:

\section{Table 3: Heat Flux Calibration Values}

\begin{tabular}{lll} 
Zone & \multicolumn{1}{c}{ A } & B \\
1 & .78724 & -3.06 \\
2 & .78436 & -2.09 \\
3 & .78106 & -2.53 \\
4 & .78631 & -1.76 \\
5 & .78426 & -0.97 \\
6 & .78328 & -1.22 \\
7 & .8014 & 0.27 \\
8 & .791 & -4.5 \\
9 & .78561 & -0.56 \\
10 & .78749 & -0.86
\end{tabular}

Using the voltage that is read from the data acquisition as $v$ in millivolts, the following equation can be used with the respective values for $A$ and $B$ substituted to arrive at the total heat flux, $Q$ in Watts, into the particular zone,

$$
Q=A \times V+B
$$

\section{Heat loss}

After the heat input calibration procedure was completed, the heat loss calibration was performed. The test section was isolated from the rest of the system and only the copper tube was heated. A vacuum was pulled on the tube so as to eliminate any heat losses due to convection within the tube. This is done so that any heat lost from the heaters is due solely to convection and radiation to the surrounding ambient and through conduction to the adjoining zones within the tube.

The heat loss calibration was performed in the following manner. The test tube was heated so as to attain an isothermal temperature 
field over the entire length of the tube. This was possible by setting the temperature controllers on the power control panel, see figure 22, to the desired temperature. Then the temperature controller while monitoring the temperature of its zone adjusts the power controller to apply the necessary heat flux so as to satisfy the temperature condition. To measure this heat loss, the heat input to each zone is merely recorded. This heat flux is the actual heat loss from each zone. This is true because for isothermal conditions the only escape for the heat is through convection and radiation to the ambient. The temperature of the tube being isothermal no heat can be conducted to the adjoining zones, except at the ends of the tube. At the end zones the heat losses noted are much higher. The above calibration process was performed for a number of wall temperatures in the range $75^{\circ} \mathrm{F}$ to $350^{\circ} \mathrm{F}$ in approximate steps of $25^{\circ} \mathrm{F}$.

The difference in wall and ambient temperatures $\Delta \mathrm{T}\left(=\mathrm{T}_{\mathrm{wall}}{ }^{-} \mathrm{T}_{\mathrm{atm}}\right)$ versus heat loss is used to arrive at a heat-transfer coefficient, $h$. Then in the actual flow boiling tests, the heat loss incurred can be calculated from the value of the measured $\Delta T$ and the respective $h$ for the zone. The value of $T_{\text {wall }}$ is taken as the average temperature over the zone.

Temperature

Approximately eighty type-E thermocouples were used in the experiment. All the thermocouples were of the 1/16" stainless steel sheathed ungrounded type. The temperature differences $\Delta T$ expected in the experiment were in the range of $4^{\circ} \mathrm{F}$ to $20^{\circ} \mathrm{F}$. In order to reduce the uncertainty in the measurement and results it was necessary to calibrate the entire lot of thermocouples in conjunction with their connectors and the data acquisition. In order to calibrate the thermocouples, a constant temperature bath of FC-70 (approximately one gallon, boiling point: $419^{\circ} \mathrm{F}$ ) was used. This particular inert ozone-safe freon was chosen for its high boiling point, but any similar liquid may be used, even well ventilated motor oil. The calibration unit consists of an integral heater-chiller with a stirrer which is submerged into the FC-70 bath. The temperature of the bath is maintained at a preset value by a temperature controller which monitors a RTD immersed in the bath. A separate calibrated platinum resistor allowed measurement of the temperature within $\pm 0.05^{\circ} \mathrm{F}$.

The four data acquisition cards are visible from the back of the data acquisition cabinet (see figures 38 and 39). On each of these cards are three sets of seven thermocouples, one set for each zone, for a total of ten zones. The bottom card, card \#4, has only one set of seven thermocouples attached, the tenth set. All of the wires and barrier strips used to connect the thermocouples on the test section to their corresponding location on the data acquisition cabinet were in their proper place at the time of the calibration. All of these connections were intact and had not changed at the time of the disassembly of the system. Calibrating 
the thermocouples with all connections made and everything properly installed, eliminated any errors due to varying junction compensation.

once all of this was done, the actual calibration could begin. only one data acquisition card was calibrated at a time to keep the FC-70 bath from becoming too crowded. only the first six thermocouples in each set of seven were calibrated, thus there were eighteen thermocouples in the bath. The calibrated platinum resistor was bundled along with the 18 thermocouples and submerged in the bath. Care was taken to align the tip of the thermocouples with the platinum resistor so as to assure that the temperature measured by all the devices were from the same region in the bath.

The temperature was set at $100^{\circ} \mathrm{F}$ and allowed to reach steady state (this took about 1.5 hours). After the fluid temperature held steady, $+/-0.05^{\circ} \mathrm{F}$, for approximately 15 minutes the temperature of the platinum resistor, the cold junction, as well as an average of 100 readings from each of the thermocouples were recorded. The above procedure was repeated for increments of approximately $25^{\circ} \mathrm{F}$. Once $350^{\circ} \mathrm{F}$ was completed, the thermocouples were removed, the FC-70 bath was cooled to $100^{\circ} \mathrm{F}$ and the 18 thermocouples on the next card were inserted into the bath. This procedure was followed for all the thermocouples. Hence, a table of standard platinum RTD temperatures versus thermocouple temperatures was created.

When the flow boiling test was run at a later time, the temperature readings from the data acquisition were interpolated with the table data to give the corrected temperatures. During the calibration with the data acquisition it was observed that the cold junction compensation at the barrier strip of the card was not sufficient. This is because the cold junction temperature was measured at the center of the card, but our measurements indicated that a temperature gradient existed in the barrier strip of the card. Hence, to reduce this temperature gradient a fan was installed so as to blow air over and through the data acquisition system. This reduced the error significantly. The calibration was then performed with the fan in place. From our analysis of the data the uncertainty in the measured temperature is not more than $\pm 1^{\circ} \mathrm{F}$.

\section{Experimental Procedure}

The variable flow pump is started and the flow rate adjusted to the desired predetermined value. The pre-heater is powered to control the inlet coolant entry temperature. The antifreeze is circulated through the cooling coils in the condenser at a flow rate such that the saturation pressure at the inlet is maintained at the desired value. Excessive cooling may cause a low pressure at the pump inlet and lead to cavitation. Too little cooling will cause the pressure to rise and force the liquid at the inlet to the 
test section to be sub-cooled. A heater on the storage tank in conjunction with a cooling coil in the storage tank is used as an accessory control for inlet temperature and pressure, see figure 5 .

The heaters are turned on and the power is ramped up in steps to the desired heat flux, making certain that steady state conditions are reached on the surface of the heater. The data acquisition is set up to measure the heat input and temperature of the heaters. With this information the heat loss from the test section to the ambient and end-plates is calculated from the earlier heat loss calibration. The balance of the heat is necessarily taken up by the fluid. Both the heat loss and the heat flux into each zone are displayed simultaneously by the data acquisition. Hence, it is possible to determine and set in real time the heat flux conditions. Steady-state thermal flow field conditions are determined by monitoring the temperatures of the various regions of the system. When the temperature transients die down ( $1{ }^{\circ} \mathrm{F}$ or less change over a 5 minute period), the system can be assumed to be in thermal equilibrium. At this juncture the:

a) temperatures at various locations

b) system pressure and region differential pressures

c) power input to the heaters

d) flow meter readings

e) flow field observations

are noted down and collected by the data acquisition unit.

The above process is followed for a step-wise increase in heat flux until CHF conditions are imminent. At this juncture the heat flux is further increased so as to span the $L / D$ ratios for the CHF study. In regions where CHF occurs the heat flux is reduced so as to maintain the wall temperatures approximately equal to the upstream zone to reduce any heat losses. This is because at CHF the heat-transfer coefficient drops significantly and the wall temperature rises correspondingly. To prevent too high a temperature in the particular zone the heat flux must be dropped.

At the end of the experimentation all heaters are turned off. The antifreeze is pumped through the heat exchanger in the storage tank. This creates a low pressure zone in the storage tank (by condensing the vapor). This induces all the vapor and the liquid in the rest of the loop to collect in the storage tank. Once the system has cooled down, all power to the loop is disconnected and the storage tank is isolated from the rest of the system by closing the valves. Small leaks do exist in the flow loop and isolation reduces loss of freon over periods of no operation.

For each fluid at a predetermined boiling temperature and mass flux, the zone heat flux is gradually increased, (i.e. constant heat flux was applied to successive zones in the direction of flow) until CHF conditions are reached in the exit heat zone. This was evidenced by a sharp rise in wall temperature at the last heated zone. As the critical condition was reached, the electrical power 
input to that particular zone was cut off or reduced.

In all runs undertaken, the flow at the inlet was slightly subcooled, the saturation temperature was maintained constant, and the surface heat flux was varied. The range of different coolant flow rates was then repeated at this surface heat flux. Once the surface heat fluxes between 5000 and $35000 \mathrm{Btu} / \mathrm{hr} / \mathrm{ft}^{2}$ were covered, a new saturation temperature was set and the entire procedure repeated. Thus, essentially, the coolant flow rate was the parameter most frequently varied, the surface heat flux the second most frequently varied, and the saturation temperature the least frequently varied. (Owing to the thermal mass of the fluid in the system, approximately 150 lbs, change in saturation conditions needed about three hours to reach steady state).

During the above tests the saturation temperature and pressure of the system are maintained steady at the predefined values. For each saturation temperature and each mass flow rate setting, the run provided data of the form:

1) Surface superheat temperature versus heat flux

2) Saturation temperature conditions

3) Pressure drop profile

4) Flow field observations

5) Pressure at the inlet of the test section

6) Pressure drop along the test section with intervals of 2, 4, 6,8 and $9.88 \mathrm{ft}$ from the inlet

7) Room temperature

8) Wall and in-stream temperatures in each of the 10 zones

9) Gross heat input; heat loss and heat flux to each of the 10 zones

10) Coolant flow rate and temperature at inlet of the test section

11) Water flow rate and temperatures at inlet and outlet of the condenser

\section{CONCLUSIONS}

A flow boiling heat transfer measurement system to study flow boiling from the internal surface of a vertical cylindrical tube was designed, fabricated and constructed. The system is capable of operating over a wide saturation temperature range of $50^{\circ} \mathrm{F}$ to 250 ${ }^{\circ} \mathrm{F}$, pressure range of 14 to $350 \mathrm{psig,} \mathrm{a} \mathrm{heat} \mathrm{flux} \mathrm{range} \mathrm{of} 0$ to $60,000 \mathrm{btu} / \mathrm{hr}$. ft $\mathrm{t}^{2}$ and a mass flux of 0 to $1.4 \mathrm{e} 6 \mathrm{lbm} / \mathrm{hr} . \mathrm{ft}^{2}$. Optical access for flow visualization is incorporated into the design. The test section is a 10 foot long, 0.78 " ID instrumented copper tube divided into 10 zones in the longitudinal direction. Facilities to measure temperature, pressure and flow rate within each region and at various locations in the system are provided. 


\section{ACKNOWLEDGEMENTS}

This work was performed for Martin Marietta Energy systems, Inc., under contract \# 11K-NR559C. Thanks are due to Dr. Shin Park, Andy Szady, David Lannom, Paul Kreitz, and Prof. Louis Chow, for their technical help and discussions. Thanks are also due to Jon Curless, Gregory Mayes, Matthew McMillin, Tom Nett, E.B. Yates, Gregory Patrick, Larry Patrick, and walter cooper for their assistance at various stages of the project.

\section{REFERENCES}

1. Collier, J.G., 1981, "Convective Boiling and Condensation", 2nd ed., McGraw-Hill.

2. Rohsenow, W.M., 1952, "A Method of Correlating Heat Transfer Data for Surface Boiling of Liquids", Trans. ASME, Vol. 74, pg. 1969 .

3. Cole, R., 1974, "Boiling Nucleation", Advances in Heat Transfer, Vol. 10, p. 85.

4. Carey, V. P., Liquid-Vapor Phase-Change Phenomena, Hemisphere Publishing Corporation, 1992 .

5. Zuber, N., 1958, "On the stability of Boiling Heat Transfer", Trans. ASME, Vol. 80, pg. 711.

6. Chen, J.C., 1963, 'A correlation for boiling heat transfer to saturated fluids in convective flow', ASME 6th Natl. Heat Transfer Conf., Boston, 63-HT-34.

7. Jallouk, P.A., 1976, "Two-phase flow pressure drop and heat transfer characteristics of refrigerants in vertical tubes", Report \# K-1864, Oak Ridge National Laboratories, Oak Ridge, TN 37831 .

8. Kandlikar, S.G., 1990, "A General Correlation for Saturated Two-Phase Flow Boiling Heat Transfer inside Horizontal and vertical Tubes", J. of Heat Transfer, Vol. 112, pp. 219-228, February.

9. Steiner, D. and Taborek, J., 1992, "Flow Boiling Heat Transfer in vertical Tubes Correlated by an Asymptotic Model", Heat Transfer Engineering, Vol. 13, No. 2, pp.43-69.

10. Webb, R.I. and Gupte, N.S., 1992, "A Critical Review of Correlations for Convective Vaporization in Tubes and Tube Banks", Heat Transfer Engineering, Vol. 13, No. 3, pp. 58-81.

11. Shah, M., 1976, 'A new correlation for heat transfer during boiling flow through pipes', ASHRAE Trans., Vol. 82, No. 2, pp. 66-86.

12. Tong, W., Bar-Cohen, A., Simon, T.W., You, S.M., 1990, "Contact Angle Effects on Boiling Incipience of Highly-Wetting Liquids", Int. J. Heat Mass Transfer, Vol. 33, No. 1, pp. 91-103.

13. Sehmbey, M.S., Pais, M.R. and Chow, 1990, "Effect of Surface Material Properties and Surface Characteristics in Evaporative Spray Cooling", to be presented at Joint ASME/AIAA Conf., June, Seattle, WA. 
14. You, S.M., Simon, T.W., Bar-Cohen, A., Tong, W., 1990, "Experimental Investigation of Nucleate Boiling Incipience with a Highly-Wetting Dielectric Fluid (R-113)", Int. J. Heat Mass Transfer, Vol. 33, No. 1, pp. 105-117.

15. Berenson, P.J., 1962, "Experiments on Pool-Boiling Heat Transfer", Int. J. Heat Mass Transfer, Vol. 5, pp. 985-999.

16. Webb, R.L., 1981, "The Evolution of Enhanced Surface Geometries for Nucleate Boiling", Heat Transfer Engrg. , Vol. 2 , No 3-4.

17. Pais, M.R. and Singh, S.N., 1988, A Fourier Analysis Approach for Surface Definition and the Effect of Roughness on the Local Convective Heat-Transfer Coefficient as Related to Ice Accretion, AIAA 26th Aerospace Sciences Meeting, Reno, AIAA-88-0117.

18. Pais, M.R., Chow, L.C., and Mahefkey, E.T., "Surface Roughness and its Effect on the Heat Transfer Mechanism in Spray Cooling", ASME Winter Annual Meeting, San Francisco, December, 1989 .

19. Bankoff, S.G., 1958, "Entrapment of Gas in the spreading of a Liquid over a Rough surface", AICHE.J., pp. 24-26.

20. Hsu, Y.Y., 1962, "On the size of Range of Active Nucleation Cavities on a Heating Surface", J. Heat Transfer, Vol. 84, pg. 204 .

21. Bankoff, S.G. and Mikesell, R.D., 1959, "Bubble Growth rates in. highly sub-cooled nucleate boiling", Chem. Engrg. Prog. Symp. Ser., No. 29, pp. 95-109.

22. Weatherhead, R.J., 1963, 'Nucleate boiling characteristics and the critical heat flux in sub-cooled axial flow water systems', ANL 6675.

23. Brown. W.T., 1967, "A study of flow surface boiling", PhD Thesis, Mech. Engrg. Dept., MIT, January.

24. Kays, W.M., Crawford M.E., 1980, "Convective Heat and Mass Transfer", McGraw-Hill, 2nd ed., pp. 313-331.

25. Chirkin, V.S. and Iukin, V.P., 1956, 'Critical point in heat removal from boiling water flowing through an annular gap', Soviet Physics, Vol. 1, No. 7, pp. 1503-1515.

26. De Bartoli, R.A., et al., 1958, 'Forced convection heat transfer burnout studies for water in rectangular channels and round tubes at pressures above 500 psia', WAPD-188.

27. Aladyev, I.L., Miropolskii, z.I., et al., 1961, 'Boiling crisis in tubes', Int. Develop. in Heat Transfer, Vol. II, paper 28, Univ. of Colorado, Boulder.

28. Vachon, R.I., 1967, "Evaluation of the constants for the Rohsenow Pool Boiling Correlation", Paper 67-HT-33, presented at U.S. Natl. Heat Transfer Conf., Seattle, August.

29. Lienhard, J.H., 1976, "Correlation of the Iimiting Super-heat" Chem. Engrg. Sci., Vol. 31, pp. 847-849.

30. Kast, W., Bankoff, S.G., 1957, "Ebullition from solid surfaces in the absence of a pre-existing gaseous phase", Trans. ASME, Vol. 79, pg. 735 .

31. Zuber, N., 1963, "Nucleate Boiling - The Region of Isolated Bubbles - similarity with Natural convection", Int. J. Heat 
Mass Transfer, Vol. 6, pg. 53 .

32. Borishanski, V.M., 1969, "Correlation of the Effect of Pressure on the critical Heat flux and Heat Transfer Rates Using the Theory of Thermodynamic Similarity", Problems of Heat Transfer and Hydraulics of Two-Phase Media, Pergamon Press, pp. 16-37.

33. Chawla, T.C., and Ishii, M., "Two-Fluid Model of Two-Phase Flow in a Pin Bundle of Nuclear Reactor", Int. J. Heat Mass Transfer, Vol. 23, pp. 991-1001.

34. Vargaftik, N.B., 1975, "Tables on the Thermophysical Properties of Iiquids and Gases", 2nd ed., John Wiley.

35. Ishii, M. and Mishima, K., 1984, "Two-Fluid Model and Hydrodynamic Constitutive Relations", Nuclear Engrg. and Designs, Vol. 82, pp. 107-126.

36. Bergles, A.E., 1981, "Two-Phase Flow and Heat Transfer in the Power and Process Industry", Hemisphere Publishing Co.

37. Katto, Y., 1978, 'A generalized correlation for critical heat flux for the forced convection boiling in vertical uniformly heated round tubes', Int. J. Heat Mass Transfer, Vol. 21, pp. 1527-1542. 

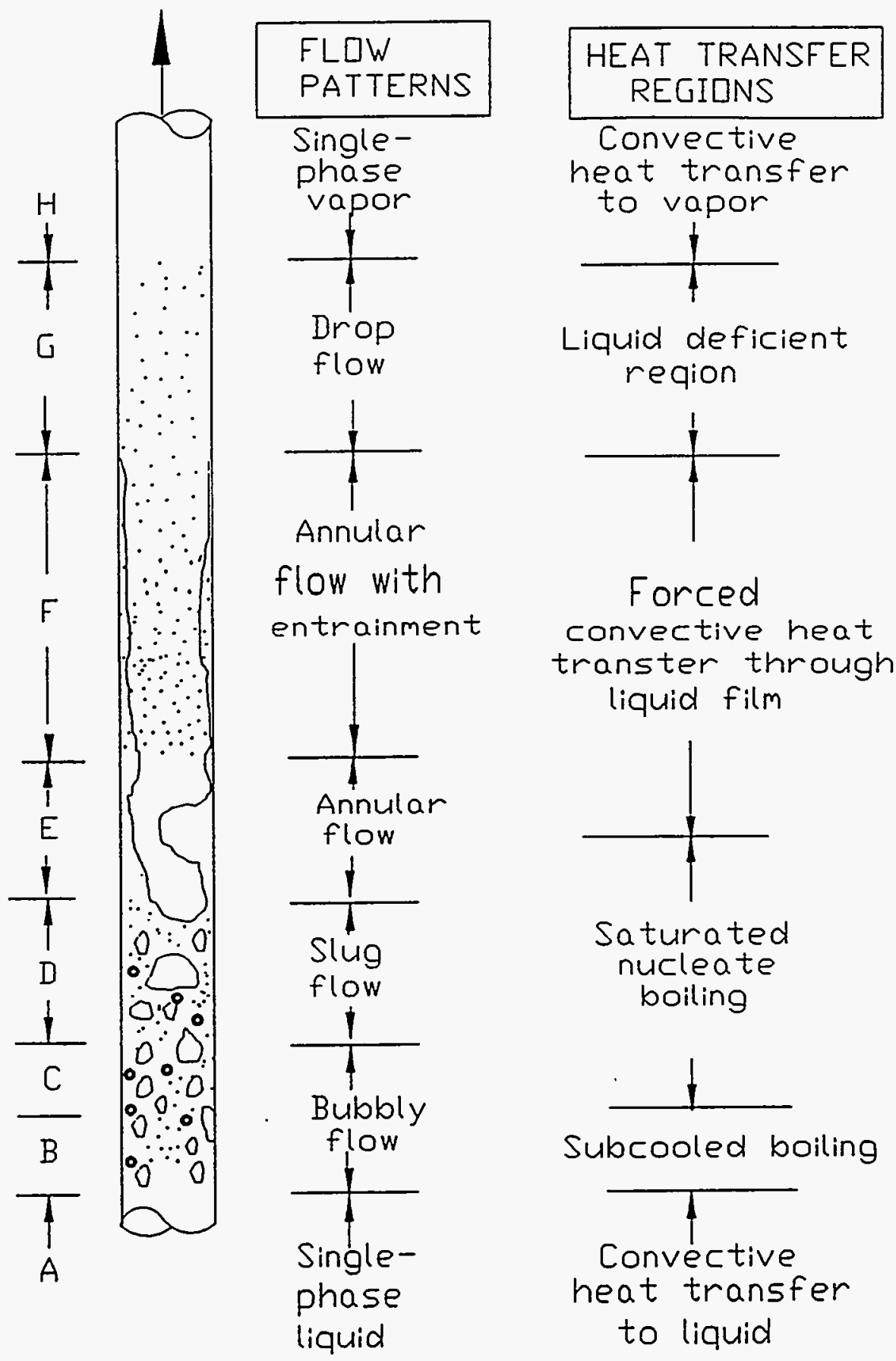

Liquid deficient reqion

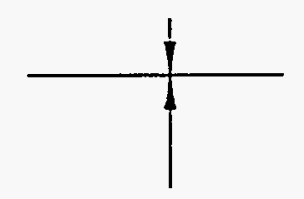

Forced convective heat transter through liquid film

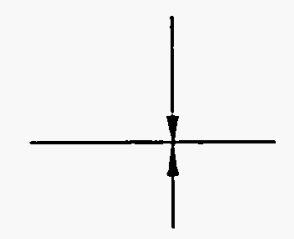

$$
\begin{gathered}
\text { Saturated } \\
\text { nucleate } \\
\text { boiling }
\end{gathered}
$$

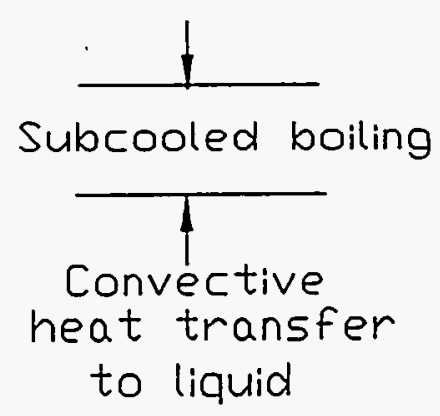

FIGURE 1 


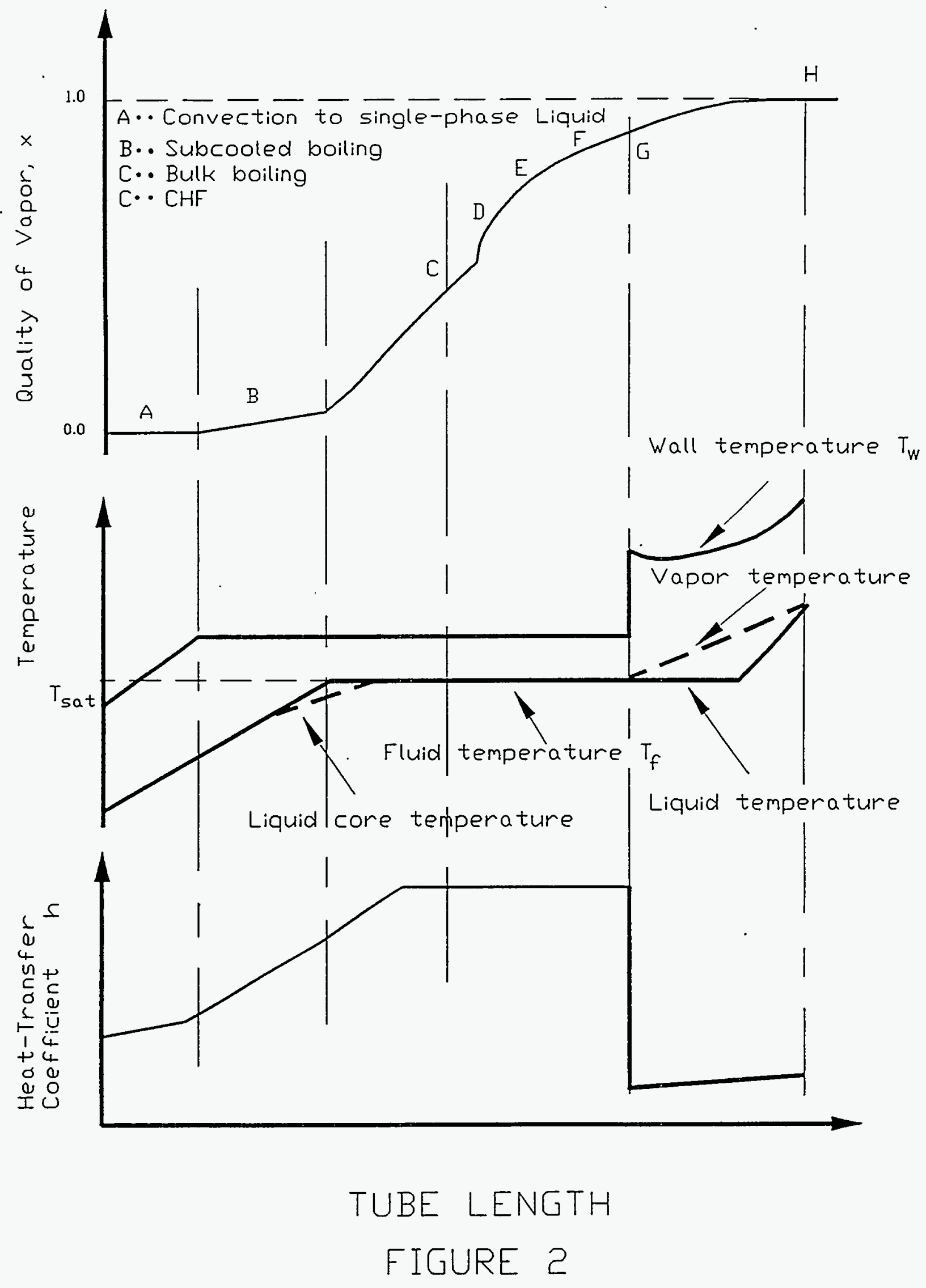




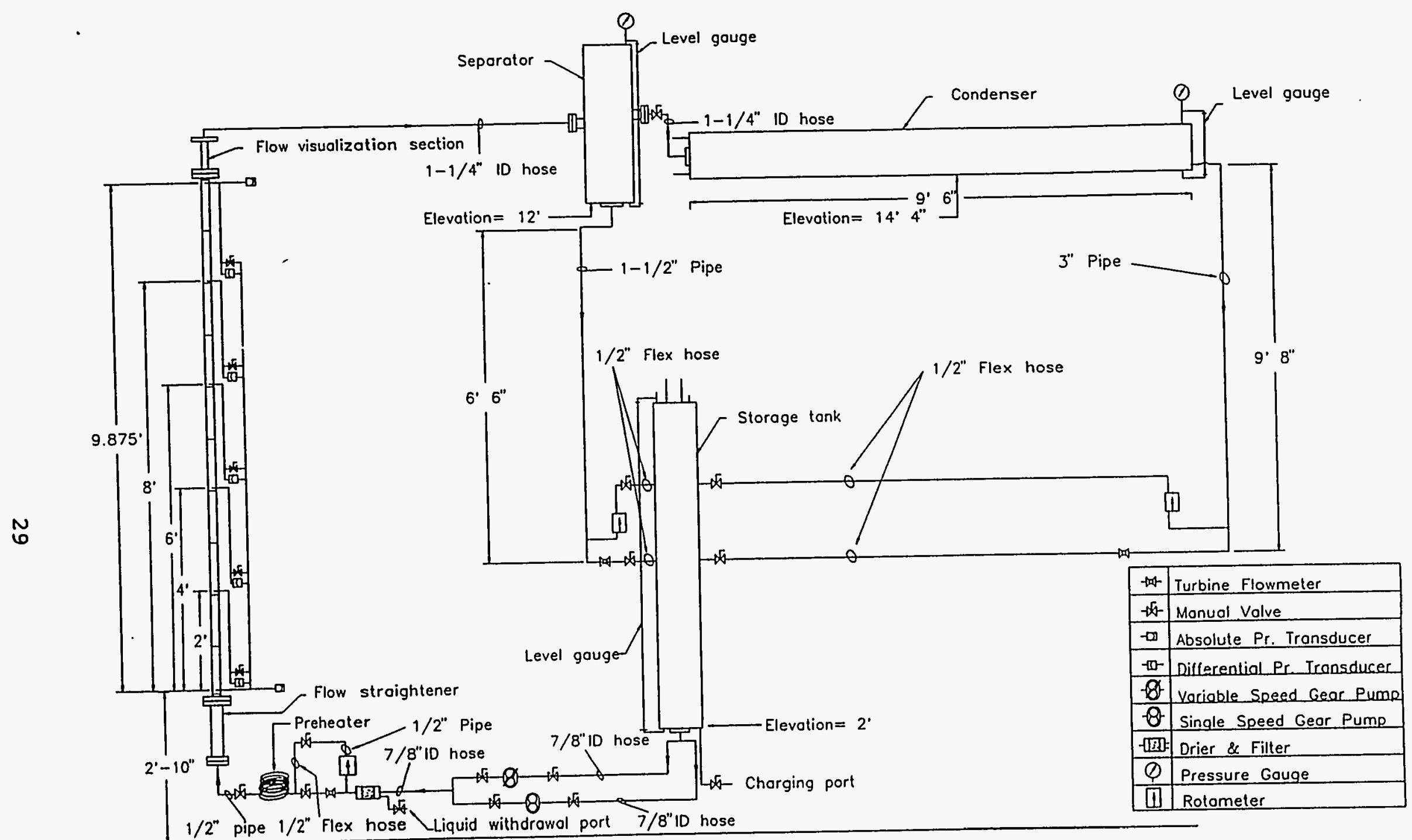

\section{TEST FLUID LOOP}

FIGURE 3 


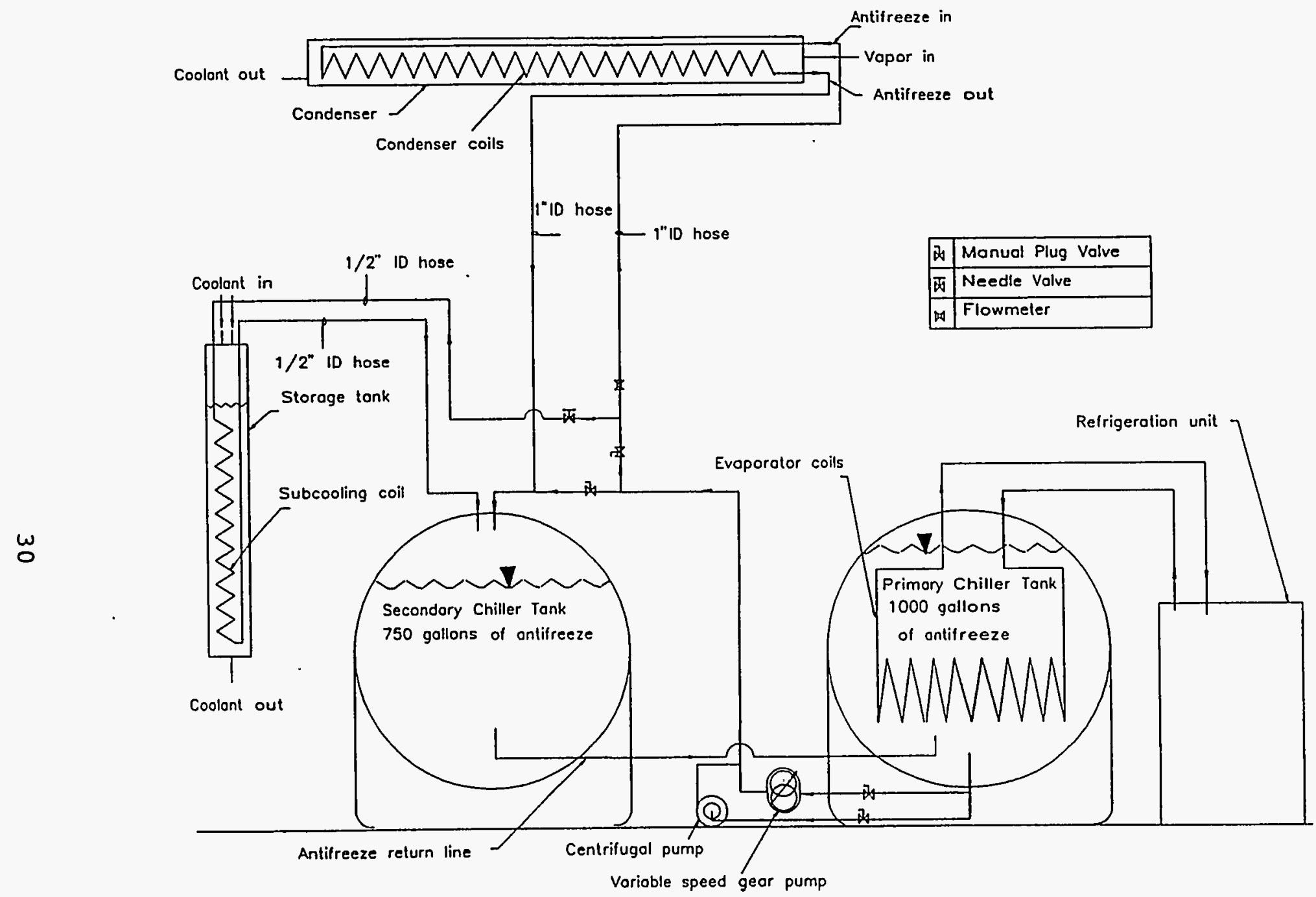

CHILLER SYSTEM

FIGURE 4 

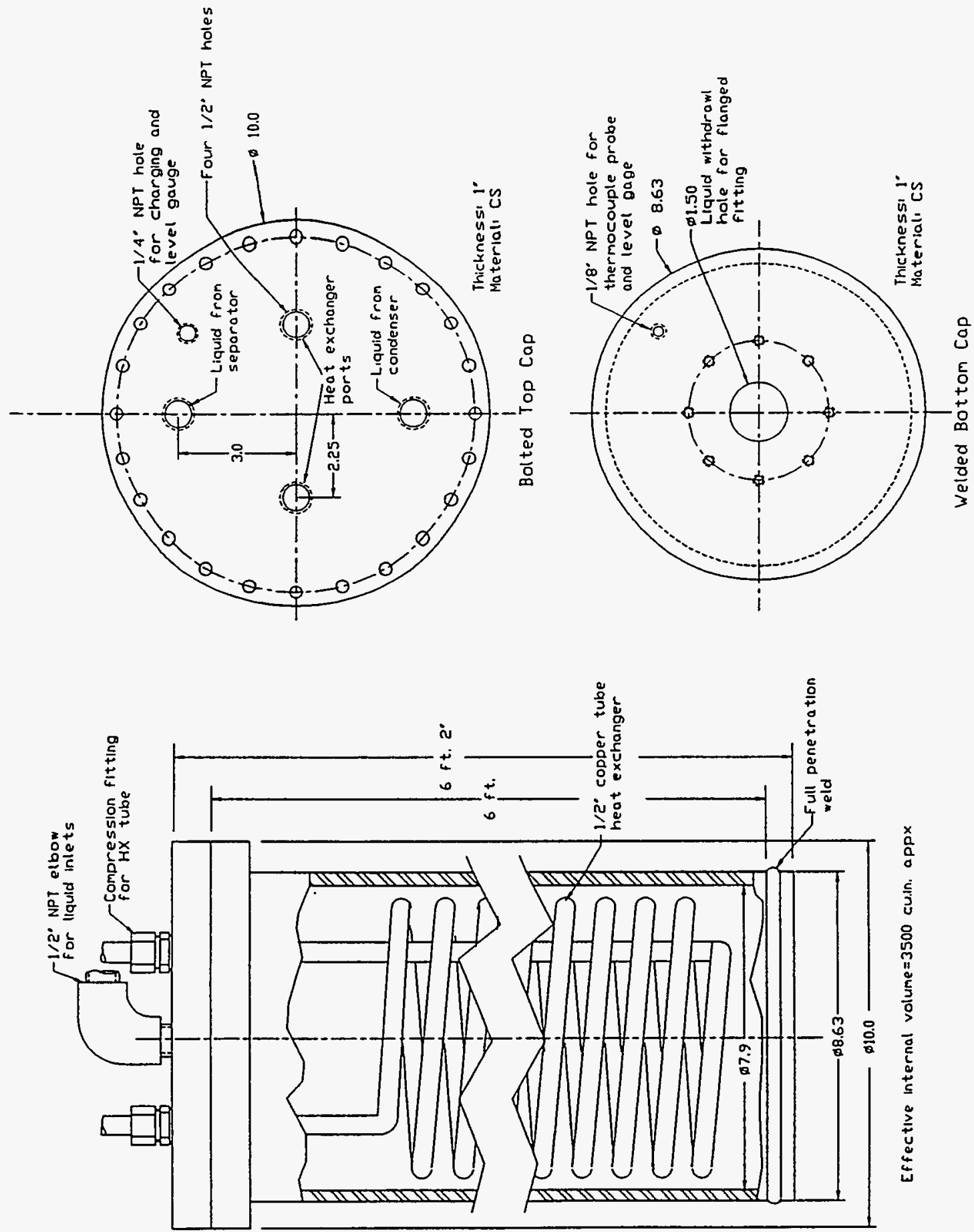

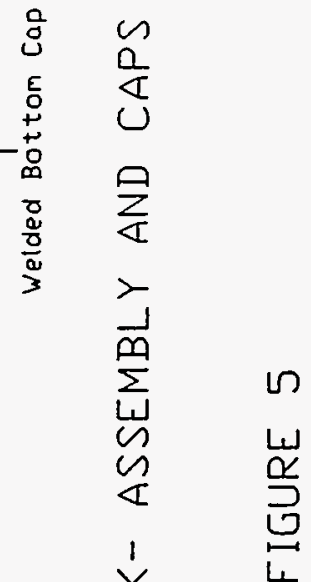

$\underset{⿱}{Z}$

岂

吕 


\section{- 0 PSID \\ 100 PSID}

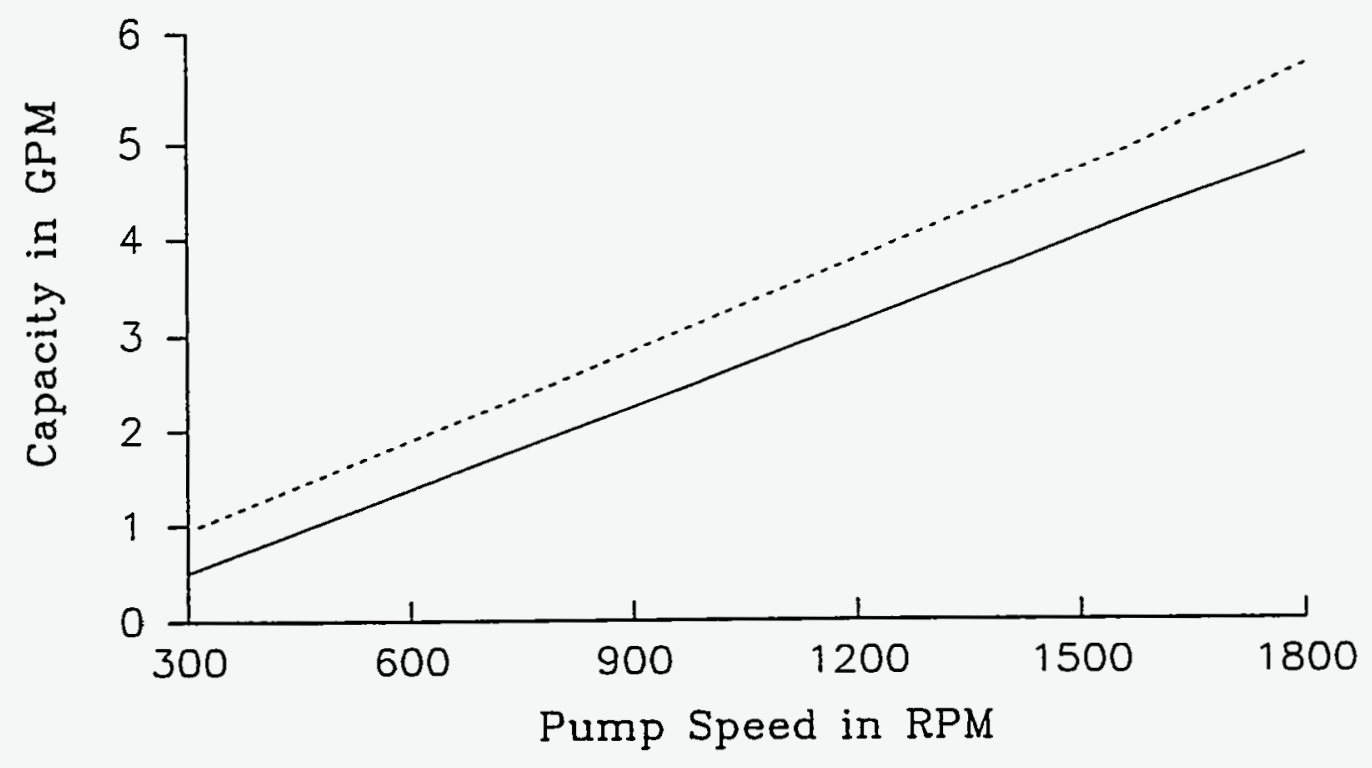

\section{PUMP CAPACITY VS. SPEED \\ FIGURE 6}




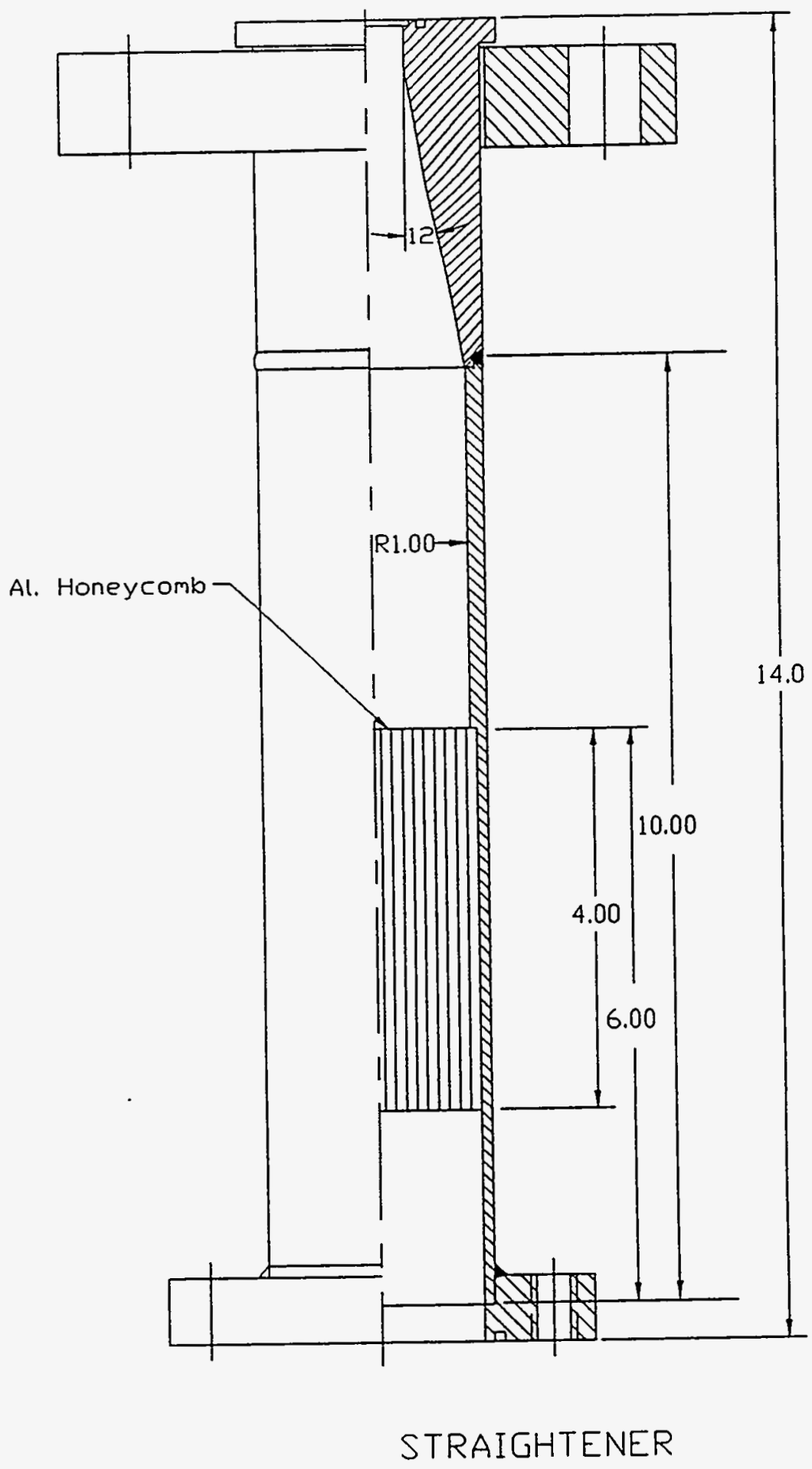

FIGURE 7 


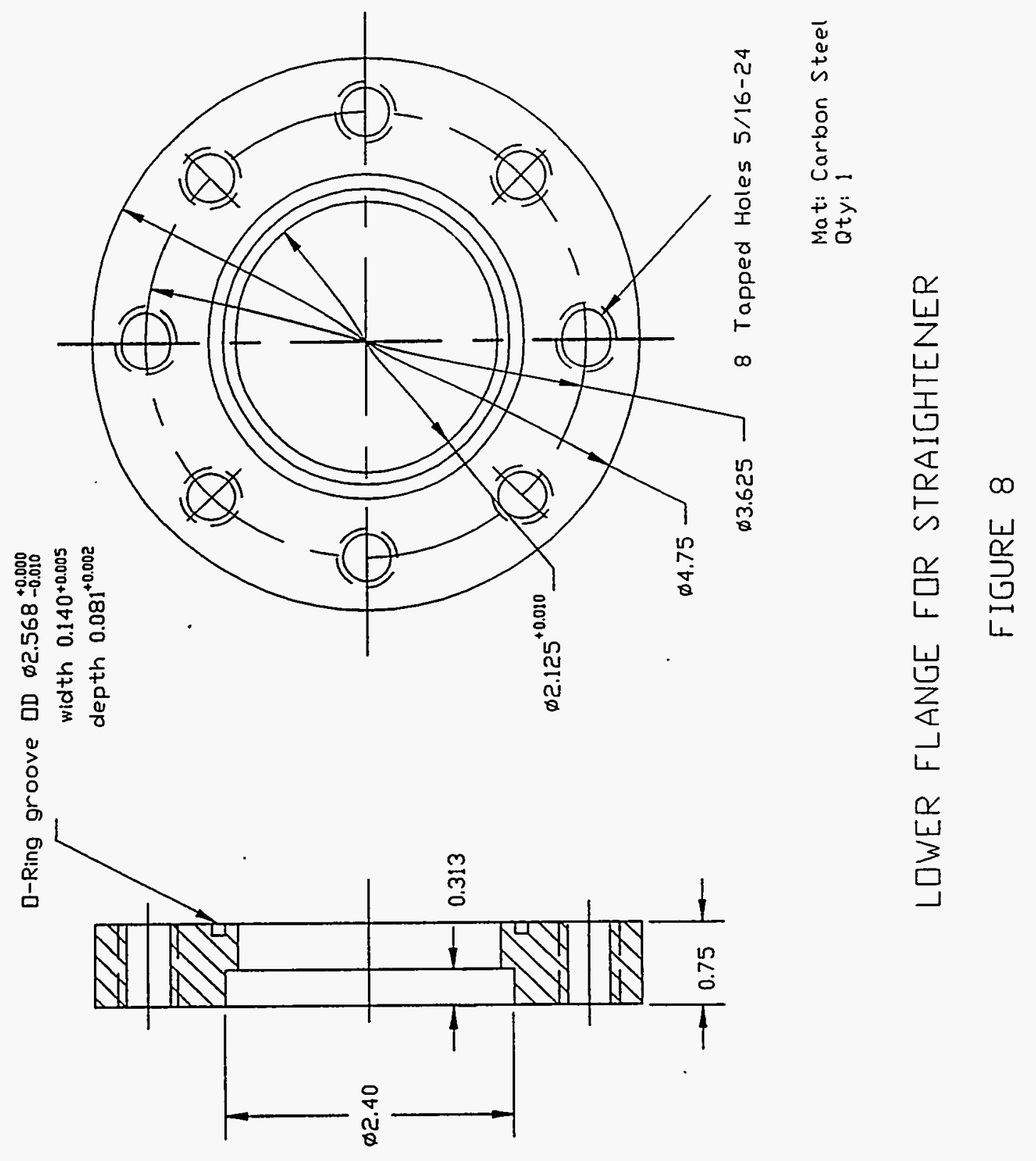




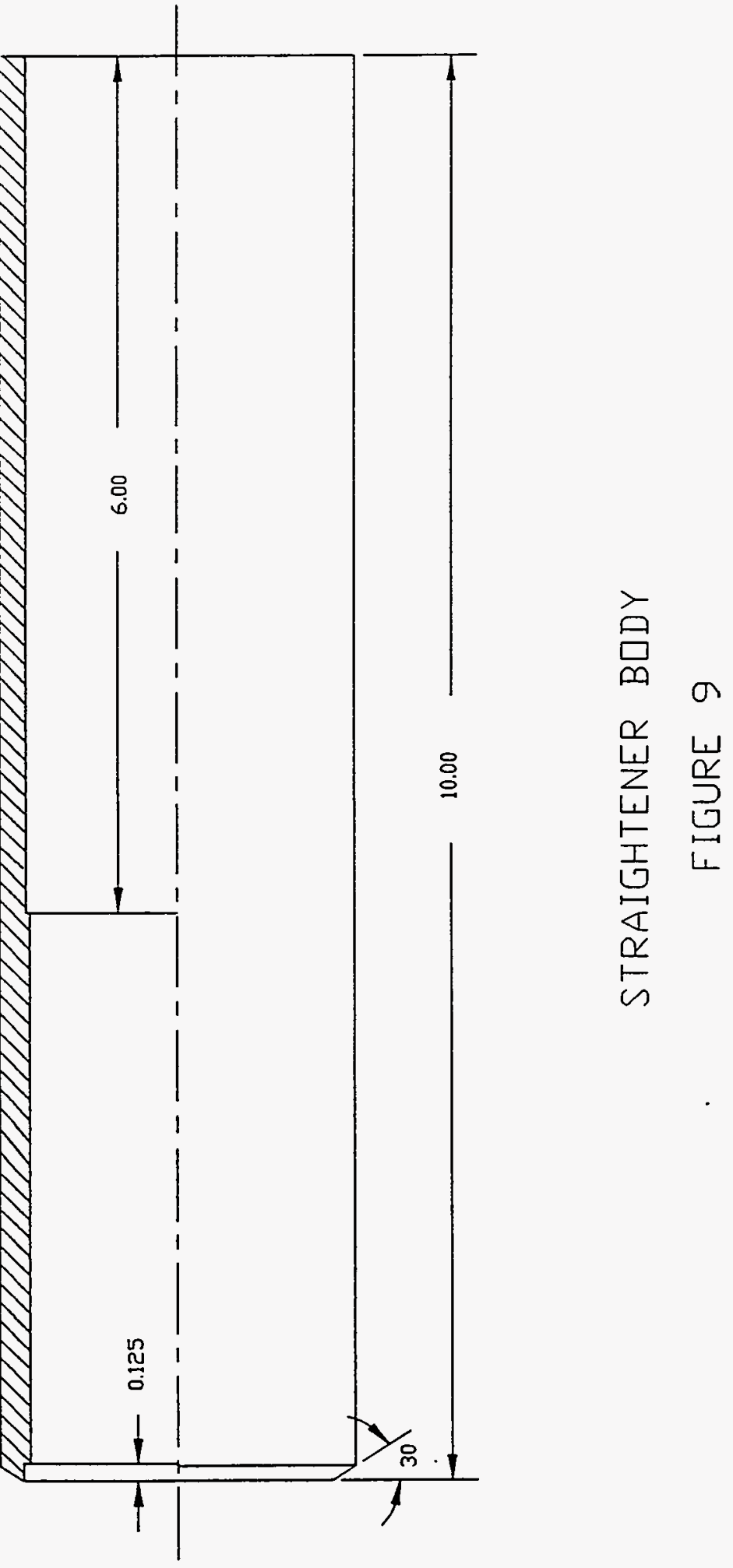




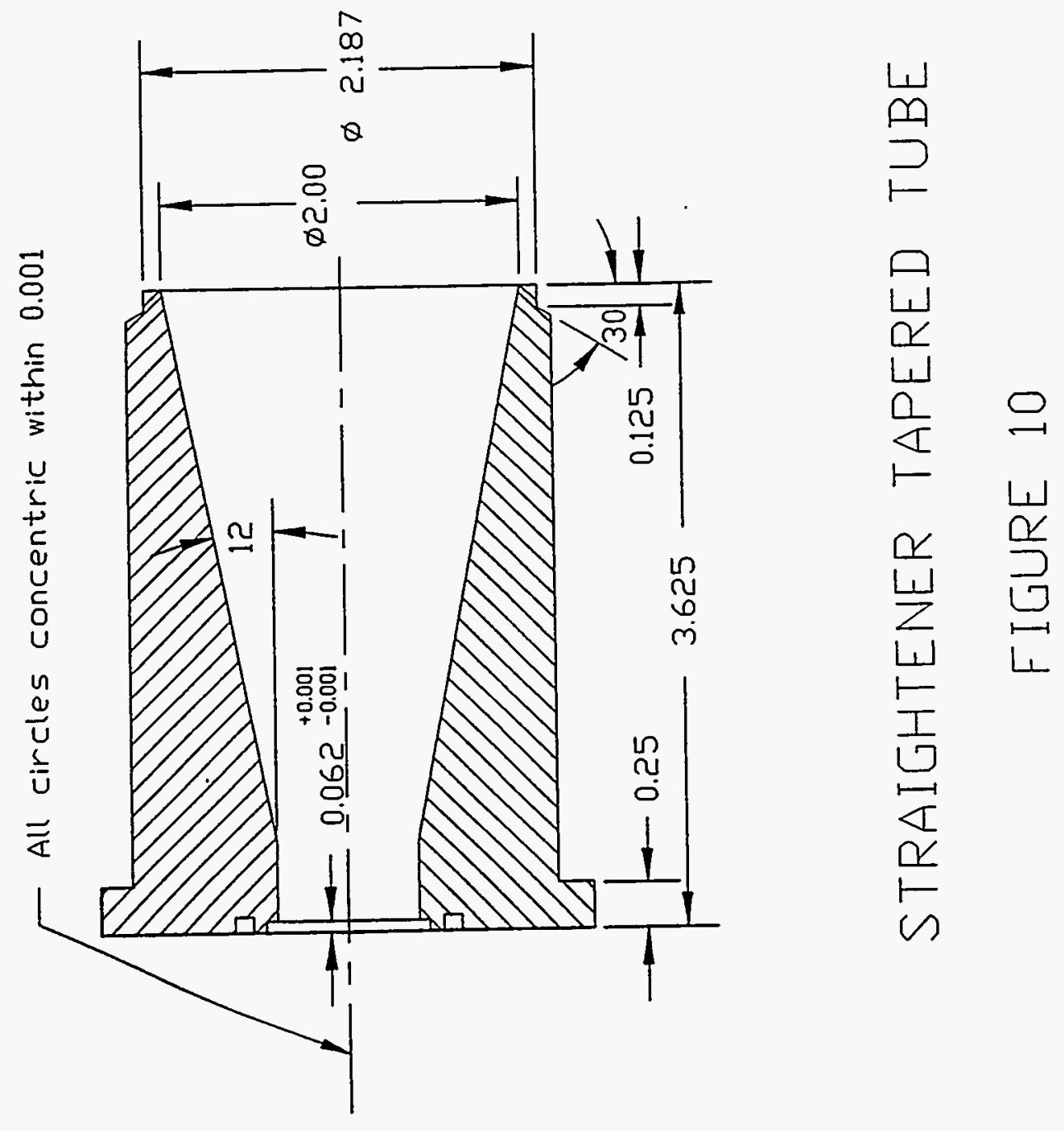




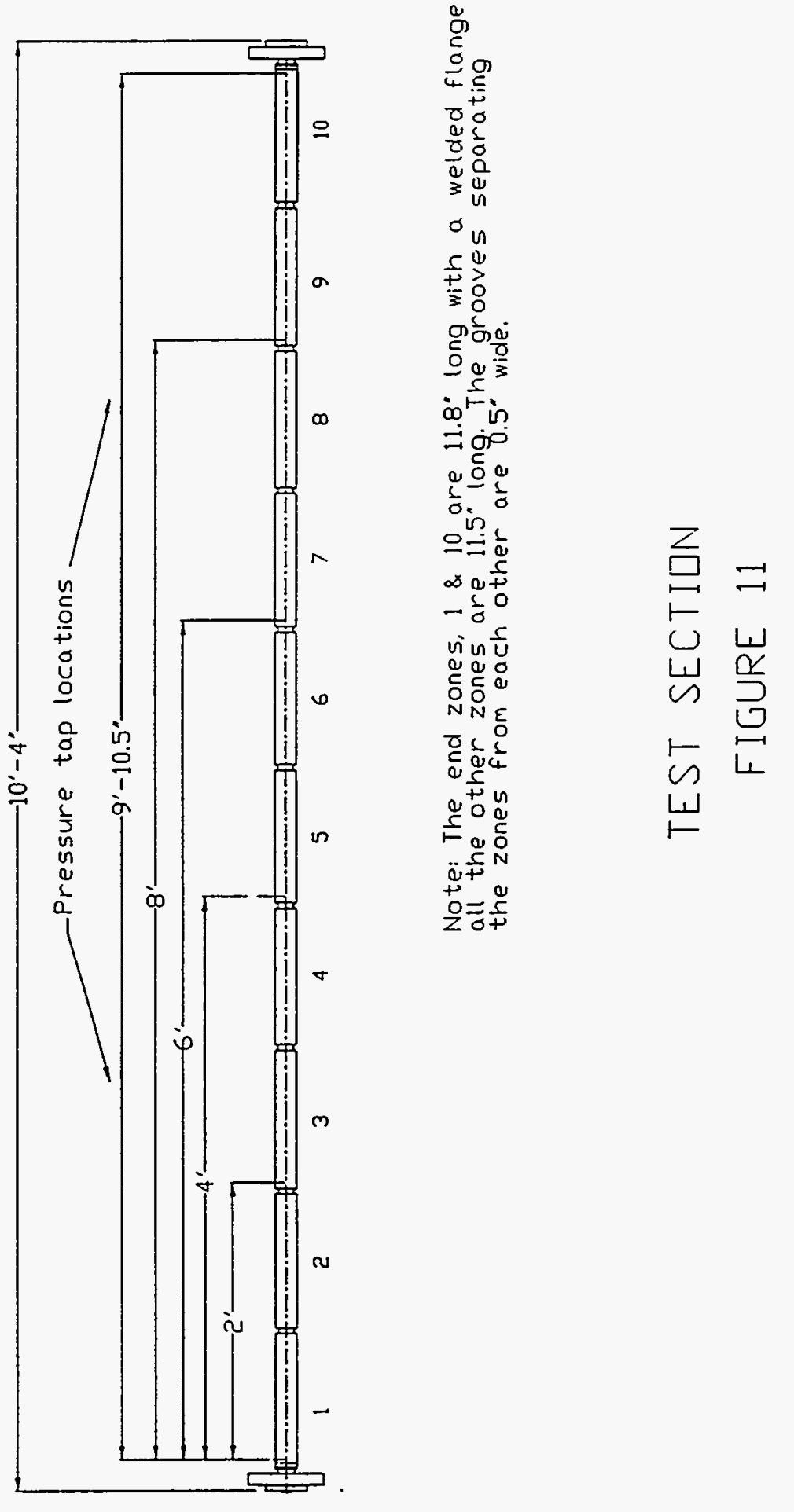




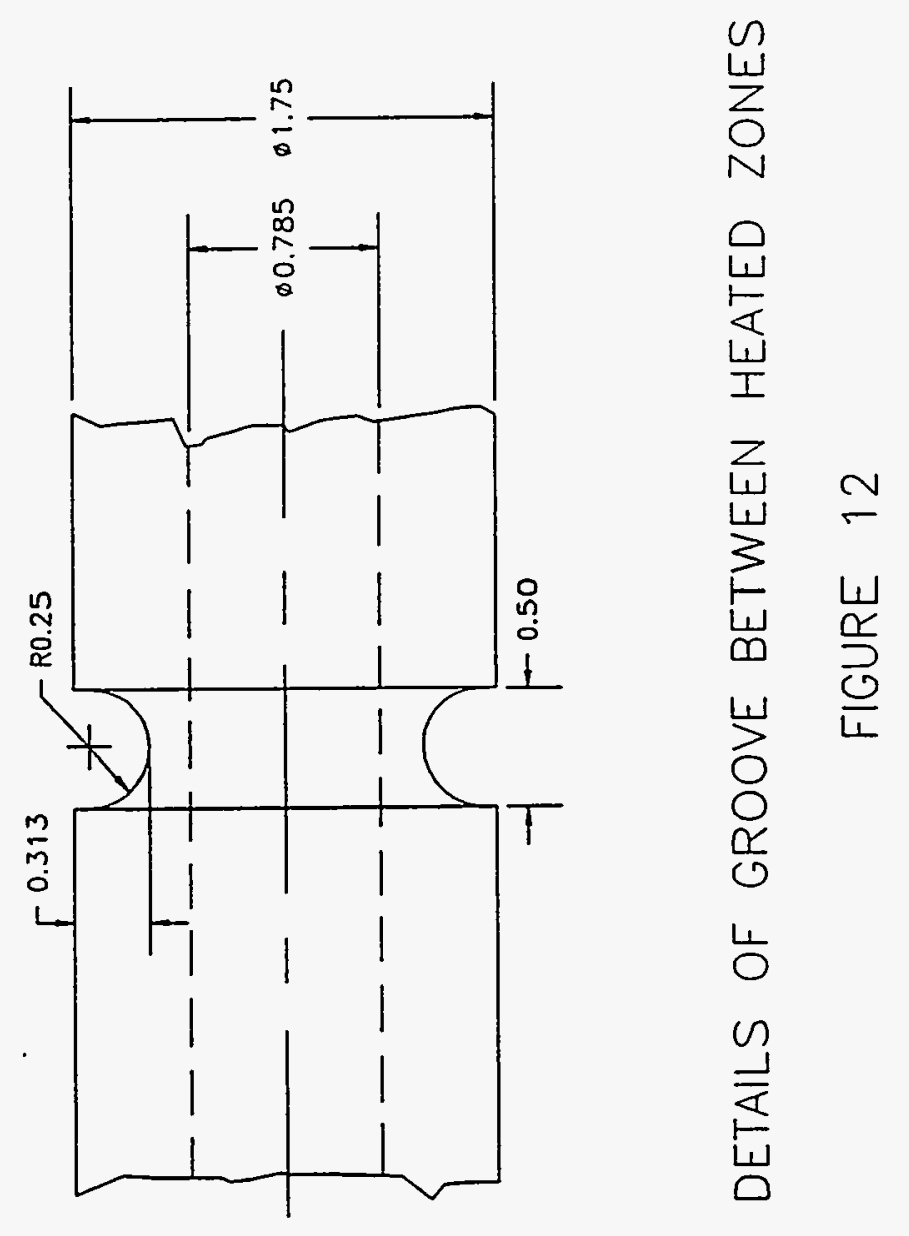




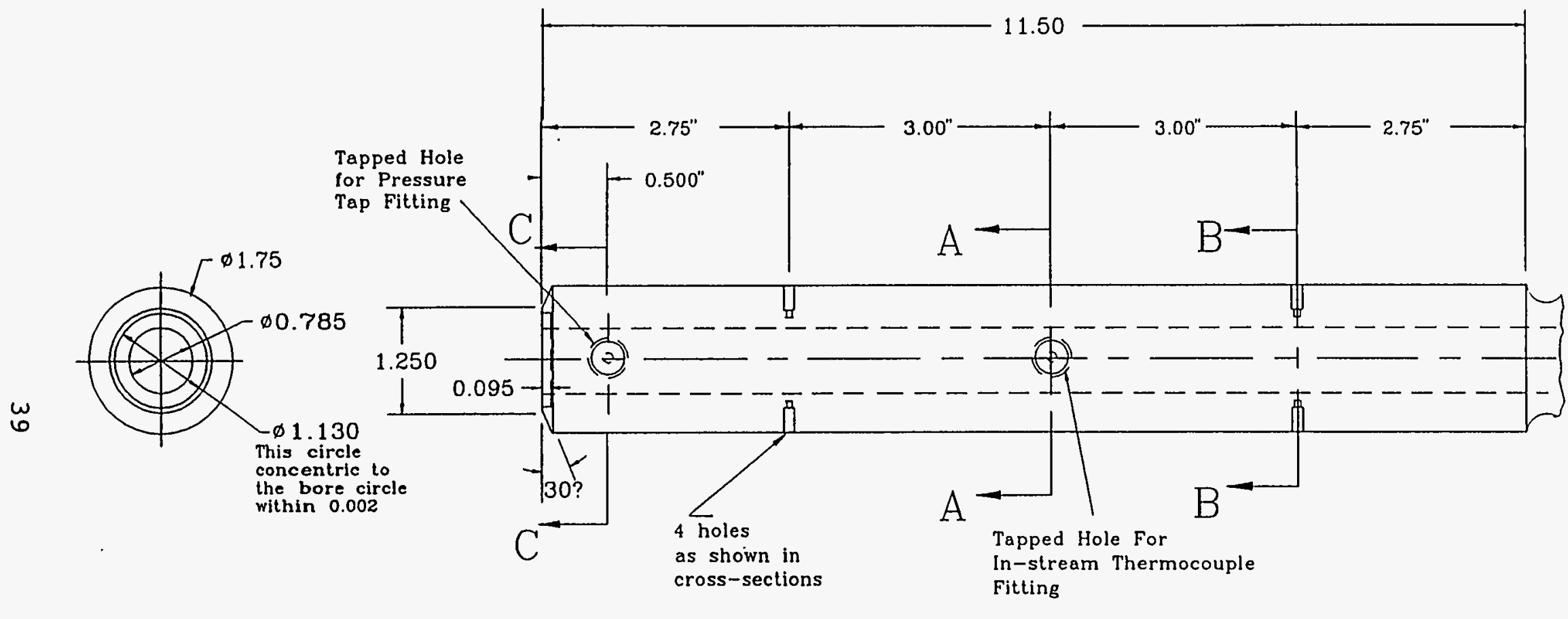

DETAILS GF ZDNE \# 1 (BEF DRE WELDING END-FLAIIGE)

FIGURE 13 


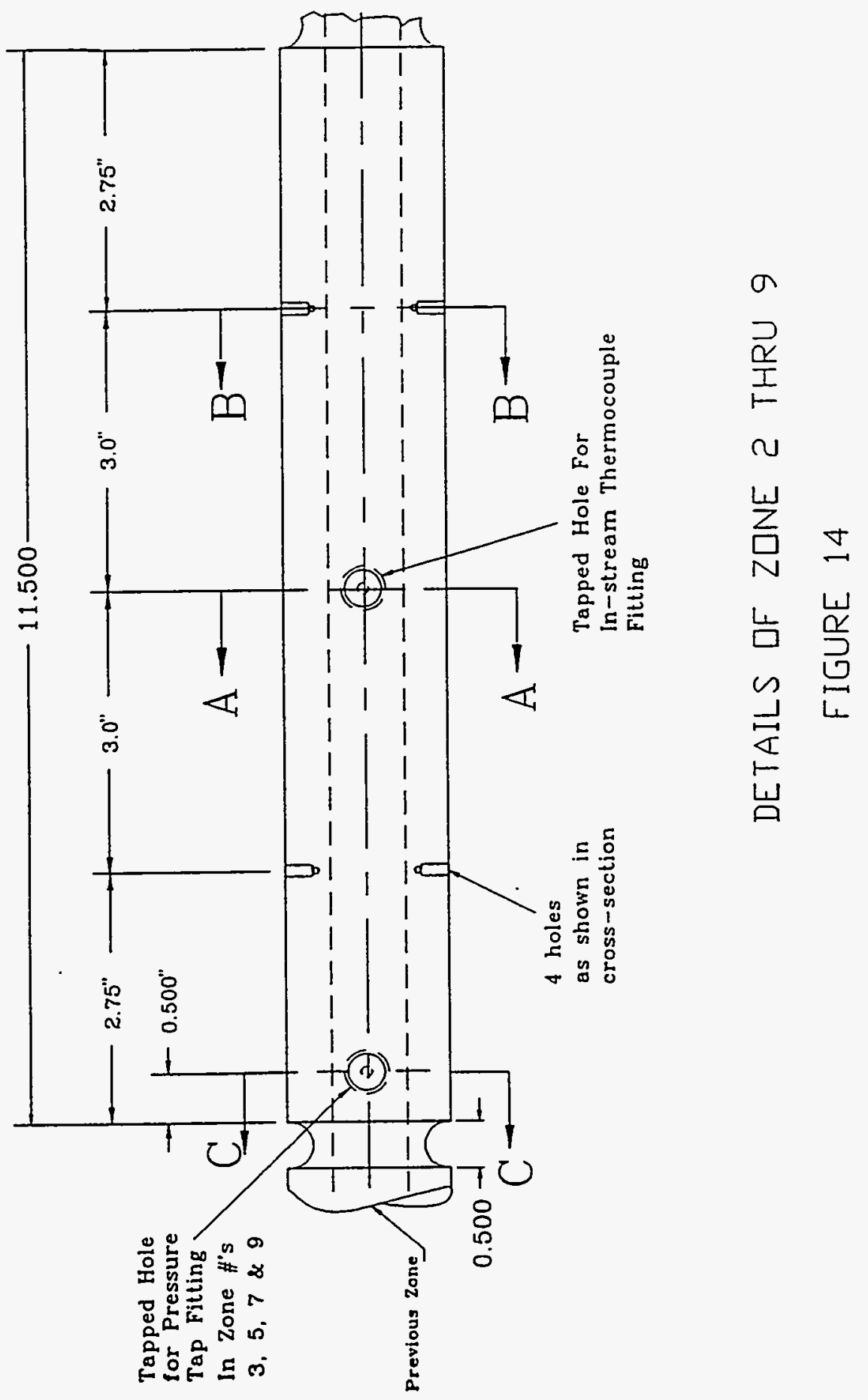




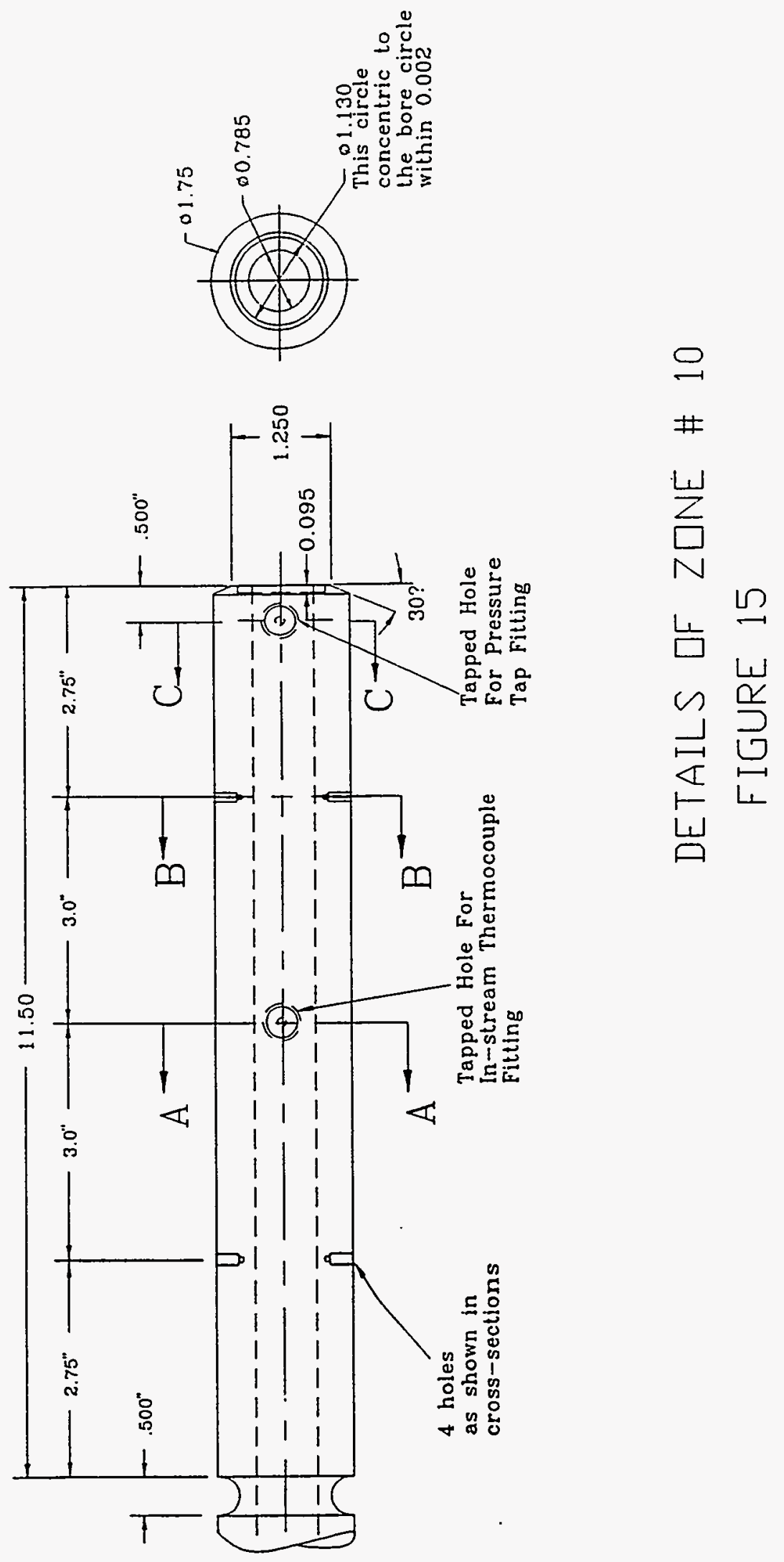




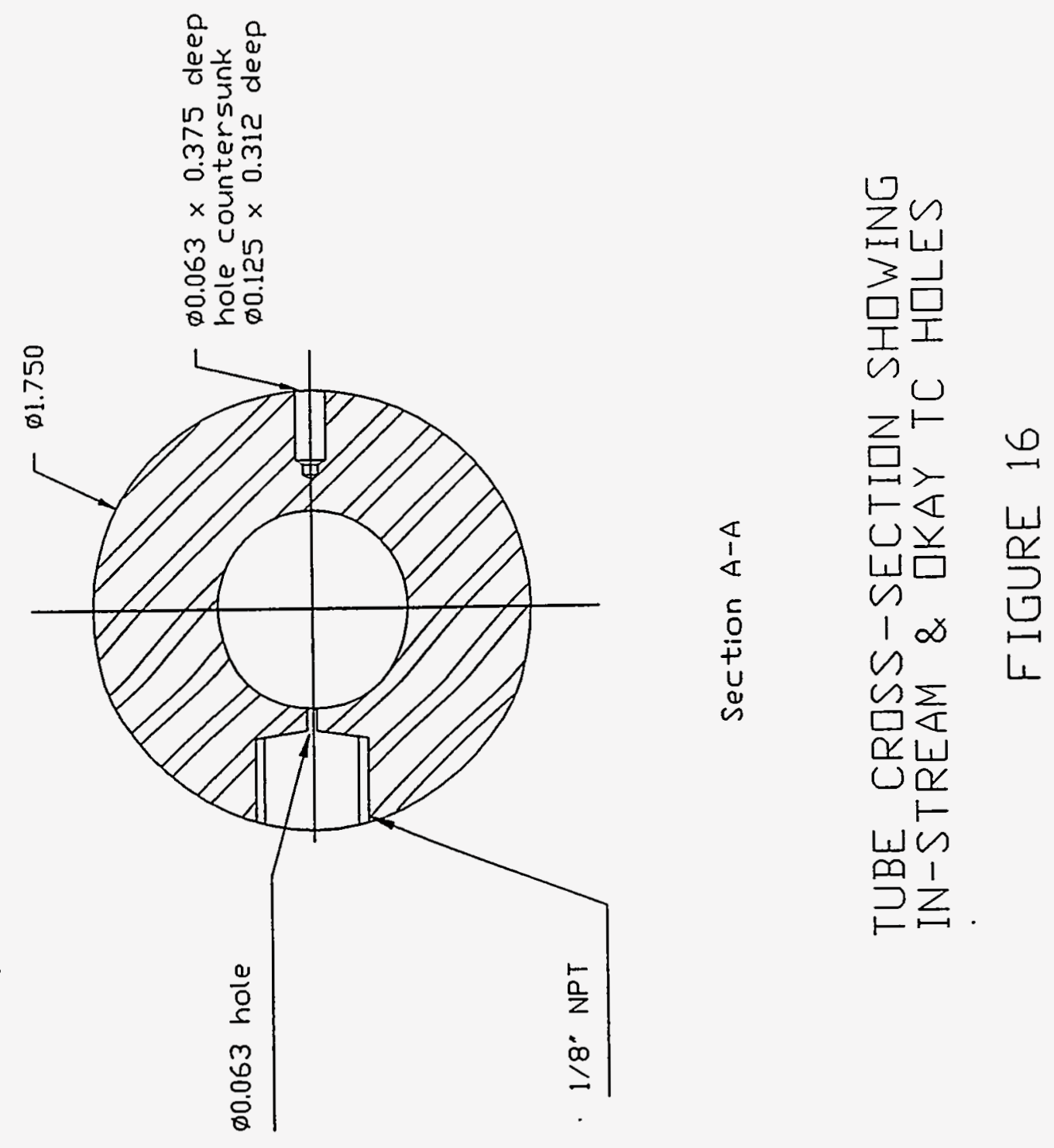



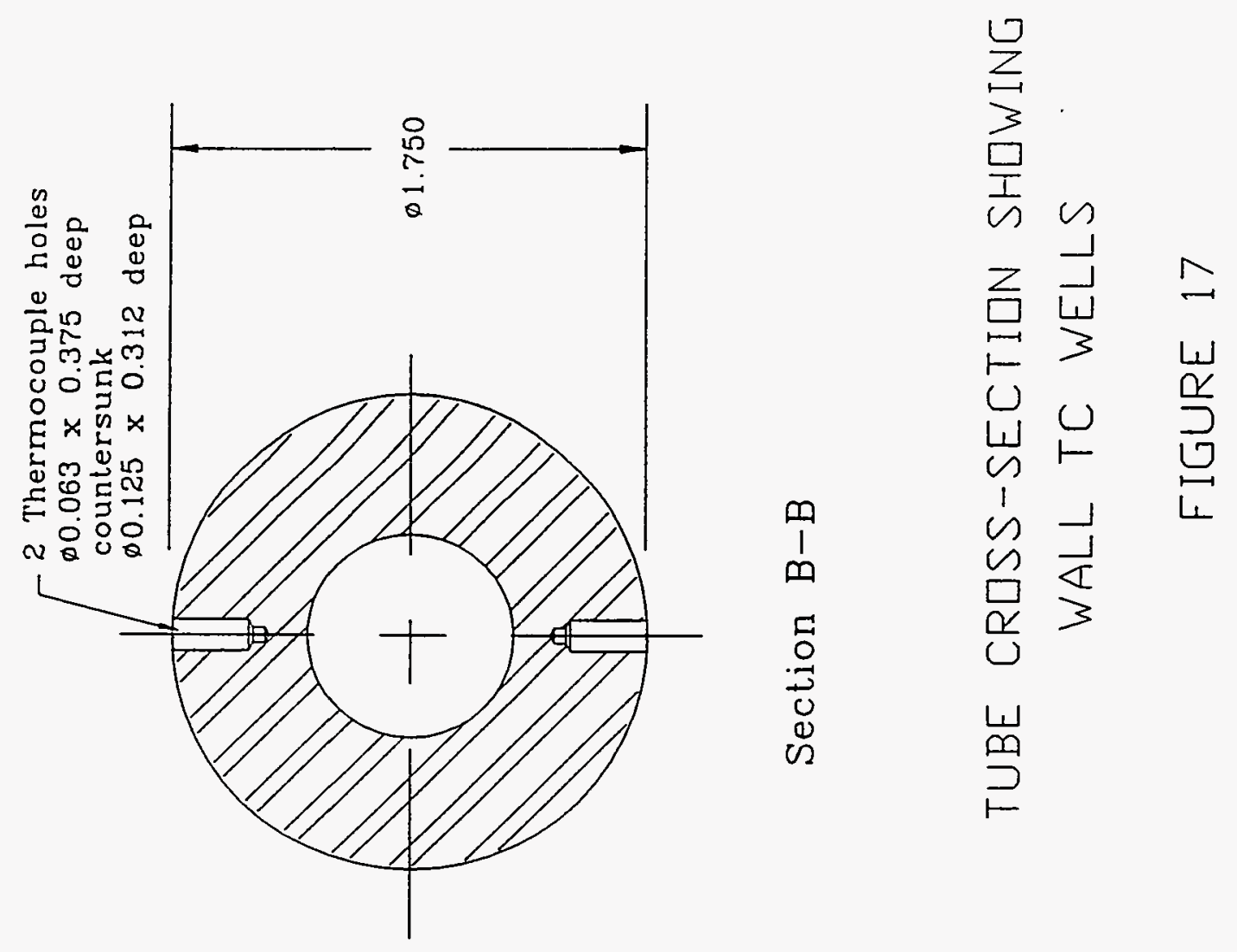

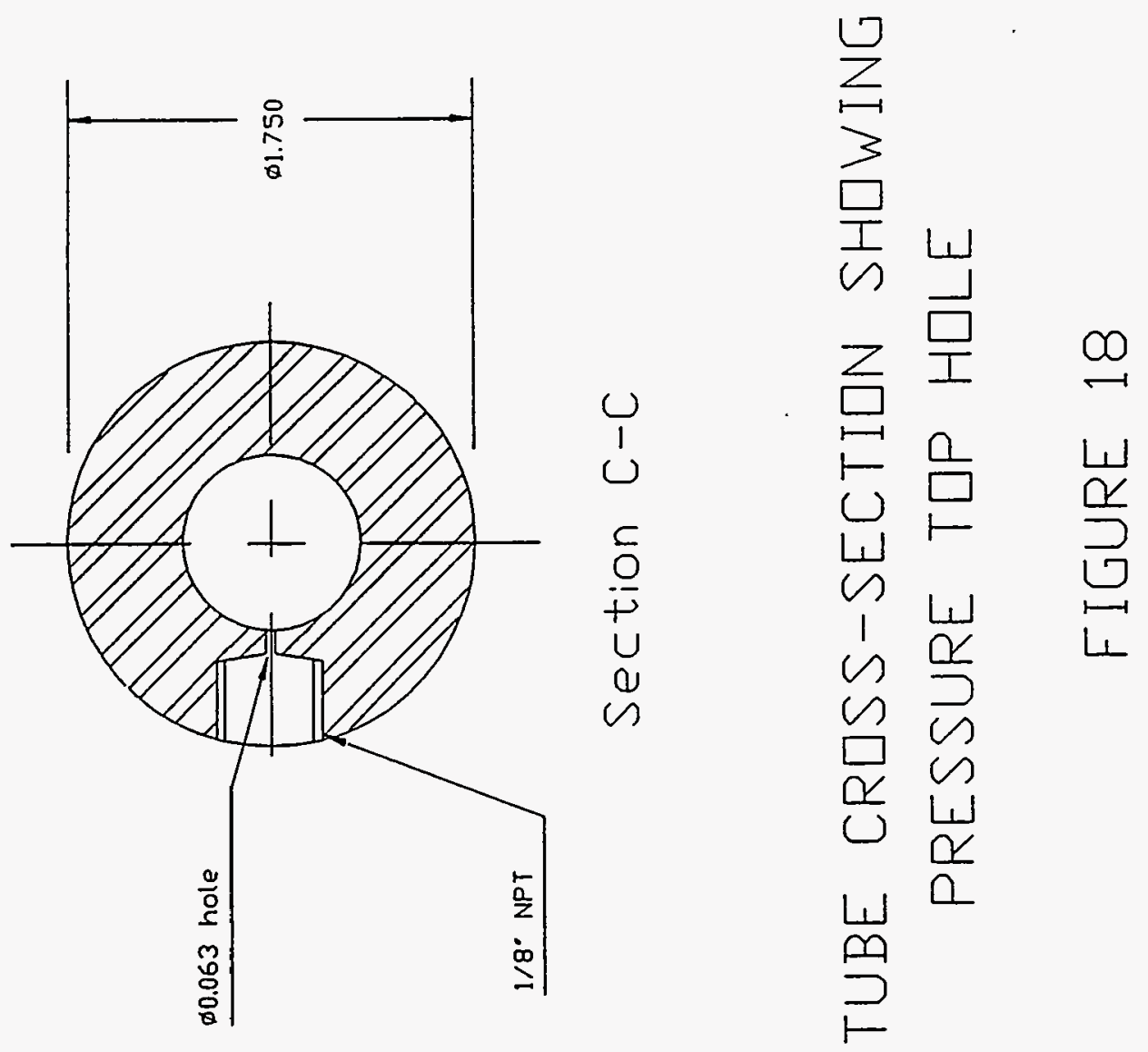

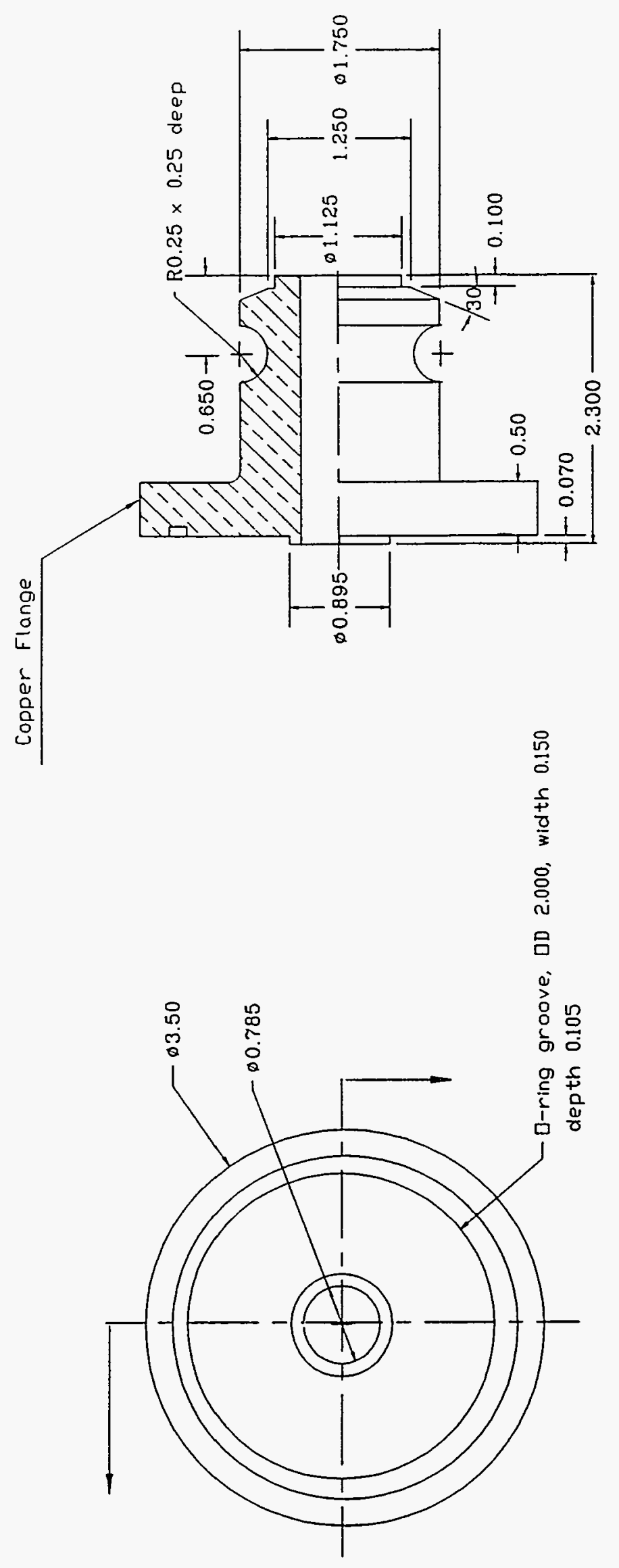


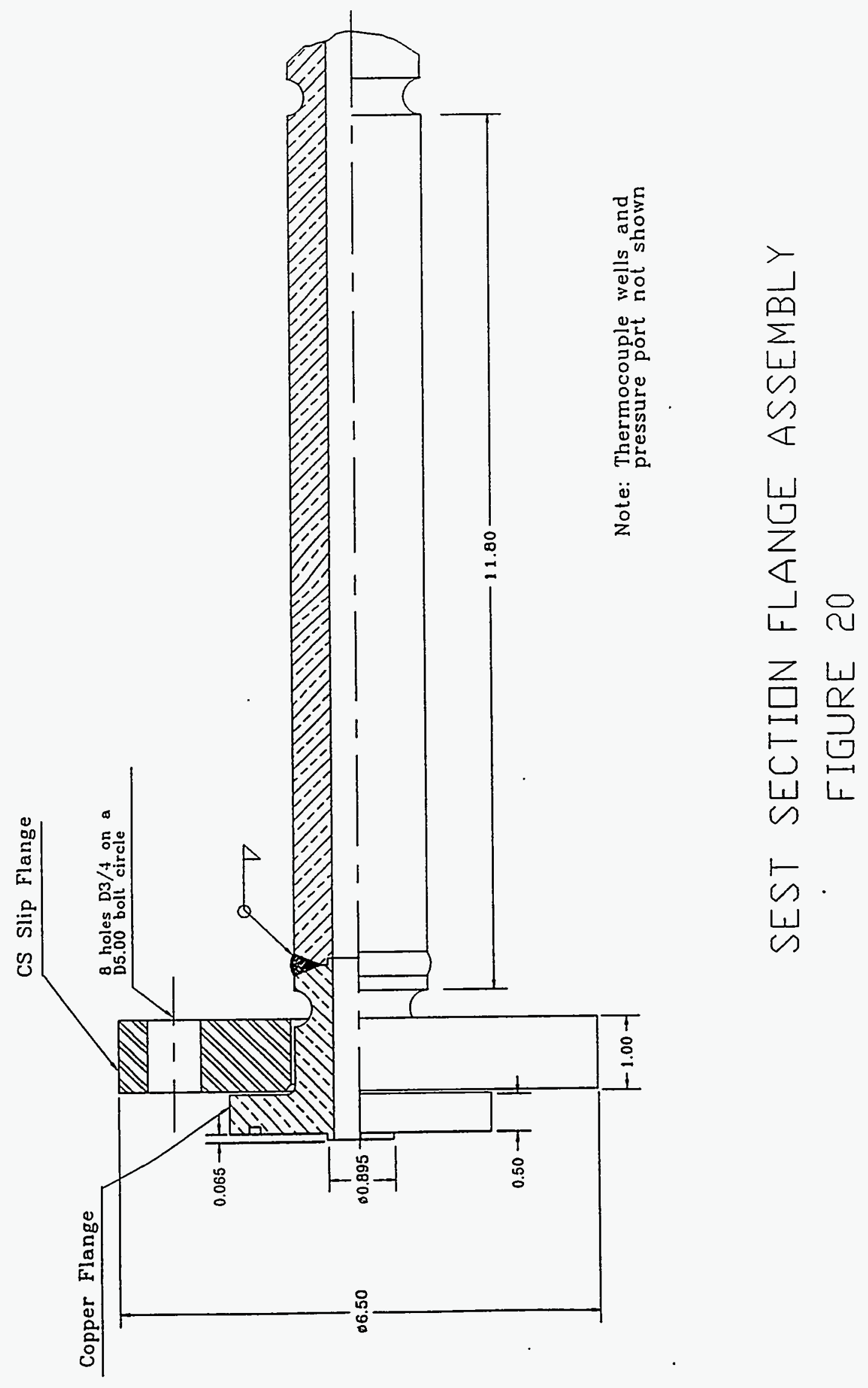




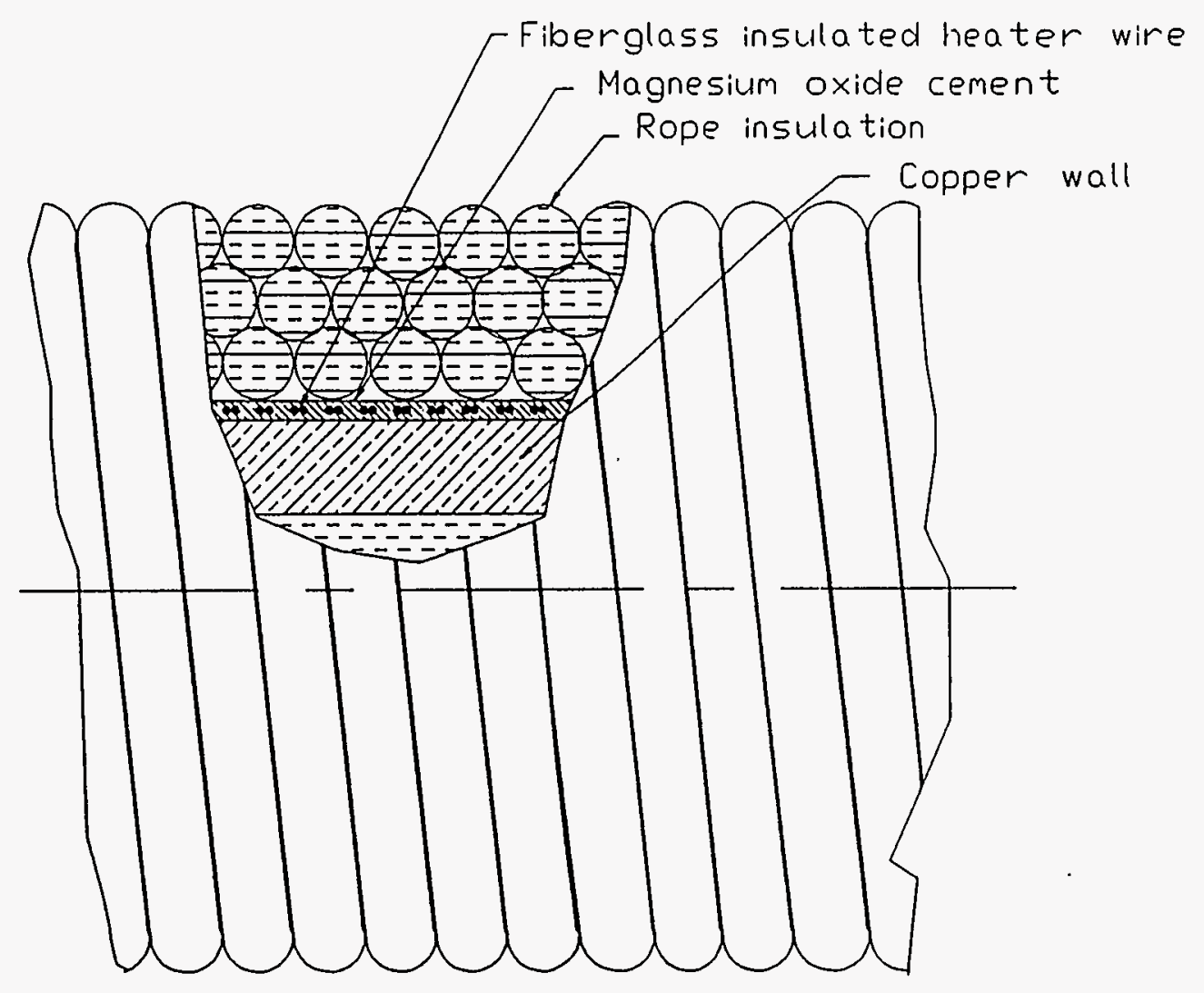

HEATER AND INSULA TION DETAILS

FIGURE 21 


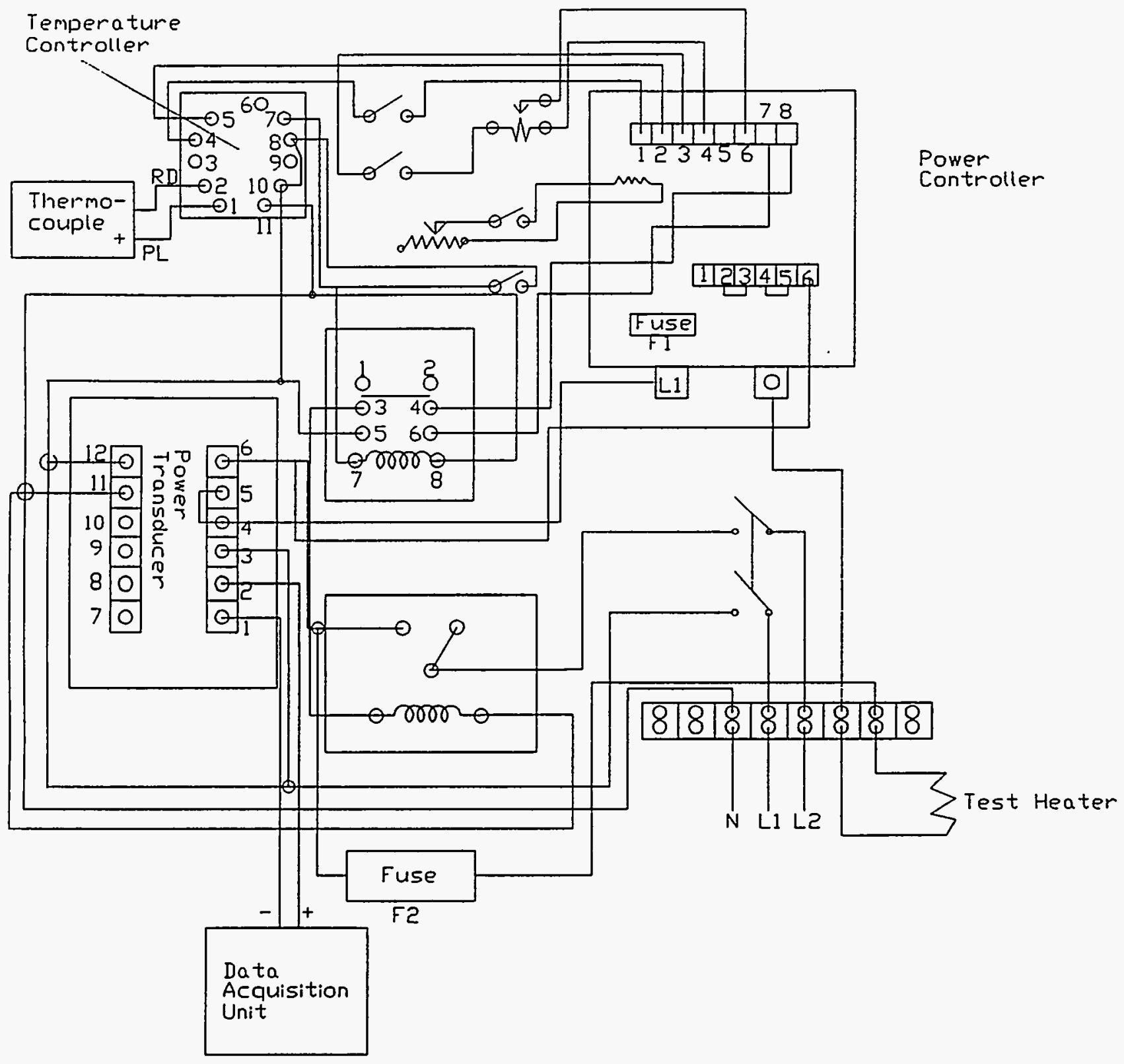

TEMPERATURE AND PQWER CINTRIL CIRCUIT FIGURE 22 

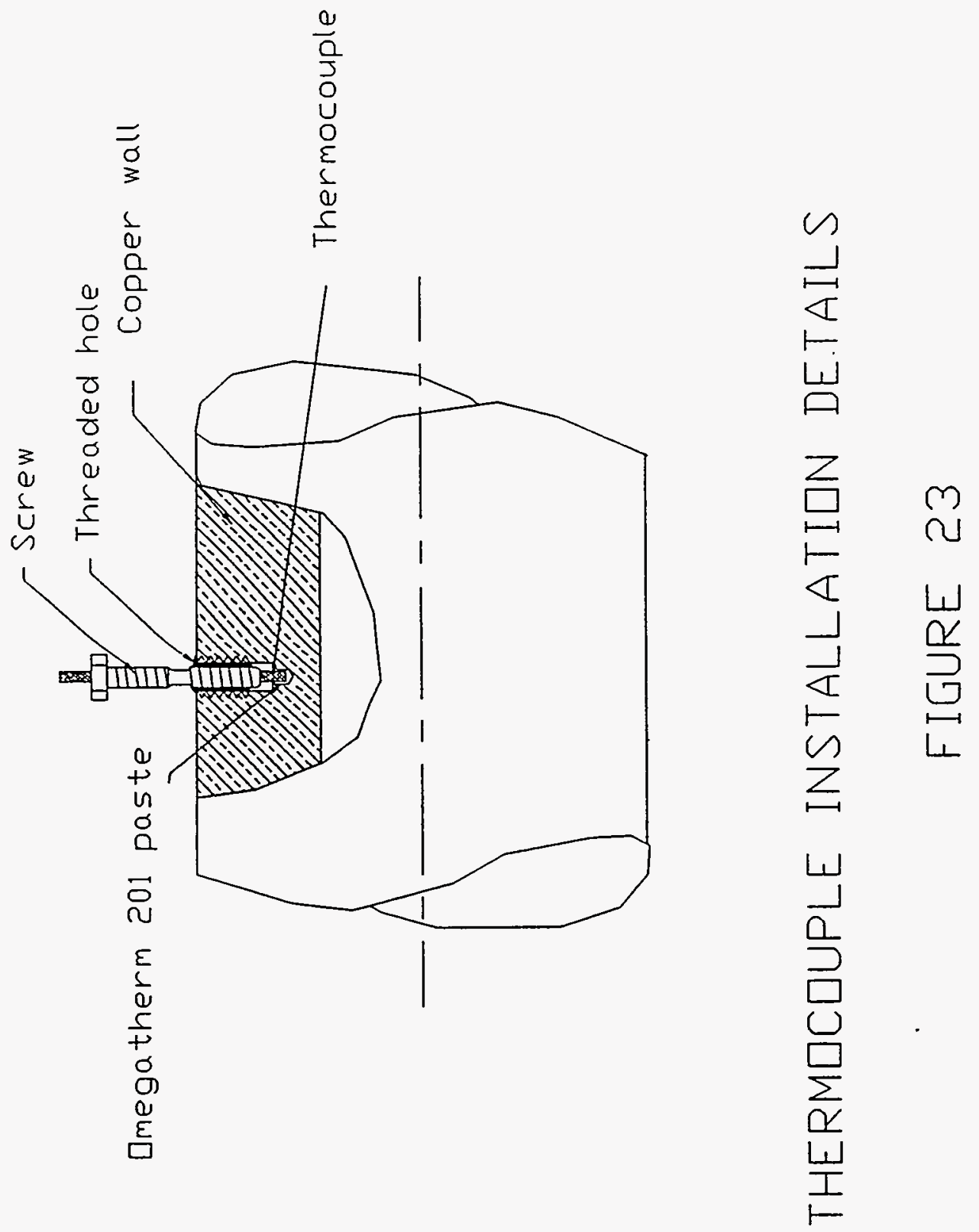


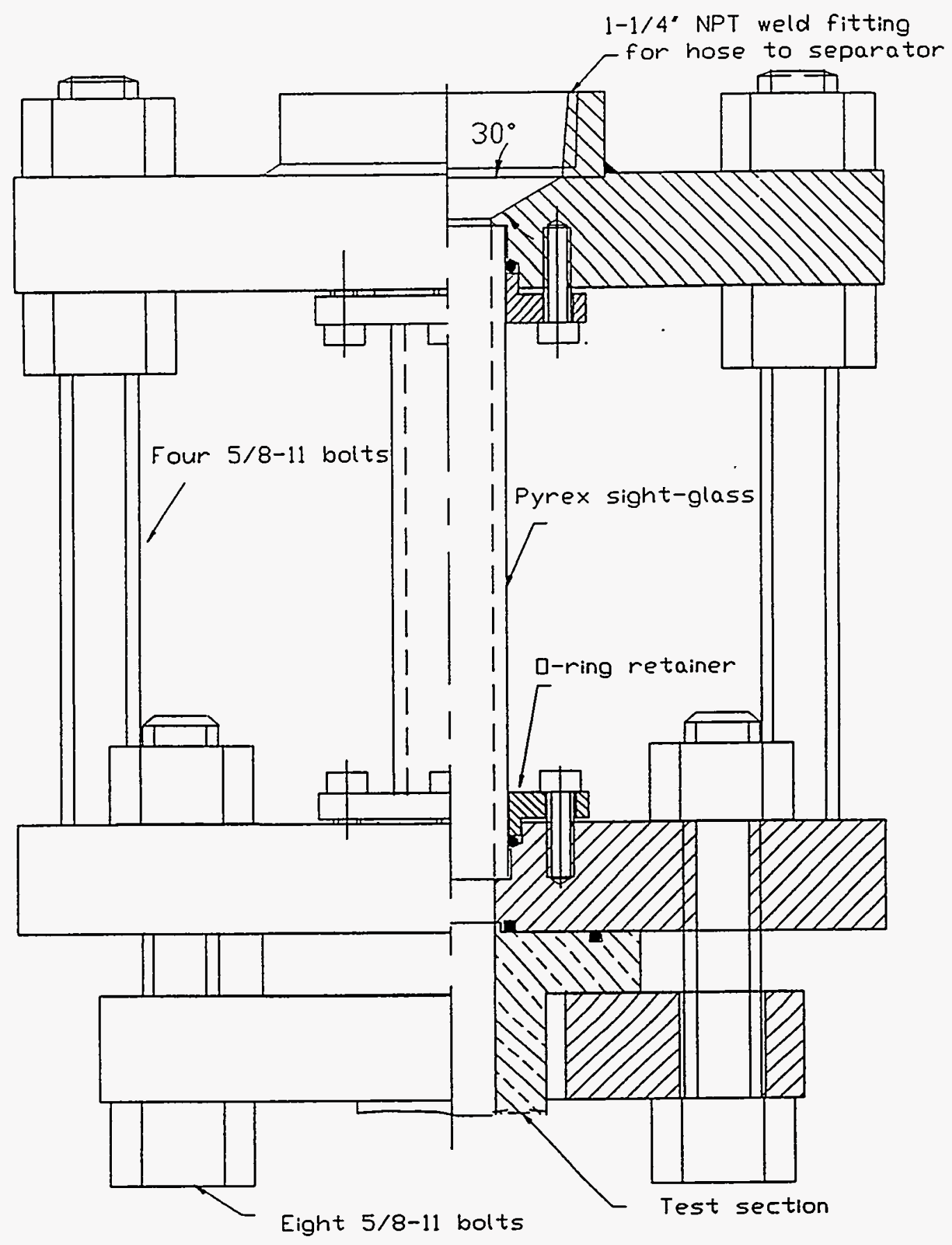

FLOW VISUALIZATION SIGHT GLASS DETAILS

FIGURE 24 


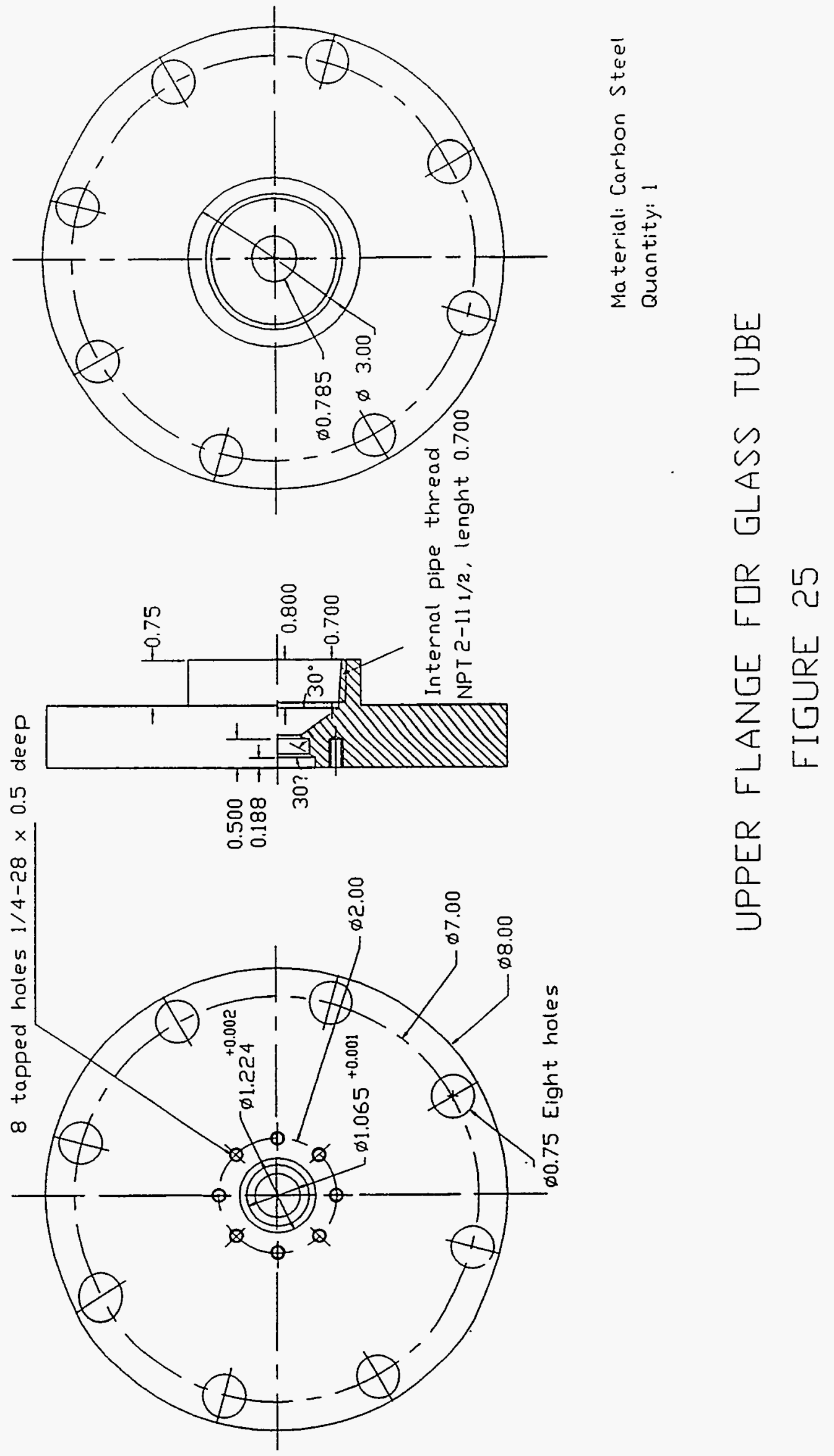




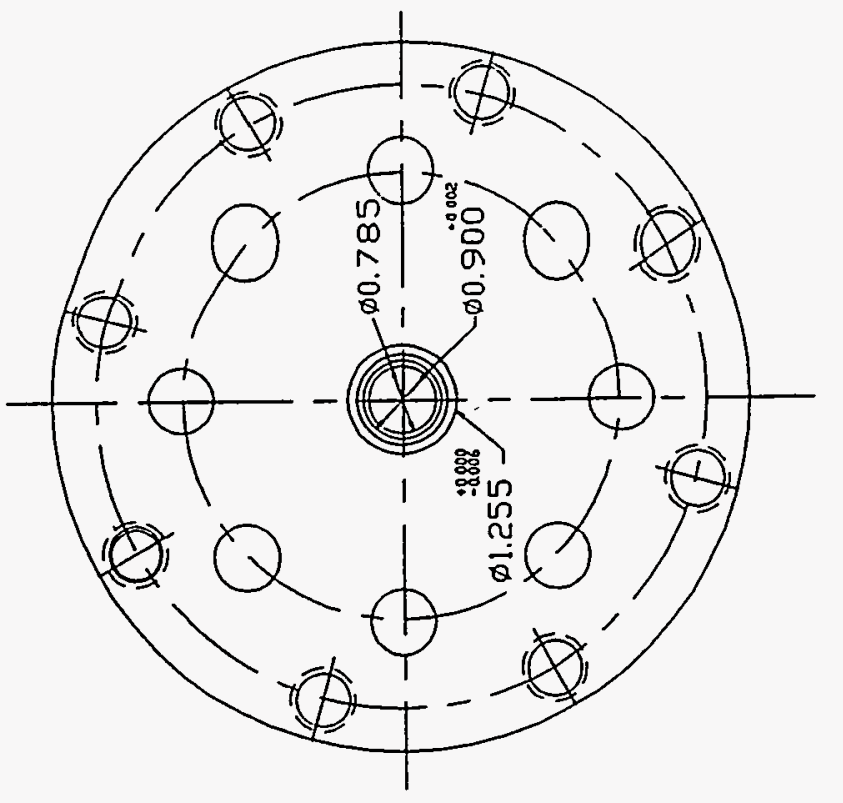

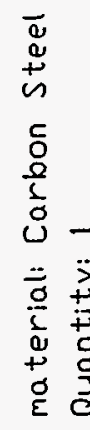
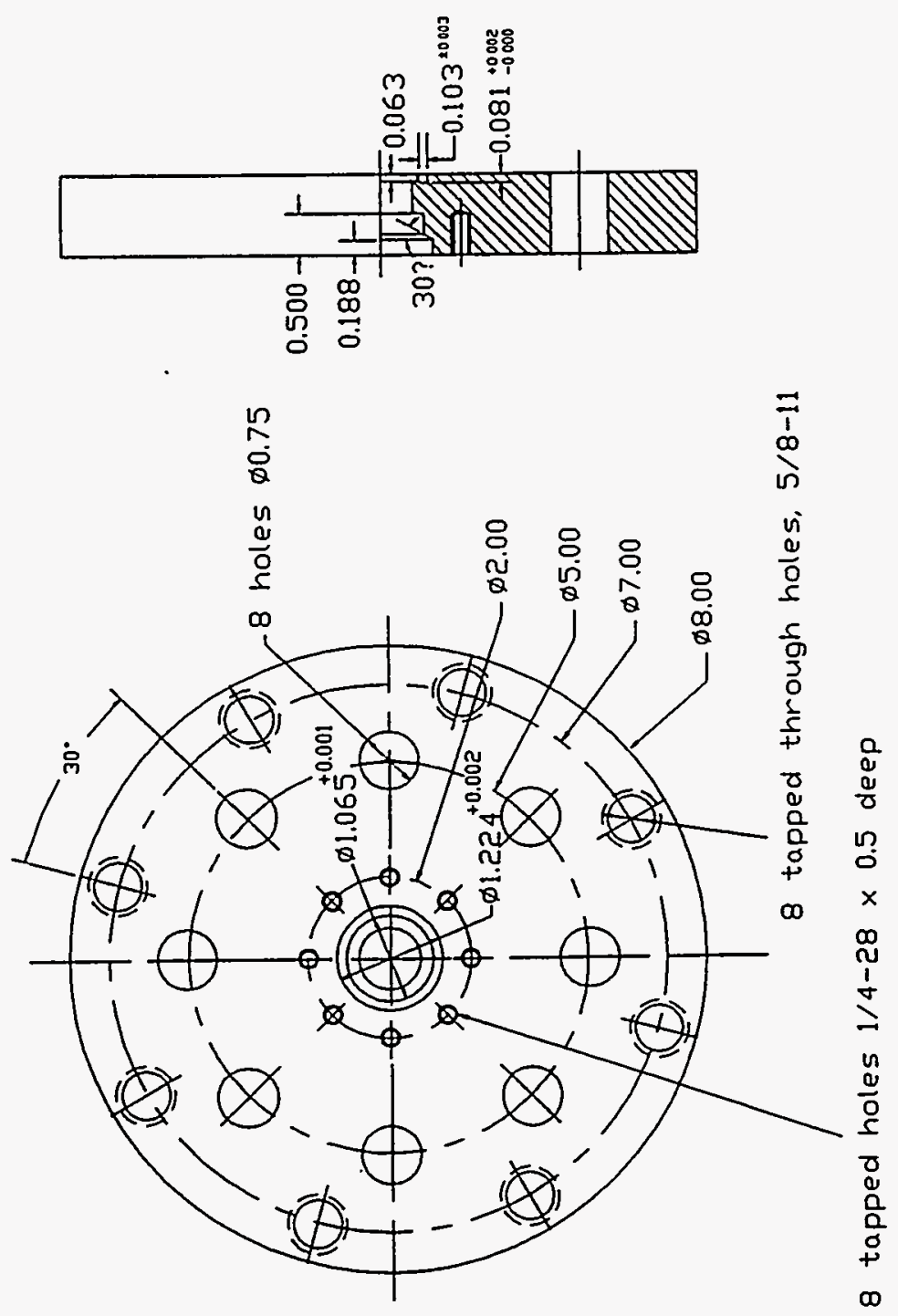

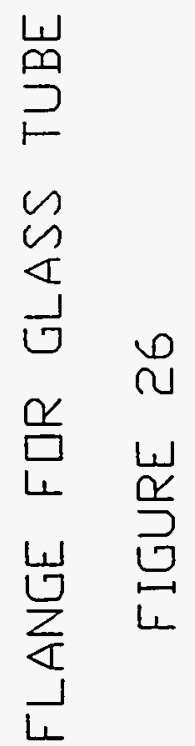




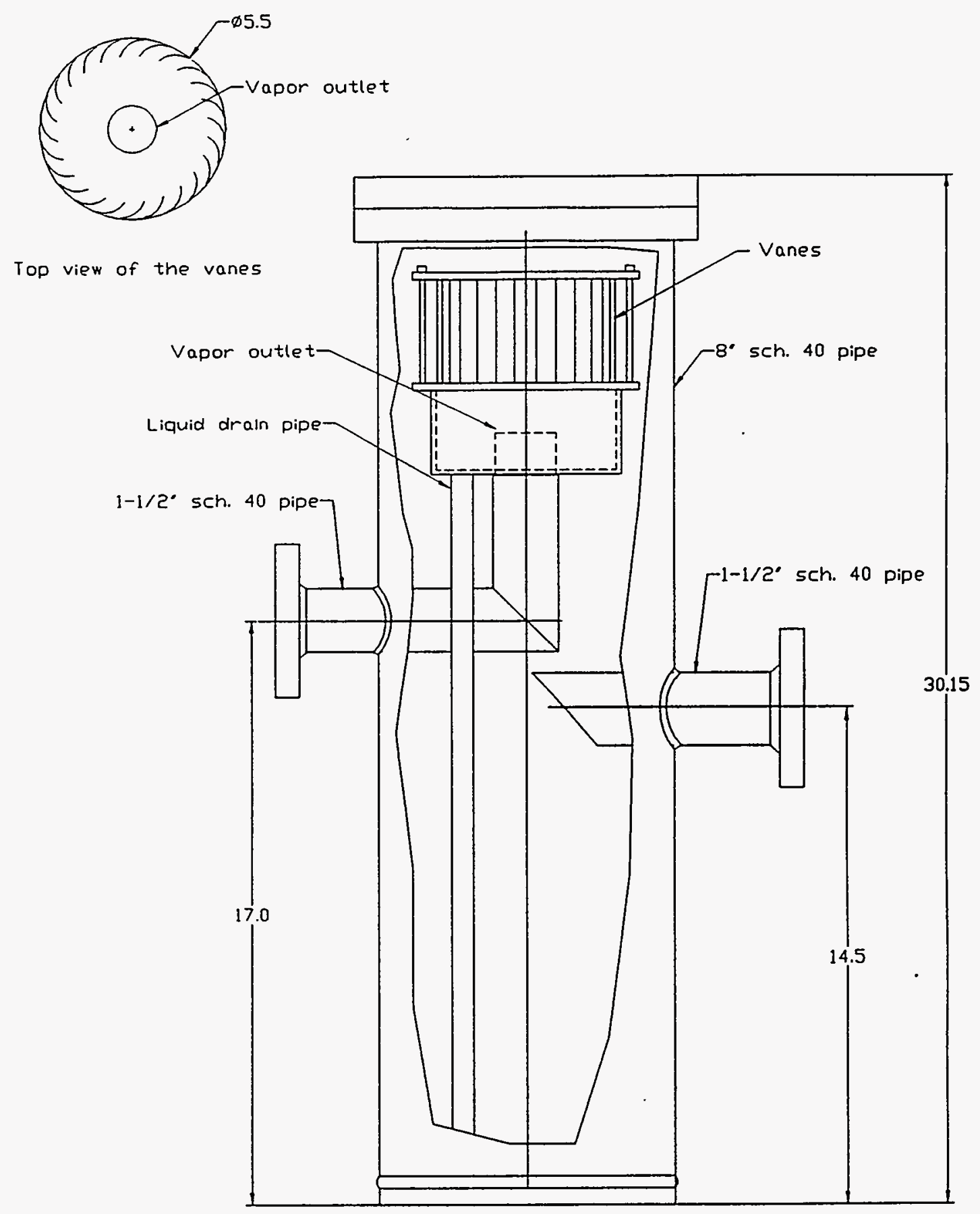

SEPARATUR

FIGURE 27 


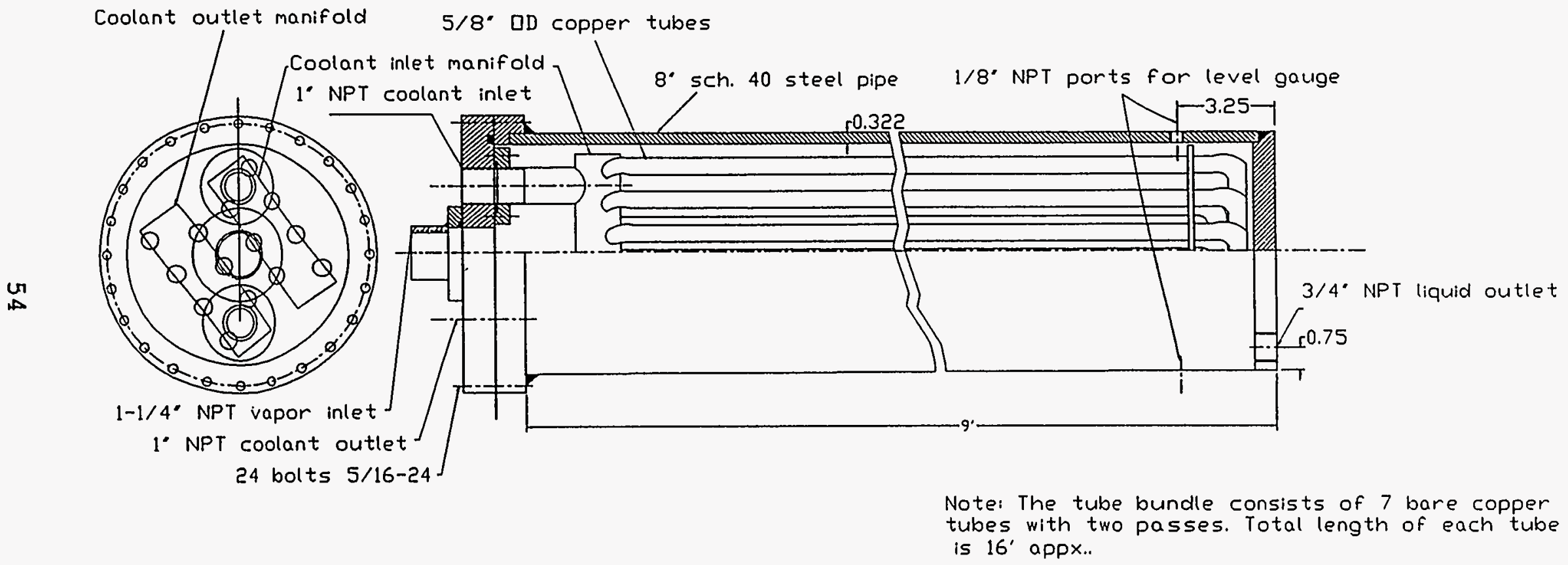

CONDENSER ASSEMBLY

FIGURE 28 


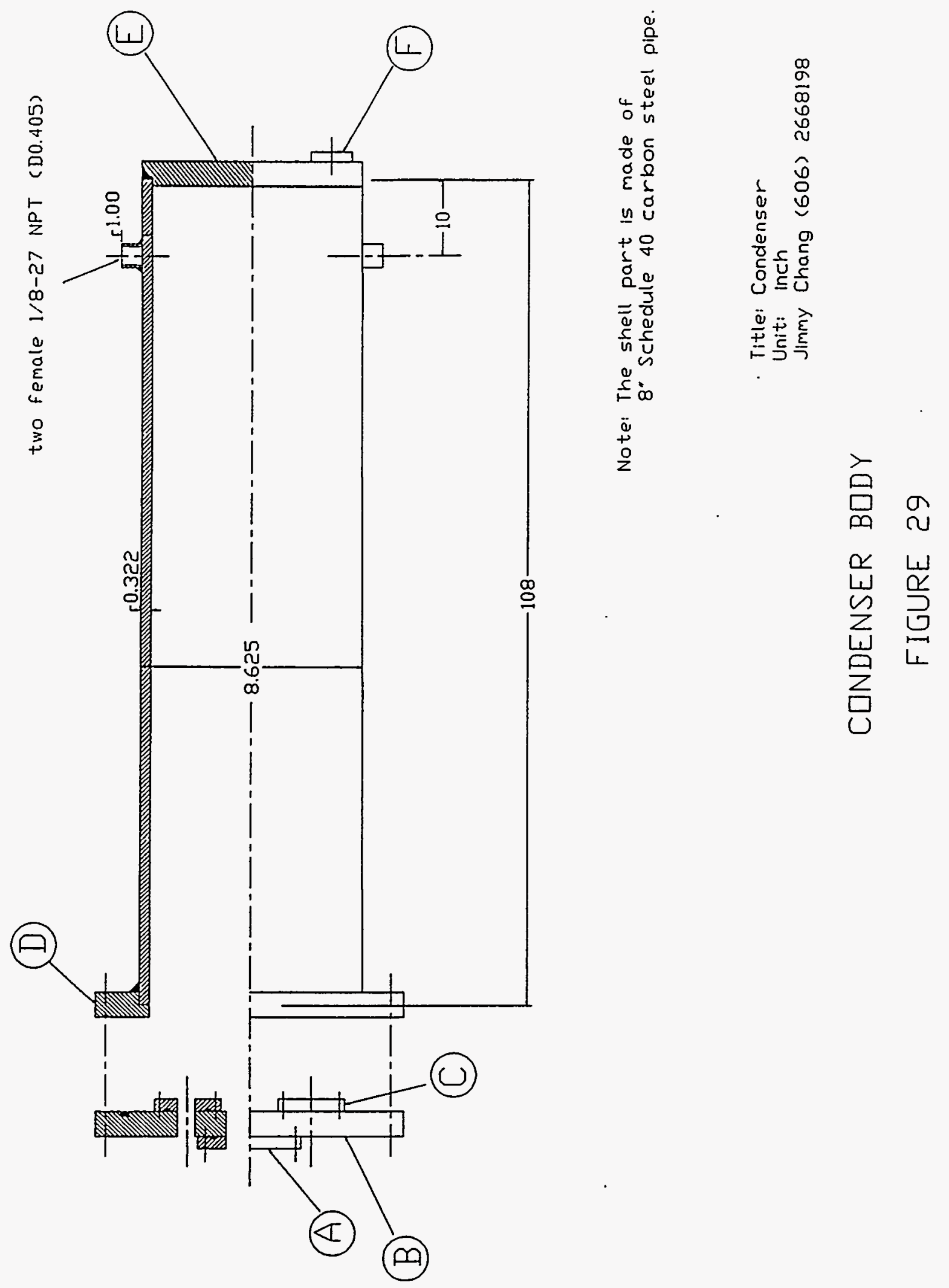




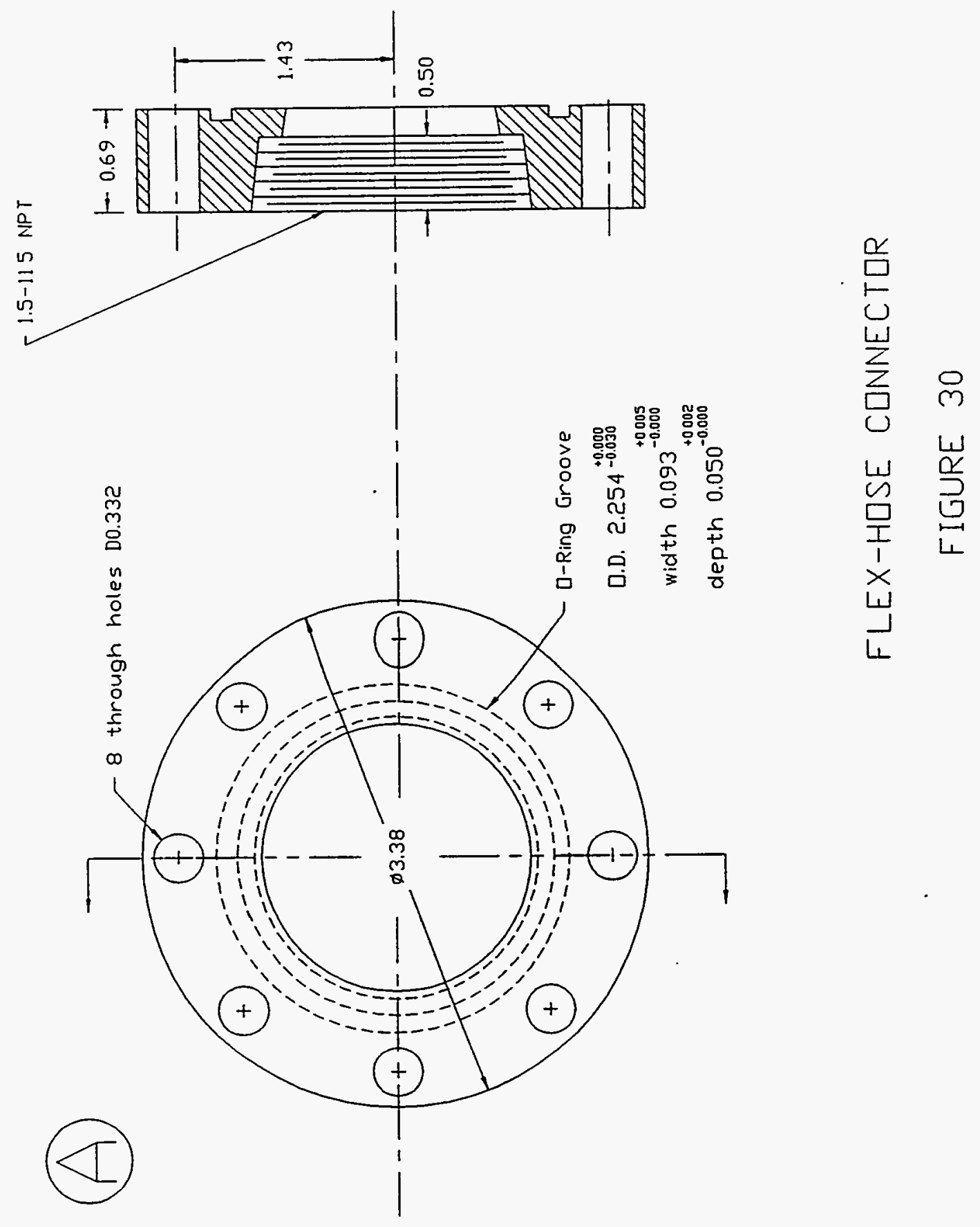




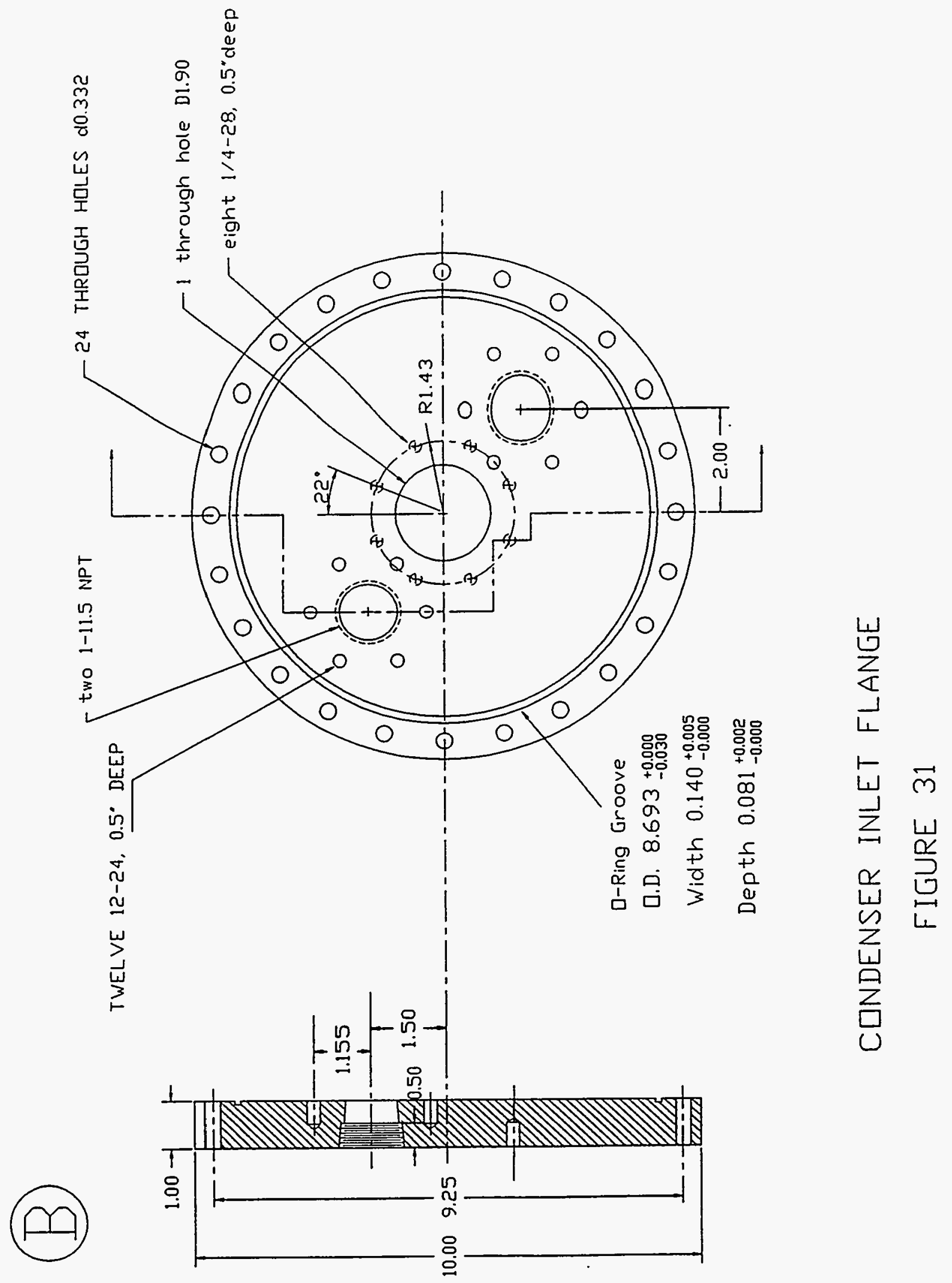




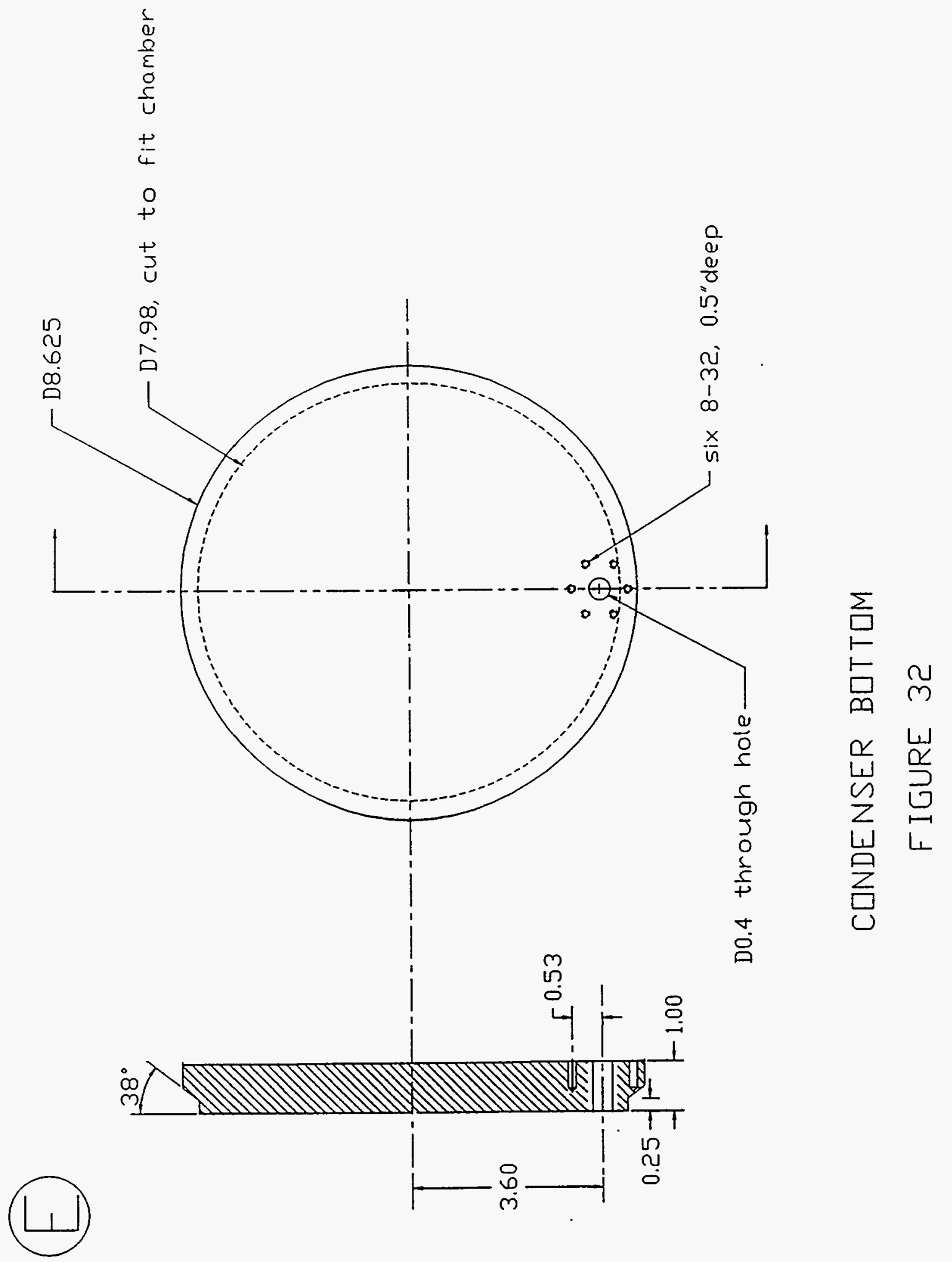




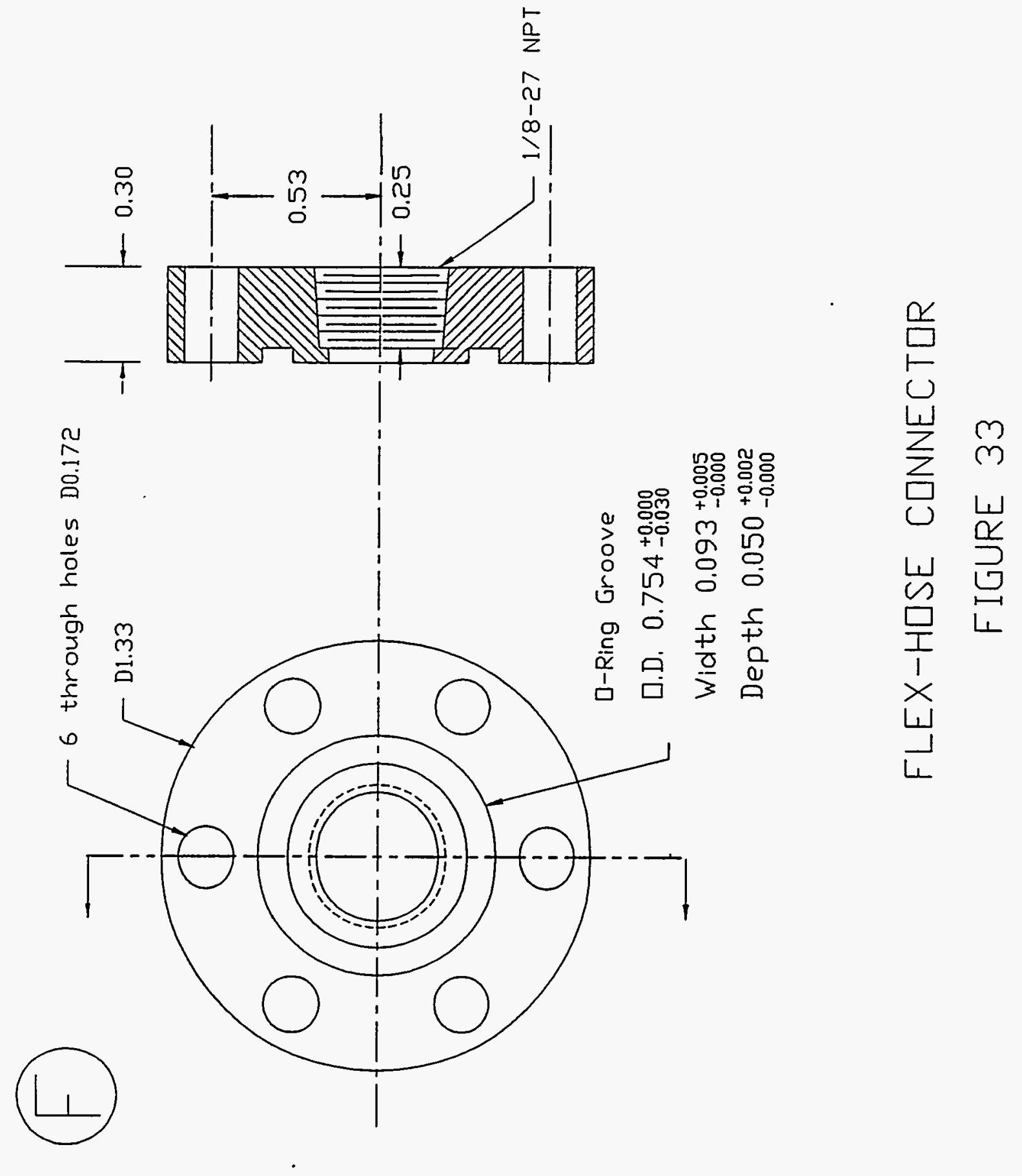




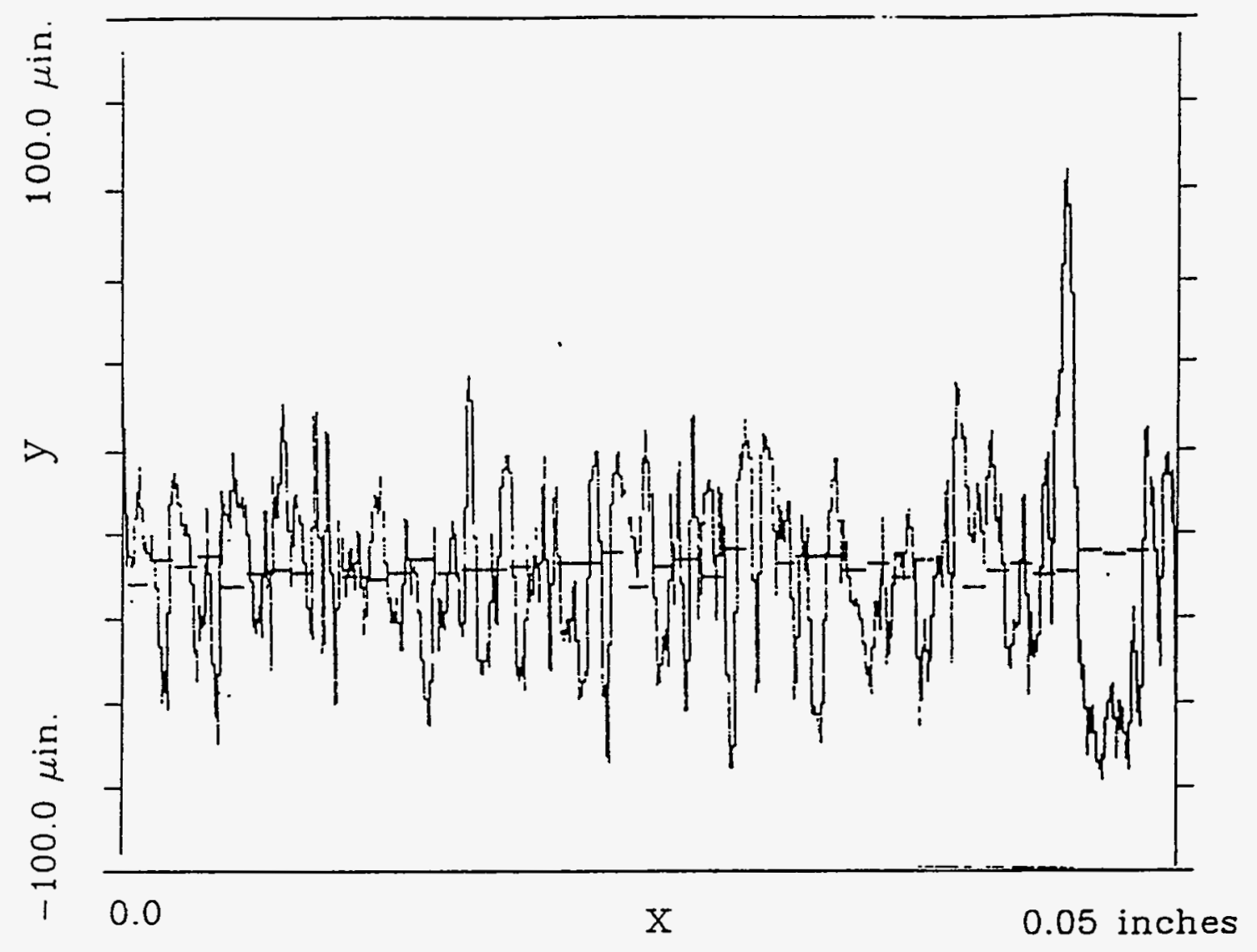

NEW TEST SECTION ROUGHNESS PROFILE

FIGURE 34 


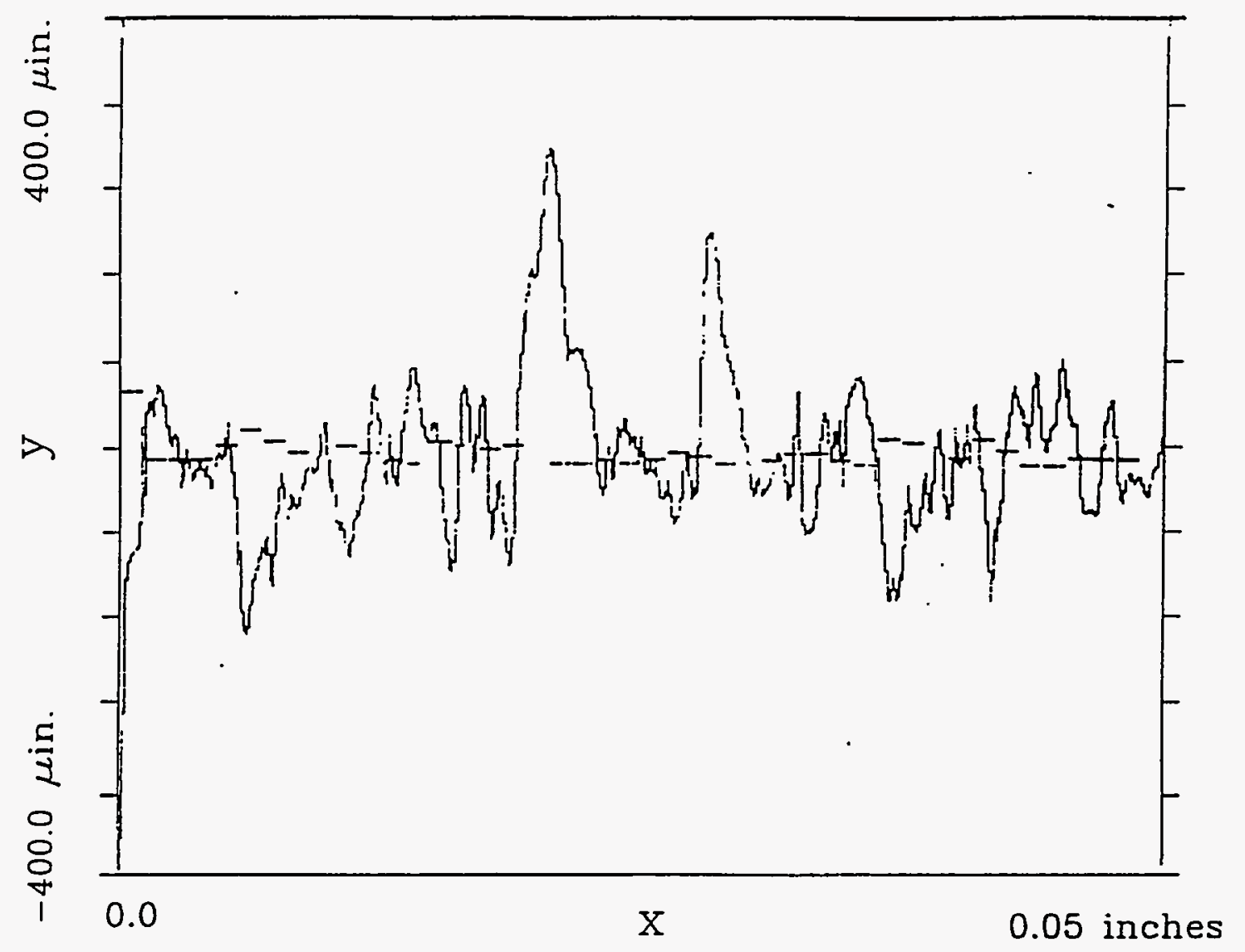

OLD TEST SECTION ROUGHNESS PROFILE [?] FIGURE 35 


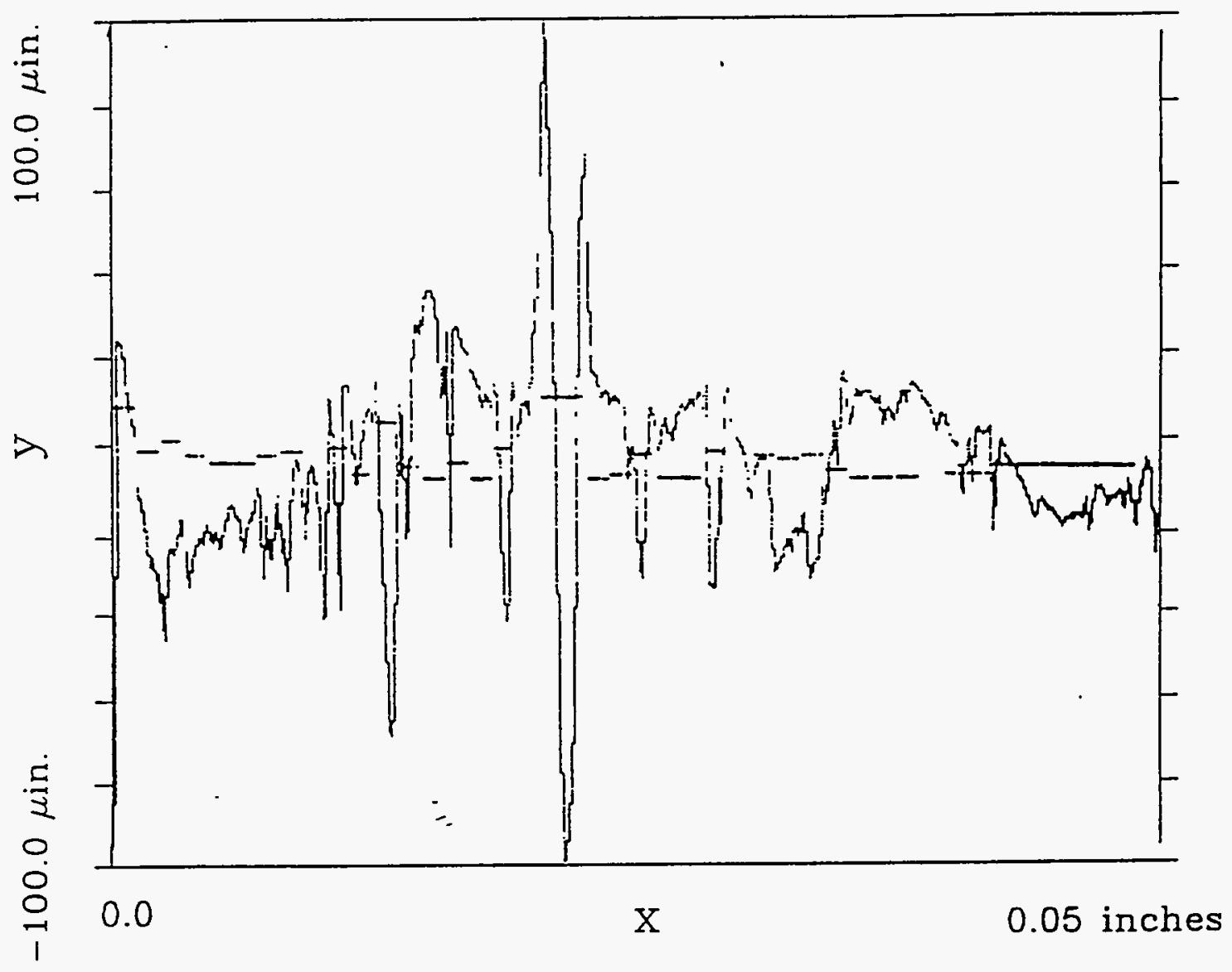

1/2" COPPER TUBE ROUGHNESS PROFILE

FIGURE 36 


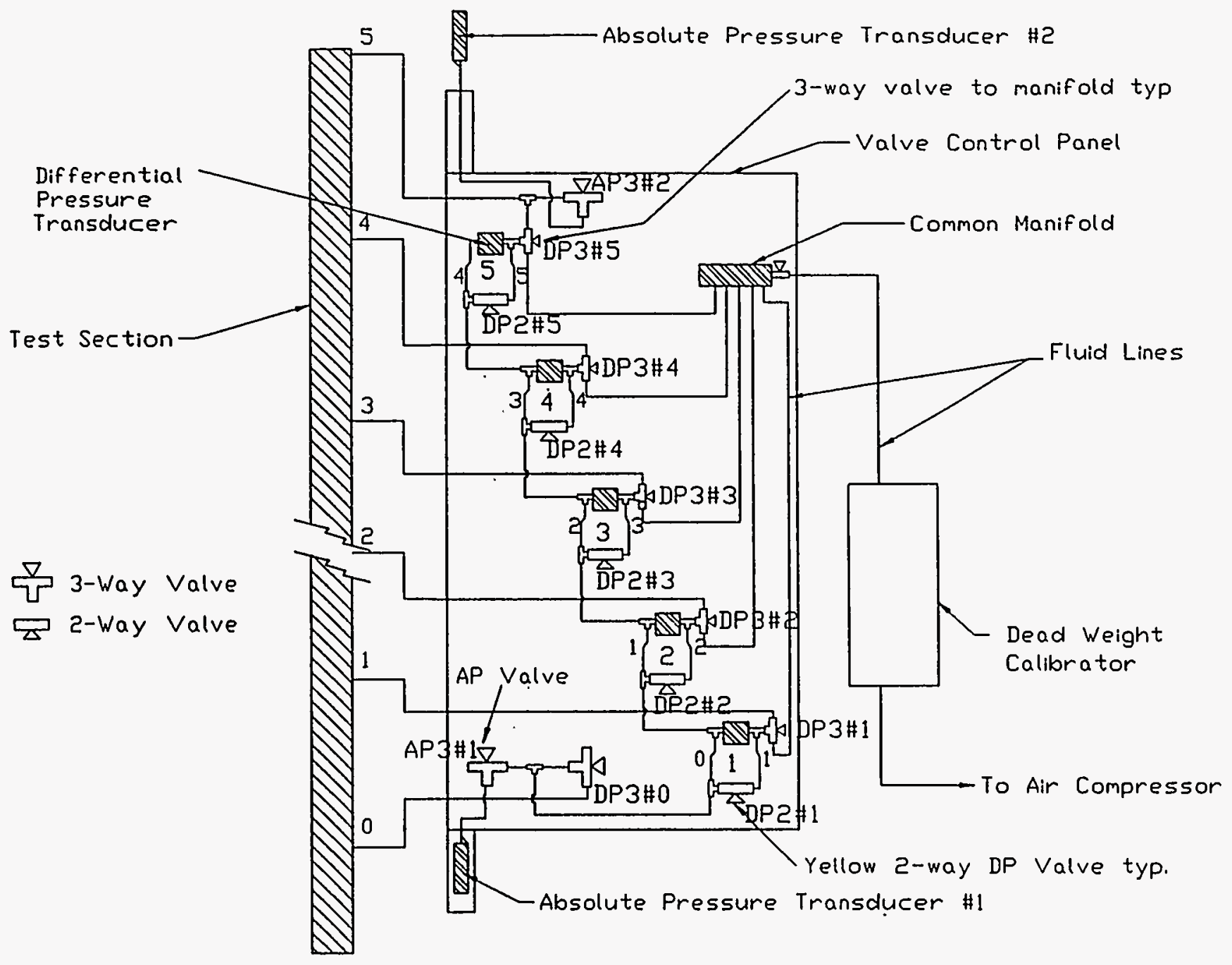

\section{PRESSURE TRANSDUCER CALIBRATIDN \\ CONTRUL PANEL}

FIGURE 37 


\section{THERMICOUPLE WIRING FOR TEST SECTIDN}

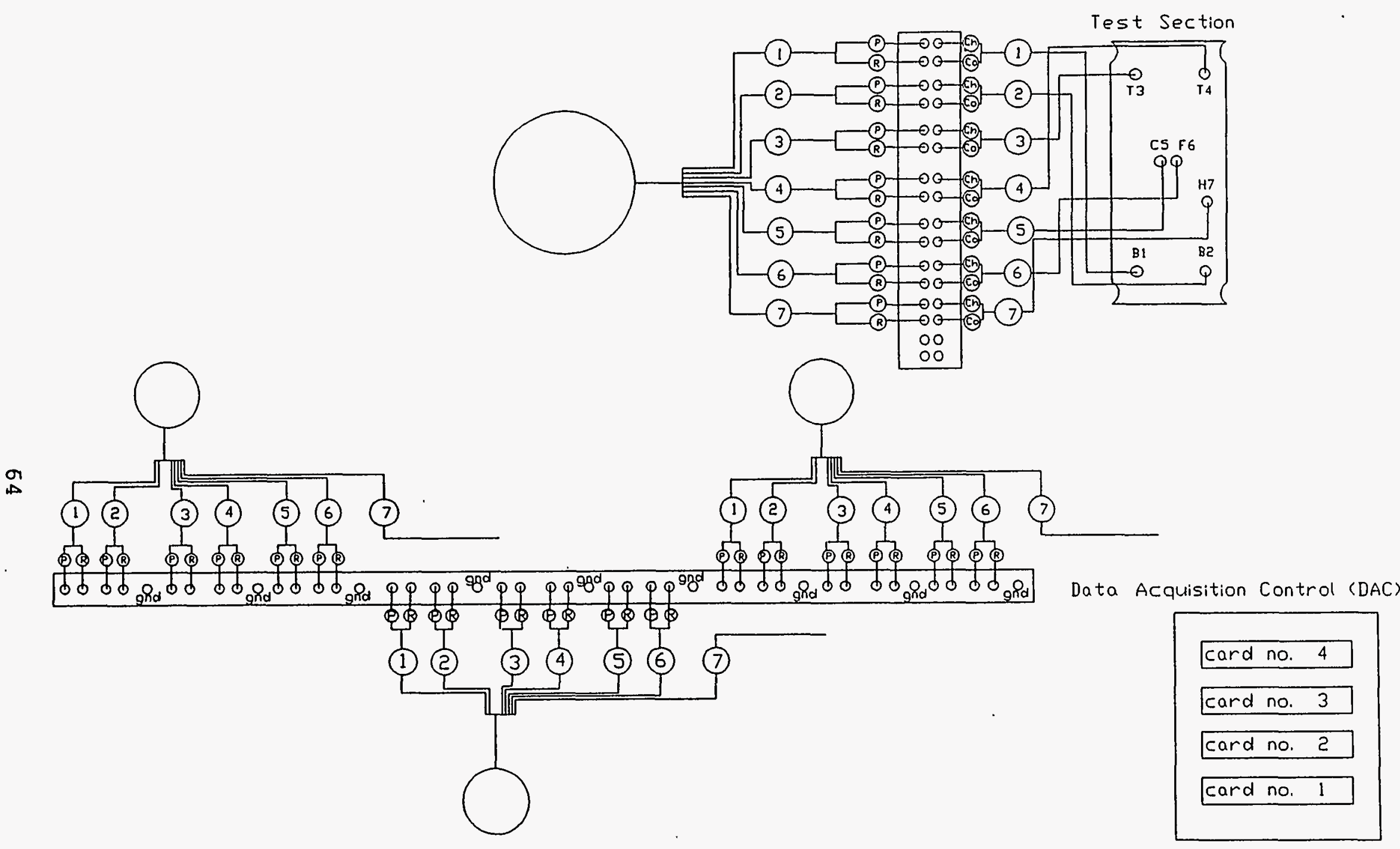

TC WIRING GN DAC

FIGURE 38 


\section{THERMICQUPLE WIRING FUR TEST SECTIDN}

Test Section

Data Acquisition Control (DAC)

TC WIRING QN DAC

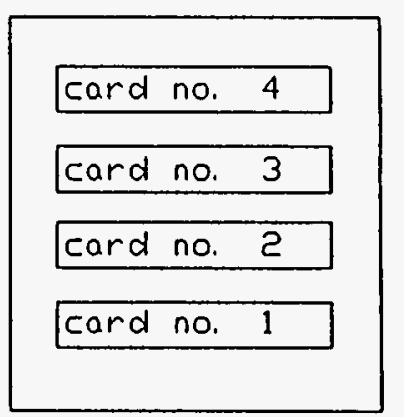

FIGURE 39 
Section 2

Study of Flow Boiling of

R-114, $C_{4} F_{10}$, and $C_{4} F_{8}$ in a Vertical Tube 
Study of Flow Boiling of R-114, $C_{4} F_{10}$ and $C_{4} F_{8}$ in a Vertical Tube

by

Cudo Technologies, Ltd.

P.O. Box 185, Lexington, KY 40584

Contract \# 11K-NR559

Report \# MMES-Cudo-NR559-2

Marine Pass

Martin R. Pais, PhD, PE

Principal Investigator

ming \&. Chang

Maniuderfelwity

Ming Jr Chang, PhD

Maninder S. Sehmbey 


\section{Study of Flow Boiling of R-114, $C_{4} F_{10}$ and $C_{4} F_{8}$ in a Vertical Tube}

by

Cudo Technologies, Ltd.

P.O. Box 185, Lexington, KY 40584

Contract \# 11K-NR559

Report \# MMES-Cudo-NR559-2

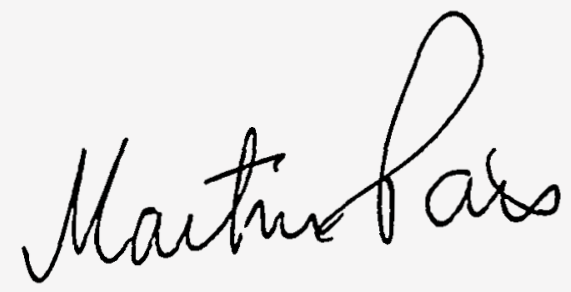

Martin R. Pais, PhD, PE

Principal Investigator

ming f. Chang

Ming Jr Chang, PhD

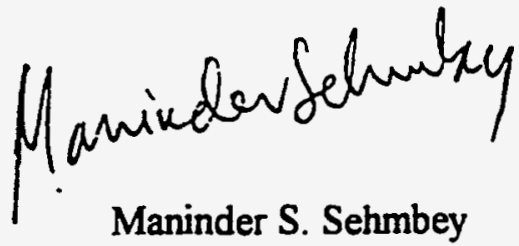




\begin{abstract}
Convective pool and flow boiling experiments were conducted for three different liquids $\mathrm{R}$ $114, \mathrm{C}_{4} \mathrm{~F}_{10}$ and $\mathrm{C}_{4} \mathrm{~F}_{8}$ to determine the heat transfer and pressure drop characteristics in a vertical tube. Experiments were run over the saturation temperature range of $125^{\circ} \mathrm{F}$ to $200^{\circ} \mathrm{F}$, heat flux range of 5000 to $25000 \mathrm{btu} / \mathrm{hr} . \mathrm{ft}^{2}$ and mass flow rate range of $0.2 \mathrm{E} 6$ to $1 \mathrm{E} 6 \mathrm{lbm} / \mathrm{hr}$. $\mathrm{ft}^{2}$. The heat-transfer coefficient for $\mathrm{C}_{4} \mathrm{~F}_{10}$ and $\mathrm{C}_{4} \mathrm{~F}_{8}$ are nearly the same and almost $80 \%$ higher than that for R-114. However, the critical heat flux supported by R-114 is almost $70 \%$ higher than that for $\mathrm{C}_{4} \mathrm{~F}_{10}$ and $\mathrm{C}_{4} \mathrm{~F}_{8}$. Correlations are presented which provide a best fit prediction of the data.
\end{abstract}


TABLE OF CONTENTS

\section{ABSTRACT}

Table of contents

Table of Figures

NOMENCLATURE

INTRODUCTION

Theory

TECHNICAL OBJECTIVES 5

Experimental Setup

Experimental Procedures

Heat Transfer

Roughness Measurement

Correlation

Convective Flow Boiling Models

Superposition Model

The Enhancement Model

Asymptotic Model

Heat Transfer Correlation

Single Phase Liquid Flow

Nucleate Pool Boiling

Convective Flow Boiling

Critical Heat Flux

Pressure Drop

Table 1. 


\section{TABLE OF FIGURES}

Figures $\quad$ Page

1. Test Fluid Loop $\quad 27$

2. Storage Tank-Assembly and Caps $\quad 28$

3. Details of Zone \# 1 29

4. Heat Flux Versus the Wall-to-instream Temperature Difference
for R-1 14 at $T_{\text {sat }}=125^{\circ} \mathrm{F}$

5. Heat Flux Versus the Wall-to-instream Temperature Difference for R-114 at $\mathrm{T}_{\text {sat }}=150^{\circ} \mathrm{F} \quad 31$

6. Heat Flux Versus the Wall-to-instream Temperature Difference
for R-114 at $\mathrm{T}_{\text {sat }}=175^{\circ} \mathrm{F}$

7. Heat Flux Versus the Wall-to-instream Temperature Difference
for R-114 at $\mathrm{T}_{\text {sat }}=200^{\circ} \mathrm{F}$

8. Heat Flux Versus the Wall-to-instream Temperature Difference
for $\mathrm{C}_{4} \mathrm{~F}_{10}$ at $\mathrm{T}_{\text {sat }}=125^{\circ} \mathrm{F}$

9. Heat Flux Versus the Wall-to-instream Temperature Difference for $\mathrm{C}_{4} \mathrm{~F}_{10}$ at $\mathrm{T}_{\text {sat }}=150^{\circ} \mathrm{F} \quad 35$

10. Heat Flux Versus the Wall-to-instream Temperature Difference
for $\mathrm{C}_{4} \mathrm{~F}_{10}$ at $\mathrm{T}_{\text {sat }}=175^{\circ} \mathrm{F}$

11. Heat Flux Versus the Wall-to-instream Temperature Difference

12. Heat Flux Versus the Wall-to-instream Temperature Difference for $\mathrm{C}_{4} \mathrm{~F}_{8}$ at $\mathrm{T}_{\text {sat }}=125^{\circ} \mathrm{F}$

13. Heat Flux Versus the Wall-to-instream Temperature Difference for $\mathrm{C}_{4} \mathrm{~F}_{8}$ at $\mathrm{T}_{\text {sat }}=150^{\circ} \mathrm{F}$

14. Heat Flux Versus the Wall-to-instream Temperature Difference

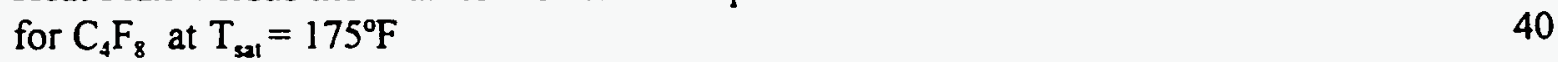

15. Heat Flux Versus the Wall-to-instream Temperature Difference for $\mathrm{C}_{4} \mathrm{~F}_{8}$ at $\mathrm{T}_{\text {sat }}=190^{\circ} \mathrm{F}$

16. New Test Section Roughness Profile $\quad 42$

17. Old Test Section Roughness Profile $\quad 43$

18. 1/2" Copper Tube Roughness Profile 44

19. (a) Superposition and Asymptotic Models in form q vs. $\Delta T$. 45

(b) Superposition and Asymptotic Models in the form of $h$ vs. q

20. Correlation of Forced Convection for R-114 46

21. Correlation of Forced Convection $\mathrm{C}_{4} \mathrm{~F}_{10}$

22. Correlation of Forced Convection $\mathrm{C}_{4} \mathrm{~F}_{8} \quad 48$

23. Correlation of Nucleate Pool Boiling for R-114 49

24. Correlation of Nucleate Pool Boiling for $\mathrm{C}_{4} \mathrm{~F}_{10}$

25. Correlation of Nucleate Pool Boiling for $\mathrm{C}_{4} \mathrm{~F}_{8}$ 
26. Comparison of Measured and Predicted Heat Transfer Coefficients for $\mathrm{R}-114$ at $\mathrm{T}_{\mathrm{sal}}=125^{\circ} \mathrm{F}$

27. Comparison of Measured and Predicted Heat Transfer Coefficients for $\mathrm{R}-114$ at $\mathrm{T}_{\text {sat }}=150^{\circ} \mathrm{F}$

28. Comparison of Measured and Predicted Heat Transfer Coefficients for $\mathrm{R}-114$ at $\mathrm{T}_{\mathrm{sal}}=175^{\circ} \mathrm{F}$

29. Comparison of Measured and Predicted Heat Transfer Coefficients for R-1 14 at $\mathrm{T}_{\text {sat }}=200^{\circ} \mathrm{F}$

30. Comparison of Measured and Predicted Heat Transfer Coefficients for $\mathrm{C}_{4} \mathrm{~F}_{10}$ at $\mathrm{T}_{\text {sal }}=125^{\circ} \mathrm{F}$

31. Comparison of Measured and Predicted Heat Transfer Coefficients for $\mathrm{C}_{4} \mathrm{~F}_{10}$ at $\mathrm{T}_{\mathrm{sal}}=150^{\circ} \mathrm{F}$

32. Comparison of Measured and Predicted Heat Transfer Coefficients for $\mathrm{C}_{4} \mathrm{~F}_{10}$ at $\mathrm{T}_{\mathrm{sal}}=175^{\circ} \mathrm{F}$

33. Comparison of Measured and Predicted Heat Transfer Coefficients for $\mathrm{C}_{4} \mathrm{~F}_{10}$ at $\mathrm{T}_{\text {sat }}=190^{\circ} \mathrm{F}$

34. Comparison of Measured and Predicted Heat Transfer Coefficients for $\mathrm{C}_{4} \mathrm{~F}_{8}$ at $\mathrm{T}_{3 \mathrm{sat}}=125^{\circ} \mathrm{F}$

35. Comparison of Measured and Predicted Heat Transfer Coefficients for $\mathrm{C}_{4} \mathrm{~F}_{8}$ at $\mathrm{T}_{32 \mathrm{i}}=150^{\circ} \mathrm{F}$

36. Comparison of Measured and Predicted Heat Transfer Coefficients for $\mathrm{C}_{4} \mathrm{~F}_{8}$ at $\mathrm{T}_{\mathrm{s21}}=175^{\circ} \mathrm{F}$

37. Comparison of Measured and Predicted Heat Transfer Coefficients for $\mathrm{C}_{4} \mathrm{~F}_{8}$ at $\mathrm{T}_{\mathrm{s21}}=190^{\circ} \mathrm{F}$

38. Comparison of Predicted Heat Transfer Coefficients vs. Heat Flux at $\mathrm{T}_{\text {sat }}=125^{\circ} \mathrm{F}$

39. Comparison of Predicted Heat Transfer Coefficients vs. Heat Flux at $T_{\text {sat }}=150^{\circ} \mathrm{F}$

40. Comparison of Predicted Heat Transfer Coefficients vs. Heat Flux at $\mathrm{T}_{\mathrm{sal}}=175^{\circ} \mathrm{F}$

41. Comparison of Predicted Heat Transfer Coefficients vs. Heat Flux at $\mathrm{T}_{\text {sas }}=190^{\circ} \mathrm{F}$

42. Critical Heat Flux versus the dry-out location $L$ for $R-114$ at $125^{\circ} \mathrm{F}$

43. Critical Heat Flux versus the dry-out location $L$ for $R-114$ at $150^{\circ} \mathrm{F}$

44. Critical Heat Flux versus the dry-out location $L$ for $R-114$ at $175^{\circ} \mathrm{F}$

45. Critical Heat Flux versus the dry-out location $L$ for $R-114$ at $200^{\circ} \mathrm{F}$

46. Critical Heat Flux versus the dry-out location $L$ for $C_{4} F_{10}$ at $125^{\circ} \mathrm{F}$

47. Critical Heat Flux versus the dry-out location $L$ for $C_{4} F_{10}$ at $150^{\circ} \mathrm{F}$

48. Critical Heat Flux versus the dry-out location $L$ for $\mathrm{C}_{4} \mathrm{~F}_{10}$ at $175^{\circ} \mathrm{F}$

49. Critical Heat Flux versus the dry-out location $L$ for $C_{4} F_{10}$ at $200^{\circ} \mathrm{F}$

50. Critical Heat Flux versus the dry-out location $\mathrm{L}$ for $\mathrm{C}_{4} \mathrm{~F}_{8}$ at $125^{\circ} \mathrm{F}$

51. Critical Heat Flux versus the dry-out location $L$ for $C_{4} F_{8}$ at $150^{\circ} \mathrm{F}$

52. Critical Heat Flux versus the dry-out location $L$ for $C_{4} F_{8}$ at $175^{\circ} \mathrm{F}$ 
53. Critical Heat Flux versus the dry-out location $\mathrm{L}$ for $\mathrm{C}_{4} \mathrm{~F}_{8}$ at $190^{\circ} \mathrm{F}$

54. Comparison of Measured and Predicted Pressure Drop for R-114 at $\mathrm{T}_{\mathrm{sat}}=125^{\circ} \mathrm{F}$

55. Comparison of Measured and Predicted Pressure Drop for R-114 at $\mathrm{T}_{\text {sat }}=150^{\circ} \mathrm{F}$

56. Comparison of Measured and Predicted Pressure Drop for R-114 at $\mathrm{T}_{\mathrm{sat}}=175^{\circ} \mathrm{F}$

57. Comparison of Measured and Predicted Pressure Drop for R-114 at $T_{\text {sat }}=200^{\circ} \mathrm{F}$

58. Comparison of Measured and Predicted Pressure Drop for $\mathrm{C}_{4} F_{10}$ at $T_{\text {sal }}=125^{\circ} \mathrm{F}$

59. Comparison of Measured and Predicted Pressure Drop for $C_{4} F_{10}$ at $T_{\text {sat }}=150^{\circ} \mathrm{F}$

60. Comparison of Measured and Predicted Pressure Drop for $\mathrm{C}_{4} \mathrm{~F}_{10}$ at $T_{\text {sat }}=175^{\circ} \mathrm{F}$

61. Comparison of Measured and Predicted Pressure Drop for $C_{4} F_{10}$ at $\mathrm{T}_{\text {sat }}=190^{\circ} \mathrm{F}$

62. Comparison of Measured and Predicted Pressure Drop for $\mathrm{C}_{4} \mathrm{~F}_{8}$ at $\mathrm{T}_{\mathbf{s 1}}=125^{\circ} \mathrm{F}$

63. Comparison of Measured and Predicted Pressure Drop for $\mathrm{C}_{4} \mathrm{~F}_{8}$ at $T_{\text {sat }}=150^{\circ} \mathrm{F}$

64. Comparison of Measured and Predicted Pressure Drop for $\mathrm{C}_{4} \mathrm{~F}_{8}$ at $\mathrm{T}_{\mathbf{s a t}}=190^{\circ} \mathrm{F}$ 


\section{NOMENCLATURE}
A Area
Bo Boiling number
c Constant
C Correction factor
Co Convection number
$\mathrm{C}_{\mathrm{p}}$ Specific heat
d Inside diameter of tube
E Enhancement factor
F Fluid dependent constant
Fr Froude number
g Gravitational acceleration
G Mass flux
h Heat-transfer coefficient
$k$ Thermal conductivity
$\mathrm{Nu} \quad$ Nusselt number $\left(=h_{\mathrm{i}} / \mathrm{k}\right)$
p Pressure
Pr Prandtl number $\left(=C_{p} \mu / k\right)$
$q \quad$ Heat flux $(=Q / A)$
Q Heat input
$R \quad$ Roughness
$\operatorname{Re} \quad$ Reynolds number (Gd; $/ \mu$ )
$S \quad$ Suppression factor
$T$ Temperature
$\mathrm{X}$. Vapor quality
$z \quad$ Coordinate in direction of flow

\section{Greek}

$\alpha \quad$ Void fraction

$\Delta \quad$ Difference

$\lambda \quad$ Latent heat of vaporization

$\mu \quad$ Viscosity

$\sigma \quad$ Surface tension

$\rho$ Density

v Specific volume

\section{Superscript}

n Exponent 


$\begin{array}{ll}\text { Subscript } \\ \mathrm{a} & \text { Average } \\ \mathrm{cv} & \text { Convection } \\ \mathrm{f} & \text { Instream } \\ \mathrm{fl} & \text { Fluid } \\ \mathrm{F} & \text { Friction } \\ \mathrm{g} & \text { Vapor phase } \\ \mathrm{i} & \text { Inside diameter } \\ \mathrm{l} & \text { Liquid phase } \\ \mathrm{nb} & \text { Nucleate boiling } \\ \mathrm{nbf} & \text { Nucleate boiling factor } \\ \text { npb } & \text { Nucleate pool boiling } \\ \mathrm{q} & \text { Root-mean-square } \\ \mathrm{tpf} & \text { Two-phase factor } \\ \mathrm{w} & \text { Wall } \\ \text { wf } & \text { Wall-fluid }\end{array}$




\section{INTRODUCTION}

In several proposed cooling applications, the use of fully fluorinated carbon compounds is being seriously considered. This is because the common refrigerants in use today (chlorofluorocarbons CFCs) have been linked to the depletion of ozone in the stratosphere. The current world plan ironed out by the Montreal Protocol calls for a total ban of all CFCs by the year 2000. The USA has unilaterally called for a ban on all CFCs by 1995. This demands that replacement refrigerant fluids be sought and tested which will not only satisfy the tougher environmental standards but also, more importantly, provide satisfactory cooling capacities within the current existing installations.

Two fluids, namely, perfluorobutane $\mathrm{C}_{4} \mathrm{~F}_{10}$ and per-fluorocyclobutane $\mathrm{C}_{4} \mathrm{~F}_{8}$ have been selected as promising replacements for R-114 in the nuclear fuel enrichment facilities. These fluids are special because they provide high heat transfer rates and do not react with the process fluids. They are also very inert, have thermophysical properties that are comparable to the currently used refrigerant R114, and can be manufactured with currently available technology.

Convective flow boiling experiments were conducted at Cudo Technologies Ltd.(Cudo) for three different liquids $\mathrm{R}-114, \mathrm{C}_{4} \mathrm{~F}_{10}$ and $\mathrm{C}_{4} \mathrm{~F}_{8}$. The experimental setup is described in detail in reference 1. Experiments were run for different saturation temperatures, heat fluxes, and mass flow rates so as to determine the heat transfer capability and pressure drop conditions. Also correlations are presented which provide a best fit prediction of the data.

\section{Theory}

The theory of nucleation and the solid-liquid-vapor relationship of forced convective boiling is addressed in detail in reference [1]. The flow regimes are generally divided into the single-phase, subcooled nucleate boiling, saturated nucleate boiling, slug flow, annular flow, single phase vapor and the critical heat flux. Depending on the heat flux and the temperature conditions at the wall, the occurrence of the intermediate regimes can be suppressed. The heat transfer capability of the fluid is dependent on a number of parameters [1]. Depending on the operating temperatures, pressures 
and heat loads envisaged, conditions within the heat exchanger system can be reached which can be deleterious to the process. Hence, it is imperative that heat transfer and pressure drop data on these fluids be obtained. 


\section{TECHNICAL OBJECTIVES}

The overall objective of this proposed study is to develop a method of measuring the heat-transfer coefficient and pressure drop on the internal surface of a cylindrical pipe under conditions of uniform heat flux in flow boiling in a vertical orientation with respect to gravity.

The overall objective of this part of the total research effort is to:

A. 1. Measure the boiling heat-transfer coefficient on the internal surface of a tube under conditions of:

- flow and free convective boiling,

- uniform heat flux, 3,000 to $60,000 \mathrm{Btu} / \mathrm{hr} . \mathrm{ft}^{2}$ per zone.

- fluid mass flux of $0.1 \mathrm{e} 6$ to $1.1 \mathrm{e} 6 \mathrm{lbm} / \mathrm{hr}^{-\mathrm{ft}^{2}}$.

- test section exit quality of 0 to 1 .

- tube saturated length to diameter ratio $\mathrm{L} / \mathrm{D}=30$ to 150 .

2. Measure the two-phase flow pressure drop.

3. Measure the critical heat flux.

4. Measure the surface temperature distribution.

5. Measure the flow temperature distribution.

6. Measure and define the surface roughness conditions.

7. Maintain the desired saturation conditions, $100^{\circ} \mathrm{F}$ to $250^{\circ} \mathrm{F}$.

B: Run tests using:

1. CFC-114; Compare the resuits with the currently existing R-114 data from the Oak Ridge Gaseous Diffusion Plant (ORGDP) [2].

2. $\mathrm{C}_{4} \mathrm{~F}_{10}$

3. $\mathrm{C}_{4} \mathrm{~F}_{8}$

C. Compare existing or determine a new cortelation for:

- the heat flux,

- the CHF, and

- the pressure drop. 


\section{Experimental Setup [1]}

Figure 1 illustrates the flow boiling system capable of operating at pressures from 0 to 350 psig and temperatures ranging from $50^{\circ} \mathrm{F}$ to $300^{\circ} \mathrm{F}$. Liquid from the storage tank is pumped by a variable flow gear pump at a predetermined flow rate. The liquid on exiting the pump passes through a filter-drier, a flow meter and a pre-heater before entering the test section. The pre-heater allows fine control of the inlet sub-cooled/saturation conditions by the use of a temperature controller. Before entering the test section the liquid is made to flow through a flow straightener consisting of a honeycomb section ahead of a converging cone which mates with no transition in geometry with the inlet of the test tube test section. The liquid on flowing through the test section takes up heat through phase change and exits as a liquid-vapor mixture. At the top and exit of the test section is a sight-glass tube allowing visualization of the exit flow. The exiting liquid-vapor mixture next passes through a cyclone separator. The settled liquid passes through a flow meter before draining into the storage tank. The vapor is condensed in the condenser. The condensate passes through a flow meter before draining into the storage tank.

The test section $[1,2]$ is primarily a 0.785 inch $\mathrm{ID}$ and 1.75 inch $\mathrm{OD}$ copper tube about 10 feet in length. At each end of the test section is a welded copper flange for mating with the other parts of the setup. The test section is divided into ten zones, each 11.5 inches long and thermally isolated from the adjacent zones by a $1 / 2$ inch wide groove. Figure 2 illustrate the positioning of the thermocouples and pressure transducers [1]. The temperature profile in each zone is provided by seven thermocouples distributed longitudinally and azimuthally. Five thermocouples are imbedded in the $1 / 2$ inch thick wall so as to be $1 / 8$ inch from the inner tube wall. A sixth thermocouple is positioned midway in the fluid stream and a seventh thermocouple is clamped to the external heater. All the thermocouples were calibrated with their respective wiring harnesses in conjunction with the data acquisition. The uncertainty in the temperature measurement is $\pm 1^{\circ} \mathrm{F}$ [1].

Seven pressure transducers were positioned along the length of the tube [1], see figure 1. One at the entrance of the tube and one at the exit measured the absolute pressures. Additionally, five 
transducers are placed equidistantly within the test region to provide the differential pressure relative to the pressure at the inlet. The pressure taps are located in zones $1,3,5,7,9 \& 10$ such that the distance between two consecutive taps is $2^{\prime}$ except for the tap in zone 10 which is at a distance of $22.5 \mathrm{l}$ from that in zone 8 . The differential pressure transducers provide the pressure drop information along the length of the tube. The uncertainty in the differential pressure is $\pm 0.05 \mathrm{psi}$ and in the absolute pressure \pm 1 psia.

Each zone of the test section is wrapped with heaters capable of providing over 60,000 $\mathrm{Btu} / \mathrm{hr} \cdot \mathrm{ft}^{2}$ to the outer surface of the tube [1]. The heater arrangement and the test section was thermally insulated from the environment by three layers of high temperature Fiberfrax insulation ( $k$ $=0.65 \mathrm{Btu} . \mathrm{in} / \mathrm{hr} \cdot \mathrm{ft}^{2} .{ }^{\circ} \mathrm{F} @ 500^{\circ} \mathrm{F}$ ) and an aluminum foil radiation heat shield. The uncertainty in the heat flux is $\pm 175 \mathrm{btu} / \mathrm{hr} . \mathrm{ft}^{2}$.

SCR power supplies regulate the power to the heaters by varying the phase angle on each $A C$ cycle proportional to the control signal [1]. This method of applying power provides a continuous uniform heat flux with fine control. A temperature controller, monitors the temperature of the outer heater surface. In the event of an overshoot in the temperature of the surface, an alarm disconnects the power to the heaters via relays. In the design of this experiment the number of devices to be measured included over eighty thermocouples, seven pressure transducers, six flow meters, and ten power transducers. To expedite on-site data analysis a PC-based data acquisition was used to measure, perform the necessary calculations, display system conditions and store the data. To reduce the uncertainty in the overall measurement, the devices were calibrated using standards in conjunction with the data acquisition.

\section{Experimental Procedure [1]}

With reference to figure 1 , the variable flow pump is started and the flow rate adjusted to the desired predetermined value. The pre-heater is powered to control the inlet coolant entry temperature. The antifreeze is circulated through the cooling coils in the condenser at a flow rate such that the saturation pressure at the inlet is maintained at the desired value. Excessive cooling may 
cause a low pressure at the pump inlet and lead to cavitation. Too little cooling will cause the pressure to rise and force the liquid at the inlet to the test section to be sub-cooled. A heater on the storage tank in conjunction with a cooling coil in the storage tank is used as an accessory control for inlet temperature and pressure. see figure 3 .

The heaters on the test section are turned on and the power is ramped up in steps to the desired heat flux, making certain that steady-state conditions are reached on the surface of the heater. The data acquisition is set up to measure the heat input and temperature of the heaters. With this information the heat loss from the test section to the ambient and end-plates was calculated from the earlier heat loss calibration [1]. The balance of the heat is necessarily taken up by the fluid. Both the heat loss and the heat flux into each zone are displayed simultaneously by the data acquisition. Hence, it is possible to determine and set in real time the heat flux conditions. Steady-state thermal flow-field conditions are determined by monitoring the temperatures of the various regions of the system. When the temperature transients die down ( $\left({ }^{\circ} \mathrm{F}\right.$ or less change over a 5 minute period), the system can be assumed to be in thermal equilibrium. At this juncture the:

- temperatures at various locations

- $\quad$ system pressure and region differential pressures

- $\quad$ power input to the heaters

- flow meter readings

- flow field observations

are noted down and collected by the data acquisition unit.

The above process is followed for a step-wise increase in heat flux until CHF conditions are imminent. At this juncture the heat flux is further increased so as to span the $\mathrm{L} / \mathrm{D}$ ratios for the CHF study. In regions where $\mathrm{CHF}$ occurs the heat flux is reduced so as to maintain the wall temperatures approximately equal to the upstream zone to reduce any heat losses. This is because at $\mathrm{CHF}$ the heattransfer coefficient drops significantly and the wall temperature rises correspondingly. To prevent too high a temperature in the particular zone the heat flux must be dropped. 
At the end of the experimentation all heaters are tumed off. The antifreeze is pumped through the heat exchanger in the storage tank. This creates a low pressure zone in the storage tank (by condensing the vapor). This induces all the vapor and the liquid in the rest of the loop to collect in the storage tank. Once the system has cooled down. all power to the loop is disconnected and the storage tank is isolated from the rest of the system by closing the valves. Small leaks do exist in the flow loop and isolation reduces loss of freon over periods of no operation.

For each fluid at a predetermined boiling temperature and mass flux. the zone heat flux is gradually increased, (i.e. a constant heat flux was applied to successive zones in the direction of flow) until $\mathrm{CHF}$ conditions are reached in the exit heat zone. This was evidenced by a sharp rise in wall temperature at the last heated zone. As the critical condition was reached, the electrical power input to that particular zone was cut off or reduced.

In all runs undertaken, the flow at the inlet was slightly sub-cooled, the saturation temperature was maintained constant, and the surface heat flux was varied. The range of different coolant flow rates was then repeated at this surface heat flux. Once the surface heat fluxes between 5000 and $35000 \mathrm{Btu} / \mathrm{hr} / \mathrm{ft}^{2}$ were covered, a new saturation temperature was set and the entire procedure repeated. Thus, essentially, the coolant flow rate was the parameter most frequently varied, the surface heat flux the second most frequently varied, and the saturation temperature the least frequently varied. (Owing to the thermal mass of the fluid in the system, approximately $150 \mathrm{lbs}$, change in saturation conditions needed about three hours to reach steady state).

During the above tests the saturation temperature and pressure of the system are maintained steady at the predefined values. For each saturation temperature and each mass flow rate setting, the run provided data of the form:

- Surface superheat temperature versus heat flux

- Saturation temperature conditions

- $\quad$ Pressure drop profile

- Flow field observations 
- $\quad$ Pressure at the inlet of the test section

- Pressure drop along the test section with intervals of 2, 4. 6, 8 and $9.88 \mathrm{ft}$ from the inlet

- Room temperature

- Wall and in-stream temperatures in each of the 10 zones

- Gross heat input, heat loss and heat flux to each of the 10 zones

- Coolant flow rate and temperature at inlet of the test section

- Water flow rate and temperatures at inlet and outlet of the condenser

Owing to the heat losses to the flanges at either end of the test section the uncertainty in the heat input calculation is difficult to determine. The temperature measurement in section 6 indicated a inexplicable departure from the rest of the test section. The difference was attributed to the temperature compensation within the data acquisition. Hence for purpose of analysis the heat transfer data from sections 1,6 and 10 are not presented or used for correlations in this study.

The range of parameters covered by these experiments are listed below:

Saturation temperature, ${ }^{\circ} \mathrm{F}$ :

$$
T=125,150,175,190 \text { (200 for R-114). }
$$

Liquid mass flux, lbm/hr- $\mathrm{ft}^{2}$ :

$$
\mathrm{G}=0.2,0.36,0.52,0.68,0.84,1.0 * 10^{6}
$$

Surface heat flux, Btu/hr/ $/ \mathrm{ft}^{2}$

$$
q=0.5,1.0,1.5,2.0,2.5 * 10^{4}
$$

\section{Heat Transfer}

The heat transfer characteristics are presented in the form of the heat flux $q$ versus the wallto-instream temperature difference $\Delta T . \Delta T$ is the temperature difference between the average inside-wall temperature and the instream temperature. The average inside-wail temperature was determined by taking the average thermocouple measurements from five different locations on the section wall [1], see figure 2, and correcting them for wall thickness to determine the internal wall temperature. Five thermocouples were imbedded in the walls of each section. Owing to the 
intermittent heating and cooling of the test section some of the thermocouples would not make good contact with the wall. The discrepancy was evident on study of the temperature field in the section in question. In such cases the incorrect temperature was not considered in the calculations. Generally at the end of the testing the thermocouple attachments were fixed.

From the results shown in Figures 4 to 15 , the following trends are observed:

- $\Delta \mathrm{T}$ increased with increase in heat flux $q$.

- As the saturation temperature increased, smaller $\Delta T$ were obtained.

- $\Delta \mathrm{T}$ for liquids $\mathrm{C}_{4} \mathrm{~F}_{10}$ and $\mathrm{C}_{4} \mathrm{~F}_{8}$ are smaller than that for $\mathrm{R}-114$, this implies that $\mathrm{C}_{4} \mathrm{~F}_{10}$ and $\mathrm{C}_{4} \mathrm{~F}_{8}$ provide higher heat-transfer coefficients than $\mathrm{R}-114$.

- $\mathrm{C}_{4} \mathrm{~F}_{10}$ and $\mathrm{C}_{4} \mathrm{~F}_{8}$ dry out earlier than R-114. In other words, $\mathrm{C}_{4} \mathrm{~F}_{10}$ and $\mathrm{C}_{4} \mathrm{~F}_{8}$ support lower critical heat flux (CHF) levels.

- The liquid mass flux does not have a strong effect on the heat-transfer coefficient.

The copper tube used as the test section for this flow boiling experiment was identical to the one used by Jallouk [2]. R-114 was also used as the test fluid in Jallouk's experiment. Hence, initially experiments were run to determine the heat transfer and flow characteristics with R-114 and the data was compared with those of Jallouk's [2]. Figures 5 and 7 show the comparisons between the flow boiling test data of Cudo and Jallouk's [2] for R-114 at saturation temperatures of $150^{\circ} \mathrm{F}$ and 200 ${ }^{\circ} \mathrm{F}$. As it can be seen, the agreement is very poor. Jallouk's data shows a much lower heat-transfer coefficient compared with the data from Cudo. A number of experiments were then run within the single phase forced convection regime to determine the heat transfer characteristics of the tube and it was determined that a good agreement existed between the experiment and previously published correlations [3] (see Correlation section). Similarly, pool boiling experiments were run with the tube in the vertical orientation. The data indicated a significant increase from that of Jallouk [2]. Such a difference may be attributable to the surface characteristics such as roughness which play a controlling part in nucieation [4]. 


\section{Roughness measurement}

The 10-foot copper tube was supplied by Martin Marietta Utility Services. Inc. (MMUS). The inner surface was successively cleaned using 400 grit sand paper, acetone. and methanol. Also supplied was a part of the test section used by Jallouk [2] in his experiments. The roughness profiles of Jallouk's [2] test section and the present one were measured using a Surtronic 3P profilometer (made by Rank Taylor-Hobson); figures 16 and 17 show sample roughness profiles and table 1 shows the values of commonly used roughness parameter $\mathbf{R}_{\mathbf{z}}$ and $\mathbf{R}_{\mathbf{q}} . \mathbf{R}_{\mathbf{2}}$ is defined as the arithmetic mean of the departure of the roughness profile from the mean line.

$$
R_{a}=\frac{1}{L} \int_{0}^{L}|y(x)| d x
$$

$R_{q}$ is defined as the root-mean-square average of the departure of the roughness profile from the mean line.

$$
R_{q}=\sqrt{\frac{1}{I} \int_{0}^{i} y(x)^{2} d x}
$$

Here $L$ is the scan length, $y$ the roughness profile in the direction parallel to the tube axis.

As shown in table 1 , three scans were carried out for each tube. The third scan for each tube is presented in figures 16 and 17. The results show a marked difference in the roughness of the two test section tubes; moreover, the resuits of the old test section, see figure 17, show a large variance. This is due to the fact that the old test section had a thick layer of oxide like deposits on the inside that made it impossible to determine its original roughness; any attempt to remove the oxide layer would inevitably result in a change in the original roughness of the surface. Therefore it is not possible to compare the roughness of the present test section with the previous one.

Since the purpose of this study is to simulate flow boiling conditions encountered in actual practice, it would be more appropriate to compare the roughness of the test section with that of a commercially available copper tube. This was carried out by measuring the internal roughness of a 
piece of $1 / 2$ " outer diameter copper tubing commonly used in the refrigeration industry; the results are presented in figure 18 and table 1 . It can be seen that the roughness values of the new test section and the commercial tube are comparable. Thus the test section can be assumed to be an appropriate representation of the real situation.

\section{Table 1 Roughness Values}

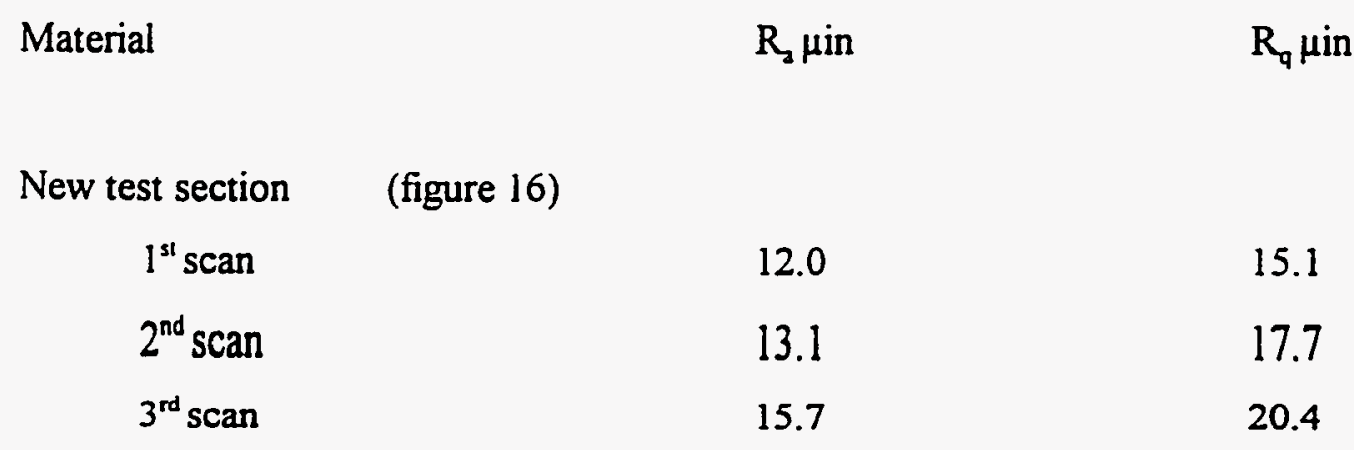

Jallouk [7] (figure 17)

$\begin{array}{lll}1^{\text {st }} \text { scan } & 29.9 & 37.7 \\ 2^{\text {nd }} \text { scan } & 59.3 & 85.2 \\ 3^{\text {rd }} \text { scan } & 52.9 & 76.1\end{array}$

Commercial $1 / 2$ " copper tubing (figure 18)

$\begin{array}{lll}1^{\text {st }} \text { scan } & 10.4 & 15.2 \\ 2^{\text {nd }} \operatorname{scan} & 14.7 & 21.5 \\ 3^{\text {rd }} \operatorname{scan} & 15.6 & 21.8\end{array}$

\section{Correlation}

The correlation for flow boiling developed by Jallouk [2] using R-114 test data was based on a superposition model first introduced by Chen [5]. In this correlation Chen [5] hypothesized that the flow boiling heat transfer could be modeled as a linear superposition of the forced convection and 
nucleate boiling components of the flow field. Since the introduction of the Rohsenow superposition model [6] several new variations have been introduced. Within this part of the study an attempt is made to analyze the efficacy of some of the correlations to model the Cudo data.

\section{Convective Flow Boiling Models}

In forced convective boiling, the forced convection, nucleate boiling and evaporation components exists in varying degrees depending on the regime of flow boiling [1], complicating the mechanisms for heat transfer and the ability to empirically model them. The two terms in convective flow boiling namely, comvection $\left(\mathbf{q}_{\mathrm{r}}\right)$ and boiling $\left(\mathbf{q}_{\mathrm{nb}}\right)$ are specific types of the heat transfer process. Convection is the transport of heat through change in the sensible heat content of the fluid, i.e. by a change in the fluid temperature. Boiling occurs when vapor bubbles are formed at the heated wall. Evaporation occurs when liquid is superheated at the wall, and vaporizes at the liquid-vapor interface. If nucleate boiling occurs on a surface, without convective flow, the process is called nucleate pool boiling. This heat flux is written as $\mathbf{q}_{\mathrm{npb}}$. If nucieate boiling and convection occur simultaneously in a flowing fluid, the coexisting heat transfer mechanisms are described as convective flow boiling.

A variety of correlations exist for convective flow boiling. Three types of phenomenological models are generally used for calculating the heat-transfer coefficient in convective flow boiling, namely, the superposition, asymptotic, and enhancement models. These three types of convective flow boiling models incorporate different techniques for combining the nucleate boiling and convection contributions. Figures 19(a) \& (b) [7] illustrate the convective flow boiling curve on the $\log$-log plot of heat flux versus wall superheat $\Delta \mathrm{T}\left(=\mathrm{T}_{\pi}-\mathrm{T}_{f}\right)$. The convective flow boiling curve is asymptotic to $q_{c v}$ at low $\Delta T$, and $q_{n b}$ at high $\Delta T$. The $q_{c v}$ asymptote has unity slope, because its heat-transfer coefficient $h_{\mathrm{cv}}$ is independent of $\Delta \mathbf{T}$. These three different models are described below.

\section{Superposition Model}

Rohsenow [6] initially proposed the superposition model and applied it to subcooled convective flow boiling. The superposition model assumes that the total heat flux $q$ is the sum of the convective and nucleate boiling components. Thus, the basic equation for the superposition model 
is.

$$
q=q_{n b}-q_{c v}
$$

The terms $q_{n b}$ and $q_{c}$ are calculated at the value of wall superheat $\Delta T$ that exists in the system. One may write the superposition model in terms of the component heat-transfer coefficients by dividing Eq. (3) by the wall superheat $\Delta T$ to obtain,

$$
h=h_{n b}+h_{c v}
$$

Chen [5] argued that as the velocity of the flow increased it suppressed the ability of the fluid to nucleate at the wall. Hence, a suppression factor $S$ was introduced which empirically had the effect of modifying the contribution of nucleate boiling, i.e., $\mathbf{q}_{\mathrm{nb}}=\mathbf{S} \mathbf{q}_{\mathrm{npb}}$

\section{The Enhancement Model}

The enhancement model was proposed by Shah [8]. For flow boiling in vertical tubes, it was described by,

$$
h=E h_{l}
$$

where $E$ is the enhancement factor which is a function of the boiling number (Bo) and the convection number (Co). The term $h_{1}$ is the single-phase heat-transfer coefficient for the liquid phase flowing alone. The Bo and Co numbers were defined as,

$$
B o=\frac{q}{\lambda G}
$$

and

$$
C o=\left(\frac{1-x}{x}\right)^{0.8}\left(\frac{\rho_{g}}{\rho_{l}}\right)^{0.5}
$$

For application to horizontal tubes, $E$ also depends on a third parameter, the Froude number $\left(F r_{1}\right)$, if $F r_{1}<0.04$. The Froude number accounts for partial wall wetting, which may occur in 
horizontal channels. Kandlikar [9] modified the Shah [8] correlation with an empirical fluiddependent parameter $F_{f \text { th }}$ which multiplies the nucleate boiling term. Kandlikar [9] updated the values of $F_{\mathfrak{f}}$, based on a large data base containing 5246 data points.

\section{Asymptotic Model}

Kutateladze [10,11] proposed a power-type asymptotic non-linear superposition model for the two boiling components in the form,

$$
q^{n}=q_{n b}^{n}+q^{n}
$$

When $n>1$, the curved portion of the convective flow boiling curve is asymptotic with the limiting straight lines $\mathbf{q}_{\mathrm{d}}$ and $\mathbf{q}_{\mathrm{nb}}$ on figure 19(a). One of the asymptotes correlates the forced convection component, where the nucleate boiling contribution is insignificant. The second asymptote predicts the nucleate boiling component, the contribution due to convective heat transfer being insignificant in this regime. The curved region between the two asymptotes reduces as the value of $\mathbf{n}$ increases. As $\mathbf{n} \rightarrow \infty$, the curved region shrinks to zero. If $\mathbf{n}=1$, the total heat flux is the sum of the two components.

Kutateladze [10] introduced the asymptotic correlation for subcooled water in plain tubes and used the exponent $\mathbf{n}=2$. There is no theoretical basis for selection of the exponent that gives the best fit of the data. Steiner and Taborek [12] have proposed a correlation for in-tube flow boiling data based on a variant of the asymptotic model. Their correlation is based on the asymptotic model Eq.(8) with $\mathbf{n}=3$, which was evaluated using the data bank from the University of Karlsruhe. The two components in this model are independent of each other, their integration being governed by the basic equation Eq. (9). Thus, the selection of methods for the nucleate and convective components is independent of the base model itself.

$$
\begin{gathered}
h^{n}=\left[h_{n b}^{n}+h_{c v}^{n}\right]^{\frac{1}{n}} \\
=\left[\left(h_{n p b} C_{n b f}\right)^{n}+\left(h_{l} C_{q f f}\right)^{n}\right]^{\frac{1}{n}}
\end{gathered}
$$


here,

$h_{n p h}$ is the local nucleate pool boiling coefficient. The coefficient itself can originate from any predictive method or data. Thus, it leaves a great freedom of choice.

$C_{n b r}$ is a nucleate boiling correction factor to $h_{n p b}$, which compensates for differences between the pool and the flow conditions.

$h_{1}$ is the local convective heat-transfer coefficient for single-phase liquid flow.

$C_{t p f}$ is the two-phase flow correction factor to the convective component $h_{1}$, accounting for enhancement of the coefficient in the liquid-vapor mixture.

\section{Heat Transfer Correlation}

The flow boiling heat transfer tests conducted at Cudo, on R-114, $\mathrm{C}_{4} \mathrm{~F}_{10}$ and $\mathrm{C}_{4} \mathrm{~F}_{8}$ has provided the necessary data to establish a new correlation. The correlation will be used in the gas cooler computer models to predict cooling water requirements under a variety of operating conditions and with different CFC replacement candidates. The existing correlation for the gas cooler computer models was developed by Jallouk [2] using R-114 test data, and was based on a superposition model. As we mentioned earlier, Figs. 5 and 7 shows poor agreement between the data from Cudo and Jallouk's [2] data. The R-114 test data from Cudo has consistently shown better heat transfer performance than that reported by Jallouk [2]; Consequently, the Jallouk [2] correlation under predicts the Cudo data by as much as $100 \%$.

The present test data was also compared with the correlation provided by Kandlikar [9], which was recommended by Carey [13] and is an expansion of Shah's model [8]. The comparison did not show consistent agreement through various test conditions and the disagreement varies from about 10 to $100 \%$. A set of constants in this correlation was established by regression analysis, using Kandlikar's [9] own data bank on water. However, when tested on refrigerants data, a fluid specific correction factor $F_{n}$ needs to be added to the correlation R-114 was one of the ten fluids tested by Kandlikar [9] and was ascribed a $F_{\mathfrak{n}}=1.24$. Even with this experimentally determined fluid parameter for R-114, the Kandlikar's [9] correlation did not predict our data well. Also, the values 
of $F_{n}$ follow no logical pattern and therefore are presumed to be data source dependent. Thus Kandlikar's [9] method can not be used for general applications. as values of $F_{n}$ are not known.

The type of model chosen in this study is the asymptotic model as proposed by Steiner and Taborek [12], which was strongly recommended by Webb and Gupte [7]. Like the superposition model, the asymptotic model relies on combining a forced convection component and a nucleate pool boiling component. However, the asymptotic model uses a power relationship as given by Eq. (9). The model is called asymptotic because the flow boiling coefficient $\mathbf{h}$ approaches the larger values of the convective coefficient $h_{c v}$ and the pool boiling coefficient $h_{n p b}$ as shown in figure 19(b). The maximum addition occurs when the two components are equal.

Steiner and Taborek [12] compared the predictive ability of their correlation with Chen [5], Shah [8], and Gungor and Winterton [14, 15] for ethanol, ammonia, and R-12. Their [12] asymptotic form avoids the abrupt transition between the nucleate boiling and convective regions and in general they showed that their correlation is significantly better than the other correlations $[6,8-11]$.

In order to use this approach one must have experimentally determined values for the two components $h_{n b}$ and $h_{c v}$ in Eq. (9) or use those published by Steiner and Taborek [12] (determined from a 13,000 point data base covering a variety of fluids). We chose to use the experimental approach, and only used the convective multiplier $C_{\mathrm{tpr}}$, which accounts for enhancement due to the liquid-vapor mixture, given in the paper. Thus, Eq. (10) can be rewritten as,

$$
h^{n}=\left[h_{n b}{ }^{n}+\left(h_{l} C_{t p f}\right)^{n}\right]^{\frac{1}{n}}
$$

From Taborek's [12] analysis, $C_{\mathrm{tpr}}$ was derived as a function of the vapor fraction $x$ and the liquid-vapor density ratio $\rho_{\mathrm{f}} / \rho_{\mathrm{g}}$.

$$
C_{\text {tff }}=\left[(1-x)^{1.5}+1.9 x^{0.6}\left(\frac{\rho_{l}}{\rho_{g}}\right)^{0.35}\right]^{1.1}
$$

This correlation was developed and validated by Taborek [12] using a large data bank containing 15 
fluids from 34 sources.

\section{Single-Phase Liquid Flow}

The forced convection component $\boldsymbol{h}_{1}$ in Eq. (11) was determined experimentally at Cudo by running subcooled liquid through the copper rube for $R-114, C_{4} F_{10}$ and $C_{4} F_{8}$, and then correlating that data with a Dittus-Boelter type equation as given below,

$$
N u=\frac{h_{1} d_{i}}{k}=c R e^{0.8} \operatorname{Pr}^{0.4}
$$

In this equation, the coefficient $\mathbf{c}$ is equal to 0.023 for singe-phase flow inside a smooth cylindrical tube. For the tube we used in our experiment, $c$ was determined from single-phase liquid flow tests for the three different liquids. The results are shown in figures 20-22. As we can see, both fluids $R-$ 114 and $\mathrm{C}_{4} \mathrm{~F}_{10}$ correlate quite well with equation (13) with $\mathrm{c}=0.028$. This is exactly what one would expect for forced convection since the equation is in terms of dimensionless numbers. The coefficient c for $\mathrm{C}_{4} \mathrm{~F}_{8}$ is slightly smailer and is about 0.025 . This could be due to change of the surface roughness inside the tube during the course of our tests.

\section{Nucleate Pool Boiling}

The nucleate pool boiling term $h_{n b}$ in Eq. (11) was also determined at Cudo by running very low flow in the tube and only using heat flux data from the bottom two zones 2 and 3 [1]. One of the most widely used nucleate boiling correlations for flow boiling presented by Rohsenow [6] was chosen for this study,

$$
\frac{C_{p_{l}} \Delta T}{\lambda P_{l}^{1.7}}=C_{w f}\left(\frac{Q / A}{\mu_{l} \lambda} \sqrt{\frac{\sigma}{g \Delta \rho}}^{0.33}\right.
$$

The coefficient $C_{w}$ is dependent on the wall-fluid material characteristics and was determined from the pool boiling experiments. 
The experimental results for the three different fluids are plotted in figures 23-25 using the Rohsenow [6] correlation Eq. (14) with a logarithmic scale. The line used by Jallouk [2] in his correlation is also shown for reference. Here, again all three fluids correlated the same, with an intercept value of 0.0038025 . This number is the experimentally determined value of the coefficient $\mathrm{C}_{\mathrm{wr}}$ in Eq. (14). It should be noted that in this coordinate system. the lower the line the higher the nucleate boiling coefficient. After the coefficient $C_{w r}$ is determined. the correlation of the nucleate boiling term $h_{n b}$ in Eq. (11) can be obtained from Eq. (14) in the form,

$$
h_{n b}=\frac{Q}{A \Delta T}=\left(\frac{k_{l}^{1.7}}{C_{w f} \mu_{l}^{1.7} C_{p_{l}}{ }^{0.7}}\right)^{3} \mu_{l}\left(\frac{\Delta T}{\lambda}\right)^{2} \sqrt{\frac{g \Delta p}{\sigma}}
$$

The fact that the predictive correlation is the same for the three different fluids does not mean that the nucleate boiling heat-transfer coefficient is the same. The difference in the physical properties of the fluids will resuit in different heat-transfer coefficients for the same temperature level and heat flow.

\section{Convective Flow Boiling}

With expressions for the convective and nucleate boiling components from Eqs. (13) and (15), one can make predictions for the flow boiling heat-transfer coefficient by employing Eq. (11). From the flow boiling tests at Cudo, the measured flow boiling heat-transfer coefficient also can be determined from the measured heat flux and $\Delta T$. A flow boiling FORTRAN code was written and used for the calculation of the predicted and measured heat-transfer coefficients. All of the experimental data and calculated results for the flow boiling tests were set up in EXCEL spreadsheets.

Figures 26-29 show the comparison between the predicted and measured flow boiling heat transfer coefficients for R-114 at $125,150,175$ and $200^{\circ} \mathrm{F}$ with heat fluxes of $10,000,15,000$, 20,000 , and $25,000 \mathrm{btu} / \mathrm{hr} / \mathrm{ft}^{2}$. An exact match would follow the 45 -degree solid line. The same 
information for $\mathrm{C}_{4} \mathrm{~F}_{8}$ and $\mathrm{C}_{4} F_{10}$ are shown in figures 30-37. Here the data was taken at $125.150,175$ and $190^{\circ} \mathrm{F}$, in the heat flux range of 5.000 to $20,000 \mathrm{btu} / \mathrm{hr} / \mathrm{ft}^{2}$. For all three fluids the liquid mass flux was varied from $0.2 * 10^{6}$ to $1.0^{*} 10^{6} \mathrm{lbm} / \mathrm{hr} / \mathrm{ft}^{2}$. Note, from these figures, that the correlation predicts the experimental results well. In general. the correlation consistently over predicts the experimental data at the lower saturation temperatures. The difference between the predicted and measured $h$ for most of the R-114 and $C_{4} F_{10}$ data fall within $\pm 25 \%$, and $\pm 30 \%$ for $C_{4} F_{8}$. All the figures clearly show that the predicted coefficient is largely unaffected by changes in liquid mass flux and axial position (local quality) along the length of the tube. Part of the horizontal spread shown in the figures is attributed to uncertainty in the experiment.

It should be noted that the flow boiling heat-transfer correlation has not been adjusted based on the actual flow boiling test data from Cudo. Only the forced convection and in-tube pool boiling data have been used. This implies that this correlation can provide good prediction for in-tube flow boiling as long as pool boiling and forced convection data are available.

Figures 38 to 41 give comparison of predicted flow boiling heat-transfer coefficients for three different liquids with saturation temperatures of $125,150,175$ and $190^{\circ} \mathrm{F}$. Heat-transfer coefficients under dry-out conditions were not presented in these figures. As we mentioned earlier, the predicted heat-transfer coefficient is largely unaffectedly by changes in liquid mass flux and axial position along the tube. Thus, we only present the coefficients with a typical mass flux of $0.52 \mathrm{lbm} / \mathrm{hr} / \mathrm{ft}^{2}$ at section 5 [1]. These figures show that the flow boiling heat-transfer coefficient is strongly dependent on the heat flux and the saturation temperature. The higher the heat flux and saturation temperature, the higher is the heat-transfer coefficient obtained. From these comparisons, we find that the heattransfer coefficients for $\mathrm{C}_{4} \mathrm{~F}_{10}$ and $\mathrm{C}_{4} \mathrm{~F}_{8}$ are about the same but much higher than that for $\mathrm{R}-114$.

\section{Critical Heat flux}

The critical heat flux (CHF) is the maximum upper limit of heat flux at which a system can be operated normaily without dryout. In exceeding this limit, the system will experience a rapid increase in wall temperature and sharp decrease in heat transfer effectiveness. The dry-out crisis 
occurs in flow boiling when the number of vapor bubbles close to the wall coalesce to form an insulating vapor blanket thus impeding the contact of liquid with the wall and dropping the heattransfer coefficient drastically. Then if the heat flux is held steady a steep increase in wall temperature is realized.

For flow boiling, CHF is strongly dependent on the mass flowrate and latent heat of the working fluid. In the present flow boiling tests, dryout was initiated within the last zone of the test section after the applied heat flux exceeded the CHF. When the heat flux was increased further, the dryout proceeded to advance upstream. In other words, dryout will occur earlier with higher heat flux. When CHF conditions were imminent the heat flux to the affected section was reduced to prevent any excessive temperatures.

A practical way to present $\mathrm{CHF}$ for the flow boiling is the critical heat flux versus the dry-out location of the tube. Figures 42-53 show the results from our CHF tests for the three different liquids. In general, dryout occurs earlier for the lower flowrate and higher heat flux. In comparing the CHF characteristics of the three different liquids, R-114 always is the one to dryout the latest and $\mathrm{C}_{4} \mathrm{~F}_{10}$ is the one to dryout the earliest under the same flow and heat flux conditions.

\section{Pressure Drop}

For two-phase flows inside a tubes, the gravitational, frictional, and acceleration components contribute to the pressure drop and all three must be taken into account. Two different models are generally used in predicting pressure drop for two-phase flows. They are the homogeneous and the separated flow models. The homogeneous model considers the two-phase flow as a single-phase flow possessing mean fluid properties. On the other hand, the separated flow model considers the twophase flow to be artificially segregated into two streams; one of liquid and one of vapor. In the homogeneous model, each stream is assumed to travel at a mean velocity. In the case when a very large percentage of the two-phase flow inside a tube is annular in nature, the application of a separated flcw model will yield results better than those obtained by the homogeneous model. 
In this study, the separated flow model prèsented by Martinelli-Nelson [16.17] was adopted for pressure drop prediction. In the Martinelli-Nelson model [16.17], the two phases are assumed to be separate. The pressure drop is defined by Equation (2-46) of Collier's [18] as given below,

$-\left(\frac{d p}{d z}\right)=-\left(\frac{d p}{d z}\right)_{F}+G^{2} \frac{d}{d z}\left[\frac{x^{2} v_{g}}{\alpha}+\frac{(1-x)^{2} v_{l}}{(1-\alpha)}\right]+g\left[\alpha p_{g}+(1-\alpha) p_{t}\right]$

The three terms contribute to the friction, acceleration, and gravitational pressure drop, respectively. These three pressure drop components are calculated using the Martinelli-Nelson model $[16,17]$ in each section of the test tube. In the experiment, pressure drops with intervals of $2,4,6,8$ and 9.88 feet from the inlet of the test section were measured by five differential pressure transducers. The pressure readings from the differential pressure transducers were corrected for elevation of the pressure tap lines. The corrected pressure drop was then converted to the equivalent elevation of the liquid column at the operating saturation temperature.

All of the measured and predicted pressure drops were listed in EXCEL spreadsheets. Figures 54 to 54 present the comparison between the predicted and measured pressure drop along the test section for the three different liquids. As we can see from these figures, the Martinelli-Nelson model $[16,17]$ consistently and slightly over predicts the measured pressure drop.

\section{CONCLUSIONS}

Forced convection, pool and flow boiling tests were run using R114, $C_{4} F_{10}$ and $C_{4} F_{8}$ to determine the heat-transfer coefficient, $\mathrm{CHF}$ and pressure drop. A correlation for $\mathrm{h}$ based on a flow convective and pool boiling model proved to predict the measured flow boiling $\mathrm{h}$ within $30 \%$. The dryout length under conditions of $\mathrm{CHF}$ increased with heat flux and reduction in flow rate. A pressure drop correlation, based on a separated flow model, slightly but consistently overpredicted the measured results. 


\section{ACKNOWLEDGEMENTS}

This work was performed for Martin Marietta Energy Systems. Inc.. under contract \# 11K-NR559C. Thanks are due to Dr. Shin Park Andy Szady, David Lannom and Prof. Louis Chow, for their technical help and discussions. Thanks are also due to Jon Curless, Gregory Mayes, Matthew McMillin. Tom Nett. E.B. Yates. Gregory Patrick. Larry Patrick. and Walter Cooper for their assistance at various stages of the project. 


\section{REFERENCES}

I. Pais, M.R.. Chang. M.J., and Sehmbey, M.S. 1994, Destgn. construction and testung of an experimem to measure the flow boiling heat transfer and pressure drop in a vertical tube. Rep. \# MMES-CTL-NR559-1, Lexington, KY.

2. Jallouk, P. A., 1976, Tw'0-Phase flow' Pressure Drop and Heat Transfer Characteristics of Refrigerants in Vertical Tubes, K-1864, Oak-Ridge Gaseous Diffusion Plant, Union Carbide Corporation, Oak Ridge, Tennessee, January.

3. Kays, W.M. and Crawford, M.E., Convective Heat and Mass Transfer, 2 ed., Chapter 8 and 13, John Wiley.

4. Berenson P.J., 1962, Experiments on pool boiling heat transfer, Int. J. Heat Mass Transfer, Vol. 5, pp. 985-999.

5. Chen, J. C., 1966, A Correlation for Boiling Heat Transfer to Saturated Fluids in Convective Flow, Industrial Engineering Chemistry Process Design and Development, Vol. 5, No. 3, pp. 322-329.

6. Rohsenow, W. M., 1952, A Method of Correlating Heat Transfer Data for Surface Boiling of Liquids, Transactions of ASME, Vol. 74, pp. 969-976.

7. Webb, R. L. and Gupte, N. S., 1992, A Critical Review of Correlations for Convective vaporization in Tubes and Tube Banks, Heat Transfer Engineering, Vol. 13, No. 3.

8. Shah, M. M., 1976, A New Correlation for Heat Transfer During Boiling Flow Through Pipes, ASHRAE Transactions, Vol. 82, Part 2, pp. 66-86.

9. Kandlikar, S. G., 1990, A General Correlation for Saturated Two-Phase Flow Boiling Heat Transfer Inside Horizontal and Vertical Tubes, J. Heat Transfer, Vol. 112, pp. 219-228.

10. Kutateladze, S.S., 1961, Boiling Heat Transfer, Int. J. Heat Mass Transfer, Vol. 4, pp. 3-45.

11. Kutateladze, S. S., 1963, Fundamentals of Heat Transfer, Edward Annold, London.

12. Steiner, D. and Taborek, J., 1992, Flow Boiling Heat Transfer in Vertical Tubes Correlated by An Asymptotic Model, Heat Transfer Engineering, Vol. 13, No. 2, pp. 43-69.

13. Carey, V. P., 1992, Liquid-Vapor Phase-Change Phenomena, Hemisphere Publishing Corporation. 
14 Gungor. K. E. and Winterton. R. H. S.. 1986. A General Correlation for Flow Boiling in Tubes and Anmull. Int. J. Heat Mass Transfer. Vol. 29. No. 3. pp. 351-358.

15 Gungor, K. E. and Winterton. R. H. S.. 1987. Simplified General Correlation for Saturated Flow' Boiling and Comparisons of Correlation with Data. Chem. Eng. Res. Dec., Vol. 65 , pp. 148-156.

16. Lockhart, R. W. and Martinelli, R. C., 1949, Proposed Correlation of Data for Isothermal Two-Phase, Two-Component Flow' Pipes, Chem. Eng. Prog., Vol. 45, p. 39.

17. Martinelli, R. C. and Nelson, D. B., 1948, Prediction of Pressure Drop During Forced Circulation Boiling of Water, Trans. ASME. Vol. 70, P. 695.

18. Collier, J. G., 1972, Convective Boiling and Condensation, McGraw-Hill Book Company, New York. 


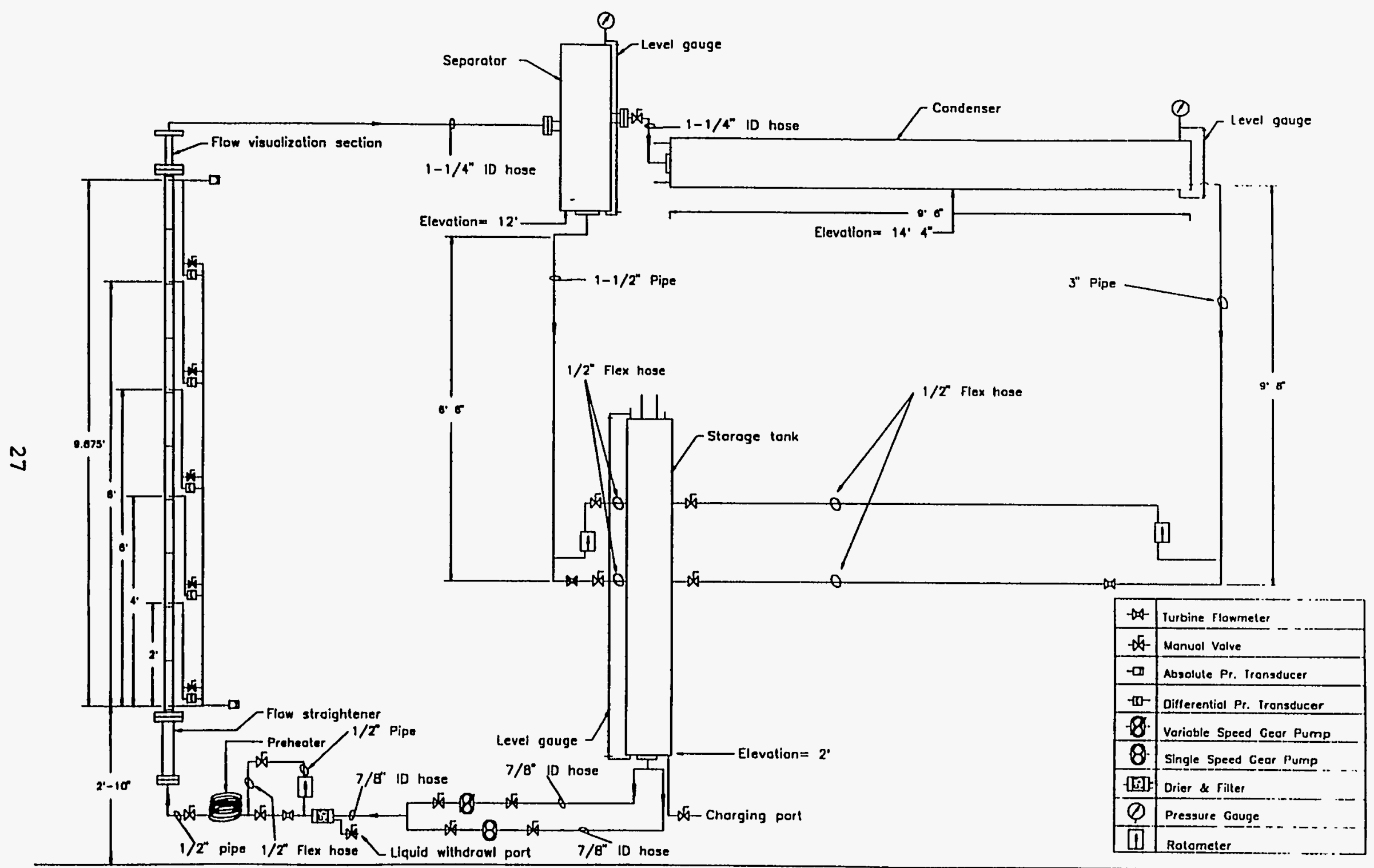

Figure 1. Test Fluid Loop 

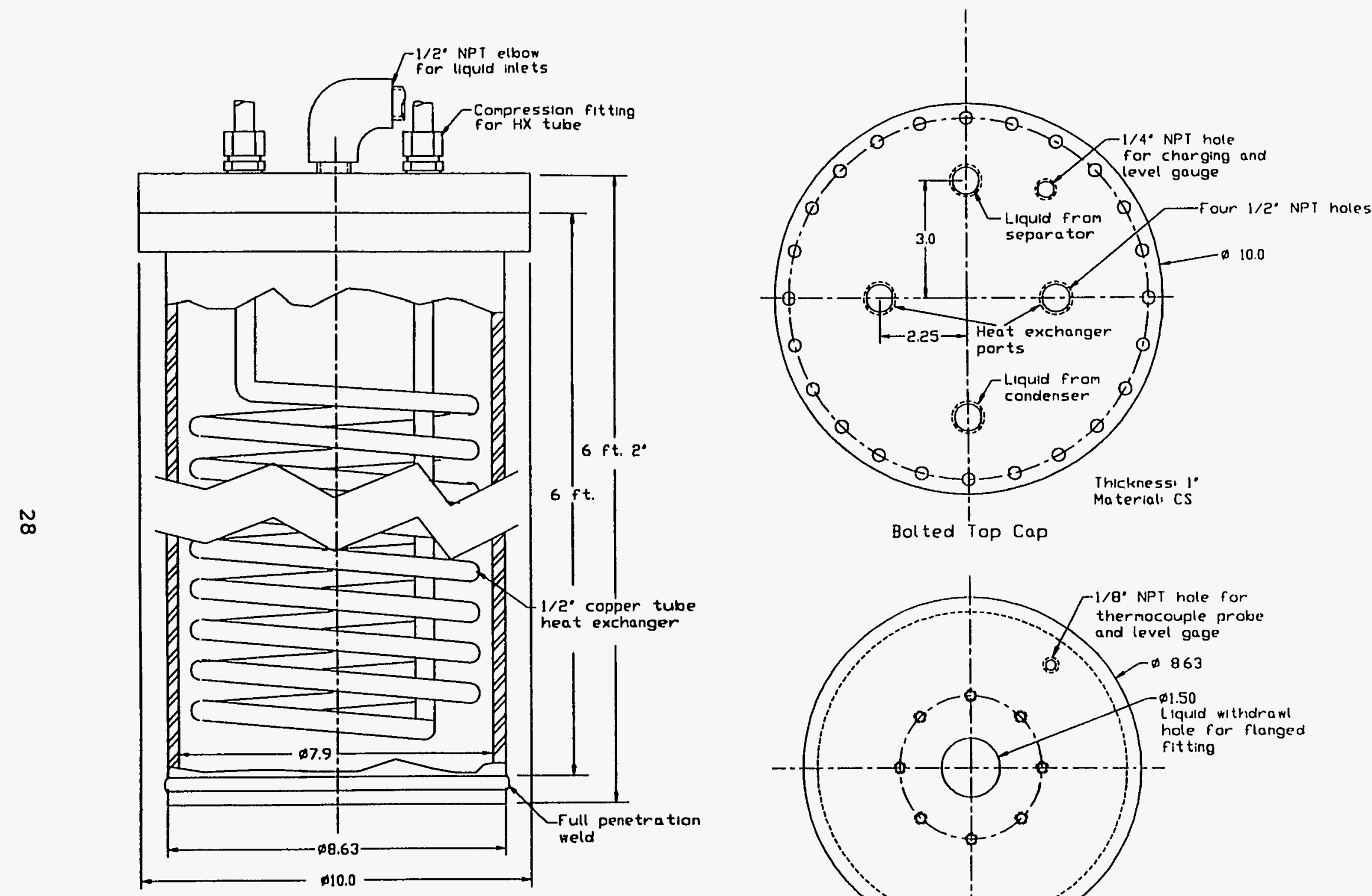

Effective internal volume $=3500$ cu.In. appx

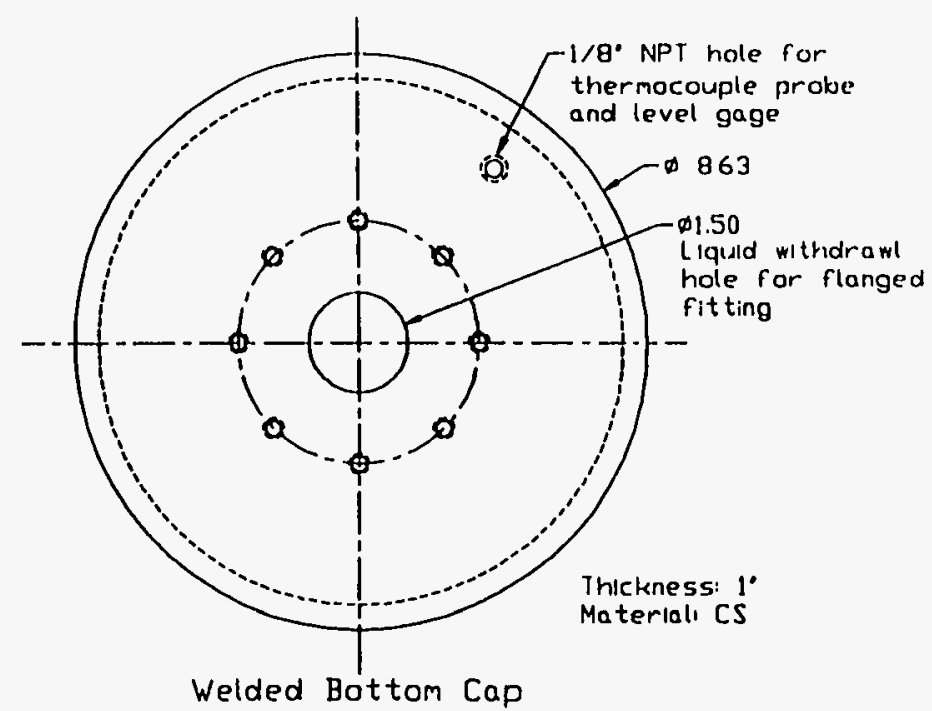

Storage Tank- Assembly and Caps

Figure 2. 


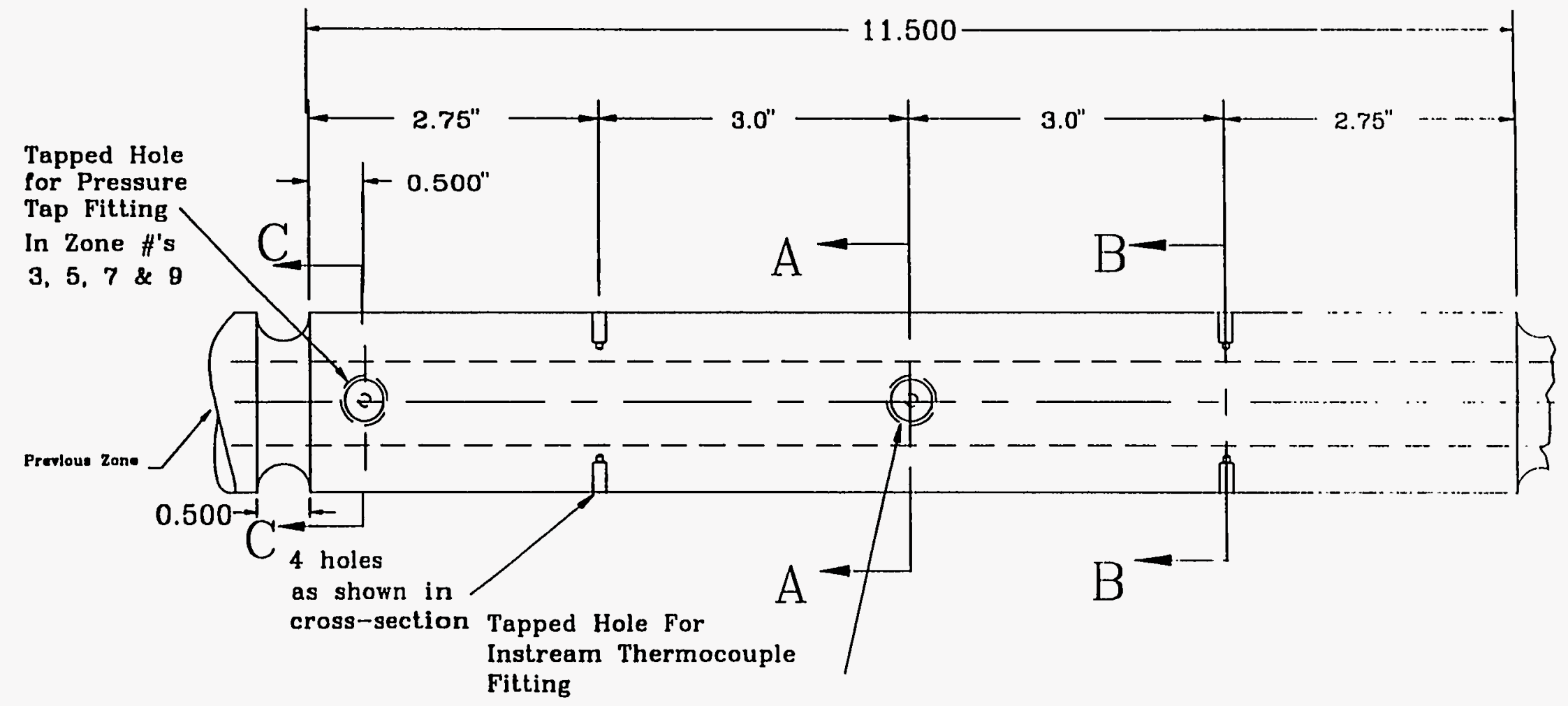

Details of Zone 2 thru 9

Figure 3. 


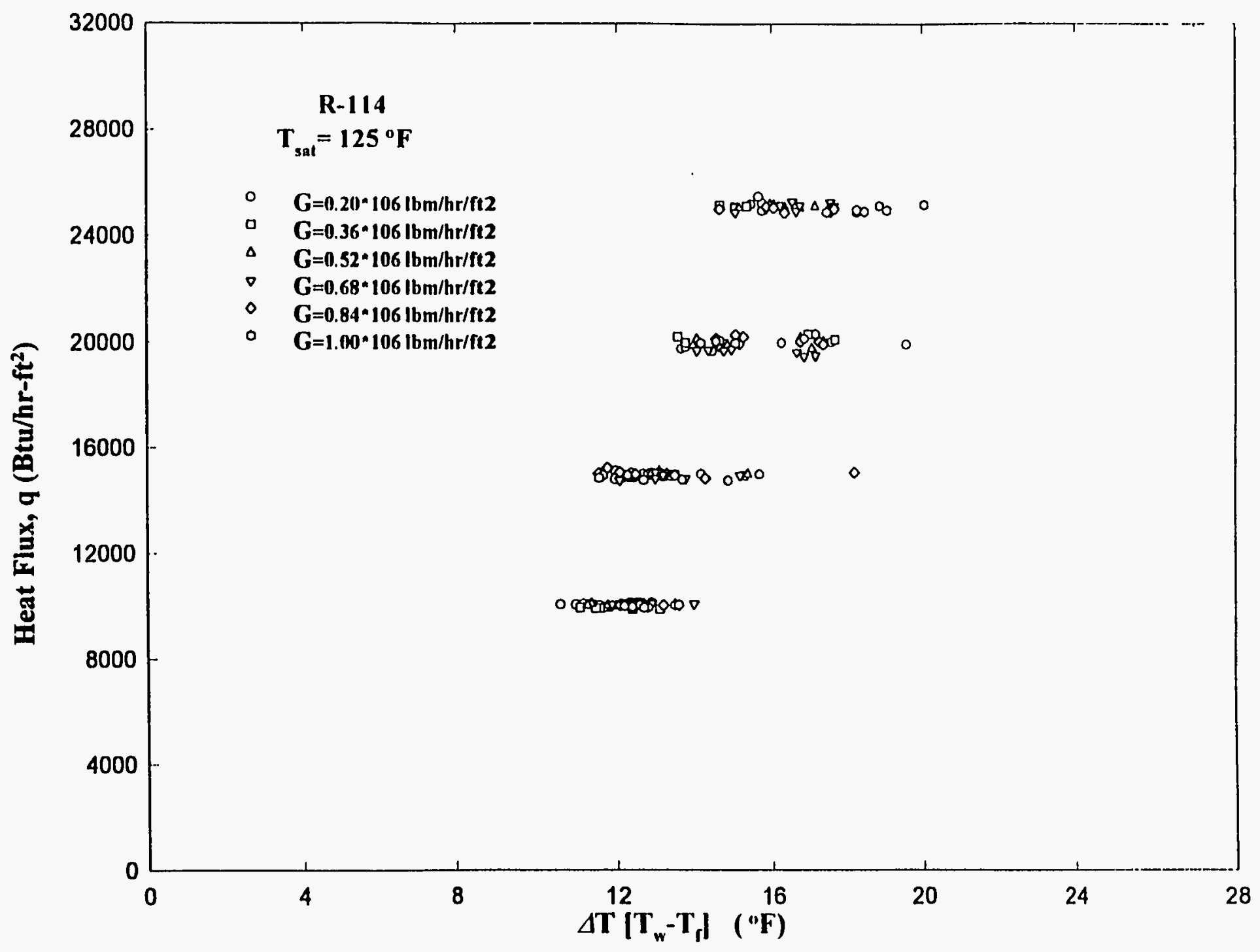

Figure. 4 Heat Flux Versus the Wall-to-instream Temperature Difference for $R-114$ at $T_{\text {smt }}=125^{\circ} \mathrm{F}$ 


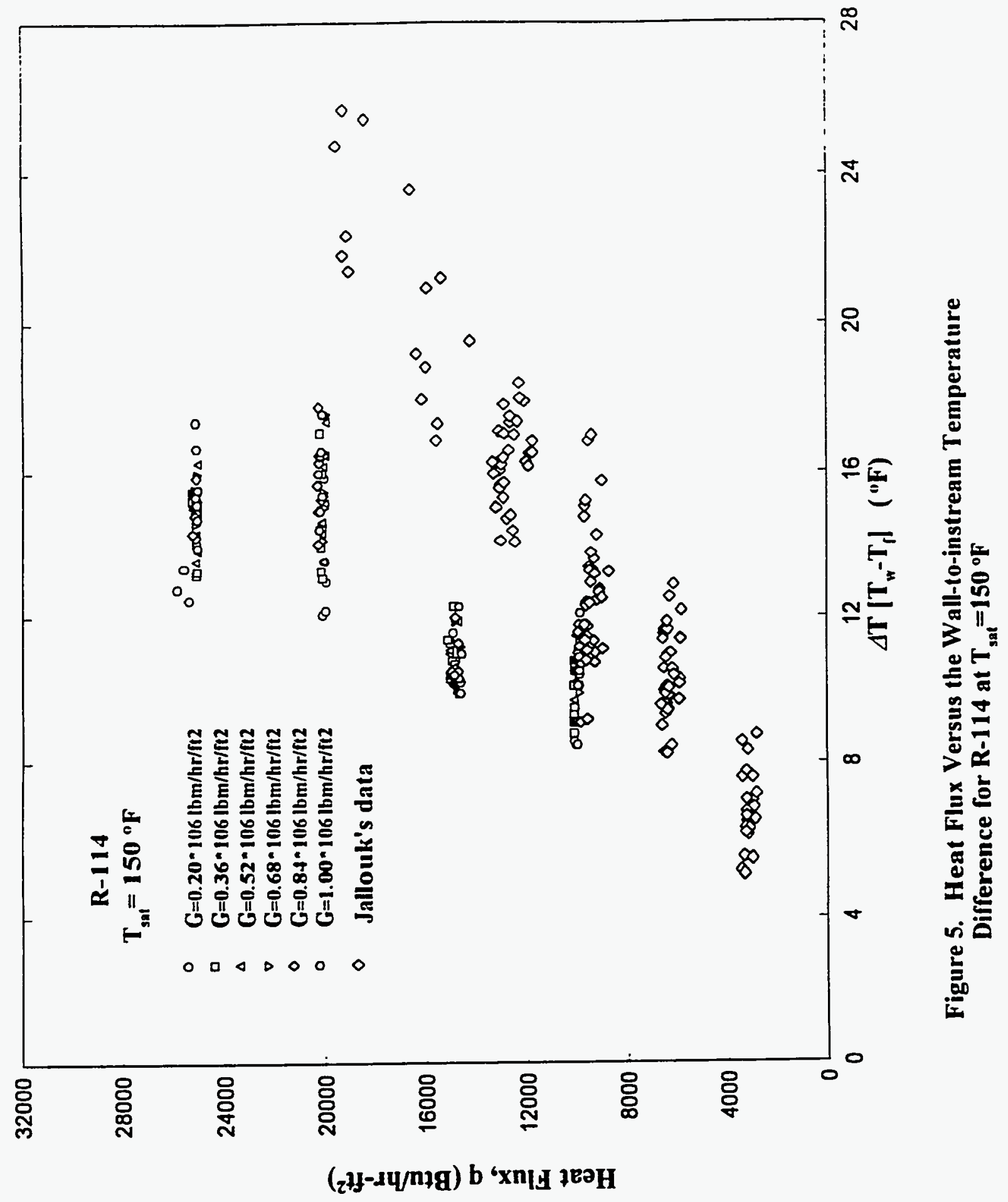




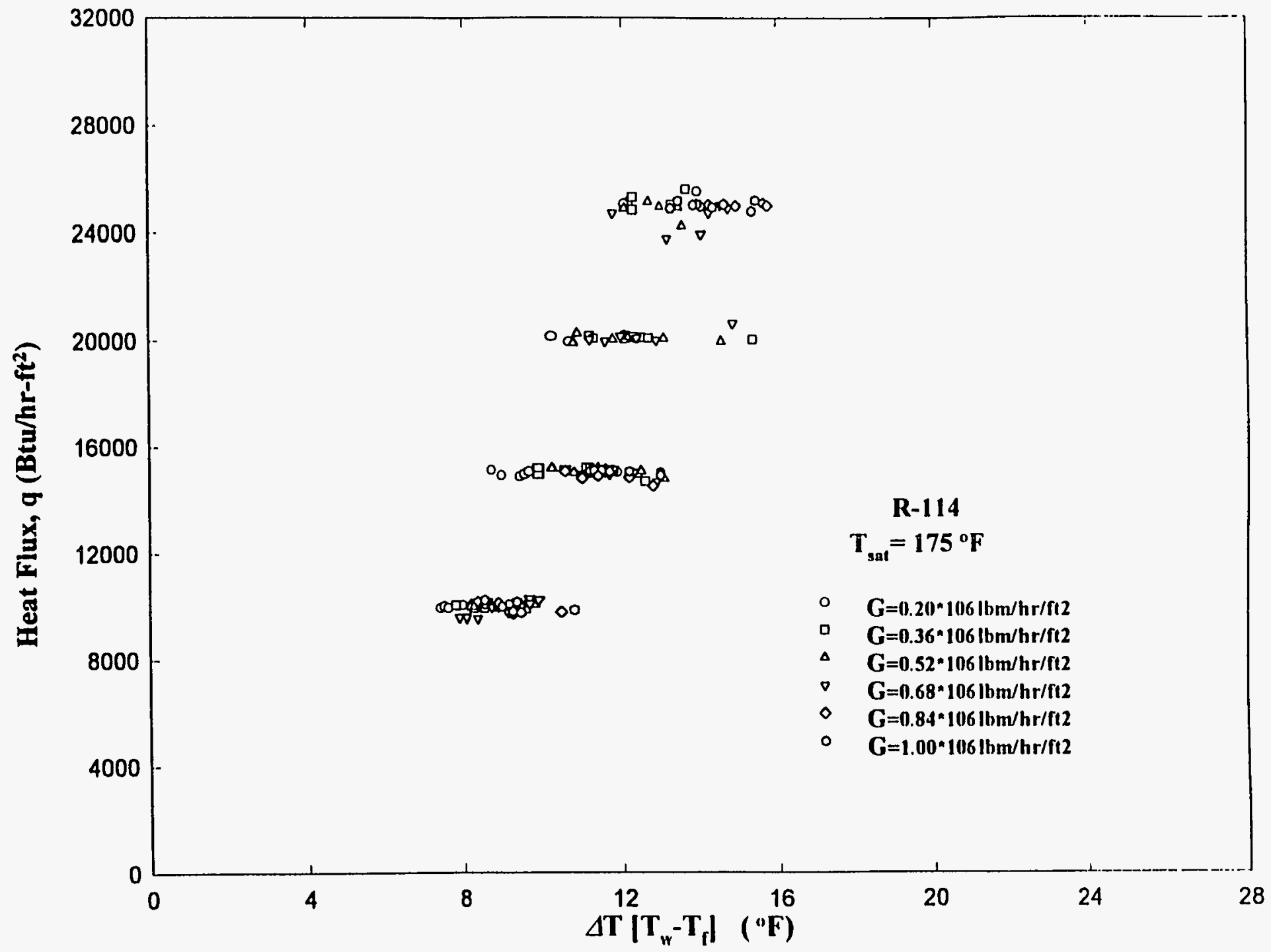

Figure 6. Heat Flux Versus the Wall-to-instream Temperature Difference for $R-114$ at $T_{\text {sat }}=175^{\circ} \mathrm{F}$ 


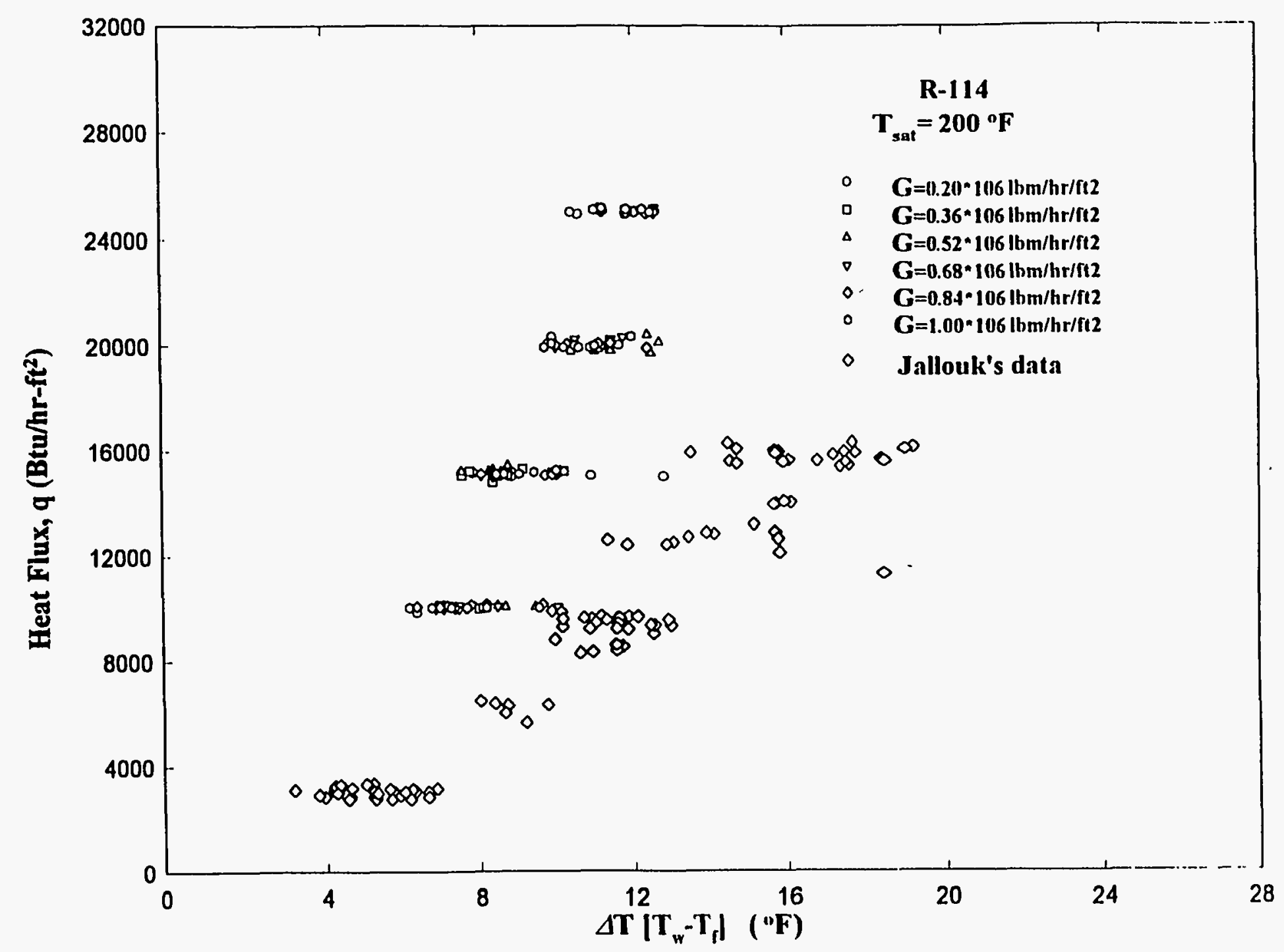

Figure 7. Heat Flux Versus the Wall-to-instream Temperature Difference for $\mathrm{R}-114$ at $\mathrm{T}_{\text {sat }}=200^{\circ} \mathrm{F}$ 


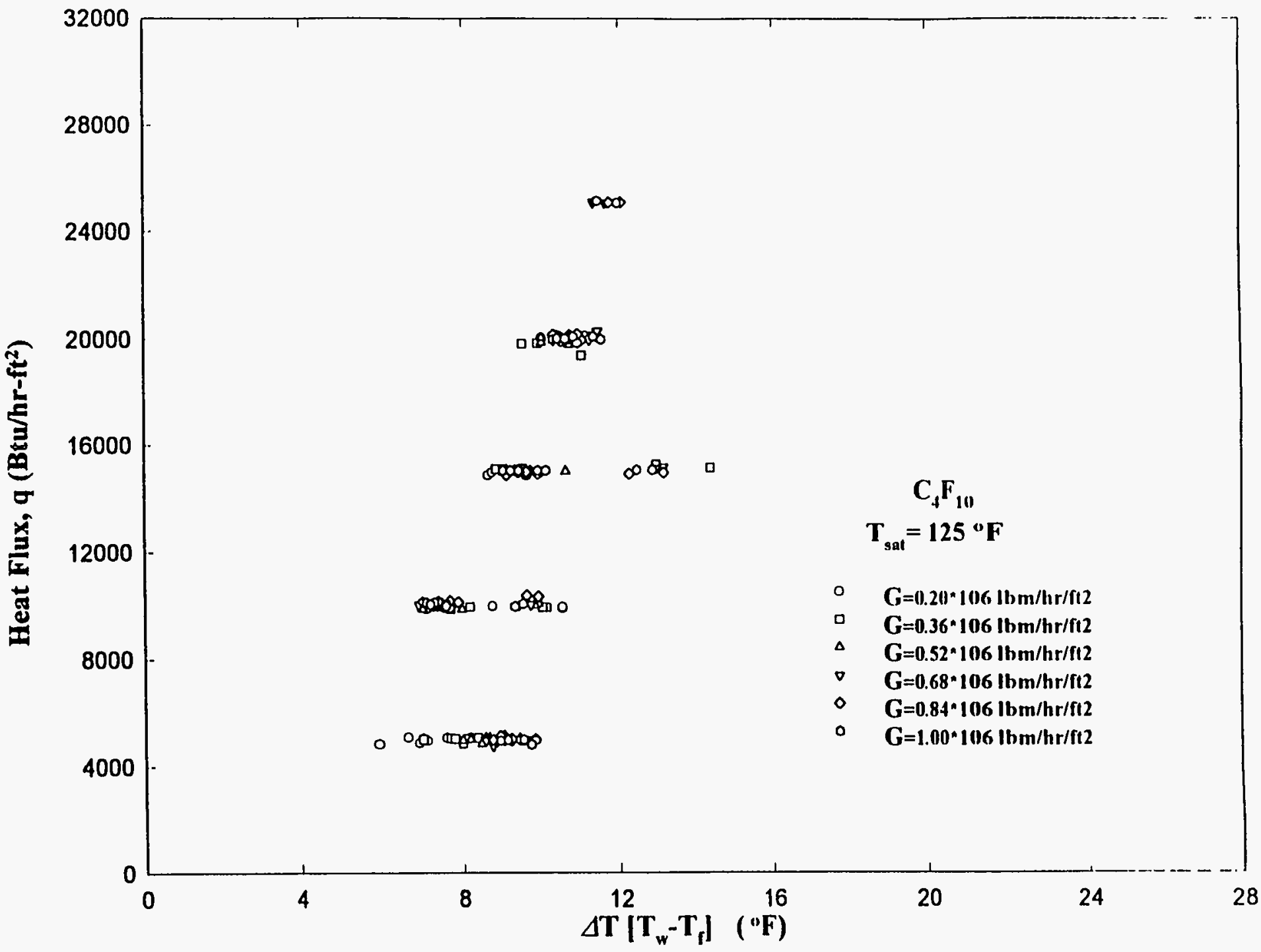

Figure 8. Heat Flux Versus the Wall-to-instream Temperature Difference for $\mathrm{C}_{4} \mathrm{~F}_{10}$ at $\mathrm{T}_{\text {sat }}=125^{\circ} \mathrm{F}$ 


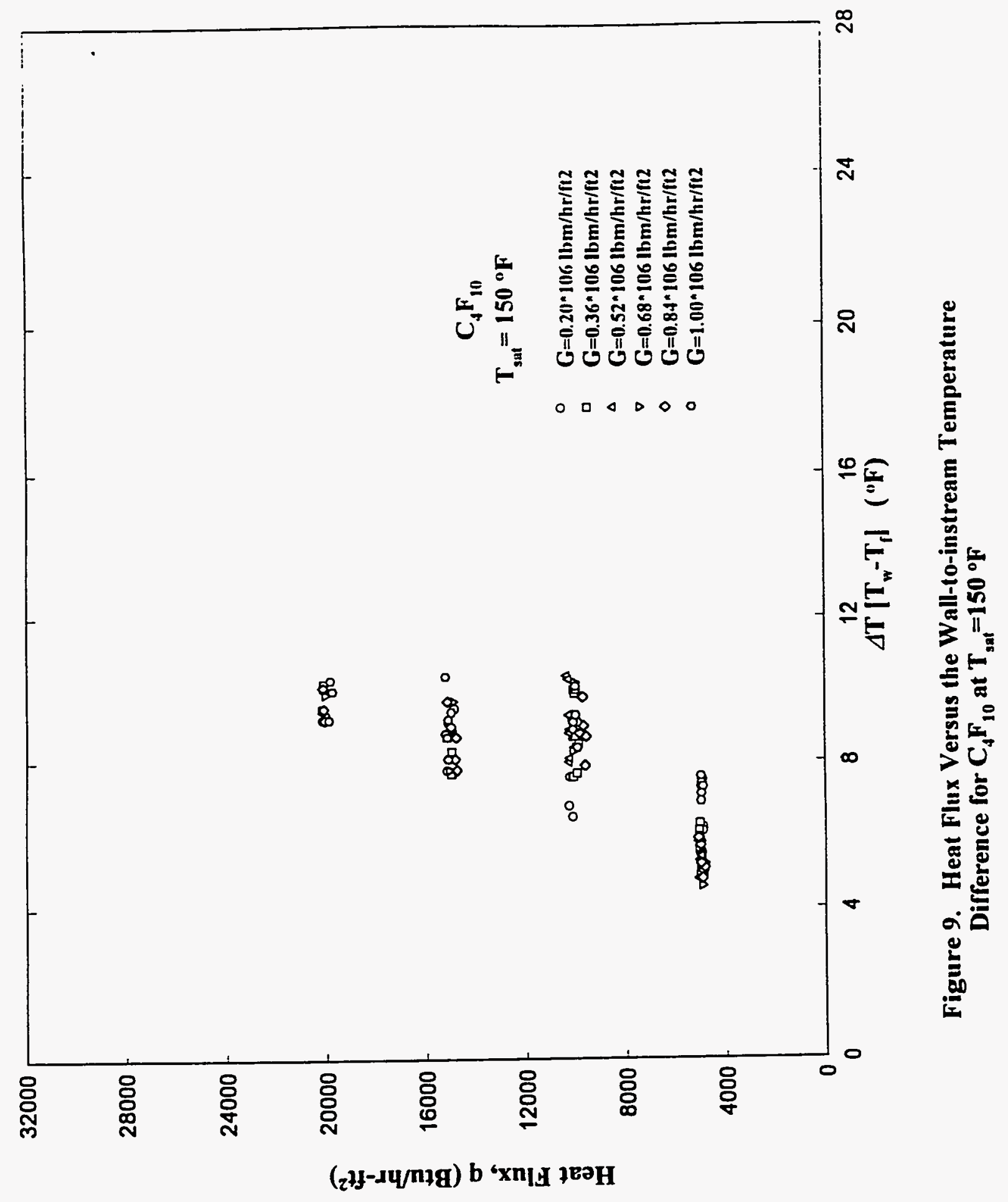




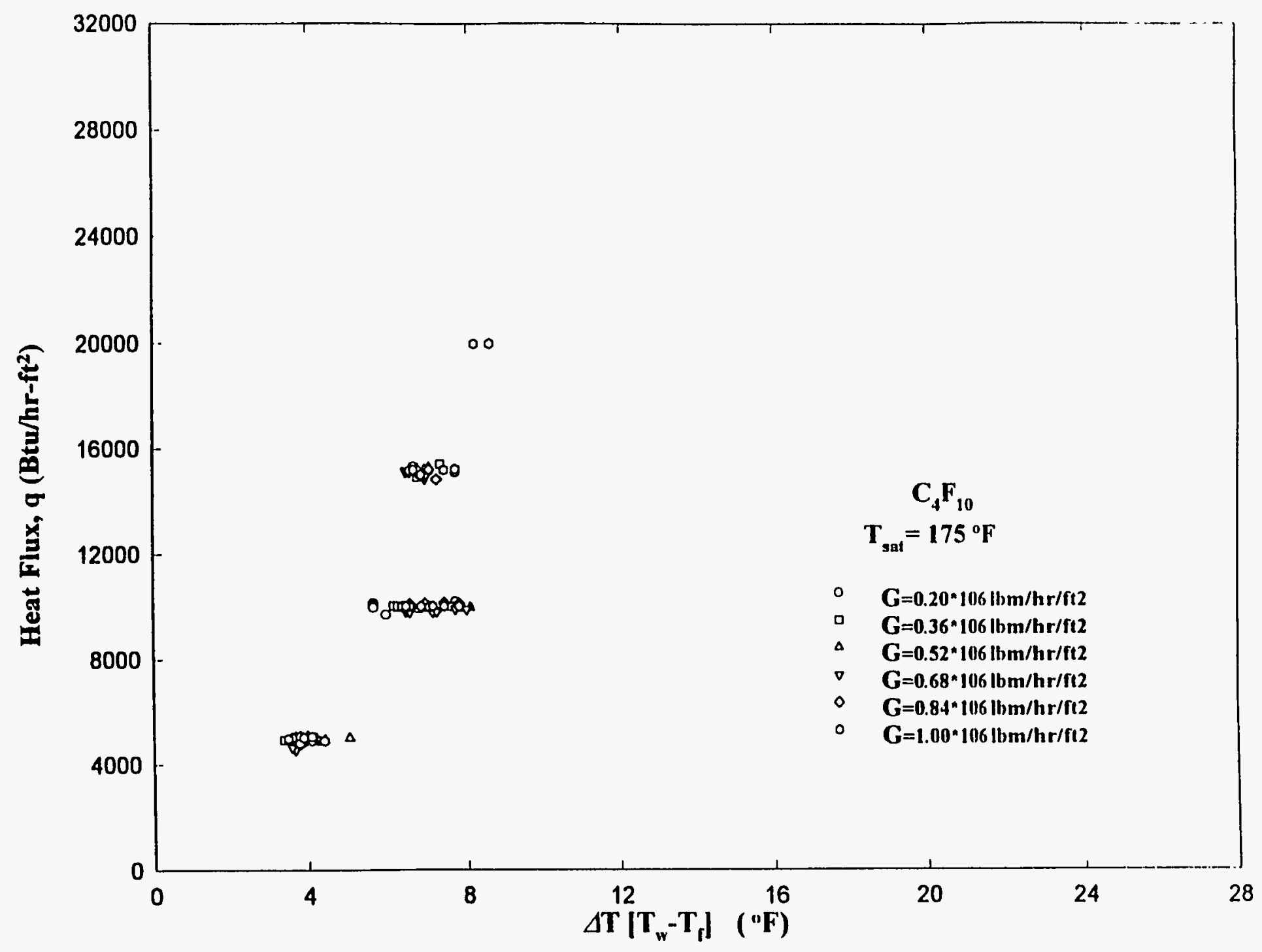

Figure 10. Heat Flux Versus the Wall-to-instream Temperature Difference for $\mathrm{C}_{4} \mathrm{~F}_{10}$ at $\mathrm{T}_{\mathrm{sat}}=175^{\circ} \mathrm{F}$ 


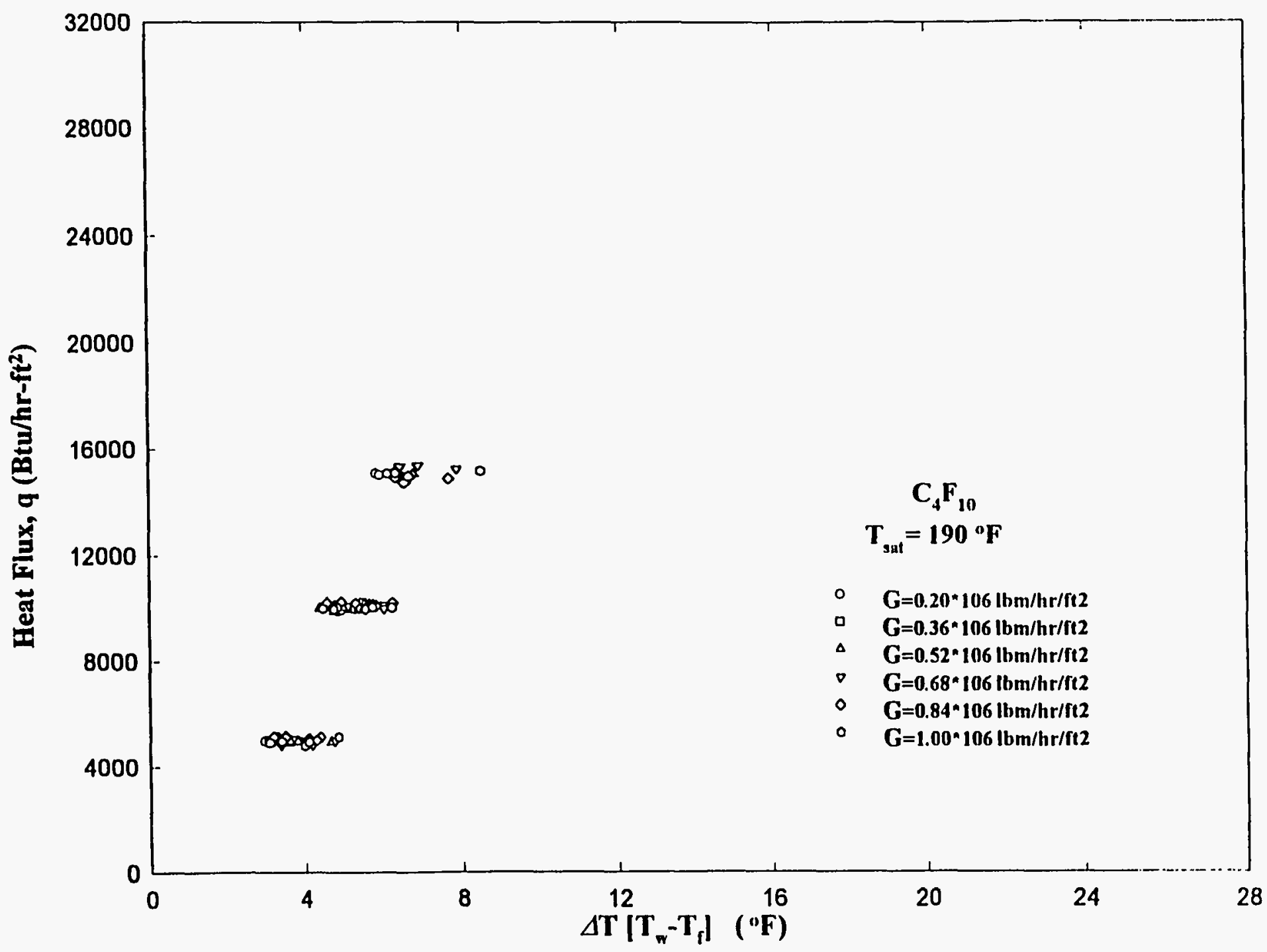

Figure 11. Heat Flux Versus the Wall-to-instream Temperature Difference for $\mathrm{C}_{4} \mathrm{~F}_{10}$ at $\mathrm{T}_{\text {sat }}=190^{\circ} \mathrm{F}$ 


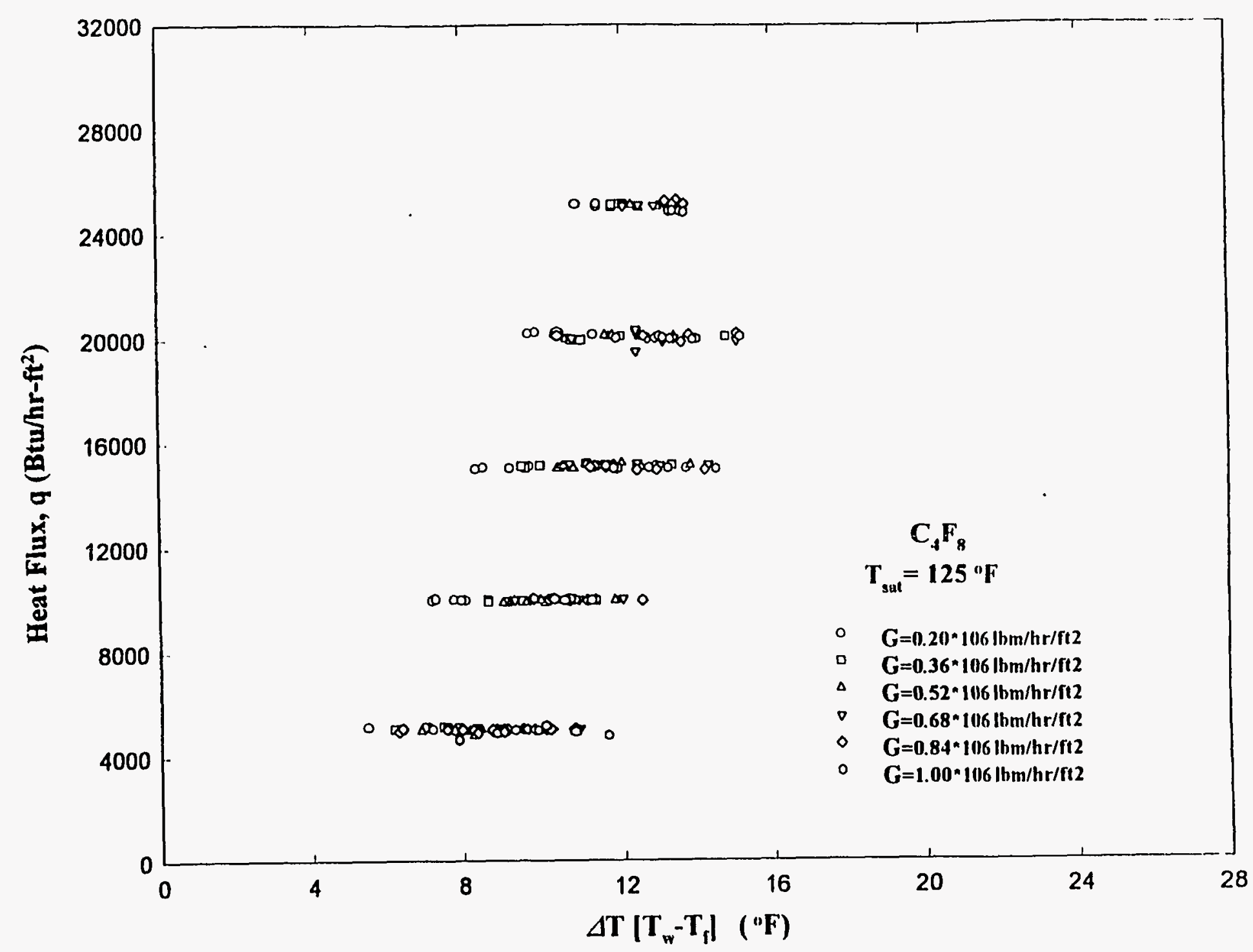

Figure 12. Heat Flux Versus the $W$ all-to-instream Temperature Difference for $C_{4} F_{8}$ at $T_{\text {sat }}=125^{\circ} \mathrm{F}$ 


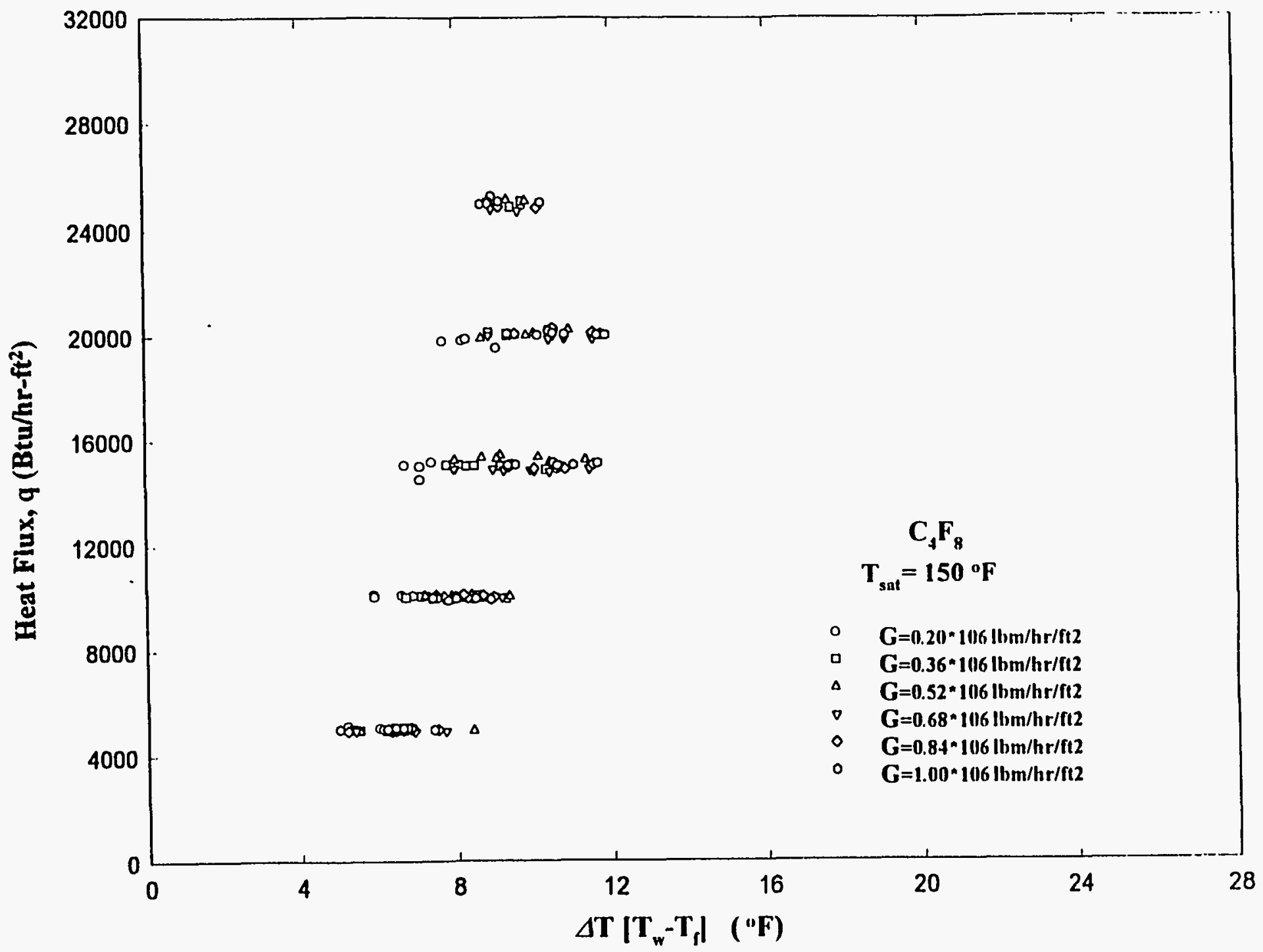

Figure 13. Heat Flux Versus the Wall-to-instream Temperature Difference for $\mathrm{C}_{4} \mathrm{~F}_{8}$ at $\mathrm{T}_{\mathrm{sat}}=150{ }^{\circ} \mathrm{F}$ 


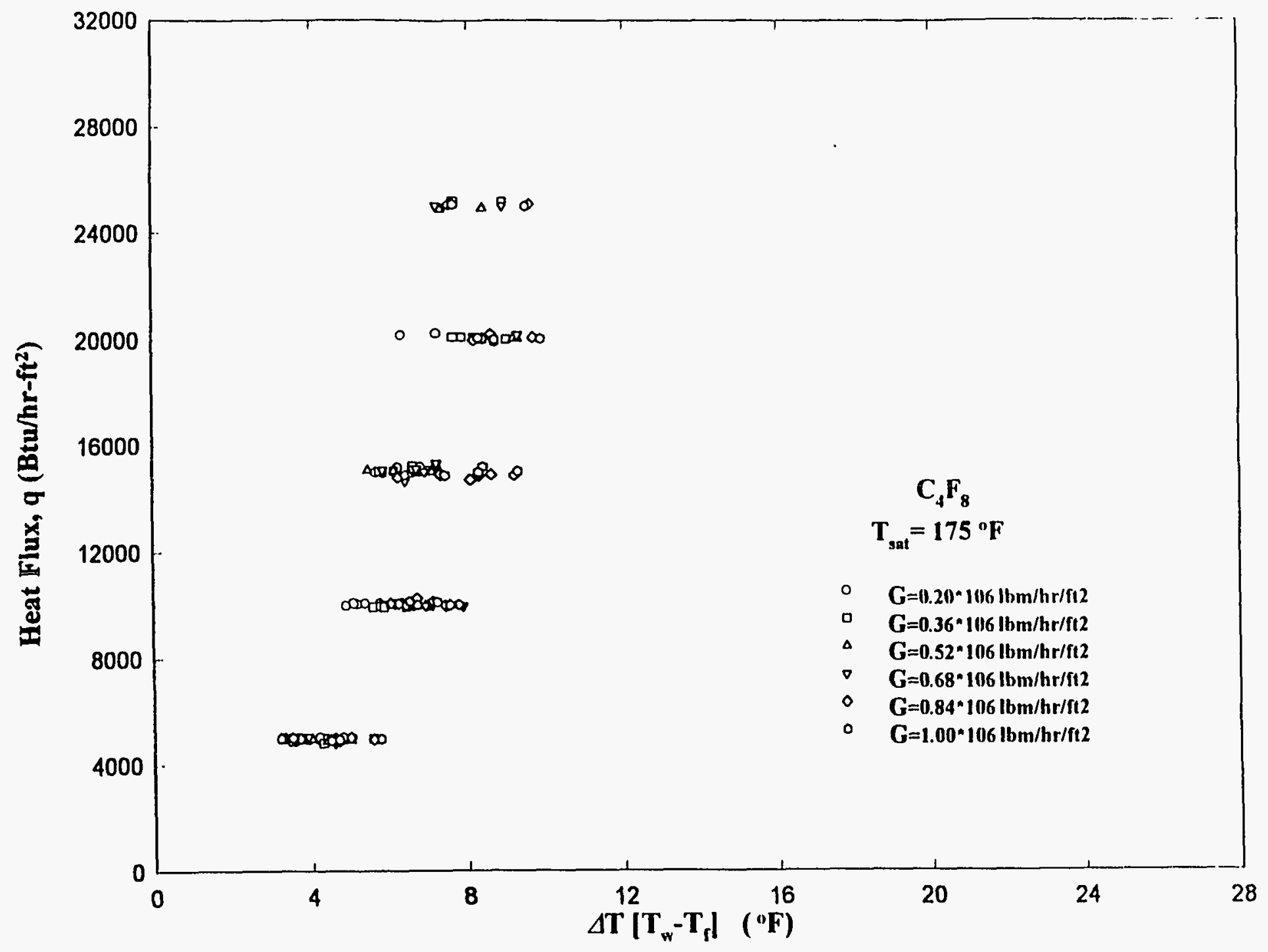

Figure 14. Heat Flux Versus the Wall-to-instream Temperature Difference for $\mathrm{C}_{4} \mathrm{~F}_{8}$ at $\mathrm{T}_{\text {sut }}=175^{\circ} \mathrm{F}$ 


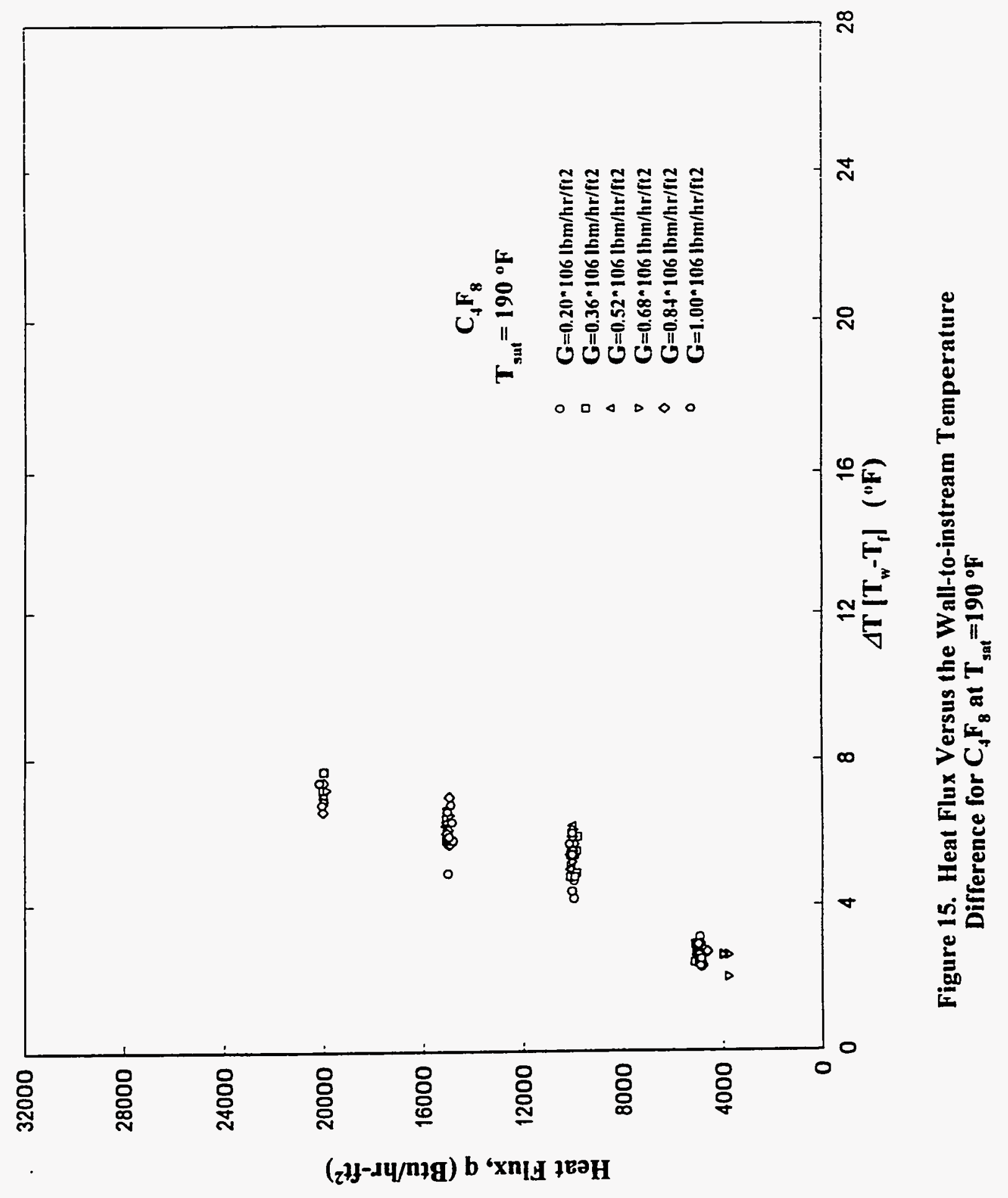




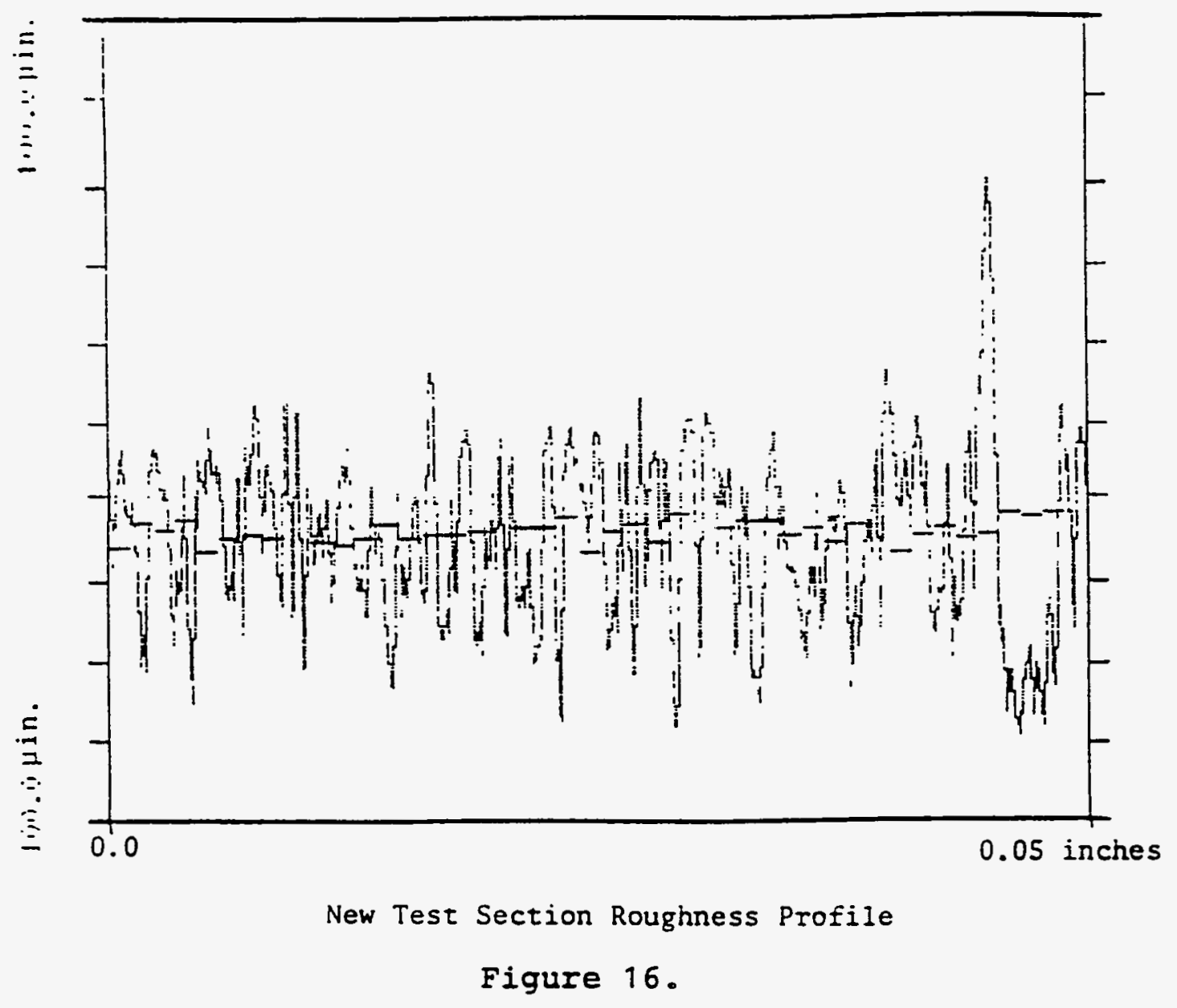




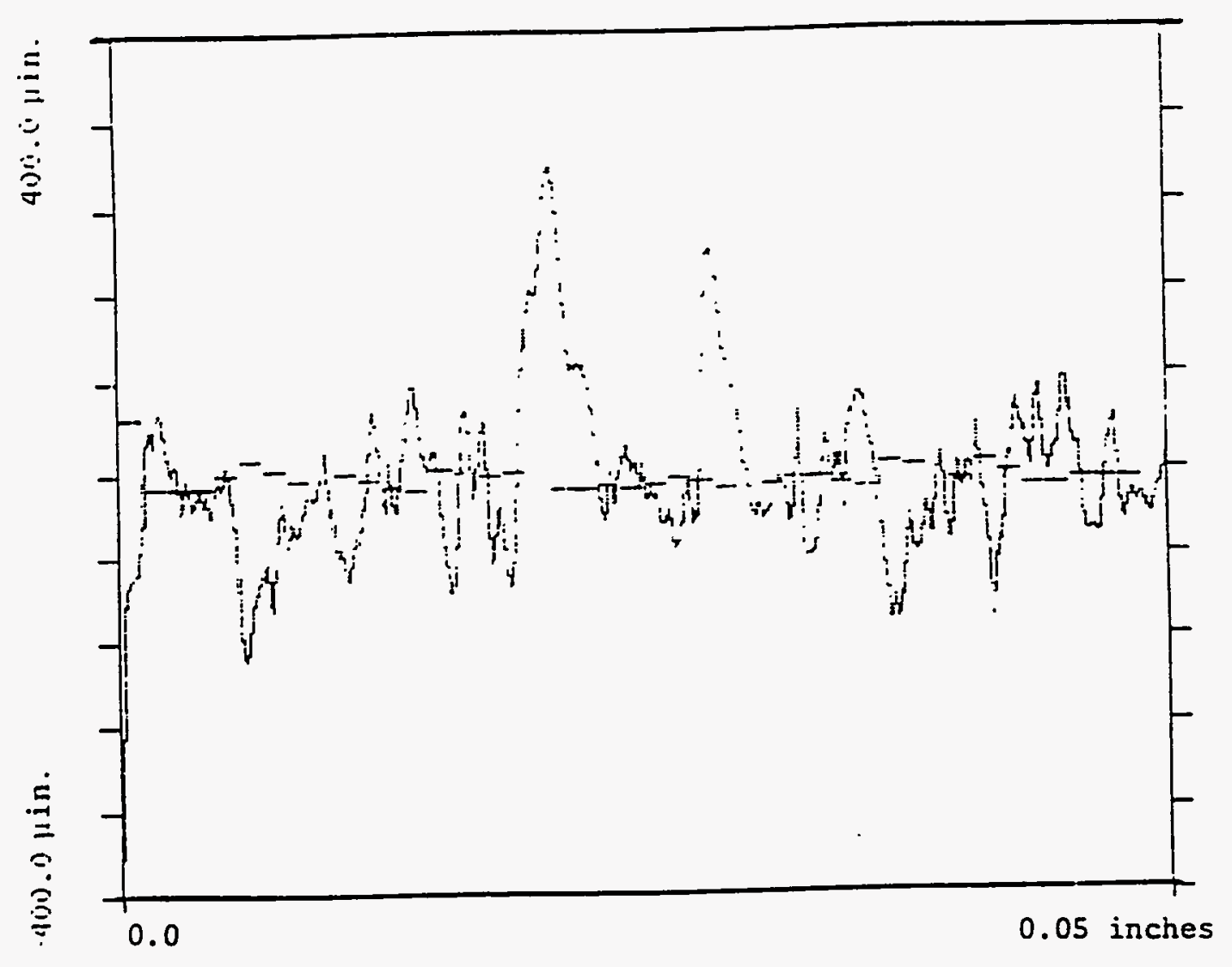

Old Test Section Roughness Profile

Figure 17. 


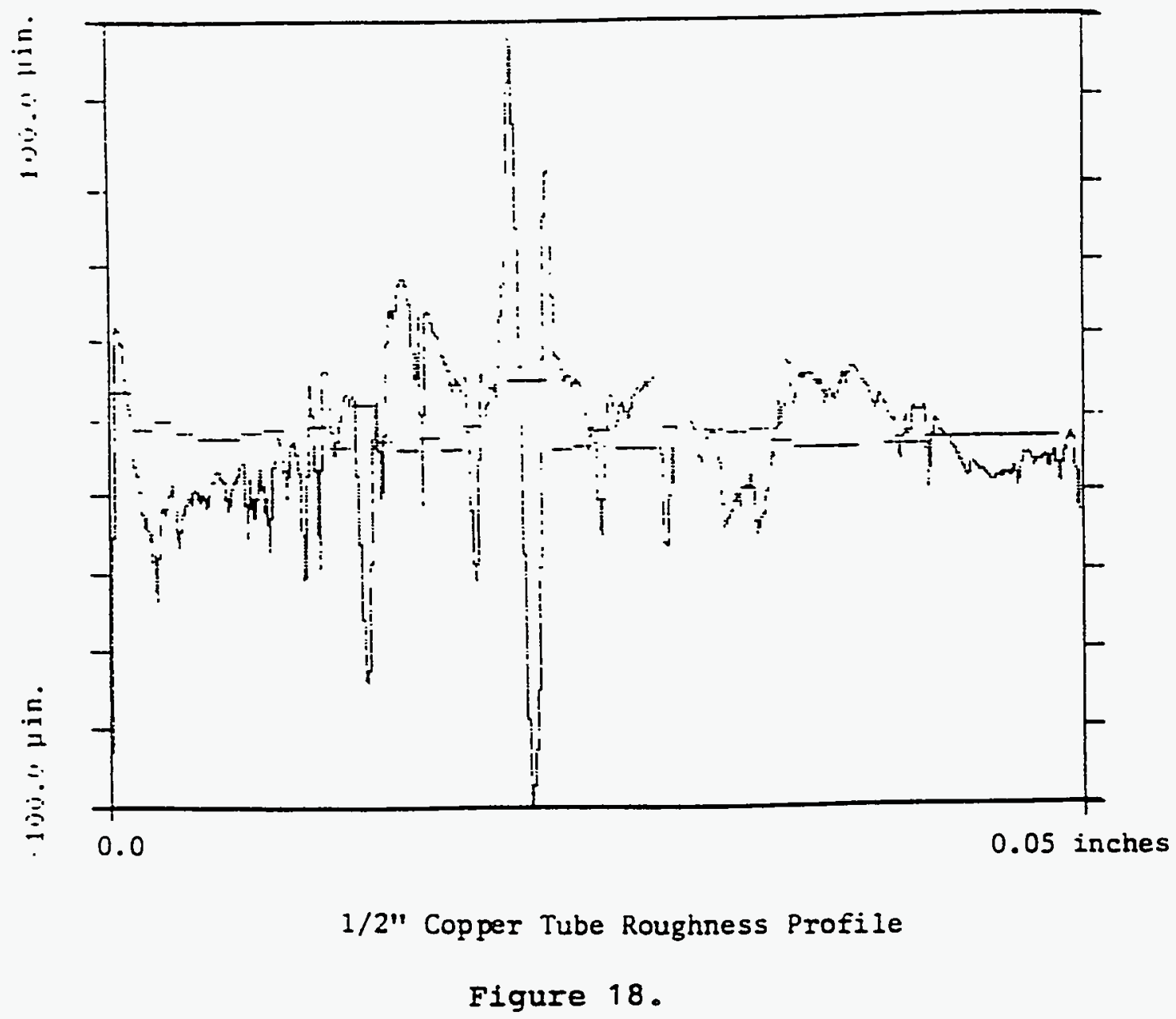




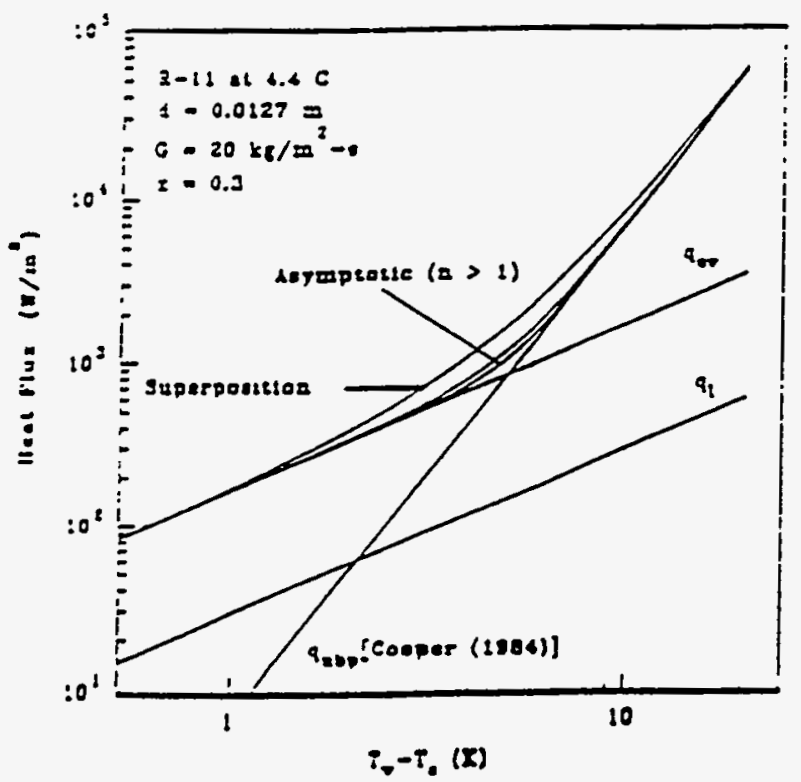

(a)

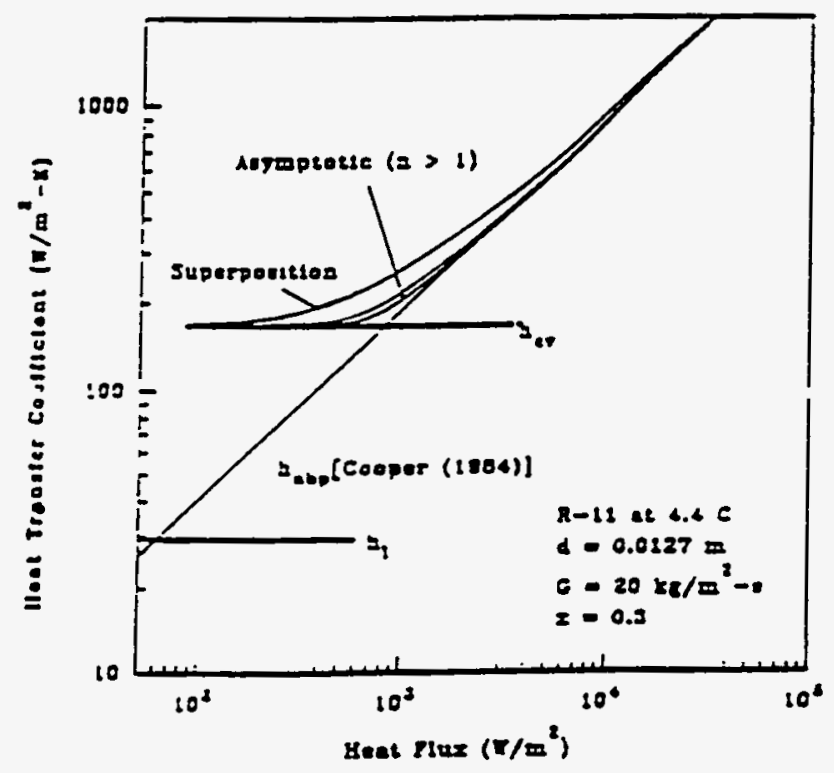

(b)

Figure 19. (a) Superposition and Asymptotic Models in the form $q$ vs. $\Delta T$.

(b) Superposition and Asymptotic Models in the form h vs. $q$ (from Ref. 7) 


\section{R-114}

के

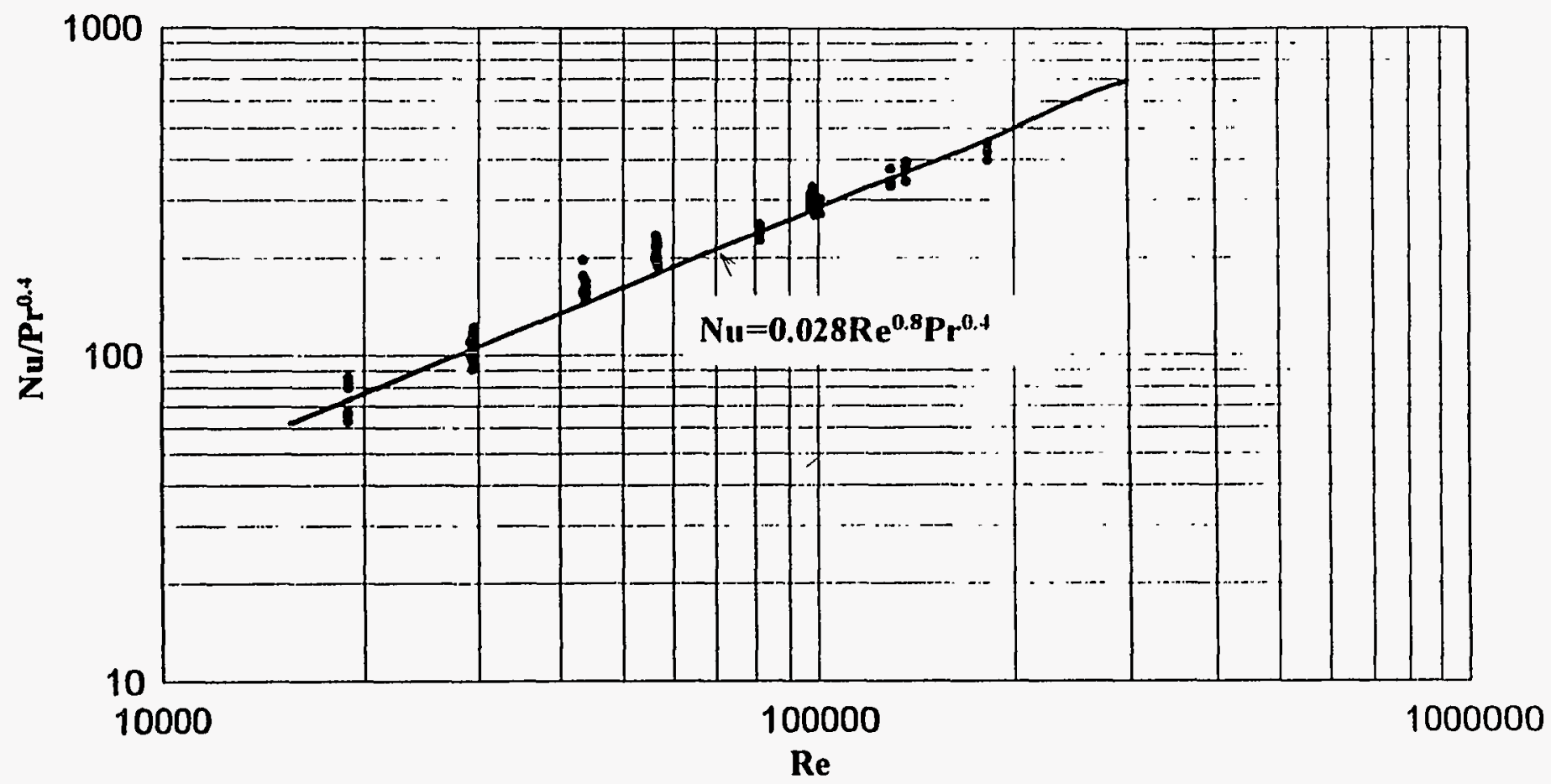

Figure 20. Correlation of Forced Convection for R-114 


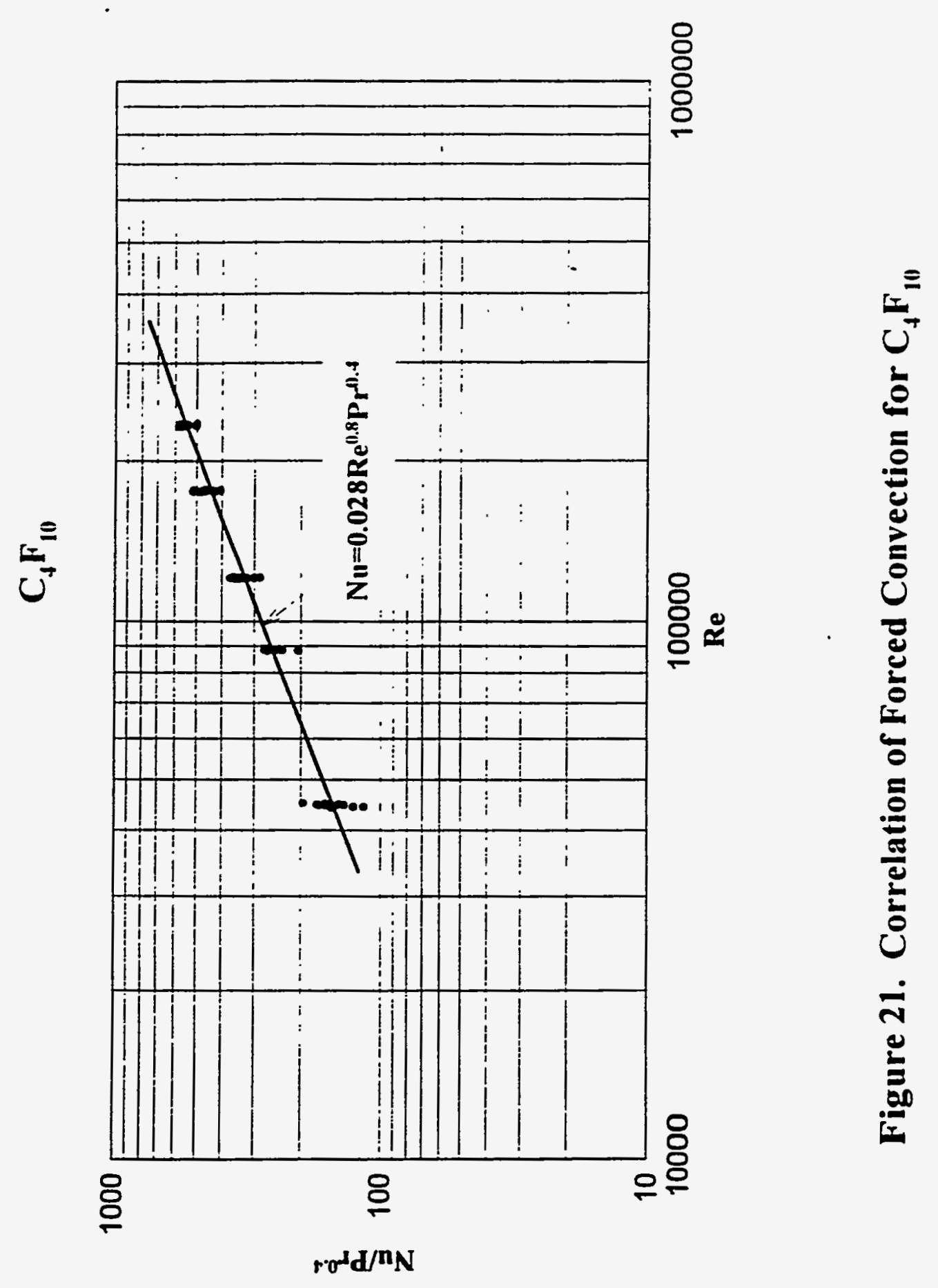




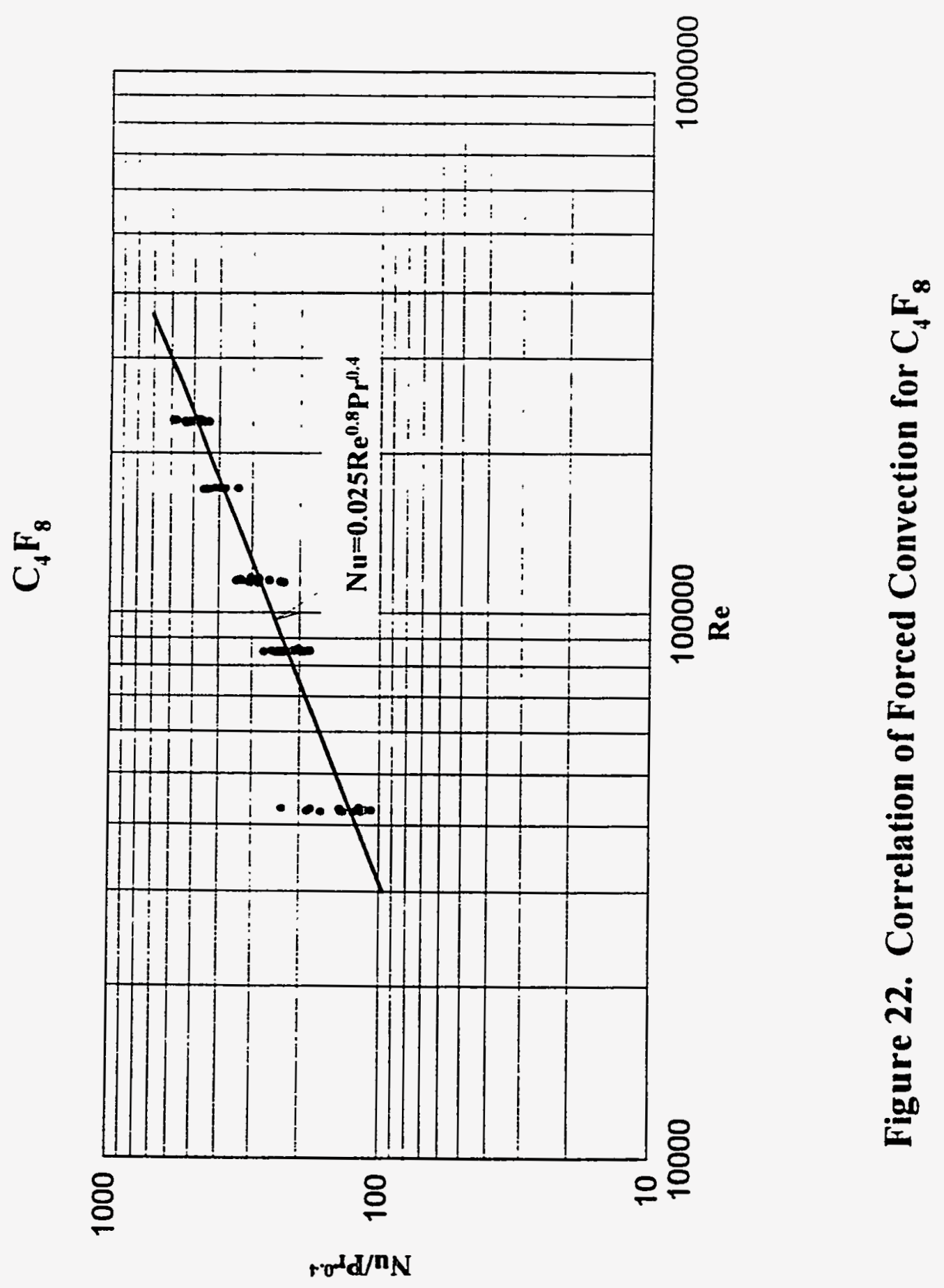




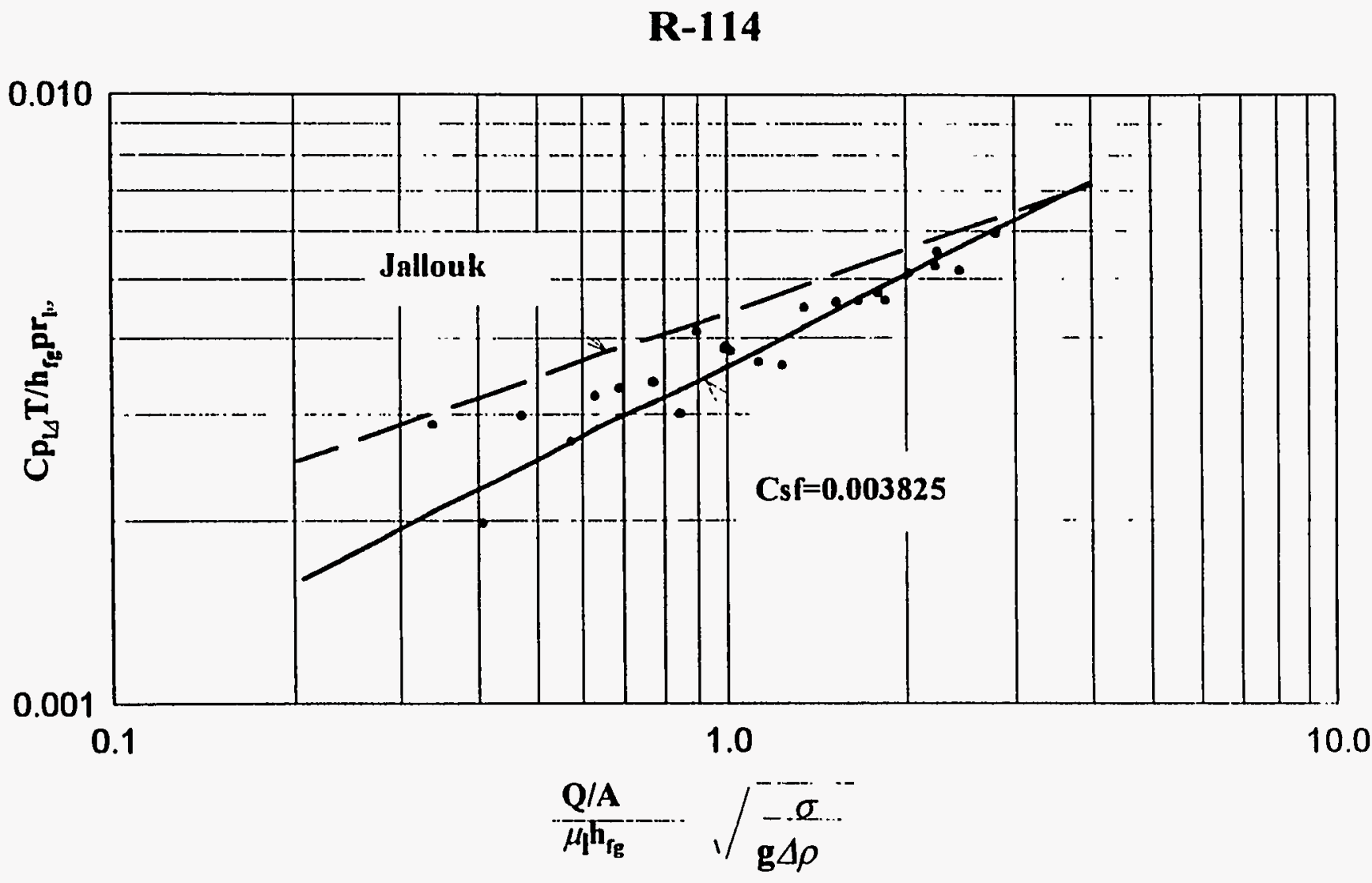

Figure 23. Correlation of Nucleate Pool Boiling for R-114 

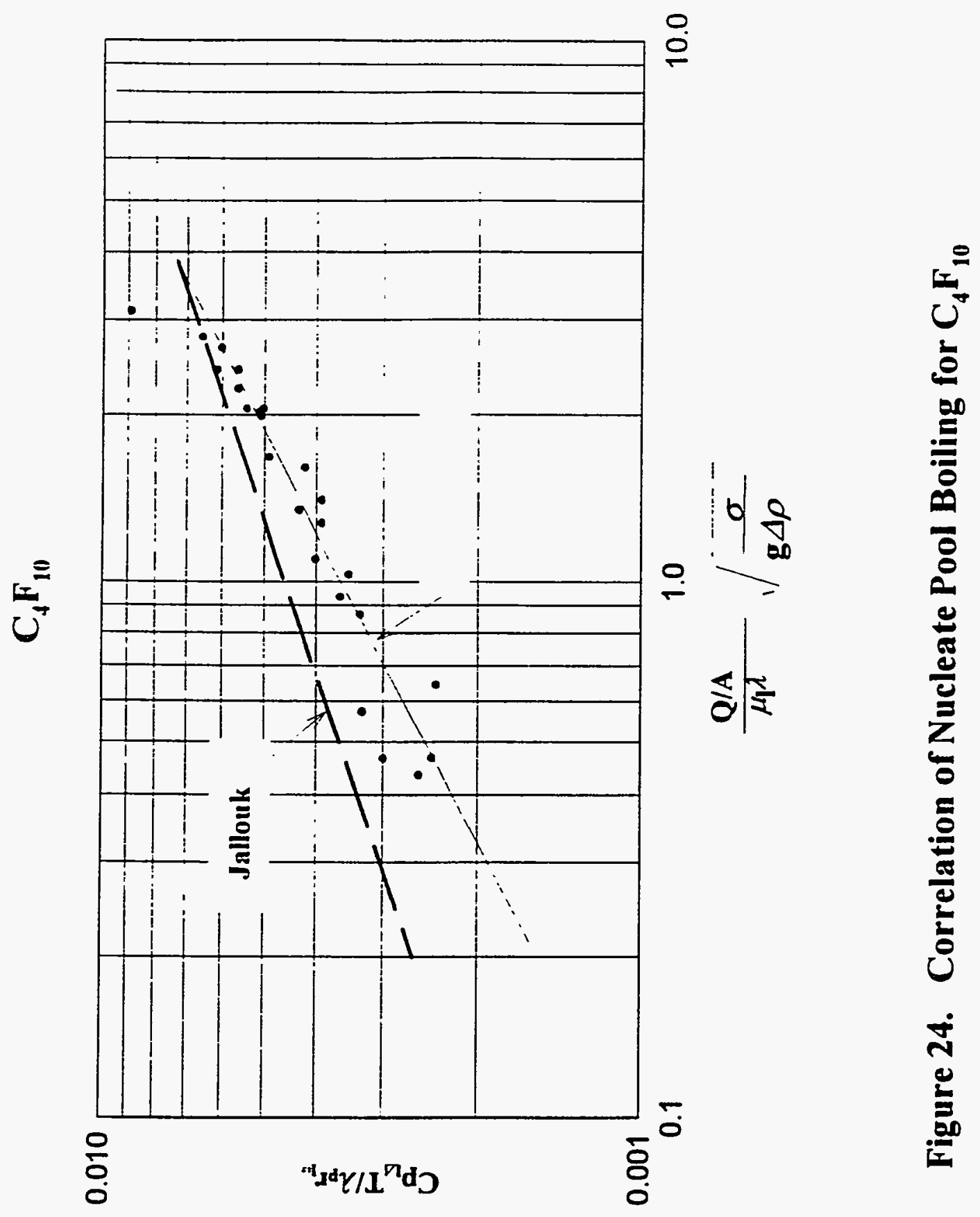


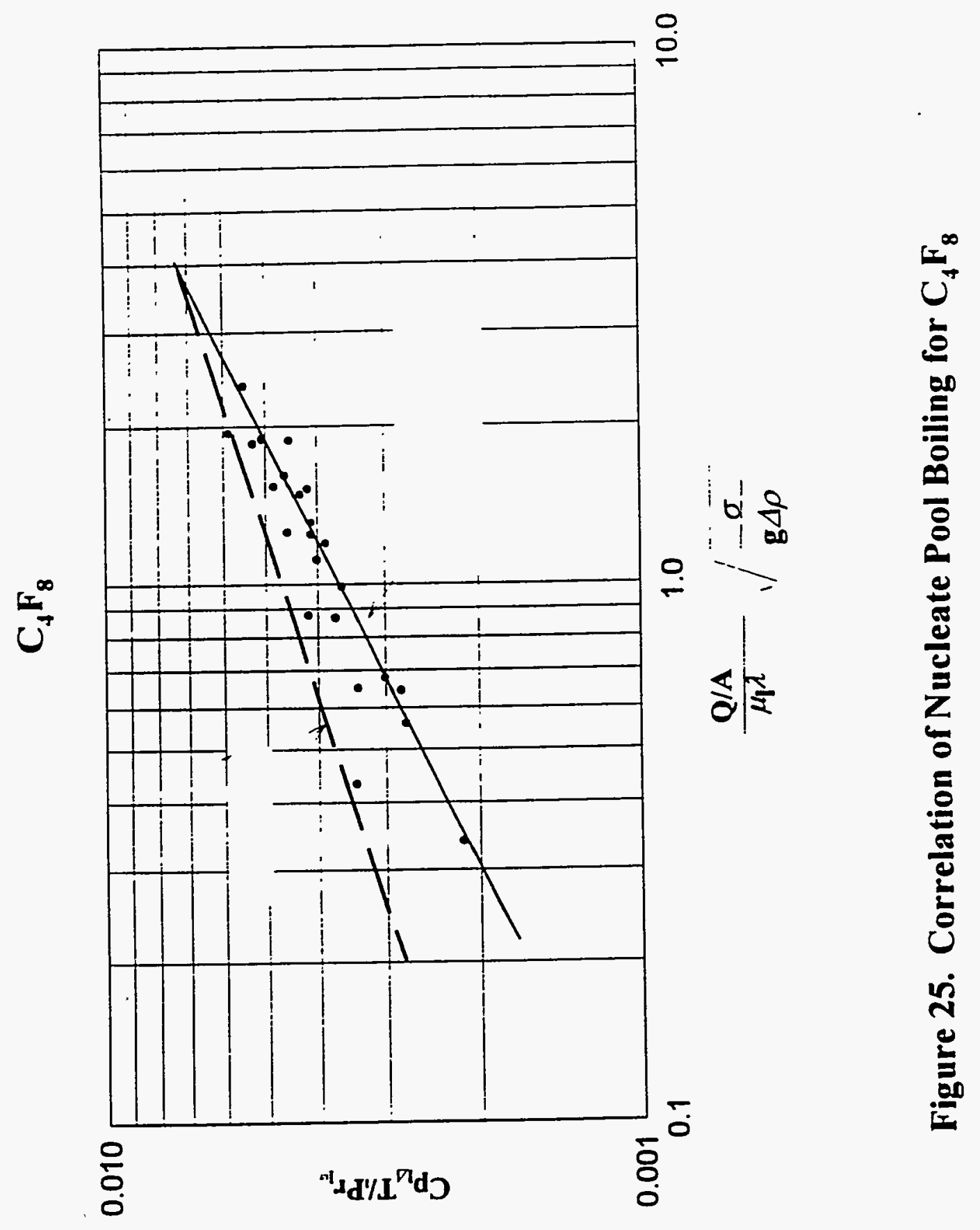




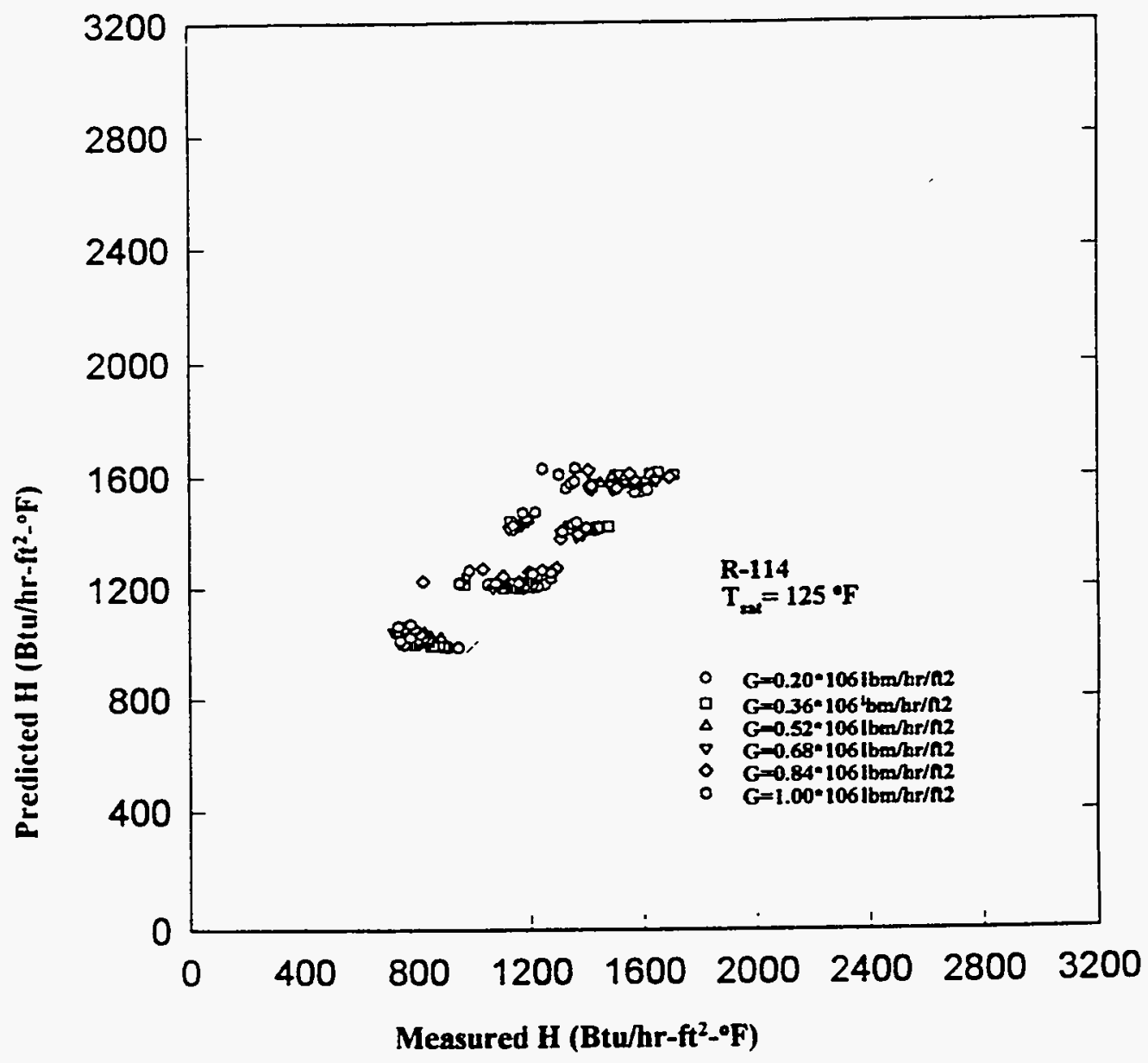

Figure 26. Comparison of Measured and Predicted Heat

Transfer Coefficients for $R-114$ at $T_{\text {sat }}=125^{\circ} \mathrm{F}$ 


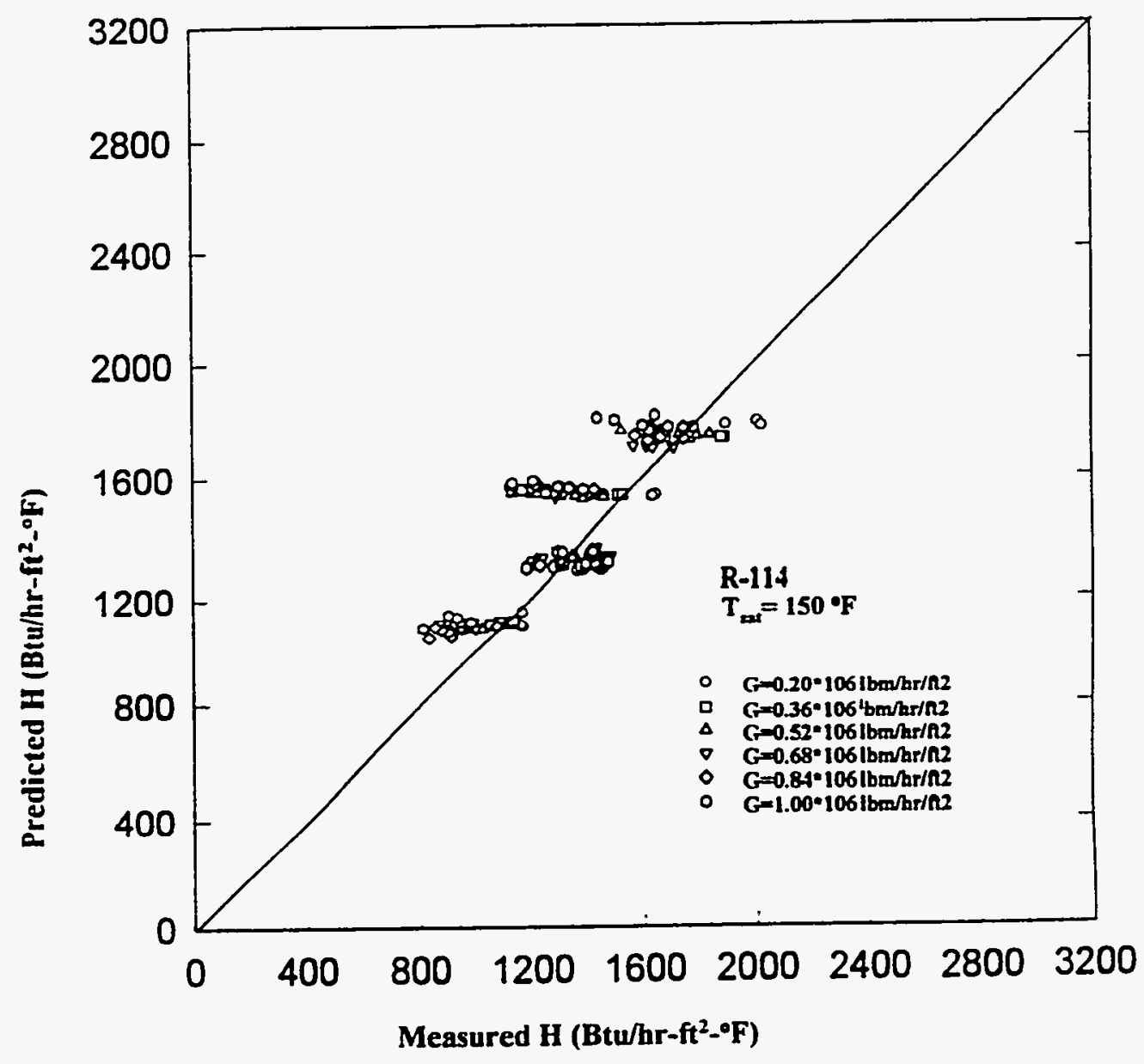

Figure 27. Comparison of Measured and Predicted Heat

Transfer Coefficients for $\mathbf{R}-114$ at $T_{\text {sat }}=150^{\circ} \mathrm{F}$ 


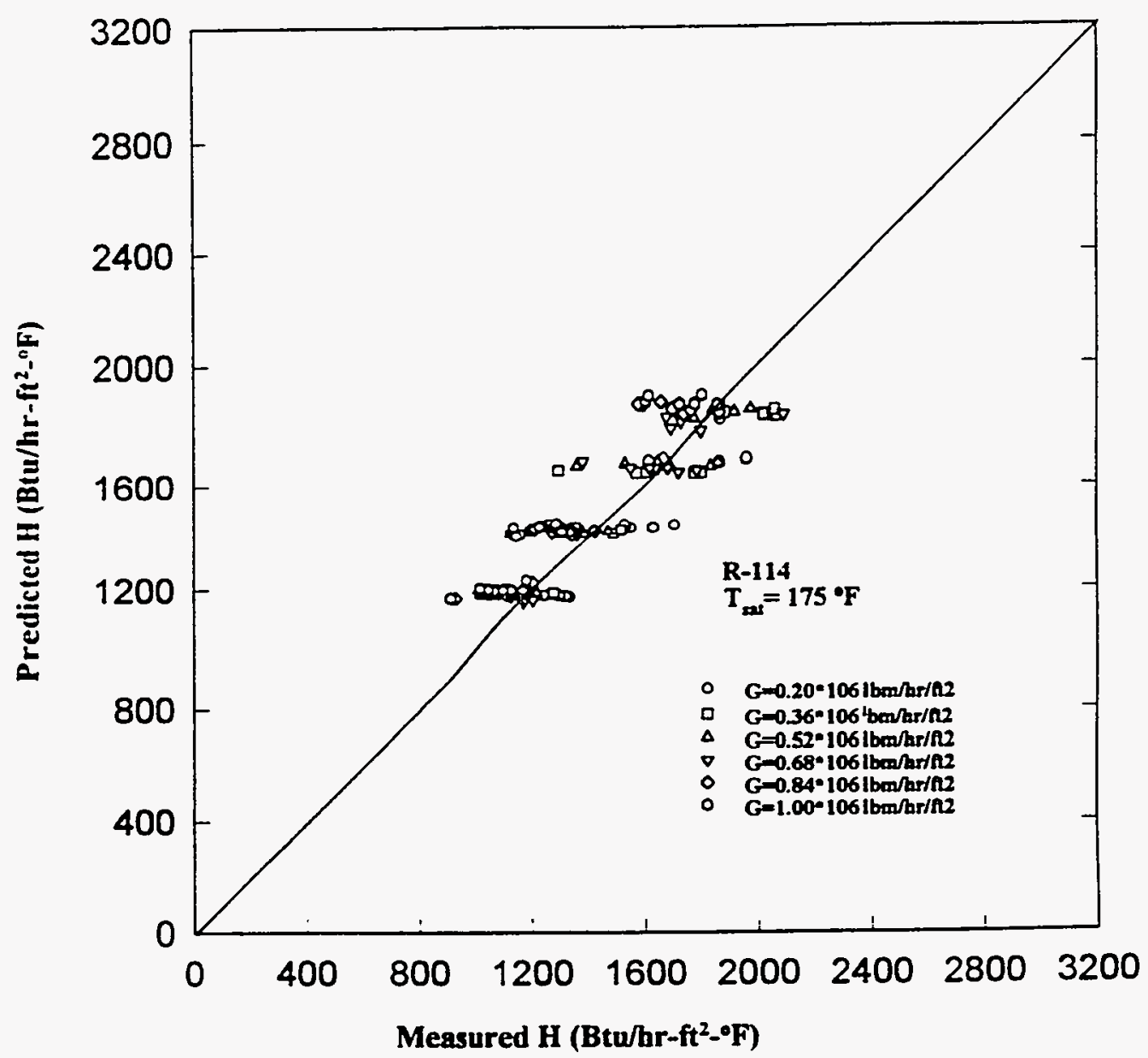

Figure 28. Comparison of Measured and Predicted Heat Transfer Coefficients for $R-114$ at $T_{\text {sat }}=175^{\circ} \mathrm{F}$ 


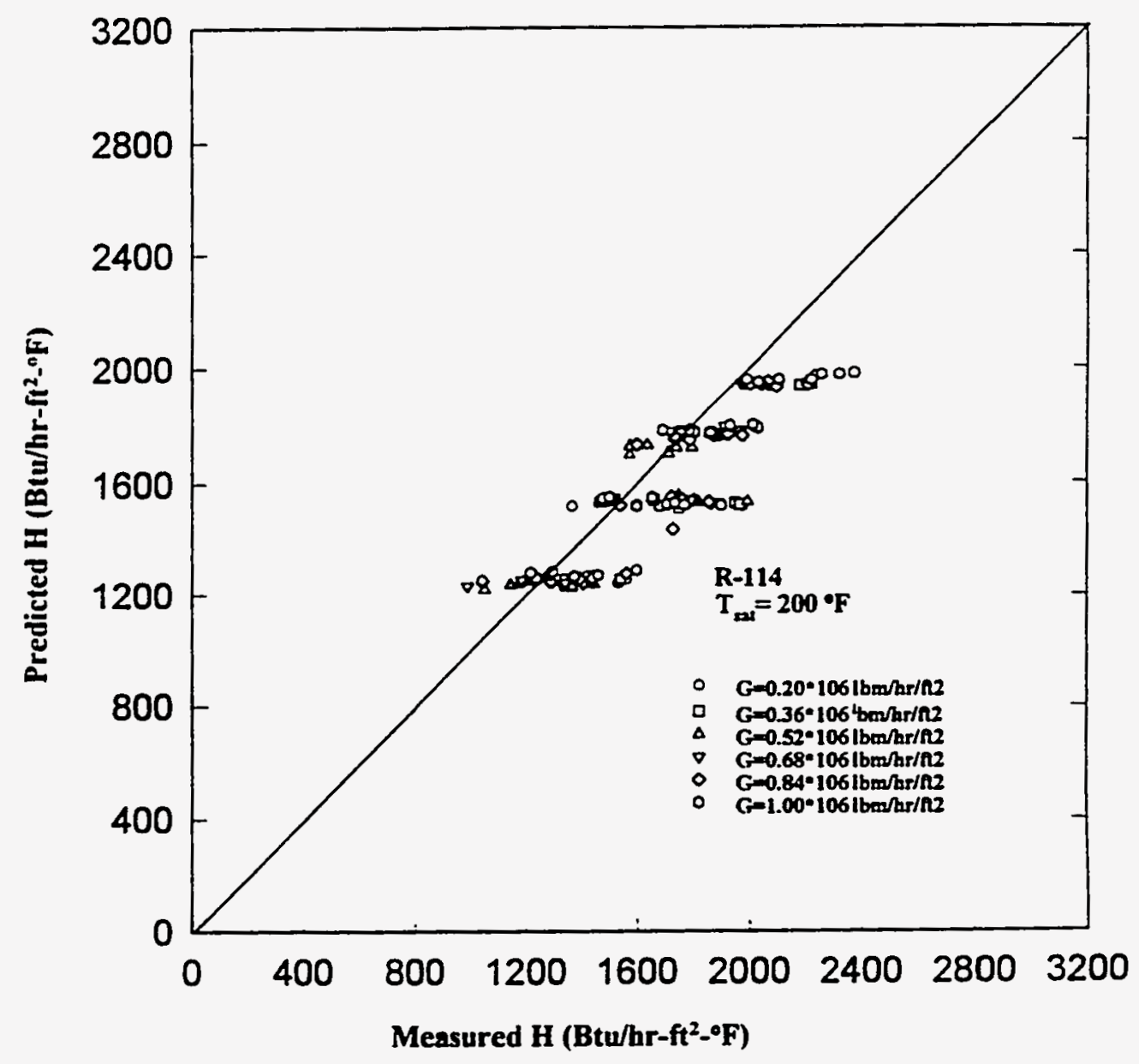

Figure 29. Comparison of Measured and Predicted Heat

Transfer Coefficients for R-114 at $T_{\text {sat }}=200^{\circ} \mathrm{F}$ 


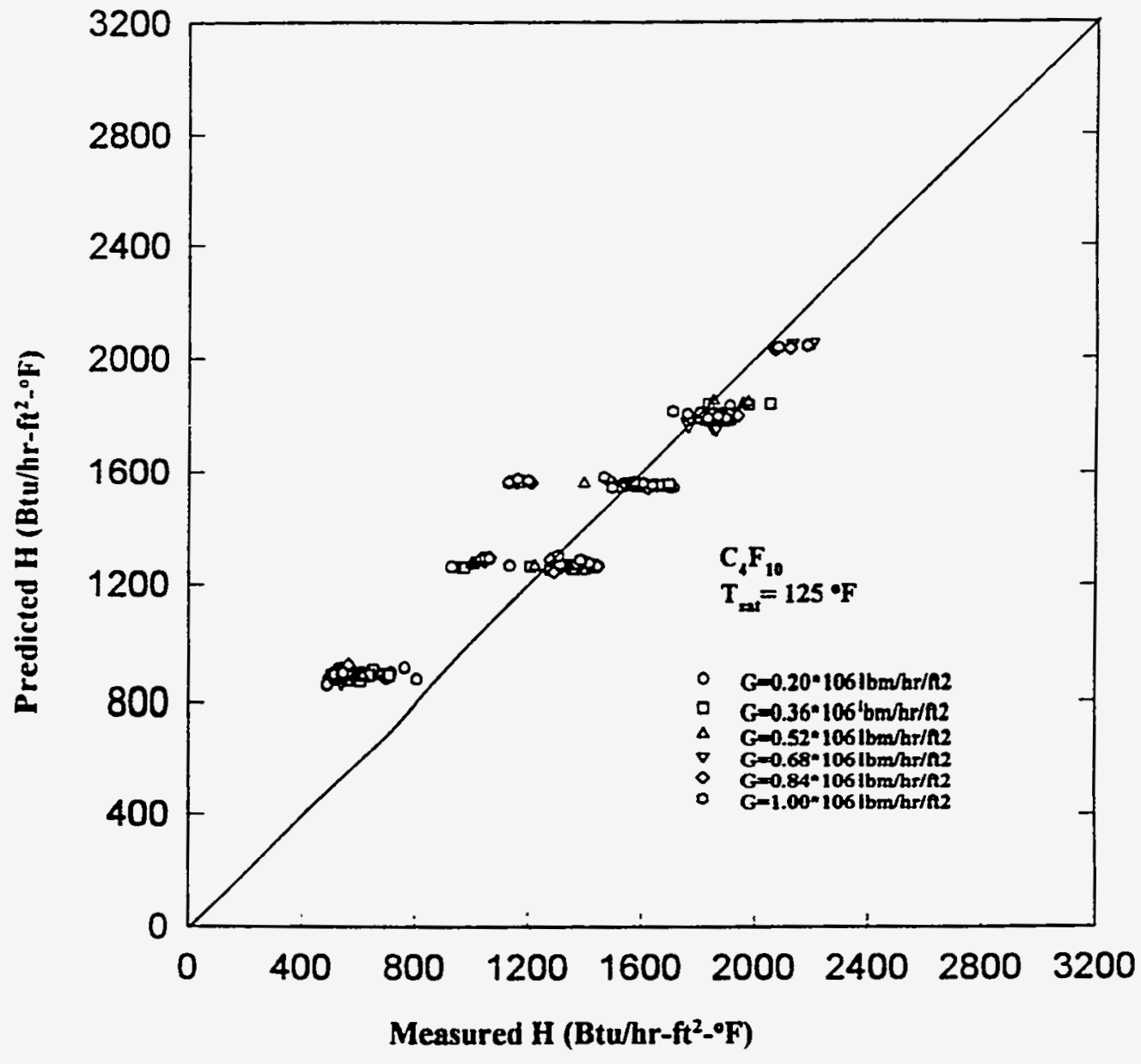

Figure 30. Comparison of Measured and Predicted Heat

Transfer Coefficients for $C_{4} F_{10}$ at $T_{\text {sat }}=125^{\circ} \mathrm{F}$ 


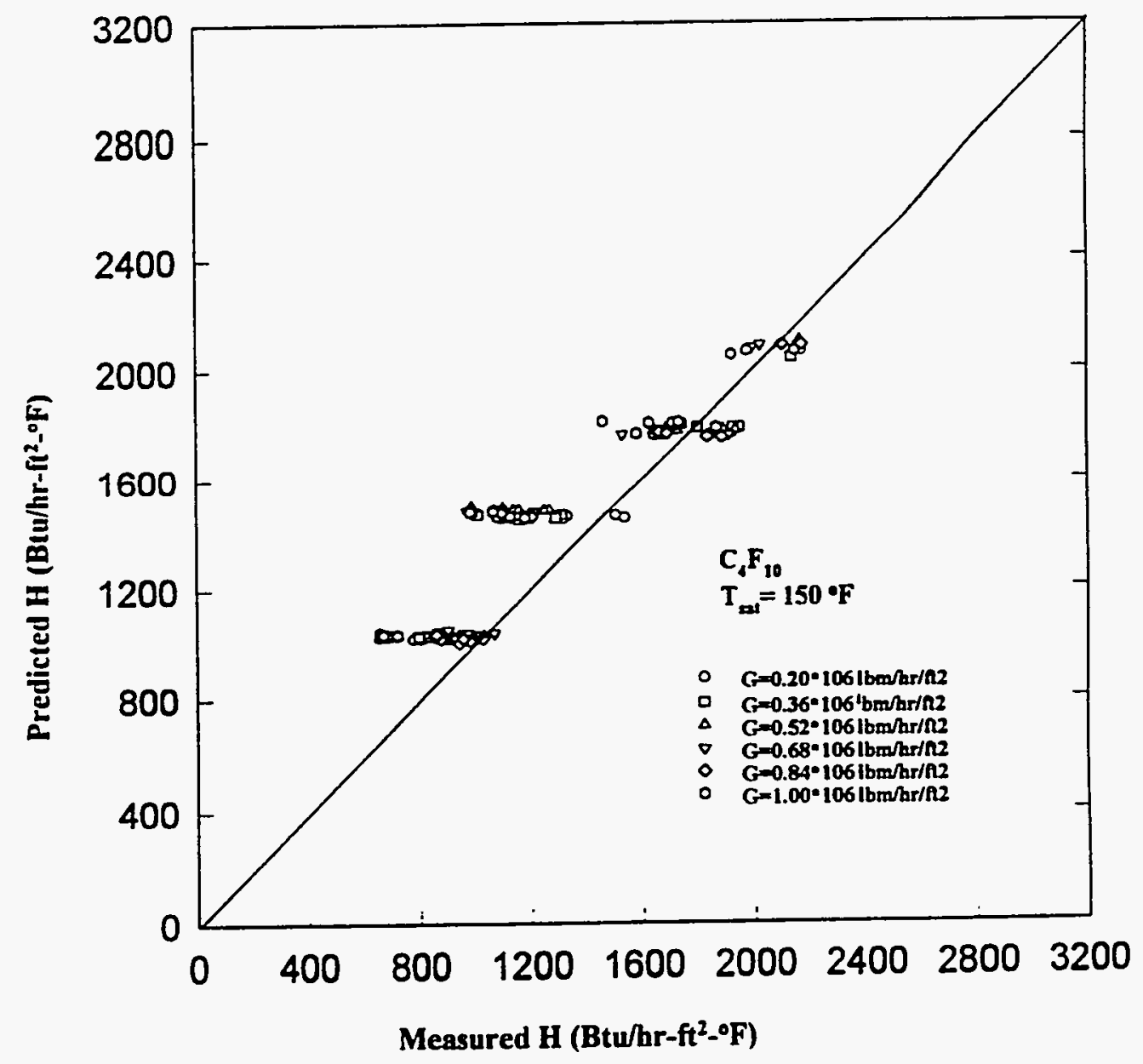

Figure 31. Comparison of Measured and Predicted Heat Transfer Coefficients for $\mathrm{C}_{4} \mathrm{~F}_{10}$ at $\mathrm{T}_{\text {sat }}=150^{\circ} \mathrm{F}$ 


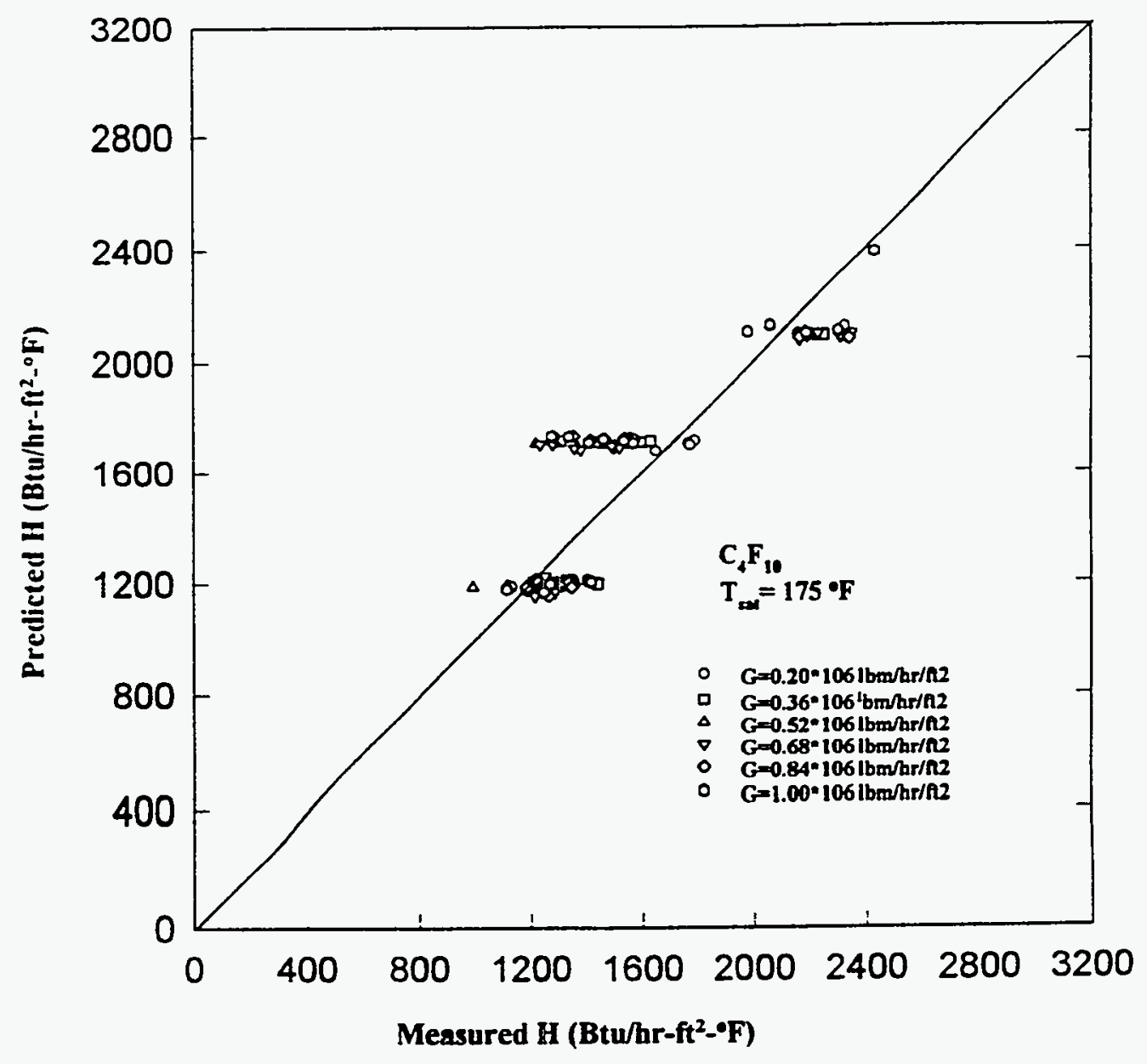

Figure 32. Comparison of Measured and Predicted Heat Transfer Coefficients for $C_{4} F_{10}$ at $T_{\text {sat }}=175^{\circ} \mathrm{F}$ 


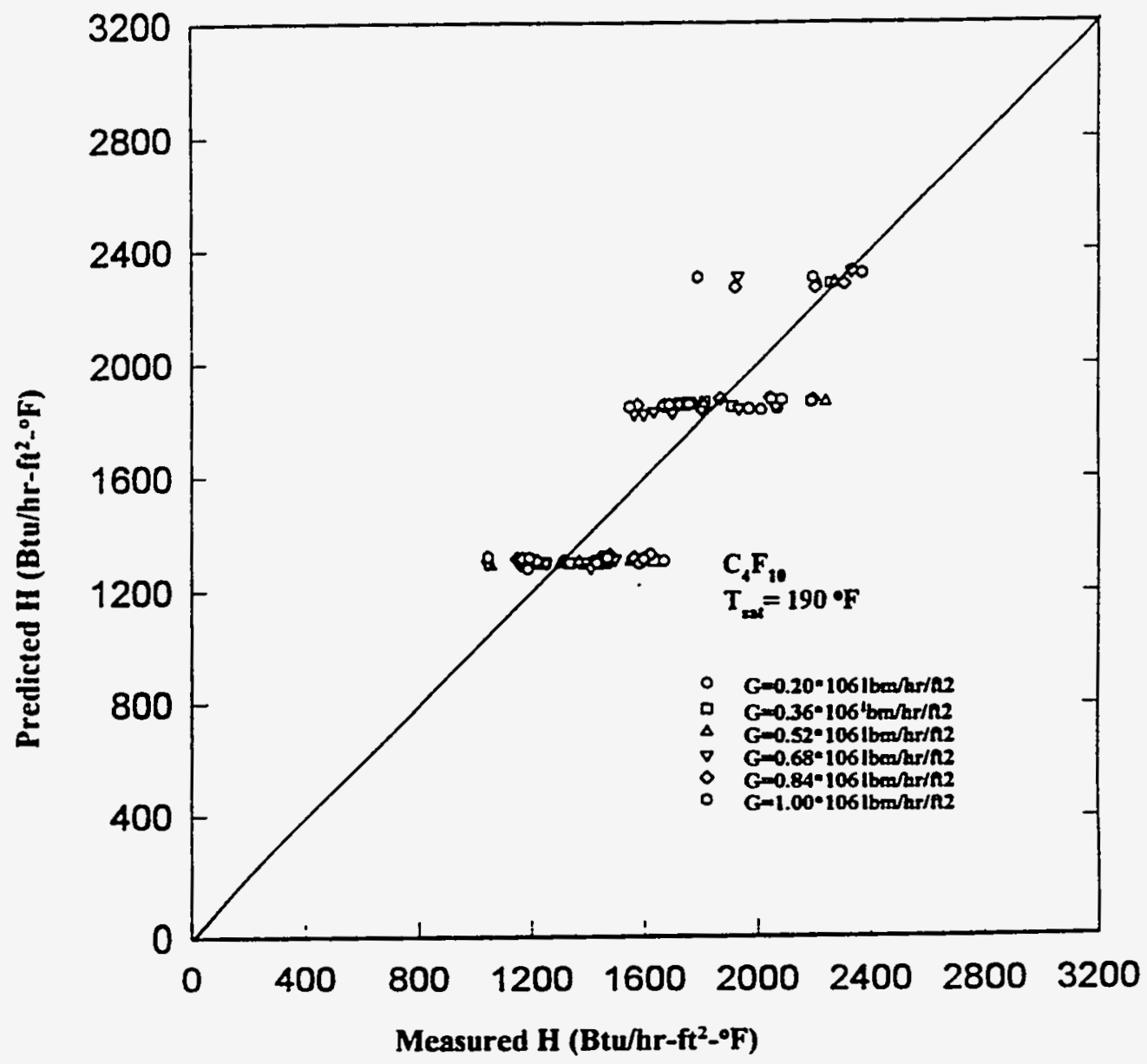

Figure 33. Comparison of Measured and Predicted Heat

Transfer Coefficients for $C_{4} F_{10}$ at $T_{\text {sat }}=190^{\circ} \mathrm{F}$ 


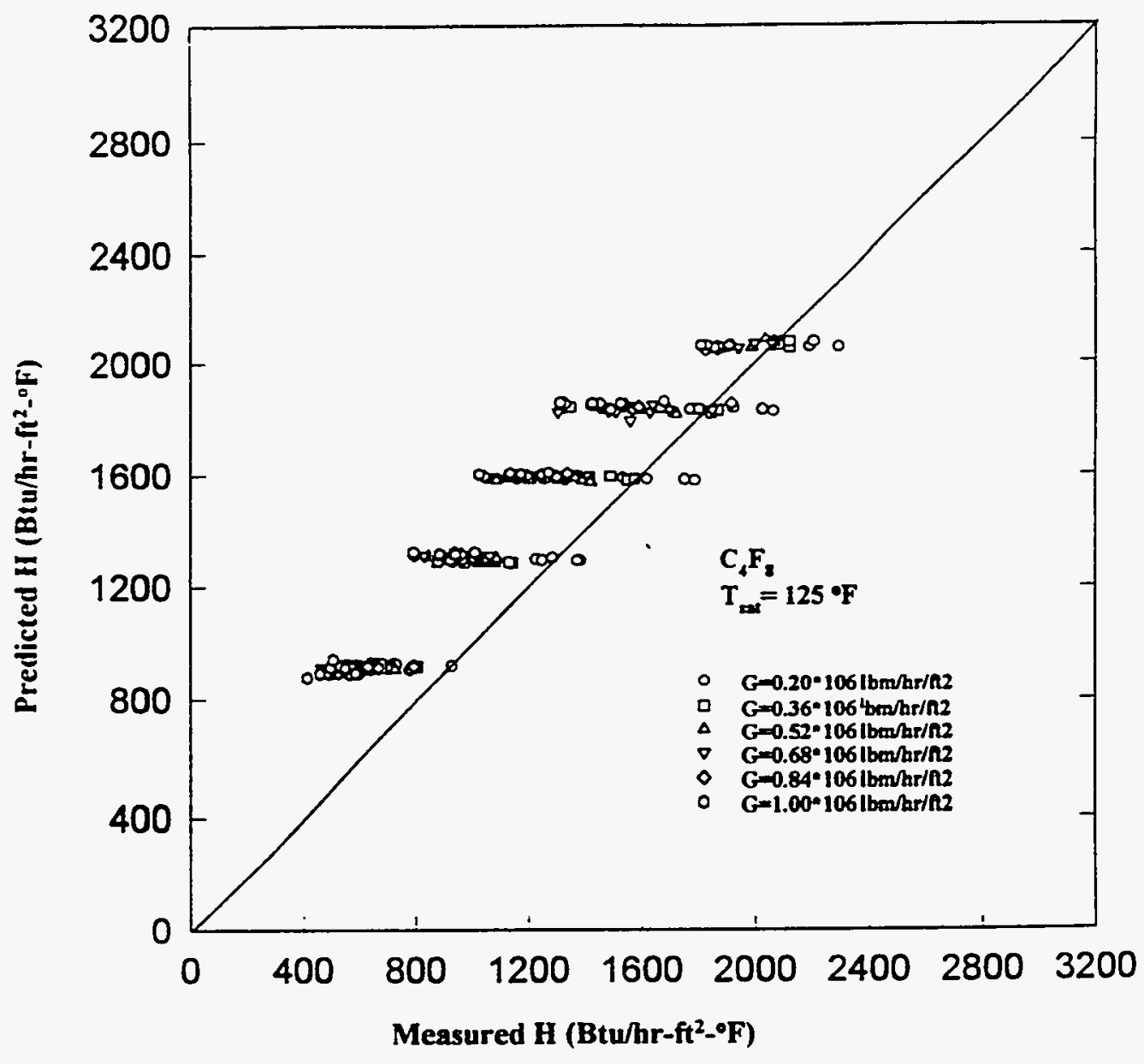

Figure 34. Comparison of Measured and Predicted Heat Transfer Coefficients for $\mathrm{C}_{4} \mathrm{~F}_{8}$ at $\mathrm{T}_{\text {sat }}=125^{\circ} \mathrm{F}$ 


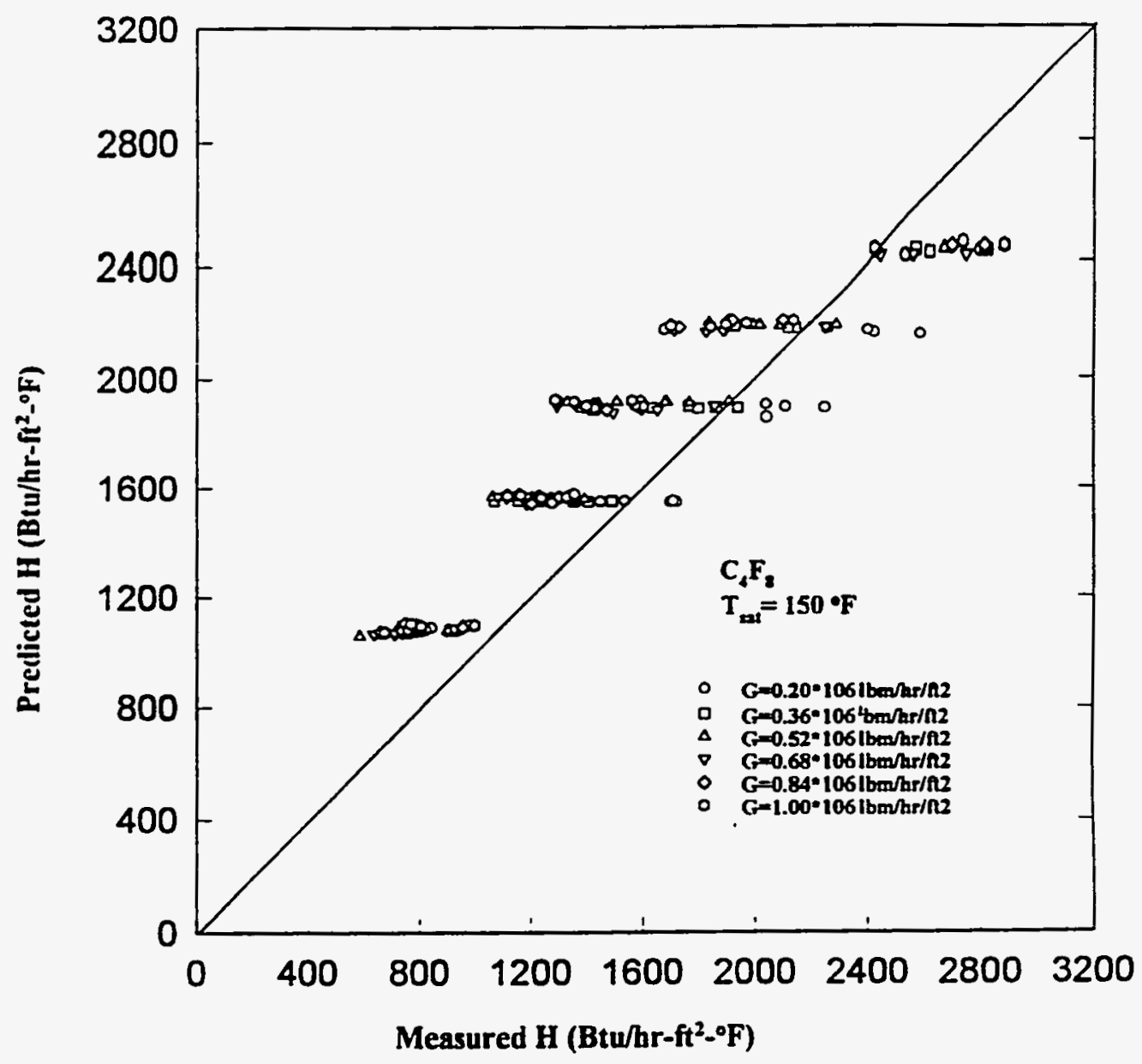

Figure 35. Comparison of Measured and Predicted Heat Transfer Coefficients for $C_{4} F_{8}$ at $T_{s a t}=150^{\circ} \mathrm{F}$ 


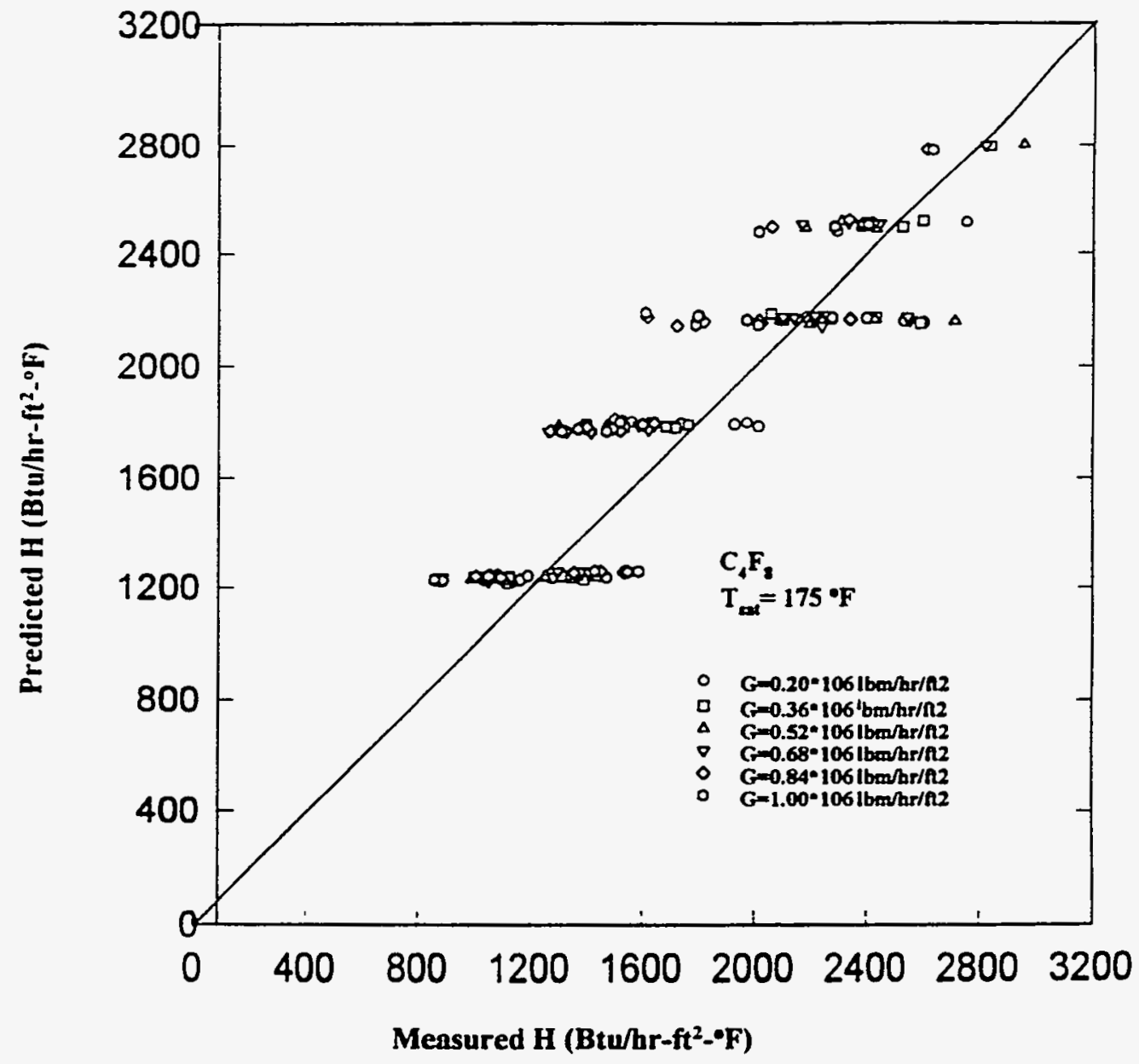

Figure 36. Comparison of Measured and Predicted Heat Transfer Coefficients for $C_{4} F_{8}$ at $T_{s a t}=175^{\circ} \mathrm{F}$ 


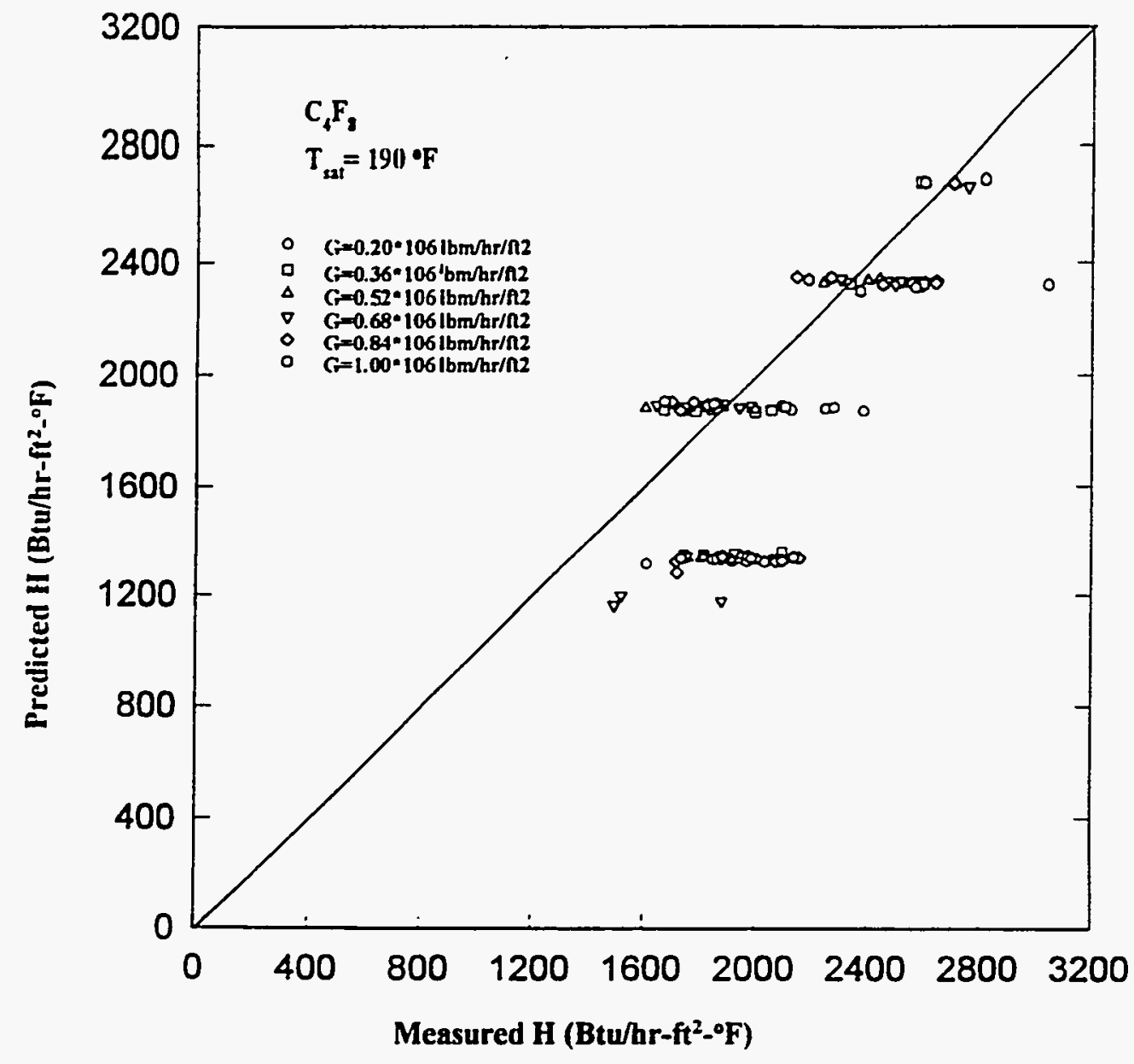

Figure 37. Comparison of Measured and Predicted Heat

Transfer Coefficients for $C_{4} F_{8}$ at $T_{s a t}=190^{\circ} \mathrm{F}$ 


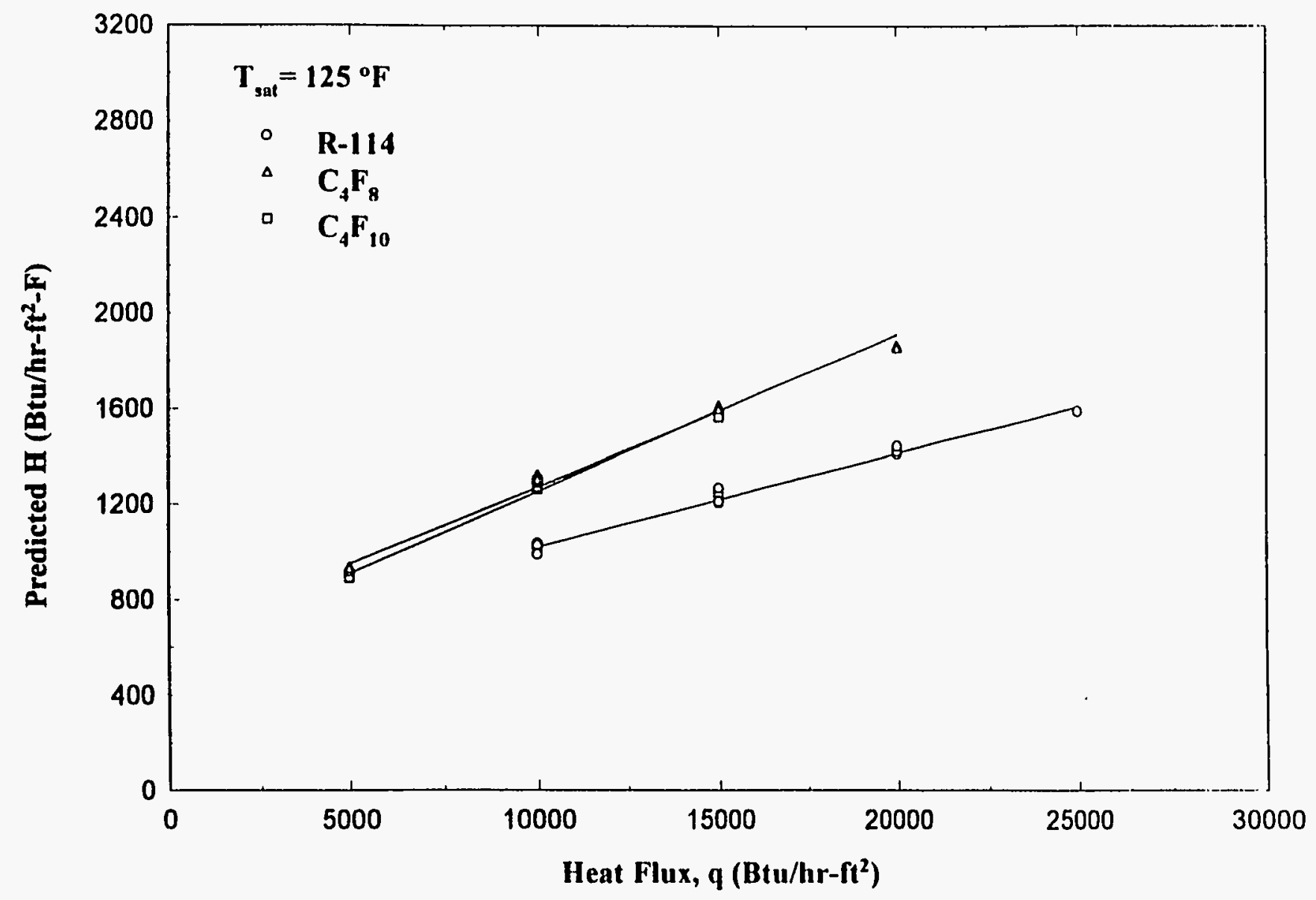

Figure 38. Comparison of Predicted Heat Transfer Coefficients vs Heat Flux at $T_{\text {sat }}=125^{\circ} \mathrm{F}$ 


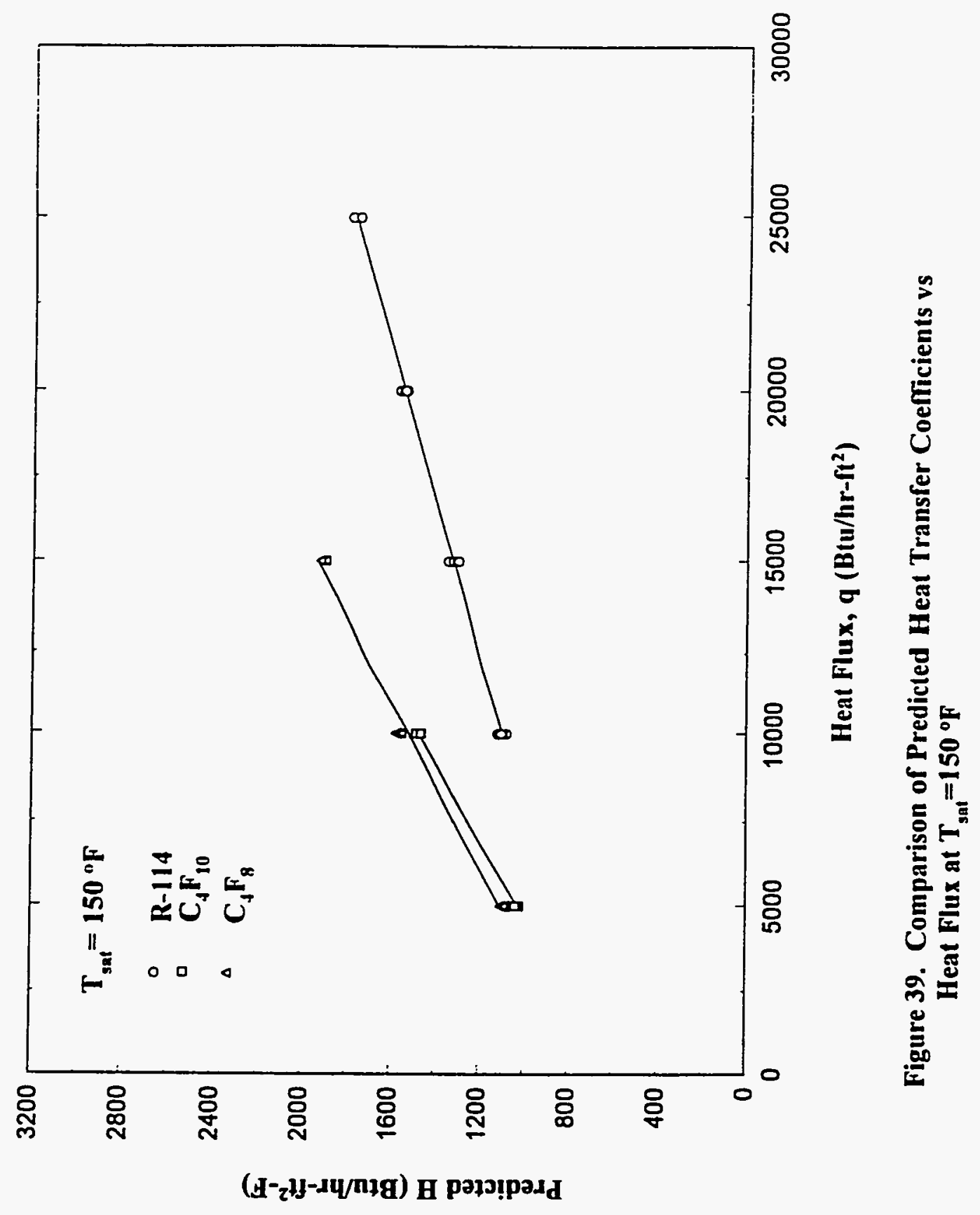




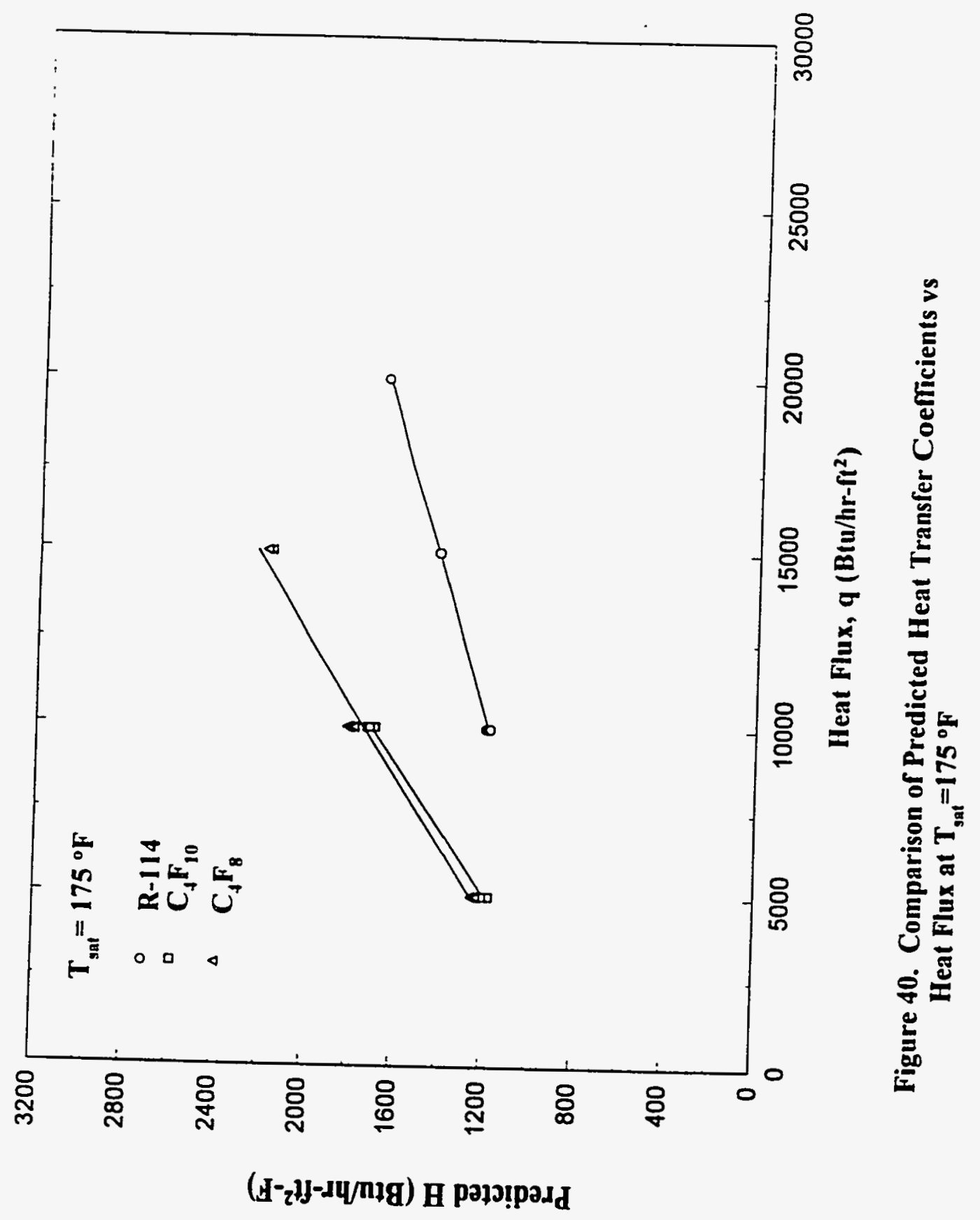




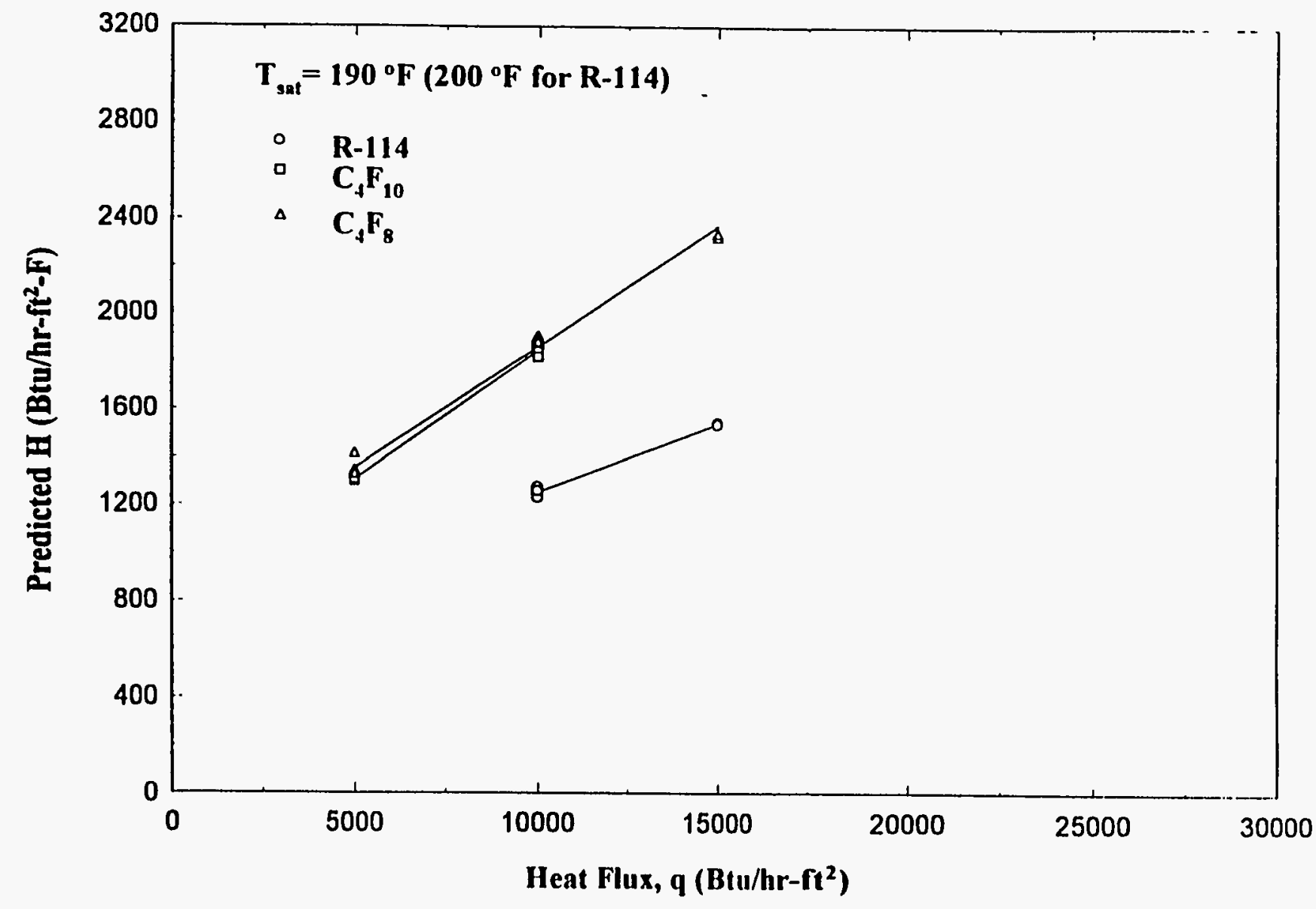

Figure 41. Comparison of Predicted Heat Transfer Coefficients vs Heat Flux at $T_{\text {sat }}=190^{\circ} \mathrm{F}$ 


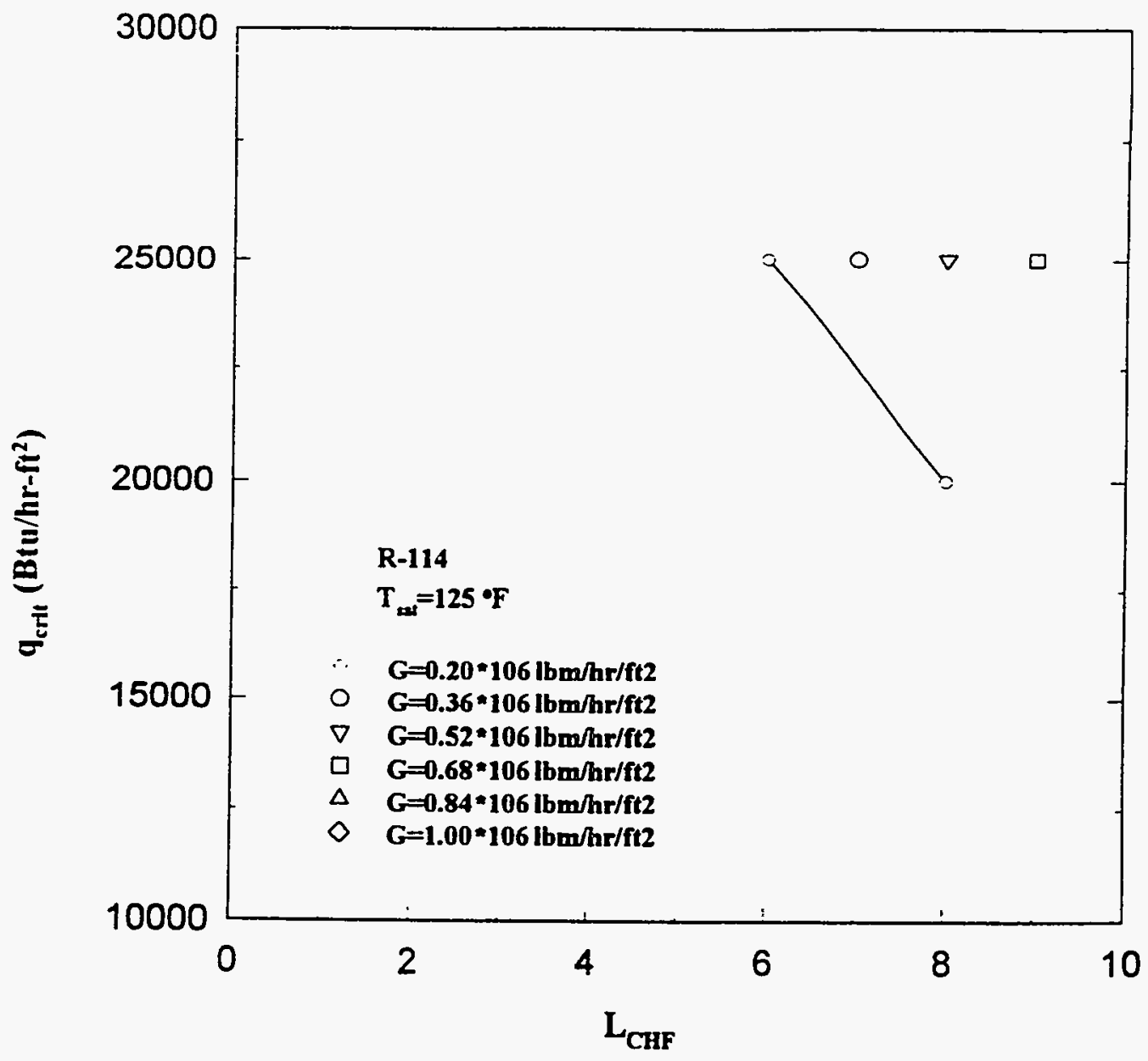

Figure 42. Critical Heat Flux versus the Dry-out Location L for R-114 at $125^{\circ} \mathrm{F}$ 


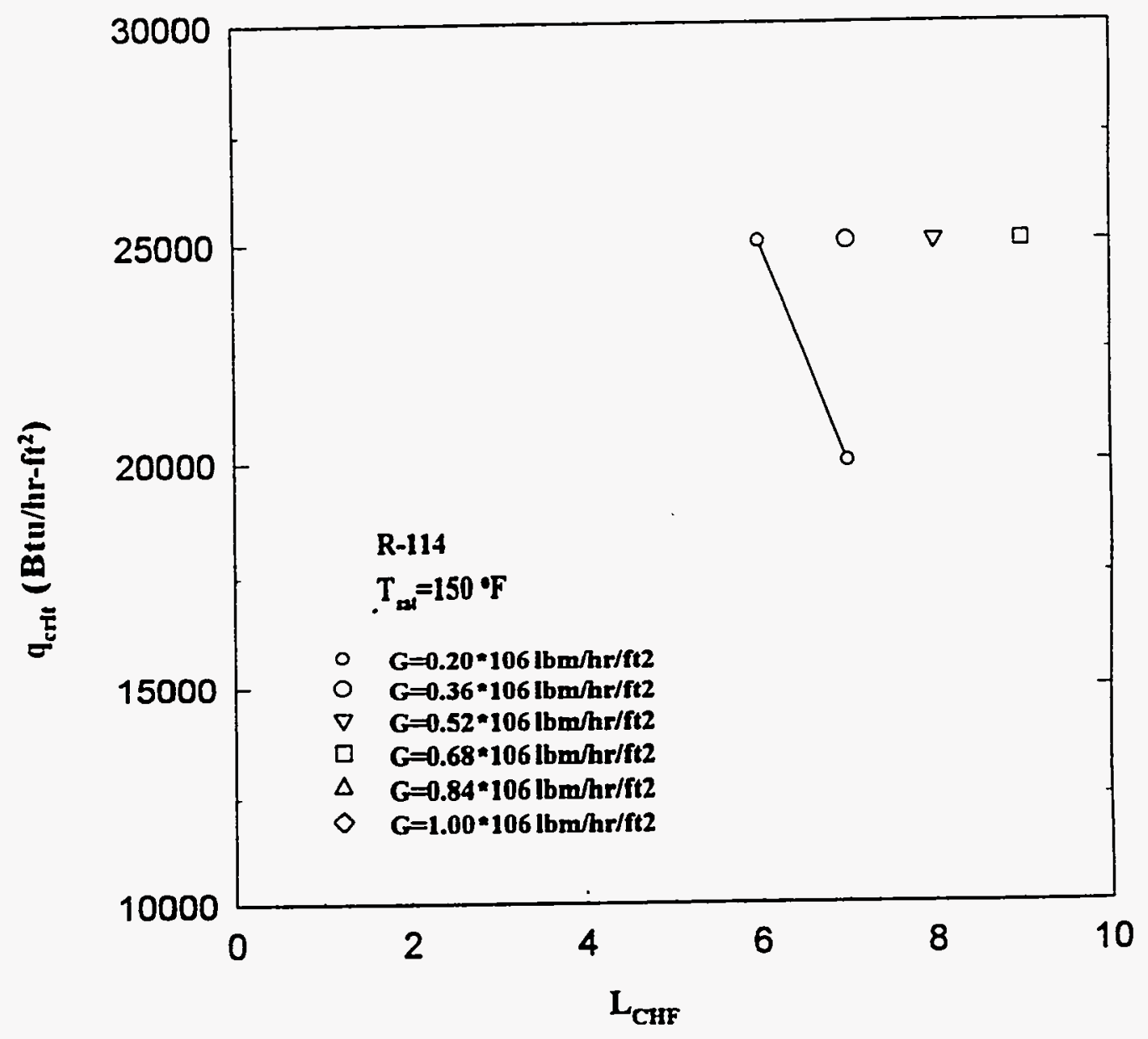

Figure 43. Critical Heat Flux versus the Dry-out Location L for R-114 at $150^{\circ} \mathrm{F}$ 


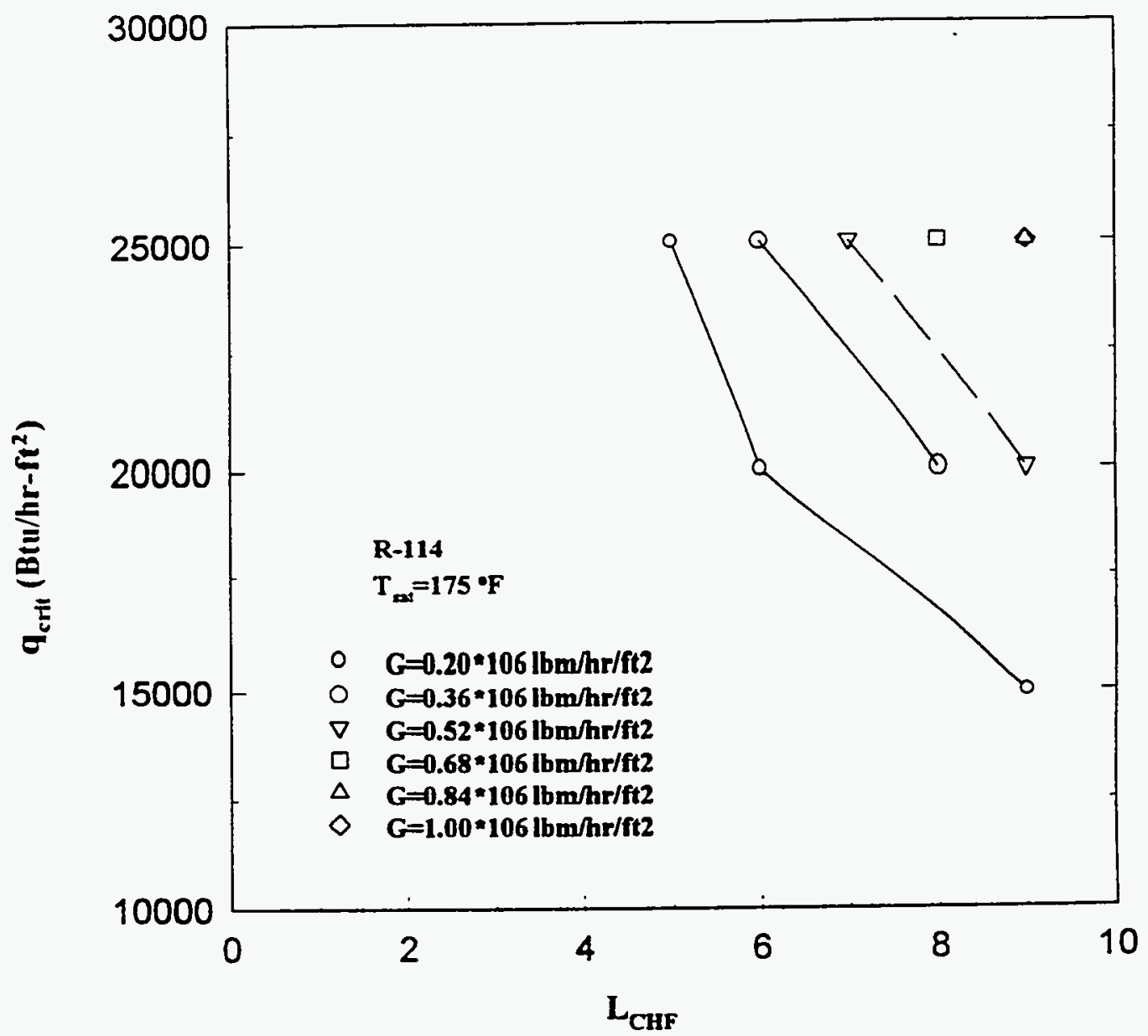

Figure 44. Critical Heat Flux versus the Dry-out Location $L$ for R-114 at $175^{\circ} \mathrm{F}$ 


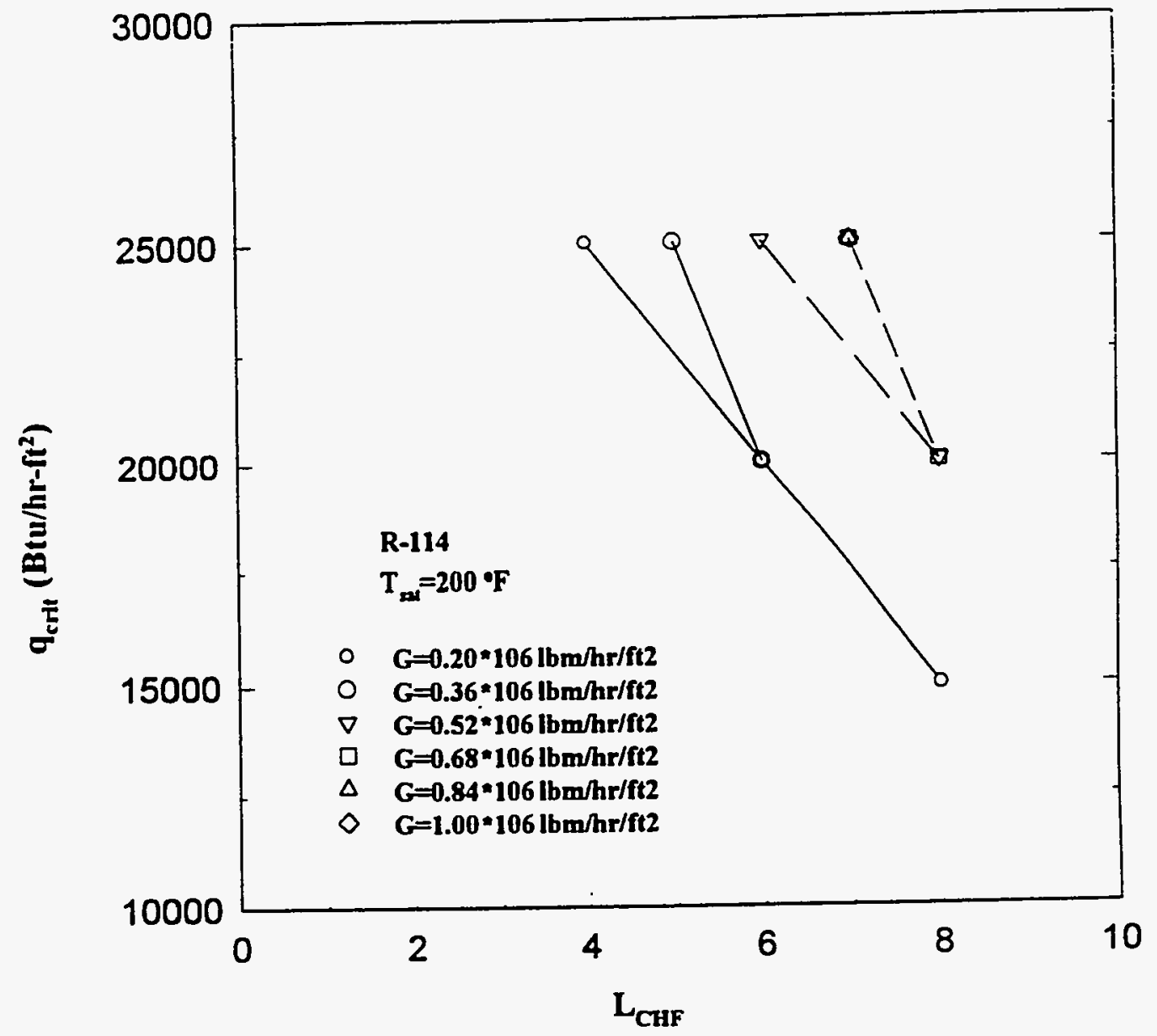

Figure 45. Critical Heat Flux versus the Dry-out

Location L for R-114 at $200^{\circ} \mathrm{F}$ 


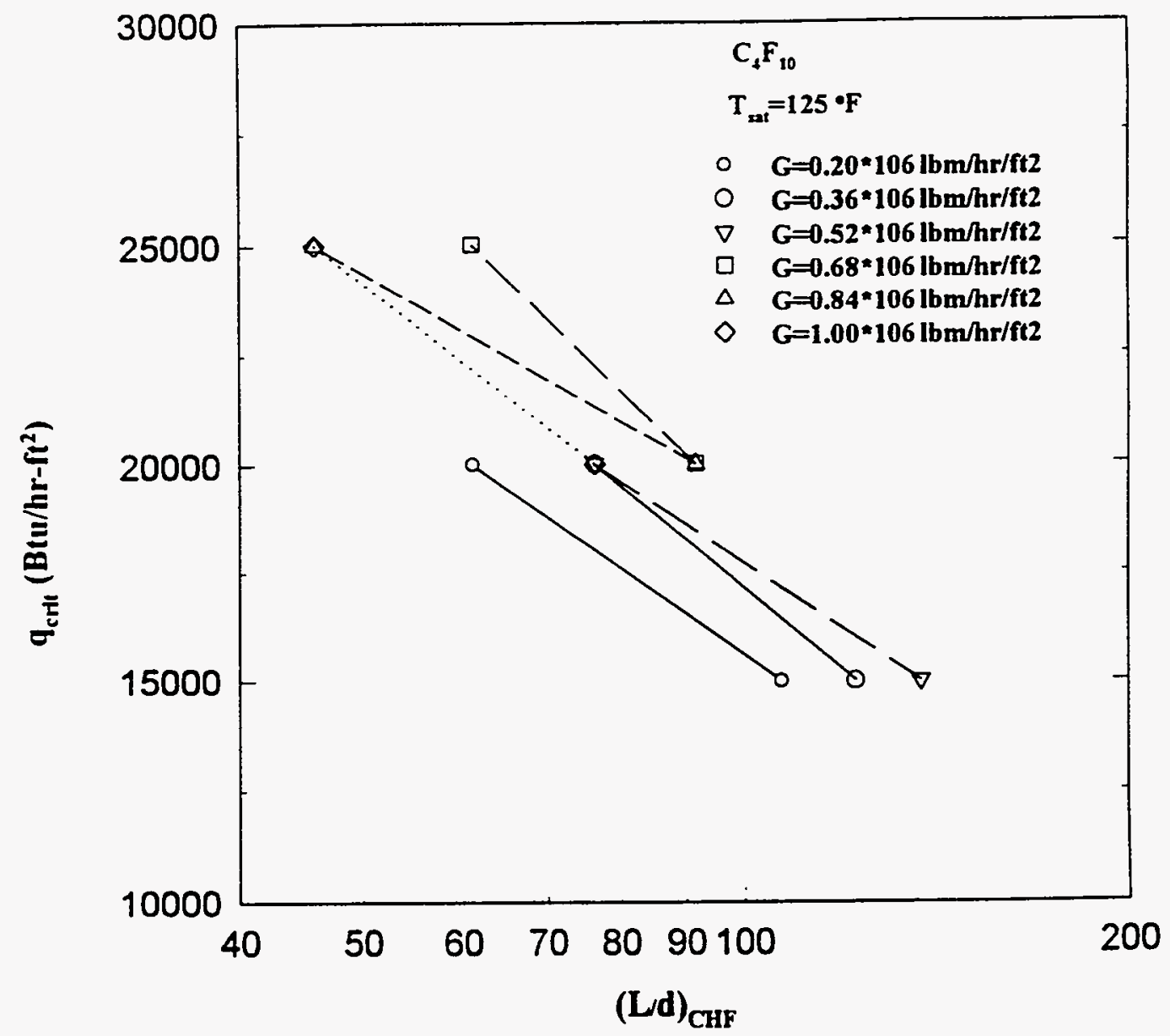

Figure 46. Critical Heat Flux versus $L / d$ Ratio for $\mathrm{C}_{4} \mathrm{~F}_{10}$ at $125^{\circ} \mathrm{F}$ 


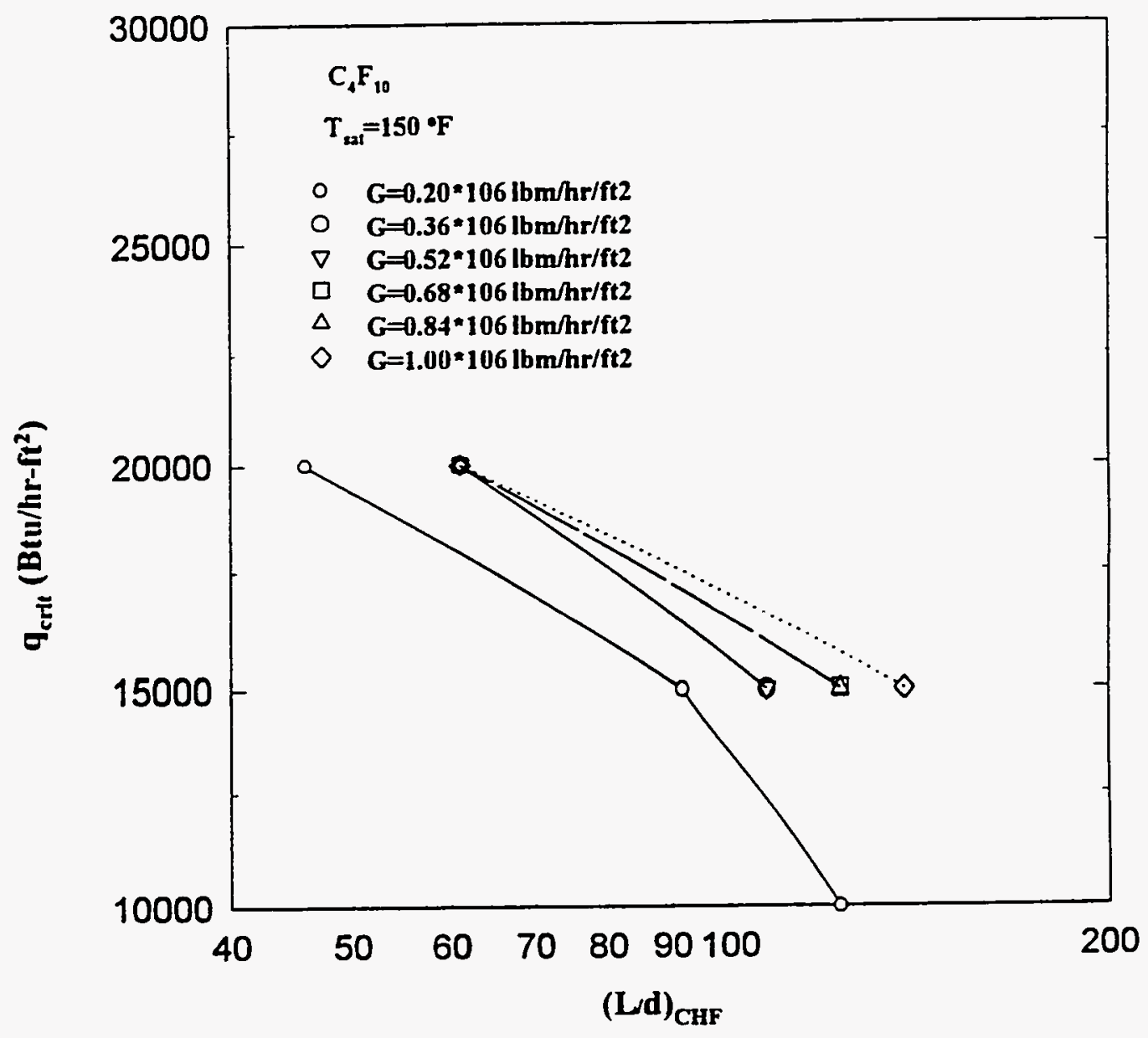

Figure 47. Critical Heat Flux versus L/d Ratio for $\mathrm{C}_{4} \mathrm{~F}_{10}$ at $150^{\circ} \mathrm{F}$ 


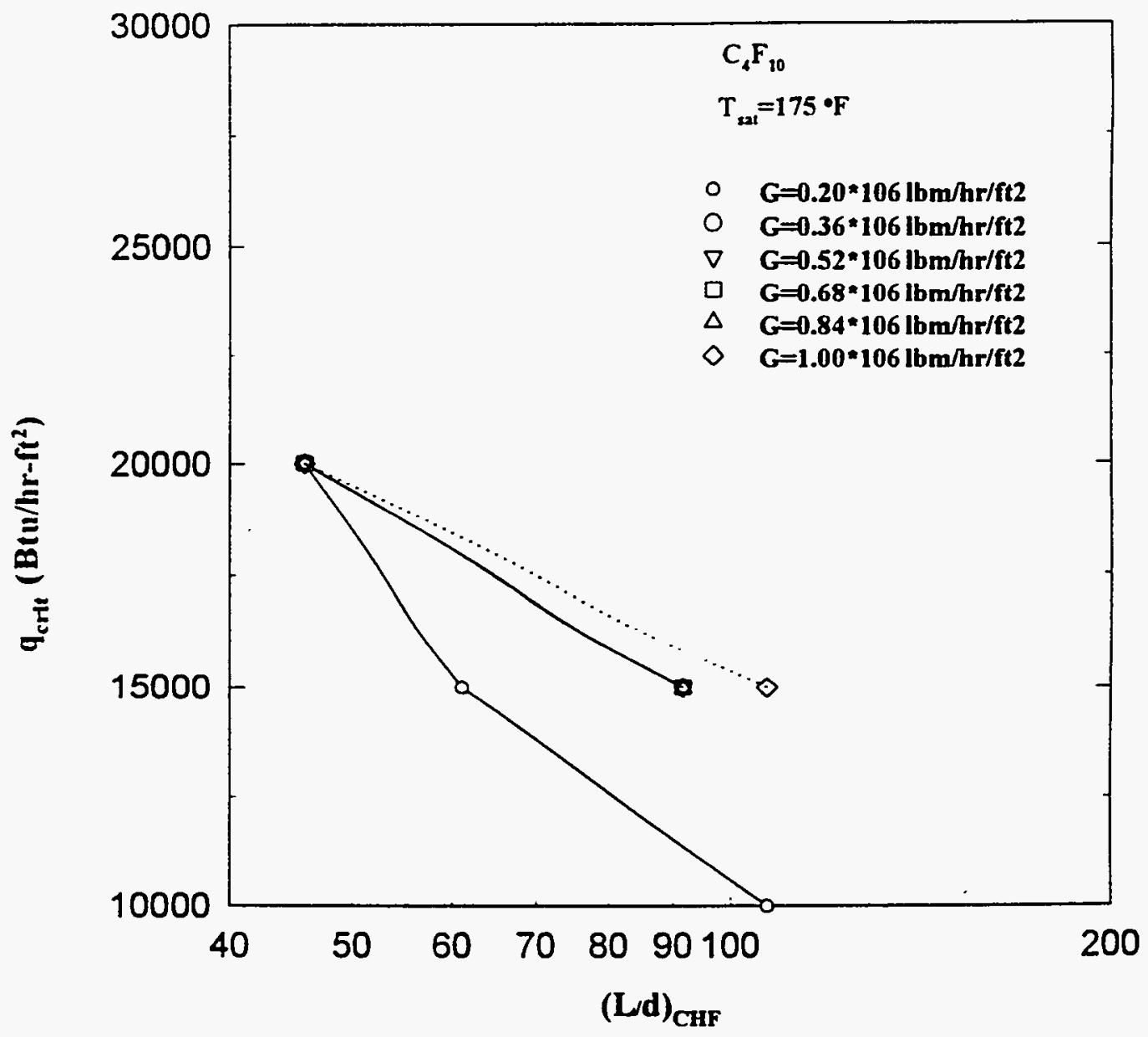

Figure 48. Critical Heat Flux versus $L / d$ Ratio for $\mathrm{C}_{4} \mathrm{~F}_{10}$ at $175^{\circ} \mathrm{F}$ 


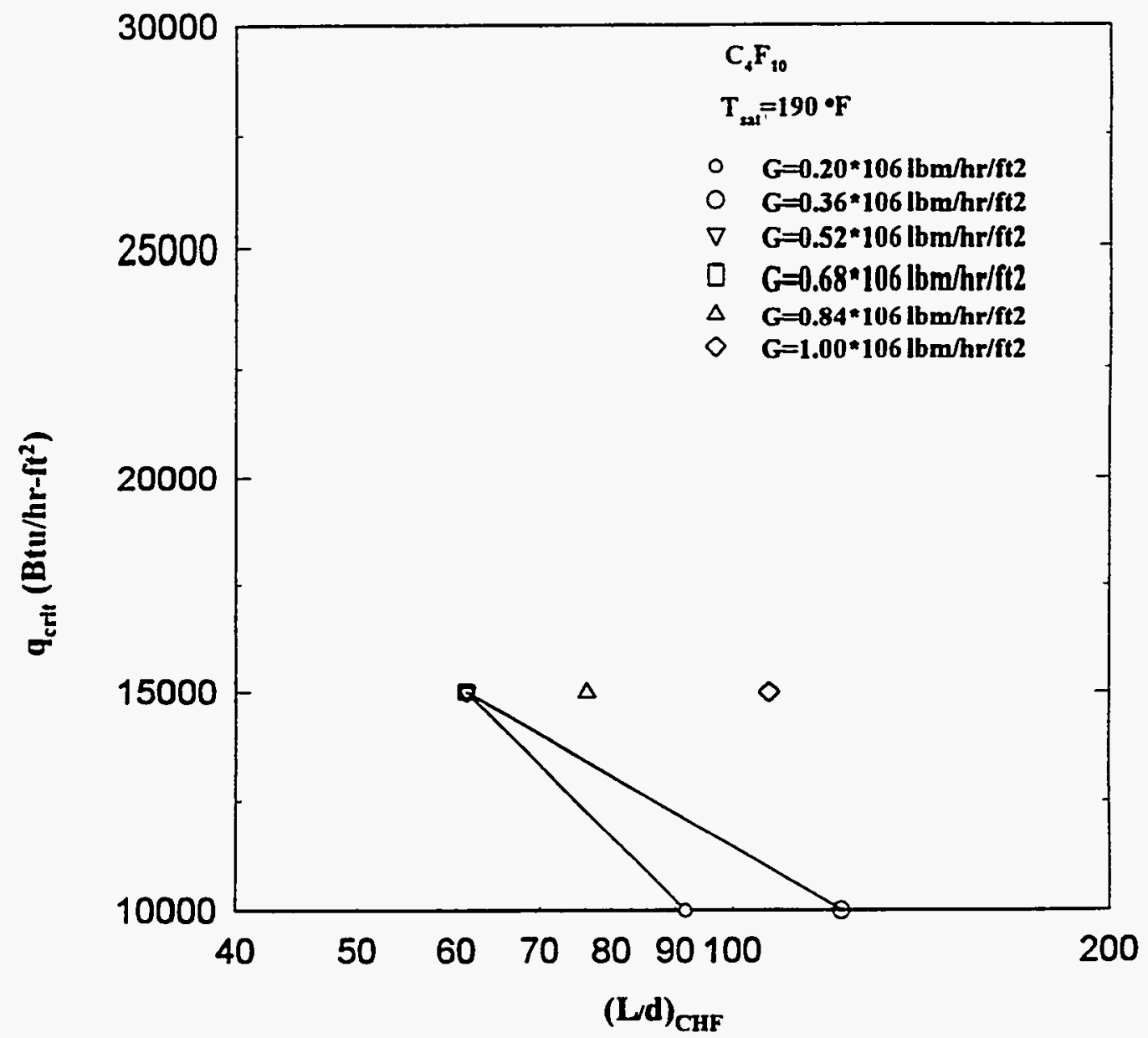

Figure 49. Critical Heat Flux versus L/d Ratio for $\mathrm{C}_{4} \mathrm{~F}_{10}$ at $190^{\circ} \mathrm{F}$ 


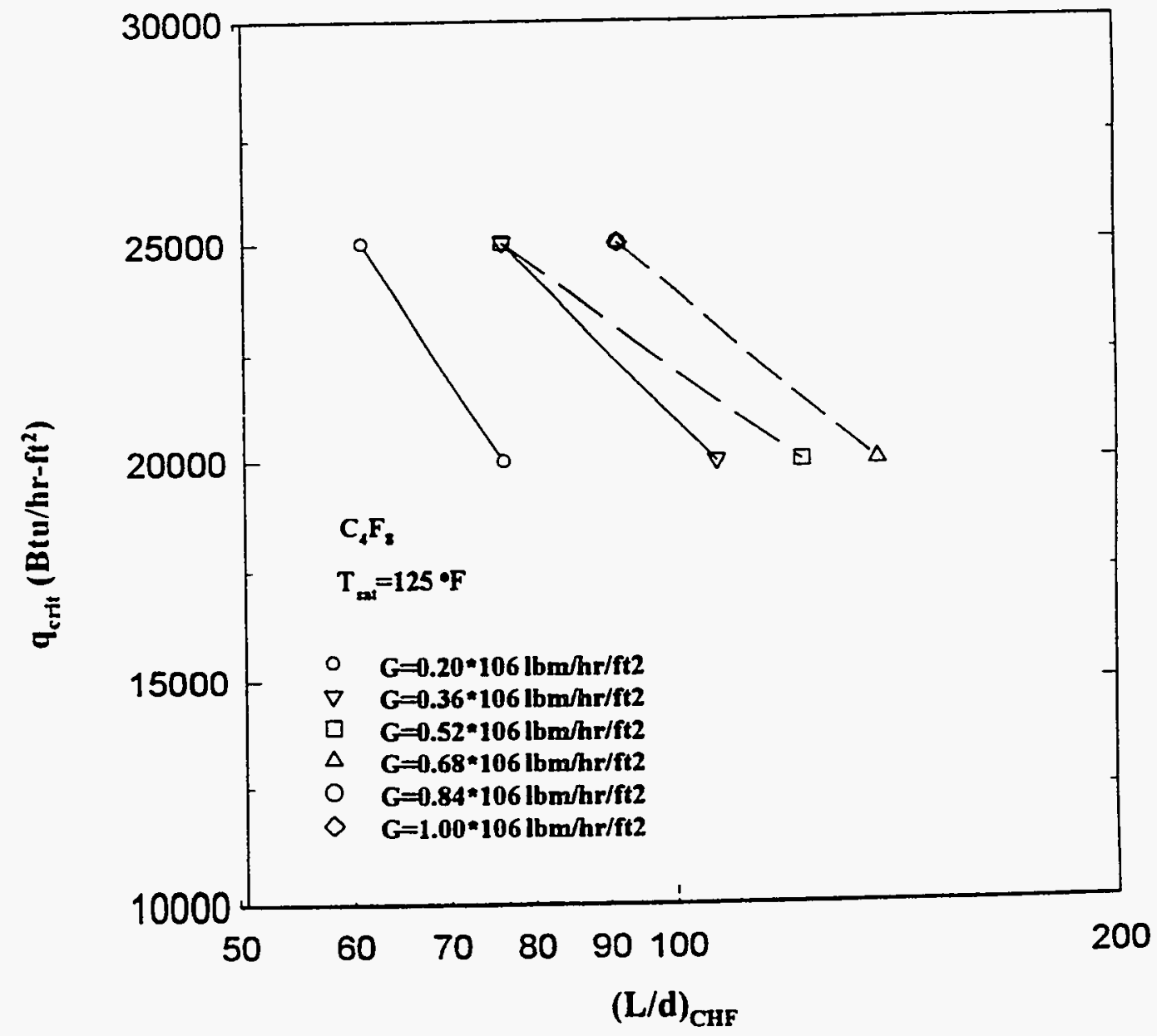

Figure 50. Critical Heat Flux versus L/d Ratio for

$\mathrm{C}_{4} \mathrm{~F}_{8}$ at $125^{\circ} \mathrm{F}$ 


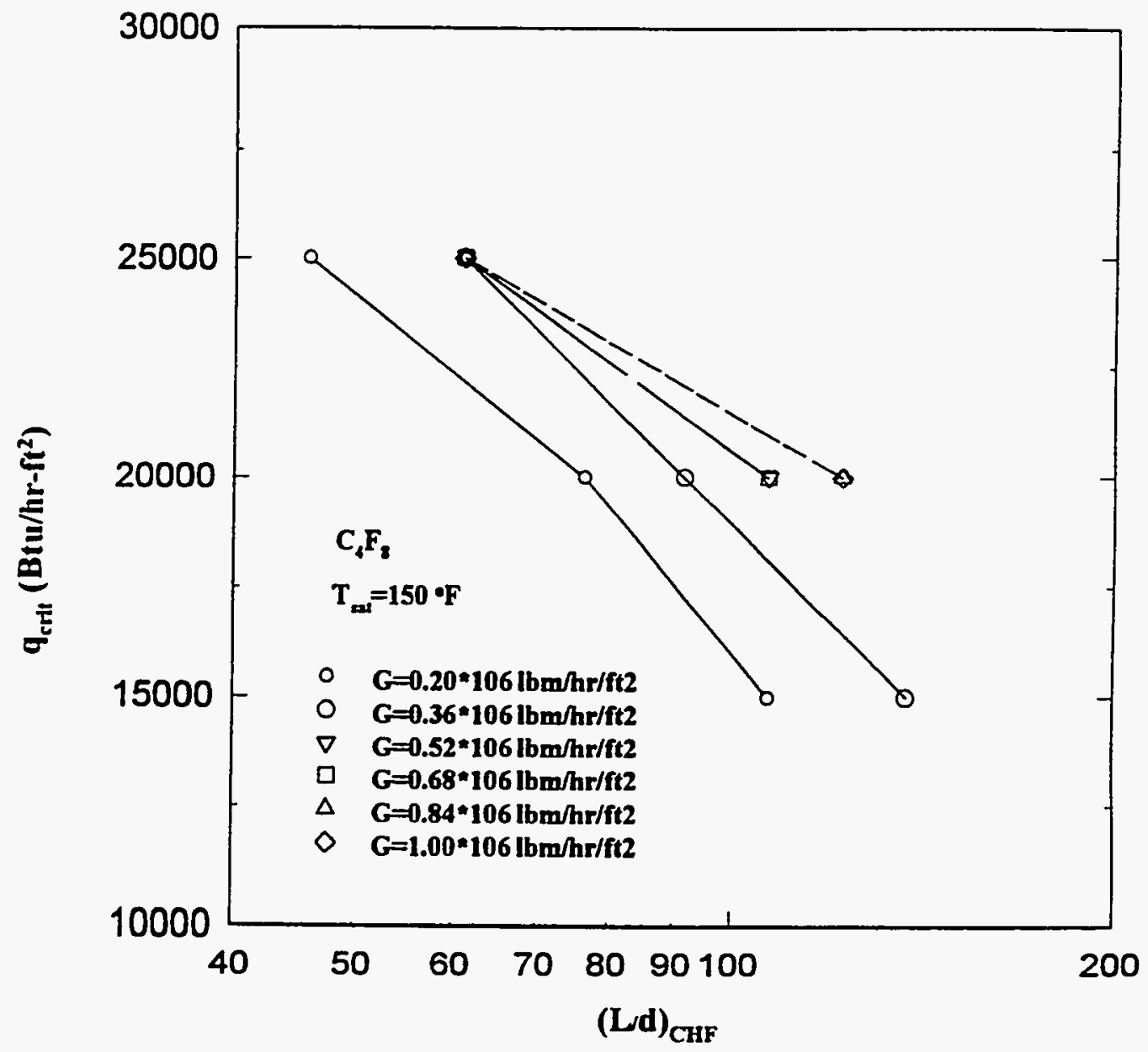

Figure 51. Critical Heat Flux versus $L / d$ Ratio for $\mathrm{C}_{4} \mathrm{~F}_{8}$ at $150^{\circ} \mathrm{F}$ 


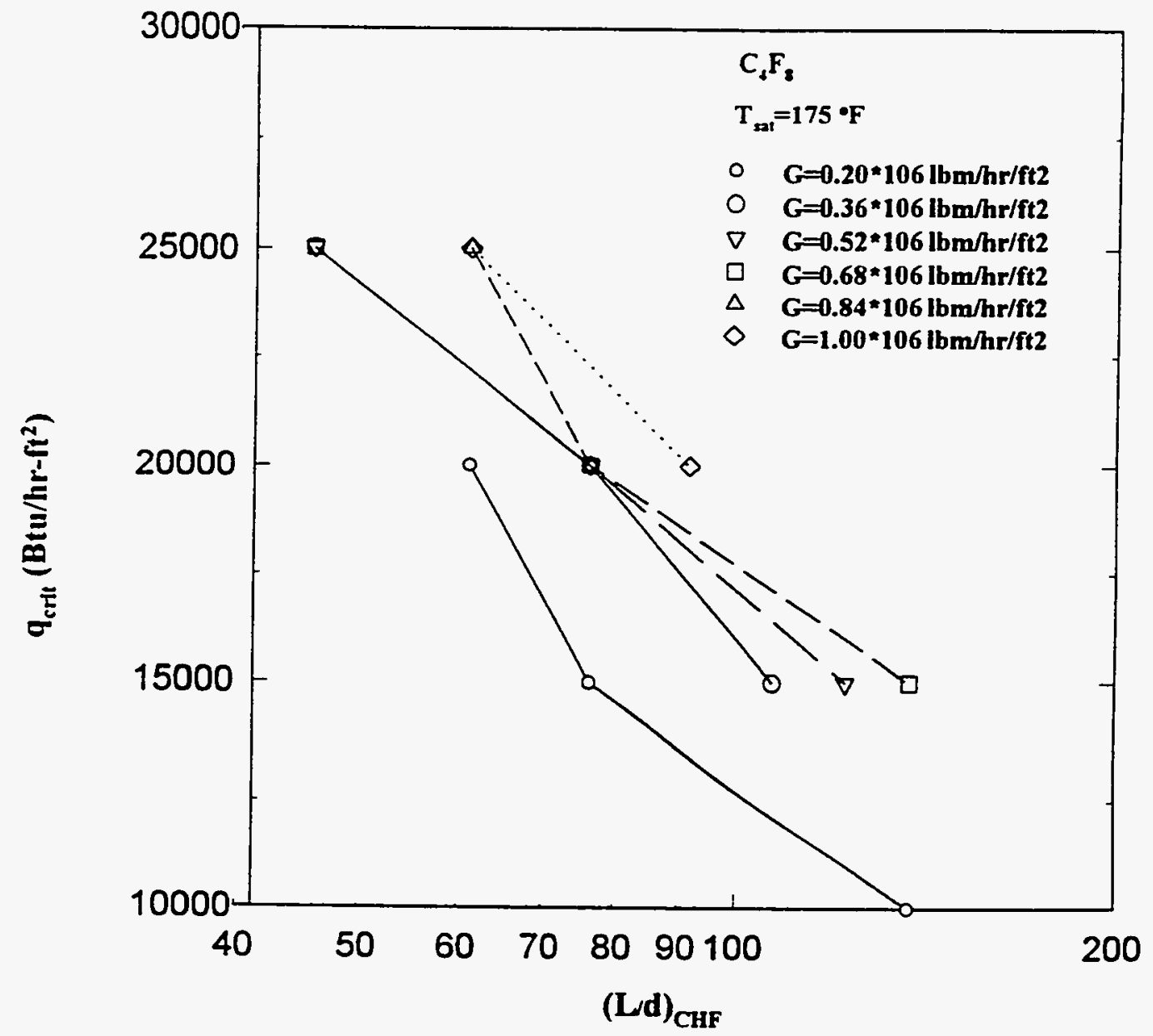

Figure 52. Critical Heat Flux versus $L / d$ Ratio for $\mathrm{C}_{4} \mathrm{~F}_{8}$ at $175^{\circ} \mathrm{F}$ 


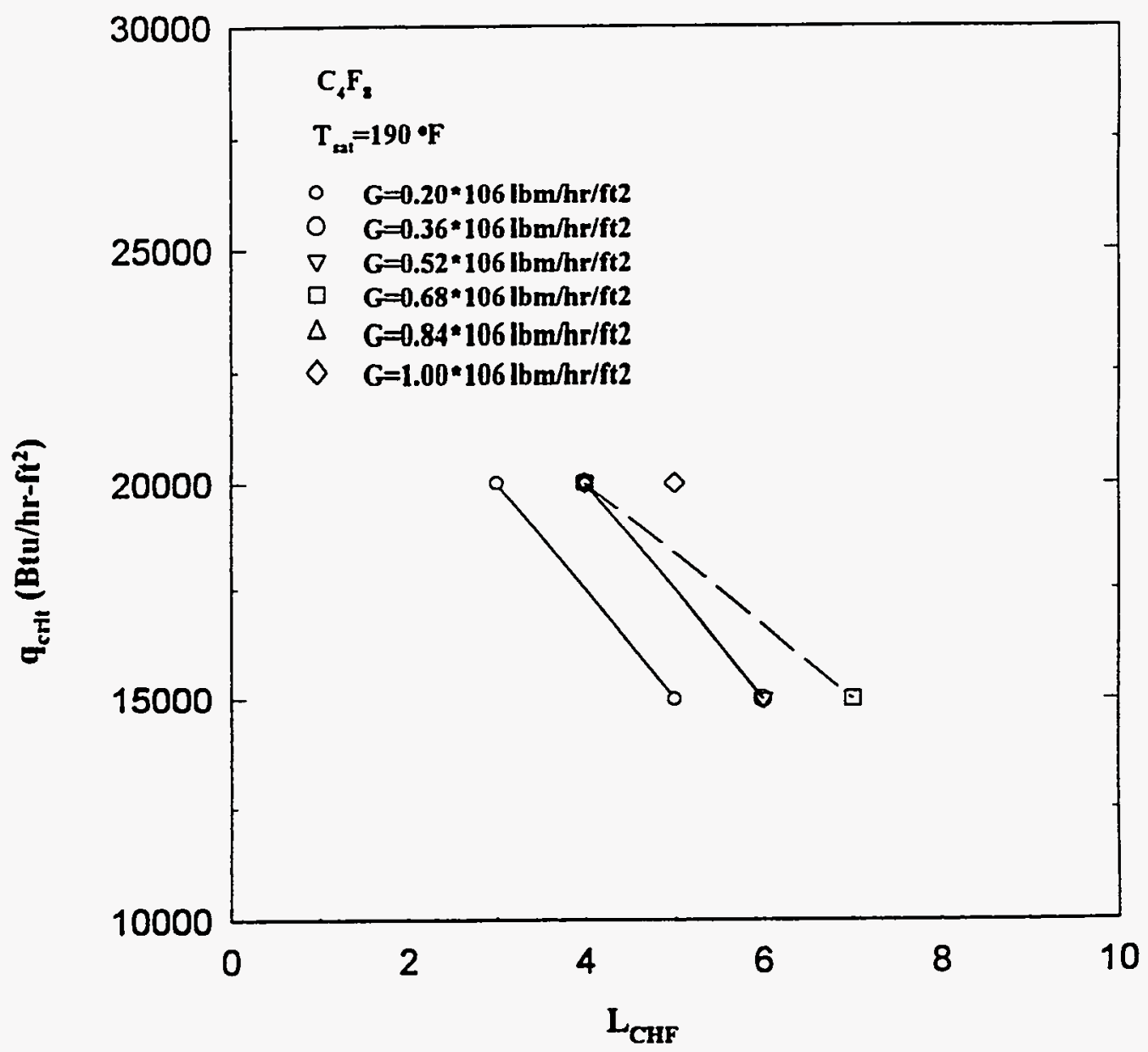

Figure 53. Critical Heat Flux versus the Dry-out Location $\mathrm{L}$ for $\mathrm{C}_{4} \mathrm{~F}_{8}$ at $190^{\circ} \mathrm{F}$ 


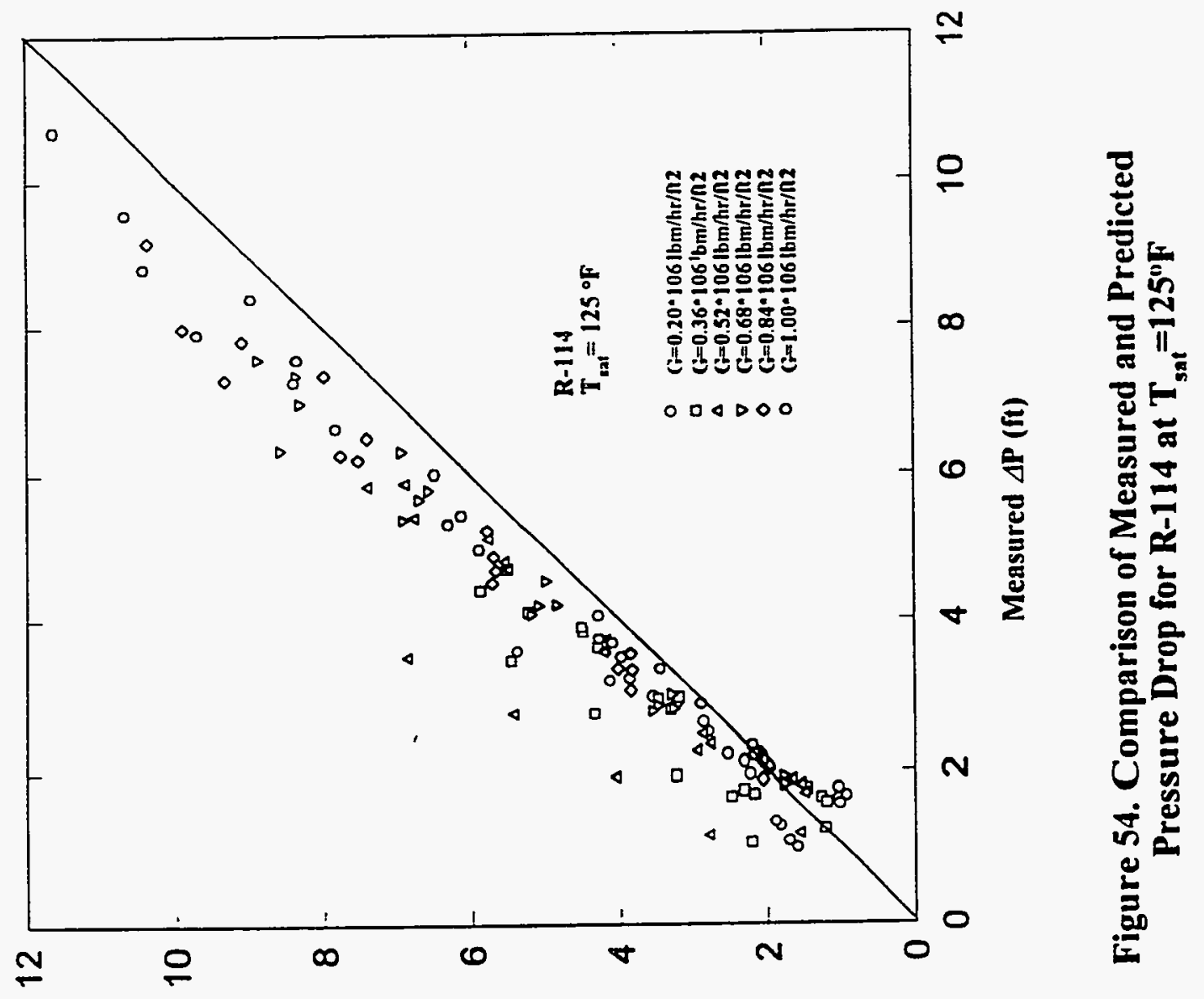

(น) dV pəџฺ!padd 


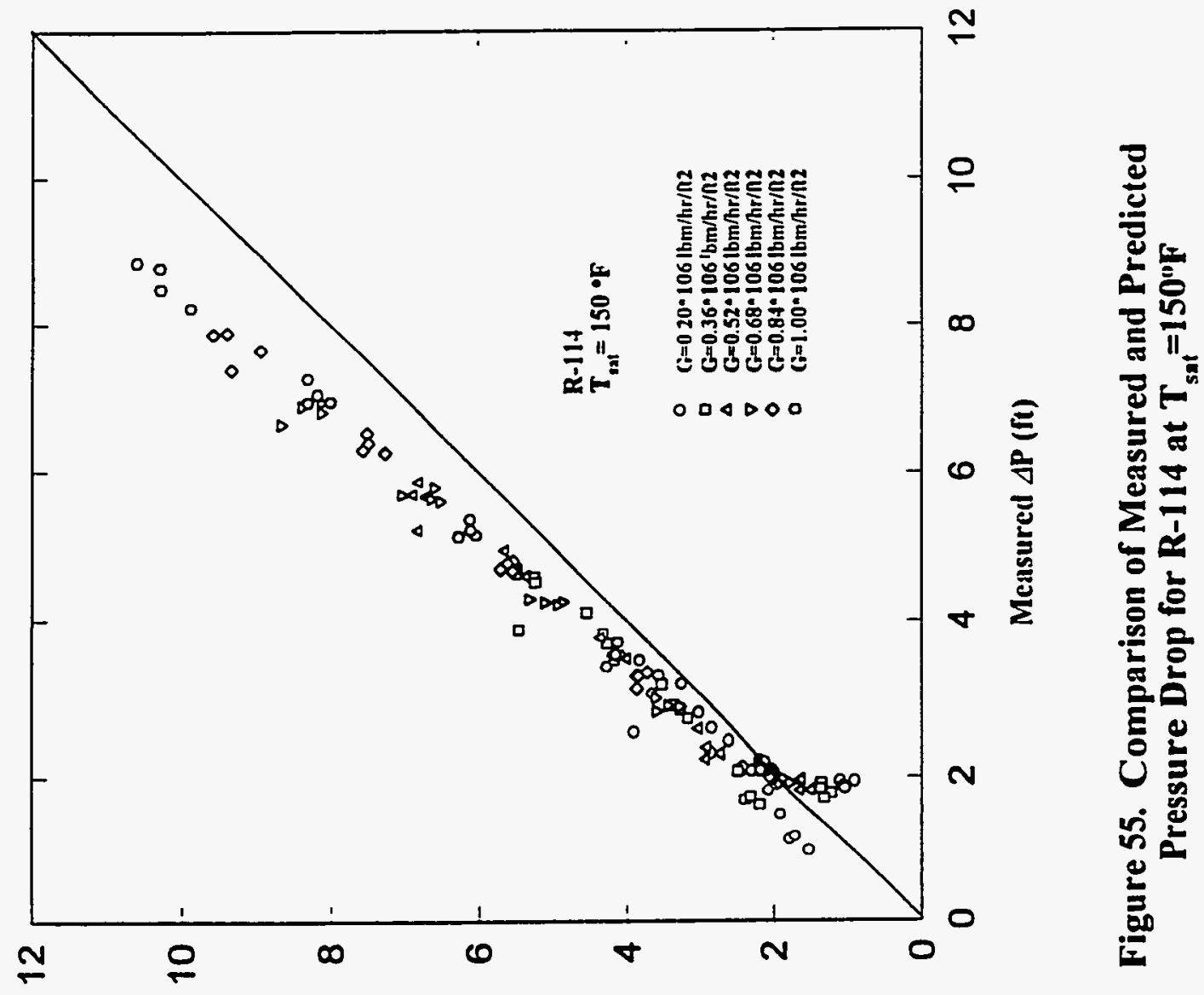

(y) dV paxכ!̣padd 


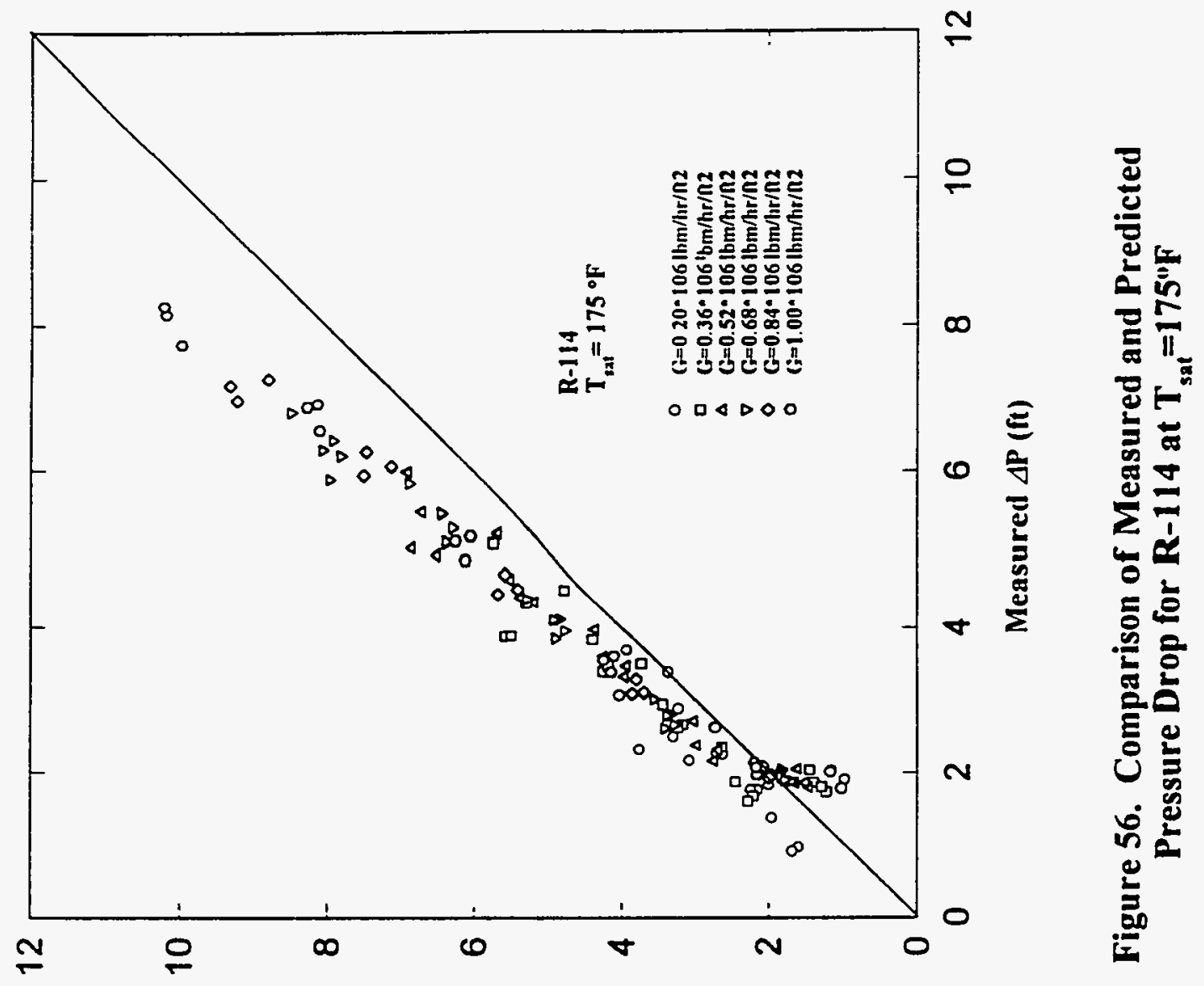

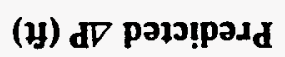




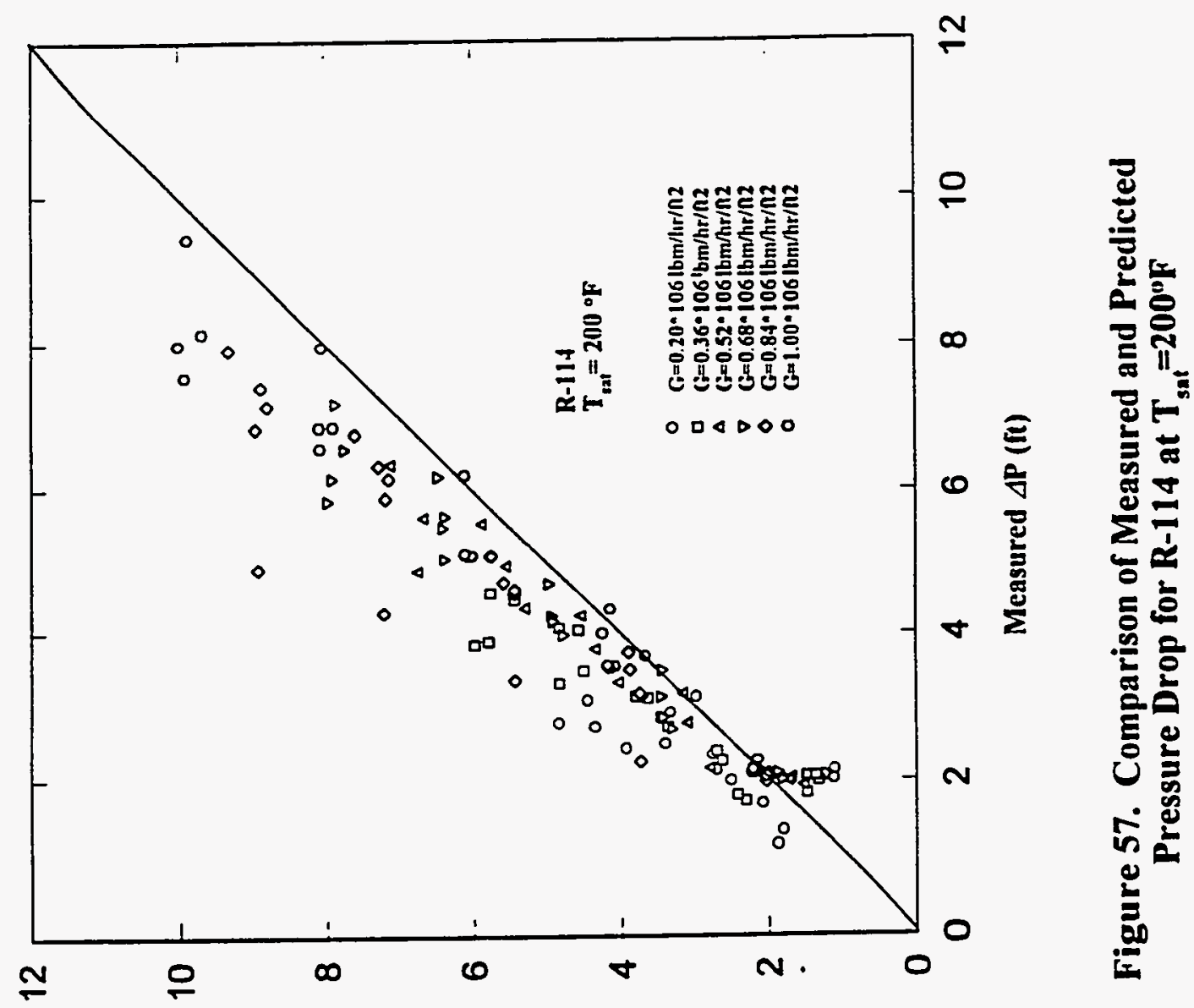

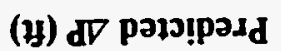




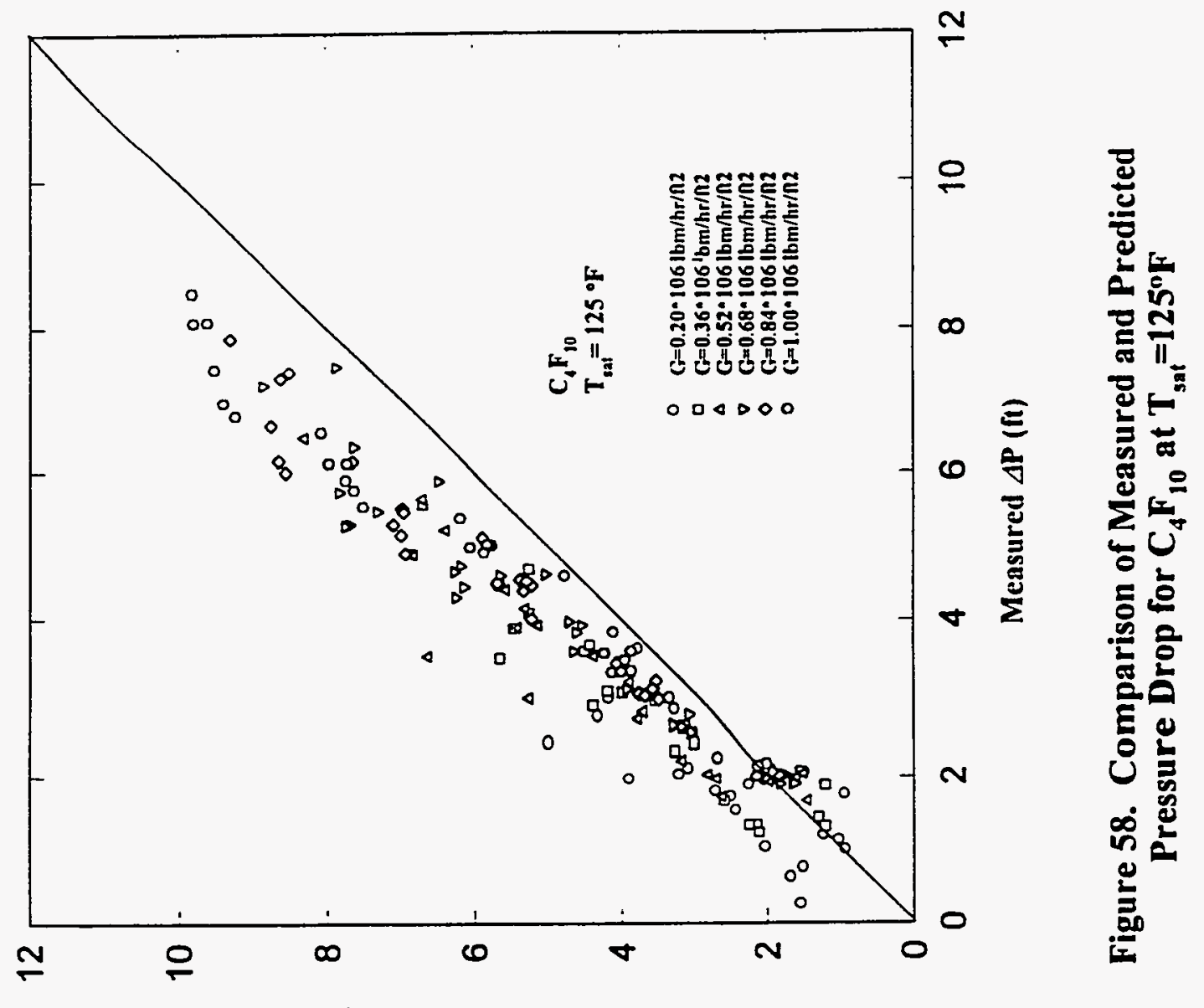

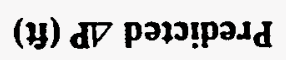




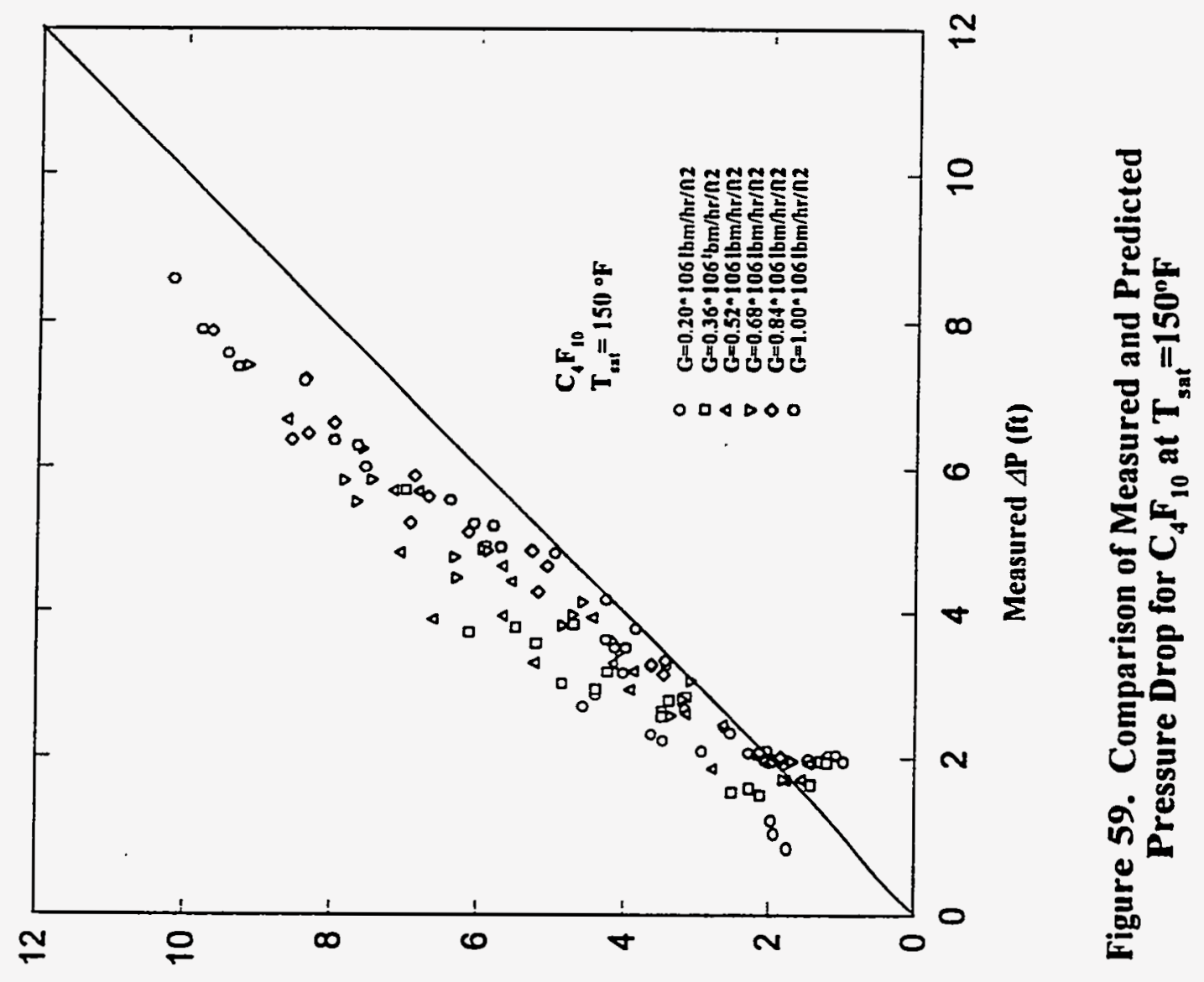

(अ) d рәтэрадd 


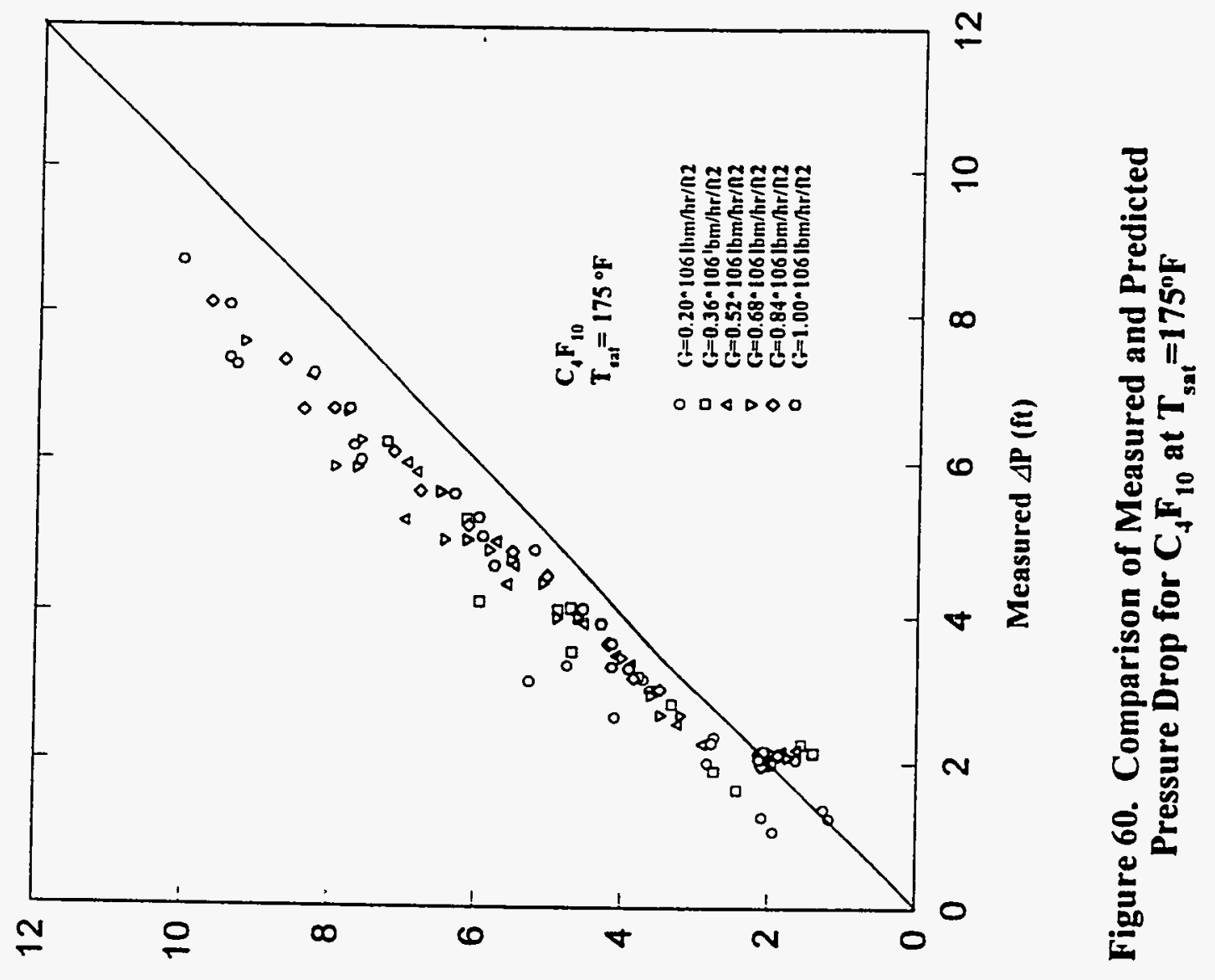

(y) $d \nabla$ porว!pวdd 


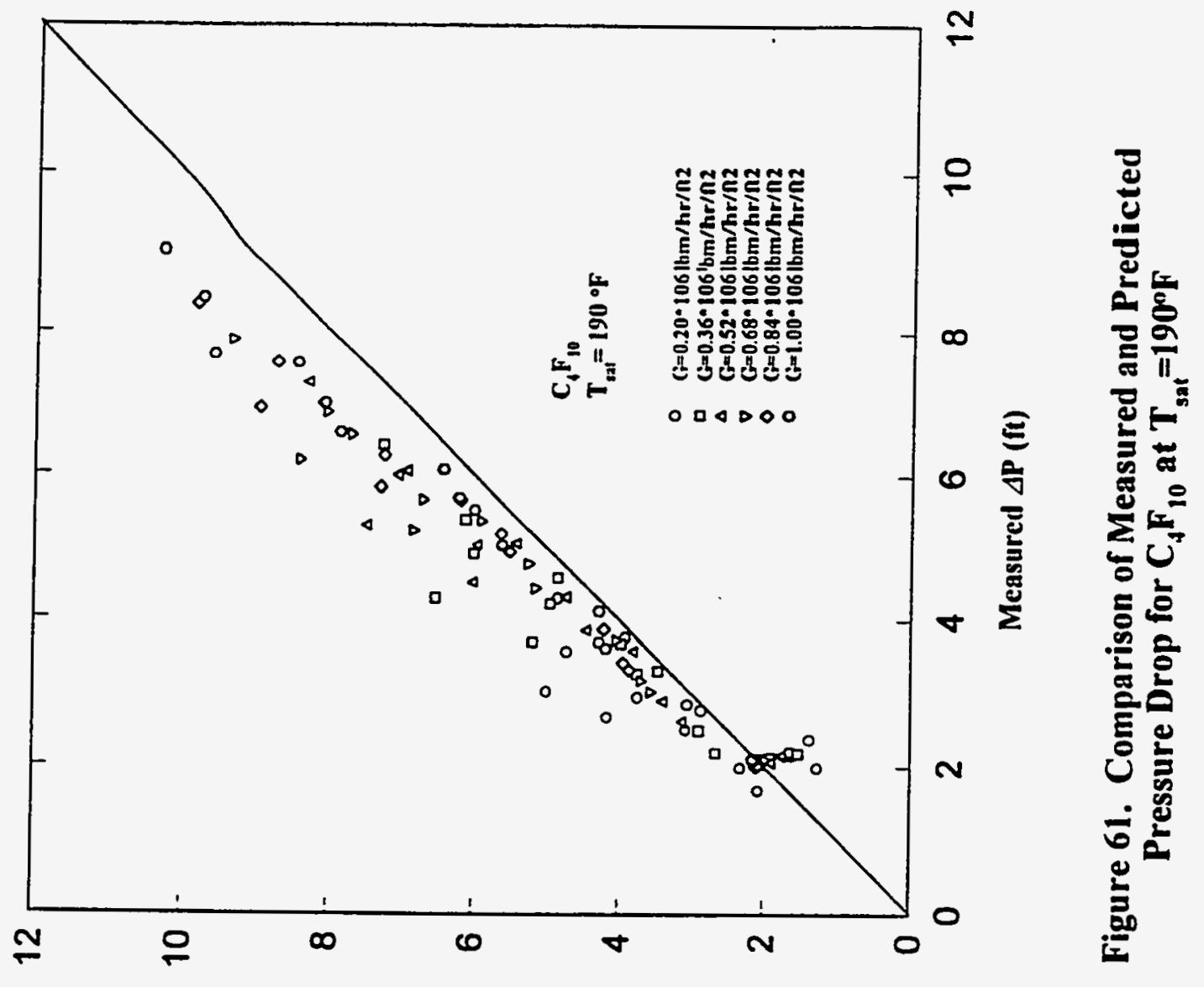

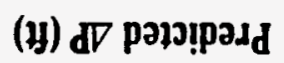




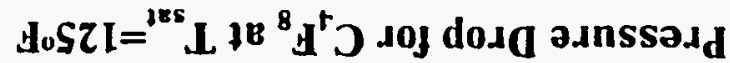

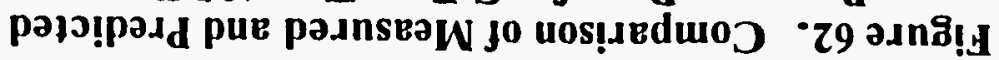

(IJ) $d \nabla$ pa.snseวw

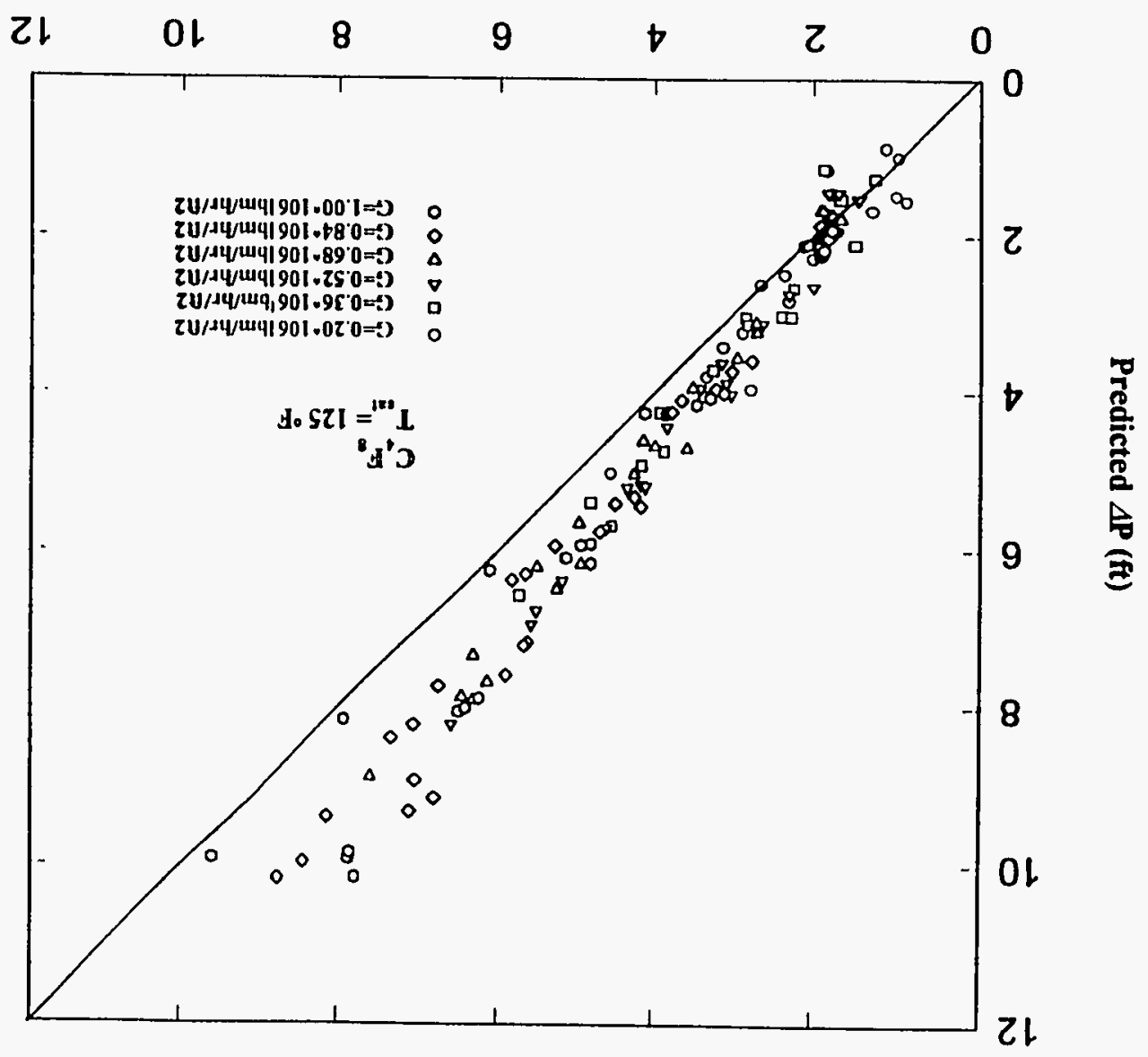




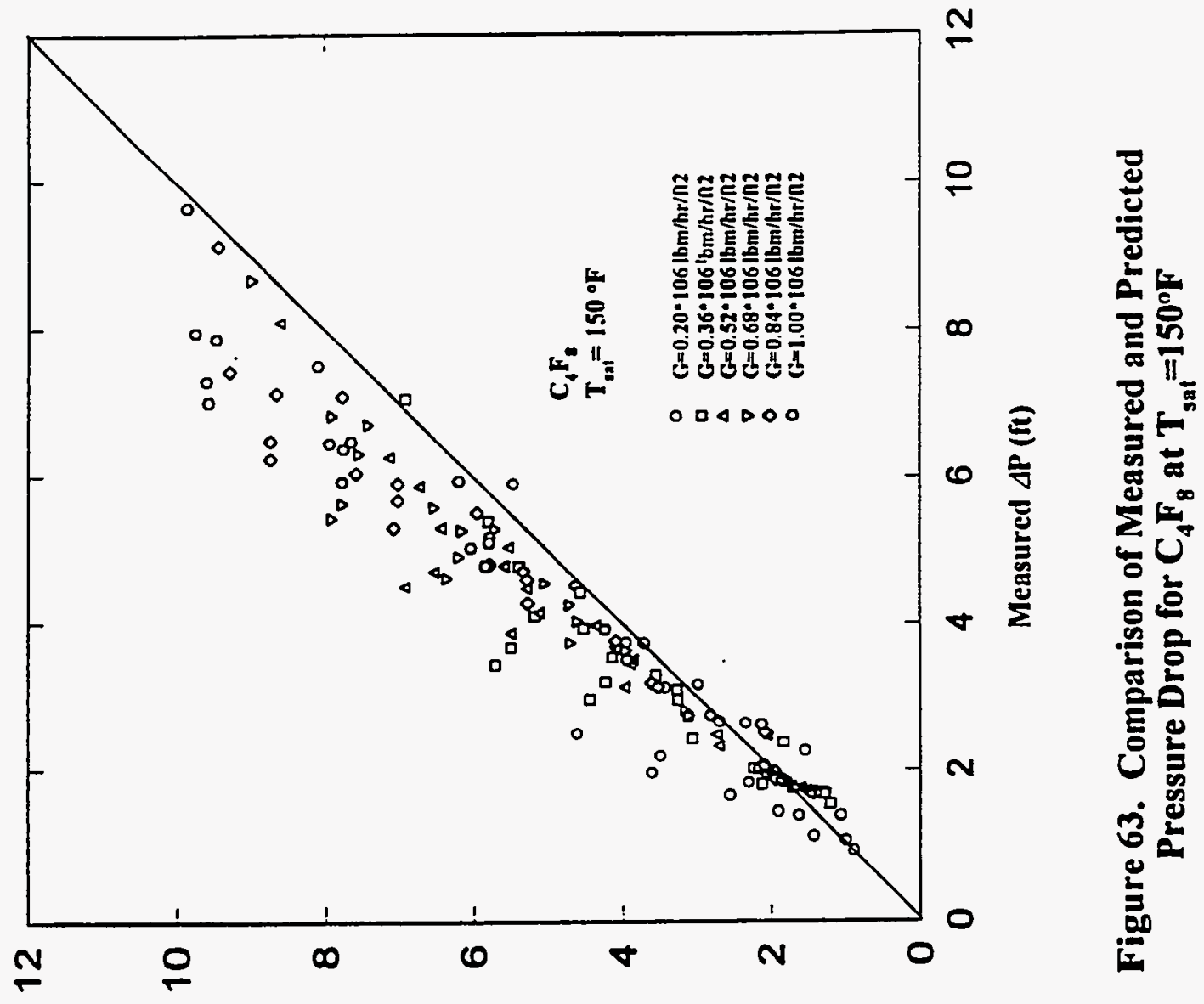

(y) dV pox!pasd 


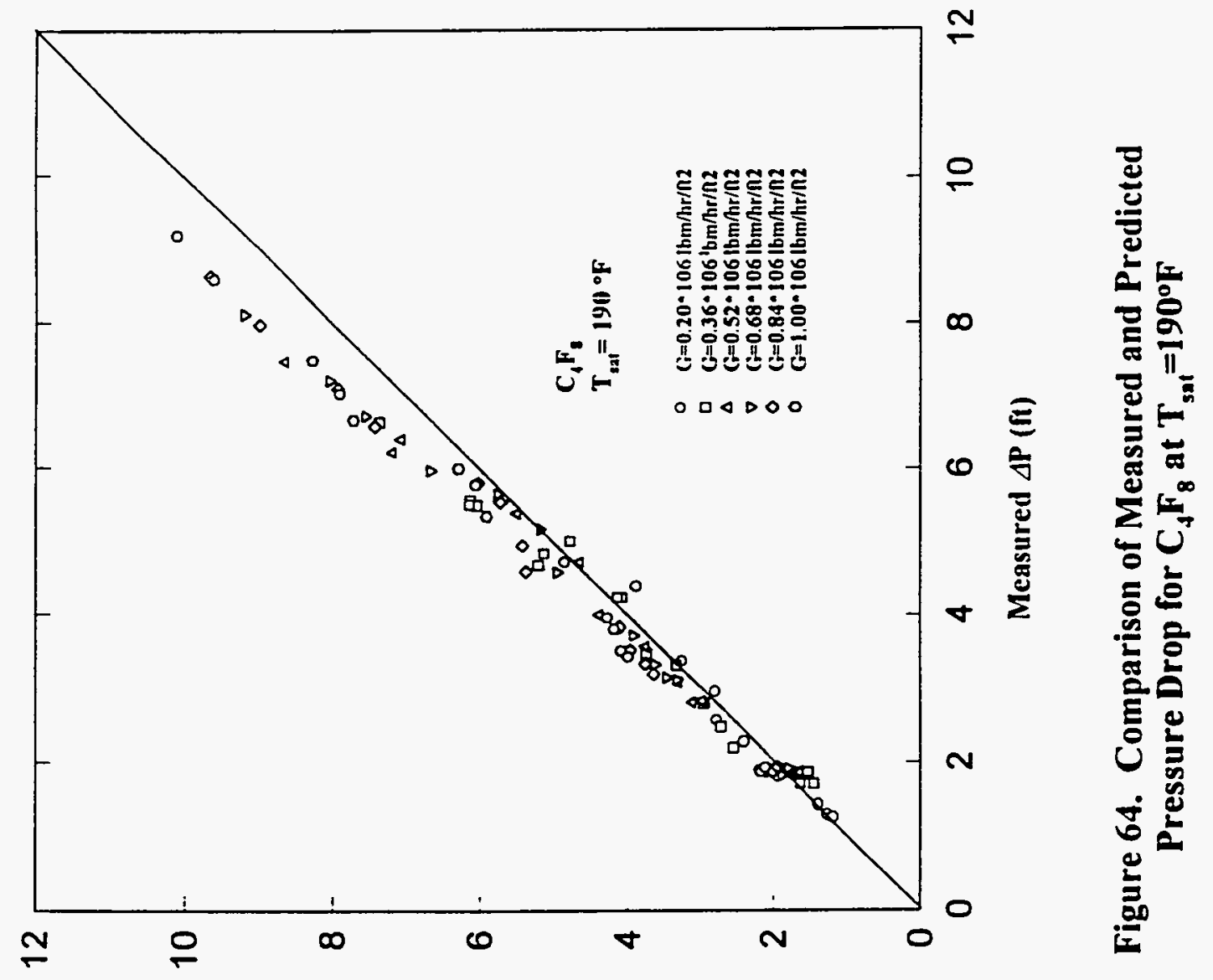

(y) dV рәтэ!радd 


\section{Section 3}

Study of Flow Boiling of Mixtures of R-114, and $\mathrm{C}_{4} \mathrm{~F}_{10}$, in a Vertical Tube 


\section{Study of Flow Boiling of Mixtures of R-114 and $\mathrm{C}_{4} \mathrm{~F}_{10}$ in a Vertical Tube}

by

Cudo Technologies, Ltd.

P.O. Box 185, Lexington, KY 40584

Contract \# 11K-NR559

Report \# MMES-Cudo-NR559-3

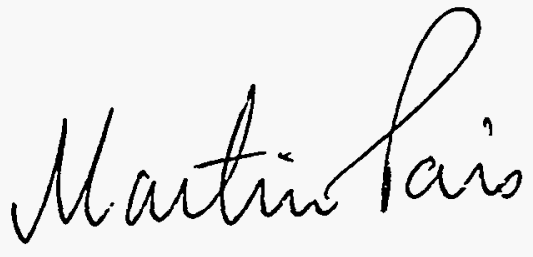

Martin R. Pais, PhD, PE

Principal Investigator<smiles>[Al]C=CC=C1C=C1</smiles>

Ming Jr Chang, $\mathrm{PhD}$ 



\begin{abstract}
Convective pool and flow boiling experiments were conducted for three different mixtures of liquid R-114 and $\mathrm{C}_{4} \mathrm{~F}_{10}$ to determine the heat transfer and pressure drop characteristics in a vertical tube. Experiments were run over the saturation temperature range of $130^{\circ} \mathrm{F}$ to $170{ }^{\circ} \mathrm{F}$, heat flux range of 5000 to $25000 \mathrm{btu} / \mathrm{hr} . \mathrm{ft}^{2}$ and mass flow rate range of $0.5 \mathrm{E} 6$ to $1 \mathrm{E} 6 \mathrm{lbm} / \mathrm{hr} . \mathrm{ft}^{2}$. Experiments were run for different saturation temperatures, heat fluxes, and mass flow rates so as to determine the heat transfer capability and pressure drop conditions. The heat-transfer coefficient of the mixture is as much as $30 \%$ lower than the ideal values under the same flow and saturation conditions. Correlations are presented which provide a best fit prediction of the data.
\end{abstract}


TABLE OF CONTENTS

Page

\section{ABSTRACT}

Table of contents

Table of Figures

ii

Nomenclature

iv

INTRODUCTION

Experimental Setup

Experimental Procedures

Experimental Program

Heat Transfer Measurement and Analysis

Heat Transfer Correlation

Single Phase Liquid Flow

Nucleate Pool Boiling

Pressure Drop Correlation

RESULTS AND DISCUSSION

Heat Transfer

Pressure Drop

CONCLUSIONS

ACKNOWLEDGEMENTS

REFERENCES 


\section{TABLE OF FIGURES}

Figures

Page

1. Test Fluid Loop $\quad 17$

2. Details of Zone \# $1 \quad 18$

3. Storage Tank-Assembly and Caps 19

4. Saturation Pressure vs Temperature for Different Compositions of $\mathrm{C}_{4} \mathrm{~F}_{10}$ and $\mathrm{R}-114$

5. Liquid Density vs Temperature for Different Compositions of $\mathrm{C}_{4} \mathrm{~F}_{10}$ and $\mathrm{R}-114$

6. Correlation of Forced Convection for R-114 22

7. Correlation of Forced Convection for $\mathrm{C}_{4} \mathrm{~F}_{10} \quad 23$

8. Correlation of Nucleate Pool Boiling for R-114 24

9. Correlation of Nucleate Pool Boiling for $\mathrm{C}_{4} \mathrm{~F}_{10}$

10. Heat Flux Versus the Wall-to-instream Temperature Difference
for the Mixture of $26 \% \mathrm{C}_{4} \mathrm{~F}_{10}+74 \% \mathrm{R}-114$ at $\mathrm{T}_{\text {sat }}=130^{\circ} \mathrm{F}$

11. Heat Flux Versus the Wall-to-instream Temperature Difference for the Mixture of $26 \% \mathrm{C}_{4} \mathrm{~F}_{10}+74 \% \mathrm{R}-114$ at $\mathrm{T}_{\text {sat }}=150^{\circ} \mathrm{F}$

12. Heat Flux Versus the Wall-to-instream Temperature Difference for the Mixture of $26 \% \mathrm{C}_{4} \mathrm{~F}_{10}+74 \% \mathrm{R}-114$ at $\mathrm{T}_{\mathrm{sat}}=170^{\circ} \mathrm{F}$

13. Heat Flux Versus the Wall-to-instream Temperature Difference for the Mixture of $51 \% \mathrm{C}_{4} \mathrm{~F}_{10}+49 \% \mathrm{R}-114$ at $\mathrm{T}_{\text {sat }}=130^{\circ} \mathrm{F}$

14. Heat Flux Versus the Wall-to-instream Temperature Difference for the Mixture of $51 \% \mathrm{C}_{4} \mathrm{~F}_{10}+49 \% \mathrm{R}-114$ at $\mathrm{T}_{\text {sat }}=150^{\circ} \mathrm{F}$

15. Heat Flux Versus the Wall-to-instream Temperature Difference for the Mixture of $51 \% \mathrm{C}_{4} \mathrm{~F}_{10}+49 \% \mathrm{R}-114$ at $\mathrm{T}_{\text {sat }}=170^{\circ} \mathrm{F}$

16. Heat Flux Versus the Wall-to-instream Temperature Difference for the Mixture of $66 \% \mathrm{C}_{4} \mathrm{~F}_{10}+34 \% \mathrm{R}-114$ at $\mathrm{T}_{\text {sat }}=130^{\circ} \mathrm{F}$

17. Heat Flux Versus the Wall-to-instream Temperature Difference for the Mixture of $66 \% \mathrm{C}_{4} \mathrm{~F}_{10}+34 \% \mathrm{R}-114$ at $\mathrm{T}_{\text {sat }}=150^{\circ} \mathrm{F}$

18. Heat Flux Versus the Wall-to-instream Temperature Difference for the Mixture of $66 \% \mathrm{C}_{4} \mathrm{~F}_{10}+34 \% \mathrm{R}-114$ at $\mathrm{T}_{\text {sal }}=170^{\circ} \mathrm{F}$

19. Flow Boiling Heat Transfer Characteristics for Mixtures of R-114 and $\mathrm{C}_{4} \mathrm{~F}_{10}$ with Various Compositions

20. Flow Boiling Heat Transfer Characteristics for Mixtures of R-114 and $\mathrm{C}_{4} \mathrm{~F}_{10}$ with Various Compositions

21. Comparison of Measured and Predicted Heat Transfer Coefficients for $26 \% \mathrm{C}_{4} \mathrm{~F}_{10}+74 \% \mathrm{R}-114$ at $\mathrm{T}_{\text {sat }}=130^{\circ} \mathrm{F}$

22. Comparison of Measured and Predicted Heat Transfer Coefficients for $26 \% \mathrm{C}_{4} \mathrm{~F}_{10}+74 \% \mathrm{R}-114$ at $\mathrm{T}_{\text {sat }}=150^{\circ} \mathrm{F}$

23. Comparison of Measured and Predicted Heat Transfer Coefficients 
for $26 \% \mathrm{C}_{4} \mathrm{~F}_{10}+74 \% \mathrm{R}-114$ at $\mathrm{T}_{\text {sat }}=170^{\circ} \mathrm{F}$

24. Comparison of Measured and Predicted Heat Transfer Coefficients for $51 \% \mathrm{C}_{4} \mathrm{~F}_{10}+49 \% \mathrm{R}-114$ at $\mathrm{T}_{\text {sat }}=130^{\circ} \mathrm{F}$

25. Comparison of Measured and Predicted Heat Transfer Coefficients for $51 \% \mathrm{C}_{4} \mathrm{~F}_{10}+49 \% \mathrm{R}-114$ at $\mathrm{T}_{\text {sat }}=150^{\circ} \mathrm{F}$

26. Comparison of Measured and Predicted Heat Transfer Coefficients for $51 \% \mathrm{C}_{4} \mathrm{~F}_{10}+49 \% \mathrm{R}-114$ at $\mathrm{T}_{\text {sat }}=170^{\circ} \mathrm{F}$

27. Comparison of Measured and Predicted Heat Transfer Coefficients for $66 \% \mathrm{C}_{4} \mathrm{~F}_{10}+64 \% \mathrm{R}-114$ at $\mathrm{T}_{\text {sat }}=130^{\circ} \mathrm{F}$

28. Comparison of Measured and Predicted Heat Transfer Coefficients for $66 \% \mathrm{C}_{4} \mathrm{~F}_{10}+34 \% \mathrm{R}-114$ at $\mathrm{T}_{\text {sat }}=150^{\circ} \mathrm{F}$

29. Comparison of Measured and Predicted Heat Transfer Coefficients for $66 \% \mathrm{C}_{4} \mathrm{~F}_{10}+34 \% \mathrm{R}-114$ at $\mathrm{T}_{\text {sat }}=130^{\circ} \mathrm{F}$

30. Comparison of Measured and Predicted Pressure Drop for $26 \% \mathrm{C}_{4} \mathrm{~F}_{10}+74 \% \mathrm{R}-114$ at $\mathrm{T}_{\text {sal }}=130^{\circ} \mathrm{F}$

31. Comparison of Measured and Predicted Pressure Drop for $26 \% \mathrm{C}_{4} \mathrm{~F}_{10}+74 \% \mathrm{R}-114$ at $\mathrm{T}_{\text {sat }}=150^{\circ} \mathrm{F}$

32. Comparison of Measured and Predicted Pressure Drop for $26 \% \mathrm{C}_{4} \mathrm{~F}_{10}+74 \% \mathrm{R}-114$ at $\mathrm{T}_{\text {sat }}=170^{\circ} \mathrm{F}$

33. Comparison of Measured and Predicted Pressure Drop for $51 \% \mathrm{C}_{4} \mathrm{~F}_{10}+49 \% \mathrm{R}-114$ at $\mathrm{T}_{\mathrm{sat}}=130^{\circ} \mathrm{F}$

34. Comparison of Measured and Predicted Pressure Drop for $51 \% \mathrm{C}_{4} \mathrm{~F}_{10}+49 \% \mathrm{R}-114$ at $\mathrm{T}_{\text {sal }}=150^{\circ} \mathrm{F}$

35. Comparison of Measured and Predicted Pressure Drop for $51 \% \mathrm{C}_{4} \mathrm{~F}_{10}+49 \% \mathrm{R}-114$ at $\mathrm{T}_{\text {sat }}=170^{\circ} \mathrm{F}$

36. Comparison of Measured and Predicted Pressure Drop for $66 \% \mathrm{C}_{4} \mathrm{~F}_{10}+34 \% \mathrm{R}-114$ at $\mathrm{T}_{\text {sal }}=130^{\circ} \mathrm{F}$

37. Comparison of Measured and Predicted Pressure Drop for $66 \% \mathrm{C}_{4} \mathrm{~F}_{10}+34 \% \mathrm{R}-114$ at $\mathrm{T}_{\text {sal }}=150^{\circ} \mathrm{F}$

38. Comparison of Measured and Predicted Pressure Drop for $66 \% \mathrm{C}_{4} \mathrm{~F}_{10}+34 \% \mathrm{R}-114$ at $\mathrm{T}_{\text {sat }}=170^{\circ} \mathrm{F}$ 


\section{NOMENCLATURE}
A Area
c Constant
C Correction factor
$C_{p} \quad$ Specific heat
$\mathrm{d}$ Inside diameter of tube; Differential operator
g Gravitational acceleration
G Mass flux
h Heat-transfer coefficient
$\mathrm{k}$ Thermal conductivity
$\mathrm{Nu} \quad$ Nusselt number $\left(=h \mathrm{~d}_{\mathrm{i}} / \mathrm{k}\right)$
P Pressure
Pr Prandtl number $\left(=C_{p} \mu / k\right)$
q Heat flux $(=Q / A)$
Q Heat input
Re Reynolds number $\left(\mathrm{Gd}_{i} / \mu\right)$
$T$ Temperature
$\mathrm{x} \quad$ Vapor quality
$z \quad$ Coordinate in direction of flow

\section{Greek}

$\begin{array}{ll}\alpha & \text { Void fraction } \\ \Delta & \text { Difference } \\ \lambda & \text { Latent heat of vaporization } \\ \mu & \text { Viscosity } \\ \sigma & \text { Surface tension } \\ \rho & \text { Density } \\ \nu & \text { Specific volume }\end{array}$

\section{Superscript}

n Exponent

\section{Subscript}

$\begin{array}{ll}\mathrm{cV} & \text { Convection } \\ \mathrm{F} & \text { Friction } \\ \mathrm{g} & \text { Vapor phase } \\ \mathrm{i} & \text { Inside diameter } \\ \mathrm{l} & \text { Liquid phase } \\ \mathrm{nb} & \text { Nucleate boiling } \\ \mathrm{nbf} & \text { Nucleate boiling factor } \\ \mathrm{npb} & \text { Nucleate pool boiling } \\ \mathrm{sf} & \text { Based on surface characteristics } \\ \mathrm{tpf} & \text { Two-phase factor }\end{array}$




\section{INTRODUCTION}

Recently, a number of studies [1-3] on forced convective flow boiling of mixtures of fluorinated carbon liquids have been conducted as part of the effort to improve the performance of heat pump systems. One of the characteristics of such mixtures is the variable temperature during phase change at constant pressure. This phenomena can be utilized to reduce the thermodynamic irreversibility in counter-current heat exchangers resulting in an increase in heat exchanger efficiency.

The flow boiling tests conducted at CUDO, on mixtures of $\mathrm{C}_{4} \mathrm{~F}_{10}$ and $\mathrm{R}-114$ provide useful information in selecting the replacement for the CFC liquid R-1 14 for gas-cooler heat exchangers. According to some experimental studies [4-7] for flow boiling using different mixtures, the heattransfer coefficient of mixtures is significantly lower than that of pure fluid. The results from this experimental study show a good agreement with this trend. This study indicates that the heattransfer coefficient of the mixture is strongly dependent on its composition and generally lower than the ideal value between the two pure liquids. The heat-transfer coefficient of the mixture is as much as $30 \%$ lower than the ideal values under the same flow and saturation conditions. References $[4,6]$ attribute the reduction in the heat-transfer coefficient to the mixture effects on nucleate boiling, non-ideal variations in physical properties and the heat transfer resistance in the vapor phase.

Convective flow boiling experiments were conducted at Cudo Technologies Ltd.(Cudo) for three different liquid mixture compositions of $\mathrm{R}-114$ and $\mathrm{C}_{4} \mathrm{~F}_{10}$. The experimental setup is described in detail in reference 1. Experiments were run for different saturation temperatures, heat fluxes, and mass flow rates so as to determine the heat transfer capability and pressure drop conditions. Also correlations are presented which provide a best fit prediction of the data. 


\section{Experimental Setup [8]}

Figure 1 illustrates the flow boiling system capable of operating at pressures from 0 to 350 psig and temperatures ranging from $50^{\circ} \mathrm{F}$ to $300^{\circ} \mathrm{F}$. Liquid from the storage tank is pumped by a variable flow gear pump at a predetermined flow rate. The liquid on exiting the pump passes through a filter-drier, a flow meter and a pre-heater before entering the test section. The preheater allows fine control of the inlet sub-cooled/saturation conditions by the use of a temperature controller. Before entering the test section the liquid is made to flow through a flow straightener consisting of a honeycomb section ahead of a converging cone which mates with no transition in geometry with the inlet of the test tube test section. The liquid on flowing through the test section takes up heat through phase change and exits as a liquid-vapor mixture. At the top and exit of the test section is a sight-glass tube allowing visualization of the exit flow. The exiting liquid-vapor mixture next passes through a cyclone separator. The settled liquid passes through a flow meter before draining into the storage tank. The vapor is condensed in the condenser. The condensate passes through a flow meter before draining into the storage tank.

The test section [8] is primarily a 0.785 inch ID and 1.75 inch OD copper tube about 10 feet in length. At each end of the test section is a welded copper flange for mating with the other parts of the setup. The test section is divided into ten zones, each 11.5 inches long and thermally isolated from the adjacent zones by a $1 / 2$ inch wide groove. Figure 2 illustrate the positioning of the thermocouples and pressure transducers [8]. The temperature profile in each zone is provided by seven thermocouples distributed longitudinally and azimuthally. Five thermocouples are imbedded in the $1 / 2$ inch thick wall so as to be $1 / 8$ inch from the inner tube wall. A sixth thermocouple is positioned midway in the fluid stream and a seventh thermocouple is clamped to the external heater. All the thermocouples were calibrated with their respective wiring harnesses in conjunction with the data acquisition. The uncertainty in the temperature measurement is \pm 1 ${ }^{\circ} \mathrm{F}[8]$.

Seven pressure transducers were positioned along the length of the tube [8], see figure 1. One at the entrance of the tube and one at the exit measured the absolute pressures. Additionally, five 
transducers are placed equidistantly within the test region to provide the differential pressure relative to the pressure at the inlet. The pressure taps are located in zones $1,3,5,7,9 \& 10$ such that the distance between two consecutive taps is $2^{\prime}$ except for the tap in zone 10 which is at a distance of $22.5^{\prime \prime}$ from that in zone 8 . The differential pressure transducers provide the pressure drop information along the length of the tube. The uncertainty in the differential pressure is \pm $0.05 \mathrm{psi}$ and in the absolute pressure \pm 1 psia [8].

Each zone of the test section is wrapped with heaters capable of providing over 60,000 $\mathrm{Btu} / \mathrm{hr} . \mathrm{ft}^{2}$ to the outer surface of the tube [8]. The heater arrangement and the test section was thermally insulated from the environment by three layers of high temperature Fiberfrax ${ }^{r}$ insulation $\left(\mathrm{k}=0.65 \mathrm{Btu} . \mathrm{in} / \mathrm{hr} . \mathrm{ft}^{2}{ }^{\circ} \mathrm{F} @ 500^{\circ} \mathrm{F}\right)$ and an aluminum foil radiation heat shield. The uncertainty in the heat flux is $\pm 175 \mathrm{btu} / \mathrm{hr} . \mathrm{ft}^{2}[8]$.

SCR power supplies regulate the power to the heaters by varying the phase angle on each AC cycle proportional to the control signal [8]. This method of applying power provides a continuous uniform heat flux with fine control. A temperature controller, monitors the temperature of the outer heater surface. In the event of an overshoot in the temperature of the surface, an alarm disconnects the power to the heaters via relays. In the design of this experiment the number of devices to be measured included over eighty thermocouples, seven pressure transducers, six flow meters, and ten power transducers. To expedite on-site data analysis a PCbased data acquisition was used to measure, perform the necessary calculations, display system conditions and store the data. To reduce the uncertainty in the overall measurement, the devices were calibrated using standards in conjunction with the data acquisition.

\section{Experimental Procedure [8]}

With reference to figure 1, the variable flow pump is started and the flow rate adjusted to the desired predetermined value. The pre-heater is powered to control the inlet coolant entry temperature. The antifreeze is circulated through the cooling coils in the condenser at a flow rate such that the saturation pressure at the inlet is maintained at the desired value. Excessive cooling 
may cause a low pressure at the pump inlet and lead to cavitation. Too little cooling will cause the pressure to rise and force the liquid at the inlet to the test section to be sub-cooled. A heater on the storage tank in conjunction with a cooling coil in the storage tank is used as an accessory control for inlet temperature and pressure, see figure 3.

The heaters on the test section are turned on and the power is ramped up in steps to the desired heat flux, making certain that steady-state conditions are reached on the surface of the heater. The data acquisition is set up to measure the heat input and temperature of the heaters. With this information the heat loss from the test section to the ambient and end-plates was calculated from the earlier heat loss calibration [8]. The balance of the heat is necessarily taken up by the fluid. Both the heat loss and the heat flux into each zone are displayed simultaneously by the data acquisition. Hence, it is possible to determine and set in real time the heat flux conditions. Steady-state thermal flow-field conditions are determined by monitoring the temperatures of the various regions of the system. When the temperature transients die down (1 ${ }^{\circ} \mathrm{F}$ or less change over a 5 minute period), the system is assumed to be in thermal equilibrium. At this juncture the:

- temperatures at various locations

- system pressure and region differential pressures

- power input to the heaters

- flow meter readings

- flow field observations

are noted down and collected by the data acquisition unit.

The above process is followed for a step-wise increase in heat flux until CHF conditions are imminent. At this juncture the heat flux is further increased so as to span the L/D ratios for the CHF study. In regions where CHF occurs the heat flux is reduced so as to maintain the wall temperatures approximately equal to the upstream zone to reduce any heat losses. This is because at $\mathrm{CHF}$ the heat-transfer coefficient drops significantly and the wall temperature rises correspondingly. To prevent too high a temperature in the particular zone the heat flux must be 
dropped.

At the end of the experimentation all heaters are turned off. The antifreeze is pumped through the heat exchanger in the storage tank. This creates a low pressure zone in the storage tank (by condensing the vapor). This induces all the vapor and the liquid in the rest of the loop to collect in the storage tank. Once the system has cooled down, all power to the loop is disconnected and the storage tank is isolated from the rest of the system by closing the valves. Small leaks do exist in the flow loop and isolation reduces loss of freon over periods of no operation.

For each fluid at a predetermined boiling temperature and mass flux, the zone heat flux is gradually increased, (i.e. a constant heat flux was applied to successive zones in the direction of flow) until CHF conditions are reached in the exit heat zone. This was evidenced by a sharp rise in wall temperature at the last heated zone. As the critical condition was reached, the electrical power input to that particular zone was cut off or reduced.

In all runs undertaken, the flow at the inlet was slightly sub-cooled, the saturation temperature was maintained constant, and the surface heat flux was varied. The range of different coolant flow rates was then repeated at this surface heat flux. Once the surface heat fluxes between 5000 and $35000 \mathrm{Btu} / \mathrm{hr} / \mathrm{ft}^{2}$ were covered, a new saturation temperature was set and the entire procedure repeated. Thus, essentially, the coolant flow rate was the parameter most frequently varied, the surface heat flux the second most frequently varied, and the saturation temperature the least frequently varied. (Owing to the thermal mass of the fluid in the system, approximately $150 \mathrm{lbs}$, change in saturation conditions needed about three hours to reach steady state).

During the above tests the saturation temperature and pressure of the system are maintained steady at the predefined values. For each saturation temperature and each mass flow rate setting, the run provided data of the form:

- Surface superheat temperature versus heat flux

- Saturation temperature conditions 
- Pressure drop profile

- Flow field observations

- Pressure at the inlet of the test section

- Pressure drop along the test section with intervals of $2,4,6,8$ and $9.88 \mathrm{ft}$ from the inlet

- Room temperature

- Wall and in-stream temperatures in each of the 10 zones

- Gross heat input, heat loss and heat flux to each of the 10 zones

- Coolant flow rate and temperature at inlet of the test section

- Water flow rate and temperatures at inlet and outlet of the condenser

Owing to the heat losses to the flanges at either end of the test section the uncertainty in the heat input calculation is difficult to determine. The temperature measurement in section 6 indicated an inexplicable departure from the rest of the test section. The difference was attributed to the temperature compensation within the data acquisition. Hence for purpose of analysis the heat transfer data from sections 1,6 and 10 are not presented or used for correlations in this study.

\section{Experimental Program}

The range of parameters covered by these experiments are listed below:

Saturation temperatures, ${ }^{\circ} \mathrm{F}$ :

$$
T=130,150,170
$$

Liquid mass fluxes, $\mathrm{lbm} / \mathrm{hr}-\mathrm{ft}^{2}$ :

$$
G=0.5,0.75,1.0^{*} 10^{6}
$$

Surface heat fluxes, Btu/hr/ $\mathrm{ft}^{2}$

$$
q=0.5,1.0,1.5 * 10^{4}
$$

Composition

Mass fraction of $25 \%$ to $75 \%$ of $\mathrm{C}_{4} \mathrm{~F}_{10}$ in $\mathrm{R}-114$ 


\section{Heat Transfer Measurement and Analysis}

The heat transfer characteristics are presented in the form of the heat flux $\mathbf{q}$ versus the wallto-instream temperature difference $\Delta \mathrm{T} . \Delta \mathrm{T}$ is the temperature difference between the average inside-wall temperature and the instream temperature. The average inside-wall temperature was determined by taking the average thermocouple measurements from five different locations on the section wall [8], see figure 2, and correcting them for wall thickness to determine the internal wall temperature. Five thermocouples were imbedded in the walls of each section. Owing to the intermittent heating and cooling of the test section some of the thermocouples would not make good contact with the wall. The discrepancy was evident on study of the temperature field in the section in question. In such cases the incorrect temperature was not considered in the calculations. Generally at the end of the testing the thermocouple attachments were fixed.

Convective flow boiling experiments were conducted at Cudo Technologies Ltd. (Cudo) for the mixture of $\mathrm{C}_{4} \mathrm{~F}_{10} / \mathrm{R}-114$ with three different compositions. The three compositions are $26 \%$, $51 \%$ and $66 \%$ of $\mathrm{C}_{4} \mathrm{~F}_{10}$ by mass fraction. Figures 4 and 5 show the saturation pressure and liquid density versus temperature for the mixture with three different compositions. Experiments were run for different saturation temperatures, heat fluxes, and mass flow rates to so as to determine the heat transfer capability and pressure drop conditions.

\section{Heat Transfer Correlation}

Convective flow boiling of mixtures is much more complicated than that for pure liquids. The complication is due to the mixture effects on nucleate boiling and the heat transfer resistance in the vapor phase. During the past decade, some correlation equations for flow boiling of mixtures have been proposed [2-7]. However, most of these correlations are not applicable to other mixtures because they also include some empirical relations or coefficients which only can be used for specific mixtures and compositions.

Pais et al. [9] performed an experimental study of flow boiling for pure liquids $R-114, \mathrm{C}_{4} \mathrm{~F}_{10}$ and $\mathrm{C}_{4} \mathrm{~F}_{8}$. They compared their heat transfer results with an asymptotic model proposed by 
Steiner and Taborek [10]. The agreement between the measured and predicted heat-transfer coefficients is very good and the differences are within $25 \%$ for three different liquids. This implies that the asymptotic model is a general correlation and can be applied to different liquids.

In this study, the flow boiling heat-transfer coefficient for the mixture $\mathrm{C}_{4} \mathrm{~F}_{10} / \mathrm{R}-114$ was assumed to be approximately equal to the mean value of that for pure liquids. Under this assumption, the heat-transfer coefficient for the mixture was correlated with its composition in mass fraction. Here, the asymptotic model [9] was used to calculate the heat-transfer coefficients for the pure liquids. Likewise, the heat transfer correlation for the mixture is written in the form,

$$
h_{(\text {midare })}=X h_{\left(C_{4} F_{10}\right)}+(1-X) h_{(R-114)}
$$

where, the heat-transfer coefficients for the pure liquids $\mathrm{C}_{4} \mathrm{~F}_{10}$ and $\mathrm{R}-114$ are calculated using the thermophysical and transport properties of each pure liquid based on the same mass flux for the mixture.

The asymptotic model proposed by Steiner and Taborek [10] for pure liquids was strongly recommended by Webb and Gupte [11]. Like the superposition model [12], the asymptotic model relies on combining a forced convection component and a nucleate pool boiling component. However, the asymptotic model uses a power relationship of the form [9],

$$
h^{n}=\left[h_{n b}^{n}+h_{c v}{ }^{n}\right]^{1 / n}
$$

or

$$
h^{n}=\left[\left(h_{n p b} C_{n b j}\right)^{n}+\left(h_{l} C_{\text {qf }}\right)^{n}\right]^{1 / n}
$$

here, 
$h_{\text {npb }} \quad$ is the local nucleate pool boiling coefficient. The coefficient itself can originate from any predictive method or data. Thus, it leaves a great freedom of choice.

$\mathrm{C}_{\mathrm{nbf}}$ is a correction factor to $h_{\mathrm{npb}}$, which compensates for differences between the pool and the flow conditions.

$h_{1} \quad$ is the local convective heat-transfer coefficient for single-phase liquid flow.

$\mathrm{C}_{\mathrm{pp}} \quad$ is the two-phase flow multiplier to the convective component $h_{\mathrm{l}}$, accounting for enhancement of the coefficient in the liquid-vapor mixture.

Steiner and Taborek [10] compared the predictive ability of their correlation with Chen [12], Shah [13], and Gungor and Winterton [14,15] for ethanol, ammonia, and R-12. Their [10] asymptotic form avoids the abrupt transition between the nucleate boiling and convective regions and in general they showed that their correlation is significantly better than the other correlations.

In order to use this approach one must have experimentally determined values for the two components $h_{n b}$ and $h_{c v}$ in Equation 2 or use those published by Steiner and Taborek [10] (determined from a 13,000 point data base covering a variety of fluids). We chose to use the experimental approach, and only used the convective multiplier $\mathbf{C}_{\mathrm{tpf}}$, which accounts for enhancement due to the liquid-vapor mixture. Thus, Equation 3 can be rewritten as,

$$
h^{n}=\left[h_{n b}{ }^{n}+\left(h_{l} C_{\text {甲Tf }}\right)^{n}\right]^{1 / n}
$$

From Taborek's [10] analysis, $\mathbf{C}_{\mathrm{tpr}}$ was derived as a function of the vapor fraction $\boldsymbol{x}$ and the liquidvapor density ratio $\rho_{\mathrm{l}} / \rho_{\mathrm{g}}$.

$$
C_{\text {tqf }}=\left[(1-x)^{1.5}+1.9 x^{0.6}\left(\frac{\rho_{l}}{\rho_{g}}\right)^{0.35}\right]^{1.1}
$$

The correlation was developed and validated by Taborek [10] using a large data bank containing 15 fluids from 34 sources. 


\section{Single-Phase Liquid Flow}

The forced convection component $h_{1}$ for pure liquids in Equation 4 was determined experimentally at Cudo by running subcooled liquid through the copper tube for $\mathrm{C}_{4} \mathrm{~F}_{10}$ and R-114, and then correlating that data with a Dittus-Boelter type equation as given below.

$$
N u=\frac{h_{l} d_{i}}{k}=c \operatorname{Re}^{0.8} \operatorname{Pr}^{0.4}
$$

In the above equation, the coefficient $c$ is equal to 0.023 for singe-phase flow inside a smooth cylindrical tube. For the tube we used in our experiment, $c$ was determined from single-phase liquid flow tests for the three different liquids. The results are shown in figures 6 and 7. Note, that when $c=0.028$ both fluids $\mathrm{C}_{4} \mathrm{~F}_{10}$ and R-1 14 correlate quite well with equation 6 .

\section{Nucleate Pool Boiling}

The nucleate pool boiling term $h_{n b}$ for pure liquids in equation 4 was also determined at Cudo by running very low flow in the tube and only using heat flux data from the bottom two zones 2 and 3. The nucleate boiling correlation for flow boiling presented by Rohsenow [16] was applied to the experimental data,

$$
\frac{C_{p_{l}} \Delta T}{\lambda P_{l}^{1.7}}=C_{55}\left(\frac{Q / A}{\mu_{l} \lambda} \sqrt{\frac{\sigma}{g \Delta p}}^{0.33}\right.
$$

The coefficient $\mathbf{C}_{\mathbf{s r}}$ is dependent on the wall-fluid material characteristics and was determined from the pool boiling experiments.

The experimental results for the two pure fluids are plotted in figures 8 and 9 using the Rohsenow [16] correlation equation 7 with a logarithmic scale. Here, again the two fluids correlated the same, with an experimentally determined value of $C_{\mathrm{sI}}=0.0038025$. It should be noted that in this coordinate system, the lower the line the higher the nucleate-boiling coefficient. After the coefficient $\mathbf{C}_{\mathbf{s}}$ is determined, the correlation of the nucleate boiling term $\mathbf{h}_{\mathbf{n b}}$ for pure 
liquids in equation 4 can be obtained from equation 7 in the form,

$$
h_{n b}=\frac{Q}{A \Delta T}=\left(\frac{k_{l}^{1.7}}{C_{s} \mu_{l}^{1.7} C_{P_{l}}^{0.7}}\right)^{3} \mu_{l}\left(\frac{\Delta T}{\lambda}\right)^{2} \sqrt{\frac{g \Delta p}{\sigma}}
$$

The fact that the predictive correlation is the same for the two different pure liquids does not mean that the nucleate boiling heat-transfer coefficient is the same. The difference in the physical properties of the fluids will result in different heat-transfer coefficients for the same temperature level and heat flow.

\section{Pressure Drop Correlation}

For two-phase flows inside a tube, the gravitational, frictional, and acceleration components contribute to the pressure drop and all three must be taken into account. Two different models are generally used in predicting pressure drop for two-phase flows. They are the homogeneous and the separated flow models. The homogeneous model considers the two-phase flow as a single-phase flow possessing mean fluid properties. On the other hand, the separated flow model considers the two-phase flow to be artificially segregated into two streams; one of liquid and one of vapor. In the homogeneous model, each stream is assumed to travel at a mean velocity. In the case when a very large percentage of the two-phase flow inside a tube is annular in nature, the application of a separated flow model will yield results better than those obtained by the homogeneous model.

In this study, the separated flow model presented by Martinelli-Nelson $[17,18]$ was adopted for pressure drop prediction for pure liquids. In the Martinelli-Nelson model [17,18], the two phases are assumed to be separate. The pressure drop for pure liquids is defined by Equation (246) of Collier's [19] as given below,

$$
-\left(\frac{d P}{d z}\right)=-\left(\frac{d P}{d z}\right)_{F}+G^{2} \frac{d}{d z}\left[\frac{x^{2} v_{g}}{\alpha}+\frac{(1-x)^{2} v_{l}}{(1-\alpha)}\right]+g\left[\alpha \rho_{g}+(1-\alpha) \rho_{l}\right]
$$


The three terms contribute to the friction, acceleration, and gravitational pressure drop, respectively. These three pressure drop components are calculated using the Martinelli-Nelson model $[17,18]$ in each section of the test tube. In the experiment, pressure drops with intervals of $2,4,6,8$ and $9.88 \mathrm{ft}$ from inlet of the test section were measured by five differential pressure transducers. The pressure readings from the differential pressure transducers were corrected for elevation of the pressure tap lines. The corrected pressure drop was then converted to the equivalent elevation of the liquid column at the operating saturation temperature.

In this study, the pressure drop for the mixture of $\mathrm{C}_{4} \mathrm{~F}_{10} / \mathrm{R}-114$ was assumed to be approximately equal to the mean value of the pure liquids. Under this assumption, the pressure drop for the mixture was correlated with liquid composition based on mass fraction by the following equation,

$$
\Delta P_{(\text {micare })}=X \Delta P_{\left(C_{4} F_{8}\right)}+(1-X) \Delta P_{(R-114)}
$$

\section{RESULTS AND DISCUSSION}

\section{Heat Transfer}

The heat transfer characteristics are presented in the form of the heat flux $q$ versus the wallto-instream temperature difference $\Delta T$. Data for the pure liquids $C_{4} F_{10}$ and $R-114$ are also compared with the results for the mixtures in Figs. 11, 14 and 17. $\Delta \mathrm{T}$ is the temperature difference between the average inside-wall temperature and the instream temperature. The average inside-wall temperature was determined by taking the average thermocouple measurements from five different locations on the section wall, see figure 2 [8], and correcting them for wall thickness to determine the internal wall temperature. From the results shown in Figures 10 to 18 , the following trends are observed:

- $\Delta \mathbf{T}$ increased with increase in heat flux $q$.

- As the saturation temperature increased, smaller $\Delta \mathbf{T}$ were obtained.

- $\Delta \mathrm{T}$ for the pure liquids $\mathrm{C}_{4} \mathrm{~F}_{10}$ and $\mathrm{R}-114$ are smaller than for the mixtures, this implies that $\mathrm{C}_{4} \mathrm{~F}_{10}$ and R-114 provide higher heat-transfer coefficients than the mixtures. $\Delta \mathrm{T}$ for the 
mixtures do not always fall in between that for the pure liquids of $\mathrm{C}_{4} \mathrm{~F}_{10}$ and $\mathrm{R}-114$

- The mixtures dry out earlier than R-114. In other words, the mixtures support lower critical heat flux (CHF) levels.

- The liquid mass flux does not have a strong effect on the heat-transfer coefficient.

Figures 19 and 20 showed the average heat transfer coefficients for the mixtures of $\mathrm{C}_{4} \mathrm{~F}_{10} / \mathrm{R}$ 114 with various compositions. Scattered data at a particular composition and heat flux are correspondent to different zones in the test section. It was found that the heat-transfer coefficients of the mixtures were strongly dependent on its composition and generally lower than the ideal value between the two pure liquids. The heat-transfer coefficient of the mixture is as much as $50 \%$ lower than the ideal values at the composition of $26 \%$ of $\mathrm{C}_{4} \mathrm{~F}_{10}$.

Figures 21 to 29 showed the comparison between the predicted and measured flow boiling heat-transfer coefficients for the mixture of $\mathrm{C}_{4} \mathrm{~F}_{10} / \mathrm{R}-114$ at 130,150 and $170^{\circ} \mathrm{F}$ with heat fluxes of $5,000,10,000$ and $15,000 \mathrm{Btu} / \mathrm{hr} / \mathrm{ft}^{2}$. The liquid mass flux was varied from $0.5 * 10^{6}$ to $1.0^{*} 10^{6}$ $\mathrm{lbm} / \mathrm{hr} / \mathrm{ft}^{2}$. The predicted values were based on the mean predicted values from the pure liquids and were calculated using the correlation equation 4 . An exact match would follow the 45-degree solid line. Note, the correlation predicts the experimental results fairly. In general, the correlation consistently over-predicts the experimental data with differences of up to $80 \%$. This implies that the mean form of the correlation equation 4 does not provide a good prediction capability of the heat transfer. Murata et al. [4] concluded that the reduction in heat-transfer coefficient of mixtures is attributed to the mixture effects on nucleate boiling and to sensible heating of the vapor phase accompanying rise of saturation in the flow direction.

\section{Pressure Drop}

Figures 30 to 38 present the comparison between the predicted and measured pressure drop along the test section for the mixtures of $\mathrm{C}_{4} \mathrm{~F}_{10} / \mathrm{R}-114$. The figures indicate that the MartinelliNelson model $[17,18]$ consistently and slightly over predicts the measured pressure drop. It seems agree with Murata's [4] conclusion that the pressure drop for mixtures is approximately 
equal to that for the equivalent pure fluid.

\section{CONCLUSIONS}

Forced convection, pool and flow boiling tests were run using mixtures of $\mathrm{R} 114$ and $\mathrm{C}_{4} \mathrm{~F}_{10}$ to determine the heat-transfer coefficient, $\mathrm{CHF}$ and pressure drop. A correlation for $\mathbf{h}$ based on a flow convective and pool boiling model is presented. The dryout length under conditions of $\mathrm{CHF}$ increased with heat flux and reduction in flow rate. A pressure drop correlation, based on a separated flow model, slightly but consistently over-predicted the measured results.

\section{ACKNOWLEDGEMENTS}

This work was performed for Martin Marietta Energy Systems, Inc., under contract \# 11K-NR559C. Thanks are due to Dr. Shin Park, Andy Szady, David Lannom and Prof. Louis Chow, for their technical help and discussions. Thanks are also due to Jon Curless, Gregory Mayes, Matthew McMillin, Tom Nett, E.B. Yates, and Hollywood Haley for their assistance at various stages of the project. 


\section{T LRENCES}

Hihara, E., Tanida, K., and Saito, T., Forced Convective Boiling Experiments of Binary Mixtures, JSME Int. J., Vol. 32, pp. 98-106, 1989.

Mishra, M.P., Varma, H.K., and Sharma, C.P., Heat Transfer Coefficients in Forced Convection Evaporation of Refrigerants Mixtures, Lett. Heat Mass Transfer, Vol. 8, pp. $127-136,1981$.

Singal, L.C., Sharma, C.P., and Varma, H.K., Heat Transfer Correlations for the Forced Convection Boiling of R12-RI3 Mixtures, Int. J. Refrigeration, Vol. 7, pp. 278-284, 1984. Murata, K. and Hashizume, K., Forced Convective Boiling of Non-azeotropic Refrigerant Mixtures Inside Tubes; Journal of Heat-Transfer, Vol. 115, pp. 680-689, August 1993.

Takamatsu, H., Momoki, S. and Fuji, T., A Correlation for forced convective boiling heat transfer of Non-azeotropic refrigerant mixture of HCFC22/CFC114 in a horizontal smooth tube, Int. J. Heat Mass Transfer, Vol. 36, No. 14, pp. 3555-3563, 1993. Jung, D.S., McLinden, M., Radermacher R. and Didion, D., Horizontal Flow Boiling Heat Transfer Experiments with a Mixture of R22/R114, Int. J. Heat Mass Transfer, Vol. 32, No. 1, pp. 131-145, 1989.

: Jung, D.S., McLinden, M., Radermacher R. and Didion, D., A Study of Flow Boiling Heat Transfer with Refrigerant Mixtures, Int. J. Heat Mass Transfer, Vol. 32, No. 9, pp. 1751$1764,1989$.

3. Pais, M.R., Chang, M.J., and Sehmbey, M.S., 1994, Design, construction and testing of an experiment 10 measure the flow boiling heat transfer and pressure drop in a vertical lube, Rep. \# MMES-CTL-NR559-1, Lexington, KY.

9. Chang, M.J., Pais, M.R., Park, S., and Szady, A., 1994, Flow boiling heat transfer characteristics of mixtures of $R-114$ and C $+F 10$ in a verticle tube, 1994 ASME WAM, HTD-Vol. 301, pp. 85-92.

10. Steiner, D. and Taborek, J., Flow Boiling Heat Transfer in Vertical Tubes Correlated by An Asymptotic Model, Heat Transfer Engineering, Vol. 13, No. 2, pp. 43-69, 1992.

11. Webb, R. L. and Gupte, N. S., A Critical Review of Correlations for Convective vaporization in Tubes and Tube Banks, Heat-Transfer Engineering, Vol. 13, No. 3, 1992. 


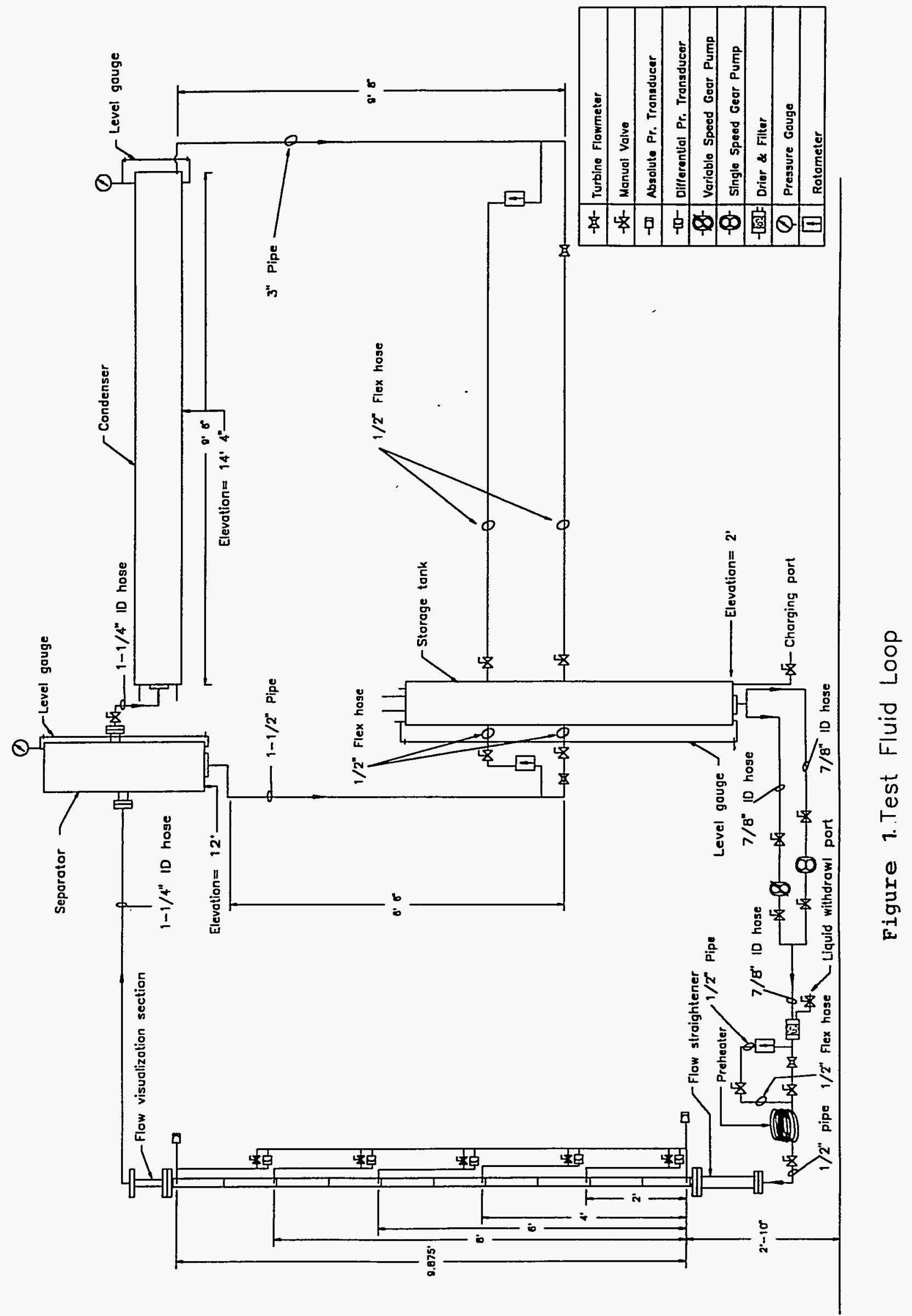




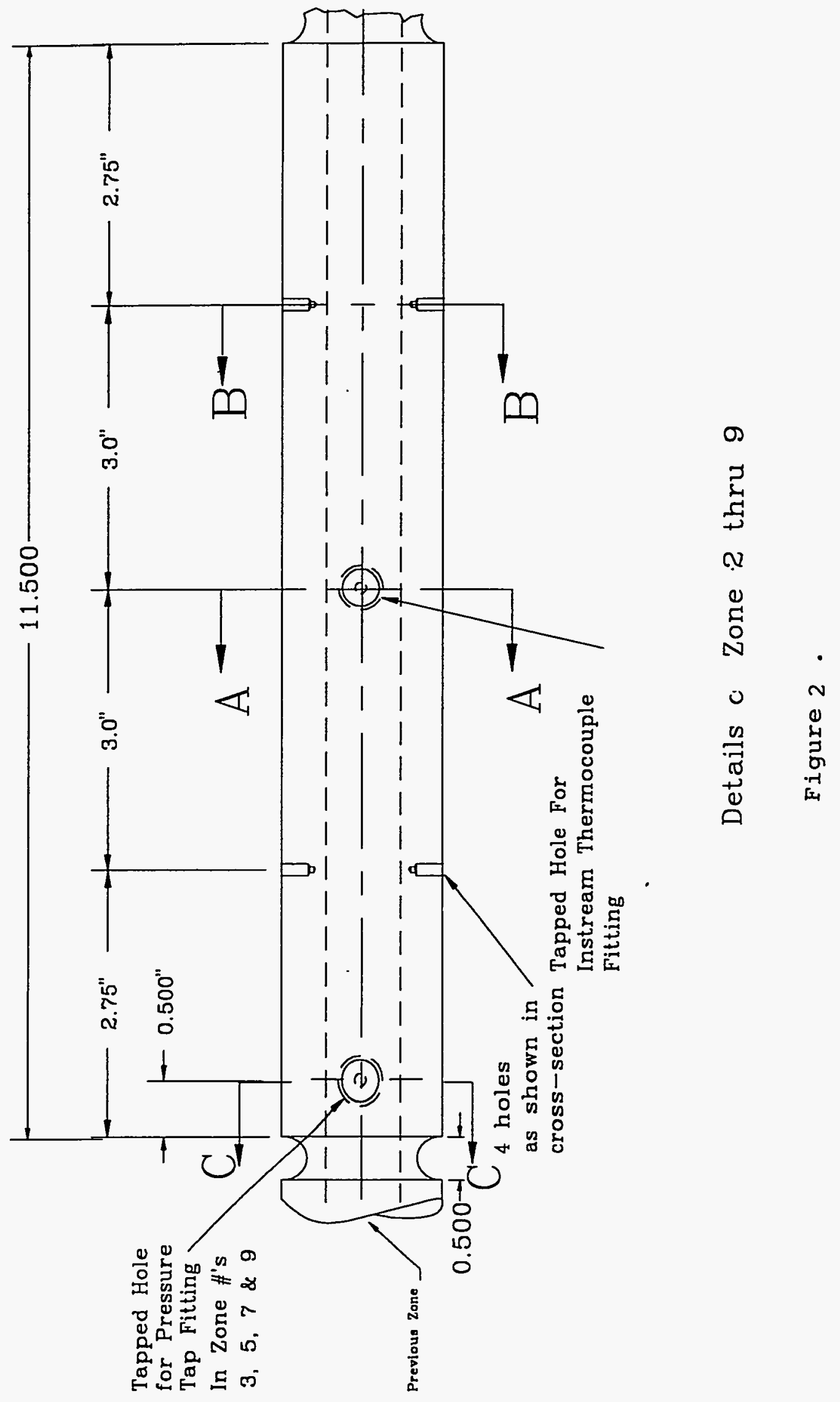



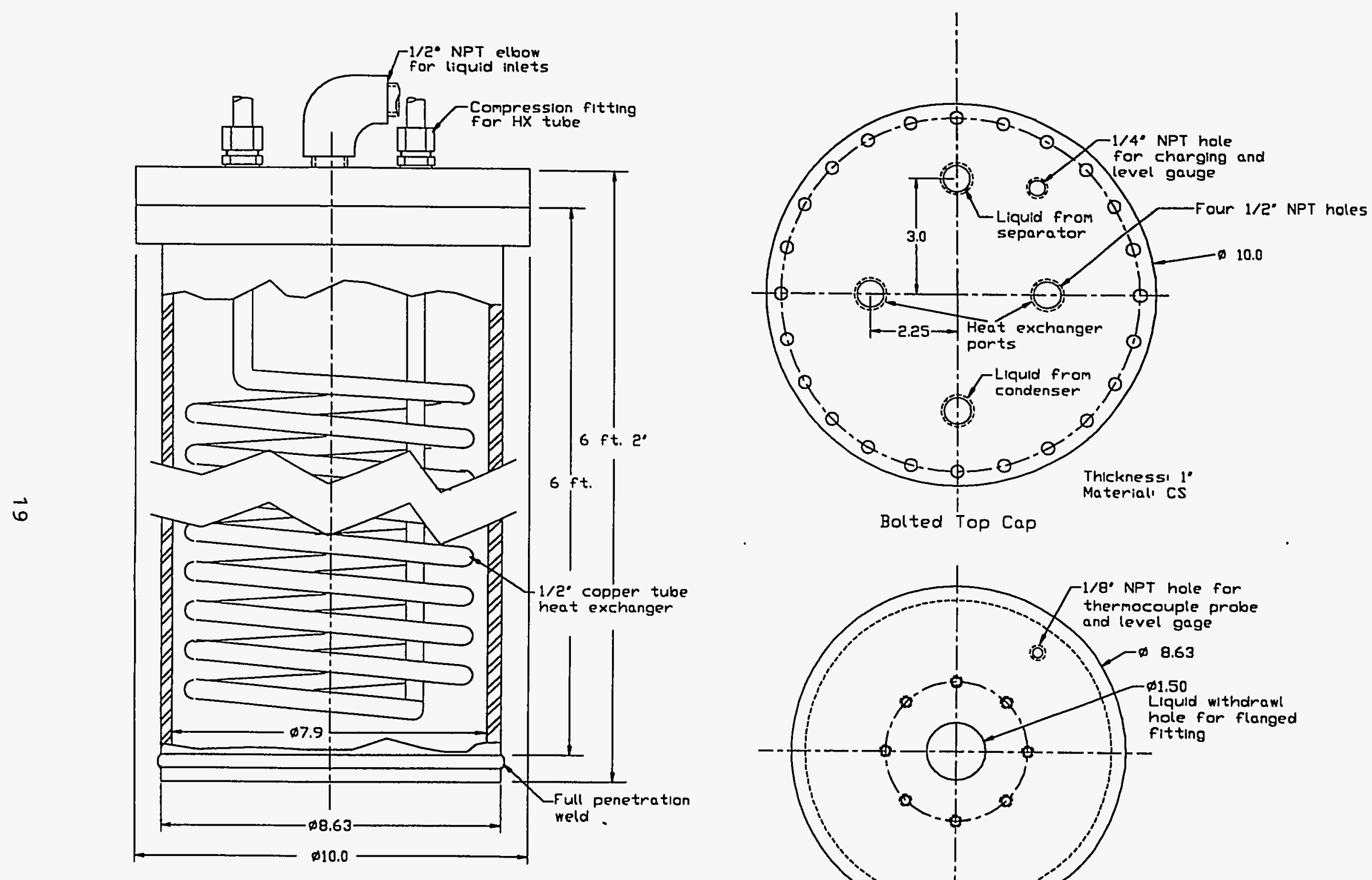

Effective internal volume $=3500$ cu.In. appx.

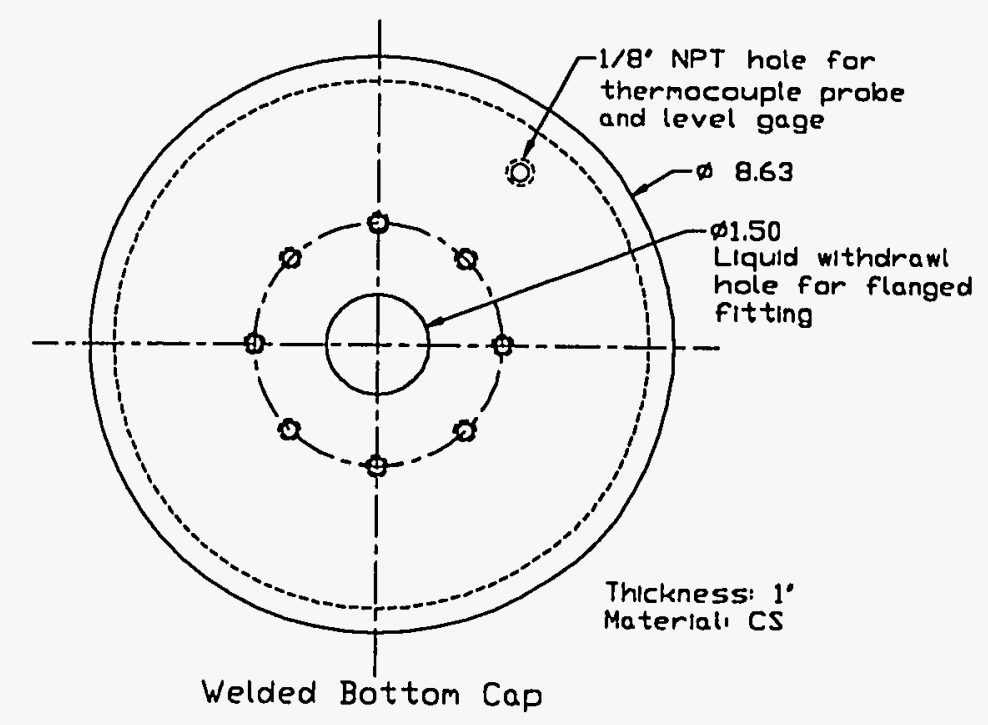

Storage Tank- Assembly and Caps

Figure 3. 


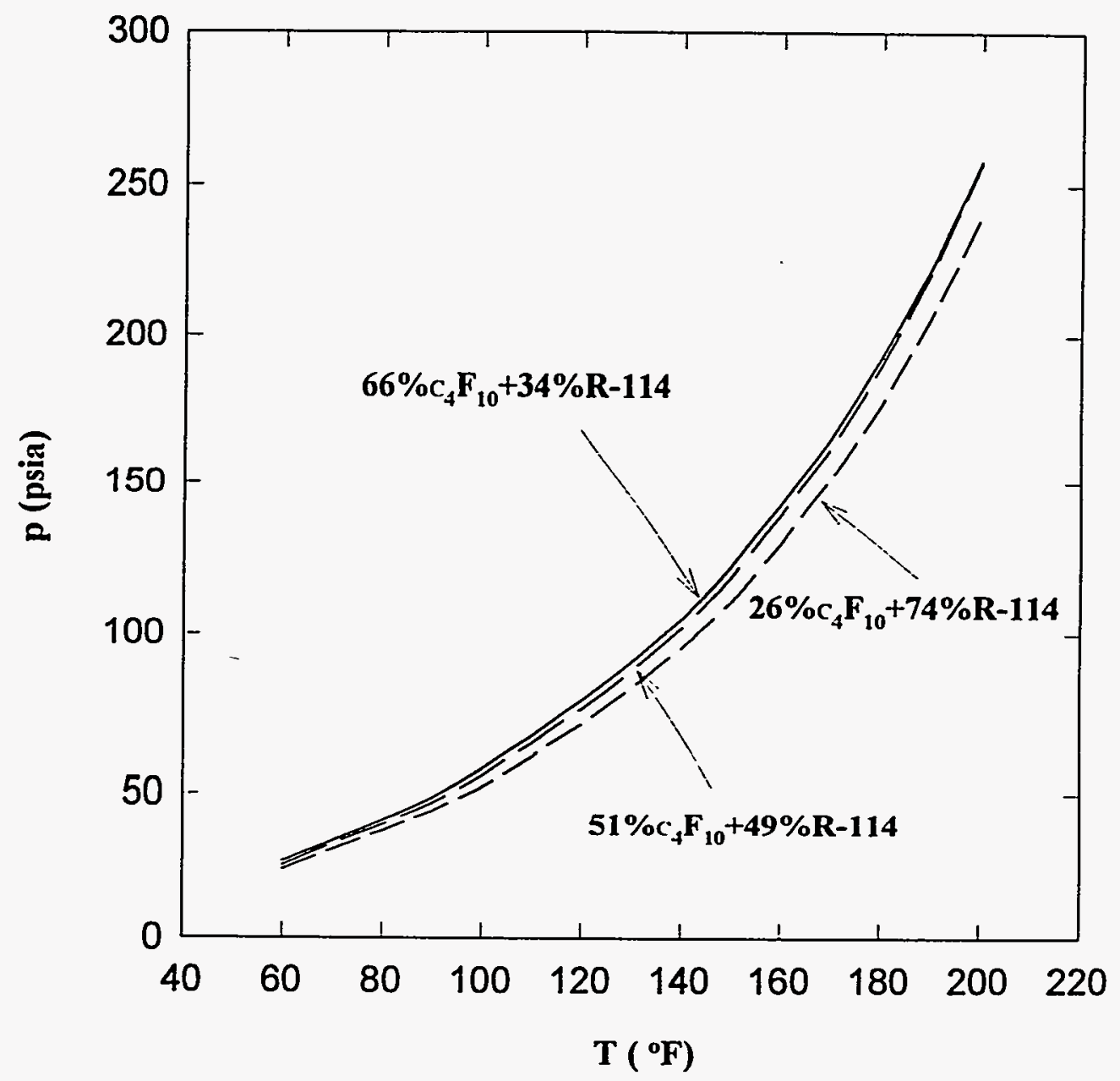

Figure 4. Saturation Pressure vs Temperature for Different Compositions of $\mathrm{C}_{4} \mathrm{~F}_{10}$ and R-114 


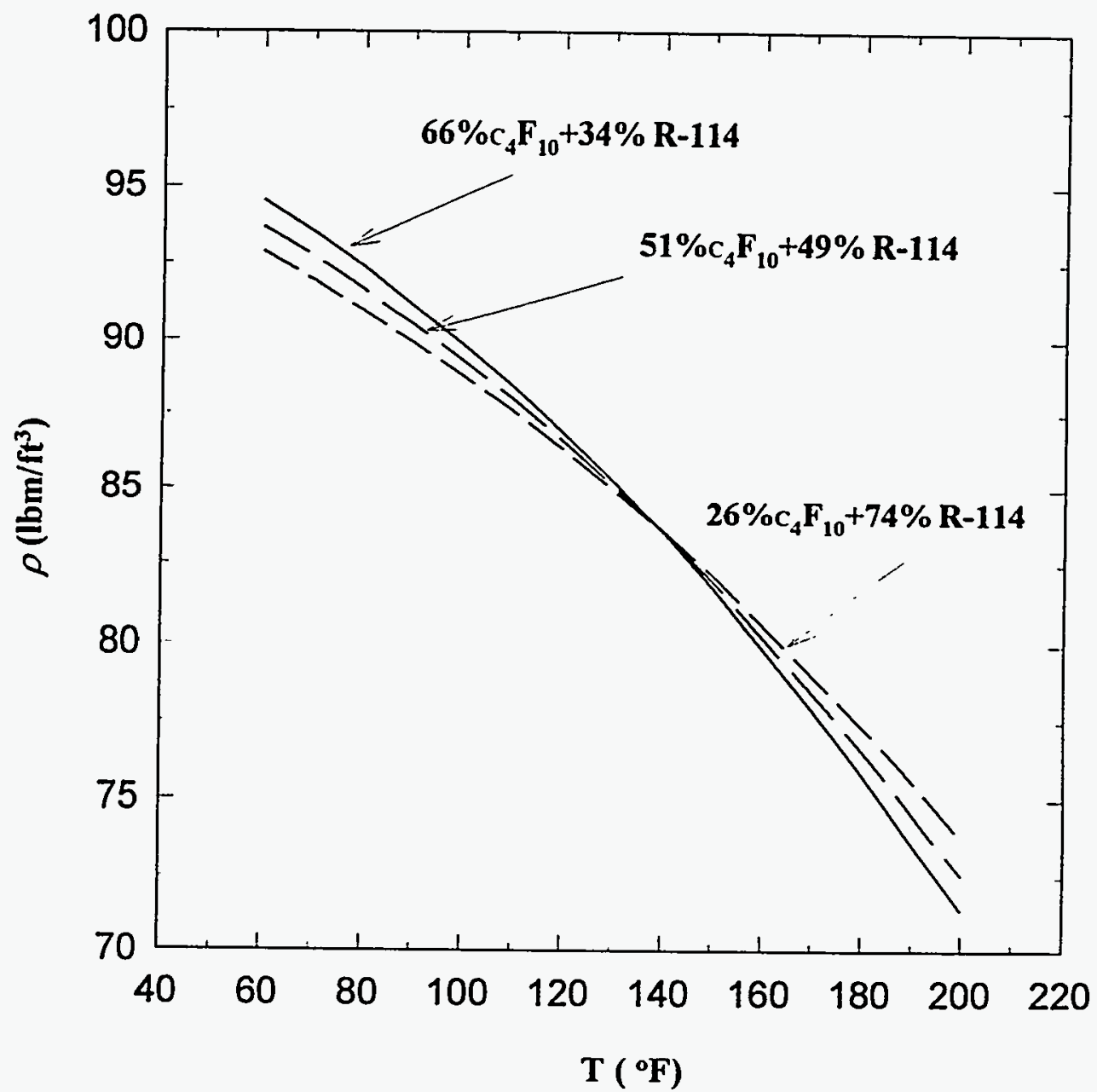

Figure 5. Liquid Density vs Temperature for Different Compositions of $\mathrm{C}_{4} \mathrm{~F}_{10}$ and R-114 


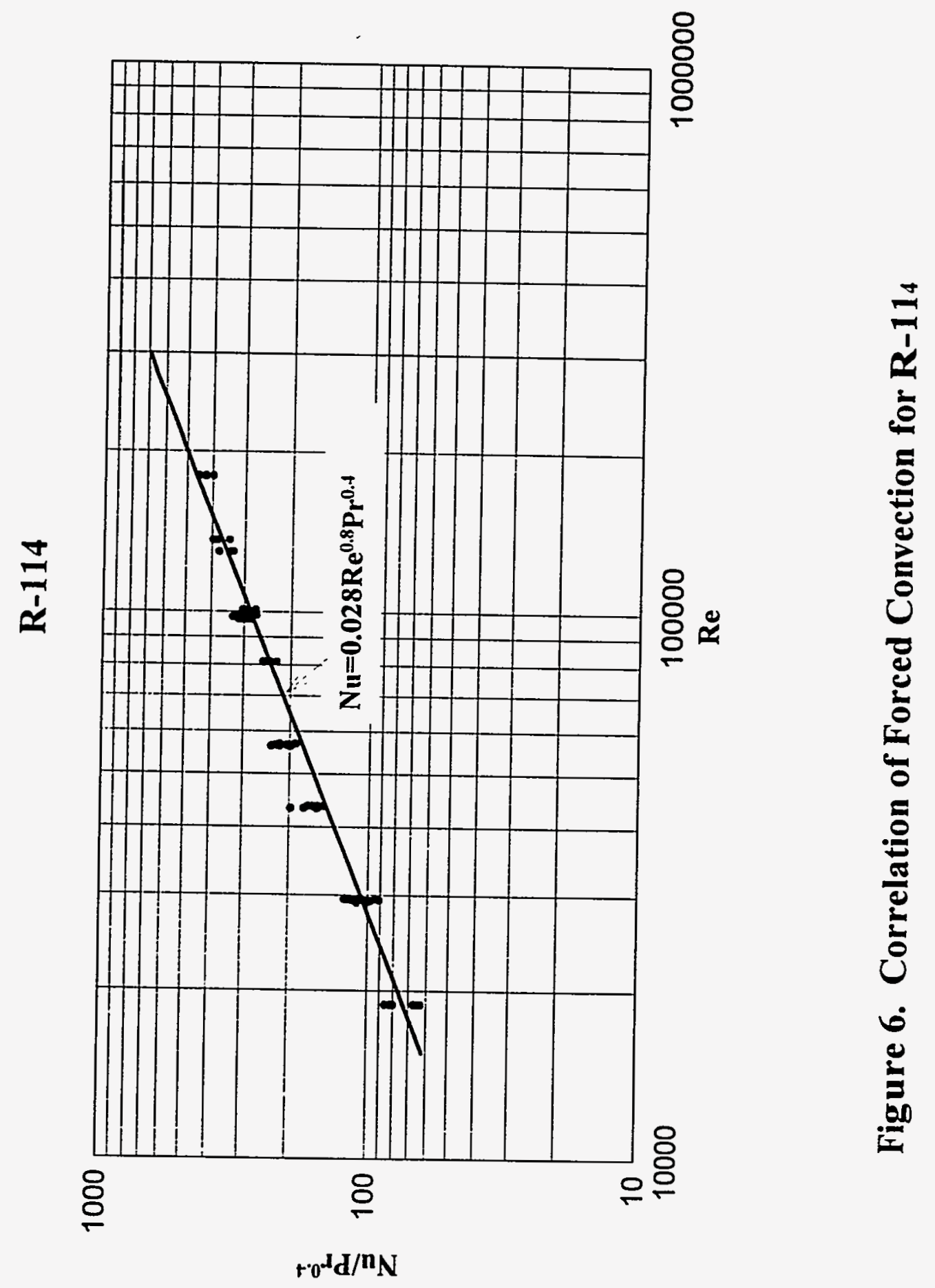




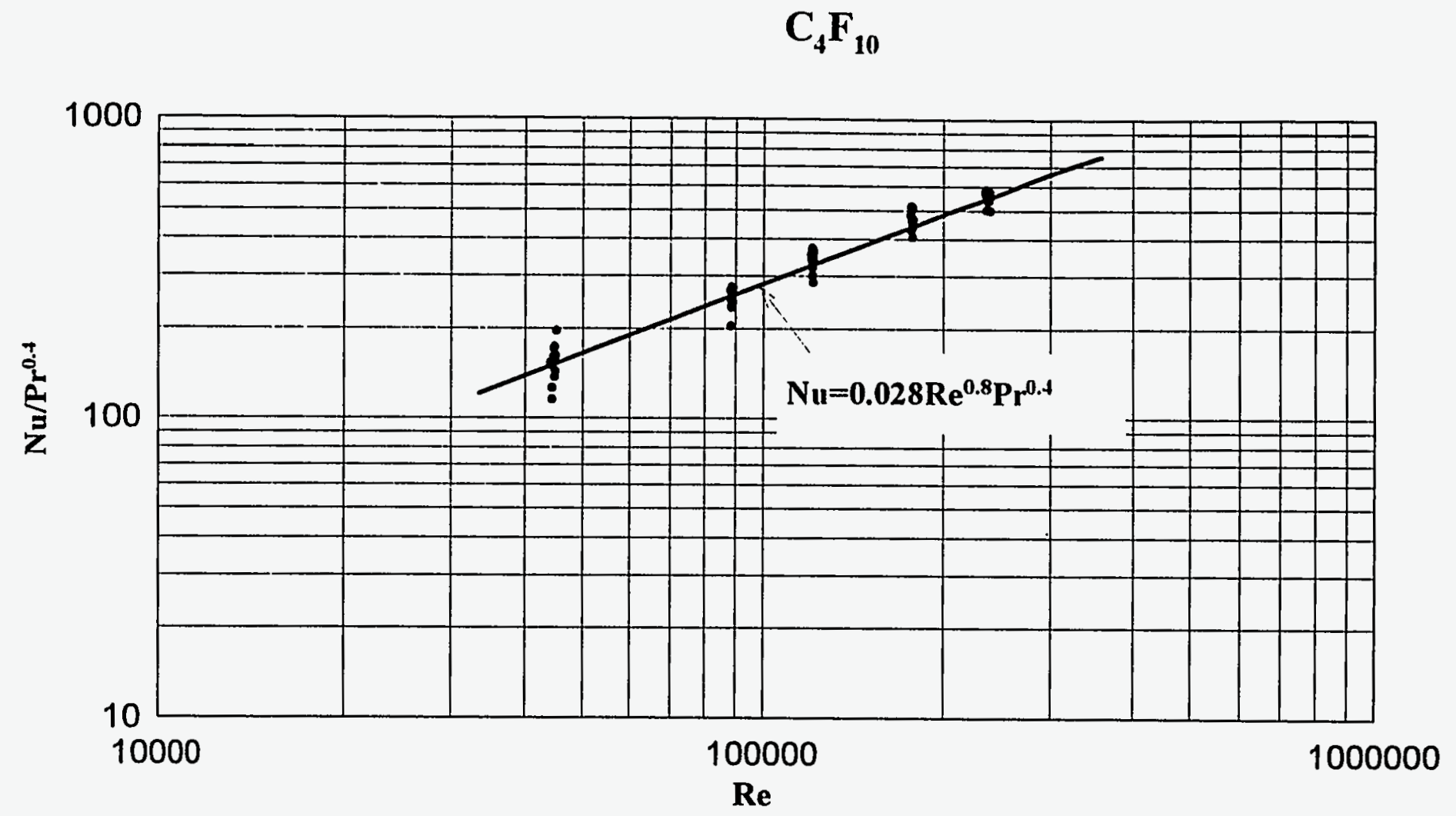

Figure 7. Correlation of Forced Convection for $\mathrm{C}_{4} \mathrm{~F}_{10}$ 


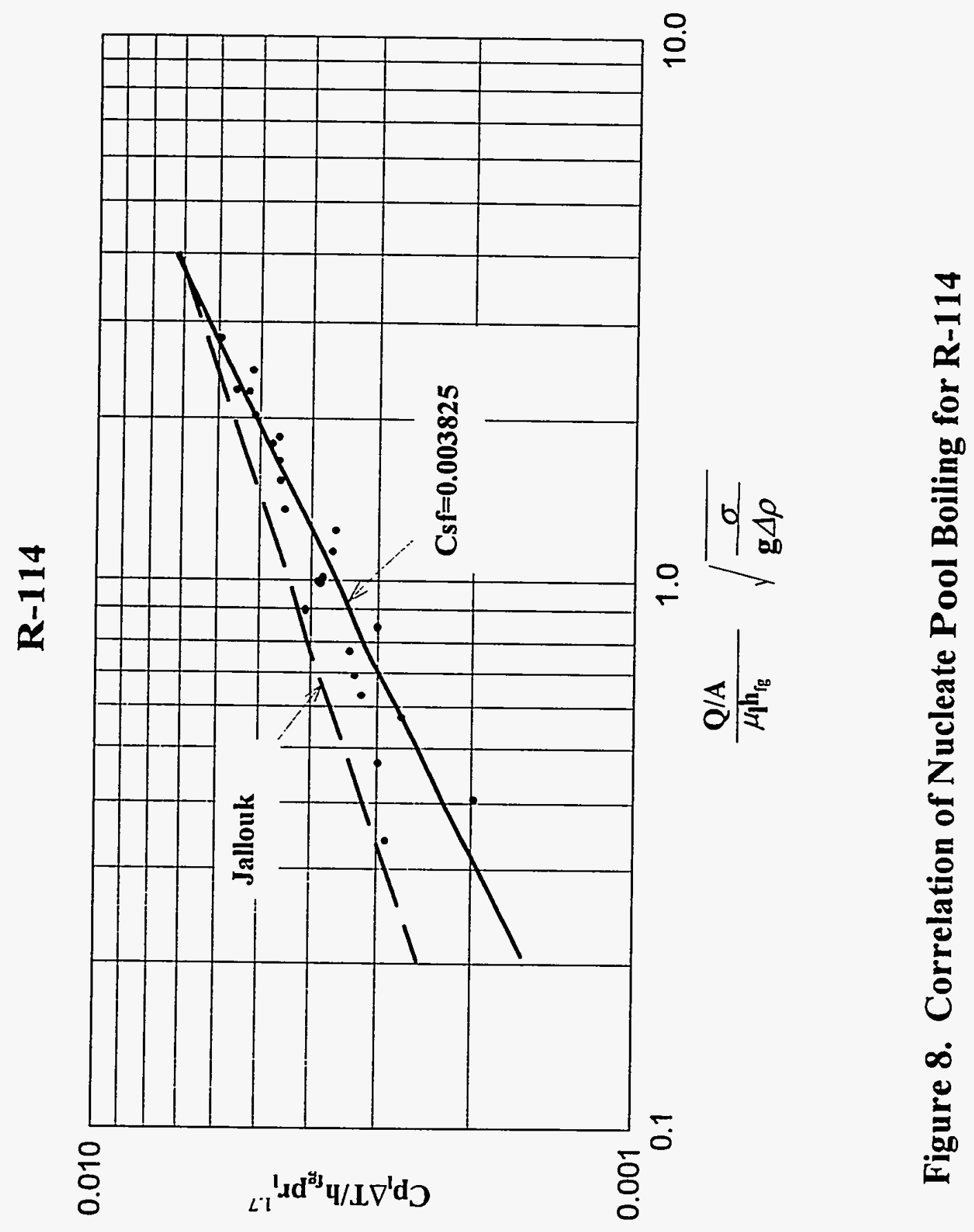




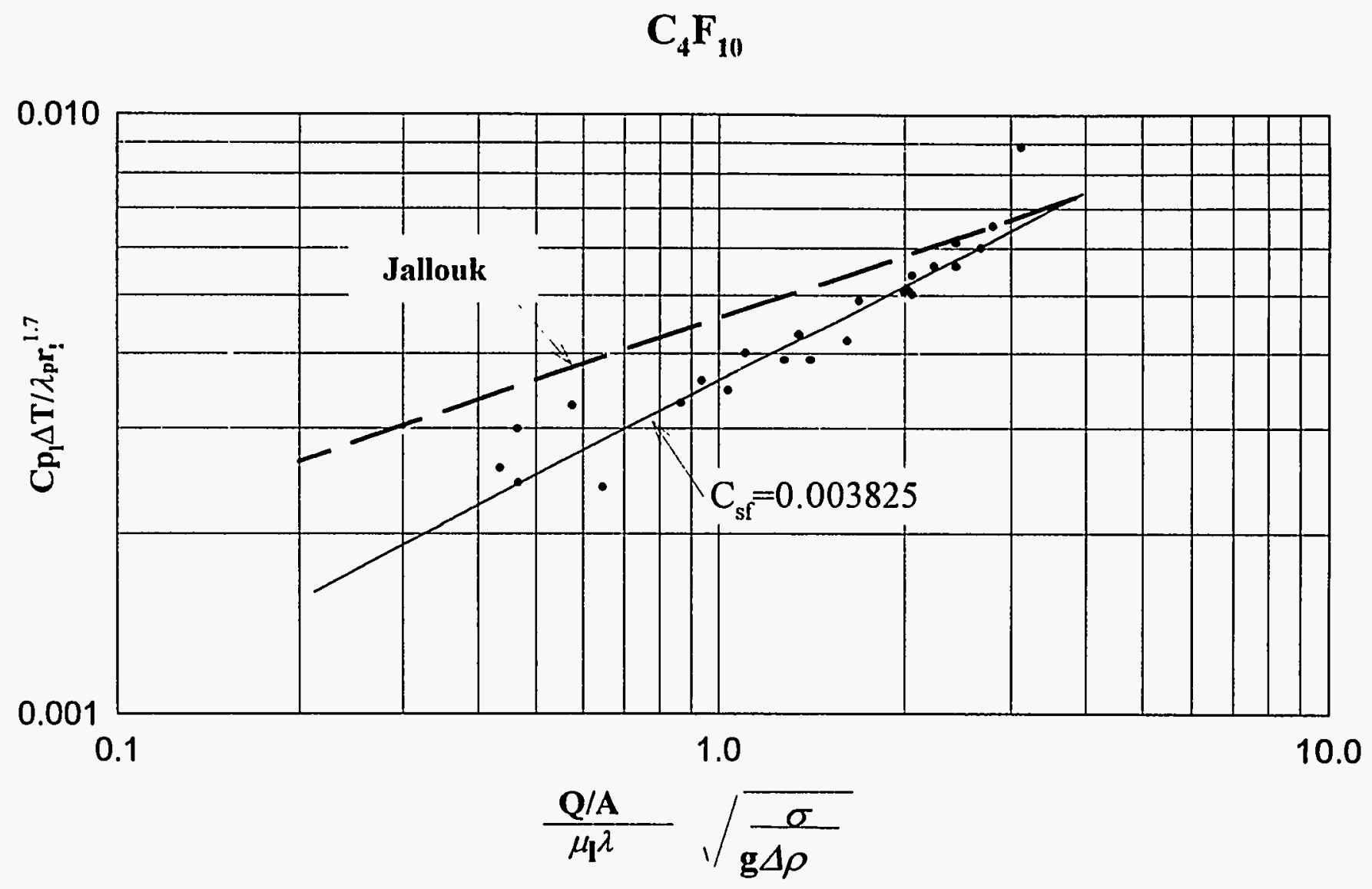

Figure 9. Correlation of Nucleate Pool Boiling for $C_{4} F_{10}$ 


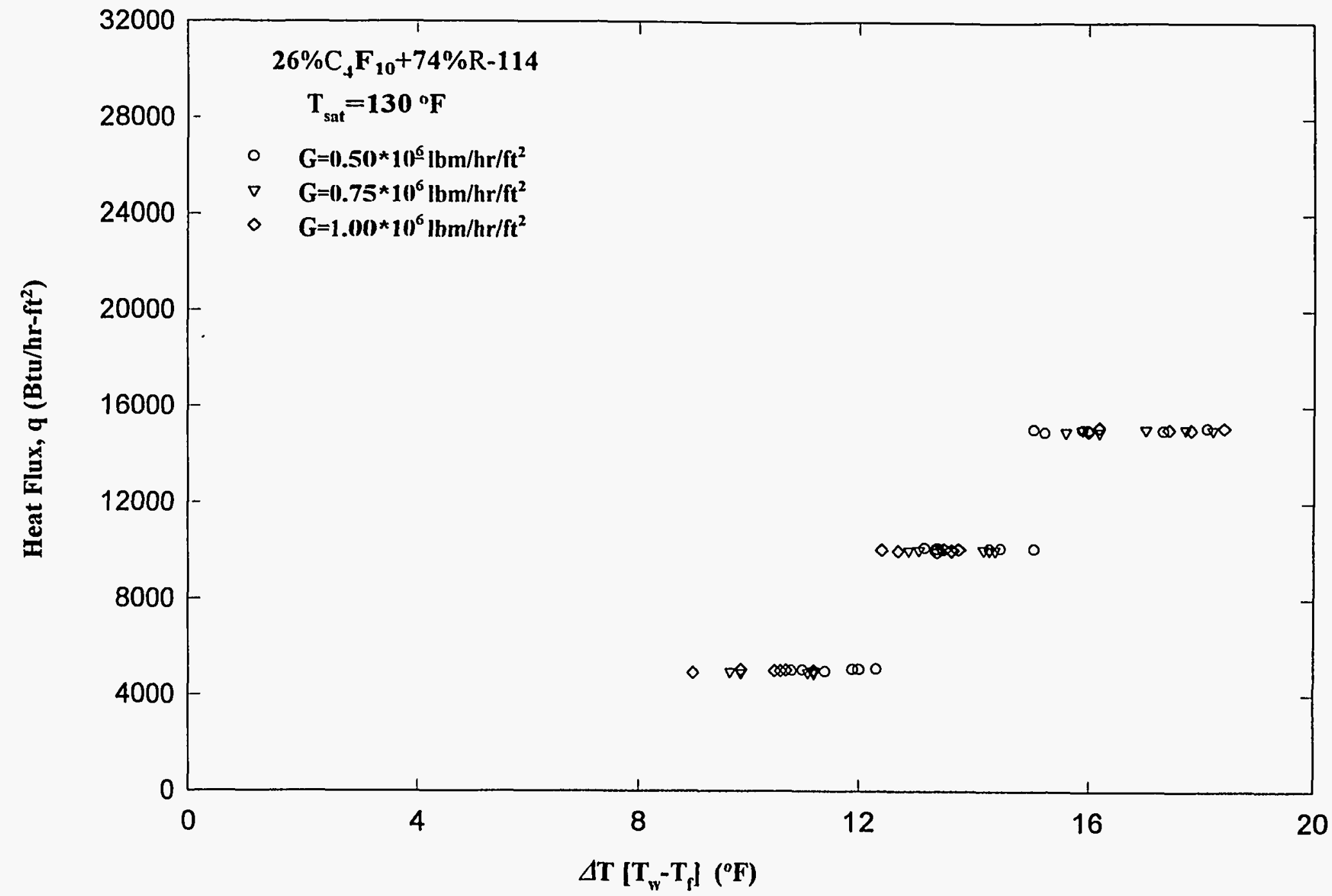

Figure 10. Heat Flux Versus the Wall-to-instream Temperature Difference for the Mixture of $26 \% \mathrm{C}_{4} \mathrm{~F}_{10}+74 \% \mathrm{R}-114$ at $\mathrm{T}_{\text {sat }}=130{ }^{\circ} \mathrm{F}$ 


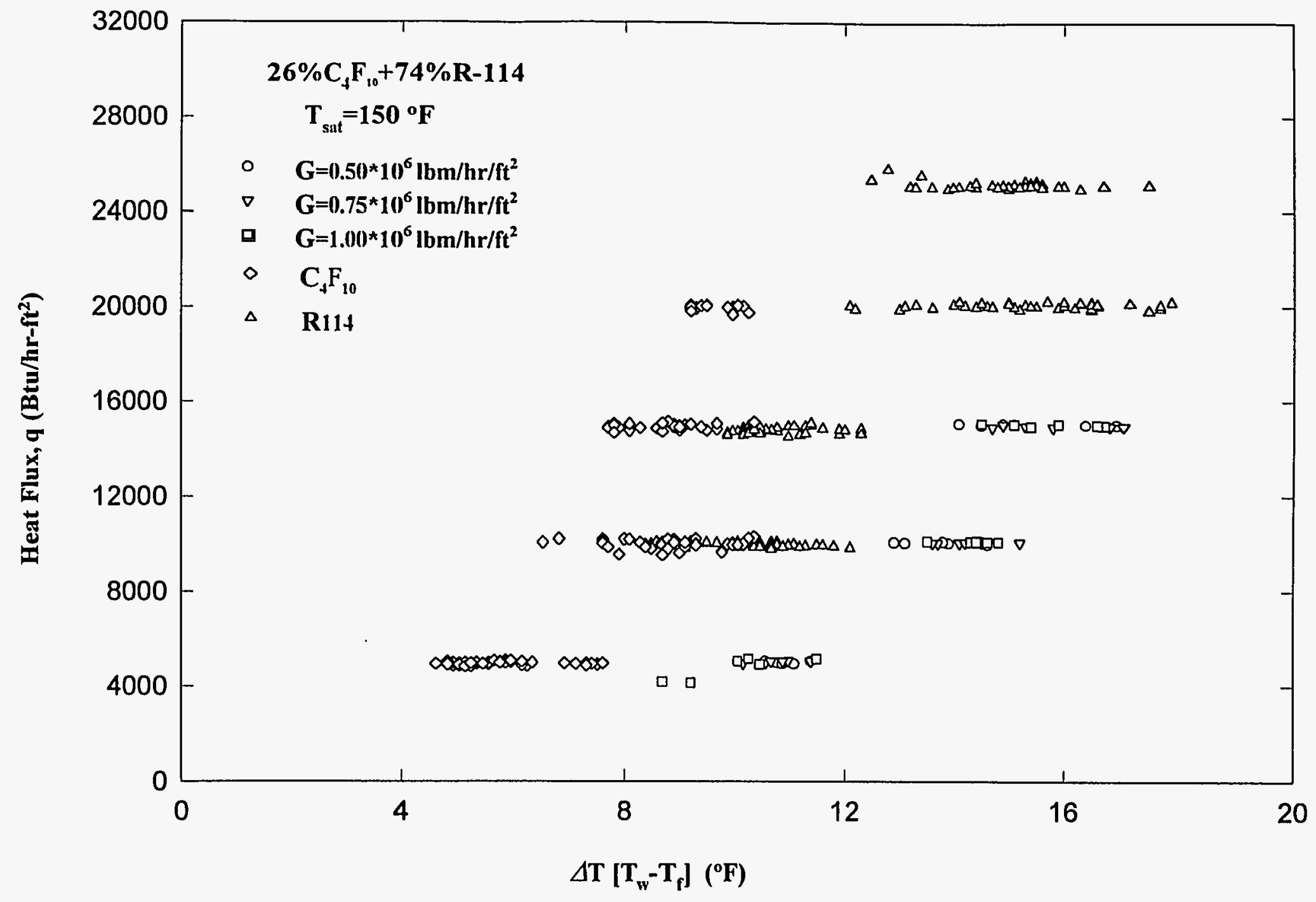

Figure 11. Heat Flux Versus the Wall-to-instream Temperature Difference for the Mixture of $26 \% \mathrm{C}_{4} \mathrm{~F}_{10}+74 \% \mathrm{R}-114$ at $\mathrm{T}_{\mathrm{sat}}=150{ }^{\circ} \mathrm{F}$ 


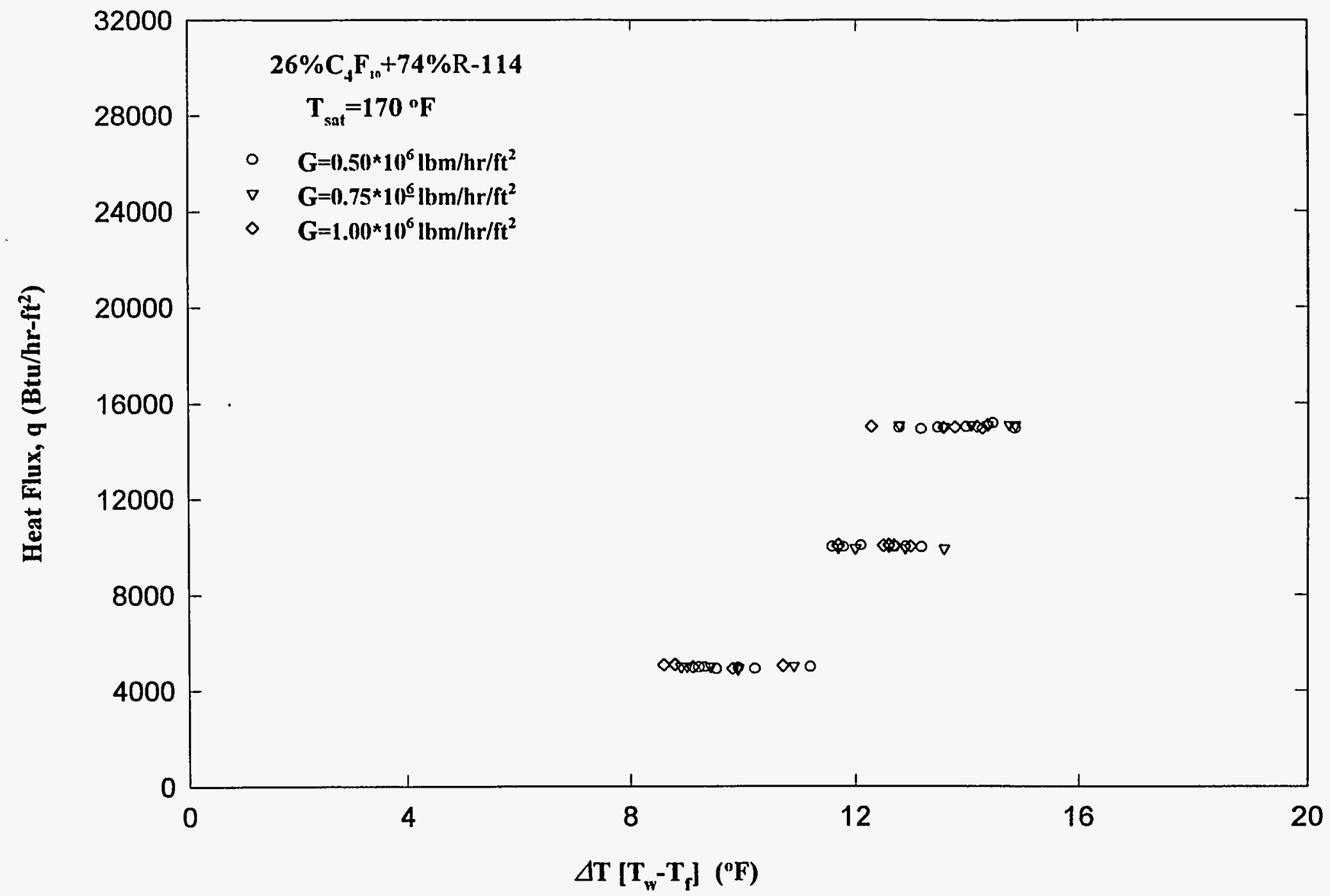

Figure 12. Heat Flux Versus the Wall-to-instream Temperature Difference for the Mixture of $26 \% \mathrm{C}_{4} \mathrm{~F}_{10}+74 \% \mathrm{R}-114$ at $\mathrm{T}_{\text {sat }}=170^{\circ} \mathrm{F}$ 


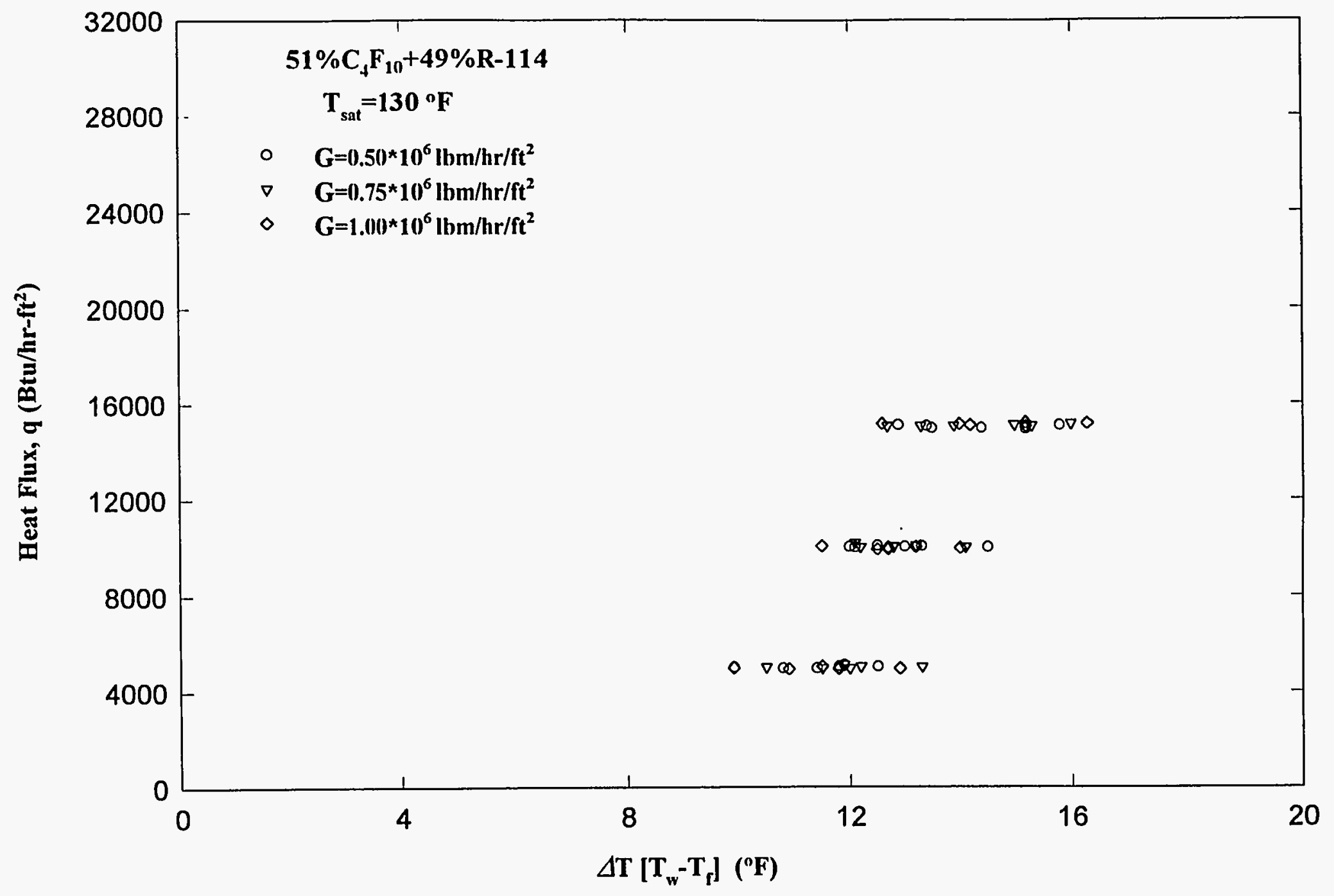

Figure 13. Heat Flux Versus the Wall-to-instream Temperature Difference for the Mixture of $51 \% \mathrm{C}_{4} \mathrm{~F}_{10}+49 \% \mathrm{R}-114$ at $\mathrm{T}_{\mathrm{sat}}=130{ }^{\circ} \mathrm{F}$ 


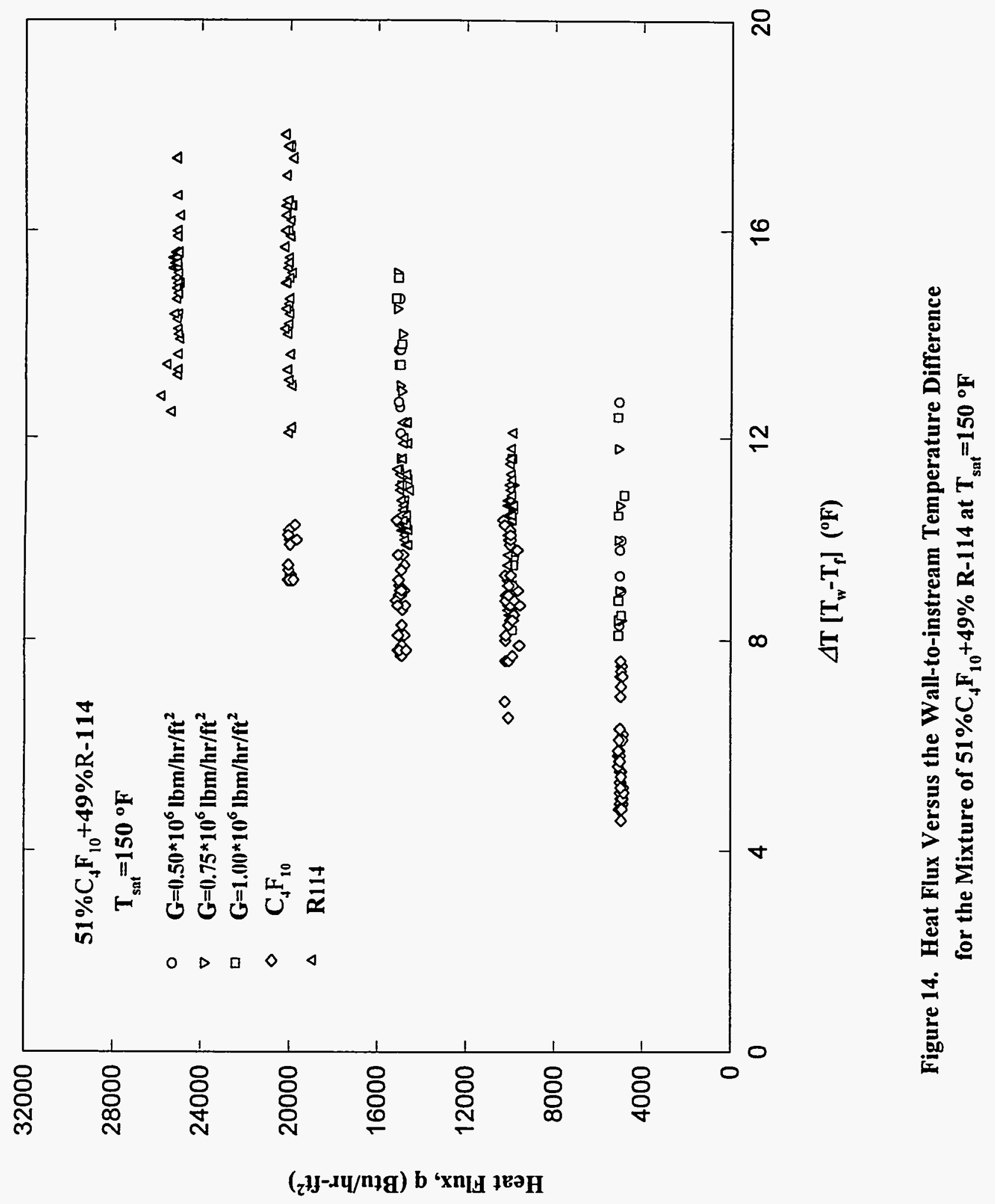




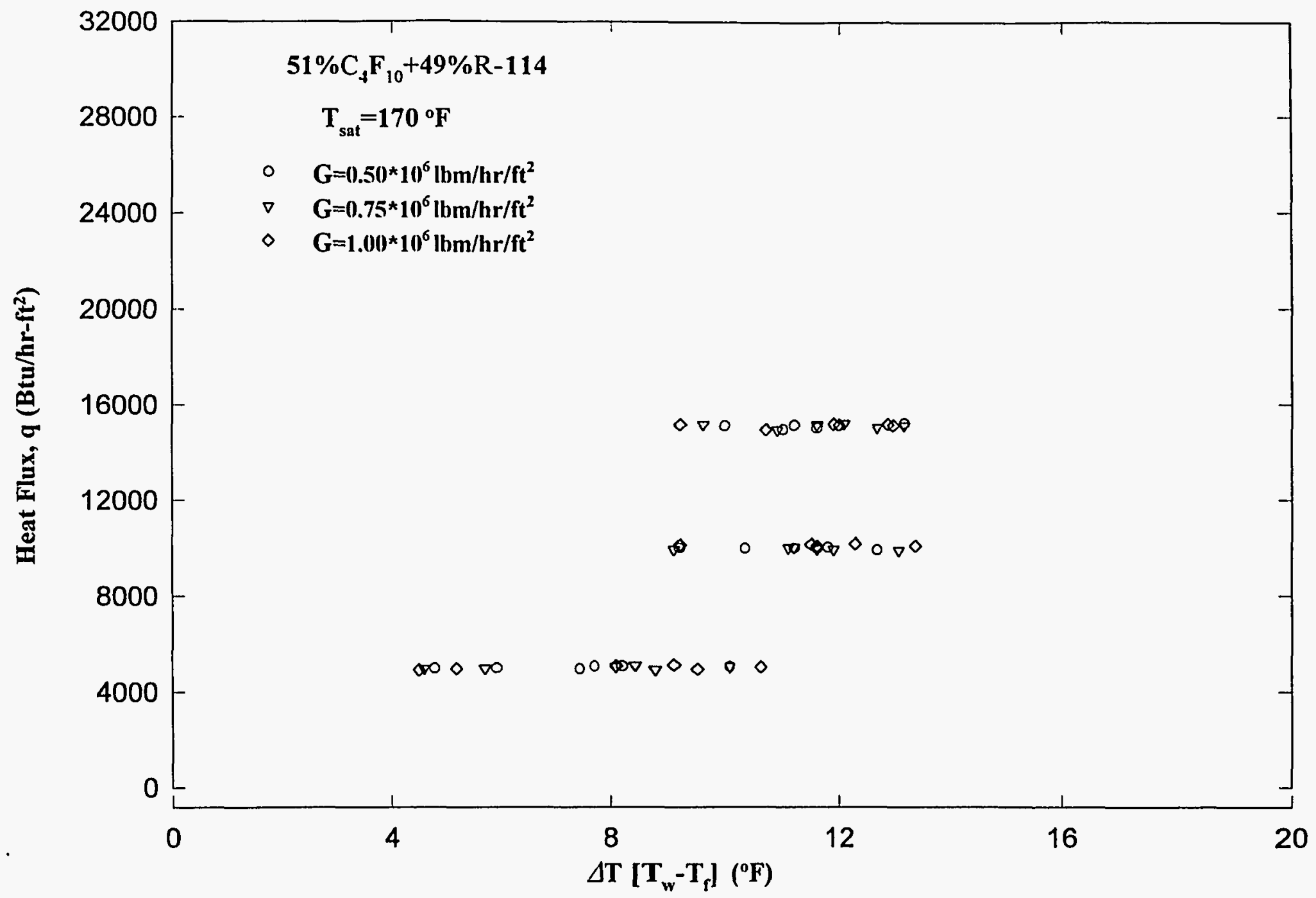

Figure 15. Heat Flux Versus the Wall-to-instream Temperature Difference for the Mixture of $51 \% \mathrm{C}_{4} \mathrm{~F}_{10}+49 \% \mathrm{R}-114$ at $\mathrm{T}_{\text {sat }}=170{ }^{\circ} \mathrm{F}$ 


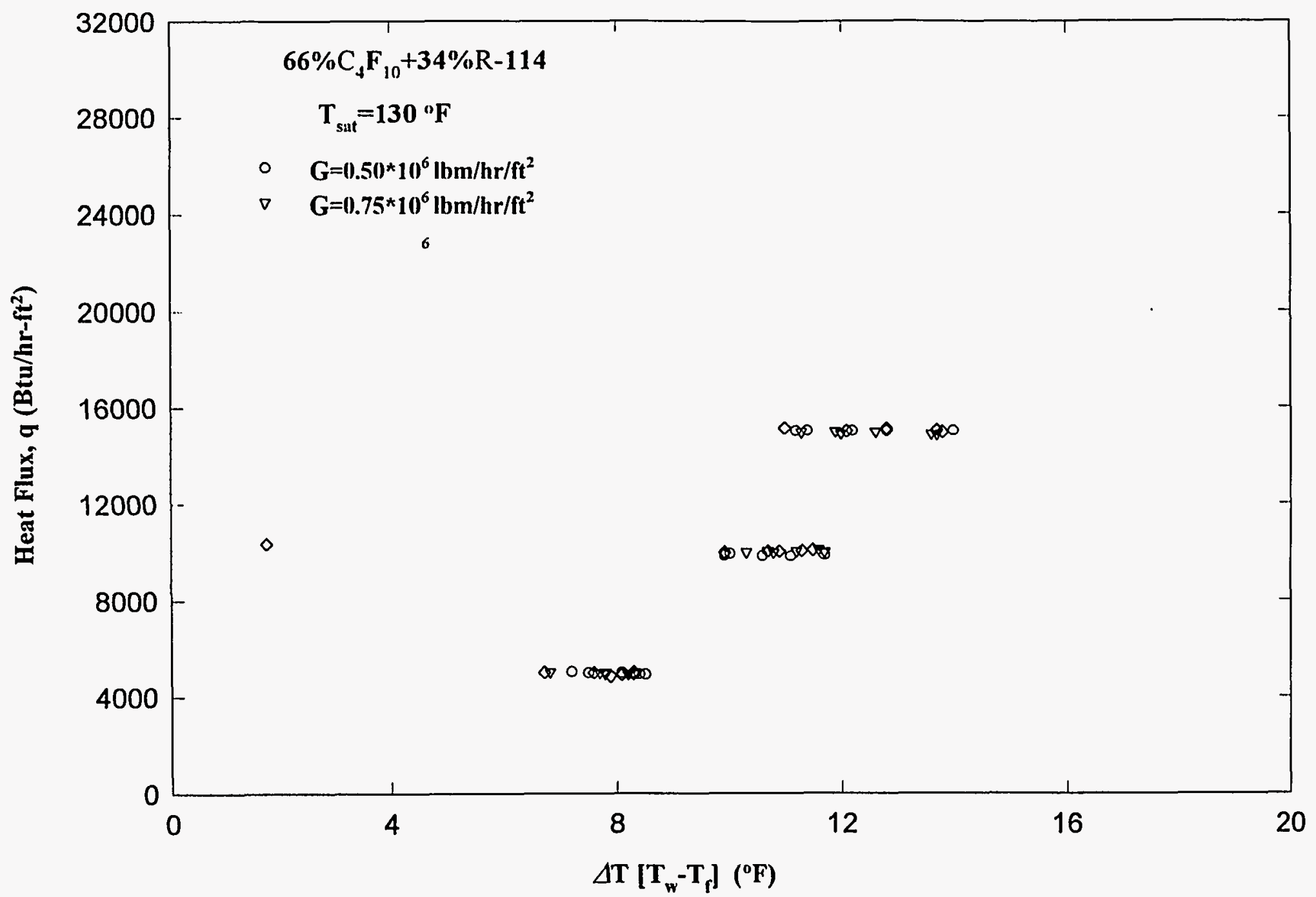

Figure 16. Heat Flux Versus the Wall-to-instream Temperature Difference for the Mixture of $66 \% \mathrm{C}_{4} \mathrm{~F}_{10}+34 \% \mathrm{R}-114$ at $\mathrm{T}_{\text {sat }}=130{ }^{\circ} \mathrm{F}$ 


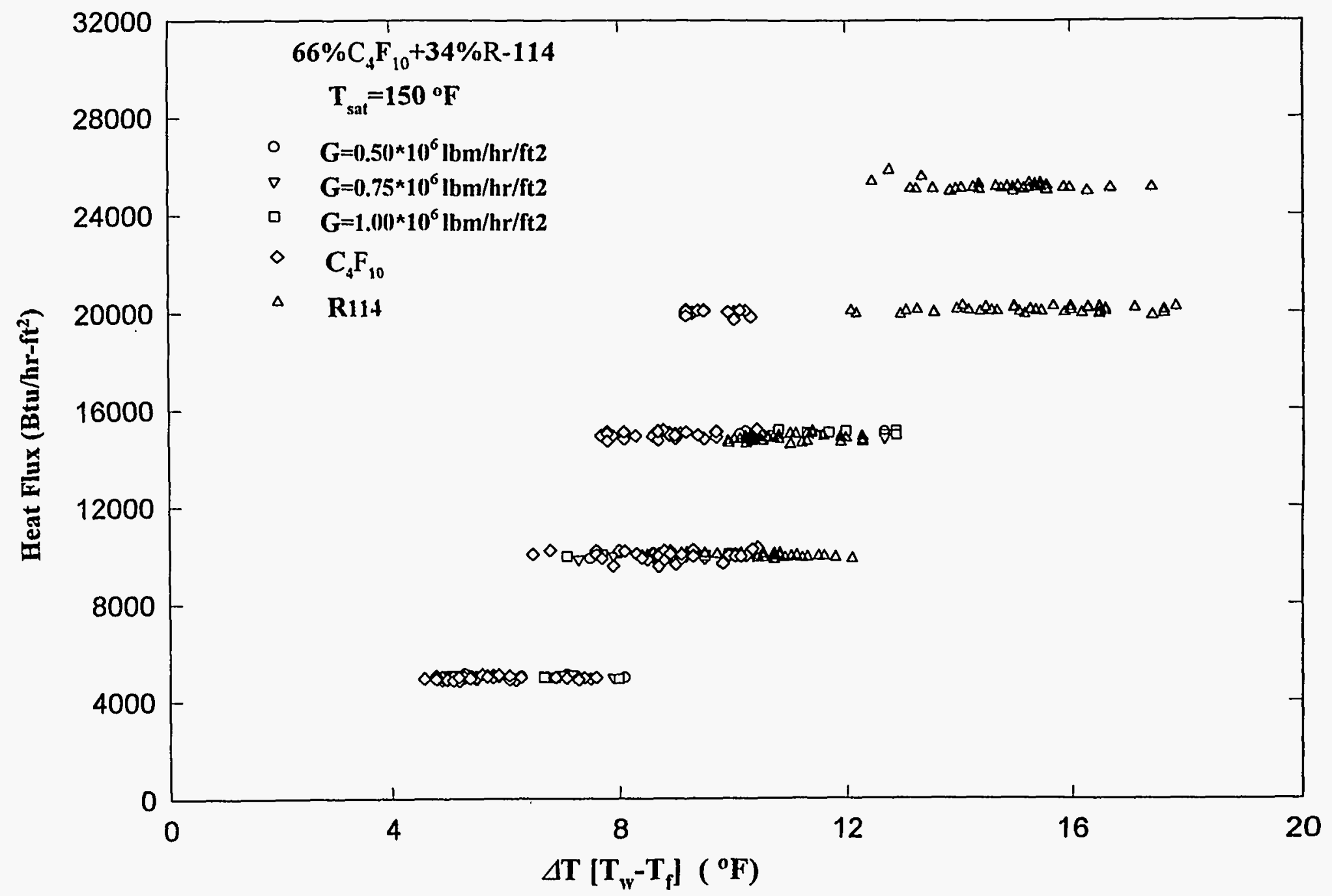

Figure 17. Heat Flux Versus the Wall-to-instream Temperature Difference for the Mixture of $66 \% \mathrm{C}_{4} \mathrm{~F}_{10}+34 \% \mathrm{R}-114$ at $\mathrm{T}_{\text {sat }}=150{ }^{\circ} \mathrm{F}$ 

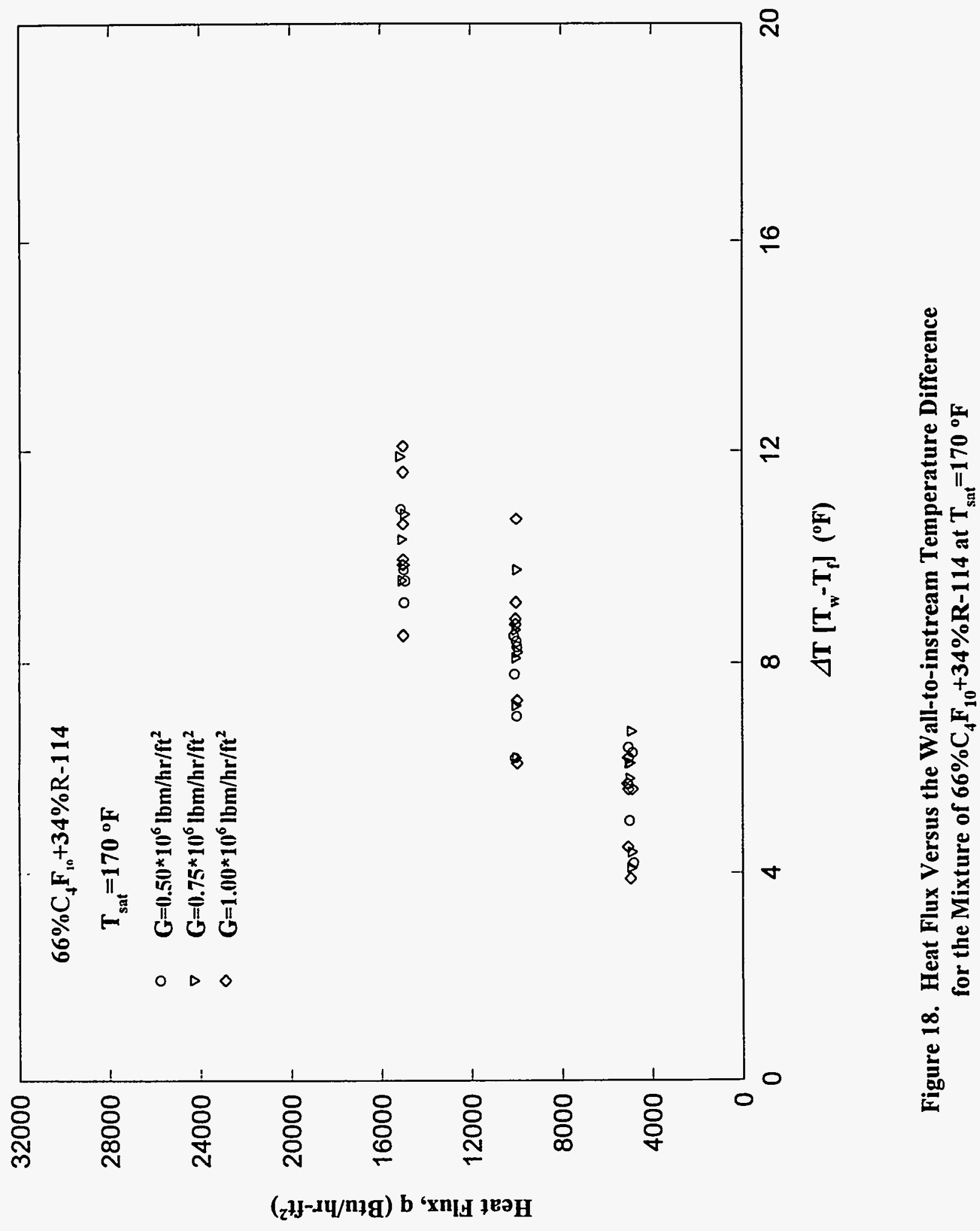


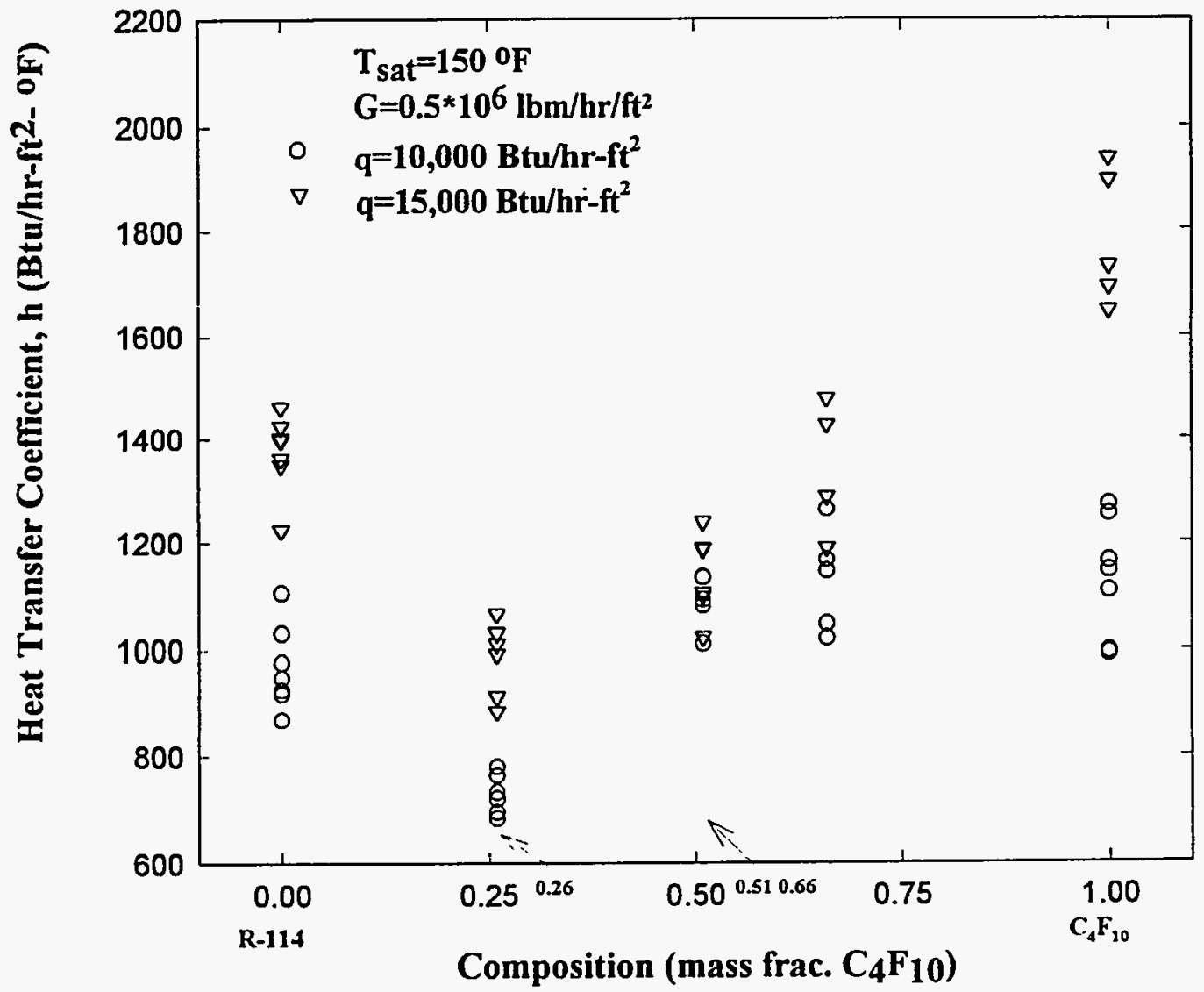

Figure 19. Flow Boiling Heat Transfer Characteristics for Mixtures of $R-114$ and $C_{4} F_{10}$ with Various.

Compositions 


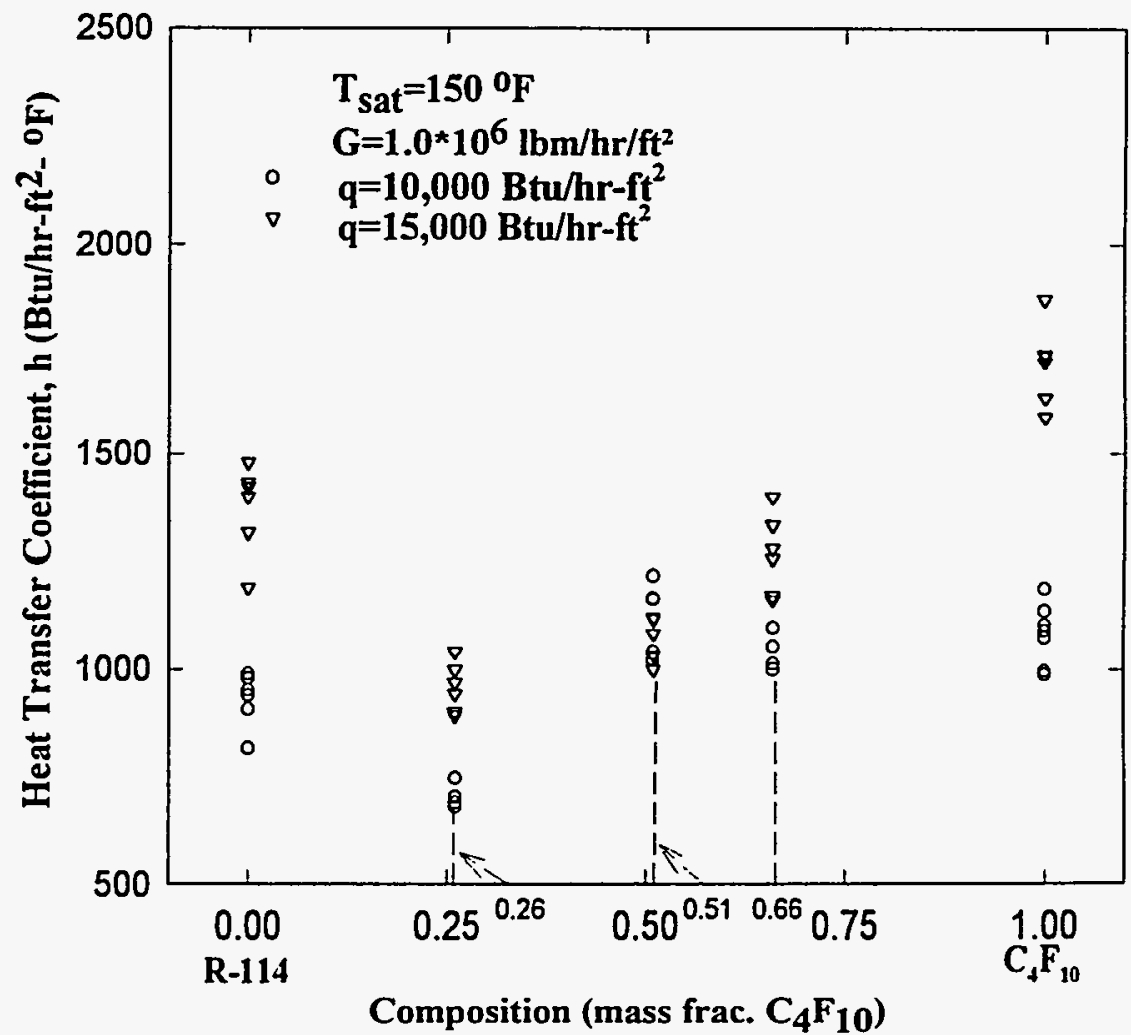

Figure 20. Flow Boiling Heat Transfer Characteristics for Mixtures of R-114 and $\mathrm{C}_{4} \mathrm{~F}_{10}$ with Various.

Compositions 


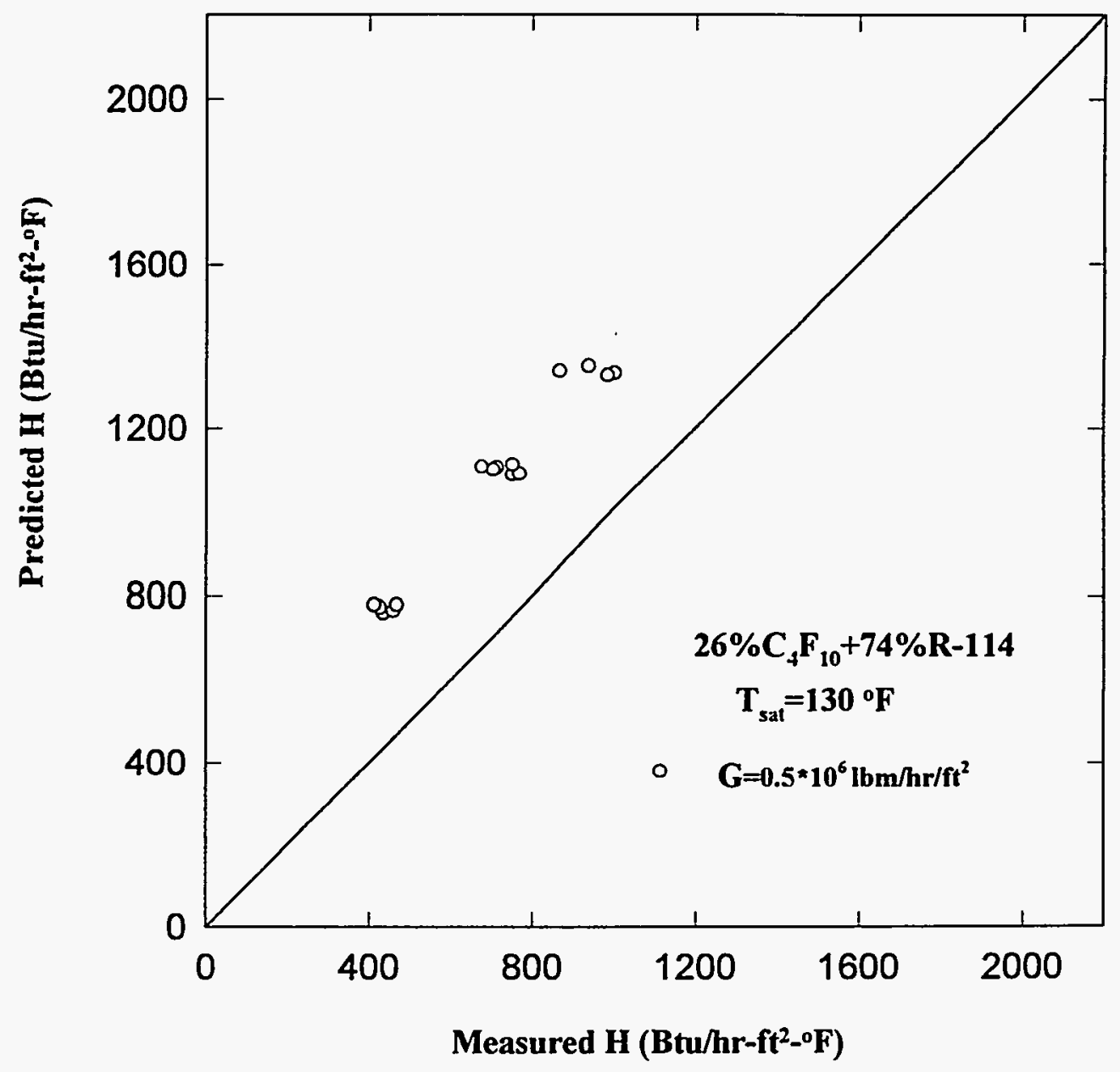

Figure 21. Comparison of Measured and Predicted Heat Transfer Coefficients for $26 \% \mathrm{C}_{4} \mathrm{~F}_{10}+74 \% \mathrm{R}-114$ at $T_{\text {sat }}=130 \cdot \mathrm{F}$ 


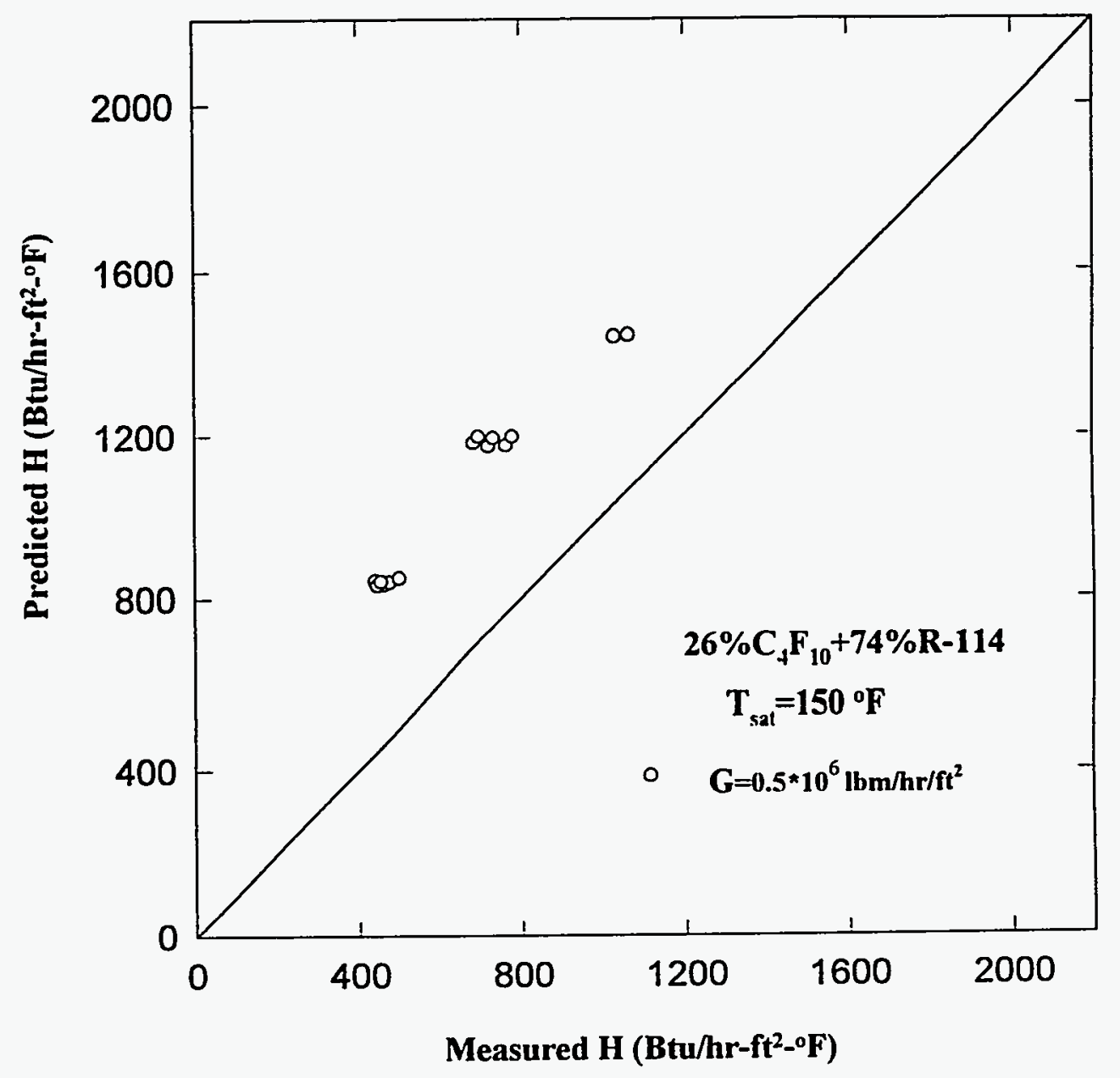

Figure 22. Comparison of Measured and Predicted Heat Transfer Coefficients for $26 \% \mathrm{C}_{4} \mathrm{~F}_{10}+74 \% \mathrm{R}-114$ at $T_{\text {sat }}=150 \cdot F$ 


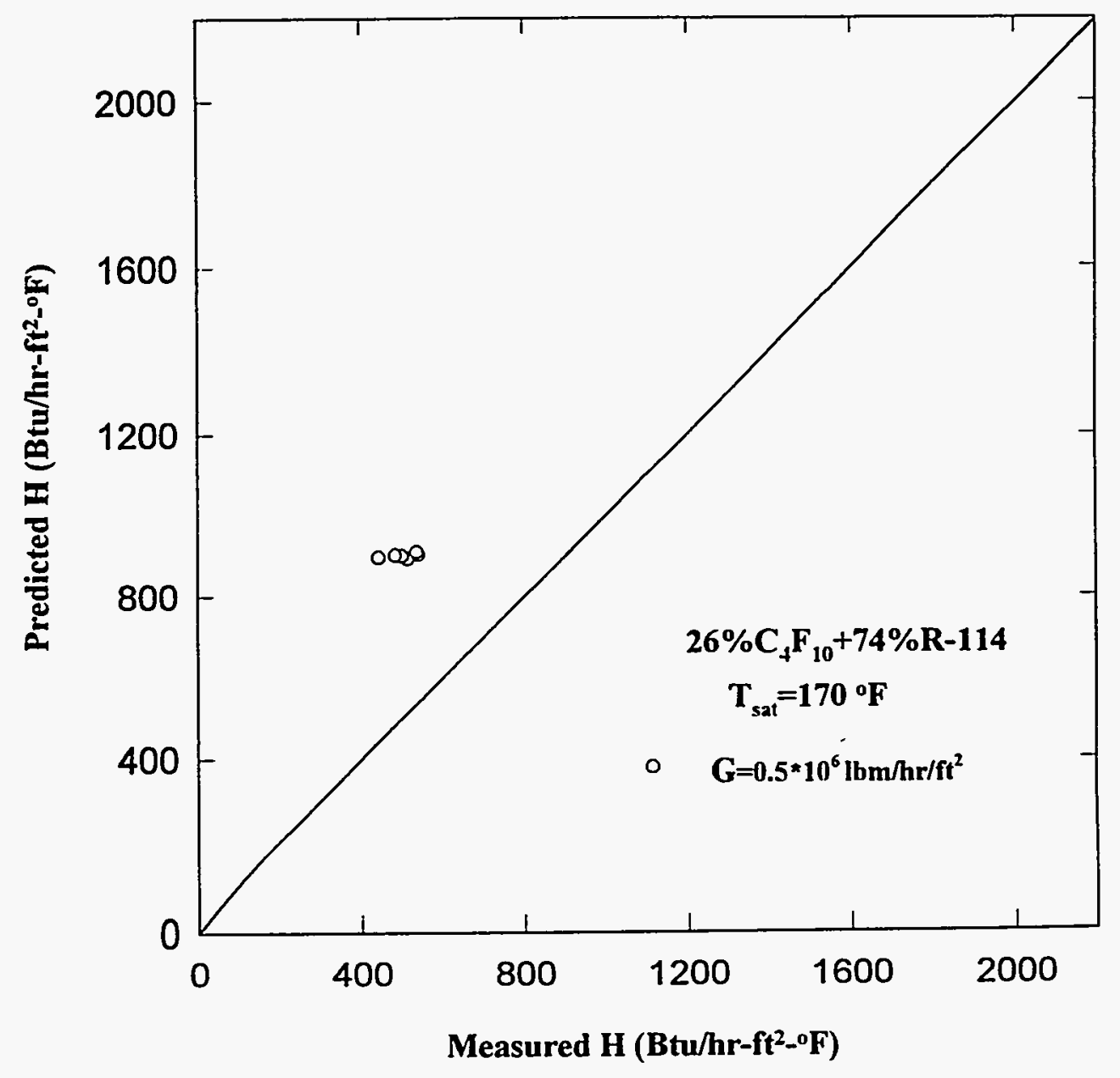

Figure 23. Comparison of Measured and Predicted Heat Transfer Coefficients for $26 \% \mathrm{C}_{4} \mathrm{~F}_{10}+74 \% \mathrm{R}-114$ at $T_{s 2 t}=170 \cdot F$ 


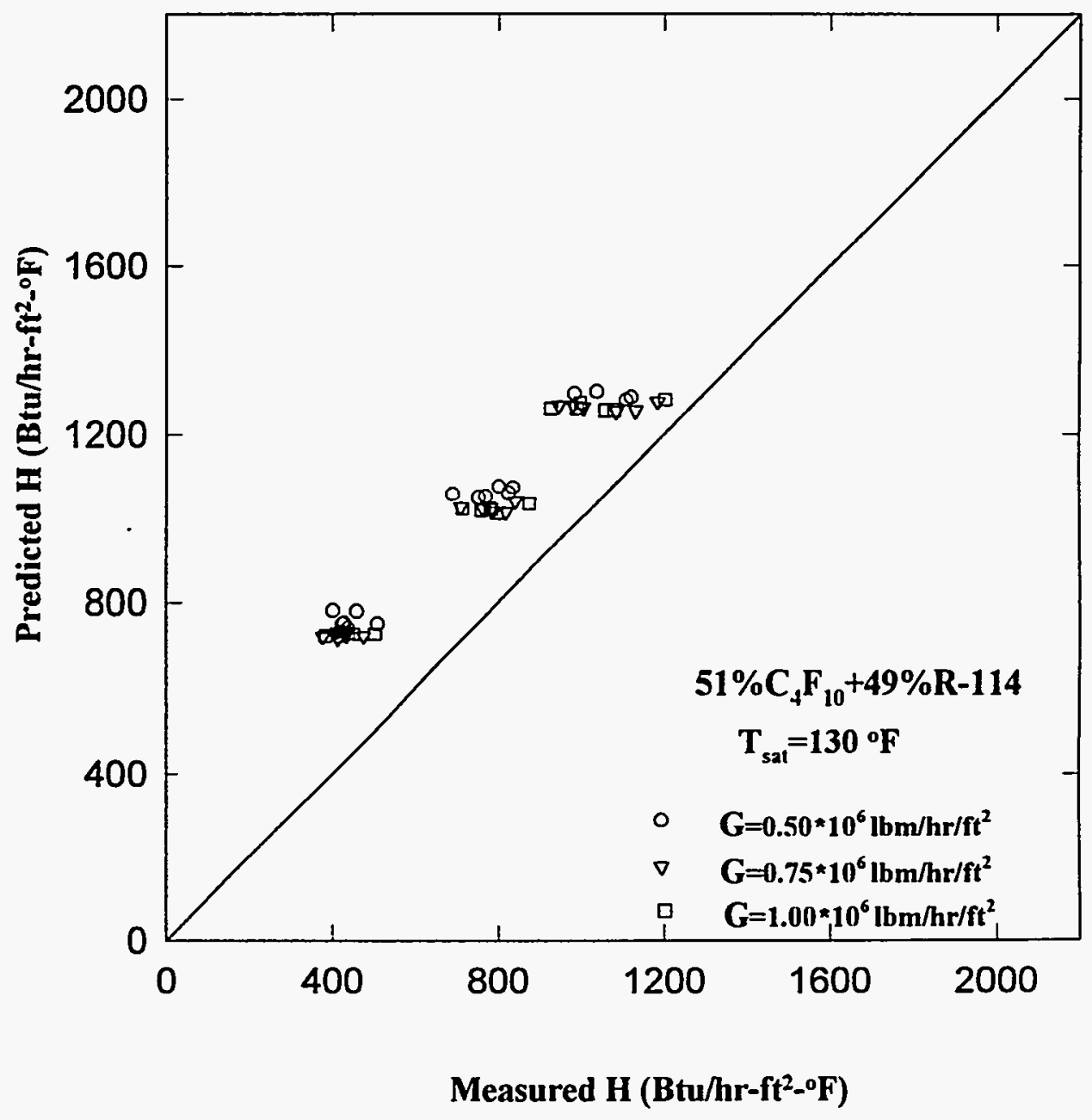

Figure 24. Comparison of Measured and Predicted Heat Transfer Coefficients for $51 \% \mathrm{C}_{4} \mathrm{~F}_{10}+49 \% \mathrm{R}-114$ at $T_{\text {sat }}=130 \cdot F$ 


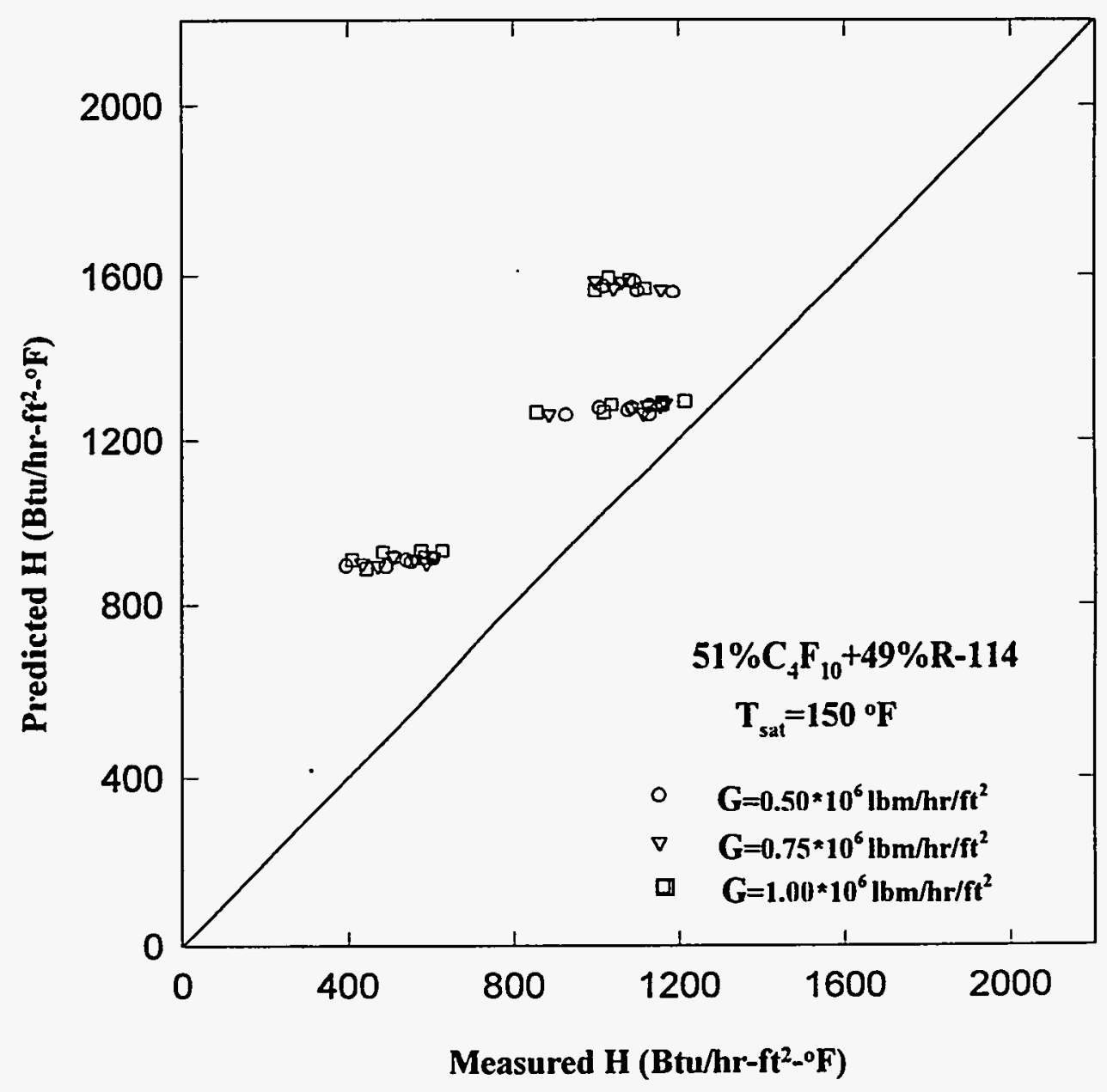

Figure 25. Comparison of Measured and Predicted Heat Transfer Coefficients for $51 \% \mathrm{C}_{4} \mathrm{~F}_{10}+49 \% \mathrm{R}-114$ at $T_{\text {sal }}=150 \cdot F$ 


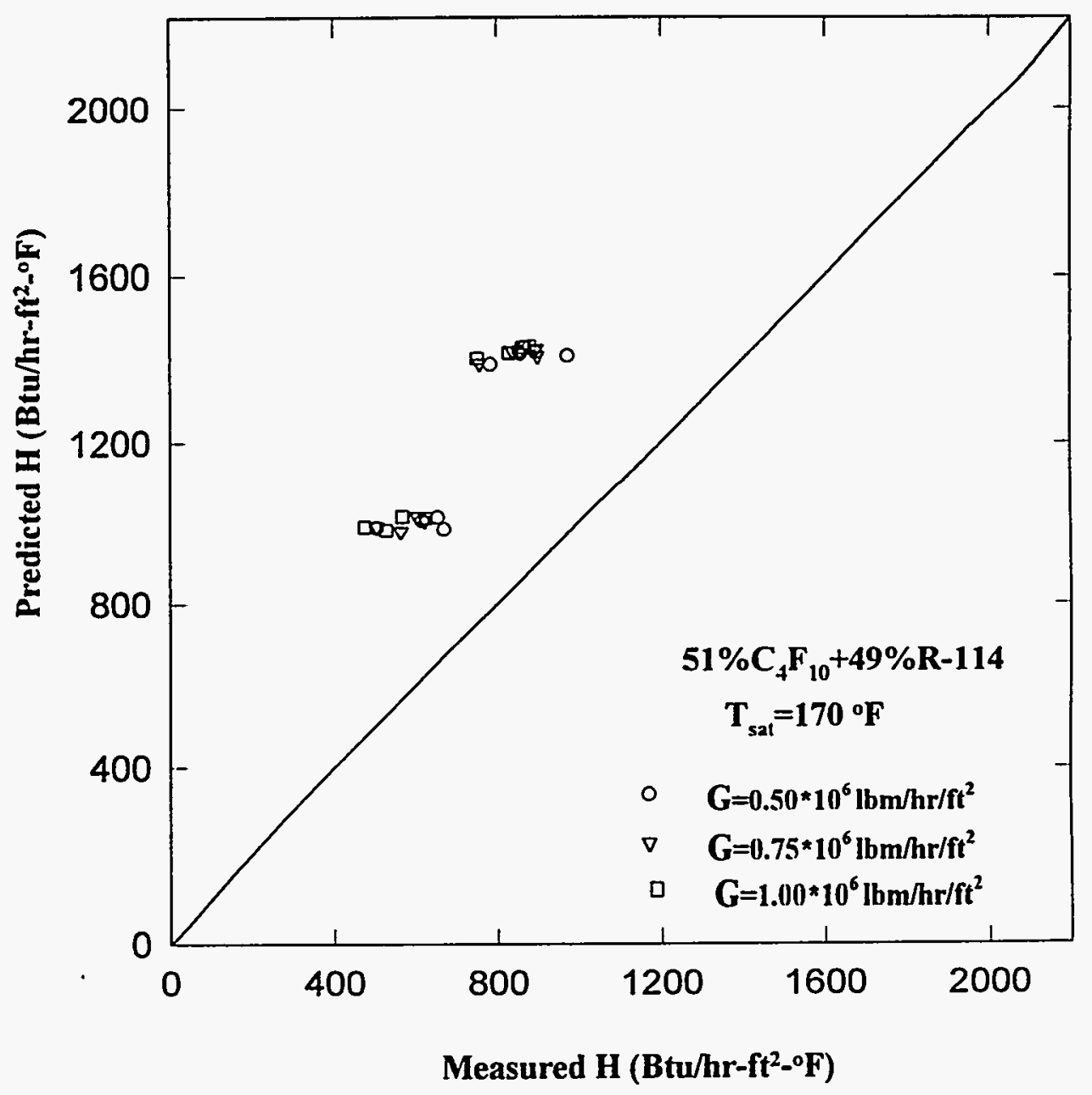

Figure 26. Comparison of Measured and Predicted Heat Transfer Coefficients for $51 \% C_{4} F_{10}+49 \% R-114$ at $T_{\text {sat }}=170 \cdot F$ 


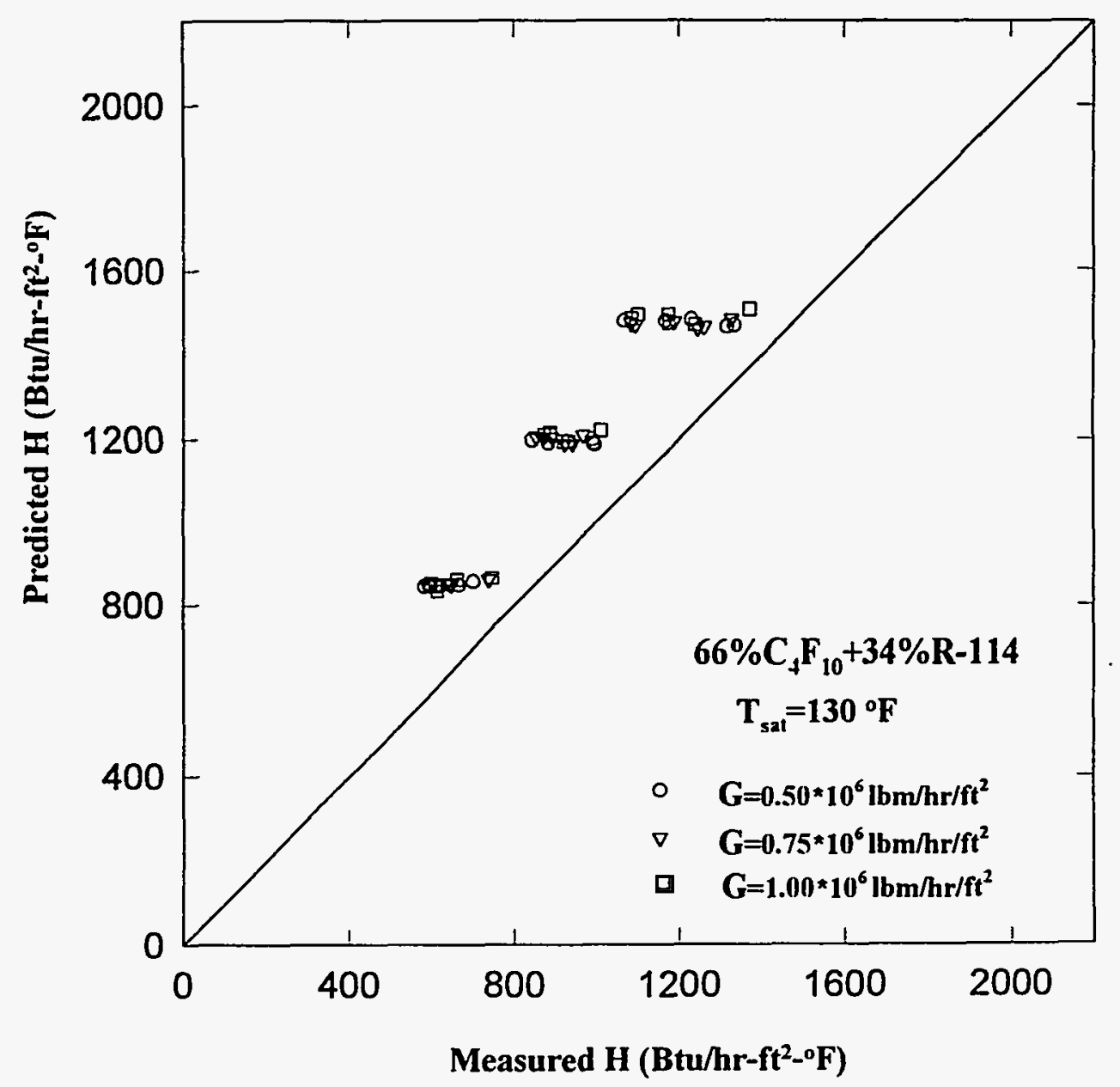

Figure 27. Comparison of Measured and Predicted Heat Transfer Coefficients for $66 \% \mathrm{C}_{4} \mathrm{~F}_{10}+34 \% \mathrm{R}-114$ at $T_{\text {sat }}=130 \cdot \mathrm{F}$ 


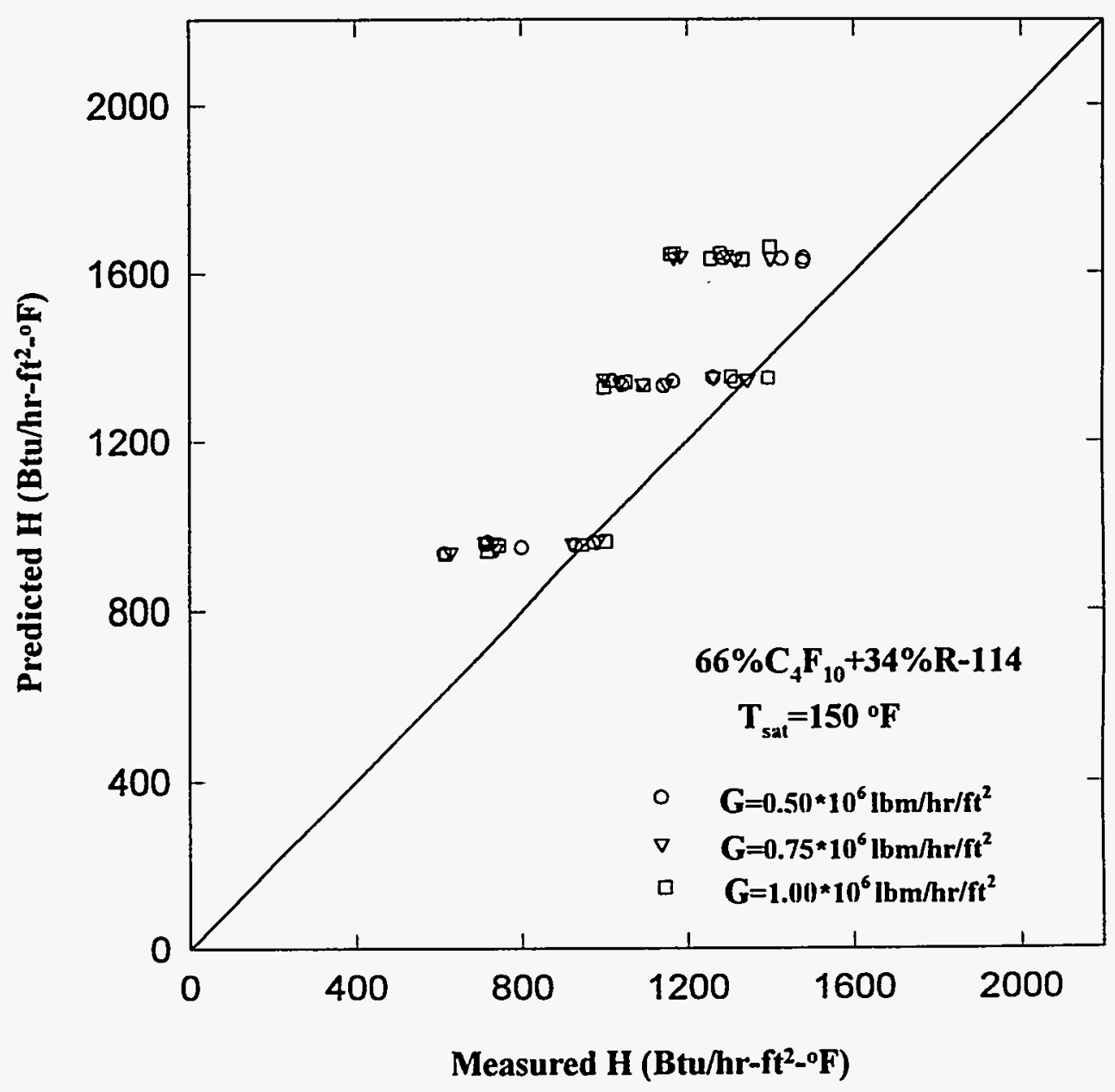

Figure 28. Comparison of Measured and Predicted Heat Transfer Coefficients for $66 \% \mathrm{C}_{4} \mathrm{~F}_{10}+34 \% \mathrm{R}-114$ at $T_{\text {sat }}=150 \cdot \mathrm{F}$ 


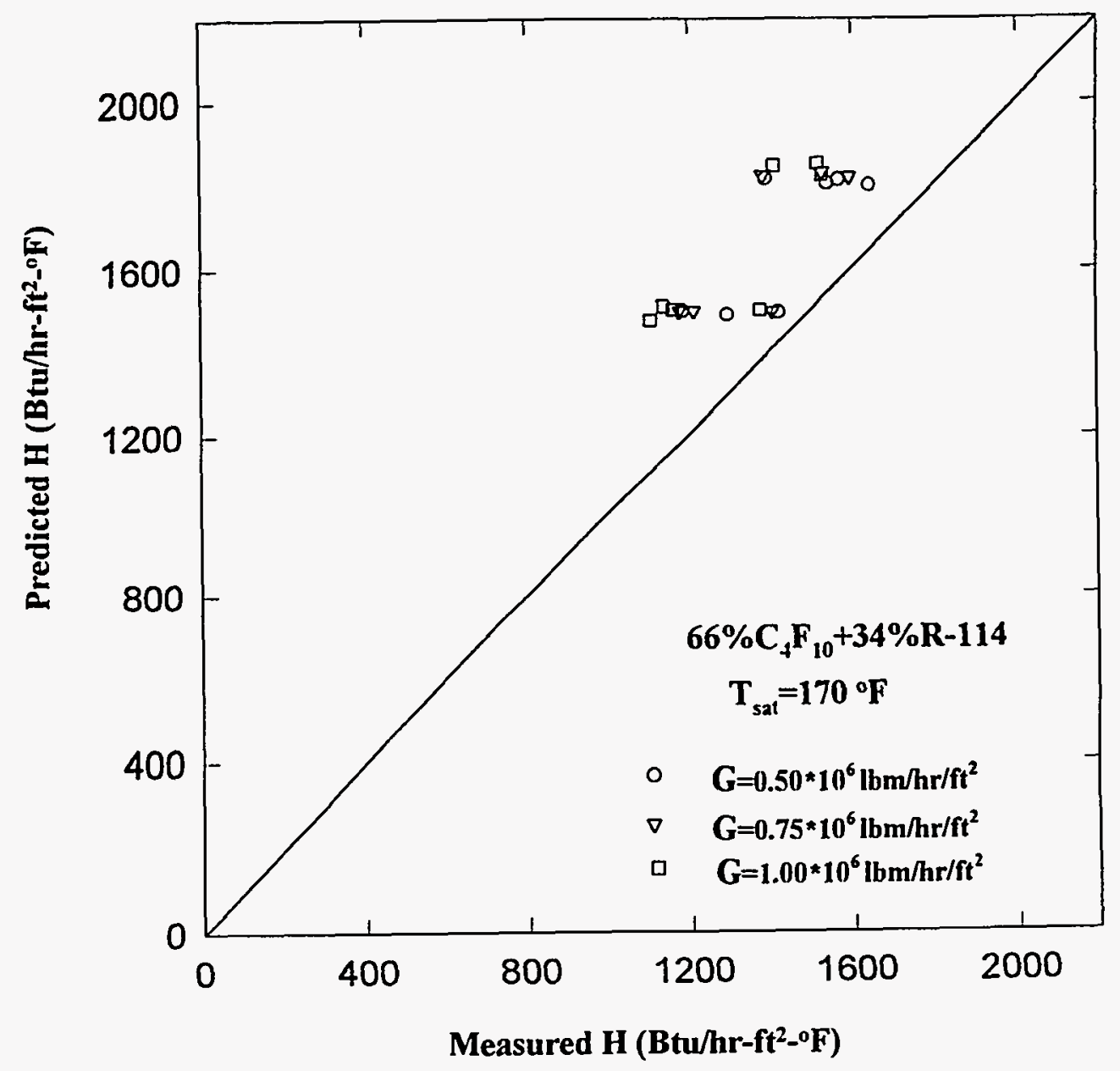

Figure 29. Comparison of Measured and Predicted Heat Transfer Coefficients for $66 \% \mathrm{C}_{4} \mathrm{~F}_{10}+34 \% \mathrm{R}-114$ at $T_{\text {sat }}=170 \cdot F$ 


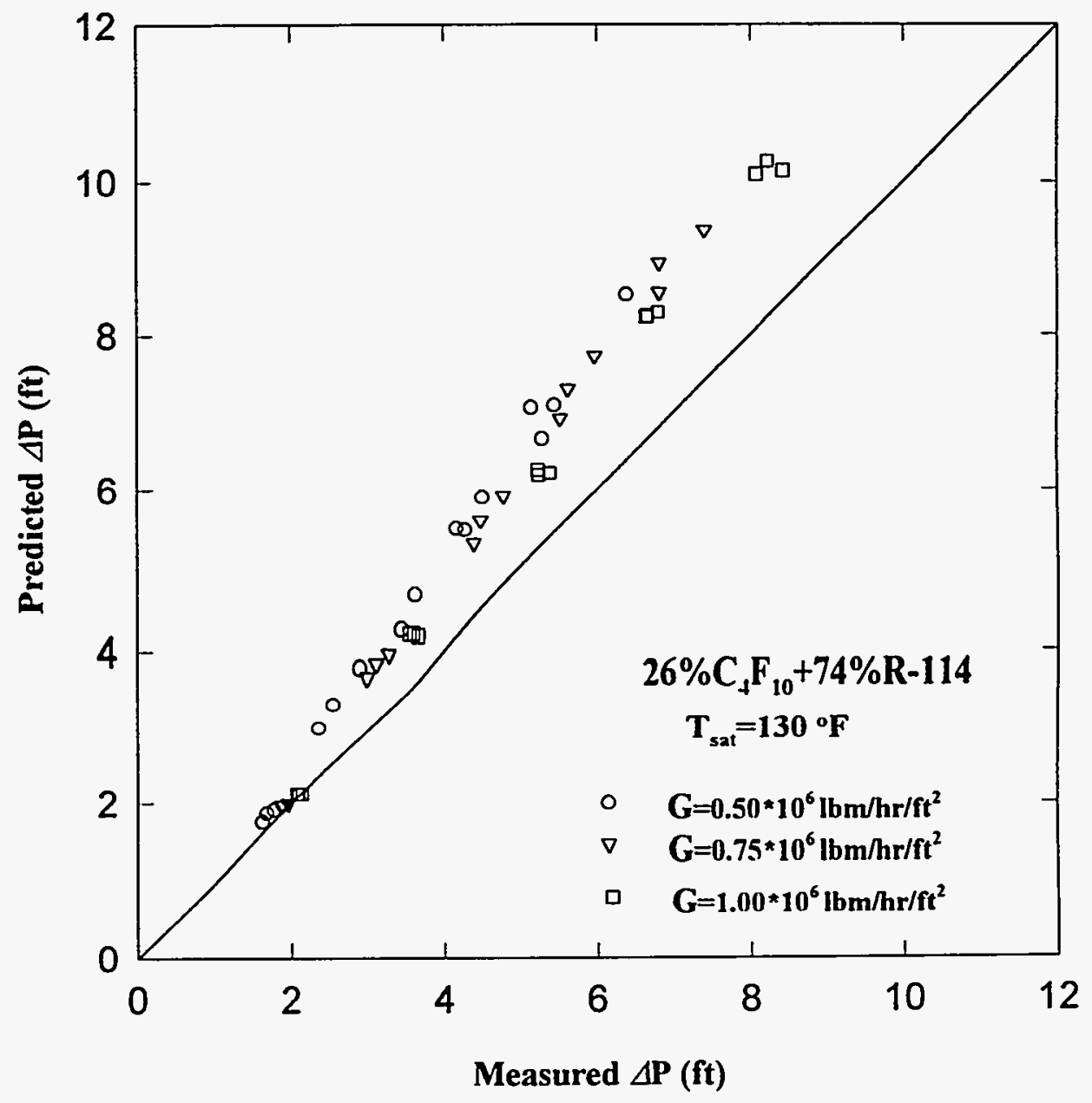

Figure 30. Comparison of Measured and Predicted Pressure Drop for $26 \% \mathrm{C}_{4} \mathrm{~F}_{10}+74 \% \mathrm{R}-114$ at $\mathrm{T}_{\text {sat }}=130 \cdot \mathrm{F}$ 


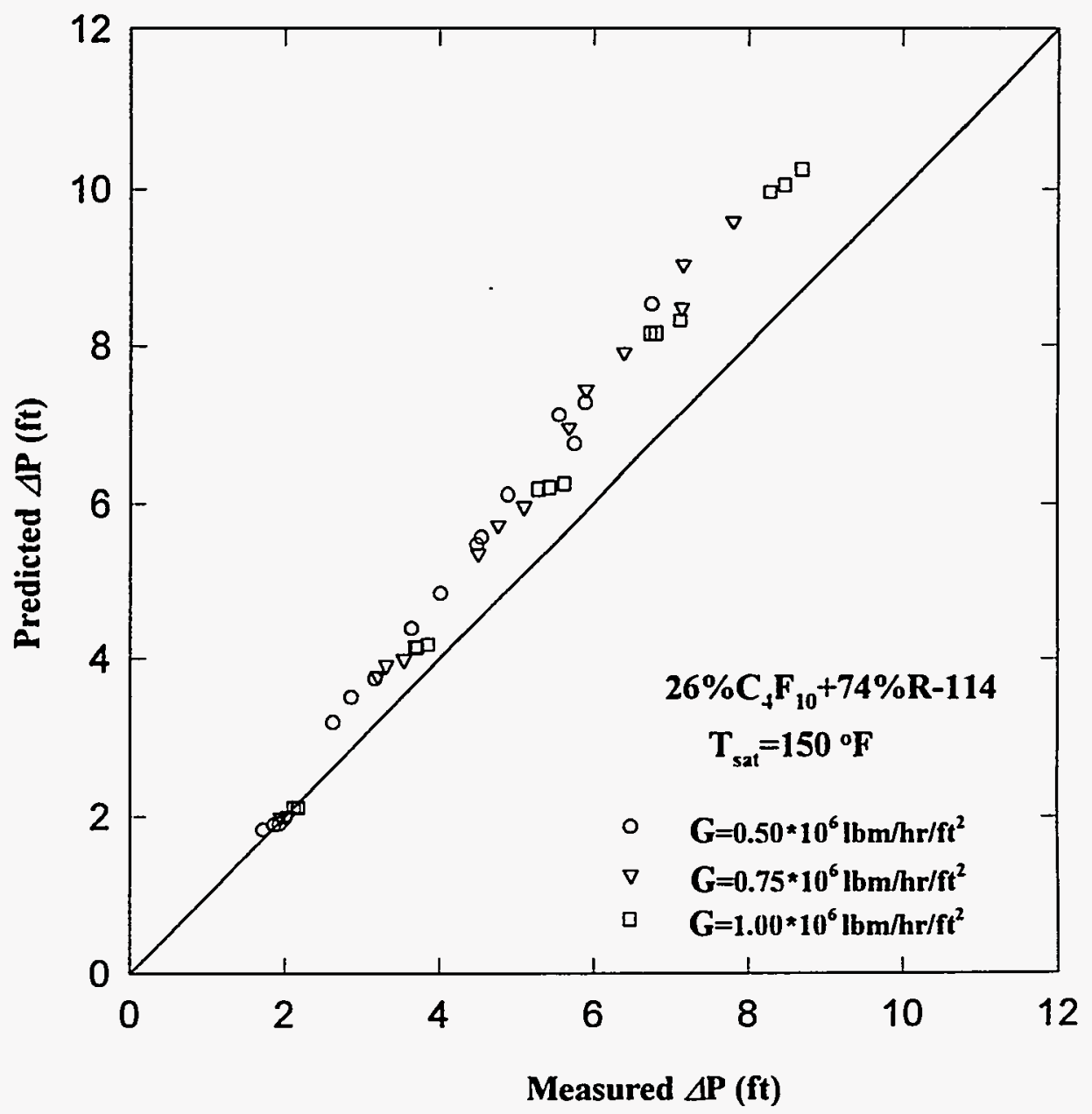

Figure 31. Comparison of Measured and Predicted Pressure Drop for $26 \% \mathrm{C}_{4} \mathrm{~F}_{10}+74 \% \mathrm{R}-114$ at $\mathrm{T}_{\mathrm{sat}}=150 \cdot \mathrm{F}$ 


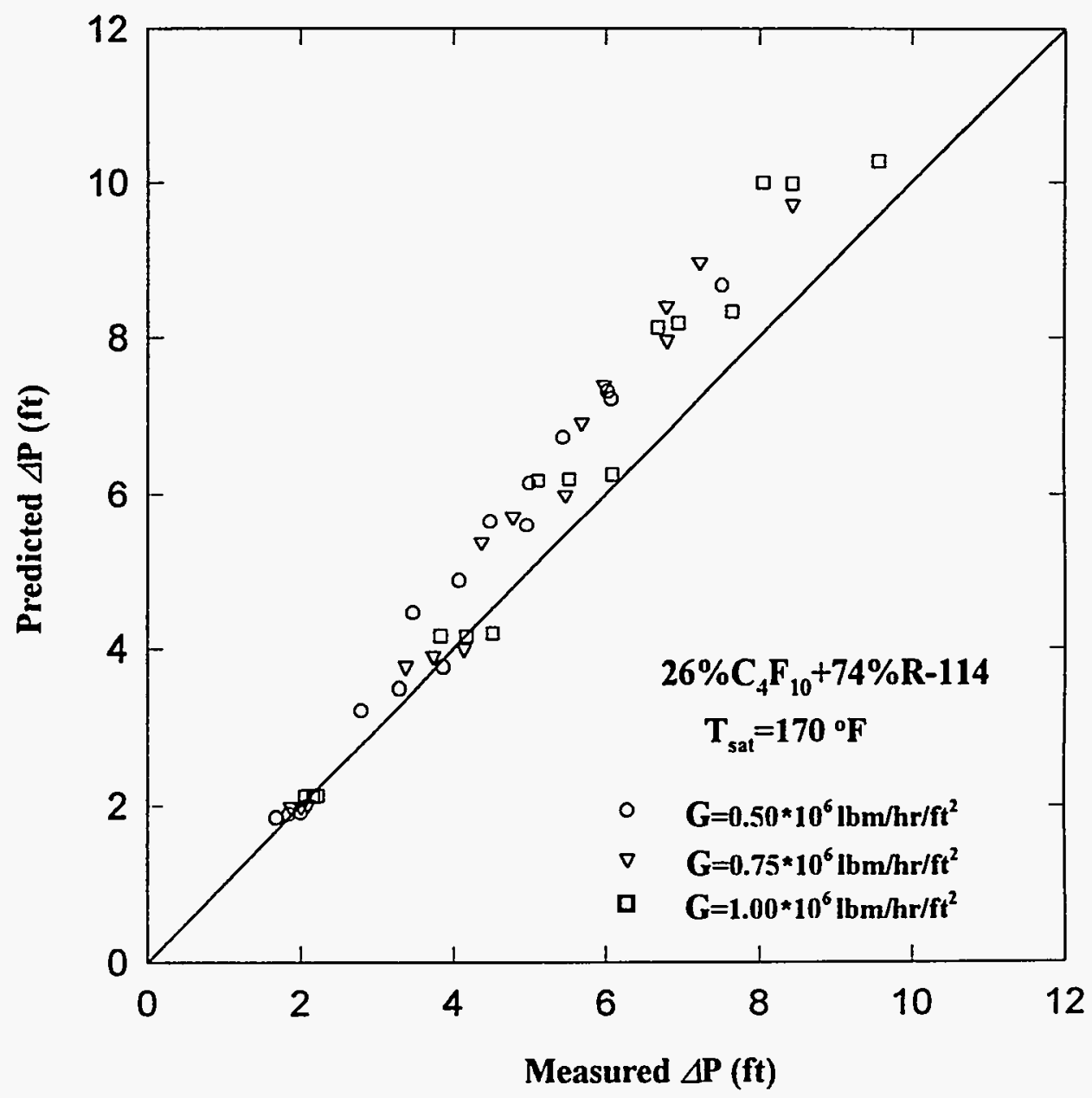

Figure 32. Comparison of Measured and Predicted Pressure Drop for $26 \% \mathrm{C}_{4} \mathrm{~F}_{10}+74 \% \mathrm{R}-114$ at $\mathrm{T}_{\text {sal }}=170 \cdot \mathrm{F}$ 


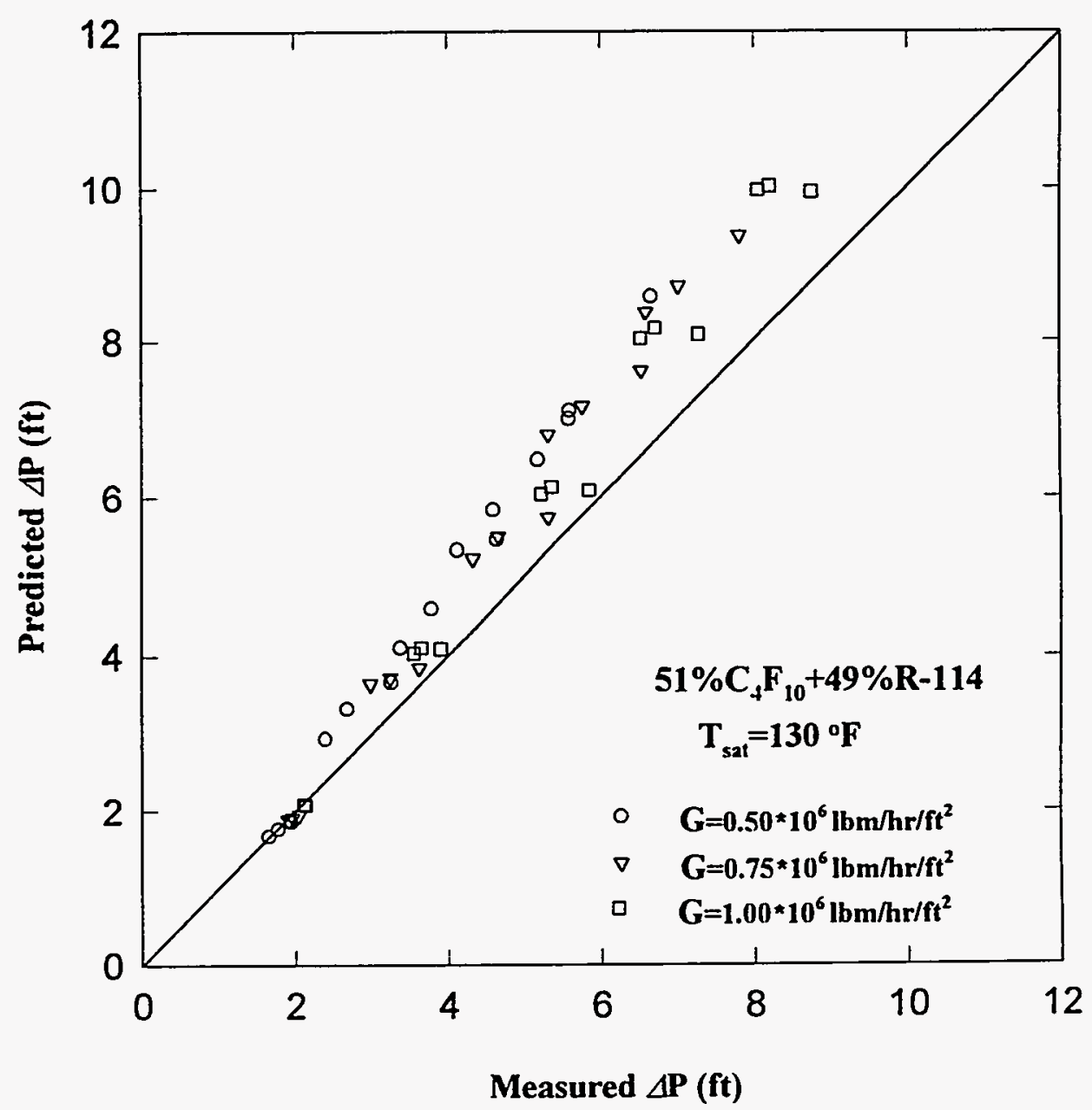

Figure 33. Comparison of Measured and Predicted Pressure Drop for $51 \% \mathrm{C}_{4} \mathrm{~F}_{10}+49 \% \mathrm{R}-114$ at $\mathrm{T}_{\mathrm{sat}}=130 \cdot \mathrm{F}$ 


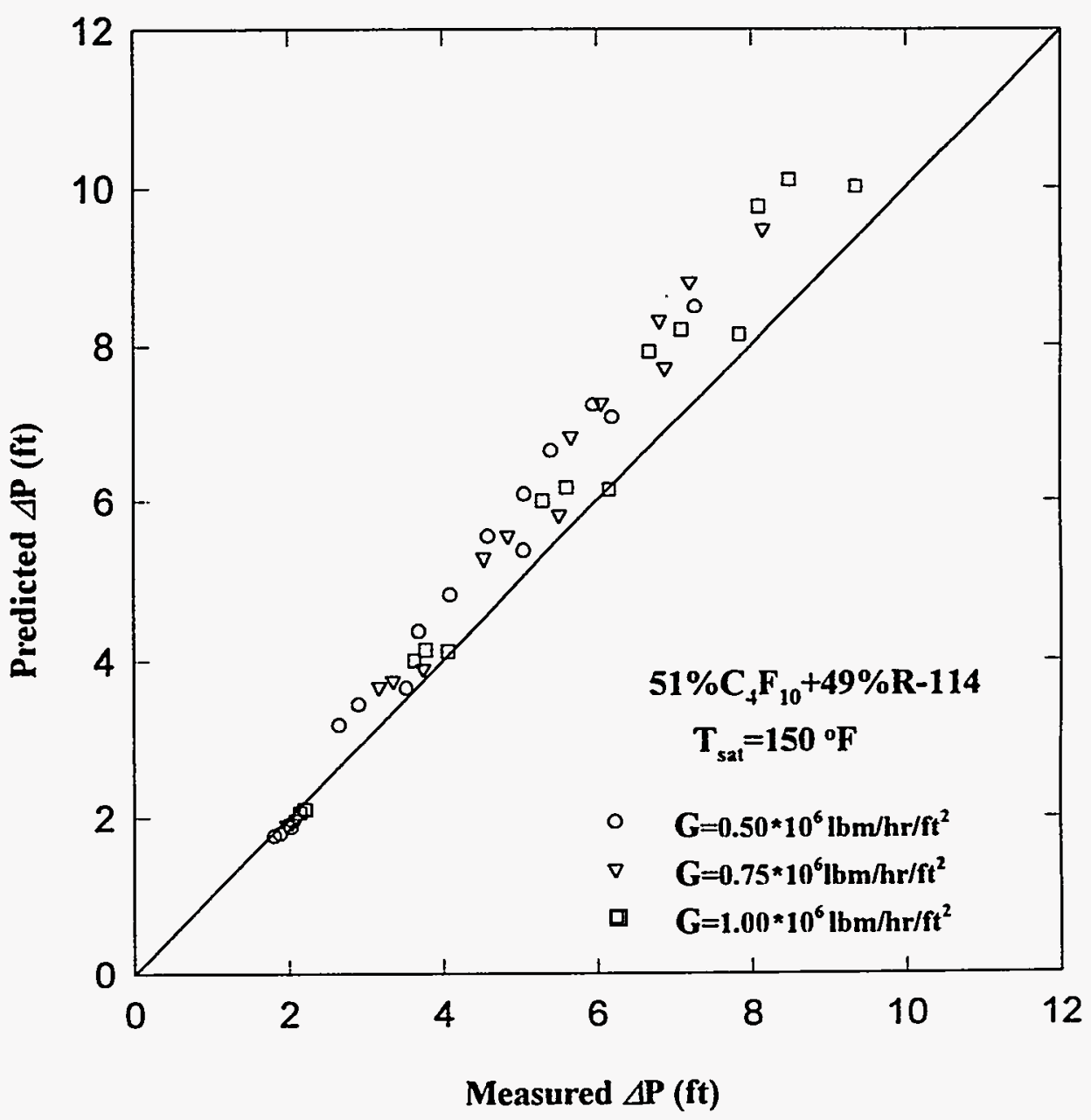

Figure 34. Comparison of Measured and Predicted Pressure Drop for $51 \% \mathrm{C}_{4} \mathrm{~F}_{10}+49 \% \mathrm{R}-114$ at $\mathrm{T}_{\text {sat }}=150 \cdot \mathrm{F}$ 


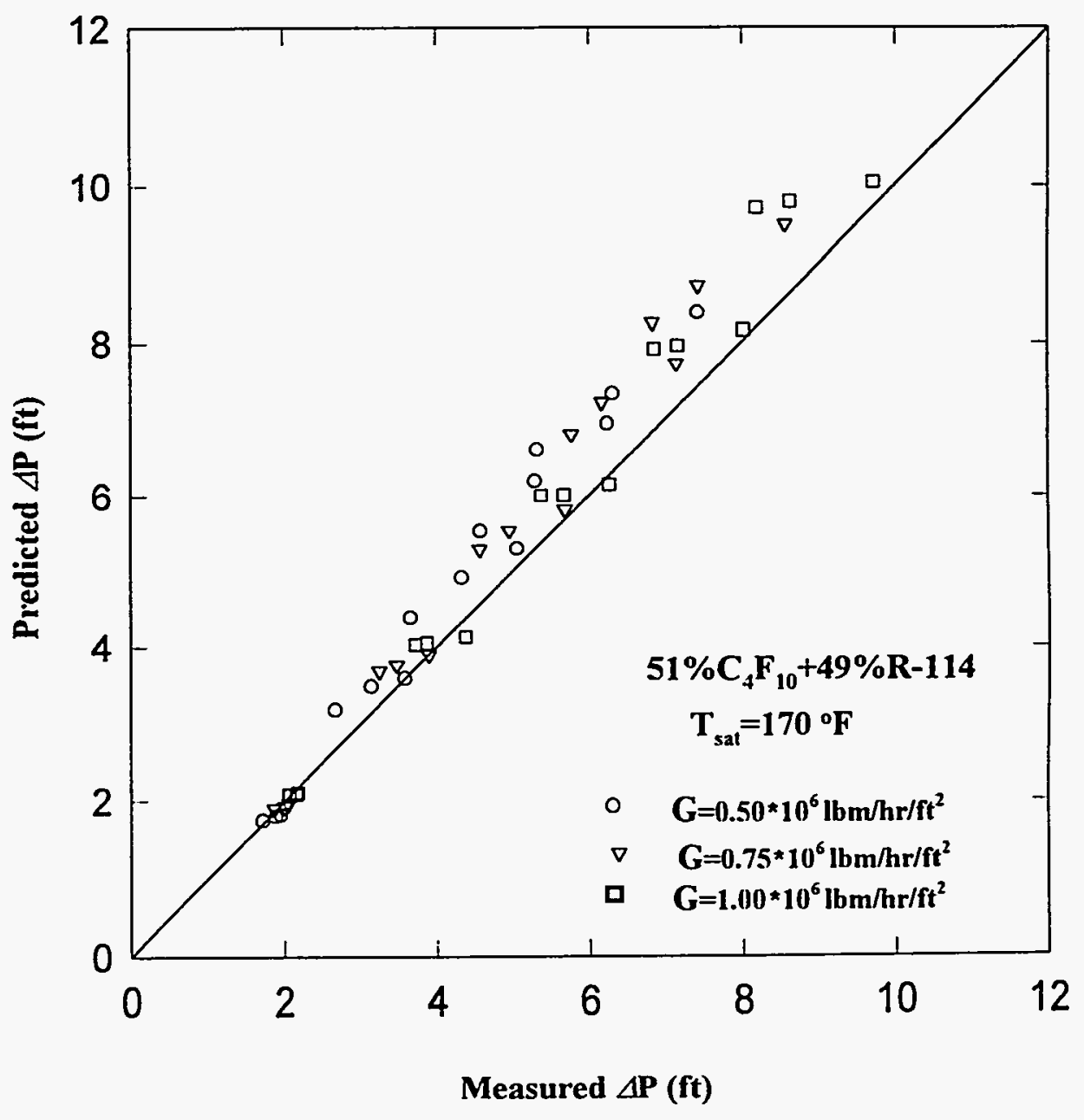

Figure 35. Comparison of Measured and Predicted Pressure Drop for $51 \% \mathrm{C}_{4} \mathrm{~F}_{10}+49 \% \mathrm{R}-114$ at $\mathrm{T}_{\mathrm{sat}}=170 \cdot \mathrm{F}$ 


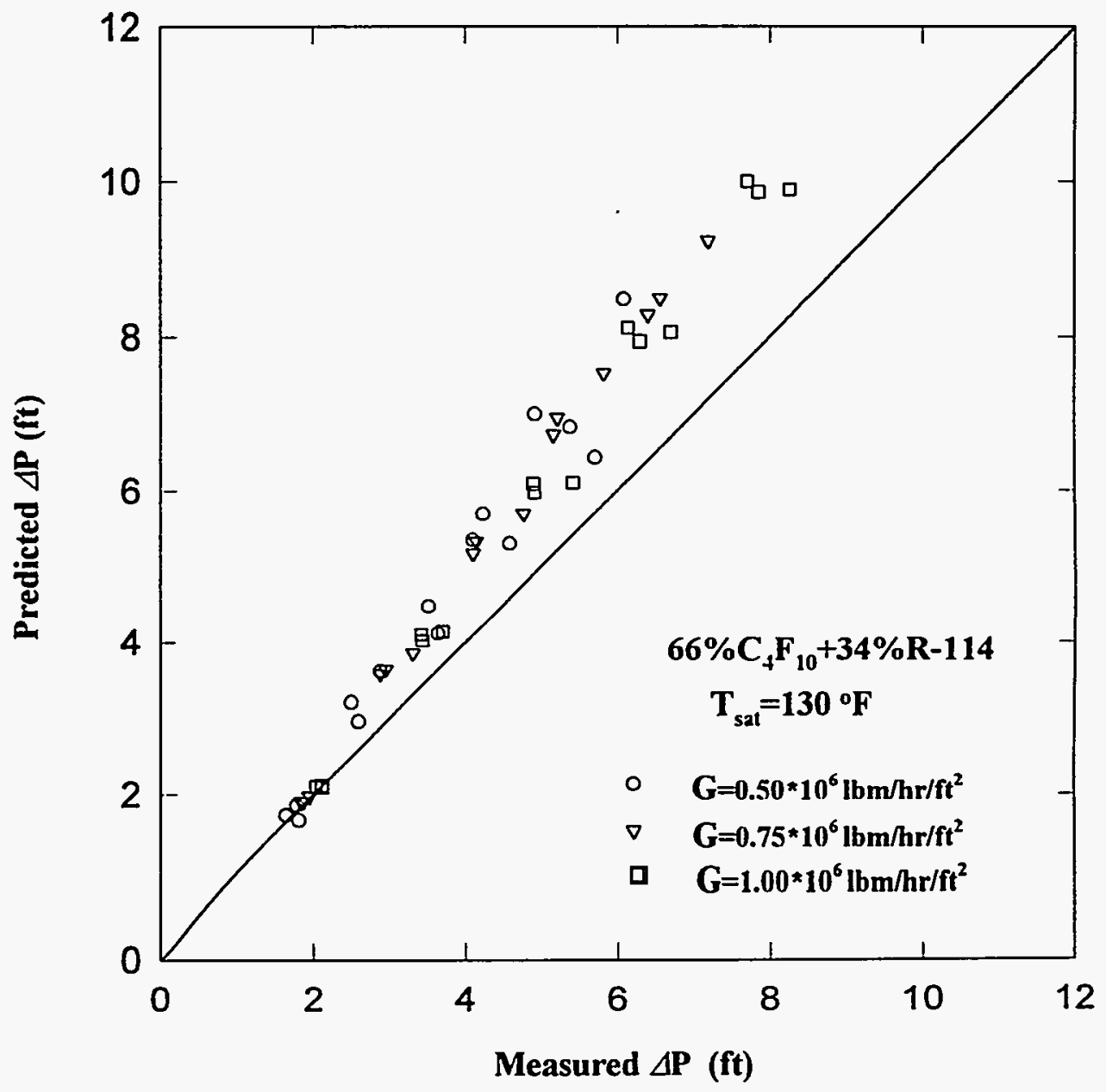

Figure 36. Comparison of Measured and Predicted Pressure Drop for $66 \% \mathrm{C}_{4} \mathrm{~F}_{10}+34 \% \mathrm{R}-114$ at $\mathrm{T}_{\mathrm{sat}}=130 \cdot \mathrm{F}$ 


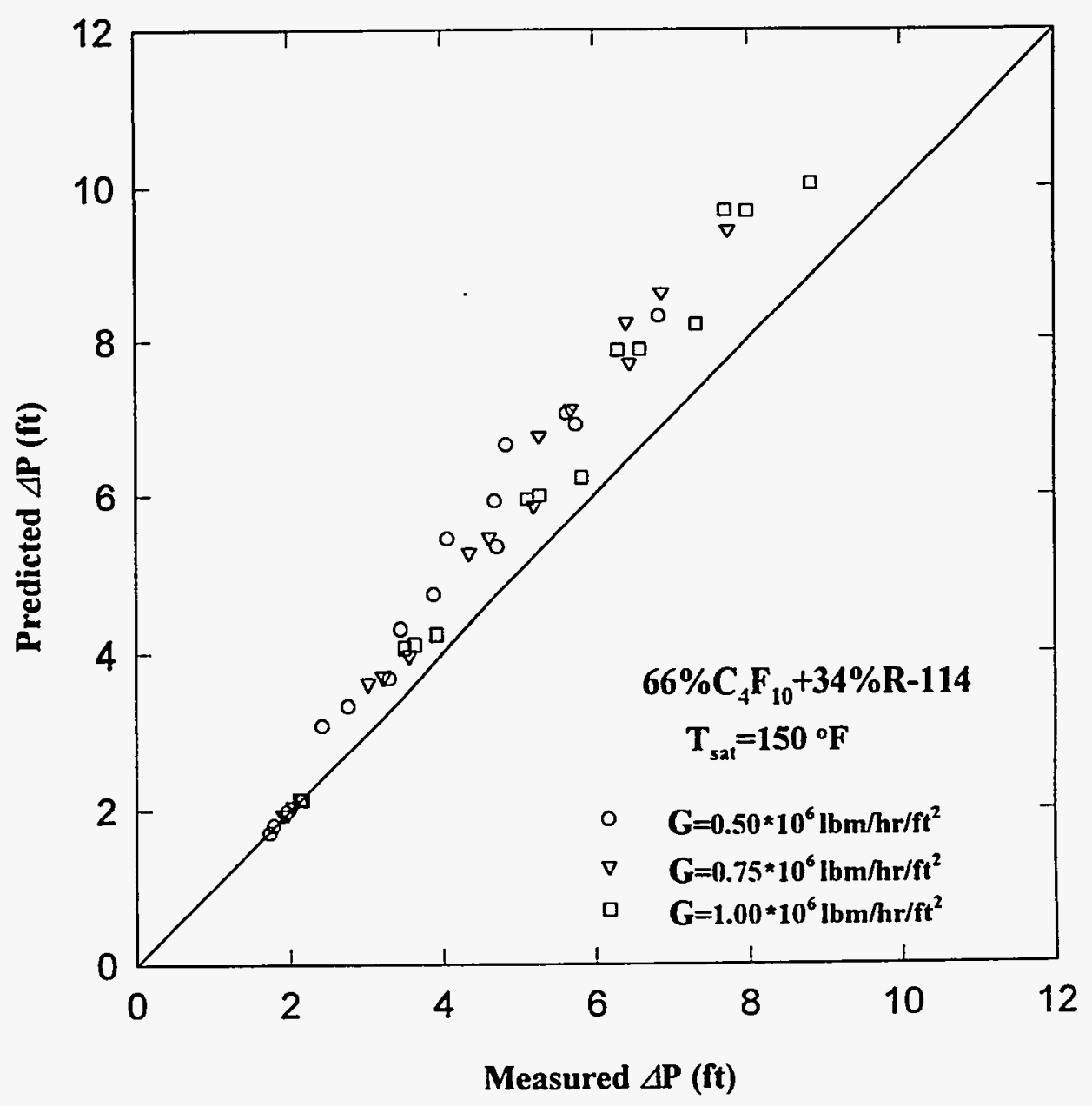

Figure 37. Comparison of Measured and Predicted Pressure Drop for $66 \% \mathrm{C}_{4} \mathrm{~F}_{10}+34 \% \mathrm{R}-114$ at $\mathrm{T}_{\text {sat }}=150 \cdot \mathrm{F}$ 


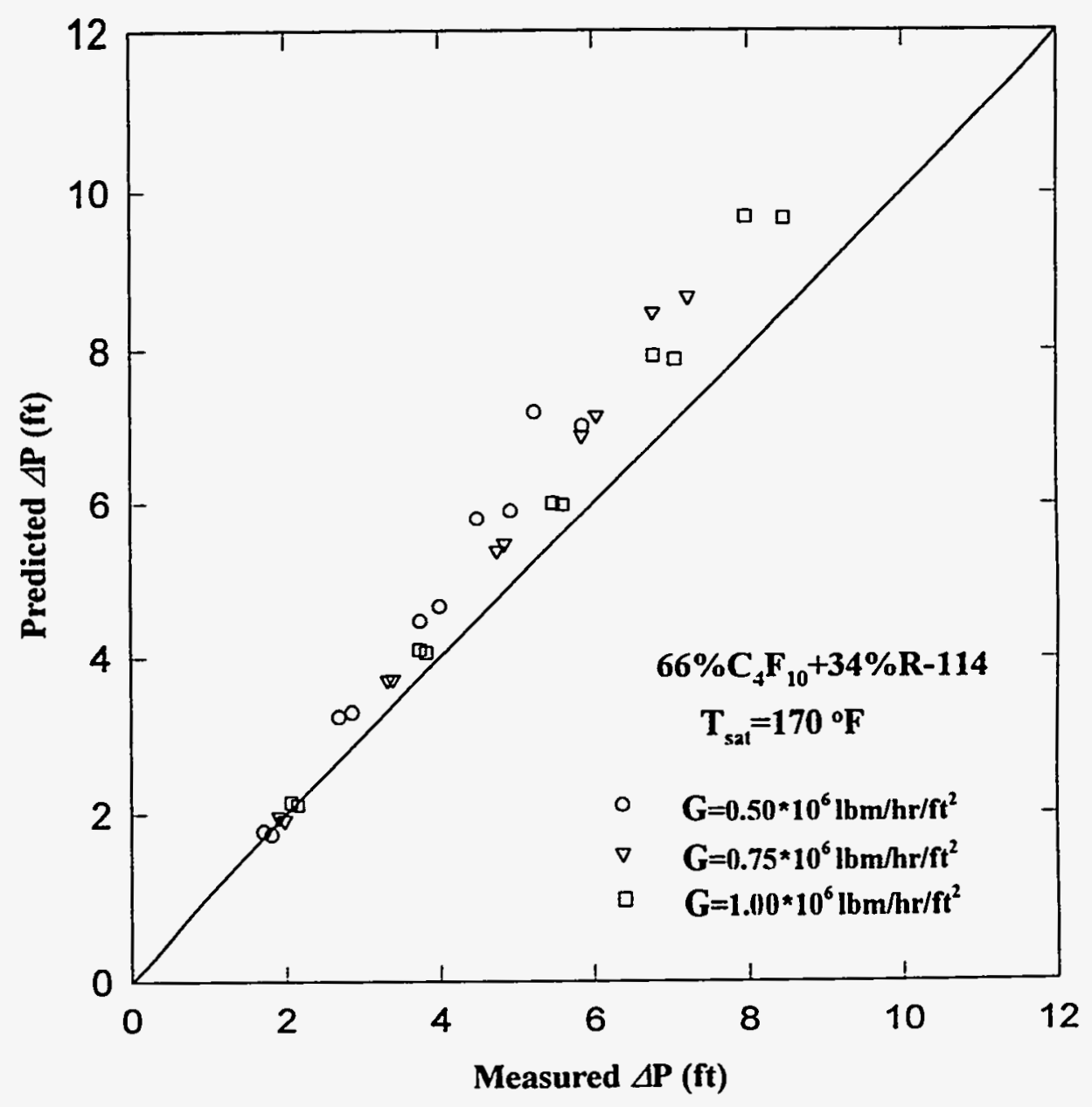

Figure 38. Comparison of Measured and Predicted Pressure Drop for $66 \% \mathrm{C}_{4} \mathrm{~F}_{10}+34 \% \mathrm{R}-114$ at $\mathrm{T}_{\mathrm{sat}}=170 \cdot \mathrm{F}$ 


\section{Section 4}

Test Data 


\section{Appendix A}

The data contains terms both measured and calculated. The definition of each term listed on the data sheets is as follows:

Run \# A sequential test number

Test Fluid Coolant tested.

Coolants tested are CFC-114, $\mathrm{C}_{4} \mathrm{~F}_{10}, \mathrm{C}_{4} \mathrm{~F}_{8}$, Mixture of CFC-114 \& $\mathrm{C}_{4} \mathrm{~F}_{10}$

Flow Area-ft ${ }^{2} \quad$ Cross-section area of test tube

Heat Flow Area- $f t^{2} \quad$ Total heating area

Sat. Pressure-psig Saturation pressure of coolant entering the test section

Sat. Temp.-F(inlet) Saturation temperature of coolant entering the test section

\section{Physical Properties of Coolant}

Liquid density Coolant liquid density based on condition listed below for each zone

Latent heat Latent heat of coolant based on condition listed below

Specific heat Specific heat of coolant based on condition lised below

Liquid viscosity Viscosity of liquid coolant based on condition listed below

Liquid thermal

conductivity

Thermal conductivity of coolant in liquid based on condition listed below

Liquid Prandtl

Number

Prandtl number of coolant in liquid based on condition listed below

Inlet Flow

Rate-GPM

Coolant flow rate into test section

Outlet Liq. Flow

Rate-GPM

Flow rate of outlet liquid coolant separated from entrainment separator

Outlet Vap. Flow

Rate-GPM

Flow rate of outlet vapor coolant separated from entichment separator

Inlet Temperature-F Temperature of coolant entering the test section 
Outlet Liq.Temp.-F

Outlet Vap. Temp.-F

Water Flow Rate-GPM

Water Inlet Temp.-F

Water Outlet Temp.-F

Inlet G-lbm/hr/ft

Subcooling, $F$

Flow Observation
Temperature of outlet liquid coolant

Temperature of outlet vapor coolant

Recirculating cooling water flow rate to condenser

Temperature of water entering the condenser

Temperature of water exiting the condenser

Coolant mass flux, [flow rate/tube cross-section area]

Target subcooling of coolant at entrance of test section

There are 10 separate heating zones on the test section. [Zone 1 - Zone 10]

Heat Input-Watts(net)

Heat Loss-Watts

Heat Flux-Btu/hr/ft

Wall Temp.,-F

$\# 1$ to \#5

Instream Temp.,-F

Ave. Wall Temp.-F

Ave.Wall DT.-F

Remaining Subcool

Exit Quality

Exp. $H$

Jallouk
Net heat input to heating zone

Heat loss from the heating zone to ambient, measured

Heat flux to test section

Five temperature sensors in each zone to measure cylinder wall temperature

One temperature sensor to measure coolant temperature

Average wall temperature from five sensors

Difference between average wall temperature and instream temperature

Difference between desired subcool and measured subcool (See Item 1 in Appendix B)

Quality of coolant exiting the test section (See Item 2 in Appendix B)

Boiling heat transfer coefficient obtained from test

Boiling heat transfer coefficient by Jaliouk's method (See Item 3 in Appendix B) 
Szady1(Cudo Tube)

Szady2

RLIQ, F, HLIQ, HBOI
Boiling heat transfer coefficient by Szady's method using pool boiling data generated from the test section at Cudo Tech. (See Item 3 in Appendix B)

Boiling heat transfer coefficient by Szady's revised method using different values for the Rohsenow correlation. (See Item 3 in Appendix B)

See Item 4 in Appendix B

Pressures were measured at five locations along test section.

DP liquid Temp., $F$

DP Liquid Density-lbm/ft $t^{3}$

Elevation-ft

Elevation of pressure tap measured from the entrance of test section

Measured DP - psid

Differential pressure measured between the entrance and 5 pressure taps

Zero Corrections - psid

Correction factor for reference pressure point

Corrected DP -psid

Corrected pressure differential between entrance and 5 pressure taps

Actual DP - $f t$

Measured pressure differential

Measured TP - psig

Total pressure measured at entrance point of test section

Measured Overall - $f t$

Overall pressure head measured

computed DP - ft

Computed pressure differential

Friction - $f t$

Pressure loss by friction

Acceleration - $f t$

Pressure loss by fluid acceleration

Elevation - $f t$

Pressure loss due to elevation variation 


\section{Appendix B}

\section{Remaining subcool}

$$
\text { Remaining Subcool }=\text { Subcooling }-\frac{q^{\prime \prime} A_{\text {HeatFlow }}}{G A_{\text {Flow }} C_{p l}}
$$

where,

$\begin{array}{lll}\mathrm{q} " & \text { Heat flux }\left[\mathrm{btu} / \mathrm{hr} / \mathrm{ft}^{2}\right] & =5011 \\ \mathrm{~A}_{\text {HealFlow }} & \text { Heat Flow Area }\left[\mathrm{ft}^{2}\right] & =0.19695 \\ \mathrm{~A}_{\text {Flow }} & \text { Flow Area }\left[\mathrm{ft}^{2}\right] & =0.00336 \\ \mathrm{G} & \text { Inlet Liquid Flow Rate }\left[\mathrm{lbm} / \mathrm{hr} / \mathrm{ft}^{2}\right] & =1021878 \\ \mathrm{C}_{\mathrm{p}} & \text { Specific Heat }\left[\mathrm{btu} / \mathrm{lbm} /{ }^{\circ} \mathrm{F}\right] & =0.312 \\ \text { Subcooling }\left[{ }^{\circ} \mathrm{F}\right] & =1.6 \\ \text { Remaining Subcool }\left[{ }^{\circ} \mathrm{F}\right] & =0.6\end{array}$

\section{Exit Quality}

$$
\begin{gathered}
x_{\text {in }}=0 \text { [only liquid entering at section 1] } \\
x_{\text {out }}=\frac{q " A_{\text {HeatFlow }}-G A_{\text {Flow }} C_{p l} \Delta T_{\text {sub }}}{G A_{\text {Flow }} H_{f g}}
\end{gathered}
$$

$\begin{array}{lll}\Delta \mathrm{T}_{\text {sub }} & \text { Subcooling }\left[{ }^{\circ} \mathrm{F}\right] & =1.6 \\ \mathrm{H}_{\mathrm{fg}} & \text { Latent Heat }[\mathrm{btu} / \mathrm{lbm}] & =31.2 \\ \mathrm{X}_{\mathrm{out}} & \text { Exit Quality } & =-0.01 \text { (within experimental uncertainty) }\end{array}$




\section{Evaluation of Experimental $H$ and Computed $H$ (Jallouk, Szady1, Szady2)}

Note eq. 14 of report ( Vol \#2)

Nucleate boiling correlation used for flow boiling presented by Rohsenow [6],

$$
\frac{C_{p l} \Delta T}{H_{f g} P_{l}^{1.7}}=C_{w f}\left(\frac{Q / A}{\mu_{l} H_{f g}} \sqrt{\frac{\sigma}{g \Delta \rho}}\right)^{n}
$$

The coefficient $\mathbf{C}_{\mathrm{wf}}$ is dependent on the wall-fluid material characteristics and was determined from the pool boiling experiments. The value of the exponent $\mathbf{n}$ changes for the different predictions.

The experimental results for the fluid were plotted using the Rohsenow [6] correlation Eq. (14) with a logarithmic scale. For the Cudo tube (Szady1) the data correlated with an intercept value of $\mathbf{C}_{\mathrm{wf}}=$ 0.0038025 and $\mathbf{n}=0.494$. This number is the experimentally determined value of the coefficient $\mathbf{C}_{\mathrm{wf}}$ in Eq. (14). After the coefficient $\mathbf{C}_{\mathrm{wf}}$ is determined, the correlation of the nucleate boiling term $\mathbf{h}_{\mathrm{nb}}$ in Eq. (11) can be obtained from Eq. (14) in the form,

$$
h_{n b}=\frac{Q}{A \Delta T}=\left(\frac{k_{l}^{1.7}}{C_{w f} \mu_{l}^{1.7} C_{p l}^{0.7}}\right)^{3} \mu_{l}\left(\frac{\Delta T}{H_{f g}}\right)^{2} \sqrt{\frac{g \Delta \rho}{\sigma}}
$$

For Jallouk and Szady2 the following values were used: $\mathbf{C}_{\mathrm{wf}}=0.0047$ and $\mathbf{n}=0.33$

4. Rliq Reynolds number of liquid

$$
R_{l i q}=\frac{\rho V D(1-x)}{\mu_{l}}
$$

$V \quad$ Mean flow velocity

$D$ Diameter of tube 


\section{5. $F, \mathbf{H}_{\text {liq }}, \mathbf{H}_{\text {boil }}$}

Steiner and Taborek proposed a correlation for in-tube flow boiling data based on a variant of the asymptotic model. Their correlation is based on the asymptotic model Eqs. $(7,8)$ with $\mathbf{n}=3$. The two components in this model are independent of each other,

$$
h^{n}=\left[H_{L i q}^{n}+H_{B o i l}^{n}\right]^{\frac{1}{n}}
$$

$$
=\left[\left(h_{n p b} C_{n b f}\right)^{n}+\left(h_{l} F\right)^{n}\right]^{\frac{1}{n}}
$$

here,

$\mathbf{h}_{\mathrm{npb}}$ is the local nucleate pool boiling coefficient. The coefficient itself can originate from any predictive method or data. Thus, it leaves a great freedom of choice.

$\mathbf{C}_{\mathrm{nbf}}$ is a nucleate boiling correction factor to $h_{\mathrm{npb}}$, which compensates for differences between the pool and the flow conditions.

$\mathbf{h}_{\ell}$ is the local convective heat-transfer coefficient for single-phase liquid flow.

F is the two-phase flow correction factor to the convective component $\mathrm{h}_{\ell}$, accounting for enhancement of the coefficient in the liquid-vapor mixture.

and $\mathbf{h}_{\boldsymbol{t}}$ is obtained from the Dittus-Boelter type equation as given below,

$$
\mathrm{N}=\frac{h_{t} d_{i}}{k}=c \operatorname{Re}^{0.8} \operatorname{Pr}^{0.4}
$$




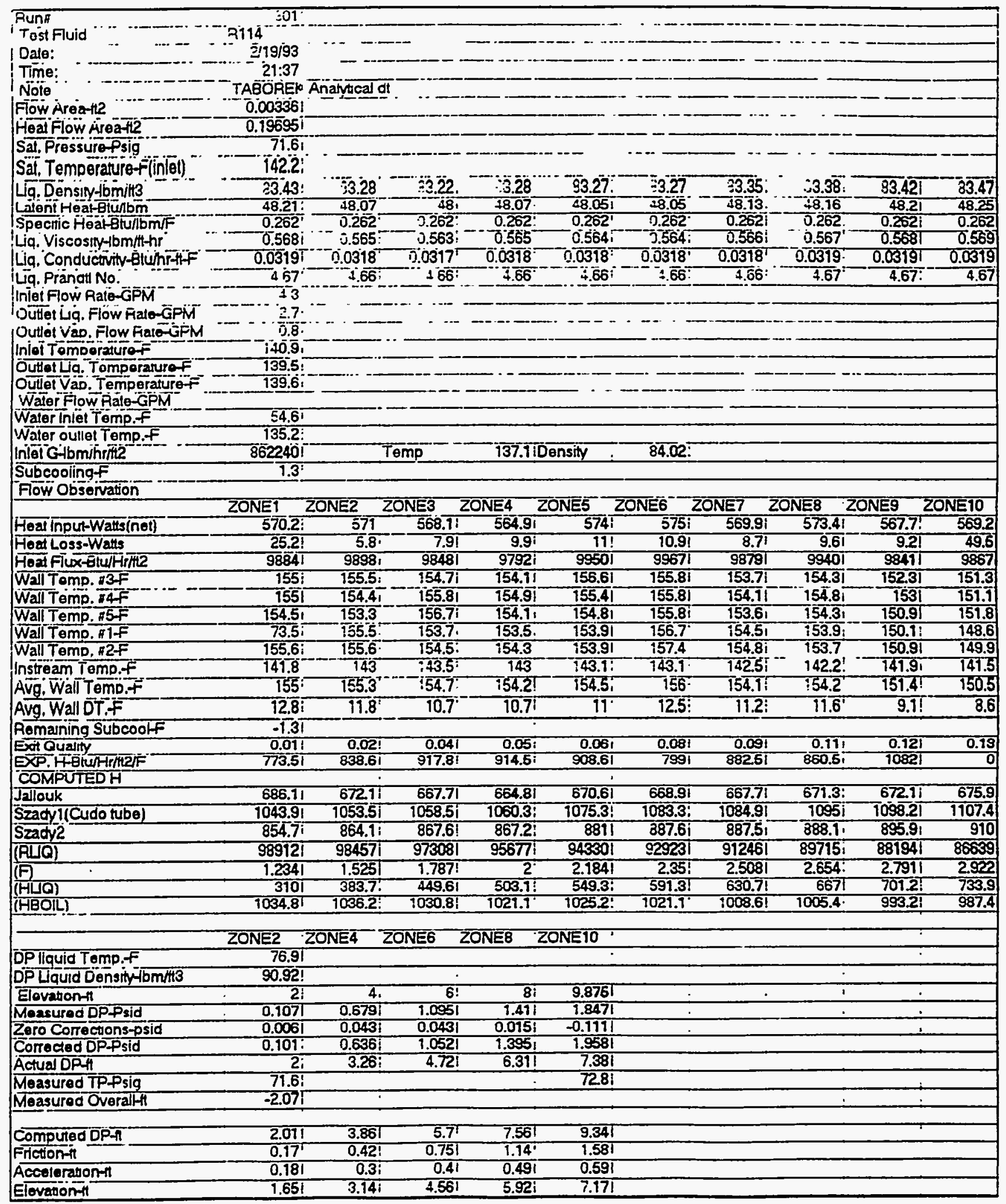




\begin{tabular}{|c|c|c|c|c|c|c|c|c|c|c|}
\hline Runt & 3021 & & & & & 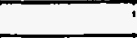 & 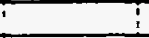 & 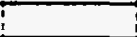 & $i$ & \\
\hline Test fuid & ip114 & & & i & 3 & 7 & 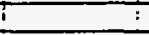 & 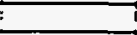 & & \\
\hline Dat: & 2/19931 & i & 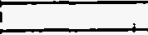 & i & $\bar{z}$ & $i$ & $i$ & 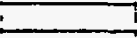 & & \\
\hline Time: & $23: 511$ & i & 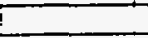 & $i$ & I & $i$ & i & & $i$ & \\
\hline Nole & TABOREK & Analutical d & dt & $!$ & & & I & I & 5 & \\
\hline Flow Aresti2 & $i \quad 0.003561$ & 1 & 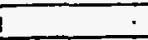 & 1 & $i$ & i & 1 & 1 & & \\
\hline Hos Fow Arest2 & 0.19505 & i & $i$ & i & 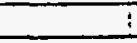 & 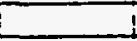 & 1 & 1 & & \\
\hline Sad. Presture Psig & 85.6 & $\frac{1}{1}$ & 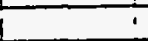 & 1 & 1 & 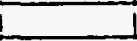 & $i$ & $i$ & 1 & \\
\hline Sal. Tempermerrof(indel) & 153.21 & 1 & 1 & 1 & 1 & +1 & 1 & I & & \\
\hline Lig. Density tom & 81.99 & 81.91 & 81.72 & 81.75 & 81.74 & 81.72 & 81.76 & 81.82 & 81.84 & 81.86 \\
\hline Letenx Heat Btulbm & 46.82 & 46.74 & 46.561 & 46.591 & 46.571 & 46.56 & 46.6 & 46.65 & 46.68 & 46.79 \\
\hline Specifo Heal Buhlbmif & 0.266 & 0.266 & 0.266 & 0.266 & 0.2661 & 0.256 & 0.2661 & 0.265 & 0.256 & 0.268 \\
\hline Wa. Viscosinfbminhr & 0.5381 & 0.5361 & 0.532 & 0.5331 & 0.5331 & 0.532 & 0.5331 & 0.534 & 0.535 & 0.537 \\
\hline Liq. Conductivity Btuhn AfF & 0.03091 & 0.0309 & 0.0308 & 0.0308 & 0.0508 & 0.0308 & 0.0308 & 0.0308 & 0.0308 & 0.0309 \\
\hline Liq. Prandi No. & 4.611 & 4.611 & 4.611 & 4.611 & 4.611 & 4.61 & 4.61 & 4.61 & 4.61 & 4.61 \\
\hline Ind Fow Rat-GPM & 5.11 & i & i & 1 & 1 & & 1 & -1 & & \\
\hline Oulot Lig. Flow Rat-GPM & 3.21 & & & t & & & & & $i$ & \\
\hline OUdA VaO. Flow RA GTPM & 0.81 & i & I & 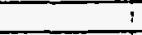 & & & & & & \\
\hline Intat Tompermuref & 15231 & 1 & 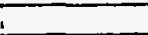 & & i & & & & & \\
\hline Outef Lug. Temparature & 157.21 & & 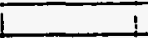 & 1 & & & & & & \\
\hline OTtat vep. Temperaturef & 151.5 & 1 & 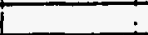 & 1 & 1 & & & & & \\
\hline Water Flow PaI-GPM & & & 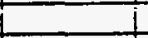 & & & & & & & \\
\hline Werer hlet Temo.f & 61.1 & & & & 1 & & & & & \\
\hline Weter outel Temo.f & 148.81 & & & & + & & & & & \\
\hline Intot Gibminntive & 10058141 & & Tomp & 148 & Density & 8264 & & & & \\
\hline Subcoolingf & 0.91 & & 工 & 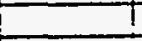 & & & & & & \\
\hline Flow Observation & $T$ & & & & & & & & & \\
\hline & ZZONET & ZONE2 & ZONES |2 & ZONET & ZONES & ZONEB & ZONET & ZONE & ZONE9 & ZONETO \\
\hline Hod hout-Wastinet) & | 561.8 & 568 & 570.6 & 573.7 & 569.8 & 568.4 & 569.8 & 570.6 & 575.6 & 5728 \\
\hline Hodlose+Wals & 31 & 6.8 & 8.9 & 10 & 10.8 & 10.5 & 9.7 & 10.8 & 10.7 & 66 \\
\hline 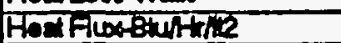 & 973 & अाठ। & ङ91 & 36 & $\$ 88$ & \$6 & 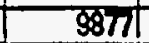 & क5: & 9976 & $\$ 8 \% 1$ \\
\hline WEITOMP. R3F & 165.5 & 165.8 & 165.8 & 765.4 & 167.7 & 167 & 1652 & 165.4 & 163.8 & 1622 \\
\hline WEITOMP. IAF & 165.8 & 165.3 & 168.5 & 168.1 & 166.7 & 167 & 165.7 & 168.8 & 164.6 & 1620 \\
\hline Wall Tomo. $85+$ & 165.3 & 164 & 167.3 & 165.2 & 165.8 & 167.2 & 165.1 & 165.6 & 1026 & 168.4 \\
\hline Wall Temp. 1 F & 731 & 166.4 & 164.8 & 164.6 & 165 & 168.1 & 168 & 165.5 & 161.8 & 169.8 \\
\hline Whi Temp. $2 F$ & 166.4 & 966.4 & 165.3 & 165.1 & 165.3 & 168.6 & 165.3 & 165.5 & 1625 & 1616 \\
\hline Instream Tomp.f & 1531 & 153.6 & 155 & 154.8 & 154.9 & 155 & 154.7 & 154.3 & 154.7 & 1532. \\
\hline Avg. Wall Tamp.f & 165.8 & 166.11 & 165.6 & 165.3 & 165.3 & 167.3 & 165.7 & 965.6 & 163.1 & 161.8 \\
\hline Avg. Wall DT.F & 12.3 & 12.1 & 10.1 & 10.1 & 10.4 & 11.9 & 10.5 & 10.9 & 8.5 & 8.3 \\
\hline Remaning Subcoolf & -1.2 & & & & & & 5 & & & \\
\hline Ext Gualy & 0.011 & 0.021 & 0.03 & 0.04 & 0.06 & 0.07 & 0.08 & 0.09 & 0.11 & 0.12 \\
\hline 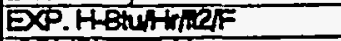 & 791.1 & 816.7 & 978 & 989.5 & 950.8 & 829.4 & 939.5 & 908 & 11724 & 0 \\
\hline COMAOUTEDH & & & & & & & & & & \\
\hline Jaliout & 523 & 689 & 690.5 & 691.7 & 688.4 & 687.3 & 689.41 & 691.7 & 658.2 & 695.7 \\
\hline Sedyilado tuba) & 1077.7 & 1091 & 1104.8 & 1113.5 & 1117 & 1124 & 11322 & 1139.9 & 1152 & 1150.7 \\
\hline $\operatorname{sen} 12$ & 870.4 & 885.4 & 900.8 & 922.8 & 922.8 & 927 & 933.4 & 940 & 94.8 & 9562 \\
\hline (R्य) & 121964 & 121147 & 1204501 & 118805 & 117329 & 115864 & 114174 & 11243 & 110766 & 10878 \\
\hline (F) & 1.214 & $1.47 \pi$ & 1.682 & 1.876 & 2.034 & 2.175 & 2.300 & 2.434 & 2561 & 2680 \\
\hline 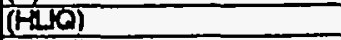 & 348.4 & 4222 & 485.8 & 539 & 584.6 & 625.2 & 6532 & 699 & 732.6 & 763.2 \\
\hline (H\&OIL) & 1055.4 & 1069.51 & 10722 & 1068.7 & 1050.9 & 1055.4 & 1050.5 & 1044.6 & 1043.4 & 10358 \\
\hline & -5 & & -1 & 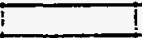 & & & & & & \\
\hline & ZZONEZ & ZONE4 & ZONEB & ZONES & ZONETO & & & & & \\
\hline DP liquid Temp.F & $1 \quad 75.4$ & & & & & & & & & \\
\hline DPLIquid Dansithm/a3 & 91.081 & & & & & & & & & \\
\hline Elevetionth & $2 !$ & 4 & 6 & 8 & 9.875 & & & & & \\
\hline Mossured DPPsid & 0.084 & 0.55 & 0.9011 & 1.12 & 1.44 & & & & & \\
\hline Zero Corrections-psid & 0.006 & 0.043 & 0.0531 & 0.015 & -0.111 & & & & & \\
\hline Correded DPPsid & 0.078 & 0.507 & 0.8581 & 1.105 & 1.553 & & & & & \\
\hline Actealofil & 200 & 3.55 & 5.18 & 6.95 & 8.5 & & & & & \\
\hline Maseurod TPFitg & 85.8 & & & & 87.6 & & & & & \\
\hline Meseured Ovarallh & 3.34 & & & & & & & & & \\
\hline & & & & & & & & & & \\
\hline Compured DPA & 2.12 & 4.09 & 6.04 & 8 & 9.88 & & & & & \\
\hline Frictiont & 0.21 & 0.52 & 0.91 & 1.36 & 7.85 & & & & & \\
\hline Aocotoption-ti & 0.22 & 0.36 & 0.47 & 0.57 & 0.68 & & & & & \\
\hline Elevation-t & 1.68 & 3.21 & 4.671 & 6.07 & 7.35 & & & & & \\
\hline
\end{tabular}




\begin{tabular}{|c|c|c|c|c|c|c|c|c|c|c|}
\hline & & & 1 & & ! & ! & & & i & ! \\
\hline Runt & 3031 & & T & $!$ & $i$ & i & & & & \\
\hline Tost Fuid & :R114 & & $i$ & $i$ & 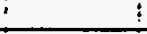 & 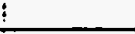 & & & & \\
\hline Dade: & $2 / 20930$ & & I & 1 & i & I & & L & & \\
\hline Timo: & $10: 30$ & & I & $!$ & 1 & 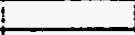 & & & & \\
\hline Note & I TABOREA & Analytion & & i & I & & & & & \\
\hline Flow Auarle & 0.00336 & & I & T & $i$ & T & & & & \\
\hline Hoal Flow Aroat? & $0.19 \% 5$ & & 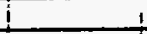 & 1 & i & I & i & & & \\
\hline S2.P7ossurefsig & 81.41 & & $\perp$ & $I$ & I & I & L & & & \\
\hline Sal. Tompereuref(intel) & 1501 & & I & 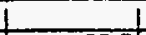 & L & L & & & & \\
\hline Liq.Densitytomnis & 82.381 & 822 & 82.11 & 822 & 8221 & 82.17 & 822 & 8224 & 82.82 & 8230 \\
\hline Letonk Hostefulom & 47.21 & 47.02 & 46.931 & 47.02) & 47.03 & 47 & $47 . \infty 2$ & 47.05 & 47.13 & 47.11 \\
\hline Speoifio Heategturbm/F & 0.2651 & 0.265 & 0.2651 & 0.265 & 0.265 & 0.265 & 0.265 & 0.265 & 0.265 & 0.200 \\
\hline Lig. Viscositylbminhr & 0.5461 & 0.542 & 0.541 & 0.542 & 0.542 & 0.541 & 0.542 & 0.543 & 0.541 & $0.5 \times 4$ \\
\hline Ud. Conductivity otuhr-fif & 0.0312 & 0.0311 & 0.0371 & 0.03111 & 0.03111 & 0.0311 & 0.0311 & 0.0371 & 0.0312 & 0.0011 \\
\hline Lig. Prandd No. & 4.63 & 4.62 & 4.621 & 4.62 & 4.62 & 4.62 & 4.02 & 4.02 & 4.22 & 4.62 \\
\hline Intetflow Rate-GPM & 3.51 & & i & 1 & 1 & I & & & & \\
\hline Outel Lia. Flow Rate-GPM & 2.51 & & $i$ & 1 & 1 & i & & & & \\
\hline Owol Vap. Flow Rade-GPM & 0.71 & & T & i & $!$ & ! & 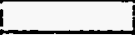 & & & \\
\hline In ot Tomperauraf & 140.71 & & I & $\therefore$ & i & 3 & & & & \\
\hline Outrelug. Temperaturof & 148.51 & & $i$ & & 1 & 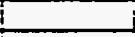 & $i$ & & & \\
\hline Outet Vap. Tomporrurof & 148.61 & & I & i & I & 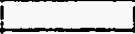 & & & & \\
\hline WEler Flow R-GOCPM & & $i$ & i & 1 & 1 & L & & & & \\
\hline Wearlntet Temp.f & 58.11 & & 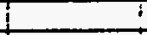 & 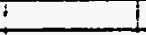 & I & 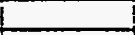 & & & & \\
\hline Weter outtal Temp.f & 146.1 & & 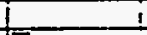 & $i$ & 1 & I & & & & \\
\hline Inlot G-lbming/ine & 695194 & & Tomp & 143.41 & Donsity & 83.23 & & & & \\
\hline Subcoolingt & 0.91 & & 1 & i & 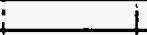 & $i$ & & & & \\
\hline \multirow{2}{*}{ Flow Observation } & $T$ & & & 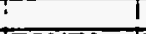 & t & 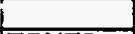 & & & & \\
\hline & IZONET & ZONE2 & ZONE3 & RONE4 T & ZONE5 & ZONE6 & ZONET & ZONE8 & IZONEB & ZORETO \\
\hline Hod hout-Wate(nel) & 1577.1 & 575.6 & 1 572 & 573.6 & 5723 & 570.8 & 573.1 & 571.7 & 5772 & 574.6 \\
\hline HonLose-Wads & 29.4 & 6.5 & 8.7 & 10 & 10.7 & 10.8 & 9.5 & 10.6 & 10.3 & 54.8 \\
\hline Hodfix-Bustrine & 10004 & 9978 & 3916 & 996| & क्षका & \$894 & 9934 & 9910 & 10000 & \$900 \\
\hline WELTOmp. BAF & 162.81 & 16.2 & 1624 & 1624 & 164.4 & 164.8 & 161.8 & 1627 & 161 & T605: \\
\hline Wal Tomp. "4F & 16281 & 162.1 & 163.4 & 1డ्2g & 163.8 & 164.8 & 1626 & 163,8 & 161.7 & 160.4 \\
\hline Well Tomo, $15 F$ & 162.1 & 160.8 & 164.8 & 1624 & 1628 & 164.8 & 162 & 1627 & 158.5 & 181 \\
\hline Wa Tomp. \#1F & 73.8 & $1 \approx 0.3$ & 161.6 & 161.4 & 1玉2 & 164.8 & $1 \approx 20$ & $1 \approx 2$ & 158.8 & 1572 \\
\hline We Tomp. I2F & 163.3 & 169.3 & 1622 & 1623 & 1623 & 186.6 & 1632 & 727 & 160.4 & 160 \\
\hline Intream Tomp.f & 150 & 151.4 & 152.11 & 151.4 & 157.3 & 151.6 & 151.4 & 151.1 & 150.5 & 150.7 \\
\hline Avg. Wall Temo.f & 1628 & 163 & 1624 & 1628 & 162.8 & 164.4 & 1625 & 1628 & 160.1 & 159.8 \\
\hline Avo. WaH DT.F & 12.3 & 11.1 & 9.91 & 10.4 & 11 & 124 & 10.7 & 11.3 & 9.1 & 8.5 \\
\hline Pomaning Subcooff & $-2.3 !$ & & 1 & 1 & & & & & & \\
\hline Exd Gus'sy & 0.01 & 0.03 & 0.05 & 0.07 & 0.08 & 0.1 & 0.12 & 0.14 & 0.16 & 0.17 \\
\hline EXP.fHethritrice & 813.4 & 896.8 & 1008.4 & 953.7 & 901.7 & 799.4 & 931 & 8792 & 1088.7 & 0 \\
\hline \multicolumn{11}{|l|}{ COMPUTEDH } \\
\hline Jallouk & 715.5 & 698.6 & 690.8 & 687.1 & 684.7 & 684.1 & 685.8 & 680.8 & 683.11 & 58.8 \\
\hline Szady 1 (Oudo tubo) & 1078.3 & 1085.2 & 1088.31 & 1091.8 & 1056 & 1099.6 & 1106.8 & 1109.8 & 1118.7 & 1728.4 \\
\hline Sacty2 & 873 & 880 & 883.4 & 888 & 905.11 & 907.6 & 912.4 & 913.9 & 921.7 & 925 \\
\hline (RLG) & 828331 & 82124 & 800005 & 79135 & 77601 & $7 \overline{220}$ & 74656 & 73058 & 71351 & Ge्य] \\
\hline (F) & 1.314 & 1.643 & 1.813 & 2.147 & 2.338 & 2.512 & 2676 & 2831 & 2.381 & 3.176 \\
\hline (HUO) & 279.8 & 350.5 & 408.3 & 456.7 & 498.6 & 535.8 & 570.7 & 603.8 & 635.4 & 68 \\
\hline \multirow[t]{3}{*}{ (HBOH) } & 1071.9 & 10729 & 1068.8 & 1064.5 & 1059.4 & 1055.5 & 1053.2 & 1046.8 & 1045.8 & 1040 \\
\hline & & & & & & & & & & \\
\hline & 1ZONE2 12 & ZONEA & ZONE 6 & ZONEB & ZONE10 & & & & & \\
\hline DP hquid Temp.F & 77.8 & & & & & & & & & \\
\hline \multicolumn{11}{|l|}{ DPLiquid Density tomm } \\
\hline Evinion't & 2 & 4 & 6 & 8 & 9.875 & & & & & \\
\hline Mosaured DPPrid & 0.139 & 0.859 & 1.359 & 1.805 & 2321 & & & & & \\
\hline Zero comectone-pate & 0.008 & 0.043 & 0.043 & 0.015 & -0.111 & & & & & \\
\hline Corrected DP P sid & 0.138 & 0.828 & 1.328 & 1.79 & 2488 & & & & & \\
\hline Acted DPA & 1.98 & 2.97 & 4.31 & 5.7 & 6.65 & & & & & \\
\hline Mosulred TPP sig & 81.4 & & & & 83.9 & & & & & \\
\hline \multicolumn{11}{|l|}{ Mereured Overall- } \\
\hline & & & & & & & & & & \\
\hline Compuned DPA & 1.88 & 3.81 & 5.32 & 7.04 & 8.67 & & & & & \\
\hline Friction- & 0.13 & 0.32 & 0.67 & 0.87 & 1.19 & & & & & \\
\hline Aocotercionnt & 0.74 & 0.22 & 0.8 & 0.37 & 0.45 & & & & & \\
\hline Elovacionth & 1.67 & 3.07 & 4.461 & 5.8 & 7.03 & & & & & \\
\hline
\end{tabular}




\begin{tabular}{|c|c|c|c|c|c|c|c|c|c|c|}
\hline Run & $304 !$ & & & & & & & & & \\
\hline Test Fuid & R114 & & & & & & & & & \\
\hline Dato: & 2201931 & & & 1 & & I & 5 & & & \\
\hline Time: & $0: 43$ & & & 1 & $:$ & $i$ & $i$ & $\therefore$ & 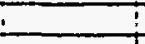 & \\
\hline Note & TABOREK & Analvical df & & $\vdots$ & 1 & & & & 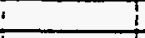 & \\
\hline Fow Area+12 & $0.00536 i$ & 1 & - & & I & & $\therefore$ & $!$ & $\vdots$ & \\
\hline Hed flow Areatle & 0.19651 & 1 & 1 & $\therefore$ & 1 & 1 & $!$ & $i$ & 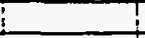 & \\
\hline Sat. Prossure psig & 801 & $i$ & 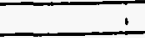 & $i$ & 1 & 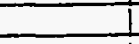 & 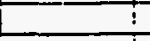 & & 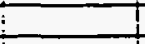 & \\
\hline Sat. Temperature f(inlet) & $148 . \overline{91}$ & 1 & & 1 & +1 & & & & & \\
\hline Lig. Density tbonftit3 & 82.551 & 8229 & 82.25 & 82.34 & 82.34 & 82.32 & 82.361 & 82.391 & 8241 & 8245 \\
\hline Lotent Hoat Bhelbm & 47.361 & 47.11 & 47.07 & 47.16 & 47.161 & 47.13 & 47.17 & 47.21 & 47.221 & 47.26 \\
\hline Speoifio Hod-stuhibmif & 0.264 & 0.265 & 0.2651 & 0.255 & 0.256 & 0.286 & 0.2651 & 0.265 & 0.255 & 0.28 \\
\hline Liq. Viscositylomithr & 0.549 & 0.544 & 0.548 & 0.545 & 0.545 & 0.5 .4 & 0.5451 & 0.548 & 0.516 & 0.547 \\
\hline Liq. Conductivity BtuhreflF & 0.0313 & 0.0312 & 0.03111 & 0.0312 & 0.0312 & 0.0312 & 0.03121 & 0.0312 & 0.0312 & 0.0312 \\
\hline Liq. Praned No. & 4.6 & 4.52 & 4.621 & 4.51 & 4.63 & 4.62 & 4.63 & 4.68 & 4.63 & 4.68 \\
\hline Intel fiow Rado-GPM & 2.61 & i & 1 & 1 & 1 & 1 & 1 & & 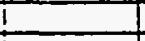 & \\
\hline Outlat Lia. Flow Reto-GPM & 2.11 & I & $!$ & 1 & 1 & i & I & & I & \\
\hline Octet Vap. Flow Rato-GPM & 0.61 & & & $\vdots$ & t & & $!$ & & & \\
\hline Inlel Tomperaxuref & 1481 & $\therefore$ & & $\bar{i}$ & $i$ & & 1 & & I & \\
\hline Outle La. Temperaure F & 147.71 & & & 1 & $!$ & & $i$ & & $i$ & \\
\hline Outor Vao. Temporárure F & 147.61 & & 1 & $i$ & $I$ & I & $i$ & 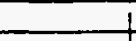 & $t$ & \\
\hline Walor Fow Rato-GPM & & & $\vdots$ & & i & & I & & & \\
\hline Wetar Inlat Tomp.F & 57.71 & & & i & $i$ & & 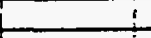 & & & \\
\hline Water out et Temp.F & 145.6 & & & & & & & & & \\
\hline Inlel Glbmhriml2 & 518240 & & Tomp & 141.110 & Density & 83.52 & & & I & \\
\hline Subooolingf & 0.91 & & 1 & 1 & - & & 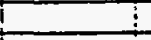 & 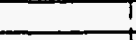 & $T$ & \\
\hline Fow Observation & $i$ & $T$ & ? & $i$ & & T & i & +2 & 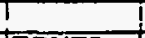 & \\
\hline & ZZONET I & ZONE2 IV & ZONE3 & ZONE4 IZ & ZONE5 12 & ZONE6 I & ZONE7 I2 & ZONEB & ZONES & ZONETO \\
\hline 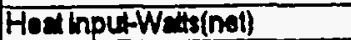 & 579.5 & 57.71 & 579.31 & 576.6 & 5801 & 574.8 & 574.8 & 574.4 & 579.5 & 577.3 \\
\hline HodLoss-Wale & 28.6 & 6.3 & 8.51 & 10 & 10.71 & 10.7 & 9.4 & 10.5 & 10.2 & 54.7 \\
\hline Honflioxbutithite & 10045 & 100014 & $100 \times 21$ & 995 & 10054 & 95041 & 9501 & $\$ 501$ & 70045 & TOCOI \\
\hline Wall Tomo. *3F & 161.31 & 162.1 & 161.21 & 161 & 163.31 & 163.2 & 160.8 & 161.8 & 160.1 & 1595 \\
\hline WE TOMP. ILF & 161.7 & 160.9 & 16231 & 161.8 & 162.81 & 163.2 & 167.6 & 1625 & 161 & 159.5 \\
\hline WEI Temp. $15 F$ & 161.91 & 159.8 & 163.3 & 181 & 162 & 1632 & 161 & 181.5 & 158.8 & 160.1 \\
\hline WDI Temp. IF & 73.51 & 161.9 & 160.3 & 150.1 & 160.7 & 16.8 & 161.8 & 161.7 & 758.1 & 156.8 \\
\hline Wall Tomp. PfF & 162.11 & 161.8 & 1611 & 161 & 160.9 & 164.6 & 162.11 & 161.7 & 158.8 & 168.2 \\
\hline Instream Tamp.f & 148.7 & 150.7 & 1511 & 950.3 & 150.3 & 150.5 & 1502 & 149.9 & 149.8 & 149.6 \\
\hline Avg. Wall Temp.F & 161.61 & 161.7 & 161.21 & 1611 & 161.6 & 163.4 & 161.5 & 167.8 & 159.4 & 158.8 \\
\hline Avg. Wall DT.F & 12.4 & 10.5 & 9.71 & 10.2 & 10.81 & 12.4 & 10.8 & 11.5 & 9.1 & 8.9 \\
\hline Remaving Subcoolf & -3.41 & & ! & & 1 & & & & I & \\
\hline Exot Guality & 0.021 & 0.04 & 0.071 & 0.091 & 0.17 & 0.14 & 0.16 & 0.19 & 0.21 & 0.20 \\
\hline Ex.HBuprnefF & 810.4 & 349.4 & 10050.3 & 977.7 & 927 & 803.5 & 920 & 869.6 & 1103.1 & 0 \\
\hline COMPUIEDH & & & & & & & & & & \\
\hline Jallouk & 720.31 & 716.8 & 701.6 & 692.5 & 6911 & 686.1 & 684.3 & 682.9 & 585.2 & 688.8 \\
\hline szady 1 (a)do tubl) & 1074.4 & 10822 & 1087.6 & 1085.7 & 100221 & 1091.8 & 1094.7 & 1097.6 & 1908.1 & 1100 \\
\hline seady2 & 869.8 & 877 & 888 & 881.7 & 889.11 & 888.8 & 904.1 & 9052 & 912.8 & 9127 \\
\hline (RLO) & 61168 & 60442 & 59032 & 57333 & 558361 & 54306 & 528501 & 51259 & 49756 & 4818 \\
\hline (F) & 1.399 & 1.795 & 2.115 & 2.385 & 2.618 & 2.825 & 3.02 & 3.204 & 3.376 & 3.54 \\
\hline (HDO) & 235.3 & 3025 & 356.4 & 401.8 & 440.9 & 475.9 & 508.8 & 539.8 & 568.4 & 598.8 \\
\hline$(1 \mathrm{BOOIL})$ & 1070.7 & 1074.3 & 1074.6 & 1057.1 & 1067.7 & 1060.8 & 1056.8 & 10523 & 1053.7 & 1047.1 \\
\hline & -1 & 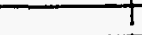 & 1 & 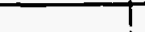 & & & & & & \\
\hline & ¿ZONE2 I & ZONEA & ZONE6 & ZONEB & ZONE10 & & & & & \\
\hline DP liguid Tomp. $f$ & 75.91 & $-\infty$ & -1 & -2 & & T & & & & \\
\hline DPLiquid Density lbm/t3 & 91.081 & & 1 & & & & & & & \\
\hline Elovetion & 21 & 41 & 6) & 8 & 9.8751 & & & & & \\
\hline Mosatured DPPsid & 0.147 & 1.083 & $1 . \overline{673}$ & 2.231 & 2.869 & & & & & \\
\hline Zero Corrections-pstd & 0.008 & 0.043 & 0.048 & 0.015 & -0.111 & & & & & \\
\hline Comected DPPsid & 0.141 & 1.05 & 1.63 & 2.218 & 2.9751 & & & & & \\
\hline Acted DPA & 1.98 & 258 & 3.78 & 4.86 & 5.71 & & & & & \\
\hline Measured TPPsig & 801 & & $\therefore$ & & $82.7 !$ & & & $i$ & & \\
\hline Masurued Ovaralih & -4.721 & & i & & & & & I & & \\
\hline & & & & & & & & & & \\
\hline Computed DPA & 1.65 & 3.02 & 4.34 & 5.64 & 6.881 & & & & & \\
\hline Fitciont & 0.11 & 0.281 & 0.5 & 0.79 & 1.12 & & & 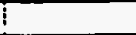 & & \\
\hline Acostercitiont & 0.11 & 0.15 & 0.21 & 0.27 & 0.34 & & & 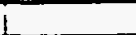 & & \\
\hline Elevetion-t & 1.451 & 2.61 & 3.69 & 4.58 & 5.431 & & & & & \\
\hline
\end{tabular}




\begin{tabular}{|c|c|c|c|c|c|c|c|c|c|c|}
\hline & & & & & & & : & : & : & 1 \\
\hline Runt: & 3051 & & & & & & & & & \\
\hline Testfuid & TR114 & & & & & & & & T & \\
\hline Dato: & 2209931 & T & & & & & & & T & \\
\hline Time: & $1: 24$ & . & & & & & & & 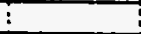 & $i$ \\
\hline Nole & T TABOREK & Analutical c & & & & 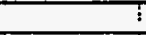 & 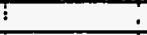 & & & ! \\
\hline Flow Arostte & 0.003361 & 1 & 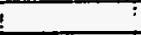 & & 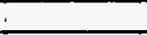 & $\bar{i}$ & 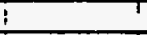 & & i & 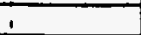 \\
\hline Hod Fow Aroath & $0.19 \% 51$ & & & & & 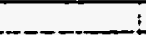 & $i_{1}$ & & & T \\
\hline Sat. Prossure Psig & 85.71 & & & & & & 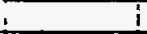 & i & & \\
\hline Set. Temperaurof (Inlel) & 153.31 & 1 & i & & & & & & & \\
\hline Lig. Denstydomnitis & 81.821 & 81.551 & 81.551 & 81.63 & 81.691 & 81.6 & 81.04 & 81.67 & 81.08 & 81.7 \\
\hline Latom Hect Btunbm & 46.65 & 46.39 & 46.391 & 46.47 & 46.471 & 46.45 & 46.481 & 46.51 & 46.52 & 46.54 \\
\hline Specifo Hastutulbm/F & 0.2661 & 0.267 & 0.267 & 0.266 & 0.2061 & 0.267 & 0.2661 & 0.256 & 0.268 & 0.256 \\
\hline UTa. Viscosity & 0.5341 & 0.5291 & 0.5291 & 0.5371 & 0.5311 & 0.53 & 0.539 & 0.531 & 0.532 & 0.532 \\
\hline Lq. Conductivity Btwhing frf & 0.03081 & 0.0307 & 0.03071 & 0.0307 & 0.0307 & 0.0307 & 0.0307 & 0.0307 & 0.0007 & 0.0508 \\
\hline Lig. Prandt No. & 4.611 & 4.61 & 4.61 & 4.6 & 4.61 & 4.61 & 4.6 & 4.61 & 4.61 & 4.81 \\
\hline Inlot flow Rede-GPM & 1.81 & 1 & I & 3 & 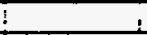 & 1 & $I$ & & & \\
\hline OuthelLtg. Flow Raio-GPM & 7.81 & i & I & & & T & I & & & \\
\hline Outtal Vap. Fow Rato-GPM & 0.41 & & & & & $!$ & T & i & & \\
\hline Inter Temporauref & 153.41 & $\mathbf{i}$ & & $i$ & i & $i$ & i & & & \\
\hline Outle/ La. Tomperanur of & 153.41 & & & & & & & & & \\
\hline Outel Vap. Tomperantef & 153.5 & i & & & & 1 & 1 & & & \\
\hline Water flow Pox-GPM & & & & & & & & & & \\
\hline Water inlel Tomp.f & 59.5 & & & & & & 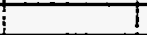 & & & \\
\hline Weder outtel Tomp.f & 150.5 & & & I & & 1 & I & & & \\
\hline Inlat Glbm/hr/h2 & $356871 !$ & & Tomp & 144.6 & Density & 83.08 & & & & \\
\hline Subcoolingf & -0.11 & & & & 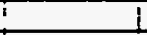 & 1 & I & & & \\
\hline Flow Observation & & & & $i$ & & i & 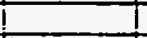 & & & \\
\hline & TZONET & ZONE2 & ZONEB & 1ZONE4 & ZONE5 & ZONE6 & ZONET & ZONE8 & ZZONE9 & ZOVEIO \\
\hline 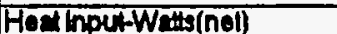 & 584.1 & 582.7 & 582.3 & 581.3 & 581.91 & 576.8 & 579.9 & 580.8 & |581.8 & \begin{tabular}{|l|l|}
378.8 \\
\end{tabular} \\
\hline Hect Lose-Wais & 30.8 & 6.7 & 8.9 & 10 & 10.6 & 10.5 & 9.7 & 10.8 & 10.8 & 58.7 \\
\hline 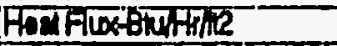 & Т0स्डा & 10णा & $1008 \pi$ & $100 \%$ & 10087 & 70000 & 10052 & $100 \%$ & To0:5 & 70003 \\
\hline Wall Tamp. BF & 168 & 166.41 & 7651 & 165.8 & 16821 & 168 & 188 & 166.1 & 165.6 & 16.7 \\
\hline Wal Tomp. IAF & $165.2 !$ & T65.5T & 167.1 & 166.3 & 167.6 & 168.1 & 168.7 & 157.3 & 168.1 & 184.5 \\
\hline WEH TOMP. $15 \mathrm{~F}$ & 165.7 & 184.3 & 168.21 & 165.9 & 168.6 & 187.8 & 180.1 & 168.1 & 104 & 105 \\
\hline Wal Tomp. NTF & 74.81 & 166.2 & 164.81 & 164.8 & 165.4 & 168.5 & 167 & 166.7 & 160.4 & 162 \\
\hline WAlTomp. "2F & 168.6 & 166.2 & 165.61 & 165.8 & 165.6 & 169.2 & 167.21 & 167 & 164 & $1 \approx .6$ \\
\hline Intraam Tomp.F & 154.31 & 156.3 & 156.31 & 155.7 & 155.7 & 155.9 & 155.6 & 155.4 & 155.3 & 155.2 \\
\hline Avg. Wall Temp.F & 166.11 & 166.11 & 165.91 & 165.7 & 166.31 & $168.1 !$ & 166.61 & 166.6 & 164.8 & 164 \\
\hline Avg. WaH DT . F & 11.41 & $9.3 !$ & 9.11 & 9.51 & 10.11 & 11.81 & 10.5 & 10.8 & 8.8 & 8.3 \\
\hline Romaining subooolf & -6.31 & & & & & & & & & \\
\hline Exot Quadisy & 0.041 & 0.071 & 0.111 & 0.141 & 0.181 & 0.211 & 0.251 & 0.29 & 0.32 & 0.36 \\
\hline EXP.HEtuHrmak & 890.7 & 1083.91 & 11101 & 1058.5 & 996.81 & 849.4 & 953.2 & 933.7 & 1140.3 & 0 \\
\hline COMPUIEDH & & & & & & & & & & \\
\hline Jalouk & 754.51 & 744.8 & 729 & 719 & 713.9 & 706.8 & 705 & 702.8 & 7012 & 697.8 \\
\hline Szady (Cudo tube) & 1097.81 & 1105 & 1106.41 & 1105.3 & 1107.91 & 1108.2 & 1110.4 & 1113.3 & 1116.8 & 1118.6 \\
\hline $\operatorname{sendy} 2$ & 8911 & 897.71 & 839.61 & 899 & 902.31 & 900.6 & 905.8 & 900.8 & 9132 & 913.4 \\
\hline (ALQ) & 429061 & 41739 & 4016 ा & 384811 & 359121 & 35365 & 3375 & 32182 & 30004 & 29081 \\
\hline$(F)$ & 1.571 & 2.0721 & 2.44 & 2.7551 & 3.0281 & 3.271 & 3.500 & 3.721 & 3.565 & 4.12 \\
\hline (स) & 196.9 & 260.2 & 306.4 & 345.8 & 380.1 & 410.7 & 439.7 & 466.9 & 4928 & 517 \\
\hline (HBOIL) & 1005.7 & 1100.2 & 1098.5 & 10033.9 & 10928 & 1087 & 1087 & 10852 & 1083.7 & 1078.3 \\
\hline & & & & & & & & & & \\
\hline & ZZONEZ & ZONE4 & ZONE6 & ZONEB & ZONE10 & & & & & \\
\hline DPliquid Tomp.f & 77.6 & & & & & & & & & \\
\hline DPLiquid Donsty-tom/tes & 90.85 & & & 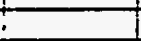 & & & & & & \\
\hline Eovetork & रां & 4 & 61 & 8 & 9.8751 & & & & & \\
\hline Mesoured DPF gid & 0.178 & 1.388 & 2.0059 & 2.725 & 3.478 & & & & & \\
\hline Zoro Corrections-psid & 0.008 & 0.048 & 0.0431 & 0.015 & -0.1111 & & & & & \\
\hline Corredod DPPsid & 0.172 & 1.345 & 1.998 & 2.71 & 3.587 & & & & & \\
\hline Actial DPA & 1.92 & 2.08 & 3.15 & 4.12 & 4.651 & & & & & \\
\hline Meanured TPPoig & 86.7 & & & : & 89.7 & & & & & \\
\hline Meseved Overalla & -7.05 & & 1 & 1 & & & & & & \\
\hline & & & & & & & & & & \\
\hline Computed DPA & 1.361 & 2.48 & 3.5. & 4.53 & 5.48 & & & & & \\
\hline Fitcion- & 0.07 & 0.19 & 0.35 & 0.56 & 0.8 & & & & & \\
\hline Acostercion-t & 0.08 & 0.1 & 0.141 & 0.19 & 0.24 & & & & & \\
\hline Elvmitiont & 1.24 & 2.19 & 3.031 & 3.78 & 4.441 & & & & & \\
\hline
\end{tabular}




\begin{tabular}{|c|c|c|c|c|c|c|c|c|c|c|}
\hline$-\ldots . . .1-\ldots$ & 306 & & & & & & & & 1 & \\
\hline Testfinid & R114 & & & & & & & & 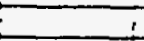 & 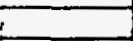 \\
\hline Date: & 220193 & & & & & & & & & \\
\hline Time: & $2: 19$ & & & & & & & & & \\
\hline Note & TABOREK & Añurvical c & & & & I & 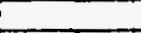 & . & & \\
\hline Flow Aroa-H2 & $-0.00336 i$ & & & & & & & & & \\
\hline Heat flow Areatit & 0.196951 & & & & & & & & & \\
\hline Sad. Pressure Psig & 85.21 & & & & & 1 & & & & \\
\hline Sat Temporaturof(inlei) & $152.9 i$ & & & & & & 1 & & T! & \\
\hline Liq. Donsity lom nits & 81.71 & 81.6 & 81.631 & 81.721 & 81.681 & 81.881 & 81.671 & 81.721 & 81.74 & 81.70 \\
\hline Lotent Hoat Bturbm & 46.541 & 46.45 & 46.471 & 46.561 & 46.521 & 46.52 & 46.511 & 46.56 & 46.57 & 46.6 \\
\hline Specino Hea BtuibmiF & $0.265 !$ & 0.2671 & $0.266 i$ & 0.266 & 0.2661 & 0.2661 & 0.2681 & 0.266 & 0.266 & 0.266 \\
\hline Liq. Viscosinybmiths & 0.5321 & 0.53 & 0.5311 & 0.5321 & 0.5321 & 0.5321 & 0.5311 & 0.5321 & 0.533 & 0.533 \\
\hline Liq. Conductivitusturns if & 0.33081 & 0.03071 & 0.03071 & 0.03081 & $0.0307 i$ & 0.03071 & 0.03071 & 0.0308 & 0.03081 & 0.0308 \\
\hline Liq. Prand No. & 4.67 : & 4.61 & 4.6 & 4.611 & 4.671 & 4.611 & 4.51 & 4.611 & 4.611 & 4.61 \\
\hline Iniot Fow Rato-GPM & -9 & & & & & 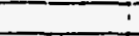 & & & & \\
\hline Outlot Liq. Flow Ralo-GPM & 1 & & & & & & & 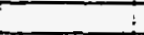 & : & \\
\hline outlet Vao. Flow Rato-GPM & $0.4:$ & & & 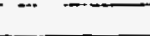 & & 1 & & $\mathrm{i}$ & & \\
\hline InTet Temperaruref & $152.4 !$ & & & & & & & & & \\
\hline Outot La. Temperaruref & $-153 i$ & & 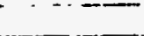 & 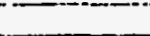 & & & & & i & \\
\hline Outel Vap. Temperaturef & 153.21 & & & & & & & 1 & & \\
\hline Wader Flow Rato-GPM & & & & & & 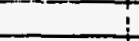 & & i & & \\
\hline Wator inlot Temo.f & 58.91 & & & & & 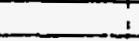 & & & & \\
\hline Wator outidet Temp.f & $151.2 \mathrm{i}$ & & & & & & & & & \\
\hline 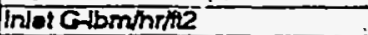 & 1996841 & & Tomp & $139.91 \mathrm{~L}$ & Density & 83.67 & & & & \\
\hline Subcoolingt & 0.51 & & & & & & I & 1 & & \\
\hline Flow Observation & & & & & & & & 1 & & \\
\hline & ZONET: & ZONE2 & $\angle O N E 3:$ & ZONE4 12 & ZONES :2 & ZONE6 & ZONE7 & ZONEE & ZONE9 & ZONETO \\
\hline Heat lnpul-Watts(net) & $5 \pi$ & 577.1 & 581.71 & 580.9 & 579.4 & 578.6 & 579.9 & 580.5 & 581 & 578.6 \\
\hline Hoe LossotVats & 30.61 & 6.7 & 8.91 & $10 !$ & 10.61 & 10.5 & 9.71 & 10.91 & 10.8 & 58.7 \\
\hline 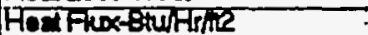 & 10001 & 10004 & 100831 & $700 \%$ & ग004का & 70001 & 700521 & उ0003 & $7007 \pi$ & 70050 \\
\hline Wall Tomp. I3F & 164.91 & 165.4 & 1651 & 164.7 & 167.3 & 167.2 & 165.8 & 165.7 & 165 & 164.1 \\
\hline WE TOMP. FAF & 1651 & 164.31 & 1661 & 165.21 & 168.7 & 1672 & 168.0 & 768.8 & 165.7 & 163.8 \\
\hline Wal Temp. PSF & 164.9 & 169 & 167.61 & 165.11 & 165.51 & 168.7 & 165.8 & 165.6 & 169.0 & 184.4 \\
\hline WNT Tomp. If & 73.11 & 164.9 & 163.81 & 16421 & 164.2 & 167.6 & 166.6 & 166,3 & 162.9 & 161.5 \\
\hline Wall Tamp. $\$ 2 F$ & 1861 & 164.9 & 164.31 & 164.81 & 164.61 & 168.3 & 166.8 & 166.8 & 163.6 & 1632 \\
\hline Insteam Tomp. $F$ & 155.21 & 155.9 & 155.71 & $155 !$ & 155.31 & 155.31 & 955.41 & 155 & 154.9 & 154.7 \\
\hline Avg. Wall Temp.F & $1652 !$ & $164.9 !$ & 164.81 & 164.81 & 165.31 & $167.2 !$ & 166.3 & 166.21 & 164.21 & 160.4 \\
\hline Avg. WaldT.F & $9.5 !$ & 8.51 & $8.6:$ & 9.31 & 9.51 & 11.41 & 10.5 & 10.7 & 8.8 & 8.3 \\
\hline Romaining Subcooff & -70.51 & & & & & & & & & \\
\hline Exat Cuality & 0.061 & 0.121 & 0.191 & 0.251 & 0.311 & 0.38 & 0.441 & 0.51 & 0.57 & 0.63 \\
\hline 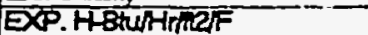 & 1047.5 & 9173.7 & 1169.9 & 1077.51 & 1057.61 & 878.11 & 958.7 & 936.51 & 9143.8 & \\
\hline COMPUTEDH & & & & & & & & & & \\
\hline Jatiouk & $7 \overline{69} . \overline{8} i$ & 764.31 & $752.7 !$ & -742.11 & 737.51 & 733.6 & 723.5 & 719.4 & 796.6 & 712.1 \\
\hline Szadvi(ado tube) & 1093.51 & 1096.81 & 1101.31 & 1099.11 & 1099.8 & 1100.41 & 1103.3 & 1104 & 1105.8 & 1104.7 \\
\hline Sacty? & 885.11 & 888.3 & $8 \overline{93 . \overline{81}}$ & 8922 & 892.91 & 833.7 & 897.11 & 898.6 & 900.7 & 899.8 \\
\hline (F्यान) & $23819 i$ & 22382 & 208001 & 1916! & 176381 & 16003 & 145341 & 12950 & 11387 & 9824 \\
\hline$(\mathrm{F})$ & 1.7651 & 2.4621 & 2.984 & 3.431 & 3.807 & 4.164 & 4.475 & 4.7891 & 5.084 & 5.37 \\
\hline (A) & 139.21 & 194.2 & 235.41 & 270.41 & 300.2 & $327.6 !$ & 3529 & 377.61 & 400.8 & 4233 \\
\hline (HBOIiL) & $10927 !$ & 1094.7 & $1097.7:$ & 1093.6 & 1092.41 & 1090.6 & 109121 & 1089.11 & 10881 & 10083.8 \\
\hline & & & & & & & & & & \\
\hline & ZONE2 & ZONE4 & ZONE6 & ZONES & ZONE10 : & & & & & \\
\hline DPFaudd Temp.f & 75.8 & & & 1 & 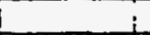 & & & & & \\
\hline DP Liquid Density lbmitis & 91.041 & & & & & & & & & \\
\hline Elevetion $\mathrm{ft}$ & $2 !$ & 4 & 61 & 81 & 9.875 & & & & & \\
\hline Measured DPP Pid & 0.1561 & 7.7221 & 2.4531 & 3.277 & 4.1561 & & & & & \\
\hline Zero Corrections-psid & $0.008 i$ & 0.0431 & 0.0431 & 0.015 & -0.111 & & & & & \\
\hline Comoctod DPP sid & 0.151 & T.679 & 2.411 & 3.262 & 4.287 & & & & & \\
\hline Actual DPA & 1.961 & 1.5 & 2.41 & 3.16 & 3.48 & & & & & \\
\hline Massurad TPFsig & 85.21 & & i & & 89.51 & & & & & \\
\hline Mosetured Ovaralih & -7.571 & & $\bar{T}$ & it & & & & & & \\
\hline & & & & & & & & & & \\
\hline Computed DF & 9.17 & 1.91 & 2.611 & 3.25 & 3.821 & & & & & \\
\hline Friction & 0.031 & 0.1 & 0.19 & 0.31 & 0.45 & & & & & \\
\hline Aoouterationt & 0.031 & 0.051 & 0.081 & 0.12 & $0.17 !$ & & & & & \\
\hline Elevation ft $^{-}$ & 9.051 & $9.76 !$ & 2.331 & 2.82 & 3.21 & & & & & \\
\hline
\end{tabular}




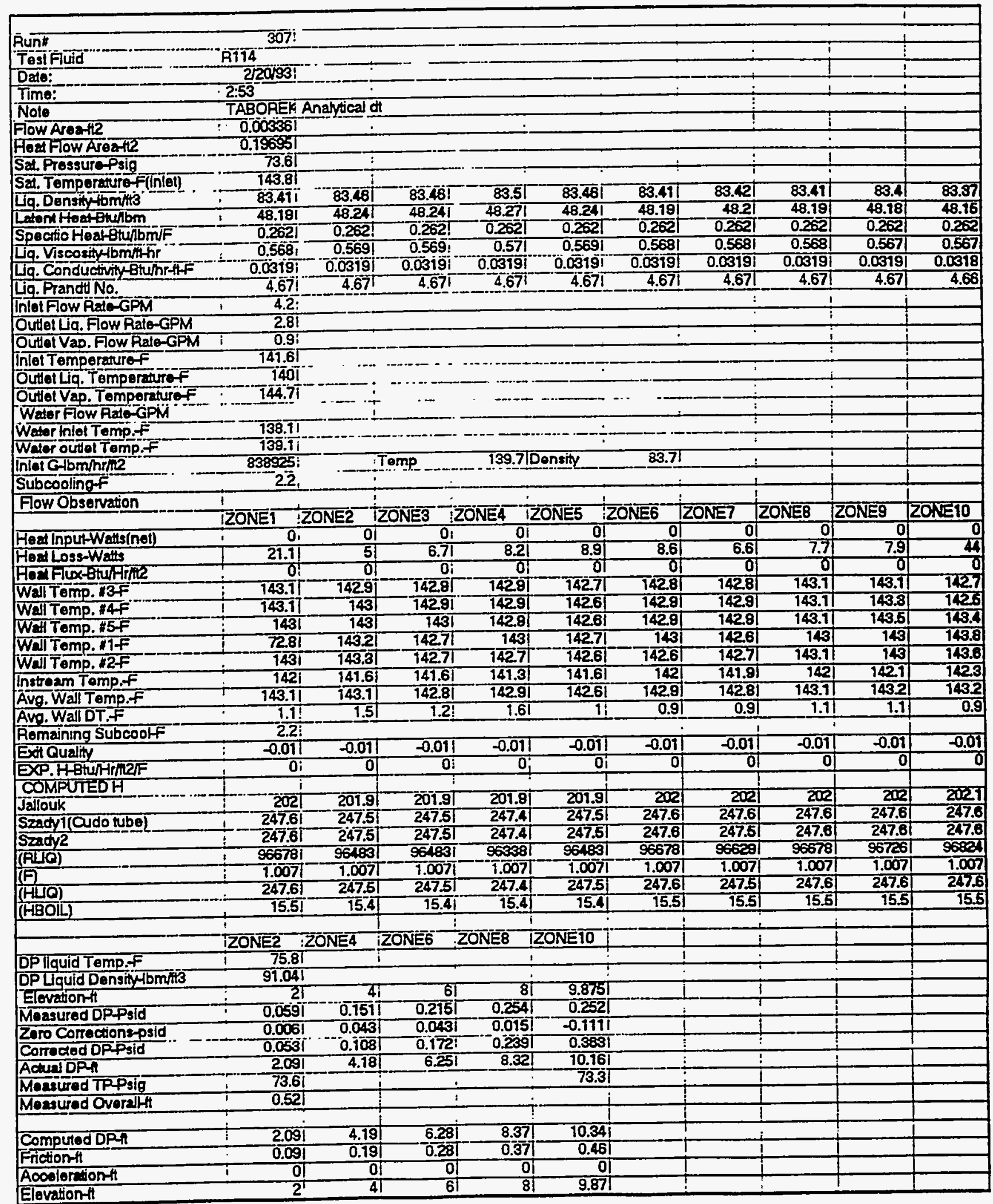




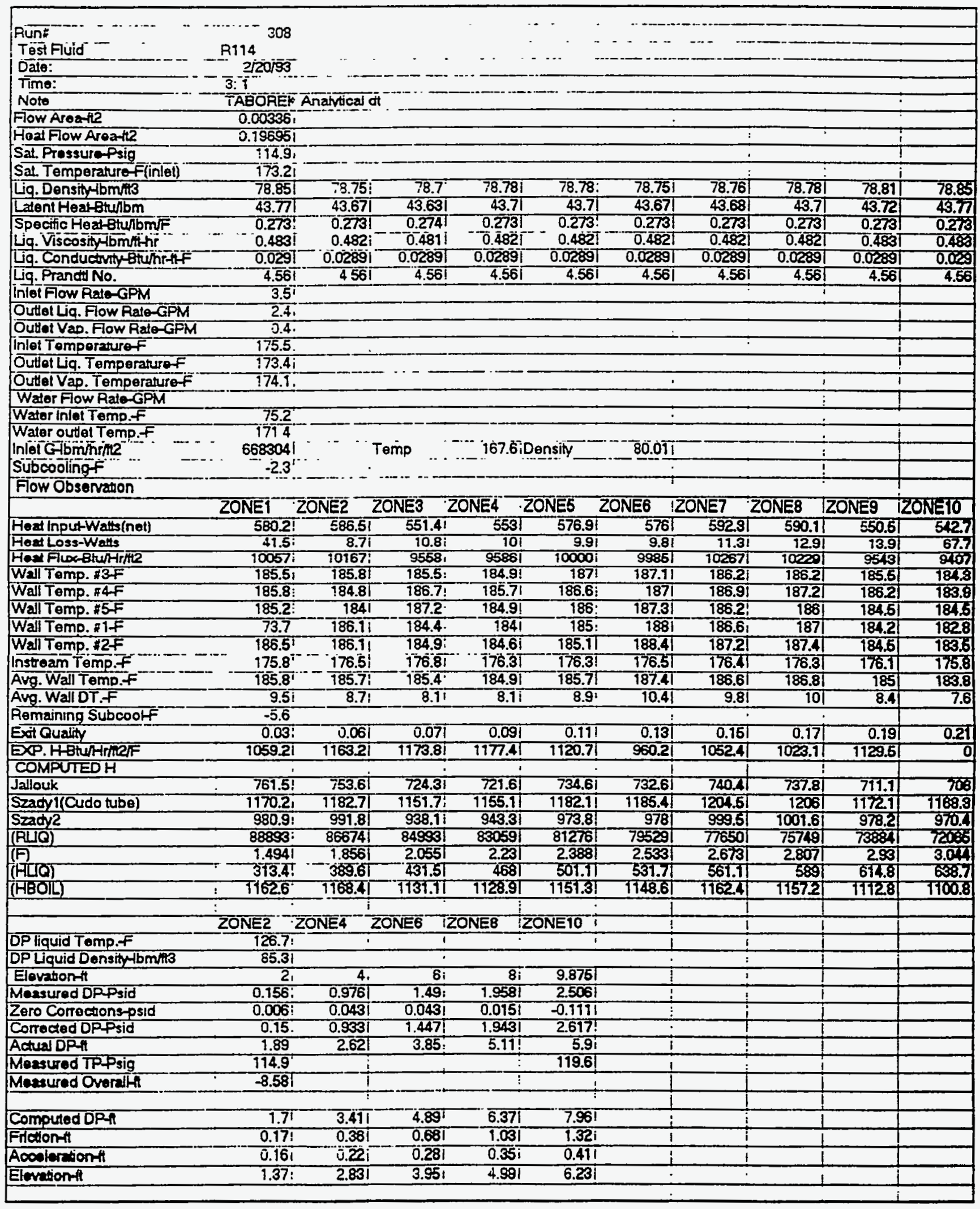




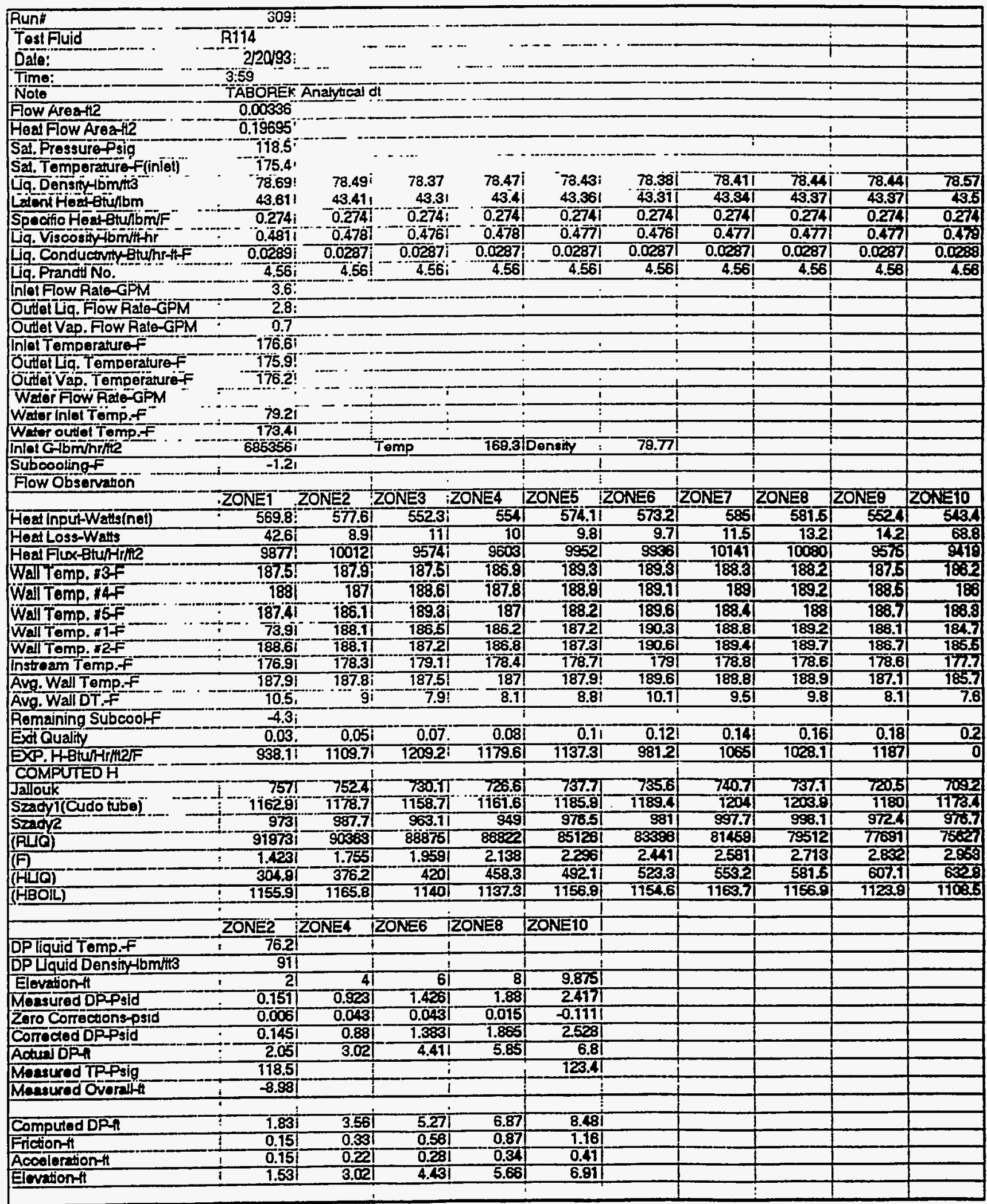




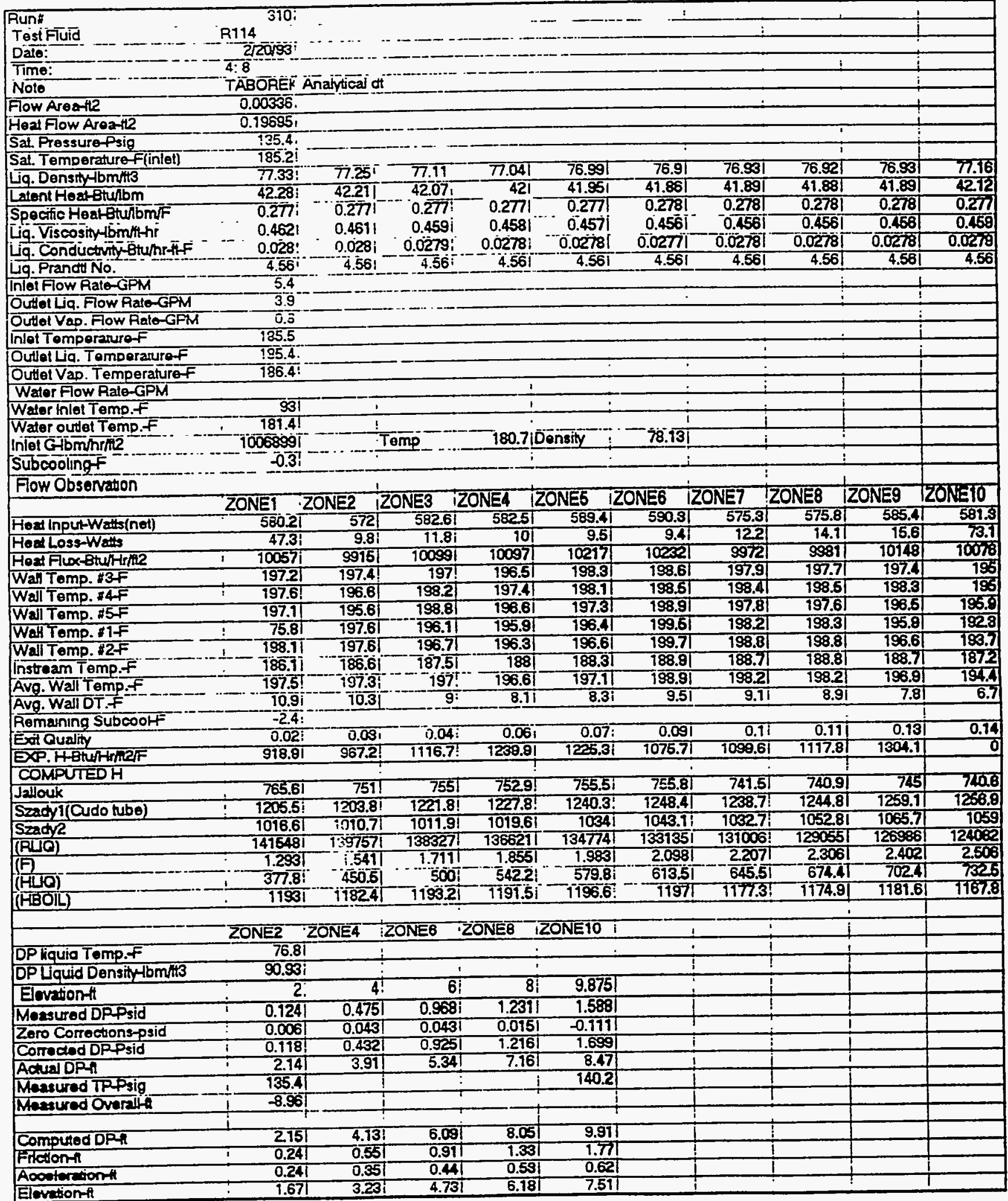




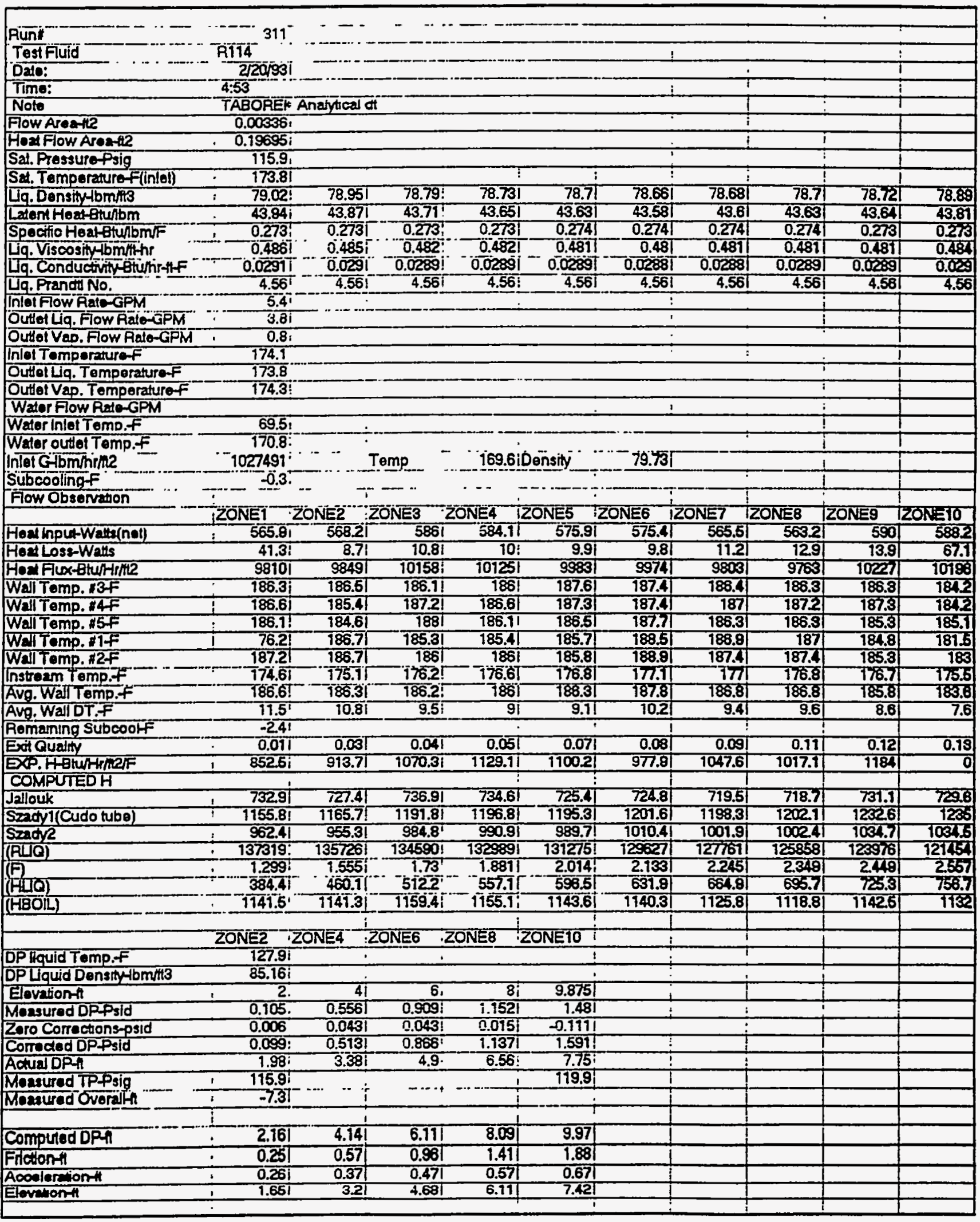




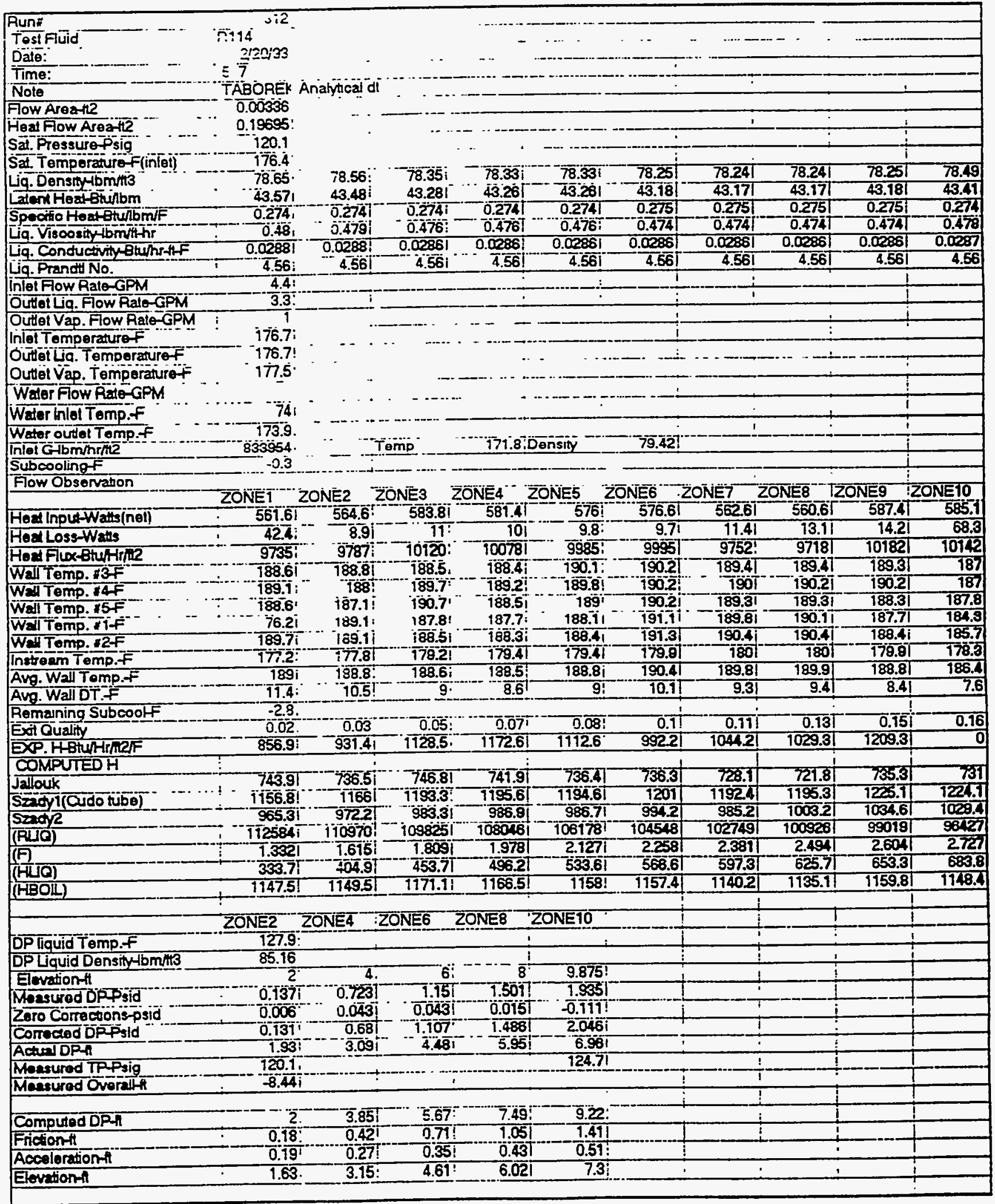




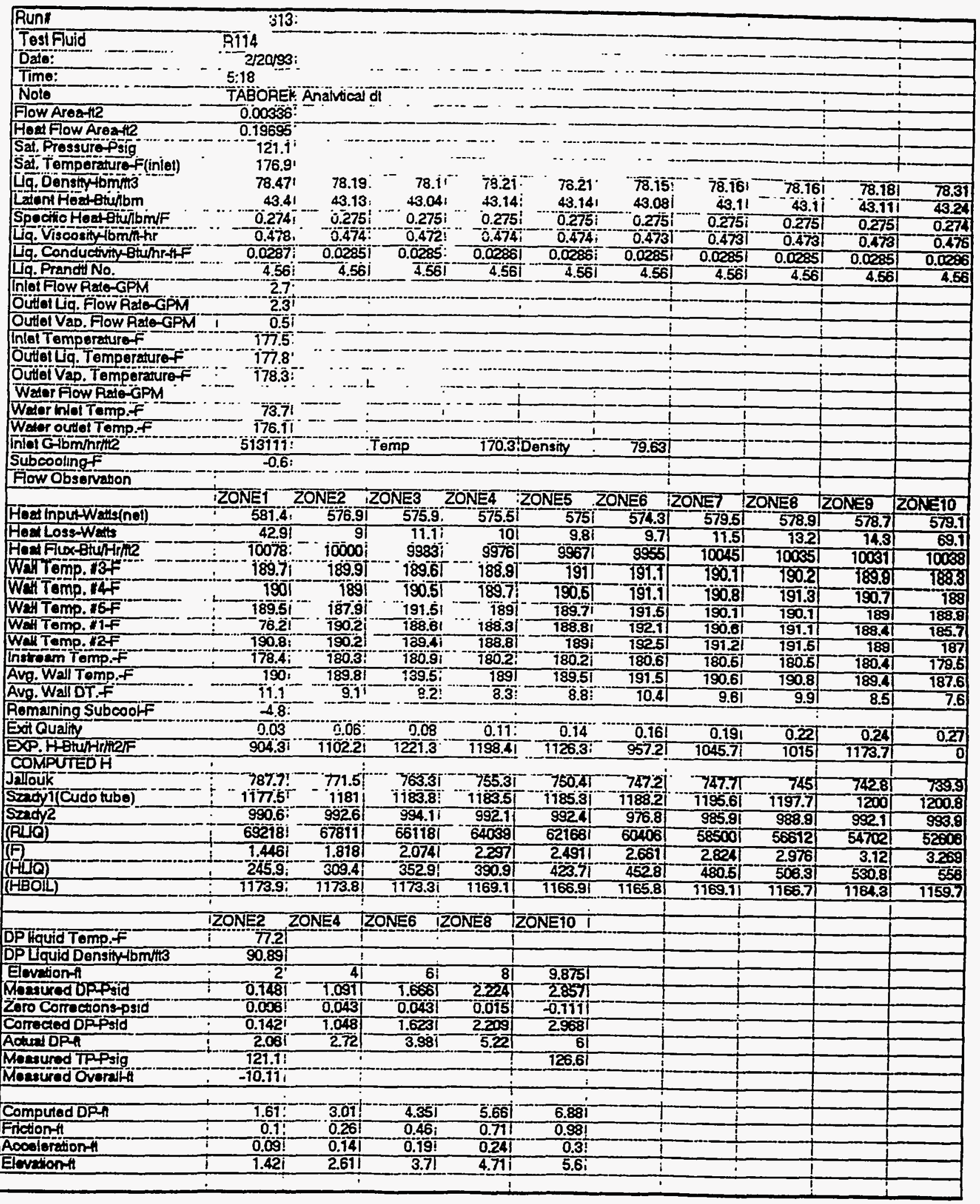




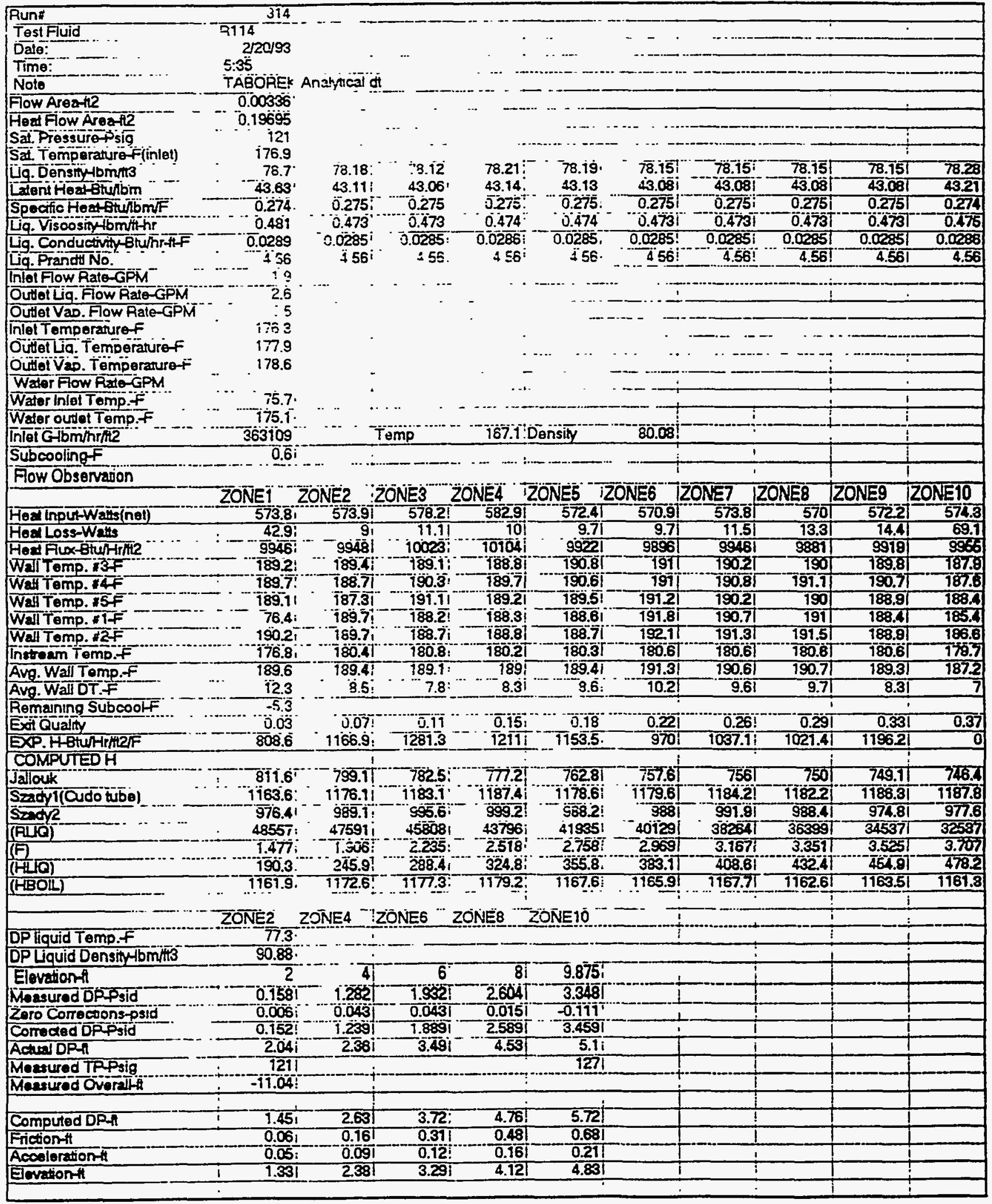




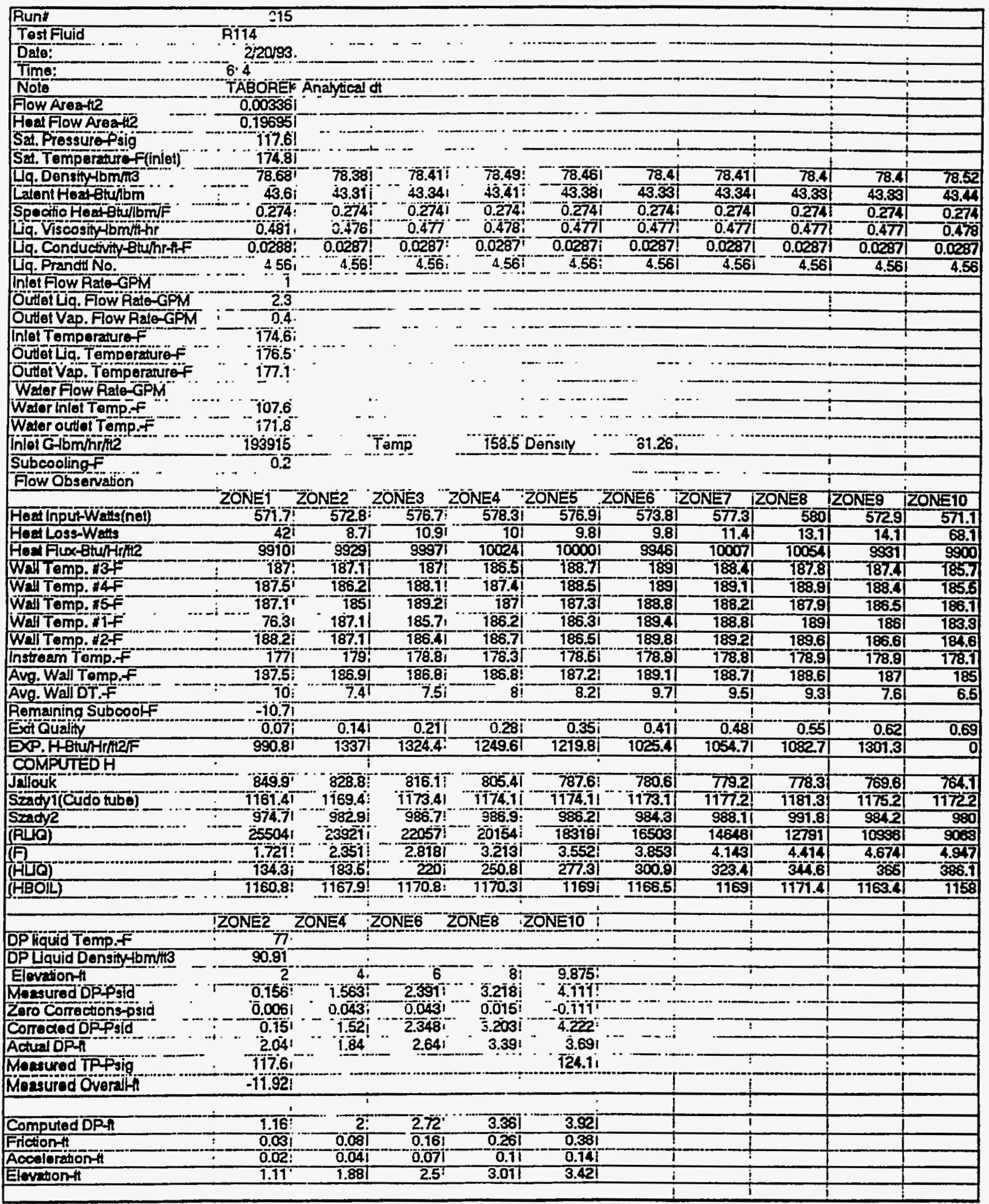




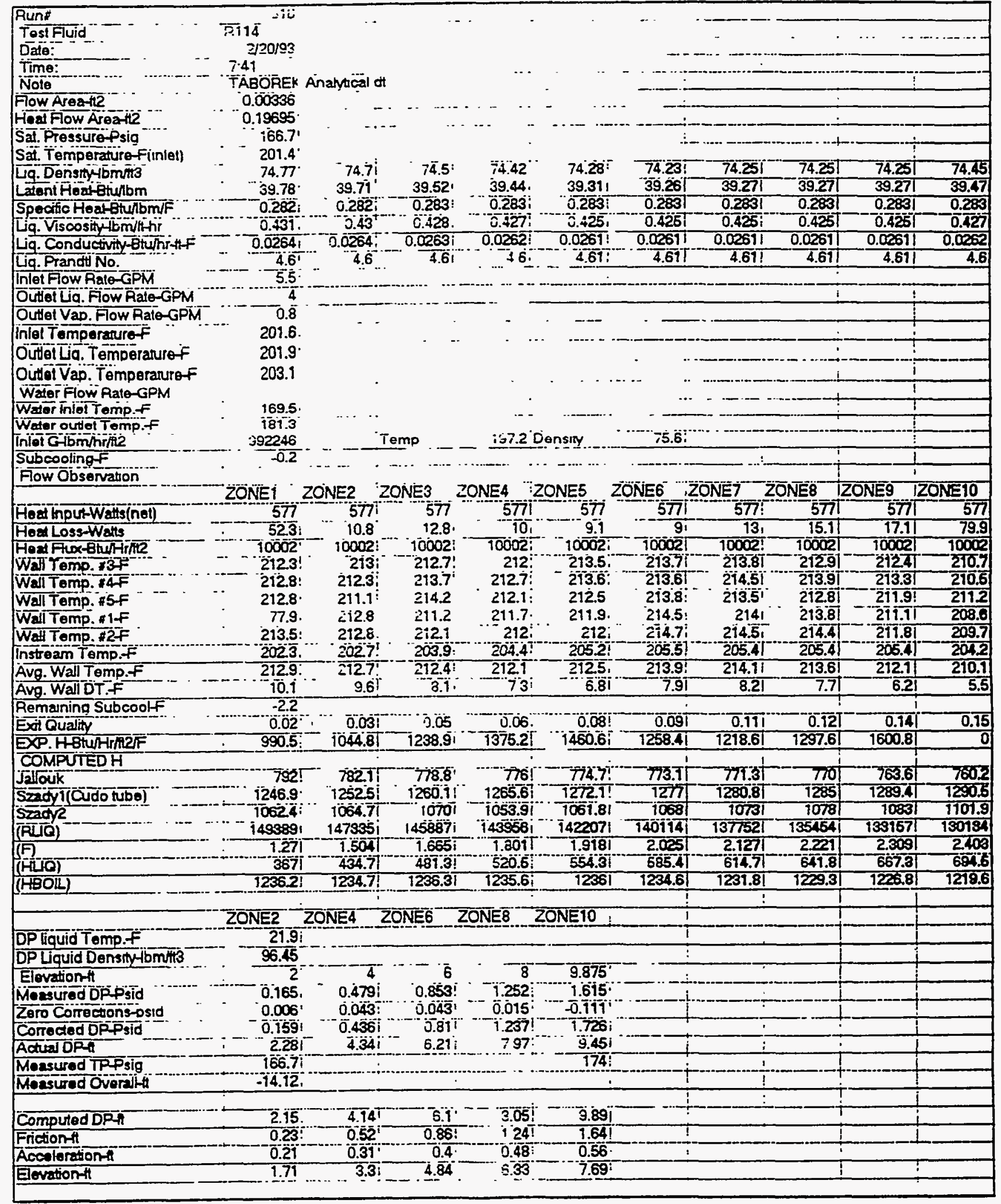




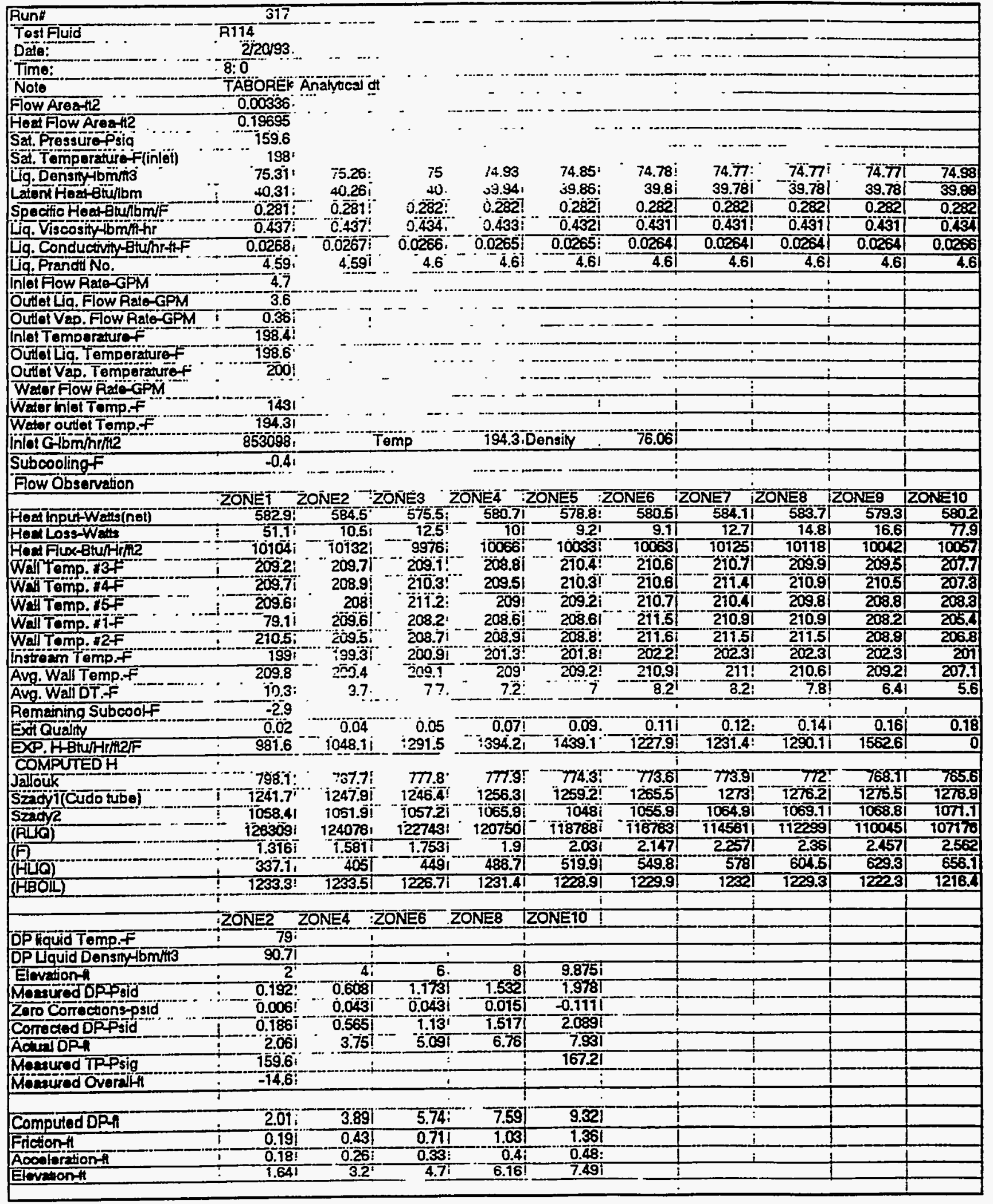




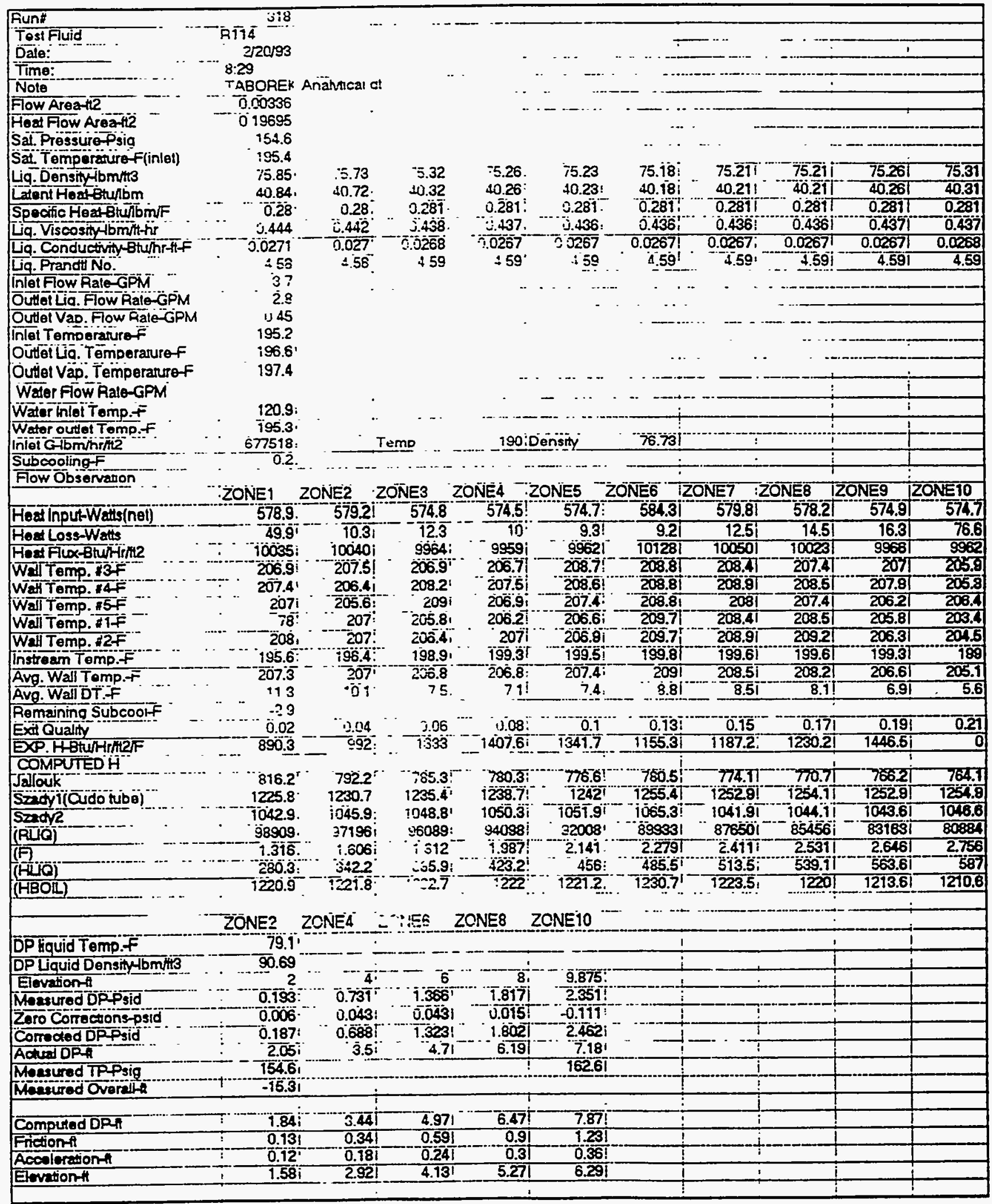




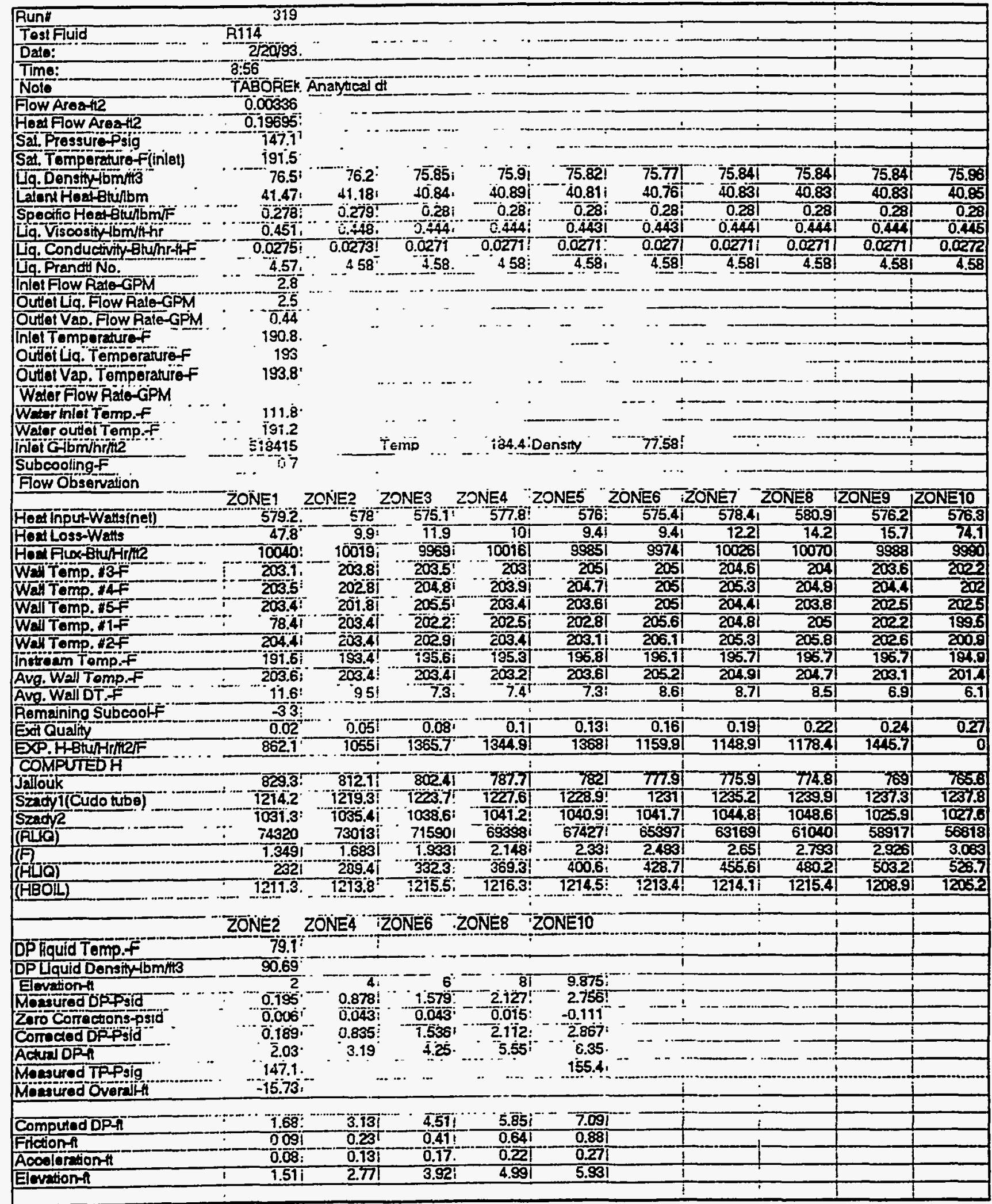




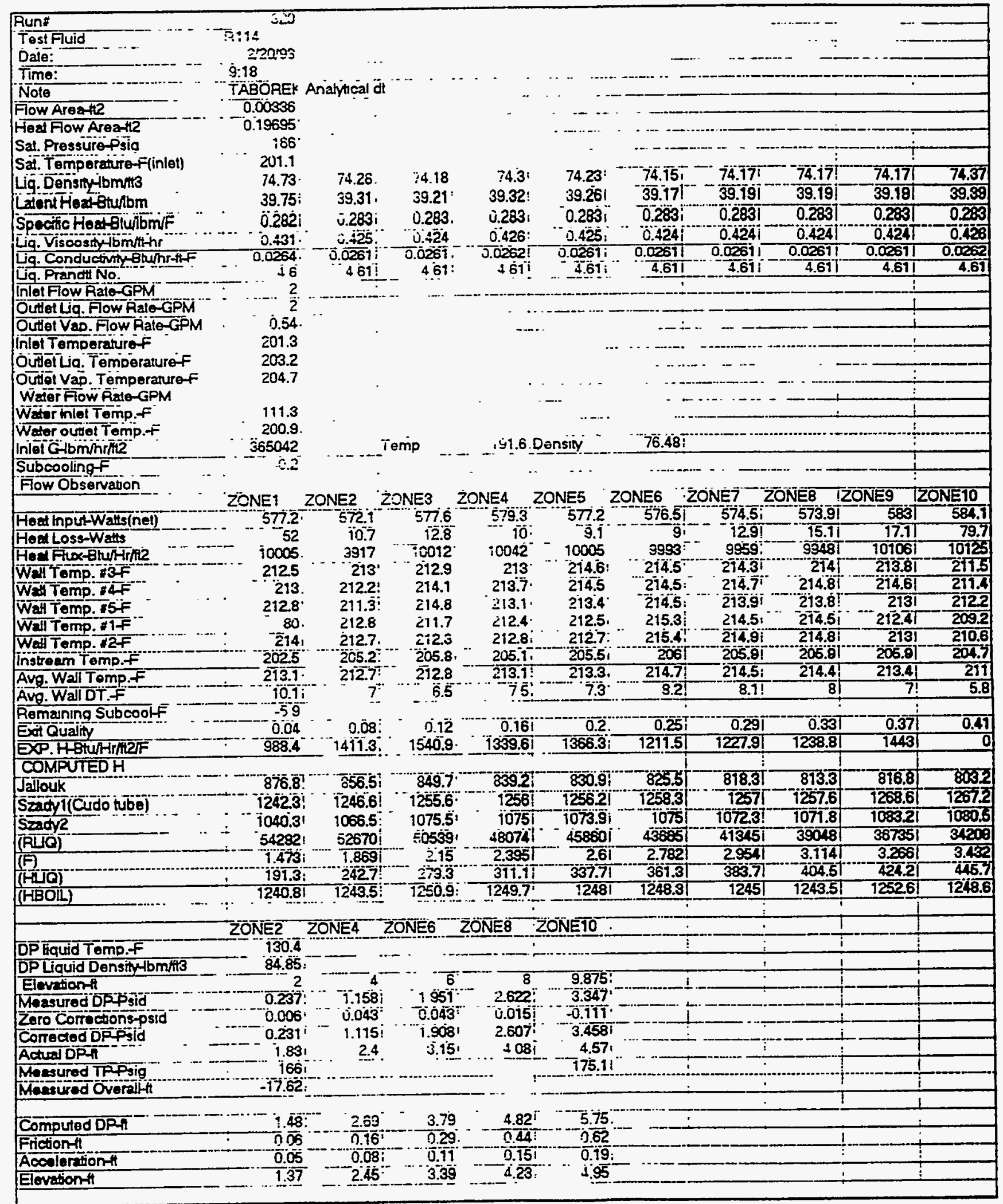




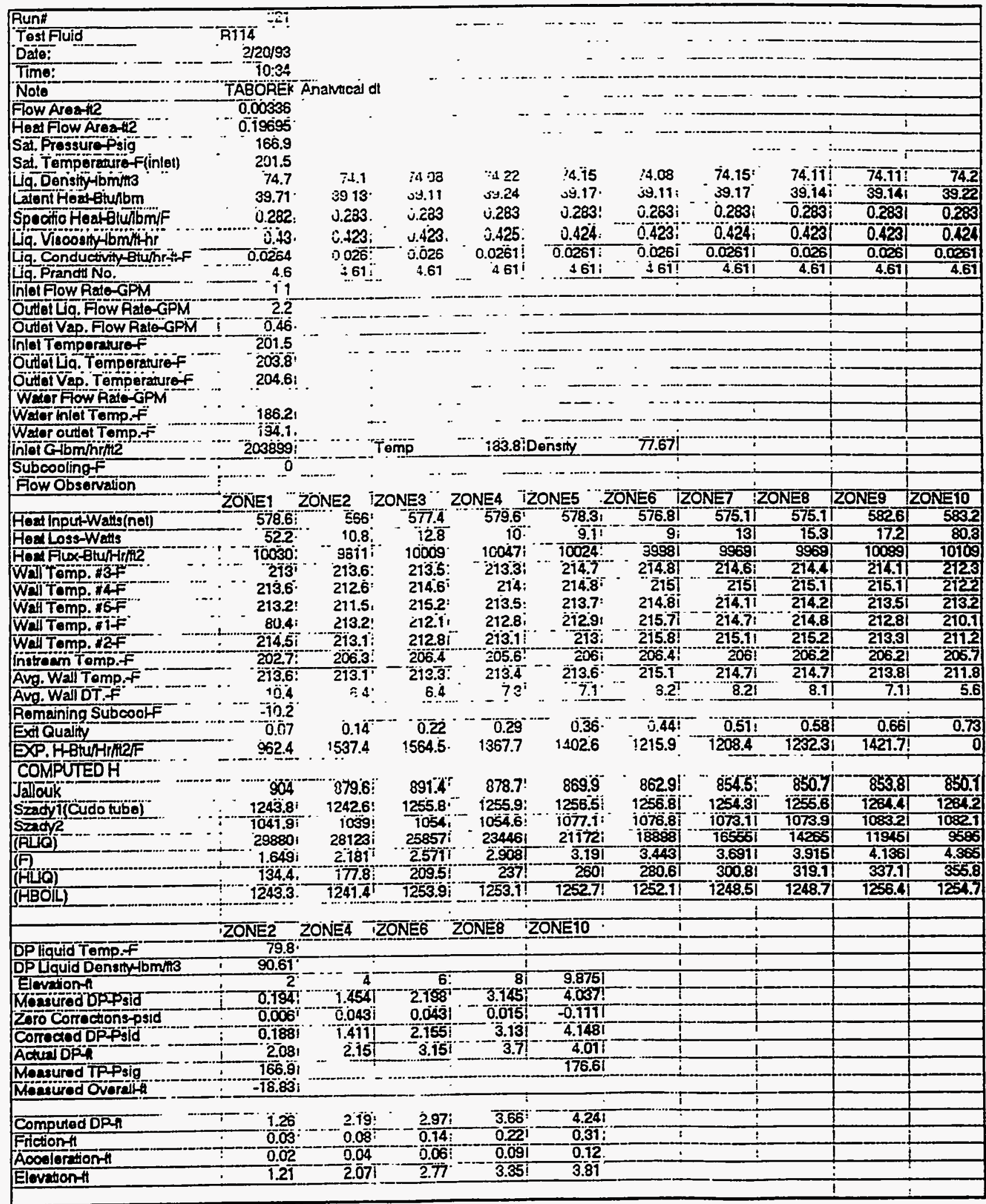




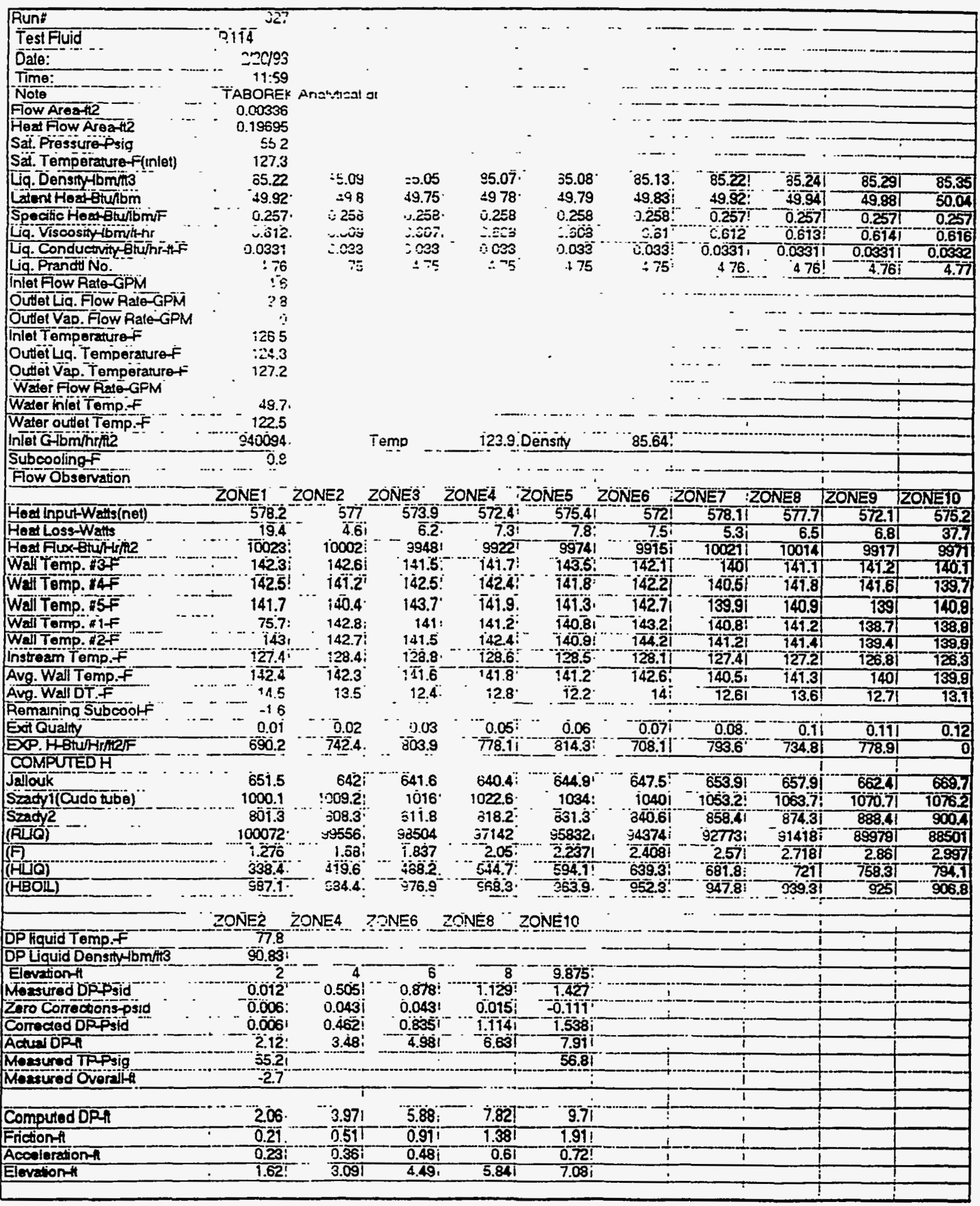




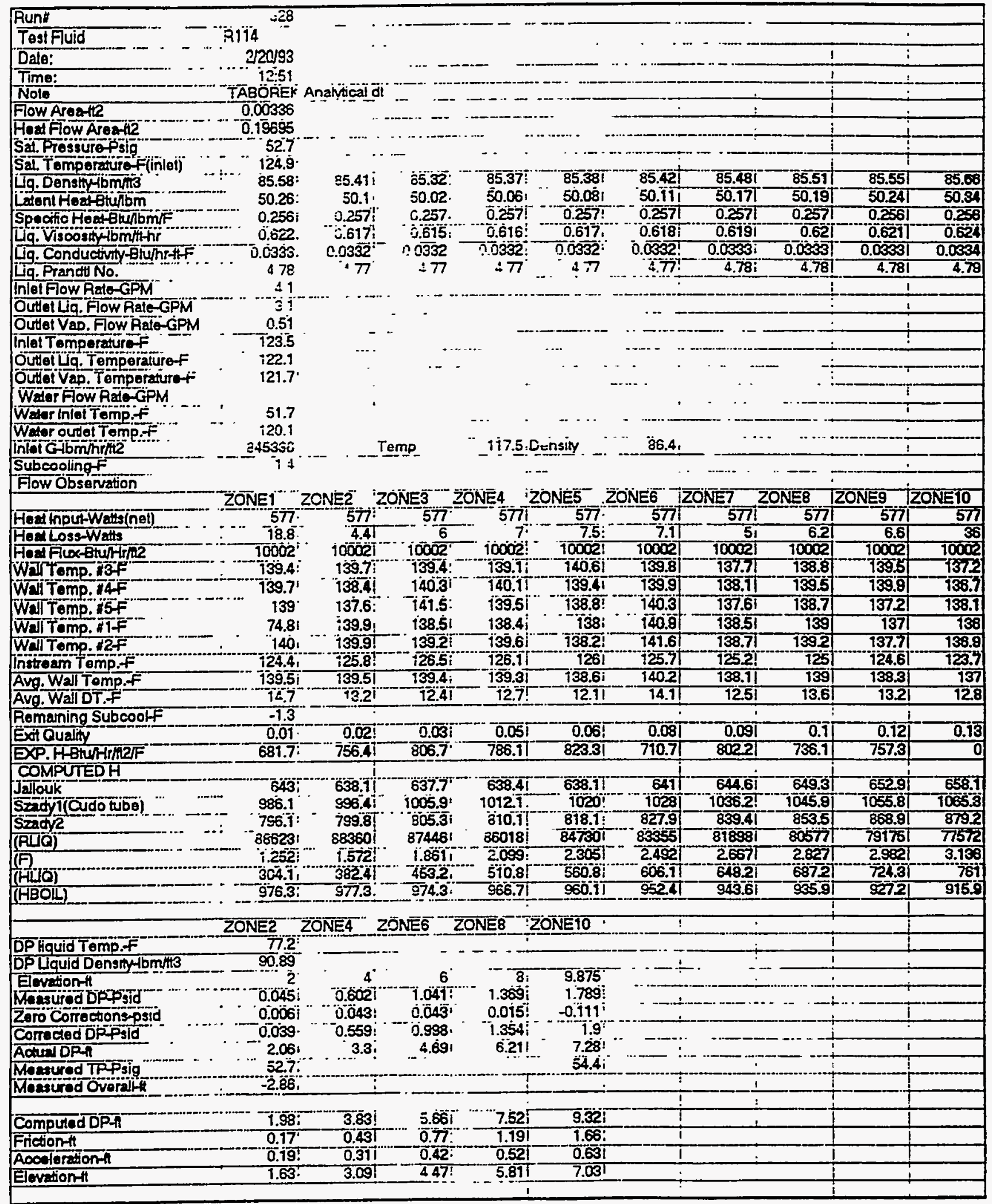




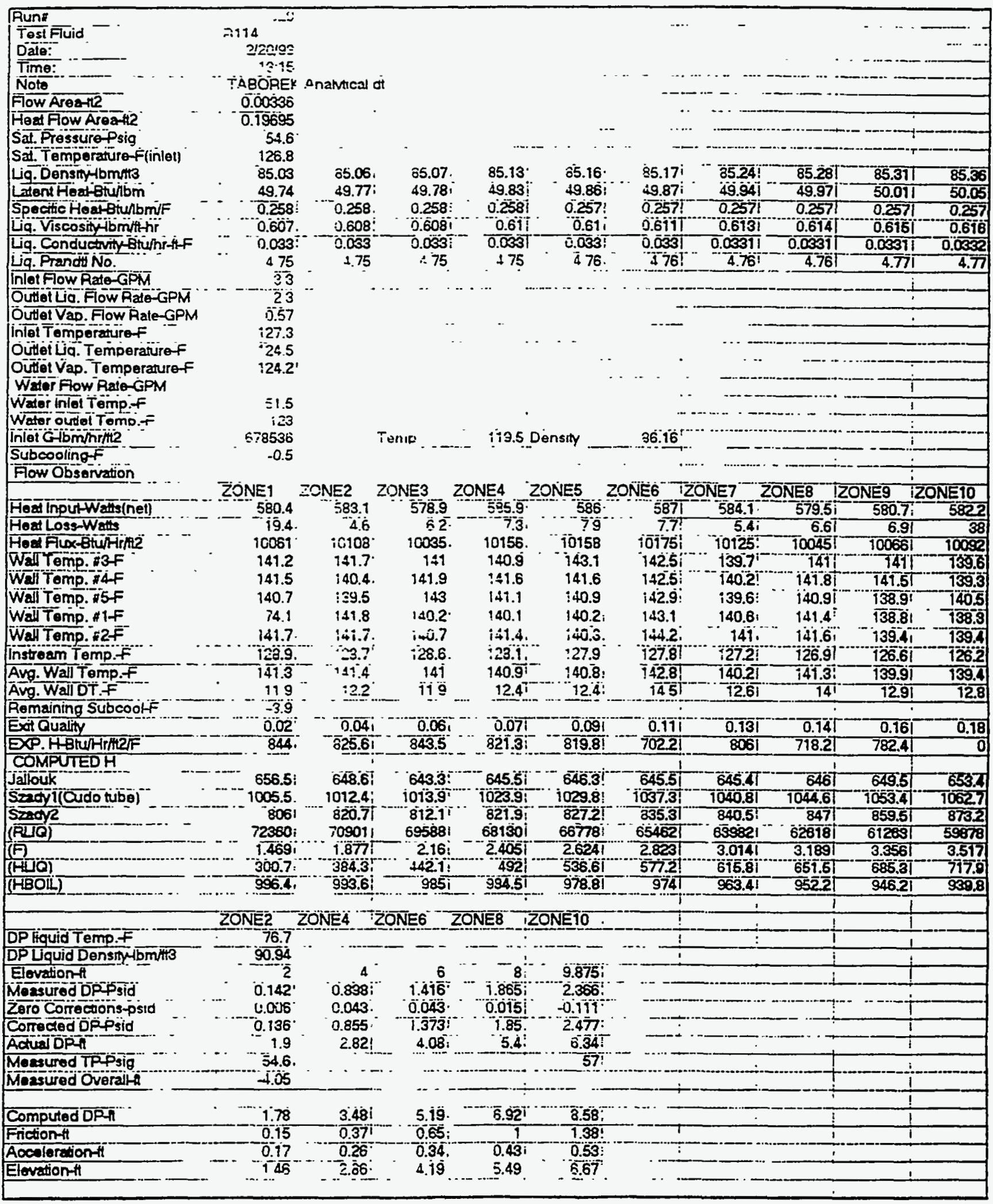




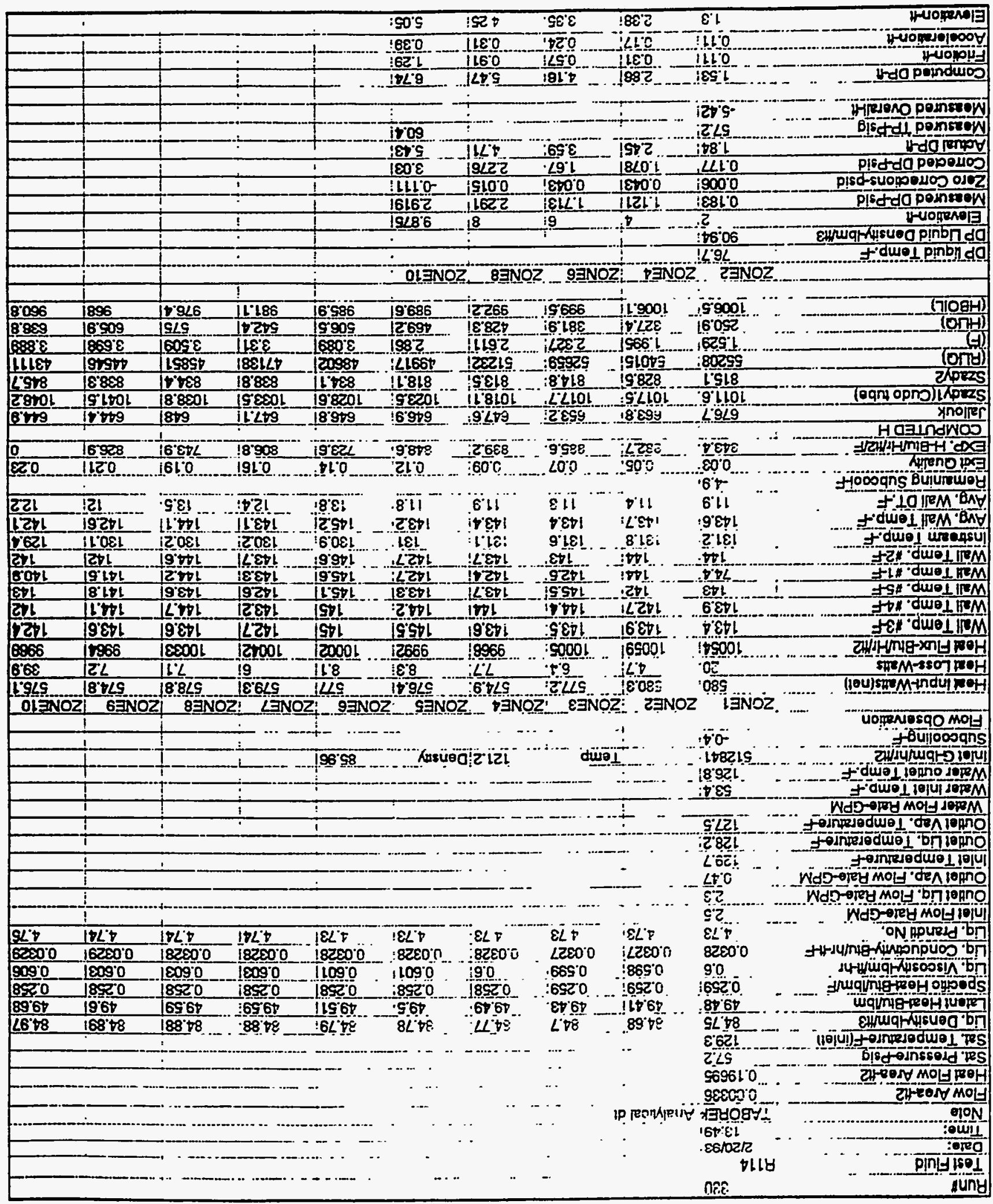




\begin{tabular}{|c|c|c|c|c|c|c|c|c|c|c|}
\hline Runt & 3311 & & & & & & & & : & \\
\hline Test Fuid & R114 & $\overline{-}$ & . & . & & & 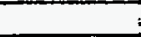 & 1 & 1 & I \\
\hline Dato: & 12299931 & 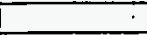 & $\rightarrow$ & & & I & \pm & & $!$ & \\
\hline Time: & 14.081 & & & 1 & & & : & & $i$ & \\
\hline Note & TABOREK & Analytacal d & & $i$ & & & & & i & \\
\hline Fow Areatl2 & 0.003361 & & & $\therefore$ & & $=$ & : & $i$ & E & \\
\hline How Flow Areatt2 & 0.190561 & & $\therefore$ & $\therefore$ & & & 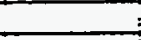 & 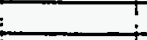 & 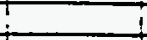 & 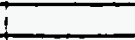 \\
\hline Sat. Pressure Psig & $52 !$ & & & & & $\vdots$ & ; & & T & \\
\hline Sat. Temporatureffinlet) & 124.11 & & 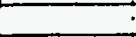 & $\square$ & & $\mathrm{i}$ & it & & ! & \\
\hline Liq. Density lbm/tis & 85.41 & 85.31 & 85.36 & 85.4 & 85.41 & 85.41 & 85.47 & 85.49 & 85.49 & 85.53 \\
\hline Latont Hex-Btunbm & 50.09 & 50 & 50.05 & 50.09 & 50.11 & 50.1 & 50.16 & 50.18 & 50.18 & 50.21 \\
\hline Specifio Heat-Bunbmif & 0.257 & 0.257 & 0.257 & 0.257 & 0.257 & 0.257 & 0.257 & 0.257 & 0.257 & 0.366 \\
\hline Uq. Viscostatbomitihy & 0.677 & 0.644 & 0.676 & 0.617 & 0.617 & 0.677 & 0.619 & 0.679 & 0.619 & 0.02 \\
\hline ija. Conductivatureta & 0.03321 & 0.0033 & 0.03521 & 0.03821 & 0.03321 & 0.0332 & 0.00331 & 0.00331 & 0.0833 & 0.0038 \\
\hline Liq. Pranes No. & 4.77 & 4.77 & 4.77 & 4.77 & 4.77 & $4 . \pi$ & 4.78 & 4.78 & 4.78 & 4.78 \\
\hline Inlot Flow Rate-GPM & 1.61 & 1 & 1 & & 1 & 1 & 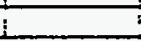 & & 1 & \\
\hline Oulet Lig. Flow Rate-GPM & 1.71 & i & $i$ & it & 1 & & 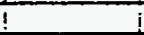 & i & i & \\
\hline Outlel Vap. Fow Rote-GPM & 0.541 & & 7 & \pm & 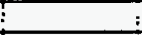 & $\therefore$ & 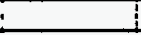 & & $i$ & \\
\hline Inlot Temporeturef & 924.4 & & $\therefore$ & - & & \& & & & & \\
\hline Outal Lia. Temporaturef & 123.4 & & & & & & & & & \\
\hline Outlel Vap. Temperatur of & 123.5 & & & $:$ & & & & & & \\
\hline Water Fow Rato-GPM & . & & & 5 & & & & & & \\
\hline Water inlet Temp.f & 50.81 & $t$ & 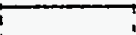 & $\bar{i}$ & i & i & ! & & T & \\
\hline Water outet Temp.F & 121.9 & $i$ & i & & $i$ & 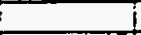 & & & & \\
\hline Inlet Glbmhint? & 3301111 & & ITomp & 117 & Donsity & 86.45 & & & & \\
\hline Subcoolingff & -0.31 & & & & 工 & $\mathrm{i}$ & i & & & \\
\hline Fow Observation & 1 & I & $\square$ & 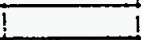 & 1 & I & L & & $!$ & \\
\hline & ZONE1 & ZONE2 & ZONE3 & ZONNE4 & ZONE5 & ZONES6 & ZONE7 & ZONE8 I & ZONE9 & $20 N=10$ \\
\hline Hed input-Watte(net) & 569.41 & 571.9 & 578.3 & 570.8 & 570.2 & 568.5 & 568.8 & 5682 & 572.8 & 575.8 \\
\hline Hoed Loss-Wats & 18.7 & 4.4 & 6 & 7 & 7.6 & 7.11 & 5.9 & 6.3 & 6.6 & 36.8 \\
\hline Hed Floc Buntrite & 8870 & 9014 & 10024 & 8686 & 384 & 382001 & 9857 & 881 & \%का & का \\
\hline Wall Tamp. +3F & 138.6 & 138.5 & 138.2 & 137.6 & 140.4 & 1392 & 137.8 & 138.1 & 138.4 & 137.7 \\
\hline Wall Tomp. $\$ 4 F$ & 138.7 & 137.31 & 139.3 & 138.5 & 139 & 139.4 & 138.3 & 139 & 138.8 & 1372 \\
\hline WAN TOMP. "5F & 138.2 & 136.4 & 140.7 & 138.4 & 137.8 & 13.2 & 137.5 & 738.2 & 138.6 & 138 \\
\hline Wall Tomp. $11 \mathrm{~F}$ & 74.2 & 138.6 & 137.1 & 137.4 & 137 & 139.7 & 138.4 & 138.7 & 138 & 136 \\
\hline Wall Temp. R2F & 139.2 & 138.4 & 137.7 & 138.6 & 137.2 & 140.8 & 138.8 & 1392 & 137 & 138.6 \\
\hline Instraam femp.F & 125.9 & 126.7 & 726.2 & 125.9 & 125.8 & 725.8 & 125.31 & 125.11 & 125.1 & 724.8 \\
\hline Avg. Wall Temp.f & 138.7 & 138.2 & 138.1 & $\mid 36$ & 137.8 & 13.4. & 138.2 & T38.81 & T37.8 & 137.1 \\
\hline AVg. Wall DT.f & 12.3 & 11.7 & 17.4 & $\pi 1.8$ & 11.5 & 13.1 & 12.4 & 13.1 & 11.8 & 17.8 \\
\hline Remaingng Subcooff & -7.1 & & & & & & & & 1 & \\
\hline Ext Quality & 0.041 & 0.07 & 0.11 & 0.14 & 0.18 & 0.21 & 0.251 & 0.28 & 0.32 & 0.96 \\
\hline EXP.HBtuHr/t2/F & 800.61 & 897 & 877.6 & 850.2 & 859.2 & 747.8 & 793.6 & 752.2 & 842.2 & 0 \\
\hline COMPUTED H & & & & & & & & & & \\
\hline Jallouk & $6 \% .1$ & $6 \infty 0.6$ & 648.6 & 643.7 & 637.1 & 6327 & 637.4 & 630.4 & 632.7 & 633.8 \\
\hline seady1(Qudo tuba) & 981.2 & 988.3 & 994.8 & 989.1 & 991.7 & 991.8 & 995.3 & 998.6 & 1006.7 & 10126 \\
\hline seady2 & 791.8 & 7961 & 789.8 & 791.5 & 799.1 & 791.2 & 794.1 & 796.6 & 804.8 & 847.5 \\
\hline (RLO) & 343711 & 33262 & 31934 & 30647 & 29404 & 28183 & 26892 & 25647 & 24428 & 23163 \\
\hline (F) & 1.674 & 2.2791 & 2.728 & 3.107 & 3.439 & 3.7391 & 4.027 & 4.2931 & 4.542 & 4.78 \\
\hline (Aिव) & 191.9 & 261.51 & 312.9 & 356.3 & 39,3 & 428.7 & 467.41 & 491.81 & 520.3 & 548.7 \\
\hline$(1 \mathrm{BOOLI})$ & 978.81 & 9822 & 983.8 & 973.4 & 970.4 & 99.5 & 961.11 & $\$ 57.11$ & 958 & 956.8 \\
\hline & 1 & 1 & 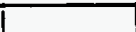 & & & & & & I & \\
\hline & ZONER I & ZZONEA & ZONE 6 & ZZONE8 & ZONETO & & & & 1 & \\
\hline DP fiquid Temp.f & 76.51 & 1 & -2 & 1 & 1 & $\vec{i}$ & & & $i$ & \\
\hline DPLiquid Densinumita & 90.07 & & & $i$ & & & & & & \\
\hline Elovation & $2 i$ & 41 & 6 & 8 & 9.875 & & & & & \\
\hline Mensurad DPPsid & 0.269 & 1.51 & 2.187 & 2.921 & 3.685 & & & & & \\
\hline Zero CorTections-psid & 0.006 & 0.043 & 0.043 & 0.015 & -0.111 & & & & & \\
\hline Corrected DPPsid & 0.253 & 1.467 & 2.144 & 2.906 & 3.706 & & & & & \\
\hline Actual DPAt & 1.68 & 1.78 & 2.77 & 3.62 & 4.71 & & & & & \\
\hline Measured TP $\phi_{s i g}$ & 521 & 1 & 1 & & 55.5 & & & & & \\
\hline Measured Overalla & -5.89 & & & & & & & & & \\
\hline & & & & & & & & & & \\
\hline Computed DPA & 1.27 & 2.371 & 3.3 & 4.28 & 5.21 & & & & & \\
\hline Filatonf & 0.07 & 0.18 & 0.38 & $0 . \overline{2}$ & 0.89 & & & & & \\
\hline Acoularationft & 0.061 & 0.1 & 0.15 & 0.21 & 0.28 & & & & & \\
\hline Elevetiont & 1.15 & 2.01 & 2.7 & 3.45 & 4.04 & & & & & \\
\hline
\end{tabular}




\begin{tabular}{|c|c|c|c|c|c|c|c|c|c|c|}
\hline Runt & 3321 & & & & 1 & & + & 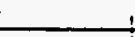 & & \\
\hline Tost Fluid & 8114 & & & & & í & $i$ & 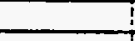 & & \\
\hline Dato: & $2 / 201931$ & & & & + & 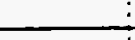 & + & & & \\
\hline Time: & $14: 371$ & & & & & 1 & 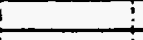 & & & \\
\hline Noto & TABOAEK & Anatytical d & & & & & I & $\therefore$ & & \\
\hline Flow Avea-tid & 0.00336 & 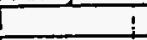 & 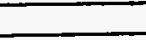 & & & & 1 & 1 & 1 & \\
\hline Hoan flow Aroati? & 0.19635 & $i$ & & & & $i$ & + & $i$ & & \\
\hline Sat. Prossurapig & 49.71 & + & & & 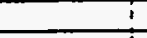 & & 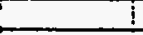 & T & & \\
\hline St. Temperauref finlet) & 121.81 & $!$ & $\underline{i}$ & 1 & 1 & $\vec{a}$ & & 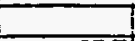 & & \\
\hline Liq. Densinytomith & 85.571 & 85.591 & 85.631 & 85.65 & 85.041 & 85.68 & 85.71 & 85.7 & 85.71 & 85.77 \\
\hline Letent Hestemulbem & 50.द्रा & 50.27 & 50.31 & 50.38 & 50.32 & 50.34 & 50.37 & 50.371 & 50.38 & 50.4 \\
\hline Speatio Hen Bth/bm/F & 0.256 & 0.256 & 0.2561 & 0.256 & 0.256 & 0.255 & 0.256 & 0.256 & 0.256 & 0.256 \\
\hline Liq. Viscosity lbmithr & 0.6211 & 0.622 & 0.6231 & 0.521 & 0.023 & 0.224 & 0.025 & 0.25 & 0.625 & $0 . \overline{27}$ \\
\hline Lig. Conductivity BtuhrffF & 0.03331 & 0.0333 & 0.0334 & 0.03341 & 0.0334 & 0.0334 & 0.0334 & 0.0334 & 0.0334 & 0.0335 \\
\hline Lig. Prandt No. & 4.781 & 4.78 & 4.791 & 4.79 & 4.791 & 4.79 & 4.79 & 4.79 & 4.79 & 4.8 \\
\hline Inlot Flow Rado-GPM & 1 & 1 & 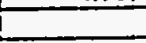 & & ! & 1 & & & & \\
\hline Outlel Lig. Fow Rate-GPM & 0.81 & 1 & 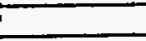 & $\therefore$ & 工 & 7 & & 1 & 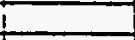 & \\
\hline Outel Vap. Fow Fra-GPM & 0.521 & 7 & 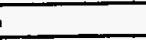 & & ? & & & & & \\
\hline Inlol Tomperaturaf & 12221 & $\therefore$ & $\vdots$ & & & $\mathbf{i}$ & I & -1 & 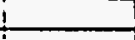 & \\
\hline Outel Lig. Temperaturef & 121.7 & & 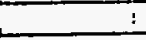 & 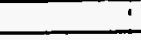 & 1 & $i$ & 1 & & & \\
\hline Outtel Vap. Temperaturof & 121.31 & 1 & 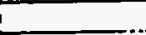 & & & & $i$ & & & \\
\hline Watur Flow Rate-GPM & & & $i$ & & $i$ & i & 1 & 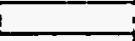 & & \\
\hline Wher indal Temp.f & 50.8 & 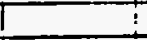 & 1 & & 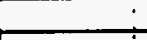 & + & $i$ & 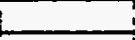 & 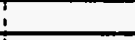 & \\
\hline Were octlat Temp.f & 120.7 & $\therefore$ & $i$ & 1 & $i$ & 1 & 1 & 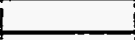 & $i$ & \\
\hline |ndol Gibmh/he & 207600 & & Tomp & 112.41 & Dansity & 86.99 & $i$ & 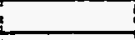 & $!$ & \\
\hline Subcoolingf & -0.41 & & & ! & 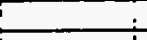 & ; & i & 工 & $i$ & 1 \\
\hline Flow Observation & & - & $\cdot$ & & & & & & & \\
\hline & ZZONE1 & ZONE2 I & ZONE3 & ZONE4 I & ZONE5 :2 & ZONE6 & ZONET : & ZONE8 & ZONE9 & ZONE10 \\
\hline Heal (nour-Watts(nef) & 580.71 & \begin{tabular}{|l|}
578.91 \\
\end{tabular} & 577.5 & 579.7 & 578.3 & 578.3 & 5825 & 581.9 & 576.6 & 579.4 \\
\hline Hed Loss-Watts & 181 & 4.21 & 5.71 & 6.61 & 7.1 & 6.71 & 4.81 & 6 & 6.3 & 352 \\
\hline Hes Floxtturtific & $700 \times 5$ & 10035 & 100ा1 & 10049 & T00241 & 10024 & 300097 & 10087 & $\$ 350$ & 1004 \\
\hline Wail Temp. WF & 135.5 & 135 & 135.71 & 135.2 & 138.2 & 137.3 & 136.2 & 136 & 136.4 & 135.5 \\
\hline Wal Tomp. 14F & 135.7 & 134.7 & 136.7 & 138.1 & 136.8 & 137.4 & 138.7 & 137 & 136.7 & 135 \\
\hline WEll Tomp. $15 F$ & 135.8 & 133.6 & 138.4 & 135.8 & 135.5 & 137 & 135.7 & 138 & 134.6 & .15 .8 \\
\hline Wall Tomp. IIF & 74.11 & 135.3 & 134.4 & 134.8 & 134.5 & 137.7 & 136.8 & 137 & 134.1 & 133.8 \\
\hline Wall Tomp. N2F & 136.8 & 135.41 & 134.9 & 136.1 & 134.7 & 138.7 & 137.1 & 137.6 & 135 & 134.7 \\
\hline Intream Tomp. $F$ & 124.51 & 124.31 & 124 & 123.8 & 1239 & 123.7 & 123.4 & 123.4 & 123.3 & 1228 \\
\hline Avg.Wall Temp.f & 135.9 & 135.4 & 135.4 & 135.5 & 135.4 & 137.4 & 136.6 & 136.7 & 135.3 & 135 \\
\hline Avo. WallDT $f$ & 10.91 & 10.6 & 111 & 11.2 & 11 & 13.2 & 12.6 & 12.9 & 11.6 & 11.7 \\
\hline Remaning Subcoolf & -11.5 & & & +1 & 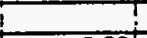 & & & & & \\
\hline Ext Quality & 0.061 & 0.111 & 0.171 & 0.23 & 0.28 & 0.34 & 0.4 & 0.45 & 0.51 & 0.57 \\
\hline EXP.H-BtuHr/M2/F & 919.71 & 9471 & 912.31 & 893.51 & 909.5 & 759.6 & 798.6 & 784.1 & 8525 & 0 \\
\hline COMPUTEDH & & & 1 & & & & & & & \\
\hline Jallouk & 690.91 & 659.8 & 85231 & 654.8 & 647.9 & 641.8 & 643.2 & 643.2 & 634.7 & 633.4 \\
\hline szadyi(cudo tube) & 985.11 & 984 & 983.71 & 988 & 987.11 & 988.6 & 993.51 & 995.7 & 99 & 997.8 \\
\hline szacty2 & 789 & 782.8 & 7829 & 797.8 & 798.2 & 785 & 7928 & 799.4 & 804.2 & 799.6 \\
\hline$($ (RIU) & 212101 & 1993 & 186741 & 17429 & 1ब्टा1 & 14971 & 1372 | & 12492 & 11264 & 10018 \\
\hline$(F)$ & 1.804 & 2.721 & 3.3161 & 3.8161 & 4.256 & 4.684 & 5.040 & 5.406 & 6.748 & 6.00 \\
\hline (मபव) & 150.41 & 214.9 & 261.71 & 3012 & 336 & 358.7 & 398.3 & 4255 & 452,4 & 480.1 \\
\hline (FIBOLL) & 983.81 & 980.51 & 976.8 & 976.5 & 974 & 971.3 & 971.7 & 958.8 & 961.6 & 959.8 \\
\hline & & & & 1 & & & & & & \\
\hline & IZONE2 & ZONE4 & ZONE6 & ZZNEB & ZONE10 & & & & & \\
\hline DFliquid Temp.f & 76.4 & & $T$ & 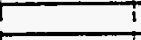 & $i$ & & & & & \\
\hline DPLquid Dentitulomin 3 & 90.98 & 1 & $T$ & I & & & & & & \\
\hline Elovation h & 2ा & 41 & हा & 8 & 9.8751 & & & & & \\
\hline Measurad DPPsid & $0.27 \pi$ & 1.79 & 2521 & 3.36 & 4.2251 & & & & & \\
\hline Zoro Cortectons-psid & 0.0001 & 0.0431 & 0.0431 & 0.0151 & -0.1111 & & & $i$ & & \\
\hline Corrected DPPsid & 0.2711 & 1.747 & 2.477 & 3.345 & 4.3361 & & & $i$ & $i$ & \\
\hline Actua DPA & 1.67 & 1.31 & 2.211 & 2.87 & 3.19 & & & $I$ & i & \\
\hline Messured TPPaig & 49.7 & & 1 & + & 63.6 & T & & & & \\
\hline Messured Overalth & -6.55 & & 1 & 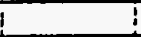 & 1 & 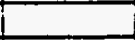 & & & & \\
\hline & $\div$ & & & I & $\vdots$ & 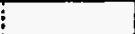 & & $i$ & & \\
\hline Computed DPAn & 1.04 & 1.82 & 2.53 & 3.27 & 3..5ा & & & & & \\
\hline Friction-t & 0.041 & 0.121 & 0.25 & 0.411 & 0.6 & & & & & \\
\hline Acosiarationtt & 0.03 & 0.07 & 0.111 & 0.16 & 0.22 & & & & & \\
\hline Elovation-th & 0.97 & 1.69 & 2.17 & 2.64 & 3.031 & & & & & \\
\hline
\end{tabular}




\begin{tabular}{|c|c|c|c|c|c|c|c|c|c|c|}
\hline \multicolumn{11}{|l|}{$\begin{array}{l}\text { Runt } \\
\text { Tost Fivid } \\
\text { Date: }\end{array}$} \\
\hline \multicolumn{11}{|c|}{ Time: $19: 53 !$} \\
\hline \multicolumn{11}{|c|}{ Nole TABOREE Anarvical dt } \\
\hline \multicolumn{11}{|c|}{ Fow Avea-A2 $\quad 0.00336 !$} \\
\hline \multicolumn{11}{|c|}{ Heat Flow A vea-t2 0.19695} \\
\hline \multicolumn{11}{|c|}{ Sat Pressure Psig $\square 98 . \overline{41}$} \\
\hline \multicolumn{11}{|c|}{ Sat. Temporaturoffinlel) } \\
\hline \multicolumn{11}{|c|}{ Lq. Density-ibmitis -81.341} \\
\hline \multicolumn{11}{|l|}{ Letent Hert-Bubm } \\
\hline \multicolumn{11}{|l|}{ Speatio Hearstuhbm/F } \\
\hline \multicolumn{11}{|l|}{ Liq. Viscosinulbmithth } \\
\hline \multirow{2}{*}{$\begin{array}{l}\text { Liq. Conductivity Bhuhr-iff } \\
\text { Lig. Prandt No. }\end{array}$} & 0.03051 & 0.0305 & 0.03041 & 0.03551 & 0.03551 & 0.03551 & 0.03051 & 0.03051 & 0.0306 & 0.0306 \\
\hline & 4.6 & 4.59 & 4.59 & 4.59 & 4.61 & 4.61 & 4.6 & 4.6 & 4.6 & 4.6 \\
\hline Inlet Flow Rate-GPM & 5.11 & & & & & 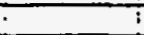 & & ! & 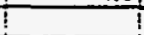 & \\
\hline Outlet Lia. Flow Rato-GPM & 2.11 & & & & & $\mathbf{i}$ & i & & i & \\
\hline Outel Vap. Fow Rado-GPM & 1.2 & & 1 & & & ; & & $!$ & $!$ & \\
\hline Inlet Temperaturaf & 155.81 & & & & & & & $\bar{i}$ & $\mathbf{i}$ & \\
\hline Outtet Lia. Temperaturef & 96.61 & & & & & & & 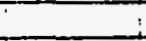 & 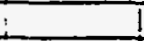 & \\
\hline Outiel Vap. Temperaturéf & $\overline{154.81}$ & & & & & & & ; & ; & \\
\hline Water Fow Raio-GPM & & & & & & & & i & 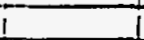 & \\
\hline Weter Inlot Temp.F & 45.4 & & & & $\therefore$ & & & 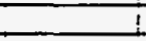 & \pm & \\
\hline Weder outel Temp.F & 148.11 & & & & & & & & & \\
\hline nat G lbm/hr/A2 & $998673 !$ & & Tomp & 15251 & IDonsity & 82.05 & & & & \\
\hline Subcoolingf & $6 \overline{6 i}$ & & & & & & & & & \\
\hline Fow Observanon & & & & & & & & & & \\
\hline & ZONE1 & ZONE2: & :ZONE3 & ZONE4 & ZZONES : & ZZONE6 : & ZONE7 & ZONE8 & ZZNE9 & ZONE10 \\
\hline Heat Input-Watts(net) & 1446.31 & 1447.3 & 1443.81 & 1444.41 & 1442.7 & 1447.8 & 1445.7 & 1447.1 & 1445 & it 1442 \\
\hline Heat Loss-Walls & 35.8 & 7.71 & 9.91 & 101 & 10.2 & 10.71 & 70.6 & 121 & 12.51 & 626 \\
\hline Heat Fiox-Bturifite & $25071 !$ & 25088 & 250271 & 250381 & 250081 & 25097 & 250601 & 250851 & 250481 & 24988 \\
\hline Wall Temp. \$3F & 1741 & 177.71 & 174 & 174.21 & 180.17 & 176.11 & 975.31 & 175.8 & 775.81 & 175.4 \\
\hline Wall Temp. $4 f$ & 173.91 & 173 & 176.41 & $176 !$ & 176.71 & 178.11 & 176.11 & 177.5 & 176.51 & 174 \\
\hline Wal Temp. 55 & 172.81 & 170.6 & 178.31 & 173.9 & $17 \overline{4} .7$ & 178.61 & 174.81 & 174.9 & 171.8 & 173.8 \\
\hline Wat Tomp. AF & 70.31 & $174.8 i$ & 172.7 & 173.5 & 173.61 & 178.8 & 177 & 176.51 & 171.2 & 967.4 \\
\hline Walt Tomp. \$2-F & 174.51 & $\overline{i 74.31}$ & 173.81 & $177.8 !$ & 174.31 & 183.61 & 177.61 & 177 & 174.1 & 771.6 \\
\hline Instram Tomp.F & 157.9 & 158.4 & $159.1 \mathrm{i}$ & 158.5 & 158.11 & 158.2 & 158.3 & 157.8 & 157.5 & 156.7 \\
\hline Ang. Wall Temp.f & 173.81 & 175 & $174.3 !$ & 174.41 & 174.8 & 176.9 & 176.1 & 176.3 & 973.8 & 9725 \\
\hline Avg. Wall DT.f & 14.81 & 15.41 & 141 & $14.8 !$ & 15.6 & 17.6 & 16.7 & 17.4 & 15.2 & 14.6 \\
\hline Remaining Subcooff & 1.1 & & & & & & & & & \\
\hline Exit Quality & $-0.01 i$ & 0.031 & 0.061 & 0.091 & $0.12 !$ & 0.751 & 0.18 & 0.221 & 0.25 & 0.28 \\
\hline 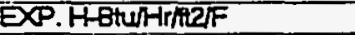 & 1697.81 & 1627.41 & 1785.2 & 1695.31 & 1603.61 & 1426.71 & 1503.51 & 1441.11 & 1647 & 0 \\
\hline COMPUTEDH & 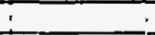 & & & & & i & & & & \\
\hline Jallouk & 1259.81 & 1185.41 & 1106.61 & 1057.4 & 1017.31 & 980.8 & 967.1 & 909.9 & 873.2 & 835.5 \\
\hline Szadyi(audo sube) & 1757.7 & 1783.51 & 1777.61 & 1775.7 & 1780.41 & 17924 & 1800.81 & 1809.1 & 1817.1 & 1810.9 \\
\hline szady2 & 1682.11 & 1688.7 & 1877 & 1680.81 & 1701.71 & 1708.5 & 1710.71 & 1694.2 & 1701.8 & 170 \\
\hline (RLG) & 1244111 & 123517 & 1199491 & 115624 & 111434 & 107518! & 1035951 & 993881 & 95285 & 9009 \\
\hline (F) & 1.0061 & 1.3831 & 1.9081 & 2.255 & 2.6681 & 2821 & 3.062 & 3.278 & 3.478 & 3.691 \\
\hline (HUO) & 288.2 & 396.1 & 345.61 & 648.81 & 734.11 & 807.8 & 874.2 & 9372 & 9958 & 10532 \\
\hline (सВOLL) & 1755.1 & 1758.8 & $1754.2 !$ & 1746.8 & 1737.7 & 1735.9 & 1729.4 & 1721 & 1771.4 & 1683.5 \\
\hline & & & & & & & & & & \\
\hline & ZZONE2 & $20 N E 4$ & ZONE6 & ZONE8 & ZONE10 & & & & & \\
\hline DP liquid Temp.F & 73.81 & & $!$ & $!$ & 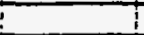 & & & & & \\
\hline DP Liquid Density-lbmiti3 & 91.251 & 1 & . & ! & $\div$ & : & & & & \\
\hline Elovation fit & $2 !$ & 41 & 61 & 8 & 9.8751 & & & & & \\
\hline Measured DPFsid & 0.0561 & 0.498 & 0.8021 & 0.998 & 1.211 & & & & & \\
\hline Zero Corrections-psid & 0.0161 & $0.059 !$ & 0.0381 & $0.036 !$ & -0.031 & & & & & \\
\hline Correctod DP Psid & 0.021 & 0.429 & $0 . \overline{784}$ & 0.9621 & 7.247 & & & t & & \\
\hline Achea DPA & 2.21 & 3.72 & 5.37 & $7.26 !$ & 8.861 & & & & & \\
\hline Messured TP Psig & 38.4 & 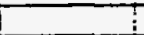 & i & i & 0.21 & & & & & \\
\hline Mossured Ovaralit & 173.511 & & & & 1 & & & & & \\
\hline & & & & & & & & & & \\
\hline Computed DPA & $2.19 !$ & 4.121 & $6.12 !$ & 8.311 & 10.59 & & & & & \\
\hline Friction-t & 0.2 & 0.721 & 1.531 & 2.6 & 3.8251 & & & & & \\
\hline Acooloration-ft & 0.251 & 0.531 & 0.8 & 1.081 & 1.421 & & & & & \\
\hline Elevation-i & 1.741 & 2.861 & 3.81 & 4.63 & 5.341 & & & & & \\
\hline
\end{tabular}




\begin{tabular}{|c|c|c|c|c|c|c|c|c|c|c|}
\hline & & & & & & $\div$ & 1 & $\perp$ & 1 & 1 \\
\hline Runt & 334 & & & & & & 1 & 1 & & \\
\hline Test Fluid - & R114 & & & & & & & & & \\
\hline Dalo: & $2 \longdiv { 2 6 1 9 3 }$ & & & & & & & & & \\
\hline Time: & $21: 15$ & & & & & & & & & \\
\hline Note & TABOREK & Analytical d & & & & & & & & \\
\hline Flow Asea-t12 & 0.00336 & & & & & & & & & \\
\hline Head Flow Areastl? & 0.19695 & & & & & & & & & \\
\hline Sat.PTessuro Psig & 89.1 & & & & & & 1 & & & \\
\hline Sat. Temperaturef $f(i n i e l)$ & 155.81 & $\overrightarrow{0}$ & & & & & 1 & 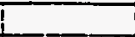 & & i \\
\hline Liq. Density lbmitt3 & - 82.15 & $82.12 !$ & $82.05 i$ & 82.081 & 82.151 & $8 \overline{2.13 i}$ & 82.181 & 82.22 & 82.261 & 82.33 \\
\hline Ladont Hoatoturibm & 46.971 & $46.94 \overrightarrow{1}$ & $46.88 \mathrm{i}$ & $46 . \overline{9} 11$ & $46 . \overline{971}$ & 46.961 & 46.981 & 47.05 & 47.08 & 47.15 \\
\hline Specifio Heat Btulbm/F & 0.2651 & 0.2651 & $\hat{0.2651}$ & 0.265 i & 0.265 & 0.2651 & 0.2651 & 0.265 & 0.265 & 0.285 \\
\hline Lig. Viscositylbmithth & 0.5491 & 0.541 & $0.539 i$ & 0.5391 & 0.5411 & 0.54 & 0.5411 & 0.542 & 0.543 & 0.54 \\
\hline Lig. Conductivity-itume fif & 0.0311 & 0.0311 & 0.0311 & 0.0311 & 0.0311 & 0.031 & 0.0311 & 0.0311 & 0.0311 & 0.0312 \\
\hline Lig. Prandtd No. & 4.62 & 4.621 & 4.621 & $4.62 !$ & 4.62 & 4.62 & $4 . \approx 2$ & 4.62 & 4.62 & 4.63 \\
\hline Inlot Fow Rado-GPM & $4.2 !$ & & & & : & 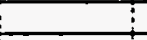 & 1 & 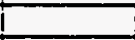 & & \\
\hline Outlol Lia. Flow Ralo-GPM & 1.61 & & & & & & & & & \\
\hline Outtel Vap. Flow Ralo-GPM & $1.4^{1}$ & & & & & $\mathrm{i}$ & i & & I & \\
\hline Inlet Temperaturef & 949.51 & & & & & T & T & & & \\
\hline Outlel Lua Temperaturo & 98.3 & & & & $\vdots$ & 1 & I & 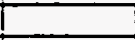 & & \\
\hline Outlel Vap. Temperafure & 147.91 & & & & 1 & $!$ & ! & $!$ & & \\
\hline Watar Flow Rato-GPH & & $\vdots$ & & & 1 & $!$ & $!$ & $!$ & & \\
\hline Weter inlot Temp.f & 43.91 & 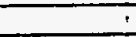 & $\div$ & & $i$ & 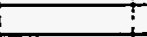 & i & i & & \\
\hline Wher outat Temp.f & 140.41 & 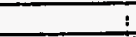 & $\perp$ & & & & & & & \\
\hline Inlol C-lbm/hr/th & 831672 & & Tomp & 145.41 & Density & 8298 & & & & \\
\hline Subcooling $F$ & 6.31 & & & & -1 & +1 & 1 & 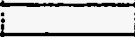 & & \\
\hline Flow Observation & & & & & & & & & & \\
\hline & ZONE1 & ZONE2 & ZONE3 I2 & ZONE4 & ZONE5 & ZONE6 IZ & ZONE7 & ZONE8 & ZONE9 & ZONETO \\
\hline Head Input-Watts(net) & 1453.4 & 1448.4 & 1447.6 & 1446 & 1446 & 1443.7 & 1449.4 & 1454.8 & 1445.6 & 149.6 \\
\hline Hoed Loss-Watts & 32.41 & 7.11 & 9.31 & 10 & 10.4 & 10.3 & 9.9 & 11.1 & 11.5 & 59.1 \\
\hline Hod Flux-BtuHint & 25194 & 25107 & 25093 & 25005 & 25055 & 25026 & 25T241 & 2520 & 25050 & $\mathbf{2 5 1 2 8}$ \\
\hline Wall Temp, $13 F$ & 167.7 & 171.5 & 167.81 & 168.8 & 174.3 & 170 & 168.5 & 167.8 & 169.8 & 169.8 \\
\hline Wa Temp. $4 F$ & 167.4 & 166.01 & 170.2 & 170 & 170.7 & 170.1 & 167 & 167.7 & 170.2 & 169.1 \\
\hline WaITomp. 15F & 166.51 & 164.2 & 172.21 & 168 & 168.7 & 170.5 & 165.8 & 168.1 & 165.5 & 187.8 \\
\hline Well Tomp. IIF & 69.71 & 168.4 & 166.81 & 167.51 & 167.7 & 1729 & 168.8 & 166.4 & 164.4 & 161.3 \\
\hline Wall Temp. WLF & 167.7 & 167.9 & 167.6 & 17221 & 168.31 & 17.7 & 170.5 & 165.8 & 167 & 165.2 \\
\hline Instroam Tomp.F & 151.8 & 152 & 1525 & 1523 & 151.8 & 151.8 & 151.7 & 151.2 & 150.9 & 150.4 \\
\hline Avg. Wall Temp, $F$ & 167.31 & 168.6 & $168 !$ & 968.51 & 168.9 & 170.9 & 167.7 & 166.8 & 167.4 & 165.7 \\
\hline Avg.Wall DT. $f$ & $14.4 !$ & 15.5 & 14.31 & $15 !$ & 15.9 & 17.8 & 14.9 & 14.4 & 15.3 & 15.1 \\
\hline Remaining Subcoolf & -0.41 & & & & & & + & 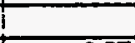 & & \\
\hline Exot Quality & 0 & 0.041 & 0.081 & $0.12 !$ & 0.15 & $0.19 !$ & 0.23 & 0.27 & 0.3 & 0.34 \\
\hline EXP.HBtu/HrmaF & 1751.21 & 1623.4 & 1749.81 & 1669.1 & 1574.71 & 1402.51 & 1688 & 1748.9 & 16327 & 0 \\
\hline COMPUTEDH & & & & & & & & & & \\
\hline Jallouk & 1263.21 & 9167.4 & 1096.51 & 10501 & 1017.31 & 985.1 & 954.7 & 926.1 & 8929 & 882.6 \\
\hline szacylicudo tub & 1727.31 & 1728.31 & 1738.11 & 1740.31 & 1744.01 & 1751.51 & 170211 & 177.8 & 1775.1 & 1785.5 \\
\hline Srady? & 1627.41 & 1628.2 & 1638.6 & 1640.8 & 1639.21 & 1683.9 & 1689.8 & 1874.5 & 1655.8 & 1600.1 \\
\hline (AL1O) & 1005001 & 985861 & 95034 & 911क्3 & 87132 & 833911 & 79527 & 75545 & 716811 & 67722 \\
\hline (F) & 1.107, & 1.63 & 2.151 & 2.538 & 2.888 & 3.154 & 3.418 & 3.67 & 3.008 & 4.120 \\
\hline (म) & 27251 & 401.5 & 520.8 & 6252 & 705.3 & 776.8 & 841.7 & 9032 & S504 & 10156 \\
\hline (HBOII) & 1725 & 1721 & 1719.5 & 17129 & 1705.1 & 1699 & 1695.6 & 1699.8 & 1676 & 1683.5 \\
\hline & & & & & & & & & & \\
\hline & ZONE2 & ZONEA & ZONE6 & ZONEB & ZONETO & & & & & \\
\hline DP liquid Tomp. $f$ & $\quad 74.5$ & -2 & & & & & & & & \\
\hline DPLiquid Denstiny-bmith & 91.181 & & & & & & & & & \\
\hline Elevation-ft & 2ा & 4 & 61 & 81 & 9.8751 & & & & & \\
\hline Moasured DPpsid & 0.009 & 0.712 & 1.0811 & 1.384 & 1.719 & & & & & \\
\hline Zoro Cortectons-psid & 0.018 & 0.069 & 0.0381 & 0.0381 & -0.03 & & & & & \\
\hline Correasd DPPsid & 0.0761 & 0.649 & 1.0481 & 1.328 & 1.749 & & & & & \\
\hline Actud DPA & 2.081 & 3.31 & 4.82 & 6.54 & 7.881 & & & & & \\
\hline Measured TPPsig & 89.11 & & & & 0.1 & & & & & \\
\hline Moasurod Overallth & 155.67| & & & & & & & & & \\
\hline & & & & & & & & & & \\
\hline Computed DP- & 2.01 & 3.72 & 5.53 & 7.51 & 9.58 & & & & & \\
\hline Friction-th & 0.18 & 0.64 & 1.35 & 220 & 3.37 & & & & & \\
\hline Accoleration-A & $0.23 !$ & 0.46 & 0.7 & 0.38 & 1.3 & & & & & \\
\hline Elevation-t & 1.61 & 2.62 & 3.48 & 4.25 & 4.9 & & & & & \\
\hline
\end{tabular}




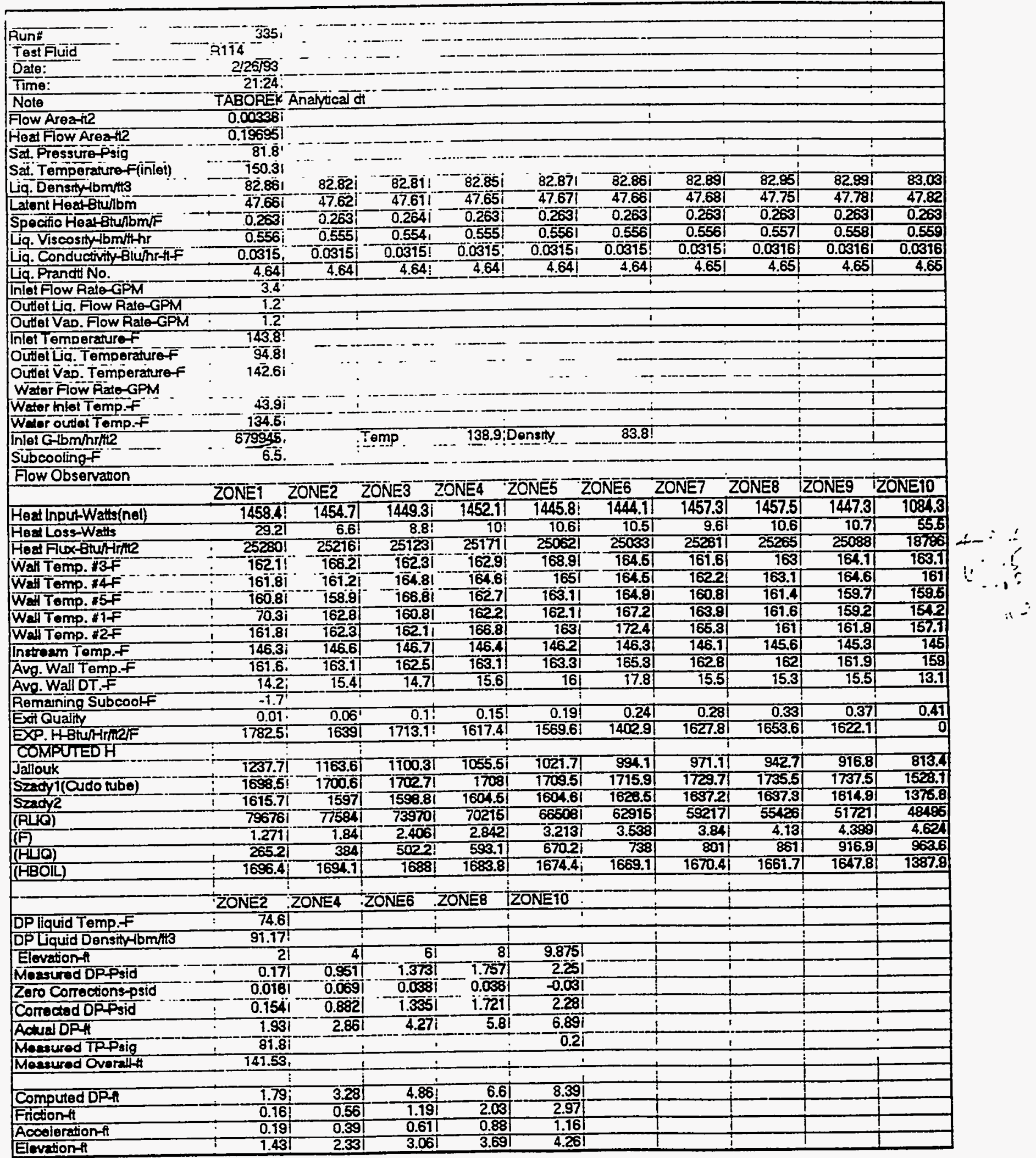




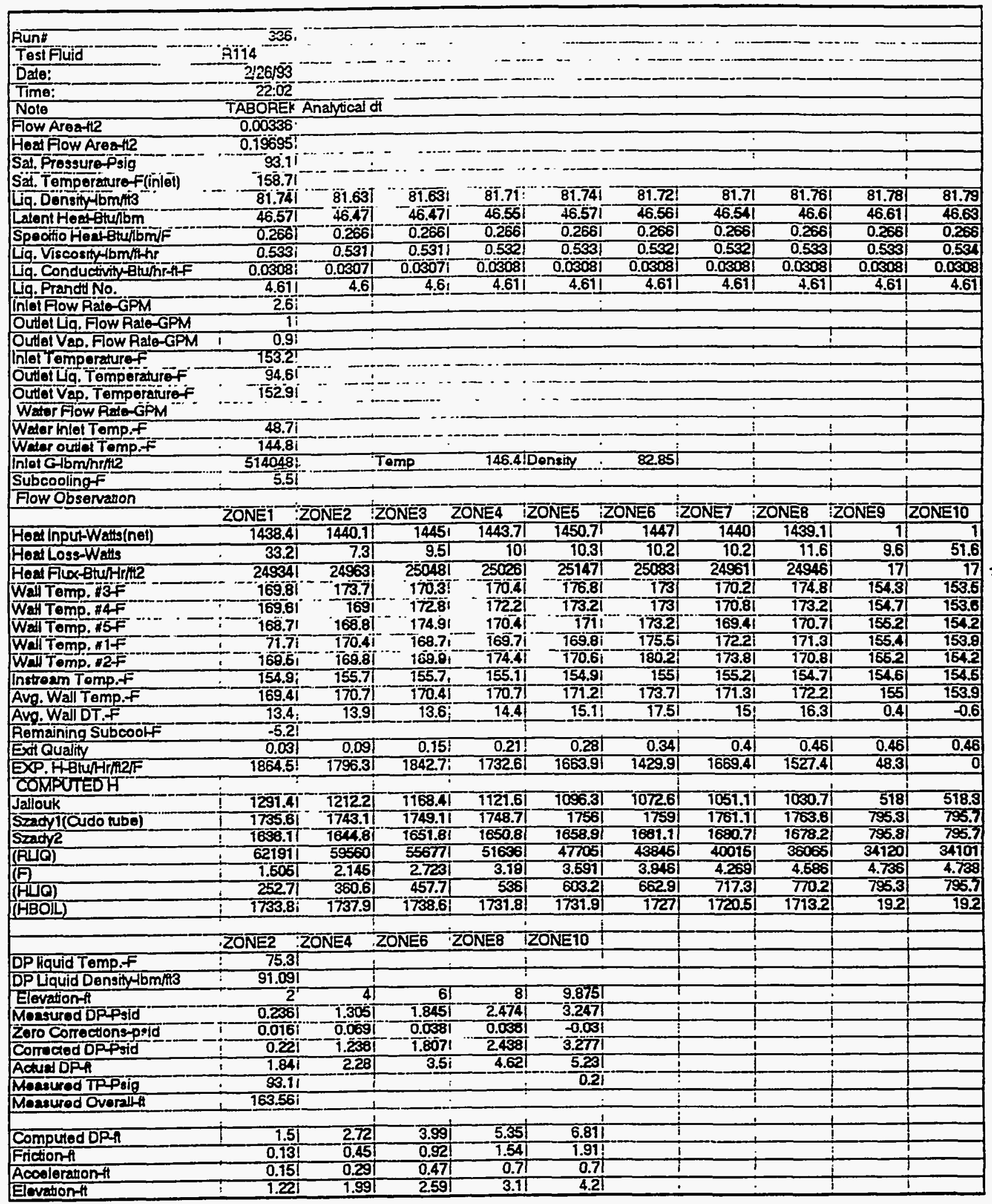




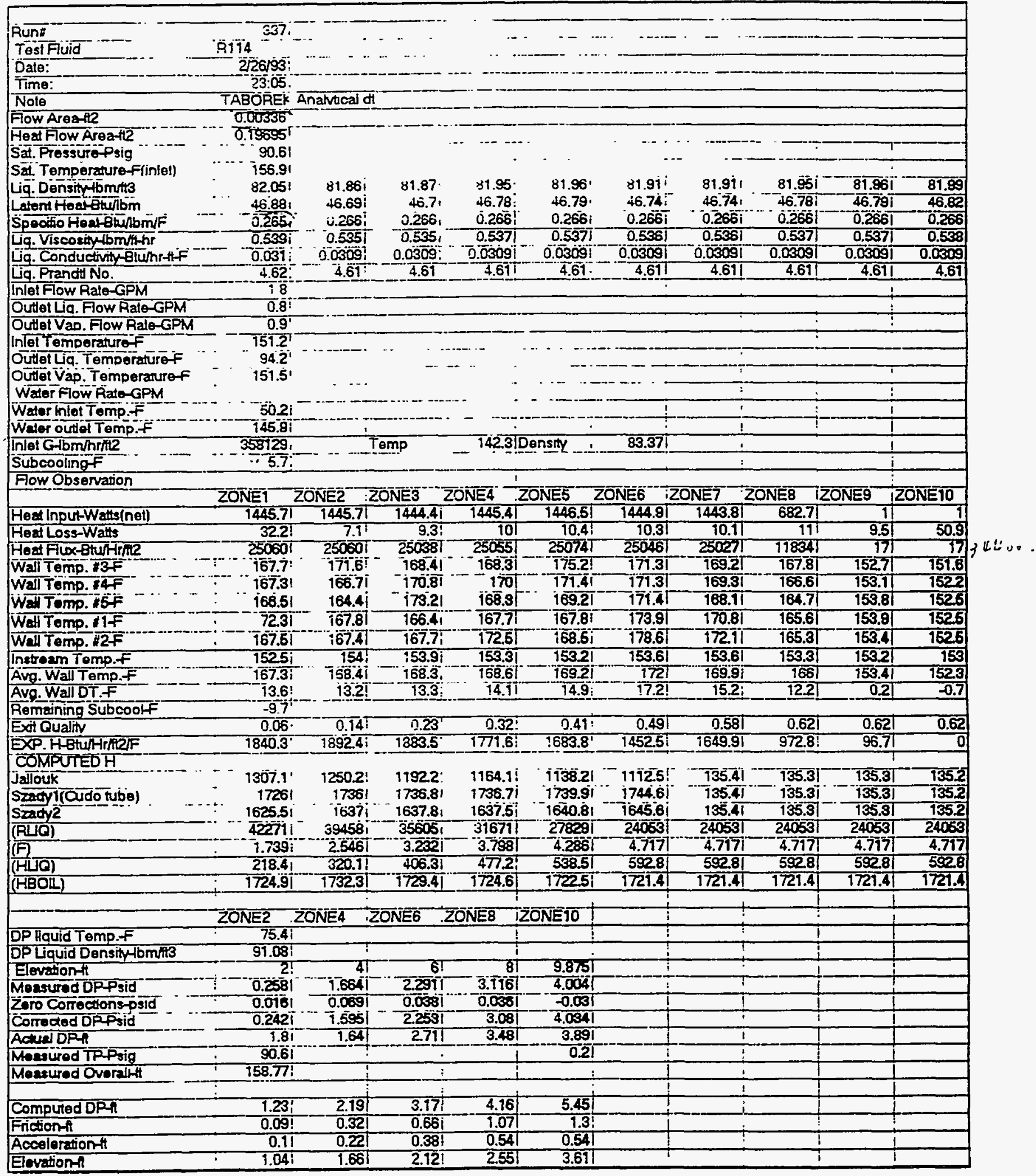




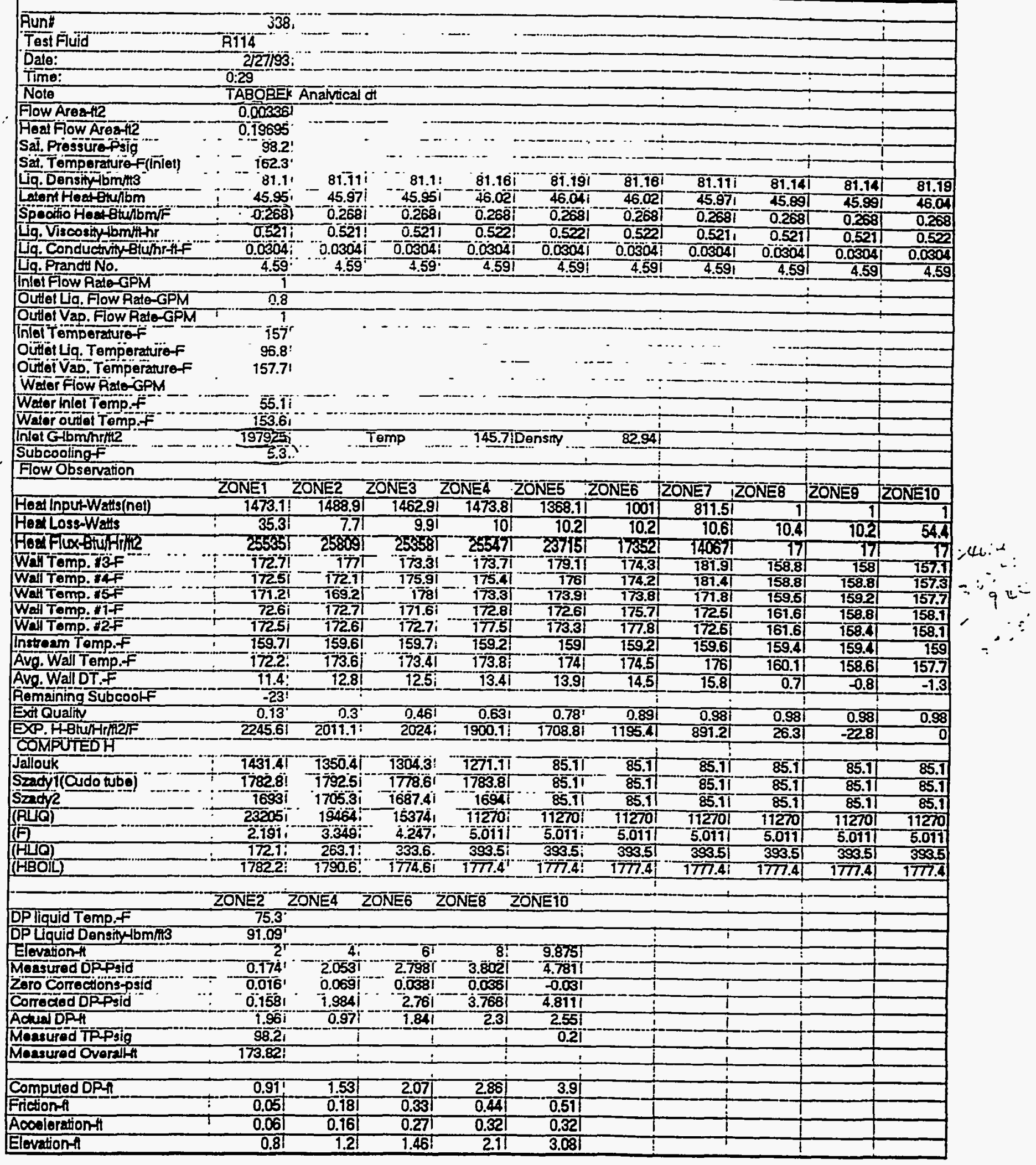




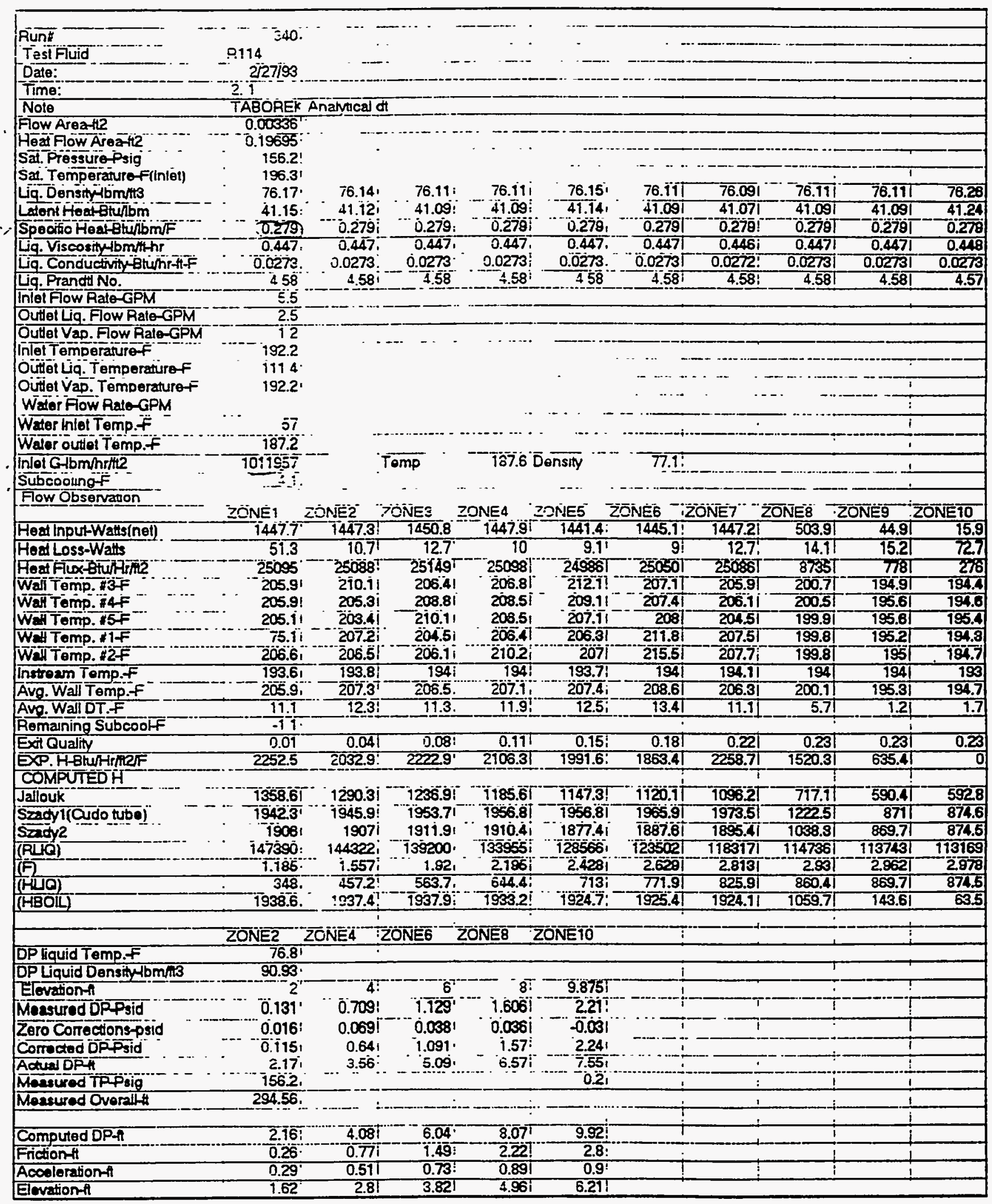




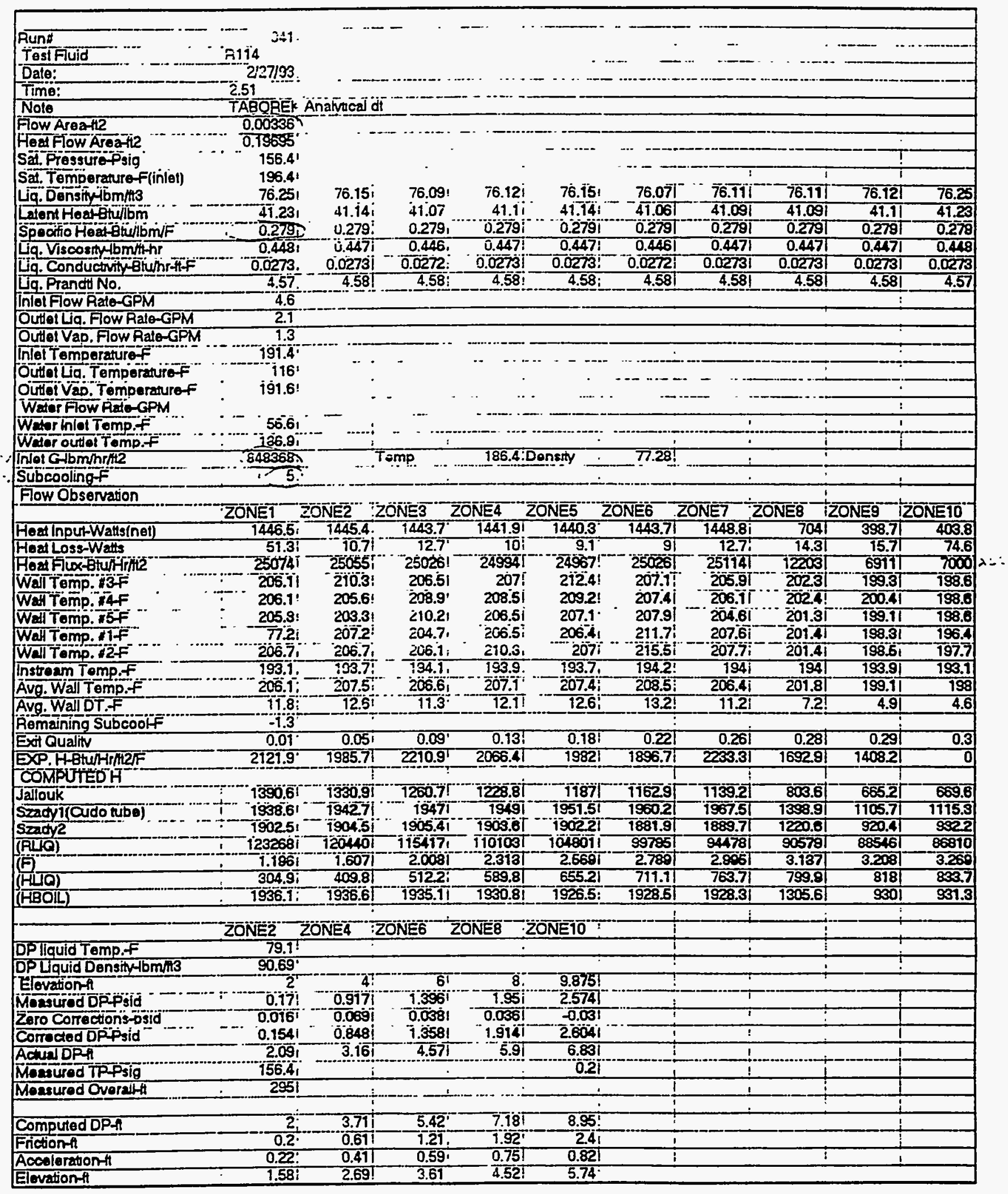




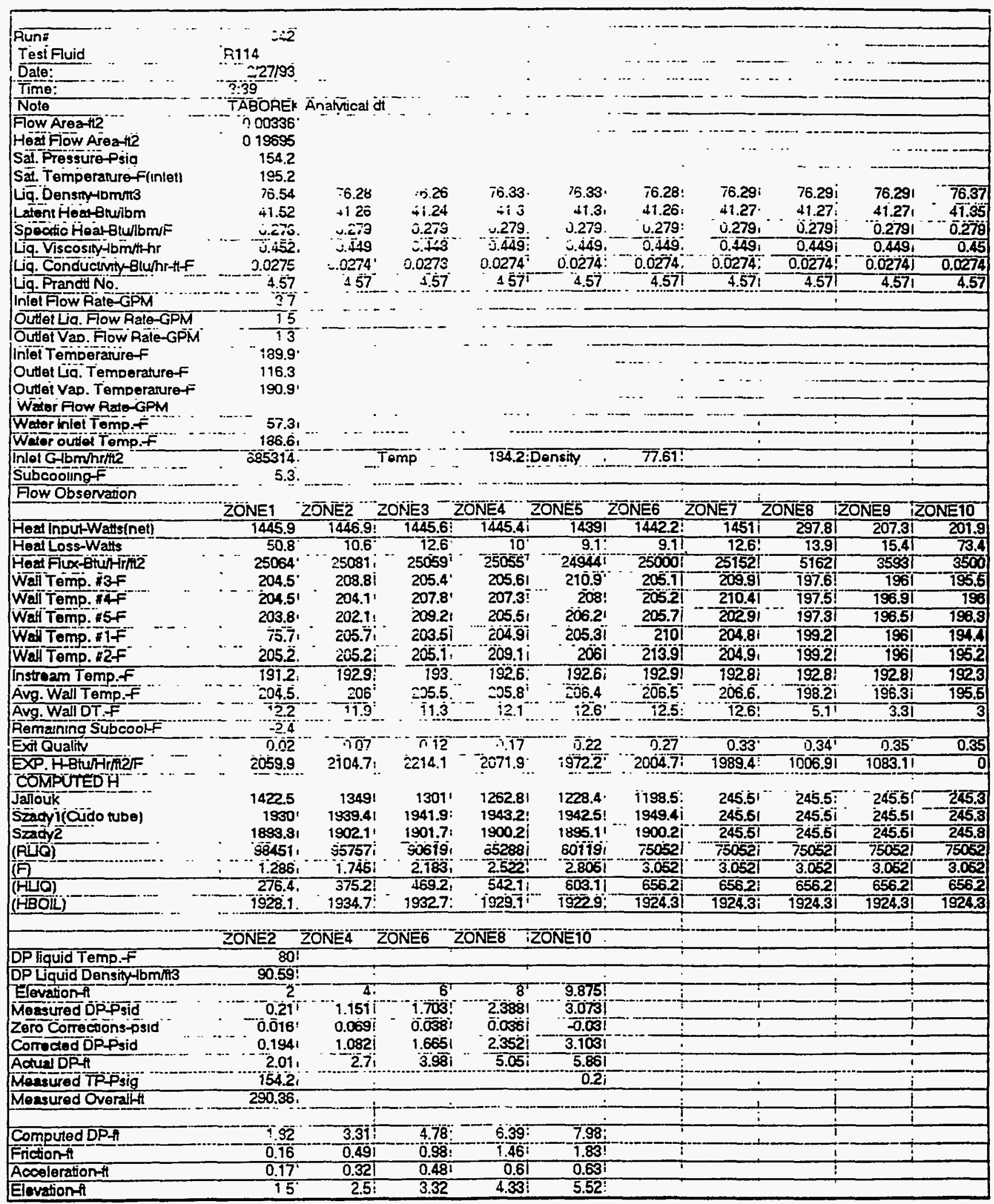




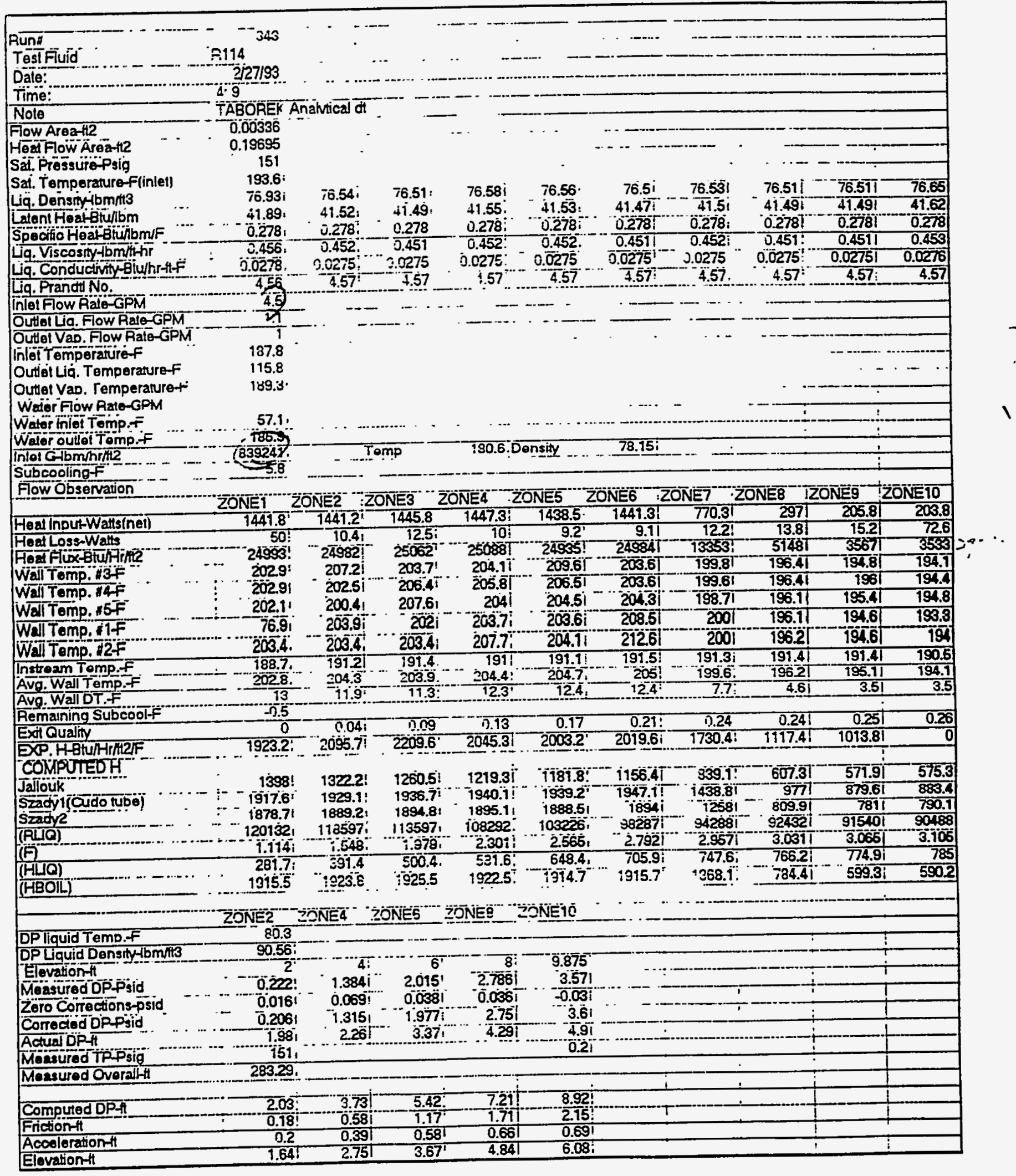




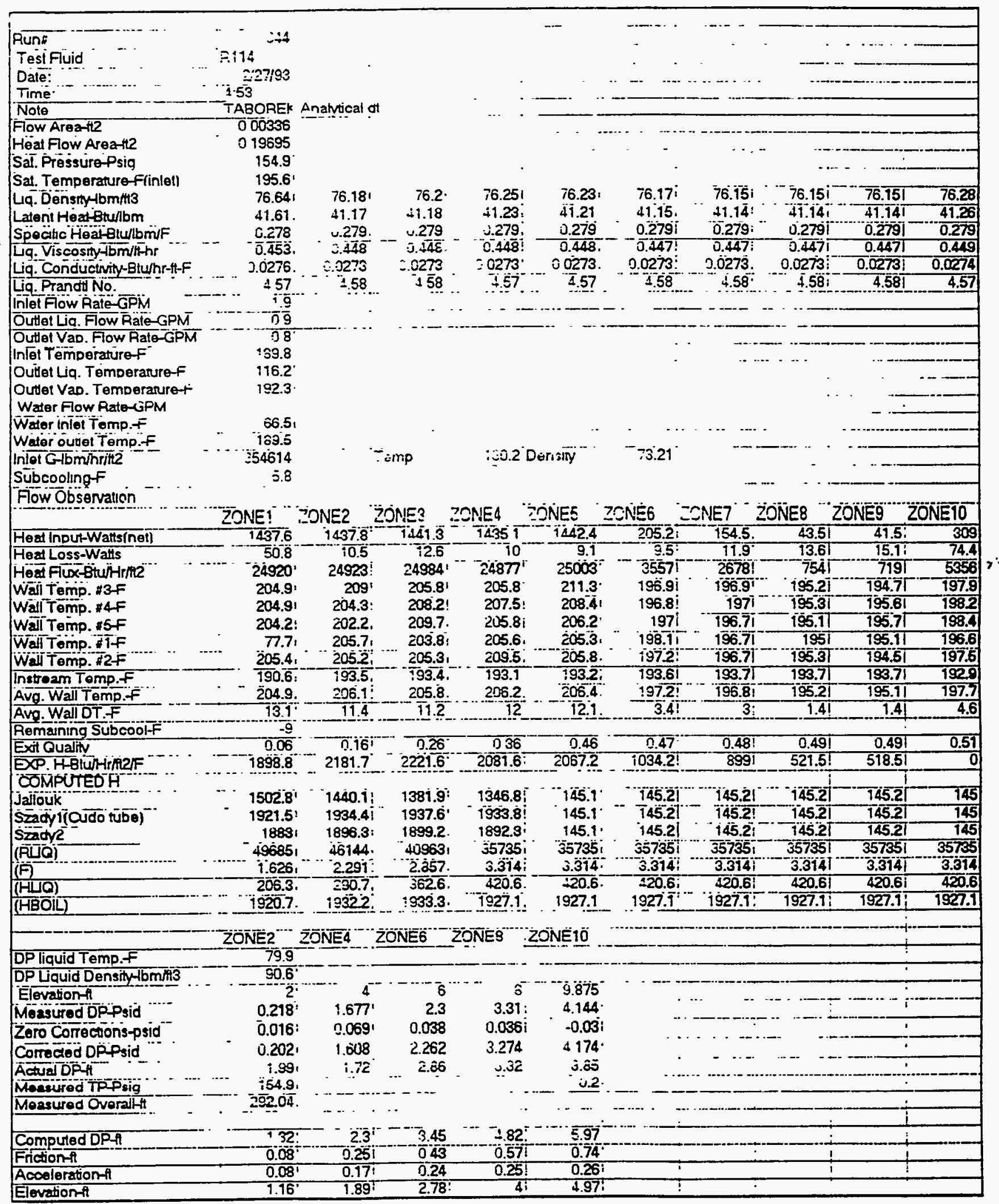




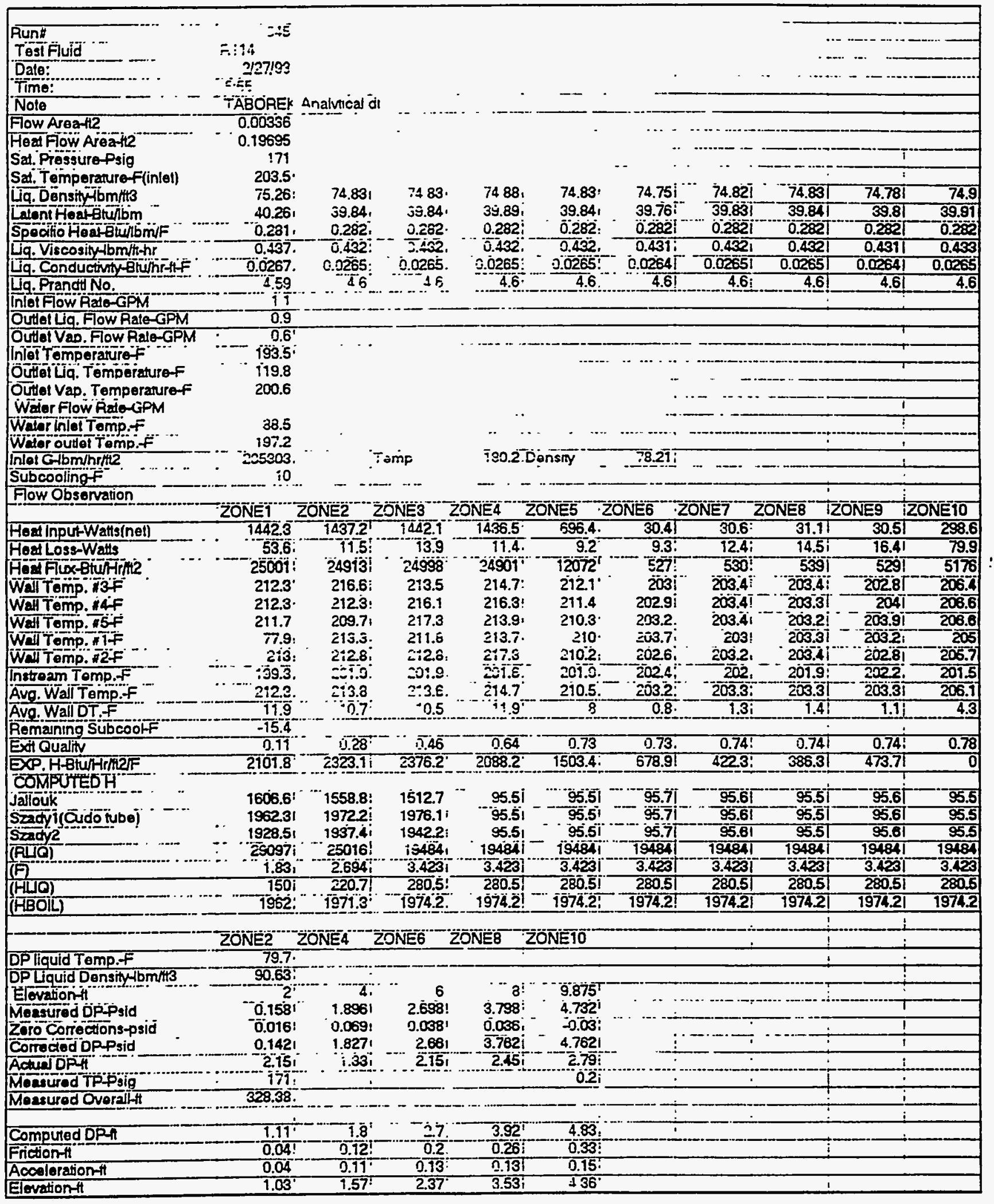




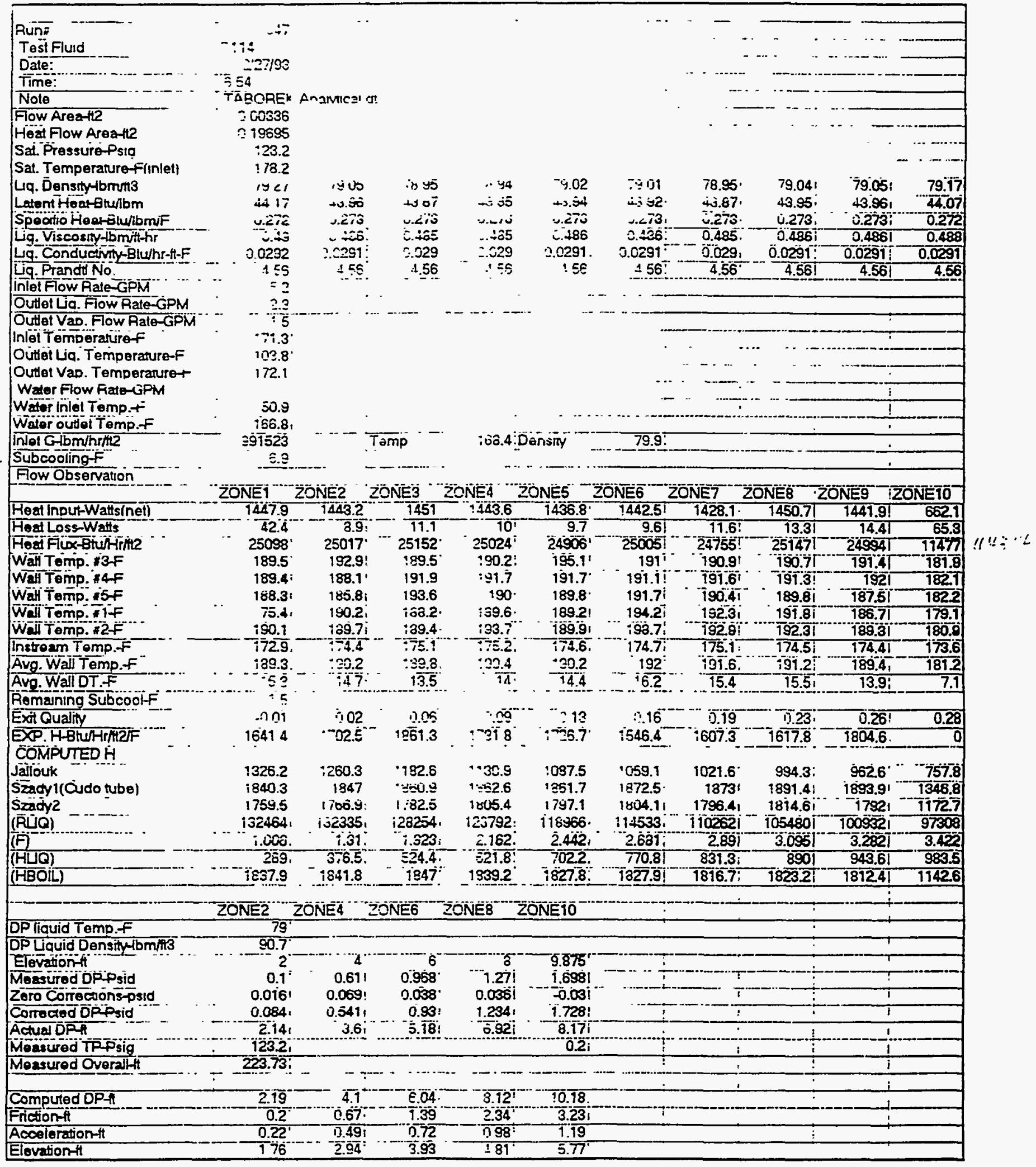




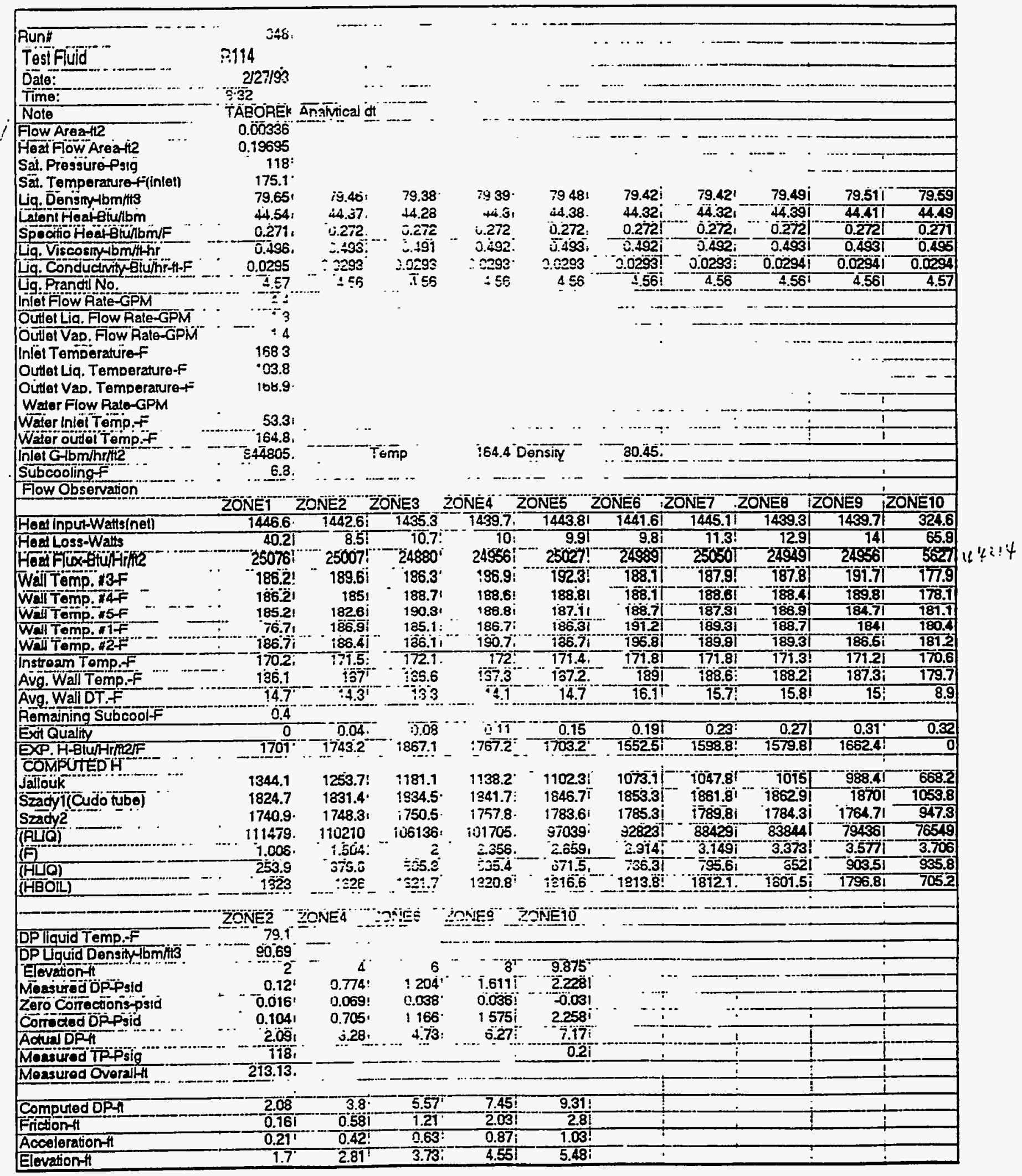




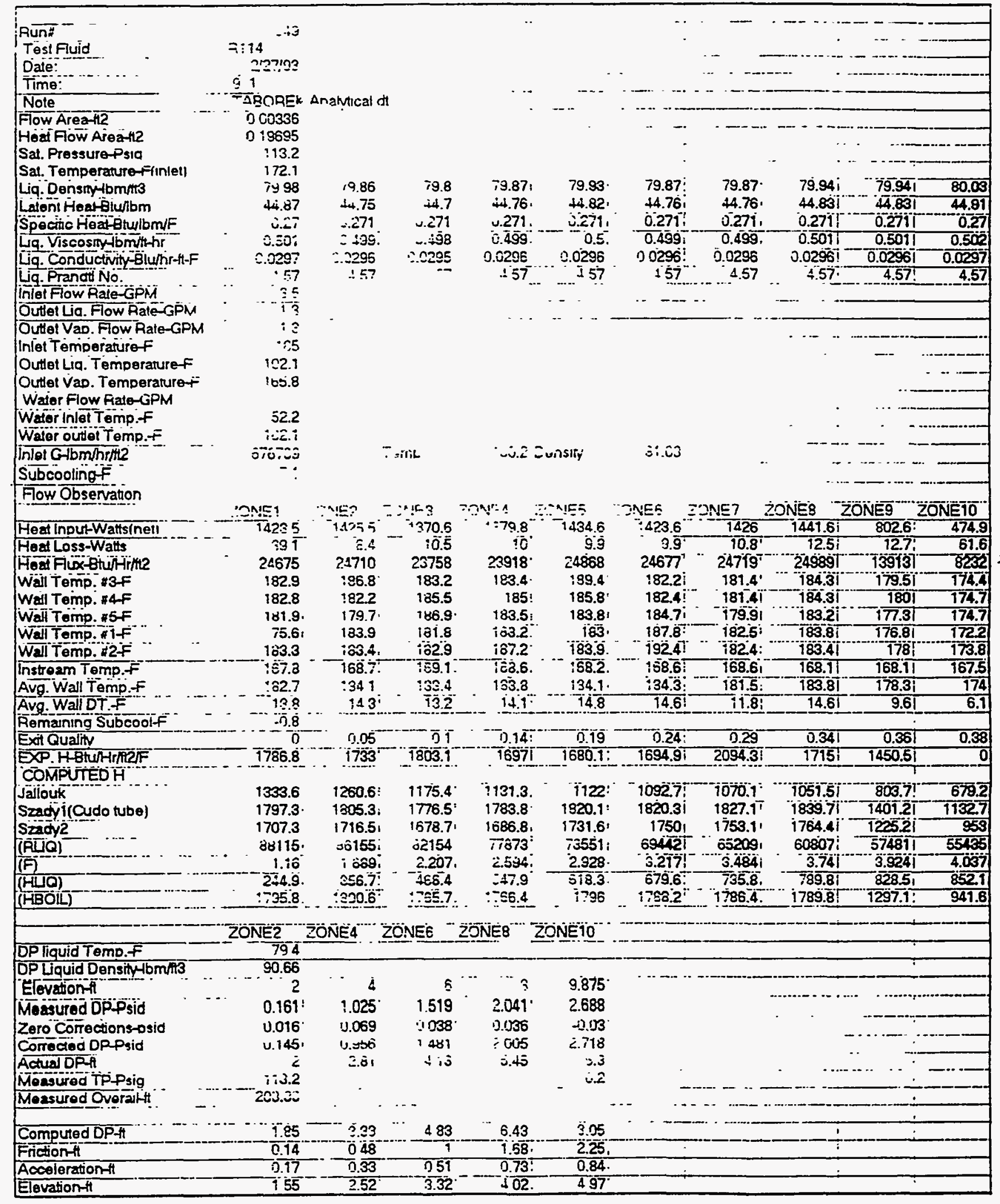




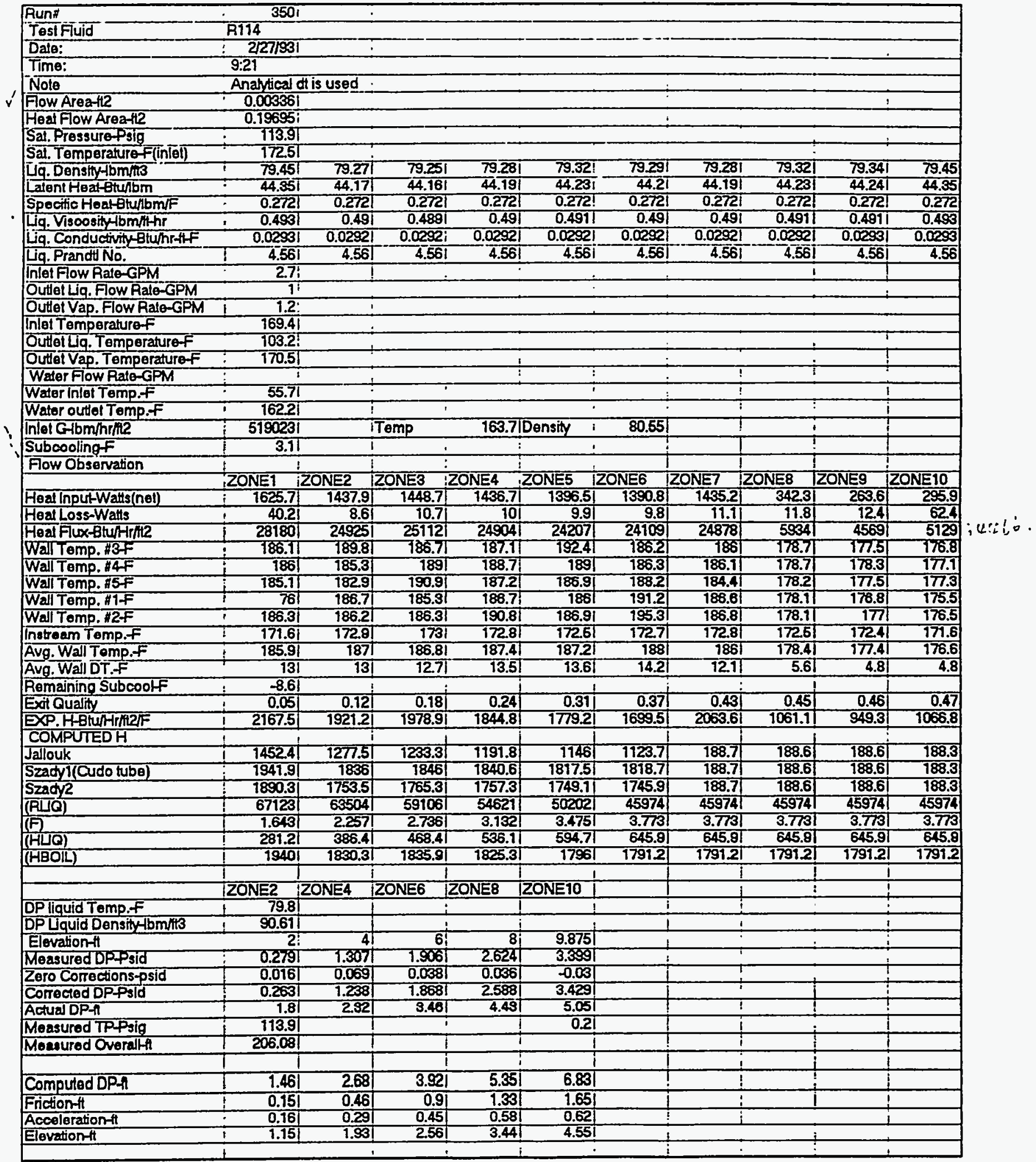




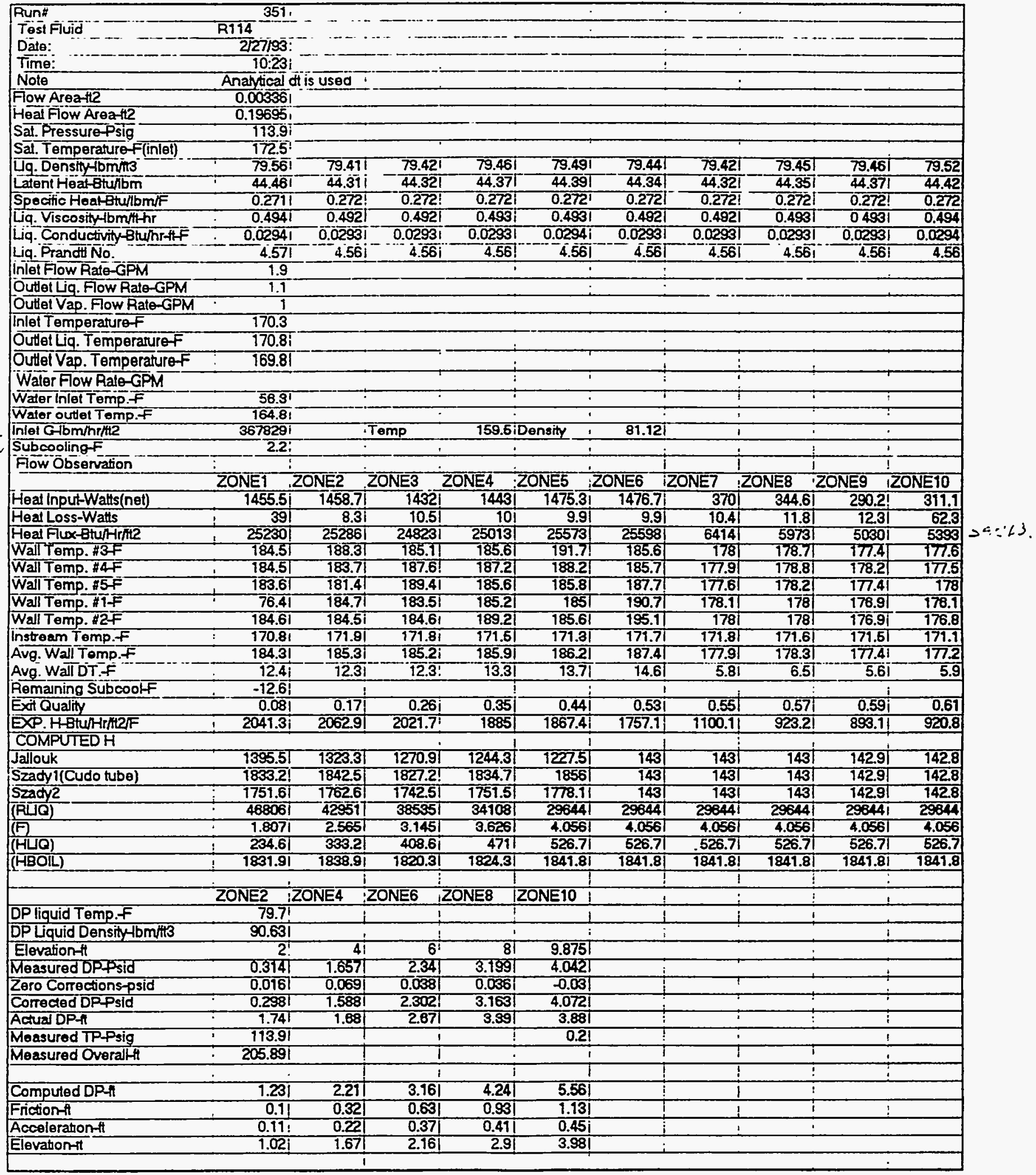




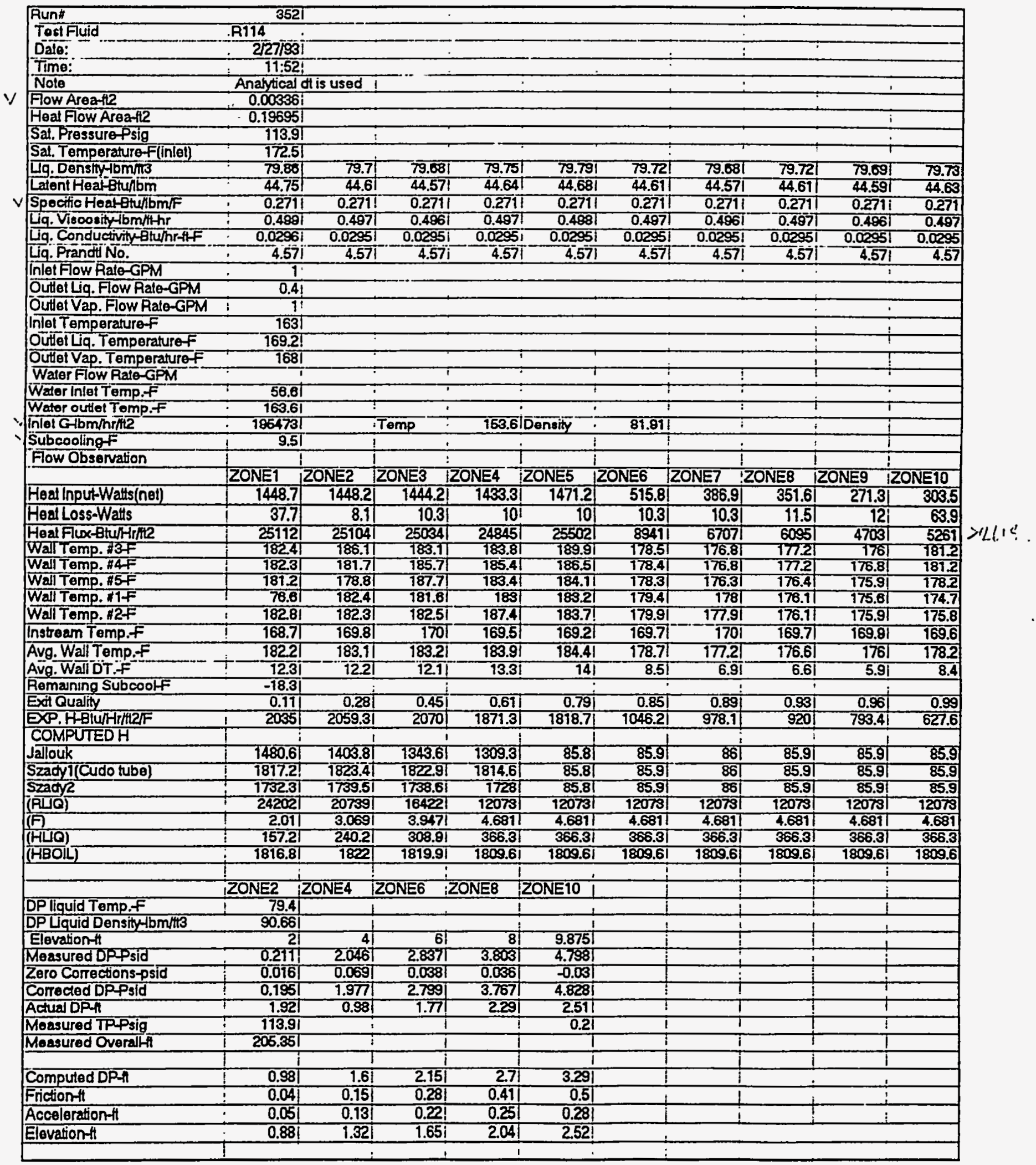




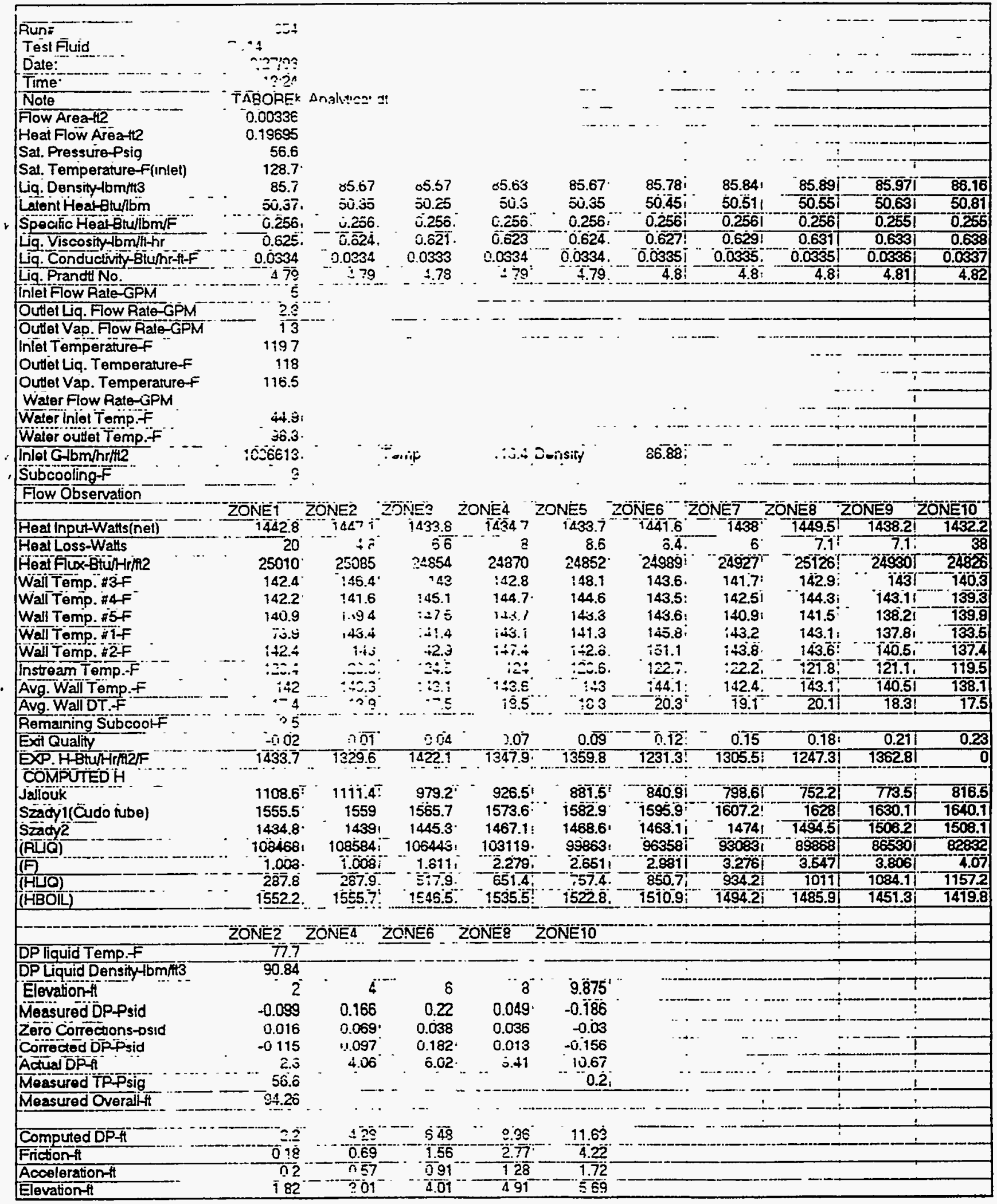




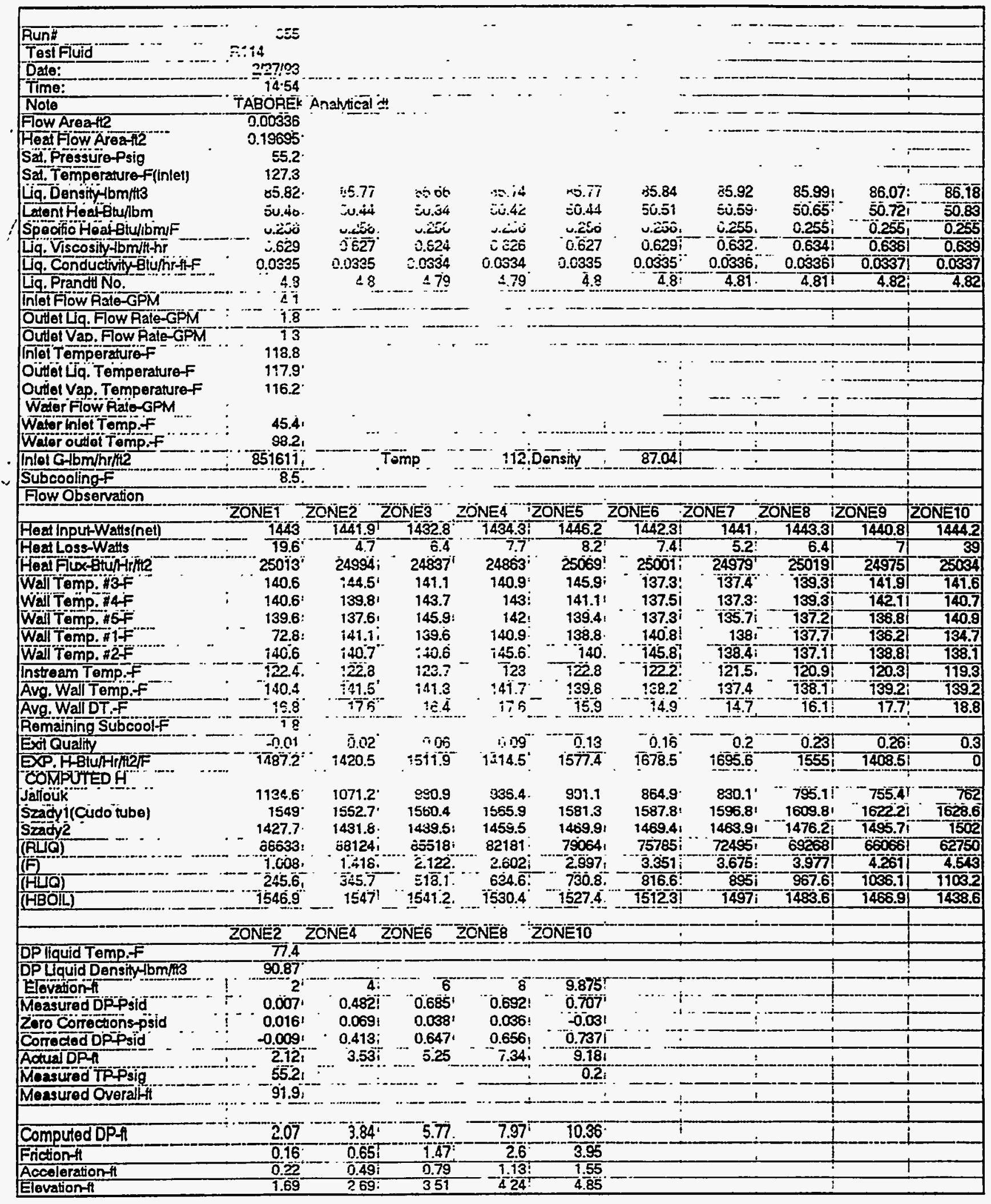




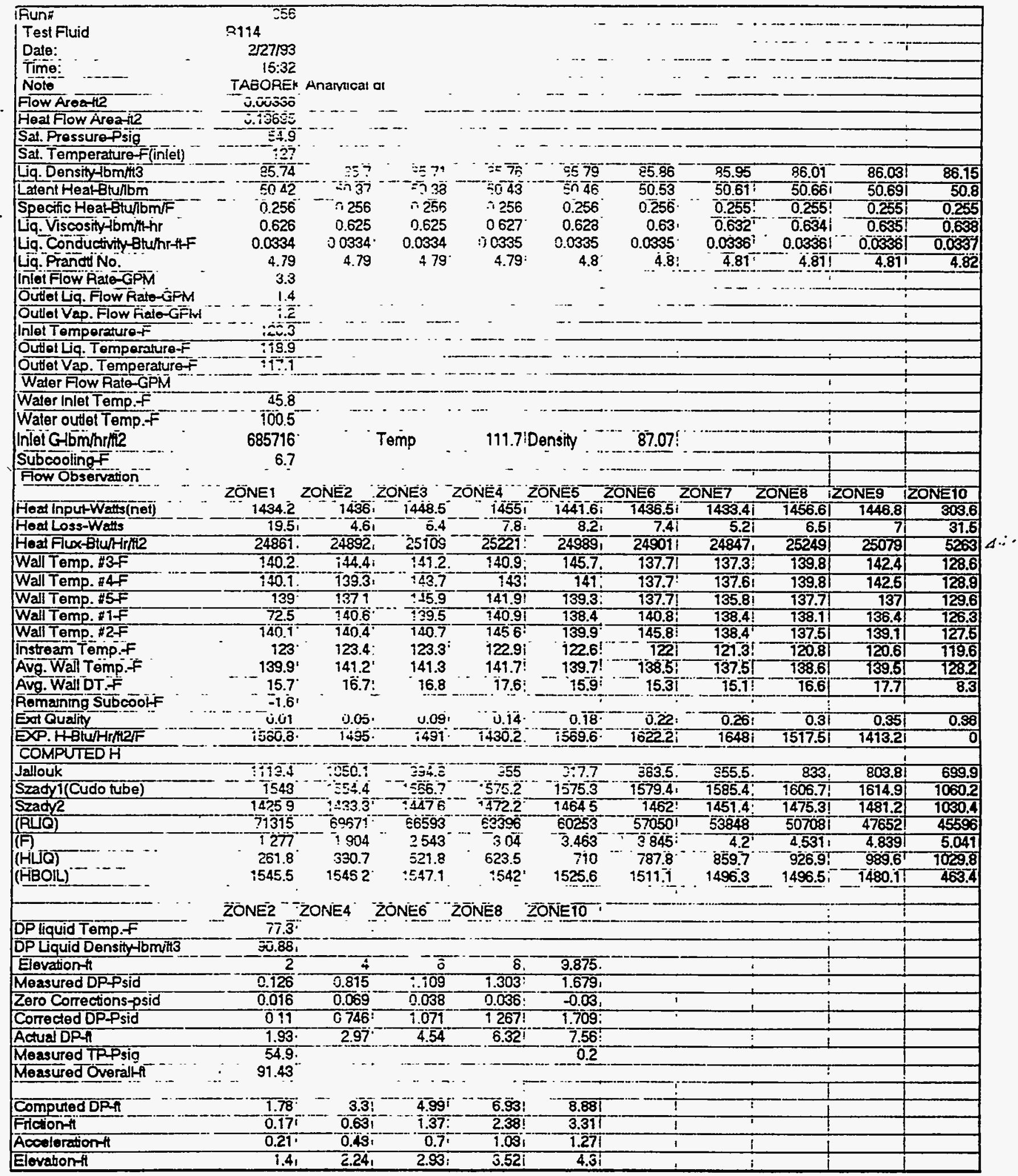




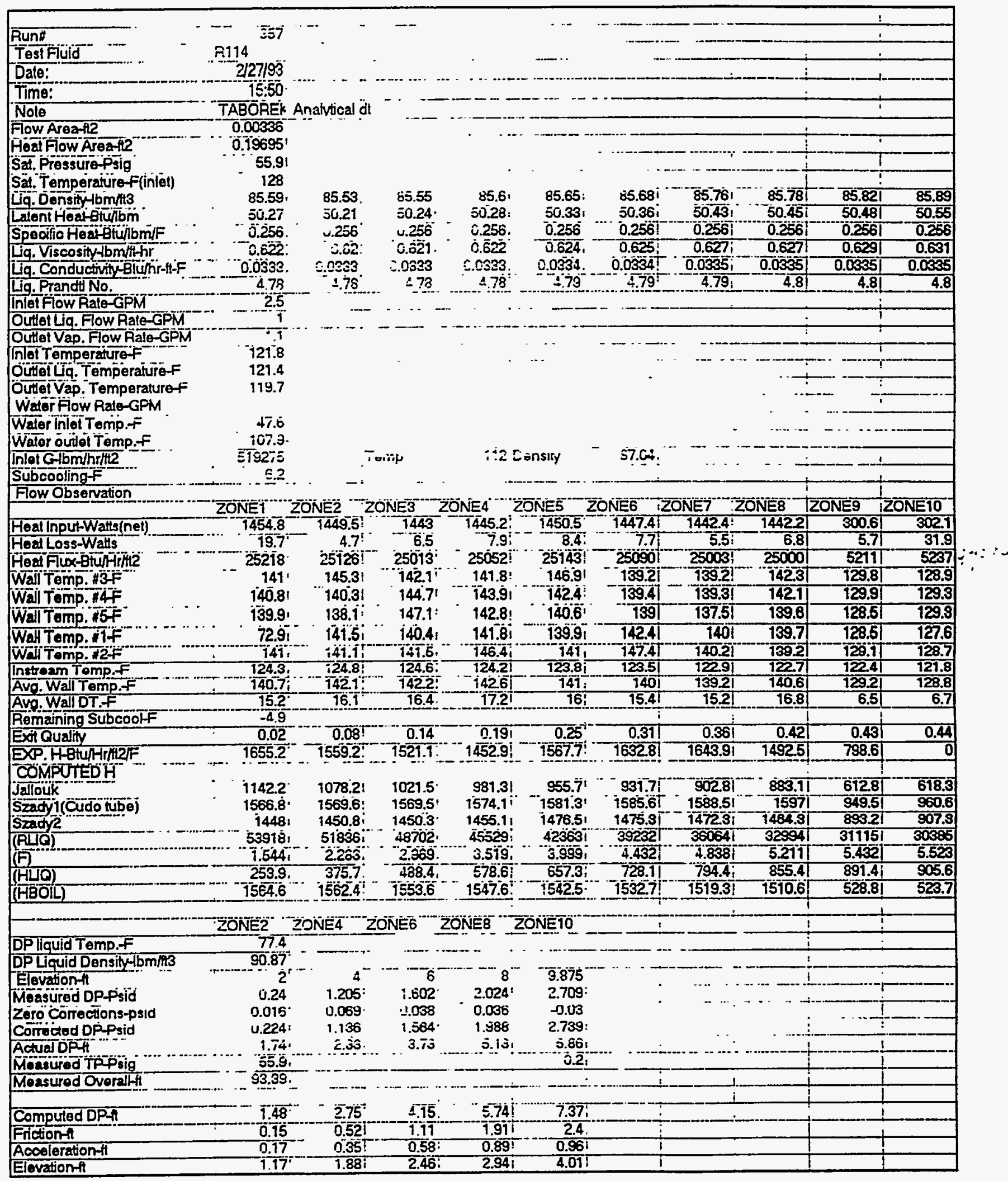




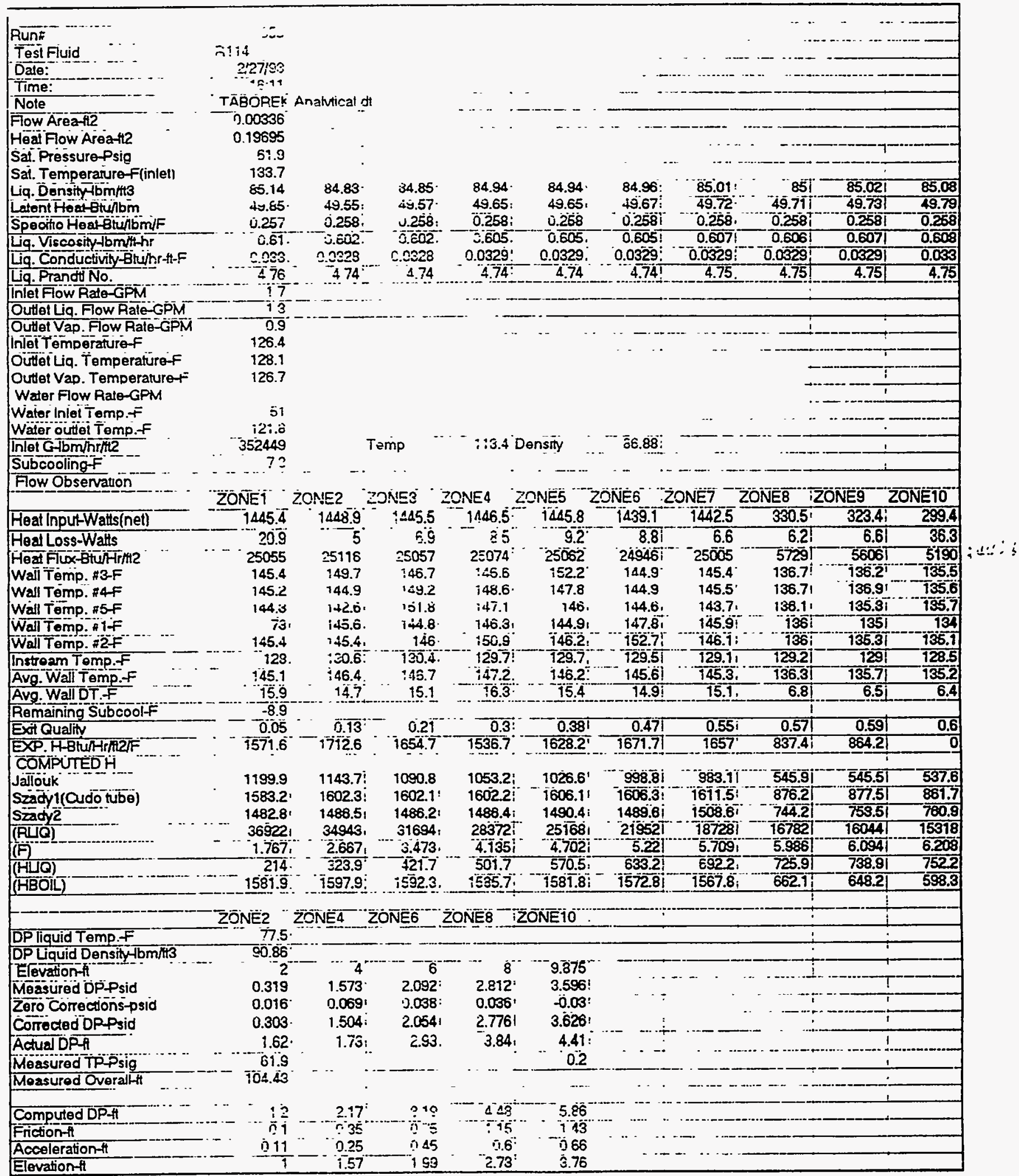




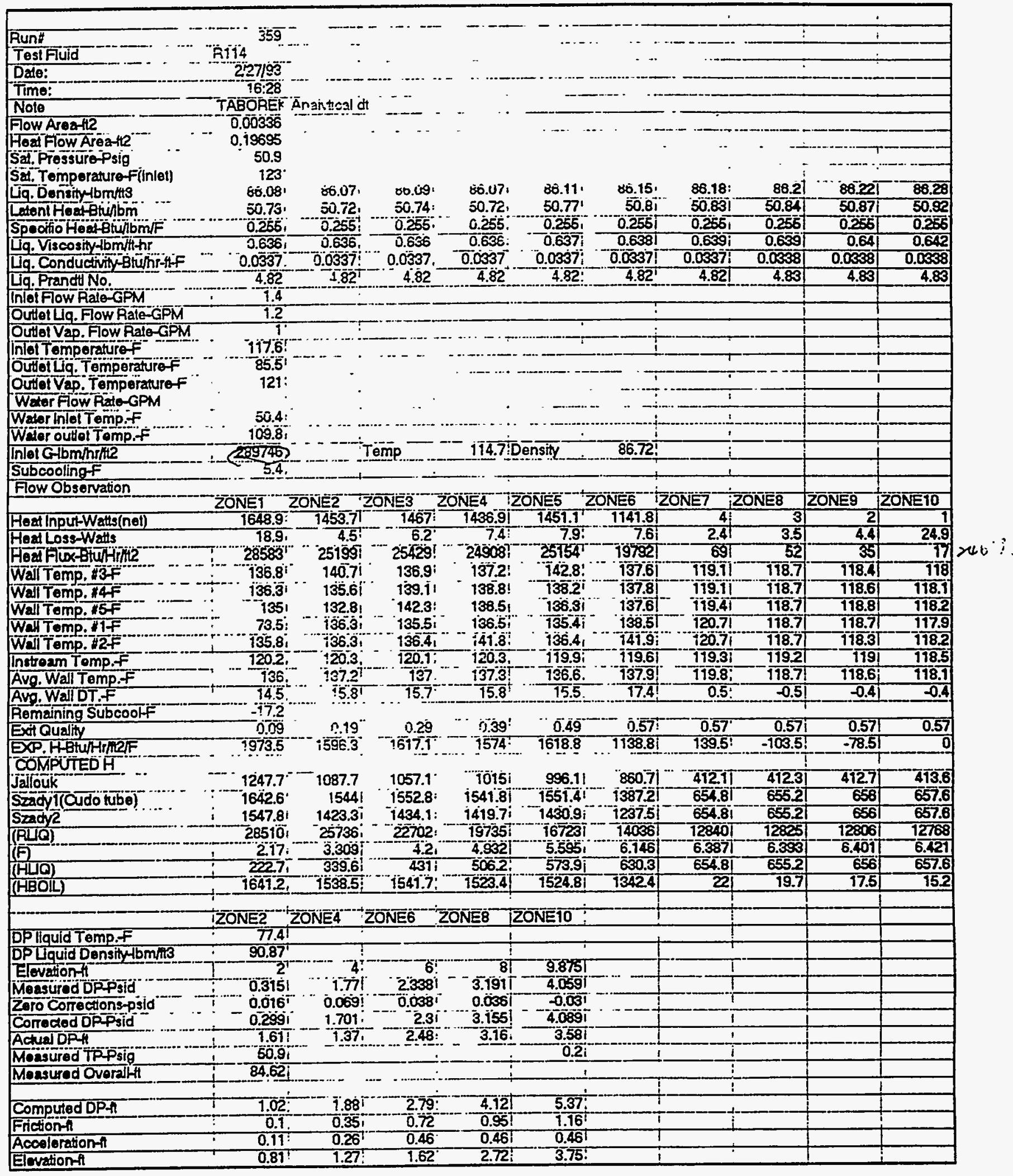




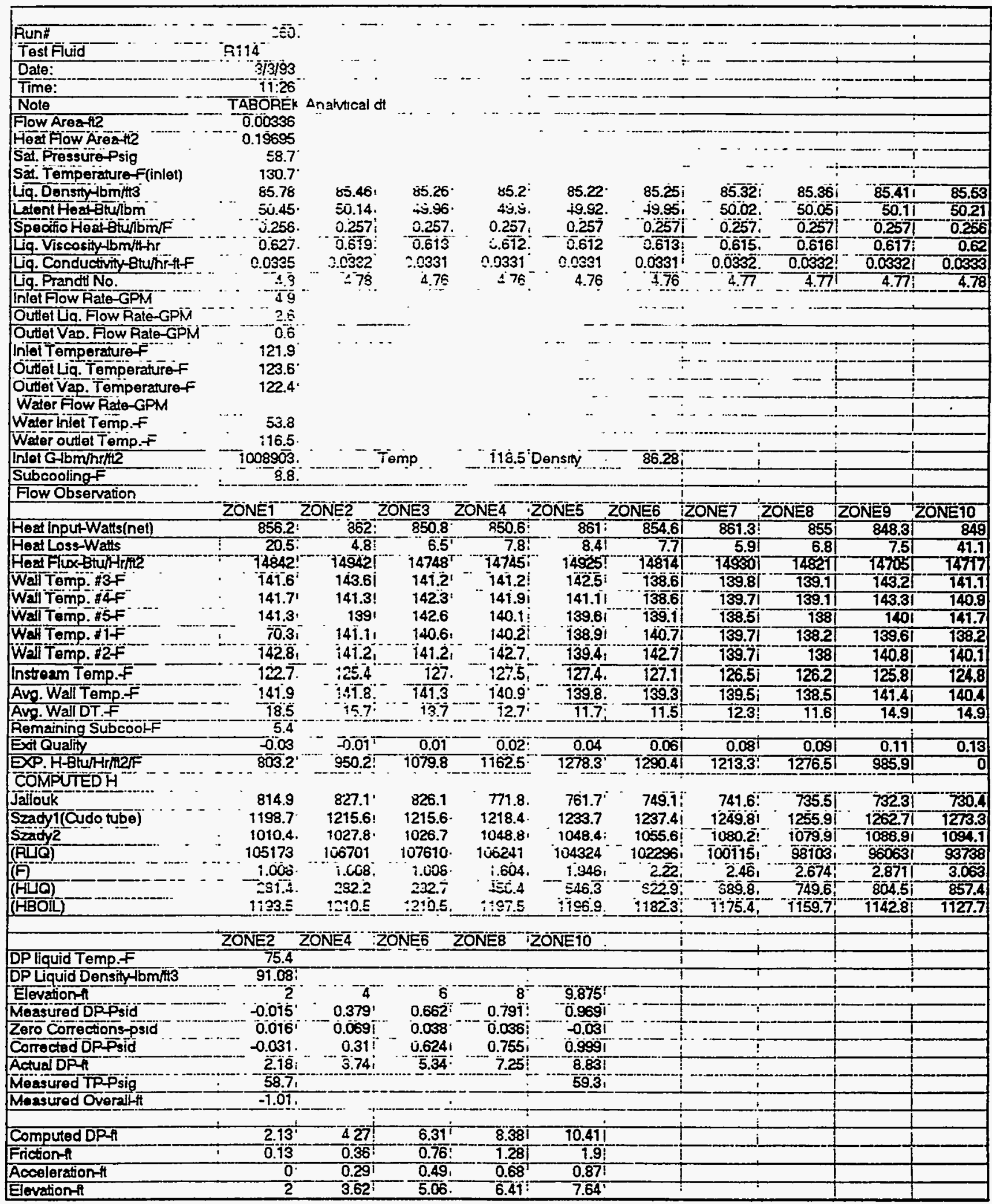




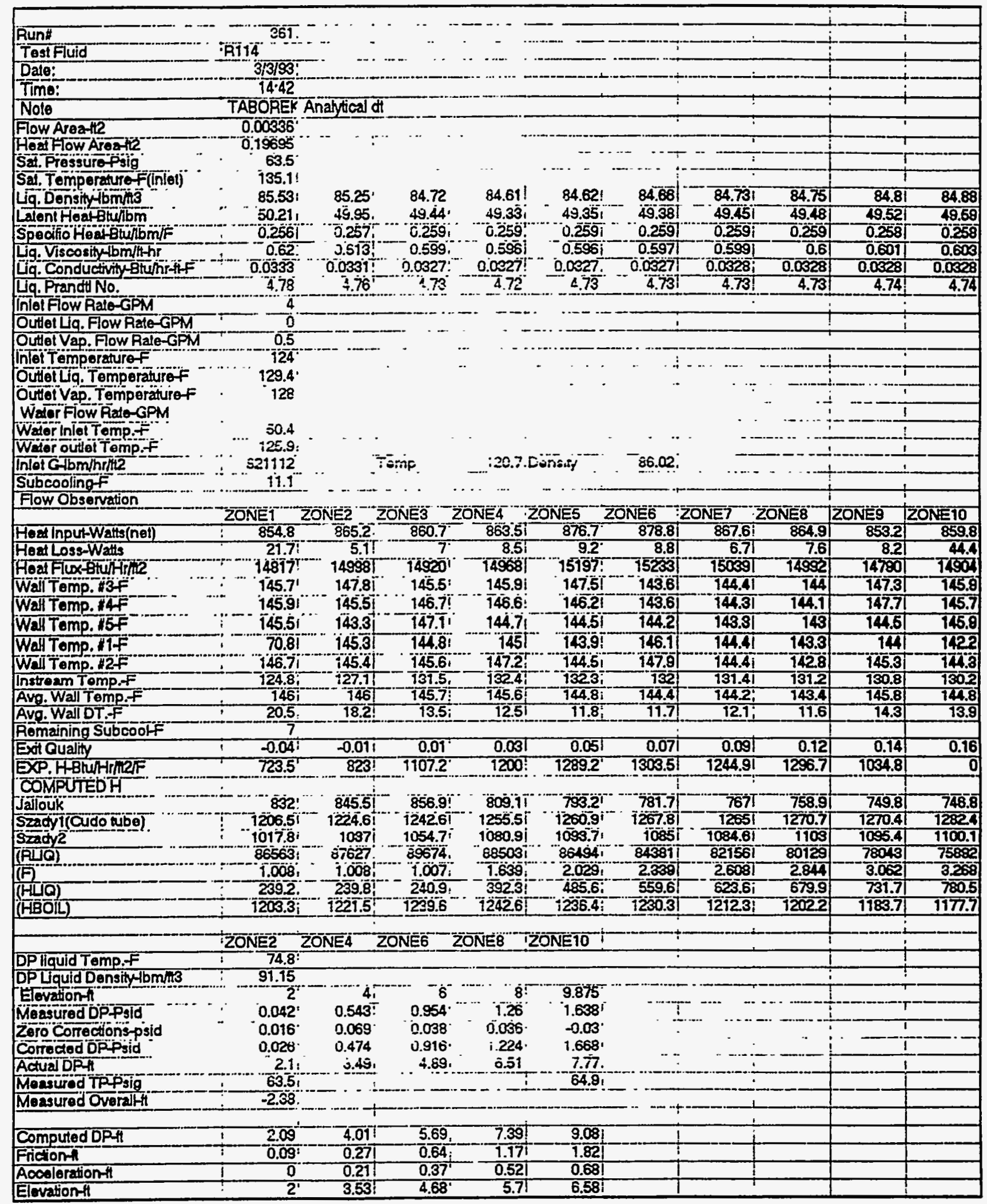




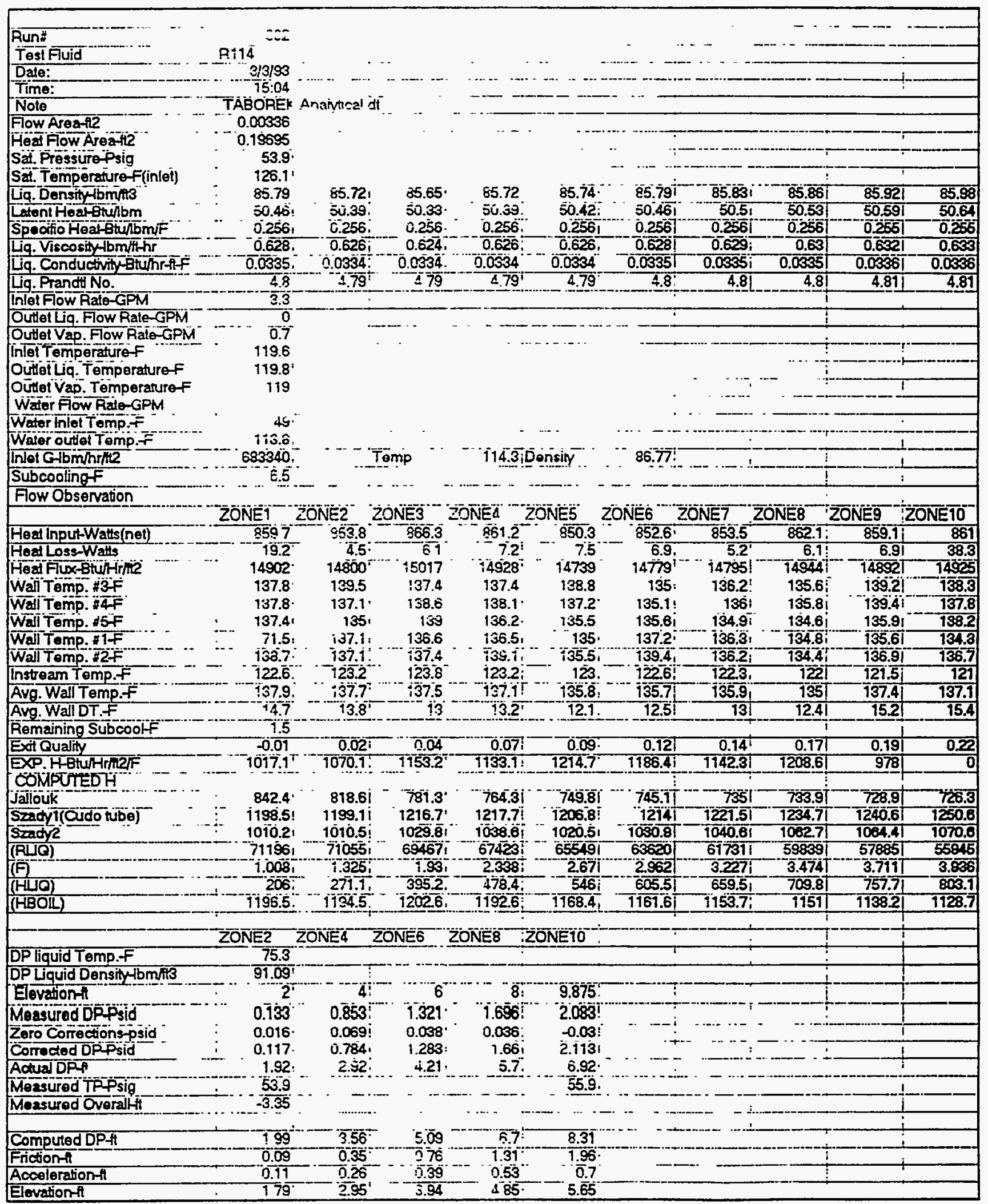




\begin{tabular}{|c|c|c|c|c|c|c|c|c|c|c|}
\hline Run: & 3631 & $\dot{-}$ & 2 & : & $\dot{.}$ &. & 1 & . & $\cdot$ & $\cdot$ \\
\hline Test Fuid & 1R114: & $i$ & $\cdot$ & 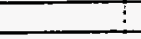 & $\dot{-}$ & : & $\dot{-}$ & 5 & 1 & 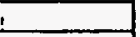 \\
\hline Date: & 3/31931 & & $\therefore$ & 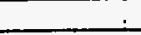 & & $i$ & $i$ & 3 & ; & ; \\
\hline Time: & $15: 271$ & & & $:$ & 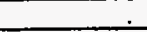 & & & & & $i$ \\
\hline Nole & Analytical d & It is used I & & $:$ & 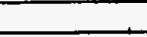 & & & $!$ & 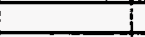 & 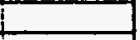 \\
\hline Fow Area-fl2 & 10.003361 & & $t$ & & & & & 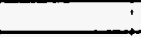 & 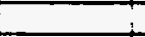 & \\
\hline Heal Flow Areatil & 0.19595 & 1 & 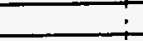 & $\therefore$ & & & & & & 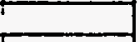 \\
\hline Sat. Pressure Psig & $52 . \pi$ & $i$ & & 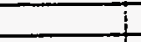 & 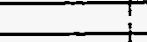 & & $i$ & 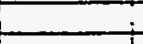 & 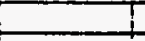 & \\
\hline Sal. Temperature-f(inlet) & 124.3 & & & & & & & & & \\
\hline La. Denstrybmntis & 86.27 & 85.891 & 85.861 & 85.89 & 85.921 & $85.99 !$ & $85.99 !$ & 88.05 & 86.081 & 86.15 \\
\hline Latent Heat-Btuibm & 50.91 & 50.55 & 50.531 & 50.55 & 50.591 & 50.651 & 50.651 & 50.71 & 50.731 & 50.8 \\
\hline Specific HoatetulbmuF & 0.2551 & 0.256 & 0.256 & 0.256 & 0.2551 & 0.2551 & 0.2551 & 0.255 & 0.2551 & 0.255 \\
\hline Liq. Viscosity $/ b m / 1$ hr & 0.642 & 0.631 & 0.63 & 0.631 & 0.632 & 0.634 & 0.634 & 0.6351 & 0.636 & 0.638 \\
\hline Liq. Conductivity BhwhrffF & 0.00338 & 0.03351 & 0.03351 & 0.0335 & 0.03361 & 0.0336 & 0.03361 & 0.0337 & 0.0337 & 0.0337 \\
\hline Ĺ. Prandt No. & 4.831 & 4.81 & 4.81 & 4.81 & 4.811 & 4.81 & 4.811 & 4.821 & $4.82 !$ & 4.82 \\
\hline Inlet Flow Rate-GPM & 251 & 1 & 1 & $i$ & i & i & $i$ & 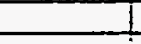 & & 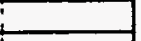 \\
\hline Outlet Lig. Flow Rate-GPM & 01 & 1 & 1 & & & 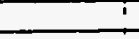 & 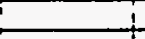 & 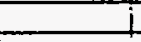 & & \\
\hline Outlet Vap. Flow Rate-GPM & 0.71 & 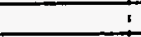 & $\therefore$ & & $i$ & & $t$ & 1 & 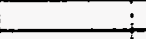 & 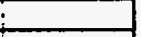 \\
\hline Inlet Temperature-F & 197.41 & 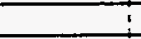 & 4 & 1 & 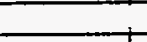 & 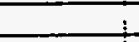 & i & 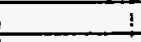 & I & I \\
\hline Outlet Liq. Temperaturo F & 118.9 & 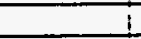 & 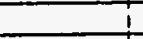 & & & & & & & \\
\hline Outlet Vap. Temperature f & 117.5 & i & & & $\vdots$ & & & 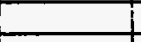 & & \\
\hline Water Flow Rato-GPM & 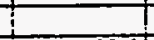 & & & & & & & & & \\
\hline Wader Inlet Temp, $F$ & 48.11 & 1 & $T$ & & 7 & & 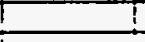 & 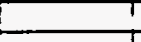 & & \\
\hline Water outlet Tomp.f & 113.4 & t & $!$ & 1 & 1 & & I & 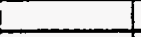 & & \\
\hline Inlot G $-1 b m / h r / i 2$ & 519275 & & Temp & $1121 \overline{1}$ & Density & 87.04 & & & & \\
\hline Subcooling $F$ & 6.9 & & I & & & & & 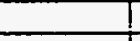 & & \\
\hline Fow Observation & & & & & & & & & & \\
\hline & ZONE1 & ZONE2 & ZONE3 IZ & ZONE4 & \begin{tabular}{|l|l} 
ZONES & 2 \\
\end{tabular} & ZONE6 & ZONE7 & ZONE8 & \begin{tabular}{|l|l} 
ZONES & 2 \\
\end{tabular} & ZONE10 \\
\hline Heal Input-Watts(nel) & 1059.1 & 855.2 & 858.7 & 864.5 & 854.3 & 857.3 & 856.9 & 864.1 & 861.3 & 865.7 \\
\hline Head Loss-Watts & 18.8 & 4.4 & 6 & 7.1 & 7.4 & 6.8 & 5 & 5.9 & 6.8 & 37.4 \\
\hline Heal Fux-Bturfiriz & 18359 & 14824 & 15058 & 14886 & 14800 & 14861 & 14854 & 14979 & 14956! & 15006 \\
\hline Wall Tomp. HF & 735.81 & 137.5 & 735.8 & 136.2 & 137.6 & 134.1 & T34.9) & 334.5 & 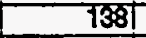 & 137 \\
\hline Wall Temp. N4F & 135.8 & 135.5 & 136.9 & 136.7 & 136.2 & 134.1 & 134.8 & 134.6 & 138.5 & 736.7 \\
\hline Wall Tomp. $5 F$ & 135.8 & 133.3 & 1372 & 135 & 134.3 & 134.6 & 133.7 & 133.6 & 134.7 & 736.7 \\
\hline Wall Temp. "1F & 71.2 & 135.2 & 134.7 & 135.11 & 133.7 & 138.2 & 135.1 & 13.6 & 134.6 & 133 \\
\hline Wall Tomp. WF & 136.9 & 135.3 & 135.7 & 137.8 & 134.3 & 138.2 & 135.1 & 133.4 & 135.7 & 135.3 \\
\hline Instroam Temp.f & 118.6 & 121.8 & 122। & 121.8 & 121.5 & 120.8 & 120.8 & 120.4 & 120.2 & 119.6 \\
\hline Avg. Wall Tomp. $F$ & 136.11 & 135.9 & 135.81 & 135.8 & 134.61 & 134.8 & 134.7 & 733.9 & 136.31 & 135.7 \\
\hline Avg. Wall DT \& & 16.6 & 13.4 & 13.1 & 13.3 & 1251 & 13.21 & 13.11 & 1291 & 15.4 & 15.5 \\
\hline Remaining SubcoofF & -1.3 & & & & 1 & & & & & + \\
\hline Exil Quality & 0.01 & 0.04 & 0.07 & 0.11 & 0.14 & 0.17 & 0.21 & 0.24 & 0.27 & 0.31 \\
\hline EXP.HBtuHr/mar & 11029 & 1105.9 & 1150 & 1129 & 1188.9 & 1127.7 & 1129.7 & 1164.5 & 967.9 & 970.5 \\
\hline COMPUIEDH & & & & & & & & & & \\
\hline Jallouk & 953.2 & 807.1 & 787.4 & 768.3 & 754.2 & 746.8 & 740 & 737.1 & 731.9 & 728.5 \\
\hline Szady1(Cudo tube). & 1306.6 & 1194.2 & 1209 & 1211 & 1199.8 & 12052 & 1211.4 & 1221.3 & 1226.5 & 1235.6 \\
\hline Szady2 & 1153.1 & 1005.9 & 10225 & 1034 & 1026.9 & 1020.4 & 1029.1 & 1041.1 & 1054.2 & 1050.4 \\
\hline$(P \| Q)$ & 52775 & 52699 & 50504 & 49042 & 471741 & 45247 & 43471 & 41577 & 39754 & 37852 \\
\hline$(\mathrm{F})$ & 1.251 & 1.798 & 2349 & 2.78 & 3.1511 & 3.485 & 3.782 & 4.07 & 4.337 & 4.601 \\
\hline (HLQ) & 204.8 & 238.0 & 385.3 & 466.2 & 516.4 & 570.8 & 619.6 & 656.8 & 709.8 & 762.6 \\
\hline (HBOIL) & 1304.9 & 1128.3 & 1195.8 & 1189.1 & 1167 & 1150.9 & 1154.8 & 11512 & 1141.5 & 1134.6 \\
\hline & & & & & & & & & & \\
\hline & ZONE2 & ZONE4 & ZONE6 & ZONE8 & ZONE10 & & & & & \\
\hline DPliquid Temp.f & $\begin{array}{r}75.3 \\
\end{array}$ & & & & & & & & & \\
\hline DPLiquid Density $1 \mathrm{bm} / \mathrm{t} 3$ & 91.09 & & & & & & & & & \\
\hline Elevation- & & 4 & 6 & 8 & 9.875 & & & & & \\
\hline MeasuredDPPsid & 0.202 & 1.749 & 1.704 & 2.215ा & 2.774 & & & & & \\
\hline Zero Corrections-psid & 0.016 & 0.059 & 0.038 & 0.036 & -0.081 & & & & & \\
\hline Corrected DPPsid & 0.188 & 1.08 & 1.655 & 2.179 & 2.744 & & & & & \\
\hline Actual DP-A & 1.8 & 2.42 & 3.58 & 4.82 & 5.881 & & & & & \\
\hline Mosasured TPPsig & 52.1 & & & & 54.8 & & & & & \\
\hline Measured Overallit & -4.61 & & & & & & & & & \\
\hline & & & & & & & & & & \\
\hline Computed DPA & 1.65 & 2.93 & 4.19 & 5.52 & 6.86 & & & & & \\
\hline Friction-t & 0.09 & 0.32 & 0.68 & 1.17 & 1.74 & & & & & \\
\hline Accoleration-ft & 0.11 & 0.21 & 0.32 & 0.45 & 0.6 & & & & & \\
\hline Elevation- & 1.45 & 2.4 & 3.19 & 3.9 & 4.51 & & 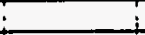 & & & \\
\hline & & & & & & & $!$ & & & \\
\hline
\end{tabular}




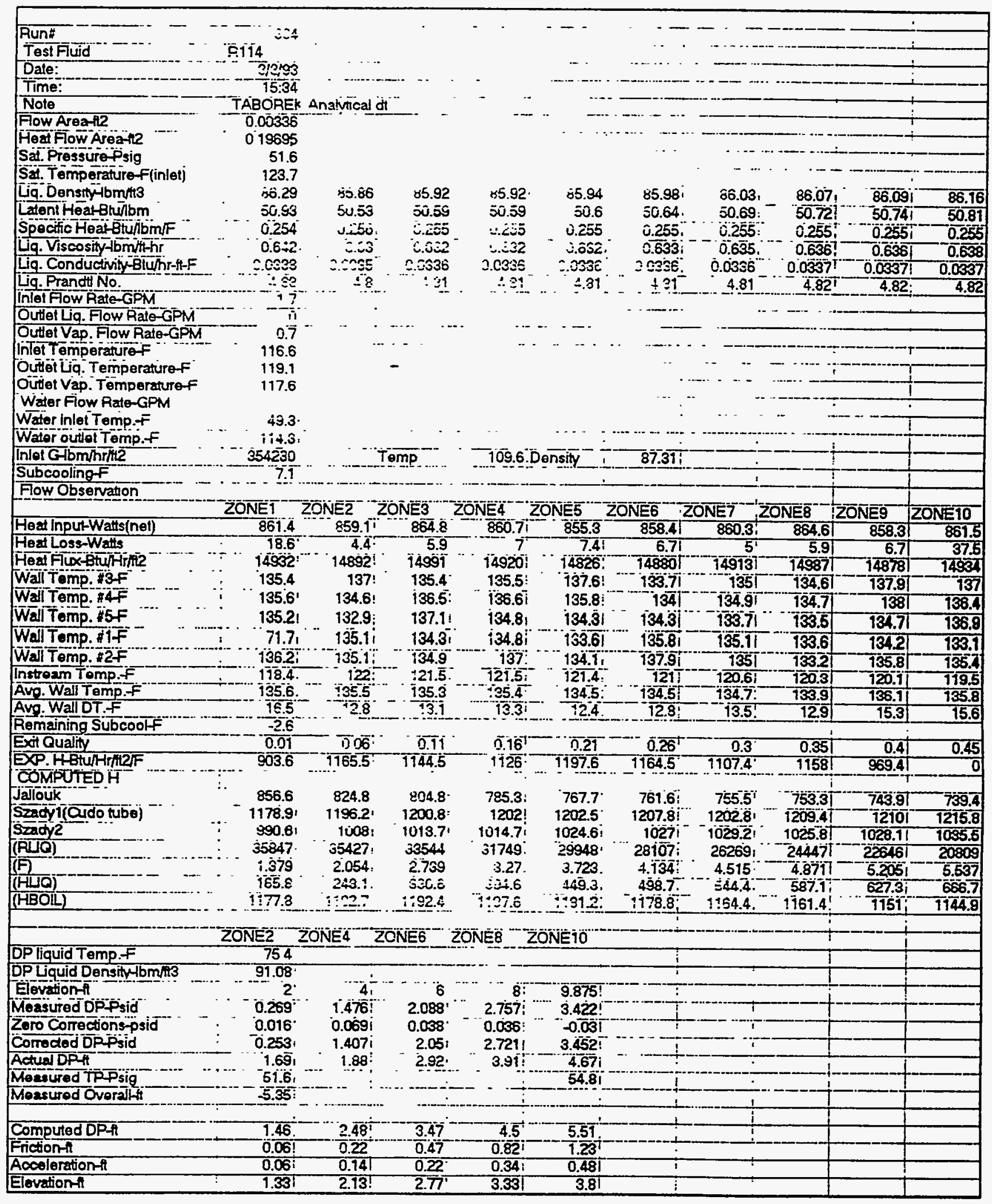




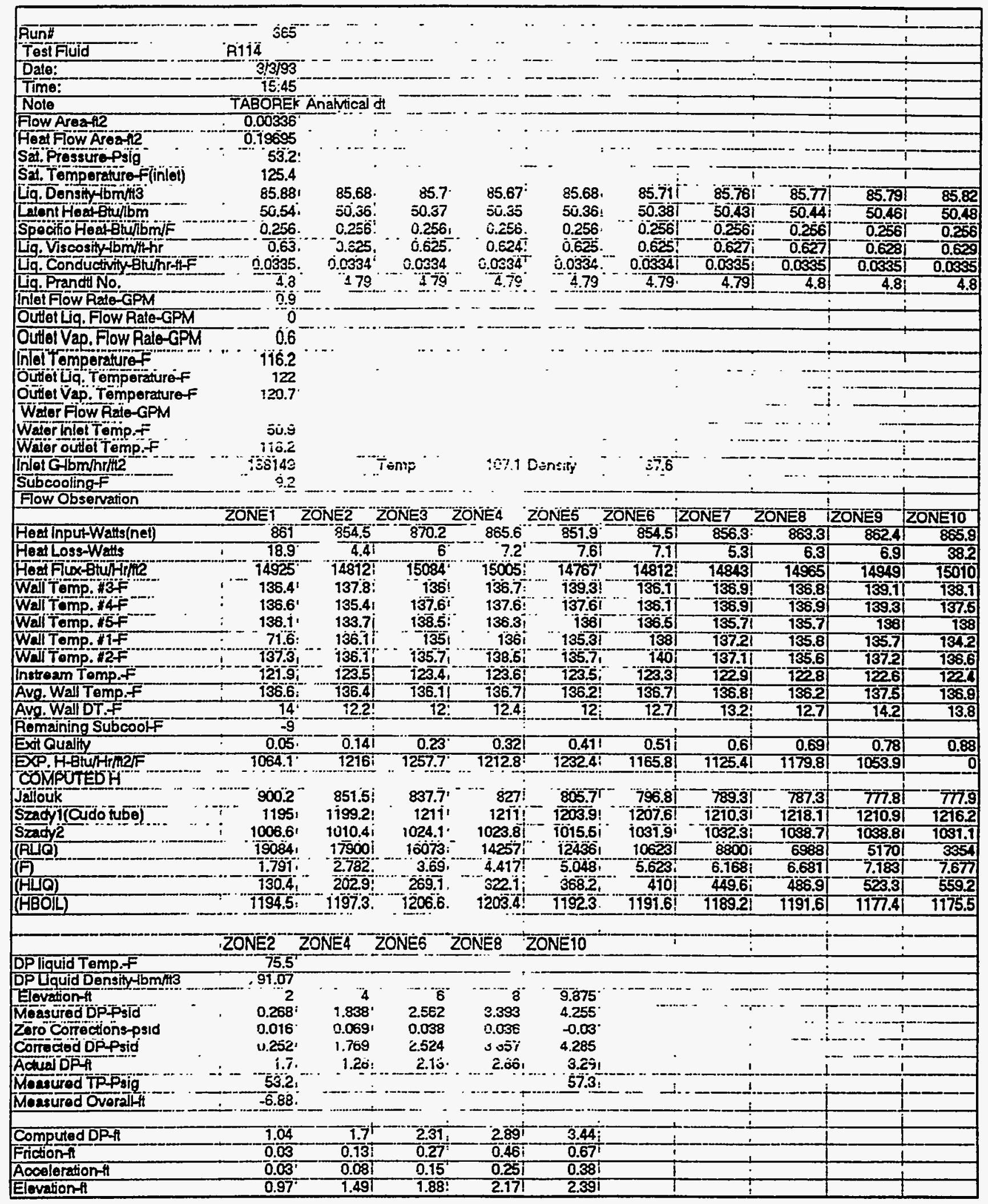




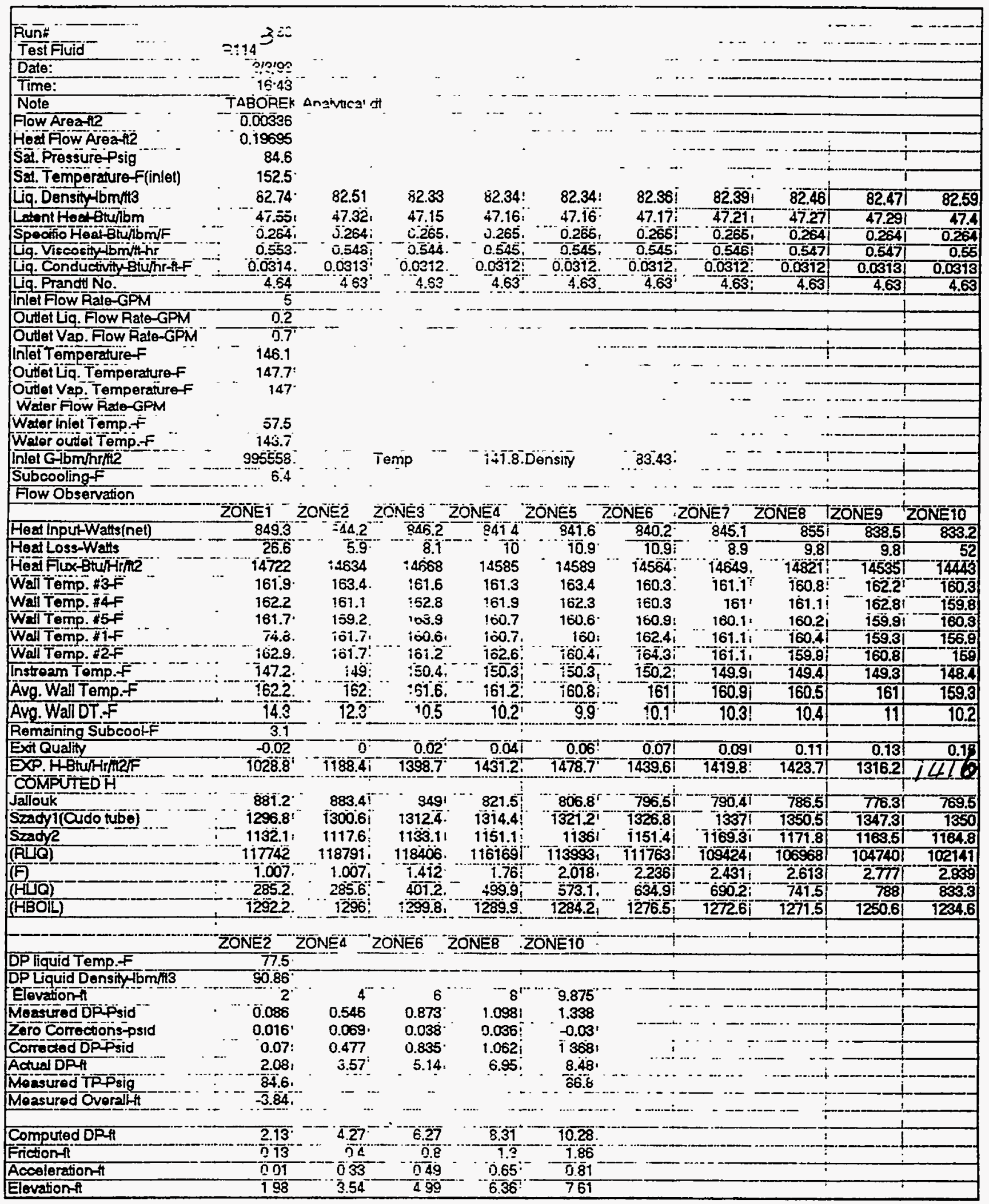




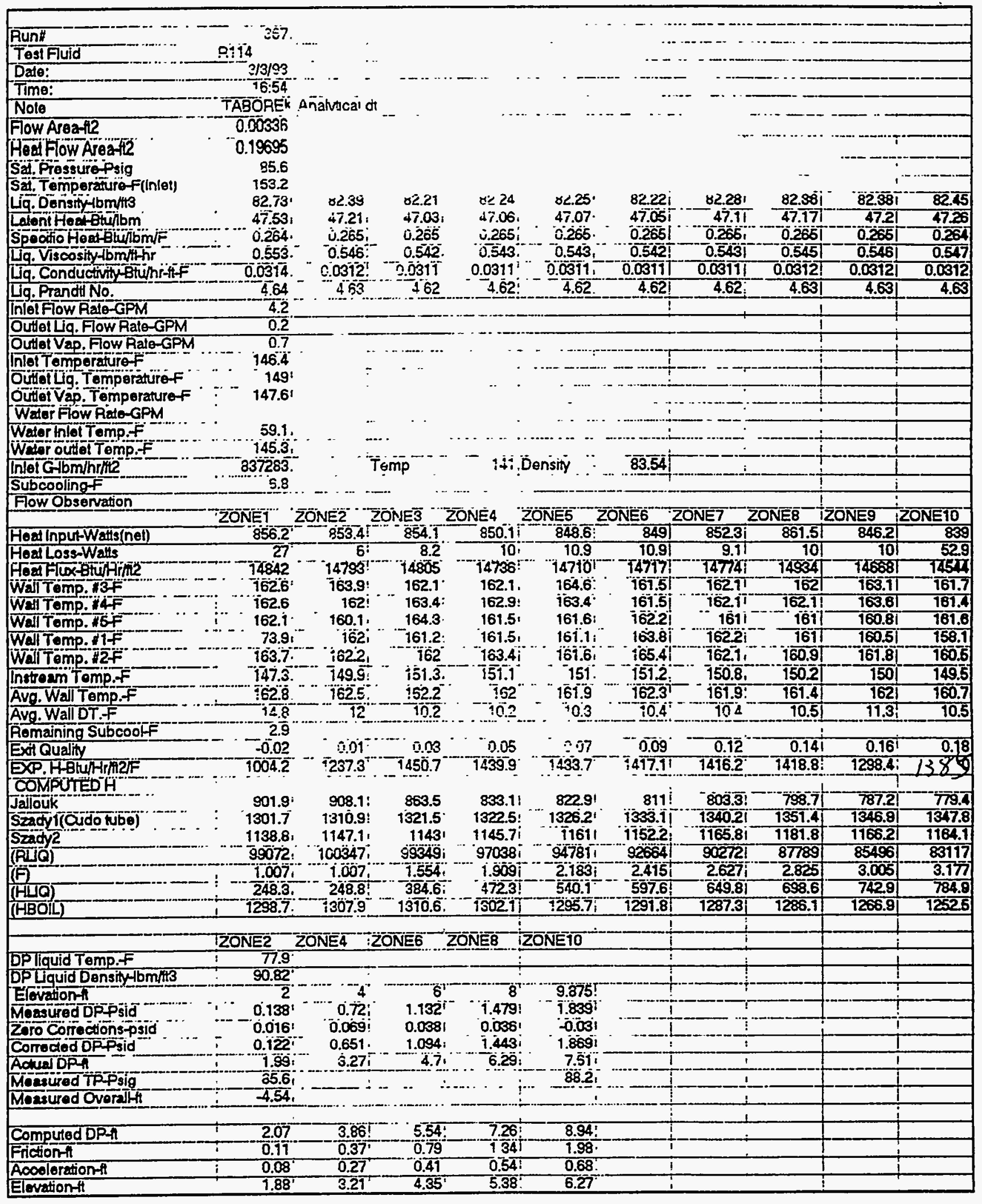




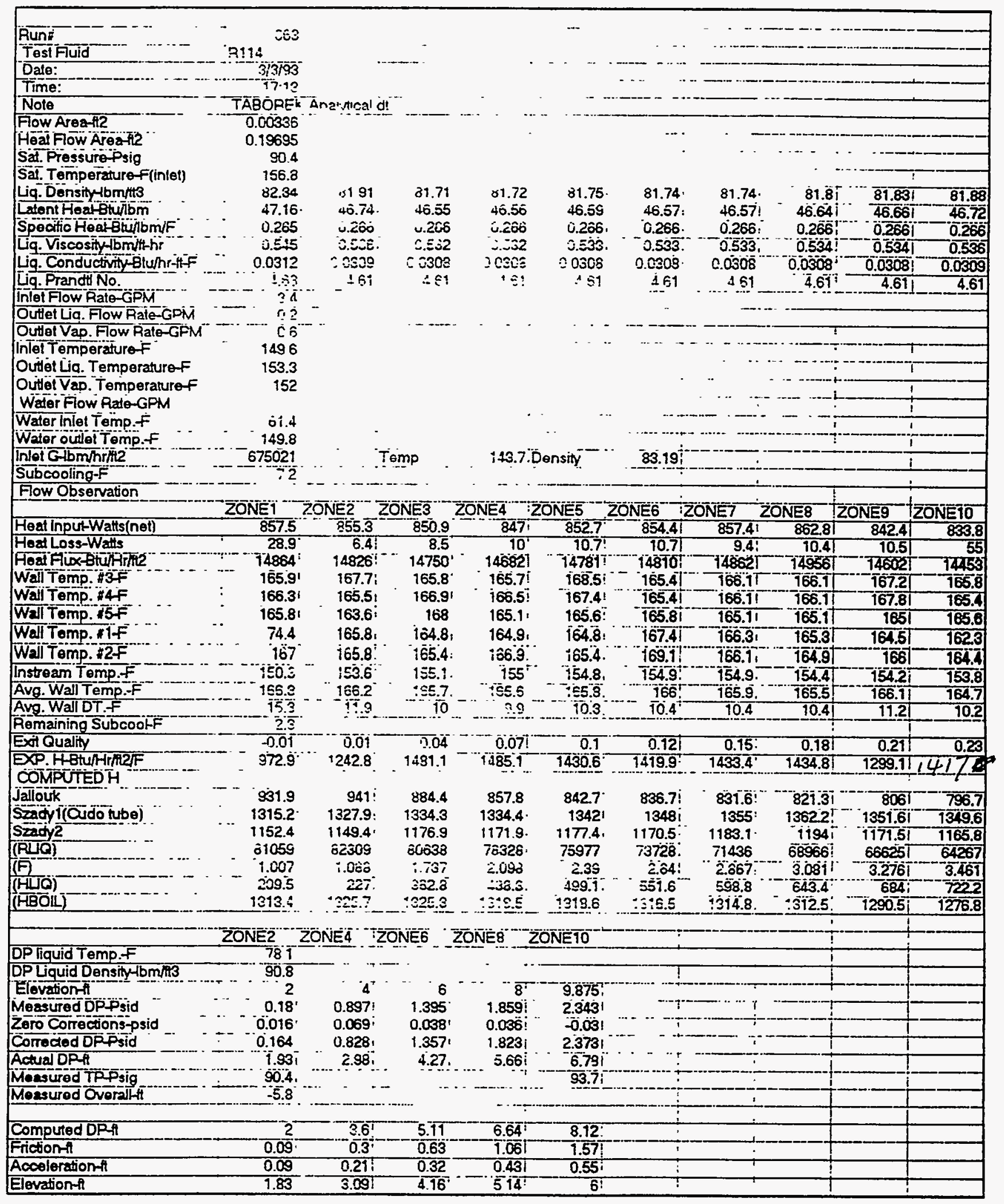




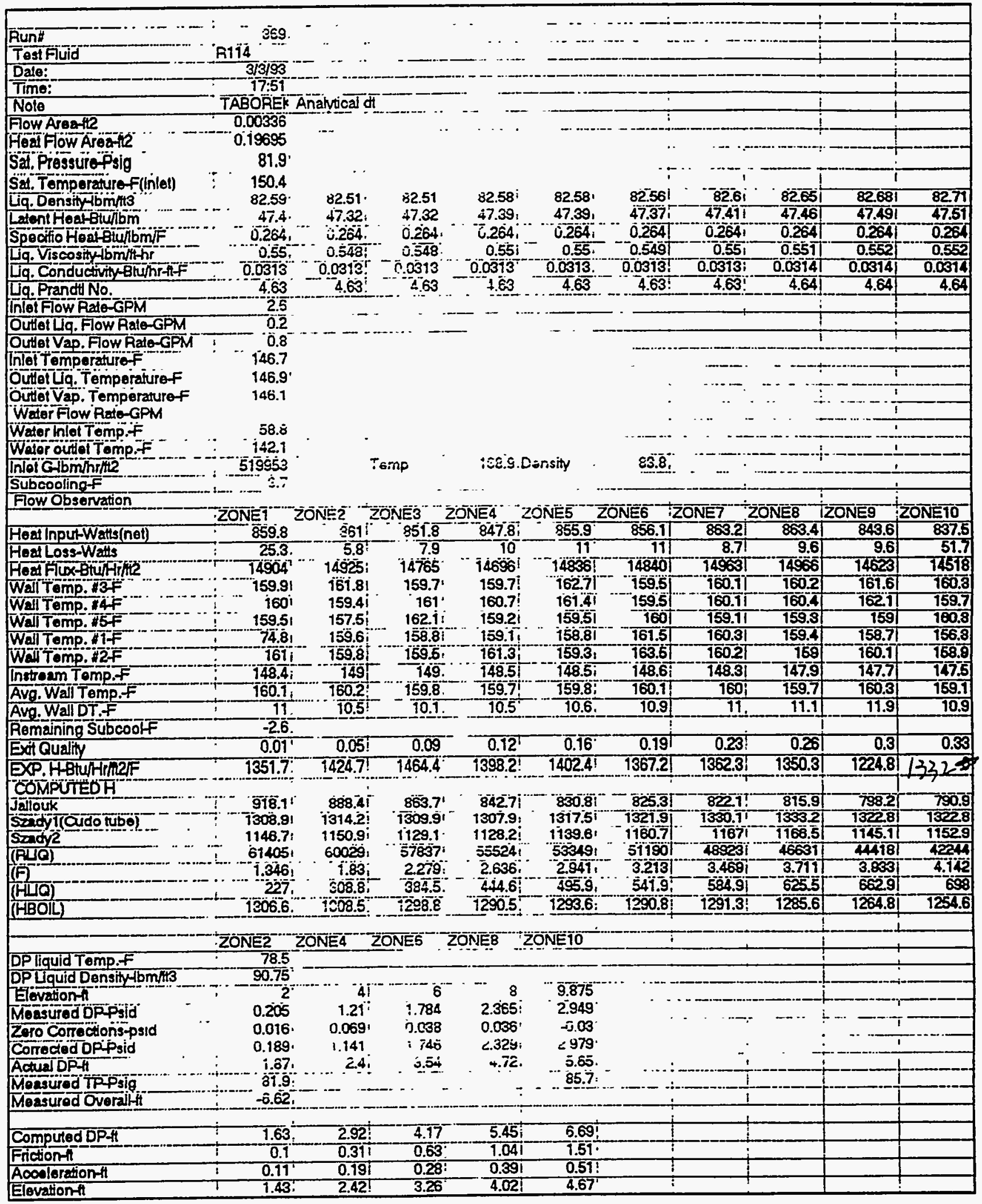




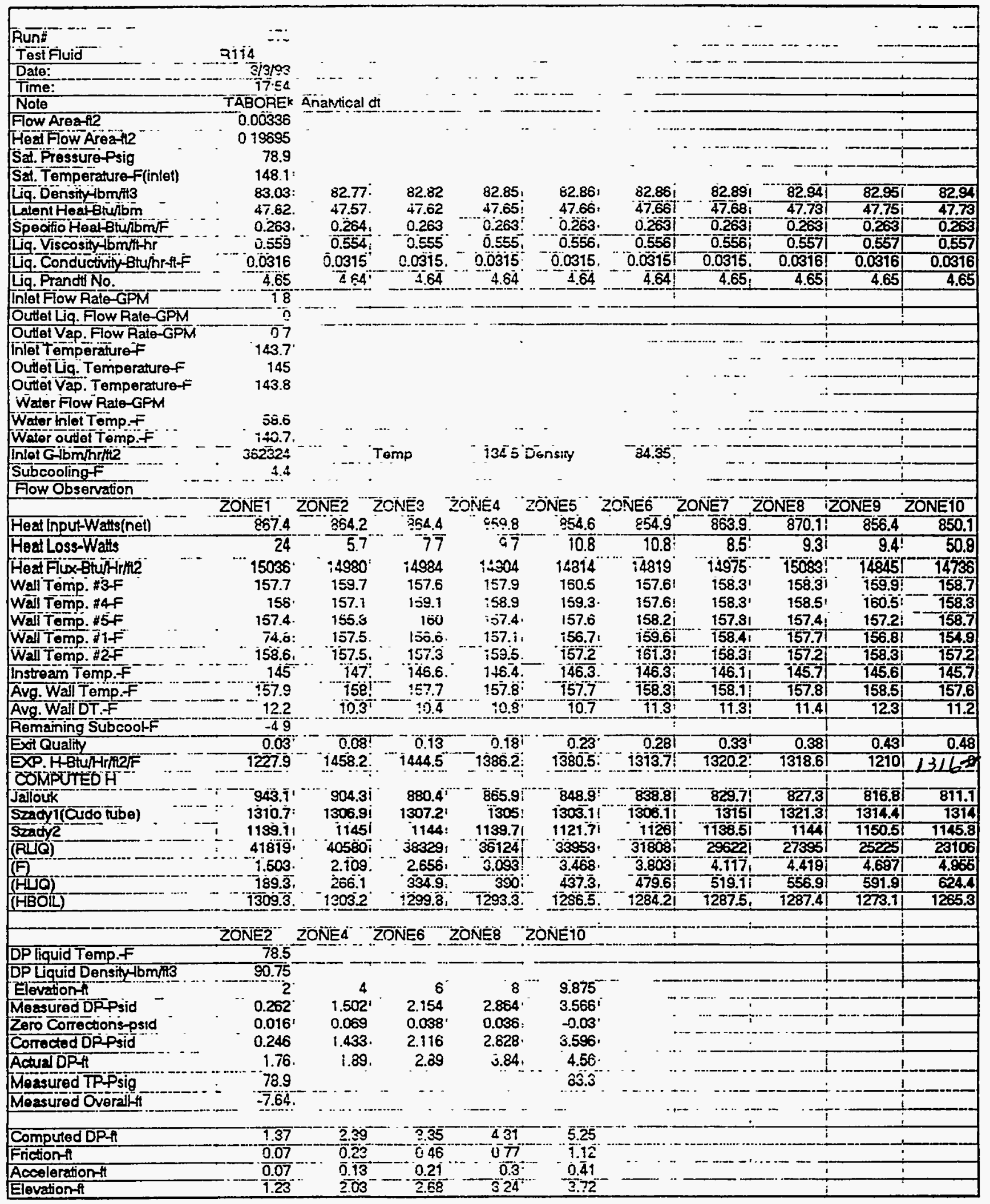




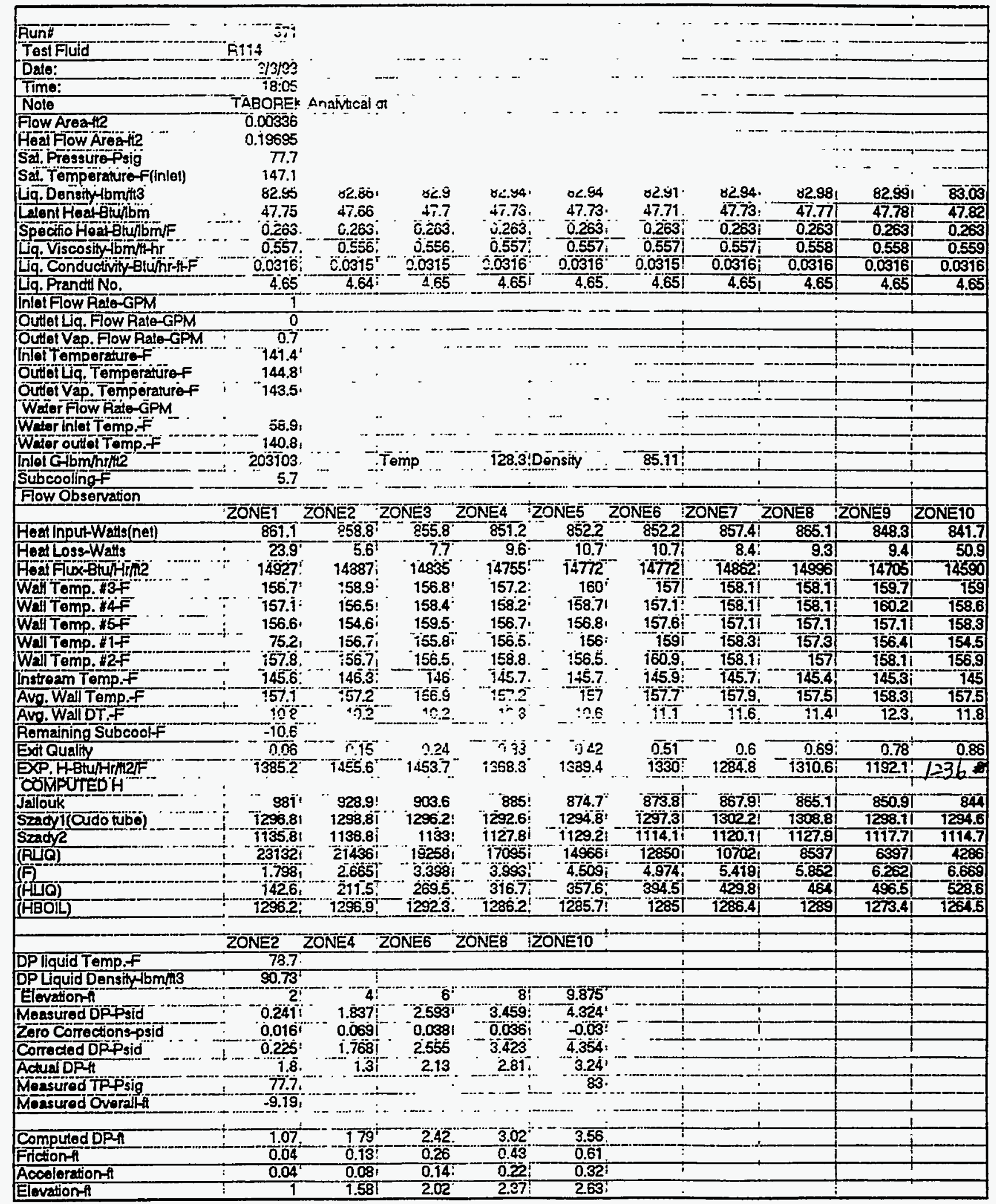




\begin{tabular}{|c|c|c|c|c|c|c|c|c|c|c|}
\hline$\frac{\text { Ruñt }}{\text { Test Fluid }}$ & $F !: 4$ & $-\cdots$ & $\cdots$ & & & $\cdots$ & $\cdots$ & & & \\
\hline Date: & $\begin{array}{r}3 / 5993 \\
-49.50\end{array}$ & & $\cdots$ & & $\cdots$ & $-\cdots$ & & & & \\
\hline Note & TABOREK & Anaktical & & & $i$ & & & & & \\
\hline Fow Areatl2 & 0.00336 & & & & 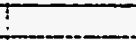 & & & & & \\
\hline Hed How Areat? & 0.19695 & & & & $\dot{\vdots}$ & & & & & \\
\hline Sat. Pressure Psig & $11 \overline{3} .7$ & & & $T^{i}$ & 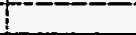 & & $T$ & & & \\
\hline Sat. Temperaturef(inlel) & 172.4 & & +7 & $i$ & 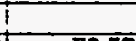 & & $\longrightarrow$ & & & \\
\hline Liq. Densitylomitas & 79.861 & 79.73 & 79.521 & 79.48 & 79.52 & 79.52 & 79.511 & 79.59 & 79.61 & 79.69 \\
\hline Lesont Houtetulbm & 44.75 & 44.63 & 44.42 & 44.38 & 44.42 & 44.42 & 44.47 & 44.49 & 44.5 & 44.60 \\
\hline Specifo Heateburbm/F & 0.271 & 0.271 & 0.272 & 0.272 & 0.272 & 0.272 & 0.272 & 0.271 & 0.271 & 0.271 \\
\hline Liq. Viscosity bomithr & 0.499 & 0.497 & $0 . \overline{494}$ & 0.493 & 0.494 & 0.494 & $0 . \overline{493}$ & $0 . \overline{485}$ & $0 . \overline{45}$ & 0.498 \\
\hline Lig. Conductivity Btuhr-hF & 0.02961 & 0.0295 & 0.0294 & 0.0293 & 0.0294 & 0.0294 & 0.0294 & 0.0294 & 0.0294 & 0.0295 \\
\hline Lig. Prandt No. & 4.57 & 4.57 & 4.56 & 4.56 & 4.56 & 4.56 & 4.56 & 4.57 & 4.57 & 4.57 \\
\hline Inlet fow Rato-GPM & 5.2 & & & & 1 & & & & & \\
\hline Outlet Lig. Fow Rate-GPM & $3 !$ & & & & T & & & & & \\
\hline Outtet Vap. Fow Ralo-GPM & 0.8 & & & & & & & & & \\
\hline Inleit Temperature & 167.41 & & & & & & & & & \\
\hline Outret Liq. Temperature $F$ & 103.6 & & 7 & T & & & & & & \\
\hline Outhet Vap. Temperaturef- & 168.11 & & & $!$ & & i & & & & \\
\hline Weder Fow Rato-GPM & $\mathrm{i}$ & i & & t & & T & & & & \\
\hline Wair hlet Temp.F & 75.2 & & & 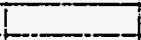 & & & & & & \\
\hline Water outdei Temp.F & 166.41 & & & & & & & & & \\
\hline Inlot G-bm/hrne & 1002821 & & Tomp & 161.8 & Density & 80.81 & & & & \\
\hline Subcoolingf & 5 & & & & & 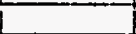 & & & & \\
\hline Fow Observation & & & & & & & & & & \\
\hline & ZONE 1 & ZONE2 & ZONEB & ZZONE4 & ZONE5 & ZONE 6 & ZONE7 & ZONE8 & ZONE9 & ZONETO \\
\hline Healnout-Wats(net) & 869.9 & 864.7 & 859.5 & 870 & 858.1 & 860.6 & 867.3 & 857.9 & 857.5 & 870.3 \\
\hline Head Loss-Watts & 40.3 & 8.6 & 10.7 & 10 & 9.9 & 9.8 & 11.2 & 12.8 & 13.7 & 67.3 \\
\hline Headfiox-buifine & 150691 & 14989 & 15072 & $15 \overline{61}$ & 14875 & 14918 & 15038 & 14871 & 15058 & 15636 \\
\hline Wall Temp. $3 F$ & 182.31 & $1 \overline{84.5}$ & 183.1 & 183.2 & 185.5 & 184.8 & 183.8 & T84 & 183.8 & 188 \\
\hline Wall Tomp. FfF & 182.7 & 182.2 & 184.3 & 184.1 & 184.4 & 184.8 & 1842 & 185 & 184.8 & 182.7 \\
\hline Wall Temp. $16 F$ & 181.8 & 180.6 & 185.2 & 183.8 & 1829 & 184.9 & 183.21 & 183.4 & 181.8 & 1827 \\
\hline Well Tomp. \#1F & 70.11 & 183.3 & 182.11 & 183.1 & 182.5 & 186.5 & 184.4 & 184.4 & 181.4 & 179.2 \\
\hline Waltemp. ILF & 183.3 & 183.3 & 182.7 & 184.6 & 182.7 & 187.8 & $\overline{184.8}$ & 784.8 & 782.6 & 181.4 \\
\hline Infteam Tomp.F & 168.71 & 169.6 & 171.11 & 171.4 & 171.1 & 177.1 & 171.2 & 170.6 & 170.5 & 160.0 \\
\hline Avg. Wall Temp.F & 182.5) & 183.3 & 183.11 & 183.6 & 983.1 & 185 & 184.1 & 184.3 & 182.8 & 181.8 \\
\hline Avo. Wall DT.F & 13.1 & 13 & 11.3 & 11.5 & 11.4 & 13.2 & 12.2 & 13 & 11.7 & 11.2 \\
\hline Remaining Subcooff & 1.8 & & & & & & & & & \\
\hline Ext Qualiny & -0.01 & 0.01 & 0.03 & 0.05 & 0.07 & 0.09 & 0.17 & 0.13 & 0.15 & 0.17 \\
\hline DP. HBtuhrnaf & 1147.3 & 1148.8 & 1337.5 & 1315 & 1310.2 & 1127.9 & 12322 & 1939.7 & 1289.6 & 0 \\
\hline COMPOTEDH & & & & & & & & & & \\
\hline Jalouk & $\mathbf{8 5 8 . 2}$ & $\$ 55.3$ & 908.7 & 885.1 & 862.7 & 852.9 & 846.8 & 833.7 & 830.2 & 823.3 \\
\hline Szadyi(audo wibo) & 1403.8 & $14 \overline{003.3}$ & 1418.71 & 1425.3 & 1419.9 & 1427.5 & 1439.11 & 1430 & 1449.7 & 1458.8 \\
\hline Szady2 & 1284.4 & 1230.5 & 1263.61 & 1270 & 12625 & 1269.1 & 12801 & 1273.8 & 1288.8 & 1291.8 \\
\hline (स्वाQ) & 131421 & 131866 & 130384 & 127930 & 125135 & 122530 & 119667 & 117030 & 114371 & 111448 \\
\hline (7) & $1.00 \overline{6}$ & 1.006 & 1.531 & 1.807 & -2.027 & 2216 & 2.383 & 2.642 & 2.686 & 2.828 \\
\hline$(1 \bar{H})$ & 291.11 & 291.2 & 443.4 & 523.5 & $5 \overline{87.3}$ & 641.7 & 690.3 & 736.2 & 777.9 & 818.8 \\
\hline (HBOIL) & 1399.7 & 1399.1 & 1404.1 & 1401.3 & 1385.5 & 1382.9 & 1384.1 & 1368.3 & 1370.8 & 1365 \\
\hline & ZONE2 & ZONE4 & ZONE & ZONE8 & ZONETO & & & & & \\
\hline DP Bquid Tomp.F & 71.5 & & & & & & & & & \\
\hline DP Lquid Density-bmit3 & 97.49 & & & & & & & & & \\
\hline Elovation & 2 & 4 & 6 & 8 & 9.875 & & & & & \\
\hline Measurod DPPsid & 0.13 & 0.529 & 1.005 & 1.296 & 1.52 & & & & & \\
\hline Zero Corrections-psid & 0.009 & 0.044 & 0.029 & 0.018 & -0.074 & & & & & \\
\hline Corrected DPPsid & 0.121 & 0.585 & 0.976 & 1.278 & 1.69 & & & & & \\
\hline Actual DPA & 2.08 & 3.54 & 5.13 & 6.88 & 8.28 & & & & & \\
\hline Mosesurod TPF & 113.7 & & $-i$ & & 115.8 & & & & & \\
\hline Mensurod Overalle & 3.79 & & & & & & & & & \\
\hline & & & & & & & & & & \\
\hline Computod DP-1 & 2.17 & 4.24 & 6.24 & 8.26 & 10.21 & & & & & \\
\hline Friction-t & 0.17 & 0.46 & 0.85 & 1.33 & 1.87 & & & & & \\
\hline Acoleration-f & 0.13 & 0.34 & 0.49 & 0.64 & 0.79 & & & & & \\
\hline Elevationt & 9.87 & 3.44 & 4.89 & 6.29 & 7.56 & & & & & \\
\hline
\end{tabular}




\begin{tabular}{|c|c|c|c|c|c|c|c|c|c|c|}
\hline & & & & I & 1 & I & & & & \\
\hline Runt & 373 & & & I & & & & & & \\
\hline Tostfluid & R114 & & & I & 1 & I & & & & \\
\hline Dado: & $3 / 593$ & & & $t$ & 1 & 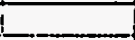 & & & & \\
\hline Tims: & $13: \overline{0}$ & & & & i & \pm & & & & \\
\hline Note & TABOREK & Analytical & & & & & & & & \\
\hline Flow Areath & 0.00356 & & & & & & & & & \\
\hline Hoal Flow Aroat? & 0.19655 & & & & & & & & & \\
\hline Sat. Prossurópsig & 713.6 & & & & & & & & & \\
\hline SA. Temperatur of (inlot) & 172.4 & & & i & & & & & & \\
\hline Lq. Density-bm/us & 79.56 & 79.46 & 79.42 & 79.41 & 79.481 & 79.45 & 79.41 & 79.48 & 79.51 & 79.68 \\
\hline Ladont Heareturibm & 44.46 & 44.37 & 44.32 & 44.31 & 44.38 & 44.35 & 44.31 & 44.38 & 44.411 & 44.53 \\
\hline Speofio Hoet attulbm/F & 0.271 & 0.272 & 0.272 & 0.272 & 0.272 & 0.272 & 0.272 & 0.272 & 0.272 & 0.271 \\
\hline Lig. Viscositylom/hhr & 0.494 & 0.493 & 0.492 & 0.492 & 0.493 & 0.493 & 0.492 & 0.493 & 0.09 & 0.005 \\
\hline Lig. Conductivity thuhr-fiF & 0.0234 & 0.0293 & 0.0293 & 0.0293 & 0.0293 & 0.0293 & 0.0293 & 0.0293 & 0.0294 & 0.0029 \\
\hline Lig. Prandt No. & 4.57 & 4.56 & 4.56 & 4.56 & 4.56 & 4.56 & 4.56 & 4.56 & 4.56 & 4.57 \\
\hline Inlot Flow Pato-GPM & 4.4 & & & & & & & & & \\
\hline Outot Lq. Flow Rato-GPM & 2.3 & & & & & & & & & \\
\hline Outlot Vap. Flow Rato-GPM & 0.9 & & & & & & & & & \\
\hline InTot temperaturot & $\mid 69.9$ & & & & & & & & & \\
\hline Outet Li Temperature $F$ & 104.3 & & & & & & & & & \\
\hline Outlot Vap. Temperaturef & 168.9 & & & i & & 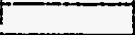 & & & & \\
\hline Wador Fow Pado-GPM & & & & $T$ & & & & & & \\
\hline Weder inlet Tomp,F & 72.9 & & & & & & & & & \\
\hline Welar outlol Tomp.F & 164.6 & & & i & & & & & & \\
\hline 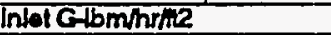 & 845670 & & Tomp & 163.8 & Donaity & 80.54 & & & & \\
\hline Subcooling F & 2.5 & & & $i$ & & & & & $\cdot$ & \\
\hline \multicolumn{11}{|l|}{ Flow Observation } \\
\hline & ZONET & ZONE2 & ZONE3 & ZONE4 & ZONES & ZONE6 & ZONE7 & ZONE8 & ZONE9 & ZONE1O \\
\hline Featlinput-Watts(nol) & 854.3 & 853.3 & 868.8 & 868.9 & 857.8 & 859.7 & 854.9 & 835.5 & 866.3 & 870.7 \\
\hline Hest Lose-Watts & 40.1 & 8.5 & 10.6 & 10 & 9.9 & 9.8 & 11.2 & 12.8 & 13.8 & 67.1 \\
\hline Hoed Fkx Bturifrime & 14800 & 14791 & 15060 & 15052 & 14869 & 14500 & 14819 & 14483 & 15017 & 15030 \\
\hline Wall Temp. HF & 1823 & 184.2 & 183 & 183.6 & 185.7 & 784.7 & 184.5 & 784.4 & 184.5 & 183 \\
\hline Wall Temp. \#4F & 1827 & 182.1 & 184.4 & 184.5 & 184.8 & 184.7 & 184.8 & 185.6 & 185.8 & 182.8 \\
\hline Wadl Tomp. & 181.7 & 180.5 & 185.2 & 189.8 & 183.21 & 185.5 & 189.8 & 183.8 & 1826| & 183.1 \\
\hline WaHTomp. IfF & 69.9 & 183.3 & 182 & 183.2 & 1827 & 186.8 & 1852 & 185 & 181.8 & 1792 \\
\hline Wall Tomp. 12 & 189.2 & 183.1 & 182.8 & 184.8 & 183.11 & 1882 & 185.5 & 185.3 & 189.1 & 181.3 \\
\hline introam Tompof & 170.8 & 177.5 & 171.8 & 171.9 & 171.4 & 171.6 & 171.9 & 171.4 & 171.2 & 170.3 \\
\hline Avg. Wall Tomp.F & 182.5 & 183.2 & 183.1 & 183.8 & 183.5. & 185.4 & 184.8 & 184.8 & 183.4 & 181.9 \\
\hline Avo. WallDT.f & 11 & 11 & 10.6 & 11.2 & 11.4 & 13.2 & 12.2 & 12.8 & 11.6 & 10.9 \\
\hline Romaining Subcoolf & -1.3 & & & & & & & & & \\
\hline Ext Quality & 0.01 & 0.03 & 0.05 & 0.08 & 0.1 & 0.12 & 0.15 & 0.17 & 0.19 & 0.22 \\
\hline EXP. HOturtrmerF & 1345.5 & 1343.8 & 1424.9 & 1345.5 & 1306.9 & 1133.1 & 1215.7 & 1134.5 & 1298.9 & 0 \\
\hline \multicolumn{11}{|l|}{ COMPUTEOA } \\
\hline Jallouk & \$47.1 & 919.8 & 903.8 & 886.9 & 8724 & 859.1 & 849.8 & 833.2 & 839.4 & 832.9 \\
\hline Szady1(Cudo ubbo) & 1399.9 & 1404.8 & 14223 & 1427 & 1420.6 & 1427.7 & 1450 & 1418.3 & 1447.3 & 1453.6 \\
\hline $\operatorname{sendy2}$ & 1243.9 & 1249.4 & 1267.9 & 1272.1 & 1263.6 & 1289.9 & 1270.7 & 1254.7 & 1285.7 & 12002 \\
\hline (स्व) & 111451 & $1100 \approx 2$ & 107595 & 104897 & 102132 & 99613 & 97139 & 94348 & 91678 & 88677 \\
\hline$(\overline{\boldsymbol{F}})$ & 1.214 & 1.648 & 1.856 & 2.103 & 2.317 & 2.501 & 2667 & 2.83 & 2976 & 3.13 \\
\hline (Hय) & 306.9 & 390 & 469.3 & 537.7 & 585.6 & 6323 & 6745 & 714.8 & 7523 & 790.7 \\
\hline \multirow[t]{3}{*}{ (HBO/L) } & 1395 & 1394.5 & 1405.1 & 1401.9 & 1385.7 & 1385.1 & 1378.1 & 1355 & 1376 & $137 \pi$ \\
\hline & & & & & & & & & & \\
\hline & ZONE2 & ZONE4 & ZONE6 & ZONEB & ZONE10 & & & & & \\
\hline DPliquid Temp.F & 72.1 & & & & & & & & & \\
\hline DPLiquid Densitylbm/13 & 91.43 & & & & & & & & & \\
\hline Elevationt & 2 & 4 & 6 & 8 & 9.875 & & & & & \\
\hline Moasured DPPsid & 0.188 & 0.884 & 7.327] & 1.738 & 2.18 & & & & & \\
\hline Zero contections-psid & 0.009 & 0.044 & 0.020 & 0.018 & -0.074 & & & & & \\
\hline Combeted DPPsid & 0.179 & 0.82 & 1.292 & 1.718 & 2.2541 & & & & & \\
\hline Actud DPA & 1.971 & 3.11 & 4.55 & 6.08 & 7.251 & & & & & \\
\hline Monoured Tppig & 173.6 & & & & 116.4 & & & & & \\
\hline \multirow{2}{*}{\multicolumn{11}{|c|}{ Meseured Overalla }} \\
\hline & & & & & & & & & & \\
\hline Computed DP-A & 1.97 & 3.69 & 5.39 & 7.11 & 8.79 & & & & & \\
\hline Frictionnt & 0.18 & 0.51 & 0.96 & 1.52 & 2.15 & & & & & \\
\hline Acooleration- & 0.19 & 0.32 & 0.45 & 0.57 & 0.7 & & & & & \\
\hline Elevation? & 1.6 & 2.86 & 3.98 & 5.02 & 5.93 & & & & & \\
\hline
\end{tabular}




\begin{tabular}{|c|c|c|c|c|c|c|c|c|c|c|}
\hline & & i & $i$ & & $\therefore$ & & & 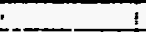 & 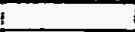 & $i$ \\
\hline Run\# & 3741 & & & & & & & 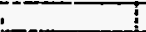 & 5 & \\
\hline Test Fuid & IR114 & 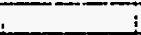 & $i$ & & 3 & & $i$ & $i$ & & i \\
\hline Date: & $3 / 5 / 93$ & & & & $i$ & & 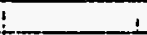 & - & & i \\
\hline Time: & $13: 361$ & $i$ & $i$ & & i. & & 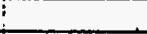 & I & I & 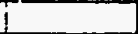 \\
\hline Note & TTABOREF & Analytical & & & ; & & 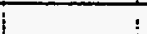 & : & $\therefore$ & 4 \\
\hline Flow Areg-t2 & 0.00336 & & 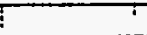 & & T & & & & & \\
\hline Headfow Arez-12 & $0 . \overline{196951}$ & I & & & & & & $\Gamma$ & & \\
\hline Sat Pressuro Psig & 177.81 & & & & & & & $T$ & & \\
\hline Sat. Temperaturo-f(inlel) & 171.2 & it & +1 & 5 & & & 1 & 1 & & \\
\hline Liq. Density fbmint3 & 79.821 & 79.68 & 79.61 & 79.69 & 79.68 & 79.69 & 79.581 & 79.65 & 79.69 & 79.78 \\
\hline Letent Hoafotunbm & 4.71 & 4.53 & 44.5 & 44.53 & 4.57 & 4.53 & 44.48 & 44.54 & 44.59 & 44.65 \\
\hline Spooito Heat envibmiF & 0.271 & 0.271 & 0.271 & 0.271 & 0.271 & 0.271 & 0.2717 & 0.271 & 0.271 & 0.27 \\
\hline Liq. Viscositulbm/hr & 0.4981 & 0.456 & 0.495 & 0.495 & $0 . \overline{496}$ & 0.455 & $0 . \overline{4051}$ & 0.4561 & 0.406 & 0.400 \\
\hline Liq. Conductivity-8tuhr-hif & 0.02961 & 0.0294 & 0.0294 & 0.0294 & 0.02951 & 0.0294 & 0.02941 & 0.0205 & 0.0295 & 0.0295 \\
\hline Lig. Prandt No. & 4.57 & 4.57 & 4.57 & 4.57 & 4.57 & 4.57 & 4.57 & 4.57 & 4.57 & 4.57 \\
\hline Inlot Fow Rate-GPM & 3.5 & & 1 & 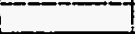 & i & & & 1 & & \\
\hline Outlel Liq. Flow Rate-GPM & 2.11 & i & t & & +1 & & & & & \\
\hline Outlet Vap. Flow Rato-GPM & 0.8 & & & & 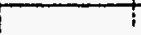 & & & & & \\
\hline inlot Temperature & 167.91 & & & & & & & & & \\
\hline Outel Liq. Temperaturof & 104.31 & & Ti & & 7 & & & & & \\
\hline Outrel Vap. Temperature-F & 167.8 & & i & i & T & & & & & \\
\hline Water Flow Rato-GPM & & & 1 & 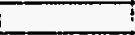 & & & & & & \\
\hline Water inlet Temp.F & 75.61 & & 1 & 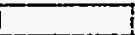 & & & & & & \\
\hline Water outel Temp.f & 164.61 & & 1 & & & & & & & \\
\hline Indet Glbm/hrite & 676337 & & Tomp & 160.6 & Donsity & 80.97 & & & & \\
\hline Subcoolingf & 3.3 & & 1 & -7 & -2 & & & & & \\
\hline \multirow[t]{2}{*}{ Flow Observation } & & & & & 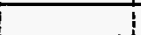 & & & & & \\
\hline & ZONET & ZONEZ & ZONE3 & ZONE 4 & ZONES & ZONE6 & ZONE7 & ZONE8 & ZONE9 & ZONE10 \\
\hline Heat Input-Watts(nel) & 865.2 & 861.5 & 873.8 & 874.1 & 861.2 & 865.9 & 863.7 & 844.5 & 871.7 & 877.9 \\
\hline Heat Loss-Walls & 39.5 & 8.4 & 10.5 & 10 & 9.9 & 9.9 & 11.1 & 127 & 13.6 & 66.8 \\
\hline 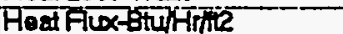 & 149881 & $14 \overline{34}$ & $1 5 \longdiv { 4 7 ! }$ & 15153 & $14 \% 28 T$ & 75010 & 14972 & 14539 & 75110 & 15218 \\
\hline Wăt Temp. BF & 181 & 182.9 & 181.8 & 182.6 & $\{84.51$ & 183.9 & 183.3 & 183.4 & 183.5 & 7823 \\
\hline Wail Temp. 4 F & 181.6 & 180.8 & 183 & 183.4 & 183.8 & 183.9 & 183.8 & 184.3 & 184.2 & 182.1 \\
\hline Wal Temp. 55 & 180.4 & 179.5 & 1841 & 182.8 & 182.11 & 184.4 & 18291 & 188 & 181.8 & 182.8 \\
\hline Well Tomp.\#1F & 70.3 & 182 & 1811 & 182.11 & 181.6 & 185.5 & 184 & 184.1 & 180.8 & 178.3 \\
\hline Wall Temp. T2F & 182 & 182 & 181.4 & 183.7 & 182 & 187 & 184.47 & 184.2 & 1822 & 980.6 \\
\hline Inetresm Tomp.f & 169 & 170.3 & 170.5 & 170.3 & 170 & 170.3 & 170.7 & 170.2 & 169.9 & 169.4 \\
\hline Avg. Wall Temp.f & 187.3 & 181.9 & 181.8 & 182.7 & 18241 & 184.4 & 183.7 & 183.8 & 182.4 & 181.1 \\
\hline Avg. Wall DT.F & 11.6 & 11 & 10.6 & 11.7 & 11.7 & 13.4 & 12.3 & 12.9 & 11.8 & 11 \\
\hline Romaining Subcooff & -1.5 & & & & 1 & & & & & \\
\hline Exct Quality & 0.011 & 0.04 & 0.07 & 0.1 & 0.13 & 0.16 & 0.18 & 0.21 & 0.24 & 0.27 \\
\hline EXP. HBtuHimęF & 1296 & 1353.8 & 1426.9 & 1296.1 & 1275.9 & 1116.2 & 1216.9 & 9131.4 & 1278.7 & 0 \\
\hline COMPUTEOH & & & & & & & & & & \\
\hline Jallouk & 971.4 & 947 & 925.4 & 905.7 & -8921 & 878.5 & -872.5 & 849.6 & 855.2 & 850.5 \\
\hline Szadyi(Oudo (ube) & 1400.6 & 1404.7 & 1418.5 & 1421.1 & 1418 & 1421.7 & 1425.31 & 1412.5 & 1437.2 & 1446 \\
\hline Szaty? & 1220.5 & 1228.9 & 1244.4 & 1245.4 & 1255.6 & 1284.4 & 1287.5 & 1251.4 & 1277.8 & 1284.5 \\
\hline (RUQ) & $883 \approx 21$ & 87201 & 84671 & 81961 & 792381 & 76748 & 742811 & 71538 & 68859 & 66000 \\
\hline (F) & 1.23 & 1.606 & 1.972 & 2258 & 2503 & 2713 & 2.9031 & 3.088 & 3.26 & 3.43 \\
\hline$\frac{1}{(H U Q)}$ & 259.7 & 339.4 & 416.6 & 47.2 & 528.7 & 573.3 & 613.61 & 6524 & 688.7 & 724.5 \\
\hline \multirow[t]{3}{*}{ (HBOLL) } & 1397.6 & 1398.1 & 1406.4 & 1402.9 & 1387.9 & 1369.9 & 1386.4 & 1364.5 & 9382.5 & 1381.5 \\
\hline & & & & & & & & & & \\
\hline & ZONE2 & ZONE 4 & ZONE 6 & ZONE8 & ZONE10 & & & & & \\
\hline DP Fquid Temp.f & 72.4 & & & & & & & & & \\
\hline DPLiquid Density $/ \mathrm{bm} / \mathrm{m}$ & 91.4 & & & & & & & & & \\
\hline Elevation-h & & -4 & का & -8 & 9.875 & & & & & \\
\hline Measured DPFsid & 0.202 & 1.038 & 1.558 & 2.074 & 2.033 & & & $T$ & & \\
\hline Zoro Contectons-psld & 0.0091 & 0.044 & 0.0291 & 0.018 & -0.074 & & & & & \\
\hline Corrocted DPPsid & 0.198 & 0.389 & 1.5291 & 2.058 & 2.707 & & & 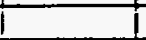 & & \\
\hline Actud DPA & 1.94 & 2.8 & 4.121 & 5.46 & 6.431 & & & & & \\
\hline Monsured TPQ ag & $\overline{119.8}$ & & & & 114.8 & & & & & \\
\hline \multirow[t]{2}{*}{ Measured Ovorallia } & 5.42 & & & & & & & & & \\
\hline & & & & & & & & & & \\
\hline Computed DP-A & 1.84 & 3.39 & 4.911 & 6.44 & 7.91 & & & & & \\
\hline Friction-A & 0.13 & 0.37 & 0.72 & 7.16 & 1.65 & & & & & \\
\hline Acooleration- & 0.14 & 0.24 & 0.34 & 0.44 & 0.57 & & & & & \\
\hline Envalion-f & 1.57 & 2.78 & 3.85 & 4.83 & 5.7 & & & & & \\
\hline
\end{tabular}




\begin{tabular}{|c|c|c|c|c|c|c|c|c|c|c|}
\hline & 7 & 1 & s. & . & i & i & 1 & $\mathbf{i}$ & i & I \\
\hline Reñit & $375 !$ & & 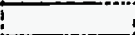 & 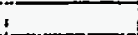 & & $i$ & T & $i$ & & i \\
\hline Test Fluid & A114 & & tis & 1 & & 1 & 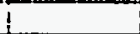 & 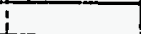 & 5 & \\
\hline Date: & अ5393 & & & $i$ & & 1 & T & I & 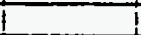 & 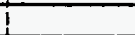 \\
\hline Time: & $13: 46$ & & & 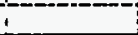 & 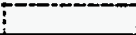 & & $\bar{i}$ & 1 & t & +1 \\
\hline Nolo & TABOREK & Analytical & dt & & 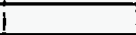 & & i & & & \\
\hline Flow Area-12 & 0.00336 & & & & & & & & & \\
\hline Heat Fow AYTOA & 0.19595 & & & & & & & ț & & \\
\hline Sat. Prossurérig & 112.5T & & & & & & & & & \\
\hline Sal, Temperature finlet) & 171.7 & & - & T & & $i$ & & i & & \\
\hline Lig. Density tomints & $79.7 \pi$ & 79.55 & 79.49 & 79.52 & 79.58 & 79.55 & 79.51 & 79.55 & 79.55 & 79.68 \\
\hline Letent Heat-Beshbm & 4.67 & 44.45 & 44.39 & 44.42 & 4.46 & 44.45 & 44.41 & 4.45 & 4.451 & 4.57 \\
\hline Speorfo Hentetiriom/F & 0.271 & 0.272 & 0.272 & 0.272 & 0.271 & 0.272 & 0.272 & 0.272 & 0.272 & 0.271 \\
\hline Uিq. Viscosity-lbmithr & 0.4981 & 0.494 & 0.493 & 0.494 & 0.494 & 0.494 & 0.483 & 0.438 & 0.49 & 0.466 \\
\hline Liq. Conductivity Btuhf-fF & 0.0255 & 0.0294 & 0.0294 & 0.0234 & 0.0294 & 0.0294 & 0.0294 & 0.0294 & 0.0294 & 0.0295 \\
\hline Lig. Prandt No. & 4.57 & 4.57 & 4.56 & 4.56 & 4.57 & 4.57 & 4.56 & 4.57 & 4.57 & 4.57 \\
\hline inlotflow Pato-GPM & $-\frac{2.7}{2}$ & & & & & 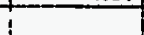 & & & & \\
\hline Outlet Liq. Flow Rato-GPM & 1.7 & & & & & & & & & \\
\hline Outel Vap. Flow Rate-GPM & 0.7 & & & & & & & & & \\
\hline intol temperaturet & -168.6 & & & & & & & & & \\
\hline Óvel Ĺa. TemperaturóF & 104.5 & & & & & & & & & \\
\hline Outlet Vap. Temperatur of & 168.81 & & & & & & & & & \\
\hline Weder Flow Rato-GPM & 1 & & & & & & & & & \\
\hline Water inlet Temp.f & $76.5 !$ & & & & & & & & & \\
\hline Weter outel Tomp.f & 165.7 & & & & & & & & & \\
\hline intel Gibminrit2 & 522704 & & Tamp & 159.5 & Density & 81.12 & & & & \\
\hline Subcoolingf & 3.1 & & & & & 1 & & & & \\
\hline \multicolumn{11}{|l|}{ Flow Observation } \\
\hline & ZONET & ZONE2 & ZONE3 & ZONE4 & ZONE5 & ZONE6 & ZONE7 & ZONEB & ZONE9 & ZONETO \\
\hline Head Input-Watts(net) & 868.2 & 855.2 & 874.7 & 874.3 & 864.5 & 867.8 & 8672 & 8502 & 871.4 & 875.9 \\
\hline Hoal Loss-Watts & 39.7 & 8.4 & 10.6 & 10 & 9.9 & 9.8 & 11.2 & 12.8 & 13.7 & 67 \\
\hline Hogl Fix & 150500 & 72988 & $151 \% 2$ & 15155 & 7485 & 15073 & 75032 & 74738 & T5105 & 75183 \\
\hline Wal Temp. WF & 181.5 & 183.2 & 782.3 & 183 & 185 & 184.3 & 184 & 184.1 & 184.2 & 1828 \\
\hline Wall Tomp. RAF & 182 & 781.3 & 983.4 & 18.8 & 184.4 & 184.3 & 184.5 & 185.2 & 184.8 & 182.8 \\
\hline Wall Tomp. $15+F$ & 181 & 179.8 & 184.6 & 189.2 & 182.6 & 184.8 & 183.4 & 183.7 & 1822 & 182.8 \\
\hline WEH Temp. IIF & 71.11 & 182.6 & 781.4 & 182.7 & 182 & 186.3 & 185 & 184.8 & 181.71 & 179 \\
\hline Wall Tomp. 政F & 1826 & 1824 & 182 & 1842 & 1824 & 887.8 & 185.2 & 185.2 & 18271 & $7 \overline{81.2}$ \\
\hline Instream Tomp.F & 169.31 & 170.9 & 171.31 & 171.1 & 170.8 & 170.9 & 171.21 & 170.9 & 170.9 & 170 \\
\hline Avg.Wall Temp.f & 181.81 & 182.4 & 18231 & 183.2 & 182.9 & 184.9 & $\overline{184.41}$ & 184.6 & 183.1 & 181.7 \\
\hline Avo. Wall DT.F & 11.8 & 10.8 & 10.3 & 11.4 & 11.4 & 13.3 & 12.5 & 13.1 & 11.6 & 11.1 \\
\hline Romaining Subcooff & -3.2 & & & & & & & & & \\
\hline Exct Quality & 0.021 & 0.06 & 0.1 & 0.13 & 0.17 & 0.21 & 0.25 & 0.28 & $0 . \overline{32}$ & 0.36 \\
\hline EXP. HBHUHAmeF & 1276 & 1389 & 1473.5 & 1330.6 & 1317.7 & 1127.2 & 1198.7 & 1129 & 1307 & 0 \\
\hline \multicolumn{11}{|l|}{ COMPUTEDH } \\
\hline Jalouk & 988.4 & 958.4 & -949.2 & $\overline{928.5}$ & 907.1 & -8921 & 887.4 & 874 & $8 \overline{87.2}$ & $8 \overline{4} \overline{2}$ \\
\hline szadvi(audo tube) & 1403.9 & 1409.4 & 1420.8 & 1422 & 1415.5 & 1421.3 & 1425 & 1413.4 & 1433.6 & 1497.5 \\
\hline Szady2 & 1233.71 & 1234.4 & 1247 & 1246.8 & 1259.6 & 1285.4 & 1268.9 & 1255 & 1276.4 & 1279.1 \\
\hline (संग) & 68033 & 66570 & 640611 & 61353 & 58537 & 55014 & 53493 & 50820 & 482 दाइ & 45384 \\
\hline (F) & 1.361 & 9.802 & 2.186 & $25 \overline{14}$ & 2.788 & 3.028 & 3.245 & 3.453 & 3.645 & 3.847 \\
\hline (HUTa) & 233.8 & 300.9 & 37.7 & 4324 & 479.5 & 520.8 & -5582 & 533.9 & 626.9 & 6612 \\
\hline \multirow[t]{3}{*}{$(1 \mathrm{BO} / \mathrm{L})$} & 1401.71 & $\overline{1404.3}$ & 1411.9 & 1408.6 & 1396.8 & 1397.6 & 135.8 & 1377.5 & 13925 & 13892 \\
\hline & & & & & & & & & & \\
\hline & ZONEZ & ZONE4 & ZONE6 & ZONEB & ZONETO & & & & & \\
\hline DPfiquid Temp.f & 72.6 & & & & & & & & & \\
\hline DPLiquid Density tbm/lit3 & 91.38 & & & & & & & & & \\
\hline Elovation $h$ & हो & 4 & $6 t$ & 8 & 9.875 & & & & & \\
\hline Moasured DPFsid & 0.245 & 1.26 & 1.8577 & $\overline{2.513}$ & 3.162 & & & & & \\
\hline Zoro Correctons-psld & 0.009 & 0.044 & 0.029 & 0.018 & -0.074 & & & & & \\
\hline Corrected DPPsid & 0.238 & 1.218 & 1.822 & 2.495 & 3.208 & & & & & \\
\hline Achual DPA & 1.87 & 2.39 & 3.59 & 4.67 & 5.48 & & & & & \\
\hline Monaured Tppag & 112.5 & & & & 116.2 & & & & & \\
\hline \multirow[t]{2}{*}{ Measured Overall-ä } & -6.69 & & & & & & & & & \\
\hline & & & & & & & & & & \\
\hline Computed DPA & 1.65 & 2.97 & 4.23 & 5.49 & 6.69 & & & & & \\
\hline Friction-h & 0.1 & 0.3 & 0.58 & 0.95 & 9.35 & & & & & \\
\hline Accolerationt & 0.1 & 0.18 & 0.26 & 0.35 & 0.46 & & & & & \\
\hline Elovation $\mathrm{At}$ & 1.45 & 2.49 & 3.39 & 4.19 & 4.88 & & & & & \\
\hline
\end{tabular}




\begin{tabular}{|c|c|c|c|c|c|c|c|c|c|c|}
\hline & I & 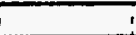 & i & $\vdots$ & 1 & 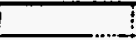 & 1 & I & I. & I \\
\hline Run: & 376 & & $\vdots$ & & $i$ & $i$ & 1 & i & $i$ & $i$ \\
\hline Testfluid & R114 & & i & & & & & 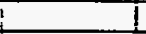 & I & 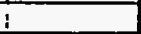 \\
\hline Date: & $3 / 5931$ & & $t$ & 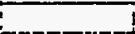 & 1 & t. & & & & 1 \\
\hline Time: & : 13.531 & & 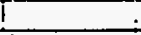 & 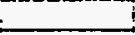 & 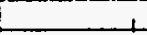 & & & & i & $i$ \\
\hline Note & TABOREM & Analytical & $d t$ & - & 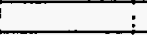 & & & 1 & 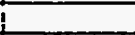 & \\
\hline Flow Area-tl2 & 0.00336 & & & & 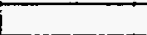 & & & 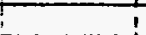 & 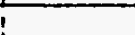 & \\
\hline Heat Fow Areanic & $0.1965 !$ & & & & & & & & 4 & \\
\hline Sat. Pressure-pig & $11 \overline{4} \mid$ & & & 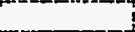 & 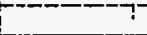 & & & & & \\
\hline Sa. Temperature-f(niel) & 172.6 & & $i$ & i & & & & & & T \\
\hline Liq. Densitylbm/n3 & 79.581 & 79.29 & 79.311 & 79.35 & 79.391 & 79.38 & 79.321 & 79.35 & 79.35 & 79.45 \\
\hline Latent Hoestibulbm & 44.481 & 44.2 & 44.21 & 44.261 & $44.3 T$ & 44.27 & 44.231 & 44.261 & 44.26 & 44.35 \\
\hline Spectio Heat Btunb m/F & 0.271 & 0.272 & 0.272 & 0.272 & 0.2721 & 0.272 & 0.272 & 0.272 & 0.272 & 0.272 \\
\hline ig. Viscositylbmithr & 0.495 & 0.49 & 0.49 & 0.491 & 0.4921 & 0.491 & $0 . \overline{4911}$ & 0.4911 & 0.491 & 0.403 \\
\hline Liq. Conductivity Btuhr-fif & 0.02941 & 0.0292 & 0.02921 & 0.0293 & 0.02331 & 0.0293 & 0.0292 & 0.0293 & 0.0293 & 0.0253 \\
\hline Lig. Prandt No. & 4.57 & 4.56 & 4.56 & 4.56 & 4.56 & 4.56 & 4.561 & $4.56 \mid$ & 4.56 & 4.56 \\
\hline Inlet Fow Rate-GPM & 1.9 & & & $-\infty$ & 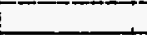 & & & & & \\
\hline Outlet Liq. Fow Rate-GPM & $1.4^{\prime}$ & & & 1 & 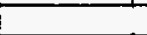 & & & & & \\
\hline Outlet Vap. Flow Rale-GPM & 0.81 & & & 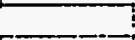 & 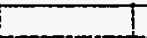 & & & & & \\
\hline Inlet Temperaturef & 16.61 & & $\bar{i}$ & i & ; & & & & & \\
\hline OutietLiq. TemporatureF & $104 . \overline{8}$ & & 7 & $\mathrm{H}^{+}$ & 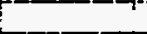 & & & ! & & \\
\hline Outel Vap. Tomperaturof & $170.2 !$ & & $i$ & $i$ & 1 & 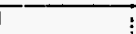 & $i$ & 1 & & \\
\hline Water Fow Pado-GPM & 1 & 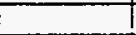 & 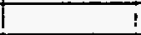 & 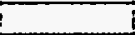 & $\mathrm{i}$ & & 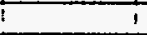 & 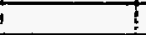 & & \\
\hline Water inlet Temp.f. & 76.81 & & T & & & & i & i & & \\
\hline Water outol Tomp.F & 167.11 & & & آ & & & & & & \\
\hline Inlat Gibmhriliz & 3589871 & & Tomp & 157.6 & Donsity & 81.381 & & I & & \\
\hline Subcooling $f$ & 3 & & 1 & 1 & & & 1 & I & & \\
\hline \multirow[t]{2}{*}{ Fow Observation } & 1 & & & & & & & 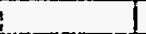 & & \\
\hline & ZONE1 & ZONE2 & ZONEB & ZONE4 & ZONE5 & $\angle O N=6$ & ZONE & ZZONEB & ZONE9 & ZONE10 \\
\hline Reat Input-Watts(nel) & 865 & 852.2 & 875.6 & 876.7 & 863.5 & 865.8 & 8525 & 845.6 & 875 & 879.1 \\
\hline Heat Loss-Walls & 40.3 & 8.5 & 10.7 & 10 & 9.9 & 9.8 & 11.3 & 12.9 & 13.9 & 67.5 \\
\hline Fout Fux-Buthritie & $\left.14694\right|^{\circ}$ & 74976 & 75178 & $75 T 971$ & 745851 & 75025 & T4\$5Tा & 746581 & 15168 & 75230 \\
\hline Wall Tomp. \#3F & 182.7 & 784.3 & 183.4 & -184 & 186.3 & 185.7 & 185.2! & $185.1 \mid$ & 185.3 & 183.9 \\
\hline WaH Temp. F4F & 183.2 & 182.5 & $184.6 !$ & 185 & 185.3 & 185.5 & 185.71 & 186.1 & 186 & 183.6 \\
\hline Wat Tomp. 佔F & 182.11 & 181 & 185.71 & 184 & 183.71 & 186.1 & 184.71 & 184.4 & 182.9 & 183.4 \\
\hline Wall Temp. BIF & $71.9 !$ & 183.6 & 182.11 & 183.4 & 183.2 & 187.3 & 186.1 & 185.9 & 1826 & 179.9 \\
\hline Wall Temp. \#2F & 183.6 & 18321 & 183 & 185 & 183.3 & 188.8 & 186.4 & 186.4 & 184 & 781.0 \\
\hline Instream Tomp.f & 170.71 & 172.7 & 17261 & 172.3 & 172 & 1722 & 1725 & 972.3 & 172.3 & 771.6 \\
\hline Avg. Wall Temp.F & 182.9 & 183.4 & 183.31 & 184.1 & $\overline{183.91}$ & 1862 & $185.6 \mid$ & 185.6 & 184.2 & 1825 \\
\hline Avg. Wall DT.F & 11.5 & 10 & 10 & 11.1 & 11.2 & 13.3 & 12.4 & 12.6 & 11.2 & 10.3 \\
\hline Remaining Subcoolf & -5.8 & & & & & & & & & \\
\hline Exat Quality & 0.04 & 0.00 & 0.14 & 0.2 & 0.25 & 0.31 & 0.361 & 0.41 & 0.47 & 0.52 \\
\hline EXP. H-Bturhrita & 1301.3 & 1490.9 & 1519.5 & 1367.5 & 1336.6 & 11322 & 1201.4 & 1161.7 & 1357.3 & 0 \\
\hline \multicolumn{11}{|l|}{ COMPUTED } \\
\hline Jahlouk & 1014.8 & \$85.2. & 971 & 954.5 & 938.11 & -929 & 917.7 & 898.8 & $\mathbf{9 0 9 . 6}$ & 897.9 \\
\hline Szady 1 (Cudo tubo) & 1406.4 & T413.1 & 1425.1 & 1426.4 & $14 \overline{16.21}$ & $\overline{1421.5}$ & 1421.21 & $14 \overline{08.9}$ & 1435 & $\overline{1438.2}$ \\
\hline seadr 2 & 1233.2 & 1280.1 & 12725 & 1273.3 & 12621 & 1287.6 & 1287.11 & 1253 & 1260.8 & 1283.1 \\
\hline (R्यव) & 479441 & 46203 & 435261 & 40785 & 380711 & 35462 & 328651 & 30224 & 27592 & 24837 \\
\hline (F) & 1.612 & 2.054 & 2.5731 & 2889 & $3.2141^{\circ}$ & 3.488 & 3.757 & 4.004 & 4.236 & 7.470 \\
\hline (HUO) & 196.81 & 267.6 & 327.41 & 376.4 & 418.61 & 455.7 & 489.41 & 521.5 & 551.8 & 583.3 \\
\hline \multirow[t]{3}{*}{ (HBOIL) } & 14052 & 1400.9 & $\overline{419.41}$ & 1417.6 & 9403.9 & 1405.8 & 1409.61 & 1384.7 & 1407.3 & 1405.6 \\
\hline & -1 & & 1 & & & & & & & \\
\hline & ZONEZ T & ZONE4 & ZZONE6 & ZONEB & ZONETO & & & & & \\
\hline DPliquid Temp.F & 72.9 & & & & & & & & & \\
\hline DPLiquid Density $/ \mathrm{bm} / \mathrm{s} 3$ & 91.35 & & & & & & & & & \\
\hline Elevation-a & 2 & & छां & $\overline{8}$ & 9.875 & & & & & \\
\hline Measured DPpsid & $\overline{0.248}$ & 1.545 & 2.2061 & 2.982 & 3.784 & & & & & \\
\hline Zero Correctons-psld & $0 . \overline{009}$ & 0.0441 & 0.0291 & 0.018 & -0.074 & & & & & \\
\hline Cortected DPPsid & 0.2391 & 1.5011 & 2.1771 & 2.964 & 3.8581 & & & 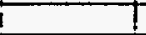 & & \\
\hline Actual DPa & 1.87 & 1.88 & 2.951 & 3.83 & 4.361 & & $i$ & i & & \\
\hline Measured TP Pag & 1141 & & 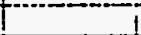 & & 178.5 & & & & & \\
\hline \multirow[t]{2}{*}{ Messured Overalit } & -8.16 & & 1 & $L$ & & & & & & \\
\hline & & & & & & & & & & \\
\hline Computed DPA & 1.39 & 2.45 & 3.43 & 4.38 & 5.28 & & & & & \\
\hline Frictionfl & 0.07 & 0.21 & 0.42 & 0.68 & 0.98 & & & & & \\
\hline Accelerationt & 0.07 & 0.12 & 0.19 & 0.26 & 0.36 & & & & & \\
\hline Elovation-f & 1.25 & 2.11 & 2.82 & 3.43 & 3.94 & & & & & \\
\hline
\end{tabular}




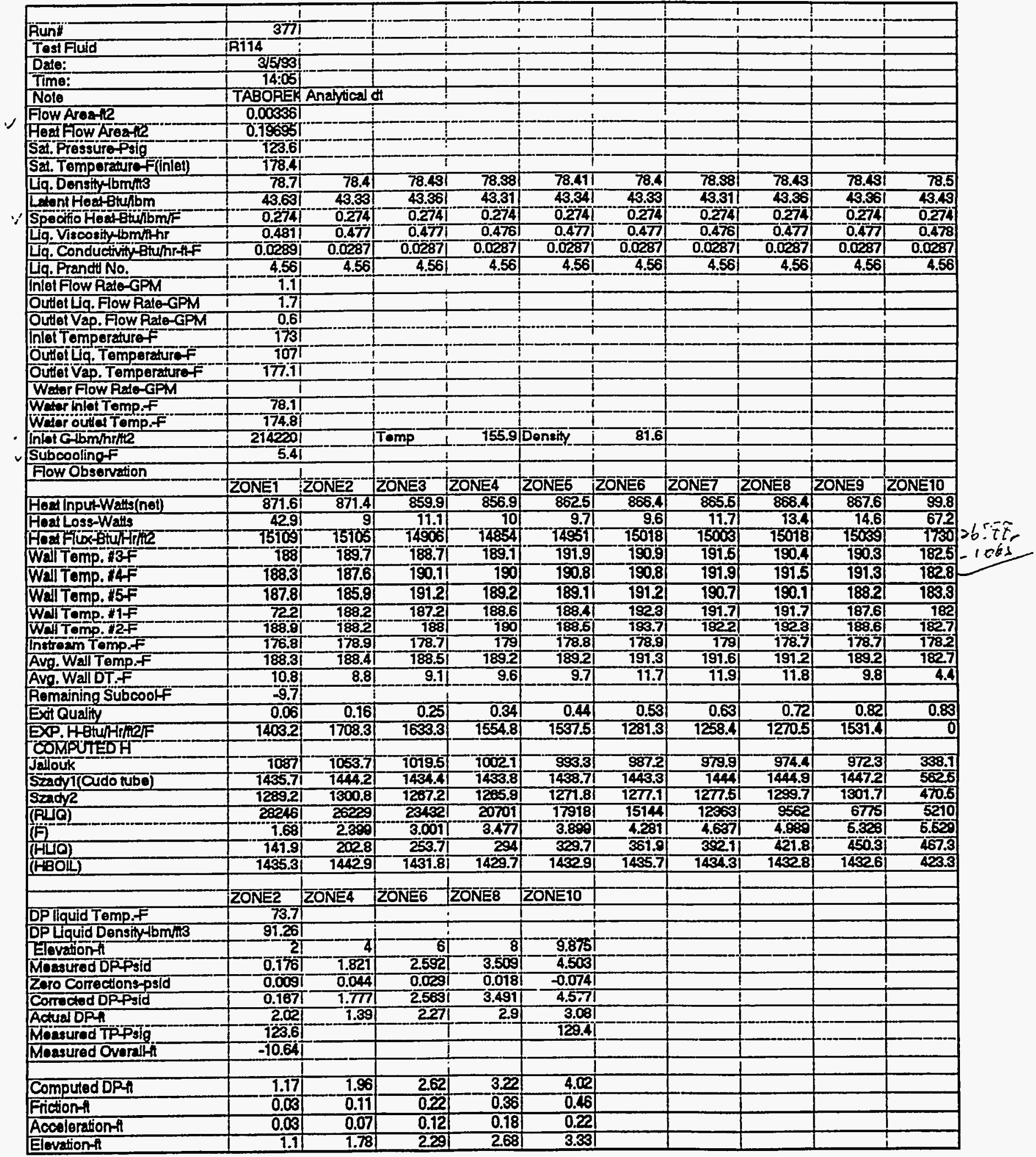




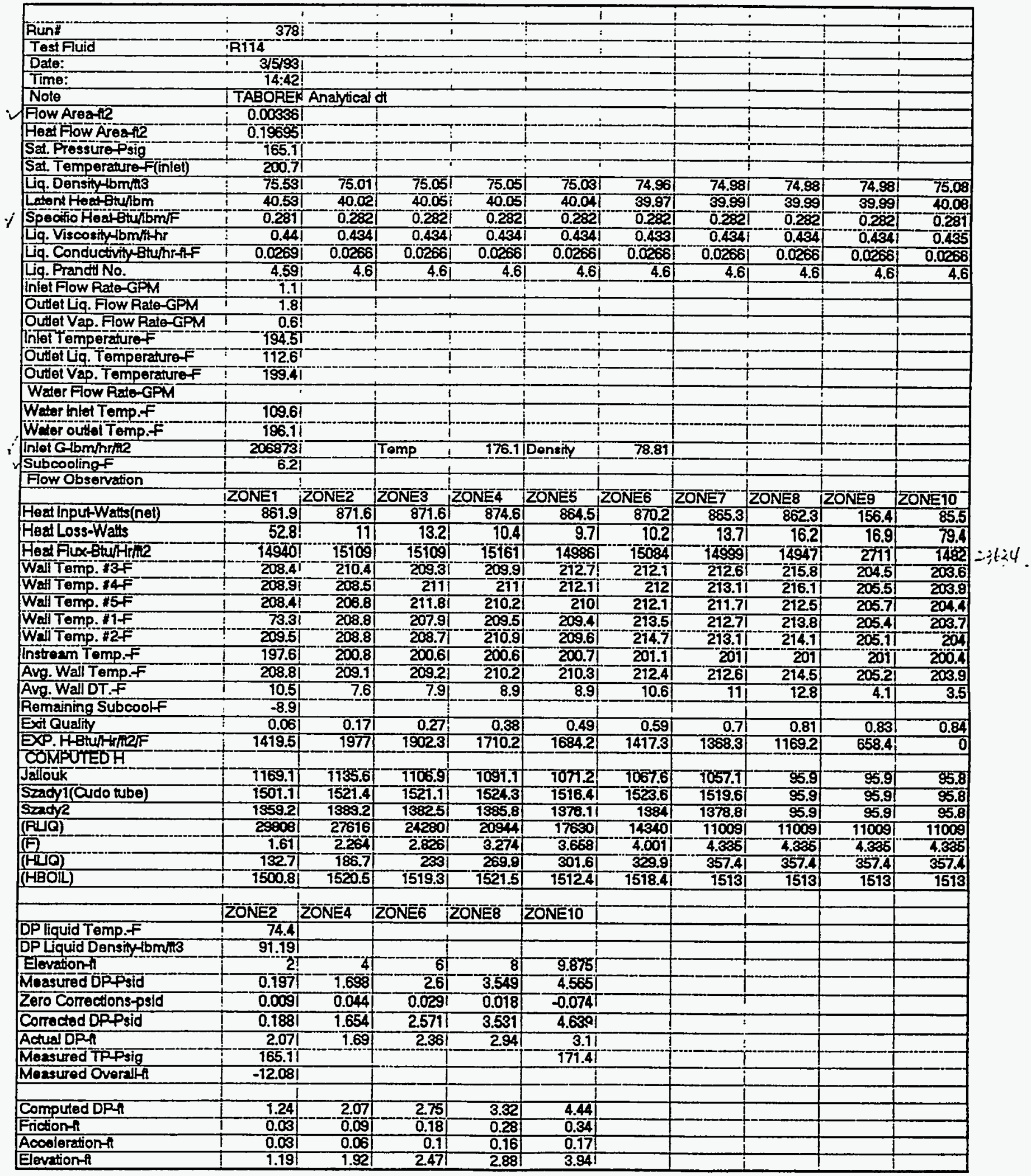




\begin{tabular}{|c|c|c|c|c|c|c|c|c|c|c|}
\hline & 1 & & & - & 1 & 1 & i. & 1 & & $i$ \\
\hline Runt & -379 & & & 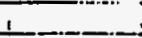 & & & & $i$ & & $i$ \\
\hline Test Fluid & R114 & & & $i$ & & & $!$ & 1 & & 1 \\
\hline Date: & 35933 & & 1 & $i$ & $i$ & i & i & 1 & & 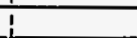 \\
\hline Time: & $15: 25$ & & 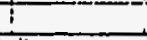 & & & & $\vdots$ & 1 & & it \\
\hline Note & TABOREK & Analytical & $d t$ & I & ; & & $!$ & + & & + \\
\hline Fow Area-12 & 0.00336 & & & & & & $i$ & 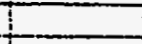 & & \\
\hline Hod How AJea-12 & 0.196951 & & & & & & & & & \\
\hline Sat. Pressure Psig & 167.51 & & & 1 & T & $T$ & & † & & t \\
\hline Sat. Temperaure-F(in|et) & 201.8 & & & 1 & ! & 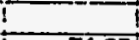 & & 1 & & \\
\hline Lig.Densitylbmuk3 & 75.241 & 74.85 & 74.831 & 74.88 & 74.881 & 74.85 & $7 \overline{4.851}$ & 74.87 & 74.र्वा & 74.85 \\
\hline Letant Host Btulbm & 40.241 & 39.86 & 39.84 & 39.89 & 39.891 & 39.86 & 39.861 & 39.88 & 39.911 & 39.86 \\
\hline 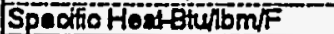 & 0.2811 & 0.282 & 0.2821 & 0.282 & 0.2821 & 0.2821 & 0.2821 & 0.282 & 0.282 & 0.282 \\
\hline Liq. Viscositylbminhr & 0.4371 & 0.432 & 0.4321 & 0.432 & 0.4321 & 0.432 & 0.432 & 0.432 & 0.433 & 0.133 \\
\hline Lig. Conductivity Btuhefflf & 0.0267 & 0.0265 & 0.0265 & 0.0265 & 0.02651 & 0.0265 & 0.0265 & 0.0265 & 0.025 & 0.0265 \\
\hline Liq. Prandt No. & 4.59 & 4.6 & 4.6 & 4.6 & 4.6 & 4.6 & 4.6 & 4.6 & 4.6 & 4.6 \\
\hline inlotflow Ralo-GPM & $2 !$ & & & $i$ & 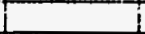 & i & & & & \\
\hline Outiof Lig. Flow Rado-GPM & 1.6 & & & & & & & & & \\
\hline Outlet Vap. Flow Rade-GPM & 0.8 & & & & & & & ? & & \\
\hline Inlet temperature & 738.4 & & & & & & & & & \\
\hline Outelluq. Temperature $F$ & 173.1 & & & & & & & & & \\
\hline öutoi vap Temporauréf & 2001 & & & i & 7 & & & & & \\
\hline Water Flow Rato-GPM & 1 & & & $T$ & 1 & & & † & & \\
\hline Wetor hilot Temp,f & 91.31 & & & & & & & & & \\
\hline Wher out l Tomp.f & 197 & & & & & & & & & \\
\hline Inlot Glbmhrnt2 & 368638 & & Tomp & 186.7 & IDonsity & 7.24 & & & & \\
\hline Subcoolingf & 3.4 & & & I & $-1-1$ & & & 1 & & \\
\hline \multirow[t]{2}{*}{ Fow Obsenvation } & & & & 1 & $i$ & & & & & \\
\hline & ZONET & ZONE2 & ZONE3 & ZONE4 & ZONNES & ZONE6 & ZONE7 & ZONE8 & ZONE9 & ZONE10 \\
\hline Hed Inpur-Watts(nei) & 874.3 & 876 & 866.7 & 851.7 & 876 & 881 & 875 & 875.1 & 880.9 & 868.7 \\
\hline Heal Loss-Watts & 531 & 11.9 & 13.3 & 10.3 & 9.7 & 10.1 & 13.7 & 15.9 & 17.7 & 83.2 \\
\hline 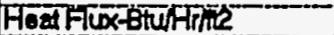 & 751555 & -75185 & 15024 & 14764 & 75785 & 15272 & 15168 & $151 ख 9$ & उ5270 & 15058 \\
\hline Wall temp. HF & 209.5 & 211.5 & 210.4 & 210.5 & 213.1 & 212.5 & टॉट.6 & 212.1 & 217.8 & 211.6 \\
\hline Wal Temp. H4F & 210.3 & 200.6 & 211.91 & 211.6 & 2125 & 2122 & 213.11 & 273.1 & 213.1 & 211.5 \\
\hline Wall Temp. IEF & 209.5 & 207.9 & 21261 & 210.5 & 210.8 & 212.5 & 212 & 211.7 & 210.6 & 210.9 \\
\hline Wall Tomp. "1F & 74.3 & 210 & 208.91 & 210.2 & 210.11 & 214 & 2131 & 213 & 210.3 & 207.9 \\
\hline Wall Tomp. $2 F$ & $210 . \overline{7}$ & 210 & 209.71 & 211.6 & 210.5 & 215.3 & 213.4 & 213.6 & 211.11 & 200.5 \\
\hline Instroan Tomp.F & 199.4 & 201.8 & 201.91 & 201.6 & 201.6 & 201.8 & 201.8 & 201.7 & 201.5 & 2012 \\
\hline Avg. Wall Temp.f & 210 & $2 \overline{10.3}$ & 210.21 & 210.7 & 2111 & 2128 & 212.8 & 212.7 & 211.4 & 210.3 \\
\hline Avo.WallDT.F & 9.91 & -7.8 & 7.6 & 8.4 & 8.7 & 10.3 & 10.3 & 10.3 & 9.2 & 8.4 \\
\hline Ramaining Subcooff & -5.7 & & & & & & & & & \\
\hline Exit Cuality & 0.04 & 0.1 & 0.16 & 0.21 & 0.28 & 0.34 & 0.4 & 0.46 & 0.52 & 0.58 \\
\hline 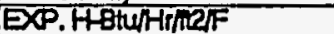 & 1528.5 & 1949.6 & 1964.9 & 1750.7 & 7747.6 & 1481.3 & 9467.7 & 1470.7 & 1661.6 & 0 \\
\hline \multicolumn{11}{|l|}{ COMPOUTEDH } \\
\hline Jallouk & 7717) & 7089.6 & 1053.6 & 1039.6 & 1043.9 & 1035.8 & 1020.9 & 7017.9 & 1007.9 & 9022 \\
\hline Szady 1 (audo tube) & 1518.7 & 1529.8 & 15229 & 1509.5 & 1552.1 & 1538.4 & 1534.4 & 1535.5 & 1541.2 & 1531.2 \\
\hline Szady2 & 1379.7 & 13925 & 1389.81 & 1887.2 & 1392.91 & 1399.5 & 1369.51 & $13 \% 0.8$ & 1378 & 1305.7 \\
\hline (RLa) & 542461 & 52148 & 488241 & 2545 & 42116 & 38766 & 35379 & 31888 & 28577 & 25175 \\
\hline (F) & 1.41 & 1.818 & 2.328 & 2.646 & 2821 & 3.169 & 3.389 & 3.618 & 3.82 & 4.021 \\
\hline (HuO) & 188.51 & 251.1 & 304.1 & 346.3 & 382.41 & 414.8 & 44.8 & 473 & 500.1 & 526.3 \\
\hline \multirow[t]{3}{*}{ (1+801L) } & 1517.7 & 1527.5 & 1518.8 & 1503.4 & 7524.9 & 1528.2 & 1521.8 & 1520.4 & 1523.5 & 1510.1 \\
\hline & & & & & & & & & & \\
\hline & ZONE2 & $\mathrm{ZONE} 4$ & ZONEE & ZONEB & ZONETO & & & & & \\
\hline DPliquid Temp.f & \begin{tabular}{|l|}
74.9 \\
\end{tabular} & & & & & & & & & \\
\hline DPLiquid Densityetbm/13 & 91.14 & & & & & & & & & \\
\hline Elevation-l & 2 & 4 & 6 & 8 & 9.875 & & & & & \\
\hline Moasured DPFsid & 0.201 & 1.394 & 2.205 & 2.972 & 3.846 & & & & & \\
\hline Zoro Corrections-osid & 0.009 & 0.044 & 0.029 & 0.018 & -0.074 & & & & & \\
\hline Corrocted DPPsid & 0.192 & 1.35 & 2.1761 & 2.554 & 3.92 & & & & & \\
\hline Actual DPA & 2.061 & 2.27 & 3.12 & 4.05 & 4.48 & & & & & \\
\hline Mensured TPPsig & 167.6 & & & & 173.3 & & & & & \\
\hline \multirow[t]{2}{*}{ Messured Overalli } & -11.14 & & & & & & & & & \\
\hline & & & & & & & & & & \\
\hline Computed DPA & 1.48 & 2.62 & 3.2 & 4.56 & 5.42 & & & & & \\
\hline Friction-t & 0.06 & 0.18 & 0.35 & 0.56 & 0.79 & & & & & \\
\hline Accolerationth & 0.06 & 0.1 & 0.16 & 0.23 & 0.31 & & & & & \\
\hline Elevation-f & 1.36 & 2.303 & 3.111 & 3.7 & 4.331 & & & & & \\
\hline
\end{tabular}




\begin{tabular}{|c|c|c|c|c|c|c|c|c|c|c|}
\hline Run\# & 380 & & & & . & & & & & \\
\hline test fluid & TR114 & & & & & & & & & \\
\hline Date: & अ593 & & 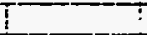 & 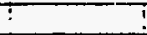 & $!$ & & & & & \\
\hline Time: & 15:38 & & & $\vec{B}$ & & $i$ & i & $!$ & & \\
\hline Note & TABOREK & K Analytical & & 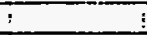 & $\overline{1}$ & 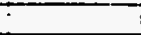 & $t$ & i & & \\
\hline Fow Areate? & 0.00336 & & 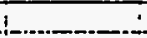 & 1 & \pm & $\because$ & $\therefore$ & $T$ & & $\because$ \\
\hline Hoat Flow Aroant & 0.19695 & & & & $:$ & $i$ & & $\mathrm{~T}$ & & $i$ \\
\hline Sat. Pressure-Psig & 169.2 & & & i & $i$ & 1 & & $T$ & & \\
\hline Sad. Temperature-f(inlel) & 202.7 & & & & & & & & & \\
\hline Ĺg. Densitylbmiti & 75.16 & 74.8 & $74.72 !$ & 74.781 & 74.781 & 74.72 & 74.72 & 74.73 & 74.73 & 74.85 \\
\hline Ladent Heat-8iulbm & 40.16 & 39.87 & $39.73 !$ & 39.8 & 39.8 & 39.73 & 39.78 & 39.75 & 39.75 & 39.86 \\
\hline Specific Head-Btu/bm/F & 0.281 & 0.282 & 0.282 & 0.2821 & 0.282 & 0.282 & 0.282 & 0.282 & 0.282 & 0.282 \\
\hline ig. Viscositylbminhr & 0.456 & 0.437 & 0.43 & $0.43 \pi$ & 0.4317 & 0.43 & 0.43 & 0.437 & 0.4311 & 0.432 \\
\hline Liq. Conductivity tumprif & 0.0267 & 0.0265 & 0.0264 & 0.02641 & 0.0264 & 0.0264 & 0.0264 & 0.0264 & $0.0 \leq 04$ & 0.0205 \\
\hline Lig. Prandt No. & 4.59 & 4.6 & 4.61 & 4.6 & 4.6 & 4.6 & 4.6 & 4.6 & 4.6 & 4.6 \\
\hline Inlet Fow Rato-GPM & 2.8 & & $i$ & 3 & 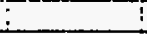 & 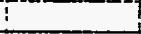 & & & & T \\
\hline Outel Lig. Fow Rato-GPM & 7.9 & & & & 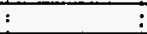 & 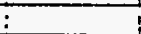 & 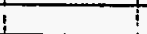 & & & $\mathrm{i}$ \\
\hline Outtel Vap. Fow Ralo-GPM & 0.9 & & & 1 & 4 & 1. & & & & \\
\hline inlai Tomporaturef & 198.6 & & & 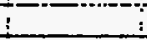 & $\mathrm{i}$ & i & 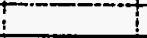 & & & \\
\hline Outel Liq. Temperaturef $F$ & 201.3 & & . & . & i & i & . & & I & T \\
\hline Outlel Vap. Temperaturef & 200.7 & & & $:$ & 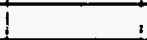 & & & & & - \\
\hline Water Flow Rato-GPM & $\vdots$ & & & & & & & & & \\
\hline Wedor hilot Temp.f & 88.8 & & & & i & & & & & \\
\hline Water outlet Temp.F & 197.5 & & & & $+\ldots$ & & & & & \\
\hline Thlat Gbmbihlite & 512407 & & Ttomp & T90.3 & Density & 76.68 & & & & \\
\hline Subcooling ff & 4.1 & & & & 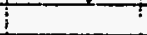 & & & & & \\
\hline \multirow[t]{2}{*}{ Fow Obsenvation } & i & & & t & ! & ! & & & & \\
\hline & ZONE1 & ZONEZ & ZONE3 & ZZONE4 & ZONES & IZONEB & ZONET & ZONEB & ZONE9 & ZOONEIO \\
\hline Hoat Input-Wats(net) & ! 875.4 & 11876.2 & 875.3 & 860.7 & 879.1 & 880.5 & 875.31 & 878.5 & 888.9 & 878.6 \\
\hline Hoat Loss-Wells & 53.2 & 11.2 & 13.4 & 10.6 & 9.8 & 10.2 & 13.7 & 16.9 & 17.7 & 83.3 \\
\hline Hoa Fux-Btunilin & $15174 !$ & 15188 & $75 \overline{173 !}$ & $14 \overline{920}$ & 152391 & 15263 & 15173 & 15184 & 154007 & 16184 \\
\hline Wall Tamp. HF & 210.4 & 2124 & 210.91 & 211.1 & 213.6 & 212.7 & 213.2 & 212.7 & 212.6 & 211.8 \\
\hline Wall Temp. \#4F & 210.9 & 270.4 & 2126 & 212.1 & 212.9 & 212.5 & 213.7 & 213.7 & 213.6 & 211.6 \\
\hline Wall Tomp. \#5F & 210.4 & 208.71 & 213.4 & 211.3 & 2111 & 213 & 212.6 & 212.2 & 211.1 & 211.3 \\
\hline Wall Temp. If & 75.3 & 210.9 & 209.8 & 210.8 & 210.7 & 214.5 & 213.7 & 213.6 & 210.7 & 208.1 \\
\hline Walf Temp. \#2F & 211.5 & 210.8 & 210.3 & 212.2 & 210.7 & 215.6 & 214.2 & 214.2 & 212 & 210.1 \\
\hline Instream Temp.f & 199.9 & 202.11 & 2026 & 2022 & 2022 & 202.61 & 20261 & 202.5 & 2025 & 201.8 \\
\hline Avg. Wall Temp.F & 210.8 & 211.11 & 210.9 & 211.3 & 217.3| & 213.2) & 273.5 & 213.3 & रारा & 210.6 \\
\hline Avg. Wall DT.F & 10.2 & 8.3 & 7.6 & 8.5 & 8.41 & 9.9 & 10.2 & 10.1 & 8.8 & 8.1 \\
\hline Romaining Subcooff & -2.11 & & & & & & & & & \\
\hline Exit Quality & 0.011 & 0.08 & 0.11 & 0.14 & $0.19 \dagger$ & $0.28 !$ & 0.28 & 0.52 & 0.381 & 0.41 \\
\hline QXP.HBtuAHREF & 1485.6 & 1821.5 & 1992.71 & 1785.61 & 1806.3 & $15+4$ & 1488.4 & 1505.3 & 1760.2 & 0 \\
\hline \multicolumn{11}{|l|}{ COMPUIEDH } \\
\hline Jallouk & 1055.8 & 1067.1 & 9052 & 1019.2 & 1017.1 & 1006.4 & 991.9 & 983.1 & 982.9 & 961.9 \\
\hline Szady 1 (Cudo tubo) & 1521.7 & 1539.61 & 1534.11 & 1521.2 & 1539 & 1543.4 & 1540.7 & 1543.3 & 1555.9 & 1544.8 \\
\hline Szady2 & 1383.2 & 1394.3 & 1396.3 & 1379.9 & 1374.8 & 1379.9 & 1376.4 & 1379.5 & 1394.9 & 1381.7 \\
\hline (RUQ) & 76374 & $\overline{74867}$ & 716541 & 68162 & 64791 & 61495 & 58087 & 54659 & 51232 & 47656 \\
\hline (F) & 1.262 & 1.6541 & 2.02 & 2305 & 2.545 & 2.755 & 2.3481 & 3.128 & 3.297 & 3.469 \\
\hline (माएO) & 214.9 & 281.71 & 344.7 & 392.7 & 435.51 & 469.2 & 50221 & 532.9 & 561.6 & 500.8 \\
\hline \multirow[t]{3}{*}{ (HBOIL) } & 1520.31 & 1528.4 & $1528.3 !$ & 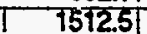 & $1527.5 !$ & 1528.8 & 1522.7 & 1521.8 & 1531.1 & 1515,5 \\
\hline & & $T$ & & & & & & & & \\
\hline & ZONE2 I & TZOONE4 & ZONE 6 & ZONE8 & ZONETO & & & & & \\
\hline DP liquid Temp.f & $i \quad 75.31$ & & & $i$ & & & & & & \\
\hline DPLiquid Densiy-lom/it3 & 91.001 & & & 1 & & & & & & \\
\hline Elevationfli & $--2 i$ & $4 \mid$ & 61 & $-\overline{8}$ & 9.875 & & & & & \\
\hline Messured DPPsid & 0.233 & 1.139 & 1.857 & 2.506 & 3.246 & & & & & \\
\hline Zero Corrections-psid & 0.009 & 0.044 & 0.029 & 0.018 & -0.074 & & & & & \\
\hline Comected DPPsid & 0.224 & 1.087 & 1.828 & 2.488 & 3.32 & & & & & \\
\hline Actual DPh & & 2.78 & 3.79 & -4.95 & 5.63 & & & & & \\
\hline Measured TPPsig & 169.2 & & & & 174.6! & & & & & \\
\hline \multirow[t]{2}{*}{ Measured Overalifi } & -10.39 & & & & & & & & & \\
\hline & & & & & & & & & & \\
\hline Compured DP्A & 1.72 & 3.07 & 4.32 & 5.52 & 6.641 & & & & & \\
\hline Friction- & 0.09 & 0.25 & 0.491 & 0.78 & 1.1 & & & & & \\
\hline Acoelerationm & 0.081 & 0.15 & 0.211 & 0.29 & 0.38 & & & & & \\
\hline \multirow{2}{*}{ Elovation } & 1.56 & 2.67 & उ.ू্ & 4.46 & 5.16 & & & & & \\
\hline & & & & & & & & & & \\
\hline
\end{tabular}




\begin{tabular}{|c|c|c|c|c|c|c|c|c|c|c|}
\hline Run: & 3811 & & & & 1 & 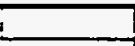 & & 1 & & i. \\
\hline Tost Fuid & TR114: & & $\therefore$ & & & & 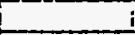 & i & & \\
\hline Date: & $1 \quad 3 / 5 / 31$ & & $\therefore$ & & + & 1 & - & $i$ & L & \\
\hline Time: & $16: 101$ & & 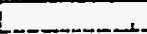 & & & 1 & $!$ & & & I \\
\hline Note & TABORER & Anarytical & dt & 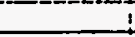 & 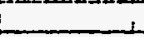 & & & & & 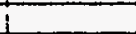 \\
\hline Fow Area-te? & 0.003361 & & & & & & & i & & \\
\hline Heat Flow Areatt2 & 0.196981 & & & & & & & & & \\
\hline Sat. Pressurepsig & 169.8 & & & & & & & & & \\
\hline Sâ. Temperaturo-f(inloi) & 202.9 & & & 1 & 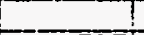 & 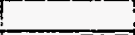 & & & & \\
\hline La .Density & 75.05 & 74.87 & 74.75 & 74.7 & 74.71 & 74.7 & 74.73 & 74.73 & 74.751 & 74.87 \\
\hline Lalont Hoareturbm & 40.051 & 39.88 & 39.781 & 39.71 & 39.711 & 39.71 & 39.75 & 39.75 & 39.76 & 39.88 \\
\hline Spocitio HoatBtu/bmiF & 0.2821 & 0.282 & 0.2821 & 0.282 & 0.282 & 0.282 & 0.2821 & 0.282 & 0.282 & 0.282 \\
\hline Lig. Visoosity & 0.434 & 0.432 & 0.4311 & 0.43 & 0.431 & 0.43 & 0.4371 & 0.431 & $0.43 i$ & 0.432 \\
\hline Liq. Conduetivity Btumr-thF & 0.02651 & 0.0265 & 0.02641 & 0.0264 & 0.02641 & 0.0264 & 0.02641 & 0.0264 & 0.02641 & 0.0265 \\
\hline Lig. Prandid No. & 4.61 & 4.6 & 4.61 & 4.6 & 4.61 & 4.6 & 4.61 & 4.6 & 4.6 & 4.6 \\
\hline Inlelflow Rato-GPM & 3.7 & & & & 1 & 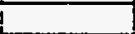 & & & & 1 \\
\hline Outel Liq. Flow Rado-GPM & 2.31 & & & & & & & & & \\
\hline Outtel Vap. Flow Rado-GPM & 0.9 & & i & i & $\div$ & 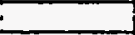 & & & & \\
\hline Inlet Temperaturef & 1991 & & & & & & & & & \\
\hline 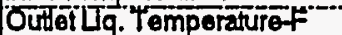 & $207.7 !$ & & & & & & & & & \\
\hline Outiet Vap. Temperaturof & 200.3 & & & & & & & & & \\
\hline Water Fiow Rato-GPM & 1 & & ! & 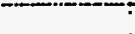 & t & $f$ & & & & \\
\hline Wator inlel Temp, $F$ & 88.91 & & 1 & 1 & $T$ & $\Gamma$ & $!$ & $!$ & & \\
\hline Water outlof Tomp, F & 197.7 & & $i$ & 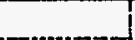 & i & 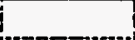 & 1 & 1 & & \\
\hline Inlat Gibmininte & 673949 & & TTemp & 192.6 & Density & 76.33 & 1 & & & \\
\hline Subooolingf & 3.91 & & 1 & $-2-1$ & 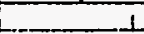 & 5 & 1 & & & \\
\hline \multirow{2}{*}{ Fow Observation } & & & & & & & & & & \\
\hline & ZONE1 & ZONE2 & ZONE3 & ZONE4 & ZONE5 & ZONE6 & ZONE? & ZONE8 & ZONE9 & ZONE10 \\
\hline Hoat Input-Watts(not) & 873.5 & 873.6 & 868.11 & 864.4 & 873.7 & 871.8 & 875.4 & 876 & 874.2 & 872 \\
\hline Heat Loss-Watts & 53.2 & 11.2 & 13.3 & 10.4 & 9.7 & 10.1 & 13.6 & 15.8 & 17.6 & 83 \\
\hline Hoat Flux-BtuhHihe & 15142 & 15143 & 15048 & 14984 & 15145 & 15112 & 15174 & 15185 & 15154 & 15116 \\
\hline Wall Temp. "3F & 210.7 & 2125 & 211.21 & 211.5 & 213.8 & 213.2 & 213.1 & 2125 & 212.4 & 211.7 \\
\hline Wal Tomp $4 \mathrm{~F}$ & 211.3 & 210.6 & 212.9 & 2124 & 213.2 & 213 & 213.7 & 213.6 & 213.4 & 211.7 \\
\hline Wall Temp. $5 F$ & 210.6 & 208.9 & 213.21 & 211.8 & 271.61 & 213.2 & 212.5 & 212 & 210.9 & 211.7 \\
\hline Wall Temp. "1F & 75.11 & 211 & 209.7 & 211.5 & 211.11 & 214.6 & 213.7 & 213.6 & 210.4 & 208.1 \\
\hline Wall Tomp. Na & 211.8 & 210.8 & 210.81 & 212.5 & 211.41 & 215.7 & 274.7 & 214.1 & 211.7 & 200.8 \\
\hline Inotroem Tomp, F & 200.61 & 201.7 & 20241 & 2027 & 2027 & 2027 & 20251 & 2026 & 202.4 & 201.7 \\
\hline Avg. Wall Tomp.F & 211.1 & 211.2 & 211.21 & 211.8 & 211.81 & 213.5 & 213.41 & 213.2 & 271.81 & 210.6 \\
\hline Avg. Wall DT.F & 9.8 & 8.8 & 8.91 & 8.4 & 8.41 & 10.1 & 10.2 & 10 & 8.71 & 8.2 \\
\hline Remaining Subcooff & -0.7 & & & & & & & & & \\
\hline Exot Quality & 0.01 & 0.04 & 0.07 & $\overline{0.11}$ & 0.14 & 0.17 & 0.2 & 0.24 & 0.27 & 0.3 \\
\hline EXP.HEtuH H & 1542.6 & 17129 & 1864.7 & 1778.9 & 1794.3 & 1493.7 & 14827 & 15225 & 1746.8 & 0 \\
\hline \multicolumn{11}{|l|}{ COMPUTEDH } \\
\hline Jalouk & 7078.3 & 1053 & 7018.9 & 986.8 & $\$ 87.6$ & 967.3 & 963.9 & 956.4 & $\$ 50$ & 935.6 \\
\hline Sżadyi(Cudo tube) & 15229 & 1528.4 & 1528.3 & 1528.3 & 1538.7 & 1539.4 & 154.2 & 1547.3 & 1548.1 & 1546.6 \\
\hline seady2 & 1384.5 & 1390.1 & 1388.5 & 7386.9 & 1373.91 & 1374.6 & 1380.5 & 1384.1 & 1384.8 & 1383.2 \\
\hline (PLQ) & 101249| & 99804 & 967581 & 93528 & s01501 & 88756 & 83284 & 798883 & 78452 & 72898 \\
\hline (F) & 1.141 & 1.485 & 1.8221 & 2072 & 2.284 & 2.471 & 2.642 & 2.799 & 2946 & 3.002 \\
\hline (H) & 241.8 & 314.8 & 386.71 & 439.5 & 484.4 & 524.1 & 560.5 & 533.6 & 624.8 & 656.8 \\
\hline \multirow[t]{3}{*}{ (1BÖIL) } & 1520.9 & 1524 & 1520 & 1516.1 & 152251 & 1518.9 & 1519.2 & 1517.6 & 1513.4 & 15062 \\
\hline & & & & & & & & & & \\
\hline & ZONE2 & ZONE4 & ZONE6 & ZONE8 & ZONE10 & & & & & \\
\hline \multicolumn{11}{|l|}{ DP liquid Temp.f } \\
\hline \multicolumn{11}{|l|}{ DPLiquid Density-lbm/th3 } \\
\hline \multicolumn{11}{|l|}{ Elevation-h } \\
\hline Measured DPF sid & 0.1901 & 0.539 & $1.617 !$ & 2.739 & $2.757 !$ & & & & & \\
\hline Zoro Corrections-psid & 0.009 & 0.074 & 0.029 & 0.018 & -0.0741 & & & & & \\
\hline CorrectodDPPald & 0.191 & 0.895 & 1.588 & 2.1211 & 28371 & & & & & \\
\hline Actual DPA & 2.07 & 3.14 & 4.241 & 5.65 & 8.501 & & & & & \\
\hline Measurod TPPsig & 169.8 & & 1 & 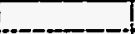 & 174.91 & & & & & \\
\hline \multirow[t]{2}{*}{ Mosaured Ovarafin } & -0.811 & & 1 & 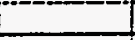 & & & & & & \\
\hline & & & & & & & & & & \\
\hline Computad DPA & 7.91 & 3.46 & 4.931 & 6.38 & 7.75 & & & & & \\
\hline Friction-t & 0.12 & 0.34 & 0.65 & 1.03 & 1.46 & & & & & \\
\hline$A$ ccoleration-t & 0.11 & 0.2 & 0.29 & 0.38 & 0.47 & & & & & \\
\hline Elovation-ft & 1.67 & 2.92 & 4 & 4.98 & 5.82 & & & & & \\
\hline & & & & & & & & & & \\
\hline
\end{tabular}




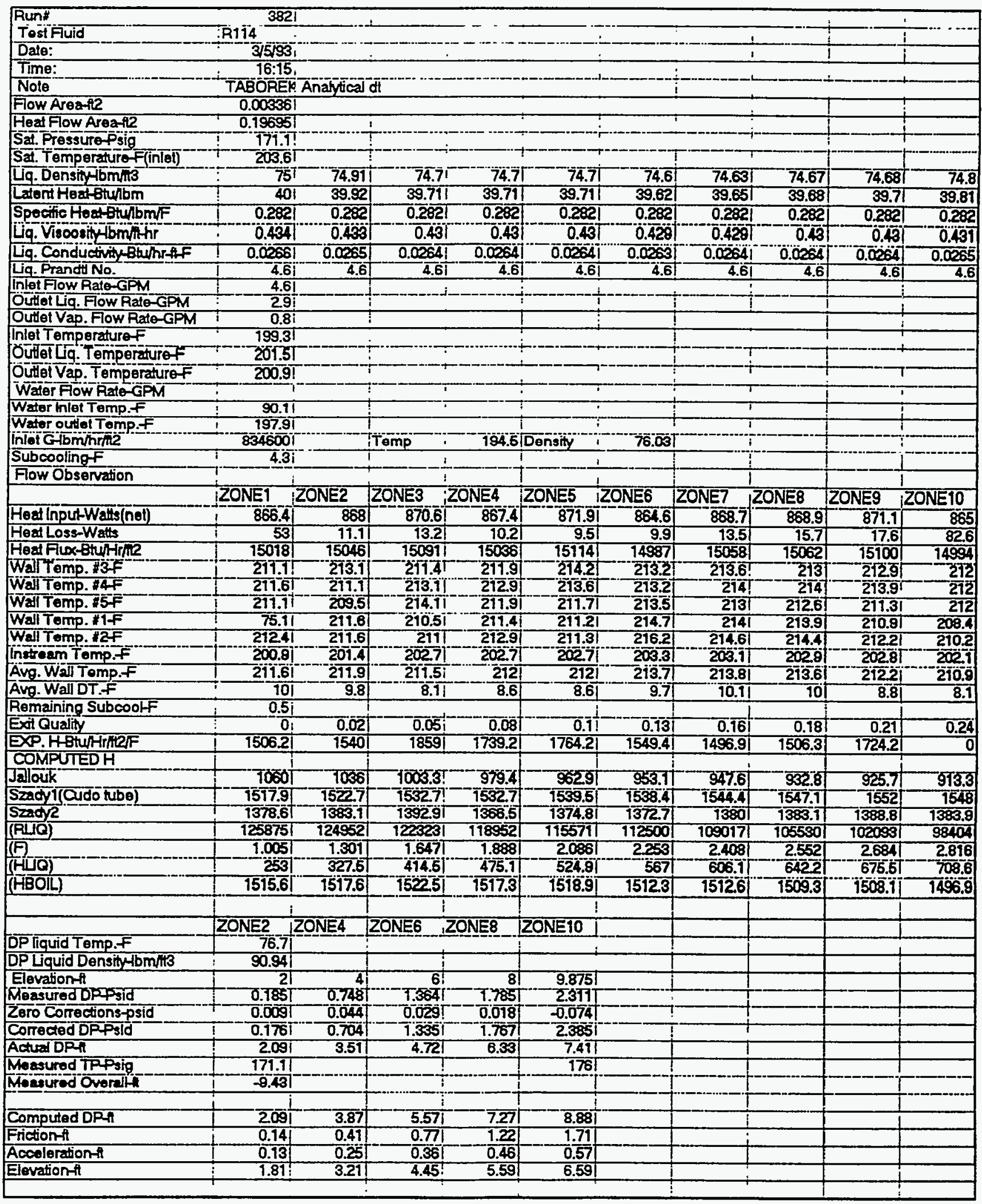




\begin{tabular}{|c|c|c|c|c|c|c|c|c|c|c|}
\hline Run & $383 !$ & ! & & & & $\therefore$ & & & & \\
\hline Test Fuid & 17114 & & & $!$ & : & $i$ & 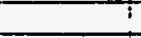 & & & \\
\hline Da10: & 35531 & $!$ & & & & $i$ & I & tis & & \\
\hline Time: & 16.57 & 1 & & & & & + & 1 & 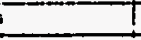 & \\
\hline Noto & ¿TABOREK & Analytical c & & & $\vdots$ & & $T$ & 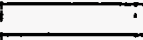 & & i \\
\hline Flow Area-tt2 & i 0.003361 & & & & ! & $i$ & I & I. & 1. & \\
\hline Heat Flow Aros & $0.19655 i$ & & 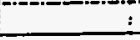 & $\perp$ & 1 & $i$ & $i$ & $i$ & & \\
\hline S2. Pressure Prig & 165.2 & & & $i$ & 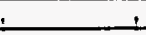 & $!$ & & 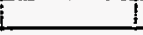 & 1 & 1 \\
\hline S21. Temperaturef(inlet) & 200.7 & & & & $!$ & 1 & 1 & $t$ & & $t$ \\
\hline Lq. Density lbmit3 & 75.34 & 75.29 & 75.14 & 75.161 & 75.16 & 75.1 & 75.1 & 75.11 & 75.16 & 75.24 \\
\hline Latent Heatetulbm & 40.34 & $40 . \overline{29}$ & 40.15 & 40.16 & 40.16 & 40.1 & 40.1 & $40.12 !$ & 40.16 & 40.24 \\
\hline Spectio Hear BtuntomiF & 0.281 & 0.2811 & 0.281 & 0.281 & 0.2811 & 0.281 & 0.2811 & 0.281 & 0.281 & 0.281 \\
\hline Lq. Viscosity & 0.4381 & 0.437 & 0.435 & 0.4365 & 0.4361 & 0.435 & 0.435 & 0.435 & 0.456 & 0.437 \\
\hline Lq. Conductivity Btunr-fiF & 0.02681 & 0.0268 & $0.0267 !$ & 0.0267 & 0.0267 & 0.0265 & 0.0265 & 0.02601 & 0.0287 & 0.0267 \\
\hline Lq. Prandt No. & 4.591 & 4.59 & 4.591 & 4.59 & 4.59 & 4.59 & 4.59 & 4.59 & 4.591 & 4.59 \\
\hline Inlot Flow Pato-GPM & 5.51 & & 7 & -1 & 1 & & & & & 1 \\
\hline Outret Lq. Flow Rato-GPM & 3.31 & & 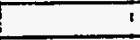 & $!$ & $!$ & & & 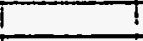 & T & I \\
\hline Outlot Vap. Flow Rato-GPM & 0.9 & & 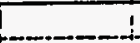 & 4 & 1 & & & & & $T$ \\
\hline Intof Tomperature & 197.21 & & & $i$ & 1 & & & & $i$ & $i$ \\
\hline Outlat Liq. Temparaturef & 198.61 & & & $i$ & $i$ & 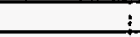 & & I & I & I \\
\hline Outtel Vap. Temperaturef & 197.7 & & & & $\perp$ & & & 1 & & \\
\hline Wader Flow Rato-GPM & $i$ & & & & & & & & $\perp$ & 4. \\
\hline Waler inlol Temp.f & 80.9 & & & & & & & & & it \\
\hline Wator outlel Temp.f & 195 & & & & & & & & & \\
\hline Infot $\mathrm{Gl} / \mathrm{bm} / \mathrm{hr} / \mathrm{L} 2$ & 1002637 & & Tomp & 192.2 & Density & 76.391 & & & & \\
\hline SubcoolingF & 3.5 & & & & & & & & & \\
\hline Flow Observation & & & & & & & & & & \\
\hline & ZONEI T & ZONE2 & ZONES & ZZONE4 & ZONE5 & ZONE 6 & ZONET I & ZONE & ZONES & TZONE10 \\
\hline Head Input-Walls(net) & 875.2 & 874.4 & 859.8 & 868.9 & 870.4 & 888 & 878.2 & 878.2 & 870.7 & $1 \quad 888.1$ \\
\hline HedLoss-Wets & 61.7 & 10.71 & 12.8 & 10 & 9.11 & 9 & 12.9 & 16.1 & 17.1 & 80.9 \\
\hline 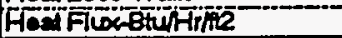 & 161719 & 961571 & 36077 & 15027 & 160881 & 16012 & 161881 & 15223 & 160031 & 16013 \\
\hline Wall Tamp. & 208.7 & 210.61 & 209.31 & 2091 & 211.3 & 210.2 & 2111 & 210.41 & 210.3 & 209.7 \\
\hline Wall Temp. d4F & 209.2 & 200.6 & 210.91 & 210.11 & 210.7 & 210.1 & 211.51 & 211.5 & 2112 & 200.1 \\
\hline Wall Temp. & 208.5 & 207.2 & 211.3 & 209.4 & 209.2 & 210.7 & 210.3 & 209.9 & 208.9 & 200.3 \\
\hline Wall Temp. ItF & 77.3 & 200 & 207.8 & 209 & 208.3 & 2122 & 211.5 & 211.4 & 208.5 & 205.7 \\
\hline Wall Temp. & 209.9 & 208.8 & 208.8 & 210.4 & 208.9 & 213.41 & 212 & 211.9 & 209.6 & 207.7 \\
\hline Instream Temp.f & 198.8 & 199.1 & 2001 & 199.9 & 199.9 & 200.31 & $200 . \overline{3}$ & 2003 & 199.81 & 199.4 \\
\hline Avg. Wal Temp.F & 209.1 & 209.31 & $203.2 !$ & 209.4 & 209.3 & 210.8 & 211.3 & 217 & 209.7 & 200.3 \\
\hline Avg.Wall DT.F & 9.61 & 9.5 & 8.51 & 8.81 & 8.7 & 9.8 & 10.3 & 10.1 & 9.1 & 8.2 \\
\hline Romaining Subcooff & 0.41 & & & & 1 & -1 & 1 & i & & \\
\hline Exat Quality & 01 & 0.02 & 0.041 & 0.061 & 0.091 & 0.111 & 0.131 & 0.15 & 0.17 & 0.2 \\
\hline 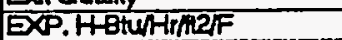 & 158211 & 1601.4 & 1768.91 & 1708.41 & 1735.61 & 1528.4 & 1478.4 & 1502.5 & 1656.3 & 0 \\
\hline COMPUTEDH & & & & & & & & & & \\
\hline Jallouk & 1037.2 & 1008.5 & 971.4 & 949.1 & 941.2 & 9232 & 918.6 & 910.3 & 898 & 887.5 \\
\hline Szady1(Cudo tubo) & 1518.7 & 1521.2 & 1524.21 & 1525 & 1531.9 & 1533.4 & 1546.2 & 1551.9 & 1549.1 & 1548.4 \\
\hline Szady2 & 1378.7 & 1355.7 & 1358.4 & 1359.2 & 1367.3 & 1358.5 & 1383.5 & 1389.7 & 1385.7 & 1384.2 \\
\hline (स्व) & 149838 & 14873 & 146056 & 142609 & 139400 & 136341 & 133025 & 129625 & 126130 & 122563 \\
\hline$(F)$ & 1.005 & 1.283 & 1.555 & 1.814 & 1.994 & 2.148 & 2.29 & 2421 & 2.546 & 2.664 \\
\hline (मीएO) & 2931 & 374 & 455 & 528.7 & 581.2 & 625.2 & 667.4 & 705.8 & 441.9 & 76.4 \\
\hline$(\mathrm{HBO})$ & 1515.11 & 1513.6 & 1509.6 & 1503.5 & 1503.5 & 1497.8 & 1503.6 & 1507.6 & 1490.2 & 1480.4 \\
\hline & & & & & & & & & & \\
\hline & ZONE2 TI & ZONE4 & 12ONEB & ZONE8 & ZONE10 & & & & & \\
\hline DP liquid Temp.F & $\pi 7.7$ & & & $!$ & & 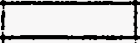 & & & & \\
\hline DPUquid Densinglbmin & 90.84 & & & $i$ & T. & & & & & \\
\hline Elovation-a & -21 & $-\frac{4}{4}$ & 61 & 8 & 9.875 & & & & & \\
\hline Masured DPPaid & 0.171 & 0.723 & 1.1491 & 1.479 & 1.8911 & & & & & \\
\hline Zero Corrections-psid & 0.009 & 0.044 & 0.029 & 0.018 & $=0.0741$ & & & I & & \\
\hline Corrected DPPsid & 0.162 & 0.679 & 1.12 & 1.4611 & 1.965 & & & & & \\
\hline Actual DP-A & 2.1 & 3.53 & 5.1 & $6 . \overline{86}$ & 8.16 & & & & & \\
\hline Measured TPPsig & 165.2 & & & & 169.7 & & & & & \\
\hline Measured overalin & $-8.6 \pi$ & & & & & & & & & \\
\hline & & & & & & & & & & \\
\hline Computed DPf & 2.22 & 4.15 & 6.01 & 7.89 & 9.69 & & & & & \\
\hline Fitcton-n & $0 . \overline{191}$ & 0.54 & 1 & 7.58 & $2.21 \dagger$ & & & & & \\
\hline Aocoleration-A & 0.171 & 0.34 & 0.46 & 0.59 & 0.72 & & & & & \\
\hline Elovation $\mathrm{ft}$ & 1.861 & 3.28 & 4.55 & 5.72 & 6.76 & & & & & \\
\hline
\end{tabular}




\begin{tabular}{|c|c|c|c|c|c|c|c|c|c|c|}
\hline Runt & 384 & & & & & & & 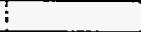 & & 1 \\
\hline Test Fivid & R114 & & i & i & & & & & & \\
\hline Date: & $3 \sqrt{53}$ & I & T! & & & $T$ & & & & \\
\hline Time: & 19:15 & & $i$ & T & & 1 & & & $i$ & \\
\hline Flow Areatll2 & 0.0038 & & 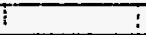 & $\vdots$ & $\vdots$ & ț & 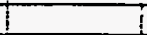 & 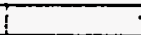 & & \\
\hline Head Flow Areaft? & 0.19655 & & 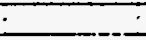 & : & & - & 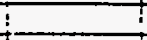 & $!$ & & I \\
\hline Sal. Prossura Dsig & 183 & & . & - & & 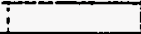 & & 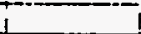 & 1 & \\
\hline Sal. Tomperaturo-f(inlel) & 213.6 & & . & . & & & & & 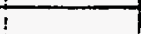 & \\
\hline Lig. Densitylbmfit3 & 75.61 & 75.711 & $75.73 i$ & 75.691 & 75.65 & 75.27 & 73.071 & 72.99 & 72.99 & 73.03 \\
\hline Latent Heat-Bturibm & 40.61 & 40.7 & 40.72 & 40.69 & 40.64 & 40.28 & 38.061 & 38.04 & 38.04 & 38.08 \\
\hline Specitic Hear-Btulbmp & 0.28 & $0 . \overline{28}$ & 0.28 & $0 . \overline{28}$ & 0.28 & 0.281 & 0.286 & 0.286 & 0.286 & 0.286 \\
\hline Liq. Viscosity lbmilithr & 0.441 & 0.442 & 0.442 & 0.442 & 0.441 & 0.437 & 0.49 & 0.41 & 0.41 & 0.41 \\
\hline Lig. Conductivity-BtuhrffF & 0.002 & 0.027 & $0 . \overline{027}$ & 0.027 & 0.027 & 0.0267 & 0.0254 & 0.0254 & 0.0254 & 0.0254 \\
\hline Liq. Prand No. & 4.59 & 4.58 & 4.581 & 4.581 & 4.59 & 4.59 & 4.62 & 4.62 & 4.62 & 4.62 \\
\hline Inlet fow Rato-GPM & 7.5 & & & & 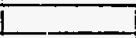 & & & & & \\
\hline Outel Lg. Fow Rato-GPM & $7 . \overline{4}$ & IT & i & i & & & 1 & & & \\
\hline Outlot Vap.Fow Rato-GPM & 0.7 & & ? & ; & & $i$ & I & 1 & & \\
\hline Inlot Temperaturof & 196.1 & & $i$ & & & $\cdot$ & $:$ & $i$ & & \\
\hline Outiot Liq. Temperatur of & 212.2 & & $\bar{i}$ & ! & & . & : & & & \\
\hline Outiet Vap. Temperaturef & 291.1 & & $\vdots$ & 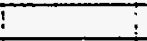 & & $\vdots$ & $i$ & & & \\
\hline Water Flow Ralo-GPM & & & & I & I & & 1 & & & \\
\hline Water hlot Temp.f & 187.5 & & 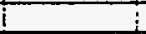 & 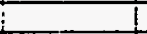 & & & & & & \\
\hline Water outlet Temp.F & 197.8 & & $i$ & & & & & & & \\
\hline Indot Glbmpritit2 & 272491 & & Temp & 193.9 & Density & 76.12 & & & & \\
\hline Subcooling & 17.5 & & :-L & 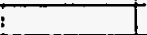 & & & & & & \\
\hline \multicolumn{11}{|l|}{ How Observation } \\
\hline & ZZONET & ZONEZ & ZONES & ZOONE4 & ZONE5 & IZONE6 & ZONET & ZONE8 & ZONE9 & ZONETO \\
\hline Heat inpur-Watts(net) & $T-1$ & $1-20$ & 20.81 & 20.5 & 20.4 & 1624.4 & 1684.8 & 1714.2 & 1686.3 & 1088.3 \\
\hline Hoat Loss-Wats & 46.2 & 9.8 & 11.81 & 10 & 9.3 & 14.2 & 181 & 18.1 & 19.7 & 93.8 \\
\hline Hoed Floretu/Hith2 & 17 & 347 & 357 & 356 & 354 & 28168 & 28868 & 29716 & 292311 & 18966 \\
\hline Wall Tomp. \#3F & 188.2 & 189.1 & 189.7 & 189.8 & 206.1 & 227.4 & 227.6 & 229.31 & 2322 & 225.6 \\
\hline Wall Tomp. \#4F & 200 & 199.7 & 99.8 & 199.8 & 207.1 & 227.3 & 228.2 & 230.5 & 233.3 & 234.9 \\
\hline Wall Temp. I5- & 198.5 & 199.5 & 199.2 & 200.2 & 202.4 & 227.9 & 226.4 & 227.2 & 224.5 & 228.3 \\
\hline Wall Temp. $1 F$ & 76.7 & 199.8 & 199.2 & 199.8 & 200.4 & 230.9 & 228.3 & 228.9 & 223.8 & 221.4 \\
\hline Wal Temp. +2F & 195.2 & 200.11 & 799.2 & 198.6 & 200.1 & 235 & 228.8 & 228.9 & 226.6 & 223.2 \\
\hline Instream Temp.F & 197.1 & 196.5 & 196.4 & 196.6 & 196.9 & 199.2 & 2126 & 212.7 & 212.7 & 212.5 \\
\hline Avg. Wall Temp.f & 198.2 & 199.7 & 199.5 & 199.91 & 202.5 & 228.4 & 227.9 & 220 & 228.1 & 228.7 \\
\hline Avg.WaldT.F & 1.1 & 3.2 & 3.11 & 3.3 & 5.6 & 27.9 & 14 & 14.9 & 14.1 & 75.3 \\
\hline Remalning Subcooff & 17.41 & & 1 & 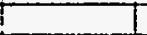 & & & & & & \\
\hline Ext Guality & 01 & 01 & 01 & 01 & 0 & 0 & of & 01 & 0 & 0 \\
\hline DP. HBtu/HTMaF & 15.41 & 109.71 & 116.7 & 108.2 & 63.3 & 1000.2 & 2067.8 & 1992 & 2079.2 & 1230.8 \\
\hline COMPUTEDH & & & 1 & $i$ & & I & & & & \\
\hline Jallouk & 84.41 & 84.41 & 84.4 & 84.4 & $84 . \overline{4}$ & 84.4 & 84.4 & 84.4 & 84.4 & 84.4 \\
\hline Szadyl(Cudo tube) & 104.91 & 106.5 & 107.3 & 108 & 108.7 & 109.2 & 109.3 & 109.8 & 110.3 & 110.7 \\
\hline Szady2 & 104.9 & 106.5 & 107.3 & 108 & 108.7 & 109.2 & 109.31 & 109.8 & 110.3 & 110.7 \\
\hline (RUQ) & 40423 & 40315 & 40294 & 40324 & 40377 & 40766 & 43418 & 43435 & 43432 & 43383 \\
\hline (F) & 1.021 & 1.0361 & 1.044 & 1.051 & 1.057 & 1.052 & 1.053 & 1.058 & 1.073 & 1.07 \\
\hline$(\mathrm{H} \perp \mathrm{LO})$ & 104.9 & 106.51 & 107.3 & 108 & 108.7 & 109.2 & 109.3 & 109.8 & 110.3 & 110.7 \\
\hline \multirow[t]{3}{*}{ (HBOIL) } & 160.5 & 4612 & 4461 & 480.7 & 828 & 4348.9 & 2304.71 & 2469 & 2323.6 & 2535.3 \\
\hline & & & 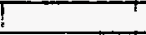 & & & & & & & \\
\hline & ZONE2 & ZONE4 & ZZONE6 & ZZONES & ZONE1O & & & & & \\
\hline DP llqudd Temp.f & 78.21 & & ! - - & T-C. & & & & & & \\
\hline DP Liquid Density & 90.79 & & & T & & i & & & & \\
\hline Elovetion-h & 2 & 41 & 61 & 81 & 9.875 & & & & & \\
\hline Measurod DPFsid & $0 . \overline{179 !}$ & $\overline{0.478}$ & 0.897 & 1.4611 & 2.336 & & & & & \\
\hline Zoro Corroctions-psid & 0.009 & 0.044 & 0.029 & 0.018 & -0.074 & & & & & \\
\hline Corrected DPPsid & 0.17 & 0.434 & 0.8681 & 1.443 & 2.41 & & & & & \\
\hline Actual DP-n & 2.15 & 4.12 & 5.75 & 7.11 & 7.52 & & & & & \\
\hline Moasured TPPsig & 193 & & & & 196.8 & & & & & i \\
\hline Measured Overallit & -7.49 & & & & & & & & & \\
\hline & & & & & & & & & & \\
\hline Computed DRA & 2.01 & 4.03 & 6.041 & 8.06 & 9.94 & & & & & \\
\hline Friction-n & 0.01 & 0.00 & 0.04 & 0.061 & 0.07 & & & & & \\
\hline Acooleration- $\mathrm{A}$ & 0 & 0 & 0 & 0 & 0 & & & & & \\
\hline \multirow[t]{2}{*}{ Elevation-it } & 2 & 4 & 6 & 8 & 9.87 & & & & & \\
\hline & & & & 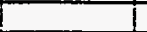 & 1 & I & & & & \\
\hline
\end{tabular}




\begin{tabular}{|c|c|c|c|c|c|c|c|c|c|c|}
\hline Run & $385 !$ & & i & 1 & & & & $i$ & $i$ & \\
\hline Test Fuid & R114 & & & & & & & & & \\
\hline Date: & $3 / 593$ & & 7 & i & & & & & & \\
\hline Time: & 19:17! & & $\vdots$ & 1 & I & 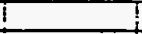 & & I & $!$ & \\
\hline Flow Aroatt? & 0.00338 & & $\overline{!}$ & t & I & & & $i$ & & \\
\hline Hoed Flow Areatic & 0.18698 & & $:$ & 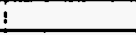 & & & 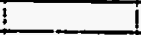 & I & & \\
\hline S. Prosecuro Prig & 203.61 & & $i$ & 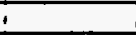 & & & i & & & \\
\hline Sad. Tomperatureff(inlal) & 218.1 & & & & & & & & & \\
\hline Ljg. Density-lbmitt3 & 75.39 & 75.52 & 75.51 & 75.45 & 75.37 & 75.03 & 72.17 & 72.15 & 72.19 & 72.22 \\
\hline Latent Hear-Btunbm & 40.39 & 40.511 & 40.5 & 40.45 & 40.37 & 40.04 & 37.24 & 37.22 & 37.25 & 37.29 \\
\hline Spectio HoatetullomiF & 0.281 & 0.281 & 0.281 & 0.281 & 0.281 & 0.282 & 0.287 & 0.287 & 0.287 & 0.287 \\
\hline Lig. Viscosity-lbmithr & 0.438 & 0.44 & 0.44 & 0.439 & 0.438 & 0.434 & 0.4 & 0.4 & 0.4 & 0.401 \\
\hline [iq. Conductivity Btumpalf & 0.02681 & 0.02091 & 0.0269 & 0.0269 & 0.0268 & 0.0266 & 0.0240 & 0.0249 & 0.0340 & 0.0269 \\
\hline Lq.PrandtNo. & 4.59 & 4.59 & 4.59 & 4.59 & 4.59 & 4.6 & 4.62 & 4.62 & 4.2 & 4.62 \\
\hline Inlolfow RAOGPM & $\mathbf{3 . 5}$ & & & & & & & & & \\
\hline Outol La. Fow Rato-GPM & 1.2 & & 1 & $!$ & & & & it & & \\
\hline Outiol Vap. Flow Rate-GPM & 0.6 & & & I & & & & & & \\
\hline Inlol Temperaturof & $197.6 !$ & & 1 & 1 & & & & & & \\
\hline Outlet Lig. Tomperaturef & 216.7 & & & $i$ & & & & 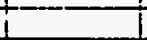 & & \\
\hline Outlet Vap. Temperaturef & 215.9 & & T! & 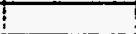 & & & & & & \\
\hline Waler Flow Rato-GPM & $i$ & & I & $i$ & & & & & & \\
\hline Water Inlot Tomp.f & 193.4 & & i & & & & & & & \\
\hline Water outlet Temp.F & 205.9 & & & & & & & & & \\
\hline Inlol G-lbmhrime & 269921 & & Temp & 198.4 & Density & 75.4 & & & & \\
\hline Suboooling & 20.51 & & & & & & & & & \\
\hline Flow Observation & & & & & & & & & & \\
\hline & ZONEI & ZONE2 & ZONES & ZONE4 & ZONES & ZONE6 & ZONE7 & IZONEB & ZONES & ZONETO \\
\hline Hoad Inpu-Wats(nel) & 0.3 & $\mid-30.6$ & 29.8 & ॐ.1 & 31.1 & 1620.2 & 1650 & 1697 & 168 & 1069.7 \\
\hline Hoar Loss-Watts & 47.31 & 10.1 & 121 & 10 & 9.2 & 15.4 & 18.8 & 18.9 & 20.8 & 100 \\
\hline Hodflow bturititie. & 5ा & 5301 & 517 & 522 & 539 & 28189 & 28602 & 29416 & 2944 & 18889 \\
\hline Wall Tomp. & 201.4 & 201.8 & 2024 & 202.7 & 200.7 & 231.6 & 232.11 & 233.91 & 245.6 & 248.0 \\
\hline Wall Tomp. 4 F & 201.9 & 20251 & 202.6 & 2027 & 210.9 & 231.4 & 2326 & 234.9 & 246.1 & 216 \\
\hline Wall Temp. $15+$ & 200.3 & 2022 & 201.9 & 202.9 & 206 & 232.1 & 230.9 & 231.8 & 230 & 230.5 \\
\hline Wall Temp."1F & 76.9 & 202.5 & 201.9 & 202.7 & 203.7 & 235 & 2325 & 233.2 & 228.9 & 23.7 \\
\hline Wall Temp. $2 f$ & 197.1 & 202.9 & 201.9 & 201.1 & 203.2 & 239.2 & 233.1 & 233.2 & 231.6 & 230.8 \\
\hline Instream Temp.f & 198.5 & 197.7 & 197.8 & 198.1 & 198.6 & 200.7 & 217.3 & 217.4 & 217.2 & 217 \\
\hline Avg. Wall Temp.f & 200.21 & 202.4 & 2022 & 2028 & 200 & 232.5 & 232 & 233.4 & 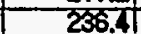 & 238.4 \\
\hline Avg.WalloT.F & 1.7 & 4.7 & 4.4 & 4.6 & 7.3 & 30.5 & 73.6 & 74.7 & 17.9 & 20.5 \\
\hline Romaining Subcoolf & 20.5 & & -1 & 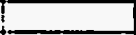 & & & & & & \\
\hline Ext Quality & of & 0! & का & 0 & 0 & 0 & of & 0 & of & 0 \\
\hline EXP. HBtüHrite & 3.11 & 112.8 & 118 & 112.8 & 73.6 & 923.4 & 2005.81 & 2005.1 & 1643.4 & 9202 \\
\hline COMPUTEDH & & & & & & & & & & \\
\hline Jallouk & 83.8 & 83.8 & 83.8 & 83.8 & 83.8 & 83.8 & 83.8 & 83.8 & 83.7 & 83.7 \\
\hline Szadyl(Cudo tubo) & 104.11 & 105.6 & 106.4 & 107.2 & 107.8 & 108.र्या & 108.3 & 108.8 & 109.3 & 100.7 \\
\hline Szacty2 & 104.1 & 105.6 & 106.41 & 107.2 & 107.8 & 108.3 & 108.3 & 108.8 & 109.3 & 109.7 \\
\hline (RLO) & 40282 & 40138 & 40151 & 40199 & 40281 & 40648 & 44038 & 44118 & 44055 & 44012 \\
\hline$(\bar{\theta}$ & 1.02 & 1.036 & 1.043 & 1.05 & 1.057 & 1.02 & 1.052 & 1.066 & 1.071 & 1.075 \\
\hline (HUO) & 104.1 & 105.6 & 106.4 & 107.2 & 107.8 & 108.3 & 108.3 & 108.8 & 109.3 & 100.7 \\
\hline (HBOLL) & 243 & 636.7 & 647.8 & 686.7 & TRO2.1 & 4803.1 & 2325.7 & 2507.4 & 307.4 & 3526.6 \\
\hline & & & & & & & & & & \\
\hline & ZONE2 & ZONEA & ZONE6 & ZONEB & ZONETO & & & & & \\
\hline DP Hquid Temp.F & 78.7 & 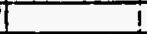 & 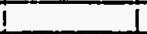 & & & & & & & \\
\hline DPLquid Donsity tbm/n 8 & 90.73 & & I & & & & & & & \\
\hline Elovationt & & 4 & 61 & 8 & 9.875 & & & & & \\
\hline Measured DPFid & 0.175 & 0.484 & 0.8961 & 1.453 & 2.308 & & & & & \\
\hline Zaro Conactions-psid & 0.009 & 0.044 & 0.0291 & 0.018 & -0.074 & & & & & \\
\hline Corrected DPPsid & 0.166 & 0.44 & 0.866 & 1.415 & 2.382 & & & & & \\
\hline Actual DPA- & 2.18 & 4.15 & 5.81 & 7.23 & 7.66 & & & & & \\
\hline Measured TPPsig & 203.6 & & & & 207.6 & & & & & \\
\hline Moasured Overallit & -7.98 & & & & & & & & & \\
\hline & & & & & & & & & & \\
\hline Computed DP- & 2.01 & 4.03 & 6.041 & 8.06 & 9.94 & & & & & \\
\hline Frictonf & 0.01 & 0.03 & 0.04 & 0.06 & 0.07 & & & & & \\
\hline Acoolerationt & of & 0 & of & 0 & 0 & & & & & \\
\hline Elevation & 2) & 41 & 81 & 8 & 9.87 & & & & & \\
\hline & & & & & & & & & & \\
\hline
\end{tabular}




\begin{tabular}{|c|c|c|c|c|c|c|c|c|c|c|}
\hline Run $\#$ & 386 & & & & & & & & & \\
\hline $\begin{array}{l}\text { Tét Fluid } \\
\text { Dato: }\end{array}$ & R114 & $-\cdots$ & $-\longrightarrow$ & $-\ldots$ & -5 & --2 & $-\infty$ & $-\infty \cdots-$ & & \\
\hline Time: & $19: 231$ & & & & & & & & & \\
\hline Fow Areant? & 0.00336 & & & & & & & & & \\
\hline Hoal Alow Áa-t2 & 0.196951 & & &. & & & & & & 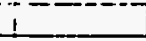 \\
\hline Sat. Prossure-Psig & $212.6 !$ & & & & & & & . & & 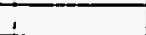 \\
\hline Sat. Temperature-f(inlel) & 221.8 & & & & & & & & & \\
\hline Liq. Densitylbmfit 3 & 74.821 & 74.981 & .5 & 74.961 & $74.88 !$ & 74.471 & 71.52 & $71.5 !$ & 71.52 & 71.56 \\
\hline Latent Heat-gtuibm & 39.831 & 39.99 & 401 & $39.97 !$ & 39.89 & 39.49 & 36.6 & 36.59 & $36.6 i$ & 36.64 \\
\hline Specific Heat-Btuntbmli & $0.282^{\prime}$ & 0.282 & $0.282 i$ & $0.282 !$ & 0.282 & 0.2831 & 0.288 & 0.288 & 0.2881 & 0.288 \\
\hline Liq. Viscosity-bmithr & $0.432^{\top}$ & 0.4341 & $0 . \overline{434 !}$ & 0.433 & $0.432 !$ & $0 . \overline{4281}$ & 0.392 & 0.3921 & $0.392 !$ & 0.393 \\
\hline Liq. Conductivity-Btuhr-ite & 0.02051 & 0.0285 & 0.02656 & 0.02801 & $0.0265 !$ & 0.0203 & 0.0245 & 0.02451 & $0.0245 !$ & 0.0245 \\
\hline Uq. Prandtino. & 4.61 & $4.6 !$ & $-\frac{-61}{4.61}$ & $4.6 !$ & 4.61 & 4.6 & 4.62 & $\frac{.621}{4.621}$ & 4.621 & 4.62 \\
\hline inlot Fow Aato-GPM & 1.51 & & 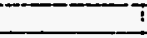 & -1 & & & & & & $i$ \\
\hline Outlot Liq. Flow Rato-GPM & 1. & T. & $:$ & 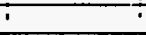 & i: & $\vdots$ & 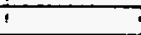 & T & T & T \\
\hline Outlot Vap. Fow Roto-GPM & 0.71 & & $\bar{\vdots}$ & $\therefore$ & & & & & & i \\
\hline Inlol Tomperaturef & 200.31 & & $:$ & $\because$ & is & 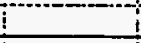 & $i$ & 1 & & I \\
\hline Outlat Lig. Tomporaturef & 220.4 & & 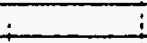 & $\vdots$ & & $\vdots$ & 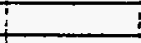 & $!$ & & 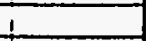 \\
\hline Outlet Vap. Temperaturef & 219.61 & & $i$ & $i$ & & & I & $!$ & & 1 \\
\hline Wator Flow Rato-GPM & $=$ & & $\bar{i}$ & 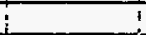 & 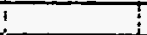 & & 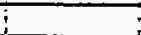 & 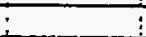 & 1 & 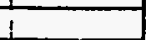 \\
\hline Water Inlet Temp.f & 206.6 & & $i$ & 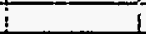 & 1 & 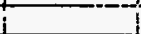 & 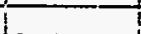 & it & ד̦ & + \\
\hline Water outiet Tomp.F & 215.9 & & & tis & & & $t$ & 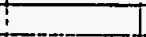 & & \\
\hline Inlet Gibminrme & 2675811 & & Temp & 202.4 & Density & 74.75 & & $\because$ & & \\
\hline Subcoolingt & 21.5 & & 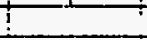 & & & & t & ti & & \\
\hline Flow Observation & & & & ! & 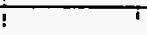 & & 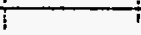 & & & \\
\hline & ¿ZONE1 & TZONE2 & ZONE3 & $\angle O N=4$ & ZONES & IZONEG & ZONE7 & ZONEB & ZONE9 & TZONE 10 \\
\hline Heat Input-Walts(net) & $\because \quad 11$ & $1 \quad 39.91$ & 41.5 & 40.1 & 39.31 & 1622.51 & 16501 & $\mid 1697.3$ & 1688.5 & 1080.8 \\
\hline Hoad Loss-Watts & $47.6 i$ & 10.41 & 12.41 & $10 !$ & 9.11 & 16.31 & 17.3 & 19.41 & $22 !$ & 105.3 \\
\hline Headfiux-BtuHrint2 & 17 & 6921 & 719 & 695 & 6811 & 28125 & 286021 & 284221 & 29269 & -17737 \\
\hline Wall Temp. $3 F$ & 202.6 & 205.71 & 207.11 & 207.11 & 294.11 & $235 i$ & 235.61 & 237.31 & 260.61 & 256.5 \\
\hline Wall Temp. $4 F$ & 203.21 & 206.81 & 207.2 & 207.11 & $215 i$ & 235 & 2361 & 238.61 & 2611 & 255.7 \\
\hline Wall Temp. $5 F$ & 201 & 2061 & 206.2 & 207.11 & 210 & 235.7 & 233.9 & 235.21 & 236.5 & 249.1 \\
\hline Wall Temp. $1 F$ & 77.1 & 205.7 & 206.2 & 206.9 & 207.6 & 238.61 & 236.1 & 236.61 & 233.1 & 236.2 \\
\hline Wall Temp. \#2F & 1981 & 206.21 & 206.21 & 205.61 & 207.5 & 243.1 & 236.6 & 236.5 & 235.6 & 238 \\
\hline Instream temp.f & 2021 & $20 \pi$ & 200.91 & 201.11 & 207.61 & 204.1 & 220.81 & -220.91 & 20.8 & $2 \% 0.6$ \\
\hline Avg. Wall Temp.f & 201.2! & 206.11 & 206.7 & 207.11 & $210 !$ & 236.1 & 235.61 & 236.8 & 245.4 & 247.1 \\
\hline Avg.WallDT.F & 01 & 5.1 & 5.7 & 5.9 & 8.41 & 30.7 & 13.5 & 14.6 & 23.2 & 25.7 \\
\hline Remaining subcooff & 21.5 & & & -1 & $T$ & -1 & T & $T$ & & \\
\hline Exit Quality & ol & 01 & of & o! & 01 & 0 & 01 & 0 & वो & $\overline{0}$ \\
\hline EXP.HBtufHrnarF & 1733.4 & 136.5 & 125.31 & 117.41 & 81.21 & 916 & 2111.2 & 2013.7 & 1259.6 & 730.4 \\
\hline COMPUIEDH & -1 & I & -1 & 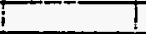 & & & -1 & - & & \\
\hline Jallouk & 83.21 & 83.2 & 83.2 & 83.21 & $83.2 !$ & 83.2 & 83.21 & 83.2 & 83.2 & 83.2 \\
\hline Szady1(Cudo tube) & 103.3 & 104.8 & 10.6 .6 & 106.3 & 106.9 & 107.5 & $107.5 !$ & 107.9 & 108.4 & 108.8 \\
\hline Szady2 & 103.3 & 104.8 & 105.6 & 106.3 & 106.9 & 107.5 & 107.5 & 107.9 & 108.4 & 108.8 \\
\hline (RUQ) & 405511 & 40355 & 40343 & 40375 & 40460 & 40916 & 44591 & 446121 & 44582 & 44525 \\
\hline (F) & 1.02 & 1.035 & 1.043 & 1.05 & 1.056 & 1.0611 & 1.06 & 1.055 & 1.059 & 1.074 \\
\hline (Hढ़Q) & 103.3 & 104.8 & 105.6 & 105.3 & 106.9 & 107.5 & 107.5 & 107.9 & 108.4 & 108.8 \\
\hline (HBOIL) & $1.3 !$ & 764.6 & 858.5 & 896.6 & 1285.8 & 4916.7 & 2371.9 & 2564.8 & 4122.21 & 4554.4 \\
\hline & & & & & & & & & & \\
\hline & ZONE2 I & ZONEA & ZONE6 & ZONE 8 & ZONETOT & & & & & \\
\hline DP liquid Tomp.f & 791 & & 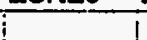 & 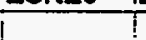 & i & & & & & \\
\hline DP Liquid Donsity lom/13 & 90.71 & & i & 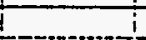 & & & & & $i$ & I \\
\hline Elovetion & 2 & 4 & 61 & 8 & $\overline{9} . \overline{8} 7 \overline{6}$ & & & & & \\
\hline Maxsurad DPPsid & 0.178 & 0.4961 & 0.912 & 1.453 & 2.3321 & & & & & \\
\hline Zero Cortections-psid & 0.000 & 0.044 & 0.029 & 0.018 & -0.074 & & & & & \\
\hline Corrected DPPsid & 0.167 & 0.452 & 0.883 & 1.435 & 2.406 & & & & & \\
\hline Actual DP- & 2.2 & 4.16 & 5.83 & 7.25 & 7.67 & & & & $t$ & \\
\hline Measured TPPsig & 212.6 & & & & 217 & & & & & \\
\hline Measured overallit & -8.85 & & & & & & & & & \\
\hline & & & & & & & & & & \\
\hline Computod DPA & 2.01 & 4.031 & 6.041 & 8.06 & 9.94 & & & & & \\
\hline Fricton-1 & 0.01 & 0.031 & 0.041 & 0.08 & 0.07 & & & & & \\
\hline Accoloration-ti & of & 0| & 01 & $\overline{0}$ & ol & & & & & \\
\hline Elevation $\mathrm{ft}$ & 2 & 41 & 61 & 81 & 9.87 & & & & & \\
\hline & & & & & & & & & & \\
\hline Run\# & 387 & t! & & & & & & & & \\
\hline Test Fluid & R114 & & & & & & & & & \\
\hline Date: & अ593 & I & I & & & & & & & \\
\hline Time: & $19: 32$ & & & & & & & & & \\
\hline Flow Aroa-te & 0.00365 & & & I & & & & & & \\
\hline Head Fow Areath & 0.1965 & & & $i$ & & & & & & \\
\hline Sat. Prossure Psig & 216.3 & & & & & & & & & \\
\hline Sat. Temperaturo f finfet) & 223.3 & & & & & & & & & \\
\hline Ug. Density & 72.831 & 72.97 & 72.97 & 73.041 & 72.941 & 72.65 & 71.45 & 71.18 & 71.24 & 71.28 \\
\hline Litent Heat-Btuntbm & 37.89 & 38.09 & 38.031 & 38.1 & 37.991 & 37.71 & 38.53 & 38.27 & $\mathbf{3 8 . 3 3}$ & 39.97 \\
\hline Specifio Heal Eth/lbm/F & 0.2861 & 0.2861 & 0.2386 & 0.2861 & $0.286 !$ & 0.285 & 0.288 & 0.289 & 0.289 & 0.289 \\
\hline 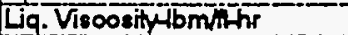 & 0.408 & $0 . \overline{41}$ & $\overline{0.411}$ & $0.419 T$ & 0.4001 & 0.4061 & 0.391 & 0.388 & 0.3801 & 0.300 \\
\hline Liq. Conductivity Btuhs-iff & 0.02531 & 0.02531 & $0.0253 i$ & 0.02541 & 0.02531 & $0.0252 !$ & 0.0244 & 0.0243 & 0.0243 & 0.0243 \\
\hline Liq. Prand No. & $4.62 i$ & $4.62 !$ & $4.62 !$ & 4.521 & $4.62 !$ & $1.52 !$ & 4.52 & 4.62 & 4.621 & 4.62 \\
\hline InlotFlow Ralo-GPM & $3 !$ & ! & & & & & & & & \\
\hline Outlet Lig. Flow Rato-GPM & 1.9 & & & & & & & & & \\
\hline
\end{tabular}




\begin{tabular}{|c|c|c|c|c|c|c|c|c|c|c|}
\hline Wader outlet Temp.F & 215.8 & & & 1 & 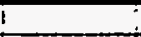 & & & & & \\
\hline Intef G-bm/nrite & 523582 & & Temp & 211.91 & Density & 73.13 & & & & \\
\hline Subcoolingf & 11 & & & & & & & & & \\
\hline \multicolumn{11}{|l|}{ Flow Obsenvation } \\
\hline & IZONET & IZONEZ2 & ZONE3 & TZONE4 - & ZONE5 -T & ZONE & ZONE7 & ZONE8 & ZONE9 & ZONETO \\
\hline Hod input-Wals (nel) & 11 & 61.2 & 60.4 & 59.2 & 59.9 & 1619.3 & 1661.3 & 1700.8 & 1564.4 & 892.8 \\
\hline Hoed Loss-Woth & 62.91 & 11.7 & 14.9 & 17.41 & 11.3 & 16.6 & 17.6 & 23.8 & 136.7 & 162.6 \\
\hline Heatfiux Bturtrme & 17 & 1061 & 1047 & 10261 & 10381 & 280701 & 28798 & 29482 & 27118 & 16476 \\
\hline Wall Tomp. \#3F & 213.8 & 217.3 & 218.4 & 218.21 & 220.6 & 236.11 & 236.8 & 233.5 & 483.71 & 307.5 \\
\hline Wall Temp. $4 F$ & 214.3 & 217.9 & 218.5 & 218.2 & 221.9 & 236.3 & 237.3 & 294 & 488.5 & 307.9 \\
\hline Wall Temp. 15F & 213.4 & 217.7 & 217.3 & 218.6 & 218.9 & 236.8 & 235.6 & 237.4 & 464.8 & 367.8 \\
\hline Wall Tomp. & $\pi 7.7$ & 217.4 & 217.4 & 218.2 & 218.1 & 239.9 & 237.8 & 237.7 & 425.1 & 402.3 \\
\hline Wall Temp. $2 F$ & 210.7 & 217.8 & 217.5 & 218.5 & 217.5 & 244.41 & 238.3 & 237.5 & 424.7 & 402.3 \\
\hline Instroam tomp. f $^{-}$ & 213.6 & 212.8 & 212.8 & 21271 & 293 & 274.6 & चृत. & 2026 & अृ2उ & C201 \\
\hline Avg. Wal Temp.F & टंड़ा। & 217.6 & 218 & 218.31 & टरव.7ा & 237.31 & 237.2 & 250 & 2501 & 250 \\
\hline Avg. WallDT.F & $0 !$ & 4.8 & 5.1 & 5.9 & 6.1 & 27.4 & 14.7 & 26.1 & 26.5 & 27.2 \\
\hline Aomalning subcoolf & 111 & & & $!$ & & & & & & \\
\hline Ext Quality & of & 0 & 0 & 0 & 0 & 0 & $\overline{0}$ & (0) & 0 & 0 \\
\hline EXP. HEtuHrherF & 1733.4 & 223.2 & 206.2 & 175.3 & 171.5 & 1311.3 & 1964.6 & 1131 & 1024.3 & 669 \\
\hline \multicolumn{11}{|l|}{ COMPUTED } \\
\hline Jallouk & 142.41 & 142.3 & 142.3 & 142.3 & 142.3 & 142.3 & 142.3 & 142.3 & 142.3 & 142.3 \\
\hline Szadyl(Cudo tubo) & 176.6 & 179 & 180.3 & 181.4 & 182.4 & 183.3 & 183.8 & 184.6 & 185.3 & 186.1 \\
\hline Szadt2 & 176.6 & 179 & 180.3 & 181.4 & 182.4 & 183.3 & 183.8 & 184.6 & 185.3 & 186.1 \\
\hline (PLO) & 83915 & 83555 & 83546 & 83366 & 83616 & 84310 & 87455 & 88175 & 88008 & 87895 \\
\hline (F) & 1.019 & 1.033 & 1.04 & 1.047 & 1.052 & 1.057 & 1.06 & 1.064 & 1.069 & 1.073 \\
\hline$(\mathrm{HLO})$ & 176.6 & 179 & 180.3 & 181.4 & 182.4 & 183.3 & 183.8 & 184.6 & 185.3 & 186.1 \\
\hline \multirow[t]{3}{*}{ (सBOLL) } & 1.4 & 765.5 & 823.4 & 945.3 & 982.11 & 3619.9 & 2579.7 & 4706.2 & 476.4 & 4855.3 \\
\hline & & & & $T$ & & & & & & \\
\hline & IZONE2 & TZONE4 & ZONE 6 & Z2ONE8 & ZONETO & & & 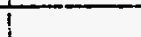 & & \\
\hline DPhquid Temp.F & i 79.9 & & 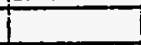 & 1 & -1 & & & 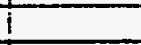 & & \\
\hline \multicolumn{11}{|l|}{ DPLiquid Densintbm/is } \\
\hline Elovetion & 2 & -4 & -6 & $\overline{8}$ & 0.876 & & & & & \\
\hline Measured DPFyid & 0.205 & 0.513 & 0.925 & 1.311 & 2.017 & & & & & \\
\hline Zero Corrections-psid & 0.009 & 0.044 & 0.020 & 0.018 & -0.074 & & & & & \\
\hline Contected DPP sid & 0.196 & 0.469 & 0.896 & 1.293 & 2.091 & & & 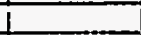 & & \\
\hline Actual DP ff & 2.15 & 4.14 & 5.82 & 7.56 & 8.33 & & & & & \\
\hline Messured TPpsig & 216.3 & & & & 20.7 & & & & & \\
\hline \multirow{2}{*}{\multicolumn{11}{|c|}{ Measured Overalli }} \\
\hline \multirow{2}{*}{\multicolumn{11}{|c|}{ Computed DP-n }} \\
\hline & & & & & & & & & & \\
\hline Fitction-a & 0.05 & 0.1 & 0.15 & 0.21 & 0.25 & & & & & \\
\hline Accolerationt & 0 & 0 & 0 & 0 & 0 & & & & & \\
\hline \multirow[t]{2}{*}{ Elovaciont } & 2 & 4 & 6 & 8 & 9.87 & & & & ! & \\
\hline & & & & & & & & & I & \\
\hline
\end{tabular}




\begin{tabular}{|c|c|c|c|c|c|c|c|c|c|c|}
\hline Run\# & 388 & & & & & & & & & \\
\hline Test Fuid & RTI4 & & & & & & & & & \\
\hline Date: & $3 / 5 \sqrt{33}$ & & & & & & & & & \\
\hline Time: & $-\frac{19: 45}{2}$ & & & & : & & $T$ & & & \\
\hline Flow Areatt2 & 0.00336 & & & & ? & 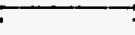 & & & & T \\
\hline Heat Flow Areatt2 & 0.19696 & & & & $\therefore$ & 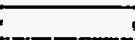 & 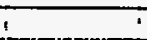 & 8 & & \\
\hline Sal. Pressuro osig & 216.6 & $T$ & . & t. & 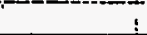 & 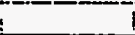 & 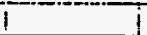 & i & & $i$ \\
\hline Sat. Temperaturof f(inlot) & 223.5 & & & & & & & & & 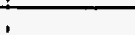 \\
\hline Liq. Density-lbmst3 & 72.78 & 72.871 & 72.871 & 72.9 & 72.83 & 72.52 & 71.45 & 71.22 & $71.22 !$ & 71.3 \\
\hline Latent Heat-Btu/hm & 37.84 & 37.921 & 37.921 & 37.96 & 37.8 & 37.68 & 36.53 & 36.31 & 36.311 & 36.38 \\
\hline Spocific Heat-Btulbmif & 0.286 & 0.286 & 0.286 & 0.286 & 0.2861 & 0.286 & 0.288 & 0.289 & 0.289 & 0.289 \\
\hline Liq. Viscosity & 0.407 & 0.4091 & $0 . \overline{4091}$ & 0.409 & 0.4081 & 0.406 & 0.3971 & 0.389 & 0.389 & 0.389 \\
\hline Ug. Conductivity Btuhn & 0.0252 & 0.02531 & 0.0253 & 0.02531 & 0.02531 & 0.02511 & 0.0244 & 0.02431 & 0.02431 & 0.0243 \\
\hline Lig.Prandt No. & 4.62 & 4.62 & $4.62 !$ & 4.621 & $4.62 !$ & 4.62 & $4.62 !$ & 4.62 & 4.62 & 4.62 \\
\hline Inlet Fow Ralo-GPM & 3.11 & & i & - & & & & $\rightarrow$ & & \\
\hline OUTOR Ua. Flow Rato-GPM & $1.9 i$ & & T & : & $\vdots$ & & 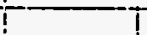 & $\bar{i}$ & $\bar{i}$ & 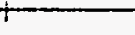 \\
\hline Outlet Vap. Flow Rato-GPM & 0.6 & & & $\because$ & 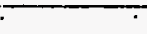 & $=$ & ! & $\mathrm{i}$ & i & T \\
\hline inlet Temperetur of & 212.21 & & & . & - & - & & i & $i$ & $i$ \\
\hline Oútollo. Tomporaturo F & 227.8 & & - & 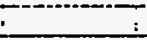 & - & 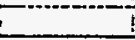 & $!$ & & 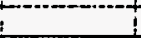 & + \\
\hline Outlel Vap. Temperaturef & 220.8 & & & $\bar{t}$ & . & . & & & & $i$ \\
\hline Water Flow Rate-GPM & $i$ & & & I & I & & $i$ & 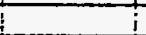 & i & 1 \\
\hline Water Inlel Temp.F & 204.6 & & i & 1 & 1 & 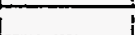 & 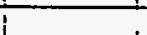 & & & \\
\hline Waler outtel Temp.F & 215.7 & & $\mathrm{i}$ & & 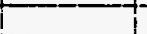 & 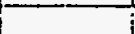 & & & & \\
\hline Inlet Glbm/hrmL2 & 540645 & & Temp & 212.211 & Density & 73.08 & & & & \\
\hline Subcooling & 11.31 & & & & & & & & & \\
\hline Flow Observation & & & & & & & & & & \\
\hline & ZONET & ZONE2 & ZONE3 & ZONE4 & ZONES TL & ZONE6 & ZONE7 & ZONEB & ZONE9 & ZONETO \\
\hline Head inpur-Watts(net) & 1 & i 40.41 & -40.7 & $40.4 T^{1}$ & 40.21 & 16321 & 1689.6 & | 1494.21 & 1012 & 743.3 \\
\hline Hoat Loss-Walls & 53.21 & 11.6 & 13.9 & 11.11 & 10.9 & 16.5 & 17.51 & 53.7 & 117.7 & 125.9 \\
\hline Hodf Flox-Btu/th/the & 171 & 7001 & 7061 & 700 & 597 & 282801 & 29238 & 25901 & 17642 & 12885 \\
\hline Wall Tomp. \$3F & $2 \longdiv { 4 . 8 ! }$ & 216.8 & 217.4 & 217.4 & 219.9 & 236.3 & 236.9 & 450.51 & 423.5 & 241.9 \\
\hline Wall Tomp. \#4F & 215.5 & 217.3 & 217.4 & 217.41 & 220.81 & 236.4 & 237.41 & 449.4 & 427 & 2422 \\
\hline Wall Temp. $\$ 5 F$ & 214.5 & 217.1 & 216.7 & 217.4 & 218.2 & 237 & 235.6 & 404.1 & 42591 & 2929 \\
\hline Wall Temp. \#1F & 77.8 & 217 & 216.8 & 217.4 & 217.3 & 240.2 & 237.9 & 308.9 & 426.3 & 351.2 \\
\hline Wall Temp. $2 f$ & 2122 & 217.3 & 216.8 & 216 & 217 & 244.6 & 238.4 & 309.8 & 425.9 & 3524 \\
\hline Instream Temp.F & 213.9 & 213.41 & 213.4 & 213.2 & 213.6 & 214.8 & 221.2 & 222.4 & 222.4 & 222 \\
\hline Avg. Wall Temp F & 214.3 & 217.11 & 217.11 & 217.4 & 218.3 & 2375 & 2373 & 2501 & 250 & 250 \\
\hline Avg. Wallot. & $0.3 !$ & 3.7 & 3.7 & 4.2 & $4.7 !$ & 21.41 & 44.7 & 26.4 & 26.81 & 27.4 \\
\hline Remaining Subcooff & 71.21 & 1 & +1 & & & & 1 & $=$ & & \\
\hline Ext Cualtiy & $0 !$ & oi & 01 & oi & 01 & 01 & of & 01 & oi & $\mathbf{0}$ \\
\hline DXP. Heturtrfiter & 49.81 & 190.9 & 192.3 & 1681 & 148.5 & 1322.2 & $1990.2 !$ & 9801 & 654.41 & 469.9 \\
\hline COMPUTEDH & & & 1 & $i$ & & & & & & i \\
\hline Jallouk & 146.11 & 146 & 146 & 146 & $-\overline{146}$ & $\overline{146}$ & 146 & 146 & 146 & 746 \\
\hline Szady1(Cudo tuba) & 181.21 & 183.61 & 184.9 & 186.1 & 187.1 & 188 & 188.6 & 189.4 & 190.21 & 190.9 \\
\hline Szady2 & 181.21 & 183.61 & 184.9 & 186.1 & 187.11 & 188 & 188.6 & 189.4 & 190.2 & 190.9 \\
\hline (RUQ) & 86785 & 86546 & 86537 & 86439 & $86610 !$ & 87150 & 903061 & 90939 & 90930 & 90705 \\
\hline (F) & 1.0191 & 1.033 & 1.04 & 1.046 & 9.052 & 1.057 & 1.051 & 1.064 & 1.059 & 1.073 \\
\hline (Hण्) & 181.21 & 183.6 & 184.9 & 186.1 & 187.1 & 188 & 188.6 & 189.4 & 190.2 & 190.9 \\
\hline (HBÓL) & 53.2 ? & 589.5 & 589.51 & 67. & 759.8 & 363.17 & 2590.11 & 4765.1 & 4834.9 & 4931.3 \\
\hline & & & & & & & & & & \\
\hline & IZONE2 & 'ZONE4 & ZONE6 & IZONEB & ZONETO & & & & & \\
\hline DP llquid Temp.f & 80.11 & 1 & $+2=$ & 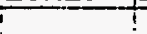 & $+i$ & & & & & $i$ \\
\hline DP Liquid Density & 90.581 & $!$ & $T$ & $i$ & 1 & & & i & 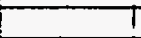 & $T$ \\
\hline Elovation-t & $2 i$ & 4 & $6 i$ & 81 & 9.875 & & & T & T & i \\
\hline Mossurod DPFid & 0.2081 & 0.5161 & 0.9281 & 1.337 & 2.008 & & & & & \\
\hline Zero Corrections-psid & 0.0001 & 0.0441 & 0.029 & 0.018 & -0.074 & & & & & $t$ \\
\hline Conrected DPPsid & 0.1991 & 0.472 & 0.899 & 1.319 & $2.08 \%$ & & & & & \\
\hline Actual DRA & 2.14 & 4.13 & 5.81 & 7.5 & 8.34 & & & & & \\
\hline Messured TPPsig & 216.61 & + & : & 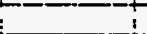 & 221.2 & & & & & \\
\hline Measured Overalli & $9.29 !$ & & & & & & & & & \\
\hline & & & & & & & & & & \\
\hline Computedor-t & 2.051 & $4 . \pi$ & 6.161 & 8.27 & 10.13 & & & & & \\
\hline Frian-n & 0.051 & 0.1 & 0.16 & 0.21 & 0.26 & & & & & \\
\hline Accoleration- & 01 & 0 & of & oो & of & & & & & \\
\hline Elevationft & 21 & 41 & 61 & 8 & 9.871 & & & & & \\
\hline & T! & $\bar{i}$ & 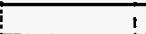 & T & 1 & 5 & i & 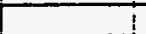 & 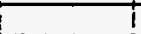 & \\
\hline
\end{tabular}




\begin{tabular}{|c|c|c|c|c|c|c|c|c|c|c|}
\hline Runt" & 3891 & & & & & & & & & \\
\hline Test Fuld & TA114 & & & . & & ? & & & & \\
\hline Date: & $3 / 5931$ & I & 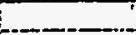 & & & & & & & \\
\hline Time: & $99: 531$ & 5 & 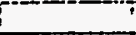 & & & i & & & ! & \\
\hline Flow Arouth & 0.003361 & I & 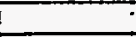 & 5 & 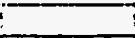 & 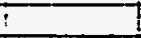 & 1 & ! & 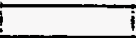 & i \\
\hline Host Fow Area-ph & 0.19686 & & 7 & 5 & & i & 1 & T & 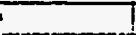 & \\
\hline Sat. Prossure prig & 217.17 & & 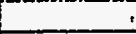 & + & & 1 & 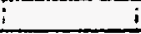 & $i$ & tis & \\
\hline Sat. Tomperature-f(inlat) & 223.7 & & & & & & & & & \\
\hline Liq. Density $16 \mathrm{~m} / 1 \mathrm{k}$ & 72.69 & 72.83 & 72.831 & 72.851 & 72.81 & 72.53 & 71.39 & 71.111 & 71.13 & 71.24 \\
\hline Latent Hoatstumbm & 37.75 & 37.89 & 37.89 & 37.911 & 37.87 & 37.59 & 36.48 & 36.21 & 36.22 & 36.33 \\
\hline Specifio Heat-Btu/bm/F & 0.286 & 0.286 & 0.2861 & 0.286 & 0.286 & 0.286 & 0.289 & 0.2891 & 0.289 & 0.289 \\
\hline Liq. Viscosinglomithr & 0.406 & 0.408 & 0.408 & 0.4081 & 0.408 & 0.404 & 0.391 & 0.387 & 0.387 & 0.389 \\
\hline Lig. Conductivity EtuhrftF & $0.0252 !$ & 0.0253 & 0.0353 & 0.0253 & 0.0253 & $0.025 \pi$ & 0.02441 & 0.0242 & 0.0242 & 0.0273 \\
\hline Lig. PranditNo. & 4.621 & 4.62 & 4.62 & 4.521 & 4.62 & 4.62 & 4.62 & 4.52 & 4.62 & 4.62 \\
\hline Inlel Flow Rato-GPM & 3.11 & & 7 & 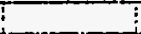 & & & 1 & & & \\
\hline Outol LT. Fów Rate-GPM & 1.91 & & $i$ & i & & $T$ & T & & & \\
\hline Outlol Vap. Flow Rato-GPM & 0.71 & & ? & 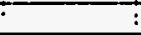 & & I & ! & & & \\
\hline Intel Temperaurof & 212.61 & & $\therefore$ & 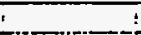 & & & I & & & \\
\hline Outolig. Tomporaturef & 2221 & & 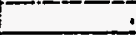 & 5 & & i & i & & & \\
\hline Outtel Vap. Temperaturof & 221.11 & & . & . & & & & & & \\
\hline Wator Flow Ralo-GPM & & & & & & & i & & 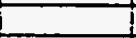 & \\
\hline Waler inlel Tomp.F & 200.4 & & i & i & & & + & & & \\
\hline Wador outlel Tomp.F & 214.4 & & & i & & & & & & \\
\hline Inlet G-lbm/hr/iz & 540254 & & Temp & 212.51 & Density & 73.03 & & & & \\
\hline Subcooling & $\{1.11$ & & & $i$ & - & & & & 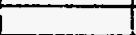 & \\
\hline \multirow[t]{2}{*}{ Fow Observation } & & & & & & & & & & \\
\hline & ZONET & ZONEZ & ZONE3 & ZONE4 & ZONES & ZZONE6 & ZONE7 & ZONE8 & ZONE9 & ZONE10 \\
\hline Hoal input-Wats(nel) & 1 & 31.1 & 30.4 & T.8. & 31.1 & 1620.3 & 1697 & 1467.7 & 8228 & 753.6 \\
\hline Hoal Loss-Walts & 53.2 & 11.5 & 13.61 & 10.8 & 10.6 & 16.5 & 17.5 & 40.7 & 106.3 & 109 \\
\hline Hod Flux Btumiriti2 & 17 & 539 & 527 & 53 & 539 & 282य1 & 2946 & क्या & 14263 & 13053 \\
\hline Wall Tomp. HzF & 215 & $\overline{2} \overline{1} \overline{6}$ & 216.71 & 216.9 & 219.6 & 235.5 & 237.4 & 412.5 & 402.9 & 2329 \\
\hline Wall Tomp. $4 F$ & 215.9 & 217 & 216.7 & 216.9 & 220.4 & 238.7 & 237.8 & 411.6 & 405.6 & 2329 \\
\hline Wall Tomp. 15f & 214.8 & 216.7 & 216.11 & 216.9 & 217.5 & 237.2 & 236.11 & 344.3 & 409.4 & 2412 \\
\hline Wall Tomp. \#1F & 78.1 & 216.6 & 216.1 & 216.91 & 216.8 & 240.4 & 238.3 & 244.1 & 409 & $\mathbf{2 9 0 . 6}$ \\
\hline Wall Tomp. $2 F$ & 212.8 & 216.9 & 216.2 & 215.3 & 216.4 & 244.9 & 238.8 & 245.3 & 408.8 & 293.4 \\
\hline Instream Temp.f & 214.4 & 213.6 & 213.6 & 213.5 & 213.7 & 215.3 & 221.5 & 223 & 222.9 & 2223 \\
\hline Avg. Wall Temp. F & $2\lceil 4.6 \mid$ & 216.6 & 216.4 & 216.9 & 217.8 & 237.7 & 237.71 & सरण1 & 250 & 250 \\
\hline Avg.Wallot. & 0.2 & 3 & 2.8 & 3.4 & 4.1 & 21.11 & 14.9 & 25.9. & 26.5 & 27.1 \\
\hline Romaining subcooff & 11.1 & & & & & & 1 & & & \\
\hline Extt Quality & 01 & 0 & $\overrightarrow{0 i}$ & $0 i$ & 0 & of & of & 01 & of & $\overline{0}$ \\
\hline EXP. H Guthmlar & 77.91 & 179.7 & 188.11 & 158.2 & 139.1 & 1397 & 1980.8 & $9842 \mid$ & 539.11 & 481.8 \\
\hline \multicolumn{11}{|l|}{ COMPUTEDH } \\
\hline Jallouk & 146 & $94 \overline{6}$ & 146 & i45.0ं & 946.8 & 145.0 & 1461 & 746 & $14 \overline{6}$ & 146.0 \\
\hline Szady1(Cudo tube) & 981.11 & 183.5 & 184.81 & 185 & 187 & 187.9 & 188.5 & 189.2 & 190 & 190.8 \\
\hline $\operatorname{szady} 2$ & 181.11 & 183.5 & 184.8 & 186 & 187 & 187.9 & 188.5 & 189.2 & 190 & 190.8 \\
\hline (RIIQ) & 86951 & 86574 & 80565 & 86511 & 85593 & 87319 & 90399 & 91201 & 91137 & 90801 \\
\hline (F) & 1.019 & 1.003 & 1.04 & 1.046 & 1.052 & 1.057 & 1.06 & 1.064 & 1.059 & 1.073 \\
\hline (H)Q & 181.1 & 183.5 & 184.81 & 186 & 187 & 187.9 & 188.5 & 189.2 & 190 & 190.8 \\
\hline \multirow{3}{*}{ (ALOIL) } & ऊ3.9 & 480.5 & 447.81 & 541.8 & 553.8 & 3588.2 & 2620.7 & 4681.8 & 4790 & 4886.7 \\
\hline & & & & & & & & & & \\
\hline & ZONE2 & ZONE4 & ZONE6 & ZONE8 & ZONETO & & & & & \\
\hline DPHquldTomp.f & 80.3 & & & 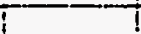 & & & & t! & & \\
\hline DिPLiquid Densinylbm/133 & $\mathbf{9 0 . 6 6 1}$ & & & & & & & t & & \\
\hline Elvation $\mathrm{A}$ & 2 & 4 & 6 & 8 & 9.875 & & & & & \\
\hline Masaured DPFPId & $0 . \overline{208}$ & $0.61 \overline{6}$ & 0.927 & -1.33 & 1.998 & & & t & & \\
\hline Zero Corrections-psid & 0.009 & 0.044 & 0.029 & 0.018 & -0.074 & & & 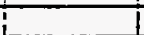 & & \\
\hline Coneded DPPsid & 0.199 & 0.472 & 0.898 & 1.312 & 2.072 & & & & & \\
\hline Actual DP-n & 2.14 & 4.13 & 5.81 & 7.52 & 8.37 & & & & & \\
\hline Measured TPPsig & 217.1 & & & 1 & 221.7 & & & & & \\
\hline \multirow[t]{2}{*}{ Measured Overalh } & -9.3 & & & & & & & & & \\
\hline & & & & & & & & & & \\
\hline Computed DP- & 2.05 & 4.1 & 6.16 & 8.टा & 10.13 & & & & & \\
\hline Fricton-h & 0.05 & 0.1 & 0.76 & 0.27 & 0.26 & & & & & \\
\hline Accoloration- & of & 0 & 0 & 0 & 0 & & & & & \\
\hline \multirow[t]{2}{*}{ Elovationft } & 2! & 4 & 61 & 8 & 9.87 & & & & & \\
\hline & & & & & & & & & & \\
\hline
\end{tabular}




\begin{tabular}{|c|c|c|c|c|c|c|c|c|c|c|}
\hline \begin{tabular}{|l} 
Runk \\
Test Fuid
\end{tabular} & $390 !$ & & & & & & & & & \\
\hline Dafe: & $3 / 5 / 331$ & & $T$ & & & & & & & ' \\
\hline Time: & $19: 591$ & & & & & & & & 1 & \\
\hline Flow Areafte & 0.003361 & & $\bar{t}$ & - & & 7 & $T$ & 7 & T & 1 \\
\hline Heat Fow Areatil & 0.196951 & & $\because$ & & & 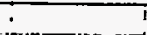 & i & & & $i$ \\
\hline Sad. Prossuro psig & 217.91 & & 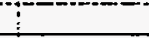 & & $i$ & & "ִ & 7 & & i \\
\hline Sat. Tomporaturo finlel) & 224 & & & & & & & & & \\
\hline Liq. Densitylbmiti3 & 72.241 & 72.42 & 72.42 & 72.42 & 72.4 & 72.15 & 71.63 & 71.17 & 71.111 & 71.13 \\
\hline Latent Head-8tuibm & 37.31 & 37.49 & 37.49 & 37.49 & 37.47 & 37.22 & 36.71 & 36.2 & 36.2 & 36.22 \\
\hline Specific Heat-BtunbmiF & 0.287 & 0.287 & 0.287 & 0.287 & 0.287 & 0.287 & 0.288 & 0.289 & 0.289 & 0.289 \\
\hline Liq. Viscosity tbmiththr & 0.4011 & 0.403 & 0.403 & 0.403 & 0.403 & 0.4 & 0.394 & 0.387 & 0.387 & 0.387 \\
\hline Liq. Conductivity Btuhr-fiF & 0.02491 & $0.0 \% 5$ & 0.025 & 0.025 & 0.025 & 0.0249 & 0.02451 & 0.0242 & 0.02421 & 0.0242 \\
\hline Lig. PrandtNo. & 4.621 & 4.62 & 4.62 & 4.62 & 4.62 & 4.62 & 4.62 & 4.62 & 4.621 & 4.62 \\
\hline Inlet Flow Rate-GPM & $4 !$ & & I & & & 1 & & & 5 & $!$ \\
\hline Outrot La. Fow Rate-GPM & 2.51 & & $T$ & & & 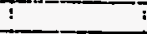 & $i$ & $i$ & i & $i$ \\
\hline Outlot Vap. Flow Rato-GPM & 0.71 & & T & & i & 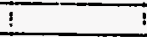 & $\vdots$ & 1 & $i$ & $i$ \\
\hline Inlot Tomperaturof & 215.31 & & & & & 1 & $\therefore$ & $\therefore$ & i & $i$ \\
\hline Outtat Liq. TomporaturoF & 222.41 & & & & & $\div$ & $:$ & - & $\vdots$ & 1 \\
\hline Outlet Vap. Temperaturof & 221.41 & & & & & & 1 & & 1 & $\perp$ \\
\hline Water Flow Rato-GPM & & & & & & & 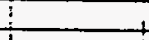 & & & \\
\hline Wator Inlet Temp.F & 198.3 & & & & $i$ & i & $!$ & $i$ & & \\
\hline Water outlet Temp.F & $215 !$ & & 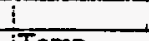 & 214 & IDensity & $\frac{:}{72641}$ & $\vdots$ & & & \\
\hline $\begin{array}{l}\text { Inlol Glbm/hrit2 } \\
\text { Subcooling }\end{array}$ & $\begin{array}{r}693375 \\
\quad 87 \\
\end{array}$ & & Temp & $214.7 !$ & IDensiny & & 7 & & & \\
\hline $\begin{array}{l}\text { Subcooling } \\
\text { Flow Observation }\end{array}$ & $\begin{array}{l}i \\
\vdots \\
\end{array}$ & & $\frac{1}{i}$ & & & & & & & \\
\hline Flow Observation & IZONEI & ZONEZ & IZONE3 & ZONE4 & ZZONE5 & IZONEG & IZONE7 & ZZONEB & ZONES & IZONETO \\
\hline Hoad Input-Wats(nol) & $1 \quad \pi$ & 38.5 & 51 39.21 & 38.31 & 39.6 & $10-1634$ & 1690.7 & 1459.7 & Tा & $T^{2017510} \frac{10}{745.6}$ \\
\hline Hoad Loss-Watts & 53.61 & 11.8 & 14.21 & 11.51 & 11.21 & 16.61 & 17.51 & 48.1 & 107.31 & 116.9 \\
\hline Hoad Floctultine & 171 & 667 & 6801 & 6641 & $686 !$ & 28324 & 293071 & 253031 & 136511 & 12024 \\
\hline Wall Tomp. $3 F$ & $216 \bar{i}$ & 218.2 & 2191 & 219.21 & 220.6 & 236.81 & 237.71 & 438.41 & $401.9 T$ & 239.1 \\
\hline Wall Tomp. \#4F & 216.51 & 218.9 & 219.11 & 219.11 & 221.91 & 235.81 & 238.11 & 437.41 & 404.51 & 239.2 \\
\hline Wall Temp. $\$ 5 F$ & 216 & 218.81 & $218.4 i$ & $219.2 !$ & 219.51 & 237.4 & 236.31 & 381.6 & 410 & 262 \\
\hline Wal Temp. \#1F & 78 & 218.5 & 218.5 & 219.2 & 218.7 & 240.5 & 238.4 & 278.41 & 414.4 & 318.3 \\
\hline Wall Temp. \&2f & $214 . \overline{3}$ & $21 \overline{8} . \overline{8}$ & 218.5 & 217.61 & 218.7 & 245.3 & 2391 & 279.5 & 414.3 & 319.1 \\
\hline Instroam Tomp.F & 216.91 & 215.9 & 215.9; & 215.91 & 216 & 217.4 & 220.2 & 2231 & 223 & 222.9 \\
\hline Avg. Wall Temp.F & 215.71 & 218.61 & $218.8 !$ & 219.2! & 219.7 & 237.91 & $237.9 !$ & 2501 & 250 & 250 \\
\hline Avg. Wall DT.F & 01 & 2.7 & $2.8 !$ & 3.2 & 3.7 & 19.2 & 16.4 & 25.91 & 26.4 & 26.5 \\
\hline Remaining Subcooff & 8.71 & & & & & & 1 & $i$ & 1 & \\
\hline Exit Quality & 01 & $0 !$ & 01 & $0 i$ & 01 & $0 !$ & oा & 01 & 01 & \\
\hline EXP. HBturinga & 1739.4 & 2501 & 238.9 & 204.61 & 187.11 & 1475.61 & 1789.7 & 978.61 & 517.4 & 487.4 \\
\hline COMPUTEDH & & & 21 & 1 & & & 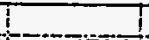 & & & \\
\hline Jallouk & 178.31 & 178.2 & -778.2 & 178.2 & 178.21 & -178.2 & 178.21 & 178.21 & 778.2 & 178.2 \\
\hline Szadyi(ardo tube) & 221.11 & 224 & 225.61 & 226.9 & 228.21 & 229.31 & 230.21 & 231.1 & 2321 & 232.9 \\
\hline Szady? & 221.1 & 224 & 225.61 & 226.9 & 2282 & 229.3 & 230.2 & 231.1 & 232 & 232.9 \\
\hline (PUQ) & 113104 & 112474 & 112463 & 112452 & 112501 & 113353 & 115146 & 117050 & 117038 & 116956 \\
\hline (F) & 1.019 & 1.032 & 1.039 & 1.046 & 1.052 & 1.056 & 1.0611 & 1.064 & 1.009 & 1.073 \\
\hline (HएO) & 221.1 & 224 & 225.6 & 226.9 & 228.2 & 229.3 & 230.2 & 231.11 & 232 & 232.9 \\
\hline ( $\mathrm{HBOIL})$ & $-1.4 !$ & $43 \overline{3.1}$ & $4 \pi 2.1$ & 528.9 & 600.2 & 3302.11 & 2866.6 & 4682.91 & 4780.7 & 48012 \\
\hline & & & & & & & & & & \\
\hline & ZONE2 1 & ZONE4 & ZONE6 & ZONE8 & ZONE 10 & & & & & \\
\hline DP llquid Temp. $F$ & 80.51 & & $i$ & I & & I & & $i$ & $i$ & \\
\hline DP Liquid Densingtomitis & 90.541 & & 1 & 7 & & & t & 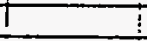 & $!$ & \\
\hline Elovation-t & 2) & $4 !$ & $6 i$ & 81 & 9.8761 & & & $i$ & $i$ & \\
\hline Measured DPPsid & 0.2161 & 0.403 & 0.8851 & 1.2161 & $1.816 \overline{1}$ & & & -1 & 5 & \\
\hline Zoro Correctionspsid & $0.009 !$ & $0.044 !$ & 0.0291 & 0.018 & -0.0741 & & I & 1 & $:$ & \\
\hline Correded DPPsid & 0.2061 & 0.449 & 0.8561 & 1.198 & 1.89 & & & & & \\
\hline Actual DPAA & 2.13 & 4.18 & 5.9 & 7.761 & $8.74_{1}$ & & & & & \\
\hline Measured TP Psig & 217.9 & & 1 & & 222.41 & & & & & \\
\hline Measured Overal- & -9.11 & & & & & & & 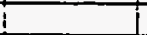 & & \\
\hline & & & & & & & & & & \\
\hline Computed DPA & 2.081 & 4.161 & 6.25 & 8.33 & 10.28 & & & & & \\
\hline Friction-l & 0.081 & 0.16 & 0.251 & 0.33 & 0.411 & & $i$ & $t$ & & \\
\hline Accoleration-1 & 01 & 01 & 01 & 01 & 01 & & $i$ & i & & \\
\hline Elovationfl & 21 & 41 & 61 & 81 & 9.87 & & $i$ & i & & \\
\hline & & & & & 1 & $!$ & & & & \\
\hline
\end{tabular}




\begin{tabular}{|c|c|c|c|c|c|c|c|c|c|c|}
\hline Run" & 3911 & & & & & & & & & \\
\hline Testfluid & IR114 & 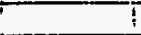 & & & & $:$ & 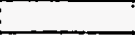 & & & $T$ \\
\hline Date: & $35 / 93$ & $!$ & & i & 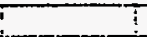 & i & 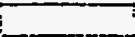 & & & \\
\hline Time: & $20: 081$ & i & & 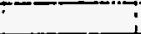 & 1 & 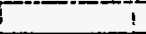 & 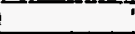 & & $i$ & $t$ \\
\hline Flow Areathe & 0.008361 & T & 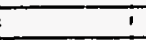 & I & $i$ & $i$ & 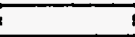 & $i$ & 1 & $i$ \\
\hline Hoat Flow Areanl & 0.196951 & I & & 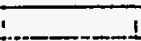 & 7 & 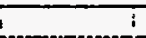 & 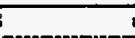 & $t$ & i & i \\
\hline Sal. Prossure prig & 217.81 & & & $\because$ & 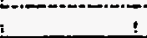 & 7 & 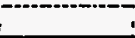 &. & it & $i$ \\
\hline Sad. Tomperature-F(inlet) & 223.91 & & & $i$ & & . & 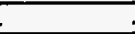 & & ! & \\
\hline Lig. Densitylbmitis & 7226 & 72.44 & $72.42 !$ & 72.42 & $72.33:$ & 72.081 & $71.52 !$ & 71.05 & 71.07 & 71.11 \\
\hline Latent Hoat-Btulbm & 37.33 & 37.5 & 37.49 & 37.49 & 37.4 & 37.15 & 36.6 & 36.14 & 36.16 & 36.2 \\
\hline Specitic Heat-Btulbmif & 0.287 & 0.287 & 0.287 & 0.287 & 0.287 & 0.287 & 0.288 & 0.289 & 0.289 & 0.289 \\
\hline Liq. Viscosity-bmitithr & 0.401 & 0.403 & 0.4031 & 0.40031 & 0.402 & 0.399 & 0.392 & 0.3861 & 0.387 & 0.387 \\
\hline La. Conductivitytuhe-iff & 0.02491 & 0.025 & 0.025 & 0.0251 & 0.0251 & 0.02481 & 0.0245 & 0.02421 & 0.02421 & 0.0242 \\
\hline Iig. Prand No. & 4.62 & 4.62 & 4.62 & 4.621 & $4.62 !$ & 4.52 & 4.62 & 4.62 & 4.62 & 4.62 \\
\hline Inlot Flow Rate-GPM & $4 !$ & & & & & -1 & & & & $t$ \\
\hline Outiof Lif. Fiow Rato-GPM & 2.51 & ti & $T$ & T & $\because$ & ti & 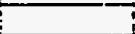 & 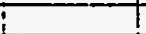 & & \\
\hline Outlot Vap. Flow Rate-GPM & 0.71 & 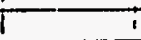 & $i$ & 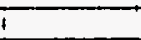 & ! & 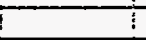 & 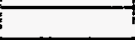 & & T & \\
\hline Inlet Temperaturef & 216.21 & 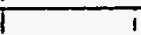 & & $!$ & $\overline{!}$ & 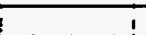 & 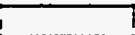 & T & & \\
\hline Outlet Lig. Temperature- & 222.41 & & & $i$ & $i$ & 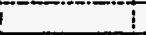 & 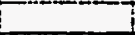 & i & & \\
\hline Outtot Vap. Tomperaturef & 221.5 & & & $i$ & $\bar{i}$ & $\bar{i}$ & 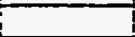 & I & & \\
\hline Waler Fow Ralo-GPM & & & & 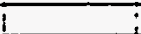 & & i & 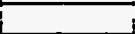 & i & i & \\
\hline Wator Inlet Temp.F & 196 & I & 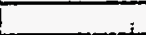 & & & I & 1 & i & & \\
\hline Water outot Temp.F & 214.1 & T & & t & & & 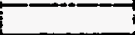 & + & & \\
\hline Inlat Glbm/hrint & 693033 & & Temp & 214.9 & Density & 72.6 & & & i & \\
\hline Subcoolingt & 8.7 & & & & & & & & & \\
\hline Flow Observation & & & & & & & & & & \\
\hline & ZONET & ZONE2 & ZONE3 $T_{2}$ & ZZONE4 & ZONES I & IZONE6 I & ZONE7 & ZONE8 & ZZNE9 & ZONE10 \\
\hline Heat Inplt-Watts(net) & $\pi$ & $1-60$ & 59.61 & $1-59.6$ & -58.11 & 1680 & 1667.3 & 1143.2 & T35.8 & 644.8 \\
\hline Heal Loss-Walls & 53.51 & 11.9 & 14.5 & 11.9 & 11.7 & 16.6 & 17.5 & 20.8 & 58.81 & 115.4 \\
\hline Hest Fionturitrite & 171 & 1040 & 1033 & 103 & 1007 & 29122 & 28902 & 19817 & 6297 & 11178 \\
\hline Wal Tomp. 35 & 216.3 & 210.6 & 220.61 & 220.8 & 222.41 & 237.2 & 237.7 & 268.71 & 330.11 & 242.7 \\
\hline WaH TOMp. H4F & 216.9 & 220.5 & 220.7 & 200.91 & 223.61 & 237.21 & 238.2 & 270.2 & 3311 & 242.7 \\
\hline Wall Temp. $\$ 5 F$ & 216 & 219.9 & 219.8 & 20.9 & 221.31 & 237.7 & 236.4 & 233.7 & 325.4 & 265.7 \\
\hline Wall Temp. \#1F & 78 & 219.9 & 219.8 & 220.9 & 220.5 & 241 & 238.4 & 231.9 & 319.4 & 306.8 \\
\hline Walt Temp. 27 & 274.5 & 220 & 220.1 & 219.2 & 220.5 & 245.8 & 238.9 & 231.4 & 320.1 & 308.5 \\
\hline Instream Temp.f & 216.8 & 215.8 & 215.9 & 215.9 & 216.4 & 217.8 & 220.8 & 223.3 & 223.2 & 223 \\
\hline Avg. Wall Tomp. $F$ & 215.9 & 2201 & 220.31 & 220.90 & 321.51 & 238.31 & 237.9 & 2472 & 2501 & 350 \\
\hline Avg.Wallot. & 0 ! & 4.2 & 4.41 & 5 & $5 !$ & 19.2 & 15.8 & खा & 26.6 & 26.5 \\
\hline Romaining Subcooff & 8.71 & & & $T$ & ! & $\div$ & 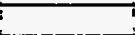 & & & \\
\hline Ext Qúainy & 01 & 01 & 01 & 01 & $0 !$ & का & 0 & 0 & 0 & 0 \\
\hline EXP.HBturtrtiar & 1733.4 & 250.4 & 237.31 & 208.6 & 200.21 & 15201 & 1827.6 & 8622 & 199.4 & 421.8 \\
\hline COMPUTEDH & & & & & & & & & & \\
\hline Jallouk & 178.2 & $17 \overline{2} .2$ & 178.11 & 178.1 & 178.11 & 178.11 & 178.1 & 178.21 & 178.11 & 178.1 \\
\hline Sradvil(Cudo hube) & 221 & 223.9 & 225.5 & 226.9 & 228.1 & 2292 & 230.1 & 230.9 & 237.9 & 232.8 \\
\hline Szady2 & 2211 & 223.9 & 225.5 & 226.9 & 228.1 & 220.2 & 230.1 & 230.9 & 237.9 & 232.8 \\
\hline (AID) & 112987 & 112358 & 112408 & 112396 & 112690 & 113548 & 115489 & 117205 & 117122 & 116969 \\
\hline (F) & 1.019 & 1.052 & 1.009 & 1.046 & 1.051 & 1.056 & 1.06 & 9.064 & 1.069 & 1.073 \\
\hline (HUQ) & 221 & 223.9 & 225.5 & 226.9 & 228.1 & 229.2 & 230.1 & 230.9 & 231.9 & 232.8 \\
\hline$(\mathrm{HBO})$ & 1.41 & 680.5 & 714.6 & 815.7 & 831.5 & 3305.5 & 27732 & 4161.5 & 4821.8 & 4801.4 \\
\hline & & & & & & & & & & \\
\hline & ZONEZ T & IZONE4 & ZONE 6 & ZZONE8 & ZONE10 & & & & & \\
\hline bPhquld Temp.f & \begin{tabular}{|l|} 
\\
\end{tabular} & & & $i$ & & & & & & \\
\hline DPLiquid Densihetbm/na & 90.511 & & & T & & & & i & & \\
\hline Elovationnt & 2) & 4 & 6! & 8 & 9.8751 & & & 1 & & \\
\hline Messured DRpid & 0.2081 & 0.498 & 0.884 & 1.19 & 1.8211 & & & & & \\
\hline Zaro Correctionse-psid & 0.0091 & 0.044 & 0.029 & 0.018 & -0.074 & & & $\frac{1}{i}$ & & \\
\hline Corrected DPPsid & 0.199 & 0.449 & 0.855 & 1.172 & 7.895 & & & $i$ & & \\
\hline ActualDP-t & 2.14 & 4.18 & 5.91 & 7.81 & 8.73 & & & 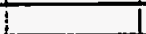 & & \\
\hline Measured TPPsig & 217.8 & & & & 2224 & & & 1 & & \\
\hline Messured Overalit & -9.32 & & & & & & & & & \\
\hline & & & & & & & & & & \\
\hline Computed DR & र.खा & 4.36 & 6.25 & 8.33 & 70.28 & & & & & \\
\hline Friction-t & 0.08 & 0.16 & 0.25 & 0.35 & 0.47 & & & & & \\
\hline Acoderation- & 01 & 0 & of & 0 & of & & & & & \\
\hline Elevation-t & 2 & 4 & 6 & 8 & 9.871 & & & & & \\
\hline & & it & & 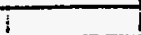 & & $i$ & & & & \\
\hline
\end{tabular}




\begin{tabular}{|c|c|c|c|c|c|c|c|c|c|c|}
\hline Run\# & $392 !$ & & & & & & & c & & \\
\hline Test Fluid & R114 & & & & & & & $T$ & & i \\
\hline Date: & आ5931 & & & & & & & & & ti \\
\hline Time: & $20: 161$ & & & & & & & 7 & & i \\
\hline Fow Areafli & 0.003831 & & $\bar{\vdots}$ & 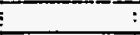 & $:$ & & $\therefore$ & $T$ & 7 & $i$ \\
\hline Hoet Flow Aroeth & 0.196861 & & 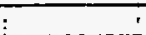 & 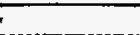 & & . & - & T & & T \\
\hline Sì. Prosecurögig & $\overline{2} \overline{1} \overline{2} . \overline{3}$ & & 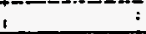 & 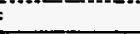 & & $\therefore$ & ? & י & 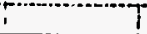 & $T$ \\
\hline S2t. Tomporature-f(inlat) & 221.71 & & & & & & & & i & 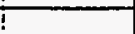 \\
\hline Liq. Density-1bmit3 & $72.52 !$ & 72.78 & 72.761 & 72.621 & 72.581 & 72.371 & 77.911 & $71.45 \mid$ & 71.451 & 71.58 \\
\hline Latent HeatBtulbm & 37.681 & 37.84 & 37.821 & 37.68 & 37.641 & 37.431 & 36.99 & 36.531 & 36.53 & 36.66 \\
\hline Specific Heat-Btunbmif & 0.286 & 0.286 & $0.286 \mathrm{i}$ & 0.2861 & 0.286 & 0.287 & 0.288 & 0.2881 & 0.288 & 0.288 \\
\hline Lig. Viscositylbmitiht & $0.406 !$ & 0.407 & 0.407 & 0.4061 & 0.405 & $0.403 !$ & 0.3971 & 0.3911 & 0.3911 & 0.393 \\
\hline La. Conduetivity-Btuhinf-il & 0.0257 & 0.02521 & $0.0252 !$ & 0.02511 & 0.02517 & $0.0 \mathrm{ZॉI}$ & 0.0247 & 0.02441 & 0.02441 & 0.0245 \\
\hline Wq. Prand No. & $4.62 !$ & 4.52 & $4.62^{\mathrm{T}}$ & 4.62 & 4.62 & 4.62 & 4.52 & 4.621 & 4.621 & 4.52 \\
\hline Inlelfow Rato-GPM & $4 !$ & & & & & & -1 & i & 7 & $i$ \\
\hline Outiot Lig. Flow Rate-GPM & $2.5 i$ & & & & & & 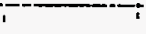 & 7 & $i$ & t \\
\hline Outlet Vap. Flow Rato-GPYM & $0 . \overline{9 i}$ & & & & & & $i$ & $i$ & $!$ & $!$ \\
\hline Inlel Temperaturof & $213.3 \mathrm{i}$ & & & & & & & 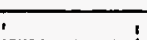 & : & 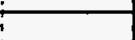 \\
\hline Outlot Lia. Temporaturaf & 220.2 & & 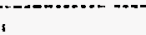 & & & 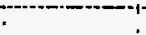 & i & $i$ & $\mathrm{j}$ & $\mathrm{i}$ \\
\hline Outlet Vap. Temperaturef & 219.11 & & $\vdots$ & $\overline{7}$ & 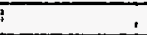 & & 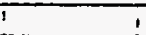 & 1 & 1 & 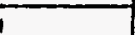 \\
\hline Water Flow Rato-GPM & & & 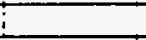 & & & & 1 & 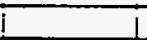 & 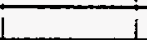 & 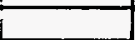 \\
\hline Water Inlet Temp.F & 197.4! & & $\vdots$ & & $\begin{array}{l}\vdots \\
\vdots\end{array}$ & & ! & 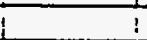 & 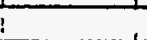 & \\
\hline Water outet Temp.F & $21 \overline{2.9 !}$ & & $i$ & & & : & T & $i$ & $i$ & \\
\hline Inlat Glbmhr/A2 & $695584 !$ & & Temp & 213.41 & Density & 72.871 & & & 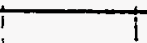 & \\
\hline Subcooling $F$ & 8.41 & & & & & & i & & 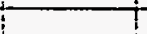 & \\
\hline Fow Observation & & & & & & & & & & \\
\hline & ZONE1 & ZONEZ I & ZONEB T & ZONE4 4 & ZONE5 12 & ZZONE6 & ZONE7 : & ZONE8 & ZONE9 & ZONETO \\
\hline Hoat input-Watis(not) & -1 & -81 & $\square \quad 80.51$ & 801 & $80.3 i$ & 1438 & T521.51 & 1015.8 & 354.2 & 305.2 \\
\hline Hoad Loss-Watts & 52.9 & 11.9 & 14.41 & 121 & 11.7 & 15.5 & 16.9 & 17.9 & 191 & 90 \\
\hline Head Flusturhitit? & 17 & 1404 & 1385 & 13871 & 13821 & 24927 & 26374 & 176051 & 6140 & 6331 \\
\hline Wall Tomp. \#̈ & 214.81 & दोव.8 & 220.71 & $\overline{221.4 T}$ & 222.61 & 234 & 2351 & 231.21 & 226.7 & 226 \\
\hline Wall Tomp. \#4F & 215.41 & 220.41 & $220.7 !$ & 229.41 & 223.71 & 2341 & 235.3 & 231.21 & 226.51 & 225.9 \\
\hline Wall Temp. \#5F & 214.5 & 219.9 & 219.9 & 221.4 & 221.61 & 234.6 & 233.7 & 229.8 & 225.5 & 226.4 \\
\hline Wall Temp. 11F & 78 & 219.6 & 219.9 & 221.1 & $220.8 i$ & 237.4 & 235.4 & 228.9 & 224.6 & 223.3 \\
\hline Wall Temp. \#2F & 213.11 & 2201 & 2201 & 219.8 & $220.6 \mathrm{i}$ & 241 & 235.91 & 228.51 & 225 & 224.7 \\
\hline Instream Temp.f & 214.8 & 213.9 & 214 & 214.8 & 215 & 216.2 & $218.7 !$ & 221.2 & $221.2 !$ & 220.5 \\
\hline Avg. Wall Temp.F & 214.5 & $-220 \mid$ & $220.3 !$ & 221.31 & $221.7 !$ & 235 & 235.11 & 229.9 & 225.5 & 25.3 \\
\hline Avg. Wallot $F$ & 01 & $6 !$ & $6.3 !$ & 6.51 & 6.61 & $77.7 !$ & $15.2 !$ & 7.9 & $4.7 !$ & 4.5 \\
\hline Remaining Subcooff & 8.41 & & & & & & & & 1 & \\
\hline Exto Quality & -01 & 01 & 0 & $0 i$ & 01 & 01 & 01 & o广 & 01 & 0 \\
\hline อXP. HEtuntriferF & 1733.41 & $233.6 !$ & 222.81 & 274.81 & $210.5 !$ & 1410.41 & 1738.81 & 2221.61 & 1511.3! & 1415 \\
\hline COMPUTEDH & & & & & & & & & & \\
\hline Jallouk & 178.7 & 178.71 & 178.71 & 178.6 & $978.6 丁$ & 178.6 & 178.61 & 178.6 & 178.6 & 178.6 \\
\hline Szady1(Cudo tube) & 221.61 & 224.61 & $226.2 !$ & 227.61 & 228.81 & 2229.9 & 230.91 & 231.7 & 232.7 & 233.7 \\
\hline Szady2 & 221.61 & 224.6 & 226.21 & 227.6 & 228.81 & 229.9 & 230.9 & 231.7 & 232.7 & 233.7 \\
\hline (RบO) & 112187L & 111640 & 111687 & 112148 & 112256 & 112971 & 114560 & 116174 & 116162 & 115678 \\
\hline (F) & 1.019 & 1.033 & 1.04 & 1.046 & 1.052 & 1.057 & 1.067 & 1.065 & 1.069 & 1.074 \\
\hline (HUQ) & 221.6 & 224.61 & 226.2 & 227.6 & 228.8 & 229.9 & 230.9 & 231.7 & 232.7 & 233.7 \\
\hline (HBOIL) & 1.41 & 981 & $1023.6 T$ & 105291 & 1089.7 & 3008.11 & 2619.8 & 1373.91 & 693 & 760.7 \\
\hline & & & & & & & & 1 & & \\
\hline & RONE2 I & ZONE4 & ZONE6 & ZONE8 & ZONE10 & & & $!$ & 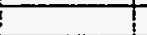 & \\
\hline DP liquid Tomp.f & 81.11 & 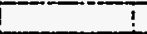 & 1 & i & $5-1$ & i & & $\mathrm{T}$ & $T$ & \\
\hline DP Liquid Densitytbm/113 & 90.48 & T & $T$ & $i$ & 1 & & t & i & T & \\
\hline Elovationft & 2ा & $4 !$ & 61 & 81 & 9.875 & & $i$ & 1 & $t$ & \\
\hline Mossured DP prid & 0.2381 & 0.483 & 0.8721 & 1.1411 & 1.6861 & & $\overline{1}$ & ! & 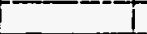 & \\
\hline Zero Corroctions-psid & 0.0001 & 0.0441 & 0.0281 & 0.0181 & $-0.074 !$ & & 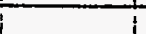 & 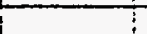 & & \\
\hline Corrected DPPsid & 0.229 & 0.439 & 0.8431 & 1.123 & 1.76 & & & i & 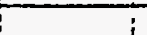 & \\
\hline Actual DPA & 2.07 & 4.17 & 5.89 & 7.851 & $8.94 !$ & & & & & \\
\hline Measured TPPsig & रा2.3 & & i & & $216.4 !$ & & & & & \\
\hline Measured Overallit & -8.25 & & & & & & & & I & \\
\hline & & & & & & & & & & \\
\hline Computed DPA & 2.081 & 4.161 & 6.ङा & 8.35 & 10.281 & & & & & \\
\hline Frictornt & 0.081 & 0.16 & 0.251 & 0.35 & 0.411 & & & & & \\
\hline Accoleration-it & 01 & 0 & 01 & of & 01 & & i & & & \\
\hline Elovation-t & 21 & 41 & 61 & 81 & 9.871 & & I & 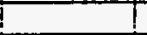 & t & \\
\hline & & & $!$ & 1 & & & 1 & 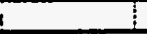 & & \\
\hline
\end{tabular}




\begin{tabular}{|c|c|c|c|c|c|c|c|c|c|c|}
\hline Runt & 3931 & & & & & & & & & \\
\hline Test Fuld & R114 & & & & & & & & & ? \\
\hline Dafe: & अ5 593 & & & & & & & & is & \\
\hline Timo: & $20: 31$ & & & & & & & & & \\
\hline Flow Areatti & 0.003361 & $!$ & 1 & $\dot{\vdots}$ & ¿ & i & $i$ & $!$ & $!$ & \\
\hline Hoed Flow Areathe & 0.18695 & $i$ & 7 & 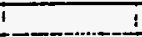 & 8 & $i$ & I & $!$ & $!$ & $i$ \\
\hline Sat. Prossure Psig & 200.4 & t! & 1 & & $i$ & $i$ & $i$ & $i$ & & $i$ \\
\hline Sal. Tomperatire f (inlet) & 216.8 & & $i$ & & 1 & i & & & 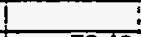 & $\vdots$ \\
\hline Lig. Density -1 om/t3 & 73.411 & 73.59 & 73.551 & 73.48 & 73.45 & 73.29 & 73.011 & 72.62 & 72.461 & 72.48 \\
\hline Letent HeatBtu/bm & 38.45 & 38.62 & 38.59 & 38.521 & 38.491 & 38.34 & 38.06 & 37.68 & 37.52 & 37.54 \\
\hline Specifio Heat-BtuhbmiF & 0.285 & 0.284 & 0.285 & 0.285 & 0.285 & 0.285 & 0.286 & 0.2861 & 0.287 & 0.287 \\
\hline Lig. Viscosity -lbmilithr & 0.415 & 0.4171 & 0.417 & 0.416 & 0.475 & 0.4141 & 0.41 & 0.4061 & 0.404 & 0.404 \\
\hline Liq. Conductivityetuhr fif & 0.02565 & 0.0257 & 0.02531 & 0.0257 & 0.025561 & 0.02551 & 0.0254 & 0.02511 & 0.025 & 0.0251 \\
\hline Lig. PranduNo. & 4.61 & 4.61 & 4.61 & 4.611 & 4.611 & 4.62 & 4.62 & 4.621 & 4.62 & 4.62 \\
\hline Inlet Fow Rato-GPM & 6 & - & $\ldots$ & & & $\ldots$ & & & & I \\
\hline Outlol La. Flow RA10-GPM & 3.61 & 3 & II & & & i & & 1 & 1 & T! \\
\hline Outlet Vap. Flow Rado-GPM & 0.91 & $\%$ & 1 & & $\vdots$ & 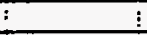 & & 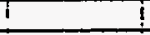 & & \\
\hline Inlet Temperaturof & 208.91 & & $\stackrel{-}{a}$ & & 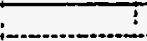 & $!$ & $\therefore$ & $\therefore$ & & I \\
\hline Outot Lig. Tomperaturof & 2131 & & 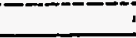 & & 1 & 1 & & $i$ & & I \\
\hline Outtet Vap. Temperaturef & 214.51 & & 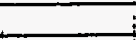 & & & & & 1 & 1 & $i$ \\
\hline \begin{tabular}{|l|} 
Water Flow Rato-GPM \\
\end{tabular} & & $\perp$ & $\perp$ & & 1 & 1 & & i. & & \\
\hline Water Inlot Temp. $F$ & 190.31 & t & & & & & & & & \\
\hline Water outtel Temp.F & 205.9 & T & 1 & 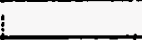 & & $\vdots$ & & & 1 & 1 \\
\hline Inlet G $16 \mathrm{~m} / \mathrm{hr} / \mathrm{th} 2$ & 1055858 & & Temp & 200.4 & Density & 73.74 & & & & \\
\hline Subcooling- & 7.91 & & & & & 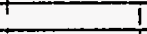 & & & & \\
\hline Fow Observation & & & & & & & & & & \\
\hline & ZONE] I & ZONE2 I & ZONE3 & ZZONEA & ZONE5 I & IZONE & ZONE7 & TZONE & ZONEO & ZONE \\
\hline Hod Input-Watts(net) & 11 & 79.41 & 80 & 80.7 & 78.81 & 1433.8 & 1515.5 & 1009.9 & $7^{-353.2}$ & 367.3 \\
\hline Hoad Loss-Walts & 51.21 & 11 & 12.9 & 10 & 9.2 & 13.9 & 15.91 & 17.3 & 18.8 & 86.8 \\
\hline Hoed Flox Etu/Hrite & 17 & 1376 & 1387 & 1309 & 13661 & 24851 & 26270 & 17506 & 6122 & 6367 \\
\hline Wall Temp. \& & 200.8 & $21 \overline{3} . \overline{1}$ & 213.9 & 214.6 & 215.31 & 2292 & 229.41 & 228.3 & 221.1 & 221.6 \\
\hline Wall Tomp. $4 F$ & 210.4 & 214 & 214 & 214.6 & 216.2 & 2022 & 2301 & 229.1 & 221.6 & 221.9 \\
\hline Wall Temp. & 209.9 & 213.8 & 213.4 & 214.6 & 214.5 & 229.61 & 228.6 & 226.7 & 220.6 & 221 \\
\hline Wall Temp. \#F & 78.7 & 213.6 & 213.5 & 214.6 & 213.9 & 232.3 & 230.6 & 224.5 & 219.6 & 218.8 \\
\hline Wall Temp. \$2F & 200.3 & 214 & 213.5 & 212.9 & 213.9 & 235 & 230.7 & 224 & 220.1 & 220.1 \\
\hline Instream Temp.f & 210.31 & 209.3 & 209.5 & 209.9 & 210.1 & 211 & 212.6 & 214.8 & 215.7 & 215.6 \\
\hline Avg. Waltemp.t & 809.91 & 213.81 & 213.7 & 214.6 & $244.6 !$ & 230.7 & 220.9 & 225.5 & 220.6 & 220.7 \\
\hline Avg.WallDT.F & $0 !$ & 4.4 & 4.2 & 4.6 & 4.51 & 18 & 16.1 & 10.9 & 4.6 & 4.8 \\
\hline Remaining Subcooff & 7.81 & & 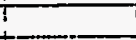 & & $:$ & & & & & T \\
\hline Ext Qualliy & 01 & 01 & 0 & 01 & 01 & - & 01 & 0 & 0 & 0 \\
\hline EXP.HEtu/Hrnea & 1733.41 & 313.71 & 333.2 & 301.71 & 3061 & 1384.5 & 1634.5 & 1601.8 & 1324.3 & 1328.8 \\
\hline COMPUTEDH & & & $\ldots$ & & & & & & & \\
\hline jallouk & 249.51 & $=249.5$ & 249.4 & 248.4 & 249.4 & 249.4 & 248.4 & 249.4 & 249.4 & 249.3 \\
\hline Szady1(Oudo tube) & 309.61 & 313.91 & 316.2 & 318.11 & 319.9 & 321.5 & 323 & 324.2 & 325.5 & 326.8 \\
\hline Szady2 & 309.6 & 313.9 & 316.2 & 318.1 & 319.9 & 321.5 & 323 & 324.2 & 325.5 & 326.8 \\
\hline (RID) & 166401 & 165555 & 165702 & 165013 & 166161 & 165893 & 168239 & 170165 & 170069 & 170861 \\
\hline (F) & 1.019 & 1.033 & $1 . \overline{41}$ & 1.047 & 1.053 & 1.059 & 1.003 & 1.067 & 1.071 & 1.076 \\
\hline (H)Q & 309.6 & 313.9 & 316.2 & 318.1 & 319.91 & 321.5 & 323 & 324.2 & 325.5 & 366.8 \\
\hline$(\mathrm{ABO})$ & 7.41 & 690.2 & 654.6 & 733 & 705.7 & 29525 & 2363.4 & 7820.7 & 759 & 788.9 \\
\hline & & & & & & & & & & \\
\hline & ZONE2 & ZONE4 & ZONE6 & ZONEB & ZONE10 & & & & & \\
\hline DP Hquld Temp.F & ! 81.4 & & ( & & & 7 & & & & \\
\hline DP Liquid Density & 90.441 & + & ! & i & & 1 & & & & \\
\hline Elovation $\mathrm{A}$ & 21 & $4 !$ & 6 & 8 & 9.875 & & & & & \\
\hline Measured DP Psid & $0 . \overline{162}$ & $0 . \overline{348}$ & $0 . \overline{664}$ & 0.76 & 1.1041 & & & & & \\
\hline Zoro Comactions-psid & 0.009 & 0.0441 & 0.029 & 0.0181 & -0.0741 & & & & & \\
\hline Conected DPPsid & 0.153 & 0.304 & 0.635 & 0.742 & 1.178 & & & $\perp$ & & i \\
\hline Actual DPA & 2.19 & 4.39 & 6.23 & 8.511 & 9.98 & & & & & \\
\hline Moasured TPPsig & 200.4 & & & & 203.4 & & & & & \\
\hline Mossured Overaltht & -5.96 & & 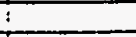 & & & & & & & \\
\hline & & & & & & & & & & \\
\hline Computed DPA & 2.17 & 4.34 & 6.52 & 8.69 & 10.73 & & & & & \\
\hline Friction-f & 0.17 & 0.34 & 0.51 & 0.69 & 0.85 & & & & & \\
\hline Accoloration-l & 01 & 0 & 0 & 0 & 01 & ot & & & & \\
\hline Elevalion-t & 21 & 4 & $\overline{6}$ & 81 & 9.871 & & & $T$ & & \\
\hline & 1 & 1 & 1 & $\overrightarrow{1}$ & 1 & i & & $\div$ & & I \\
\hline
\end{tabular}




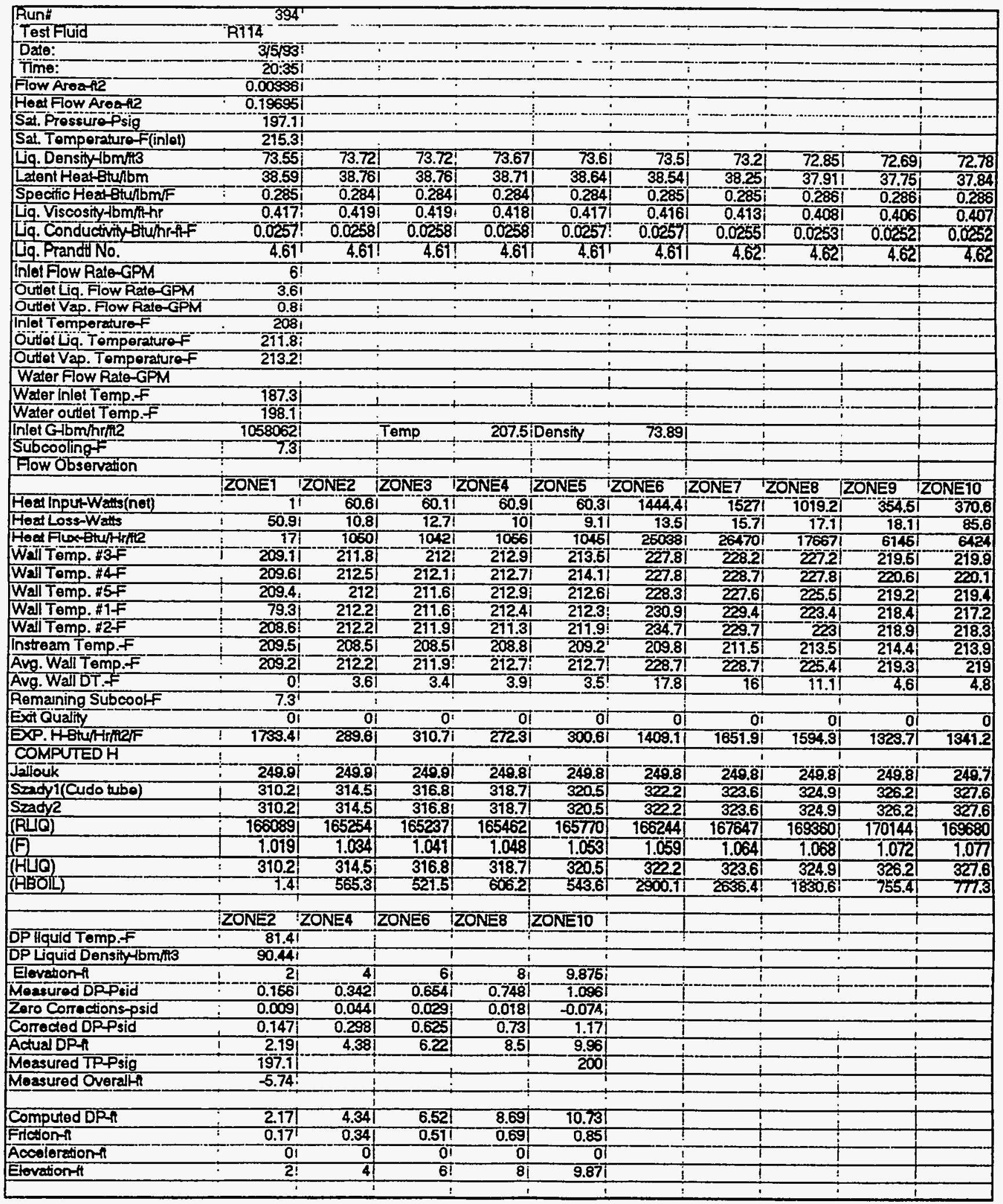




\begin{tabular}{|c|c|c|c|c|c|c|c|c|c|c|}
\hline Runt & उभा & $\Gamma$ & 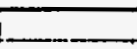 & & & & & & & \\
\hline Test Fuid & TATा4 $\rightarrow$ & & & & & & & & & \\
\hline Date: & $i \quad 35931$ & 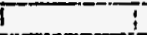 & 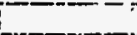 & & & & & & & \\
\hline Timo: & $20: 401$ & & & & 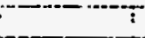 & & & & $\bar{\vdots}$ & 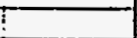 \\
\hline how Areatil & 0.003361 & 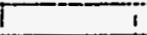 & $i$ & & 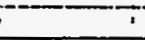 & 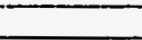 & & 5 & & 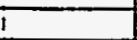 \\
\hline Hoad Flow Aroe-12 & 0.196961 & i. & 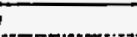 & $\therefore$ & $\therefore$ & $\therefore$ & : & 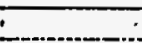 & & $!$ \\
\hline Sal. Pressurópsig & 194.81 & $\because$ & 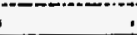 & : & $i$ & 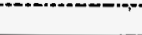 & & 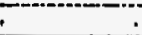 & & 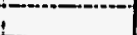 \\
\hline Sal. Tomparature-F(inlet) & 214.31 & : & & & & $!$ & +1 & & & 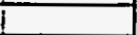 \\
\hline Lig. Density-lbmit3 & 73.71 & 73.88 & $73.83 !$ & $73.79 !$ & 73.71: & 73.55 & 73.261 & 72.961 & 72.811 & 72.92 \\
\hline Latont Heat Bturbm & 38.74 & 38.911 & $38.86 !$ & 38.831 & 38.74 & 38.59 & 38.3 & 38.01 & 37.87 & 37.97 \\
\hline Spocifio Heat-stulb m/F & 0.284 & 0.284 & 0.284 & 0.284 & $0.284 i$ & 0.2851 & 0.285 & 0.286 & 0.286 & 0.286 \\
\hline Lig. Viscositylbm/ithr & 0.4191 & 0.421 & 0.42 & 0.42 & 0.4191 & 0.417 & $0.413 i$ & 0.41 & 0.408 & 0.409 \\
\hline Uq. Conductivity stuhinttF & 0.02581 & 0.0259 & 0.0259 & 0.0258 & 0.02581 & 0.02571 & 0.02551 & 0.0253 & 0.0253 & 0.0253 \\
\hline Ug. Prand No. & 4.611 & 4.61 & 4.611 & 4.611 & 4.61 ! & 4.61 & $4.62 !$ & 4.52 & 4.62 & 4.62 \\
\hline Inlet flow Rato-GPM & $6 !$ & 1 & 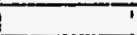 & & & & & & & \\
\hline Out of La. Fow Ralo-GPM & 3.71 & 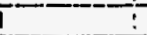 & 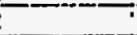 & & & i & 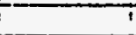 & & & 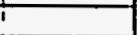 \\
\hline Outtet Vap. Flow Ralo-GPM & 0.81 & $!$ & 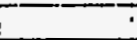 & 5 & 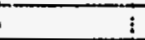 & 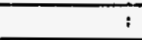 & 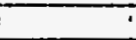 & t & & 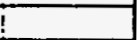 \\
\hline Inlet Tomperaturof & 206.91 & 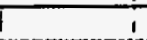 & 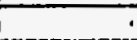 & i & 1 & ? & 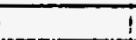 & 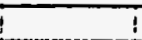 & & $!$ \\
\hline Outtet Lug. Tomporaturof & 211.21 & 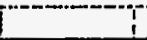 & 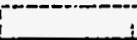 & 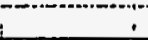 & 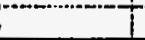 & 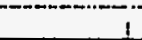 & 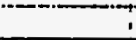 & 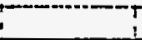 & & \\
\hline Outlat Vap. Tomperaturof & 212.11 & & 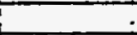 & 1 & 1 & & & 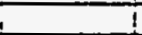 & & 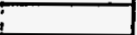 \\
\hline Water Flow Rato-GPM & & & & & & 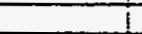 & & & & 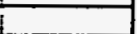 \\
\hline Water Inlot Temp.F & 185.1 & & 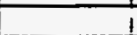 & 4 & 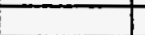 & $i$ & & & & t \\
\hline Waler outel Temp.f & 193.6 & it & - & -1 & & & & & & 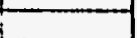 \\
\hline Inlet G-lbm/hrme & 1059524 & & Temp & $206.91 \mathrm{~L}$ & Density & 74 & & & & \\
\hline Subcoolingt & 7.41 & & & & & & & & & \\
\hline Flow Observation & & & & & & & & & & \\
\hline & ZONET & ZONE2 & ZONE3 & ZONE4 I2 & ZONE5 & ZONE6 & ZONET & ZONE8 & ZONE9 & ZONE10 \\
\hline Hoal Input-Wats(net) & 1 & 1001 & 1001 & -99.7 & 99.8 & 1441.81 & 1519.11 & 10127 & 353.31 & 370 \\
\hline HeadLoss-Watts & 50.51 & 10.9 & 12.81 & 10 & 9) & 13.3 & 15.51 & 17 & 181 & 85.2 \\
\hline Heed Floxetu/hint? & 171 & 1733 & 17331 & 17281 & 17301 & 24903 & 263531 & 17564 & 6124 & 6414 \\
\hline Wall Tomp. \#3F & 208.61 & 212.6 & 213.61 & $213.8 \Gamma^{-1}$ & 214.6 & 226.7 & 227.41 & 226.61 & 218.6 & 218.1 \\
\hline Wal Tomp. \#4F & 209.11 & 213.3 & 213.61 & 213.81 & 215.31 & 226.7 & 227.91 & 226.9 & 219.81 & 219.4 \\
\hline Wall Temp. $15 F$ & 208.61 & 212.8 & 212.61 & 213.9 & 213.91 & 27.5 & 226.8 & 224.8 & 218.41 & 218.7 \\
\hline Wall Temp. "IF & 79.3 & 212.61 & 212.5 & 213.71 & 213.2 & 230.31 & 228.5 & 222.51 & 217.6 & 216.3 \\
\hline Wall Temp. $2 F$ & 207.6 & 213 & 212.71 & 212.41 & 213 & 233.7 & 228.8 & 222 & 217.8 & 217.6 \\
\hline Instream Temp.F & 208.6 & 207.6 & 207.91 & 208.11 & 208.6 & 209.51 & 2917 & 212.9 & 213.7 & 213.1 \\
\hline Avg. Wall Temp.F & 208.51 & 212.91 & $213.1 !$ & 213.81 & 213.9 & 227.8 & 27.91 & 224.6 & 218.4 & 218.2 \\
\hline Avg. Wall DT.f & $0^{1}$ & 5.21 & 5.11 & 5.61 & 5.2 & 17.2 & 15.5 & 10.9 & 4.51 & 4.8 \\
\hline Romaining subcooff & 7.41 & & : & 1 & & & +1 & & & T \\
\hline Extt Quality & oi & 0 & $0 i$ & 01 & of & को & 01 & 0 & of & T \\
\hline EXP. HAtuHrnerF & 1733.41 & 39.6 & 340.11 & 307.4 & \$4.51 & 1455.61 & 17001 & 1812.5 & 137221 & 1327.8 \\
\hline COMPUTEDH & & & & & & & & & & 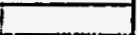 \\
\hline Jallouk & 250.21 & 250.21 & 250.11 & 250.11 & 250.7 & $260.1 \dagger$ & 250.11 & $250 . \overline{1}$ & 2501 & 250 \\
\hline Szady1(Oudo bubo) & 310.5 & 314.9 & $317.1 i$ & 319.11 & 320.91 & 322.6 & 324 & 325.3 & 326.6 & 320 \\
\hline szady2 & 310.5 & 374.9 & 317.1 & 319.1 & 320.9 & 322.6 & 3241 & 325.3 & 326.6 & 328 \\
\hline (स्पि) & 165588 & 164765 & 164986 & 165129 & $165513 \mid$ & 166227 & 167624 & 169067 & 169754 & 169208 \\
\hline (i) & -1.019 & 1.034 & 7.0411 & 1.048 & 1.054 & 1.059 & 1.064 & 1.0 .58 & 1.072 & $\frac{-07}{1.07}$ \\
\hline (HपDQ) & 310.5 & 314.9 & 397.11 & 399.1 & 320.9 & 322.6 & 324 & 325.31 & 326.61 & 328 \\
\hline (HBOLL) & 1.4 & 812.6 & 7981 & 883.3 & 813,31 & 279.1 & 2541.7 & 7790.51 & 722.11 & 779.9 \\
\hline & & & & & & & & & & \\
\hline & ZONE2 & ZONE4 & ZONE6 & ZONE8 & ZONETO & & & & & \\
\hline DPHquld Temp.f & 81.4 & i & - & & ! & & & & & \\
\hline DPLiquid Density bmitt3 & 90.44 & 1 & 1 & & & & & & & \\
\hline Elovetion-t & 2 & $4 !$ & $6 i$ & 8 & 9.8751 & & & & & \\
\hline$\vec{M}$ Easured DopPsid & 0.157 & 0.338 & 0.6471 & 0.743 & 1.006 & $t$ & & & & \\
\hline Zoro Conrections-psid & 0,000 & 0.044 & 0.0291 & 0.0181 & -0.0741 & & & & & \\
\hline Corrocted DPFsid & 0.148 & 0.294 & 0.618 & 0.725 & 1.17 & & & & & \\
\hline Actual DPA & 2.19 & 4.38 & 6.22 & 8.49 & 9.94 & & & & & \\
\hline Measured TRPsig & 194.8 & -1 & & & 197.8 & & & & & \\
\hline Measured Overalth & 5.92 & & & & & $t$ & 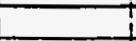 & & & \\
\hline & & & & & & & & & & \\
\hline Computed DPh & 2.17 & 4.34 & 6.521 & 8.69 & 10.731 & & & & & \\
\hline Frition-a & 0.17 & 0.341 & 0.511 & 0.59 & 0.851 & & & & & \\
\hline Accoloration- & of & 0 & 01 & $0 !$ & $0.0 \pi$ & & & & & it \\
\hline Elevation- & 2) & 4 & 61 & 8 & 9.871 & & 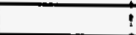 & & & i \\
\hline & & & & & & & & & & \\
\hline
\end{tabular}




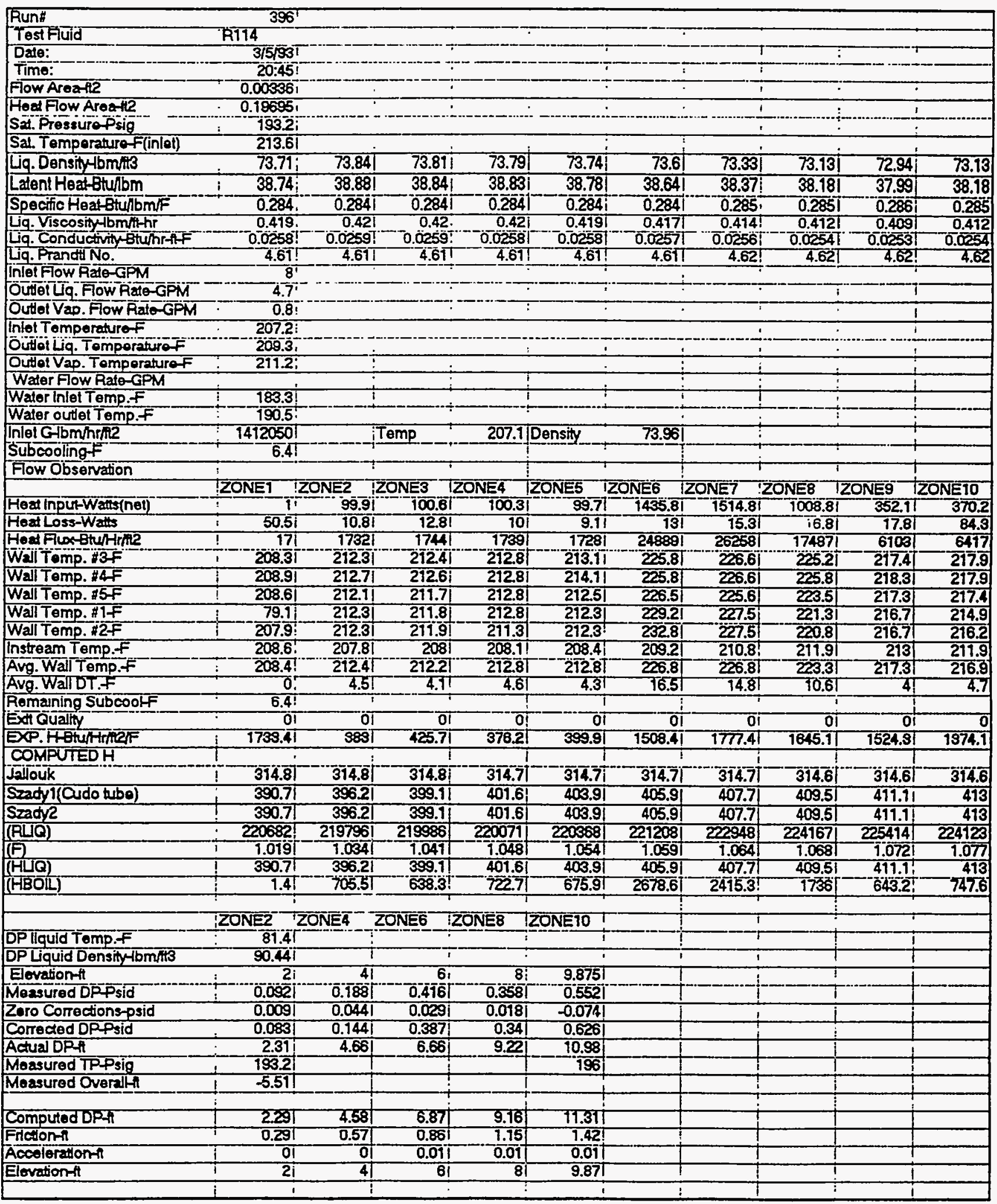




\begin{tabular}{|c|c|c|c|c|c|c|c|c|c|c|}
\hline Run\# & 397 & & & & & & & & & \\
\hline Test Fuld & :R714 & & & & & & & & & \\
\hline Date: & अ अं5क्31 & $!$ & & & & : & & I & & \\
\hline Timo: & 20.501 & iٓ & & & $\bar{Z}$ & $!$ & & $\overline{1}$ & $!$ & $!$ \\
\hline Fow Areaft2 & 0.003361 & I & & & $i$ & $!$ & 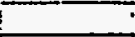 & $\div$ & & T \\
\hline Hodflow Areatt2 & $0.19695 i$ & $i$ & & & i & 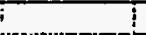 & 1 & 1 & & T \\
\hline Sä. Pressurópgig & 191.51 & & $\therefore$ & $i$ & $\because$ & ! & ! & 7 & & 1 \\
\hline Sal. Tamperatureff(inlat) & 212.9 & & & & 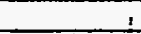 & $\leq$ & & 1 & & 1 \\
\hline Lig. Density-bm/it3 & 73.77 & 73.971 & 73.881 & 73.911 & $73.83 i$ & 73.721 & 73.46 & 73.221 & 73.13 & 73.24 \\
\hline Latent Heal-Btulbm & 38.81 & 38.941 & 38.91 & 38.941 & 38.861 & 38.78 & 38.51 & 38.27 & 38.18 & 38.28 \\
\hline Specifio Heat-BtunimiF & 0.284 & 0.284 & 0.284 & 0.284 & $0 . \overline{84 !}$ & 0.284 & 0.285 & 0.285 & 0.285 & 0.285 \\
\hline Lig. Viscositylbmithr & 0.419 & 0.4211 & 0.4211 & 0.4211 & 0.42 & 0.4191 & 0.416 & 0.413 & 0.412 & 0.413 \\
\hline Lig. Conduetvity Btuhe-if & 0.0258 & 0.0259 & 0.0259 & 0.0259 & $0.0259 !$ & 0.02581 & 0.0256 & 0.0255 & 0.0254 & 0.0255 \\
\hline Lja.PTandd No. & 4.611 & -4.611 & 4.611 & 4.611 & $4.67 !$ & 4.611 & 4.61 & 4.52 & 4.62 & 4.62 \\
\hline Inlel Flow Ralo-GPM & 81 & & & 1 & : & ț & & & & 1 \\
\hline Outef La. Fow Ralo-GPM & 4.71 & i & T & $\mathbf{i}$ & 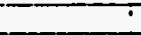 & 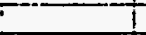 & & $i$ & & 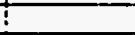 \\
\hline Outlet Vap. Flow Ralo-GPM & 0.81 & $T$ & 1 & $i$ & 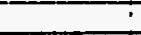 & - & & T & & i \\
\hline inlol Tomperaturof & $206.6 i$ & $\bar{i}$ & & & $T$ & I & & 1 & & $i$ \\
\hline Outtol Lq. Tomperaturef & 209.21 & & $\because$ & 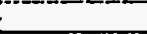 & $\div$ & $\underline{!}$ & & $i$ & & \\
\hline Outlat Vap. Tomporature F & 210.11 & & & & & $i$ & 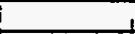 & & & I \\
\hline Wader Flow Rato-GPM & & ! & & & $\div$ & \pm & & 1 & & \\
\hline Water Inlet Tomp.F & 181.3 & & & 1 & i & I & & $i$ & & \\
\hline Water outel Temp.F & 1881 & & & & & & & & & \\
\hline Inlet Glbminrin2 & 1414642 & & Temp & 206.311 & Density & 74.11 & & & & \\
\hline Subcooling & 6.31 & T & & & & & & T & & \\
\hline \multirow[t]{2}{*}{ Fow Observation } & & & & & & & & & & \\
\hline & ZONEI & ZONE2 & ZONE3 & ZONE4 & ZONES I & ZONE6 II & ZONE? & ZONE 8 & ZONEG & ZZONETO \\
\hline Heat inpun-Watts(net) & $i-11$ & 180.9 & 81.21 & 80.51 & 79.2 & 14332 & 1512.5 & 1005.81 & 354 & 370.8 \\
\hline Hod Loss-Watts & 50.11 & 10.7 & 12.61 & $100^{\circ}$ & 9.11 & 12.7 & 15.21 & 16.6 & 17.7 & 88.6 \\
\hline Heat Flox-Btu/Hrm? & 171 & 1402 & 14081 & 1395 & 1373 & 24844 & 26218 & 174351 & 61351 & 6428 \\
\hline Wall Temp. & 207.7 & 210.7 & 211.21 & 211.51 & 211.8 & 25.21 & 225.9 & 224.51 & 216.7 & 217 \\
\hline Wall Tomp. \#4F & 2008.21 & 211.31 & 211.41 & 217.51 & 212.6 & 225.31 & 226.1 & 224.91 & 217.8 & 217.4 \\
\hline Wall Tomp. \#6F & 208.2 & 210.7 & 210.6 & 271.5 & 211.2 & 2261 & 225 & 2229 & 216.7 & 216.5 \\
\hline Wall Temp. "\#1F & 79.2 & 210.7 & 210.6 & 211.5 & 210.9 & 228.51 & 226.7 & 220.8 & 215.8 & 274.5 \\
\hline Wall Temp. "2F & 207.3 & 211.2 & 210.7 & 209.9 & 210.9 & 231.9 & 227 & 20.3 & 216.2 & 215.5 \\
\hline Instream Temp.F & 208.2 & 207.4 & 207.6 & 207.41 & 207.9 & 208.5 & 210 & 211.4 & 211.9 & 211.3 \\
\hline Avg. Wa TTemp.F & उ०7.9! & 217 & हाना & 217.51 & 217.41 & 220.31 & 22.1 & 22271 & 216.61 & 216.2 \\
\hline Avg.WalloT. & $0 !$ & 3.5 & 3.3 & 41 & $3.4 !$ & 16.6 & 15 & 10.5 & 4.5 & 4.6 \\
\hline Remaining Subcooff & 6.31 & & & & & & & & & \\
\hline Ext Quallity & 01 & 0 & of & 01 & 01 & 0 & 0 & 01 & of & \\
\hline EXP. HBtultrilerF & 1738.4 & \$99.8 & 425.11 & 345.71 & 399.31 & 1494.1 & 1753.7 & 1681.7 & 18751 & 1400.5 \\
\hline COMPUTEDH & & & & & 1 & 4 & & & & \\
\hline Jallouk & 316.3 & 315.2 & $31 \overline{6.21}$ & 315.2 & 316.21 & 315.1 & 316.1 & 316.1 & 316.11 & 316 \\
\hline Szady1(Cudo bube) & 391.31 & 396.81 & 399.7 & 4022 & 404.51 & 406.6 & 408.4 & 410.11 & 411.9 & 413.7 \\
\hline szady 2 & 391.3 & 396.8 & 399.71 & 40221 & 404.5 & 406.6 & 408.41 & 410.1 & 411.9 & 413.7 \\
\hline (RLQ) & 220658 & 21977 & 219966 & 21973 & 220240 & 220858 & 222468 & 224010 & 224556 & 223852 \\
\hline (F) & 1.019 & 1.034 & 1.041 & 1.048 & 1.054 & -1.059 & 1.064 & 1.068 & 1.073 & 1.078 \\
\hline (HUQQ) & 391.31 & 366.8 & 399.7 & 402.21 & 404.5 & 406.61 & 408.4 & 410.1 & 411.9 & 413.7 \\
\hline \multirow[t]{3}{*}{ (FBOIL) } & 1.3 & 543.3 & 51ट2ा & 626.7 & 533.1 & 2688.5 & 2433.7 & 1707.5 & 713.6 & 731.6 \\
\hline & & & & & & & & & & \\
\hline & ZONE2 & ZONE4 & ZONE6 & ZONE8 & ZONE10 T & & & & & \\
\hline DP llqud Tomp.F & 81.7 & & & & & T & I & & & \\
\hline DPLiquid Density 4bm/t3 & 90.411 & 1 & 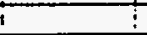 & 1 & 1 & 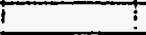 & 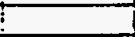 & & & \\
\hline Elovation $\mathrm{A}$ & 21 & $4 !$ & 61 & 81 & 9.8751 & $i$ & & & & \\
\hline Mesured DPFsid & 0.089 & 0.183 & 0.4091 & 0.349 & 0.5381 & & & & & \\
\hline Zaro Cornections-psid & 0.000 & 0.0441 & 0.029 & 0.0181 & -0.0741 & & & & & \\
\hline Corrected DPPsid & 0.08 & 0.139 & 0.381 & $0.331 !$ & 0.612 & & & & & \\
\hline Actual DPA & 2.31 & 4.66 & 6.66 & 9.23 & 10.99 & & & & & \\
\hline Measured TPPsig & 191.5 & & & & 194.2 & & & & & \\
\hline \multirow[t]{2}{*}{ Measured Overallf } & -5.31 & & & & & & & & & \\
\hline & & & & & & & & & & \\
\hline \multirow{5}{*}{$\begin{array}{l}\text { Computed DP } \\
\text { Friction-f } \\
\text { Acouleration-1 } \\
\text { Elevation-t }\end{array}$} & 2.29 & 4.581 & 6.87 & 9.16 & नर.उा & & & & & \\
\hline & 0.20 & 0.57 & 0.861 & 1.15 & 7.43 & & & & & \\
\hline & 0 & 0 & 0.011 & 0.011 & 0.011 & & & & & \\
\hline & 2 & 4 & $6 i$ & 81 & 9.871 & & i & I & & I \\
\hline & 7 & T & $?$ & 1 & i & & & T & & I \\
\hline
\end{tabular}




\begin{tabular}{|c|c|c|c|c|c|c|c|c|}
\hline Runt & 398 & & & & & & & \\
\hline Test Fiud & R114 & & & & & & & \\
\hline Date: & $3 / 5 / 93$ & & & & & & & \\
\hline Time: & $21: 01$ & & & & & & & \\
\hline Flow AJea-12 & 0.00336 & & & & & & & \\
\hline Heal Flow Area-ti2 & $0.19695:$ & & & & & & & \\
\hline Sat. Pressure Psig & 190.11 & & & & & & & \\
\hline Să Temperaturo-(inlet) & 212.31 & i & & & & 7 & 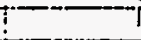 & $\Gamma$ \\
\hline Wq. Density $4 \mathrm{bm} / \mathrm{m}$ & 73.961 & 74.061 & $73 . \overline{911}$ & $73 . \overline{81}$ & 73.531 & 73.331 & $73.22 !$ & 73.34 \\
\hline Latent Heat-Btulbm & 38.991 & 39.091 & 38.941 & 38.84 & 38.571 & 38.37 & 38.271 & 38.89 \\
\hline Spocitic Hoaterubmit & $0.284 i$ & 0.2841 & 0.2841 & 0.2841 & 0.2851 & 0.2851 & 0.285 & 0.285 \\
\hline iq. Visoosit ibm/ithr & 0.4221 & 0.4231 & 0.4217 & 0.421 & 0.4761 & 0.414 & 0.413 & 0.414 \\
\hline Ig. Conductivy Btumt-4f & 0.02591 & 0.0261 & 0.02591 & 0.02591 & 0.02571 & 0.02561 & 0.02551 & 0.0256 \\
\hline Liq. Prandit No. & $4.61:$ & 4.611 & 4.611 & 4.61 & $4.67 !$ & 4.621 & 4.62 & 4.62 \\
\hline Inlet Fow Rate-GPM & 8 & 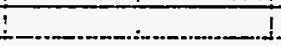 & & & & & & i- \\
\hline Outlel Lig. Flow Rato-GPM & 4.7. & & & & & 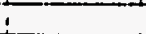 & & \\
\hline Outlol Vap. Flow Rato-GPM & $0.8:$ & & & & $i$ & & & \\
\hline Inlet Temperature $\mathrm{F}$ & $206 !$ & & & & & & & \\
\hline Outert iq. Temperaturef & $208.4 !$ & & & & & & & \\
\hline Outiel Vap. Temperaturo-F & $209.1 !$ & & & & T & $T$ & & \\
\hline Water Fow Rato-GPM & 1 & $i$ & 1 & & & $i$ & $i$ & I \\
\hline Water inlet Temp.F & 180.11 & $T$ &. & & + & $i$ & & $T$ \\
\hline Water outlot Tomp.F & $186 !$ & & $\therefore$ & & & T & & $\div$ \\
\hline Inlet $\mathrm{G}$ bmihrine & 14746421 & Temp & Donsity & 74.1 & & $i$ & & ? \\
\hline Subcooling $F$ & $6.3 !$ & {$[-. .11$} & 1 & & & 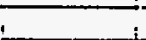 & & 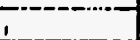 \\
\hline Fow Observation & & & & & & & & 1 \\
\hline & ZONE1 ZONE2 : & ZONE3 ZONE4 I & ZONE5 & ZONE6 - & ZONE7 & ZONE8 & ZONE9 & ZONE10 \\
\hline Heat Input-Watts(nel) & $1 ! \quad 1201$ & $120 i \quad 120$ & 120 & 1420.5 & 1464.9 & $\quad 343.4$ & 203.6 & 246.9 \\
\hline Heat Loss-Watts & $49.6 i$ & $12.7 i$ & 9.1 & 12.5 & $15:$ & 16.5 & 17.6 & 83.2 \\
\hline Hoat Flux-Btúfinit2 & 20801 & 20801 & 20301 & 24623 & 25393 & 16353 & 3529 & 4280 \\
\hline Wall Temp & 207.11 & 211.8 ! & 21271 & 224.4 & 225.11 & 234.1 & 216.21 & 26.5 \\
\hline Wall Temp. HF & $207.7 !$ & 212.6 & 213.5 & 224,3 & 225.6 & 224.47 & $216 . \overline{9}$ & 216.6 \\
\hline Wail Temp. & 207.91 & $211.2 !$ & 212.31 & 225.41 & 224.5 & $2222 !$ & 215.91 & 276 \\
\hline Wal Tomp. IF & 211.3 & $211.2 !$ & 211.91 & 227.9 & 226.21 & 220.2 & 215.31 & 218.8 \\
\hline Wall Temp. & $205.8 !$ & $211 . \overline{2}$ & $211.7 i$ & 231.4 & 226.51 & 2201 & 215.31 & 214.7 \\
\hline Inetroam Tomp. $F$ & 207.17 & 206.51 & 207.41 & 208 & 209.61 & 210.81 & 211.41 & 210.7 \\
\hline Avg. Wal Tomp.F & $207.2 i$ & 211.6 & $212.4 !$ & 225.5 & 225.61 & 22221 & $2 \overline{15.9 i}$ & 215.5 \\
\hline Avg. Wall DT.F & $0 . \overline{1}$ & $5.5 !$ & $4.9 !$ & 16.41 & 14.81 & 10.6 & $4 . \overline{4 !}$ & 4.6 \\
\hline Remaining Subcoolf & 6.31 & & & & & & & \\
\hline at Quality & $0 !$ & 0 & $0_{i}$ & 0 & $\overline{0}$ & of & 01 & \\
\hline EXP.HBtuHriterF & $233.6 i$ & 419.7 & $428.4 i$ & 1502.6 & 1712 & 1536.8 & 809.41 & 921.1 \\
\hline COMPUTEDH & & & & & & & & \\
\hline Jallouk & 315.3 & 375.21 & 315.2 & 315.11 & $315.1 t$ & 375.11 & 315.11 & 315 \\
\hline Szady I(Cudo tubs) & 391.41 & 399.81 & 404.6 & 406.7 & 408.5 & 410.21 & 411.9 & 413.8 \\
\hline szady2 & $391.41-396.9$ & 399.81 & 404.61 & 406.7 & 408.51 & -410.2 & -411.91 & 413.8 \\
\hline (RLO) & $2194951 \quad 218838$ & 2188181 & 2197111 & 220324 & 2220281 & 223334 & 2239381 & 223178 \\
\hline$(F)$ & 1.034 & 1.0471 & 1.054 & 1.059 & 1.064 & 1.009 & 1.0731 & 1.078 \\
\hline 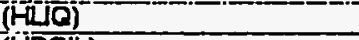 & 391.41 & 399.81 & 404.61 & 406.71 & 408.51 & 410.2 & 411.9 & 413.8 \\
\hline ( $\mathrm{HBOLL})$ & 789.1 & 769.21 & $7572 !$ & 2641 & 2407.41 & 1725.9 & 694.71 & 7338.2 \\
\hline & & & & & & & & \\
\hline & ZONE2 ZONE4 & ZONE6 LONE8 & ZONE10 & & & & & 1 \\
\hline DPliquid Temp.F & $81 . \overline{1}$ & & i & & & & & $i$ \\
\hline DPLiquid Densitylbmit3 & $90.41 i$ & & & & & & & \\
\hline Elevation-t & $2 !$ & & 9.875 & & & & & \\
\hline MeasuredDPPSid & 0.087 & $0.402 !$ & 0.5335 & & & & & \\
\hline Zoro Corrections-psid & 0.009 & 0.029 & $=0.074$ & & & & & \\
\hline Corrected DP pild & 0.0781 & 0.3731 & 0.6071 & & & & & \\
\hline Actual DPA & 2.311 & 6.681 & 10.981 & & & & i & i \\
\hline Moasured TPPSig & 190.11 & $i$ & 198.11 & & & & & \\
\hline Measured Overalin & -5.891 & 1 & 1. & & & & & \\
\hline & & & & & & & & \\
\hline Computod DP-1 & 4.58 & 9.16 & $11.3 i$ & & $i$ & & & 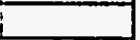 \\
\hline Friction-f & 0.57 & 0.86 & $1.42 !$ & & & & & \\
\hline Acceleration- & 0 & 0.011 & $0.01 !$ & & & & & \\
\hline Elevation-t & 41 & 6. & 9.87 & & & & & \\
\hline
\end{tabular}




\begin{tabular}{|c|c|c|c|c|c|c|c|c|c|c|}
\hline Pun" & 399 & & & & & & & & & \\
\hline Testfluid & R194 & & & $:$ & & & ! & T & t & \\
\hline Date: & अ5931 & 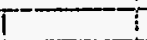 & & & & & 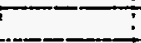 & 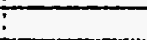 & & t \\
\hline Time: & i-—-21:19i & & & & & & $!$ & $!$ & & \\
\hline Note & TABORER & Anaryicald & & & & 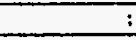 & & : & & $!$ \\
\hline Fow Area-fl2 & 0.003361 & & $\therefore$ & 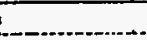 & & 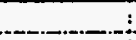 & $\therefore$ & 1 & 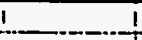 & 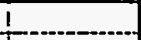 \\
\hline Hoat Flow Aroz-H2 & 0.18685 & & $i$ & & 1 & & 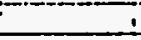 & I & 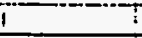 & 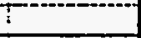 \\
\hline Sal. Pressurofasig & $172.7 !$ & &. & 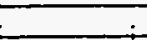 & 1 & $\therefore$ & 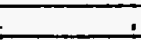 & $i$ & 1 & 1 \\
\hline Sat. Temperature-f(inlet) & 204.31 & 1 & & 1 & & 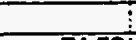 & & ! & 1 & 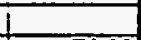 \\
\hline La. Density 1 bmitis & 74.77 & 74.7 & 74.58 & 74.531 & 74.55 & 74.52 & 74.47 & 74.51 & 74.52 & 74.68 \\
\hline Laten I Hoat Btulbm & 39.78 & 39.71 & 39.6 & 39.551 & 39.57 & 39.54 & $39.49 i$ & 39.52 & 39.54 & 39.7 \\
\hline Specifio Heat-BhulbmiF & 0.282 & 0.282 & 0.282 & 0.2831 & 0.2831 & 0.2831 & 0.2831 & 0.283 & 0.283 & 0.282 \\
\hline L ia. Viscosity & 0.431 & 0.431 & $0.429 !$ & $0.428 !$ & 0.4281 & $0.4 \times 2$ & 0.428 & 0.4281 & 0.4881 & 0.43 \\
\hline Lq. Conductivity stuheft & $0.0264 T$ & 0.0864 & 0.0263 & $0.0253 !$ & $0.0263 !$ & 0.02631 & 0.02631 & 0.0233 & 0.02631 & 0.0264 \\
\hline Iq. Prandt No. & $-\frac{1.61}{4.61}$ & 4.6 & 4.61 & 4.61 & 4.61 & 4.6 & 4.6 & 4.61 & 4.6 & 4.6 \\
\hline In & 5.7 & & & & -1 & 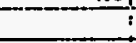 & & 1 & 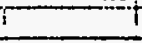 & \\
\hline Outlot Lig. Flow Rato-GPM & $2.9 !$ & & 7 & & 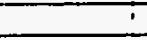 & 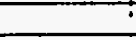 & 1 & i & 1 & 1 \\
\hline Outiot Vap. Flow Rato-GPM & 1.5 & & & & 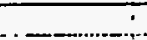 & $\therefore$ & 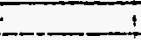 & 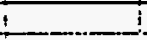 & 1 & \\
\hline Inlot Tomporeaurof & $200 . \overline{6}$ & & & & $\because$ & 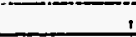 & & \pm & & \\
\hline Outrol Lua. Temperaturef & 202.11 & & & & $i$ & $\overline{1}$ & i & $i$ & & $i$ \\
\hline Outtel Vap. Temporaturef & 201.9 & & $i$ & 1 & $i$ & $\vec{i}$ & & & & \\
\hline Water Flow Rate-GPM & 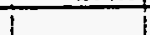 & & & & $i$ & $i$ & i & $!$ & & \\
\hline Water inlet Temp.f & 65.6 & & & 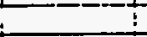 & + & 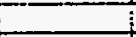 & & 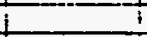 & 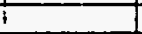 & \\
\hline Wader outlet Temp.f & 198.8 & & & $i$ & I & 1 & & & & \\
\hline |ntat Glbm/hr/n2 & 1027453 & & Temp & $197.6]$ & Density & 75.53 & & 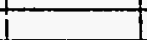 & & \\
\hline Subcooling & 3.7 & & & 1 & (2) & & & & & \\
\hline Fow Observation & & & & & & & & & & \\
\hline & ZONET & TZONE2 & ZONE3 - & ZONE $4-\overline{12}$ & ZONE5 T2 & ZONE & ZONET & ZONE8 & ZZONEE I & IZONETO \\
\hline Hoallnpu-Watts(nef) & 1162.6 & 1170.1 & 1147.21 & $\mid 1147$ & 1150.5 & 1151.5 & 1147.9 & 1158 & 1148.1 & 11147.8 \\
\hline Hoed Losg-Walls & 52.51 & 111 & 13 & 10 & 9.21 & 9.6 & 12.91 & 15.11 & 17.3 & 81.6 \\
\hline 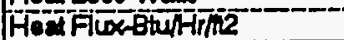 & 20163! & 20283 & 18886 & 18883 & $18943 i$ & 18061 & 18388 & 20039 & $1 \% 02$ & $198 \% 3$ \\
\hline Wall Tomp. 3 3F & 214.5 & 217.2 & 214.91 & 215.3 & 218.71 & 215.6 & 214.31 & 216.11 & 216.11 & 217.2 \\
\hline Wall Temp. $4 F$ & 214.9 & 214.1 & 216.81 & 216.6 & $217.3 !$ & 215.6 & 214.11 & 216.11 & 217.4 & 216.3 \\
\hline Wall Temp. $15 F$ & 214.11 & 212.6 & 217.5 & 215.4 & 215.3 & 216.3 & 213.3 & 214.31 & 213.6 & 215.3 \\
\hline Wall Temp. TF & 80.5 & 215.7 & 213.5 & 215.1 & 214.9 & 218.6 & 216.2 & 213.9 & 213.1 & 210.5 \\
\hline Wall Temp. \#2F & 215.7 & 215.41 & 214.71 & 217.31 & 215.1 & 221.1 & 216.1 & 213.41 & 214.8 & 213.3 \\
\hline Instream Temp. $F$ & 202.3ी & 202.71 & 203.41 & 203.71 & 203.6 & 203.8 & 204.11 & 203.9 & 203.8 & 202.8 \\
\hline Avg. Wall Temp.t & $214.8 !$ & 215.61 & 215ा & 215.61 & 215.7 & 216.5 & 2) & 214.81 & 215 & 214.5 \\
\hline Avg.WallDT.F & 11.61 & $\frac{12}{12}$ & 70.71 & $11 !$ & 11.11 & 11.8 & 9.81 & 10 & 10.3 & 10.8 \\
\hline Romaining subcootf & -0.41 & -1 & & $!$ & $\mathrm{T}$ & & & $T$ & & \\
\hline Ext Quality & 01 & 0.08 & 0.081 & 0.09 & 0.121 & 0.15 & 0.18 & 0.21 & 0.291 & 0.28 \\
\hline EXP.HBluHrhe/F & 1738.81 & 1692.6 & 185261 & 1807.31 & 1788.81 & 1688.8 & 2030.31 & 2013 & 1932.1 & 0 \\
\hline COMPUTEDH & & & & & $-\bar{i}$ & & & & & \\
\hline Jallouk & 1233.2 & 1176.91 & 1127.4 & 1097.31 & 1068.51 & 1053 & 1036.5 & 1023.6 & 1004.6 & 984.9 \\
\hline Szady1(Cudo tube) & 1767.9 & 1777.7 & 1766.7 & 1771.5 & 177.5 & 1783 & 1785.6 & 1795.11 & 1793.2 & 1733.5 \\
\hline $\begin{array}{l}\text { Szady2 } \\
\end{array}$ & 1658 & 1668.5 & 1681.5 & 1686.11 & 1691.4 & 1696.4 & 1697.9 & 1706.8 & 1702.1 & 1699.1 \\
\hline$(R L Q)$ & 155738 & 153552 & 149523 & 1452411 & 140673 & 136286 & 131956 & 127308 & 1227 & 117679 \\
\hline$(a)$ & 1.09 & 1.42 & 1.74 & 1.974 & 2.172 & 2.343 & 2.496 & 2.642 & 2.77 & 2915 \\
\hline$\frac{(1)}{(H-1) Q)}$ & 324.1 & 421.9 & 517.31 & 586.7 & 645.5 & 696.4 & 741.9 & 785.2 & 825.4 & 866.5 \\
\hline (HBDIL) & 1764.3 & 1759.7 & 7757.81 & 1749.81 & 1748.6 & 1746.8 & 1747.8 & 1743.5 & 1732.9 & 1723.4 \\
\hline & & & & & & & & & & \\
\hline & ZONE2 T & TZONE4 & ZONE & ZONEB & ZONETO & & & & $\frac{1}{4}$ & \\
\hline DP liquid Tomp. $F$ & 82.2 & & & & & & & & & \\
\hline DPLquid Density bmem & 80.36 & & & & & & & & & \\
\hline Elovation-t & 21 & $-4 \mid$ & - & -81 & $\overline{8.876}$ & & & & & \\
\hline Mossured DPpsid & 0.151 & 0.702 & 1.14 & 1.4851 & 1.975 & & $i$ & & & \\
\hline Zero Corrections-psid & 0.009 & 0.044 & 0.029 & 0.018 & -0.074 & & & & & \\
\hline Conrected DPPsid & 0.142 & 0.658 & 1.111 & 1.468 & 2.049 & & & & & \\
\hline Actual DRी & 2.15 & 3.57 & 5.12 & 6.851 & $8:$ & & 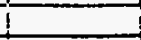 & $!$ & & \\
\hline Measured TPPsig & 172.7 & & & & 178.11 & & & & & \\
\hline Measured Overallin & -10.41 & & & & & & & & & \\
\hline & & & & & & & & & & \\
\hline Computed DPA & 2.रा & 4.17 & 6.11 & 8.081 & 70.071 & & i & & & \\
\hline Friction-li & 0.23 & 0.841 & 1.21 & $1.89]$ & 2.681 & & $i$ & I & & \\
\hline Acooleration $\mathrm{At}$ & 0.231 & 0.42 & 0.591 & 0.781 & 0.95 & & $T$ & i & 1 & t \\
\hline Elovation $-1 t$ & 1.75 & 3.1 & 4.371 & 5.43 & 6.411 & & $i$ & 1 & T & \\
\hline
\end{tabular}




\begin{tabular}{|c|c|c|c|c|c|c|c|c|c|c|}
\hline Run & $400 i$ & & 8 & & ( & & & & & \\
\hline Test Fuid & $: \operatorname{Ri14}$ & & & & & & & & & \\
\hline Dade: ....... & 35931 & & & & & & & & & \\
\hline Time: & $21: 34 i$ & & & & & & & & & \\
\hline Note & TABOREK & Analytical & & & & & $\dot{s}$ & & & \\
\hline Fow Area-tl2 & $\therefore \quad 0.003361$ & & & & & & & & & \\
\hline Heat Flow Aveatle & -0.195957 & & & & & & & & & \\
\hline Sat. Pressure psig & 158.41 & & & & & & & & & \\
\hline sat. Temperature-F(iniet) & 197.41 & & & & & & & $i$ & & $i$ \\
\hline Lq. Densitybmitis & $76 !$ & 75.84 & 75.71 & 75.611 & $75.63 !$ & $75.61 !$ & 75.521 & 75.55 & 75.551 & 75.77 \\
\hline Latent Hoar Btunbm & 40.981 & 40.831 & 40.71 & 40.611 & 40.621 & 40.611 & 40.511 & 40.54 & 40.541 & 40.76 \\
\hline Specifo Hea & 0.28 & 0.281 & 0.281 & $0 . \overline{28}$ & $0 . \overline{281}$ & 0.28 & 0.2871 & $0 . \overline{28}]$ & 0.281 & 0.28 \\
\hline Liq. Viscosity-lbm $\overline{f i t h r}$ & $0.445 \mid$ & 0.444 & $0.442 i$ & $0.441 i$ & 0.4411 & 0.441 & 0.441 & 0.441 & 0.441 & 0.443 \\
\hline Iq. Conductivity Btuhr fif & 0.0272 & 0.02711 & $0.027 i$ & 0.027 & 0.027 & 0.027 & 0.02591 & 0.0269 & 0.02691 & 0.027 \\
\hline Liq. Prandt No. & $4.58 !$ & 4.581 & 4.58 & 4.59 & 4.59 & 4.59 & 4.59 & 4.59 & 4.59 & 4.58 \\
\hline Inlot Flow Ralo-GPM & $4.6 !$ & & & & & & & & & \\
\hline Outlet Liq. Flow Rate-GPM & 2.5 & & & & & & & & 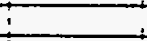 & \\
\hline Outlet Vap. Flow Rato-GPM & $1.4^{\circ}$ & & & & & & & & & \\
\hline Inlet Temperaturef & 193: & & & & & & & & & $T$ \\
\hline Ōtlet Liq. Temperaturef & 195.5 & & & & & & & & & \\
\hline Oútet Vap. Temperaturer & $194.9 i$ & & & & & & & & & T \\
\hline Water Flow Rato-GPM & -1 & & & : & 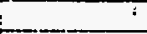 & $T$ & I & $\div$ & $\mathrm{i}$ & 1 \\
\hline Water Inlot Temp.F & 58.7 & & & & & $\bar{t}$ & 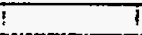 & $T$ & & $T$ \\
\hline Wator outtel Temp.F & 1927 & & & & $\bar{t}$ & 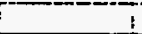 & 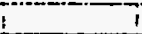 & 7 & & $i$ \\
\hline Inlot Glbm/hr/h2 & 8424901 & & Tomp & 189.91 & Donsity & 76.75 & & $i$ & & i \\
\hline Subcooling $F$ & $4.4 !$ & & & 5 & & & 1 & $i$ & 1 & \\
\hline Flow Observation & & & & & & & & & & 1 \\
\hline & IZONE 1 & ZONE2 & ZONE3 3 & ZONE4 & ZONE5 & ZONE6 I & ZONET & ZONE 8 & ZŌNĒ & ZONE10 \\
\hline Heal Input-Watts(nel) & 11146.9 & 1144.9 & 1155.1 & 1156 & 1156.1 & 1148.7 & 1153.11 & 1155.8 & $1151.4 i$ & i $\quad 1149.6$ \\
\hline Head Loss-Watts & 49.9 & 10.3 & 12.4 & 101 & 9.2 & 9.2 & 12.5 & 14.5 & 16.5 & 77.5 \\
\hline Heal Flux-BtuHinz & 19881 & 19846 & 200331 & 20039 & 30540 & 19912) & $19888 !$ & 20035 & T9556 & T9928 \\
\hline Wall Tomp. 3 3F & 2081 & 210.71 & 208.51 & 209.31 & 2127 & 209.4 & 208.11 & 210.1 & 210.3 & 210.2 \\
\hline Wall Temp. \$4F & 208.61 & 207.51 & 210.51 & 210.61 & 211.1 & 209.31 & 2081 & 210.1 & 217.41 & 209.5 \\
\hline Wall Tomp. $5 F$ & 207.51 & 205.8 & 211.21 & 209.3 & 208.91 & 2101 & 207.31 & 208.3 & 207.81 & 208.7 \\
\hline Wall Temp. If & 80.41 & 209.1 & 207 & 2091 & 208.71 & $212.1 !$ & 210.11 & 207.9 & 2071 & 203.5 \\
\hline Wall Temp: ${ }^{2} \mathrm{~F}^{-}$ & 209.11 & 208.7 & 208.41 & 211.5 & 208.91 & $214.6 !$ & 210.1 & 207.6 & 208.8 & 206.6 \\
\hline Instream Tomp.F & 194.71 & 195.71 & 196.51 & 197.11 & $197 !$ & 197.11 & 197.7 & 197.5 & 197.5 & 196.1 \\
\hline Avg. Wall Temp. $f$ & 208.31 & 209 & $208.6 i$ & 209.61 & 209.41 & $210.2 !$ & 208.7 & 2008.81 & 209 & 207.7 \\
\hline Avg. Wall DT.F & 127 & 12.41 & 11.21 & 11.5 & 11.5 & 12.2 & 10.1 & 10.41 & 10.6 & 10.7 \\
\hline Remaining Subcooff & -0.6 & & & & & & & & & \\
\hline Exot Quality & 01 & 0.04 & $0.07 i$ & 0.11 & 0.141 & 0.17 & $0.21 !$ & 0.24 & 0.28 & 0.31 \\
\hline EXP.HBtuHH & 1565.21 & 1600.1 & 1788.51 & 1735.8 & 1743.5 & 1632.1 & 1975.8 & 1927.5 & 1876.2 & \\
\hline COMPUIEDH & & & & & & & & & & \\
\hline Jallouk & 1223.71 & 1168.5 & 1142.51 & 1112.8 & $1087.7 !$ & 1058.3 & 1048.5 & 1035.5 & 1020.31 & 1005.8 \\
\hline Szadyl(Cudo tube) & 1724.41 & 1728.4 & 17421 & 1748 & 1750.81 & 1749 & 1758 & 1762.9 & 1763.6 & 1761.6 \\
\hline Szady? & 1627.21 & 1638.4 & 1658.61 & 1697.3 & 1637.91 & 1630.8 & 1638.61 & 1642.4 & 1685.31 & 1660.2 \\
\hline (RעQ) & 1234961 & $12 \overline{16681}$ & 1178511 & 113878 & 1095461 & 105317 & 1013041 & 96921 & 926241 & 87820 \\
\hline (F) & 1.1251 & 1.7911 & -1.8541 & 2.122 & 2.3491 & 2.547 & 2.72 & 2.887 & 3.042 & 3.207 \\
\hline (H) & $285 \overline{2 i}$ & 378.11 & $470.1 \%$ & 5381 & 55.71 & 645.91 & 689.71 & 7322 & 77.31 & 8132 \\
\hline (HBOIL) & $\$ 721.8 i$ & 17224 & $1730.5 i$ & 1730.8 & $1727.5 i$ & 1719.1 & 1721.91 & 1719.8 & 1712.91 & 1701.8 \\
\hline & & & & 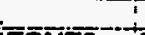 & & & & & & \\
\hline & ZONEZ : & ZONE4 & ZONEE' & ZONE & ZONE10 & & & & & \\
\hline DP liquid Temp.F & $\quad 82.81$ & $i$ & & & & & & & & \\
\hline DP Liquid Density-lbmit3 & 90.29 & & & & & & & & & \\
\hline Elevation-A & $2 !$ & 4 & 6 & 81 & 9.87 & & & & & \\
\hline Measured DPPsid & 0.787 & 0.876 & 1.365 & 1.795 & -2.361 & & & & & \\
\hline Zero Corrections-psid & 0.0091 & 0.044 & $0.029 !$ & 0.0181 & -0.0741 & & & & & \\
\hline Corrected DPPsid & 0.172 & 0.832 & 1.3361 & $1.77 \pi$ & 2.4341 & & & $t$ & & \\
\hline Actual DPA & 2.061 & $3.19 !$ & 4.611 & 6.161 & 7.141 & & & $\therefore$ & & \\
\hline Mensured ip psig & 158.41 & & 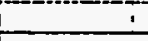 & 1 & 164.2 & & & & & \\
\hline Moasured OveralfA & $-11.02 !$ & & 1 & & & & & $i$ & & \\
\hline & & & & & & & & & & $\perp$ \\
\hline Computed DRA & 2.031 & 3.74 & 5.421 & 7.13 & 8.79 & & & & & \\
\hline Frictionft & 0.171 & 0.52 & $1:$ & 1.61 & 2.3 & & & & & \\
\hline Acceleration-t & 0.18 & 0.33 & $0.48 !$ & 0.63 & 0.81 & & & 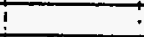 & & \\
\hline Elovation-ft & 1.671 & 2.891 & 3.94: & 4.881 & $5.7 !$ & & & & & \\
\hline
\end{tabular}




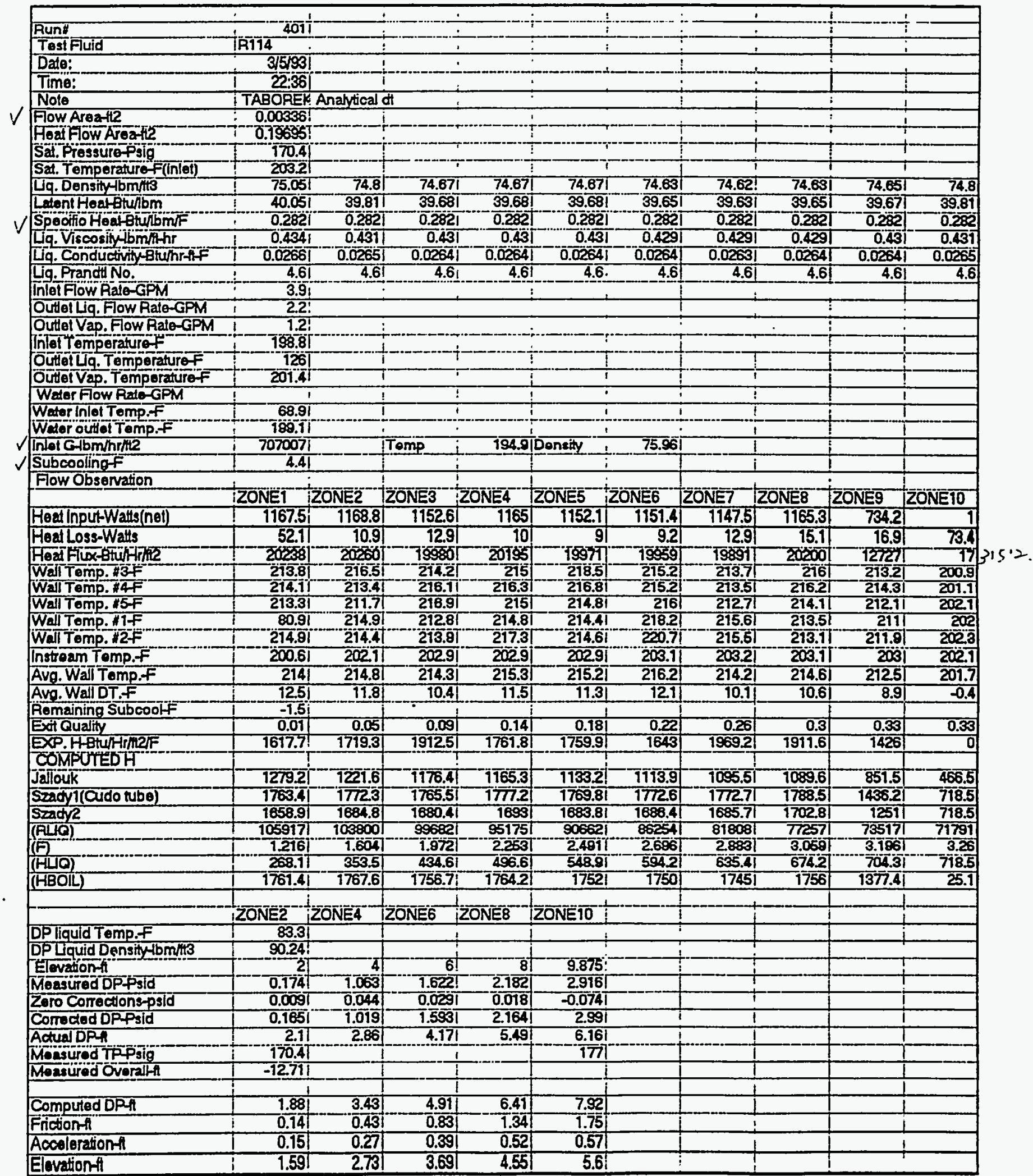




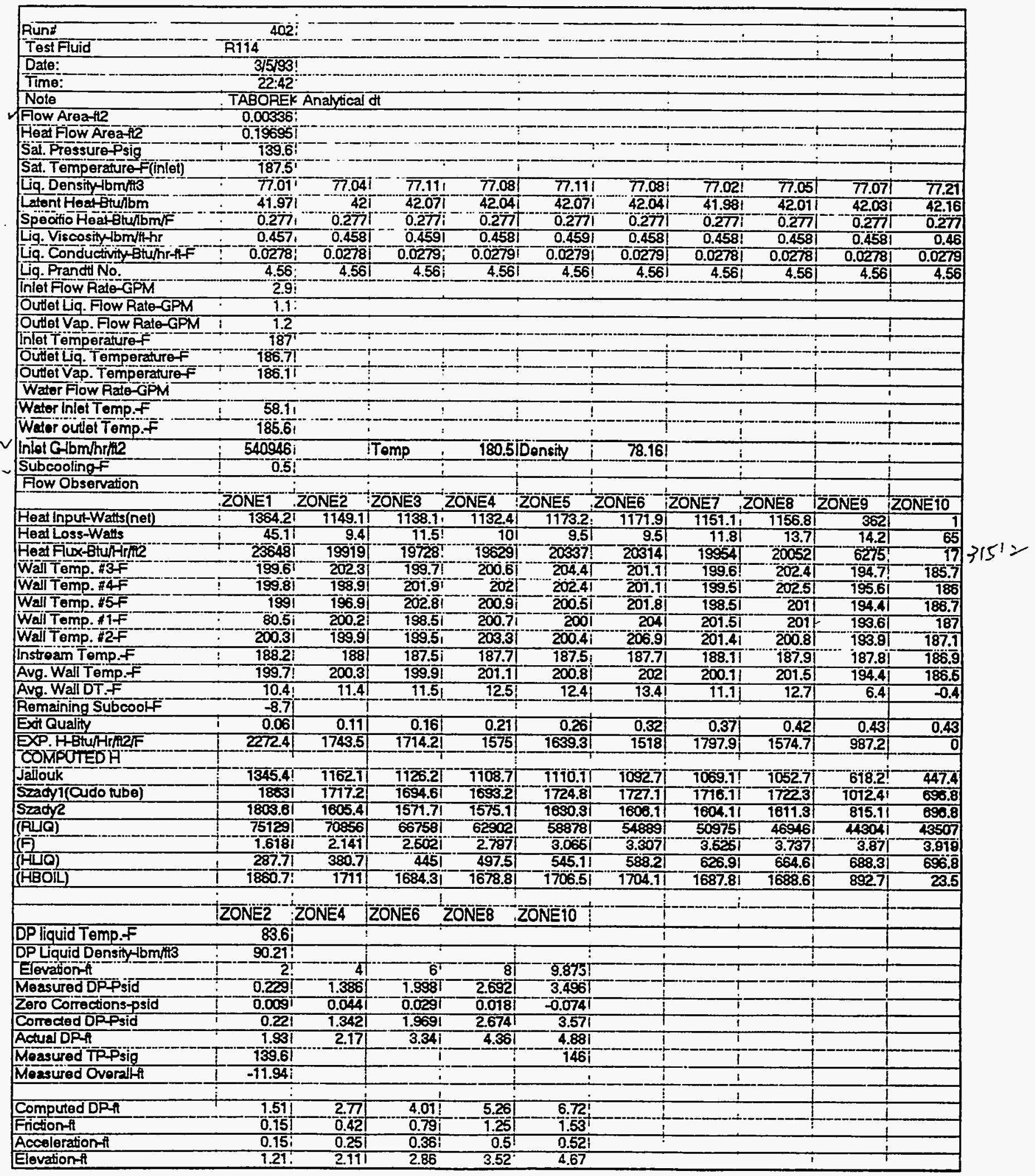




\begin{tabular}{|c|c|c|c|c|c|c|c|c|c|c|}
\hline & . & & & & - & $\therefore$ & & & $\therefore$ & - \\
\hline Rünit & 4031 & $!$ & & & & & & & $\because$ & 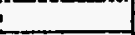 \\
\hline Tostfluid & Rि114 & & & & & & & & $i$ & i \\
\hline Dale: & $3 / 5931$ & & & & & & & & & \\
\hline Time: & $23: 17 i$ & & & & & & & & & \\
\hline Note & ITABOREK & Anahtical c & & & & & & 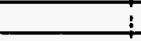 & 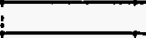 & \\
\hline Flow Areant & $0.00336 !$ & i & & & & $\vdots$ & 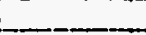 & & & \\
\hline Heal Fow Areante & 0.1955 & & & & & $i$ & 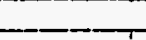 & & & \\
\hline Saf. Pressure Psig & 169.61 & & & & & & & & & \\
\hline Sat. Temperaturef (intel) & 202.81 & -1 & & $-\bar{\vdots}$ & & -1 & & 1 & $i$ & i \\
\hline Liq. Density $4 b m$ itt3 & 74.71 & 74.671 & $74.72 i$ & 74.681 & 74.71 & 74.631 & 74.61 & 74.621 & 74.681 & 74.78 \\
\hline Latent Hoat Btun bm & 39.711 & 39.68 & $39.73 i$ & 39.71 & 39.711 & 39.651 & 39.621 & 39.61 & 39.65 & 39.8 \\
\hline Specitic Heat BtutibmiF & 0.282 & 0.282 & 0.2821 & 0.2821 & 0.282 & 0.2821 & 0.282 & 0.282 & 0.2821 & 0.282 \\
\hline Liq. Viscosity lom/thr & 0.431 & 0.43 & 0.431 & 0.43 & 0.431 & 0.4291 & 0.4291 & 0.4291 & 0.4291 & 0.431 \\
\hline Lig. Conductivity Btumi-ftF & 0.02641 & 0.0264 & 0.0264 & 0.0264 & 0.02641 & 0.0268 & $0 . \overline{02631}$ & 0.0253 & 0.0264 & 0.0264 \\
\hline Lig. Prandt No. & 4.6 & $4.6 !$ & 4.6 & $4.6 !$ & 4.6 & 4.61 & 4.61 & 4.6 & 4.6 & 4.6 \\
\hline Inlet Flow Rato-GPM & 2 & & & $\cdots$ & : & & & 1 & 1 & 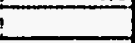 \\
\hline Outlol Lig. Flow Rato-GPM & 1.1 & & & & & & & & & \\
\hline Outtel Vap. Flow Rato-GPM & 1.1 & & & & & & & & & \\
\hline Inteltemperature & 2011 & & & & & & & & T & \\
\hline Oútel Líg. Tomporaturef & 202.1 & & & & & & & & tra & \\
\hline Outei vap. Temperaturef & $201.3 !$ & $\mathrm{i}$ & & & 1 & 1 & 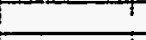 & & & \\
\hline Water Flow Rate-GPM & 1 & & & & 1 & 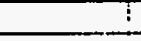 & 1 & & 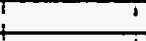 & 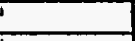 \\
\hline Wator Inlol Tomp.F & 661 & & 1 & & 1 & 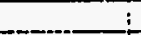 & $\therefore$ & & i & $i$ \\
\hline Wator out of Tomp.F & 180.11 & & & -1 & 1 & & $\therefore$ & & $i$ & $i$ \\
\hline Inlet Glbmhrhit2 & 3655611 & & Tomp & 190.9 & Donsty & 76.59 & $\dot{.}$ & & 1 & $\perp$ \\
\hline Subcoolingf & 1.81 & & & $i$ & $i$ & 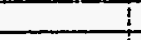 &. & & $i$ & $\dot{-}$ \\
\hline Flow Observation & & & & & $j_{1}$ & & & & 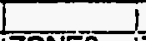 & $10=0$ \\
\hline & IZONEET & $Z O N=2$ & ZONE3 & ZONE & $\angle O N E S$ & ZONE6 6 & ZONET & ZONE8 & ZONE9 & ZONE1O \\
\hline Heaf Input-Watts(net) & $1 \quad 1128.7 i$ & 1150.3 & 1141.9 & 1140.11 & 1144.5 & 1147.6 & 939.2 & 154.9 & 139.31 & 116.2 \\
\hline Heaf Loss-Watts & 521 & 10.8 & 12.9 & 10 & 91 & 9.5 & 12.9 & 14.3 & 16.11 & 76.9 \\
\hline Hest flux $x$ Btur & 19565 & 199401 & 19794 & 19763 & 13839 & 79833 & $16280 !$ & 2885 & 2415 & 2014 \\
\hline Wal Tomp. \#F & 213.9 & $216.7]$ & 214.11 & 214.5 & 218.3 & 215.91 & 214 & 207.3 & 206.4 & 206.6 \\
\hline Wall Temp. "4F & 213.51 & 213 & 216.11 & 215.7 & 216.71 & 215.8 & 2141 & 207.3 & 207.31 & 207 \\
\hline Wall Temp. H5F & 212.81 & 211.1 & 216.81 & 214.61 & 214.41 & 216.41 & 21251 & 206.8 & 2071 & 207.3 \\
\hline Wall Tomp. 1F & 81.11 & 214.1 & 212.31 & 214.4 & 213.91 & 218.6 & 215.11 & 207 & 206.31 & 206.4 \\
\hline Wall Tomp & 213.91 & 213.6 & 213.51 & 216.71 & 214.21 & 2211 & 2151 & 2072 & 2051 & 206.8 \\
\hline Instoam Tomp.f & 2027 & 2029 & 20261 & 20281 & 20271 & 203.11 & 203.31 & 203.2 & 203.11 & 2022 \\
\hline Avg. Wall Temp.F & 213.31 & 214.21 & $214 i$ & 214.81 & $214.8 !$ & 216.7 & 214.11 & 207.11 & 206.61 & 206.8 \\
\hline Avg. Wall DT .F & 9.71 & 10.4 & 10.5 & 11.1 & $112 \mathrm{~L}$ & 127 & 10.11 & 3.8 & 3.41 & 4.5 \\
\hline Romaining Subcooff & -9.31 & & & & & & & & $i$ & \\
\hline Ext Quality & 0.071 & 0.15 & 0.231 & 0.311 & 0.39 & 0.47 & $0.53 i$ & 0.54 & 0.55 & 0.56 \\
\hline 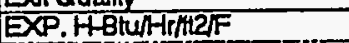 & 2008.61 & 1917.5 & 1884.2 & 1779.3 & 1770.8 & 1569.3 & $1614.4 i$ & 706.9 & 712.11 & 0 \\
\hline COMPUIEDH & & & & & & & & & & \\
\hline Jailou $\bar{k}$ & 1322.7 & $\overline{1285.8}$ & 1234.41 & 1212.9 & $1198.7 !$ & -1789 & 152.11 & 752 & 152 & 151.7 \\
\hline Szady 1 (cudo tube) & 174211 & 1760.5 & 1753.61 & 1754.2 & 1758.31 & 1763.7 & 152.11 & 152 & 1521 & 151.7 \\
\hline Sady2 & 1855 & 1675.6 & 1667.21 & 1687.9 & 1672 & 1678.8 & 152.11 & 152 & 1521 & 151.7 \\
\hline (PUQ) & 537501 & 49728 & 452041 & 40805 & 36345 & 319451 & 31945 & 31945 & 31945 & 31945 \\
\hline$(A)$ & 1.6151 & 2.186 & 2647 & 2.986 & 3.31 & 3.587 & 3.5871 & 3.587 & 3.587 & 3.587 \\
\hline (सएव) & 209.91 & 285.5 & 343.3 & 389.6 & 430.31 & 466.4 & 466.41 & 466.4 & 465.41 & 466.4 \\
\hline (HBOIL) & 1741.11 & 1758 & 1749.2 & 1747.7 & 1749.61 & 1752.7 & 175271 & 1752.71 & 17527 & 17527 \\
\hline & $1 \ldots$ & & & & $i$ & i & $\perp$ & $\perp$ & 1 & 1 \\
\hline & ZONE2 & ZONE4 & ZONE 6 & ZONEB & ZONE 10 ; & & 1 & L & & \\
\hline DP liquid Temp.f & 183.8 & & & $\overrightarrow{4}$ & $i$ & & & & & \\
\hline DP Liquid Densith-lbmplits & 90.191 & & 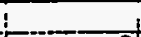 & & & & & & & \\
\hline Elevation-ft & & 4 & 6 & 8 & 9.8751 & & & & & \\
\hline Mossured DPFsid & 0.192 & 1.674 & 2.3711 & 3.217 & 4.083 & & & & & T \\
\hline Zoro Cortections-psid & $0 . \overline{009}$ & 0.044 & $0 . \overline{0291}$ & 0.018 & -0.0741 & & & & it & 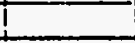 \\
\hline Corrocled DPPsid & 0.188 & 1.57 & 2.3421 & 3.199 & 4.157 & & & t & & \\
\hline Actual DPA & 2.061 & 1.8 & 2.78 & 3.49 & 3.91 & & $i$ & i & & 1 \\
\hline Meceuted Tp sig & 169.6 & & 7 & & 177.31 & & i & I & & T \\
\hline Mossured Ovorallit & -14.831 & & 1 & & $\ldots$ & & 1 & 1 & & I \\
\hline & & & & & & & i & i & & 1 \\
\hline Computed DPA & 1.36 & 2.41 & 3.35 & 4.49 & 5.78 & & $i$ & & & $i$ \\
\hline Friction-fl & 0.081 & 0.23 & 0.43 & 0.621 & 0.76 & & $i$ & 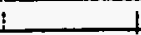 & $\perp$ & $\perp$ \\
\hline Accoleration-t & 0.07 & 0.14 & 0.23 & 0.27 & 0.28 & & & & & \\
\hline Elovationft & 1.21 & 2.04 & 2.69 & 3.61 & 4.741 & & & & & \\
\hline
\end{tabular}




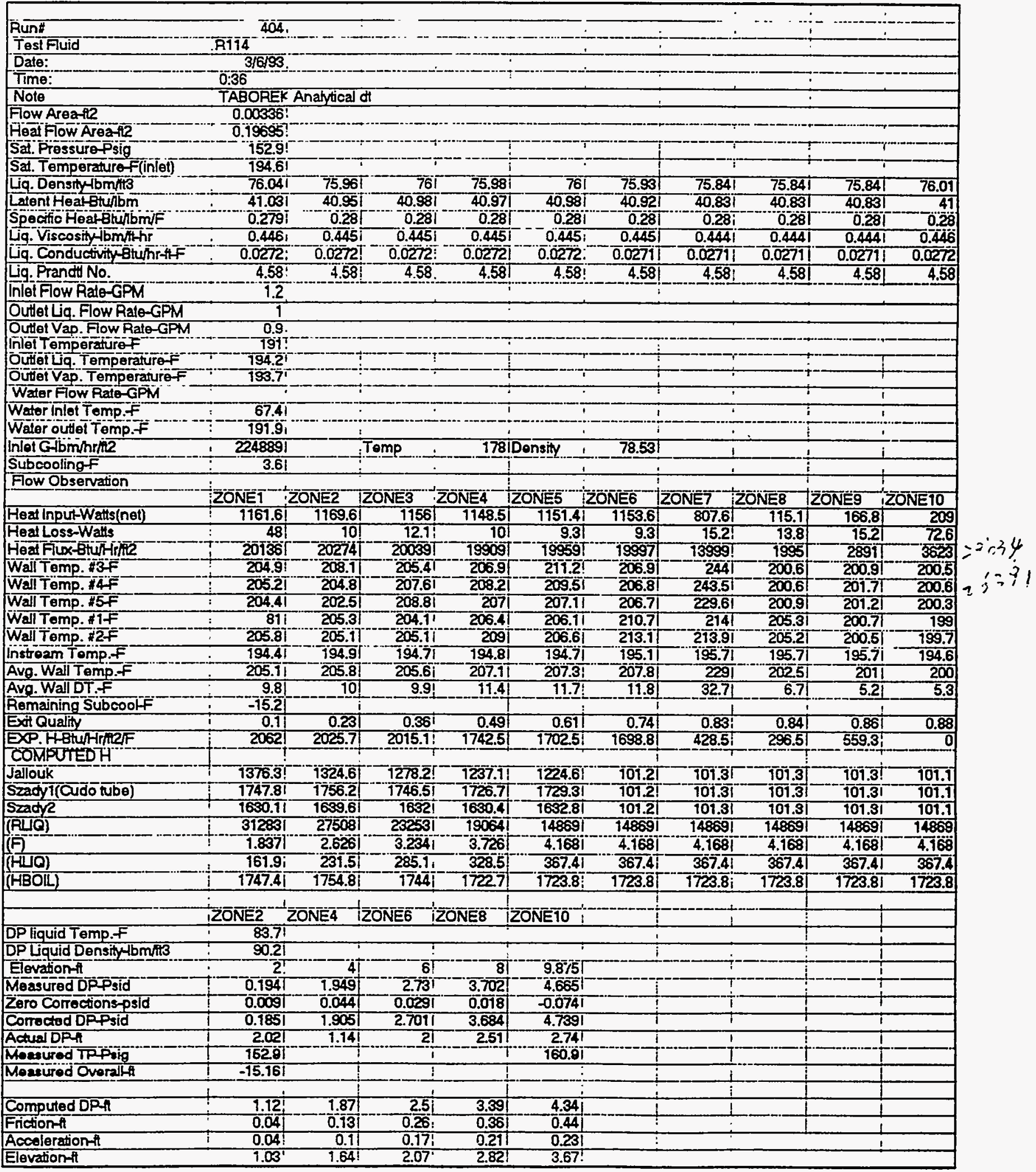




\begin{tabular}{|c|c|c|c|c|c|c|c|c|c|c|}
\hline & 1 & & & & . & $\therefore$ & $\therefore$ & $:$ & - & \\
\hline Runt: & 4051 & & & 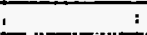 & : & & $\because$ & $\because$ & $i$ & \\
\hline Tost Fluid & R114 & & & & & &. & & 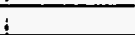 & \\
\hline Date: & $3 \longdiv { 9 9 3 }$ & & & & + & + & & $!$ & 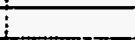 & \\
\hline Time: & $0: 55$ & & & & it & 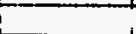 & & & t & \\
\hline Nole & ITABOREK Analytical C & & & & 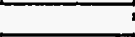 & 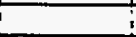 & 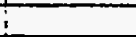 & 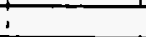 & & \\
\hline Fow Areatt? & 0,00336 & & & & & & : & 1 & & \\
\hline 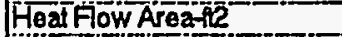 & 0.19695 & & & & & & & & $T$ & \\
\hline Sä. Prossure Psig & 125.91 & & & & & T & $T$ & T & ! & \\
\hline Sal. Temperature finlet) & $\left.179.8\right|^{-}$ & & & & & & & 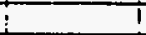 & I & \\
\hline Liq. Donsity-lbmitio & 78.191 & 78.211 & 78.18 & 78.221 & 78.18 & 78.091 & 78.1 & $78.12 !$ & 78.22 & \\
\hline Lotent Hoat-Btunbm & 43.131 & 43.141 & 43.111 & $43.16 !$ & 43.11 & 43.031 & 43.04 & 43.061 & 43.16 & \\
\hline Specifo Hoat BtuibmiF & $0.275 i$ & 0.275 & 0.275 & 0.2751 & 0.275 & 0.275 & 0.276 & 0.275 & 0.276 & \\
\hline Liq. Viscositylbmithir & $0 . \overline{4741}$ & $0.474 !$ & 0.4731 & 0.4741 & 0.473 & $0.472 !$ & 0.472 & 0.473 & $0 . \overline{474}$ & \\
\hline Liq. Conductivityetiuhr-fif & $0.0285 !$ & 0.0286 & 0.0285 & 0.02861 & 0.0285 & $0.0<851$ & 0.0285 & 0.0285 & 0.0286 & \\
\hline ig. Prandt No. & $4.56 \mid$ & 4.56 & 4.56 & 4.561 & 4.56 & 4.561 & 4.56 & 4.56 & 4.56 & \\
\hline Inletflow Rato-GPM & 1.031 & & & (1) & & & & 1 & 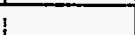 & \\
\hline Outlol Lig, Flow Rato-GPM & 1 & & & & & & & 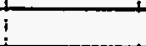 & & \\
\hline Outtol Vap. Fow Ralo-GPM & $0.9 !$ & & & & & & & 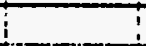 & $\vdots$ & \\
\hline Inlot Tomperature & $776.3 !$ & & & & & & & & & \\
\hline Outel Lq. Temperaturef- & $120.4 !$ & & & & & & & & & \\
\hline Outlel Vap. Temperaturef & 179.41 & & & T & t & $T$ & 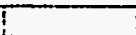 & $t$ & 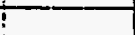 & \\
\hline Waler Flow Alade-GPM & - & & & 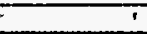 & - & & $t$ & ! & ! & \\
\hline Water Inlet Tomp.F & $64.8 i$ & & & $\therefore$ & 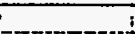 & 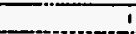 & $T^{2}$ & & ! & \\
\hline Wador out tomp.F & 177.7 & & & $\because$ & 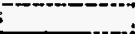 & & $\because$ & & $i$ & \\
\hline Inlat G-lbm/hr/h2 & $1982 \times 3 i$ & Tomp & 163! & IDonsity & 80.65 & $i$ & $i$ & t & & \\
\hline Subcoolingt & 3.51 & & & & & $\bar{t}$ & I & $i$ & & \\
\hline \multirow[t]{2}{*}{ Flow Observation. } & & & & 1 & 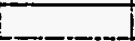 & & & 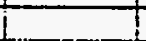 & & \\
\hline & ZONEI ZONE2 : & $\angle O N E 3$ & ZONE4 & ZONES & ZONE6 & ZONE7 & ZONE8 & ZZONEO & ZONETO & \\
\hline Heat Input-Watts(nel) & 1161.2! 1162.2 & 1149.8 & 11624 & 1154.4 & 1160.8 & 759 & 118.4 & 186 & 174.4 & \\
\hline HeatLoss-Watts & 40.7 & 10.8 & 10 & 9.8 & 9.8 & 12.2 & 13.1 & 16.81 & 85.8 & \\
\hline Heaf FLx-BtuHing & $20129 !$ & 1993iा & 20149 & 20011 & 20122 & 13157 & 2052 & 3224 & 3023 & 123634. \\
\hline Wall Tomp. HF & $191.2 !$ & 191.61 & 193.1 & 197.4 & 192.6 & 2228 & 193.4 & 275.4 & 214.7 & \\
\hline Wall Temp. $4 \bar{F}$ & 191.51 & $183.7 \pi$ & 194.6 & 195.51 & 192.6 & 222.41 & 193.1 & 216.5 & 215 & \\
\hline Wall Tomp. \#5F & 190.81 & 195.31 & 193.2 & 193.11 & 192.51 & 197.31 & 192.5 & 210.7 & 2220 & \\
\hline Wall Temp."1f & 191.41 & 190.51 & 192.61 & 19221 & 196.3 & 1927 & 197.4 & 204.31 & 221.7 & \\
\hline Wal Tomp. 政F & 192.21 & 191.31 & 185.61 & 182.71 & 189.2 & 192.7 & 197.3 & 203.81 & 221.8 & \\
\hline Instroàn Tomp.f & 180.31 & 180.21 & 180.4 & 180.11 & 180.4 & -1811 & 180.9 & 180.81 & 180.1 & \\
\hline Avo. Wall Temp.F & 191.41 & 191.81 & 193.4 & 193.41 & 193.5 & 205.61 & 194.7 & 210.11 & 219.2 & \\
\hline Avo.Wall DT, $F$ & 10.2 & 10.7 & 12.1 & 12.41 & 12.2 & 24 & 13.7 & 29.2 & 38.9 & \\
\hline Romaining Subcooff & -18.2 & & & & & & & 1 & 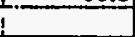 & \\
\hline Ext Quality & 0.121 & 0.39 & 0.53 & 0.67 & 0.8 & 0.891 & 0.91 & 0.93 & 0.95 & \\
\hline EXP. HBtuAHril2/F & $1970.4 !$ & 1867.2 & 1670.2 & 1617.6! & 1650.6 & 548.51 & 149.3 & 110.4 & 0 & \\
\hline COMPUTEDH & & & & & & & & & & \\
\hline Jallouk & 7331.5 & 12201 & 1200.9 & $1981.2 !$ & 88.7 & 88.8 & 88.8 & 88.81 & 88.6 & \\
\hline Szzady 1 (Cudo tubo) & 1686.31 & 1679.41 & 1690.2 & 1684.41 & 88.7 & 88.81 & 88.8 & 88.81 & 88.6 & \\
\hline Szady2 & 1550.71 & 1542.61 & 1555.1 & 1548.11 & 88.7 & 88.81 & 88.8 & 88.81 & 88.0 & \\
\hline (RUQ) & 25796 & 185501 & 14802 & 11017 & 11017 & 11017 & 11017 & 11017 & 11017 & \\
\hline 年 & 1.569 & 3.577 & -4.149 & 4.6741 & 4.674 & 4.6741 & 4.674 & $4.674 T$ & 4.674 & \\
\hline (मบQ) & 156.61 & 284.41 & 320.91 & 371.61 & 371.6 & 371.6 & 371.6 & $371.6 i$ & 371.6 & \\
\hline \multirow[t]{3}{*}{ (HBOIL) } & 1685.91 & 1676.7 & 1686 & 1678.41 & 1678.4 & 1678.4 & 1678.4 & 1678.41 & 1678.4 & \\
\hline & & & & & 1 & & & $\ldots$ & I. & \\
\hline & ZONE2 ZONE 4 & ZONEE & ZONE8 & ZONE10 : & 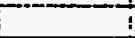 & & & 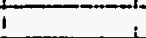 & & \\
\hline DPliquid Temp, $F$ & 83.6 & & & & 7 & & & 1 & 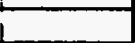 & \\
\hline DPLiquid Densitufbmat3 & क0.21 & & $i$ & & & & & & & \\
\hline Elovation $\mathrm{At}$ & $2 !$ & 6 & 8 & 9.8751 & & & & T & & \\
\hline Móasured DPFsid & 0.2871 & $2825 !$ & 3.844 & 4.844 & & & & & & \\
\hline Zero Controctions-psida & 0.0091 & 0.0291 & 0.0181 & -0.0741 & & $\bar{\vdots}$ & ! & 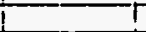 & $\Gamma$ & \\
\hline Corrected DP Psid & 0.2781 & 2.7981 & 3.8287 & 4.9181 & 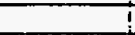 & & & $T$ & T & \\
\hline Actual DPA & 1.791 & 1.77 & 2.18 & 2.30 & 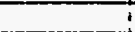 & I & ! & T & T & \\
\hline Meneured TPPsig & 125.91 & & & 134 & 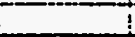 & $\mathrm{T}$ & $T$ & T & I & \\
\hline \multirow[t]{2}{*}{ Mossured Ovorallft } & -14.911 & $i$ & & 1 & 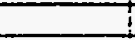 & I & 1 & 1 & 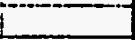 & \\
\hline & & & & & & 1 & $i$ & 1 & 1 & \\
\hline Computed DPA & 1.02 & 2.241 & 3.07 & 3.75 & & +1 & & $i$ & 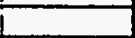 & \\
\hline Friction-t & 0.041 & 0.25 & 0.34 & $0.43 i$ & 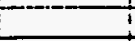 & & & 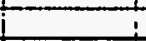 & 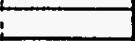 & \\
\hline Accoleration-f & 0.041 & 0.18 & 0.22 & 0.241 & & & 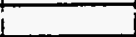 & 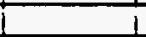 & 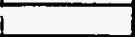 & \\
\hline Elevation-At & 0.94 & 1.81 & 251 & 3.081 & & & & $i$ & & \\
\hline
\end{tabular}




\begin{tabular}{|c|c|c|c|c|c|c|c|c|c|c|}
\hline Run & 406. & & 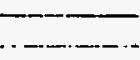 & $-\ldots$ & .......... - & & $-\ldots$ & & & $\dot{-}$ \\
\hline Test Fuid & Ri14 & & - & & & & & & & \\
\hline Date: & 3/6/93: & & & & & & & & & \\
\hline Time: & $1: 16$ & & & & & & & & & \\
\hline Note & TABOREK & Analytical of & & & & & & & & \\
\hline Fow Area-12 & $0.00336 !$ & & & & & & & & & \\
\hline Heat Flow Area-t? & $0.19605 !$ & & & & & & & & & \\
\hline Sal. Pressure Fsig & 111.8 & & & & & & & & & \\
\hline Sat Temperature-f(iniet) & $171.2^{1}$ & -7 & & & & 7 & 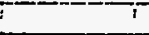 & & & $\mathrm{i}$ \\
\hline Liq. Density-16min & 79.631 & 79.521 & 79.561 & 79.551 & 79.611 & 79.581 & 79.481 & 79.531 & 79.551 & 79.68 \\
\hline Lotent Hear Bhinbm & 74.531 & 44.42 & $74 . \overline{461}$ & 44.45i & 44.51 & $44.48 i$ & 44.381 & 44.431 & 44.451 & 44.56 \\
\hline Speodio Heal Btullbmif & 0.2711 & 0.272 & $0.271 \mathrm{i}$ & 0.2721 & 0.2711 & $0.27 \overline{1}$ & 0.27201 & 0.272 & 0.272 & 0.271 \\
\hline Liq. Viscositylbmilihr & $0.45 i$ & 0.4941 & $0.49 \overline{41}$ & 0.4941 & $0.495 !$ & $0 . \overline{4951}$ & $0.493 !$ & 0.4941 & 0.004 & 0.496 \\
\hline Liq. Conductivity 8 tuphifi-f & 0.02941 & 0.0296 & 0.02941 & 0.02941 & 0.02941 & 0.02941 & 0.02931 & 0.0294 & 0.02941 & 0.0295 \\
\hline Lia. Prandt No. & 4.57 & $4.56 !$ & 4.57 & 4.57 & 4.57 & 4.57 & 4.56 & 4.56 & 4.57 & 4.57 \\
\hline Inlet Flow Ralo-GPM & 1.9 & & & & & & & & & \\
\hline Outtet Lig. Flow Rato-GPM & $0.9 !$ & & & & & & & & & 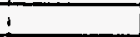 \\
\hline Outel Vap. Flow Ralo-GPM & 1.9 & & & & & & & & & \\
\hline Inlet Temperaturef & $168.5 !$ & & & & & & & & $!$ & \\
\hline Outret La. Tomperaturef & $970.1^{\top}$ & & & & & & $T$ & & & \\
\hline Out et vap. Temperature F & 169.11 & & & & & & & & & $T$ \\
\hline Water Flow Rate-GPM & & & & & & & 1 & & & $!$ \\
\hline Water Inlet Tomp.F & $60 !$ & & & 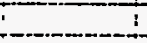 & & & I. & & 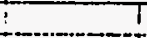 & $T$ \\
\hline Water outel Temp.F & 167.2 & & & & & & $\dot{-}$ & & $\therefore$ & I \\
\hline Inlat G-lbminrmile & 3674001 & & Temp & 160.21 & Density & 81.03 & $T$ & & i & I \\
\hline Subcooling $F$ & 2.7 & & & $-\infty$ & 1 & -3 & & & & $I$ \\
\hline Fow Observation & & & & & & & & & & \\
\hline & ZONE1 2 & ZONE2 : & ZONE3 & $20 N E 4$ & $2 O N E 5$ & ZONEG is & ZONET iZ & ZONEB & ZONET & ZONETO \\
\hline Heat Input-Watts(nel) & 1155.31 & 1161.7 & $1156.3 !$ & 1158.3 & $1156.3 \mathrm{i}$ & 1157.5 & $1157.8 i$ & 1152.2 & 318 & 204.8 \\
\hline Heat Loss-Watts & $36.4 !$ & 7.8 & 10 & 10 & 10.91 & 10.11 & $10.6 !$ & 12.3 & 11.8 & 59.5 \\
\hline Heat Fux-BturArfe & 200261 & 20137 & 200941 & 20078 & 200044 & 20055 & $20070 !$ & 19973 & 55121 & 3550 \\
\hline Wall Temp. $3 \mathrm{~F}$ & $182.8 !$ & 185.31 & 182.81 & 184 & 1881 & 182.8 & 184.81 & 187.4 & 177.31 & 175.8 \\
\hline Wall Temp. 44 & 1831 & 182.11 & 184.81 & 185.3 & 1861 & 182.8 & 184.81 & 188.41 & 177.91 & 175.9 \\
\hline Wall Temp. $\# 5 F$ & 182.21 & $179.7 i$ & 186.51 & 184.2 & 183.91 & 1831 & 183.21 & 188.1 & 177.41 & 178.5 \\
\hline Wall Tomp. \#1f & 79.51 & $1827 \pi$ & $181.7 i$ & 183.71 & 183.11 & 186.7 & 1851 & 187.2 & 177.31 & 174.5 \\
\hline Womp Ta & $183.7 i$ & 182.61 & 182.61 & 186.6 & 183.61 & 189.6 & $186 i$ & 187.31 & 177.61 & 176.4 \\
\hline Instroam Tomp.F & 170.31 & 171.71 & 170.8: & 170.91 & 970.5 & 170.71 & $17 \overline{1.4}$ & 1711 & 170.91 & 170.1 \\
\hline Avg. Wall Temp.F & 182.9 & $183.2 !$ & 1831 & 184.31 & 184.2 & 183.8 & 184.61 & 187.3 & $177.5 i$ & 175.6 \\
\hline Avg.Wall DT .F & $11.7 !$ & $11.2 !$ & 11.3 & 12.5 & 12.7 & 122 & $12.3 !$ & 15.4 & 6.41 & 5.4 \\
\hline Remaining SubcoolfF & $9 i$ & & & & & & & & & \\
\hline Exat Quality & $0.06 !$ & 0.13 & 0.2 & 0.27 & 0.34 & 0.42 & 0.49 & 0.56 & 0.58 & 0.59 \\
\hline EXP. HBhuHr/i2/F & 1708.7 & 1803.6 & 1778.6 & 1607.2 & 1572.8: & 16422 & $1637.9 !$ & 1298.8 & 858 & 0 \\
\hline COMPUTEDH & & & & & & & & & & \\
\hline Jallouk & $1217^{7}$ & 7159 & 11231 & 1701.8 & $1085.4 !$ & 1071 & 1000.7 & 1045.T & 560.11 & 485.4 \\
\hline Szadyloudo tubo) & 1634.71 & 1644.41 & $1641.2 !$ & T645.2! & $1644.4 !$ & 1648.9 & 1655.3 & 1653 & 908.4 & 775.5 \\
\hline Szady2 & 1521.21 & 1531.2 & 1525.11 & 1527.8 & $1524.5 !$ & 1527.31 & 1509.71 & 1508.5 & 718.7 & 647.7 \\
\hline (ALQ) & 47167 & 442431 & 405801 & 371961 & 3634 & 301701 & 267601 & 23214 & 20976 & 20111 \\
\hline (i) & 1.6671 & 2.3311 & 2.8671 & 3.29 & 3.6721 & 4.008 & 4.3081 & 4.674 & 4.7991 & 4.884 \\
\hline$(H \cup Q)$ & 216.21 & 302.4 & 371.11 & 426.71 & 476.21 & 519.91 & 5591 & 598.61 & 2251 & 633.3 \\
\hline (HBOIL) & $9633.4 i$ & 16411 & 1034.81 & 1635.6 & 16311 & 1631.5 & 1633.8 & 1626.4 & $798.2 !$ & 596.7 \\
\hline & & & & & & & 1 & & & \\
\hline & ZONE2 Z & ZONE4 & ZONEG :Z & ZONE 8 & TONEIO! & & & & & \\
\hline DP liquid Temp.f & $83.3 i$ & & & & & & & & & \\
\hline DP Liquid Density $/ \mathrm{bm} / \mathrm{m} 3$ & $90.24 !$ & & & & & & & & & \\
\hline Elevatont & & & & 81 & 9.8751 & & & & & \\
\hline Measured DPFsid & 0.2631 & 1.66 & 2.3331 & 3.111 & 3.9521 & & & & & \\
\hline Zero Contections-psld & 0.0091 & 0.044 & 0.0291 & 0.018 & $-0.074 !$ & & $i$ & & & \\
\hline Corrected DPPsid & 0.2541 & 1.6161 & 2.3041 & 3.092 & 4.0381 & I & $T$ & & & \\
\hline Actuad DPA & 1.81 & 1.611 & $2.63 !$ & 3.47 & 3.891 & & $T$ & & & \\
\hline Measured TPPsig & 111.8 & & 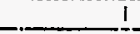 & 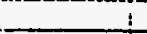 & 118.21 & & $T$ & & & \\
\hline Messured Ovoraltht & -11.57 & & & & & & $\therefore$ & & & \\
\hline & & & & & & & & & & \\
\hline Computed DPA & $1.29 !$ & $2.28 !$ & 3.23 & 4.17 & $5.48 !$ & & & 1 & $L$ & \\
\hline Friction-t & 0.09 & $0.27 !$ & $\frac{0.53 !}{0.77}$ & 0.871 & $1.05 !$ & & & & $i$ & L \\
\hline $\begin{array}{l}\text { Acceleration-ft } \\
\text { Elevation-f }\end{array}$ & $\frac{0.091}{1.12^{\prime}}$ & $\frac{0.17 i}{i .85 !}$ & $\frac{0.27}{2.42}$ & $\frac{0.4 !}{2.9 !}$ & $\frac{0.43 i}{3.98}$ & & & & & \\
\hline
\end{tabular}




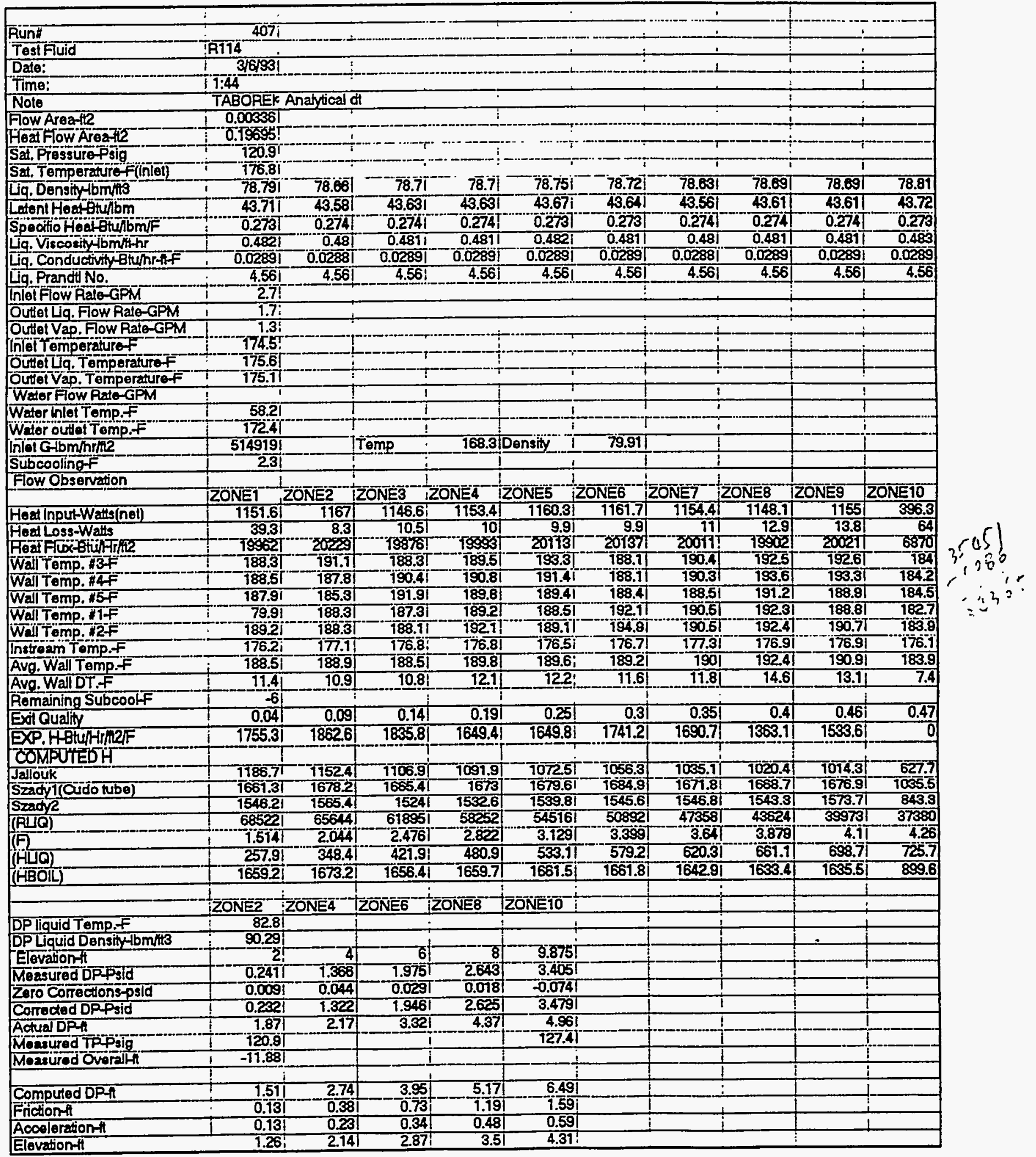




\begin{tabular}{|c|c|c|c|c|c|c|c|c|c|c|}
\hline Run" & 4081 & & & & - & $\therefore$ & & $\therefore$ & & \\
\hline Tost Fluid & .8114 & & & $\therefore$ & 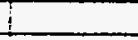 & 1 & & $\dot{1}$ & $i$ & $\vdots$ \\
\hline Date: & $3 / 6 \sqrt{33}$ & & & & i & $\cdot$ & $\div$ & & $:$ & \\
\hline Time: & $2: 13$ & & & $\dot{\vdots}$ & $i$ & $i$ & $i$ & & $!$ & \\
\hline Nole & Analytical & dit is used & 1 & & i & 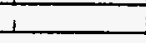 & $\vdots$ & & 1 & \\
\hline $\begin{array}{l}\text { Fow Areatt2 } \\
\text { Heat Alow Area-t2 }\end{array}$ & i- 0.00336 & & & & & $\div$ & $\therefore$ & & & \\
\hline \begin{tabular}{|l|} 
Heat Flow Area-fl \\
Sat. Pressuro-Psig \\
\end{tabular} & $\frac{0.195951}{114.61}$ & & $\therefore$ & & & & & & 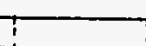 & \\
\hline Sal. Temperature finlet) & 173 & & & & & & & & & \\
\hline प9. Densiny bmint3 & 79.42 & 79.37 & 79.29 & 79.31 & 79.36 & 79.35 & 79.28 & 79.35 & 79.35 & 79.51 \\
\hline Latent Heat-Btulbm & 44.321 & 44.21 & 44.2 & 44.21 & 44.27 & 44.26 & 44.19 & 44.26 & 44.26 & 44.41 \\
\hline Specific Hoat-Btunbmif & 0.272 & 0.272 & 0.272 & 0.272 & 0.272 & 0.272 & 0.272 & 0.272 & 0.272 & 0.272 \\
\hline Liq. Visoositylbm/tithr & 0.4921 & 0.491 & 0.49 & 0.48 & 0.481 & 0.491 & 0.481 & 0.491 & 0.491 & 0.408 \\
\hline Liq. Conductivity Btu/hr-ftF & 0.0293 & 0.02921 & 0.0292 & 0.0292 & 0.0293 & 0.0293 & 0.0292 & 0.02931 & 0.0293 & 0.0294 \\
\hline Liq. Prandt No. & 4.561 & 4.56 & 4.56 & 4.56 & 4.56 & 4.56 & 4.56 & 4.56 & 4.56 & 4.56 \\
\hline Inlot Flow Rate-GPM & 3.51 & & 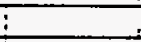 & 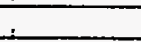 & & 1 & 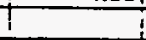 & in & 0 & \\
\hline Outel Lig. Flow Rato-GPM & 2.8 & & t & & i & 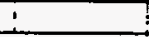 & 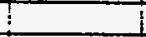 & & 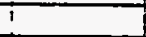 & \\
\hline Outlet Vap. Flow Rale-GPM & 1.2 & & $\therefore$ & & 1 & 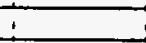 & $\therefore$ & & j & \\
\hline Inlet Temperaruref & 169 & & & & & & 5 & & 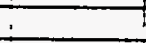 & \\
\hline Outiet Liq. Temperaturef & $171 !$ & & & & & & & & 7 & \\
\hline Outtet Vap. Temperaturoff & 770.11 & & & & & & & & & \\
\hline Water flow Ralo-GPM & 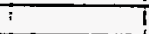 & & & & & & & & & \\
\hline Water Inlet Temp.f & 58.71 & & $\frac{1}{1}$ & $i$ & & tis & : & t & & \\
\hline Wetor outlot Tomp.f & 1671 & & 7 & $\cdot$ & $t$ & t & \pm & I & & \\
\hline Inlol Glbm/ht/ti2 & 6726821 & & Temp & 163.8 & Denaity & 80.54 & & i & & \\
\hline Subcooling $f$ & 41 & & & $i$ & & 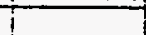 & & & & \\
\hline \multirow{2}{*}{ Flow Observation } & $i$ & & & $i$ & & & & & & \\
\hline & ZONE1 & ZONE2 & ZONE3 & IZONE4 & ZONE5 & ZONE6 & ZONET & ZONE8 & ZONE9 & ZONETO \\
\hline Heat Input-Walts(net) & 1175.11 & 1150.4 & 1153.9 & $\quad 1162.6$ & 1157.1 & 1160.2 & 1161.2 & 1187.1 & 1151.8 & 1159.5 \\
\hline Heat Loss-Watts & 37.5 & 81 & 10.1 & 10 & 10.1 & 10.1 & 10.7 & 12.4 & 13.1 & 64.6 \\
\hline Head Flux-Btu/Hiffle & 20370 & 19941 & 20002 & 20153 & 20058 & 20111 & 20129 & 20578 & 19966 & 20099 \\
\hline Wall Temp. & 184.8 & 187.1 & 784.6 & 185.6 & 189.1 & 183.9 & 185.8 & 188.1 & 787.5 & 186.6 \\
\hline Wall Temp. "4F & 185.1 & 184.1 & 186.5 & 186.9 & 187.2 & 183.9 & 185.8 & 189.2 & 188.2 & 186 \\
\hline Wall Temp. \#5F & 184.3 & 181.6 & 187.9 & 185.6 & 185.3 & 184.2 & 184.11 & 187.1 & 184.31 & 185.9 \\
\hline Wall Temp. $1 F$ & 78.81 & 184.8 & 983.6 & 185 & 184.4 & 187.8 & 188.3 & 188 & 184.11 & 181 \\
\hline Wall Tomp. $2 F$ & 185.61 & 184.51 & 184.51 & 188.1 & 185 & 190.7 & 186.3 & 188.1 & 186.21 & 183.8 \\
\hline Instream Temp.f & 171.8 & 172.6 & 172.71 & 172.6 & 172.2 & 172.3 & 172.8 & 172.3 & 172.31 & 171.2 \\
\hline Avg. Wall Temp.F & 185 & 185.1 & 184.81 & 185.8 & 185.5 & 185 & 185.7 & 188.11 & 186.1 & 184.7 \\
\hline Avg. Wall DT.f & 12.21 & 11.61 & 11.21 & 12.3 & 12.4 & 11.7 & 12 & 14.9 & 12.9 & 126 \\
\hline Romaining Subcooff & -2.5 & 1 & 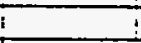 & -1 & & 1 & i & 1 & 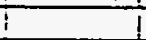 & \\
\hline Exit Quality & 0.02 & 0.05 & 0.09 & 0.13 & 0.17 & 0.21 & 0.25 & 0.29 & 0.33 & 0.37 \\
\hline EXP.HBtu/Hr/i2, F & 1655.6 & 1723 & 1786.5 & 1643.2 & 1621.7 & 1712.9 & 1684.4 & 1383.8 & 1552.8 & 1601.3 \\
\hline \multicolumn{11}{|l|}{ COMPUTEDH } \\
\hline Jallouk & 1170.3 & 1108.9 & 1077.8 & 1052.3 & 10225 & 1012.5 & 965.3 & 989.6 & 9क्ष.4 & $\$ 45.4$ \\
\hline Szady1(Cudo tube) & 1656.4 & T645.9 & 1652.51 & 1662 & 1650.9 & 1654.8 & 1661.7 & 1681.7 & 1652 & 1667.8 \\
\hline Szady2 & 1547 & 1528.4 & 1532.91 & 1541.5 & 1516.2 & 1524.2 & 15329 & 1555.8 & 1552.31 & 1555.8 \\
\hline (Rप्र) & 887231 & 88589 & 83099 & 79511 & 75817 & 72307 & 68915 & 65105 & 615881 & 57701 \\
\hline$(\mathrm{F})$ & 1.315 & 1.76 & 2.173 & 2.5 & 2.782 & 3.029 & 3.249 & 3.469 & 3.668 & 3.875 \\
\hline (HUQ) & 276.91 & 370.7 & 467.71 & 526.6 & 685.8 & 638 & 684.4 & 730.6 & 772.5 & 816.6 \\
\hline \multirow[t]{3}{*}{ (HBOIL) } & 1653.8 & 1639.6 & 1640.71 & 1644.2 & 1636.2 & 16226 & 162 & 1634.4 & 1604.4 & 1600 \\
\hline & & & & & & & & & & \\
\hline & ZONE2 T & ZONE4 & ZONE6 & ZONE 8 & ZONE10 & & & & & \\
\hline DP liquid Temp.f & 82.6 & 1 & 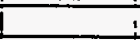 & 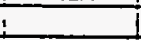 & & & & & & \\
\hline DP Liquid Density lbmsit3 & 90.311 & & & & & & & & & \\
\hline Elovation-ft & 2 & 4 & 6 & 8 & 9.875 & & & & & \\
\hline Moasured DPPsid & 0.274 & 1.078 & $1.606 \mid$ & 2.12 & 2.6851 & & & & & \\
\hline Zero Corrections-psid & 0.000 & 0.044 & 0.029 & 0.018 & -0.074 & & & & & \\
\hline Corrected DPPsid & 0.205 & 1.034 & 1.577 & 2.102 & 2.76 & & & & & \\
\hline Actual DPA & 1.9 & 2.67 & 3.981 & 5.28 & 6.22 & & & & & \\
\hline Moasured TPPsig & 114.61 & & 1 & 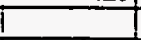 & 120.1 & & & & & \\
\hline \multirow[t]{2}{*}{ Moesured Overall-t } & -9.861 & & 1 & 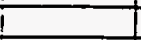 & & & & & & \\
\hline & 1 & & & & & & & & & \\
\hline Computed DP-n & 1.791 & 3.28 & 4.76 & 6.28 & 7.8 & & & & & \\
\hline Friction-t & 0.15 & 0.46 & 0.99 & 1.49 & 2.141 & & & & & \\
\hline Accoleration-t & 0.16 & 0.3 & 0.43 & 0.59 & 0.78 & & & & & \\
\hline \multirow[t]{2}{*}{ Elovation-ft } & 1.48 & 2.53 & 3.42. & 4.19 & 4.88 & & & & & \\
\hline & $\longrightarrow$ & & & 1 & & & & & & \\
\hline
\end{tabular}




\begin{tabular}{|c|c|c|c|c|c|c|c|c|c|c|}
\hline & 1 & & ' & 1 & ; & & & ! & ' & ' \\
\hline Runi & 4421 & & & & 1 & & & & & \\
\hline Tost Fiuid & R114 & & & & & & & & & \\
\hline Dale: & t. $3 / 27993$ & & & & & $i$ & & & & \\
\hline Time: & 2.26 & & & & & & & & & \\
\hline Note & TABOREY & Anabytical d & & & $\div$ & i & & 1 & & \\
\hline Fow Area-112 & $0.00336 !$ & 1 & & & : & & & & & \\
\hline Healflow ATOZALE & $\because 0.15655 T$ & & & 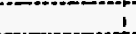 & & $i$ & & & & \\
\hline Sat. Pressure Psig & $87.1^{1}$ & & & 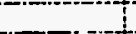 & & & & & & \\
\hline Sat. Temperature f(iniei) & 154.31 & & -7 & & & & & & & \\
\hline Liq. Density tbm nila & 82.541 & 82.341 & $82 \overline{21}$ & $82.17 !$ & 82.211 & 82.2 & 82.21! & 82.28 & 82.381 & 82.38 \\
\hline Laront Hoatetunbm & 47.351 & 47.16 & $47.02 !$ & 47 & 47.031 & 47.02 & 47.031 & 47.11 & 47.151 & 47.2 \\
\hline Speotio Heat Gtúbmil & 0.2641 & 0.265 & 0.2661 & 0.2651 & 0.2651 & 0.2651 & 0.255 & 0.2651 & $0 . \overline{561}$ & 0.265 \\
\hline Lig. Viscosity-lbm/ithr & 0.549 & 0.545 & 0.5421 & $0.541 i$ & 0.5421 & 0.5421 & 0.542 & 0.5431 & 0.5441 & 0.546 \\
\hline Liq. Conductivity $\mathrm{Btu}$ hroff & 0.03131 & $0.0312 !$ & $0.0311 i$ & 0.03111 & 0.03111 & 0.03111 & 0.03111 & $0.0 \overline{311}$ & $0.0312 !$ & 0.0312 \\
\hline Liq. Prande No. & 4.631 & 4.63 & 4.62 & 4.62 & 4.62 & 4.62 & 4.62 & 4.62 & 4.63 & 4.63 \\
\hline Inlolfflow Rato-GPM & $5.1:$ & & & & & & & -1 & & \\
\hline Outlet Lia. Flow Rato-GPM & $2.6 i$ & $:$ & & & & & & 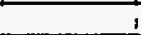 & & \\
\hline Outlet Vap. Fow Rato-GPM & 1.1 & & & & & & & & & \\
\hline Inlet Temperaturo & 147.41 & & & $\therefore$ & & & & & & \\
\hline Outtet Lia. Temperaturef & 148.91 & & & 7 & 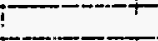 & & & & & \\
\hline Outlet vap. Temperaruro-f & 14251 & 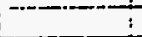 & & 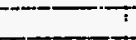 & $t$ & & & 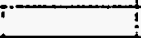 & & \\
\hline Water Fow Palo-GPM & -7 & 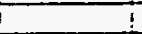 & & $\therefore$ & 5 & 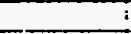 & & I & & $\vdots$ \\
\hline Water inlet Tomp.t & 69.41 & $i$ & $i$ & 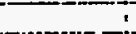 & $\div$ & $\therefore$ & & $i$ & & I \\
\hline Water outlet Temp.F & 130.17 & & $i$ & & $\because$ & & & I & & $i$ \\
\hline Inlol Glbm/hr/ht2 & 10112901 & & Tomp & 144.51 & ibonsity & 83.09 & & 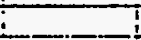 & & 1 \\
\hline Subcooling $F$ & 6.91 & & & & i & & & 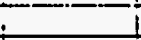 & 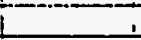 & $\perp$ \\
\hline \multirow[t]{2}{*}{ Fow Observation } & & & & & & & & & & \\
\hline & ZONET & ZONE2 : & ZONE 3 - & ZONE4 & IZONES 2 & ZONEE & ZOONET & ZONE $8-1$ & ZONE & ZONE10 \\
\hline Heat Input-Watts(net) & 1159.6 & 1164.7 & $11 \approx 3$ & 1961 & 1155.7 & 1153.6 & 1160.3 & 1156.2 & 1158.6 & 1158.2 \\
\hline Heat Loss-Walts & 32.8 & 7.2 & 9.41 & 101 & 10.4 & 10.3 & 10.2 & 11.5 & 11.9 & 6.1 \\
\hline 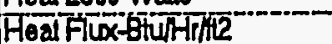 & 20101 & 20189 & ¿णढ! & टेटा & 200331 & 10907 & 20ंगाओ & 20042 & 20084 & 2007 \\
\hline Wall Tomp. H3F & 166.5 & 969.11 & 165.8 & 167.2 & 173.6 & 168.7 & 168.3 & 16.2 & 170.2 & 169.7 \\
\hline Wal Temp. \#AF & 166.7 & 166 & 168.7 & 768.5 & $16.2^{1}$ & 168.8 & 168.81 & 170.4 & 169.91 & 168 \\
\hline Wal Temp. $5 F$ & 165.81 & 163.8 & 169.71 & 167.61 & 167.61 & 168.9 & 167.21 & 167.8 & 165.71 & 167.6 \\
\hline Wall Temp. \#F & 69.41 & 167.1 & 165.31 & 166.81 & 166.61 & 170.51 & 1691 & 169.3 & 165.51 & 162 \\
\hline Wall Temp. HLF & $167.11^{\circ}$ & 166.81 & $166.4 i$ & 168.7 & 167 & 174 & 170.21 & 169.7 & 168 & $-\overline{165.6}$ \\
\hline instroam Tomp.F & 148.8 & 150.3 & 151.41 & $151.6 !$ & 157.31 & 151.41 & 151.31 & 150.8 & 150.41 & 150 \\
\hline Avg. Wall Temp.F & 166.41 & 167.31 & 166.8 & 167.5 & 167.6 & 169.2 & 168.71 & 169.3 & 167.91 & 166.6 \\
\hline Avg. WallDT.F & 16.7 & 16 & 14.5 & 15 & 15.4 & 16.9 & 16.5 & 17.6 & 16.6 & 15.7 \\
\hline Romaining SubcooffF & $-\frac{25}{2.5}$ & & & & & & & & & \\
\hline Exot Quality & -0.011 & 0.011 & 0.041 & 0.06 & 0.08 & 0.11 & 0.13 & 0.16 & 0.18 & 0.21 \\
\hline EXP. H-BtuntrmedF & 1204.31 & 1258.9 & 1391.41 & 1340.3 & 1301.31 & 1181.8 & 1219.6 & 1140.4 & 1213.3 & 0 \\
\hline COMPUTEDH & & & & & & & & & & \\
\hline Jälouk & 1068.2 & 1076.4 & $\overline{988.6 !}$ & \$50 & 914.9 & 893.4 & 874.1 & 853.9 & $\overline{834.8}$ & 816.5 \\
\hline Szadyl(Cudo tube) & 1529.51 & 1540.6 & 1552.91 & 1560.4 & 1564.21 & 1561.4 & 7573.4 & 1577.6 & 1587 & 1595.4 \\
\hline Szady? & 1384.8 & 1397.5 & 14101 & 1431.5 & 1427.91 & 1418.8 & $1428.6 !$ & 1483.5 & 1458.8 & 1460 \\
\hline$(\overline{R U Q})$ & 1205491 & 121439 & 119295 & 11638 & 113192 & 110235 & 107166 & 103893 & 100689 & 97490 \\
\hline (a) & 1.0071 & 1.007 & 1.6671 & 2.0051 & 2.3121 & 2.655 & $27 \pi$ & 2.881 & 3.1731 & 3.356 \\
\hline (HUO) & 289.21 & 289.51 & 479.99 & 583.11 & 665.31 & 735.4 & 798.5 & 857.7 & 912.61 & 964.5 \\
\hline \multirow[t]{3}{*}{ (HBOIL) } & 1526.11 & -1537.21 & 1537.5 & 1532.8 & 15231 & 1505 & 1501.6 & 1488.1 & 1479.2 & 1468 \\
\hline & & -1 & & 1 & 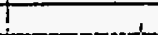 & & & $i$ & & 1 \\
\hline & IZONE2 & ZONEA & ZOONE & ZONE & ZONETEO I & & & i & & \\
\hline DP liquid Temp,f & $1 \quad 71 i$ & $i$ & & & & & & & & \\
\hline DP Liquid Density-lbm/n & 97.551 & & & & & & & & & \\
\hline Elevation-t & & -4 & $-6 !$ & -8 & 9.875 & & & & & \\
\hline Moasured DPFsid & 0.089 & 0.576 & 0.844 & 1.108 & 1.220 & & & T! & & \\
\hline Zoro Cortections-psid & 0.0151 & 0.066 & 0.021 & 0.0531 & -0.0211 & & & & & \\
\hline Comeded DPpsid & 0.0741 & 0.51 & 0.8241 & 1.05 & 1.251 & & & $T$ & & 1 \\
\hline Actual DPA & $2.09 i$ & 3.55 & 5.231 & 7.05 & 8.791 & & & $T$ & & \\
\hline Measurod tppig & 87.11 & $\cdots$ & i & 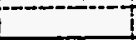 & 802 & & & I & & i \\
\hline \multirow[t]{2}{*}{ Moasured Ovorallit } & 12.051 & & $i$ & 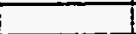 & $\bar{i}$ & $\bar{i}$ & & 1 & & I \\
\hline & T & 1 & ! & & & & I & I & & 1 \\
\hline ComputedDP-A & 2.181 & 4.15 & $6.11 \mathrm{i}$ & 8.18 & 10.29 & & $i$ & 1 & & $!$ \\
\hline Friction-ft & 0.17 & 0.58 & 1.22 & 2.08 & 3.07 & & 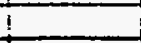 & $i$ & & \\
\hline Accoleration-A & 0.16 & 0.44 & 0.66 & 0.89 & 1.14 & & $i$ & 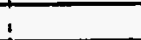 & & 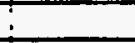 \\
\hline Elevation-ft & 1.84 & 3.13 & 4.2द & 5.22 & $6.09 !$ & & $\bar{\vdots}$ & & & \\
\hline
\end{tabular}




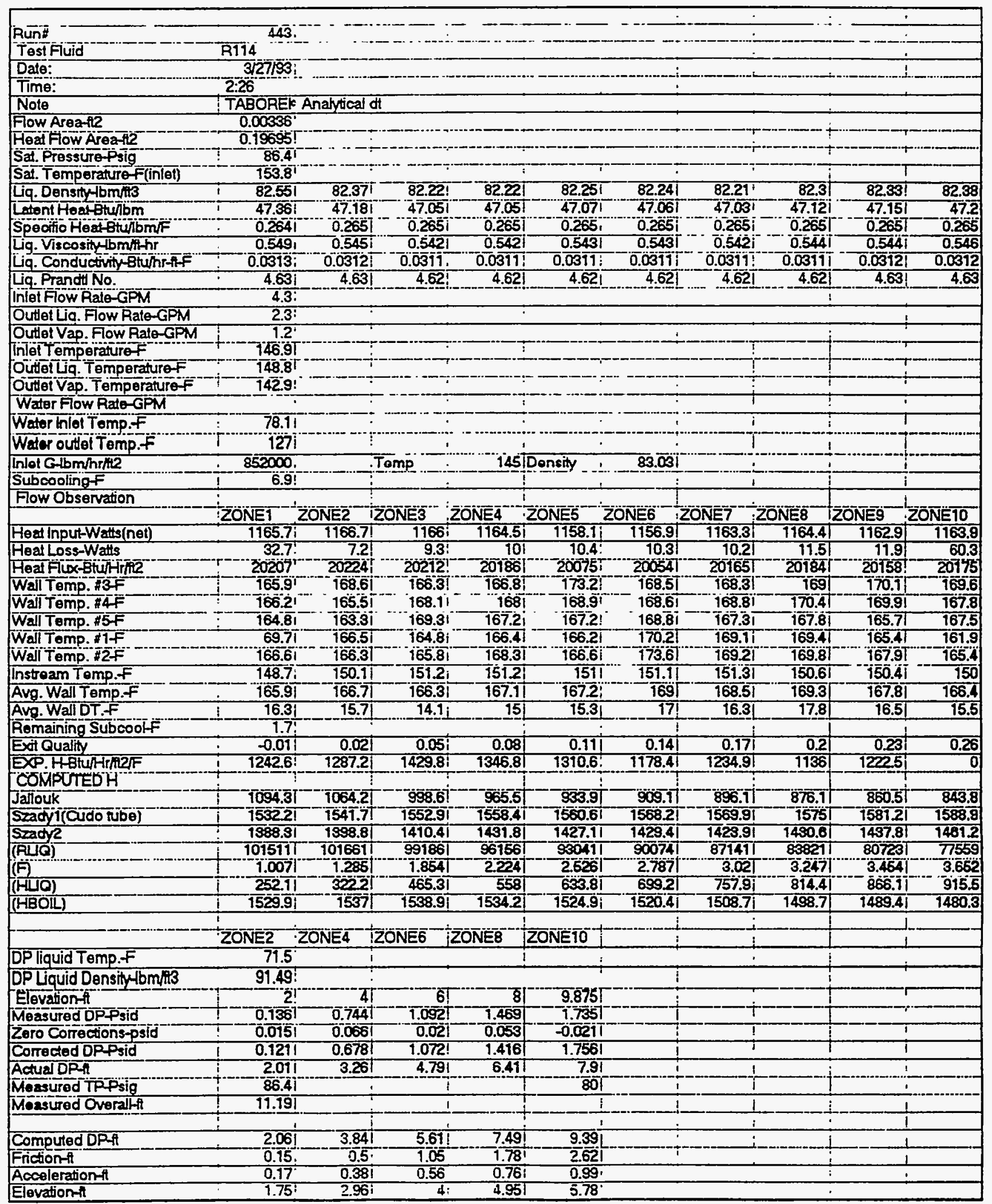




\begin{tabular}{|c|c|c|c|c|c|c|c|c|c|c|}
\hline Aun" & 441 & & $T$ & & $\therefore$ & $\dot{-}$ & 1 & 1 & + & ' \\
\hline Tostfluid & IR114: & & I & $\dot{-}$ & 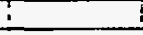 & $!$ & $!$ & $i$ & & 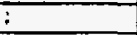 \\
\hline Date: & 13271931 & & $\mathrm{i}$ & $i$ & \pm & $i$ & I & & & . \\
\hline Time: & 3.49 & & i & $i$ & & & $!$ & 7 & 1 & \\
\hline Nole & I Analytical c & dt is used & $i$ & & $\therefore$ & & 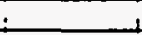 & & & 1 \\
\hline Flow Area-12 & 0.003361 & & i & 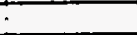 & 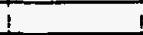 & 1 & i & & & \\
\hline Heat Fow Aseatl2 & 0.196951 & & & 5 & & & & & & \\
\hline Sat. Pressure Psig & 84.51 & & & & $i$ & & & & & \\
\hline Sal. Temperaturof(inlel) & 152.4 & & & & $\therefore$ & & & & & \\
\hline La. Denstiy-bm/n3 & 82.69 & 82.52 & 82.38 & 82.42 & 82.43 & 82.411 & 82.411 & 82.49 & $82.51 !$ & 82.55 \\
\hline Latont Hoat Btulbm & 47.5 & 47.34 & 47.2 & 47.23 & 47.25 & 47.22 & 47.22 & 47.3 & $47.32 !$ & 47.38 \\
\hline Spocifio Hoos-btulbmif & 0.264 & 0.264 & 0.265 & 0.265 & 0.2641 & 0.265 & 0.2651 & 0.264 & 0.264 & 0.264 \\
\hline Lig. Viscosity-lbm/lithr & 0.552 & 0.548 & 0.546 & 0.546 & 0.547 & 0.546 & 0.546 & 0.548 & 0.5481 & 0.549 \\
\hline Lg. Conductivity Btu/hr-th-F & 0.03141 & 0.03131 & 0.0312 & 0.0312 & 0.0312 & 0.0312 & 0.0312 & 0.0313 & 0.03731 & 0.0313 \\
\hline Liq. Prandt No. & 4.641 & 4.63 & 4.63 & 4.63 & 4.63 & 4.631 & 4.531 & 4.63 & 4.631 & 4.53 \\
\hline Inlel Flow Rate-GPM & 3.41 & & & & +1 & I & & & 1 & $\mathbf{i}$ \\
\hline Outlet Lig. Flow Rato-GPM & 2 & & & ; & $t$ & 1 & $t$ & i & & $\bar{i}$ \\
\hline Outlel Vap. Flow Rado-GPM & 11 & & & 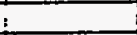 & $i$ & $\mathbf{i}$ & & i & 1 & 1 \\
\hline Inlel Temperaturef & 1451 & & 1 & & $i$ & & i & i & ?. & ?. \\
\hline Outleflua. Temperaturer & 747.81 & & & & $i$ & & & & & \\
\hline Outle! Vap. Temperaturef & 149.7| & & & & & & & & & \\
\hline Water Fow Rato-GPM & & & & & I & $T$ & & & & \\
\hline Water Inlet Tomp.F & 80.11 & & & & 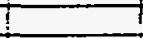 & I & & & & \\
\hline Water outhol Temp.F & 13.11 & & & & & 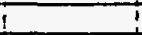 & & I & & $\bar{i}$ \\
\hline Inlet Glbm/hr/iti & 677390 & & Temp & 141.4 & Donsity & 83.48 & & i & & \\
\hline Subcooling $F$ & 7.41 & & & & & & & i & & \\
\hline \multirow[t]{2}{*}{ Flow Observation } & & & & & & & & & 1 & 1 \\
\hline & ZONE1 & ZONE2 & ZONE3 & ZONE4 & TZONE5 & ZONE6 & ZONE7 & ZONEB & ZONE9 & ZONEIO \\
\hline Head Input-Watts(nol) & 1143 & 1154 & 1156 & 1153 & 1154 & 1156 & 1154 & 1149 & 1150 & 1158 \\
\hline Heal Loss-Watts & अ.1 & 6.7 & 8.9 & 10 & 10.5 & 10.5 & 9.8 & 11.1 & 11.2 & 57.7 \\
\hline Heat Flox Btuhtrhtit & 19813 & 20004 & 20039 & 19987 & 20004 & 20039 & 20004 & 19917 & 19935 & 20073 \\
\hline Wall Temp. मnऊF & 164.4 & 167.2 & 165.1 & 165.5 & 172.1 & 167.4 & 166.9 & 167.4 & 168.7 & 168.3 \\
\hline Wall Temp. H4F & 164.6 & 164 & 167.1 & 166.7 & 167.6 & 167.5 & 167.4 & 168.7 & 168.4 & 166.8 \\
\hline Wall Temp. H5F & 163.3 & 162 & 168.1 & 165.8 & 165.9 & 167.7 & 166.1 & 166.2 & 164.4 & 166.3 \\
\hline Wall Temp. IF & 72.3 & 165.1 & 163.5 & 164.8 & 164.9 & 16.11 & 167.8 & 787.8 & 164.11 & 160.6 \\
\hline Wall Temp. KLF & 164.9 & 164.8 & 164.6 & 166.9 & 965.3 & 172.5 & 168.4 & 168.3 & 166.61 & 164.3 \\
\hline Instream Temp.f & 147.6 & 148.9 & 150 & 149.7 & 149.6 & 149.8 & 149.8 & 149.2 & 149 & 148.7 \\
\hline Avg. Wall Tomp.f & 164.3 & 165.3 & 165.1 & 165.7 & 165.9 & 167.9 & 167.31 & 167.7 & 166.41 & 165.3 \\
\hline Avg. Wall DT, f & 15.81 & 15.5 & 14.2 & 15.1 & 15.41 & 17.21 & 16.6 & 17.6 & 16.51 & 15.7 \\
\hline Remaining Subcooff & 0.91 & & & & & & & & & \\
\hline Exct Quality & -0.01 & 0.03 & 0.07 & 0.1 & 0.14 & 0.18 & 0.21 & 0.25 & 0.29 & 0.32 \\
\hline EXP.HEtu/Hrit2/F & 1253.6 & 1290.9 & 1414.2 & 1321.7 & 1297.2 & 1163.7 & 1203.9 & 1133 & 1205.3 & 12824 \\
\hline \multicolumn{11}{|l|}{ COMPUTEDH } \\
\hline Jallouk & T15.6 & 10.521 & 7005.4 & 976.7 & 953.6 & 933.9 & $913.4 \mid$ & 895.9 & 881.7 & 869.9 \\
\hline Szadyil(Cudo tube) & 1511.2 & 1520.9 & 1538.4 & 1540 & 1545.9 & 1554.4| & 1560 & 7550.1 & 7556.4 & 15672 \\
\hline szadyz & 1353.4 & 1381.5 & 1393.9 & 1413.4 & 1414.7 & 1419.2 & 1420 & 1401.4 & 1409.41 & 1421.9 \\
\hline (RUQ) & 80271 & 79725 & 7789 & 74105 & 71009 & 68194 & 65219 & 62068 & 550591 & 56020 \\
\hline (F) & 1.007 & 1.488 & 2.06 & 2.467 & 2.808 & 3.099 & 3.36 & 3.674 & 3.8471 & 4.07 \\
\hline (HiLO) & 209.6 & 310.1 & 429.8 & 514.5 & 584.6 & 645.5 & 700.81 & 753.7 & 802.11 & 848.4 \\
\hline \multirow[t]{3}{*}{ (HBOIL) } & 1509.81 & 15227 & 1527.1 & 1520.6 & 1517.5 & 1516.4 & 1511.4 & 1488.2 & 1481.9 & 1479.5 \\
\hline & & & & & & & & & & \\
\hline & ZONE2 & ZONE4 & ZONE6 & ZZONE8 & ZONETO & & & i & & \\
\hline DP liquid Temp.f & 71.5 & & & & & & 1 & 1 & & \\
\hline DPLiquid Density 4 bm/1t3 & 91.49 & & & & & & & & & \\
\hline Elovation & 21 & 4 & 6 & 8 & 9.875 & & & & & \\
\hline MeasuredDPFsid & 0.18 & 0.962 & 1.406 & 1.919 & 2.275 & & & & & \\
\hline Zero Corrections-psid & $\overline{0.015}$ & 0.066 & 0.02 & 0.053 & -0.021 & & & & & \\
\hline Conectod DPPaid & 0.168 & 0.896 & 1.388 & 1.866 & 2.296 & & & & & \\
\hline ActualDP-A & 1.92 & 2.87 & 4.23 & 5.61 & 6.94 & & & & & \\
\hline Mossured TPPsig & 84.51 & & & & 79.11 & & & & & \\
\hline \multirow[t]{2}{*}{ Messured Overallit } & 8.421 & & & & & & & & & \\
\hline & & & & & & & & & & \\
\hline Computed DPA & 1.93 & 3.44 & 4.93 & 6.52 & 8.12 & & & & & \\
\hline Friction-t & 0.111 & 0.41 & 0.88 & 1.52 & 2.25 & & & & & I \\
\hline Accoleration-A & 0.14 & 0.29 & 0.44 & 0.62 & 0.83 & & & & & \\
\hline Elevation-t & 1.68 & 2.74 & 3.61 & 4.38 & 5.04 & & & & & \\
\hline & & & & & & & & & & \\
\hline
\end{tabular}




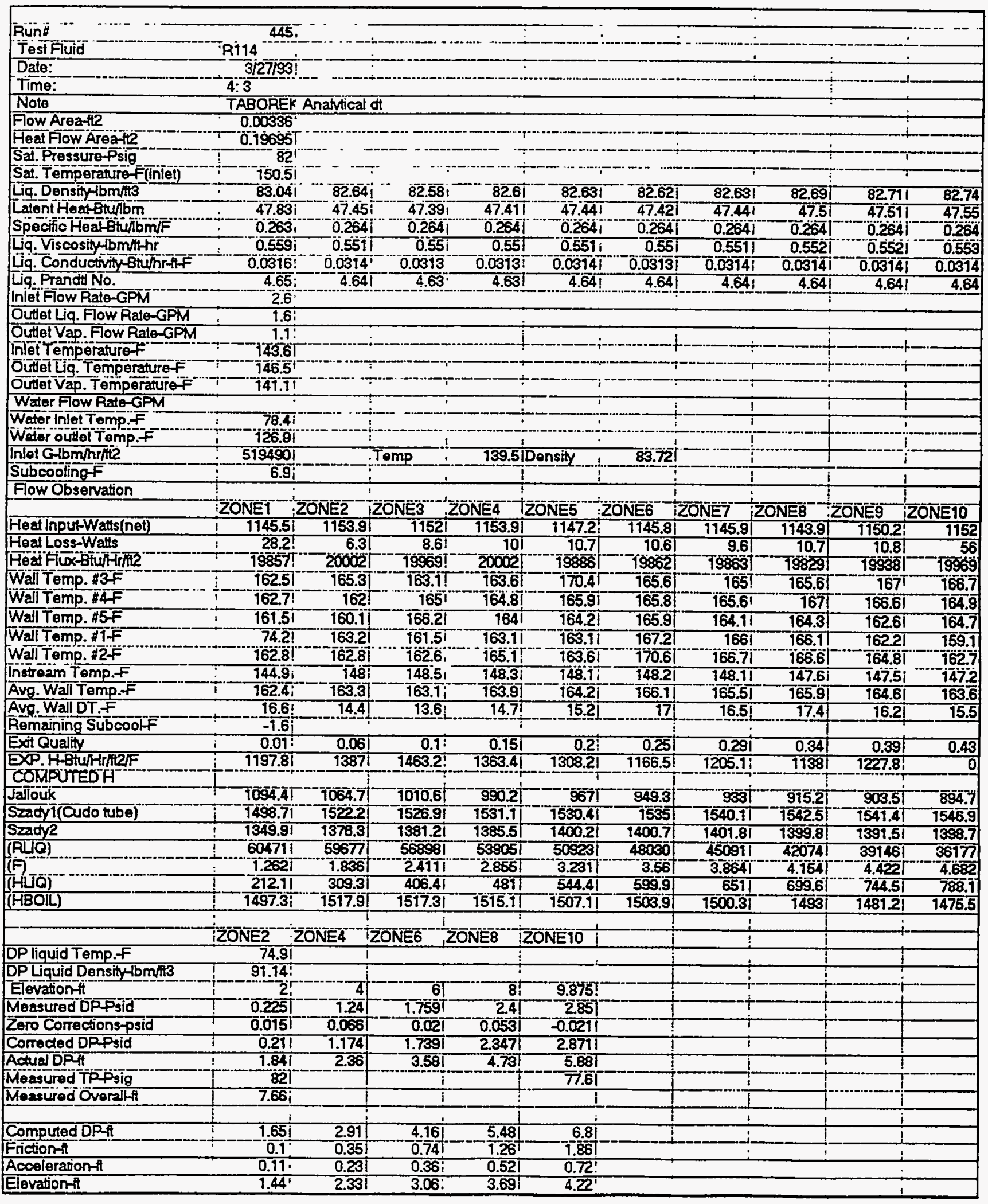




\begin{tabular}{|c|c|c|c|c|c|c|c|c|c|c|}
\hline & & & & & . & & . & & & \\
\hline Ruñ & $4 \overline{46}$, & & $\therefore$ & & & & $\because$ & 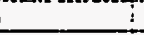 & - & \\
\hline Test Fuid & RTiT4 & & 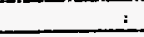 & & - & & 1 & 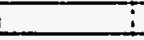 & \pm & \\
\hline Date: & 327193 & & & & $!$ & 1 & 1 & 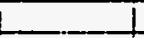 & & \\
\hline Time: & $4: 3$ & & & & & & & & & \\
\hline Note & TABOREK & Analyical ot & it & & & $i$ & \pm & L & $\therefore$ & \\
\hline Flow Area-12 & $0.00336 !$ & i & & $!$ & & 1 & + & 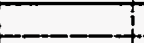 & & \\
\hline Heaf How Area & $0.196951^{\circ}$ & & T & 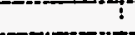 & & & $T$ & & & \\
\hline Sat. PTossuro $\overline{p s i g}$ & 83.11 & & & tִ & 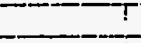 & $\bar{\vdots}$ & $T$ & 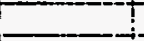 & & \\
\hline Sat. Temperäure-F(inlel) & 151.31 & & -1 & -1 & -1 & -1 & -7 & & & \\
\hline Lig. Density lbmitt 3 & 82.911 & 82.421 & 82.411 & 82.431 & 82.451 & 82.431 & 82.411 & 82.451 & 82.491 & 82.51 \\
\hline Latent Heat Btuibm & 47.711 & 47.23 & 47.221 & 47.251 & 47.261 & 47.251 & 47.221 & 47.261 & 47.31 & 47.32 \\
\hline Spectio Hear Gtútbmi & 0.2631 & 0.265 & 0.2651 & 0.264 & 0.2641 & $0 . \overline{2641}$ & 0.2651 & 0.264 & 0.2641 & 0.264 \\
\hline Liq. Viscositivibmifthr & $0.557 i$ & 0.5461 & 0.5461 & 0.547 & 0.5471 & 0.547 & 0.5461 & 0.5471 & 0.5481 & 0.548 \\
\hline 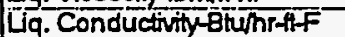 & 0.0315 & $0.0312:$ & 0.0312 & 0.0312 & 0.0312 & 0.03121 & 0.03121 & 0.03121 & 0.0313 & 0.0313 \\
\hline Lig. Prandd No. & 4.65 & 4.631 & 4.63 & 4.63 & 4.631 & 4.63 & 4.631 & $4 . \approx 3$ & $4.63 \mathrm{~L}$ & 4.63 \\
\hline Inlol Flow Palo-GPM & 1.8 & & & & & 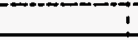 & & & & \\
\hline Outlet Lig. Flow Rato-GPM & $0.7^{\circ}$ & & & & & & & & & \\
\hline Outlat Vap. Flow Rato-GPM & 1.2 & & & & & & & & & \\
\hline Títeltemperature & -144.61 & & & & & & & & & \\
\hline Outet Uq. Temporaturef & 148.8 & & 1 & & & & & & & \\
\hline Outlet vap. Temperaturef & 144.51 & & & $!$ & & & 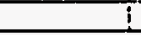 & i & & \\
\hline Water Fiow Rato-GPM & & & 1 & & $\dot{\square}$ & & 1 & 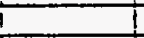 & & \\
\hline Water Inlot Temp.F & 81.21 & & $\therefore$ & & $\therefore$ & & T & $T$ & & \\
\hline Water outat Temp.F & 130.61 & & $i$ & & $i$ & & I & I & & \\
\hline InIol Gibminrit2 & 3594851 & i7 & Tomp & 139.8 & Density & 83.69 & 1 & $I$ & $i$ & \\
\hline Subcooling F & 6.71 & + & $\therefore$ & & i & & 1 & 1 & & \\
\hline Fow Observation & & & & & $!$ & & & & & \\
\hline & ZONET & ZONE2 IZ & ZONES & ZONE4 & ZONE5 12 & ZONE6 & ZONET & ZONE8 & ZONE9 & ZONE10 \\
\hline Head Input-Watts(net) & 1156.8 & 1158.61 & 1155.5 & 1159.2 & 1155.7 & 1158.9 & 1160.6 & 1167.1 & \begin{tabular}{|l|}
1156.3 \\
\end{tabular} & 1154.4 \\
\hline HeatLoss-Watts & 27.8 & 6.2 & $8.5 !$ & 10 & 10.71 & 10.6 & 9.61 & 10.7 & 10.7 & 56.7 \\
\hline Heâfilux-Bturifhe & 20052 & 20084 & 200301 & 20094 & $20033 !$ & 2008 & 20118 & $201 \overline{27}$ & 20044 & 20011 \\
\hline Wall Tomp. $3 F$ & 163.3 & $=-166$ & 164 & 164.3 & 171.31 & 165.8 & 166.4 & 167.2 & 168.41 & 171.9 \\
\hline Wal Temp. & 163.41 & 162.8 & 165.81 & 165.4 & 166.91 & 166.9 & 967 & 168.6 & 1681 & 169.2 \\
\hline Wall Temp. \#SF & 162.31 & 160.9 & 167.21 & 164.6 & 165.11 & 168.8 & 165.51 & 165.9 & 1691 & 168.1 \\
\hline Wall Tomp. 11 & $7.3 i$ & 163.41 & 16231 & 163.9 & 163.8 & 168.4 & 167.7 & 167.8 & 163.7 & 160.6 \\
\hline Wail Tomp. & 163.71 & 163.3 & $163.1 \mathrm{i}$ & 165.8 & 164.31 & 171.81 & 168.2 & 168.2 & 166.31 & 164.3 \\
\hline Instroam Tomp.f & 145.91 & 149.7 & $149.8 i$ & 149.6 & 149.5 & 149.6 & 149.81 & 149.51 & 149.21 & 149 \\
\hline Avg. Wall Tamp.F & 163.21 & 163.9 & 163.81 & 164.6 & 165 & 167.2 & 167 & 167.5 & 166.11 & 166.4 \\
\hline Avg. Wall DT F & 16.4 & 13.3 & 13.1 & 14 & 14.6 & 16.71 & 16.3 & 17.1 & 161 & 16.5 \\
\hline Remaining SubcoofF & -5.7 & & - & & & & & & -1 & \\
\hline Exot Quality & 0.03 & 0.1 & $0.17 !$ & $0.24 !$ & 0.311 & 0.38 & 0.451 & 0.52 & 0.581 & 0.65 \\
\hline EXP. H HBtuHRril2/F & 1225 & 1513.8 & 1529.61 & 1431 & 1370.3 & 1201.7 & 1238 & 1174.9 & 1254.8 & \\
\hline COMPUTESH & & & & & & & & & & \\
\hline Jalouk & 11322 & 1086.2 & $1054.3 !$ & 7018.81 & 1005.41 & 992 & 9821 & $97 \overline{0.61}$ & 957.61 & 948 \\
\hline Szady 1 (Cudo rube) & -15117 & 1533.4 & 1534.21 & 1538.3 & 1538.7 & 1544.8 & 1550.71 & 1553.8 & 1554.11 & 1557.1 \\
\hline$\frac{1}{\text { Szadty? }}$ & 1384.31 & 1389.11 & $7389.6 !$ & 1394.11 & 1393.91 & 1418 & 1421.21 & 1421.1 & 14171 & 1418.3 \\
\hline (RLQ) & 415811 & 40217 & 372711 & 342551 & 312611 & 28299 & 253411 & 22317 & 19309 & 16328 \\
\hline (F) & 1.551 & 2.25 & -2.8971 & 3.4141 & 3.861 & 4.257 & 4.621 & 4.974 & 5.312 & 5.635 \\
\hline$($ H】Q & 194.11 & 2827 & 3641 & 429 & 4851 & 534.9 & 580.71 & 224.91 & 6672 & 707.7 \\
\hline$(H B O I L)$ & 1500.9 & 1530.2 & 1527.41 & 1527.11 & 1522.51 & 1523.1 & 1523.11 & 1519.41 & 1512 & 1506.8 \\
\hline & & & & & & & & & i. & \\
\hline & ZZONE2 & ZONNE4 & ZONEE'-Z & ZONENE & ZOONE10 & & & 1 & 1 & \\
\hline DP liquid Temp.F & 78.4 & & & 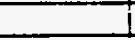 & 2 & & & 1 & & \\
\hline DP Liquid Density $4 \mathrm{bm} / \mathrm{t} 3$ & 90.76 & & & & & & $\cdot$ & & & \\
\hline Elevation & $2 !$ & 4 & $6 !$ & 8 & 9.875 & & & & & \\
\hline Measured DRpsid & 0.2831 & 7.585 & 2.18 & 2.9751 & $3 . \overline{20}$ & & & & & \\
\hline Zero Conections-psid & 0.0151 & 0.0601 & $0.02 !$ & 0.053 & -0.0211 & & & $T$ & T & \\
\hline Comrocted DPPsid & 0.268 & 1.52 & 2.181 & 2.922 & 3.6231 & & & I & T & \\
\hline Actual DPA & 1.731 & 1.75 & 2.831 & 3.7 & 4.54 & & & & & \\
\hline Monoured TFFig & 83.17 & & - & & 80.4 & & & I & & \\
\hline Meseured Ovorall & 4.791 & & 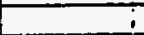 & & & & & & & \\
\hline & & & 1 & 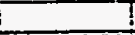 & & & I & & 1 & \\
\hline Computed DPA & 1.321 & 2.311 & 3.271 & 4.26 & 5.23 & & & 1 & 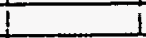 & \\
\hline Friction-fl & 0.081 & $0 . \overline{26}$ & 0.55 & 0.92 & 1.33 & & & 1 & $i$ & \\
\hline Acceleration-" & 0.081 & 0.17 & 0.281 & 0.43 & 0.02 & & & i. & 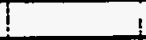 & \\
\hline Elovation-ft & $1.16 i$ & 1.88 & 2.44 & 2.91 & 3.281 & & 1 & & & \\
\hline
\end{tabular}




\begin{tabular}{|c|c|c|c|c|c|c|c|c|c|c|c|}
\hline Runt & 4471 & & & - & & & & ; & : & & \\
\hline Tost Fluid & R114 & & $:$ & & & & & & & & \\
\hline Date: & 327193 & & & & . & & & & 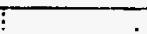 & & \\
\hline Time: & $4: 3$ & & . & & & & & & & & \\
\hline Note & Änalytical c & dt is used & 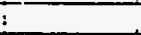 & & & & & & & & \\
\hline Flow Areatt2 & 0.003361 & & 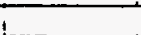 & & & & & & ? & & \\
\hline Heat Fow Area-ti2 & 0.19595 & & 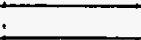 & & $!$ & & & & & & \\
\hline Sad. PressurePsig & 83.4 & & 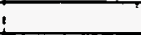 & & 1 & & & & & & \\
\hline Sat. Temperaturef (inlet) & 951.6! & & & & + & & & & & & \\
\hline Wq. Densitylbmn3 & 82.81 & 82.33 & $82.34 !$ & 82.341 & 82.361 & $82.36 !$ & 82.331 & $82.37 !$ & $82.37 !$ & 82.42 & \\
\hline Latent Heat-Btuflbm & 47.61 & 47.15 & 47.161 & 47.16 & 47.171 & $47.17 !$ & 47.15 & 47.18 & 47.18 & 47.23 & \\
\hline Specific Hear-stulbmfF & 0.2641 & 0.265 & 0.2651 & 0.2651 & 0.265 & 0.2651 & 0.2651 & 0.2651 & 0.2651 & 0.265 & \\
\hline Lig. Viscosity-lbmithr & 0.5541 & 0.5441 & 0.5451 & 0.545 & $0 . \overline{445}$ & 0.545 & 0.544 & 0.545 & 0.5451 & 0.546 & \\
\hline Lig. Conductivity BtuhrttfF & 0.03151 & 0.03121 & 0.03121 & 0.0312 & 0.0312 & 0.0312 & 0.0312 & 0.0312 & 0.03121 & 0.0312 & \\
\hline Liq. Prandi No. & 4.64 & 4.631 & 4.631 & 4.63 & 4.631 & 4.63 & $4 . \approx 31$ & $4 . \infty 8$ & $4 . \approx 31$ & 4.63 & \\
\hline Inlet Fow Rale-GPM & 11 & : & 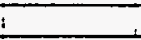 & & 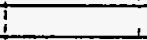 & & 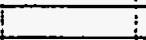 & 1 & 1 & 1 & \\
\hline Oútiot Lia. Fow Ralo-GPM & 0.71 & & 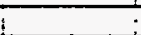 & & 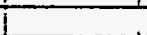 & & & & $\div$ & 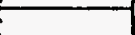 & \\
\hline Outet Vap. Flow Rate-GPM & 0.8 ! & & & & $\div$ & & + & 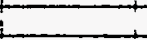 & $\div$ & 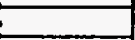 & \\
\hline Inlet Temporaturef & 140.91 & & & & $i$ & & & 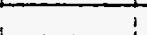 & 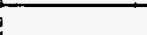 & & \\
\hline Outhef Liq. Temperaturef & 349.61 & & & & : & & & & $T$ & 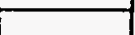 & \\
\hline Outtel Vap. Temperature $F$ & 146.6 & & & & $T$ & & & & t! & 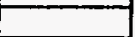 & \\
\hline Waler Flow Rate-GPM & & & 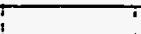 & T & $T$ & & $t$ & t & Tं & + & \\
\hline Water inlel Temp.F & 83.71 & & 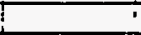 & & 1 & & $T$ & t & $i$ & 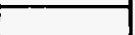 & \\
\hline Water outlel Temp.F & 141.11 & & 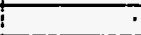 & & $\overline{1}$ & & $!$ & 1 & $T$ & i & \\
\hline Inlet Glb $\bar{m} / \overline{h r}$ & 2010251 & & Temp & 135.4 & Density & $84.24 !$ & $i$ & 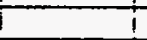 & $i$ & 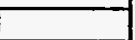 & \\
\hline Subcoolingf & 10.71 & & 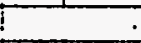 & & 2 & & $i$ & T & 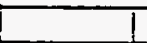 & 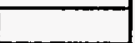 & \\
\hline Fow Obsenvation & & 1 & & & 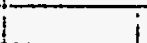 & & I & 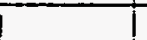 & & & \\
\hline & ZONEI 1 & ZONE2 & ZONE3 & ZONE4 & ZONE5 & ZONE6 & ZONE7 & ZONE8 & ZONE9 & ZONE10 & \\
\hline Hoat input-Watts(net) & $i \quad 1150.11$ & 1156 & 1148.4 & 1147.4 & 1150.3 & 1155.4 & 1159 & 749.5 & 0 & 573.6 & \\
\hline Hoad Loss-Watts & 27.7 & 6.2 & 8.51 & 101 & 10.7 & 10.6 & 9.6 & 10.31 & 8.6 & 53.9 & \\
\hline Hoaf Fux-Btunhriti2 & 19936 & 20039 & 19907 & 19889 & 19940 & 20028 & 19952 & 12992 & 01 & 9943 & $15 \div$ \\
\hline Wall Tomp. & TE2.91 & 165.5 & 163.51 & 164 & 171.2! & 166.8 & 166.4 & 163.5 & 150.5 & 16 & \\
\hline Wall Temp. & 162.9 & 152.6 & 165.1 & 765.3 & 166.8 & 166.8 & 167.5 & 163.3 & 151 & 162.4 & \\
\hline Wall Temp :5F & 1621 & 100.1 & 165.8 & 164.1 & 164.7 & 166.8 & 166 & 162.2 & 150.5 & 161.5 & \\
\hline Wall Temp. \#1F & 76.9 & 162.8 & 162.11 & 163.5 & 163.4 & 168.4 & 167.71 & 163.8 & 151 & 158.6 & \\
\hline Wall Temp. H2F & 163.51 & 162.9 & 162.7 & 165.31 & 164 & 171.6 & 168.5 & 164.3 & 150.51 & 160.7 & \\
\hline Instream Temp.f & 146.8 & 150.4 & 150.3 & 150.3 & 150.21 & 150.2 & 150.41 & 150.11 & 150.91 & 148.7 & \\
\hline Avg. Wall Tomp.F & 162.81 & 163.5 & 163.4 & 164.2) & 164.71 & 1672 & 167.2 & 163.4 & 150.71 & 161.2 & \\
\hline Avg. Wallot.f & 15.11 & 12.11 & 12.21 & 131 & -13.6 & 16.11 & 15.91 & 12.7 & 0.61 & 11.1 & \\
\hline Remaining SubcoofF & -11.4 & & & & & & & & & & \\
\hline Exit Quality & 0.06 & 0.19 & 0.311 & 0.43 & 0.56 & 0.681 & 0.8 & 0.881 & 0.88 & 0.94 & \\
\hline EXP. HBtuHTrnarF & 1318.2 & 1650 & 1638.4 & 1526.9 & 1453.6 & 1244.4 & 1253.4 & 1020.4 & 0 . & 896.5 & \\
\hline COMPUTEDH & 1 & & & & & & & & & & \\
\hline Jallouk & T188. Tा & Tन44.6| & $1092 !$ & T059.11 & $10 \% 2$ & 7050.6 & 84.81 & 84.7 & 84.71 & 84.7 & \\
\hline Szadyl(Cudo tube) & 15111 & 1534.3 & 1530.1 & 1531 & 1534.2 & 1539.6 & 84.8 & 84.7 & 84.7 & 84.7 & \\
\hline szady2 & 1353.8 & 1389.9 & 1384.6 & 1385.4 & 1389 & 1395.2 & $84.8 !$ & 84.7 & 84.7 & 84.7 & \\
\hline (RUQ) & 229801 & 21147 & 181701 & 15201 & 122281 & 9249 & 92431 & 9243 & 92481 & 9248 & \\
\hline (F) & 1.8271 & 2.806 & 3.6881 & 4.394 & 5.015 & 5.586 & 5.586 & 5.586 & 5.5861 & 5.586 & \\
\hline (HUQ) & 143.81 & 221.6 & 291.21 & 347 & 3861 & 441.1 & 441.11 & 441.1 & 441.1 & 441.1 & \\
\hline (HBOIL) & 1510.61 & 153281 & 1526.6 & 1525 & 1525.31 & 1527.4 & $1527.4 !$ & 1527.4 & 1527.4 & 1527.4 & \\
\hline & & & & & & & & & & & \\
\hline & ZONE2 & ZONE4 & ZONE6 & ZONE 8 & ZONE10 & & & & i & & \\
\hline $\bar{D} \bar{P}$ liquid Temp. $F$ & 77.7 & + & 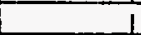 & & & & & & i & & \\
\hline DP Liquid Densitylbm/t3 & 90.841 & $i$ & & & & & & & + & 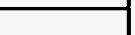 & \\
\hline Elevation-f & 21 & 4 & 61 & 8 & 9.875 & & & & 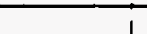 & & \\
\hline easured DPPsid & 0.208 & 1.918 & 2.6111 & 3.609 & 4.472 & & & & & & \\
\hline Euro Corrections-psid & 0.015 & 0.066 & 0.02 & 0.053 & -0.021 & & 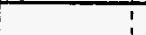 & & T & & \\
\hline Corrected DP Psid & 0.193 & 1.852 & 2.5911 & 3.556 & 4.493 & & t & & & & \\
\hline Actual DPA & 1.871 & 1.17 & 2.001 & 28 & 3.001 & & $i$ & 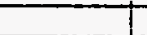 & it & & \\
\hline Mossured TPPsig & 83.4 & 1 & 1 & & 80.91 & & i & & $T$ & & \\
\hline Mossured Ovaralli & 4.371 & & 1 & & 1 & & T & 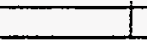 & $T$ & & \\
\hline & & & 1 & & & & & & I & & \\
\hline Computed DPA & $1.04 !$ & $1.71 !$ & 2.31 & 284 & 3.65 & & 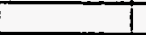 & & I & 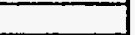 & \\
\hline Friction-At & 0.041 & 0.141 & 0.31 & 0.48 & 0.6 & & 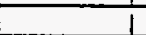 & 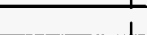 & 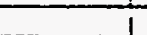 & - & \\
\hline Accoleration-ft & 0.041 & 0.1 & 0.2 & 0.311 & 0.35 & & & & 1 & 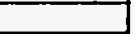 & \\
\hline Elovation-tt & $0.96 i$ & 1.461 & 1.8 & 2.06 & 2.71 & & & & $i$ & & \\
\hline
\end{tabular}




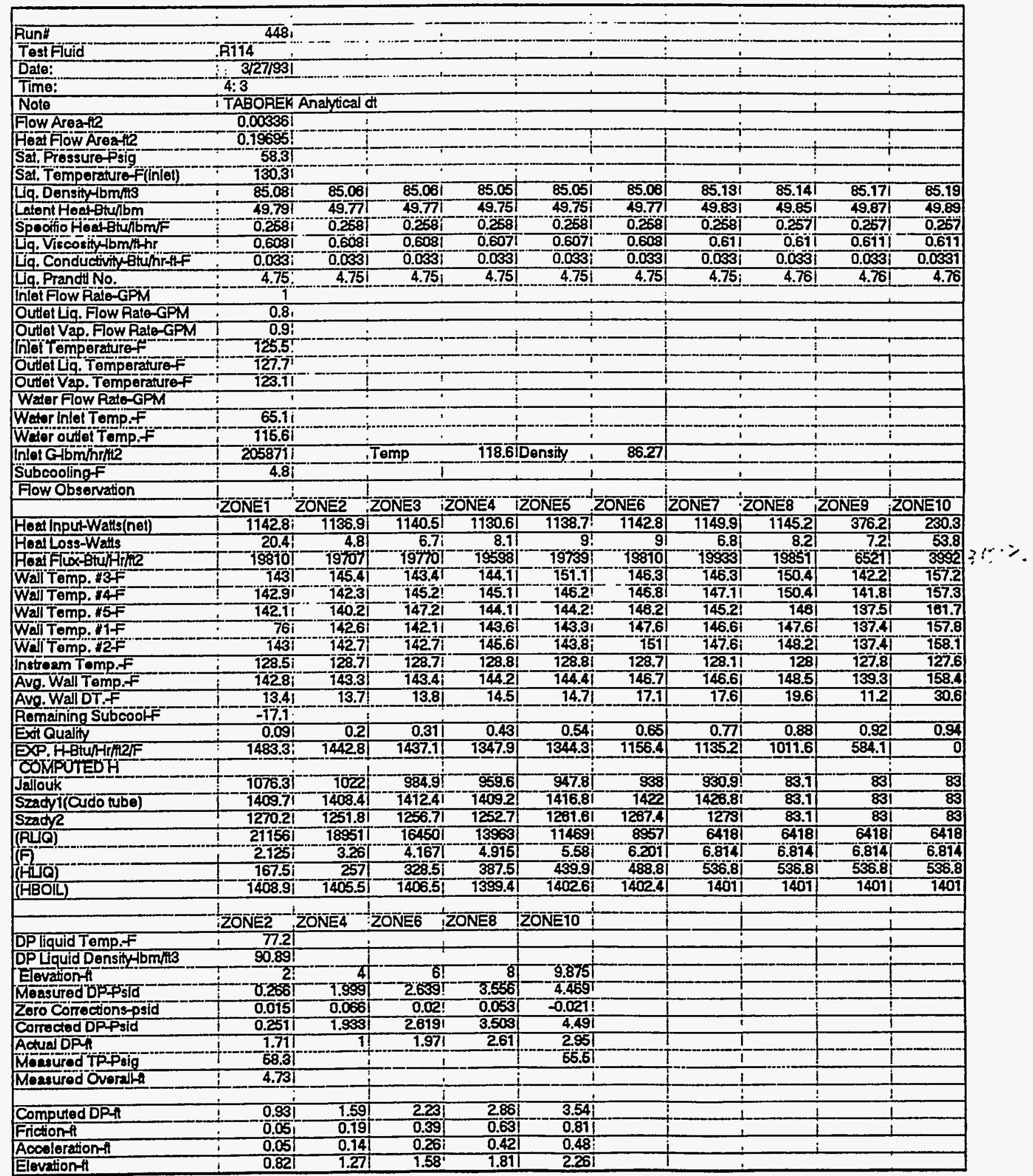




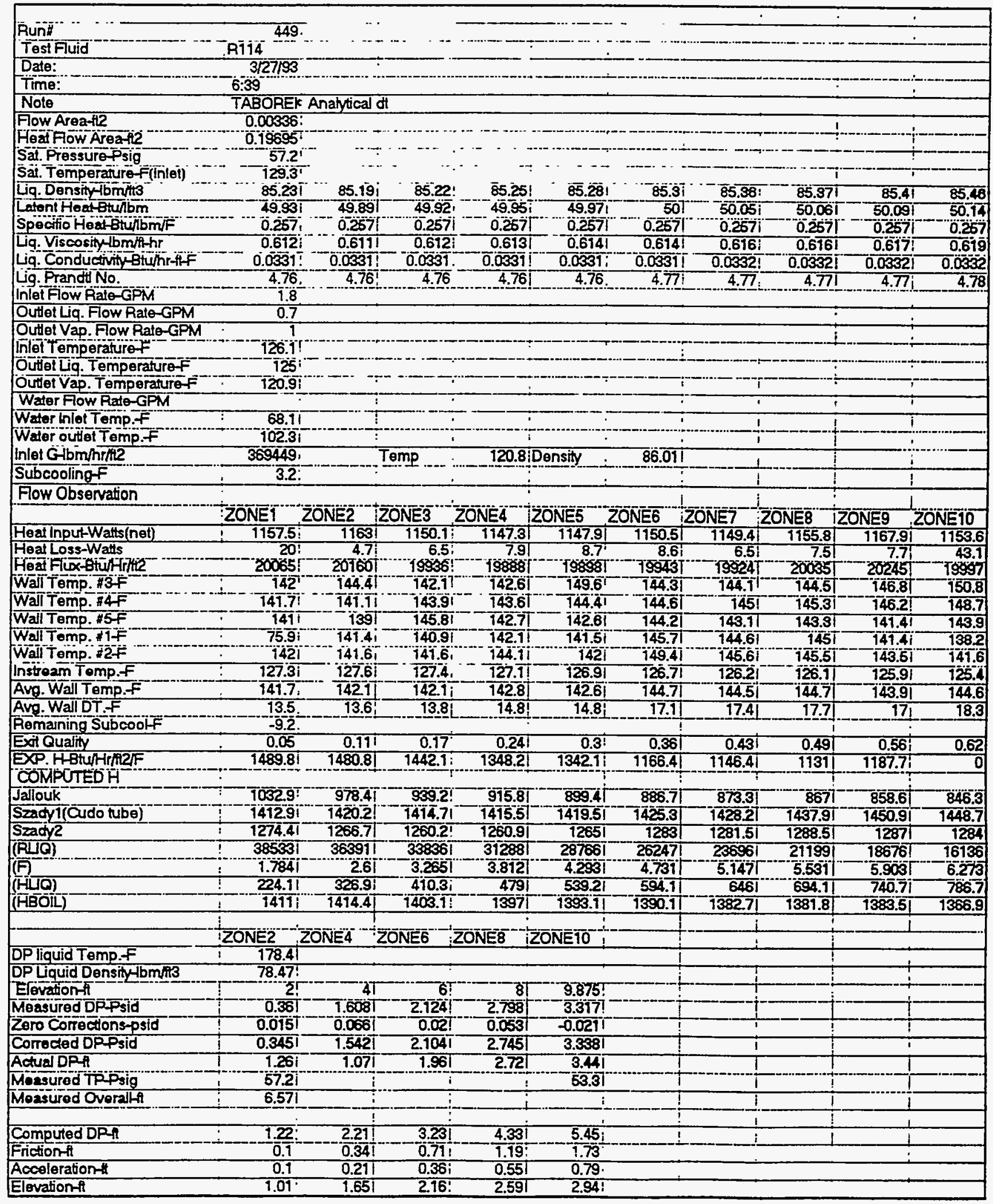




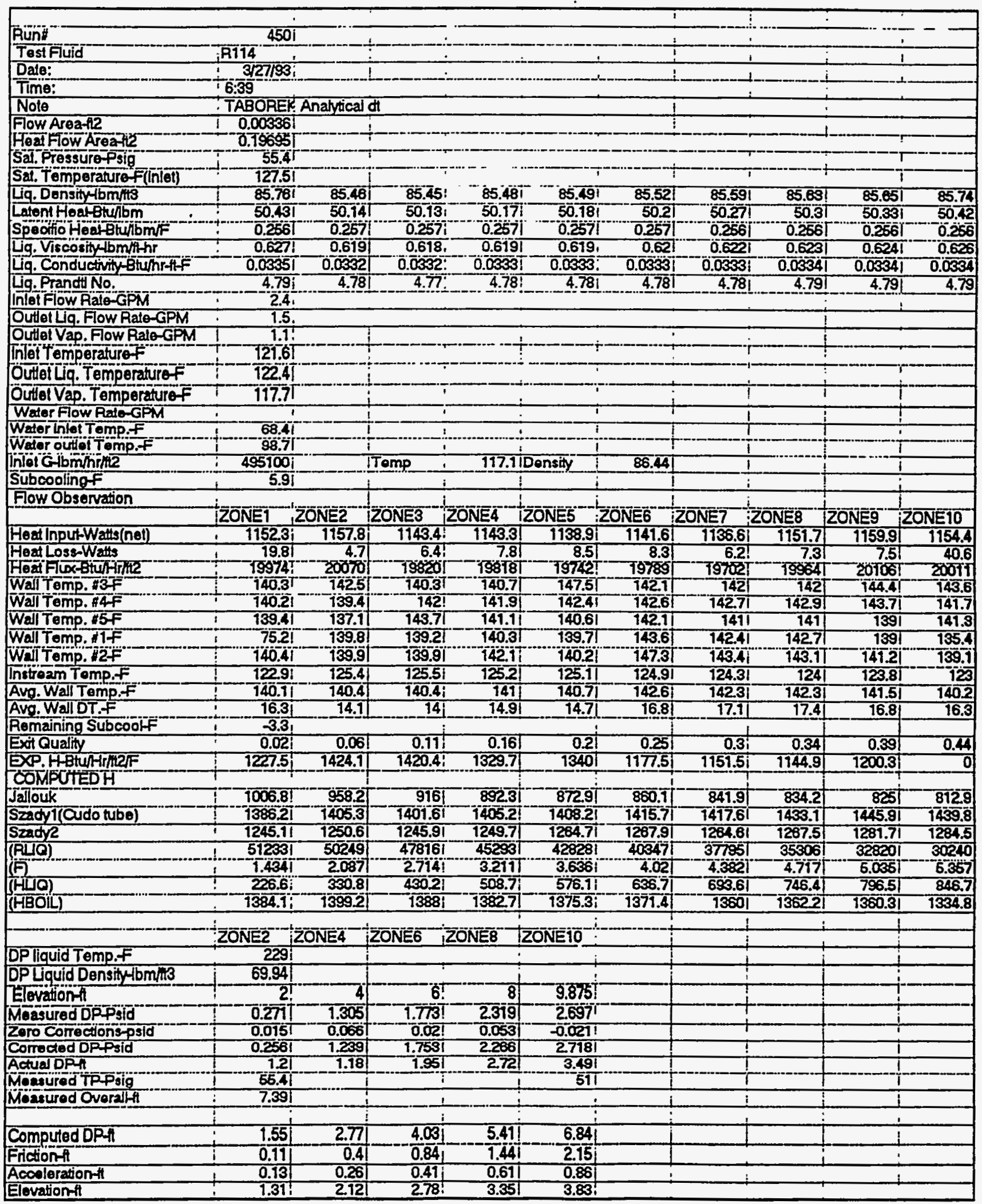




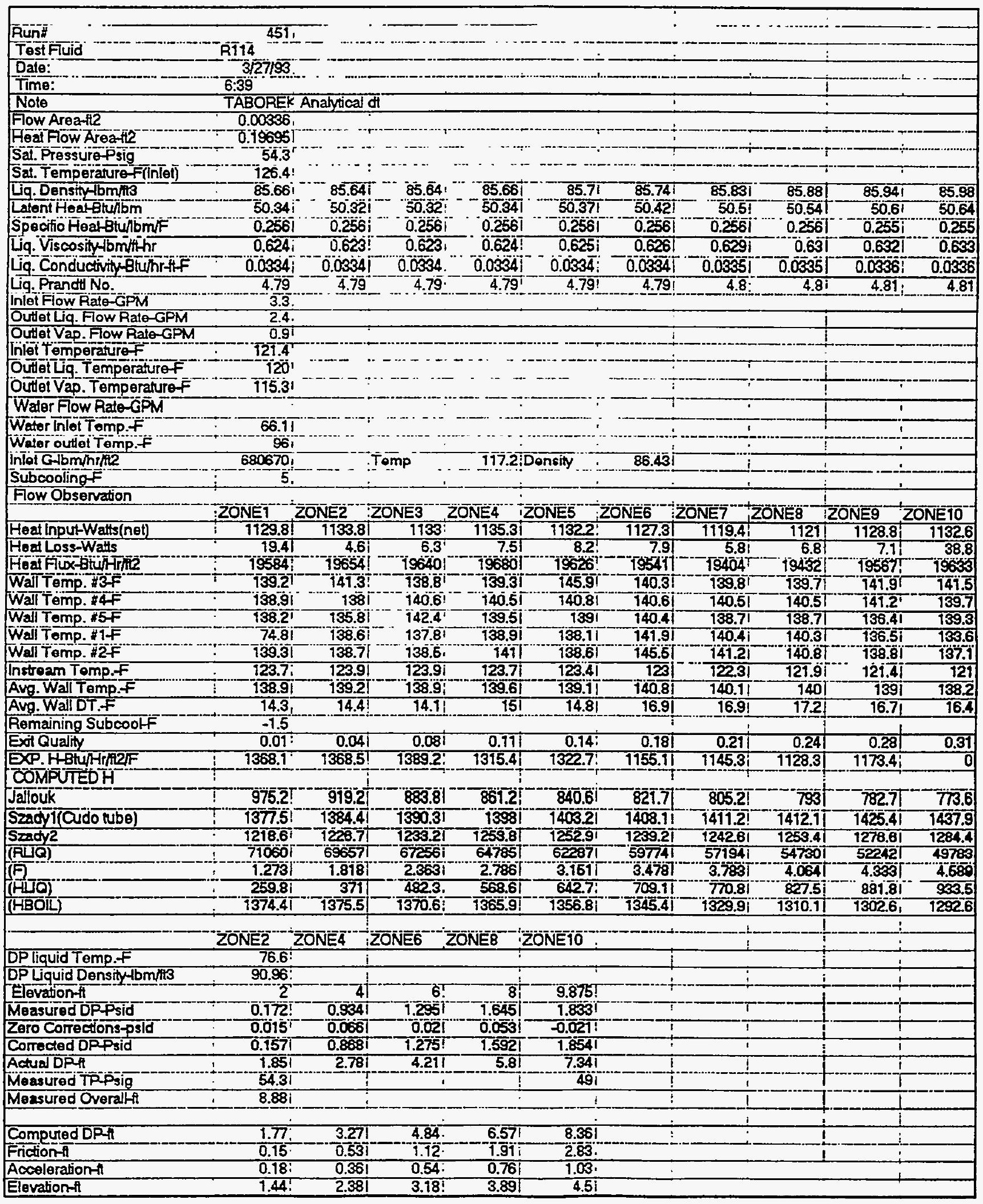




\begin{tabular}{|c|c|c|c|c|c|c|c|c|}
\hline Runt & $452 !$ & & & & & & & \\
\hline Tost Fluid & :R114 & & & & & & & \\
\hline Dafe: & $3 / 27193$ & & & & & & & \\
\hline Time: & $6: 39$ & & $i$ & i & & & & \\
\hline Noto & TABOREK Anatytical d & & & & & & & \\
\hline Flow Areat12 & $0,00336 !$ & & & & & & & \\
\hline Heat Flow Areatte & 0.190951 & & & & & & & \\
\hline Sal. Pressuro + sig & 51.91 & & & & & & & \\
\hline Sat. Temperature-F(n|lel) & $124 !$ & & & 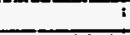 & $!$ & $!$ & T & i \\
\hline Liq.Density-bm/13 & 85.991 & 85.961 & 86.031 & 86.11 & 86.181 & 8.23 & 86.291 & 86.37 \\
\hline Ledent Hoed Giullbm & 50.651 & $50.62 !$ & 50.69 & 50.751 & 50.831 & 50.88 & 50.931 & 51.01 \\
\hline Spoófio Heat Gtunbm/t & 0.2551 & 0.2551 & $0.256 i$ & 0.2551 & $0.2551^{\circ}$ & 0.255 & 0.2541 & 0.254 \\
\hline Ljg. Viscosity $4 \mathrm{bm} / \mathrm{ithr}$ & $0.634 !$ & 0.6331 & 0.635 & 0.6371 & 0.6391 & 0.647 & 0.6421 & 0.645 \\
\hline Liq. Conductivite btumr-af & 0.03361 & 0.03361 & $0.0356 i$ & 0.0337 & 0.03371 & 0.03381 & 0.03381 & 0.0339 \\
\hline Lig. Prandt No. & 4.81 & 4.81 & $4.81 i$ & 4.82 & 4.82 & 4.831 & 4.83 & 4.84 \\
\hline inlelfilow Ralo-GPM & 4.11 & & & & & & & \\
\hline Outlet Lig. Flow Rato-GPM & 2.51 & & & & & & & \\
\hline Outlet Vap. Fow Rate-GPM & 1.2 & & & & & & & \\
\hline The tomperaturef & $718.2 !$ & & & & & & & \\
\hline Outtel Lig. Temperaturef & 116.3T & & 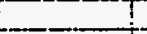 & & & & & \\
\hline Outol Vap. TemperanteF & 109.61 & & & & & & & \\
\hline Water Fow Rato-GPM & -1 & I & 1 & & & I & & i \\
\hline Water inlol Tomp, $F$ & 62.91 & $\therefore$ & 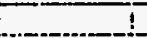 & & & 1 & & I \\
\hline Water outlot Tomp.F & 86.11 & $i$ & 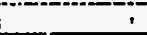 & & & $T$ & & $i$ \\
\hline Intol & 848999 & Tomp & Densiy & 86.77 & & 1 & & 1 \\
\hline Subcooling & $5.8 i$ & &. & 1 & & 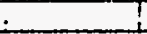 & & 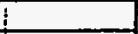 \\
\hline Fow Observation & & & & & & & & 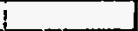 \\
\hline & IZONE1 ZONE2 & $\angle O N=3$ ZZONE4 & ZONE5 & ZONE6 & ZONE & ZZONE8 & ZONE9 & ZONE10 \\
\hline Heat Input-Watts(net) & $11141.5_{1}^{1} \quad 1147.41$ & $1158.91 \quad 1162.4$ & 1166.91 & 1159.5 & 1151.7 & 1145.6 & 1152 & 1152.8 \\
\hline Heat Loss-Watts & 18.8 & 6.1 & 7.71 & 7.3 & 5.3 & 6.4 & 6.7 & 36.6 \\
\hline Heat Fix $x$ tunhriti & 19787 & $20089 !$ & $2022 !$ & 20090 & 19964 & 198581 & 1965 ! & 19983 \\
\hline Wall Temp. $3 F$ & 137.1 & $136.6^{1}$ & 143.51 & $1 \overline{3} \overline{7} . \overline{1}$ & 137.3 & $-137 !$ & 138.91 & $13 \overline{8} .5$ \\
\hline Wal Temp A4F & $135.9 !$ & $138.31^{\circ}$ & 138.11 & 137.9 & 137.91 & 137.71 & 138.31 & 136.7 \\
\hline Wall Temp, $15 F$ & 133.71 & 140.11 & 136.61 & $1 \overline{3} 7 . \overline{6}$ & 1361 & 135.81 & 133.51 & 136.8 \\
\hline Wall Tomp. \#1F & 136.61 & 135.61 & 135.5 & 139.2 & 137.81 & 137.51 & 133.61 & 130.5 \\
\hline Waltomp. Hz & 137.21 & $136.4 i$ & 136.11 & 143 & 138.61 & 137.81 & 1365 & 134.2 \\
\hline Instroam Tomp. $F$ & 120.91 & 121.21 & 120.61 & 1201 & 119.3 & 118.91 & 118.41 & 117.7 \\
\hline Avg. Wall Temp.F & $136.9 !$ & $136.7 !$ & 136.61 & 138.11 & 137.5 & 137.2 & 136.11 & 135.2 \\
\hline Avg. Wall DT . F & 15.11 & 14.6 & 15.11 & 17.2 & 17.3 & 17.4 & 16.8 & 16.6 \\
\hline Remaining Subcoolf & 0.5 & & & & & & & \\
\hline Exot Quality & 0.02 & 0.05 & 0.11 & 0.13 & 0.16 & 0.19 & 0.22 & 0.24 \\
\hline EXP. HEtuntrnterF & 1312.1 & 1374.3 & 1343 & 1170.8 & 11528 & 1143.7 & 1191.7 & 0 \\
\hline COMPUTEDH & & & & & & & & \\
\hline Jailouk & $983.2 !$ & 873.91 & 825.7 & 805.1 & 785.7 & 77.3 & 758.7 & 745.8 \\
\hline Szadyt(Cudo tube) & 1369.31 & 1392.81 & 1414.5 & 1419.8 & $1417.2^{1}$ & 1425.4 & 1441.11 & 1454.6 \\
\hline Szady? & $1210.8 !$ & 1258.4 & 1264.61 & 1255 & 1280.61 & 1270 & 1287.31 & 1288.9 \\
\hline (RLQ) & 876501 & 844501 & 793491 & 75656 & 74037 & 715301 & 68999 & 66403 \\
\hline & $7.0081^{-1}$ & 2.0811 & 2.8381 & 3.140 & 3.434 & 3.694 & 3.847 & 4.182 \\
\hline (स्मिक) & 244.6 & $505.3 i$ & 688.6 & 763.5 & 831.81 & $894.4 !$ & 953.71 & $1011 . \overline{1}$ \\
\hline (HBOLI) & 1366.7 & 1370.31 & 1357.9 & 1342 & 1394.41 & 1296.7 & 1285.71 & 1269.1 \\
\hline & & & & & & 1 & & \\
\hline & ZONE2 ZONEA & ZONE6 $Z$ ZONE 8 & ZONE 10 & & & 1 & & \\
\hline DP liquid Temp.f & 127.31 & $i$ & & & & 1 & & 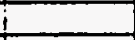 \\
\hline DPLiquid Density fbmits & 85.231 & & 1 & & & ; & & \\
\hline Elovation-i & $2 !$ & 6! & 9.875 & & & & & \\
\hline Mosasurad DPPsid & $0 . \overline{619}$ & 0.8531 & 1.03 & & & & & \\
\hline Zoro Corrections-psald & 0.0151 & 0.053 & -0.0211 & & & 1 & & I \\
\hline Comeded DPPsid & 0.045 & 0.8431 & 1.0511 & & & $i$ & & i \\
\hline ActualDPA & 3.03 & 4.521 & 7.991 & & & I & & I \\
\hline Moasurad TPPsig & 51.91 & $-i$ & 461 & & & I & & 1 \\
\hline Moasured Overallin & 9.841 & $\perp$ & 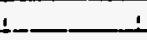 & & $\perp$ & 1 & & I \\
\hline & & & & & & 1 & & 1 \\
\hline Computed DP-At & 3.84 & 5.71 & 9.891 & & 1 & $i$ & & $i$ \\
\hline Friction-t & 0.16 & $1.26 !$ & 3.241 & & I & & & 1 \\
\hline Acooleration-ft & $0.21 !$ & 0.67 & $1.23 i$ & & I & & & \\
\hline Elevation-1 & $1.69 !$ & 3.771 & 5.42 & & & & & \\
\hline
\end{tabular}




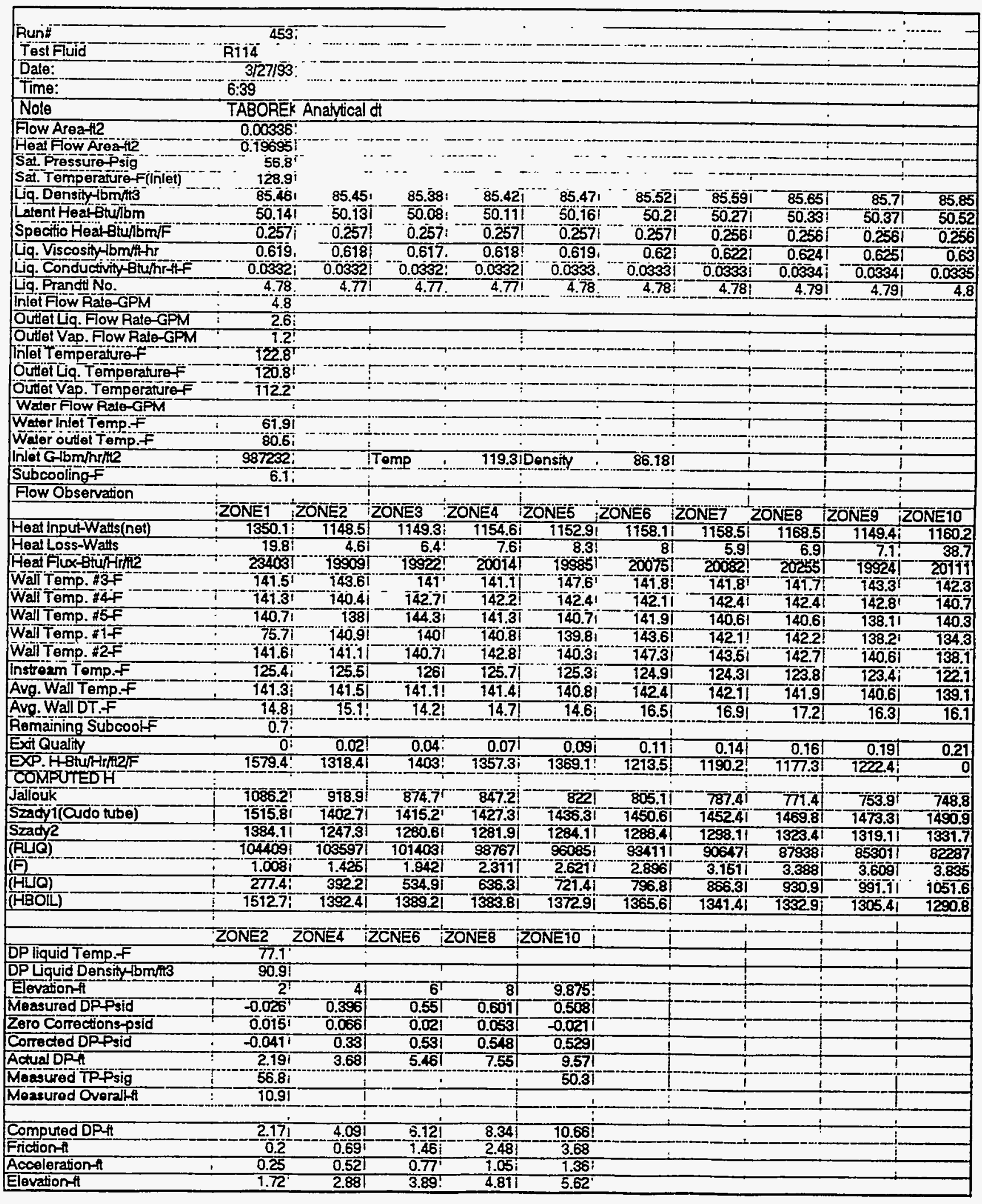




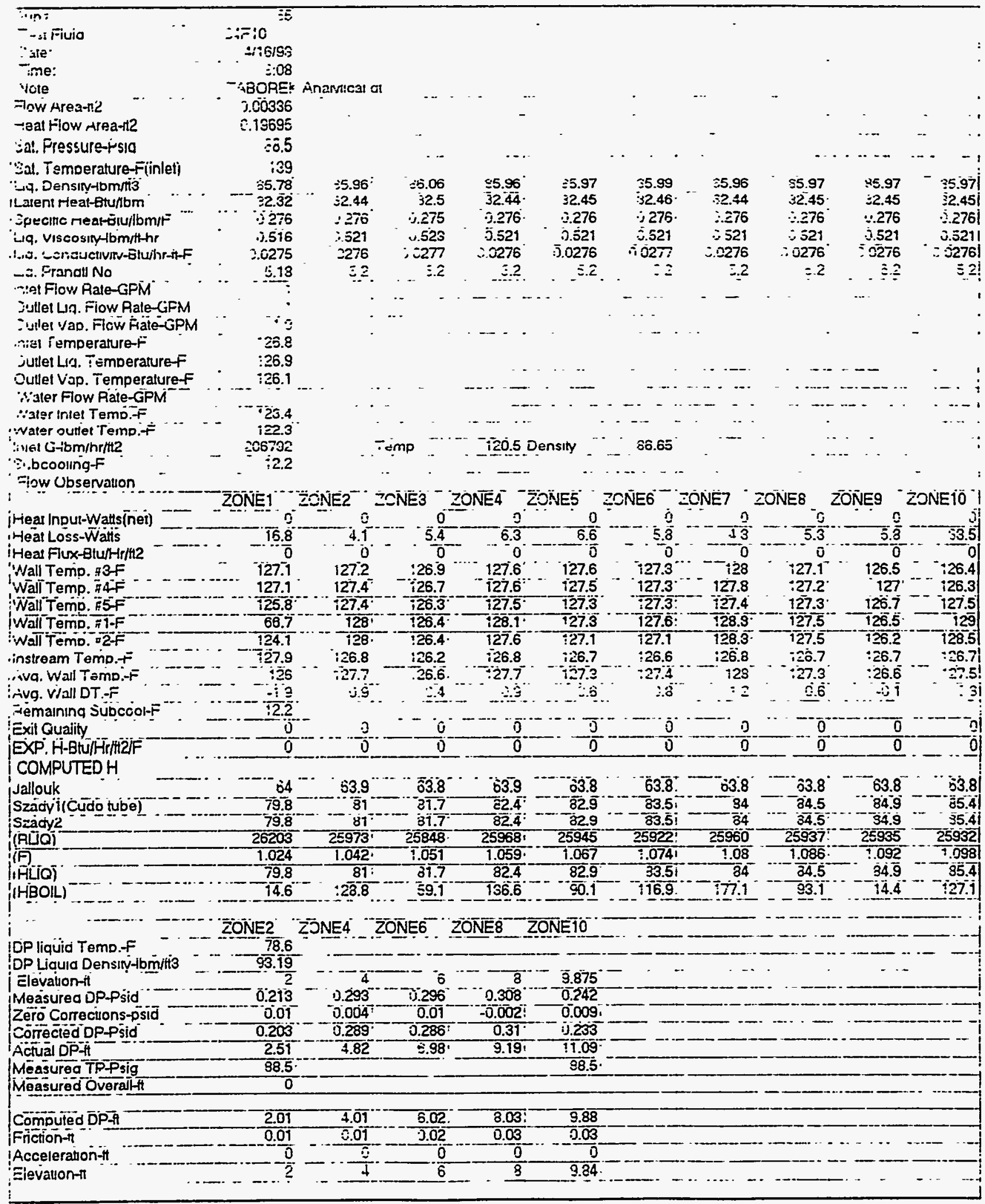




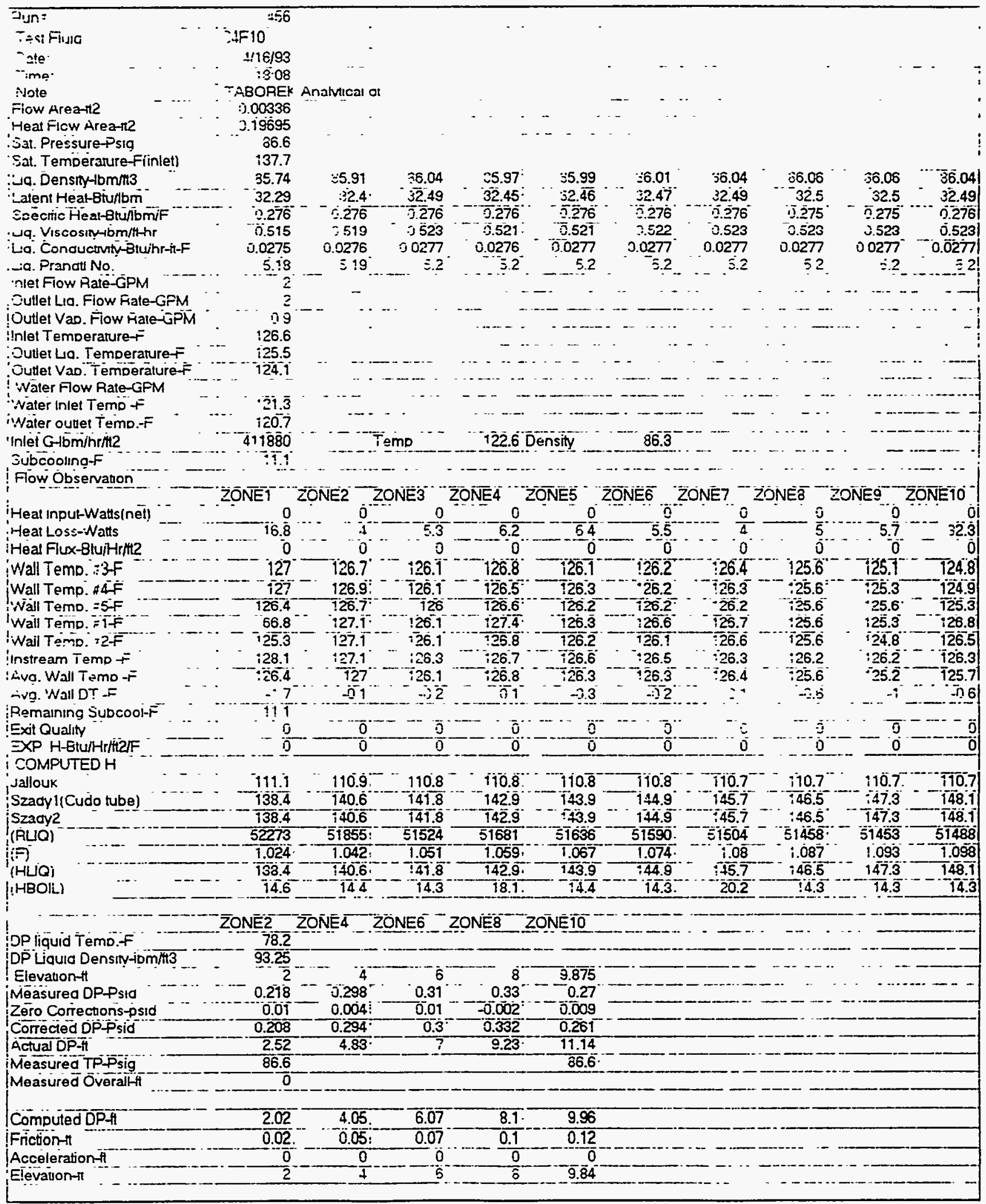




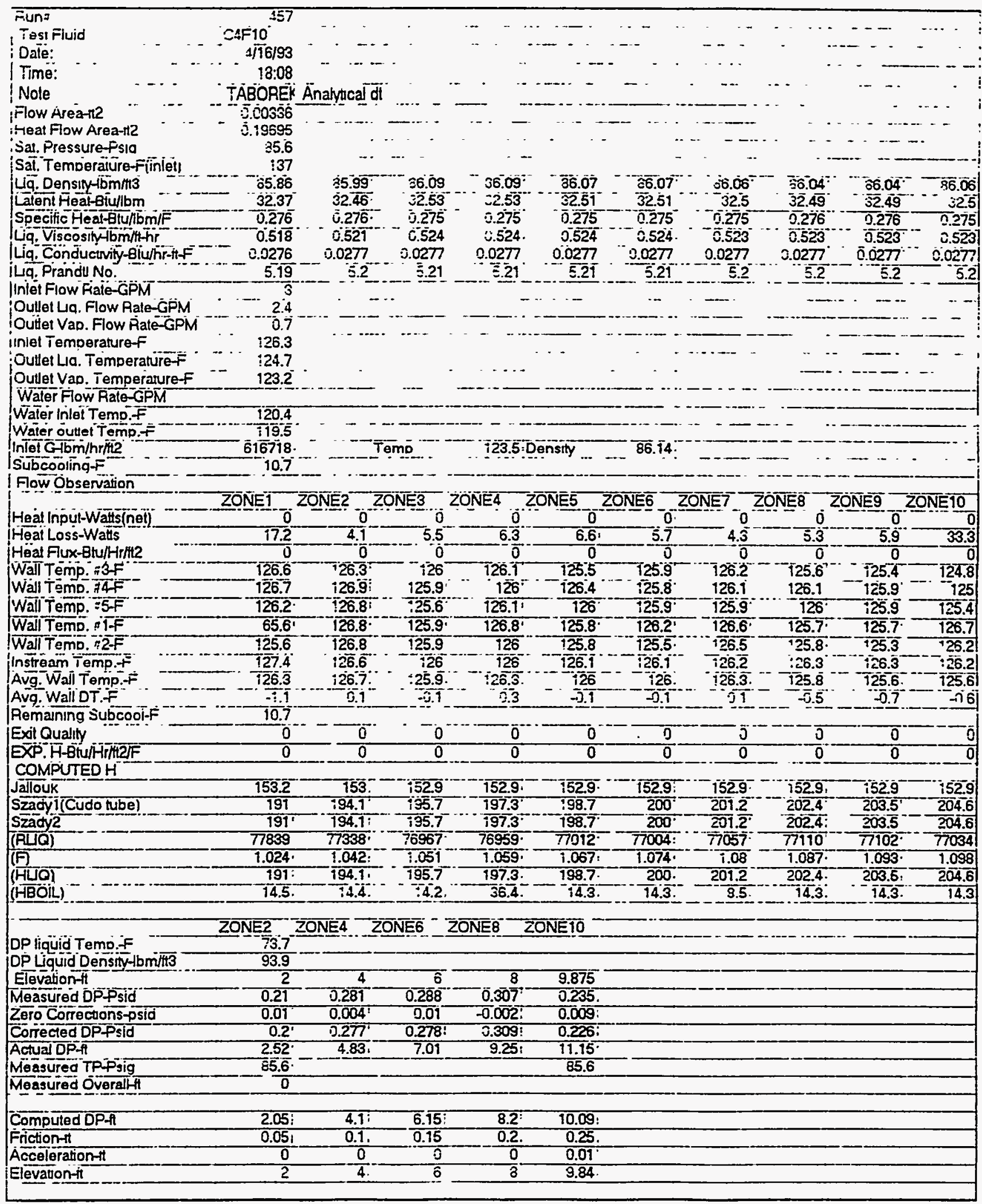




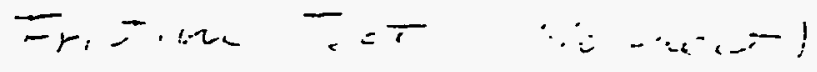

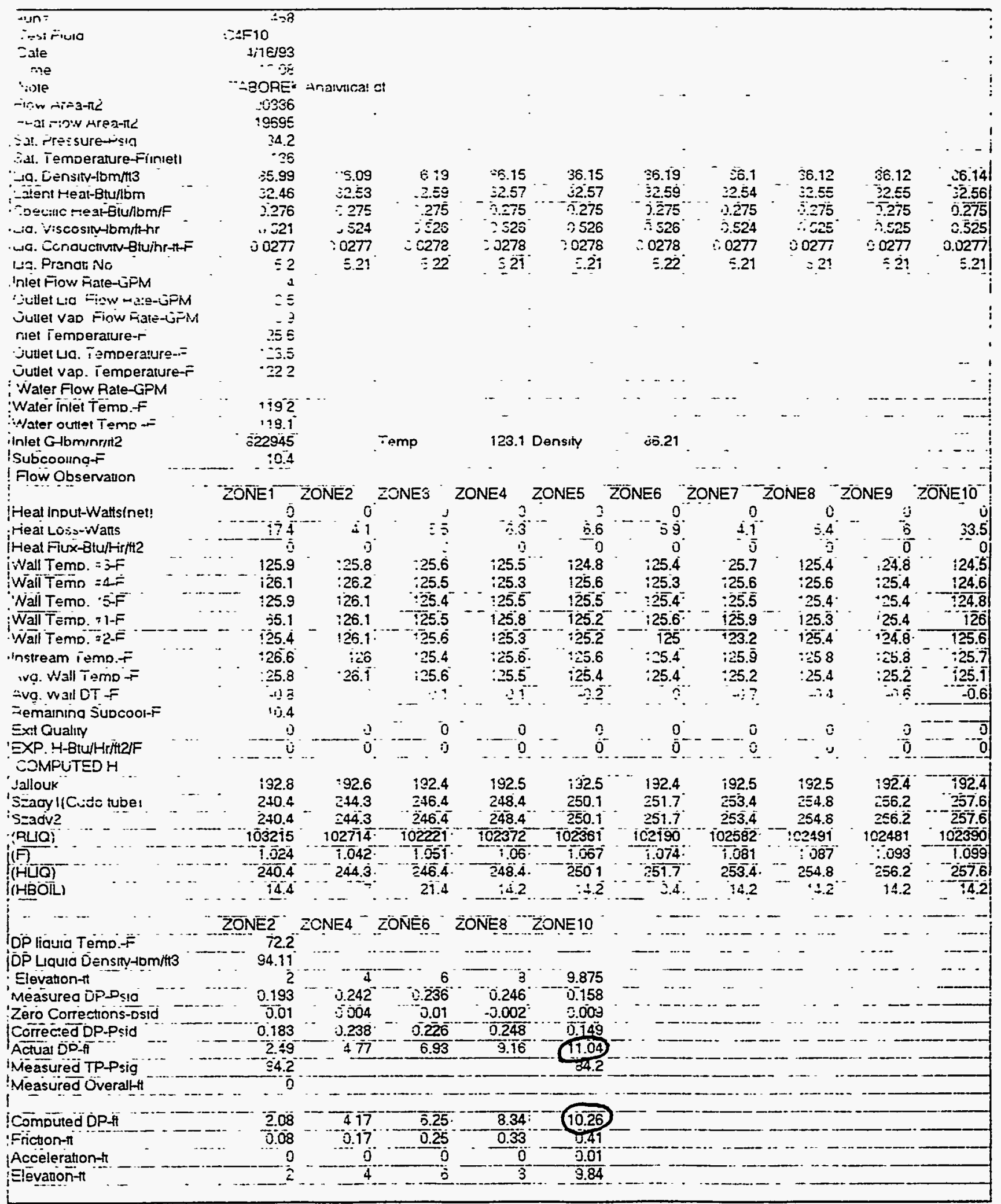




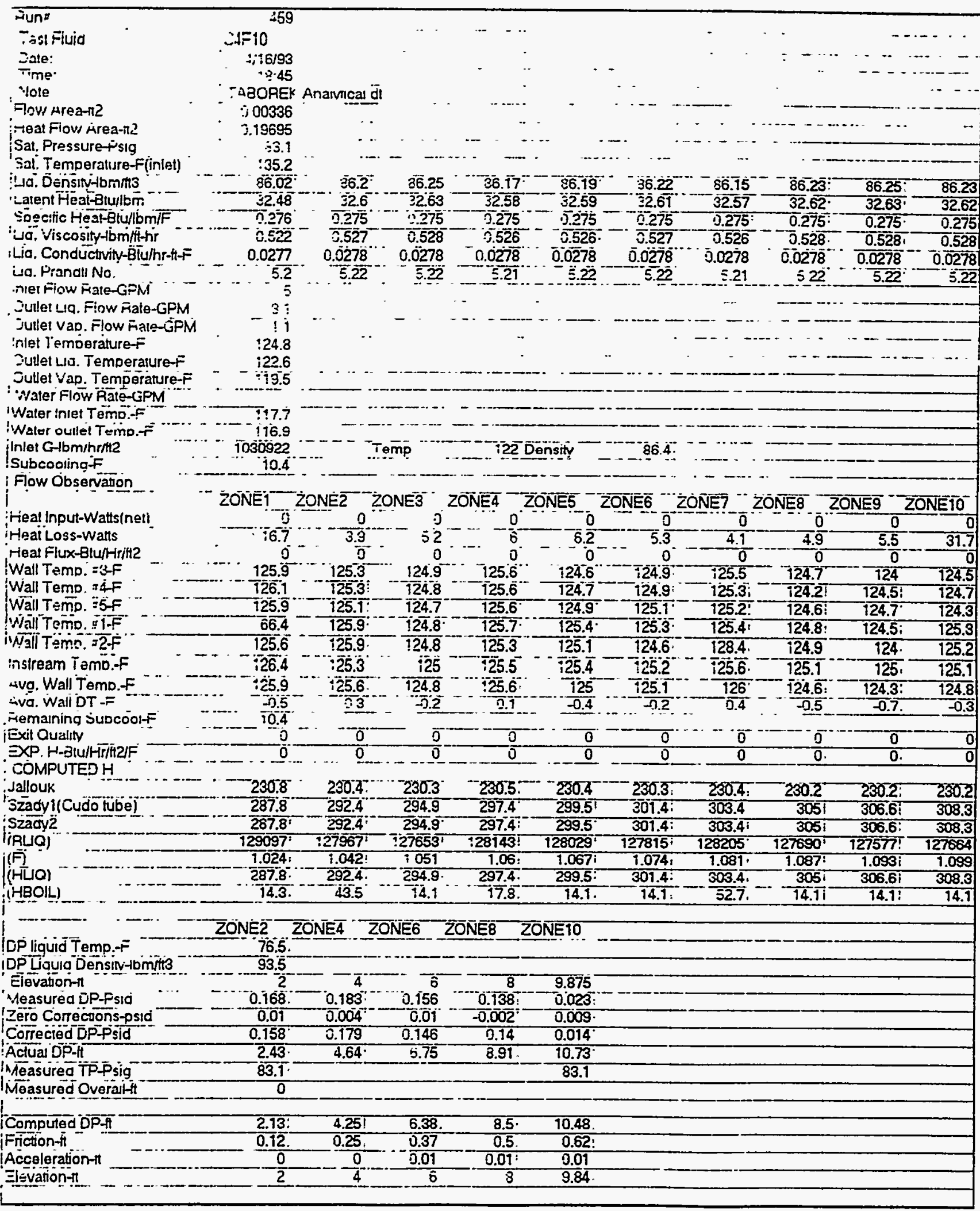




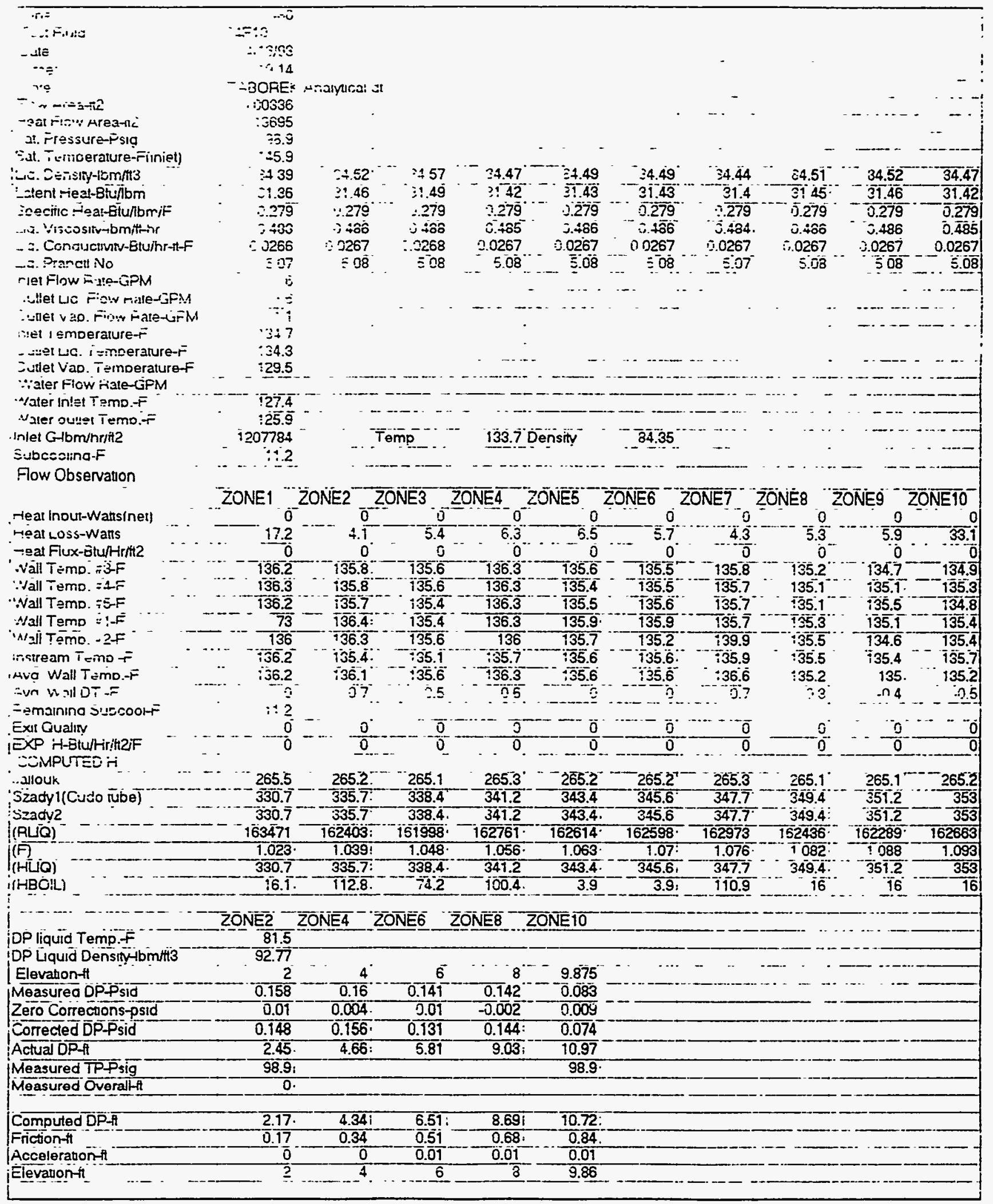




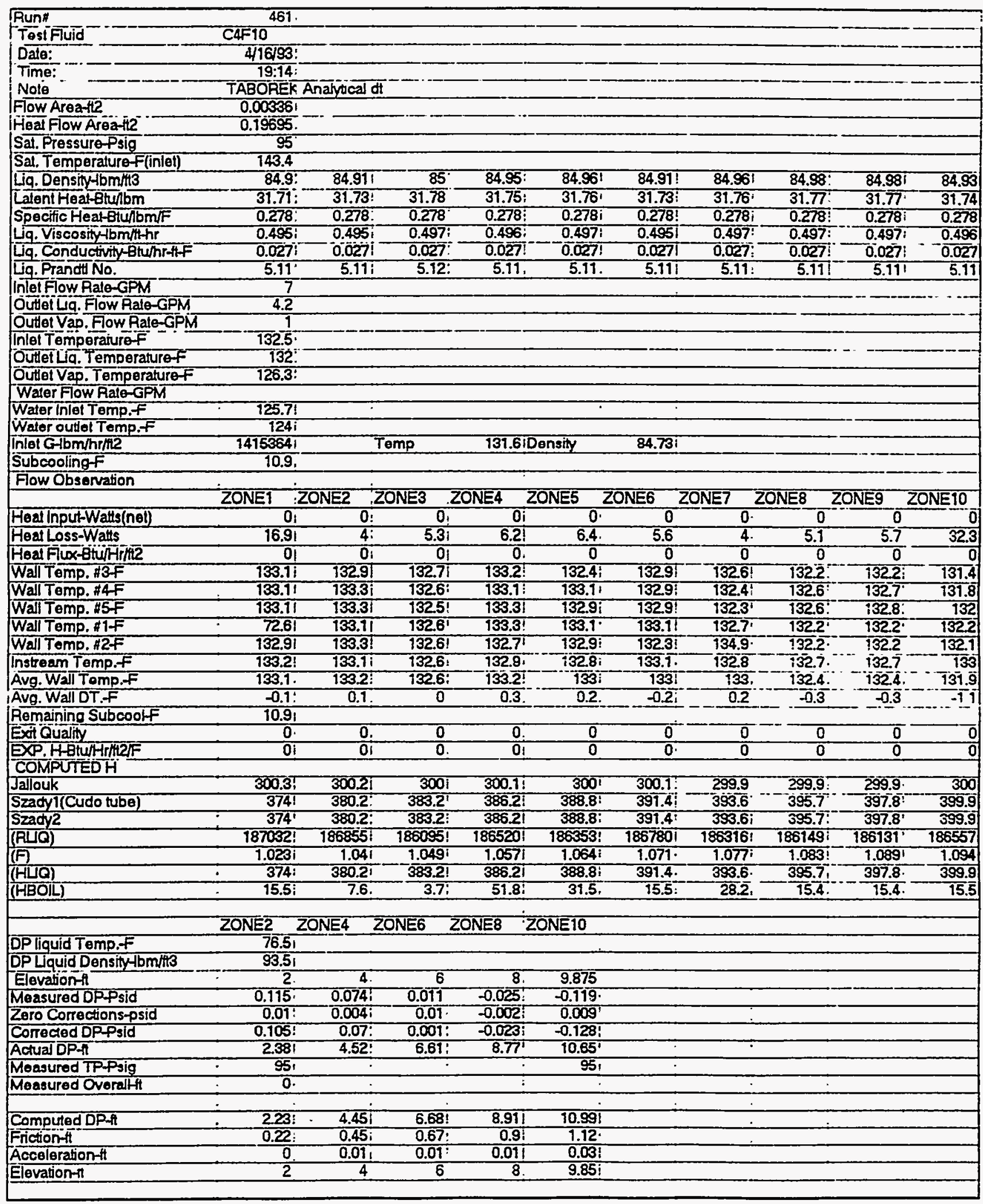




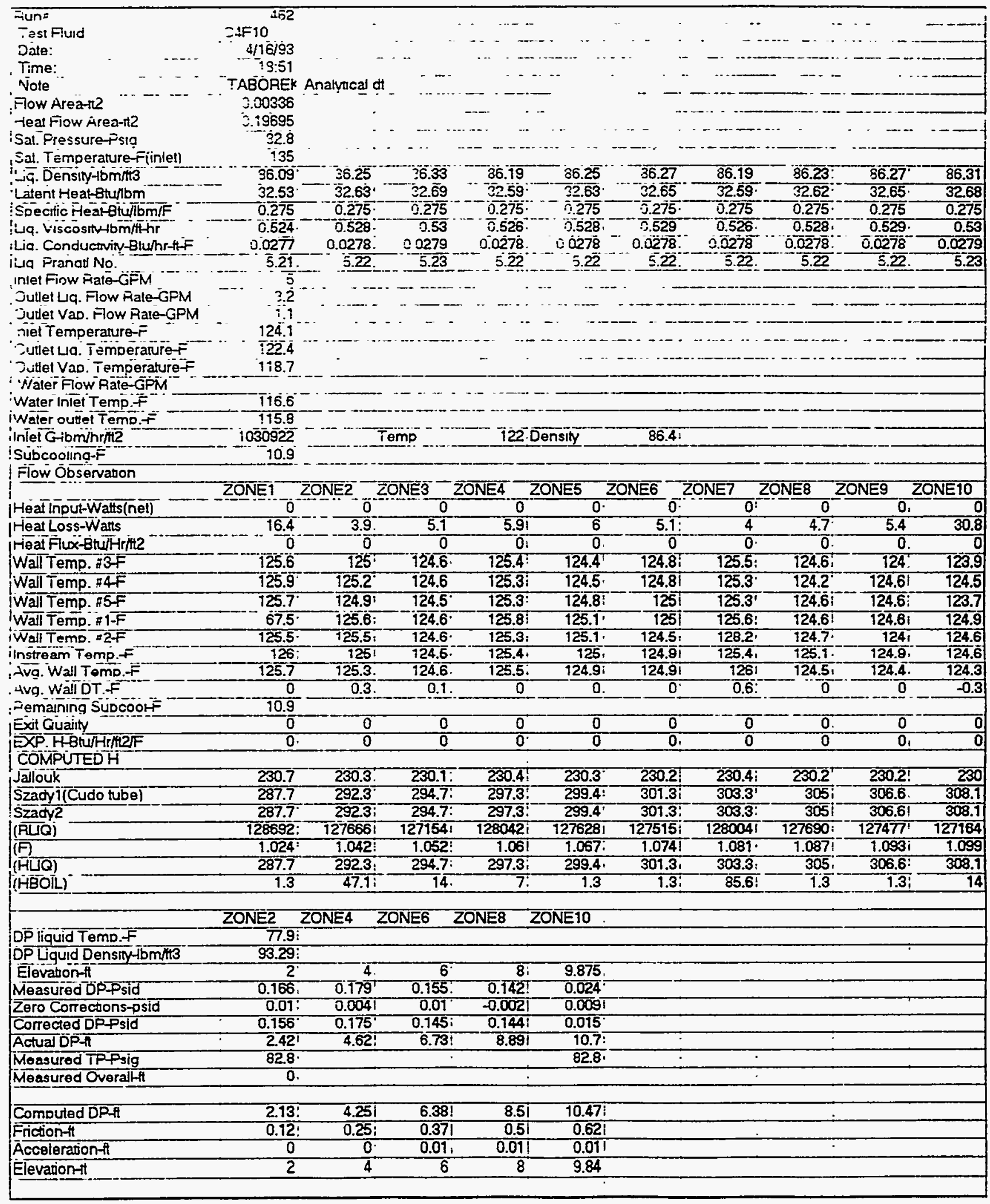




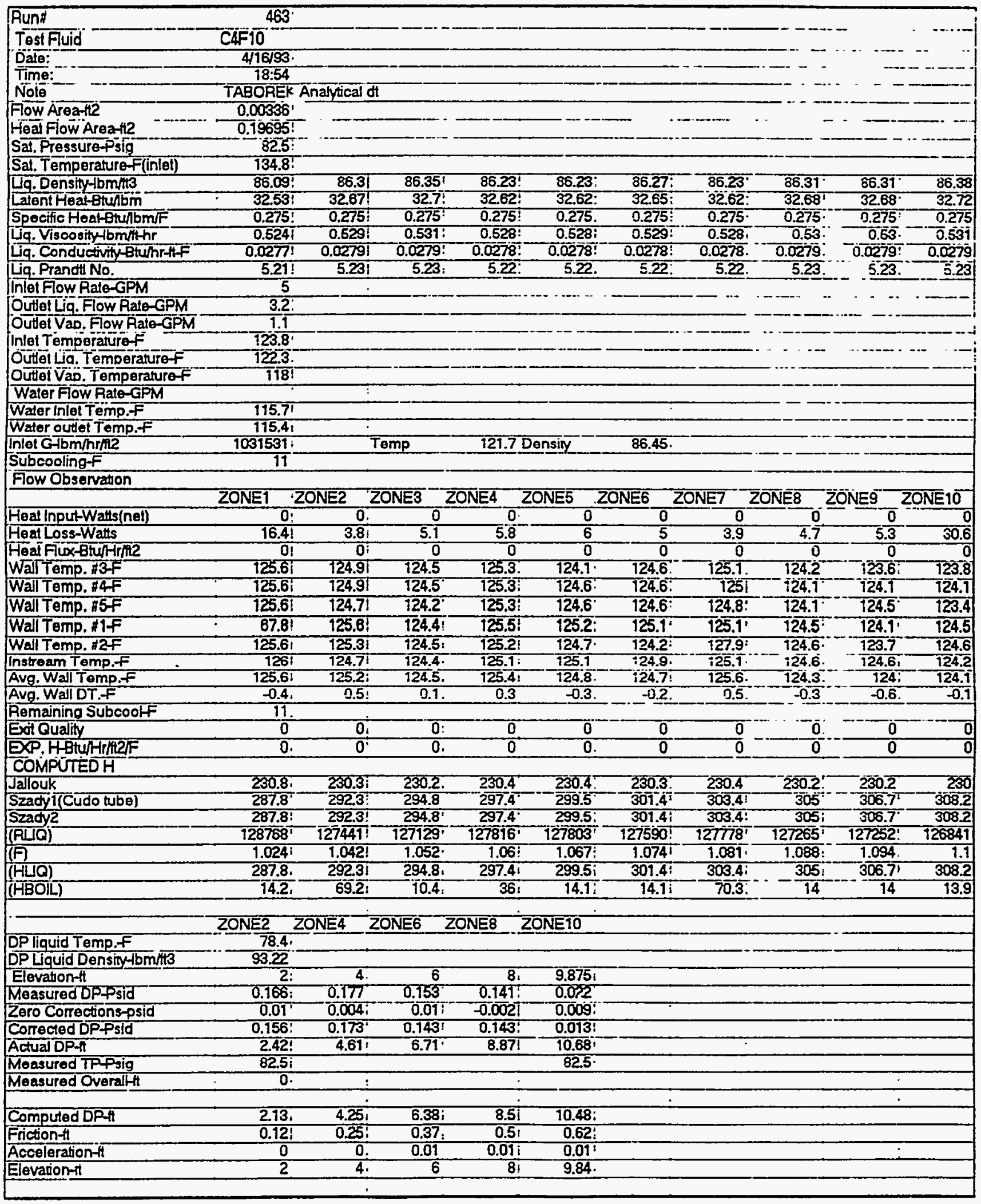




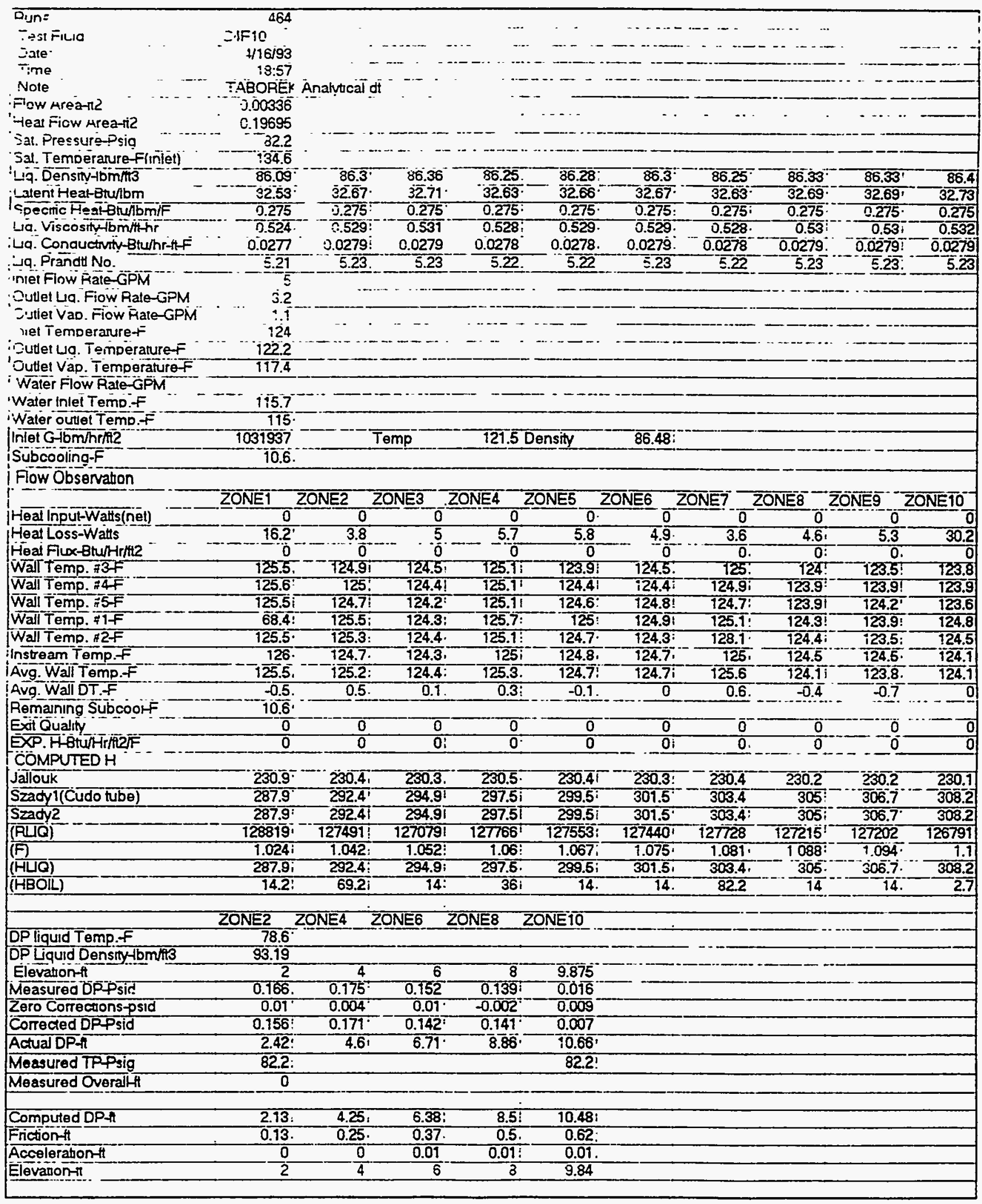




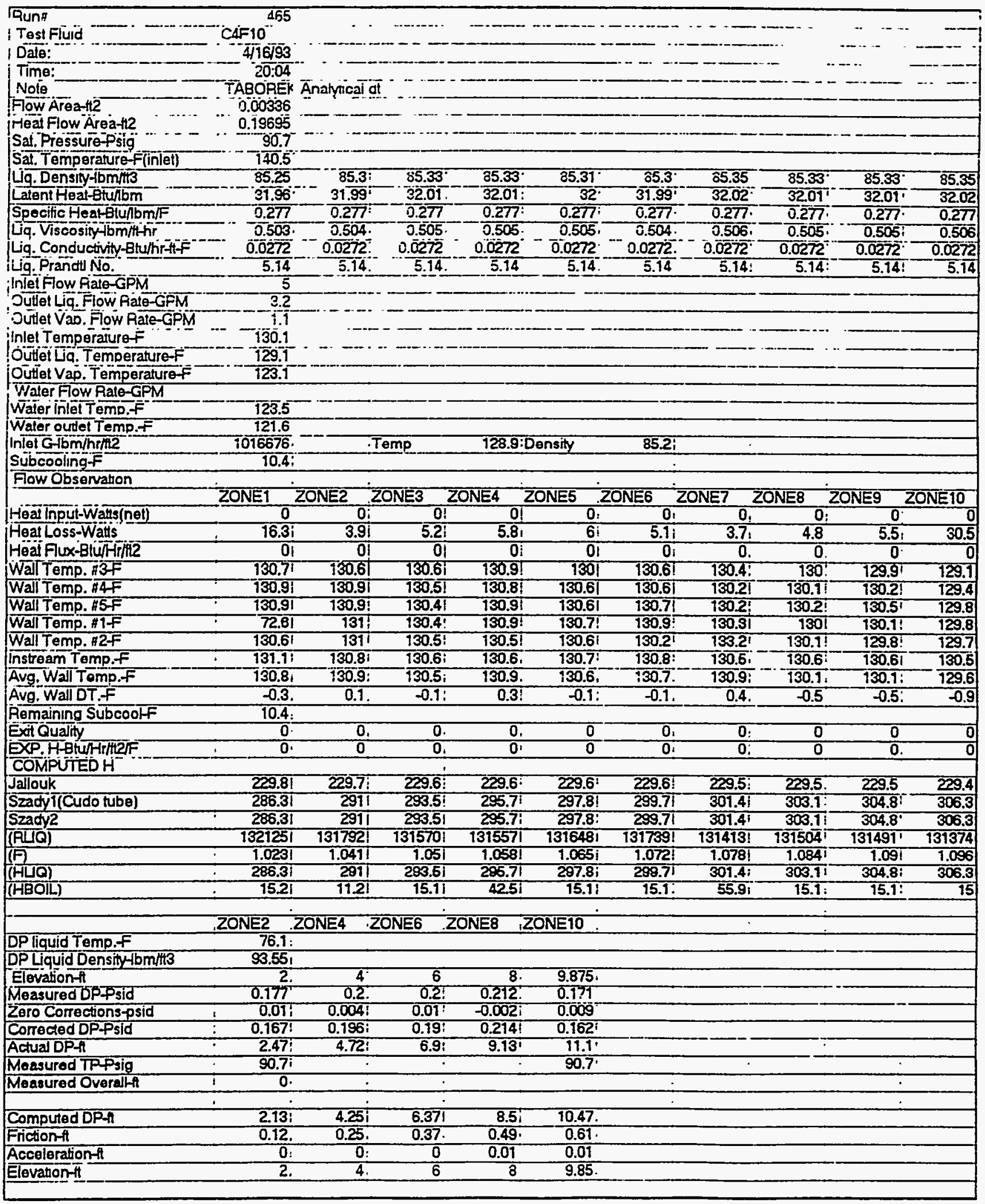




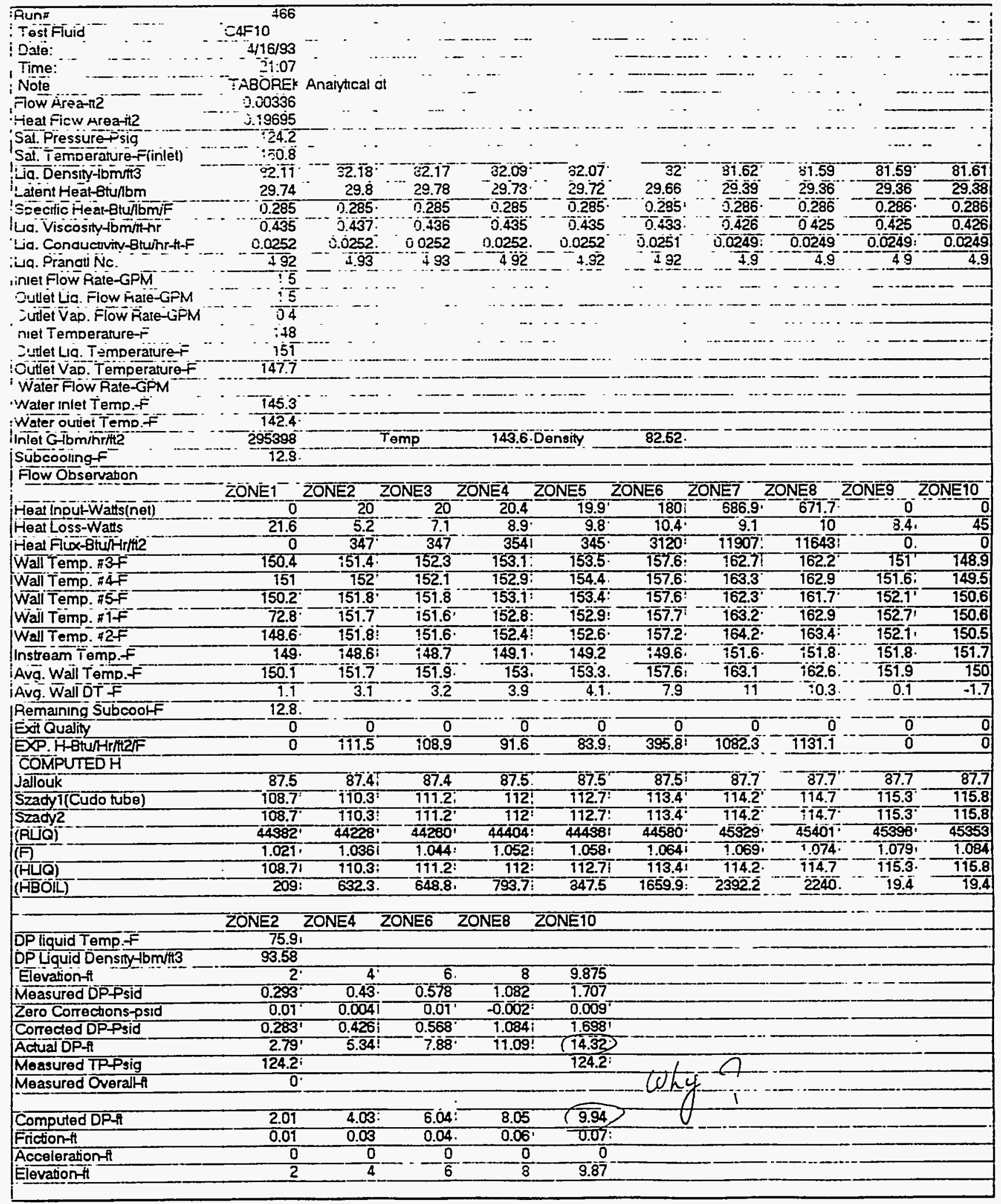




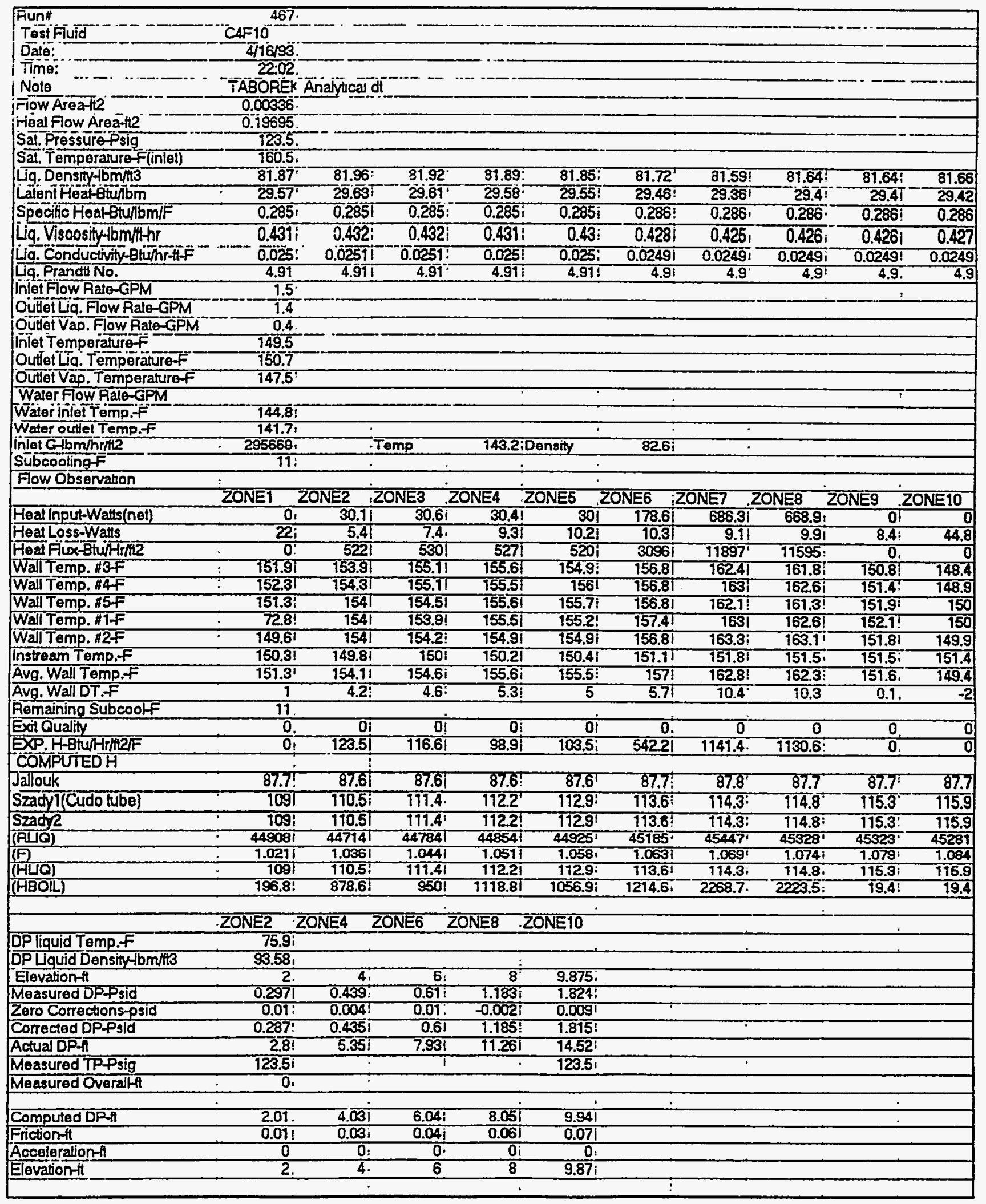




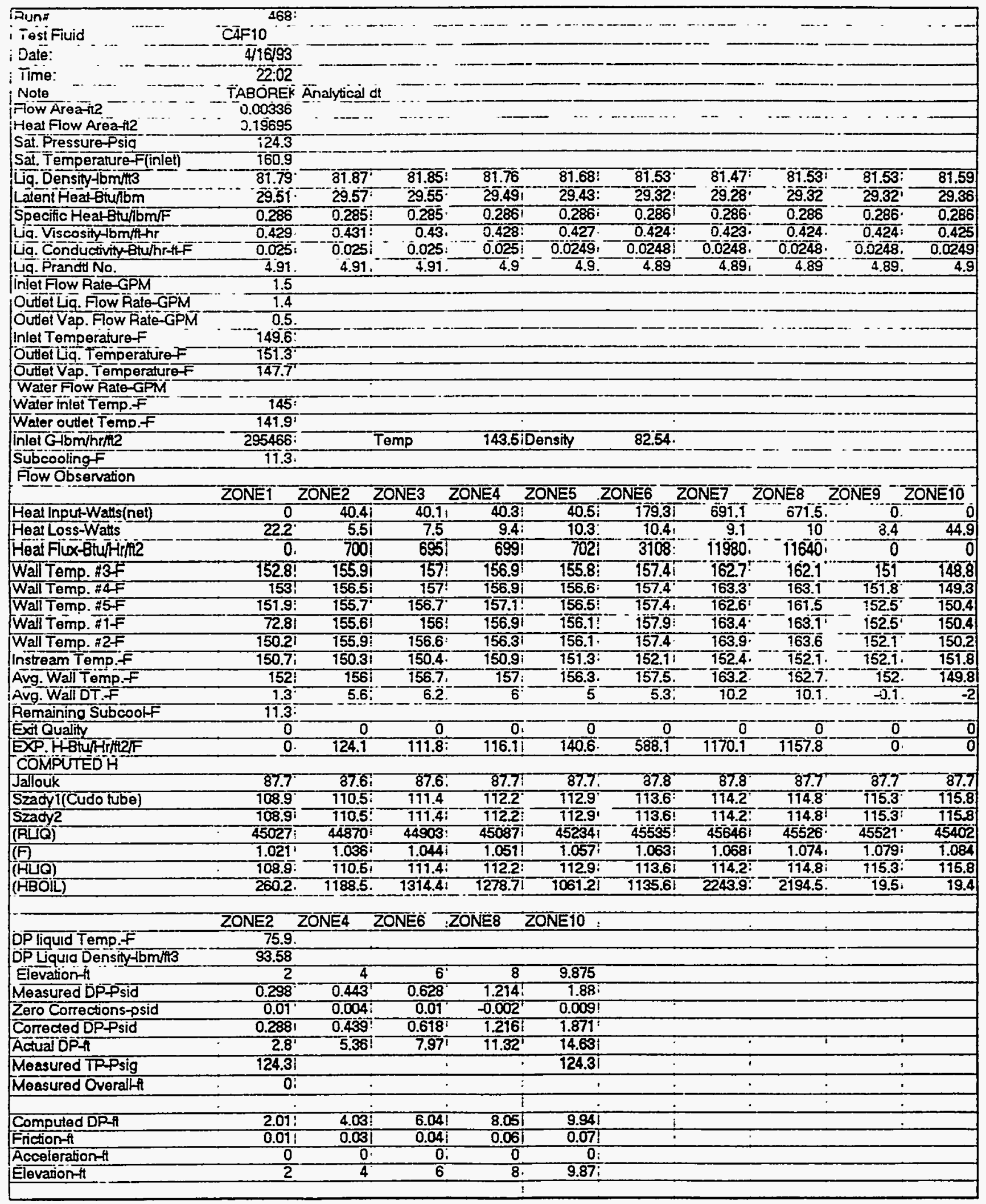




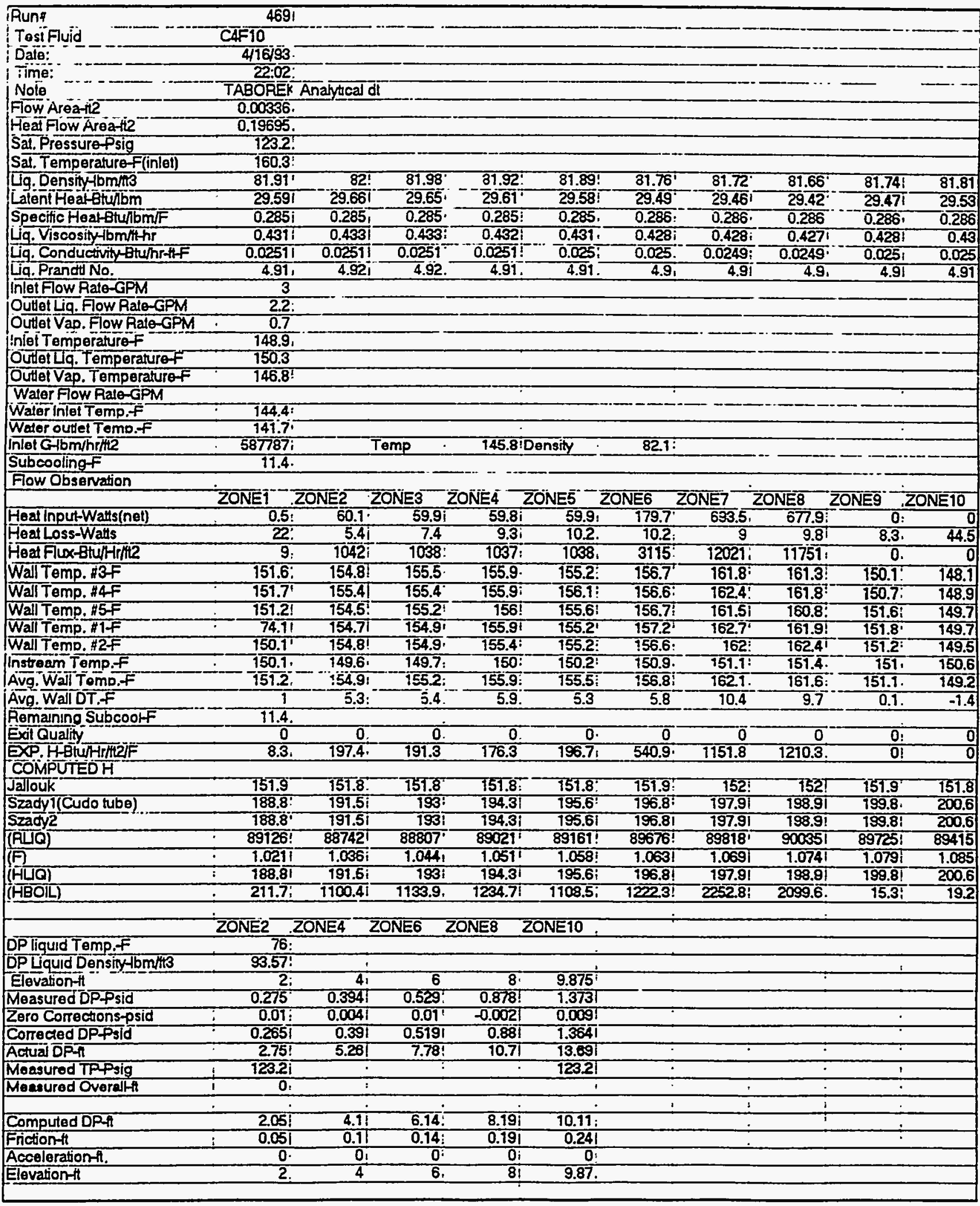




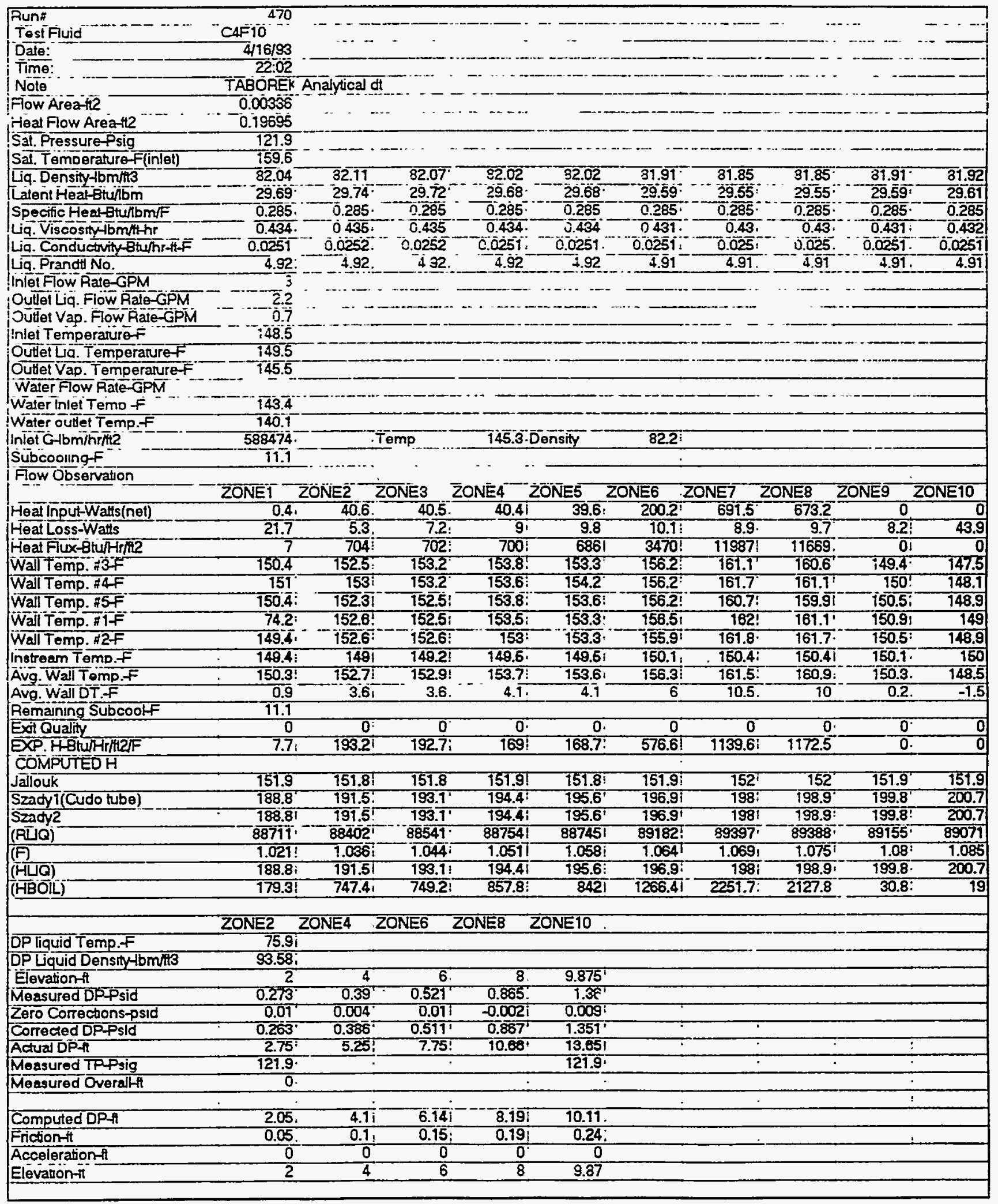




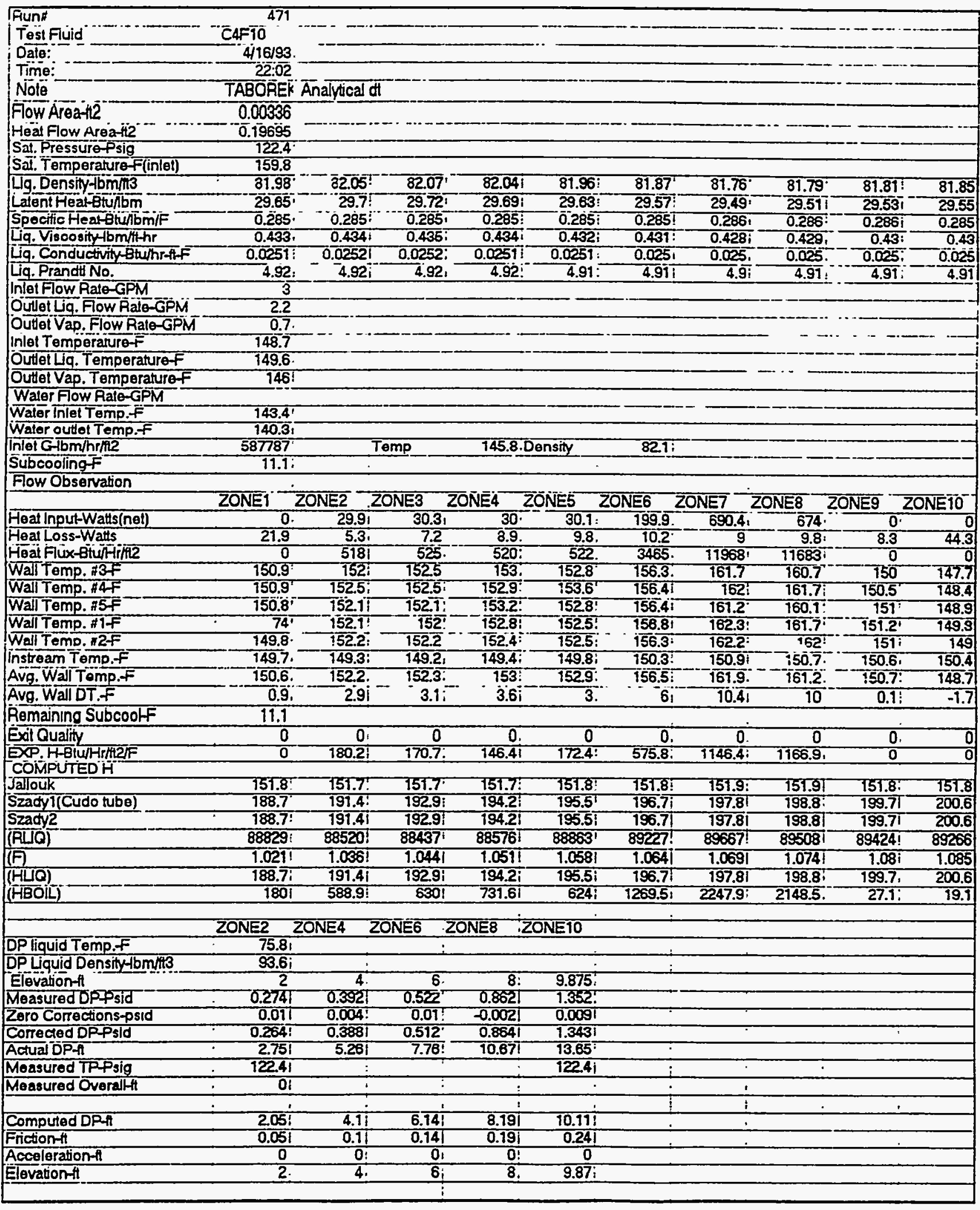




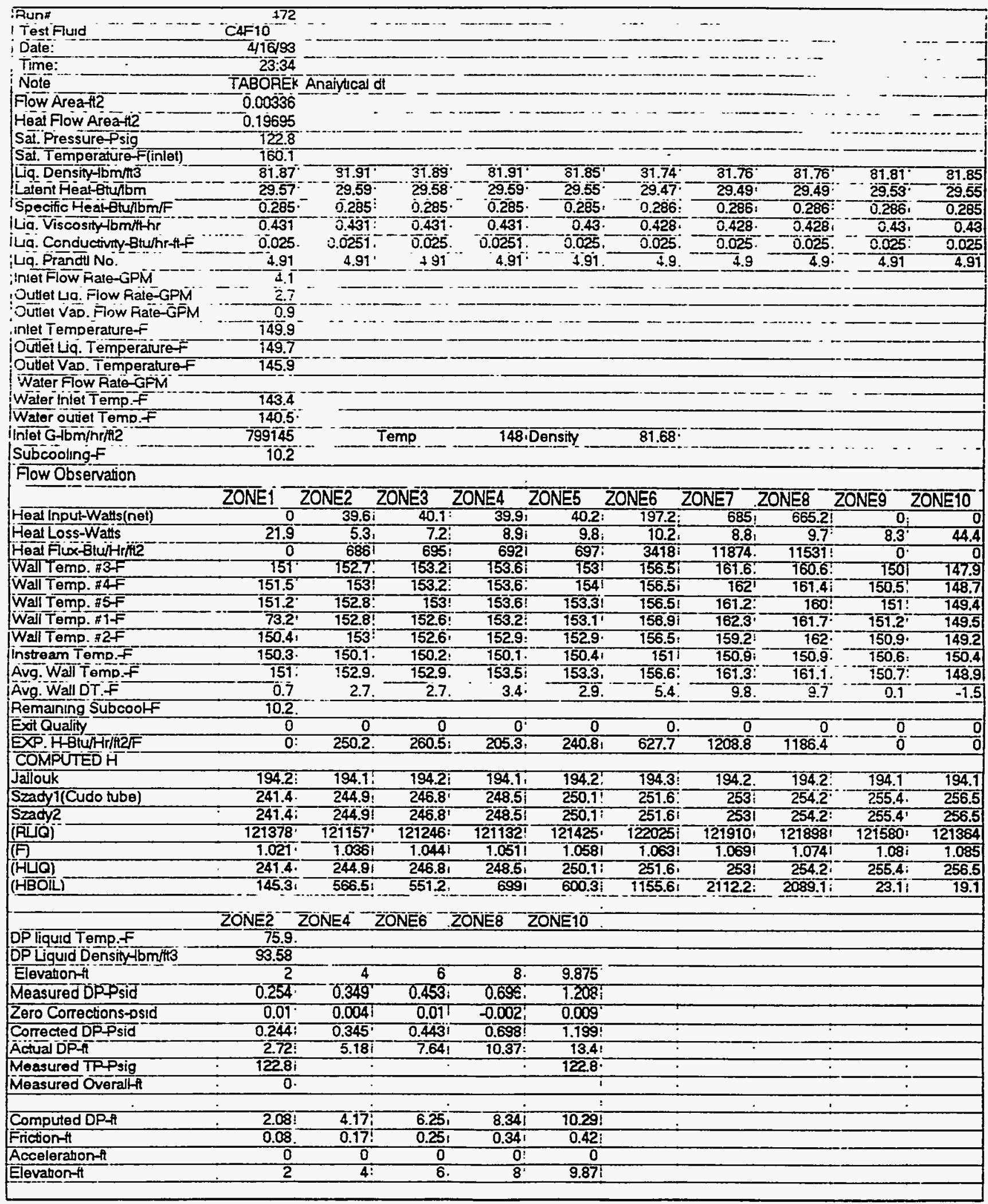




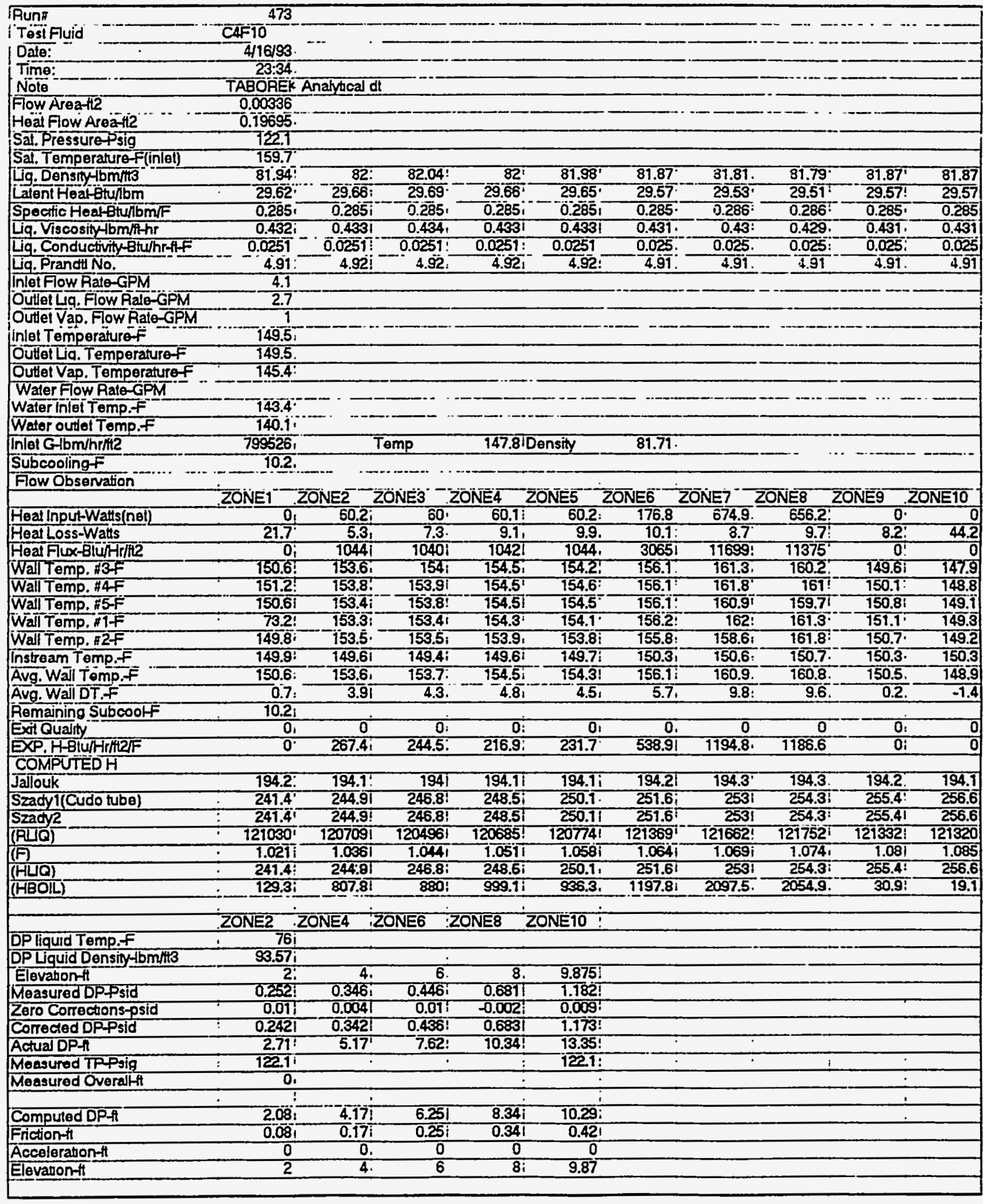




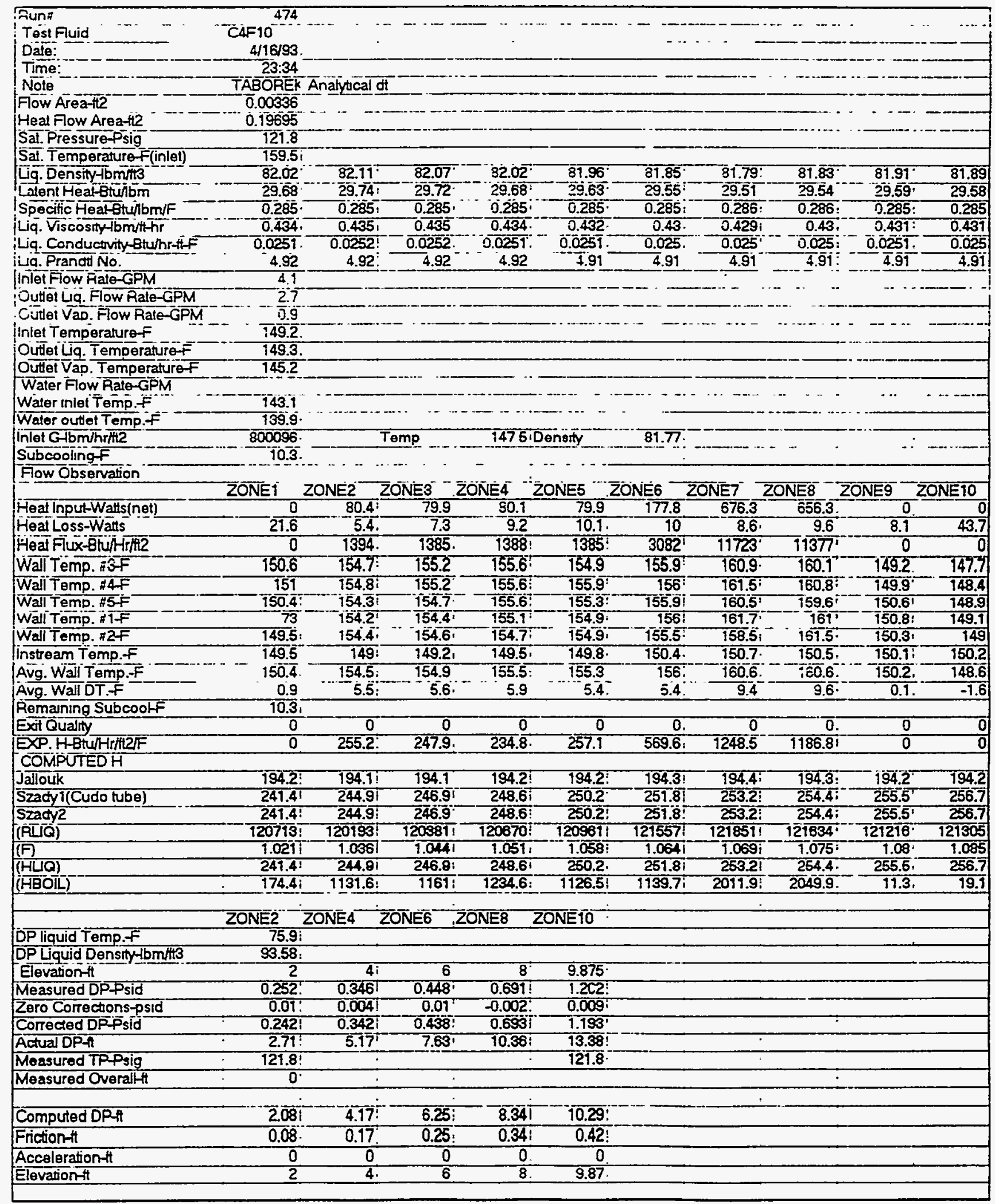




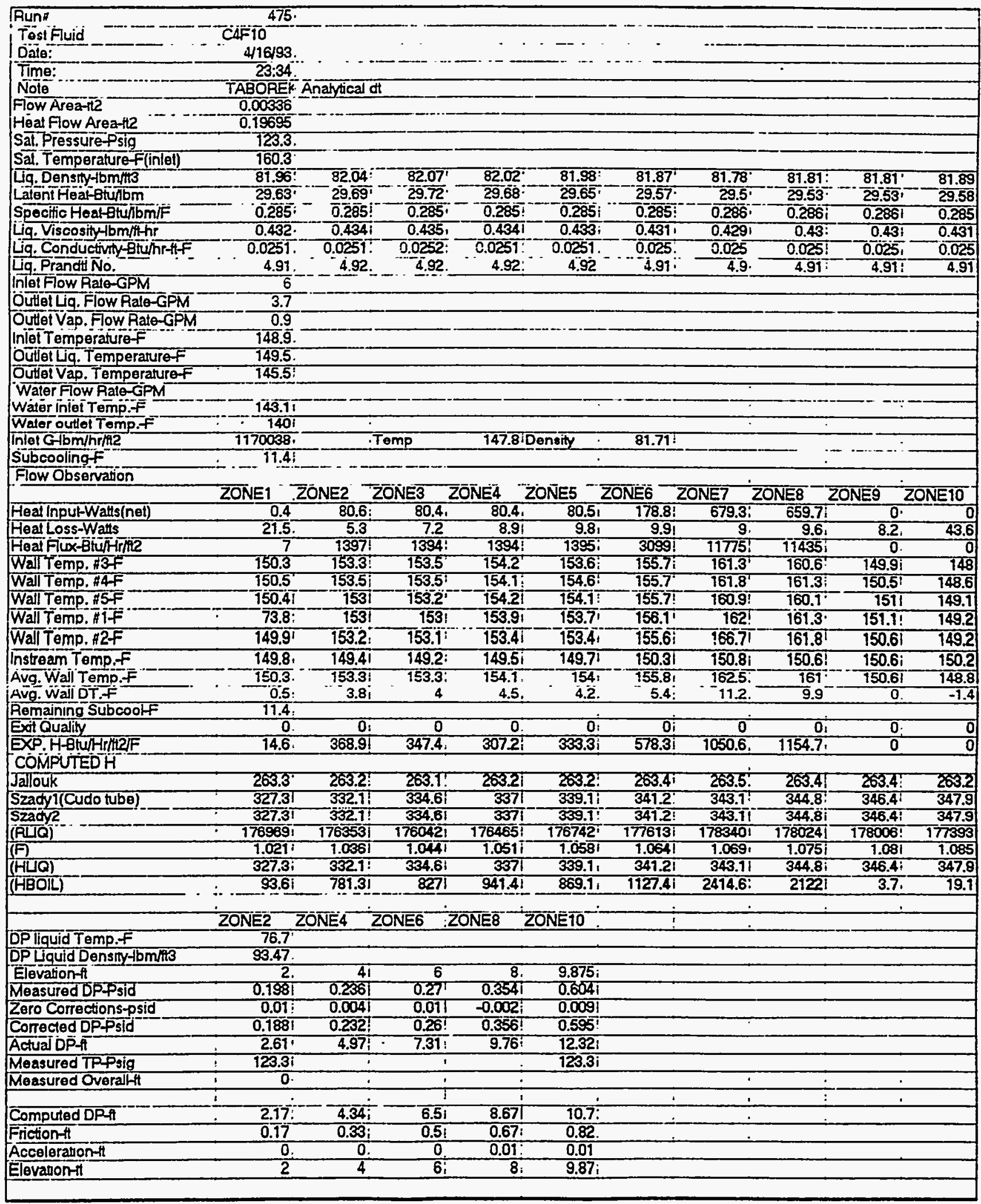




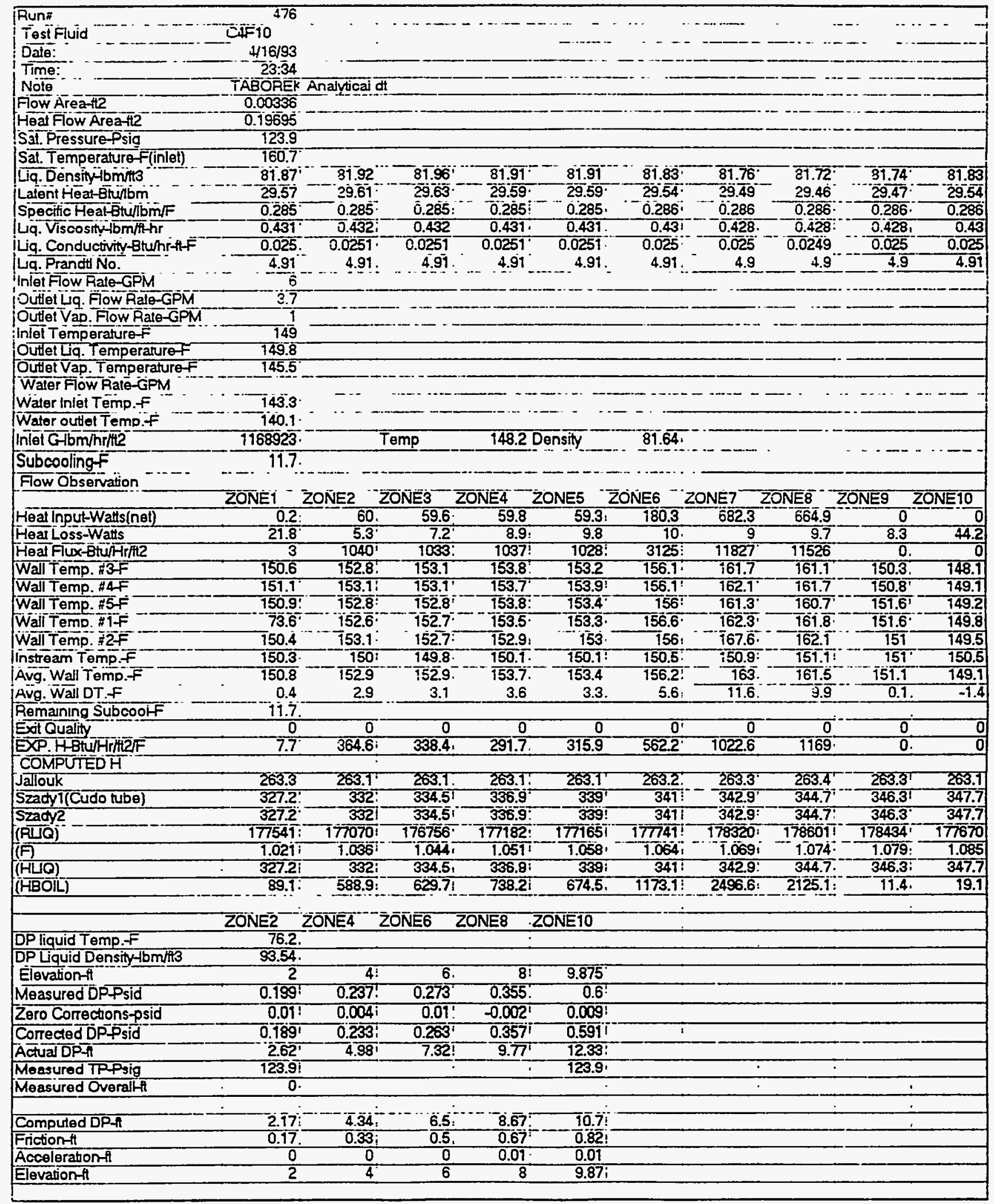




\begin{tabular}{|c|c|c|c|c|c|c|c|c|c|c|}
\hline Run & $683 !$ & & & & & & & & & \\
\hline Test fivid & C4 $=10$ & & & & & & & & & \\
\hline Date: & $7 \pi 1931$ & & & & & & & & & \\
\hline Timo: & $15: 281$ & & ‘ & & T & i & & & 1 & T \\
\hline Noto & - Anatyical o & dt is used t & $t$ & & & 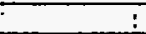 & 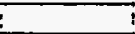 & $i$ & & 7 \\
\hline Flow Areafie & 0.003361 & & 1 & $\cdot$ & $:$ & T. & T. & 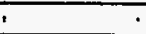 & & 7 \\
\hline Heat Fow Arost2 & 0.186851 & & $\cdot$ & 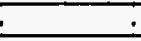 & - & 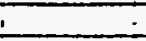 & 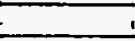 & 1 & 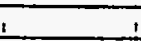 & 1 \\
\hline Sat. Pressure $P_{s i g}$ & $190.7 !$ & & 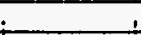 & & I & + & - & 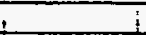 & $\mathrm{i}$ & 1 \\
\hline Sat. Temperature f(inlet) & $192.1 !$ & & & & & & & & & \\
\hline Uq. Density & 72.881 & 72.631 & $72.57 i$ & $72.54 \mid$ & $72.54 \mid$ & 72.431 & $72.32 !$ & $72.26 !$ & 72.231 & 72.54 \\
\hline Latent Heat Etulbm & 22.75 & 22.55 & $22.51 t$ & 22.49 & 22.49 & $22.4 !$ & 22.31 & 2227 & 22.241 & 22.49 \\
\hline Specifio Heat-Etulbm/F & 0.313 & 0.3141 & 0.394 & 0.315 & 0.395 & 0.315 & 0.315 & 0.3961 & $0.316 i$ & 0.315 \\
\hline Lig. Viscosity $4 b \mathrm{~m} / \mathrm{h} h \mathrm{~s}$ & 0.2361 & 0.283 & $0.292 !$ & $0.203 !$ & $0.202 !$ & 0.29 & 0.289 & -0.2881 & $0.288 \mathrm{~T}$ & 0.292 \\
\hline Lig. Conductivity-8tuhr-fF & $0.0155 !$ & 0.0193 & $0.0183 !$ & 0.01931 & 0.0193 & 0.0192 & 0.0192 & 0.0191 & 0.01911 & 0.0183 \\
\hline La. Prandt No. & $4.75 T$ & 4.75 & 4.761 & 4.761 & 4.761 & 4.761 & 4.76 & 4.76 & 4.761 & 4.76 \\
\hline Inlet Flow Rato-GPM & $9.1 !$ & & $T$ & & $-T$ & $i$ & 1 & -1 & 1 & $T$ \\
\hline Outlot Lig. Flow Reto-GPM & 0.91 & & 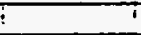 & & $T$ & 1 & 1 & 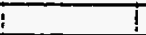 & 7 & i \\
\hline Outlel Vap. Flow Rato-GPM & $0.7 \mathrm{i}$ & & $i$ & & $\cdot$ &. & 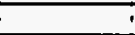 & i & $i$ & $T$ \\
\hline Inlet Temperaturef & $188 . \overline{11}$ & & ! & & $\bar{i}$ & $\bar{i}$ & & 1 & & - \\
\hline Outiol Lig. Tomparaturef & 1921 & & i & & $i$ & 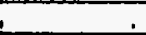 & & 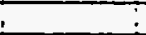 & & i \\
\hline Outtel Vap. Temperaturef & 189.61 & & & & & & & & & $\perp$ \\
\hline Water Flow Rato-GPM & & & & & & & & & & \\
\hline Walor Inlel Temp.f & 120.81 & & & & & & & & - & \\
\hline Water outlel Temp.F & 179.91 & & & & & & & & & \\
\hline Inlet Glbmhrnie & 1969201 & & Temp & 178.71 & TDensity & 75.011 & & & & \\
\hline Subcoolingt & 41 & & & & & & & & & T \\
\hline FOW ODservation & & & i & & & & & & & \\
\hline & ZOONET: & ZONEQ ! & !ZOONE3 & ZONE4 : & ZOONES T/2 & $\angle Z O N E B$ IR & ZONET & ZONEB & ZONE9 & ZZONETO \\
\hline Hoad InputWatts(nel) & 805.61 & 887.41 & 889.91 & 058.71 & 248.51 & 85.21 & 81.81 & 87.7 & 971 & 1111.2 \\
\hline Heal Losg-Walls & 19.41 & 4.61 & 6.31 & 7.51 & 7.51 & 6.61 & 5.11 & 6.31 & 6.81 & 37.2 \\
\hline 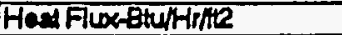 & 160051 & 150036 & 140751 & 173831 & 43081 & $14 \pi 7$ & 14141 & 15201 & 1681 & 1828 \\
\hline Wall Tomp. "3F & 196.71 & 199.31 & 198.3 & 199.31 & 195 & 133.51 & 1951 & 194.8 & 195.21 & 195 \\
\hline Wall Temp. 4 FF & 197.41 & 197.3 & 2001 & 199.7 & 195.5 & 194.41 & 195 & $194.8 i$ & 195.4 & 195.7 \\
\hline Wall Tomp. \#5F & $197.4 !$ & 196 & 199.21 & 197.6 & 194.7! & 193.5 & 195 & 194.8 & 195.3 & 195 \\
\hline WallTemp. & 95.31 & 197.41 & 196.8 & 196.31 & 194.71 & 193.9 & 194.1 & 194.8 & 194.8 & 192.7 \\
\hline Wall Temp. \#2F & 197.81 & 997.61 & 197.71 & 197 & 194.8 & 193.2 & 194.4 & 195.11 & 194.8 & 193.5 \\
\hline Instream temp.f & T90.3! & 391.2! & 191.4! & 197.5 & 191.5 & |S1.9| & 192.31 & 1925 & 192.6 & 191.5 \\
\hline Avg. Wall Temp. & $797.3 !$ & 797.9 & $198.2 !$ & Tझ.र्टो & T94.9! & 193.81 & 794.7 & उक्. & $1 \% 5.1$ & T94.4 \\
\hline Avg. Wall DT.F & 6.31 & 6 & 6.11 & 6.21 & 3.21 & 7.9 & 2.31 & 2.31 & 2.4 & 2.8 \\
\hline Romalning Subcooff & -10.31 & 1 & 7 & 7 & 1 & : & 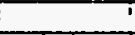 & $T$ & 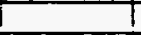 & t \\
\hline Exit Quadity & 0.141 & 0.341 & 0.541 & 0.691 & 0.741 & 0.761 & 0.781 & 0.81 & 0.821 & 0.85 \\
\hline 巨XP.HEtuhHRA/F & 2364.11 & 2497.41 & $2446.6 i$ & 1832.91 & $1333.6 i$ & 794.81 & 605.61 & 653.51 & 693.71 & 690.2 \\
\hline COMPUTEDH & $i$ & & 1 & & 1 & 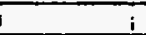 & & & 1 & 1 \\
\hline Jallouk & 183241 & 77.91 & 77.91 & 77.91 & $77.9 !$ & 7.91 & $77.9 !$ & 7.91 & 77.91 & 77.9 \\
\hline Szady 1 (Cudo tube) & $2283.2 i$ & 77.91 & 77.91 & 77.9 & 77.9 & 77.9 & 77.91 & $\pi .91$ & 77.9 & 77.9 \\
\hline Szadt2 & $2162.7 !$ & $\pi .91$ & 7.9 & $\pi .9$ & 77.9 & 77.9 & 77.9 & 77.9 & $\pi .9$ & 77.9 \\
\hline (RUO) & 40483 & 40493 & 40493 & 40493 & 404931 & 40493 & 40493 & 40493 & 40493 & 40493 \\
\hline (F) & 1.735 & 1.735 & 1.735 & 1.7351 & 1.735 & 1.735 & 1.735 & 1.7351 & 1.735 & 9.735 \\
\hline (FIIO) & 73.9. & 38.9 & 138.9 & 138.9 & 738.9 & 738.91 & 738.91 & 138.91 & 138.9 & T38.9 \\
\hline (सEOIL) & 2283 & 2283 & 2283 & 2263 & 2283 & 2783 & 238 & 22831 & 238 & 2283 \\
\hline & & & & & & & & & 1 & $T$ \\
\hline & ZONE2 I & ZONE4 & ZONEB & $\angle O N=8$ & ZZONETO & $T$ & & & & \\
\hline DP liquid Temp.F & 97.7 & & $\square$ & & 1 & i & & & 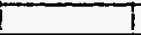 & $T$ \\
\hline DPLiquid Density $+b m+13$ & 80.391 & & i & & & I & 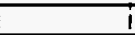 & & 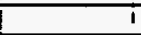 & $i$ \\
\hline Elovation & 21 & 41 & 61 & 8 & 9.8751 & I & 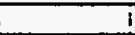 & & 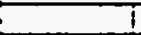 & I \\
\hline Mensured DPpaid & 0.3691 & 1.7471 & 2.632 & 3.685 & 4.521 & I & & & & \\
\hline Zero Corrections-psid & 0.122 & 0.089 & 0.139 & 0.02 & -0.044 & 1 & 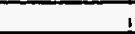 & & 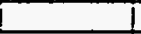 & I \\
\hline Compcted OPPsid & 0.247 & 1.658 & 2.493 & 3.655 & 4.665 & & & & & \\
\hline Actual DPA & 2 & 1.69 & 2.52 & 2.69 & 3.04 & & & & & \\
\hline Measured TPPsig & 190.7 & & & & 300.11 & & & & & \\
\hline Measured Overalth & I & & & & -7 & 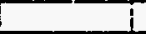 & & & & \\
\hline & ! & & & & & & & & & \\
\hline Computed DPA & 1.27 & 2.07 & 3.071 & 4.161 & 4.991 & & & & & \\
\hline Friction-h & 0.021 & 0.08 & 0.11 & 0.141 & 0.171 & & i & & 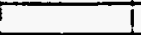 & I \\
\hline Acooleration- & $0.0 \boldsymbol{0} !$ & 0.06 & 0.071 & 0.07 & 0.081 & & i & & 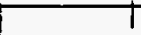 & t \\
\hline Elovation-l & 1.21 & 1.94 & 291 & 3.851 & 4.78 & & $i$ & & & I \\
\hline
\end{tabular}




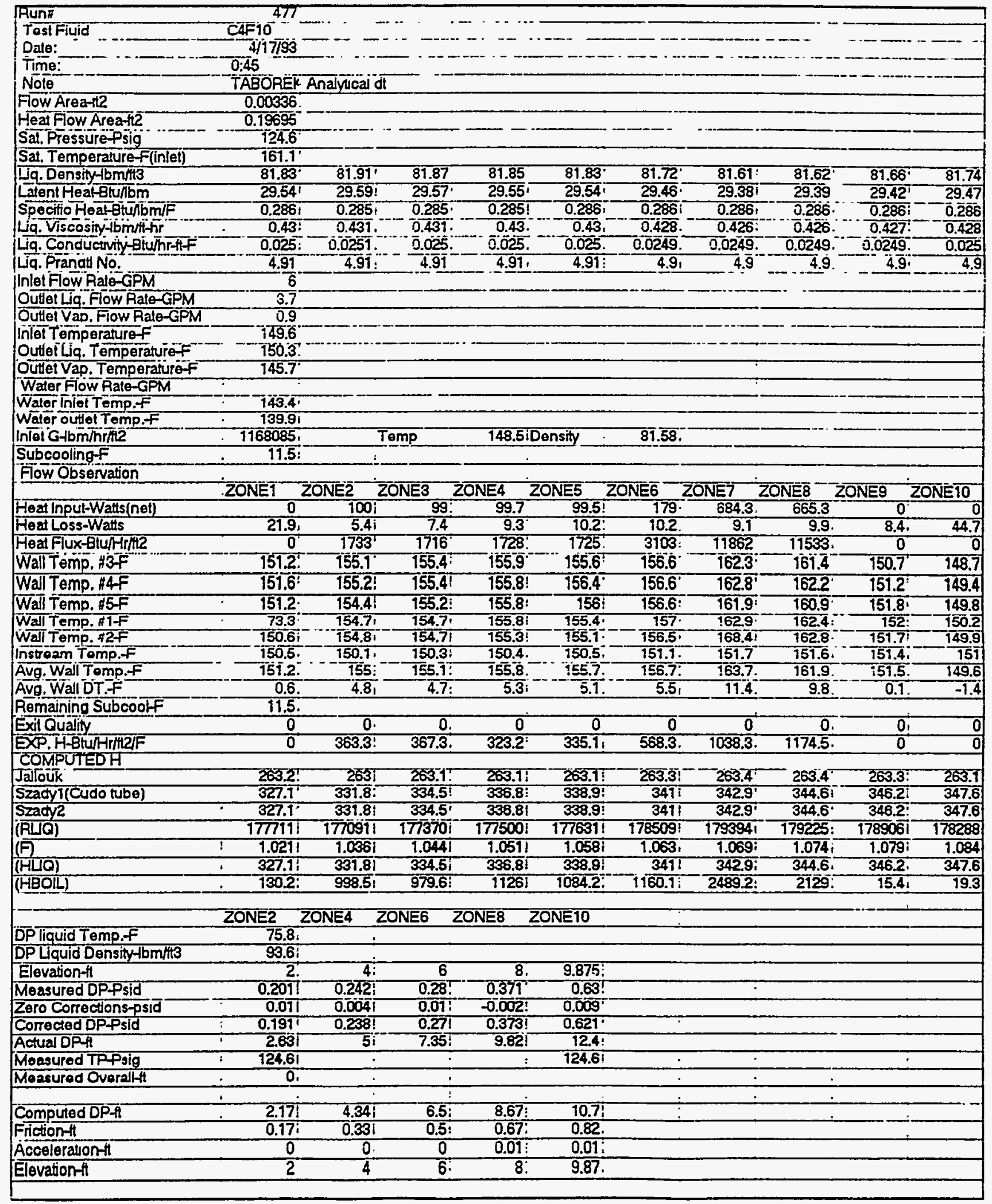




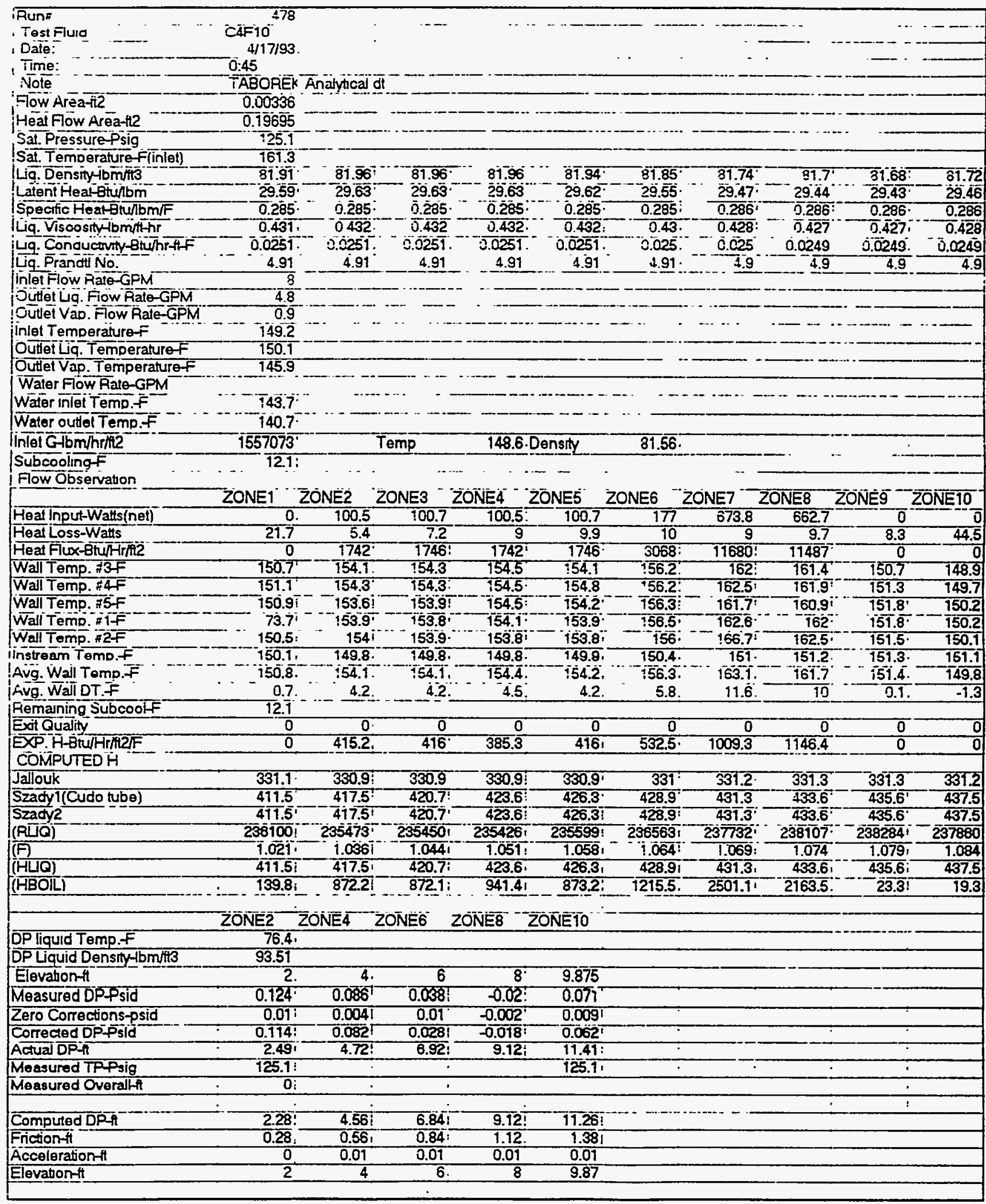




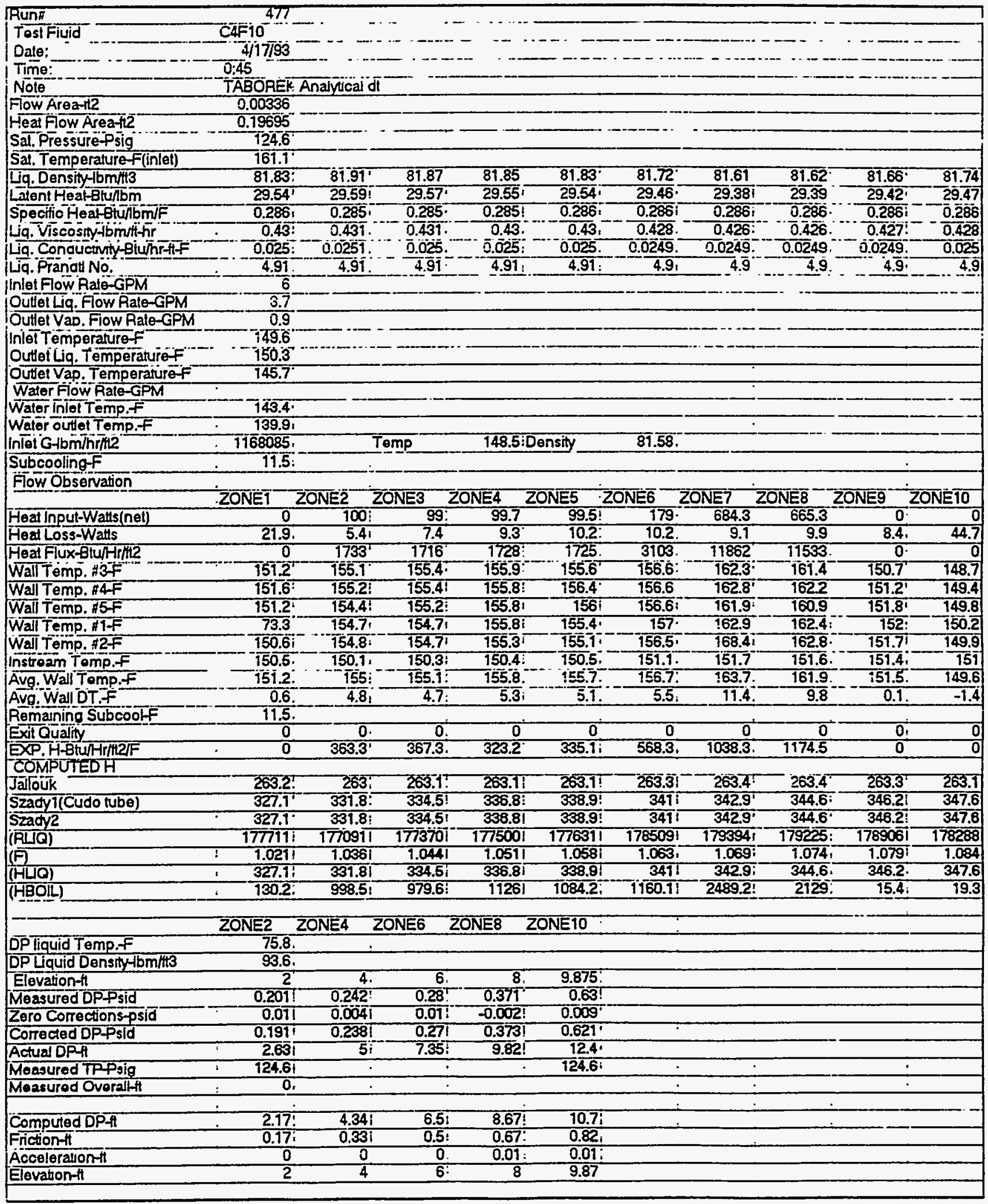




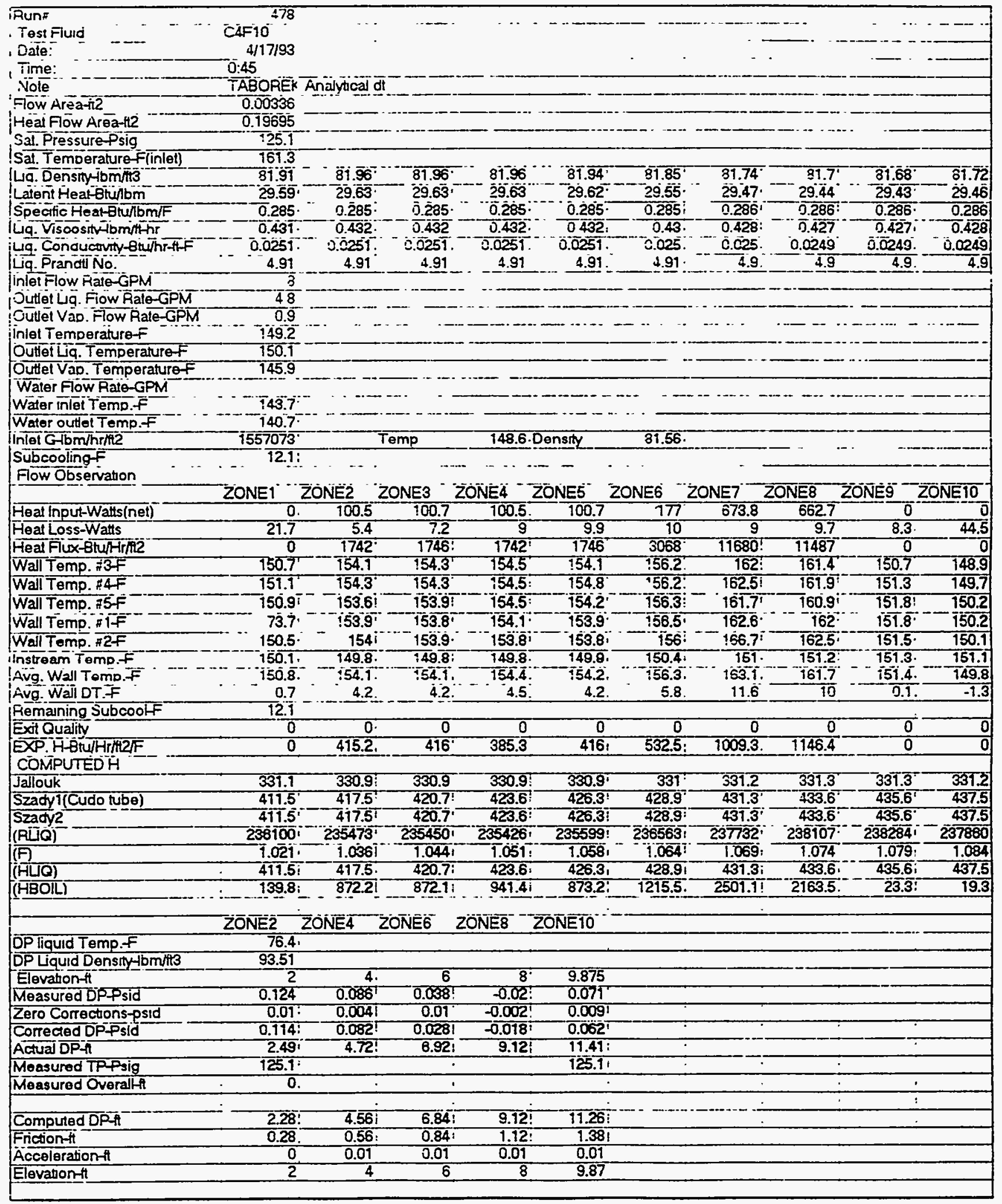




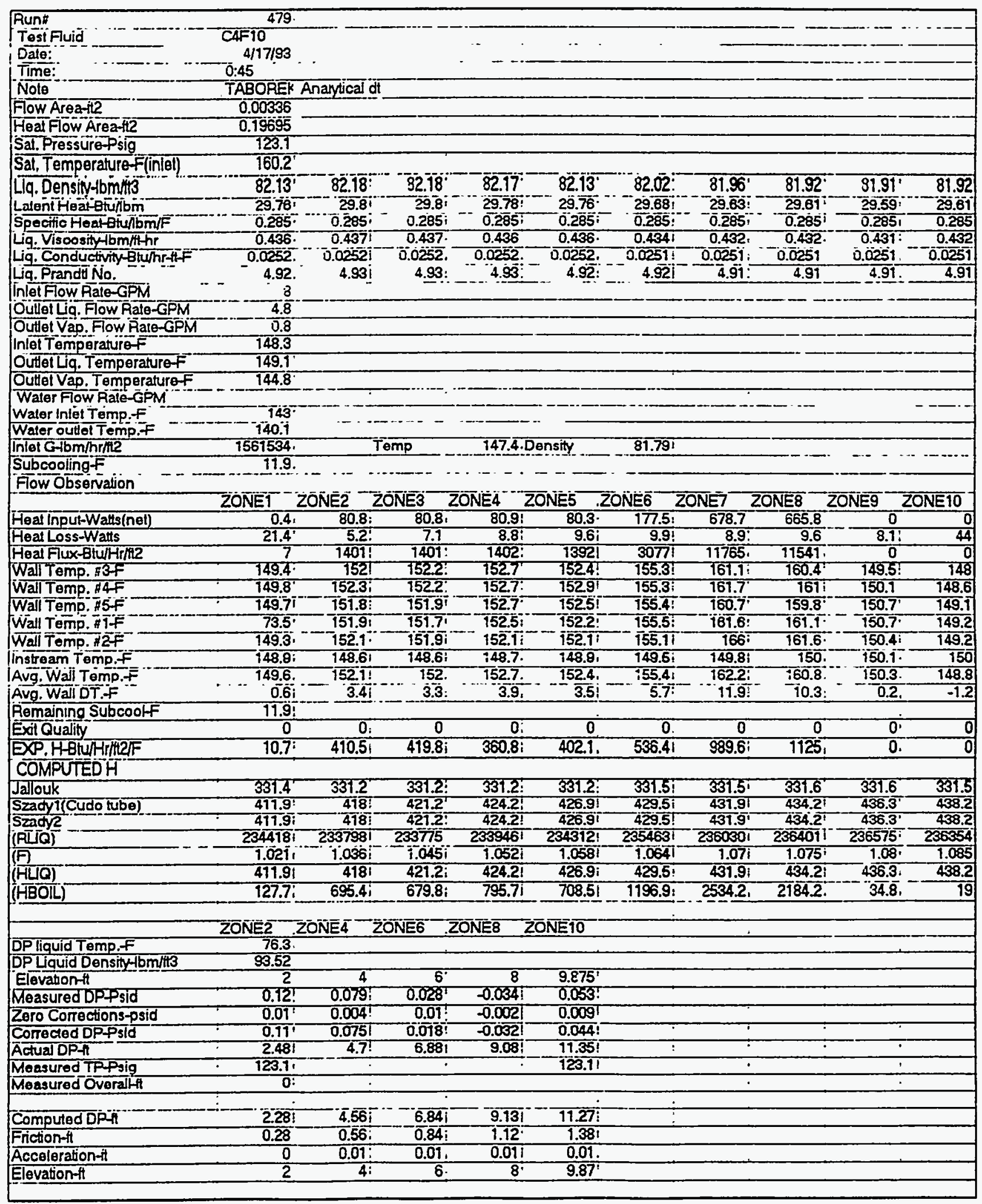




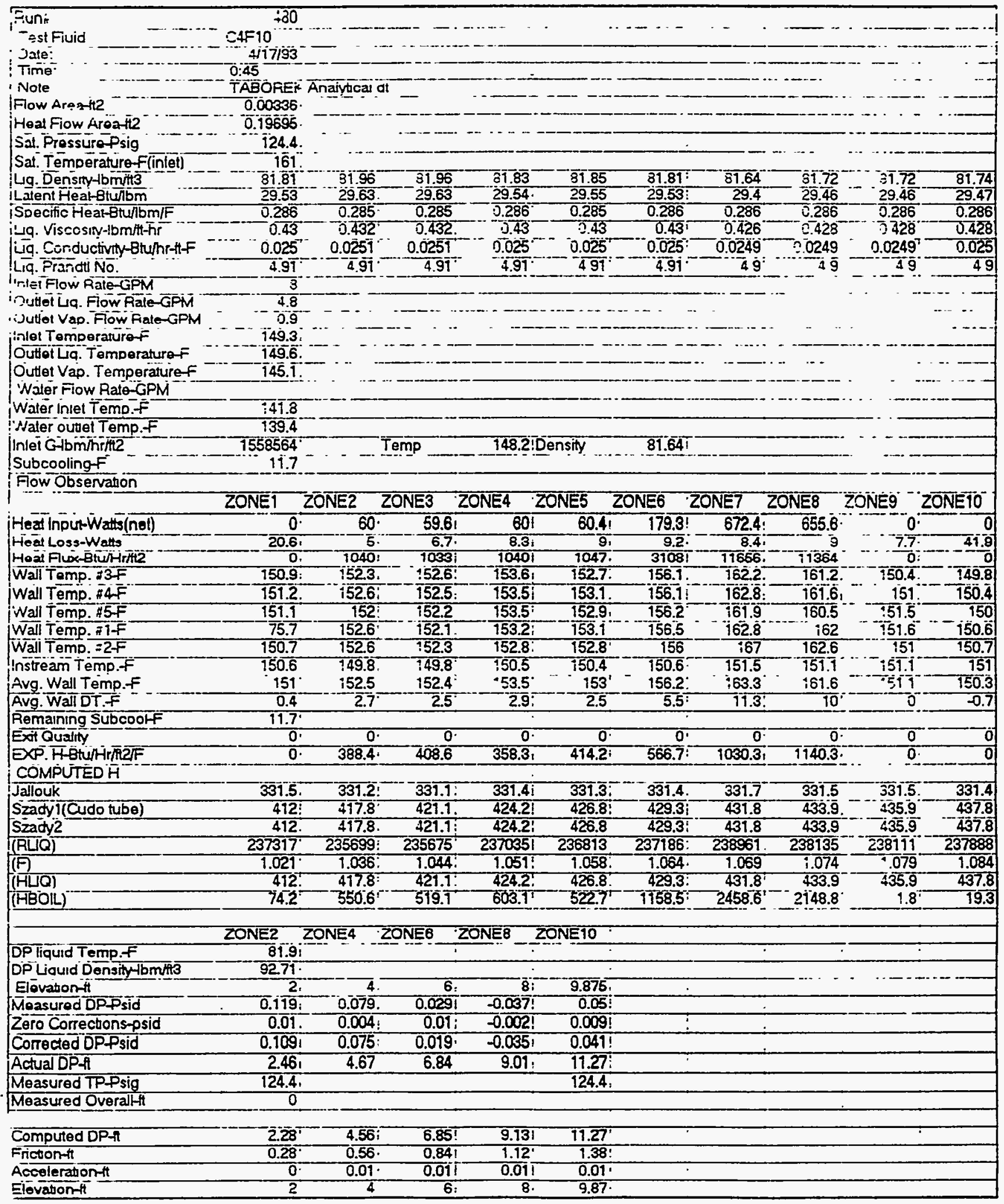




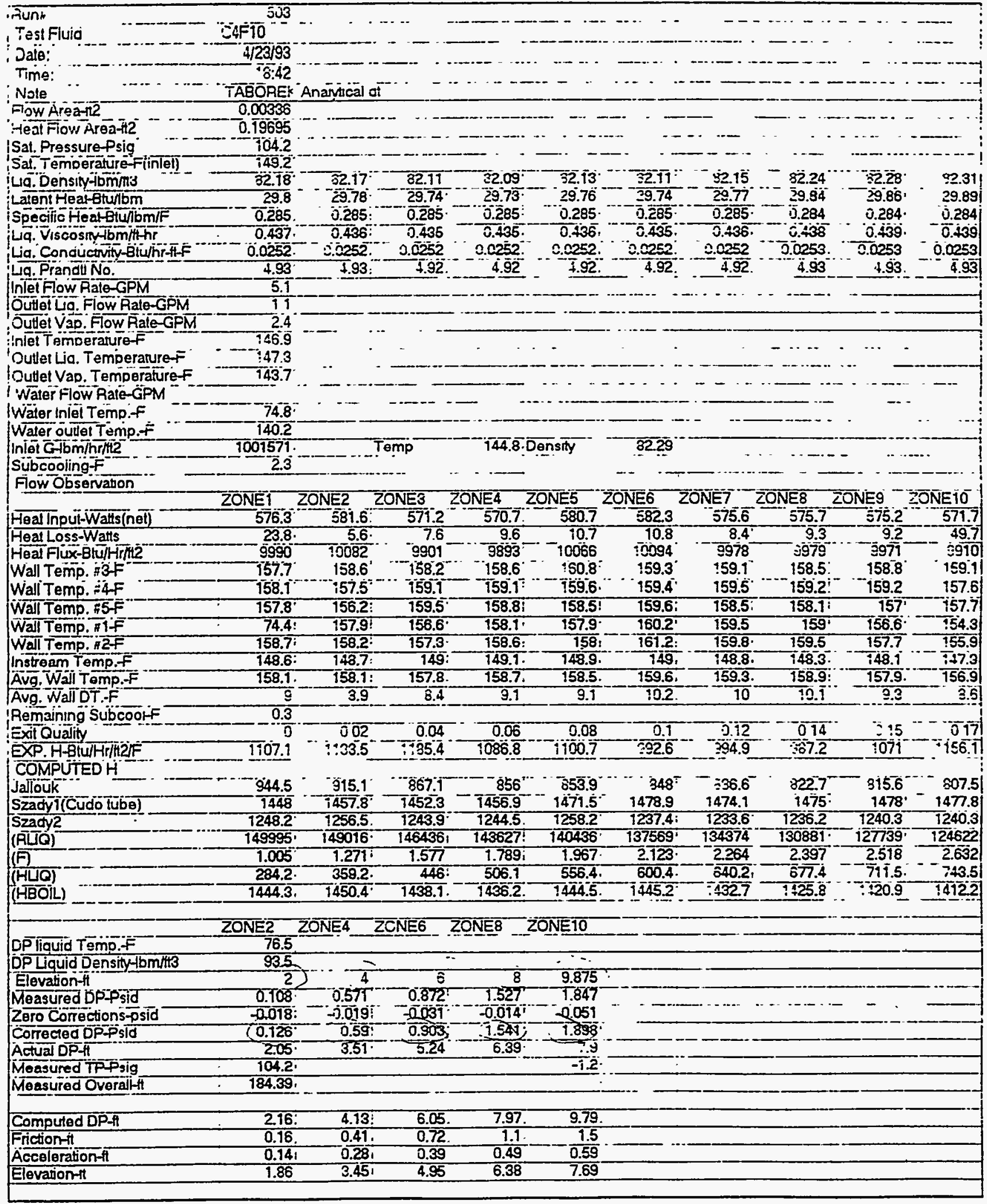




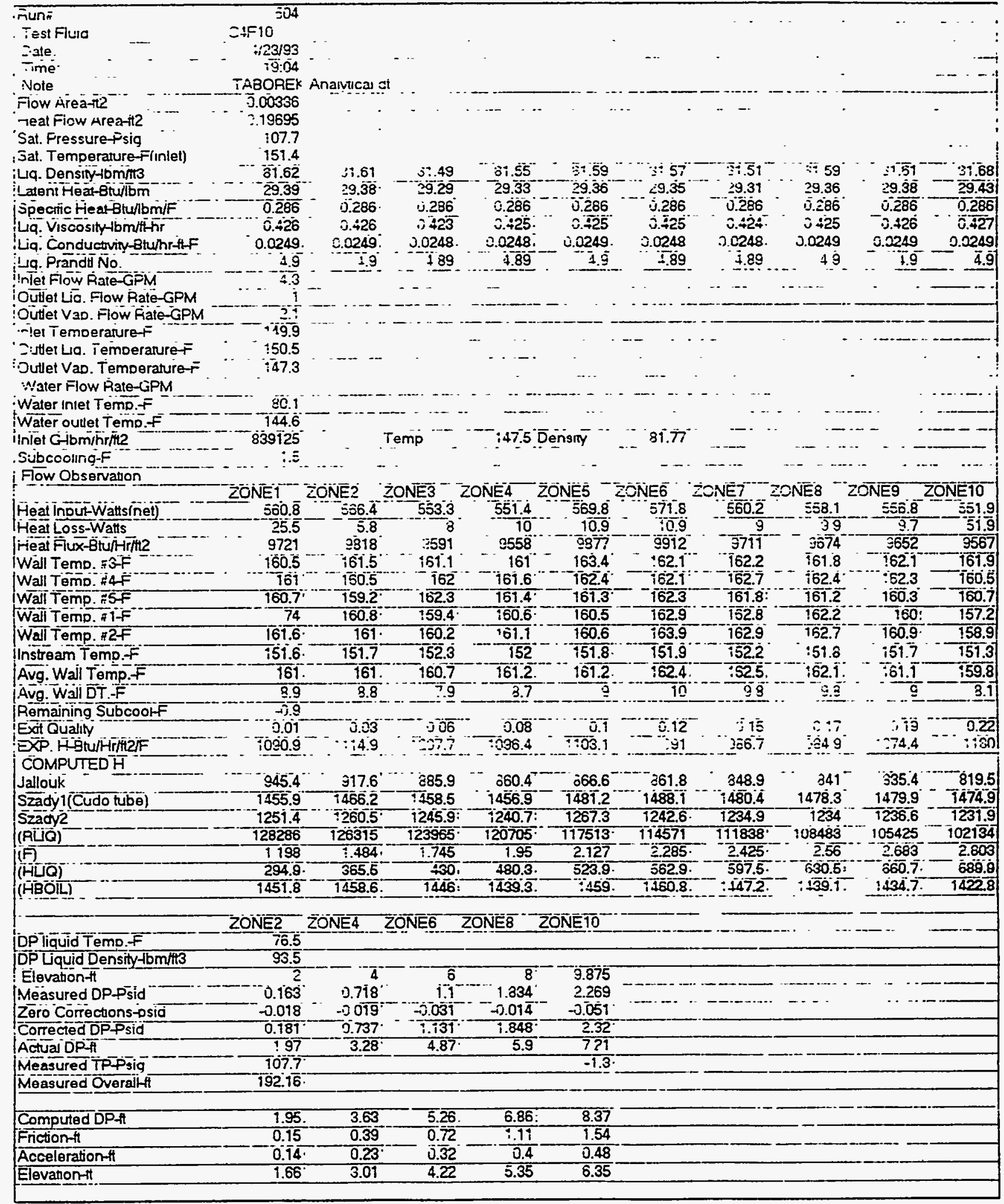




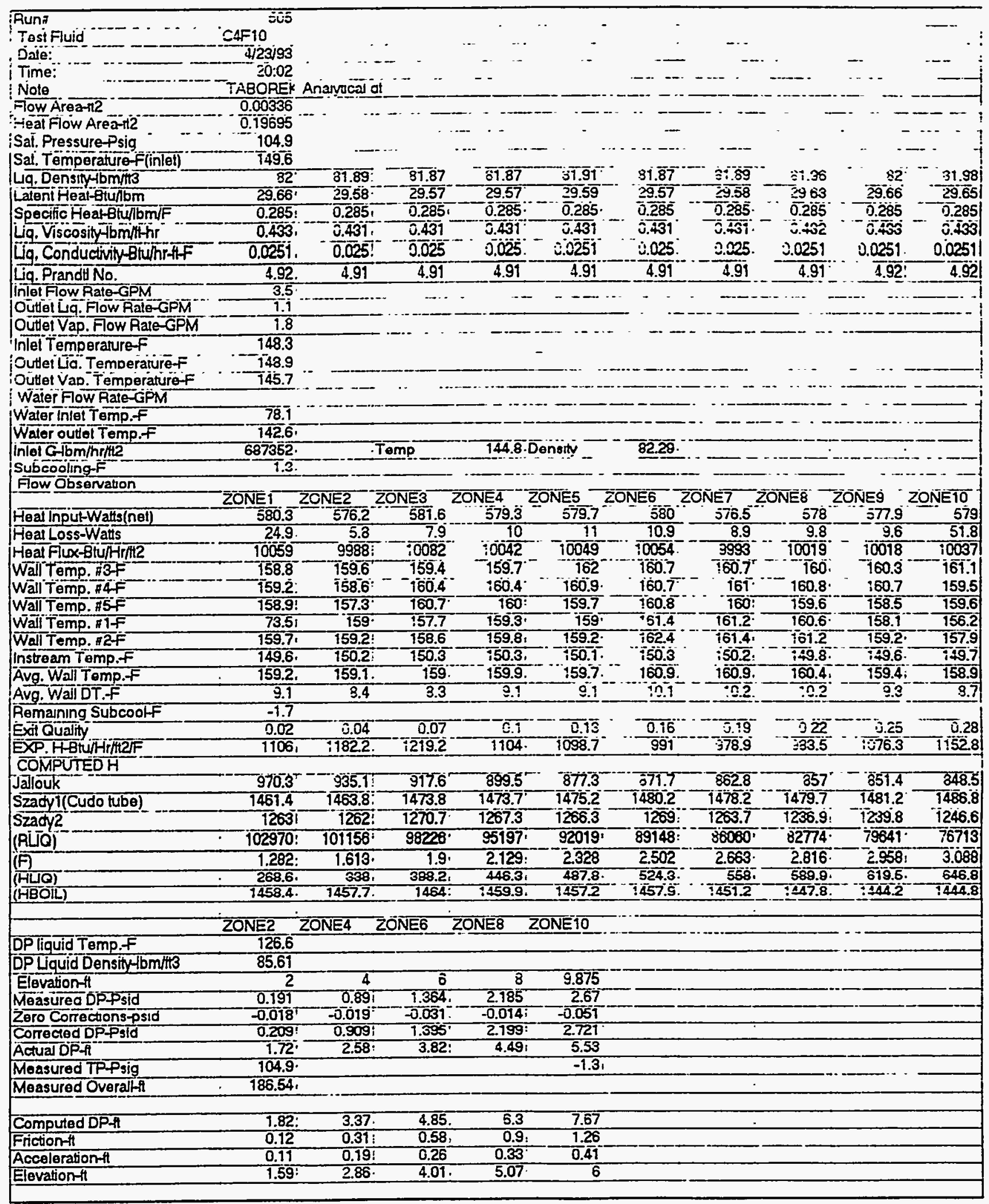




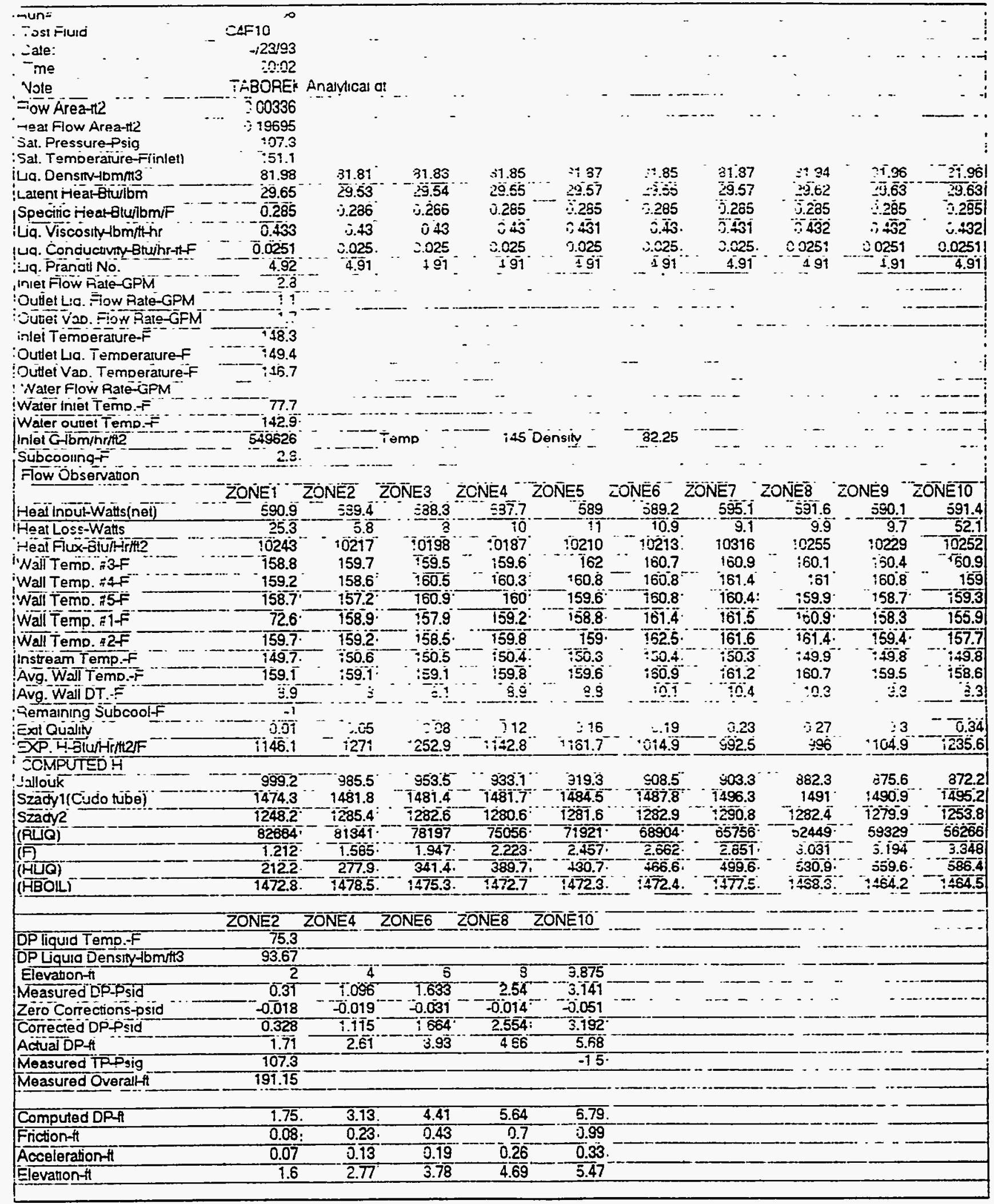




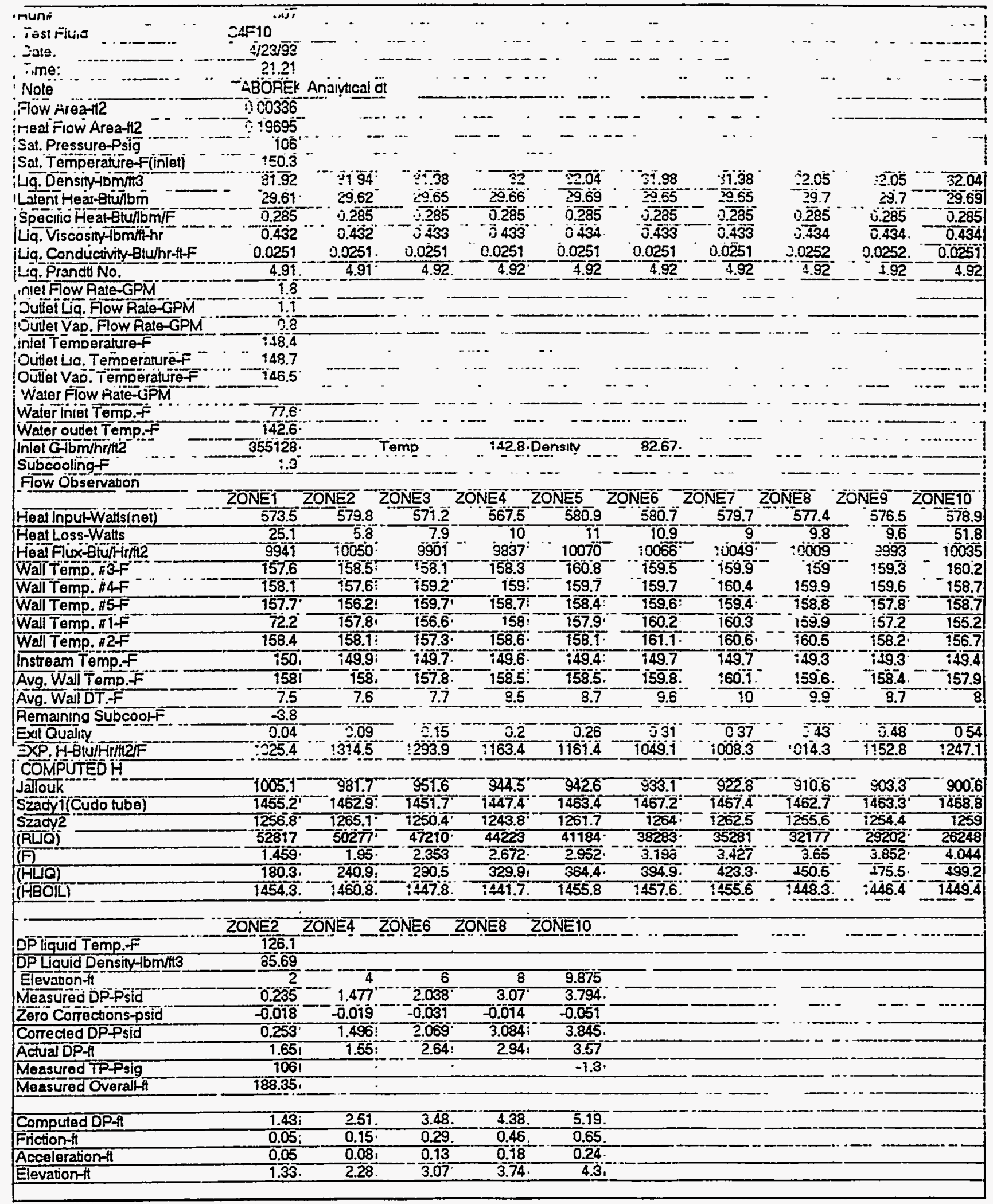




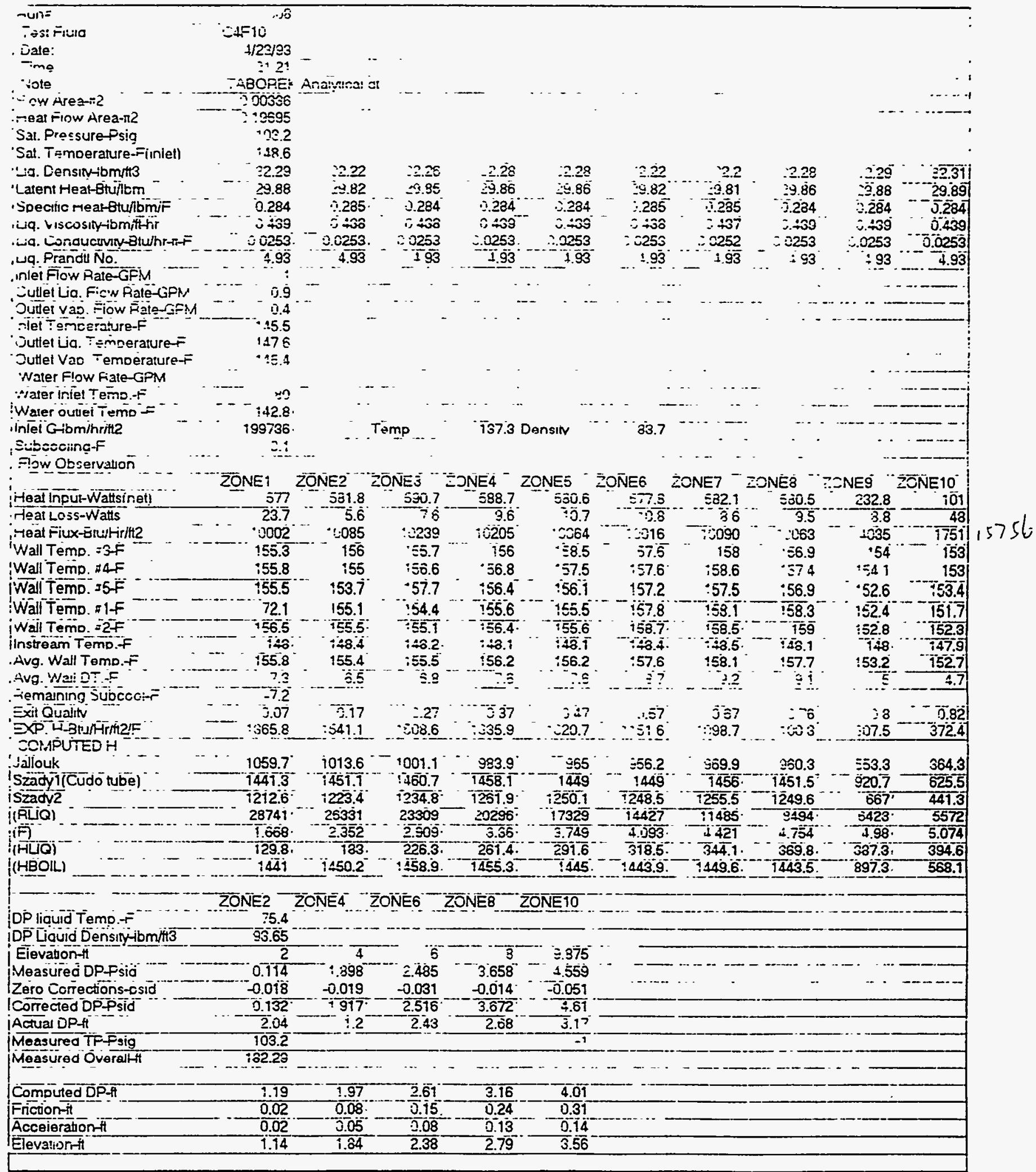




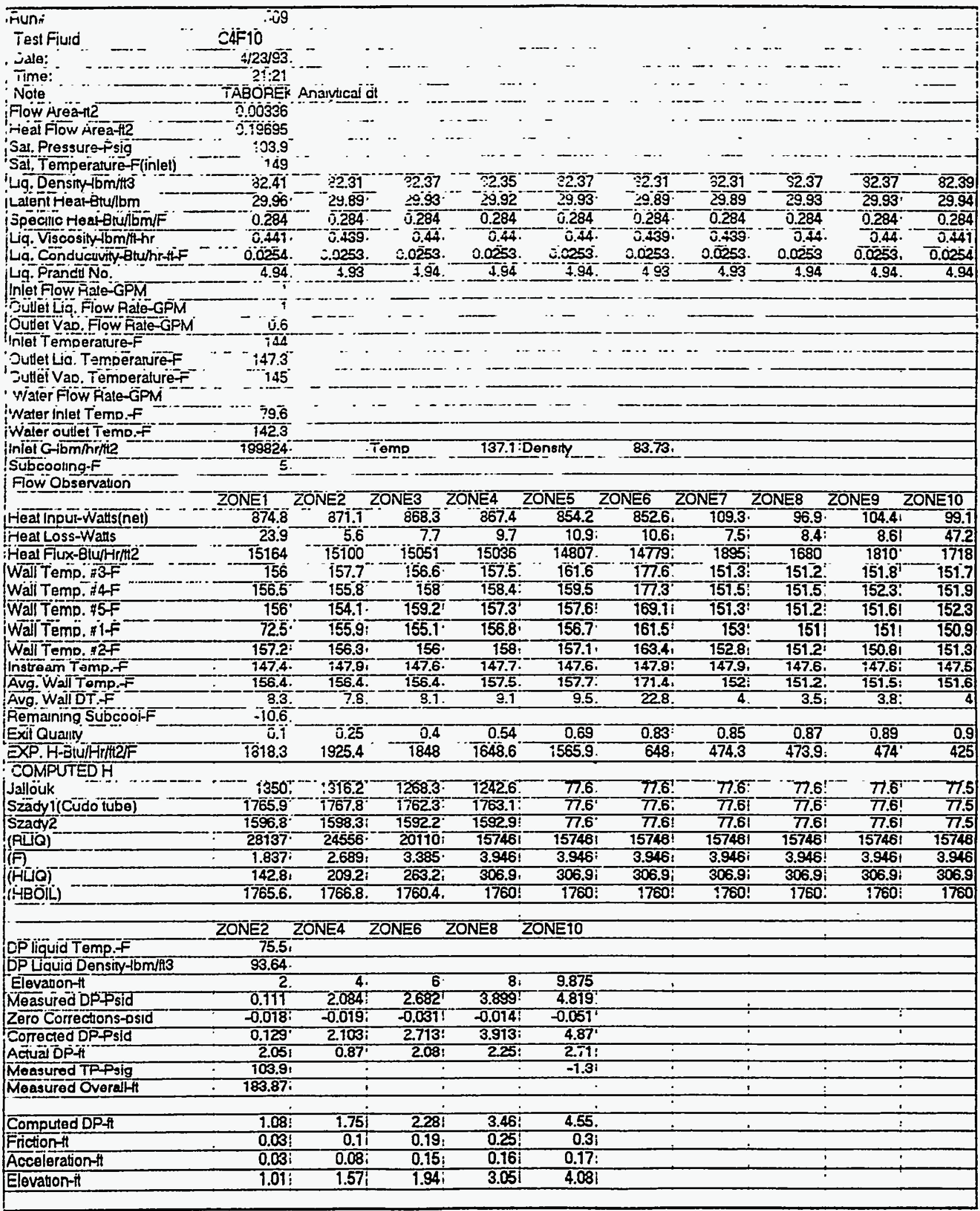




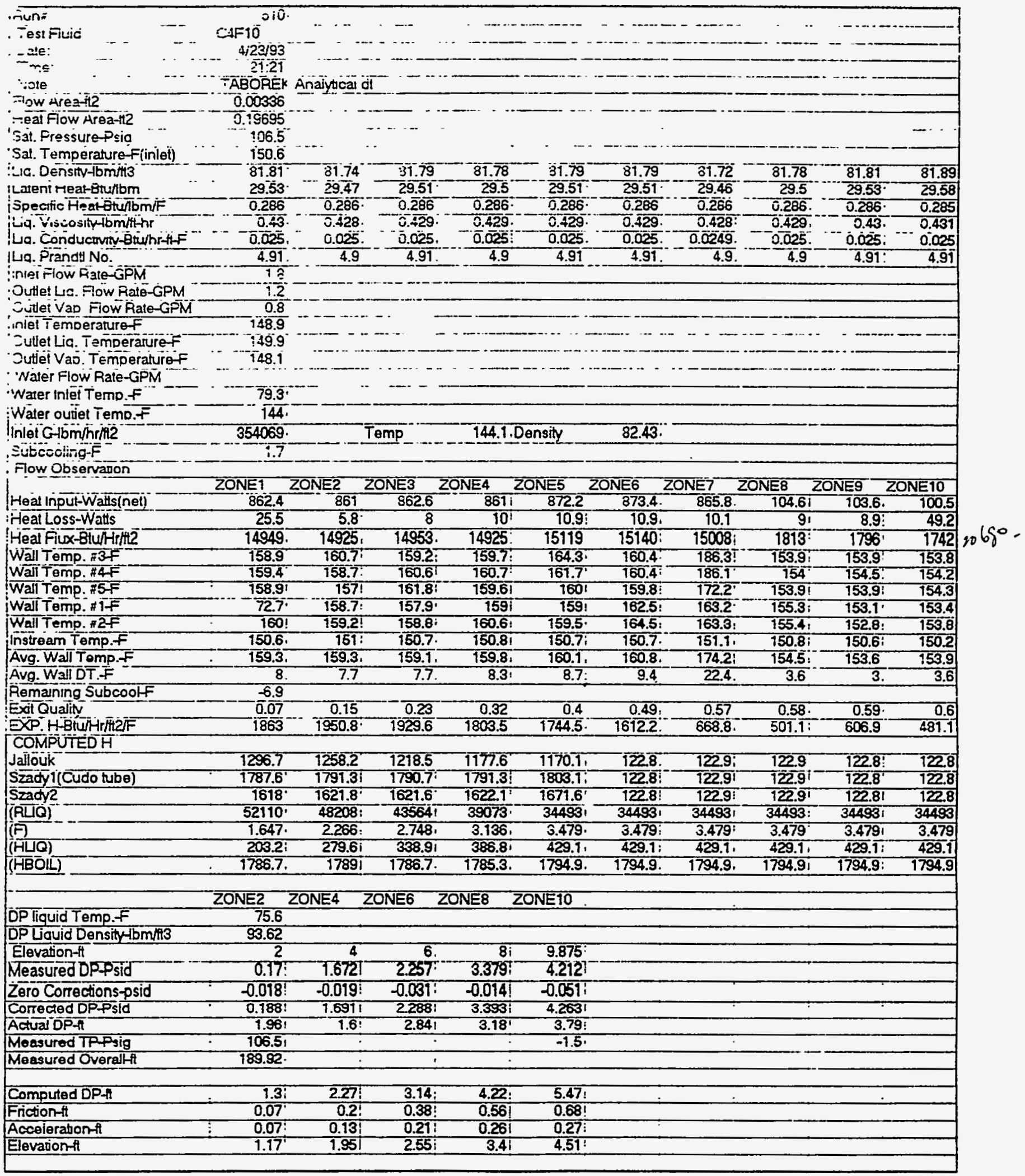




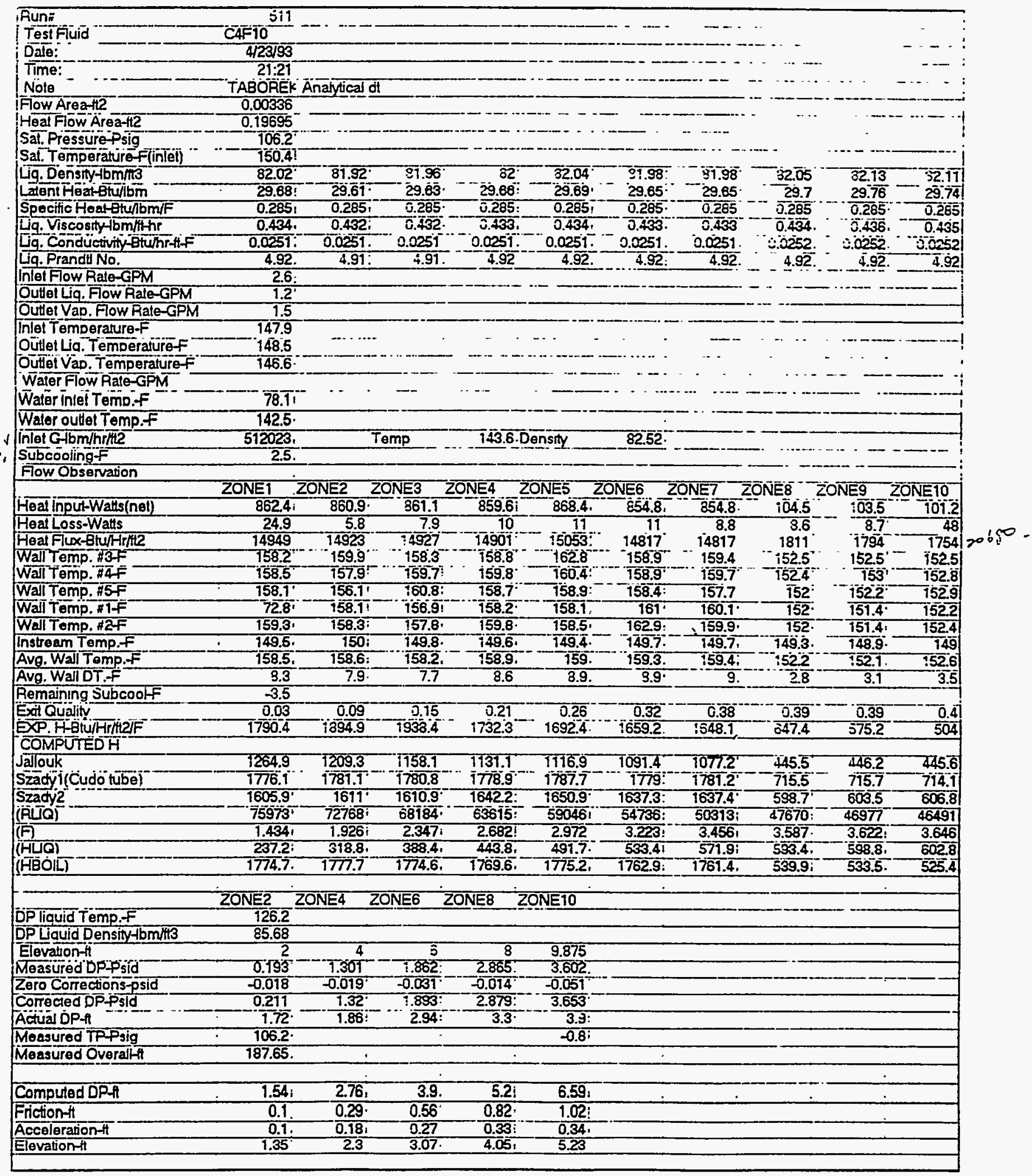




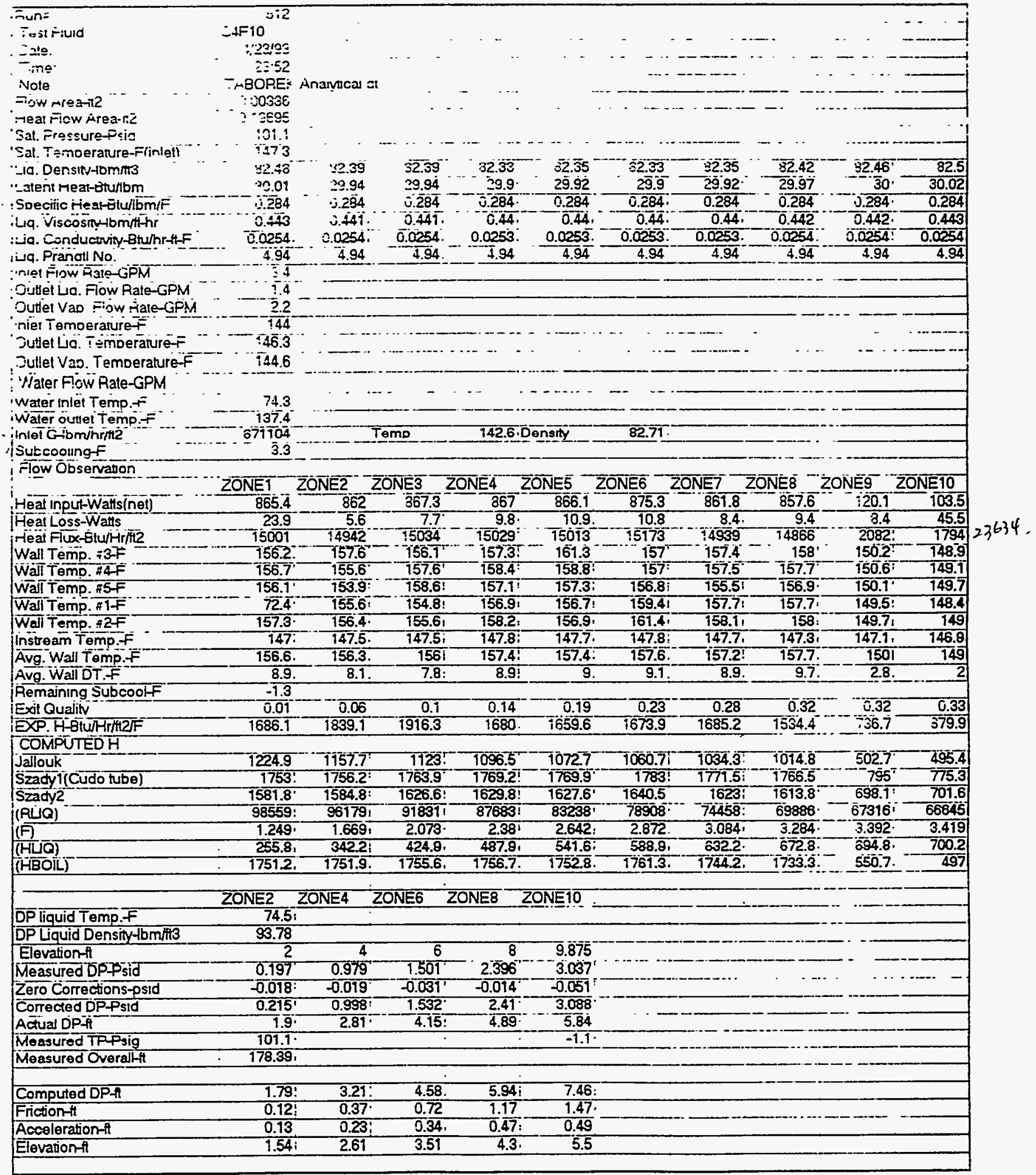




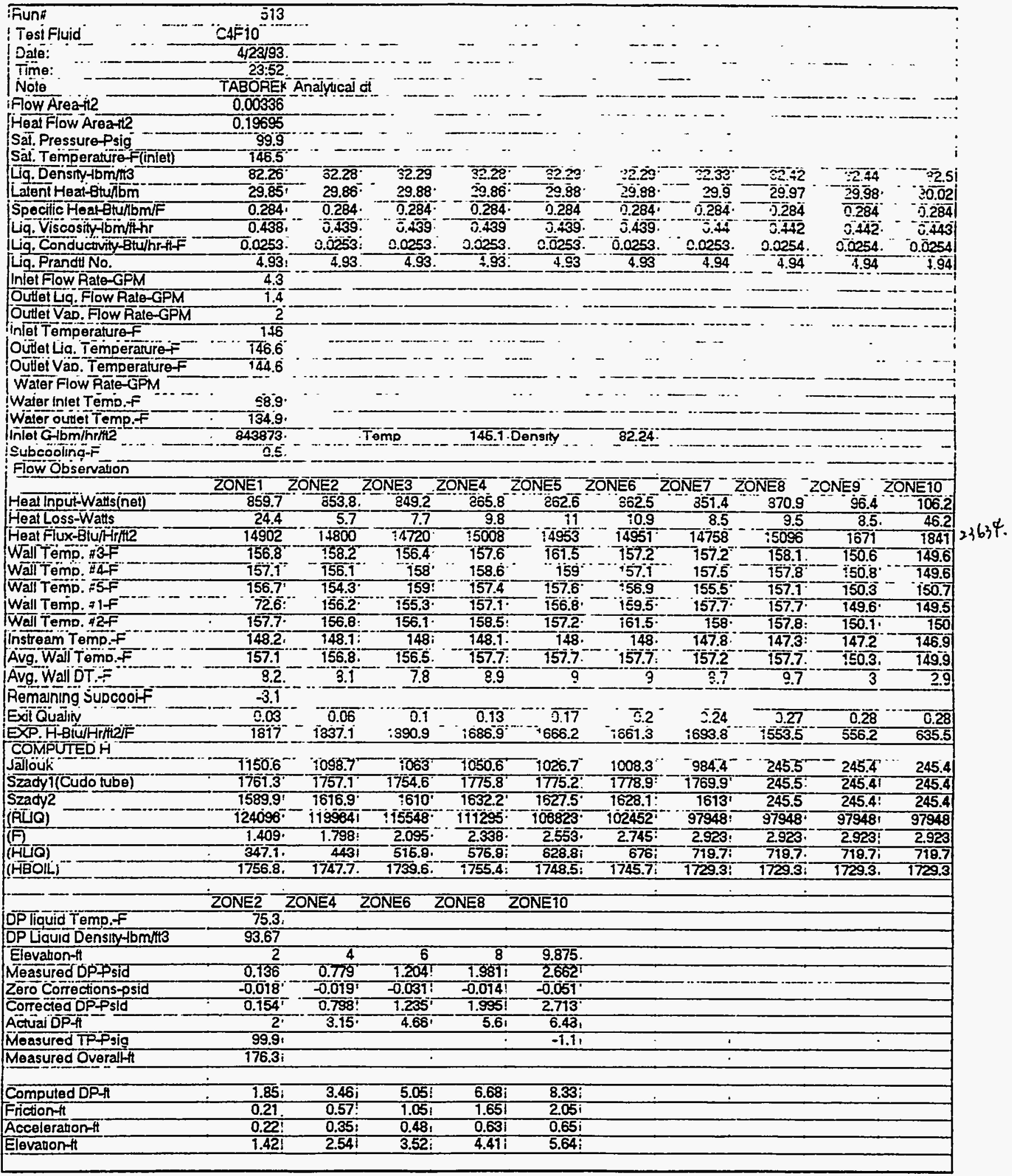




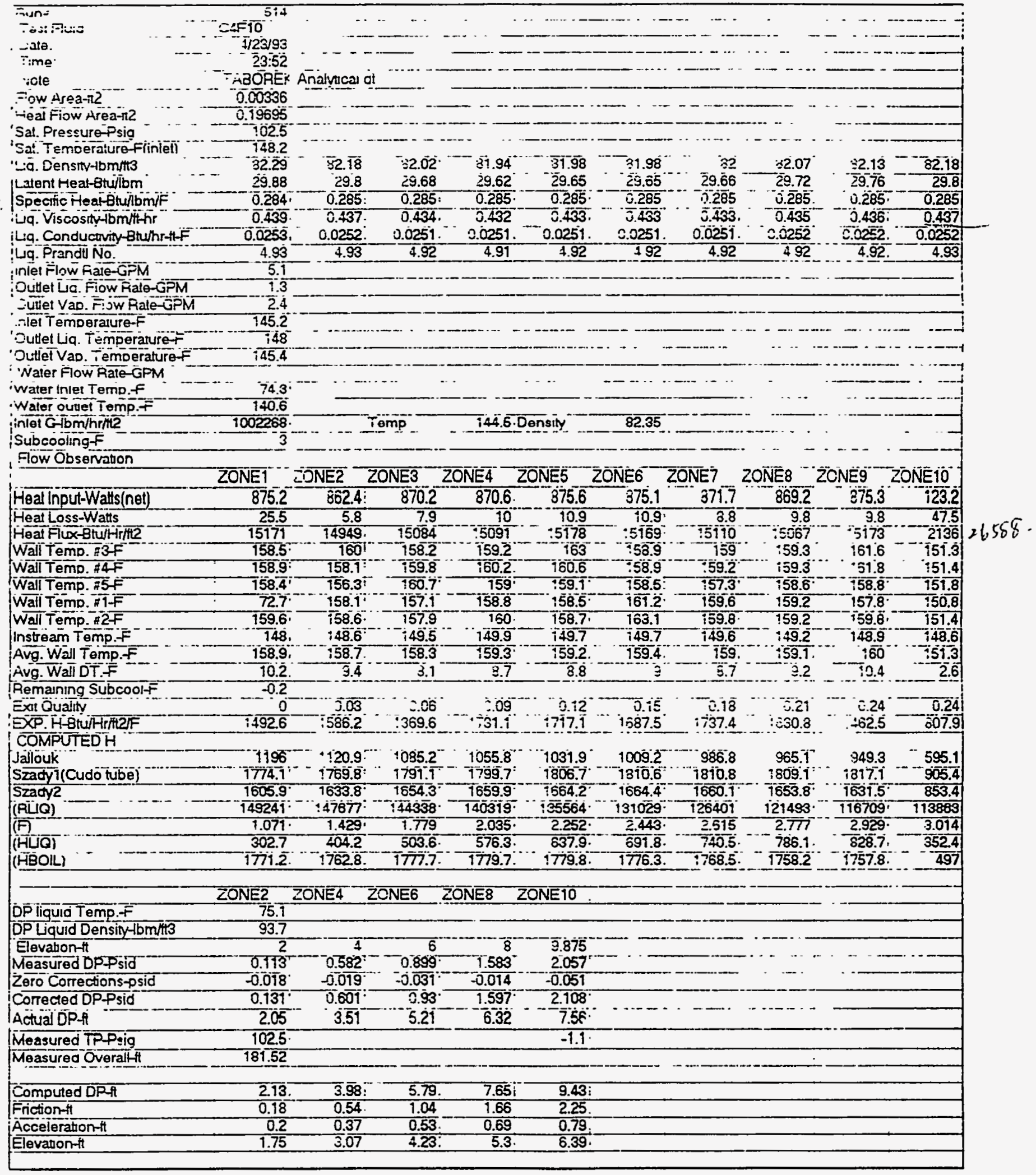




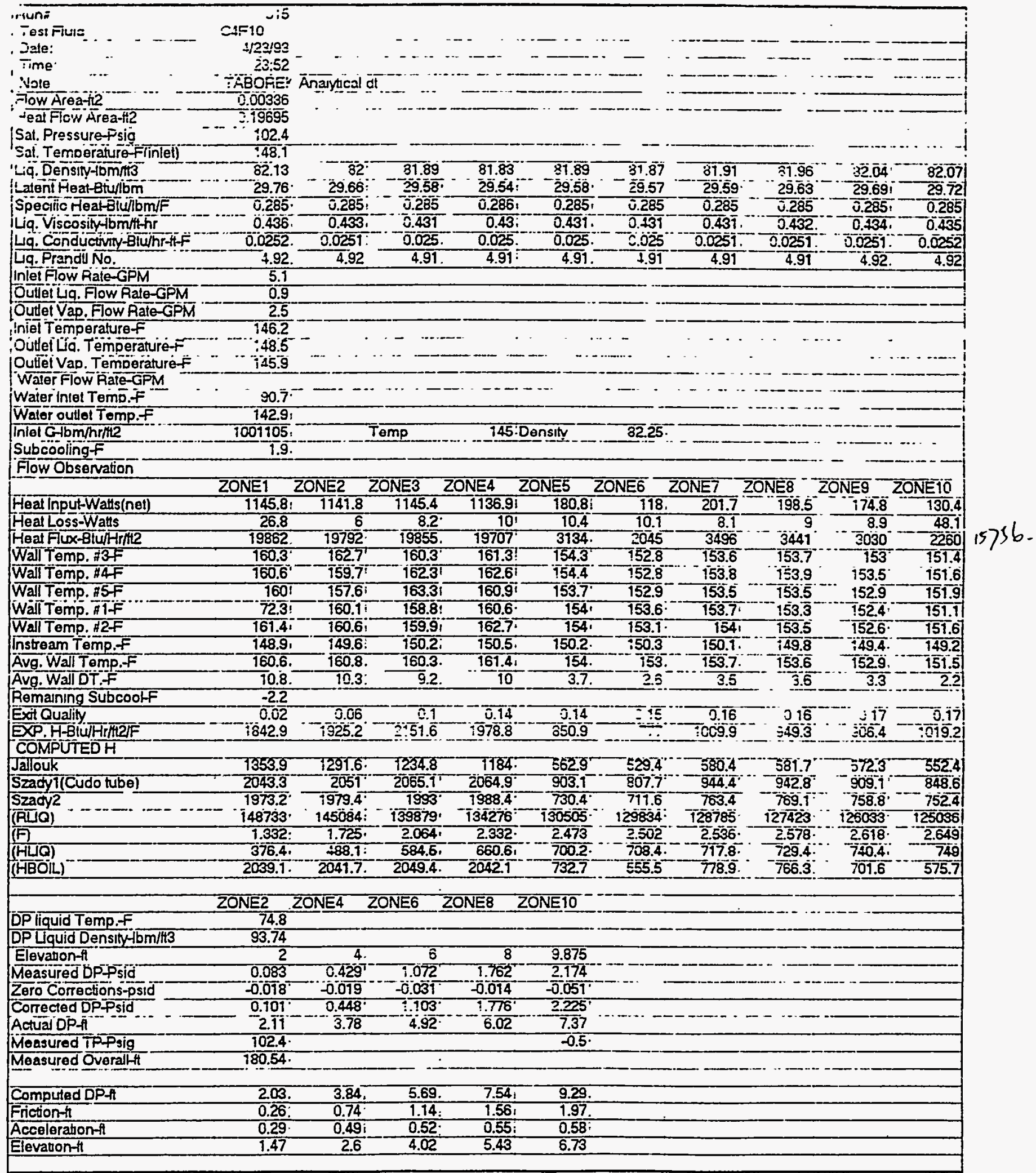




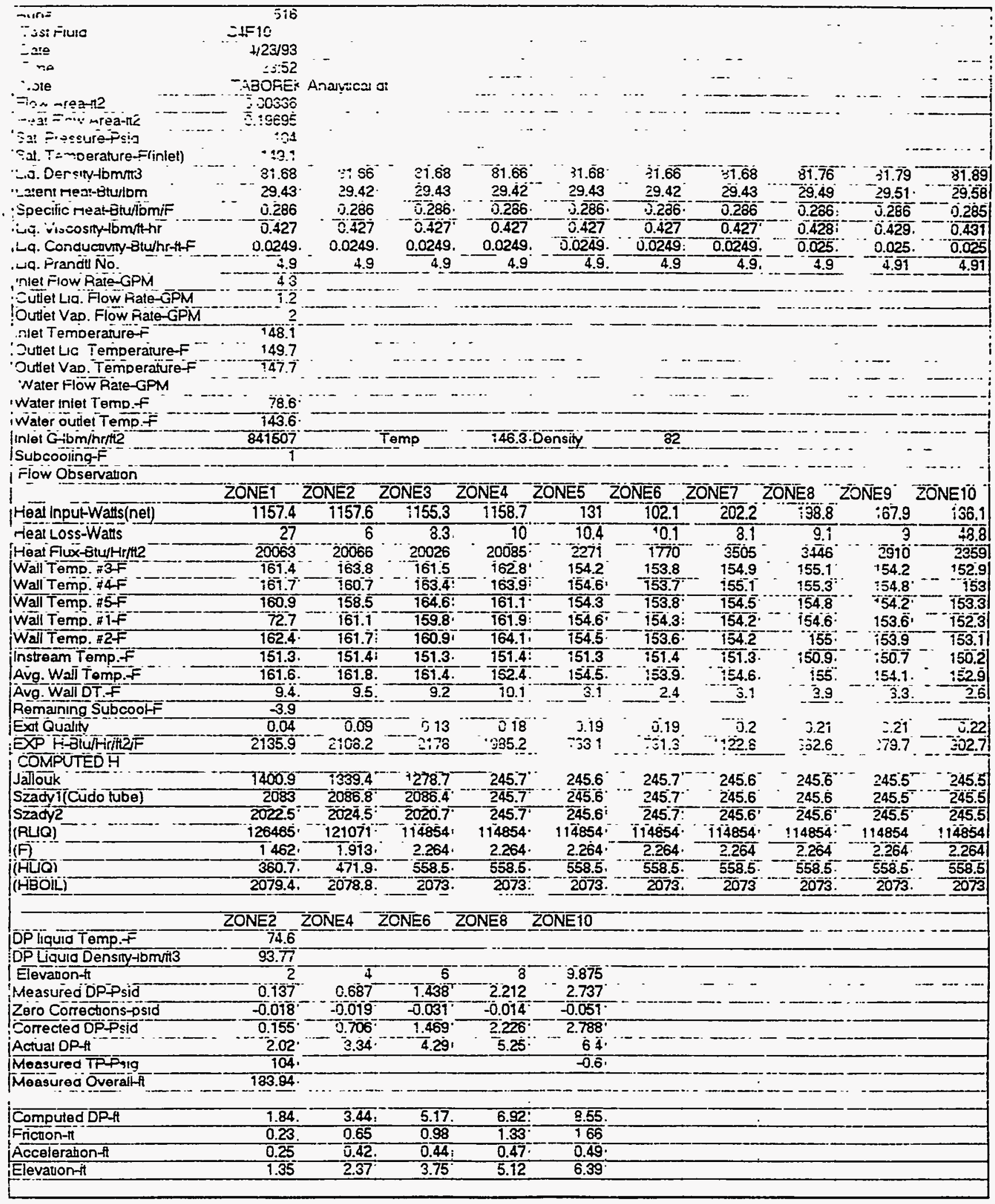




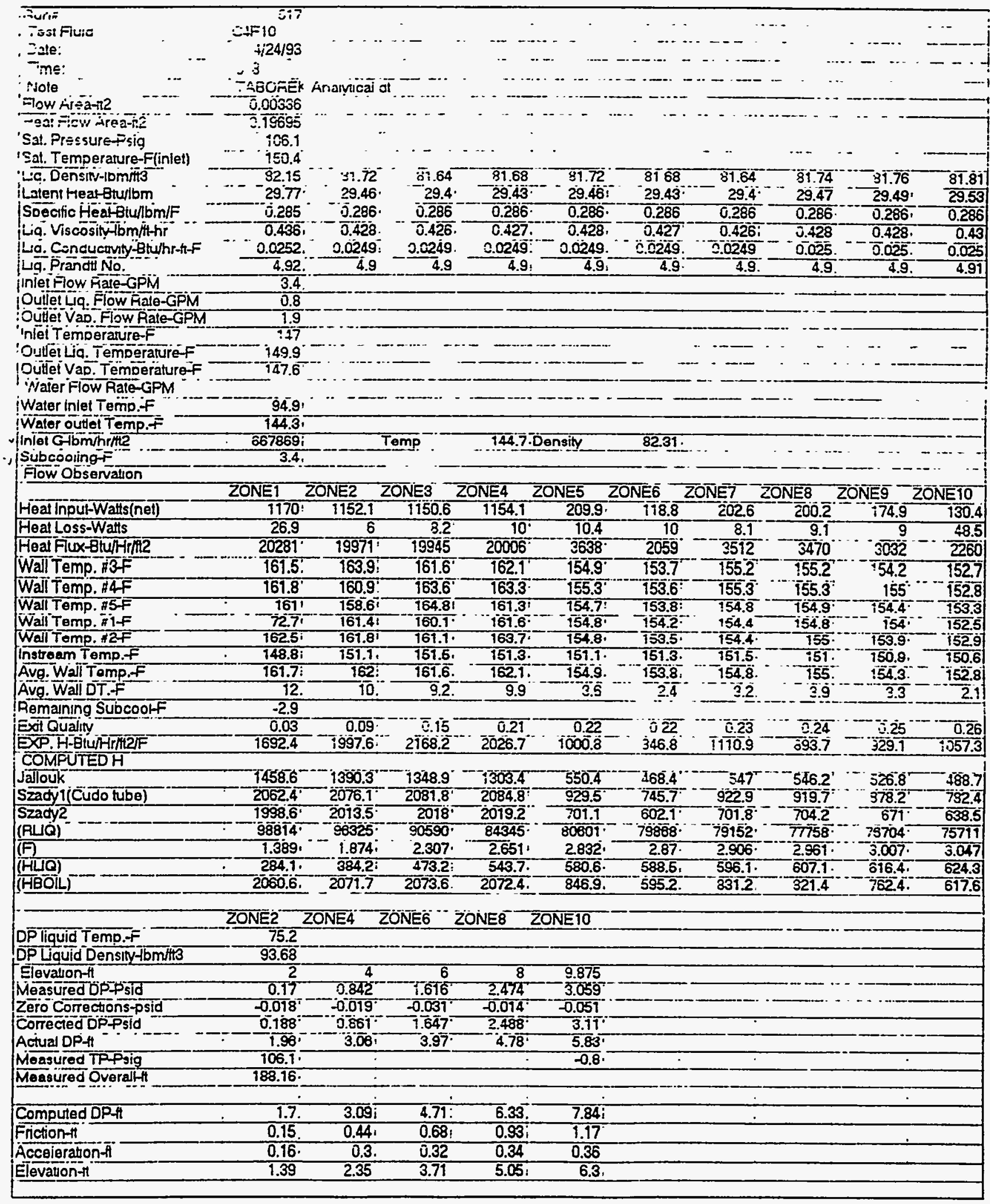




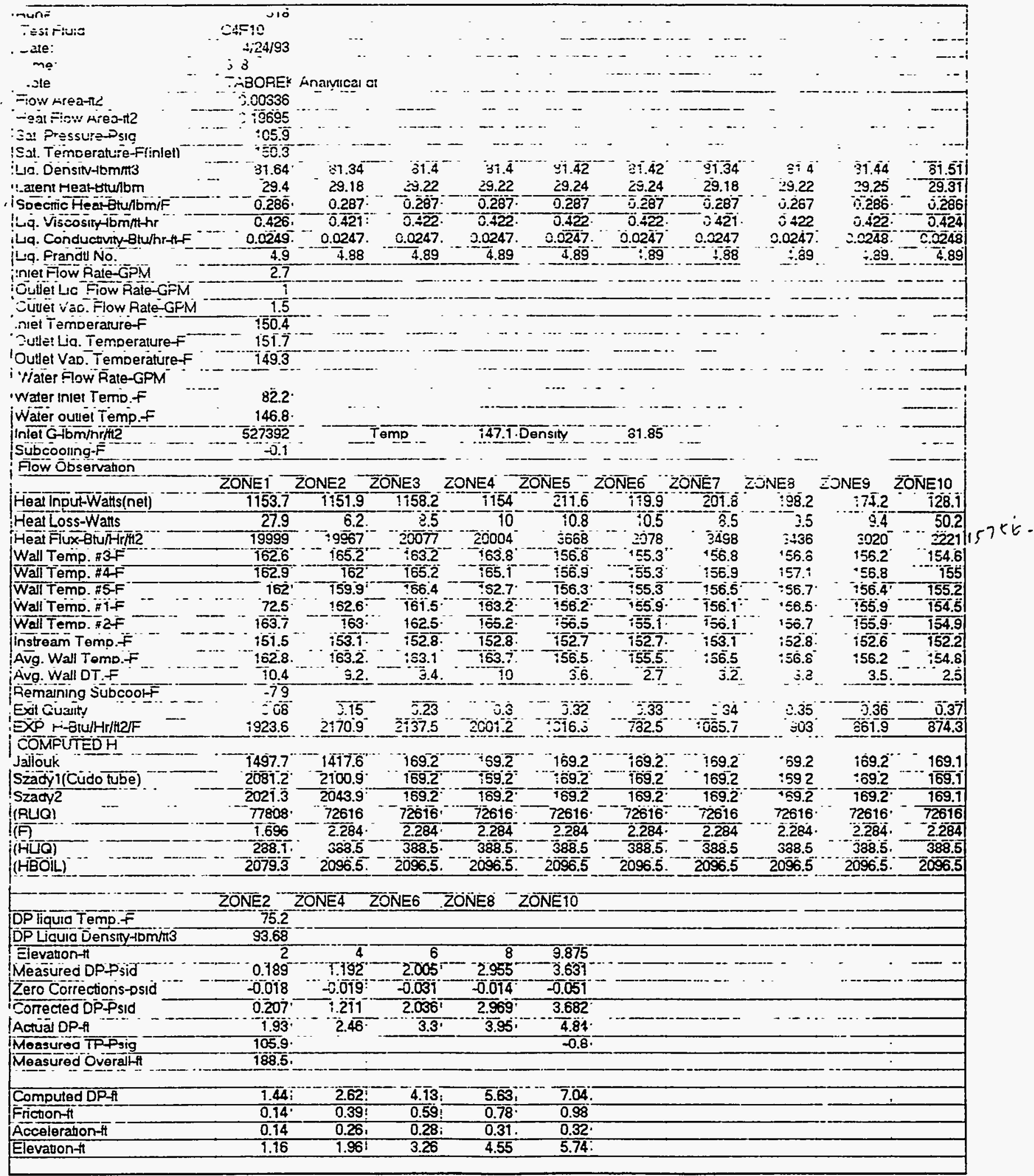




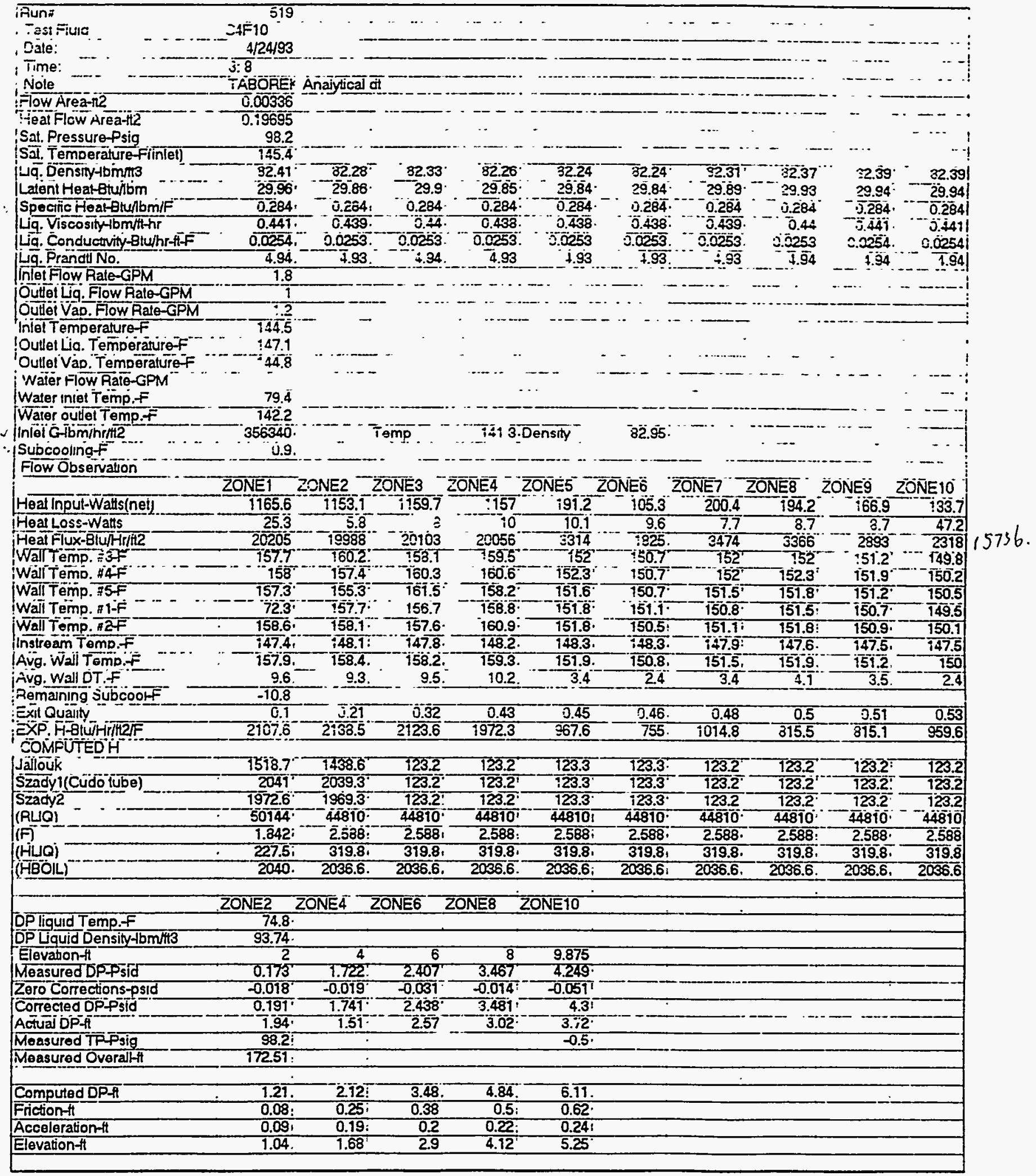




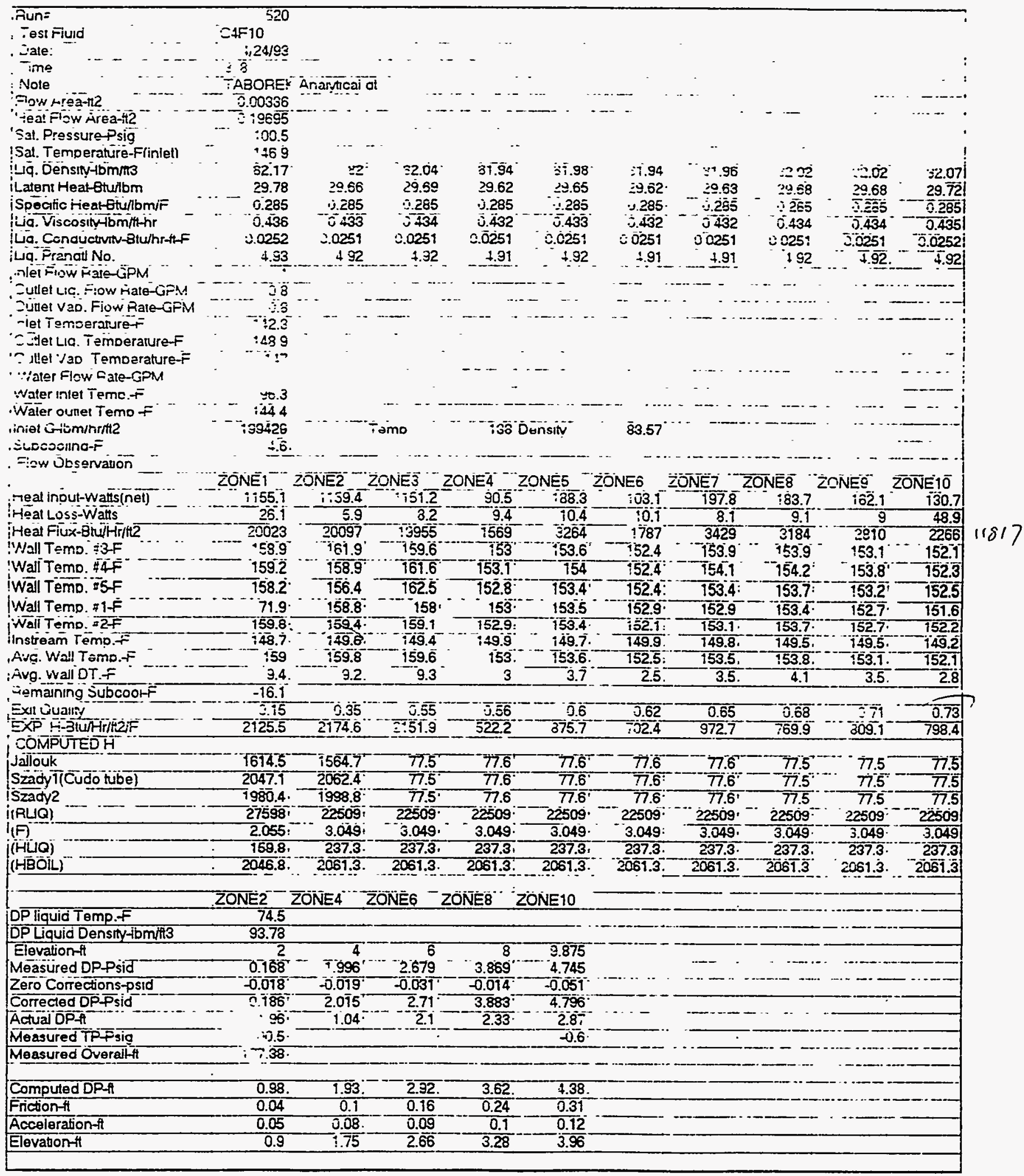




\begin{tabular}{|c|c|c|c|c|c|c|c|c|c|c|c|}
\hline Runt & $5 \quad 521$ & & 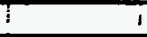 & 1 & & 1 & 7 & 1 & $I$ & 1 & \\
\hline TostFluid & CAF10 & 1 & I & 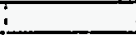 & & $\perp$ & & $i$ & $T$ & $i$ & \\
\hline Date: & $4 \quad 4 / 2493$ & & 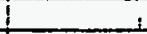 & $i$ & & 1 & & i & I & 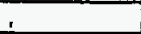 & \\
\hline Time: & $6: 1$ & & T & ; & & & & i & & 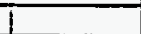 & \\
\hline Nole & Anahyical & dit is used & I & i & & $I$ & & i & I & $\perp$ & \\
\hline Fow A reafte & 0.00336 & & 1 & & & i & & $t$ & L & $i$ & \\
\hline Heat Fow Area-t? & 0.19695 & & 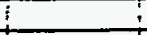 & & & & & $i$ & $i$ & & \\
\hline Sad. Prossuro Psig & 77.4 & & 1 & & & & & $T$ & & & \\
\hline Sat. Temperature $F($ inlel) & 131.7 & & & & & & & 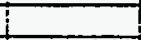 & & & \\
\hline Wa. Density-lbmint3 & 85.53 & 85.36 & 85.431 & 85.4 & 85.4 & 85.4 & 85.41 & 85.41 & 85.47 & 85.5 & \\
\hline Lotent Heat Eturibm & 32.15 & 32.04 & 32.08 & 32.06 & 32.06 & 32.06 & 32.07 & 32.07 & 32.07 & 32.13 & \\
\hline Specifio Heat Btulbm/F & 0.277 & 0.277 & 0.277 & 0.27 & 0.277 & 0.277 & 0.27 & 0.277 & 0.277 & 0.277 & \\
\hline isg. Viscositytbm/ithr & 0.57 & 0.506 & 0.5081 & 0.507 & 0.507 & 0.507 & 0.507 & 0.507 & 0.507 & 0.500 & \\
\hline Lig. Conductivity Bhu/hraftf & 0.0274 & 0.0273 & $0.0273 !$ & 0.0273 & 0.0273 & 0.0273 & 0.02731 & 0.0273 & 0.0273 & 0.0273 & \\
\hline iq. Prandt No. & 5.16 & 5.15 & 5.15 & 5.15 & 5.15 & 5.15 & 5.15 & 5.15 & 5.15 & 5.16 & \\
\hline Inlet Fow Rato-GPM & 0.9 & & & 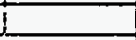 & & & & I & & & \\
\hline Outtel Lig. Flow Rato-GPM & 0.7 & & 1 & 1 & & & & & & & \\
\hline Outtel Vap. Flow Ralo-GPM & 0.4 & & 1 & 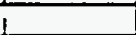 & & & & 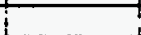 & & & \\
\hline Inlet Temperature of & 121.8 & & & & & & & & & & \\
\hline Outertiq. Temperaturef & 729.7 & & & & & & & I & & & \\
\hline Outlel Vap. Temperaturef & 127.5 & & & & & & & & & & \\
\hline \multicolumn{11}{|l|}{ Water Fow Rate-GPM } & \\
\hline \multicolumn{11}{|l|}{ Water tnlet Tormp.f } & \\
\hline Weder outlot Temp.f & 126.1 & & & 1 & & & & I & & & \\
\hline Intat G-lbm/h/m2 & 1868001 & & Tomp & 118.1 & Donsity & 87.06 & & I & & & \\
\hline Subcooling $f$ & 9.3 & & & 1 & & & & & & & \\
\hline \multicolumn{11}{|l|}{ Flow Observation } & \\
\hline & ZONEI & ZONE2 & ZONE3 I & ZONE4 & ZONE5 & ZONE6 & ZONE7 & ZONE8 & ZONE9 & ZONETO & \\
\hline Hoad input-Watts(nol) & 1154 & 1154 & 1154 & 1154 & 100 & 100 & 100 & 100 & 100 & 100 & \\
\hline HoadLoss-Watts & 202 & 4.8 & 6.6 & 8.1 & 7.2 & 6.6 & 5.2 & 6 & 6.6 & 36.5 & \\
\hline Healflux-BtuHirite & 20004 & 20004 & 20004 & 20004 & 1733 & 173 & 1733 & 1733 & 1733 & 1730 & \\
\hline Wall Temp. \#3F & 140.9 & 143.6 & 141.31 & 942.8 & 73.2 & 134 & 135.4 & 134 & 13 & 134 & \\
\hline Wall Temp. 14F & 141.2 & 140.4 & 143.2 & 144.1 & 133.6 & 134 & 135.3 & 134 & 134.5 & 734.1 & \\
\hline Wall Temp. & 139.8 & 138 & 144.3 & 141.7 & 133.3 & 133.7 & 135 & 133.9 & 134 & T34.1 & \\
\hline WaAt Tomp. "1F & 70.11 & 140.4 & 140 & 141.8 & 133.4 & 133.8 & 135 & 133.5 & 133.6 & 133.1 & \\
\hline Wall Tomp. HCF & 140.7 & 141.2 & 140.9 & 144.2 & 133.4 & 133.4 & 135.3 & 134 & 133.6 & 133.6 & \\
\hline Instroam Tomp.f & 128.4 & 130.4 & 1301 & 130.2 & 13.2 & 130.2 & 130.1 & 130.1 & 130.11 & 129.6 & \\
\hline Avg. Wall Tomp.F & 140.71 & 741.4 & 141.4 & 142.6 & 133.4 & 133.9 & 135.2 & 133.9 & 133.9 & 133.8 & \\
\hline Avg. Wall DT, F & $10.3 \mid$ & 10.1 & 10.41 & 11.5 & 3.11 & 3.6 & 5 & 3.7 & 3.7 & 4.1 & \\
\hline Remaining Subcoolf & -13.41 & & & & & & & & & & \\
\hline Ext Quality & 0.12 & 0.37 & 0.51 & 0.7 & 0.72 & 0.74 & 0.75 & 0.7 & 0.79 & 0.8 & \\
\hline EXP.HEHUHRRZF & 1933.5 & 1981.4 & 1915 & 1736.3 & 550.9 & 478.6 & 345.2 & 468.3 & 463.3 & 422.6 & \\
\hline \multicolumn{11}{|l|}{ COMPUTEDH } & \\
\hline Jallouk & 1454.4 & 7385.2 & 1333.1 & 72.4 & 72.4 & 72.4 & 72.4 & 72.4 & 72.4 & 72.4 & \\
\hline Szady 1(Cudo tube) & 1824.1 & 1835.4 & 1831.8 & 72.4 & 72.4 & 72.4 & 72.4 & 72.4 & 724 & 724 & \\
\hline Szadple & 1723.9 & 1705.7 & 1702.1 & 724 & 724 & 72.4 & 72.4 & 724 & 72.4 & 724 & \\
\hline (RLO) & 22594 & 19024 & 14257 & 14257 & 14257 & 14257 & 14257 & 14257 & 14257 & 14257 & \\
\hline (F) & 2.0231 & 3.149 & 4.122 & 4.122 & 4.122 & 4.122 & 4.122 & 4.122 & 4.122 & 4.122 & \\
\hline (HUAO) & 145.7 & 227.1 & 297.1 & 297.1 & 297.1 & 287.1 & 207.11 & 297.1 & 297.1 & 297.1 & \\
\hline \multirow[t]{3}{*}{ (HBOIL) } & 1823.8 & 1834.3 & 1829.2 & 1822.2 & 1822.2 & 18202 & 182921 & 1829.2 & 18292 & 1829.2 & \\
\hline & & & & & & & & & & & \\
\hline & ZONE2 & ZONE4 & ZONE6 & ZONE8 & ZONE10 & & & & & & \\
\hline DPliquid Temp.f & 73.6 & & & & & & & & & & \\
\hline DPLiquid Donsity /bm/it3 & 93.91 & & & & & & & & & & \\
\hline Elevation-f & 2 & 4 & 6 & 8 & 9.875 & & & & & & \\
\hline Measured DPPsid & 0.228 & 2.126 & 2.802 & 4.021 & 4.341 & & & & & & \\
\hline Zero Contections-psid & -0.018 & -0.018 & -0.031 & -0.014 & -0.051 & & & & & & \\
\hline Corrected DPsald & 0.246 & 2.145 & 2.853 & 4.035 & 4.992 & & & & & & \\
\hline Actual DPA & 1.78 & 0.78 & 1.82 & 1.99 & 2.44 & & & & & & \\
\hline Mensured TPPsig & 77.4 & & & & 0.6 & & & & & & \\
\hline \multirow{2}{*}{\multicolumn{11}{|c|}{ Meseured Overnith }} & \\
\hline & & & & & & & & & & & \\
\hline Computed DPA & $\frac{0.55}{0.04}$ & 1.52 & 271 & 3.89 & 4.99 & & & & & & \\
\hline Friction & 0.04 & 0.13 & 0.181 & 0.24 & 0.3 & & & & & & \\
\hline Acouleration-ft & 0.04 & 0.12 & 0.13 & 0.14 & 0.15 & & & & & & \\
\hline Elevation-tt & 0.87 & 1.27 & 2.39 & 3.5 & 4.53 & & & & & & \\
\hline & & & & & & & & & & & \\
\hline
\end{tabular}




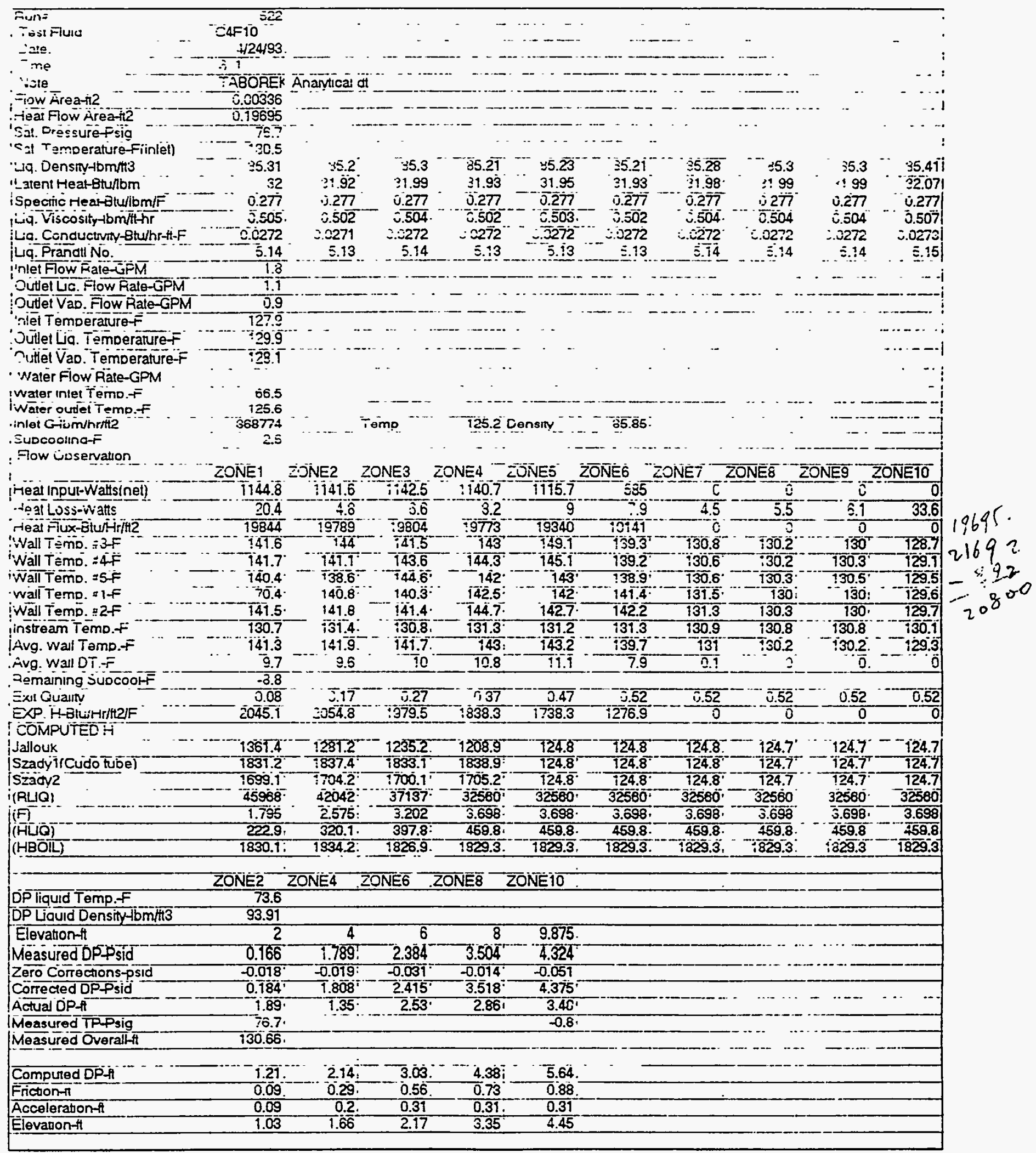




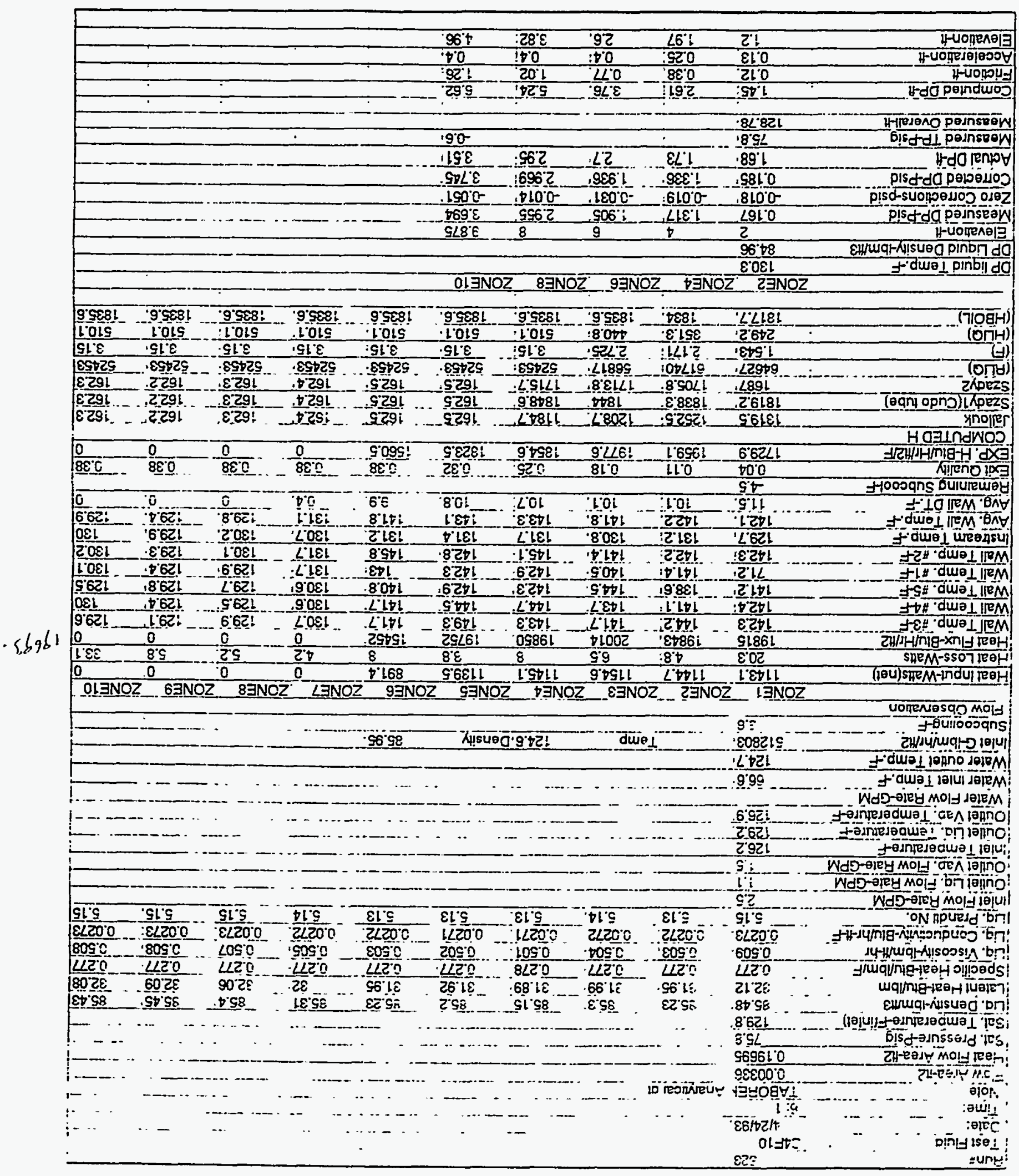




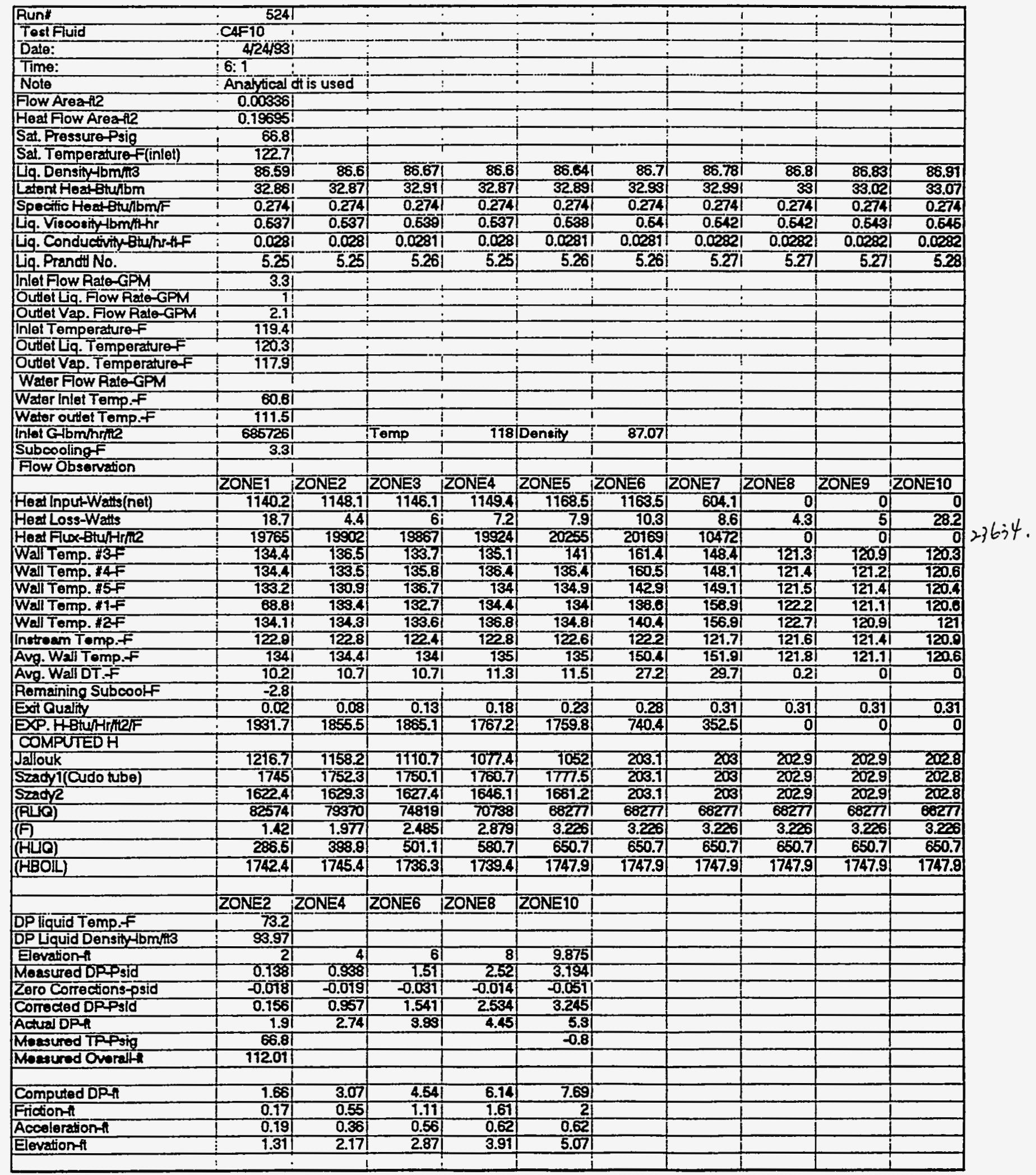




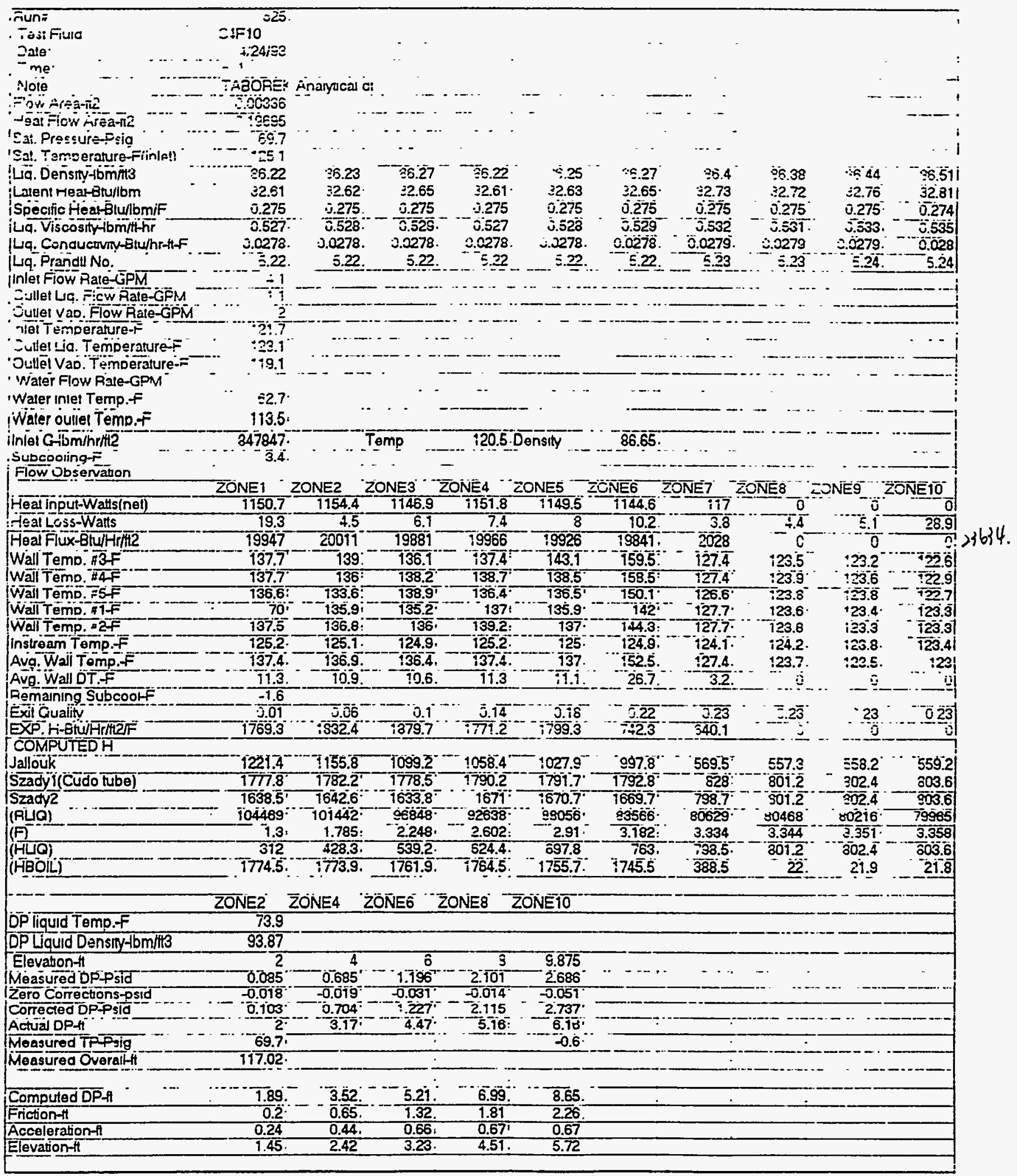




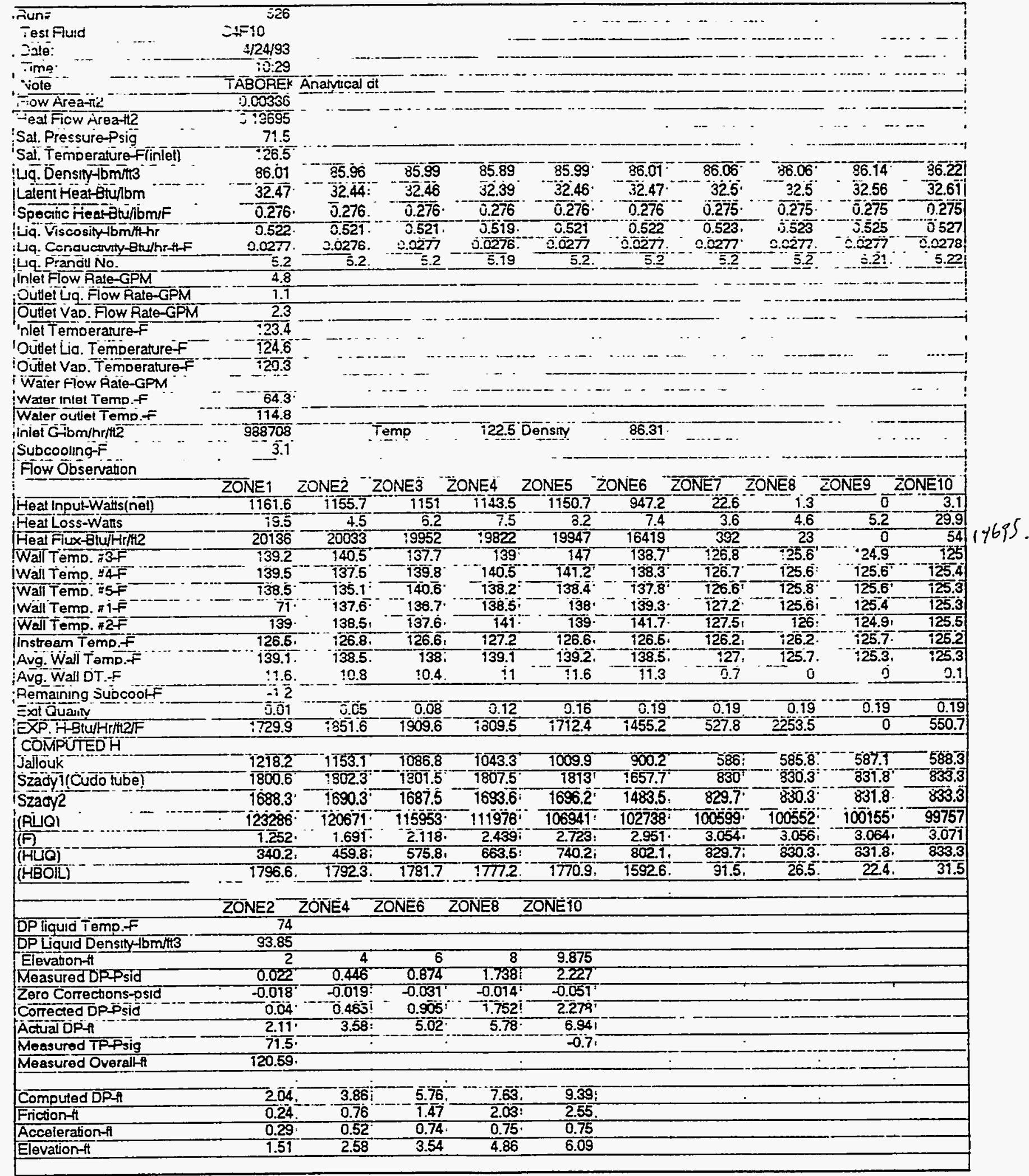




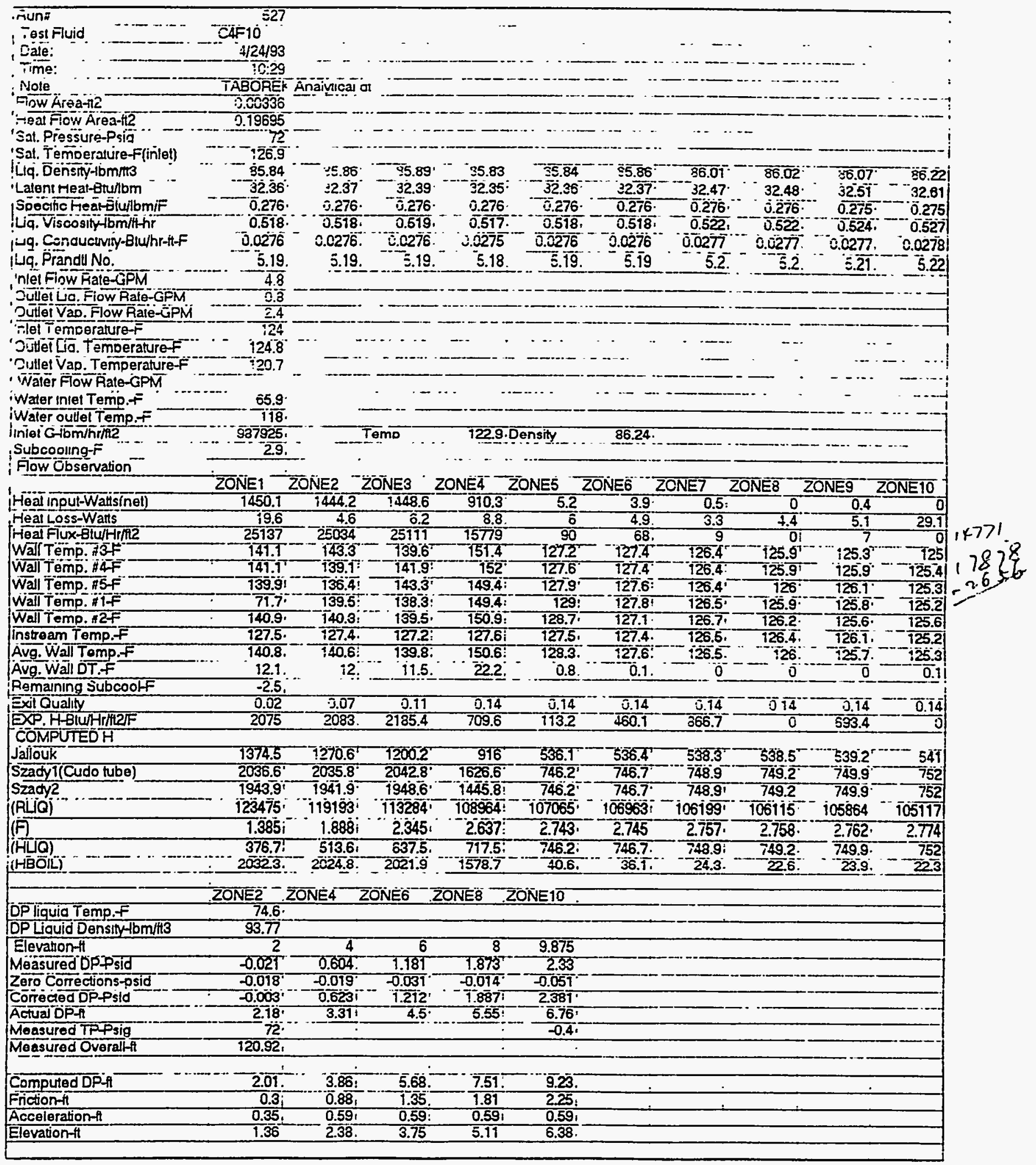




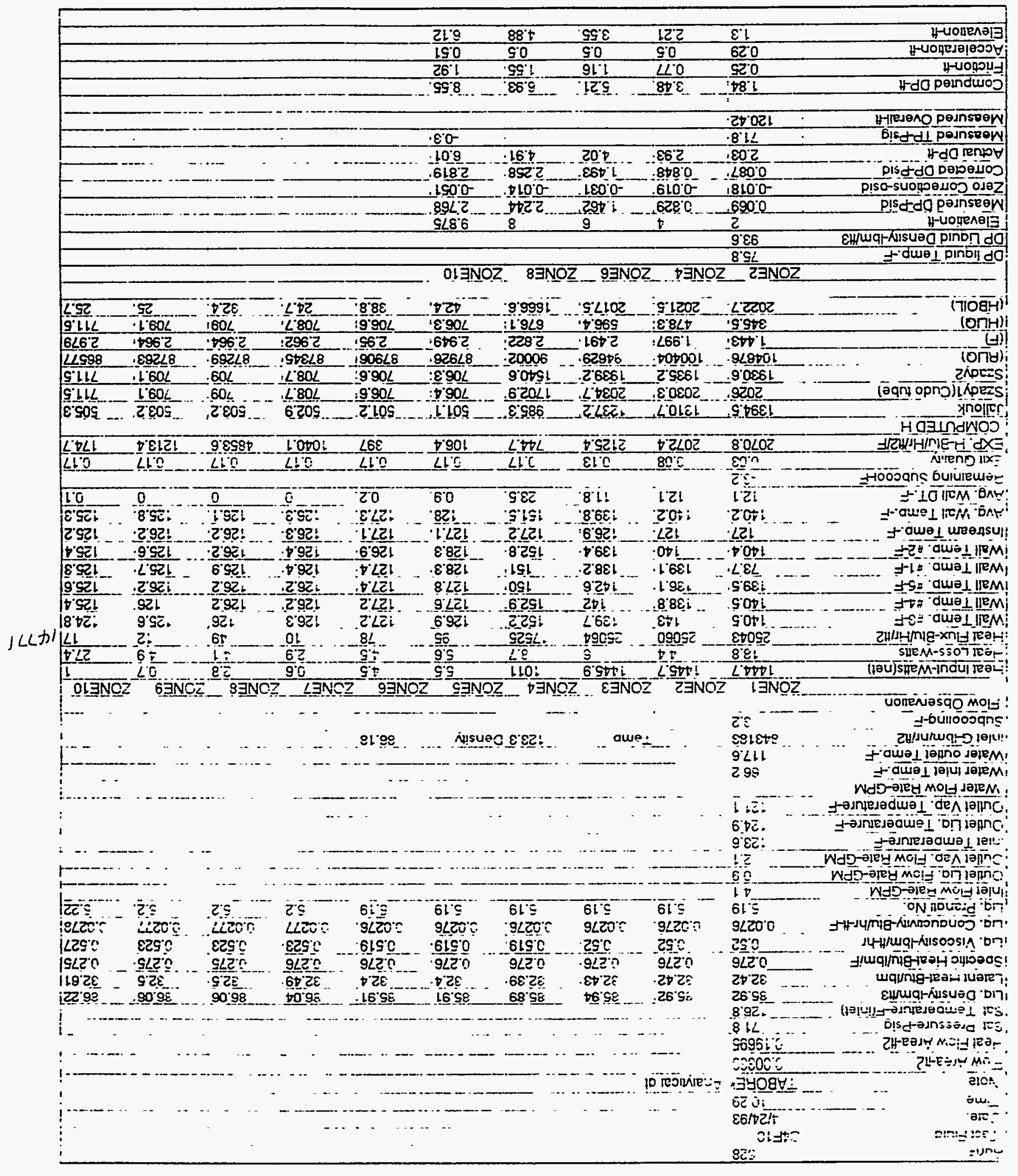




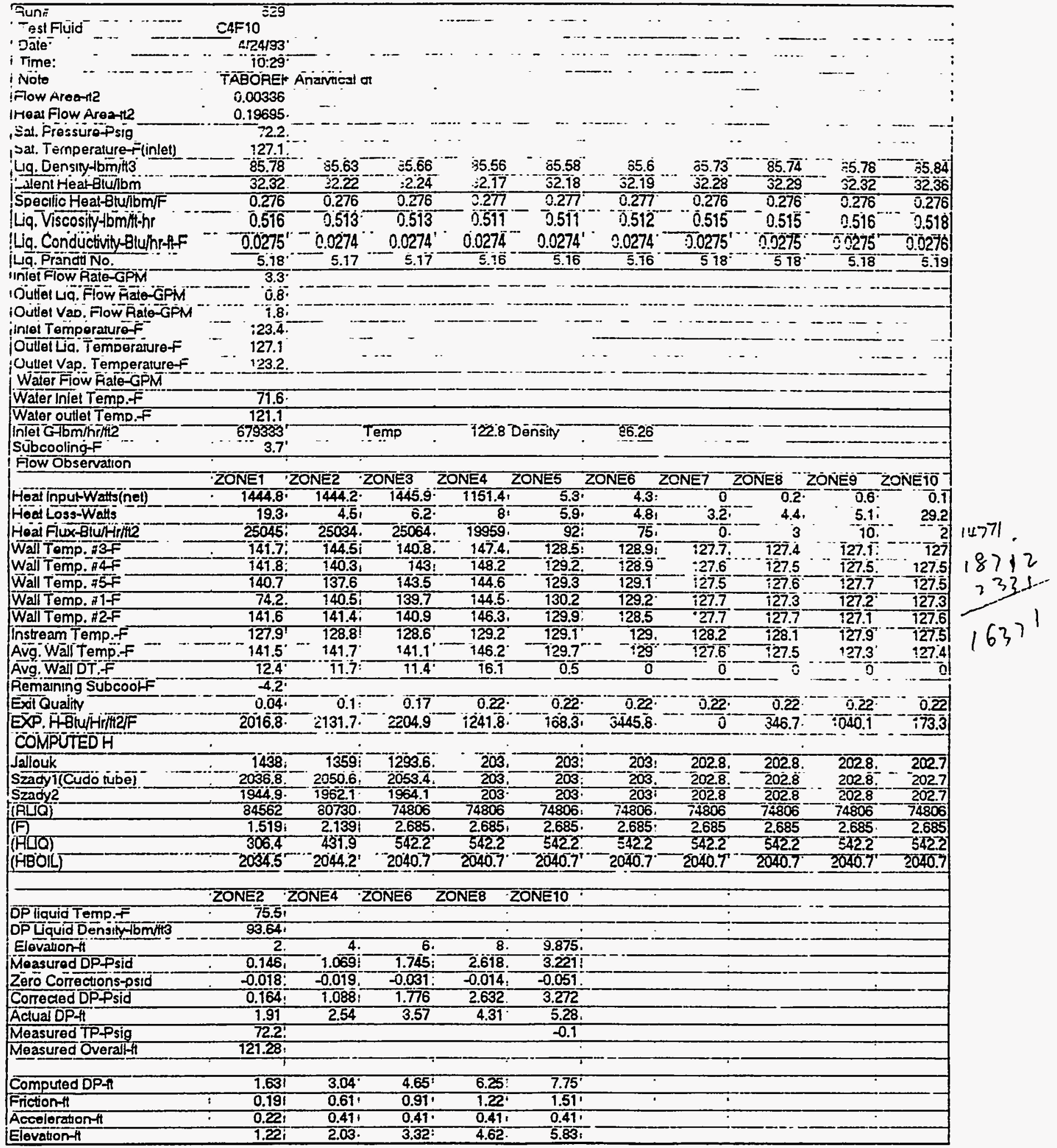




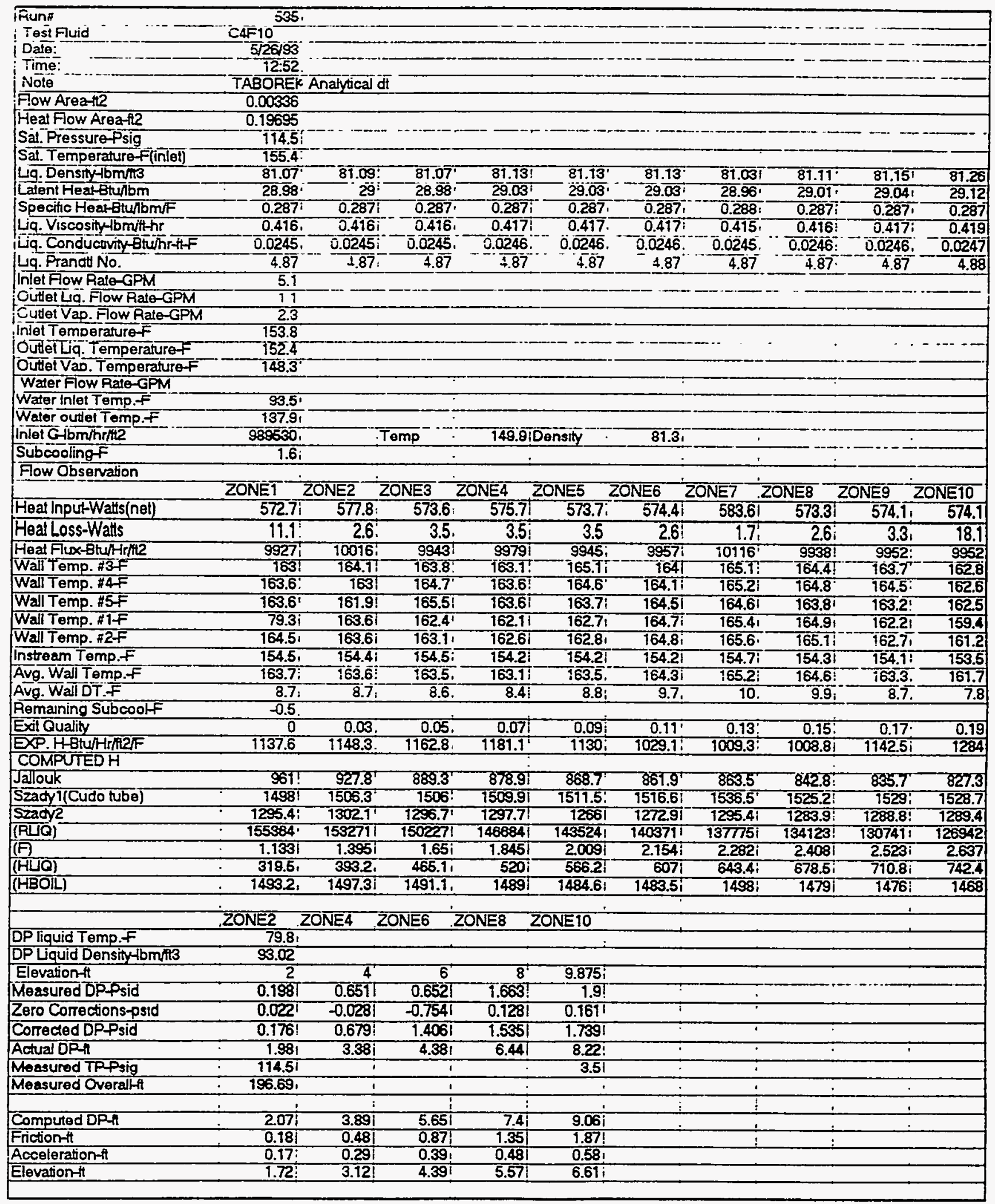




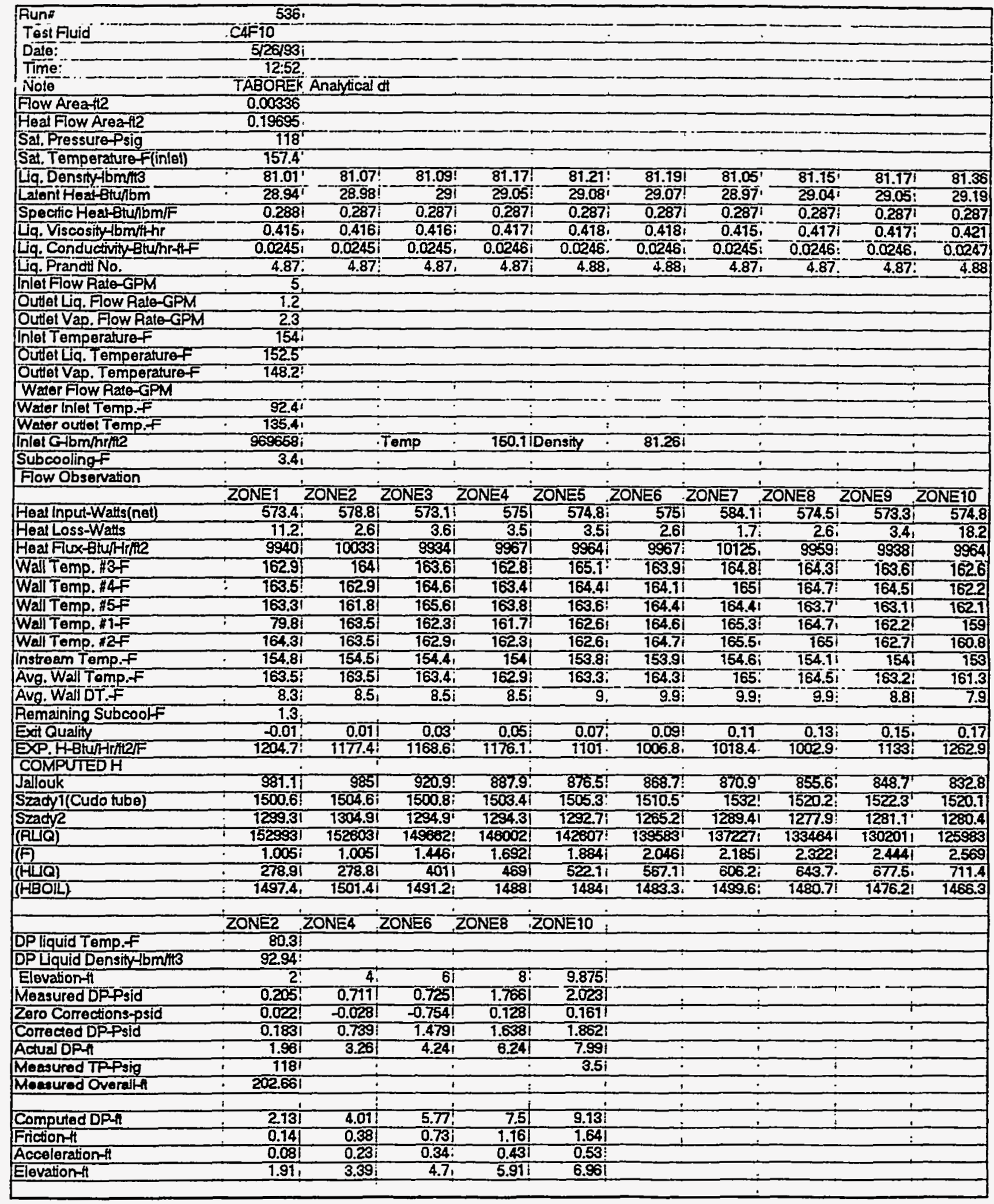




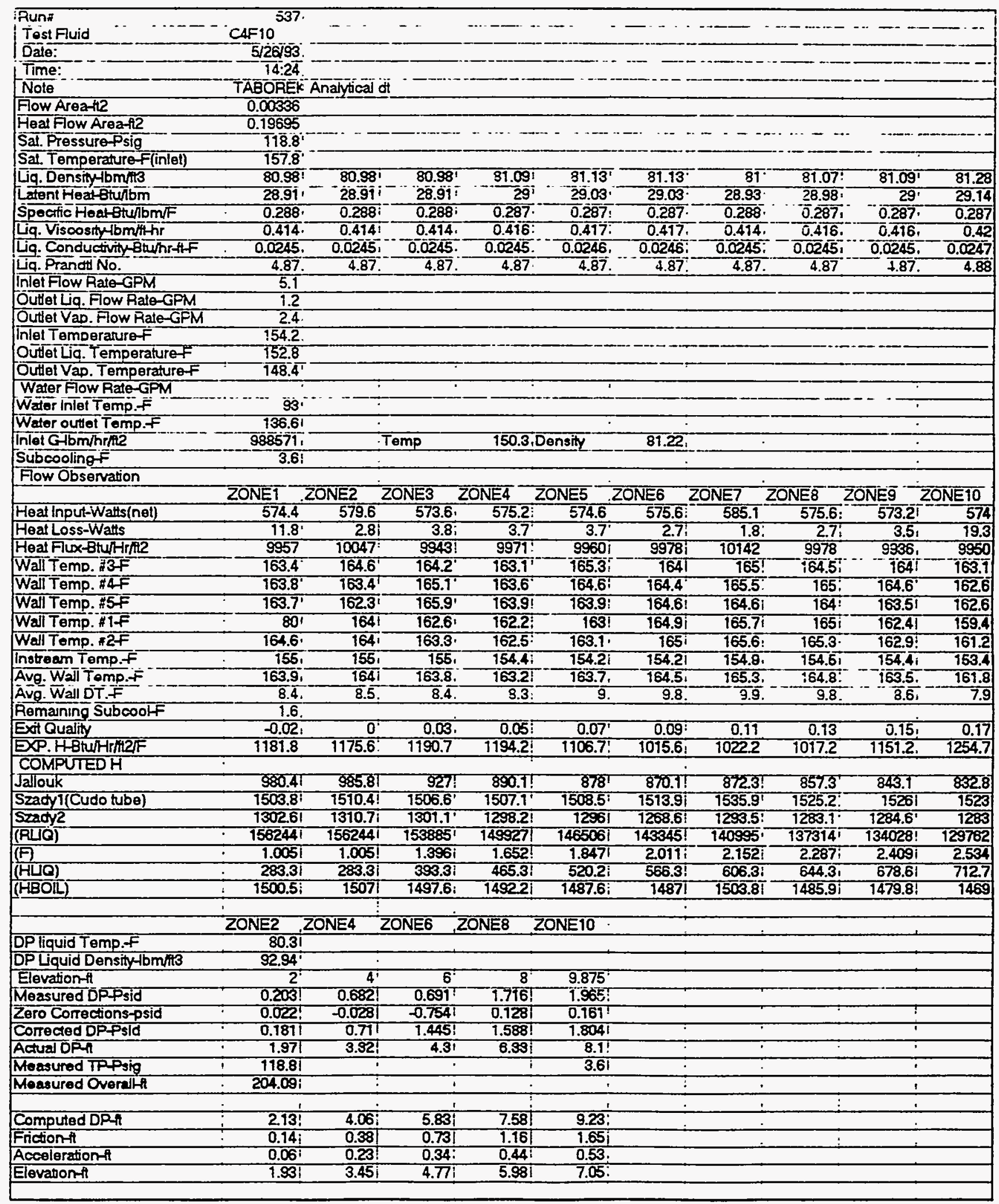




\begin{tabular}{|c|c|c|c|c|c|c|c|c|c|c|}
\hline Run\# & 538. & & & & & & . & & & \\
\hline Tost Fiuid & CAF 10 . & & & & & & & & & . \\
\hline Dale: & $5 \sqrt{2693}$ & & & & & & & & & \\
\hline Time: & $14: 24$ & & & & & & & & & \\
\hline Nolo & TABOREK & Anahytical & & & & & & & & \\
\hline Flow Area-t2 & 0.00336 & & & & & & & & & \\
\hline Heat Flow Areatil & 0.19695 & & & & & & & & & \\
\hline Sal. PressurePsig & $117.7^{r}$ & & & & & & & & & \\
\hline sal. Temperature-f(inlel) & 157.2! & & & & & & & & & \\
\hline Lq. Density-lompli3 & 80.841 & 80.88 & $80.88 !$ & $80.98 !$ & 811 & 80.961 & $80.84^{\prime}$ & 80.941 & $80.98 !$ & 81.15 \\
\hline Latent Heat-tuibm & 28.811 & 28.84 & 28.841 & $28.91 i$ & 28.93 & $28.9 !$ & 28.81 & $28.88 !$ & 28.91 & 29.04 \\
\hline Spocific Heatetu/bm/F & 0.2881 & 0.288 & $0.288 i$ & $0.288 !$ & 0.288 & 0.2881 & 0.2881 & 0.2881 & $0.288 i$ & 0.287 \\
\hline Lig. Visoositylbm/tithr & 0.4111 & 0.412 & 0.4121 & 0.4141 & 0.4141 & 0.4141 & $0.411 i$ & $0.413 !$ & 0.414. & 0.417 \\
\hline iq. Conductivitutuhr al & 0.02441 & 0.0244 & 0.0244 & $0.0245 i$ & 0.0245 & 0.0245 & 0.0244 & 0.0244 & 0.0245 & 0.0246 \\
\hline Uq. Prandt No. & 4.861 & 4.86 & 4.86 & 4.87. & 4.87 & $4.86:$ & 4.86 & $4.86:$ & 4.87 & 4.87 \\
\hline Inlelflow Rate-GPM & 5.1 & & & & & & & & & \\
\hline Outlel Lig. Flow Ralo-GPM & 1.2 & & & & & & & & & \\
\hline Outlel Vap. Flow Ralo-GPM & 2.4 & & & & & & & & & \\
\hline Inlel Temperaturef & 154.91 & & & & & & & & & \\
\hline Outlel Liq. Temperaturef & $153.3 !$ & & & & & & & & & \\
\hline Outtel Vap. Temperaturef & 149! & & & & & & & & & \\
\hline Water Flow Rato-GPM & & & & & $T$ & t! & & & & T \\
\hline Wator Inlet Temp.f & $94.2 !$ & & 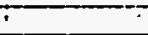 & $\because$ & $T$ & $\because$ & $T$ & & & $\div$ \\
\hline Wador outtol Temp.f & 137.4 & & & : & & $\because$ & $\div$ & & 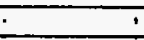 & $\div$ \\
\hline Inlet Glbm/hrit2 & $987126 \mathrm{i}$ & & Temp & 150.911 & Density & $81.17:$ & F & ? & 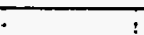 & $\overline{:}$ \\
\hline Subcooling $F$ & 2.31 & & & & & ? & 1 & & & 7 \\
\hline Flow Observation & & & & & & & & & & \\
\hline & ZONE1 & ZONE2 & ZONE3 & ZONE4 & ZONE5 & ZONE6 & ZONE7 2 & ZONE8 : & .ZONE9 & ZONE10 \\
\hline Heat Input-Watts(net) & 573.3 & 579.2 & 573.6 & $575.6 i$ & $576.8 i$ & $577.5 i$ & 5851 & 574.81 & 574.31 & 572.9 \\
\hline Heat Loss-Watts & $12.2 !$ & 2.9 & $3.9 \mathrm{i}$ & 3.81 & $3.8 \mathrm{i}$ & 2.81 & 1.91 & 2.91 & 3.7 & 20.1 \\
\hline Heat Flux-Bturirhite & 9938 & 10040 & 99431 & 99781 & 99981 & T00111 & 101411 & 99641 & 9955 & 9931 \\
\hline Wallemp. & 163.81 & 165.17 & 164.7 & $163.9 !$ & 165.1 & 164.91 & 165.8 & 165.21 & $764.5 !$ & 103.3 \\
\hline Wall Temp. H4F & 164.41 & 163.91 & 165.6 & 164.5! & 165.51 & 164.9 & $166 !$ & 165.41 & 165.41 & 163.2 \\
\hline Wall Temp. & 164.31 & 762.81 & 165.41 & 164.8! & 164.41 & $165.5 !$ & 165.2 & 164.71 & 163.81 & 163.3 \\
\hline Wall Temp. \#1F & $80.4 !$ & 104.5 & $163.2 !$ & 162.7 & 169.41 & 165.41 & $168.3 i$ & 185.71 & $183.1 !$ & 180.3 \\
\hline Wall Tomp. REF & $165.3 i$ & 164.51 & 163.91 & $163.2 i$ & $163.5 i$ & 165.4 & 166.41 & 1661 & 163.61 & 161.8 \\
\hline instream Temp.F & $155.7 i$ & 155.61 & 155.51 & 1551 & $154.9 i$ & 155.11 & 155.71 & $155.2 i$ & 1561 & 164.1 \\
\hline Avg. Wall Tomp.f & 164.5: & 164.51 & $164.4 i$ & 163.9 & 164.21 & 165.2 & $165.9 !$ & $165.4 i$ & 164.1 & 1624 \\
\hline Avg.Wall DT.F & $8.3 !$ & $8.5 !$ & 8.41 & 8.51 & 8.81 & $9.6 i$ & 9.81 & 9.7 & $8.6 !$ & 7.8 \\
\hline Romaining Subcoolf & $0.3 !$ & & & & & & & & & \\
\hline Ext Quality & of & 0.02 & 0.04 & 0.061 & 0.08 & 0.11 & 0.12 & $0.14 i$ & $0.16 i$ & 0.18 \\
\hline EXP. HBtuHARt2F & 1197.2! & 1174.8 & $1183.6 i$ & 1977.4 & 1130 & $1040.3 !$ & $1036.7 i$ & 1022 & $1153.5 i$ & 1268.1 \\
\hline COMPUTEDH & $\vdots$ & & & $i$ & & 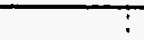 & & $i$ & . & \\
\hline Jallouk & 383.5 & 951.31 & 912 & 8861 & $877.1 !$ & 870.5 & 871.1 : & 855.81 & 841.8 & 830.9 \\
\hline Szadyl(Cudo tube) & $1509 !$ & $7516.8 !$ & $1513.8 !$ & 1515.6 & $1520.2 !$ & 1527 & 1545.8 & 1533.31 & $1535.5 !$ & 1530.8 \\
\hline szadyz & $1307.3 !$ & 1314.41 & 1305.71 & 1304.61 & 1305.31 & 1282.91 & 1304.21 & $1291.6 !$ & 1294.81 & 1290.8 \\
\hline (PШQ) & $156951 !$ & 1554791 & 1522555 & 1484081 & 14508001 & 142122 & 1396051 & 1357781 & 1323491 & 128176 \\
\hline (F) & 1.0051 & 1.266 & 1.5671 & $1.779 !$ & 1.9531 & 2.102 & 2.2341 & 23531 & 2.4811 & 2.6 \\
\hline (HบO) & 283.11 & 356.5 & 441.41 & 500.81 & 549.71 & 591.91 & 629.21 & $665.4 i$ & 698.31 & 731 \\
\hline (HBOIL) & 1505.61 & 1510.2 & 1501.21 & 1497.2 & $1495.9 i$ & 1496.81 & $1510.2 !$ & 1490.41 & 1485.81 & 1473.1 \\
\hline & 1 & & & 4 & & 1 & 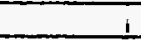 & 1 & 1 & \\
\hline & ZONE2 & ZONE4 & ZONE6 & ZONE8 & ZONE10 & & & & & \\
\hline DP liquid Temp.F & 80.8 & & & & & & & & & \\
\hline DPLiquid Density-lbm/it3 & 92.87 & & & & & T. & & & & \\
\hline Elevation-f & & $4 !$ & & 81 & $9.875 i$ & & & & & \\
\hline MeasuredDPFsid & $0.202 !$ & 0.6771 & 0.6851 & $1.709 !$ & $7.55 \pi$ & & & & & \\
\hline Zero Corrections-psid & 0.0221 & -0.028 & -0.7541 & 0.1281 & 0.161 & $T$ & Tt & T & $T$ & \\
\hline Corrected DPPsid & 0.181 & 0.705 & 1.4391 & 1.5871 & $7.796 !$ & $\bar{i}$ & & & & t \\
\hline Actual DPA & 1.97 & 3.38 & $4.31 !$ & 6.351 & 8.111 & $i$ & $\overline{!}$ & & $\because$ & \\
\hline Moasured TPPsig & $117.7 !$ & 1 & $T$ & i & 3.61 & $T$ & T! & ! & T & $T$ \\
\hline Measured Overallft & 202471 & : & 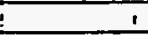 & 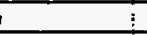 & 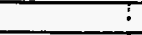 & $i$ & + & 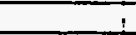 & $\bar{i}$ & $\bar{i}$ \\
\hline & I & $i$ & & 1 & & $i$ & $i$ & 1 & $\bar{i}$ & $i$ \\
\hline Computad DP- & 215 & 41 & 5.761 & 7.511 & 9.16 & & 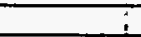 & & & \\
\hline Friction-tt & 0.15 & 0.43 & 0.81 & 1.27 & 1.77 & & $:$ & & 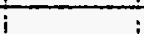 & \\
\hline Accoleration-t & 0.14 & 0.27 & 0.371 & 0.461 & $0.566_{1}$ & & : & & & $\frac{1}{i t}$ \\
\hline Elevation-t & $1.86 \mathrm{i}$ & 3.31 & 4.591 & 5.781 & $6.83 i$ & 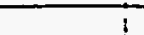 & & & ! & \\
\hline & & & & & &. & & & & $!$ \\
\hline
\end{tabular}




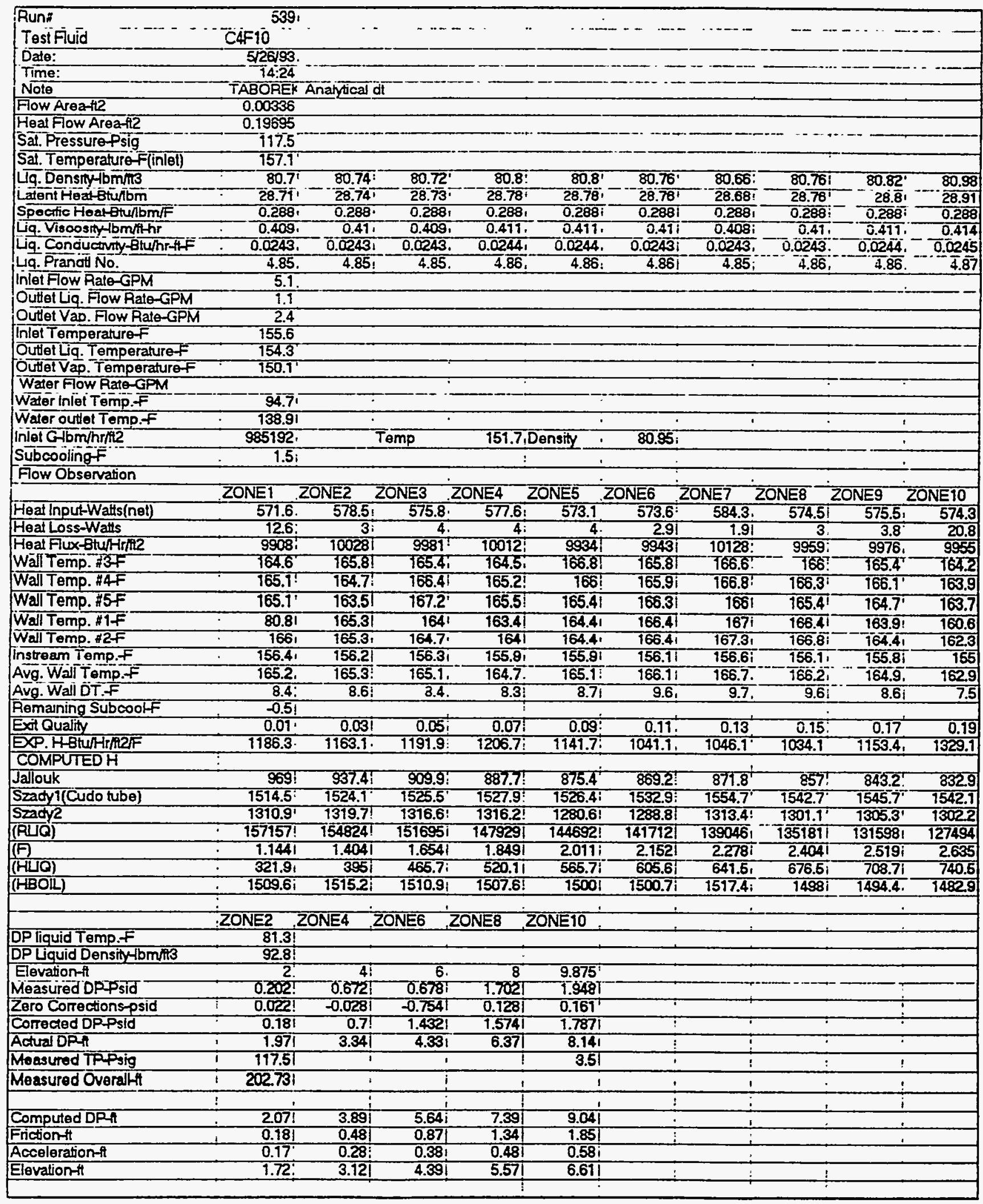




\begin{tabular}{|c|c|c|c|c|c|c|c|c|c|c|}
\hline Run" & 5401 & & & 1 & i & & & 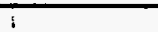 & & \\
\hline Test Fuid & प्F=TO & I & & & & 1 & & $i$ & & T \\
\hline Date: & $5 \sqrt{261931}$ & $i$ & & $i$ & $T$ & T & ! & $T$ & & $T$ \\
\hline Time: & $14: 241$ & 2 & & 1 & i & $i$ & i & $T$ & & \\
\hline Noto & I TABOREK & Anahytical c & $d t$ & 1 & t & f & & 1 & i & I \\
\hline Flow Areant? & 0.00336 & $i$ & 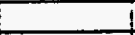 & 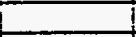 & $i$ & 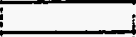 & & 1 & & i \\
\hline Heal Flow Aroat 2 & 0.18605 & & & & & & & $i$ & & + \\
\hline S2!. Ptessure Psig & 143.9 & & & & & & I & $i$ & & \\
\hline Sat. Temperaturo f (inlet) & 171.1 & & & & & & & & & \\
\hline Lig. Density -lbmitis & 78.75 & 78.67 & 78.35 & 78.11 & 78 & 77.94 & 77.72 & 77.8 & 77.83 & 78.11 \\
\hline Latent Hoat-Btulbm & 27.25 & 27.19 & 26.95 & 26.76 & 26.68 & 26.63 & 26.46 & 26.53 & 26.55 & 26.76 \\
\hline Specific Heat Btulbmif & 0.294 & 0.294 & 0.295 & 0.205 & 0.296 & 0.296 & 0.296 & 0.296 & 0.296 & 0.205 \\
\hline Lig. Viscosity bmithr & 0.376 & 0.3741 & 0.369 & 0.356 & 0.364 & 0.363 & 0.36 & 0.3511 & 0.361 & 0.366 \\
\hline Uta. Conductivity Btuhr-fiF & 0.02311 & 0.023 & 0.0238 & 0.0227 & 0.02261 & 0.02261 & 0.0224 & 0.00251 & 0.0225 & 0.0227 \\
\hline Lq. Prandd No. & 4.781 & 4.78 & 4.77 & 4.76 & 4.76 & 4.76 & 4.75 & 4.761 & 4.76 & 4.76 \\
\hline Inlet Fow Rate-GPM & 5.3 & & 1 & 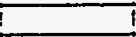 & 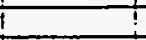 & & & 1 & & \\
\hline Outlot Lig. Flow Rate-GPM & 0.8 & 7 & 1 & 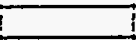 & + & 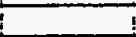 & $!$ & $T$ & & \\
\hline Outlel Vap. Flow Redo-GPM & 2.91 & & & & $\mathrm{i}$ & $i$ & & 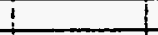 & & 1 \\
\hline Inle! Temperaturef & 125.1 & & & & I & & & + & & \\
\hline Outtat Liq. Tomperaturef & 168.6 & & & & I & & & & & \\
\hline Outlot Vap. Temperaturef & 165.8 & & & 1 & 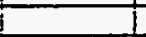 & & & & & \\
\hline Wader Flow Rade-GPM & & & & 4 & 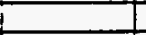 & & & 1 & & \\
\hline Water Inlet Temp. f & 113.4 & & & & & & & & & \\
\hline Wader outet Temp.f & 160.9 & & & & & & & & & \\
\hline |nlat Glominini2 & \$91275| & & Tomp & 764.7 & Density & 78.37 & & & & \\
\hline Subcoolingt & को & & & & & & & & & \\
\hline Flow Observation & & & & & & & & & & \\
\hline & ZONE1 & ZONE2 I & ZONE3 & ZONE4 & ZONES & ZONEO & ZONET & ZONE8 & ZONE9 & ZONETO \\
\hline Hoal Input-Watts(nel) & 568.1 & 578.7 & 605.6 & 603.7 & 620.7 & 618.8 & 581.5 & 5728 & 600.4 & 608.6 \\
\hline Hoad Loss-Watts & 16.6 & 3.7 & 5 & 6.4 & 5.7 & 4.8 & 3.6 & 4.7 & 5.2 & 28.3 \\
\hline 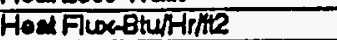 & 8948 & 9997 & 10498 & 10465 & 10769 & 10723 & 10080 & 8966 & 10564 & 10515 \\
\hline Wall Tamp. $3 F$ & 177.7 & 178.7 & 178.9 & 97.7 & 180.1 & 179.2 & 180.1 & 179.4 & 179 & 177.7 \\
\hline Wal Temp. $44 F$ & 178.2 & 177.8 & 179.7 & 178.4 & 179.6 & 179.3 & 180.3 & 179.8 & 179.9 & 177.4 \\
\hline Wall Tomp. " 5 F & 178.2 & 176.9 & 180.5 & 178.5 & 178.7 & 179.7 & 179.6 & 178.8 & 178.6 & 177.3 \\
\hline Wall Temp. $1 F$ & 81.4 & 178.6 & 177.5 & 176.7 & 177.8 & 179.9 & 180.4 & 179.8 & 17.7 & 174.3 \\
\hline Wall Temp, \#2F & 179 & 178.7 & 178.1 & 177.2 & 177.91 & 180 & 180.7 & 180.2 & 178.2 & 175.9 \\
\hline Instroam Temp.F & 166 & 166.4 & 167.9 & 169 & TE.5 & 769.8 & 770.8 & $\{70.4$ & 770.3 & 169 \\
\hline Avg. Wall Temp.t & $\mid 78.3$ & T78.5 & 778.6 & 177.8 & T78.5 & 179.5 & 780.2 & 179.81 & 78.7 & 176.5 \\
\hline Avo. Wall DT, $F$ & 11.8 & 11.6 & 10.2 & 8.4 & 8.5 & 9.2 & 9 & 8.81 & 7.9 & 7 \\
\hline Romalning Subcooff & 4 & & & & & & & & & \\
\hline Ext Quality & -0.04 & -0.02 & 0 & 0.02 & 0.05 & 0.07 & 0.09 & 0.12 & 0.14 & 0.16 \\
\hline EXP.HABUH Hrit2/F & 832.4 & 861.8 & 1031.7 & 1253 & 1263.8 & 1160.5 & 1124.4 & 11342 & 1336.7 & 14926 \\
\hline COMPUTEDH & & & & & & & & & & \\
\hline Jallouk & 1020.3 & 1031.8 & 1071.8 & 1043.3 & 1026.6 & 10025 & 948.7 & 920.21 & 949.4 & 931.8 \\
\hline Szady1(Cudo tube) & 16031 & 1618.5 & 1672.5 & 1684.2 & 1715.2 & 1718.8 & 1683.9 & 1671.2 & 1721.6 & 1708.1 \\
\hline Szady2 & 1395.1 & 1413.5 & 1477.4 & 1445.1 & 1480.7 & 1485.5 & 1445.8 & 1431.6 & 1489.4 & 1474.8 \\
\hline (R山Q) & 172525 & 173150 & 175526 & 175052 & 171699 & 167937 & 165337 & 160723 & 156473 & 150444 \\
\hline (F) & 1.005 & 1.005 & 9.005 & 1.325 & 1.592 & 1.787 & 1.938 & 2.0751 & 2.199 & 2.320 \\
\hline (FIDO) & 287.11 & 287.2 & 287.6 & 379.8 & 456.31 & 5725 & 555.3 & 595.31 & 630.9 & 867.5 \\
\hline (HIBOIL) & 7599.9 & 7615.5 & 1669.6 & 1677.8 & 7704.3 & 1703.5 & 1653.4 & 1845.6 & T692.9 & 1673.4 \\
\hline & & & & & & & & & & \\
\hline & ZONE2 & ZONE4 & ZONE6 & ZONE8 & ZONE10 & & & & & \\
\hline DP tiquid Tomp,f & 81.9 & & & & & & & & & \\
\hline DPL'quid Density-lbm/t3 & 82.71 & & & & & & & & & \\
\hline Elovation-h & 2 & 4 & 6 & 8 & 9.876 & & & & & \\
\hline Measured DPFsid & 0.213 & 0.53 & 0.447 & 1.349 & 1.602 & & & & & \\
\hline Zero Corrections-psid & -0.018 & 0.016 & -0.17 & -0.102 & -0.027 & & & & & \\
\hline Cortected DPPsid & 0.231 & 0.514 & 0.617 & 1.451 & 9.629 & & & & & \\
\hline Actual DP-n & 1.95 & 3.8 & 5.98 & 6.82 & 8.72 & & & & & \\
\hline Monsured TPPsig & 143.9 & & & & 3.6 & & & & & \\
\hline Measured Overallih & 258.65 & & & & & & & & & \\
\hline & & & & & & & & & & \\
\hline Computed DPA & 2.13 & 4.27 & 6.14 & 7.93 & 9.59 & & & & & \\
\hline Filctionth & 0.13 & 0.3 & 0.58 & 0.94 & 1.34 & & & & & \\
\hline Acoelerationth & $0 \mid$ & 0.14 & 0.26 & 0.38 & 0.45 & & & & & \\
\hline Elovationft & 21 & 3.831 & 6.3 & 6.68 & 7.79 & & & & & $\perp$ \\
\hline
\end{tabular}




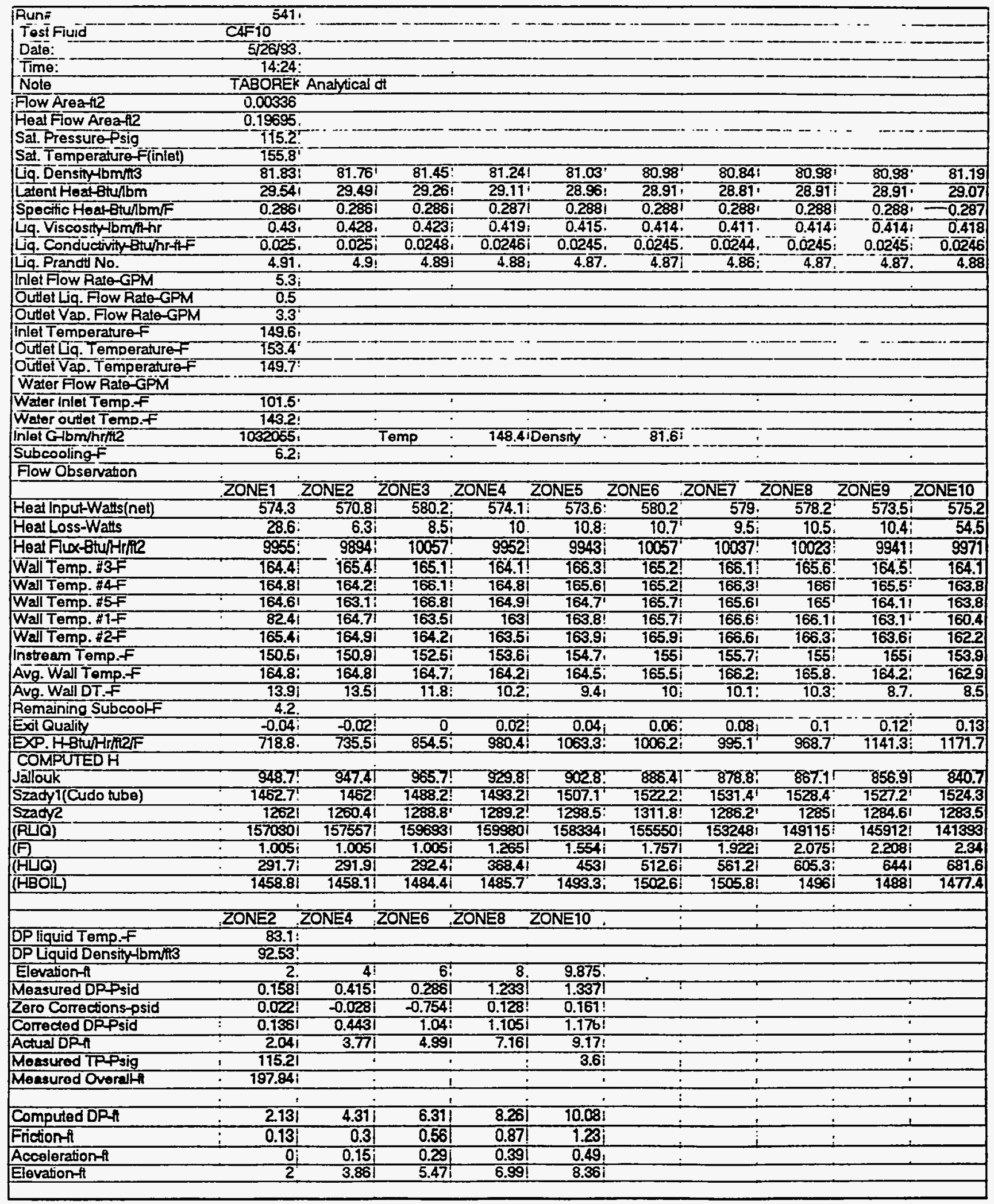




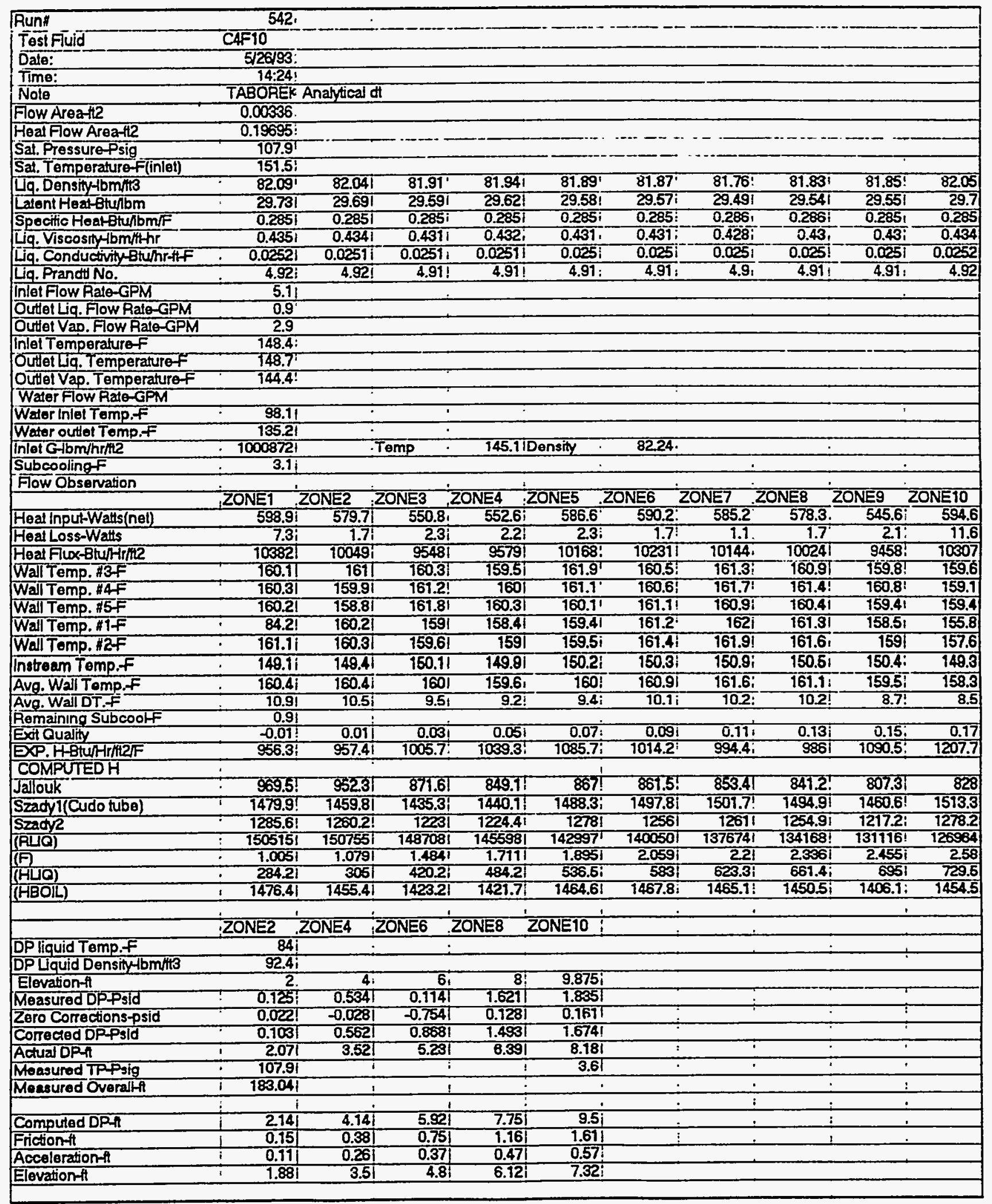




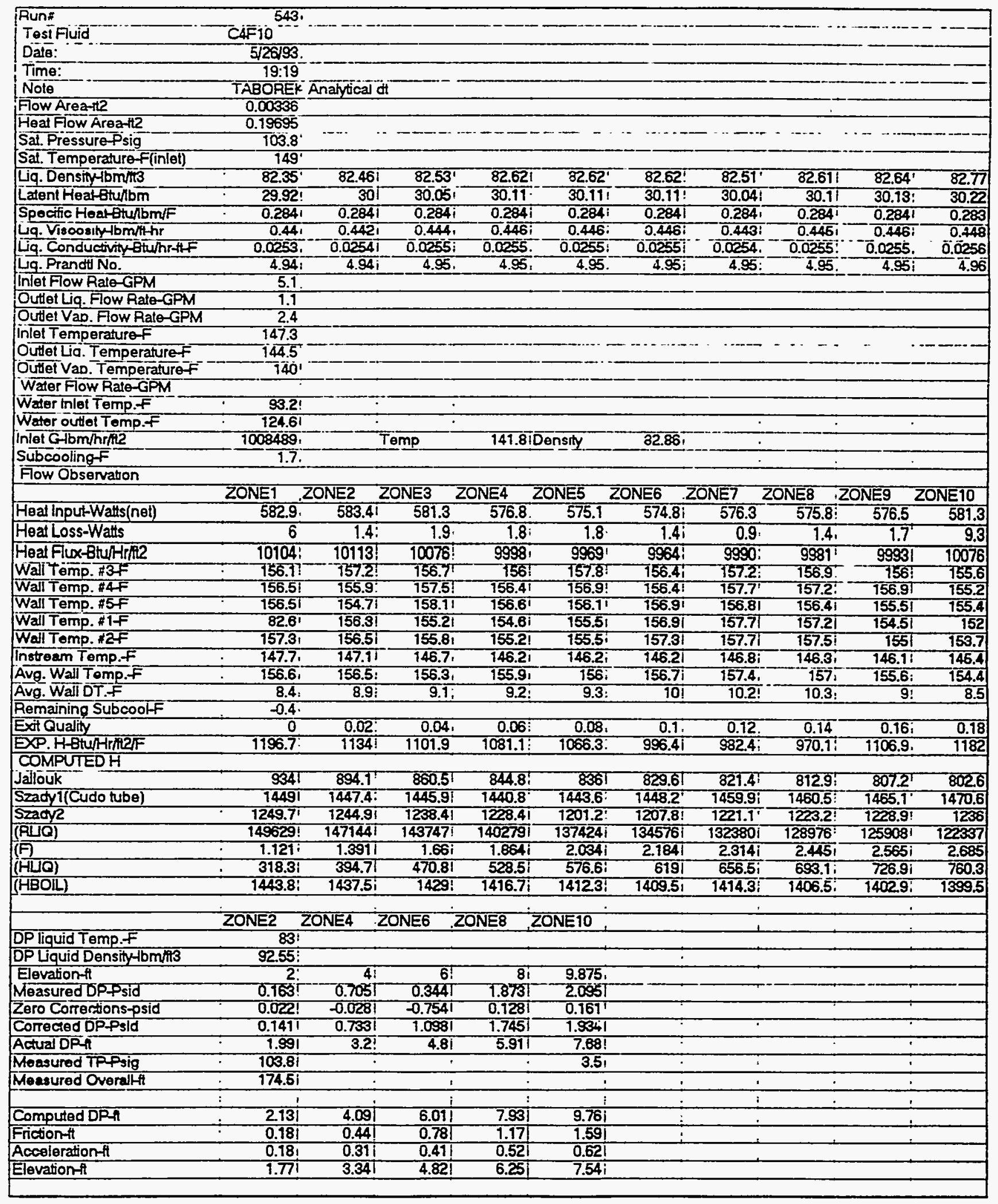




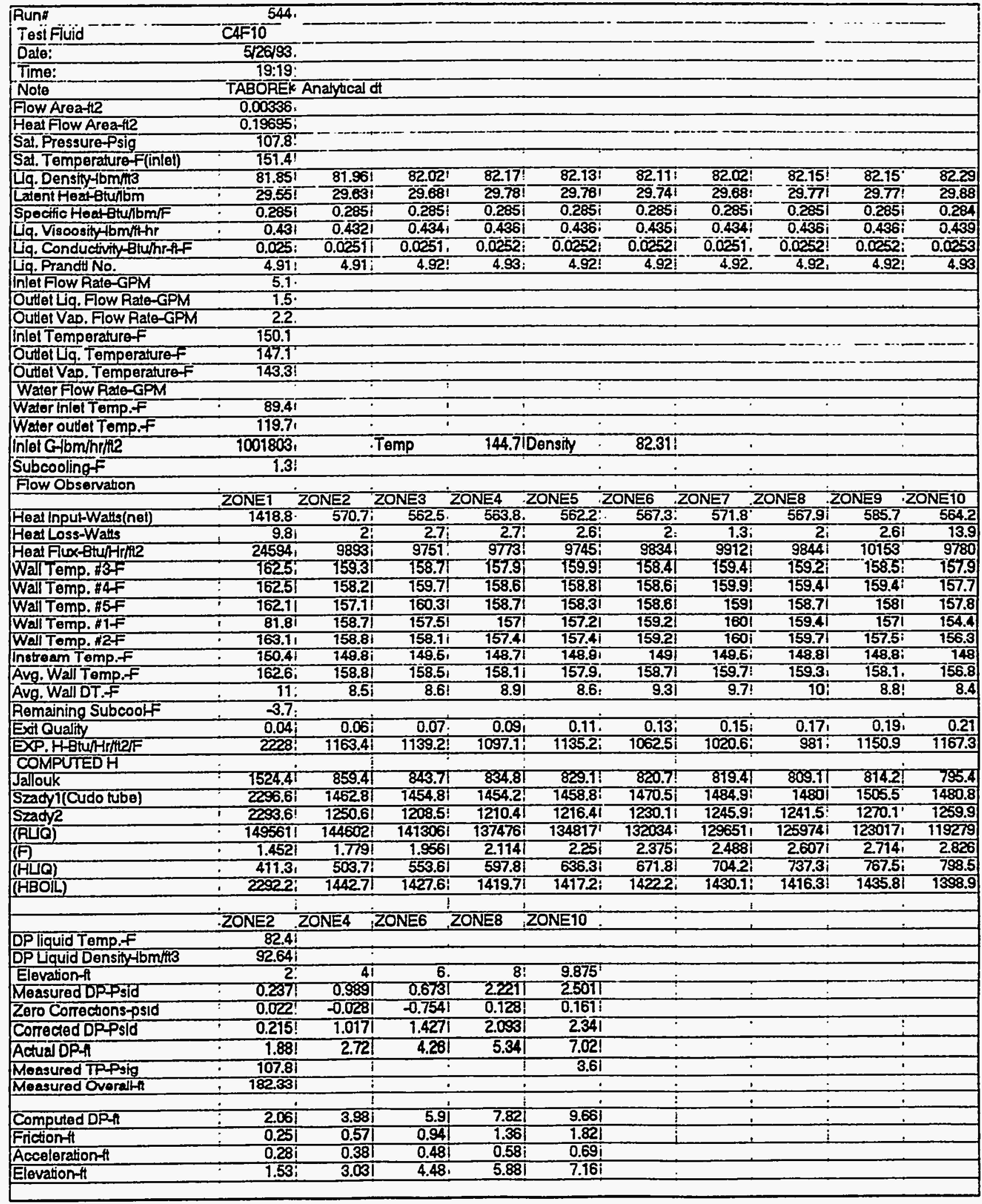




\begin{tabular}{|c|c|c|c|c|c|c|c|c|c|c|}
\hline Runn: & $545_{1}$ & & & - & - & : & & & & \\
\hline Tost Fuid & CAF 10 & & & & & & & & - & - \\
\hline Date: & $5 \longdiv { 2 6 9 3 1 }$ & & & & & . & & & & \\
\hline Time: & $19: 19 !$ & & & $:$ & & & & 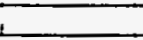 & & \\
\hline Note & TABOREK & Anatyical 0 & & & & & & & & \\
\hline Flow Area-112 & $0.00336^{\circ}$ & & & & & & & & & \\
\hline Heal Fow Areatt2 & 0.19695 & . & & & & & 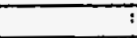 & & & \\
\hline Sat. Pressure Psig & 1051 & & & & & & & & & \\
\hline Sat. Temperature F(inlet) & 150.31 & & & & & & & & 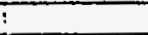 & \\
\hline iq. Density-lbm/n3 & 81.891 & 82.071 & $82.13 !$ & 82.131 & $82.15 !$ & 82.15 & 82.021 & 82.07 & 82.151 & 82.22 \\
\hline Latent Hest-8tulism & 29.581 & 29.72 & 29.761 & 29.76 & $29.7 i$ & 29.77 & 29.681 & 29.72 & 29.71 & 29.82 \\
\hline Spocitic Heat-Btuhbm/F & 0.2851 & 0.285 & 0.2851 & 0.2851 & 0.2851 & 0.2851 & 0.2851 & 0.2851 & 0.285 & 0.285 \\
\hline Liq. Viscositylbmithr & 0.4311 & 0.436 & 0.4361 & 0.436 & $0.436 i$ & 0.4361 & 0.4341 & 0.4351 & $0.436 i$ & 0.438 \\
\hline Liq. Conductivity $B$ tuhr-ftF & 0.0251 & 0.02521 & 0.0252 & 0.02521 & 0.02521 & 0.02521 & 0.02571 & 0.02521 & 0.02521 & 0.0253 \\
\hline Liq. Prandt No. & 4.911 & 4.921 & 4.921 & 4.921 & 4.921 & 4.921 & 4.921 & 4.921 & 4.921 & 4.93 \\
\hline Inlet Fow Rato-GPM & $5 i$ & +1 & & & & $\vdots$ & & & - & i \\
\hline Outlet Liq. Flow Rato-GPM & 1.61 & 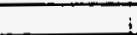 & & $i$ & $i$ & 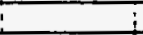 & 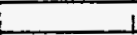 & & i & \\
\hline Outlel Vap. Flow Rato-GPM & 2.2 & & & & & 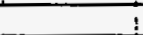 & 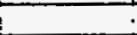 & & & \\
\hline Inlef Temporaturof & 150 & & & & $:$ & : & & & & \\
\hline Outtel Liq. Temperaturef & 147.5i & $i$ & & . & & 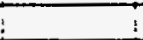 & & & & \\
\hline Outiot Vap. Temperature-F & 143.11 & ti & & 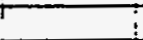 & & $t$ & $\vec{i}$ & & i & I \\
\hline Wader Flow Faio-GFMi & & 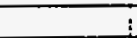 & 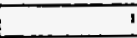 & 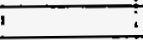 & & & $\bar{\vdots}$ & & 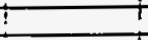 & \\
\hline Water tnlet Temp.F & 87.71 & & & $\because$ & & & 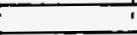 & & & \\
\hline Water outlet Temp.F & $118.2 !$ & I & & $!$ & \pm & $i$ & & & & \\
\hline Inlot Glbm/hr/it2 & 9810191 & & Temp & 145.2. & Density & 82.22] & & & 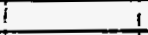 & 1 \\
\hline Subcooling $f$ & 0.31 & I & ( & 7 & 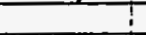 & 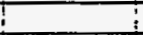 & & & 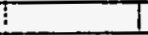 & \\
\hline Flow Observation & $\mathrm{i}$ & $i$ & & $i$ & & 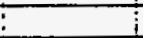 & & & 1 & \\
\hline & ZONET & ZONE2 & ZONE3 & ZONE4 & ZONE5 12 & ZONE6 : & ZONE7 & ZONE8 & ZONE9 & ZONE10 \\
\hline Head Input-Watts(nei) & 864.11 & 866.2 & 867.3 & 868.2 & 876.3 & 877.11 & 861.8 & 859.2 & 115.4 & 46.4 \\
\hline Head Loss-Watts & $9.2 !$ & 2.21 & $3 i$ & 3 & 31 & 2.2) & 1.5 & 2.8 & 2.1 & 10.7 \\
\hline Heal Fix-Btu/Hr/A2 & 14979 & 15015 & 15034 & 15050 & 15190 & 15204 & 14509 & 14894 & 2000 & 804 \\
\hline Wall Temp. \#3F & 159.21 & 161.61 & 160.4 & 159.8 & 163.5 & 161 & 161.6 & 174.4 & 151.6 & 749.1 \\
\hline Wall Temp. \#4F & $159.5 !$ & 159.3 & 161.9 & 160.9 & 161.7 & 161.11 & 162.1 & 172.5 & 151.7 & 149.7 \\
\hline Wall Temp. \#5F & 159.31 & 157.8 & 162.6 & 160.9 & 160.71 & 161.6 & 161.3 & 162.4 & 151.7 & 150 \\
\hline Wall Temp. ATf & 81.2 & 160.2 & 158.7 & 158.4 & 159.7 & 161.8 & 162.91 & 159.81 & 152 & 149.1 \\
\hline Wall Temp. $2 F$ & 159.7 & 160.2 & 159.7 & 159.3 & 160 & 162.3 & 162.9 & 160.2 & 152 & 948.4 \\
\hline Instream Temp.F & 160.21 & 148.2 & 148.9 & 148.0 & 148.81 & 148.8 & 149.6 & 949.2 & 148.8 & 148.4 \\
\hline Avg. Wall Tomp.F & 159.41 & 160.3! & 160.2 & 160 & 160.5 & 161.3 & 1622 & 165.8 & 951.8 & 140.6 \\
\hline Avg. Wall DT F & 8.5 & 10.4 & 10.6 & 10.4 & 11 & 91.8 & 12 & 15.9 & 2.9 & 1 \\
\hline Remaining Subcoolf & -2.8 & & & & & & & & & $!$ \\
\hline Exot Quality & 0.03 & 0.061 & 0.09 & 0.12 & 0.15 & 0.18 & $0.2]$ & 0.24 & 0.24 & 0.24 \\
\hline EXP.HBtu/HritifF & 1752.31 & 1437.3 & 1418.9 & 1444.3 & 1376.1 & 1284.3 & 1246.5 & 9341 & 687.5 & 785.7 \\
\hline COMPUTEDH & & & & & & & & & & \\
\hline Jallouk & 1142.31 & 1089.3 & 1055.7 & 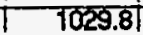 & 7011.91 & 992.31 & 970.1 & 277.3 & 277.2 & 277.1 \\
\hline Szadyi(Cudo tube) & 1788 & 1783.1 & 1784.9 & 1789.8 & 1801.21 & 1806.5 & 1803.2 & 277.3 & 277.2 & 277.1 \\
\hline szadyz & 1619.11 & 1645.7 & 1643.6 & 1645.1 & 1654.8 & 1656.9 & 1647.5 & 27.3 & 277.2 & 27.1 \\
\hline (RLG) & 146874 & 141414 & 136603 & 132162 & 127576 & 123087 & 119381 & 119831 & 119331 & 119331 \\
\hline (F) & 1.383 & 1.747 & 2.02 & 2.244 & 2.44 & 2.617 & 2.769 & 2.769 & 2.769 & 2.769 \\
\hline (HบO) & 385.31 & 486.1 & 561.8 & 624.9 & 678.7 & 728 & 770.8 & 770.8 & 770.8 & 770.8 \\
\hline \multirow[t]{3}{*}{ (HBOIL) } & 1782.11 & 1770.9 & 1766.1 & 1764.2 & 1768.5 & 1766.2 & 1755 & 1755 & 1755 & 1755 \\
\hline & $i$ & & & & & & & & & I \\
\hline & ZONE2 & ZONE4 & ZONE6 & ZONE8 & ZONE10 & & & & & \\
\hline DP liquid Temp.F & 82.1 & 1 & 1 & 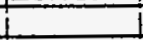 & -1 & 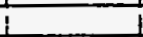 & & & & \\
\hline DP Liquid Density lbment3 & 92.681 & & & & 1 & & & & & I \\
\hline Elevation-t & 21 & 4 & 6 & 8 & 9.8751 & & & & & $i$ \\
\hline Measured DPP sid & 0.227 & 0.920 & 0.62 & 2.300 & 2.780 & & & & & \\
\hline Zero Corrections-psid & 0.022 & -0.028 & -0.754 & 0.128 & 0.1611 & & & & & \\
\hline Cotrected DPPsid & 0.2051 & 0.957 & 1.383 & 2.181 & 2.621 & & & & & \\
\hline Actual DP- & 1.9 & 2.83 & 4.84 & 5.2 & 6.54 & & I & I & & \\
\hline Measured TPP sig & 1061 & & & 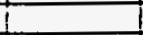 & 3.4 & & & & & \\
\hline \multirow[t]{2}{*}{ Measursd OveralfA } & 178.69 & & & I & & & I & & & \\
\hline & & & & & & & & & & \\
\hline Computed DPA & 1.98 & 3.73 & 5.48 & 7.33 & 9.08 & & & & & \\
\hline Frictionft & 0.26 & 0.7 & 1.28 & 1.94 & 2.42 & & & & & \\
\hline Accoleration-t & 0.27 & 0.42 & 0.58 & 0.74 & 0.76 & & & & & \\
\hline \multirow[t]{2}{*}{ Elovation-1 } & 1.451 & 2.6 & 3.62 & 4.65 & 5.9 & & & & & \\
\hline & & & & & & & & & & \\
\hline
\end{tabular}




\begin{tabular}{|c|c|c|c|c|c|c|c|c|c|c|}
\hline Runt & 5461 & & $i$ & $i$ & 8 & T & 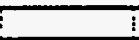 & 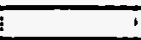 & - & $i^{2}$ \\
\hline Tost Fluid & ICAF10 : & & i & 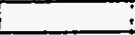 & 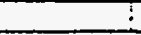 & $!$ & 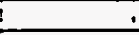 & - & & \\
\hline Dale: & $5 \longdiv { 2 6 9 3 1 }$ & & & & & & & & $\stackrel{t}{\prime}$ & $!$ \\
\hline Time: & $19: 19$ & ! & ! & & & & 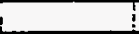 & & & \\
\hline Note & ITABOREK & Analyical d & & & it & & & & & 1 \\
\hline Flow Area-f12 & 0.00336 & & & 1 & $\therefore$ & & & & 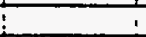 & . \\
\hline Heat Flow Areante & 0.19695 & & & & 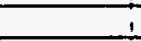 & 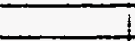 & 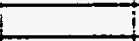 & & 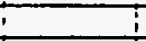 & $i$ \\
\hline Sat. Pressuro Psig & 114.5 & & & 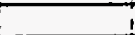 & & & & & $:$ & \\
\hline Sal. Temperature $f($ inlel) & 155.4 & 1 & 1 & T & 1 & 1 & i & & i & \\
\hline Lq. Density-tom/nit3 & 81.81 & 81.741 & 81.511 & 81.53 & 81.531 & 81.531 & 81.45 & 81.531 & 81.57 & 81.06 \\
\hline Latent Heat-Btuibm & 29.531 & 29.47 & 29.311 & 29.92 & 29.521 & 29.32 & 29.28 & 29.32 & 29.35 & 29.42 \\
\hline Specific Heat-otu/bm/F & 0.2861 & 0.286 & 0.286 & 0.286 & 0.286 & 0.286 & 0.286 & 0.286 & 0.286 & 0.286 \\
\hline Liq. Visoosity /bm/ithr & 0.48 & 0.428 & 0.424 & 0.424 & 0.424 & 0.424 & 0.423 & 0.424 & 0.425 & 0.427 \\
\hline Liq. Conductivity Btuphseffe & 0.025 & 0.025 & 0.0248 & 0.0248 & 0.0248 & 0.0248 & 0.0248 & 0.0248 & 0.0248 & 0.0240 \\
\hline Liq. Prandt No. & 4.911 & 4.91 & 4.89 & 4.89 & 4.891 & 4.89 & 4.891 & 4.89 & 4.89 & 4.9 \\
\hline Inlot Flow Rate-GPM & 5.1 & & & & & & & & & \\
\hline Outtot Lig. Fow Rato-GPM & 1.5 & & & & & & & & & \\
\hline Outtet Vap. Fow Rato-GPM & 2.3 & & & & & & $L$ & & $!$ & \\
\hline Inlat Temperature $F$ & 149.8 & & & & & & 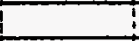 & & 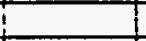 & \\
\hline Out ef Liq. Temperaturef & 150.7 & & & & & & & & & \\
\hline Outlet Vap. Temperaturef & 146.4 & & & & & & & & & \\
\hline Waler Fow Rato-GPM & & 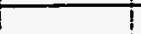 & $i$ & 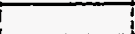 & & & & & i & i \\
\hline Water Inlot Tomp.f & 88.11 & & 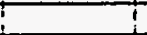 & 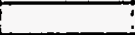 & 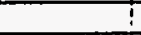 & & & & $!$ & 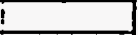 \\
\hline Water out ot Tomp.F & 121.91 & & 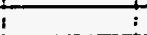 & $=$ & $!$ & & & T & 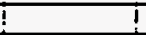 & i \\
\hline Inlet G-bm/hrithe & 9968501 & & Tomp & 147.2 & Density & 81.831 & & & & \\
\hline Subcoolingf & 5.6 & & 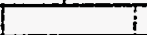 & 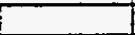 & & & & & & \\
\hline \multirow[t]{2}{*}{ Fow Observation } & & & & & & & & & & \\
\hline & ZONE1 I2 & ZONE2 & ZONE3 & ZONE4 & ZONE5 & ZONE6 & ZONE7 & ZONEB & ZONE9 & ZONE10 \\
\hline Hoat Input-Walts(net) & 868.4 & 874.6 & 867.31 & 864.8 & 866.5 & 868.5 & 869.6 & 872.1 & 874 & 856.5 \\
\hline Heat Loss-Watts & 9.61 & 2.3 & 3.11 & 3 & 3.11 & 2.3 & 1.5 & 2.1 & 2.8 & 30.8 \\
\hline Hoot Flox-BturHritt2 & 15053 & 151बा & 15034 & 14991 & 150201 & 15055 & 15074 & 15117 & 15150 & 14847 \\
\hline Wall Temp. H3F & 162.4 & 164.8 & 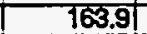 & 163 & 166.7 & 164.4 & 164.4 & 162.5 & -1611 & 207.2 \\
\hline Wall Temp. :4F & 162.9 & 162.8 & 165.2 & 163.7 & 165 & 964.4 & 165.2 & 161.9 & 1627 & 200.7 \\
\hline Wal Temp. $15 f$ & 162.81 & 161.4 & 168 & 163.9 & $1 \approx 3.9$ & 164.9 & 164.3 & 161 & 161 & 180 \\
\hline WaH Temp. "1F & 78.6 & 169.8 & 102.1 & 101.5 & 182.9 & 105.1 & 165.9 & 161.9 & 100.4 & 157.0 \\
\hline Wall Tomp. HaF & 963.2 & 163.6 & 162.9 & 162.2 & 16 & 165.7 & 166.2 & 162.6 & 161.3 & 100.4 \\
\hline Instroem Tomp.F & 160.6 & 151 & 1622 & 162.1 & 152.1 & 152.1 & 162.6 & 1621 & 151.8 & 151.4 \\
\hline Avg. Wall Tomp.F & 1628 & 153.8 & $1 \approx 3.5$ & 16 & 163.7 & 164.7 & 165.2 & 162 & 161.3 & 183.6 \\
\hline Avg. Wall DT tf & 11.5 & 12.1 & 10.6 & 10.2 & 10.9 & 11.9 & 12 & 9.2 & 8.7 & 31.5 \\
\hline Remaining Subcoolf & 2.5 & & & & & & & & & \\
\hline Exit Quality & -0.02 & 0.011 & 0.04 & 0.07 & 0.1 & 0.13 & 0.16 & 0.19 & 0.22 & 0.25 \\
\hline EXP.HBtu/Hr/h2/F & 1303.9 & 1256.6 & 14122 & 1452.9 & 1375.3 & $12 \approx 3$ & 1254.2 & 1643.8 & 17423 & 471.2 \\
\hline \multicolumn{11}{|l|}{ COMPUTEDH } \\
\hline Jallouk & T223.45 & T231.6 & T137.8 & 10्91.9 & 705241 & 1038.6 & 7019.81 & 998.9 & $\$ 00.3$ & $\$ 1.7$ \\
\hline Szadyiloudo tubo) & 1794.7 & 1805.4 & 1814 & 1814 & 1819.5 & 1825.6 & 1835.11 & 7837.8 & 7842.2 & 1824.6 \\
\hline seacty 2 & 1020.7 & 1639.1 & 1683.9 & 1679.3 & 1681.5 & 1684.5 & 1691.4 & 1650.7 & 1692 & 1055.8 \\
\hline (RLO) & 151688 & 152173 & 150434 & 145692 & 141086 & 1384911 & 132239 & 127131 & 1222581 & 117160 \\
\hline (F) & 1.005 & 1.005 & 1.493 & 1.819 & 2.056 & 2.274 & 2.453 & 2.624 & 2781 & 2.931 \\
\hline (HบQ) & 283.6 & 283.7 & 422 & 514.1 & 583.8 & 642.6 & 633.6 & 741.6 & 785.7 & 827.6 \\
\hline \multirow[t]{3}{*}{ (HBOIL) } & 17923 & 1803.1 & 1806.3 & 1800.1 & 1799.3 & 1798.6 & 1801.5 & 1796.6 & 1793.3 & 1766 \\
\hline & & & & & & & & & & \\
\hline & TZONE2 & ZONE4 & ZONE6 & ZONE8 & ZONE10 & & & & & \\
\hline DPliquid Tomp.F & 80.7 & & & & & & & & & \\
\hline DPLiquid Density $1 \mathrm{bm} / \mathrm{tit}$ & 9289 & & & & & & & & & \\
\hline Elovation-ft & 2 & & 6 & 8 & 9.875 & & & & & \\
\hline MeasuredDPPsid & 0.1281 & $0.5 \%$ & 0.231 & 7.655 & 2.135 & & & & & \\
\hline Zero Corrections-psid & 0.022 & -0.028 & -0.754 & 0.128 & 0.161 & & & & & \\
\hline Corrected DPPsld & 0.101 & 0.623 & 0.9851 & 1.527 & 1.974 & & & & & \\
\hline Actual DPA & 2.1 & 3.45 & 5.09 & 8.41 & 7.75 & & & & & \\
\hline Mossured TPPogig & 114.5 & & & & 3.51 & & & & & \\
\hline \multirow{2}{*}{\multicolumn{11}{|c|}{ Measured Overallita }} \\
\hline & & & & & & & & & & \\
\hline Computad DPA & 213 & 4.04 & 5.791 & 7.56 & 9.31 & & & & & \\
\hline Friction-f & 0.14 & 0.44 & 0.9 & 1.49 & 2.15 & & & & & \\
\hline Accoleration-l & 0.08 & 0.3 & 0.45 & 0.6 & 0.77 & & & & & \\
\hline Elevation-f & 1.91 & 3.3 & 4.44 & 5.46 & 6.38 & & & & & \\
\hline & & & & & & & & & & \\
\hline
\end{tabular}




\begin{tabular}{|c|c|c|c|c|c|c|c|c|c|c|}
\hline Runz & 547 & & & & & & & & $\therefore$ & . \\
\hline Tost Fluid &. CAF10 & & & & & & & & & \\
\hline Date: & 522693 & & & & & & & & $T$ & \\
\hline Time: & $19: 19$ & & & & & & & & !! & \\
\hline Note & TABOREK & Analytical & & & & & & & & \\
\hline Flow Asea-tl2 & $\therefore .00336$ & & & & & & & & & \\
\hline Heat Fow Area-th & 0.19695 & & : & & & & & & . & , \\
\hline Sad. Pressure Psig & 108.1 & & $i$ & & & & & & & 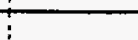 \\
\hline Sat. Temperature $F$ (inleil) & 151.6 & & 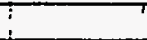 & & & & & & & \\
\hline La. Denstitybmilis & 81.78 & 81.92 & 81.981 & 82.04 & 82.05 & 82.04 & 81.981 & 82.09 & 82.13 & 82.13 \\
\hline Lant Heatstufibm & 29.5 & 29.811 & 29.651 & 29.69 & 29.7 & 29.69 & 29.651 & 29.73 & 29.78 & 29.78 \\
\hline Specific Hoed-Bthbm/F & 0.286 & 0.285 & 0.2851 & 0.285 & 0.285 & 0.285 & 0.2851 & 0.285 & 0.285 & 0.285 \\
\hline Liq. Visoosity lbmithr & 0.428 & 0.4321 & 0.4331 & 0.434 & 0.434 & 0.434 & 0.4331 & 0.436 & 0.4351 & 0.436 \\
\hline iq. Conductivity Btuhr-af & 0.025 & 0.0251 & 0.02511 & 0.0251 & 0.0252 & 0.0251 & 0.02591 & 0.0252 & 0.0252 & 0.0252 \\
\hline Liq. Prandit No. & 4.9 & 4.91 & 4.921 & 4.92 & 4.92 & 4.92 & 4.92 & 4.92 & 4.92 & 4.92 \\
\hline Inlet Flow Rate-GPM & 4.3 & & i & $i$ & & & & $\begin{array}{l}1 \\
\vdots \\
\vdots\end{array}$ & & I \\
\hline Outlet Lig. Flow Rato-GPM & 1.5 & & $i$ & t & 7 & $t$ & & & & + \\
\hline Outlel Vap. Flow Rato-GPM & 2 & 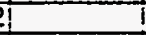 & I & 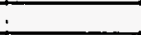 & & & & & & i \\
\hline Inlet Temperaturef & 150.3 & & $\bar{i}$ & & & & & & & \\
\hline Outel Liq. Temperaturef & T47.9 & & $i$ & $!$ & & & & & & \\
\hline Outlet Vap. Temperature-F & 143.7 & & it & & & & & & & \\
\hline Warer Fow Rafo-GPM & & & $i$ & : & & & & & & 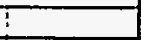 \\
\hline Wader Inlet Temp.f & 83.8 & & $\begin{array}{l}t \\
\vdots\end{array}$ & 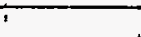 & & & & T & $\bar{i}$ & $i$ \\
\hline Wator outel Temp.F & 118.2 & & $T$ & $i$ & 1 & 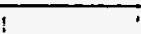 & & & 7 & 1 \\
\hline Inlat Glbm/hrin2 & 843686 & & Temp & 145.5 & Density & 82.161 & & $\mathrm{i}$ & & $T$ \\
\hline Subcoolingf & 1.3 & & 1 & 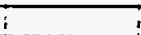 & 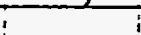 & -1 & & 7 & & \\
\hline \multirow[t]{2}{*}{ Flow Observation } & 1 & & i & $T$ & & & & & & \\
\hline & ZONET & ZONE2 & ZONE3 & ZONE4 & ZONE5 & ZONE6 & ZONE & ZONE8 & ZZONE9: & ZONE1O \\
\hline Heat Input-Watts(net) & 8688.5 & 869.8 & 879.2 & 873.4 & 846.4 & 854.5 & 876.41 & 875.6 & 466.2 & 331.3 \\
\hline Hoat Loss-Watts & 8.7 & 2.1 & 29 & 2.8 & 2.81 & 2.1 & $1.4 i$ & 2 & 2.2 & 11.9 \\
\hline Hoat Flox-Bturitrit2 & 15055 & 15077 & 15240 & 151401 & 14672 & 14812 & 15192 & 15178 & 8081 & 5743 \\
\hline Wall Temp. HF & 159.8 & 1624 & 161.2 & 160.6 & 163.61 & TE1.ा & 161.81 & 161.1 & 156.31 & 154.8 \\
\hline Wall Temp. $14 F$ & 160.3 & 160.7 & 162.6 & 161.6 & 162.7 & 767.41 & 162.6 & 160.6 & 156.8 & 754.7 \\
\hline Wall Temp. H5F & 100.1 & 158.6 & 165.3 & 767.61 & 161.11 & 162 & 161.61 & 158.7 & $155.7 !$ & 154.5 \\
\hline Watt Temp. * IF & 77.9 & 161 & 159.5 & 159 & 960 & $16 \hat{2}$ & 169.21 & 159.4 & 154.91 & 152.7 \\
\hline Wall Temp. " \#ZF & 160.6 & 1611 & 160.4 & 159.9 & 160.5 & 162.4 & 163.2 & 160.5 & 155.1 & 153.7 \\
\hline Instream Tomp.f & 160.8 & 150 & 148.71 & 149.41 & 149.3 & 198.4 & 149.71 & 148.1 & 148.8 & 148.0 \\
\hline Avg. Wall Tomp.F & 160.2 & 167.7 & 160.9 & 160.7 & 160.9 & 161.6 & 162.51 & 160.1 & 155.8 & 154.1 \\
\hline Avg. Wall DT.F & 8.71 & 10.4 & 10.5 & 10.6 & 111 & 11.6 & 12.1 & 10.3 & 6.5 & 4.8 \\
\hline \multicolumn{11}{|l|}{ Remaining Subcooff } \\
\hline Exot Quality & 0.02 & 0.06 & 0.09 & 0.13 & 0.16 & 0.2 & 0.23 & 0.27 & 0.2 & 0.3 \\
\hline EXP. HBtuAHFit2/F & 1726.6 & 1443.7 & 1446.5 & 1426.2 & 1338.4 & 1281.8 & 1256.2 & 1477.3 & 1244.31 & 1167.1 \\
\hline \multicolumn{11}{|l|}{ COMPUTEDH } \\
\hline Jallouk & 1182.2! & नाओं। & 1100.61 & 1057 & 9026.4 & 1014 & 1012.2 & 245.6 & 245.61 & 245.6 \\
\hline Szadyl(Cudo tube) & $1797.8 !$ & 1793 & 1802.41 & 1796.61 & 9779.8 & 1784.7 & 1813.6 & 245.6 & 245.6 & 245.6 \\
\hline Szady2 & $1 \approx 50.11$ & 1680 & 1668.11 & 1657.3 & 1622.4 & 1634.4 & 1666.4 & 245.6 & 245.6 & 245.6 \\
\hline (RUQ) & 127127 & 122514 & 117694 & 112870 & 1083411 & 104046 & 998331 & 99833 & \begin{tabular}{l|l|}
98839 \\
\end{tabular} & 99838 \\
\hline (F) & 1.3461 & 1.727 & 2.0451 & 2.301 & 2517 & 27061 & 2.8791 & 2.879 & 2.879 & 2.879 \\
\hline (HLQ) & 332.51 & 426.11 & 504.31 & 567.3 & 620.5 & 6672 & 710.1 & 710.11 & 710.1 & 710.1 \\
\hline \multirow[t]{3}{*}{ (HBOLL) } & 1794 & 1784.9 & 1789.21 & 177.6 & 1746 & 1753 & 1776.51 & 1776.5 & 1776.5 & 1776.5 \\
\hline & & & & & & & 1 & & & \\
\hline & IZONE2 & ZONE4 & ZONE6 & ZONEB & ZONE10 & & & & & \\
\hline DP liquid Temp.F & 79.5 & & & & & & & 1 & & \\
\hline DP Liquid Densitylbm/t3 & 93.061 & & & & & & & & & \\
\hline Elevation-t & & 4 & & 8 & 9.875 & & & & & \\
\hline Measured DPYSid & 0.1821 & 0.351 & 0.729 & 2.308 & 2.981 & & i & $i$ & & \\
\hline Zero Contections-psid & 0.0221 & -0.028 & -0.754 & 0.128 & 0.167 & & & & & \\
\hline Contected DPPsid & 0.16 & 0.989 & 1.483 & 2.18 & $2 . \overline{8081}$ & & i & it & & \\
\hline Actual DPA & 1.991 & 2.8 & 4.2 & 5.24 & 5.27 & & + & t & & \\
\hline Moasurod TPPsig & 108.1 & & & & 3.5 & & & i & & \\
\hline \multirow{2}{*}{ Mesusured Overali } & 183.4 & & T & & & & T & $\mathrm{T}$ & & \\
\hline & & & & & & & 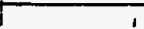 & 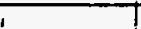 & & \\
\hline Computed DPA & 1.87 & 3.48 & $5.07 !$ & 6.7 & 8.41 & & $i$ & i & & \\
\hline Friction-f & 0.2 & 0.54 & 1.01 & 1.59 & 2 & & & & & \\
\hline Accoleration-fl & 0.2 & 0.33 & 0.46 & 0.611 & 0.68 & & & & & \\
\hline \multirow[t]{2}{*}{ Elevation-At } & 1.47 & 2.61 & 3.6 & 4.5 & 5.72 & & + & $t$ & & \\
\hline & & & & & & & & & & \\
\hline
\end{tabular}




\begin{tabular}{|c|c|c|c|c|c|c|c|c|c|c|}
\hline Run\# & 5481 & & & & & & & & & \\
\hline Test Fuid & . CAF 10 & & & & $\dot{-}$ & & & & & \\
\hline Date: & 55261931 & $i$ & & & & & 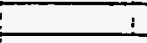 & & & \\
\hline Timo: & 19:19 & & & & & & & & & \\
\hline Nole & - TÁBOREK & Analytical o & & & & I & & & & \\
\hline Flow Area-tL2 & 0.00336 & & & & & ! & 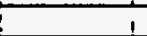 & & & \\
\hline Heat Flow Area-tL2 & 0.19695 & & & & & & & & & \\
\hline Sat. Pressurepsig & $|2| 2 \mid$ & & & & & & & & & \\
\hline Sat. Temperature of (inlel) & 159.2 & & & & & & & & & \\
\hline Lg. Denshythomitis & 80.981 & 80.851 & 80.081 & 80.08 & 80.721 & 80.7 & 80.611 & 80.72 & 80.74 & 80.76 \\
\hline Ladent Heal-Btulbm & 28.911 & 28.891 & 28.71 & 28.7 & 28.791 & 28.71 & 28.04 & 28.78 & 28.74 & 28.78 \\
\hline Spocifio Hoot-Btulbmif & 0.2881 & 0.288 & 0.288 & 0.288 & 0.288 & 0.288 & 0.289 & 0.288 & 0.288 & 0.288 \\
\hline Lig. Visoosity $1 \mathrm{bm} / \mathrm{ithr}$ & 0.4141 & 0.412 & 0.4091 & 0.400 & 0.4091 & 0.408 & 0.4071 & 0.400 & 0.0011 & $\frac{0.000}{0.41}$ \\
\hline Liq. Conductivity eturhatif & 0.02451 & 0.02441 & 0.02431 & 0.0243 & 0.02431 & 0.0243 & 0.0242 & 0.0243 & 0.0243 & 0.0243 \\
\hline Liq. Prandt No. & $4.87 \mid$ & 4.86 & 4.851 & 4.85 & 4.85 & 4.85 & 4.85 & 4.85 & 4.851 & 4.86 \\
\hline Inlet Fow Rato-GPM & 4.3 & & & & 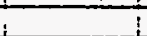 & & & & & \\
\hline Outlet Lig. Fow Rado-GPM & 1.2 & & & & 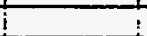 & & $\frac{1}{1}$ & & & \\
\hline Outtel Vap. Fow Rato-GPM & 2.11 & $\frac{1}{1}$ & 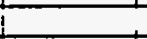 & & & & & & & \\
\hline Inlei Temperaturef & 154.41 & & & & & & & & & \\
\hline Outlef Liq. Temperaturef & 155.21 & & & & & & & & & \\
\hline Outlol Vap. Temperaturef & 151.2 & & & & & & & & & \\
\hline Water Fow Rato-GPM & & & & & & & $i$ & & & \\
\hline Water Inlel Temp.F & 90.41 & & & & & & $!$ & & & \\
\hline Water outhel Temp.F & 128.51 & & & & & & $i$ & & & \\
\hline Inlol Glbm/hriti2 & 831876 & & Temp & 151.11 & Density & 81.071 & i & & & \\
\hline Subcoolingf & 4.8 & & & & & & I & & & \\
\hline How Observation & & & & & & & & & & \\
\hline & ONE 1 & ONE2 I & ZONE3 & EE4 & & VE6 I & NE7 I & NE8 I & NE9 12 & ONE10 \\
\hline Head lnput-Watts(net) & 877.6 & 876 & 890.1 & 893.8 & 854.6 & 862.91 & 876.5 & 872.8 & 495.11 & 350.6 \\
\hline Heat Loss-Watts & 10.4 & 2.5 & 3.4 & 3.3 & 3.4 & 2.51 & 1.6 & 2.4 & 28 & 15.1 \\
\hline Head Flox-BtuHirfite & 15213 & 15185 & 154291 & 154931 & $14814 \mid$ & 14958 & 15194 & 15129| & 85821 & 607 \\
\hline Wall Temp. & 766.3 & T68.6 & $167.6 T$ & 167.11 & 770.41 & 788.1 & 168.2 & 166.6 & 162.4 & 767.6 \\
\hline Wall Temp. "4tF & 166.9 & 166.6 & 169.1 & 168 & 168.9 & 168.3 & 168.8 & 166.7 & 163.61 & 761.6 \\
\hline Wall Temp. $85+$ & 186.6 & 165.1 & 169.8 & 168 & $167.8 !$ & 168.8 & 168 & 165.11 & 162.1 & 161.2 \\
\hline Wall Temp. If & 7.41 & 167.5 & 188 & 165.7 & 168.7 & 169 & 16.7 & 165.9 & 181.8 & 159.6 \\
\hline Wall Tomp. WF & 167.11 & 167.41 & 166.8 & 166.31 & 966.91 & 169.5! & 169.9 & 166.8 & 162 & 160.6 \\
\hline Inetroam Temp.F & 965 & 155.6 & 156.6 & 156.5 & 156.3 & 156.4 & 156.8 & 156.3 & $\frac{156.21}{156}$ & 166.1 \\
\hline Avg.Wall Tomp.F & 165.71 & 167.5 & 167.4 & 167.2 & 167.61 & 168.6 & 168.9 & 166.2 & 162.31 & 160.9 \\
\hline Avg. Wall DT.F & 111 & 11.2 & 10.21 & 10 & 10.6 & 11.5 & 11.3 & 9.2 & 5.7 & 4.5 \\
\hline Remaining Subcooff & 1.1 & & & & & & & & & \\
\hline Ext Quality & -0.011 & 0.03 & 0.06 & 0.11 & 0.14 & 0.18 & 0.21 & 0.25 & 0.27 & 0.20 \\
\hline 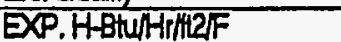 & 1378.31 & 1351.1 & $1516 \mid$ & 1549.4 & 1396.8 & 1303.6 & 1340.6 & 1638 & 1507.7 & 1337.1 \\
\hline COMPUTEDH & & & & & & & & & & \\
\hline Jalouk & 1303.21 & 1253.6 & 1188.31 & 1155 & 7056.21 & $108 \pi$ & 1073.91 & 7049.21 & 790.81 & 687.6 \\
\hline Szadyl(Cudo tube) & 1852.1 & 1858 & 1885.71 & 1892.3 & 1851.7 & 1864.6 & 1887.6 & 1880.5 & 1429.7| & 1228.4 \\
\hline szady2 & 1689.81 & 1696 & 1728.41 & 1772.9 & 17181 & 1730.4 & 1755.21 & 1743 & 1214.5 & 885.1 \\
\hline (RUQ) & 1314781 & 131008 & 127139 & 122096 & 116942 & 112182 & 1077241 & 102218 & 982811 & 95782 \\
\hline & $\frac{1.005}{1.005}$ & 1.272 & 1.74 & 2.045 & 2.289 & 2.498 & 2678 & 2.853 & 298 & 3.056 \\
\hline (सिपQ) & 246.71 & 312.4 & 427.7 & 5028 & 56261 & 613 & 667.6 & 701.31 & 732.51 & 761.1 \\
\hline (HBOLL) & 1850.6 & 1855 & 1878.3 & 1880.4 & 1834.2 & 18423| & 1800.6 & 1847.5 & 132.5 & 1126.6 \\
\hline & & & & & & & & & & \\
\hline & ZONE2 & ZONE4 & ZONE6 & ZONE8 & ZONE10 & & & & & \\
\hline DP liquid Tem & 79.2 & & & & & & & & & \\
\hline DPUquid I & 93.1 & & & & & & & & & \\
\hline Elevatior & & 4 & & & 9.875 & & & & & \\
\hline Mersured DPPsid & 0.151 & 0.795 & 0.556 & 2.044 & 2.720 & & & & & \\
\hline Zero Comections-psid & 0.022 & -0.028 & -0.754 & 0.128 & 0.1611 & & & & & \\
\hline DPPSild & 0.129 & 0.823 & 1.31 & 1.916 & 2.0011 & & & & & \\
\hline Actual OP-A & $\frac{0.081}{2.081}$ & 3.14 & 4.58 & 5.81 & 6.751 & & & & & \\
\hline Moasurod TPPsig & 12121 & & & & 3.61 & & & & & \\
\hline Moesurad overalth & 200.68 & & & & & & & & & \\
\hline & & & & & & & & & & \\
\hline puted DP-n & 2.05 & 3.72 & 5.311 & 6.89 & 8.48 & & & & & \\
\hline Fii & 0.12 & 0.39 & 0.78 & 1.27 & 1.69 & & & & & \\
\hline Accolerationft & 0.12 & 0.26 & 0.38 & 0.52 & 0.59 & & & & & \\
\hline Elovation-th & 1.8 & 3.07 & 4.15 & 5.11 & 6.2 & & & & & \\
\hline & & & & & & & & & & \\
\hline
\end{tabular}




\begin{tabular}{|c|c|c|c|c|c|c|c|c|c|c|}
\hline Runt & $549 i$ & & & & & & & $\cdot$ & $:$ & . \\
\hline Tostfluid & . CAF 10 & & 5 &. & & & & 1 & & \\
\hline Date: & $5 / 2793$ & & 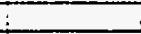 & 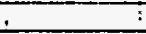 & 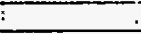 & & & & & \\
\hline Time: & $1: 43$ & & & & & & & & & \\
\hline Note & TABOAEK & Analytical & dt & & & & & & & \\
\hline Flow Area-fl2 & 0.00336 & & & & & & & & & - \\
\hline Heal Flow Area-t2 & $0.19695 i$ & & & & & & & & & \\
\hline Sat. Prossuro sig & $117.6 !$ & & & & & & & & & \\
\hline Sat. Temperature-f(inlet) & 157.2! & & & & & & & $T$ & 7 & T \\
\hline Üg. Densty-lbm/133 & $81 . \overline{3 !}$ & 81.17 & 81.27 & 81.24 & $81 . \overline{261}$ & 81.261 & 81.211 & $81.32 !$ & 81.321 & 81.24 \\
\hline atenl Heat-Btunbm & $29.15 i$ & 29.05 & 29.08 & 29.11 & 29.121 & 29.121 & 29.081 & 29.17 & 29.17 & 29.11 \\
\hline Spocific Hoet-Btu/bm/F & 0.287 & 0.287 & 0.287 & 0.287 & 0.2871 & 0.2871 & 0.287 & 0.287 & 0.2871 & 0.287 \\
\hline Liq. Viscosity lomithr & 0.421 & $0 . \overline{417}$ & 0.418 & 0.419 & $\overline{0.4191}$ & $0.4 \overline{19}$ & 0.418 & 0.421 & 0.421 & 0.419 \\
\hline Lig. Conductivity Btuhr-fif & 0.02471 & 0.0246 & 0.0246 & 0.0246 & 0.02471 & 0.02471 & 0.02461 & 0.0247 & 0.02471 & 0.0246 \\
\hline Liq. Prandt No. & 4.881 & 4.87 & 4.88 & 4.88 & 4.881 & 4.881 & 4.88 & 4.88 & 4.881 & 4.88 \\
\hline Inlet Flow Rado-GPM & 3.2 & & $i$ & . & 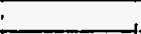 & 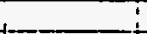 & i & $i$ & I & $!$ \\
\hline Outlet Liq. Flow Rato-GPM & $1.2_{i}^{i}$ & & & $i$ & i & & & & 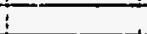 & \\
\hline Outlet Vap. Flow Ralo-GPM & $2.1 !$ & & $\therefore$ & $i$ & i & & 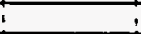 & 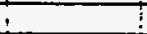 & $\begin{array}{l} \\
\vdots\end{array}$ & 1 \\
\hline Inlet Temperature-F & $152.7^{1}$ & & & 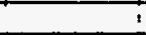 & 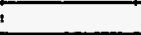 & & & & $:$ & \\
\hline Outlet Lia. Temperaturef & $152.5 !$ & & & & & & & & T & t \\
\hline Outlet Vap. TemperaturefF & 148.8 & & & & & & & & $!$ & 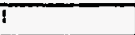 \\
\hline Water Flow Rato-GPM & & & & & & $\bar{\vdots}$ & 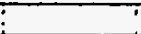 & & $T$ & $T$ \\
\hline Water inlet Temp.f & 88.71 & & i & ; & - & it & it & & T & $t$ \\
\hline Water outtet Temp.F & 134.41 & & $:$ & i & T & $\because$ & $:$ & T. & ti & $t$ \\
\hline Inlot G $16 \mathrm{~m} / \mathrm{hr} / \mathrm{hl}$ & 6249101 & & Temp & 147.21 & IDensity & 81.891 & & i & i & 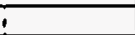 \\
\hline Subcooling & 4.51 & & & & - & 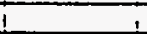 & & 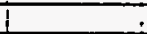 & & $i$ \\
\hline \multirow[t]{2}{*}{ Flow Observation } & 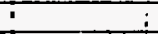 & & & 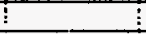 & 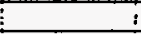 & $:$ & & 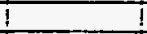 & 1 & \\
\hline & ZONEI & ZONE2 & ZONE3 & ZONE4 & ZONE5 & ZONE6 & ZONE7 & ZONE8 & ZONE9 & ZONEIO \\
\hline Heat Input-Watts(nel) & 876.41 & 865.2 & 880.2 & 885.9 & 871.8 & 8867 & 852.6 & 560 & 46.5 & 69.9 \\
\hline Heaf Loss-Watts & $11.2 !$ & 2.7 & 3.6 & 3.6 & $3.6 \mathrm{i}$ & 2.6 & 9.6 & 2.41 & 2.61 & 14.8 \\
\hline Heat Fux-bturHrifz & 15192 & 14998 & 15258 & 15357 & 151121 & 15358 & 14953 & 9707 & 8061 & 1212 \\
\hline Wall Temp. Fr & 163.51 & 165.8 & 764.8 & 1ब्या & T67.4 & 164.91 & 162.7 & 162.7 & 154.9 & 155.1 \\
\hline Wall Temp. "4F & 163.9 & 163.7 & 166.2 & 1651 & 165.8 & 165.4 & 162.7 & 162.11 & 155.3. & 155.1 \\
\hline Wall Temp. 45 F & 163.91 & 152.2 & 167 & 165 & 64.91 & 165.8 & 161.81 & $161.1 !$ & 155.31 & 15.4 \\
\hline Wबil Temp. & 73.91 & 164.5 & 163 & 162.51 & 163.8 & 165.91 & 163.61 & 161.11 & 154.81 & 155.2 \\
\hline Wall Tomp. *2F & 164.31 & 164.5 & 164 & 163.61 & 163.91 & 766.51 & 163.31 & 167.11 & 154.91 & 155.3 \\
\hline Introam Temp.F & 163.31 & 154 & 153.8 & 163.6 & 153.61 & 163.61 & 153.81 & 163.21 & 153.2 & 163.6 \\
\hline Avg. Wall Tomp.F & 163.91 & 164.6 & 164.5 & 164.11 & 164.6 & 165.5 & 162.8 & 161.5 & 155 & 1552 \\
\hline Avg. Wall DT f & 9.91 & 9.9 & 10 & 9.81 & 10.41 & 11.3 & 8.31 & 7.9 & 1.8 & 1.6 \\
\hline Remaining Subcoolf & -0.5 & & & & & & & & & \\
\hline Exit Quality & 0.01 & 0.05 & $\overline{0.1}$ & 0.15 & 0.21 & 0.25 & 0.3 & 0.35 & 0.33 & 0.34 \\
\hline EXP.H-BtüHrhterF & 1532.41 & 1507.7 & 1524.2 & 15521 & 1450.7 & 1358.4 & 1792 & 1234.8 & 446.9 & 74.1 \\
\hline COMPUTEDH & & & $\mathrm{i}$ & & & & & & & \\
\hline Jallouk & 1325.61 & 1243.9 & 1190.1 & 1163.6 & 1127.6 & 1118.6 & 1086 & 853.1 & $435.6 !$ & 448.2 \\
\hline Szady 1(Cudo tube) & $1839.7 !$ & 1828.8 & 1844.2 & 1849.9! & 1836.4 & 1853.4 & 1834.91 & 1493.3 & 665.21 & 693.1 \\
\hline szady2 & 1667.61 & 1652.8 & 1681.8 & 1725.4 & 1705.71 & 1724.1 & 1697.5 & 1248.9 & 646.2 & 647.9 \\
\hline (RUQ) & 971071 & $\$ 507$ & 90159 & 85179 & 803191 & 75534 & 709561 & 68739 & 650851 & 64989 \\
\hline (F) & 1.131 & 1.583 & 2.066 & 2.356 & 2.629 & 2.867 & 3.07 & 3.249 & 3.3161 & 3.321 \\
\hline (HंजO) & 2221 & 308.8 & 385.7 & 460.3 & 612.41 & 668.0 & 689.81 & 633.11 & 646.11 & 647.4 \\
\hline \multirow[t]{3}{*}{ (HBOIL) } & 1830.61 & 1825.8 & 1838.1 & 1840.4 & 18231 & 1836.3 & 1813.3 & 1454.4 & 200.61 & 324.8 \\
\hline & & & & & & & & & & \\
\hline & ZONE2 & ZONE4 & ZONE6 & ZONE8 & ZONE10 & & & & & \\
\hline DP liquid Temp.f & $7 \pi .4$ & & & & & & & & & $i$ \\
\hline DP Liquid Density-lbm/ti3 & $93.37 i$ & & & & & & & & & + \\
\hline Elevation-t & $2 i$ & 4 & 6 & 81 & 9.8751 & & & & & \\
\hline Measured DPPsid & 0.1481 & 1.121 & 0.388 & 2.6781 & 3.36 & & & & & \\
\hline Zero Corrections-psid & 0.0221 & -0.028 & -0.754 & 0.128 & 0.1611 & & & & & \\
\hline Cortected DPPsid & 0.1261 & 1.149 & 9.742 & 2.49 & 3.1991 & & & & & \\
\hline Actual DPA & 2.081 & 2.58 & 3.81 & 4.78 & 5.68 & & & T! & i & i \\
\hline Measured TPPsig & 117.61 & & 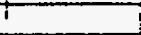 & 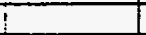 & 3.51 & & & $T$ & i & $i$ \\
\hline \multirow[t]{2}{*}{ Measured Overallift } & 202231 & & & & & & & I & & i \\
\hline & $i$ & & & & & & & $I$ & & \\
\hline Computed DPA & $1.82 !$ & 3.23 & 4.55 & 5.851 & 7.31 & & & I & & \\
\hline Friction-th & 0.09 & 0.3 & 0.61 & 0.99 & 1.23 & & & i & & i \\
\hline Acceleration-f & 0.1 & 0.2 & 0.3 & 0.39 & 0.4 & & & & i & i \\
\hline \multirow[t]{2}{*}{ Elevation-H } & 1.63 & 2.73 & 3.64 & 4.47 & 5.68 & & & & & \\
\hline & & & & & & & & & t & ; \\
\hline
\end{tabular}




\begin{tabular}{|c|c|c|c|c|c|c|c|c|c|c|}
\hline Run" & 6501 & $\therefore$ & 1 & - & $\therefore$ & $\dot{-}$ & $\therefore$ & 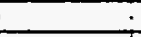 & : & \\
\hline Tost Fluid & CAFIO : & 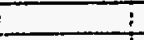 & $\dot{3}$ & $\therefore$ & 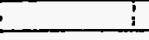 & $!$ & 1 & 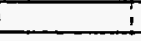 & 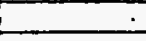 & \\
\hline Date: & 527193 & $i$ & $\therefore$ & 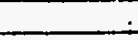 & 1 & 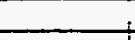 & - & & & \\
\hline Time: & $1: 43$ & 5 & 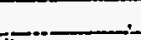 & $!$ & 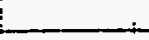 & & & & & \\
\hline Nole & TABOREK & Analytical d & & 1 & $L$ & ? & & & & \\
\hline Flow Areatt2 & 10.00336 & $\div$ & 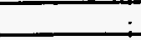 & $i$ & 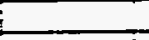 & & & $\therefore$ & :- & \\
\hline Heat Flow Areatt2 & $0.19595 ;$ & 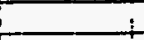 & $\div$ & : & $i$ & 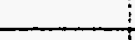 & & & & \\
\hline Sat. Pressure Psig & 109.51 & 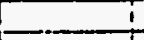 & & & & & & & & \\
\hline Sat. Temperature f $($ inlet) & 152.4 & & & $i$ & & & $?$ & & & \\
\hline Lq. Densitylbm/ns & 81.941 & 82.021 & 8211 & 82.111 & 82.051 & 82.04 & 82.021 & 82.13 & 82.181 & 82.17 \\
\hline Latont Heartumbm & 29.621 & 29.68 & 29.741 & 29.74 & $29.7 !$ & 29.691 & 29.681 & 29.761 & 29.81 & 29.78 \\
\hline Specitic Heat-Bu/bmiF & 0.2851 & 0.2851 & 0.2851 & 0.285 & 0.285 & 0.285 & 0.2851 & 0.2851 & 0.285 & 0.285 \\
\hline Liq. Visoositylbmithith & 0.432 & 0.4341 & 0.4351 & 0.455 & 0.4341 & 0.434 & 0.4341 & 0.4361 & 0.437 & 0.436 \\
\hline Lg. Conductivy Btuhr-fif & 0.02511 & 0.0251 & 0.02521 & 0.02521 & 0.0252 & 0.02511 & 0.02511 & 0.0252 & 0.02521 & 0.0252 \\
\hline Liq. PrandtNo. & 4.911 & 4.92 & $4.92 !$ & 4.921 & 4.921 & 4.921 & 4.921 & 4.92 & 4.931 & 4.83 \\
\hline Inlet Flow Rato-GPM & 3.2 & & $\therefore \quad$ i & 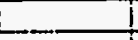 & 1 & & & 1 & 1 & 1 \\
\hline Outlet Lig. Fow Rafo-GPM & 1.31 & i & 1 & $L$ & & 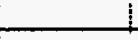 & 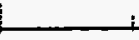 & + & 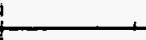 & \\
\hline Outlet Vap. Flow Ralo-GPM & 1.91 & & $\therefore$ & 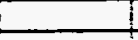 & & $i$ & : & I & $\therefore$ & - \\
\hline Inlet Temperature $\mathrm{F}$ & 146.91 & & & & 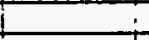 & & & & $\frac{1}{3}$ & \\
\hline Outlet Liq. Temperaturof & 749.41 & I & & $\therefore$ & 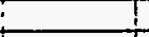 & & & & $T$ & \\
\hline Outlot Vap. Temperaturef & 144.11 & & & + & 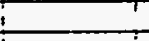 & & & & & \\
\hline Water Flow Rado-GPM & 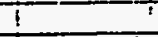 & & & $-i$ & t & & & & & \\
\hline Wader Inlet Tomp.f & 83.21 & & $i$ & $\bar{i}$ & $!$ & 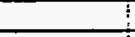 & ; & & I & I \\
\hline Wator outt of Temp.f & 124.21 & & I & 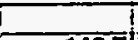 & i & 1 & $\therefore$ & 5 & 1 & I \\
\hline Intot Glbminring & 6300371 & & Temp & 143.7 & Density & 82.51 & 1 & + & $i$ & 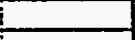 \\
\hline Suboooling $F$ & 5.51 & I & 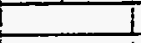 & 1 & 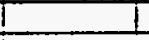 & 4 & i & & & \\
\hline \multirow[t]{2}{*}{ Fow Observation } & & & 10 & 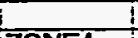 & 1 & & & & & 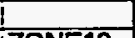 \\
\hline & ZONE1 & ZONE2 & ZONE3 & ZONE4 & ZONE 5 & ZONE6 & ZONET & ZONE8 & ZONE9 1 & ZONE10 \\
\hline Heat Input-Wats(nel) & 1882.31 & 877.6 & 891.5 & 894.6 & 877 & 889.5 & 879.5 & 563.1 & 46.7 & 70.7 \\
\hline Heat Loss-Watts & 11 & 2.6 & 3.61 & 3.5 & 3.61 & 26 & 1.61 & 2.3 & 2.5 & 14.5 \\
\hline 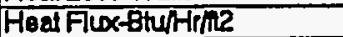 & 15294 & 15213 & 15454 & 15507 & 152021 & 15419 & 15246 & 9761 & 810 & 1226 \\
\hline Wall Tomp, & 159.21 & 661.5ा & $160.2 !$ & 159.9 & 163.5 & 16.6 & 158.41 & 157.6 & 750.61 & 150 \\
\hline Wall Tomp 44 & 159.6 & 159.2 & 161.7 & 160.9 & 161.71 & 161.2 & 158.5 & 157.9 & 150.61 & 150.6 \\
\hline Wall Temp. $\# 5 F$ & 159.81 & 157.9 & 162.81 & 160.9 & 160.5 & 161.6 & 157.61 & 157 & 151 & 151.1 \\
\hline Wall Tomp. AT & 72.91 & 160 & 158.5 & 158.7 & 159.7 & 181.7 & 159.31 & 158.5 & 150.61 & 150.5 \\
\hline Wall Tomp. \#F & 159.9 & 160 & 159.51 & 159.31 & 159.9 & 162.3 & 159.1 & 157 & 150.61 & 150.7 \\
\hline Instream Temp, $F$ & 149.8 & 149.6 & 149 & 149 & 149.3 & 149.4 & 149.51 & 148.8 & 149.61 & 148.7 \\
\hline Avg. Wall Tomp. $F$ & 159.6 & 160.2 & 1601 & 160.1 & 160.5 & 161.3 & 158.61 & 157.2 & 150.71 & 150.6 \\
\hline Avg.Wall DT. $f$ & 9 & 10 & 10.3 & 10.4 & 10.5 & 11.2 & 8.41 & 7.91 & 21 & 1.8 \\
\hline Romaining Subcooff & 0.5 & & & & & & & & 1 & 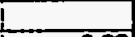 \\
\hline Ext Quality & 0.011 & 0.04 & 0.09 & 0.14 & 0.19 & 0.24 & 0.28 & $0.31 !$ & 0.32 & 0.32 \\
\hline EXP.HBtuHriner & 1702.41 & 1523.2 & 1503.81 & 1491.2 & 1453 & 1379.4 & 1816.9 & 1242 & 396.2 & 671.7 \\
\hline COMPUTEDH & & & & & & & & & & \\
\hline Jallouk & 13320.4 & Tस्253.4] & 1174.4 & 1743.8 & 1100.7 & 105 & 1071.61 & 837.8 & $441.8 !$ & 453.9 \\
\hline Szadyi(Cudo tubo) & 1800.5 & $17 \overline{9} 2.3$ & 1803.11 & 1808.4 & 1796.2 & 18123 & 1806 & 1459.8 & 667 & 692.8 \\
\hline szadyz & 1034.91 & 1625.6 & 1639.41 & 1678.7 & 1000.41 & 1077.4 & 1000.3 & 1217.6 & 650.71 & 653.3 \\
\hline (Aएव) & 95382 & 93297 & 88955 & 89787 & 794201 & 74955 & 70432 & 6037 & 848471 & 84401 \\
\hline (F) & 1.0051 & 1.45 & 1.972 & 2.326 & 2.6091 & 2.857 & 3.081 & 3.261 & 3.335 & 3.346 \\
\hline (HUQ) & $186 . \overline{4}$ & 285.8 & 384.8 & 453.9 & 609.61 & 557.8 & 601.6 & 636.4 & 650.7 & 653 \\
\hline \multirow[t]{3}{*}{ (HBOIL) } & 1799.71 & 1789.9 & 1797.21 & 1798.8 & 178241 & 1794.5 & 1783.41 & 1418.41 & 276.9 & 3782 \\
\hline & & & & & & & & & & \\
\hline & ZONE2 & ZONE4 & ZONE6 & ZONE8 & ZONE10 & & & & & \\
\hline DP liquid Temp.F & 76 & & & & & & & & & \\
\hline DP Liquid Density-lbm/it3 & 93.57 & & i & & & & & & $\perp$ & \\
\hline Elovation- & 21 & 4 & 6 & 8 & 9.875 & & & & I & \\
\hline Mossured DP\$sid & 0.145 & 1.225 & $\{.059$ & ह7गा & 3.4561 & & & & & \\
\hline Zero Corrections-psid & $0.0 \mathrm{c} 2$ & -0.028 & $=0.754$ & 0.128 & 0.1611 & & & & & \\
\hline ComectedDPFsid & 0.120 & 1.253 & 1.823 & 2.583 & 3.295 & & & & & \\
\hline Actual DPR & 2.081 & 2.38 & 3.64 & 4.58 & 5.47 & & & & I & \\
\hline Measured TPPrig & 109.5 & & & & 3.3 & & & & 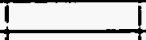 & \\
\hline \multirow{2}{*}{ Messured Overalle } & 185.6 & & & & & & & & & \\
\hline & & & & & & & & & & \\
\hline Computed DPA & 1.9 & 3.32 & 4.64 & 5.96 & 7.43 & & & & & \\
\hline Friction-t & 0.08 & 0.29 & 0.6 & 0.99 & 1.25 & & & & & t \\
\hline Accoleration-n & 0.00 & 0.2 & 0.3 & 0.4 & 0.47 & & & & & \\
\hline Elovation-1 & 1.73 & 2.83 & 3.74 & 4.56 & 5.77 & & & & 1 & 1 \\
\hline & & & & & & & & & & \\
\hline
\end{tabular}




\begin{tabular}{|c|c|c|c|c|c|c|c|c|c|c|}
\hline Run\# & $551 !$ & & & & & & $\cdot$ & & & \\
\hline Tost Fluid & C4F10 & & & & & & \pm & - & : & \\
\hline Dafe:-- & $5 / 2793$ & & & & & & - & \pm & \pm & \\
\hline Time: & $1: 43$ & & & & & & & & & \\
\hline Noté & TABOAREK & Anatytical c & & & & & & & & \\
\hline Flow Areatt2 & $0.00336 i$ & & & & & & & & & \\
\hline Heat Fow Avea-ll2 & 0.196951 & & & & & & $\therefore$ & : & : & \\
\hline Sat.Pressure psig & $113.2 !$ & & & & & & & & & \\
\hline Sal. Temperaturo-f(inlet) & 754.67 & & & & & & & & & \\
\hline Lq. Density & 81.791 & 81.591 & $81.52 !$ & $81.64 !$ & $81.62 !$ & 81.611 & $81.61^{1}$ & $81.7^{-}$ & $81.72 !$ & 81.72 \\
\hline atent Heat-Btunbm & $29.5 \pi$ & $29.36 !$ & 29.391 & 29.41 & 29.391 & $29.38 !$ & 29.381 & $29.44 !$ & 29.461 & 29.46 \\
\hline Spectic Hoat-Btufbmif & 0.2861 & 0.286 & 0.2861 & $0.286 !$ & 0.28561 & 0.2861 & 0.2861 & 0.2861 & 0.2861 & 0.286 \\
\hline iq. Viscositybmithr & 0.4291 & 0.425 & 0.4261 & 0.4261 & 0.4261 & 0.4261 & 0.4261 & 0.427 & 0.4281 & 0.428 \\
\hline Lig. Conductivity-Btuhr-itF & 0.0251 & 0.0249 & 0.02491 & 0.02491 & 0.02491 & 0.0249 & 0.02491 & 0.02491 & 0.02491 & 0.0249 \\
\hline Liq. Prandtl No. & 4.911 & 4.91 & $4.9 i$ & 4.9 & 4.91 & 4.91 & $4.9 i$ & 4.91 & 4.91 & 4.9 \\
\hline Inlet Fow Rate-GPM & 2.61 & & & & & & & & 1 & 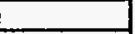 \\
\hline Outlet Lig. Flow Rate-GPM & $9.1 !$ & & & $i$ & i & & i & 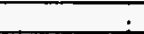 & - & 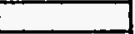 \\
\hline Outtet Vao. Fow Ralo-GPM & $9.6 i$ & & & & & & $\div$ & 1 & 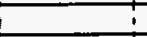 & 5 \\
\hline Inlet Temperaturef & 147.1 & & & & & & 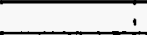 & & & \\
\hline Outet Liq. Temperaturof & $1525 !$ & & & & & & & & & \\
\hline Outet vap. Temperaturef & 746.6! & & & & & & & & & \\
\hline Waler Fow Ralo-GPM & & & & & & & & & & \\
\hline Water Inlet Temp.F & 87.71 & & 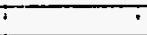 & & ; & & & & & \\
\hline Water outlet Temp.F & $131.2 !$ & & $\therefore$ & & : & & 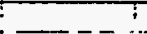 & 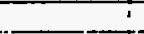 & $T$ & 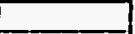 \\
\hline inlot G G mhrine & 51225881 & & Tomp & $\overline{143.410}$ & Density & 82.56 & $i$ & $\overrightarrow{.}$ & $\therefore$ & 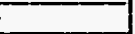 \\
\hline Subcoolingf & $7.5 i$ & & \pm & 1 & 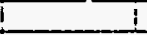 & 1 & I & 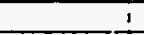 & $I$ & $L$ \\
\hline Flow Öbservation & !̇ & & $\mathrm{i}$ & $i$ & & & 1 & & $\begin{array}{ll} \\
\end{array}$ & 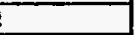 \\
\hline & ZONE1 & ZONE2 & $\angle O N E 3$ & ZONE4 & ZONES & ZONE6 :2 & ZONE7 : & ZONE8 & ZONE9 & ZONE10 \\
\hline Heat Input-Watts(nel) & $873.6 i$ & $86 \overline{6}$ & $1-864.1$ & \begin{tabular}{|l|}
867.71 \\
\end{tabular} & 851.71 & 859.5 & 877.8 & 547.11 & 461 & 67.9 \\
\hline Heat Loss-Watts & 12.81 & 31 & 4.11 & 4.11 & 4.11 & 31 & 1.81 & 2.81 & 3.11 & 17.6 \\
\hline Heat Flux-Bturhrfit2 & 151431 & 150121 & 14979 & 15041 & 14764 & 14899 & 15216 & 9484 & 797 & 117 \\
\hline Wall Temp. & $161.3 !$ & 163.4 & 162.21 & 161.7 & 165.11 & 162.8 & 160.41 & 1601 & 752.81 & 7523 \\
\hline Wail Temp. \#4-F & 767.91 & 161.31 & 163.7 & 162.61 & 163.5 & 163.2 & 160.71 & 160.31 & 1537 & 152.8 \\
\hline Wall Temp. \#5-F & $161.9 i$ & $160 !$ & 164.91 & 1626 & 16221 & 163.31 & 159.91 & 159.11 & 153.21 & 153.3 \\
\hline Wall Temp. \#1F & 72.11 & 161.9 & 160.41 & 160.4 & 161.81 & 163.8 & 161.31 & 158.6 & 152.61 & 152.8 \\
\hline Wall Temp. \#2F & 162.11 & 1621 & 161.41 & 161.2 & 161.91 & 164.31 & 161.41 & 158.5 & 152.7 & 153 \\
\hline Instream Temp.F & 150.71 & $151 . \overline{8}$ & 151.61 & 151.6 & 151.6 & 151.7 & 151.71 & 151.2 & 151.11 & 151.1 \\
\hline Avg. Wall Temp.f & 161.81 & 1622 & 161.91 & 161.8 & 152.31 & 163.3 & 160.71 & 159.3 & $152.9 !$ & 152.8 \\
\hline Avg. Wall DT.f & 10.41 & 9.7 & 9.6 & 9.6 & 101 & 10.91 & 8.41 & 7.71 & 1.71 & 1.7 \\
\hline Remaining Subcoolf & $1.5 !$ & & & & & & & & & \\
\hline Ext Quality & -0.011 & 0.04 & 0.1 & 0.161 & 0.22 & 0.28 & 0.34 & 0.37 & 0.38 & 0.38 \\
\hline EXP.H-BtunHr/i2/F & 1453.91 & $1552.1 !$ & 1552.5 & 1559.41 & 1471.5: & 1366.7 & 1821.81 & 1236.21 & $462.5 !$ & 697.8 \\
\hline COMPUIEDH & $\therefore$ & & & & & & 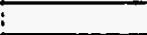 & & & \\
\hline Jallouk & T官 & 7295.5ा & र2161 & 1169.91 & TTझ्3.41 & Тसरत.81 & T119.7! & 848 & 395.41 & 411.2 \\
\hline 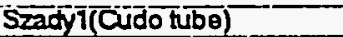 & 18001 & $180 \overline{4.61}$ & 1801.9 & 9806.11 & 1792.31 & 1803.41 & 1824.21 & 1452.87 & 600 & 641.7 \\
\hline Szady2 & 1033.17 & $1 6 \longdiv { 5 6 . 8 ! }$ & $1 \% 53.81$ & 1639 & 1656.41 & 1657.5 & 1691 & 1204.81 & 584 & 587 \\
\hline (Rण्) & 780721 & 77615 & 729011 & 68245 & 63747 & 59250 & 546351 & 506351 & 490251 & 48725 \\
\hline (F) & 1.0051 & 1.403 & 2.0071 & 2.404 & 2.7211 & 2.941 & 3.244 & 3.4431 & 3.5221 & 3.536 \\
\hline (संप्रि) & 966.61 & 232.8 & $353 i$ & 398.8 & 467.41 & $4 \overline{86.71}$ & 538.21 & $57 \pi$ & 688.91 & 686.3 \\
\hline (HBOIL) & 1799.61 & 1803.3 & 1798.11 & 1799.61 & 1782.71 & 1790.7 & 1808.41 & 1422.71 & 299.11 & 397.1 \\
\hline & 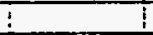 & & & & 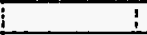 & 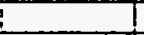 & 1 & 1 & 1 & 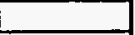 \\
\hline & ZONE2 & ZONE4 & ZONE6 & ZONE8 & ZONE10 & & I & 1 & $t$ & 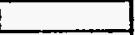 \\
\hline$\overline{D P}$ liquid Temp.F & : 74.9 & & 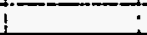 & & & & & 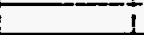 & 1 & \\
\hline DP Liquid Density-lbm/tis & 93.73 & & + & & 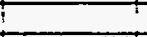 & & & & & \\
\hline Elevation-ft & 21 & 4 & 61 & $8 !$ & 9.8751 & & t & & 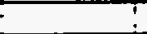 & \\
\hline Measured DP\$sid & 0.133 & 1.3दा! & 1.19 & 2.835 & 3.679 & & & & 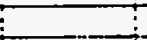 & \\
\hline Zero Corrections-psid & $0.022 !$ & -0.028 & $-0.754 !$ & 0.728 & 0.167 & & $t$ & & $?$ & ! \\
\hline Corrected DPPsid & 0.176 & 1.34 & 1.9531 & 2.7551 & 3.518 & & & & $\mathrm{~T}$ & $\Gamma$ \\
\hline Actual DPA & 2.091 & 2.231 & 3.44 & 4.31 & 5.13 & & & & i & t \\
\hline Measured TPPsig & 113.21 & & & & 3.7 & & $t$ & f & 1 & 1 \\
\hline Messured Overalfit & 193.13 & & $i$ & & 1 & I & ! & 1 & 1 & 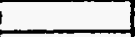 \\
\hline & $i$ & & i & & & & $\bar{i}$ & $i$ & I & \\
\hline Computed DPA & 9.851 & 3.151 & 4.311 & 5.46 & 6.841 & & & & I & I \\
\hline Friction-ft & 0.051 & 0.21 & 0.44 & 0.74 & 0.93 & & & & 1 & $L$ \\
\hline Accoleration-f & 0.061 & 0.14 & 0.23 & 0.31 & 0.32 & & & i & 1 & 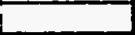 \\
\hline Elevation-f & 1.73 & 2.79 & 3.641 & 4.41 & 5.6 & & & & 1 & 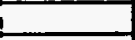 \\
\hline & $i$ & & & & & & & & $i$ & 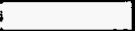 \\
\hline
\end{tabular}




\begin{tabular}{|c|c|c|c|c|c|c|c|c|c|c|}
\hline Runit & $\quad 652$ & & - & 1 & $i$ & & $\div$ & & 1 & ' \\
\hline TostFluid & C4F 10 & & 8 & & 1 & & i & & & \\
\hline Data: & $-5 / 27193$ & & & & $i$ & & & & & \\
\hline Time: & $1: 43$ & & & & & & & & & \\
\hline Noto & TABOAEK & Anatyical d & & & 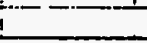 & & & & & \\
\hline Fow Areatil & 0.00336 & 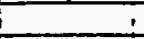 & 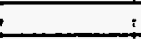 & & & & & & & \\
\hline Head Fow Areafl & 0.19695 & 4 & 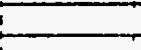 & & & & & & & \\
\hline $\begin{array}{l}\text { Sat. Pressure- } \\
\text { Sat. Tomperag }\end{array}$ & $\begin{array}{l}108.97 \\
1521\end{array}$ & & & & & & & & & \\
\hline 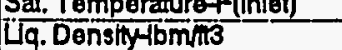 & $-8 \frac{102.1}{81.87}$ & 81.961 & 82.041 & $81.96 !$ & 81.981 & 81.98 & $81.92 !$ & 82.04 & 82.04 & 82.00 \\
\hline Latent Heat-Btulbm & 29.57 & 29.63 & 29.691 & 29.631 & 29.651 & 29.65 & 29.611 & 29.691 & 29.691 & 29.73 \\
\hline Spectio Hear-Btudthmf & 0.285 & 0.285 & 0.2851 & 0.2851 & 0.2851 & 0.285 & 0.2851 & 0.2851 & 0.2851 & 0.285 \\
\hline Lig. Viscositylom hithr & 0.4311 & 0.4321 & $0.434 T$ & 0.4321 & 0.4331 & 0.433 & 0.4321 & 0.4341 & 0.434 & 0.435 \\
\hline 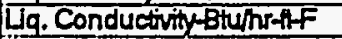 & 0.025 & 0.02511 & 0.02511 & 0.02511 & 0.02511 & 0.02511 & 0.02511 & 0.02511 & 0.02511 & 0.0252 \\
\hline İg. Prandtt No. & 4.91 & 4.911 & 4.921 & 4.911 & 4.921 & 4.921 & 4.911 & 4.921 & 4.921 & 4.92 \\
\hline InlatFlow Rato-GPM & 2.6 & & 1 & & -1 & & $i$ & & & \\
\hline Out lof Liq. Fow Ralo-GPM & 1.3 & & & & 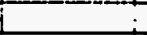 & & ; & & & \\
\hline Outlet Vap. Flow Rato-GPM & 1.71 & i & is & & 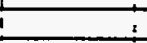 & & & & & \\
\hline Inlet TemperatureF & 146.9 & & & & 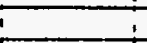 & & & & & \\
\hline Outet Lq Temporatura & 150.4 & & $T$ & & $r$ & & & & & \\
\hline Outuet vap. Temporafurét & 144.8 & & & & & & & & & \\
\hline Water Flow Rato-GPM & & & & & $\bar{\vdots}$ & & $!$ & & & \\
\hline Water inlot Tomp.F & 85.21 & $i$ & $i$ & $\bar{t}$ & 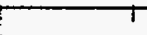 & & $T$ & & & $t$ \\
\hline Wator out let Tomp.F & 126.2 & & $=$ & $i$ & 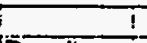 & & & & & \\
\hline InTot G- & 512728 & & Tomp & 143 & Do Density & 82.21 & & & & \\
\hline Subcoolingf & 5.21 & & & 1 & 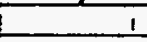 & & I & & & \\
\hline Fow Observation & & & & & & & & & & \\
\hline & & & NEs & NE4 I & NNE5 i2 & NE6 & NE7 j & ZONE 8 & ZONES iR & ZONE10 \\
\hline Heat Input-Watts(nei) & 870.2 & $853.4 !$ & 864 & 863.61 & 864.1 & 868.41 & 867.71 & 545.3 & 46.3 & $\begin{array}{r}67.5 \\
\end{array}$ \\
\hline Hoat Loss-Watts & 12.8 & & 4.1 & 4.11 & 4.2 & 3.1 & 1.91 & 28 & 3.1 & 17.7 \\
\hline Head Flux-BturfirmL & 15084 & 14966 & 1497 & 14970 & 14979 & 15053 & 150411 & 3452 & 803 & 1170 \\
\hline Wall Temp. H3F & 759.2 & 161.11 & 159.8 & 759.7 & 163.5 & 161 & 158.8 & 157.7 & 151.1 & 150.4 \\
\hline Wall Temp. $4 \mathrm{~F}$ & 159.5 & 159.11 & i61.4 & 160.7 & 161.6 & 961.3 & 159.3 & 158.21 & 151.3 & 750.9 \\
\hline Wall Tomp. \#SF & 159.71 & 157.9 & 16261 & 160.7 & $\frac{160.51}{160.1}$ & 161.6 & 158.31 & 157.14 & 151.31 & 151.5 \\
\hline Wall Tomp. IIF & 71.81 & 159.8 & 158 & 158.6 & 159.61 & 16211 & 159.71 & 156.4 & & 150.8 \\
\hline Wall Tomp. WF & 159.9 & 159.8 & 1591 & 759.7 & 160.1 & 16241 & 159.91 & 156.41 & 150.91 & 151.1 \\
\hline Instream Tomp.F & 160.3 & 149.8 & 840.4 & 149.81 & 149.7 & 149.7 & 1501 & 149.41 & 149.4 & 149.1 \\
\hline Avg. Wall Tomp. $F$ & 159.6 & 160 & 959.61 & 159.8 & 160.5 & 161.5 & 159.21 & 157.21 & 151.1 & 150.9 \\
\hline Avg. WallDT.F & 8.6 & 9.5 & 9.51 & 9.4 & 10.1 & 11.11 & 8.51 & 7.31 & 1.7 & 1.8 \\
\hline Aomaining Subcoolf & -0.9 & & & & & & & & & \\
\hline Exit Quality & 0.01 & 0.07 & 0.12 & 0.18 & 0.24 & 0.3 & 0.36 & 0.39 & 0.39 & 0.4 \\
\hline EP. HETURHRLIF & 1755.4 & 1579.8 & 15811 & 1584.4 & 1487 & 1353.7 & 1765.3 & 1289.1 & 482.41 & 654.7 \\
\hline COMPUIEDH & & & & & & & & & & \\
\hline Jallouk & 7299.5 & 7235.1 & 7170.41 & 7145.41 & 1123.31 & 7108.5 & 7094.41 & 837 & 403.61 & \\
\hline Szady' (Cudo tubo) & 17924! & 1780.9 & 1778.8 & 1784.3 & 1785.6 & 1792 & $1796.8 \Gamma$ & 1437 & 62.81 & \\
\hline Szadty2 & 1624.41 & 1611.2 & 1609.21 & 1649.81 & 1649.11 & iE54.5 & $1657.3 !$ & 1190.3 & 6011 & 604.1 \\
\hline (सUQ) & 775581 & 74688 & 699511 & 657101 & 611841 & 56898 & 523341 & 48421 & 468921 & $\overline{484}$ \\
\hline (i) & 1.192 & 1.692 & 2.1861 & 2.5451 & 2.851 & 3.118 & 3.3571 & 3.554 & 3.6281 & 3.6 \\
\hline (HLO) & 187.67 & 280.31 & 3621 & 421.7 & 472.11 & 516.6 & 556.3 & 588.61 & 600.81 & 604.1 \\
\hline (HBOIL) & 1791.6 & 1778.6 & 9773.81 & 1776.5 & 1774.61 & 1777.6 & 1778.7 & 7403.31 & 290.7 & 382.4 \\
\hline & & & & & & & & & & \\
\hline & ZONE2 & ZONE4 & ZONE6 6 & ZONE8 & ZONE10 & & & & & \\
\hline Sp liquid Temp. $F$ & 74.5 & & 1 & & & & & & & \\
\hline DPLiquid Density-lbmitt3 & 93.78 & & & & & & & & & \\
\hline Elevation- & & 41 & 6 & 8 & 9.875 & & & & & \\
\hline Measured DPPsid & 0.135 & 1.469 & 1.33 & 3.045 & 3.839 & & & & & \\
\hline Źoro Cortections-psid & 0.022 & -0.028 & -0.754 & $\left.0.128\right|^{-1}$ & $0.761 \Gamma$ & & & & & \\
\hline Comectod DPP id & 0.1131 & 1.497 & 2.084 & 2.917 & 3.6781 & & & & & \\
\hline Actual DPA & 2.091 & 1.941 & 3.21 & 4.021 & $4.83 !$ & & & & & \\
\hline Measured TPPsig & 108.91 & & 1 & & 3.51 & & & & & \\
\hline Mossured Overaliff & 184.89 & & & & $T$ & & & & & \\
\hline & & & & & & & & & & \\
\hline Computed DPA- & $1.71 \mathrm{i}$ & 2.96 & 4.111 & 5.26 & 6.641 & & & & & \\
\hline Friction-f & 0.07 & 0.24 & 0.5 & 0.81 & 1.01 & & & & & \\
\hline Accoleration-t & 0.08 & 0.16 & 0.2 & 0.34 & 0.34 & & & & & \\
\hline Elevation-tt & 1.56 & 2.56 & 3.36 & 4.111 & 5.281 & & & & & \\
\hline & & & & & & & & & & \\
\hline
\end{tabular}




\begin{tabular}{|c|c|c|c|c|c|c|c|c|c|c|}
\hline Run\# & $553 i$ & & & & & & + & 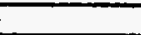 & ' & ' \\
\hline Tost Fuid & CAF10 & & $i$ & & $:$ & 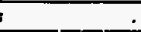 & 1 & & & \\
\hline Dale: & $5 \sqrt{27193}$ & & & & & & & ; & ; & \\
\hline Time: & $1: 43$ & & & & & 1 & & & & \\
\hline Note & TABOREK & Anahyical & & & & & & & & \\
\hline Flow Area-ti2 & $0.00336 i$ & & & & & & & & & \\
\hline Heat Fow Areatt2 & 0.19695 & & $\therefore$ & & & & & & & \\
\hline Sal Pressure-psig & $116.6 !$ & & $i$ & & & & & & 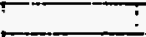 & \\
\hline Sal. Temperaturo-finlet) & $156.6^{7}$ & & 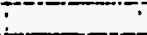 & & & -1 & & & & \\
\hline Lq.Density ibmims & 81.381 & 81.17 & $81.23 !$ & $81.17 !$ & $81.17 !$ & 81.15 & 81.091 & 81.171 & 81.231 & 81.23 \\
\hline Latent Hear-Bturbm & 29.211 & 29.051 & 29.11 & 29.051 & 29.051 & 29.041 & 291 & 29.051 & 29.11 & 29.1 \\
\hline Specitic Heet-Btuntb $/ \mathrm{F}$ & 0.287 & 0.287 & 0.2871 & 0.2871 & 0.287 & 0.2871 & 0.2871 & 0.2871 & 0.287 & 0.287 \\
\hline Liq. Vieooeth ismithr & 0.421 & 0.4171 & 0.4181 & 0.417 & 0.497 & 0.4171 & $0 . \overline{416 i}$ & $0.417 \pi$ & 0.4181 & $0 . \overline{4} \overline{8}$ \\
\hline Lg. Conductivity Btuhr-fF & 0.02471 & 0.0246 & 0.02461 & 0.02461 & 0.02461 & 0.02461 & 0.02451 & 0.02461 & $0.0246 i$ & 0.0246 \\
\hline Liq. Prandi No. & 4.881 & 4.87 & 4.881 & 4.87 & 4.871 & 4.87 & 4.871 & 4.871 & 4.881 & 4.88 \\
\hline Inlet Fow Rate-GPM & (1.8) & & $i$ & -1 & & -1 & & 1 & $\square$ & \\
\hline Outet Liq. Fow Rato-GPM & 1 & & 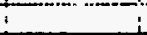 & $i$ & & & & & & \\
\hline Outlet Vap. Flow Rate-GPM & 0.9 & & - & 1 & 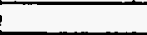 & & & & & \\
\hline Inlet Temperaturaf & $149.4 i$ & & & 1 & & & & & & \\
\hline Outet Liq. Temperature- & $155 !$ & & & 1 & & & & & & \\
\hline Outlat Vap. Temperaturef- & 149.91 & & i & & & & & & & \\
\hline Water Fow Rato-GPM & & & $T$ & 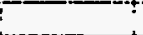 & & $\mathrm{i}$ & & & & \\
\hline Water Inlet Temp.F & 91.71 & & $i$ & 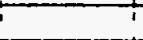 & T & 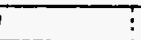 & $T$ & & ' & ' \\
\hline Water outlet Temp.F & $\overline{131.1 !}$ & & 1 & 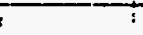 & 7 & T & $\because$ & & T & 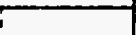 \\
\hline Inlet Gibm/nrate & 3535781 & & iTemp & 144.7 & Donsity & 82.311 & T & & & i \\
\hline Subcoolingf & $7.2 i$ & & & 1 & -2 & 1 & $i$ & I & i & 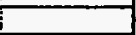 \\
\hline Flow Observation & & & & & & & & $\mathrm{i}$ & $i$ & \\
\hline & ZONE 1 & ZONE2 & ZONES & ZONE4 4 & ZONES & ZONE6 & ZONE7 & ZONE8 & ZONE9 I & ZONETO \\
\hline Heat Input-Watts(net) & 863.9 & 857.31 & 852 & 860.17 & 863.6 & 368.4 & $576 !$ & 56.3 & 45.6 & 64.7 \\
\hline Heat Loss-Watts & 14 & 3.3 & $4.5 i$ & 4.81 & $5 i$ & 4.1 & 2. & 2.8 & $3.6 i$ & 20.7 \\
\hline Hoat Flux-Btu/Hrifi2 & 14975 & 148611 & 14942 & 145091 & 14970 & 15053 & 9985! & 976 & 7901 & 1122 \\
\hline Wall Temp. $3 F$ & 162.3. & 164.9 & 163.1 & 163.3 & 166.9 & 164.2 & 161.67 & $955.7 !$ & 955.5 & 154.9 \\
\hline Wall Temp. \#4F & 152.91 & 162,2 & 164.7 & 164.2 & 165.4 & 165.1 & $161.4 !$ & 155.9 & $155.7 !$ & 155.4 \\
\hline Wall Temp. $5 F$ & 162.9 & 161.31 & 166.11 & 164 & 164 & 165.41 & 1601 & 156 & $155 \overline{81}$ & 156 \\
\hline Wadl Temp. 1F & 79.41 & 162.8 & 161.4 & 181.9 & 168.3 & 168 & 161.11 & 955.5 & 155 & 155.5 \\
\hline Wall Tomp. $12 F$ & 163.3 & 162.8 & 162.21 & 162.8 & 163.41 & 166.5 & 161.11 & $155.8 !$ & 1551 & 155.7 \\
\hline Instroem Tomp.f & 162.91 & 164 & 168.7 & 164 & 164 & 164.1 & 164.41 & 164 & 163.71 & 163.7 \\
\hline Avg. Wall Tomp.F & 162.91 & 1631 & 1629 & $1 E-4$ & 164 & 165.2 & 1611 & 155.8 & 155.41 & 155.5 \\
\hline Avg. Wall DT $F$ & 9.31 & 8.3 & 8.51 & 8.7 & 9.3 & 10.4 & 6.21 & 1.7 & 1.71 & 1.7 \\
\hline Remaining SubcoofF & -1.51 & & & & & & & & & \\
\hline Exot Quality & 0.01 & 0.11 & 0.181 & 0.27 & 0.351 & 0.44 & 0.5 & 0.5 & 0.51 & 0.51 \\
\hline 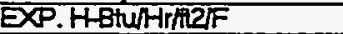 & $1614.9 i$ & 1789.7 & $17 \overline{63.1 i}$ & 1718.4 & $9601.3 !$ & 1448.2 & $1613.3 i$ & 562.21 & $474.9 i$ & 641.1 \\
\hline COMPUIEDH & 5 & & & & & & & & & \\
\hline Jallouk & 1358.2! & $1 \overline{299.81}$ & $1267.6 !$ & 1234.7 & 72731 & न184.9ं & $933.4 !$ & उद्वा & उ32.2! & 356.9 \\
\hline Szadyi(Cudo tubs) & 1813.81 & 1819.4 & 1822 & 1824.3 & 7829.1 & 7835.5 & 1508.37 & 565.1 & 533.2 & 586.2 \\
\hline szady2 & 1646.31 & 1651.41 & $1655 !$ & 1657.2 & $1 6 \longdiv { 3 ! }$ & 1700 & 1299.51 & 483.61 & 485.51 & 489.6 \\
\hline (RUQ) & 544971 & 522701 & 474511 & 42858 & 38140! & 334231 & 29538 & 27704 & 273551 & 27054 \\
\hline$(\mathrm{F})$ & 1.2611 & 1.855 & 2.4381 & 2.8651 & 3.2261 & 3.5461 & 3.787 & 3.91 & 3.9241 & 3.942 \\
\hline$(\overline{H L O})$ & 165.81 & 229.4 & 301.41 & 354.31 & 399 & 438.61 & 468.51 & 482.31 & 485.11 & 487.3 \\
\hline (HBOIL) & 1813.41 & 1818.2 & 1819.2 & 1819.8 & $1822.7 !$ & 1828.11 & 1493.11 & 408.61 & 334.41 & 440.9 \\
\hline & & & & & $=0$ & & 1 & 1 & 1 & \\
\hline & ZONE2 & ZONE4 & ZONE6 & ZONE8 & \begin{tabular}{|l|} 
ZONE10 \\
\end{tabular} & & 1 & & 1 & 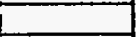 \\
\hline DPliquid Temp.F & 74 & & & & 1 & 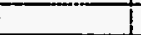 & & & 1 & 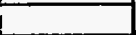 \\
\hline DP Liquid Densitylbm/tis & 93.851 & & & & & & & & 1 & \\
\hline Elevation-t & 2 & 4 & 6 & $8 \mid$ & 9.875 & & & & & \\
\hline Measured DPPsid & 0.084 & 1.59 & 1.612 & 3.415 & 4.261 & & & & & \\
\hline Zero Corrections-psid & $0 . \overline{022 !}$ & -0.028 & -0.754 & 0.128 & 0.1611 & & $T$ & & & \\
\hline Conected DPFid & $0.042 !$ & 1.718 & 2.3661 & 3.287 & 4.11 & & I & & t & \\
\hline Actual DPA & 2.241 & 1.58 & 2.741 & 3.421 & 4.141 & & $i$ & & i & \\
\hline Mosured TPPsig & 116.61 & & & & 3.11 & & & & & \\
\hline Messured Overallit & 200.69 & & & & 1 & t & $T$ & & & \\
\hline & 1 & 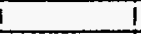 & $I$ & 1 & 1 & I & $i$ & 1 & I & 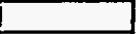 \\
\hline Computed DP-I & 9.571 & 26 & 3.5 & 4.611 & 5.871 & & & & 1 & \\
\hline Friction-t & 0.04 & 0.15 & 0.321 & 0.47 & 0.581 & & 1 & & $i$ & 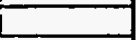 \\
\hline Accoleration-ft & 0.05 & 0.1 & 0.171 & 0.211 & 0.21 & i & & 1 & L & \\
\hline Elevation-t & 1.48 & 2.35 & 3.01 & 3.93 & 5.08 & ! & 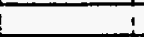 & i & 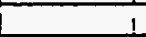 & \\
\hline
\end{tabular}




\begin{tabular}{|c|c|c|c|c|c|c|c|c|c|c|}
\hline Run: & $554 i$ & & & & & & & & , & \\
\hline Test Fluid & .CAF10 & & & $\dot{-}$ & & & $\therefore$ & - & + & \\
\hline Dale: & $15 / 27 / 931$ & & 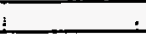 & 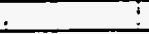 & & & & & $i$ & \\
\hline Time: & $5: 28$ & & $\vdots$ & & & & & & & \\
\hline Note & ¿TABOREK & Analytical c & & & & $\vdots$ & - & 1 & $\perp$ & \\
\hline Fow Area-t12 & 0.00336 & & & & & - & - & & & \\
\hline Hoal Flow Arearti2 & 0.19695 & & $i$ & & & 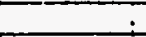 & . & & & \\
\hline Sat. Pressura Psig & $106.3 !$ & & & & & & & & & \\
\hline Sat. Temperaturof(inlet) & 150.5 & & & 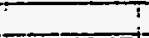 & & & $\longrightarrow$ & I & $i$ & \\
\hline Lg. Densfin $1 \mathrm{bm} / \mathrm{s}$ & 81.981 & 82.051 & 82.131 & $82.07 !$ & 82.091 & 82.091 & 81.941 & 82.05 & 82.071 & 82.07 \\
\hline Latont Hoar-Btufbm & 29.651 & 29.7 & 29.761 & 29.721 & 29.731 & 29.731 & 29.621 & 29.7 & 29.721 & 29.72 \\
\hline Speoctio Heaf Btulibm/F & 0.285 & 0.2851 & 0.2851 & 0.285 & 0.2861 & 0.2851 & 0.2851 & 0.285 & 0.2851 & 0.285 \\
\hline Liq. Visoositylbm/fithr & 0.4331 & 0.434 & 0.4361 & 0.435 & 0.4351 & 0.4361 & 0.4321 & 0.434 & 0.4351 & 0.436 \\
\hline Liq. Conductivity the $h$ hrfif & 0.02511 & 0.02521 & $0.0252 i$ & 0.0252 & $0.0252 i$ & 0.02521 & 0.02511 & 0.0252 & 0.02521 & 0.0252 \\
\hline Lig. Prandt No. & 4.92 & 4.92 & 4.92 & 4.921 & 4.92 & 4.921 & 4.911 & 4.92 & 4.921 & 4.92 \\
\hline Inlet Flow Rato-GPM & 11 & & j & 1 & 1 & 1 & 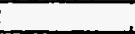 & 1 & 1 & \\
\hline Outlet Lia. Flow Rate-GPM & 1.41 & & & & $i$ & 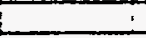 & & it & 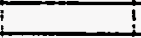 & \\
\hline Outtel Vap. Flow Rate-GPM & 1.5 & & 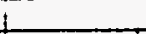 & & : & : & & :- & & \\
\hline nnlel temperature & 145.4t & & 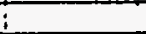 & $\therefore$ & & & & 1 & & \\
\hline OutletLiq. Temperature & $150.3 !$ & & & & & & & & & \\
\hline Outlef Vap. Temperaturef F & 145.71 & & & 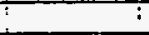 & ! & ! & 1 & & it & \\
\hline Watop Fow Rato-GPM & $i$ & & : & : & : & 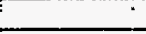 & 1 & & & \\
\hline Water trlol Tomp.F & 92.11 & & 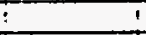 & i & : & : & i & i & 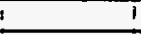 & \\
\hline Water outtol Temp.F & 133.3 & & 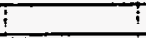 & 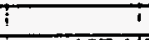 & $\dot{\square}$ & I & 5 & ! & - & \\
\hline Inlol Glbm/hrit2 & 1998241 & & Tomp & 137.11 & Density & 83.73 & & & 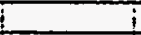 & \\
\hline Subcooling $F$ & 5.11 & & & 1 & $i$ & $i$ & $!$ & 1 & I & \\
\hline Flow Observation & & & & $i$ & $i$ & 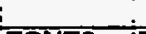 & & & 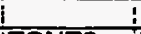 & \\
\hline & ZONE1 & ZONE2 & ZONE3 1 & ZONE4 & ZONE5 & ZONE6 & ZONE? & ZONE8 & ZONE9 & ZONETO \\
\hline Heal input-Watts(net) & 875 & 863.2 & 858.5 & 857 & 857.2 & 650.4 & 77.61 & 55.8 & 46.5 & 65.2 \\
\hline HoatLoss-Watts & 13.7 & 3.2 & 4.4 & $4.6 i$ & 4.91 & 4.81 & 1.81 & 2.8 & 3.6 & 20.9 \\
\hline Hozf Flux-BTu/Hrite & 15168 & 1453 & 14882 & 148561 & 148591 & 11274 & 7345 & 967 & 806 & 1130 \\
\hline Wall Temp. \#3+ & 157.11 & 159.2 & 157.31 & 158.41 & 161.7 & 165.8 & 152.2 & 151.8 & 751.3! & 751 \\
\hline Wall Temp. H4F & 157.6 & 157.1 & 1591 & 159.4 & 1601 & 165.6 & 152.3 & 151.8 & $151.7 !$ & 151.5 \\
\hline Wall Tomp. H5F & 157.4 & 155.6 & 160.61 & 158.7 & 1591 & 160.3 & 1525 & 151.9 & 151.71 & 152.1 \\
\hline Wall Tomp. IF & 70.8 & 157.1 & 156.11 & 156.5 & 158.11 & 159.4 & 159.2 & 151.4 & 150.81 & 151.6 \\
\hline Wall Tomp. IZF & 158.2 & 157.4 & 156.9 & 167.6 & 158.61 & 169.4 & 153.4 & 961.8 & 150.8 & 161.7 \\
\hline Inetroam Tomp.f & 149.7 & 149.3 & 148.9 & 1492 & $149.1 \Gamma$ & 149.1 & 140.01 & 148.8 & 140.2 & 140.2 \\
\hline Avg. Wall Tamp. $F$ & 157.6 & 157.7 & 157.3 & 158.31 & 158.9 & 162.81 & 152.7 & 151.71 & $151.3 !$ & 151.6 \\
\hline Avg. Wall DT.F & 7.21 & 7.71 & 7.8 & 8.4 & 9.21 & 13.2 & 2.8 & 2.4 & $2 !$ & 2.3 \\
\hline Romaining Subcooff & -10.5 & & & & & & & & & \\
\hline Exot Quality & 0.1 & 0.25 & 0.4 & 0.54 & 0.69 & 0.8 & 0.81 & 0.82 & 0.83 & 0.84 \\
\hline 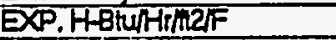 & 2109.7 & 1937.3 & 1919.6 & 1773 & 1623.3 & 856.3 & 487.5 & 403.6 & 398.3 & 489.5 \\
\hline COMPOTEDH & & & & & & : & & & & \\
\hline Jallouk & 369.9 & 73्30.11 & T272.61 & 1248.2 & 77.61 & 77.61 & 77.7 & 7.7 & 77.6 & 77.6 \\
\hline szady 1(Cudo tube) & 9790.6 & 1774.6 & 17661 & 1768.3 & 77.61 & 77.61 & 7.7 & 7.7 & 77.61 & 77.6 \\
\hline $\operatorname{szady}^{2}$ & 1623.2 & 1604.3 & 1594.51 & 1590.8 & 77.01 & 77.01 & 7.7 & 7.7 & 77.6 & 7.0 \\
\hline (FLO) & 28877 & 248301 & 2003291 & 15939 & 15969 & 15939 & 15969 & 15939 & 15969 & 15960 \\
\hline (F) & 1.824 & 2.673 & 3.361 & 3.908 & 3.9081 & 3.9081 & 3.908 & 3.908 & 3.9081 & 3.908 \\
\hline (सLOC) & 142.2] & 208.31 & 261.71 & 304,4 & 304.4 & 304.4 & 304.4 & 304.4 & 304.41 & 304.4 \\
\hline (HBOIL) & 1790.31 & 1773.6 & 1764.11 & 1765.31 & 1765.31 & 1765.31 & 1765.3 & 1765.31 & 1765.3 & 1765.3 \\
\hline & & & & $!$ & & i & +2 & & & \\
\hline & ZONE2 & ZONE4 & IZONE6 & ZONE8 & ZONETO & $i$ & & & & \\
\hline DPliquid Temp.f & 74.1 & & 1 & $i$ & 1 & $i$ & i & & & \\
\hline DPLiquid Density-bmins & 93.84 & & & & & & & & & \\
\hline Elovation-h & & & $6 i$ & 81 & 9.875 & & i & & & \\
\hline Measured DPPsid & 0.5371 & 2.258 & 2.7581 & 4.054 & 5.003 & & & & & \\
\hline Zero Contoctions-psld & 0.002 & -0.0281 & -0.7541 & 0.128 & 0.161 & & 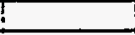 & & & \\
\hline Comected DPPsid & $0.515 !$ & 22861 & 2.912 & 3.9281 & $4 . \overline{842}$ & & & & & \\
\hline Actual DPA & 1.38 & 0.58 & 1.751 & 2.28 & 2.8 & & & & & \\
\hline Measured TPPSig & 106.3 & & & -1 & 3.31 & $\bar{\vdots}$ & 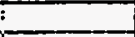 & & I & \\
\hline Measured Overalin & 180.18 & & & & 1 & I & & & & \\
\hline & & & & & & & & & & \\
\hline Computed DP- & 1.09 & 1.77 & 2.31 & 3.481 & 4.591 & & & & I & \\
\hline Friction-t & 0.03 & 0.1 & 0.19 & 0.24 & 0.29 & & & & & \\
\hline Accoleration-t & 0.03 & 0.08 & 0.13 & 0.14 & 0.15 & & & & & \\
\hline Elovation-ti & 1.02 & 1.59 & 1.98 & 3.1 & 4.15 & & & & & \\
\hline & & & & & & & & & & \\
\hline
\end{tabular}




\begin{tabular}{|c|c|c|c|c|c|c|c|c|c|c|}
\hline Run\# &.$\quad 5551$ & & & $\dot{-}$ & & & $\dot{-}$ & $\dot{-}$ & ' & 1 \\
\hline Tost Fluid & C4F10: & & & & & & & $\therefore$ & & $\dot{.}$ \\
\hline Date: & $5 \sqrt{27} 193$ & & & & & & & & & \\
\hline Time: & $5: 28$ & & & & & & & & & \\
\hline Note & TABÖREK & Anahytical o & & & & & & & & 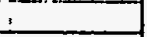 \\
\hline Fow Area-t2 & $0.00336:$ & & & & & & & & & \\
\hline Heat Fow Area-t? & $0.19595:$ & & & & & & & & & \\
\hline Sad. Pressuropsig & $101.4^{\circ}$ & & & & & & & & & \\
\hline Sat. Temperaturerfinlet) & $\overline{47.5 !}$ & & & & & & & & & \\
\hline Uq. Densiny-binm & 82.391 & 82.5 & $8257^{\prime}$ & $82.51 !$ & $8 \overline{2.51}$ & 82.481 & 82.39 & $82.48 !$ & $82.59^{\prime}$ & 825 \\
\hline Latent Hear-sturibm & 29.941 & 30.021 & 30.071 & 30.041 & 30.021 & 30.071 & $29.94 !$ & 30.011 & 30.041 & 30.02 \\
\hline Spocific Hod tuflbmif & $0.284 i$ & 0.284 & 0.2841 & 0.2841 & 0.2841 & 0.2841 & 0.284 & 0.284 & 0.284 & 0.284 \\
\hline Lia. Visoosit ibmithr & 0.4411 & 0.448 & $0 . \overline{441}$ & $0 . \overline{4431}$ & 0.4431 & $0 . \overline{4431}$ & 0.441 & 0.4431 & 0.4431 & 0.448 \\
\hline Liq. Conductivity-Btu/hr-flf & 0.0254 & 0.0254 & 0.02551 & 0.02541 & 0.02541 & 0.02541 & 0.02541 & 0.02541 & 0.0254 & 0.0254 \\
\hline iq. Prandit No. & 4.941 & 4.94 & 4.551 & 4.951 & 4.941 & 4.941 & 4.941 & 4.94 & 4.95 & 4.94 \\
\hline Inlet Flow Rate-GPM & & & & & & & & & & \\
\hline Outlel Liq. Fow Rato-GPM & 1.7. & & & & & & & i & & \\
\hline Outlet Vap. Flow Rate-GPM & 2.61 & & & & & & & & & \\
\hline Inlet Temperaturef & 144 & & & & & & & & & \\
\hline Outlel Liq Temperature- & 148.7? & & & & & & & & & \\
\hline Out & $943.5^{\circ}$ & & & & & & & & & \\
\hline Water Fow Ralo-GPM & & & & & & & & $i$ & & \\
\hline Wader Inlet Temp.F & 89.71 & & & & & & & t & & $\bar{T}$ \\
\hline Water outet Temp.F & 129.4 & 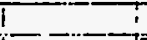 & 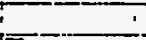 & & 1 & 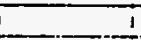 & 1 & $\cdot$ & 1 & 1 \\
\hline InTot Gibmhritiz & 2003031 & & Temp & 13610 & Density & 83.331 & & $i$ & i & $i$ \\
\hline SubcoolingF & 3.5 & & 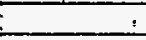 & 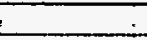 & $i$ & 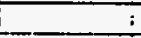 & & 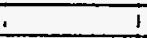 & 1 & 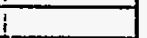 \\
\hline Flow Observation & & & & & & & & & & \\
\hline & ZZONE1 & ZONE2 I & $Z O N E 3$ & ZONE4 & ZONE5 & ZONE6 & ZONE7 & ZONE8 & ZONE9 & ZZONETO \\
\hline Hoat Input-Watts(not) & $1 \quad 863.3 i$ & 8527 & 848 & 843.41 & 847.2 & 642.7 & 76.3 & 55.2 & 45.4 & $1-\overline{64.6}$ \\
\hline Heat Loss-Watts & 13.1: & $3.1 !$ & $4.2 i$ & 4.41 & $4.6 !$ & 4.41 & 1.7 & 2.61 & 3.51 & 19.8 \\
\hline Heat Flx-Bturhrnit & 14965 & 147811 & 147001 & 14620 & 14686 & 11141 & 1323 & 957 & 787 & 1120 \\
\hline Wall Temp. $\# 3=$ & 754.81 & 156.7 & T55! & 756.41 & 759.81 & 768.11 & 749.9 & 749.4 & $\lceil 49.31$ & 140 \\
\hline Wall Temp. \#4F & 155.3 & 154.51 & 156.81 & 157.31 & 157.8 & 167.9 & 1501 & 149.51 & 149.4 & 149.3 \\
\hline Wall Temp. & 155.11 & 155.2 & 158.31 & 156.5 & 156.71 & 161.1! & $150.2 !$ & 149.5 & $149.4 T$ & 149.9 \\
\hline Wall Tomp. "1F & $79 !$ & 154.7 & 153.7 & 154.01 & 156.11 & 157.9 & 1511 & 149.4 & 148.71 & 149.1 \\
\hline Wall Temp. HF & 156.11 & 155.11 & 154.61 & 155.4 & 156.41 & 157.7 & 151.11 & 149.4 & 148.71 & 149.4 \\
\hline Instroam Temp.t & 147.61 & $14 \overline{6.81}$ & 146.61 & $1 \overline{46.81}$ & 146.01 & $1 \overline{47}$ & 147.6 & 147 & 146.81 & 146.0 \\
\hline Avg. Wall Tomp.F & 155.3 & 155.31 & 1551 & 15621 & 156.81 & 163.8 & 150.4 & 149.4 & 149.1 & 149.3 \\
\hline Avg. Wall DT.F & 7.11 & 7.7 & 7.91 & $8.7 !$ & $9.2 !$ & 16.21 & 2.9! & 2.41 & 231 & 24 \\
\hline Remaining Subcooff & -11.91 & & & & & & & & & \\
\hline Ext Quality & 0.11 & 0.261 & $0.4 j$ & 0.54 & 0.691 & 0.791 & 0.81 & 0.821 & 0.821 & 0.84 \\
\hline EXP. H-BtuAHRT2 & 2003.3 & $1924.1 !$ & 1870 & 1672.9 & $1598.6 i$ & 685.71 & 459.21 & 399.2 & $347.5 i$ & 468.7 \\
\hline COMPUTEDH & 7650 & & & & & & & & & \\
\hline Jallouk & $1334.4 !$ & 1284.81 & $1237.9 !$ & $1212.7 !$ & $77.7 !$ & 7.7 & 7.7! & 71.71 & $\pi 7.7 !$ & 77.7 \\
\hline Szady (Cudo tubo) & 1755.2! & 1738.6 & 1730.2 & 1729.3! & 7.71 & $\pi .7$ & 77.7 & 77.7 & 77.7 & 77.7 \\
\hline Seady2 & 1583.7 & 1564.5 & 1555 & 1553.4 & 77.71 & $\pi .71$ & 77.7 & 77.7 & $77.7 !$ & $\pi .7$ \\
\hline (RLO) & 280431 & 24094 & 19774 & 15605 & 156051 & 15605 & 156051 & 15605 & 156051 & 15605 \\
\hline$(\bar{P}$ & 1.894 & 276 & 3.434 & 3.974 & 3.9741 & 3.9741 & 3.9741 & 3.974 & 3.9741 & 3.974 \\
\hline (HUQ) & 147.61 & 214.8 & 26721 & 300.41 & 300.41 & $30 \overline{9.41}$ & 300.41 & 309.41 & 309.41 & 309.4 \\
\hline (HBOIL) & 1754.9! & 1737.51 & 1728.11 & 1726 & 17261 & 1726 & 17261 & 17261 & 17261 & 1726 \\
\hline & & & & & $i$ & & $i$ & $\mathrm{i}$ & 1 & 1 \\
\hline & ¿ZONE2 & ZONE4. & ZONE6 & ZONE8 & ZONE10_i & & & & 1 & \\
\hline DP liquid Temp.F & $1 \quad 74.71$ & & & & & & & & & 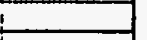 \\
\hline DP Liquid Density-lbm/tit3 & 93.75 & 1 & & & & & & & $\mathrm{i}$ & i \\
\hline Elovation-f & & $4 i$ & 6 & 81 & 9.875 & & & & & \\
\hline Measured DPAsid & 0.744 & $2.382 !$ & 2.2381 & 4.7451 & 5.1041 & & & & & \\
\hline Zero Corrections-psid & $0.022 !$ & -0.0281 & $-0.754 !$ & 0.128 & 0.167 & & & & & \\
\hline Corrected DPFsid & $0.722 !$ & 2.411 & 2.992 & $4.017 !$ & 4.9431 & t & $t$ & & & \\
\hline Actual DPA & 1.011 & 0.34 & 1.61 & 2.081 & 2.591 & 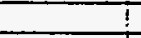 & it & 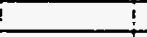 & i & i \\
\hline Mossured TPPsig & 101.4 & & 1 & 1 & 3.01 & & $i$ & i & & \\
\hline Measured OveralH & 170.711 & & I & 1 & $-i$ & $\vdots$ & $!$ & $T$ & i & \\
\hline & 1 & & $i$ & 1 & & & I & i & I & I \\
\hline Computed DPA & 1.051 & 1.72 & 2.261 & 3.44 & 4.541 & & 1 & 1 & 1 & $L$ \\
\hline Friction-ft & 0.03 & 0.1 & 0.21 & 0.25 & 0.3 & & & & $\perp$ & $L$ \\
\hline Accoleration-t & 0.03 & 0.08 & $0 . \overline{14}$ & 0.14 & 0.15 & & & & & 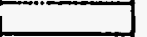 \\
\hline Elevationft & 0.99 & 1.54 & 1.92 & $3.04 !$ & 4.09 & & & & & \\
\hline & & & & & & & & & & \\
\hline
\end{tabular}




\begin{tabular}{|c|c|c|c|c|c|c|c|c|c|c|}
\hline Run: & 5561 & $\div$ & ? & & 3 & & $\therefore$ & : & & \\
\hline Tost Fuid & CAF 10 & & & & $\dot{i}$ & $:$ & & & . & - \\
\hline Dalo: & $5 \sqrt[5]{271931}$ & & $\therefore$ & & 1 & $\dot{s}$ & & & , & \\
\hline Time: & $5: 28$ & & & & & & & & & \\
\hline Note & TĀBOOAEK & Añantical d & & & & 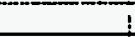 & & & & \\
\hline Fow Area-t12 & 0.003361 & & & : & : & + & 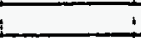 & \pm & & \\
\hline Heal Flow Areatt? & 0.196951 & & & & & & & & & \\
\hline Sat. Pressuro & $112.8 !$ & & & & & & & & & \\
\hline Sat. Temporature-f(intel) & $15 \overline{4.4} 1$ & & & & & & & & & \\
\hline Uq. Densthy -1bmins & 82.131 & $81.42 !$ & $81.49 i$ & $81.32 !$ & $81 . \overline{4 !}$ & 81.451 & $81.19 !$ & 81.351 & 81.471 & 81.38 \\
\hline Latent Hoat Btufibm & 29.781 & 29.241 & 29.291 & 29.17 & 29.221 & 29.28 & $29.07 !$ & 29.191 & 29.281 & 29.21 \\
\hline Specific Heat Btunbm/F & 0.2851 & 0.2871 & 0.2861 & 0.287 & $0.287 !$ & 0.2881 & 0.2871 & 0.2871 & 0.2861 & 0.287 \\
\hline Liq. Viscosituibmithr & 0.4361 & 0.4221 & 0.4231 & 0.42 & 0.4221 & 0.423 & 0.418 & 0.4211 & 0.4231 & 0.421 \\
\hline Liq. Conductivity Btu/hr-ft $F$ & 0.02521 & 0.0247 & 0.02481 & 0.02471 & 0.02471 & 0.02481 & 0.0246 & 0.0247 & 0.02481 & 0.0247 \\
\hline íg. Prandt No. & 4.921 & 4.89 & 4.891 & 4.881 & 4.891 & 4.89 & 4.881 & 4.88 & 4.891 & 4.88 \\
\hline Inlet flow Rate-GPM & 1 & 1 & & & $\ldots$ & $\ldots 1$ & +1 & $i$ & in & \\
\hline OutdotLig. Fow Rato-GPM & 0.9 & 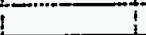 & & & $i$ & $\bar{i}$ & $i$ & 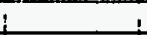 & & \\
\hline Outtet Vap. Flow Ralo-GPM & $0.7 i$ & & ; & $?$ & 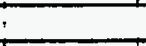 & $i$ & & & & \\
\hline Inlet Temperahuref & 142.4 & i & & 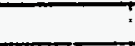 & & 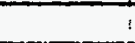 & & & . & . \\
\hline Outetlqu. Temperaturef & $153.3 !$ & & & & & & & & & \\
\hline Outlel vap. Tomperaturof & $149.5^{\top}$ & & & & & & & & & \\
\hline Weter Fow Rate-GPM & & & & & 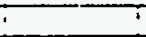 & & $\because$ & & $\because$ & \\
\hline Wader nlet Temp.F & 97.21 & & 5 & & $\dot{5}$ & & $i$ & 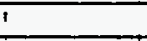 & i & 5 \\
\hline Water outlot Tomp.f & 138.11 & & $\div$ & & $\therefore$ & & & i & $i$ & $\bar{i}$ \\
\hline Thlat $\mathrm{G} \mathrm{bm} / \mathrm{hr} / \mathrm{LL}$ & $19007 \pi$ & & Temp & 138.8 & Density & 83.42 & $\mathbf{i}$ & 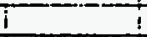 & $\bar{i}$ & $\mathrm{i}$ \\
\hline Subcoolingf & 121 & & 7 & ; & 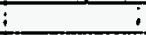 & & T & i & 1 & I \\
\hline Flow Observation & & & & & & & & & $i$ & \\
\hline & ZONE 1 & $2 O N E 2$ & ZONE3 & ZONE4 & ZONE & ZONE 6 & ZONE? & ZONE8 & ZONE9 I & ZONE10 \\
\hline Hoaf Input-Watts(net) & 8726 & $882 !$ & 874.3 & 868.11 & 871.6 & 665.5 & $=-76.9$ & 56.7 & 44.21 & 55.6 \\
\hline Heal Loss-Watts & $14.4{ }^{\circ}$ & 3.41 & $4.6 i$ & 5 & $5.3 !$ & 4 & 1.91 & 2.91 & 3.81 & 22.8 \\
\hline Hoal Fux-Btu/Hr/i2 & $15126 !$ & 15289 & 15155! & 15048 & 151001 & 11536 & 13331 & 983 & $766 !$ & 964 \\
\hline Wall Temp. \#3F & 160.5 & 162.61 & $160.6 !$ & 1624 & $765.3 !$ & 761.5 & $756.2 !$ & 155.6 & 154.87 & 755.1 \\
\hline Wal Tomp. $4 F$ & 1611 & 160.5 & 1623 & 763.3 & $1 \overline{3} .3$ & 767.5 & 156.3 & 155.51 & 154.9 & 155.4 \\
\hline Wall Temp. is F & 160.91 & 158.91 & 163.7 & 162.4 & $1622 !$ & 1611 & 158.31 & 155.41 & 154.81 & 155.4 \\
\hline Wall Tomp. & 73.11 & 160.5 & 159.51 & 160.5 & 161.91 & 1621 & 158.61 & 155.51 & 154.31 & 155.6 \\
\hline Wall Temp. W2F & 161.71 & $160.9 !$ & 160.31 & 161.6 & 162.2 & $1 ब$ & 156.6 & 155.6 & 154.41 & 155.7 \\
\hline Inetrieam Temp. $F$ & 148.81 & 162.7 & 152.31 & 153.2 & 152.81 & 162.6 & 153.9 & 1531 & 152.41 & 1520 \\
\hline Avg. Wall Tomp.F & 161 & 161.1 & 160.71 & 1622 & 162.41 & 161.5 & 156.41 & 155.51 & $154.6 !$ & 155.4 \\
\hline Avg. Wall DT.F & 11.4 & 7.7 & $7.7 !$ & 8.3 & 8.9 & 8.5 & 2.41 & 251 & 221 & 2.5 \\
\hline Remaining Subcooff & 3.71 & & & & & & & 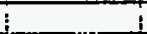 & & \\
\hline Ext Quality & 0.03 & 0.19 & $0.34 !$ & 0.49 & 0.64 & 0.76 & 0.77 & 0.78 & 0.79 & 0.8 \\
\hline EXP. H. Btu/HR/f2/F & 1322 & 1976.8 & $1970.8 \mathrm{i}$ & 1819.6 & $1694.3 i$ & 1360.6 & $546.4 i$ & 3971 & 347.4 & 386.1 \\
\hline COMPUTEDH & & & & & & & & & & \\
\hline Jallouk & $13922 !$ & 73722 & $1375.4 !$ & 1307.7 & $1285 . \pi$ & 7.6 & 77.7 & 77.61 & $\pi .61$ & 77.6 \\
\hline Szadyi(Cudotubo) & 1779.5 & 1830.6 & 1818.7 & 18226 & $18226 !$ & 7.6 & $\pi .71$ & 77.6 & 77.6 & 77.6 \\
\hline Szady? & 1610.91 & 1667.2 & $1653.2 !$ & 1656.6 & 1657.71 & $\pi .6$ & 77.7 & 77.61 & 77.61 & 77.6 \\
\hline (ALQ) & 293661 & 27445 & 226811 & 18147 & 13396 & 13396 & 13356 & 13395 & 133961 & 13396 \\
\hline$(F)$ & 1.4481 & 2.261 & 3.051 & 3.525 & 4.145 & 4.1451 & 4.1451 & 4.145 & 4.1451 & 4.145 \\
\hline (Hंपa) & 112.61 & 176.31 & 237.81 & 2828 & 323.41 & 323.4 & 323.4 & 323.41 & 323.41 & 323.4 \\
\hline (HBOIL) & 179.31 & 1830.1 & 1817.31 & 1820.3 & 1819.21 & 1819.2 & 1819.21 & 1819.21 & 1819.21 & 1818.2 \\
\hline & & 1 & $i$ & & & & & 1 & $i$ & $i$ \\
\hline & ZONE2 & ZONE4 & ZONE6 & ZONEB & IZONE1O & & & 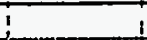 & & \\
\hline DPliquid Tomp.f & i 80.91 & & 1 & 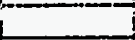 & $i$ & & & 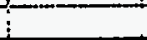 & 1 & 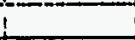 \\
\hline DP Liquid Densitylbmiti3 & 92.85 & & & & & & & + & & \\
\hline Elevation-ft & & 4 & 6 & 8 & 9.875 & & & & & \\
\hline Measured DPF Sid & 0.585 & 1.939 & 1.9231 & 3.78 & $4 . \% 51$ & & & & & \\
\hline Z̈́ro Comections-psid & 0.022 & -0.028 & -0.754 & 0.128 & 0.167 & & & & & \\
\hline Comected DPPSild & 0.5631 & 1.557 & 2.677 & 3.658 & 4.547 & & & 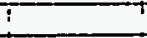 & & \\
\hline Actual DPA & 1.291 & 1.08 & 2.111 & 2.68 & $3.22 !$ & & & $i$ & & 1 \\
\hline Measured TPPsig & 112.8 & & -1 & & 3.71 & & & T & & I \\
\hline Measured Overalift & 183.051 & & 1 & 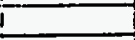 & -1 & & & i & & I \\
\hline & 1 & & & & & & & I & 1 & I \\
\hline Computed DR-A & 1.291 & 203 & 2.591 & 3.78 & 4.91 & & & i & & \\
\hline Friction-th & 0.021 & 0.08 & 0.16 & 0.21 & 0.26 & & & 1 & & I \\
\hline Accoleration-t & 0.02 & 0.06 & 0.12 & 0.12 & 0.13 & & & 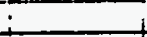 & & I \\
\hline Elovation-t & 1.25 & 1.88 & 231 & 3.45 & 4.511 & & & & 1 & 1 \\
\hline
\end{tabular}




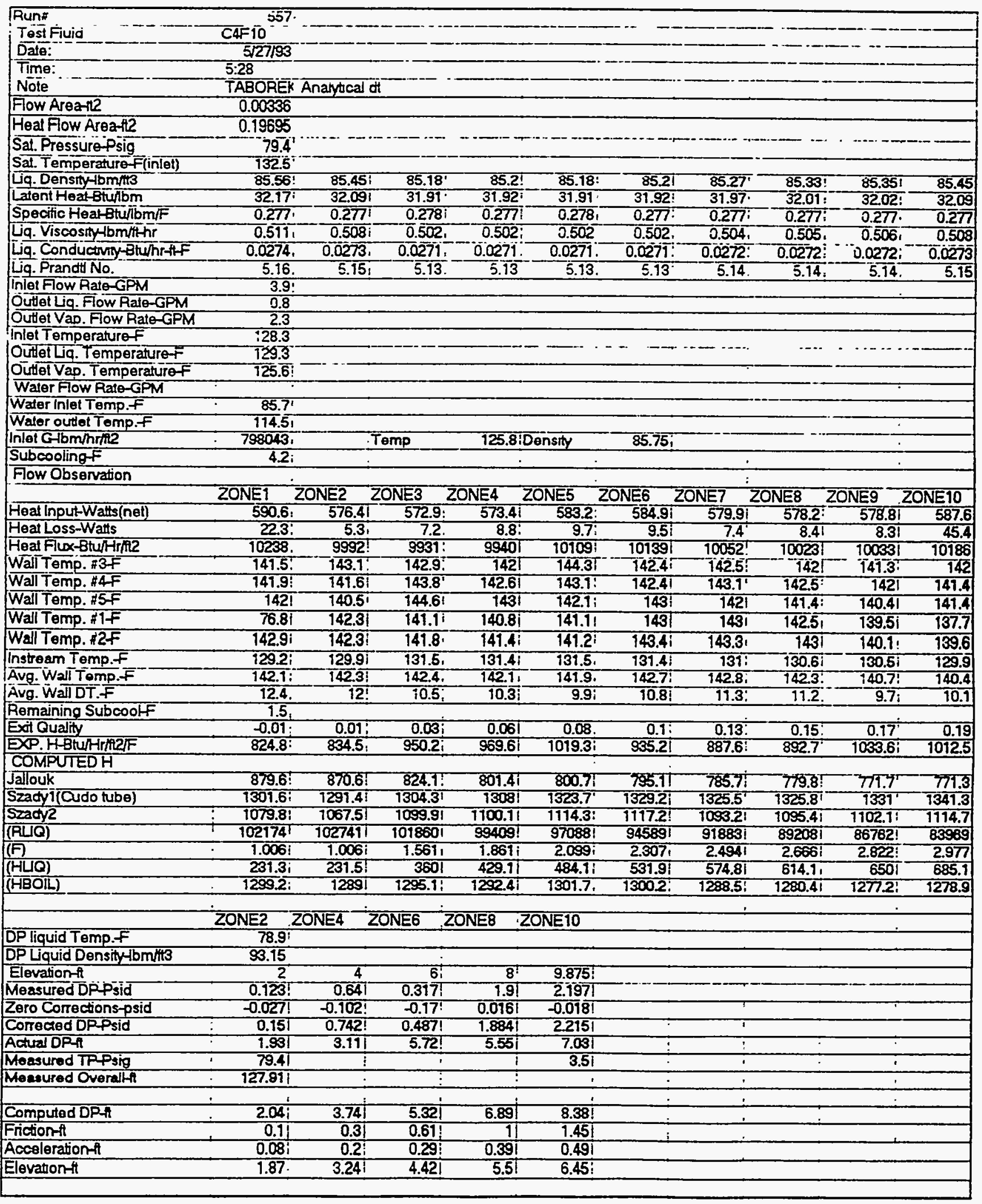




\begin{tabular}{|c|c|c|c|c|c|c|c|c|c|c|}
\hline Run" ..... & 558, & & & & & & & & & \\
\hline Tost Fluid & CAF10: & & & & & & & & & \\
\hline Dale: & $527193:$ & & & & & & & & & \\
\hline Time: & $9: 54$ & & & & & & & & & \\
\hline Note & TABOREK & Analytical o & & & & & & & & \\
\hline Flow Area-tl2 & $0.00336 i$ & & & & & & & & & \\
\hline Hoat Flow Area-th2 & 0.19695 & & & & & & & & & \\
\hline Sat.PTessure-Psig & 80.11 & & & & & & & $--\infty$ & & \\
\hline Saf. Temperature-f(inlet) & 133.11 & & & & & & & & & \\
\hline Liq. Densitylbmitt3 & 85.711 & 85.61 & $85.3 !$ & $85.3 i$ & 85.31 & 85.311 & 85.351 & 85.361 & $85.36 !$ & 85.53 \\
\hline Lalent Hoat-Btunibm & $32.27 !$ & 32.191 & $31.99 t$ & $31.99 !$ & 31.99! & 32 & $32.02 !$ & 32.041 & $32.04 !$ & 32.15 \\
\hline Specifio Heartululbm/F & $0.276 !$ & $0.27 \pi$ & 0.277 & 0.2771 & $0.277 \mathrm{i}$ & $0.27 !$ & 0.277 & $0.277 i$ & $0.277 i$ & 0.277 \\
\hline Liq. Viscosity -bmifihr & 0.5151 & 0.512 & $0.504 i$ & 0.5041 & $0.504 i$ & 0.505. & $0.506 i$ & $0.505:$ & $0.506 i$ & 0.51 \\
\hline Liq. Conductivity Btuhr-ffF & $0.0275 i$ & 0.02741 & 0.02721 & 0.0272 & 0.0272 & $0.0272 !$ & $0.0272 !$ & $0.0273 !$ & 0.02731 & 0.0274 \\
\hline Liq. Prandt No. & $5.17 !$ & 5.161 & 5.14 & $5.14 !$ & $5.14 i$ & $5 . \overline{14 i}$ & $5.14 !$ & $5.15:$ & 5.15 & 5.16 \\
\hline Inlet Flow Rato-GPM & $3.9 !$ & & & & & & & & & \\
\hline Outlel Lig. Flow Ralo-GPM & $0.8:$ & & & & & & & & & \\
\hline Outtel Vap. Flow Rate-GPM & 2.3. & & & & & & & & & \\
\hline Inlet Temperaturef & 127.5 & & & & & & & & & \\
\hline Outlel Lg. Temperaturef & $128.7 i$ & & & & & & & & & \\
\hline Outel Vap. Temperaturef & $125.5 !$ & & & & & & & & & \\
\hline Wader Flow Pado-GPM & & & & 1 & & & & & & \\
\hline Water inlot Tomp.F & 86.31 & & & & & & & & & \\
\hline Wator outlot Tomp.F & 114.41 & & & i & & & & & & \\
\hline Inlet Gibmihrift2 & 7986881 & & Temp & 125,41 & Density & $85.81 i$ & & : & - & - \\
\hline Subcooling $f$ & $5.6 i$ & & 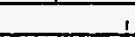 & $\longrightarrow$ & $\bar{i}$ & 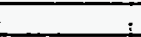 & & & & \\
\hline Flow Observation & & & & & & & & & & \\
\hline & ZONE : : & ZONE2 & ZONE3 : & ZONE4 & ZONE5 .2 & ZONE6 & ZONE7 Z & ZONE8 & ZONE9 & ZONE10 \\
\hline Heatlnput-Watls(net) & 588.2 & $573.5 i$ & 574.81 & 574.5 & 581.11 & $582.8 !$ & $577.6 i$ & 576 & 580.1 & 589 \\
\hline Heat Loss-Watts & $22 !$ & 5.2 & 7.11 & 8.71 & $9.6 !$ & $9.3 i$ & $7.3:$ & $8.2 \mathrm{i}$ & 8.2 & 44.6 \\
\hline Hoat Flux-Btu/Hi/t2 & 10196 & 9941 & 99641 & 9959 & 100731 & 10102 & $10012:$ & 99851 & 10056 & 10210 \\
\hline Wall Temp. "HF & 140.9 & 142.41 & 1422! & $141.8 !$ & 144.11 & 941.8 & $1424 !$ & $141.7 !$ & $1 \overline{41.1 T}$ & 141.5 \\
\hline Wall Temp. \#4F & 141.41 & 140.9 & 143.11 & 142.3! & 142.81 & 142.31 & $142.9 !$ & $141.9 i$ & $141.9 !$ & 140.7 \\
\hline Wall Temp. \#5-F & 141.41 & 139.91 & 143.91 & $1426 !$ & $141.7 !$ & 142.41 & 141.61 & 141.2! & 140.11 & 140.7 \\
\hline Wall Temp. \#1F & $77.2 !$ & 141.61 & $140.6 \mathrm{i}$ & 140.2 & 140.9 & 142.61 & 142.51 & 142.41 & 1391 & 137 \\
\hline Wall Temp. N2F & $1422 i$ & 141.71 & $141.1 !$ & 140.91 & 140.91 & $142.8 i$ & 142.91 & 142.71 & $139.6 !$ & 138.8 \\
\hline Instroam Temp.F & 128.31 & 1291 & 130.81 & 130.81 & 130.81 & 130.7 & $\overline{130 . \bar{i}}$ & 130.41 & $130.4 i$ & 129.4 \\
\hline Avg. Wall Tomp.F & $141.5 \mathrm{i}$ & $141.7 !$ & 141.8, & $141.7 !$ & $141.6 !$ & $1423 i$ & $142.5 i$ & $142 !$ & 140.3! & 139.7 \\
\hline Avg. Wall $\bar{D} T$ T & $127 !$ & 122 & $10.5 i$ & 10.5 & $10.3 !$ & 11.11 & $11.5 !$ & 11.11 & $9.5 ;$ & 9.9 \\
\hline Remaining Subcooff & 2.81 & & & & & & & & & \\
\hline Ext Quality & -0.02 & $0 !$ & 0.02 & 0.041 & $0.07 t$ & $0.09 i$ & 0.11 & $0.14 i$ & $0.16 !$ & 0.18 \\
\hline EXP.HBtuHHRM2/F & 801.91 & 814.81 & $949 i$ & $\$ 50.7 !$ & 976.11 & 908.61 & 870.11 & 897.2 & $1000.1 i$ & 1033.5 \\
\hline COMPUIEDH & 1 & $i$ & & & & & & & & \\
\hline Jallouk & 872.61 & 83.11 & 837.81 & 805.11 & $800.1 !$ & $793.8 !$ & 784.51 & $779.3 !$ & $778.7 i$ & 772 \\
\hline Szady1(Cudo tube) & $1291.9 i$ & 1281.2 & $1298.9 !$ & $13025 !$ & $1313.9 !$ & 1319.31 & 1316.91 & $1319.4 !$ & 1328.9: & 1356.3 \\
\hline szady2 & 1070.21 & 1057.31 & 1096.7 & 1006.51 & 1105.51 & 1108.2 & $1083.7 !$ & 1088.11 & 1099.51 & 1100.7 \\
\hline (FUO) & 101533 & 102095 & 1025651 & 1002061 & 978271 & 953561 & $92822 !$ & 903891 & 880141 & 84944 \\
\hline (F) & 1.0061 & 1.0061 & 1.3551 & 1.724 & $1.99 i$ & 2.212 & 2.4071 & 2.5831 & 2.7431 & 2.909 \\
\hline (HULQ) & 231.11 & 231.31 & 312.41 & 397.6 & $458.7 i$ & 509.81 & $554.8 \mathrm{i}$ & 595 & 632.11 & 669.2 \\
\hline (HBOLL) & 1289.41 & 1278.7 & 1292.9! & 1290.11 & 12951 & $1293.4 !$ & 128321 & $1277.7:$ & $\overline{1279.4}$ & 127.8 \\
\hline & 1 & & & & : & $\quad:$ & & & & \\
\hline & ZONE2 . 2 & ZONE4 & ZONE6 6 & ZONE8 & ZONE10 : & & & & & \\
\hline DPliquid Temp.F & $79.2 !$ & $i$ & +1 & & & & & & & \\
\hline DPLiquid Density-lbm/1t3 & 93.11 & & & & & & & & & \\
\hline Elevation-t & & 41 & $6:$ & $8 !$ & 9.875 & & & & & \\
\hline Messured DPFsid & 0.1221 & 0.628 & 0.3011 & 1.8761 & 2.13 & & & & & \\
\hline Zoro Comections-psid & -0.027 & -0.1021 & -0.171 & 0.016 & -0.0181 & & & & 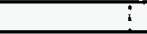 & \\
\hline Corrected DPPsld & 0.1491 & 0.731 & 0.4711 & 1.861 & 2.198 & & ! & & + & \\
\hline ACtual DPA & 1.991 & $3.19 !$ & $5.74^{1}$ & 5.58 & $7.05 !$ & & & & $i$ & \\
\hline Measured TPFig & 80.11 & i & 1 & $:$ & $3.6 !$ & & & 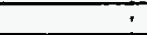 & $\bar{\vdots}$ & \\
\hline Measured Overallin & 128.81 & $!$ & $!$ & 1 & 1 & :- & $\therefore$ & & $i$ & \\
\hline & 1 & & & $i$ & & & 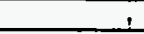 & & $\vdots$ & \\
\hline Computed DPA & 2.081 & 3.89 & 5.481 & 7.05 & 8.541 & & & & $!$ & \\
\hline Friction-ft & 0.081 & 0.261 & 0.541 & 0.911 & 1.34 & & - & & & \\
\hline Accoleration-fl & $0 i$ & $0.18 \mathrm{i}$ & $0.27 i$ & 0.361 & 0.461 & & 1 & & & \\
\hline Elevation-t & $2 !$ & 3.461 & $4.67 !$ & 5.771 & 6.731 & & & & & \\
\hline & 1 & & & & & & & & & \\
\hline
\end{tabular}




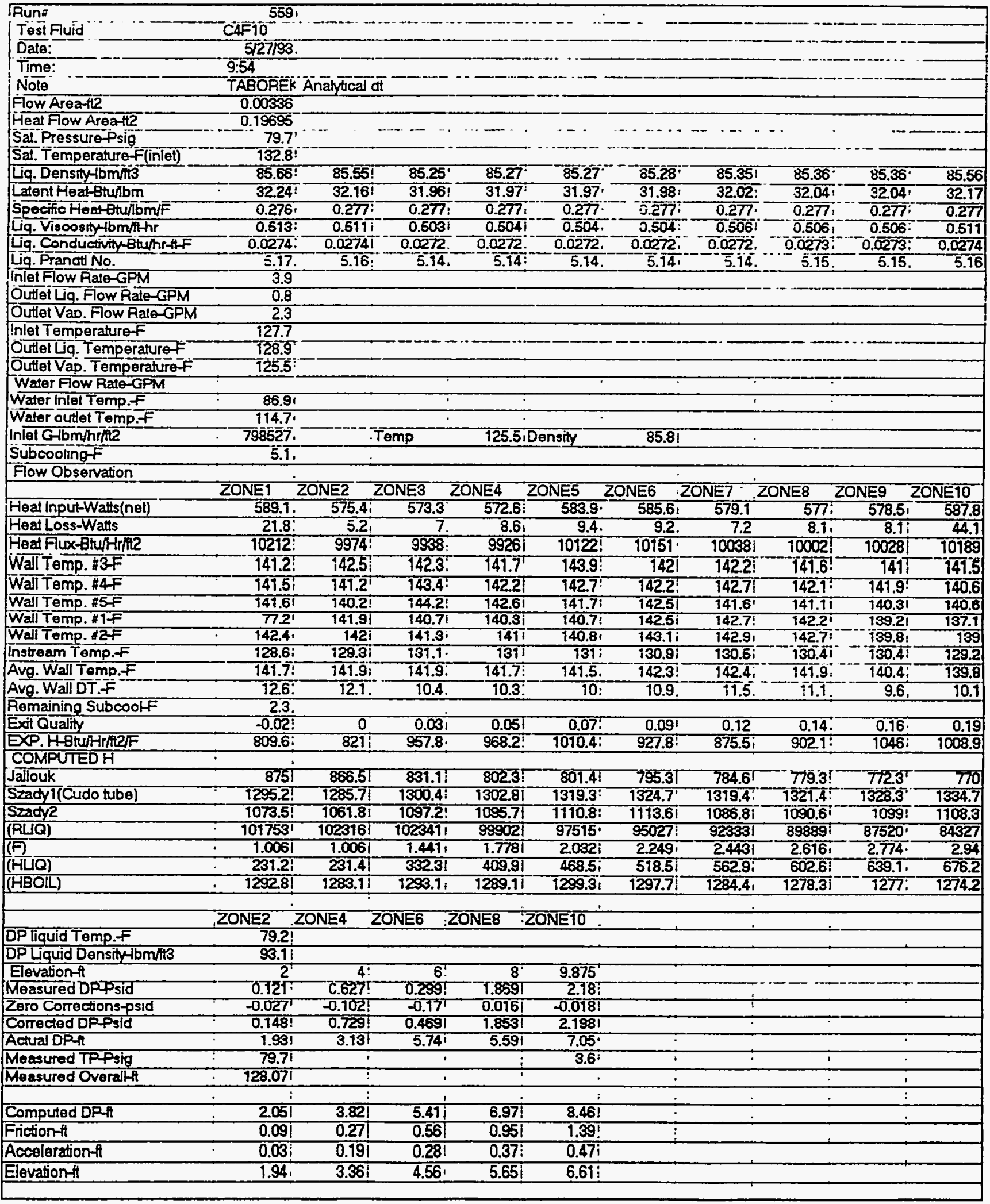




\begin{tabular}{|c|c|c|c|c|c|c|c|c|c|c|}
\hline Runt & 560 & & & & & & & & & \\
\hline Test Fiuid & CAF10 & & & & & & & & & \\
\hline Dade: & $5 / 27 / 93_{i}$ & & & & & & & & & \\
\hline Time: & $9: 54$ & & & & & & & & & \\
\hline Note & TABOREK & Analytical dt & & & & & & & & \\
\hline Flow Asea-th & 0.00336 & & & & & & & & & \\
\hline Heal Flow Areatt2 & $0.19695:$ & & & & & & & & & \\
\hline Sat.Pressurepsig & $823 !$ & & & & & & & & & \\
\hline Sat. Temperaturo f(inlet) & 934.71 & & & & & & & & & \\
\hline एq. Densing-ibmitis & $85.28 !$ & 85.161 & 851 & $84.95 !$ & 84.961 & 84.981 & $85.79 !$ & $85.11 i$ & $85.15 !$ & 85.37 \\
\hline Ladent Heat-Bturibm & 31.981 & 31.91 & $31.78 !$ & 31.75 & 31.781 & $31.7^{1}$ & 31.881 & 31.861 & 31.891 & 32 \\
\hline Specific Hool thu/bm/f & $0.2 \pi 7$ & 0.2781 & $0.278 !$ & 0.278 & $0.278 !$ & $0.278 i$ & 0.278 & 0.2781 & 0.2781 & 0.277 \\
\hline Lig. Viscosity lbm/thr & 0.5041 & 0.5011 & 0.4971 & 0.496 & $0.497 i$ & $0.497 i$ & 0.51 & $0.5 i$ & $0.501 i$ & 0.505 \\
\hline Lig. Conductivity BtwhreftF & 0.02721 & 0.0279 & $0.027 !$ & 0.027 & $0.027 !$ & $0.027:$ & $0.0271:$ & 0.02711 & $0.0271:$ & 0.0272 \\
\hline Liq. Pranctd No. & $5.14:$ & $5.13 !$ & 5.12. & 5.11 & $5.11 i$ & 5.11 & 5.12 & $5.12 !$ & 5.13 & 5.14 \\
\hline Inlet flow Rato-GPM & $3.9 !$ & & & & & & & & & \\
\hline Outlol Lg. Flow Ralo-GPM & $0.8_{1}$ & & & & & & & & & \\
\hline Outlel Vap. Flow Rale-GPM & 2.4 & & & & & & & & & \\
\hline Inlel Temperature-f & $129.7:$ & & & & & & & & & \\
\hline Outlet Liq. Temperature & 130.61 & & & & & & & & & \\
\hline Outet Vap. Temperaturof & 127.21 & & & & & & & & & \\
\hline Water Fow Rato-GPM & & & & & & & & & & \\
\hline Water Inlet Temp.f & $88.1 !$ & & & & & & & & & \\
\hline Waler outlel Temp.f & 115.81 & & & & & & & & $\cdot$ & 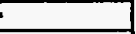 \\
\hline Inlot Glbmhrith & $796427 i$ & & Temp & 126.81 & Density & $85.57 !$ & & & & \\
\hline Subcooling & $5 i$ & & 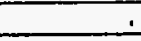 & 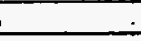 & 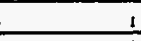 & & & & & \\
\hline \multicolumn{11}{|l|}{ Fow Observation } \\
\hline & ZONE1 . & ZONE2 2 & ZONE3 & ZONE4 & ZONE5 & ZONE6 & ZONE7 .2 & ZONE9 & ZONE9 & .ZONE10 \\
\hline Heat Input-Watts(net) & 593.11 & $578.6 i$ & 569.11 & $569.4 ;$ & $579.3^{\circ}$ & $580.6 \mathrm{i}$ & 581.5 & $579.6 i$ & 573.1 & 580.7 \\
\hline Heat Loss-Watts & 21.7 & 5.1 & $7 !$ & 8.51 & $9.4 i$ & 9.21 & $7.1 i$ & $8 !$ & 8 & 43.8 \\
\hline Heat Flux-Btu/Hrine & 10281 & 10030 & $9865 i$ & 9870 & 100421 & 10054 & 100801 & 100471 & 99341 & 10066 \\
\hline Wall Temp. H3 & 142.41 & 743.8 & 743.61 & 143.21 & 145.5: & 143.31 & 143.6 & $742.9 !$ & 742.2! & 742.6 \\
\hline Wall Temp. $44 F$ & 1431 & $742.4 !$ & 144.61 & 143.81 & 144.2! & 143.7! & 143.9 & 143.61 & 142.9 & 747.8 \\
\hline Wall Temp. H5F & 1431 & $141.7 !$ & 145.5 & 143.91 & 143.21 & 1441 & $143 !$ & 142.31 & 141.81 & 141.8 \\
\hline Wall Temp. $1 F$ & $78.2 !$ & 143.31 & 141.91 & $141.8 \mathrm{i}$ & 142.41 & 144.21 & 143.91 & 143.71 & 140.71 & 138.2 \\
\hline Wall Temp. Haf & $143.7 i$ & $143.3 i$ & 142.51 & 142.21 & $142.4 i$ & 144.21 & $144.5 i$ & 143.91 & 141.31 & 140.2 \\
\hline Instroam Tomp.F & 130.81 & 137.61 & $132.6 t$ & 132.91 & $132.8 i$ & 132.71 & $131.9 i$ & $131.9 i$ & 131.71 & 130.7 \\
\hline Avg.Wall Tomp.F & $143 i$ & 143.21 & 143.21 & 143.21 & 743.11 & 143.81 & 143.8 & $143 . \overline{3 !}$ & 141.81 & 140.9 \\
\hline Avg. Wall DT. $f$ & $11.7 !$ & $11.3 !$ & 10.1 & $9.8 !$ & $9.8 !$ & $10.6 \mathrm{i}$ & 11.44 & $10.9 !$ & 9.61 & 9.8 \\
\hline Pomaining SubcoofF & $2.2 !$ & & & & & & & & & \\
\hline Ext Quality & -0.02 & 01 & 0.03 & 0.05 & 0.07 & 0.11 & 0.12 & $0.14 !$ & 0.17 ! & 0.19 \\
\hline DXP.HEtu/HRlterF & $881.7^{\prime}$ & 899.81 & 976.31 & 1004.2 & 1025.11 & $945.4 i$ & 882.31 & $919.6 !$ & 1031.5 & 1030.8 \\
\hline COMPUTEDH & & & & & & & 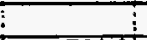 & & & \\
\hline Jallouk & 891.31 & 8821 & 833.51 & 807.41 & 805.31 & 7901 & 791.31 & 786.21 & 773.6 & 77 \\
\hline Szadyl(Cudo tubo) & 1317.8 & $1307.3 i$ & 1307.61 & 1374.21 & 1328.4 & 1333.31 & 1332.7 & 1335.6: & $13323^{2}$ & 1338.4 \\
\hline Szady2 & 1095.8 & 10831 & 1103.81 & 1106.71 & 1119.41 & 1121.5 & 1099.31 & $1103 . \overline{81}$ & $1107.6 !$ & 1110.3 \\
\hline (PUQ) & 103347 & 1039231 & 1031611 & 1010141 & $98514 i$ & $\$ 5994$ & 92955 & 905311 & 879881 & 84912 \\
\hline (ค) & 1.0061 & 1.0061 & 1.46 & 1.7841 & 2.00331 & 2.2461 & 2.4411 & 2.6121 & $2.771 i$ & $2 . \$ 31$ \\
\hline$(H \cup Q)$ & 231.41 & 231.61 & 336.5 & 411.51 & 458.91 & 517.9 & 56231 & 601.7 & 638.1 & 674.1 \\
\hline (HBOIL) & $1315.4 \mathrm{i}$ & 1304.81 & 7300.11 & 1300.61 & $1308.6 i$ & $1306.8 \mathrm{i}$ & 1298.5 & $1293.6 \mathrm{i}$ & 1281.6 & 1278.8 \\
\hline & 1 & & & 1 & 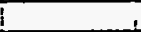 & $\vdots$ & $i$ & $!$ & : & \\
\hline & ZONE2 & ZONE4 & ZONE6 & ZONE8 & ZONE10 & & $i$ & & & \\
\hline DPPliquid Temp.F & $\therefore \quad 80.2$ & & & & & & i & & & \\
\hline DPLiquid Density $1 \mathrm{bm} / \mathrm{th}$ & 92.96 & & & & & & & & & \\
\hline Elevation-ft & & & & & 9.875 & & & & & \\
\hline Moasured DPFsid & 0.1281 & 0.6641 & 0.3481 & $1.333 !$ & 2.237 & & & & & \\
\hline Zero Cortections-psid & -0.027 & -0.1021 & -0.171 & 0.016 & -0.018 & & & $i$ & & \\
\hline Corrected DP Psld & 0.155 & 0.766 & 0.5181 & $1.917 !$ & $2255 !$ & & i & i & & \\
\hline Actual DPA & $1.92 !$ & 3.07 & 5.681 & 5.481 & $6.95 !$ & & & 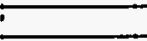 & & \\
\hline Meesured TPPsig & 82.31 & & $!$ & \pm & $3.6 !$ & & 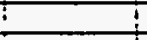 & $\div$ & $\bar{i}$ & \\
\hline Measured Overaltiti & 132.891 & & 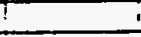 & - & . & & $\therefore$ & $\therefore$ & 1 & 1 \\
\hline & . & & & & & & $\vdots$ & 1 & & $i$ \\
\hline Computed DP- & 2.05ा & 3.81 & 5.391 & 6.951 & 8.441 & & $i$ & & & 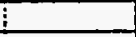 \\
\hline Friction-tit & 0.091 & 0.281 & 0.56 & 0.94 & 1.381 & & & & & 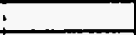 \\
\hline Accaleration-ft & 0.041 & $0.19 !$ & 0.281 & 0.37 & $0.47 i$ & & & & & \\
\hline Elovation-th & $1.92:$ & 3.341 & 4.54 & 5.64 & 6.591 & & & & & \\
\hline
\end{tabular}




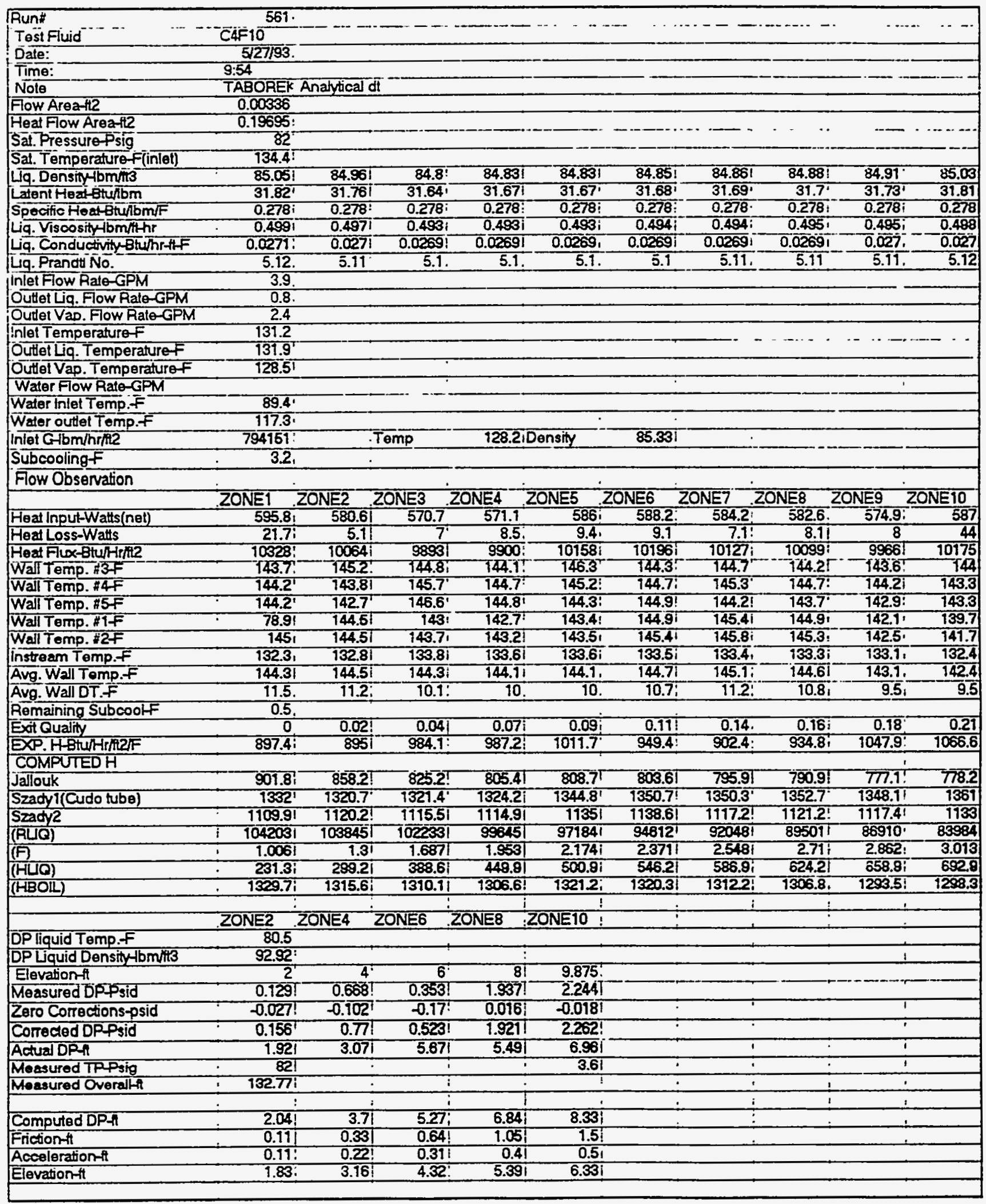




\begin{tabular}{|c|c|c|c|c|c|c|c|c|c|c|}
\hline Runt & 5621 & & & & & & & & & \\
\hline Tost Fluid &. CAF 10 & & & & & & & & & \\
\hline Date: & $5 / 27 / 93 !$ & & & & & & & & & \\
\hline Time: & $9: 54$ & & & & & & & & & \\
\hline Noto & TABOREK & Analytical ot & & & & & & & & \\
\hline Flow Area-fle & 0.003361 & & & & & & & & & \\
\hline Heat Flow Area-fi2 & 0.196951 & & & & & & & & & \\
\hline Sal. Pressure Sig & 85.81 & & & & & & & & & \\
\hline Sal. Temperaturof (inlet) & $137.1 !$ & & & & & & & & & \\
\hline Lq. Densiny 4 bm/tit & $84.91 !$ & 84.871 & 84.641 & 84.681 & 84.681 & $84.69 !$ & 84.661 & $84.74 !$ & $84.76^{\prime}$ & 84.91 \\
\hline Latent Heat Burbm & $31.73 !$ & $31.68 !$ & 31.541 & $31.56 !$ & 31.561 & $31.57^{1}$ & 31.551 & $31.61^{i}$ & 31.621 & 31.73 \\
\hline Specific Hear-otulbmir & 0.2781 & 0.2781 & $0.279 i$ & $0.279 !$ & $0.279 i$ & $0.279:$ & $0.279 !$ & 0.2791 & 0.2781 & 0.278 \\
\hline iq. Viscositylbm/ithr & $0 . \overline{435 i}$ & 0.4931 & 0.4891 & 0.491 & 0.491 & 0.491 & 0.4891 & $0.491 i$ & $0.492 i$ & 0.485 \\
\hline Lig. Conductivity Btuhr-thF & $0.027 !$ & 0.02691 & 0.02681 & 0.02681 & 0.02681 & 0.02081 & 0.02681 & 0.02591 & 0.02691 & 0.027 \\
\hline iq. Prandt No. & $5.11:$ & $5.1 !$ & $5.09 !$ & 5.09 & $5.09 \mathrm{i}$ & $5.09 !$ & 5.091 & $5.1 !$ & 5.1. & 5.11 \\
\hline Inlet Flow Rato-GPM & 3.9 & & & & & & & & & \\
\hline OutletLig. Flow Rato-GPM & 0.8 & & & & & & & & & \\
\hline Outlet Vap. Flow Rate-GPM & 2.4 & & & & & & & & & \\
\hline Inlet Temperaturef & 1321 & & & & & & & & & \\
\hline Outlet Liq. Temperaturef & 132.5! & & & & & & & & & \\
\hline Outlet Vap. Temperaturof & $129.6 !$ & & & & & & & & & \\
\hline Water Flow Rato-GPM & 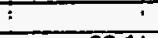 & & & & $!$ & & I & & & \\
\hline Wator Inlet Temp.f & 90.11 & & I & & i & & 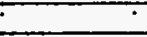 & & & \\
\hline Water outlot Temp.F & 1181 & & $\dot{-}$ & 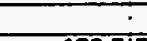 & $!$ & & - & . & & $\therefore$ \\
\hline Inlot Glbm/hr/i2 & 7920241 & & Temp & 129.610 & Density & 86.11 & $\dot{-}$ & & & 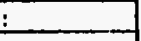 \\
\hline Subcooling & $5.1 i$ & & $\cdot$ & $:$ & $\therefore$ & ; & $\dot{-}$ & . & 1 & 1 \\
\hline Flow Observation & & & & & & & & & & \\
\hline & ZONE1 :2 & ZONE2 : & ZONE3 & ZONE4 : & ZONE5 :Z & ZONE6 & ZONE7 & ZONE8 & ZONE9 & ZONE10 \\
\hline Heat Input-Watts(nel) & 598.7 & 583.41 & $574.1 !$ & $574.4 !$ & 591.2 & $592.8 !$ & $586.9_{1}$ & $586.1 i$ & 578.4 & 589.7 \\
\hline Hoat Loss-Walts & 21.81 & $5.2 !$ & $7 !$ & 8.51 & 9.41 & 9.2 & $7.3 i$ & $8.2 !$ & $8.1 i$ & 44.1 \\
\hline Heat Flox-BturHrite & 10378 & 10113 & $9952 !$ & 9957 & 102481 & 10276 & $10174 !$ & 10160: & $10026 !$ & 10222 \\
\hline Wall Temp. म3F & 144.6 & 146.11 & 145.8 & $145 !$ & $147.4 i$ & 145.5 & 146.1 & 145.41 & $144.6 !$ & 145.1 \\
\hline Wall Tomp. \#4F & 144.9 & 144.51 & 146.61 & 145.61 & 746.11 & 145.61 & 146.5! & 145.8! & 145.51 & 144 \\
\hline Wall Temp. \#5- & 145.11 & 143.6 & 147.51 & 145.81 & 1451 & 146.11 & 145.41 & 144.91 & $143.9 !$ & 144 \\
\hline Wall Tomp. "1F & 79.81 & 145.3 & 1441 & 143.5 & $144.2 !$ & 146.11 & $146.5 i$ & 146.1 & 142.81 & 140.6 \\
\hline Wall Tomp. \#2F & $145.8 \mathrm{i}$ & 145.31 & 144.71 & 1441 & $144.2 !$ & $146.3 \mathrm{i}$ & $146.6 i$ & $146.5 !$ & 143.51 & 1424 \\
\hline Instream Tomp.F & $133.1 !$ & 133.7 & 134.71 & 134.51 & 134.5i & 134.41 & $134.6 !$ & $134.1 !$ & $134 i$ & 133.1 \\
\hline Avg. Wall Tomp.F & $145.1 !$ & 145.31 & 145.31 & $145 !$ & 144.91 & 145.81 & $146.2 i$ & $145.7 !$ & 144.1. & 143.2 \\
\hline Avg. Wall DT.f & $11.5:$ & 11.1 & 10.1 & 10: & $9.9 i$ & $11 ;$ & 11.2 & 11.2 & 9.6. & 9.7 \\
\hline Remaining Subcoolf & 2.41 & & & & & & & & & \\
\hline Exot Quality & -0.02 & 01 & 0.031 & $0.05 i$ & 0.071 & 0.11 & 0.12 & 0.151 & 0.17 & 0.19 \\
\hline EXP.H-BtuHHrhle F & 9001 & 907.61 & 982.91 & 993.21 & $1033.9 \mathrm{i}$ & 937.51 & 911.6 & $908.7 !$ & $1043.6 !$ & 1058.4 \\
\hline COMPUTEDH & & & & & & & & & & \\
\hline Jallouk & $909.2 !$ & $898.5 T$ & 8501 & $-818.7 \pi$ & $821.5:$ & 814.51 & 805.51 & 798.41 & $789.9 !$ & 784.6 \\
\hline Szadyi(Cudo tubo) & 1341.7! & 1329.41 & $1329.7 i$ & 13322 & 7354.81 & 1359.9! & 1359.41 & 1359.3! & 1355.11 & 1365.5 \\
\hline $5 z a d y 2$ & 1119.81 & $1105.1 !$ & 1127.21 & 1126 & 1148.31 & $1150.6 !$ & 1724.31 & 1126.51 & 1123.21 & 1136.7 \\
\hline (PLLO) & 1045871 & 105088 & 1044091 & 10177 & $99272 !$ & 968501 & 942681 & 913811 & $88817 !$ & 85704 \\
\hline (F) & 1.0061 & 1.0061 & 1.4391 & 1.772 & $2.024 i$ & 2.24 & $2.427 !$ & 2.6021 & 2761 & 2.919 \\
\hline (HUQ) & 237.11 & 231.2 & $331.2 i$ & 407.81 & 465.81 & 515.5 & 558.61 & $588.6 !$ & 634.81 & 670.5 \\
\hline (HBOLL) & 1339.41 & 1327 & $13228 !$ & 1319.31 & 1326.21 & 1334.7 & $13272 !$ & 1319.41 & 13071 & 1309.4 \\
\hline & & & & & & & 1 & 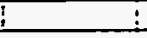 & 1 & $\vdots$ \\
\hline & ZONE2 .2 & ZONE4 & ZONE6 & ZONE8 & ZONE10! & & I & 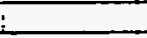 & & \\
\hline DP liquid Temp.F & $80.3 i$ & & & & $i$ & $\vdots$ & $\vdots$ & & & \\
\hline DP Liquid Density lbmitt3 & $92.94 !$ & & & & & & & & & \\
\hline Elevation-1 & & 4 & 6 & $8 i$ & $9.875 !$ & & & & & \\
\hline MeasuredDPPsid & 0.131 & 0.6761 & 0.353 & 1.557 & 2.2631 & & & & & \\
\hline Zero Comections-psid & -0.027 & -0.1021 & -0.17 & 0.016 & -0.018 & & I & 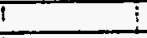 & 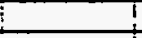 & ! \\
\hline Conected DPPsid & 0.1571 & 0.778 & 0.5331 & 1.9411 & $2.281 !$ & & 1 & I & & \\
\hline Actual DP- & 1.92 & 3.001 & 5.681 & 5.471 & 6.941 & & I & I & & I \\
\hline Moasured TRPsig & 85.81 & 1 & 1 & 1 & 3.6 & & $!$ & 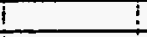 & ! & $!$ \\
\hline Mossured Overalth & 139.41 & $\therefore$ & \pm & \pm & 1 & & 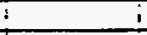 & $\div$ & $i$ & $i$ \\
\hline & 1 & & 1 & $!$ & 1 & I & 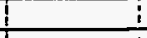 & $i$ & 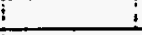 & $i$ \\
\hline Computed DP-n & 2.051 & 3.821 & 5.411 & $6.97 !$ & 8.45 & & $\vdots$ & I & I & \\
\hline Friction-ft & $0.09 j$ & 0.27 & 0.55 & $0.92 !$ & 1.35 & & 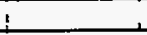 & is & $i$ & \\
\hline Acceleration-ft & 0.031 & $0.18 !$ & $0.27 !$ & 0.361 & $0.46 i$ & & & & & \\
\hline Elevation-At & $1.94 !$ & $3.37 !$ & $4.58 !$ & 5.68 & 6.641 & & & & & \\
\hline
\end{tabular}




\begin{tabular}{|c|c|c|c|c|c|c|c|c|c|c|}
\hline $\begin{array}{l}\text { Run } \vec{\pi} \\
\text { Test Fuid }\end{array}$ & $-c 4=10^{563}$ & & & & & & & & & \\
\hline Date: & $5 / 27 / 93^{\circ}$ & & & & & & & & & \\
\hline Time: & $12: 14$ & & & & & & & & & \\
\hline Noto & TABOREK & Anatytical d & & & & & & & & \\
\hline Fow Areaft2 & 0.00336 & & & & & & & & & \\
\hline 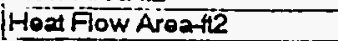 & 0.19695 . & & & & & & & & & \\
\hline Sal. PressuroPsig & $95.3:$ & & & & & & & & & \\
\hline Sal. Temperarure-finlet) & 136.8: & & & & & & & & & \\
\hline Lig. Density-lbmytit3 & 84.761 & $84.64 !$ & 84.491 & 84.511 & 84.52 & 84.52 & 84.52 & $84.54 !$ & $34.54:$ & 84.69 \\
\hline Latent Heat-Btu/bm & 31.52 & 31.54 & 31.43 & 37.45 & 31.46: & $31.46:$ & 31.46 & 31.47 & 31.47 . & 37.57 \\
\hline Specific Heat-Btufibmif & 0.278 & 0.2791 & 0.2791 & 0.2791 & 0.279 & $0.279:$ & 0.279 & 0.279 & 0.279 & 0.279 \\
\hline iq. Viscosity-bmithr & $0.492:$ & 0.489 ! & $0.486 !$ & $0.486 \Gamma$ & 0.485 & 0.486 & $0.486:$ & $0.487^{\prime}$ & 0.487 & 0.49 \\
\hline Liq. Conductivity-Bhuhr-HtF & 0.0259 & 0.02681 & $0.0267^{1}$ & 0.0267 & 0.0267 & 0.0267 & 0.0267 & $0.0267^{\circ}$ & 0.0267 & 0.002 \\
\hline Iug. Pranat No. & $5.1^{1}$ & $5.09 !$ & 5.081 & $5.08^{\circ}$ & 5.08 & $5.08 !$ & 5.08 & $5.08^{1}$ & 5.08 & 5.09 \\
\hline Inlet filow fate-GPM & $3 . \overline{9}$ & & & & & & & & & \\
\hline Outlet Lug. Fow Ralo-GPM & 0.8 & & & & & & & & & \\
\hline Outlot Vap. Flow Rato-GPM & $2 . \overline{4}$ & & & & & & & & & \\
\hline Inlel Temperature- & 133.4 & & & & & & & & & \\
\hline Outel Lq. Jomperaturof & 133.6. & & & & & & & & & \\
\hline Outer vap. Temperaturef & 130.6: & & & & & & & & & \\
\hline Water Flow Rato-GPM & & & & & & & & & & \\
\hline Water Inlet Temp.F & 91 & & & & & & & & & \\
\hline Waler outel Temp.F & 119.1 & & & & & & & & & \\
\hline Inet G-Bbm/hroth & $790708^{\circ}$ & & Temp & 130.3! & Density & $84.95 !$ & & & & \\
\hline Subcooling F & 3.4 & & & & & & & & & \\
\hline Flow Observation & & & & & & & & & & \\
\hline & ZONEI & 'ZONE2 $\cdot 2$ & ZONE3: & ZONE4 & ZONE5 & ZONE6 & ZONE7 & ZONE8 & ZŌNE9 & ZONE10 \\
\hline Hoal Input-Watts(nel) & 600.41 & 584.61 & $573.7 i$ & 5741 & $588 \mathrm{i}$ & $589.8 \mathrm{i}$ & 587.3 & 586.4 & 577.61 & 1589.1 \\
\hline Hoed Loss-Wadts & 21.91 & 5.21 & $7.7 i$ & 8.61 & $9.5 i$ & $9.2 !$ & 7.31 & $8.2 !$ & 8.21 & 44.4 \\
\hline Heat Flox-8turiritiz & 104081 & $10 \overline{134}$ & $9945 i$ & 99501 & 101931 & $10224 !$ & $10180 i$ & $10165 !$ & 100121 & 10212 \\
\hline Wall Tomp. \#3F & $145.4 !$ & $147 !$ & 146.6: & $146.1 !$ & $148.2 !$ & 146.2! & 147 & 146.41 & 145.5 ? & 145.9 \\
\hline Wall Temp. \#4F & 145.91 & 145.5 & 147.5 & $146.5 !$ & $147 !$ & 146.61 & $147.4 !$ & $146.7 ;$ & 96.4 & 145.1 \\
\hline Wall Temp. 55 & $145.9_{i}$ & 144.5 & 148.31 & 146.71 & $146 !$ & $146.8 !$ & $146.3 !$ & $145.8 !$ & 945.11 & 145.1 \\
\hline Wall Temp. $\$ 1 F$ & $79.8 i$ & $146.2 !$ & 144.91 & $144.5 !$ & 145.2 & 146.9 & 147.2 & 147.1 & 143.9 & 141.5 \\
\hline Wall Temp. $\$ 2-F$ & 146.8 & 146.2 & 145.5. & 145.11 & 145.2 & 147 & 147.8 & 147.4: & 144.5: & 143.5 \\
\hline Instream Temp.F & 134 & 134.7 & $135.6^{?}$ & 135.5 & $135.4 !$ & 135.41 & 135.4 & 135.3 & 135.3 & 134.4 \\
\hline Avg. Wall Temp.F & $146^{!}$ & $946.2^{\top}$ & $\$ 46.9^{i}$ & $946^{5}$ & $145.9^{\prime}$ & $946.6^{\prime}$ & 147.1 & 146.7 & $145.1^{\top}$ & 144.2 \\
\hline Avg.Wall DT.F & $17.5^{5}$ & $11.1 i$ & $10.1 \mathrm{i}$ & $10^{\circ}$ & $10 !$ & $10.8 i$ & $11.3 !$ & $10.9 !$ & $9.3^{\prime}$ & 9.4 \\
\hline Remarning Subcooff & $0 . \overline{6} !$ & & & & & & & & & \\
\hline Exot Qually & -0.011 & $0.02 !$ & 0.041 & 0.071 & 0.091 & $0.11 !$ & $0.14 !$ & $0.16^{:}$ & 0.18 & 0.21 \\
\hline EP. HBtuntr/n2/F & 902.71 & 915.7 & 9871 & 9951 & 1020.31 & $949.9 i$ & $902.5 i$ & 930.8 & 1073.41 & 1091.2 \\
\hline $\bar{C} \bar{C} \bar{M} \bar{P}$ UIEDH & & & & & & & & & & \\
\hline Iallouk & $916 !$ & $874.5 !$ & $837.2 !$ & $816 !$ & $817.9 !$ & $812.6 !$ & $805.8 !$ & 800.7 & $786.9:$ & 786.5 \\
\hline Szady1(Cudo tube) & 1350.9 & $1340.3 i$ & 1338.9 & 1342.3 & 1361.1 . & 1367.2 & $1368.9 !$ & $1371.9 !$ & 367.3 & 1377.9 \\
\hline Szady2 & $1129 !$ & 11411 & 1134 & 1133.9 & 1152.11 & 1155.8 & 1134.61 & 1139.31 & $1135.2 !$ & 1148.8 \\
\hline (RעQ) & 105165; & $105066 \mathrm{i}$ & 103316 & 100741 & 981361 & $95578 !$ & 93022 & 90401 & 37875 & 84733 \\
\hline (F) & 1.006 & $1.275 i$ & $1.572 !$ & 1.941 & $2.162 i$ & 2.3581 & 2.5331 & $2.695 i$ & 2.843 & 2.996 \\
\hline (FणOQ) & 2311 & 293.2 & $384.9^{i}$ & 446.41 & $497.5 !$ & $542.5 !$ & 582.9 & 620.11 & 654.2 & 588.5 \\
\hline (HBOIL) & T348.6! & $1335.6 !$ & 328.3: & $\mid 325.61$ & $1338.5^{5}$ & 1338.11 & $13328 !$ & 1328.3 & $\sqrt{315.4}$ & 1378.1 \\
\hline & ZONE2 'Z & ZONE4 & ZONE6 & ZONE8 & ZONE10 : & & $T$ & & & \\
\hline DP liquid Temp.f & 80.81 & : & & & 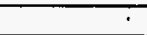 & & $=$ & & & \\
\hline DPLiquid Density $4 \mathrm{bm}$ /tt3 & $92.87 i$ & $\cdot$ & . & . & 7 & , & 7 & & 7 & \\
\hline Elovation-4t & 2 & $4 i$ & 6. & $8:$ & 9.876 . & & & & 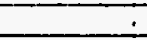 & 2 \\
\hline Moasured DPPsid & 0.133 & $0.68_{1}$ & 0.37 & $1.92 !$ & 2.271 & $i$ & . & & & \\
\hline Zero Corrections-psid & -0.027 & -0.1021 & $-0.17 !$ & 0.0161 & -0.018 & $i$ & & & & \\
\hline Corrected DPPsid & 0.16 & $0.782 !$ & $0.54 !$ & 1.9041 & $2.289 !$ & & & & & \\
\hline Actual DPA & 1.92 & 3.061 & $5.66 i$ & $5.54 \mathrm{i}$ & $6.94 i$ & 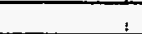 & & & & \\
\hline Measured TPPsig & 85.31 & 1 & & & 3.6 & 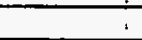 & - & & & \\
\hline Measured Overalift & 138.91! & & & & & & & & & \\
\hline & & & & & & I! & & & T & \\
\hline Computed DPA? & $2.04 !$ & $3.7 !$ & 5.271 & 6.841 & 8.321 & $\div$ & & & : & \\
\hline Frietion-1 & $0.1^{\circ}$ & 0.321 & 0.631 & 1.031 & 1.471 & & $i$ & & & \\
\hline Accoieration-tit & $0.1 !$ & $0.21 !$ & 0.31 & $0.39 i$ & 0.491 & 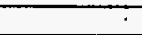 & & & & \\
\hline Elovation $\bar{t}$ & $1.84 i$ & $3.17 !$ & $4.34 i$ & $5.42 !$ & 6.361 & - & 7 & & - & \\
\hline
\end{tabular}




\begin{tabular}{|c|c|c|c|c|c|c|c|c|c|c|}
\hline Runt & 5641 & & $\therefore$ & 4 & • & & 1 & $!$ & $!$ & 1 \\
\hline Tost Fuid & C4F10 i & & $\dot{-}$ & 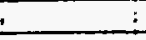 & 1 & 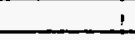 & 1 & 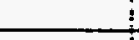 & 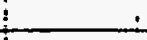 & $\therefore$ \\
\hline Dalo: & $5 / 27 / 931$ & & & & & & $\div$ & & & \\
\hline Time: & $12: 14$ & & $i$ & i & & & & & & \\
\hline Note & TABOREK & Analytical c & & $!$ & $\therefore$ & 1 & I & & & \\
\hline Flow Avea-fl2 & 0.00336 & & & $\vdots$ & & & & & & 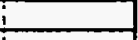 \\
\hline Head Fow Areaft? & 0.195951 & & & & $\therefore$ & & & & 1 & \\
\hline Sat. Prossure Psig & 84 & & & & & & & & 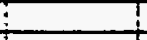 & \\
\hline Sat. Temperatur of (inlel) & 135.9 & & & & & & 1 & & & \\
\hline La. Densthy & 84.641 & 84.52 & 84.371 & 84.391 & 84.371 & 84.371 & 84.41 & 84.42 & 84.441 & 84.57 \\
\hline Latent Heat-Bturibm & 31.541 & 31.46 & 31.35 & 37.361 & 31.35 & 37.351 & 31.37 & 31.39 & 31.4 & 31.49 \\
\hline Spocific Hoartuntbm/F & 0.2791 & 0.279 & 0.279 & 0.2791 & 0.279 & 0.279 & 0.279 & 0.279 & 0.279 & 0.279 \\
\hline Liq. Viscositylbm/thr & 0.4891 & 0.485 & 0.4831 & 0.4831 & 0.4831 & 0.483 & 0.484 & 0.484 & 0.484 & 0.488 \\
\hline Lig. Conductivity Btuhrefif & 0.0268 & 0.0267 & 0.02661 & 0.02561 & 0.02651 & 0.02651 & 0.02661 & 0.0267 & 0.0287 & 0.0258 \\
\hline Liq. Prandt No. & 5.09 & 5.08 & 5.071 & 5.071 & $5.07 !$ & 5.07 & 5.071 & 5.071 & 5.07 & 5.08 \\
\hline Inlet Flow Rate-GPM & 3.9 & & 1 & 1 & & : & i & & & \\
\hline Outlet Lig. Flow Rate-GPM & 0.9 & & & & & & & & & \\
\hline Outlot Vap. Flow Rate-GPM & 2.41 & & & 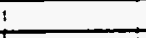 & & $\dot{-}$ & : & & & 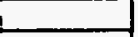 \\
\hline Inlel Tomperaturef & 133.7 & & & 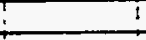 & 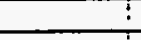 & 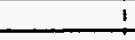 & 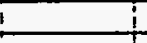 & & i & 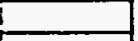 \\
\hline Outle! Lq. Temperafuref & 134.4 & & & 3 & & & & & & \\
\hline Outlot Vap. Temperaturef & 731.31 & & & $!$ & & & & & & \\
\hline Water Flow Rato-GPM & & & & 1 & I & & $\mathrm{T}$ & & & \\
\hline Water Inlot Tomp.F & 91.61 & & & I & $\overline{!}$ & & 1 & 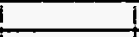 & & \\
\hline Water outtel Tomp.f & 119.41 & & & 1 & \pm & & I & L & & \\
\hline Indol Glbm/hr/iti & 7886631 & & Temp & 1311 & Density & 84.83 & I & i & & \\
\hline Subcoolingf & 22 & & & 1 & I & & 1 & 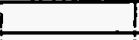 & & \\
\hline Flow Observation & & & & $i$ & & & 1 & 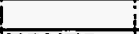 & & \\
\hline & ZONE1 I & ZONE2 & ZONE3 & ZONE4 & ZONE5 & ZONE6 & ZONE7 & ZONEB & ZONE9 & ZONE10 \\
\hline Hoef input-Watts(nel) & I599.7 & 584.2 & 576.7 & 577.2 & 587 & 588.7 & 586.6 & 586.11 & 581.9 & 5925 \\
\hline Hoat Loss-Walts & 21.9 & 5.2 & 7.11 & 8.6 & 9.5 & 9.2 & 7.31 & 8.2 & 8.2 & 44.4 \\
\hline Heat Flox-Bturfrrit2 & 10395 & 10127 & 9997 & 10005 & 10175 & 10205 & 10168 & 10160 & 100087 & 10271 \\
\hline Wall Temp. \#3F & 1461 & 147.4 & 747.21 & 146.6 & 148.91 & 147 & 147.4 & 146.9 & T45.4 & 146.3 \\
\hline Wall Temp, HAF & 746.5 & 146.1 & 148.1 & 147.1 & 147.6 & 147.11 & 148 & 147.4 & 147 & 145.8 \\
\hline Wall Temp. & 146.5 & 145.1 & 149 & 147.4 & 148.8 & 147.5 & 147 & 146.4 & 145.7 & 145.7 \\
\hline Wall Tomp. If & 80.21 & 146.8 & 145.51 & 145.3 & 145.8 & 147.5 & 148.1 & 147.4 & 144.7 & 142.2 \\
\hline Wall Tomp. "Naf & 147.31 & 146.81 & 1461 & 145.8. & 146 & 147.91 & 148.4 & 148 & 145.31 & 144 \\
\hline Inetream Temp.F & 134.7 & 735.4 & 136.81 & 136.2 & 136.31 & 736.31 & 136.11 & 135 & 135.81 & 735.1 \\
\hline Avg. Wall Tomp.F & 146.61 & 146.8 & 146.7 & 146.6 & 146.6 & 147.3 & 147.81 & 147.2 & 145.8 & 144.8 \\
\hline Avg.Wall DT of & $\$ 1.41$ & 10.9 & 9.91 & 9.91 & 9.8 & 10.5 & 112 & 10.8 & 9.5 & 9.2 \\
\hline Remaining Subcoolf & -0.6 & & & & & & & & & \\
\hline Exit Quality & 0.01 & 0.03 & 0.05 & 0.08 & 0.1 & 0.12 & 0.15 & 0.17 & 0.2 & 0.22 \\
\hline EXP.HBtunHrnter & 911.5 & 927.6 & 1004.9 & 1005.8 & 1039.3 & 970.6 & 906.2 & 944.1 & 1055.8 & 1112 \\
\hline COMPUTEDH & & & & & & & & & & \\
\hline Jallouk & 902.4 & 858.6 & 826.5 & 897.5 & 817.8 & 813 & 806.11 & 801.5 & 789.8 & 789.2 \\
\hline Szady1(Cudo tube) & 1356.6 & 7347.2 & 1349.7 & 1353.2 & 1359 & 7375.1 & 7375.7 & 7379.1 & 7378.7 & 1389.1 \\
\hline szady2 & 1134.6 & 1148.7 & 1143.7 & 1144.1 & 1158.7 & 1139.1 & 1741.6 & 1146.6 & 1147.2 & 1160.3 \\
\hline (RLQ) & 105317 & 104858 & 102577 & 99939 & 974941 & 94915 & 92187 & 89547 & 86922 & 83818 \\
\hline (F) & 1.163 & 1.487 & 1.8011 & 2.048 & 2.240 & 2.43 & 2.6031 & 2.759 & 2.909 & 3.053 \\
\hline (स्मिO) & 267 & आ1.7 & 494.41 & 470.1 & 617.61 & 560 & 588.9 & 634.7 & 668 & 701.6 \\
\hline (FBOL) & 1353.11 & 1339.8 & 1336.5 & 1334 & 13439 & 1343.4 & 1336.8 & 1332.7 & 1324.31 & 1326.7 \\
\hline & & & & & & & & & & \\
\hline & ZONE2 & ZONE4 & ZONE6 & ZONE8 & ZONE10 & & & & & \\
\hline DP liquid Temp. $f$ & $\begin{array}{l}81.3 \\
\end{array}$ & & & & & & & & & \\
\hline DPLiquid Density $1 \mathrm{bm} / \mathrm{t} 3$ & 92.8 & & & & & & & & & \\
\hline Elevation-ft & $2 !$ & 4 & 6 & 8 & 9.875 & & & & & \\
\hline Moasured DPPSId & 0.133 & 0.679 & 0.37 & 7.\$51 & 2.259 & & & & & \\
\hline Zero Conections-psid & 0.022 & -0.028 & -0.7341 & 0.128 & 0.167 & & & & & \\
\hline Correctod DPPsid & 0.171 & 0.707 & 1.124 & 1.823 & 2.108 & & & & & \\
\hline Actual DPf & 2.01 & 3.19 & 4.67 & 5.67 & 7.251 & & & & I & 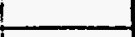 \\
\hline Measured TPP Prg & 84 & & & & 3.61 & & & & & I \\
\hline Mesucued Overalth & 136.89 & & & & & & & & & i \\
\hline & & & & & & & & & & \\
\hline Computed DPA & 1.911 & 3.54 & 5.1 & 6.66 & 8.15 & & & & & \\
\hline Friction-ft & 0.13 & 0.37 & 0.7 & 1.11 & 1.57 & & & & & \\
\hline Acceleration-li & 0.13 & 0.23 & 0.32 & 0.41 & 0.51 & & & & i & \\
\hline Elevation-t & 1.65 & 2.94 & 4.09 & 5.15 & 6.08 & & 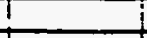 & & $i$ & \\
\hline & & & & & & & & & & \\
\hline
\end{tabular}




\begin{tabular}{|c|c|c|c|c|c|c|c|c|c|c|}
\hline Run & $565 i$ & & & & & & & & & \\
\hline Test Fuid & CAFTO & & & & & & & & & \\
\hline Date: & $527133^{1}$ & & & & & & & & & \\
\hline Time: & $12: 141$ & & & I & & i & $t$ & I & 1 & 1 \\
\hline Note & TABOREK & Analytical c & & $\div$ & $\div$ & ? & & & & \\
\hline Fow Areatl2 & $: 0.003351$ & & $\cdot$ & . & F & $\cdot$ & 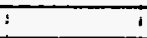 & $T$ & $\rightarrow$ & - \\
\hline Hoad Flow Aroatt2 & 0.196551 & & $\cdot$ & 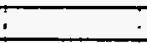 & $\dot{.}$ & 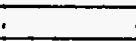 & . & $!$ & &. \\
\hline Sat. Pressura Psig & 96.71 & & 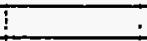 & 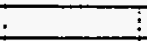 & $\vdots$ & 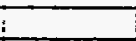 & I & & 7 & 1 \\
\hline Sat. Temperaturo - (inlet) & $144.5 i$ & & 1 & 1 & $i$ & & $!$ & & 2 i & $\frac{1}{i}$ \\
\hline Lq. Density-lbm/t3 & $84.61 i$ & 84.491 & 84.09 & 83.781 & $83.31:$ & 82.91 & 82.821 & $82.88 !$ & 82.91 i & 83.04 \\
\hline Latent Heatbiulbm & $31.52 !$ & 31.43 & $31.16 i$ & 30.941 & 30.61 & 30.32 & $30.26 i$ & 30.3 & 30.32 & 30.41 \\
\hline Specific Heat-Btunbmif & 0.2791 & 0.2791 & 0.281 & 0.2811 & 0.2821 & 0.283 & 0.2831 & 0.283 & 0.2831 & 0.283 \\
\hline Lq. Viscosity lbmithr & 0.4881 & 0.4861 & $0.477 !$ & 0.47 & 0.461 & 0.451 & 0.45 & 0.4511 & 0.4511 & 0.454 \\
\hline Liq. Conductivity stuhr-fif & 0.0258 & 0.0267 & 0.02641 & 0.02621 & 0.02591 & 0.0257 & 0.02356 & 0.02257 & 0.0257 & 0.0258 \\
\hline Liq. Prander No. & $5 . \infty 9$ & 5.081 & 5.051 & 5.031 & $4.99 !$ & 4.97 & 4.961 & 4.97 & 4.971 & 4.98 \\
\hline Inlet Fow Rate-GPM & 3.91 & & & & & 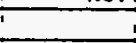 & 1 & & & $i$ \\
\hline Outel Liq. Flow Rato-GPM & 1.71 & & 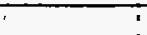 & $:$ & 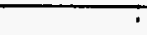 & $\cdot$ & & & & $\div$ \\
\hline Outlet Vap. Flow Rato-GPM & 3.91 & & T & 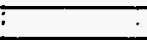 & 7 & 7 & & & $T$ & $T$ \\
\hline Inlet Temperaturef & 133.91 & & . & $\dot{.}$ & 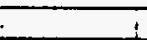 & 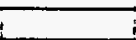 & $i$ & $?$ & $\because$ & 1 \\
\hline Outlet Liq. TemperaturefF & 143.21 & & $i$ & ! & $!$ & & 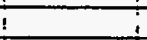 & & T & \\
\hline Outlet Vap. Temperaturef & 140.41 & & $i$ & & & & $i$ & & & \\
\hline Wator Fow Rato-GPM & & & & & ! & & & & + & i \\
\hline Water Inlet Temp.F & 104.2 & & & & & & 1 & & 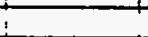 & \\
\hline Water outlet Temp.F & 137.7! & & & & 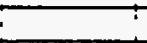 & & & & 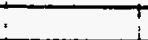 & \\
\hline Inlet Glbmhrike & $785 \% 98$ & & Temp & 132.61 & Density & 84.55 & & & $i$ & \\
\hline Subcooling & 10.61 & & & & & & & & & \\
\hline Flow Observation & & & & & & & & & & + \\
\hline & ZONE1 12 & ZONEE I & ZONE3 & ZONE4 & ZONES : & ZONE6 & ZONE7 I2 & ZONEB & ZONES & IZONE1O \\
\hline Hoat tnput-Wats(net) & 607.1 & 594.2 & 591.9 & 590.31 & 588.9 & 588.8 & 595.11 & $5 \$ 5.1$ & 597.2 & 598.4 \\
\hline Hoal Loss-Watls & 26.31 & 61 & 8.21 & 10 & 10.9 & 10.9 & 9.2 & 10.11 & 9.8 & 52.5 \\
\hline Hoad FLCE-8Hu/Hifi2 & $10524 !$ & 10300 & 102501 & 10232 & 10174 & 10206 & 10316 & 10316 & 103521 & 10373 \\
\hline Wall Tomp. \#3F & 154.6 & 156 & 155.6 & 154.91 & 156.91 & 155.8 & 156.3 & 155.7 & 155.11 & 155.2 \\
\hline Wall Temp. \#4F & $154.9 !$ & 154.6 & 156.8 & 155.6 & 156.1 & 155.8 & 156.8 & 156.3 & 155.7 & 154.4 \\
\hline Wall Temp. $\$ 5 F$ & 154.9 & 153.5 & 157.8 & 155.61 & 155.2 & 156.1 & 155.8 & 155.4 & 154.1 & 154.3 \\
\hline Wall Temp. \#1F & 81.31 & 155.21 & 154.2 & 153.5 & 154.3 & 156.11 & 157 & 156.3 & 153.4 & 1508 \\
\hline Wall Temp. $\# 2 F$ & 155.21 & 155.21 & 154.7 & 1541 & 154.31 & 156.11 & 157.31 & 156.81 & 154 & 152.7 \\
\hline Instream Temp.f & 134.91 & $135.6 !$ & $137.9 !$ & 139.71 & 142.41 & 144.6 & 145.11 & $144.8 !$ & 144.61 & 143.9 \\
\hline Avg. Wall Temp. $F$ & 154.91 & 155.31 & 155.31 & 154.91 & 1551 & 156 & 156.6 & 756.11 & 154.51 & 153.5 \\
\hline Avg. Wall DT.F & 19.5 & $19.2 !$ & $17 !$ & 14.7 & 12.1 & 10.9 & 17.11 & 10.8 & 9.41 & 9.1 \\
\hline Remaining Subcooff & 7.81 & & 1 & 1 & 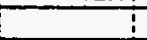 & & & & i & i \\
\hline Exit Quality & -0.071 & -0.04 & -0.021 & of & 0.081 & 0.05 & 0.081 & 0.11 & 0.18 & 0.18 \\
\hline DXP.HBWUHriarF & 5391 & $536.9 !$ & 604.91 & 694.31 & 839.7 & 937.3 & 931.6 & 95221 & 11022 & 1138.6 \\
\hline COMPUTEDH & $i$ & & ${ }^{\circ}$ & & & & & & & \\
\hline Jallouk & 927.91 & 919.9 & 930.9 & 939.81 & 899.4 & 887.21 & 870.21 & 859.91 & 853.2 & 844.7 \\
\hline Szady1(Cudo tube) & 1365.7 & 1357 & 1373.4 & 1386.6 & 1408.3 & 1432.7 & 1447.7 & 1448.6 & 1453 & 14523 \\
\hline Szady2 & 1144.6 & 1133.8 & 1149.3 & 1161.8 & 1209 & 1230.5 & 1243.9 & 1241.6 & 1243.6 & 1214.6 \\
\hline (RUQ) & 105412 & 106004 & 107979 & 109558 & 110126 & 100312 & 105890 & 103730 & 100652 & 97200 \\
\hline (F) & 1.006 & 1.006 & 1.006 & 1.006 & 9.454 & 1.76 & 1.996 & 2.201 & 2.381 & 2.552 \\
\hline$(\mathrm{HLQ})$ & $230.4 !$ & 230.6 & 231.31 & 237.81 & 336.4 & 408.3 & 463.2 & 570.6 & 552.4 & 591.4 \\
\hline (HBOIL) & 1363.5 & 1354.8 & Tउना.2! & T384.5 & 1401.9 & 7421.5 & 1431.7| & 4427.1 & $\{425.91$ & 7478.9 \\
\hline & 1 & 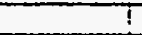 & $!$ & & & & & & & \\
\hline & ZONE2 I & ZONE4 & ZONE6 & ZONE8 & ZONE10 & & & & & \\
\hline DP liquid Tomp.F & 82.21 & $i$ & 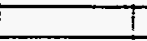 & 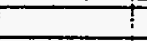 & -1 & i & i & $i$ & 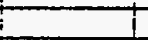 & \\
\hline DP Liquid Densitylbm/tit3 & 92.671 & $!$ & 1 & 1 & & 1 & & $i$ & 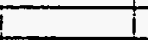 & \\
\hline Elovation-ft & 2. & 41 & 61 & 81 & 9.876 & I & t & & & \\
\hline Measured DPPsid & $0.108 i$ & 0.347 & -0.216 & 1.13 & 1.499 & & 1 & & & \\
\hline Zoro Conections-psid & 0.0221 & -0.028 & -0.7541 & 0.128 & 0.161 & & & & & \\
\hline Corrected DPPsid & 0.086 & 0.375 & 0.538 & 1.002 & 1.338 & & & & & \\
\hline Actual DPA & 2.08 & 3.811 & $5.76 !$ & 7.19 & 8.7 & & & & & \\
\hline Measured TPPsig & 96.71 & & & & 3.5 & & & & & \\
\hline Measured Cveralli & 161.62 & & & & & & & & & \\
\hline & & & & & & & & & & \\
\hline Computed DP-A & 2.081 & 4.13 & $5.89 !$ & 7.48 & 8.95 & & & & + & \\
\hline Fricton-t & 0.081 & 0.17 & 0.351 & 0.62 & 0.941 & & & & $i$ & 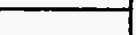 \\
\hline Acooleration-ft & 01 & 0.04 & 0.18 & 0.28 & 0.351 & & T & & & \\
\hline Elovationft & 21 & 3.821 & 6.361 & 6.61 & 7.661 & & $T$ & $t$ & & \\
\hline
\end{tabular}




\begin{tabular}{|c|c|c|c|c|c|c|c|c|c|c|}
\hline Runit & 5661 & $\Gamma$ & & 1 & : & : & $:$ & & 1 & $i$ \\
\hline TestFuid & CAF10 & . & & 1 & $t$ & 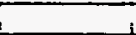 & . & 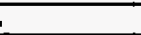 & 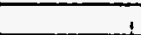 & 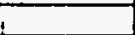 \\
\hline Dala: & $527 \mid 331$ & & & - & & $i$ & & & & \\
\hline Time: & 13.231 & & & & 1 & 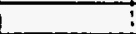 & $\vdots$ & & & \\
\hline Note & TTABOREK & Analytical o & & $i$ & 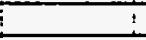 & 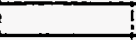 & $!$ & & i & i \\
\hline Fow Area-fl2 & 0.003361 & $i$ & 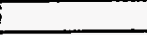 & & & & & 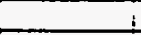 & ; & ; \\
\hline Heal Flow Area-tt2 & 0.196951 & $\Gamma$ & & . & 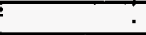 & & & & & 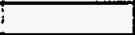 \\
\hline Sat. Pressure Psig & 103.4 & & & & $i$ & & & & & \\
\hline Sad. Temporaturof(inlet) & 748.7 & & & & & & & $i$ & 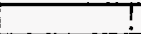 & \\
\hline Lg. Densty-1bmam3 & 84.31 & 84.161 & $83.82 !$ & 83.391 & 82.931 & 82.611 & 82.481 & 82.57 & 82.641 & $82.7 \pi$ \\
\hline Latent Hoat-butiom & 31.31 & 31.21 & 30.981 & 30.681 & 30.391 & 30.11 & 30.011 & 30.07 & $\$ 0.13$ & 30.18 \\
\hline Specific Hoes Gtu/bm/F & 0.281 & 0.281 & 0.2811 & 0.2821 & 0.2831 & 0.284 & 0.2841 & 0.284 & 0.284 & 0.283 \\
\hline Lig. Visoositylbm/ithr & 0.4871 & 0.478 & $0.47 \pi$ & 0.462 & 0.4621 & 0.446 & 0.4431 & 0.444 & 0.446 & 0.447 \\
\hline Lig. Conductivity Btumh-thF & 0.02661 & 0.02651 & 0.02631 & 0.0261 & 0.02571 & 0.02551 & 0.0254 & 0.02551 & 0.02551 & 0.0256 \\
\hline Liq. Prandt No. & 5.061 & 5.051 & 5.031 & 5 & $4.97 !$ & 4.95 & 4.941 & 4.55 & 4.55 & 4.96 \\
\hline Inletflow Rato-GPM & 3.91 & & & & & & & & & \\
\hline Outlel Liq. Flow Rato-GPM & 1.6 & & & & & & & & & \\
\hline Outtel Vap. Flow Ralo-GPM & 3.11 & & $\cdot$ & $\cdot$ & $\vdots$ & - & 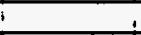 & & $\div$ & 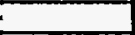 \\
\hline Inlel Temperaturef & 136.11 & & & : & & F & 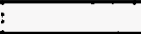 & & & \\
\hline Outilug. Temperaturer & T45.21 & & & & I & 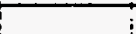 & $\bar{i}$ & & & \\
\hline outlel vap. Temperature-f & 142.2! & ! & 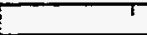 & & 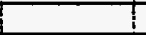 & 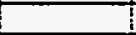 & & & ! & ! \\
\hline Water Flow Rala-GPM & & & . & & & 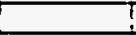 & I & 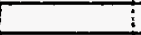 & & 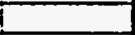 \\
\hline Water Inlet Temp.F & 102.51 & & & I & i & 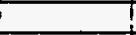 & i & & & 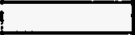 \\
\hline Water outtet Temp.F & 1361 & & & T & 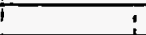 & 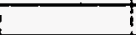 & 1 & 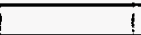 & & 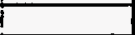 \\
\hline Inlat G-lbm/hr/at2 & 7832111 & & Temp & 134.8 & Density & 84.15 & T & 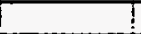 & T & T \\
\hline Subcoolingf & 1261 & T & 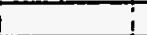 & $i$ & -2 & $\overrightarrow{1}$ & 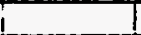 & i & 1 & 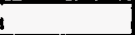 \\
\hline \multirow{2}{*}{ Flow Obsenvation } & 1 & 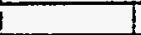 & & I & 1 & 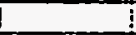 & & I & & \\
\hline & ZONE1 & ZONE2 & ZONE3 & ZONE4 & ZONE5 & ZONE6 & ZONE7 & ZONE8 & ZONE9 & ZONE10 \\
\hline Heat Input-Watts(nel) & 575.5 & 575.9 & 579.6 & 580.3 & 573.5 & 576.8 & 582.2 & 578 & 575.5 & 1155.2 \\
\hline Head Loss-Watts & 26.1 & 5.9 & 8.1 & 10 & 10.9 & 10.9 & 9.2 & 10.1 & 9.8 & 55.1 \\
\hline Head Flox-Btu/Hint & 9976 & 9988 & 10047 & 10059 & 99411 & 9998 & 10002 & 10019 & 9976 & 20025 \\
\hline Wall Temp. \#34 & 355.7 & 757.2 & 157 & 756.5 & 158.7 & 757.41 & 158.11 & 15T.21 & 756.61 & 162.6 \\
\hline Wail Temp. "\#4F & 156 & 155.8 & 757.81 & 157.1 & 957.8 & 157.6 & T58.2 & 157.7 & 157.2 & 100.5 \\
\hline Wall Temp. "5F & 156.2 & 154.6 & 758.81 & 157.4 & $156.7 !$ & 157.7 & 157.51 & 156.61 & $156 !$ & 161 \\
\hline Wall Temp. "1F & 81.51 & 156.4 & 155.21 & 155.3 & $158 !$ & 157.7 & 158.51 & 158 & 1551 & 154.6 \\
\hline Wall Tomp. "arF & 156.21 & $156.4 !$ & 155.91 & 155.8 & 1561 & 158.11 & 158.81 & 158.31 & 155.61 & 158.3 \\
\hline Instream Tomp.f & 136.7 & 137.6 & 139.5 & 141.9 & 144.61 & 146.3 & 147 & 146.6 & 146.11 & 145.7 \\
\hline Avg. Wall Tomp,F & 156 & 156.5 & 156.5 & 156.6 & 156.61 & 157.6 & 158.2 & 157.6 & 156.11 & 159.4 \\
\hline Avg. Wall DT.f & 18.9 & 18.5 & 16.5 & 14.2 & 11.7 & 10.8 & 10.8 & 10.61 & 9.5 & 12.8 \\
\hline Romaining Subcoolf & 101 & & & & & & & & & \\
\hline Exit Quality & -0.00 & -0.07 & -0.04 & -0.02 & 0.01 & 0.03 & 0.06 & 0.08 & 0.11 & 0.16 \\
\hline EXP. HEtulHrnterF & 528.5 & 539.6 & 608.1 & 707.4 & 851.4 & 921.7 & 937.6 & 944.6 & 1046.9 & 1567.5 \\
\hline \multicolumn{11}{|l|}{ COMPUTEDH } \\
\hline Jallouk & 909.11 & 913.9 & 928.9 & 929.2 & \$6्36.11 & 907.7 & 892.1! & 85 & 850.41 & T2334 \\
\hline Szadyi(Cudo tubo) & 1344.71 & 1351.7 & 13725 & 1393.5 & 1407.6 & 1430.3 & $1446 \mid$ & 1439.9 & 1437 & 2019 \\
\hline Szady2 & 1118.3 & 1125.2 & 1146.11 & 1166.7 & 1179.2 & 1228.5 & 12422 & 1232 & 1225.4 & 1937.7 \\
\hline (RLA) & 1064411 & 107128 & $1088 \% 81$ & 111007 & 113389 & 112790 & 110565 & 107223 & 104010 & 99399 \\
\hline (F) & 1.0061 & 1.006 & 1.0061 & 1.006 & 1.005 & 1.492 & 1.784 & 20151 & 2.2121 & 2.466 \\
\hline (सUQ) & 230.11 & 230.3 & 230.8 & 231.61 & 232.31 & 345.6 & 413.6 & 466.8 & 512 & 570.7 \\
\hline \multirow[t]{3}{*}{ (HBOIL) } & 1342.4 & 1349.4 & 1370.3 & 1391.41 & 1405.5 & 1423.5 & 9434.71 & 1423.41 & 1415 & 2003.6 \\
\hline & & & & 1 & & & & & & \\
\hline & ZONE2 & ZONE4 & ZONE6 & ZONE8 & ZONE10 & & & & & I \\
\hline DPliquid Temp.f & 82.6 & & & 5 & $i$ & & & & & 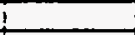 \\
\hline DPLiquid Density & 92.611 & & & & & & & & & \\
\hline Elovation-t & & 4 & 6ा & 8 & 9.875 & & & & & \\
\hline MoasuredDPYsid & 0.113 & 0.351 & -0.1931 & 1.159 & 1.4851 & & & & & I \\
\hline Zero Comections-psid & 0.022 & -0.028 & -0.754 & 0.128 & 0.161 & & & & & \\
\hline Corrected DPPsld & 0.0911 & 0.389 & 0.5611 & 1.031 & 1.324 & & & & & t \\
\hline Actual DPA & 2.08 & 3.8 & 5.79 & 7.16 & 8.75 & & & & & i \\
\hline Moasurod TPPsig & 100.4 & & & I & 3.6 & & $i$ & & & \\
\hline \multirow{2}{*}{ Measured Overallf } & 173.741 & & & & & & 1 & 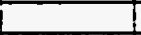 & & I \\
\hline & & & & & & & & & & $i$ \\
\hline Computed DPAt & 2.08 & 4.16 & 6.07 & 7.71 & 9.171 & & & & & \\
\hline Friction-ll & 0.08 & 0.16 & 0.20 & 0.52 & 0.83 & & 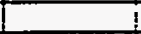 & 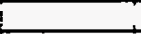 & & \\
\hline Accoleration-tl & 0 & 01 & 0.13 & 0.22 & $0.34 \mid$ & & & & & $\dot{t}$ \\
\hline \multirow[t]{2}{*}{ Elovation-th } & 2 & 41 & 5.651 & 6.97 & 81 & & & & & 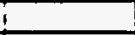 \\
\hline & & & & & : & & $i$ & & & \\
\hline
\end{tabular}




\begin{tabular}{|c|c|c|c|c|c|c|c|c|c|c|}
\hline Runt & $567 i$ & & & & & - & & $\therefore$ & & \\
\hline Tost Fluid & CFF10. & & $:$ & & & & & 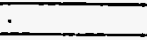 & & \\
\hline Date: & $5 \sqrt{27} / 93 !$ & & & & & & & & & \\
\hline Time: & $13: 23$ & & & & & & & & & \\
\hline Note & TABOREK & Analytical & & & & & & & & \\
\hline Fow Area-fl2 & 0.00336 & & & & & & & & & \\
\hline Hoad Flow Areafle & 0.196951 & & & & & & & & & \\
\hline Sat. Pressure Psig & 97.91 & & & & & & & & & \\
\hline Sat. Temperature f(inlet) & 145. $\overline{31}$ & & & & & & & & & \\
\hline Lg. Densty-1bm/lis & 84.681 & 84.571 & 84.22 & 83.87 & 83.41 & 83.11 & 82.97 & 83.061 & 83.071 & 83.2 \\
\hline Latont Head-Btulbm & 31.581 & 31.49 & 31.24 & 91 & $\$ 0.68$ & $\$ 0.48$ & 30.38 & 30.42 & 30.441 & 30.53 \\
\hline Specitic Heat-Btu/bm/F & 0.2791 & 0.2791 & 0.28 & 0.281 & 0.282 & 0.282 & 0.2831 & 0.2831 & 0.282 & 0.282 \\
\hline Lig. Viscosity lbm/ithr & 0.491 & 0.4881 & 0.478 & 0.472 & 0.462 & 0.456 & 0.4631 & 0.4541 & 0.4661 & 0.457 \\
\hline ig. Conductivity Btwhr-iff & 0.02681 & 0.0268 & 0.0265 & 0.0263 & 0.026 & 0.0258 & 0.02571 & 0.0258 & 0.02581 & 0.0259 \\
\hline Liq. Prandt No. & 5.091 & 5.081 & 5.06 & 5.03 & 5 & 4.98 & 4.971 & 4.98 & 4.98 & 4.99 \\
\hline Inlot fow Ralo-GPM & 3.9 & & & & & & $!$ & & & \\
\hline Outlet Liq. Flow Ralo-GPM & 1.7 & & & & & & & 5 & $\vdots$ & \\
\hline Outiel Vap. Fow Rate-GPM & 3.1 & & & & & & & & & \\
\hline Inlet Temperahurof & 133.7 & & $i$ & & & & $\bar{t}$ & & & \\
\hline Outlet Liq. Temperaturef & 142.2 & & & & & & i & & & \\
\hline Outlet Vap. Temperaturef & 139.3 & & & & & & & ! & & \\
\hline Waler Fow Ralo-GPM & & & $\bar{\vdots}$ & & $i$ & & 7 & T & & \\
\hline Water Inlet Temp.F & 99.11 & & & 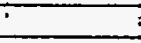 & & & $i$ & $i$ & 1 & $i$ \\
\hline Water outel Temp.f & 131.51 & & $\vec{z}$ & 7 & 7 & & $i$ & 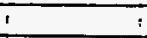 & $i$ & $\bar{i}$ \\
\hline Inlel Glbm/hrit2 & 7877301 & & Temp & 132.1 & Density & 84.64 & & 1 & 1 & $i$ \\
\hline Subcoolingf & 11.61 & & $\tau$ & 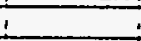 & + & & . & 4 & $i$ & $i$ \\
\hline Flow Observation & & & & 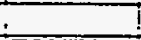 & & & & 3 & i & I \\
\hline & ZZONE1 i & ZZONE2 ! & ZONE3 & ZONE4: & ZONES & ZONE6 & ZONE7 & ZONE8 : & ZONE9 2 & ZONETO \\
\hline Heat Input-Walts(net) & 567.6 & 582.4 & $\begin{array}{l}579.7 \\
\end{array}$ & 581.7 & 579 & 579.3 & 580.7 & 573.4 & 1152.9 & 1153.4 \\
\hline Heat Loss-Watts & 24.9 & 5.8 & 7.9 & 9.9 & $11 !$ & 11 & $9 !$ & 9.7 & 9.9 & 53.4 \\
\hline Heat Flux-Btu/Hriti & 9839 & 10096 & 10049 & 10083 & 10037 & 10042 & 10066 & 99401 & 199851 & 19993 \\
\hline Wall Temp. \#3F & 753.41 & 1551 & 154.7 & 154 & 156.3 & 154.7 & 755.6 & 154.71 & 157.5 & 159.8 \\
\hline Wall Temp. \#4f & 153.81 & 753.51 & 155.6! & 154.6 & 155.11 & 154.8 & 155.9 & 155.7 & 1591 & 157.8 \\
\hline Wall Temp. $\# 5 F$ & 153.91 & 152.4 & 156.61 & 155 & 154.2 & 155.1 & 155 & 154.3 & 156.31 & 158.4 \\
\hline Wall Temp. $1 F$ & 81.91 & 154.11 & 1531 & 152.7 & 153.31 & 155.1 & 158.11 & 155.5 & 155.11 & 151.7 \\
\hline Wall Tomp. & 1541 & 154.31 & 153.51 & 153.4 & 153.41 & 155.5 & 156.41 & 155.91 & 956.41 & 155.8 \\
\hline Instroam Temp.F & 134.5 & 135.1 & 137.21 & 139.2 & 141.81 & 143.5 & 144.31 & 143.81 & 143.71 & 143 \\
\hline Avg. Wall Tomp.F & $153.8 i$ & 154.21 & 154.21 & 154.11 & 1541 & 154.9 & 155.81 & 155.11 & 156.9 & 156.7 \\
\hline Avg. WallDT.F & 18.81 & 18.7 & 16.51 & $14.4 !$ & $11.7 !$ & 111 & 111 & $10.9 i$ & 12.3 & 12.8 \\
\hline Remaining Subcooff & 8.91 & & & & & & i & & 1 & \\
\hline Ext Quality & -0.08 & -0.06 & -0.03 & -0.011 & 0.02 & 0.04 & 0.07 & 0.09 & 0.14 & 0.19 \\
\hline EXP. HBtuHriflef & 522.5 & 540.8 & 607.3 & 699.3 & 854.4 & 915.3 & 911.4 & 916 & 9630.5 & 15624 \\
\hline COMPUTEDH & & & & & & & & & $!$ & \\
\hline Jallouk & $888.7 !$ & 906 & 915.2 & 928.5 & 914.41 & 883.7 & 870 & 843.2 & Z23.1! & 11828 \\
\hline Szady1(Cudo tube) & 1317.8 & 1339.6 & 1353.6! & 7372.5 & 1392.2! & 1410.41 & 1422.21 & 1412.9 & 1992.5 & 7988.1 \\
\hline szady2 & 10091.11 & 1714.7 & 1127.81 & 1146.4 & 1165.5 & 1207.7 & 1216.6 & 1202.4 & 1908.21 & 1898 \\
\hline (RLIQ) & 105187 & 105693 & 1074861 & 109231 & 111029 & 109839 & 10775 & 104560 & 1003261 & 94249 \\
\hline$(F)$ & 1.0061 & 1.0061 & 1.0061 & 1.006 & 1.2181 & 1.624 & 1.884 & 2.1011 & 2.373 & 2.687 \\
\hline (HULO) & 230.61 & 230.7 & 231.31 & 231.81 & 281.81 & 376.6 & 437.31 & 487.4 & 650.21 & 522.6 \\
\hline (HBOLL) & 1315.51 & 1337.3 & 1351.41 & 1370.3 & 1388.41 & $1401.4 !$ & 1408.3 & 1398.31 & 1978.4 & 1967.5 \\
\hline & & & & & 1 & & 1 & 1 & $!$ & \\
\hline & ZONE2 & ZONE4 & ZONE6 & ZONE8 & ZONE10 & & & 1 & 1 & \\
\hline DP liquid Temp.F & 83.2 & & & & & & & $\frac{i}{i}$ & & \\
\hline DPLiquid Densitylbmiti3 & 92.521 & & & $i$ & & & & & & \\
\hline Elevation-ft & & 4 & 61 & 81 & 9.875 & & & & & \\
\hline Measured DPPsid & 0.106 & 0.348 & -0.207 & 1.157 & 1.456 & & & & i & \\
\hline Zero Cortections-psid & $0.022 !$ & -0.0281 & -0.754 & 0.128 & 0.1611 & & & & t & \\
\hline Corrected DPPsid & 0.0841 & 0.376 & 0.547 & 1.020 & 1.2951 & & & & $T$ & \\
\hline Actual DPA & 2.081 & 3.8 & 5.731 & 7.12 & 8.741 & & & & i & \\
\hline Moasured TPPsig & 97.91 & & 1 & & 3.61 & & & & i & \\
\hline Messured Overaltek & 163.21 & & & & 1 & & & & it & \\
\hline & 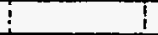 & & & & $\mathrm{T}$ & & & & $T$ & \\
\hline Computed DPA & 2.08 & 4.161 & 6.03 & 7.64 & 9.11 & & & & & \\
\hline Friction-ft & 0.08 & 0.16 & 0.31 & 0.56 & 0.94 & & & & & \\
\hline Accoleration-ft & 0 & of & 0.15 & 0.241 & 0.47 & & & & & \\
\hline Elevation-ti & 2 & 41 & 5.56 & 6.84 & 7.74 & & & & & \\
\hline & & $T$ & & & & & & & & \\
\hline
\end{tabular}




\begin{tabular}{|c|c|c|c|c|c|c|c|c|c|c|}
\hline Run: & $5 \longdiv { 5 8 }$ & & & & & & & & & \\
\hline Test Fluid & CAF 10 & & & & & & & & & \\
\hline Date: & $5 / 271931$ & & & & & & & & & \\
\hline Time: & $14: 15$ & & & & & & & & & \\
\hline Noto & TABOREK & Anadytical c & & & I & E & $!$ & & & $i$ \\
\hline Fow Aroatt2 & | & & : & 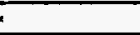 & 7 & 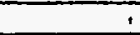 & $\vec{i}$ & & & $\therefore$ \\
\hline Ho2f Flow Aro2tL2 & 0.196851 & & & i & 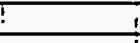 & & $I$ & & & $I$ \\
\hline S21. Prossura-Psig & $\quad 82.21$ & & $i$ & & $i$ & & & & & 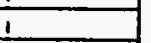 \\
\hline Sal. Temperaturof(inlel) & 134.61 & & & & & & & & & \\
\hline Liq. Density-lbmilt3 & 85.86 & 85.74 & 85.411 & $85 i$ & 84.88 & 84.861 & 84.86 & 84.9 & 84.9 & 85.03 \\
\hline Latent Heat-Biufibm & 32.37 & 32.291 & $32.07 i$ & 31.781 & $31.7 \mathrm{i}$ & 31.69 & 31.691 & 31.711 & 31.711 & 31.81 \\
\hline Specific Heat-Btulbm/F & 0.2761 & 0.276 & 0.277 & 0.2781 & 0.278 & 0.278 & 0.278 & 0.278 & 0.2781 & 0.278 \\
\hline Lq. Viscosity-libm/ithr & 0.5181 & 0.515 & 0.507 & 0.497 & 0.455 & 0.494 & 0.494 & 0.495 & 0.405 & 0.488 \\
\hline 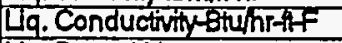 & 0.02761 & 0.02751 & 0.0273 & $0.027 !$ & $0.0209 !$ & 0.0269 & $0.0269 !$ & 0.0271 & 0.0271 & 0.027 \\
\hline L9. Pranatt No. & $5.19 !$ & 5.18 & 5.151 & $5.12 !$ & 5.111 & 5.111 & 5.111 & 5.171 & 5.171 & 5.12 \\
\hline Inlet Fow Rado-GPM & 3.91 & & & & & & & & & \\
\hline Outtel Ug. Flow Rate-GPM & 1.51 & & 7 & & & & $\cdot$ & & & $T$ \\
\hline Outlol Vap. Fow Ralo-GPM & 2.61 & i & $I$ & & $\therefore$ & & & & & $i$ \\
\hline Inlol Tomporaturof & 126.51 & & & & 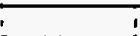 & & & & 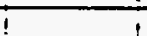 & \\
\hline Outlof Liq, Tomporatur of & 131.5 & & & & & & & & & $i$ \\
\hline Outel Vap. Temperaturef & 127.9 & & & & & & & & & i \\
\hline Water Flow Ralo-GPM & & & & & & & & & & \\
\hline Waler inlet Temp.f & 93.11 & & & & & & & & & \\
\hline Water outdet Temp.F & 118.9 & & & & & & & & & it \\
\hline Intet G $1 \mathrm{Bm} / \mathrm{hr}$ & 7996521 & & Temp & 124.8 & Density & 85.92 & & & & $i$ \\
\hline Subcoolingt & 8.11 & & & & & & & & & I \\
\hline \multirow[t]{2}{*}{ Fow observation } & & & & & & & & & & \\
\hline & ZONE1 12 & ZONE2 & ZONE3 IS & ZONE4 & ZONE5 & ZONE6 & ZONET & ZONE8 & ZONE9 & ZZONE10 \\
\hline Heat inpurtWatts(nel) & 554.8 & 568.7 & 570.8 & 584.8 & 568.7 & 568.8 & 561.4 & 559.8 & 562.7 & 569.5 \\
\hline HodLoso-Watts & 21.21 & 51 & 6.91 & 8.2 & 9.11 & 8.8 & 6.9 & 7.7 & 7.7 & 42.1 \\
\hline Hox Floc-Bluhts shtt & 96141 & 88231 & 98911 & 9700 & 98231 & 28251 & 97321 & 9700 & 9754 & 8872 \\
\hline Wall Tamp. 43 F & 143.8! & 145.11 & 144.91 & 144.2 & 746.51 & 144.7 & 145.21 & $144.3 !$ & 14281 & 1427 \\
\hline Wall Temp. & 144.1 & 143.8 & 146 & 144.8 & 145.1 & 144.9 & 145.7 & 144.7 & 143.7 & 141.8 \\
\hline Wall Temp. $\# 5 F$ & 144.2 & 142.7 & 146.7 & 145.2 & 144.2 & 145.11 & 144.7 & 143.8 & 142.4 & 142.7 \\
\hline Wall Temp. \#1F & 82.2 & 144.4 & 143.41 & 142.9 & 143.4 & 145.1 & 145.71 & 145.1 & 141.4! & \\
\hline Wall Temp. \#2F & 144.7 & 144.41 & 143.9 & 143.5 & 143.5 & 145.5 & 146.11 & 145.4 & 1421 & 141.5 \\
\hline Instream Temp. F & 127.41 & 128.11 & 130.11 & Tस्32.61 & 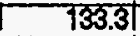 & 733.41 & 133.41 & T3.3.21 & 133.2 & 732.4 \\
\hline Avg. Wall Temp.F & 144.2 & 144.4] & 144.61 & 144.31 & 144.1 & 145 & 145.5. & 144.7 & 142.5 & 741.5 \\
\hline Avg. Wall DT.F & 16.4 & 15.91 & 141 & 11.2 & 10.3 & 11.11 & 11.6 & 111 & $8.8 !$ & 8.7 \\
\hline Romaining Subcooff & 5.5 & & & & & & & & & \\
\hline Exti Quality & -0.05 & -0.02 & 01 & 0.02 & 0.041 & 0.071 & 0.09 & 0.111 & 0.18 & 0.10 \\
\hline 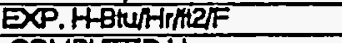 & 587.41 & 618.6 & $706.3 !$ & 871.6 & 953.2 & 884.71 & 8361 & 880.11 & 1106 & 1138.1 \\
\hline COMPUTEDH & & & & & & & & & & \\
\hline Jallouk & 837.6 & 852.21 & 866.21 & 839.7 & 810 & 800.81 & 790.31 & 783.6 & 781.9 & 780.1 \\
\hline Szactil(Cudo tube) & 1248 & 1266.7 & 1286.6 & 1301.7 & 1313.2) & 1318.11 & 1316.3 & 1317.2 & 1325.3 & 1331 \\
\hline Szady2 & 1022.3 & 1042.3 & 1061.8 & 1098.3 & 1106.3 & 1107.8 & 1101.9 & 1079.9 & 1090.3 & 1090 \\
\hline (सШQ) & 100937 & 101495 & 1031711 & $104251 !$ & 102452 & 100133 & 977411 & 95211 & 92835 & 898 \\
\hline (F) & 1.006 & 1.006 & 1.006 & 1.333 & 1.699 & 1.9581 & 2.173 & 2.3611 & 2.531 & 2.6 \\
\hline (H) & 231.1 & 231.31 & 231.9 & 308.4 & 393.3 & 453.51 & 503.2 & 546.7 & 585.9 & 623.7 \\
\hline \multirow{3}{*}{ (ABOL) } & 9245.31 & 7264.21 & 12841 & 7295.9 & 1301.3 & T209.9) & 7291.3 & 1725.1 & 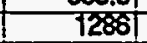 & 1284.4 \\
\hline & & & & & & & & & & \\
\hline & ZONEZ 12 & ZONE4 & ZONE6 & ZONEB & ZONE10 & & & & & \\
\hline DPliquid Temp.F & 83.4 & & & & & & & & & \\
\hline DPLquid Density-bmint3 & 92.401 & & & & & & & & & \\
\hline Elovation $f$ t & 21 & 4 & 61 & 8 & 9.875 & & & & & \\
\hline Measured DPPsid & 0.08 & 0.4031 & 0.039 & 1.5171 & 1.7681 & & & & & \\
\hline Zero Corrections-psid & 0.022 & -0.028 & -0.754 & 0.128 & 0.1611 & & & & & \\
\hline Corrected DP-Psid & 0.058 & 0.431 & 0.715 & 1.389 & 1.607 & & & & & \\
\hline Actual DPA & 2.08 & 3.62 & 5.32 & 6.35 & $8.02 !$ & & & & & \\
\hline Measured TPPsig & 8221 & & & & 3.6 & & & & & \\
\hline \multirow{2}{*}{ Moasured Overallit } & 133.11 & & & & & & & & & \\
\hline & & & & & & & & & & \\
\hline Computed DPA & 2.08 & 4.13 & 5.78 & 7.36 & 8.831 & & & & & \\
\hline Friction & 0.081 & 0.19 & 0.41 & 0.731 & 1.111 & & & & & \\
\hline & 01 & 0.11 & 0.221 & 0.311 & 0.41 & & & & & $T$ \\
\hline trom & & & 5.151 & & & & & & & \\
\hline
\end{tabular}




\begin{tabular}{|c|c|c|c|c|c|c|c|c|c|c|}
\hline Runf & 5691 & & & & & & & & & \\
\hline Tost Fluid & CAF10 & & & & & & & & & \\
\hline Dale: & $5 / 27193$ & & & & & & & & & \\
\hline Time: & $15: 03$ & & & & & & & & & \\
\hline Nole & TABOREK & Analytical d & & & & & & & & \\
\hline Fow Area-fl2 & 0.003361 & & & & & & & & & \\
\hline Heat Fow Area-t2 & 0.19595 & & & & & & & & & \\
\hline Sat. Pressure & 94.81 & & & & & & & & & \\
\hline Sat. Temperature f(in|el) & 143.21 & & & & & & & & & \\
\hline Lig. Densty-bm/n3 & 84.081 & 84.731 & $84.78^{\prime}$ & 84.741 & 84.591 & 84.181 & 83.75 & 83.751 & 83.781 & 83.89 \\
\hline Latent Hoat-Btulbm & 31.551 & 31.61 & 31.631 & $31.61 !$ & 31.51 & 31.211 & 30.91 & 3.911 & 30.931 & 39.01 \\
\hline Spoofílo Hoat-gturibm/F & 0.2701 & 0.2791 & 0.2781 & 0.2781 & $0.278 i$ & 0.281 & 0.281 & $0.289 !$ & 0.2811 & 0.281 \\
\hline Lig. Visoositylbmithr & $0.489 \mathrm{j}$ & 0.4891 & $0.492 i$ & 0.4911 & 0.4881 & 0.4781 & 0.469 & 0.4691 & $0.469 i$ & 0.472 \\
\hline Lig. Conductinty-etuhs-iff & 0.02681 & 0.02381 & $0.0269 \mathrm{i}$ & 0.02691 & 0.02681 & 0.02651 & 0.0262 & 0.02621 & 0.0262 & 0.0263 \\
\hline Liq. Prandt No. & 5.09 & $5.09 !$ & 5.1 & 5.11 & 5.081 & $5.05 !$ & 5.02 & 5.021 & 5.02 & 5.03 \\
\hline Inlelfiow Rato-GPM & 3.91 & & & & & & & & & \\
\hline Outlet Lig. Flow Rate-GPM & $1.7^{\circ}$ & & & & & & & & & \\
\hline Outlel Vap. Flow Rade-GPM & $2.9^{\circ}$ & & & & & & & & & \\
\hline Inlet Temperature of & $13 \times 3.71$ & & & & & & & & & \\
\hline Outlel Lia. Temperaturef & $138.9 !$ & & & & & & & & $i$ & \\
\hline Outlel Vap. Temperaturef & $135 !$ & & & & & & & $\bar{t}$ & & $T$ \\
\hline Water Fow Rado-GPM & 7 & $i$ & 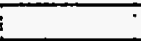 & & 5 & i & 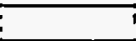 & $i$ & 7 & 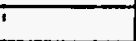 \\
\hline Water Inlet Temp. $F$ & 1181 & tis & 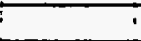 & T & 7 & 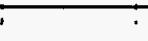 & 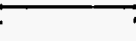 & 7 & . & 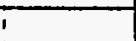 \\
\hline Waler out of Temp.F & 128.9 & 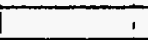 & 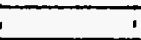 & & 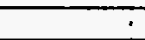 & 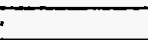 & & 7 & & 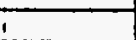 \\
\hline Inlet G-bm/hr/il2 & 7865641 & & Temp & $132.8 \mathrm{iL}$ & Density & 84.511 & & $\div$ & & \\
\hline Subcoolingf & 9.51 & & & & 2 & 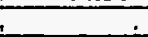 & . & 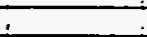 & & \\
\hline Flow Observation & & & & & & $!$ & & & & \\
\hline & ZONE 1 & ZONE2 2 & ZONE3 & ZONE4 & ZONE5 & ZONE6 & ZONE7 & ZONEB & IZONEG & ZONE10 \\
\hline Heat Input-Watts(net) & of & 0 & -2 & $571.1 !$ & 578.2 & 573.91 & 573.4 & 576.5 & 573.2 & 565.1 \\
\hline Heat Loss-Watts & 17.31 & 4.11 & 5.61 & 8.61 & 9.61 & 9.41 & 7.4 & 8.3 & 8.11 & 44 \\
\hline Heat Fiux-btumhifit & $0 !$ & वा & 01 & 99001 & 100231 & 99481 & 99401 & \$303 & क965 & 9796 \\
\hline Wal Temp. His & 733.51 & T33.2। & 135.7 & 150.71 & $\mid 531$ & 151.41 & 151.9 & 151.11 & 749.5 & 148.8 \\
\hline Wall Temp. $4-F$ & 133.61 & 733.71 & 135.61 & 751.31 & $152 !$ & 757.51 & 152.3 & 151.71 & 150.3 & 748.1 \\
\hline Wall Temp. $\$ 5 F$ & 133.81 & 133.7 & 134.31 & 151.41 & 1511 & 157.81 & 151.4 & 150.61 & 149.2 & 148.7 \\
\hline Wall Temp. $1 F$ & 81.71 & 139.7 & 138.61 & 149.11 & 1501 & 151.9 & 152.41 & 1521 & 148.2 & 145.7 \\
\hline Wall Temp. KzF & 133.91 & 133.8 & 133.61 & 149.6 & 150.11 & $152.2 !$ & 152.7 & 152.31 & 148.81 & 147.5 \\
\hline Instream Tomp.F & 134.61 & 934.21 & 133.81 & 134.9 & 1351 & 137.5 & 139.81 & 139.91 & 139.81 & 139.1 \\
\hline Avg. Wall Tomp.f & 133.5 & 133.61 & 134.61 & $150.6 i$ & 150.81 & 151.71 & 152.91 & $151.5 \mathrm{i}$ & $149.2 !$ & 147.8 \\
\hline Avo. Wall DT.F & $0 !$ & 0 & 0.7 & $16.1 i$ & 15.31 & 13.71 & 11.81 & 11.2 & $9 !$ & 8.2 \\
\hline Remaining Subcoohf & 9.5 & & & & & & & & & \\
\hline Exit Quality & -0.08 & -0.08 & -0.081 & -0.061 & -0.04 & -0.011 & $0.01 \mathrm{i}$ & 0.08 & 0.061 & 0.08 \\
\hline EXP. HBBUARRILA & 01 & 01 & $0 i$ & 615.7 & 654.11 & 726.1 & 843 & 893.21 & 1710.11 & 1192.1 \\
\hline COMPUIEDH & 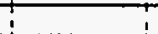 & & & i & & & & & & \\
\hline Jallouk & T88.2! & 188.1 & 1881 & 8501 & 907.6 & 971.51 & 924.6! & 869.91 & 837.31 & 819.3 \\
\hline Szadyi(Oudo tube) & 230.31 & 230.2 & 230.11 & 1318.61 & 1334 & 1349.4 & 1358.61 & 1375.91 & 1374.8 & 1363.4 \\
\hline Szady2 & 230.21 & 230.11 & 2301 & $1092.5 !$ & 1108.5 & 1122.61 & 1141.11 & 1173.31 & 1168 & 1151.6 \\
\hline (RLQ) & 105116 & 1047811 & 1045301 & 104697 & 105452 & 107586 & 109688 & 1072811 & 1045621 & 101382 \\
\hline$(F)$ & 1.0061 & 1.006 & 1.0061 & 1.0061 & 1.0061 & 1.006 & 1.0061 & 1.5441 & 1.8361 & 2.068 \\
\hline (रणपQ) & 230.21 & 230.11 & 2301 & 230.1 & 230.41 & 239.11 & 231.8 & $355.8 i$ & 423.2 & 476.3 \\
\hline (HBOLL) & $25 i$ & 24.8 & 24.8 & 1316.31 & $1331.7 !$ & $1347.9 !$ & 1366.41 & 1367.91 & 1361.3 & 1343.8 \\
\hline & 1 & 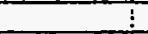 & & & & & & & & \\
\hline & ZONE2 & ZONE4 & ZONE6 & ZONE8 & ZONE10 & & & & & \\
\hline DP liquid Temp.f & 83.3 & $i$ & & $i$ & & & & & & \\
\hline DPLiquid Densitylbmit3 & 92.51! & & & & & & & & & \\
\hline Elevation-h & 2 & 41 & & 81 & 9.8751 & & & & & \\
\hline Measured DPPsid & 0.11 & 0.281 & -0.3891 & 0.961 & 1.171 & & & $T$ & & i \\
\hline Zero Corrections-psid & $0.02 !$ & -0.028 & -0.754 & 0.1281 & D.JETI & & & !t & & \\
\hline Conected DPpsid & 0.0781 & 0.308 & 0.3551 & 0.8881 & 1.0001 & 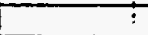 & & i & i & \\
\hline Actual DPA & 2.071 & 3.881 & 5.991 & 7.391 & 9.18 & $!$ & & T & & \\
\hline Measured TPPsig & 94.81 & 1 & 1 & & 3.51 & 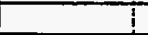 & & $!$ & & \\
\hline Messured Overalla & 156.73 & $i$ & & & & $i$ & & $i$ & & \\
\hline & & & & & & & & $i$ & 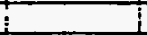 & \\
\hline Computed DPA & $2.08 !$ & 4.16 & 6.28 & 8.121 & 9.65 & & & & & \\
\hline Frictionft & 0.08 & 0.16 & 0.24 & 0.37 & 0.6 & & & T & & \\
\hline Accoleration-fi & 0 & of & 0 & $0.14 !$ & 0.23 & & & & & \\
\hline Elovation-t & $2 !$ & $4 !$ & 61 & 7.61 & 8.811 & & & 1 & & \\
\hline & 1 & & & 1 & & & & & & \\
\hline
\end{tabular}




\begin{tabular}{|c|c|c|c|c|c|c|c|c|c|c|}
\hline Run\# & $570 i$ & 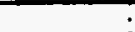 & . & & . & 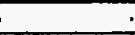 & 7 & . & . & \\
\hline Tost Fuid & CAF 10 & . & $\bar{i}$ & & 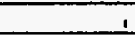 & 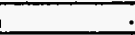 & $\bar{i}$ & . & . & \\
\hline Date: & $527193 !$ & & & & $\perp$ & $\dot{:}$ & . & & $i$ & \\
\hline Time: & $15: 03$ & & & & $!$ & 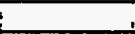 & + & & i & \\
\hline Note & ITABOREK & Analytical c & & & + & 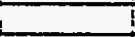 & I & & & \\
\hline Flow Area-t12 & 0.00336 & & & & & & & & & \\
\hline Heal Flow Areaft2 & 0.196951 & 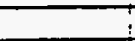 & & & & $\therefore$ & 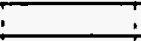 & & & \\
\hline Sat. Pressute Psig & $97.4 !$ & & : & & & & & & & \\
\hline Sat. Temperature-f(inlel) & 144.91 & & & & & & & $T$ & $T$ & \\
\hline Llg. Denstrylbmints & 84.711 & 84.76 & $84.81 !$ & 84.851 & 84.81 & 84.63 & 84.16 & 83.761 & 83.681 & 83.75 \\
\hline Lalont Hoat-Btulbm & 31.591 & 31.62 & 31.661 & 31.681 & 31.641 & 31.53 & 37.211 & 30.93 & 30.861 & 30.91 \\
\hline Spoctio Hoat Btu/hm/F & 0.2791 & 0.278 & 0.278 & 0.278 & 0.2781 & 0.279 & 0.28 & 0.2811 & 0.2811 & 0.281 \\
\hline Liq. Visoosity lbmithr & 0.4911 & 0.492 & 0.4931 & 0.4941 & 0.4981 & 0.489 & 0.478 & 0.469 & 0.4681 & 0.469 \\
\hline Liq. Conductivity Btuhr-ftF & 0.02681 & 0.0259 & 0.02691 & 0.0269 & 0.02691 & 0.0268 & 0.02651 & 0.0252 & 0.02621 & 0.0262 \\
\hline Liq. Prandth No. & 5.091 & 5.1 & $5.1 !$ & 5.11 & 5.11 & 5.091 & 5.051 & 5.021 & 5.021 & 5.02 \\
\hline Inlet Flow Ralo-GPM & 3.91 & & & & 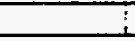 & & 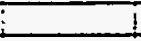 & : & : & \\
\hline Outlot Lig. Flow Rato-GPM & $1.7:$ & & & & & & + & 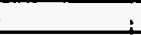 & 1 & \\
\hline Outlot Vap. Flow Ralo-GPM & $3 i$ & & & & & 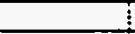 & & & 4 & \\
\hline Inlel Temperature of & 133.11 & & & & & & & $\div$ & 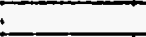 & \\
\hline Outlet Liq. Temperaturef & 139 & & & & & & & & ! & \\
\hline Outlet Vap. Temperaturef & 135.7 & & & & & & & & & \\
\hline Waler Flow Rato-GPM & 1 & & 1 & & & 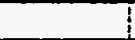 & & t & T & \\
\hline Water inlet Temp.F & 108.3 & i & $i$ & 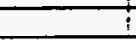 & $\frac{1}{1}$ & 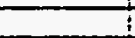 & & $i$ & 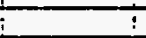 & \\
\hline Water outlol Tomp.f & $133.5 i$ & & 1 & 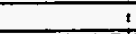 & 1 & +1 & $!$ & I & 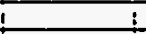 & \\
\hline Inlot Glbm/hrm2 & 788726 & & Tomp & 137.61 & Density & 84.74 & & T & $i$ & \\
\hline Subcooling & 11.81 & & 工 & 1 & 资 & & 1 & $!$ & $i$ & \\
\hline \multirow[t]{2}{*}{ Flow Observation } & 1 & 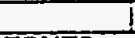 & & & & & & $!$ & 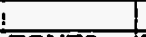 & \\
\hline & ZONEI & ZONE2 & ZONE3 & ZONE4 & ZONE5 & ZONE6 & ZONE7 & ZONE8 & ZONE9 & ZONE10 \\
\hline Heat input-Watts(net) & 0 & o & 0 & 01 & 572 & 5727 & 567.9 & 569.5 & 560.2 & 569.8 \\
\hline Heal Loss-Watts & 20.3 & 4.8 & 6.5 & 8 & 10.9 & 10.9 & 92 & 10 & 9.6 & 51.7 \\
\hline Hoal A $(x-B$ tuntintit & 01 & 0 & 01 & oi & 5915! & 99271 & \$844 & 98721 & 9711 & 9877 \\
\hline Wall Temp. \$ & 334.3 & 734.11 & 734.41 & 736.6 & 153.71 & 15टा & 1530 & 151.9| & 750.3 & 750.1 \\
\hline Wall Temp. & 134.5 & 134.4 & 134.41 & 736.6 & 152.5 & 152.7 & 153.3 & $152.4 \mid$ & 151.9 & 149.2 \\
\hline Wall Tomp. 16f & 134.71 & 134.6 & 134.6 & 135 & $151.6 !$ & 152.51 & 152.31 & 151.41 & 149.8 & 149.8 \\
\hline Wall Tomp. $1 F$ & 82.7 & T34.5 & 134.3 & 134.1 & 150.21 & 1525 & 153.51 & 1529 & 1491 & 148.8 \\
\hline Wall Tomp. "LF & 134.11 & 734.6 & 134.31 & 134.4 & 150.4 & 152.9 & 153.81 & 153.21 & 149.51 & 148.7 \\
\hline Instroem Temp.f & 134.3 & 134 & 733.7 & 133.6 & 133.81 & 134.8 & 137.51 & 139.81 & 140.31 & 139.8 \\
\hline Avg, Wall Tomp. $F$ & 134.41 & 134.41 & 134.41 & 135.61 & 151.2 & 152.3 & 153.21 & 152.4 & 149.91 & 148.9 \\
\hline Avg. Wall DT.F & 0.1 & 0.41 & 0.71 & $2 \pi 1$ & 16.9 & 17 & 15.2 & 12.11 & 9.21 & 8.6 \\
\hline Remaining Subcooff & 11.8 & & & & & & & & & \\
\hline Ext Quality & -0.1 & -0.1 & $-0.1 !$ & -0.11 & -0.08 & -0.051 & -0.03 & -0.011 & 0.01 & 0.04 \\
\hline EXP.HBtu/Hrifter & 0 & 0 & 0 & 0] & 585.8 & 583.1 & 646.2 & 814.91 & 1055.4 & 1152 \\
\hline COMPUTEDH & i & & & & & & & & $i$ & \\
\hline Jalouk & 788.6 & 188.4 & 188.3 & 188.3 & $888.9 \mid$ & 895 & 905.41 & 979.91 & 913.5 & 851.4 \\
\hline Szady1(Cudo tube) & 230.8 & 230.7 & 230.6 & 230.5 & 1317.3 & 1326.1 & 1342.4 & 13532 & 1356.6 & 1358.5 \\
\hline Szady? & 230.7 & 230.6 & 230.51 & 230.4 & 1091.41 & 1099.9 & 1114.7 & 1135.11 & 1127 & 1164.2 \\
\hline$(P \amalg Q)$ & 105152 & 104901 & 104051 & 104484 & 104734 & 105573 & 107882 & 1099011 & 1102871 & 107340 \\
\hline (F) & 1.006 & 1.006 & 1.006 & 1.006 & 1.006 & 1.006 & 1.006 & 1.006 & 1.061 & 1.574 \\
\hline (सिएQ) & 230.7 & 230.6 & 230.61 & 230.4 & 230.5 & 230.8 & 231.6 & 232.31 & 245.3 & 363.7 \\
\hline \multirow[t]{3}{*}{ (HBOIL) } & 24.9 & 24.8 & 24.7 & 24.6 & 1314.9 & 1323.7 & 1340.1 & 1361 & 1353.9 & 1359.8 \\
\hline & & & & & & & & $2+$ & ! & \\
\hline & ZONE2 & ZONE4 & ZONE6 & ZONE8 & ZONE10 & & & i & $i$ & \\
\hline DP liquid Temp.F & $\quad 83.9$ & & 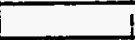 & 2 & & & & 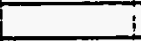 & 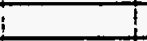 & \\
\hline DPLiquid Density-bm/it3 & 92.42 & & . & 1 & & & & 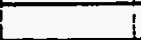 & 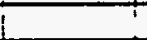 & \\
\hline Elevation $\mathrm{A}$ t & 2 & 4 & 6ा & 8 & 9.875 & & & 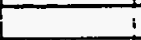 & 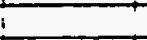 & \\
\hline Measured DPFasd & 0.097 & $0.27 \pi$ & -0.474 & 0.8071 & 0.84 & & & $i$ & i & \\
\hline Zero Corrections-psid & 0.022 & -0.028 & -0.754 & 0.1281 & 0.1611 & & & & & \\
\hline Cotrected DPPsild & 0.075 & 0.299 & 0.28 & 0.673 & 0.738 & & & & & \\
\hline Actual DP- & 2.08 & 3.9 & 8.141 & 7.67 & 9.04 & & & & $!$ & \\
\hline Moasured TPPsig & 97.4 & +1 & 1 & 3 & 3.6 & & & $I$ & $!$ & \\
\hline \multirow[t]{2}{*}{ Mensured Overalla } & 161.28 & & I & & 1 & & & & & \\
\hline & i & & & 1 & & & & & & \\
\hline Computed DPA & 208 & 4.16 & 6.24 & 8.31 & 10.00 & & & 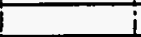 & i & \\
\hline Friction-th & 0.08 & 0.16 & 0.24 & 0.32 & 0.45 & & $\frac{1}{1}$ & L & & \\
\hline Acceleration-ft & 0 & D & 0 & 01 & 0.14 & & & 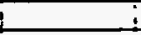 & & \\
\hline \multirow[t]{2}{*}{ Elovation-t } & 2 & 4 & 6 & 8 & 9.5 & & & & & \\
\hline & & & & & & & & $i$ & & \\
\hline
\end{tabular}




\begin{tabular}{|c|c|c|c|c|c|c|c|c|c|c|}
\hline Runt & $571:$ & & $\cdot$ & - & & 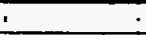 & - & - & & \\
\hline Tost Fluid & CAF 10 & & . & - & 1 & & & $\dot{-}$ & & - \\
\hline Date: & $527793 !$ & & & & & 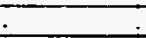 & & & & \\
\hline Time: & $15: 03$ & & & & & & & & & \\
\hline Note & TABOREK & Analytical d & & & & & & & & \\
\hline Flow Area-fi2 & $0.00336 !$ & & & & & & & & & \\
\hline Heal Flow Area-f2 & 0.196951 & & & & & & & & & \\
\hline Sal. Pressure $\$$ sig & $89.8 !$ & & & & & & & & & \\
\hline Sat. Temperatureff(inlet) & $139.9 !$ & & & & & & & & & \\
\hline Lq. Density-bm/ms & 85.531 & 85.61 & 85.611 & 85.561 & $85.51 !$ & 85.51 & 85.311 & 84.931 & 84.591 & 84.69 \\
\hline Latent Heat-Btulbm & $32.15 !$ & $32.19 !$ & $32.2 !$ & $32.17 !$ & 32.141 & 32.131 & 321 & 31.741 & $31.5 !$ & 31.57 \\
\hline Specific Heat-BtuntbmfF & $0.27 !$ & $0.2 \pi !$ & $0.277 i$ & 0.277 & 0.277 & 0.277 & 0.277 & $0.278 !$ & 0.2791 & 0.279 \\
\hline Liq. Viscosity-lbm/ithr & 0.611 & 0.5121 & 0.5121 & 0.5111 & 0.511 & 0.6091 & 0.5051 & 0.4961 & 0.4881 & 0.49 \\
\hline iq. Conductivity-BtuhrfftF & 0.02741 & 0.02741 & 0.02741 & 0.02741 & $0.0274 i$ & 0.02731 & $0.0272 ;$ & 0.0271 & 0.02681 & 0.0258 \\
\hline Liq. Prandt No. & $5.16 !$ & $5.16 i$ & 5.17 & 5.16 & $5.16 !$ & $5.16 i$ & 5.14 & 5.111 & $5.08 !$ & 5.09 \\
\hline Inlet Fow Rate-GPM & $3.9 !$ & & & & & & & & & \\
\hline Outlet Liq. Fow Rate-GPM & 1.61 & & & & & & & & & \\
\hline Outlet Vap. Flow Rate-GPM & $2.9 !$ & & & & & & & & & \\
\hline Inlet Temperaturef & 128.51 & & & & & & & & & \\
\hline Outlet Liq. Temperaturef & 134 & & & & & & & & & \\
\hline Outtel Vap. Temperatureff & $130.3 !$ & & & & & & & & & \\
\hline Water Fow Rato-GPM & $-i$ & $i$ & $i$ & & & 7 & $\overline{!}$ & ! & & \\
\hline Water tnlet Temp.F & 99.81 & & & & 7 & $i$ & T & ' & ? & \\
\hline Water outtot Tomp.F & 128.21 & & \multirow[b]{2}{*}{ Temp } & $\div$ & . & $\cdot$ & ; & $i$ & 7 & - \\
\hline Inlot Glbm/hrme & 7861031 & & & 127II & Density & 85.541 & 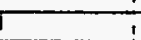 & 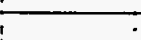 & ? & 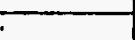 \\
\hline \multirow{3}{*}{$\begin{array}{l}\text { Subcooling } F \\
\text { Fow Observation }\end{array}$} & $11.4_{1}$ & & 7.1 & & $2=1$ & 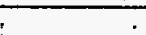 & 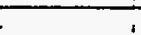 & & 7 & . \\
\hline & & & & & & & & & & \\
\hline & ZONE1 & ZONE2 & ZONE3 & ZONE4 & ZZONE5 & ZONE6 & ZONE7 & ZONE8: & ZZONE9 & ZONE1O \\
\hline Hoat lnput-Watts(net) & 01 & 01 & 01 & 01 & 0 & 589.7 & 566.6 & 5681 & 561.2 & 5724 \\
\hline Heat Loss-Watts & $19.3 i$ & $4.6 !$ & 6.2. & 7.41 & $8.2 !$ & 10.81 & 8.71 & 9.51 & 9.11 & 49.4 \\
\hline Heat Flux-Btuntinfiz & $0 i$ & $0 !$ & $0 !$ & 0 & 01 & 10222 & 9822 & 9846 & 97281 & 9922 \\
\hline Wall Tomp. \#3F & 729.51 & T29.3 & $\mid 29.71$ & 130.1 & 133.11 & 147.5 & 148.11 & 147.11 & 145.41 & 745.2 \\
\hline Wall Temp. \#4F & 129.61 & 129.71 & 129.6 & 130.1 & $132.9 !$ & 147.7 & 148.5 & 147.71 & 146.3 & 144.3 \\
\hline Wall Temp. \#5F & 1301 & 129.91 & $129.9 !$ & 130.11 & 131.21 & 148 & 147.61 & 146.7 & 145.11 & 145 \\
\hline Wall Tomp. \#F & 82.21 & 129.61 & 129.41 & 129.81 & 130.51 & 147.91 & 148.7 & 148.11 & 144.11 & 141.8 \\
\hline Wall Temp. \#2f & 129.51 & 129.81 & $129.5 i$ & 129.91 & $130.5 i$ & 148.2 & 149 & 148.41 & 144.71 & 143.7 \\
\hline Instream Temp.F & 129.41 & 1291 & 128.91 & 129.2! & 129.51 & $\mid 29.6$ & 130.71 & 133 & 135 & 134.4 \\
\hline Avg. Wall Temp.f & 129.7i & 129.61 & 129.6 & 1301 & 131.31 & 147.8 & 148.41 & 147.6 & 145.11 & 144 \\
\hline Avg. Wall DT.F & 0.3 & 0.61 & 0.7 & 0.8 & $1.8 !$ & $17.7:$ & 17.2 & $14.2 !$ & 9.71 & 9.2 \\
\hline Remaining Subcooff & $11.4 !$ & & & & & & & & & \\
\hline Exit Quality & -0.1 . & $-0.1 i$ & $-0.1_{1}$ & -0.11 & $-0.1 !$ & -0.07 & -0.05 & -0.031 & 0.011 & 0.02 \\
\hline EXP.HBtuHrft2IF & $0 i$ & 01 & 0 & $0 !$ & 0 & 577.11 & 569.81 & 695.6 & 1004.91 & 1084.2 \\
\hline COMPUTEDH & & & & $i$ & & & 7 & & & \\
\hline Jallouk & 188.61 & 188.51 & 188.51 & 188.6 & $188.6 !$ & $81.2 !$ & $866.2 !$ & 879.81 & $884.2 !$ & 856.8 \\
\hline Szady1(Cudo tube) & $230.9 !$ & 230.81 & 230.81 & 230.9 & 2311 & 1303.8 & 1286.7 & 1306.41 & 7314.5 & 1323.8 \\
\hline szady2 & 230.91 & 230.71 & 230.71 & 250.81 & 230.91 & 1087.71 & 1051.21 & 1080.21 & 1086.71 & 11227 \\
\hline (RUQ) & 1020871 & 1017841 & 1016841 & 1019251 & 1021671 & 102248 & 1081421 & 1050421 & 1087311 & 105725 \\
\hline (F) & 1.0061 & 1.0061 & 1.0061 & 1.006 & 1.0061 & 1.006 & 1.006 & 1.006 & 1.0061 & 1.227 \\
\hline (HUQ) & 230.81 & 230.7 & 230.7 & 230.8 & 230.81 & 230.91 & 231.31 & 232 & 232.61 & 283.4 \\
\hline (HBOIL) & 23.51 & 23.31 & 23.3 & 23.41 & 23.51 & 1301.31 & 1284.21 & 1304 & 1312.11 & 1319.4 \\
\hline$'$ & $i$ & $i$ & 1 & & $i$ & i & 1 & 1 & 1 & \\
\hline & ZONE2 :2 & ZONE4 & ZONE6 & ZONE8 & ZONE10 & 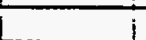 & 1 & 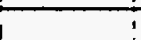 & $!$ & \\
\hline DP liquid Temp.f & $83.6 i$ & & & & & & & 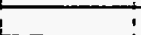 & 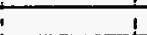 & \\
\hline DPLiquid Density-lbmiti3 & $92.46 i$ & & i & I & 1 & t! & $\because$ & i & $\rightarrow$ & \\
\hline Elevation-ft & 21 & $4 !$ & $6 !$ & 81 & 9.875 & $i$ & $i$ & & & \\
\hline Measured DPPsid & 0.0851 & 0.71 & -0.5381 & 0.611 & 0.7131 & & $T$ & $r$ & $T$ & \\
\hline Zero Corrections-psid & 0.0221 & -0.0281 & -0.7541 & 0.1281 & $0.16 i$ & & & & & \\
\hline Corrected DP-Psid & 0.0641 & 0.1281 & 0.2161 & 0.5531 & 0.5521 & & T! & ! & ! & ! \\
\hline Actual DPA & 2.071 & 4.15 & 6.18 ! & 7.79 & 9.841 & & i & $t$ & $i$ & 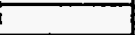 \\
\hline Measured TPPsig & 89.81 & 1 & i & $i$ & $3.5 !$ & 1 & & & 1 & \\
\hline Messured Overalift & 146.731 & & & $i$ & 1 & 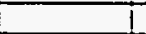 & i & T & 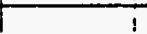 & 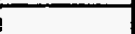 \\
\hline & & 1 & & 1 & $i$ & $i$ & $i$ & 1 & $\mathrm{i}$ & \\
\hline Computed DP- & 2081 & 4.16 & 6.241 & 8.321 & $10.25 !$ & 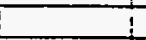 & I & L & & \\
\hline Friction-A & 0.081 & 0.16 & 0.24 & 0.32 & 0.41 & & & & & \\
\hline Accelerationft & 0 : & 01 & O & of & 0.1 & $\div$ & 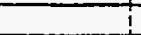 & it & 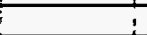 & 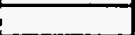 \\
\hline Elevation-ft & $2 !$ & 4 & 6) & $8 !$ & 9.741 & 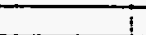 & & & & \\
\hline & & & & & & & & & & \\
\hline
\end{tabular}




\begin{tabular}{|c|c|c|c|c|c|c|c|c|c|c|}
\hline $\begin{array}{l}\text { Run } \\
\text { Test Fuid }\end{array}$ & TAR? & & & & & & & & & \\
\hline Date: & $5 \sqrt{271931}$ & & & & & & & & & \\
\hline Time: & $15: 031$ & & ' & & $\dot{\square}$ & - & & $i$ & & $\cdot$ \\
\hline Note & TABOPEM & Analytical c & dt & & $\dot{5}$ & 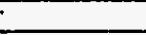 & & I & 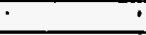 & - \\
\hline Fow Areaft? & 0.003361 & . & \pm & $:$ & $\because$ & - & & 1 & 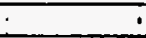 & $\dot{\square}$ \\
\hline Hoad Flow Aroath2 & 0.18685 & $\dot{\square}$ & 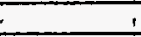 & . & 7 &. & & $\therefore$ & & ?. \\
\hline Sat. Prossuro-Pgig & 80.5 & $!$ & 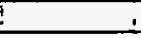 & & i & $i$ & & $\therefore$ & $\vdots$ & $i$ \\
\hline Sat. Temperaturef(inlel) & 133.3 & 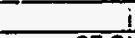 & & & & & & & & \\
\hline Liq. Densitylbmit3 & 85.27 & 85.31 & 85.311 & 85.381 & $85.36 !$ & $85.45 !$ & 85.431 & $85.45 i$ & $85.53 \mid$ & 85.66 \\
\hline Ladent Heaf Btunbm & $31.97 !$ & 31.99 & 32 & 32.051 & 32.041 & 32.091 & 52.081 & 32.091 & 32.15 & 32.24 \\
\hline Specifio Heat Btulbm/F & 0.277 & 0.277 & 0.277 & 0.277 & 0.277 & 0.277 & 0.277 & 0.277 & $0.27 \pi$ & 0.276 \\
\hline 19. Viscosity-1bmilithr & 0.504 & 0.504 & 0.5051 & 0.5061 & 0.5061 & 0.5081 & 0.5081 & 0.5081 & 0.511 & 0.513 \\
\hline Liq. Conductivity-Btuhr-flF & 0.02721 & 0.02721 & $0.0272 !$ & 0.02731 & $0.0273 !$ & $0.0273 !$ & $0.0273 !$ & $0.0273 !$ & $0.0274 !$ & 0.0274 \\
\hline Lig. Prandt No. & $5.14 !$ & 5.141 & 5.141 & $5.15 !$ & 5.151 & 5.151 & 5.15 & 5.15 & 5.161 & 5.17 \\
\hline Inlelflow Rato-GPM & 3.91 & $i$ & $i$ & $?$ & $?$ & ". & & ' & i & T \\
\hline Outtel Lig. Fow Aato-GPM & 1.21 & 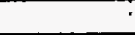 & : & - & $!$ & 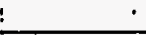 & & ' & - & $\vdots$ \\
\hline Outtot Vap. Flow Rate-GPM & 2.11 & $\dot{-}$ & E & & $\dot{-}$ & - & & $\therefore$ & - & $\therefore$ \\
\hline Inlel Tomperature of & 1301 & 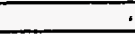 & - & & $\div$ & . & & $\therefore$ & & 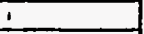 \\
\hline Outlot Liq. Tomperaturaf & 128.11 & . & $\cdot$ & & $\mathrm{i}$ & $i$ & & $i$ & $\cdot$ & + \\
\hline Outtel Vap. Temperaturef & 123 & 1 & $\underline{1}$ & $\vdots$ & & $!$ & & 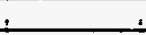 & & 1 \\
\hline Water Flow Rato-GPM & $i$ & & $!$ & $i$ & $\vdots$ & $\vdots$ & & $\vdots$ & i & $i$ \\
\hline Water Inlet Temp. $\mathrm{F}$ & 85.8 & & 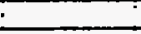 & & & & & & & \\
\hline Water outlet Temp.F & 113.8 & $:$ & & & $i$ & 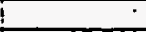 & & & : & \\
\hline Inlet Glbm/hink & 7961031 & & Temp & 1ट̆| & Density & 85.541 & & $i$ & & 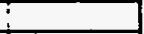 \\
\hline Subcooling $F$ & 3.3 & & & & & & & $i$ & & + \\
\hline Flow Observation & & $i$ & & & t! & i & & + & & $T$ \\
\hline & IZONET I & ZONE2 & ZONE3 & ZONE4 & ZONES I & IZONE6 IR & ZONET I & 'ZONEB ! & IZONE9 I & ZONE10 \\
\hline Hoatinput-Watts(net) & ! $\quad$ ol & 0 & $3 i$ & 0.5 & 587.3 & 583.7 & 576.41 & 580.2 & 581.21 & $1 \quad 580.9$ \\
\hline Healloss-Walts & 4.9 & 1.21 & 7.51 & 1.61 & 2.6 & 1.9 & 1.2 & 1.91 & 2.3 & 12.3 \\
\hline Heatflux-Btunhrfin2 & 0 & 01 & 521 & 91 & 101801 & 10118 & 9982 & 10067 & 10075 & 10174 \\
\hline Wall Tamp. \#3F & 130.8| & 130.71 & 131.11 & 732.51 & 143.81 & 741.5 & 142.51 & 741.51 & 139.8 & 139.1 \\
\hline Wall Temp. "4F & 130.9 & 130.9 & 1311 & 132.51 & 142.8 & 141.5 & 142.8 & 142 & 140.6 & 138.4 \\
\hline Wall Temp. \#5F & 131.2 & 131.3 & 131.1 & 131.11 & 141.5 & 142.11 & 141.8 & 1411 & 139.2 & 139.1 \\
\hline Wall Temp. $1 F$ & 83.31 & 1311 & 130.8 & 130.31 & 141.1 & 142.11 & 1431 & 142.5 & 138.31 & 135.9 \\
\hline Wall Temp. \#2F & 130.7 & 1311 & 130.91 & 130.5 & 141.8 & 142.41 & 143.1 & 142.61 & 138.9 & 137.7 \\
\hline Instroam Temp.F & 131 & 130.81 & 130.7 & 130.31 & 130.4 & 729.91 & 1301 & 129.9 & 129.4 & 128.6 \\
\hline Avg. Wall Tomp.F & 130.9 & 130.91 & 131 & 131.61 & 141.81 & 141.81 & 142.61 & 141.9 & 139.4 & 138 \\
\hline Avg. Wall DT.F & $0 !$ & 0.11 & $0.2 !$ & 1.31 & 10.91 & 11.41 & 12.21 & 11.61 & 9.51 & 9 \\
\hline Romaining Subcooff & 3.31 & & 1 & & & ! & & 1 & ; & $i$ \\
\hline Ext Quality & -0.031 & $-0 . \infty$ & -0.081 & -0.081 & -0.011 & 0.021 & 0.041 & 0.06 & 0.09 & 0.11 \\
\hline EXP.HOHUHine/F & 01 & 0 & 2101 & 6.7 & 830.61 & 884.21 & 810.8 & 859.6 & 1060 & 1132.0 \\
\hline COMPUTEDH & 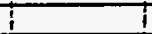 & & & & & 1 & & I & & I \\
\hline Jallouk & 1891 & 189 & 189.7 & 188.91 & 828.41 & 848.81 & 805.31 & 797 & 788.51 & 783.8 \\
\hline Szady1(Cudo tube) & 231.5 & 231.4 & 2323 & 231.31 & 1307.4 & 13011 & 1297.8 & 1305.31 & 1307 & 1311.7 \\
\hline Szadyz & 231.4 & 231.3 & 231.3 & 231.1 & 1084.8 & 1100.7 & 10025 & 1007.2 & 1095.9 & 1080.6 \\
\hline (RLQ) & 103387 & 103224 & 103142 & 102816 & 102897 & 1018631 & 99576 & 97141 & 94400 & 91452 \\
\hline$(F)$ & 1.006 & 1.006 & 1.006 & 1.0061 & 1.006 & 1.274 & 1.686 & 1.952 & 2.194 & 2.401 \\
\hline (HDQ) & 231.4 & 231.31 & 231.31 & Z3.II & 231.2 & 292.61 & ऊ7.2 & 450.7 & 503.51 & 550.5 \\
\hline (ABOOI:L) & 23.9 & 23.9 & 55.11 & 28.9 & 1305! & 120. & T286.2. & T287.2 & 1281.61 & T278.5 \\
\hline & & & & & & i & & T & & \\
\hline & ZZONE2 I & ZONE4 & ZONEB & ZONE8 & ZONETO & i & & $i$ & & \\
\hline DP liquid Temp.f & 1841 & & & + & & i & & I & $i$ & \\
\hline DPLquid Densitylbmitio & 82.41 & & & 1 & & $T$ & & $!$ & 1 & i \\
\hline Elovation-tt & 2 & 41 & 6 & 8 & 9.8751 & I & & $i$ & 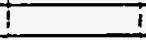 & $i$ \\
\hline Mossured DPPsid & 0.089 & 0.262 & -0.4001 & 1.1211 & 1.344 & it & & 1 & $\mathrm{i}$ & $i$ \\
\hline Zaro Conections-psid & 0.022 & -0.028 & -0.754 & 0.128 & 0.161 & i & & $i$ & $!$ & \\
\hline Corrected DPPsid & 0.067 & 0.29 & 0.351 & 0.993 & 1.183 & $i$ & & I & 1 & \\
\hline Actual DPA & 2.04 & 3.83 & 5.88 & 6.951 & 8.66 & & & & & \\
\hline Moasured TPPsig & 80.5 & & & & 3.61 & & & & I & 1 \\
\hline Measured Overallit & 129.27 & & & & & & & 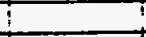 & & \\
\hline & & & & & & & & & & \\
\hline Computed DPAn & 2.081 & 4.16 & 6.21 & 7.861 & 9.341 & & & I & 1 & 1 \\
\hline Fricton-ti & 0.081 & 0.18 & 0.201 & 0.49 & 0.791 & & & $T$ & 1 & \\
\hline Accoleration-A & 01 & 0 & 0.11 & 0.22 & 0.311 & & & $i$ & I & I \\
\hline Elovition & $2 i$ & 41 & 5.891 & 7.161 & 8.231 & I & & $t$ & $t$ & 1 \\
\hline
\end{tabular}




\begin{tabular}{|c|c|c|c|c|c|c|c|c|c|c|}
\hline Run\# & 573 & & 1 & 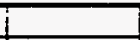 & & ! & & & & \\
\hline Tost Fluid & CAF10 & & & & & & & & & \\
\hline Dafe: & ब/193 & & & & & & & & & \\
\hline Time: & $8: 30$ & & & & & & & & & \\
\hline Nole & TABOREK & Analytical & dो & & & & & & & \\
\hline Flow Areaftl & 0.00336 & & & & & & & & & \\
\hline Head Flow Areatt? & 0.1955 & & & & & & & & & \\
\hline Sat. Prossuro Psig & 153.4 & & & & & & & & & \\
\hline Sat. Temperature F(inlet) & 175.7 & & & & & & & & & \\
\hline Lig. Densitylbmint3 & 76.77 & 76.7 & 76.72 & 76.67 & 76.7 & 76.63 & 76.58 & 76.7 & 76.7 & 76.98 \\
\hline Latent Heatstu/bm & 25.74 & 25.74 & 25.7 & 25.67 & 25.68 & 25.63 & 25.59 & 25.68 & 25.68 & 25.80 \\
\hline Specific Hoat Btulbm/F & 0.299 & 0.299 & 0.299 & 0.3 & 0.3 & 0.3 & 0.3 & 0.3 & 0.3 & 0.299 \\
\hline Liq. Visoosity 1 bm/nht & 0.346 & 0.346 & 0.346 & 0.344 & 0.345 & 0.344 & 0.343 & 0.346 & 0.345 & 0.948 \\
\hline Liq. Conductivity Btuhr-ffF & 0.0218 & 0.0218 & 0.0218 & 0.0218 & 0.0218 & 0.0218 & 0.0217 & 0.0218 & 0.0218 & 0.0219 \\
\hline Lig. Prandit No. & 4.74 & 4.74 & 4.74 & 4.74 & 4.74 & 4.73 & 4.78 & 4.74 & 4.74 & 4.74 \\
\hline Intet Fow Rate-GPM & 5.4 & & & & & & & & & \\
\hline Outtel lig. Fow Rato-GPM & 1.1 & & & & & & & & & \\
\hline Outlel Vap. Fow Rato-GPM & 2.6 & & & & & & & & & \\
\hline Inlet Tomperaturef & 172.1 & & & & & & & & & \\
\hline Outel Lig. Temperaturef & 775.7 & & & & & & & & & \\
\hline Outiet Vap. Tomperaturef & 171.9 & & & & & & & & & \\
\hline \multicolumn{11}{|l|}{ Water Flow Rato-GPM } \\
\hline Water inlel Tomp.f & 107.8 & & & & & & & & & \\
\hline Water outiel Tomp.f & 757 & & & & & & & & & \\
\hline Intet Glbmhnnte2 & 8574 & & Tomp & 769.1 & Density & 77.27 & & & & \\
\hline Subcooling $F$ & 3.6 & & & & & & & & & \\
\hline \multicolumn{11}{|l|}{ Flow Observation } \\
\hline & ZONE1 & ZONE2 & ZONE3 & ZONE4 & ZONE5 & ZONE6 & ZONE7 & ZONE8 & ZONE9 & ZONE10 \\
\hline Head Input-Watts(nel) & 577 & 57 & 57 & 57 & $5 \pi$ & 57 & 57 & 577 & 57 & 57 \\
\hline Hean Loss-Walts & 17.9 & 4.2 & 5.7 & 6.5 & 7 & 6.4 & 4.8 & 5.8 & 6.2 & 33.7 \\
\hline Hosd Flux-Btulfinte & 10002 & 10002 & 10002 & 10002 & 10002 & 10002 & 10002 & 10002 & 10002 & 10002 \\
\hline Wall Temp. FF $^{\prime}$ & 181.8 & 182.6 & 1824 & 182 & 183.7 & 183.2 & 183.9 & 182.9 & 1824 & 181.4 \\
\hline Wall Tomp. If & 182.5 & 182 & 183.2 & 182.8 & 183.6 & 183.2 & 184.2 & 183.4 & 183.2 & 181 \\
\hline Wall Temp. PFF & 182.8 & 181.1 & 184 & 182.7 & 182.5 & 183.5 & 183.4 & 1824 & 7821 & 181 \\
\hline Wall Tomp. & 73 & 182.8 & 180.8 & 181.1 & 182.1 & 184 & 184.2 & 183.4 & 181.1 & 178 \\
\hline Wall Tomp. \#2F & 183.3 & 182.7 & 182.11 & 181.7 & 182.1 & 183.7 & 184.6 & 183.9 & 181.8 & 179.7 \\
\hline Instream Tomp.F & 175 & 176 & 175.2 & 175.4 & 175.81 & 175.6 & 176.8 & 175.3 & 976.8 & 174.3 \\
\hline Avg: Wall Tomp of & 182.6 & 1825 & 1822 & 1822 & 182.6 & 183.6 & 184.1 & 183.2 & 182.1 & 1802 \\
\hline Avg. Wall DT F & 7.1 & 7.1 & 6.5 & 6.3 & 6.8 & 7.4 & 7.8 & 7.4 & 6.4 & 5.5 \\
\hline Remaining Subcooff & 1.6 & & & & & & & & & \\
\hline Exit Quality & -0.02 & 0 & 0.03 & 0.05 & 0.07 & 0.1 & 0.12 & 0.14 & 0.16 & 0.18 \\
\hline EXP. HBtuHr/R2/F & 1399.3 & 1414.1 & 15392 & 1575.6 & 9465.9 & 1347.4 & 1281 & 13429 & $15 \% 7$ & 1829.2 \\
\hline \multicolumn{11}{|l|}{ COMPUTEDH } \\
\hline Jallouk & Tस्81.3 & T0्81.3 & Ta17.8 & 7016.4 & 99:र्टा & $\$ 57.8$ & 959 & 947.6 & 939.2 & 985.5 \\
\hline Szady (Cudo tube) & 1710.7 & 1710.7 & 7715.8 & 9721.1 & 1728 & 1729.3 & 7734.6 & 1732.2 & 1707 & 1699.7 \\
\hline seady2 & 1475.7 & 1475.7 & 1526.5 & 1520 & 1523.5 & 15252 & 1526.4 & $15 \not 2$ & 1521.8 & 1513.3 \\
\hline (P्याO) & 188415 & 188416 & 185908 & 181888 & 177481 & 173599 & 169578 & 164422 & 1600084 & 15म27 \\
\hline (F) & 1.005 & 1.005 & 1.346 & 9.585 & 1.764 & 7.917 & 2.04 & 2.162 & 2.27 & 2.382 \\
\hline (1)O & 200.5 & 280.6 & 389.2 & 468.4 & 610.1 & 652.8 & 680.1 & 625.3 & 658.6 & 688.8 \\
\hline \multirow[t]{2}{*}{ (HBOIL) } & 1707.8 & 1707.8 & 1709.1 & 17102 & 1708 & 1710.3 & 1711.4 & 1704.7 & 1673.9 & 16812 \\
\hline & ZONE2 & ZONE4 & ZONE6 & ZONEB & ZONEIO & & & & & \\
\hline \multicolumn{11}{|l|}{ DP fiquid Temp.f } \\
\hline \multicolumn{11}{|l|}{ DPLiguid Denstiy lbm/th } \\
\hline Elevation-ft & 2 & 4 & 6 & 8 & 9.875 & & & & & \\
\hline Measured DPPsid & 0.213 & 0.6 & 0.352 & 1.757 & 2.18 & & & & & \\
\hline Zoro Cortections-psid & 0.039 & -0.054 & -0.741 & 0.111 & 0.077 & & & & & \\
\hline Comected DPPald & 0.174 & 0.054 & 9.103 & 1.62 & 2.103 & & & & & \\
\hline Actued DPA & 2.12 & 3.68 & 5.28 & 6.72 & 8.11 & & & & & \\
\hline Meserured TPPsig & 153.4 & & & & 3.5 & & & & & \\
\hline \multicolumn{11}{|l|}{ Mesosured Overall- } \\
\hline & & & & & & & & & & \\
\hline ComputodDPA & 2.14 & 4.14 & 5.97 & 7.75 & 9.47 & & & & & \\
\hline Friction-t & 0.14 & 0.37 & 0.69 & 1.07 & 1.49 & & & & & \\
\hline Acouterations & 0.04 & 0.2 & 0.3 & 0.39 & 0.47 & & & & & \\
\hline Elevation-th & 1.96 & 3.56 & 4.88 & 6.20 & 7.44 & & & & & \\
\hline & & & & & & & & & & \\
\hline
\end{tabular}




\begin{tabular}{|c|c|c|c|c|c|c|c|c|c|c|}
\hline Runt & 674 & & $i$ & - & $i$ & & 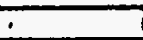 & 1 & & $!$ \\
\hline Tostfluid & CAF 10 & & & 1 & $i$ & & 1 & $i$ & & \\
\hline Dato: & ब1/93 & & & i & $!$ & & & & & \\
\hline Time: & $11: 33$ & & & $\vdots$ & I & & \pm & & & \\
\hline Nolo & TABOREA & Analytical & dt & 5 & $i$ & & $i$ & $\dot{4}$ & & \\
\hline Flow Arearte & 0.00335 & & + & $i$ & 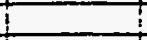 & & + & & & \\
\hline Hoat Fow Aroati? & 0.196951 & & & & & & & & & \\
\hline Sat. Prossulo Prig & 152 & & & & & & & & & \\
\hline Sat. Tomporatur of (intel) & 175 & & & & & & & & & \\
\hline Ua. Donstityibminz & 76.72 & 76.7 & 76.67 & $76 . \pi$ & 76.78 & 76.7 & 76.7 & 76.77 & 76.74 & 78.9 \\
\hline LEonk Hoatehunbm & 25.7 & 25.68 & 25.67 & 25.74 & 25.78 & 25.68 & 25.68 & 25.74 & 25.72 & 25.84 \\
\hline Spectic Hoat-buhtbm/F & 0.299 & 0.3 & 0.31 & 0.299 & 0.299 & 0.3 & 0.3 & 0.299 & 0.299 & 0.299 \\
\hline Lig. Visoosintbm/ithr & 0.345 & 0.35 & 0.344 & 0.346 & 0.346 & 0.345 & 0.375 & 0.346 & 0.345 & 0.348 \\
\hline Lig. Conductivity ButhreffF & 0.0218 & 0.0218 & 0.0218 & 0.0218 & 0.0219 & 0.0218 & 0.0218 & 0.0218 & 0.0218 & 0.0219 \\
\hline Liq. Prandeno. & 4.741 & 4.74 & 4.74 & 4.74 & 4.74 & 4.74 & 4.74 & 4.74 & 4.74 & 4.74 \\
\hline Inlolfiow Rato-GPM & 4.5 & & & & & & & & & \\
\hline Outlol Liq. Fow Rato-GPM & 1.1. & & & & & & & & & \\
\hline Outtel Vap. Flow Ralo-GPM & 2.2 & & & & & & & & & \\
\hline Inlel Temperaturef & 172.3 & & & & & & & & & \\
\hline OutetLa. Temperaturaf & T75.7 & & & & & & & & & \\
\hline Outlet Vap. Tempereture F & 171.5 & & & & & & & & & \\
\hline \multicolumn{11}{|l|}{ Welar Fow Rato-GPM } \\
\hline Wer hlot Temo,f & 109.4 & & & & & & & & & \\
\hline Wew oull ot Tamp.f & 160.3 & & & & & & & & & \\
\hline Intal Glominume & 828816 & & Tomp & 169.5 & Donsity & 77.18 & & & & \\
\hline Subooolingf & 2.7 & & & & & & & & & \\
\hline \multicolumn{11}{|l|}{ Fow Observation } \\
\hline & ZONEI & ZONE2 & ZONE3 & ZONE 4 & ZONE5 & ZONE6 & ZONE7 & ZONEB & ZONE9 & ZONE10 \\
\hline Hoed Input-Watts(net) & 582.3 & 582.5 & 581.7 & 576.9 & 575.9 & 577.8 & 581.7 & 583.9 & 575.2 & 583 \\
\hline Hor Loss-Waits & 17.9 & 4.2 & 5.7 & 6.5 & 7 & 6.4 & 4.8 & 5.8 & 6.2 & 34 \\
\hline Heat Flux Bturtrite & 10094 & 10097 & 10083 & 10000 & 9983 & 10016 & 10033 & 10122 & $\$ 97$ & 10108 \\
\hline WalTomp. BF & T81.81 & 1828 & 1828 & 181.8 & 183.7 & 183.2 & 183.3 & 182.6 & 1822 & 181.6 \\
\hline Wal Temp. If & 1825 & 182.1 & 783.6 & 182.4 & 183.3 & 783.2. & 183.7 & 183.1 & 183.1 & 181.2 \\
\hline Wal Tomp. 16-F & 182.8 & 181.3 & 184.2 & 1823 & 1822 & 183.3 & 1829 & 1821 & 182 & 187.4 \\
\hline Wall Tamp. MTF & 73.2 & 1828 & 181.2 & 180.8 & TE2 & 183.8 & 183.8 & 183.1 & 180.8 & 177.8 \\
\hline Waf Tamp. WLF & 183.2 & 182.8 & 1822 & 181.3 & 1821 & 189.7 & 184.1 & 183.6 & 181.5 & 179.8 \\
\hline Introam Tomp.f & 176.2 & 176.8 & 176.4 & 175 & 174.0 & 176.2 & 175.3 & 176 & 175.1 & 174.4 \\
\hline Avg. Wall Tomp.f & 182.6 & 182.6 & 1824 & 181.9 & 182.4 & 183.4 & 183.6 & 182.9 & 181.9 & 180.4 \\
\hline Avo.WelDT. $f$ & 6.8 & 6.9 & 6.5 & 6.4 & 7 & 7.6 & 7.8 & 7.4 & 6.4 & 5.6 \\
\hline Remaining Subooolf & 0.4 & & & & & & & & & \\
\hline Ext Qually & 0 & 0.02 & 0.05 & 0.08 & 0.11 & 0.13 & 0.16 & 0.19 & 0.22 & 0.24 \\
\hline 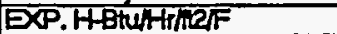 & 1458.9 & 1470 & 1540.8 & 1563 & 1421.3 & 1314 & 1288.7 & 1359.8 & 1560.5 & 1836.3 \\
\hline \multicolumn{11}{|l|}{ COMPUTEDH } \\
\hline Jallouk & Tन16.4 & 105.2 & 1057.5 & 7028.6 & 1008.4 & 997.7 & \$8:2 & 967.1 & $\$ 53.2$ & 949.2 \\
\hline Szadri(Cudo ubb) & 1795.5 & 1722 & 1724.7 & 1716 & \{715.9 & 1725.3 & 173 & 1735.3 & 1727.2 & 17320 \\
\hline szady? & 1485.7 & 1488.9 & 1535.7 & 1521.9 & 1518.4 & 1524.9 & 1532.3 & 1633.9 & 1516.8 & 1520.4 \\
\hline (FIJU) & 157130 & 155746 & 151525 & 146008 & 142161 & 138888 & 134048 & 129297 & 125089 & 119948 \\
\hline (F) & 1.004 & 1.267 & 1.58 & 1.794 & 1.967 & 2113 & 2247 & 2.373 & 2.488 & 2.601 \\
\hline (H) & 250.8 & 318.6 & 394.6 & 48 & 401.2 & 627.7 & 6612 & 682.6 & 620.6 & 640 \\
\hline \multirow[t]{3}{*}{ (HBOAL) } & 1717.7 & 1718.7 & 1717.8 & 1705.8 & 17024 & 1708.7 & 1713.2 & 1711.9 & 1700.1 & 1702 \\
\hline & & & & & & & & & & \\
\hline & ZONE2 & ZONEA & ZONE 6 & ZONE8 & ZONETO & & & & & \\
\hline DP Hquid Temp.f & 73.8 & & & & & & & & & \\
\hline DPLuquid Density tomit3 & 93.88 & & & & & & & & & \\
\hline Elevation & & & 6 & 8 & 9.875 & & & & & \\
\hline Measured DPF sId & 0.215 & 0.846 & 0.606 & 2.055 & 2582 & & & & & \\
\hline Zoro Corrections-psid & 0.039 & -0.054 & -0.741 & 0.111 & 0.077 & & & & & \\
\hline Corroced DPapild & 0.178 & 0.9 & 1.344 & 7.944 & 2.505 & & & & & \\
\hline Actual DPA & 2.11 & 3.2 & 4.81 & 6.18 & 7.38 & & & & & \\
\hline Mosulured TPPrig & 162 & & & & 3.6 & & & & & \\
\hline \multicolumn{11}{|l|}{ Messured Overal't } \\
\hline & & & & & & & & & & \\
\hline Computed DPA & 2.06 & 3.84 & 5.51 & 7.14 & 8.65 & & & & & \\
\hline Frictionft & 0.12 & 0.32 & 0.59 & 0.91 & 1.25 & & & & & \\
\hline Accolerationt & 0.08 & 0.18 & 0.26 & 0.33 & 0.41 & & & & & \\
\hline Elevation-h & 1.85 & 3.34 & 4.67 & 5.9 & 6.99 & & & & & \\
\hline & & & & & & & & & & \\
\hline
\end{tabular}




\begin{tabular}{|c|c|c|c|c|c|c|c|c|c|c|}
\hline Runt & 675 & & I & 1 & $!$ & $!$ & 1 & 1 & $i$ & $i$ \\
\hline Tert Fuid & tCAF 10 & I & I. & I. & T & I & & & $\because$ & 1 \\
\hline Dade: & $\begin{array}{ll} & 6 / 1 / 93 \\
\end{array}$ & & 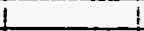 & 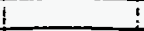 & 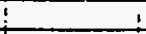 & 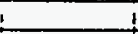 & & & $i$ & 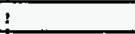 \\
\hline Time: & $12: 45$ & & t & & & 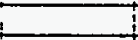 & & & & \\
\hline Note & TABOREK & Analytical o & $d t$ & & I & 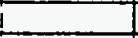 & & & & \\
\hline Flow Area-4t & 0.00336 & & & & & & & \pm & & \\
\hline Hoat Fow Area-th & 0.19595 & & & & & & & & & \\
\hline Sat. Pressure & 150.8 & & & & & & & & & \\
\hline Sad. Tomperature F(inlel) & 174.5 & & & & & & & & & \\
\hline Lig. Densinytbmin3 & 77 & 76.95 & 76.88 & 76.931 & 76.97 & 76.9 & 76.9 & 77 & 76.97 & 77.71 \\
\hline Latent Hear-Bturbm & 25.91! & 25.88 & 25.831 & 25.861 & 25.91 & 25.841 & 25.84 & 25.91 & 25.9 & 28 \\
\hline Specific Hoed Btulbm/F & 0.299 & 0.299 & 0.2901 & 0.299 & 0.299 & 0.299 & 0.299 & 0.299 & 0.299 & 0.299 \\
\hline Lig. Visoosity tom/ahr & 0.349 & 0.348 & 0.347 & 0.348 & 0.349 & 0.348 & 0.348 & 0.349 & 0.349 & 0.351 \\
\hline Liq. Conductivity Buhreff & 0.022 & 0.021 & 0.0219 & 0.0218 & 0.022 & 0.0219 & 0.0219 & $0 . \infty 2$ & 0.022 & 0.0021 \\
\hline Liq. Prandi No. & 4.741 & 4.74 & 4.74 & 4.74 & 4.741 & 4.74 & 4.74 & 4.74 & 4.741 & 4.74 \\
\hline Inlet fow Rato-GPM & 3.6 & & & & & & & & & \\
\hline Outiot Liq. Fow Rate-GPM & 1.11 & & & & & & & & & \\
\hline Oulel Vap. Flow Plate-GPM & 9.9 & & & & & & & & & \\
\hline Indet Temperature F & 170.8 & & & & & & & & & \\
\hline Oufef Lu. Temperaturef & $1 \%$ & & & & & & & & & \\
\hline Outhel Vap. Temperaturef & 170.7 & & & & & & & & & \\
\hline \multicolumn{11}{|l|}{ Water Fow Rate-GPM } \\
\hline Wator finld Tomp.F & 109.1 & & & & & & & & & \\
\hline Wetar ouled Temp.f & 1562 & & & & & & & & & \\
\hline Intal G-bminrin2 & 665756 & & Tomp & 168.1[ & Donsiny & 77.49 & & & & \\
\hline Subcoolingf & 3.71 & & & & & & & & & \\
\hline \multicolumn{11}{|l|}{ Fow Observition } \\
\hline & ZONE1 & ZONE2 & ZONE3 & ZONE4 & ZONE5 & ZONE6 & ZONE7 & ZONEB & ZONE9 & ZONE10 \\
\hline Heat lnputWatts(nel) & 556.6 & 553.9 & 565 & 565.4 & 564.7 & 571.5 & 569.9 & 571.6 & 564.4 & 569.5 \\
\hline Head Loss-Watts & 17.2 & 4 & 5.5 & 6.2 & 6.6 & 6 & 4.4 & 5.4 & 5.9 & 32.1 \\
\hline Hedficox-Bturtifite & 9822 & 9775 & 9811 & 9801 & 9789 & 9907 & 9879 & 9908 & 9784 & 9872 \\
\hline Wall Tomp. 134 & $78 \pi$ & 181.8 & 781.8 & 381.2 & TE्3 & 182.4 & 782.6 & 781.8 & 181.3 & 80.9 \\
\hline Wall Tomp. $14 F$ & 881.6 & 181.2 & 182.5 & 181.7 & 1827 & 182.5 & 182.9 & 7824 & 182.2 & 180.4 \\
\hline WE: Temp. $75 F$ & 181.8 & 180.4 & 183.4 & 181.7 & 181.7 & 182.7 & 1824 & 181.3 & 181.1 & 780.8 \\
\hline Wad Tomp. I1F & 78.4 & 181.8 & 180.3 & 180.1 & 181.8 & 183.1 & 182.8 & 182.4 & 180.1 & 178.9 \\
\hline Wal Tomp. raF & 1822 & 182 & 181.3 & 180.6 & 181.2 & 188.1 & 183.3 & 1820 & 180.7 & 170 \\
\hline Intreasm Temp. $F$ & 174 & 174.2 & 174.5 & 174.3 & 174.1 & 174.4 & 174.4 & 174 & 174.1 & 178.5 \\
\hline Avg.Wall Temp.F & 181.7 & 181.7 & 181.5 & 1812 & 181.7 & 1827 & 1828 & 1822 & 181.1 & 179.6 \\
\hline Avg. Wall DT.F & 7.2 & 7.1 & 6.5 & 6.4 & 7.2 & 7.8 & 8 & 7.7 & 6.5 & 5.7 \\
\hline Romaining Subcoolf & 0.8 & & & & & & & & & \\
\hline Ext Quality & -0.01 & 0.02 & 0.06 & 0.09 & 0.12 & 0.16 & 0.19 & 0.23 & 0.26 & 0.20 \\
\hline EXP.HBtuhHrna & 1363 & 1384.9 & 15021 & 1523.7 & 13528 & 1265.6 & 1239 & 1284.8 & 1496.4 & 1746 \\
\hline \multicolumn{11}{|l|}{ COMPUTEDH } \\
\hline Jillouk & 1120.4 & 1083.3 & 1000.2 & 1050.1 & 7028.5 & T022: & 7007.1 & 934.5 & 977.9 & 970 \\
\hline Seadyt(Cudo tubl) & 1684 & 1683.1 & 1691.1 & 1689.9 & 1688.6 & 1708 & 77025 & $7 \pi 2.6$ & 7695.9 & 7693.0 \\
\hline sendy2 & 1445.2 & 1444.3 & 1497 & 1493.7 & 1450.2 & 1500.9 & 1502.1 & 1502 & 1487.6 & 14928 \\
\hline (सएव) & 124778 & 124057 & 120039 & 115892 & 111446 & 107509 & 10395 & \$8750| & 94007 & 89982 \\
\hline (F) & 1.005 & 1.234 & 1.613 & 1.86 & 2.0581 & 2.225 & 2.377 & 2.52 & 2.647 & 2.775 \\
\hline (संIO) & 210.3 & 258.4 & 337.8 & 389.4 & 430.81 & 468 & 497.8 & 627.7 & 654.2 & 580.7 \\
\hline \multirow[t]{3}{*}{$(1+3014)$} & 16829 & 1681.7 & 1686.6 & 1683 & 1679.1 & 1691.3 & 1688.4 & 1685.6 & 1675.9 & 1675.6 \\
\hline & & & & & & & & & & \\
\hline & $\angle O N=2 \quad 12$ & ZONEL & ZONE 6 & ZONES & ZONE1O & & & & & \\
\hline DP liquid Temp.f & 76.4 & & & & & & & & & \\
\hline \multicolumn{10}{|l|}{ DPLiquid Density tompit3 } & \\
\hline Elevationf & 2 & 4 & 6 & 8 & 9.875 & & & & & \\
\hline Mossured DPPSid & 0.274 & 0.933 & 0.796 & 2.3. & 2.91 & & & & & \\
\hline Zero Corrections-psid & 0.039 & -0.054 & -0.741 & 0.171 & 0.07 & & & & & \\
\hline Corrected DPPSild & 0.175 & 1.017 & $1.537 !$ & 2.199 & 2.888 & & & & & \\
\hline Actual DPA & 2.7 & 2.55 & 4.41 & 5.59 & 6.68 & & & & & \\
\hline Measured TPPsig & 150.8 & & & & 3.6 & & & & & \\
\hline \multirow{2}{*}{\multicolumn{11}{|c|}{ Moseured Overall }} \\
\hline & & & & & & & & & & \\
\hline Computad DPA & 1.99 & 3.61 & 5.09 & 6.5 & 7.79 & & & & & \\
\hline Friction-ft & 0.08 & 023 & 0.44 & 0.0 & 0.97 & & & & & \\
\hline Acouleration & 0.06 & 0.13 & 0.19 & 0.25 & 0.32 & & & & & \\
\hline ElovationA & 1.85 & 3.25 & 4.47 & 5.56 & 6.5 & & & & & \\
\hline & & & & & & & & & & \\
\hline
\end{tabular}




\begin{tabular}{|c|c|c|c|c|c|c|c|c|c|c|}
\hline Runt: & $1 \quad 676$ & & 1 & ! & & & & & & \\
\hline Tetf Flud & TCAF & & & i & & i & & & & \\
\hline Dexte: & - $1 / 1 / 33$ & & & & & $i$ & & & & \\
\hline Time: & 12.45 & & & & & & & & & \\
\hline Noto & TABOREK & Analytical d & dt & & & & & & & \\
\hline Flow Areaft2 & 0,00356 & & & & & & & & & \\
\hline Hosl Fow Areat? & 0.19695 & & & & & & & & & \\
\hline Sot. Prossuratig & 150 & & & & & & & & & \\
\hline Set. Temperature f(intal) & 174.11 & & & & & & & & & \\
\hline Uq.Densthyom/ins & 77.04 & 76.9 & 76.9 & 76.95 & 76.85 & 78.9 & 76.86 & 76.93 & 76.93 & 77.11 \\
\hline Letent Henturibm & 25.85 & 25.84 & 25.84 & 25.88 & 25.88 & 25.84 & 25.81 & 25.86 & 25.86 & 28 \\
\hline Specito How Bunlbm/F & 0.298 & 0.289 & 0.299 & 0.299 & 0.29 & 0.299 & 0.29 & 0.299 & 0.299 & 0.298 \\
\hline Lig. Viscosity tominth & 0.35 & 0.348 & 0.348 & 0.348 & 0.348 & 0.348 & 0.347 & 0.348 & 0.348 & 0.351 \\
\hline Itg. Conductivit Btwhrth & 0.022 & 0.0219 & 0.0219 & 0.022 & 0.02 & 0.0218 & 0.0219 & 0.0218 & 0.0218 & 0.0221 \\
\hline Lq. Prandt No. & 4.74 & 4.74 & 4.74 & 4.74 & 4.74 & 4.74 & 4.74 & 4.74 & 4.74 & 4.74 \\
\hline Inlet Fow Pade-GPM & 2.8 & & & & & & & & & \\
\hline Outlol Liq. Flow Rato-GPM & 1.2 & & & & & & & & & \\
\hline Outed Vap. Fow Ralo-GPM & 1.7 & & & & & & & & & \\
\hline Intel Tomperaturef & 171 & & & & & & & & & \\
\hline Outet Lig. Tempericher & 175 & & & & & & & & & \\
\hline Oudelvep. Temporature F & 171.3 & & & & & & & & & \\
\hline Waler Fow REG-GPM & & & & & & & & & & \\
\hline Water hild Tomp,f & 109.4 & & & & & & & & & \\
\hline Water oullet Temp.f & 166.1 & & & & & & & & & \\
\hline Inlet Glbm/nrine & 619446 & & Tomp & 167 & Denuity & 77.74 & & & & \\
\hline Suboodingf & 3.1 & & & & & & & & & \\
\hline Flow Observation & & & & & & & & & & \\
\hline & ZONE1 & ZONE2 & ZONE3 & ZONE4 & ZONE5 & ZONE 6 & ZONET & ZONE8 & ZONE9 & ZONE10 \\
\hline Hoat lnput-Watts(net) & 570.1 & 577.2 & 571.7 & $5 \% 0.9$ & 538.1 & 571.4 & 573.7 & 574.6 & 570.3 & 575.3 \\
\hline Hod Loss-Watts & 17.1 & 4 & 5.5 & 6.2 & 6.7 & 6 & 4.5 & 5.5 & 5.9 & 325 \\
\hline Hox Fiox-Btuhrhe & 9882 & 10005 & 9910 & 9896 & 9848 & 9906 & 9945 & $\$ 960$ & 9886 & 9972 \\
\hline Wât Tremp. & 180.8 & 181.8 & 787.7 & 78ा.8 & 183 & 9824 & 1820 & 7825 & 781.5 & 7812 \\
\hline Walt Tomp. $14 \mathrm{~F}$ & 1815 & 187 & 182.8 & 181.7 & 182.8 & 1824 & 783.3 & 1827 & 182.6 & 180.7 \\
\hline Wall Tomp. $15 f$ & 181.6 & 180.2 & 183.4 & 181.7 & 181.7 & 1827 & 182.6 & 181.5 & 181.5 & 180.8 \\
\hline Wall Tomp. ift & 7.7 & 181.8 & 180.2 & 1802 & 181.4 & 188.1 & 183.4 & 182.7 & 180.4 & 177.6 \\
\hline Well Temp. IRF & 182 & 181.8 & 181.2 & 180.7 & 181.8 & 183 & 189.2 & 183.1 & 181.1 & 179.4 \\
\hline Instreem Tomp. $f$ & 173.8 & 174.4 & 974.4 & 1742 & 174.2 & 174.4 & 174.6 & 174.3 & 174.3 & 173.5 \\
\hline Avo. Wall Tomp.f & 181.5 & 181.7 & 181.4 & 181.2 & 181.8 & 1827 & 183.2 & 182.4 & 181.4 & 179.9 \\
\hline Avo. WEHDT, F & 7.2 & 6.8 & 6.6 & 6.6 & 7.2 & 7.8 & 8.1 & 7.7 & 6.7 & 6 \\
\hline Pemsining Subooof & -0.7 & & & & & & & & & \\
\hline Ext Qual'y & 0.01 & 0.05 & 0.00 & 0.14 & 0.18 & 022 & 027 & 0.31 & 0.35 & 0.4 \\
\hline 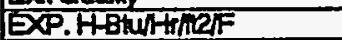 & 13672 & 1477.8 & 1506.7 & 1504.5 & 1376.3 & 1259.5 & 1223.1 & 1295.3 & 1481.4 & 1670.6 \\
\hline COMPUTEDH & & & & & & & & & & \\
\hline Jallouk & 7741.7 & 7178.4 & 7084.4 & 1053.8 & $10 \% 0.7$ & 1050.9 & 7042 & T020.6 & 7015.3 & 7000.2 \\
\hline Szady (Cudo tubo) & 1686.6 & 1703.8 & 1697.4 & 155.4 & 16928 & 1700.9 & 1707.6 & 1707.1 & I7ण्ट.7 & 17026 \\
\hline sadt? & 1447.9 & 1467.6 & 1400.5 & 1458.2 & 1498.4 & 1508.3 & 1512.1 & 1511.2 & 1502 & 15055 \\
\hline$(F \perp \cdot Q)$ & 86807 & 94808 & 90009 & 86240 & 82089 & 77989 & 73898 & 6942 & 65:212 & 60521 \\
\hline (F) & 7.154 & 1.507 & 1.848 & 2.101 & 2.308 & 2.489 & 2.652 & 2.800 & 2.551 & 3.000 \\
\hline$(\mathrm{HLC})$ & 188.1 & 258.8 & 317.3 & 360.7 & 386.5 & 427.5 & 455.4 & 4828 & 506.7 & 631.8 \\
\hline (FBOIL) & 1685.6 & 1701.8 & 1633.7 & 1689.9 & 1685.5 & 1691.8 & 1656.7 & 1694.1 & 1587.6 & 165.1 \\
\hline & & & & & & & & & & \\
\hline & ZONE2 & ZONE4 & ZONE6 & ZONE8 & ZONE10 & & & & & \\
\hline DPliquid tomp. $F$ & 7.1 & & & & & & & & & \\
\hline DPLiquid Densithetom/it3 & $\$ 3.41$ & & & & & & & & & \\
\hline Eovetion & 2 & 4 & 6 & 8 & 9.875 & & & & & \\
\hline Moasured DPFST & 0.18 & 1.178 & 9.055 & 2.653 & 3.3 .6 & & & & & \\
\hline Zoro Corrections-psid & 0.039 & -0.054 & -0.741 & 0.111 & 0.07 & & & & & \\
\hline Corrocted DPPyid & 0.141 & 1.232 & 1.795 & 2.542 & 3.259 & & & & & \\
\hline Aotus DPA & 2.16 & 264 & 3.87 & 4.84 & 5.86 & & & & & \\
\hline Moasured TPPsig & 150 & & & & 3.5 & & & & & \\
\hline Measured Overill & 273.69 & & & & & & & & & \\
\hline & & & & & & & & & & \\
\hline Comoutad DP: & 1.811 & 3.28 & 4.51 & 5.71 & 6.8 & & & & & \\
\hline Friction & 0.06 & 0.18 & 0.35 & 0.55 & 0.77 & & & & & \\
\hline Accoleretion-h & 0.06 & 0.1 & 0.15 & 0.2 & 0.26 & & & & & \\
\hline Elovationnt & 1.69 & 2.94 & 4.01 & 4.96 & 5.7 & & & & & \\
\hline & & & & & & & & & & \\
\hline
\end{tabular}




\begin{tabular}{|c|c|c|c|c|c|c|c|c|c|c|}
\hline Runt & i $\quad 577$ & & $i$ & 1 & & 1 & & $!$ & - & ! \\
\hline Tostfluid & TCAF10 I & i & I & $i$ & I & 1 & $\vdots$ & 1 & T & I \\
\hline Date: & ब1/1931 & & I & I & & $I$ & 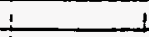 & 1 & & 1 \\
\hline Time: & $12: 45$ & & 1 & $i$ & & $I$ & 1 & & & I \\
\hline Nolo & TABOREK & Analytical & $d t$ & & & & $\dot{i}$ & & & \\
\hline Fow Areat? & 0.00336 & & $\frac{1}{\vdots}$ & & & & 1 & & & \\
\hline Hoat Flow Areatil & 0.15695 & & & & & & $t$ & & & \\
\hline Sat. Pressuro Psig & 457.81 & & & & & & & & & \\
\hline Sad. Temperature f(inlei) & 174.9 & & & & & & & & & \\
\hline Liq. Density -bm/118 & 76.72 & 76.67 & 76.77 & 76.72 & 76.72 & 76.67 & 76.58 & 76.67 & 76.63 & 76.88 \\
\hline Latent Hest-Btutbm & 25.7 & 25.67 & 25.74 & 25.7 & 25.7 & 25.67 & 25.59 & 25.67 & 25.63 & 25.78 \\
\hline Specific Hoalsurbmif & 0.299 & 0.3 & 0.29 & 0299 & 0.29 & 0.3 & 0.3 & 0.3 & 0.3 & 0.299 \\
\hline Liq. Viscosingtomathr & 0.345 & 0.34 & 0.346 & 0.345 & 0.345 & 0.34 & 0.34 & $0.3 M$ & 0.344 & 0.347 \\
\hline Lig. Conduchisy BtwhrffF & 0.0218 & 0.0218 & 0.0218 & 0.0218 & 0.0218 & 0.0218 & 0.0217 & 0.0218 & 0.0218 & 0.0219 \\
\hline Liq. Prand No. & 4.74 & 4.74 & 4.741 & 4.74 & 4.74 & 4.74 & 4.79 & 4.74 & 4.73 & 4.74 \\
\hline Intet Fow Rate-GPM & 1.9 & & & & & & & & & \\
\hline Outlet Lig. Flow Rato-GPM & 1.31 & & & & & & & & & \\
\hline Outlet Vap. Fow Rate-GPM & 0.9 & & & & & & & & & \\
\hline Inlet Temperaturef $F$ & 1722 & & & & & & & & & \\
\hline Oudatlig. TomperatureF & 176.5 & & & & & & & & & \\
\hline Outel Vap. Tomperaturef & 1725 & & & & & & & & & \\
\hline Waor Fow Rato-GPM & & & & & & & & & & \\
\hline War inlat Tomp.F & 109.5 & & & & & & & & & \\
\hline Wew r outhet Temp.f & 154.3 & & & & & & & & & \\
\hline Inder G fomprife & 3524811 & & Tomp & 167 & Density & 77.74 & & & & \\
\hline Suboodingtf & 2.7 & & & & & & & & & \\
\hline Fow Observation & & & & & & & & & & \\
\hline & ZONE1 & ZONE2 & ZONEB & ZONEA & ZONES & ZONE6 & ZONE? & ZONE8 & ZONE9 & ZONEIO \\
\hline Heal Input-Watts(net) & 5724 & 577.3 & 575.3 & 574.6 & 575.4 & 576.5 & 573.7 & 575.9 & 576.8 & 561.8 \\
\hline Head Loss-Watts & 17 & 4 & 5.5 & 6.2 & 6.6 & 5.9 & 4.51 & 5.5 & 6 & 46.8 \\
\hline Hoat Flox-Btuthrice & 9022 & 10007 & 9972 & 9960 & 9974 & 9993 & 9945 & 998 & 998 & 9738 \\
\hline Wall Temp. BF & 181.3 & 18ट2. & 1821 & नांर्श & 188.9 & 183.4 & 183.8 & 183.2 & 1827 & 200.4 \\
\hline WEII Temp. ThF & 181.9 & 981.5 & 783 & 182.5 & 183.5 & 183.4 & 184.4 & 189.6 & 783.8 & 209.4 \\
\hline Wad Tomp. $15 F$ & 182.1 & 180.6 & 183.9 & 182.5 & 1825 & 183.4 & 183.3 & 1826 & 182.2 & 205 \\
\hline Wall Temp. ITF & 79.5 & 182 & 180.8 & 180.6 & 182.1 & 183.9 & 184.9 & 189.8 & 181.1 & 188.2 \\
\hline WNI Temp. HaF & 182.6 & 1822 & 781.8 & 187.3 & 182.1 & 188.8 & 184.4 & 184.2 & 181.8 & 189.6 \\
\hline Instream Tomp. $f$ & 1762 & 175.4 & 176 & 175.2 & 175.2 & 175.4 & 175.8 & 175.4 & 176.6 & 174.7 \\
\hline Ang.Well Temp.F & 182 & 182 & 181.8 & 181.8 & 182.8 & 183.5 & 184 & 183.4 & 1823 & 200.3 \\
\hline Avg. Wal DT If & 6.3 & 6.1 & 6.4 & 62 & 6.9 & 7.7 & 7.8 & 7.6 & 62 & 252 \\
\hline Remaining Subcooff & -2.8 & & & & & & & & & \\
\hline Ext Quality & 0.03 & 0.1 & 0.18 & 0.23 & 0.29 & 0.36 & 0.4 & 0.48 & 0.55 & 0.61 \\
\hline EXP.HBtuHAnar & 1558.3 & 1634.4 & 1564.5 & $1 \approx 0.1$ & 146.7 & 13023 & 1283.1 & 1315.5 & 1605.4 & 388.8 \\
\hline COMPUTEDH & & & & & & & & & & \\
\hline Jallouk & 1183.1 & 1157.1 & Tद27 & TTा0.5 & 1008 & T06.6. & 1080 & T06्8.5. & Тद्या & TZ्ठ3.8 \\
\hline Sendyi(audo hube) & 7704.8 & $1 \pi 45$ & 1708 & 1710 & 1711.9 & 1716.5 & 177.8 & 7717.5 & 124 & 120.8 \\
\hline $\operatorname{send} 2$ & 14692 & 1480.1 & 1472.6 & 1475 & 1521.7 & 15252 & 1522 & 1523.4 & 124 & 120.6 \\
\hline (स्थि & 65748 & 62000 & 58076 & 53880 & 49575 & 45346 & 41184 & 38089 & 36099 & 90000 \\
\hline (F) & 1.358 & 1.788 & 2.16 & 2.446 & 2689 & 2.908 & 3.093 & 3.284 & 3.284 & 3.284 \\
\hline (HUO) & 171.17 & 225.3 & 2728 & 308.8 & 38.0 & 356.8 & 389.8 & 413.8 & 413.8 & 413.8 \\
\hline (HBOL) & 1704.3 & 17132 & 175.7 & 1706.6 & 1707.5 & 1710.9 & 1711.1 & 1709.4 & 1703.4 & 1700.4 \\
\hline & & & & & & & & & & \\
\hline & \begin{tabular}{|l|l} 
ZONE2 & 2 \\
\end{tabular} & ZONEA & ZONE6 & ZONEB & ZONE10 & & & & & \\
\hline DP lquid Tomp.F & 78.1 & & & & & & & & & \\
\hline DPLiquid Densing $16 \mathrm{~m} / \mathrm{k}$ & $\$ 3.28$ & & & & & & & & & \\
\hline Elovation & 2 & 4 & 6 & 8 & 9.875 & & & & & \\
\hline Mossured DPpsid & 0.127 & 1.521 & 1.425 & 3.12 & 3.955 & & & & & \\
\hline Zero Corrections-psid & 0.039 & -0.054 & -0.741 & 0.171 & 0.07 & & & & & \\
\hline Comedod DPPsid & 0.088 & 1.575 & 2.168 & 2.890 & 3.8881 & & & & & \\
\hline Actual DP & 2.28 & 1.8 & 3.22 & 4.1. & 4.7 & & & & & \\
\hline Moatured TPPsig & 151.8 & & & & 3.6 & & & & & \\
\hline Maseured Overalli & 277.7 & & & & & & & & & \\
\hline & & & & & & & & & & \\
\hline Computed DPA & 1.56 & 2.74 & 3.77 & 4.7 & 5.51 & & & & & \\
\hline Friction-t: & 0.04 & 0.12 & $0 \approx$ & 0.36 & 0.5 & & & & & \\
\hline Accoleration & 0.04 & 0.07 & 0.11 & 0.16 & 0.2 & & & & & \\
\hline Elevation & 1.48 & 2.55 & 3.43 & 4.18 & 4.8 & & & & & \\
\hline & & & & & & & & & & \\
\hline
\end{tabular}




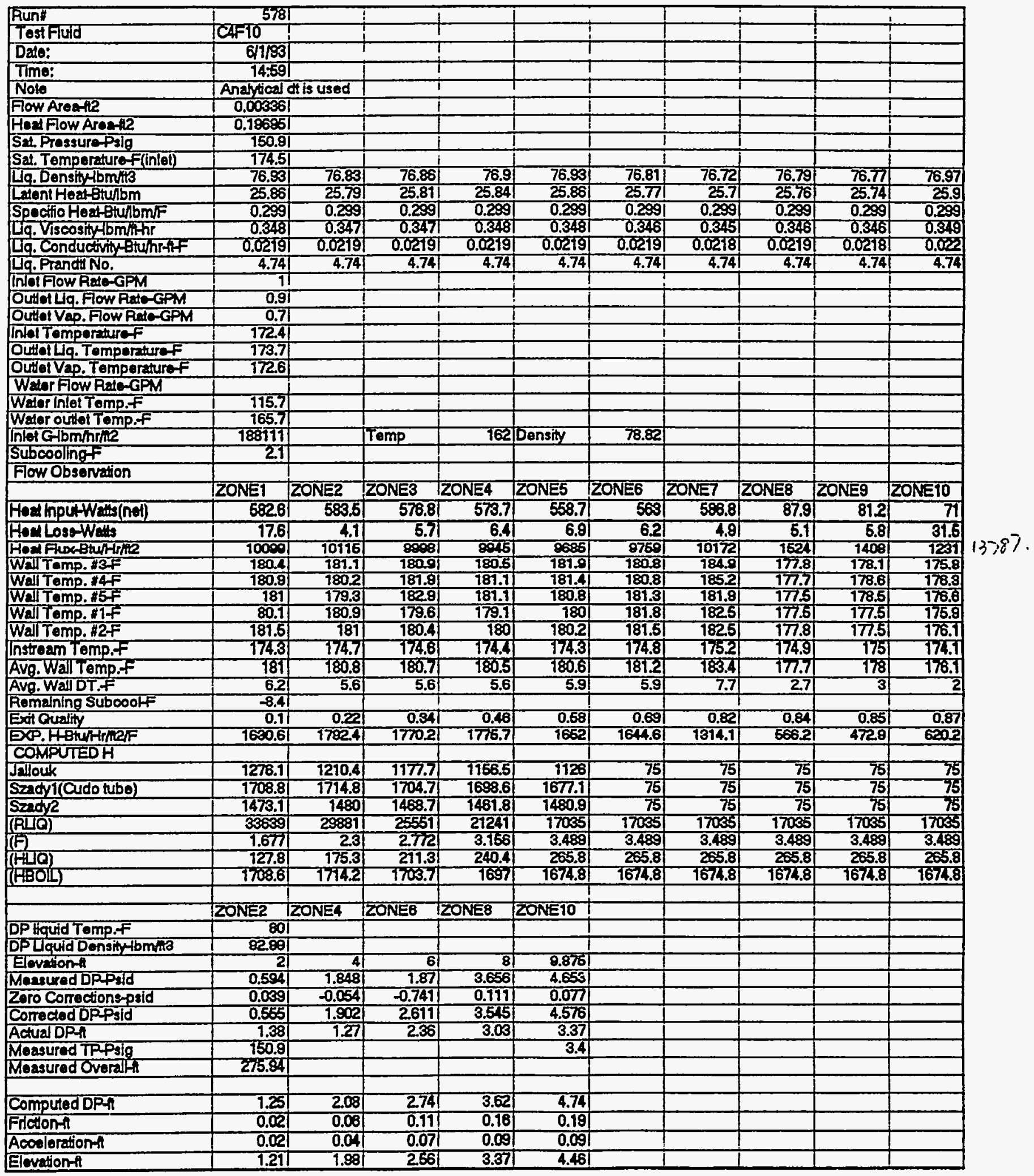




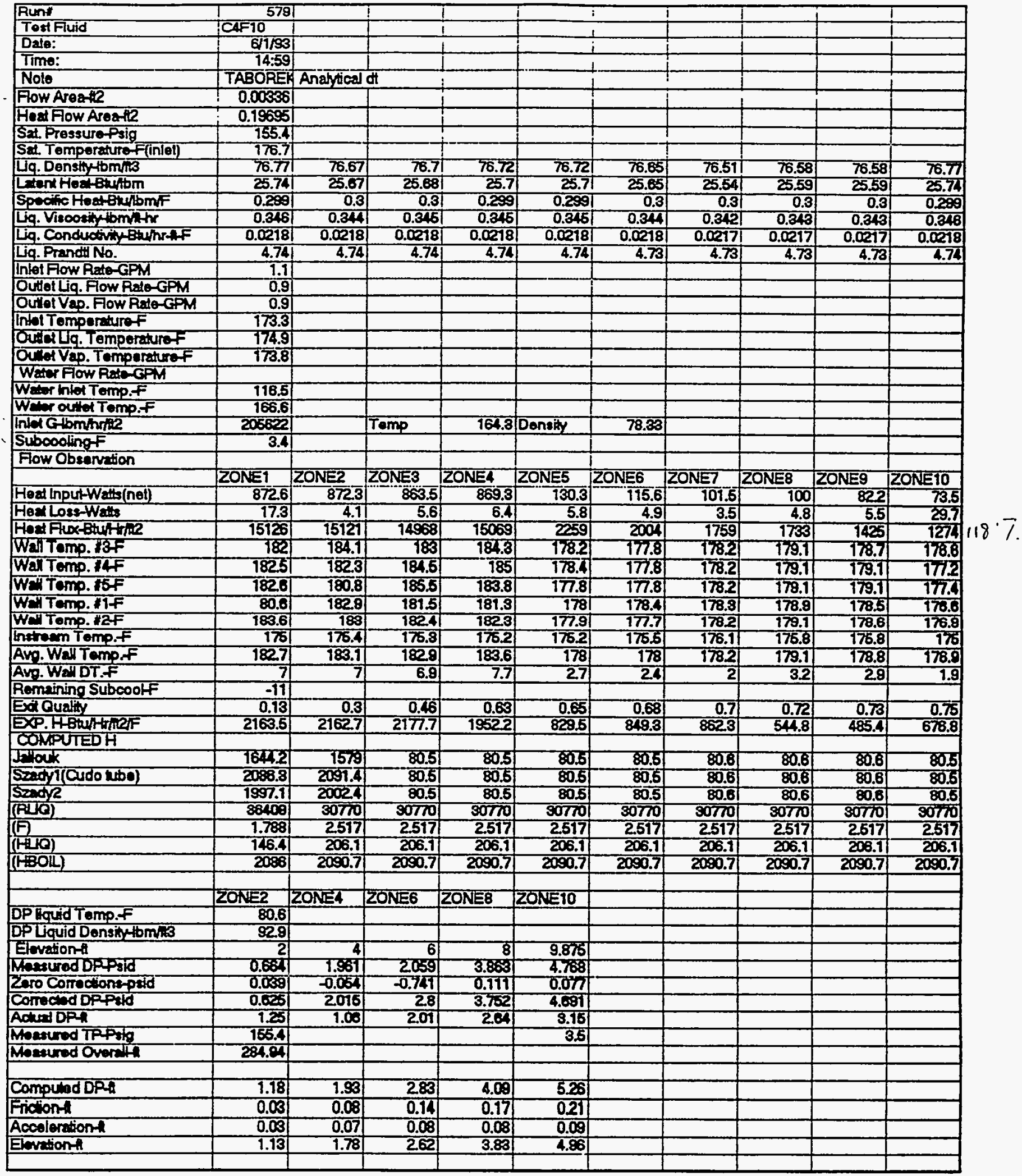




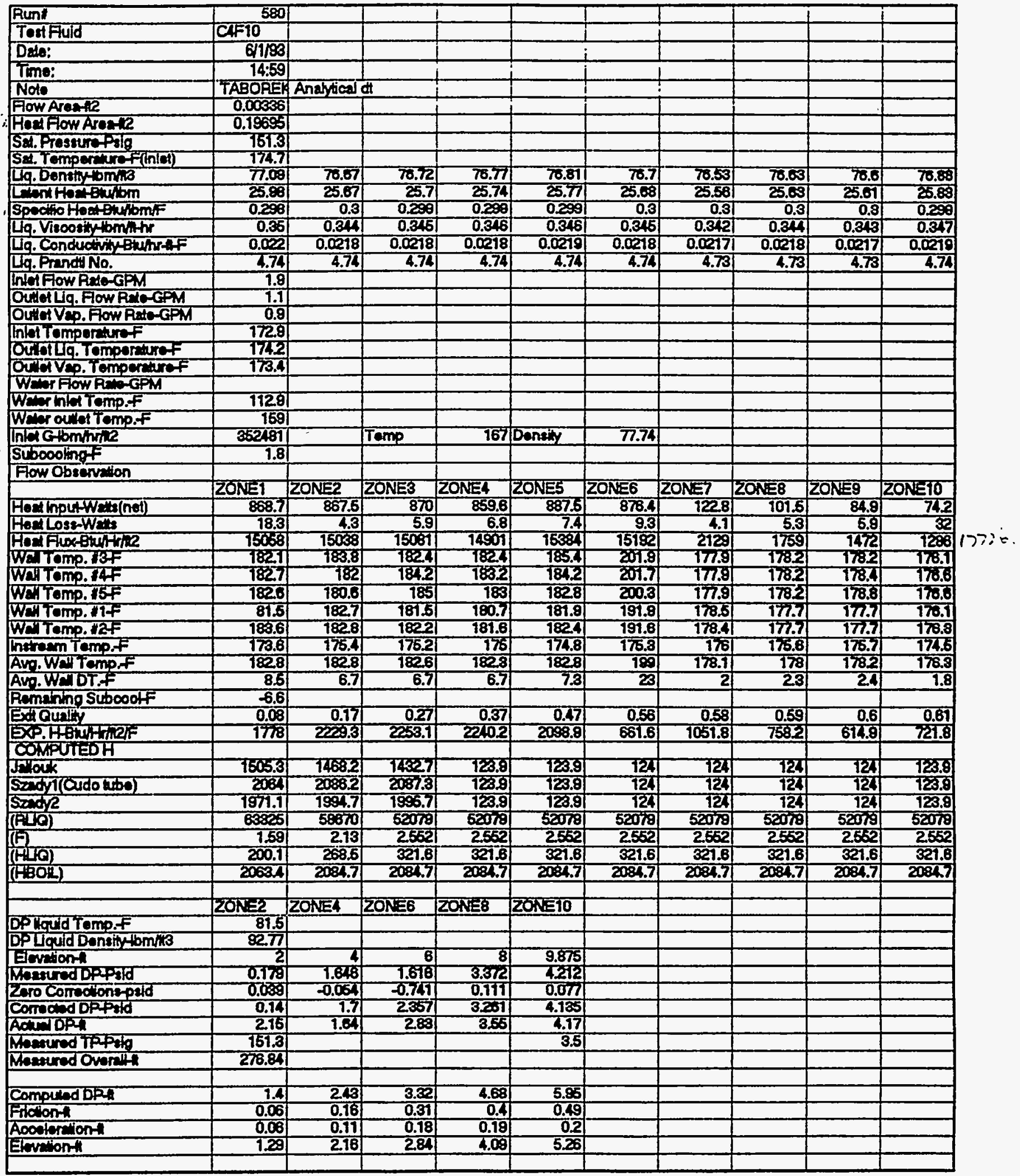




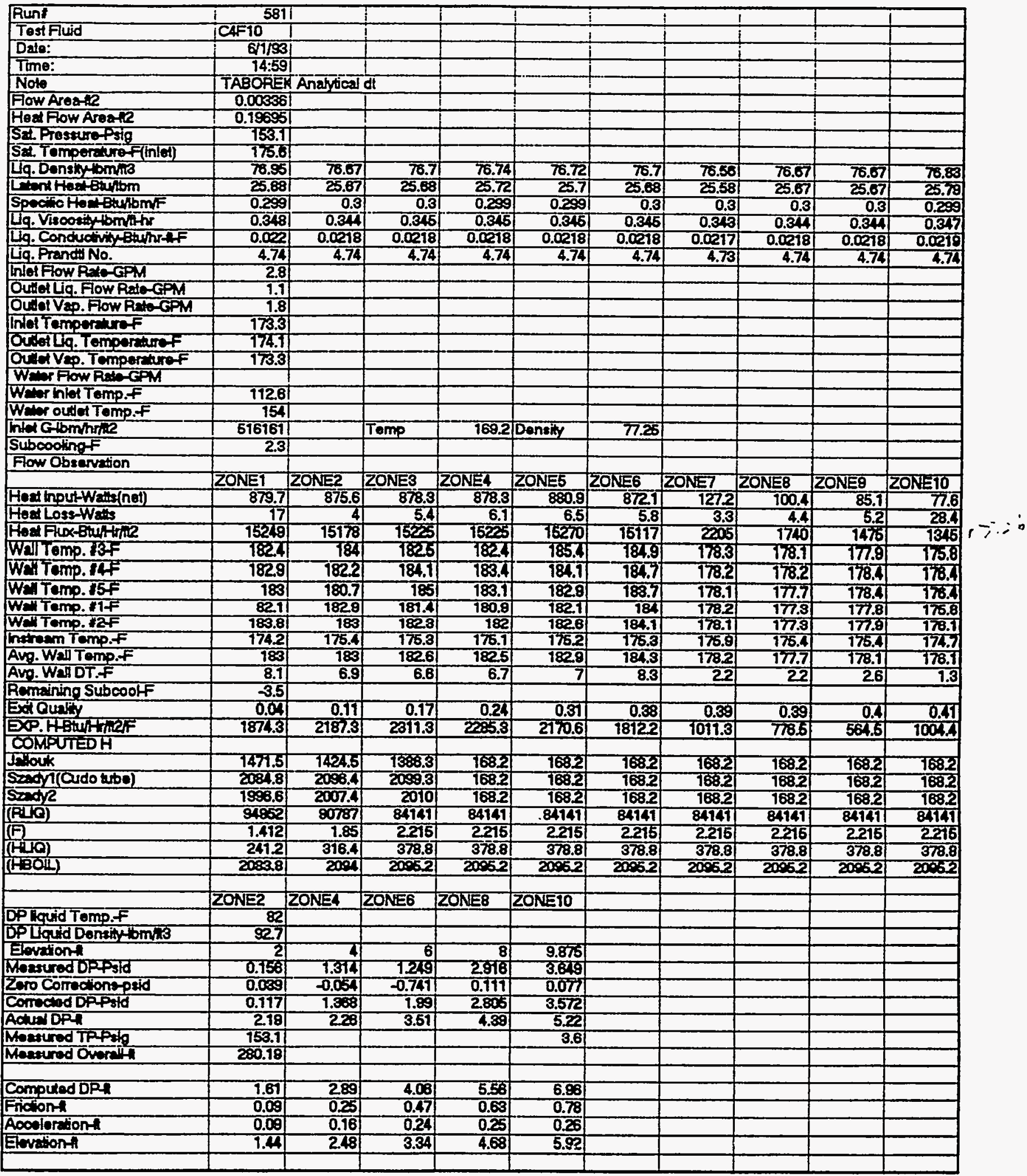




\begin{tabular}{|c|c|c|c|c|c|c|c|c|c|c|}
\hline Aunl & 5821 & & 1 & 1 & $\perp$ & 1 & I & 1 & $\perp$ & \\
\hline ToAfluid & CAF 10 & & & 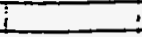 & 1 & $i$ & & 1 & 1 & \\
\hline Deds: & ब1183 & & & & 1 & i & $i$ & $\perp$ & & $L$ \\
\hline Time: & 14:591 & & & & & 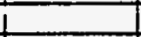 & & 1 & & \\
\hline Noto & TABOFEA & Analyiol & di & & & & & & & \\
\hline Flow A roaile & 0.00356 & & & & & & & & 1 & \\
\hline Hect Fow Areshe & 0.1955 & & & & & & & & & \\
\hline S1 Prosere Plg & 100.31 & & & & & & & & & \\
\hline Sat Tempervire f (iniel) & 178.9 & & & & & & & & & \\
\hline Llg. Densing $46 m / 13$ & 76.7 & 76.6 & 76.5 & 76.77 & 76.81 & 76.79 & 76.56 & 76.07 & 76.67 & 76.9 \\
\hline Lienithestawbm & 25.68 & 25.61 & 25.05 & 25.74 & 25.77 & 25.78 & $\mathbf{2 5 . 5 8}$ & 25.67 & 25.67 & 25.84 \\
\hline Specino Heate'wibm/F & 0.3 & 0.3 & 0.3 & 029 & 0.299 & 0.20 & 0.3 & 0.3 & 0.31 & 0.29 \\
\hline 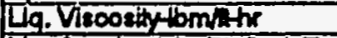 & 0.345 & 0.343 & 0.34 & 0.346 & 0.345 & 0.346 & 0.34 & 0.34 & 0.314 & 0.398 \\
\hline Dq. Conductivinghnraf & 0.0218 & 0.0217 & 0.0218 & 0.0218 & 0.0219 & 0.0219 & 0.0217 & 0.0218 & 0.0218 & 0.0219 \\
\hline Lig. PrandWNo. & 4.74 & 4.73 & 4.78 & 4.74 & 4.74 & 4.74 & 4.78 & 4.74 & 4.74 & 0.74 \\
\hline Inlet Fow Rade-GPM & 3.7 & & & & & & & & & \\
\hline Guld Uq.Fow Rale-CPM & 1.3 & & & & & & & & & \\
\hline Otalvap. Fow Raso-GPM & 1.9 & & & & & & & & & \\
\hline Intut Temperaturef & 174.6 & & & & & & & & & \\
\hline Ortetug. Tomportures & 773.8 & & & & & & & & & \\
\hline Dritet vep. Temperauref & 1727 & & & & & & & & & \\
\hline Went Fow RateGPM & & & & & & & & & & \\
\hline Wetrintertomp. & 1122 & & & & & & & & & \\
\hline Werer oulet Temp.f & 163.7 & & & & & & & & & \\
\hline Intat G bominrine & $67 \% 869$ & & Temp & 170.8 & Densiny & 77 & & & & \\
\hline Subcooling ff & 4.3 & & & & & & & & & \\
\hline Fow Obsenvion & & & & & & & & & & \\
\hline & ZONEI & ZONE? & ZONE3 & ZONE4 & ZONES & ZONE6 & ZONE? & ZONEB & ZONES & ZONEIO \\
\hline Hocl InputWatis(nol) & 860 & 868.8 & 872.4 & 874.8 & 858.8 & 856.7 & 493.2 & 82 & 85.7 & 79.7 \\
\hline FortLoss-Whits & 16.5 & 3.9 & 5.3 & 5.8 & 6.2 & 5.3 & 3.6 & 4.1 & 4.8 & 27.1 \\
\hline Hed flox-Bufint & 14908 & 15052 & 16122 & 15155 & 14852 & 14850 & 8519 & 1421 & T485 & 1382 \\
\hline Weiromp. BF & 1825 & 184.1 & 182.8 & 18:1 & 784.8 & 1824 & 181.8 & 777.1 & 177.7 & 1755 \\
\hline WeTTamp. I4F & 1829 & 1822 & 1842 & 18321 & 183.5 & 1824 & 181.9 & 377.1 & 178.1 & 176.1 \\
\hline Weitemp. 15f & 183.1 & 180.7 & 185.1 & 1827 & 1824 & 1828 & 180.4 & 177.1 & 178.1 & 176.1 \\
\hline WETTOmp. IfF & 83.4 & 183 & 181.7 & 1805 & 181.5 & 183.8 & 181 & 178.7 & 176.6 & 170.6 \\
\hline WN Tomp. HEF & 183.8 & 1832 & 1829 & 181.6 & 1821 & 183.8 & 180.9 & $17 \pi .1$ & 176.7 & 175.7 \\
\hline Introem tamp, $\mathrm{F}$ & 175.3 & 176.7 & 1756 & 175 & 174.8 & $17 \overline{4.8}$ & 175.9 & 176.4 & 175.4 & 174.4 \\
\hline Avq, Wall Temp, f & 183.1 & 183.1 & 182.7 & 182.1 & 1823 & 1828 & 181.2 & 177 & 177.4 & 175.8 \\
\hline Avq.Wali DT, F & 7.1 & 6.7 & 6.5 & 6.4 & 6.9 & 72 & 4.9 & 1.6 & 2 & 1.3 \\
\hline Romaining Subcooff & 0 & & & & & & & & & \\
\hline ExtQualy & 0 & 0.05 & 0.1 & 0.16 & 02 & 0.35 & 0.38 & 0.28 & 0.29 & 0.29 \\
\hline DAP. HBtuHhlaF & 2039.8 & 233,3 & 230.6 & 2353.3 & 2167 & 2058.6 & 17828 & 913.6 & 753 & 1078.6 \\
\hline COMPUIEDF & & & & & & & & & & \\
\hline Jisouk & 1457:21 & 1408.11 & 35727 & TE27.3 & T278.2 & 203.6 & 209.7 & 209.71 & 209.7 & 298.6 \\
\hline Sady (ado tube) & 2075.6 & 20023 & 2055.4 & 20928 & $20 \pi 1.4$ & 209.6 & 200.7 & 209.7 & 203.7 & Z0.6. \\
\hline Sadre & 1881.6 & 20012 & 2008.6 & 19502 & 19.58 .8 & 209.6 & 200.7 & 209.7 & 209.7 & 200.6 \\
\hline (RIA) & 125010 & 120009 & 115510 & 112089 & 10571 & $105 \pi 11$ & 1057II & 105711 & 1057il & $1007 \pi$ \\
\hline$(\mathbf{F})$ & 1.004 & 1.458 & 1.850 & 2.147 & 2.88 & 238 & 2.38 & 2.38 & 238 & 238 \\
\hline (F) & 214.1 & 310.6 & \%5.8 & 467.6 & 607.1 & E07.1 & 507.1 & 607.1 & 507.1 & 607.1 \\
\hline (Fioli) & 2074.8 & 2000 & 2000.8 & 2085.5 & 2061.2 & 20512 & 20512 & 2061.2 & 20512 & 20513 \\
\hline & & & & & & & & & & \\
\hline & $20 N 32$ & ZONE4 & ZONE6 & 20NE8 & ZONEIO & & & & & \\
\hline DP iquid Tomp.t & 832 & & & & & & & & & \\
\hline 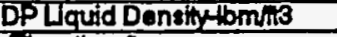 & 9252 & & & & & & & & & \\
\hline Elovation- & 2 & 4 & 6) & 8 & 9.875 & & & & & \\
\hline Whasured Dprald & 0.182 & 1.09 & 0.985 & 2.012 & 3252 & & & & & \\
\hline Zero Comoctions-psid & 0.059 & -0.054 & -0.761 & 0.111 & 0.077 & & & & & \\
\hline Comectod DPPsid & 0.148 & 1.144 & 1.727 & 2.491 & 3.185 & & & & & \\
\hline Ach:1DPh & 2.14 & 267 & 3.88 & 4.88 & 5.92 & & & & & \\
\hline Mesurred TPpip & 160.3 & & & & 3.6 & & & & & \\
\hline Nesured Owith & 283.42 & & & & & & & & & \\
\hline & & & & & & & & & & \\
\hline ComputedDPh & 1.97 & 3.48 & 4.8 & 6.4. & 7.96 & & & & & \\
\hline Fitciont & 0.1 & 0.3 & 0.58 & 0.83 & 1.04 & & & & & \\
\hline Acoulertion & 0.00 & 0.19 & 0.28 & 0.32 & 0.33 & & & & & \\
\hline Elovation & 1.78 & 3 & 4.04 & 5.29 & $6 . \overline{0} 8$ & & & & & \\
\hline & & & & & & & & & & \\
\hline
\end{tabular}




\begin{tabular}{|c|c|c|c|c|c|c|c|c|c|c|}
\hline Runt & 588 & & 1 & 1 & & I & $!$ & & | & \\
\hline \begin{tabular}{|l} 
Tost fluid \\
\end{tabular} & CAF 10 & & & I & & 1 & & & 1 & \\
\hline Dada: & 61183 & & & 1 & & $:$ & & & & \\
\hline Time: & $18: 44$ & & & 1 & & 1 & & & & \\
\hline Note & TABOREK & Analytical & dt & & & & & & & \\
\hline Fow Areatle & 0.00536 & & & & & 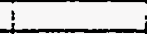 & & & & \\
\hline Hedi Fow A veat & 0.19695 & & & & & & & & & \\
\hline S*1 Prossurepsig & 152.2 & & & & & & & & & \\
\hline S-at. Temperature-finlet) & $175.1]$ & & & & & & & & & \\
\hline Lig. Densthybmin & 76.861 & 76.79 & 76.831 & 76.86 & 76.9 & 76.9 & 76.74 & 76.79 & 76.79 & 77.02 \\
\hline Lentent Hoad Btwhom & 25.811 & 25.78 & 25.79 & 25.81 & 25.84 & 25.84 & 25.72 & 25.78 & 25.78 & 25.88 \\
\hline 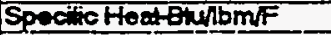 & 0.2991 & 0.299 & 0.291 & 0.299 & 0.29 & 0.299 & 0.299 & 0.299 & 0.299 & 0.299 \\
\hline Liq. Viscosity lomithe & 0.347 & 0.346 & 0.347 & 0.347 & 0.348 & 0.398 & 0.345 & 0.346 & 0.346 & 0.340 \\
\hline Liq. ConductivitstunfAF & 0.0219 & 0.0219 & 0.0279 & 0.0219 & 0.0219 & 0.0219 & 0.0218 & 0.0218 & 0.0219 & 0.02 \\
\hline Lig. Prand No. & 4.74 & 4.74 & 4.74 & 4.74 & 4.74 & 4.74 & 4.74 & 4.74 & 4.74 & 4.74 \\
\hline Indet Fow Rato-GPM & 3.7 & & & & & & & & & \\
\hline Outet Liq. Fow Rato-GPM & 1.3 & & & & & & & & & \\
\hline Oubet Vap. Flow Rate-GPM & 1.9 & & & & & & & & & \\
\hline Indat Temperaturef & 173.9 & & & & & & & & & \\
\hline Outet Líg. Temperatura & 1732 & & & & & & & & & \\
\hline Oufot Vap. TomperaturaF & 1723 & & & & & & & & & \\
\hline Wator Fow Rato-GPM & & & & & & & & & & \\
\hline Water hilet Temp.F & 9122 & & & & & & & & & \\
\hline Werer outtot Temp.f & 155.1 & & & & & & & & & \\
\hline Intat G bompinthe & 680872 & & ITemp & 169.8 & Dansity & 7.11 & & & & \\
\hline Subcooling ff & 1.2 & & & & & & & & & \\
\hline Fow Obsarvation & & & & & & & & & & \\
\hline & ZONE1 & ZONE2 2 & ZONE3 & ZONEA & ZONES & ZONE6 & ZONE7 & ZONE8 & ZONE9 & ZONETO \\
\hline Hed hput-Watt (net) & 857.1 & 859.7 & 869.8 & 871.6 & 850.6 & 860.1 & 494.2 & 82.1 & 85.8 & 79.9 \\
\hline Hoc Loss-Walls & 16.4 & 3.9 & 5.3 & 5.8 & 6.2 & 5.3 & 3.5 & 4 & 4.8 & 27 \\
\hline Hed Fox BtuhHrhe & 14857 & 15076 & 1507 & 15109 & 14918 & 14900 & 8567 & 1423 & 1487 & 1365 \\
\hline WA Tomp. \#3F & 181.8 & 183.4 & 181.9 & 181.6 & 184.5 & 181.9 & 181.4 & 176.4 & 177.3 & 175.1 \\
\hline WN Tomp. \$4F & 182.3 & 181.5 & 183.4 & 182.7 & 1829 & 181.9 & 781.8 & 776.4 & 777.3 & 776.6 \\
\hline Wal Tomp. $15 F$ & 1828 & 180.3 & 184.3 & 1822 & 182 & 182.4 & 180.2 & 176.4 & 17.3 & 175.7 \\
\hline Wa Tomp. IIF & 83.8 & 182.4 & 180.7 & 180.2 & 1812 & 182.8 & 180.4 & 178.4 & 178.4 & 175.2 \\
\hline Wall Tomp. 12F & 183.1 & 1825 & 181.6 & 181.1 & 181.6 & 182.8 & 180.4 & 176.4 & 176.4 & 175.4 \\
\hline Intruam Tomp.f & 174.6 & 174.8 & 174.7 & 174.6 & 174.4 & 174.4 & 175.1 & 174.8 & 174.8 & 973.8 \\
\hline Aug. Wall Tomp.f & 182.4 & 1825 & 181.9 & 181.7 & 181.9 & 1823 & 180.7 & 176.4 & 776.9 & 175.4 \\
\hline Avg. Wall DT.f & 7.1 & 6.9 & 6.5 & 6.4 & 6.9 & 72 & 5.3 & 1.4 & 2 & 1.4 \\
\hline Remaining Subcooff & 3 & & & & & & & & & \\
\hline Exa Quality & 0.04 & 0.00 & 0.14 & 0.19 & 0.24 & 0.29 & 0.31 & 0.32 & 0.32 & 0.33 \\
\hline EXP.HBtuHTHER & 2091.5 & 2194.8 & 2313 & 2353.6 & 2177.5 & 2077.6 & $1 \approx 0.9$ & 991.8 & 753.8 & 963.6 \\
\hline COMPUEDH & & & & & & & & & & \\
\hline Jallouk & 1398 & 7379.6 & उउ्3.4. & 200.9 & 209.8 & 209.8 & 200.9 & 209.9 & 200.9 & 209.8 \\
\hline Szady1(Oudo trbo) & 2053.9 & 2084 & 2083.2 & 209.8 & 200.8 & 200.8 & 203.9 & 209.9 & 200.8 & 209.8 \\
\hline sadty & 1967 & 1990.8 & 1987.9 & 203.8 & 200.8 & 209.8 & 200.8 & -209.9 & 200.8 & 200.8 \\
\hline (स्सिख) & 120087 & 120951 & 114254 & 114254 & 114254 & 114254 & 114254 & 114254 & $17 a 25 \mid$ & 11625 \\
\hline (F) & 1.378 & 1.761 & 2.069 & 2.069 & 2.059 & 2059 & 2.009 & 2.059 & 2.068 & 2.069 \\
\hline (FiLO) & 203.8 & 376.6 & 41.2 & 441.2 & 441.2 & 447.2 & 44.2 & 41.2 & 441.2 & 44.2 \\
\hline (HBOIL) & $20 \approx 2$ & 2079.9 & 2076.6 & 2076.6 & 2076.6 & 2075.6 & 2076.6 & 2076.6 & 2076.6 & 2078.6 \\
\hline & & & & & & & & & & \\
\hline & ZONE2 & ZONE4 & ZONE6 & ZONE8 & ZONE10 & & & & & \\
\hline DP lquid Temp.F & 832 & & & & & & & & & \\
\hline DPLiguid Denshy lbmil3 & 92.52 & & & & & & & & & \\
\hline Elovation-n & & (4) & 6 & 8 & 9.875 & & & & & \\
\hline Measured DPF्sidd & 0.207 & 1.087 & 0.982 & 2538 & 3.252 & & & & & \\
\hline Zero Corrections-psid & 0.039 & -0.054 & -0.761 & 0.111 & 0.07 & & & & & \\
\hline Corrocted DP pald & 0.165 & 9.141 & 1.723 & 2487 & 3.185 & & & & & \\
\hline Actual DPA & 2.09 & 287 & 3.99 & 4.98 & 5.91 & & & & & \\
\hline Mhasured TPPsig & 1522 & & & & 3.4 & & & & & \\
\hline Messured Overedth & 27821 & & & & & & & & & \\
\hline & & & & & & & & & & \\
\hline Computed DPA & 1.75 & 3.21 & 4.61 & 6.13 & 7.65 & & & & & \\
\hline Frtationt & 0.14 & 0.37 & 0.68 & 0.95 & 7.17 & & & & & \\
\hline Acoderationt & 0.18 & 0.22 & 0.32 & 0.36 & 0.37 & & & & & \\
\hline Elovation & 1.49 & 2.22 & 3.61 & 4.88 & 6.11 & & & & & \\
\hline & & & & & & & & & & \\
\hline
\end{tabular}




\begin{tabular}{|c|c|c|c|c|c|c|c|c|c|c|}
\hline Runt & 6841 & & I & I & & & & & & \\
\hline Toof Flid & CAF1O I & & 1 & I & & & & & & \\
\hline Date: & 61/93 & & 1 & L & & & & & & \\
\hline Timo: & $18: 44$ & & & & & & & & & \\
\hline Nole & TABOREA & Anatioa d & & & & & & & & \\
\hline Fow Ares-12 & 0.00336 & & $i$ & $L$ & & & & & & \\
\hline Heat Flow Areare & 0.19595 & & & 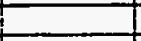 & & & & & & \\
\hline Sat. Pressure o ing & 148.4 & & t & 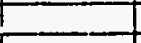 & & & & & & \\
\hline Sil temperaver finta) & $17 \overline{3}, 3$ & & & & & & & & & \\
\hline Lq. Denstytomin & 77.06 & 77.06 & 77.06 & 77.15 & 72. & 77.11 & $\pi$ & 77.00 & 77.00 & 77.3 \\
\hline Lenent Hostewh/tom & 25.97 & 25.97 & 25.97 & 26.04 & 28.07 & 28 & 25.91 & 25.97 & 25.97 & 26.17 \\
\hline Specifo HenteruibmiF & 0.298 & 0.298 & 0.298 & 0.298 & 0.298 & 0.298 & 0299 & 0.25 & 0.298 & 0.309 \\
\hline Lq. Visoosits bominht & 0.35 & 0.35 & 0.35 & 0.351 & 0.352 & 0.351 & 0.349 & 0.35 & 0.35 & 0.354 \\
\hline 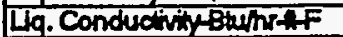 & 0.022 & 0.022 & 0.002 & 0.0221 & 0.0221 & 0.0221 & 0.022 & 0.002 & 0.022 & 0.0022 \\
\hline Liq. Prandino. & 4.741 & 4.74 & 4.74 & 4.74 & 4.74 & 4.74 & 4.74 & 4.74 & 4.74 & 4.75 \\
\hline Indet Fow RA-GPM & 4.5 & & & & & & &.$\quad 1$ & & \\
\hline Quidefla. Fow Rag-GPM & 1.2 & & & & & & & & & \\
\hline Oullat Vep. Fow Rato-GPM & 22 & & & & & & & & & \\
\hline Intal Tompereturef & 172.1 & & & & & & & & & \\
\hline Outet Liq. Tamporature & 172 & & & & & & & & & \\
\hline octat Vop. Tomperaturef & 770.9 & & & & & & & & & \\
\hline Wato FoW REDA-GPM & & & & & & & & & & \\
\hline Weter hiflet Tomp.F & 111.5 & & & & & & & & & \\
\hline Water outet Tamp.F & 152.9 & & & & & & & & & \\
\hline Intat G 16 mhnhic & 828816 & & Temp & 160.6 & Dencity & 77.18 & & & & \\
\hline Suboooling f & 0.6 & & & & & & & & & \\
\hline Fow Observetion & & & & & & & & & & \\
\hline & ZONE & ZONE2 & ZONES & ZONE & ZONE5 & ZONES6 & $20 N=7$ & ZOKES & ZOKEO & $\angle O N=10$ \\
\hline HoxlnoutWate(nol) & 871.3 & 877 & 874.3 & 876.2 & 855 & 855.1 & 2128 & 83.2 & 88.4 & 81.6 \\
\hline Heal Lose-We"ts & 16.1 & 3.8 & 5.1 & 5.6 & 5.9 & 5 & 2.8 & 3.8 & 4.7 & 25.8 \\
\hline 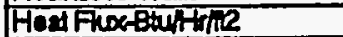 & 16100 & 15200 & 15155 & 15188 & 14821 & 7483 & 360 & 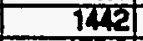 & 160 & 7474 \\
\hline Wall Tomp. B广 & 180.7 & 18्2द & 180.8 & 780.6 & 183.3 & T80.4 & 776.9 & T752 & 775.9 & 78.7 \\
\hline We Temp. Af & 1812 & 180.4 & 1824 & 181.7 & 181.8 & 180.6 & 78.8 & 1752 & $1 \% 5.2$ & 7773 \\
\hline Wa T'TOmp. & 181.2 & 179.2 & 180.3 & 181.8 & 181 & 181.1 & 178.6 & 175.1 & 776.2 & $77 \pi .6$ \\
\hline WaITomp. IIF & 827 & 181.4 & 179.8 & 178.2 & 180.8 & 187.6 & $7 \pi$ & 175.2 & T75.2. & 178.7 \\
\hline Wall Temp. I2F & 1821 & 181.4 & 180.4 & 180.8 & 180.6 & 181.8 & 176.9 & 1752 & 1762 & 174.1 \\
\hline Thatern Tomp.f & 178.7 & 173.7 & 173.7 & 173.8 & 178.1 & 173.5 & 174 & 178.7 & 173.7 & 1726 \\
\hline Avo. Wall Tomp fF & 181.3 & 181.4 & 1809 & 180.7 & 180.9 & 1800 & 778.8 & 1752 & 175.7 & T74.1. \\
\hline Ang. Wall DT.f & 6.9 & 7 & 6.5 & 6.7 & 7.2 & 6.8 & 27 & 1.4 & 2 & 1.5 \\
\hline Remining Subcootf & 3 & & & & & & & & & \\
\hline Exd Quality & 0.08 & 0.08 & 0.12 & 0.18 & 0.2 & 0.24 & 0.5 & 0.30 & 0.28 & 020 \\
\hline EXP.HBtuhnaf & 2182.8 & 2167.7 & 23442 & 2623 & 207.3 & 218.3 & \$376.4 & 108.4 & 759.4 & 8150 \\
\hline COMPUTEDH & & & & & & & & & & \\
\hline Jallouk & T3880.5 & Tउ्दा.4 & T275.7 & 245.5 & 275.5 & 245.5 & 245.6 & 2455 & 245.5 & 215. A \\
\hline sandy (Cudowbo) & 203.7 & 2078.5 & 2077.5 & 2355 & 215.5 & 245.6 & 246.6 & 245.5 & 245.5 & 245.4 \\
\hline seady2 & 1975.8 & 1984.8 & 1980.4 & 225.5 & 245.5 & 245.5 & 245.6 & 245.6 & 245.6 & 2154 \\
\hline (FLO) & 152253 & 146402 & 140000 & 940000 & 140000 & 140000 & 140000 & 140000 & 140000 & 140000 \\
\hline$(F)$ & 1.374 & 1.728 & 1.987 & 1.897 & 1.897 & 1.997 & 1.907 & 1.897 & 1.807 & 1.807 \\
\hline$(H \cup Q)$ & 3426 & -430.8 & 488 & 488 & 48 & 48 & 48 & 498 & 48 & 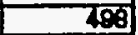 \\
\hline (HBOL) & 2008.6 & 20723 & 2087.9 & 2067.9 & 20579 & 2067.9 & 20579 & 2067.0 & 2057.9 & 20635 \\
\hline & & & & & & & & & & \\
\hline & ZONE2 & ZONE4 & ZONis6 & ZONES & ZONETO & & & & & \\
\hline 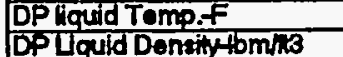 & 83.4 & & & & & & & & & \\
\hline DPLquid Densily fom/la & 9249 & & & & & & & & & \\
\hline Elevalion & & & 6 & 8 & 9.875 & & & & & \\
\hline Moavered DPPald & 0.188 & 0.884 & 0.689 & 2218 & 2824 & & & & & \\
\hline Zero Correctionspeid & 0.089 & -0.054 & -0.741 & 0.117 & $0.07 \pi$ & & & & & \\
\hline Corrected DP Psid & 0.147 & 0.838 & 1.485 & 2.182 & 2.747 & & & & & \\
\hline Actud DPA & 2.12 & 3.04 & 4.5 & 5.6 & 6.7 & & & & & \\
\hline Mroaured tP Prig & 148.4 & & & & 3.4 & & & & & \\
\hline Mescured Overall & 268.89 & & & & & & & & & \\
\hline & & & 508 & 6.7 & 8.38 & & & & & \\
\hline Compuled DPA & $\frac{1.87}{0.19}$ & $\frac{3.48}{0.5}$ & $\frac{5.03}{0.8}$ & $\frac{0.71}{1.2}$ & $\frac{0.00}{1.49}$ & & & & & \\
\hline Frolion-l & 0.19 & 0.5 & 0.81 & 0.48 & 0.49 & & & & & \\
\hline Acoolerction-a & 0.18 & 0.29 & 0.41 & 0.48 & & & & & & \\
\hline Elovitiont & 1.5 & 2.68 & 3.72 & 5.18 & 6.45 & & & & & \\
\hline & & & & & & & & & & \\
\hline
\end{tabular}




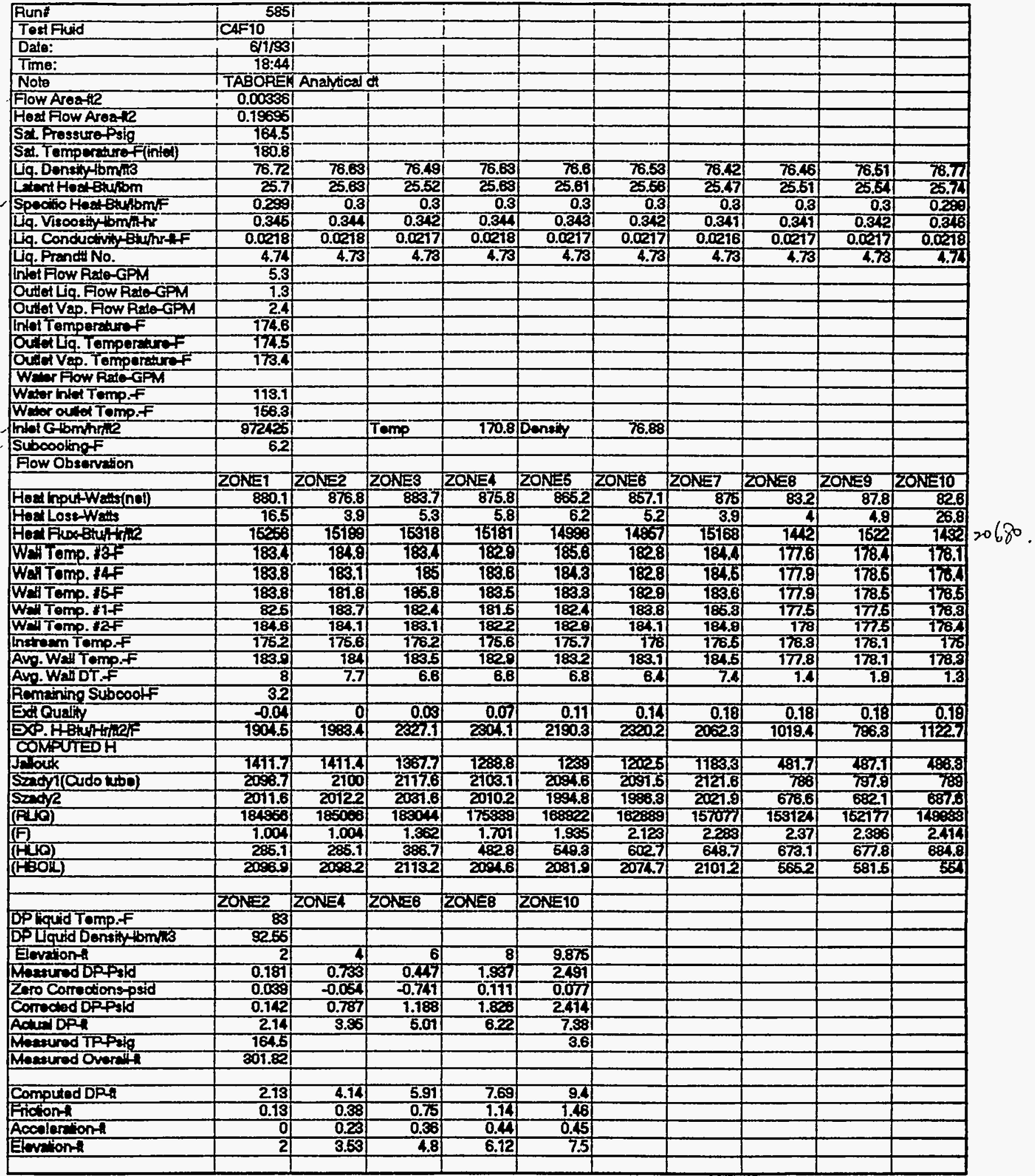




\begin{tabular}{|c|c|c|c|c|c|c|c|c|c|c|}
\hline Run: & 586 & I & T & 1 & & 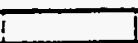 & I & I & & \\
\hline Tort Fuid & CAFTO & & & & & & & 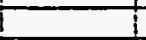 & & \\
\hline Doul: & बा/क्छा & & & & & & & I & & \\
\hline Time: & $18: 44$ & & & & & & & & & \\
\hline Note & TABOPEK & Analicalo & di & & & & & & & \\
\hline Flow Aseate & 0.003851 & & I & & & & & & & \\
\hline Hex Fow Arent? & 0.1865 & & & & & & & & & \\
\hline Set.Prossurepig & 156 & & & & & & & & & \\
\hline Set. Tomperatureffind) & $7 \%$ & & & & & & & & & \\
\hline La. Densitytomus & 76.88 & 76.95 & 77.0. & 77.18 & 77.22 & 7.15 & 76.97 & 7.06 & $\pi .60$ & 77.31 \\
\hline Letend Hestethrom & 25.83 & 25.88 & ङ.55 & 26.05 & 26.09 & 26.04 & 25.9 & 25.87 & 25.88 & 26.16 \\
\hline Spectio Hecishribmi & 0.289 & 0.299 & 0.209 & 0.238 & 0.298 & 0.288 & 0.299 & 0298 & 0.258 & 0.298 \\
\hline Le. Visoosintomithr & 0.347 & 0.348 & 0.35 & 0.352 & 0.352 & 0.351 & 0.349 & 0.35 & 0.35 & 0.354 \\
\hline 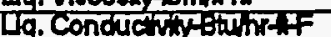 & 0.0028 & 0.022 & 0.002 & $0.002 \pi$ & $0.02 \pi$ & 0.0221 & 0.028 & 0.028 & 0.002 & 0.0222 \\
\hline LG. Prand Ko. & 4.74 & 4.74 & 4.74 & 4.74 & 4.74 & 4.74 & 4.74 & 4.74 & 4.74 & 4.70 \\
\hline InteIFOW RT-GPM & 5.3 & & & & & & & & & \\
\hline OdILlth. Flow $A=-G P M$ & 0.9 & & & & & & & & & \\
\hline OUIAV Vep. Flow PUA-GPM & 2.6 & & & & & & & & & \\
\hline Intel Tomoperterof $=$ & 174.2 & & & & & & & & & \\
\hline ButliLa. Tempermiref & 771.8 & & & & & & & & & \\
\hline Outatveo. Tempereturef & 170.6 & & & & & & & & & \\
\hline Wear flow Reto-gPM & & & & & & & & & & \\
\hline Wer hlot Temp. $F$ & 118 & & & & & & & & & \\
\hline Weter outer Tomp.F & 164.1 & & & & & & & & & \\
\hline Intot Glomintic & 980424 & & Tomp & 168 & Densing & 77.62 & & & & \\
\hline Subcoolingt & 2.7 & & & & & & & & & \\
\hline Fow Observen & & & & & & & & & & \\
\hline & ZONE1 & ZONEQ & ZONE3 & ZONE 4 & ZONES & ZONES & ZONE7 & ZORES & ZONES & $\angle O N=10$ \\
\hline 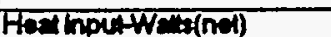 & 1181 & 1151.2 & 1151.8 & 105.8 & 71.7 & 71.8 & 725 & 74.4 & 81 & 708 \\
\hline Hon Lore-Wat & 16.8 & 4 & 5.4 & 5.2 & 6.7 & 4.1 & 28 & 4 & a.0 & 6.7 \\
\hline Host Fhoreterthe & 20125 & 1क\$ & 19067 & 1825 & 1249 & 1241 & 1571 & 1290 & Tक्म & 122 \\
\hline Wa Tomp. WF & 1822 & 185 & 183.1 & 175.4 & 174.1 & 174.8 & 175.3 & 174.6 & 775.6 & 7732 \\
\hline WeIT Tomp. IfF & 1828 & 1823 & 185.1 & 175.4 & 175 & 174.6. & 775.3 & 178 & 775.8 & 783.8 \\
\hline Waltomo $16+$ & 182.5 & 180.3 & 783.8 & 975.4 & 774.7 & 174.7 & 775.4 & T75.11 & T75.9 & 174 \\
\hline WeT Tomp. It & 80.7 & 183 & 182 & 175.5 & T74.6 & 175.1 & 775.2 & 174.7 & 174.8 & 173.6 \\
\hline WellTomp.IRt & T83.5 & 1829 & 182. & 175.6 & 174.4 & 174.4 & 775.2 & 175 & 174.8 & 773.7 \\
\hline Intrann tomp.t & 774.6 & 774.2 & 773.8 & 778.2 & 173 & 773.8 & 774.1 & 173.7 & 778.6 & 7726 \\
\hline Avp. Wai tomp. & T827 & 780.8 & 183.8 & T75.4 & 774.7 & 774.7 & 175.8 & 174.9 & 175.4 & 73.1 \\
\hline Avg.WEDT.F & 7.3 & 8.2 & 8.6 & 2.7 & 1.8 & 1.4 & 7.1 & दू & 1.7 & \\
\hline Praming siboodf & -1.8 & & & & & & & & & \\
\hline Ed Guthy & 0.01 & 0.08 & 0.11 & 0.17 & 0.11 & 0.12 & 0.12 & 0.12 & 0.78 & 0.18 \\
\hline 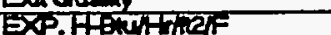 & 27506 & रम्बा & 23202 & 8723 & 787.8 & 806.7 & T118.8 & Tर्कामी & 806.5 & श्ट्रान \\
\hline COMPUTESH & & & & & & & & & & \\
\hline Jalouk & 1608.5 & 1536.2 & 30.8 & 280.8 & 20.8 & 2008 & 3000 & 30.8 & 3500 & 2308 \\
\hline Sadyil(Cudo ubo) & 2398.8 & 238 & 280.8 & 200.8 & 200.8 & 200.8 & 200.8 & 20.8 & 280.8 & 200.8 \\
\hline Sadve & 23524 & 2355.6 & 260.8 & 280.8 & 280.8 & 280.8 & 280.8 & 280.8 & 280.0 & 380.8 \\
\hline (RLSO) & 183650 & 177068 & 177056 & 77008 & $17 \pi 05$ & 17 isos & 177008 & $17 \%$ & 17503 & 17 LS8 \\
\hline & 1.28 & 1.56 & 1.59 & 1.59 & 1.59 & 1.59 & 1.59 & 1.59 & 1.59 & 7.50 \\
\hline (सDO) & STर & 453.7 & 253.7 & 453.7 & 253.7 & 753.7 & 253.7 & 463.7 & 258.7 & 153.7 \\
\hline (स्O) & 2303 & 2800.8 & $\mathbf{2 5 0 . 6}$ & 200.6 & 2380.6 & 2द्20.6 & 2300.6 & 2380.8 & 2380.6 & 2380.0 \\
\hline & & & & & & & & & & \\
\hline & $280 N=2$ & $\angle O N E 2$ & $20 N=6$ & ZONE8 & ZOTETO & & & & & \\
\hline DPRgudd Temp, F & 81.5 & & & & & & & & & \\
\hline DPLquid Dentity tomin & gem & & & & & & & & & \\
\hline Ereviont & रो & 7) & 6) & 8 & 9.875 & & & & & \\
\hline Whesured Dpsatd & $0.1 \%$ & 0.735 & 0.65 & टण्या & 2.617 & & & & & \\
\hline Źro Corrections-prd & 0.06 & -0.054 & -0.741 & 0.113 & 0.077 & & & & & \\
\hline Comodopty & 0.72 & 0.78 & 1.37 & 1.52 & 2.4ी & & & & & \\
\hline ACtuld DP & 217 & 3.30 & 4.68 & 8.02 & 7.3 & & & & & \\
\hline Mesured tprasla & 158 & & & & 3.6 & & & & & \\
\hline Whasured oventlit & 283.85 & & & & & & & & & \\
\hline & & & & & & & & & & \\
\hline Compund DP & टर्णा & 3.91 & 6.76 & 7.59 & 9.3 & & & & & \\
\hline Fraton-h & 0.22 & 0.64 & 0.84 & 1.18 & 1.4. & & & & & \\
\hline Acooteretions & 0.22 & 0.32 & 0.39 & 0.34 & 0.58 & & & & & \\
\hline Everiont & 1.6 & 3.04 & 468 & 6.11 & 7.64 & & & & & \\
\hline
\end{tabular}




\begin{tabular}{|c|c|c|c|c|c|c|c|c|c|c|}
\hline Runt & 687 & & & & & & & & & \\
\hline Test Find & CAF10 & & & & & & & & & \\
\hline Den: & $61 / 93$ & & & & & & & & & \\
\hline Time: & $23: 10$ & & & & & & & & & \\
\hline Not & Analyical & Pin pasat i & 3 assumed & & & & & & & \\
\hline Fow Areate & 0.00356 & & & & & & & & & \\
\hline Hod Fow Arest? & 0.19695 & & & & & & & & & \\
\hline Sat. Prossure Prig & 623 & & & & & & & & & \\
\hline 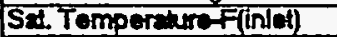 & 119 & & & & & & & & & \\
\hline Uq.Denstitiomis & 88.59 & 86.59 & 86.59 & 88.54 & 88.59 & 88.05 & 88.72 & 80.70 & 88.81 & 80.84 \\
\hline Lelont Hoct Etwibm & 32.80 & 32.88 & 32.88 & 32.83 & 32.83 & 329 & 32.85 & 32.28 & 33.01 & 93.00 \\
\hline 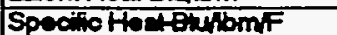 & 0.274 & 0.274 & 0.274 & 0.274 & 0.274 & 0.274 & 0.274 & 0.274 & 0.274 & 0.278 \\
\hline Uig. Visoositiomithx & 0.637 & 0.537 & 0.537 & 0.536 & 0.637 & 0.539 & 0.54 & 0.541 & 0.56 & 0.616 \\
\hline Lig. Conductivat Bthrstif & 0.028 & 0.028 & 0.028 & 0.028 & 0.028 & 0.0281 & 0.0281 & 0.0281 & 0.0282 & 0.0288 \\
\hline Liq. Prandi No. & 5.25 & 5.25 & 5.25 & 5.25 & 5.25 & 5.26 & 5.26 & 5.27 & 5.27 & 6.29 \\
\hline Intalfow Rato-GPM & 4.8 & & & & & & & & & \\
\hline Oriet Liq. Fow Rato-GPM & 1.1 & & & & & & & & & \\
\hline Outa Vap. Flow Rato-GPM & 25 & & & & & & & & & \\
\hline Inlet Temperaturef & 119.7 & & & & & & & & & \\
\hline Outollig. Tomporturef & T21.4 & & & & & & & & & \\
\hline Outet Vep. Tomperaturef & 118.1 & & & & & & & & & \\
\hline Water Fiow Rato-GM & & & & & & & & & & \\
\hline Weler inlat Temp.f & 77.2 & & & & & & & & & \\
\hline Wer outhol Temp.F & 107.8 & & & & & & & & & \\
\hline intalominne & $\$ 97086$ & & Tamp & $.118,2$ & Dentiy & 87.04 & & & & \\
\hline Subooolingt & 0.7 & & & & & & & & & \\
\hline Flow Observelion & & & & & & & & & & \\
\hline & ZONET & ZONE2 & ZONE3 & ZONE & ZONE5 & ZONE6 & ZONE? & ZONE8 & ZONES & ZONE10 \\
\hline HealmpltWathef & 289.7 & 286.7 & 27.1 & 2848 & 2862 & 288 & 287.7 & 287.1 & 285.1 & 2824 \\
\hline Hed Lose-Watis & 9.6 & 2.1 & 2.9 & 2.8 & 2.8 & 2 & 1.3 & 2 & 2.6 & 13.8 \\
\hline Hod Fix - Buthin? & 5022 & $45 \%$ & 4800 & 453 & 4981 & 4658 & 487 & $49 \pi$ & 492 & 7805 \\
\hline 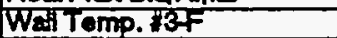 & 73.8 & 733.1 & 133 & T329 & T33.7 & 731.9 & उ3. & अ32 & 137.8 & 1205 \\
\hline Wa Tomp. I4F & 733.8 & 1326 & 733.4 & 133.3 & 133.8 & 131.8 & 132 & 131.3 & 131.7 & 720.4 \\
\hline WNTTEMP. I6F & 135.8 & 1324 & 134.4 & 133.4 & 132.8 & 1328 & 131.3 & 1350.8 & 130.8 & 726.8 \\
\hline Wall Temp. $\$ 1 \mathrm{~F}$ & $7 \pi .4$ & 138.4 & 1328 & 1328 & 132.5 & 183 & 132 का & 131.8 & 120.8 & T27.8 \\
\hline Well Tomo. 12F & 135.1 & 133.1 & 1328 & 1328 & 1326 & 133 & 1322 & 131.6 & 130 & 120 \\
\hline Intreen Tomp.f & 1228 & 1228 & 1220 & 1232 & 122.8 & 2225 & 122.1 & 121.8 & 127.5 & TE.7.7 \\
\hline Avq. Wa Tomp.F & 134.6 & 133 & 1328 & 139 & 132.8 & 1328 & 132 & 131.8 & 130.7 & Eद. \\
\hline Avo. WaIDT.F & 11.4 & 9.9 & 9.8 & 9.8 & 9.6 & 9.6 & 8.8 & 9.2 & 9 & 82 \\
\hline Romaining Subcooff F & -1.8 & & & & & & & & & \\
\hline Ext Cuatioy & 0.02 & 0.02 & 0.08 & 0.04 & 0.05 & 0.05 & 0.07 & 0.08 & 0.00 & 0.00 \\
\hline EXP. HBushther & 439.6 & 502 & 491 & 517 & 515.4 & 519.1 & 517.6 & 538.8 & 548.1 & 698.6 \\
\hline COMPUTEDH & & & & & & & & & & \\
\hline Jillok & 546.4 & 549.9 & 578.1 & 559.9 & 561.8 & 565.8 & 57T.4. & 570.1 & 580.3 & 368.8 \\
\hline Fad 1 (CUdo tho) & 882.6 & 885.6 & 8712 & 896.5 & 907.8 & $\mathbf{9 0 5 . 9}$ & 9टठ & 917.4 & 920.6 & 827.8 \\
\hline $\sin d x$ & 6525 & 868.1 & 648.7 & 6727 & 688.7 & 690.8 & 701.4 & $\pi i$ & 7202 & 7632 \\
\hline (F०) & 120567 & 119108 & 118047 & 117203 & 113912 & 114475 & 118015 & $111 \pi 0$ & 110385 & 106001 \\
\hline (F) & 1.324 & 1.568 & 1.706 & 1.824 & 1.988 & 2.04 & 2.189 & 2281 & 2310 & 2.407 \\
\hline (FUO) & 360.4 & 426.8 & 464.3 & 496.8 & 627 & E55.1 & 681.5 & 608.8 & E2.8 & 653.1 \\
\hline (सBOI) & 852 & 8512 & 831.5 & 841.8 & 837.8 & 8502 & 85.1 & 818.9 & 80.63 & 756 \\
\hline & & & & & & & & & & \\
\hline & ZONE2 & ZOEEA & ZONE6 & ZONEB & ZONET10 & & & & & \\
\hline DPliqud Temp.F & 35 & & & & & & & & & \\
\hline 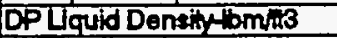 & $\mathbf{9 3 . 7 1}$ & & & & & & & & & \\
\hline Eevation & & 4 & & 8 & 9.875 & & & & & \\
\hline Messured DPF & $0.0 \%$ & 0.381 & 0.102 & 7.382 & 1.57 & & & & & \\
\hline Zaro Cortoctions-psid & 0.037 & 0.087 & -0.75 & 0.127 & 0.273 & & & & & \\
\hline Cormeciod DPPatd & 0.051 & 0.458 & 0.648 & 1.281 & 1297 & & & & & \\
\hline Acteal DPA & 2.07 & 3.56 & 5.39 & 6.58 & 8.5 & & & & & \\
\hline Measured TPPsig & 62.8 & & & & 8.7 & & & & & \\
\hline Mestured Overallh & 87.08 & & & & & & & & & \\
\hline & & & & & & & & & & \\
\hline Computed DRA & 205 & 3.98 & 5.78 & 7.64 & 9.38 & & & & & \\
\hline Frictont & 0.21 & 0.46 & 0.78 & T.1 & 1.46 & & & & & \\
\hline Acooterationt & 0.21 & 0.20 & 0.34 & 0.47 & 0.47 & & & & & \\
\hline Elevationt & 1.84 & 3.19 & 4.68 & 6.18 & 7.46 & & & & & \\
\hline & & & & & & & & & & \\
\hline
\end{tabular}




\begin{tabular}{|c|c|c|c|c|c|c|c|c|c|c|}
\hline Runt & 688 & & & & & & & & & 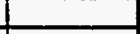 \\
\hline Tastfud & $C 4=10$ & & & & & & & & & \\
\hline Dets: & 62293 & & & & & & & & & \\
\hline Time: & $0: 16$ & & & & & & & & & \\
\hline Now & Anstycica & Pinspret is & assumed & & & & & & & \\
\hline Fow Areatic & 0.00356 & & & & & & & & & \\
\hline Host Flow Aroate & 0.18595 & & & & & & & & & \\
\hline Selpresturetig & 65.3 & & & & & & & & & \\
\hline 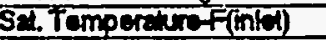 & 122.3 & & & & & & & & & \\
\hline La. Dentinthomiro & 88.28 & 88.28 & 88.17 & 88.14 & 86.14 & 88.22 & 86.23 & 86.81 & 88.91 & 8..4. \\
\hline Lent Hostoturom & 3268 & 3268 & 3268 & 3256 & 3266 & 32.61 & 32.2 & 32.68 & 32.68 & $327 \%$ \\
\hline Specilio Hostereliomf & 0.275 & 0.275 & 0.275 & 0.275 & 0.275 & 0.275 & 0.275 & 0.275 & 0.276 & 0.275 \\
\hline La. Visoosity & 0.629 & 0.528 & 0.526 & 0.525 & 0.525 & 0.627 & 0.528 & 0.63 & 0.63 & 0.538 \\
\hline 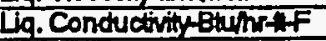 & 0.0278 & 0.0278 & 0.0078 & 0.027 & 0.027 & 0.0278 & 0.0278 & 0.0279 & 0.027 & 0.0279 \\
\hline Lig.Prandeno. & 522 & 52 & 5.21 & 521 & 521 & 52 & 522 & 5.23 & 5.28 & 5.2. \\
\hline Inteiflow Rato-GPM & 4.1 & & & & & & & & & \\
\hline Oulot Lq. Flow Rato-CPM & 1 & & & & & & & & & \\
\hline Oulet Vap. Flow RateGPM & 22 & & & & & & & & & \\
\hline Indet tomporaturef & 1227 & & & & & & & & & \\
\hline OutaLg. Tomperatiof & 124 & & & & & & & & & \\
\hline Ouflvap. Tomperturef & 718.5 & & & & & & & & & \\
\hline WHA Flow RaAO-GPM & & & & & & & & & & \\
\hline Wrich hlet Tomp.F & 79.2 & & & & & & & & & \\
\hline Welor oull Tomp.f & 110 & & & & & & & & & \\
\hline Intat Ginhme & 84652 & & Temp & 121.8 & Dentily & 86.42 & & & & \\
\hline Subooding F & -0.4 & & & & & & & & & \\
\hline Fow Obsenvation & & & & & & & & & & \\
\hline & ZONET & ZONE2 & ZONE 8 & ZONE 4 & ZONIE5 & ZONE6 & $20 N=7$ & ZONE8 & ZONE9 & $20 N=10$ \\
\hline Hen InputWereinel) & 200.6 & 287.5 & 285.1 & 205.2 & 286.5 & 288.7 & 287.7 & 289.2 & 2940 & 288.8 \\
\hline Hos'Lose-We"ts & 10.5 & 24 & 3.3 & 3.2 & 3.2 & 28 & 1.5 & 28 & 3 & 76 \\
\hline 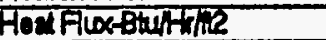 & 6037 & 4987 & किमाद & 5117 & 7668 & 455 & 4987 & 5018 & 51द2 & 5008 \\
\hline Weitromp. B子 & 335.1. & 135 & 735.1 & 134.8 & 135.8 & 134.7 & 13.6 & 133.8 & आ.8 & 7327 \\
\hline Whis Tomp. IfF & 135.8 & 33.7 & 135.6 & $1 \% 2$ & 185.4 & 134.6 & 135 & 134 & 137.6 & 1826 \\
\hline WEITamp. $16 F^{-5}$ & 136.2 & 134.8 & 138.6 & 135.7 & 134.7 & 13.7 & 734.6 & 138.8 & 733.8 & 1327 \\
\hline WeTtemp. I1f & $\pi$ & 185.1 & 134.1 & 134.5 & 184.4 & 135.8 & 185.2 & 134.8 & 138.2 & 180.8 \\
\hline Well Tomp. :2F & 136.8 & 13.1 & 135.1 & 134.7 & 7845 & 135.8 & 185.1 & 134.8 & 183.6 & 181.8 \\
\hline Intreen Temp.F & 124,8 & 124.8 & 125.5 & 125.7 & 726.7 & 1252 & I25.1 & 124.8 & 124.6 & 128.8 \\
\hline 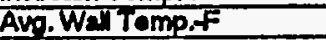 & 135.9 & 135 & 135 & 135.1 & 734.8 & 134.8 & 1340 & 134.1 & 133.6 & 1821 \\
\hline AYO.WNDT.F & 10.8 & 9.9 & 9.8 & 9.1 & 8.8 & 9.4. & 95 & 9.3 & g) & 8.1 \\
\hline Remainho Subcootf & -1.6 & & & & & & & & & \\
\hline Exd Qualty & 0.01 & $0 . \infty 2$ & 0.08 & 0.05 & 0.06 & 0.07 & 0.08 & 0.00 & 0.11 & 0.11 \\
\hline EXP.HBturar $=$ & 465.5 & 500.8 & 534.2 & 559.6 & 5527 & 527.5 & 5zo. & 538.8 & 5652 & 618.5 \\
\hline COMPUTEDH & & & & & & & & & & \\
\hline Jelouk & 556 & 549.5 & 551.8 & 568.8 & 550.5 & 563.1 & BSA. & 567.9 & 575.5 & 574.5 \\
\hline Sach I (Cudo wbo) & 889.8 & 890.1 & 894.2 & 914.4 & $\$ 0012$ & 907.8 & 914.5 & 918.8 & 9328 & 8283 \\
\hline $5 \sin \sqrt{2}$ & 65.1 & $\$ 58.7$ & 670.2 & 694.6 & 673 & 68.1 & 698.8 & 711 & 715.8 & $7 \pi .7$ \\
\hline (FUA) & 100848 & 10557 & 102002 & 10003 & 89837 & 9868 & 87252 & $85 \pi 2$ & 91810 & 8080 \\
\hline (F) & 1.300 & 1.563 & 1.712 & 1.852 & 1.97 & 2094 & 22 & 2.303 & 230 & 2488 \\
\hline (स) & 811.8 & 375 & 410 & 46.6 & 478.6 & 5012 & 526.5 & 551 & 573.8 & EE8.7 \\
\hline (सBOL) & 877 & 858 & 8815 & $8 \pi 2$ & 800.8 & 853.7 & 852.1 & 848 & 858.4 & 830 \\
\hline & & & & & & & & & & \\
\hline & $\angle O N=2$ & ZONE4 & $201: 56$ & ZONE8 & ZONEIO & & & & & \\
\hline DP Houdd Tomo.f & 74.2 & & & & & & & & & \\
\hline DPUlquid Denstytbmiz & 33.83 & & & & & & & & & \\
\hline Eloviont & & 4 & & 8 & 9.875 & & & & & \\
\hline Kontured DPPFid & 0.122 & 0.5 & 0.088 & 7.6 & 1.898 & & & & & \\
\hline Zoro Comotions-prd & 0.0 .87 & -0.087 & -0.75 & $0.12 \pi$ & 0.278 & & & & & \\
\hline Corrected DPFidd & 0.005 & 0.567 & 0.883 & 1.519 & 1.713 & & & & & \\
\hline AChual DPA & 2.08 & 3.4 & 5.12 & 0.15 & 7.88 & & & & & \\
\hline Meevered TPPh & 66.8 & & & & 3.7 & & & & & \\
\hline Mesecured over"in & T0128 & & & & & & & & & \\
\hline & & & & & & & & & & \\
\hline Computed DPA & 7.55 & 3.75 & 5.51 & 7.26 & 8.91 & & & & & \\
\hline Fifcton- & 0.16 & 0.34 & 0.57 & 0.84 & 1.12 & & & & & \\
\hline Aoculartion- & 0.15 & 0.21 & $02 \mathbf{3}$ & 0.31 & 0.36 & & & & & \\
\hline Elovetion- & 1.65 & 3.18 & 4.67 & 6.11 & 7.42 & & & & & \\
\hline & & & & & & & & & & \\
\hline
\end{tabular}




\begin{tabular}{|c|c|c|c|c|c|c|c|c|c|c|}
\hline Runt & 589 & & & & & & 1 & & & \\
\hline Toet Flid & CAF10 & & & & & & & & & \\
\hline Date: & 62193 & & & & & & & & & \\
\hline Time: & $0: 16$ & & & & & & & & & \\
\hline Not & Analyian & Pinupsad is & assumed & & & & & & & \\
\hline Flow Aroat? & 0.00338 & & & & & $\cdot$ & & & & \\
\hline Hosl Flow Ares-2 & 0.19595 & & & & & & & & & \\
\hline Sad Pressur Paig & 64.7 & & & & & & & & & \\
\hline Sal Tempere urof(inten) & 121 & & & & & & & & & \\
\hline Le. Densty-tom/13 & 86.81 & 86.28 & 86.28 & 86.8 & 86.8 & 88.35 & 86.41 & 86.48 & 86.48 & 8657 \\
\hline Lient Hoad-8hibm & 32.68 & 32.66 & 32.66 & 32.67 & 32.67 & 3269 & 32.74 & 32.75 & 327 & 3285 \\
\hline Specite thentelubmfr & 0.275 & 0.275 & 0.275 & 0.275 & 0.275 & 0.275 & 0.275 & 0.275 & 0.275 & 0.274 \\
\hline Liq. Visoosity tomphr & 0.68 & 0.528 & 0.529 & 0.528 & 0.629 & 0.63 & 0.682 & 0.638 & 0.634 & 0.683 \\
\hline Lig. Condectivit Bump of & 0.0279 & 0.0278 & 0.0278 & 0.0279 & 0.0279 & 0.0278 & 0.0270 & 0.0020 & 0.028 & 0.028 \\
\hline Lig. Prand No. & 5.23 & 5.22 & 5.22 & 523 & 528 & 5.23 & 5.24 & 521 & 5.24 & 5.25 \\
\hline In hat Flow Rato-GPM & 3.3 & & & & & & & & & \\
\hline Outh Liq. Fow Rate-GPM & 0.9 & & & & & & & & & \\
\hline Outet Vap. Fow Rato-GPM & 1.9 & & & & & & & & & \\
\hline Indel Temperaturef & 121.4 & & & & & & & & & \\
\hline 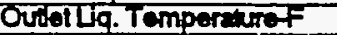 & T24.1 & & & & & & & & & \\
\hline Outelvap. Tempersteref & 177.5 & & & & & & & & & \\
\hline WHar FOW REN-GPM & & & & & & & & & & \\
\hline Weler nlet Temp.f & 78.8 & & & & & & & & & \\
\hline Werer outed Tomp.f & 107.6 & & & & & & & & & \\
\hline Indat Gibmhrhe & 684740 & & Tomp & 118.2 & Density & 86.87 & & & & \\
\hline Suboodingf & -0.4 & & & & & & & & & \\
\hline Flaw Obsenvion & & & & & & & & & & \\
\hline & ZONET & ZONES & ZONE3 & ZONE4 & ZONEE & ZONE6 & ZONET & ZONE8 & ZONEO & ZOTETO \\
\hline 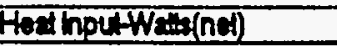 & 289.8 & 287.1 & 276 & 282.8 & 288.6 & 288.2 & 2872 & 288.7 & 285.8 & 288 \\
\hline Hod Loss-Walt & 10.3 & 2.4 & 32 & 3.2 & 3.1 & 23 & 1.5 & 22 & 2.9 & 15.8 \\
\hline 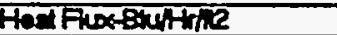 & 6028 & 497 & 4750 & 4902 & 4988 & 4961 & (575 & 5004 & 4916 & 4858 \\
\hline Wal Tomp. BF & 738.8 & 134.1 & 134 & 733.8 & 734.8 & 138.7 & 133.8 & 1327 & 1327 & 13.7. \\
\hline WE:TEMP. IfF & 734.3 & 133.8 & 134.4 & 13.8 & 134.8 & 138.7 & 133.8 & 1332 & 1332 & निक्ठ। \\
\hline WALTOMP. 155 & 134.7 & 733.5 & 135.8 & 1342 & 733.5 & 738.7 & 133.8 & 1327 & 1826 & 137.7 \\
\hline WDTomp. IIF & 68.8 & 134.8 & 1828 & 138.8 & 138.1 & 134.8 & 184.4 & 1382 & 181.9 & 129.7 \\
\hline WElTomp. IRF & 135.8 & 134.8 & 133.8 & 138.8 & 16 & 134.8 & 1315 & 138.6 & 131.8 & 120.7 \\
\hline Intreem Tomp.F & 124.6 & 124.8 & 124.8 & 124.7 & 124.र्ग & $12 \pi 5$ & 124 & 120.0 & 120.8 & 12 \\
\hline Ang. Wal Tomp.f & 134.7 & 134.7 & 933.8 & 133.8 & 133.5 & 138.0 & 134 & 135.1 & 1324 & 1आ.1 \\
\hline Avp. Wall DT.F & 9.8 & 9.1 & 8.8 & 8.9 & 8.8 & 9.1 & 9.8 & 8 & 8.8 & 7.8 \\
\hline Remaining Subcooff & -2 & & & & & & & & & \\
\hline Exit Quality & 0.02 & 0.08 & 0.04 & 0.05 & 0.07 & 0.08 & 0.09 & 0.11 & $0 . \overline{2}$ & 0.13 \\
\hline ExP.HBturtineh & 510.1 & 549.8 & 5422 & 550.6 & 581 & 548.6 & 509.8 & 558.8 & 574 & 6327 \\
\hline COMPUIESH & & & & & & & & & & \\
\hline Jellowk & 5502 & 5542 & 536.11 & 545.7 & 551.1 & 5528 & 655.3 & 5352 & 559.8 & 558 \\
\hline 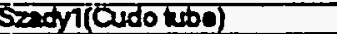 & 885.8 & 886.7 & 858.91 & 885 & 894 & 898 & 898.8 & 804.4 & 90208 & 904.0 \\
\hline $50 d \sqrt{2}$ & 688.1 & $\$ 51.7$ & 639.8 & 868.7 & 675.6 & 670 & 668.7 & 678.9 & 683.5 & 6500 \\
\hline (Pप्रि) & 8379 & 82659 & 81600 & 8046 & 79851 & 78128 & 78727 & 76509 & 74907 & 72875 \\
\hline (F) & 1.388 & 1.618 & 9.8 & 1.955 & 2.088 & 2.227 & 2.851 & 2.465 & 2575 & 2.683 \\
\hline (1) & 2702 & 328.8 & 363.5 & 39.7 & 4282 & 49.6 & 474.8 & 487.8 & 610.2 & 540.6. \\
\hline (1-3014) & 878.8 & 870.6 & 847.1 & 858 & 8612 & 856.8 & 8524 & 8512 & 800 & 8621 \\
\hline & ZONE2 & ZONE4 & ZONE6 & ZOKE8 & 20NET0 & & & & & \\
\hline Dptquid Temp.f & 728 & & & & & & & & & \\
\hline DPLquid Density tom/13 & 99.05 & & & & & & & & & \\
\hline Elovation-h & & & B & 8 & 9.875 & & & & & \\
\hline Whanured Dpprid & 0.725 & 0.708 & 0.100्टी & 2.052 & 2.38 & & & & & \\
\hline Zero Comscions-prid & 0.067 & -0.087 & -0.75 & 0.1रा & 0.273 & & & & & \\
\hline Correcled DFFyd & 0.108 & 0.7 & 1.152 & 1.931 & 2125 & & & & & \\
\hline Aoles DP & 1.80 & 3.00 & 4.6 & 5.48 & 7.18 & & & & & \\
\hline Hearured TPPsig & 64.7 & & & & 3.7 & & & & & \\
\hline Meapured Overall & 101.46 & & & & & & & & & \\
\hline & & & & & & & & & & \\
\hline Compulad bPa & 1.80 & $3.5 n$ & 5.21 & 6.88 & 8.4 & & & & & \\
\hline Frictiont & 0.11 & 0.25 & 0.42 & 0.68 & 0.89 & & & & & \\
\hline Acooleration-n & 0.11 & 0.16 & 0.18 & 0.24 & 0.28 & & & & & \\
\hline Envitiont & 1.द्2 & 3.14 & 4.58 & 6 & 738 & & & & & \\
\hline & & & & & & & & & & \\
\hline
\end{tabular}




\begin{tabular}{|c|c|c|c|c|c|c|c|c|c|c|}
\hline Run: & 5901 & & & & & & & & & \\
\hline Tatflid & CAFIO & & & & & & & & & \\
\hline Dets: & 6यक3 & & & & & & & & & \\
\hline Time: & 0.16 & & & & & & & & & \\
\hline Nob & Anatrical & Pin $=p_{\text {sad }}$ is & 5 assumed & & & & & & & \\
\hline Fow Areath & 0.00336 & & & & & & & & & \\
\hline Houflow Aroate? & 0.19695 & & & & & & & & & \\
\hline Sa. Pressurepsig & 63.7 & & & & & & & & & \\
\hline SA, Temperatrof (InL) & 1202 & & & & & & & & & \\
\hline 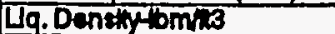 & 88.88 & 86.36 & 86.38 & 88.55 & 86.85 & 86.38 & 86.48 & 86.48 & 86.50 & 86.02 \\
\hline 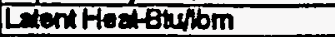 & 3272 & 327 & 327 & 327 & 327 & 32.72 & 327 & 3278 & 3284 & $\$ 2.88$ \\
\hline 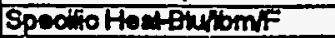 & 0.275 & 0.275 & 0.275 & 0.275 & 0.275 & 0.275 & 0.275 & 0.275 & 0.274 & 0.274 \\
\hline 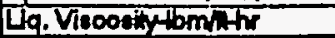 & 0.631 & 0.691 & 0.531 & 0.53 & 0.631 & 0.631 & 0.635 & 0.634 & 0.686 & 0.589 \\
\hline Lq. Conductivy BunthF & 0.0278 & 0.0278 & 0.0279 & 0.0279 & 0.0279 & 0.0279 & 0.080 & 0.028 & 0.038 & 0.0281 \\
\hline Wq. Prand" No. & 520 & 5.23 & 5.23 & 5.23 & 5.28 & 5.23 & 523 & 5.24 & 5.25 & 5.28 \\
\hline InGAFIOW R-GOGM & 2.5 & & & & & & & & & \\
\hline OLALLI. Fow RAC-GPM & 0.6 & & & & & & & & & \\
\hline Outel Vap. Flow HESGPM & 2 & & & & & & & & & \\
\hline Indat Tomperaturet & 120.7 & & & & & & & & & \\
\hline Otraldig. Temperturef & T23.7 & & & & & & & & & \\
\hline 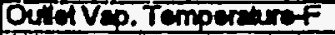 & 117.3 & & & & & & & & & \\
\hline Whar Flow RaW-GPM & & & & & & & & & & \\
\hline Water hifot Temp.f & 78.8 & & & & & & & & & \\
\hline Werrocllot Temp,f & 107.7 & & & & & & & & & \\
\hline 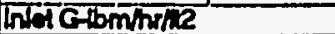 & 518000 & & Temp & 118.2 & Densiy & 87.04 & & & & \\
\hline Suboosilngf & -0.5 & & & & & & & & & \\
\hline Flow Obsorvation & & & & & & & & & & \\
\hline & ZONET & ZONE2 & ZONE3 & ZONE 4 & ZONE5 & ZONE6 & ZONET & ZONE8 & ZONES & ZON:S10 \\
\hline Healhort-We"indi) & 291.8 & 289 & 278.4 & 297 & 287.4 & 287.1 & 288.8 & 200 & 285.8 & 289.1 \\
\hline Herilose-WES & 102 & 2.8 & 32 & 3.1 & 3.1 & 2.3 & 1.5 & 22 & 2.8 & 75.8 \\
\hline Henfinoxthithice & 5058 & 5010 & 4828 & 5014 & 4986 & 4977 & 50000 & 5027 & क्ST & हणना \\
\hline WET Tomp. BF & 733.3 & Tख్హ. & Tद्ध一, & 133.3 & T34.T & Tस्3.1 & 738.8 & उद्ध一2 & 731.7 & 135.4 \\
\hline We'Tomp. IAF & 738.6 & 1320 & 735.8 & 133.8 & 130.8 & 1332 & 133.5 & 1324 & 1324 & 131.8 \\
\hline WCITCMp. $16 F$ & 134,2 & 1324 & 134.8 & 130.9 & 1328 & 1829 & 1329 & 182. & 1312 & किस \\
\hline Whitomp. IIF & 69 & 138.5 & 182 & 1920 & 1824 & 188.6 & 188.8 & 132.8 & 180.7 & 128.1 \\
\hline WeT Tomp. मRF & 18.7. & 138.4 & 1828 & 13 & 1328 & 188.5 & 133.7 & 138.1 & 950.7 & 150.1 \\
\hline hofreen Temp.f & 124.2 & 121.8 & 124.8 & 124.4 & 124.4 & 124,2 & 123.7 & 128.8 & 123.1 & 1227 \\
\hline Avg. Wall Temp ff & 134 & 1332 & 133 & 183.9 & 1820 & 1332 & 133.5 & 1325 & 131.3 & 1300 \\
\hline AY. WIIDT.F & 9.5 & 8.7 & 8.5 & 8.6 & 8.2 & 8.8 & 8.5 & 8.7 & 8 & 7.7 \\
\hline Remaining Suboooff & -26 & & & & & & & & & \\
\hline Ext Quality & 0.02 & 0.04 & 0.06 & 0.07 & 0.09 & 0.11 & 0.12 & 0.14 & 0.16 & 0.18 \\
\hline 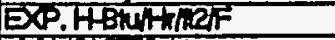 & 531.2 & 575.8 & $5 \% 0.6$ & 583.4 & 603.8 & 568.8 & 525.8 & $58 \pi$ & 617.6 & 651.4 \\
\hline COM:PUIEOH & & & & & & & & & & \\
\hline Jefolk & 5612 & 548.7 & 5427 & 556.6 & 547.4 & 547.4 & 549 & 55121 & 548.2 & 5521 \\
\hline Szedyil(cudo wob) & 899.8 & 883 & 860 & 890.0 & 887.9 & 889 & 89i. & 895.8 & 890.1 & 896.4 \\
\hline Seath & 663.9 & 845.2 & 605.7 & $\$ 8.1$ & 657.8 & 673.6 & 674.7 & 677.2 & 670.6 & 600.8 \\
\hline (F्थि) & 63218 & 62011 & 60828 & 69888 & 58778 & 57586 & 56204 & 55120 & 63876 & 52560 \\
\hline (F) & 1.401 & 1.782 & 1.847 & 2.181 & 2208 & 245 & 2586 & 27 & 2.867 & 2.805 \\
\hline$(1+10)$ & 225.8 & 280.4 & 315.2 & 345 & 372 & 396.6 & 418.8 & 44.4 & 4623 & 482.7 \\
\hline (HBOL) & 884.4 & 873.5 & 855 & 873.8 & 865.6 & 861.9 & 859.7 & 858.6 & 846.4 & 8472 \\
\hline & & & & & & & & & & \\
\hline & ZONE2 & ZONE4 & ZONE: & ZONE8 & ZONETO & & & & & \\
\hline DPhoud Tomp.F & 72.9 & & & & & & & & & \\
\hline DP Llquid Densint"om/3 & 9.01 & & & & & & & & & \\
\hline Elovionst & 2 & 4 & 6 & 8 & 9.875 & & & & & \\
\hline Mosaured DPPमd & 0.176 & $0.87 \pi$ & 0.686 & 2.385 & 2883 & & & & & \\
\hline 20"o Comedons-petd & 0.087 & -0.067 & -0.75 & 0.121 & 0.278 & & & & & \\
\hline Comeand DPpild & 0.189 & 0.938 & 1.416 & 2274 & 250 & & & & & \\
\hline Actur DPA & 1.84 & 27 & 4.10 & 4.8 & 6.46 & & & & & \\
\hline Mhaurred TPPidg & 6.7 & & & & 3.7 & & & & & \\
\hline Mecoured Overallh & 99.75 & & & & & & & & & \\
\hline & & & & & & & & & & \\
\hline Computed DPA & 1.68 & 3.28 & 4.85 & 6.38 & 7.82 & & & & & \\
\hline Ffictions & 0.07 & 0.12 & 0.3 & 0.4 & 0.8 & & & & & \\
\hline Aocolerctiond & 0.07 & 0.11 & 0.14 & 0.17 & 0.2 & & & & & \\
\hline Elovionth & 1.58 & 3.01 & 4.41 & 5.78 & 7.0. & & & & & \\
\hline & & & & & & & & & & \\
\hline
\end{tabular}




\begin{tabular}{|c|c|c|c|c|c|c|c|c|c|c|}
\hline Runt & 591 & & & & & & & & & \\
\hline Torf Fluid & CAF10 & & & & & & & & & \\
\hline Dalo: & 6293 & & & & & & & & & \\
\hline Time: & $0: 16$ & & & & & & & & & \\
\hline Note & Anatylical & Pin $=p_{\text {sed }}$ is & 5 assumed & & & & & & & \\
\hline Fow Areat? & 0.00336 & & & & & & & & & \\
\hline Had Flow Arante & 0.19395 & & & & & & & & & \\
\hline Sat. Presture Psig & 64.9 & & & & & & & & & \\
\hline Sat. Tomperedure findel) & 121.2 & & & & & & & & & \\
\hline LG.Densistoming & 86.23 & 86.15 & 88.251 & 8.23 & 86.25 & 86.28 & 88.3 & 86.85 & 88.38 & 80.6 \\
\hline Litent Hed Ethiom & 32.62 & 32.57 & 32.68 & 32.02 & 32.63 & 32.60 & 32.87 & 32.7 & 32.72 & 32.75 \\
\hline 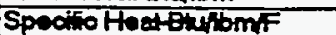 & 0.275 & 0.275 & 0.275 & 0.275 & 0275 & 0.275 & 0.275 & 0.276 & 0.275 & 0.275 \\
\hline Lig. Visooshthem & 0.628 & 0.626 & 0.638 & 0.528 & 0.528 & 0.620 & 0.620 & 0.631 & 0.631 & 0.639 \\
\hline Lig. Conductivity the th & 0.0278 & 0.0278 & 0.0278 & 0.0278 & 0.0278 & 0.0278 & 0.0279 & 0.0270 & 0.0279 & 0.025 \\
\hline Lig. Prend No. & 5.22 & 5.21 & 5.22 & 5.22 & 5.22 & 5.22 & 5.23 & 5.23 & 5.28 & 5.20 \\
\hline Intelflow Rato-GPM & 1.7 & & & & & & & & & \\
\hline Oúlelig. Fow Rato-GPM & 0.4 & & & & & & & & & \\
\hline Oubet Vap. Flow Rate-GPM & 1.2 & & & & & & & & & \\
\hline Inde Tempereduref & 121.3 & & & & & & & & & \\
\hline OCAllLg. Tamperaturef & 124.9 & & & & & & & & & \\
\hline OWet Vep Tomperdurof & 118.3 & & & & & & & & & \\
\hline Whr Fow Rato-GPM & & & & & & & & & & \\
\hline Wader intet Temp.f & 79.5 & & & & & & & & & \\
\hline Weref oullet Temp.f & 108.7 & & & & & & & & & \\
\hline nhat Gibmhnti2 & 363888 & & Tamp & 117.8 & Donsity & 87.11 & & & & \\
\hline Subooolingf & -0.1 & & & & & & & & & \\
\hline Flow Observation & & & & & & & & & & \\
\hline & ZONE1 & ZONE2 & ZONE3 & ZONE4 & ZONE5 & ZONE6 & ZONET & ZONEB & ZONES & $\angle O N=10$ \\
\hline Hoat inputwata(net) & 2926 & 289.7 & 279.3 & 289.1 & 288.8 & 288.7 & 289.6 & 291.1 & $=287.7$ & 200.4 \\
\hline Haclloss-We'ts & 102 & 2.3 & 3.1 & 3.11 & 3 & 22 & 1.5 & 22 & 2.8 & 15.8 \\
\hline 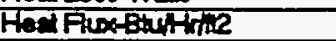 & E072 & 502 & 4841 & 5017 & 5003 & 5004 & 5020 & 5046 & 4087 & 3003 \\
\hline We Temp. B & 733.8 & 733.5 & 733.5 & 733.4 & 734.3 & 733.6 & 34.1 & 132.3 & 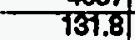 & $T$ स्ट्ट \\
\hline WE Temp. 14F & 135.5 & 133.1 & 73 & 138.4 & 134.1 & 133.7 & 134 & 13 & 13523 & 737.7 \\
\hline WAITAmp.16F & 134.7 & 132.6 & 135.6 & 13.1 & 133 & 1382 & 335.5 & 1323 & 137.4 & 735.2 \\
\hline WE Tomp. IIF & 6 & 183.6 & 1324 & 132.8 & 1827 & 138.8 & 1352 & 189.6 & 130.8 & T20.0 \\
\hline WNT Tamp. I2F & 135 & 133.8 & 132.8 & 1320 & 1324 & 138.7 & 1342 & 133.8 & 130.0 & 180.7 \\
\hline Instresm Tomp.f & 125.1 & 25.6 & 125 & 125.1 & 125 & 124.8 & 124.7 & 124.4 & 1242 & 120.0 \\
\hline Avg. Wall Tomp.F & 134.6 & 133.5 & 1332 & 733.4 & 733.1 & 133.6 & 13 & 133 & 131.4 & 131 \\
\hline Avo. WaY DT.F & 8.2 & 7.7 & 8 & 8.1 & 7.8 & 8.6 & 9.1 & 8.4 & 7 & 6.9. \\
\hline Remaining Suboooff & 3.2 & & & & & & & & & \\
\hline Ext Quality & 0.08 & 0.05 & 0.08 & 0.1 & 0.18 & 0.15 & 0.18 & 0.2 & 0.23 & 0.28 \\
\hline EXP.HBLuthar & 553.1 & 65.5.5 & 608.5 & 618.8 & 639.9 & 583.7 & 553.3 & 602.7 & $7 \pi$ & 728.2 \\
\hline COMPUIEDH & & & & & & & & & & \\
\hline Jalouk & 581.2 & 556.8 & 547.3 & 550 & 555.1 & 5523 & 554.6 & 356.8 & 547 & 549.1 \\
\hline Szadyil(addotube) & 903.6 & 904.1 & 870.4 & 887.4 & 887.8 & 888.8 & 890.8 & 898.3 & 888.7 & 693.6 \\
\hline sady? & 689.8 & 687 & 648.8 & 649.1 & 651.8 & 654.8 & 658.6 & 6827 & 674.5 & 677.7 \\
\hline (स्थि) & 43219 & 42203 & 40910 & 39874 & 38729 & 37058 & 3315 & अ516 & 34054 & 32872 \\
\hline (i) & 1.449 & 1.84 & 2.127 & 2861 & 257 & 2767 & 285 & 3.115 & 3.274 & 3.458 \\
\hline (H) & 172.6 & 219.8 & 253.4 & 281.8 & 306.4 & 329.4 & 350.5 & 370.7 & 389.5 & 407.6 \\
\hline (18O4) & 901.5 & 898.8 & 853.2 & 877.8 & 875.5 & 872.8 & 8723 & 871.5 & 863.5 & 864.4 \\
\hline & & & & & & & & & & \\
\hline & ZONE? & ZONE4 & ZONE6 & ZONE8 & ZONE1O & & & & & \\
\hline DP Fquid Tomp.f & 72.7 & & & & & & & & & \\
\hline DPLiquid Density 1 bm/3 & 94.04 & & & & & & & & & \\
\hline Eievations & & & (6) & 8 & 9.875 & & & & & \\
\hline Whasured DP्म sid & 0.120 & 7.147 & 1.017 & 2.050 & 3.378 & & & & & \\
\hline zero Corroctions-pid & 0.037 & -0.037 & -0.75 & 0.127 & 0.273 & & & & & \\
\hline Comeand DPpsid & 0.002 & 1.214 & 1.707 & 2538 & 3.1 & & & & & \\
\hline Achel DPh & 2.02 & $2 \%$ & 3.58 & 4.48 & 5.58 & & & & & \\
\hline Moaured TPPsig & 64.8 & & & & 3.7 & & & & & \\
\hline Measured Overall' & 101.97 & & & & & & & & & \\
\hline & & & & & & & & & & \\
\hline Computad DP: & 7.48 & 278 & 3.9 & 5.02 & 6.05 & & & & & \\
\hline Frictiont & 0.05 & 0.12 & 0.22 & 0.34 & 0.47 & & & & & \\
\hline Accalerationth & 0.04 & 0.08 & 0.08 & 0.1 & 0.13 & & & & & \\
\hline Elovionth & 1.39 & 256 & 3.61 & 4.58 & 5.45 & & & & & \\
\hline & & & & & & & & & & \\
\hline
\end{tabular}




\begin{tabular}{|c|c|c|c|c|c|c|c|c|c|c|}
\hline Punt & 582 & & & & & & & & & \\
\hline Tostfivid & C\&F10 & & & & & & & & & \\
\hline Dena: & 6238 & & & & & & & & & \\
\hline Time: & 0.18 & & & & & & & & & \\
\hline Now & Anaticas & Pinspesati & astumed & & & & & & & \\
\hline Fow ATEAR & 0.00538 & & & & & & & & & \\
\hline Hest Flow Arente & $0.18 \% 5$ & & & & & & & & & \\
\hline SE. PTesture pilg & 6.4 & & & & & & & & & \\
\hline 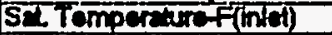 & 121.8 & & & & & & & & & \\
\hline Hg. Denothem/3 & 88.09 & 88.01 & 80.1 & 88.02 & 88.04 & 80.00 & 88.00 & 80.10 & 80.16 & 0032 \\
\hline 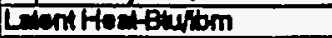 & $\$ 238$ & 3247 & 8264 & 32.48 & 82.49 & 325 & 5268 & 3267 & 3257 & s2.61 \\
\hline Specinc Hosteruriom, & 0.275 & 0.276 & 0275 & 0.276 & 0.276 & 0.275 & 0.275 & 0.275 & 0.275 & 0.275 \\
\hline Lig. Viocotifytom/ hr & 0.524 & 0.622 & 0.524 & 0.522 & 0.528 & 0.62 & 0.524 & $0.5 \overline{28}$ & 0.526 & 0.527 \\
\hline Lla. Conductivit Buthrfof & 0.027 & 0.027 & 0.027 & 0.027 & $0.02 \pi$ & 0.0277 & 0.027 & 0.027 & 0.028 & 0.0278 \\
\hline Liq. PrandtiNo. & 5.21 & 5.2 & 5.21 & 52 & 52 & 5.2 & 5.21 & 5.21 & 5.21 & 5.22 \\
\hline In R Fow RE-GPM & 0.9 & & & & & & & & & \\
\hline 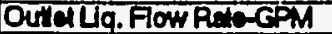 & 0.4 & & & & & & & & & \\
\hline Oulet Vep. Flow PL-GPM & 0.8 & & & & & & & & & \\
\hline Intat tompercheref & 122 & & & & & & & & & \\
\hline Ot'AlLg. Temperature & 738.6 & & & & & & & & & \\
\hline 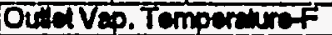 & 120.1 & & & & & & & & & \\
\hline Whar Fow R WAM & & & & & & & & & & \\
\hline Whar hinet Temp.f & 81.8 & & & & & & & & & \\
\hline Werer ocll Temp.f & 110.8 & & & & & & & & & \\
\hline Fnd Glbm/hnt2 & 788017 & & Temp & 115.2 & Dentidy & 87.54 & & & & \\
\hline Subooolingf & -0.4 & & & & & & & & & \\
\hline Flow Obsenvetion & & & & & & & & & & \\
\hline & ZONE1 & ZONE2 & ZONE3 & ZONEA & ZONES & ZONE6 & ZONE & $20 N=8$ & ZONE9 & $20 N E 10$ \\
\hline Haclinoutwate(nol) & 29.8 & 291.6 & 2802 & 28.8 & 288.8 & 288.8 & 200.8 & 291.4 & 27.8 & 285.4 \\
\hline Honlose-Weas & 10.4 & 2.3 & 3.2 & 3.2 & 3.11 & 2.3 & 1.5 & 23 & 2.8 & 16.8 \\
\hline 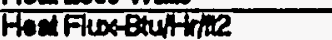 & हार्ण & 500 & 4857 & 4915 & 5008 & 5000 & 50सा & 505 & 4807 & 494 \\
\hline We Tromp. B & 134.1 & 1352 & 133.3 & 723.8 & T34.8 & अमद्या & 13.2 & 1328 & Iस्ट्ट & 1328 \\
\hline WCITTemp. IfF & 73्यम, & 133 & 134 & 133.8 & 134.4 & 1342 & 734.7 & 1332 & 7327 & 1822 \\
\hline WE TEmp. $16 \mathrm{~F}$ & 135.1 & 1323 & 135.6 & 134.1 & 130.5 & 138.8 & 738.8 & T328 & 137.7 & 1822 \\
\hline We'Temp. HTF & 68 & 188.5 & 182.1 & 189.2 & 138.1 & 139.4 & 134.6 & 138.7 & T80.8 & 180.0 \\
\hline WeITemp. WL & $135 \mathrm{~A}$ & 138.8 & 1827 & 139.8 & 18 & 13.8 & 134.7 & 1348 & 131.5 & 181.8 \\
\hline Introem Temp. f $^{\prime}$ & 125 & 126.5 & 125.6 & 126.4 & 120.8 & 1282 & 126 & 126.6 & 125.8 & 1252 \\
\hline Avo. Wall Tomp.f & 734.8 & 133.3 & 133 & 133.8 & 133.5 & 7342 & 134.4 & 133.4 & 131.8 & T3.8 \\
\hline AU. WNDT.F & 8.5 & 6.6 & 6.8 & 7.1 & 7 & 7.7 & 8.2 & 7.6 & 5.9 & 8.4 \\
\hline Remaning Subcooff & -62 & & & & & & & & & \\
\hline Ext Qually & 0.05 & 0.1 & 0.15 & 0.19 & 0.24 & 0.28 & 0.34 & 0.30 & 0.43 & 0.48 \\
\hline EXP.HAtur Hrile, & 598.8 & 708.8 & 703.4 & 69 & 718.1 & 645.8 & 618.8 & 658.9 & 803.9 & 778.8 \\
\hline COMPUTEDH & & & & & & & & & & \\
\hline Jaloed & उद्या & 581.8 & 568.6 & $5 \% 0.8$ & $5 \% 0.5$ & 568.7 & 565.5 & 5627 & 530.8 & 576.8 \\
\hline Szady 1 (Oudo tho) & 910.8 & 910.1 & 874.3 & 885.5 & 897.8 & 8916 & 8912 & 893.8 & 873.2 & 887.7 \\
\hline $501+2$ & 678.2 & 671.2 & 658.8 & 668.7 & 660 & 668.7 & 67.1 & 655.7 & 638.4 & 800.1 \\
\hline (R्य) & 2058 & 21760 & 20518 & 19517 & 1887 & TदES & 18077 & Tद885 & 13700 & 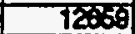 \\
\hline (6 & 1.667 & 2.28 & 2681 & 2054 & 3.50 & 3.5्रा & 3.775 & 4.0ना & 4.21 & 4.468 \\
\hline (FU) & T18.6 & 760.7 & 189.4 & टाटठ & 23 & 253.5 & टं1.8 & 289.8 & 305 & 3200 \\
\hline$\left(\mathrm{F} 3 \mathrm{O}_{4}\right)$ & 9006 & 008.6 & 871.3 & 8 & 8860 & 896 & 8.8 & 883.7 & 850.7 & 875 \\
\hline & ZONES & ZOKS4 & $20 N=6$ & $20 N=8$ & $\angle O N=10$ & & & & & \\
\hline DPIqud Temp.f & $\pi$ & & & & & & & & & \\
\hline DPIIquid Dentit bomik8 & 94.14 & & & & & & & . & & \\
\hline Eiention- & 2) & & B & 8 & 9.875 & & & & & \\
\hline Meatured DPFold & $0.10 \mathrm{~T}$ & 7.480 & $7.38 \%$ & 3.049 & 3.867 & & & & & \\
\hline Zoro Contectione-padd & 0.037 & -0.067 & -0.75 & 0.121 & 0.278 & & & & & \\
\hline Cornead DPPAd & 0.064 & 7.565 & 2.169 & 2.968 & 3.654 & & & & & \\
\hline Actud DPA & 2.08 & 1.75 & 2.80 & 8.84 & 4.61 & & & & & \\
\hline Measured TPpotg & 65.4 & & & & 3.7 & & & & & \\
\hline Menered Overallt & 108.05 & & & & & & & & & \\
\hline & & & & & & & & & & \\
\hline Complad DPA & 12 & 291 & 2क्2 & 3.65 & 439 & & & & & \\
\hline Frotion- & 0.02 & 0.06 & 0.11 & 0.18 & 0.25 & & & & & \\
\hline Acoeterationth & 0.02 & 0.08 & 0.04 & 0.00 & 0.08 & & & & & \\
\hline Evverion-l & 1.18 & 208 & 2.76 & 3.41 & 3.86 & & & & & \\
\hline & & & & & & & & & & \\
\hline
\end{tabular}




\begin{tabular}{|c|c|c|c|c|c|c|c|c|c|c|}
\hline Run: & 5931 & & & & 1 & & & & & \\
\hline Tesffivid & CAF10 & & & & & & & & & \\
\hline Dats: & 62233 & & & & & & & & & \\
\hline Time: & $5: 13$ & & & & & & & & & \\
\hline Noto & Antylicad & Pin psad is & assumed & & & & & & & \\
\hline How Areate & 0.00336 & & & & & & & & & \\
\hline Hoal Fow Areat? & 0.1955 & & & & & & & & & \\
\hline St Presture Pirg & 62.7 & & & & & & & & & \\
\hline Sed Tomporauref(iniei) & 119.8 & & & & & & & & & \\
\hline Wg. Densthem $6 \mathrm{~m} / \mathrm{s}$ & 86.15 & 86.02 & 88.08 & 86.02 & 86.04 & 86.06 & 86.03 & 86.1 & 86.12 & 80.22 \\
\hline 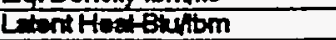 & 32.57 & 32.48 & 32.51 & 32.48 & 32.49 & 32.5 & 32.5 & 32.54 & 32.55 & 32.61 \\
\hline Specitio Hort Bullomif & 0.275 & 0.276 & 0.275 & 0.276 & 0.276 & 0.275 & 0.275 & 0.276 & 0.275 & 0.275 \\
\hline Lig. Visoosithomitr & 0.526 & 0.622 & 0.628 & 0.622 & 0.523 & 0.623 & 0.520 & 0.624 & 0.625 & 0.621 \\
\hline Lig. Conductivis Bupsth & 0.0278 & 0.0277 & 0.027 & 0.027 & 0.027 & 0.027 & 0.0277 & 0.0277 & 0.0277 & $0.0 \mathrm{c7}$ \\
\hline Liq. Prand No. & 5.21 & 5.2 & 5.2 & 5.2 & 5.2 & 5.2 & 5.2 & 5.21 & 5.21 & 522 \\
\hline In A Fow Rato-GPM & 1 & & & & & & & & & \\
\hline Outlat Liq. Fow Rate-GPM & 0.9 & & & & & & & & & \\
\hline Oullot Vap. Fow Rato-GPM & 0.6 & & & & & & & & & \\
\hline Ind Temporaturef & 120.2 & & & & & & & & & \\
\hline Ov'Alig. Tomperturef & 127.1 & & & & & & & & & \\
\hline Outeiveo. Tempereluref & 1224 & & & & & & & & & \\
\hline Wat Fow Rat-GPM & & & & & & & & & & \\
\hline Water klet Temp.f & 78.41 & & & & & & & & & \\
\hline Water oullel Temp.f & 107.1 & & & & & & & & & \\
\hline Intat Glbmhrhi2 & 207876 & & Temp & 117.8 & Donsity & 87.11 & & & & \\
\hline Subosolingf & -0.9 & & & & & & & & & \\
\hline Fow Observation & & & & & & & & & & \\
\hline & ZONE1 I & ZONE2 & ZONE3 & ZONEA & ZONE5 & ZONE6 & ZONE7 & ZONE8 2 & ZONES & ZONETO \\
\hline Hod houtWest(nal) & 674.2 & 538.2 & 580.6 & 570.2 & 573 & 573.9 & 559.4 & 571.2 & 574.2 & 571.1 \\
\hline HedLose-Wals & 11.5 & 2.7 & 3.6 & 3.7 & 3.7 & 28 & 1.8 & 28 & 3.6 & 20.2 \\
\hline 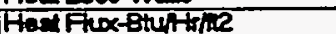 & 9958 & 9849 & 10058 & 9884 & 9938 & 9948 & 9870 & 9901 & 9953 & 9800 \\
\hline Wa Tomp. Ba & 134 & 734.8 & 133.6 & 734.8 & 736.8 & 136.4. & 136.8 & 136.3 & 735.7 & 738.8 \\
\hline WE Temp $14 \mathrm{~F}$ & 134.3 & 133.7 & 135.6 & 135.8 & 135.7 & 786.4 & 137.8 & 137.3 & 156.1 & 1350 \\
\hline WalTomp. $\$ 67$ & 134 & 1325 & 136 & 134.6 & 134.4 & 1862 & 136.2 & 138.2 & 134.6 & 155 \\
\hline Weil Tomp. IfF & 67.1 & 188.4 & 1328 & 138.2 & 183.8 & 136.8 & 137.8 & 137.8 & 194 & 1323 \\
\hline Wal Temp. IFF & 133.8 & 134 & 133.5 & 133.8 & 134 & 136.4 & 137.7 & 137.8 & 184.6 & 738.9 \\
\hline Intrean Temo.f & 125.6 & 128.4 & 1262 & 126.4 & 126.8 & 126.2 & 1282 & 126.9 & 125.8 & 125.2 \\
\hline Avo. Wall Temp of & 134 & 134 & 132.6 & 134.5 & 134.5 & 136.3 & 1372 & 137 & 135 & 13.7 \\
\hline Avg. Wat DT F & 8 & 7.1 & 7 & 7.6 & 7.7 & 9.7 & 10.6 & 10.6 & 8.8 & 8 \\
\hline Remaining Suboooff & -11.1 & & & & & & & & & \\
\hline Ext Quality & 0.09 & 0.18 & 0.27 & 0.35 & $0 . \overline{4}$ & 0.52 & 0.61 & 0.7 & 0.78 & 0.87 \\
\hline EXP.HBuH & 1248 & 1381.4 & 144.6 & 1295.7 & 1285.6 & 1028.2 & 935.2 & 931.2 & 1137.6 & 1098.4 \\
\hline CONPUTEDH & & & & & & & & & & \\
\hline Jallouk & 889.3 & 870.8 & 851.7 & 840.8 & 833.3 & 828.8 & 817.1 & 813 & 812.8 & 78.4 \\
\hline sady1(cudo tubo) & 1251.7 & 1255.1 & 1267.8 & 1259.2 & 12627 & 1234.2 & 1280.8 & 126211 & 203.4 & 78.4 \\
\hline Seady2 & 1001.9 & 1031.4 & 1057.8 & 1058.3 & 1059 & 1059.5 & 10542 & 1054.4 & 1057.8 & 78.4 \\
\hline (P.DO) & 24600 & 2487 & 20008 & 17568 & 15787 & 13488 & 11249 & 89991 & 67021 & 6702 \\
\hline$(\bar{F})$ & 1.89 & 275 & 3.278 & 3.787 & 4.152 & 4.585 & 4.882 & 5.349 & 5.5811 & 5.581 \\
\hline (HDO) & 160.6 & 211.9 & 256.4 & 31.7 & 24 & 353.0 & 381.7 & 4001 & 496.8 & 406.8 \\
\hline (1.ंत्या & 125 & 1253 & $1264 a$ & 1254 & 1255.5 & 12540 & 1209 & 1227.6 & 1200 & 1200 \\
\hline & & & & & & & & & & \\
\hline & ZONE2 & ZONE4 & ZONE6 & ZONES & ZONETO & & & & & \\
\hline DP tquid tamp.F & 70.7 & & & & & & & & & \\
\hline 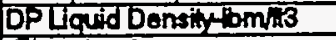 & 94.82 & & & & & & & & & \\
\hline Eovitiond & & 4 & 6) & 8 & 9.875 & & & & & \\
\hline Meanured DPPsid & 0.615 & 3.917| & 3.828 & 3.672 & 7.573 & & & & & \\
\hline Zero Comedions-psid & 0.087 & -0.067 & -0.75 & $0.12 \pi$ & 0.278 & & & & & \\
\hline Corroded DP Patd & 0.578 & 1.934 & 2578 & 3.551 & 4.3 & & & & & \\
\hline Ached DPA & 1.22 & 1.00 & 220 & 2.82 & 8.02 & & & & & \\
\hline Nhomured TPP & $\approx .7$ & & & & 3.7 & & & & & \\
\hline Magatured Overdil' & 88.64 & & & & & & & & & \\
\hline & & & & & & & & & & \\
\hline Compled DP-A & 1.08 & 1.76 & 239 & 296 & 3.44 & & & & & \\
\hline Filctionth & 0.04 & 0.19 & 0.21 & 0.39 & 0.48 & & & & & \\
\hline Acoteretion- & 0.03 & 0.08 & 0.11 & 0.16 & 0.23 & & & & & \\
\hline Evertion & 0.97 & 1.58 & 2.08 & 246 & 2.75 & & & & & \\
\hline & & & & & & & & & & \\
\hline
\end{tabular}




\begin{tabular}{|c|c|c|c|c|c|c|c|c|c|c|}
\hline Run: & 694 & & & 1 & & & & & & \\
\hline Tatflid & CAF 10 & & & & & & & & & \\
\hline Den: & 6233 & & & & & & & & & \\
\hline Time: & $5: 13$ & & & & & & & & & \\
\hline Now & Anstion & Pinspsadis & 3 essumed & & & & & & & \\
\hline Flow ATOLR & 0.00836 & & & & & & & & & \\
\hline Hen flow Areste & 0.18695 & & & & & & & & & \\
\hline SEl.Pressure PII & 842 & & & & & & & & & \\
\hline S2. Tomperientefinde) & 120.6 & & & & & & & & & \\
\hline 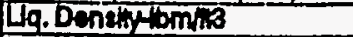 & 88.80 & 88.17 & 86.18 & 88.15 & 88.16 & 88.18 & 88.2 & 88.27 & 88.28 & 88.80 \\
\hline Leink Hoarbuhbm & $32 \pi$ & 32.68 & 3259 & 32.57 & 32.57 & 32.59 & 32.6 & 3265 & 3260 & उटत \\
\hline Speoifo Hod aturomp & 0.275 & 0.275 & 0.275 & 0.275 & 0.275 & 0.275 & 0.275 & 0.276 & 0.275 & 0.275 \\
\hline Lq. Visoogithom/hri & 0.531 & 0.625 & 0.626 & 0.625 & 0.620 & 0.628 & 0.527 & 0.620 & 0.620 & 0.651 \\
\hline 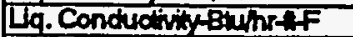 & $0.0<79$ & 0.0278 & 0.0278 & 0.0278 & 0.0278 & 0.0278 & 0.0278 & 0.0278 & 0.028 & 0.0270 \\
\hline Liq.Prandino. & 6.23 & 5.21 & 5.22 & 5.21 & 521 & 5.22 & 52 & 52 & 52 & 5.28 \\
\hline IntAFlow RWo-GPM & 1.7 & & & & & & & & & \\
\hline Outellig. Flow Ra-GPM & 0.9 & & & & & & & & & \\
\hline Oull Vep. Fow Rato-GPM & 0.8 & & & & & & & & & \\
\hline Intel Tomperviuref & 120.8 & & & & & & & & & \\
\hline 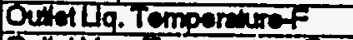 & T25.7 & & & & & & & & & \\
\hline dudvep. Temperminef & 120.8 & & & & & & & & & \\
\hline 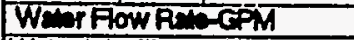 & & & & & & & & & & \\
\hline Weler inlet Tomp.f & 78 & & & & & & & & & \\
\hline Wator outh Temp.f & 1062 & & & & & & & & & \\
\hline Indel Gbmhrie & 262845 & & Temp & 118.6 & Dentity & 86.07 & & & & \\
\hline Siboodingt & -0.3 & & & & & & & & & \\
\hline Flow Observition & & & & & & & & & & \\
\hline & ZONET & ZONE2 & ZONE8 & ZONE4 & ZONE5 & ZONE6 & ZONET & ZONE8 & ZONES & $20 N=10$ \\
\hline 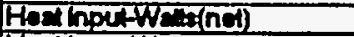 & 574.9 & 572.8 & 578 & 567.5 & 570 & 570.8 & $5 \% .7$ & 571.3 & 572 & 57.8 \\
\hline ForLLose-Wete & 11.3 & 26 & 3.5 & 3.6 & 3.6 & 27 & 1.8 & 27 & 3.4 & 18.6 \\
\hline 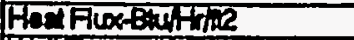 & 9968 & 9928 & 1000 & 9887 & छा & 9894 & 9893 & 8900 & 9918 & 8008 \\
\hline Wa'Tamp. 13 & 7352 & 134 & 1327 & 13 & 135.9 & 135.2 & 735.8 & 135.1 & T33.8 & 135 \\
\hline WET Tomp. If & 188.7 & -133 & T38.8 & 134.6 & 13.7 & 135.8 & 136.4 & 135.6 & $\mid 34.7$ & 1345 \\
\hline WeTtamp.15F & 133,8 & 131.8 & 138.8 & 130.9 & 133.5 & 135.2. & 135.1 & 134.5 & 135.1 & रुआ, \\
\hline Wa'Temp. IIF & 672 & 182.8 & क्टा & 1326। & 18 & 135,2 & 18 & 135.8 & ISEd & 181.1 \\
\hline WeIT Temp.lat & 138.8 & 133.2 & 1328 & 183 & 138.8 & 1355 & 1365 & 163.4 & 188.1 & TE28 \\
\hline Invirem Temp. fe & 124.8 & दर्56 & 125.4 & $\sqrt{25.6}$ & 125.6 & 125. & 20.8 & 1220 & 1246 & 122.8 \\
\hline Awo. Wal Temp.F & 133.4 & 133.3 & 1320 & 133.7 & 133.8 & 1352 & 186 & 1355 & 133.4 & $73 \mathbf{3} .6$ \\
\hline Avo.WalDT.A & 8.8 & 3.3 & 7 & 7.7 & 7.8 & 9.4 & 10.2 & 70.1 & 8.2 & 8.8 \\
\hline Remaining Suboooff & -6.3 & & & & & & & & & \\
\hline Exat Quality & 0.06 & 0.1 & 0.15 & 0.2 & 0.28 & 0.31 & 0.86 & 0.41 & 0.48 & 0.51 \\
\hline DXP.HBu/hma & 1155.5 & 1359.4| & 1431.8 & 1280.8 & 1300.8 & 1055. & 968.8 & 977.4 & स्रिठठ & 1123.8 \\
\hline CONPUIEDH & & & & & & & & & & \\
\hline Jalock & 865.8 & 836.4 & Eख.8. & 804.8 & 790.11 & 784.6 & 779.8 & 7752 & 7725 & 767.0 \\
\hline इExd & 12762 & T25.3. & 1301 & 1233 & 1257.7 & 1258.8 & 12008 & 1281.2 & 126.1 & Z2:20 \\
\hline seangle & 1024.2 & 1052.3 & 1033.1 & 1047.1 & 1050.5 & 9000.5 & 1050.4 & 109.1 & 10883 & 1058.5 \\
\hline (सLO) & 4809 & 20456 & \$817 & Sort7 & 89810 & \$15:7| & 25978 & 27019 & 2478 & 2030 \\
\hline$(F)$ & 1.675 & 2.256 & 2.675 & उ.ण्टा & 3.827 & 3.600 & 3.689 & 4.12 & 4.855 & 4.501 \\
\hline (मिक) & 1की & 2685 & 378.4 & ङ27 & 38 & 4205 & 450.4 & 4002 & 674.8 & 645.5 \\
\hline ( & 12146 & 12502 & 12512 & 12द3. & 12याड & 1224 & 1220.1 & 120 & 12345 & 127.7 \\
\hline & & & & & & & & & & \\
\hline & ZONE2 & ZONE4 & ZONE6 & ZONES & ZONETO & & & & & \\
\hline Dp laud tomp.f & 70.9 & & & & & & & & & \\
\hline DPLiguid Donsity & 94.3 & & & & & & & & & \\
\hline ENonton & 2 & 4 & 6 & 8 & 9.875 & & & & & \\
\hline Mesured DPY Fd & 0.1 & 1.547 & 7.428 & 3.162 & उ.ख्यम & & & & & \\
\hline zoro Comections-pid & 0.087 & -0.057 & -0.75 & O.Jटा| & 0.278 & & & & & \\
\hline Comend DPfid & 0.008 & 1.614 & 2179 & 3.041 & 3.651 & & & & & \\
\hline Ached DP- & 2.08 & 9.8 & 28 & 3.60 & 4.68 & & & & & \\
\hline Moasured TPphe & 64.2 & & & & 3.7 & & & & & \\
\hline Meanured Overalit & 100.88 & & & & & & & & & \\
\hline & & & & & & & & & & \\
\hline Compuled DP, & 1.27 & 226 & 3.17 & 4.05 & 4.87 & & & & & \\
\hline Frotion & 0.07 & 0.19 & 0.37 & 0.59 & 0.84 & & & & & \\
\hline Aocolortionth & 0.08 & 0.1 & 0.18 & 0.2 & 0.20 & & & & & \\
\hline Envetont & 1.14 & 1.86 & 2.83 & उ.2. & 3.8 & & & & & \\
\hline & & & & & & & & & & \\
\hline
\end{tabular}




\begin{tabular}{|c|c|c|c|c|c|c|c|c|c|c|}
\hline Runt & 685 & & & & & & & & & \\
\hline Tot Fuid & CAF10 & & & & & & & & & \\
\hline Dato: & बथक्3 & & & & & & & & & \\
\hline Time: & $5: 13$ & & & & & & & & & \\
\hline Nots & Anstical & $P$ in $=p_{\text {sat }}$ is & s assumed & & & & & & & \\
\hline Fow Areste & 0.00336 & & & & & & & & & \\
\hline Hoat Fow Arear? & 0.19695 & & & & & & & & & \\
\hline Set. Piossure Pasig & 64.7 & & & & & & & & & \\
\hline Sat Tomperature f(inla) & 121 & & & & & & & & & \\
\hline Lag. Denaty-tomins & 86.27 & 88.18 & 86.22 & 86.09 & 86.12 & 86.18 & 88.22 & 88.3 & 86.81 & 86.4 \\
\hline Latent Hos ow & 32.65 & 32.59 & 3261 & 3253 & 3255 & 32.59 & 32.81 & 32.67 & 32.68 & $\$ 2.78$ \\
\hline Specifo thentexwhomif & 0.275 & 0.275 & 0.275 & 0.275 & 0.275 & 0.275 & 0.275 & 0.275 & 0.275 & 0.275 \\
\hline Liq. Visoositymith & 0.520 & 0.526 & -0.527 & 0.524 & 0.525 & 0.526 & 0.627 & 0.529 & 0.68 & 0.632 \\
\hline Lo. Conductivitu Btwers if & 0.0278 & 0.0278 & 0.0278 & 0.0277 & 0.027 & 0.0278 & 0.0278 & 0.0279 & 0.0279 & 0.0278 \\
\hline Liq. Prande No. & 5.22 & 5.22 & 5.22 & 5.21 & 5.21 & 5.22 & 522 & 5.28 & 5.28 & 5.20 \\
\hline Indolflow Rato-GPM & 2.5 & & & & & & & & & \\
\hline Outlot Lig. Flow Rato-GPM & 0.9 & & & & & & & & & \\
\hline Outet Vap. Fow Rato-GPM & 1.6 & & & & & & & & & \\
\hline Inlel Temperaturef & 121.6 & & & & & & & & & \\
\hline Oret La Tompor tura & 725.5 & & & & & & & & & \\
\hline Ou'd vap. Tomporuter & 120.3 & & & & & & & & & \\
\hline 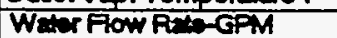 & & & & & & & & & & \\
\hline Water hilet Temp.F & 77.8 & & & & & & & & & \\
\hline Water outet Tomp.f & 106 & & & & & & & & & \\
\hline IntaGomin? & 518700 & & Tamp & 118.7 & Denaity & 86.06 & & & & \\
\hline Suboodingt & -0.6 & & & & & & & & & \\
\hline Fow Observition & & & & & & & & & & \\
\hline & ZONE1 & ZONE? & ZONE3 & ZONE4 & ZONE5 & ZONE6 & ZONE7 & ZONEB & ZONE9 & $20 K=10$ \\
\hline Hed InputWatts(net) & 574.6 & 578.9 & 538.2 & 569.5 & 573.7 & 571.9 & 579.5 & 575.2 & 567 & 588.4 \\
\hline Hoct LossotWat's & 11.4 & 2.6 & 3.5 & 3.6 & 3.6 & 2.7 & 1.8 & 2.7 & 3.4 & 19.4 \\
\hline Heathix-buturtits & 9960 & 10035 & 3849 & S872 & 9934 & 9914 & 10045 & 997 & 8880 & 9868 \\
\hline Wah Temp. Bł & 仿 3.5 & 134.3 & 32.7 & 13 & 135.8 & 1352 & \$35.5 & 734.6 & 133.4 & 734.6 \\
\hline Wat Tomp : 14t & 733.8 & 1328 & 733.6 & 734.6 & 134.6 & 735.2 & 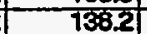 & 135.2 & 134.2 & 138.7 \\
\hline WEN Temp. $85 F$ & 133.5 & 131.9 & 134 & 134 & 733.6 & 134.8 & 134.6 & 134.1 & 133 & 134 \\
\hline WE Tomp. If & 68.1 & 138 & 137.8 & 138 & 1382 & 135.2 & 135.8 & 135.5 & 131.8 & 190.8 \\
\hline Wa' Tomp. हLF & 133.5 & 133.6 & 1325 & 133.5 & 1332 & 135.8 & 136.2 & 135.8 & 1328 & 1325 \\
\hline Intrasm Tamp.f & 124.9 & 125.4 & 1252 & 126 & 125.8 & 125.4 & 1252 & 124.7 & 124.8 & 124.1 \\
\hline Avg. Wal Temp.F & 138.6 & 133.4 & 1327 & 133.9 & 133.7 & 135.1 & 135.7 & 135 & 133.1 & 133.1 \\
\hline Avp. Wa DT.F & 8.2 & 7.5 & 7 & 7.5 & 7.4 & 9.3 & 10 & 9.9 & 8 & 8.8 \\
\hline Remaining Subcooff & -4.7 & & & & & & & & & \\
\hline Ext Cually & 0.04 & 0.07 & 0.11 & 0.14 & 0.18 & 0.21 & 0.25 & 0.28 & 0.37 & 0.56 \\
\hline DXP.HBturfnef & 1211 & 1329.7 & 7401.1 & 1324.4 & 1342 & 1071.5 & 1001.8 & 10082 & 1226.1 & 1149.1 \\
\hline COMPUTEDH & & & & & & & & & & \\
\hline Jahouk & 834.7 & 874.7 & 784.8 & 781.6 & 777.9 & $7 \pi$ & 771.81 & 764.3 & 756.8 & 7642 \\
\hline Fodvicudotibl & 12515 & 12025 & 72521 & 12028 & 7257.8 & TFE8:8 & 127 & $72 / 2.8$ & 1207.7 & T270 \\
\hline sady? & 1000 & 10.59 .2 & 1045.1 & 1063.4 & 1057.8 & 1003.8 & Ta17.4 & 104.6 & 1090.1 & 1048.8 \\
\hline (R्य) & 62312 & 60788 & 58485 & 60041 & 5489 & 51840 & 20081 & 47218 & 44802 & कि:56 \\
\hline (F) & 1.564 & 2048 & 2.374 & 2.64 & 2887 & 3.176 & 3.327 & 3.53 & 3.716 & 3.0 \\
\hline (स) & 2532 & 331 & 38.5 & 428.1 & 468 & 504.8 & 538.8 & 677.5 & 601.3 & 650.6 \\
\hline$(1+3014)$ & 1248 & 1254.8 & 1239.9 & 125.7 & 12462 & 1239.6 & 1242 & 1233.1 & 122.8 & Tदा5. \\
\hline & & & & & & & & & & \\
\hline & ZONE2 & ZONE4 & ZONE6 & ZONE8 & ZORETO & & & & & \\
\hline DPlogd Tomo $f$ & 70.8 & & & & & & & & & \\
\hline DPLiquid Density Hom, & 94.38 & & & & & & & & & \\
\hline Elovationt & & & 6 & 8 & 9.875 & & & & & \\
\hline Messured DPFid & 0.165 & 1.25 & 7.075 & 277 & 3.352 & & & & & \\
\hline Zero Corrections-prid & 0.087 & -0.087 & -0.70 & 0.127 & 0.278 & & & & & \\
\hline CompadDP & 0.129 & 1.269 & 1.865 & 2.598 & 3.078 & & & & & \\
\hline Actud DPA & 1.87 & 22 & 3.51 & 4.41 & 5.68 & & & & & \\
\hline Mereured TPF & 64.7 & & & & 3.7 & & & & & \\
\hline Mesured Overath & 107.67 & & & & & & & & & \\
\hline & & & & & & & & & & \\
\hline Computed DP: & 9.47 & 2.72 & 3.91 & 5.1 & 6.23 & & & & & \\
\hline Fiation-h & 0.11 & 0.3 & 0.58 & 0.88 & 1.35 & & & & & \\
\hline Acoserertion- & 0.17 & 0.17 & 0.23 & 0.31 & 0.39 & & & & & \\
\hline Elevationt & 1.26 & 225 & 3.12 & 3.91 & 4.59 & & & & & \\
\hline & & & & & & & & & & \\
\hline
\end{tabular}




\begin{tabular}{|c|c|c|c|c|c|c|c|c|c|c|}
\hline Runt & 686 & & & & & & & & & \\
\hline Teaffid & CAF 10 & & & & & & & & & \\
\hline Dats: & 6च93 & & & & & & & & & \\
\hline Time: & $5: 13$ & & & & & & & & & \\
\hline Nob & Andycien & Pin $=$ pad is & 8 assumed & & & & & & & \\
\hline Flow AYOAR2 & 0.00356 & & & & & & & & & \\
\hline Hod Fow Areat? & 0.19695 & & & & & & & & & \\
\hline SA.PTessurepsig & 6.8 & & & & & & & & & \\
\hline Sel. Temperainofinde) & 122 & & & & & & & & & \\
\hline La. Denstatomin & 86.14 & 88.09 & 86.07 & 85.87 & 85.99 & 88.08 & 88.1 & 86.17 & 862 & 86.8 \\
\hline Letent Hos Buritom & 3266 & 3249 & 3251 & 3245 & 32.46 & 325 & 32.54 & 3258 & 32.8 & 32.87 \\
\hline 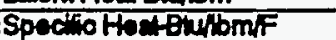 & 0.275 & 0.276 & 0.275 & 0.276 & 0.276 & 0.275 & 0.275 & 0.275 & 0.275 & 0.275 \\
\hline Lig. Visoositutbmithr & 0.525 & 0.528 & 0.524 & 0.521 & 0.521 & 0.523 & 0.524 & $0.5 \%$ & 0.527 & 0.529 \\
\hline La. Conductury BterthrfF & 0.027 & 0.0277 & 0.027 & 0.0276 & 0.027 & 0.027 & 0.027 & 0.0278 & 0.0278 & 0.0278 \\
\hline Lq.PrandidNo. & 5.21 & 5.2 & 5.21 & 5.2 & 5.2 & 5.2 & 5.21 & 6.21 & 5.2 & 5.23 \\
\hline InlafFow Rak-CPM & 3.3 & & & & & & & & & \\
\hline Ou'PlLla. Fow ReAG GM & 0.9 & & & & & & & & & \\
\hline OutalVap. Fow Pala-GPM & 24 & & & & & & & & & \\
\hline Intel Tempersure of & 1223 & & & & & & & & & \\
\hline 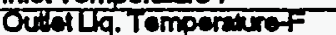 & 126 & & & & & & & & & \\
\hline Ou'N Vap. Tomperauref & 720.8 & & & & & & & & & \\
\hline WET Fow RAR-GPM & & & & & & & & & & \\
\hline Water thet Temp.t & 78.2 & & & & & & & & & \\
\hline Water octsel Tomp.F & 106.6 & & & & & & & & & \\
\hline Inta Glbmhwhe & 685070 & & Temp & 120 & Densily & 86.74 & & & & \\
\hline Surooolingf & -0.3 & & & & & & & & & \\
\hline Flow Observition & & & & & & & & & & \\
\hline & 20ास & ZONE2 & ZONE3 & ZONE & ZONE5 & ZONEE & ZONET & ZONE8 & ZONES & ZONETK \\
\hline HealmputWals(nel) & 57 & 577 & $5 \pi$ & 577 & 57 & 577 & 57 & $5 \pi$ & 57 & 577 \\
\hline HenLose-We"s & 71.8 & 2.7 & 3.8 & 3.7 & 3.7 & 2.8 & 1.8 & 28 & 3.5 & 9.8 \\
\hline HocfFux-Bturth/i2 & 100002 & 10000 & 1000 & 70000 & 1000 & Tote & 10000 & 1000 & Tote & 10000 \\
\hline Wetromp. & 33.8 & उद्य & 133.8 & 134.8 & 138.4 & 135.6 & 135.1 & \$S.1 & 13्य & 130 \\
\hline WतTtomp. If & 13.7 & 1383.8 & T34.6 & 125.8 & 135.2 & 135.7 & 38.8 & 75.6 & 13.7 & रुम \\
\hline Wa'tomp. I5F & 134.4 & 1328 & 134.8 & 734.6 & 134.1 & 135.8 & 1352 & 737.8 & 1303 & 7345 \\
\hline WCTtemp. IfF & 68 & 138.8 & 1328 & 183.6 & 133.7 & 135.7 & 188.8 & 155.8 & 1324 & का \\
\hline We't Temp. 12t & 13.3 & 1342 & 133.8 & 134 & 134 & 1358 & 138.7 & 186.4 & 138.1 & 188 \\
\hline Instroen Temp.f & 125.7 & 128.8 & 128.1 & [28.3 & 128.6 & 128.2 & 125.8 & 1255 & 125.8 & 124.7 \\
\hline Avo. Wall Tomp.F & 134.4 & 1342 & 133.5 & 134.6 & 134.3 & 135.6 & 1382 & 135.6 & 133.5 & 135.5 \\
\hline Avo. Wall DT. $F$ & 8.8 & 7.4 & 6.9 & 7.4 & 7.2 & 8.9 & 9.8 & 9.5 & 7.7 & 8.3 \\
\hline Remaining Subcootf & 3.4 & & & & & & & & & \\
\hline Ed Cuatity & 0.03 & 0.08 & 0.08 & 0.17 & 0.13 & 0.16 & 0.19 & 0.21 & 0.24 & 0.27 \\
\hline EXP. HBturthner, & 1209 & 1342.9 & 144.7 & 1352 & 1389.5 & 7120.8 & 1017.7 & 1047.5 & 12042 & 1198.1 \\
\hline COWNUTEH & & & & & & & & & & \\
\hline Jallok & हल5 & 7933.8 & 783.4 & 778.8 & 774 & 768.8 & $7 \% 5$ & 761.5 & 753.8 & 748.8 \\
\hline 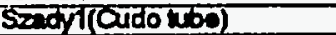 & 1201.4 & $7 \mathbf{6 0 . 5}$ & 12ก.7 & 7200 & T2835 & 7205 & 7287.6 & 1200.8 & 7283.7 & $\$ 250.8$ \\
\hline soady & 10502 & 10042 & 1038.5 & 1068.8 & 1051.8 & 305.6 & 1000 & 1063.7 & 100.8 .6 & TDES.1 \\
\hline (सB) & 8896 & 8185 & 78505 & 7817 & $755 \% 8$ & 7278 & 70876 & 67814 & 65578 & Esth \\
\hline (F) & 1.468 & 1.87 & 2.168 & 2389 & 2.602 & $2 \% 8$ & 288 & 3.133 & 3.313 & 8.474 \\
\hline (HW) & 296.2 & 377.0 & K5.6. & 488.3 & 528.1 & 665.6 & 6021 & $\approx 8.6$ & 658.8 & 700.6 \\
\hline (महु) & 12550 & $\left\{\begin{array}{c}258,3 \\
\end{array}\right.$ & 125a4| & 12567 & 12538 & 1247.4 & 12421 & 1265.9 & 12312 & $\sqrt{228}$ \\
\hline & & & & & & & & & & \\
\hline & $2015=2$ & $\angle O N E$ & ZONE6 & ZONE8 & ZONEIO & & & & & \\
\hline DPtiqudTemp.f & 126 & & & & & & & & & \\
\hline 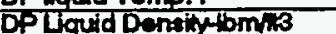 & 101.85 & & & & & & & & & \\
\hline Eevetionth & & 4 & 6 & 8 & 9.875 & & & & & \\
\hline 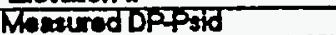 & 0.162 & 0.888 & 0.727 & टयक & 2787 & & & & & \\
\hline Zero Comections-prdd & 0.087 & -0.087 & -0.75 & ठ.गट & 0.273 & & & & & \\
\hline Corredad Dapild & 0.135 & 0.885 & 1.47 & 2.128 & 253 & & & & & \\
\hline Actid DPA & 2.15 & 3.07 & 4.62 & 5.8 & 7.45 & & & & & \\
\hline Whesured tP्phop & 65.9 & & & & 3.7 & & & & & \\
\hline Meedured Over"ll & 106.78 & & & & & & & & & \\
\hline & & & & & & & & & & \\
\hline Computed DPA & 9.69 & 3.17 & 7..22 & 6.08 & 7.49 & & & & & \\
\hline Froton-t & 0.15 & 0.4 & 0.72 & 1.73 & 1.57 & & & & & \\
\hline Aocoter mion? & 0.15 & 0.28 & 0.52 & 0.4 & 0.5 & & & & & \\
\hline Elovation't & 1.38 & 2.54 & 3.58 & 4.56 & 5.47 & & & & & \\
\hline & & & & & & & & & & \\
\hline
\end{tabular}




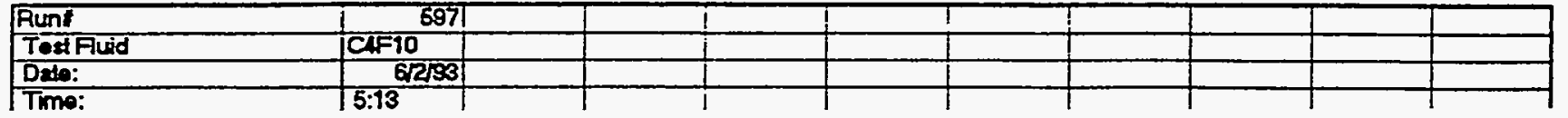

\begin{tabular}{|c|c|c|c|c|c|c|c|c|c|c|}
\hline Punt & 599 & & & & & & & & & \\
\hline Test Fuid & CAFIO & & & & 1 & 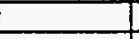 & & & & \\
\hline Dato: & $6 \sqrt{233}$ & & & & & & & & & \\
\hline Time: & $7: 14$ & & & & & & & & & \\
\hline Now & Analytion & Pin=Psat is & assumed & & & & & & & \\
\hline Fow Areate & 0.00365 & & & & & & & & & \\
\hline Hoat Fow Areatil & 0.1955 & & & & & & & & & \\
\hline Sat Pressure psig & 64.7 & & & & & & & & & \\
\hline S2 Tomperaire F(ntal) & 127 & & & & & & & & & \\
\hline LGa. Danthy & 86.25 & 86.15 & 88.11 & 86.00 & 80.1 & 88.16 & 88.27 & 88.28 & 88.31 & 86.4. \\
\hline Linin Heat Bhwbm & 3203 & 3257 & 3264 & 32.53 & 32.54 & 32.57 & 32.05 & 32.68 & 32.8 & 3270 \\
\hline Specife too Eufitomn & 0.275 & 0.275 & 0.275 & 0.275 & 0.275 & 0.275 & 0.275 & 0.275 & 0.275 & 0275 \\
\hline Lig. Vieoositibm/ntr & 0.623 & 0.535 & 0.624 & 0.523 & 0.624 & 0.526 & 0.629 & $0.52 \%$ & 0.68 & 0.53 \\
\hline Lig. Conduching Buhrthe & 0.0278 & 0.0278 & 0.0277 & 0.0277 & 0.0277 & 0.0278 & 0.0278 & 0.0278 & 0.0279 & 0.0279 \\
\hline Liq. PrandidNo. & 522 & 5.21 & 5.21 & 5.21 & 5.21 & 5.21 & 522 & 5.22 & 5.23 & 524 \\
\hline Inlet Flow Rato-GPM & 4.8 & & & & & & & & & \\
\hline Outet Liq. Fow Rato-GPM & 1.4 & & & & & & & & & \\
\hline Outet Vso. Flow Rate-GPM & 2.2 & & & & & & & & & \\
\hline Inlat Temperature of & 121.4 & & & & & & & & & \\
\hline Cutset Lig. Temperaturef & 124.6 & & & & & & & & & \\
\hline Outel Vep. Temperature f & 118.4 & & & & & & & & & \\
\hline Welp Fow Pale-GPM & & & & & & & & & & \\
\hline Wertar hied Temp.F & 74.6 & & & & & & & & & \\
\hline Weter outlel Tomp.F & 97.8 & & & & & & & & & \\
\hline Enhat Gibm/nrin & 890501 & & Temp & 120.5 & Densify & 86.65 & & & & \\
\hline Subcooting F & -0.4 & & & & & & & & & \\
\hline Flow Observation & & & & & & & & & & \\
\hline & ZONEI & ZONE2 & ZONE3 & ZONE & ZONES & ZONE6 & ZONE7 & ZONE8 & ZONEO & ZONETO \\
\hline HadthpltWais(nel) & 868 & 855 & 858 & 883 & 866 & 865 & 868 & 863 & 868 & 868 \\
\hline HedLose-Walts & 9.1 & 21 & 28 & 28 & 2.9 & 2.2 & 1.4 & 2.2 & 2.7 & 15.4 \\
\hline Fad Finestuttine & 15012 & 15012 & 15012 & 15012 & 15012 & 15012 & 15012 & 15012 & 15012 & 15012 \\
\hline Wal Tomp. BF & 13.8 & 137.6 & 135.5 & 136.3 & 730.6 & 137.4 & 137.8 & 138.1 & 136.1 & 138.1 \\
\hline What Tomp. IfF & 138.8 & 135.8 & 137.7 & 737.1 & 137.4 & 137.7 & 138.5 & 138.5 & 137.4 & TE3.7 \\
\hline Whil Temp. \&5 & 130 & 134 & 157 & 130.8 & 130.1 & 137.7 & 150.9 & 137.3 & 134.9 & 137 \\
\hline Well Tomp. IF & 73.1 & 1S5.0 & 184.8 & 134.4 & 1352 & 138 & 138.4 & 138.7 & 184.2 & 181.4 \\
\hline Wall Tomp. & 138 & 1682 & 135.5 & 135.8 & 135.7 & 138.7 & 138.7 & 139.2 & 134.8 & 134.6 \\
\hline Intraam Tamp.f & 125 & 125.6 & 125.8 & 126 & 125.8 & 125.8 & 124.9 & 124.8 & 124.8 & 123.8 \\
\hline Avg. Wal Tomp.F & 1362 & 136.3 & 135.7 & 136 & 136.1 & 137.7 & 138.1 & 138.4 & 1356 & 135.5 \\
\hline Av. Wall DT.F & 10.5 & 10 & 9.1 & 9.3 & 9.5 & 11.4 & 125 & 129 & 102 & 11.1 \\
\hline Femaining SubcoofF & 3.6 & & & & & & & & & \\
\hline Ext Qualiy & 0.03 & 0.08 & 0.08 & 0.11 & 0.14 & 0.17 & 0.19 & 0.22 & 0.25 & 0.28 \\
\hline EXP.HBturinaf & 1483.6 & 1497.8 & 1641.2 & 1606.1 & 1576.6 & 1314.3 & 1202.7 & 1165.8 & 1468.6 & 1357.1 \\
\hline COMPUTEDH & & & & & & & & & & \\
\hline Jalouk & $\$ 87.1$ & 947.7 & 927 & 90.1.1 & 887.6 & 8722 & 855.3 & 843.8 & 831.4 & 815.4 \\
\hline Sady 1 (Cudo wbe) & $1 5 \longdiv { 1 5 }$ & 1552.5 & 1560.8 & 1587.8 & $15 \% 5$ & 1577.8 & 1579.6 & 1588.8 & 1533.8 & 1553.8 \\
\hline $5 x+\frac{1}{2}$ & 137.9 & 13621 & 1377.3 & 13782 & 1385.5 & 13005 & 74723 & 1414.8 & 1417.8 & 1416.8 \\
\hline (R्DO) & 121074 & 118082 & 1149S5 & 11772 & 108251 & 104881 & 100718 & 97297 & 93818 & 85821 \\
\hline$(\mathrm{F}$ & 1.487 & 1.898 & 2185 & 248 & 2.644 & 2.813 & 3.094 & 3.208 & 3.37 & 3.588 \\
\hline$($ (1)0 & 403.8 & 517 & 555.3 & 681.4 & 7002 & 7742 & 8255 & 872 & 916.9 & 860.8 \\
\hline (18O4) & I5622 & 15332 & 1531.4 & 1527.6 & 1521.5 & 15132 & 1500.6 & 1493.8 & 1485.7 & 14712 \\
\hline & ZONNE2 & ZONE4 & ZONE6 & ZONER & ZONETO & & & & & \\
\hline DP Fquid Temp.f & 74.7 & & & & & & & & & \\
\hline DP Lquid Dansivestom/3 & 93.75 & & & & & & & & & \\
\hline Elevation & 2 & 4 & 6 & 8 & 9.875 & & & & & \\
\hline Whesured DPFsid & $0.73^{7}$ & 0.555 & 0.139 & 1.65 & 3.837 & & & & & \\
\hline ZYo Correctioneoedo & 0.037 & -0.057 & -0.75 & ర.T2ী & 0.278 & & & & & \\
\hline
\end{tabular}




\begin{tabular}{|c|c|c|c|c|c|c|c|c|c|c|}
\hline $\operatorname{Run}:$ & 600 & & & & & i & & & & \\
\hline Tostfind & CAFIO & & & & & & & & & \\
\hline Den: & $6 \sqrt{233}$ & & & & & & & & & \\
\hline Tims: & $3: 14$ & & & & & & & & & \\
\hline Noto & Anstyocal & Pinepsatis & assumed & & & & & & & \\
\hline Flow A & 0.00356 & & & & & & & & & \\
\hline Hos flow Aresde & 0.1869 & & & & & & & & & \\
\hline SE. Prossuro orig & 64.7 & & & & & & & & & \\
\hline Sat Tempordur find) & दा & & & & & & & & & \\
\hline Wq.Densintomis & 88.2 & 86.12 & 88.14 & 86.12 & 88.12 & 86.15 & 8.2.2 & 88.8 & 88.8 & 86.80 \\
\hline Lelent Hesterestom & 32.6 & 3255 & 32.58 & 3255 & 32551 & 3257 & 328 & 3267 & 32.67 & 327 \\
\hline 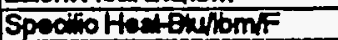 & 0.275 & 0.276 & 0.275 & 0.275 & 0.275 & 0.275 & 0.275 & 0.275 & 0.275 & 0.275 \\
\hline 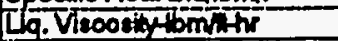 & 0.527 & 0.535 & 0.525 & 0.65 & 0.625 & 0.628 & 0.627 & 0.520 & 0.620 & 0.681 \\
\hline 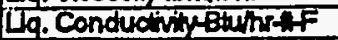 & 0.0278 & 0.027 & 0.027 & 0.027 & 0.027 & 0.0278 & 0.0278 & 0.0279 & 0.0279 & 0.0278 \\
\hline Lig. Prand No. & 5.22 & 5.21 & 5.21 & 5.21 & 5.21 & 5.21 & 522 & 5.23 & 5.28 & 5.28 \\
\hline Int Fow PWD-GPM & 4.1 & & & & & & & & & \\
\hline OUtAlla. Fow RELGPM & 1.4 & & & & & & & & & \\
\hline OU:ATVap. Fow hato-GFM & 1.9 & & & & & & & & & \\
\hline Intel Tompereuref & 121.8 & & & & & & & & & \\
\hline Outlug. TemperatureF & 1252 & & & & & & & & & \\
\hline Otlavep. Tempertiref & 118.5 & & & & & & & & & \\
\hline Werer Flow Rato-GPM & & & & & & & & & & \\
\hline Were intel Temp, ff & 75.11 & & & & & & & & & \\
\hline We ter ovilet Tomp.f & 98.5 & & & & & & & & & \\
\hline IndaGbm/hrit2 & 84681 & & Temp & 120.6 & Density & 88.64 & & & & \\
\hline Subooolingf & 0.6 & & & & & & & & & \\
\hline Fow Observetion & & & & & & & & & & \\
\hline & ZONE 1 & ZONE2 & ZONE3 & ZONEQ & ZONES & ZONE6 & ZONET & ZONES & ZONE9 & ZONETO \\
\hline HoalnoutWeitnet) & 857.1 & 864.8 & 857.4 & 8642 & 8623 & 859.7 & 8582 & 860.8 & 859.1 & $\mathbf{8 8 5 . 6}$ \\
\hline Factlose-We'ts & 8.6 & 2 & 27 & 2.7 & 2.7 & 2.1 & 1.4 & 21 & 25 & 74.7 \\
\hline Horl Fix Buwthin? & 14057 & 14992 & 14852 & 14500 & 1494 & 14902 & 1487 & 14921 & 14\%8 & 15000 \\
\hline We Tromp. Tअ & T38.8 & 137.9 & 1352] & 735.4 & T39.8 & T375 & T37.8 & T38.8 & 735.8 & TS8.1 \\
\hline WE Tomp.14 & 136.8 & 135.4. & 137.1 & 137.5 & 137.5 & 737.8 & 138.8 & 138.8 & 137.2 & 783.8 \\
\hline Wa'Tomp.15F & 135.6 & 134 & 137.1 & 130.4 & 135.9 & 1378 & 130.8 & 137.3 & 135 & 187.1 \\
\hline WeITomp. It & 78 & 195.8 & 134.6 & 13a., & 185.1 & 138 & 188.6 & 188.8 & 184.1 & 181.7 \\
\hline WhTomp. MAF & 135.7 & 136.8 & 1352 & 135.8 & 135.6 & 138.6 & 139 & 139.4 & 135 & 185 \\
\hline hotrom Temp.f & 125.3 & 125.8 & 125.7 & 125.8 & 125.8 & 125.6 & 125.8 & 124.7 & 124.7 & 1228 \\
\hline Avo. Wa Temp.F & 136 & 136.3 & 1355 & 1362 & 156 & T37.8 & 138.2 & 138.5 & 135.4 & 135.7 \\
\hline Avo. WaIDT F & 10 & 9.8 & 9.2. & 9.7 & 9.5 & 11.6 & 12.3 & 13.2 & 10 & 10.8 \\
\hline Rempining Subooff & -4.3 & & & & & & & & & \\
\hline Exot Quatiny & 0.04 & 0.07 & 0.1 & 0.13 & 0.16 & 0.2 & 0.20 & 0.26 & 029 & 0.52 \\
\hline DXP.HBWWHhe & 14852 & 1520.3 & 1623.7 & 154.7 & 1565.8 & 1230.1 & 12126 & 1133.4 & 1488.2 & 1394.2 \\
\hline COMPUEDOH & & & & & & & & & & \\
\hline Jalouk & 1002 & $\$$ & 9382 & 9द्व.8 & 90032 & 887.8 & 874 & 867.8 & 8512 & 8422 \\
\hline Seavicudotwe) & 765.1 & T55.5 & 1518.2 & 75602 & 1684 & 1585.8 & 7588.4 & 75724 & 1578.2 & 1587.8 \\
\hline sadte & 1338.3 & 1360 & 1858.7 & 1370.7 & 1874.8 & 1377.1 & 1379.7 & 14045 & 1405.8 & 14सटर \\
\hline (Rि) & 108328 & 100118 & 96085 & $83 \sqrt[2]{ }$ & 80005 & $855 \%$ & 88018 & 79347 & FED| & T्यद्ध \\
\hline (F) & 1.537 & 1.902 & 2308 & 2.569 & 2008 & 3.018 & 3218 & 3.4T4 & 3.588 & 3.783 \\
\hline (समक) & 868.9 & 778.4 & -563.6 & 678.8 & 678 & 724.4 & $\pi 2.3$ & 818.8 & 860.1 & $80 \pi 5$ \\
\hline (HBOL) & 1538 & 1555.5 & 152य.3 & 15274 & 1521.8 & 15124 & 1503.3 & 140.7 & 14879 & Thent5 \\
\hline & & & & & & & & & & \\
\hline & ZONE2 & ZONE4 & ZONE6 & $201=8$ & ZONE10 & & & & & \\
\hline DPT huid tomp.f & 74.7 & & & & & & & & & \\
\hline DPLlguld Density omin & 93.75 & & & & & & & & & \\
\hline Eortions & & 4 & & 8 & 9.875 & & & & & \\
\hline Moatured opy Jid & 0.161 & 0.7169 & 0.485 & 2051 & 23\% & & & & & \\
\hline Zero con actions-psid & 0.087 & -0.067 & -0.75 & 0.127 & 0.27 & & & & & \\
\hline Comedopold & 0.104 & 0.818 & 1.188 & 1.98 & 2058 & & & & & \\
\hline Achentoph & 2 & 2.98 & 4.58 & 5.47 & 720 & & & & & \\
\hline Wheoured TPFig & 64.7 & & & & 3.7 & & & & & \\
\hline Meatured Overall & 101.7 & & & & & & & & & \\
\hline & & & & & & & & & & \\
\hline Computad DPA & 1.8 & 3.4 & 5.05 & 6.78 & 8.51 & & & & & \\
\hline Ficton-h & 0.26 & 0.68 & 1.20 & 203 & 2.86 & & & & & \\
\hline Acosteration-l & 0.27 & 0.42 & 0.58 & 0.75 & 0.86 & & & & & \\
\hline Elevation & 1.28 & 2.29 & 3.18 & 3.99 & 4.69 & & & & & \\
\hline & & & & & & & & & & \\
\hline
\end{tabular}




\begin{tabular}{|c|c|c|c|c|c|c|c|c|c|c|}
\hline Runt & 6011 & & 1 & I & & & & I & & \\
\hline Tosfivid & CAF10 & & & & & & & & & \\
\hline Dato: & 52193 & & & & & & & & & \\
\hline Time: & $7: 14$ & & & & & & & & & \\
\hline Noto & Analytical & Pintopat is & sassumed & & & & & & & \\
\hline Fow Aroal? & 0.00336 & & & & & & & & & \\
\hline Hod flow Areate & 0.19595 & & & & & & & & & \\
\hline Sa PTossure Pig & 65.1 & & & & & & & & & \\
\hline Sa. Tomperatro-f(inia) & 121.3 & & & & & & & & & \\
\hline Lq. Densing & 86.12 & 88.09 & 86.12 & 86.09 & 86.1 & 86.12 & 86.27 & $86 . \overline{27}$ & 86.27 & 80.80 \\
\hline Lend Hexteribm & 32.55 & 32.53 & 32.55 & 32.53 & 32.54 & 3256 & 32. 65 & 32.65 & 32.65 & ख27 \\
\hline Spocilothod Benthom & 0.275 & 0.275 & 0.275 & 0.275 & 0.275 & 0.275 & 0.275 & 0.275 & 0.276 & 0.275 \\
\hline Lq. Visoosh tom/hhr & 0.625 & 0.524 & 0.525 & 0.624 & 0.524 & 0.525 & 0.629 & 0.520 & 0.520 & 0.531 \\
\hline Lig. Conductivin Burfff & 0.0277 & 0.027 & 0.0277 & 0.027 & $0.02 \pi$ & 0.0277 & 0.0278 & 0.0278 & 0.0278 & 0.0279 \\
\hline Uq. Prandt No. & 5.21 & 5.21 & 5.21 & 5.21 & 5.21 & 5.21 & 522 & 5.22 & 5.22 & 5.23 \\
\hline Inlet Fiow Rate-GPM & 3.3 & & & & & & & & & \\
\hline Oulet Lig. Fow Rado-GPM & 1.4 & & & & & & & & & \\
\hline OU pet Vap. Fow Rate-GPM & 2 & & & & & & & & & \\
\hline Inlat Temperauref & 121.7 & & & & & & & & & \\
\hline Oubet Lq. TemperatureF & 125.5 & & & & & & & & & \\
\hline Oulat vep. Tomperaturof & 119.1 & & & & & & & & & \\
\hline Walor Fow Rate-GPM & & & & & & & & & & \\
\hline Water inlot Tomp.f & 75.9 & & & & & & & & & \\
\hline Weror odvet Tomp.F & 100 & & & & & & & & & \\
\hline Ena G bminn? & 682680 & & Tomp & 120.3 & Denty & 88.69 & & & & \\
\hline Subooding & -0.4 & & & & & & & & & \\
\hline Flow Observition & & & & & & & & & & \\
\hline & ZONE1 & ZONE2 & ZONE3 & ZONE4 & ZONE5 & ZONE6 6 & ZONE7 & ZONEB & ZONES & ZONETO \\
\hline HodinputWatnot) & 888.5 & 871.4 & 855.8 & 872.6 & 851.3 & 859.9 & 869.2 & 872.4 & 865.2 & 878.8 \\
\hline Hod Loss Watis & 8.5 & 2 & 27 & 2.7 & 27 & 2.1 & 1.4 & 2.1 & 25 & 14.6 \\
\hline 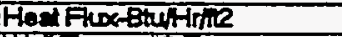 & 15055 & 15105 & 15008 & 15126 & 14930 & 14506 & 15087 & 15122 & 14938 & 15147 \\
\hline Wai Temp. BF & 130 & 137.6 & 735.4 & 136.6 & 139.7 & 137.7 & 138.2 & 78.5 & 735.7 & 138.2 \\
\hline WEITOmp. ThF & 135.8 & 135.6 & 137.2 & 137.4 & 187.7 & 138 & 139.2 & 139.1 & 737.4 & 7372 \\
\hline WEH Tomp. IEF & 135.9. & 134 & 138.8 & 138.6 & 138.1 & 137.9 & 737.4 & 137.4 & 135.4 & 137.0 \\
\hline WD Tomp. HIF & 72.7 & 135.4 & 134.8 & 134.4 & 19.4 & 138,2 & 138.8 & 139.1 & 1345 & 191.0 \\
\hline Wal Tomp. IaF & 135.8 & 138.2 & 135.4 & 135.6 & 135.7 & 138.8 & 139.2 & 139.6 & 135.4 & 125 \\
\hline Intresm Temp.f & 125.8 & 126 & 125.8 & 126 & 125.0 & 125.8 & 124.0 & 124.0 & 124.0 & 124.8 \\
\hline Avg. Wall Temp.f & 136 & 136.2 & 135.7 & 136.8 & 136.2 & 138 & 138.6 & 138.7 & 135.7 & 136 \\
\hline Aug. WaH DT $F$ & 9.5 & 9.5 & 9.2 & 9.6 & 9.7 & 11.5 & 18 & 13.2 & 10.1 & 11 \\
\hline Remaining subcooff & -5.1 & & & & & & & & & \\
\hline Exat Qusality & 0.04 & 0.08 & 0.12 & 0.16 & 0.2 & 0.24 & 0.28 & 0.32 & 0.36 & 0.4 \\
\hline EXP. HBunthnar & 1677.3 & 1587.1 & 1627.5 & 15812 & 1547.1 & 1298.8 & $11 \approx 0.8$ & 1149.4 & 1484.6 & 1300.1 \\
\hline COMPUTEDH & & & & & & & & & & \\
\hline Jaliouk & $10 \times 29$ & 997.8 & 960.4 & $\$ 51.6$ & 928.8 & 9124 & 901.1 & 82.3 & 879.8 & 2028 \\
\hline Sedvi(ado ube) & 1548.6 & 7558.3 & 1553 & 1564.7 & 1568.1 & 7560.6 & 1585.8 & 1578.9 & 1573.5 & 2028 \\
\hline $\operatorname{sen} 2$ & 1384.7 & 13912 & 13622 & 1375.8 & 1368 & 137 & 1378.5 & 1387.4 & 1380 & 3028 \\
\hline (स्थि) & 89278 & 79887 & 78382 & 73114 & 6900 & 68278 & 62469 & 59111 & 55705 & 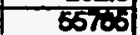 \\
\hline (F) & 1.687 & 2.000 & 2.481 & 2762 & 3.09 & 3.272 & 3.511 & 3.728 & 3.228 & 3.60 \\
\hline (1) & 320.6 & 420.0 & 497 & 657.8 & 617.8 & 660.5 & 708.11 & 760.8 & \$1.1 & क01.1 \\
\hline ( $\mathrm{BBO4})$ & 1544 & 1546.7 & 1635.8 & 1540.7 & 1526 & 1520.1 & 1516 & 1514.7 & 1503.9 & 15020 \\
\hline & & & & & & & & & & \\
\hline & ZONE2 & ZONE 4 & ZONEE & ZONEB & ZONETO & & & & & \\
\hline DP Tquid Temp.F & 74.8 & & & & & & & & & \\
\hline DP Liquid Density bmik & 93.74 & & & & & & & & & \\
\hline Elevetion- & 2 & 4 & B & 8 & 9.875 & & & & & \\
\hline Whesured DPPSid & 0.201 & 0.358 & 0.77 & 2.488 & 2.85 & & & & & \\
\hline Zaro Comections-psid & 0.037 & -0.057 & -0.75 & 0.121 & 0.273 & & & & & \\
\hline Correctod DPpild & 0.165 & 9.055 & 1.52 & 2.357 & 2.620 & & & & & \\
\hline Acter DPR & 7.9 & 262 & 8.88 & 4.74 & 8.34 & & & & & \\
\hline Mosetured TPPsig & 65.11 & & & & 3.1 & & & & & \\
\hline Measured Overalt & 102.38 & & & & & & & & & \\
\hline & & & & & & & & & & \\
\hline Computad DPI & 9.52 & 3.03 & 4.48 & 5.98 & 7.471 & & & & & \\
\hline Ficion-1 & 0.18 & 0.52 & 0.99 & 7.57 & 222 & & & & & \\
\hline Acoelertion-1 & 0.18 & 0.32 & 0.45 & 0.62 & 0.8 & & & & & \\
\hline Exvation-1 & 1.28 & 22 & 3.04 & 3.79 & 4.4 & & & & & \\
\hline & & & & & & & & & & \\
\hline
\end{tabular}




\begin{tabular}{|c|c|c|c|c|c|c|c|c|c|c|c|}
\hline Run! & 602 & & & & & & & & & & \\
\hline Tatfild & CFF10 & & & & & & & & & & \\
\hline Date: & 62293 & & & & & & & & & & \\
\hline Time: & $7: 14$ & & & & & & & & & & \\
\hline Note & Anstycal & Pin psat is & assumed & & & & & & & & \\
\hline Flow Areath & 0.00365 & & & & & & & & & & \\
\hline Hent Fow Areath & 0.18555 & & & & & & & & & & \\
\hline SEl prossurepigig & 64.9 & & & & & & & & & & \\
\hline 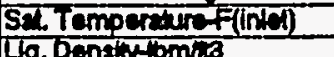 & 1212 & & & & & & & & & & \\
\hline 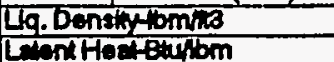 & 88.25 & 86.16 & 86.18 & 86.12 & 86.12 & 86.12 & 86.21 & 86.27 & 68.27 & 86.50 & \\
\hline 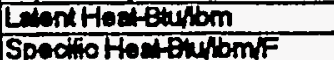 & 32.63 & 3257 & 3259 & 3256 & 32.55 & 3255 & 32.611 & 32.85 & 32.65 & $32 \pi$ & \\
\hline Specifo HenturbmF & 0.275 & 0.275 & 0.275 & 0.276 & 0.275 & 0.275 & 0.275 & 0.275 & 0.275 & 0.275 & \\
\hline Lo. Viroosh & 0.628 & 0.625 & 0.626 & 0.525 & 0.625 & 0.625 & 0.627 & 0.520 & 0.620 & 0.631 & \\
\hline Wq. Conductivit Buhw-Af & 0.0278 & 0.0278 & 0.0278 & 0.0277 & 0.027 & 0.027 & 0.0278 & 0.0278 & 0.0278 & 0.0279 & \\
\hline Liq. Prandtuno. & 622 & 5.21 & 5.22 & 5.21 & 5.21 & 5.21 & 5.22 & 5.22 & 5.22 & 520 & \\
\hline Inlot Fow Pate-GPM & 2.5 & & & & & & & & & & \\
\hline OutélLiq. Flow Palo-GPM & 1.3. & & & & & & & & & & \\
\hline Outel Vap. Fow Ral-GPM & 1.4 & & & & & & & & & & \\
\hline Inta Tomperamer & 121.6 & & & & & & & & & & \\
\hline Ousellug. Temperative & 126 & & & & & & & & & & \\
\hline OTClVep. Tomper Tref & 119.9 & & & & & & & & & & \\
\hline Wa' Flow PAO-GPM & & & & & & & & & & & \\
\hline Wer hlet Temp.f & 77.8 & & \pm & & & & & & & & \\
\hline Wecer ow Tomp.F & 103.2 & & 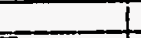 & & & & & & & & \\
\hline Intabminthe & 677282 & & Temp & 120.2 & Denciny & 86.7 & & & & & \\
\hline Suboolingf & -0.4 & & & & & & & & & & \\
\hline Flow Oberenvition & & & & & & & & & & & \\
\hline & ZONE1 1 & ZONE? & ZONE3 & ZONEA & ZONE5 & ZONE6 & ZONE7 & $20 N=8$ & ZONE9 & ZONEIO & \\
\hline Healinoutwathel & 863.5 & 861.2 & 862.6 & 884 & 858.7 & 852.5 & 868.5 & 868.7 & 853.6 & 1028 & \\
\hline Henlose-Wels & 9.7 & 2.3 & 3.1 & 3.1 & 3.1 & 24 & 1.6 & 24 & 3 & 127 & 88. \\
\hline Hod Flox-Bulfhice & 14858 & 14928 & 1458 & 1497 & 14855 & 14S5I & 15055 & 15024 & 1490 & 778 & \\
\hline Wal Tomp. 13F & T55.4. & T3T.1 & 73 & 136.1 & 739.6 & 737.8 & 138.5 & 138.3 & 737.1 & $72] .8$ & .933 \\
\hline Weil Temp. Wh & 735.8 & 135 & 136.7 & 137.1 & 737.5 & 138.2 & 130.2 & 139.11 & 188 & 127.7] & \\
\hline WE Tomp. I5F & 135.4 & 138.5 & 1360 & 138.4. & 130.1 & 1382 & 137.4 & 187.4 & 135.5 & 128.8 & +171 \\
\hline WhTomp. 11F & 79.5 & 134.8 & 1842 & 184.4. & 1852 & 138.2 & 189.1 & 139.4 & 134.8 & 127.1 & \\
\hline WEITomp.12F & 135.4 & 135.6 & 134.8 & 135,8 & 135.7 & 138.8 & 138.5 & 139.8 & 135.8 & 127.7 & $2715^{\circ}$ \\
\hline Intrean Tomp, $F$ & 125 & 125.6 & 125.4 & 125.8 & 125.8 & 125.8 & 125 & 124.0 & 124.0 & T2.8. & \\
\hline Avq.WUI Tomp.F & 135.5 & 135.7 & 1522 & 138 & 136.1 & 138.1 & 138.7 & 138.8 & 186,8 & 127.8 & \\
\hline Avo.WallDT.F & 9.8 & 9.4 & 9.1 & 9.5 & 9.7 & 11.8 & 129 & 13.2 & 10.7 & 3.2 & \\
\hline Romaining Subcootf & -6.6 & & & & & & & & & & \\
\hline Exaquey & 0.06 & 0.11 & 0.16 & 0.21 & 0.26 & 0.32 & 0.37 & 0.42 & 0.47 & 0.48 & \\
\hline EXP. HETURMRRF & 1523.7 & 1592.8 & 1638.8 & 1572.7 & 1542.1 & 1283.4 & 1170.7 & 1156.8 & 1398.6 & 350.1 & \\
\hline COMPUTEDH & & & & & & & & & & & \\
\hline Jelouk & 7057.3 & 1018.8 & 891.1 & $\$ 5.6$ & \$526 & 946.6 & \$s]| & 9192 & 1625 & 1624 & \\
\hline 5adylCudowb & 1655,8 & 754t.5 & T53.5 & 1551? & 156.6. & 1556.4 & 7560.1 & 7669.8 & 1625 & T626 & \\
\hline Sodye & 1870.8 & 1375.0 & 1351.7 & 13.0 .1 & 1358.2 & 1300.8 & 1371.8 & 137.8 & 1025 & To24 & \\
\hline (Rंख) & 60294 & 59180 & 55701 & 52527 & 29178 & 46825 & 4258 & 38871 & \$881 & \$851 & \\
\hline (F) & 1.680 & 2280 & 2708 & 3.059 & 3.365 & 3.647 & 3.824 & 4.18 & 4.18 & 4.18 & \\
\hline (सUA) & 2728 & 360.1 & 477.4 & -79 & 5+42 & 6890 & 63 & 675.1 & 675.1 & 675.1 & \\
\hline$(\mathrm{H} 304)$ & 15320 & 15344 & 1531.7 & 153.8 & 15350 & 1527.6 & 1522.8 & 1516.6 & 1516.8 & 1516.8 & \\
\hline & & & & & & & & \begin{tabular}{l|l}
+ \\
\end{tabular} & & & \\
\hline & $20 N=2$ & ZONE4 & ZONEB & $20 \mathrm{~N}=8$ & ZONESTO & & & & & & \\
\hline DPTguid Temp.f & 75.4 & & & & & & & & & & \\
\hline DPLguid Dentity tomil3 & 93.85 & & & & & & & & & & \\
\hline Elovent & & 4 & 6) & 8 & 9.875 & & & & & & \\
\hline MoasuredDPpaid & 0.138 & 1.300 & T.15 & 2.967 & 3.5611 & & & & & & \\
\hline Zero Correctionspaid & 0.087 & $=0.067$ & -0.75 & 0.121 & 0.278 & & & & & & \\
\hline Cormoded DPpild & 0.301 & 1.376 & 1.804 & 2846 & 3.258 & & & & & & \\
\hline Actiol & 2 & 2.04 & 3.38 & 3.98 & 5.28 & & & & & & \\
\hline Mestured Tipsig & 64.8 & & & & 3.7 & & & & & & \\
\hline Mesouredoverall & 10204 & & & & & & & & & & \\
\hline & & & & & & & & & & & \\
\hline Computed DPA & 1.4 & 258 & 3.72 & 4.9 & 6.17 & & & & & & \\
\hline Fiflon- & 0.14 & 0.39 & 0.75 & 1.21 & 1.61 & & & & & & \\
\hline Aocaternionth & 0.18 & 0.28 & 0.35 & 0.48 & 0.58 & & & & & & \\
\hline Elev'ion-1 & 1.18 & 1.84 & 2.62 & 3.21 & 3.80 & & & & & & \\
\hline & & & & & & & & & & & \\
\hline
\end{tabular}




\begin{tabular}{|c|c|c|c|c|c|c|c|c|c|c|}
\hline Rinn: & 603 & & I & & & & & & & \\
\hline Tost Fuid & CAF10 & & I & & & & & & & \\
\hline Data: & $6 \sqrt{2 / 931}$ & & & & & & & & & \\
\hline Time: & $7: 14$ & & & & & & & & & $L$ \\
\hline Note & Anajyicas & Pin $=$ psat is & assumed & & & & & & & \\
\hline Fow Aroatle & 0.00336 & & & & & & & & & \\
\hline Hod flow Aroa-R & 0.15595 & & & & & & & & & \\
\hline Sa Pressure psig & 65.1 & & & & & & & & & \\
\hline SI. Temper urof(inter) & 122.2 & & & & & & & & & \\
\hline Hg. Denstytbinis & 86.12 & 86.02 & 86.1 & 86.02 & 85.99 & 88.01 & 86.08 & 88.07 & 86.00 & 86.2 \\
\hline Leterd Hagitemibm & 3256 & 32.48 & 32.54 & 32.48 & 32461 & 32.47 & 32.5 & 3251 & 32.58 & 326 \\
\hline Specino Hon Burbmr & 0.275 & 0.276 & 0.275 & 0.276 & 0.276 & 0.276 & 0.275 & 0.275 & 0.275 & 0.275 \\
\hline Lig. Viscosithminh & 0.525 & 0.52 & 0.5231 & 0.522 & 0.627 & 0.522 & 0.623 & 0.524 & 0.624 & 0.527 \\
\hline Liq. Conductivity Buhr-ifF & $0.02 \pi$ & 0.027 & 0.0277 & $0.02 \pi$ & 0.027 & 0.027 & 0.027 & $0.02 \pi$ & $0.02 \pi$ & 0.0278 \\
\hline Liq. Prand No. & 5.21 & 5.2 & 5.21 & 52 & 5.2 & 5.2 & 5.2 & 5.21 & 5.21 & 5.22 \\
\hline Inlet Fow Pato-GPM & 1.7 & & & & & & & & & \\
\hline \multicolumn{11}{|l|}{ Outh Liq. Fow Rato-GPM } \\
\hline Oudot Vap. Flow Rato-GPM & 0.7 & & & & & & & & & \\
\hline Inlat Temperaluref & 1222 & & & & & & & & & \\
\hline Oulat Liq. Tamperaturef & T27 & & & & & & & & & \\
\hline \multirow{2}{*}{\multicolumn{11}{|c|}{$\begin{array}{l}\text { Oulot Vep. Tomperaturef } \\
\text { Weter fow Rate-GPM }\end{array}$}} \\
\hline & & & & & & & & & & \\
\hline \multicolumn{11}{|l|}{ Water hitet Tamp.f } \\
\hline Ware oultet Temp.f & 106.6 & & & & & & & & & \\
\hline Indabommire & 351762 & & Temp & 120.2 & Donsiy & 86.7 & & & & \\
\hline Subooolingf & 이 & & & & & & & & & \\
\hline \multicolumn{11}{|l|}{ Fow Observation } \\
\hline & ZONE1 & ZONE2 & ZONE3 & ZONE4 & ZONE5 & ZONE6 & ZONE7 & ZONE8 & ZONEQ & ZONETO \\
\hline Heat hout-Wats(ned) & 872 & $=85.6$ & 809.4 & 870.9 & 864.1 & 865.6 & 877.9 & $8 \pi 0.4$ & 108.8 & 101.1 \\
\hline Hod Los:-Wats & 9.5 & 2.2 & & 3.1 & 3.1 & 24 & 1.6 & 2.6 & 2.3 & 12.8 \\
\hline 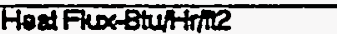 & 15116 & 15072 & 15070 & 15006 & 14970 & 15005 & 15218 & 16088 & 1853 & 1753 \\
\hline Wat Temp. 134 & 135.8 & - 137.6 & T35.3 & 137 & 740.4 & 130 & 139.8 & 1428 & 729.5 & T28.4 \\
\hline Wol Tomp.IfF & 136.1 & 135.2 & 137.8 & 138.1 & 138.2 & 739.1 & 140.4 & 142 & 1208 & T28.7 \\
\hline WA TOMP. I5F & 135.7 & 133.9 & 137.3 & 137 & 736.8 & 139.1 & 738.7 & 139.1 & 129.4 & 723.8 \\
\hline WalTomp. HF & 73.7 & 135.2 & 134.7 & 134.8 & 138.1 & 139.1 & 140 & 140.8 & 128.8 & 120 \\
\hline WN Tomp. ILF & 135.8 & 135.8 & 135.8 & 135.8 & 136.7 & 139.6 & 140.6 & 1442 & 128.8 & 1385 \\
\hline Entream Tomp.f & 125.8 & 126.4 & 125.8 & 126.4 & 126.6 & 126.5 & 126.2 & 128.1 & 126 & 125.8 \\
\hline Ayp. Well Temp.f & 135.8 & 136 & 135.7 & 136.7 & 137 & 139.1 & 139.8 & 1472 & 129.3 & 128.6 \\
\hline Avo. WaIDT.F & 9.4 & 8.9 & 9.1 & 9.8 & 9.7 & 11.9 & 13 & 14.4 & 3.2 & 3.2 \\
\hline Romaining Subooolf & 9.2 & & & & & & & & & \\
\hline Exal Quatily & 0.08 & 0.15 & $0 . \overline{23}$ & 0.31 & 0.39 & 0.48 & 0.54 & 0.62 & 0.69 & 0.62 \\
\hline EP. HBhuHRneE & 1613.7 & 169.5 & 1651.8 & 1565.6 & 1544.5 & 1261.2 & 1169.5 & 1046.4 & 572.6 & 5476 \\
\hline \multicolumn{11}{|l|}{ COMPUTEDH } \\
\hline Jilouk & T105 & 10732 & 10639.5 & 1002 & 1001.6 & 988.7 & T19.6 & 719.5 & TT9.5 & 718.4 \\
\hline 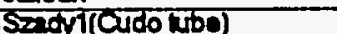 & 7500 & 1554.7 & T1551.8 & 1559.4 & 1557.2 & T550.8 & 719.6 & 118.6 & 119.5 & 718.4 \\
\hline 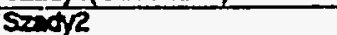 & 1387.6 & 1391.8 & 13872 & 1384.7 & 1390.6 & 1308.8 & 118.6 & 118.51 & 119.5. & 718.4 \\
\hline$(\mathrm{P} \cup \mathrm{O})$ & क्राम & 38985 & 35399 & 92128 & 2879 & 25505 & 25565 & 25805 & 25006 & 2535 \\
\hline (F) & 1.852 & 2565 & 3.103 & 3.588 & 3.920 & 4.28 & 428 & 4.28 & 4.28 & 4.28 \\
\hline (संயa) & 217.7 & 304.8 & 368.6 & 120.6 & 465.6 & 600 & 600 & 609 & 600 & 600 \\
\hline \multirow[t]{3}{*}{ (1BOL) } & 1548.6 & 1550.8 & 154.6 & 1549.1 & 156.1 & 15द1.7 & 1541.7 & 1541.7 & 1541.7 & T65.7 \\
\hline & & & & & & & & & & \\
\hline & ZONE2 & ZONE4 & ZONE8 & ZONE8 & ZONE10 & & & & & \\
\hline \multicolumn{11}{|l|}{ DP liquid Tomp.f } \\
\hline OPLiquid Density tom/13 & \$3.58 & & & & & & & & & \\
\hline Eleveliont & 2 & 4 & 6 & 8 & 9.875 & & & & & \\
\hline Weasured DPpsid & 0.485 & T.722 & 7.597 & 3.515 & 4.214 & & & & & \\
\hline Zero Corractions-psid & 0.037 & -0.067 & -0.75 & 0.121 & 0.23 & & & & & \\
\hline Corroctad DPP gid & $0.4 \overline{2}$ & 1.789 & 2.341 & 3.384 & 3.97 & & & & & \\
\hline Actuad DPA & 9.45 & 1.85 & 2.8 & 3.02 & 4.09 & & & & & \\
\hline Measured TPPsig & 66.11 & & & & 3.7 & & & & & \\
\hline \multicolumn{11}{|l|}{ Mesaured Overalli } \\
\hline & & & & & & & & & & \\
\hline Computad DRA & 1.19 & 2.1 & 297 & 3.84 & 5.09 & & & & & \\
\hline Friction-t & 0.08 & 0.25 & 0.49 & 0.78 & 0.86 & & & & & \\
\hline Accollerationt & 0.08 & 0.16 & 0.25 & 0.38 & 0.4 & & & & & \\
\hline Ebvation- & 1.02 & 1.7 & 223 & 2.67 & 3.73 & & & & & \\
\hline & & & & & & & & & & \\
\hline
\end{tabular}




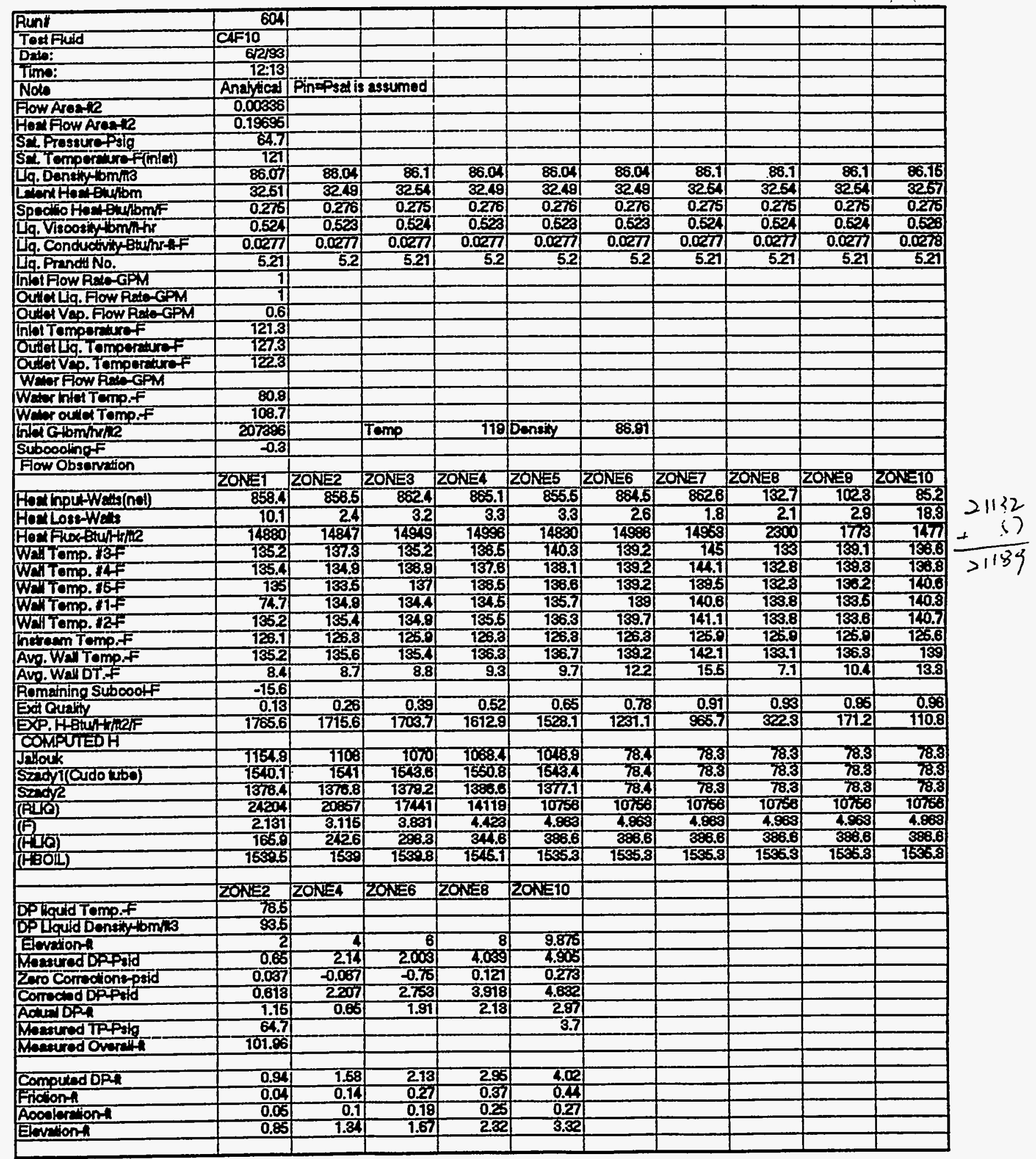




\begin{tabular}{|c|c|c|c|c|c|c|c|c|c|c|}
\hline Runt & \multicolumn{2}{|l|}{6051} & \multicolumn{2}{|c|}{ i } & & & & & & \\
\hline Tost Fuid & CAFIO & & & & & & & & & \\
\hline Data: & 6293 & & & & & & & & & \\
\hline Time: & $12: 13$ & & & & & & & & & \\
\hline Noto & Anarycal & Pin $=$ part is & $s$ assumed & & & & & & & \\
\hline Fow Areat2 & 0.00336 & & & & & & & & & \\
\hline Hoalfow Area-ti2 & 0.19695 & & & & & & & & & \\
\hline Sal Prosture sig & 64.3 & & & & & & & & & \\
\hline SA T Tomperature f(inlel) & 120.7 & & & & & & & & & \\
\hline एq. Density-bmatas & 86.25 & 86.19 & 86.22 & 86.17 & 86.2 & 86.23 & 86.31 & 86.31 & 86.33 & 88.48 \\
\hline Lrent Heartuthom & 32.63 & 3259 & 32.611 & 32.58 & 32.6 & 32.02 & 32.68 & 3268 & 32.69 & 32.79 \\
\hline Specifor Hoal BunbmiF & 0.275 & 0.275 & 0.275 & 0.275 & 0.275 & 0.275 & 0.275 & 0.275 & 0.275 & 0.275 \\
\hline Ug. Vieooshybminhr & 0.628 & 0.628 & 0.627 & 0.526 & 0.527 & 0.628 & 0.63 & 0.63 & 0.53 & 0.684 \\
\hline Liq. Condudivit Bthr-fF & 0.0278 & 0.0278 & 0.0278 & 0.0278 & 0.0278 & 0.0278 & 0.0279 & $0 . \infty 29$ & 0.0279 & 0.028 \\
\hline Liq. Prand No. & 5.22 & 5.2 & 5.22 & 5.21 & 5.22 & 5.22 & 5.23 & $5 \not 3$ & 5.23 & 5.24 \\
\hline Inlat Fow Rate-GPM & 4.8 & & & & & & & & & \\
\hline Oubet Lig. Fow Rato-GPM & 7.7 & & & & & & & & & \\
\hline Oubat Vap. Fow Rato-GPM & 2.3 & & & & & & & & & \\
\hline Inlat Tomperaturef & 121.3 & & & & & & & & & \\
\hline Outat Liq. Temperature & 124.4 & & & & & & & & & \\
\hline Outat Vep. Tomperaturef & 119 & & & & & & & & & \\
\hline Wale Flow Rato-GPM & & & & & & & & & & \\
\hline Water infet Tamp.f & 79.6 & & & & & & & & & \\
\hline Wer outel Temp.f & 104.9 & & & & & & & & & \\
\hline Finfl Gom/nnte & 891825 & & Temp & 120.9 & Deneity & 86.58 & & & & \\
\hline Suboooling & -0.6 & & & & & & & & & \\
\hline Flow Observation & & & & & & & & & & \\
\hline & ZONEI & ZONE2 & ZONES & ZONE4 & ZONE5 & ZONE6 & ZONEZ7 & $20 N=8$ & ZONEQ & ZONE10 \\
\hline Heat hout-Watsinat) & 1161.6 & 1156.8 & 1153 & 1152.5 & 1156.9 & 535.1 & 109.6 & 1012 & 120.1 & 107.8 \\
\hline Hoat Loss-Watts & 12 & 28 & 3.8 & 3.8 & 3.9 & 2.4 & 1.3 & 2.1 & 27 & 15 \\
\hline 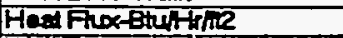 & 20736 & 20052 & 19987 & 19978 & 20054 & 9278 & 1900 & 1754 & 2082 & 1860 \\
\hline Wa Tamp & 137.1 & 33.6 & 136.4 & T31.2 & 735.4 & 131.8 & 127.6 & 127.2 & 127.3 & $\sqrt{20.8}$ \\
\hline Wall Tomp. IfF & 137.4 & -138 & 138.5 & 138.5 & 740.3 & 132 & 127.5 & 127.2 & 127.6 & 128.4 \\
\hline WM Tomp. $15 \mathrm{~F}$ & 138.6 & 134.3 & 138.4 & 137.5 & 138.8 & 132 & 127.4 & 127.3 & 127.3 & 120.0 \\
\hline Wallomp. PlF & 78.1 & 138.8 & 135.8 & 135.1 & 186.1 & 188 & 127.8 & 127.2 & 128.8 & T25.6. \\
\hline Wall Tomp. HEF & 187.1 & 137 & 736.8 & 133.1 & 137 & 133.1 & 127.6 & 1272 & 126.8 & 126.1 \\
\hline Instreem Temp.f & 126 & 125.4 & 1252 & 1255 & 135.3 & 125.1 & 124.6 & 124.6 & 124.6 & 120.6 \\
\hline Avg.Wall Tomp F & 137.1 & 1372 & 136.6 & 137.1 & 137.6 & 1322 & 127.6 & 127.2 & 1272 & 1262 \\
\hline Avo. Wall DT.F & 11.1 & 10.9 & 10.5 & 10.7 & 11.4 & 6.7 & 2.9 & 2.5 & 26 & 25 \\
\hline Remaining Subcoolf & -5 & & & & & & & & & \\
\hline Ext Quarty & 0.04 & 0.08 & 0.11 & 0.16 & 0.19 & 0.2 & 0.29 & 0.21 & 0.21 & 0.22 \\
\hline EXo.HBtumtmâ & 1807.5 & 1836.5 & 1899.6 & 1872 & 1764 & 1388,4 & 656.4 & 690.4 & 811.8 & 745.2 \\
\hline COMPUTEDH & & & & & & & & & & \\
\hline Jalouk & Tन1.8 & 7087.8 & 7039.6 & 273.8 & 273.7 & 273.7 & 273.5 & 273.5 & 273.5 & 2732 \\
\hline Szady $1($ Cudo wbo) & 1788.7 & 17927 & 1738.4 & 273.8 & 273.7 & 273.7 & 273.5 & 273.5 & 273.5 & 27.2 \\
\hline Sendy 2 & 1650.3 & 1648.8 & 1673.8 & 273.8 & 273.7 & 273.7 & 273.6 & 2735 & 2735 & 273.2 \\
\hline (R्यिa) & 120289 & 115855 & 111211 & 11121 & 111211 & 11121t & 111211 & 111211 & T1121T| & Tा1टT1 \\
\hline (F) & 1.582 & 2.075 & 2416 & 2.416 & 2416 & 2416 & 2.416 & 2.418 & 2.478 & 2418 \\
\hline (स्थि) & 750.3 & 564.6 & 657.1 & $\$ 57.1$ & 657.1 & 657.1 & 657.1 & 657.1 & 667.1 & 657.1 \\
\hline (1- & 1778.4 & 1773.8 & 1753.5 & 1765.5 & $17 \approx 2.6$ & 1736.6 & 1753.6 & $17 \approx 3.5$ & 1763.5 & 7763.6 \\
\hline & ZONE2 & ZONET & ZONE6 & ZONE8 & ZONETO & & & & & \\
\hline DP houid Tomp.f & 77.6 & & & & & & & & & \\
\hline DPLiquid Density 1 bm/13 & 53.34 & & & & & & & & & \\
\hline Elavelonth & & 4 & 6 & 8 & 9.875 & & & & & \\
\hline Mestured DP्यमid & ס.0.री & 0.475 & 0.183 & 1.758 & टदां & & & & & \\
\hline Zero Comectons-perd & $\overline{0.037}$ & -0.067 & -0.75 & 0.127 & 0.273 & & & & & \\
\hline Compad DFPaid & 0.004 & 0.512 & 0.858 & 1.635 & 1.943 & & & & & \\
\hline Achua DPA & 2.15 & 3.48 & 4.82 & 5.81 & 7.42 & & & & & \\
\hline Measured TPFig & 64.3 & & & & 3.7 & & & & & \\
\hline Meseured Overait & 100.91 & & & & & & & & & \\
\hline & & & & & & & & & & \\
\hline Compuled DPA & 2 & 3.85 & 5.88 & 7.75 & 9.57 & & & & & \\
\hline Friotion-t & 0.36 & 1 & 1.7 & 2.3 & 2.88 & & & & & \\
\hline Aoceleration- & 0.4 & 0.63 & 0.82 & 0.85 & 0.87 & & & & & \\
\hline Elevationt & 1.26 & 2.22 & 3.31 & 4.61 & 5.82 & & & & & \\
\hline & & & & & & & & & & \\
\hline
\end{tabular}




\begin{tabular}{|c|c|c|c|c|c|c|c|c|c|c|}
\hline \multirow{3}{*}{$\begin{array}{l}\text { Aunt } \\
\text { Togtfoid } \\
\text { Drto: }\end{array}$} & 606 & & & & & & & & & \\
\hline & CAF10 & & & & & & & & & \\
\hline \multirow{2}{*}{\multicolumn{6}{|c|}{ Timn: }} & & & & & \\
\hline & & & & & & & & & & \\
\hline \multirow{2}{*}{\multicolumn{11}{|c|}{\begin{tabular}{|c|} 
Ansylyeat Pin sosat is assumed \\
0.00336
\end{tabular}}} \\
\hline & & & & & & & & & & \\
\hline \multicolumn{11}{|l|}{ Hosl Flow Arosele } \\
\hline \multicolumn{11}{|l|}{ SA Prossure Psig } \\
\hline \multicolumn{11}{|l|}{ Se Temper the finlel) } \\
\hline \multirow{2}{*}{\multicolumn{11}{|c|}{ 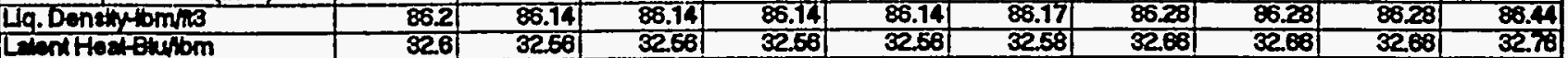 }} \\
\hline & 32.6 & 32.56 & 32.58 & 3256 & & & & & & \\
\hline 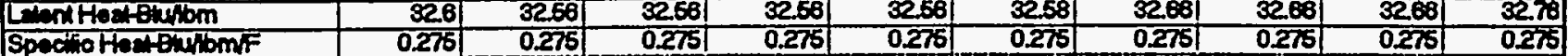 & 0.276 & 0.276 & 0.275 & 0.275 & 0.276 & 0.275 & 0.275 & 0.275 & 0.275 & 0.276 \\
\hline \multicolumn{11}{|l|}{ Liq. Vieoosintominhe } \\
\hline \multirow{2}{*}{\multicolumn{11}{|c|}{ 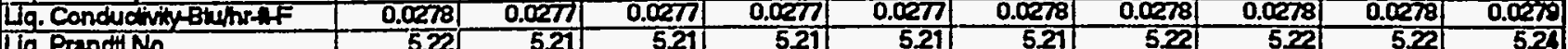 }} \\
\hline & 5.22 & 5.21 & 5.21 & 5.21 & 5.21 & 5.21 & 5.22 & 5.22 & 5.22 & 5.24 \\
\hline Intot flow Rato-GPM & 4.1 & & & & & & & & & \\
\hline 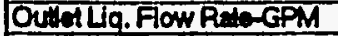 & 1 & & & & & & & & & \\
\hline Oullot Vho. Flow Rato-GPM & & & & & & & & & & \\
\hline Intal Tomporiurof & 121.2 & & & & & & & & & \\
\hline Outat Lq. Tomporatura & 124.8 & & & & & & & & & \\
\hline Outat vap. Tomporturiof & 119 & & & & & & & & & \\
\hline WeM Fow RaL-GPM & & & & & & & & & & \\
\hline Weir intel Tomp.F & 80.2 & & & & & & & & & \\
\hline Whor ould Tomp.f & 106.3 & & & & & & & & & \\
\hline Thlol Glominitie & 847616 & & Tramp & 120.7 & Donsiky & 86.62 & & & & \\
\hline Subooolingt & -0.4 & & & & & & & & & \\
\hline Flow Observation & & & & & & & & & & \\
\hline & ZONET & ZONE2 & ZONE3 & ZONE 4 & ZONES & ZONE6 & ZONET & ZONEB & ZONE9 & ZON:STO \\
\hline 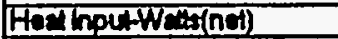 & 1184.5 & 11622 & 1161.3 & 1160.2 & 1165.3 & 542.6 & 112.3 & 104.8 & 121.5 & 113.8 \\
\hline 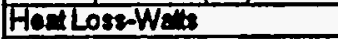 & 10.5 & 2.5 & 3.3 & 3.3 & 3.4 & 2.1 & 1.11 & 1.7 & 2.3 & 12.7 \\
\hline Hos Flox-Btuhtrint & 20186 & 20146 & 20130 & 20111 & 20006 & 9406 & 1947 & 1817 & 2106 & 1964 \\
\hline Wall Tomp. 13F & 737.2 & -940 & 738.7 & 137.4 & 1423 & 73्32 & 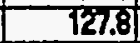 & 127.3 & 727.6 & 128.7 \\
\hline WE Tomp. If & 737.8 & 138.4 & 138.8 & 139 & 138.5 & 1322 & T27.7 & 127.6 & 120.1 & Tएटा? \\
\hline WNITomp. $75+$ & 153.7 & 134.5 & 738.8 & 737.8 & 138.8 & 1322 & 1270 & 127.6 & 127.0 & 127.1 \\
\hline Wal Tamp. IIF & 74.4 & 190.7 & 195.6 & 195.2 & 150.8 & 1882 & 127.8 & 127.1 & 121 & 128.1 \\
\hline Wal Tomp. & 137.2 & 137.2 & 136.7 & 136.8 & 1372 & 1332 & 127.8 & 227,4 & 121 & 128.7 \\
\hline Indrean Temp.F & 125.3 & 125.7 & 125.7 & 125.7 & 125.7 & 125.6 & 124.8 & 124.8 & 124.8 & 128.8 \\
\hline Avg. Wall Tomp.F & 137.1 & 137.6 & 137 & 137,4 & 1372 & 132.5 & 127.8 & 127.4 & 1275 & 126.8 \\
\hline AUD. WEITT.F & 10.9 & 11 & 10.4 & 10.8 & 10.8 & 6.5 & 2.8 & 2.5 & 2.8 & 2.8 \\
\hline Remaining Subooff & 5.4 & & & & & & & & & \\
\hline Exi Qu2'ty & 0.05 & 0.09 & 0.13 & 0.17 & 0.22 & 0.24 & 0.24 & 0.24 & 0.25 & 0.25 \\
\hline 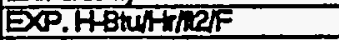 & 1854 & 1837.4 & 7942.1 & 1868 & 1885.7 & 1441.6 & 677.8 & 733.1 & 8212 & 684 \\
\hline COMPUTEDH & & & & & & & & & & \\
\hline Jallowk & 7185 & T125. & 7078.8 & दूt.5 & 241.5 & 2दा.4 & 24.2 & 2413 & 241.2 & या7 \\
\hline 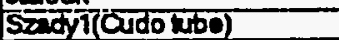 & 1791 & 1797.7 & $180 t .6$ & 247.5 & 261.5 & 241.4 & 267.2 & $24 \sqrt{3}$ & 261.2 & $24 \pi$ \\
\hline seady? & 7656.4 & 1050.4 & 1605.2 & 221.5 & 241.5 & 241.4 & 241.2 & 241.2 & 261.2 & \\
\hline$(P \square)$ & 102014 & 98462 & 83961 & 98951 & 93951 & 93951 & 839511 & 96951 & 93561 & 98351 \\
\hline (F) & 1.614 & 2.147 & 2534 & 2.524 & 2.524 & 2.524 & 2524 & 2524 & 2524 & 2.524 \\
\hline (1)Da) & 387.1 & 515.8 & 605.75 & 605.7 & 605.7 & 605.7 & 605.7 & 605.7 & 605.7 & 606.7 \\
\hline 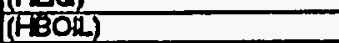 & 1785 & 7783.5 & 7778.6 & 7778.5 & 1778.5 & 177.5 & 1778.5 & 1778.5 & 1778.5 & \\
\hline & & & & & & & & & & \\
\hline & ZONE2 & ZONEA & ZONE6 & ZONE & ZONETO & & & & & \\
\hline DP Rqudd Tomp.F & 76.2 & & & & & & & & & \\
\hline DPLTuid Densitytomit3 & 93.64 & & & & & & & & & \\
\hline Elevaliont & & & & & 9.875 & & & & & \\
\hline Whanured DPPSid & 0.082 & 0.697 & 0.578 & 2.153 & 2712 & & & & & \\
\hline zero Comectons - sid & 0.037 & -0.007 & -0.70 & 0.121 & 0.278 & & & & & \\
\hline Comond DPF ind & 0.055 & 0.704 & 1203 & 2.012 & 2.438 & & & & & \\
\hline Adtel DPA & 2.07 & 3.00 & 4.38 & 5.3 & 6.22 & & & & & \\
\hline Maciured TPuig & 64.6 & & & & 3.7 & & & & & \\
\hline Maspured Overali & 901.28 & & & & & & & & & \\
\hline & & & & & & & & & & \\
\hline Comoltad DPA & 1.8 & 3.44 & 52.24 & 7.08 & 8.78 & & & & & \\
\hline Fitotiont & 0.20 & 0.83 & 1.4.2 & 1.92 & 2.4 & & & & & \\
\hline Acosterniont & 0.32 & 0.52 & 0.69 & 0.71 & 0.74 & & & & & \\
\hline Exvation-1 & 1.19 & 2.00 & 3.13 & 4.4 & 5.59 & & & & & \\
\hline & & & & & & & & & & \\
\hline
\end{tabular}




\begin{tabular}{|c|c|c|c|c|c|c|c|c|c|c|}
\hline Runt & \multicolumn{2}{|l|}{607} & & I & & & & 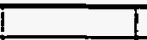 & I & 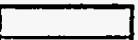 \\
\hline Test Fuid & CAF10 & & & 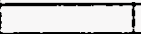 & & & & & & \\
\hline Dato: & 62 93 & & i & L & & & & & & \\
\hline Time: & $12: 13$ & & & & & & & & & \\
\hline Noto & Analytical & Pind pat is & assumed & & & & & & & \\
\hline Forw Area-12 & 0.00356 & & & & & & & & & \\
\hline Head Fow Areate & 0.1965 & & & & & & & & & \\
\hline Sa. Prossure 3 sig & 63.7 & & & & & & & & & \\
\hline Sat. Tomperaturef(inlet) & 120.2 & & & & & & & & & \\
\hline Ug. Densty tbmins & 88.2 & 86.1 & $8 \overline{6.14}$ & 86.12 & 88.12 & 86.15 & 88.2 & 86.23 & 86.25 & 80.38 \\
\hline Lent fles buthm & 32.8 & 32.54 & 32.68 & 32.55 & 32.50 & 32.57 & 32.6 & 32.82 & 32.68 & 32.72 \\
\hline Specifo thatbutbmf & 0.275 & 0.275 & 0.275 & 0.275 & 0.275 & 0.275 & 0.275 & 0.275 & 0.275 & 0.275 \\
\hline La. $\sqrt{1}=00 \mathrm{~s}$ ibomithr & 0.627 & 0.624 & 0.625 & 0.525 & 0.525 & 0.625 & 0.627 & 0.628 & 0.628 & 0.591 \\
\hline 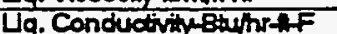 & 0.0278 & 0.027 & $0 . \infty \pi$ & 0.027 & $0.02 \pi$ & 0.0278 & 0.0278 & 0.0278 & 0.0278 & 0.0270 \\
\hline Lig. Prandt No. & 5.22 & 5.21 & 5.21 & 5.21 & 521 & 5.21 & 522 & 5.22 & 5.22 & 520 \\
\hline Inlet Fow Rato-GPM & 3.3 & & & & & & & & & \\
\hline Outel Lig. Fow Rato-GPM & 1.2 & & & & & & & & & \\
\hline Outel Vap. Fow Rate-GPM & 2.1 & & & & & & & & & \\
\hline Inlal Temporaturef & 120.8 & & & & & & & & & \\
\hline Oubat Lig. Temperaturef & 125.7 & & & & & & & & & \\
\hline Outel Vap. TomperatureF & 119.7 & & & & & & & & & \\
\hline WEH Fow Ra-GPM & & & & & & & & & & \\
\hline Water inted Temp.f & 80.2 & & & & & & & & & \\
\hline Wew outel Tomp.F & 104.6 & & & & & & & & & \\
\hline Inlel Glominine & 682413 & & Temp & 120.6 & Density & 86.65 & & & & \\
\hline Subcooling $F$ & -0.7 & & & & & & & & & \\
\hline Fow Obsorvation & & & & & & & & & & \\
\hline & ZONE1 & ZONE2 & ZONE3 & ZONE & ZONE5 & ZONE6 & ZONE7 & ZONE8 & ZONE9 & ZONE 10 \\
\hline Healingutwatsinat) & 1155.2 & 1153 & 1148.8 & 1149.8 & $=1158.4$ & 1855.4 & 268.4 & 107.8 & 124.5 & 118.5 \\
\hline Hoal LosetWats & 10.1 & 2.4 & 32 & 3.2 & 3.2 & 2.4 & 1.2 & 1.6 & 22 & 12.0 \\
\hline Had Fix-Bhenthile & 20005 & 19987 & 19915 & 1993 & 20080 & 20003 & 405 & 1865 & 2158 & 2054 \\
\hline Wa TOmp. Tअ & 7372 & 140.1 & 736.7 & 137.7 & 141.9 & 738.6 & 13.4 & 727.7 & 128.3 & T27.4 \\
\hline Wa Tomp. If & 737.3 & 136.4 & 139 & 139.1 & 138.4 & 138.2 & 131.2 & 127.7 & 728.7 & 127.8 \\
\hline WE TEmp. ISF & 136.7 & 134.6 & 139 & 138.1 & 137 & 137.5 & 130.6 & 127.7 & 128.3 & 1282 \\
\hline WE Tomp. IIF & 78.4 & 198.5 & 135.7 & 135.6 & 1385 & 138.8 & 130.5 & 127.4 & 127.4 & 120.0 \\
\hline Wal Tomp. IIF & 1372 & 137.2 & 136.71 & 136.8 & 137.6 & 139.6 & 130.5 & 127.7 & 127.7 & 127.8 \\
\hline hetreen Temp. $f$ & 125.3 & 125.8 & 25.7 & 125.8 & 125.8 & 125.6 & 125.8 & 125.1 & दूळ & 1242 \\
\hline Avg. Wall Tomp.F & 137.1 & 137.6 & 137 & 137.6 & 137.4 & 138.2 & 130.8 & 127.6 & 928.1 & 127.5 \\
\hline Avg. Wall DT. $f$ & 10.9 & 10.8 & 90.4 & 10.9 & 10.7 & 91.7 & 5.3 & 2.5 & 3 & 3.2 \\
\hline Remaining Subcooff & -7 & & & & & & & & & \\
\hline Exat Cuaty & 0.06 & 0.11 & 0.16 & 0.22 & 0.27 & 0.32 & 0.34 & 0.34 & 0.35 & 0.35 \\
\hline EXP. H-8tuthrierF & 1838 & 1851.2 & 1910.4 & 1828.8 & 18824 & 1708.9 & 987.5 & 767 & 723.6 & 648.8 \\
\hline COMPUTEDH & & & & & & & & & & \\
\hline Jatouk & 7208.9. & T157.2 & T1क. & 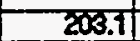 & 203.1 & 2003 & 203 & zoe.91 & स्20.9 & 3027 \\
\hline sendvicudo rbal & T7828 & 7790.8 & 178.1 & 203.1 & 200.1 & 200 & 203 & छट्वा & 2029 & 2027 \\
\hline Szady? & $18+5.6$ & 1675.1 & 167.9 & 203.1 & 200.1 & 203 & 203 & 202.9 & 2029 & 202.7 \\
\hline (RL) & 82028 & 77834 & $732 \pi 1$ & $732 \pi$ & 7327 & $732 \pi$ & 7827 & $782 \pi$ & $73 \mathrm{C}$ & 7527 \\
\hline (I) & 1.712 & 2.316 & 2736 & 2.738 & 2.736 & 2.766 & 2.796 & 2736 & 2.756 & 2.786 \\
\hline (1) & 346.2 & 467.3 & 5521 & 552.1 & 552.1 & 8521 & -552.1 & 552.1 & 552.1 & 6521 \\
\hline (HOIL) & 1778.6 & 17802 & 177.4 & 177.4 & 1771.4 & 1771.4 & 177.4 & 1771.4 & 177.4 & $77 \pi .4$ \\
\hline & & & & & & & & & & \\
\hline & ZONE2 & ZONE4 & ZONEE6 & ZONE8 & ZONE10 & & & & & \\
\hline DP liquid Tomp.f & 75.4 & & & & & & & & & \\
\hline DPUouid Dentinglom/3 & 93.65 & & & & & & & & & \\
\hline Erevitiont & & 4 & & & 9.875 & & & & & \\
\hline Mbanured DPPsid & 0.119 & 0.973 & 0.854 & 2.520 & 3.24 & & & & & \\
\hline Zero Com cions-psid & 0.087 & -0.057 & -0.70 & 0.127 & 0.278 & & & & & \\
\hline Comected DP psid & 0.002 & 1.04 & 1.601 & 2.408 & 2.974 & & & & & \\
\hline Achul DPA & 2.08 & 2.8 & 3.83 & 4.88 & 5.75 & & & & & \\
\hline Whasured TPPadg & 68.7 & & & & 3.7 & & & & & \\
\hline Meaured Overelt & 100.02 & & & & & & & & & \\
\hline & & & & & & & & & & \\
\hline Computed DPan & 9.58 & 2.99 & 4.4.17 & 6.16 & 7.78 & & & & & \\
\hline Finctiont & 0.23 & 0.6 & 1.26 & 1.68 & 2.08 & & & & & \\
\hline Accolereiont & 0.24 & 0.49 & 0.62 & 0.66 & 0.68 & & & & & \\
\hline Elevetiont & 1.12 & 1.5 & 2.59 & 3.82 & 4.97 & & & & & \\
\hline & & & & & & & & & & \\
\hline
\end{tabular}




\begin{tabular}{|c|c|c|c|c|c|c|c|c|c|c|}
\hline Runt & 608 & & & & & & & & & \\
\hline Test Fuxd & CAF10 & & & & & & & & & \\
\hline Dato: & $6 / 2 / 93$ & & & & & & & & & \\
\hline \multicolumn{11}{|l|}{ Time: } \\
\hline Now & Anstytical & \multicolumn{2}{|c|}{ Pinepied is assumed } & & & & & & & \\
\hline Fow Areane & 0.00356 & & & & & & & & & \\
\hline Head Fow Areat? & 0.19656 & & & & & & & & & \\
\hline Set. Pioseturetsig & 624 & & & & & & & & & \\
\hline Sal. Tomperciurof(n) & 719 & & & & & & & & & \\
\hline Lq. Denthethom/is & 80.49 & 86.22 & 88.23 & 86.22 & 8.21 & 88.2 & 86.8 & 86.81 & 86.31 & 86.4 \\
\hline Lent Heaterusiom & 328 & 32.61 & 32.62 & 32.01 & 320 & 32.8 & s267 & 3268 & 3268 & 3278 \\
\hline Specino Hot Eutromp & 0.275 & 0.275 & 0.275 & 0.275 & 0.275 & 0215 & 0.275 & 0.275 & 0.275 & 0.275 \\
\hline ía. Visoosintom Thr & 0.534 & 0.621 & 0.628 & 0.627 & $0.5 \overline{2}$ & 0.627 & 0.529 & 0.68 & 0.63 & 0.692 \\
\hline 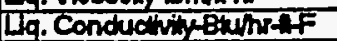 & 0.08 & 0.0278 & 0.028 & 0.0278 & 0.0278 & 0.0278 & 0.0270 & $0.02 \pi$ & 0.0279 & 0.0270 \\
\hline Lla. Pranded No. & 5.24 & 5.22 & 5.22 & 5.22 & 5.22 & 522 & 5.23 & 5.23 & 5.20 & 5.28 \\
\hline Inter Flow Re-GPM & 25 & & & & & & & & & \\
\hline Outhlla. Fow Rete-GPM & 1.1 & & & & & & & & & \\
\hline Outel Vap. Flow Rade-GPM & 1.5 & & & & & & & & & \\
\hline Indet Temperduret & 120 & & & & & & & & & \\
\hline outille. Tempermitref & 725.4 & & & & & & & & & \\
\hline otaivep. Tomperature F & 178.3 & & & & & & & & & \\
\hline Weir Flow PAY-GPM & & & & & & & & & & \\
\hline Wenter the Temp.f & 81.2 & & & & & & & & & \\
\hline War ould Tomp, & 108.3 & & & & & & & & & \\
\hline Intakiomnrie & 518489 & & Temp & 118: & Densihy & 86.81 & & & & \\
\hline Subooding ff & -1 & & & & & & & & & \\
\hline Flow Observalion & & & & & & & & & & \\
\hline & ZONET & ZONE2 2 & \begin{tabular}{|l|l|} 
ZONE & 2 \\
\end{tabular} & ZONEA & ZONE5 & ZONE6 & $20 \mathrm{~K}=7$ & ZON:E8 & ZON=S & CONEIO \\
\hline 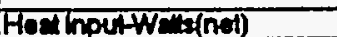 & 1155.4 & 1154.8 & 1140.6 & 1150.8 & 11524 & 568.7 & 205.1 & 108 & 118.5 & 1152 \\
\hline FanLose-Wests & 10 & 2.3 & 3.2 & 3.2 & 3.2 & 2.1 & 1.2 & 1.6 & 22 & 12.8 \\
\hline How Flo thrtide & 20038 & 20000 & 13928 & 19950 & 19978 & 67il & 4962 & 1872 & कीता & 1997 \\
\hline WE TOMP B & 138.6 & 73.4 & 138.1 & T87.2 & 747.8 & 133.6 & 128.8 & T27.3 & 121.8 & 1272 \\
\hline WETTOMp.14F & 138.6 & 135.8 & 738.6 & 138.6 & 187.8 & 133.4 & 120.7 & 127.8 & 128.1 & 727.5 \\
\hline We Tamp.15F & 180.1 & 134 & 138.6 & 737.5 & 788.2. & 138.1 & 129.1 & 127.3 & 127.8 & 127.6 \\
\hline Wh" Tomp. IIF & 7.1 & 158.1 & 135 & 139.8 & 135.8 & T88.0 & 181.1 & स्टत & 128.8 & 120.8 \\
\hline WET'Temp. 12 & 136.6 & 138.6 & 186.1 & 165.1 & 137 & 183.7 & 737.1 & 127.3 & 127.1 & 1280 \\
\hline Instreen tomp. & 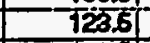 & 125.2 & 125.1 & 125.2 & 135.8 & 126.5 & 124.7 & 124.6 & 124.6 & T24. \\
\hline AVo. We Tomp.F & 138.6 & 137 & 138.5 & 137.1 & 136.7 & 133.4 & 1302 & 1272 & 127.6 & सE. \\
\hline Avo. WATDT F & 12 & 10.9 & 10.4 & 10.8 & 10.5 & 7.7 & 52 & 2.6 & 2.9 & 29 \\
\hline Reminino subooof & -9.2 & & & & & & & & & \\
\hline Ext Quarity & 0.08 & 0.15 & 0.27 & 0.28 & 0.35 & 0.39 & 0.4 & 0.41 & 0.42 & 0.42 \\
\hline 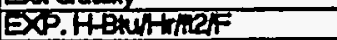 & 16528 & 1840.6 & 1907 & 1822 & 1898.5 & $12 \pi .7$ & 943.7 & 7326 & 7227 & 683.8 \\
\hline COMPUTEDH & & & & & & & & & & \\
\hline Jatouk & ख्या & T1822 & 1149.8 & 1629 & 1620 & TE्रु & TE:8 & 1528 & 1628 & 1627 \\
\hline sady (Cudo ubs) & 7708.2 & 17827 & 1780.8 & 15:0 & 1620 & 1620 & 1628 & 1628 & 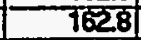 & 1627 \\
\hline Sradty & TEम & 1643.1 & 167.6 & 1620 & 1620 & 1620 & 1ह28 & 1628 & 1828 & 1627 \\
\hline (FDO) & 81081 & 57168 & 52676 & 52570 & 52878 & 52675 & 5287 & 5287 & 5257 & 62076 \\
\hline (F) & $1.8 \pi$ & 2541 & 3.083 & 3.03 & 3.088 & 3.038 & 3.038 & 3.083 & 3.088 & 8.088 \\
\hline (1) & 297.8 & 411.3 & 780.0 & 400.0 & 700.01 & 4000 & 780.0 & 400.0 & 400.0 & 480.5 \\
\hline (मBOH) & $1760 \mathrm{~A}$ & 1775.8 & 1758.1 & 1768.1 & 7768,1 & 178.1 & $77 \approx \pi$ & 17521 & 1768.1 & TZ3.1 \\
\hline & & & & & & & & & & \\
\hline & ZONE2 & ZOREA & ZONE6 & $201=8$ & ZONIETO & & & & & \\
\hline bopqud tamofF & 75.6 & & & & & & & & & \\
\hline 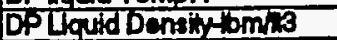 & $\$ 3.62$ & & & & & & & & & \\
\hline Eloventiont & & & & & 9.675 & & & & & \\
\hline Moetured DPFald & 0.703 & T.34 & 1.258 & 293 & 3.761 & & & & & \\
\hline Zero Comections-peld & 0.037 & -0.087 & $=0.75$ & 0.121 & 0.27 & & & & & \\
\hline Comeand DF ald & 0.077 & 7.411 & 2.008 & 2808 & 3.488 & & & & & \\
\hline Acted DPa & 205 & 1.88 & 3.16 & 3.8 & a69 & & & & & \\
\hline Moatured Tppos & E24 & & & & 3.7 & & & & & \\
\hline Wheuredoventil & 87.84 & & & & & & & & & \\
\hline & & & & & & & & & & \\
\hline Compeled DPa & 9.36 & 253 & 3.74 & 5.20 & 6.88 & & & & & \\
\hline Filont & 0.16 & 0.48 & o.gi & 12 & 1.48 & & & & & \\
\hline Acosterniont & 0.17 & 0.37 & 0.45 & 0.48 & 0.5 & & & & & \\
\hline Envationth & 1.03 & 1.74 & 238 & 3.58 & 4.7 & & & & & \\
\hline & & & & & & & & & & \\
\hline
\end{tabular}




\begin{tabular}{|c|c|c|c|c|c|c|c|c|c|c|}
\hline Rent & 609 & & & & & & & & & \\
\hline Toufind & CAF10 & & & & & & & & & \\
\hline Date: & 6293 & & & & & & & & & \\
\hline Time: & $15: 17$ & & & & & & & & & \\
\hline Now & Anatitial & Pincipsat is & $\$$ assumed & & & & & & & \\
\hline Flow Area-12 & 0.00336 & & & & & & & & & \\
\hline Hest Fow Arest? & 0.19695 & & & & & & & & & \\
\hline SA. Piessurahigh & 63 & & & & & & & & & \\
\hline SI Temperatur f f(ndC) & Tा9.8T & & & & & & & & & \\
\hline Lg.Dent & 86.48 & $8 \overline{825}$ & 88.3 & 88.28 & 86.28 & 88.27 & 88.38 & 86.91 & 86.35 & 86.48 \\
\hline Lend Hed exprom & 32.79 & 32.88 & 32.7 & 3268 & 32.68 & 32.85 & 3289 & 32.68 & 32.7 & 327 \\
\hline Spocine Hasterulom/t & 0.275 & 0.275 & 0.275 & 0.275 & 0.275 & 0.275 & 0.275 & 0.275 & 0.275 & 0.275 \\
\hline Lig. Viscostyomithr & 0.634 & 0.628 & 0.520 & 0.620 & 0.6201 & 0.620 & 0.63 & 0.68 & 0.637 & 0.639 \\
\hline Lig. Conductivatyshraf & 0.028 & 0.0278 & 0.0279 & 0.0278 & 0.0278 & 0.0278 & 0.0279 & 0.0279 & 0.0279 & 0.028 \\
\hline Lq. Prendil No. & 5.24 & 5.22 & 5.23 & 52 & 5.22 & 5.22 & 528 & 5.28 & 5.23 & 5.24 \\
\hline Intof Fow Rato-GPM & 1.7 & & & & & & & & & \\
\hline Oullilig. Fow Roto-GPM & 9.1 & & & & & & & & & \\
\hline Orint Veo. Fow Rado-GPM & 0.8 & & & & & & & & & \\
\hline 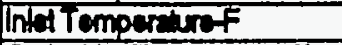 & 120.1 & & & & & & & & & \\
\hline Outel Liq. Tomperaturef & 125.5 & & & & & & & & & \\
\hline 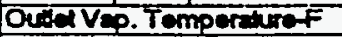 & 119.8 & & & & & & & & & \\
\hline Wala flow Ran-GPM & & & & & & & & & & \\
\hline Wher hilet Temp.f & 81.2 & & & & & & & & & \\
\hline Weiter ould Temp.f & 108 & & & & & & & & & \\
\hline Inder G tomminic & 353504 & & Tomp & 118.1 & DDentiny & 86.80 & & & & \\
\hline Subcooling & -0.5 & & & & & & & & & \\
\hline Fonw Obsenvation & & & & & & & & & & \\
\hline & ZONET & ZONE2 & ZONES & ZONE4 & ZONE5 & ZONES & ZONET & ZONE8 & ZONES & $20 N=10$ \\
\hline Hed hpetwathed & 1161.4 & 1158.5 & 1155.8 & 1158.5 & 1167.3 & 584.8 & 287.5 & 109.6 & 121.1 & 116.7 \\
\hline HedLose-Wets & 10.1 & 24 & 3.2 & 3.2 & 3.8 & 27 & 1.2 & 1.7 & 2.3 & 128 \\
\hline Hed Fiox Buththe & 20132 & 20082 & 20035 & 20082 & $2008 \pi$ & 9792 & 4834 & 1898 & 209 & 202 \\
\hline We Themp. Bf & 135.8 & 138.8 & 135.8 & $\mid 38.7$ & 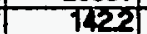 & 73.1 & Fo.1 & 127.3 & T282 & T272 \\
\hline WET Tomp. If & 138.11 & 1352 & 738.8 & 738.2 & 738.6 & 73 & 720.8 & 127.5 & T28.8 & 127.9 \\
\hline W:T Tomp. T5F & 135.5 & 138.0 & 138.1 & 137.3 & 157.8 & 13 & 120.6 & 127.5 & 128.1 & 127.9 \\
\hline WE Tomp. $81 \mathrm{~F}$ & 72.5 & 1356 & 134.8 & 134.6 & 193 & 134 & 130.2 & 127 & 127.1 & 120,6 \\
\hline WElTemp. I2F & 135.8 & 736.1 & 135.8 & 135.7 & 138.8 & 132.2 & 130.1 & 127.1 & 127.1 & 127 \\
\hline Intreem Temp.F & 120.6 & 125 & 124.7 & 124.8 & 124.8 & 124.0 & T24.5 & 124.6 & 124.4 & T28.7 \\
\hline Avq.Wall Tomp.f & 135.8 & 136.4 & 1362 & 136.7 & 1372 & 133.3 & 130 & 127.3 & 127.8 & 1272 \\
\hline Avo. WaSDT.F & 11.3 & 10.5 & 10.5 & 11 & 11.4 & 7.9 & 5.3 & 2.6 & 3.3 & 3.4 \\
\hline Remaining Subcooff & -12.7 & & & & & & & & & \\
\hline Exi Guality & 0.11 & 0.21 & 0.31 & 0.41 & 0.51 & 0.58 & 0.59 & 0.6 & 0.61 & 0.62 \\
\hline EXP.HBuhthas & 1783.2 & 1913.9 & 1900 & 1826.8 & 1753.1 & 1234.4 & 948.4 & 731.7 & 642. & 597 \\
\hline CONAUTEDH & & & & & & & & & & \\
\hline J'lock & Fु5.3 & Tद्आ.ा & TIG. & $7 \pi 8.8$ & 779.8 & 119.8 & T19.5 & $T 19.6$ & 719.5 & Tाध.4 \\
\hline Fedri(Oudo wbe) & 7 & 17822 & 778.7 & Tाब.6 & Tिद्ध & 798.6 & 118.6 & T19.8 & 719.5 & नादू. \\
\hline $\operatorname{sen} 2$ & 1677.8 & 1648.4 & 1641.6 & 178.6 & 119.6 & 119.8 & 118.5 & 119.6 & 118.5 & 178.4 \\
\hline (सम्) & 20887 & 86700 & 32208 & 32008 & 32000 & 32203 & 32200 & 32208 & 32203 & \$2द्208 \\
\hline (F) & 2016 & 2682 & 3.503 & 8.508 & 3.508 & 3.508 & 3.508 & 3.508 & 3.508 & 3.606 \\
\hline (HUO) & 28.1 & 325 & 418.8 & 478.8 & 418.8 & 418.8 & 496.8 & 418.8 & 478.8 & 416.8 \\
\hline$(\mathrm{HO}$ & 17587 & 1778 & $17 \pi$ & 177 & $17 \pi$ & $17 \pi$ & $17 \pi$ & 177 & $17 \pi$ & Thi \\
\hline & & & & & & & & & & \\
\hline & 20 NiE2 & ZONEA & $20 N=6$ & ZONE8 & ZONETO & & & & & \\
\hline DPRguid Tomp.f & 75.2 & & & & & & & & & \\
\hline DPUlavid Density & 83.68 & & & & & & & & & \\
\hline Elevelion- & & 4 & 6 & 8 & 8.875 & & & & & \\
\hline Wemurd DPPsid & 0.539 & 1.781 & 7.650 & 3.600 & 7.35T & & & & & \\
\hline Zero Contuctions-pald & 0.087 & -0.037 & -0.75 & 0.721 & 0.273 & & & & & \\
\hline Corrected DP Pad & 0.502 & 1.854 & 2वर & $\mathbf{3 . 8 8}$ & 2.088 & & & & & \\
\hline Achor DPa & 1.38 & 120 & 262 & 3.04 & 3.89 & & & & & \\
\hline Meavered TPpsig & 6 & & & & 3.7 & & & & & \\
\hline Wherered Overath & 88.5 & & & & & & & & & \\
\hline & & & & & & & & & & \\
\hline Computed bPan & 1.12 & 2.01 & 20 & 4.08 & 6.81 & & & & & \\
\hline Fickon- & 0.1 & 0.82 & 0.8 & 0.82 & 0.80 & & & & & \\
\hline Acosterentiont & 0.11 & 0.22 & 0.34 & 0.88 & $0 . \$$ & & & & & \\
\hline Eloveliont & 0.91 & 1.47 & 1.85 & 2.89 & 3.85 & & & & & \\
\hline & & & & & & & & & & \\
\hline
\end{tabular}




\begin{tabular}{|c|c|c|c|c|c|c|c|c|c|c|}
\hline Runt & 670 & & & & & & & & & \\
\hline TaAlFId & CAFIO & & & & & & & & & \\
\hline Des: & 6233 & & & & & & & & & \\
\hline Timo: & $15: 17$ & & & & & & & & & \\
\hline Nots & Andriod & Pinspratis & essumed & & & & & & & \\
\hline Flow Aroti? & 0.00836 & & & & & & & & & \\
\hline Heal fow Areale & 0.1835 & & & & & & & & & \\
\hline Silprosture Paig & $\approx$ & & & & & & & & & \\
\hline S1. Temperciure f(inlol) & 119.6 & & & & & & & & & \\
\hline La.Dentivetomin & 862 & 86.19 & 86.23 & 86.17 & 8.1 & 86.12 & 8620 & 86.2 & 88.2 & 86.83 \\
\hline Lenthestokntibm & 32.6 & 32.59 & 32.62 & 32.68 & 32.5 & 3265 & 32.62 & 326 & 326 & 32.69 \\
\hline 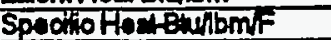 & 0.275 & 0.275 & 0.275 & 0.275 & 0.275 & 0.275 & 0.275 & 0.215 & 0.275 & 0.270 \\
\hline Lo. Vecosingtomith & 0.627 & 0.526 & 0.588 & 0.528 & 0.524 & 0.525 & 0.508 & 0.527 & 0.627 & 0.63 \\
\hline 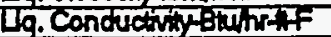 & 0.0278 & 0.0278 & 0.0278 & $0.0<18$ & 0.027 & $0.027 \pi$ & 0.0278 & 0.0078 & 0.028 & 0.0279 \\
\hline If. Prindil $\mathrm{No}$. & 5.2 & 5.22 & 5.2 & 5.21 & 5.21 & 5.21 & 5.22 & 5.22 & 52 & 528 \\
\hline In FFow RE-GP & 1 & & & & & & & & & \\
\hline 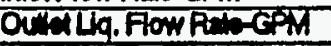 & 0.8 & & & & & & & & & \\
\hline 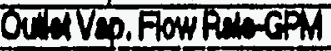 & 0.7 & & & & & & & & & \\
\hline Intat Tomper & 1201 & & & & & & & & & \\
\hline Oclatlq. Temperature F & 126.6 & & & & & & & & & \\
\hline Oollet Vep. Temperaluref & 121.6 & & & & & & & & & \\
\hline Whar How RAO-GPM & & & & & & & & & & \\
\hline Werer hilet fomp.t & 84.1 & & & & & & & & & \\
\hline Wher outh Tremp.f & 110.8 & & & & & & & & & \\
\hline Intasom/12 & $201 / 58$ & & Temp & T\}8.1L & Densiny & 87.06 & & & & \\
\hline secoolinge & -0.6 & & & & & & & & & \\
\hline Fow Obrovitan & & & & & & & & & & \\
\hline & 20NET & $\frac{20123}{11567}$ & $\frac{201: 3}{1159.2}$ & $\frac{20 N E 4}{1157,2}$ & $\frac{20 \times 35}{470.7}$ & $\frac{20 N=6}{1028}$ & $\frac{20 N=7}{144.7}$ & $\frac{\text { ZONES }}{149.5}$ & ZONE9 & $20 N=10$ \\
\hline 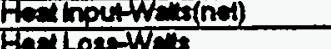 & $\begin{array}{r}1151.8 \\
111\end{array}$ & \begin{tabular}{|r|}
1168.7 \\
2.8 \\
\end{tabular} & $\frac{1158.2}{3.6}$ & $\begin{array}{r}1157.4 \\
3.7\end{array}$ & $\begin{array}{r}470.7 \\
3.2\end{array}$ & -14291 & \begin{tabular}{r|}
74.8 .1 \\
1.4
\end{tabular} & \begin{tabular}{|l|}
741.0 \\
22
\end{tabular} & $\begin{array}{r}118.21 \\
2.81\end{array}$ & $\begin{array}{r}1127 \\
18\end{array}$ \\
\hline 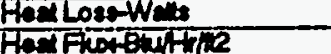 & $\frac{11.1}{100 \%}$ & 2005 & 2000 तो & 20063 & 8160 & 1724 & 2508 & 2158 & का & \\
\hline 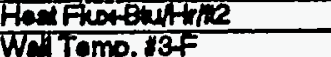 & 10000 & & & 137.8 & & & & & & 1054 \\
\hline Wh Tomp. 13F & 738.1 & 139.8 & 136.2 & 137.8 & 135 & 128.8 & 130.1 & 130.8 & 130.3 & T282:2 \\
\hline We'tamp. IfF & 138.1 & 135.8 & 138.5 & 1392 & 134 & 128.7 & 120.8 & 130.8 & 130.8 & 13.6 \\
\hline We tramp. $16 F$ & 135.4. & 133.7 & 138.7 & 1382. & 1320 & 128.8 & 129.8 & 130.3 & 130.8 & T30., \\
\hline WET Tomp. IT & $7 \pi .8$ & 135.8 & 135.2 & 135.8 & 1326 & 120.7 & 129.6 & 129.7 & 1202 & 728.8 \\
\hline Wa Tomp. ILF & 735.7 & 188.4 & 136.2. & 135.8 & 132.8 & 129.1 & 120.5 & 130 & 120.8 & 1232 \\
\hline hotream tomp.t & T20.3 & Tि. & TE.I & T255 & T25.8 & Tि.8.8 & 725.1 & 725.8 & 125.8 & T2256) \\
\hline Avo. Wall Tomp.F & $\{55.8$ & 156.8 & 133.5 & 137.6 & 133.1 & 1艾 & 120.7 & 130.3 & 130 & T29:5 \\
\hline AVO.WADT.F & 9.8 & 10.5 & 10.5 & 112 & 6.8 & 3.1 & 4.5 & 4.8 & 4.6 & 4.8 \\
\hline Remaining suboooff & -21.1 & & & & & & & & & \\
\hline Exdoustiny & 0.18 & 0.35 & 0.53 & 0.7 & $0.7 \pi$ & 0.78 & 0.81 & 0.83 & 0.85 & 0.88 \\
\hline 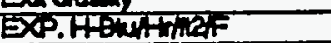 & 2074.8 & 1801.6 & 1910.6 & T78 & 1188.8 & 56.4. & 661.8 & 608.1 & 448.8 & 407 \\
\hline COADUTED & & & & & & & & & & \\
\hline Ielock & $7 \overline{3}$ & 13060 & 78. & 784 & 78.4 & 784 & 78.4 & 78.4 & 78x & 78.8 \\
\hline Frady1(Cudothe) & 7778.4 & 1784 & 78.4 & 78.4 & 78.a & 78.4 & 78.4 & 78.4 & 78.4. & 78.8 \\
\hline seadr & 7810.1 & 1646.6 & 78.4 & 78.4 & 78.4 & 78.4 & 78.4 & 78.4 & 78.4 & 78.8 \\
\hline (AL्म) & उद्ध一 & 18960 & 1860 & 18850 & 18SB⿹ & 18960 & 18900 & 18980 & 18800 & 18800 \\
\hline (1) & 2,358 & 3.550 & 3.655 & 3.525 & 3625 & 3.55 & 3.585 & 3.525 & 3.625 & 3.625 \\
\hline (F. & 183.8 & 274.6 & 27.6 & 274.8 & 274.6 & 274.6 & 274.8 & 276.6 & 274.6 & 274.6 \\
\hline$(4: 0,5)$ & $17 \pi / .7$ & 1781.9 & 7781.8 & T78.9 & 1781.8 & 7781.8 & 1781.8 & 7781,9 & T781.8 & 7787.8 \\
\hline & & & & & & & & & & \\
\hline & $20 N=3$ & $28 N=4$ & ZONE6 & $\angle O C=6$ & $\angle O N=10$ & & & & & \\
\hline DP tquid Tomp.f & 752 & & & & & & & & & \\
\hline DPUladdonsty tomit3 & 83.68 & & & & & & & & & \\
\hline Evarion & 3 & 4 & 6 & 8 & 0.875 & & & & & \\
\hline Meacured DFFad & 0.727 & 2.31 & टसता & $4.0 \%$ & 5.035 & & & & & \\
\hline Zaro Comedions-psid & 0.037 & -0.037 & -0.75 & 0.1 ता & 0.28 & & & & & \\
\hline Comeand OPPId & 0.69 & 2.37 & 2.961 & 3.871 & 4.782 & & & & & \\
\hline ActualDan & 1.0 & 0.3 & 1.58 & 2.06 & 2.74 & & & & & \\
\hline Measured TPFidg & 68 & & & & 3.7) & & & & & \\
\hline Mexured Overatith & 88.91 & & & & & & & & & \\
\hline & & & & & & & & & & \\
\hline Compited ban & 0.8 & T.47 & 235 & 3.1 & 7.2. & & & & & \\
\hline Ficlont & 0.00 & 0.17 & 0.28 & 0.89 & 0.46 & & & & & \\
\hline Aoosterniont & 0.08 & 0.16 & 0.19 & 0.21 & 0.8 & & & & & \\
\hline Everiont & 0.70 & 1.18 & 1.86 & 26 & 3.511 & & & & & \\
\hline
\end{tabular}




\begin{tabular}{|c|c|c|c|c|c|c|c|c|c|c|}
\hline Run & 6251 & & $i$ & 1 & & 1 & i & $i$ & 1 & i \\
\hline Toet Fuid & CAF & & i & 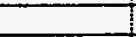 & & 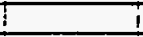 & & $i$ & 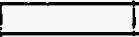 & \\
\hline Dalo: & 6/933 & & 1 & 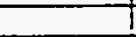 & & I & & i & $i$ & I \\
\hline Time: & $8: 48$ & & $i$ & 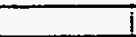 & & $i$ & & 1 & 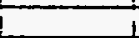 & 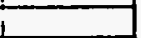 \\
\hline Note & Anatyical o & Af is used & 1 & & & & & & & \\
\hline Fow Area-12 & 0.003561 & & 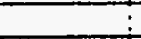 & 1 & & + & & i & 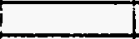 & \\
\hline Head Fow Area-i2 & 0.1955 & & 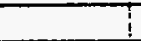 & & & & & & & \\
\hline Sal. Piessure Psig & 107.4 & & $?$ & 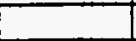 & & & & & 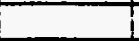 & \\
\hline Sat Temperature F(inlel) & 151.21 & & & & & & & & & \\
\hline Lq. Densthybm/tis & 8204 & 81.79 & 81.831 & 87.89 & 81.91 & 81.83 & 81.76 & 81.81 & $81.81 !$ & 81.98 \\
\hline Latent Hoat-Btuhbm & 29.69 & 29.51 & 29.54 & 29.58 & 29.59 & 29.54 & 29.49 & 29.53 & 29.53 & 29.65 \\
\hline Spechic Heaf BtulbmiF & 0.285 & 0.286 & 0.286 & 0.285 & 0.285 & 0.286 & 0.286 & 0.286 & 0.286 & 0.285 \\
\hline Liq. Visoosity fbm/1ht & 0.434 & 0.429 & 0.43 & 0.431 & 0.431 & 0.43 & 0.428 & 0.43 & 0.43 & 0.433 \\
\hline Lig. Conductivity BtumiffF & 0.0251 & 0.005 & 0.025 & 0.025 & 0.0251 & 0.025 & 0.025 & 0.025 & 0.025 & 0.0651 \\
\hline Liq. Prandt No. & 4.92 & 4.91 & 4.91 & 4.91 & 4.91 & 4.91 & 4.9 & 4.91 & 4.91 & 4.82 \\
\hline Inlot Fow Rado-GPM & 11 & & & & & & & & & \\
\hline Outlot Liq. Flow Rate-GPM & 0.8 & & & & & $t$ & & & & \\
\hline Outlet Vap. Fow Rate-GPM & 0.7 & & & & & & & & & \\
\hline Inlet Temperature F & 148.5 & & & & & & & & & \\
\hline Outlet Lig. Temperaturef- & 749.31 & & & & & & & & & \\
\hline Outuet Vap. Temperaturef & 147.8 & & & & & & & & & \\
\hline Waterflow Rafe-GPM & & & & & & & & & & \\
\hline Water inlot Temp.F & 108.1 & & & & & & & & & \\
\hline Weter outtat Temp.F & 145 & & & & & & & & & \\
\hline Intel Glbmprine & 188056 & & Temp & 141.1 & Density & $82 \%$ & & & & \\
\hline Subcoolingf & 27 & & & & & & & & & \\
\hline Aow Observation & & & & & & & & & & \\
\hline & ZONEI & ZONE2 & ZONE3 & ZONE4 & ZONES & ZONE6 & $\mathbf{Z O N}^{x}=7$ & ZONE8 & ZONEO & ZONE10 \\
\hline Heat Input-Wafls(not) & 289.3 & 285.5 & 285.9 & 284.7 & 288.9 & 209 & 281.2 & 2828 & 281.2 & 288.4 \\
\hline Hoat Loss-Waths & 14.1 & 3.3 & 4.5 & 4.7 & 4.8 & 3.8 & 2.6 & 3.7 & 4.4 & 24.7 \\
\hline Head Fix-Btuhrie & 5015 & 4919 & 4556 & 4965 & 5008 & 5010 & 4874 & 4902 & 4874 & 4909 \\
\hline Wall Temp. HßF & 155.9 & 1552 & 156.4 & 155.8 & 156.8 & 156.7 & 157.3 & 155.4. & 755.7 & $75 \pi .8$ \\
\hline Wall Temp. $4 \mathrm{~F}$ & 156.3 & 155.9 & 157 & 156.8 & 156.8 & 156.9 & 157.6 & 158.8 & 156.8 & 754.6 \\
\hline Wafl Temp. 155 & 156.3 & 155.5 & 157.9 & 156.4 & 156.1 & 157 & 157 & 156.8 & 155.9 & 155.1 \\
\hline Wall Temp. IfF & 82.3 & 158.1 & 155.4 & 155.8 & 155.2 & 157 & 157.5 & 157.1 & 1552 & 159.4 \\
\hline Wah Temp. T2F & 156.9 & 156.2 & 155.7 & 155.4 & 155.3 & 158.9 & 157.4 & 157.3 & 155.4 & 154.1 \\
\hline Instream Tomp.F & 149.4 & 150.7 & 150.5 & 150.2 & 150.1 & 150.5 & 160.9 & 150.6 & 160.6 & 149.7 \\
\hline Avg. Wall Tomp.F & 156.4 & 156.1 & 156.1 & 156 & 155.9 & 156.9 & 157.4 & 156.8 & 155.7 & 154.4 \\
\hline Avg. Wall DT.f & 6.7 & 5.2 & 5.4 & 5.5 & 5.5 & 6.2 & 62 & 6.1 & 4.9 & 4.4 \\
\hline Remaining Subcooff & -25 & & & & & & & & & \\
\hline Exi Quality & 0.02 & 0.07 & 0.12 & 0.17 & 0.2 & 0.27 & 0.32 & 0.37 & 0.42 & 0.47 \\
\hline EPP. HBtuHr/nar & 745.9 & 956.1 & 917.6 & 8928 & 905.6 & 811.5 & 7812 & 809.1 & 998.8 & 1127.5 \\
\hline COMPVIEDH & & & & & & & & & & \\
\hline Jallouk & 705.5 & 685.9 & 674 & 6021 & 65T.8) & 658.5 & 633.1 & 6324 & 630.1 & 633.1 \\
\hline Szady1(Cudo tube) & 1020.5 & 1021.9 & 1027.8 & 1018.3 & T025.6 & 1028.7 & 1017.7 & 1018.3 & 1077 & 7005.1 \\
\hline Srady2 & 707.7 & $\pi 7.1$ & 770.8 & $\pi 3.8$ & 778.9 & 784.3 & 781.3 & 781.8 & 781.8 & 7828 \\
\hline (RLA) & 2948 & 28708 & 27168 & 25600 & 24092 & 22604 & 21245 & 19718 & 18235 & 16017 \\
\hline$(\mathbf{F})$ & 1.352 & 1.805 & 2201 & 2.514 & 2.782 & 3.014 & 320 & 3.425 & 3.671 & 3.8 \\
\hline (HUC) & 105.4 & 139.8 & 170.5 & 194.7 & 215.4 & 208.5 & 249.7 & 265.4 & 278.8 & 284.0 \\
\hline (HBOIL) & 1020.1 & T0द्रा & 10202 & 1015.9 & 1022.4 & 1024.7 & 10126 & 1013.3 & 10099 & 1018.9 \\
\hline & & & & & & & & & & \\
\hline & ZONEZ & ZONE4 & ZONE6 & ZONE8 & ZONE10 & & & & & \\
\hline DP Hquid Temp.F & 83.5 & & & & & & & & & \\
\hline DP Liquid Density $4 \mathrm{bm} / 13$ & 9248 & & & & & & & & & \\
\hline Elovation-h & 2 & 4 & 6 & 8 & 9.875 & & & & & \\
\hline Mensured DPFsid & 0.184 & 1.792 & 1.246 & 2.912 & 3.73 & & & & & \\
\hline Zero Corrections-psid & 0.052 & -0.04 & -0.734 & 0.191 & 0.148 & & & & & \\
\hline Corrected DPPsid & 0.152 & 1.232 & 1.98 & 2.751 & 3.586 & & & & & \\
\hline Actud DPh & 1.89 & 2.35 & 3.28 & 4.18 & 4.84 & & & & & \\
\hline Measured TPPsig & 107.4 & & & & -1.6 & & & & & \\
\hline Mesesured Overallh & 191.46 & & & & & & & & & \\
\hline & & & & & & & & & & \\
\hline Computed DPA & 9.46 & 258 & 3.41 & 4.24 & 4.9 & & & & & \\
\hline Friction & 0.02 & 0.5 & 0.09 & 0.14 & 0.2 & & & & & \\
\hline Accolertion-h & 0.01 & 0.02 & 0.03 & 0.05 & 0.06 & & & & & \\
\hline Elovetion-ft & -1.43 & 2.46 & 3.29 & 4.05 & 4.67 & & & & & \\
\hline & & & & & & & & & & \\
\hline
\end{tabular}




\begin{tabular}{|c|c|c|c|c|c|c|c|c|c|c|}
\hline Runt & 628 & & & & & ! & 1 & I & 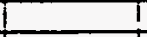 & I \\
\hline Teetfluid & CAF10 & & & & & & T & & & \\
\hline Date: & 6\%93 & & & & & & & & & \\
\hline Time: & $11: 28$ & & & & & & & & & \\
\hline Note & Analyticald & df is used & 1 & $L$ & & & & & & \\
\hline Flow A veatic & 0.003361 & & & & & & $\perp$ & & & \\
\hline Hosd Fow Aroant & 0.1969 & & & & & & & & & \\
\hline Sat. Pressure Prig & 111 & & & & & & & & & \\
\hline Sat. Temperature f(inlel) & 153.3 & & & & & & & & & \\
\hline Lq.Densthy lbmins & 81.61 & 81.62 & 81.57 & 81.53 & 81.53 & 81.47 & 81.51 & 81.59 & 81.61 & 81.7 \\
\hline Lelont Hestbtulbm & 29.38 & 29.89 & 29.5 & 29.82 & 29.32 & 29.28 & 29.31 & 29.38 & 29.38 & 29.4 \\
\hline Spectio HeatexuhtomiF & 0.286 & 0.286 & 0.286 & 0.286 & 0.286 & 0.286 & 0.286 & 0.286 & 0.286 & 0.286 \\
\hline Lig. Visoosity thom/liht & 0.426 & 0.426 & 0.4251 & 0.424 & 0.424 & 0.428 & 0.424 & 0.43 & 0.426 & 0.427 \\
\hline Ug. Conductivy stuhrofff & 0.0249 & 0.020 & 0.0248 & 0.0248 & 0.0248 & 0.0248 & 0.0248 & 0.0249 & 0.0240 & 0.020 \\
\hline Liq. Prandt No. & 4.91 & 4.9 & 4.89 & 4.89 & 4.89 & 4.89 & 4.89 & 4.9 & 4.9 & 4.9 \\
\hline Inlet Flow Rado-GPM & 3.41 & & & & & & & & & \\
\hline Outol Liq. Flow Rato-GPM & 0.8 & & & & & & & & & \\
\hline Outtol Vap. Flow Rato-GPM & 2 & & & & & & & & & \\
\hline \multicolumn{11}{|l|}{ Inlat Temperaturef } \\
\hline Outeltig. Temporatur of & 15231 & & & & & & & & & \\
\hline Outlet Vap. Temporadure F & 148 & & & & & & & & & \\
\hline \multicolumn{11}{|l|}{ Water Flow PRate-GPM } \\
\hline Water hilet Tomp.f & 103.2 & & & & & & & & & \\
\hline Wetrot outet Tomp.f & 145.2 & & & & & & & & & \\
\hline Inlel G-lbm/nrti2 & 6646861 & & Tomp & $146.8 \mathrm{D}$ & Density & 81.87 & & & & \\
\hline Suboodingf & 4.91 & & & & & & & & & \\
\hline \multicolumn{11}{|l|}{ Fow Observation } \\
\hline & ZONE1 & ZONE2 2 & ZONE3 & ZONEA 12 & ZONE5 & ZONE6 T2 & ZONE7 Z & \begin{tabular}{|l|} 
ZONE8 \\
\end{tabular} & ZONE9 & ZONE10 \\
\hline Hoad Input-Walts(net) & 291 & 288.7 & 281.8 & 279.3 & 289 & 289.9 & 291.7 & 294.2 & 285.8 & 285 \\
\hline Head Loss-Wats & 12.7 & 3 & 4 & 4 & 3.9 & 29 & 1.9 & 29 & 3.7 & 20.6 \\
\hline Host Fiox-Bturtim & $50 \times 4$ & 5004 & 4885 & 4847 & 5010 & 5025 & 5056 & 5100 & 4954 & 490 \\
\hline Wal Tomp. B⿸尸 & 157.8 & 157.7 & 757.3 & 757.7 & 158.11 & 157.9 & 158.1 & 157.3 & 756.6 & 755.4 \\
\hline WaH Tomp. T4F & 158.3 & 157.5 & 157.8 & 157.7 & 158.2 & 157.9 & 158.4 & 157.7 & 157.2 & 155.8 \\
\hline Wall Tomp. $\$ 5+$ & 158.3 & 157 & 158.8 & 157.7 & 157.4 & 157.9 & 157.8 & 157.5 & 156.6 & 155.8 \\
\hline WEH Tomp. IF & 80.2 & 157.7 & 156.8 & 156.8 & 157.1 & 158.5 & 158.1 & 157.7 & 150 & 159.8 \\
\hline WaH Tomp. IFF & 158.7 & 157.8 & 156.9 & 1572 & 157.1 & 158.4 & 158.11 & 158.1 & 156.8 & 154.8 \\
\hline Introem Tomp,f & 161.7 & 161.6 & 161.9 & 1621 & 1621 & 1624 & 1622 & 151.8 & 151.71 & 151.2 \\
\hline Avg. Wall Tomp, f & 158.3 & 157.7 & 157.1 & 157.5 & 157.5 & 158.1 & 158.1 & 157.7 & 156.5 & 155.2 \\
\hline Avg. WallDT.f & 6.3 & 5.8 & 5 & 5.2 & 5.11 & 5.4 & 5.7 & 5.6 & 4.6 & 3.7 \\
\hline Romaining Subcooff & 3.4 & & & & & & & & & \\
\hline Ext Quality & -0.03 & -0.02 & 0 & 0.01 & 0.08 & 0.04 & 0.08 & 0.07 & 0.09 & 0.1 \\
\hline EXP.HBturhnerF & 794.7 & 855.6 & 986 & 934.4 & 977.8 & 926.7 & 891.6 & 905.9 & 1073.2 & 13221 \\
\hline \multicolumn{11}{|l|}{ COMPUTEDH } \\
\hline Jalouk & 600.7 & 607 & 637.7 & 633.5 & दा.8 & E34.1 & 6325 & 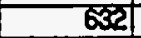 & б्टा.3 & 618.5 \\
\hline Szady I(Cudo tubo) & 1003 & 1034,2 & 1023.6 & 1021.8 & 1041 & 1046.4 & 1050.4 & 1054.4 & 1041.2 & T039.1 \\
\hline Sadnd & 790.4 & 7928 & 788.4 & 790.7 & 807.4 & 817 & 822.0 & 827.1 & 789.4 & 787.6 \\
\hline (P.य) & क्राम4 & 102058 & 10 काष। & 102008 & 100549 & 99258 & 97550 & 5639 & 94015 & कारण \\
\hline (F) & 1.005 & 1.005 & 1.005 & 1.188 & 7.46 & 1.645 & 7.798 & 1.935 & 2.05 & 2.168 \\
\hline (1) & 205.4 & 205.4 & 205.5 & 2429 & 298.5 & 336.4 & 357.7 & 395.4 & 418.9 & 424 \\
\hline \multirow[t]{3}{*}{ (1+BOIL) } & 1036.3 & 1031.6 & 1020.8 & 1016.7 & 10327 & 1034.7 & 1035.1 & 1035.5 & 1017.9 & 1011.6 \\
\hline & & & & & & & & & & \\
\hline & ZONE2 & ZONE4 & ZONE6 & ZONE8 & ZONETO & & & & & \\
\hline DP liquid Temp.F & 79.7 & & & & & & & & & \\
\hline \multicolumn{11}{|l|}{ DPLiquid Density 4 bmint3 } \\
\hline Elevation-h & & 4 & 6 & 8 & 9.875 & & & & & \\
\hline Monsured DPPSid & 0.193 & 0.587 & 0.379 & 1.802 & 2337 & & & & & \\
\hline Zro Conoctions-psid & 0.032 & -0.04 & -0.734 & 0.181 & 0.748 & & & & & \\
\hline Comoctod DPFild & 0.161 & 0.027 & 9.113 & 1.671 & 2.189 & & & & & \\
\hline Actul DPA & 1.99 & 3.45 & 4.87 & 0.27 & 7.39 & & & & & \\
\hline Meaured TPFstg & III & & & & -1.6 & & & & & \\
\hline \multirow{2}{*}{\multicolumn{11}{|c|}{ Meceured Overall }} \\
\hline & & & & & & & & & & \\
\hline Computad DPA & 206 & 4.07 & 5.87 & 7.59 & 9.16 & & & & & \\
\hline Frictionfl & 0.06 & 0.13 & 0.24 & 0.37 & 0.511 & & & & & \\
\hline Acosloration- & 0 & 0.05 & 0.1 & 0.14 & 0.17 & & & & & \\
\hline Elevation- & 2) & 3.88 & 5.53 & 7.08 & 8.48 & & & & & \\
\hline & & & & & & & & & & \\
\hline
\end{tabular}




\begin{tabular}{|c|c|c|c|c|c|c|c|c|c|c|}
\hline Run: & 629 & & i & 1 & I & I & 1 & i & 1 & I \\
\hline Toat Fluid & CAF10 & & 1 & 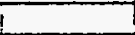 & $\Gamma$ & i & & $i$ & $T$ & 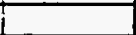 \\
\hline Date: & 69993 & & & & T & 1 & 1 & 1 & 1 & 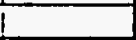 \\
\hline Time: & $11: 28$ & & 5 & & $!$ & 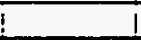 & i & & $i$ & i \\
\hline \begin{tabular}{|l|} 
Note \\
\end{tabular} & Analytical & dt is used & & t & 1 & I & $\perp$ & & $i$ & $i$ \\
\hline Flow Area-ti2 & 0.003361 & & & (1) & 1 & 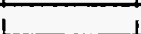 & 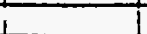 & & 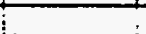 & \\
\hline Heat Fow Areat2 & 0.19695 & & & & & & & & & \\
\hline Sat. Pressure Psig & 1061 & & & & & & & & & \\
\hline Sad. Temperature-f(inlet) & 150.3 & & & & & & & & & \\
\hline Lq. Denstiny lomins & 82.11 & 82.13 & 82.07 & 82.09 & 82.11 & 82.11 & 82.171 & 8224 & 8226 & 8220 \\
\hline Latent Heat-Btulbm & 29.74 & 29.70 & 29.72 & 29.78 & 29.74 & 29.74 & 29.781 & 29.84 & 29.85 & 29.89 \\
\hline Spectio Heal-gtuflbmif & 0.2851 & 0.205 & 0.285 & 0.285 & 0.2851 & 0.285 & 0.285 & 0.284 & 0.284 & 0.284 \\
\hline Lig. Viecosity tbm/ahr & 0.435 & 0.436 & 0.435 & 0.435 & 0.485 & 0.485 & 0.436 & 0.438 & $0.4 \%$ & 0.439 \\
\hline Liq. Conductivity Btuhr-tfF & 0.0252 & 0.02521 & 0.0252 & 0.0252 & 0.02521 & 0.0252 & 0.02521 & 0.0253 & 0.0253 & 0.0250 \\
\hline Liq. Prandt No. & 4.92 & 4.92 & 4.921 & 4.92 & 4.92 & 4.92 & 4.93 & 4.93 & 4.931 & 4.99 \\
\hline Inlet Fow Rato-GPM & 4.3 & & & t & & & & & 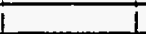 & \\
\hline \multicolumn{11}{|l|}{ Outtet Liq. Fow Rato-GPM } \\
\hline Outlet Vap. Flow Rale-GPM & 2.3 & & $t$ & & & 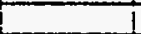 & 1 & 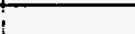 & i & \\
\hline Inlet Temperaturof & 145.7 & & & & 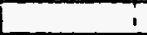 & 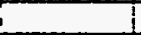 & & 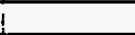 & $\vdots$ & \\
\hline Outlet Liq. Temperaturef & 148.91 & & & & & & & & 1 & \\
\hline Outlet Vap. Temperaturef & 144.2! & & & & & & & & & \\
\hline \multicolumn{11}{|l|}{ Wader Fow Rado-GPM } \\
\hline Water inlot Temp.F & 102.9 & & & & & & & & & \\
\hline Water outiot Temp.F & 147.31 & & & & & & & & & \\
\hline Inlet Glbminritiz & 845832 & & Temp & 144.9 & Density & 82.43 & & & & \\
\hline Subcoolingf & 4.6 & & & & & & & & & \\
\hline \multicolumn{11}{|l|}{ Fow Observation } \\
\hline & ZONE1 & ZONE2 & ZONE3 I & ZONE4 & ZONE5 & ZONE6 & ZONE7 & ZONE8 & ZONE9 & ZONE10 \\
\hline Heat Input-Wats(nel) & 291.5 & 289.1 & 283.4 & 280.3 & 288.1 & 288.3 & 291.9 & 294.5 & 284.3 & 288.3 \\
\hline Head Loss-Watts & 12.1 & 2.8 & 3.7 & 3.7 & 3.7 & 2.7 & 1.8 & 27 & 3.5 & 19.3 \\
\hline Hoat Flox-Bturithl & 5053 & 5011 & 4913 & 4859 & 4994 & 4997 & 5050 & 5105 & 4928 & 4907 \\
\hline Wall Temp. H35 & 154.8 & 754.8 & 154.5| & 154.6 & T55.1 & 154.9 & 154.5 & 354.1 & 153.3 & 7525 \\
\hline Wall Tomp. 4 F & 155.2 & 158.7 & 15.11 & 154.7 & 155 & 154.8 & 155.1 & 154.5 & 154 & 152.6 \\
\hline Wall Temp. $15 f$ & 155.5 & 154.4 & 156.1 & 154.7 & 154.3 & 154.9 & 154.6 & 154.1 & 153.4 & 152.9 \\
\hline Wed Tomp. $\# 1 F$ & 79.3 & 154.8 & 153.7 & 158.8 & 154.2 & 155.4 & 154.7 & 154.5 & 152.4 & 150.8 \\
\hline Wall Temp. I2F & 155.6 & 155 & 154.81 & 154.4 & 154.2 & 154.9 & 155.1 & 155 & 152.0 & 152.1 \\
\hline instoem Temp.F & 149 & 148.0 & 140.2 & 149.1 & 749 & 140 & 948.7 & 148.3 & 148.2 & 148 \\
\hline Avg. Wall Tomp.F & 155.3 & 154.8 & 154.4 & 154.5 & 154.4 & 155 & 154.8 & 154.4 & 153.2 & 1522 \\
\hline Avg. Wall DT.F & 6 & 5.7 & 5 & 5.1 & 5.2 & 5.8 & 5.9 & 5.9 & 4.8 & 4 \\
\hline Remaining Subcooff & 3.4 & & & & & & & & & \\
\hline Exot Quality & -0.03 & -0.02 & -0.01 & 0 & 0.01 & 0.031 & 0.04 & 0.05 & 0.06 & 0.07 \\
\hline DXP.HBtu/Hr/ma/F & 835.7 & 879.4 & 986.9 & 947.1 & 960.5 & 861.8 & 861.8 & 863.8 & 1031.6 & 1263.9 \\
\hline \multicolumn{11}{|l|}{ COMPUTED H } \\
\hline Jahlouk & 632.6 & 629.9 & 625.5 & 621 & 630.1 & 617.6 & 679.11 & 620.1 & 6102 & 674.4 \\
\hline Szady1(Cudo tube) & 1024.5 & 1019.6 & 1011.3 & 1006.2 & 1021.4 & 1024.8 & 1032.2 & 1037.2 & 10224 & 10312 \\
\hline $5 \operatorname{sad} 2$ & 774.1 & 770.5 & 767.2 & 7529 & 779.1 & 788.4 & 797.9 & 805.6 & 775.7 & 785.1 \\
\hline (RUO) & 127094 & 12638 & 127305 & 127199 & 128113 & 124633 & 122838 & 120035 & 119363 & 117713 \\
\hline (F) & 1.005 & 1.000 & 1.0051 & 1.005 & 1.278 & 1.476 & 1.628 & 1.758 & 1.871 & 1.974 \\
\hline (1:DO) & 248.4 & 248.3 & 248.4 & 248.4 & 315.7 & 364.6 & 402 & 434 & 461.0 & 487 \\
\hline \multirow[t]{3}{*}{ (1-18OL) } & 1019.6 & 1014.7 & 1006.3 & 1000.1 & 10112 & 10092 & 1011.4 & 1011.3 & 990 & 983.6 \\
\hline & & & & & & & & & & \\
\hline & ZONE2 & ZONE4 & ZONE6 & ZONE8 & ZONE10 & & & & & \\
\hline DP liquid Temp.f & 79.5 & & & & & & & & & \\
\hline DP Lquid Density thm/113 & 93.06 & & & & & & & & & \\
\hline Elevation-A & 2 & 4 & 6 & 8 & 9.875 & & & & & \\
\hline Measured DPF'sid & 0.177 & 0.494 & 0.29 & 1.577 & 2000 & & & & & \\
\hline Zero Combctions-psid & 0.052 & -0.04 & -0.734 & 0.191 & 0.148 & & & & & \\
\hline Corrected DPPsld & 0.145 & 0.534 & 0.953 & 1.386 & 1.878 & & & & & \\
\hline Actual DPA & 2.01 & 3.59 & 5.12 & 8.62 & 7.88 & & & & & \\
\hline Wesured fppsig & 106 & & & & -1.6 & & & & & \\
\hline \multicolumn{11}{|l|}{ Messured Overallit } \\
\hline & & & & & & & & & & \\
\hline Computed DPA & 2.09 & 4.17 & 6.13 & 7.97 & 9.65 & & & & & \\
\hline Friction-A & 0.09 & 0.18 & 0.33 & 0.51 & 0.71 & & & & & \\
\hline Acosoleration- & 0 & 0.02 & 0.13 & 0.18 & 0.28 & & & & & \\
\hline Elovation $f$ & 2 & 3.97 & 5.57 & 7.27 & 8.71 & & & & & \\
\hline & & & & & & & & & & \\
\hline
\end{tabular}




\begin{tabular}{|c|c|c|c|c|c|c|c|c|c|c|}
\hline Run: & 626 & & 1 & & & & & & & 1 \\
\hline Teet Fluid & CAF10 & & 1 & & & & & & & \\
\hline Defo: & 6/933 & & & I & & & & & & \\
\hline Tima: & $11: 28$ & & 1 & 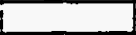 & & & & & & \\
\hline Note & Analyical & oft is used & & & & & & & & \\
\hline Flow Areat? & 0.00336 & & & & & & & & & \\
\hline Hod Fow Aroed2 & 0.19695 & & & & & & & & & \\
\hline Sa. Pressure Psig & 106.2 & & & & & & & & & \\
\hline Sal. Temperature ff(inlel) & 150.4 & & & & & & & & & \\
\hline Da. Densthytbmit3 & 8207 & 81.98 & 82.04 & 82.04 & 82.04 & 81.98 & 82.02 & 82.07 & 82.07 & 82.17 \\
\hline Latont Hoad Btulbm & 29.72 & 29.65 & 29.69 & 29.69 & 29.69 & 29.65 & 29.68 & 29.72 & 29.72 & 29.78 \\
\hline Spectic Heat Btuhbmir & 0.285 & 0.285 & 0.285 & 0.285 & 0.265 & 0.285 & 0.285 & 0.285 & 0.285 & 0.285 \\
\hline Jjg. Visoositylbm/iht & 0.435 & 0.433 & 0.434 & 0.434 & 0.434 & 0.438 & 0.434 & 0.435 & 0.436 & 0.456 \\
\hline Ua. Conductivatubturntif & 0.0252 & 0.0251 & 0.02511 & 0.0251 & 0.0251 & 0.0251 & 0.0251 & 0.0252 & 0.0252 & 0.0652 \\
\hline Lu. Prandt No. & 4.92 & 4.92 & 4.92 & 4.82 & 4.82 & 4.92 & 4.92 & 4.22 & 4.92 & 4.93 \\
\hline Inlot Flow Pate-GPM & 1.8 & & & & & & & & & \\
\hline Outhet Liq. Fow Ralo-GPM & 0.8 & & & & & & & & & \\
\hline Outlet Vap. Flow Rato-GPM & 1.3 & & & & & & & & & \\
\hline Inlet Tomperaturef & 146.1 & & & & & & & & & \\
\hline OuthetLiq. Tomperature & 149.8 & & & & & & & & & \\
\hline Outet Vap. Temperaturef & 145.9 & & & & & & & & & \\
\hline Watof Fow Rale-GPM & & & & & & & & & & \\
\hline Water hilet Tomp.F & 102.4 & & & & & & & & & \\
\hline Wath outlet Temp.f & 141.8 & & & & & & & & & \\
\hline Inlot G-16m/hr/me & 356128 & & Tamp & 1428 & Density & 82.87 & & & & \\
\hline Suboodingf & 4.3 & & & & & & & & & \\
\hline Fow Observation & & & & & & & & & & \\
\hline & ZONE1 & ZONE2 & ZONEB & ZONE4 & ZONES & ZONE6 & ZONET & ZONE8 & ZONE9 & ZONETO \\
\hline Hoal input-Watts(nel) & 289.2 & 2872 & 287.9 & 289.1 & 286.3 & 288.7 & 289.5 & 291.7 & 288.9 & 29.3 \\
\hline Hoat Loss-Watts & 14.9 & 3.5 & 4.7 & 5.1 & 5.2 & 4.3 & 2.9 & 4.1 & 4.7 & 26.3 \\
\hline Hos Flox-Btulturite & 5013 & 4978 & 4991 & 5011 & 4963 & 5004 & 5018 & 5056 & 5008 & 5119 \\
\hline Wall Tomp. "B- & 155 & 155 & 155.j & 1552 & 755.9 & 755.8 & 755.9 & 155 & 754.5 & 753.5 \\
\hline Wall Temp. IAf & 15.4 & 754.8 & 155.6 & 155.8 & 155.9 & 155.8 & 156.3 & 155.5 & 155 & 153.6 \\
\hline Wall Tomp. $15 F$ & 155.6 & 954.4 & 156.8 & 155.5 & 155 & 155.9 & 155.6 & 1552 & 154.5 & 153.8 \\
\hline Wal Tomp. IF & 79.1 & 155 & 154.2 & 154.4 & 154.5 & 156.2 & 158 & 155.8 & 153.7 & 151.6 \\
\hline WalTomp. ILF & 156.1 & 155 & 154.5 & 154.6 & 154.4 & 155.8 & 156.2 & 158.1 & 154 & 153.1 \\
\hline Introan Tomp.F & 149.2 & 149.7 & 149.4 & 148.4 & 149.4 & 149.7 & 149.6 & 149.2 & 148.2. & 148.7 \\
\hline Avg. Wall Tamp.F & 155.5 & 155 & 154.8 & 155.1 & 155 & 156 & 156 & 155.5 & 154.8 & 153.1 \\
\hline Avg. Wat DT, F & 6.1 & 5 & 5.2 & 5.5 & 5.3 & 6 & 6.3 & 6.1 & 4.9 & 4.2 \\
\hline Romaining subcooff & 1.4 & & & & & & & & & \\
\hline Exat Quality & -0.01 & 0.01 & 0.04 & 0.07 & 0.1 & 0.12 & 0.15 & 0.18 & 0.21 & 0.24 \\
\hline EP. HEtuH-142,F & 822 & 990.7 & 952 & 915.6 & 931.9 & 827.3 & 800 & 835.6 & 1019.2 & 12221 \\
\hline COMPUTEDH & & & & & & & & & & \\
\hline Jallouk & 669.6 & 688.1 & 655.7 & 648 & 629.7 & 6327 & 2324 & 63.4 & अा & 635.5 \\
\hline szadyi(a)do tubo) & 1019.3 & 9018.7 & 1019.1 & T0221 & 1018.1 & 5025.1 & 1026.3 & 1029.4 & 1025.6 & 1034.8 \\
\hline szady2 & 766.1 & 768.7 & 769.6 & 73.0 & 773.3 & 781.6 & 783.6 & 788.3 & 788.5 & 792 \\
\hline (स्व) & 53450 & 53871 & 52058 & 50564 & 49080 & $47 \pi 7$ & 46144 & 4450 & 43008 & 41801 \\
\hline$(\mathrm{F})$ & 1.005 & 1.014 & 1.585 & 1.878 & 21 & 2292 & 2.468 & 2.691 & 2.778 & 2.925 \\
\hline (स) & 124.1 & 1252 & 195.7 & 231.2 & 259.8 & 283 & 304.7 & 324.7 & 342.9 & 360.8 \\
\hline (1-18OL) & 1018.6 & 1018.1 & 1016.7 & 1018.2 & 1012.4 & 1017.8 & 10172 & 1018.5 & 10126 & 1000 \\
\hline & & & & & & & & & & \\
\hline & ZONE2 & ZONE4 & ZONE6 & ZONE8 & ZONE10 & & & & & \\
\hline DPliquid Temp.f & 80.4 & & & & & & & & & \\
\hline DPLiquid Density tomnit3 & 92.83 & & & & & & & & & \\
\hline Elevation-l & & & 6 & 8 & 9.875 & & & & & \\
\hline Measurod DPFaid & 0.193 & 0.970 & 0.947 & 2.547 & 3.268 & & & & & \\
\hline Zoro Contections-psid & 0.032 & -0.04 & -0.734 & 0.191 & 0.148 & & & & & \\
\hline Corrected DPPsid & 0.181 & 0.989 & 1.681 & 2.356 & 3.12 & & & & & \\
\hline Actual DPA & 1.98 & 279 & 3.84 & 4.92 & 5.7 & & & & & \\
\hline Mosaured Tppoig & 106.2 & & & & -1.6 & & & & & \\
\hline Mossured Overalth & 188.83 & & & & & & & & & \\
\hline & & & & & & & & & & \\
\hline Computed DPA & 1.91 & 3.38 & 4.68 & 5.89 & 6.98 & & & & & \\
\hline Fiction-t & 0.02 & 0.07 & 0.14 & 0.23 & 0.33 & & & & & \\
\hline Accoloration-a & 0.02 & 0.04 & 0.06 & 0.08 & 0.1 & & & & & \\
\hline Elovation-fi & 1.87 & 3.27 & 4.48 & 5.59 & 6.56 & & & & & \\
\hline & & & & & & & & & & \\
\hline
\end{tabular}




\begin{tabular}{|c|c|c|c|c|c|c|c|c|c|c|}
\hline Runt & 627 & & & & $!$ & & & & $!$ & T \\
\hline Teat Fuid & ICAFTO & & & & & & & & & \\
\hline Date: & $6 \sqrt{993}$ & & & & & & & & & \\
\hline Tima: & $91: 28$ & & & & & & & & & \\
\hline Noto & Analytical & dt is used & & & & & & & & \\
\hline Fow Area-ti & 0.00336 & & & & & & & & & \\
\hline Head Row Areande & 0.19695 & & & & & & & & & \\
\hline Sat. Pressure Psig & 108.7 & & & & & & & & & \\
\hline Sal. Temperaturof(inlet) & 152 & & & & & & & & & \\
\hline Lag. Density-hmins & 81.87 & 81.83 & 81.76 & 81.83 & 81.85 & 81.78 & 81.81 & 81.87 & 81.87 & 81.92 \\
\hline Litend Hear Btunbm & 29.57 & 29.54 & 29.49 & 29.54 & 29.55 & 29.5 & 29.59 & 29.57 & 29.57 & 29.61 \\
\hline Specifio Heat-Btulbm/F & 0.285 & 0.286 & 0.286 & 0.286 & 0.2851 & 0.286 & 0.286 & 0.285 & 0.285 & 0.285 \\
\hline Liq. Visoosity & 0.431 & 0.48 & 0.4281 & 0.43 & 0.43 & 0.420 & 0.49 & 0.439 & 0.431 & 0.432 \\
\hline Lig. Conduetivit Btu/hstif & 0.025 & 0.025 & 0.025 & 0.025 & 0.025 & 0.025 & 0.025 & 0.025 & 0.0251 & 0.0251 \\
\hline Liq. PrandtiNo. & 4.91 & 4.91 & 4.9 & 4.91 & 4.91 & 4.9 & 4.91 & 4.91 & 4.91 & 4.91 \\
\hline Inlot Flow Rato-GPM & 2.6 & & & & & & & & & \\
\hline Outlet Liq. Fow Rade-GPM & 0.9 & & & & & & & & & \\
\hline Outlet Vap. Flow Rato-GPM & 2.1 & & & & & & & & & \\
\hline Inlet Temperaturef & 147.1 & & & & & & & & & \\
\hline Outhet Lq. Temperaturef & 750.7 & & & & & & & & & \\
\hline Outet Vap. Temperaturef & 146.5 & & & & & & & & & \\
\hline Wator Flow Rato-GPM & & & & & & & & & & \\
\hline Wator Inlot Tomp.f & 104.2 & & & & & & & & & \\
\hline Water oustel Temp.f & 143.4 & & & & & & & & & \\
\hline Intol Glbmhwhit? & 510605 & & Temp & 144.8 & Density & 8229 & & & & \\
\hline Subcoalingt & 4.9 & & & & & & & & & \\
\hline Flow Observation & & & & & & & & & & \\
\hline & ZONEI & ZONE2 & ZONE3 & ZONEA & ZONE5 & ZONE6 & ZONE7 & ZONE8 & ZONES & ZONE10 \\
\hline Head (nput-Watts(nei) & 290.3 & 288.8 & 289.3 & 289.2 & 286.1 & 288.2 & 290.8 & 293.2 & 291.7 & 294.4 \\
\hline Heat Lose-Walls & 15.3 & 3.6 & 4.8 & 5.2 & 5.4 & 4.5 & 3.1 & 4.2 & 4.8 & 27 \\
\hline Hogd flox-BtuAHr/n2 & 5032 & 5006 & 5015 & 5013 & 4959 & 4996 & 5041 & 5082 & 5056 & 5100 \\
\hline Wall Temp. BF & 756.4 & 156.4 & 756.2 & 156.1 & 756.7 & 756.7 & 156.6 & 756 & 755.4 & 754.6 \\
\hline Wall Tomp. \#4F & 156.7 & 156.1 & 156.8 & 156.3 & 956.8 & 156.7 & 157.1 & 156.5 & 156 & 154.7 \\
\hline Wall Temp. $15 F$ & 158.8 & 155.6 & 157.8 & 158.3 & 158 & 158.6 & 156.3 & 156 & 155.5 & 155.2 \\
\hline Wak Temp. \#F & 78.8 & 156.2 & 155.2 & 155.4 & 155.6 & 157.1 & 158.7 & 158.5 & 154.6 & 159.1 \\
\hline Wall Tomp. I2f & 157.4 & 156.4 & 155.7 & 155.7 & 155.6 & 157 & 157.1 & 156.8 & 154.9 & 154.2 \\
\hline Instresm Tomp.F & 160.8 & 150.6 & 960.8 & 160.6 & 160.4 & 160.8 & 160.8 & 150.3 & 150.8 & 160 \\
\hline Avg. Wall Tomp.F & 156.9 & 156.3 & 156 & 156 & 158 & 156.8 & 156.8 & 156.4 & 155.3 & 154, \\
\hline Avg. Wall DT.f & 6.3 & 5.5 & 4.8 & 5.3 & 5.4 & 5.7 & 5.9 & 5.8 & 4.8 & 4.1 \\
\hline Remaining Subcooff & 2.8 & & & & & & & & & \\
\hline Exot Cuality & -0.03 & -0.01 & 0.01 & 0.03 & 0.05 & 0.07 & 0.0 & 0.11 & 0.13 & 0.15 \\
\hline EXP.HBtu/H?m2/F & 795.8 & 902.2 & 1034.3 & 946.1 & 9225 & 868.8 & 849.7 & 871.7 & 1054.2 & 1235.8 \\
\hline COMPUTEDH & & & & & & & & & & \\
\hline Jallouk & 671.6 & 670.1 & 667.4 & 638.8 & 52.6 & 63.1 & 682 & 60.1 & E26.5 & 627.3 \\
\hline Srady) (Cudo tubo) & 1028.4 & 1026.9 & 1050.6 & 1029.5 & 1024.8 & 10324 & 100372 & 1041.2 & 1040.1 & 10.7 \\
\hline sady2 & 779.4 & 779.8 & 785.2 & 785.8 & 785.8 & 798.1 & 8012 & 800.4 & 808.4 & 813.2 \\
\hline (स्थां) & 77560 & 77690 & 7807 & 76082 & 74469 & 73207 & 71568 & 69662 & 68334 & 668688 \\
\hline$(F)$ & 1.005 & 1.005 & 9.118 & 1.498 & 1.725 & 1.904 & 2.053 & 2.207 & 2.337 & 2.481 \\
\hline$(H-1 Q)$ & 166.1 & 166.1 & 184.9 & 247.7 & 285.1 & 314.8 & अवा & 364.7 & अ98.2 & 408.6 \\
\hline (1BOIL) & 1026.9 & 1005.5 & 1028.6 & 9024.7 & 1017.4 & 10225 & 1024.8 & 1026 & 1022.1 & 1023.8 \\
\hline & & & & & & & & & & \\
\hline & ZONE2 & ZONE4 & ZONE6 & ZONE8 & ZONE10 & & & & & \\
\hline DP kquid Tomp.F & 79.6 & & & & & & & & & \\
\hline DPLiquid Density $4 \mathrm{bm} n 13$ & 93.05 & & & & & & & & & \\
\hline Eovation-l & 2 & 4 & 6) & 8 & 9.875 & & & & & \\
\hline Measured DPPPid & ठ..2त्रा & 0.720 & 0.676 & रास्ट & 2.734 & & & & & \\
\hline Zoro Corrections-psid & 0.032 & -0.04 & -0.734 & 0.191 & 0.148 & & & & & \\
\hline Corrected DPPSld & 0.187 & 0.76 & 1.35 & 1.931 & 2.586 & & & & & \\
\hline Achald DPA & 1.94 & 3.19 & 4.41 & 5.69 & 6.67 & & & & & \\
\hline Meseurod TPPsig & 108.7 & & & & -1.6 & & & & & \\
\hline Moe:ured Overallt & 193.88 & & & & & & & & & \\
\hline & & & & & & & & & & \\
\hline Computed DRA & 2.04 & 3.85 & 5.52 & 7.13 & 8.6 & & & & & \\
\hline Friction-t & 0.04 & 0.1 & 0.18 & 0.28 & 0.38 & & & & & \\
\hline Acosleration-t & 0 & 0.05 & 0.08 & 0.11 & 0.13 & & & & & \\
\hline Eovationft & 2 & 3.71 & 5.26 & 6.74 & 8.08 & & & & & \\
\hline & & & & & & & & & & \\
\hline
\end{tabular}




\begin{tabular}{|c|c|c|c|c|c|c|c|c|c|c|}
\hline Runt & 6301 & & i & i & T! & 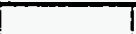 & ( & I & i & 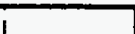 \\
\hline Tost Fluid & CAF10 & & 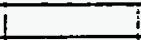 & 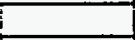 & & & & & & i \\
\hline Date: & 69993 & & I & 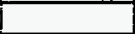 & I & 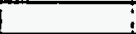 & & & & \\
\hline Time: & $11: 28$ & & 1 & L. & I & 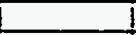 & & & & \\
\hline Noto & Analyticalc & of is used & 1 & 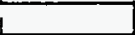 & 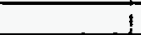 & 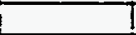 & & & & \\
\hline Flow Areati2 & 0.00336 & & 1 & 1 & 1 & 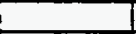 & & & i & \\
\hline Heat Flow Areat2 & 0.19695 & & 1 & I & & & & & & \\
\hline Sad.PTossure + sig & 1012 & & 7 & T & & & & & & \\
\hline Sa. Temperaturo f(inlot) & 147.3 & & & & & & & & & \\
\hline La. Denstivyomins & 82.44 & 82.5 & 82.55 & 82.02 & 82.02 & 82.02 & 82.64 & 82.71 & 82.7 & 82.80 \\
\hline Lotent Hoat Etulbm & 29.98 & 30.02 & 30.08 & 30.11 & $\$ 0.111$ & 30.11 & 30.18 & 30.18 & 30.22 & 50.28 \\
\hline Specitio Hod tulbmit & 0.284 & 0.284 & 0.284 & 0.284 & 0.2841 & 0.284 & 0.284 & 0.283 & 0.283 & 0.288 \\
\hline Liq. Visoositybm/ithr & 0.442 & 0.448 & 0.444 & 0.446 & 0.4461 & 0.446 & 0.46 & 0.447 & 0.448 & 0.46 \\
\hline Wq. Conductivity stump-tif & 0.0254 & 0.0254 & 0.0255 & 0.0255 & 0.02551 & 0.0255 & 0.0255 & 0.0256 & 0.0256 & 0.0257 \\
\hline Liq. Prandt No. & 4.941 & 4.94 & 4.951 & 4.95 & 4.551 & 4.95 & 4.95 & 4.96 & 4.96 & 4.97 \\
\hline Inlet Fow Ralo-GPM & 4.21 & & 1 & 1 & & & & & & \\
\hline Outet Lig. Flow Rate-GPM & $1+$ & & 1 & 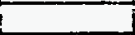 & 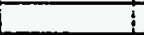 & & & & & \\
\hline Outlet Vap. Flow Rate-GPM & 2.3 & & 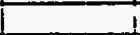 & 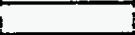 & 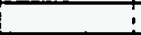 & & & & & \\
\hline inlet Tomperaturef & 144.41 & & 1 & & & & & & & \\
\hline Outlet Lq. Temperaturef & 145.Tा & & & & & & & & & \\
\hline Outlat Vap. Temperaturof & 142 & & & & & & & & & \\
\hline Welor Flow Rato-GPM & & & & & & & & & & \\
\hline Wader Inlet Temp. F & 101.6 & & & & & & & & & \\
\hline Water outlet Temp.F & 139 & & & & & & & & & \\
\hline inlat Glbmhrne2 & 829956 & & Temp & 142.1 & Density & 82.8 & & & & \\
\hline Subcooling $F$ & 29 & & & & & & & & & \\
\hline Flow Observation & & & & & & & & & & \\
\hline & ZONE1 & ZONE2 & ZONE3 & ZONE4 & ZONE5 & ZONE6 & ZONE7 & ZONE8 & ZONE9 & ZONE10 \\
\hline Hoat Input-Watts(net) & 288.1 & 0 & 293.8 & 0 & 292 & 0 & 294.7 & 0 & 288.5 & 0 \\
\hline Hoad Loss-Watts & 11.2 & 2.3 & 3.5 & 3 & 3.3 & 2.1 & 1.6 & 2.1 & 3.2 & 14.8 \\
\hline Host Flox-BtuhHrite & 4994 & 0 & 5033 & 0 & 5052 & 0 & 5908 & 0 & 5001 & 0 \\
\hline Wall Tomp. HF & 1524 & 747.6 & 152.1 & T47.2 & 752.4 & T46.6 & 1522 & 746 & 150.8 & T4 \\
\hline Wall Tomp. *4F & T520 & 148 & 152.6 & 147.7 & 152.3 & 746.5 & 152.6 & 746.7 & 151.3 & 744.3 \\
\hline Wall Tomp. $15 F$ & 1529 & 148 & 153.7 & 147 & 151.7 & 146.4 & 752 & 148.1 & 150.8 & 144.8 \\
\hline WAHTOmp. \#IF & 78.9 & 148.3 & 151.8 & 147.4 & 151.2 & 147 & 1522 & 148.4 & 150 & 94.6 \\
\hline Wall Tomp. HaF & 153.8 & 148.4 & 151.8 & 147.5 & 15121 & 146.6 & 1526 & 148.6 & 150.4 & 144.7 \\
\hline Inttrom Tomp.f & 147.2 & 146.8 & 146.6 & 146.2 & 146.2 & 146.2 & 146.1 & 145.7 & 145.4 & 144.9 \\
\hline Avg. Wall Temp.f & 152.9 & 148.1 & 152 & 147.2 & 151.6 & 146.6 & 152.3 & 146.2 & 150.7 & 144.5 \\
\hline Avg. Wall DT.f & 5.41 & 1.2 & 5.1 & 1 & 5.21 & 0.4 & 6 & 0.5 & 5 & 0 \\
\hline Ramaining Subcooff & 1.7 & & & & & & & & & \\
\hline Ext Quality & -0.02 & -0.02 & 0 & 0 & 0.01 & 0.01 & 0.02 & 0.02 & 0.00 & 0.03 \\
\hline EXP.HBHUhthlif & 916.5 & 0 & 994.7 & 0 & 978.8 & 0 & 855.8 & 0 & 993.4 & 0 \\
\hline COMPUTEDH & & & & & & & & & & \\
\hline Jallouk & E225. & 199.5 & 625.8 & 199.4 & Eट2या & 246.7 & 617.9 & 281.4 & 606.5 & 305.6 \\
\hline Szady1(Cudo tubo) & 1007.5 & 244.2 & 1013.8 & 244 & 1009.3 & 311.1 & 1016.7 & 361.4 & 1005.1 & 399 \\
\hline szady? & 753.4 & 244 & 7826 & 243.8 & $\pi .1$ & 311 & 764.6 & 361.4 & 761.5 & 398.8 \\
\hline (R्वि) & 122859 & 122554 & 1222511 & 121848 & 121694 & 120011 & 120082 & 118961 & 117959 & 116770 \\
\hline (F) & 1.005 & 1.005 & 1.005 & 1.005 & 9.118 & 1.282 & 1.396 & 1.491 & 1.572 & 1.647 \\
\hline (सUO) & 244.11 & 244 & 241 & 243.8 & 271.2 & 311 & 338.5 & 361.4 & 381 & 398.8 \\
\hline (HBOIL) & 90027 & 2 & 1000.11 & 28.7 & 10027 & 28.7 & 1004.1 & 285 & 986.6. & 28.8 \\
\hline & & & & & & & & & & \\
\hline & ZONE2 & ZONE4 & ZONE6 & ZONE8 & ZONE10 & & & & & \\
\hline DP liquid Temp. $F$ & 79.9 & & & & & & & & & \\
\hline DPLiquid Densitubm/t3 & 93 & & & & & & & & & \\
\hline Elovation-t & 2 & 4 & 6 & 8 & 9.875 & & & & & \\
\hline Mossured DPFsid & 0.191 & 0.482 & 0.16 & 1.468 & 7.87 & & & & & \\
\hline Zoro Corrections-psid & 0.032 & -0.04 & -0.734 & 0.191 & 0.148 & & & & & \\
\hline Cortectod DPPsid & 0.159 & 0.522 & 0.894 & 1.27 & 1.723 & & & & & \\
\hline Actuad DPA & 1.97 & 3.58 & 5.18 & 8.78 & 8.09 & & & & & \\
\hline Mosoured TPPsig & 101.2 & & & & -1.6 & & & & & \\
\hline Mosisured Ovoralth & 178.65 & & & & & & & & & \\
\hline & & & & & & & & & & \\
\hline Computed DPA & 200 & 4.18 & 6.15 & 8.03 & 9.74 & & & & & \\
\hline Friction-h & 0.09 & 0.18 & 0.29 & 0.43 & 0.58 & & & & & \\
\hline Acosleration- & 0 & 0 & 0.06 & 0.11 & 0.14 & & & & & \\
\hline Elevation $\mathrm{At}$ & 2 & 4 & 5.8 & 7.49 & 9.02 & & & & & \\
\hline & & & & & & & & & & \\
\hline
\end{tabular}




\begin{tabular}{|c|c|c|c|c|c|c|c|c|c|c|}
\hline Run: & $1 \quad$ का1 & $T$ & 1 & $T$ & 1 & $T$ & & & $i$ & 1 \\
\hline Test Fluid & TCAF10 & 1 & $T$ & ! & $!$ & T & & & & $i$ \\
\hline Date: & 69 & & $!$ & 1 & I & I & & 1 & & $i$ \\
\hline Time: & $16: 34$ & & I & 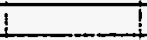 & $!$ & 1 & & & & 1 \\
\hline Note & Analytical c & of is used I & $t$ & & $t$ & 1. & & & & 1 \\
\hline Flow Areatt2 & 0.00335 & & & & & & & & & $\frac{1}{1}$ \\
\hline Hod Fiow Area-tl2 & 0.19695 & & & 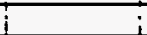 & & & & & & \\
\hline Sa. Pressuro srig & 116.61 & & & + & ! & & & & & \\
\hline Sat. Temperature finlet) & 756.61 & & T & $T$ & & & & & & \\
\hline Lig. Density & 80.91 & 80.94 & $80.92 !$ & 80.92 & 80.96 & 80.96 & 80.85 & 80.98 & 80.98 & 81.17 \\
\hline Latent Heat Btufibm & 28.881 & 28.88 & 28.87 & 28.87 & 28.9 & 28.9 & 28.83 & 28.91 & 28.91 & 29.05 \\
\hline Specitic Heat-Btulbm/F & 0.2881 & 0.238 & 0.288 & 0.288 & 0.288 & 0.288 & 0.288 & 0.288 & 0.288 & 0.287 \\
\hline Liq. Visoosity & 0.4121 & 0.413 & 0.413 & 0.413 & 0.474 & 0.414 & 0.412 & 0.414 & 0.414 & 0.417 \\
\hline Lig. Conductivity-Btuhr-ffF & 0.02441 & 0.0244 & 0.0244 & 0.0244 & 0.0245 & 0.0245 & 0.0244 & 0.0245 & 0.0245 & 0.0246 \\
\hline Liq. Prandt No. & 4.861 & 4.86 & 4.86 & 4.86 & 4.86 & 4.86 & 4.86 & 4.87 & 4.87 & 4.87 \\
\hline Inlet Flow Rate-GPM & 5.1 & & $i$ & 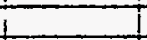 & & & & & & \\
\hline Outlef Lig. Fow Rate-GPM & 0.9 & & $t$ & i & & & & & & \\
\hline \multicolumn{11}{|l|}{ Outlet Vap. Fow Rate-GPM } \\
\hline \multicolumn{11}{|l|}{ Inlel Temperature F } \\
\hline Outle! Liq. Temperaturef & 154.7 & & & & & & & & & \\
\hline Outel Vap. Temperaturof & 950.71 & & & & & & & & & \\
\hline \multicolumn{11}{|l|}{ Water Flow Rato-GPM } \\
\hline Water Inlet Temp.f & 108.91 & & & & & & & & & \\
\hline Water outel Temp.f & 147.11 & & & & & & & & & \\
\hline Inlet Glbm/hrint & 8882811 & & Tomp & 1501 & IDensity & 81.28 & & & & \\
\hline Subooolingf & 4.9 & & i & 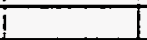 & & & & & & \\
\hline \multicolumn{11}{|l|}{ Aow Obsarvation } \\
\hline & ZONE1 & ZONE2 & ZONE3 & ZONE4 & ZONE5 & ZONE6 & ZONE7 & ZONE8 & ZONE9 & ZONE10 \\
\hline Head input-Watts(not) & 291.3 & 294 & 288.5 & 290.4 & 294.8 & 294 & 2924 & 295 & 289.7 & 290.3 \\
\hline Hoat Loss-Wats & 12.6 & 3.11 & 4.1 & 4.2 & $4 !$ & 3 & 1.9 & 3 & 3.7 & 21.2 \\
\hline Head ALX Bturtrite & 5049 & 5096 & 5001 & 50034 & 5710 & 5005 & 5069 & 5174 & 5017 & 5032 \\
\hline Wall Temp. \#З & 160.7 & 152.4 & 761.61 & TE.6 & 162.5 & $1 E 2.9$ & 161.6 & TEL.3 & 159.8 & $7 \infty 0.5$ \\
\hline Wal Temp. & 161.2 & 1622 & 1621 & 162.8 & 162.4 & 762.9 & 161.8 & 162.6 & 160.1 & 165.5 \\
\hline Wall Temp. $5 F$ & 161.3 & 161.8 & 163.3 & 163.2 & 161.8 & $1 \sqrt{3}$ & 161.4 & 102.3 & 159.8 & 160.5 \\
\hline Wall Temp. IF & 80.21 & 182.3 & 160.8 & 181.8 & 181 & 768.2 & 161.7 & 162.3 & 159.1 & 158.5 \\
\hline Wall Tomp. \#2F & 161.6 & 162.3 & 161.4 & $1 \pi$ & 160.9 & 163.1 & 161.8 & 1628 & 159.4 & 159.7 \\
\hline Instreem Tomp.f & 155.4 & 155.2 & 155.3 & 165.3 & 165.11 & 165.1 & 165.61 & 155 & 1551 & 164 \\
\hline Avg. Wall Tomp.f & 161.2 & 152.3 & 161.5 & 1526 & 161.5 & 163 & 161.7 & 122.5 & 959.6 & 159.0 \\
\hline Avg. Wall DT.f & 5.6 & -6.9 & 5.9 & 7 & $6.2 !$ & 7.7 & 5.81 & 72 & 4.4 & 5.7 \\
\hline Remaining Subcooff & 3.8 & & & & 1 & 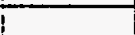 & & & & \\
\hline Exit Quality & -0.04 & -0.03 & -0.02 & -0.01 & 0 & 0.01 & 0.02 & 0.03 & 0.04 & 0.06 \\
\hline EXP. HBturAriter F & 906.3 & 741.9 & 840.6 & 714.3 & 825 & 664.5 & 869.3 & 707.4 & 1135.5 & 880.8 \\
\hline \multicolumn{11}{|l|}{ COMPUIEDH } \\
\hline Jallouk & 656.8 & 658.6 & 653.7 & 655.5 & 658.9 & 642.8 & 63.21 & 639.1 & 633.1 & 6324 \\
\hline Szadyil(Cudo tube) & 1065.1 & 1068.7 & 1059.3 & 10628 & 1059.5 & 10721 & 1075.9 & 1080.2 & 1073.2 & 10728 \\
\hline $5 \operatorname{sen} 1 / 2$ & 800.61 & 814.8 & 800.1 & 807.3 & 816.2 & 818.6 & 817.9 & 823.6 & 815.4 & 817.4 \\
\hline (स्थाण) & 156892 & 158624 & 156758 & 158758 & 156491 & 155171 & 154188 & 151778 & 150152 & 147205 \\
\hline (F) & 1.005 & 1.005 & 1.005 & 1.005 & 1.005 & 1.281 & 1.488 & 1.581 & 1.692 & 1.786 \\
\hline$(+140)$ & 288.6 & 283.6 & 283.61 & 283.6 & 283.6 & 387.4 & 408.7 & 44.0 & 477.2 & 505.8 \\
\hline \multirow[t]{3}{*}{ (1BOIL) } & 1058.3 & 1002 & 10525 & 1068 & 1062.8 & 10582 & 1055.0 & 10542 & 1040.8 & 1035.9 \\
\hline & & & & & & & & & & \\
\hline & ZONE2 & ZONE4 & ZONE6 & ZONE8 & ZONETO & & & & & \\
\hline DP faquid Temp.f & 79.5 & & & & & & & & & \\
\hline DP Liquid Density $-1 \mathrm{bm} / 13$ & 93.05 & & & & & & & & & \\
\hline Elevation-t & & 4 & 6 & 8 & 9.875 & & & & & \\
\hline Measured DPFisid & 0.169 & 0.482 & $0.03 \pi$ & T.392 & T.794 & & & & & \\
\hline Zero Corrections-psid & 0.052 & -0.04 & $=0.734$ & 0.191 & 0.148 & & & & & \\
\hline Comected DPPSid & 0.137 & 0.522 & 0.825 & 1.201 & 1.648 & & & & & \\
\hline Actur DPA & 2.05 & 3.68 & 5.42 & 7.04 & 8.4 & & & & & \\
\hline Measured TPPrig & 116.6 & & & & -1.6 & & & & & \\
\hline \multirow[t]{2}{*}{ Masasurad Overalla } & 200.7 & & & & & & & & & \\
\hline & & & & & & & & & & \\
\hline Computed DP-A & 2.13 & 4.25 & 6.35 & 8.31 & 10.09 & & & & & \\
\hline Friction-t & 0.13 & 0.ङ्ञा & 0.41 & 0.62 & 0.84 & & & & & \\
\hline Acoeleration- & 0 & 0 & 0.12 & 0.2 & 0.26 & & & & & \\
\hline Elevation-th & 2) & 4 & 5.83 & 7.5 & 8.99 & & & & & \\
\hline & & & & & & & & & & \\
\hline
\end{tabular}




\begin{tabular}{|c|c|c|c|c|c|c|c|c|c|c|}
\hline Runt & $632 !$ & & 1 & 1 & 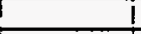 & 1 & & & & \\
\hline Teqf Fuid & ICAF10 & & & I & 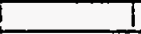 & & & & & I \\
\hline Date: & 6993 & & I & I & & & & & & \\
\hline Time: & 16.341 & & $i$ & $i$ & & & & & & \\
\hline Nole & Anatical o & dis used & 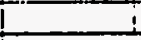 & 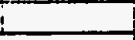 & & & & & & \pm \\
\hline Fow Areate & 0.003361 & & & 1 & & i & & & & \\
\hline Hout Flow Areati2 & 0.15695 & & 1 & & & & & & & \\
\hline Sa. Pressuro + sig & 110.91 & & & & & & & & & \\
\hline SA. Temporatuto f(inlei) & 153.3 & & & & & & & & & \\
\hline Lq.Densthy-1bmins & 81.68 & 81.68 & 81.64 & 81.68 & 81.66 & 81.62 & 81.59 & 81.66 & 81.72 & 81.85 \\
\hline Latemt Hos Etulibm & 29.43 & 29.43 & 29.4 & 29.43 & 29.421 & 29.39 & 29.36 & 29.42 & 29.48 & 29.55 \\
\hline Specifo Hoet Btwlbm/F & 0.286 & 0.286 & 0.286 & 0.286 & 0.2881 & 0.286 & 0.286 & 0.286 & 0.286 & 0.285 \\
\hline Lig. Visoosintomints & 0.427 & 0.427 & 0.426 & 0.427 & 0.427 & 0.426 & 0.425 & 0.427 & 0.428 & 0.48 \\
\hline Wa. Conductivity Btuhw-AfF & 0.0249 & 0.0249 & 0.0249 & 0.0249 & 0.0249 & 0.0249 & 0.0249 & 0.0249 & 0.0249 & 0.025 \\
\hline Lig. Prandtt No. & 4.9 & 4.9 & 4.91 & 4.9 & 4.8 & 4.9 & 4.9 & 4.9 & 4.9 & 4.91 \\
\hline Inlet Flow Rato-GPM & 5.11 & & 1 & 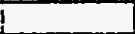 & & & & & & \\
\hline OutlotLiq. Fow Rate-GPM & 1 & & $i$ & $i$ & & & & & & \\
\hline Outlet Vap. Flow Rate-GPM & 2.7 & & & & & & & & & \\
\hline Inlet Temperaturef & 147.8 & & & & & & & & & \\
\hline Outlet Lg. Tomperaturef & 151 & & & & & & & & & \\
\hline Outlot Vap. Temperateref & 146.5 & & & & & & & & & \\
\hline Wador Fiow Pato-GPM & & & & & & & & & & \\
\hline Water hlet Tomp.F & 104.1 & & & & & & & & & \\
\hline Wer outet Temp.f & 149 & & & & & & & & & \\
\hline Ind G-16mhine & 9078221 & & Temp & 146.4 & Density & 87.90 & & & & \\
\hline Subooding f & 5.5 & & & 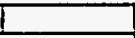 & & & & & & \\
\hline \multicolumn{11}{|l|}{ Fow Observetion } \\
\hline & ZONE1 & ZONE2 & ZONE3 & ZONE4 & ZONE5 & ZONE6 & ZONE7 & ZONE8 & ZONE9 & ZONETO \\
\hline Head Input-Watts(net) & 281.5 & 284.6 & 286.9 & 287.8 & 287.8 & 288.8 & 287.8 & 286.7 & 2827 & 287.7 \\
\hline Hoad Loss-Watts & 11.9 & 2.7 & 3.7 & 3.7 & 3.6 & 2.7 & 1.7 & 2.7 & 3.5 & 19 \\
\hline Hoat Flox-BtuHirine & 4880 & 4933 & 4978 & 4889 & 4989 & 5006 & 4989 & 4970 & 4900 & $4 \% 97$ \\
\hline Wall Tamp. BH & 759 & 159.2 & 159 & 758.7 & 159.2 & T59.7 & 159.4 & 158.8 & 158.8 & 157 \\
\hline Wall Tomp. "4f & 959.5 & 158.8 & 159.6 & 759.1 & 159.3 & 159.2 & 159.8 & 159.2 & 159.3 & 757.1 \\
\hline Waft Tomp. . 55 & 160 & 158.5 & 160.5 & 159.8 & 158.4 & 159.4 & 159 & 158.8 & 158.8 & 1572 \\
\hline WAH TOmp. TIT & 80.51 & 159 & 158 & 158.1 & 158.8 & 159.7 & 159.7 & 159.1 & 157.9 & 155.2 \\
\hline WAH TOMP. 12F & 160.8 & 159 & 158.8 & 158.5 & 158.1 & 159.4 & 159.8 & 159.4 & 158.2 & 158.2 \\
\hline Instreem Temp.F & 151.3 & 167.3 & 151.6 & 151.3 & 161.4 & 151.6 & 151.8 & 151.4 & 151.1 & 150.4 \\
\hline Avg.Wal Temp.F & 159.7 & 159 & 158.9 & 158.8 & 158.5 & 159.4 & 159.6 & 159.1 & 158.6 & 156.6 \\
\hline Avg. Wall DT.f & 8.2 & 7.5 & 7.1 & 7.3 & 6.91 & 7.51 & 7.6 & 7.4 & 7.3 & 5.9 \\
\hline Romaining subcooff & 4.5 & & & & & & & & & \\
\hline Est Quality & -0.04 & -0.03 & -0.02 & -0.01 & 0 & 0.01 & 0.02 & 0.03 & 0.04 & 0.05 \\
\hline 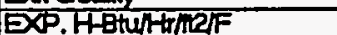 & 596.6 & 657.6 & $\$ 8$ & 685.8 & 723.11 & 655.4 & 660.4 & 668.4 & 673.3 & 843.2 \\
\hline \multicolumn{11}{|l|}{ COMPUTEDH } \\
\hline Jallouk & ख्ञा.7 & 64.6 & 637.5 & 637.6 & 6381 & 63.8 & 6242 & 520.7 & 616.4 & 620.5 \\
\hline Szady1(Cudo tube) & 1022 & 1027.5 & 10329 & 1033.3 & 1033.9 & 1038.1 & 1041.8 & 1041.2 & 1063.2 & 1044.4 \\
\hline szady 2 & 789.5 & 7928 & 797.3 & 790.3 & 797.3 & 803.8 & 791.4 & 790.1 & 784.8 & 794.5 \\
\hline$(P \perp O)$ & 152845 & 152845 & 153102 & 152845 & 152078 & 155039 & 151758 & 149720 & 147844 & 145489 \\
\hline (F) & 1.005 & 1.005 & 1.006 & 1.006 & 1.0051 & 1.005 & 1.341 & 1.494 & 1.618 & 1.728 \\
\hline (संLa) & 2842 & 2842 & 284.8 & 284.2 & 284.81 & 309.8 & 378.3 & 4226 & 457.3 & 488 \\
\hline \multirow[t]{3}{*}{ ( } & 1014.6 & 10202 & 1025.7 & 1025 & 1020.7 & 1028.8 & 1024.9 & 1017.5 & 1005.6 & 1007.6 \\
\hline & & & & & & & & & & \\
\hline & ZONE2 & ZONEA & ZONE6 & ZONE8 & ZONE10 & & & & & \\
\hline DPliquid Temp.f & 79.5 & & & & & & & & & \\
\hline DPLlquid Density tbm/13 & 93.06 & & & & & & & & & \\
\hline Elevation- & 2 & 4 & 6 & 8 & 9.875 & & & & & \\
\hline Measured DPPsid & 0.138 & 0.487 & -0.016 & 1.273 & 1.65 & & & & & \\
\hline Zoro correctionepsid & 0.032 & -0.04 & -0.734 & 0.181 & 0.148 & & & & & \\
\hline Corrected DPPsid & 0.106 & 0.521 & 0.778 & 1.082 & 1.502 & & & & & \\
\hline Actur DPR & 2.09 & 3.68 & 5.56 & 7.18 & 8.59 & & & & & \\
\hline Measured TPPsig & 110.8 & & & & -1.6 & & & & & \\
\hline \multirow[t]{2}{*}{ Messured Overalli } & 197.82 & & & & & & & & & \\
\hline & & & & & & & & & & \\
\hline Computed DPA & 2.13 & 4.25 & 6.38 & 8.38 & 10.18 & & & & & \\
\hline Friction-th & 0.13 & 0.25 & 0.39 & 0.59 & 0.81 & & & & & \\
\hline Acosleration-h & 0 & 0 & 0.08 & 0.18 & 0.24 & & & & & \\
\hline Elevation-t & 2 & 4 & 5.91 & 7.61 & 9.13 & & & & & \\
\hline & & & & & & & & & & \\
\hline
\end{tabular}




\begin{tabular}{|c|c|c|c|c|c|c|c|c|c|c|}
\hline Run: & i 6331 & $i$ & + & 1 & ' & 1 & 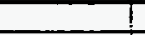 & & 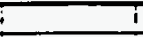 & $i$ \\
\hline Toet fluid & ICAF10 i & I & $!$ & & $i$ & 1 & $i$ & - & & I \\
\hline Date: & ! ब933! & 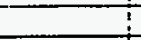 & $\perp$ & & 1 & $i$ & $i$ & - & & $i$ \\
\hline Time: & $19: 51$ & & $\vdots$ & & 1 & 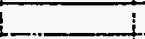 & $i$ & . & & 1 \\
\hline Nole & I Analytical d & th is used & $\div$ & - & 1 & $L$ & 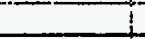 & & & 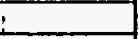 \\
\hline Flow Area-4t2 & 0.003361 & & $!$ & 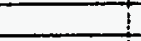 & $\dot{i}$ & 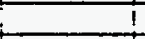 & + & 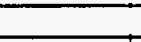 & & 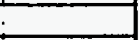 \\
\hline Head Fow Area-ll & 0.19551 & & 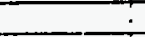 & 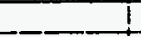 & 1 & 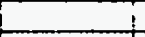 & 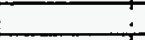 & & & 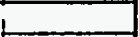 \\
\hline Sat Pressure Psig & 755.91 & & $\dot{i}$ & 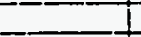 & $i$ & 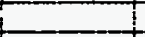 & & & & \\
\hline Sat. Temperature F(inlet) & 176.9 & & & & & & & & & \\
\hline La. Densindbm/13 & 76.441 & 76.34 & 76.391 & 76.39 & 76.39 & 76.27 & 76.3 & 76.34 & 76.34 & 76.49 \\
\hline Latent Hoar-Btulbm & 25.491 & 25.411 & 25.45 & 25.45 & 25.451 & 25.88 & 25.381 & 25.47 & 25.41 & 25.52 \\
\hline Specific Heat-Btulbmif & 0.31 & 0.301 & 0.31 & 0.3 & 0.3 & 0.301 & 0.3011 & 0.301 & 0.3011 & 0.3 \\
\hline Liq. Viscosity & 0.3411 & 0.34 & 0.34 & 0.34 & 0.341 & 0.339 & 0.339 & 0.34 & 0.34 & 0.342 \\
\hline Liq. Conductivity BtuminffF & 0.02161 & 0.0216 & 0.02161 & 0.0216 & 0.02161 & 0.0215 & 0.0216 & 0.0216 & 0.0216 & 0.0217 \\
\hline Liq. Prandil No. & 4.731 & 4.73 & 4.731 & 4.73 & 4.731 & 4.73 & 4.73 & 4.73 & 4.73 & 4.73 \\
\hline Inlet Flow Rafo-GPM & 0.9 & 1 & 1 & 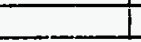 & 1 & 1 & 1 & 1 & 1 & $L$ \\
\hline Outlet Liq. Flow Rate-GPM & 0.8 & & 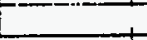 & & 1 & 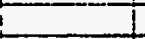 & 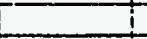 & 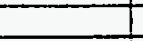 & & $L$ \\
\hline Outlet Vap. Flow Rale-GPM & 0.9 & 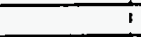 & $i$ & & $i$ & $\perp$ & 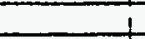 & - & & 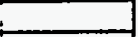 \\
\hline Inlat Temperaturef & 172.5 & & ti & 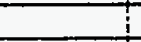 & 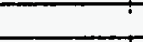 & i & 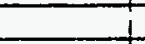 & & & 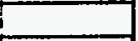 \\
\hline Outlet Liq. TemperatureF & 176 & & $T$ & & & 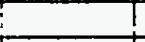 & & & & 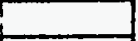 \\
\hline Outiet vap. Temperaturef & 173.9 & & $T$ & . & & 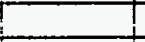 & & 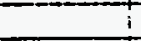 & 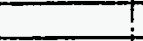 & \\
\hline Walor flow Rato-GPM & & & $T$ & & & & & & & \\
\hline Water inlot Temp.F & 151.8 & & 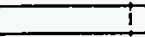 & & & 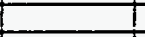 & & & & 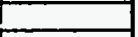 \\
\hline Weter outet Temp,F & 167.41 & & $i$ & & & $i$ & $\Gamma$ & 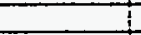 & $T$ & $T$ \\
\hline Indet Glbmist/R2 & 170785 & & Temp & 158.71 & Density & 79.62 & & & & \\
\hline SubooolingF & 4.41 & & 1 & 7. & & & & & & \\
\hline \multicolumn{11}{|l|}{ Fow Observation } \\
\hline & ZONEI & ZONE2 & ZONEB IZ & ZONEA & ZONE5 & ZONE6 & ZONE7 I & ZONE8 & ZONE9 & ZONETO \\
\hline Hoat Input-Watts(nel) & 282.9 & 280.8 & 279.3 & 286 & 288.8 & 289.5 & 280.4 & 281 & 282 & 279.5 \\
\hline Heat Loss-Walls & 151 & 3.5 & 4.7 & 5.1 & 5.2 & 4.2 & 28 & 4.1 & 4.8 & 26.6 \\
\hline Heat Flex-BtuhHifit2 & 4904 & 4867 & 4811 & 4958 & 5006 & 5018 & 4861 & 4871 & 4888 & 4846 \\
\hline Wal Temp. H3 & 180.8 & 180.5 & 880.9 & 180.9 & T81.11 & 181 & 181.11 & 181.3 & 180.8 & 779.6 \\
\hline Wall Temp. $4 F$ & 181.5 & 180.6 & 181.4 & 181.2 & 181.4 & 781 & 181.3 & 181.4 & 181.5 & 179.7 \\
\hline Wall Temp. $\# 5 F$ & 187.9 & 180.1 & 183 & 180.9 & 180.9 & 180.9 & 180.9 & 181.1 & 181.1 & 179.8 \\
\hline Wall Temp. "1F & 77.9 & 180.9 & 179.8 & 180.9 & 180.7 & 181.6 & 180.9 & 181.3 & 180.2 & 177.9 \\
\hline Wall Temp. "2F & 182 & 180.8 & 180.3 & 180.8 & 180.7 & 181.2 & 181.11 & 181.5 & 180.4 & 178.8 \\
\hline Instream Temp.f. & 176.4 & 176.8 & 176.6 & 176.6 & 176.61 & 177.1 & 177 & 176.8 & 176.8 & 176.2 \\
\hline Avg. Wall Tomp.fF & 181.6 & 180.7 & 180.6 & 180.8 & 180.9 & 181.1 & 181.11 & 181.3 & 180.8 & 179.2 \\
\hline Avg. Wall DT.f & 4.9 & 3.7 & 3.81 & 4 & 4.11 & 3.8 & 3.8 & 4.3 & $3.8 !$ & 2.8 \\
\hline Remaining SubcoofF & -1.2 & & 1 & 5 & & & & & & $L$ \\
\hline Exat Quality & 0.01 & 0.08 & 0.15 & 0.21 & 0.28 & 0.35 & 0.41 & 0.48 & 0.54 & 0.61 \\
\hline EXP. HBtuHriter & 995 & 1313.8 & 1280.4 & 1239.1 & 1221.4 & 1321.2 & 1265.7 & 1132.8 & 1293.5 & 1754.8 \\
\hline \multicolumn{11}{|l|}{ COMPUTED H } \\
\hline Jallouk & 844.2 & 818.5 & 775.4 & 7812 & 780.5 & 779 & 7572 & 7542. & 753.2 & 743.7 \\
\hline Szady I(Cudo tube) & 1187.8 & 1185.4 & 1182 & T195.3 & 12024 & 1207.7 & 1188.2 & 1188.3 & 1190.7 & 11815 \\
\hline Sexdr2 & 914.5 & 912.4 & 9002 & 923.8 & \$50.5ा & 935.7 & 9151 & 915.6 & 918.4 & $\mathbf{9 0 9 . 7}$ \\
\hline (RLO) & 325231 & 31398 & 29129 & 26900 & 247581 & 22848 & 20428 & 18224 & 16067 & 13810 \\
\hline$(F)$ & 1.221 & 1.652 & 2.052 & 2.358 & 2.628 & 2.845 & 3.049 & 3.239 & 3.476 & 3.6 \\
\hline$(1+\mathrm{H})$ & 86.3 & 116.8 & 146.7 & 167.3 & 185.6 & 201.1 & 215.5 & 228.9 & 241.4 & 257.8 \\
\hline \multirow[t]{3}{*}{$(1+B O I L)$} & 1187.7 & 1186.1 & 1181.3 & 11952 & 1200.8 & 1205.9 & 1185.9 & 1185.5 & 1187.4 & 1177.6 \\
\hline & & & & & & & & & & L \\
\hline & ZONE2 & ZONE4 & ZONE6 & ZONEB & ZONE1O & & & & & 1 \\
\hline DP liquid Temp.F & -80 & & & & & & & & & 1 \\
\hline DP Liquid Density hbmint3 & 929 & & & & & & & & & \\
\hline Elevation- & 2 & 4 & 6 & 8 & 9.875 & & & & & \\
\hline Measured DPFsid & ర.ा8्डा| & 1.304 & 1.35 & 3.0.53 & 3.919 & & & & & \\
\hline Zero Correctons-psid & 0.032 & -0.04 & -0.734 & 0.191 & 0.148 & & & & & \\
\hline Corrected DPPsid & 0.157 & 1.344 & 2.069 & 2.902 & 3.771 & & & & & \\
\hline Actued DPA & 2.14 & 2.33 & 3.381 & 4.28 & 4.911 & & & & & \\
\hline Measured TPPsig & 155.9 & & & & -1.6 & & & & & \\
\hline Messured Overalli & 295.531 & & & & & & & & & \\
\hline & & & & & & & & & & \\
\hline Computed DPA & 1.5 & 277 & 3.71 & 4.53 & 52 & & & & & \\
\hline Friction-t & 0.01 & 0.08 & 0.06 & 0.09 & 0.13 & & & & & \\
\hline Acosleration-t & 0.01 & 0.02 & 0.02 & 0.03 & 0.05 & & & & & \\
\hline Elevation-fi & 1.61 & 2.73 & 3.68 & 4.4 & 5.02 & & & & & \\
\hline & & & & & & & & & & \\
\hline
\end{tabular}




\begin{tabular}{|c|c|c|c|c|c|c|c|c|c|c|}
\hline Runt & 63 & & 1 & 1 & 1 & 1 & 1 & I & T & i \\
\hline Tost Fuid & CAF10 & & $i$ & $i$ & & & & & & $i$ \\
\hline Date: & 69938 & & $i$ & $i$ & & & $i$ & & & $i$ \\
\hline Timo: & $19: 51$ & & & t & & & I & & & 1 \\
\hline Note & Anartical & th is used & & 1 & & & $\perp$ & & & \\
\hline Flow AYOOA-12 & 0.00336 & & 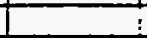 & 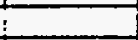 & & & 1 & & 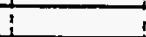 & + \\
\hline Head Flow Areatil & 0.1955 & & & & & & & & & \\
\hline Sa. Pressure trig & 155.1 & & & & & & & & & \\
\hline Sal. Tomperature finlot) & 176.5 & & & & & & & & & t! \\
\hline Lq. Densthy & 76.63 & 76.46 & 76.49 & 76.49 & 76.49 & 76.44 & 76.32 & 76.34 & 76.37 & 76.67 \\
\hline Ladent Hos Btwhibm & 25.69 & 25.51 & 25.521 & ¿5.52 & 25.52 & 25.49 & 25.4 & 25.41 & 25.43 & 25.67 \\
\hline Specifio HeatbtulbmiF & 0.3 & 0.3 & 0.31 & 0.3 & 0.31 & 0.3 & 0.301 & 0.301 & 0.3011 & 0.3 \\
\hline Liq. Viscosity dbminti & 0.344 & 0.341 & 0.3421 & 0.342 & 0.342 & 0.341 & 0.338 & 0.34 & 0.34 & 0.344 \\
\hline Lig. Conductivity Btuhrif- & 0.0218 & 0.0217 & 0.0217 & 0.0217 & 0.0217 & 0.0216 & 0.0216 & 0.0216 & 0.0216 & 0.0218 \\
\hline Lq. Prandt No. & 4.73 & 4.73 & 4.781 & 4.73 & 4.73 & 4.73 & 4.73 & 4.73 & 4.73 & 4.74 \\
\hline Inlot Flow Rate-GPM & 2 & & 1 & $\perp$ & 1 & $\perp$ & & & & \\
\hline Outtel La. Fow Rato-GPM & 0.8 & & 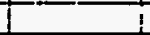 & 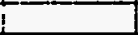 & $i$ & i & & & & \\
\hline OutdeI Vap. Flow Rado-GPM & 1.4 & & & & & & & & & \\
\hline Inlet Temperature $F$ & 172.2 & & & & & & & & & \\
\hline Outelug. Temperature & 175.2 & & & & & & & & & \\
\hline Outolvap. Temperaturof & 773.4 & & & & & & & & & \\
\hline Wrep fow Rate-GPM & & & & & & & & & & \\
\hline Wador inlet Tomp.F & 129.2 & & & i & & & & & & \\
\hline Water outlel Temp.f & 169.6 & & & & & & & & & \\
\hline Infet Glbm/hrhe & 371350 & & Tomp & 166.7 & Deneivy & 77.8 & & & & \\
\hline Suboooling $f$ & 4.3 & & & & & & & & & \\
\hline Flow Observation & & & & 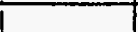 & & & & & & \\
\hline & ZONE1 & ZONE2 & ZONES & ZONE4 & ZONE5 & ZONE6 & ZONET & ZONE8 & ZONE9 & ZONE10 \\
\hline Hoatinput-Watts(nel) & 283.2 & 286.7 & 286.7 & 281 & 288.8 & 290.3 & 284.5 & 283.7 & 283.2 & 2823 \\
\hline Hood Loss-Wedts & 14.7 & 3.4 & 4.6 & 4.9 & 5 & 3.9 & 2.7 & 3.9 & 4.7 & 25.3 \\
\hline Heat Flox Etuntrine & 4900 & 4970 & 4970 & 4877 & 5006 & 5032 & 4332 & 4918 & 4900 & 4898 \\
\hline Wall temp. B⿸尸 & 780.5 & 180.2 & 180.4 & 180.4 & 180.5 & 180.3 & 780.8 & TE्ष.7 & 1802 & 778.6 \\
\hline Wall Tomp. $4 F$ & 181.1 & 180.3 & 181 & 180.5 & $180 . \overline{8}$ & $180 . \overline{3}$ & 180.8 & 181.2 & 180.8 & 978.7 \\
\hline Wed Tomp. " 5F & 181.7 & 180 & 1824 & 180.5 & 180.3 & 180.3 & 180.9 & 180.6 & 180.6 & 178.9 \\
\hline Wall Tomp. \#1F & 80.6 & 180.5 & 179.4 & 179.7 & 180.1 & 181 & 180.6 & 180.8 & 179.7 & 177.1 \\
\hline Wal Temp. & 181.8 & 180.6 & 179.9 & 180.1 & 180 & 180.7 & 180.8 & 181.3 & 180.2 & 177.9 \\
\hline Instroam Tomp.f & 175.6 & 176.3 & 176.21 & 176.2 & 176.2 & 176.4 & 176.9 & 176.8 & 176.7 & 176.4 \\
\hline Avg. Wall Tomp.f & 181.3 & 180.4 & 18021 & 180.3 & 180.3 & 180.5 & 180.8 & 180.9 & 180.3 & 178.2 \\
\hline Avg. WallDT.f & 5.5 & 3.9 & 3.8 & 3.9 & 3.91 & 3.8 & 3.7 & 3.9 & 3.4 & 2.6 \\
\hline Remaining Subcooff & 1.7 & & & & & & & & & \\
\hline Exit Quality & -0.02 & 0.01 & 0.04 & 0.07 & 0.1 & 0.13 & 0.16 & 0.19 & 0.22 & 0.26 \\
\hline EXP. HBtuTHREF & 9002 & 12824 & 1325.1 & 1260.6 & 1284 & 1307.9 & 1348.5 & 1255.2 & 1444.6 & 1858.6 \\
\hline COMPUTEDH & & & & & & & & & & \\
\hline Jallouk & 778 & 7825 & 760.3 & 740 & 745.6 & $74 \times .2$ & 732.1 & 726.5 & 721 & 722 \\
\hline Szeady (cudo tube) & 1182.8 & 1195.3 & 1155.2 & 118 & -200.8 & 1205 & 1198.3 & 7186.6 & 1195.5 & 1185.1 \\
\hline szady2 & 910.7 & 922.9 & 923.5 & 911.8 & 930.4 & 938.1 & 927.5 & 926.3 & 925.7 & 917.1 \\
\hline$(R L L)$ & 70078 & 71152 & 69258 & 67000 & 64918 & 62835 & 60035 & 58692 & 56455 & 55005 \\
\hline (F) & 1.004 & 1.004 & 1.465 & 1.724 & 1.9281 & 2.001 & 2.2351 & 2.368 & 2491 & 2.22 \\
\hline (1) & 122 & 132.1 & 192.61 & 226.7 & 2528 & 275 & 294 & 311.6 & 327.7 & 34.6 \\
\hline (HBOIL) & 1182.3 & 1194.7 & 1138.5 & 1181.2 & 1197.1 & 12012 & 11924 & 1189.5 & 1187.3 & 1175.4 \\
\hline & & & & & & & & & & \\
\hline & ZONE2 & ZONE4 & ZONE6 & ZONE8 & ZONE10 & & & & & \\
\hline DP liquid Tomp.F & 80.6 & & & & & & & & & 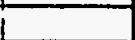 \\
\hline DPLquid Density -bm/ís & क्2.9 & & & & & & & & & \\
\hline Elevationa & 2 & 4 & 6 & 8 & 9.875 & & & & & \\
\hline Measured DPPPSId & 0.216 & $0.900 \mid$ & 0.875 & 2.476 & 3.748 & & & & & \\
\hline Zero Corrections-psid & 0.032 & $=0.04$ & -0.734 & 0.191 & 0.148 & & & & & \\
\hline Comected DPPsid & 0.184 & 0.948 & 1.609 & 2.285 & 3 & & & & & \\
\hline Actud DP & 2.08 & 3.07 & 4.25 & 5.4 & 8.381 & & & & & \\
\hline Moasured TPPsig & 155.11 & & 1 & & -1.6 & & & & & \\
\hline Mosucuedoverifith & 28.8 & & & & 1 & & & & & \\
\hline & & & & & & & & & & \\
\hline Computed DP-n & 7.96 & 3.52 & 4.88 & 6.13 & 7.23 & & & & & \\
\hline Friction-t & 0.03 & 0.07 & 0.14 & 0.22 & 0.31 & & & & & \\
\hline Accolerationn & 0.01 & 0.03 & 0.05 & 0.07 & 0.08 & & & & & \\
\hline Elevation-ft & 1.92 & 3.41 & 4.59 & 5.85 & 6.84 & & & & & \\
\hline & & & & & & & & & & \\
\hline
\end{tabular}




\begin{tabular}{|c|c|c|c|c|c|c|c|c|c|c|}
\hline Run: & i 6351 & 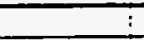 & $:$ & i. & $\dot{-}$ & - & $\therefore$ & 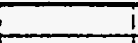 & & $i$ \\
\hline Tost Fuid & CAF 10 & $!$ & 1 & I & 1 & 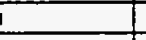 & T & 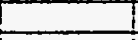 & & $i$ \\
\hline Dale: & i $\quad$ 99931 & $i$ & 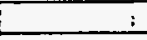 & 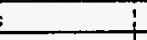 & $i$ & i & 1 & 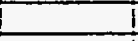 & & I \\
\hline Time: & $19: 511$ & $!$ & 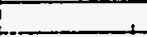 & & & & i. & in & & I. \\
\hline Note & Anatytical 0 & His used! & $i$ & 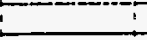 & 1 & L & 1 & 1 & & $i$ \\
\hline Flow Areatt2 & 0.003361 & 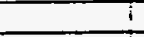 & 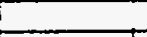 & & $\dot{T}$ & 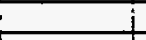 & & 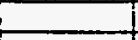 & & $\perp$ \\
\hline Hoat Flow Areatil & 0.19595 & $i$ & $\therefore$ & 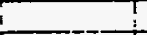 & $\underline{I}$ & I. & & & & \\
\hline Sat Pressute Psig & 153.5 & & & & & & & & & \\
\hline Sat. Temperaturo of (iniol) & $\overline{175 . \overline{8}}$ & & -1 & t & & & & & & \\
\hline Ug.Density $/ \mathrm{bm} / \mathrm{i}$ & 76.95 & 76.88 & 76.671 & 76.6 & 76.67 & 76.63 & 76.58 & 76.71 & 76.71 & 76.88 \\
\hline Latent Heat-Btulbm & 25.88 & 25.83 & 25.67 & 25.61 & 25.67 & 25.63 & 25.59 & 25.68 & 25.681 & 25.83 \\
\hline Specifio HeatBtulbmfF & 0.2991 & 0.299 & 0.31 & 0.3 & 0.31 & 0.3 & 0.3 & 0.3 & 0.3 & 0.299 \\
\hline Lia. Visos & 0.348 & 0.347 & 0.3441 & 0.343 & 0.344 & 0.341 & 0.343 & 0.345 & 0.345 & 0.347 \\
\hline Lig. Conductivity tuhr-itf & 0.0221 & 0.0219 & 0.02181 & 0.0217 & 0.0218 & 0.0218 & 0.0217 & 0.0218 & 0.0218 & 0.0219 \\
\hline Liq. Prandet No. & 4.74 & 4.74 & 4.74 & 4.73 & 4.741 & 4.78 & 4.73 & 4.74 & 4.741 & 4.74 \\
\hline Inlot Flow Rato-GPM & 2.8 & & 1 & $!$ & & 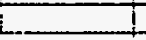 & 1 & & & \\
\hline Outet Lig. Flow Rate-GPM & 0.9 & & $i$ & $i$ & i. & 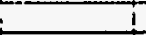 & 1 & $L$ & & \\
\hline Outlel Vap. Fow Rate-GPM & 2.2 & & $\therefore$ & + & $i$ & $i$ & $i$ & 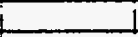 & & \\
\hline Inlel Temperaturof & $171.1 !$ & $i$ & $i$ & 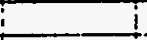 & & & & $i$ & & \\
\hline Oudtet Liq. Temperaturer & 774.31 & & & & & & & & & \\
\hline Outlet Vap. Temperaturef & 172.4 & & t & r & & & & & & \\
\hline Wator Fow Rato-GPM & -21 & 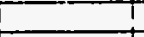 & ? & $T$ & T & T & & i & & \\
\hline Water tnlet Temp.f & 125.21 & & & i & & t & ! & & & $T$ \\
\hline Water outel Temp.t. & 169.31 & & & I & & & & $i$ & & I \\
\hline InIGlbmhrnL & 5182581 & & Temp & 167.81 & IDensity & 77.56 & & & & \\
\hline Subcoolingf & 4.71 & & $\perp$ & 1 & & & & $i$ & & \\
\hline \multicolumn{11}{|l|}{ Flow Observation } \\
\hline & ZONE1 1 & ZONE2 I & ZONE3 L & ZONE4 & ZONE5 & ZONE6 & ZONE7 & ZONE8 & ZONE9 & ZONE10 \\
\hline Heat Input-Watts(net) & 287.6 & 288.2 & 290.4 & 291.3 & 285.6 & 284.7 & 285.8 & 284.7 & 290.6 & 294.6 \\
\hline Heat Loss-Watts & 14.3 & 3.3 & 4.5 & 4.7 & 4.7 & 3.5 & 2.4 & 3.6 & 4.4 & 24.4 \\
\hline Heal Flox-Btuhtrine & 4985 & 4996 & 5034 & 5049 & 4851 & 4935 & 4954 & 4935 & 5037 & 5105 \\
\hline Wall Temp. & T79.51 & 179.5 & 179.5 & 779.8 & 179.9 & 179.5 & 179.9 & 179.8 & 779.2 & 778.3 \\
\hline Wall Tomp. \$4F & 180.5 & 179.7 & 180.2 & 180.3 & 180.2 & 179.5 & 979.9 & 180.1 & 179.8 & 178.4 \\
\hline Wadl Temp. $5 F$ & 181.11 & 179.3 & 181.6 & 180.1 & 179.6 & 179.5 & 179.8 & 179.7 & 179.61 & 178.4 \\
\hline WEH TEmp. \#1F & 82.21 & 179.9 & 178.81 & 179.5 & 179.4 & 180.1 & 179.9 & 179.8 & 178.61 & 778.5 \\
\hline Wall Tomp. Ha & 181.31 & 179.9 & 179.3 & 179.8 & 179.31 & 179.7 & 179.91 & 180.3 & 178.9 & 177.5 \\
\hline inetream Temp.f & 174.2 & 174.5 & 175.41 & 176.71 & 176.4 & 175.6 & 175.81 & 175.3 & 176.31 & 174.6 \\
\hline Avg.Wall Tomp.F & 180.71 & 179.8 & 179.5 & 179.8 & 179.6 & 179.7 & 179.9 & 179.9 & 179.2 & 977.8 \\
\hline Aviv. Wall DT.F & 6.3 & 5 & 3.8 & 4 & 41 & 3.8 & 3.9 & 4.4 & 3.71 & 3.1 \\
\hline Remaining Subcooff & 2.8 & & & $i$ & & & & & & \\
\hline Exit Quality & -0.03 & -0.011 & 0.01 & 0.03 & 0.06 & 0.081 & 0.11 & 0.12 & 0.14 & 0.17 \\
\hline EXP. HBturHriter & 794.6 & 994.3 & 1316.9 & 1263.4 & 1237.3 & 1289.6 & 1284.8 & 1117.3 & 1364.3 & 16525 \\
\hline \multicolumn{11}{|l|}{ COMPUTEDH } \\
\hline Jallouk & 755.1 & 757.4 & 764.8 & 744.31 & 725 & 718.5 & 76.4 & 708.9 & 714.3 & 713.8 \\
\hline Szadyil(Cudo tube) & 1182.3 & T185.7 & 1996.9 & 1202.2 & 1789.3 & 7189.8 & 1194.6 & 7189.9 & 1203 & 1206.2 \\
\hline Szady 2 & 912.7 & 915.9 & 928.6 & 932.6 & 919.9 & 920.9 & 9262 & 9225 & 937 & 942.1 \\
\hline (R्व) & 97318 & 97597 & 98557 & 96256 & 94008 & 92037 & 90081 & 87482 & 85319 & 82489 \\
\hline (F) & 1.005 & 1.005 & $1.0 \% 5$ & 1.438 & 1.648 & 1.802 & 1.937 & 2.053 & 2.175 & 2.287 \\
\hline (सUQ) & 172.1 & 1722 & 1827 & 246.7 & 281.8 & 300.2 & 332.5 & 363.9 & 373 & 3921 \\
\hline \multirow[t]{3}{*}{ (HBOIL) } & 1181.1 & 1184.5 & 1195.5 & 1198.7 & 1184 & 11829 & 1186 & 1179.3 & 1190.9 & 11922 \\
\hline & & & & & & & & & & \\
\hline & ZONE2 & ZONE4 & ZONE6 & ZONE8 & ZONE10 & & & & & \\
\hline DPliguid Temp.f & 81.7 & & & 1 & & $\perp$ & & & & \\
\hline DP Liquid Density lbmit3 & 92.74 & & & & & & & & & \\
\hline Elevation-a & & 4 & 6 & 8 & 9.875 & & & & & \\
\hline Messured DPFsid & 0.216 & 0.702 & 0.557 & 2.068 & 2.659 & & & & & \\
\hline Zoro Corrections-psid & 0.052 & 0.04 & -0.734 & 0.191 & 0.1481 & & & & & \\
\hline Colrocted DPpsidd & 0.184 & 0.742 & 1.297 & 1.877 & 2.511 & & & & & \\
\hline Ackud DPA & 2.07 & 3.44 & 4.82 & 6.13 & 7.21 & & & & & \\
\hline Moesured TPPsig & 153.51 & & & I & -1.6 & & & & & \\
\hline \multirow[t]{2}{*}{ Mogured Overell } & 290.511 & & & & & & & & & \\
\hline & & & & & & & & & & \\
\hline Computed DPA & 204 & 3.87 & 5.46 & 6.94 & 8.27 & & & & & \\
\hline Friction-fi & 0.04 & 0.1 & 0.19 & 0.3 & 0.42 & & & & & \\
\hline Accolerationt & 0 & 0.04 & 0.07 & 0.09 & 0.12 & & & & & \\
\hline Elovation-1 & 2 & 3.72 & 5.19 & 6.54 & 7.78 & & & & & \\
\hline & & & & & & & & & & \\
\hline
\end{tabular}




\begin{tabular}{|c|c|c|c|c|c|c|c|c|c|c|}
\hline Runk & $1 \quad 6361$ & I & I & 1 & 1 & $i$ & & I & 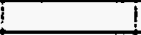 & 1 \\
\hline Teat Fluid & ¡CAF10 & L & & 1 & L & $\perp$ & & 1 & & \\
\hline Dale: & 69931 & & & 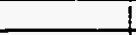 & 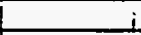 & $i$ & & I & & I \\
\hline Time: & $19: 51$ & & 1 & 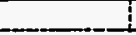 & & j. & & & & 1 \\
\hline Nolo & Anatyical & dt is used & $i$ & + & L & L & & & & \\
\hline Flow Aveatti2 & 0.00336 & & & & & & & 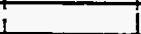 & & 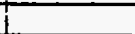 \\
\hline Head Flow Area-d2 & 0.19695 & & & & & & & & & 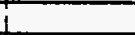 \\
\hline Sat.Pressuro Psig & $15 \overline{6} .1$ & & & & & & & & & \\
\hline Sal. Tomporatur of (inlet) & $17 \pi$ & & & & & & & & & \\
\hline Lq.Denstiybmn3 & 76.44 & 76.39 & 76.42 & 76.3 & 76.37 & 76.37 & 76.25 & 76.3 & 76.37 & 76.85 \\
\hline Latent Heat Btubm & 25.49 & 25.45 & 25.47 & 25.38 & 25.491 & 25.43 & 25.34 & 25.38 & 25.431 & 25.65 \\
\hline Spectrio Hed tulbmiF & 0.31 & 0.3 & 0.31 & 0.3011 & 0.3011 & 0.301 & 0.301 & 0.301 & 0.3011 & 0.3 \\
\hline Lq. Visooshytb m/ahr & 0.3411 & 0.34 & 0.347 & 0.330 & 0.34 & 0.34 & 0.338 & 0.389 & 0.34 & 0.344 \\
\hline Lig. Conductivity Btump-hf & 0.02161 & 0.0216 & 0.0216 & 0.0216 & 0.02161 & 0.0216 & 0.0215 & 0.0216 & 0.0216 & 0.0218 \\
\hline Lig. Prandt No. & 4.731 & 4.73 & 4.73 & 4.73 & 4.731 & 4.73 & 4.73 & 4.73 & 4.73 & 4.73 \\
\hline Intet Flow Rato-GPM & 3.7 & & & & & I & & & & \\
\hline Outtellig. Flow Rate-GPM & 0.8 & & & & & & & & & \\
\hline Outtet Vap. Flow Rate-GPM & 2 & & & & & & & & & \\
\hline Inlet Temperaturef & 173.7 & & & & & & & & & \\
\hline OuthetLq. Temperature & 175.41 & & & & & & & & & \\
\hline ÖUTeI Vap. Temperaturef & 173.6 & & & & & & & & & \\
\hline Watop Flow Rate-GPM & & & & & & & & & & \\
\hline Water hlot Temp, F & 124.4 & & & & & & & & & \\
\hline Weter outle! Temp.F & 169.6 & & & & & & & & & \\
\hline Intet Glbmintine & 678671 & & Temp & 170.6 & Donsity & 78.85 & & & & \\
\hline Subcooling & 3.3 & & $=$ & 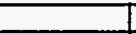 & & & & & & \\
\hline Flow Obsenvation & & & & & & & & & & \\
\hline & ZONEI I & ZONE2 & ZONES & ZONE4 & ZONE5 & ZONE6 & ZONE7 & ZONEB & ZONE9 & ZONE10 \\
\hline Hout Input-Watts(net) & 288.8 & 286.5 & 294.5 & 263.3 & 282.5 & 281.4 & 293.4 & 285.4 & 267.8 & 286.6 \\
\hline Head Loss-Watts & 14.2 & 3.3 & 4.4 & 4.7 & 4.6 & 3.5 & 2.3 & 3.6 & 4.3 & 24 \\
\hline Heat Flox-BtuhHI/R2 & 5006 & 4960 & 5105 & 4564 & 4897 & 4878 & 5086 & 4947 & 4642 & 4568 \\
\hline WalTemp. Wh & 781.1 & 180.6 & 780.6 & 180.9 & $18 \pi$ & 180.5 & 181.3 & 181.2 & 880.5 & 179 \\
\hline Woll Temp. Th & 181.7 & 180.7 & 187.2 & 181.2 & 181.2 & 180.6 & 181.7 & 181.5 & 181.1 & 179.2 \\
\hline Warl Tomp. $15 F$ & 182.3 & 180.4 & 182.8 & 181.1 & 180.6 & 180.6 & 181.8 & 181.1 & 180.7 & 179.2 \\
\hline WaH Tomp. ITF & 82.3 & 181 & 180 & 180.0 & 180.0 & 181.7 & 181.4 & 181.2 & 180 & 177.5 \\
\hline Wall Tomp. WF & 182.8 & 181.2 & 180.4 & 180.9 & 180.6 & 180.7 & 181.7 & 181.5 & 180.2 & 178.4 \\
\hline Inetroan Tomp. $f$ & 176.4 & 976.6 & 176.6 & $17 \pi$ & 176.7 & 176.7 & 177.2 & 177 & 176.7 & 176.6 \\
\hline Avg. Wall Temp.F & 181.9 & 180.8 & 180.6 & 181 & 180.8 & 180.7 & 181.5 & 181.3 & 180.5 & 178.7 \\
\hline Avg. Wall DT.F & 5.21 & 4.1 & 3.8 & 3.7 & 3.8 & 3.8 & 4.1 & 4.1 & 3.6 & 29 \\
\hline Romaining Subcooff & 1.8 & & & & & & & & & \\
\hline Exit Quadity & -0.02 & 0 & 0.01 & 0.03 & 0.04 & 0.06 & 0.08 & 0.1 & 0.11 & 0.13 \\
\hline EXP.HBtu/HrmerF & 958.3 & 1226.1 & 1365.6 & 1219.1 & 1279 & 1290.6 & 1255.7 & 1213.6 & 1293 & 16924 \\
\hline COMPUTEDH & & & & & & & & & & \\
\hline Jalouk & 746.8 & 743.6 & 749.2 & 587.7 & 710.1 & 703.8 & 718.8 & 703.7 & 670.5 & $\$ 6.2$ \\
\hline szady 1 (Oudo tube) & 1201.4 & 7798.1 & 7214.6 & 9154.3 & 1194.8 & 7190.5 & 1223.3 & 1207 & 1769.8 & 1201.8 \\
\hline szadyz & 930.1 & 926.2 & 949.7 & 880.8 & 924.6 & 924.7 & 956.4 & 940.2 & 9027 & 938 \\
\hline (PLO) & 130814 & 130567 & 129948 & 128434 & 125968 & 123794 & 122179 & 119708 & 117228 & 113770 \\
\hline$(9)$ & 1.004 & 1.004 & 1.152 & 1.402 & 1.569 & 1.697 & 1.811 & 1.917 & 2012 & 2.707 \\
\hline (स्थव & 214.2 & 2142 & 245.7 & ळ9 & 330.3 & 361.9 & 386.4 & 400.1 & 420.11 & 49 \\
\hline (HBOLL) & 1199.1 & 1195.8 & 1211.2 & 1147.6 & 1185.6 & 11823 & 1210.3 & 1191.1 & 1150.3 & 11805 \\
\hline & & & & & & & & & & \\
\hline & ZONE2 & ZONE4 & ZONE6 & ZONE8 & ZONE1O & & & & & \\
\hline DP liquid Temp.F & 82.4 & & & & & & & & & \\
\hline DPLquid Density 4 bmitiz & 92.64 & & & & & & & & & \\
\hline Elovationt & 2 & & & 8 & 9.875 & & & & & \\
\hline Mossured DPF PId & 0.256 & 0.655 & 0.458 & 1.906 & 242 & & & & & \\
\hline Zoro Corrections-psid & 0.032 & -0.04 & -0.734 & 0.191 & $0.748+$ & & & & & \\
\hline Corrected DP Psld & 0.204 & 0.095 & 1.192 & 1.715 & 2.272 & & & & & \\
\hline Actual DPan & 2.00 & 3.53 & 5.011 & 6.45 & 7.67 & & & & & \\
\hline Moesurod TPPsig & 156.2 & & & & -1.61 & & & & & \\
\hline Menaured Overalla & 206.27 & & & & & & & & & \\
\hline & & & & & & & & & & \\
\hline Computed DPA & 207 & 4.01 & 5.831 & 7.6 & 921 & & & & & \\
\hline Friction-t & 0.07 & 0.16 & 0.28 & 0.41 & 0.55 & & & & & \\
\hline Accelorationft & 0 & 0.07 & 0.1 & 0.14 & 0.16 & & & & & \\
\hline Elovation & 2 & 3.78 & 5.45 & 7.05 & 8.5 & & & & & \\
\hline & & & & & & & & & & \\
\hline
\end{tabular}




\begin{tabular}{|c|c|c|c|c|c|c|c|c|c|c|}
\hline Run\# & 637 & I & $\cdot$ & $\cdot$ & : & 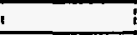 & $\mathbf{i}$ & i & $i$ & \\
\hline Tost Fluid & ICAFIO & I & + & + & 1 & & $I$ & I & 1 & \\
\hline Date: & 6/9/331 & & 1 & $i$ & 1 & & 1 & 1 & 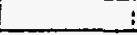 & \\
\hline Time: & $19: 51$ & & & ذin & 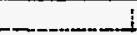 & $\vdots$. & 1 & $t$ & & \\
\hline Nole & Analytical & dt is used & & $\therefore$ & 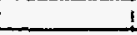 & & $\div$ & 5 & & \\
\hline Flow Areatt2 & 0.00336 & & & & & & & & & \\
\hline Hoat Fow Asea-fl & 0.19595 & & & $\dot{-}$ & & & & & & \\
\hline Sat. PTessure & 950.7 & & & & T & & & & & \\
\hline Sad. Temperature $f($ in $T$ t) & 974.41 & & & & & & & & & \\
\hline Lg. Denstifybunitis & $7 \pi$ & 7 & $\pi i$ & 76.93 & 77 & 76.97 & 76.83 & 76.95 & 77 & $\pi .29$ \\
\hline Latent Heat-Bufibm & 25.911 & 25.91 & 25.911 & 25.88 & 25.91 & 25.9 & 25.79 & 25.88 & 25.91 & 26.14 \\
\hline Specific Hoatbulbmif & 0.299 & 0.299 & 0.299 & 0.299 & 0.29 & 0.239 & 0.299 & 0.299 & 0.299 & 0.298 \\
\hline Lig. Visoosity lom/inhr & 0.349 & 0.349 & 0.3401 & 0.348 & 0.3797 & 0.349 & 0.347 & 0.348 & 0.340 & 0.353 \\
\hline Liq. Conductivit Btuhthf & 0.0221 & 0.022 & 0.0221 & 0.0219 & 0.0221 & 0.02 & 0.02191 & 0.022 & 0.022 & 0.0222 \\
\hline Liq. Prandt No. & 4.741 & 4.74 & 4.741 & 4.74 & $4 . \overline{741}$ & 4.74 & 4.741 & 4.74 & 4.74 & 4.74 \\
\hline Inlet Fow Rate-GPM & 4.5 & & & & & & & & {[} & \\
\hline Outet Liq. Flow Rafo-GPM & 0.91 & & & ; & & & & & i & \\
\hline Outiet Vap. Flow Rate-GPM & 2.41 & & $\therefore$ & $:$ & & & & & & \\
\hline Inlel Temporaturef & 170.9 & & & & & & & & & \\
\hline Outlet Lig. Temperaturer & $172.7 !$ & & & & & & & & & \\
\hline Outlet Vap. Temperaturef & 170.8 & & & & 1 & & & & & \\
\hline Water fow Rato-GPM & & & & & T & & $T$ & t & & \\
\hline Water inlet Temp.f & 124.11 & & & I & I & & & & I & \\
\hline Water outfet Tomp.F & 165.21 & & $T$ & $T$ & 7 & & & & & \\
\hline Intet GTbmintine & 832195 & & Tomp & 168.1 & Density & 77.49 & & & & \\
\hline Subcoolingf & 3.51 & & & i & $i$ & & & & & \\
\hline \multirow[t]{2}{*}{ Fow Observation } & & & & & $i$ & & & & & \\
\hline & ZONE1 & ZONE2 & ZONE3 & ZONE 4 & ZONE5 & ZONE6 & ZONET & ZONE8 & ZONE9 & ZONE10 \\
\hline Hoal input-Watts (net) & 287.2 & 285.1 & 275.1 & 287.6 & $280 . \overline{4}$ & 279.2 & 291.9 & 283.9 & 283.6 & 296.3 \\
\hline Heat Loss-Walts & 13.61 & 3.2 & 4.2) & 4.4 & 4.3 & 3.1 & 2 & 3.2 & 41 & 22.4 \\
\hline Head Flux-Btunhrine & 4978 & 4942 & 4769 & 4985 & 4861 & 4840 & 50501 & 4921 & 4916 & 5136 \\
\hline Wall Temp. H广 & 778.51 & 778.1 & 778.11 & 778.6 & 78.4 & 777.8 & 778.7 & 778.5 & 77.9 & 776.3 \\
\hline Wai Tomp. $14 F$ & 779.2 & 778.2 & 178.6 & 178.7 & 778.6 & 177.8 & 178.7 & 778.6 & 178.5 & 176.4 \\
\hline WaH Temp. & 179.8 & 177.9 & 179.9 & 178.7 & 178 & 177.8 & 178.6 & 178.3 & 178 & $17 \overline{6} .5$ \\
\hline Wall Temp. :1F & 89.01 & 178.5 & 177.4 & 178.1 & 1781 & 178.5 & 178.8 & 178.6 & 177.3 & 174.8 \\
\hline Wath Tomp. Nat & 179.8 & 178.7 & 177.81 & 178.3 & 177.91 & 178.1 & 178.7 & 178.8 & 177.6 & 775.7 \\
\hline Inetream Tomp.F & -734i & 174 & -174 & 174.8 & 174 & 174.1 & 174.7 & 174.2 & 174 & 172.7 \\
\hline Ang. Wall Tomp.F & 179.3 & 178.4 & 178 & 178.5 & 178.11 & 178 & 178.7 & 178.6 & 177.9 & 175.9 \\
\hline Avg. Wall DT.F & 5.11 & 4.2 & 3.81 & 4 & 3.9 & 3.7 & 3.8 & 4.1 & 3.6 & 3 \\
\hline Remaining Subcooff & 2.31 & & & & & & 1 & & & \\
\hline Ext Quality & -0.03 & -0.01 & of & 0.01 & 0.03 & 0.04 & 0.05 & 0.07 & 0.08 & 0.09 \\
\hline EXP. HBtuHr/L2 & 976.2 & 1790.4 & 1258.4 & 1246.4 & 1244.6 & 1323.7 & 1341.7 & 1189.4 & 1351.4 & 1707.6 \\
\hline \multicolumn{11}{|l|}{ DOMPUTEDH } \\
\hline Jallouk & 721 & 717.7 & 701.8 & $-\pi 2 \pi$ & 691.3 & 685.4 & 700.7 & 685 & 6829 & 621 \\
\hline Szadyi(Cudo tube) & 1181.6 & 1177.3 & 1156.6 & 1186.2 & 1171.6 & 1172 & 12041 & $118 \overline{6} . \overline{4}$ & 1186.5 & 1204.6 \\
\hline Szady2 & 913.2 & 908.6 & 886.51 & 918.7 & 905 & 906.3 & 940.11 & 923.2 & 954.2 & 970.7 \\
\hline$(R \perp Q)$ & 155972 & 155972 & 1559721 & 155466 & 152934 & 151019 & 149741 & 146936 & 144565 & 140582 \\
\hline (F) & 1.005 & 1.005 & 1.005 & 1.205 & 1.402 & 1.541 & 1.655 & 1.761 & 1.854 & 1.949 \\
\hline (HLO) & $\overline{\mathbf{E}} \mid \overline{1.4}$ & उ5i.4 & 251.4 & 301.5 & अ1 & 385.7 & $\overline{414.5}$ & $\overline{440.8}$ & 464 & 487.2 \\
\hline \multirow[t]{3}{*}{ (HOL) } & 1977.8 & 1173.5 & 1152.6 & 1179.7 & 1161.11 & 1157.9 & 1187.31 & 1165.8 & 11623 & 1177.4 \\
\hline & & & & & & & & & & \\
\hline & ZONE2 & ZONE4 & ZONE6 & ZONE8 & ZONE10 & & & & & \\
\hline DP hquid Tomp.F & $1-827$ & & & & & & & & & \\
\hline DP Liquid Density-lbm/173 & 92.59 & & & & & & & & & \\
\hline Elevation- & 2 & 4 & 6 & 8 & 9.875 & & & & & \\
\hline Mroasured DPYsid & 0.219 & 0.54 & 0.202 & 7.643 & 2.092 & & & & & \\
\hline Zero Corractions-psid & 0.032 & -0.04 & -0.734 & $0 . \overline{1911}$ & $0.148 !$ & & & & & \\
\hline Corrected DPF Fild & 0.187 & 0.58 & 0.996 & 1.452 & 1.9441 & & & & & \\
\hline Actual DPA & 2.051 & $3.7 \pi$ & 5.39 & 6.88 & 8.21 & & & & & \\
\hline Measurod TPFig & 150.7 & & & & -1.6 & & & & & \\
\hline \multirow[t]{2}{*}{ Moasured Overalli } & 283.75 & & & & & & & & & \\
\hline & & & & & & & & & & \\
\hline Computed DP-A & 2.11 & 4.18 & 6.111 & 7.97 & 9.671 & & & & & \\
\hline Friction-ft & 0.1 & 0.21 & 0.36 & 0.53 & 0.71 & & & & & \\
\hline Accoleration-l & 0 & 0.07 & 0.13 & 0.17 & 0.21 & & & & & \\
\hline \multirow[t]{2}{*}{ Elovation $A$} & 2. & 3.91 & 5.63 & 7.27 & 8.75 & & & & & \\
\hline & & & & & & & & & & \\
\hline
\end{tabular}




\begin{tabular}{|c|c|c|c|c|c|c|c|c|c|c|}
\hline Run & 638 & & & & & & & & & \\
\hline Tost Fuld & CAF $=10$ & & & & & & & & & \\
\hline Dade: & 61093 & & & & & & & & & \\
\hline Time: & $2: 3$ & & i & i & & t & $T$ & & & I \\
\hline Note & Analytical & dt is used & & $i$ & & $T$ & $\bar{i}$ & & & $i$ \\
\hline Fow Areathe & 0.003661 & & & $i$ & & $i$ & & & & $T$ \\
\hline Heat Flow Arae-12 & 0.10605 & & 7 & $T$ & & $\pi$ & & & & \\
\hline Sat. Pressuropsig & 152.9 & & & I & & & & & & \\
\hline Sat. Temperaturef(inlel) & 175.5 & & & 1 & & & & & & \\
\hline Liq. Density 4 bmitt3 & 76.81 & 76.81 & 76.79 & 76.74 & 76.86 & 76.81 & 76.58 & 76.72 & 76.77 & 77.02 \\
\hline Latent Hoes-Btulbm & 25.77 & 25.77 & 25.76 & 25.72 & 25.81 & $25 . \pi$ & 25.59 & 25.7 & 25.74 & 25.33 \\
\hline Spectio Heat-Btulbmif & 0.299 & 0.299 & 0.299 & 0.299 & 0.299 & 0.299 & 0.3 & 0.239 & 0.299 & 0.299 \\
\hline Lq. Viscosity bm/ithr & 0.346 & 0.346 & 0.346 & 0.345 & 0.347 & 0.346 & $0.343 \mid$ & 0.345 & 0.346 & 0.349 \\
\hline La. Conductivity stumprif & 0.0219 & 0.0219 & 0.0219 & 0.0218 & 0.0219 & 0.0219 & 0.0217 & ठ.0्टाषा & 0.0218 & 0.002 \\
\hline Uf. Prandt No. & 4.74 & 4.74 & 4.74 & 4.74 & 4.741 & 4.74 & 4.73 & 4.74 & 4.74 & 4.74 \\
\hline Inlol Flow Rate-GPM & 5.4 & & & & & $T$ & & & & \\
\hline Outlol Liq. Flow Rato-GPM & 0.8 & & 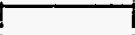 & T & & T & & & & t \\
\hline Outlel Vap. Flow Rolo-GPM & 2.7 & & & I & & & & & & $\mathrm{i}$ \\
\hline Inlal Tomporaturaf & 172.5 & & & & & & & & & \\
\hline Outlof Lia. Tamporaturef & 174 & & & & & i & & I & & I \\
\hline Outlot Vap. Tomperatur of & 171.4 & & & & & & & 1 & & \\
\hline Weder Flow Rato-GPM & & & & & & & & & & \\
\hline Water Inlet Temp.F & 119.6 & & & & & & & & & \\
\hline Water outtel Temp.f & 167 & & & & & & & & & \\
\hline Inhat Glbmhrme & 99487 & & Temp & 169.4 & Density & $\pi .2$ & & & & \\
\hline Subcoolingt & 3 & & & & & & & & & \\
\hline Flow Observeltion & & & & & & & & & & \\
\hline & ZONE 1 & ZONE2 & ZONE3 & |ZONE4 & ZONES & ZONEO & ZONE & ZONE8 & ZONE9 & ZONETO \\
\hline Fon houtWalts(nel) & 284.81 & 282 & 278.7 & 288.8 & 2321 & 2822 & 288.9 & 291.8 & 288.4 & 287.7 \\
\hline Heal Loso-Wat & 13.8 & 3.1 & 4.1 & 4.2 & 4.11 & 3.3 & 1.9 & 3) & 3.8 & 21.6 \\
\hline Hedfloxbuthlite & 4939 & 466 & 476 & 6003 & -6055 & 4802 & 6008 & ह000 & 465 & 4087 \\
\hline Wall Temp. "IF F & 179.5 & 179.1 & 179.2 & 179.4 & 179.3 & 180.1 & 179.7 & 179.5 & 178.8 & 177.4 \\
\hline Wall Tomp. N4F & 180.11 & 179.1 & 179.5 & 179.4 & 179.4 & 180.3 & 179.9 & 179.7 & 179.5 & 177.3 \\
\hline Wall Tomp. $15 F$ & 180.2 & 178.8 & 180.7 & 179.4 & 178.8 & 182.1 & 179.7 & 179.41 & 178.9 & 177.3 \\
\hline Wall Tomp. 11F & 84.2 & 179.6 & 178.4 & 178.8 & 178.81 & 182.71 & 179.2 & 179.5 & 178 & 175.7 \\
\hline WallTomp. "WCF & 180.8 & -479.8 & 178.7 & 178.8 & 178.8 & 182.9 & $179.4 \mid$ & 179.7 & 178.4 & 176.8 \\
\hline Instroan temp.F & 174.8 & 174.8 & 174.9 & 175.1 & $T 74.6$ & 774.8 & 775.8 & 975.2 & 175 & 173.9 \\
\hline Avg. Wall Temp.F & 180.2 & $5+4$ & 779 & 779.30 & 179 & 781.3) & 178.61 & 779.6 & 178.7 & 776.8 \\
\hline Avg. Wat DT.F & 5.1 & 4.4 & 3.8 & 3.9 & 4.7 & 6.3 & 3.6 & 4.1 & 3.5 & 28 \\
\hline Romaining suboootf & 2 & & & & & $\rightarrow$ & & & & \\
\hline Exatality & -0.0 (1) & -0.01 & 0 & 0.01 & 0.021 & 0.08 & 0.041 & 0.08 & 0.07 & 0.08 \\
\hline 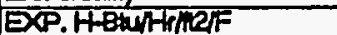 & $8 \approx 3.81$ & 1116.3 & 1251.8 & 1274.9 & 120.11 & T70.1 & 1400.2 & 1222.1 & 14202 & 1797.4 \\
\hline COMPUTEDH & & & & & & & & & & \\
\hline Jallouk & 708.5 & 704.1 & 696.61 & 706.8 & का1 & 684.8 & 698.51 & 692.2 & 684.4 & 681.6 \\
\hline Szadyl(Cudo tube) & 1183.8 & 1177.9 & T167.6 & 1195.6 & 12023 & T186.3 & 1200.5 & 1212.6 & 1204.1 & 1201.8 \\
\hline Szadve & 915.1 & 908.7 & 897.6 & 928.5 & 937.9 & 9201 & 946.1 & 980.3 & 968.5 & 953.8 \\
\hline (PUQ) & 187890 & 187800 & 188059 & 187358 & T8450द & 18्2777 & 182355 & 1791111 & 176500 & 172636 \\
\hline$(F)$ & 1.005 & 1.005 & 1.005 & 1.173 & 1.36 & 1.488 & 1.59 & 1.686 & 1.771 & 1.853 \\
\hline (HLO) & 200.2 & 290.2 & 2902 & 339 & उक्र & 429.9 & 459.6 & 487.3 & 511.9 & 535.6 \\
\hline (F:OL) & 1178 & 1172 & T161.6 & T186.5 & T188.2 & TI67.2 & T186.9 & $T 185.8$ & 11724 & 11652 \\
\hline & & & & & & & & & & \\
\hline & ZONEZ I & ZZONE 4 & ZONEO & ZONE & ZONE1O & & & & & \\
\hline DP hquid Tomp.f & 89.41 & & & 1 & & & & & & \\
\hline DPLiquid Density tomins & 02.481 & & & & & & & & & \\
\hline Elovition- & 2 & 4 & 6 & 8 & 8.8751 & & & & & \\
\hline Messurod DPpald & 0.174 & 0.474 & 0.046 & 1.401 & 1.787 & & & & & \\
\hline Zaro Corrections-psid & 0.032 & -0.04 & -0.734 & 0.191 & 0.148 & & & & & \\
\hline Compcled DPPsid & 0.142 & 0.454 & 0.78 & 1.21 & 1.60 & & & & & \\
\hline Actual DPA & 2.14 & 3.95 & 5.75 & 7.34 & 8.78 & & & & & \\
\hline Measured TP्PSIg & 152.9 & & & & -1.6 & & & & & \\
\hline Messured Overalth & 288.87 & & & & & & & & & \\
\hline & & & & & & & & & & \\
\hline Computed DPA & 2.14 & 4.29 & 6.3 & 8.25 & 70.06 & & & & & \\
\hline Fricton-1 & 0.14 & 0.20 & 0.48 & 0.71 & 0.95 & & & & & \\
\hline Acooleretion-h & of & 0.08 & 0.16 & 0.22 & 0.27 & & & & & \\
\hline Elevationt & 21 & 3.92 & 5.66 & 7.33 & 8.841 & & & & & \\
\hline
\end{tabular}




\begin{tabular}{|c|c|c|c|c|c|c|c|c|c|c|}
\hline Run:" & 6661 & & . & t. & & 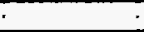 & & & 1 & 1 \\
\hline Tost Fuid & C4F10 & & . & . & & & & 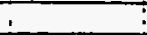 & . & . \\
\hline Date: & $7 / 293 i$ & & & & & & & & & \\
\hline Time: & $11: 31$ & & & & & & & & 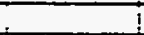 & 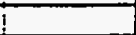 \\
\hline Note & Analyticad c & this used & & & & & & & & \\
\hline Fow Areartl2 & 0.00361 & & & & & & & + & & \\
\hline Head Flow Area-fl & 0.1955 & & & & & & & & & \\
\hline Sat. Pressure Psig & काजा & & & & & 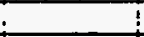 & & 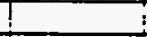 & $i$ & \\
\hline Sat. Temperatureff(inlet) & 192.2 & & & & & & & & & \\
\hline Liq. Density 4 bm/ns & 73.13 & 72.97 & 72.881 & 72.8 & 72.8 & 72.741 & 72.6 & 72.6 & 72.6 & 73 \\
\hline Latent Heat-Btulbm & 22.941 & 22.81 & 22.751 & 22.68 & 22.681 & 22.64 & 22.53 & 2253 & 2259 & 22.89 \\
\hline Speocitic Heat-Btuhtomit & 0.312 & 0.313 & 0.313 & 0.313 & 0.3131 & 0.314 & 0.3741 & 0.314 & 0.314 & 0.313 \\
\hline Lig. Viscosity lbm/ithr & 0.299 & 0.297 & 0.296 & 0.255 & 0.295 & 0.2391 & 0.292 & 0.292 & 0.292 & 0.297 \\
\hline Lig. Conductivaty Btuhs-AF & 0.01961 & 0.0195 & 0.019 & 0.0194 & 0.0194 & 0.01941 & 0.0193 & 0.0193 & 0.0193 & 0.0196 \\
\hline Lig. Prandt No. & 4.741 & 4.75 & 4.75 & 4.75 & 4.75 & 4.75 & 4.75 & 4.75 & 4.75 & 4.75 \\
\hline Inlet Flow Rato-GPM & 5.61 & & & & & & & & & \\
\hline Outtel Liq. Fow Rato-GPM & 11 & & i & 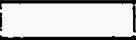 & & 1 & & & & \\
\hline Outlel Vap. Flow Rato-GPM & 31 & & $\cdot$ & & & it & & i & 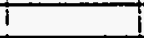 & \\
\hline Inlet Temperaturof & 187.8 & & & & & t! & & & & \\
\hline Outel Liq. Temperaturef & 189.41 & & & & & & & & & \\
\hline Outiel Vap. Temperaturef & 786.1 & & & & & I & & & & \\
\hline \multicolumn{11}{|l|}{ Weter Fow Rado-GPM } \\
\hline Wator hlet Temp.F & $1 \$ 0.8$ & & & & & & & & & \\
\hline Wetar outlel Temp.F & 182.1 & & & & & & & & & \\
\hline Inth Glbm/hr/ne & 978751 & & Temp & 185.7 & Density & 73.24 & & & & \\
\hline Subcooling $f$ & 4.4 & & & & & & & & & \\
\hline \multicolumn{11}{|l|}{ Fow Observation } \\
\hline & ZONE1 & ZONE2 & ZONE3 & ZONE4 & ZONE5 & ZONE6 & ZONE7 & ZONE8 & ZONE9 & ZONE10 \\
\hline Head Input-Walts(nel) & 298.3 & 295.8 & 276.7 & 283.8 & 283.9 & 269.3 & \begin{tabular}{|l|}
286.4 \\
\end{tabular} & 284.2 & 285.1 & 295.2 \\
\hline Heat Loss-Watts & 16.9 & 4 & 5.4 & 6 & 6.3 & 5.6 & 4 & 5.1 & 5.9 & 31.5 \\
\hline Hoad Fux-Btuntrin? & 5179 & 5128 & 4796 & 4919 & 4921 & 5015 & 4965 & 4926 & 4942 & 5117 \\
\hline Wall Temp. "3+ & क्य, & 155.1 & 194.6 & 194.4 & $\$ 93.9$ & 194.4 & 194.81 & 194.7 & 795.8 & $7 \$ 3.2$ \\
\hline Wall Temp. \#4F & 195.1 & 194.8 & 194.9 & 194.6 & 194.3 & 155.5 & 195.2 & 194.6 & 196.2 & 193.4 \\
\hline Wall Tomp. 15 F & 155.2 & 194.2 & 194.7 & 199 & 193.7 & 194.6 & 194.7 & 194.6 & 195.9 & 1928 \\
\hline Wall Tamp. K1F & 86.8 & 195.3 & 194.2 & 194 & 193.8 & 195.8 & 194.8 & 194.8 & 195.1 & 191 \\
\hline Wall Temp. *2F & 195.3 & 195.3 & 194.5 & 194.1 & 993.9 & 194.6 & 19 & 194.6 & 195.3 & 1922 \\
\hline Instroam Tomp.f & 189.4 & 190 & 190.3 & 190.6 & 190.6 & 190.8 & 191.3 & 191.3 & 191.3 & 189.9 \\
\hline Avg. Wall Temp.F & 195 & 195.1 & 194.6 & 194.3 & 193.9 & 195 & 194.9 & 194.6 & 195.7 & 1925 \\
\hline Avo. Wall DT.F & 5.41 & 4.9 & 4 & 3.4 & 3.1 & 3.9 & 3.4 & 3.1 & 4.1 & 24 \\
\hline Remaining Subcooff & 3.4 & & & & & & & & & \\
\hline Ext Guality & -0.05 & -0.08 & -0.02 & -0.01 & 0.01 & 0.02 & 0.03 & 0.05 & 0.06 & 0.07 \\
\hline EXP.HBtuHFla & 963.6 & 1047.9 & 1189.2 & 1435.2 & 1586.2 & 1278.2 & 1470.7 & 1600.9 & 1194.7 & 21422 \\
\hline \multicolumn{11}{|l|}{ COMPUTEDH } \\
\hline Jallouk & 782.5 & 780.5 & 750.3 & 763 & 7632 & 757.8 & 748.11 & 740.1 & 737.6 & 7429 \\
\hline Szadyl(Cudo tube) & T302.1 & 1321.3 & 1280.9 & 1299.2 & 1299.4 & 136.1 & 1315.7 & 1313 & 1317 & 1830.6 \\
\hline seady? & 1043.3 & 1041.7 & 997.9 & 1017.2 & 1017.4 & 1057.8 & 1058.7 & 10037.2 & 10429 & 1059.8 \\
\hline (RULO) & 214459 & 215870 & 210588 & 217301 & 217301 & 215114 & 213425 & 210554 & 207882 & 201614 \\
\hline (F) & 1.004 & 1.004 & 1.004 & 1.004 & 1.004 & 1.272 & 1.411 & 1.52 & 1.613 & 1.704 \\
\hline (HบO) & 289.7 & 289.8 & 289.8 & 289.8 & 289.8 & 367.8 & 407.7 & 438.1 & 466.8 & 491.8 \\
\hline \multirow[t]{3}{*}{ (HBOIL) } & 1317.4 & 1316.6 & 1275.9 & 1204.4 & 129.6 & 1306.5 & 13026 & 1296.4 & 1297.3 & 1307.8 \\
\hline & & & & & & & & & & \\
\hline & ZONE2 & ZONE4 & ZONE6 & ZONE8 & ZONE10 & & & & & \\
\hline DP liquid Temp.F & 89.7 & & & & & & & & & \\
\hline DPLiquid Density-lbm/13 & 91.55 & & & & & & & & & \\
\hline Elovebon-ft & 2 & 4 & 6 & 8 & 9.875 & & & & & \\
\hline Measured DPPsid & 0.347 & 0.563 & 0.884 & 7.31 & 1.67 & & & & & \\
\hline Zero Corrections-psid & 0.144 & 0.111 & 0.156 & 0.051 & 0.032 & & & & & \\
\hline Correctod DPPsid & 0.200 & 0.452 & 0.728 & 1.259 & 1.638 & & & & & \\
\hline Actid DPh & 211 & 4.13 & 6.09 & 7.55 & 375ा & & & & & \\
\hline Measured TFPsig & 1911 & & & & 300.1 & & & & & \\
\hline \multicolumn{11}{|l|}{ Manured Overallh } \\
\hline & & & & & & & & & & \\
\hline Computed DRA & 2.14 & 4.28 & 6.42 & 8.44 & 10.29 & & & & & \\
\hline Friction-t & 0.14 & 0.28 & 0.45 & 0.65 & 0.86 & & & & & \\
\hline Accolerdion-t & 0 & 0 & 0.09 & 0.15 & 0.2 & & & & & \\
\hline Elevation- & 2) & 4 & 5.87 & 7.64 & 9.24 & & & & & \\
\hline & & & & & & & & & & \\
\hline
\end{tabular}




\begin{tabular}{|c|c|c|c|c|c|c|c|c|c|c|}
\hline Run" & 6671 & & $:$ & 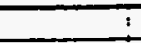 & - & 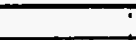 & & & i & \\
\hline Teat Fuid & CAF 10 i & 1 & I & 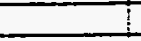 & 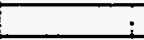 & 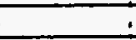 & i & & & $i$ \\
\hline Dato: & $17 / 21931$ & I & & $!$ & 1 & 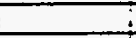 & 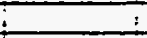 & & & - \\
\hline Time: & $11: 47 !$ & & 1 & & 1 & 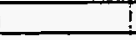 & ! & $!$ & $!$ & \\
\hline Note & Analytical d & th is used & & 1 & 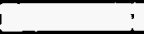 & & & & 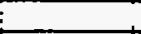 & \\
\hline Flow Areattl2 & 0.00336 & 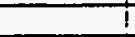 & 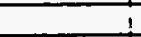 & & 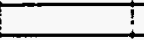 & 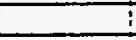 & i & & $i$ & $!$ \\
\hline Hoat Flow Areafl2 & 0.19655 & & & $i$ & 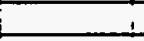 & $!$ & $!$ & & 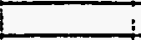 & $i$ \\
\hline Sat. Pressuro Psig & 187.31 & & & $t$ & 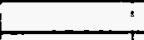 & 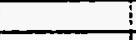 & 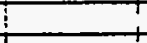 & & & \\
\hline Sed. Temperature flinlel) & 190.71 & & & & & & +1 & & & \\
\hline Lug. Density $/ \mathrm{bm} / \mathrm{mi} 3$ & 73.351 & 73.16 & 73.19 & 73.25 & 73.22 & 73.16 & 73.021 & 73 & 72.971 & 73.33 \\
\hline Latont Heat-Btuhbm & 23.111 & 2298 & 2298 & 23.03 & 23.011 & 22.96 & 22.86 & 22.89 & 22.811 & 23.09 \\
\hline Spectio Hoat-Btulbm/F & 0.311 & 0.312 & 0.312 & 0.312 & 0.312 & 0.312 & 0.313 & 0.313 & 0.313 & 0.311 \\
\hline Lig. Visoositylominhr & 0.3011 & 0.299 & 0.299 & 0.3 & 0.3 & 0.299 & 0.297 & 0.297 & 0.297 & 0.301 \\
\hline Lig. Conductivity Gtu/hr-iff & 0.0198 & 0.0197 & 0.0197 & 0.0197 & 0.0197 & 0.0197 & 0.0196 & 0.0196 & 0.015 & 0.0198 \\
\hline Lig. Prandt No. & 4.741 & 4.74 & 4.74 & 4.74 & 4.741 & 4.74 & 4.741 & 4.75 & 4.75 & 4.74 \\
\hline Inlet flow Rate-GPM & 4.8 & & & & & & i & & 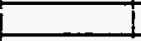 & \\
\hline Outtet Liq. Flow Rato-GPM & 1 & & & & & & & & & \\
\hline Outlet Vap. Flow Rato-GPM & 2.5 & & & & i & & 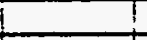 & & & \\
\hline Inlel Temperaturof & 187.2 & 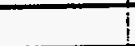 & & & & & & & & \\
\hline OutitLq. Temperaturer & T88.2 & & & & & & $T$ & & & \\
\hline Outlet Vap. Temperaturof & 184.6 & & & & & & $t$ & & & \\
\hline Walor Fow Rato-GPM & & & & & & & & & & \\
\hline Water inlot Temp.f & 925.3 & & & & & & i & E & & \\
\hline Water outlot Tomp.f & 180.9 & & & & & & & & & \\
\hline Inlot G-lbm/hrin? & 843714 & & Tomp & 184.1[ & Density & 73.68 & & & & \\
\hline Subcooling-F & 3.5 & & & & & & i & & & \\
\hline \multirow[t]{2}{*}{ Flow Observation } & & & & & & & & & & \\
\hline & ZONE1 2 & ZONE2 & ZONE3 & ZONE4 & ZONE5 & ZONE6 & ZONE7 I2 & ZONEB & ZONE9 & ZONE10 \\
\hline Heat Input-Watts(net) & 298.9 & 295.8 & 291.9 & 297.1 & 295.3 & 296.3 & $2 \% 5.9$ & 296.8 & 288.7 & 296.8 \\
\hline Heat Loss-Watts & 16.4 & 3.9 & 5.2 & 5.7 & 5.9 & 5.1 & 3.7 & 4.8 & 5.7 & 30.1 \\
\hline Heat Flox-BtuhHitit2 & 5181 & 5128 & 5050 & 5150 & 5119 & 5136 & 5129 & 5145 & 5004 & 5145 \\
\hline Walt Tomp. \#3 & 193.61 & 1903.9 & 193.6 & 193 & 192.7 & 193.1 & Tड्ड.5. & $1 \$ 3.41$ & 194.5 & 1920 \\
\hline Wall Temp. N4F & 194.2 & 193.6 & 194.1 & 193.1 & 193.2 & 194 & 193.7 & 193.3 & 195.1 & 1924 \\
\hline Wall Temp. $15 F$ & 194.2 & 193.2 & 193.6 & 192.5 & 1927 & 193.2 & 133.3 & 193.4 & 194.7 & 191.8 \\
\hline Wall Temp. "1F & 87.3 & 194.2 & 193 & 1924 & 1922 & 193.8 & 193.3 & 193.2 & 194.1 & 1902 \\
\hline Wall Temp. HCF & 194.2 & 194.2 & 193.6 & 192.9 & 1923 & 193.3 & 193.7 & 193.2 & 194.1 & 191.5 \\
\hline Intream Temp.f & 188.6 & 189.8 & 189.2 & 189 & 189.1 & 189.3 & 189.8 & 189.8 & 180 & 188.7 \\
\hline Avg. Wall Tomp.F & 194.1 & 194 & 199.6 & 192.8 & 192.6 & 193.5 & 193.5 & 193.3 & 794.5 & 191.6 \\
\hline Avg. Wall DT.F & 52 & 4.4 & 4.1 & 3.5 & 3.3 & 4 & 3.5 & 3.2 & 4.3 & 27 \\
\hline Remaining Subcooff & 2.3 & & & & & & & & & \\
\hline Ext Quality & -0.03 & -0.02 & 0 & 0.01 & 0.03 & 0.05 & 0.06 & 0.08 & $0 . \infty$ & 0.11 \\
\hline EXP.HBtuHrriefF & 993.4 & 1154 & 1220.3 & 1464.2 & 1566 & 1286.3 & 1478.9 & 1624.3 & 1170.9 & 1914.4 \\
\hline \multicolumn{11}{|l|}{ COMPUTEDH } \\
\hline Jallouk & 7933.3 & 790.61 & 783.5 & 780.4 & 767 & 702.7 & 759 & 756.41 & 742 & 745.3 \\
\hline Szady (cudo tubo) & 1316.3 & 1315 & 7305.60 & 7316.9 & 1315.7 & 13:टार & 1325.8 & 1330.2 & 1315 & 1324.4 \\
\hline seady? & 1037.2 & 1034.9 & 1024.9 & 1038.7 & 1038.9 & 1045.8 & 1051.4 & 1057.1 & 1041.9 & 10542 \\
\hline (स山Q) & 183272 & 184689 & 184468 & 182741 & 180084 & 17618 & 175711 & 173008 & 170327 & 185009 \\
\hline (F) & 1.004 & 1.004 & 1.004 & 1.203 & 1.392 & 1.526 & 1.634 & 1.729 & 1.814 & 1.902 \\
\hline (HLOA) & 257.1 & 257.2 & 2572 & 308.1 & 356.6 & 300.8 & 418.7 & 443.2 & 464.8 & 487 \\
\hline \multirow[t]{3}{*}{ (HBOIL) } & 1313 & 1311.7 & 1302.3 & 1311.3 & 1307 & 1309.7 & 1311.8 & 1313.6 & 129.4 & 1302.1 \\
\hline & & & & & & & & & & \\
\hline & ZONEZ 2 & ZONE4 & ZONE6 & ZONEB & ZONETO & & & & & \\
\hline \multicolumn{11}{|l|}{ DPliquid Tomp.F } \\
\hline DPLquid Density bm/13 & 97.45 & & & & & & & & & \\
\hline Elovation & & 4 & 6 & 8 & 9.875 & & & & & \\
\hline Measured DPFsid & 0.384 & 0.658 & 7.075 & 1.583 & 2.026 & & & & & \\
\hline Zero Comections-psid & 0.144 & 0.111 & 0.156 & 0.051 & 0.052 & & & & & \\
\hline Corrected DPPsid & 0.24 & 0.557 & 0.919 & 1.532 & 1.994 & & & & & \\
\hline Acturad DPA & 2.021 & 3.89 & 5.68 & 6.97 & 8.4 & & & & & \\
\hline Measured TPPsig & 187.3 & & & & 300.11 & & & & & \\
\hline \multirow{2}{*}{\multicolumn{11}{|c|}{ Messured Overalth }} \\
\hline & & & & & & & & & & \\
\hline Computad DPA & 2.11 & 4.21 & 6.18 & 8.08 & 9.88 & & & & & \\
\hline Friction-t & 0.11 & 0.23 & 0.38 & 0.55 & 0.73 & & & & & \\
\hline Accoleration-h & 0 & 0.06 & 0.12 & 0.16 & 0.19 & & & & & \\
\hline Elevation-th & 2 & 3.92 & 5.68 & 7.37 & 8.9 & & & & & \\
\hline & & & & & & & & & & \\
\hline
\end{tabular}




\begin{tabular}{|c|c|c|c|c|c|c|c|c|c|c|}
\hline Run\# & E58! & & & & & & & & & \\
\hline Test Fuid & CAF10. & & . & & $\overline{1}$ & & $\underline{\vdots}$ & $i$ & $\mathbf{i}$ & $\mathrm{i}$ \\
\hline Dato: & $7 / 2 / 33$ & & $i$ & 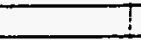 & $i$ & $\vdots$ & $i$ & 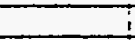 & 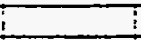 & 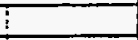 \\
\hline Time: & $111: 47$ & & & 1 & 1 & 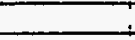 & & & & \\
\hline Note & Analytical c & th is used & & & & + & & $\bar{\vdots}$ & 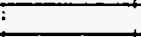 & \\
\hline Flow Aveafle & 0.00366 & & $i$ & 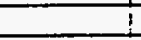 & 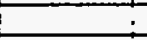 & it & & 1 & 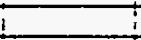 & \\
\hline Heat Fow Area-t2 & 0.196951 & & + & 1 & 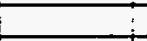 & & $i$ & & 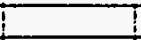 & \\
\hline Sat. Pressure Psig & 183.31 & $i$ & $i$ & 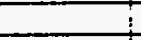 & 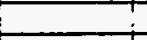 & $i$ & T! & & & \\
\hline Sat. Temperaturo f(inlel) & 991.11 & & & 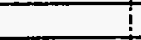 & 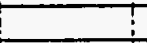 & $?$ & 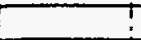 & & i & \\
\hline Lq. Density-Abmitis & 73.251 & 73.13 & 73.02 & 73.05 & 73 & 72.94 & 72.861 & 72.861 & 72.86 & 73.13 \\
\hline Latent Heat-atulbm & 23.031 & 22.94 & 22.88 & 22.88 & 22.831 & 22.79 & 227 & 22.73 & 2273 & 22.94 \\
\hline Spocitic Hoat-Btulibmif & 0.312 & 0.312 & 0.3131 & 0.312 & 0.313 & 0.313 & 0.313 & 0.373 & 0.313 & 0.312 \\
\hline Lig. Visoositylomphr & 0.3 & 0.289 & 0.297 & 0.298 & 0.297 & 0.296 & 0.295 & 0.296 & 0.295 & 0.209 \\
\hline Lig. Conduativity Btumpr tF & 0.01971 & 0.0196 & 0.0196 & 0.0196 & 0.0196 & 0.079 & 0.0195 & 0.0195 & 0.0195 & 0.0196 \\
\hline Lig. Prandt No. & 4.74 & 4.74 & 4.741 & 4.74 & 4.75 & 4.75 & 4.75 & 4.75 & 4.75 & 4.74 \\
\hline Inlet Flow Rato-GPM & 3.8 & & 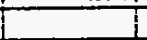 & & & & & & & \\
\hline Outlel Lig. Flow Rato-GPM & 1 & & $!$ & 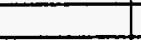 & & & & & & \\
\hline Outhet Vap. Fow Rate-GPM & 2.1 & & 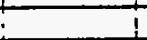 & & & & & & & \\
\hline Inlot Temperaturef & 187.6 & & t & & & & & & & \\
\hline Outlet Lia. Temperaturef & 189.1 & & & & & & & & & \\
\hline Outtet Vap. Temperature-F & 185.4 & & & & & & & & & \\
\hline Water Fow Rate-GPM & & & t & 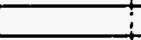 & & & $\mathrm{E}$ & & & \\
\hline Water inlet Temp.f & 124.9 & & $i$ & 1 & & & I & & & \\
\hline Weter outlot Tomp.F & 181.3 & & 1 & 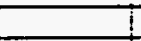 & & & & & & \\
\hline $\ln 1 \mathrm{e} \mathrm{G}+\mathrm{bm} h \mathrm{~h} / \mathrm{R}$ & 667470 & & Tomp & 184.3 & Density & 73.6 & & & & \\
\hline Subcooling & 3.5 & & 工 & & & & & & & \\
\hline \multicolumn{11}{|l|}{ Fow Observation } \\
\hline & ZONE1 & ZONE2 & ZONE3 & ZONE4 & $\begin{array}{lll}\text { ZONE5 } & 2 \\
\end{array}$ & ZONE6 & ZONE7 & ZONE8 & ZONE9 & ZONE10 \\
\hline Heat Input-Walts(net) & 287.3 & 288.1 & 287.3 & 279.41 & 288.4 & 291.7 & 289.5 & 289.7 & 279.3 & 289.4 \\
\hline Heal Loss-Walls & 16.4 & 3.9 & 5.2 & 5.8 & 6 & 5.3 & 3.7 & 4.8 & 5.7 & 30.3 \\
\hline Head Fl(x-BtuHTrnL & 4980 & 4994 & 450 & 4843 & 4999 & 5056 & 5018 & 5022 & 4841 & 5017 \\
\hline Wall Temp. H3 & 193.6 & T94.3 & T34 & 793.41 & 193.4 & 194 & $\$ 3.9$ & अख्ड.9 & 794.8 & 192.6 \\
\hline Wall Temp. $4 F$ & 194.3 & 194.1 & 194.4 & 193.8 & 193.8 & 155 & 194.3 & 193.7 & 15.4 & 1926 \\
\hline Wall Temp. 15F & 194.3 & 193.6 & 194.1 & 193.2 & 193.2 & 194.1 & 193.8 & 193.7 & 195 & 192.4 \\
\hline Wall Tomp. IIF & 87.9 & 194.6 & 199.6 & 198 & 198 & 194.7 & 199.8 & 199.7 & 194.2 & 190.0 \\
\hline Wall Tomp. ILF & 194.4 & 199.6 & 194 & 133.4 & 193.2 & 194.1 & 194.1 & 199.7 & 194.5 & 191.8 \\
\hline Insteam Temp.F & 189 & 189.4 & 189.8 & 189.7 & 189.9 & 190.1 & 180.4 & 190.4 & 190.4 & 189.4 \\
\hline Avg. Wall Tomp.F & 194.2 & 194.4 & 194 & 193.4 & 193.3 & 194.5 & 194 & 193.7 & 194.8 & 192 \\
\hline Avg. WallDT.F & 4.9 & 4.8 & 4 & 3.4 & 3.2 & 4.1 & 3.4 & 3.1 & 4.2 & 2.4 \\
\hline Remaining Subcooff & 2.1 & & & & & & & & & \\
\hline Exit Quality & -0.03 & -0.01 & 0.01 & 0.03 & 0.05 & 0.07 & 0.09 & 0.11 & 0.12 & 0.14 \\
\hline EXP. HBtu/HPR2/F & 1011.2 & 1046 & 1252.9 & 1411.6 & 1575 & 1226.8 & 1496.6 & 1613.1 & 1163.5 & 2113.8 \\
\hline \multicolumn{11}{|l|}{ COMPUTEDH } \\
\hline Jallouk & 7926 & 795.5 & 795.5 & 763.6 & 772.2 & 772.5 & 76.4 & 761.7 & 942.5 & 750.8 \\
\hline Szadyi(Cudo bube) & 1293 & 1297.8 & 1299.2 & 1282.4 & 1305.31 & 1315.3 & 1313.9 & 1315.6 & 1293.7 & 1309.8 \\
\hline seady? & 1054.21 & 1059.5 & 1000.8 & 1000 & 1025.5 & 1037.1 & 1038.2 & 10088.9 & 1016.5 & 10055.4 \\
\hline$(R \cup Q)$ & 145017 & 140252 & 146858 & 149934 & 141471 & 139988 & 130520 & 133685 & 130849 & 120697 \\
\hline$(F)$ & 1.004 & 1.004 & 1.028 & 1.354 & 1.522 & 1.655 & 1.768 & 1.8691 & 1.958 & 2.05 \\
\hline (सU⿺辶) & 2182 & 213.3 & 218.31 & 287.6 & 323.3 & 351.8 & 376.8 & 387.2 & 416.3 & 435.5 \\
\hline \multirow[t]{3}{*}{ (1BOL) } & 129 & 1205.9 & 1297.1 & 1277.6 & 1208.7 & 1306.9 & 1303.6 & 1303.4 & 1279.2 & 1293.5 \\
\hline & & & & & & & & & & \\
\hline & ZONE2 & ZONE4 & ZONE6 & ZONEB & ZONE10 & & & & & \\
\hline DP liquid Temp.f & 90.9 & & & & & & & & & \\
\hline DPLiquid Density-lbm/1t3 & 91.37 & & & & & & & & & \\
\hline Elevation $\mathrm{A}$ & & 4 & 6 & 8 & 9.875 & & & & & \\
\hline Measured DPPSid & 0.389 & 0.75 & 7.227 & 1.803 & 2खा & & & & & \\
\hline Zoro Corrections-psid & 0.144 & 0.117 & 0.156 & 0.057 & 0.032 & & & & & \\
\hline Corrected DPPsild & 0.245 & 0.639 & 1.071 & 1.7521 & 2250 & & & & & \\
\hline Actual DPR & 2.02 & 3.74 & 5.39 & 6.55 & 7.89 & & & & & \\
\hline Moasurod TPPsig & 188.3 & & & & 300.1 & & & & & \\
\hline \multirow[t]{2}{*}{ Measured Overalht } & fexs: & & & & & & & & & \\
\hline & & & & & & & & & & \\
\hline Computed DPA & 2.07 & 4.05 & 5.91 & 7.7 & 9.35 & & & & & \\
\hline Friction- & 0.07 & 0.16 & 0.27 & 0.39 & 0.52 & & & & & \\
\hline Acceleration- & 0 & 0.05 & 0.09 & 0.12 & 0.15 & & & & & \\
\hline Elevationt & 2 & 3.83 & 5.55 & 7.19 & 8.69 & & & & & \\
\hline & & & & & & & & & & \\
\hline
\end{tabular}




\begin{tabular}{|c|c|c|c|c|c|c|c|c|c|c|}
\hline Runt: & 0291 & & I & $\cdot$ & - & 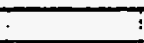 & 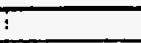 & & & , \\
\hline Tost Fluid & TCAF 10 & & i & $i$ & i & 1 & 1 & 1 & $i$ & . \\
\hline Date: & $17 / 2 / 931$ & & $\mathrm{i}$ & 1 & T & 1 & 1 & 1 & $\bar{i}$ & . \\
\hline Time: & $11: 47$ & & I & $!$ & $I$ & $!$ & t & 1 & & 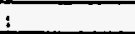 \\
\hline Note & Analytical & oth is used & i & 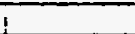 & & $\bar{l}$ & 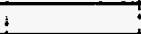 & ?t & 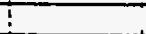 & \\
\hline Fow Area-tL2 & 0.00336 & & i & : & ! & I & 1 & 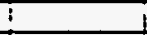 & 1 & i \\
\hline Hoat flow Avea-fl & 0.1955 & & & . & $i$ & $i$ & . & & $;$ & 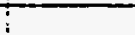 \\
\hline Sal. Pressure + sig & 187.3 & & $i$ & i & i & & $i$ & & $\mathrm{i}$ & $i$ \\
\hline Sal. Temperature F(inlel) & 190.7 & & 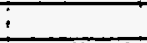 & 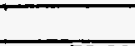 & ! & $i$ & $i$ & & $!$ & \\
\hline Lag. Density-ibmitis & 73.381 & 73.22 & 73.081 & 73.08 & 73.08 & 73 & 72.971 & 72.94 & 72.881 & 73.13 \\
\hline Latont Hoan-Btuhbm & 23.18 & 23.01 & 229 & 2.9 & 22.9 & 22.831 & 22.811 & 2279 & 2275 & 22.94 \\
\hline Spocitio Hoat-Btu/bm/F & 0.391 & 0.312 & 0.3121 & 0.312 & 0.312 & 0.313 & 0.313 & 0.313 & 0.313 & 0.312 \\
\hline Lig. Visoosinylbm/hhr & 0.301 & 0.3 & 0.238 & 0.298 & 0.298 & 0.297 & 0.297 & 0.286 & 0.286 & 0.299 \\
\hline Luq. Conductivity BumprfF & 0.0198 & 0.0197 & 0.0196 & 0.0196 & 0.0196 & 0.0196 & 0.0195 & 0.0195 & 0.0195 & 0.0186 \\
\hline Liq. Prandt No. & 4.74 & 4.74 & 4.74 & 4.74 & 4.74 & 4.75 & 4.75 & 4.75 & 4.75 & 4.74 \\
\hline Inlot Flow Plate-GPM & 2.9 & & & 1 & & $I$ & & & & \\
\hline Outtet Lig. Fow Rate-GPM & $\overline{1.1}$ & & & & & & & & & \\
\hline Outtet Vap. Flow Rade-GPM & 1.9 & & I & & & & & & & \\
\hline Inlet Temperaturof & 187.4 & & & & & & & & i & \\
\hline Outlet Liq. Temperaturef & 188.91 & & & & & & & & & \\
\hline Outlet Vap. Temperaturef & 185.5 & & & & & & & & & \\
\hline Water Fow Ralo-GPM & -1 & t & T & I & & 1 & & i & 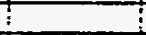 & \\
\hline Water Inlet Temp. $F$ & 127.9 & & 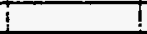 & t! & i & I & & I & 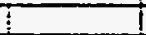 & \\
\hline Water outlot Temp.f & 182.1 & & & & 1 & $!$ & & & $i$ & \\
\hline Inlot G-bminnitie & 513113 & & Temp & 1822 & [Density & 74.14 & & & 1 & \\
\hline Subcooling $f$ & 3.3 & & & & $i$ & I & & & & \\
\hline Fow Observation & & & & & & & & & & \\
\hline & ZONE1 & ZONE2 & ZONE3 & ZONE4 & ZONE5 & ZONE6 & ZONE7 & ZONE8 & IZONE9 & ZONE10 \\
\hline Hoat Input-Walts(nel) & 284 & 285.7 & 286.8 & 295.9 & 288.4 & 291.6 & 287.1 & 287.1 & $1 \quad 285.1$ & 284.7 \\
\hline Heat Loss-Watts & 16.9 & 4 & 5.4 & 6.1 & 6.3 & 5.6 & 4.1 & 5.2 & 6 & 31.7 \\
\hline Hoal Flox-BturHrile & 4923 & 4952 & 4971 & 5129 & 4999 & 5055 & 4977 & 4977 & 4942 & 4935 \\
\hline Wall Temp. Ho & $9 \% 3.5$ & T33.9 & 193.71 & 793.6 & Tक्ड.4 & 7\$3.8 & T\$3.8 & T36.7 & 194.8 & T\$26 \\
\hline Wall Tomp. & 194 & 193.8 & 194 & 193.7 & 193.9 & 194.7 & 194.1 & 193.4! & 795.3 & 192.8 \\
\hline Wall Temp. \#5F & 194 & 193.4 & 193.91 & 193.1 & 193.4 & 193.9 & 193.6 & 193.6 & 194.8 & 1924 \\
\hline Wall Tomp. :1F & 88.6 & 194.1 & 193.3 & 193.1 & 192.9 & 194.3 & 193.7 & 199.5 & 194 & 190.8 \\
\hline Wall Tomp. W & 194.1 & 1942 & 193.5 & 193.3 & 193 & 194 & 194.1 & 193.4 & 194.3 & 191.7 \\
\hline Instroam Temp.f & 188.5 & 189.1 & 189.6 & 189.6 & 189.6 & 189.8 & 190 & 190.1 & 190.3 & 189.4 \\
\hline Avg. Wall Temp.f & 193.9 & 194 & 193.6 & 193.4 & 193.3 & 194.2 & 193.9 & 193.5 & 194.6 & 192.1 \\
\hline Avg. Wall DT.f & 52 & 4.7 & 3.8 & 3.5 & 3.5 & 4 & 3.6 & 3.2 & 4.1 & 24 \\
\hline Remaining Subcooff & 1.5 & & & & & & & & & \\
\hline Ext Qualify & -0.02 & 0 & 0.03 & 0.05 & 0.08 & 0.11 & 0.13 & 0.15 & 0.18 & 0.2 \\
\hline DXP. HBtuHhriter & 950.8 & 1059.1 & 1308.2 & 1447.6 & 1439 & 1249.1 & 1369.1 & 1557.6 & 1200.5 & 2025.1 \\
\hline COMPUTEDH & & & & & & & & & & \\
\hline Jallouk & 829.2 & 835.3 & 7956.8 & 8003.7 & 783.5 & 785 & 773.4 & 770 & 764.2 & 756.8 \\
\hline Szady 1(Cudo tube) & 1281.2 & 1289.6 & 1296.7 & 1317.8 & 1302 & 13122 & 1303.9 & 1305.4 & 1300.3 & 1296.6 \\
\hline sady2 & 10422 & 1051.2 & 1014.2 & 1037.8 & 1021.5 & 10329 & 1024.4 & 1020.7 & 1024.9 & 1018.4 \\
\hline (RLPa) & 111398 & 112004 & 1107031 & 107991 & 105085 & 102507 & 99874 & 97163 & 94557 & 90894 \\
\hline (F) & 1.004 & 1.004 & 1.3311 & 1.554 & 1.72 & 1.85 & 1.978 & 2.078 & 2.173 & 2.273 \\
\hline (HUO) & 172.7 & 172.8 & 220.11 & 267.6 & 296.1 & 378.3 & 338.6 & 357.8 & 374.2 & 391.1 \\
\hline (HBOIL) & 12802 & 1288.6 & 1294.4 & 1314.1 & 1296.9 & 1305.9 & 1256.1 & 1296.4 & 1293 & 1284.6 \\
\hline & & & & & i & & & & & \\
\hline & ZONE2 & ZONE4 & ZONE6 & ZONE8 & ZONE1O & & & & & \\
\hline DP liquid Temp.f & 91.5 & & & & & & & & & \\
\hline DP Liquid Density-bm/n3 & 91.28 & & & & & & & & & \\
\hline Elevation- $t$ & 2 & 4 & 6 & 8 & 9.875 & & & & $i$ & \\
\hline Moasurod DPPSid & 0.359 & 0.8251 & T.3.38 & 2.038 & 2.6 & & & & & \\
\hline Zoro Contections-psid & 0.144 & 0.111 & 0.156 & 0.051 & 0.032 & & & & & \\
\hline Conected DPPsid & 0.225 & 0.714 & 1.2261 & 1.987 & 2568 & & & & & \\
\hline Actual DPA & 2.05 & 3.59 & 5.07 & 6.07 & 7.27 & & & & & \\
\hline Moesured TPPsig & 187.3 & & & & 300.1 & & & & & \\
\hline Measured Overallit & कारूम & & & & & & & & & \\
\hline & & & & & & & & & & \\
\hline Computad DPA & 2.02 & 3.79 & 5.4 & 6.91 & 8.28 & & & & & \\
\hline Friction-h & 0.05 & 0.11 & 0.2 & 0.31 & 0.42 & & & & & \\
\hline Accaloration-A & 0.01 & 0.05 & 0.07 & 0.09 & 0.11 & & & & & \\
\hline Elevation-f & 1.96 & 3.68 & 5.12 & 6.51 & 7.74 & & & & & \\
\hline & & & & & & & & & & \\
\hline
\end{tabular}




\begin{tabular}{|c|c|c|c|c|c|c|c|c|c|c|}
\hline Run\# & 5701 & & & & . & & $\dot{-}$ & & - & \\
\hline Tost Fluid & CAF10 & & & $\leq$ & & . & : & 1 & $\therefore$ & \\
\hline Date: & 7721931 & $\vdots$ & 1 & 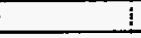 & $i$ & $!$ & - & & $\dot{-}$ & \\
\hline Time: & $11: 471$ & 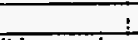 & 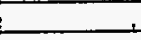 & $!$ & & $i$ & & & $\dot{-}$ & \\
\hline Note & Anatytical d & tis used i & $i$ & \pm & $i$ & : & 2 & & & \\
\hline Fow Area-t2 & 0.00336 & & & 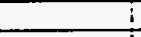 & & i & & & $\vdots$ & \\
\hline Heat Flow Areatil2 & 0.19695 & & & 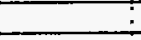 & & 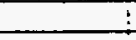 & 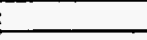 & & & \\
\hline Sat. PTessuro Psig & 186.81 & 5 & 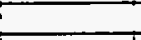 & $\dot{5}$ & & ; & & & & \\
\hline Sat. Temperaturef (inlet) & 190.51 & 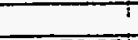 & 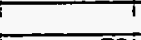 & & & : & & & & \\
\hline Ug. Density fbmntit3 & 73.351 & 73.051 & 731 & 73.221 & 73.161 & $73.08 !$ & 72.941 & 72.911 & 72.911 & 73.08 \\
\hline Latent Heat-Bturlbm & 23.111 & 22.881 & 22.831 & 23.011 & 22.961 & 2291 & 22.791 & 22.77 & 2277 & 229 \\
\hline Speofic Heat-Btulbmif & 0.3111 & 0.3121 & 0.3131 & $0.312 \mid$ & $0.312 !$ & 0.3121 & 0.3131 & 0.313 & 0.313 & 0.312 \\
\hline Lig. Visoosity bm/ithr & 0.3011 & 0.2981 & 0.297 & 0.3 & 0.299 & 0.2981 & $0.296 i$ & 0.296 & 0.2961 & 0.293 \\
\hline Lig. Conductivity Btumr-fff & 0.0198 & 0.01961 & 0.01961 & 0.01971 & 0.01971 & 0.01961 & 0.01951 & 0.0195 & 0.01951 & 0.0196 \\
\hline Liq. Prandt No. & 4.741 & 4.741 & 4.751 & 4.74 & 4.741 & $4.74 !$ & 4.751 & 4.75 & 4.751 & 4.74 \\
\hline Inlet Flow Rato-GPM & 2 & & 1 & L & 1 & & 1 & : & 1 & \\
\hline Outlet Liq. Flow Rato-GPM & 1 & & 1 & 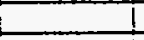 & 1 & 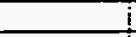 & $i$ & I & L & \\
\hline Outet Vap. Fow Rato-GPM & 1.5 & & & 1 & $\div$ & $i$ & $\div$ & & $\therefore$ & \\
\hline Inlat Temperaturof & 187.41 & & & $i$ & & 1 & 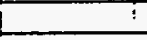 & 1 & 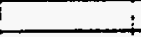 & \\
\hline Outlet Liq. Temperature F & 188.91 & & & 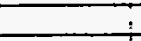 & & $\bar{z}$ & 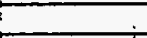 & $i$ & $i$ & \\
\hline Oưle! Vap. Temperaturef & 185.71 & i & t & $!$ & $!$ & 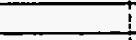 & $!$ & $\vdots$ & 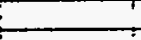 & \\
\hline Water flow Rato-GPM & 1 & $\bar{\vdots}$ & 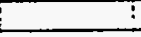 & & & & & & & \\
\hline Water Inlet Temp.F & 127 & 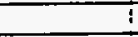 & & $:$ & & & & $\vdots$ & $\vdots$ & \\
\hline Water outlot Temp.F & 182.21 & it & 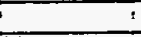 & ; & $!$ & 1 & ; & & 1 & \\
\hline Inlet G-lbm/hr/tt2 & 3568611 & & Temp & 179.71 & Density & 74.77 & $i$ & - & 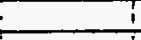 & \\
\hline Subcooling $f$ & 3.11 & 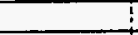 & & & 1 & & 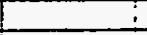 & & I & \\
\hline \multirow[t]{2}{*}{ Flow Observation } & & & 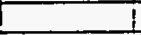 & & & & & & & \multirow[b]{2}{*}{ ZONE10 } \\
\hline & ZONE 1 & ZONE2 & ZONE3 & ZONE4 I & ZONE5 & ZONE6 1 & ZONE7 & ZONEB : & ZZONE9 & \\
\hline Heat Input-Watts(nel) & 285.8 & 287.4 & 285.9 & 289.2 & 287.61 & 290.2 & 288.8 & 288.31 & 284.8 & 284.4 \\
\hline Heat Loss-Watts & 16.8 & 4 & 5.3 & 6ा & 6.21 & 5.5 & 4 & 5.1 & 5.9 & 31.7 \\
\hline Heat Flux-8tunilini & 4954 & 4982 & 4956 & 5013 & 4685 & 5030 & 5006 & 4997 & 4937 & 4930 \\
\hline Wall Temp. & \$\$3.4 & 193.9 & 193.8 & 153.1 & 1929 & 193.5 & 193.7 & 193.5 & 194.5 & 192.7 \\
\hline Wall Temp. \#4F & 194 & 193.8 & 194.1 & 193.4 & 193.4 & 994.4 & 193.9 & 193.5 & 195 & 193.1 \\
\hline Wall Temp. "IFF & 194.2 & 193.3 & 193.8 & 192.9 & 192.8 & 193.8 & 193.5 & 193.4 & 194.6 & 192.5 \\
\hline Wall Tomp. $\# 1 F$ & 89.11 & 194.2 & 193.31 & 192.9 & 192.7 & 194.1 & 193.7 & 193.5 & 193.7 & 190.7 \\
\hline Wall Temp. "LF & 194.21 & 134.2 & 193.6 & 193 & 1928 & 193.8 & 193.7 & 193.5 & 194.1 & 191.7 \\
\hline Instream Tomp.F & 188.61 & 189.7 & 189.8 & 189.11 & 189.3 & 189.6 & 190.1 & 190.2 & 190.2 & 189.6 \\
\hline Avg.Wall Tomp.F & 194 & 194 & 193.7 & 193.1 & 19291 & 194 & 193.7 & 193.5 & 194.4 & 192.1 \\
\hline Avg. Wall DT. $f$ & 5.11 & 4.1 & 3.6 & 3.7 & 3.4 & 4.1 & 3.4 & 3.1 & 4 & 2.3 \\
\hline Remaining Subcooff & 0.5 & & & & & & & & & \\
\hline Exot Quality & -0.011 & 0.03 & 0.06 & 0.11 & 0.14 & 0.17 & 0.21 & 0.24 & 0.28 & 0.32 \\
\hline EXP.HABuHring/F & $966.5 i$ & 1215.1 & 1385.9 & 1337.4 & 1466.4 & 1220.2 & $1483.9 i$ & 1636.3 & 1247.7 & 2127.5 \\
\hline COMPUTEDH & & & & & & & & & & \\
\hline Jallouk & 870.1 & 860.81 & 839.2 & 8291 & 798.5 & 739.8 & 795.2 & 791.11 & 781.5 & 775.3 \\
\hline Szady1(Cudo tubo) & 1285.6 & 1297.9 & 1296.6 & 1298.31 & 1296.8 & 1305.4 & 1306.6 & 1306.7 & 1239.5 & 1294.6 \\
\hline szady2 & $1047.4 !$ & 1060.3 & 1058.2 & 1016.6 & 1015.2 & 1024.5 & 1025.7 & 1026.2 & 1018.9 & 1014.8 \\
\hline$(F \cup \cup)$ & 77517 & 77545 & 749251 & 71483 & $68841 !$ & 6623 & 63795 & 61090 & 582051 & 55098 \\
\hline (F) & 1.004 & 1.265 & 1.588 & 1.81 & 1.984 & 2.131 & 2.2591 & 2.378 & 2.489 & 2602 \\
\hline (HบO) & 128.2 & 162.8 & 204.5 & 2328 & 255.31 & 274.3 & 290.8 & 306.8 & 320.6 & 334.8 \\
\hline (HBOIL) & 1285.2 & 1297 & 1294.9 & 1295.8 & 1293.5 & 1301.3 & 1301.8 & 1301.1 & 1292.9 & 1287.1 \\
\hline & & & & & & & 1 & & & \\
\hline & ZONE2 & ZONE4 & ZONE6 & ZONE8 & ZONE10 & & 1 & & & \\
\hline DP liquid Temp.F & \begin{tabular}{|r|}
91.9 \\
\end{tabular} & & & & 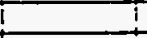 & & $i$ & & $\vdots$ & \\
\hline DPLiquid Density lbm/ti3 & 91.21 & & & & & & & & $\perp$ & \\
\hline Elevation-t & 21 & 4 & 6 & 81 & 9.875 & & & & & \\
\hline Measured DPPsid & 0.351 & 0.952 & 1.625 & 2379 & 3.036 & & & & & \\
\hline Zoro Corrections-psid & 0.144 & 0.117 & 0.156 & 0.057 & 0.032 & & I & & & \\
\hline Corrected DPPsid & 0.187 & 0.851 & 1.469 & 2.328 & 3.004 & & I & & 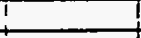 & \\
\hline Actual DPA & 2.13 & 3.32 & 4.591 & 5.4 & 6.411 & & & & & \\
\hline Moasured TPPsig & 186.8 & & & & 300.11 & & 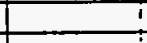 & & $!$ & \\
\hline Measured Overath & $\sin$ & & & & & & & & 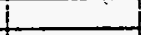 & \\
\hline & & & & & & & & & & \\
\hline Computed DPA & 1.91 & 3.46 & 4.84 & 6.12 & 7.25 & & & & 1 & \\
\hline Friction-li & 0.03 & 0.07 & 0.13 & 0.2 & 0.28 & & i & & & \\
\hline Accoloration-t & 0.02 & 0.03 & 0.05 & 0.06 & 0.08 & & & & $!$ & \\
\hline Elevation-t & 1.87 & 3.36 & 4.66 & 5.85 & 8.89 & & & & 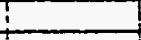 & \\
\hline & & & & & & & & & i & \\
\hline
\end{tabular}




\begin{tabular}{|c|c|c|c|c|c|c|c|c|c|c|}
\hline \multirow{2}{*}{$\begin{array}{l}\text { Runk } \\
\text { Testflicid } \\
\end{array}$} &.$\quad 671 i$ & $71 i$ & \pm & $\cdot$ & -2 & 1 & - & . & \multicolumn{2}{|c|}{3} \\
\hline & CAF10 & & - & $\dot{-}$ & + & 1 & - & 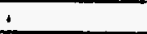 & & . \\
\hline Date: & $1 \quad 7 / 2 \sqrt{331}$ & & $\leq$ & . & $\overline{-}$ & 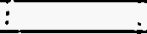 & 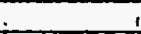 & 1 & & . \\
\hline Time: & $11: 471$ & & & & 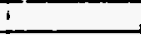 & & 1 & $!$ & $:$ & \\
\hline Nole & Analytical c & ot is used ! & $!$ & & & & : & & & \\
\hline Fow Areatt? & 0.003361 & & & & 1 & & 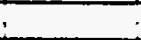 & ; & & \\
\hline Hoal Flow Area-ti2 & $! 0.196951$ & & & & & & i & 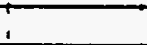 & & \\
\hline Sat. Prossure Psig & 188.8 & & ? & : & $\div$ & 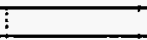 & & & $\cdot$ & \\
\hline Sat. Temperaturof (inlel) & 191.31 & & & & $\vec{i}$ & & 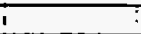 & & & \\
\hline 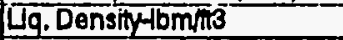 & $7 \overline{3} 1$ & 72.77 & 72.81 & 72.881 & 72.77 & 72.51 & 72.61 & 72.541 & 72.541 & 72.8 \\
\hline Lalont Hoat-Btunbm & 22.831 & 22.661 & 22.681 & 22.751 & 22.66 & 22.61 & 22.531 & .22 .491 & 22.491 & 22.68 \\
\hline Specifio Hear-btuhbm/F & 0.3131 & 0.3141 & 0.3131 & 0.3131 & 0.314 & 0.314 & 0.3141 & 0.315 & 0.315 & 0.313 \\
\hline Lig. Viscositylbm/ithr & 0.297 & 0.234 & 0.2551 & 0.2061 & 0.294 & 0.2931 & 0.2921 & 0.2521 & 0.2921 & 0.296 \\
\hline Liq. Conductivity - Btuhr fof & 0.01961 & 0.01941 & 0.07941 & 0.0195 & 0.0194 & 0.01941 & 0.01931 & 0.01931 & 0.01931 & 0.0194 \\
\hline Lig.Prandt1No. & 4.751 & 4.75 & 4.751 & 4.75 & 4.75 & 4.751 & 4.751 & 4.761 & 4.761 & 4.75 \\
\hline Inlelflow Ralo-GPM & 1.1 & & 1 & 7 & $!$ & 1 & 1 & 1 & 1 & 1 \\
\hline Outtel Lig. Flow Pato-GPM & 0.9 & & i & 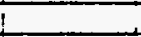 & & 1 & 1 & $i$ & i & $i$ \\
\hline Outlol Vap. Flow Ralo-GPM & 0.7 & & $t$ & 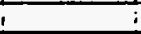 & 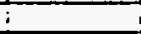 & 1 & 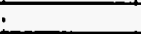 & & $i$ & 1 \\
\hline Inlet Temperaturof & 188.8 & & i & & 1 & $i$ & 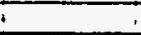 & 7 & : & \\
\hline Outlel Lia. Temperahure & T90.31 & & $\div$ & ? & & & & & & ! \\
\hline Outhel vap. Temperaturef & 187.8 & & T & T & $t$ & $?$ & T & $t$ & t & ! \\
\hline Water fiow Rate-GPM & : & 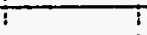 & T & 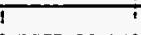 & it & 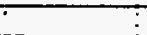 & $i$ & $i$ & $t$ & \\
\hline Water Inlel Temp.F & 128.41 & & ! & T & 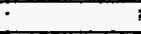 & $i$ & 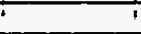 & i & - & \\
\hline Water outlel Temp,f & 184.21 & & $T$ & i & & i & 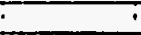 & 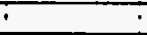 & 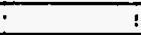 & $!$ \\
\hline Inlot Glbmhrnt2 & 199135: & & ITemp & 175.2. & Density & 75.861 & & 1 & 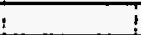 & $!$ \\
\hline Subcooling $F$ & 2.51 & & & & 1 & & 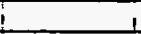 & 1 & : & . \\
\hline \begin{tabular}{|l|} 
Flow Observation \\
\end{tabular} & & $i$ & & 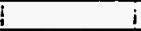 & & $\mathrm{i}$ & $i$ & 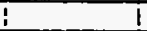 & I & i \\
\hline & ZONE1 & ¿ZONE2 & ZONEO & ZONE4 & ZONE5 & ZZONE6 & ZONET & ZONE8 & ZONE9 & ZONE10 \\
\hline Hoal Input-Walts(nel) & 284.9 & 286.9 & 287.3 & 294.5 & 287.1 & 290.31 & 288.21 & 287.6 & 284.3 & 283.7 \\
\hline Heaf Loss-Watts & 17.2 & 4.1 & 5.5 & 6.2 & 6.5 & 5.9 & 4.31 & 5.41 & 6 & 32.8 \\
\hline Hoat Fux-su/Hirit2 & 4939 & 4973 & 49801 & 5105 & 497 & 5032 & 49961 & 4985 & 4928 & 4918 \\
\hline Wall Temp. $3+$ & 794.11 & 194.7 & 194.41 & 194.11 & 194 & 194.5 & 195 & 194.8 & 195.2 & 193.6 \\
\hline Wall Temp. "4F & 194.71 & 194.3! & 194.81 & 194.4! & 194.3! & 195.6 & 195.11 & 194.8 & 195.9 & 134 \\
\hline Wall Temp. "H5F & 194.81 & 194 & 194.5 & 194 & 193.7 & 194.7 & 194.71 & 194.7 & T55.3| & 193.5 \\
\hline Wall Tomp. \#1F & 89.51 & 194.9 & 193.81 & 199.7 & 193.81 & 195.21 & 194.7 & 194.8 & 194.4 & 191.8 \\
\hline Wall Tomp. WFF & 194.9 & 194.9 & 194.31 & 194 & 193.8 & 194.71 & $1 \$$ & 194.8 & 194.8 & 192.7 \\
\hline Instroem Temp,f & 189.8 & 190.7 & 180.61 & 180.31 & 180.7 & 1911 & 191.31 & 191.5 & 191.6 & 190.6 \\
\hline Avg. Wall Tomp.F & 194.7 & 194.7! & 194.31 & 194.1 & 193.9 & 1951 & 194.91 & 194.8 & 195.1 & 193.1 \\
\hline Avg. Wall DT.f & 4.5 & 3.8 & 3.5 & 3.5 & 3 & 3.8 & 3.41 & 3.1 & 3.4 & 2.3 \\
\hline Romaining Subcoolf & -2.11 & & & & & & & & & \\
\hline Exit Quality & 0.03 & 0.09 & 0.16 & 0.22 & 0.29 & 0.35 & 0.421 & 0.48 & 0.55 & 0.61 \\
\hline EXP. HEtUHRMLF & 1091 & 1317.3 & 1422.91 & 1450.6 & 1672.8 & 1333.9 & 1480.61 & 1632 & 1450.6 & 2140.2 \\
\hline COMPUTEDH & & & & & & & & & & \\
\hline Jallouk & 916.4 & 9031 & 886.21 & 885.61 & 865.91 & 866.51 & 858.71 & 853.91 & 843.81 & 8303.5 \\
\hline Szady1(Cudo tube) & 1293.4 & 7304.2 & 7304.51 & 1318.5 & 1305.3 & 1315 & 1312.8 & 1313.31 & 1306.1 & 1298.2 \\
\hline szady2 & 1055.9 & 1057.41 & 1057.51 & 1082.51 & 1057.81 & 1078.1 & 1075.41 & 1075.5 & 1022.6 & 1015.4 \\
\hline (PपQ) & 432201 & $41543 \mid$ & 386511 & 35639 & 32007 & 30138 & 273911 & $24488 \mid$ & 215921 & 18520 \\
\hline$(F)$ & 1.304 & 1.678 & 2.004 & 2.259 & 2.465 & 2.645 & 2.008 & 2.961 & 3.107 & 3.269 \\
\hline (मीUO) & 105.3 & 135.6 & 161.81 & 182.6 & 180.21 & 213.7 & 226.8 & 23.3 & 251.11 & 264.1 \\
\hline (HBOIL) & 7293.21 & 1303.7 & 1303.7 & 1317.4 & 1303.81 & 1313.11 & 1310.6 & 1310.6 & 1300 & 1234.6 \\
\hline & & & 1 & 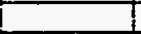 & & & & & & \\
\hline & ZONE2 & ZONE4 & ZONE6 & ZONE8 & ZONE10 & & & i & & \\
\hline DPliquid Temp.F & 92.6 & & & & & $t$ & 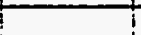 & & & \\
\hline DPLiquid Density & 91.12 & & & & & 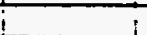 & 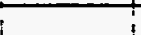 & & & 1 \\
\hline Elovation-ft & 2) & 4 & 61 & 8 & 9.875 & & & & & \\
\hline Measured DPFPid & ठ.उस्दा & T.य3। & 20431 & 2.366 & 3.728 & & & & & \\
\hline Zero Conections-psid & 0.078 & -0.044 & 0.008 & 0.009 & 0.018 & & & & & \\
\hline ComededDPPsid & 0.234 & 1.274 & 2.00351 & 2.897 & $3.7 \pi$ & & & & & \\
\hline Actual DPA & 2041 & 2.49 & 3.481 & 4.281 & 5.02 & & & & & \\
\hline Measurod TPPsig & 188.8 & & 1 & $T$ & 300.1 & & & & & \\
\hline Mecsured Overalth & Fond | & & $T$ & 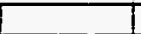 & & T & † & & & \\
\hline & & I & & 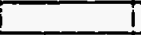 & & & & & & \\
\hline Computed DPA & 1.62 & 2.861 & 3.911 & 4.841 & 5.61 & & & & & \\
\hline Friction-ft & 0.01 & 0.04 & 0.07 & 0.11 & 0.15 & & & & & \\
\hline Accolerationt & 0.01 & 0.02 & 0.03 & 0.04 & 0.05 & & & & 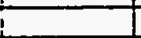 & \\
\hline Elevation-t & 1.6 & 2.81 & 3.82 & 4.69 & 5.47 & & & & & \\
\hline & & & & & & & & & & \\
\hline
\end{tabular}




\begin{tabular}{|c|c|c|c|c|c|c|c|c|c|c|}
\hline Runt & 672 & & i & : & & & 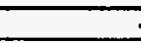 & $\therefore$ & & - \\
\hline Tost Fluid & .CAF10: & & I & , & . & $i$ & . & $i$ & 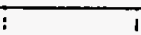 & $T$ \\
\hline Date: & 776931 & & 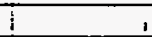 & 1 & 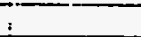 & $i$ & : & $T$ & i & \\
\hline Time: & $8: 29$ & & $!$ & & & & $:$ & 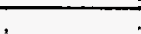 & & \\
\hline Nole & Analytical & dt is used & 1 & & . & & $i$ & & & \\
\hline Fow Areafle & 0.003561 & & & & 1 & 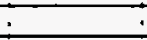 & 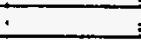 & $:$ & & \\
\hline Heat Flow Area-t? & 0.19695 & & & & & & $i$ & & & \\
\hline Sat. Pressure Psig $_{\text {sig }}$ & 191.91 & & 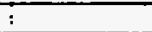 & & & & T & & & \\
\hline Sat. Temperaturof(inlel) & $192.6 !$ & & 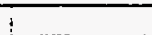 & & . & & ti & T & & $\frac{t}{\vdots}$ \\
\hline Lig. Density $-1 b m$ min & 73.131 & 73 & 72.881 & 72.8 & 72.74 & 72.661 & 72.61 & 72.57 & 72.521 & 72.94 \\
\hline Latent Heat-Buflbm & 22.941 & 22.83 & 22.751 & 22.68 & 22.64 & 22.571 & 22.53 & 22.511 & 22.471 & 22.79 \\
\hline Spocitic Hoat-Btu/bmif & 0.312 & 0.313 & 0.3131 & 0.313 & 0.314 & 0.3141 & 0.314 & 0.314 & 0.315 & 0,313 \\
\hline Lig. Viscosiky $/ \mathrm{bm} / \mathrm{hth}$ & 0.298 & 0.297 & 0.2661 & 0.296 & 0.224 & 0.283 & 0.282 & 0.292 & 0.281 & 0.286 \\
\hline Lia. Conductivity-Bturnstif & 0.01961 & 0.0196 & 0.01951 & 0.0194 & 0.0194 & 0.0194 & 0.0193 & 0.0193 & 0.0193 & 0.0195 \\
\hline Liq. Prandt No. & 4.74 & 4.75 & 4.751 & 4.75 & 4.75 & 4.75 & 4.75 & 4.76 & 4.76 & 4.75 \\
\hline Inlet fow Rate-GPM & 5.7 & & $i$ & $=$ & i & it & 1 & & & \\
\hline Outlot Lig. Fow Rato-GPM & 1.2 & & 1 & 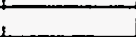 & 1 & 1 & & & & \\
\hline Outlet Vap. Flow Ralo-GPM & 2.8 & & 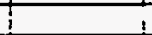 & 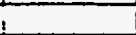 & $i$ & ? & & & & \\
\hline inlel Temporaturo & 187.3 & & & 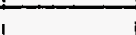 & i & i & & & & \\
\hline Outlel Lq. Temperaturef & 790.8 & & i & 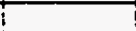 & i & & $t$ & & & \\
\hline Outlet Vap. Temperaturef & 186.6 & & I! & 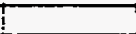 & & t & + & & & \\
\hline Water Fow Rato-GPM & & ! & 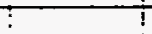 & t & 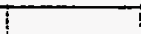 & $t$ & t & & & \\
\hline Water Inlet Temp.F & 114.41 & & 1 & $i$ & i & ! & 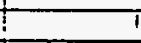 & & & \\
\hline Wator outhet Tomp.f & 169.4 & & $T$ & i & it & $i$ & $i$ & ? & & \\
\hline Inlot Gibminhme & 8980161 & & Tomp & 185.2 & Density & 73.37 & & & & \\
\hline Subcooling ff & 5.3 & & & 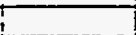 & & 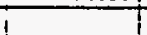 & $i$ & $i$ & & \\
\hline \multirow[t]{2}{*}{ Flow Obsenvation } & & $\frac{1}{1}$ & & & & & & & & \\
\hline & ZONEI & ZONE2 & ZONE3 & ZONE4 & ZONE5 & ZONE6 & ZONE7 : & ZONE8 & ZONE9 & ZONE10 \\
\hline Head Input-Walts(net) & 579.5 & 576.8 & 577.8 & 575.7 & 573.6 & 575.5 & 575.1 & 577.9 & 574.2 & 5712 \\
\hline Heat Loss-Watts & 18.31 & 4.3 & 5.9 & 6.8 & 7.3 & 6.6 & 4.9 & 6.1 & 6.6 & 35.7 \\
\hline Hoat Fix-BtuHrinz & 10045 & 9988 & 10016 & 9979 & 9943 & 9976 & 9059 & 10018 & 9953 & 9001 \\
\hline Wall Temp. H3 & TS.8 & $\lceil 97.2$ & 156.81 & \$S. & 997.7 & |Sका & 196.3 & 197.1 & โका. & 196.5 \\
\hline Wall Temp. $4 F$ & 195.3 & 196.2 & 197.6 & 197.3 & 197.7 & 196.9 & 1969 & 1972 & 1989 & 1959 \\
\hline Wall Temp. 5 F & 198.7 & 195.8 & 198.4 & 197.2 & 197 & 196 & 195.9 & 196.6 & 196.8 & 195.8 \\
\hline Wall Tomp. "NF & 88.8 & 196.9 & 195.7 & 195.9 & 190.4 & 197 & 198.1 & 196.2 & 105.7 & 1923 \\
\hline Woll Tamp. IRF & 197 & 196.8 & 196.6 & 196.3 & 196.5 & 196.5 & 196.3 & 196.6 & 196.4 & 194.4 \\
\hline Instream Tomp.F & 189.41 & 789.8 & 180.8 & 180.6 & 980.8 & 181.1 & 191.8 & 181.4 & 181.6 & 180.1 \\
\hline Avg. Wall Tomp.F & 196.5 & 196.8 & 156.7 & 196.8 & 196.9 & 196.5 & 196.31 & 196.7 & 186.8 & 195 \\
\hline Avg. Wall DT tF & 6.6 & 6.4 & 5.9 & 5.7 & 5.7 & 4.9 & 4.5 & 4.9 & 4.8 & 4.4 \\
\hline Remaining Subcooff & 3.4 & & & & & & & & & \\
\hline Ext Quality & -0.05 & -0.02 & 0.01 & 0.03 & 0.06 & 0.08 & 0.11 & 0.14 & 0.16 & 0.19 \\
\hline EXP. HBtu/HrAt2/F & 15229 & 1550.6 & 16912 & 1751.1 & 1759.6 & 2025.9 & 2191.2 & 2049.7 & 2086.61 & 2233.8 \\
\hline \multicolumn{11}{|l|}{ COMPUTEDH } \\
\hline Jallouk & Tरक्.2 & 716.9 & $1173.5 \mid$ & 1140 & 904.9 & 1085.41 & 1057.11 & 1054.7 & 1036.8 & 1003.1 \\
\hline Szadyi(Oudo tubo) & 1843.71 & 1844.1 & 1850 & 1851.6 & 18522 & 1850.2 & 1863.3 & 1870.6 & 1868.5 & 1850 \\
\hline $5 z a d y 2$ & 1632.2 & 1688.6 & 1603.6 & 16989 & 7699.4 & 1708.5 & 1711.8 & 1719.3 & 1716.7 & 1690.2 \\
\hline (RUO) & 2188801 & 219878 & 220848 & 217485 & 212249 & 207207 & 201884 & 196203 & 190898 & 182041 \\
\hline (F) & 1.004 & 1.004 & 1.004 & 1.35 & 1.564 & 1.726 & 1.8611 & 1.98 & 2.086 & 22 \\
\hline (HपQ) & 294.21 & 294.3 & 294.4 & 394.4 & 458.8 & 506.5 & 546.21 & 581.1 & 612.1 & 644.0 \\
\hline \multirow[t]{3}{*}{ ( } & 1840.6 & 1841.6 & 1847.5 & 9845.7 & 1842.8 & 1847.6 & 1847.6 & 1851.7 & 1846.3 & 1823.5 \\
\hline & 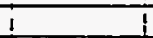 & & & & & & & & 1 & \\
\hline & ZONE2 & ZONE4 & ZONE6 & ZONEB & ZONE10 & & & & & \\
\hline DPliquid Temp.fF & i 88.5 & & + & 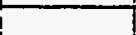 & & & t & & & \\
\hline DPLjquidDensitylbm/1/3 & 91.73 & & & & & & & & & \\
\hline Elevation-t & & 4 & को & 8 & 9.875 & & & & & \\
\hline MoasuredDPFsid & 0.344 & 0.758 & T.07ा & 1.598 & 2082 & & & & & \\
\hline Zero Corrections-psid & 0.733 & 0.101 & 0.139 & 0.036 & 0.086 & & & & & \\
\hline Cortected DPPsid & 0.217 & 0.687 & 0.932 & 1.563 & 1.998 & & & & & \\
\hline Actuad DRA & 2.11 & 3.71 & 5.711 & 6.98 & 8.48 & & & & & \\
\hline Moesured TPPsig & 191.9 & & & & 300.11 & & & & & \\
\hline \multirow[t]{2}{*}{ Moasured Overalth } & presen & & & & & & & & & \\
\hline & & & & & & & & & & \\
\hline Computed DPA & $2.15 !$ & 4.28 & 6.211 & 8.06 & 9.75 & & & & & \\
\hline Friction-t & 0.15 & 0.33 & 0.6 & 0.92 & 127 & & & & & \\
\hline Accoleration-i & 0 & 0.13 & 0.23 & 0.31 & 0.30 & & & & & \\
\hline Elevation-ft & 2) & 3.82 & 5.39 & 6.83 & 8.09 & & & & & \\
\hline & & & & & & & & & & \\
\hline
\end{tabular}




\begin{tabular}{|c|c|c|c|c|c|c|c|c|c|c|}
\hline Aunt & 6731 & - & • & $:$ & . & 1 & 1 & 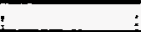 & & $!$ \\
\hline Tost Fuid & TCAFIO I & & 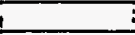 & 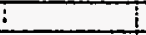 & 1 & 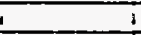 & I & I & & $\bar{i}$ \\
\hline Date: & $7 / 693$ & & & $i$ & $i$ & $i$ & $i$ & 0 & $i$ & $i$ \\
\hline Time: & $11: 111$ & & 5 & $!$ & 1 & 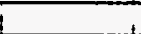 & & i & & 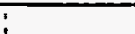 \\
\hline Nole & Analyticald & $t$ is used I & & 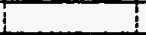 & + & 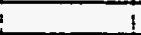 & i & 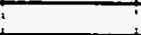 & & \\
\hline Flow Areaft2 & 0.00336 & $i$ & $i$ & 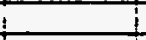 & $\therefore$ & 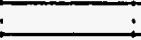 & $:$ & 1 & & \\
\hline Heat Flow Area-t2 & 0.19695 & i. & t. & & & 7 & 7 & & & \\
\hline Sat. Pressure Psig & 187.9 & & 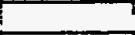 & $T$ & $T$ & T & 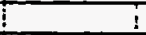 & $\vdots$ & & T \\
\hline Sal. Temperature & 190.91 & & & $T$ & & & t! & ! & & T \\
\hline Uq. Densty y bm/its & 73.331 & 73.191 & 73.081 & $73.76 !$ & 73.131 & 73.051 & 731 & 72.941 & 72.881 & 73.22 \\
\hline Latont Hoateturibm & 28.091 & 22.98 & 22.91 & 22901 & 22941 & 22881 & 22891 & 227 & 22.75 & 29.01 \\
\hline Spectio Hod thelbm/F & 0.3111 & 0.312 & 0.312 & 0.312 & 0.312 & 0.3121 & 0.3131 & 0.313 & 0.313 & 0.312 \\
\hline Liq. Visoosity tominthr & 0.3011 & $0.2 \%$ & 0.298 & 0.299 & $0.2 \% 1$ & 0.2981 & 0.227 & 0.296 & 0.2961 & 0.3 \\
\hline Lig. Conductivity BumptfF & 0.0198 & 0.0197 & 0.0196 & 0.0197 & 0.01861 & 0.0196 & 0.01961 & 0.0155 & 0.0195 & 0.0197 \\
\hline Liq. Prandty No. & $4.74 !$ & 4.74 & 4.74 & 4.74 & 4.741 & 4.74 & 4.751 & 4.75 & 4.75 & 4.74 \\
\hline Intot fow Rate-GPM & 4.7 & 1 & L & 4 & $i$ & +1 & 1 & $i$ & & \\
\hline OutlolLig. Flow Rato-GPM & 1.3 & 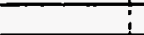 & 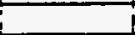 & 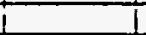 & 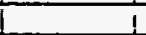 & 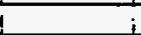 & $i$ & 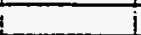 & & \\
\hline Outlel Vap. Fow Ralo-GPM & 2.2 & I & L & 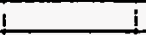 & $i$ & 1 & I & & & \\
\hline Inlet Temperaturef & 187.1 & $i$ & $i$ & & $i$ & 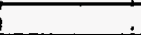 & & 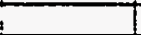 & & \\
\hline Outtel Lig. Temperaturef & 189.7 & & & & & & & & & \\
\hline Outlot Vap. Temperaturof & 185.7 & & : & $T$ & $T$ & $\Gamma$ & & & & \\
\hline Water Fow Pag-GPM & $!$ & $\mathrm{i}$ & 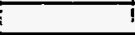 & t & I & it & & & & \\
\hline Water inlot Temp,f & 100.71 & $\overline{:}$ & 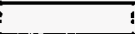 & $!$ & $!$ & i & $i$ & i & & \\
\hline Water outel Temp.f & 167.41 & 1 & 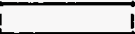 & i & 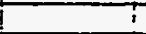 & 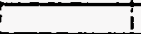 & 1 & $i$ & & 1 \\
\hline Inlot Glbmhrnt2 & 8223361 & & Temp & 185.4 & Donsity & 73.32 & & 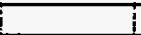 & & \\
\hline Suboooling $F$ & 3.8 & 1 & 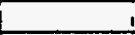 & 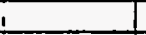 & $i$ & 1 & I & I & & \\
\hline \multicolumn{11}{|l|}{ Flow Observation } \\
\hline & ZONE1 & ZONE2 & ZONE3 & ZONE4 & ZONE5 & ZONE6 & ZONE7 & ZONE8 & ZONE9 & ZONETO \\
\hline Heat Input-Watts(nel) & 588.5 & 586 & 584.3 & 584.8 & 580.4 & 583 & 588.1 & 590.4 & 587.1 & 585.4 \\
\hline Head Loss-Watts & 18.3 & 4.3 & 5.9 & 6.8 & 7.3 & 6.6 & 4.8 & 6 & 6.6 & 35.7 \\
\hline Hoad Flux-BtuHiniti2 & 10201 & 10158 & 10128 & 10137 & 100611 & 10106 & 10194 & 10234 & 10177 & 10148 \\
\hline Wall Temp. HЗ & T\$5.2 & 796.5 & T\%5.1 & $7 \% .7$ & 796.8 & T\$5.31 & T\$ & $|\$ 5.9|$ & 185.4 & $7 \$ 5.8$ \\
\hline Wall Temp. f4F & 195.61 & 795.5 & 156.7 & 196.1 & 796.61 & 196.2 & $795.5 !$ & 156.2 & 197.3 & 195.3 \\
\hline Wall Tomp. "I5F & 195.61 & 195 & 197.7 & 198.1 & 195.81 & 195.3 & 194.4 & 195.5 & 190.6 & 195.8 \\
\hline Wall Tomp. N1F & 89.31 & 198.2 & 195 & 194.8 & 195.51 & 195.7 & 194.8 & 195.1 & 195.1 & 191.6 \\
\hline Wh Temp. KaF & 196.21 & 196.2 & 195.7 & 195.2 & 195.6 & 155.6 & 195.3 & 15.1 & 195.7 & 193.8 \\
\hline Intream Tomp.F & 188.7 & 189.2 & 189.6 & 189.8 & 189.4 & 189.7 & 180.0 & 180.1 & 180.8 & 189.1 \\
\hline Avg. Wall Tomp.f & 195.7 & 196.1 & 195.8 & 195.6 & 195.9 & 195.6 & 185 & 155.6 & 196.2 & 194.4 \\
\hline Avp. Wall DT. 5 & 6.5 & 6.4 & 5.8 & 5.9 & 6) & 5.5 & $4.6 !$ & 5 & 5.4 & 4.8 \\
\hline Remaining Subcooff & 7.5 & & & & & & & & & \\
\hline Exát Quality & -0.02 & 0.01 & 0.04 & 0.07 & 0.11 & 0.14 & 0.17 & 0.2 & 0.23 & 0.26 \\
\hline DXP.HEtuntrinaf & 1572.1 & 1577.1 & 1741.1 & 1727.9 & 1671.1 & 1848.1 & 2197.4 & 2047.9 & 1870.7 & 2096 \\
\hline \multicolumn{11}{|l|}{ COMPUTEDH } \\
\hline Jallouk & र213.1 & 1213.2 & 7159.6 & 1746.4 & T178.2 & 770.41 & 7005.2 & 7084.41 & 1008.11 & 244.5 \\
\hline Szadyl(Cudo wbo) & 1849.5 & 1850.9 & 1854 & -9853 & 1848.4! & 1857.1 & 1858.6 & 1875.8 & 1874.1 & 244.6 \\
\hline Szady2 & 1647.5 & 1645.4 & 1845.11 & T644.7 & 1636.21 & 1645 & 7658.6 & 1658.1 & 1719.2 & 24.5 \\
\hline (RिO) & 1788211 & 179734 & 175713 & 169474 & 1640041 & 158888 & 1535711 & 148066 & 142559ी & 142591 \\
\hline$(\boldsymbol{F})$ & 1.004 & 1.004 & 1.4311 & 1.667 & 1.845 & 1.991 & 2.121 & 2.238 & 2.345 & 2345 \\
\hline (1-थO) & 251.9 & 252 & 358.2 & 418.4 & 45 & 499.8 & 532.6 & 562 & 588.9 & 588.8 \\
\hline \multirow[t]{3}{*}{ (HBOIL) } & 1847.9 & 1849.3 & 1849.5 & 1845.9 & 1838.7 & 1844.8 & 1854.1 & 1858.8 & 1854.5 & 1854.5 \\
\hline & & & & & & & & & & \\
\hline & ZONE2 & ZONE4 & ZONE6 & ZONE8 & ZONE10 & & & & & \\
\hline DPliquid Temp.F & 90.5 & & & & & & & & & \\
\hline \multicolumn{11}{|l|}{ DPLiquid Density 1 bm/13 } \\
\hline Elevation-h & & 41 & 6 & 8 & 9.875 & & & & & \\
\hline MeasuredDPPsid & 0.30 & 0.898 & 1.3 & T.924 & 2511 & & & & & \\
\hline Zero Corrections-psid & 0.133 & 0.101 & 0.130 & 0.056 & 0.085 & & & & & \\
\hline Corrected DPPsid & 0.2081 & 0.797 & 1.187 & 1.888 & 24241 & & & & & \\
\hline Actual DPA & 2.001 & 3.48 & 5.211 & 8.28 & 7.56 & & & & & \\
\hline Moesured TPPsig & 187.8 & & & & 300.11 & & & & & \\
\hline \multicolumn{11}{|l|}{ Measured Overalth } \\
\hline & & & & & & & & & & \\
\hline Computed DPA & 2.1 & 3.95 & 5.631 & 7.24 & 8.72 & & & & & \\
\hline Friction-1 & 0.11 & 0.29 & 0.53 & 0.83 & 1.14 & & & & & \\
\hline Accoloration-th & 0.05 & 0.75 & 0.22 & 0.29 & 0.36 & & & & & \\
\hline Elevation-t & 7.93 & 3.51 & 4.88 & 6.13 & 7.22 & & & & & \\
\hline & & & & & & & & & & \\
\hline
\end{tabular}




\begin{tabular}{|c|c|c|c|c|c|c|c|c|c|c|}
\hline run\# & 6741 & & & & & & & & & \\
\hline Tost Fluid & CAF10 & & $i$ & $\vdots$ & I & I & : & $!$ & & \\
\hline Date: & $7 / \sqrt{2931}$ & Tा & 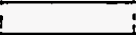 & $i$ & & & $\div$ & & & \\
\hline Time: & $11: 191$ & & & $i$ & $i$ & $i$ & & & & \\
\hline Note & Analytical c & dtis used & & & & 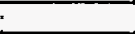 & & & & \\
\hline Flow Areatil & 0.003361 & & & 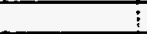 & & & & & & \\
\hline Heat Fow Area-fl2 & 0.19695 & & & & & & ; & & & \\
\hline Sal. Prossuro Psig & 782.31 & & & 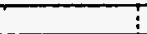 & & & & 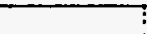 & & \\
\hline Sal. Temperature F(inlet) & 788.61 & & $\square$ & ! & & & & & & \\
\hline 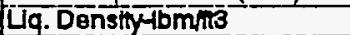 & $\overline{73.921}$ & 73.791 & 73.081 & 73.71 & 73.851 & 73.6 & 73.571 & 73.55 & 73.521 & 73.73 \\
\hline Latent Hoar-Btulbm & 23.551 & 23.44 & 23.381 & 29.38 & 23.341 & 23.3 & 23.281 & 23.28 & 23.241 & 23.4 \\
\hline Specific Hot-Bunbmit & 0.3001 & 0.3091 & 0.31 & 0.311 & 0.311 & 0.31 & 0.311 & 0.31 & 0.3111 & 0.31 \\
\hline Liq. Visoosity bin/ithr & 0.3081 & 0.306 & 0.3006 & 0.305 & 0.3051 & 0.304 & 0.3041 & 0.3031 & 0.3031 & 0.306 \\
\hline Lig. ConductivityBtuhr-fFF & 0.0201 & 0.02 & 0.021 & 0.02 & 0.021 & 0.0199 & 0.0199 & 0.0199 & 0.01991 & 0.02 \\
\hline Lig. Prand No. & 4.731 & 4.73 & 4.731 & 4.73 & 4.731 & 4.73 & 4.741 & 4.741 & 4.74 & 4.78 \\
\hline Inlet Flow Rate-GPM & 3.8 & & 1 & 1 & 1 & 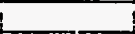 & 1 & + & & 1 \\
\hline Outiel Lig. Flow Rato-GPM & 9.41 & & 1 & 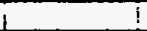 & : & 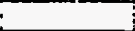 & & 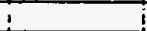 & $i$ & 1 \\
\hline Outlet Vap. Flow Rato-GPM & 1.91 & & & 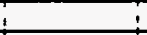 & 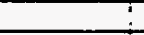 & 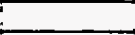 & & & & \\
\hline Inlat Temperaturef & 184.61 & T & 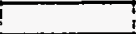 & $i$ & $i$ & 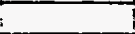 & 1 & $?$ & & \\
\hline Outlet Liq. Temperaturof & 787.91 & & & & & & & & & \\
\hline Outlet Vap. Temperaturef & 783.61 & & & & 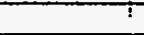 & : & $i$ & & & $T$ \\
\hline Water Flow Rato-GPM & ? & 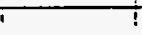 & & $\mathrm{T}$ & $\because$ & : & & $i$ & & $i$ \\
\hline Water hlot Temp.F & 907.71 & $i$ & 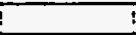 & 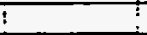 & : & 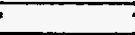 & & '. & & i \\
\hline Water outlet Temp.f & 166.31 & & I & ! & $\cdot$ & 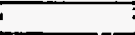 & & 1 & & i \\
\hline Inlot Glomint/12 & 6718951 & & Tomp & 182.41 & Density & $74 . \overline{01}$ & & & & \\
\hline Subcoolingf & 41 & L & & 1 & 1 & 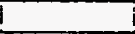 & i & & & i \\
\hline \multirow[t]{2}{*}{ Flow Observation } & & & & 1 & 1 & 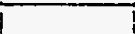 & & & & \\
\hline & ZONE1 & ZONE2 & ZONE3 & ZONE4 & ZONE5 IZ & ZONE6 & ZONE7 & ZONE8 & ZONE9 & IZONETO \\
\hline Hoat Input-Watts(net) & 578.5 & 580.4 & 579 & 582.7 & 574.61 & $5 \pi . \overline{2}$ & 586.7 & 581.3 & 577.3 & 579.8 \\
\hline Heat Loss-Watts & 17.6 & 4.2 & 5.7 & 6.5 & 6.91 & 6.1 & 4.41 & 5.6 & 6.3 & 33.8 \\
\hline Heat Flox-8turtrmit & 10028 & 10061 & 100037 & 10101 & 99601 & 10005 & 101701 & 10076 & 10007 & 10050 \\
\hline Wall Temp. \#3-F & 1929 & 194.3 & 193.8 & 193.8 & 1\$.ग & 193.4 & 793.2 & Tभ.ी & 193.9 & 193.7 \\
\hline Wall Temp. FfF & 193.6 & 793.4 & 194.8 & 194.6 & 194.8 & 194.3 & 793.8 & 794.7 & 195.1 & 193.3 \\
\hline Wall Tomp. $15 f$ & 793.7 & 192.8 & 195.6 & 194.4 & 194.3 & 193.4 & 192.6 & 193.3 & 194.2 & 193.4 \\
\hline Wall Tomp. IF & 89.51 & 198.0 & 1927 & 198 & 193.7 & 194.2 & 192.9 & 193 & 199.1 & 189.8 \\
\hline Well Tomp. \#2F & 194.11 & 193.8 & 199.7 & 193.4 & 193.81 & 193.8 & 193.4 & 193.3 & 193.71 & 191.8 \\
\hline Instream Temp.f & 186.5 & 187 & 187.4 & 187.3 & 187.5 & 187.7 & 187.8 & 187.8 & 188 & 187.2 \\
\hline Avg. Wall Temp.F & 193.6 & 193.9 & 193.8 & 193.9 & 194.2 & 193.8 & 193.2 & 193.6 & 194 & 1924 \\
\hline Avg. Wall DT.f & 6.6 & 6.4 & 5.9 & 6.2 & 6.2 & 5.7 & 4.9 & 5.2 & 5.5 & 4.7 \\
\hline Remaining SubcoofF & 1.2 & & & & & & & & & \\
\hline Exit Quality & -0.02 & 0.02 & 0.06 & 0.1 & 0.13 & 0.17 & 0.211 & 0.25 & 0.28 & 0.32 \\
\hline 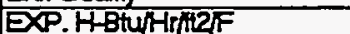 & $1514.4 \mid$ & 1567 & 1702.1 & 1637.5 & 1600.1 & 1763.7 & 2066.91 & 1936.11 & 1803.8 & 2126.7 \\
\hline \multicolumn{11}{|l|}{ COMPUIEDH } \\
\hline Jallouk & Тि19.7| & 1213.8 & T164.3! & 1143.9 & T133.61 & 1120,3 & 1716.5 & 1097.2 & 1085.र & 208 \\
\hline Szady 1 (Cudo tube) & 1809.7 & 1818.3 & 1821.5 & 1827.4 & 1817.7 & 1825 & 1842.2 & 1835.8 & 1831.7 & 200 \\
\hline Szacty2 & 1609.31 & 1617.2 & 1617.2 & 1625.2 & 1608.81 & 1616.6 & 1639 & 1628.2 & 1620.61 & 208 \\
\hline (RLV) & 1427081 & 142394 & 138281 & 132683 & 1275681 & 122454 & 117127 & 111761 & 1064281 & 106423 \\
\hline (F) & 1.004 & 1.131 & 1.560 & 1.797 & 1.985 & 2.144 & 2286 & 2.415 & 2.5331 & 2.533 \\
\hline (1)O & 214 & 241 & 337.17 & 383 & 423.3 & 457.2 & 487.5 & 515.1 & 540.21 & 5402 \\
\hline \multirow[t]{3}{*}{ (HBOIL) } & 1808.7 & 9816.9 & 1817.9 & 9821.7 & 1810 & 1815.4 & 1830.71 & 18222 & 1815.91 & 1815.9 \\
\hline & & & & & & & & & & 1 \\
\hline & ZONE2 & ZONE4 & ZONE6 & ZONE8 & ZONE10 & & & & & \\
\hline DP liquid Temp.F & 90.8 & & & & & & & & & \\
\hline DP Liquid Densitylbmitis & 91.39 & & & & & & & & & \\
\hline Gevation-A & & 4 & 6 & 8 & 9.875 & & & & & \\
\hline Messured DPPsid & 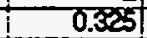 & 7.005 & 7.497 & 2.205 & 2.847 & & & & & \\
\hline Zero Corrections-psid & 0.133 & 0.1011 & 0.139 & 0.035 & 0.088 & & & & & \\
\hline CorTected DPPSId & 0.192 & 0.904 & 1.358 & 2.169 & 2.7611 & & & 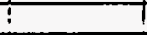 & & \\
\hline Actual DPA & 2.11 & 3.18 & 4.78 & 5.68 & 6.851 & & & & & \\
\hline Measured TPPsig & 1823 & & & t & 300.11 & & & & & \\
\hline \multirow[t]{2}{*}{ Messured Overal ht } & exton & & & i & 1 & $\Gamma$ & & & & \\
\hline & & & & & & & & & & \\
\hline Computad DPA & 2.02 & 3.71 & 5.25 & 6.71 & 8.04 & & & & & \\
\hline Frictiont & 0.08 & 0.22 & 0.41 & 0.64 & 0.89 & & & & & \\
\hline Accoleration-t & 0.05 & 0.12 & 0.17 & 0.23 & 0.3 & & & & & \\
\hline Elevation-fi & 1.88 & 3.37 & 4.67 & 5.84 & 6.85 & & & & & \\
\hline & & & & & & & & & & \\
\hline
\end{tabular}




\begin{tabular}{|c|c|c|c|c|c|c|c|c|c|c|}
\hline Runt" & 675 & & $:$ & $!$ & - & & & - & & 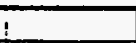 \\
\hline Test Fuid & ¿CAF10 & & & 1 & 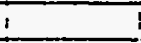 & $!$ & . & & . & $?$ \\
\hline Date: & $17 / 6193$ & & $i$ & $!$ & 8 & 1 & & - & $\bar{i}$ & \\
\hline Time: & $i \quad 11: 11$ & & 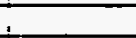 & 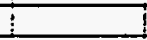 & & $i$ & 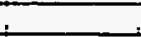 & & $!$ & \\
\hline \begin{tabular}{|l} 
Nolo \\
\end{tabular} & Anaytical & dis used & i & & $\because$ & & $\therefore$ & & $\div$ & \\
\hline Flow Area-th & 0.00336 & & 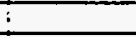 & $\therefore$ & & & & & $\div$ & $i$ \\
\hline Heat Flow Area-12 & 0.19695 & & $:$ & 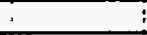 & 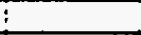 & & t & ? & $T$ & \\
\hline Sat. Pressure psig & 189.41 & & $T$ & $\vdots$ & 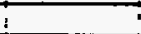 & ' & & & & \\
\hline Sat. Temperature finlet) & 191.51 & & 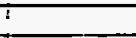 & $T$ & & & & & + & \\
\hline Lg. Density-hm/iz & 73.111 & 72.881 & 72.88 & 72.911 & 72.77 & $72.69 !$ & 72.61 & 72.6 & 72.571 & 72.83 \\
\hline Ladent Hertetulbm & 22.921 & 22.751 & 22.73 & 22.77 & 22.681 & 22.6 & $22.53 !$ & 2253 & 22.511 & 22.7 \\
\hline Specifio Hoat-Btu/bm/F & 0.3121 & 0.313 & 0.313 & 0.313 & 0.314 & 0.3141 & 0.314 & 0.314 & 0.314 & 0.313 \\
\hline Liq. Viscosity & 0.298 & 0.206 & 0.255 & 0.206 & 0.2291 & 0.298 & 0.282 & 0.292 & 0.292 & 0.205 \\
\hline Lig. Conductivity- BtuhrffF & 0.0196 & 0.019 & 0.0185 & 0.0195 & 0.0194 & 0.0194 & 0.0193 & 0.0193 & 0.01831 & 0.0195 \\
\hline Liq. Prandt No. & 4.741 & 4.75 & 4.75 & 4.75 & 4.75 & 4.75 & 4.75 & 4.75 & 4.76 & 4.75 \\
\hline Inlel Fow Rato-GPM & 2.9 & & & $i$ & I & 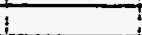 & 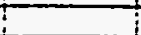 & 1 & $=1$ & $x_{0}$ \\
\hline Outlel La. Flow Rato-GPM & 7.3 & & & 1 & $\perp$ & I & 1 & & i & \\
\hline Outlet Vap. Flow Rato-GPIM & 1.8 & & $t$ & + & 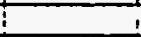 & 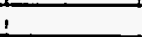 & 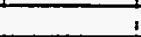 & & 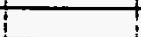 & \\
\hline Inlol Temperaturef & 188.11 & & I & 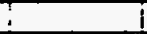 & $i$ & $i$ & 7 & & ! & \\
\hline Outtel Liq. Temperaturef & 191.1 & & T & $T$ & $i$ & ! & & & ;- & \\
\hline Outei Vap. Tomperaturof & 187.5 & & i & $!$ & $T$ & + & $T$ & & & \\
\hline Water Flow Ralo-GPM & 1 & $i$ & 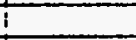 & $i$ & 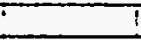 & $!$ & & & I & \\
\hline Wator hlot Tomp.f & 111.41 & & ! & $\overline{1}$ & ! & 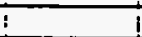 & 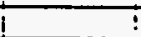 & & 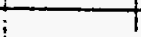 & \\
\hline Water outiot Tomp.F & 164.31 & & $i$ & T & ! & i & i & & 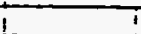 & \\
\hline Inlet Glbmprhthe & 5104591 & & Temp & 183.7 & Donsity & 73.76 & $T$ & & $i$ & \\
\hline Suboooling $F$ & 3.41 & & & 1 & & 1 & 1 & & $i$ & \\
\hline \multirow{2}{*}{ Flow Obsenvation } & & & & & & & & & 1 & \\
\hline & ZONEI & ZONE2 & ZONE3 & ZONE4 & ZONE5 & ZONE6 & ZONE7 & ZONE8 & ZONE9 & ZONE10 \\
\hline Heal Input-Wats(nel) & 576.6 & 579.6 & 577.2 & 576.11 & 576.3 & 578 & 575.4 & 578.5 & 570 & 5728 \\
\hline Heat Loss-Watts & 18.6 & 4.4 & 6 & 7 & 7.6 & 6.9 & 5.1 & 6.3 & 6.7 & 40.1 \\
\hline Hoat Flox Etu/HPII & 99951 & 10047 & 10005 & 9986 & 99901 & 9930 & 9974 & 10028 & 9881 & 9920 \\
\hline Wall Tomp. H & 195.5 & TS6.8 & 196.5 & 196.5 & $\$ 8.11$ & 196.31 & 196.4 & 1972 & 156.8 & 2053 \\
\hline Wall Tomp. $14 F$ & $\overline{196.3}$ & 196.2 & 197.4 & 797.1 & 197.8 & 197.2 & 195.8 & 197.5 & 197.8 & 205.9 \\
\hline Wall Tomp. $15 F$ & 196.31 & 195.4 & 198.3 & 196.9 & 197 & 196.3 & 195.51 & 196.5 & 196.6 & 197.1 \\
\hline Wall Tomp. $11 F$ & 91.61 & 198.4 & 195.5 & 195.7 & 196.5 & 197 & 195.9 & 198.6 & 195.7 & 198.1 \\
\hline Wall Tomp. WLF & 196.71 & 196.5 & 196.1 & 196.2 & 996.61 & 196.9 & 196.4 & 196.4 & 196.8 & 195.3 \\
\hline Introem Tomp.f & 189.6 & 100.3 & 190.4 & 190.2 & 190.7 & 1911 & 791.3 & 191.3 & 191.41 & 180.5 \\
\hline Av . Wall Tamp.f & 196.2 & 196.5 & 196.4 & 156.6 & 197 & 196.7 & 196.2 & 196.8 & 196.6 & 199.5 \\
\hline Avg. Wall DT.F & 6.2 & 5.7 & 5.5 & 5.9 & 5.81 & 5.3 & 4.4 & 5.1 & 4.8 & 8.6 \\
\hline Romaining Subcooff & -0.2 & & & & & & & & & \\
\hline Exot Quality & 0 & 0.05 & 0.1 & 0.15 & 0.2 & 0.20 & 0.31 & 0.36 & 0.41 & 0.46 \\
\hline EXP.HBtun & 1599.6 & 1756.2 & 1811.6 & 1633 & 1715.4 & 1891.5 & 2241.81 & 1971.4 & 2061.3 & 1155.7 \\
\hline \multicolumn{11}{|l|}{ COMPUTEDH } \\
\hline Jallouk & 1284 & 1246.31 & 12122 & 1188.3 & 1176.2 & 1160.8 & 1174.2 & 166.9 & 166.91 & 165.9 \\
\hline Szady1(audo tube) & 1838.61 & 1852.4 & 1850.2 & 1847 & 1853.3 & 1851.8 & 1859.6 & 165.9| & 165.9 & 166.9 \\
\hline szacty2 & 1625.8 & 1699.3 & 1697.2 & $1 \% 27$ & 1701.21 & 1700.5 & 1709.81 & 166.9 & 165.91 & 106.9 \\
\hline (सएव) & 1118001 & 109769 & 104180 & 98207 & $\$ 90911$ & 87871 & 821981 & 82198 & 82198 & 82190 \\
\hline (F) & 1.082 & 1.44 & 1.779 & 2.021 & 2.213! & 2379 & 2.5271 & 2.527 & 2.527 & 2.527 \\
\hline (HUQ) & 185.41 & 246.8 & 305.1 & 346.7 & 379.7 & 408.21 & 433.61 & 433.6 & 438.61 & 43.6 \\
\hline \multirow[t]{3}{*}{ (HBOLIL) } & 1837.91 & 1850.9 & 1847.5 & 18429 & 1848 & 1845.2 & 1851.7 & 1851.71 & 1851.7 & 1851.7 \\
\hline & & & & & & & & & & \\
\hline & ZONE2 & ZONE4 & ZONE6 & ZONE8 & ZONETO & & & & & \\
\hline DPliquid Temp.F & 93.4 & & & & & & & & & \\
\hline DPLquid Density-lomint3 & 90.99 & & & & & & & & & \\
\hline Elevation- & & 4 & 6 & 8 & 9.875 & & & & & \\
\hline Measured DPPsid & 0.357 & 7.156 & 1.748 & 25386 & 3.269 & & & & & \\
\hline Zero Comections-psid & 0.133 & 0.707 & 0.139 & 0.036 & 0.085 & & & & & \\
\hline Corrected DPPsid & 0.218 & 1.055 & 1.609 & 2.502 & 3.2003 & & & & & \\
\hline Actual DPA & 2.071 & 2911 & 4.32 & 5.05 & 6.011 & & i & & & \\
\hline Mecoured TPPSig & 189.4 & & & $\mathrm{i}$ & 300.11 & & & & & \\
\hline \multirow[t]{2}{*}{ Mesaured Overalf } & Exton 1 & & & i & & & & & & \\
\hline & & & & & & & & & & \\
\hline Computed DPA & 1.89 & 3.38 & 4.71 & 5.94 & 7.031 & & & & & \\
\hline Friction-At & 0.06 & 0.16 & 0.3 & 0.47 & 0.66 & & & & & \\
\hline Accoleration- & 0.05 & 0.09 & 0.13 & 0.18 & 0.24 & & & & & \\
\hline Elevation-t & 1.79 & 3.13 & 4.27 & 5.28 & 6.13 & & & & & \\
\hline & & & & & & & & & & \\
\hline
\end{tabular}




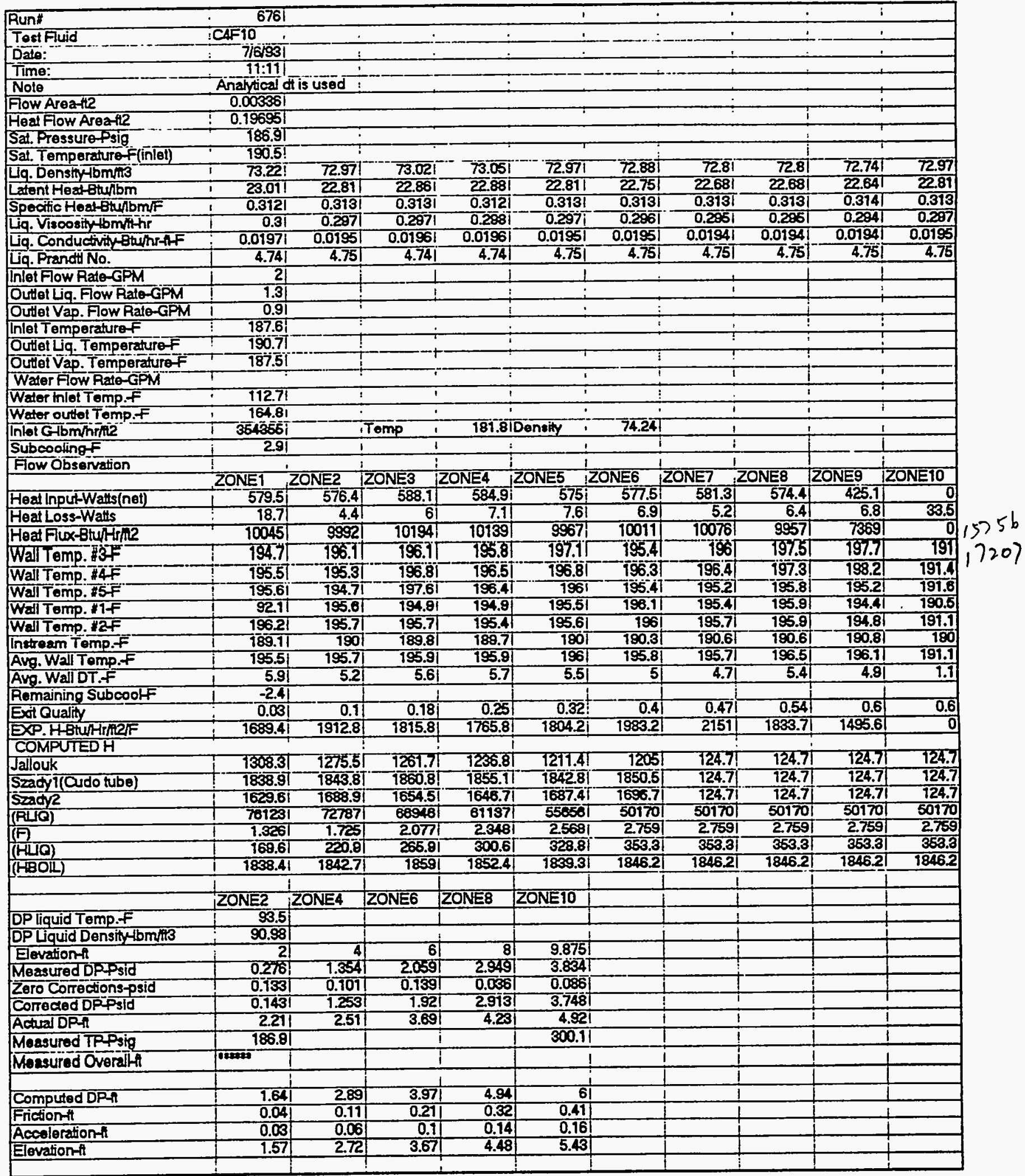




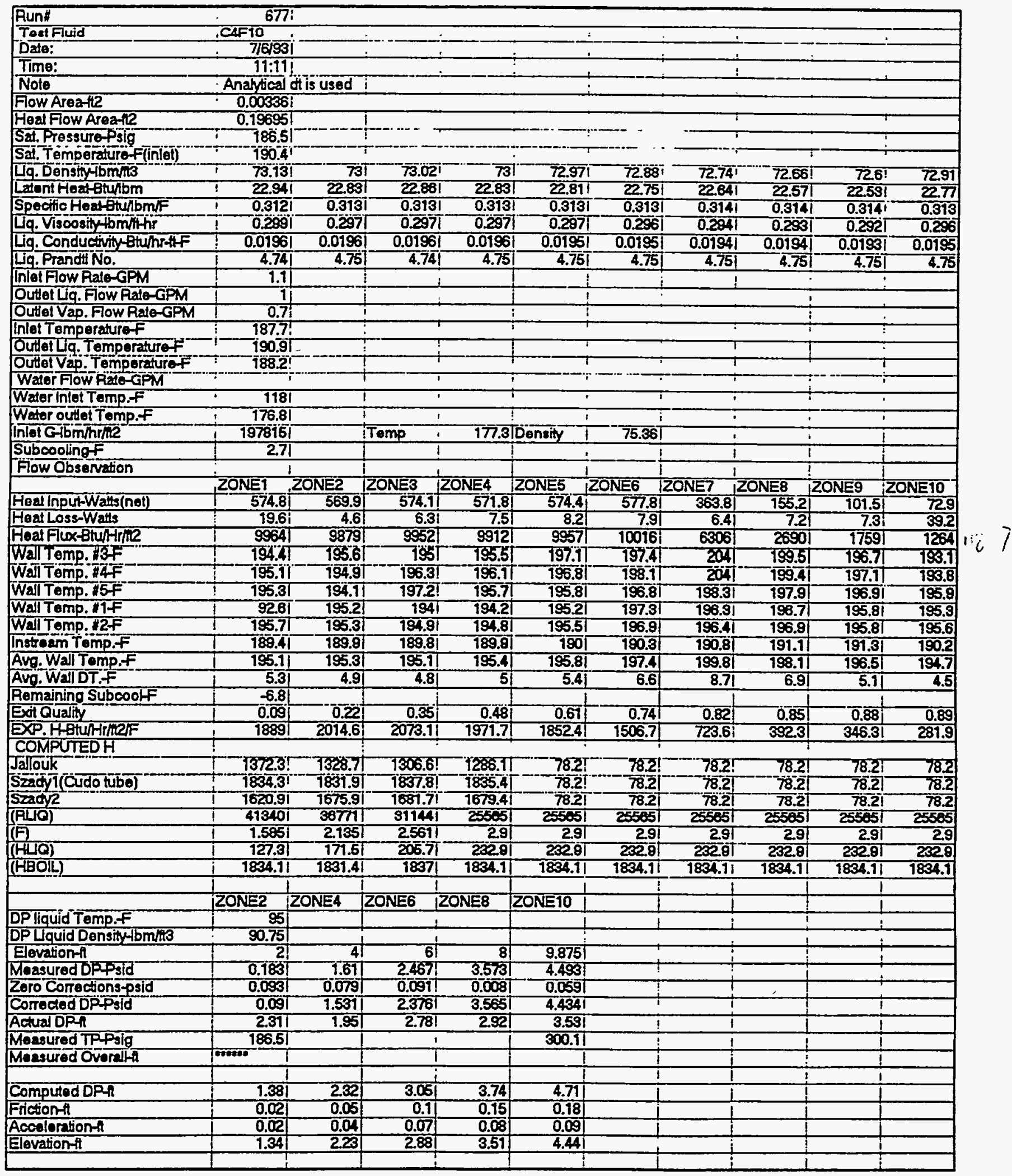




\begin{tabular}{|c|c|c|c|c|c|c|c|c|c|c|}
\hline Run: & 6781 & & : & L. & & & 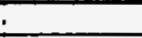 & & & - \\
\hline Tost Fluid & .C4F10 I & & 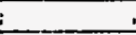 & $i$ & 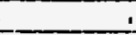 & $\therefore$ & & & & \\
\hline Date: & $7.7 / 7 / 931$ & & & & & & & & & \\
\hline Time: & $8: 40$ & & 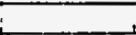 & & & & & & & \\
\hline Note & Analytical & dt is used & 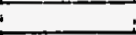 & & & & & & & \\
\hline Fow Areatt2 & 0.003361 & & 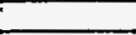 & & & & & & & \\
\hline Heat Flow Area-ti2 & 0.19695 & & : & 1 & & & & & & \\
\hline Sat Prossure Psig & $794.7 !$ & & & & & & T & & & \\
\hline Sat. Temperature & 193.71 & & & & & & & & & \\
\hline पq. Densinglbmilis & 72.941 & 72.7 & $72.52 !$ & 72.41 & 72.341 & 72.321 & 72.141 & 72.11 & 72.08 & 72.49 \\
\hline Latent Headetulbm & 22.791 & 2266 & 22.471 & 22.381 & 22.331 & 22.311 & 22.181 & $22.15 !$ & 22.13 & 22.44 \\
\hline Spectic Heat-Btulbm/F & 0.3131 & 0.314 & 0.3151 & 0.315 & 0.3151 & 0.3151 & 0.3161 & 0.3161 & 0.317 & 0.315 \\
\hline Lig. Viscosidylominthr & 0.2961 & 0.294 & 0.291 & 0.281 & 0.2891 & 0.289 & $0.287 \pi$ & $0.287 i$ & 0.286 & 0.291 \\
\hline Lig. Conductivity Btuhr-thF & 0.0195 & 0.0194 & 0.0193 & 0.01921 & 0.0192 & 0.01921 & 0.0191 & $0.019 !$ & 0.019 & 0.0193 \\
\hline Lig. Pranditi No. & 4.751 & 4.75 & 4.76 & 4.76 & 4.761 & 4.76 & 4.77 & 4.77 & $4 . \overline{7}$ & 4.76 \\
\hline Inlot Flow Ralo-GPM & 5.7 & & $-i$ & 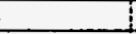 & & $i$ & & & & $i$ \\
\hline Outet Lg. Flow Rato-GPM & $7.4 i$ & & 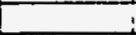 & 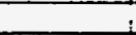 & & & & & & 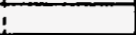 \\
\hline Outlet Vap. Fow Rate-GPM & $2.7 i$ & & + & $-2+$ & & & & $i$ & & \\
\hline Inlet Temperaturof & 188.3 & & 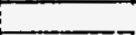 & 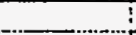 & & & & i & & \\
\hline Outot Lig- Temporaturer & 191.81 & & 7 & & & & & & & \\
\hline Outel Vap. Temperaturer & $788.7 !$ & ! & i & & & & & & & \\
\hline Water Fow Pato-GPM & & & & & & & & ! & $!$ & \\
\hline Water hilet Tomp.F & 109.7 & T & 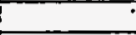 & & & 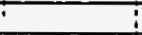 & & 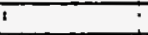 & & \\
\hline Water outlol Temp.f & 162.31 & I & 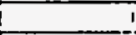 & 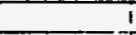 & & $\therefore$ & & $\cdot$ & T & i \\
\hline Intet Gibminrin2 & 8915381 & & Tomp & 187 & Densizy & 72.891 & & 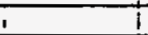 & 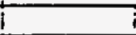 & $i$ \\
\hline SubooolingF & 5.4 & & 5 & 1 & & 1 & & $I$ & & $i$ \\
\hline Fow Observation & $I$ & & 1 & 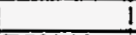 & & & & 1 & & $i$ \\
\hline & ZONE1 & ZONE2 & ZONE3 & ZONE4 & ZONE5 & IZONE6 & ZONE? & ZONE8 & ZONE9 & ZONE10 \\
\hline Head Input-Watts(nel) & 875.2 & 874.1 & 861.8 & 868.1 & 871.1 & 873.4 & 868.6 & 420.3 & 94.3 & 100.4 \\
\hline Heal Loss-Watts & 19.4 & 4.6 & 6.2 & 7.3 & 81 & 7.5 & 5.71 & 6.31 & 6.4 & 35.3 \\
\hline Hodf Flux-Btulirme & 15171 & 15152 & 14939 & 15048 & 151001 & 15140 & 150571 & 72861 & 1635 & 1896 \\
\hline Wall Temp. \#3F" & 198.8 & 201.1 & 190 & 199.11 & 201.51 & 199.2 & 199.91 & 197.51 & 194.3 & 193.4 \\
\hline WalT Temp. A4 & $\mathbf{1 9 9 . 3}$ & 198.9 & 200.31 & 199.9 & 200.4 & 200.11 & 200.31 & 197.2 & 794.7 & 194.2 \\
\hline Wall Tamp. $15 F$ & 199.3 & 198 & 200.11 & 199.11 & 199.21 & 199.71 & 199.21 & 197 & 195 & 194.1 \\
\hline Wall Temp. IF & 89.21 & 199.7 & 1981 & 198 & 198.7 & 200.4 & 200.31 & 196.91 & 194 & 192.4 \\
\hline Wall Temp. $\quad 2 F$ & 199.91 & 199.7 & 1991 & 198.6 & 199.1 & 200,41 & 200.71 & 1971 & 1941 & 193.1 \\
\hline Instream Tomp.f & 190.11 & 190.7 & 191.61 & 192 & 192.21 & 1923 & 192.8 & 193 & 193.11 & 191.7 \\
\hline Avg. Wall Tomp.f & 199.31 & 199.9 & 199.11 & 199 & 199.4 & 199.9 & 200.11 & 197.1 & 994.4 & 193.4 \\
\hline Avg. Wall DT F & 8.51 & 8.5 & 6.81 & 6.31 & 6.5 & 6.9 & 6.51 & 3.8 & 1.2 & 1.7 \\
\hline Romaining Subcooff & 25 & & & & & & & $\longrightarrow$ & & \\
\hline Exat Quality & -0.03 & 0 & 0.04 & $0.08 !$ & 0.12 & 0.16 & 0.2 & 0.22 & 0.23 & 0.23 \\
\hline EXP.HBtuhHrm2h & 1776.6 & 1789.9 & 2196.9 & 2371.6 & 2334.7 & 2205.1 & $2316.6 i$ & 1922 & 1333.2 & 1146.3 \\
\hline COMPUIEDH & & & & & & & & & & \\
\hline Jallouk & 7517.41 & $7520.8 !$ & 74371 & 74091 & 1369.31 & उ83.9ं। & 283.81 & 283.81 & 283.8 & 283.9 \\
\hline Szady (Cudo hube) & 2233.6 & 2300.5 & 2299 & 2314.7 & 2323.3 & 283.9 & 283.81 & 283.81 & 283.8 & 283.9 \\
\hline Szady2 & 2774.81 & 2184.5 & 2184.71 & 2204.3 & 2215ा & 283.9 & 283.81 & 283.81 & 283.8 & 283.9 \\
\hline (RLO) & 2189311 & 200989 & 2172281 & 209419 & 2009981 & 200988 & 2009881 & 200988 & 200988 & 200988 \\
\hline (F) & 1.0041 & 1.004 & 1.3981 & 1.682 & 1.889 & 1.889 & 1.8891 & 1.889 & 1.889 & 1.889 \\
\hline (स्यव) & 232.81 & 233 & 408.31 & 491.3 & 551.81 & 551.81 & 561.81 & 557.81 & 559.8 & 651.8 \\
\hline (HBOLL) & 222921 & 2298.9 & 2294.7 & 2307.3 & 23129 & 2312.9 & 23129 & 23129 & 23129 & 2312.9 \\
\hline & 1 & & & & & 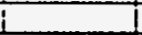 & & -1 & & i. \\
\hline & iZONE2 & ZONE4 & ZONE6 6 & ZONE8 & ZONE10 & & & I & & L \\
\hline DP liquid Temp.F & i 92.3 & & & & & & & 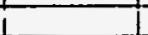 & & 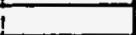 \\
\hline DP Liquid Density $1 \mathrm{bm} / \mathrm{it3}$ & 91.16: & & & & & & & 1 & $i$ & 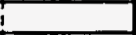 \\
\hline Elevation- & $2 !$ & 4 & 6 & 81 & 9.875 & & & & & \\
\hline Measured DPFsid & 0.319 & 0.794 & 1.148 & 1.71 & 2.345 & & & & & ! \\
\hline Zero Corrections-psid & 0.122 & 0.089 & 0.139 & 0.001 & -0.0441 & & & t & & \\
\hline Corrected oppsid & 0.197 & 0.705 & 1.009 & 1.75 & 2.3891 & & & I & & $t$ \\
\hline Actured OPA & 2.12 & 3.68 & 5.54 & 6.58 & 7.871 & & & $T$ & & \\
\hline Moasured IPP & 194.71 & & 1 & & 300.11 & & & $\mathrm{i}$ & & i \\
\hline Mesutred Overalls & pente 1 & & & & & & & I & & 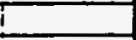 \\
\hline & & & 1 & & & & & i & & 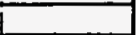 \\
\hline Computed DPA & 215 & 4.18 & 61 & 7.85 & 9.59 & & & 1 & & $L$ \\
\hline Friction-t & 0.15 & 0.4 & 0.761 & 1.13 & 1.44 & & & 1 & & $i$ \\
\hline Accoleration- $\mathrm{A}$ & 0.04 & 0.22 & 0.34 & 0.44 & 0.45 & & & & & \\
\hline Elovation-A & 1.961 & 3.55 & 4.89 & 6.28 & 7.7 & & & $i$ & & 1 \\
\hline & & & & & & & & & & \\
\hline
\end{tabular}




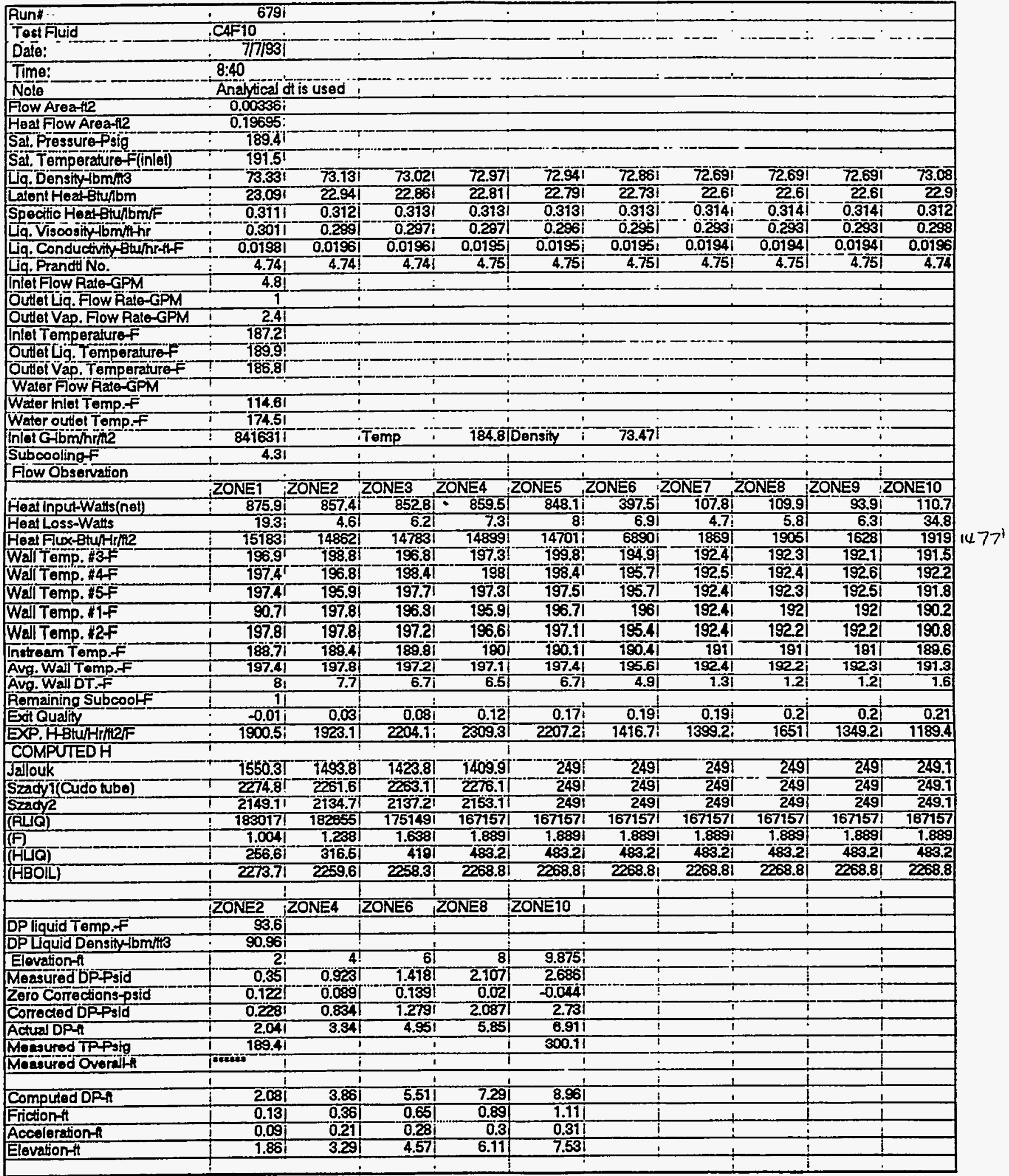




\begin{tabular}{|c|c|c|c|c|c|c|c|c|c|c|}
\hline Runt & 680. & & & & & & & & & - \\
\hline Tost Fluid & CAF10. & & & 1 & & & & & & \\
\hline Date: & $7 \pi / 1931$ & & & & & & & & & \\
\hline Time: & $8: 40$ & & & & & & & & & \\
\hline Note & Anahicalc & dit is used & & & & & & & & \\
\hline Flow Area-t2 & $0.00336 i$ & & & & & & & & & \\
\hline Heat Flow Area-fle & 0.19595 & & & & & & & & & \\
\hline Sa. Pressure Psig & $191.8^{\top}$ & & & & & & & & & \\
\hline Sat. Temperature & $192.5 !$ & & & & & & & & & \\
\hline Liq. Density $/ b m / 113$ & 73.16 & 72.86 & 72.66 & 72.691 & 72.61 & 72.547 & 72.431 & 72.41 & 72.371 & $72.7 \overline{4}$ \\
\hline Ladent Hoat-Btutibm & 22.961 & 22.73 & 22.57 & 22.61 & 22.531 & 22.491 & 22.41 & 22.38 & 22351 & 22.64 \\
\hline Specific Hodtuinbmit & 0.3121 & 0.313 & $0.314 !$ & 0.3141 & 0.3141 & 0.3151 & $0.315 !$ & 0.315 & 0.3151 & 0.314 \\
\hline Lq. Visoositylbmilihr & 0.2391 & 0.205 & 0.2031 & 0.2031 & $0.202 i$ & 0.2021 & $0.29 j$ & 0.29 & 0.281 & 0.204 \\
\hline Lig. Conductivitytuhr-fif & $0.0197 !$ & 0.019 & 0.0194 & 0.01941 & 0.0193 & 0.01931 & 0.0192 & 0.0192 & 0.0192 & 0.0194 \\
\hline Lig. Prandt No. & 4.74 & 4.75 & 4.75 & 4.75 & 4.75 & 4.761 & 4.76 & 4.76 & 4.76 & 4.75 \\
\hline Inlet Fow Rato-GPM & 3.8 & & $t$ & $i$ & & & & & : & ; \\
\hline Outet Lq. Fow Rato-GPM & 11 & & j & . & : & 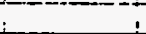 & 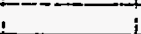 & & + & + \\
\hline Outhet Vap. Fow Rato-GPM & 2 & & $\therefore$ & i & & & & & 之 & \\
\hline Inlet Temperaturef & $187.9 !$ & & i & & 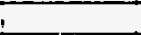 & & & & i & \\
\hline Out et Liq. Temperaturef & 191.1! & & ! & 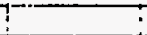 & & & & & & \\
\hline Outet Vap. Temperature & 987.9 ! & & $?$ & & & & & & & \\
\hline Water Fiow Rate-GPM & $T$ & & $t$ & & & & & & & \\
\hline Water inlot Tomp.F & 117.2 & & 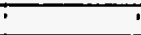 & 'T & i & & ' & & $i$ & $\mathrm{i}$ \\
\hline Water outlot Tomp.F & $176.2 !$ & & t & i & 1 & & . & & T & , \\
\hline Inlot Gibminint & 6653431 & & TTemp & 185.21 & Density & 73.371 & 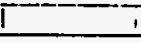 & & $\bar{i}$ & 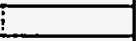 \\
\hline Subcooling f & 4.61 & & & t & & . & 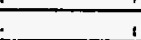 & & $\frac{1}{1}$ & 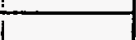 \\
\hline Fow Observation & & & & & & & & & & tat \\
\hline & ZONE1 & ZONE2 & ZONE3 & ZONE4 & ZONE5 & ZONE6 & ZONET & ZONE8 & ZONE9 & ZONE10 \\
\hline Heat Input-Walts(net) & 877.1 & 877.4 & 883 & 885.9 & 482.1 & 2021 & 106.8 & 97.7 & 95.5 & 110.9 \\
\hline Heat Loss-Watts & 19.1 & 4.5 & 6.1 & 7.2 & 7.81 & 6.5 & 4.6 & 5.7 & 6.2 & 34.7 \\
\hline Heat Flu-Btu/Hith2 & $15204 i$ & 15209 & 15305 & 15357 & 8357 & 3502 & 18511 & 1694 & 1655 & 1922 \\
\hline Wal tamp. H3F & $197.9 !$ & 205 & 198.11 & 1991 & $200.7 !$ & 194.41 & $1 \$ 3.3$ & 193.2 & 193.2 & 9826 \\
\hline Wall Temp. $4 \mathrm{~F}$ & $798.4 !$ & 198 & $799.7 !$ & 199.97 & 199.5 & 995.2 & 193.51 & 193.5 & 193.7 & 193.3 \\
\hline Wall Temp. & $198.5 !$ & 197.1 & 199.31 & 198.6 & 190.4 & 194.9 & 193.51 & 193.4 & 193.8 & 193 \\
\hline Wall Tomp. IF & 91.11 & 198.9 & 197.91 & 197.11 & 197.41 & 195.8 & 193.21 & 192.8 & 1991 & 191.6 \\
\hline Wath Tomp. \$2F & 1991 & 198.8 & 198.31 & 197.0 & 1971 & 1951 & 193.31 & 193.11 & 190.11 & 192.1 \\
\hline Intream Temp. $F$ & 189.31 & 190.4 & 191.11 & 181 & 181.31 & 191.61 & 191.81 & 1021 & 182.1 & 100.8 \\
\hline Avg. Wall Tomp.F & 198.51 & 199 & 198.41 & 198.7 & 198.31 & $195.1 \mathrm{i}$ & 193.41 & 193.2 & 193.41 & 192.5 \\
\hline Ávg. Wall DT.F & 8.51 & 7.9 & 6.61 & 7 & 6.61 & 3.41 & 1.4 & 1.1 & 1.2 & 1.6 \\
\hline Remaining Subcooff & 0.3 & & & & & & & & & \\
\hline Exot Quality & 0 & 0.05 & 0.11 & 0.17 & 0.21 & 0.221 & 0.23 & $0.23 !$ & 0.24 & 0.25 \\
\hline EXP. HBtuHriter & 1796.5 & 1934.4 & 2333.9 & 2207.7 & 1257.2 & 1024.8 & 1345.1 & 1507.5 & 1396.81 & 1191.7 \\
\hline COMPUTEDH & & & & & & & & & & \\
\hline Jallouk & $1613.5 \mathrm{~T}$ & 1557.8 & 7512.9 & 205.31 & 206.37 & 206.3 & 206.31 & 206.3 & 206.31 & 206.3 \\
\hline Szady i(Cudo tubo) & 2284.31 & 2300.6 & 23318.61 & 206.31 & $\overline{206} \overline{\mathbf{3}}$ & 206.3 & 206.3 & 206.31 & 206.31 & 206.3 \\
\hline szadiz & 2161.81 & 2183.9 & $2207.2 !$ & 206.31 & 206.31 & 206.3 & $206.3 \Gamma$ & 200.3 & 206.31 & 206.3 \\
\hline (RUQ) & 1456281 & 143741 & 1381211 & 1381211 & 1381211 & 1381211 & 1381211 & 1381211 & 1361211 & 138121 \\
\hline (F) & 1.0041 & 1.408 & 1.801 & 1.801 & 1.8011 & 1.8011 & 1.8011 & 1.801 & 1.8011 & 1.801 \\
\hline (Hџब) & 212.71 & 298.6 & 381.9 & 381.8 & 381.81 & 387.91 & 381.91 & 381.9 & $381.9 !$ & 381.8 \\
\hline (HBOIL) & 2283.71 & 2298.9 & 2315.1 & 2315.11 & 2315.11 & 2315.11 & 2315.11 & 2315.1 & 2315.1 & 2315.1 \\
\hline & 1 & & & & 1 & 1 & 1 & & 1 & \\
\hline & ZONE2 & ZONE4 & ZONE6 & ZONE8 & ZONE10 & & & & & \\
\hline$\overline{D P}$ liquid Temp. $F$ & 94.1 & & & & & 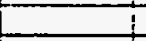 & & & $i$ & \\
\hline DP Liquid Density-lbmits & 90.89 & & & & & $\div$ & & & & \\
\hline Elovation-ft & & 4 & 6 & 8 & 9.8751 & & & & & \\
\hline Measured DPFsid & $0.318 !$ & 1.077 & 1.684 & 2.419 & 3.0571 & & & & & \\
\hline Zero Contrections-psid & $0.122 !$ & 0.089 & 0.139 & 0.02 & $=0.044 T$ & & & & & \\
\hline Comected DPFild & 0.158 & 0.988 & 1.545 & 2.399 & 3.0051 & $i$ & T & & 1 & \\
\hline Actual DPA & 2.111 & 3.04 & 4.44 & 5.25 & 6.211 & $i$ & $!$ & & T & \\
\hline Moesured TPPsig & ! 191.81 & 3 & 1 & i & 300.11 & $T$ & $i$ & & I & \\
\hline Measured Overallth & ind & & I & i & & i & $i$ & & $\div$ & \\
\hline & & & & & & 1 & $i$ & & 1 & \\
\hline Computed DPA & $1.99 !$ & 3.57 & 5.16 & 6.84 & 8.411 & I & 1 & $i$ & $i$ & \\
\hline Frictiont & 0.09 & 0.27 & 0.451 & 0.611 & 0.77 & & 1 & & $i$ & \\
\hline Accoleration- & 0.08 & 0.16 & 0.2 & 0.211 & 0.22 & $t$ & 4 & & $\frac{1}{1}$ & \\
\hline Elevation-At & 9.821 & 3.14 & 4.511 & 6.02 & $7.42 i$ & & $i$ & & 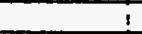 & 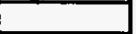 \\
\hline & $i$ & 1 & & & & & & & $i$ & \\
\hline
\end{tabular}




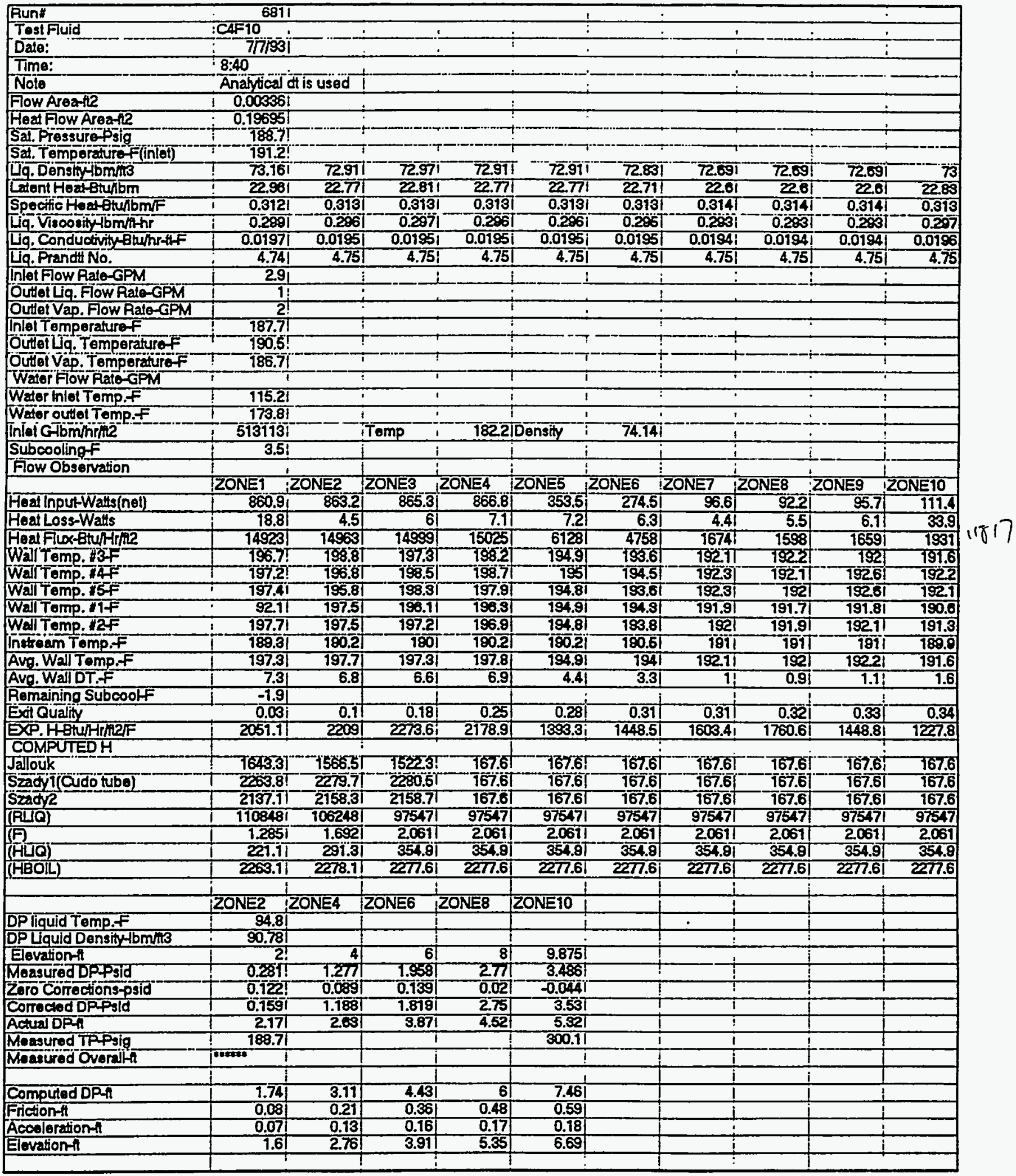




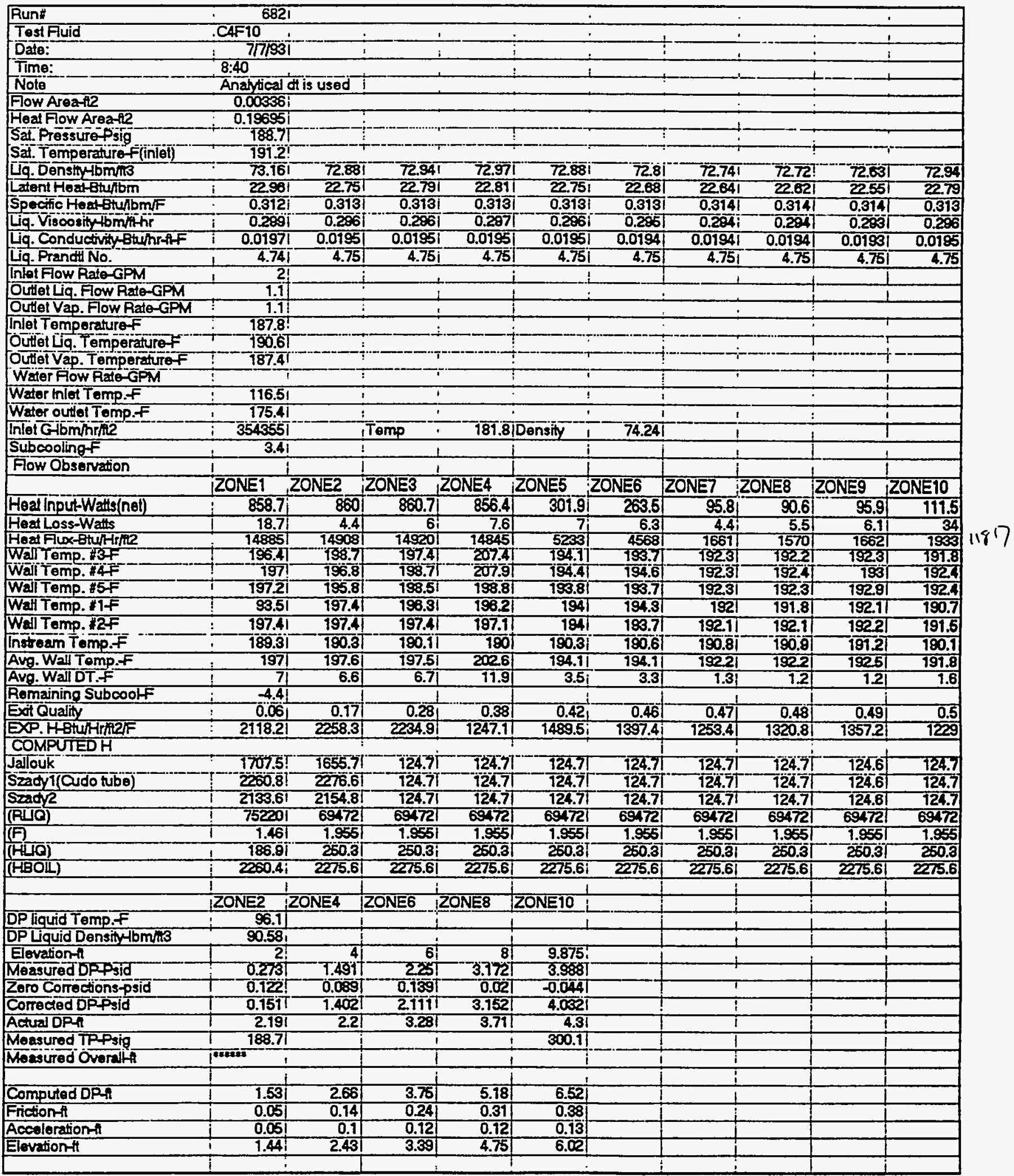




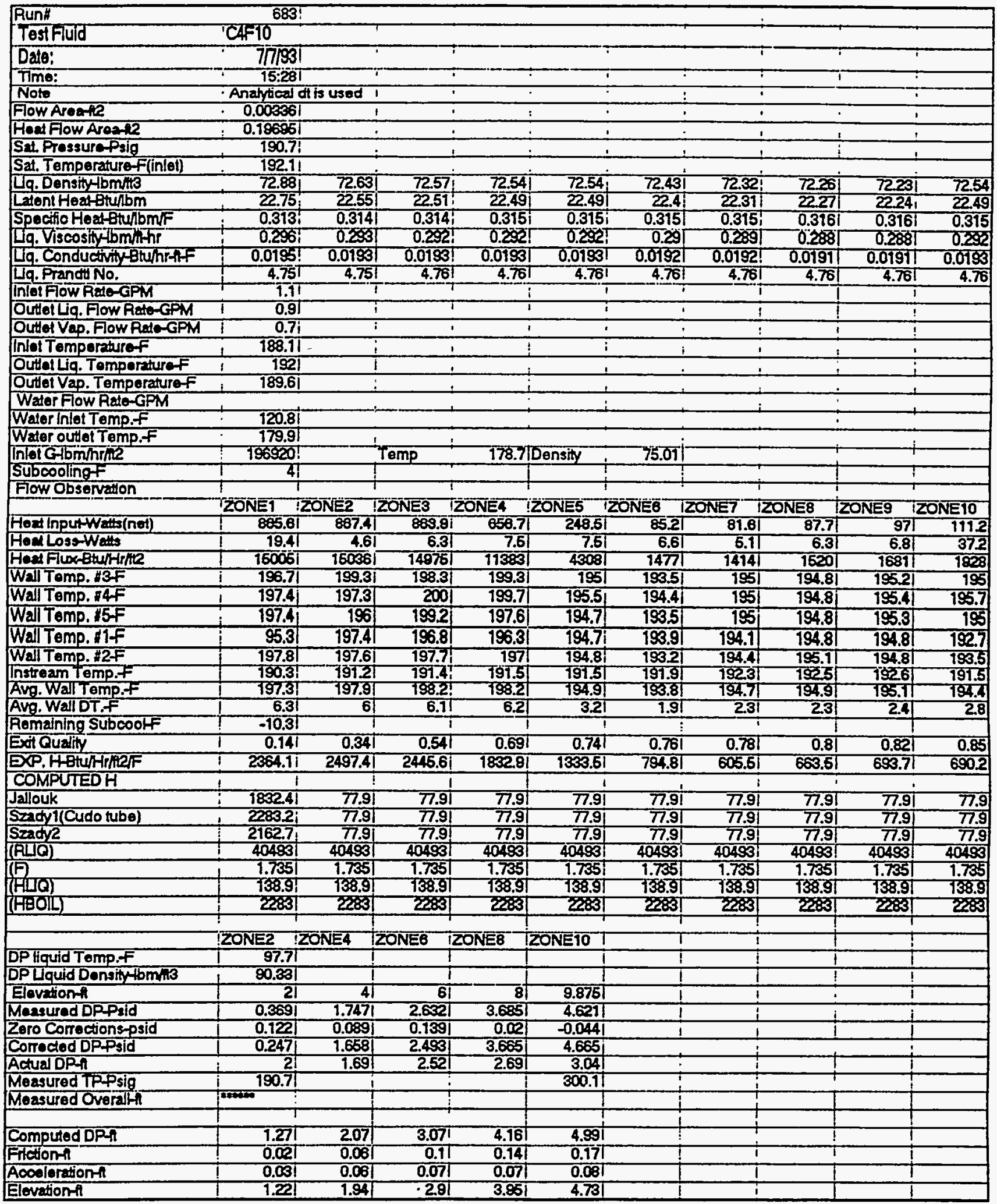




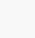




\begin{tabular}{|c|c|c|c|c|c|c|c|c|c|c|}
\hline Run: & 6851 & ; & i & & & & $:$ & : & & \\
\hline Tast Fuid & CAF 8 & & & & & & is & ; & & \\
\hline Data: & $7 / 1933$ & & & & & & $\frac{1}{1}$ & $!$ & & \\
\hline Tima: & $13: 47$ & $\vdots$ & $\vdots$ & & & & + & $\dot{5}$ & : & \\
\hline Fow Areatti2 & 0.009581 & 1 & $\therefore$ & & & 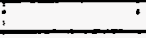 & $i$ & $\div$ & 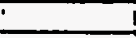 & 1 \\
\hline How Fiow Aroathe & 0.186051 & & $\therefore$ & 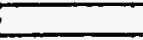 & 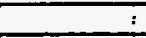 & $\Xi$ & $i$ & $\bar{i}$ & 5 & $I$ \\
\hline Sa.Proseuroforig & 4.2 & 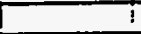 & $i$ & 3 & $i$ & $i$ & . & 1 & $i$ & \\
\hline Sal Tomperaturef(inlot) & 301 & & & & & 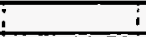 & i & 5 & 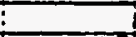 & I \\
\hline Liq. Density 1 bmitis & 93.65 & 93.65 & \$3.55 & 93.581 & $\$ 3.59$ & 93591 & 93.71 & 93.7 & 93.7 & 93.6 \\
\hline Latent Hos Btulbm & 44.881 & 44.881 & 44.88 & 44.83 & 44.84 & 44.84 & 44.92 & 44.92 & 44.92 & 44.85 \\
\hline Specifio Heat etwhbmiF & 0.261 & 0.261 & 0.261 & 0.261 & 0.2611 & 0.261 & 0.261 & 0.261 & 0.26 & 0.261 \\
\hline Liq. Viscosintomihhr & 0.879 & 0.879 & 0.879 & 0.876 & 0.877 & 0.877 & 0.882 & 0.882 & 0.882 & 0.877 \\
\hline Liq. Conductivty BtuhtafF & 0.0378 & 0.03781 & 0.03781 & 0.03781 & 0.03781 & 0.0381 & 0.03791 & 0.03791 & 0.0379 & 0.0378 \\
\hline Liq. Prande No. & 6.05 & 6.05 & 6.051 & 6.04 & 6.041 & 6.04 & 6.061 & 6.061 & 6.051 & 6.05 \\
\hline Inlet Flow Pat-C्CPM & 11 & & & & & 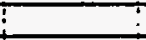 & 1 & 1 & 5 & \\
\hline OUtet Lg. FOW RथG-GPM & OI & & & & & & 1 & I & & \\
\hline Outtot Vap. Flow Rat-GPM & 0.91 & & $\therefore$ & 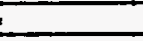 & & $\dot{-}$ & ! & $\vdots$ & $\vdots$ & I \\
\hline Intel Temperaurof & 75.31 & & $\div$ & - & & 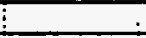 & i & 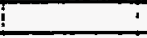 & 5 & \\
\hline Outellig. Tompertiuref & 78.7 & $i$ & $\div$ & 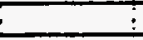 & $i$ & i & $!$ & $i$ & 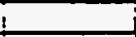 & \\
\hline Outhat Vap. Tomporaturef & 78.71 & & $i$ & $!$ & 1 & i & 1 & $i$ & i & \\
\hline Walor Fow Pate-GPM & & & 1 & 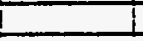 & & & I & I & I & \\
\hline Water hlot Temp, f & 81.31 & & & I & 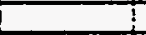 & ! & ! & $i$ & i & \\
\hline Wator outiol Temp.f & 81.4 & & & & & 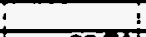 & 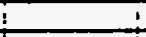 & 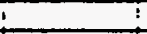 & ton & \\
\hline Intet G-bm/hr/ne & 223712 & & Temp & 76.71 & Donsity & $93.74 !$ & 1 & 1 & L & \\
\hline Subcoolingt & 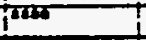 & & & & & & & 1 & 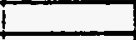 & \\
\hline Flow Observition & & & & & & & & I & I & \\
\hline & ZONET & ZONEZ & ZONE3 & ZONEA & ZONES & ZONEO & ZONET & ZONEB & ZONES & TZON=10 \\
\hline Honlnputwernat) & ol & 0 & of & 0 & 0.11 & T. 0 & 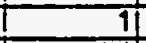 & of & 0.0 & 3 \\
\hline HedLoss+W" & -24 & -0.6 & -0.81 & -0.7 & -0.7 & -0.51 & -0.41 & -0.81 & 0.7 & 3.5 \\
\hline 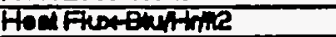 & of & of & of & 01 & हा & of & 17 & of & 10 & 65 \\
\hline Wal'temp. $13 F$ & 76.0 & 78.01 & 76.91 & 77.5 & 77.5 & 7.51 & 76.91 & 76.01 & 76.2 & 78.4 \\
\hline War Tomp. $14 F$ & 76.9 & $76.9 !$ & 76.9 & 77.5 & $\pi 7,3$ & 77.5 & 78.9 & 76.5 & 76.8 & 8. \\
\hline WeIT Tomp. $15 F$ & 76.7 & 76.4 & 76.41 & $\pi .11$ & 76.9 & 7.1 & 76.4 & 76.41 & 76.4 & 77.8 \\
\hline Wal Temp. "If & E0.1 & 76.9 & 76.9 & 77.5 & 77.5 & 77.5 & 76.8 & 76.9 & 76.2 & 782 \\
\hline Wah Temp. 12F & 76.8 & 76.9 & 76.9 & 77.5 & 77.51 & 77.51 & 76.71 & 76.91 & 78.8 & 77.8 \\
\hline Instream Temo.f & 76.8 & 76.8 & 76.81 & 77.31 & 7.21 & 77.2 & 76.41 & 76.41 & 76.4 & $\pi .1$ \\
\hline Avg. Wall Tomp.t & 76.9 & 76.91 & 76.91 & 77.41 & 77.31 & 77.4 & 76.7 & 76.7 & 76.8 & 78.1 \\
\hline Rug.Wallot, & 0.11 & 0.1 & 0.11 & 0.11 & 0.11 & 0.21 & 0.31 & 0.3 & 0.4 & \\
\hline Remaining Suboooff & -45.3 & & & & & & & & & \\
\hline Exh Cully & of & of & 0 & 0 & of & of & of & 01 & 0 & 0 \\
\hline 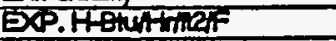 & of & 01 & का & of & 17.31 & 0) & 51.ाI & of & 28 & 51.1 \\
\hline COMPUIEDH & & & & & & & & & & \\
\hline Jallowk & 65.1 & $65 . \pi$ & 65.1 & 65.2. & 65.21 & E5.1 & 65 & $6 \mathbf{6 5}$ & 6 & 6.5. \\
\hline Sradyi(Cudotubo) & 81.9 & 83.81 & 84.81 & 85.81 & 86.61 & 87.4 & 88 & 88.6 & 89.3 & 50 \\
\hline sendtr & 81.9 & 83.81 & 89.8 & 85.81 & 86.8 & 87.4 & 88 & 88.8 & 89.8 & 90 \\
\hline (RLO) & 16658 & 16883 & 1653 & 16701 & 16583 & 16684 & |65ta) & $165 \pi$ & 16569 & 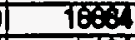 \\
\hline & 1.033 & 1.057 & 1.07 & 1.08 & 1.091 & 1.101 & 1.11 & 1.119 & 1.127 & 1.33 \\
\hline (H) & 81.8 & 83.8 & 84.8 & 85.8 & 86.6 & 87.4 & 88 & 88.8 & 89.8 & 80 \\
\hline$(\mathrm{ABO}) \mathrm{L})$ & 3.5 & 7 & 7 & 7.1 & 7.1 & 74.4. & 24.5ी & 23.1 & 20 & 762 \\
\hline & & & & & & & & & & \\
\hline & $20 \mathrm{kE}$ & ZONEA & $\angle O N=6$ & $\angle O N=8$ & ZONETO & & & & & \\
\hline DPTgud tomo.f & 80 & & & & & & & & & \\
\hline DPU doud Donshybming & क्टका & & & & & & & & & \\
\hline Ejomiont & 2 & 4 & 61 & 8 & 9.875 & & & & & \\
\hline Meceured DPpild & 0.052 & 0.065 & -0.4601 & -0.378 & 0.0311 & & & & & \\
\hline Zero Cortectons-psid & ס.717 & 0.088 & 0.0461 & 0.001 & 0.094 & & & & & \\
\hline Correcod DPpid & $-0.0 \mathrm{E}$ & 0.021 & -0.504 & -0.316 & -0.028 & & & & & \\
\hline Actud DPA & 2.01 & 3.98 & 6.7 & 8.42 & 9.78 & & & & & \\
\hline Mesured tPrpig & 4.2 & & & & 300.1 & & & & & \\
\hline Mesured over lith & $x=\infty$ & & & & & & & & & \\
\hline & & & & & & & & & & \\
\hline Computed DP & 2.01 & 4.07 & $5.9 \pi$ & 7.881 & 9.60 & & & & & \\
\hline Filuont & 0.01 & 0.07 & 0.0 . & 0.03 & 0.09 & & & & & \\
\hline Acostertilon & of & 0 & of & 0 & of & & & & & \\
\hline Eloviont & 2 & 4 & 5.94 & 7.85 & 9.62 & & 1 & & & \\
\hline & & & & & & & 1 & & & \\
\hline
\end{tabular}




\begin{tabular}{|c|c|c|c|c|c|c|c|c|c|c|}
\hline Run" & 685 & & & & & & . & 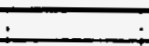 & $\div$ & \\
\hline Test Fuid & :C4F8 & & & & & & & $\because$ & $\div$ & \\
\hline Date: & $7 / 1933$ & 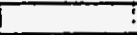 & t & & & & $!$ & $i$ & t & \\
\hline Time: & $13: 47$ & & & & & & & $i$ & $t$ & \\
\hline Fow Areaft2 & 0.00986 & i & 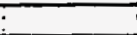 & - & $\cdot$ & $\cdot$ & 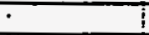 & i & 7 & $\vdots$ \\
\hline Hoed Fow Arooth2 & 0.18695 & 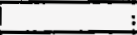 & 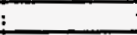 & & 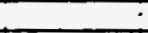 & E & & : & 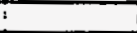 & 7 \\
\hline Sed. Prossuraphig & 4.2 & $i$ & 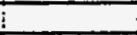 & & $i$ & I & דit & $+!$ & ! & $T$ \\
\hline Sal Temporaturo F(inlat) & 301 & 1 & 1 & & $i$ & 1 & i & $!$ & $!$ & $!$ \\
\hline Liq. Density lbmitis & 93.4 & 93.491 & 93.49 & 93.37 & 93.471 & 93.42 & 93.55 & $\$ 3.55$ & 93.55 & 93.60 \\
\hline Latent Hoat Btulbm & 44.72 & 44.76 & 44.76 & 44.66 & 44.69 & 44.71 & 44.8 & 44.8 & 44.8 & 74.78 \\
\hline Spectic Hoaf-BtulbmiF & 0.281 & 0.261 & 0.261 & 0.261 & 0.261 & 0.281 & 0.261 & 0.261 & 0.261 & 0.261 \\
\hline Lig. Viscosinybmithr & 0.859 & $0.87 \pi$ & 0.871 & 0.885 & 0.867 & 0.8681 & 0.8741 & 0.8741 & 0.874 & 0.871 \\
\hline Wg. Conduetivity 6 tuhr-fif & 0.0377 & 0.0377 & 0.0377 & 0.0376 & 0.0377 & 0.0377 & 0.03781 & 0.0378 & 0.0378 & 0.0377 \\
\hline Tig. Prand No. & 6.07 & $6.02 !$ & 6.02 & 6 & 6.011 & 6.071 & 6.031 & 6.03 & 6.03 & 6.02 \\
\hline Inter Fow Ralo-GPM & 2 & & & & 1 & $\square$ & : & 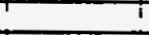 & 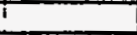 & T \\
\hline Outel Ua. Flow Rat-GPM & 0 & & & & 1 & $i$ & t & 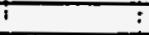 & $\vdots$ & $i$ \\
\hline Outlat Vap. Flow Rato-GPM & 1.8 & I & & & $i$ & $i$ & $i$ & t & $\mathfrak{t}$ & $t$ \\
\hline Inlot Temporaturef & 76.5 & & & & i & 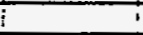 & 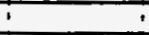 & - & - & T \\
\hline Outallig. Tempereture ff & 78.11 & i & 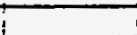 & i & F. & - & $i$ & 7 & . & i \\
\hline Outel Vap. Tomparaturef & 78.6 & $i$ & i & $i$ & 1 & tT & 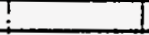 & I & I & $i$ \\
\hline Water Flow Rato-GPM & 1 & & I & 1 & : & 1 & 1 & I & I & I \\
\hline Water inlet Temp.f & 81.3 & & I & $i$ & 1 & L & $I$ & I & I & I \\
\hline Water outtel Temp.f & 81.5 & 1 & 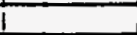 & & 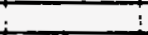 & 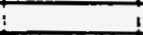 & 1 & I & I & \\
\hline Inlet Glbmhrith & 1446566 & & Tomp & 77.4 & Density & 93.651 & & $\bar{i}$ & i & \\
\hline Subcooling & & & & & & & $\mathrm{I}$ & i & & \\
\hline \multicolumn{11}{|l|}{ Fow Obsenvion } \\
\hline & ZONET & ZONEZ & ZZONE3 & ZZONEA & ZONES & ZOONE6 & ZZONET & ZONE & TZONES & ZONEETO \\
\hline Heal Inptw Wats(net) & 0 & 01 & 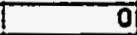 & 0 & 0.11 & of & 1 & of & $0 ! \quad 0.6$ & $51-2-3$ \\
\hline Hod Loss-Walts & -22 & -0.5 & -0.7 & -0.6 & -0.81 & -0.51 & -0.81 & -0.51 & -0.7 & \begin{tabular}{|r|r|} 
& 35 \\
& 35
\end{tabular} \\
\hline Hoelfionstuptrine & 0 & 0 & 0 & 0 & 2) & 01 & 171 & & 0) & 62 \\
\hline Wa Tomp. \#3F & 78 & $\pi .7$ & $\pi .7$ & 78.0 & 78.2 & 781 & 77.6 & 77.6 & 77.6 & 78.7 \\
\hline Wall Tamp. HF & 78 & 77.7 & 7.7 & 78.8 & 78 & 781 & 7.5 & 7.2. & 77.5 & 78.7 \\
\hline WaH Tomo. $15 \mathrm{~F}$ & 77.7 & 77.7 & 77.7 & 78 & $\pi .9$ & $\pi .8$ & $\pi .1$ & 7.2 & 77.1 & 77.8 \\
\hline Wall Tamp. \#F & 80.5 & 7.7 & $\pi .7$ & 79.1 & 78.3 & 78 & 77.5 & 77.5 & 77.6 & 785 \\
\hline Wall Tamp. $2 F$ & $\pi .7$ & 7.71 & 77.7 & 78.9 & 78.21 & 78.21 & 77.5 & 7.5 & 77.5 & 78.1 \\
\hline Instram Tomp.f & 78.3 & $\pi .9$ & 77.9 & 78.8 & 78.51 & 78.41 & 77.5 & 7.51 & 7.5 & 77.9 \\
\hline Avg. Wall Temp.F & 77.8 & 7.7 & 77.7 & 78.7 & 78.11 & 78 & 77.4 & 77.41 & 77.4 & 78.4 \\
\hline Avg. WallDT.f & 0 & 0 & 0 & 0 & of & of & of & of & 0 & 0.5 \\
\hline Remainung Subcodf & -46.5 & & & & & & & & & \\
\hline Exct Quality & 0 & 0! & 0 & 0 & of & of & of & of & $\overline{0}$ & 0 \\
\hline EXP.HEWUARER & 01 & 0 & 0 & 0 & 173.3 & 01 & 1733.4 & 이 & 868.7 & 1680 \\
\hline COMPUTEDH & 1 & & & & & & & & & \\
\hline Jallouk & 113.6 & 113.6 & 1135 & T13.7 & 113.6 & 913.5. & 713.3 & 173.3 & 913.3 & T173.4 \\
\hline Szady1(Oudo uubo) & 7428 & 146! & 147.7 & 140.5 & 150.8 & $152 \pi$ & 1532 & 154.3 & 1556 & 1538 \\
\hline seady2 & 1428 & 148 & 147.7 & 749.5 & 150.8 & 152.11 & 153.2. & 154.3 & 155.5 & 758.6 \\
\hline (ALb) & 3650 & 33513 & 33510 & 33750 & 33872 & 33870 & 35355 & 333का & 33378 & 3546 \\
\hline (F) & 1.033 & 7.057 & 1.059 & 1.08 & 1.09 & 1.1 & 1.11 & 1.118 & 1.128 & 1.134 \\
\hline (HLO) & 142.8 & 748 & 147.7 & 149.5 & 150.8 & 152.1 & 153.2 & 154.3 & 155.5 & 756.8 \\
\hline (मBOUL) & 0.7 & 0.7 & 0.7 & 0.7 & 0.7 & 0.7 & 0.7 & 0.7 & 0.7 & 355 \\
\hline & & & & & & & & & & \\
\hline & $20 N=2$ & ZONEA & ZOKE 6 & ZONES & $\angle O N=10$ & & & & & \\
\hline DP ligudd tomp.F & 88.5 & & & & & & & & & \\
\hline DPLquid Density tom & क2.37 & & & & & & & & & \\
\hline Elovion & 2 & 4 & 6 & 8 & 9.875 & & & & & \\
\hline Mazured Dppid & 0.08 & 0.046 & -0.37 & $\$ .350$ & -0.0381 & & & & & \\
\hline Zero Correctians-psd & 0.177 & 0.086 & 0.045 & 0.027 & 0.06 & & & & & \\
\hline Corrected DPPsid & -0.037 & -0.043 & -0.468 & -0.35 & -0.122 & & & & & \\
\hline Actud DPA & $2 \infty$ & 4.02 & 6.6 & 8.51 & 9.84 & & & & & \\
\hline Mesured Tpping & 4.2 & & & & 300.11 & & & & & \\
\hline Mescured Overath & $=\infty$ & & & & & & & & & \\
\hline & & & & & & & & & & \\
\hline Computed Das & 203 & 4.05 & 6.03 & 7.97 & 9.77 & & & & & \\
\hline Frictont: & 0.03 & 0.05 & 0.08 & 0.17 & 0.14 & & & & & \\
\hline Acosterisiont & 0 & 0 & 0 & 0.01 & 0.01 & & & & & \\
\hline Elevitionth & 2] & का & 5.94 & 7.85 & 9.62 & & & & & \\
\hline & & & & & & & & & & \\
\hline
\end{tabular}




\begin{tabular}{|c|c|c|c|c|c|c|c|c|c|c|}
\hline Run: & 6871 & & & & & & & & & \\
\hline Testfuid & ICAF & & & & & & $\vdots$ & 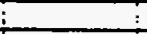 & & \\
\hline Date: & $7 / 79301$ & 3 & & & & & I & 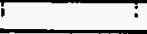 & : & : \\
\hline Time: & $13: 47$ & & & $\vdots$ & $\vdots$ & & $\overline{!}$ & 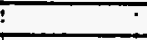 & & \\
\hline Flow Areatt2 & 0.003581 & TI & $\vdots$ & - & $\div$ & ; & i & 7 & i & 1 \\
\hline Hoed Fow Aroanth & 0.19695 & 3 & $i$ & $T$ & i & i & $!$ & $i$ & i & \\
\hline Sal. Proseure paig & 4.21 & T) & T & 1 & $T$ & T & 1 & $i$ & $i$ & i \\
\hline S21. Tomporaturaf(inlaf) & 301 & T & I & & & i & $i$ & 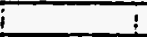 & 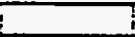 & \\
\hline Liq.Density 4 bm/t3 & 93.37 & 93.31 & 93.33! & 93.21 & 93.261 & 93.24 & 93.42 & \$3.42 & 93.42 & 93.3 \\
\hline Ladon Heat Btuhbm & 44.62 & 44.62 & 44.68 & 44.53 & 44.57 & 44.56 & 44.7 & 44.7 & 44.7 & 44.61 \\
\hline Specitio Hod-otulbmiF & 0.281 & 0.2611 & 0.261 & 0.261 & 0.261 & 0.281 & 0.2611 & 0.2611 & 0.261 & 0.251 \\
\hline Lq. Viscosity & 0.852 & 0.852 & 0.853 & 0.8561 & 0.859 & 0.858 & 0.858 & 0.8681 & 0.858 & 0.861 \\
\hline 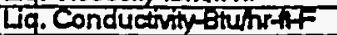 & 0.0376 & 0.0376 & 0.03761 & 0.03751 & 0.03751 & 0.03751 & 0.0377 & 0.0377 & 0.0377 & 0.0376 \\
\hline La. Prandt No. & 5.99 & 5.99 & 5.99 & 5.971 & 5.981 & 5.381 & 6.011 & 6.011 & 6.01 & 5.98 \\
\hline Inlot Fow Rato-GPM & $3 !$ & 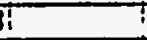 & 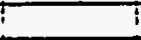 & 1 & 1 & ! & 1 & 1 & 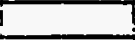 & \\
\hline Outot Lig. Fow Rato-GPM & 11 & II & 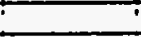 & $\div$ & $i$ & $i$ & $\vdots$ & I & I & I \\
\hline Outel Vap. Fow Rate-GPM & 2.2 & I & 5 & $\div$ & 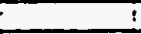 & $\vdots$ & $i$ & $i$ & 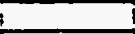 & I \\
\hline Inlet Tomperenturef & $\pi .7$ & & 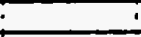 & 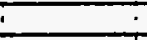 & $i$ & $i$ & i & $\bar{i}$ & 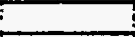 & \\
\hline OuterLiq. Tomperaturef & 79.6 & T & i & T & $\mathbf{i}$ & i & 1 & $i$ & $i$ & \\
\hline Outlat Vap. Tomparateref & 78.6 & $\pi$ & i & $i$ & $i$ & $i$ & 1 & 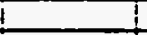 & & \\
\hline Walor Flow Ralo-GPM & I & & I & I & $\bar{\vdots}$ & $\bar{\vdots}$ & I & I & I & \\
\hline Water Inlet Temp.F & 81.3 & 3 & 1 & 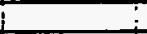 & & & $i$ & $i$ & 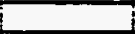 & \\
\hline Water outlat Tomp.F & 81.5 & & & & & & 1 & $!$ & 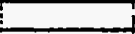 & \\
\hline Inter Glbmhinite & 668855 & & Temp & 78.41 & Density & 93.42| & & 1 & 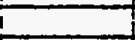 & \\
\hline Subcoolingt & Tाम & & & & & & & I & 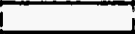 & \\
\hline \multicolumn{11}{|l|}{ Fow Obsorvation } \\
\hline & ZOWNE1 & TZONEZ & ZONE3 & ZOONE 4 & ZONES & $120 N=6$ & ZONET & ZONE8 & ZONEO & $201=10$ \\
\hline Hoxinputwasinel) & 0 & 0 & 01 & of & 01 & 101 & 1 & ol & 0.5 & 25 \\
\hline HeAloss-Wath & -1.9 & -0.5 & -0.6 & -0.51 & -0.61 & -0.41 & -0.81 & -0.51 & -0.8 & 3 \\
\hline 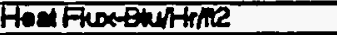 & 0 & 01 & 0 & 01 & 0 & 01 & 171 & of & g & 43 \\
\hline WeI Tomp. \$ $3 F$ & 79 & 78.81 & 78.01 & 801 & 79.6 & 79.6 & 78.41 & 78.4 & 78.4 & 80 \\
\hline Wall Tamp. *4F & 79.2 & 78.91 & 78.91 & 79.71 & 79.8 & 79.81 & 78.41 & 78.41 & 78.4 & 80 \\
\hline Wall Tomp. $15 f$ & 78.9 & 78.9 & 78.91 & 79.81 & 79.8 & 79.81 & 78.41 & 78.41 & 78.3 & 78.8 \\
\hline WNT Tomp. HF & 81.7 & 78.9 & 78.9 & 80.1 & 79.6 & 79.6 & 78.4 & 78.4 & 78.4 & 79.5 \\
\hline Wath Temp. I2f & 78.9 & 78.9 & 78.9 & 80.1 & 79.6 & 79.8 & 78.41 & 78.4 & 78.4 & 79.4 \\
\hline Instroam Tomp.F & 79.2 & 79.2 & 79.11 & 801 & 79.6 & 79.71 & 78.41 & 78.41 & 78.4 & 79.3 \\
\hline Avg. Wall Tomp. F & 79 & 78.91 & 78.81 & 79.81 & 79.8 & 79.61 & 78.41 & 78.4 & 78.4 & 79.6 \\
\hline Avg. Wall DT. & 0 & 0 & 01 & 01 & of & 01 & 01 & 01 & 0 & 0.3 \\
\hline Pemaining Suboooff & -47.7 & & 7 & 1 & & 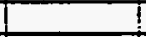 & 1 & 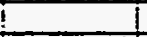 & & \\
\hline Exd Cuallity & 01 & 01 & 01 & of & की & 01 & 01 & 01 & 0 & 0 \\
\hline DXP.HBWh hllaF & 0 & 0 & 0 & 01 & 01 & 01 & 1738.4 & 0) & 868.7 & 167.8 \\
\hline \multicolumn{11}{|l|}{ COKFUTEDH } \\
\hline Jehiouk & Tह7.21 & 16721 & 167.11 & 157.41 & 157.31 & 157.31 & 168.01 & 166.8 & 168.8 & 767.1 \\
\hline Szadyil(Oudo kuba) & 197.6 & $202 \pi$ & 204.51 & 20301 & 2038 & 210.8 & 271.91 & 213.51 & 215 & 2188 \\
\hline sendy 2 & 197.6 & 202.7 & 204.5 & 208.9 & 208.8 & 210.81 & 211.91 & 213.51 & 215 & 218.8 \\
\hline (सLC) & 50753 & 50746 & 50098 & $5 t 0801$ & 50902 & 500901 & 508301 & 50375 & 50570 & 50748 \\
\hline (i) & 1.082 & 1.056 & 1.059 & 1.078 & 1.00 & 1.099 & 1.109 & 1.117 & 9.125 & 7.132 \\
\hline (FLO) & 197.6 & 2021 & 204.6 & 200.8 & 208.8 & 210.61 & हा1.9 & 213.5 & 275 & 216.8 \\
\hline \multirow[t]{3}{*}{ (ABOL) } & 0.7 & 0.7 & 0.7 & 0.7 & 0.7 & 0.7 & 0.7 & 0.7 & 0.7 & 19.3 \\
\hline & & & & & & & & & & \\
\hline & ZONEZ2 & TZONE & ZONEE & ZOEE & ZONETO & & & & & \\
\hline DPTqud Temp.f & 88.6 & & & & & & & & & \\
\hline DPLiquid Donsityom/3 & क्2.21 & & & & & & & & & \\
\hline Elentiont & 2 & 4 & $6 !$ & 8 & 9.8751 & & & & & \\
\hline Measured DPPyid & 0.061 & 0.008 & 0.181 & -0.485 & -0.18 & & & & & \\
\hline Zero Contetionsepsid & 0.757 & 0.088 & 0.055 & 0.027 & $0.08 \pi$ & & & & & \\
\hline Comated DPP sid & 20.053 & -0.078 & -0.230 & -0.482 & -0.244 & & & & & \\
\hline Actor opt & 2.06 & 4.08 & 6.28 & 8.68 & 10.14 & & & & & \\
\hline Measured Tprpig & 4.2 & & & & 300.1 & & & & & \\
\hline \multicolumn{11}{|l|}{ Mecoured Overalle } \\
\hline & & & & & & & & & & \\
\hline Computed DPII & 2.08 & 4.7t & 6.72 & 8.00 & 9.82 & & & & & \\
\hline Fretiont & 0.06 & 0.17 & 0.17 & 0.22 & 0.28 & & & & & \\
\hline Acoterion-1 & 0 & 0 & 0.01 & 0.01 & 0.021 & & & & & \\
\hline Elevionth & 2 & 4 & 5.951 & 7.881 & 9.681 & & & t & & \\
\hline & & & & & & & $T$ & & & \\
\hline
\end{tabular}




\begin{tabular}{|c|c|c|c|c|c|c|c|c|c|c|}
\hline Run: & 6881 & & & & & & & & & \\
\hline Test Fuid & CAF & & & & & & & & & \\
\hline Date: & $7 \pi 9331$ & & & & & $\ldots$ & & & & \\
\hline Time: & $13: 47$ & $\therefore$ & 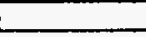 & $\therefore$ & & & & & & \\
\hline Fow Avea-li? & 0.003861 & 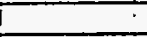 & $\div$ & & & & & $\cdot$ & - & $\cdot$ \\
\hline Hoed Flow Aroedt? & 0.106951 & 7 & $\because$ & & $\dot{-}$ & & & $\div$ & & $i$ \\
\hline Sal Prosaurefsig & 4.31 & 7 & $\therefore$ & $\dot{-}$ & $\therefore$ & & $\therefore$ & $\therefore$ & & 1 \\
\hline Sat Tomperature F(inlel) & 3021 & 1 & . & & & & & 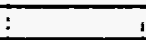 & & 1 \\
\hline Lig. Densth 4 bmitio & 92.981 & 93.021 & 931 & 92.991 & 92.991 & 92.991 & 93.171 & 93.17 & 33.171 & 93.09 \\
\hline Lotont Heateturibm & 44.351 & 44.38 & 44.371 & 44.361 & 44.361 & 44.361 & 44.671 & 44.511 & 44.611 & 44.44 \\
\hline Spectic Heat-Btulbmif & 0.2021 & 0.262 & 0.262 & 0.2521 & 0.2521 & 0.2521 & 0.2521 & 0.2521 & 0.252 & 0.262 \\
\hline Liq. Viscositylbmithr & 0.8451 & 0.8471 & 0.8461 & 0.845 & 0.845 & 0.8451 & 0.855 & 0.855 & 0.8551 & 0.85 \\
\hline Lig. Conductivity Btuhr-tif & 0.03731 & 0.03741 & 0.0374 & 0.03731 & 0.03731 & 0.03731 & 0.03751 & 0.03751 & 0.03751 & 0.0374 \\
\hline Lig. Prandid No. & 5.93 & 5.93 & 5.93 & 5.931 & 5.39 & 5.931 & 5.96 & 5.961 & 5.961 & 5.35 \\
\hline Inlet Fow Rate-GPM & $4:$ & $\vdots$ & 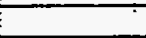 & & & & & $i$ & & $!$ \\
\hline Outiof La. Fow Rate-GPM & 1.41 & & & & & & & & & \\
\hline Outet Vap. Flow Rato-GPM & 2.41 & $\because$ & 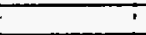 & & & & & & & $I$ \\
\hline in isi Tompermuref & 79.31 & & $\therefore$ & & & & & & & $i$ \\
\hline Outhel Lig. Tomperaturef & 891 & i & T. & $\therefore$ & $\cdot$ & & & $\div$ & & 1 \\
\hline Outlel Vap. Tamperatiuse F & 79.31 & & 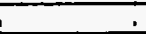 & 1 & $\bar{i}$ & & & 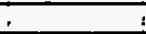 & T & T \\
\hline Water Fow Pate-GPM & & & 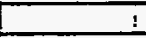 & 1 & . & $i$ & 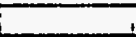 & $i$ & 1 & 1 \\
\hline Water inlet Temp.f & 81.41 & - & - & 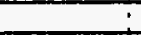 & & & & 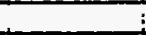 & & $i$ \\
\hline Water outel Temp.F & 81.8 & $\perp$ & $L$ & & & & & $!$ & & $\therefore$ \\
\hline Inlot Glbmhnnt2 & . 889410 & & Tomp & 80.21 & Density & 93.171 & & & & \\
\hline Subcooling F & sकen & i & 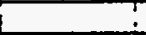 & & & & & & & 7 \\
\hline \multirow[t]{2}{*}{ Fow Observation } & $i$ & & & & & & & & & \\
\hline & ZONE1 T: & ZONE2 & ZONE3 & ZONE4 I8 & ZONES & ZONE6 & ZONET & TZONEB & ZONE9 & ZONETO \\
\hline Hesingur-Wathot) & $\therefore \quad 01$ & 01 & 01 & 01 & Oा & $0 !$ & $0.9 !$ & 0 & 0.31 & 12 \\
\hline Hod'Loss-Walls & -1.41 & -0.31 & -0.5 & -0.41 & -0.41 & $-0.3 i$ & 0.2 & -0.4 & -0.51 & -24 \\
\hline How Finos Burthrin & 01 & 01 & 01 & $0 !$ & $\eta^{1}$ & 01 & 16 & $0 !$ & हा & 36 \\
\hline Well Tomp. BF & 81.31 & 80.81 & 80.81 & 81... & $\because$ : בा! & $91.3 !$ & 80.21 & 80.2 & 8021 & 81.1 \\
\hline Wall Temp. " $4 F$ & 81.31 & 80.81 & 80.81 & 81.51 & 81.71 & 81.31 & 8021 & 801 & 80.21 & 81.1 \\
\hline Wall Temp. 15 F & 80.51 & 80.51 & 80.51 & 81.31 & 81 & $8 \pi$ & 79.7 & 79.6 & 79.5 & 80.6 \\
\hline WAH Temp. IF & 81.71 & 81.11 & 80.8 & 81.5 & 81.5 & 81.31 & 80.2 & 80.2 & 80.2 & 81.1 \\
\hline Wall Temp. ILF & 811 & 811 & 80.9 & 81.5 & 87.5 & 81.4 & 802 & 80.2 & 80.2 & 81.1 \\
\hline Instream Temp.F & 81.61 & 81.31 & 81.4 & 81.5 & 81.51 & 81.51 & 80.2 & 80.2 & 80.2 & 80.8 \\
\hline Avg. Wall Temp.F & 811 & 80.91 & 80.81 & 81.51 & 87.31 & $81.2 !$ & 80.7 & 801 & 80.11 & 81 \\
\hline Avg. WaH DT.F & $0 !$ & 01 & $0 !$ & $0 !$ & $0 !$ & 01 & 0 & 0 & ठा & 0.2 \\
\hline Remaining SubcoofF & -49.71 & & & & & & & & & t \\
\hline Ext Qualky & ol & of & 01 & 01 & of & 01 & 0 & 01 & 01 & 0 \\
\hline 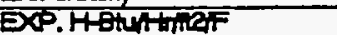 & o1 & 01 & oा & 01 & of & of & 1560.1 & 0] & 5201 & 194.8 \\
\hline COMPUTEDH & & & & & 1 & 1 & & & & \\
\hline Jallouk & क्रूस & 198,3 & 198.4 & 1994! & 188.4 & 189.41 & 197.8 & 197.8 & 197.7 & 180 \\
\hline Sradvi(Oudo tube) & 2494. & 25x & 257.91 & 25081 & 2631 & 255.31 & 256.91 & 268.9 & 270.81 & 2728 \\
\hline $5 \pi d \sqrt{2}$ & 249.41 & 254.81 & 257.91 & 260.6 & 263 & 255.31 & 268.9 & 268.9 & 270.8 & 2729 \\
\hline (R्णि) & 68879 & 68553 & 68744 & 68796 & 68790 & 68783 & 68018 & 68017 & 68005 & 68346 \\
\hline (F) & 1.032 & 1.055 & 9.068 & 1.079 & 1.089 & 1.038 & 1.108 & 1.116 & 1.124 & 1.137 \\
\hline (HLC) & 249.4 & 254.9 & 257.9 & 200.6 & 263 & 255.31 & 268.9 & 268.9 & 270.8 & 2729 \\
\hline \multirow[t]{3}{*}{ (HBOL) } & 0.7 & 0.7 & 0.7 & 0.7 & 0.71 & 0.7 & 0.7 & 0.7 & 0.7 & 395 \\
\hline & $i$ & 1 & 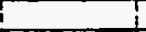 & & & & & & & \\
\hline & ZONE2 12 & ZONEA 4 & ZONEB & ZONEE & ZONETO & & & & & \\
\hline DPliqud Temp.f & $1 \quad 87.21$ & 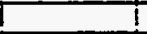 & 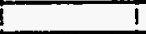 & & 1 & & & 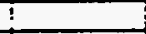 & & \\
\hline DPLiquid Density formith & 82181 & I & i & & 1 & & & & & \\
\hline Eovisionth & 21 & 4 & 61 & 8) & 9.876 & & & & & \\
\hline Meatured DPPsid & 0.0351 & -0.041 & -0.1341 & -0.578 & -0.290 & & & 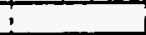 & & \\
\hline Zaro Corrections-psid & 0.1171 & 0.0851 & 0.045 & 0.0221 & 0.0841 & & & $i$ & $i$ & $I$ \\
\hline Corrected DPPsid & -0.0821 & -0.128 & -0.1791 & -0.605 & -0.3831 & & & & & \\
\hline Actud DPA & 2.17 & 4.16 & 6.32 & 8.88 & 10.37 & & & & & \\
\hline Meatured ip Psig & 4.3 & & 1 & & 300.1 & & & & & \\
\hline \multirow[t]{2}{*}{ Meatured Overall } & ixtom & & 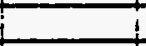 & & & & & & & \\
\hline & i & & & & & & & & & \\
\hline ComputedDRn & 2.091 & 4.78 & ठ.या & 8.281 & 70.13 & & & & & \\
\hline Fichond - & 0.001 & 0.18 & 028 & 0.38 & 0.47 & & & & & \\
\hline Acosterwont & of & 0.07 & 0.011 & 0.02 & 0.091 & & & & & \\
\hline Efrviontt - & 2) & 4 & 5.351 & 7.88 & 9.69 & & & t & & \\
\hline & $i$ & & 1 & & & & & & & \\
\hline
\end{tabular}




\begin{tabular}{|c|c|c|c|c|c|c|c|c|c|c|}
\hline Run & 6891 & & & & & & & & & \\
\hline Test Fuid & $\mathrm{C} 4=8$ & & & & & & & & & \\
\hline Dats: & 71933 & & & & $T$ & & 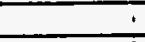 & ; & & \\
\hline Time: & $14: 411$ & & & & $\div$ & $\div$ & 5 & & & \\
\hline Fow Aroart? & 0.003531 & $\div$ & & 1 & : & 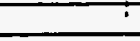 & $\div$ & * & $\div$ & - \\
\hline Hest Fow Arenti2 & 0.196851 & $\div$ & 4 & $\therefore$ & 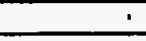 & $\div$ & $\div$ & 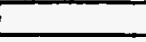 & - & 5 \\
\hline Sal Prossure Psig & 4.41 & : & 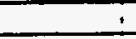 & $\dot{2}$ & $i$ & $\div$ & 1 & 1 & . & : \\
\hline Sy Temperature f(inlol) & 30.51 & & 1 & ! & ! & $\div$ & . & : & . & . \\
\hline Lag.Density-lbm/133 & 92.85 & 92.55 & 92951 & 92.891 & 92891 & 92.891 & 93.11 & 93.11 & 93.11 & 9 \\
\hline Laten Hoat Buifbm . & 44.331 & 44.331 & 44.331 & 44.28 & 44.28 & 44.281 & 44.45 & 44.451 & 44.45 & 44.31 \\
\hline Spectio Heaf Btu/hom/F & 0.262 & 0.202 & 0.262 & 0.252 & 0.2621 & 0.252 & 0.252 & 0.252 & 0.262 & 0.25 \\
\hline Ga. Viscosth-lbm/ithr & 0.843 & 0.8481 & 0.848 & 0.84 & 0.84 & 0.841 & 0.8511 & 0.851 & 0.8511 & 0.816 \\
\hline [ia. Conductivity thulhr-if & 0.037 & 0.03731 & 0.0373 & 0.05731 & 0.03731 & D.037 & 0.0574 & 0.0374 & $0.0374 !$ & 0.00574 \\
\hline Liq. Prandt No. & 5.92 & 5.92 & 5.921 & 5.911 & 5.911 & 5.911 & 5.55 & 5.\$5ा & $5 . \$ 51$ & 5.93 \\
\hline Inlot Fow Rede-GPM & $4 !$ & $\div$ & & : & 1 & & & : & 1 & 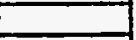 \\
\hline 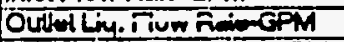 & 1.41 & 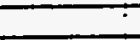 & 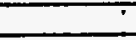 & $\div$ & & $\div$ & - & & $T$ & 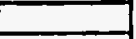 \\
\hline Outlot Vap. Flow flato-GPM & 2.41 & $\div$ & $\dot{-}$ & $i$ & $\because$ & - & 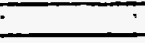 & $=$ & 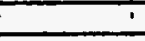 & 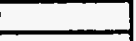 \\
\hline Inlet Tomperaturef & 80.2 & $!$ & 1 & i & $T$ & $\vdots$ & - & $\dot{-}$ & $\div$ & 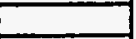 \\
\hline 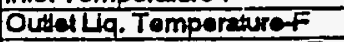 & 82.11 & $\therefore$ & 1 & $!$ & $!$ & $i$ & $i$ & $\dot{-}$ & 1 & $L$ \\
\hline Outict Vap. Temperature of & 79.81 & 1 & $i$ & 1 & $i$ & - & $\overline{1}$ & $i$ & 1 & 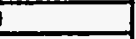 \\
\hline Water Flow Rate-GPM & . & 1 & $i$ & I & 1 & $!$ & 1 & 1 & $i$ & 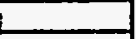 \\
\hline Water Inlet Temp. $F$ & 81.6 & $!$ & & 1 & $\dot{5}$ & $i$ & 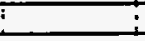 & : & 1 & $L$ \\
\hline Wader outsol Temp.F & 81.7 & $i$ & $i$ & & 1 & + & 1 & 1 & 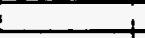 & \\
\hline Inlet Gibm/hritt2 & 888741 & i & Temp & 80.71 & Density & 93.11 & $T$ & 1 & 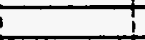 & \\
\hline Subcoolingf & $\sin$ & $\div$ & & & 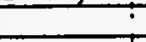 & 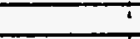 & $t$ & $!$ & $!$ & \\
\hline Fow Observation & $i$ & T & ! & & + & $!$ & $!$ & ! & 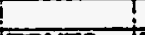 & \\
\hline & iZONET & ZONEZ & ZONEB T & ZONE & ZONES IZ & ZONEG I & IZONE7 & ZONEB & ZONES I & LON:ETO \\
\hline Hortinput-Watnot) & ol & o| & ol & 01 & 01 & 01 & 0.9 & 01 & 0.31 & 1.7 \\
\hline Hod Loso-Wates & -1.2 & $0.0 !$ & ב.:! & $\mathbf{0 . 3 1}$ & -0.31 & -0.81 & $-02 !$ & -0.41 & -0.51 & -21 \\
\hline Hed fhex-Burthte & oा & 01 & $0_{1}$ & $\mathbf{0}_{1}$ & 01 & $0 !$ & 161 & 01 & SI & की \\
\hline Wall Temp. BßF & 81.81 & 81.81 & 87.8 & 8231 & 8221 & 8221 & 80.11 & 80.7 & 80.7 & 82 \\
\hline Wall Tomp. $4{ }^{4}$ & 81.81 & 81.81 & 81.8 & 8221 & 8231 & 8221 & 80.71 & 80.7 & 80.7 & 82 \\
\hline Wall Tomp, $16 F$ & 81.7 & 81.6 & 81.7 & 8221 & 82.11 & 92.1 & 80.51 & 80.7 & 80.7 & 81.4 \\
\hline Wall Temp. IfF & 81.6 & 81.8 & 81.8 & 822 & 822 & 822 & 80.7 & 80.7 & 8..1 & 81.8 \\
\hline Wall Temp. $2 f$ & 89.8 & 81.8 & 81.8 & 823 & 82.2 & 822 & 80.71 & 80.7 & 80.7 & 81.4 \\
\hline Instrosm Tomp.f & 81.81 & 81.8 & 81.8 & 822 & 822 & 8221 & 80.7 & 80.7 & 80.7 & 81.4 \\
\hline Avg. Wall Tomp.t & 81.81 & 81.8 & 81.81 & 8231 & 822 & 823 & 80.7 & 8.7 & 80.7 & 81.7 \\
\hline Avg. Wall DT + & 0! & 01 & 01 & वां & $0 !$ & 01 & 01 & 01 & ol & 0.3 \\
\hline Remuining Subcooff & -49.71 & & 1 & $\mathrm{i}$ & $!$ & : & 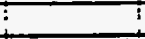 & : & 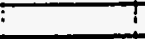 & \\
\hline Exacualy & of & of & of & 0! & $0 !$ & of & 01 & 01 & of & 6 \\
\hline Dx.HBufrines & 01 & of & of & 01 & of & of & 1500.1 & 01 & 5201 & 92. \\
\hline COMPUTEDH & T & 1 & & 1 & 1 & & & ! & -1 & \\
\hline Jallouk & 188.41 & 198.41 & 188,4 & 188.8 & 188.81 & 188.5 & 197.9 & 197.01 & 197.81 & \\
\hline Sradyiloudo the) & 249.3 & ङडा & 257.91 & 260.8 & 253.21 & 205.11 & 266.91 & 25801 & 270.81 & 2720 \\
\hline seady2 & 249.31 & 255 & 257.91 & 250.81 & 2532 & 255.4 & 266.9 & 268.9 & 270.8 & 2728 \\
\hline (RLO) & 58976 & 68535 & 6\%281 & 69168 & 69152 & 59145 & 68058 & 680501 & 68213 & $68 \times 15$ \\
\hline$(\theta)$ & 1.032 & 1.055 & 1.058 & 1.078 & 1.088 & 1.088 & 1.107 & 1.116 & 1.124 & 1.13 \\
\hline$\left(F^{\prime} D\right)$ & 249.81 & 255 & 257.91 & 200.8 & 263.2 & 265.4 & 268.8 & 268.9 & 270.81 & 2729 \\
\hline$(\mathrm{P} \cdot 30 \mathrm{IL})$ & 0.7 & 0.7 & 0.7 & 0.7 & 0.7 & 0.7 & 0.71 & 0.7 & 0.7 & 24.7 \\
\hline & & & & & & & & & & \\
\hline & ZONEE & ZONES & $\angle O N E 6$ & ZOTVE & ZONETO & & & & & \\
\hline DPT Tqud Tomp,t & 87 & & & 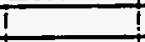 & & 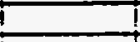 & & & 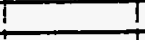 & \\
\hline 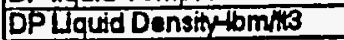 & करा। & 7 & & $T$ & ! & 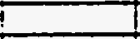 & & I & 1 & \\
\hline Elevion-1 & 21 & 4 & 6ा & 81 & 9.873 & & & 1 & 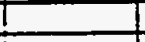 & \\
\hline Measured DPPstd & 0.035 & -0.057 & 0.132 & -0.689 & -0.308 & & $i$ & 1 & 1 & \\
\hline Zaro Corrections-osid & 0.117 & 0.0861 & 0.055 & $0.0 \times 7$ & 0.034 & & & $i$ & $i$ & \\
\hline Corructed DPPair & -0.022 & -0.123 & -0.177 & -0.616 & -0.39 & & & I & i & \\
\hline Actura DPA & 2.11 & 4.16 & 6.22 & 8.89 & 10.39 & & 1 & 1 & 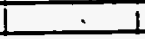 & \\
\hline Messured TPPsig & 4.4 & 1 & & + & 300.1 & & & 1 & 1 & \\
\hline Mes:ured Overallh & 1 & & & & & & & & 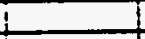 & \\
\hline & & & & & & & & & & \\
\hline Computed DPA & 201 & 4.791 & 6.27 & 8.23 & 70.78 & & & & & \\
\hline Fitcion- & 0.09 & 0.781 & 0.281 & 0.38 & 0.47 & & & & & \\
\hline Aocalertiont & 0 & 6.01 & D.011 & 0.02 & 0.031 & & & 1 & & \\
\hline Elevetion-1 & 2 & 4 & 5.95 & 7.88 & 9.691 & & & 1 & & \\
\hline & & & & & & I & & $T$ & & \\
\hline
\end{tabular}




\begin{tabular}{|c|c|c|c|c|c|c|c|c|c|c|}
\hline Run & 6901 & & & & & & & & & \\
\hline Test Fiuld & CAF & & & & & & & & & \\
\hline Date: & $\pi 193$ & & $\vdots$ & & & & & $\cdot$ & & \\
\hline Time: & $14: 45$ & & 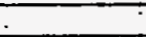 & & & & & & & 7 \\
\hline Fow Aroat? & 0.00338 & & : & $\because$ & : & & $\dot{7}$ & ; & $\dot{2}$ & ' \\
\hline Hoad Flow Aroa-il & 0.19595 & & $\vdots$ & & $\cdot$ & - & & 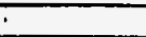 & & : \\
\hline Sat. Prossuropsig & 4.4 & & 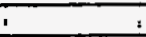 & $:$ & & & & & & $\bar{i}$ \\
\hline Sal Tomperature F(iniot) & 30.5 & & . & 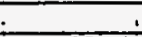 & 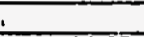 & & & 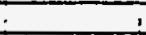 & 1 & i \\
\hline Lig. Densitylbm/ti3 & 92.85 & 92.851 & 92851 & 92.821 & 92821 & 92.821 & 93.031 & 93.021 & 93.021 & 92.92 \\
\hline Latont Heat Btunbm & 44.25 & 44.251 & 44.251 & 44.231 & 44.23 & 44.231 & 44.391 & 44.381 & 44.38 & 44.81 \\
\hline Specitic Heat-BtulbmiF & 0.262 & 0.2621 & 0.252 & 0.252 & 0.252 & 0.252 & 0.2621 & 0.262 & 0.262 & 0.252 \\
\hline Lig. Viscoshthbm/thr & 0.838 & 0.838 & 0.8381 & 0.837 & 0.837 & 0.8371 & 0.847 & 0.847 & 0.847 & 0.842 \\
\hline Lq. Conductivity-BtuhroffF & 0.0372 & 0.03721 & $0.0372 !$ & 0.06321 & 0.0372 & 0.03721 & $0.0374 !$ & 0.03741 & 0.03741 & 0.0370 \\
\hline Lq. Ptand No. & 5.9 & 5.91 & 5.91 & $5.9 !$ & 5.9 & 5.91 & 5.941 & 5.931 & 5.931 & 5.98 \\
\hline inlet Flow Rate-CPM & $-i$ & & & & & & & & & \\
\hline Oumet Ua. Fow Rate-GPM & $1 . \overline{41}$ & & & & & & & & $=$ & ; \\
\hline Outlet Vap. Flow Rato-GPM & $2.4 !$ & & & 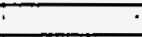 & & - & $\div$ & ; & $;$ & 1 \\
\hline Inlet Temperesuref & 80.31 & & 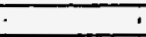 & $\cdot$ & & : & & - & & $\overline{1}$ \\
\hline Outlet Liq. Tomperature $F$ & 8221 & & . & 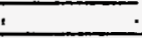 & $\therefore$ & 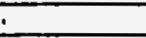 & & $\cdot$ & $\dot{-}$ & 1 \\
\hline Outlot Vap. Tomperaturef & 802 & & 1 & 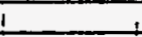 & 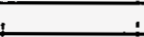 & 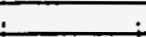 & 1 & 1 & . & $i$ \\
\hline Wator Fow Rato-GPM & 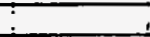 & & 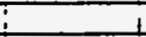 & 1 & & & & 1 & $i$ & $i$ \\
\hline Water Inlet Temp.F & 81.6 & & 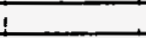 & & & & & & $i$ & $i$ \\
\hline Water outlel Temp.f & 81.9 & & 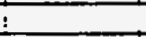 & & & & & & 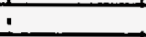 & \\
\hline Inlet G-lbm/hrfite & .888473 & & Temp & 80.91 & Density & 53.071 & . & , & 1 & 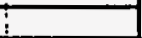 \\
\hline Subcooling & $\sin$ & & 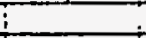 & & & & & & $\bar{\vdots}$ & 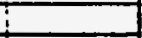 \\
\hline Fow Observation & 1 & & 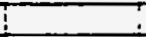 & & & & $T$ & $!$ & 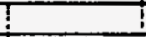 & 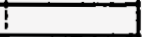 \\
\hline & ZONET & ZONEZ & ZZONES & ZONE4: & ZONES & ZONE6 I & ZONE? & ZONE & ZONE9 I & 1ZONIETO \\
\hline Heat inputhatts(net) & $1 \quad 01$ & 101 & 01 & 101 & 101 & 01 & $5.9 i$ & $i \quad 3$ & 3.3: & 1.8 \\
\hline Hed Loss-Wals & -1.2 & -0.31 & 0.41 & -0.41 & -0.41 & -0.31 & -0.21 & -0.41 & $0.5 i$ & -2 \\
\hline Hod FRoc-8huthrie & 01 & 01 & $0_{1}$ & 01 & 01 & 01 & 161 & 01 & 5 & 28 \\
\hline Wall Tomp. HF & 82.3 & 82 & 8221 & 82.61 & 8231 & 8241 & 80.7 & 80.91 & 81.71 & 826 \\
\hline Wall Temp. & 82.31 & 8211 & 8221 & 824 & 824 & 8241 & 80.81 & 80.51 & 8121 & 826 \\
\hline Wall Temp. $55 F$ & 81.81 & 81.81 & 81.81 & 8221 & 8221 & 82.21 & 80.51 & 80.51 & 80.51 & 81.4 \\
\hline Wall Temp. I1F & 81.8 & 822 & 8221 & 8271 & 82.3 & 82.41 & 80.5 & 80.7 & 81 & 82 \\
\hline Wall Temp. $2 F$ & 81.81 & 82.11 & 8231 & 82.61 & $82.3 i$ & 82.41 & 80.61 & 80.81 & 81.1 & 82 \\
\hline Instroam Temp.f & 82.5 & 8251 & 8251 & 82.71 & 82.71 & 82.71 & 81.21 & 81.31 & 81.31 & 82 \\
\hline Avg. Wall Temp.f & $82 \pi$ & 82.11 & 8221 & 82.51 & 82.31 & 824 & 80.61 & 80.71 & 811 & 822 \\
\hline Avg. Wall DT.F & 0? & 01 & $0 !$ & 01 & 0 & $0 !$ & 01 & $0 !$ & 01 & 0.2 \\
\hline Romaining Subcooff & -49.81 & 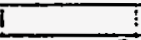 & $\vdots$ & 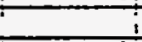 & 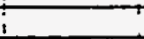 & & & & & \\
\hline Exi Cualty & 01 & 01 & 01 & 01 & 01 & of & 01 & 01 & 01 & 0 \\
\hline 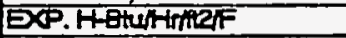 & 01 & 01 & 01 & of & 01 & 01 & 1560.11 & 01 & 5201 & 774.1 \\
\hline CONAUTEDH & 1 & 1 & 1 & +1 & & 1 & 1 & 1 & 1 & 1 \\
\hline ímod & 188.7 & 198.7 & 198.7 & 198.7 & $198.7 !$ & 198.7 & 1981 & 198.11 & 1881 & 198.2 \\
\hline Sedvi(Cudo tube) & 249.61 & 25521 & 25821 & 2600 & 263.31 & 255.61 & 267 & 269.11 & 27ा & 273 \\
\hline Sady2 & 249.61 & 2552 & 2582 & 260.9 & 263.31 & 265.61 & 267 & 239.11 & $2 \pi$ & 273.1 \\
\hline (AUC) & 693701 & 69350 & 69523 & 69861 & 69429 & 69422 & 68527 & 68579 & 68572 & 68978 \\
\hline $\mathbf{F}$ & 1.032 & 1.055 & 1.057 & 1.078 & 1.088 & 1.007 & 1.107 & 1.115 & 1.123 & 1.13 \\
\hline (FinO) & 249.61 & 25521 & 258.21 & 250.8 & 263.3 & 265.6 & $26 \pi$ & 269.11 & 271 & 273.1 \\
\hline (ABOIL) & 0.71 & 0.7 & 0.71 & 0.7 & 0.7 & 0.7 & 0.7 & 0.7 & 0.7 & 122 \\
\hline & 1 & & 1 & & & & & & & \\
\hline & ZONNE? & ZONE & ZONE6 & ZONES & ZONETO & ! & t & i & & \\
\hline DP tquid Tomp.F & ! 87.31 & & 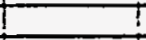 & 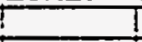 & & i & i & $\frac{1}{\vdots}$ & & \\
\hline DPLiquid Density tom/133 & 92.171 & & 1 & 7 & & 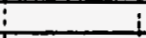 & $i$ & 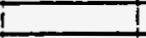 & & i \\
\hline Elevition-h & 21 & 41 & 61 & $8 !$ & 9.8761 & 1 & $i$ & $i$ & & 1 \\
\hline Manturad DPPsid & 0.038 & -0.0851 & -0.131 & -0.6531 & -0.311 & T & I & 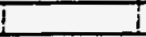 & & \\
\hline Zero Correctonspesid & 0.117 & $\overline{0.085 !}$ & AM:Et & 0.0?! & 0.024 & I & ; & $\vdots$ & & 1 \\
\hline Corrected DPPsid & -0.0811 & -0.122 & -0.170 & -0.681 & -0.394 & $I$ & $i$ & 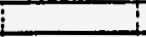 & & \\
\hline Actual DPA & 2.11 & 4.161 & 6.2.2 & 8.99 & 10.41 & 1 & $i$ & $i$ & & \\
\hline Macured fopsig & 4.4 & & & & 300.11 & $\frac{1}{1}$ & 1 & & & \\
\hline Merested Overilta & $x=0$ & & i & 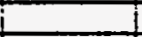 & & 1 & $i$ & & & \\
\hline & 1 & & & & & & & & 1 & \\
\hline Computed DP-A & 209 & 4.78 & 6.24 & 835 & 70.74I & & + & 1 & $i$ & \\
\hline Ficton-h & 0.091 & 0.18 & 0.28 & 0.38 & 0.47 & & & & & \\
\hline Acosleration & 01 & 0.011 & 0.011 & 0.02 & 0.001 & & & i & $i$ & \\
\hline Eloviondt & 21 & 41 & 5.551 & 7.88 & 9.841 & & i & 1 & 1 & \\
\hline & $i$ & & 1 & 1 & 1 & 1 & 1 & 1 & $i$ & I \\
\hline
\end{tabular}




\begin{tabular}{|c|c|c|c|c|c|c|c|c|c|c|}
\hline Test Fluid -- & 'C41. & & & & & & & & : & i \\
\hline Date: & $7 / 19931$ & & & & & & & & $T$ & 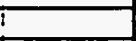 \\
\hline Time: & $14: 49$ & & & & & & & & & \\
\hline Flow Aroath2 & 0.003361 & & & : & 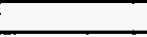 & & & I & $i$ & 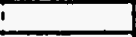 \\
\hline Hexthow Areatic & 0.196961 & & 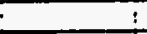 & 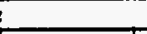 & $\therefore$ & 3 & - & $\therefore$ & $i$ & 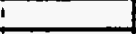 \\
\hline Sed. Proseurephig & $4.5 i$ & $\div$ & $i$ & 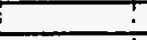 & $\dot{-}$ & 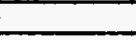 & $\therefore$ & $i$ & i & $i$ \\
\hline Sat. Temperatureffinlal) & 30.81 & 1 & 1 & 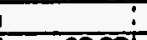 & & : & $\bar{\vdots}$ & 1 & I & 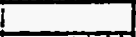 \\
\hline Liq. Densitylbmitis & 92.781 & 92.79 & 92791 & 92.821 & 92.82 & 92.82 & 92.99 & क्खा & $92 \% 1$ & 92.80 \\
\hline Latent Hoat-Btulbm & 44.19 & .44 .2 & 44.2 & $44.23 !$ & 44.23 & 44.231 & 44.361 & 44.37 & 44.35 & 44.26 \\
\hline Specifio Hoat-8tulbmif & 0.252 & 0.262 & 0.2521 & 0.252 & 0.262 & 0.252 & 0.262 & 0.202 & 0.202 & 0.252 \\
\hline Lig. Viscositylbmithr & 0.834 & 0.835 & 0.8351 & 0.837 & 0.837 & 0.837 & 0.845 & 0.8461 & 0.845 & 0.830 \\
\hline Gq. Conductivityethraf & 0.03721 & 0.0372 & $0.0372 !$ & 0.03721 & $0.0372 !$ & 0.03721 & 0.03737 & 0.03741 & 0.03731 & 0.00372 \\
\hline Lig. Pranat No. & $5.80 !$ & 5.89 & 7.89 & $-\frac{2.91}{101}$ & $5.9 !$ & $5.9 !$ & 5.93: & 5.331 & 5.931 & 5.9 \\
\hline Intot FOW Redo-GPM & $4 !$ & 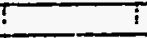 & - : & & & & 1 & $\square$ & $T$ & I \\
\hline Outot LIG. Flow RAO-GPM & $1.4 !$ & & $\dot{-}$ & & & & 1 & 2 & $i$ & 3 \\
\hline Outlot Vap, Fow Rato-GPM & 2.41 & & $\dot{\square}$ & - & 1 & $\dot{5}$ & 1 & : & $i$ & i \\
\hline Inlat Tempernturef & 81.31 & & $\dot{5}$ & $\therefore$ & $\therefore$ & $:$ & $\therefore$ & I & $!$ & $\vdots$ \\
\hline Outet Lia. Tempereturef & 82.51 & $\cdot$ & 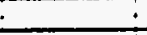 & - & $i$ & : & $\therefore$ & 1 & 1 & 1 \\
\hline Outlat vap. Tomparaturef & 80.41 & 1 & 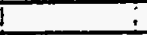 & 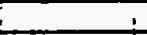 & $i$ & $\bar{l}$ & $i$ & 1 & i & i \\
\hline Wator Flow Rato-GPM & 1 & $I$ & $\therefore$ & $i$ & $i$ & 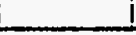 & 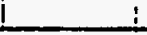 & 1 & 1 & $L$ \\
\hline Water Inlol Temp.f & 82 & $i$ & $i$ & 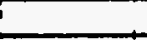 & & & $i$ & $i$ & 1 & $L$ \\
\hline Walor outdel Temp.F & 82.2 & & & & & $:$ & & & 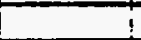 & 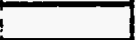 \\
\hline InlatGlbm/hrhe & 8878031 & & Tomp & $81.41 t$ & Density & \$3) & $i$ & $\bar{i}$ & 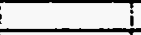 & \\
\hline Subcooling & rant & 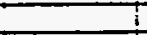 & 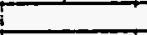 & & & & 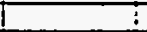 & $\overline{\mathbf{i}}$ & i & 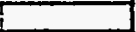 \\
\hline \multirow{2}{*}{ Fow Obsarvation } & & & $T$ & & & & & & & \\
\hline & ZONET & ZONE2 & ZONE3 & ZONEA & ZONE5 & ZONE6 & ZZONET ? & ZONEO & Zivice & Esteit \\
\hline Host tonpur-Wats(nat) & oा & $T$ & 01 & 01 & 01 & 01 & 0.91 & 01 & 0.31 & $1 \quad 1.2$ \\
\hline Hod LosstWaus & -1.11 & -0.31 & -0.41 & -0.31 & -0.31 & -0.21 & -0.21 & -0.31 & -0.41 & -1.7 \\
\hline Hest fluxbluhtinile & 01 & $0 !$ & 01 & 01 & 01 & 01 & 161 & 01 & 61 & दा \\
\hline Wall Tomp. 13F & 82.71 & क25ा & $825 T$ & 8271 & 827 & 827 & 81.4 & 81.4 & 81.4 & 83.4 \\
\hline Wall Tomp. "N4F & 82.81 & 2251 & 8261 & 8271 & 827 & 8271 & 81.41 & 89.4 & $89 ., 4$ & 824 \\
\hline WaH Tomp. $5 F$ & 82.51 & 825. & 82.5ा & 82.7 & 8281 & 82.81 & $81.4 !$ & 81.41 & 87.4 & 823 \\
\hline WaH Tamp. "IF & 81.8 & 8251 & 825 & 827 & 827 & 827 & 81.4 & 81.4 & 81.4 & 83.8 \\
\hline Wall Tomp. ILF & 82.51 & 82.5 & 82.51 & 827 & 82.7 & 82.7 & 87.41 & 81.41 & 81.4 & 82.8 \\
\hline Instream Temp.f & 83 & 82.91 & 8291 & 82.71 & 82.71 & 82.71 & 81.51 & 81.41 & 81.61 & 824 \\
\hline Avg. Wall Temp.f & 82.61 & 82.51 & 8251 & 82.7 & 82.71 & 82.7 & 81.41 & 81.41 & 81.4 & 83 \\
\hline Avg. Wallot. & 01 & 01 & $0^{\prime}$ & $0 !$ & 01 & 0 & 01 & $0 !$ & 01 & 0.6 \\
\hline Romaning suboooff & 50.51 & 7 & 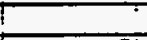 & $i$ & $i$ & 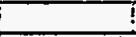 & & i & 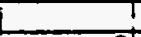 & T \\
\hline Extoualty & of & 01 & 01 & 01 & 01 & 01 & 01 & of & 01 & 0 \\
\hline 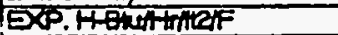 & 01 & 01 & of & 01 & 01 & 01 & 1560.11 & of & 5001 & 3 \\
\hline Diverifosh & 1 & & 1 & 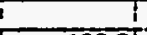 & 1 & 1 & & 1 & & गया \\
\hline Jallouk & 188.81 & 188.71 & 188.7 & 789.6 & 198.6 & 188.8 & 1981 & 198 & 188.11 & 788.4 \\
\hline Szady1(Cudo tubo) & 249.71 & 255.31 & 258.21 & 260.81 & 253.21 & 265.41 & 2571 & 2091 & 271 & 273 \\
\hline Szadt/2 & 249.7 & 255.8 & 258.2 & 250.81 & 2532 & 265.4 & 267 & 269 & $2 \pi 1$ & 273.1 \\
\hline (RLA) & 69587 & 69517 & 695101 & 69383 & 69375 & 65389 & 68852 & 685861 & 68697 & 69163 \\
\hline & 1.032 & 1.055 & 1.087 & 1.078 & 7.088 & 1.097 & 1.107 & 1.115 & 1.122 & 1.13 \\
\hline (AIS) & 249.7 & 255.31 & 258.21 & 200.8 & 2632 & Z5.4. & 267 & 269 & $27 \pi$ & 273.1 \\
\hline \multirow[t]{3}{*}{ (HBOUL) } & 0.7 & 0.7 & 0.71 & 0.71 & 0.7 & 0.7 & 0.7 & 0.7 & 0.7 & 51.1 \\
\hline & & & & & & & & & & \\
\hline & ZONE2 & ZONE4 & $\angle O N=6$ & ZONE 8 & ZONE10 & & & I & & \\
\hline DP tqudd Tomp.t & 87.11 & & 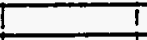 & 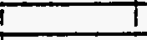 & 1 & & & $t$ & & \\
\hline DPUquid Dematy fomnis & 92.2 & & I & 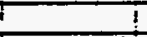 & 1 & 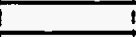 & & I & & \\
\hline Eroviont & 21 & 21 & 61 & 81 & 9.8761 & I & & I & & \\
\hline Mecmured DPPsid & 0.0381 & $=0 . \overline{055 !}$ & c.700? & 0.74 & -0.3261 & 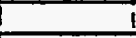 & & I & & \\
\hline Zuro Comections-peid & 0.117 & 0.0881 & Q.CI5] & 0.027 & 0.095 & & & i & & \\
\hline Comaded DPpid & -0.079 & -0.121 & -0.1741 & -0.767 & -0.4 & & & 1 & & \\
\hline Actuld DPA & 2.11 & 4.18 & 6.23 & 9.13 & 10.42 & & & $t$ & L & \\
\hline Mescurod TPSPIg & 4.5 & & & & 300.11 & & & 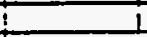 & & \\
\hline \multirow[t]{2}{*}{ Measured Overalle } & $\lim$ & & & & & & & I & L & \\
\hline & & & & & & & & & & \\
\hline computad DPA & 2.001 & 4.18 & 6.241 & 8.281 & 10.14 & & & & & \\
\hline Fitcton-h & 0.091 & 0.181 & 0.281 & 0.37 & 0.47 & & & & & \\
\hline Aconleration & 01 & 0.011 & 0.011 & 0.021 & 0.081 & & & I & & \\
\hline \multirow[t]{2}{*}{ Elevationt } & 21 & 4 & 5.951 & 7.881 & 9.641 & & & I & t & \\
\hline & & & 1 & 1 & 1 & & & 1 & $i$ & \\
\hline
\end{tabular}




\begin{tabular}{|c|c|c|c|c|c|c|c|c|c|c|}
\hline Runt & $692:$ & & & & & & & & & \\
\hline Test Fluid & C4F8 & & & & & & & & & \\
\hline Date: & 719393 & & & & & & & & & - \\
\hline Time: & $-14: 54 !$ & & & & & & & & & \\
\hline Flow Areatl2 & $0.003336 i$ & & & $\cdot$ & & & \pm & & & \\
\hline Hoad Flow Aroarti2 & $0.19695 \mathrm{i}$ & & & $\div$ & $\dot{\square}$ & & : & & $\div$ & $:$ \\
\hline Sal. Pressure Psig & $4.6 i$ & & & $\div$ & & $\therefore$ & & & $\div$ & 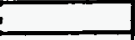 \\
\hline Sat. Tomperatura-finlef) & 30.81 & & & & & & & & $\vec{i}$ & i \\
\hline Lig. Density-bmitis & 92.721 & 92.74 & 92.721 & 92.741 & 92741 & 92.741 & $92.89 !$ & 92.89 & 92.891 & 9274 \\
\hline Latont Hoat-Btuhbm & 44.151 & 44.161 & $44.15 i$ & 44.16 & 44.161 & $44.16 !$ & 44.281 & 44.281 & 44.281 & 44.16 \\
\hline Specifio Heat-Btulbmif & $0.2 \approx 1$ & 0.253 & 0.263 & 0.2631 & 0.253 & 0.263 & 0.262 & 0.262 & 0.262 & 0.263 \\
\hline Liq. Viscostity lbm/thr & 0.832 & 0.832 & $0.832 !$ & 0.8321 & $0.832 !$ & 0.8321 & $0.84 !$ & 0.84 & 0.84 & 0.832 \\
\hline 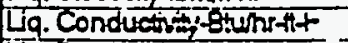 & $\therefore \quad 15 \pi$ & $0.0372 i$ & 0.0371 & $0.0572 !$ & 0.0372 & $0.0372 !$ & $0.0373 !$ & 0.0373 & 0.03731 & 0.05872 \\
\hline Lig. Prandt No. & 5.881 & $5.88 !$ & $5.88 !$ & 5.881 & 5.881 & 5.881 & $5.91 !$ & 5.911 & 5.911 & 5.88 \\
\hline Inlot Fow Rate-GPM & $4 !$ & & & 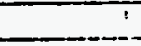 & & 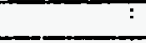 & & & 1 & 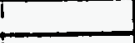 \\
\hline Outal La. Flow Rato-GPM & $1.4 i$ & & & & & & & & i & i \\
\hline Outiet Vap. Flow Rate-GPM & $2.4 !$ & & & & & & & & $T$ & \\
\hline Iniel Tomperanuref & 81.61 & & & & & $\dot{-}$ & $\therefore$ & & \pm & i- \\
\hline Outfet La. Temporedurof & $82.8 \mathrm{t}$ & & & & $\dot{5}$ & & . & & $\dot{-}$ & $\dot{-}$ \\
\hline Outtel Vap. Temparature F & $81.1:$ & & & & $=$ & $\therefore$ & 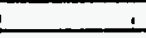 & & 1 & \\
\hline Water Flow Rato-GPM & & & & & & $!$ & 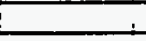 & & 1 & I \\
\hline Water iniet Temp.f & $82.2 !$ & & & & & & & & 1 & 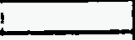 \\
\hline Wator outtet Temp.f & 82.41 & & & & & $\therefore$ & & & & \\
\hline Inlot Glbminhit2 & 886997 & & Temp & 8215 & Density & 92.921 & & & & \\
\hline Subcoolingf & trm-1. & & & & & : & 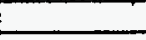 & & & \\
\hline Fow Obsorvation & & & & & & & & & & \\
\hline & ZONEI & ZONE2 : & ZONES & ZONE & ZONES & ZONEE T2 & ZONE7 : & ZONE 8 & 'ZONE9 I & ZONE 10 \\
\hline Hoa inpurwatts(net) & 01 & 01 & $0^{\circ}$ & $0 !$ & $0 !$ & 01 & 0.91 & 01 & 10.31 & 1.2 \\
\hline Heas Loss-We & -11 & -0.21 & -0.31 & -0.31 & $-0.3 !$ & -0.21 & -0.21 & -0.31 & -0.41 & -1.7 \\
\hline Hoed FhorbtuAttrite & 0, & 01 & oi & 01 & 01 & 01 & 161 & 01 & 61 & टा \\
\hline Wall Tomp. \$3F & 83.11 & 83.11 & 83.71 & 83.3 & 83.31 & 83.31 & 82.21 & 82.21 & 8221 & 83.7 \\
\hline Wall Tamp. \#4F & 83.11 & 83.11 & 83.11 & 83.31 & 83.31 & 83.31 & 8221 & 81.91 & 8221 & 83.7 \\
\hline Wall Temp. F5F & 82.91 & 8291 & 82.91 & 83.31 & 83.21 & 83.11 & 81.81 & 81.91 & 81.81 & 89 \\
\hline Wall Tomp. \#1F & 82.11 & 83.11 & 83.11 & 83.31 & 83.31 & $83,3 !$ & 81.91 & 82.21 & 82.11 & 89.7 \\
\hline Wall Temp. $2 f$ & 83.11 & 83.11 & 83.11 & 83.31 & $83.3 i$ & 83.31 & 822 & 82.2! & 8221 & 83.8 \\
\hline Instraam Temp.F & 83.41 & 83.31 & 83.41 & 83.31 & 83.31 & 83.31 & 82.2 & 82.21 & 82.21 & 83.3 \\
\hline Ang. Wall Temp.F & $83.1 !$ & 83.11 & $83.1 !$ & 83.3 & $83.3 !$ & 83.31 & $82.1 !$ & 82.11 & $82 \pi$ & 835 \\
\hline Avg. Wallot.F & $0 !$ & oा & $0 !$ & $0 !$ & 0 & $0 ;$ & 0 & 01 & 81 & 0.2 \\
\hline Romaning subcooff & -50.81 & & & & & & & & & \\
\hline Ext Quatioy & 01 & of & 01 & of & oi & 01 & $0 !$ & $0 !$ & of & 0 \\
\hline 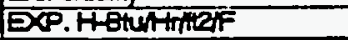 & Di & 01 & 01 & of & 01 & 01 & 1560.11 & 01 & 5201 & 118.2 \\
\hline COMPUTEDH & i & 1 & $\overline{1}$ & & 1 & + & 1 & 7 & 1 & \\
\hline Jallouk & 198.81 & 198.8 & 198.81 & 198.7 & 198.71 & 198.71 & 198.21 & 198.2 & 198.2 & 188.6 \\
\hline Srady1(Cudo tube) & 249.71 & 255.31 & 25231 & 252.81 & $2632 !$ & 265.51 & 267.11 & 269.11 & 2711 & 233 \\
\hline srady2 & 249.71 & 255.3 & 258.31 & 250.81 & $2632 i$ & 265.51 & 267.1 & 259.1 & 271 & 278.3 \\
\hline (RLQ) & 69765 & 69694 & 69748 & 695801 & 69673 & 65661 & 69002 & 68995 & 68989 & 69638 \\
\hline (1) & 1.032 & 1.0551 & 1.067 & 1.078 & 1.088 & 1.097 & 1.106 & 1.115 & 1.122 & 1.128 \\
\hline (HШठ) & 249.7 & 255.31 & 258.31 & 250.81 & 263.2 & 265.51 & 267.11 & 269.11 & $2 \pi$ & 273.3 \\
\hline (HOIL) & 0.7 & $0.7 !$ & 0.71 & 0.71 & $0.7 !$ & 0.7 & $0.7 !$ & 0.7 & 0.71 & 74.1 \\
\hline & & & & & & & & & & \\
\hline & IZONES is & ZONE4 & ZZONE 6 & ZONE8 & ZONETO & & & & & \\
\hline DP Hauld Temp.F & 87.81 & & I & & 1 & I & I & \pm & 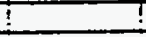 & \\
\hline DPLiquid Density tom/43 & 92.11 & 1 & : & & & 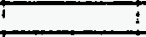 & I & I & 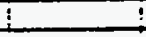 & $!$ \\
\hline Crivition & 21 & 4 & 61 & 81 & 9.876 & & & $t$ & & \\
\hline Magesured OPPsid & 0.0381 & -0.0031 & -0.127 & -0.691 & -0.3231 & & 1 & $i$ & $!$ & \\
\hline Zaro Corrections-psid & 0.1871 & $0.086 !$ & 0.0451 & 0.027 & 0.084 & & $i$ & & $I$ & \\
\hline Corrected DPPsid & -0.079 & -0.719 & -0.1721 & -0.717 & -0.407 & & $\perp$ & & 1 & \\
\hline Acters DP & 2.11 & 4.16 & 628 & 9.061 & 10.44 & & & & & \\
\hline Mensured Tpusig & 4.51 & & & & 300.1 & & & & 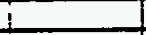 & \\
\hline Messured Overall & +8 & & & & & & & & & \\
\hline & & & & & & & & & & \\
\hline Computed DPAT & 2.05 & 4.191 & 6.24 & 8.28 & 70.14 & & & & & \\
\hline Frtetion & 0.091 & 0.18 & 0.281 & 0.37 & 0.47 & & & & & \\
\hline Acosteratonn & 01 & 0.071 & 0.01 & 0.02 & 0.081 & & & I & $i$ & \\
\hline Elevetion & 21 & 41 & 5.951 & 7.871 & 9.64 & & \pm & i & 2 & \\
\hline & 1 & & 1 & & & 1 & & $?$ & $!$ & \\
\hline
\end{tabular}




\begin{tabular}{|c|c|c|c|c|c|c|c|c|c|c|}
\hline Rund & 6931 & & & & & & & & & \\
\hline Test Flud & C4F & & & & & & & & & \\
\hline Date: & $7 / 19931$ & & & & & & & & & \\
\hline Tims: & $15: 011$ & & & & & & & & & I \\
\hline Fow Arvatte & 0.005981 & & & & T. & - & & F & T & $T$ \\
\hline Hoad Fow Aroati2 & 0.186861 & & - & - & $\cdot$ & $\div$ & : & $\div$ & T & T \\
\hline Sat. Proseure Psig & 4.61 & t. & 7 & 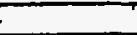 & . & $i$ & $\bar{i}$ &. & $\bar{\vdots}$ & $\bar{i}$ \\
\hline Sal. Tomperature ff(inlal) & 30.81 & & $\square$ & & 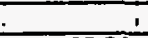 & 1 & 1 & 1 & . & . \\
\hline Lig. Densitylbm/173 & 92.621 & 92.651 & 92651 & 92.621 & 92.841 & 92641 & 92.81 & 92.821 & 92811 & 92.64 \\
\hline Latend Hesteturbm & 44.07 & 44.091 & 44.09 & 44.071 & 44.081 & 44.081 & 44.22 & 44.201 & 44.22 & 44.08 \\
\hline Spectic Hoatstuhibm/F & 0.2681 & 0.2631 & 0.251 & 0.2631 & 0.2631 & 0.2501 & 0.262 & 0.2521 & 0.262 & 0.263 \\
\hline Lig. Viscosity lbmintir & 0.827 & 0.8281 & 0.8281 & 0.827 & 0.827 & त.527 & 3.336 & $0.837 !$ & 0.8361 & 0.821 \\
\hline Liq. Conductivity btuhraf & 0.0371 & 0.03711 & 0.0371 & 0.0371 & 0.03715 & 0.03711 & 0.0372 & 0.03721 & 0.0372 & ర.037 \\
\hline Liq. Prandt No. & $5.86 !$ & 5.861 & 5.861 & 5.861 & 5.881 & 5.86 & 5.8 & 5.9 & 5.89 & 5.86 \\
\hline Inlot Flow Rato-GPM & $4^{\prime}$ & & & & & & & T & ! & \\
\hline Outet La. Flow Rato-GPM & 7.41 & & $\because$ & & : & & & & & t \\
\hline Outlot Vap. Flow Rade-GPM & 2.41 & & T & i & ". & & & - & $\because$ & $\div$ \\
\hline Intet Tempermureff & 82.31 & & I & 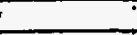 & & & : & : & - & - \\
\hline Outtet Liq. Temperaturef & 83.61 & & $\therefore$ & - & $\cdot$ & - & & i & + & $i$ \\
\hline Outtal Vap. Tomperaturef & $81.4 !$ & & F & . & . & & & $i$ & 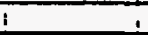 & 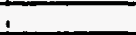 \\
\hline Wator Fow Aalo-GPM & & ti & $\bar{i}$ & & $i$ & ! & ! & I & 1 & 1 \\
\hline Waterinlot Temp.F & 82.91 & & & & & & & & $\therefore$ & \\
\hline Water outlot Temp.F & 83 & & & $\vdots$ & & & & & & + \\
\hline Inlot Glbm/nrmi2 & 8861901 & & Temp & 82.61 & Density & 92.841 & & & $!$ & \\
\hline \multirow{3}{*}{$\begin{array}{l}\text { Subcoolingt } \\
\text { Flow Observation }\end{array}$} & & & & & & & & & $:$ & \\
\hline & $!$ & & & 1 & t & 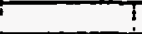 & 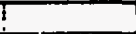 & & & \\
\hline & ZZOMEI & ZONEZ & ZZONEB & ZONE4 & ZONES & ZZONEG & IZONE7 & TZONEB & ZONEO & T2ONi=10 \\
\hline Hexinpur-Waratnoil) & of & of & o1 & 01 & of & 01 & $1 \quad 0.9$ & oा & $1 \quad 0.31$ & 11.1 .2 \\
\hline HonLLoss-Wats & -1.11 & -0.31 & -0.41 & -0.31 & -0.31 & -0.21 & -0.2 & -0.31 & -0.41 & -1.8 \\
\hline How Flowenuthrite & 01 & oi & oा & 01 & 01 & 01 & 16 & oi & हा & दा \\
\hline Wal Tomp. B.F & 83.9 & 83.61 & $83.6 T$ & 84.7 & 841 & 841 & 827 & 8261 & $82 \pi$ & 84.3 \\
\hline Wall Tamp. $4 \mathrm{~F}$ & 83.91 & 83.81 & 83.61 & 841 & 8था & 841 & 82.7 & 82.61 & 827 & 848 \\
\hline Wall Tomp. $15 F$ & 83.6 & 83.61 & 83.61 & 841 & 841 & 841 & 82.5 & 8251 & 8251 & 83.8 \\
\hline Wall Tomp. & 823 & 83.81 & 83.8 & 84.31 & 84 & 841 & 82.6 & 8261 & 828 & 84.3 \\
\hline Wall Temp. $2 F$ & 83.8 & 83.81 & 83.61 & 84.21 & 841 & 841 & 82.7 & 82.71 & 82.61 & 84.2 \\
\hline Instraam Temp.f & 84.1 & 83.91 & 83.91 & 84.11 & 84! & 841 & 82.8 & 82.7 & 82.81 & 84 \\
\hline Avg. Wall Temp.t & 83.8 & 83.7 & $83.6 !$ & 84.11 & $84 !$ & 84 & 82.8 & 82.61 & 82.61 & 842 \\
\hline Avg. Wallot.F & $\because$ & $\mathrm{J}$ & $0^{\circ}$ & 0 & 0 & $0 !$ & 0 & $0 !$ & $0 !$ & 0.2 \\
\hline Remaning subcoolf & -51.51 & : & ' & & & & & & T & $T$ \\
\hline Ext oually & 01 & 01 & 01 & 01 & 01 & गा & 0 & 01 & of & 0 \\
\hline DXP. HQturther & 01 & of & $0 t$ & 01 & 01 & of & 1560.1 & का & 5201 & 118.2 \\
\hline COMPUTEDH & $T$ & 1 & 1 & i & 1 & 1 & 7 & I & 1 & $T$ \\
\hline Jallouk & 1991 & 198.9 & 198.91 & 198.8 & 188.81 & 188.91 & 198.3 & 198.21 & 198.81 & 198.8 \\
\hline Szadv)(Cudo tubo) & 249.8 & 255.41 & 258.31 & 2611 & 263.41 & 265.61 & 2672 & 269.11 & राग & 273.3 \\
\hline sudte & 249.8 & 255.4 & 258.31 & 2611 & 203.4 & 265.8 & 267.2 & 269.11 & 2III & 273.8 \\
\hline (स्मे) & 70128 & 69994 & 69837 & 70101 & 700331 & 700261 & 69206 & 69291 & 65282 & 696 \\
\hline (F) & 1.037 & 1.054 & 1.067 & 1.077 & 1.087 & 7.0061 & 1.108 & 0.1141 & 1.122 & 1.128 \\
\hline (HDO) & 249.91 & 255.4 & 258.31 & 261 & 263.4 & 265.81 & 2672 & 269.11 & 271 & 273.3 \\
\hline \multirow[t]{3}{*}{ (HEOLI) } & 0.7 & 0.71 & $0.7 !$ & 0.7 & 0.7 & 0.7 & 0.7 & 0.7 & 0.71 & 74.2 \\
\hline & & & & & & & & & & \\
\hline & TZONE2 & ZONEG & ZONE & ZONEO & ¿GKEIŨ: & & $\vdots$ & & & \\
\hline DPTgud Tomp.f & $T \quad 881$ & & $T$ & - & $1 \quad-\frac{1}{1}$ & 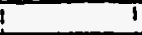 & T & 1 & & \\
\hline DPLiquid Donsity tom/13 & 92.071 & & $T$ & T & 1 & I & $!$ & $!$ & I & 1 \\
\hline Elowiont & 21 & 41 & 61 & 81 & 9.8751 & & & & & \\
\hline Mesaured OPFid & 0.0381 & -0.0311 & -0.1231 & -0.728 & -0.331 & & i & $i$ & I & I \\
\hline Zero Cortectons-osid & 0.117 & 0.0861 & 0.0251 & 0.027 & 0.084 & & i & $T$ & 1 & 1 \\
\hline Cometed DPPsid & -0.079 & -0.117 & -0.168 & -0.755 & -0.4141 & & i & $i$ & 1 & $I$ \\
\hline Acturiopa & 2.11 & 4.16 & 6.22 & 9.12 & 10.48 & & 1 & & 1 & $i$ \\
\hline Masured TPpsig & 4.5 & & & & 300.1 & & 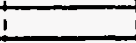 & + & 1 & 1 \\
\hline \multirow{2}{*}{ Mosaured Overalha } & Then & & & & & & 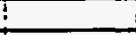 & & 1 & \\
\hline & & & & & & & & & & \\
\hline ComoutedDPA & 209 & 4.191 & ह.या & 8.28 & T0.1का & & & & & \\
\hline Findiont & 0.09 & 0.18 & 0.281 & 0.37 & 0.47 & & ! & & i & t \\
\hline Acostertiont & 01 & $0.01 !$ & 0.01 & 0.02 & $0.03 i$ & & & & & ! \\
\hline \multirow{2}{*}{ Eleventiont } & 2) & 41 & 5.51 & 7.877 & 9.641 & & & 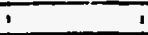 & i & 1 \\
\hline & & & & & & & & & I & T \\
\hline
\end{tabular}




\begin{tabular}{|c|c|c|c|c|c|c|c|c|c|c|}
\hline Run: & 694 & & & & & & & & & \\
\hline Tost Fuid & CAF8 & & & & & & & & & \\
\hline Date: & $7 / 19931$ & & & & & & & & & \\
\hline Time: & $15: 12 !$ & & & & & & & & & \\
\hline Tow Arazthe & 0.003961 & & & & & & & & & $\cdot$ \\
\hline Hoed Fow Areate? & $0.186 \overline{85 i}$ & & 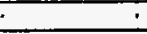 & & & & & & & - \\
\hline S21. Prossuraprig & 4.61 & & 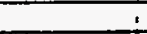 & · & & & & & &. \\
\hline Sal. Tomporeturaf (inlel) & 311 & & & & & & & & & 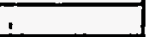 \\
\hline Liq. Density-lbmitt3 & 92411 & 92.44 & 92.441 & 92.451 & 92.451 & 92.451 & $9254 !$ & 92.541 & 92.541 & 924 \\
\hline Latent Heat-Btulbm & 43.91 & 43.92 & $43.92 !$ & 43.931 & $43.33_{1}$ & 43.931 & 44 & 4 & -4 & 13.5 \\
\hline Specific Heat-Bu/lbm/F & $0.2 \approx 5$ & 0.2631 & 0.263 & 0.2531 & $0.263 i$ & 0.2631 & 0.2531 & 0.2631 & 0.253 & 0.250 \\
\hline Lq. Viscosinylbmithr & $0.816 !$ & 0.817 & $0.817 !$ & $0.818 !$ & $0.818 !$ & $0.818 !$ & 0.822 & $0.822 !$ & 0.822 & 0.817 \\
\hline [ig. Conductivity Btumplif & $0.0369 !$ & 0.0359 & $0.0369 !$ & 0.03691 & $0.0359 !$ & 0.03691 & $0.037 !$ & 0.037 & 0.037 & 0.0359 \\
\hline Sa. Prande No. & $5.82 !$ & 5.82 & $5.82 !$ & 5.831 & 5.831 & 5.83 & 5.841 & 5.841 & 5.84 & 5.82 \\
\hline Inlet Fow Rato-GPM & $5^{\circ}$ & & & & & & & & & \\
\hline Outlot La. Fow Rata-GPM & 1.31 & & & & & & & & & i \\
\hline Outlot Vap. Flow Rate-GpiM & 2.81 & & & & & & & & & 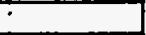 \\
\hline Inlet Tompereturef & 84.41 & & & & $\dot{-}$ & 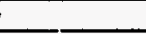 & & & & - \\
\hline Outlet Lia. Temparaturef & 84.51 & & & & . & & $\cdot$ & 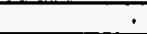 & & . \\
\hline Outlat Vap. Temporature of & $82.3 i$ & & & & . & & . & $i$ & 7 & 7 \\
\hline Wader flow Ralo-GPM & & & & & & & & & & $i$ \\
\hline Water Inlet Temp.F & $84 !$ & & & & & & & & & \\
\hline Wator oudet Temp.F & $84 i$ & & & & & & & & & \\
\hline Inlol Glomprin2 & 1104188: & & Temp & 84.71 & Density & 92.541 & & & & \\
\hline sübcoolingf & inter - & & & & & & & & & \\
\hline Fow Obsenvation & & & & & & & & & & \\
\hline & ZONE & ZONE2 & ZONES & ZONE4 & ZONES & ZONEE 12 & ZONE7 ' & ZONE8 :2 & ZONE9 & TZONETO \\
\hline Hoat Input Watts(nol) & oi & $1-0$ & ! $0 !$ & $0 !$ & $0 !$ & $0 !$ & $0.8 !$ & ol & 0.21 & $\begin{array}{ll}1 & 0.9 \\
\end{array}$ \\
\hline Foed LossotWaths & -0.71 & -0.2 & -0.31 & -0.21 & -0.21 & -0.21 & -0.11 & $-0.2 !$ & -0.81 & -1.2 \\
\hline 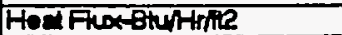 & ol & 0 & 01 & 01 & 01 & 01 & 141 & of & 3 & 98 \\
\hline Wall Tomp. F3F & 85.41 & 85.2 & $85.2 i$ & 85.31 & 86.31 & 86.31 & 84.71 & 84.7 & $84.7 \pi$ & 86.7 \\
\hline Wail Tomo. DAF & 85.41 & 95.2. & $85.1 !$ & 85.31 & 85.31 & 85.31 & 84.71 & $84.7 !$ & 84.71 & 85.7. \\
\hline Wall Temp. $55 F$ & $85.2 !$ & 85 & 851 & 85.31 & 85.31 & 85.31 & 84.71 & 84.61 & 84.7 & 852 \\
\hline Wall Tomp. \#1F & $82.7 !$ & 85.4 & 85.1 & 85.51 & 85.3 & 85.3 & $84.7 !$ & 84.71 & 84.7 & 85.7 \\
\hline Wall Temo. $2 F$ & $85.4 i$ & 85.3 & $85.1 i$ & $85 . \overline{3 !}$ & 85.31 & 85.31 & 84.71 & 84.71 & 84.7 & 85.8 \\
\hline Instroam Temp.F & 85.61 & 85.4 & 85.4 & 85.31 & 85.31 & 85.31 & $84.7 i$ & 84.7 & 84.7 & 85.4 \\
\hline ivg. Wall Tomp. & $85.4 !$ & 85.3 & 85.11 & 85.31 & $85.3 !$ & 85.31 & $84.7 !$ & 84.7 & 84.7 & 85.6 \\
\hline Avg. Wall DT.F & $0^{7}$ & 0 & 0 & $0^{\prime}$ & 01 & $0^{\prime}$ & 0 & 01 & 0 & 0.2 \\
\hline Remaining Subcooff & $-53.4 !$ & & & & & & & & & $?$ \\
\hline Ext Guality & 01 & 0 & 01 & 01 & 01 & ô & 01 & 01 & 01 & 0 \\
\hline EXP. HBtuntritas & 01 & 01 & of & 01 & 01 & 01 & 1386.71 & 01 & 348.7 & 87 \\
\hline COMPUTEDH & 1 & & 1 & & . & 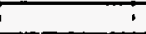 & 1 & 1 & & $i$ \\
\hline Jallouk & 238.11 & 237.8 & 237.81 & 237.8 & 237.81 & 237.81 & 237.51 & 237.51 & 237.41 & 2372 \\
\hline Szeqdy (Qdo tubo) & 298.91 & 305.4 & 308.81 & $311.9 !$ & 314.71 & 397.41 & 319.5 & 321.91 & 324.11 & 3286 \\
\hline Seady2 & 298.91 & 305.4 & 308.8 & 371.9 & 314.71 & 317.4 & 319.51 & 321.91 & 324.11 & 328.8 \\
\hline (RUQ) & 88527 & 88359 & 88350 & 88264 & 88255 & 882461 & $87 m$ & 87768 & 87759 & 88209 \\
\hline (F) & 1.031 & 1.054 & 1.056 & 1.077 & 1.087 & 1.0961 & 1.105 & $1.118 i$ & 9.12 & 1.127 \\
\hline (HLिO) & 298.91 & 305.4 & 308.81 & 311.9 & 314.7 & 317.41 & 319.5 & 321.81 & 324.71 & $3 \mathbf{2 6 0}$ \\
\hline (ABOIIC) & 0.81 & 0.8 & 0.81 & 3.91 & 0.81 & 0.81 & 0.7 & 0.71 & 0.7 & 76.5 \\
\hline & ZZONEZ & ZONEA & ZZONEE & ZONEB & ZONETOT & $\dot{i}$ & $T$ & & & i \\
\hline DPligud Tomp F & 88.41 & 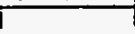 & 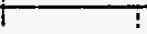 & 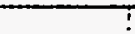 & $\div$ & $\frac{t}{t}$ & it & & & $T$ \\
\hline DPLiquid Density bomitis & 9201 & & 1 & & 1 & $!$ & $T$ & $T$ & & $i$ \\
\hline Elovaliont & 21 & 4 & 61 & 81 & 9.8761 & t & $!$ & 1 & & 1 \\
\hline Whazured DPPaid & 0.0001 & -0.09 & -0.2111 & -0.3471 & -0.4821 & t! & 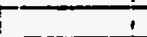 & $i$ & & i \\
\hline Zero Carrections-psid & 0.1171 & 0.085 & 0.0451 & 0.0271 & 0.0841 & $!$ & $\div$ & 1 & & I \\
\hline Correded DPPsid & -0.108 & -0.176 & -0.256 & -0.374 & -0.565 & 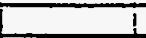 & 1 & & & I \\
\hline Actud DPA & 2.16 & 4.26 & 6.37 & 8.551 & 10.71 & $\perp$ & 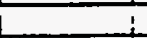 & & & \\
\hline Measured TPPsig & 4.61 & - & i. & & 300.1 & & & & & \\
\hline Mesured Overalt & In & & & & & & & & & \\
\hline & 7 & & & & & & & & & \\
\hline Computed DPh & र.141 & 4.28 & 6.381 & 8.461 & 10.36 & & & & & \\
\hline Fincions & $0.13 !$ & 0.27 & 0.411 & $0.58 !$ & 0.71 & & t & T & & \\
\hline Acostereviont & U.v11 & U.जा & 0.021 & 0.081 & 0.041 & $t$ & & 7 & & $T$ \\
\hline Envitiont & $2 \mathrm{i}$ & 4 & 5.951 & 7.871 & 9.851 & : & & & & T \\
\hline & 1 & $\longrightarrow$ & 1 & 1 & 1 & i & 1 & I & & 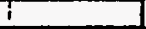 \\
\hline
\end{tabular}




\begin{tabular}{|c|c|c|c|c|c|c|c|c|c|c|}
\hline Runt & 695. & & & & & & & & & \\
\hline Test Fuid & CAf & & & & & & & & & \\
\hline Date: & $7 / 1993$ & & & & & & & & & \\
\hline Time: & $15: 35$ & & & & & & & & & \\
\hline Fow Arearte & 0.003361 & & & & $\dot{\square}$ & & & & & \\
\hline Hos flow Areatil & 0.196951 & & & & & & $\therefore$ & & & \\
\hline S21. Prossuroftig & 4.81 & & - & & - & & $\therefore$ & & & \\
\hline S2t. Temporaturef(inlel) & $31.6:$ & & & & & & & & & \\
\hline Lug. Density-lomstos & 92.12 & 20 15: & $\infty 12$ & 92.12: & $92.12:$ & $32.12 !$ & $92.25 !$ & 92.251 & $9225 !$ & 92.07 \\
\hline Latont Hea-blunbm & 43.67 & 43.671 & $43.67 !$ & $43.67 !$ & $43.67 !$ & 43.671 & 43.77 & 43.71 & 43.77 & 43.63 \\
\hline Spectic Hoar BtulibmiF & 0.264 & 0.264 & 0.264 & 0.2641 & 0.264 & 0.264 & $0.264 i$ & $0.264 i$ & 0.264 & 0.264 \\
\hline Lig. Viscosity & 0.8021 & 0.8021 & $0.802 !$ & 0.802 & 0.802 & 0.8021 & $0.808 !$ & 0.8081 & 0.808 & 0.793 \\
\hline Lq. Conductivityetumath & $0.0367 !$ & 0.0357 & $0.0367 !$ & 0.0557 & $0.0367 !$ & $0.0667 !$ & 0.03581 & 0.03581 & 0.03681 & 0.065 \\
\hline Lq. Prandt' No. & $5.76 !$ & 5.761 & $5.76 !$ & 5.761 & 5.761 & 5.761 & 5.791 & 5.791 & $5.79 !$ & 5.75 \\
\hline Inlot Flow AREGPM & $6 !$ & & & & & & & & & \\
\hline Outtel Lig. Fow Rate-GPM & $0.6 !$ & & ¿ & & & & \pm & & & \\
\hline Outlet Vap. Flow Rate-GPM & $3.2 !$ & & $\div$ & & i & - & $\dot{-}$ & $\dot{-}$ & $\vdots$ & \\
\hline Intel Temperature F & 86.91 & & $\therefore$ & & 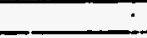 & ? & $\therefore$ & . & \pm & \\
\hline Outel Lia. Tomperaturef & 86.31 & - & $\div$ & & $\therefore$ & & $\therefore$ & $\because$ & $\div$ & \\
\hline Outtal Vap. Tomperaturof & $84.9 i$ & i & & & $\dot{-}$ & & $\dot{-}$ & & $i$ & \\
\hline Wader Flow Rato-GPM & & $!$ & 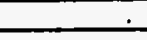 & & & & $\vdots$ & & 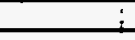 & \\
\hline Water Inlet Tomp.f & 85.8 & & & & & & & & & \\
\hline Wader outtel Temp.t & 85.8 & & & & & & & & & \\
\hline Intat G-lbmhrnt2 & 13209401 & & Temp & 86.715 & Dansity & 92.251 & & & & \\
\hline Subcooling $f$ & & & & & & & & & & \\
\hline \multirow[t]{2}{*}{ Fow Observition } & & & & & & & & & & \\
\hline & TZONET & ZONE2 & ZONE3 & TZONEA & ZONES 12 & ZONE6 & ZONE & ZONEB 12 & ZZONE9 & TONETO \\
\hline Hoal input-Watis(not) & ! 01 & o! & 01 & 0 & 01 & 01 & 0.81 & 01 & 0.11 & 0.4 \\
\hline Hod LoSs-Wets & $=0.71$ & -0.2 & -0.21 & -0.21 & -0.21 & -0.11 & 0.11 & -0.21 & -0.31 & -0.8 \\
\hline 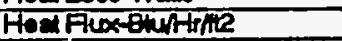 & 01 & 01 & 01 & 01 & 01 & 01 & 141 & ol & 2 & \\
\hline Wall Tomp. d3F & 87.51 & 8721 & 87.21 & 87.61 & 87.5 & 87.61 & 86.71 & 86.71 & 86.71 & 88 \\
\hline Wall Tomp. d4f & 87.51 & 872! & 87.21 & 87.61 & 87.61 & 87.61 & 86.51 & 86.71 & $86.7 !$ & 88 \\
\hline Wall Temp. $75 f$ & 87.41 & $87.2 !$ & 87.21 & 87.61 & 87.51 & 87.61 & 86.71 & 86.71 & $86.7 !$ & 87.8 \\
\hline Wall Temp. $1 F$ & 83.11 & 87.41 & 87.2! & 87.81 & 87.6 & 87.61 & 88.7 & 86.71 & 86.7 & 68.2 \\
\hline Wa Temp. 24 & 87.31 & 87.31 & 87.2 & 87.71 & $87.8:$ & 57.8 & 36.7 & $6 \overline{6.7 .}$ & 86.7 & 88 \\
\hline Instream Temp.f & 87.61 & 87.61 & 87.61 & 87.61 & $87.6 i$ & 87.61 & 86.71 & 85.71 & 86.71 & 88 \\
\hline Avg.Wall Temp.F & $87.4 !$ & 87.31 & $87.2 !$ & $87.6 !$ & $87.6 !$ & 87.61 & $86.7 !$ & 86.71 & 86.71 & \\
\hline Avg. WalloT. & $0^{1}$ & 01 & 0 & $0^{\prime}$ & 01 & 01 & 0 & $0^{\prime}$ & $0 !$ & 0 \\
\hline Remaining Subcooff & -55.31 & & & & & & & & & \\
\hline Ext Cuality & oi & 01 & 01 & $0 !$ & 01 & 01 & 01 & 0 & of & 0 \\
\hline EXP.HQturtriner & 01 & 0 & 01 & 01 & 01 & 01 & 1388.7 & 01 & 179.31 & 693.4 \\
\hline COMPUTEDH & i & 1 & 7 & 1 & $T$ & $T$ & 1 & 1 & & \\
\hline Jallowk & 2761 & 276 & $2761^{-1}$ & 276.91 & 276.01 & 275.91 & 276.31 & 275.31 & 275.31 & 276.1 \\
\hline Szadvilordo tube) & 306.41 & 353.9 & 357.91 & 361.51 & 364.71 & 357.71 & 3701 & 37271 & 375.31 & 3725 \\
\hline Szactre & 346.4 & 353.9 & 357.91 & 361.51 & 364.71 & 357.71 & 3701 & 372.71 & 375.31 & 378.5 \\
\hline (RUQ) & 107787 & $1077 \%$ & 107/60I & 107749 & 10738 & 107727! & 106864 & 106853 & 106812 & 100087 \\
\hline (F) & 1.031 & 1.053 & 1.065 & 1.076 & 1.085 & 1.094 & 1.108 & 1.1111 & 1.119 & 1.125 \\
\hline (Hua) & 346.4 & 353.91 & 357.81 & 357.51 & 364.71 & $367.7 !$ & $3 \%$ & $3727:$ & $375.3 !$ & 3786 \\
\hline (ABOIL) & 0.81 & 0.81 & 0.81 & 4.7 & 0.81 & 0.81 & 0.81 & 0.81 & 0.81 & 0.8 \\
\hline & & & & & & ! & & 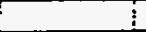 & & \\
\hline & ZONE2 & ZONEA & ZZNEB & ZONEB & ZZONETO! & T! & T & I & & \\
\hline DP Wqudd Temp.f & 88.81 & & 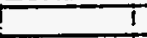 & $\square$ & $1-1$ & I & 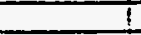 & I & & \\
\hline DPLiquid Density tom/ha & 91.951 & & 7 & & 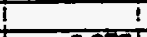 & $\underline{!}$ & & 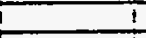 & & \\
\hline Elovetiont & $2 !$ & 4 & $6 !$ & 8 & 9.8761 & $\bar{\vdots}$ & I & I & & \\
\hline Measured DP P gid & 0.0201 & -0.1631 & -0.307 & $-0 . \overline{441}$ & -0.7331 & 1 & 1 & 1 & 1 & \\
\hline Zero Cortections-perd & 0.1171 & 0.0861 & 0.0451 & 0.027 & 0.0841 & $!$ & 1 & $i$ & i & \\
\hline Comeded DPpsid & -0.141 & -0.239 & -0.352 & -0.477 & -0.817 & $i$ & $!$ & 1 & & \\
\hline ACsual DPA & 222 & 4.37 & 6.54 & 8.73 & 11.14 & $i$ & & $\therefore$ & & \\
\hline Mestured TPFsig & 4.8 & & 6 & & 300.1 & $\div$ & $i$ & $i$ & & \\
\hline Mesexured Ovarali & Intsen & & & & & & & & & \\
\hline & & & & & & & $i$ & : & & \\
\hline ComputedDPh & $2.19 !$ & 4.381 & 6.551 & 8.91 & 10.681 & & I & I & & \\
\hline Friclom & 0.191 & 0.37 & 0.571 & 0.77 & 0.981 & & $!$ & $i$ & & \\
\hline Acosileriton-i & 0.011 & 0.011 & 0.031 & 0.051 & 0.081 & I & 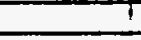 & 1 & & \\
\hline Elevionth & $2 !$ & 41 & 5.951 & 7.881 & 9.681 & i & 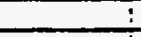 & 1 & & 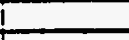 \\
\hline & & 7 & $i$ & 1 & $i$ & $i$ & I & \pm & & \\
\hline
\end{tabular}




\begin{tabular}{|c|c|c|c|c|c|c|c|c|c|c|}
\hline Run & $70-0$ & & & & & & & & & \\
\hline Test fiud & C4F8 & & & & & & & & & \\
\hline Dafo: & $7 / 791931$ & & & & & & & & & \\
\hline Time:- & $15: 351$ & & & & & & & & & \\
\hline Fow Areathe & $0.00336 i$ & & & & & & & & i & \\
\hline Hoat Flow Aroa-tl2 & $0.19605:$ & & & & & & 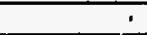 & & & \\
\hline Sed. Pressurepsig & 5. & & . & & & . & & & 1 & \\
\hline Sat Temperatureff(inlel) & $32.1 \mathrm{i}$ & & & & & & & & 1 & \\
\hline Lig. Densitylbmilt3 & 91.911 & 91.911 & $91.91 !$ & 91.841 & 91.851 & 91.861 & $91.99 i$ & 92.041 & 92.011 & 91.82 \\
\hline Latent Heat-Btunbm & 43.51 & 43.51 & 43.5 & 43.441 & $43.45 i$ & $43.47 !$ & $43.57 i$ & 43.61 & 43.581 & 43.43 \\
\hline Spocíic Heat-otulbmif & 0.264 & 0.2641 & 0.264 & $0.265 !$ & $0.264 !$ & 0.2641 & $0.264 i$ & 0.264 & 0.264 & 0.265 \\
\hline Lig. Viscosity -bm/ithr & 0.799 & 0.7991 & $0.791:$ & 0.7871 & $0.788:$ & 0.7891 & 0.795 & $0.797 !$ & 0.796 & 0.787 \\
\hline Lig. Conduetivity-8tums-itF & $0.0365 !$ & 0.0365 & 0.0365 ? & 0.03651 & $0.0365 !$ & $0.0365 !$ & $0.0366 !$ & 0.03661 & $0.0366 !$ & 0.0365 \\
\hline Lig. Prandti No. & 5.72 ? & $5.72 !$ & 5.72 & 5.79 & 5.791 & $5.72 !$ & $5.74 !$ & $5.75 i$ & 5.74 & 5.71 \\
\hline Inlet Fow Ralo-GPM & $7^{1}$ & & & & & & & & & \\
\hline Outel Lia. Fow Ralo-GPM & $0^{\prime}$ & & & & & & & & & \\
\hline Outet Vap. Flow Rato-GPM & $3.6:$ & & & & & & & & & \\
\hline Infel Tompermuref & 68.81 & & & & & & & & & \\
\hline Oülé Liq. Tomperzturar & $8 \overline{8}$ & & & & & & & & & \\
\hline Outlat Vap. Tomporateref & $86.3 j$ & & & & & & & & & \\
\hline Water Flow Rate-GPM & & & & & & & & & & \\
\hline Water Inlet Temp.F & $87.3 ;$ & & & & & & & & & \\
\hline Walor octiot Temp.f & $87.2 i$ & & & & & & & & & \\
\hline Inlat Glbmhritiz & 1537740 & & Temp & 88.111 & Density & 92.051 & & & & \\
\hline Subcooling $f$ & ताहा - - & & & & & & & & & \\
\hline Fow Observation & & & & & & & & & & \\
\hline & ZONET & 'ZONE2 & ZONE3 & ZONE4 & ZONE5 & ZONE6 & ZONE & ZONE8 & ZONE9 2 & ZONE10 \\
\hline Hodinpui-Watts(net) & 01 & $T-0 !$ & $\overline{01}$ & $0 !$ & 01 & 01 & $0.8 !$ & 01 & $0.1 \pi$ & 0.1 \\
\hline Hod Loss-Walls & -0.51 & -0.11 & -0.11 & -0.11 & -0.11 & -0.11 & -0.11 & -0.21 & -0.21 & -0.4 \\
\hline 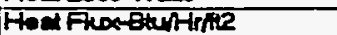 & 0, & 01 & $0 i$ & $0:$ & $0 i$ & 01 & 141 & 01 & 21 & 2 \\
\hline Wall Tomp. 3F & 89.11 & 891 & 88.91 & 89.61 & $89 i$ & 89.21 & 88.11 & 88.11 & 88.11 & 89.5 \\
\hline Wall Tomp. \$4F & 89.11 & 891 & 8881 & 8941 & 89.11 & 89.21 & क्ष8 11 & 88.11 & 88.11 & 89.7 \\
\hline Wall Temp. $15 f$ & 89.11 & 88.81 & 38.8 & 39.2 & $89 i$ & 89 & 88.11 & 88.11 & 88.11 & 89.4 \\
\hline Wall Temp. "\#1F & 83.41 & 89.11 & 88.91 & 89.61 & 89.31 & 89.11 & 88.11 & 88.11 & 88.11 & 89.8 \\
\hline Wall Temp. & 89.71 & 89.11 & 891 & 89.61 & 89.2 & 89.21 & 88.11 & 88.11 & 88.1 & 89.5 \\
\hline Instream Tomp.F & 89.11 & 89.11 & 89.1 & 89.61 & $89.5 \mathrm{i}$ & 89.41 & $88.5 i$ & 88.21 & 88.41 & 89.7 \\
\hline Avg. Wall Temo.f & 89.11 & $89.1 !$ & $38.9 !$ & 89.41 & 89.2 & $89.1 i$ & $88.7 !$ & 88.11 & 88.11 & 89.6 \\
\hline Avg. Wall DT.F & $0^{\circ}$ & $0^{1}$ & 0 & 0 & 0 & 0 & $0 !$ & $0 !$ & $0 ?$ & 0 \\
\hline Remaning subcooff & $-56.7 \pi$ & & & & & & & & & \\
\hline Exth cuality & $0 !$ & 01 & 01 & $0 !$ & of & DI & $0^{\prime}$ & oा & 01 & 0 \\
\hline DP. HBtuntriafF & 01 & 01 & 01 & $\mathbf{0}_{1}$ & $0_{1}$ & 01 & 1388.71 & 01 & 173.81 & 179.8 \\
\hline COMPUTED H & $\cdot$ & & & 1 & 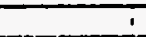 & 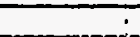 & . & & +1 & \\
\hline Lallouk & 372.81 & 3127 & 31271 & 373 & 3120 & 312.81 & उदूरा & $\overline{311.8 T}$ & $312 . \pi$ & 313 \\
\hline Sradyl(Cudo bubo) & 302.41 & 400.91 & $405.3 i$ & $400.7 i$ & 413.31 & 416.61 & 419.11 & 421.91 & 424.91 & 428.5 \\
\hline szady? & 392.41 & 400.91 & 405.31 & 409.7 & 413.31 & 416.61 & 419.11 & 421.91 & 424.91 & 428.5 \\
\hline (RLR) & $12716 T_{1}$ & $12714 i$ & 127129 & $127585:$ & $\overline{2} 758$ & 1274311 & 1264011 & 126052 & 126263 & 127720 \\
\hline (F) & 1.03 & 1.053 & 1.064 & 1.075 & 1.084 & 1.093 & 1.102 & 1.17 & 1.118 & 1.124 \\
\hline (HUQ) & 392.4 & 400.91 & 405.3 & 409.7 & 413.3 & 416.6 & 419.11 & 421.9 & 424.9 & 4285 \\
\hline (FiBOIL) & 0.81 & 0.81 & 0.81 & 0.8 & 0.8 & 0.81 & $0.8 !$ & 0.81 & 0.81 & 0.8 \\
\hline & & & & & & & & & & \\
\hline & IZONEZ & ZONEL & ZONE & ZONE & ZONE10 & $!$ & + & & & \\
\hline DP nquid Temp.F & i 89.11 & $T-2$ & $+\infty$ & & & & $i$ & & T & \\
\hline DPLquid Densitybom/nes & 91.911 & 1 & T. & $:$ & $!$ & T & 1 & I & 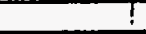 & \\
\hline Elevation & 21 & 41 & 61 & $8 i$ & 9.8761 & $i$ & $i$ & + & $i$ & \\
\hline Masaurod D̄pasid & $=0 . \overline{0611}$ & $-0 . \overline{23} 1$ & 0.422 & -0.5531 & 0.891 & $:$ & $i$ & $\overrightarrow{5}$ & I & \\
\hline Zero Corrections-psid & 0.1171 & 0.0861 & 0.0 .5 & 0.027 & 0.084 & 1 & 1 & $\therefore$ & $\perp$ & L \\
\hline Correded DPPsid & -0.178 & -0.316 & -0.467 & -0.62 & -0.9741 & + & + & $!$ & 1 & \\
\hline Actual DPA & 2.28 & $4.5 i$ & 6.74 & 8.991 & 11.41 & $i$ & & $\therefore$ & & \\
\hline Mossured TPPsig & 5 & & & & 300.1 & & $i$ & 1 & & \\
\hline Mesured overalth & $\therefore \operatorname{sensen}$ & & & & & & & & 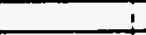 & \\
\hline & 1 & & & & & 1 & & & & \\
\hline Computed DPR & 2251 & 4.51 & 6.74 & 8.95 & T1.01! & 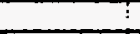 & & & & \\
\hline Fricton- & 0.241 & 0.49 & 0.75 & 7.07 & $1.27 !$ & $\bar{\vdots}$ & $T$ & & & \\
\hline Accolerationth & 0.071 & 0.021 & 0.04 & 0.001 & 0.081 & & T? & & I & \\
\hline Elevetion & 21 & 41 & 5.901 & 7.891 & 9.681 & T & $!$ & 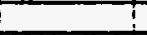 & I & 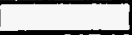 \\
\hline & 1 & 1 & & & & & $i$ & 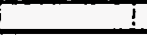 & $i$ & \\
\hline
\end{tabular}




\begin{tabular}{|c|c|c|c|c|c|c|c|c|c|c|}
\hline Runt & $697^{\prime}$ & & & & & & & & & \\
\hline Test Fuid & CAF 8 & & & & & & & & & \\
\hline Dato: & $7 / 19 / 93^{1}$ & & & & & & & & & \\
\hline Timo: & $15: 351$ & & & & & & & & & \\
\hline Flow Arsatle & 0.003381 & & & & $\dot{-}$ & $!$ & & $i$ & 5 & \\
\hline Hod Flow Aros-42 & $0.18695 i$ & & & $\therefore$ & $\therefore$ & & & $\therefore$ & 7 & 7 \\
\hline Sat piosour porig & $5.2 \mathrm{i}$ & & & $\because$ & $\because$ & 7 & ? & ?. &. & 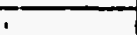 \\
\hline Sx. Temporatureffin[el) & $325 i$ & & & &. & $i$ & & 1 & 5 & \\
\hline Liq.Densty-bmitts & 91.65 & 91.661 & 91.651 & 99.61 & 91.591 & $91.59 \mid$ & 97.691 & 91.69 & 91.681 & 91.8 \\
\hline Latont Hear etubm & 43.291 & $4 \mathbf{3 . 3 1}$ & $43.29 !$ & 43.261 & 43.251 & 43.251 & 43.331 & 43.331 & $43.32 !$ & 40.20 \\
\hline Specitio Hear Bunbmik & 0.265 & 0.265 & 0.265 & 0.265 & $0.265 i$ & 0.2651 & 0.265 & 0.2651 & 0.265 & 0.265 \\
\hline Lq. Viscositylbmithr & $0.778 i$ & 0.7791 & $0.778 !$ & $0.776 !$ & 0.7761 & 0.776 & 0.781 & 0.78 & $0.78 i$ & 0.776 \\
\hline Lg. Conduetivity tuhrfir & 0.0353 & 0.03531 & $0.0363 !$ & 0.03651 & $0.0365 !$ & 0.03531 & 0.03641 & 0.0351 & 0.0364 & 0.063 \\
\hline Liq. Prandt No. & 5.681 & 5.68 & 5.68 & 5.67 & $5.66 !$ & 5.651 & 5.681 & $5.68 !$ & 5.68 & 5.67 \\
\hline Inletfow Rata-GPM & $7.6^{!}$ & & & & & & & & & \\
\hline 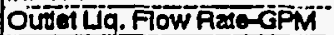 & -ii & & & & & 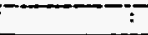 & $\because$ & $\div$ & 7 & $\bar{t}$ \\
\hline Outhot Vap. Fow Rata-GPM & 3.91 & & & & & & & & 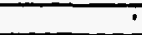 & $\overline{5}$ \\
\hline Indet Tomperturef F & 90.31 & & : & & & 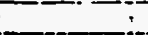 & . & - & $\bar{F}$ & 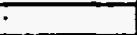 \\
\hline Outellig. Tomperature & $90.2 i$ & & & & $\because$ & $\because$ & . & - & . & . \\
\hline Outlet Vap. Tomparduref & $88.7 i$ & $\bar{i}$ & $i$ & & 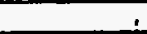 & $!$ & $!$ & $!$ & $\bar{i}$ & $\bar{i}$ \\
\hline Water Fow Ralo-GPM & & & & & $i$ & 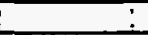 & & 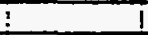 & I & \\
\hline Wator Inlet Tomp.F & $88.6 !$ & & & & & & & & & \\
\hline Water outel Temp.F & 881 & & & & & & & & & \\
\hline Inlet $\mathrm{G}-1 \mathrm{bm} / \mathrm{m} / 2$ & $1662729^{\prime}$ & & Temp & 90.71 & Density & 91.681 & & & & \\
\hline Subcoolingf & :760 & & & & & & & & & \\
\hline Flow Observation & 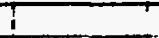 & & & & & & & & & \\
\hline & ZONE1 & ZONEE2 & 'ZONE3 & ZONE4 & IZONES & ZONE6 & ZONET & ZONE 8 & IZONES & ZZONETO \\
\hline Foed hour-Watnot) & 01 & 01 & 01 & 01 & oi & 01 & 0.71 & 01 & 01 & 0 \\
\hline Hoet Loss-Wald & -0.21 & -0.11 & -0.11 & 01 & -0.11 & 01 & -0.71 & -0.11 & 0.11 & 0.8 \\
\hline How Fin eturtint? & 0, & $0 \mathrm{i}$ & $0 i$ & o1 & तू & nू & $12 !$ & 01 & $0 !$ & 0 \\
\hline WAIT Temp. BOF & $90.6 i$ & 90.51 & 90.61 & 91.11 & 90.81 & $31 i$ & $50.8 i$ & 90.21 & 90.81 & 900 \\
\hline Wall Temp. 44 & क0.6i & 90.61 & $90.6 i$ & 911 & 91.11 & 91.11 & 90.21 & 90.21 & 90.31 & 509 \\
\hline Wall Temp. 155 & 90.61 & क0.61 & 90.61 & 971 & 911 & 911 & 90.11 & 90.21 & 90.31 & 90.8 \\
\hline Wall Tomp. \#1F & 83.21 & 90.61 & $90.6 \mathrm{i}$ & 9121 & 911 & 991 & 90.11 & 90.11 & 90.8 & 912 \\
\hline Wal Temp. 27 & 90.6 & 90.61 & $90.6 \mathrm{i}$ & $91.1 !$ & 91! & 911 & 90.11 & 90.21 & 90.3 & 90.8 \\
\hline Instream Temp.f & $90.9 !$ & 90.81 & 90.91 & 91.2 & 91.31 & 91.31 & 90.61 & 90,81 & 90.7 & 91.2 \\
\hline Avg. Wall Temp. & $90.6 !$ & 90.61 & 90.61 & 91.11 & 91! & 911 & 90.71 & 90.21 & 90.31 & 30.9 \\
\hline Ava.WalloT, $=$ & 0! & $0 !$ & $0^{\prime}$ & $0 !$ & $0^{\prime}$ & $0^{1}$ & $0^{\prime}$ & 01 & $0 !$ & 0 \\
\hline fomaining subcoolf & $-57.7 !$ & & & & & & & & 7 & T \\
\hline Ext Qualty & 01 & o! & 01 & 01 & 01 & oi & 01 & वां & 01 & 0 \\
\hline EXP.HEtuthlar & oा & of & DI & of & ol & oi & 1218.41 & 01 & 0 it & 0 \\
\hline COMPUTEDH & 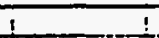 & & & & $i$ & $!$ & & 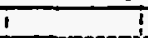 & 1 & \\
\hline JNouk & 33.31 & अ3.21 & उद्या & 33.5 & 33.61 & खरा हा & xa्a! & 33050 & 33301 & 336 \\
\hline Sendilodo tubo) & 419.3 & 428.21 & 4331 & 437.41 & 441.3, & 44497 & 187.7. & $50.9 i$ & $45 \pi$ & 457.8 \\
\hline senty2 & 419.31 & 428.2 & 4331 & 437.4 & 447.31 & 444.8 & 447.71 & 450.91 & 454 & 457.3 \\
\hline (RDO) & 139730 & 1395841 & 130595 & 140069 & 9401711 & 140157 & 139284 & 1392501 & 1393611 & 139875 \\
\hline & 1.031 & 1.052 & 1.084 & 1.074 & 9.089 & 1.092 & 1.1011 & $1.109 !$ & 1.116 & 1.123 \\
\hline (1) & 419.3 & 428.21 & 4331 & 437.41 & 441.3 & 444.8 & 447.7 & 450.91 & 454 & 457.3 \\
\hline$($ (FiBOIL) & 0.81 & 0.81 & 0.8 & 0.8 & 0.8 & 0.81 & 0.81 & 0.8 & 0.81 & 0.8 \\
\hline & & & & & & : & & $?$ & & \\
\hline & TZONE2 2 & ZONEA & ZONEB & 120NE8 & ZONETO & $i$ & & $T$ & & \\
\hline DPlqud Temp.f & I 88.41 & $t$ & 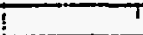 & & & $\frac{1}{1}$ & & I & 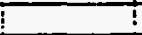 & $\vdots$ \\
\hline DPLiquid Dentherm/13 & 92011 & $T$ & T & T & 1 & 1 & & i & I & i \\
\hline Everctiont & 21 & 41 & 61 & 81 & 9.8751 & $T$ & & i & $\mathbf{T}$ & i \\
\hline Menured DF Frid & -0.0851 & $-0 . \overline{2781}$ & -0.4971 & -0.6911 & $=0.875$ & $\mathrm{i}$ & & 1 & & i \\
\hline Zaro Corrections-psid & 0.1751 & 0.088 & 0.0451 & 0.027 & 0.08341 & 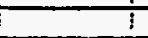 & & 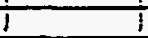 & $\frac{1}{;}$ & $\frac{1}{5}$ \\
\hline CorrectedDPrsid & $-0.202 !$ & -0.365 & -0.542 & -0.7881 & $-0.899_{i}$ & & & & I & i \\
\hline ActuA DPA & 2.33 & 4.59 & 6.88 & 9.16 & 19.33 & $i$ & & 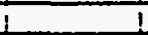 & I & \\
\hline Meraured TPFsig & $5.2 !$ & & & & $300.1 !$ & 1 & 1 & 1 & & \\
\hline Mesaured Overallh & 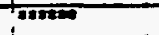 & & & & & 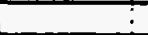 & & 1 & 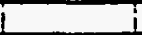 & \\
\hline & & & & & & & & + & 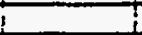 & \\
\hline Computed DRA & $2.29 !$ & 4.581 & 6.881 & 9.721 & T1.22! & & & & & \\
\hline Fictors & 0.281 & 0.561 & 0.881 & 9.181 & 7.461 & $T$ & & $T$ & & \\
\hline Acotorion & 0.017 & 0.021 & 0.0 .1 & 0.011 & 0.091 & t! & & $T$ & $\mathrm{~T}$ & \\
\hline Elovationth & 21 & 4 & 5.981 & 7.89 & 9.871 & ! & & I & $!$ & \\
\hline & $\therefore$ & & i & $i$ & & I & & 1 & & \\
\hline
\end{tabular}




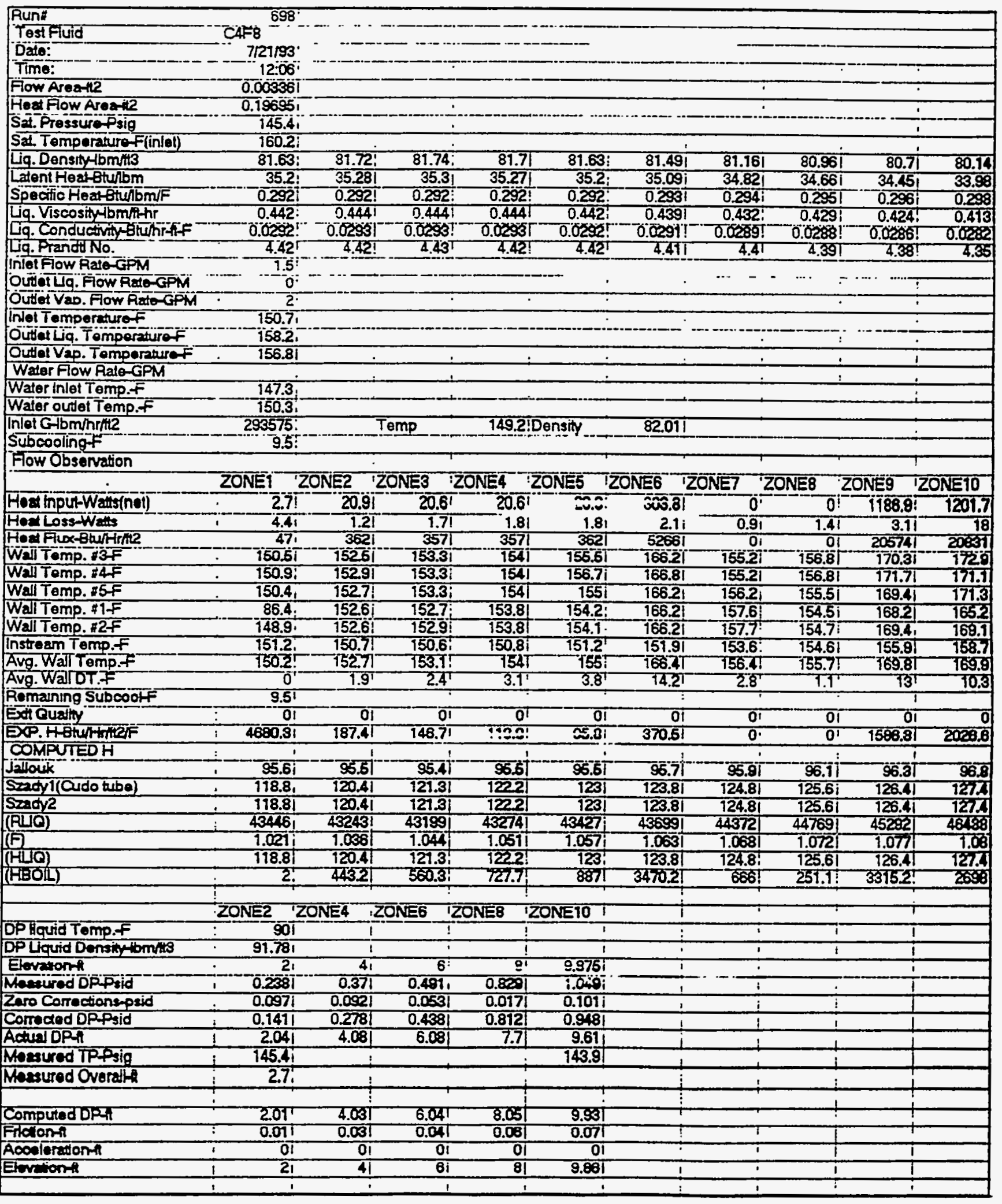




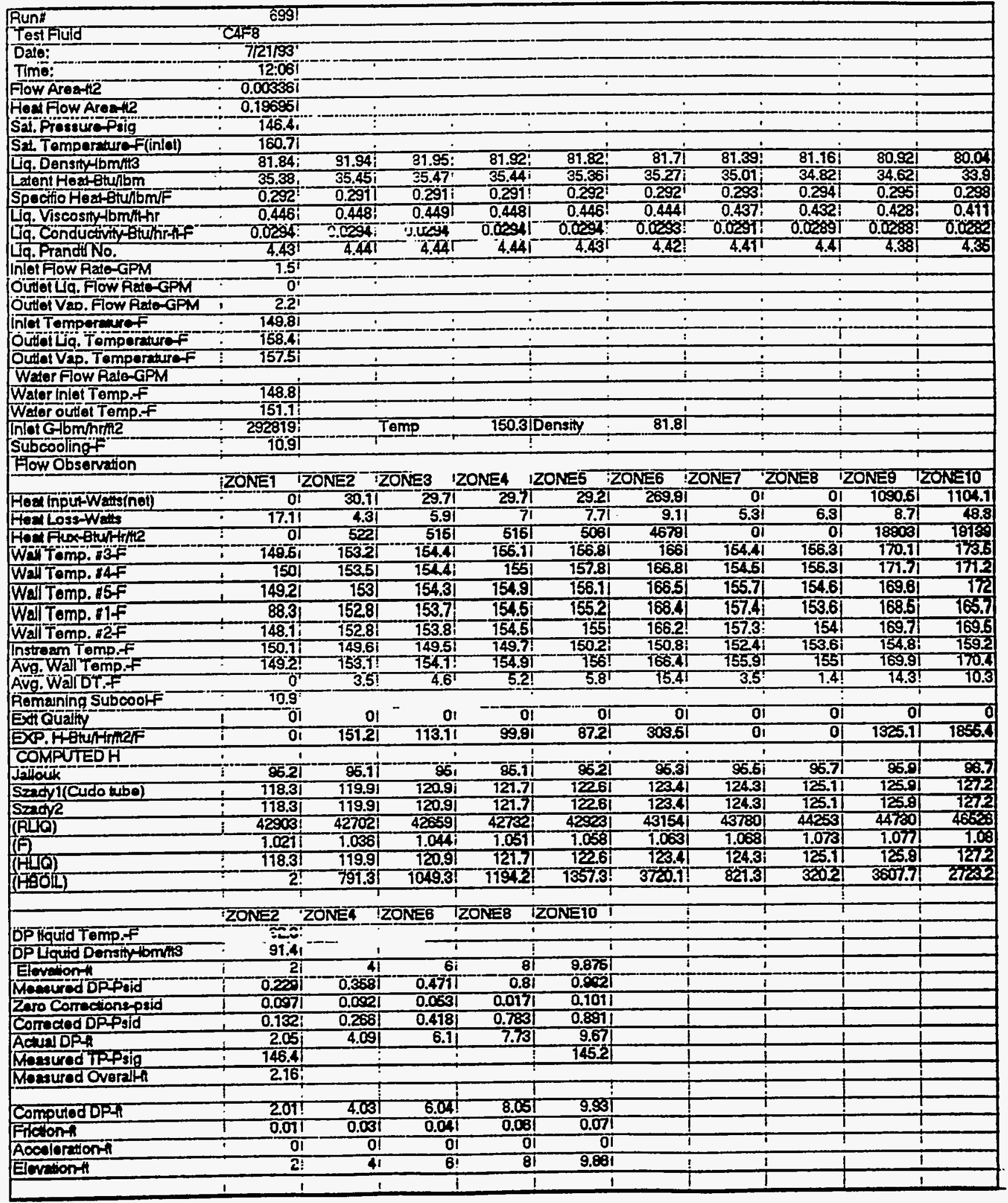




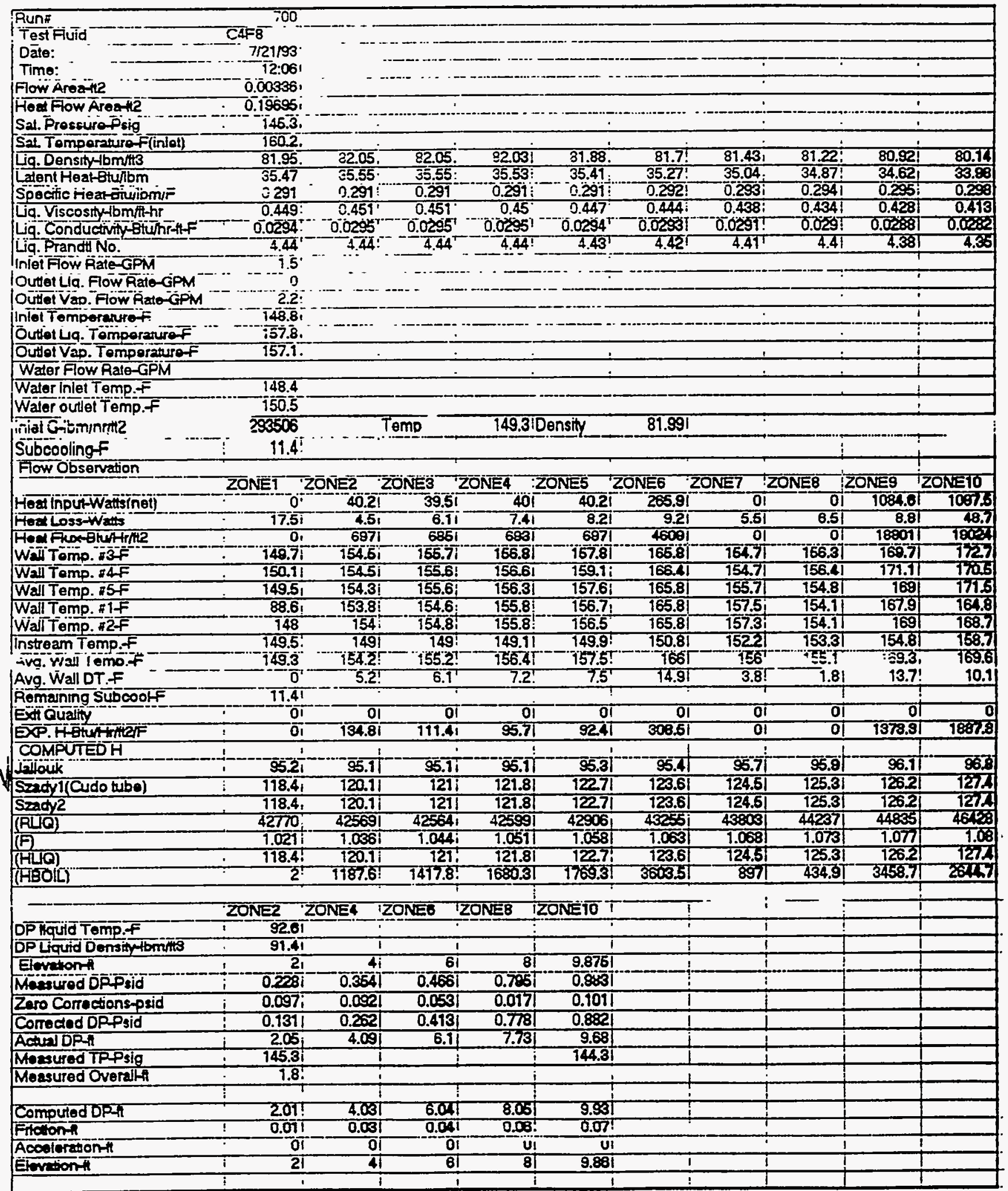




\begin{tabular}{|c|c|c|c|c|c|c|c|c|c|c|}
\hline \}Un̈" & 701 & & & & & & & & & \\
\hline Test Fluid & CA $F$ & & & & & & & & & \\
\hline Date: & $7 \sqrt{1} 193$ & & & & & & & & & \\
\hline Timo: & $94: 34 !$ & & & & & & & & & $\bar{T}$ \\
\hline Flow Areante & 0.003981 & & & & & & $\therefore$ & $\therefore$ & i & $i$ \\
\hline Hod Fow A Poath & 0.186951 & $\therefore$ & & & $\therefore$ & - & 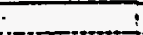 & 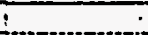 & - & $!$ \\
\hline SaA. Prossura psig & $136 i$ & & & & $\therefore$ & - & & $\therefore$ & $=$ & $\therefore$ \\
\hline Sat Tompereutre-F(inlel) & 155.7: & & & & & & & $i$ & i & \\
\hline Liq. Densitylomit3 & 82.24 & 82.241 & 3226. & 52.70 & OS ia: & 25n? & 81.91 & 81.76 & 81.59 & 81.2 \\
\hline Latent Heatetuibm & 35.7 & 35.7 & 35.72 & 35.691 & 35.61 & $35.56 i$ & 35.42 & 35.311 & 35.17 & 34.85 \\
\hline Specitic Heat Btuhb & 0.29 & 0.291 & $0.29 i$ & $0 . \overline{29}$ & 0.291 & 0.2911 & 0.291 & 0.292 & 0.292 & 0.294 \\
\hline Lig. Viscosity fbm/nhr & 0.455 & 0.4551 & 0.455 & $0.455 !$ & 0.4531 & 0.4511 & 0.4481 & $0.445 \mid$ & 0.441 & 0.433 \\
\hline [ig. Conduetwivetuhrof & 0.028 & 0.0261 & 0.0297 & 0.02561 & 0.0296 & $0.0 \times 5$ & 0.0294 & 0.02331 & 0.029 & 0.0089 \\
\hline Lig. Ptandóno. & 4.46 & 4.461 & $4.46 !$ & $4.45 T$ & 4.451 & $4.44 !$ & $4.43 !$ & 4.431 & 4.42 & 4.4 \\
\hline Iniet Fow Paro-GPM & 3 & & & & & & & & & \\
\hline OÚtel Uq. Fow Ra & 0.81 & & & & & & & $\overline{3}$ & 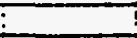 & $!$ \\
\hline Oütol Vad. Flow RateGPM & $2.7 !$ & & & & & & 7 & F & 5 & i \\
\hline Intat Tempormuret & 147.81 & & & & & & & & 1 & 1 \\
\hline Outlel Lia. Tomperatur of & $153.4 i$ & & & & & & . & - & T & I \\
\hline Outtel vap. Tomperaturef & 151.6 & & & & - & 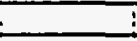 & $\bar{i}$ & $i$ & 1 & $\frac{1}{1}$ \\
\hline Water Flow Rato-GPM & & & & & & : & & it & 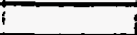 & $i$ \\
\hline Water Inlet Temp.f & 144.8 & & & & & & & & & \\
\hline Waler outel Temp.f & 145.31 & & & & & : & & $i$ & 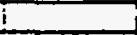 & \\
\hline Inlet Glbmhrnt2 & 592023 & & Temp & 145.611 & Density & 82.91 & & 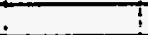 & ! & 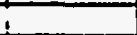 \\
\hline Sübcoolingt- & 7.9 & & & & & & & & & \\
\hline Flow Observation & & & & & & & & & & \\
\hline & IZONET & ZZONE2 & ZONES & ZONE 4 & ZONES & ZONE6 & IZONE7 & ZZNE8 & ZONES & LONEIO \\
\hline Hod npor-Wato(nol) & $0 i$ & $1-21.81$ & 31.4 & 31.91 & 129.01 & 345.81 & $0 i$ & $0 !$ & 1317.7 & 1292 \\
\hline Hed Loss-Wen & 22.81 & 5.41 & $7.4 \pi$ & 9.41 & 10.41 & 10.71 & 8.11 & 8.91 & 11.11 & 58.8 \\
\hline 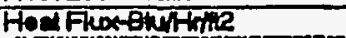 & & 3781 & 544 & 6581 & 6131 & 5966 & 01 & $0 i$ & 2287 & ב⿸\zh14𠃋 \\
\hline W개 Temp. 位F & i70.21 & 149.61 & $15 \overline{0.81}$ & $\overline{157.81}$ & 1521 & 961.91 & 150.71 & 151.8 & $1 \% 6.61$ & 170.7 \\
\hline Wall Tomp d4F & $149.7 !$ & 150.71 & 150.81 & 151.71 & 1531 & $1 \approx 29 !$ & 150.71 & $152 \pi$ & 168.11 & 167.8 \\
\hline Wall Temp. $15 \mathrm{~F}$ & 149.7 & 1501 & 151.1! & $151.7 !$ & 151.81 & 1625 & 151.6 & 151 & 165.4 & 1692 \\
\hline Wall Tomp. If & $89.9 !$ & $1502 !$ & 150.3: & 151.31 & 151.41 & 162.41 & 152.8 & 150.11 & 164.8 & 161.2 \\
\hline Wall Tomp. Rf & 1486. & $750 . \overline{2}$ & $150.6=$ & 151.31 & $151.1 !$ & 16231 & 152.8 & 150.4 & 165.4 & 15.8 \\
\hline Instream Temp.f & 148 & 1481 & $147.9 i$ & 148.11 & 148.51 & 148.8 & 149.81 & 150.51 & 151.4 & 753.4 \\
\hline Avg. Wall Tomp.F & 749.3! & 1501 & $150.8 !$ & 151.61 & $751.8 !$ & 762.41 & 157.71 & 151.71 & 165.9 & 167 \\
\hline Avg. Wall DT. & 1.3 & $2 !$ & $2.7^{1}$ & 3.51 & 3.31 & 13.31 & 1.9 & 0.61 & 13.5 & 125 \\
\hline Romaining Suboooff & 7.91 & & & & & $\overline{1}$ & & 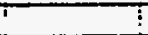 & 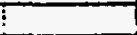 & \\
\hline Ext Qually & 01 & of & 01 & ol & 01 & 0ा & 01 & 01 & 0 & \\
\hline DP. Hetumtmar & 01 & 18821 & 201.61 & 1581 & 155.41 & 451.8 & 01 & 01 & 1691 & 7784.8 \\
\hline COMPUTEOH & 1 & & & & & & 1 & 51 & 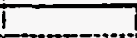 & \\
\hline Jallouk & 168.5 & $168.6 !$ & 166.41 & 166.51 & 766.61 & 768.71 & 167 & 167.2 & 767.5 & 168.1 \\
\hline Sadyi(Cudo tube) & 207 & 210.1 & 217.71 & 21321 & 214.7 & 216.11 & 217.51 & 218.81 & 220.11 & 22.7 \\
\hline Srady 2 & 207 & 210.1 & 211.7 & 2132 & 214.71 & 216.11 & 217.51 & 218.8 & 220.1 & בृ21. \\
\hline (RाQ) & 85096 & 85031 & 84997 & 85144! & 85477 & 85751 & 8649 & 869331 & 87699 & 8925 \\
\hline (F) & 1.021 & 1.0361 & 1.045 & 1.052 & 1.058 & 1.080 & 1.059 & 1.074 & 1.079 & $1.0 \overline{83}$ \\
\hline$\frac{1}{1+250}$ & 207 & $210 . \pi$ & 211.7! & टाउदा & 274.71 & 216.71 & 217.51 & 218.8 & 220.1 & 221.7 \\
\hline (1-30L) & 2852 & 4452 & $6023 !$ & 787.61 & 745.71 & 37122 & 435.11 & 728.8 & 3274 & 3170.8 \\
\hline & & & & & & & & & & \\
\hline & TZONEZ & TZONE4 & ZONE6 & ZONE8 & ZONETOT & & & $T$ & & \\
\hline DPT जuld Temp. $f$ & 93.1 & & 1 & $!$ & 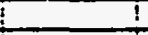 & !- & & 1 & 5 & \\
\hline DPLquid Donsity 1 bm/13 & 91.39 & & $i$ & i & 1 & & & $t$ & 1 & \\
\hline Elovion- & $2 !$ & 4 & 61 & 81 & 9.8751 & $\Gamma$ & & T & T & I \\
\hline Mecuired DFFid & 0.100 & 0.28 & 0.381 & 0.6781 & 0.507 & 1 & & ! & T! & 1 \\
\hline Zaro Corrections-prid & 0.0971 & 0.0021 & 0.053 & 0.077 & 0.1011 & 1 & & 1 & I & $i$ \\
\hline Comected DPPsid & 0.102 & 0.206 & 0.327 & 0.6611 & 0.5561 & 1 & & $i$ & i & \\
\hline Acual DPA & 2.07 & 4.13 & 6.17 & 7.831 & 10.051 & I & & & & \\
\hline hersured tipstig & 136 & & & 1 & 135.7 & $i$ & & 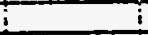 & $i$ & \\
\hline Messured Overall & 0.531 & & & & & : & & & & \\
\hline & & & & & & $i$ & & & & \\
\hline Computed DPA & 2.05 & $4 . \pi$ & 6.151 & 8.2. & T0.1! & 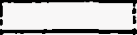 & & !! & & \\
\hline Fitowion-l & 0.05 & 0.11 & 0.151 & 0.2 & 0.261 & & & T & t & \\
\hline Acooleratont & 0 & of & 01 & of & 01 & & & T & & \\
\hline Elve'ion- & 21 & a1 & 61 & 81 & 9.851 & T & & $i$ & $i$ & $T$ \\
\hline & & & & $T$ & 1 & I & & 1 & $T$ & \\
\hline
\end{tabular}




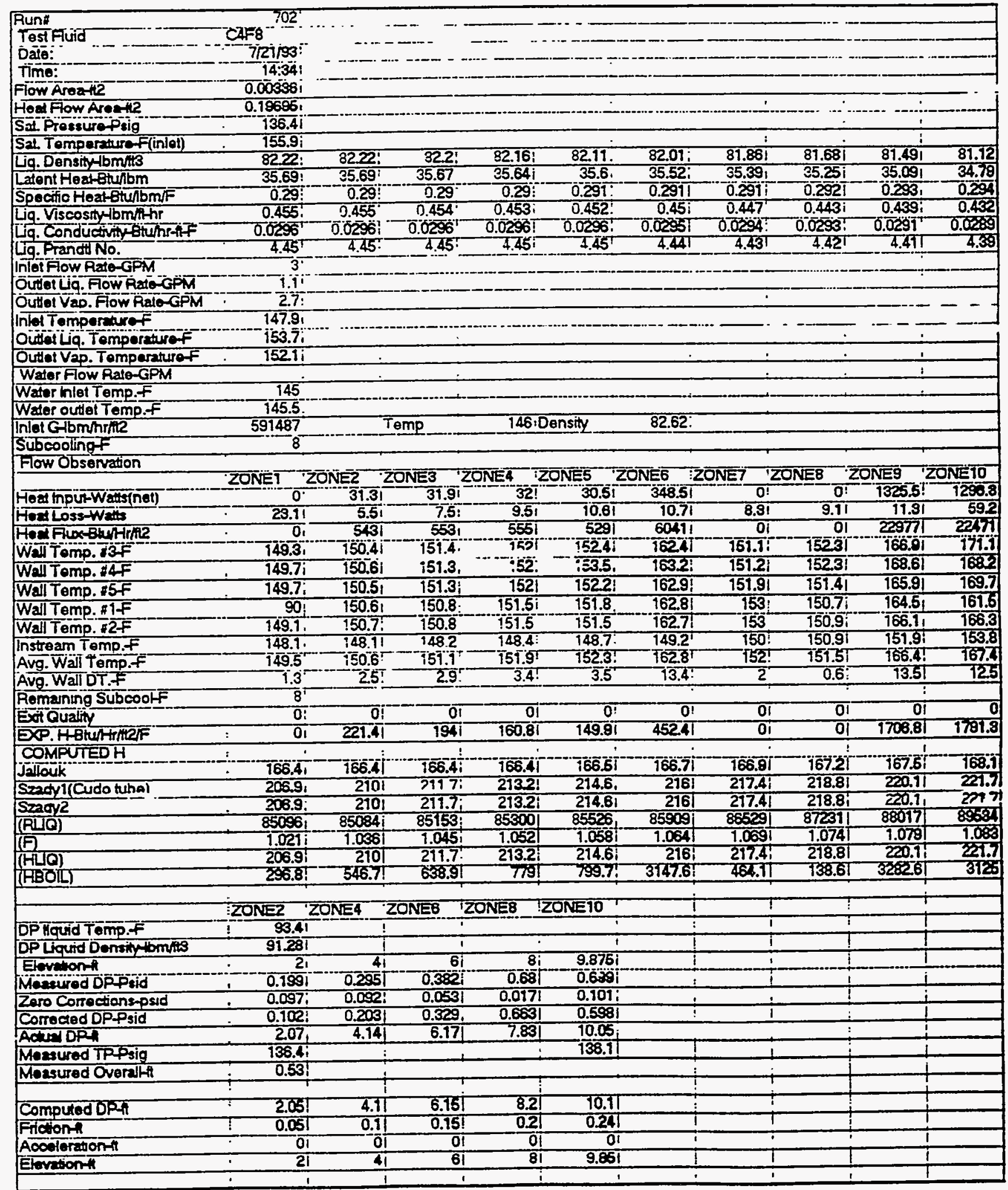




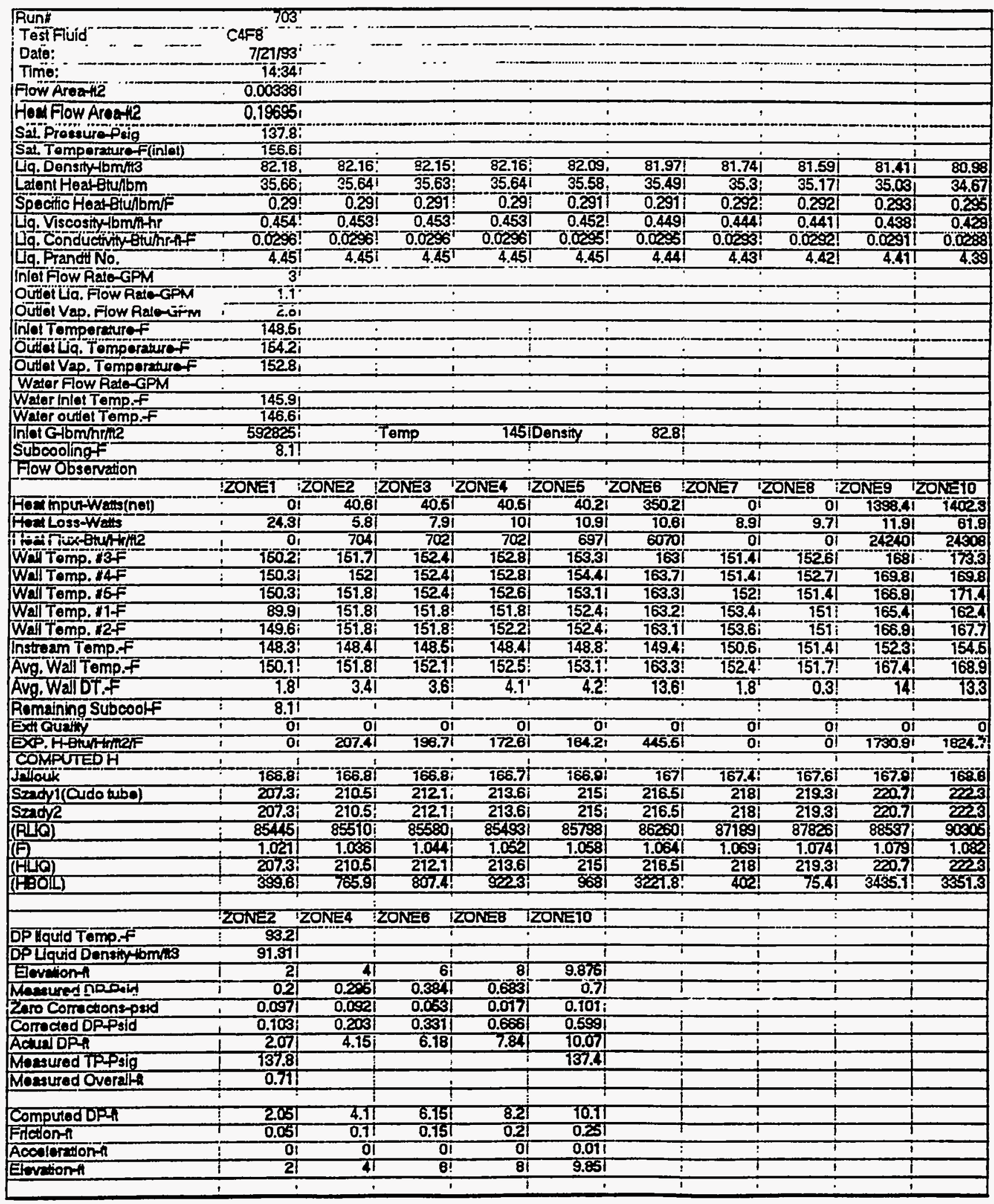




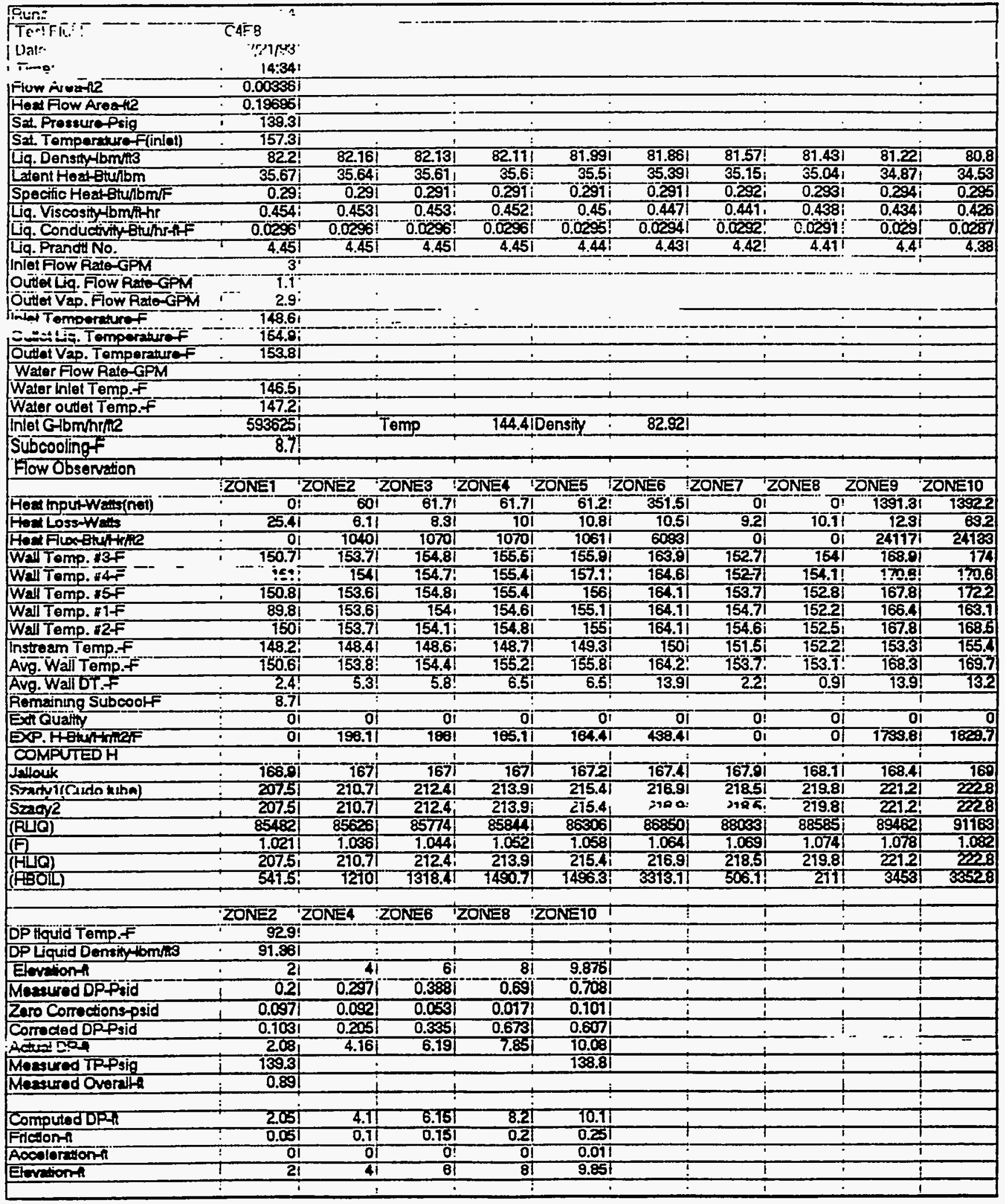




\begin{tabular}{|c|c|c|c|c|c|c|c|c|c|c|}
\hline Bun: & $705 !$ & & & & & & & & & \\
\hline TestFiud & C4F8 & & & & & & & & & \\
\hline Daio: & $-8=15=0$ & - & & & & & & 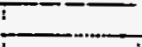 & & \\
\hline Time: & $\frac{8.14}{0.003981}$ & & & & & & & & & \\
\hline $\begin{array}{l}\text { Fow Aroath } \\
\text { How Fow Aroatk? }\end{array}$ & $\begin{array}{r}0.003981 \\
0.06051\end{array}$ & & - & $\dot{-}$ & . & & & - & $\div$ & $:$ \\
\hline 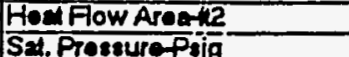 & - 0.186961 & 1 & - & - & & $\dot{5}$ & & - & & \\
\hline Sat. Prossure-psig & 134.21 & . & - & ?. & - & & & & - & $:$ \\
\hline Sad. Temperature finlel) & 154.81 & & & & & & & & & \\
\hline Lia. Densitylbm/103 & $82.22 !$ & 82.241 & 82.261 & 82.21 & 82.161 & 82.001 & 81.881 & 81.81 & 81.651 & 81.57 \\
\hline Ladent Heat-Bturbm & 35.69 & 35.71 & 35.72 & 35.67 & 35.641 & $35.58 !$ & 35.411 & 35.341 & 35.221 & 35.15 \\
\hline Speodio Heat-gh/bm/F & 0.291 & 0.291 & 0.29 & 0.291 & $0.29 \mathrm{i}$ & $0.291 i$ & $0.291 i$ & 0.2921 & 0.2921 & 0202 \\
\hline Lq. Viscositylom/hh & 0.455 & 0.455 & 0.455 & 0.454 & $0.453 !$ & 0.4521 & $0.447 i$ & $0.445 i$ & 0.4421 & 0.441 \\
\hline 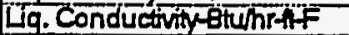 & 0.02961 & $0.0206 !$ & $0.0297 !$ & 0.0203 & $0.0206 !$ & 0.02051 & $0.0 \times 4 !$ & 0.02031 & 0.0025 & 0.0002 \\
\hline Lig.Prandd No. & 4.451 & 4.451 & 4.46 & 4.451 & $4.45 T$ & 4.451 & 4.431 & 4.431 & 4.421 & 4.42 \\
\hline intetfow Rato-GPM & $4^{1}$ & & & & & & & & & \\
\hline 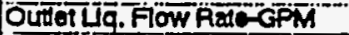 & $7.2 !$ & & & $\mathrm{i}$ & $T$ & & & & $\cdot$ & \\
\hline 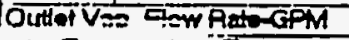 & 3.31 & $\vdots$ & 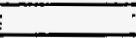 & $\cdot$ & - & ت & $\div$ & :- & ; & \\
\hline Intel Tolingen ing & 146.31 & 7 & 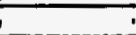 & 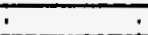 & . & . & T. & 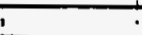 & $\div$ & 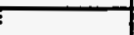 \\
\hline Out ll Lig. Tomporature & 162.11 & $\div$ & 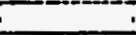 & - & I & 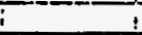 & I & I & . & \\
\hline Oudat Vap. Temperatur of & 150.11 & 1 & 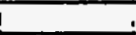 & 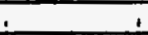 & $i$ & 1 & i & i & $\overline{1}$ & \\
\hline Walar Flow Ralo-GPM & & & & 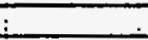 & 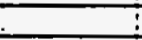 & $\vdots$ & $!$ & $\vdots$ & 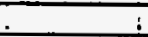 & 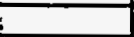 \\
\hline Water inlot Temp. F & 141.3 & & & & & & & & & \\
\hline Water outlet Tomp.f & 141.7 & & & & $i$ & & $i$ & 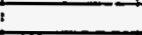 & & \\
\hline Inlel G-bmhrnte & $791322_{1}$ & & Temp & 144.51 & Donsity & 82.9 & & & & \\
\hline Subcoolingf & $8.5 !$ & & & & & & & & $i$ & \\
\hline \multicolumn{11}{|l|}{ Fow Observation } \\
\hline & ZONET & ZONE2 & ZONES & ZZONEA & IZONES & ZZONEB & ZONE7 & ZZONEB & ZONES & ZONEIO \\
\hline HoThporwang & 01 & 39.81 & -39.91 & I 39.31 & 39.31 & 340 & $0 !$ & - 01 & 13136.61 & 13528 \\
\hline Hed Loss-Wets & 291 & 5.61 & $7.8 !$ & 9.81 & 10.61 & 10.81 & 8.21 & 9.11 & 11.31 & 592 \\
\hline Hodffix-Btuthine & ol & 6921 & 692 & 6811 & 6871 & 59981 & $0 !$ & 01 & 233411 & 23150 \\
\hline Wall iemp. 73 ? & 148.31 & $150.6 \overline{1}$ & 1511 & 152 iा & 157.61 & 160.51 & 150.51 & TE!.A! & 166.81 & $1 \pi .1$ \\
\hline Wall Tomp. I4F & 948.61 & 1511 & 157.11 & 151.91 & 15291 & 161.11 & 150.61 & 157.51 & 168 & $16 \% .6$ \\
\hline Wall Temp. $15 F$ & 1491 & 1511 & 151.51 & 151.8 & 1521 & 160.5 & 151.31 & 150.7 & 16521 & 158.8 \\
\hline Wall Temp. $1 F$ & 81.3 & $150.7 i$ & 150.61 & 751.61 & 151.61 & 160.51 & 152 & 150.11 & 163.81 & 160.7 \\
\hline Wall Temp.ref & $148.2 i$ & 150.81 & $150.8 i$ & 151.71 & 151.4 & 160.61 & $151.9 i$ & 150.31 & 165.21 & 165.4 \\
\hline Instream Tomp.f & 148.1 & 1481 & 147.91 & 148.2. & 148.41 & 148.81 & 149.9 & 150.31 & 151.11 & 151.5 \\
\hline Avg. Wai Temp.t & $748.5 T$ & 756.81 & $150.9 !$ & 151.91 & $152 !$ & 160.71 & $151.3 !$ & $150.8 !$ & 965.7 & 166.7 \\
\hline Avg.Wall DT.F & 0.4 & 2.71 & 2.91 & 3.61 & 3.51 & T1.6T & 1.41 & 0.51 & 13.51 & 74.2 \\
\hline Romainting subcooff & 8.51 & & & : & : & 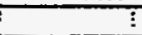 & & 1 & $i$ & \\
\hline Extoualty & 01 & 01 & ठा & oा & oा & 01 & oi & 01 & of & \\
\hline EXP. HQthenthar & oi & 25211 & 205 & 188.8 & 19221 & 518 & o1 & 이 & 172321 & 1050 \\
\hline COMPUTEDH & : & & & 1 & 1 & 1 & 1 & 1 & 1 & \\
\hline Jallouk & 2101 & 2101 & 200.01 & 2701 & 210.71 & 21021 & 270.61 & 210.8 & 211.11 & $2 \pi 2$ \\
\hline Sandy (Cudo bubo) & 261.11 & $255 i$ & 菏 & 2EO? & 2309 & $2725:$ & 274.44 & 275.9 & 2751 & 278 \\
\hline Szacty2 & 261.11 & 265 & 2671 & 2091 & 270.81 & 27251 & 274.41 & 275.9 & 27.51 & 278.9 \\
\hline (स्वि) & $11 F 348$ & 113725 & 113610 & 1139711 & 114108 & 114514 & 115657 & 1160671 & 116900 & 117316 \\
\hline (F) & 1.021 & 1.066 & 1.045 & 1.052 & 1.058 & 1.059 & 1.059 & 1.075 & 1.079 & 1.084 \\
\hline (सायक) & 261.11 & 265 & 267 & 269 & $270.8:$ & 2726 & 274.41 & 275.91 & 277.51 & 278.8 \\
\hline (FBOTL) & 90.91 & 6731 & 6581 & छ्दो.9ा & $800.8 !$ & 2706.4 & 301 & 710.31 & उदुव.1 & 340.8 \\
\hline & & & & & & & & & & \\
\hline & ZONE2 2 & ZOME & ZONEB & ZONE8 & ZONE10 & & T & & $T$ & \\
\hline DPT & $i \quad 83.41$ & $T$ & $-\infty$ & 1 & 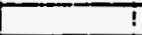 & & $t$ & T & i & \\
\hline DPLquid Density tompin & 9272 & $T$ & T & $\mathrm{T}$ & T & $T$ & $T$ & $T$ & $T$ & \\
\hline Elevationth & 21 & 41 & $6 i$ & 8 & 9.8751 & I & I & ! & $!$ & \\
\hline Masturad DPPaid & $0.309]^{\circ}$ & $0 . \overline{28} 41$ & 0.3711 & 0.549 & 0.6271 & 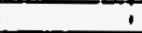 & I & & & \\
\hline 7am Corrartionsensid & (10031 & 0.0761 & 0.081 & 0.027 & 0.0881 & 1 & & $\frac{1}{1}$ & 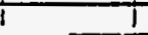 & \\
\hline Sirveted DPpid & $0.106 !$ & 0.2008 & 0.291 & 0.5161 & $0.541_{1}$ & & & & & \\
\hline Actul DPh & 2.09 & 4.18 & 6.311 & 8.19 & 10.21 & & & & 1 & \\
\hline Messured TPFsig & 1342 & & & & 132 & & $\frac{1}{\vdots}$ & & & \\
\hline Mozsurad Overalth & 3.88 & & + & 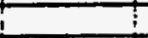 & & & 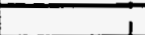 & & $i$ & \\
\hline Campedrats & & & & & & & & & & \\
\hline Computed DPA & 2.081 & 4.77 & 6.251 & 8.33 & $\frac{10.2 .1}{0.75}$ & & & & & \\
\hline Fraton-1 & 0.081 & 0.171 & 0.251 & 0.331 & 0.471 & & & & & \\
\hline Aocolortion & o1 & of & 01 & 01 & 0.071 & 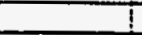 & i & 1 & I & \\
\hline Eloviont & 2ा & का & 81 & 81 & 9.851 & $T$ & $T$ & I & $T$ & \\
\hline & $=$ & & $i$ & $i$ & $\longrightarrow$ & & $t$ & $T$ & 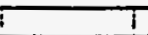 & \\
\hline
\end{tabular}




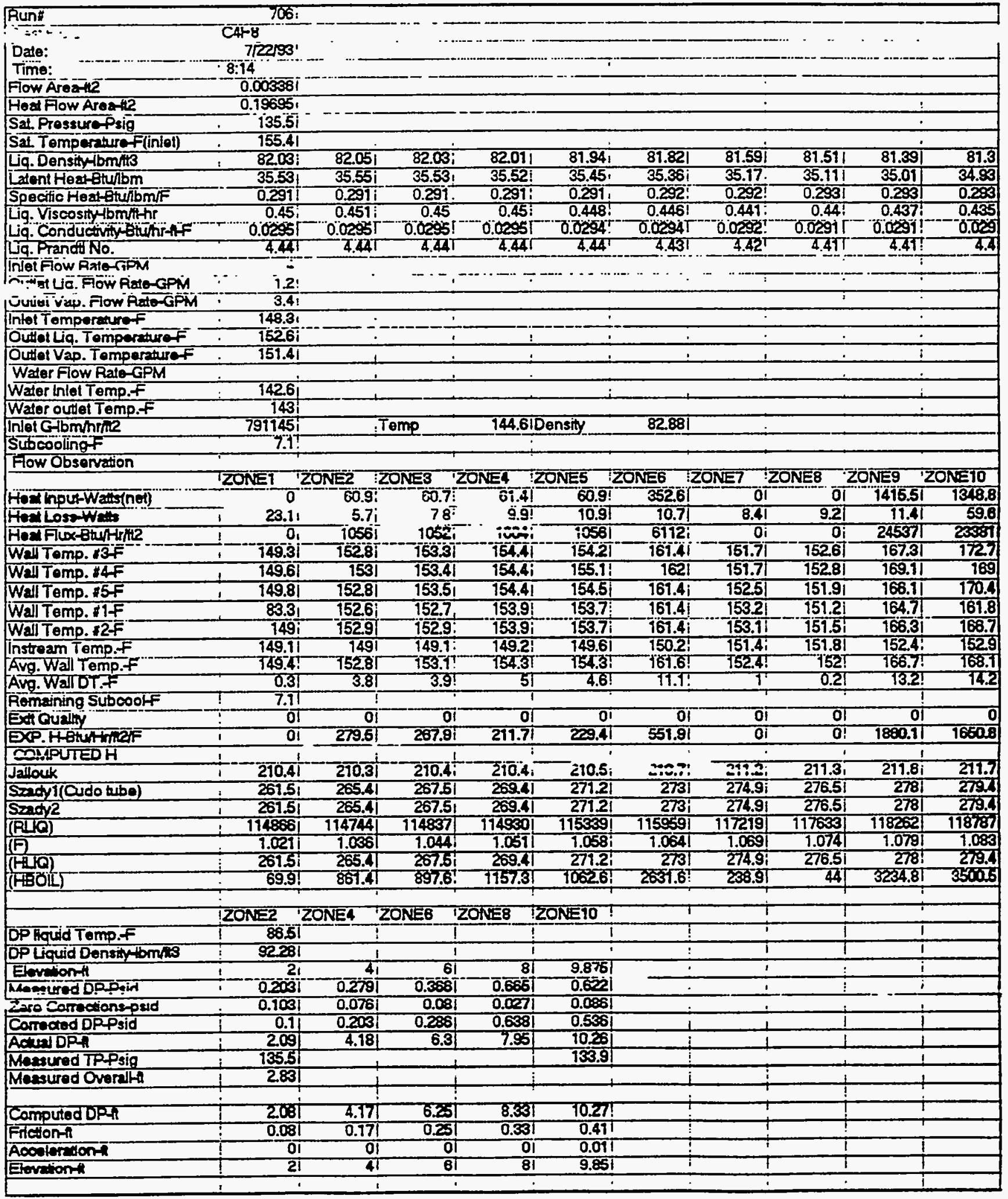




\begin{tabular}{|c|c|c|c|c|c|c|c|c|c|c|}
\hline$\frac{\text { P.unf }}{\text { Test Fiva }}$ & CAF8 & & & & & & & & & \\
\hline Dato: & $7 \sqrt{2} 293^{1}$ & & & & & & & & & \\
\hline Time: & $8: 14$ & & & & & & 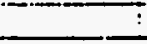 & : & & \\
\hline Flow Areanit & - 0.003261 & & & & ! & ? & ' & ' & : & \\
\hline Hodflow Areath? & 0.19255 & & & & 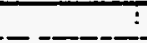 & 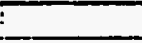 & & & ' & \\
\hline S2t. Proseure Psig - & 137.47 & $\because$ & & & $\because$ & $=$ & . & - & 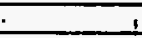 & \\
\hline S21. Temporatureffinlet) & 156.41 & & & & & & 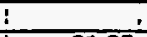 & i & $i$ & \\
\hline Lag. Density 4 bmitis & 81.921 & 81.941 & 81.92. & 81.781 & $81.7 !$ & 81.531 & $81.35 i$ & 81.31 & 81.181 & 81.12 \\
\hline Laten Hear Btulbm & 35.44 & $35.45 i$ & $35.44 !$ & 35.331 & 35.27 & 35.21 & $34.38 ;$ & $34.93 !$ & 34.83 & 34.79 \\
\hline Specitic Hear Btulbm/F & 0.291 & 0.2911 & 0.291 & 0.292 & 0.292 & 0.2921 & 0.293 & 0.2931 & 0.294 & 0.294 \\
\hline Liq. Viscosity-bminthr & 0.448 & 0.448 & 0.448 & 0.445 & 0.444 & 0.442 & 0.4361 & $0.435 !$ & $0.433 !$ & 0.432 \\
\hline Liq. ConductivitytührtiF & $0.0294 !$ & 0.02941 & 0.0254 & 0.02931 & 0.0251 & 0.020 & 0.0291 & 0.0281 & $0.0209 !$ & 0.0289 \\
\hline Ug. Prandt No. & $4.44^{\top}$ & $4.44 !$ & 148 &.+12 & $\triangle Q 2$ & $4.23 !$ & $\$ .47$ & $4.4 !$ & $4.4^{\circ}$ & 4.39 \\
\hline IñetAow PalOGPM & $4 !$ & & & & & & & & & \\
\hline OUtôUg. Fow RAT-GPM & $1.1^{T}$ & & & & $\cdot$ & & 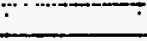 & & it & \\
\hline Outhot Vap. Flow Rato-GPM & 3.41 & & & & 1 & & & & & \\
\hline Inlet Tempentareff & 148.61 & $\therefore$ & & $\therefore$ & $i$ & 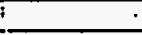 & $-\dot{ }$ & - & $\dot{-}$ & \\
\hline Outhet Lig. Temperature & 153.91 & 2 & $\therefore$ & $\therefore$ & $\because$ & . & $\therefore$ & $\therefore$ & $\vdots$ & \\
\hline Outhol Vap. Tomporaturef & 15221 & 1 & 1 & i & 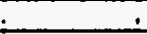 & 1 & $i$ & 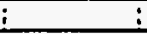 & $i$ & \\
\hline Water Flow Aalo-GPM & & $:$ & & & & & i & : & & \\
\hline Water hlat Tomp.f & 1432 & & & & & & & & & \\
\hline Water outel Tomp.f & $143.7 i$ & & & & & & i- & 5 & & \\
\hline Intot Glbm/hr/th & 789721: & & Temp & 145.4 & Density & 8273 & & & & \\
\hline Subcooling & $7.8 !$ & & & & & & $T$ & & & \\
\hline Flow Obsenvation & $\cdot$ & & & & & & ! & $T$ & $T$ & \\
\hline & ZZONET & IZONEZ :2 & ZONE8 & IZONEA & ZONES & ZONE & :ZONE & Poiveo & ESives & ZONEIO \\
\hline Hoxhpuntwatnat) & o1 & 81.2 & 81.2 & $1 \quad 81.11$ & 80.71 & 538.71 & 101 & 1 01 & $140 \overline{9.11}$ & $1-140.1$ \\
\hline Howlooss-WEA & 23.91 & 5.81 & 81 & $10 !$ & 10.81 & 10.71 & 8.71 & 9.51 & 11.81 & 60.7 \\
\hline Homflox Qhuthite & 01 & 1408 & 1408 & 14061 & 13901 & 6218 & 01 & OI & 24501 & 24409 \\
\hline Wall Temp. Bf & 16721 & 164.81 & 756.4 & 957.11 & $16 \overline{8} . \overline{1}$ & $152 \overline{2}$ & 15281 & 153.61 & 168.11 & 173.7 \\
\hline W.ll Temp. \#4F & 151.71 & 1551 & 155,4 & 158.8! & 157.61 & 16291 & 15281 & 153.81 & 1701 & 169.6 \\
\hline Wall Temp. $15+$ & 151.71 & 154.81 & 155.6 & 156.5 & 158.71 & 1625 & 153.51 & 1528 & 166.8 & 171.4 \\
\hline Wall Temp. 11F & 84 & 154.61 & 154.6 & 156 & 156.1 & 1624 & 154.11 & 152.3 & 165.4 & 1624 \\
\hline Wall Temp. RF & 150.6 & 154.8 & 154.7 & 156 & 155.91 & 16221 & 154.31 & 152.61 & 167.11 & 167.5 \\
\hline Instranm Temp. $f$ & 149.71 & 149.61 & 149.7 & 150.4 & 150.8 & 15121 & 15261 & 15291 & $153.5 !$ & 153.8 \\
\hline Avg. Wail Temp.t & -151,3! & 154.81 & 155 & $756.6:$ & $-156.6 !$ & T62.5! & 153.5 & 153 & TE7.5! & 168.9 \\
\hline Avg.Wallot. & $4.6 !$ & 5.11 & 5.3 & 6.1 & 5.7 & III & 0.91 & 0.11 & T29! & 74 \\
\hline Romaning subcooff & 7.8 & & & & & & & & & \\
\hline Exaunity & of & 01 & 0 & 01 & 01 & 01 & 01 & $0 !$ & $0^{\prime}$ & \\
\hline SXP.Heturher & 01 & 274 & 267.5 & 220.9 & 244.81 & 564.81 & of & 이 & 1894 & 1746.4 \\
\hline WNF & -1 & & & & -1 & $\ldots 1$ & 1 & -1 & 1 & \\
\hline Jellouk & 2iōal & $210 . \overline{31}$ & 210.3 & $2 i 0.5$ & 210.71 & 210.81 & 217.4 & 211.61 & 211.7 & 217.8 \\
\hline Szadv1(Cudo uba) & 251.41 & 265.31 & 267.4 & 2695 & 271.31 & 2731 & 2751 & 276.51 & 278 & 27.4 \\
\hline Szady2 & 261.41 & 265.3 & 257.4 & 269.5 & 271.31 & 273 & 275 & 276.51 & 278 & 279.4 \\
\hline (RLA) & 115288 & 115168 & 115269 & 115964 & 116355 & 116807 & 118287 & 1185971 & 1192301 & 11S5 \\
\hline & 1.0211 & 1.0381 & 1.044 & 1.051 & 7.057 & $1.0 \% 3$ & 1.058 & 1.073 & 1.078 & 1.083 \\
\hline (HIIC) & 267.41 & 265.31 & 257.4 & 2095 & 271.31 & LTI & 275 & 276.51 & 278 & 2724 \\
\hline$($ (Hisolit) & उ50.5ा & 7189.11 & 1202 & 7441.1 & 1345.71 & 2657 & 207.31 & 26.4 & $32032 !$ & 35012 \\
\hline & & & & & $T$ & $\mathrm{~T}$ & & & & \\
\hline & ZONEe i & ZONE4 & ZONEB & TZONEB & ZZONETO & & 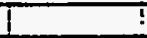 & $!$ & & \\
\hline DPFrad Tomp.F & $! \quad 88.91$ & & & 1 & & i & & 1 & 1 & \\
\hline DP Liquid Density-bm/B & 92क्या & ! & ! & i & 1 & t & & 1 & $i$ & \\
\hline Elemionth & 2 & 41 & 6 & 8 & 9.8761 & & & $i$ & & \\
\hline Mamurod DPFcia & (1; & $0.309 !$ & 0.373 & 0.663 & 0.681 & & & $\perp$ & & \\
\hline Zaro Corractions-osid & 0.1031 & 0.0761 & 0.08 & 0.027 & 0.0881 & & & $i$ & & \\
\hline Cortecled DP Pidd & 0.1011 & 0.2071 & 0.293 & 0.631 & 0.5521 & & 1 & 1 & & \\
\hline Achun Dल & 200 & 4.18 & 6.3 & 7.98 & 10.25 & & & 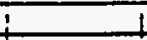 & 1 & \\
\hline Mozsurod TPPSig & 137.4 & & & & 135.8 & & 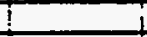 & $\perp$ & 1 & \\
\hline Messurod Overallh & 2.84 & & I & I & & & $\perp$ & $\overrightarrow{1}$ & 1 & \\
\hline & & & & & & & & & & \\
\hline computad DPसा & 2.081 & 4.17T & 6.25 & 8.33 & 10.27 & & & & & \\
\hline Fiction-f & 0.081 & 0.17 & 0.25 & 0.38 & 0.47 & & & & & \\
\hline Acoiteriont & 01 & 01 & 0 & 0 & 0.071 & & $i$ & & & 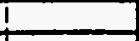 \\
\hline Erevtionn & 21 & का & 8 & 8 & 9.851 & & $!$ & 1 & 1 & \\
\hline & 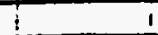 & $!$ & ! & & 1 & i & $i$ & - & i & 1 \\
\hline
\end{tabular}




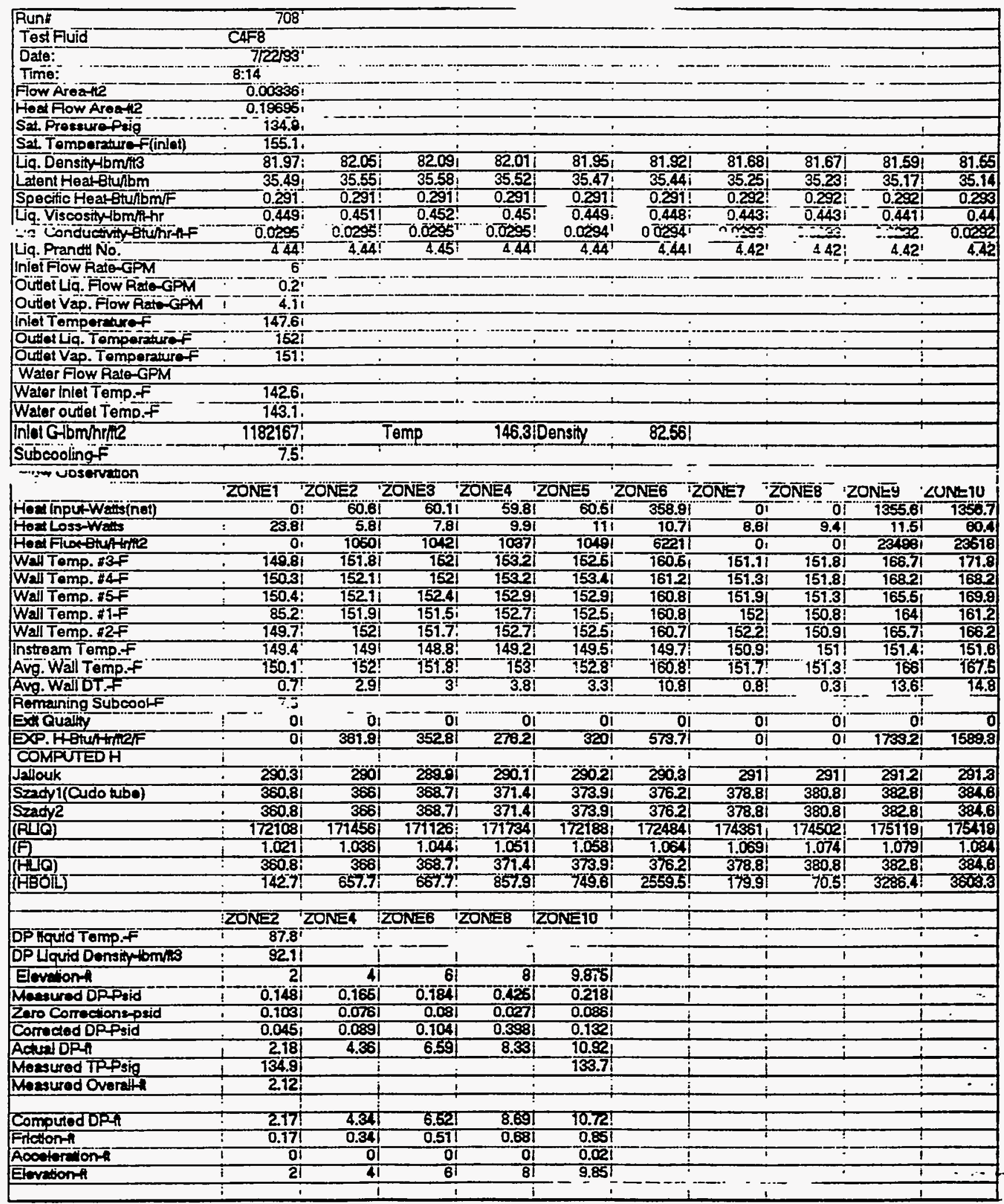




\begin{tabular}{|c|c|c|c|c|c|c|c|c|c|c|}
\hline Run: & 709 & & & & & & & & & \\
\hline Testflud & CAF & & & & & & & & & \\
\hline Dale: & $7 \sqrt{2253}$ & & & & & & & & & \\
\hline Time: & $13: 10 i$ & & & & & & & & & \\
\hline Flow Areatic & $: 0.00350 \mathrm{i}$ & - & 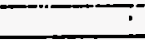 & & 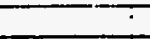 & $\cdot$ & 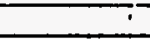 & 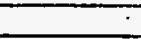 & & \\
\hline Hecl fow Arean"? & 0.185851 & $\therefore$ & $\therefore$ & & & $\dot{5}$ & 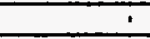 & $\div$ & $\cdot$ & - \\
\hline Sat. Preenurepig & $134 i$ & $\therefore$ & 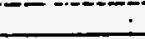 & $\therefore$ & $\therefore$ & $\dot{-}$ & $\therefore$ & $\dot{-}$ & $\therefore$ & 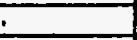 \\
\hline Sat. Temperave F(inlot) & 154.71 & 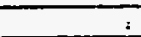 & 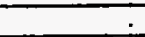 & &. & : & : & : & . & . \\
\hline Lia. Densty 4 bm/it3 & 82.131 & 82.16 & $82.15 !$ & 82.03 & 82.031 & 81.921 & $81.72 !$ & 81.71 & 81.591 & 81.57 \\
\hline Lalent Hear-Bturbm & 35.611 & 35.64 & $35.53 i$ & 35.531 & 35.531 & $35.44 !$ & 35.28 & $35.27 i$ & 35.17 & 35.15 \\
\hline Specific Heaf-btu/bm/F & 0.297 & 0.29 & 0.2911 & 0.2911 & 0.291 : & 0.2911 & 0.292 & 0.202 & U.s. & $=$ \\
\hline$\approx=$ iscoshyibmithr & $0.453 i$ & 0.4531 & 0.4531 & 0.45 & 0.451 & 0.4481 & 0.444 & 0.4441 & 0.4411 & 0.441 \\
\hline Lig. Conductivity BhíhrafF & 0.0ट⿺s! & $0.0295 \Gamma$ & 0.02051 & 0.02051 & 0.0285 & 0.02941 & 0.0293 & 0.0293 & 0.0295 & 0.0292 \\
\hline La.Piandi No. & 4.451 & 4.451 & 4.451 & 4.441 & 4.441 & 4.441 & $4.42 !$ & $4.42 !$ & 4.421 & 4.42 \\
\hline inlet Fow Pade & $6^{1}$ & & & & & & & & & \\
\hline oútei tia. Fiow Rato-GPM & 0.11 & & 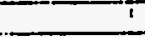 & & & i & 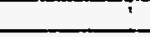 & & & \\
\hline Out i Vap. Flow Rate-GPM & $4.2 !$ & $\because$ & 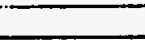 & & - & - & $\dot{-}$ & & & \\
\hline Inlet Tomperaurof & 147.31 & $!$ & 2 & & $\therefore$ & $\therefore$ & $\therefore$ & $\cdot$ & $:$ & $:$ \\
\hline Outlot Lig. Tomperstere & 157.81 & 1 & 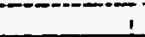 & i & 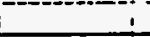 & 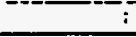 & $\therefore$ & 1 & 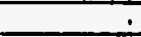 & $\therefore$ \\
\hline Outtet Vap. Temperature F & 150.41 & . & 1 & 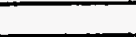 & $\vdots$ & & 1 & - & 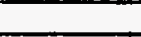 & \\
\hline Water flow Rale-GPM & . & & 1 & 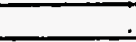 & . & & & 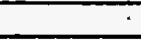 & 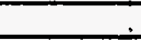 & . \\
\hline Wator Inlot Tomp.F & 142.5 & & & & & & & & & \\
\hline Water outtet Temp.F & 142.6 & & & & & & & & & \\
\hline Indat Glbm/hrits? & : 11835101 & & Temp & 145.8 & Density. & 82.651 & $\therefore$ & 1 & 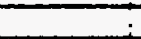 & \\
\hline Subcoolinaf" & 7.4 & & & & & : & 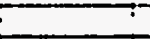 & & & \\
\hline Fow obbsonvation & & & & & & & & 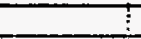 & & \\
\hline & TZONET & ZONEZ & ZONES & ZONES I & ZONES 12 & ZONEO I & ZONE & ZONTE & ZONEQ IS & TZONET0 \\
\hline 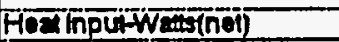 & $0 i$ & 8021 & 801 & 80.31 & 81.11 & 348.51 & 01 & ol & 1337.2 & 1334.4 \\
\hline Hed Loss-Wous & 23.81 & 5.81 & 7.81 & 101 & 111 & 10.71 & 8.41 & 9.91 & 11.4 & $\mathbf{5 9 . 9}$ \\
\hline Hect Fub-Bwhine & $0_{1}$ & 13901 & 1387 & 1382 & 14061 & 6047 & 01 & 01 & 23170 & 23131 \\
\hline Wall Temp. I3 & $7405 \mathrm{i}$ & 16231 & 15261 & 1538 & 16331 & 1601 & 150.71 & 161.4 & 1661 & 171.4 \\
\hline Wall Tamp. s4f & $: \quad 149.81$ & 15241 & $1525 !$ & 15381 & 154.41 & 160.21 & 150.81 & 151.6 & 167.9 & 167.9 \\
\hline Wall Temp. 16+ & 15021 & 1522 & $1529 !$ & 153.8 & 153.61 & 160.4 & 151.31 & 151 & 164.8 & 169.4 \\
\hline Wall Tomp. IfF & 85.8 & 152.1 & 151.9 & 153.31 & 153.2 & 160.31 & $151.9 !$ & 150.51 & 163.51 & 161.1 \\
\hline Wall Temp. ILF & 149.21 & 1524 & 1522 & 153.31 & 153.11 & $160.3 !$ & 151.8 & 150.71 & 164.9 & 165.8 \\
\hline Instream Temp.f & $148.6 i$ & 148.41 & $148.5 !$ & 149.7 & $149.1 !$ & 149.7 & 150.7 & 150.81 & 151,4 & 151.5 \\
\hline Avg. Wall Temp.t & 149.7? & $1523 i$ & $1523 !$ & $153.7 !$ & 153.6! & 160.41 & $151.3 !$ & 151 & 165.41 & 167.1 \\
\hline Avg. Wall DT & 1.1 & 3.8 & 37 & $\$ .5$ & $4.4 !$ & 10.41 & 0.61 & 0.21 & $|3|$ & 14.6 \\
\hline Remaining Subcoolf & 7.41 & & $T$ & & & & 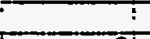 & $i$ & $?$ & \\
\hline Exil Qualin & 01 & of & 01 & 01 & ô & 01 & 01 & 01 & 01 & 0 \\
\hline DP. FEUW & 01 & 352.91 & 373.81 & 308.51 & 318.71 & 579.41 & ol & 01 & 1784.1 & 1587.1 \\
\hline COMPUTEDH & -1 & 1 & 1 & -1 & 1 & -1 & 1 & 1 & $i$ & $i$ \\
\hline Jallouk & 200.11 & ж) & 201 & 20031 & 200.31 & 290.61 & 291.11 & 291.11 & 291.41 & 291.5 \\
\hline Sendvi(Cudo tube) & 350.61 & 365.91 & 368.81 & 371.7 & 3741 & 376.51 & 379.11 & 3811 & 383.11 & 3819 \\
\hline Stady2 & 380.8 & 355.9 & 368.81 & 371.7 & 3741 & 376.5 & 379.11 & 3811 & 383.1 & 384.9 \\
\hline (FाO) & 171050 & $1707 / 21$ & 170851 & $1717 / 3$ & 17175s & 172580 & 174221 & 1743831 & 175318 & 175460 \\
\hline$(F)$ & $1.021 i$ & 1.036 & 9.0441 & 1.051 & 1.058 & 1.004 & 1.059 & 1.0741 & 1.079 & 1.084 \\
\hline (HLA) & 350.61 & 365.91 & 358.8 & 371.7 & $374 !$ & 378.5 & 379.1 & 381 & 383.11 & 384.8 \\
\hline (FiBOLL) & 236.61 & 838.71 & 840.81 & T034.71 & TOT.1 & 2458.81 & 733.71 & 52.41 & 3146.1! & 353.4 \\
\hline & & & & & & & 1 & t & T & T \\
\hline & ZON=2 & ZONEA & ZONiE & ZONIEB & ZONETO! & & 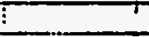 & & & \\
\hline DPlavd Temp.f & 881 & -2 & 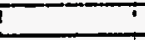 & 2 & 1 & & 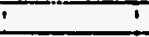 & $\div$ & : & $\div$ \\
\hline DPLquid Density & 92.071 & 1 & $T$ & 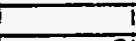 & 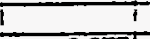 & $\div$ & i & 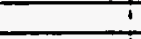 & 1 & 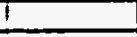 \\
\hline Elevimont & 21 & $4 !$ & 61 & $8 !$ & 9.8 .51 & & $\bar{i}$ & $i$ & 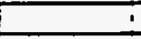 & 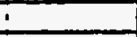 \\
\hline 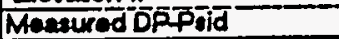 & 0.7461 & 0.1611 & 0.1791 & $0 . \overline{4} \mid \overline{8} 1$ & 0.2051 & & $i$ & 1 & 1 & 1 \\
\hline Zero Correctonspeid & 0.103 & 0.0751 & 0.081 & 0.027 & 0.0881 & & 1 & $!$ & 1 & 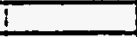 \\
\hline Corrected DPPsid & 0.0421 & 0.085 & 0.0991 & 0.391 & $0.12 !$ & & $i$ & $\dot{.}$ & + & + \\
\hline Acted DPh & .2 .181 & 4.361 & 6.6 & 8.34 & 10.83 & & ! & $i$ & 1 & 1 \\
\hline Mosured TPpsig & $134:$ & & : & is & $\mid 32.7$ & & 4 & 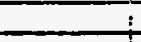 & $i$ & $i$ \\
\hline Measured Overalle & 230 & & 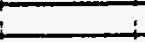 & 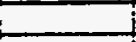 & $\div$ & & 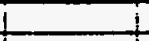 & 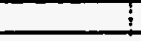 & 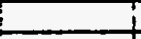 & \\
\hline & & & & & & & 1 & : & ; & \\
\hline Computed DPAT & 277 & 4.34 & $6.52 !$ & $8.69 !$ & 10.721 & & 1 & 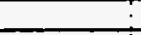 & 4 & \\
\hline Ficton-h & 0.771 & 0.341 & 0.511 & 0.69 & 0.851 & & $T$ & & 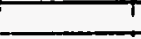 & \\
\hline$\therefore$ indind & 0 & 01 & oí & 0 & 0.021 & & 1 & 2 & 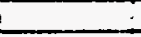 & \\
\hline Elevinon & 21 & 41 & 81 & 8 & 9.851 & & 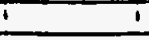 & 1 & i & 1 \\
\hline & 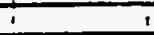 & $i$ & $i$ & . & 1 & ' & 1 & ; & $i$ & 1 \\
\hline
\end{tabular}




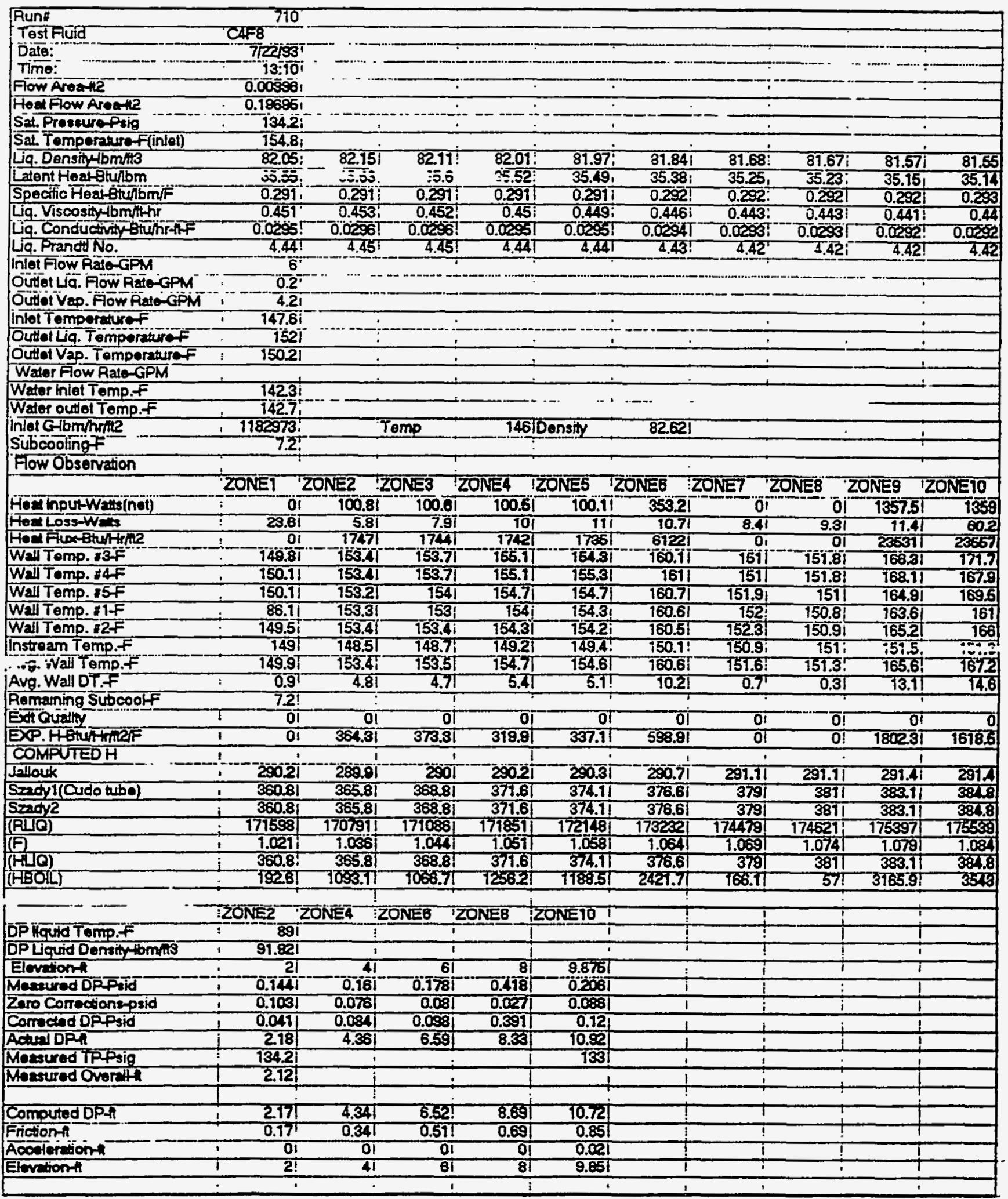




\begin{tabular}{|c|c|c|c|c|c|c|c|c|c|c|}
\hline $\begin{array}{l}\text { Run: } \\
\text { Test Fiud }\end{array}$ & CAES & & & & & & & & & \\
\hline Date: & $7 / 22193$ & & & & & & & & & \\
\hline Time: & $13: 101$ & & & & $\because$ & & . & & $\overline{7}$ & i \\
\hline Fow Arátie & 0.003831 & i & 3 & & & & & & " & \\
\hline Hod flow Arearte & 0.19686 & & $\therefore$ & $\therefore$ & $\therefore$ & $\therefore$ & $\therefore$ & $\therefore$ & $\dot{-}$ & - \\
\hline S2l. Pressuro poig & 1325 & & $\dot{-}$ & $\because$ & 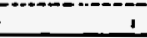 & 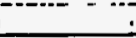 & 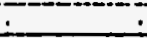 & $i$ & 7 & . \\
\hline Sa. Tomperaturef $F(i n i a l)$ & 153.91 & & 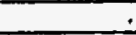 & & $i$ & 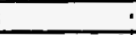 & - & $i$ & F & 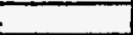 \\
\hline Lq. Consiig & $2 \longdiv { 7 9 }$ & $82.18 !$ & $82.2 !$ & 82.111 & $82.13 !$ & 82.051 & 81.86 & 81.841 & 81.781 & 8178 \\
\hline Latent Heat-Btunibm & 35.6 & 35.661 & 35.67 & 35.61 & 35.61 & 35.55 & 35.39 & 35.38 & 35.331 & 35.31 \\
\hline Specitic Hear-etunbmit & 0.291 & 0.291 & 0.29 & $0.291 !$ & 0.2911 & 0.2911 & 0.2911 & 0.2921 & 0.2921 & 0.292 \\
\hline Ig.Viscosithlomphith & $0.452 !$ & 0.4541 & $0.454 !$ & 0.452 & 0.453 & 0.4511 & 0.447 & $0.446 i$ & 0.4451 & 0.445 \\
\hline 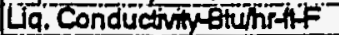 & 0.0296 & 0.0201 & 0.02056 & 0.0201 & 0.0286 & 0.02051 & 0.02941 & 0.0294 & $0.0233 !$ & 0.023 \\
\hline Lig.Prandi No. & 4.45 & 4.451 & 4.45 & 4.451 & 4.451 & 4.441 & 4.431 & 4.431 & 4.431 & 4.43 \\
\hline Iñlet Fow Rato-GPM & $8^{\circ}$ & & & & & & & & & \\
\hline Outat Lia. Flow RaAOGPM & 01 & & & & I & & & & it & E. \\
\hline Outlo! Vap. Flow Rato-GPM & 4.21 & & · & - & & & - & & & \\
\hline Inlot Temporaturef & $147.6 i$ & $\therefore$ & $\therefore$ & $\therefore$ & - & · & - & & $!$ & 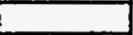 \\
\hline Outel Liq. Tomporeture F & $150 . \overline{6}$ & $:$ &. & 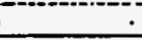 & 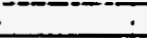 & $\dot{-}$ & $\dot{-}$ & - & $\bar{i}$ & 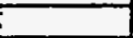 \\
\hline Outlat Vap. Tomporaturef & $149.8 i$ & & . & & 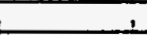 & & 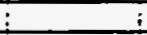 & 1 & 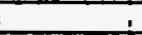 & \\
\hline Walor Flow Rato-GPM & & & & & $\div$ & & $:$ & + & & \\
\hline Wader Intal? añétF & - & & & & & & & & & \\
\hline Water outtel Temp.F & 142.1: & & & & & & & & & \\
\hline Inlot Glbmhrne & 1575223 & & Temp & 146.311 & Density & 82.561 & i & & i & \\
\hline Subcoolingf & $6.4^{1}$ & & & & & & & & & \\
\hline Fow Obsenvation & & & & & & & : & - & & \\
\hline & ZONEI & ZONE2 : & ZONE3 & ZONE4 : & ZONES I2 & ZONE6 & IZONET & ZONE & ZONE9 & ZONETO \\
\hline Healnp(n-Watongt) & 01 & 00.4 & 61.21 & 60.51 & 6051 & 352.91 & it & al & 1386.51 & 1388 \\
\hline Hod Lossowals & 23.7 & 5.71 & 7.7 & 9.81 & 10.81 & 10.81 & 8.41 & 9.91 & 11.41 & 60.2 \\
\hline Had Fior Biwhthe & 01 & 1047 & 1061 & 10481 & 10481 & 69171 & 01 & 01 & 236871 & 23713 \\
\hline Wal Tomp. 13f & 140.21 & $150.6 \bar{i}$ & 150.81 & 161.81 & 151.11 & 158.71 & 16001 & 160.51 & 165.31 & 17 \\
\hline Wall Tomp. $14 F$ & 969.41 & 1511 & 150.81 & 151.81 & 15211 & 159.51 & 150.11 & $150.6 i$ & 167.1 & 167.4 \\
\hline Wall Temp d5F & 149.7 & 150.91 & $151.1 !$ & $151.8 !$ & 151.41 & 159.1 & 150.6 & 150.11 & 1642 & 168.9 \\
\hline Wal Tomp. & 86.71 & 150.81 & 150.4 & $151.3 i$ & 151.2 & $158.9 !$ & 151 & 1501 & 162.7 & 160.3 \\
\hline Wall Temo, & 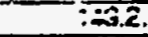 & $150.8 \mathrm{i}$ & 150.7 & 151.31 & 151.11 & 158.81 & $151 i$ & 150 & 1642 & 165.4 \\
\hline Instream Temp.f & 148.7: & 148.31 & 148.2 & 148.71 & 148.61 & 1491 & 1501 & 150.11 & 150.4 & 150.5 \\
\hline Avg. Wall Temp.f & $149.4^{\top}$ & 150.81 & $150.7 !$ & 151.7! & 151.5 & 159.11 & $150.5 i$ & 150.21 & $164.7 !$ & 166.8 \\
\hline Avg. Wall DT.F & $0.7^{\prime}$ & $2.5 !$ & 2.41 & $2.9 !$ & 2.81 & 9.81 & $0.5 !$ & 0.11 & $13.2 !$ & 15 \\
\hline Remaining Subcooff & 6.41 & 1 & & $T$ & 1 & & $i$ & & 1 & \\
\hline Exon aually & 01 & $0 i$ & ol & 01 & 01 & 01 & 01 & o1 & of & 0 \\
\hline EXP. HERAThla & 01 & 428.91 & 437.11 & 358.21 & 374.21 & 65.91 & of & 01 & 1790.51 & 1577.9 \\
\hline COMPUTEDH & $!$ & $\ldots 1$ & & & $\ldots 1$ & & $!$ & 4 & -1 & -1 \\
\hline Jallouk & 364.91 & 36461 & 36461 & 36431 & 367.71 & 3651 & 366.61 & 365.71 & 365.81 & 3650 \\
\hline Sadyl(ado ube) & 453.61 & 460.11 & $4 \sqrt{261}$ & 46721 & 4701 & 4731 & 476.31 & 478.71 & $481.2 !$ & 4824 \\
\hline Sendy2 & $453.6]$ & 460.1 & 460.61 & 4672 & 4701 & 4731 & 476.31 & $478.7 i$ & 481.21 & 483.4 \\
\hline (सU]0) & 228016 & 227150 & $230920_{1}$ & 227351 & 227706 & 2205161 & 230586 & 230772 & 231380 & 231567 \\
\hline (F) & 1.021 & 1.038 & 1.0451 & 1.052 & 1.058 & 1.064 & 1.059 & 1.075 & 1.081 & 1.034 \\
\hline (Hयa) & $753.6 i$ & $460.1 !$ & 463.61 & 467.21 & 470 & 473 & 476.31 & $478.7 !$ & 4812 & 483.4 \\
\hline (FiBOIL) & $147.1 !$ & $548.6 \Gamma$ & $542 !$ & हात & 61.31 & 2280.8 & 118.91 & 29.91 & 3165.41 & $36 \pi 7.4$ \\
\hline & & & & & & & $?$ & $:$ & & \\
\hline & TONE2 & ZONEA & ZONEB & ZONE8 & ZONEIO 1 & & & & & \\
\hline DP liquid tomp.F & $T-89.21$ & 1 & -2 & $!$ & 1 & & $T$ & $\stackrel{t}{t}$ & I & \\
\hline DP Liquid Density-bmith & 91.891 & 1 & 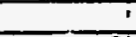 & 7 & 1 & & i & $\overline{3}$ & i & 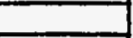 \\
\hline Elevetiont & 21 & 4 & 61 & 81 & $8.376 i$ & & 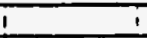 & 1 & - & 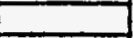 \\
\hline Mesecured DPpid & 0.067 & 0.0031 & -0.067 & $0.0 \%$ & -0.2731 & & i & $i$ & I & \\
\hline Zaro Contections-psid & $0.103 !$ & 0.0781 & 0.081 & 0.0271 & 0.0881 & & $\div$ & $i$ & $i$ & \\
\hline Corrected DPPsid & -0.036 & -0.073 & -0.147 & 0.072 & -0.3591 & & 1 & & $i$ & \\
\hline Achuloph & 2.31 & 4.62 & 7 & 8.861 & 11.73 & & 1 & 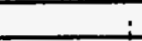 & 4 & 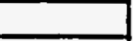 \\
\hline Mossured if posig & 132.5 & & 1 & & 131.5 & & & 1 & 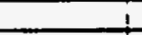 & \\
\hline Mesured Overath & $1 . \overline{81}$ & $i$ & & & & & I & 1 & & \\
\hline & & & & & & & ; & & & \\
\hline Computed DPA & 2.291 & 4.581 & 6.851 & 9.151 & 71.31 & & & 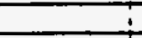 & & \\
\hline Fricton-h & 0.291 & 0.571 & 0.881 & 1.151 & 1.431 & & & & & \\
\hline Acostorilont & 01 & 01 & 0.011 & 0.017 & 0.031 & & 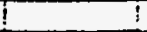 & 1 & 1 & 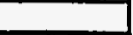 \\
\hline Elovetionth & 21 & 41 & 81 & 81 & 9.851 & & 1 & $\overline{:}$ & $i$ & 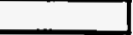 \\
\hline & $\mathbf{i}$ & $\div$ & & \pm & . & & $\therefore$ & . & 1 & \\
\hline
\end{tabular}




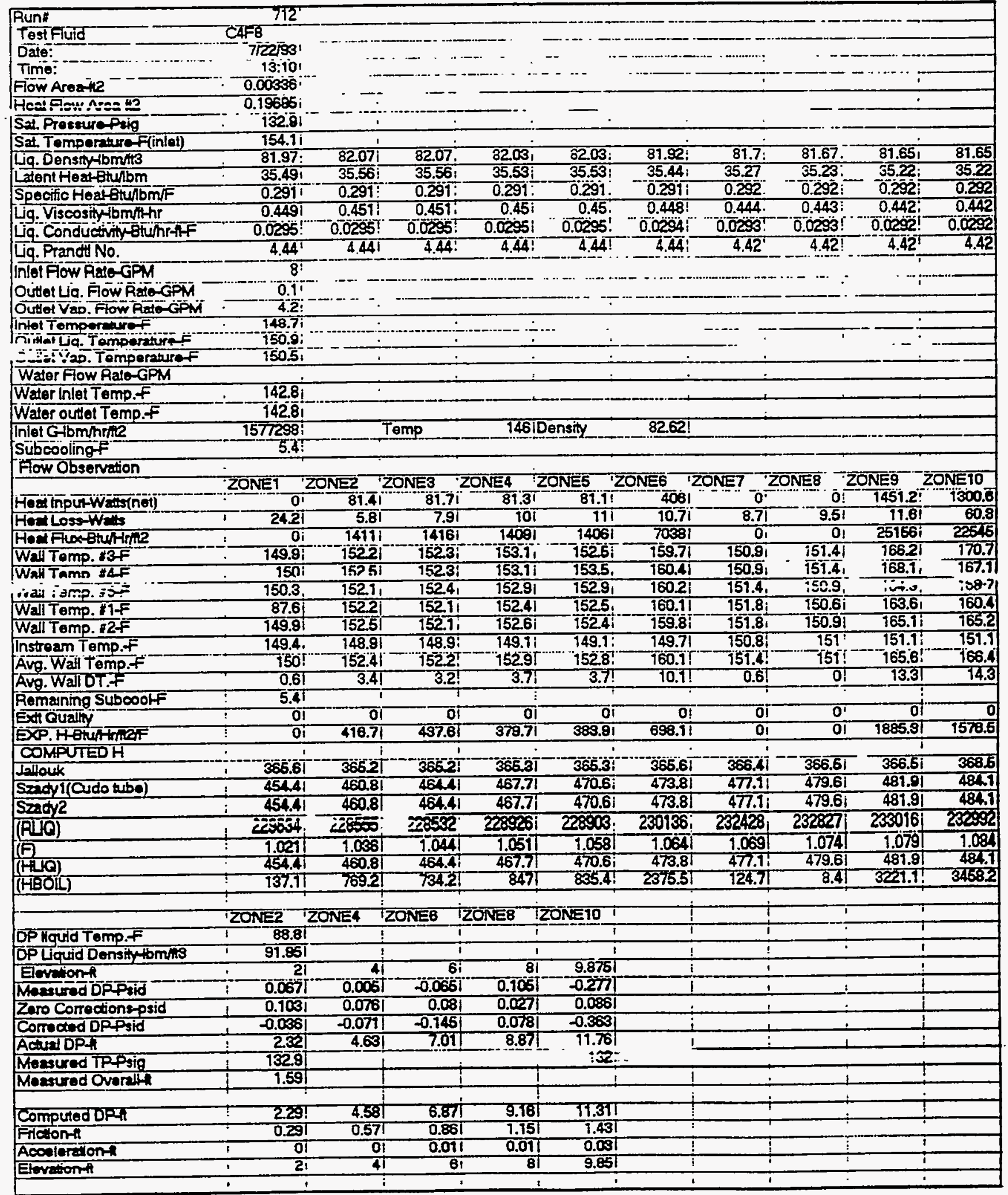




\begin{tabular}{|c|c|c|c|c|c|c|c|c|c|c|}
\hline Runt & 713 & & $\therefore$ & & & & & & I & . \\
\hline Test Fluid & C4F 8 & & & & & : & & & & \\
\hline Dato: & 71221931 & & & & & & & & & \\
\hline Tima: & $13: 10$ & & & & & & & & & \\
\hline Flow Aroz-12 & $0.00536:$ & & & & & & & & & \\
\hline 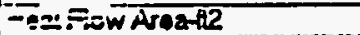 & 0.19695 & & & & & & & & & \\
\hline Sad. Prossure-Psig '“ & $\$ 34.21$ & & & & & & & 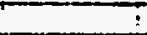 & 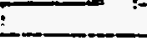 & \\
\hline Sal. Temporaturo f $($ inlotit & 154.81 & & & & & & & & $T$ & $T$ \\
\hline Lig. Densitytbmin & 81.91 & 81.941 & 81.941 & 81.91 & 81.881 & $81.76 !$ & 81.551 & 81.531 & 81.49 & 81.49 \\
\hline Latont Hoat Btutbm & 35.421 & 35.451 & 35.451 & 35.421 & 35.411 & 35.311 & 35.141 & 35.121 & 35.091 & 35.09 \\
\hline specitio Heaf-Buibmif & 0.2911 & 0.291 & 0.291 & 0.291 & 0.291 & 0.292 & 0.2331 & 0.2931 & 0.2831 & 0.293 \\
\hline Liq. Viscostribmithi & 0.4481 & 0.4481 & 0.4481 & 0.4481 & 0.447 & 0.461 & $0.44 i$ & 0.441 & 0.4391 & 0.439 \\
\hline Liq. Conductity Bturit & 0.02041 & 0.0294 & 0.0244 & 0.02091 & 0.02041 & 0.02931 & 0.02321 & 0.0021 & 0.02911 & 0.0291 \\
\hline Liq. PrasiatiNo. & 4.431 & 4.441 & $4.44 i$ & $4.43 !$ & 4.431 & $4.43 i$ & $4.42 !$ & 4.411 & 4.411 & -4.41 \\
\hline Inlot Flow Rafo-GPM & 8 & & & & & & & & & \\
\hline Outlot Lig. Fow Ralo-GPM & 0.1 . & & & & & & & & & \\
\hline Outlet Vap. Fow Rate-GPM & 4.2. & & & & & & & & & : \\
\hline Inlol Temperatur of & 149.4! & & & & & & & $i$ & & \\
\hline 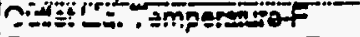 & 89.85 & & & & & & & & & \\
\hline Outtet Vap. Temperaturef & $750.6^{\circ}$ & & & & & & & & 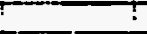 & 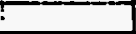 \\
\hline Wador Fow Rato-GPM & $--\rightarrow$ & 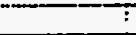 & I & & & $!$ & 1 & & T & i \\
\hline Wator inlet Temp.f & 1431 & & 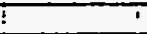 & & 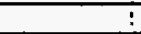 & $i$ & & 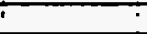 & 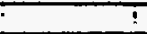 & 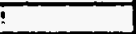 \\
\hline Wat out of Tomp.f & 143.21 & 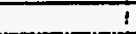 & $T$ & & I & 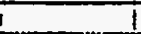 & & $:$ & 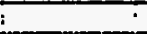 & - \\
\hline Intal Gibmhince & .1676864 & & Temp & 146.4 & Dentiky & 82641 & $\therefore$ & $\dot{1}$ & 1 & 1 \\
\hline Subcoolingf & 5.41 & & + & : & 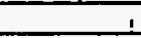 & 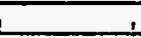 & $\dot{-}$ & 1 & \pm & \pm \\
\hline Fow Obsenvation & $!$ & & & & $!$ & & & $\vdots$ & $i$ & $i$ \\
\hline & ZONE1 & ZONE2 & ONE3 & ZONE4 & ZONE5 & ZONE 6 & ZONE? & ZONE 8 & ZONES & $20 N=10$ \\
\hline Heat (nput-Watis(net) & 0 & 98.11 & 98.11 & 98.3 & $97.5 i$ & 455.5 & $0 i$ & $0 i$ & 1483.5 & 1294.8 \\
\hline Hoalooss-Watts & 24.41 & 5.9 & 8.11 & 10 & 10.9 & 10.6 & 8.91 & 9.7 & 11.8 & 61 \\
\hline Hoat Flix-BtunHr/R2 & $0^{\circ}$ & 77001 & $7 \% 001$ & 1704 & 1600 & 78961 & of & 01 & 25761 & 2215 \\
\hline Wail temp. I3 & 349.6 & $15332]$ & 153.4 & 154.7 & 154 & T61 & T51.8! & 751.91 & 167.11 & 171 \\
\hline W & 150.31 & 153.4 & 753.41 & 154.7 & 154.91 & 161.91 & 751.81 & 15231 & 168.81 & 1675 \\
\hline Wall lemp. axof & 150.31 & 1531 & 153.31 & 154.4 & 154.31 & 161.4 & $152.2 !$ & 151.8 & 165.54 & 169 \\
\hline WaH Tomp. IF & 88.61 & 153.31 & 152.91 & 153.61 & 154 & 161.7 & 1525 & 151.11 & 164.8 & 160.8 \\
\hline Wat Tomp. WaF & 149.61 & 153.31 & 153.21 & 153.8 & 153.81 & 161.31 & 15251 & 151.7 & 105.61 & 165.6 \\
\hline Inotroem Tomo.f & $149.8 i$ & 148.6 & 149.6 & 149.81 & 149.01 & $150 . \overline{6}$ & 151.61 & -151.71 & 151.01 & 751.9 \\
\hline Avg. Wall Tamp.f & $150 !$ & 153.31 & 15321 & 154.41 & 154.31 & 161.61 & 15221 & 151.71 & 16281 & 168.8 \\
\hline Avo. WallDT.f & $0.1 i$ & 3.61 & 3.51 & 4.51 & 4.31 & 10.7 & 0.61 & $0 \bar{i}$ & 13.2 & 13.9 \\
\hline Romaining Subcooff & 5.41 & & & & & & & & 1 & \pm \\
\hline Exct Cuality & $0^{\circ}$ & 01 & of & of & of & $0 i$ & 01 & 01 & $0 !$ & \\
\hline EXP. H-BtuMritefF & oi & 469.3 & 479.31 & 380.9 & 395.51 & 738.4 & of & $0_{1}^{1}$ & 1948.5! & 1676.4 \\
\hline COMPUTEDH & & & & & & & & & & \\
\hline 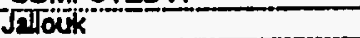 & 365.61 & 365.71 & 365.4 & 3355 & 35535 & $365.9 !$ & 358.71 & 356.7 & 338.81 & 368.8 \\
\hline Szadyi(Cudo tubo) & 454.41 & 4671 & 464.61 & 467.8 & $470.9 !$ & 4741 & $4 \pi .3$ & 479.7 & 482.11 & 484.8 \\
\hline $5=0$ & $454.4 T$ & 4611 & 484.81 & 467.81 & 470.81 & 4741 & 47.31 & 479.71 & $482 \pi$ & 484.8 \\
\hline (RUO) & 2302531 & 229809 & 2297881 & 23018्31 & 2306391 & 2310071 & 233901 & $2340 \mathrm{~s} 31$ & 2350001 & 20070 \\
\hline (F) & 1.0211 & 1.056 & 1.041 & 1.0511 & 1.0581 & 1.0581 & 1.0091 & 1.074 & 1.0781 & 1.084 \\
\hline (Hivio) & 464.41 & $46 \mathrm{il}$ & 464.61 & 467.0 & 470.91 & 474 & 47.31 & 479.7 & $482 \pi$ & 485.8 \\
\hline (HBOLL) & 31.9 & 831.7 & 8141 & 1034.61 & 988.61 & 2548.51 & 125.91 & 8.41 & $3216.8 i$ & 3338.6 \\
\hline & & & & & & & & & -1 & \\
\hline & ZONE2 & ZONE4 & ZZONE6 & ZONE8 & ZONETO & & & i & & \\
\hline DPliquid Temp.F & $: \quad 89.91$ & & + & & & & & $i$ & $i$ & $i$ \\
\hline DPUTquid Density tom/th & 91.791 & & & & & & & & & \\
\hline Elevation- & $2 !$ & 4 & $6 i$ & 8 & 9.8751 & & & & & \\
\hline Mesuured DFFsid & 0.057 & 0.007 & 0.0031 & $0.1 \pi 7$ & $=0.2751$ & & & & & \\
\hline Źrocorioctions-psid & $0.103 !$ & 0.076 & 0.081 & 0.027 & 0.0881 & & & & & \\
\hline Contecid DP+ald & -0.0307 & 0.009 & -0.1431 & 0.084 & -0.3611 & & \pm & $i$ & & 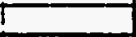 \\
\hline Reted OPI & 2.821 & 4.68 & 7.071 & 8.801 & 11.701 & & 1 & I & I & I \\
\hline 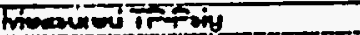 & 134.21 & & 1 & & 13951 & & & 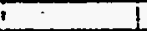 & & \\
\hline Macured Overeli & 1.24 & & I & 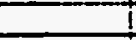 & & & I & T & I & I \\
\hline & $I$ & & 1 & & 1 & 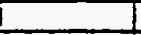 & & $i$ & $i$ & $i$ \\
\hline Computed DPA & $2.20 !$ & 4.58 & 6.871 & 9.16 & 11.311 & & 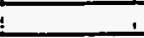 & & & \\
\hline Friction-t & 0.291 & 0.57 & 0.861 & 1.15 & 1.43 & & & & 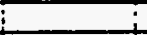 & \\
\hline Acouleration & $0 i$ & of & 0.071 & 0.01 & 0.03 & & & & & \\
\hline Elovation-ft & $2 !$ & $4 !$ & $6 i$ & 8 & 9.851 & & & & & \\
\hline
\end{tabular}




\begin{tabular}{|c|c|c|c|c|c|c|c|c|c|c|}
\hline Runt: & 771 & 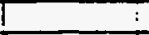 & : & $!$ & $!$ & 1 & : & 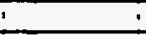 & $\therefore$ & \\
\hline Test Fuid & C4F8 & & & & $\vdots$ & & & & & \\
\hline Data: & $8 / 1693$ & & & & 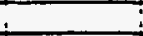 & & 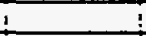 & 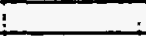 & & \\
\hline Time: & $11: 411$ & $!$ & 5 & & & 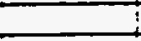 & E & 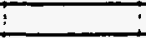 & 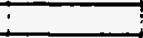 & \\
\hline Note & Anatylicad o & dis used I & I & & $\mathrm{i}$ & 1 & 1 & i & i & \\
\hline Fow Ares & 0.00336 & & & & i & & I & 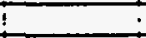 & 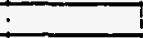 & \\
\hline Hod Fow Areatil & 0.15595 & & & & i & & 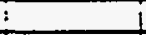 & 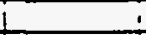 & $\div$ & \\
\hline Sat. PressurePsig & 85.6 & & & & 1 & & & & $T$ & \\
\hline Sat. Tomperature F(intel) & $\mid 26.8$ & & & & 1 & & I & & $T$ & \\
\hline Lq. Densiny & 86.32 & 86.25 & 86.3 & 86.24 & 86.29 & 86.3 & 86.24 & 86.31 & 86.32 & 85.78 \\
\hline Latent Hoet-Btulbm & 39.02 & 38.98 & 39.01 & 38.95 & 38.99 & 3.01 & 38.96 & 39.011 & 39.02 & 3868 \\
\hline 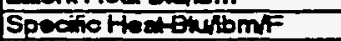 & 0.278 & 0.278 & 0.278 & 0.278 & 0.278 & 0.278 & 0.278 & 0.278 & 0.278 & 0.28 \\
\hline Lig. Visosethornthr & 0.581 & 0.662 & 0.664 & 0.687 & 0.563 & 0.564 & 0.667 & 0.564 & 0.562 & 0.647 \\
\hline Lig. Conductivin throf & 0.0325 & 0.0324 & 0.0525 & 0.0324 & 0.0324 & 0.00251 & 0.0224 & 0.0325 & 0.00325 & 0.002 \\
\hline Liq. Prand No. & 4.83 & 4.82 & 4.88 & 4.82 & 4.89 & 4.88 & 4.82 & 4.831 & 4.88 & 2.7 \\
\hline Indet Fow Pat-GPM & 9.11 & & 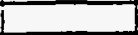 & & & & & & & \\
\hline OU:Al Lig. Fow Rato-GPM & 0.4 & 7 & & & & & & & & \\
\hline Outel Vap. Fow Rac-GPM & 0.8 & + & 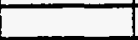 & & & & & & $!$ & \\
\hline Intet Temper inver & 1225 & & & & & & & T & $i$ & \\
\hline Oulatig. Temperaluref & 724.81 & & & & & & & & & \\
\hline Outet Vap. Tomper turef & 1221 & & & & & & & & & \\
\hline Wator Flow Rato-GPM & & & & & & & & & & \\
\hline Wher inde Tomo.f & 8201 & & & & & & & & & \\
\hline Welor ontet Tomp.F & 111.6 & & & & & & & & & \\
\hline Inlet Giom/nrhe & 227820 & & Tomp & 1223 & Density & 86.79 & & & & \\
\hline Subcoaling $f$ & 4.3 & & & & & & & & & \\
\hline Fow Observation & & & & & & & & & & \\
\hline & ZONE1 & ZONE2 & ZONE3 & ZONE4 & ZONE5 & ZONE6 & ZONE7 & ZONE8 & ZONE9 & $20 N=10$ \\
\hline Hed inputwasinet) & 289.6 & 2927 & 287.8 & 288.7 & 288.8 & 287.7 & 285 & 2925 & 289.4 & 20.8 \\
\hline Heal Loss-Wat's & 5.3 & 1.2 & 1.5 & 1.6 & 1.5 & 1.1 & 0.7 & 1.2 & 1.5 & 8.4 \\
\hline Heal Flox Buthrhe & 5020 & 5063 & 499 & 4970 & 4978 & 4987 & 4090 & 5070 & 5017 & 5011 \\
\hline WE TOMP. औ & 731.3 & 13.5 & उख्वा & 131 & 130.7 & 130.1 & उउदा. & उदा.2 & T3. 0.6 & 130.2 \\
\hline WE Tomp. IA & 135.8 & 130.9 & 130 & 13t.2. & 130.7 & 130.7 & 131.3 & 131.8 & 13.. & 73.9 \\
\hline WE Tomp. $15+$ & 731.7 & 730.6 & 135.3 & 130.8 & 1302 & 130 & 130.8 & 131.2 & 7302 & 730.1 \\
\hline WE Tomp. IIF & 88.8 & 131.31 & 190.3 & 150.3 & 1902 & 130.4 & 1312: & 131.6 & 129.7 & T20.0 \\
\hline WE" Tomp. I2F & ब死 & 131.51 & 130.8 & 130.5 & 1302 & 130.3 & 130.9 & 137.9 & 130.1 & 100.4 \\
\hline hetreen Temp.f & 125. & 75.61 & TE5:2 & 125.6 & 125.8 & 125.2 & 125.8 & 125.2 & 126.1 & 128.8 \\
\hline Avg. Ws Tomp F & 131.7 & 131.3 & 730.2 & 130.8 & 130.8 & 130.3 & 131 & 131.5 & 1308 & 930.4 \\
\hline AVg. WEDT.F & 6.3 & 5.6 & 4.7 & 5 & 4.8 & 4.9 & 5.2 & 6.1 & 5 & 1.9 \\
\hline Remaining subcooff & -0.3 & & & & & & & & & \\
\hline Ext Cualy & 0 & 0.09 & 0.07 & 0.1 & 0.13 & 0.17 & 0.2 & 0.23 & 0.27 & 0.3 \\
\hline EXP.H8tuHThEF & 793.8 & 908.8 & 1058.3 & 988.8 & 1005 & 1023.1 & 947 & 829.7 & 1000.7 & 2684 \\
\hline COMPUIESH & & & & & & & & & & \\
\hline Jeok & 60032 & 595.3 & 588 & 580.8 & 575.2 & 564 & 564.7 & 571.8 & 570.4 & 385.7 \\
\hline 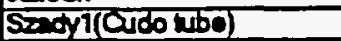 & 907.6 & 9|5.5 & 906.8 & 907.6 & 50.4 & 907.6 & 906.7 & 916.5 & 911.7 & \$60.4 \\
\hline sendr & 608.01 & 674.7 & 6032 & 60.6 & 6052 & 685.8 & 682.1 & 673.81 & 6528 & 670.8 \\
\hline$(\mathrm{F} 2 \mathrm{~b})$ & 26368 & 20017 & 25000 & 24209 & 23952 & 22400 & 2107 & 20721 & 19622 & 1953 \\
\hline (F) & 1.100 & 1.5.60 & 1.894 & 2.36 & 2.593 & 2.898 & 3.048 & 3.258 & 3.465 & 3.57 \\
\hline (H) & 88.6 & 12451 & 1602 & 188.2 & 208.8 & 227.8 & 246.1 & 261.7 & 2712 & 288.6 \\
\hline$(\mathrm{H} B \mathrm{OH})$ & 907.8 & 913.8 & 904.6 & 905 & 9028 & 902.8 & $\mathbf{9 0 0 . 7}$ & 909.3 & 908 & 9212 \\
\hline & & & & & & & & & & \\
\hline & $\angle O N=2$ & 2OE=4 & ZONE & $20 N 38$ & ZONE=10 & & & & & \\
\hline DP iquid Temp.f & $92 B$ & & & & & & & & & \\
\hline DPLopid Dentithom 13 & 91.4 & & & & & & & & & \\
\hline Eievetiont & & 4) & & 8 & 9.875 & & & & & \\
\hline Whated op & 0.203 & 1.30 & 278 & 2ब्या & 3.3. & & & & & \\
\hline Zero Comecions-psid & -0.000 & 0.00 तो & 0.06 & 0.008 & -0.08 & & & & & \\
\hline Correcod DPFidd & 0.216 & 1.283 & 278 & 2077 & 3.400 & & & & & \\
\hline Actud DPa & 1.71 & 2.11 & 2.78 & 4.18 & 4.81 & & & & & \\
\hline 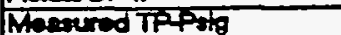 & 8.61 & & & & 84.7 & & & & & \\
\hline Moepured Overdil & $1.6 \pi$ & & & & & & & & & \\
\hline & & & & & & & & & & \\
\hline Compend beth & T.दा & $2 \%$ & $3 . \pi$ & 4.61 & 5.2. & & & & & \\
\hline Frotont & 0.02 & 0.05 & 0.11 & 0.18 & 0.28 & & & & & \\
\hline Acosterention & 0.01 & 0.18 & 0.04 & 0.05 & 0.07 & & & & & \\
\hline Eleviont & 1.6 & 2E & 3.68 & 4.38 & 5.06 & & & & & \\
\hline & & & & & & & & & & \\
\hline
\end{tabular}




\begin{tabular}{|c|c|c|c|c|c|c|c|c|c|c|}
\hline Run: & 772 & & & & 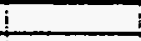 & $i$ & I & & 1 & I \\
\hline Torf Fluid & CAFB & & & & & $i$ & & & $i$ & \\
\hline Dala: & श1633 & & & & & & & & I & \\
\hline Time: & $11: 41$ & & & & & & & & I & \\
\hline Now & Anshioal & dis used & & & & & & & t & \\
\hline Fow Aronte & 0.00355 & & & & & & & & & \\
\hline Hod Flow Aros? & 0.18685 & & & & & & & & & \\
\hline Sal. Prossure pigh & 85.6 & & & & & & & & & \\
\hline Se.Temperturefind) & 728.8 & & & & & & & & & \\
\hline La. Denatym & 80.3 & 80.20 & 88.3 & 80.20 & 80.32 & 86.37 & 8020 & 80.58 & 88.80 & 8532 \\
\hline Letent flo butiom & 39.07 & 38.83 & 39.01 & \$8.99| & 39.02 & 39.00 & 38.901 & 39.05 & 39.05 & 38.94 \\
\hline Specinothenterwomif & 0.278 & 0.278 & 0.278 & 0.278 & 0.278 & 0.281 & 0.278 & 0.278 & 0.278 & 0.278 \\
\hline Dq. Visoosivitominhe & 0.689 & 0.662 & 0.664 & 0.668 & 0.684 & 0.5651 & 0.665 & 0.665 & 0.686 & 0.681 \\
\hline Lq. Conductingyeshreff & 0.0325 & 0.0324 & 0.0285 & 0.0324 & 0.0325 & 0.0055 & 0.0324 & 0.0525 & 0.0525 & 0.0024 \\
\hline Liq. Prand" No. & 4.88 & 4.82 & 4.83 & 4.83 & 4.83 & 4.84 & 4.88 & 4.88 & 4.84 & 4.82 \\
\hline Inter Fow PDI-CPM & 1.7 & & & & & & & & & \\
\hline 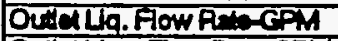 & 0.5 & & & & & & & & & \\
\hline OWet Vap. Fow RalogPM & 1.3 & & & & & & & & & \\
\hline Intet Temperélireff & 1227 & & & & & & & & & \\
\hline Ou'CLLG. Tomperritref & T24.3 & & & & & & & & & \\
\hline Outavep. Temperauref & T21.8 & & & & & & & & & \\
\hline WLPF Flow PE:CFM & & & & & & & & & & \\
\hline Wher hlel Temo.f & 829 & & & & & & & & & \\
\hline Wher outh Tomp.f & 110.2 & & & & & & & & & \\
\hline Inlet Gbommine & 3574801 & & Tomp & 123.2 & Dentiy & 85.6य1 & & & & \\
\hline Subooolingt & 4.1 & & & & & & & & & \\
\hline Flow Observetion & & & & & & & & & & \\
\hline & ZONET & IZONES & ZON=8 & ZONE4 & ZONES & ZON=6 & ZON: & $2 O K=8$ & 2ON=9 & $201=10$ \\
\hline Hathou-We"tad) & 2829 & 289 & 291.7 & 290.2 & 288.1 & 288.8 & 256 & 289.8 & 291.7 & 25 \\
\hline Heclose-We"s & 4.8 & 1.1 & 1.4 & 1.4 & 1.3 & & 0.6 & 1.1 & $=1.3$ & 7.4 \\
\hline Hex FWx- & क्विय & 4968 & 5008 & $50<0$ & 45 & 497i & $4 \$ 3$ & 5001 & 505 & 5ारक \\
\hline We Tamp. 134 & उउस्यो & T31.4 & 1295 & 730.6 & 730.8 & T29:8 & 730.8 & 30.8 & 730.1 & T29.7 \\
\hline WeTramp.14 & 737.6 & 130.8 & 130 & 730.7 & 730.8 & 7301 & 7306 & अ.ता & 73008 & T3., \\
\hline We' Tamp. 15F & 73. & 1300 & Iउस & 730.4 & Z29.8 & 129.7 & 70.7 & स्जog & ध一ा & Tर्व: \\
\hline We' Tomp. IfF & 89.61 & 197.1 & 130.2 & 130.11 & 129.7 & 150 & 190.5 & 150.01 & 120.4 & T्र.1 \\
\hline Well Tomp. 12F & 137.8 & 131.4 & 130.7 & 130.11 & 129.8 & 120.9 & 130.1 & 131.11 & 1298 & 12.8 \\
\hline Instrean Temo.f & 125: & 125.6 & 126.2 & 125.8 & 125.11 & 124.8 & T25.3 & 124.0 & 124.0 & T5.7. \\
\hline Avg. Wall Tomp.f & 131.6 & 131.2 & 130.1 & 130.6 & 129.9 & 129.0 & 130.31 & 131 & 130 & T20.9 \\
\hline AVo.WAIDT.F & 6.2 & 5.5 & 4.7 & 4.8 & 4.8 & 4.8 & 4.8 & 5.8 & 4.9 & 4 \\
\hline Ramining Suboolf & 12 & & & & & & & & & \\
\hline Extounty & $-0 . \overline{1}$ & 0.01 & 0.03 & 0.06 & 0.08 & 0.1 & 0.12 & 0.14 & 0.18 & 0.18 \\
\hline EXP.HEthrthe & 758.8 & 905.8 & 10824 & Tष्टा. & Tơr7. & 1074.5 & 924.6 & 86t2 & 10425 & 1282 \\
\hline COTPUIEDH & & & & & & & & & & \\
\hline JThok & 368.2 & 385.8 & 5883.8 & 575.8 & 562.8 & 6567.8 & 533.8 & 538.31 & 570.61 & $57 \pi .4$ \\
\hline sedyi(Cudotwe) & 897.8 & 5032 & 912ह & 97T.8 & 905 & Sos & 855.6 & 9124 & 9ाठ.. & F्ट8.1 \\
\hline sed 2 & 858.4 & 663.8 & 6723 & 670.4 & 6028 & 68201 & 603.11 & 655.81 & हुना & 8728 \\
\hline (F⿻) & 40\%5 & 40820 & 3980 & 38507 & 3000 & $3 \% 0511$ & 3588 & 3567II & 34601 & 3395 \\
\hline & 7.008 & 1.148 & $1.6 \infty$ & 7.872 & 2176 & ट348 & 2518 & 2.675 & 289 & 2968 \\
\hline (सय) & Tाद.8 & 130.6 & विक्षी & 217.4 & 24.7 & 256.5 & 285.6 & 308.7 & उटाइ & 337.8 \\
\hline (FEOU) & 807.1 & $\operatorname{sen} 1$ & 910.2 & 9078 & 85.11 & 897 & 81 & 901 & S्रदा & 913 \\
\hline & & & & & & & & & & \\
\hline & 20132 & ZONET & $20 \%=36$ & ZON'38 & ZOREIO & & & & & \\
\hline Dpligud tomp.f & \$3.1 & & & & & & & & & \\
\hline DPI tasid Densitutiom/3 & 91.3 & & & & & & & & & \\
\hline Gevelion- & & & & & 9.875 & & & & & \\
\hline Wearurediplarid & $0.18 \pi$ & 1.048 & 7.703 & 23:2 & -2850 & & & & & \\
\hline Zcro Corroctom-pidd & -0.000 & -0.001 & -0.03 & 0.003 & -0.08 & & & & & \\
\hline Comend DPsyd & 0.188 & 1.067 & 1.700 & 230 & 296 & & & & & \\
\hline Acter DP & 1.8 & 240 & 3.6ा & 4.78 & 5.35 & & & & & \\
\hline Weavired iptong & \$5.6 & & & & 84.0 & & & & & \\
\hline Meneded Over"lith & 1.17 & & & & & & & & & \\
\hline & & & & & & & & & & \\
\hline Compulad DPl & 1.88 & 3.5.5 & 2.48 & 5.78 & 6.83 & & & & & \\
\hline Frotont & 0.02 & 0.08 & 0.18 & 0.28 & 0.38 & & & & & \\
\hline Aooterniont & 0.02 & 0.05 & 0.08 & $0 . \overline{0.1}$ & 0.1 & & & & & \\
\hline Exveriont & 1.84 & 3.10 & 4.25 & 5.41 & 6.53 & & & & & \\
\hline & & & & & & & & & & \\
\hline
\end{tabular}




\begin{tabular}{|c|c|c|c|c|c|c|c|c|c|c|}
\hline Runt & 773 & . & . & & ; & i & $i$ & & & ! \\
\hline Test Fuid & CAF : & $\div$ & & I & $i$ & & $!$ & & T & 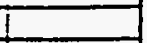 \\
\hline Dalo: & 8/683 & & & $i$ & $\vdots$ & t & I & & + & \\
\hline Time: & $11: 41$ & & & 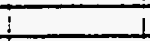 & $\perp$ & $i$ & $i$ & T & $i$ & \\
\hline Note & Anation of & dis used & & & & 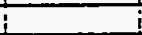 & $i$ & I & i & \\
\hline Flow Areatt2 & 0.00336 & & & & & & 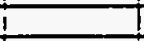 & & & \\
\hline Heal Fow Areat? & 0.195951 & & & & & & & & & \\
\hline Sat PTessure psig & 87 & & & & & & & & & \\
\hline Sad. Temperature f(inlel) & 127.81 & & & & & & & & & \\
\hline Lq. Densinytomns & 86.391 & 86.32 & 8824 & 88.081 & 86.16 & 86.17 & 86.16 & 86.25 & 86.20 & 86.17 \\
\hline Letent Hectshitom & 39.07 & 39.02 & 38.55 & 38.881 & 38.88 & 38.9 & 38.88 & 38.96 & 38.89 & 38.8 \\
\hline Specido HexteribmiF & 0.278 & 0.2781 & 0.278 & 0.279 & 0.279 & 0.278 & 0.279 & 0.278 & 0.278 & 0.278 \\
\hline Lig. Visoositybminthe & 0.5661 & 0.6641 & 0.6671 & 0.657 & 0.560 & 0.669 & 0.658 & 0.552 & 0.563 & 0.550 \\
\hline Liq. Conductivity & 0.03251 & 0.0325 & 0.00224 & 0.00201 & 0.03231 & 0.03241 & 0.03281 & 0.0328 & 0.0324 & 0.0326 \\
\hline Lig. Prandeno. & 4.84 & 4.83 & 4.82 & 4.81 & 4.81 & 4.81 & 4.811 & 4.821 & 4.88 & 4.87 \\
\hline Inlet fiow Rate-GPM & 2.5 & & & & & & & & & I \\
\hline Outel Lia. Fow Rate-GPM & 0.8 & 1 & 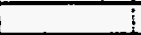 & 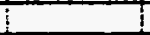 & $t$ & & & & & \\
\hline Outtol Vap. Fow Pato-GPM & 1.11 & & & 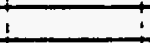 & $\vdots$ & & & & & \\
\hline Inlat Temperabureff & 1224 & & & i & & & 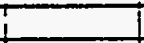 & & & \\
\hline Outet Lig. Temparahref & 124.81 & & & & & & & & & \\
\hline Outal Vap. Tempersirof & 1223 & & & & & & & & & \\
\hline Water Flow Pata-GPM & & & & & i & & & & & \\
\hline Wertide Tomo.F & 83.81 & & & & ! & & & & & \\
\hline Weter outtel Tomp.F & 110.8 & & & & $i$ & & & & & \\
\hline Intet G-ibmhnthe & 5165:5ा & & Tomp & 123.5 & Density & 86.69 & & & & \\
\hline Suboodingf & 5.4 & & & & & & & & & \\
\hline \multirow{2}{*}{ Fow Obsanvion } & & 1 & 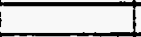 & & & & & & & \\
\hline & ZONE1 T & ZONE2 & ZONE3 & ZONE & ZONES & ZONE6 & ZONE7 & ZONE8 & ZONES & ZOK:ETO \\
\hline Has input-Watinget) & 287 & 288 & 293.2 & 2925 & 296.2 & 2902 & 289 & 286.7 & 289.9 & 291.8 \\
\hline HeI Loss $+W=$ & 5 & 1.1 & 1.5 & 1.5 & 1.4 & 9 & 0.7 & 1.1 & 1.3 & 7.5 \\
\hline Hed Flox-Bulftring & 4975 & $4 \sin$ & 5003 & 5070 & 513 & 5050 & 5010 & 4959 & $50 \times 1$ & 5040 \\
\hline Wall Temp. I34 & T31.9| & स्द्2! & T30.31 & $|3| .7$ & 731.1 & 130.8 & T3. & 131 & T3013 & 180 \\
\hline We Temp. 144 & 1325 & 731.7 & 130.8 & 131.7 & 131.31 & 130.9 & 137.8 & 131.5 & 131 & 737.8 \\
\hline WNT Temp.15f & 13251 & T31.1 & 131.91 & 131.3 & 130.8 & 130.2 & 130.5 & 137.3 & 130.4 & 130.1 \\
\hline Wal Tomp. fif & 89.51 & 131.8 & 130.9 & 137.1 & 130.7 & T30.9 & 131.6 & 137.3 & 129.8 & 129.5 \\
\hline WEll Tomp. ILF & 13251 & 132.71 & 131.4 & 131.31 & 130.9 & 730.8 & 131.11 & 131.5 & 130 & 130.2 \\
\hline Instream Temp.F & 124.71 & 125.1 & 125.6 & 126.5 & 126.1 & 128 & 126.11 & 125.6 & 725.8 & 126 \\
\hline Avg. Wall Temp.f & 13241 & 132 & 130.9 & 131.5 & 137 & 130.7 & 131.2 & 137.3 & 730.3 & 130.3 \\
\hline Avo. WallDT.F & 7.4 & 6.6 & 5 & 4.7 & 4.6 & 4.5 & 4.8 & 5.6 & 4.8 & 4.1 \\
\hline Remaning Subcooff & 3.3 & & & & & & & & & \\
\hline Exi Qualy & -0.02 & -0.01 & 0.01 & 0.02 & 0.04 & 0.5 & 0.06 & 0.08 & 0.00 & 0.11 \\
\hline 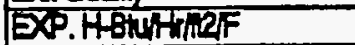 & $6 \pi$ & 750.8 & 1007.4 & 1074 & Tा1.8 & 1125.7 & 9053.4 & 888.3 & 1018.6 & 12662 \\
\hline \multicolumn{11}{|l|}{ COMPUTEDH } \\
\hline Jallouk & 585.7 & 588.7 & 597.7 & 574.8 & 581.3 & 572.8 & 572.6 & 56.2 & 574.1 & 579.9 \\
\hline Sedvi (Cudo tube) & Sot.4 & 905.7 & 917.4 & 924 & 928.4 & 975.8 & gæ0.t & 913.1 & 919.4 & 927.0 \\
\hline send? & E़ा.8 & 685.8 & 67 & $6 \pi .2$ & 684.8) & 6612 & ES3.7 & 659.7 & 6512 & 678.0 \\
\hline (P्DC) & 69678 & 59001 & 6018: & 5004 & 58785 & 5788 & 5708 & 55818 & 54883 & 535 \\
\hline (F) & 1.006 & 9.008 & 9.008 & 1.436 & 1.685 & 1.88 & 2.04 & 2.196 & 2.332 & 2451 \\
\hline (स) & 156.41 & 165.6 & 766.7) & स्26 & 251.1 & 201.2 & 318.8 & 369.7 & 350.71 & 37.7 \\
\hline \multirow[t]{3}{*}{ (सBOUL) } & 8008 & क्वा2] & 916.0 & 919.7 & 9215 & 910 & 907.4 & 897.2 & 900.5 & 9063 \\
\hline & & & & & & & & & & \\
\hline & ZONES & ZONEL & $\angle O N=6$ & ZONiEB & $201=10$ & & & & & \\
\hline 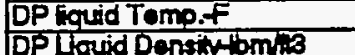 & 93.5 & & & & & & & & & \\
\hline DPLquid Density 4 mi3 & 91.2] & & & & & & & & & \\
\hline Elevation 1 & 2 & 4 & 6 & 8 & 9.875 & & & & & \\
\hline Meatured Despid & 0.178 & 0.727 & T.Z2 & T.728 & 2सा3 & & & & & \\
\hline Zero Corrections-pid & -0.000 & $0.0 \mathrm{il}$ & -0.08 & 0.028 & -0.08 & & & & & \\
\hline Correctod DP Pald & 0.127 & 0.746 & 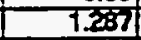 & 1.703 & 233 & & & & & \\
\hline Actud DPa & 1.91 & 289 & 4. & 5.8 & 8.6. & & & & & \\
\hline Whenred Tान्यक & 87 & & & & 88.8 & & & & & \\
\hline \multirow{2}{*}{\multicolumn{11}{|c|}{ Meapured Over"lt }} \\
\hline & & & & & & & & & & \\
\hline Compulad DPA & 204 & 3.84 & 5.48 & 7.0 & 8.51 & & & & & \\
\hline Fridont & 0.04 & 0.1 & 0.18 & 0.3 & 0.4 & & & & & \\
\hline Accolervion & 0 & 0.06 & 0.00 & 0.12 & 0.15 & & & & & \\
\hline Enevitiont & 2) & 3.69 & 5.2 & 6.63 & 7.88 & & & & & \\
\hline & & & & & & & & & & \\
\hline
\end{tabular}




\begin{tabular}{|c|c|c|c|c|c|c|c|c|c|c|}
\hline Runi & 774 & & 1 & & & & & & & \\
\hline \begin{tabular}{|l|} 
Toaffuid \\
\end{tabular} & ICAF & & $i$ & & & & & i & $\mathrm{i}$ & \\
\hline Data: & $8 / 17 / 93$ & & I & & & & & & & \\
\hline Time: & $11: 17$ & & & & & & & & 5 & \\
\hline Nole & Anstrical & dis used & & & & & & & & \\
\hline Flow A Meatle & 0.00356 & & & & & & & & & \\
\hline Hoxt Fow Areale & 0.19696 & & & & & & & & & \\
\hline Sat. Prossuro Psig & 84.41 & & & & & & & & & \\
\hline 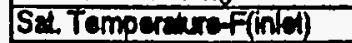 & 128 & & & & & & & & & \\
\hline Lig. Deneliytomniz & 88.18 & 86.24 & 88.24 & 86.22 & 88.28 & 86.201 & 88.37ा & 86.41 & 86.44 & 86.15 \\
\hline Laton Hoal Btullom & 38.87 & 38.56 & 38.85 & 38.81 & 3.99 & 38.99 & 39.06 & 39.09 & 3.17 & 39.13 \\
\hline 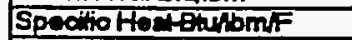 & 0.278 & 0.278 & 0.278 & 0.278 & 0.278 & 0.278 & 0.278 & 0.278 & 0.278 & 0.278 \\
\hline 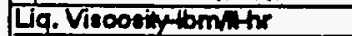 & 0.56 & 0.6611 & 0.5811 & 0.6671 & 0.669 & 0.658 & 0.668 & 0.667 & 0.668 & 0.568 \\
\hline 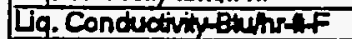 & 0.0324 & 0.0324 & 0.0324 & 0.0324 & 0.0324 & $0.032 \pi$ & 0.0325 & 0.0058 & 0.0025 & 0.0028 \\
\hline Ug. Pranditno. & 4.821 & 4.82 & 4.82 & 4.82 & 4.83 & 4.83 & 4.84 & 4.841 & 4.85 & $\frac{0.0200}{4.85}$ \\
\hline Intaf Fow Rato-GPM & 4.8 & & $\frac{1}{1}$ & & & & $\frac{7.001}{1}$ & 4.001 & 9.001 & 9.00 \\
\hline Outhl Lig. How RatoGPM & 1.2 & & & & & & & & & \\
\hline Quthl Vap. Fow Rate-GPM & 2.3 & & & & & & & & & \\
\hline Inlat Tompereturef & 123.1 & & & & & & & & & \\
\hline 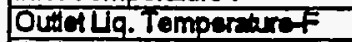 & 123.9 & & & & & & & & & \\
\hline Outa Vap. Tomperatrof & 120.9 & & & & & & & & & \\
\hline WW Flow RAtG-GPM & & & & & & & & & & \\
\hline Water inlet Temp.F & 82.9 & & & & & & & & & \\
\hline Water oultet Temp.f & 112 & & & & & & & & & \\
\hline Indactompanth & Diga! & & Temp & 123.6 & senoing & 86.601 & & & & \\
\hline Subooding f & 2.9 & & & & & & & & & \\
\hline Fow Obsenvion & & & & & & & & & & \\
\hline & ZONE & ZONE2 & ZONE3 & ZONE4 & ZONES & ZONEE & ZONE7 & ZONEB & ZONES & $20 \pi=10$ \\
\hline 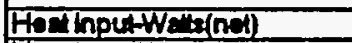 & 460 & 286.1 & 290.6 & 289.4 & 284,2 & 288.7 & 281.5 & 285.4 & 291.8 & \\
\hline Hox Loss-Wes & 5.5 & $\frac{0.04}{1.2}$ & 1.6 & 1.6 & 1.6 & $\frac{1.21}{1.21}$ & $\frac{0.7}{0.7}$ & $\frac{20.91}{1.11}$ & $\frac{20.00}{1.44}$ & \\
\hline Hox Flox Exwhthe & 8320 & 4960 & 5037 & 5017 & 4928 & यदां। & 4800 & $49 \pi$ & 5058 & $5 \times 12$ \\
\hline Wal Tomp. WF & 733.3 & 1326 & 7322 & T32.6. & 733.3 & 1327 & 7र्ड2.6 & अंजी & र्ञा & 12016 \\
\hline Wa'Tamp. 14F & 733.7 & 132.11 & 132.6 & 132.8 & 133.3 & 133.6 & 132.5 & 737.81 & 731.8 & 735.1 \\
\hline Wal Tomp.15F & 734.8 & 131.71 & 133.3 & 138.11 & 132.5 & 132.7 & 13211 & 137.51 & 130.9 & रू: \\
\hline WA Temp. ItF & 85.9 & 132.2 & 131.4 & 1821 & 732.7 & 132.7 & 13281 & 131.5 & 150.2 & 128,8 \\
\hline WAIITOMP. WRF & 134.11 & 132.6 & 131.9 & 132.11 & 132 2 & 132.7 & 13271 & 13211 & 130.8 & 129.7 \\
\hline Intrean Temp.f & 125.90 & 725.6 & 725.6 & 125.71 & 125.31 & 725.31 & 124.81 & 124.6 & 124.4 & 124.8 \\
\hline AV. We Tomp F & 733.9 & 132.41 & 132 & 7328 & 1325 & 1329 & 1325 & 131.61 & 130.8 & 129.7 \\
\hline AND. WENDT.F & 7.6 & 6.5 & 6.2 & 6.81 & 7 & 7.41 & 7.5 & 6.8 & 6.2 & 5.2 \\
\hline Rempining Suboodf & 1.2 & & & & & & & & & \\
\hline Exol Cualy & -0.01 & का & 0.01 & 0.01 & 0.02 & 0.03 & 0.04 & 0.04 & 0.05 & 0.06 \\
\hline 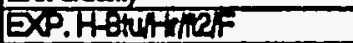 & 7098.6 & 700 & 812.8 & 754.6 & 703.5 & 666.6 & 648.9 & 730.11 & 819.6 & 364.8 \\
\hline COMIPUIEOH & & & & & & & & & & \\
\hline Jnlouk & 703.8 & 570.5 & 5724 & 577.8 & 579.4 & 588.4 & 585.5 & 55र.5ी & 6001 & 610.5 \\
\hline Szadyi(cudo whol & 7188.6 & 909.21 & 918.8 & SCEST & 914.4 & 917.4 & 914.6 & 9231 & 336.1 & 938 \\
\hline $5 \sin y^{2}$ & 940.2 & 8.9 .4 & कor. & 65.3 & 87r.4 & 677.9 & 674.31 & 675.6 & E. & 70.5 \\
\hline (स्था) & 116898 & 7156011 & 1152003 & 11448 & 113128 & 112308 & 11089 & 109018 & 108774 & \\
\hline & 1.006 & 1.000 & 1.788 & 1.382 & 7.530 & 1.650 & 1.708 & 1.868 & 1.9301 & 20 \\
\hline (स) & उEटा & 2624 & 300.0 & 363.2 & 400.9 & 42211 & 460.7) & 484.8 & 50050 & 630.7 \\
\hline (HBOA) & 11822 & 901.8 & 903.9 & 9623 & 880 & 82 तो & 874.9 & 878.11 & 882 & 875.3 \\
\hline & & & & & & & & & & \\
\hline & ZONE2 & ZONEA & ZONEB & ZONES8 & $Z O N=10$ & & & & & \\
\hline DP Lould Tomp. $F$ & 88.8 & & & & & & & & & \\
\hline 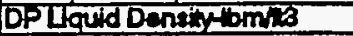 & 9202 & & & & & & & & & \\
\hline Eevilont & & & & & 9.875 & & & & & \\
\hline 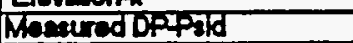 & 0.06 & 0.518 & 0.301 & 7.153 & 7.42. & & & & & \\
\hline Zero Compotiona-psid & -0.006 & -0.016 & -0.006 & 0.022 & -0.082 & & & & & \\
\hline 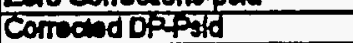 & 0.057 & 0.034 & 0.856 & 7.131 & 1.474 & & & & & \\
\hline Acter DPA & 2.02 & 3.37 & 4.60 & 6.61 & 8.06 & & & & & \\
\hline Momered TPung & हम4 & & & & ह2⿺ & & & & & \\
\hline Maselured Overesth & 3 & & & & & & & & & \\
\hline & & & & & & & & & & \\
\hline Compland DPa & 212 & 4.17 & 6.07 & 7.89 & 9.68 & & & & & \\
\hline Frictionth & 0.12 & 0.3 & 0.54 & 0.891 & 1.14 & & & & & \\
\hline Acosteritionth & of & 0.17 & 0.25 & 0.32 & 0.38 & & & & & \\
\hline Env'iont & & 3.7 & 5.28 & 6.7 & 8.18 & & & & & \\
\hline & & & & & & & & & & \\
\hline
\end{tabular}




\begin{tabular}{|c|c|c|c|c|c|c|c|c|c|c|}
\hline Runt & $776 !$ & & \pm & $\because$ & : & $i$ & $!$ & ! & $!$ & \\
\hline Togt Fuid & ICAF8 & $!$ & 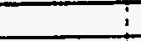 & $i$ & $i$ & & $!$ & $i$ & $i$ & \\
\hline Dala: & i $8 / 17 / 33$ & $!$ & & 1 & I & & $i$ & $!$ & $!$ & \\
\hline Time: & $15: 16$ & & & & i & & $I$ & $i$ & $i$ & \\
\hline Not & jAnalyioed d & As used I & & & & & & & & \\
\hline Fow Areante & 0.00355 & & & & & & I & 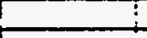 & ! & \\
\hline Hex fFow Aroate & 0.195551 & & & & & & & & 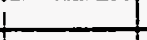 & \\
\hline Sal Pressure Psig & 84.41 & & & & & & i & 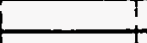 & $T$ & \\
\hline Sat. Tempereturef(iniot) & 126 & & & & & & 7 & & & \\
\hline Ua. Denstny & 86.201 & 86.29 & 86.27 & 86.22 & 88.25 & 88.201 & 86.351 & 86.391 & 86.4 & 86.42 \\
\hline Latan Heat Ethibm & 38.98 & 38.891 & 38.98 & 38.84 & 38.89 & 38.991 & 39.00 & 39.07 & 39.09 & 39.1 \\
\hline Specifo Hertelubmit & 0.278 & 0.2781 & 0.278 & 0.278 & 0.278 & 0.278 & 0.278 & 0.2781 & 0.278 & 0.279 \\
\hline Liq. Visooshylomhth & 0.562 & 0.5631 & 0.552 & 0.5611 & 0.5521 & 0.5601 & 0.5651 & 0.566 & 0.567 & 0.587 \\
\hline Lig. Condustivity Btwhrff & 0.0324 & 0.0324 & 0.0524 & 0.0324 & 0.0324 & 0.03291 & 0.0525 & 0.00851 & 0.0825 & 0.0325 \\
\hline Lig. Prand No. & 4.82 & 4.83 & 4.89 & 4.82 & 4.82 & 4.881 & 4.84 & 4.841 & 4.84 & 4.84 \\
\hline Inlot flow Ratu-GPM & 4.11 & +1 & & & & 1 & I & & 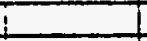 & \\
\hline Outet Liq. Fow Reto-GPM & 1.2 & & & & & & $i$ & I & I & \\
\hline Qubat Vap. Fow Rale-GPM & 1.9 & 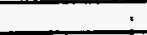 & & & & & & & 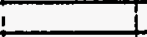 & \\
\hline Inlot Temperaturof & 1225 & & & & & & & & & \\
\hline Outelíq. Temporturef & 124.5 & & & & & & & & & \\
\hline Outer Vap. Temporatef & 120.61 & 1 & & & & & & & & \\
\hline WEN FOW RAT-GPM & 1 & $T$ & & & & & 1 & & & \\
\hline Wher hilot Tomp.F & 84.8 & & & & & & i & & & \\
\hline Wator outhot Tomp.f & 113.8 & & & & 1 & & 1 & & 1 & \\
\hline Intox Gbomphrie & 848837 & & Temp & 122.81 & Densiy & 86.7 & & & & \\
\hline Suboodingf & 3.5 & & & & & & & & & \\
\hline \multirow[t]{2}{*}{ Flow Observation } & 1 & & & & & & & & & \\
\hline & ZONE1 & ZONE2 IL & ZONE3 & ZONE4 12 & ZONES & ZONE6 & ZONE7 & ZONE8 & ZONE9 & $20 N=10$ \\
\hline Hed Input-Watts(not) & 285.7 & 288.4 & 2924 & 291.8 & 285.4 & 284.8 & 283.8 & 287.4 & 294.1 & 252 \\
\hline Heat Loss-Wa'ts & 5.2 & 1.1 & 1.5 & 1.5 & 1.5 & 1.7 & 0.7 & 1 & 1.3 & \\
\hline Hed fiox buritrine & \$क्षे & 4599 & 5059 & 5058 & 4947 & 4587 & 4918 & 4928 & 5003 & 5005 \\
\hline Wall Tomp. & T33.8 & T32.81 & T321 & T32] & 733.3 & 1332 & T32, & T312 & $13+2$ & 129.8 \\
\hline We Tomp. $14 F$ & 133.6 & 1324 & T328 & 13 & 130.6 & 133.8 & 133.1 & 152 & 737.8 & 13.3 \\
\hline Wa Temp. I5f & 134.5 & 131.7 & 133.6 & 133 & 13261 & 133 & 1325 & 131.81 & 1312 & 129.8 \\
\hline WE Tomp. HF & 88.9 & 132.3 & 131.51 & 132.1 & 132.4 & 139.1 & 133.4 & 131.8 & 130.9 & 729.1 \\
\hline WEI Temp. REF & 134.8 & 132.7 & 132.7 & 1322 & 132.3 & 1332 & 133.81 & 132.31 & 187 & 130.1 \\
\hline Intrean Temp.f & 125.6 & 125.31 & 125.41 & 125.7 & 125.5 & 125.3 & 124.91 & 124.7 & 124.6 & 124.6 \\
\hline Avg. Wall Temp.f & 134 & 132.61 & 1322 & 132.71 & 1327 & 135.3 & 1331 & 131.8 & 1312 & 130 \\
\hline Avo. Wall DT.F & 8.31 & 71 & 6.61 & 6.81 & 7 & 7.81 & 7.9 & 6.91 & 6.4 & 5.3 \\
\hline Remaining Subcoolf & 23 & & & & & & & & & \\
\hline Ext Oualdy & -0.02 & -0.01 & 0 & 0.01 & 0.02 & 0.00 & 0.09 & 0.05 & 0.05 & 0.08 \\
\hline EXP.HBtuHARE & 598.4 & 711.7 & $77 \pi .4$ & 747 & 706.6 & 63.8 & 621.3 & 7226 & 797.6 & $\$ 53.7$ \\
\hline COMPUTEOH & & & & & & & & & & \\
\hline Jallouk & 5352 & 568.8 & 570.8 & 573.51 & 5701 & 573.8 & 577.4 & 585.4 & 301.81 & 5958 \\
\hline Frady1(Qudo kube) & 906.6 & 900.6 & 976.6 & 920.4 & 912 & 9126 & 917.4 & 918.7 & 9312 & 831 \\
\hline $\operatorname{sen} \sqrt{2}$ & E04.01 & 607.6 & 674.51 & 6.2 & 658.7 & 60201 & 674.6 & 6802 & 691.7 & Wo:7 \\
\hline (RIIA) & 88747 & 985581 & 99038 & 983401 & 97282 & 962881 & 95003 & 939081 & 95000 & 92008 \\
\hline$(\vec{A})$ & 1.008 & 1.0081 & 1.006 & $1.2 \pi$ & 1.478 & 1.620 & 7.754 & 1.809 & 1.975 & 2074 \\
\hline (स्थि) & 201.6 & 231.41 & 231.6 & 294.11 & 3396 & 373.0 & 403.1 & 429.8 & 463.6 & 7782 \\
\hline \multirow[t]{3}{*}{$(\mathrm{BBO}$} & sot.8 & son. & 911.8 & 910.31 & 86 & 891.2 & 88.8 & 886.8 & 893.6 & 887.5 \\
\hline & & & & & & & & & & \\
\hline & ZONES & ZONET & ZONE6 & ZONE8 & ZONET & & & & & \\
\hline DP rquid Tomp.f & 188.5 & & & & & & & & & \\
\hline \multicolumn{11}{|l|}{$\begin{array}{l}\text { DP iquid Tomp.f } \\
\text { DPLiquid Densivem/3 }\end{array}$} \\
\hline Eevationt & & 4 & 6 & 8 & 9.875 & & & & & \\
\hline Mestured op prid & 0.002 & 0.578 & T.09 & T.319 & 7.657 & & & & & \\
\hline Zero Correctons-pesd & -0.006 & -0.018 & -0.000 & 0.082 & -0.0821 & & & & & \\
\hline Correand DP 4 id & 0.068 & 0.5821 & 1.074 & 1.297 & 1.690 & & & & & \\
\hline Actup DP & 2का & 3.2ग & 4.6 & 6.50 & 7.68 & & & & & \\
\hline moscured TPusg & 84.4 & & & & 820 & & & & & \\
\hline \multirow{2}{*}{\multicolumn{11}{|c|}{ Masured Overall }} \\
\hline & & & & & & & & & & \\
\hline Comperad Dan & 209 & 4.111 & 5.88 & 7.74 & 9.4 & & & & & \\
\hline Fricion-h & 0.09 & 0.21 & 0.38 & 0.8 & 0.84 & & & & & \\
\hline Acodterionth & 0 & 0.1 & 0.18 & 0.20 & 0.29 & & & & & \\
\hline Elevitiont & 2 & 3.8. & 5.30 & 6.9 & 8.27 & & & & & \\
\hline & & & & & & & & & & \\
\hline
\end{tabular}




\begin{tabular}{|c|c|c|c|c|c|c|c|c|c|c|}
\hline Runt: & 776 & 1 & $:$ & $\dot{\square}$ & & $i$ & & & $\dot{-}$ & $i$ \\
\hline Tastfluid & CAFB & 7 & $i$ & is & & $i$ & & 1 & $I$ & \\
\hline Dete: & $8 / 17 / 33$ & 1 & 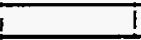 & 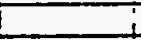 & & 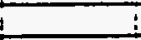 & 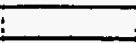 & & $\therefore$ & 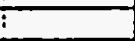 \\
\hline Tims: & $15: 16$ & 1 & $L$ & i & $i$ & & & $!$ & & 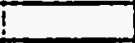 \\
\hline Nob & Inticalo & dis used i & i & 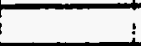 & & & & 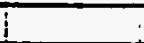 & : & 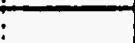 \\
\hline FlOW ANOLAL & 0.00336 & & 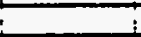 & & & & & - & & \\
\hline Hes Fow Arear & 0.1955 & & & & & & & 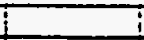 & $i$ & \\
\hline SE. PTessure का/g & 84.5 & & & & & & & & & \\
\hline SEl Tompermerte $($ (n) & 126.1 & & & & & & & & & \\
\hline Lg. Dentity fomins & 86.25 & 88.261 & 86.181 & 88.2 1 & 85ट्या & 86.251 & 8620 & 86.841 & 88.87 & 88.12 \\
\hline 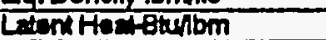 & 38.881 & अ.55| & 38.91 & 38.841 & 38.5्डा & 38.981 & 38.89 & 39.031 & 39.00 & 38.80 \\
\hline Specifo Hen Bupburf & 0.278 & 0.278 & 0.2781 & 0.2781 & 0.278 & 0.278 & 0.278 & 0.278 & 0.278 & 0.279 \\
\hline Lig. Viscotihtominhr & 0.562 & 0.5611 & 0.68 & 0.5611 & 0.6611 & $0.5 \approx 2$ & 0.653 & 0.555 & 0.568 & 0.658 \\
\hline Wa. Conductivingturht F & 0.0324 & 0.0324 & 0.03241 & 0.0324 & 0.0324 & 0.00224 & 0.0524 & 0.05651 & 0.0525 & 0.0523 \\
\hline Lq. Prand $\mathrm{No}$. & 4.82 & 4.821 & 4.82 & 4.821 & 4.82 & 4.821 & 4.83 & 4.831 & 4.84 & 4.81 \\
\hline Intelfow Rate-GPM & 3.3 & & & & & 1 & & & $i$ & \\
\hline OutaLLG. Flow RDE-CPM & 1.1 & & & & & & & 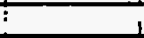 & 1 & \\
\hline 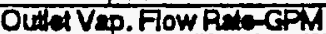 & 1.6 & & & & & & & - & & \\
\hline Intel Tomperculuref & 1226 & & & & & & & & & \\
\hline 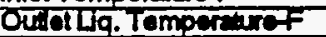 & 124.6 & & & & & & & & & \\
\hline OUAV Vep. Tomperatiof & 127 & & & & & & & & & \\
\hline WMP FOW REA-GPM & $\mathrm{t}$ & & & & & & & & & \\
\hline Weter hlet Tomo.f & 84.7 & $\mathrm{i}$ & i & & t & & & & & \\
\hline We'er oftilet Temp.f & 114.2 & $T$ & & $T$ & $T$ & 7 & & & & \\
\hline Intat Gibmhrtie & 68677 & & Tomp & 12281 & IDonsity & 86.691 & & & & \\
\hline Suboodingf & 3.5 & & & & & 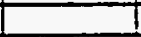 & & & & \\
\hline Fow Observion & & & & & & Tर & & & & \\
\hline & ZONEA & ZONE2 & ZONE3 & ZONER & ZONES & ZONE6 & ZONE? & ZONEB & ZONEO & $\angle O N=10$ \\
\hline Hadlnputwatud & 287.1 & 289.7 & 297.4 & 26.2 & 288.1 & 288 & 285.1 & 289.7 & 297.8 & $\mathbf{2 0 . 6}$ \\
\hline Heallosghts & 5.2 & 1.1 & 1.5 & 1.5 & 1.5 & 1.11 & 0.7 & 1.1 & 1.4 & 7.3 \\
\hline 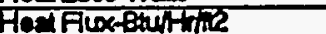 & 4971 & 5002 & उाइ & 51ओ & 78 & 482 & 496 & $500 \pi 1$ & 5TEम & 5ादा \\
\hline WE Temp. 1अ & 735.6 & 13201 & 13226 & 73 & 133.7 & 733.6 & 133.4 & 73.8 & T31.8 & 13005 \\
\hline Wad Tomp. 14F & 133.8 & 132.3 & Fु3.11 & T33.4. & 133.8 & 134.3 & 133.6 & 1325 & 1328 & स्द्2:2 \\
\hline We Tomp. 15F & 13., & 131.8 & 33.6 & 13.5| & 133.1 & 133.6 & 133 & 13281 & 131.8 & 1305 \\
\hline Wall Tamp, IIF & 88.7 & 132.5 & 131.6 & 1324 & 132.8 & 133.0 & 139.8 & 1324 & 131.2 & 1296 \\
\hline Well Temo. IIF & 134.8 & 1325 & 132 & 1324 & 1327 & 133.61 & 133.7 & 133 & 131.8 & 130.6 \\
\hline Intreen Tomp.f & T25.5| & 125.61 & 125.91 & 125.7 & 125.6 & 2255 & 125.9 & 125 & 124.8 & 128.3 \\
\hline Avg. Wall Tomp.f & 13 & 1326 & 1323 & 13.3 .11 & 133.1 & 133.8 & 133.5 & 132.4 & 131.8 & 130.7 \\
\hline Aug. Well DT.F & 8.3 & 6.7 & 6.2 & 7.1 & 7.3 & 81 & 8 & 721 & 6.7 & 4.1 \\
\hline Remaning Suboooff & 2 & & & & & & & & & \\
\hline Exd Cual'y & 0.01 & 0 & 0.01 & 0.0 .2 & 0.08 & 0.04 & 0.05 & 0.06 & 0.08 & 0.09 \\
\hline EXP. HBturkntar & 599.6 & 746.0 & 85.9 & 778.8 & 6812 & क्या & 618 & 697.6 & 75.2 & 1226.6 \\
\hline COMPUTEDH & & & & & & & & & & \\
\hline Jalouk & 577.4 & 581 & $57 / .3$ & 588.8 & 570.8 & 5729 & 573.1 & 579.7 & 589.0 & 397.1 \\
\hline इzactilcudotuse) & 907.8 & 912.6 & 9EX.7 & 928.7 & 9165 & 916.8 & 913.2 & 918.1 & 934. & 947.0 \\
\hline $5 \pi y^{2}$ & E.0.7 & 87. & 685.4 & 80.0 .0 & 00.0 & 803.8 & 003.1 & 683.2 & 69:2 & 7040 \\
\hline (Pाa) & क्रमण्रो & 795101 & 79510 & 78क्रा & $7 \sqrt{521}$ & 78578 & 75505 & 74482 & 73467 & 75.10 \\
\hline$(\mathbf{F})$ & 1.0061 & 1.0061 & ..177 & 1.462 & 7.65 & 1.809 & 1.969 & 2002 & 2.178 & 2212 \\
\hline (1) & 184.61 & 184.6 & 227.8 & 2828 & 310.8 & 348.91 & 374.8 & 383.4 & 420.5 & 402 \\
\hline$(\mathrm{H} 3012)$ & S048 & 900.8 & $\operatorname{ses.1}$ & 917.8 & 9023 & 899.8 & 891.6 & 893.1 & S04.8 & 911.6 \\
\hline & & & & & & & & & & \\
\hline & 20152 & $2 O N=4$ & $\angle O N E B$ & ZONE⿱一𫝀口 & $\angle O N=10$ & & & & & \\
\hline DP Lquid Tomp.f & 89.2 & & & & & & & & & \\
\hline DPLlquid Densityomiz & 91.89 & & & & & & & & & \\
\hline Eeviontiont & & 4 & 6 & 8 & 9.875 & & & & & \\
\hline Meatured Dpustd & $0.100 \mid$ & 0.7iा & T.2यो & 7.605 & 2.003 & & & & & \\
\hline Zero Combcions-pedd & -0.006 & -0.016 & $=0.00$ & 0.02 & $0.0 \times 2$ & & & & & \\
\hline Comectad DP prda & 0.108 & 0.721 & 130 & 1.588 & 205 & & & & & \\
\hline ACTDDP & 1.56 & 3.01 & A.2. & 5.69 & 7.5 & & & & & \\
\hline Whesured Thpolg & 845 & & & & 826 & & & & & \\
\hline Mecoured Overalth & ट6्ठ & & & & & & & & & \\
\hline & & & & & & & & & & \\
\hline Comperad DPA & 208 & 3.82 & 5.84 & 7.32 & 8.87 & & & & & \\
\hline Frictiont & 0.08 & 0.18 & $0 . \hat{3}$ & 0.47 & 0.68 & & & & & \\
\hline Aocelertion- & 01 & 0.1 & 0.14 & 0.18 & 0.22 & & 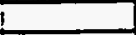 & & & \\
\hline Elertiont & दा & 3.57 & 5.2. & 6.68 & 7.99 & I & i & & & \\
\hline & & & & & & & & & & \\
\hline
\end{tabular}




\begin{tabular}{|c|c|c|c|c|c|c|c|c|c|c|}
\hline Runt & $\pi 71$ & & & & & & - & & & ! \\
\hline Toetfivid & CAF 8 & - & & $\therefore$ & & & - & 1 & I & L \\
\hline Date: & 8 19893 & $i$ & & L. & & & \pm & & & 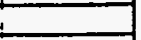 \\
\hline Timo: & $8: 34$ & & & $\therefore$ & & & \pm & & & 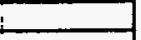 \\
\hline Note & Anatical d & is used & & & :- & 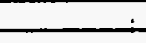 & & & & \\
\hline Fow Aros-A2 & 0.003361 & 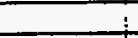 & & & & & & & & i \\
\hline Hedfow Areatl2 & 0.19351 & & & & & & $\vdots$ & & & 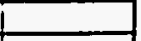 \\
\hline Sat. ProsslutePsig & 86.31 & & & & & & & & & \\
\hline Sa Tomperzuref(inde) & 127.31 & & & & & & & 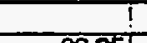 & & \\
\hline La.Donstintomn & 86.18 & 86.151 & 86.18 & 86.18 & 86.24 & 86.24 & 86.22 & $86.25 !$ & 86.3 & 85.80 \\
\hline Latern Hoat Bunbm & 38.91 & 38.88 & 38.91 & 3.911 & 38.55 & 38.95 & 38.94 & 38.961 & 39.01 & 38.78 \\
\hline Spocifio Hestoulbmif & 0.278 & 0.279 & 0.278 & 0.278 & 0.2781 & 0.2781 & 0.2781 & 0.278 & 0.278 & 0.279 \\
\hline Liq. Visoosth & 0.56 & 0.5691 & 0.56 & 0.561 & 0.5671 & 0.5611 & 0.5671 & 0.5621 & 0.56 & 0.550 \\
\hline Lig. Condustivity ethrtaf & 0.0324 & 0.03231 & 0.0324 & 0.03241 & 0.03241 & 0.03291 & 0.05241 & 0.0324 & 0.00251 & 0.0522 \\
\hline Liq. Prendeno. & 4.82 & 4.811 & 4.82 & 4.821 & 4.821 & 4.821 & 4.82 & 4.821 & 4.88 & 4.79 \\
\hline Intet Fow Ralo-GPM & 4.8 & & & & i & $:$ & 1 & $!$ & $\perp$ & L \\
\hline Outet Lig. Fow Rato-GPM & 1.2 & & $i$ & 1 & & & 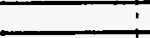 & 1 & L & \\
\hline Outlet Vap. Flow Rato-GPM & 2.11 & & & & & & & $\therefore$ & I & 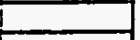 \\
\hline Intat Tempereturef & 121.7 & & & & & & & & & \\
\hline Outat Liq. Temperaturef & 124 & & & & & & & & & \\
\hline Out of Vao. Temper throf & 727.8 & & & & & & & & & \\
\hline Wador Fow Rato-GPM & $!$ & & & I & & & $i$ & I & I & \\
\hline Water hilat Tamp.f & 75.81 & & & i & & I & i & $i$ & 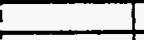 & \\
\hline Weter outlet Temp.F & 106.8 & & & 1 & & 1 & 5 & I & 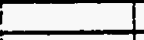 & \\
\hline Intal Gomhine & 89684 & & Temp & 121.111 & Density & 86.881 & 1 & $\mathrm{i}$ & 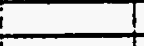 & \\
\hline Subooolingf & 5.6 & 1 & & ¡ & & & 1 & 1 & I & \\
\hline Flow Obsarvation & $i$ & 1 & & 1 & & 1 & 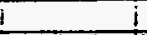 & 1 & 1 & \\
\hline & ZONE1 & ZONE2 & ZONES TZ & ZONE4 12 & ZONES II & ZONE6 & ZONE & ZONE8 12 & IZONE9 I & ZONE10 \\
\hline Horlhout-Wets(net) & 584.7 & 586.8 & 589.5 & 584.8 & 583.3 & 582.9 & 589.2 & 587.1 & 586.7 & 585.8 \\
\hline HeAl Loss-WE't: & 7.9 & 1.8 & 25 & 25 & 25 & 1.9 & 1.2 & 1.9 & 23 & 124 \\
\hline 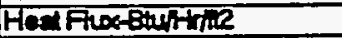 & 10135 & 10172 & 10219 & 10137 & 10117 & 10104 & 10213 & 10177 & 10770 & 10146 \\
\hline WaI Tomp. ABF & 734.7 & 136.1 & 135.8 & 135.7 & 137.4 & $137.9 !$ & 137.7 & 135.6 & 135 & 3320 \\
\hline WeHTomo.t4f & 135.7 & 134.7 & 138.8 & 138.2 & 137.31 & 138.1 & 137.9 & 136.8 & 135.8 & 138.5 \\
\hline WedT Temp. \$5F & 135.91 & 133.9 & 1372 & 1382 & 138.2 & 137.31 & 138.8 & 135.8 & 134.4 & 73्र21 \\
\hline WEHTemp. IIF & 831 & 1351 & 134.2 & 134.01 & 135.5 & 137.91 & 137.9 & 138.4 & 138.9 & 131 \\
\hline WEITOMo. HF & 136.5 & 135.2 & 135.8 & 135.1 & 135.91 & 137.8 & 138.1 & 137.31 & 134.81 & 1327 \\
\hline Inetraam Temp.ff & 125.01 & 126.1 & 125.0 & 125.9 & 125.81 & 125.61 & 125.7 & 125.6 & 12621 & 127.2 \\
\hline Avg. Wall Tomp.f & 135.7 & 135.3 & 135.6 & 135.7 & 136.2 & 137.71 & 137.7 & 136.4 & 134.8 & 133 \\
\hline Ain. WaDT.F & 9.31 & 8.71 & 32 & 9.31 & 10.2 & 11.61 & 11.5 & 10.4 & 9.1 & 5.4 \\
\hline Remaining Suboooff & 3.5 & & & & & & & & & \\
\hline Ext Qualis & 0.02 & -0.01 & 0.01 & 0.02 & 0.04 & 0.05 & 0.07 & 0.08 & 0.11 & 0.11 \\
\hline EX. H-Bulnthar & 1084.9 & 17705 & I1121 & 1088 & 994.4 & 871.5 & 8852 & 974.8 & 1115.ी1 & 1885.3 \\
\hline COMPUTEDH & & & & & & & & & & \\
\hline Jilouk & 8572 & 880.31 & 861.3 & 815.4 & 808.4 & 803.81 & 800.61 & 797.2 & $7 \% 5.8$ & 8020 \\
\hline Sady (C)do tab) & T307. & 1311.6 & 13126 & 1317.8 & 1377.2 & 1316 & 1327.I & 7327.51 & 1320.1 & 7351.6 \\
\hline seadyz & 1078.3 & 10829 & 1084.1 & 1078.7 & 1074.8 & 1075.8 & 1068.8 & 1071.4 & 1074.8 & 108.010 \\
\hline (स्थि) & T184\%5 & 116847 & 116450 & 114827 & 112722 & 110948 & $105 \mathrm{E} / 3$ & 107279 & 105185 & $105+17$ \\
\hline (F) & 1.0081 & 1.0081 & 1.006 & 1.458 & 1.705 & 1.902 & 2.072 & 2.3261 & 2.369 & 247 \\
\hline (1) & 250.61 & 203.7 & 263.6 & 380.71 & 446.8 & 408 & 5द2.6 & 582.7 & 610.61 & 650.7 \\
\hline (130) & 1302.6 & 13081 & 1300 & 13011 & 1203.7 & 1290.7 & 12962 & 1289 & 1282.6 & 1298 \\
\hline & & & & & & & & & & \\
\hline & ZONE2 & ZONE & $2 O N=B$ & ZONEB & ZONE10 & & & & $\perp$ & \\
\hline DP Rquid Tomp.f & 85.2 & & & & & & & $L$ & $i$ & \\
\hline DPLlquid Density 13 & 92.47 & & & & & & & & & \\
\hline Everntion & 2 & 4 & 6 & 8 & 9.875 & & & & & \\
\hline Meavered DPFid & 0.065 & 0.538 & 1.137 & 1.322 & 7.828 & & & & & \\
\hline zero Correctonsend & 0.008 & 0.057 & 0.178 & 0.063 & 0.06 & & & & & \\
\hline 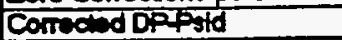 & 0.057 & 0.520 & 0.838 & 1.20 & 1.61 & & & & & \\
\hline Ached DPa & 200 & 3.42 & 4.85 & 0.48 & 7.98 & & & & & \\
\hline Mecoured TFFi & 86.8 & & & & 84.8 & & & & i & \\
\hline Meenered over"ith & 2.85 & & & & & & & & I & \\
\hline & & & & & & & & & & \\
\hline Computed DPQ & 2.12 & 4.21! & 6.16 & 8.07 & 9.9 & & & & & \\
\hline Froion-t & 0.12 & 0.31 & 0.61 & 1 & 1.44 & & & & \lfloor & \\
\hline Acostereliont & 0 & 0.21 & 0.35 & 0.46 & 0.57 & & & & 1 & \\
\hline Eleveriont & 2 & 3.68 & 5.18 & 6.61 & 7.9 & & & & 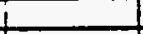 & \\
\hline & & & & & & & & & & \\
\hline
\end{tabular}




\begin{tabular}{|c|c|c|c|c|c|c|c|c|c|c|}
\hline Runt & 7781 & & & & & & & & & \\
\hline Touffivid & ICAF8 & & $i$ & & \pm & & 1 & & & \\
\hline Oata: & $818 / 83$ & & & & & & 1 & & & \\
\hline Tims: & $8: 34$ & & & & & & & & & \\
\hline Not & Anstiond & dis used & & & & & & & & \\
\hline Flow AYORAR & 0.003361 & & & & & & & & & \\
\hline Hod Fow AVASAR & 0.19656 & & & & & & & & & \\
\hline Set. Prossure trig & 86.1 & & & & & & & & & \\
\hline 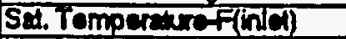 & 727.21 & & & & & & & & & \\
\hline Lq. Densty-tom/13 & 86.201 & 86.221 & 86.18 & $86.2 \pi$ & 86.24 & 88.241 & 8857 & 86.20 & 8620 & 85.26 \\
\hline Lentend Hom Exulbm & 38.99 & 38.94 & 38.81 & 38.94 & 38.85 & 38.95 & 38.98 & 38.991 & 38.99 & 38.16 \\
\hline 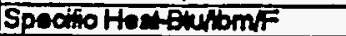 & 0.2781 & 0.278 & 0.278 & 0.2781 & 0.278 & 0.278 & 0.278 & 0.278 & 0.278 & 0.281 \\
\hline Lig. Vieosolyttommhr & 0.669 & 0.6611 & 0.66 & 0.68911 & 0.5611 & 0.6871 & 0.562 & 0.6681 & 0.653 & 0.532 \\
\hline Lig. Conductivity he th & 0.0324 & 0.0324 & $0.032 \pi$ & 0.03221 & 0.03241 & 0.02281 & 0.03221 & 0.0824 & 0.0324 & 0.0317 \\
\hline Liq. Prend No. & 4.89 & 4.821 & 4.82 & 4.821 & 4.821 & 4.821 & 4.83 & 4.83 & 4.83 & 4.71 \\
\hline Inlot Fow Rac-GPM & 4.1 & & & & & & & & & \\
\hline Outel Liq. Fow Ruto-GPM & 1.1 & & & & & & & & & \\
\hline Outal Vap. Flow Rate-GPM & 1.8 & & & & & & & & & \\
\hline Inlet femporaturet & 122.2 & & & & & & & & & \\
\hline Outel La. Tomperdurof & T24.3 & & & & & & & & & \\
\hline Outal Vap. Temporituref & Tटा.7 & & & & & & & & & \\
\hline WCS HOW RDA-CPPM & & & & & & & & & & \\
\hline Werer inlet Tomp, & 78.6 & & $i$ & & I & & & & & \\
\hline Wer orret Tomp.f & 108,4 & & & & & & & & & \\
\hline nhelolbmprithe & 840634 & & Tomp & 1221 & IDenaty & 86.841 & & & & \\
\hline Suboodingf & 5 & & & & & & & & & \\
\hline Flow Obsenvition & & & & & & & & & & \\
\hline & ZONE & ZONE2 & ZONE3 & 20124 & $20=5$ & ZONE6 & ZONE I & ZONE8 & ZONES & $20 \pi=10$ \\
\hline Hadhoutwata(nel) & 568.8 & 579.1 & 578 & 575.4 & 570.1 & 5724 & 579.5 & 574.7 & $5 \pi .6$ & 572. \\
\hline HOE LOSSWEW & 8.8 & & 28 & 28 & 28 & 2.2 & 1.4 & $2 i$ & 26 & 142 \\
\hline 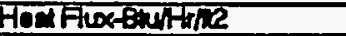 & 9818 & 10038 & 1007 & 9974 & & एक्सा & 70025 & 99020 & 5897 & 9951 \\
\hline Wall Tome. $13+$ & 135.6 & 136.2 & 735.81 & 135.8 & 737.5 & 737.9 & 737.5 & 7క5.4I & 135 & 733.3 \\
\hline Wh Tomp. 14t & 735.4 & 134.7 & 137.1 & 138.4 & 137.6 & 138.8 & 137.7 & 138.7 & 735.7 & 738.8 \\
\hline 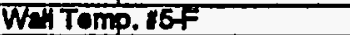 & 135.6 & 1391 & 137.6 & 130.4 & 138.49 & 137.51 & 786.81 & 135.7 & $134 \delta$ & 1325 \\
\hline WeV Tomp.IIF & 89.8 & 134.91 & 134 & 134.8 & 135.6 & 137.5 & 197.7 & 135.91 & 133.8 & 131.4 \\
\hline WAHTOmp. Iaf & 138.1 & 1351 & 796 & 135.21 & 135.81 & 137.91 & 937.71 & 137.31 & 134.8 & 139.1 \\
\hline Introem Temp.f & 125.3 & 125.71 & 125.0 & 125.7 & 125.8 & 125.6 & 125.4 & 125.81 & 125.8 & 131.3 \\
\hline Avg. Wall Tomp.F & 135.4 & 135.2 & 135.6 & 135.91 & 136.3. & 137.8 & 137.6 & 136.21 & 134.8 & 139.4 \\
\hline ANO. WADT.F & 9.7 & 9! & 9.11 & 9.71 & 10.3 & 11.81 & 11.6 & 10.4 & 9ो & 1.7 \\
\hline Rempining Suboooff & 25 & & & & & & & & & \\
\hline Ext Cual'y & -0.02 & & 0.02 & 0.04 & 0.05 & 0.01 & 0.09 & 0.11 & 0.12 & 0.14 \\
\hline EXP.HAtuthinalF & 1016.6 & 1100.7 & 1098.8 & 10023 & 963.8 & 844.30 & 864 & 553.3 & 1050 & 5352 \\
\hline COMPUTEDH & & & & & & & & & & \\
\hline Jallouk & 851.3 & $8 \times 5.3$ & 8327 & 807.6 & 788.71 & 798.11 & 7952 & 790.21 & 787.4 & 871.4 \\
\hline Szady 1 (Cudo urbo) & 1200.1 & 7298.31 & १र्खा.री & 7Z6.61 & 128.11 & 13022 & 1371.6 & 1300.6 & 1308.21 & 1378 \\
\hline $\sin y 2$ & 9049.9 & 1009.6 & 100.1 & 10.0 .31 & 1058.6 & 100201 & $10 \leq 2$ & $1051.6 \mid$ & 10526 & 1717.4 \\
\hline (Pसto) & 98700 & 99087 & 984दी & 98484 & 94651 & 92916 & 909011 & 891501 & 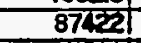 & 90781 \\
\hline & 1.008 & 1.008 & 1.348 & 7.681 & 1.897 & 20911 & 2.2051 & 2.4281 & 2560 & 26 \\
\hline (सIO) & 231.7 & 231.9 & 30.7 & 388.81 & 4372 & 781.0 & हद्टा.ग & 557.0 & 5900 & 613.3 \\
\hline$(1+304)$ & 1277.6 & 1225,8 & 7256.8 & 1208.4 & 125.4 & 1270.8 & 12035 & 1274.81 & 1257.8 & 73500 \\
\hline & & & & & & & & & & \\
\hline & ZONEE 12 & 2ONE4 & ZONE 6 & $20 \pi=6$ & ZONE10 & & & & & \\
\hline DPliguid Tamp.F & 862 & & & & & & & & & \\
\hline DPUguid Densty & 3252 & & & & & & & & & \\
\hline Elevetiont & & a & 6 & 8 & 9.875 & & & & & \\
\hline Mosulred DP4 & 0.086 & 0.744 & T.30 & T.578 & 2008 & & & & & \\
\hline zero Comectionspsd & 0.006 & 0.067 & 0.178 & 0.053 & 0.018 & & & & & \\
\hline Compond DP4 Ifld & 0.08 & 0.647 & 1.150 & 1.5806 & 2.णर्यो & & & & & \\
\hline Acter DP & 208 & $3.2 \mathrm{n}$ & 4.5.5ी & 0.001 & 7.30 & & & & & \\
\hline hasurrod TPP & 66.7 & & & & 8,6 & & & & & \\
\hline heacured overeste- & 2.68 & & & & & & & & & \\
\hline ComoradpD- & 200 & 408 & 5 कर्श & उर्बा & gat & & & & & \\
\hline Fonplont & $\frac{c 00}{0.09}$ & -..20 & 0.036 & 0.87 & 1.ंख्यो & & & & & \\
\hline Aoceler tiont & of & 0.2 & 0.3 & 0.39 & 0.48 & & & & & \\
\hline Enctionth & 2 & 3.58 & 5.04 & ह.दूरी & 7.0 & & & & & \\
\hline & & & & & & & & & & \\
\hline
\end{tabular}




\begin{tabular}{|c|c|c|c|c|c|c|c|c|c|c|}
\hline Runt & 779 & & - & $\therefore$ & 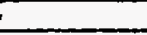 & 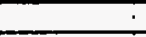 & $i$ & $\cdot$ & - & \\
\hline Test Fuid &.$C 4 F 8$ & $\dot{-}$ & C. & . & & & 1 & $\therefore$ & - & \\
\hline Data: & 81893 & & & & & & 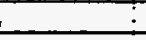 & 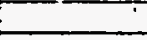 & & \\
\hline Time: & $8: 34$ & & & & & & $\dot{.}$ & 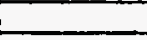 & & - \\
\hline Noto & Analyicalo & dis used I & 1 & & & $\therefore$ & :- & & & \\
\hline Flow Areate & 0.003361 & & & & & & & & & \\
\hline Heat Fow Areatil2 & 0.19555 & & & & & & - & & & \\
\hline Sat Pressure-Psig & 85.8 & & & & & & & & & \\
\hline Sat. Temperatur finlet) & 127! & & & & & 1 & I & ! & & \\
\hline Uq. Denstrytominis & 86.251 & 88.241 & 88.22 & 85.24 & 86.24 & 86.251 & 88.201 & 86.31 & 86.32 & 85.19 \\
\hline Latent Hoad Guthom & $\mathbf{3 8 . 9 8 1}$ & 38.951 & 38.94 & 38.55 & 38.95 & 38.981 & 38.88 & 39.011 & 39.02 & 38.71 \\
\hline Spocitiothetewhom/: & 0.2781 & 0.2781 & 0.278 & 0.278 & 0.278 & 0.278 & 0.278 & 0.2781 & 0.278 & 0.281 \\
\hline 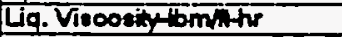 & 0.6621 & 0.667 & 0.6611 & 0.567 & 0.5611 & 0.6521 & 0.652 & 0.6641 & 0.664 & 0.63 \\
\hline iq. Conductivitheffef & 0.0324 & 0.00241 & 0.0324 & 0.03241 & 0.0324 & 0.03241 & 0.03241 & 0.03251 & 0.03251 & 0.0317 \\
\hline Liq. Prand No. & 4.821 & $4.82 !$ & 4.821 & 4.821 & 4.821 & 4.821 & 4.82 & 4.831 & 4.831 & 4.7 \\
\hline Inlat flow Rato-GPM & 3.31 & & 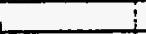 & & & $!$ & 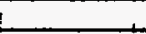 & 1 & 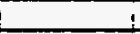 & $!$ \\
\hline Outlot Lig. Fow Palo-GPM & 1.11 & & & & & & & i & 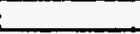 & \\
\hline Outiet Vap.Flow Rate-GPM & $1.3 \mathrm{t}$ & & & & & & & & & \\
\hline Inlat Tomporatus of & 121.7 & & & & ! & 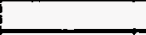 & & $?$ & & \\
\hline Outat Liq. Temperaturef & 724.8 & & & & 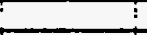 & 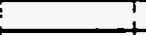 & & & & \\
\hline Outat Vap. Temperzuref & 121.8 & & & ! & $t$ & F & T!t & & & \\
\hline Water Fow Roto-GPM & & I & & i & + & 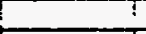 & $!$ & 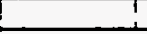 & & \\
\hline Weter thlet Tomp.F & 77.3 & 1 & 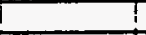 & 1 & $T$ & 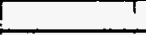 & i & & & \\
\hline Water outhat Temp.f & 109.81 & I & I & i & i & 1 & $!$ & 1 & & \\
\hline Intat Gbminwhe & 6850211 & & Tomp & 121.11 & IDensiny & 86.881 & T & $T$ & i & \\
\hline Subooolingf & 5.3 & 1 & 1 & i & 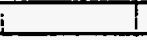 & & I & $I$ & I & \\
\hline Fow Obsenvation & & & 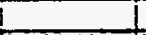 & & 1 & 1 & \pm & 1 & 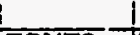 & \\
\hline & ZONE1 & ZONE2 : & ZONE3 I & ZONE 4 & ZONES & ZONE6 & ZONE7 IS & ZONE8 I & ZONE9 & ZONE 10 \\
\hline Hodinput-Wats(nd) & 586.8 & 584.3 & 578.1 & 578.7 & 587.8 & 587.4 & 589.2 & 581.4 & 580.2 & 576.7 \\
\hline Heat Loss-We's & 7.2 & 1.7 & 2.3 & 23 & 2.3 & 1.81 & 1.2 & 1.71 & 22 & 11.7 \\
\hline Horfflox Ehentrine & 10172 & 10128 & 10021 & 10031 & 10180 & 10182 & 10213 & 10078 & 10057 & 9997 \\
\hline WनII Temp. \& & 734.4 & 135.8 & 135.6 & 135.51 & 737.3 & 138 & T37.7 & 135.4 & 735.3 & 133.6 \\
\hline WA Temp.14F & 135.11 & 134.4 & 136.7 & 1381 & 737.7 & 738.51 & 137.8 & 138.8 & 135.8 & 938.7 \\
\hline WESTOMP. I5F & 135.61 & 133.9 & 137.81 & 130.2! & 138.4 & 737.71 & 138.8 & 135.91 & 734.7 & 1328 \\
\hline Way Temp. \#1F & 84.3 & 134.01 & 138.8 & 134.4 & 155.21 & 137.51 & 137.9 & 130.31 & 139.8 & 131.4 \\
\hline WEH Temp. ACF & 135.71 & 134.81 & 134.9 & 1351 & 135.61 & 137.9 & 138.1 & 137.2 & 134.8 & 733.1 \\
\hline Inetroem Temp.fF & 125.6 & 125.6 & 125.71 & 125.81 & 125.61 & 125.51 & 125.5 & 12521 & 125.1 & 131.7 \\
\hline Avg. Wall Tomp.F & 135.2 & 134.9 & 135.3 & 135.51 & 136.2 & 137.91 & 137.71 & 136.31 & 134.9 & 139.5 \\
\hline AvT Wall DT. & 9.2 & 8.81 & 9.1 & 9.51 & 10.11 & 121 & 11.7 & $10.7 !$ & 9.41 & 1.4 \\
\hline Remaining Suboooff & 2.1 & & & & & & & 1 & & \\
\hline Extcually & -0.02 & 0.01 & 0.03 & 0.05 & 0.07 & 0.1 & 0.12 & 0.14 & 0.18 & 0.18 \\
\hline EXP.HBturthan & 1100.8 & 1145.5 & 1101.6 & 050.1 & 1004.8 & 851 & 877.6 & 943.2 & 1071.6 & 7303.2 \\
\hline COMPUTESH & & & & & & & & & & \\
\hline Jalouk & 891.I & 889.6 & 8362 & 873.7 & 874.3 & 80.9 .61 & 8088.41 & 801.11 & 793.41 & 818.7 \\
\hline Szed11(Odowbo) & 130.2. & 13025 & 1298.9 & 1301 & 1373.5 & 1314.8 & 1310.7 & 1311.61 & 13125 & 1375.7 \\
\hline $5 \operatorname{sen} \sqrt{2}$ & 1053.9 & 1051.81 & 10682 & 1038.11 & 1079.6 & 1078.8 & $1082 \pi$ & 105221 & 1054.8 & 1711.8 \\
\hline$(\mathrm{R}, \mathrm{O})$ & 79737 & 79818 & 78454 & 766211 & 74801 & 72991 & 71208 & 6se34 & 67418 & 69005 \\
\hline (i) & 1.008 & 1.0051 & 1.582 & 1.85 & 2.0891 & 2.3741 & 2505 & 2.6811 & 2.842 & 2.898 \\
\hline$(\mathbf{H}$ (a) & 195.1 & 195.2. & 297.2 & 358.8 & 407.2 & 448.81 & 485.81 & 519.61 & 550.61 & 56.8 \\
\hline (1-3014) & 13028 & 1301 & 1233.7 & 1291.81 & 1300.8 & 12972 & 1297.4 & 1283.7 & 1278.4 & 1342 \\
\hline & 1 & & & & & & & & & \\
\hline & ZZONE2 & ZONEA & ZOTE6 & ZONE8 & ZONE & & & & & \\
\hline DP liquid Temp.f & 186.7 & & & - & $1+2$ & \pm & L & & & \\
\hline DP Lquid Density-bmias & 925 & & & & & & & & & \\
\hline Envetiont & & & 61 & 8 & 9.8751 & & & & & \\
\hline Masured DPPSid & 0.14 & 0.916 & 7.551 & $1.9 \% 51$ & 2444 & & & & & \\
\hline Zero Comections-orid & 0.008 & 0.067 & 0.1791 & 0.0531 & 0.0161 & & & & & \\
\hline Cormeced DP pild & 0.134 & 0.891 & 1.370 & 1.9181 & 2.438 & & & & & \\
\hline Actud DPQ & 1.84 & 281 & 4.17 & 5.481 & 6.59 & & & & & \\
\hline 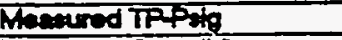 & 85.8 & & & & 84.8 & & & & & \\
\hline Masoured Overall- & 2.08 & & & & & & & & & \\
\hline & & & & & & & & & & \\
\hline Compund DPA & 2 & 3.611 & 5.08 & 6.551 & 7.94 & & & & & \\
\hline Frictiontl & 0.07 & 02 & 0.51 & 0.87 & 1.28 & & & & $i$ & \\
\hline Acoularciont & 0.05 & 0.16 & 0.24 & 0.31 & 0.38 & & & & & \\
\hline Everiont & 1.87 & 3.2 & 4.34 & 5.37 & 6.28 & & & & i & \\
\hline & & & & & & & & & & \\
\hline
\end{tabular}




\begin{tabular}{|c|c|c|c|c|c|c|c|c|c|c|}
\hline Runt & 780 & & $\therefore$ & - & . & " & & & & \\
\hline Tostfluid & CAFB & & $i$ & $\dot{.}$ & $\dot{-}$ & & & $\therefore$ & 2 & $!$ \\
\hline Date: & $i \quad 21893$ & & $i$ & & I & 1 & & & & \\
\hline Time: & $8: 34$ & i & $i$ & $i$ & & & & & & \\
\hline Note & Anavicad & dis used! & 1 & + & $\perp$ & $\perp$ & & 1 & & \\
\hline Flow Areatt2 & 0.00336 & & & & $\therefore$ & & & 1 & & \\
\hline Hogl flow Aroath & 0.1909 & & & & & & & $I$ & & \\
\hline Sal Pressure Psig & 85.2 & & & & & & & & & \\
\hline SEl Tomperedirof(inde) & 726.6 & & & & ! & & & +1 & & \\
\hline Lg.Denstintomins & 86.3 & 85.221 & 86.22 & 86.27 & 86.291 & 86.201 & 86.321 & 86.351 & 86.35 & 85.09 \\
\hline Letenk Hostaturtom & 39.01 & 38.941 & 38.89 & 38.981 & 39.99 & 38.991 & 39.02 & 39.051 & 39.05 & 38.02 \\
\hline Specifio then Euritomif & 0.278 & 0.278 & 0.278 & 0.278 & 0.278 & 0.278 & 0.2781 & 0.2781 & 0.278 & 0.281 \\
\hline Lig. Viscositylbmihith & 0.664 & $0.6 \overline{611}$ & 0.661 & 0.662 & 0.660 & 0.5631 & 0.664 & 0.6851 & 0.666 & 0.627 \\
\hline Lig. Conductinyeturing fF & 0.0325 & 0.03241 & 0.0324 & 0.0324 & 0.05241 & 0.0324 & 0.05251 & 0.03251 & 0.0025 & 0.0316 \\
\hline Lig. Prende No. & 4.83 & 4.821 & 4.82 & 4.831 & 4.831 & 4.831 & 4.831 & 4.841 & 4.84 & 4.59 \\
\hline Inlat Flow Rade-GPM & 2.5 & & $i$ & & 1 & 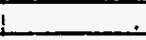 & & 1 & 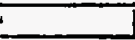 & \\
\hline Outot Lí. Flow RatoGPM & 0.9 & & + & & & & & $!$ & 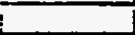 & \\
\hline Outiol Vap. Fow Rato-GFM & 1 & & & & & & & $\dot{-}$ & : & \\
\hline Inlat Tomperaturef & 121.8 & & i & & . & - & & & & \\
\hline Oututug. Temper tref & 724.9 & & & & & & & & & \\
\hline OUtat Vap. Tomperiaref & 121.8 & & T & & & 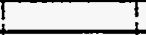 & & $?$ & & \\
\hline W Fow RET-GPM & $\square$ & & T & & & T & & $T$ & & \\
\hline Weter mlet Temp.f & $\pi .7$ & & $!$ & & T & I & & $T$ & 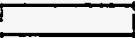 & \\
\hline Waler outtel Tomp.f & 100.4 & & $i$ & I & I & T & & + & 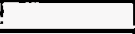 & \\
\hline Inter Gibminrine & 515053 & & Tomp & 1211 & IDensity & 871 & & $i$ & 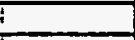 & \\
\hline Swooolingt & 4.8 & & & & 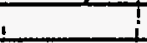 & 1 & & $!$ & 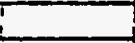 & \\
\hline \multirow{2}{*}{ Flow Obsenvation } & i & & & & 1 & 1 & & 1 & $L$ & \\
\hline & ZONEI & ZONE2 & ZONE3 & ZONE4 & ZONE5 & ZONE6 : & ZONE7 & ZONEB & ZONEO & ZONETO \\
\hline Horlmpu-Wath(nel) & 584.7 & 5827 & 576.5 & 576.1 & 5822 & 580.4 & 587.5 & 580.8 & 578.2 & 573.8 \\
\hline 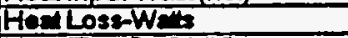 & 7.6 & 1.8 & 2.4 & 24 & 25 & 1.9 & 12 & 1.81 & 22 & 12.3 \\
\hline Hoal Flux-bturitrie & 10135 & 10001 & 9998 & 9986 & 1000 & 10061 & 10184 & 10068 & 1000 & 346 \\
\hline WaITomp. B3 & 13.1. & 735.7 & 13521 & 735.1 & 1372 & 737.51 & 737.41 & 735.31 & 135 & 7332 \\
\hline WE Temp.147 & 135.1 & 134.81 & 138.5 & 135.8 & 137.1 & 138.11 & 737.51 & 138.5 & 735.7 & 738.8 \\
\hline WeV Tomp. $15 \mathrm{~F}$ & 135.6 & 133.71 & 137.41 & 1301 & 135.8 & 13721 & 130.6 & 135.71 & 734.5 & 132.7 \\
\hline Wal Tomp. DfF & 85.4 & 134.51 & 133.51 & 134.1 & 135 & 137 & 137.61 & 150 & 133.6 & 1312 \\
\hline Wall Tomp. I2f & 135.7 & 134.61 & 134.61 & 134.7 & 135 & 137.41 & 137.61 & 136.91 & 134.2 & 1328 \\
\hline Introm Tomp.fF & 125.2 & 125.7 & 125.71 & 125,4 & 125.8 & 125.3 & 125.11 & 124.0 & 124.9 & 1323 \\
\hline Avg. Wall Temp.f & 135.11 & 134.81 & 135 & 135.31 & 135.81 & 137.51 & 137.3 & 136.11 & 134.8 & 133.3 \\
\hline Avg.WALT. $F$ & 9.5 & 8.61 & 8.8 & 9.41 & 101 & $11.7 !$ & 17.8 & 10.7 & 9.2 & 0.6 \\
\hline Rempining Subcooff & 0.6 & & & & -7 & & & & & \\
\hline Exat Qualdy & 0 & 0.02 & 0.05 & 0.08 & 0.11 & 0.14 & 0.17 & 0.2 & 0.23 & 0.28 \\
\hline 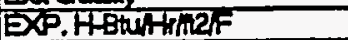 & 1070.6 & 1172 & 1135.8 & 10525 & 1000.8 & 86021 & 868 & 938.7 & 1088.8 & (5) \\
\hline \multicolumn{11}{|l|}{ COMPUTËDH } \\
\hline Jalouk & 89.4 & $875.9 !$ & 840.8 & 895.7 & 813.8 & 808.1 & 809 & 801.41 & 798.6 & 830 \\
\hline Szadri(Cudo wbe) & Tz8.5. & 13021 & 120.81 & 1295.11 & 13027 & 13028 & 1310.8 & 1303.8 & 1303.6 & 13782 \\
\hline Szady & 1047.8 & 1051.11 & 1080.81 & 108281 & 1069.5 & 1007.71 & 1074.8 & 1065.7 & 1048.6 & 7क्ज़ \\
\hline$(P \operatorname{La})$ & 600215 & 599281 & 58167 & $562 \sqrt{21}$ & 5444 & 5252 & 50820 & 489621 & 47217 & 48002 \\
\hline (F) & 1.008 & 1.376 & 1.838 & 2.181 & 24281 & 2.6681 & 2.872 & 3.0591 & 3.276 & 3.287 \\
\hline$(1+4)$ & 166.2 & 213.0 & 285.7 & 336.4 & 378.81 & 4127 & 46.7 & 478.11 & 604 & 618.8 \\
\hline \multirow[t]{3}{*}{ (HBOL) } & 1297.3 & 1300.11 & 12321 & 1287.61 & 1202 & 12899 & 12924 & 12824 & 1278 & 136.1 \\
\hline & & & 1 & & & & & & & \\
\hline & ZONE2 & ZONEA & ZONE 6 & ZONEB & ZONEID & & & & & \\
\hline \multirow{2}{*}{\multicolumn{11}{|c|}{ 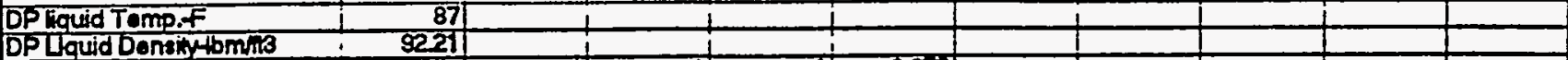 }} \\
\hline & & & & & & & & & & \\
\hline \multicolumn{11}{|l|}{ Erationt } \\
\hline Meaured DP Psld & 0.188 & T.15 & T.907 & 23701 & 293 & & & & & \\
\hline Zero Conectong-pid & 0.000 & 0.067 & 0.179 & 0.053 & 0.076 & & & & & \\
\hline Corructed op psid & 0.18 & 1.0881 & 1.728 & 2.351 & 2.85 & & & & & \\
\hline Achul DPh & 1.80 & 261 & 3.58 & 4.74 & 5.78 & & & & & \\
\hline Meacured tppoig & 82 & & & & 84 & & & & & \\
\hline Meopured Overnili & 203 & & & & & & & & & \\
\hline & & & & & & & & & & \\
\hline Comound DP & 7.85 & 3.281 & 4.56 & 5.881 & 7.07 & & & & & \\
\hline Frictant & 0.06 & 0.2 & $0.4 \pi$ & 0.69 & II & & & & & \\
\hline Acoulerion & 0.06 & 0.18 & 0.18 & 0.24 & 0.3 & & & & & \\
\hline Elovitiont & 9.73 & 2.53 & 3.58 & 4.39 & 5.71 & & & & & \\
\hline & & & & & & & & & & \\
\hline
\end{tabular}




\begin{tabular}{|c|c|c|c|c|c|c|c|c|c|c|}
\hline Run: & 7811 & i & 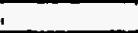 & 1 & 1 & $!$ & $!$ & 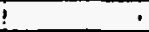 & · & 1 \\
\hline Tost Fuid & .0458 & $\check{-}$ & . & $\therefore$ & $i$ & & \pm & I & $i$ & \\
\hline Date: & 8181931 & & & & & & $i$ & & + & 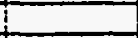 \\
\hline Timo: & $8: 34$ & 1 & 1 & & & & & 1 & $i$ & \\
\hline Nolo & Analyicalc & dtis used! & I & & & & & & & 1 \\
\hline Flow Areatt? & 0.00336 & & & & & & & 1 & 1 & \\
\hline Heat Fow Area-d2 & 0.19595 & & i. & & & & & 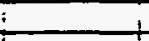 & & \\
\hline Sat. Prossure sig & 85.71 & & 7 & & & & & i & & \\
\hline Sat Tomperatur folinet) & 726.71 & & $T$ & & & & & & & \\
\hline La. Densintomint & 88.291 & 86.18 & 86.18 & 88.21 & 86.2 & 86.21 & 86.24 & 86.24 & 86.24 & 84.8 \\
\hline Latork Heatetulbm & 38.99 & 38.911 & 38.97 & 38.921 & 38.921 & 38.821 & 38.95 & 38.851 & 38.85 & 37.87 \\
\hline Spocitic Hoetstubinth & 0.278 & 0.278 & 0.278 & 0.278 & 0.278 & 0.2781 & 0.2781 & 0.2781 & 0.278 & 0.282 \\
\hline ig. Viscosth & 0.663 & 0.661 & 0.65 & 0.661 & 0.68 & 0.561 & 0.6891 & 0.6611 & 0.561 & 0.521 \\
\hline Lig. Conductinutuhr-if & 0.0324 & 0.05241 & 0.0324 & 0.00324 & 0.0324 & 0.05241 & 0.0324 & 0.0524 & 0.0324 & 0.00315 \\
\hline Lig. Prandit No. & 4.83 & 4.82 & 4.82 & 4.821 & 4.82 & 4.821 & 4.82 & 4.821 & 4.82 & 4.67 \\
\hline Inlot Flow Rado-CPM & 1.8 & & & ! & 1 & & 1 & 1 & 1 & \\
\hline Outillig. Fow Rade-GPM & 0.7 & & 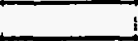 & 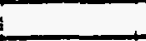 & & & 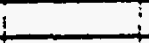 & & & \\
\hline Outle! Vap. Fow Rate-GPM & 0.71 & & & & i & & 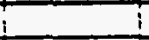 & 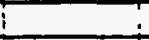 & 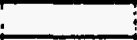 & \\
\hline Inlat Temperature fF & 1224 & & & & & & & 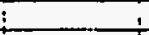 & & \\
\hline Outet Lia. Tomporartef & 125.7 & & & & & & & & & \\
\hline OUtat vap. Temperaturaf & 1225 & I & $T$ & & & & & & & \\
\hline Wator F ow Rato-GPM & 7 & T & i & T & t & & i & & & \\
\hline Water thiet Termp.F & 78.81 & $!$ & 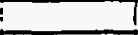 & 1 & 1 & & $!$ & $I$ & 1 & \\
\hline Watr outel Temp.f & 110.1 & T & 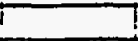 & 1 & T & & i & i & $i$ & \\
\hline niet G ibmhrint & 374212 & & Temp & 120.81 & Denaity & 87.121 & & 1 & 1 & \\
\hline Suboolingf & 4.3 & & & & & & i & $i$ & L & \\
\hline Flow Obsarvation & & & & & & & $i$ & & & \\
\hline & ZONET & ZONE2 & ZONE3 & ZONEA 1 & ZONE5 & ZONE6 & ZONE7 & ZONE8 & ZONE9 & ZONE10 \\
\hline HodhoutWath(net) & 580.6 & 576.7 & 574.7 & 57.1 & 579.3 & 578.6 & 573.2 & 577.4 & 579.3 & 5782 \\
\hline HeCloss-Wats & 6.7 & 1.6 & 2.1 & 2.11 & 2.2 & 1.7 & 1.11 & 1.6 & 2 & 11.1 \\
\hline Healfix-Buhtril2 & 1008 & 907 & 9962 & 10004 & 1004 & 100301 & 9968 & 10000 & 10062 & 90023 \\
\hline Wa tomp. I3 & T342 & 135.81 & T35.1 & $735.2 !$ & 138.8 & 137.51 & T37.31 & 735.21 & 135.1 & $73 \sqrt{2}$ \\
\hline Wa tomp. 14F & 135.T & 133.8 & 138.2 & 735.7 & 137 & 1381 & 137.7 & 136.51 & 735.8 & 737.5 \\
\hline Walt Tomp. I5F & 735.4. & 133.3. & 137.3 & 1381 & 735.8 & 1371 & 130.5 & 135.61 & 133.6 & 13.6 .6 \\
\hline WAH TOMP. IIF & 85.8 & 134.3 & 139.9 & 139.11 & 134.8 & 158.81 & 197.4 & 1351 & 139.6 & 139.8 \\
\hline Wal Tomp. taF & 135.6 & 134.51 & 134.2 & 134.41 & 1351 & 137.31 & 137.7 & 137.11 & 134.5 & 133.6 \\
\hline Indream Temo,f & 125.31 & 125.01 & 125.9 & T25.81 & 125.8 & 125.8 & 125.81 & 125.81 & T25.6. & 133.4 \\
\hline Avg. Wall Tomp.f & 135.11 & 134.5 & 134.7 & 135.3 & 135.7 & 137.31 & 137.31 & 136.11 & 134.7 & 136.1 \\
\hline AUD. Wall DT F & 9.3 & 8.11 & 8.3 & 91 & 9.4 & 11.11 & 11.31 & 101 & 8.7 & 0.3 \\
\hline Romaining subcootf & -1.4 & & & & & & & का & & \\
\hline Ecoualy & 0.01 & 0.05 & 0.09 & 0.13 & 0.17 & 0.211 & 0.25 & 0.29 & 0.33 & 0.37 \\
\hline DXP.HBturfiteF & 1078.8 & 1230.61 & 1200.3 & 1117.8 & 103.1 & 505.9 & 881.6 & 998.1 & 1158.7 & \\
\hline COMPUTEDH & & & & & & & & & & \\
\hline Jallouk & 89.3 & 878.51 & 8525 & 839.71 & 831.51 & 896.5 & 807.6 & 807.91 & 807 & 845.2 \\
\hline Szadyi(Cudo tubs) & T2948 & $1297 !$ & $12^{30}$ & T298.91 & 13027 & 1303.41 & 1291.11 & 1303.4 & 13007.2] & 133.6 \\
\hline Sadve & 104.81 & 1045.61 & 10.0 .1 & 10.81 & 1070.8 & $10 \% .11$ & 1081.81 & 1067.81 & $10 \% .1$ & 1147 \\
\hline (RIO) & 482881 & स2द्टा & 40.03 & 398781 & उतााा & 3597 & 355281 & 317801 & 30028 & 3010 \\
\hline (F) & 1.24 & 1.भाजा & 2.1741 & 2527 & $28 \pi$ & 3.009 & 3.387 & 3.581 & $3.7 \pi$ & 3.77 \\
\hline (1) & 140 & 205.21 & 20021 & 30281 & 3392 & 37 & 300.1 & 425.81 & 467 & 4621 \\
\hline (स्OपL) & |ख्याया & 1255. & 12925 & 12036 & 1205. & $12 \times 34$ & $1282 a$ & 1288 & 1259.7 & 3672 \\
\hline & & 1 & $i$ & 1 & & 1 & 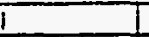 & I & i & \\
\hline & ZONE2 & ZONEQ & ZONE6 & ZONE8 & ZONE10 & & $I$ & 1 & L & \\
\hline DP liquid Tomp.f & 88.3 & \pm & $\square$ & & & & & 1 & & \\
\hline DPLiquid Densit + bmin & छूप्रा & & & & & & & & & \\
\hline Elevetiont & & 4 & 6 & 81 & 9.875 & & & & & \\
\hline Measured DPpsid & 0.216 & $1.3 \%$ & 2.88 & 28001 & 3.150 & & & & & \\
\hline Zero Corrections-psid & 0.006 & 0.057 & 0.179 & 0.0531 & 0.0181 & & & & & \\
\hline Correand DPP sid & 0.21 & $1.35 \pi$ & 2.107 & 27501 & 3.479 & & & 1 & & \\
\hline ACte DPA & 1.87 & 2.081 & 289 & 4 & 4.81 & & T & T & 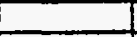 & \\
\hline Mo:ured TPFig & 85. & & & & 84.81 & & & & 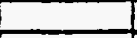 & \\
\hline Meatured Overellit & 1.87 & & & & & & & & & \\
\hline & & & & & & & & & & \\
\hline Computed DPA & 1.55 & $2 \mathbf{B}_{1}$ & 3.68 & 4.65 & 5.56 & & & I & E & \\
\hline Frictionth & 0.05 & 0.18 & 0.34 & 0.57 & 0.82 & & & I & L & \\
\hline Acoeleretionth & 0.05 & 0.09 & 0.13 & 0.18 & 0.28 & & & $i$ & 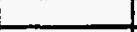 & \\
\hline Eovation-t & 7.46 & 2.41 & 3.18 & 3.91 & 4.5 & & & & L & \\
\hline & & & & & & & & & & \\
\hline
\end{tabular}




\begin{tabular}{|c|c|c|c|c|c|c|c|c|c|c|}
\hline Runt: & 782 & & $i$ & I & 1 & 1 & 1 & 1 & 1 & 1 \\
\hline Tost Fuid & CAFB & & $\therefore$ & & \pm & & 1 & $\stackrel{\vdots}{-}$ & : & $i$ \\
\hline Dalo: & : 8/8933 & & 1 & & & & 1 & 1 & 1 & I \\
\hline Time: & $i \quad 14: 44$ & & i- & & & & I & i & ; & I \\
\hline Note & Analytical & dis used & 1 & & & & & & $\vdots$ & 1 \\
\hline Flow Areati2 & 0.00356 & & 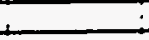 & & & & & 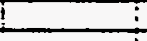 & 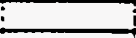 & i \\
\hline Hoad flow Area-12 & 0.19695 & & & & & & & $\mathbf{i}$ & $\mathrm{i}$ & 1 \\
\hline Sat pressure phig & 84.41 & & & & & & & + & 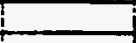 & \\
\hline Sat. Tomporatu finla) & 126 & & & & & & & & & \\
\hline Wq. Densiny & 86.39 & 86.24 & 86.27 & 86.29 & 8629 & 86281 & 86.25 & 88.291 & 862 & 84.82 \\
\hline Latont Hog Etubm & 39.03 & 38.851 & 38.981 & 38.99 & 38.991 & 38.991 & 38.981 & 38.991 & 38.99 & 37.88 \\
\hline Spectio Hoetentibmif & 0.278 & 0.278 & 0.278 & 0.278 & 0.278 & 0.278 & 0.278 & 0.278 & 0.278 & 0.282 \\
\hline Liq. Viscositylomihtr & 0.665 & 0.5611 & 0.662 & 0.563 & 0.6631 & 0.5681 & 0.662 & 0.6591 & 0.568 & 0.522 \\
\hline Liq. Conduching Bhefff & 0.0325 & 0.0324 & 0.0324 & 0.0324 & 0.0524 & 0.00241 & 0.0024 & 0.02241 & 0.0024 & 0.0315 \\
\hline Liq. Prand" No. & 4.83 & 4.82 & 4.83 & 4.831 & 4.83 & 4.831 & 4.82 & 4.831 & 4.83 & 4.67 \\
\hline Inlotfow Rate-CPM & -11 & & 1 & & & 1 & I & $i$ & $i$ & \\
\hline Outel Lig. Fow RateGPM & 0.4 & & 1 & & 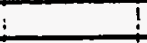 & i & i & 4 & 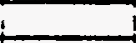 & \\
\hline Outlot Vap. Fow Rade-GPM & 0.5 & & & & & 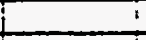 & $i$ & 1 & $t$ & \\
\hline Inlet Temporaturef & 120.6 & & & & & & tor & $t$ & 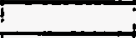 & \\
\hline Outot Lig. Temperaturef & 125.4 & & & & & & $T$ & $!$ & 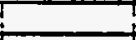 & \\
\hline Outet Vap. Tompereluref & 122.0 & & & & & & & & & \\
\hline Wer Fow Palo-GPM & T & & $!$ & & I & 1 & 3 & & & \\
\hline Weter kilet Tomp.f & 80.7 & & i & & t & 1 & $i$ & I & i & \\
\hline Wher outel Temp.f & 110.6 & & 1 & & $i$ & 1 & $\vdots$ & I & I & \\
\hline Intac-bminne & 204811 & & TTemp & 118.8 & IDoneiny & 87.281 & & & & \\
\hline Suboodingf & 5.4 & & $I$ & & i & 1 & L & 1 & I & \\
\hline \multirow[t]{2}{*}{ Fow Observion } & & & 1 & & 1 & & $\perp$ & & & \\
\hline & ZONET & ZONE2 & ZONE3 & ZONE 4 & ZONES & ZONE6 i & ZONE? & ZONE 8 & ZONE9 & ZONE=10 \\
\hline 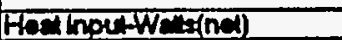 & 571.6 & 571.6 & 570.7 & 569.9 & 564.7 & 568.2 & 575.1 & 577.3 & 573.6 & 568.8 \\
\hline HenLLose-We"s & 8.4 & 1.9 & 2.6 & 2.6 & 2.6 & 2 & 1.3 & $2 i$ & 2.6 & 14.4 \\
\hline 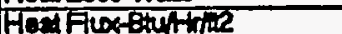 & 9908 & Sobiा & 3888 & 9879 & 97801 & \$846 & 96 & 10007 & 997 & 550 \\
\hline Wentomp. & 7327 & 133.7 & Tस्B! & 133.4 & 735.61 & 138.11 & 135.91 & 134.61 & 34 & 733.6 \\
\hline WeT Tomo. $14 \mathrm{f}$ & 739.0 & 132.2] & 134.17 & 133.9 & 1351 & 138.81 & 136.5. & 139 & 135.11 & 738.8 \\
\hline WCITOmp. 16 F & 133.8 & 131.7 & 135.7 & 134 & 133.9 & 135.2 & 135.2 & 1351 & 133.6 & 132.6 \\
\hline WEITEMP. IIF & 87.6 & 132.61 & 131.61 & 13221 & 1381 & 1351 & 150 & 185.81 & 132.0 & 1312 \\
\hline WE TOMO.12F & 134.8 & 13291 & 132.3 & 13281 & 133.11 & 135.61 & 138.41 & 136.21 & 133.5 & 1328 \\
\hline Thetreen Temp.F & 125ा & 125.81 & 125.4 & 125.3 & 125.31 & 125.31 & 125.5 & 125.8 & 125.8 & 133.8 \\
\hline Avg. Wall Temp,F & 133.6 & 132.91 & 1326 & 133.4 & 133.8 & 135.71 & 136 & 135.41 & 133.7 & 133.4 \\
\hline Avo. WallDT. $f$ & 8.2 & 6.81 & 6.8 & 7.61 & 8 & 9.91 & 10 & 9.71 & 8 & 0 \\
\hline Remaining Subcooff & -4.6 & & & & & & & & & \\
\hline Exat Qual'y & 0.03 & 0.1 & 0.18 & 0.25 & 0.32 & 0.39 & 0.46 & 0.53 & 0.6 & 0.68 \\
\hline Bxp.Hsturthen & 1211.7 & 1458.6 & 7458.5 & 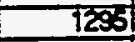 & 12324 & 99.4. & $\operatorname{sen}$ & 1055.1 & 1247.4 & \\
\hline \multicolumn{11}{|l|}{ COMPUTEDH } \\
\hline Jalouk & 913.61 & 881 & 876 & 857.8 & 8422 & 837 & 837.6 & 8328 & 825.6 & 870.7 \\
\hline Szadj 1 (Oudo nbo) & T281.71 & T28\%! & 7285.51 & 1287.81 & 1279.21 & 1284 & T295.51 & 7250.8 & 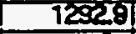 & 7367.6 \\
\hline sadr & 1069.7 & $7 \overline{035.81}$ & 1003.01 & 10542 & 1047.9 & $1052 \pi$ & 10020 & 1003.4 & 1059 & 1133.1 \\
\hline (FLO) & 287001 & 220001 & 20030 & 19108 & 17झ्ञा & 150001 & 13978 & 122021 & 10460 & Gस्सा \\
\hline (i) & 1.508 & 2.162 & 2.7681 & 3.225 & 3.5्21 & 3.9891 & 4.371 & 4.6371 & 4.882 & 2.858 \\
\hline$(\mathrm{HWO})$ & 1128 & 161.9 & 203.81 & 247.4 & 271.9 & 288.11 & 3228 & 38.8 & 369.1 & 377.7 \\
\hline \multirow[t]{3}{*}{ (1301) } & 1281.4 & 1287.11 & 12838 & 12815 & 12752 & 1278.6 & 1287.8 & 1287.6 & 12820 & 13572 \\
\hline & & & & & & 1 & ! & & & \\
\hline & ZONE2 & ZONES & ZOTE6 & ZONE8 & $20 N^{\prime}=10$ & & 1 & & L & \\
\hline DPpquid Tomp.f. & i 90.5 & 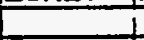 & 1 & & & & & & & \\
\hline DP L Lquid Densits thom/33 & 91.71 & & & & & & & & & \\
\hline Elovation- & & 4 & & & 9.875 & & & & & \\
\hline Masured DP Prid & 0.201 & 1.678 & 272 & 3.3.3ㄱ & 4.15 & & & & & \\
\hline Zero Comactionspid & 0.0081 & 0.057 & $0.1 \% 8$ & 0.053 & 0.076 & & & & & \\
\hline Comected DPfist & 0.201 & 1.6ता & 2.5ा & 3.208 & 4.134 & & & & & \\
\hline Achel DPa & 1.82 & 1.591 & 237 & 3.11 & 3.60 & & & & & \\
\hline Macmod TPPoh & 84.41 & & & & 83.9 & & & & & \\
\hline Mesectred Overallh & 0.85 & & & & & & & & & \\
\hline \multicolumn{11}{|l|}{ Molatredution } \\
\hline Compered DPa & 1.21 & 2.01 & 269 & 3.37 & 3.87 & & & & & \\
\hline Frictiont & 0.08 & 0.00 & 0.2 & 0.35 & 0.48 & & & & & \\
\hline Aocelerationt & 0.02 & 0.05 & 0.07 & 0.11 & 0.15 & & & & & \\
\hline Eleviont & 1.16 & 1.87 & $2 \sqrt{21}$ & 2.87 & 3.2. & & & & & \\
\hline & & & & & & & & & & \\
\hline
\end{tabular}




\begin{tabular}{|c|c|c|c|c|c|c|c|c|c|c|}
\hline Runs: & 7831 & & & & & 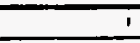 & 1 & 1 & & \\
\hline Togf Fuid & CAF 8 & & & & & & & $\mathrm{i}$ & & \\
\hline Dät: & $.8 / 19931$ & & & & & & & & & \\
\hline Time: & $8: 17$ & & & & & & & & & \\
\hline Note & - Anatical d & $\#$ is used & & & & & & & & \\
\hline Fow Areatll & 0.00336 & & & & & & & & & \\
\hline Head Fow Areat2 & 0.19695 & & & & & & & & & \\
\hline Sal Pressure Psig & 65.4 & & & & & & & & & \\
\hline SE. Temperetur of (intet) & 127.4 & & & & & & & & & \\
\hline La. Density bomitio & 86.49 & 86.351 & 86.25 & 86.241 & 86.271 & 86.271 & 86.29 & 86.81 & 86.351 & 85.98 \\
\hline Lotert Hoafotulibm & 39.16 & 39.051 & 38.981 & 38.551 & 38.98 & 38.981 & 38.99 & 39.011 & 39.05 & 38.7 \\
\hline Specifo Hoverwhomp & $1 \quad 0.2781$ & 0.2781 & 0.2781 & 0.2781 & 0.2781 & 0.2781 & 0.278 & 0.2781 & 0.2781 & 0.278 \\
\hline Liq. Visoosity ibminhr & 0.571 & 0.5651 & 0.562 & 0.5611 & $\frac{0.6621}{0.3211}$ & $0.6 \overline{21}$ & 0.6631 & 0.5641 & 0.0651 & $\frac{0.652}{0.0525}$ \\
\hline Liq. Conductity Btump- ff & $\therefore \quad 0.03261$ & 0.03251 & $\frac{0.03241}{4821}$ & $\frac{0.03241}{4.821}$ & $\frac{0.0324 !}{4.83 !}$ & $\frac{0.03241}{4.831}$ & $\begin{array}{r}0.03241 \\
.831\end{array}$ & $\frac{0.03251}{4.831}$ & 0.0325 & $\frac{0.0322}{4.79}$ \\
\hline Liq. Prandu No. & $\begin{array}{r}1 \quad 4.85 \\
4.81\end{array}$ & & & & & & & & & \\
\hline Outet Lig. Fow Rato-GPM & -0.31 & & & & & & & & & \\
\hline Outlel Vap. Fow Rato-GPM & 2.11 & & & & & & & & & \\
\hline Inlet Tomporaturof & 120.2 & & & & & & & & & \\
\hline Outot Liq. Temporicuref & 123.7 & & & & & & & & & \\
\hline Outut Vap. Tomperalof & 1213 & & & & & & & & & \\
\hline Wrat Fow Rasto-GPM & & & & & & & & & & \\
\hline Water hilot Temp.f & 73.11 & & & & & & & & & \\
\hline Water outh Tomp.F & 105.6 & & & & & & 1 & T & & \\
\hline Intei G tbm & 10031261 & & Tomp & 117.611 & Donety & $87.67 \pi$ & i & & & \\
\hline Subooding $F$ & $1 \quad 721$ & & & & & & & 1 & & \\
\hline Flow Observation & & & & & & & & & & \\
\hline & IZONE1 & & & & & 8509 & & ZONES & 870 G1 & 877 \\
\hline Heal hput-Walts(not) & 865.91 & 871.9 & 8729 & 870.91 & 866.31 & 858.8 & 867.3 & 873 & $\frac{879.6}{35}$ & 877.1 \\
\hline Hoal Loss-Watts & 8.31 & & 27 & 2.7 & 27 & 2.11 & 1.3 & 2 & 2.5 & 13.5 \\
\hline Hod Flox-Bulthile & 150101 & 15114 & 15731 & 15096 & 15017 & 14887 & 15034 & 15133! & 15247 & 15234 \\
\hline WN Tomp. $13 F$ & 735.31 & $738.9 T$ & T37.1 & $-13 \pi$ & 738.8 & 139.7 & 738.9 & 136.8T & 158.6 & 734.5 \\
\hline W T-Tomp. T4F & 935.7 & $138.7 T$ & 138.7 & 137.81 & 139.11 & 1401 & 139.4 & 138.8 & 137.5 & 739.8 \\
\hline Wal Tomp. 15 F & 138.6 & 135.11 & 139.1 & 138.1T & 137.61 & 138.91 & 138 & 137.2 & 135.51 & 133.1 \\
\hline WaH Tomp. \#1F & 81.31 & 1371 & 134.9 & 135.61 & 136.7 & 138.61 & 139.4 & 197.7 & 134.6 & 137.8 \\
\hline Wall Temp. I2F & 137.11 & 1371 & 736.21 & 13621 & 136.9 & 139.71 & 139.7 & 138.81 & 135.8 & 134.1 \\
\hline Instream Temp. F & 124.11 & 124.91 & 125.5 & 125.6 & 125.4 & 125.4 & 125.3 & 125.21 & 124.9 & 127.4 \\
\hline Avg. Wall Temp.F & 136.4 & 137.31 & 136.7 & 137.11 & 137.6 & 139.31 & 139.1 & 137.91 & 138 & 134.8 \\
\hline Ang. Wal DT.F & 11.8 & 11.71 & 10.51 & 10.81 & 11.5 & 13.2 & 13.1 & 12 & 10.4 & 6.6 \\
\hline Remaining Subcooff & & & & & & & & & & \\
\hline Exi Quality & -0.03 & -0.01 & 0.02 & 0.04 & 0.06 & 0.08 & 0.11 & 0.13 & 0.15 & 0.18 \\
\hline EP. HBtulHimer & 1288.8 & $129.4_{1}^{1}$ & 1435.4 & 73923 & 1306.2 & 1125.5 & 1147.6 & 1261.5 & 1464.6 & 2300.2 \\
\hline COMPUTEOH & & & & & & & & & & \\
\hline Jalouk & 10732 & 70821 & 7045.2 & \%9.71 & क्षाता & 943.81 & 934.8 & 9821 & 914.3 & 974.8 \\
\hline szady1(audo bibo) & -7569 & 7584.7 & 7503.5 & 7536.81 & 1553.8 & 1591.T & 1602 & 1610.8 & 1677.8 & 1649.6 \\
\hline $\sin y^{2}$ & 1369.81 & 7380.21 & 1390.8 & 1350.91 & 1388.7 & 1350.81 & 1378 & 7369.81 & 1399.2 & 1420.6 \\
\hline (RDa) & 115218 & T160971 & $116180 \mid$ & 1195681 & 1107म6| & 1081801 & 105614 & 1026821 & 997461 & 9946 \\
\hline (F) & 1.006 & 1.0081 & 1.269 & 1.601 & 1.977 & 22001 & 2612! & 2.596 & 2.77 & 289 \\
\hline (F) & 203.01 & 234.41 & 332.8 & 765.11 & 520.11 & 6811 & 634.81 & 68271 & 727.9 & 764.8 \\
\hline (1-BOLL) & 1568.6 & 1581.6 & 1588.7 & 15851 & 1575.2 & 1564.91 & 15682 & 1568.61 & 15672 & 15920 \\
\hline & & & & & & & & & & \\
\hline & ZONE2 & ZONEA & ZONE 6 & ZONESB & ZONE10 & & & & & \\
\hline DP liquid Temp.F & 83.7 & & & & & & & & & \\
\hline 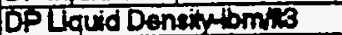 & 9268 & & & & & & & & & \\
\hline Elovition- & & & & & 9.875 & & & & & \\
\hline Mosaurud DF Psid & 0.049 & 0.584 & 1.115 & 1.279 & 7.348 & & & & & \\
\hline Zero Comoctions-psid & -0.005 & 0.062 & 0.176 & $0.07 \pi$ & -0.052 & & & & & \\
\hline Commech DP Pild & 0.049 & $0.522 !$ & 0.960 & 1.268 & 1.8 & & & & & \\
\hline Acheal DPA & 2071 & 3.44 & 4.9 & 6.5 & 7.97 & & & & & \\
\hline Wesoured TPP & 86.4 & & $T$ & & 852 & & & & & \\
\hline Magtured Overely & 201 & & 1 & & & & & & & \\
\hline & & & & & & & & & & \\
\hline Computed Dp-n & 2121 & 4.181 & 6.01 & 7.89 & 9.75 & & & & & \\
\hline Frictiont" & 0.12 & 0.391 & 0.88 & 1.56 & 234 & & \pm & & & \\
\hline Accoleretionth & of & 0.3 & 0.47 & 0.53 & 0.79 & & & & & \\
\hline Elevitiont" & 2 & 3.48 & 4.66 & 5.7 & 6.ळ. & & & & & \\
\hline
\end{tabular}




\begin{tabular}{|c|c|c|c|c|c|c|c|c|c|c|}
\hline Run & 7841 & 1 & $:$ & $\dot{-}$ & - & $\cdot$ & - & & $\dot{-}$ & : \\
\hline ToAf Fuid & CAF8 & $\therefore$ & $i$ & & $i$ & \pm & 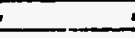 & 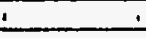 & 1 & 1 \\
\hline Date: & +819931 & i & $i$ & & $i$ & 1 & 4 & & $\dot{\vdots}$ & \\
\hline Time: & $8: 17$ & & 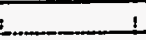 & & & & & & $:$ & \\
\hline Note & Analicalo & dis used & & & & 1 & $L$ & & & \\
\hline Fow Areante & 0.003561 & & & & & & & & & \\
\hline Hoat Fow Aroa-12 & 0.1965 & & & & & & & & & \\
\hline Sat. Prossure pig & 86.21 & & & & & & & & & \\
\hline Sa. Temporateref(ina) & 127.2 & & & & & & & & & \\
\hline La. Densinyomin & 86.451 & 86.31 & 86.251 & 85.251 & 88.27 & 86.31 & 86.52 & 86.321 & 85.85 & 85.78 \\
\hline Lathin Heat Etulbm & 39.18 & 39.011 & 38.981 & 38.981 & 38.98 & 39.011 & 39.02 & 39.021 & 39.05 & 38.5 \\
\hline Spectio Hoateribmit & 0.2781 & 0.278 & 0.278 & 0.2781 & 0.2781 & 0.278 & 0.278 & 0.278 & 0.279 & 0.28 \\
\hline Liq. Visoosity tominhr & 0.6681 & 0.5601 & 0.5621 & 0.5621 & 0.552 & 0.564 & 0.564 & 0.564 & 0.5551 & 0.546 \\
\hline Lq. Condualivy BtwhrtfF & 0.03261 & 0.03251 & 0.03241 & 0.03241 & 0.0524 & 0.03251 & 0.0525 & 0.00251 & 0.0325 & 0.002 \\
\hline Liq. Prandt No. & 4.85 & 4.831 & 4.821 & 4.82 & 4.83 & 4.831 & 4.83 & 4.831 & 4.84 & 4.76 \\
\hline Inlet Fow Rato-GPM & 4.11 & & & & & -1 & $L$ & & & \\
\hline Outot Lq. Fow Rate-GPM & 0.4 & & & & & & & & 1 & \\
\hline Outlot Vap. Flow Rato-GPM & 1.6 & & & & 4 & + & & & $\perp$ & \\
\hline Inlot Temperaturef & 120.4 & & & & & & & & & \\
\hline Out of Lfa. Temperturef & 124.21 & & & & & & & & & \\
\hline Ourat Vep. Temperen & 121.4 & & & & & & & & & \\
\hline WES FOW ALOCDM & i & & & & & & $=$ & & & \\
\hline What inlet Tomp.f & 78.11 & & I & & & T & & & & \\
\hline Weter ouliet Tomp.F & 105.7 & I & 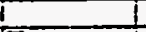 & & & 1 & & & & \\
\hline 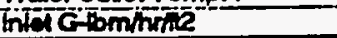 & 856000 & & Tomp & 118.6 & Densiny & 87.391 & & & & \\
\hline Suboodingf & 6.81 & & & & & -1 & & & & \\
\hline Flow Obsenvion & 1 & & & & & 1 & 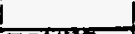 & & & \\
\hline & ZONET & ZONE2 & ZONEB & ZONEX & ZONEE & ZONE6 & ZONE? & ZONE8 & IZONEO & $Z O N=10$ \\
\hline Hoelhotwate(nel) & 1 8626 & 870.1 & 870.6 & 864.8 & 860.2 & 857.4 & $8 \pi 29$ & 870.3 & 8712 & 888.4 \\
\hline HonLose-We:" & 7.9 & 1.9 & 28 & 26 & 2.6 & 21 & 1.3 & 1.9 & 2.4 & 13 \\
\hline Hest Fioxetuhtrin & 74563 & 15031 & $150 \% 1$ & T4G9न & 14971 & 14862 & 74688 & 15000 & 15203 & 15053 \\
\hline WE Tomg. 134 & 1352 & 138.8 & 737.1 & T鹿.81 & 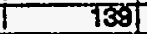 & 139.7 & 138.8 & T35.8 & T355 & 737.8 \\
\hline WriTomo. 44 & 736.6 & 138.11 & 739.11 & 737.81 & 139.2 & $140.1 T$ & 139.3 & 138.71 & 137.61 & 740.3 \\
\hline WEITOMp. $15 F$ & 138.6 & 135.11 & 139.8 & 138.11 & 137.7 & 139.11 & 138.2 & 137.11 & 135.4 & 133.4 \\
\hline WEITomo. 11F & 82 & 138.81 & 134.9 & 135.51 & 138.01 & 138.81 & 939.4 & 137.61 & 135.7 & 131.8 \\
\hline WATTEmo. REF & 137 & 137.11 & 136.4 & 136.2! & 137 & 139.71 & 139.6 & 138.7 & 135.81 & 1342 \\
\hline hintrem Tomp.F & 124.3 & 125.21 & 125.61 & 125.61 & 125.41 & 125.21 & T25.1 & 125.11 & 124.5 & 128.6 \\
\hline Avg. Wall Tomp.F & 136.31 & 137.31 & 136.9 & 137.11 & 137.6 & 139.4 & 739.7 & 337.81 & 1361 & 134.9 \\
\hline AVD. WallDT.f & 11.3 & 11.4 & 10.71 & 10.9 & 17.8 & 13.61 & 13.3 & 121 & 10.4 & 5.6 \\
\hline Romaining subosoff & 3.1 & & & & & & & & & \\
\hline Ext Cuality & -0.02 & 0 & 0.03 & 0.06 & 0.08 & 0.11 & 0.14 & 0.16 & 0.18 & 0.22 \\
\hline 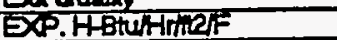 & |317.5 & 1326.7 & 7411.3 & 1378.8 & 1250.9 & 1008.6 & 1125 & 1257.4 & 1457.5 & 2578.6 \\
\hline COMPUIEDH & & & & & & & & & & \\
\hline Jisouk & 1033.51 & 7708.61 & Tण्टाय & 988.71 & 967.61 & 949.71 & 940.1 & 933.51 & 926.4 & $\$ 328$ \\
\hline seadil(Cudo wbe) & 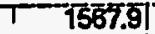 & 1585.61 & 1591.8 & 1588.61 & 7587.4 & 15881 & $15 \$ 3.6$ & 9604.11 & T612.1 & 1651.4 \\
\hline Satar & 1368.41 & 13882 & 1393.7 & 13681 & 1301.1 & 13.00 .81 & 1365 & 13821 & 1391.7 & 720 \\
\hline$($ PLO) & 983981 & 992481 & 97800 & 951741 & 924081 & COAn & 87010 & 843981 & 816000 & 8186 \\
\hline$(\mathrm{F})$ & 1.0061 & 1.008 & 1.522 & 1.897 & 2.18 & 24181 & 2.681 & 2.865 & 3.008 & 3.117 \\
\hline (H) & 25241 & 23281 & 3524 & 769.91 & EOA.7 & 560.01 & 600.8 & 653.61 & 68.01 & 727 \\
\hline$(\mathrm{FBOLL})$ & 156621 & 15830 & 1589.1 & 1578.3 & 15702 & 15524 & 1563.3 & 1567.11 & 1557.7 & 1600.9 \\
\hline & T & & & & 1 & 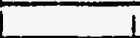 & 1 & & & \\
\hline & ZZONE2 & ZONE & ZONE6 & ZONES & ZONEIO & 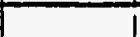 & & & & \\
\hline DP tiquid Tomp.f & 842 & & & & & & & & & \\
\hline DPUquid Denth bom/13 & \$261 & & & & & & & & & \\
\hline Elevitionth & & & & & 9.875 & & & & & \\
\hline Mostured DFPsid & 0.103 & 0.758 & 1.464 & T.6.2. & 2083 & & & & & \\
\hline Zero Comecionsprid & -0.005 & 0.062 & 0.179 & 0.णा & $=0.052$ & & & & & \\
\hline Comected Dp pastd & 0.1081 & 0.7051 & 1205 & 1.691 & 2078 & & & & & \\
\hline 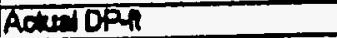 & 1.98 & 3.14 & 4.62 & 5.81 & 7.181 & & & & & \\
\hline Mravured TPPig & 882 & & & & 8.11 & & & & & \\
\hline Meavenred over"lit & 1.851 & & & & & & & & & \\
\hline & I & & & & & & & & & \\
\hline Compitsd DPA & 2.06 & 3.881 & $5.5 \mathrm{n}$ & 723 & 8.88 & & & & & \\
\hline Frictionft & 0.1 & 0.35 & 0.8 & 1.39 & 207 & & & & & $=$ \\
\hline Accolerationt & 0.06 & 0.21 & 0.61 & 0.5 & 0.69 & & & & & \\
\hline Eleverionth & 1.9 & 3.22 & 4.38 & 5.33 & 6.211 & & & & & \\
\hline & & & & & & & & & & \\
\hline
\end{tabular}




\begin{tabular}{|c|c|c|c|c|c|c|c|c|c|c|}
\hline Runt: & 7851 & & & & 1 & + & - & & 5 & \\
\hline Tost Fuid & . 458 & & 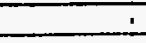 & 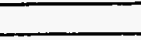 & $\div$ & - & 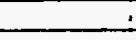 & & - & \\
\hline Date: & .8919331 & & & 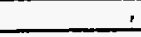 & \pm & & 1 & 1 & 1. & \\
\hline Tume: & $8: 17$ & & & & & & & & & \\
\hline Note & Analyicald & dis used ! & & & & & & & & \\
\hline Flow Areate & 0.003361 & & & & & & & & & \\
\hline Headflow Areatl? & 0.19655 & & & & & & & & & \\
\hline SAd Pressure Psig & 85.41 & & & & & & & & & \\
\hline Sat. Temperature F(inlet) & 26.7 & & & 70 & & & & & & \\
\hline Lq. Densiy tomatis & 88.46 & $86.3 \$$ & 86.31 & 88.31 & 86.32 & 86.31 & 86.351 & $86.37 !$ & 86.4 & 84.80 \\
\hline Latent Heatohubm & 39.131 & 39.091 & 39.011 & 39.011 & 39.021 & 39.011 & 39.051 & 39.061 & 39.09 & 37.91 \\
\hline 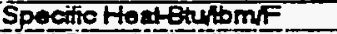 & 0.278 & 0.2781 & 0.278 & 0.2781 & 0.278 & 0.2781 & 0.2781 & 0.2781 & 0.278 & 0.282 \\
\hline Lia. Visoosif $4 \mathrm{lom} / \mathrm{hr}$ & 0.5681 & 0.6651 & 0.5641 & 0.6641 & 0.5691 & 0.564 & 0.565 & 0.5661 & 0.667 & 0.523 \\
\hline Liq. Conductivity Buhret IF & 0.08251 & 0.03251 & 0.03251 & 0.00251 & 0.03251 & 0.03251 & 0.0325 & 0.03251 & 0.0325 & 0.0315 \\
\hline Liq. Prand No. & 4.851 & 4.831 & 4.831 & $\overline{4.831}$ & 4.83 & 4.831 & 4.84 & 4.841 & 4.84 & 4.68 \\
\hline Inlet Fow RateGPM & 3.2 & & & & & & & & & \\
\hline Outlet Lig. Fow Rato-GPM & 0.31 & & & & & & & & & \\
\hline Outlet Vap. Flow Ralo-GPM & 1.2 & & & & & & & & & \\
\hline Inlat Temperaturef & 199.9 & & & & & & & & & \\
\hline Outet La temperaturef & 724.31 & & & & & & & & & \\
\hline Outet Vap Temporature & 121.27 & & & & & & & & & \\
\hline Water Fow Rate-GPM & -1 & it & 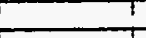 & & t & i & & & $!$ & \\
\hline Water kilet Tomp.f & 73.21 & T & $T$ & 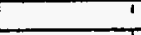 & & $I$ & & & & \\
\hline Wetroutet Temp.F & 107.7 & 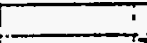 & $T$ & 1 & $i$ & 1 & & & 1 & \\
\hline Intat G tbingrine & 6683801 & & Tomp & 117.81 & Density & 87.521 & & & i & \\
\hline Suboooling $F$ & 6.81 & 1 & 4 & 1 & & $i$ & 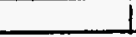 & & 1 & \\
\hline Fow Observation & 1 & 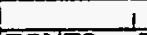 & 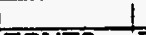 & 1 & & 1 & & & $i$ & \\
\hline & ZZONE1 ; & ZONE2 I & ZONE3 3 & ZONE4 & ZONE5 & ZONES jJ & ZONE7 & ZONE8 & ZONE9 & ZONE10 \\
\hline Hed trout-Wads(net) & 867.4 & 867.3 & $867 . \overline{6}$ & 8221 & 8628 & 861.2 & 866.6 & 869.6 & 875.9 & 867 \\
\hline Heat Loss-Watts & 7.8 & 1.81 & 2.5 & 25 & 2.6 & 21 & 1.3 & 1.9 & 2.3 & 12.9 \\
\hline 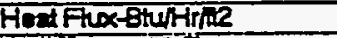 & 150351 & 150301 & 15039 & 74971 & 14958 & 14528 & 1502 & 15074 & 15183 & 15029 \\
\hline Wal Temp. $13 F$ & 735.1 & 138.31 & 136.9 & 735.71 & 138.7 & 139.7 & 138.7 & 136.8 & 136.4 & 1352 \\
\hline Wa Temp. $14 F$ & 136.4 & 135.8 & 138.01 & 137.6 & 138.8 & 140 & 139.2 & 138.5 & 137.6 & 140.6 \\
\hline Wat Temp. $15 \mathrm{f}$ & 136.4 & 134.8 & 139.21 & 137.8 & 137.4 & 1391 & 138.3 & 138.9 & 135.4 & 134 \\
\hline Wa Tomp. IIf & 89.11 & 138.61 & 134.7 & 135.71 & 138.61 & 138.61 & 139.8 & 137.61 & 135.0 & 1324 \\
\hline Well Tomp. IRF & 136.8 & 136.9 & 135.9 & 136.21 & 138.91 & 139.6 & 139.7 & 138.7 & 135.8 & 134,6 \\
\hline hoteam Tomp.F & 724.8 & 1251 & 125.2 & 1252 & $125 . \pi$ & 12521 & 124.0 & 124.8 & 124.6 & 133.11 \\
\hline Ave. Wall Tomp.f & 136.2 & 136.81 & 136.5 & 1371 & 137.4 & 139.31 & 139 & 137.71 & 136 & 135.4 \\
\hline Avo. Wall DT.F & 11.2 & 11.11 & 10.6 & 11.11 & 11.61 & 13.41 & 13.5 & $122 !$ & 10.7 & 1.6 \\
\hline Remaning Subcootf & & & & & & & & & & \\
\hline Exot Qualiny & -0.01 & 0.0 & 0.05 & 0.00 & 0.12 & 0.15 & 0.19 & 0.22 & 0.28 & 0.29 \\
\hline DP.HBHAtilite & 136 & 1351.9 & 14128 & 1348.8 & 1286.8 & 1112 & 1116 & 1237.7 & 14225 & 507.1 \\
\hline COMFUTEDH & & & & & & & & & & \\
\hline Jatalk & T129:2 & TनF2.5ा & 1038.4 & 7003.3 & 981.61 & 966.31 & $\$ 5.51$ & 946.41 & 939.7 & 976.6 \\
\hline sandy1(Cudotube) & 157.9 & 1580.51 & 1585.81 & 158256 & $75 \overline{8.51}$ & 1586.81 & 1591.1 & $1595.7 \pi$ & 16023 & 16972 \\
\hline $\operatorname{sendy2}$ & 1373.1 & 13828 & 7380.51 & 1387.81 & 1358.4 & 1361.11 & 1357.11 & 1372.91 & 13812 & T483.7 \\
\hline (RLO) & 76918 & 772521 & 74782 & 72169 & 69401 & 65957 & 64762 & 614881 & 587511 & 60805 \\
\hline (F) & 1.008 & 1.159 & 1.8002 & 2178 & 2.479 & 2.7881 & 2.975 & 3.1821 & 3.397 & 3.424 \\
\hline (स्) & 180.81 & 2201 & 324 & 413.81 & 471 & $620.3 T$ & 5651 & 6081 & 6446 & 6528 \\
\hline (1-3014) & 1571 & 1572.11 & 1580 & 1578 & 1570.5 & 1568 & 1567 & 1566.11 & 1566.8 & 16820 \\
\hline & 1 & 1 & 2 & 1 & & 1 & & & 1 & \\
\hline & ZONE2 & ZONE4 & ZONE 6 & ZONE 8 & ZONE10 & & & & $\perp$ & \\
\hline DP lquid Temp.f & 85 & & 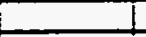 & & & & & & & \\
\hline DPLiquid Densty bm/is & 92.5 & & & & & & & & & \\
\hline Elevation-a & & $4 !$ & & 8 & 9.875 & & & & & \\
\hline MeasuredDPYsid & 0.185 & 0.991 & 1.64 & 2.055 & 2542 & & & & & \\
\hline Zero Correctionsosid & -0.0051 & $0.062 !$ & 0.179 & 0.017 & 0.053 & & & & & \\
\hline Corructod DPPstd & 0.19 & 0.901 & 7.485 & 2.054 & 2394 & & & & & \\
\hline Actiol DP & 1.801 & 2781 & 4.021 & 5.281 & 6.85 & & & & 1 & \\
\hline Mrepured TPPaig & 66.4 & & & & 84.41 & & & & $i$ & \\
\hline Mecured oventill & 1.71 & & & & & & & & & \\
\hline & I & & & & & & & & & \\
\hline Computad DFa & 1.98 & 3.481 & 4.87 & 6.31 & 7.72 & & & & & \\
\hline Frictiont & 0.08 & 0.311 & 0.6 & 1.21 & 1.79 & $\perp$ & & & $t$ & \\
\hline Acoclereliont & 0.09 & 0.21 & 0.32 & 0.48 & 0.5 & & & & & \\
\hline Eevationt & 1.81 & 2.98 & 3.85 & 4.67 & 5.38 & & & & & \\
\hline & & & & & & & & & & \\
\hline
\end{tabular}




\begin{tabular}{|c|c|c|c|c|c|c|c|c|c|c|}
\hline Runl & 7861 & - & 1 & $\div$ & 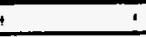 & 1 & 1 & 1 & & $i$ \\
\hline Tesfluid & CAF8 & $\therefore$ & 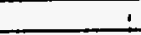 & \pm & $i$ & 1 & & 1 & & $i$ \\
\hline Date: & 819993 & - & & i & $\perp$ & 1 & 1 & $i$ & & \\
\hline Time: & $8: 17$ & & & & $\perp$ & $\perp$ & I & i & & \\
\hline Noto & inatycalo & tis used ! & & & & 1 & 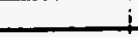 & i & & 1 \\
\hline Fow Areatt2 & 0.003361 & & & & & $i$ & 1 & I & & $\perp$ \\
\hline Heat Fow Areare & 0.19595 & & & & & & & & & \\
\hline Sat. Prossure Psig & 86.21 & & & & & & & & & \\
\hline 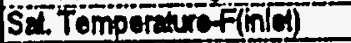 & 127.21 & 1 & 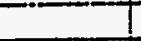 & & i & 1 & 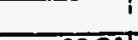 & 670 & & \\
\hline Lla.Denstingtom/i33 & 86.39 & 88.21 & 86.22 & 86241 & 86.21 & 86.24 & 8624 & 86.25 & 86.25 & 84.7 \\
\hline Letunt Hostetuhbm & 39.07 & 38.94 & 38.941 & 38.851 & 38.921 & 38.851 & 38.85 & 38.861 & 38.86 & 37.7 \\
\hline Spectio Honemibm/F & 0.278 & 0.2781 & 0.278 & 0.278 & 0.278 & 0.2781 & 0.278 & 0.2781 & 0.278 & 0.289 \\
\hline 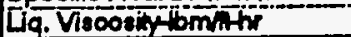 & 0.668 & $0.66 \pi$ & 0.5617 & 0.6811 & 0.661 & 0.5611 & 0.6611 & $0 . \overline{6521}$ & 0.662 & $0 . \overline{61} \overline{6}$ \\
\hline Lig. Conductiving BhrsfF & 0.03251 & 0.0524 & 0.0024 & 0.0324 & 0.00291 & 0.0024 & 0.03241 & 0.03241 & 0.00241 & 0.0318 \\
\hline Ua. Prander No. & 4.89 & 4.821 & 4.82 & 4.82 & 4.821 & 4.821 & 4.82 & 4.821 & 4.821 & 4.65 \\
\hline inlet Fow Ade-GPM & 2.5 & -1 & 1 & $\perp$ & & $\perp$ & & i & $i$ & \\
\hline OutotLig. Fow RaA GPM & 0.11 & & I & 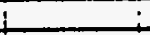 & i & & & & & \\
\hline Outet Vap. Flow Rate-GPM & 0.8 & i & 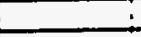 & 1 & 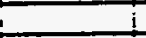 & & & & \pm & $t$ \\
\hline Inlet Tomporauref & 121.2 & & & & & & & & & \\
\hline Outát Lq. TomperatureF & $25.4 \mid$ & & & & & & & & & \\
\hline Outel Vap. Tomperaiver & T221 & $T$ & 5 & & & & & & & \\
\hline WET Flow Rato-GPM & 7 & 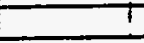 & r & & & 7 & & T & & \\
\hline Water mlot Temp.F & 72.4 & I & T & t & i & ! & & T & i & \\
\hline Wer oullet Temp.f & 10821 & $i$ & I & & I & 1 & & & & \\
\hline Hin G ibminm/L2 & 5230201 & & Tamp & 118.71 & Deneing & 87.71 & & I & & \\
\hline Subooolingf & 61 & 1 & 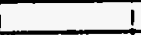 & 1 & 1 & 1 & & & $i$ & \\
\hline Fow Obsenstion & & 1 & & & & $\perp$ & & & & \\
\hline & ZONEI & ZONE2 is & ZONE3 & ZONE4 & ZONES & ZONE6 L 1 & ZONE7 & ZONE8 & ZONES & $20 N=10$ \\
\hline Hodholdtwata(ned) & 884.6 & 875.3 & 875.3 & 868.6 & 880.2 & \begin{tabular}{|l|}
878.8 \\
\end{tabular} & 858.3 & 856.3 & 8680 & 867.6 \\
\hline HenLoss-Wats & 7.7 & 1.8 & 2.5 & 25 & 2.6 & $2 i$ & 1.3 & 1.9 & 2.3 & 12.8 \\
\hline 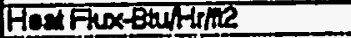 & 1533 & 15173 & 15173 & 7502 वो & 15358 & 752301 & 14878 & 14898 & $7502 \mathrm{ej}$ & 15038 \\
\hline Walt Temo. 137 & TS5.4 & 736.4 & 138.9 & 73721 & 73.4 & 740.61 & 139.8 & T37.3. & 1372 & 435.1 \\
\hline Wat Tomp. I4 & 138,81 & 735.71 & 138,8 & 737.71 & 739.61 & 140.8 & 139.87 & 139.11 & 1383 & 761.1 \\
\hline WR TOMP. $16 F$ & 135.8 & 134.81 & 139.6 & 138.2 & $138 . \pi$ & 139.8 & 738.7 & 137.61 & 130.2 & 134.4 \\
\hline WENTOMD. HF & 83.7 & 156.71 & 134.8 & 135.71 & 197.1 & 139.21 & 159.8 & 138.11 & 1S6.01 & TS20 \\
\hline Wall Tomp. I2F & 137.1 & 138.81 & 136.2 & 136.41 & 137.41 & 140.81 & 140.2 & 139.4 & 198.4 & 135 \\
\hline thitreem tomo.f & 124.7 & 125.71 & 125.71 & 125.6 & 125.8 & 125.61 & 125.6 & 125.6 & 125.5 & 13.5 \\
\hline Avg. Wall Tomp F & 136.6 & 136.9 & 136.7 & 13721 & 138.11 & 140.11 & 139.8 & 138.31 & 138.7 & 135.7 \\
\hline Aug. Wall DT.F & 11.2 & 10.51 & 10.3 & 10.9 & 11.61 & 13.81 & 13.3 & $12 \pi$ & 10.5 & 0.8 \\
\hline Rempining Suboogff & -0.1 & & & & & & & & & \\
\hline Ext Qualdy & & 0.04 & 0.09 & 0.18 & 0.18 & 0.22 & 0.26 & 0.3 & 0.35 & 0.39 \\
\hline EXp.HEtuhtrmat & 1374.4. & 7443.9 & 1471 & 1375.51 & अंक.8 & 11029 & 1118 & 1223.8 & 1433.8 & \\
\hline COMPUTESH & & & & & & & & & & \\
\hline Jilouk & 1177.1 & 7009.81 & 7053.7 & 1018 & 10123 & 9328 & $\$ 3.6$ & \$57.4 & 350 & 1005.6 \\
\hline Szadyi(cudowbe) & 15322 & 1593.67 & 1558.2. & 7590.7 & 1607.51 & 6003. & 1589.8 & 1589 & 1601? & 770.8 \\
\hline Sady2 & 385.1 & 1400\$ & $1401 . \pi$ & 139.51 & 14092 & 138231 & 13032 & 13003 & 1377.0 & 1516.6 \\
\hline (Fसी & 604101 & 5ड्डा & 5698 & 54टता & $51 \pi 01$ & 489:1 & 46801 & 48657 & 4102 & सा810 \\
\hline (F) & 1.008 & 1.6111 & 214 & 25381 & 2818 & $3.185 !$ & 3.3911 & $3 . \approx 21$ & 3.847 & 3.851 \\
\hline$(1+40)$ & 768.91 & 252 & 334.8 & 355! & 245.81 & 400.21 & 53021 & 5671 & 601.4 & 6142 \\
\hline$($ PBOI $)$ & 159.6 & 1594.61 & 1533.3 & 15826 & T5SI & 15905 & 1560.6 & 1564.61 & 15724 & 1688.7 \\
\hline & & & & & & & & & & \\
\hline & ZONE2 & ZONEA & 20NE6 & ZONE8 & ZONE10 & & & & & \\
\hline DP liquid Tomp. $F$ & 86.1 & & & & & & & & & \\
\hline DPLlquid Densitutomin & 923 & & & & & & & & & \\
\hline Elovetion & & & & 8 & 9.875 & & & & & \\
\hline Measured DPFida & 0.301 & 7.287 & $2.09 \pi$ & 25651 & 3.155 & & & & & \\
\hline Zro Corructions-pird & -0.000 & 0.032 & $0.1 \%$ & $0.017 !$ & -0.0521 & & & & & \\
\hline Corrond DP Pitd & 0.500 & 1.222 & 7.9751 & 2534 & 3.217 & & & & & \\
\hline ACter DPA & 1.00 & 2251 & 8.201 & 2.881 & 5.31 & & & & & \\
\hline 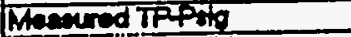 & 862 & & I & & 85.1 & & & & & \\
\hline Menoured Oven & 9.87 & & & & & & & & & \\
\hline & & & & & & & & & & \\
\hline Computed of & 1.77 & 3.0. & 421 & 5.481 & 6.87 & & & & & \\
\hline Frotion & 0.07 & 028 & 0.61 & 1.05 & 1.53 & & & & & \\
\hline Acoulerationt & 0.09 & 0.17 & 0.28 & 0.38 & 0.47 & & & & & \\
\hline Exwationt & 1.61 & 2.57 & 3.34 & 4.02 & 4.61 & & & & & \\
\hline & & & & & & & & & & \\
\hline
\end{tabular}




\begin{tabular}{|c|c|c|c|c|c|c|c|c|c|c|}
\hline Runt & 7871 & & - & $=$ & & $\dot{-}$ & & - & & 1 \\
\hline Tost Fuid & CAF8 & & . & & & & $:$ & $i$ & & $\bar{i}$ \\
\hline Dato: & $8 / 993$ & $\perp$ & 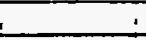 & - & & $\perp$ & 1 & 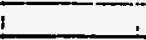 & & $i$ \\
\hline Timo: & $8: 17$ & & & 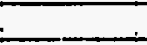 & & & & & & 1 \\
\hline Note & Ananticad & dit is used: & 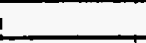 & & & 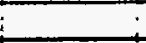 & & & & 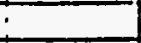 \\
\hline Fow Areatt2 & 0.003361 & & & & & & & & & \\
\hline Hoat Fow Area-t2 & 0.19695 & & & & & & & & & \\
\hline 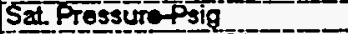 & 85.7 & & & & & & & & & \\
\hline Sat Temporaturof (intet) & $126 . \overline{9}$ & & & & & & & & & \\
\hline Ug. Densty & 86.37 & 86.241 & 86.21 & 86.21 & 85.2 & $86.22 !$ & 86.221 & 85.251 & 86.27 & 85.18 \\
\hline Latent Hostotutbm & 89.001 & 38.551 & 38.94 & 38.821 & \$8.82 & 38.941 & 38.941 & \$8.881 & 39.89 & 38.09 \\
\hline Specifichealsulbmit: & 0.278 & 0.2781 & 0.278 & 0.278 & 0.278 & 0.278 & 0.278 & 0.2781 & 0.278 & 0.281 \\
\hline Lig. Viecosh $1 \mathrm{bm}$ hh & 0.6661 & 0.681 & 0.5611 & $0.6 \overline{6}$ & $0.66 \mathrm{~T}$ & 0.6611 & 0.6611 & 0.6621 & 0.662 & 0.53 \\
\hline 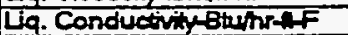 & 0.00251 & 0.08241 & 0.03281 & 0.0324 & 0.03241 & 0.0324 & 0.03241 & 0.0324 & 0.0329 & 0.0317 \\
\hline Eq. Prendot No. & 4.84 & 4.821 & 4.82 & 4.821 & 4.821 & 4.821 & 4.82 & 4.821 & 4.83 & 4.7 \\
\hline Inlat Fow Rate-GPM & $1.7 !$ & & & & $-\ldots$ & \pm & 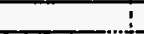 & $-5:$ & 1 & $i$ \\
\hline Outét Lig. Fow Ructo-GPM & $0 i$ & & & 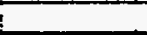 & & & & & & 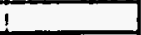 \\
\hline Outtot Vap. Flow Rate-GPM & 0.4 & & & & & & & & - & 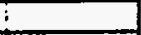 \\
\hline Inlet Temporaturef & 121.51 & & & & & & & & & \\
\hline Outet Liq. TemperzureF & 125.8 & & & & & & & & & \\
\hline Outa Vep.TomperefureF & 1225 & & ! & & & & & & I & \\
\hline Walar flow Rate-GPM & $T$ & & 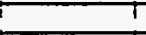 & $\pi$ & & 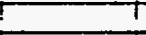 & & & & \\
\hline Wator hiot Temp.F & 73.21 & $T$ & $t$ & 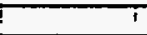 & T & $T$ & & ti & & \\
\hline Wetor outet Temp.F & 108.51 & $i$ & 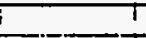 & $T$ & T! & ! & & 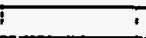 & 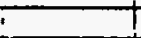 & \\
\hline IntatG+bonhrine & $35567 \pi 1$ & & ITomp & 117.21 & DDensiny & 87.621 & & T & $t$ & \\
\hline Suboooling & 5.4 & 1 & 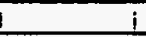 & $i$ & i & 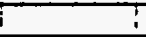 & & T & $\bar{i}$ & \\
\hline Flow Obsanvation & 1 & & 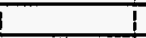 & 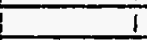 & $i$ & 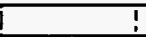 & & $i$ & 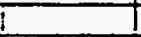 & \\
\hline & ZONET I & ZONE2 & ;ZONE3 T & ZONE4 II & ZONE5 I & ZONE 6 & ZONE? I2 & ZONES & ZONE9 & ZONE10 \\
\hline Hod inpu-Wate(nol) & 873.4 & 858.2 & 891.6 & 8824 & 8923 & 885.1 & 8723 & 871.4 & 8825 & 896.1 \\
\hline Heat Loss-Wal's & 7.3 & 1.7 & 2.4 & 2.41 & 2.5 & $2 i$ & 1.3 & 1.9 & 22 & 124 \\
\hline 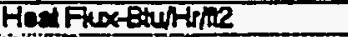 & 15140 & 150501 & 15456 & 15261 & 15467! & 1536! & ग5121 & 95105 & 15288 & 15533 \\
\hline Will Temp. B & T3.3. & $13 \pi$ & 135.7 & 36.41 & T38.5 & 140.2 & 130.ा & 137.11 & 735.8 & 1352 \\
\hline WETOMp. $14 \%$ & 135.8 & 136.2 & 737.81 & $\{37.71$ & 138.7 & 140.4 & -1001 & 1301 & 137.4 & T41.2 \\
\hline WEY TOMm. $15 F$ & 135.7! & 733.41 & 138.7 & 137.21 & 137.3 & 739.31 & 738.5 & 137.41 & 134.8 & T33.8. \\
\hline WE TOMP. ITF & 84. & 134.81 & 133.0 & 134.81 & 150.4 & 188.7 & 139.7 & 138.11 & 134.7 & T32.1 \\
\hline WE Tomp. & 136 & 135.21 & 135.21 & 135.5 & 135.5 & 139.7 & 140.21 & 139.51 & 134.7 & 134.7 \\
\hline Inetrem Temp.F & 124.81 & $\overline{125.65}$ & 125.7 & 125.81 & 125.81 & 125.71 & 125.71 & 125. $\overline{5}$ & $125 . \mathrm{A}$ & 137.8 \\
\hline Avg. Well Tomp.F & 135.51 & 135.31 & 135.8 & 136.41 & 137.2 & 139.71 & 139.5 & 138.21 & 135.4 & 135.4 \\
\hline Ang. Wall DT.F & 10 & $9 !$ & 9.2 & 9.91 & 10.7 & 13.31 & 13.91 & 121 & 9.3 & 2.9 \\
\hline Remeining Subcooff & -3.6 & & 1 & & & & & & & \\
\hline ExQualty & 0.03 & 0.09 & 0.15 & 0.22 & 0.28 & 0.351 & 0.411 & $0 . \overline{88}$ & 0.54 & 0.61 \\
\hline EXP.HBtuHrile & 1519.2 & 1668.5 & 16812 & 1533.8 & 1442. & 1157.4 & 1152.81 & 1254.8 & 16505 & 5560 \\
\hline COMPUTEDH & & & & & & & & & & \\
\hline Jallouk & 1157.9 & Tक्ष.11 & T091. & 1058.51 & 1076.2 & T0्टा.1 & 1007.4 & 999.5 & 90.8 & T03.7.7 \\
\hline Szady ( Oudo tubo) & 7583.2 & $7583.7 !$ & T612.3 & 1656.81 & 1646.67 & 1610.3 & 1600.3 & 1598.8 & 1600.51 & 7700.8 \\
\hline $\operatorname{sen} \sqrt{2}$ & 1380 & 1391.7 & 1417.7 & 1410.11 & 1420.81 & 1412.81 & 14002 & 1374.31 & 1387.4 & 1510.3 \\
\hline (PथG) & 40578 & 39096 & 36ाIा & 33745 & 31042 & 285081 & 25684 & 229381 & 20251 & 18001 \\
\hline (F) & 1.437 & 2047 & 2.618 & 3.057 & 3.452 & 3.7881 & 4.117 & 4.4081 & 4.687 & 4.787 \\
\hline (H) & 164.81 & 2351 & $300.6 \Gamma$ & 35221 & 388.8 & 4581 & 471.97 & 505.61 & 537.7 & 554.1 \\
\hline (13014) & 1582.6 & 1587 & 1608.8 & 1600.71 & 1608.6 & 1599.61 & 1588.61 & 1581.81 & 1589.8 & 16200 \\
\hline & & 1 & 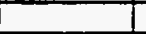 & 1 & & $i$ & & 1 & 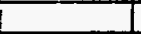 & \\
\hline & ZONE2 & ZONE4 & ZONE6 & ZONE8 & ZONE10 & 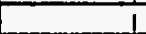 & & & & \\
\hline DP rquid Tomo.f & 86.8 & + & 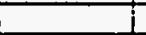 & +2 & & 1 & & & 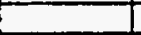 & \\
\hline DPLiquid Densik & 9232 & & & & & & & & & \\
\hline Elevetiond & & 4 & 6) & 81 & 9.875 & & & & & \\
\hline Masuludod DPFid & -0.265 & T.57ा & 2519 & 3.051 & 3.789 & & & & & \\
\hline Zero Corrections-psid & -0.005 & 0.052 & 0.179 & ठ.0111 & $-0.002 \pi$ & & & & & \\
\hline Cortecind DP Pald & 0.431 & 1.5001 & 2.3 & 3.049 & 3.8501 & i & & & & \\
\hline Ached DPA & 1.46 & 1.781 & 2.541 & 3.511 & $4.2 \pi$ & & & & & \\
\hline Maceurod TPFig & 86.7 & 1 & & & 84.61 & & & T & $\Gamma$ & \\
\hline Meeured Overill & 1.881 & I & 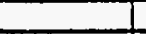 & & & & & & & \\
\hline & $\perp$ & & & & & & & $I$ & E & \\
\hline Campund DPA & 1.38 & 238 & 324 & 4.151 & 5.021 & & & I & 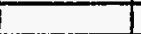 & \\
\hline Friotiont & 0.081 & 0.22 & 0.46 & 0.781 & 1.14 & & & & 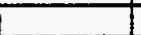 & \\
\hline Acoderetiont & 0.06 & 0.12 & 0.19 & 0.28 & 0.38 & & & & 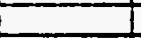 & \\
\hline Elevation-k & 1.24 & 1.99 & 2.53 & 3.09 & 3.5 & & & & & \\
\hline & & & & & & & & & & \\
\hline
\end{tabular}




\begin{tabular}{|c|c|c|c|c|c|c|c|c|c|c|}
\hline Run: & $788 !$ & & & & 1 & 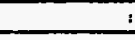 & 1 & \pm & o & \\
\hline Tost Fuid & CAF8 & 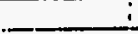 & 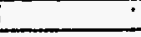 & $\therefore$ & 1 & & $\dot{s}$ & 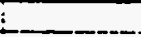 & & $\leq$ \\
\hline Data: & 81993 & $\div$ & 1 & 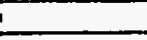 & 1 & & $!$ & $!$ & & I \\
\hline Time: & $13: 421$ & & & i & i & & 1 & $i$ & & I \\
\hline Note & Anatyical a & tis used ! & & $\dot{i}$ & 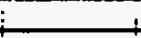 & & $i$ & $i$ & & $!$ \\
\hline Flow Arest? & 0.00336 & & i & L & 1 & 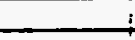 & $\div$ & $\perp$ & \pm & \\
\hline Hodf Fow Aroand & 0.19655 & & & & & & & $\perp$ & & \\
\hline Sat. Pressure & -85.31 & & & & & & & & & \\
\hline Sad Tomperaturef(ina) & 726.61 & & & & & & & & & \\
\hline L. Densinyomits & 86.291 & 85.221 & 86.24 & 80.24 & 86.181 & 86.171 & 86.24 & 86.21 & 86.18 & 86.15 \\
\hline Letark Hoof Btubm & 38.99 & 38.94 & 38.851 & अ.851 & 38.911 & 38.8 & 38.95 & 38.921 & 38.91 & 38.68 \\
\hline Spectio Hontewhibmif & 0.279 & 0.278 & 0.279 & 0.278 & 0.278 & 0.278 & 0.278 & 0.278 & 0.278 & 0.279 \\
\hline La. Visooning & 0.663 & 0.6611 & 0.687 & 0.6611 & 0.68 & 0.6591 & 0.6611 & 0.681 & 0.56 & 0.650 \\
\hline Lag. Conductivity etwhr-ar & 0.0324 & 0.0324 & 0.0324 & 0.0324 & 0.0324 & 0.03241 & 0.0024 & 0.05241 & 0.0524 & 0.0023 \\
\hline Liq. Prende $\mathrm{No}$ & 4.83 & 4.821 & 4.82 & 4.821 & 4.82 & 4.811 & 4.82 & 4.82 & 4.82 & 4.81 \\
\hline Inlot Flow Rate-GPM & 11 & i & & & 1 & 1 & 1 & 1 & 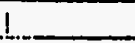 & \\
\hline Outat Lig. Fow Roto-GPM & 0.31 & & & & & & & & $\because$ & \\
\hline Outla Vap. Flow Rate-GPM & 0.5 & & & & $\dot{-}$ & & & & $\therefore$ & $!$ \\
\hline Inlot Temperaturef & 119.7 & & & & & & & & & 1 \\
\hline OutatLig. Temperatur & 726.2 & & & & & & & & & \\
\hline 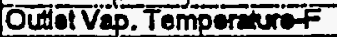 & 723.4 & T & & & & & i & 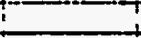 & & \\
\hline Wator Fow Rato-GPM & -7 & t & & 1 & I & & I & i & 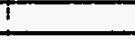 & \\
\hline Werer hlot Temp.f & 78.61 & $i$ & I & 1 & i & & 1 & 1 & 1 & \\
\hline Werer outlet Temp,F & 111.91 & $T$ & & $i$ & $i$ & & I & i & 1 & \\
\hline 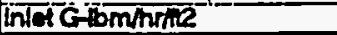 & 200907 & & Temp & 117.71 & IDonsity & 87.541 & & & $i$ & \\
\hline Subcoolingf & 6.9 & i & 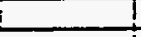 & 1 & 1 & & & 1 & I & \\
\hline Flow Obsenvation & $i$ & $!$ & 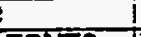 & 1 & 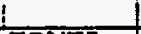 & & 1 & & & \\
\hline & ZONE1 & ZONE2 : & ZONE3 & ZONEA 1 & ZONE5 & ZONE6 & ZONE? & ZONES & ZONEO & $20 N=10$ \\
\hline Hoat lnpol-Watis(net) & 879.1 & 878 & 887.8 & 887.5 & 8829 & 878.5 & 880.6 & 878.3 & 203.9 & 207 \\
\hline HenLoss-Wats & 8.4 & & 2.7 & 2.7 & 28 & 22 & 1.4 & 2.1 & 2.1 & 124 \\
\hline Hod Fho Exhrine & 75269 & 152201 & 153391 & 15388 & 15304 & 15194 & 15255 & 1525! & 3534 & 3588 \\
\hline Walt Tomp. P3 & $\{30.6$ & T3., & 134.4. & T35.5T & 137.3 & 739.8 & 38.7 & 137.4 & 130.3 & T30: \\
\hline WNTTOMP. 147 & T35.11 & 133.7 & 135.61 & 1562] & 737.7 & 739.7 & 139.5 & 137.4 & 130.8 & 131.6 \\
\hline Wat Tomp.15F & 134.61 & 13261 & 137.7 & 1301 & 130.1 & 138.4 & 138.1 & 138.41 & 130 & 739.1 \\
\hline WaH Tomp. IIF & 87.2 & 133.91 & 133.11 & 135.61 & 1352 & 137.8 & 138.9 & 158.8 & 129.2 & 129.8 \\
\hline Well Tomp.taf & 135.11 & 134.51 & 134.2 & 134.61 & 135.5 & 138.81 & 139.8 & 138.2 & 129.6 & 130.5 \\
\hline Letroum Temp.F & 125.81 & 125.71 & 125.61 & 125.61 & 125.9 & 1261 & 125.81 & 125.8 & 125.9 & 128.1 \\
\hline Avg. Wall Tomp.f & 134.6 & 134.61 & 134.61 & 135.31 & 138.7 & 138.81 & 138.0 & 137.31 & 130 & 130.6 \\
\hline Avg.Wall DT. $f$ & 8.61 & 8.21 & 8.3 & 91 & 9.5 & 12.11 & 12.61 & 10.81 & 3.9 & 4.4 \\
\hline Rempining suboooff & 8.4 & & & & & & & & & \\
\hline Ext Qual'y & 0.061 & 0.17 & 0.28 & 0.39 & 0.5 & 0.61 & 0.72 & 0.83 & 0.86 & 0.88 \\
\hline EXP.HEth'ther & $17 / 4.8$ & 1847.7 & 18522 & 1703.9 & 1505.4 & 1254.8 & 1210.5 & 1413.4 & 897 & 823.4 \\
\hline COMPUIEDH & & & & & & & & & & \\
\hline Jilouk & 1198.8 & 7354.71 & 1124.81 & 110261 & 1083.3 & 1055.8 & 10562 & 105.1 & 40 & 498.3 \\
\hline Srovil(Cudo tubo) & T5खा.31 & 1538.51 & T60.21 & 1607,3 & 1607.4 & 1603.7 & 1608.61 & 1605.1 & 795.1 & 8028 \\
\hline $\operatorname{sen} 2 y$ & 1398.6 & 14081 & 1400 & 14121 & 1411.71 & 1403.8 & 146.1 & 1406.9 & 5655 & 5728 \\
\hline (स्य) & 235401 & 215681 & 18865 & 161721 & 135 पा & 108691 & 8185 & 54721 & 3868 & 367 \\
\hline$(\mathrm{P})$ & 1.7211 & 2.5681 & 3.302 & 3.889 & 4.398 & 4.88 & 5.5181 & 5.7231 & 5.989 & 6.08 \\
\hline (संख) & 129 & 18251 & 247.7 & 2921 & 30.7 & 3640 & 398.8 & 430.2 & 449.6 & 458.7 \\
\hline (H30) & 7504 & 1597.6 & 160221 & 1604.9 & 16028 & 1597.4 & 15.521 & 15948 & 7463 & 7503 \\
\hline & & & & & $i$ & & & & 1 & \\
\hline & ¿ZNEE & ZONE4 & ZONE6 & $20 \sqrt{2} 8$ & IZONEIO & & & & 1 & \\
\hline DP Rquid Tomp.F & 190.5 & & 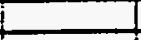 & & 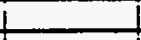 & & $i$ & & 1 & \\
\hline 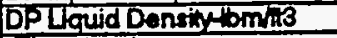 & 91.71 & & & & & & & & + & \\
\hline Eleventiont & & & & 81 & 9.875 & & & & & \\
\hline Mesulred DPP SId & 0.548 & 1.875 & 253 & $3.67 \pi$ & 4.538 & & & & & \\
\hline Zerocomedonspsid & $=0.005$ & 0.0521 & 0.178 & 0.011 & -0.002 & & & & 1 & \\
\hline Comeand DPrid & 0.358 & 1.8181 & 2ह17 & 3.01 & 4.69 & & & & I & \\
\hline Achet op & 7.2 & 1.28 & 9.681 & 201 & 2.84 & & & & 1 & \\
\hline Whenred TPFin & 85.8 & & & & 85.11 & & & & I & \\
\hline Melaured overal'- & 0.33 & & & & & & & & & \\
\hline & & & & & & & & & 1 & \\
\hline Competad DPA & 1.07 & 1.751 & 235 & 291 & 3.64 & & & & $\perp$ & \\
\hline Friatont & 0.04 & 0.13 & 0.8 & 0.46 & 0.6 & & & & $\perp$ & \\
\hline Aocolerctionth & 0.03 & 0.08 & 0.14 & 0.22 & 0.24 & & & & 1 & \\
\hline Elevionth & 0.9 & 1.541 & 1.83 & 231 & 2.81 & & & & $\perp$ & \\
\hline & & & & & & & & & $\perp$ & \\
\hline
\end{tabular}




\begin{tabular}{|c|c|c|c|c|c|c|c|c|c|c|}
\hline Runt & 7891 & . & . & . & & . & $\cdot$ & $!$ & & $\mathrm{I}$ \\
\hline Tast Fuid & . CAF8 & $\therefore$ & $\therefore$ & . & & $\therefore$ & $\therefore$ & 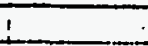 & & i \\
\hline Date: & $8 / 201931$ & & . & ; & & 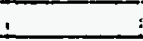 & $\vdots$ & $!$ & & 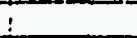 \\
\hline Tume: & $7: 31$ & & & & & & & $\div$ & & \\
\hline Noto & Anavion & dis used & & 1 & & & & 1 & & 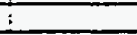 \\
\hline Flow Areat2 & 0.003361 & & & $i$ & & & & & & $!$ \\
\hline Hod Fow Avea-d2 & $0.1555 i$ & & & & & & & & & \\
\hline Sat. Prossuratopig & 85.61 & & & & & & & & & \\
\hline Sat Tomperature finlet) & โ27.5ा & & & -1 & & & & & & \\
\hline Ua. Denstypmins & 88.521 & 86.371 & 85.25 & 86.241 & 86.20 & 86.291 & 86.31 & 86.3ा & 86.37 & 85.61 \\
\hline Lalont Hodsturbm & 39.181 & 39.081 & 38.88 & 38.851 & 38.98 & 38.991 & 39.011 & 39.011 & 39.06 & 38.44 \\
\hline Specitio Hoa Brubm/F & 0278 & 0.2781 & 0.278 & 0.2781 & 0.278 & 0.2781 & 0.2781 & 0.278 & 0.278 & 0.28 \\
\hline Liq. Visoosiny tominfr & 0.577 & 0.5661 & 0.662 & 0.6611 & 0.562 & 0.6631 & 0.654 & 0.5641 & 0.668 & 0.642 \\
\hline Liq. Conductivity BtuhrffF & 0.03261 & $0.0325 i$ & 0.0022 & 0.03241 & 0.0524 & 0.03241 & 0.03251 & 0.03251 & 0.03251 & 0.032 \\
\hline Liq. Prand No. & 4.861 & 4.841 & 4.82 & 4.821 & 4.82 & 4.831 & 4.831 & 4.831 & 4.84 & 4.75 \\
\hline Inlot Fow Rato-GPM & 4.81 & 1 & 1 & & & & & & & I \\
\hline Outol Lig. Flow Rato-GoM & 0.31 & 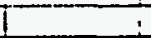 & $i$ & & & 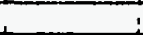 & & I & i & \\
\hline Outlet Vap. Flow Rato-GPM & $2 !$ & & . & & & 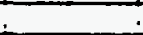 & & & & \\
\hline Inlet Temperature of & 120.71 & & & & & & & & & \\
\hline Outet Lig. Temperatrof & 123.7 & & & & & & & & & \\
\hline OU OT Vap. Temporatur F & 121.11 & & & & & & & & & \\
\hline Wator Flow Ralo-GPM & $i$ & $T$ & $T$ & $i$ & & $i$ & & T & i & \\
\hline Wator hilet Temp.F & 70.31 & $\mathrm{~T}$ & $\mathrm{~T}$ & T & $\cdot$ & $T$ & & $\mathrm{i}$ & $\mathrm{i}$ & \\
\hline Weter outlol Temp.F & 104.61 & $\mathrm{i}$ & i & $T$ & & 7 & & $\mathbf{i}$ & $\mathrm{i}$ & \\
\hline Intat Gibininine & 309741 & & iTomp & 119.31 & Donstiny & $8 \overline{87.28}$ & & T & T & \\
\hline Subcoolingt & 7.4 & $T$ & $i$ & 1 & & 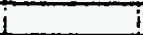 & & & $\bar{i}$ & \\
\hline \multirow{2}{*}{ Fow Obsanvion } & & 1 & 1 & 1 & & 1 & & & i & \\
\hline & ZONEI & ZONE2 : & ZONE3 & ZONE4 & ZONE5 & ZONE6 & ZZONE7 & ZONE8 & ZONE9 & ZONETO \\
\hline Heal input-Walls (not) & 1169 & 1172.1 & 1178.7 & 1174.9 & 1167 & 1164.1 & 1768.8 & 1163.8 & 1174.5 & 1172.2 \\
\hline HenLose-Wath & 8.2 & 1.9 & 2.7 & 27 & 2.7 & 2.11 & 1.2 & 1.8 & 2.3 & 13.1 \\
\hline Hoat Fiox-Buthine & 2028 & 203181 & 20152 & 20566 & 2020 & 20179 & 2060 & 20174 & 20359 & 20408 \\
\hline Walt Temp. B⿺ & T37.2 & $-74 T .81$ & 13.2 & [39.8] & 74.2 & T4T.9T & 736.91 & $\mid 38$ & 737.6 & $T 35.8$ \\
\hline Wail Tomp. ThF & 139 & \{37.5! & 747.6 & 140.8 & 947.6 & 142.71 & $|36 . \overline{8}|$ & 132 & 139.8 & 14.2 \\
\hline WEI TEmp. TEF & 138.41 & 138.31 & 141.5 & 140.9 & 139.81 & 141.11 & 735.1 & -137.71 & $13 \%$ & 134.5 \\
\hline Wal Tomp. $11 F$ & 84.81 & 138.61 & 138.8 & 137.01 & 1391 & 140.8 & 138.6 & 138.61 & 135 & T32.6 \\
\hline Wall Temp. HaF & 139.2 & 138.81 & 138.8 & 138.61 & 139.5 & 142.41 & 140 & 137.51 & 136 & 135.7 \\
\hline thatoem Tomp.F & 723.01 & 124.81 & 125.5 & 125.61 & 125.6 & 125.31 & 125.21 & 12521 & 124.8 & 129.3 \\
\hline Avo. Wall Tomp.f & 138.51 & 13921 & 139.1 & 139.51 & 1400 & 141.51 & 137.5 & 137.81 & 136.8 & 136.5 \\
\hline Avg.WaI DT. & 13.6 & 13.51 & 127 & 13 & 13.61 & 15.31 & 11.4 & 11.71 & 11.7 & 6.3 \\
\hline Remaning Suboooff & 3.1 & & & & & & & & & \\
\hline Exacuary & -0.02 & 0.01 & 0.04 & 0.07 & 0.1 & 0.13 & 0.16 & 0.19 & 0.22 & 0.25 \\
\hline DP. HBHAThR2/F & 14862 & 1509.8 & 1608.6 & 1568.1 & 1491.7 & 132211 & 17828 & 1726 & 1840.8 & 3220.8 \\
\hline \multicolumn{11}{|l|}{ COMPUTEDH } \\
\hline Järouk & 1274.81 & T285.61 & 1190 & T1220! & 1087.2 & प055ा & 708.6 & $\$$ & 9724 & $\$ 3.8$ \\
\hline Szadyi(Cudo hubo) & 18226 & 1837.6 & 1856.8 & 1857.57 & 1853.8 & 1853.7 & 1880 & 1851 & 18692 & 1938.7 \\
\hline $\sec d y 2$ & 1869.61 & 1655.4 & 1679.6 & 1705.71 & 1700.5 & 16981 & 7704.2 & 1703.7 & 1710.5 & 7739.6 \\
\hline (RUa) & 1146001 & 115589 & 113627 & 1101601 & 100507 & 10273 & 99147 & 95627 & 91735 & 90005 \\
\hline$(\mathrm{F})$ & 1.0081 & 1.0061 & 1.025 & टना & 2.316 & 25731 & 2.8 & 3.0071 & 3.205 & 3.307 \\
\hline (H.Q) & 250.11 & 208.61 & 428.4 & 5301 & 607.81 & 674.81 & 734.41 & 789.61 & 839.7 & 875.8 \\
\hline \multirow[t]{3}{*}{ (BB엔) } & 182081 & 183581 & 1842.7 & 184.11 & 1831.8 & 18227 & 1821 & 1812.6 & 1810.9 & 1875.1 \\
\hline & 1 & & & 1 & & 1 & & 1 & & \\
\hline & ZONE2 & ZONE4 & ZONE 6 & ZONE8 & ZONEIO & & & & & \\
\hline DP rguid Tomo.f & 1 87.6 & 1 & & 1 & & 1 & & 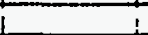 & & \\
\hline DPUquid Densty 4 bm/3 & \$2.12! & & & & & & & & & \\
\hline Eevation-t & & & & 8 & 9.875 & & & & & \\
\hline Messured DPFard & -0.074 & ర.4891 & 0.9587 & 7.191 & 7.485 & & & & & \\
\hline Žro Controctions-psid & -0.007 & 0.0671 & 0.769 & 0.021 & -0.0 iो & & & & & \\
\hline Correcied DPPsid & -0.007 & 0.4881 & 0.787 & 1.169 & 7.456 & & & & & \\
\hline Actual DPA & 2.18 & $3.5 \pi$ & 5.18 & 8.0ा & 8.171 & I & & & & \\
\hline Meapurod TPPsig & 88.8 & 1 & & I & 84.8 & & & & & \\
\hline \multirow[t]{2}{*}{ Mesured Overnllth } & 3.08 & I & & & & & & & & \\
\hline & $I$ & & & & & & & & & \\
\hline Computed DP: & 2.14 & 4.041 & 5.91 & 7.94 & 10.01 & $\mathrm{t}$ & & ! & & \\
\hline Frictiont & 0.16 & 0.561 & 1.25 & ह2 & 3.37 & 1 & & 1 & & \\
\hline Accoleration- & 0.13 & 0.421 & 0.68 & 0.85 & 1.08 & & & & & \\
\hline \multirow{2}{*}{ Elevation-l } & 1.86 & 3.061 & 4.08 & 4.88 & 5.क्या & & & & & \\
\hline & & & & & & & & & & \\
\hline
\end{tabular}




\begin{tabular}{|c|c|c|c|c|c|c|c|c|c|c|}
\hline Runi: & 7901 & & & & 1 & I & $\therefore$ & & & \\
\hline Test Fuid & C4F 8 & & & & & & & & & \\
\hline Date: & 820031 & & & & \pm & & & & & \\
\hline Time: & $\therefore \quad 11: 04$ & & & & & & & & & \\
\hline Note & Anatyicad d & dit is used & & & & & & & & \\
\hline Flow AYOathe & 0.003551 & & & & & & & & & \\
\hline Hoan How Aroatt2 & 0.19695 & & & & & & & & & \\
\hline S2. Prosguropig & 86.3 & & & & & & & & & \\
\hline 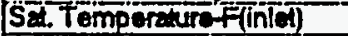 & T2'T.3T & & & & & & & & & \\
\hline tua. Denz'ytamita & 88.501 & 88.341 & 86.251 & 85.21 & 86.23 & 86.24 & 86.201 & 86.291 & 86.35 & 85.68 \\
\hline Latent Hos buibm & 39.211 & 39.031 & 38.96 & 38.52 & 38.9n & 38.95 & 38.99 & 38.991 & $\frac{\infty .001}{39.05}$ & $\frac{\infty 000}{38.48}$ \\
\hline Spocitio Hos ftuthmf & $1 \quad 0.27$ & 0.278 & 0.2781 & 0.278 & 0.278 & 0.278 & 0.278 & 0.278 & 0.278 & 0.28 \\
\hline Liq. Visosithomith & 0.5721 & 0.6661 & 0.5621 & 0.661 & 0.6671 & 0.561 & 0.5631 & 0.5631 & 0.665 & 0.54 \\
\hline Liq.Conductingethrof & 0.0326 & 0.03251 & 0.0324 & 0.03221 & 0.03221 & 0.00324 & 0.03221 & 0.02221 & 0.0525 & 0.032 \\
\hline Wq. Prandil No. & 4.861 & 4.831 & 4.821 & 4.82 & 4.821 & 4.82 & 4.831 & 4.831 & 4.84 & 4.75 \\
\hline Intot Fow Rate-GPM & 4.1 & & & & & & & 1 & & \\
\hline Outhol Liq. Fiow Rato-GPM & 0.41 & & & & & & & & & \\
\hline Outlot Vep. Fow Aate-GPM & 7.5 & & & & & & & & & \\
\hline Inflat Tomperaterof & 120.5 & & & & & & & & & \\
\hline Oufot Ĺ. Tempormor & 124.6 & & & & & & & & & \\
\hline 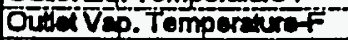 & T21.67] & & & & & & & & & \\
\hline Wan Flow A2to-GPM & & & & & & & & T & & \\
\hline Water hilat Tomp.F & 70.7 & & t & $t$ & & & & T & & \\
\hline 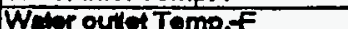 & 1048 & & $\mathbf{i}$ & T & & & & & & \\
\hline Into Gilominthe & 854612 & & iTemp & 198.01 & Denenty & 87.34 & & & & \\
\hline Subooding ff & 6.8 & & 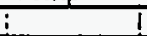 & 1 & & & & T & & \\
\hline Flow Obsenvion & & & & & & & & & & \\
\hline & RONEI i2 & ZONE2 & ZONE3 & ZONEA & ZONES & ZONE6 & ZONE7 & ZONE8 & ZONE9 & ZONE10 \\
\hline Hox lnputwets(nel) & 1149.4 & 1155.6 & $11 \overline{2.5}$ & 1758.4 & 1150.4 & 1150.2 & 1154.11 & 11432 & 1160.8 & 1164.6 \\
\hline Hox Loss-Wats & 7.41 & 1.8 & 2.5 & 24 & 2.5 & 1.9 & 1.11 & 1.7 & 21 & 11.8 \\
\hline 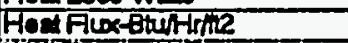 & 19924 & 200000 & 20151 & 20000 & 19961 & 19938 & 20006 & 19817 & 20173 & 20188 \\
\hline Wartomp. $13=$ & 730.8. & $14\langle .4|$ & T5:2 & 139.11 & 7271 & 742.1 & $736.9 \%$ & -137.81 & 136.3 & 755.8 \\
\hline Wen Tomp. 14 & 138.8 & 737.31 & 741.61 & 746.11 & 749.51 & 142.2 & 738.81 & 730.11 & 138.5 & 143.7 \\
\hline Wal temp. $15 f$ & 738.81 & 138.21 & 141.5 & 140.8 & 140.81 & 141.2 & 135.11 & 137.6 & 135.8 & 134.1 \\
\hline WE Tomp. IIf & 85.6 & 138.41 & 138.8 & 137.7 & 139.10 & 140.8 & 138.6 & 138.61 & 135 & 737.8 \\
\hline WAH TOmp. VIF & 738.81 & 138.71 & 138.71 & 738.61 & 139.7 & 1429 & 140.11 & 137.31 & 138.1 & 135.5 \\
\hline In troem Temp.F & 123.31 & 1251 & 125.51 & 125.81 & 125.7 & 125.6 & 125.8 & 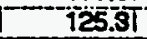 & 124.91 & 120 \\
\hline Avg. Wall Tomp.F & 138.21 & 1391 & 139.91 & 139.41 & 140.2 & 141.6 & 137.5 & 137.7 & 138.89 & 136.2 \\
\hline Avg. WallDT.F & 13.6 & $13 !$ & 12.71 & 12.71 & 13.5 & 15.11 & 11.31 & 11.51 & 10.5 & 6.3 \\
\hline Romining Suboooff & 1.9 & & & & & & & & & \\
\hline Exa Qualy & -0.011 & 0.02 & 0.06 & 0.09 & 0.13 & 0.16 & 0.2 & 0.2 & 0.27 & 0.3 \\
\hline 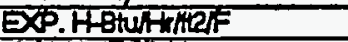 & 1465.9 & 1535.6 & 1591.2 & 158.8 & 1471.8 & 13227 & $177 \pi$ & 1725.5 & 1900.8 & 3 \\
\hline COMPUTEDH & & & & & & & & & & \\
\hline Jeouk & 3282 & 1255.1 & 1183.8 & 7134.7 & 7094.11 & 1068.9 & Tom.31 & 1017 & 998.8 & $7 \infty 005$ \\
\hline Szadyi(aido ubo) & 780.1 .1 & 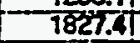 & 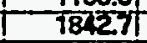 & 1846.6 & 18422 & 784.8 & 7847.2 & 18429 & 1855.4 & 1918 \\
\hline $\sin y^{2}$ & 185201 & 967.71 & 1689.81 & $169 \pi .61$ & 1080.7 & 7608.71 & 1600.8 & 1084.6 & 1658.4 & 1774 \\
\hline (सบa) & 97750 & 986601 & 95334 & 925001 & 88700 & 85118 & 81883 & 779001 & 74185 & 73400 \\
\hline & 1.008 & 1.227 & 1.841 & 223 & 2.5271 & 2.782 & 3.068 & 3.25 & 3.46 & 3.572 \\
\hline (स्थ) & 232 & 283.81 & 48.01 & 674.6 & 586.8 & 648.4 & 701.81 & 7621 & 700.8 & 839.8 \\
\hline 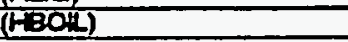 & 780281 & 1865.11 & 1835.11 & 18322 & 18223 & 1817.41 & 18128 & 1800.21 & 1804.5 & 1834.1 \\
\hline & & & & & & & & & & \\
\hline & ZONE2 12 & ZONES & 2ONE6 & ZOK=8 & ZONE 10 & & & & & \\
\hline DP kquid Tomp.f & 88.6 & & & 4 & & & & & & \\
\hline DPLiquid Density tom/in & 91.90 & & & & & & & & & \\
\hline Elovationt & & 4 & & 81 & 9.875 & & & & & \\
\hline Mosacred DPF sid & 0.061 & б.75t & $T .282$ & -1.81 & 2.018 & & & & & \\
\hline Zoro Combetions-psid & -0.007 & 0.0311 & 0.168 & $0.02)^{2}$ & $=0.01$ & & & & & \\
\hline Comacod DP PSla & 0.0081 & 0.6591 & 1.118 & 1.578 & 20, & & & & & \\
\hline Achen DP & 2.08 & 3.21 & 4.57 & 5.81 & 7.21 & I & & & & \\
\hline Moctured TPFig & 66.8 & & & & 84.8 & $T$ & & & & \\
\hline Meaured Overeth & 2.62 & & & & & & & & & \\
\hline & & & & & & & & & & \\
\hline Compued DPR & टूा & 3.73 & 5.43 & 7.281 & 9.18 & 1 & & & & \\
\hline Friciont & 0.14 & 0.511 & 1.13 & 1.86 & 2क्का & & & & & \\
\hline Acosterationt & 0.16 & 0.36 & 0.54 & 0.74 & 0.96 & & & & & \\
\hline Eleverionth & 1.74 & 2.85 & 3.75 & 4.56 & 5.26 & & & & & \\
\hline & & & & & & & & & & \\
\hline
\end{tabular}




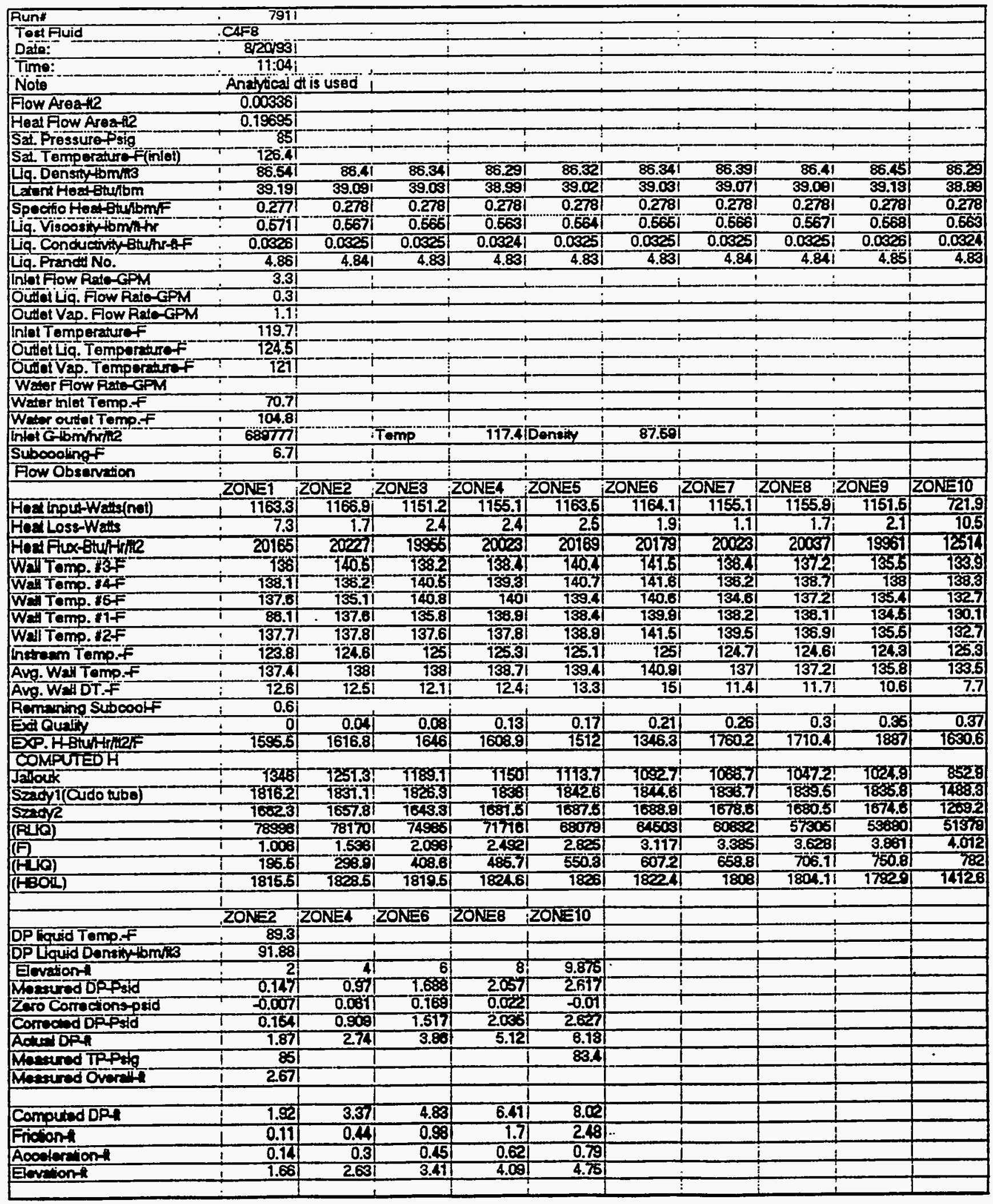




\begin{tabular}{|c|c|c|c|c|c|c|c|c|c|c|}
\hline Run! & 7921 & 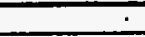 & $\div$ & $\therefore$ & 1 & $\cdot$ & $i$ & 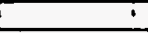 & 1 & i \\
\hline Test Fuid & ,CAFB & $\therefore$ & $\perp$ & 1 & $\therefore$ & $\dot{-}$ & $!$ & 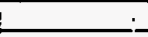 & ; & 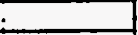 \\
\hline Data: & $18|20 \times 3|$ & , & 1 & 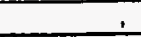 & . & - & 1 & . & ? & 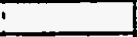 \\
\hline Time: & $11: 04$ & & & & & $\overline{-}$ & : & 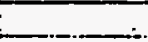 & & 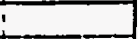 \\
\hline Not & Antyicad d & tis used 1 & & & & & & & & \\
\hline Fow Areant2 & 0.003561 & & & & & & & ' & i & \\
\hline Hoslfow Arosth & 0.1959 & & & & $i$ & $\vdots$ & $i$ & i & & \\
\hline Sat. Prossure plig & 861 & & & & + & & & + & & \\
\hline Sat Temperature finlet) & 127.1T & & & & 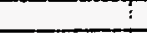 & & & & & \\
\hline Lg. Density & 88.35 & 86.21 & 86.241 & 85251 & 86241 & 86.251 & 86.27 & 86.291 & 86.29 & 86.34 \\
\hline Latent Hoctotubm & 39.211 & 38.92 & 38.95 & 38.981 & 38.S51 & 38.831 & 38.981 & 38.89 & 38.891 & 39.03 \\
\hline Specitio Hestetuhinfi & 0.277 & 0.2781 & 0278 & 0.278 & 0.2781 & $0 . \overline{278}$ & 0.2781 & 0.278 & 0.278 & 0.278 \\
\hline Lig. Visoosintomithe & -0.572 & 0.561 & 0.5611 & 0.5621 & 0.5611 & 0.5621 & 0.5521 & 0.5601 & 0.585 & 0.565 \\
\hline Liq. Conductivis Bturnrth & 0.03251 & 0.05241 & 0.05241 & 0.00241 & 0.0524 & 0.03241 & 0.03241 & 0.03241 & 0.03211 & 0.0525 \\
\hline Uq. Prand No. & 4.861 & 4.821 & 4.821 & 4.821 & 4.821 & 4.821 & 4.831 & 4.831 & 4.83 & 4.83 \\
\hline Inlet Fow Ralo-GPM & 2.51 & & 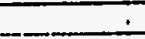 & & $i$ & & ! & $i$ & 1 & 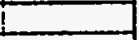 \\
\hline Outlel Lig. Flow Rate-GPM & 01 & & & & $i$ & & 1 & $i$ & 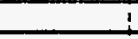 & 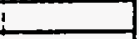 \\
\hline Outlel Vap. Flow Rate-GPM & 0.71 & & 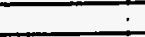 & & $\therefore$ & & 1 & $\therefore$ & 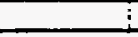 & $\vdots$ \\
\hline Inlet Temperturef & 120.91 & & $t$ & & & & i & 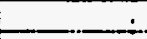 & . & \\
\hline OUtölia. Tomperaure & 125.51 & & & & & & 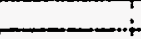 & 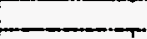 & & \\
\hline outel vap. Tomperture & 122.7 & & & - & & & & 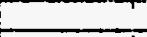 & & \\
\hline Warer Flow Rare-GPM & & $T$ & - & $T$ & $T$ & t & F & 1 & - & \\
\hline Water inlof Tomp,f & 72.3 & $\mathrm{~T}$ & $i$ & 1 & $!$ & 1 & 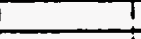 & $i$ & 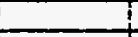 & \\
\hline Water outet Temp.f & 105.61 & $T$ & $T$ & $i$ & -................... & - & 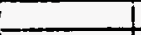 & 1 & 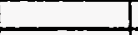 & \\
\hline Intat G-lbininhe & 620075 & & Temp & 177.81 & Denisy & 87.511 & 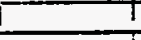 & i & 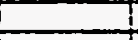 & \\
\hline Subooding & 6.2 & 1 & $I$ & 1 & 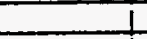 & 1 & 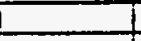 & 1 & 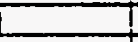 & \\
\hline Fow Observation & 1 & i & 1 & 1 & 1 & $i$ & 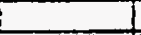 & 1 & 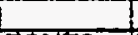 & \\
\hline & IZONET IZ & ZONE2 iC & ZONEB 2 & ZONE4 IC & ZONES I2 & ZONES I & ZONE & ZONES & ZONES & $\angle 0 N=10$ \\
\hline 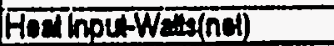 & 1153 & 1159.1 & 1170.5 & 1168 & 1135.3 & 1138.7 & 1150.7 & 1159 & 498.9 & 358.9 \\
\hline Hoct Lose-Watus & 7.4 & 1.7 & 25 & 24 & 2.5 & 1.91 & 1.1 & 1.7 & 1.8 & 9.6 \\
\hline Hestfoxemptrie & कosil & 20002 & 2000 & 20251 & 19800 & 19739 & 1997 & 20091 & 858 & 6187 \\
\hline Wall tomp. 35 & 136.1 & 7405 & 13821 & -381 & Tat & 14T.7 & 137.11 & 13821 & 133 & 731.7 \\
\hline We Temp. $4 F$ & 13:3 & 135.3 & 140.81 & 1391 & 140.61 & 1421 & 157 & 139.7 & 133.7 & T35.8 \\
\hline Wal Tomp. $15+5$ & 137.7 & $135 \lambda$ & 1471 & 139.8 & 139 & 140.81 & 135.6 & 138 & 13्या & 131 \\
\hline Wed Tomp. B1F & 86.41 & 137.41 & 135.61 & 138.61 & 137.8 & 140.81 & 139.11 & 138.8 & 131 & 1205 \\
\hline WUITomp. 12F & 137.8 & 137.7 & 137.7 & 137.61 & 138.4 & 141.51 & 140.81 & 137.81 & 131.81 & 130.7 \\
\hline Instrean Temp.f & 123.7 & 125.81 & 25.6 & 135.61 & 125.61 & $125 \overline{6}$ & 125.4 & 125. & 125.9 & 125 \\
\hline Avg. Wall Tomp.f & 137.5 & 138 & 138.11 & 138.4 & 139 & 141.21 & 137.81 & 138.11 & 132.3 & 131.3 \\
\hline Avo, Wall Dt.F & 128 & 11.3 & 11.6 & 11.91 & 1251 & 14.81 & 11.5 & 11.91 & 6.6 & 6.1 \\
\hline Remaning SubooofF & -1.9 & & & & 1 & 1 & 1 & & 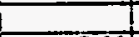 & \\
\hline Exat Oully & 0.01 & 0.07 & 0.18 & 0.19 & 0.24 & 0.3 & 0.56 & 0.2 & 0.4 & 0.46 \\
\hline DXP.HBtuntinerF & 155.8 & 1783.3 & 1755.5 & 1695.4 & 156.21 & 1333 & 1731.7 & 1686.6 & 1302.8 & 1020.8 \\
\hline COMPUTEDH & & & & & & & & & & \\
\hline J日likouk & 1320.31 & $7285.4 !$ & Tदा8.1! & T18.6! & Ұ138.3 & Tार & 1098.8 & 7087.7 & & 679.1 \\
\hline Sadyi(ado wbe) & 1808.7 & 7847.5 & 1878.4 & 1847.81 & $18 \% 5.1$ & 1828.7 & 1830.3 & $18472 !$ & 1248.4 & 1057 \\
\hline $5 x d y$ & Tर्डा & 1600.1 & 1070.0 & 1007.4 & $16 \% 0.01$ & 1673.6 & 108.8 & 1052 & 9912 & 8527 \\
\hline (स्य) & 53385 & 58579 & 5778 & 511831 & 478031 & 441801 & 40573 & उत7411 & 34882 & इ5272 \\
\hline$(F)$ & 1.3 & 1.8751 & 2.471 & 2883 & 3249 & 3.572 & 3.880 & 4.148 & 4.387 & 4.44 \\
\hline$(F D C)$ & कृा & 29287 & 38 & $450 . \overline{1}$ & 50721 & 567.4 & 608.61 & 647.11 & 678.8 & 6925 \\
\hline (H3OIL) & 18050 & 18391 & 18439 & 185881 & 1811.9 & 1811.31 & 1817.4 & 182031 & 1176.1 & 9501 \\
\hline & & & & & 1 & 1 & 1 & 1 & 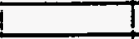 & \\
\hline & ZONV? & ZONEA & ZONE6 & ZONEB & $\angle O N=10$ & & $i$ & 1 & $L$ & \\
\hline DP liquid Temp.f & 188.8 & & & & 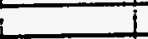 & & 1 & $L$ & $t$ & \\
\hline DPLiquid Densiny tom/3 & 91.84 & & & & & & & 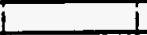 & & \\
\hline Elevation-a & & & 6 & & 9.875 & & & & & \\
\hline Whesured DPFsid & 0.288 & 1.361 & 217 & 2.5351 & 3316 & & & & & \\
\hline Zoro Correctionsepid & -0.007 & 0.031 & 0.189 & 0.022 & -0.01 & & $T$ & & & \\
\hline Cometed DPpsld & 0.381 & 1.2081 & 2.001 & 2.57 & 3.3 .31 & & $T$ & T & & \\
\hline Actul DPA & 1.61 & 2.24 & 3.05 & 4.31 & 4.971 & & 1 & 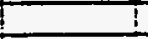 & 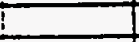 & \\
\hline 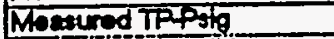 & 88 & & & 1 & 84.5 & & $i$ & 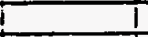 & & \\
\hline Mecured overnil & 2.5 & & & & & & $T$ & & & \\
\hline & & & & & & & & & & \\
\hline Computed DP, & 1.6 & 2.811 & 4.02 & 5.31 & 6.721 & & 1 & & 1 & \\
\hline Frictond & 0.1 & 0.57 & 0.8 & 1.37 & 1.82 & & 1 & & 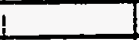 & \\
\hline Acaleration & 0.12 & $0 . \overline{231}$ & 0.35 & 0.51 & 0.58 & & 1 & 1 & 1 & \\
\hline Eleviont & 1.39 & 2दा & 2.87 & 3.43 & 4.32 & & 1 & & & \\
\hline & & & & & & & 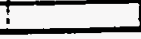 & & & \\
\hline
\end{tabular}




\begin{tabular}{|c|c|c|c|c|c|c|c|c|c|c|}
\hline Run: & 7931 & & 1 & 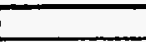 & & & $:$ & 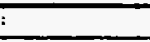 & & \\
\hline TostFluid &. . AF8 & & - & & & & & & & $:$ \\
\hline Date: & 820331 & & 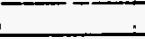 & & 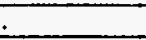 & & & & & \\
\hline Time: & $11: 04$ & & & & & & & & & : \\
\hline Note & Anabycal d & dt is used & 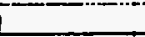 & & & & : & & & \\
\hline Fow Area-t2 & 0.005361 & & & & & & & 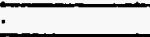 & & 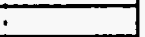 \\
\hline Heal Fow Area-42 & 0.19351 & & & & & & & 4 & & $\therefore$ \\
\hline Sat Pressure & $-84.61^{-}$ & & & & & & & & & $i$ \\
\hline Sat Temperature finiot) & 125.2 & & & 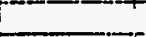 & & & & 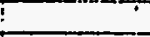 & & 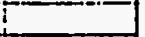 \\
\hline Liq. Density $40 \mathrm{n} / 13$ & $86.55 T$ & 86.351 & $86.34 !$ & 86.31 & 86.31 & 86.321 & 86.341 & 86.341 & 86.34 & 86.23 \\
\hline Latent Hear-Błuhbm & 39.21 & 39.051 & 39.031 & 39.011 & 39.01 & 3.021 & 39.031 & 39.001 & 39.031 & 38.95 \\
\hline Specitio Hos Q Qtunbm/ & 0.277 & $0.278 !$ & 0.2781 & 0.278 & 0.2781 & 0.2781 & 0.2781 & 0.278 & 0.2781 & 0.278 \\
\hline Liq. Visoosh ibminh & 0.5721 & $-0.565 i$ & 0.5651 & 0.5641 & 0.6641 & 0.5641 & 0.6851 & 0.5851 & 0.5651 & 0.561 \\
\hline Lig. Conductivitheshrth & $\quad 0.00251$ & 0.00351 & 0.03251 & 0.03251 & 0.05251 & 0.03251 & 0.00251 & $0.0<251$ & 0.06251 & 0.0024 \\
\hline Lig. Prand No. & 4.881 & 4.841 & 4.831 & 4.831 & $4.83 !$ & 4.831 & 4.881 & 4.831 & 4.831 & 4.82 \\
\hline Inlet Fow Rade-GPM & 1.71 & & & & & & & & & 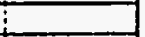 \\
\hline Outal Lig. Fow Rate-GPM & 0 & & $\div$ & - & : & & & 1 & 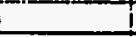 & 1 \\
\hline Outtel Vap. Flow Rate-GPM & $0.4 i$ & & 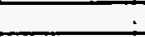 & . & 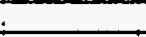 & & & & & 1 \\
\hline Inlet Temperaturef & 120.61 & & & & & & & & & $\therefore$ \\
\hline Oütê Lq Temporaturer & 135.1T & & & & & & & & & \\
\hline Outot vap. Temperdurer & $\overline{122}$ & & & & & & & & & \\
\hline Waer Fow Rate-GPM & $T$ & & ! & T & T & & : & T & $T$ & ! \\
\hline Water inlet Tomp.f & 72.01 & F & 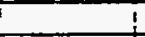 & : & : & & 1 & 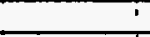 & 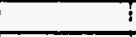 & 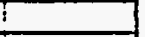 \\
\hline Welor ound Tomp.F & 105.91 & 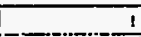 & 1 & $\ldots 1$ & 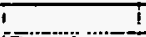 & $\ldots$ & 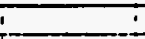 & $i$ & 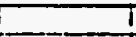 & $T$ \\
\hline IntotGibmhrme & $356582 \mathrm{i}$ & & Temp & 115.601 & iDensity & 87.891 & & $i$ & 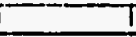 & $T$ \\
\hline Subooolingf & 5.61 & & + & 1 & 1 & $i$ & $i$ & $\therefore$ & 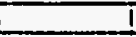 & I \\
\hline Fow Observation & 1 & 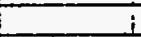 & 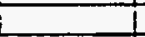 & & $i$ & & 1 & 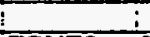 & 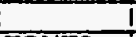 & 1 \\
\hline & ZONET & ZONE2 & ZONE3 & $20 N E 4$ & ZONES & ZONE6 & ZONE7 & ZONE8 & ZONE9 & ZONE10 \\
\hline Hoal input-Wats(nal) & 1141 & 1141.2 & 1156.5 & $1145.6 i$ & 1148.4 & 1150.3 & 1167.21 & 806.1 & 3926 & 130.6 \\
\hline Hent Loss-Wats & 7 & $1.6 i$ & 2.3 & 231 & 24 & $1.9 j$ & $1.1 i$ & $1.5 i$ & 1.7 & 9 \\
\hline Had Fox-Bunthine & 19778 & $19782 !$ & 20017 & 19858 & 19907 & 19940 & 20129 & 13973 & 6805 & 5278 \\
\hline Wall Temp. $3 F^{5}$ & 134.6 & 138.5 & 136.31 & $136.8 !$ & 139 & 141.5 & -136.71 & 136.11 & 132 & 129.9 \\
\hline Wail Tomp. B4F & 138.8 & 134.81 & 138.9 & $137.9^{1}$ & 139.5 & $141.3 !$ & 138.61 & 136.91 & 132.9 & 132 \\
\hline Wail Temp. $15 F$ & 136.2 & 133.71 & 139.7 & 138.11 & 137.9 & $140.2 !$ & 135.51 & $135.4 !$ & 131.8 & 130.3 \\
\hline Wail Temp. IfF & 871 & 135.71 & 134 & 1352 & 138.8 & 139.4 & 138.8 & 133.61 & 130.7 & 128,8 \\
\hline Wey Temp. 12F & 136.61 & 136.11 & 135.7 & 13621 & 137.51 & 140.91 & 140.31 & 134.21 & 130.8 & 130 \\
\hline Intrenm Temp.f & 123.71 & 124.91 & IEST & 12621 & 12521 & 125.11 & 125 & 1251 & 1251 & 125.6 \\
\hline Avg. Wall Tomp. $F$ & 136.11 & 136.31 & 13621 & $137 !$ & 137.91 & 140.61 & 137.6 & 135.21 & 137.81 & 130.2 \\
\hline Avg. Wali DT.F & 11.5 & $10 . \overline{51}$ & 10.31 & 10.91 & 11.8 & 14.61 & 11.7 & $9.6 i$ & 6.3 & 4.4 \\
\hline Remining SubcootF & -6.21 & & & & & & & 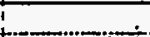 & & \\
\hline Exd Cualdy & 0.04 & 0.13 & 0.21 & $0 . \overline{29}$ & $0 . \overline{381}$ & 0.45 & 0.55 & 0.67 & 0.63 & 0.68 \\
\hline EX. HBtuHinter & 1720.5 & 1887.4 & 19427 & 1821.4 & 1683.4 & 1355.8 & 1724.8 & 1454.3 & 1078.1 & 12102 \\
\hline COMPUTED H & 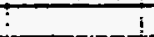 & & & & & & & & & \\
\hline Jallouk & T33.81 & $1269.2 !$ & 1256.11 & $1200.6 !$ & T185.81 & $11 \% 3.9$ & 7151.5 & 935.4 & -67.51 & 615.3 \\
\hline sedyil(Cudo ube) & 7797.11 & 7814.4 & 1820.1 & 1824.4 & 1828 & 1820.7 & 1838.8 & 1537.51 & 1000.2 & 980.2 \\
\hline $\operatorname{send} 2$ & 1600 & Tб:0.1 & 1649.1 & 16091 & 16727 & 1670.9 & 1656.2 & 1304.51 & 848 & 763.8 \\
\hline$(\mathrm{R} / \mathrm{K})$ & 39910 & 377001 & 34341 & $30900 !$ & 27464 & 23987 & 20458 & 17492 & 15685 & 16718 \\
\hline (F) & 1.6081 & 2.321 & 2561 & 3.4571 & 3.9051 & 4.304 & 4.678 & 4.9751 & 5.752 & 5.234 \\
\hline$(\overline{\mathrm{F}} \mathrm{C})$ & 188.7 & 2571 & 340.8 & 3986 & 48.0 & 494.61 & 537.3 & 571.7 & 582 & 6023 \\
\hline (19011) & 1796.1 & 181251 & 1825.1 & 1818 & 1818.9 & 1817.61 & 1823.3 & 1510.71 & 10285 & 897.7 \\
\hline & 1 & & & & $i$ & & $i$ & & 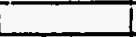 & \\
\hline & ZONES & ZONE4 & ZONE6 & ZONE8 & ZONEIO & & 1 & 1 & L & \\
\hline DP rguid Temp.F & 89.9 & & 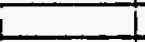 & & 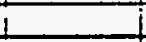 & & 1 & 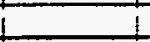 & 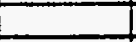 & \\
\hline DPLuqud Densivy tomins & 91.79 & & 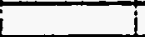 & & 1 & & 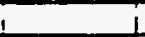 & 1 & 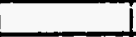 & \\
\hline Elevation-t & 2 & & & 81 & 9.875 & & 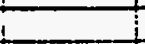 & & & \\
\hline Messured DPFsid & 0.421 & 1.591 & 26. & 3.165 & 3.37 & & & & & \\
\hline Zero Cotroctions-psid & $=0.007$ & 0.051 & 0.100 & 0.02 & 0.07 & & & & & \\
\hline Corruad DPPsid & 0.428 & 1.331 & 2474 & 3.12ा & उ.S91 & & ! & & & \\
\hline Actud DPA & $1.4 \pi$ & 1.71 & 228 & 3.31 & 3.881 & & I & & 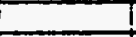 & \\
\hline Meatured TP्यig & 848 & & & & 832 & & $T$ & 1 & 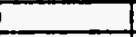 & \\
\hline Wherued overlil & 2.31 & & & & & & $T$ & & 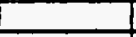 & \\
\hline & $i$ & & & & & & 1 & & 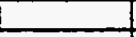 & \\
\hline Computed DPa & 124 & 2.17 & 3.08 & 4.031 & 5.05 & & 1 & 1 & $L$ & \\
\hline Friciont & 0.08 & 0.3 & 0.59 & 0.93 & 1.32 & & 1 & 1 & L & \\
\hline Acosleration & 0.08 & 0.16 & 0.27 & 0.39 & 0.44 & & 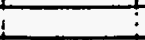 & & 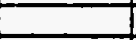 & \\
\hline Elevtion "k & 1.001 & 1.7 & 222 & 2651 & 329 & & 1 & 1 & 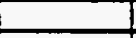 & \\
\hline & . & 1 & 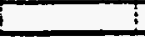 & ! & ! & & 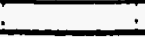 & 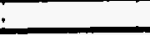 & & \\
\hline
\end{tabular}




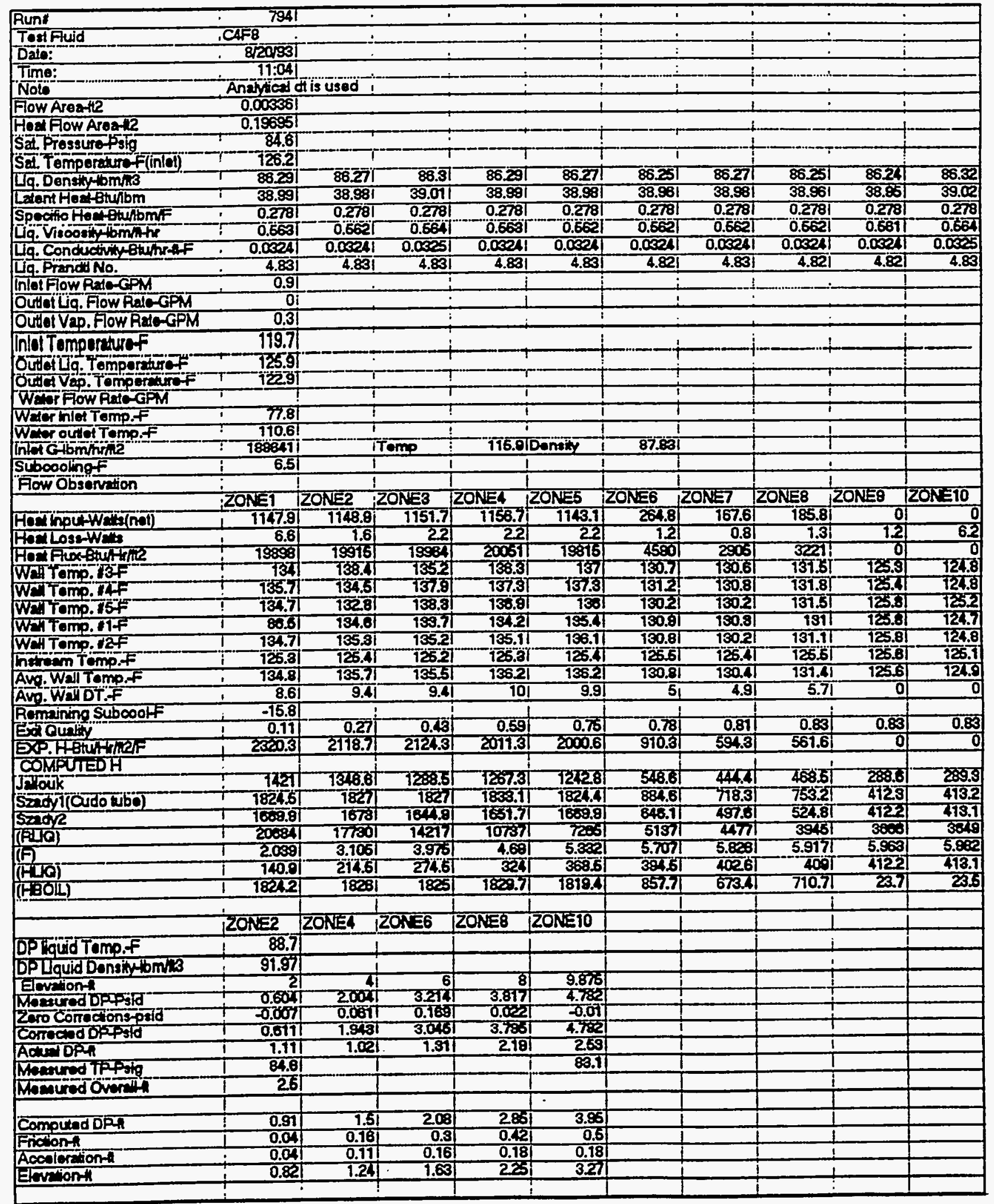




\begin{tabular}{|c|c|c|c|c|c|c|c|c|c|}
\hline Runt & 7951 & & & & & & & & \\
\hline $\begin{array}{l}\text { Tost Fuid } \\
\text { Date: }\end{array}$ & $\begin{array}{l}. \text { C4F8 } \\
. \quad 8 / 2493 i\end{array}$ & & $i$ & & & & & $\frac{1}{3}$ & \\
\hline Time: _. & $7: 35$ & & & & & & & & \\
\hline Note - - & Anarycaic & dt is used & & & & & & & \\
\hline Fow Areatl? & 0.003361 & & & & & & & & \\
\hline Hod Fow Aroat? & 0.196951 & & & & & & & & \\
\hline Sa. Pressurepsig & -88.3 & & & & & & & $\ldots \ldots$ & \\
\hline Se Temperaturofiniol) & 128.61 & & & & & & & & \\
\hline Ug. Densinytomitis & 86.41 & 86.31 & $\frac{86.181}{38.911}$ & $\frac{86.151}{38.881}$ & $\begin{array}{l}86.241 \\
38.951\end{array}$ & $\frac{86.251}{38.961}$ & $\frac{86.2 !}{38.921}$ & $\frac{86.251}{38.91}$ & \\
\hline Latent Hearterenbm & 39.09 & 39.011 & 38.91 & 38.881 & & & 38.921 & 38.981 & 39.14 \\
\hline Spogifio Hostethomf & 0.278 & 0.278 & 0.278 & 0.279 & 0.278 & 0.278 & 0.2781 & 0.2781 & $\frac{0.27}{0.56}$ \\
\hline Hq. Viroos & 0.667 & 0.6641 & 0.66 & 0.6601 & 0.6617 & 0.6621 & 0.661 & 0.5621 & \\
\hline 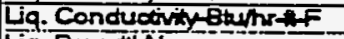 & 0.08251 & 0.03251 & 0.03241 & 0.03231 & 0.0524 & 0.03221 & 0.00241 & 0.00201 & $\frac{0.0326}{4.86}$ \\
\hline Uiq. Prantid No & 4.841 & 4.831 & 4.82 & 4.811 & 4.82 & $4.82 !$ & 4.821 & 4.821 & 4.85 \\
\hline 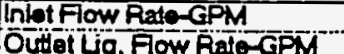 & $-\frac{4.81}{0.31}$ & & & & & & & -1 & \\
\hline $\begin{array}{l}\text { Outel ug. Fow Rato-GPM } \\
\text { Outet Vap. Fow Rato-GPMM }\end{array}$ & $\frac{0.3 i}{2.2 i}$ & & & & & & & & $\div$ \\
\hline Inhal Temporaturef & 119.61 & & & & & & & & \\
\hline Outót iq Temperaturof & 124.3 & & & & & & & & \\
\hline Outal Vap Temperauref & 121.81 & & & & & & & & \\
\hline Water Fow Rato-GPM & & & & & & & & T & \\
\hline Wath inlot Temp.F & 71.61 & & & & & & & & \\
\hline ortel Tomp.f & 108.81 & & & & & & & $\div$ & \\
\hline nnle Gomhine & $997899 i$ & & -Temp & $20.3 \pi$ & TDensy & 87.121 & & 7 & \\
\hline Subcoolingf & 91 & & & & & & & $T$ & \\
\hline Fow Obsarvation & & & & & & & & & \\
\hline & ZONE 1 i & NE2 & NE3 & NES4 & ZONES & ZONE6 & ZONE7 & ZONE8 RONES & ZONE1C \\
\hline Heal input-Walts (not) & 1441.2 & $1441.8_{j}$ & 1437.11 & 144 & 1440.61 & 1112.6 & 673.5 & 566.5 & 24 \\
\hline Hex Loss-Wats & 7.6 & 1.8 & 2.5 & $2.5 i$ & 2.3 & 1.5 & 0.8 & $1.2 i$ & \\
\hline Hodfux-Ruthrng & 24982 & 24993 & $\frac{24911}{14 \pi 6}$ & 250091 & 24972 & 192881 & 71675 & 98201 & 4240 \\
\hline Veftomp: 137 & $\begin{array}{l}-138.11 \\
-140.61\end{array}$ & & $\begin{aligned} & 140.6 \\
-143.6 & \end{aligned}$ & & $\frac{13.21}{139.41}$ & $\frac{737.91}{7375 !}$ & $\begin{array}{l}-133.61 \\
133.21\end{array}$ & $\frac{729.35}{1298}$ & 128 \\
\hline 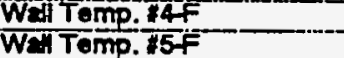 & 739.8 & $\frac{138.7 !}{137.31}$ & & $\frac{141.1 !}{142 !}$ & $\frac{139.41}{1381}$ & $\frac{737.51}{135.81}$ & $\frac{133.2}{132.01}$ & $\frac{129.8}{129.1}$ & \\
\hline WEH TOmp. RIF & & 1401 & 1381 & 998.81 & 137.4 & 130.91 & & 131.81 & \\
\hline NEA Tomp. . & $\begin{array}{ll}740.41 \\
912.65\end{array}$ & 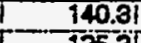 & 140.1 & 139.71 & 139.4 & 137.81 & 134 & 131.7 & 128.5 \\
\hline an Tomp. & 124.6 & 125.21 & 125.01 & 126.11 & 125.61 & 125.61 & 125.8 & $125.6 \mathrm{~T}$ & 724. \\
\hline Ang. Wall Tomp. F & 139.7 & 940.81 & 140.6 & 140.41 & 138.61 & 136.61 & 133.6 & 13211 & \\
\hline AUg. Wall DT . & 14 & 14.51 & 13.5 & 13.21 & 11.8 & 10.3 & 7.21 & 6.11 & \\
\hline Romaning Subcooff & 3.7 & & & & & & & & \\
\hline Exa Quality & -0.03 & 0.01 & 0.05 & 0.09 & 0.12 & 0.15 & 0.17 & 0.19 & \\
\hline EXP. HEturtine,F & 1788.2 & 17242 & 1838.8 & 1897.2 & 2112. & 1889 & 1614.2 & $1605.6 i$ & $107 \overline{8}$ \\
\hline $\begin{array}{l}\text { COMPUTEDH } \\
\text { JIFOUK }\end{array}$ & 7474.61 & & Tर्यद्यो & & & 1017 & & 7128 & \\
\hline Szadyi(cudo unbo) & 207.75 & 2057.91 & 2070 & 2082.6 & 2077.31 & 1818.8 & 1440.3 & 1343.5 & $\frac{15.0}{94.3}$ \\
\hline Szidy? & 1933.4 & 1943.61 & 1926.8 & 1940.71 & 193291 & 1858.7 & 1219 & 816.5 & 833. \\
\hline (PLQ) & 1151611 & 11582011 & 113145 & 10896 a & 104056 & 1000781 & 978 & 94283 & \\
\hline & 1.000 & 1.0061 & 1.712 & 2.146 & 2488 & 2.745 & 2.909 & 3.015 & 3.1 \\
\hline (1) & 253.11 & 253.61 & 440.11 & 56321 & 652.1 & 719.31 & 761.41 & 790.11 & \\
\hline (1BOL) & 2046.31 & 2058.51 & 20629 & 2068.81 & 2055.6 & 9780.31 & 1370 & 1245.4 & 774.5 \\
\hline & & & & & & & & & \\
\hline & ZONE2 IS & ZONE4 & ZONE6 & ZONE & ZONE10 & & & & \\
\hline OP Rquid Tomp. $F$ & $\begin{array}{l}\quad 86.8 \\
\end{array}$ & & & & & & & & \\
\hline DP Lquid Density tom/tit3 & 92.24 & & & & & & & & \\
\hline Elovation: & & 4 & & & 9.875 & & & & \\
\hline Mesured DPPsid & $=0.065$ & 0.469 & $\frac{7.074}{0.11}$ & 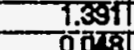 & 7.904 & & & & $\because$ \\
\hline $\begin{array}{l}\text { Zoro corrections-psid } \\
\text { Comecod DPPSld }\end{array}$ & $\begin{array}{l}\frac{7.03 \pi}{0.021} \\
.012\end{array}$ & $=0.074$ & 0.31 & 0.048 & $\begin{array}{l}-0.0283 \\
1.9321 \\
\end{array}$ & & & $\frac{1}{1}$ & \\
\hline$P_{n}$ & 2.11 & 3.51 & 4.98 & 8.31 & 7.32 & & & T & \\
\hline arened TP & 88.8 & & & & 85.8 & & & & \\
\hline 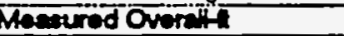 & 4.161 & & & & & & & & \\
\hline & & & & & & & & 1 & \\
\hline ODPA & 2.15 & 4. & 6. & & 10. & & & $i$ & \\
\hline ond & 0.15 & 0.671 & 1.36 & 2.011 & 2.66 & & & $i$ & \\
\hline lerwion- & 0.15 & 0.471 & 0.71 & 0.82 & 0.87 & & & & \\
\hline devetiont & 1.84 & 3 & 3.97 & 5.27 & 6.48 & & & & \\
\hline
\end{tabular}




\begin{tabular}{|c|c|c|c|c|c|c|c|c|c|c|}
\hline Runt: & 7961 & & & - & - & & : & & & \\
\hline Testfuid & . $44{ }^{8}$ & $\therefore$ & 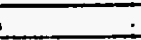 & 1 & $!$ & 1 & $\bar{\vdots}$ & 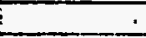 & 1 & L \\
\hline Dafo: & . 824331 & & 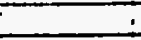 & 1 & 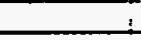 & & & & & \\
\hline Time: & $7: 35$ & & & & & & i & & & \\
\hline Noto & Analicical d & this used I & & & & \pm & 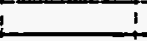 & $i$ & $:$ & - \\
\hline Flow Areatl2 & 0.003351 & & & & $\therefore$ & & 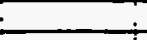 & & 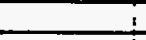 & 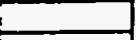 \\
\hline Host Fow Aron-4L & 0.19695 & & & & & & & . & $\therefore$ & \\
\hline SäPiessure & 86.31 & & & & & & & & & \\
\hline Sa. Tomporature fiñol) & 127.31 & & & & & & & & & \\
\hline La. Denstintom/13 & 86.52 & 86.421 & 86.31 & 86.321 & 80.37 & 86.301 & 86.4 & 86.44 & 86.79 & 86.55 \\
\hline Latent Hoa etudbm & 39.181 & 39.11 & 3.031 & 39.021 & 39.08 & 39.071 & $39.09 !$ & 39.111 & 39.111 & 39.21 \\
\hline Spectio Hos onutbmi & 0.278 & 0.278 & 0.278 & 0.2781 & 0.278 & 0.2781 & 0.2781 & 0.2781 & 0.278 & $0.2 \pi$ \\
\hline Lia. Visoositiomintr & 0.6711 & 0.6671 & 0.666 & 0.6641 & 0.6651 & 0.666 & 0.5671 & 0.6681 & 0.6687 & 0.572 \\
\hline Liq. Conductivity tulhreff & 0.03261 & 0.03251 & 0.03251 & 0.02351 & 0.0025 & 0.03251 & 0.00251 & 0.00251 & 0.0025 & 0.0326 \\
\hline Lig. Prand No. & 4.861 & 4.84 & 4.831 & 4.831 & 4.84 & 4.841 & 4.841 & $4.85 i$ & 4.85 & 4.86 \\
\hline Intetfow Pate-GPM & 4.1 & & 1 & & & & & & 1 & $L$ \\
\hline OứCLLG. Flow Rate-GPM & 0.9 & 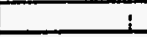 & $\dot{-}$ & 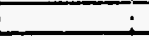 & & & & & & \\
\hline Outtet Vap. Flow Rate-GPM & 1.7 & & & & & & & & 2 & 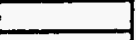 \\
\hline Inlot Tomperaturef & 119.5 & & & & & & & & & \\
\hline Outotlig Temperatere $F$ & $123.9 T$ & & & & & & & & & \\
\hline Outel Vap.Tomper rutof & 120.8 & & & & & & & & & 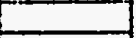 \\
\hline WEN FOW RLAOGPM & $T$ & 1 & 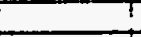 & 1 & & & & & & 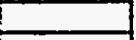 \\
\hline Water inlot Tomp.f & 73.7 & T & T & 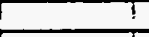 & i & ' & i & $!$ & $!$ & 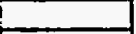 \\
\hline Weter octel Tomp.f & 108.11 & 1 & & 1 & & & i & 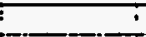 & & \\
\hline Inlot $G$ sbminure & $853814]^{-}$ & & Temp & T19.4iL & Densiy & 87.261 & & $i$ & $i$ & \\
\hline Subcoolingf & 7.8 & $I$ & 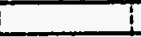 & 1 & 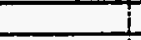 & & I & 1 & 1 & \\
\hline \multirow{2}{*}{ Fow Obsenvation } & $i$ & $I$ & $=$ & $i$ & -2 & & $!$ & 1 & $i$ & \\
\hline & ZONEI I2 & ZONE2 12 & ZONE3 & ZONEA 12 & ZONES i2 & ZONE6 & ZONET & ZONEB & ZONES & ZONE10 \\
\hline HerlnputWeis(nd) & 1445.1 & 144.11 & 1445.6 & 14532 & 14324 & 1438.4 & 403.2 & 50.5 & 42.6 & 178.3 \\
\hline Hes Loss-We't: & 6.6 & $1.6 i$ & 2.2 & 22 & 2 & 1.5 & 0.61 & 0.61 & 0.8 & 5.7 \\
\hline Hon Flox-turtane & 25050 & 25033 & 25050 & ऊ5ाइ0। & 24850 & 24871 & 698 & 875 & 73 & 压1 \\
\hline Waltomp. $13 \mathrm{~F}$ & 736.81 & 743 & 139.1 & 1381 & Fis.t & 7321 & 729.7 & 125.61 & 125.6 & 727.2 \\
\hline Wa Tomp. O4F & 730.11 & 137.61 & 1428 & 17502 & 138.61 & 737.51 & (129.6! & $125.6 !$ & 125.6 & 128.2 \\
\hline Wall Tomp. ISF & 738.8 & 136.21 & 141.7 & 141.8 & 137.01 & 137 & 129 & 125.81 & 125.6 & 127.6 \\
\hline WaH Tomp. ITF & 88.61 & 138.7 & 130.7 & 137.81 & 136.7 & 137.61 & 129.9 & 125.01 & |ळ| & 120.6 \\
\hline 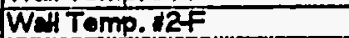 & 138.8 & 1391 & 139.1 & 138.7 & 138.81 & 139.61 & 129.71 & 125.81 & 125.1 & 127.3 \\
\hline instrean Tomo.f & 123.01 & $124.6 T$ & 1251 & 125.11 & 124.81 & 124.71 & $124.8 T$ & 124.71 & 124.4 & 123.7 \\
\hline Avg. Wall Tomp.f & 138.4 & 139.61 & 139.31 & 139.6 & 137.9 & 137.91 & 129.61 & 125.71 & 1250 & 127.4 \\
\hline Avg. Wail DT F & 13.4 & 13.91 & 1321 & 13.3 & 121 & 12.11 & 4.71 & 1.21 & 0.91 & 3.5 \\
\hline Remaining Subcooff & 1.6 & & & & & & & 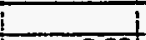 & & \\
\hline ExQ Oug & -0.011 & 0.08 & 0.08 & 0.12 & 0.16 & 0.21 & 0.21 & 0.22 & 0.22 & 0.20 \\
\hline EXP.HBtuAther & 7873.9 & $1798.5 i$ & 1903.1 & 1888.8 & 2008.6 & 2053.11 & 1498.5 & 705.7 & 780.1 & 873 \\
\hline \multicolumn{11}{|l|}{ COMPUTEDH } \\
\hline siak & T503.9| & 740.6. & 7308.8 & 12द्. & 1130 & $7143.8 !$ & -428.81 & 649.51 & 650.4. & 673 \\
\hline Szadij(C)dotubo) & 2038.7 & 2048.81 & 200.7 & 20125 & 2058.21 & 208231 & 7135.4 & 765.7 & 76 & 859.2 \\
\hline $\sec \sqrt{2}$ & T925.91 & 1934.7 & 19151 & 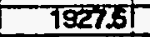 & 1900.61 & 1913.4 & 90t.31 & 761.81 & 76.8 & 7728 \\
\hline (PLㄴ) & 97876 & 974021 & 935241 & 89241 & 848461 & 802581 & 774 & 765891 & 764601 & 75521 \\
\hline (F) & 1.008 & 1.3911 & 2.021 & 241 & 2.784 & 3.08 & 3.255 & 3.31 & 3.308 & 3.34 \\
\hline$(140)$ & 231.8 & 321.11 & 467.1 & 658.97 & 64201 & 717.21 & 751.4 & 761.31 & 763.81 & 769.4 \\
\hline \multirow[t]{3}{*}{ (BOIL) } & 2057.7 & 2048.31 & 2053.7 & 20565 & 2007.11 & 2003.71 & 1013 & 196.81 & 1689 & 588 \\
\hline & 1 & & & 1 & & & $i$ & 1 & $\vdots$ & \\
\hline & ZONE2 & ZONE4 & ZONE6 & $201: 58$ & ZONETOI & & $i$ & & I & \\
\hline DP [quid Tomp $f$ & 89.5 & & & 1 & & & $t$ & I & 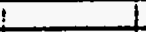 & \\
\hline DPLiquid Densityomin 3 & 91.85 & & & & & & & I & & \\
\hline Elevation & & & & $8 i$ & 9.875 & & & i & & \\
\hline Mosured bplas & 0.05 & 0.685 & 7.389 & $1 . \$ 81$ & 274 & & & & & \\
\hline Zcro comearomperd & -0.037 & -0.074 & 0.37 & 0.0781 & -0.02 & & ! & & & \\
\hline ComadDPPsid & 0.087 & 0.7031 & 1.179 & 7.751 & 2.468 & & I & & I & \\
\hline ACU:DDPA & 1.981 & 3.081 & 4.411 & 5.581 & 8.37 & & I & i & 1 & \\
\hline Mecoured TPPsh & 86.91 & 1 & 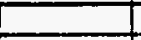 & 1 & 8421 & & T! & & 1 & \\
\hline Moesured Oviorilit & 3.461 & I & & & & & I & & T & \\
\hline & & & & & & & i & & I & \\
\hline Competad DPh & 2.03 & 3.781 & 5.571 & 7.41 & 9.15 & & I & & $\perp$ & \\
\hline Friction-1 & 0.15 & 0.61 & 1.38 & 1.96 & 2.51 & & $\perp$ & & I & \\
\hline Acoulorison-1 & 0.2 & 0.44 & 0.67 & 0.71 & 0.73 & & & & & \\
\hline Elev"ions" & 1.59 & 2.68 & 3.48 & 4.78 & 5.92 & & & i & & \\
\hline & & & & & & & & & & \\
\hline
\end{tabular}




\begin{tabular}{|c|c|c|c|c|c|c|c|c|c|c|}
\hline Runt & 7971 & & & & & & & & & \\
\hline Tost Fuid & CAF8 & & & $\therefore$ & - & & & & & $i$ \\
\hline Dato: & 824931 & & & & & & & & & \\
\hline Time: & $7: 35$ & & & & & & & & & \\
\hline Note & Analytical c & dt is used 1 & z & & & & & & & \\
\hline Fow Area-12 & 0.003361 & & & & & & & & & \\
\hline Heat Fow Area-tl2 & 0.19551 & & & & & & & & & \\
\hline Sat Pressure sig & 86.51 & & & & & & & & & \\
\hline Să Tomperaturo f(inloi) & 127.41 & & & & & & & & & \\
\hline Ja. Densityibmin & 86.61 & 86.351 & 86.201 & 86.291 & 86.341 & 86.341 & 86.391 & $86.39 !$ & 86.391 & 86.44 \\
\hline Latont Hoat Bturibm & 39.25 & 39.051 & 38.99 & 38.991 & 39.031 & 39.031 & 39.071 & 39.071 & 39.071 & 39.71 \\
\hline Spoctic Hoot BtubiniF & 0.27 & 0.278 & 0.278 & 0.2781 & $0.278 !$ & 0.2781 & 0.278 & 0.278 & 0.278 & 0.278 \\
\hline Lig. Viscostitbmithr & $0.573 !$ & 0.6651 & 0.5601 & 0.5631 & 0.565 & 0.5651 & 0.5661 & $0.566 i$ & 0.6661 & 0.568 \\
\hline Lig. Conductivitustuhr-thf & 0.0327 & 0.05251 & 0.0324 & 0.03241 & 0.03251 & 0.03251 & 0.05251 & $0.0325 !$ & 0.0325 & 0.0025 \\
\hline Liq. Prandt No. & $4.87 i$ & 4.841 & 4.831 & 4.831 & 4.831 & 4.831 & 4.841 & 4.841 & 4.84 & 4.85 \\
\hline Inlet Flow Rate-GPM & 3.3 & & & & & & & & & 1 \\
\hline Outel Lq. Fow Rado-GPM & 0.81 & & & & & & & & & \\
\hline Outlel Vap. Flow Rate-GPM & $1.4 !$ & & & & & & & & & . \\
\hline Inlot Temperaturef & 118.7 & & & & & & & & & \\
\hline Outiet Liq. Temporaturef & 124.71 & & & & & & & & & \\
\hline Outiol Vap. Tomperaturof & 121.31 & & & & & & & & & \\
\hline Water Flow Rate-GPM & & & I & 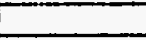 & & & & i & & \\
\hline Water hilet Temp.f & 74.71 & & 1 & 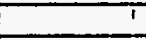 & & & & I & & \\
\hline Water outet Temp.F & 109.91 & & it & 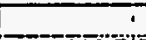 & & & & I & & \\
\hline Iniot G GbmMinh2 & 688837il & & Tomp & $198.51 \mathrm{~L}$ & Density & $87.41 i$ & + & - & & \\
\hline Suboodingf & 8.71 & & & 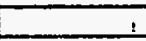 & 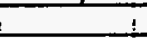 & $i$ & 5 & $i$ & & \\
\hline Alow Observation & & & & & & & & & & \\
\hline & ZONE1 & ZONE2 & ZONE3 & ZONE4:2 & ZONE5 I2 & ZONE6 2 & ZONE? I & ZONE8 :2 & ZONES & ZONE 10 \\
\hline Hoat input-Watts(net) & 1438 & $1441.8 \mathrm{i}$ & 1430.61 & 1445.5 & 1433.81 & 1441.8 & 404.71 & 50.8 & 36.2 & 177.7 \\
\hline Heal Loss-Wats & 7.4 & $1.8 \mathrm{i}$ & 2.51 & $2.4 i$ & 2.2 & 1.7 & 0.7 & $0.8 i$ & & -7.1 \\
\hline Hoad ALx-Bturtinle & 24921 & $24993 !$ & 24799 & $25057 !$ & 24854 & 249931 & 7016 & 8811 & 628 & 350 \\
\hline Wall Temp. \#3F & 736.91 & $143 \mathrm{~T}$ & 130.4 & $138.8 !$ & 138.4 & $3392 !$ & T30! & $25.8 !$ & 725.8 & 727.7 \\
\hline Wal Temp. $4 F$ & 139.4 & 137.61 & 142.4 & $740.2 !$ & 138.81 & $138.2 !$ & 129.8 & 125.9 & 125.8 & 128.8 \\
\hline Wall Tomp. $75 F$ & 738.7 & 138.11 & 142 & 741.5 & 137.81 & $137.7 T$ & 129.2 & 128.91 & 125.81 & 128.1 \\
\hline Wall Temp. If & 88.71 & 138.81 & 136.91 & 137.7 & 136.9 & 1381 & 130.1 & 125.81 & 125.3 & 127.2 \\
\hline Wall Temp. Ia & 138.9 & $139 !$ & 139.11 & 138.81 & 138.91 & 139.8 & 129.9 & 126.31 & 125.8 & 127.7 \\
\hline Introen Tomp. $F$ & 923.4 & $124.8 i$ & $125.3 T$ & 125.31 & -1351 & 1261 & 124.71 & 124.71 & 124.7 & 124.4 \\
\hline Avg. Wall Tomp.f & 138.5 & 139.61 & 139.51 & 139.61 & 138.11 & 138.31 & 129.81 & $126 !$ & 125.6 & 127.9 \\
\hline Aing Wall DT F & 13.91 & $13 . \overline{61}$ & 131 & 13.11 & $12 !$ & $12 \pi$ & 4.81 & 1.31 & 0.9 & 3.4 \\
\hline Romauning Subcooff & 1.11 & & & & & & & & & \\
\hline Ecat Qually & -0.07 & 0.05 & 0.11 & 0.16 & 0.21 & 0.261 & 0.28 & 0.28 & 0.28 & 0.29 \\
\hline DXP.HBtuHAh2F & 7787.1 & 1841.7 & 1903.3 & 19102 & 20752 & 2057.81 & 1466.7 & 687.8 & 719.9 & 916.5 \\
\hline COMPUTEDH & & & & & & & & & & \\
\hline Jallouk & 1530.2 & 1424.2! & 1338, & 1276.5 & 723502 & $1197.3 !$ & 710.4 & 594.11 & 59 & 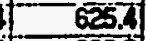 \\
\hline Sactyi(Oudo tubo) & 2025.51 & 2053.6 & 2055.2 & 20585 & 2059.31 & 2068.71 & 713.7 & -711.21 & 7093 & 853.2 \\
\hline szady2 & 1911.2 & 1938.81 & 1908 & 1923.3 & 1911.61 & 192211 & 883.4 & 7051 & 70.75 & 717.6 \\
\hline (PID) & 78584 & 781191 & 74089 & 697401 & 65185 & 608581 & 579001 & 57224 & 5709 & 56610 \\
\hline (F) & 1.0081 & 1.561 & 2.2141 & 2.6711 & 3.0511 & 3.3871 & 3.5811 & 3.527 & 3.688 & 3.68 \\
\hline (संO) & -1061 & 303.31 & 400.01 & 619.71 & 583.41 & 66751 & 681 & - & 708.7 & 777.6 \\
\hline (HBOL) & 20240 & 2051.31 & 2048,81 & $2057.5 i$ & 2042.81 & 2046,1 & 100861 & 211.11 & 157.11 & 607.7 \\
\hline & 1 & & & & & 1 & 1 & $\vdots$ & & \\
\hline & ZONE2 & ZONE4 & ZONE 6 & ZONE8 & ZONEIO & $\perp$ & I & $\perp$ & & \\
\hline DP Fquid Tamp.f & $1 \quad 88.8$ & ن & 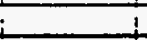 & & & 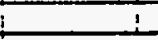 & 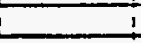 & 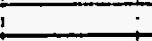 & & \\
\hline DP Liquid Densinutbm/n & 91.94 & & & & & 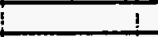 & 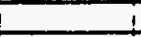 & $i$ & & \\
\hline Elevation & & & 6ा & 81 & 9.875 & & & & & \\
\hline Mestured DF pidd & 0.1451 & 0.3571 & 1.744 & $2305 !$ & 2.5051 & & & & & \\
\hline Zaro Contectons-psid & $=0.087$ & -0.0141 & 0.टा। & 0.0481 & -0.0281 & & & & & \\
\hline Comend DPpild & 0.1831 & 0.9681 & 9.534 & 2.158 & 2.9301 & & I & & & \\
\hline Achul DPA & 1.82 & 284 & 3.88 & 4.911 & 5.62 & $i$ & 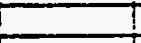 & $I$ & & \\
\hline Mosourod TPPsig. & 86.5 & 1 & I & & 84.8 & & & & & \\
\hline Moabured over & 3.17 & & & & & & & $T$ & & \\
\hline & $I$ & & & & & & & & & \\
\hline Competed DPA & 1.911 & 3.371 & 4.89 & 6.ळ21 & 8.23 & & L & 1 & & \\
\hline Frictionf & 0.12 & 0.5 & 1.13 & 1.591 & 2.03 & $\perp$ & L & 1 & & \\
\hline Aocaleraiont & 0.16 & 0.34 & 0.54 & 0.58 & 0.59 & & & & & \\
\hline Elevation & 1.64 & 253 & 3.2 .2 & 4.461 & 5.611 & & & 4 & & \\
\hline
\end{tabular}




\begin{tabular}{|c|c|c|c|c|c|c|c|c|c|c|}
\hline Runt & 7981 & $\cdot$ & - & & ! & 1 & i & . & & \\
\hline To\&fluid & ICAF8 & & & & $i$ & 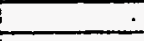 & $i$ & $i$ & 1 & 1 \\
\hline Dato: & $.8 / 24931$ & & & & 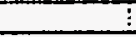 & $\div$ & 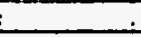 & & : & E \\
\hline Time: & $7: 35$ & & & & & & & $!$ & $i$ & \\
\hline Note & - Anaibicald & In is used I & & & & & & & 1 & 1 \\
\hline Flow Areart? & 0.003361 & & & & & & & ; & & \\
\hline Hoat Fow Areart? & 0.19595 & & & & & & & & & \\
\hline Sat. Pressuro Psig & 87.4 & & & & & & & & & \\
\hline Sat Tomperanue f(iniel) & 128 & & & & & & & & & \\
\hline Lg. Densiny tomnt3 & 86.931 & 86.221 & 86.21 & 86.21 & 88.2 & 86.21 & 86.22 & 86.2 ! & 86.25 & 86.39 \\
\hline Latant Hoad stunlom & 39.521 & 38.81 & 38.82 & $38.92 !$ & 38.921 & 38.941 & 38.84 & 38.921 & 39.96 & 39.07 \\
\hline Specitio Hestewhín' & 0.2761 & 0.278 & 0.278 & 0.2781 & 0.278 & 0.2781 & 0.278 & 0.2781 & 0.278 & 0.278 \\
\hline To. Visoosh $16 \mathrm{minh}$ & 0.6861 & 0.6611 & $0.66]$ & 0.661 & 0.66 & 0.567 & 0.6671 & 0.661 & 0.5621 & 0.656 \\
\hline Liq. Conductivity oturhe of & 0.0329 & 0.03241 & 0.03241 & 0.03241 & 0.0324 & 0.00241 & 0.0324 & 0.00241 & 0.00324 & 0.0025 \\
\hline Liq. Prand No. & 4.911 & 4.821 & 4.821 & 4.821 & 4.82 & 4.821 & 4.82 & 4.821 & 4.82 & 4.84 \\
\hline Intotflow Rale-GPM & 2.5 & & & & $\square$ & & $\ldots$ & 1 & 1 & 1 \\
\hline Outlol Lq. Flow Rato-GPM & 0.5 & & & & & $\therefore$ & 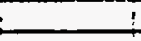 & - & 1 & $!$ \\
\hline Outlat Vap. Flow Rato-GPM & $1:$ & & & & & & & & & \\
\hline Inlat Tomperauref & 117.5 & & & & & & i & i. & & \\
\hline ÖutletLq. Tempormerof & 125.1 & & & & & & & & & \\
\hline Outet Vop. Tempor turef & 122 & & & & & & & & & \\
\hline Wator Pow Pato-GPM & & T & & & & & & \pm & & \\
\hline Weter kilot Tomp.f & 751 & I & & $i$ & & I & 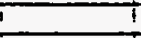 & 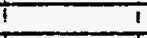 & & \\
\hline Weter ouldet Temp.f & 1102 & $!$ & $I$ & i & $\ldots$ & $=\ldots$ & $i$ & I & & \\
\hline 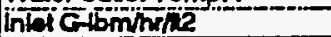 & 621687 & & $\operatorname{Ten} p$ & $178.3 i$ & Densiy & 87.44 & & $i$ & & \\
\hline Subooolingf & 10.51 & $i$ & 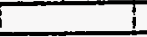 & & & $\bar{i}$ & I & I & & I \\
\hline Fow observaton & & & & 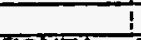 & & & & 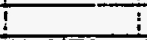 & & \\
\hline & ZONE1 & ZONE2 $\quad 12$ & ZONES I & ZONEA L & ZONES & ZONE6 & ¿ZONE & ZZNEB & ZONE9 & $20 N=10$ \\
\hline 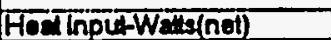 & 1451.2 & 1450.4 & 14552 & 1457.7 & 1436.4 & 14325 & 397.5 & 51.11 & 35.9 & 175.9 \\
\hline HedLLoss-Wa's & 7.2 & 1.7 & 24 & 24 & 22 & 1.91 & 0.7 & $0.8 i$ & 1.5 & 72 \\
\hline 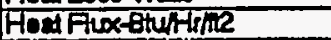 & 25156 & 25162 & 25325 & 253681 & 24899 & $24831:$ & 6800 & 886 & 622 & 3019 \\
\hline Wail tomp. 仿F & 736.8 & $\{429]$ & 1321 & 139.1 & 138.8 & $747.8 !$ & 730.8 & TES:8! & T安.7 & 128.3 \\
\hline Wal Tomo. IAF & $73 . .1$ & 137.31 & 1427 & 1403 & 139.01 & 145.81 & 730.8 & 126.91 & 128.8 & 129.3 \\
\hline We Tomp. 55 & 138.7 & 136.11 & T4231 & 141.11 & 13 & 138.61 & 150 & 128.91 & 120.4 & 128.2 \\
\hline Wall Tomp. IIF & 84.7 & 138.7 & 138.71 & 137.71 & 137.2 & 138.7 & 131.81 & 126.81 & 120.3 & TET.B \\
\hline WW Tomp.t2F & 139 & 139 & 139.21 & 138.81 & 1392 & 140.61 & 130.8 & 126.9 & 126.8 & 128.1 \\
\hline instroem Tomp, f & 121.4 & 125.7 & 125.81 & 125.81 & 125.8 & 125.71 & 125.7 & $125.8 T$ & 125.51 & 124.7 \\
\hline Avg. Wall Tomp.f & 138.5 & 139.51 & 139.51 & 139.51 & 138.5 & $9426 !$ & 130.8 & 126.91 & 1265 & 128.3 \\
\hline Avo.Wal DT.F & 15.9 & 12.81 & 12.5 & 1281 & 17.8 & 15.81 & 4.8 & 11 & 1 & 3.5 \\
\hline Remaining Subcooff & 0.3 & & & & & & & & & \\
\hline Ext Qualiy & 0 & 0.07 & 0.14 & 0.21 & 0.20 & $0 . \overline{36}$ & 0.38 & 0.38 & 0.38 & 0.39 \\
\hline EXP. HBtwhthelF & 1580.8 & $\$ 39.3$ & 2016.4 & 2008.11 & 21512 & 1573.8 & 1438.8 & 868.4 & 6408 & 880.7 \\
\hline COMMUTEDH & & & & & & & & & & \\
\hline Jallouk & $15 \% .4$ & -14881 & $|386,4|$ & 1359 & 1274.9 & 7250.7 & 6.7 & 5 & 588.2 & 574.8 \\
\hline Srady (cudo wbe) & 2001 & 2012n! & 2019.7 & 208.11 & 2071.3 & 2010 & 7108.8 & 651.9 & 65.8 & 801.8 \\
\hline Srater & T89.6। & 1539.7 & 1957 & 1951.01 & 19212 & $150 \mid$ & 865.8 & 650.7 & 63 & 600 \\
\hline (Pमी) & 386001 & 5880001 & 54404 & 500281 & 45020 & काटना & अवक्षा & 378001 & 37501 & 36535 \\
\hline$(F)$ & 1.008 & 1.787 & 2491 & 3.074 & 3.48 & 3.8051 & 4.0481 & 4.099 & 4.118 & 4.78 \\
\hline$(+\sqrt{a})$ & 1656 & 275.71 & 369.8 & 470.41 & 637.87 & $60 \% .91$ & 681.7 & 639.7 & 642 & 647.7 \\
\hline (म301L) & 201.7 & 2050.61 & 20745 & 2070.11 & 2052.1 & 2053.31 & 1055.7 & 248.11 & 1700 & क्यूर \\
\hline & & 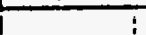 & 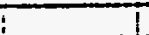 & & & : & 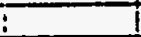 & & & \\
\hline & ZONE? & ZONEA & ZONE6 & ZONE8 & $20 N=10$ & & 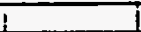 & & & \\
\hline DP ligud Tomp.f & 87.3 & & & & & & $\perp$ & + & & \\
\hline DPLLquid Donsty bmik3 & 92.17 & & & & & & 1 & & & \\
\hline Elovation- & & 4 & & 81 & 9.875 & & & & & \\
\hline 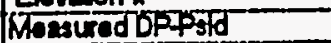 & 0.2891 & 1.2561 & 2749 & 27 & 3.505 & & & & & \\
\hline Zero Corrocionsosid & $=0.037$ & -0.074 & 0.27 & 0.078 & $=0.028$ & & & & & \\
\hline Corroced DPFild & 0.318 & 127 & 1.968 & 2.872 & 3.53 & & & & & \\
\hline Actuld DPA & 1.8 & 2.161 & 3.17 & 4.081 & 4.65 & & $i$ & $i$ & & \\
\hline Meaured TPFig & 87.4 & 1 & & & 85.8 & & I & & & \\
\hline Mesured Overiti & -3.51 & & T & & & & & & & \\
\hline & $i$ & & & & & & & & & \\
\hline Computed DP & 7.78 & 2981 & 4.22 & 5.791 & 7.25 & & & & & \\
\hline Friction & 0.08 & 0.38 & 0.87 & 1.22 & 1.55 & & 1 & 1 & & \\
\hline Acodercion-1 & 0.11 & 0.25 & 0.43 & 0.46 & 0.47 & & & 4 & & \\
\hline Elevationt & 1.58 & 235 & 2.92 & 4.12 & $5.2 \pi$ & & & 1 & & \\
\hline & & & & & & & & & & \\
\hline
\end{tabular}




\begin{tabular}{|c|c|c|c|c|c|c|c|c|c|c|}
\hline Run: & 7991 & 1 & 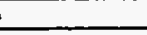 & & & & 2 & & & \\
\hline Todf Fuid & C4F 8 & 1 & 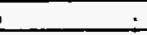 & & & & i & & & \\
\hline$-\cdots$ & $82493 i$ & & & & & & & & & \\
\hline Time: & $7: 35$ & & & & & & & & & \\
\hline Note ${ }^{-\cdots}$ & Analyical o & dis used: & & & & & & & & \\
\hline Flow Arez-ti2 & 0.003361 & & & & & & & & & \\
\hline Hoad Flow Areaft2 & 0.196551 & & & & & & & & & \\
\hline Sat Pressure osig & 84.61 & & & & & & & & 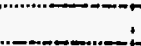 & \\
\hline Sad. Temperature finlei) & 126.2ं & & & & & & & & & \\
\hline Lg. Densityibminz & 87.11 & 86.451 & 86.491 & 86.51 & 86.791 & 86.541 & 86.471 & 86.971 & 86.471 & 88.65 \\
\hline Latent Hoot-Btulibm & 39.651 & 39.131 & 39.151 & 39.171 & 39.151 & 39.191 & 39.141 & 39.141 & 39.141 & 39.29 \\
\hline Specific Heaturibmif & 0.2761 & 0.2781 & 0.2781 & 0.2781 & 0.278 & 0.277 & 0.2781 & 0.2781 & 0.278 & 0.277 \\
\hline Lig. Viscosity & 0.501 & $0.568 i$ & $0.57 \mathrm{I}$ & 0.571 & 0.57 & 0.5711 & $0.568 \mathrm{i}$ & 0.5691 & 0.5691 & 0.575 \\
\hline Liq. Conductivity BuhrtfF & 0.0331 & 0.03261 & 0.03261 & 0.05261 & 0.03281 & 0.05261 & 0.03261 & 0.03261 & 0.03261 & 0.0327 \\
\hline ن & 4.931 & 4.851 & 4.851 & 4.851 & 4.851 & 4.861 & 4.851 & $4.85 i$ & 4.85 & 4.87 \\
\hline Inlet Fow Rate-GPM & $1.7 !$ & & & & & & & & 1 & i \\
\hline Oưtêt Lu. Flow Rate-GPM & $0 . \overline{4 i}$ & & & & & & & & & \\
\hline Outlel Vap. Flow Rate-GPM & 0.51 & & & & & & & & & \\
\hline Inlot Temporaturef & 115.81 & & & & & & & & & \\
\hline Oütet Lq. Temperaturof & T23.6] & & & & & & & & & \\
\hline OUti vap. Tempor & 120.81 & & & & & & & & ti & \\
\hline Water Fow Rato-GPM & & & & & & & & & $!$ & 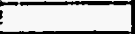 \\
\hline Wader hilet Termp.F & 75.11 & 5 & $i$ & 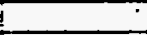 & - & I & t & I & I & I \\
\hline Weter outlet Temp. $F$ & 109.31 & $!$ & & ' & . & 7 & 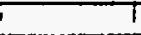 & $i$ & T & t. \\
\hline Intor Gibmhinit2 & 365798 & & iTemp & 116.71 & IDeneity & 87.71 & I & 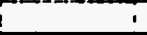 & I & i \\
\hline Subcoalingf & 10.4 & $\div$ & - & $\ldots$ & & 1 & $i$ & $i$ & I & I \\
\hline Fow observation & & & & & & 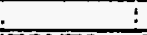 & 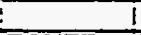 & & 1 & 1 \\
\hline & ZONE1 & ZONE2 i & ZONE3 & ZONE 4 & ZONE5 & ZONE6 12 & ZONE7 & ZONE8 & ZONE9 & ZONETO \\
\hline Heatlnpul-Watto(net) & 1449.8 & 14392 & 1455.2 & 1447.5 & 1446.5 & $698.8 \mathrm{i}$ & 393.2 & 50.5 & 36.6 & 175.2 \\
\hline Hoaloss-Wats & 6.8 & $9.6 i$ & 2.3 & $22 i$ & 2.2 & $1.3 !$ & 0.71 & 0.81 & 1.11 & 6.8 \\
\hline Head Flox-Btu/tr/il2 & 25131 & 24948 & ट5251 & 250911 & 250741 & $12113 !$ & 6816 & 8751 & 6381 & 3037 \\
\hline Wall Temp. & 134.5T & 140.51 & 135.71 & $136.9 !$ & 737.5 & $733.5 !$ & 129.6 & 725.71 & 125.4T & T20.7 \\
\hline WAI Tomp. B4F & 137.2 & 135.71 & 140.2 & $738.2 !$ & $\mid 38$ & 133.4 & 129.6 & 125.81 & 125.8] & T27.8 \\
\hline Wall Temp. $15 F$ & 136.2 & 133.7 & 140.2 & 138.41 & 136.41 & 132.11 & 128.8 & 125.81 & 125.3 & 128.9 \\
\hline Wall Temp. If & 84.11 & 136.11 & 134.8 & 134.91 & 135.71 & 132.91 & 129.7 & 125.7 & 125.1 & 120 \\
\hline Wall Tomp. & 136.81 & 136.71 & 136.61 & 136.21 & 137.21 & 133.51 & 129.4 & 125.8 & 12521 & 128.5 \\
\hline Instram Temp.f & 120.41 & 124.31 & 124.11 & 1241 & 124.11 & 123.81 & 124.2 & 124.21 & 124.21 & 123.1 \\
\hline Avg. Wall Tomp.f & 136.21 & 137.11 & 1371 & $137.1 i$ & 136.8 & 1331 & 129.4 & 125.81 & 125.41 & 126.8 \\
\hline AVG. WAHDT.F & 14.61 & $11.7 i$ & 11.7 & $12:$ & 11.61 & 8.61 & 4.91 & 1.51 & 1.11 & 3.5 \\
\hline Romaining SubcooffF & -4.61 & & & & & & & & & \\
\hline Ect Qualy & 0.03 & 0.14 & 0.24 & 0.35 & 0.45 & 0.5 & 0.53 & 0.54 & 0.54 & 0.56 \\
\hline EXP.HBtuHPREF & 7516.71 & 2137.3 & 2154.11 & 2005.81 & 2163.11 & 1404 & 1399 & 575.71 & 559.21 & 857.2 \\
\hline COMPUTEDH & & & & & & & & & & \\
\hline Jallouk & 7535.51 & $7474.9 !$ & 1401.5 & - & 7328.8 & $869.4 !$ & -668.21 & $444.3 !$ & -341.1 & 5749 \\
\hline Szadyi(cudotubo) & 1985.21 & $2041.5^{\top}$ & 2056.61 & 2075.41 & 2048.31 & T418.11 & 107t.41 & 571.9 & 563.4 & 767.5 \\
\hline $\operatorname{sen} 2$ & 1845.31 & 192.71 & 1958.8 & $1 \overline{950}, 31$ & 1931.1 & $\pi 71.2$ & 839.8 & 55251 & 554 & 58.1 \\
\hline (Rण्O) & 38808 & 374991 & अंकण & 28787 & 24504 & 21247 & 19701 & 190401 & 189101 & 18989 \\
\hline (F) & 1.5171 & 2.3211 & 3.107 & 3.719 & 4.267 & 4.597 & 4.757 & 4.8661 & 4.899 & 4.905 \\
\hline (HUO) & 17221 & 265.81 & 356.7 & 425.61 & 4851 & ह23. & -544.7 & 55261 & 6641 & 660.2 \\
\hline ( $\mathrm{HBOLL})$ & 19848 & 2010 & 207 & 20302 & 20392 & 1393.61 & 1028.9 & 264.11 & 206.11 & 651.4 \\
\hline & $i$ & & & & & & 1 & & 1 & \\
\hline & ZONE2 & ZONEA & $20 \mathrm{~N}=6$ & ZONE8 & ZONE10 & & L & $i$ & & \\
\hline DP Equd Tomp.F & 87.71 & -1 & & & & & 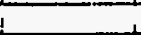 & & & \\
\hline DPLiquid Density & 92.111 & & 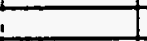 & & & & 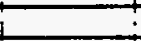 & $i$ & 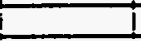 & 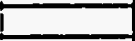 \\
\hline Elevation-1 & $2 !$ & & & & 9875 & & & & 1 & 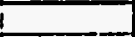 \\
\hline Messured DPF prid & 0.435 & -1.612 & 2E्या & 3.23 & 4.09 & & & & & \\
\hline Zero Correctons-psid & $=0.037$ & $=0.0141$ & $0.2 \pi$ & 0.0481 & $=0.0281$ & & & & & \\
\hline Corrected DPpard & 0.468 & $1 . \overline{6281}$ & 2.417 & 3.1851 & 4.118 & I & I & & & \\
\hline Acted DPA & 1.36 & 1.551 & 2.38 & $32 \pi$ & 3.5ा & & T & & & \\
\hline Moserured TPPig & 84.81 & & T & & 82.31 & & I & T & i & \\
\hline Mósured Overilta & 3.821 & i & I & & & & & I & I & \\
\hline & $i$ & & & & & & i & & I & \\
\hline Computed DPI & 1.28 & $22 !$ & 3.18 & 4.361 & 5.68 & & $\perp$ & $i$ & 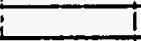 & \\
\hline Friction-ti & 0.081 & 0.31 & 0.65 & 0.92 & 1.14 & $i$ & 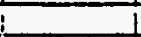 & $i$ & $i$ & \\
\hline Aooulerion- & 0.081 & 0.2 & 0.31 & 0.33 & 0.35 & 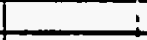 & 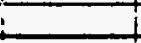 & & 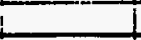 & \\
\hline Elovation: & 1.12 & 1.71 & 2.17 & 3.111 & 4.18 & & 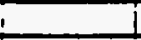 & $:$ & 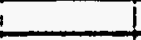 & \\
\hline & 1 & & & & & & & & & \\
\hline
\end{tabular}




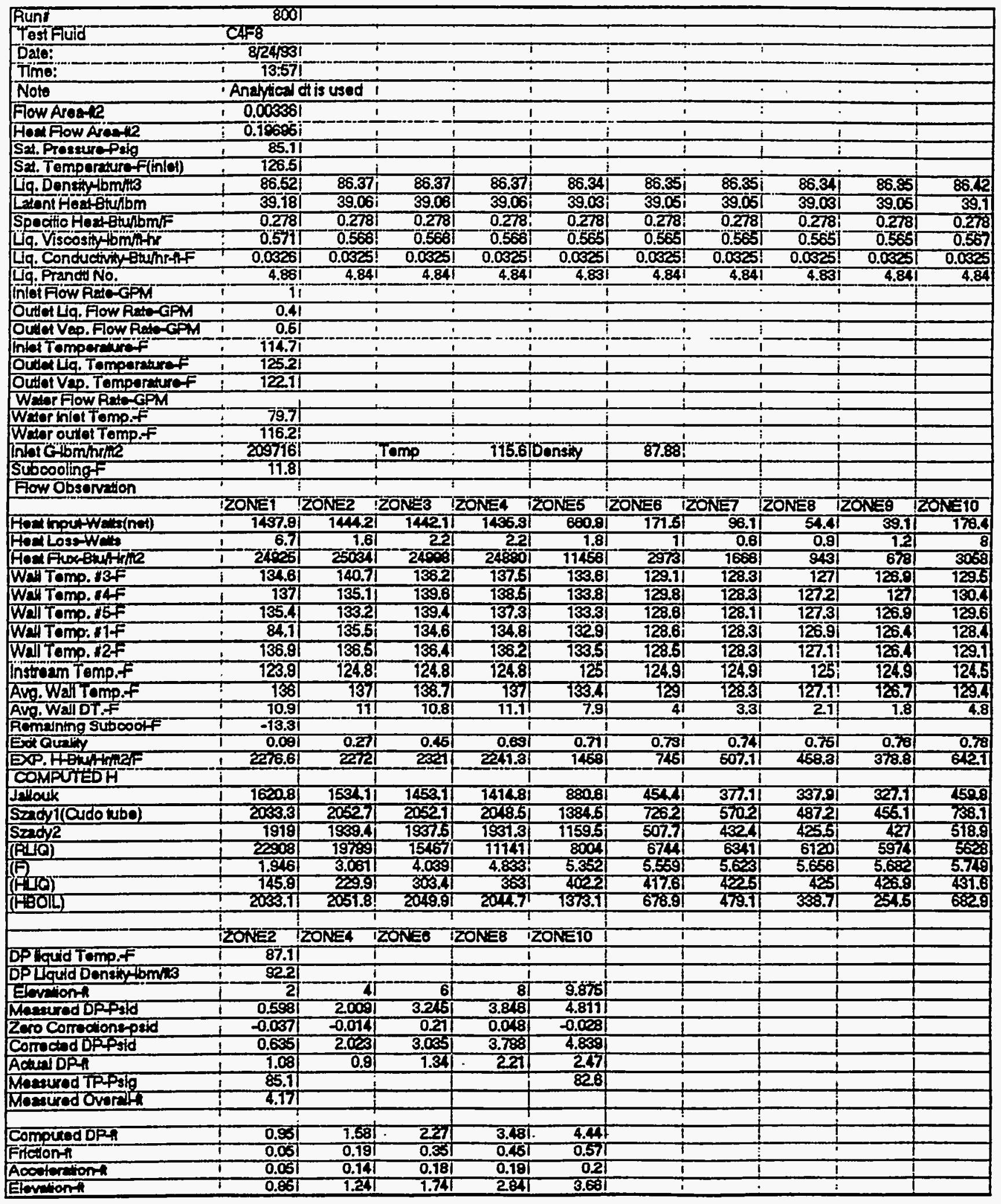




\begin{tabular}{|c|c|c|c|c|c|c|c|c|c|c|}
\hline Run: & 8011 & & & I & I &. & . & & & . \\
\hline Test Fuid & CAF8 & $\therefore$ & : & 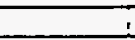 & 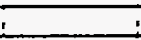 & 1 & 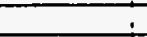 & 1 & $\perp$ & $\perp$ \\
\hline Date: & . 91593 & + & 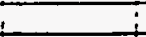 & - & 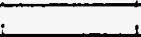 & . & $\vdots$ & $i$ & i & \\
\hline Time: & $: 8: 53$ & & & $i$ & $i$ & & & & & \\
\hline Note & Analyicalo & dt is used i & i & & & & & & & \\
\hline Fow Areant & $\mid 0.00336$ & & $i$ & : & : & & & & & \\
\hline Hoat Fow Area-12 & 0.1965 & & & . & : & 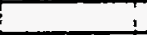 & & $i$ & 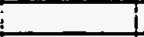 & \\
\hline Sat Pressure Psig & 127.31 & $T$ & : & & 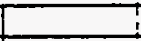 & 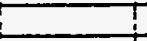 & & & & \\
\hline Sad. Temperature f(inld) & 151.3 & $\longrightarrow$ & $T$ & & 1 & i & & & & \\
\hline Lig. Densiny fbmint & 81.99 & 82.071 & 82.011 & 81.721 & 81.8 & 81.81 & 81.74 & 81.92 & 81.94 & 81.74 \\
\hline Latent Hest Bustom & 35.5 & $35.56 !$ & 35.62 & 35.281 & 35.84 & 35.34 & 35.8 & 35.44 & 35.461 & 35.8 \\
\hline Specifo Hestelubmf & 0.291 & 0.291 & 0.291 & 0.282 & 0.252 & 0.202 & 0.2921 & 0.291 & 0.291 & 0.292 \\
\hline Lig. Visoosity tominhr & 0.45 & 0.4671 & 0.45 & 0.441 & 0.446 & 0.4461 & 0.441 & 0.4481 & 0.448 & 0.44 \\
\hline Lig. Conductivity Bhth-if & 0.0005 & $0.0 \times 5$ & 0.0295 & 0.0203 & 0.0203 & 0.0023 & 0.0003 & 0.02041 & 0.0391 & 0.0203 \\
\hline Liq. Prand No. & 4.44 & 4.44 & 4.44 & 4.42 & 4.43 & 4.431 & 4.43 & 4.44 & $4.4 \mathrm{n}$ & 4.43 \\
\hline Inlet Fow Rata-GPM & 5.1 & & 1 & & & & -1 & 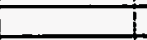 & & \\
\hline Outet Lig. Fow Rad-GPM & 1.11 & & T & & $i$ & & 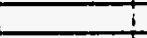 & I & & \\
\hline Outhat Vap. Fow Ruto-GPM & 2.2 & & 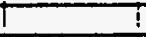 & & 1 & I & - & & & \\
\hline Inlet Tompereture f & 148.7 & 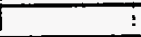 & $\bar{i}$ & $?$ & ? & T & & & & \\
\hline Outet Lia. Temperaturaf & T48.81 & & $T$ & T & $T$ & 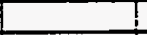 & & & & \\
\hline OutaVap. Temperaturaf & 146.1 & $T$ & 1 & 7 & $T$ & & 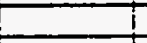 & & & \\
\hline 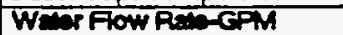 & 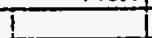 & T & i & $i$ & i & I & 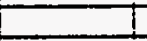 & I & & \\
\hline Water thel Tomp.f & 101.8 & & i & & t & I & I & $T$ & T & \\
\hline Werer outlel Temp.f & 144.1 & & 1 & & & 1 & 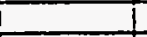 & 1 & 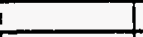 & \\
\hline Inlet G-bminna? & 1005211 & & TTemp & 145.71 & Dansity & 8267 & & & & \\
\hline Subooding ff & 2.6 & & & & & & & & & \\
\hline \multicolumn{11}{|l|}{ Fow Observation } \\
\hline & ZONE1 & ZONE2 & ZONE3 & ZONEA & ZONE5 & ZONE6 & ZONE7 2 & ZONE8 & ZONE9 & $20 N=10$ \\
\hline Hed inputWatand) & 291.4 & 288.2 & 2925 & 2927 & 292.3 & 290.7 & 291.9 & 289.9 & 289.3 & 290.1 \\
\hline Hod Loss-Wats & 7.1 & 1.6 & 22 & 2.3 & 2.2 & 1.61 & 1.1 & 1.6 & दा & 10.9 \\
\hline Hod flox-Busting & 5051 & 4988 & 5070 & 5074 & 5057 & 50039 & 5050 & 5025 & 5015 & 502 \\
\hline Wa' Tomo. औF & 158.7 & 156.7 & 158.8 & 157.8 & 157.6 & 157.41 & 157.31 & 155.51 & 954.7 & 154 \\
\hline Wal Tomp. Ift & 156.8 & 158.5 & 158.8 & 157.6 & 157.7 & 157.6 & 157.4 & 158.2 & 155.8 & 7552 \\
\hline WA Tomp. 85 F & 157.4 & 158 & 157.7 & 157.4 & 156.8 & 158.8 & 157 & 155.8 & 155.8 & 1542 \\
\hline WEITOmp. If & 148.7 & 158.8 & 155.2 & 158.9 & 158.8 & 157.2 & 157.8 & 158.41 & 1542 & 758.8 \\
\hline Wal Tomp. tat & 157.8 & 156.5 & 158 & 157 & 156.6 & 156.0 & 157.7 & 156.9 & 154.7 & 154.4 \\
\hline Inctream Temp.f & 149.8 & 148.9 & 140.2 & 150.7 & 160.3 & 150.3 & 150.6 & 149.71 & 149.6 & 160.6 \\
\hline Avo. Wall Tomph & 157.1 & 156.5 & 155.1 & 157.3 & 157 & 157.3 & 157.4 & 1562 & 154 & 1542 \\
\hline Avg. WaH DT.F & 7.5 & 7.4 & 6.7 & 6.4 & 6.4 & 6.7 & 6.6 & 6.2 & 5 & 3.5 \\
\hline Romaining Suboooff & 1.6 & & & & & & & & & \\
\hline Exa Cuathy & -0.01 & 0 & 0 & 0.01 & 0.02 & 0.03 & 0.04 & 0.05 & 0.05 & 0.06 \\
\hline 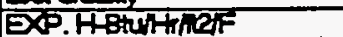 & 671.6 & 6775 & 760.11 & 796.4 & 786 & 749.61 & $\pi$ & 808.2 & 1000.3 & 1439.8 \\
\hline \multicolumn{11}{|l|}{ COMIRTEDH } \\
\hline Jellouk & 664 & 658.4 & 80.1 & 6875 & 6032 & 653.41 & 657.8 & $6 \% 8.51$ & 6827 & 688.8 \\
\hline Sadvi(cudo twb) & 1081.71 & T0̛73.11 & 1083.1 & 1097.2 & 1093.6 & 1096.8 & 1703.11 & 1006.8 & 1097.8 & 7107.9 \\
\hline 5andy? & 807.9 & 799.4 & 800.8 & 818.0 & 818.8 & 817.4 & 823.8 & 818.8 & 800.4 & 82.8 \\
\hline (स्व) & 14687 & 1458881 & 146287 & 147104 & 145887 & 144108 & 143208 & 140878 & 139688 & 13900 \\
\hline (F) & 1.006 & 1.005 & 1.005 & 1.274 & 1.422 & 1.5301 & $1 . \approx 7$ & 1.73 & 1.812 & 7.861 \\
\hline$(110)$ & 278.8 & 278 & 278.7 & 3544 & 3952 & 427.51 & 456.11 & 400.31 & 5020 & 5221 \\
\hline \multirow[t]{3}{*}{ (स:30L) } & 1075.5 & 1088.8 & 10789 & 10347 & 10792 & 10742 & 1076.6 & 1024.7 & 10000 & 1057.6 \\
\hline & & & & & & & & & & \\
\hline & ZONE2 & ZONE 4 & ZONE6 & ZOINES & ZONE10 & & & & & \\
\hline OP hquid temp.f & 79.2 & & & & & & & & & \\
\hline DP Llquid Density bmhs & $\mathbf{9 3 . 3 1}$ & & & & & & & & & \\
\hline Everationt & 2 & & (6) & 8 & 9.875 & & & & & \\
\hline Moserrod DP4 SId & -0.177 & 0.353 & 0.488 & 0.92 & 0.911 & & & & & \\
\hline Zero Corroctions-psd & 0.019 & -0.07 & -0.018 & -0.007 & $-0 . \overline{507}$ & & & & & \\
\hline Cormend DPPatd & 0.180 & 0.308 & 0.512 & 0.918 & 0.978 & & & & & \\
\hline Achar OPA & 208 & 3.89 & 5.80 & 7.51 & 9.00 & & & & & \\
\hline 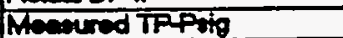 & 127.8 & & & & 1265 & & & & & \\
\hline \multicolumn{11}{|l|}{ Mecoured Oventh } \\
\hline & & & & & & & & & & \\
\hline Compled DF & 2.13 & $2 \sqrt{2}$ & 6.78 & 8.08 & 9.88 & & & & & \\
\hline Fiatonnt & 0.13 & 0.3 & 0.52 & 0.78 & 1.08 & & & & & \\
\hline Acolterion & 0 & 0.18 & 0.22 & 0.28 & 0.33 & & & & & \\
\hline Eleviont & 2 & उ.दी & 5.45 & 7.08 & 8.67 & & & & & \\
\hline & & & & & & & & & & \\
\hline
\end{tabular}




\begin{tabular}{|c|c|c|c|c|c|c|c|c|c|c|}
\hline Runt & $1 \quad 8021$ & & 1 & : & : & - & 1 & $i$ & 1 & 1 \\
\hline Tostfuid & CAFB & & & & 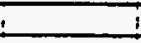 & 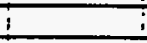 & $i$ & $i$ & 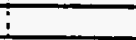 & \\
\hline Dalo: & (91593 & & & & & & & & & \\
\hline Time: & $8: 53$ & & & & & & & & 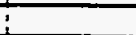 & \\
\hline Note & Anahyical c & dt is used & & & & & & & & \\
\hline Fow Aveatil? & 0.003561 & & & & & : & & & $!$ & \\
\hline Hoad Flow Arozitl & 0.19555 & & & & & 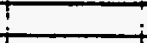 & & 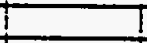 & & \\
\hline Sat. Ptessurepig & 128.5 & & T & & & ! & & t & t! & \\
\hline Sal. Temporature $f(i n i e t)$ & 151.9 & & 1 & & & $!$ & t & $!$ & $T$ & \\
\hline Lq. Dentintomns & 81.9 & 81.951 & 81.8 & 81.63 & 81.87 & 81.721 & 81.69 & 81.761 & 81.78 & 81.61 \\
\hline Lend Hescintom & 35.42 & 35.471 & 35.42 & 35.2 & 35.281 & 35.281 & 35.17 & 35.\$1। & 35.58 & $\$ 5.18$ \\
\hline 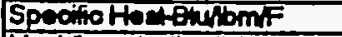 & 0.291 & 0.2911 & 0.2911 & 0.292 & 0.292 & 0.2021 & 0.28 & 0391 & 0.292 & 0.20 \\
\hline Lig. Vieoos ybmilith & 0.448 & 0.440 & 0.448 & 0.42 & 0.491 & 0.44 & 0.447 & 0.4461 & 0.446 & 0.40 \\
\hline La. Conduotivy etumath & 0.0004 & 0.020 & 0.000 & 0.002 & 0.0003 & 0.0023 & 0.002 & 0.0031 & 0.0003 & 0.0002 \\
\hline Liq. Prand No. & 4.43 & 4.44 & 4.43 & 4.42 & 4.42 & 4.42 & 4.42 & 4.43 & 4.43 & 4.42 \\
\hline \multicolumn{11}{|l|}{ Inlet Flow Rade-GPM } \\
\hline Outot Lig. Flow Aate-GPM & 1.2) & & & & & & + & & & \\
\hline Outet Vap. Flow Rate-GPM & 1.9 & & & & & & & & & \\
\hline Inlal Tomporeture & 749.11 & & & & & & & & & \\
\hline Outot Liq. Tompereturof & 149.71 & & $T$ & & & & & & t! & \\
\hline Outlet Vap. Temperautef F & 147 & & & & & & $T$ & & & \\
\hline Wat Fow RaG-CPM & & & 1 & & & & i & & $i$ & \\
\hline Waser inlet Temp.f & 1028 & & i & 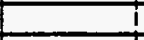 & 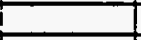 & & & & I & \\
\hline Ware outh Tomp.f & 144.8 & & & & & & & & & \\
\hline Inle G Gomprine & 846847 & & Temp & 146.6 & Densing & 82.61 & & & & \\
\hline Suboodingf & 28 & & & & & & & & & \\
\hline \multicolumn{11}{|l|}{ Fow Obsenvetion } \\
\hline & ZONEI & $2 O N=2$ & ZOKES & ZOKE & ZONES & ZONE6 & ZONE 7 & ZONE8 & ZONE9 & $2 O K=10$ \\
\hline 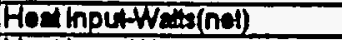 & 291.8 & 288.5 & 289.11 & 290.5 & 288.9 & 299.1 & 291.8 & 289.7 & 285. & 287.8 \\
\hline HedLosg-Walts & 7.3 & 1.71 & 22 & 2.3 & 2.3 & 1.7 & 1.1 & 7.8 & 2.1 & 11.3 \\
\hline 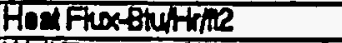 & 506 & 50011 & 501वा & $50<31$ & 5008 & 5185 & 5058 & 5021 & 4957 & 789 \\
\hline Wall Tomp. $13 \mathrm{~F}$ & 157.3 & T57.5T & 157 & 757.8 & 158.1 & 7581 & 758.2 & $|50.5|$ & 15.7 & 154.8 \\
\hline WETTomp.14F & 757.6 & 157.II & 157.8 & 757.8 & 758.11 & 158.61 & 158.4 & 158.9 & 1582 & 150.1 \\
\hline We Tomo. 16f & 157.9 & 158.6 & 158.4 & 157.8 & 157.4 & 157.61 & 158 & 158.8 & 1582 & 150 \\
\hline WE"T Tomg. \#1F & 149.1 & 157.21 & 150.8 & 157.41 & 1572 & 157.9 & 158.6 & 157.5| & 15ख्रो & 75स.8 \\
\hline WCIIT Temp. VaF & 158.7 & 157.8 & 156.7 & 757. & 1573] & 157.7 & 158.7 & 157.8 & 155.7 & 165.4 \\
\hline Intrean Temp.f & 149.8 & 1496 & 149.8 & 161.2 & 151 & 150.7 & 157.4 & 760.51 & $160 \lambda$ & 161.8 \\
\hline Aug. Wall Tomo $f$ & 157.7 & 157.3 & 156.8 & 757.8 & 157.6 & 158 & 158.4 & 157.11 & 1555 & 1552 \\
\hline Avo. WallDT.f & 7.7 & 7.5 & 6.8 & 6.3 & 6.2 & 7.11 & 6.8 & 6.3 & 5.2 & 3.7 \\
\hline Romaining SubcoofF & 1.6 & & & & & & & & & \\
\hline Ext Quality & -0.07 & 0) & 0.011 & 0.02 & 0.031 & 0.09 & 0.05 & 0.061 & 0.07 & 0.08 \\
\hline BXP.HBtuphar & 65821 & 6526 & 737.1 & 733.8 & 801.4 & 733.8 & 749.2. & 7929 & \$5.7 & 7350.8 \\
\hline \multicolumn{11}{|l|}{ COMPUIEDH } \\
\hline Jalouk & 679.8 & 675.7 & 677.8 & 285.11 & 687.1 & 67.4. & 608.71 & 683.41 & 68011 & 667.8 \\
\hline Fedy1(Cudo wbo) & 1088.7 & T078.6 & 7080.4 & 1635.8 & 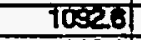 & 1777.4 & 1104.7 & 1097.1 & 7000.8 & T108.1 \\
\hline sady2 & 809.11 & 801.8 & 804.0 & 814.7 & 8121 & 8341 & 828.6 & 817.51 & 810.8 & 8ह21 \\
\hline (PUG) & 1ख्धारा & 1233881 & 12050| & 123997 & 122या & 1206581 & 120507 & 118164 & 116800 & 716007 \\
\hline (F) & 1.005 & 1.005 & 1.100 & 1.341 & 1.492 & 1.618 & 9.720 & $1.88 \pi$ & 7.811 & 1.865 \\
\hline (स्मि) & रद्वा & 24201 & 258.8 & 335 & 361.7 & 3910 & 477.8 & 44.61 & 4EDB & 487.4 \\
\hline \multirow[t]{2}{*}{ (BOIL) } & 7079.6 & 10723 & 107.9 & 1085.1 & 10792 & T0849 & 70844 & TOFट? & 1003 & 107.6 \\
\hline & ZOREZ2 & ZONET & 20NE6 & ZONE8 & ZONETO & & & & & \\
\hline DPlevid Tomp.f & 79.8 & & & & & & & & & \\
\hline \multicolumn{11}{|l|}{ 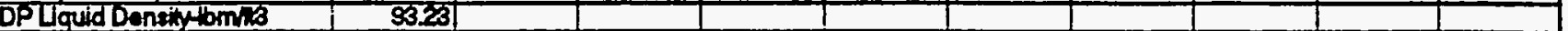 } \\
\hline \multirow{2}{*}{\multicolumn{11}{|c|}{ Elovion-h }} \\
\hline & \multicolumn{10}{|c|}{ Mosuured DPFSTI } \\
\hline Zero Cometionaperid & 0.08 & -0.01 & -0.0101 & -0.007 & -0.007 & & & & & \\
\hline \multicolumn{11}{|l|}{ Cormolid DPpidd } \\
\hline \multicolumn{11}{|l|}{ Actud DPA } \\
\hline & 128.5 & & & & 127.6 & & & & & \\
\hline \multicolumn{11}{|l|}{ Meacured Over"llit } \\
\hline \multirow{2}{*}{\multicolumn{11}{|c|}{ Comptred Dat }} \\
\hline & & & & & & & & & & \\
\hline Frotont & 0.09 & 03 & 0.4 . & 0.61 & 0.83 & & & & & \\
\hline Acouleration- & 0 & 0.71 & 0.71 & 0.2 & 0.3 & & & & & \\
\hline Elevationt & टा & 3.75 & 5.38 & 6.8 & 8.34 & & & & & \\
\hline & & & & & & & & & & \\
\hline
\end{tabular}




\begin{tabular}{|c|c|c|c|c|c|c|c|c|c|c|}
\hline Runt & 8031 & , & $\div$ & $\dot{-}$ & $\therefore$ & 1 & 1 & & & $!$ \\
\hline Tost Fuid & . CAF8 & $\dot{\dot{ }}$ & & : & & \pm & 1 & 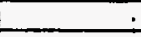 & & I \\
\hline Date: & 915/931 & & & & & & & & & \\
\hline Time: & $8: 53$ & & & & & & & & & \\
\hline Note & Anatytical & ott is used & & 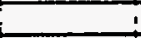 & & & $\div$ & & & \\
\hline Fow Areath2 & 0.003636 & $\square$ & & & $i$ & 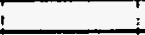 & & & & \\
\hline Hoat flow Area-12 & 0.19695 & & & & & & ? & & & \\
\hline Sat Pressure Psig & 128.4 & & & & & & & & & \\
\hline Sad. Temperature f(inlet) & 151.9 & & & & 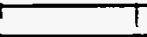 & T & $T$ & & & \\
\hline Ug. Densty tommb & 82.011 & 82.051 & 81.84 & 81.71 & 81.721 & 81.71 & 81.67 & 81.821 & 81.84 & 81.59 \\
\hline 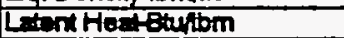 & 35.52 & 35.551 & 35.45 & 85.27 & 35.28 & 35.27 & 3.231 & 35.301 & 35.881 & $\$ 5.17$ \\
\hline Specisothew EurbmF & 0.291 & 0.2911 & 0.291 & 0.2021 & 0.292 & 0.2921 & 0.252 & 0.2921 & 0.322 & 0.202 \\
\hline Lig. Viscosithomshth & 0.46 & 0.4611 & 0.448 & 0.444 & 0.444 & 0.444 & 0.448 & 0.448 & 0.446 & 0.441 \\
\hline Lig. Conductivathr f & 0.000 & 0.0025 & 0.0024 & 0.02031 & 0.0203 & 0.0023 & $0.0 \times 91$ & 0.020 & 0.000 & 0.0002 \\
\hline Lig. Prandi No. & 4.44 & 4.441 & 4.44 & 4.42 & 4.42 & 4.421 & 4.421 & 4.43 & 4.43 & 4.42 \\
\hline Inlet flow Rate-GPM & 3.4 & & & & & + & & & 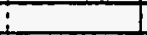 & \\
\hline Outlet Lig. Fow Rate-GPM & 1.11 & & & & & & 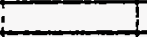 & & + & \\
\hline Outtet Vap. Fow Rate-GPiM & 1.61 & T & $i$ & ! & : & + & 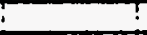 & & 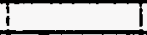 & \\
\hline Inlot Temperaturef & 148.41 & & & & & & 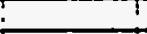 & & & \\
\hline Outel Lia. Temperaturef & 750.3 & & & & & ? & & & & \\
\hline Outlet Vap. Temperaturef & 146.5 & & & & & & & & & \\
\hline Wat Fow Rat-GPM & i & $i$ & & $T$ & 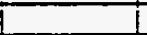 & 1 & 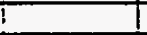 & & T & I \\
\hline Water trat Tomp.f & 103.0 & 1 & $i$ & 1 & 1 & i & I & & i & \\
\hline Wato outat Temp.f & 144.7 & 1 & 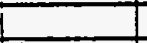 & 1 & 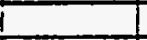 & 1 & 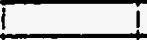 & I & I & \\
\hline InLl G-lbmhilite & 672524 & & Temp & 144.6 & Density & 82.81 & & & i & \\
\hline Subcoolingf & 3.5 & 1 & 1 & i & i & 1 & i & & i & \\
\hline \multirow[t]{2}{*}{ Fow Observation } & & & & $i$ & & & & & & \\
\hline & ZONE 1 & ZONE2 & ZONES & ZONE4 & ZONE5 & ZONE6 & ZONEA L & ZONEB & ZZONE9 & ZONETO \\
\hline 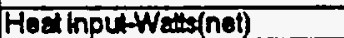 & 281.8 & 285.7 & 284 & 284.4 & 285.2 & 286.9 & 287.4 & 284.1 & 285.7 & 286.7 \\
\hline Head Loss-Wats & 7.2 & 1.7 & 2.2 & 2.3 & 2.2 & 1.7 & 1.11 & 1.6 & 2 & $\pi 1.3$ \\
\hline Hest Fix bultrine & 483 & 4942 & 4528 & 49501 & 4941 & 49731 & 4982 & 4925ा & 4932 & 1959 \\
\hline WE TOMP. 13F & 157 & 757.3 & 157 & 757.41 & 757.91 & 758.31 & 157.4 & 756.11 & 755.7 & 754.7 \\
\hline Wa Tomp. IfF & 757.2 & 158.8 & 157.4 & 757.51 & 158.21 & 158.4 & 1581 & 756.91 & 156 & 155.9 \\
\hline Wal Tomp. $15 \%$ & 757.6 & 156.6 & 158.8 & 15751 & 157.8 & 157.7 & 757.5 & 156.5 & 155.9 & 1540 \\
\hline WETTOMP. \#F & 148.4 & 150.8 & 150 & 156.8 & 150.8 & 157.7 & 1582 & 150.9 & 156.2. & TER, \\
\hline WCIT Tomp. ULF & 157.8 & 157 & 1565 & 157 & 156.8 & 157.7 & 158 & 157.4 & 155.6 & 165 \\
\hline Intraam Temp.f & 1492 & 149 & 149.6 & 150.81 & 160.7 & 150.81 & 167 & 15021 & 150.1 & 761.4 \\
\hline Avo. Wall Tomp.f & 157.4 & 157 & 156.51 & 157.3 & $157.3 !$ & 1581 & 157.8 & 156.81 & 155.7 & 155 \\
\hline Ave. WallDT.f & 8 & 7.7 & 6.9 & 6.3 & 6.4 & $7 !$ & 6.6 & 6.31 & 5.4 & 3.4 \\
\hline \multicolumn{11}{|l|}{ Remaining Subcoolf } \\
\hline Ext Quality & -0.02 & 0 & 0.07 & 0.02 & 0.03 & 0.04 & 0.06 & 0.07 & 0.08 & 0.00 \\
\hline EXP.HBuHARE & 610.3 & 639.6 & 773.2 & 785.4 & 778.4 & 710.4 & 755.4 & 777.11 & 926.8 & 7477.8 \\
\hline \multicolumn{11}{|l|}{ CIMPUEDH } \\
\hline In ouk & 253.4 & $6 \pi .7$ & 6725 & 680.9 & 658 & 658.81 & 659.5 & 653.31 & 655.40 & 68001.5 \\
\hline Szedvi(cudo tho) & 1080.8 & 7085.8 & 7068.2 & T078.7 & T081 & T0.83.8T & 100.1 & 7080.81 & 10082 & $7005 \%$ \\
\hline $\sin / 2$ & 785.8 & 791.8 & 791.8 & 798.8 & 800.8 & 8001 & 809.1 & 800.31 & 80.7 .7 & 8120 \\
\hline (R्म) & 9778 & $976 \pi$ & 97888 & 97810 & 86515 & 95389 & 94840 & 92191 & 91168 & 91000 \\
\hline$(\boldsymbol{F})$ & 1.005 & 1.005 & 1.118 & 1.3871 & 7.558 & 1.697 & 1.817 & 1.831 & 2.009 & 2.111 \\
\hline (HQ) & 202 & 201.8 & 224.8 & 279.6 & 313.9 & 341.9 & 3662 & 388.4 & 408.4 & 750 \\
\hline \multirow[t]{3}{*}{ (HBO년) } & T0585 & 1053 & 1054.8 & 10724 & 10729 & 1074.8 & 1076.1 & 1053.3 & 10645 & Tở \\
\hline & & & & & & & & & & \\
\hline & ZONE2 & ZONEA & ZONE & ZONEB & $20 N=10$ & & & & & \\
\hline \multicolumn{11}{|l|}{ DPhquid Temp.f } \\
\hline \multicolumn{11}{|l|}{ DPLlquid Density boniti } \\
\hline \multicolumn{11}{|l|}{ Elevation } \\
\hline Meacured DPPsid & -0.107 & 0.523 & $0.87 \%$ & 1.3881 & 7.4881 & & & & & \\
\hline Zero Corrections-psid & 0.018 & -0.01 & 20.018 & -0.001 & 0.007 & & & & & \\
\hline Cormand DPpydd & -0.120 & 0.538 & 0.883 & 7.389 & 1.492 & & & & & \\
\hline Actul DP & 2.51 & 3.64 & 5.8 & 6.721 & 8.68 & & & & & \\
\hline Mexoured TPFsig & 128.4 & & & & 128.8 & & & & & \\
\hline \multirow{2}{*}{\multicolumn{11}{|c|}{ Mosesured Overalle }} \\
\hline & & & & & & & & & & \\
\hline Compund DPA & 2.06 & 3.98 & 5.72 & 7.43 & 8 & & & & 1 & \\
\hline Fictiont & 0.06 & 0.15 & 0.28 & 0.43 & 0.56 & & & & & \\
\hline Acoulerationt & 0 & 0.08 & 0.12 & 0.16 & 0.19 & & & & & \\
\hline Ex tion- & दो & 3.73 & 5.32 & 6.85 & 8.23 & & & & & \\
\hline & & & & & & & & & & \\
\hline
\end{tabular}




\begin{tabular}{|c|c|c|c|c|c|c|c|c|c|c|}
\hline Run: & $1 \quad 804$ & & & - & & & & & $\dot{\square}$ & \\
\hline Tastfluid & . CAF8 & & &. & . & & . & & $:$ & \\
\hline Dalo: & $9 / 5 / 93$ & & & & & & & & 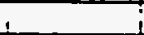 & \\
\hline Time: & $8: 53$ & & & & & & & & & \\
\hline Note & Anatytical & at is usad & & & & & & & & \\
\hline Flow AreatL & 0.003361 & & & & & & & 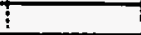 & T & \\
\hline Head Fow Areathe & 0.15951 & & & & & & & $i$ & & \\
\hline Sat.Pressure prig & $|27.3|$ & & & T & $\Gamma$ & & 1 & & & \\
\hline Sat. Temporture fincl) & 151.3 & T) & & 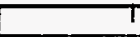 & $\Gamma$ & & & & T & \\
\hline Ug. Donaty-tomns & 8224 & 8231 & 82.01 & 81.071 & 81.701 & 81.78 & 81.67 & 81.84 & 81.8 & 87.59 \\
\hline Lotent Honteratom & 35.7 & 35.751 & $\mathbf{5 5 . 5 2}$ & 35.281 & 35.51 & 35.38 & 35.23 & $\$ 5.88$ & $\$ 5.42$ & $\$ 5.17$ \\
\hline Speciothenteribm: & 0.29 & 0.291 & 0.281 & 0.2021 & 0.202 & 0.292 & 0.292 & 0.232 & 0.2911 & 0.292 \\
\hline Lig. Viscosityominth & 0.466 & 0.466 & 0.45 & 0.443 & 0.445 & 0.446 & 0.44 & 0.446 & 0.48 & 0.41 \\
\hline 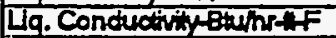 & 0.0065 & 0.029 & 0.0205 & 0.0009 & 0.003 & 0.0063 & 0.003 & 0.0001 & 0.0001 & 0.0002 \\
\hline Uq. Prandeno. & 4.46 & 4.46 & 4.44 & $4.42 !$ & 4.43 & 4.43 & 4.42 & 4.43 & 4.43 & 4.42 \\
\hline Inlat Flow RadG-GPM & 26 & & & 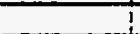 & & & 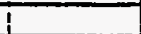 & & 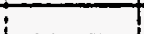 & \\
\hline Outet Lia. Flow Rate-GPM & 0.8 & & & & & & & & & \\
\hline Outtot Vap. Flow Rato-GPM & 1.41 & & & & & & & & & \\
\hline Inlat Temperauref & 147.2 ! & & & & & & & & & \\
\hline Outhet Lia. TompermureF & 150.21 & & & I & & & $T$ & & ! & \\
\hline Out TVap. Tempershref & 146.2 & & $!$ & $T$ & & & T & & $\frac{1}{\vdots}$ & \\
\hline Watop Flow ADOGPM & 1 & i & & $T$ & & & $T$ & & T & \\
\hline Werer hitet Tomp.F & 103.4 & & & i & & & i & & $\mathrm{I}$ & \\
\hline Wewer ovid Tomp.f & 144.1 & & & & & & & & & \\
\hline Inla Gibmmwhe & 516196 & & Tomp & 142.9 & Density & 83.19 & & & i & \\
\hline Suboodingt & 4.11 & & & $i$ & & & & & i & \\
\hline \multicolumn{11}{|l|}{ Fow Obsenvition } \\
\hline & ZZONEI & ZONE2 & ZONE3 & ZONEA & ZONE5 & ZONE6 & IZONET & ZONE8 & ZONES & $201 N=10$ \\
\hline Honlnpertwatnot) & 281.9 & 285.8 & 283.6 & 283.9 & 286.8 & 289.3 & 288.8 & 285 & 2852 & 2852 \\
\hline HontLoss-Wets & 7.6 & 1.7 & 2.3 & 2.4 & 2.4 & 1.8 & 1.2 & 1.7 & 23 & 12 \\
\hline Hos Fiow Euthrie & 4887 & 4SDA & 4916 & 4द्ध一ा & 497 & 5015 & 5008 & 4910 & 4an & 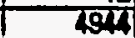 \\
\hline WeTremp. BF & 758.51 & 756.61 & 756.8 & 757.4 & 757.8 & 158 & 757.9 & 756. & 155.5 & T5\$.7 \\
\hline WETTOMo.tAF & 158.7 & 158.81 & 158.6 & 157.6 & -158 & 158.8 & 158.1 & 156.8 & 158.0 & 155.8 \\
\hline WM Tomp. 15 F & 157.81 & 155.8 & 157.5 & 157.7 & 157.8 & 157.6 & 157.8 & 150.81 & 156.8 & 754.8 \\
\hline WalTemp. म1F & 147.2 & 158.2 & 155.2 & 150.8 & 158.8 & 157.7 & 158.4 & 156.81 & 154.0 & 154.4 \\
\hline WCIT Tamp. \&2F & 157.4 & 156.8 & 155.71 & 1571 & 156.7 & 157.6 & 158.4 & 157.51 & 155.4 & 155.1 \\
\hline Instresm Temp.f & 148 & 147.7 & 1402 & 151 & 150.5 & 150.4 & 151 & 150.11 & 100. & 161.4 \\
\hline Avo. Wall Temp.f & 157 & 156.4 & 156 & 157.4 & 1572 & 157.9 & 158.1 & 156.7 & 1556 & 155 \\
\hline Aup.WallDT.F & 8.8 & 8.41 & 6.5 & 6.21 & 6.5 & 7.3 & 6.9 & 6.4 & 5.5 & 3.4 \\
\hline Rommining Subcoolf & 2.2 & & & & & & & & & \\
\hline Ext Quality & -0.02 & 0 & 0.01 & 0.00 & 0.05 & 0.06 & 0.08 & 0.00 & 0.11 & 0.13 \\
\hline EXP.HEthran: & 55821 & 588 & 753.11 & 796.6 & 765.11 & 689.5 & 7262 & $\pi \sqrt{23}$ & 9027 & 7464.1 \\
\hline \multicolumn{11}{|l|}{ COMPUIEOH } \\
\hline Jisock & 658.7 & -67.1 & 67.51 & 678.61 & 65.7 & 6652 & 653.6 & 657.6 & 63.1 & 658.3 \\
\hline Frady (Cudo tube) & 70524 & 1057.7 & 1084.81 & 1071.81 & 10812 & 1008.1 & 1000.2 & 1078.3 & 107.8 & 7069.4 \\
\hline $\sin d y^{2}$ & 8028 & 803.81 & 789.8 & 797.21 & 80201 & 808.1 & 810.1 & 799.21 & 79.8 & 808.8 \\
\hline (स्य) & 742001 & 740001 & 745811 & 746101 & 73007 & 71790 & 70054 & ब165 & $67 / 78$ & 67858 \\
\hline (F) & 1.005 & 7.0051 & 1.266 & 1.4981 & 7.680 & 1.846 & 1.98 & 2100 & 2231 & 2.38 \\
\hline (स्व) & 1ब্তা & $1 \approx$ & 2000 & 24,A1 & 2762 & 300.8 & 320 & 343.5 & 35 & 377.8 \\
\hline \multirow[t]{3}{*}{ (HEOL) } & 1051.5 & 1058.4 & 1061.9 & 1073.6 & 10752 & 1078.3 & 1080.6 & 10565 & $10 \hat{a}$ & 1074 \\
\hline & & & & & & & & & I & \\
\hline & ZONEC & ZONE & ZONE6 & ZONE8 & $201=10$ & & & & & \\
\hline \multicolumn{11}{|l|}{ DP iquid tomp.f } \\
\hline \multicolumn{11}{|l|}{ DPT quid Dansitubmit3 } \\
\hline \multicolumn{11}{|l|}{ Eention-h } \\
\hline Thenured Dppord & -0.091 & 0.591 & 7.0081 & 7.6781 & 7.7981 & & & & & \\
\hline Zro comectionepid & 0.019 & -0.01 & -0.018 & -0.0071 & $=0.007$ & & & & & \\
\hline Compand DPPald & -0.11 & 0.01 & 1.029 & 7.025 & 98081 & & & & $T$ & \\
\hline ACWeD DPA & 2.48 & 3.521 & 5.00 & 8.28 & 8.711 & & & & $i$ & \\
\hline Mecured TfPhig & 127.8 & & & & 120.2 & & & & & \\
\hline \multicolumn{11}{|l|}{ Meacured Overell } \\
\hline & & & & & & & & & & \\
\hline Computed DPA & 204 & 3.83 & 5.5 & 7.3 & 8.58 & & & & & \\
\hline Fratonf & ర.04 & 0.1 & 0.19 & $0 . \mathbf{2}$ & 0.41 & & & & & \\
\hline Acoulerniom & of & 0.06 & 0.09 & 0.11 & 0.13 & & & & & \\
\hline Etorationt & दा & 3.57 & 5.यद्यो & 6.7 & 8.04 & & & & & \\
\hline & & & & & & & & & & \\
\hline
\end{tabular}




\begin{tabular}{|c|c|c|c|c|c|c|c|c|c|c|}
\hline Run: & 3051 & 1 & & . & & - & - & 1 & 1 & \\
\hline Tosffluid & . CAF 8 &. & 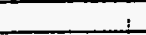 & 1 & & 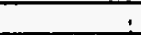 & 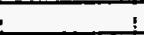 & 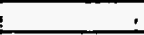 & & \\
\hline Dato: & $915993 !$ & & & & & & & & & \\
\hline Time: & $13: 35$ & & & & & & & & & \\
\hline Note & Anatyical d & this used & & & & & & & & \\
\hline Fow Area-H2 & 0.003361 & & & & & & & 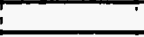 & & 1 \\
\hline Head Fow Aroard? & 0.19655 & & & & & & & & & \\
\hline Sad Ptessura prig & 32721 & : & & & & & & & & \\
\hline Sad. Temperaturof(inlel) & 751.2 & & & +1 & & 1 & & & & \\
\hline Lq. Densiny bmis & $81.99 !$ & 81.841 & 81.761 & 81.681 & 81.781 & 81.81 & 81.05 & 81.821 & 81.821 & 81.28 \\
\hline Leterd Hextevitiom & 35.51 & 35.581 & 35.311 & 35.251 & 35.31 & 35.341 & 35.22 & 35.981 & 35.441 & 34.82 \\
\hline Specilio Hos Eurbmif & 0.2911 & 0.291 & 0.292 & 0.2921 & 0.292 & 0.2921 & 0.29 & 0.2921 & 0.291 & 0.283 \\
\hline Lig. Visooshelom/hhr & 0.45 & 0.446 & 0.446 & 0.448 & 0.445 & 0.4461 & 0.442 & 0.4461 & 0.48 & 0.435 \\
\hline Lig. Conduativity & 0.0205 & 0.02341 & 0.0093 & 0.02031 & 0.0233 & 0.0238 & 0.0202 & 0.00241 & 0.02041 & 0.000 \\
\hline Liq. Prande No. & 4.44 & 4.431 & 4.43 & 4.42 & 4.43 & 4.43 & 4.42 & 4.431 & 4.44 & 4.4 \\
\hline Inlet Fow Rato-GPM & 1.9 & & & & & $\rightarrow$ & & & & \\
\hline Outol Lig. Fow Rato-GPM & 0.41 & i. & & & & & & & & \\
\hline Outlet VaD. Flow Rato-GPMM & 1.31 & & & & & & & & & \\
\hline Inlet Temperaturef & 148.7T & & & & & & & & & \\
\hline Outiet Lig. Temperaturof & 750.41 & & & T & & & & & & \\
\hline Outel Vap. Temparauref & 146.51 & $i$ & & $T$ & & & & & & \\
\hline WEM FOW RSH-GPM & $\square$ & I & 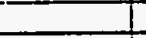 & 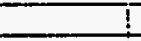 & & i & i & I & & \\
\hline Werer hital Tomp.f & 10211 & i & 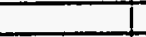 & I & & I & I & 1 & 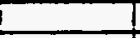 & \\
\hline Welor outed Temp.f & 144.8 & 1 & 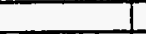 & 1 & & 1 & 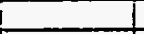 & 1 & 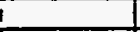 & \\
\hline 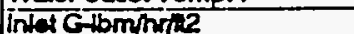 & 3781861 & & Temp & $741.8 \mathrm{II}$ & Donsity & 83.4 & & $i$ & 5 & \\
\hline Subcoolingf & 251 & $i$ & & $i$ & 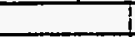 & +1 & I & $\perp$ & $L$ & $\perp$ \\
\hline \multirow[t]{2}{*}{ Fow Observation } & & & & & & & & & & \\
\hline & ZONE1 & ZONE2 2 & ZONE3 & ZONEA : & ZONES & ZONE 6 & ZONE & ZONEB & ZONE9 & ZONie10 \\
\hline Head Input-Walls(net) & \begin{tabular}{|l}
$\mid 287.6$ \\
\end{tabular} & 289.6 & 288.8 & 287 & 285.7 & 288.7 & 285.8 & 284.8 & 288.2 & 288.2 \\
\hline Hoal Loss-Wats & 7.21 & 1.6 & 2.2 & 2.3 & 2.2 & 1.7 & 1.1 & 1.6 & रो & 11.3 \\
\hline Hoal Finethelftine & 4985 & 50201 & 4973 & 49751 & $4 \overline{521}$ & 50041 & 4551 & 49391 & 4531 & 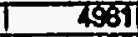 \\
\hline WE TOMP. B 35 & 756.81 & 756.81 & 756.41 & 757.2! & 757.7 & 757.6 & 757.7 & 155.9] & 755.41 & 155 \\
\hline Wal Tomo. ThF & 156.91 & 756.5 & 158.8 & 157.4 & 153.7 & 158.1 & 158.11 & $156.4 T$ & 755.8 & 768 \\
\hline We Tomp. I5F & 157.4 & 158.2 & 157.8 & 157.71 & 157 & 157.4 & 157.6 & 156.81 & 155.61 & 165 \\
\hline WEA TOmp. E1F & 148.7 & 150.61 & 155.4 & 158.8 & 150.0 & 157.6 & 158.8 & 157 & 154.8 & 154.6 \\
\hline W" Tomp. \#2F & 158 & 156.61 & 156 & 156.81 & 156.4 & 157.4 & 158.1 & 157.51 & 155.8 & 155.1 \\
\hline Introem Temp.F & 149.3 & 150.1 & 150.6 & 150.91 & 150.5 & 150.3 & 151.1 & 150.2 & 149.7 & 163 \\
\hline Avg. Wall Temp.f & 1572 & 156.6 & 1562 & 157.31 & 156.8 & 157.7 & 158 & 156.6 & $155 . \mathrm{A}$ & 155.1 \\
\hline Avo. Wallot.F & 7.7 & 6.3 & 5.5 & 6.21 & 6.2 & 7.1 & 6.6 & 6.21 & 5.5 & 1.8 \\
\hline \multicolumn{11}{|l|}{ Romarning Subcooff } \\
\hline Ext Quality & of & 0.02 & 0.01 & 0.07 & 0.09 & 0.11 & 0.73 & 0.15 & 0.18 & 0.2 \\
\hline 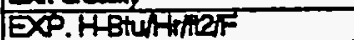 & 647.5 & 800.2 & 9125 & 808.9 & 798.6 & 3025 & 7461 & 797 & 909.3 & 2618.8 \\
\hline \multicolumn{11}{|l|}{ COMPUTEDH } \\
\hline Jallouk & 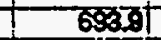 & 687 & 68.8 & 681.6 & 671.8 & 6872 & 63.6 & 657.51 & 6559 & 657 \\
\hline Sady (ado tube) & 107.5 & 1081.11 & T078.3 & 10828 & 1078.4 & 1083.4 & 1088.6 & 107.21 & 107.11 & 10895 \\
\hline $\operatorname{sen} 2$ & 8295 & 806.81 & 801.61 & 8003.81 & 799.6 & 8059 & 80261 & 798.21 & 799.8 & 8426 \\
\hline (RUO) & 64000 & 547481 & 53789 & 5हता & 51808 & 50001 & 49115 & 475401 & 46124 & 46001 \\
\hline$(\mathbf{F})$ & 1.054 & 1.355 & 1.640 & 1.8021 & 2044 & 2.2051 & 2.34 & 2481 & 2.608 & 268 \\
\hline (1) & 138.6 & 172 & 2005.5 & 236.7 & 250.7 & 280 & 2975 & 314.9 & 3000 & 32र \\
\hline \multirow[t]{3}{*}{$(\mathrm{HBO})$} & 10508 & 10726 & 1075.7 & 1079 & 10734 & 1072 & 1076.1 & 1058.11 & 10586 & 1006 \\
\hline & & & & & & & & & & \\
\hline & ZONEZ & ZONEA & ZONEG & ZONIE8 & ZONETO & & & & & \\
\hline DP requid Tomo.f & 79.4 & & & & & & & & & \\
\hline DPLlquid Dentintbmins & 933 & & & & & & & & & \\
\hline Elevetiont & & 4 & & 81 & 9.875 & & & & & \\
\hline Wocured of Psid & 0.028 & 0.827 & 7.365 & 2727 & 2.407 & & & & & \\
\hline Zoro Comections-psid & 0.018 & -0.01 & -0.016 & -0.007 & -0.007 & & & & & \\
\hline correctud DP pited & -0.047 & 0.897 & 1.381 & 2.139 & 2.714 & & & & & \\
\hline Acted DPA & 2.381 & 3.111 & 4.44 & 5.41 & 7.06 & & & & & \\
\hline Wheured ippsig & 1272 & & & & 126.8 & & & & & \\
\hline Aneceured Overall & 1.60 & & & & & & & & & \\
\hline & & & & & & & & & & \\
\hline Computed DPA & 1.83 & 3.26 & 4.56 & 5.79 & 6.9 & & & & & \\
\hline Fictiont & 0.08 & 0.05 & 0.17 & 0.28 & 0.39 & & & & & \\
\hline Accoleration- & 0.03 & 0.05 & 0.07 & 0.08 & 0.1 & & & & & \\
\hline Elevetion l & 1.77 & 3.121 & 4.32 & 5.631 & 6.41 & & & & & \\
\hline & & & & & & & & & & \\
\hline
\end{tabular}




\begin{tabular}{|c|c|c|c|c|c|c|c|c|c|c|}
\hline Runt & 8051 & $i$ & • & & & . & $\cdot$ & : & & \\
\hline Tasfluid & CAFB & 1 & 1 & & 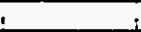 & & & E & - & \\
\hline Date: & ब/593 & & & & & & & & & \\
\hline Time: & $13: 45$ & & & & & & & & & \\
\hline Nole & Analyical & ofis used: & 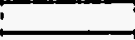 & & & & & & & \\
\hline Flow Aroath & 0.00356 & & & & & & & & & 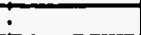 \\
\hline Hod Fow AYarde & 0.19655 & & & & & & & & & \\
\hline Sa. Prossurepsig & -126.6 & & & & $T$ & & 7 & & & 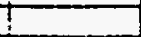 \\
\hline Sait. Temperatur of (inlet) & 150.91 & -1 & T & & & & $\mathrm{T}$ & I & I & T \\
\hline Dg.Densinytomines & 82.071 & 81.71 & 81.82 & 81.68 & 81.78 & 81.81 & 81.681 & 81.861 & 81.8 & 81.16 \\
\hline Letert Hed exphom & 35.58 & 35.271 & 35.58 & 35.25 & 35.39 & 35.341 & 35.25 & 35.391 & 35.42 & 34.82 \\
\hline Speciso Hectenthemf & 0.291 & 0.292 & 0.292 & 0.292 & 0.282 & 0.2921 & 0.292 & 0.2911 & 0.291 & 0.291 \\
\hline Lug. Visoosin/bin/hir & 0.451 & $0 . \overline{441}$ & 0.446 & 0.44 & 0.445 & 0.4461 & 0.443 & 0.447 & 0.448 & 0.432 \\
\hline Lq. Conduetiventhr fF & 0.0255 & 0.00031 & 0.029 & 0.0203 & 0.0003 & 0.0231 & 0.020 & 0.020 & 0.0201 & 0.0000 \\
\hline Lig.Psanatt No. & 4.44 & 4.42 & 4.43 & -4.42 & 4.43 & 4.431 & 4.42 & 4.43 & 4.43 & 4.4 \\
\hline Inlot Flow Rate-GPM & 1.1 & & & & & - i & \pm & & & \\
\hline OutlolLg. Flow Rate-GPM & 0.4 & & & & & & & & & \\
\hline Outlol Vap. Flow Rate-GPM & 0.91 & & 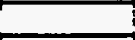 & 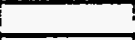 & 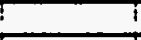 & $i$ & & & 1 & \\
\hline Inlot Tomperaturef & 148.3 & & & & & & & & & \\
\hline Oifollle. Temparatur of & 150.4 & & & & 1 & & 1 & 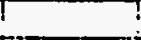 & & \\
\hline Outol Vap. Tompereturof & 146.71 & $T$ & & 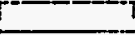 & $T$ & $I$ & T & & & \\
\hline WDA PlOW RAOGPM & & 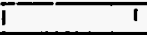 & $\pi$ & I & i & ! & i & & & i \\
\hline Wein intet Tompoff & 1021 & 1 & $\mathrm{I}$ & 1 & 1 & 1 & 1 & L & I & \\
\hline Wabr ovited Temp,f & 144.8 & i & 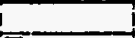 & 1 & 1 & I & I & 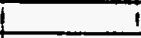 & 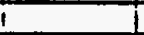 & \\
\hline Thet Glbminth2 & 2202561 & & Temp & 139 & Densidy & 83.91 & & & & \\
\hline Subooding $F$ & 26 & 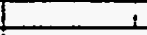 & & & 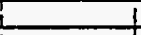 & 1 & I & $i$ & & \\
\hline \multirow[t]{2}{*}{ Flow Observation } & & & & & & & & & & \\
\hline & ZONE 1 & ZONE2 & ZONE3 & ZONEA & ZONES & ZONE6 & ZONE 7 & ZONE8 & ZONES & LONETO \\
\hline Hoal Inpor-Watis(not) & 293.5 & 255.3 & 288.4 & 290.4 & 288.8 & 280.7 & 291.5 & 290.4 & 287.8 & 288.8 \\
\hline Healloss-Wats & 7.1 & 1.61 & 2.2 & 2.2 & 22 & 1.6 & 1.11 & 1.6! & 2 & 11.2 \\
\hline 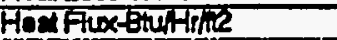 & 5080 & 5ारा & 4930 & 5034 & 5003 & 5021 & 50531 & 5034 & 450 & 5006 \\
\hline Wartemp & $158.6]$ & 158.4 & 15621 & 757.1 & 757.6 & 757.61 & 757.8 & 156.11 & 755.4 & 155 \\
\hline WRTomp. IfF & 156.7 & $15 \overline{6}$ & 756.67 & 157.1 & 157.61 & 758.7 & 158.1 & 158.6] & 155.8 & 150 \\
\hline WCITEmp. $15 F$ & 157.4 & 155.51 & 158.1 & 157.7 & 150.7 & 1571 & 157.4 & 158.81 & 155.6 & 154.8 \\
\hline WEN Tomp. IF & 148.8 & 158.21 & 155.1 & 158.5 & 158.8 & 157.4 & 158 & 1571 & 164.7 & 157.2 \\
\hline WeITomp. & 157.8 & 156.41 & 155.6 & 156.5 & 1562 & 157 & 158.2 & 157.71 & 1552 & 154.0 \\
\hline Intrean Tompof & 148.8 & 150.8 & 1502 & 150.8 & 150.4 & 150.31 & 150.8 & 1501 & 149.8 & 153.6 \\
\hline Avg. Wall Tomp.f & 157.11 & 156.3 & 155.9. & 157.1 & 156.7 & 157.5 & 157.91 & 156.71 & 155.5 & 155 \\
\hline Avo. Wall DT.F & 8 & 5.2 & 5.4 & 6 & 6.1 & $7 !$ & 6.8 & 6.5 & 5.3 & 1.2 \\
\hline \multicolumn{11}{|l|}{ Remaining Subcooff } \\
\hline Exot Oualizy & 0.02 & 0.051 & 0.09 & 0.13 & 0.17 & 0.21 & 0.24 & 0.28 & 0.32 & 0.36 \\
\hline 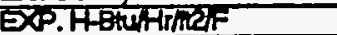 & 636.81 & 980.81 & 917.4 & 8428 & 82431 & 717.6 & 746.21 & $773 !$ & 937 & 4330 \\
\hline \multicolumn{11}{|l|}{ COMPUTEDH } \\
\hline Jizouk & 735.81 & 728.81 & 689.0 & 69.41 & 6821 & 68291 & 688.81 & 600.4 & 678.11 & 636 \\
\hline इadyi(ado itbo) & 1079.6 & 10001 & $70 \%$ & 90883 & 10852] & 1008.61 & 1091.3 & 1088.7 & $10 \pi$ & 1105.8 \\
\hline $\operatorname{sen} 2$ & 824.0 & 8211 & 803.6 & 810.6 & 8054 & 80721 & 813.7 & 8081 & 800.7 & 80 \\
\hline (PUO) & 31050 & 313201 & 2952 & 28894 & 276009 & 202021 & 25182 & 237011 & 20309 & 2078 \\
\hline (F) & 1.289 & 1.669 & 2.018 & 2381 & 2515 & 2.721 & 2.898 & 3.0051 & 3.5 & 3.300 \\
\hline$(1+10)$ & 106 & 137.7 & 168.9 & 1882 & 207.3 & 2242 & 230.1 & 254.11 & 267.6 & 278.7 \\
\hline \multirow[t]{3}{*}{ (1BOL) } & 10792 & 10558 & 1078. & T0683.3 & 1079.6 & 1080.4 & 1087.5 & 105 & 107.5 & 1096 \\
\hline & & & & & & & & & & \\
\hline & ZONEZ & ZONEA & ZONE 6 & ZONES8 & ZONETO & & & & & \\
\hline DPliqud temp.f & 79.7 & & & & & & & & & \\
\hline DPUquid Denahy & $932 \pi$ & & & & & & & & & \\
\hline Elovion & & 4 & 6 & 8 & 9.875 & & & & & \\
\hline Meamuredopysh & 0.083 & T.077 & 3.757 & 2617 & 3.076 & & & & & \\
\hline Zero conedions-psid & 0.079 & -0.011 & $=0.06$ & -0.007 & $=0.007$ & & & & & \\
\hline Cormeand DP Pridd & 0.014 & 1.087 & 1.773 & 2.024 & 3.250 & & & & I & \\
\hline Acter DPA & 2.27 & 2.871 & 3.75 & $4.6 n$ & 5.22 & & 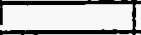 & & I & \\
\hline Meaned TPSpiq & 126.6 & 1 & & & 125.9 & & & & & \\
\hline Masured Overall- & 1.24 & & & & & & & & & \\
\hline & & & & & & & & & & \\
\hline ComputedDPI & 1.54 & 2601 & 3.7 & 4.62 & 5.46 & & $L$ & & & \\
\hline Frotiont & 0.02 & 0.06 & 0.1 & 0.17 & 024 & & & & & \\
\hline Acoster ion- & 0.01 & 0.03 & 0.04 & 0.05 & 0.081 & & & & & \\
\hline Eloviont & 7.51 & 2.87 & 3.56 & 4.4.11 & 5.151 & & & & & \\
\hline
\end{tabular}




\begin{tabular}{|c|c|c|c|c|c|c|c|c|c|c|}
\hline Runt & 8071 & i & - & 1 & 1 & ' & & & . & •• \\
\hline Toet Fluid &. $\mathrm{CAF8}$ & & & & • & 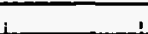 & & & & \\
\hline Date: & $9 \sqrt{20} 93 !$ & & & & & & & & & \\
\hline Time: & $9: 53$ & & & & & & & & & \\
\hline Note & Analytical c & dt is used & & & & & & & & \\
\hline Flow Areatl2 & 0.003651 & & & & & & & & & \\
\hline Hod Fow Area-te & 0.1965 & & & & & & & & & \\
\hline SaI. Pressurełsig & 128.31 & & & & & & & & & \\
\hline Sall Tomperatro finlet) & 751.81 & $\cdots+\cdots$ & $1^{-}$ & & 1 & די-- & 1 & $\mathbf{T}$ & & \\
\hline Lq. Density & 81.721 & 81.741 & 81.7 & 81.611 & 81.051 & 81.631 & 81.591 & 81.871 & 81.08 & 81.61 \\
\hline Latent Hexthitom & 35.281 & 35.81 & 35.271 & 35.191 & अ.21 & 35.21 & $\$ 5.17$ & 35.231 & 35.25 & 35.18 \\
\hline 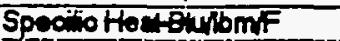 & 0.202 & 0.2921 & 0.2321 & $0.292 ;$ & 0.2921 & 0.221 & 0.2821 & 0.2921 & 0.282 & 0.292 \\
\hline Lig. Viscosidylomithr & $0 . \overline{441}$ & 0.444 & 0.44 & 0.442 & 0.442 & 0.442 & 0.441 & 0.4431 & 0.443 & 0.442 \\
\hline Liq. Conduating Btu/hr-afF & 0.02031 & 0.02031 & 0.0233 & $0.0202 !$ & 0.0202 & 0.0029 & 0.0202 & 0.0038 & $0.0 \times 23$ & 0.0292 \\
\hline Lio. Prand No. & 4.42 & 4.431 & 4.42 & 4.42 & 4.421 & $4.42 !$ & 4.42 & $4.42 !$ & 4.42 & 4.42 \\
\hline Inlot flow Rato-GPM & $5 !$ & & & & & & & & & \\
\hline Outlat Liq. Fow Roto-GPM & 0.9 & & & & & & & & & \\
\hline Outlet VaD. Flow Rate-GPM & 2.41 & & & & & $!$ & & & & \\
\hline Inlet Temporaturef & 149.81 & & & & & & & & & \\
\hline OutetLg. Temporaturef & 150.21 & & & & & & & & & \\
\hline OUtol Vap. TomperaureF & 147.81 & i & t! & & $T$ & & & 1 & 1 & \\
\hline $\bar{W}$ Lior Fiow REA GPM & $\overline{1}$ & $\mathrm{~T}$ & i & 1 & it & $T$ & & $T$ & T & \\
\hline We tor hiot Temp. f & 911 & $i$ & i & $i$ & $i$ & T & i & ! & ! & \\
\hline Welorout Tome.f & 140.5 & I & 1 & & i & 1 & & i & I & \\
\hline Intet Gibmintate & $882214 \bar{i}$ & & Tomp & $147.6 ; \mathrm{C}$ & iDonsity & $82.32 i$ & & 1 & 1 & \\
\hline Subcoolingf & 2 & & & & & +1 & & i & i & \\
\hline Flow Observation & & & & & & & & & & \\
\hline & ZONET & ZONE2 & ZONE3 & ZONE4 L & ZONE5 & ZONE6 & ZONE7 & ZONE & ZONE9 & ZONE $=10$ \\
\hline Heat input-Walls(not) & 574.6 & 571.4 & 580.8 & 576.4 & 575.4 & 579.4 & 573.2 & 575.7 & 576.8 & 580.2 \\
\hline Hoal Loss-Watts & 8.61 & $2 !$ & 27 & 2.71 & 2.7 & 2.11 & 1.3 & $2 !$ & 2.5 & 13.8 \\
\hline Hod flox-BtuHrnL & 99:0! & 99051 & 10050 & 99921 & 9974 & 10044 & 99381 & 979! & 9988 & 90057 \\
\hline 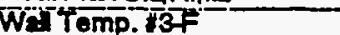 & 158.31 & 150.81 & 159 & 559.3 & 16021 & 16121 & iEO5 & 1593 & 158,0 & 156 \\
\hline Wa Temp. Iff & 159.21 & 158.81 & 160.1 & 159.8 & 160.7 & 167.81 & 160.9 & 160.51 & 159.6 & 760.1 \\
\hline Wal Temp. 15 F & 159.31 & 157.61 & 161.7 & 159.81 & 159.61 & 1502 & 160.2 & 159.61 & 158.6 & 156.8 \\
\hline Well Tomp. IF & 149.8 & 158.6 & 157.6 & 1582 & 159.2 & 160.61 & 167.1 & 159.81 & 157.8 & 165.2 \\
\hline Well Tomp. IRF & 160.1 & 158.61 & 158.8 & 158.8 & 159 & 160.61 & 161 & 160.81 & 158.8 & 156.6 \\
\hline Inteam Tomp.f & 150.7 & 150.6 & 150.8 & 157.3 & 151.11 & 15121 & 151.4 & 151 & 150.9 & 151.3 \\
\hline Avg. Wall Tomp.F & 1592 & 158.8 & 158.7 & 159.31 & 159.6 & 160.81 & 160.7 & 9601 & 158.7 & 157 \\
\hline Avg. Wall DT.F & 8.1 & 7.8 & 7.5 & 7.5 & 8 & 9.21 & 8.9 & 8.5 & 7.4 & 5.3 \\
\hline Aomaining Subcoof $F$ & 0 & & & & & & & & & \\
\hline Ext Qualay & of & $0 . \infty 2$ & 0.03 & 0.05 & 0.07 & 0.081 & 0.11 & 0.12 & 0.14 & 0.15 \\
\hline Exp. HBturtiner & 1233.51 & 1277.7 & 3346.5 & 1328 & 1239.1 & 1005.1 & 1117.5 & 1167.3 & 1357 & 1910.1 \\
\hline COMIRUIEDH & & & & & & & & & & \\
\hline Jaraxk & 7008,3 & S51.4 & 935.8 & 935.91 & 915.2 & 9ारता & \$5.8 & 8881 & 883.81 & 8827 \\
\hline Sedvi(Cudotubo) & 1546.3 & 1543.31 & 1559.7 & 1562.79 & 756274 & 157.5 & 1588.81 & 1571.24 & $15 \% .17$ & 7586.6 \\
\hline $5 \operatorname{sod} 2$ & 136.7 & 1300.61 & $1325 \sqrt{4}$ & 1324.41 & 1321.81 & 1297.31 & 1205.8 & 1299.41 & 1304.4 & 13173 \\
\hline (FU) & 144085 & 1439391 & 141160 & 1398251 & 136017 & 1342751 & 1920041 & 1291251 & 120584 & 1245W \\
\hline (7) & 1.036 & 1.293 & 1.5611 & 1.787 & 1.894 & 2.0311 & 2.153 & 2.2711 & 2.379 & 2475 \\
\hline (1) & 275 & 35261 & 4201 & 474.21 & 517 & 554.51 & 588.11 & 610.71 & 640 & 675.7 \\
\hline (HOIL) & 15462 & 1537.11 & 15023 & 1548 & 1543. & 1548.2 & T540.8 & $1538.6 !$ & 1537.5 & T6766 \\
\hline & & & & & & & & & & \\
\hline & $20 N i=2$ & ZONE4 & ZONE6 & ZONES & ZONETO & & & & & \\
\hline DP hquid Tomp.F & 80 & & & & & & & & & \\
\hline DPLquid Denaly tom/1/3 & 93.2 & & & & & & & $\mathrm{I}$ & & \\
\hline Elovition- & 2ा & का & 6) & 81 & 9.875 & & & & & \\
\hline Whaned ofysid & 0.108 & 0.0351 & 0.938 & 1.5011 & 7.9391 & & & & & \\
\hline Zero Correctionoped & -0.06 & 0.083 & -0.0531 & -0.01 & 0.031 & & & & & \\
\hline Corrocted DPpid & 0.148 & $=0.001$ & 1.021 & 1.5111 & 1873 & $T$ & & f & & \\
\hline Acten DPA & 200 & $4 \pm 0$ & 5.05 & 6.47 & 7.87 & $\mathrm{i}$ & & i & & \\
\hline Masured TPPsig & 128.8 & & & & 128.1 & & & I & 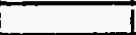 & \\
\hline Matured overallt & 0.35 & & & & & & & 1 & 1 & \\
\hline & 1 & & & & & & & & & \\
\hline Computad DP, & 2.16 & 4.12 & 6.03 & 7.94 & 9.75 & & & & & \\
\hline Friction-1 & 0.16 & 0.47 & 0.73 & 9.11 & 7.52 & & & & & \\
\hline Accaterationt & 0.15 & 0.28 & 0.38 & 0.67 & 0.55 & & & & & \\
\hline Everion-t & 1.85 & 3.43 & 4.33 & 6.37 & 7.68 & & & + & & \\
\hline & & & & & & & & $T$ & & \\
\hline
\end{tabular}




\begin{tabular}{|c|c|c|c|c|c|c|c|c|c|c|}
\hline Runt & $808 \mathrm{i}$ & $\mathrm{i}$ & t & 1 & - & . & & - & - & \\
\hline Tost Fluid &. CAF8 & & & 4 & a & 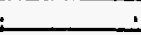 & & & & \\
\hline Dato: & 92013 & & & & & & & & & \\
\hline Time: & $12: 311$ & & & & & & & & & \\
\hline Note & Analytical d & dis used & & & & & & 5 & & \\
\hline Flow Aroa-ti2 & 0.003561 & & & & & & & & & \\
\hline Hoal Fow Aro:-12 & 0.19695 & & & & & & & & & \\
\hline Sot.Prosiure pig & - T27.9i & T & & & T & 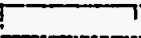 & & & & \\
\hline Sar. Temporatur fininat) & 751.6 & - i & & & & $r$ & & \rceil & $\tau$ & \\
\hline Liq. Dentivitomino & 81.9 & 81.881 & 81.78 & 81.031 & 81.871 & 81.741 & 81.57 & 81.681 & 81.7 & 81.47 \\
\hline Lent Her exprom & 35.42 & 35.411 & 35.31 & 35.21 & 35.28 & 35.31 & 35.151 & 35.251 & 35.27 & $\$ 5.08$ \\
\hline Specino Horlotulibmf & 0.291 & 0.2911 & 0.292 & 0.2221 & 0.252 & 0.2921 & 0.282 & 0.3 & 0.282 & 0.203 \\
\hline Liq. Viscosity tbmithr & 0.418 & $0 . \overline{471}$ & $0 . \overline{44}$ & $0.442]$ & 0.443 & 0.44 & 0.449 & 0.4401 & 0.44 & 0.169 \\
\hline 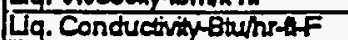 & 0.02341 & 0.0294 & 0.0293 & 0.0292 & 0.02331 & 0.02031 & 0.002 & 0.023 & 0.003 & 0.0091 \\
\hline Liq. Prandll No. & 4.43 & 4.43 & 4.43 & 4.421 & 4.42 & 4.43 & 4.42 & 4.421 & 4.42 & 4.41 \\
\hline Inlot Flow Pato-GPM & 4.3 & & & & & & & i & & \\
\hline Outhol Lig. Fow Rate-GPM & 0.9 & & & & 1 & & & & & \\
\hline Outlet Vap. Fow Rato-GPM & 2.11 & & & & & & & t & & \\
\hline Inlot Temperaturef & 149 & & & & & & & & & \\
\hline Oif ot Cla. Tompanturer & 150.31 & & & & 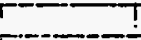 & $?$ & & $T$ & $T$ & \\
\hline 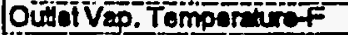 & $747.7 \pi$ & & & & I & I & & & & \\
\hline WASP FOW PAT-GPM & 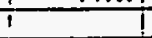 & 7 & $i$ & $i$ & $i$ & $i$ & & $\mathrm{~T}$ & $\mathbf{i}$ & \\
\hline Wher hrot Tomp.F & 90.51 & 1 & $I$ & 1 & $I$ & 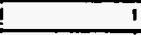 & & I & I & \\
\hline Wher outer Temp.f & 140.5 & 1 & 1 & -1 & 1. & -1 & 1 & I & I & \\
\hline Inlot Glomh & $8470<7$ & & Temp & 146.41 & Doneity & 8264 & & $\mathbf{I}$ & i & \\
\hline Subcooling $f$ & 2.61 & & & & & $\mathrm{i}$ & & 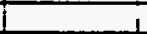 & 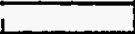 & \\
\hline \multirow{2}{*}{ Flow Obsenvation } & & & & & & & & & & \\
\hline & :ZONEI & ZONE2 & ZONE3 & ZONE4 12 & ZONE5 & ZONE6 & ZONE? & ZONE8 & ZONE9 & $20 N=10$ \\
\hline 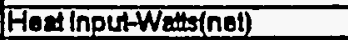 & 579.8 & 576.9 & 583.6 & 580.7 & 584 & 585.9 & 579.2 & 5826 & 580.8 & 5929 \\
\hline Heat Loss-Watts & 8.51 & 1.9 & 2.7 & 2.71 & 27 & 2 & 1.3 & 2 & 2.5 & 13.7 \\
\hline Hest Finestulthine & 100501 & 100001 & 10116 & 10058 & 10123 & 10756 & $100 \%$ & 10009 & 10068 & 90009 \\
\hline War remo. 35 & -15821 & -759.81 & -158.81 & 753.41 & 780.41 & नाया & 760.7 & 759.31 & 758.9 & -157.1 \\
\hline WeTtomp.tif & 159.11 & โ58.21 & 159.81 & 155.8] & 760.8 & 16-4. & 161 & T60.01 & 750.7 & 160 \\
\hline WeV Tomp. $15 f$ & 159.8 & 157.81 & 160.8 & 159.91 & 159.8 & 160.81 & 160.8 & 159.51 & 158.7 & 156.8 \\
\hline WhITEmo. IIF & 149 & 158.81 & 157.11 & 158.4 & 1592 & 160,4 & 161.8 & 160 & 157.7 & 155.4 \\
\hline Well Temo. I2F & 160 & 158.81 & 158.2 & 158.91 & 159.11 & 160.81 & 161.4 & 160.91 & 158.6 & 157.1 \\
\hline Instreem Tomo,f & 749.8 & 949.81 & 150.5 & $1512 \mathrm{~T}$ & 1511 & 750.61 & 151.51 & 150.9 & 150.8 & 152 \\
\hline Avg. Wall Tomo.F & 159.2 & 158.7 & 158.5 & 159.41 & 159.7 & 160.8 & 160.9 & 160.11 & 158.7 & 157.3 \\
\hline Avo. Wall DT. & 8.9 & 8.3 & 7.5 & 7.71 & 8.2 & 9.8 & 9 & 8.71 & 7.5 & 4.8 \\
\hline \multicolumn{11}{|l|}{ Remaining Subcooff } \\
\hline Exot Quality & of & 0.02 & 0.04 & 0.06 & 0.08 & 0.1 & 0.12 & 0.14 & 0.16 & 0.18 \\
\hline EXP. HEturAmer = & 1129.8 & 1205.1 & 934.6 & 13025 & 1231.9 & 1040 & 1117.3 & 1160.3 & 134.1 & $20 \leq 25$ \\
\hline \multicolumn{11}{|l|}{ COMPUIEDH } \\
\hline Jalouk & T017.81 & \$8.81 & "S5T.6I & \%5.5. & 929.ర1 & 92281 & $9\{6.8]$ & उसा.8 & 850 & 897.8 \\
\hline Szadit(Cudo & 1549.81 & 154211 & 7559.11 & 15.21 & 1568.4 & 1570.41 & 1573.4 & 7574.61 & 7574.1 & 1590.4 \\
\hline $\operatorname{sen} 1 / 2$ & 1315.17 & 7377.41 & 7327.5 & 13291 & 13332 & 133251 & 1331.5 & 1300.01 & 13021 & 138.0 \\
\hline (म्थि) & 123778 & 1228991 & 121122 & 1192:11 & 110727 & 1138201 & 112204 & 1091811 & 106007 & 105270 \\
\hline (F) & 1.005 & 1.282 & $1.5 \mathrm{rg}$ & 1.785 & 1.96 & 2176 & 2215 & 23761 & 2456 & 250 \\
\hline (F) & 243.81 & 340.11 & 3828 & 43201 & 476 . & 5126 & Eकम.8 & 57. & 6045 & $\approx 280$ \\
\hline \multirow[t]{3}{*}{ (FOOL) } & 1541.3 & 1537.9 & 1551.4 & 15528 & 1554.8 & 1552 & 1551.8 & 15484 & 15400 & 15580 \\
\hline & & & & & & & & & & \\
\hline & 2ONE2 & ZONET & ZONE 6 & ZONE8 & ZONE10 & & & & & \\
\hline \multicolumn{11}{|l|}{ DSTlould Tomp.f } \\
\hline \multicolumn{11}{|l|}{ DPUlquid Densith tomit3 } \\
\hline \multicolumn{11}{|l|}{ Elevention } \\
\hline \multicolumn{11}{|l|}{ Measured Dop pald } \\
\hline \multicolumn{11}{|l|}{ żero corredions psta } \\
\hline \multicolumn{11}{|l|}{ Comecied DPpsid } \\
\hline Actal OPA & 1.97 & 4.01 & 4.88 & 8.081 & 7.44 & T. & & $\mathrm{T}$ & I & \\
\hline Mesucured if Proth & 127.8 & & & 1 & 127.8 & & & & & \\
\hline Maneured Overallit & 0.18 & 1 & $\perp$ & 1 & 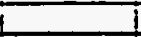 & & & & & \\
\hline & & & & & & & & & & \\
\hline ComoutedDPs & 2. & 3.96 & 5.78 & 7.58 & 9.20 & & & & & \\
\hline Fretiont & 0.12 & 0.32 & 0.58 & 0.9 & 1.24 & & & & & \\
\hline Accoleration & 0.12 & 0.3 & 0.31 & 0.38 & 0.48 & & & & & \\
\hline Elevation-t & $7.8 \pi$ & उ.दा & 4.89 & 6.31 & 7.6 & & & & I & \\
\hline & & & & & & & & & & \\
\hline
\end{tabular}




\begin{tabular}{|c|c|c|c|c|c|c|c|c|c|c|}
\hline Run: & $1 \quad 8091$ & $i$ & - & . & - & & . & . & . & \\
\hline Toslfuid & . C4F 8 & & & & & & & & & \\
\hline Date: & 920931 & & & & & & & & & \\
\hline Time: & $13: 39 !$ & & & & & & & & & \\
\hline Note & Analytical & dt is used & & & & & & & & \\
\hline Fow Area-12 & 0.003651 & & & & & & & & & \\
\hline Heat flow Areaft? & 0.1595 & & & & & & & & & \\
\hline Sa. Pressuro Psig & 127.4 & 4 & T & ? & & ! & & & & \\
\hline Sed Temperaur fo(intot) & 151.31 & & 7 & $i$ & & 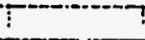 & & & T & \\
\hline Liq. Densintomins & 82011 & 81.921 & 81.76 & 81.71 & 81.74 & $81.72 !$ & $81 . \overline{81}$ & 81.76 & 81.78 & 81.18 \\
\hline 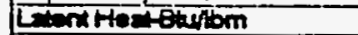 & 35.521 & 35.441 & 35.31 & 35.271 & 35.3 & 35.281 & 35.19 & 35.31 & 35.31 & 34.82 \\
\hline 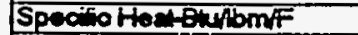 & 0.2811 & 0.2911 & 0.252 & 0.2921 & 0.292 & 0.2921 & 0.292 & 0.252 & 0.292 & 0.239 \\
\hline Lig. Viscostiom/hr & 0.46 & $0.448 i$ & 0.445 & $0 . \overline{444 i}$ & 0.444 & $0 . \overline{444 i}$ & 0.442 & 0.446 & 0.446 & 0.482 \\
\hline Lig. Conductivitstums-af & 0.0295 & 0.0294 & 0.0293 & 0.02931 & 0.0293 & 0.02931 & 0.0292 & 0.0203 & 0.0009 & 0.00209 \\
\hline Lig. Prandi No. & 4.44 & $4.44 i$ & 4.43 & 4.42 & 4.43 & 4.42 & 4.42 & 4.43 & 4.43 & 4.4 \\
\hline Inlot Fow Rato-GPM & 3.5 & & & & & & & & & \\
\hline Outed Liq. Fow Reto-GPM & 0.8 & & & & it & + & & & & \\
\hline Outiet Vap. Fow Rato-GPM & 1.9 & & & & & & & & & \\
\hline Inlot Tompereduref & 148.3 & & & & & & & & & \\
\hline Oúfollig Temperstrof & 750.61 & & & & & & & & & \\
\hline Oufl Vap. Tomperefor & 147.7 ! & & & & & & & & & 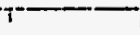 \\
\hline WaOP FIOW RET-GPM & -1 & $i$ & $i$ & $i$ & $i$ & & & & $\mathrm{~T}$ & i \\
\hline Wan hite Tomp.f & 92.11 & 1 & 1 & 1 & 1 & 1 & 1 & T & $T$ & T \\
\hline Weitr outel Tomp.f & 140.7 & 1 & 1 & -1 & $1 \ldots$ & & $i$ & $i$ & 1 & 1 \\
\hline 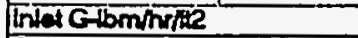 & 690537 & & Tomp & $946.7 i$ & Donsidy & $82.67 i$ & &. & 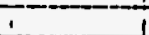 & 1 \\
\hline Subcooling $f$ & 31 & + & 1 & 1 & & & 1 & & & + \\
\hline \multirow[t]{2}{*}{ Alow Observation } & & & & & & & & & & \\
\hline & ZONET & ZONE2 & ZONE3 & ZONEA & ZONE5 & ZONE6 & ZONE7 & ZONE8 & :ZONE9 & ZONETO \\
\hline Heat Input-Watts(nel) & 57.7 & 576.7 & 578 & 576.6 & 58.2 & 585.8 & 578.1 & 1) 580.7 & it 575.5 & 575 \\
\hline Hoat Loss-Wats & 10.3 & 2.41 & 3.2 & $3.3 !$ & 3.3 & 2.51 & 1.6 & 24 & 3.1 & 17.1 \\
\hline 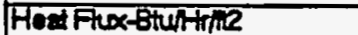 & 10014 & 9997 & 10018 & 90051 & 101871 & $1015 \times 1$ & 10021 & 100561 & ख़ा & 967 \\
\hline Wh Temp. BF & 157.91 & 159.6 & 158.8 & 159.11 & 160.6 & 161.31 & 160.7 & 15921 & -159 & 957.5 \\
\hline W'TTemp. $14 \mathrm{~F}^{-}$ & 1591 & 1581 & 159.8 & 159.7 & 160.87 & 167.21 & 760.8 & $7 \overline{60.81}$ & 759.51 & TBD.5. \\
\hline WEITEmp. $15 F$ & 159.11 & $15 \overline{31}$ & 760.8 & 159.71 & 159.6 & 160.81 & 160.7 & 159.81 & 758.6 & 157 \\
\hline WEITOM. AIF & 148.81 & 158.81 & 157 & 158.21 & 1591 & 100.4 & 1612 & 159.91 & 157.8 & 155.8 \\
\hline WEIT Temp. I2F & 159.8 & 158.41 & 157.8 & $158.6 i$ & 159 & 160.51 & 161.5 & 100.81 & 158.5 & 1672 \\
\hline Intresn Tomp.f & 148.2 & 148.7 & 150.5 & 150.8 & 150.6 & $150.7 i$ & 167.3 & 150.51 & 165.51 & 1636 \\
\hline AYg. Wall Tomp.F & 150 & 158.61 & 158.3 & 1592 & 159.6 & 160.8 & 1671 & 9601 & 158.7 & 157.6 \\
\hline AYo. Wn DI DT.F & 9.3 & 8.41 & 7.4 & 7.9 & 8.6 & 9.6 & 92 & 9 & 7.7 & 3.5 \\
\hline Remeining Subcooff & 0.11 & & & & & & & & & \\
\hline Ex Quality & $0 !$ & 0.02 & 0.05 & 0.07 & 0.1 & 0.12 & 0.14 & 0.17 & 0.19 & 0.22 \\
\hline EXP. HBWUATner & 1074.2 & 1186.81 & 1359.1 & 1267.5 & 1186.3 & 10532 & 1083.7 & 1114.1 & 1287.4 & 2808 \\
\hline \multicolumn{11}{|l|}{ COMPUTEDH } \\
\hline Jafor & TOCथIT & 7007.81 & 978.8 & 357.81 & $\$ 50.8$ & \$35.51 & 925.9 & -378 & -910.7 & क्टि13 \\
\hline 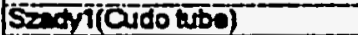 & TE5 & $15 \overline{38} .81$ & 1550.4 & 1553.4 & 1585.6 & 15681 & 1566.21 & $7563.1 T$ & -1559.5 & 1500.8 \\
\hline $\sin 2$ & 1300.1 & 1300.21 & 1318.9 & 1319.81 & 135251 & 13331 & 132721 & 132281 & 1284.6 & 1317.2 \\
\hline (F्यंत) & 1100859 & 997181 & 98010 & 958341 & 931881 & 907851 & 88810 & 857111 & 83208 & 83182 \\
\hline$(\boldsymbol{F})$ & 1.005 & 1.3391 & 1.65 & 1.8821 & 2.087 & 2.257 & 2.4081 & 2.5521 & 2.682 & 2.760 \\
\hline$(\mathrm{H}, \overline{0}$ & 206.3 & 275 & 347.7 & 3828 & 420.8 & 764.4 & 45 & 524.81 & $551.5 \mid$ & 570.8 \\
\hline \multirow[t]{3}{*}{$(\mathrm{HBOI})$} & 15328 & 1535 & 1540 & 154521 & 1554.7 & 1554.4 & 15496 & 1543.11 & 15362 & 1568 \\
\hline & & & & & & & & & & \\
\hline & ZONES & ZONE4 & 20186 & ZONE8 & ZONETO & & & & & \\
\hline \multicolumn{11}{|c|}{ DP hquid Tomp.F } \\
\hline \multicolumn{11}{|c|}{ DPLhuid Density bmin $\quad 93.171$} \\
\hline \multicolumn{11}{|l|}{ Envationa } \\
\hline Masured bPFsid & $0.772]$ & 0.0351 & 1.251 & दणठउ। & 2558 & & & & & \\
\hline Zaro Comections psid & -0.042 & 0.0831 & -0.0531 & $0.0 \mathrm{~T}$ & 0.067 & & $?$ & & & \\
\hline Cormend Dipidd & 0.टाबा & -0.0011 & 1.304 & 20031 & 2535 & & $i$ & & & \\
\hline Acterom & 1.92 & 4द3! & 4.57 & 5.501 & 6.84 & & i & & $!$ & \\
\hline Meinoured Tfupig & 127.4 & & & 1 & 127.6 & & $\mathrm{i}$ & & & \\
\hline \multirow[t]{2}{*}{ Mreoured Overall } & -0.35 & 1 & & & & & I & & & \\
\hline & & & & & & & & & & \\
\hline Comeuted DPA & 1.97 & 3.56 & 5.06 & 6.54 & 7.92 & & & & & \\
\hline Friction-t & 0.09 & 3.27 & 0.53 & 0.85 & 1.21 & & & & & \\
\hline Acoslerstion & 0.08 & 0.77 & 0.23 & 0.20 & 0.35 & & & & & \\
\hline Eovationt & 1.79 & 3.12 & 2.31 & 5.39 & 6.381 & & & & & \\
\hline & I & & & & & & & & & \\
\hline
\end{tabular}




\begin{tabular}{|c|c|c|c|c|c|c|c|c|c|c|}
\hline Run" & 8101 & . & 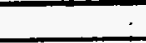 & 1 & 1 & 1 & 1 & 1 & ' & 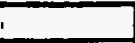 \\
\hline Tostfluid & CAF8 & - & i & 1 & 1 & 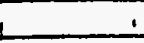 & $\div$ & 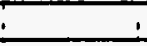 & . & - \\
\hline Dalo: & $9 / 20193$ & & & & & $\therefore$ & & & & \\
\hline Timo: & $13: 31$ & & & & & & & & & \\
\hline Note- & Anatyicald & tis used & & & & & & & & \\
\hline Flow Areatl2 & 0.00336 & & & & 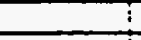 & it & - & & : & : \\
\hline Head Fow Areate? & 0.150051 & & & & & & & & & \\
\hline SAt. Piossurépsig & 127 & & ? & t & 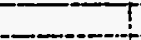 & 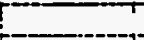 & & & & \\
\hline Sï. Tomperarof(nte) & 151.11 & -7 & 1 & in & 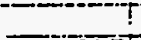 & -1 & & & & \\
\hline Lig.Donatyemins & 81.91 & 81.721 & 81.741 & $81.68 \mathrm{i}$ & 81.71 & 81.71 & 81.611 & 81.671 & 81.671 & 80.58 \\
\hline 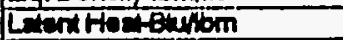 & 35.421 & 35.281 & 35.81 & 35.251 & 35.27 & 35.271 & 35.18 & 35.231 & 35.281 & 34.68 \\
\hline Specifo Hea Eurbmi & $0.297 i$ & 0.2021 & 0.292 & 0.221 & 0.292 & 0.2321 & 0.32 & 0.2921 & 0.2821 & 0.295 \\
\hline Lq. Vieco oh & 0.448 & 0.444 & 0.44 & 0.443 & 0.441 & 0.441 & 0.45 & 0.461 & 0.451 & 0.430 \\
\hline Lig. Conductivity hith & 0.0204 & 0.02031 & 0.0003 & 0.00031 & 0.0203 & $0.0 \times 3$ & 0.02021 & 0.02031 & $0.00 \times 3$ & 0.008 \\
\hline Lig. Prandt No. & 4.43 & 4.42 & 4.43 & 4.421 & 4.42 & 4.42 & 4.42 & $4.42 !$ & 4.42 & 4.39 \\
\hline Inlotflow Pato-GPM & 2.7 & & & & & & & & & 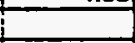 \\
\hline Outtot Lig. Fow Rate-GFM & 0.61 & & & & & & & & & 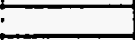 \\
\hline Outtel Vap. Flow Rate-GPM & 1.8 & & $i$ & 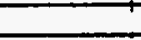 & & & & & $\bar{i}$ & 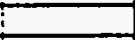 \\
\hline Inlot Tomperaturef & 148.8 & & & & & & 3 & 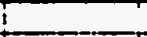 & I & 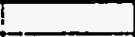 \\
\hline Outol La. Tomperiture & 150.61 & & $T$ & 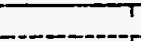 & $?$ & ti & $t$ & & & \\
\hline out â Vap. Tomporiuré & 147.9 & T & 1 & $!$ & $I$ & ? & I & T & & \\
\hline WEOT FoW RATEM & 1 & $i$ & $i$ & I & 1 & $I$ & ! & $!$ & & \\
\hline WE-r that Tomp.f & 91.21 & I & I & 1 & I & I & I & i & & I \\
\hline Wer ollat Tomp.f & 139.2 & 1 & I. & 1 & I & 1 & I & I & & 1 \\
\hline Intel G-lominh/L2 & 531612 & & Tamp & 146.61 & Densiny & ह25ा & & 1 & & \\
\hline Subcooling & 2.3 & $\perp$ & & 1 & 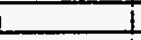 & 1 & & $\perp$ & & \\
\hline \multirow[t]{2}{*}{ Fow Observation } & & & & & & & & & & \\
\hline & ZZNE & ZONE2 : & ZONE3 2 & ZONE 4 & ZONE5 & ZONE6 & ZONE7 & ZONE8 & ZONE9 & ZONETO \\
\hline Heat Input-Walls(nef) & 584.4 & 578.3 & 5822 & 580.9 & 583.5 & 582 & 579.3 & 580.7 & 579.7 & 581.1 \\
\hline Heat Loss-Watts & 8.7 & द! & $2.7 !$ & 2.81 & 281 & 2.11 & 1.4 & 2.11 & 2.6 & 14.3 \\
\hline Had Floxturtring & 101301 & $1002 \pi 1$ & 10002 & 100701 & 101751 & 100801 & 100 |21 & 10058 & $100 \times 1$ & $700 \%$ \\
\hline WET Tomp. & -157.91 & 159.71 & 758.7 & -159.3T & 160.81 & 16.5T & 160.81 & 159.41 & 150.81 & 157.4 \\
\hline WCTTOmp. II & 159 & 158.11 & 159.6 & 159.7 & 160.8 & 16151 & โ61.1 & 160.01 & 158.0 & 160.0 \\
\hline Wh Tomp. $16+$ & 1592 & 15721 & 160.8 & 159.71 & 159.0 & 16051 & 160.81 & 159.81 & 158.8 & 150.8 \\
\hline WEIT Tomp. If & 148.8 & 158.91 & 157 & 158.81 & 1592 & 160.01 & 181.81 & 1601 & 158.1 & 155.8 \\
\hline W"ll Tomp. RF & 159.7 & 158.11 & 157.9 & 158.81 & 158 & 160.8 & 161.6 & 161.11 & 158.8 & 1572 \\
\hline Inetream Temp.F & 148.8 & 160.7 & 150.6 & $-1500 \mid$ & 150.8 & 150.81 & 151.31 & $15 \pi$ & 151 & 164.6 \\
\hline Avo. Wall Temp f & 159 & 158.5 & 158.3 & 159.3 & 159.7 & 161 & 1612 & 1602 & 159 & 157.6 \\
\hline Avo. WallDT $f$ & 8.7 & 7.3 & 7.2 & 7.9 & 8.4 & 9.8 & 9.4 & 8.71 & 7.5 & 25 \\
\hline Femaining Subcooff & -1.5 & & & & & & & & & \\
\hline Exit Quality & 0.01 & 0.04 & 0.08 & $0.11 !$ & 0.141 & 0.17 & 0.2 & 0.23 & 0.26 & 0.3 \\
\hline EXo.HBturtrnerF & 1165.4 & 1364.4 & 1330.2 & 1275.4 & 1205.11 & 10327 & 1065.31 & 1151.9 & 1335.2 & 389.7 \\
\hline COMTPUIEDH & & & & & & & & & & \\
\hline Jatouk & Tos & To्2या & $\$ 98.11$ & 980.11 & 987.81 & 95.7 & \$8.T & अ? & 928.4 & 9621 \\
\hline Sedvi & 1548.71 & 1550.7 & 15581 & 1558.7 & 13824 & 1581.8 & 1567.9 & 1586.4 & 1565.81 & 1604 \\
\hline $\operatorname{sen} 12$ & 13223 & 1327.61 & 7320.61 & 1327.31 & 1350.81 & 13281 & 1328.81 & 1328.11 & 1328.8 & 13:4.4 \\
\hline (AUA) & 772007 & 781371 & 73014 & 718441 & 688071 & $6889 \pi 1$ & 641651 & 615101 & 59037 & 5825 \\
\hline (F) & 1.212 & 1.5811 & 1.897 & 2.14 & 2.51 & 25561 & 268 & 2857 & 3.000 & 3.079 \\
\hline (1म-2) & 206.0 & 263.8 & 316.5 & 357.3 & 324 & 420.4 & 450.8 & 472.2 & 501.5 & 517.6 \\
\hline \multirow[t]{3}{*}{ (HBOL) } & 1547.4 & 15482 & 1551.6 & 15524 & 1554.11 & 15515 & 15524 & 15504 & 1548.4 & 15858 \\
\hline & & & & & & & & & & \\
\hline & ZZOKE? & ZONE4 & ZONES & ZONEB & ZONETO & & & & & \\
\hline DPF quid Tomp.f & 81.2 & & & & & & & & & \\
\hline \multicolumn{11}{|l|}{ DPU Uuid Density } \\
\hline \multicolumn{11}{|l|}{ yrev'on-h } \\
\hline Thatured Spostd & -0.277 & 0.0361 & 7.5831 & 27521 & 3.135 & & & & & \\
\hline Zero Comoótonspad & -0.04 & 0.083 & -0.053 & $=0.011$ & 0.081 & & I & & & \\
\hline Colvénd DP Pild & 0329 & 0 & 1.050 & 2.4021 & 3.074 & & i & i & & \\
\hline Actud DP- & 1.78 & 591 & 3.80 & 4.811 & 5.88 & & I & 1 & & \\
\hline Mecoured TPPhig & 127 & & & & 127.4 & & & 1 & & \\
\hline \multicolumn{11}{|l|}{ Meesured Ouverifa } \\
\hline & & & & & & & & & & \\
\hline ComputadDPA & $9 . \pi$ & 3.07 & 4.33 & 5.56 & 6.7 & & & & & \\
\hline Fictiont & 0.08 & 0.28 & 0.44 & 0.71 & 1.01 & & & & & \\
\hline Acosloration & 0.08 & 0.13 & 0.18 & 0.22 & 0.28 & & & & & \\
\hline Elevation-1 & 1.5 & $2 . \pi$ & 3.71 & 4.E. & 5.41 & & & & & \\
\hline & & & & & & & & & & \\
\hline
\end{tabular}




\begin{tabular}{|c|c|c|c|c|c|c|c|c|c|c|}
\hline Run: & 8111 & & 1 & - & 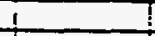 & & 1 & $\vdots$ & 1 & $\therefore$ \\
\hline Testfisid & CAF8: & & $\therefore$ & 1 & & 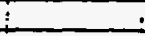 & $\cdot$ & ? & & \\
\hline Date: & isaras & & & & & & & 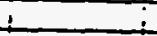 & & \\
\hline Time: & 13:31 & & & & & & & $i$ & $i$ & \\
\hline Nob & Analycalo & dis used & & & & & & 1 & & \\
\hline Fow Areare & 0.005361 & & & & $t$ & & $i$ & $i$ & 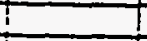 & \\
\hline Hent fow Aroare & 0.19555 & & 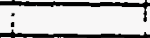 & + & & & & + & & \\
\hline Saf. Pressurepsig & 125.7 & & & & & 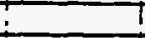 & I & 1 & & \\
\hline Sat Temperamefinglot) & $\{50.5$ & & & & $i$ & 1 & T & i & & \\
\hline Lq. Densinthomins & 81.841 & 81.74 & 81.83 & 81.78 & 81.821 & 81.84 & 81.74 & 81.81 & 81.8 & 80.58 \\
\hline Lenthentexhbm & 35.45 & 35.8 & 35.39 & 35.39 & 35.581 & 35.381 & 35.9 & 35.34 & $\$ 5.87$ & 34.85 \\
\hline Speciro Hocterubm/F & 0.2911 & 0.292 & 0.2911 & 0.292 & 0.292 & 0.292 & 0.292 & 0.221 & 0.29 & 0.206 \\
\hline Liq. Visooenthom/hrs & 0.4481 & $0.4 \times 1$ & 0.447 & 0.461 & 0.446 & 0.4461 & 0.44 & 0.446 & 0.46 & 0.427 \\
\hline 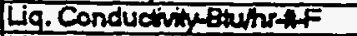 & 0.002 & 0.02031 & 0.0209 & 0.02931 & $0.02 \%$ & $0.0 \times 091$ & 0.02031 & 0.0203 & 0.0003 & 0.0285 \\
\hline Liq. Pranoty No. & 4.46 & 4.43 & 4.43 & 4.43 & 4.43 & 4.43 & 4.43 & 4.4 & 4.43 & 4.37 \\
\hline Intot Fow Rate-GPM & 1.8 & & & & i & & $\dot{3}$ & 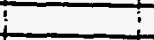 & & \\
\hline Outal Liq. Fow Rato-GPM & 0.4 & & & & & & & & & \\
\hline OunVap. Fow Rate-GPM & 1 & & & & & & $\bar{i}$ & 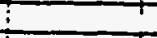 & & \\
\hline Inlat fompersturef & 148.6 & & I & I & & & i & $i$ & & \\
\hline 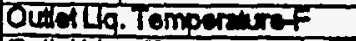 & 150.21 & & $T$ & t & & & $T$ & & & \\
\hline OtuV Vo. Temperminof & 147.8 & & $T$ & & & I & I & & & \\
\hline Wen Fow Rancom & & & 1 & & & ! & $\frac{1}{6}$ & & & \\
\hline Wererind Temp.f & $\mathbf{9 0 . 5}$ & & & & & & 1 & & & \\
\hline Wereuter Tomp.F & 138.1 & & & & & & i & I & I & \\
\hline Interominne & अ6053 & & Temp & 145.8 & Densily & 8265 & & & & \\
\hline Subcoolinge & 1.9 & & & & i & & & & & \\
\hline \multicolumn{11}{|l|}{ Fow Obsenvation } \\
\hline & ZONET & ZONEZ & ZONEB & ZONE4 & ZONES & ZOKES & ZONET & ZONE & ZON=8 & ZZONETO \\
\hline HerthputWatalnal) & \begin{tabular}{|r|}
57.9 \\
\end{tabular} & 577.7 & 579.7 & 579.2 & 577.8 & 5828 & 575 & 577.8 & 5755 & 578.5 \\
\hline HontLoss-Waks & 8.7 & 21 & 27 & 28 & 2.8 & 211 & 1.4 & 2.11 & 26 & 14.4 \\
\hline 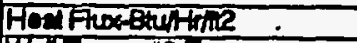 & T0018 & 100141 & 1009 & 100101 & 10018 & 10Tकe & 9987 & 10018 & 9973 & 903 \\
\hline Wa remp. iBf & 157.4 & 158.91 & 157.8 & 1582 & 159.7 & 160.61 & 160.2 & 158.8 & 158.0 & 158.8 \\
\hline WaiTomp. 14F & 158.8 & 157.8 & 158.9 & 158.81 & 160.1 & 160.8 & 1605 & 159.8 & 159.1 & 159.9 \\
\hline We Tomp. 15F & 158.4 & 15.6.6 & 160.5 & 158.81 & 159 & 159.0 & 159.8 & 159.11 & 158.7 & 156.2 \\
\hline WEMTEmp. HF & 148.6 & 157.4 & 150.3 & 1572 & 168.1 & 159.7 & 100.5 & 159.2 & 157 & 154.8 \\
\hline WeVTomp, dEF & 158.8 & 157.5 & 157.8 & 157.7 & 158.11 & 160.1 & 160.8 & 160.8 & 157.8 & 156.4 \\
\hline Inetrean Temp.fF & 149.8 & 1606 & 160 & 160.4 & 1602 & 160.1 & 160.6 & 160.9 & 160.0 & 166.6 \\
\hline Av. Wathemp.F & 1582 & 157.8 & 157.B & 158.3 & 158.8 & 1602 & 1604 & 1594 & 158.1 & 156.8 \\
\hline Avo. WallDT.F & 82 & 6.7 & 7.1 & 7.4 & 82 & 9.6 & 9.3 & 8.7 & 7.4 & 0 \\
\hline \multicolumn{11}{|l|}{ Acmaining SubcoolF } \\
\hline ExQuaty & 0.03 & 0.08 & 0.12 & 0.17 & 0.2 & 0.27 & 0.31 & 0.56 & D.दा & 0.45 \\
\hline Exp. Hotwhiner & 1225.8 & 1489.8 & 4052 & 1357.4 & 12258 & 1050.3 & 1070.8 & 1155.81 & 135.71 & 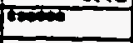 \\
\hline \multicolumn{11}{|l|}{ COMPUTEDH } \\
\hline Jenok & 7001 & Tosibl & 7008.81 & 100821 & \$od. & $982 \pi$ & 958.4 & 950.81 & S5T.5 & 988.4 \\
\hline Sedvilcudo ibe) & 1538 & 1548.8 & 15.5.6 & 1548.8 & 1547 & 1553.2 & 75492 & 1551 & 156 & 1671.5 \\
\hline $5 z d y$ & 1310.4 & 1318.7 & 1316.8 & 1318.9 & IST6.8 & 13224 & 1316.1 & 1310.7 & 137 & 1374.0 \\
\hline (PLO) & 50078 & 49100 & 4670 & 4428 & 41810 & उन्दा & 37100 & 34000 & अंको & 31478 \\
\hline (F) & 1.418 & 1.858 & 2230 & 251 & $2 \pi 3$ & 2.39 & 3.186 & 3.382 & 3.56 & 3.585 \\
\hline$(+\bar{D})$ & 171 & 22द्र & 2682 & 303.1 & 333.6 & 350.9 & 385 & 408.4 & 40 & 439.1 \\
\hline \multirow[t]{3}{*}{ (Hon) } & 1537.2 & 15.3 .2 & $15 \times 29$ & 15.5.8 & 1547.8 & 1546.7 & 15.12 & 1541.6 & 1537.9 & 1600.6 \\
\hline & & & & & & & & & & \\
\hline & LONiE2 & ZONE4 & $20 K=6$ & 20128 & $\angle O A=10$ & & & & & \\
\hline DPtauid Temp.f & 80.8 & & & & & & & & & \\
\hline Df LguddDenthe"om/3 & 93.08 & & & & & & & & & \\
\hline Elovion- & दा & & 6 & 8 & 9.875 & & & & & \\
\hline 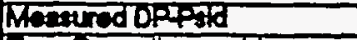 & 038:5 & 0.085 & 3.972 & 2.85 & 3.750 & & & & & \\
\hline Zero Comecioneped & 0.012 & 0.056 & 20.003 & 20.011 & 0.001 & & & & & \\
\hline Corroad DPFid & 0.887 & -0.001 & 2003 & 28001 & 3.000 & & & & & \\
\hline Aolu DPh & 1.71 & $4: 2$ & 3.31 & 3.94 & 4.8 & & & & & \\
\hline Mosecred TPping & 125.7 & & & & 125.0 & & & & & \\
\hline Heened Overelith & -0.63 & & & & & & & & & \\
\hline & & & & & & & & & & \\
\hline Comprted Dpen & $1 . \pi$ & 255 & 3.65 & 4.51 & 5.38 & & & & & \\
\hline Fricionn & 0.05 & 0.15 & 0.3 & 0.48 & 0.68 & & & & & \\
\hline Acoulerion & 0.05 & 0.08 & 0.11 & 0.151 & 0.19 & & & & & \\
\hline Elovation & 1.34 & 232 & 3.74 & 3.871 & 4.51 & & $i$ & & & \\
\hline-1 &.$i$ & $-i$ & & & i & & & & & \\
\hline
\end{tabular}





\begin{tabular}{|c|c|c|c|c|c|c|c|c|c|c|}
\hline Runt: & 8121 & 1 & & & & 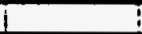 & 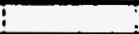 & & & \\
\hline Tert Fud & CAF & & & & & & & & & \\
\hline Deqa: & $9 \sqrt{20183}$ & & & & & & & & & \\
\hline Time: & 13.81 & & & & & & & & 1 & \\
\hline Now & Anstycalc & dit is used & & & & 1 & 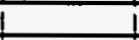 & & 1 & \\
\hline Flow Araat? & 0.00536 & & & & & i & i & & & \\
\hline Hod Flow Arest'2 & 0.18696 & & & & & & & & & \\
\hline Sat Prossure psig & 124.7 & & & & & 1 & L & & & \\
\hline Sat Temperatureff(inat) & 149.9 & & & & & & & & & \\
\hline Lq. Densitylbm/13 & 82.41 & 81.88 & 81.94 & 81.84 & 81.86 & 81.84 & 81.78 & 81.84 & 81.82 & 80.72 \\
\hline Latond Heat-Bhubm & 35.84 & 35.47 & 35.451 & 35.38 & 35.39 & 35.38 & 35.33 & 35.38 & 35.35 & 34.46 \\
\hline Spectio Hoetetultomf & 0.29 & 0.2911 & 0.2911 & 0.292 & 0.2911 & 0.2321 & 0.252 & 0.2921 & $0 . \mathbf{E}_{21}$ & 0.23 \\
\hline Da. Viscosing tomphr & 0.459 & 0.447 & 0.4481 & 0.4461 & 0.447 & 0.4461 & 0.4451 & 0.4461 & 0.4481 & 0.424 \\
\hline Liq. Conductiviteturntaf & 0.0208 & 0.02041 & 0.0024 & 0.001 & 0.0294 & 0.0281 & $0.0 \times 31$ & 0.0201 & $0.02 \pi 1$ & 0.0888 \\
\hline La. Prandeno. & 4.47 & 4.431 & 4.44 & 4.431 & 4.43 & 4.48 & 4.43 & 4.43 & 9.48 & 4.38 \\
\hline InteI FOW REA-GPM & 11 & & & & & & & & I & \\
\hline Outel Lig. Fow Ruto-GPM & 0.41 & $\frac{1}{1}$ & & & & & & & $i$ & \\
\hline 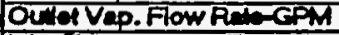 & 1.4 & & & & & & & & I & \\
\hline Inted Tomperaturef & 144.4 & & & & & & & & $i$ & \\
\hline 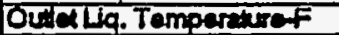 & 150.6 & & & & & & & & $i$ & \\
\hline Outlat Vap. Temperatureff & 147.6 & & & & & & & & & \\
\hline Whar Fow RateGPM & & & & & & & & & & \\
\hline Wear hilat temo.f & 912 & & & & & & & & $i$ & \\
\hline WEW outer Temp.F & 73721 & & & & & & & & & \\
\hline Inter Gibmherine & 785685| & & Temp & T405:L & Donaty & 83.63 & & & & \\
\hline Subooding & 5.5 & & & & & & & & & \\
\hline Fow Obsenvion & & & & & & & & & & \\
\hline & ZONEI & TZOKEE I & ZONE8 & ZON=4 is & 201:36 & $120 \mathrm{~N}=6$ & ZONE' & ZONEB T & 120N59 & $205=70$ \\
\hline 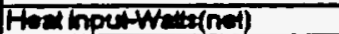 & 581.2 & 580.8 & 583.2 & 5822 & 580.8 & 588.7 & 57.8 & 580.8 & 578.6 & 581.8 \\
\hline Heal Loss-Wild & 8 & 1.8 & 2.5 & 2.5 & 2.5 & 1.9 & 1.3 & 1.9 & 2. & 13.3 \\
\hline Hox Fibethithin? & 10075 & 10068 & 10100 & 10002 & 10070 & 101201 & 10018 & 10084 & 10000 & 10078 \\
\hline Wa Tamp. $13 F$ & 155.9 & 157.3 & 155 & 157.1 & 158.1 & 159.5 & 158.8 & 157.8 & 1535 & 158 \\
\hline Wal Temp. $4 \mathrm{f}$ & 157.1 & 155.8 & 157.2 & 157.6 & 158.6 & 159.71 & 159.4 & 159.1 & 157.7 & 159.2 \\
\hline We"1 Tamp.15F & 157.5 & 155.1 & 159.11 & 157.6 & 157.4 & 158.6) & 158.4 & 157.9 & 156.4 & 15.5 \\
\hline Wall Tomp. It & 144.4 & 155.9 & 15R.81 & 156.2 & 756.8 & 758.5 & 759.2. & 158.4 & 155.6 & T54.2 \\
\hline Wertomo.l2t & 158.1 & 158.11 & 155.7 & 156.6 & 158.8 & 158.8 & 159.4 & 159.51 & 758.4 & 756.6 \\
\hline hortsem tomp. $7=$ & 747.1 & 149.8 & 748.81 & 750.11 & 750 & $750 . \pi$ & 750.41 & 150.11 & 75021 & 750.8 \\
\hline Avg. Wall Tomp.F & 7572 & 758.8 & 75.8 & 757.11 & 757.4 & $159 . \pi$ & 7591 & 758.51 & 15635 & 756.7 \\
\hline Avo. WAMT.f & 9.8 & 5.9 & 5.9 & 6.01 & 6.8 & 8.51 & 8.2 & 8 & 5.8 & 0 \\
\hline Romining swooootf & -4.7 & & & & & & & & & \\
\hline Ext Guaty & 0.04 & 0.12 & 0.2 & 020 & 0.57 & 0.48 & 0.64 & 0.62 & 0.7 & 0.78 \\
\hline 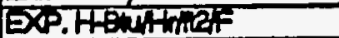 & 1050 & 1700.6 & T78 & 1633.8 & 14400 & 1184.8 & 1233 & 1260.4 & 170.6 & \\
\hline CONPUTEDH & & & & & & & & & & \\
\hline Jallouk & T176.4 & 1078.6 & $10 \cos 2$ & Tर्ख3 & 100 & 10्या & 1021.4 & T013.8 & $100 \mathrm{a}, 1$ & 10x7.4 \\
\hline Szady1(Cudo tro) & I516.7 & 154.6 & 1576 & 1549.1 & 7546.8 & 7556.8 & 1548 & 1549.2 & 7548.8 & 1607.4 \\
\hline szadyz & 1290.8 & 7317.8 & 1318.5 & 1321.8 & 1318.8 & 1328.7 & 1317.7 & 1319.1 & 1376.8 & 1375.6 \\
\hline (RLO) & 27917 & 20885 & $245 \pi$ & జ205 & 15583 & 171ము & 14746 & 12257 & $\$ 858$ & 777 \\
\hline$(\theta)$ & 1.474 & 2.052 & 2597 & 3.008 & 3.368 & 3.687 & 3.869 & 4.350 & 4,521 & 4.604 \\
\hline (1) & 171.8 & 156.8 & 197.5 & 208.9 & 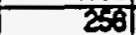 & 28.81 & 3024 & 323.8 & उ4द2 & 357.3 \\
\hline (FBOLL) & 1516.5 & 157 & 1548 & 1547.6 & 157.8 & 75328 & 154.11 & 154.61 & 15428 & TE01.8 \\
\hline & & & & & & & & & & \\
\hline & $\angle O N=2$ & ZONEZ4 & ZONE6 & $20 \pi \equiv 8$ & $201=10$ & & & & & \\
\hline Dptaqd temp.f & 80.6 & & & & & & & & & - \\
\hline DPUgud Dentit tomis & अ.1द्यो & & & & & & & & & \\
\hline Elovition- & या & 4 & & (8) & 8.876 & & & & & \\
\hline Maseured Dphapd & 0.3 & 0.03 & 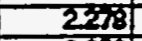 & उक्. & दुखा & & & & & \\
\hline Zaro Comections-psid & $-0.0 k$ & 0.03 & -0.068 & -0.01 & $0.0 \% 1$ & & & & & \\
\hline Compotod DP sida & 0.34 & -0.003 & रुआ & 3.400 & 4.28 & & & & & \\
\hline Actual DPA & 1.7 & 4.2 & 278 & 3.16 & 3.78 & & & & & \\
\hline Wextured tPFistg & T2द.7 & & & & 124.8 & & & & & \\
\hline Mexared Ovintith & -0.18 & & & & & & & & & \\
\hline & & & & & & & & & & \\
\hline Compled DPI & 1.28 & 2.121 & 281 & 3.4 & 3.94 & & 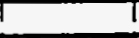 & & & \\
\hline Frictonn & 0.021 & 0.071 & 0.161 & 0.51 & 0.381 & $!$ & ! & & $!$ & \\
\hline & $\infty$ & & & $\infty$ & 0.13 & & & & & \\
\hline
\end{tabular}




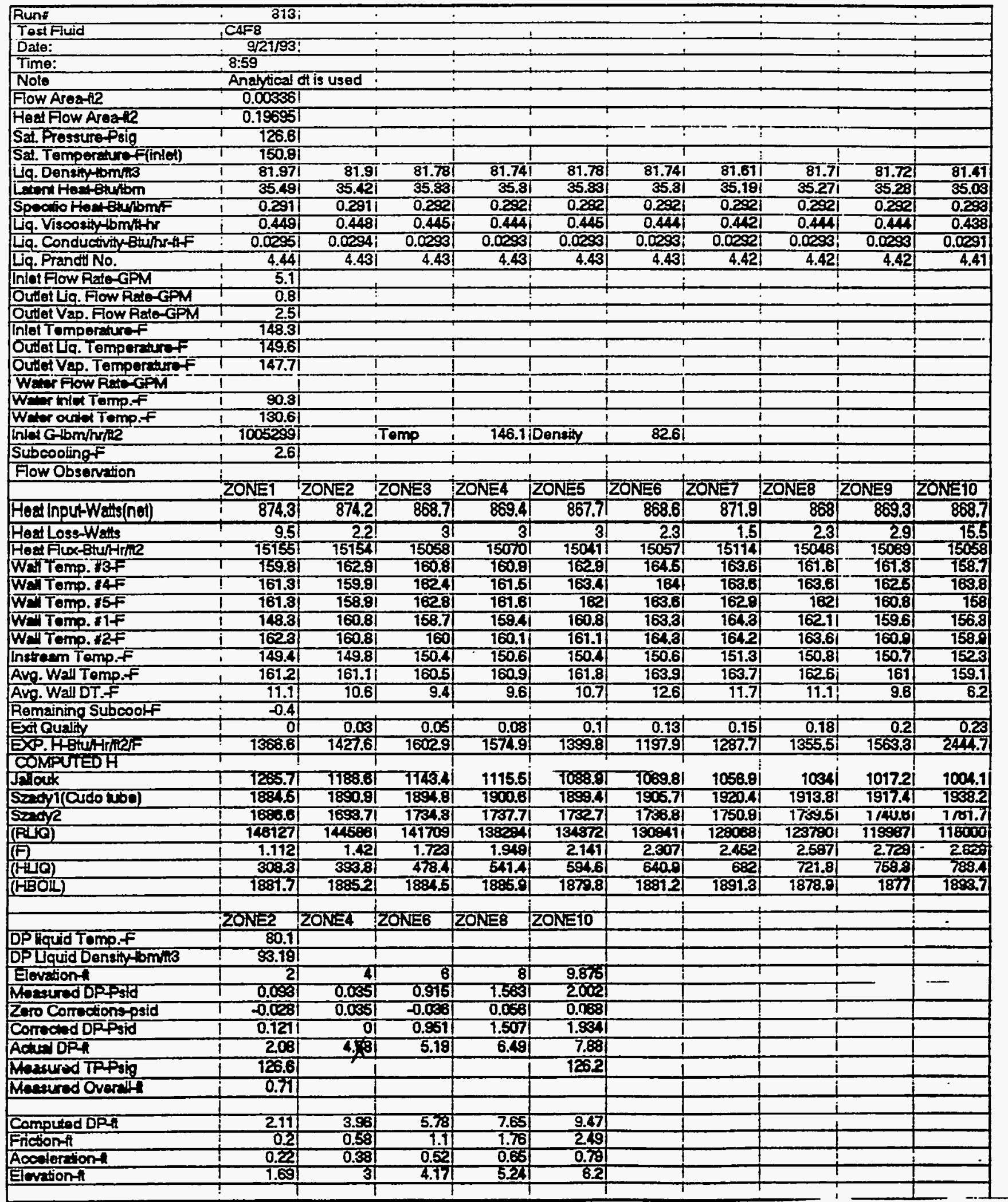




\begin{tabular}{|c|c|c|c|c|c|c|c|c|c|c|}
\hline Rund & $314 i$ & - & - & $\therefore$ & - & 1 & 1 & - & & \\
\hline Test Fluid &. CAF & $\div$ & - & 0 & & 4 & 2 & & & \\
\hline Dale: & 9/21/93: & & & & & & & & & \\
\hline Time: & $8: 59$ & & & & & & & & & \\
\hline Note & Analytical c & dt is used & & & & & & & & \\
\hline Fow Areatt2 & 0.003361 & & & & & - & & & & 1 \\
\hline Hod Fow Areat? & 0.15551 & T! & 7 & & i & i & & & & \\
\hline Sat. Pressure Psig & T27.21 & $T$ & & $T$ & & $!$ & i & & & \\
\hline Sat. Temportureff(inlel) & 151.21 & 1 & I & 1 & ! & 1 & i & $!$ & & 1 \\
\hline Uig. Densinytomits & 81.92] & 81.81 & 81.72 & 81.681 & 81.71 & 81.71 & 81.55 & 81.651 & 81.51 & 87.28 \\
\hline Lefori Hectevitom & 35.4 & 35.34 & 3528 & 35251 & 35.27 & 35.27 & 35.14 & 35.21 & 5ह2या & 3द. $=2$ \\
\hline Specifo Hestatulbmf & 0.291 & 0.2021 & 0.292 & 0.292 & 0.292 & 0.282 & 0.2331 & 0.2321 & $0.25 \mathrm{St}_{1}$ & ה..25 \\
\hline Lig. Visoosity + bmintr & 0.48 & 0.4461 & 0.444 & 0.443 & 0.444 & 0.44 & 0.49 & 0.42 & C..14n & 0.435 \\
\hline 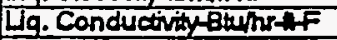 & 0.0294 & 0.0239 & 0.0203 & 0.0253 & 0.0293 & 0.02931 & 0.0202 & 0.02021 & 0.0292 & n.meg \\
\hline Lig. Prandt' No. & $4 . \overline{44}$ & 4.431 & 4.42 & 4.42 & 4.42 & 4.42 & 4.42 & 4.421 & 4.42 & 4.4 \\
\hline Inlel Flow Ralo-GPM & 4.3 & & & & & & & & & \\
\hline Outlat Lq. Fow RatoGPM & 0.9 & & & & & & & & & \\
\hline Outfet Vap. Flow Rate-GPM & 2.2 & & & & & & & & & \\
\hline Inlot Tomperaluef & 148.7 & T & & & T & 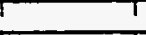 & & & & \\
\hline Outlet La. Temperature & 150.21 & & & 7 & T & $T$ & $T$ & 1 & & i \\
\hline OUtrot Vap. Tomporaturef & 148.21 & $T$ & 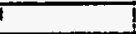 & 1 & T & I & $T$ & I & & \\
\hline Wha Flow PEo-GPi & $I$ & I & I & $!$ & I & I & i & I & & \\
\hline Wren inlet Temp.f & 90.2 & I & I & 1 & 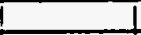 & 1 & 1 & 1 & & $\perp$ \\
\hline Welp oulat Tomp.f & 132 & $i$ & i & 1 & & 1 & i & 1 & & \\
\hline 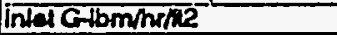 & 848566 & & Temp & 145.6 & Density & 82.60 & & 1 & & \\
\hline Subcoolingf & 2.5 & + & & +1 & & & & i & & \\
\hline \multirow[t]{2}{*}{ Fow Observation } & & & & & & & & & & \\
\hline & ZONET & ZONE2 & ZONE3 & ZONE & ZONES & ZONE6 & ZONET & ZONEB & ZONES & $\angle O N=10$ \\
\hline Hod Inpul-Watts(not) & 869.7 & 860.7 & 865.2 & 864.4 & 868.7 & 874.61 & 858.9 & 859.9 & 869.4 & 8605 \\
\hline HenLose-Wath & 8.81 & 2 & 2.8 & 2.8 & 281 & 22 & 1.4 & $2 \pi$ & 2.7 & 74.2 \\
\hline 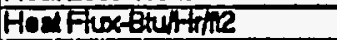 & 15078 & 14\$द्या & 14998 & 14981 & 15058 & 151611 & 15032 & 14908 & 150701 & TRG \\
\hline Wa Tomp. HF & 159.8 & 162.9 & 161.2 & 160.8 & 163.1 & 164.6 & 163.71 & 161.8 & 161.01 & 159 \\
\hline WE Tomp.14F & 161.8 & 160.11 & 1628 & 161.8 & 163.6 & 164.4 & 16.91 & 163.6 & 1820 & 163.8 \\
\hline WE Tomp. $15 F$ & 181.1 & 158.81 & 168 & 161.81 & 1628 & 163.81 & 168 & 162.1 & 181 & 158.1 \\
\hline WEIT Tomp. IIF & 148.7 & 160.7 & 158.8 & 159.7 & 161 & 163.51 & 164.4 & 16241 & 158.9 & 168.6 \\
\hline WCIVTOMP. IRF & 1621 & 160.7 & 160.8 & 160.4 & 161.4 & 164.81 & 164.4 & 163.71 & 161.1 & 168. \\
\hline Instram Tomp.f & 949.7 & 150.31 & 150.7 & 150.9 & 150.8 & 150.8 & 151.6 & 151.1 & 157.1 & 150 \\
\hline Avg. Wall Tamp.f & 161.1 & 167.11 & 160.8 & 1611 & 152.2 & 164.11 & 163.9 & 1627 & 1612 & 159.3 \\
\hline Avo. Wall DT.F & 10.7 & 10.1 & 9.4 & 9.4 & 10.8 & 12.6 & 11.6 & 10.91 & 9.5 & 5.6 \\
\hline Remaining Subcooff & -1 & & & & & & & & & \\
\hline Ext Quality & 0.01 & 0.04 & 0.07 & 0.1 & 0.131 & 0.16 & 0.18 & 0.21 & 0.24 & 0.27 \\
\hline ExP.HEturner & 1405.5 & 7473.4 & 1591.7 & 1538.6 & 7425.7 & 12042 & 1298.5 & 1361.7 & 1533.3 & 250.3 \\
\hline \multicolumn{11}{|l|}{ COMPUIEDH } \\
\hline Jallouk & T275.51 & 7 7दा.ग & 71632 & 3738.3 & 11181 & 7099.8 & 1083.6 & 1058.91 & 106.8 & 1051.8 \\
\hline Szady (cudo sube) & 188281 & 1881.81 & 1893.3 & 1897 & 190281 & T917.61 & 1977.3 & 7904.61 & $19 \overline{17.51}$ & 15320 \\
\hline $\sin 2 y^{2}$ & 168421 & 1681.81 & 1733.8 & 1735.2 & 17395 & 1747.81 & 1750.01 & 173211 & 1745. II & 1758.8 \\
\hline$(\mathrm{P}+\mathrm{O})$ & 120302 & 1216801 & 118460 & 115034 & 111249 & 1075461 & 104600 & 100489 & 98740 & 94078 \\
\hline (if) & 1.185 & 1.5211 & 1.838 & 2078 & 2.278 & 2.481 & 2.615 & 2.7721 & 2918 & 3.016 \\
\hline (सIO) & 2803 & 368.81 & 44.8 & 6032 & 5520 & 687 & 635.6 & 678.11 & 707.4 & $\$ 34.7$ \\
\hline \multirow[t]{3}{*}{ ( $\mathrm{BBOI}$ ) } & 1890.5 & 1876.9 & 18851 & 1885.1 & 1887.7 & 1892 & 1893.7 & 1876.1 & 1880 & 1632 \\
\hline & & & & & & & & +1 & & \\
\hline & ZONNE2 & ZONE4 & ZONE6 & ZONEB & ZONETO & & & 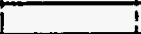 & & \\
\hline \multicolumn{11}{|l|}{ DPFiqud Temp.f } \\
\hline \multicolumn{11}{|l|}{ DPU Uuid Density 4 bmin 3} \\
\hline Elovetiont & & & 6 & 8 & 9.8751 & & & $T$ & & \\
\hline Mesaured DFpy & 0.12 (1) & 0.055 & $1.1 \pi$ & 1.897 & 2.431 & & & & & \\
\hline Zero Correctionsprid & -0.038 & 0.0351 & -0.088 & 0.058 & 0.0681 & T & 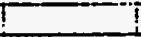 & I & & \\
\hline Colmond DPPitd & 0.17 & of & 1.207 & $1 . \overline{8411}$ & 2.300 & & I & & & \\
\hline Aatual DPA & 1.88 & $4 \times 3$ & 4.74 & 5.91 & 7.13 & $\mathrm{I}$ & I & T & & \\
\hline Mreoured TP pig & 1273 & & & & 127.11 & & I & I & I & \\
\hline \multirow[t]{2}{*}{ Mreaured Overelle } & 0.18 & & & & & & I & i & i & \\
\hline & & & & & & & & 1 & & \\
\hline \multirow[t]{5}{*}{ Camputed DPA } & 1.96 & 3.66 & 5.33 & 7.02 & 8.58 & & & I & $i$ & \\
\hline & 0.17 & 0.48 & 0.91 & 7.46 & 2.08 & & & $i$ & $i$ & \\
\hline & 0.18 & 0.3 & 0.42 & 0.53 & 0.68 & & & i & 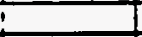 & \\
\hline & 7.67 & 2.87 & 3.99 & 5.03 & 5.97 & & & & & \\
\hline & & & & & & & & t & & \\
\hline
\end{tabular}




\begin{tabular}{|c|c|c|c|c|c|c|c|c|c|c|}
\hline Runt & 8151 & & & & & & & & & \\
\hline Testfluid & CAf & & & & & & & & & \\
\hline Dato: & $9 \sqrt{21 / 93}$ & & & & & & & & & \\
\hline Time: & $8: 59$ & & & & & & & & & \\
\hline Note & Analytical of & th is used & & & & & & & & \\
\hline Fow Areatll & 0.003361 & & & & & & & & & \\
\hline Hoat flow Aroath & 0.1965 & & & & & & & & & \\
\hline Sal Prossute Psig & 126.1 & & & & & & & & & 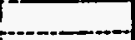 \\
\hline Sal Temper & 150.7 & -1 & -1 & & ! & & & 1 & & \\
\hline Eq. Densin 4 omnins & 82.071 & 81.881 & 81.761 & 81.681 & 81.71 & 81.741 & $81.67 i$ & 81.741 & 81.741 & 81.39 \\
\hline Ltant Hed Blifom & 35.56 & 35.411 & 35.81 & 35.251 & 35.27 & 35.31 & 35.281 & 35.31 & 35.81 & 35.01 \\
\hline Specilo Hed Btubmif & 0.2911 & 0.2911 & 0.292 & 0.2521 & 0.2521 & $0.292 !$ & 0.292 & 0.2921 & 0.2921 & 0.283 \\
\hline Lig. Visoosity lomithis & 0.457 & $0.447 \mathrm{i}$ & 0.4451 & 0.4431 & 0.441 & $0.444 i$ & 0.4431 & 0.4491 & $0 . \overline{441}$ & 0.437 \\
\hline Liq. Conductivity Btwhr-4h & 0.0255 & $0.0234 !$ & 0.02031 & 0.0293 & 0.02831 & $0.0203 ;$ & $0.0203 !$ & $0.0203:$ & 0.02031 & 0.0201 \\
\hline Liq. Prandt No. & 4.441 & 4.431 & 4.43 & 4.42 & 4.42 & 4.431 & 4.42 & 4.431 & $4.49 !$ & 4.47 \\
\hline Inlat Fiow Rato-GPM & 3.5 & & & & & & & & & \\
\hline Offot Lig. Fow Rato-GPM & 0.91 & & & & & & & & & \\
\hline Outlet Vap. Flow Rate-GPM & 1.81 & & & & & & & & & \\
\hline Inlat Tomperature $F$ & 147.91 & & & & & & & & & \\
\hline Oüflíg. Temperatur & $150.4 T$ & & & & & & & & & \\
\hline Oün vap Temperaturef & 147.71 & & & & & & & & & \\
\hline Whe Fow RA-CPM & -1 & 1 & 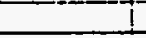 & 1 & i & $I$ & 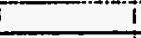 & 1 & i & 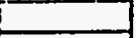 \\
\hline Weler intel Tomp.f & 89.81 & 1 & 1 & $i$ & I & $I$ & 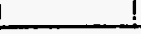 & 1 & 1 & 1 \\
\hline Water ovilet Temp.f & 131.71 & 1 & 1 & 1 & 1 & 1 & 1 & $\vdots$ & 1 & 1 \\
\hline Inlat Glbm/nx/A2 & 692351 & & Temp & 144.610 & Density & 82.881 & & 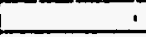 & & \\
\hline Subcoolingf & 2.81 & 1 & & & & & & $\perp$ & & 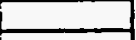 \\
\hline Flow Obsenvation & & & & & & & & & & \\
\hline & ZONE1 & ZONE2 : & ZONE3 & ZONEA 2 & ZONE5 & ZONE6 is & ZONE7 & ZONE 8 & ZONE9 & ZONETO \\
\hline Heal Input-Walls(net) & 859.9 & 857.7 & 859 & 856.9 & 856.2 & 861.31 & 859.8 & 853.4 & 860.1 & 852.6 \\
\hline Hed Loss-Walls & 91 & 2.11 & 2.8 & 2.9 & 2.91 & $2.2 !$ & 1.41 & $2.1 !$ & 2.61 & 14.3 \\
\hline Hed Fux-Bushline & 14906 & 14888 & 14850 & 14854 & 14812 & 14950 & 14904 & 14783! & 140091 & 74778 \\
\hline WeT Temp. BF & 155.31 & 1625 & 160.61 & 160.7 & 16261 & 164.7 & 16żg] & 160.81 & $|59|$ & 1582 \\
\hline Womp. ThF & -161 & 159.91 & 1628 & 161.6 & 16.11 & $1 \approx .71$ & 968.81 & $162 \overline{8}$ & 760.01 & 16 \\
\hline WaM Tomp. $15 F$ & 161 & 158.61 & 1ब्201 & $\overline{161,6 !}$ & 167.81 & $1 \overline{6.11}$ & 18251 & 161.11 & 158.8 & 157.1 \\
\hline Well Tomp. I1F & 147.8 & 160.11 & 158.8 & 159.71 & 160.8 & 1631 & 169.8 & 161.51 & 158.6 & 155.1 \\
\hline WCI Tamp daf & 161.4 & 160.21 & 159.6 & 160.2 & 160.8 & 163.61 & 163.6 & 1628 & 159.7 & 157.7 \\
\hline Instram Tomp. $F$ & 148.01 & 149.9 & 150.6 & 160.8 & 150.8 & 150.61 & 151 & 150.61 & 150.81 & 1624 \\
\hline Avg. Wall Tomp ff & 160.7 & $1605 i$ & 1602 & 160.9 & 161.5 & 163.5 & 1632 & 169.8 & 15921 & 158.2 \\
\hline Avo. WallDT of & 11.11 & 10 & 9! & 9.3 & 10.1 & $122 !$ & 11.5 & $10.5 !$ & 8 & 5.2 \\
\hline Remaining Subcooff & -1.6 & & & & & & & & & \\
\hline Ext Quality & 0.011 & 0.05 & 0.08 & 0.12 & 0.16 & 0.19 & 0.23 & 0.26 & 0.31 & 0.33 \\
\hline EXo.HBtuhnef & 1342.7 & 1493.8 & 1654.11 & 1596.51 & 1476.2 & 1223.7 & 1295.3 & 1407.3 & 1857.61 & 2838.6 \\
\hline COMPUTEOH & & & & & & & & & & \\
\hline Jalouk & 7289.1 & 7235 & IIs6.71 & 7157.31 & T134.81 & 7788.9 & 1104.8 & 70828 ! & 1073.81 & 7088.7 \\
\hline 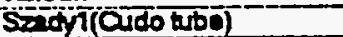 & 18628 & 18729 & 1882.9 & 1887.11 & 1887 & 1892.17 & 9897.11 & 1887.81 & 1897.2 & 1911.8 \\
\hline $\operatorname{sendy}$ & 1680.8 & 1672 & 1683.31 & 1725.91 & 17228 & 1728.91 & 1730.2 & 1718.41 & 1725.51 & $17 \overline{30.4}$ \\
\hline (R्O) & 99881 & 981681 & 950821 & 917911 & $880 \pi$ & 842001 & 80010 & 769801 & 739581 & 70870 \\
\hline (i) & 1.219 & 1.6151 & 1.354 & 2216 & 2.441 & 2.6231 & 2.818 & 2.9821 & 3.140 & 3.250 \\
\hline (1) & 256.7 & 3251 & 4027 & 466.9 & 503.3 & 544.7 & 581.5 & 616.71 & 649 & 674.9 \\
\hline (1.8014) & 8607 & 1852.4 & 1876.8 & 1878.11 & 1875 & 1876.9 & 1878.7 & 1865.6 & 1871.5 & 1883.4 \\
\hline & & & & & & & & & & \\
\hline & ZONE2 & ZONE4 & ZONE6 & ZONE8 & ZONETO & & \pm & & & \\
\hline DP Rquid Tomp.f & 80.9 & & & & & & & & & \\
\hline DPLiquid Density & 93.07 & & & & & & & & & \\
\hline Elevetion & 2 & & & 81 & 9.875 & & & & & \\
\hline Mosured DPsistd & 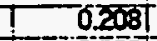 & 0.034 & 1.428 & 23421 & 28817 & & & & & \\
\hline Źro Contolionapid & -0.028 & $0.005 T$ & $=0.003$ & 0.0581 & 0.098 & & & & & \\
\hline Comeond Depald & 0.250 & -0.0011 & 1.450 & 21801 & 2818 & & 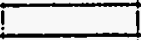 & 1 & & \\
\hline Ach $D^{2}$ & 1.87 & 4601 & 4.27 & 5.281 & 6.32 & & $i$ & 1 & & \\
\hline Mamured TPPAR & 126.1 & $N 1$ & & -1 & 1258 & & 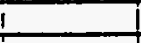 & 1 & & \\
\hline Meceured Ovarall & 0.35 & & & 1 & & 1 & $i$ & $\mathbf{i}$ & & \\
\hline & & & & & & & & & & \\
\hline Compulad DPA & 1.81 & 3.29 & 4.72 & 6.17 & 7.56 & & & & & \\
\hline Friesontt & 0.13 & 0.39 & 0.75 & 1.2 & 7.76 & & & & & \\
\hline Acouterctiont & 0.14 & 0.23 & 0.38 & 0.42 & 0.53 & & & & & \\
\hline Enetiont & 1.541 & 2.67 & 3.64 & 4.511 & $5.2 \pi$ & & & & & \\
\hline
\end{tabular}




\begin{tabular}{|c|c|c|c|c|c|c|c|c|c|c|}
\hline Auny & $816 !$ & - & $:$ & 1 & 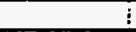 & 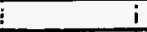 & $:$ & 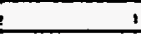 & $\cdot$ & - \\
\hline To:Aनuid & iCAF8 & 1 & & 1 & & $\perp$ & L & 1 & $i$ & i \\
\hline Date: & $921 / 93 !$ & 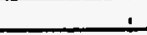 & & $\perp$ & & $\perp$ & $L$ & & i & L \\
\hline Time: & $8: 59$ & & & $i$ & & & & & & \\
\hline Nolo & Anatyical d & At is used ! & & & & & & & 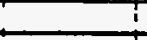 & 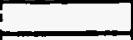 \\
\hline Fow Areatin & 0.003561 & & & & & & 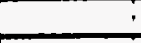 & ; & 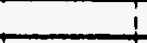 & 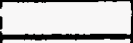 \\
\hline Hed Fow Areate & 0.1955 & & & & & $!$ & & 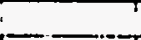 & $T$ & T \\
\hline Sä Pressuretifg & 125.4 & +1 & T & $T$ & & 7 & 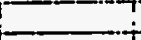 & & 1 & \\
\hline Sel Tomper fre & 150.3 & & & - T & & & & & & \\
\hline 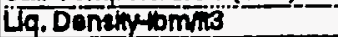 & 82.09 & 81.81 & 81.81 & 81.701 & 81.781 & 81.781 & 81.681 & 81.761 & 81.701 & 81.02 \\
\hline Litent Hothenchom. & 85.681 & 35.341 & 35.34 & 35.811 & 35.38 & 3.831 & 35.25! & 35.811 & 35.81 & $34 . \pi$ \\
\hline 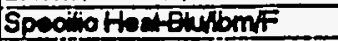 & 0.2811 & 0.2021 & 0.321 & 0.292 & 0.292 & 0.2921 & 0.21 & 0.292 & 0.2821 & 0.29 \\
\hline Liq. Visoositulbmihh & 0.462 & 0.4461 & 0.446 & 0.451 & 0.465 & 0.4451 & 0.4431 & 0.445 & 0.4461 & 0.40 \\
\hline Lig. Conductivity thr-uF & 0.0205 & 0.02031 & 0.0233 & 0.02931 & 0.0203 & 0.02931 & 0.003 & 0.02031 & 0.0023 & 0.0258 \\
\hline Lg. Prandt No. & 4.45 & 4.43 & 4.43 & 4.431 & 4.43 & 4.431 & 4.42 & 4.43 & 4.49 & 4.39 \\
\hline Inlet Fow Rate-GPM & 2.7 & & & & & i & & & & \\
\hline Oưtel Liq. Fow Rado-GPM & 0.7 & & & & 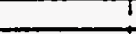 & 5 & 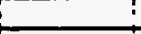 & & & \\
\hline Outlet Vao. Flow Rato-GPM & 1.7 & & & & It & 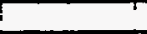 & : & & & \\
\hline Inlat Tomperatura & 747.8 & & & & $T$ & & & & & 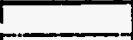 \\
\hline 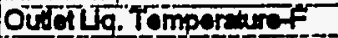 & 150.2 & $T$ & & $T^{\top}$ & T & & & & $!$ & \\
\hline OU A vap. Temper & 947.61 & $T$ & & $T^{\top}$ & 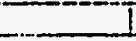 & $T$ & $r$ & & $\tau$ & \\
\hline 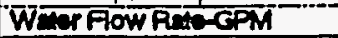 & 1 & $!$ & & 1 & 1 & $I$ & I & & I & \\
\hline Welor mila Tomp.f & 87.8 & 1 & & 1 & E & 1 & $I$ & & $i$ & E \\
\hline Werer oulder Temp.f & 130.91 & $i$ & & $L$ & & 1 & 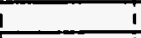 & & 8 & L \\
\hline In G G & 5353371 & & Temp & $943.6 \pi$ & Danity & 83.081 & & & 1 & \\
\hline Subcoolingf & 2.5 & & & 1 & & & I & & 1 & \\
\hline \multirow{2}{*}{ Fow Observation } & & & & & & & & & & \\
\hline & ZONE1 & ZONE2 I & ZONES & ZONE4 & ZONE5 & ZONE6 & ZONE? & ZONEB & IZONES & ZONETO \\
\hline Hoalhou-Walls(not) & 900 & 890.8 & 885.8 & 883.8 & 888.2 & 891.7 & 878.1 & 873.6 & 878.4 & 874.9 \\
\hline Hort Lose-Watts & 3.51 & 2द & & 31 & 3.1 & 24 & 1.51 & 2.31 & 2.81 & 15.6 \\
\hline How Foo-thithic & 155011 & 15435 & 153461 & 1537ा & 15352 & $1545 \pi$ & $15 x=1$ & 157131 & T52या & 15168 \\
\hline Wertomp. Ba & 158.8 & TE2 & 759.81 & 765.31 & 1628 & 1641 & 1627 & IEDJI & 758.0 & 7582 \\
\hline WeTtomo.t4F & $16 \overline{0.4}$ & 1591 & 767.7 & 160.01 & 1628 & 763.81 & TE8.T & 16261 & 980.5 & 963 \\
\hline WET Tomp. I5F & 160.4 & 1581 & 1022 & 161.1 & T01.9 & 100.21 & 1021 & 1611 & 158.01 & 163.9 \\
\hline WeTtamp. IIF & 147.8 & 159.81 & 157.8 & 158.91 & 100.8 & 1628 & 100.4 & 161.51 & 158.01 & 156.1 \\
\hline WDT Temp.12F & 1600 & 159.81 & 158.8 & 159.7 & 160.6 & 163.8 & 169.7 & 1627 & $150 \mathrm{Al}$ & 1575 \\
\hline ingtream Tomp.f & 148.8 & 150.31 & 150.3 & 150.51 & 150.4 & 150.41 & 150.8 & 150.61 & 150.5 & 154.3 \\
\hline Avg. Wall Tome.f & 160.1 & 1602 & 159.7 & 1603 & 161.3 & 163.5 & $1 \approx$ & 161.7 & 15922 & 158.7 \\
\hline Avo. Wall DT. & 10.6 & 9.2 & 8.7 & 9.11 & 10.2 & 12.4 & 11.4 & 10.5 & 8 & 3.2 \\
\hline Remaining Subcooff & 3.4 & & & & & & & & & \\
\hline Exct Qualiy & 0.03 & 0.08 & 0.12 & 0.17 & 0.21 & 0.27 & 0.31 & 0.381 & 0.45 & 0.45 \\
\hline 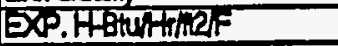 & 1469 & 1686.2 & 1767.7 & 1681.11 & 1502.8 & 1251.4 & 15ं.5 & 1428 & 1907.6 & 4807.6 \\
\hline \multicolumn{11}{|l|}{ COMPUIEDH } \\
\hline Jelok & T350.A1 & 729231 & 124.4 & स्था & -1787.81 & 7967.9 & 1775.81 & 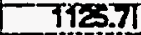 & 171774 & 9750.7 \\
\hline Sindri (cucolute) & 1808.7 & 7912.3T & 1900.1 & 1900.01 & 191291 & 19202 & 1918.7 & 1900.3 & 19782 & 1963.7 \\
\hline $5 x d r^{2}$ & 1710.8 & 17181 & 1713.9 & 1716.4 & 1757.6 & 1784.7 & 1753.4 & 7767.91 & 1749.7 & 1797.9 \\
\hline (F) & 78462 & 745681 & 70828 & 672141 & 63416 & 590601 & 58175 & 52201 & 483681 & 4612 \\
\hline (F) & 1.38 & 1.827 & 230 & 24971 & 2.753 & 2.9821 & 3.182 & 3.381 & 3.5621 & 3.60 \\
\hline (HपO) & 324 & 306.4 & 3692 & 418.01 & 461.8 & 5002 & 5342 & 56721 & 597.5 & 6165 \\
\hline \multirow[t]{3}{*}{ (1.60) } & 19026 & 1909.7 & 1903.5 & 19029 & 19030 & 1908.8 & 1899.1 & 1888.4 & 18936 & 1933.1 \\
\hline & & & & & & & & & & \\
\hline & CONE2 & ZONEA & ZONE6 & ZONE8 & ZONE10 & & & & & \\
\hline \multicolumn{11}{|l|}{ DP hqud Tomp.f } \\
\hline \multirow{2}{*}{\multicolumn{11}{|c|}{ DPUguid Density bmins }} \\
\hline & & aा & 61 & 81 & 9.875 & & & & & \\
\hline \multicolumn{11}{|l|}{ Mentred } \\
\hline Zero cormocione phad & -0.068 & 0.035 & -0.083 & 0.0561 & \multicolumn{6}{|c|}{ 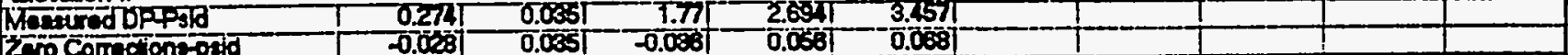 } \\
\hline \multicolumn{11}{|l|}{ Comedied DPpild } \\
\hline \multicolumn{11}{|l|}{ Aation DP: } \\
\hline & 1254 & & & 1 & 125.8 & & & & 1 & \\
\hline \multicolumn{11}{|l|}{ Meavered Overalli } \\
\hline & & & & 53.1 & $6<1$ & & & & & \\
\hline Computed DP & $\frac{1.58 \mid}{6.11}$ & $\frac{2.85}{0.31}$ & $\begin{array}{ll}4.08 \\
0.62\end{array}$ & $\frac{\pi}{11}$ & $\frac{0.41}{1.43}$ & & & & & \\
\hline Fricton-h & 0.11 & 0.37 & 0.02 & 0.34 & n., & & & & & \\
\hline Acoalerrionth & 0.11 & 0.18 & 0.28 & $0.3,4$ & 0.46 & & & & & \\
\hline Elovation-t & 1.37 & 2.35 & 3.18 & 3.951 & 4.54 & & & & & \\
\hline & & & & & & & & & & \\
\hline
\end{tabular}




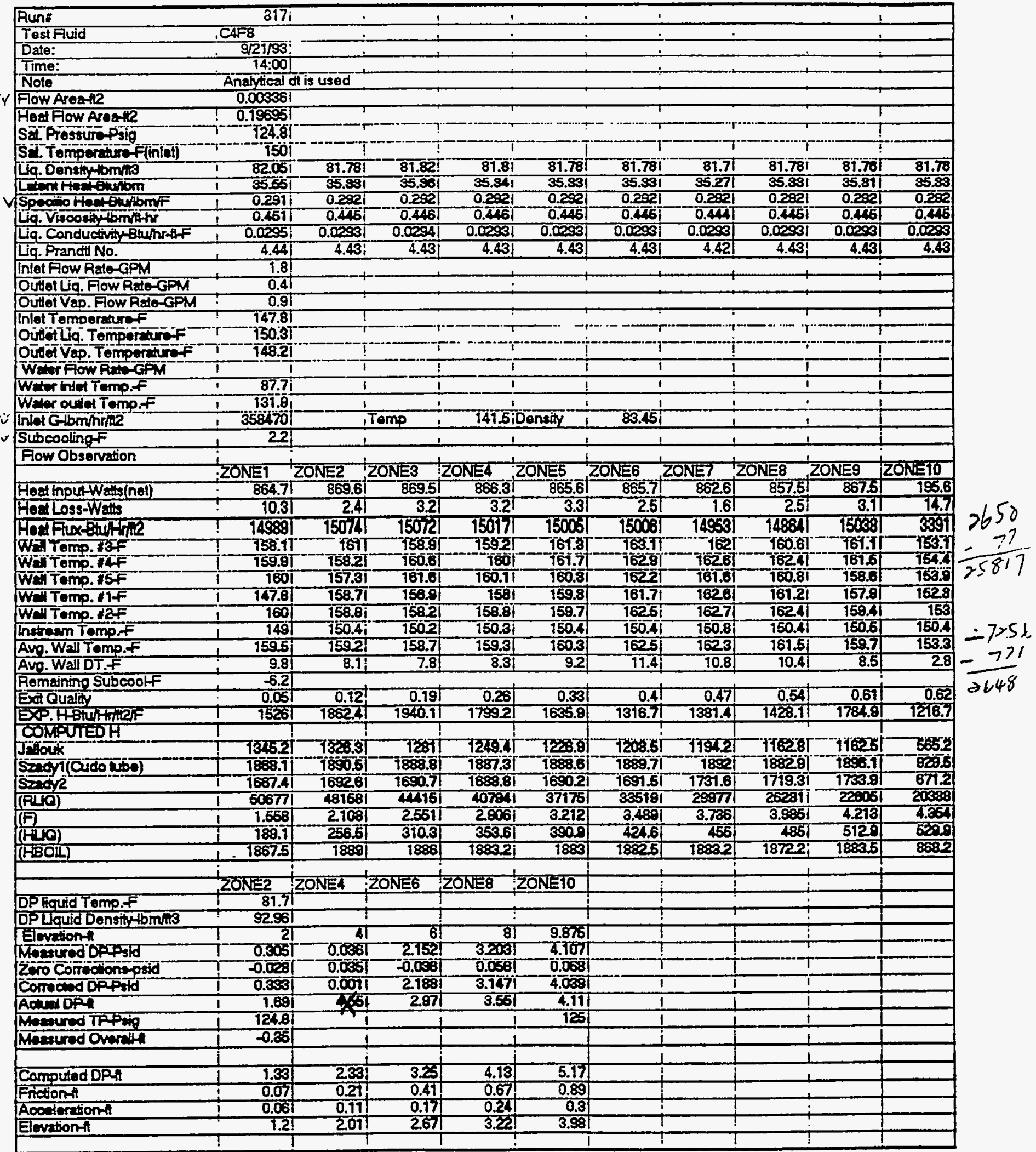




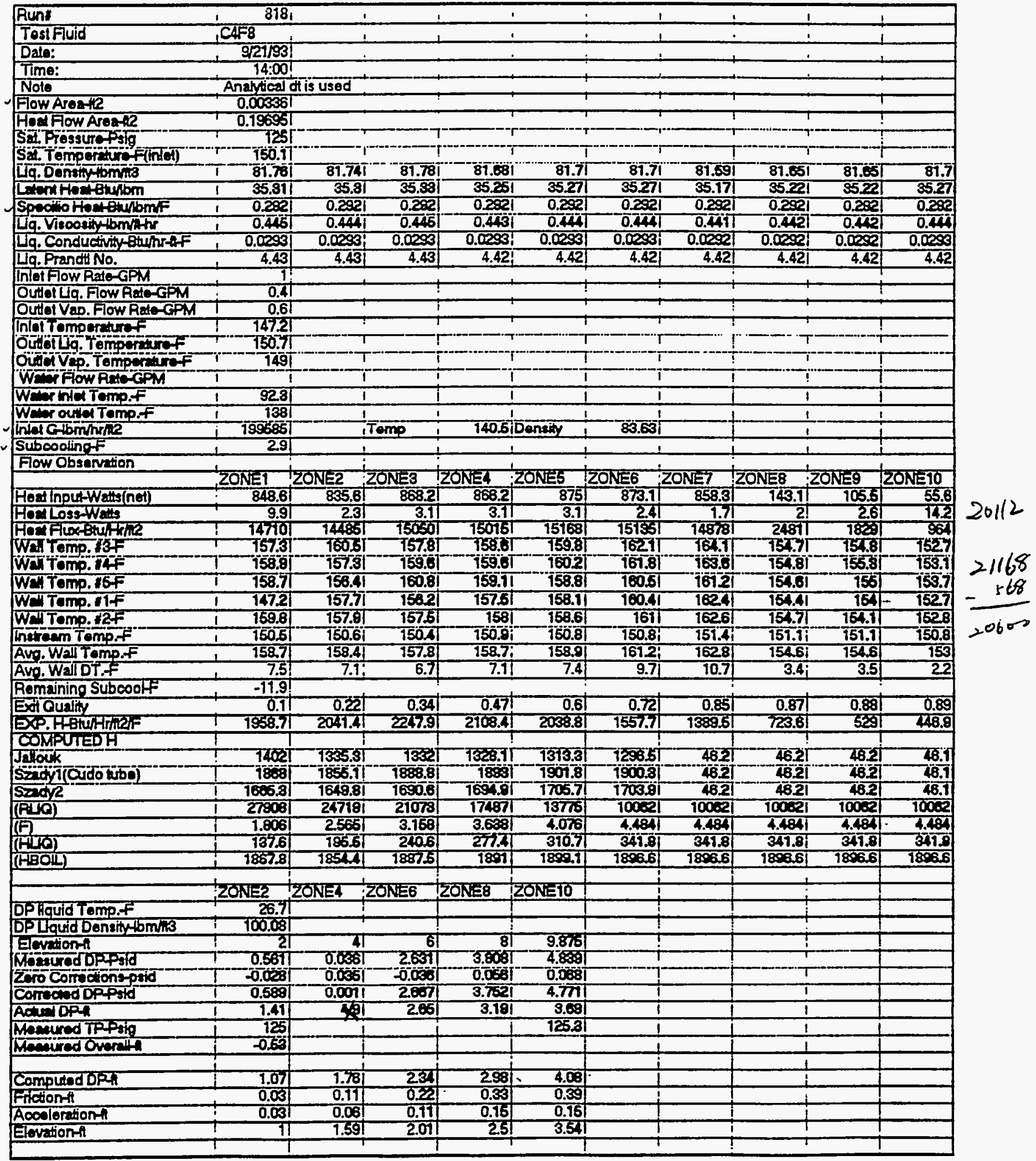




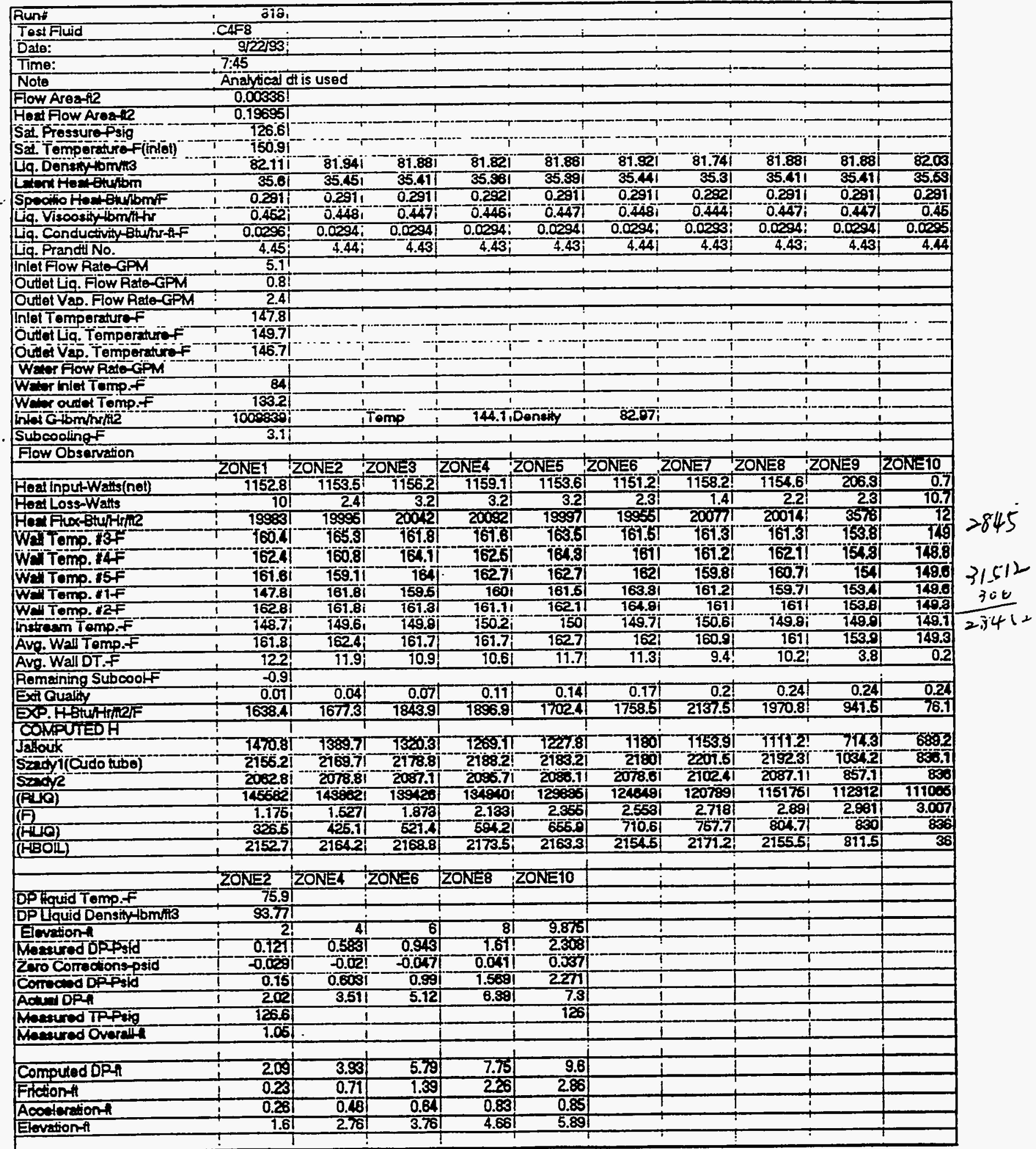




\begin{tabular}{|c|c|c|c|c|c|c|c|c|c|c|c|}
\hline Run: & 8201 & & & & & & & & & & \\
\hline Test Fund & TCAF8 & & & & & & $T$ & & & 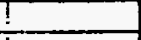 & \\
\hline Dex: & 19201931 & & $\bar{\vdots}$ & $\therefore$ & ! & & \pm & 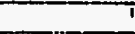 & T & I & \\
\hline Time: & $17: 45 \quad 1$ & $i$ & $!$ & 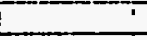 & $i$ & 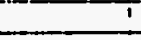 & 4 & & & $\because$ & \\
\hline Now & 1 Antriond & dis used 1 & 1 & 1 & 1 & 1 & 1 & & $i$ & 1 & \\
\hline Flow ANoath? & $10.00336 i$ & $i \quad 1$ & 1 & 1 & 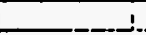 & & 1 & & 1 & $\therefore$ & \\
\hline Heafflow Areatt2 & 0.18685 & 1 & 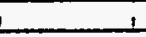 & 1 & $\perp$ & $\perp$ & 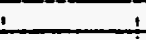 & 1 & \pm & 1 & \\
\hline Sol Prossuro Phig & 127 & $\div$ & $\perp$ & $\perp$ & 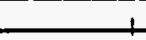 & & 1 & & & i & \\
\hline Sat. Temperature $f$ (inlel) & 151.1 & & & & & & & & & & \\
\hline Lq. Density tbmura & 82.09 & 81.88 & 81.82! & 81.72 & $81 . \%$ & 81.76 & 81.681 & 81.8 & 81.78 & 81.88 & \\
\hline Letent Hosterentbm & 35.58 & 35.451 & 35.361 & 35.28 & 35.331 & 35.31 & 35.251 & 35.34 & 35.33! & 35.39 & \\
\hline Spectio Hestanehbmir & 0.291 & 0.291 & 0.2921 & 0.29 & 0.251 & 0.29 & 0.2921 & 0.292 & $0 . \mathrm{X}^{2}$ & 0.291 & \\
\hline Lq. Viscosiny & 0.4521 & -0.447 & $0.446 \mathrm{~T}$ & 0.444 & 0.445 & 0.4451 & 0.4431 & 0.4461 & 0.475 & 0.447 & \\
\hline La. Conductiviy Etuhrsf & 0.0255 & 0.02341 & $0.029 \pi$ & 0.02831 & 0.02531 & 0.02931 & 0.02831 & 0.023 & 0.0031 & 0.028 & \\
\hline Lq.Prander No. & 4.461 & 4.431 & 4.431 & $4 . \overline{21}$ & 4.431 & 4.491 & 4.42] & 4.431 & 4.43 & 4.43 & \\
\hline Intorfow RotG-GPM & 4.31 & -1 & 1 & $i$ & 1 & & -1 & -1 & & i & \\
\hline 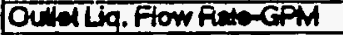 & 0.91 & 1 & 1 & 1 & 1 & 1 & $i$ & 1 & I & 1 & \\
\hline Outer Vap. Flow Palo-GPM & 2.1 & 1 & $\perp$ & 1 & 1 & $!$ & $!$ & & & 1 & \\
\hline Intat Temperaturef & 147.5 & $\perp$ & 1 & 1 & 1 & $\perp$ & $\perp$ & & & $i$ & \\
\hline Outel Lig. Tomporaturef & 149.61 & & + & 1 & L & & $\perp$ & 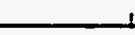 & & $\perp$ & \\
\hline Oudet Vap. Temporature of & 147.5 & & & & & & & & & & \\
\hline Water fiow Rate-GPM & & & & & & & & & & & \\
\hline Water inlel Tomp.f & 85 & & & & & & & & & & \\
\hline Wator outla tomp.F & 133.11 & & $:$ & 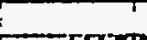 & 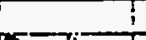 & 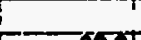 & 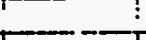 & & & & \\
\hline 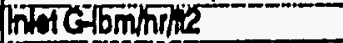 & 250671 & & Temp & 14.5T & Donsiny & 82.91 & T & & & & \\
\hline Suboooling & 3.61 & $\mathbf{T}$ & 5 & 1 & 2 & & $T$ & & & & \\
\hline Flow Observation & ! & $I$ & 1 & $T$ & 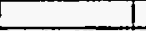 & & 1 & & 1 & & \\
\hline & ZONE 1 & $20 \mathrm{~N}=2 \quad 1$ & ZOONES & ZONEA 12 & ZONEE 12 & ZONE 6 & IZONET I & ZONEE & TCONES I & I2ONE10 & \\
\hline Heathointwatent) & 11168.61 & 11591 & $116 \overline{5} .81$ & 117021 & 1165 & 1166.7 & 1167.81 & 1168.01 & 206.6 & 197.4 & \\
\hline Hoed Loentwath & $9.1 j$ & $2 I_{1}$ & 2.91 & $20 i$ & 291 & 21 & 1.31 & 2 & & 10 & \\
\hline 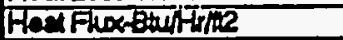 & 20170 & 200981 & 2000 & 20285 & 20021 & 20051 & 2005 & 20175 & 3567 & 82 & \\
\hline Wall Tamp. 84 & 160,4 & 165.3 & 161.7 & 152. & 1642 & IE211 & 161.8 & 1529 & $15 \mathrm{BS}$ & 150.8 & \\
\hline Wa Tomp. I4 & 1623 & 160.7 & 164.2 & 1631 & 164.7 & 161.7 & 161.7 & 162.5 & 154.8 & 150.9 & \\
\hline WaH Tomp. $15+$ & 161.8 & 159.3 & 164.2 & 163.3 & 163.1 & 162.4 & 160.3 & 167.3 & 1545. & 151.8 & \\
\hline Wall Tomp. ITF & 147.61 & 161.8 & 159.61 & 160.61 & 162 & 164 & 161.61 & $1 \notin 0.4$ & 753.11 & 151 & $\psi$ \\
\hline Wail Tomp. & 1627 & 161.8! & 161.31 & 161.61 & 15251 & 165.31 & 161.4 & $161.5 !$ & 153.7 & 351 & \\
\hline Instream Tomp. $F$ & 148.81 & 149.81 & 15021 & $150.7 !$ & 150.41 & 15051 & 150.91 & 150.3 & 150.41 & 150 & \\
\hline Ävg. Wa Temp.tF & 161.81 & 16241 & 161.71 & 16231 & 163.11 & 16281 & 161.4 & 161.7 & 754.1 & 151.1 & \\
\hline Avo.WallDT.F & 12.11 & 11.81 & 10.61 & 10.81 & 11.81 & 11.71 & $\overline{9.8} \mid$ & 10.5 & 3.8 & & \\
\hline Romining subcootf & -1.11 & 1 & 1 & -1 & 1 & 1 & I & & & 1 & \\
\hline Excianty & 0.011 & 0.051 & 0.091 & 0.181 & 0.17 & 0.211 & 0.21 & 0.28 & 0.29 & 0.20 & \\
\hline Exp.HEWH & 16686 & 173271 & 181251 & 1807.71 & 1701 & 1789.31 & 21005 & 1820 & 1001,4 & 8030 & \\
\hline COMPUTED & 1 & & & & & & & & & & \\
\hline Jallouk & 1573.6 & 14333. & 135871 & 13243 & 1257.9 & 1239.1 & 1205.9 & 1173.2 & 67.9 & $\$ 7.7$ & \\
\hline Szadv1(Cudo tube) & 2168.3 & 2178.2 & 2190.2 & 2203.6 & 2187.5 & 2193 & 2201.6 & 2202.3 & 1010.4 & 787.8 & \\
\hline Sady2 & 2077.1 & 2000,4 & 21029 & 2147.3 & 2094.2 & 2009.7 & 2106.2 & 2104.1 & 818.2 & 78.9 & \\
\hline (RLO) & 1220081 & 120809 & 11629 & 71185 & 105665 & 101871 & 973501 & 91835 & 8500 & 8822 & \\
\hline & T.20 & 1.555 & 1.972 & 2.255 & 2.51 & 2.7ता & $28 \pi$ & 3.0851 & 3.Tह्या & 3.208 & \\
\hline$(F(J a)$ & खक्ये & $387.1 !$ & 478.91 & 548.31 & 607.4 & 658.7 & 704.51 & 749.31 & 773.31 & 778.8 & \\
\hline (FBOL) & 2184.51 & 2174.11 & 218251 & 2TES31 & 2171.9 & 21731 & 2177.31 & 2973 & 828.61 & 250.7 & \\
\hline & & -4 & 1 & ! & +1 & 1 & I & & & I & \\
\hline & ZONE2 & 1ZONEA & 120N38 & ZONEB & ZONE10 & I & I & & $I$ & & \\
\hline DP hquid Temp.f & $1 \quad 77.21$ & $T$ & 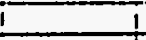 & 1 & 1 & 1 & 1 & & 1 & & \\
\hline DP Lequid Densit $16 \mathrm{~m}^{2} 3$ & 8359 & & $t$ & $\frac{1}{21}$ & 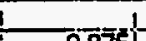 & $1-$ & 1 & & 1 & & \\
\hline Elewhiont & $2 i$ & & 61 & 8 & 9.876 & & 1 & & & & \\
\hline Mesured DPspsid & 0305 & 0.755 & 1.228 & 2008 & 276 & & 1 & & & & \\
\hline Zoro Contections-psid & -0.06 & -0.02 & -0.047 & 0.047 & 0.037 & & & & & & \\
\hline Comedod DPs fld & 0.234 & 0.775 & 1.275 & 1.587 & 2.723 & & & & & & \\
\hline Achel DPA & 1.88 & 3.21 & 4.62 & 5.69 & 6.51 & & & & & & \\
\hline Maseved TP & स्टा & & & & 126.8 & & & & & & \\
\hline Meanerod Overalt & 0.351 & & & & & & & & & 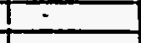 & \\
\hline & & & & & & & & & & & \\
\hline Comptred DPA & 7.851 & 3.011 & $5.28 !$ & 7.021 & 8.74 & & 1 & & $I$ & & \\
\hline Filong & 0.181 & 0.67 & 1.18 & 1.851 & 2341 & & 1 & & 1 & & \\
\hline Aoosterciontl & $0.2 \pi$ & 0.37 & 0.62 & 0.691 & 0.711 & & 1 & & $I$ & & \\
\hline Eloviont & $1.56 \mathrm{i}$ & 2.691 & 3.52 & 4.481 & 6.69 & & 1 & & 1 & $i$ & \\
\hline
\end{tabular}




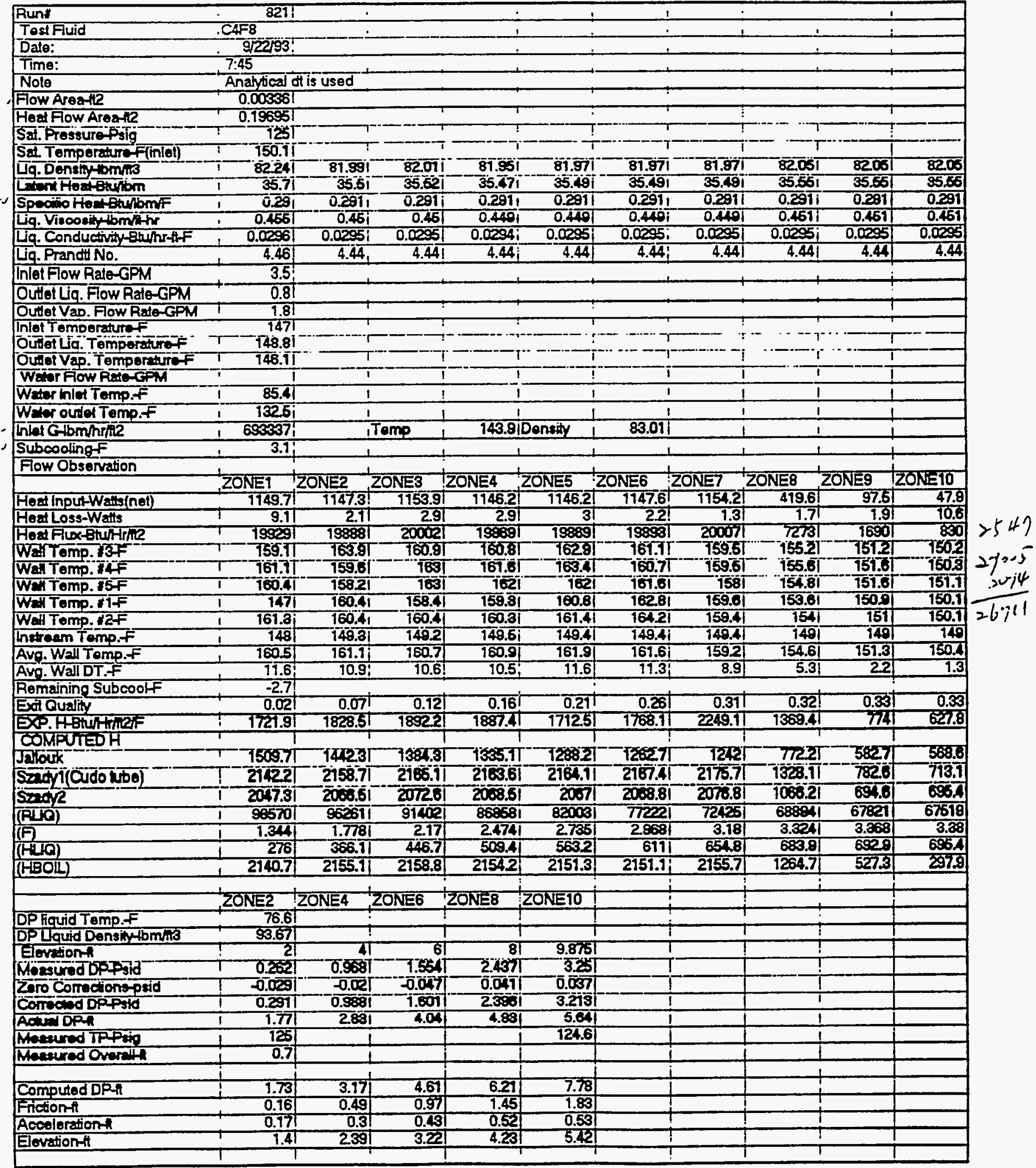




\begin{tabular}{|c|c|c|c|c|c|c|c|c|c|c|c|}
\hline Run: & $222 i$ & $i$ & - & 1 & & - & $\cdot$ & 1 & & 1 & \\
\hline Tostfuid & CAF & $\therefore$ & & & & 1 & 1 & & 1 & 1 & \\
\hline Dale: & $922193 !$ & & & & & & & & & & \\
\hline Time: & $7: 45$ & & & & & & & & & & \\
\hline Note & Analyical c & this used & & & & & & & & & \\
\hline Flow Aroath & 0.003361 & & & & & & & & & & \\
\hline Hod Flow Areatl & 0.1955 & & & & & $!$ & $\mathrm{T}$ & $T$ & & & \\
\hline Sa. Piossure prig & 126.95 & $T$ & $\Gamma$ & $?$ & & & $T$ & T & 7 & & \\
\hline 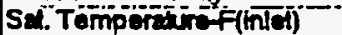 & $-15 \overline{1.11}$ & T & $\mathbf{T}$ & $-\infty-\cdots$ & & T & 1 & & 1 & & \\
\hline Ljg. Denanytomms & 82241 & 81.741 & 81.70 & 81.741 & 81.781 & 81.701 & 81.72 & 81.781 & 81.781 & 81.00 & \\
\hline 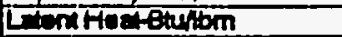 & 35.71 & \$.31 & $35.81 i$ & 35.81 & 35.31 & 35.811 & 35.28 & 35.381 & 35.89 & 85.89 & \\
\hline 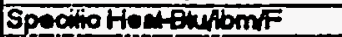 & 0.291 & $0.252 !$ & 0.292 & 0.2921 & 0.282 & 0.2321 & 0.282 & 0.282 & 0.25 & 0.291 & \\
\hline Lig. Visoosiflom/her & 0.465 & $0.44 i$ & 0.446 & $0.444 i$ & 0.4461 & $0.445 i$ & 0.444 & 0.445 & 0.475 & 0.467 & \\
\hline Lig. Conductivity-Btwhraf & 0.02061 & 0.02931 & 0.0239 & 0.02931 & 0.0233 & 0.0283 & 0.0233 & 0.0203 & 0.0023 & 0.0204 & \\
\hline Lig. Prandtt No. & 4.46 & 4.43 & 4.43 & 4.43 & 4.43 & 4.43 & 4.42 & 4.431 & 4.43 & 4.43 & \\
\hline Inletflow Rate-GPM & $2.6^{\prime}$ & & & & & & & & & & \\
\hline Outlet La. Fow Rato-GPM & 0.61 & & & & & & t & 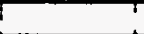 & & & \\
\hline Outlot Vas. Fow Rade-GPM & 1.71 & $T$ & r & & & & & & ?t: & T & \\
\hline Inlot femporaturef & 147.31 & & & & & $T$ & i & & & ! & \\
\hline Oư & $750.4 T$ & & & ᄀ' & & $T$ & T & $\Gamma$ & & & \\
\hline 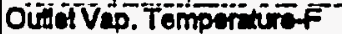 & 147.71 & & & ו & - & $i$ & 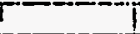 & $T$ & $T$ & & \\
\hline WAOP FOW PAOGPM & -1 & i & i & I & E & i & 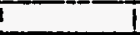 & L & I & & \\
\hline Werknet Temp.f & 87.6 & 1 & 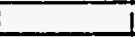 & 1 & 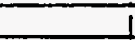 & 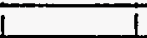 & 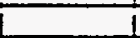 & 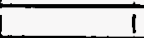 & 1 & & \\
\hline Were outed Tomp.f & 137.11 & 1 & 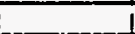 & 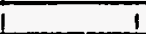 & 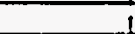 & 1 & 1 & & $i$ & & \\
\hline inter Gibm/hritiz & 515050 & & Temp & 743.81 & Density & 83.011 & & & $i$ & & \\
\hline Subcoolingf & 3.8 & & & i & & 1 & 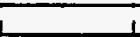 & + & 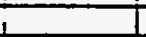 & & \\
\hline \multirow{2}{*}{ Flow Obsenvation } & & & & & & & & & & & \\
\hline & ZONE1 T & ZONE2 2 & ZONE3 & ZONEA & ZONE5 & ZONE6 & ZONE7 & ZONEB & ZONEG & $201=10$ & \\
\hline Hoed Input-Wats(not) & 1159 & 1158.1 & 1154.7 & 1154.7 & 1164.8 & 17क्. & 1147.8 & 418.2 & 96.8 & 45 & \\
\hline Hogt Loss-Walls & 9.8 & 2.31 & 3.2 & 3.2 & 3.2 & 2.4 & 1.4 & 1.81 & 22 & 12 & 25659 \\
\hline Hoa Flox-8tuHrme & 20001 & 200751 & 20003 & 20003 & 20191 & zoess! & 19898 & 72491 & 1601 & 㟧 & 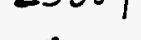 \\
\hline WETtemp 10F & 359.71 & 167.41 & 1811 & 16131 & 10321 & T62 & 760.81 & 155.51 & 1524 & T51.8 & $2900 \mathrm{c}$ \\
\hline Wh Tomp. T4F & 181.7 & $160.1 T$ & 163.81 & 16 हूरा & 163.8 & 162 & 160 & 155.61 & 15201 & 75t.5 & 1007 \\
\hline We Tomp. $15 \%$ & 101.31 & 158.91 & 168.8 & 10281 & $102 \mathrm{C}$ & 10201 & 759.8 & 155.11 & 1520 & 1524 & \\
\hline WE TOMP. HF & 147.8 & 160.91 & 158.8 & 160 & 181.4 & 163.51 & 181.1 & 154.61 & 152 & 151.8 & 190.13 \\
\hline Wall Tomp. NaF & 161.6 & 160.01 & 160.8 & 160.8 & 161.8 & 164.8 & 160.8 & 154.0 & 152. & 151.6 & \\
\hline Lnetream Tomp.F & 148 & 150.6 & 150.6 & 150.61 & 150.5 & 150.5 & 150.7 & 150.4 & 150 aो & 160 & \\
\hline Avg. Wall Tomp.F & 161.1 & 161.6 & 161 & 161.4 & 1524 & 1626 & 160.3 & 155.11 & 1525 & 151.6 & \\
\hline Avo. WallDT.F & 122 & 10.1 & 9.6 & 9.9 & 11 & 11.21 & 8.7 & 4.4 & 2 & 1.5 & \\
\hline Romaining subcooff & -4.11 & & & & & & & & & & \\
\hline Ext Quality & 0.031 & 0.1 & $0.16 !$ & $0.23 !$ & 0.29 & 0.36 & 0.42 & 0.44 & 0.45 & 0.45 & \\
\hline EXP.HHETUHIMLIF & $1651.2 !$ & 1994| & 2000.2 & 2018.5 & 1837.61 & 1800.8 & 2288.9 & 1642.8 & 82451 & 511.6 & \\
\hline CoMPUIEDH & & & & & & & & & 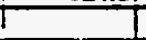 & & \\
\hline jouk & 1562 & $74972 !^{-}$ & $74503]$ & 7407.51 & $7378.6]$ & 6351.61 & 13035 & 778.91 & 5ट्राइ & 297.8 & \\
\hline Szady (cudo subo) & $2150.81^{\circ}$ & $218 \overline{5.81}$ & 2181.7 & 2184.21 & 2194.91 & 2030.71 & 2183.9 & 1320.6 & 737.8 & QM.0 & \\
\hline $\sin \sqrt{2}$ & 2068.1 & 2056.81 & 2094.7 & 20030.41 & 2107.4 & 2118.81 & 2090.7 & 1051.81 & Еटі.1 & 6005 & \\
\hline (FILO) & 72007 & 70637 & 65881 & 611101 & 56148 & 512081 & 46889 & 429581 & 41805 & दT4ा6 & \\
\hline$(F)$ & 1.438 & 1.9471 & 2.388 & 2751 & 3.058 & 3.3821 & 3.577 & 3.7481 & $3 . \pi^{\prime}$ & 3.818 & \\
\hline (HUD) & 2328 & 316.8 & 390 & 447.6 & 497.4 & 542 & 5821 & 608.81 & 617.1 & E24 & \\
\hline \multirow[t]{2}{*}{ (FBOII) } & 2149.7 & 21835 & 2175 & 2177.9 & 2985.7 & 2189.7 & 2170 & 12824 & 5404 & 307.1 & \\
\hline & ZONEEZ & ZONE4 & $\mathrm{ZON}=6$ & ZONE8 & ZOKETO & & & & & & \\
\hline DP fiquid Temp.f & 77.8 & - & & 然 & & & & & & & \\
\hline DPUquid Density $4 \mathrm{~m} / 13$ & 93.511 & $T$ & & & & & & & & & \\
\hline Elevation-h & & 41 & 6 & 81 & 9.8751 & & & & & & \\
\hline Nhenured DP & 629 & T.I6) & 1.8871 & 2.8781 & 3.781 & & & & & & \\
\hline Zoro Correctionssostd & 0.0091 & $0.02 \Gamma$ & -0.047 & 0.04 & 0.057 & & & & & & \\
\hline Coménd DP Pid & 0.3191 & 1.1831 & 1.928 & 28321 & 3.72 & & & & & & \\
\hline Actua DPA & 1.72 & 2481- & 3.48 & 4.101 & 4.78 & & L & & & & \\
\hline Necoured TPPsig & 1260 & $i$ & - & -1 & 126.6 & $t$ & 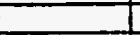 & & & & \\
\hline \multirow[t]{2}{*}{ Maneured Overalle } & 0.53 & $i$ & & & & & & & & & \\
\hline & & & & & & & & & & & \\
\hline Computed DPA & 7.51 & $2 \pi$ & 3.87 & 5.09 & 6.5 & & & & & & \\
\hline Frictionft & 0.11 & 0.55 & $0 . \pi 1$ & 9.13 & 1.39 & & & & & & \\
\hline Accalorationt & 0.11 & 0.21 & 0.32 & 0.4 & 0.4ा & & & & & & \\
\hline Elevecion-h & 1.2 & 2.75 & 284 & 3.561 & 4.ता & & & & & & \\
\hline
\end{tabular}




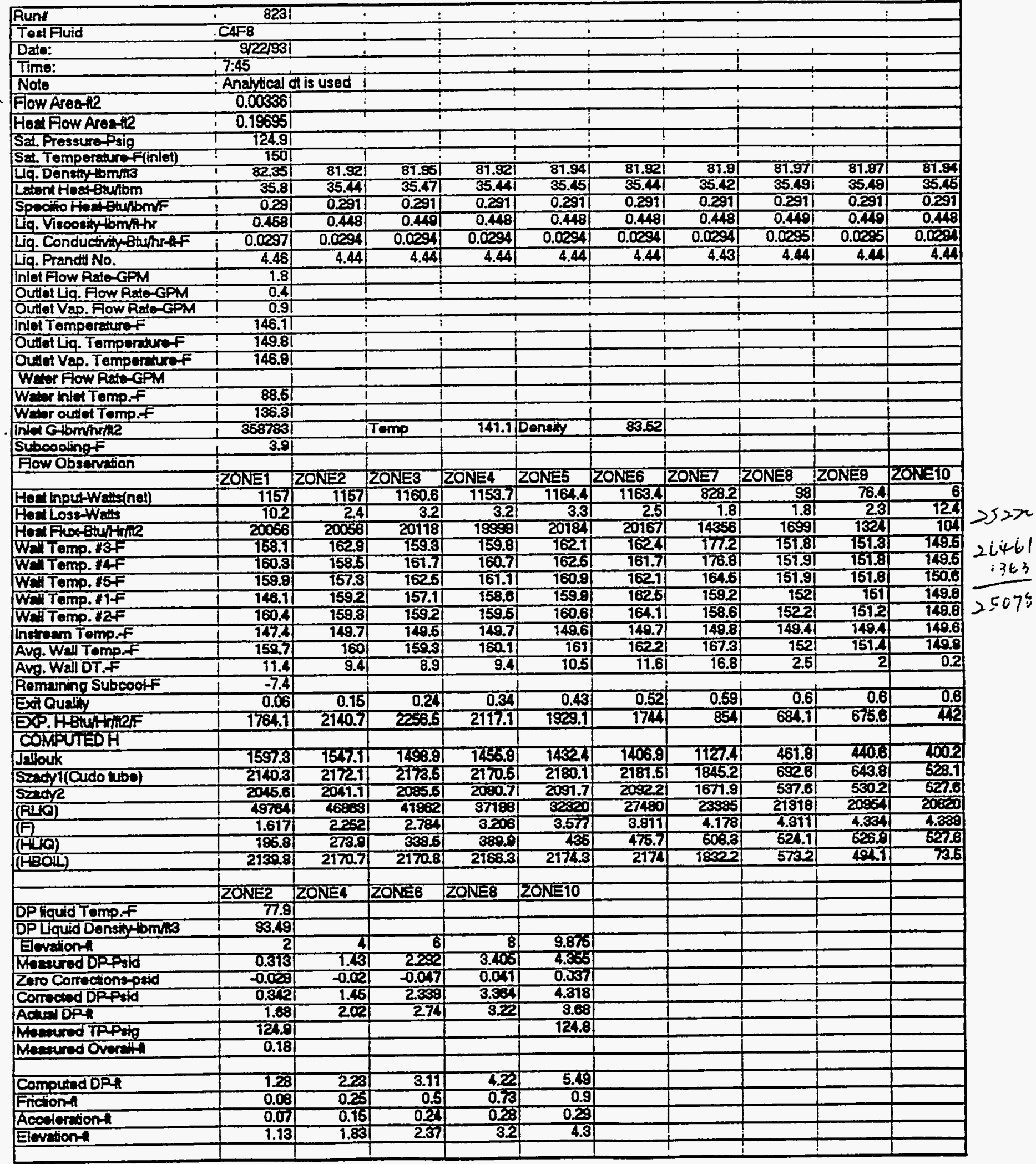




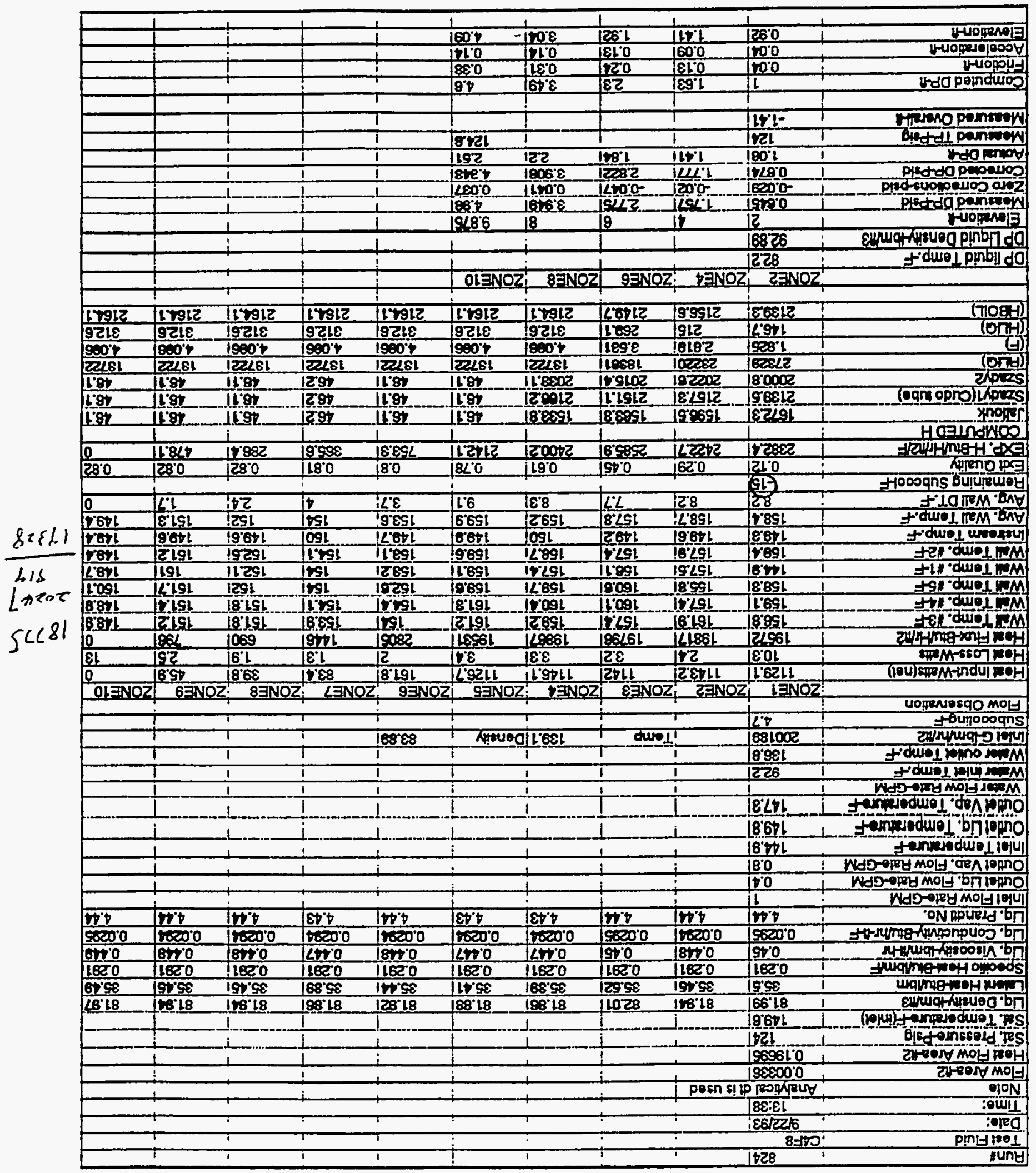




\begin{tabular}{|c|c|c|c|c|c|c|c|c|c|c|c|}
\hline Funn & 025. & $\dot{-}$ & - & - & 1 & - & & . & 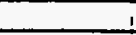 & 1 & \\
\hline TestFluid & CAf 8 & $\therefore$ & 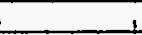 & & & & & + & 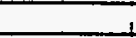 & & \\
\hline Date: & 212290: & & & & & & & & & & \\
\hline Time: & $13: 38$ & & & & & & & & & & \\
\hline Note & Analyticald & dt is used: & 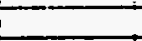 & & & & & & & & \\
\hline Fow Areatle & 0.003361 & & & & & & & & & & \\
\hline Hoat Fow Areatil & 0.196951 & & & & & & & & & & \\
\hline Sat. Prossure-psiq & 127.51 & & & & & & & & & & \\
\hline Sat. Temperature F(inlet) & $1 \quad 151.4 !$ & & & & $!$ & & -1 & $\frac{1}{0-1}$ & & & \\
\hline Lq. Dentry $10 \mathrm{~m} / \mathrm{s}$ & 81.991 & 81.841 & 81.78 & 81.71 & 81.781 & 81.781 & 81.631 & $81.74 !$ & 81.781 & 81.88 & \\
\hline Lent Hon-Buritom & $3 \overline{5.51}$ & $\$ 5.881$ & 35.311 & 35271 & 35.381 & 35.311 & 35.21 & 35.31 & 35.811 & $\$ 5.41$ & \\
\hline Specito thatenthom/f & $0.291 \mathrm{i}$ & 0.2921 & 0.292 & $0.292 \mathrm{i}$ & 0.2921 & 0.2921 & 0.292 & 0.2921 & 0.292 & 0.281 & \\
\hline íg. Viscosingibm/thr & 0.45 & 0.946, & $0.4 \overline{5}$ & $0.494_{1}$ & 0.445 & $0.446 i$ & 0.442 & 0.4441 & 0.451 & $0 . \overline{47}$ & \\
\hline 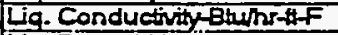 & 0.0295 & 0.0294 & 0.02931 & 0.0293 & 0.0293 & 0.0293 & 0.0292 & $0.0293 i$ & 0.0293 & 0.0294 & \\
\hline Liq. Prandeno. & 4.44 & 4.43 & 4.43 & 4.42 & 4.43 & 4.431 & 4.42 & $4.43 !$ & 4.43 & 4.43 & \\
\hline Inlet Flow Rate-GPM & 5.1 & & & & & & & & & & \\
\hline Outlat Lig. Fow Rate-GPM & 11 & & & & & & & $\therefore$ & 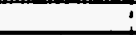 & I & \\
\hline Outlet Vap. Flow Rate-GPM & $2.6 !$ & & & & & & & & & & \\
\hline Inlot Temperaturef & 148.31 & & & & & & & & & & \\
\hline Outet Liq. Temporanuref & 149.91 & & & 17 & & & & T & & & \\
\hline Oullet Vap. Tömpor turotr & 147.8i & & i & & & & i & T & & & \\
\hline Waur fiow PIOGPM & $i^{-}$ & 1 & 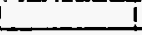 & $i$ & 1 & 1 & 1 & 1 & I & & \\
\hline Water intet Tomp.f & 90.01 & 1 & 1 & I & 1 & 1 & 1 & 1 & 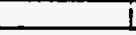 & & \\
\hline Weroutet tempef & 133.81 & & & -1 & & & $I$ & $i$ & 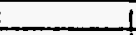 & & \\
\hline 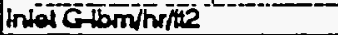 & 10064391 & & $\operatorname{Tomp}$ & $145.6 \mathrm{i}$ & Donsity & 82.691 & 1 & 1 & & & \\
\hline Subcoolingf & 3.1 & $\perp$ & 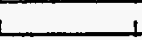 & 1 & i & 1 & + & 1 & $L$ & & \\
\hline \multicolumn{12}{|l|}{ Flow Observation } \\
\hline & ZONE9 & ZONE2 Z & ZONE3 & ZONEA I & ZONES 2 & ZONE6 I & ZONET & ZONEB & ZONE9 & ZONETO & \\
\hline Hoed input-Watts(nel) & 1447.6 & 1445.1 & 1442 & 1447.6 ! & 1077.5 & 420.1 & 210.2 & 191.8 & 117.5 & 87.5 & 2029 \\
\hline Head Loss-Walls & 10.81 & 2.51 & 3.41 & 3.41 & 3.2 & 2.11 & 1.3 & 2.11 & 2.5 & 73.9 & \\
\hline Hoed Flx 8 BuH & 25033 & 250501 & 249981 & 25093 & 18678 & 72821 & 384 & 3325 & 2037 & 1517 & \\
\hline Wa Temp. FiF & 15921 & 165.91 & 159.61 & $161.3 !$ & 160.1 & T55.81 & 155.17 & 156.11 & 15201 & 751.8 & 2337 \\
\hline We Tomp. & -16221 & 150.51 & 1628 & 1024 & 160.5 & 1581 & 1552 & 158.21 & 15361 & $15 \overline{2}$ & $3 \cdot 50$ \\
\hline Wa Tomp. $76 \mathrm{~F}$ & 160.8 & 157.81 & 16281 & 167.71 & 758.81 & 155.61 & 154.1T & 158.21 & 153.5 & 1520 & \\
\hline Wel Tomp. If & 148.81 & $160.1 \mathrm{t}$ & 158.41 & 1591 & 159 & 158.11 & 1542 & 155.71 & 152. & 161.6 & $20<21^{\prime}$ \\
\hline Woll Temp. \$2F & $\overline{1} \overline{2} .31$ & $160 . \overline{81}$ & $160 . \overline{1 !}$ & 16021 & 160.8 & 156.11 & 154.1 & 155.21 & 1520 & 151.7 & \\
\hline Instroam Temp.F & 149.3 & $750.1 i$ & 150.5 & 750.81 & 150.4 & 150.51 & 151.2 & 150.61 & 150.6 & 949.0 & \\
\hline Ang. Wall Tomp.F & 161.1 & $161.6 i$ & 160.3 & $167.7 i$ & 159.7 & 155.91 & 154.5 & 155.11 & 153.2 & 151.9 & \\
\hline Avg. Wall DT. ${ }^{\prime}$ & 10.7 & 10.31 & 8.7 & $9.2 !$ & 8.4 & 5 & 3.2 & 5.3 & 2.5 & 2 & \\
\hline Remaning Subcoolf & -1.9 & & & & & & & & & & \\
\hline Ext Quality & 0.021 & 0.061 & 0.11 & 0.14 & 0.17 & 0.18 & 0.19 & 0.19 & 0.2 & 0.2 & \\
\hline EXP.HBturtrine & 2347.11 & 2421.91 & 28829 & 2737.61 & 2221 & 1443.2 & 1147.5 & 623.8 & 799.4 & $7 m 2$ & \\
\hline COMPUTEOH & & & & & & & & & & & \\
\hline Jallouk & 678.7 & $-1578.7 \Gamma$ & 746721 & 137.51 & Tद48.5T & 780.91 & 6721 & 674.87 & 654.81 & 651.4 & \\
\hline SzadYT(Cudokubo) & 2428.71 & 2438.4 & $24 \pi, 2$ & 24581 & 2179.11 & "36.71 & 1030.11 & 98201 & 8658 & 818.2 & \\
\hline $\sin \alpha \sqrt{2}$ & 2404.81 & 2418.41 & 243.41 & 2435.81 & 2001 & $11 \overline{04.41}$ & 8061 & 793.91 & 769.8 & 773.8 & \\
\hline (ADO) & 145248 & 1421161 & 130048 & 1307971 & 124968 & 121927 & 121839 & 1190511 & 119087 & 118007 & \\
\hline (F) & 1.281 & 1.679 & 2046 & 2.338 & 2546 & 2.6671 & 2.680 & 2.7361 & 2.76 & 2.78 & \\
\hline (स्थि) & 3652 & 466.61 & 568.6 & 6475 & 207.7 & 738.9 & 751.8 & 760.81 & 767.3 & 728 & \\
\hline \multirow[t]{3}{*}{ (मBOीL) } & 24242 & 24327 & 24539 & 24408 & 20025 & 1271.II & 874.6 & 798.11 & 581.1 & 445.3 & \\
\hline & 1 & 1 & & & & & & & & & \\
\hline & ZONE2 & ZONE 4 & ZONE 6 & 20158 & ZONETO & & & & & & \\
\hline DP hquid Temp.F & \begin{tabular}{|r|} 
\\
\end{tabular} & 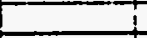 & 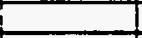 & & & & & & & & \\
\hline DPLquid Density bmins & 92.85 & & t & & & & & & & & \\
\hline Elovetiont & & 4 & & 81 & 9.875 & & & & & & \\
\hline MessuredDf4hid & 0.09 & 0.551 & 3.086 & T.82.1 & 2415 & $T$ & & & & & \\
\hline Zero Cormediong-pid & -0.009 & -0.021 & $-0 . \overline{0} 7$ & 0.051 & 0.037 & 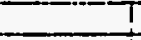 & & & & & \\
\hline Correciod Dpprid & 0.119 & 0.577 & 1.138 & 1.7831 & 2378 & 1 & & 1 & 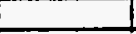 & & \\
\hline Ached DPM & 2.08 & 3.52 & 4.81 & 5.841 & 7.02 & I & & 1 & 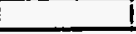 & & \\
\hline Moaved TPFiq & 127.5 & & & 1 & 1282 & I & & $\perp$ & & & \\
\hline \multirow[t]{2}{*}{ Mhasured overall } & -1.20 & & & 1 & & 1 & & & & & \\
\hline & & & & & & & & 1 & $L$ & & \\
\hline Computed DPA & 200 & 3.94 & 5.84 & $7.7 !$ & 9.57 & & & & & & \\
\hline Friction-t & 0.28 & 0.85 & 1.47 & टना & 254 & & & & & & \\
\hline Acoiterdionth & 0.32 & 0.55 & 0.67 & 0.7 & $0 . \pi$ & & & & & & \\
\hline Elovatont & 1.49 & 254 & 3.7 & $5.06 !$ & 6.321 & & & & & I & \\
\hline
\end{tabular}




\begin{tabular}{|c|c|c|c|c|c|c|c|c|c|c|}
\hline Runt & 8861 & . & 1 & - & - & 1 & 1 & $\cdot$ & 1 & 1 \\
\hline Toslfluid & . CAF8 & 7 & 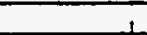 & 1 & & & & & & \\
\hline Dats: & $9 / 2293$ & & & & & & & & & \\
\hline Tims: & $13: 38$ & & & & & & & & & \\
\hline Nolo & Analyical of & It is used I & & & & & & & & \\
\hline Flow Aroati2 & 0.003361 & & & & & & & & & \\
\hline Hest Fow Arost & 0.1565 & & & & & & 1 & the & $T$ & T \\
\hline Sot Piessuro sig & -127.35 & & & & ! & 1 & $T$ & & T & T \\
\hline SA Tomperam & 151.31 & 7 & & -1 & - & -1 & -1 & & -1 & \\
\hline Lq.Donstiytomnis & 81.921 & 81.81 & 81.761 & 81.7 & 81.741 & $81 . \overline{701}$ & 81.671 & 81.741 & 81.741 & 81.82 \\
\hline Lenthedetribm & 35.41 & 35.841 & 35.811 & 35271 & 35.81 & 35.811 & 3521 & 35.81 & 35.8 & 35.88 \\
\hline Speciso Herrestomf & 0.2911 & 0.2921 & $0.292 \mathrm{i}$ & $0.292 i$ & 0.2921 & 0.2921 & 0.292 & 0.2921 & 02921 & 0.22 \\
\hline Lig. visoos & 0.4481 & $0.446 i$ & 0.4451 & 0.4441 & 0.444 & $0.445 i$ & 0.4431 & $0.444 i$ & 0.441 & 0.446 \\
\hline La. Conductivity Btuhis & 0.0294 & 0.0293 & 0.02031 & 0.0093 & 0.0233 & $0.0293 i$ & 0.02331 & 0.02931 & 0.0283 & 0.0204 \\
\hline Lig. Prandt No. & 4.44 & 4.43 & 4.43 & $4.42 !$ & 4.43 & 4.43 & 4.42 & 4.43 & 4.43 & 4.43 \\
\hline Inlat Fow Rato-GPM & 4.31 & & & & & & & & & \\
\hline Outot Lig. Fow Rate-GPM & 0.91 & & & & & & & & & \\
\hline Outlet Vao. Flow Rate-GPM & 2.11 & & & & & & & & & \\
\hline Inlet Tamperturef & 148.51 & & & & & & & & & \\
\hline 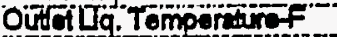 & -1501 & & & & & & & & $T$ & 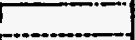 \\
\hline Ou Vop. Tomporacrof & $\overline{147.81^{-}}$ & & $T$ & [" & I & T & T & $!$ & T & $T$ \\
\hline WE FOW ADOGM & 1 & i & 1 & $i$ & $i$ & IT & $i$ & $i$ & & 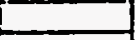 \\
\hline Wesor hilet Tomp.fF & \$0.81 & 1 & 1 & 1 & 1 & 1 & 1 & 1 & $\perp$ & L \\
\hline Werer oulat Temp ff & 133.8 & 1. & 1 & $\ldots-1$ & & -1 & $!$ & 1 & $\perp$ & L \\
\hline Inta $G$ lomming & 1848768 & & Tomp & $14 \overline{5.6 i \bar{i}}$ & Density & $827 i$ & $i$ & $\perp$ & + & $L$ \\
\hline Subcoolingf & 2.8 & 1 & & 1 & $i$ & i & i & 1 & $L$ & \\
\hline \multirow{2}{*}{ Flow Obsenvation } & & & & & & & & & \multirow[b]{2}{*}{ ZONES } & \\
\hline & ZONET & ZONEE : & ZONES T & ZONE4 & ZONE5 & ZONEB & ZONET & ZONEB & & \\
\hline Hoad Input-Watts(nol) & \begin{tabular}{|l|l|}
1637.7 \\
\end{tabular} & 1433.8 & 1443.55 & 1436.4 & 757.4 & 415.6 & 199 & 188.4 & 110.4 & $\begin{array}{r}201 .=10 \\
81.7\end{array}$ \\
\hline HenLoss-Watts & 10.91 & 2.61 & 3.4 & 3.51 & 3.11 & $2.1 !$ & 1.31 & 2.2 & 2.61 & 74.2 \\
\hline Hod Fiox-Bturtine & 233881 & 248511 & 25021 & 24891 & 130051 & 72011 & 34501 & 32681 & 1974ा & 7216 \\
\hline Wertomp. B & 158.81 & 165.97 & 959.81 & 161.81 & $758.2 !$ & $158.1 T$ & $-15,7,9]$ & 156.87 & $753.1 T$ & 7527 \\
\hline WeTtomp. In & 162.31 & 159.71 & 103 & 16241 & 158.5 & 150.81 & 155.81 & 158.41 & 753.81 & 752.0 \\
\hline WCTEMP. $16 F$ & 161 & 157.81 & 163.8 & 161.81 & 157.81 & 155.81 & $153.8 !$ & 158.41 & 153.8 & 159.2 \\
\hline Wal Temp. IIF & 148.5 & 160.21 & 158.71 & 159.11 & 15751 & 158.11 & 154 & 155.81 & 1526 & 162.1 \\
\hline Wलll Tomp. idF & 1624 & 160.8 & 1602 & 160.61 & 1584 & 156.11 & 164 & 156.91 & 169.1 & 1620 \\
\hline Inetroen Tamp. $f$ & 149.7 & $150.3 i$ & 15051 & 750.81 & 150.6 & 150.51 & 1511 & 150.61 & 150.6 & 1502 \\
\hline Avg. Wall Temp. $F$ & 161.3 & 161.7 & 150.5 & $1612 i$ & 157.9 & 156.1 & 154.4 & 156.3 & 153.1 & 1525 \\
\hline Avg. Wall DT $f$ & 10.3 & 1021 & 8.9 & $9.2 !$ & 6.7 & 5.2 & 3.31 & 5.51 & 24 & 22 \\
\hline Remaining Subcooff & -3.9 & & & & & & & & & \\
\hline Ext Quality & 0.03 & 0.08 & 0.13 & 0.18 & 0.2 & 0.22 & 0.21 & 0.23 & 0.23 & 0.24 \\
\hline EXP. Hotur Hrite $=$ & 2705 & 2430.3 & 2813.31 & 259.21 & TS33.5 & 13721 & 1056.8 & 5924 & $\$ 8 ., 1$ & 6.1.8 \\
\hline COMPUIEDH & $T$ & & & & & & & & & \\
\hline Jal ouk & 1793.81 & $1562 \pi$ & $15022]$ & TLF & 998.21 & 774.91 & GPAI & $-6 म$ & 61021 & 6044 \\
\hline 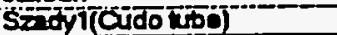 & 2589.11 & $2435.5 !$ & 249.81 & $24 \times 5.41$ & 1779.41 & $13 \overline{3} \times 4.41$ & $989.9 T$ & $\$ 5 \$, 4 T$ & 890.91 & 769.7 \\
\hline sendy & 2874 & 24101 & 2424.81 & 2428.41 & 1570.91 & 1078.81 & 761.81 & 756.41 & 362 & 78.4 \\
\hline (PUO) & 121812 & $1175 \overline{151}$ & 111680 & 1058681 & 101000 & 884071 & 970391 & 984041 & $\mathbf{9 5 8 0 0}$ & 9507 \\
\hline (F) & 1.426 & $1.876 i$ & 22का & 2.5871 & 2.787 & 2.886 & 2870 & 2916 & 2801 & 2861 \\
\hline$(\mathrm{H} U \overline{\mathrm{CO}})$ & 345.4 & 45461 & 542 & 615.81 & 664 & 688 & $6 m$ & $707 A$ & 7132 & 777.8 \\
\hline (1.30IL) & 2587.1 & 24262. & 2465.8 & 24द्2.31 & 1748 & 127021 & 8555 & 803.11 & $5 / 32$ & 447.7 \\
\hline & & & & & & & & & & \\
\hline & ZONE2 & $20 N=4$ & ZONEE & $20 \mathrm{~N}=8$ & ZONEETO & & & & & \\
\hline DP hquid Tomp.f & 82.5 & & & & & & & & & \\
\hline DPLavidDensity 16 min 3 & 92.851 & & & & & & & & & \\
\hline Eovetiont & 2 & 4! & 81 & 81 & 9.875 & & & & & \\
\hline Mécured 6pF5ld & 0.199 & 0.781 & T.365 & 2.174 & 2.8781 & & & & & \\
\hline Zoró Contoctions-peld & -0.029 & $-0.02 !$ & -0.047 & 0.047 & 0.037 & & & & & \\
\hline Cormond opprid & 0.228 & $0 . \overline{7881}$ & 1.432 & 21831 & 2.800 & & 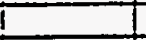 & $T$ & 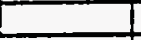 & \\
\hline Actiol OPA & 1.87 & $3.15 i$ & 428 & 5.821 & 6.28 & & I & 1 & 1 & \\
\hline Mreved TPFig & 127.3 & 1 & \pm & -1 & 1282 & & \pm & 1 & 1 & \\
\hline Meaured overall & -1.58 & & & & & & $L$ & & & \\
\hline & & & & & & & & & & \\
\hline Computed DPA & 1.86 & 3.52 & 5.27] & 7.07 & 8.74 & & & & & \\
\hline Friction-t & 0.25 & 0.75 & Tद्या & 1.5 & 2.72 & & & & & \\
\hline Accoleration & 0.28 & 0.47 & 0.54 & 0.57 & 0.58 & & & & & \\
\hline Elevationt & 1.33 & 2.31 & 3.491 & 4.81 & 6.04 & & & & & \\
\hline & & & & & & & & & & \\
\hline
\end{tabular}




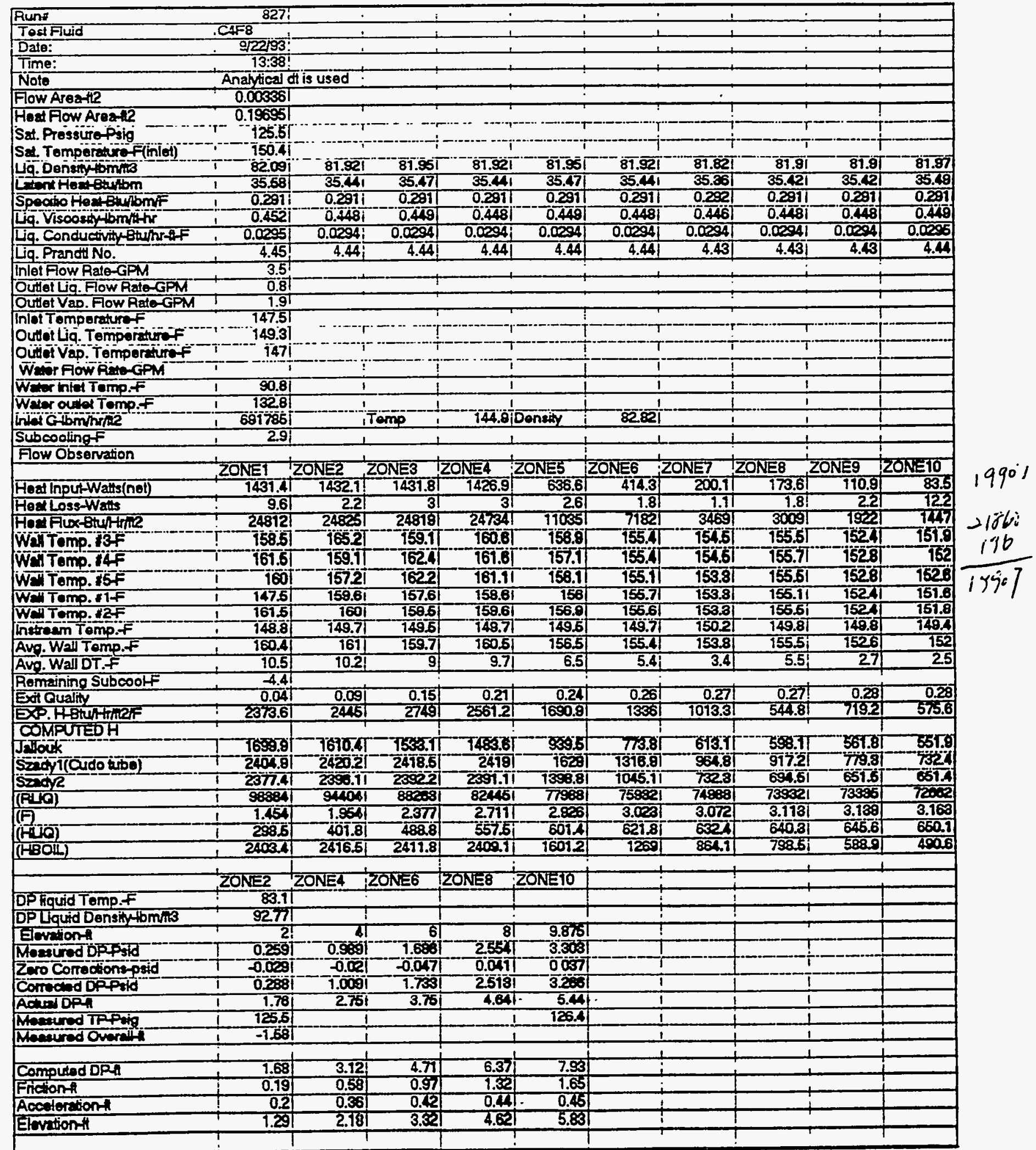




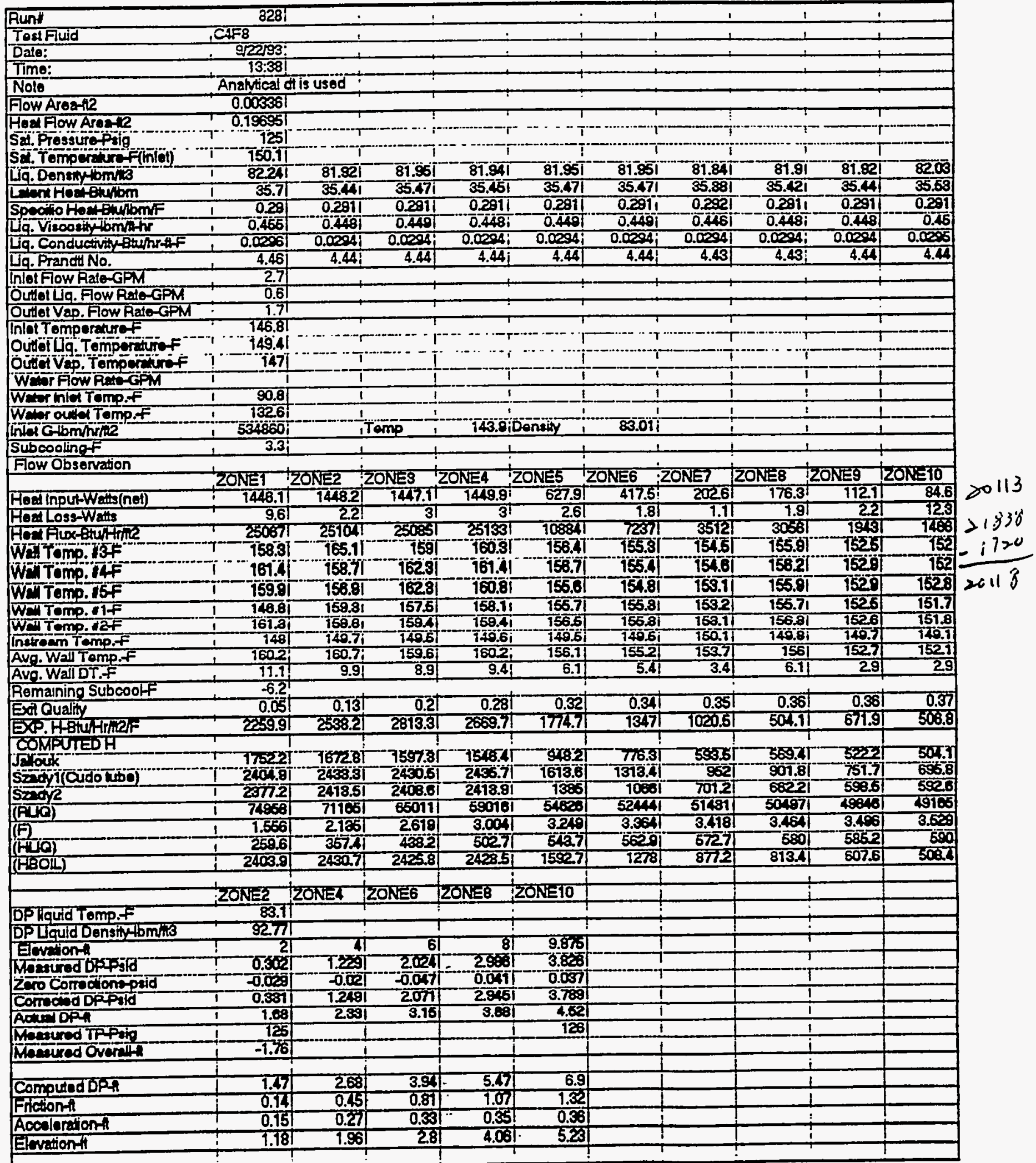




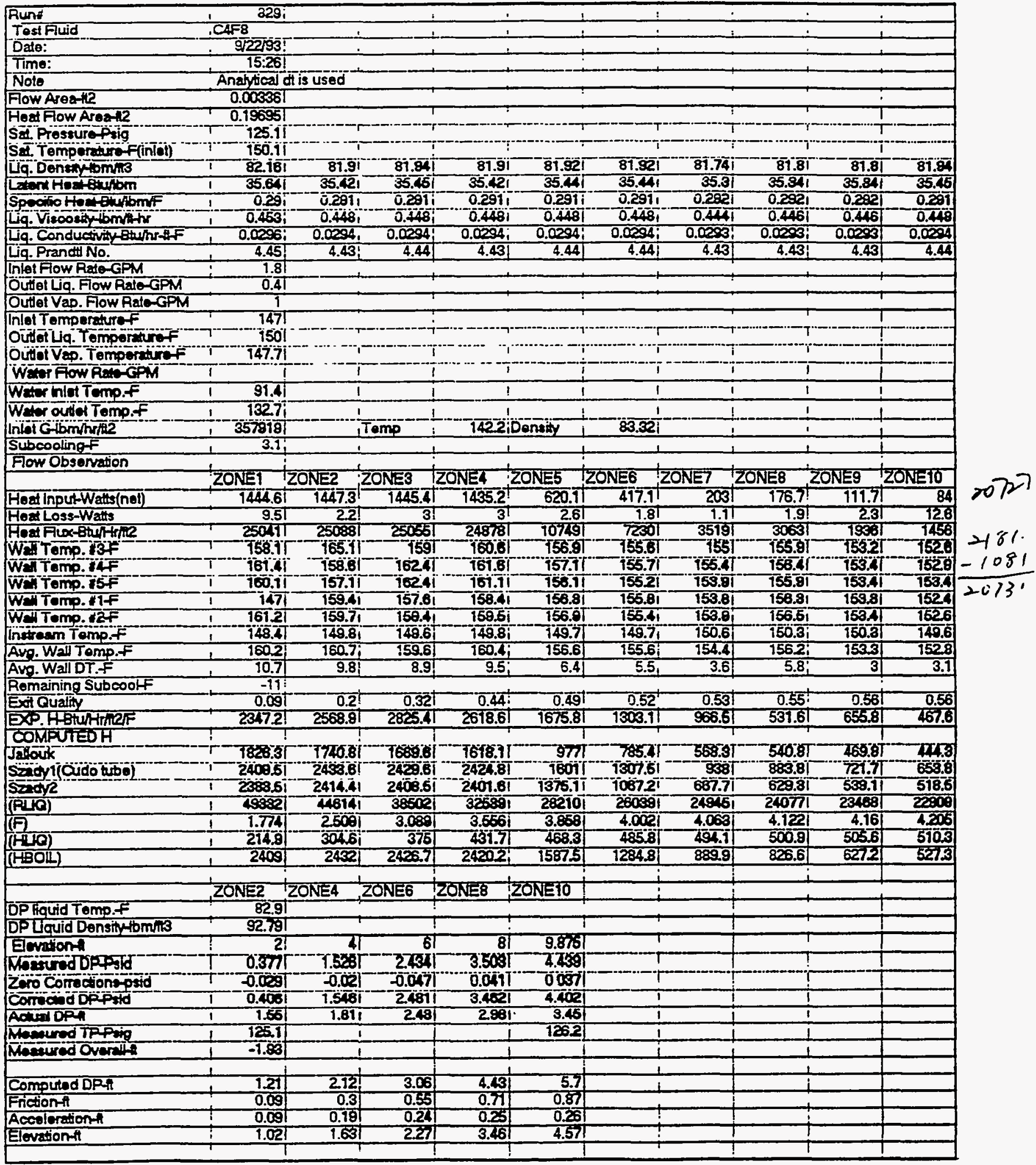




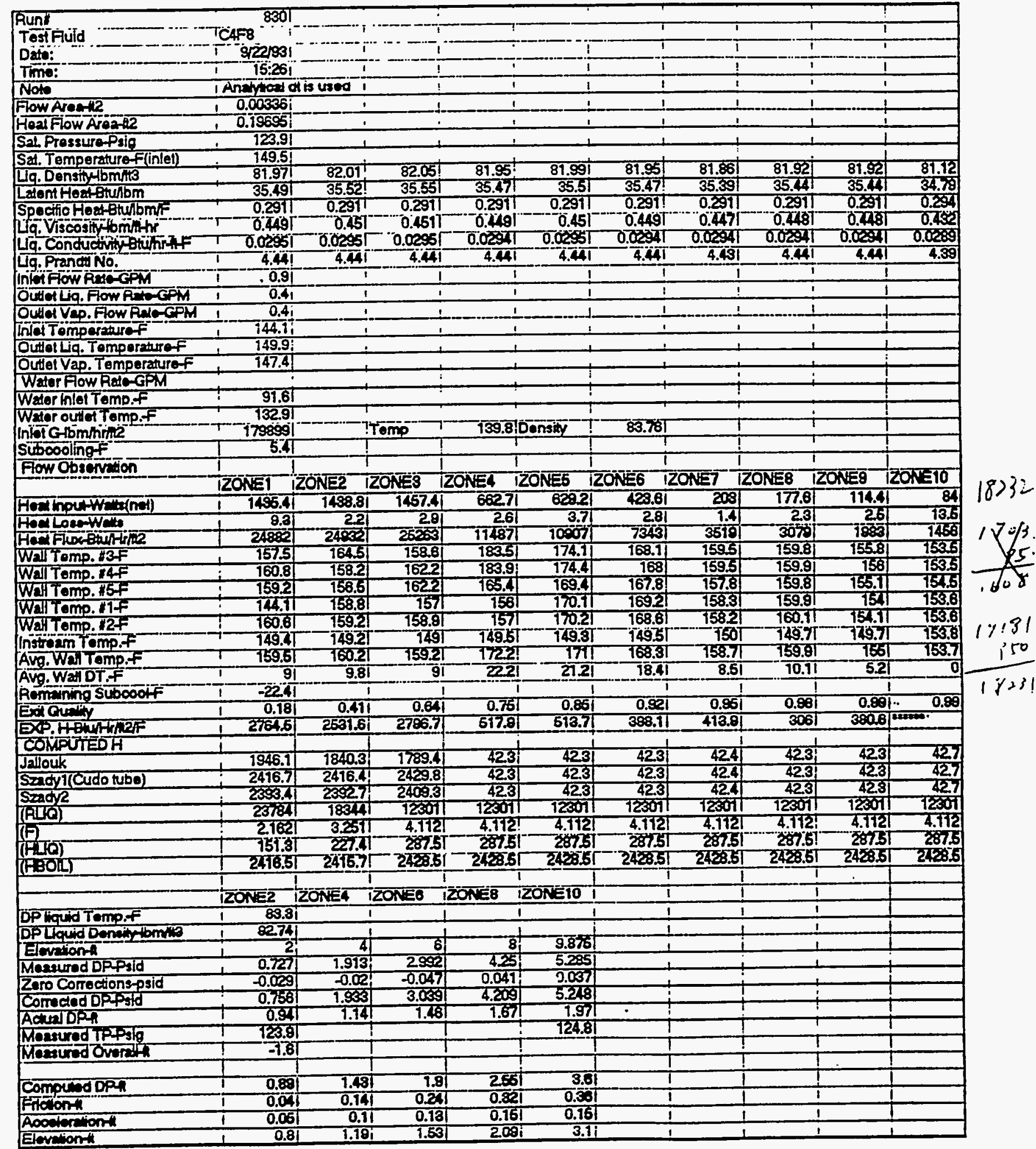




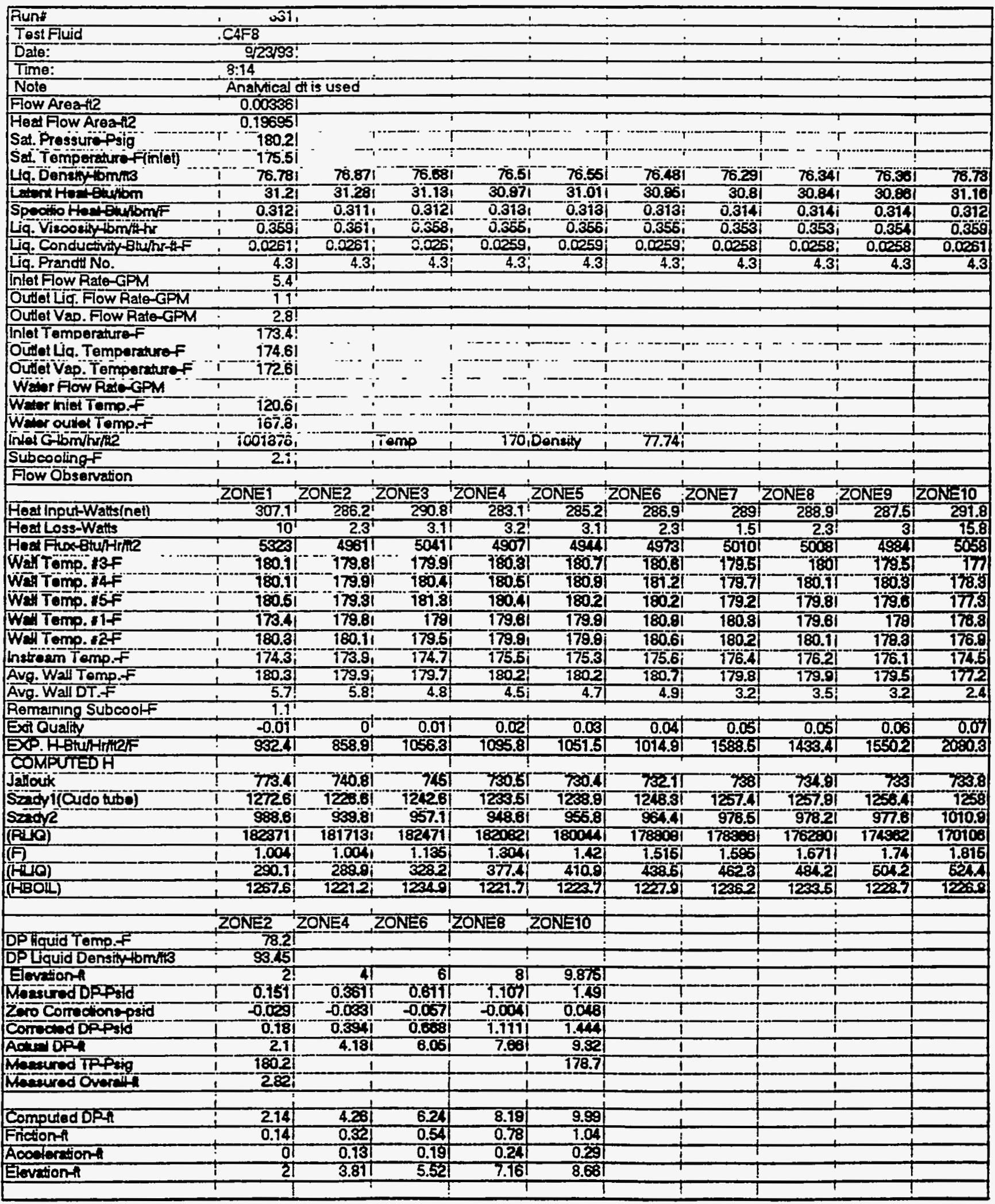




\begin{tabular}{|c|c|c|c|c|c|c|c|c|c|c|}
\hline Runy & $232 i$ & - & $:$ & 1 & i & 1 & : & . & 1 & 1 \\
\hline Tost Fluid & CAF 8 &. & 1 & 1 & & 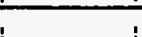 & . & 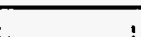 & 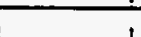 & \\
\hline Dalo: & 92393 & & & & & & & & & \\
\hline Time: & $8: 14$ & & & & & & & & & \\
\hline Note & Anahytical d & dis used & & & & & & & & \\
\hline Flow Avesth & 0.003561 & & & & & & ! & & ! & ! \\
\hline Hout Fow Arest? & 0.19695 & & & & & & & & & \\
\hline Sat. Piossurotoig & $-180.11^{\circ}$ & $\mathrm{T}$ & ( & 5 & & & & & $T$ & \\
\hline 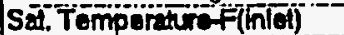 & 775.51 & ו & $-\infty$ & -7 & T & ז' & $-\cdots$ & & $T$ & T \\
\hline Uaq.Denstinyomins & 76.781 & 76.821 & 76.71 & 76.571 & 76.481 & 76.51 & 76.311 & 76.381 & 76.411 & 76.08 \\
\hline Loent heatexubm & 31.16 & 31.241 & $31.15 i$ & 31.031 & 30.851 & 30.971 & 30.821 & 30.881 & 300 & 31.18 \\
\hline 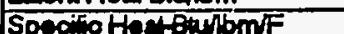 & 0.312 & 0.312 & 0.312 & 0.3131 & 0.3181 & 0.3131 & 0.314 & 0.3141 & 0.3141 & 0.312 \\
\hline 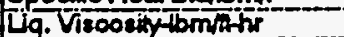 & 0.350 & $0.36 i$ & 5.358 & 0.3561 & 0.355 & $0.355 i$ & 0.353 & 0.3591 & 0.354 & 0.358 \\
\hline Liq. Conductivty-Btuhr-afF & 0.0251 & $0.0261 i$ & 0.025 & $0.025 ;$ & 0.0259 & 0.0259 & 0.0258 & 0.0258 & 0.0250 & 0.028 \\
\hline La. Prandt No. & 4.3 & $4.3 !$ & 4.3 & $4.3 !$ & 4.3 & 4.3 & 4.3 & 4.3 & 4.3 & 4.3 \\
\hline Inlot Flow Rate-GPM & 4.6 & & & & & & & & & \\
\hline Outlot Lig. Fow Rate-GPM & 1.1 & & & & & & & & & \\
\hline Out of Vap. Fow Ralo-GPM & 2.41 & & ! & & & & $T$ & & & \\
\hline Inlet Temperater of & 173.51 & & ! & & & & & & & \\
\hline Oứle Lig. TempertureF" & 174.71 & & $T$ & T & & & & & T & \\
\hline OüG Vap. Temperatiof & $-172.6 i$ & i & i & $T$ & 1 & 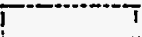 & i & $\mathrm{i}$ & T! & Iإ \\
\hline WD FOW PLE GPM & $\cdots-1$ & i & $T$ & $i$ & $i$ & it & 1 & $T$ & $t$ & 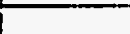 \\
\hline Weter hniet Tomp.f & 120.81 & T & 1 & 1 & 1 & 1 & 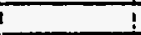 & 1 & 1 & 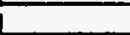 \\
\hline Wher outed Temp.f & $1 \quad 168.2$ & 1 & & 7 & & 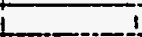 & 1 & 1 & & 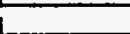 \\
\hline 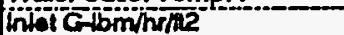 & $854662 i$ & & Tomp & $-160 \overline{6} i \mathrm{it}$ & Deneity & 77.85, & 1 & 1 & $i$ & 1 \\
\hline Subcoolingf & 2 & + & & 1 & m & 1 & & + & & $t$ \\
\hline Flow Obsenvation & & & & & & & & & & \\
\hline & ZONET & NE2 & DNE3 & ZONE4 2 & ZONE5 & ZONE6 & ZONE7 & ZONE8 & ZONE9 I & $Z O N=10$ \\
\hline Heat Input-Watts(nel) & 2991 & 285.6 & 2901 & 2824 & 287.6 & २89.2 & 288.7 & 288.11 & 289.9 & 289.2 \\
\hline Healloss-Wats & 111 & 251 & 3.51 & 3.51 & 3.4 & $2.5 !$ & 1.61 & 2.51 & 3.31 & 17.4 \\
\hline Hout floxeturthrite & 51831 & 49511 & $502 \pi$ & 48551 & 4965 & 50131 & 50011 & क्राप & 50025 & 5013 \\
\hline WE Tomp. T3F" & -180 & $779.7 \pi$ & 186.1I & 18.11 & $780.5 \mathrm{~T}$ & 780.4 & 174.4 & 979.81 & $7 \% .01$ & 777.3 \\
\hline Wétomo. & $180.2 i$ & 179.91 & 180.61 & १क्ठ5! & i81.11 & 18121 & 179.61 & 180.21 & 180.81 & 178.4 \\
\hline WE Tomp. 15F & 180.51 & $179.5 i$ & 181.81 & 180.11 & 180.41 & 180.81 & 1792 & 179.61 & 179.7 & 177.3 \\
\hline WEI Tomp. If & 178.61 & $-\overline{1801}$ & 179.11 & 178.61 & 180.11 & 1811 & $180 \mathrm{Al}$ & 179.71 & 170 & 176.8 \\
\hline WलIT Tomp. RT & $180.6 i$ & 180 & 178.71 & $178.8 \mathrm{i}$ & 180.1 & 180.41 & 180.81 & 18021 & 179.8 & 17 \\
\hline ingtroum Tomp.f & 774.51 & $774.7 i$ & 174.6 & $1762 i$ & 776.6 & $775.5 i$ & 176.3 & 176 & 175.01 & 174.9 \\
\hline Avo.Wall Tomp.f & 130.3 & 179.9 & $: 79.8$ & 180.1 & 180.4 & 180.7 & 179.8 & 179.9 & 179.6 & 177.3 \\
\hline Avo. WallDT.F & 5.6 & $5.6 !$ & 5 & 4.7 & 4.6 & 5 & 3.3 & 3.7 & 3.5 & 2.3 \\
\hline Remaining Subcoolf & 0.81 & & & & & & & & & \\
\hline Exot Cuality & -0.01 & & 0.011 & 0.021 & 0.041 & 0.05 & 0.061 & 0.07 & 0.081 & 0.06 \\
\hline EXo. HBturtriter & 927.11 & 887.81 & 1005.81 & 1051.9 & 1083.8 & 10020 & 15381 & 13592 & 1455.4 & 2748.4 \\
\hline COKGXTEDH & & & & & & & & & & \\
\hline गत्रouk & 77.5 & $748.8 T$ & 745.81 & 73.71 & $7 \overline{3} 7.21$ & 738.91 & -737.31 & 75.T & 735.81 & 737.4 \\
\hline sédyi(cudotsbo) & 208.11 & 22055 & 1200.81 & 12081 & 1274 & 1248.4 & 1258.6 & $12 \cos 21$ & 1250.81 & 12501 \\
\hline $5=02$ & 9701 & 937.61 & 958.81 & 9 321 & 958.8 & 960.4 & 971.31 & 970.81 & 970.6 & 97 \\
\hline (RLO) & 1668501 & 1552931 & 1547001 & 1638481 & 152008 & 1500091 & 150150 & 1478921 & 146100 & Tिद्टा10 \\
\hline (F) & 1.004 & 1.0041 & 1.212 & 1.301 & 1.505 & 1.609 & 1.687 & 1.7681 & $1.84 \pi$ & 1.918 \\
\hline (सUO) & 2566 & 255.4 & 378 & 3546 & 388 & 40301 & 450.7 & $450.0 i$ & 469.6 & 788.1 \\
\hline (HBOIL) & 12526 & 12218 & 12020 & 121211 & 1231.7 & 12336 & 12354 & 12329 & 1236.7 & 12210 \\
\hline & & & & & & & & & & \\
\hline & ZONEZ & ZONE 4 & ZONE 6 & ZONES & ZONEETO & & & & & \\
\hline DP hquid Tomp.f & 78.51 & & & +1 & & & & & & \\
\hline DPulaudDensity tomink & क्ष.4ा & & & & & & & & & \\
\hline Elevition & $\frac{-11}{21}$ & & 6ा & 81 & 9.875 & & & & & \\
\hline 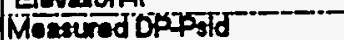 & 0.1881 & 0.4581 & 0.781 & 7.351 & 1.783 & & & & & \\
\hline Zero Comitionsotd & -0.0291 & -0.0331 & $=0.057$ & -0.0011 & 0.046 & & & & & \\
\hline Comóa DPpad & 0.217 & 0.4891 & 0.888 & 1.3501 & 1.787 & & & & & \\
\hline Actury & 2.03 & 3.861 & 5.74 & 7281 & 8.77 & f & 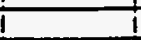 & I & & \\
\hline Whencured ip phig & 180.1 & & 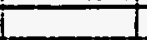 & 1 & 178.8 & i & & & & \\
\hline Meaured overelli & 2.41 & & i & 1 & & & & & & \\
\hline & & & & & & & & & & \\
\hline Computed DPA & 21 & 4.091 & 5.99 & 7.84 & 9.56 & & & & & \\
\hline Fretion-t & 0.171 & 0.26 & 0.4 & $0 . \varpi$ & 0.84 & & & & & \\
\hline Aocelerationt & 0.081 & 0.17 & 0.161 & 0.2 & 0.24 & & & & & \\
\hline Elovetion-ft & 1.961 & $3.72 !$ & 5.11 & 7.071 & 8.48 & & & & & \\
\hline & & & & & & & & & & \\
\hline
\end{tabular}




\begin{tabular}{|c|c|c|c|c|c|c|c|c|c|c|}
\hline Run & $233 i$ & I & 1 & 1 & 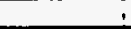 & 1 & 1 & & & \\
\hline Testfuid & $. \mathrm{CAF}=8$ &. & & & & 1 & 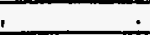 & & & \\
\hline Date: & 92393 & & & & & & & & & \\
\hline Time: & $8: 14$ & & & & & & & & & \\
\hline Noto & Analyical of & th is used & & & & & & & & \\
\hline Flow Avea-fl2 & 0.00336 & & & & & & & & $T$ & \\
\hline Hod Fow Aroa-t2 & 0.19695 & & & & & & & & & \\
\hline Sat Pressurefosig - & $1800^{\circ}$ & $T$ & . & & & & & & & \\
\hline Sed Tomporatirofiniel) & 1775.4 & & $\ldots$ & 1 & & & & & 1 & \\
\hline Liq. Densityiomis & 76.78 & 76.821 & 76.681 & 76.51 & 76.451 & 76.481 & 76.471 & 76.451 & 78.451 & 78.64 \\
\hline Ltent Hoat buthom & 31.21 & 31.241 & 31.181 & 30.971 & 30.831 & 30.951 & 30.91 & 30.831 & 30.ख्रा & 31.09 \\
\hline Specrio the thunbmif & 0.312 & 0.312 & $0.312 i$ & $0.313 !$ & 0.3131 & $0.313_{1}$ & $0.314_{1}$ & 0.3181 & 0.3121 & 0.318 \\
\hline Ha. Visoosit $1 \mathrm{bm} / \mathrm{ath}$ & 0.3691 & $-0.36 i$ & 0.3581 & 0.3561 & 0.3561 & 0.356, & 0.3541 & $0.355 i$ & 0.365 & 0.357 \\
\hline Lia. Conductivity & 0.02619 & $0.0261 ;$ & $0.026 ;$ & $0.0259 \mathrm{i}$ & 0.0259 & c.0259i & 0.02591 & 0.0259 & 0.0259 & 0.028 \\
\hline La. Prandt No. & 4.3 & 4.31 & 4.3 & 4.3 & 4.3 & 4.3 & 4.3 & 4.31 & 4.3 & 4.3 \\
\hline Inlot Flow Rato-GPM & 3.7 & & & & & & & & & \\
\hline Outiot Lig. Flow Rate-GPM & $1 !$ & & & & & & & & & \\
\hline Outtet Vap. Flow Rate-GPM & 2.11 & & T & & & & 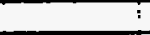 & 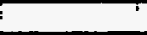 & & \\
\hline Inlot Temperature $F$ & 173.4 & & & & & & & & & \\
\hline Out o Liq. Tomporature $F$ & 174.91 & . & & 1. & & & I & & & \\
\hline Out $\mathrm{V}$ Vap. Tomperiturof & 172.21 & $\because$ & “ & $-i$ & I & IT & $I$ & 1 & & \\
\hline WAOP AOW AETOGPM & -1 & $T$ & i & 1 & & & $i$ & 1 & $i$ & \\
\hline Weter ind Tomp.f & 118.71 & 1 & & 1 & I & & $i$ & 1 & 1 & \\
\hline Wer ountemo.f & 169.11 & 1 & & $t$ & 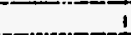 & 1 & 1 & 1 & 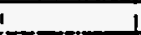 & \\
\hline 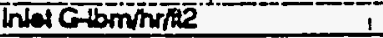 & $688416 i$ & & Tomp & $160 \mathrm{i}$ & Donsily & $77.86 i$ & 1 & 1 & 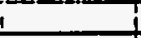 & \\
\hline Subcooling f & $2 !$ & 1 & & 1 & 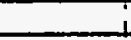 & 1 & 1 & 1 & 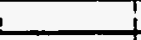 & \\
\hline \multicolumn{11}{|l|}{ Flow Observation } \\
\hline & ZONE1 & ZONE2 & DNE3 & ZONE4 & ZONES & ZONE 6 & ZONET & ZZONE8 & ZONE9 & $\angle O N=10$ \\
\hline Hoet (nput-Wata(nel) & 303.5 & 289.3 & 291 & $2 \pi .9$ & 289.2 & 290.6 & 292.11 & 292.2 & 289.4 & 200 \\
\hline Head Loss-Watts & 10.51 & 2.41 & 3.31 & 3.31 & 3.31 & 2.4 & 1.5 & 2.31 & 3.1 & 16.7 \\
\hline Hoot hox etuffinte & 5261 & 50151 & 5074 & 48171 & 50131 & 50361 & 50531 & $50 \approx 5$ & 5017 & 5027 \\
\hline Wal Temp. 13F & 180.11 & 749.81 & 18031 & $-180.4 !$ & {$[180.8]$} & 180.81 & 179.41 & 179.71 & 13951 & 177.7 \\
\hline WE Tomp. R4F & 780.21 & 179.9 & 180.6 & 180.51 & 181.11 & 181.61 & 179.51 & 180.2 & 180.2 & 778.8 \\
\hline WeVTomp. $15 F$ & 180.71 & $179.8 T$ & 187.61 & 180.41 & 180.61 & 180.61 & 170.11 & 179.61 & 179.6 & 178 \\
\hline Wall Temp. IIf & 173.41 & 180.11 & 178.21 & 179.81 & 180.81 & 181.11 & 180.61 & 179.61 & 178.9 & 778.8 \\
\hline WutTomo. IZF & $180.7 i$ & $180 . \overline{1}$ & $178.8_{1}$ & 1801 & $180,4 !$ & $180.8_{1}$ & 180.41 & 1801 & 179.2 & 177.6 \\
\hline Instoem Tomp.F & 74.31 & 174.91 & 174.71 & 175.51 & 175.7 & $175.6 i$ & 175.9 & $175.7 i$ & 175.7 & 774.9 \\
\hline Avo. Wall Temp.F & $180.4 i$ & 180 & 180 & 180.3 & 180.6 & 181 & 179.8 & 179.8 & 179.5 & 177.8 \\
\hline Avg. Wall DT.F & $5.9 !$ & 5.6 & 5 & $4.6 i$ & 4.7 & $5.2 i$ & 3.7 & $3.9 !$ & 3.6 & 2.7 \\
\hline Remaining Subcoolf & 0.61 & & & & & & & & & \\
\hline Ext Quality & -0.011 & 0.01 & 0.02 & 0.03 & 0.05 & 0.05 & 0.08 & 0.09 & 0.11 & 0.12 \\
\hline PXo.HBtutine & 83.61 & 887.81 & 1004.4 & 1051.31 & 107271 & 973.61 & 1386.81 & 1301.7 & T417.8 & 1880.8 \\
\hline COMPUTEDH & & & & & & & & & & \\
\hline Jabouk & $792 \mathrm{c} 41^{\circ}$ & $767.41^{-}$ & -754.81 & $732]$ & 745.81 & 7ATAT & -745.31 & -3742.91 & 437.81 & 735.1 \\
\hline 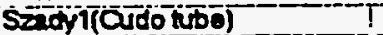 & 1263.41 & 123271 & 7247.41 & 1219.51 & $1246.9 \mathrm{i}$ & 1249.21 & 2255.51 & 255.71 & Toil & 1278.8 \\
\hline $\sin x^{2}$ & $97 \pi .51$ & 34.81 & 954.71 & 937.71 & 900.41 & 964.71 & 971.81 & 97291 & 908.7 & 9070 \\
\hline (RाO) & 1253121 & $124850 !$ & 1239081 & 1231041 & 121618 & 1197561 & 118317 & 1169821 & 114571 & 112000 \\
\hline (F) & 1.004 & $1.074 i$ & 1.309 & 1.488 & 1.600 & 1.716 & 1.009 & 1.897 & 1.87 & 2000 \\
\hline (HUS) & $1 \quad 214.8$ & 2297 & 2866 & 370 & 345 & 357.91 & 388.11 & 406.91 & 423 & 7400 \\
\hline \multirow[t]{3}{*}{$(-1)=14$} & 1251.3 & $1230.1 i$ & 12363 & 12122 & 1237.1 & 1235.5 & 1243.11 & $1247.3 i$ & 1234.6 & 12302 \\
\hline & & & & & & & & & & \\
\hline & ZONE2 & ZONE4 & 2ONEE & ZONE8 & ZONETO & & & & & \\
\hline DP Fquid Temp. $F$ & 78.8 & & & & & & & & & \\
\hline DP Louid Density tomitio & $\$ 3.37$ & & & & & & & & & \\
\hline Elovation-t & & & & 81 & 9.875 & & & & & \\
\hline Meavured DP Psid & 0.219 & 0.5761 & 0.034 & 1.5741 & $\sum 0005$ & & & & & \\
\hline Zero Comections-pe id & -0.0 .01 & $=0.003$ & $=0.057$ & $=0.004$ & $0 . \overline{0.86}$ & & & & & \\
\hline Correand DPpiold & 0.248 & 0.5791 & 1.021 & 1.5781 & 2.040 & & $T$ & & i & \\
\hline AChen DPA & 1.97 & 3.781 & 5.39 & 6.781 & 8.18 & & I & & 1 & \\
\hline Mracured TPPig & 180 & -1 & 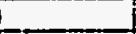 & 1 & 178.8 & & $i$ & & 1 & \\
\hline Meavered Overalli & 225 & $i$ & 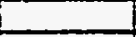 & & & & & & i & \\
\hline \\
\hline CompLted DPA & 204 & 3.92 & $5 . \pi$ & 7.46 & 9.08 & & & & & \\
\hline Friction-t & 0.08 & 0.19 & 0.32 & 0.46 & 0.62 & & & & & \\
\hline Accaloration & 0.04 & 0.091 & 0.12 & 0.15 & 0.18 & & & & & \\
\hline Elovetion-t & 1.92 & 3.641 & 5.27 & 6.851 & 8.28 & & & & & \\
\hline & & & & & & & & & & \\
\hline
\end{tabular}




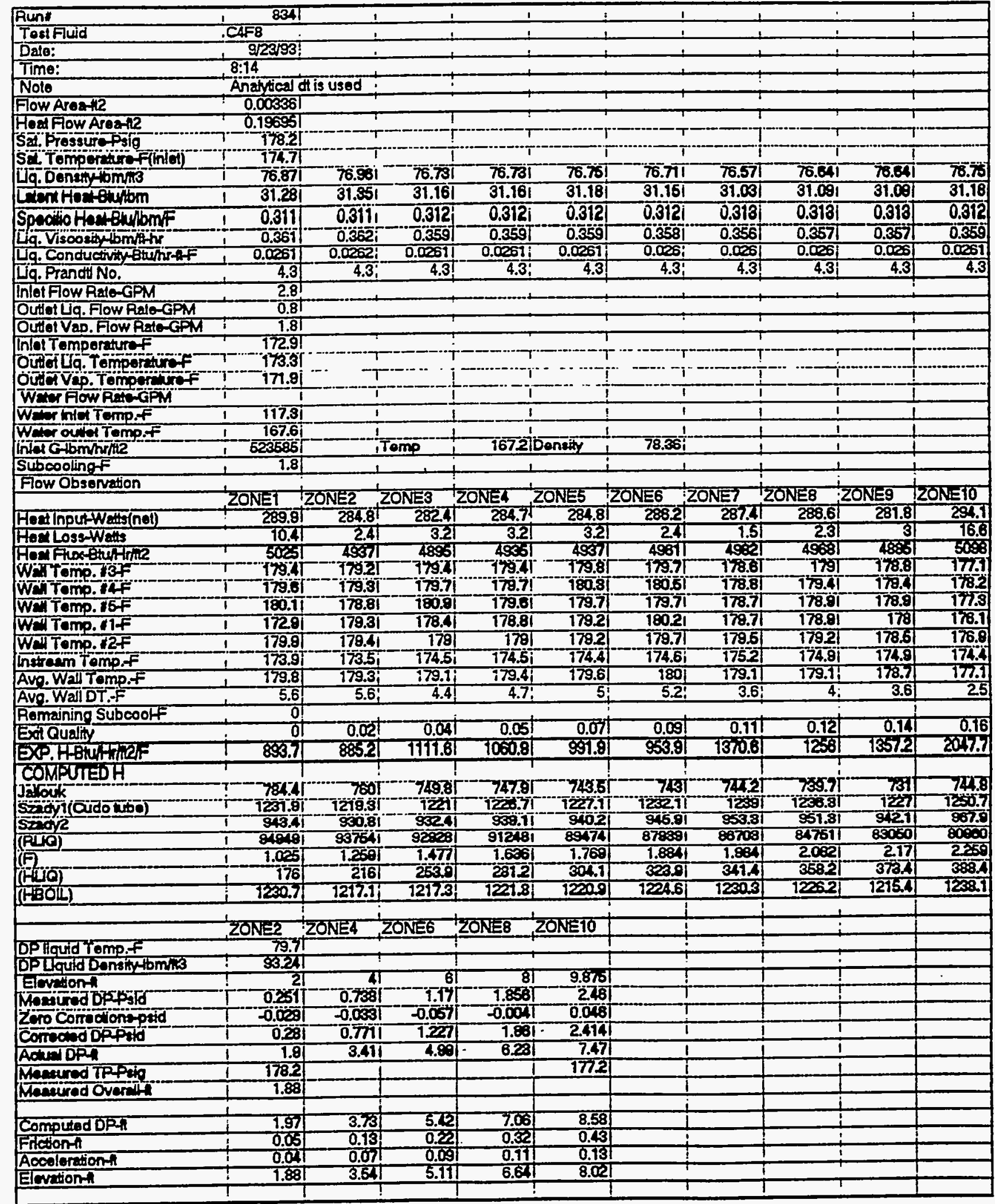




\begin{tabular}{|c|c|c|c|c|c|c|c|c|c|c|}
\hline Runt & $335 !$ & $\cdot$ & & $:$ & 1 & 1 & 1 & 1 & : & 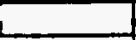 \\
\hline Tosfluid & Uf=8 & & & & & & 1 & & - & \\
\hline Date: & $9 / 23 \sqrt{93}$ & & & & & & & & & \\
\hline Time: & $12: 51$ & & & & & & & & & \\
\hline Note & Analytical d & th is used & & & & & & & & \\
\hline Flow Areafl2 & 0.003361 & & & & & & & ! & & \\
\hline Hod Fow Areant & 0.19551 & & & & & & & & & \\
\hline Sat Piessure Psig & 178.91 & & & & & & $T$ & 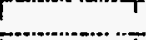 & & \\
\hline 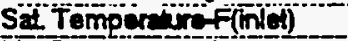 & 175 & & & & 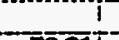 & & $=-1$ & $T$ & & \\
\hline Uq. Dansing imins & $77.12 !$ & 76.941 & 76.641 & 76.611 & 76.611 & 76.611 & 76.481 & 76.521 & $\overline{76} .21$ & 76.62 \\
\hline Lent Hed Btwhom & 31.491 & 31.341 & 31.09 & 31.071 & 31.071 & 31.071 & 30.851 & 30.991 & 30.09 & 30.80 \\
\hline Spectio Hod Bubmf & $0.31 i$ & 0.3111 & 0.313 & $0.313_{1}$ & 0.318 & 0.3131 & 0.3731 & 0.3131 & 0.313 & 0.313 \\
\hline Lig. Viscosit lom athr & $0.364 !$ & $0.362 i$ & 0.357 & 0.357 & 0.357 & 0.357 & 0.3551 & $0.356 i$ & 0.356 & 0.356 \\
\hline Liq. Conductivity-Btuhs-dfF & 0.0253 & 0.0252 & $0.026 !$ & 3.026. & 0.026 & 0.026 & $0.0259 !$ & $0.0259 ;$ & 0.0259 & 0.0259 \\
\hline Liq. Prandt No. & 4.3 & 4.3 & $4.3 !$ & 4.3 & $4.3 !$ & 4.3 & 4.31 & 4.3 & 4.3 & 4.3 \\
\hline Inlet Fow Rate-GPM & 1.9 & & & & & & & & & \\
\hline Outet Lia. Fow Rato-GPM & $0.4 !$ & & & & & & & & & \\
\hline Outlet Vab. Flow Rade-GPM & 1.5 & & & & & & & & & \\
\hline Inlot Tomporaturef & 171.8 & & & & & & & & & \\
\hline $\begin{array}{l}\text { Outa Liq. Tomporatof } \\
\text { Outo }\end{array}$ & $\begin{array}{l}174.11 \\
17221\end{array}$ & & -........... & & -1 & & & & T' & \\
\hline 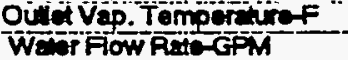 & 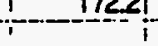 & & $-\cdots$ & $-i$ & -1 & $\frac{1}{1}$ & $T$ & & 1 & \\
\hline & 119.61 & 1 & $i$ & i & $\frac{1}{1}$ & & 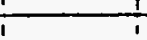 & 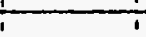 & 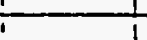 & 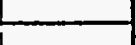 \\
\hline $\begin{array}{l}\text { Wader iniet Temp.F } \\
\text { Weter avid Temo.f }\end{array}$ & 168.61 & $\frac{1}{t}$ & 1 & -1 & 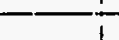 & & i & 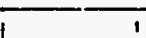 & 1 & \\
\hline & & & & & & & & & & \\
\hline Intet Gibminrib2 & 3578761 & & Tomp & $164.6,0$ & onsity & $78.91 i$ & $i$ & $i$ & 1 & \\
\hline Subcooling $F$ & 32 & & & 1 & + & & 2 & 1 & 1 & \\
\hline \multicolumn{11}{|l|}{ Flow Observation } \\
\hline & ZONE 1 & ZONE2 & ZONE3 IS & ONE 4 & NE 5 Z & ZONE 6 & ZONET & ZONE8 & ZONE9 & ZONE 10 \\
\hline Hoal Inp(t-Watts(nef) & $290.8 !$ & 285 & $27.7^{1}$ & 287.4 & $2861^{\circ}$ & 287.2 & 288.6 & 287.7 & 281.6 & 294.6 \\
\hline HoadLoss-Wats & 10.7 & 2.5 & 3.41 & 3.41 & 3.31 & 2.51 & 1.51 & 2.41 & 3.2. & 17.1 \\
\hline Hod Flx-buritile & 50411 & 49581 & 48141 & सE21 & 4551 & 6978 & 5003 & 4581 & 6का & 5105 \\
\hline Wait Temo. 13F & 179.51 & 979.31 & 179.7T & 179.7 & 180.8 & 78021 & 199 & $1 \% 9.3$ & $1 \% .11$ & 1772 \\
\hline WET Tomp TAF & 179.8 & 179.51 & 180.1 & 1801 & 180.81 & 181.11 & 179 & 179.81 & 179.81 & 178.0 \\
\hline Wa Tomp. $5 F$ & 180.81 & 17921 & 181.4 & -1801 & 18021 & 18021 & 178.81 & 179.81 & 179.9 & 177.3 \\
\hline WEl Tomp. IIF & 171.81 & 179.51 & 178.7 & 178.91 & 179.4 & 180.81 & 180 & 1791 & 178.6 & 176.4 \\
\hline Well Temp. 12F & 180.11 & $179.6 \mathrm{i}$ & 179.2 & 179.41 & 179.4 & 18021 & 179.7 & 178.71 & 178.8 & 177.4 \\
\hline instram Tomp. $F$ & 1728 & $i 73 . \overline{6 i}$ & $174.81^{\circ}$ & $176 i$ & 1751 & $-17 \bar{i}$ & 175.61 & $175 . \overline{4 i}$ & 175.4 & 176.4 \\
\hline Avg. Wall Tomp.F & 179.9 & 179.5 & 179.4 & 779.7 & 180 & 180.6 & 179.3 & 179.4 & 179.11 & 177.5 \\
\hline Avg. Wall DT.F & 6.9 & 5.7 & 4.3 & $4.4 i$ & 4.8 & 5.4 & 3.5 & 3.81 & 3.51 & 1.8 \\
\hline Remaining Subcooff & 0.5 & & & & & & & & & \\
\hline Ext Quality & & 0.02 & 0.05 & 0.07 & 0.11 & 0.12 & 0.15 & 0.18 & 0.2 & 0.23 \\
\hline EXp.HBturner & 730.91 & 877.31 & T117.51 & T125.96 & 70.5 .51 & \$30.5 & 1440.11 & 1314.3 & 136.9. & 2780.7 \\
\hline \multicolumn{11}{|l|}{ COMPUTEH } \\
\hline ]a ouk & 864.71 & 785.71 & $761 . \pi$ & 772.35 & 724.11 & 761.71 & $7525 !$ & 757 & 744.61 & 7824 \\
\hline Szadyi (Cudo zubo) & 1220.71 & $1221.81^{\circ}$ & $1212]$ & 1233.91 & iिखा & 1234.41 & 1247.5| & 2338.81 & 120.81 & $123 \sqrt{2}$ \\
\hline $\operatorname{sen} 2 \sqrt{2}$ & 938.7 & 93251 & 921.4 & 946.11 & 9428 & 940.41 & \$58.71 & 951.51 & 938.6 & 968.0 \\
\hline$(\mathrm{R}$ (2) & 64257 & 642071 & 632081 & 616711 & 69654 & 582381 & 568201 & 54834 & 53268 & 61652 \\
\hline (F) & 1.005 & 1.2381 & 1.54 & 1.7451 & 1.914 & 2081 & 2.185 & 2.3061 & 2414 & 2517 \\
\hline (1) & -127 & 156.81 & 15EA & 221A & 2420 & 267.41 & 277.5 & 292.81 & 306.6 & 315.7 \\
\hline \multirow[t]{3}{*}{ (HBOL) } & 12253 & 1220.8 & 1210.3 & 12315 & 12282 & 12305 & 1235.8 & 1233.4 & 1219.9 & ह2432 \\
\hline & & & & & & & & & & \\
\hline & ZONE? & ZONE4 & 20 NE6 & ZONE8 & SNETO & & & & & \\
\hline DP hquid Tomp.F & 79.3 & & & & $i$ & & & & & \\
\hline DPUlquid Density tom/it3 & \$3.3ा & & & & & & & & & \\
\hline Elovation & & 4 & & 81 & 9.8751 & & $T$ & & 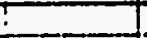 & \\
\hline Neastured DPFald & 0.2581 & 0.781 & 1.308 & 2.0761 & 27601 & & $T$ & & ! & \\
\hline 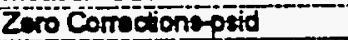 & -0.021 & 0.00351 & -0.057 & -0.0001 & 0.0481 & & $T$ & & i' & \\
\hline Comeard DPP & 0.287 & 0.8001 & 1.365 & $2.081=$ & 2.777 & & 1 & & I & \\
\hline AChNDPA & 1.8 & 3.321 & 4.55 & 5.841 & 6.881 & & 1 & $\frac{1}{1}$ & T & \\
\hline Mesoured TPPaig & 178.0 & 1 & & $!$ & 177.7 & & 1. & & $I$ & \\
\hline \multirow[t]{2}{*}{ We-nured over lil- } & 2361 & 1 & & 1 & & & 1 & & 1 & \\
\hline & & & & & & & & & 1 & \\
\hline Computed DPA & 1.89 & 3.42 & 4.81 & $6.12 !$ & 7.29 & & & & + & \\
\hline Frictont & 0.03 & 0.07 & 0.14 & 0.22 & 0.3 & & & & & \\
\hline Acosloration-t & 0.02 & 0.04 & 0.05 & 0.06 & 0.08 & & & & 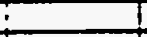 & \\
\hline Elevationt & 1.841 & उ.3ा & 4.62 & 5.84 & 6.9ा & & & & & \\
\hline & & & & & & & & & & \\
\hline
\end{tabular}




\begin{tabular}{|c|c|c|c|c|c|c|c|c|c|c|}
\hline Runt - & 8361 & & & & & & & & & \\
\hline Tost Find & CAFB & & & & & & & $!$ & $\overline{!}$ & $\overline{!}$ \\
\hline D番: & $i-823 \sqrt{13}$ & 1 & $!$ & & & T & & $i$ & $T$ & T \\
\hline Timo: & $1257 i$ & $i$ & 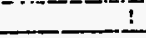 & 1 & $i$ & $i$ & I & $I$ & 1 & 1 \\
\hline Now & i Aratycaio & as usea 1 & 1 & 1 & 1 & 1 & I & 1 & I & 1 \\
\hline Flow Aresth & 0.00336 & -............. & 1 & 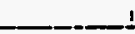 & 1 & ! & $!$ & 1 & I & \\
\hline Hoa flow Aroe 62 & 0.19595 & 1 & 1 & 1 & 1 & 1 & & 1 & 1 & \\
\hline Sat. Pressure pyig & 1772 & . & $\perp$ & 1 & $\perp$ & $\perp$ & $\perp$ & $\perp$ & $\perp$ & \\
\hline Sat. Temperature finlel) & 174.3. & & & & & & & & & \\
\hline Lig. Densitylbmins & 77.121 & 76.71 & 76.71 & 76.68 & 76.751 & 76.68 & 76.5़्रा & 76.61 & 76.57 & 76.48 \\
\hline Laen Hextsulbm & 31.49 & 37.15! & 31.151 & 31.13 & 39.181 & 31.13 & 31.05 & 31.071 & 31.08 & 30.50 \\
\hline Spectio Har Btulbmi & $0.3 \pi$ & 0.3121 & 0.312 & 0.312 & 0.3121 & 0.312 & 0.3131 & 0.3131 & 0.31 & 0.313 \\
\hline Lg. Viscosiny 6 m/nhp & $0.38 \pi$ & 0.3581 & 0.3581 & 0.3581 & 0.356 & 0.3581 & $0.35 \% 1$ & 0.357 & $0.356 \Gamma$ & 0.355 \\
\hline Lq. Conductiviy buhreffF & 0.02631 & $0.028 T$ & 0.0281 & 0.0281 & 0.08811 & 0.0261 & 0.0281 & 0.081 & 0.028 & 0.0250 \\
\hline Lig. Prano No. & 4.31 & 4.31 & $4.3 i^{-}$ & 4.31 & 4.31 & 4.31 & 4.31 & 4.31 & 4.31 & 4.3 \\
\hline InlOFFow RAOCPM & 1.11 & 7 & 1 & $-i$ & 1 & 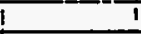 & & $i$ & 1 & I \\
\hline Oulliliq. Flow PIECFM & 0.41 & 1 & 1 & 1 & 1 & \pm & I & 1 & $I$ & 1 \\
\hline Octel Vep. Flow Pula-GPM & $0.8 i$ & 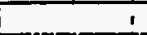 & 5 & 1 & 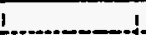 & 1 & \pm & 1 & 1. & i \\
\hline InLe Temperauref & i71.9! & 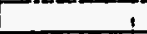 & 1 & & 1 & 1 & 1 & $i$ & 1 & $\perp$ \\
\hline Outel Liq. Tomperaturef & 174.31 & & & & & + & i & $i$ & 1 & \\
\hline Outtel Vap. Temperaturef & 172.1 & & & & & & & i & $i$ & \\
\hline Wator Fow Rato-GPM & & & & & & & & 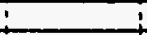 & $i$ & \\
\hline Water Inlel Temp. $f$ & 116.91 & & & & : & 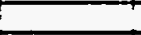 & & 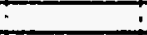 & 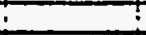 & \\
\hline Wer outel Tomp.t & 168.4 & & & & T & ? & I & I & 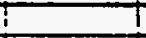 & \\
\hline Infor Glominrite & $2033552 !$ & & Tomp & 760.81 & IDenstity & 79.751 & & & & \\
\hline Scboooling & 2.4 & 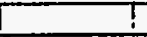 & & & $!$ & ! & $!$ & & $t$ & \\
\hline Fow Observition & $T$ & $T$ & I & 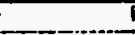 & 1 & 1 & 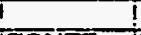 & $!$ & & \\
\hline & TZONET I & ZONE2 12 & ZONE 8 & ZONE & 12ONE5 I & $\angle O N=6-1$ & ZONET & IZONEB & ZONES & 1ZONE $=10$ \\
\hline Hoa houtwath(n) & i 288.0 & 284.81 & 28051 & 250.81 & 128451 & 28561 & 280.5 & 285.21 & 2850 & 294 \\
\hline HonLos:WE"s & 10.4 & 2.41 & 3,3 & 3.3 & 321 & 24 & 1.5 & 23 & 3.1 & 16.9 \\
\hline Heaflixelurine & 5008 & 49281 & 4852 & $50 \overline{04}$ & 4ड्श2 & 496 & 4856 & 494 & 4958 & 5103 \\
\hline Wall Tomp. $35 F$ & 179.1 & 179 & 179.41 & 179 & 179.8 & 179.8 & 178.6 & 178.9 & 178.8 & 177.3 \\
\hline Wall Tomp. I4F & T79.3 & 179.1 & 179.9 & 179.5 & 180 & 180.6 & 178.8 & 179.4 & 179.4 & 178.4 \\
\hline WE TOMP. & 179.91 & 178.7 & 180.8 & 177.5 & 779.5 & 179.8 & 178.5 & 178.91 & 178.9 & 777.3 \\
\hline Wall Tomp. IT & 171.91 & $178.9 !$ & 778.31 & 178.6 & 179 & 180.2 & 179.7 & 178.8 & 178.4 & 178.3 \\
\hline Wal Tomp. 27 & 179.7 & 179.2! & 178.8 & 178.9 & 179 & 179.81 & 179.5 & 179.4 & $|78.4|$ & $\{7$ \\
\hline ln̈stom tomp.t & 17281 & 174.61 & $174.61^{\circ}$ & 174.7 & 774.41 & 474.71 & 175.91 & 1751 & 175.21 & $\lceil 75.6$ \\
\hline Avg. Wall Tomp.F & 779.51 & 779.11 & 179.71 & 179.2 & 179.4 & 780.11 & 1791 & $\{79.11$ & 778.81 & 177.3 \\
\hline Avo.WalDT $f$ & 6.51 & 4.21 & $4.3 !$ & 421 & 4.81 & 5.21 & 3.71 & 3.91 & 3.41 & 1.4 \\
\hline Reminning sivocolf & -2.21 & 1 & 1 & +1 & -1 & 5 & 1 & 1 & I & \\
\hline Ext Cun & 0.021 & 0.071 & 0.111 & 0.15 & 0.21 & 0.21 & 0.201 & 0.391 & 0.38 & 0.42 \\
\hline 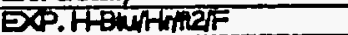 & 773.61 & 1165.81 & 1136 & 11830 & 10378 & 966.11 & 13439 & 1281.8 & 1476.7 & 35703 \\
\hline CONAUTEDH & 1 & & & & & & & & & \\
\hline Jallouk & 845.8 & 826.5 & 805.81 & 793.1 & 777.41 & 77 & 75.5 & 769.4 & 768.5 & 7823 \\
\hline Szady1(Cudotube) & 12226 & 1323.8 & 1215.8 & 1238.7 & 1223.7 & 1227.9 & 1232.6 & 1229.5 & 12325 & 72532 \\
\hline $5 z a d y 2$ & क्3.1 & 933.7 & 925.3 & 946.8 & क्खा.दा & 938.7 & 96.7 & 940.6 & 963.8 & 55.8 \\
\hline (AILO) & उ7FG। & उEख & 34850 & 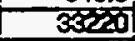 & उसक्षा & 200 & 28201 & 2647 & 24804 & 23146 \\
\hline & 1.2631 & $1.6 \% 8$ & 1.560 & ट.tक्ठ & 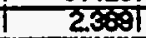 & 2.5421 & 268 & 2.8481 & 2982 & 3.108 \\
\hline (FCO) & 105.7 & Tअ.3T & 759.1 & 778.7 & 735.51 & 다이 & 20201 & -235.31 & 2786.51 & $\mathbf{2 5 . 1}$ \\
\hline$(\mathrm{FBOH})$ & T25231 & 13232 & Tटाद.9 & 1237.4 & 1201 & Tर25.81 & T2031 & 128.61 & 220.21 & 1226.8 \\
\hline & $!$ & 1 & 1 & -1 & 1 & & -1 & 1 & $I$ & I \\
\hline & ZONE2 i & ZONE4 & ZONE6 & 12ONE8 & 120NE10 & & $t$ & i & $i$ & \\
\hline DPlovid Tomp.f & 179.2 & 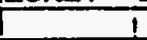 & 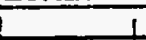 & 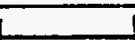 & 1 & & 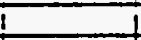 & + & I & \\
\hline 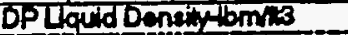 & 83.31 & 1 & 1 & 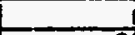 & $t$ & & i. & & $\perp$ & \\
\hline Eevalon & $\overline{2}$ & $4 i$ & 6 & 8 & 9.875 & & & & $\perp$ & \\
\hline Mensured DPFaid & 0.269 & 0.972 & 9.735 & 2638 & 3.458 & & & & $\perp$ & \\
\hline Zero Corrections-psid & -0.029 & -0.033 & 0.057 & -0.004 & 0.078 & & & & $i$ & \\
\hline Comected DP Psid & 0.318 & 1.005 & 9.7331 & 2.642 & 3.31 & & & 1 & \pm & \\
\hline ACU:DD? & 1.84 & 2.99 & 3.9ा & 4.78 & 5.681 & & & & $i$ & \\
\hline 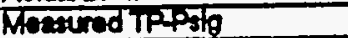 & T7h.21 & 1 & 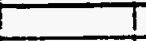 & & 178.31 & & & & & \\
\hline Weavured over is & 7.69 & T & 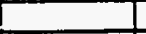 & & 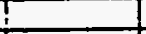 & & & & & \\
\hline & $T$ & & & & T! & & & & & \\
\hline Comperisd DPA & 7.671 & $2 \pi \pi$ & 3.82) & 4.7 & 5.61 & & & & I & \\
\hline Friolong & 0.021 & 0.04 & 0.08 & 0.18 & 0.19 & & I & I & I & \\
\hline Aocoler ion-t & 0.011 & 0.021 & 0.05 & 0.04 & 0.051 & & I & 1 & 1 & \\
\hline Eleverionth & 1.64 & 2.791 & $3.7 \pi$ & 4.61 & 5.371 & & $i$ & $I$ & $I$ & \\
\hline
\end{tabular}




\begin{tabular}{|c|c|c|c|c|c|c|c|c|c|c|}
\hline Runt & 8371 & & & & & & 1 &. & & \\
\hline Test Fluid & CAF8 & 1 & 1 & 1 & 1 & & . & 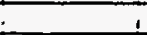 & 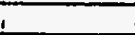 & $!$ \\
\hline Date: & $9 \sqrt{2493}$ & & & & & 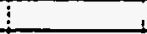 & & & & \\
\hline Time: & $8: 20$ & & & & & & $\dot{z}$ & & & \\
\hline Nole & Analitical d & dt is used ! & 1 & it & & $:$ & ti & & & $i$ \\
\hline Fow Areatl2 & 0.00336 & $\square$ & T & I & & & + & & & 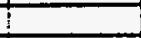 \\
\hline Heat Fow Areati? & 0.1935 & & ! & $T$ & $T$ & & 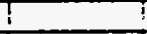 & $T$ & T & $!$ \\
\hline Sat. Pressuro Psig & $\mid 78.6\}$ & TI & 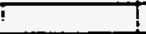 & T & i & & $\mathbf{i}$ & 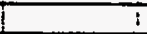 & I & \\
\hline Sat. Tomperauref(inlet) & 174.8 & 1 & $T$ & & & & & & & \\
\hline La. Densiny + bmins & 77.03 & 77.031 & 76.891 & 78.081 & 76.08 & 76.681 & 76.52 & 76.611 & 76.64 & 76.48 \\
\hline Letent Hoat Bturitom & 31.41 & 31.411 & 31.81 & 81.181 & 81.18 & 91.181 & 30.99| & 31.07 & 31.00 & 30.85 \\
\hline 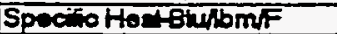 & 0.311 & 0.3111 & 0.3111 & 0.312 & 0.312 & 0.312 & 0.313 & 0.313 & 0.313 & 0.319 \\
\hline Lig. Viscosintbmithr & 0.359 & 0.3631 & 0.3611 & 0.368 & 0.358 & 0.358 & 0.358 & 0.357 & 0.357 & 0.355 \\
\hline 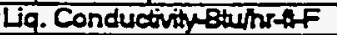 & 0.0225 & 0.0222 & 0.0252 & 0.026 & 0.026 & 0.026 & 0.0259 & 0.0261 & 0.026 & 0.0259 \\
\hline Liq. Prandt No. & 4.3 & 4.3 & 4.3 & 4.3 & 4.3 & 4.3 & 4.3 & 4.3 & 4.3 & 4.3 \\
\hline Inilot Fow Pato-GPM & 5.5 & & 1 & + & 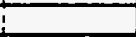 & & & $i$ & $\mathrm{i}$ & \\
\hline Outlet Liq. Flow Rato-GPM & 0.9 & & $i$ & i & & & & & & \\
\hline Outlet Vap. Fow Rato-GPM & 2.71 & & i & T & t & & t & tit & 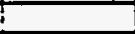 & \\
\hline Inlet Tomperatureff & 172.51 & & $i$ & i & i & ! & $\mathrm{i}$ & $T$ & t & \\
\hline Outel Líg. Temperaturef & 173.91 & T & T & ! & $!$ & I & T & T & t & \\
\hline Outel Vap. Temperaturef & 971.7 & T & T & t! & ! & & & & I & \\
\hline Wat Flow RAG-GPM & 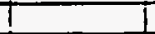 & I & I & I & I & & & & & \\
\hline Water inld Temp.f & 105.7 & $t$ & 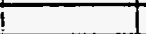 & $\mathrm{i}$ & i & & T & T & 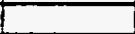 & \\
\hline Water oudet Temp.f & 165.6 & i & 1 & 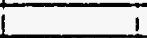 & I & & 1 & 1 & 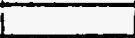 & \\
\hline Intet Glbminrine & 1024185 & & Tomp & 168.71 & Density & 78.08 & & & & \\
\hline Suboodingf & 2.3 & & & $i$ & i & & & i & 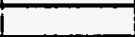 & \\
\hline \multicolumn{11}{|l|}{ Fow Observetion } \\
\hline & ZONEI & ZONE2 & ZONE3 & ZZNE4 & IZONE5 & IZONEG & ZONET & ZONE8 & IZONES & ZONETO \\
\hline Head input-Walls:(net) & 575.3 & 575.8 & 576.1 & 579.4 & 533.2 & 580.6 & 575.9 & 582.3 & 576.1 & 575.7 \\
\hline HealLoss-WE's & 10.3 & 2.41 & 3.2 & 3.2 & 3.2 & 24 & 1.6 & 2.5 & 3.2 & 17.1 \\
\hline Heal fiox bhut thine & 9972 & 99811 & 9996 & 100साi & 10109 & $1008 \mathrm{Ba}$ & 99881 & 10094 & 9968 & 9979 \\
\hline W: Tomp. BF & 380.7 & 181.7 & T812 & 789.81 & 1828 & 181.4 & 783.51 & 1824 & 78224 & 780.2 \\
\hline WDT TOMP. IIF & 181.3 & 180.6 & 1822 & 181.8 & 182.8 & 181.7 & 783.5 & 188.8 & 1832 & 3828 \\
\hline WEI TOMP. $15 F$ & 781.6 & 179.8 & 1828 & 181.9 & 181.8 & 181.9 & 183.1 & 182.3 & 187.0 & 179.7 \\
\hline WDITEmp. & 1725 & 1812 & 178.6 & 180.6 & 1812 & 1828 & 183.8 & 1826 & 781.8 & 778.8 \\
\hline Well Tamp. HEF & 181.8 & 1812 & 180.7 & 1812 & 181.8 & 1827 & 183.5 & 183.4 & 181.8 & 179.5 \\
\hline Inftreen Tomp.f & 173.2 & 178.2 & 173.8 & 174.7 & 174.7 & 174.7 & 175.4 & 175 & 174.0 & 176.6 \\
\hline Avg. Wall Tomp.f & 181.4 & 181.2 & 180.0 & 181.4 & 181.8 & 182 & 183.4 & 182.8 & 1821 & 980.5 \\
\hline AVg. Wall DT.F & 7.7 & 7.5 & 6.7 & 6.3 & 6.6 & 6.8 & 7.6 & 7.3 & 6.8 & 4 \\
\hline Remaining Subcooff & 0.5 & & & & & & & & & \\
\hline Exat Qualiy & $0 \mid$ & 0.01 & 0.03 & 0.05 & 0.07 & 0.09 & 0.11 & 0.12 & 0.14 & 0.16 \\
\hline EXP.HBturinLF & 12052 & 1328.6 & 1498.4 & 1601.6 & 1521.8 & 1481.1 & 1315.5 & 1374.6 & 1475.4 & 2464.6 \\
\hline \multicolumn{11}{|l|}{ CONANTEDH } \\
\hline Jellouk & TFस्द्ध一ा & 7705,41 & 7063.8 & 7055.8 & T028 & 1018.5 & 1007.7 & 1003 & 360.4 & 974.6. \\
\hline Szadyil(Cudo tube) & 178.6 & 1763.4 & 17R्टा & 1787.51 & 1795.2 & 17620 & 1763.8 & $17 \pi .5$ & 1780.1 & 7770.1 \\
\hline Sradre & 1539.9 & 1540.7 & 154.6 & 1519.81 & 1528.3 & 15288 & 1510.8 & 1545 & 1541.2 & 1556.1 \\
\hline (RLa) & 184689 & 183828 & 18140 & 179518 & 176057 & 172587 & 170218 & 168181 & 168510 & 160000 \\
\hline (F) & 1.005 & 1.168 & 1.432 & 1.604 & 1.748 & 1.850 & 1.978 & 2.076 & 2.160 & 2317 \\
\hline (सम) & 294.7 & 3472 & 40.8 & 77.8 & 513.6 & 549.0 & 581.1 & 610.8 & 632 & EE21 \\
\hline \multirow[t]{3}{*}{$(1-8014)$} & 1758.8 & 77502 & 17842 & 776.4 & 1781 & 17440 & 175 & 1746.9 & 17329 & $17 \$ 6$ \\
\hline & & & & & & & & & & \\
\hline & ZONE2 & ZONEA & ZOKEB & ZONE & ZONETO & & & & & \\
\hline DPFovid Temp.f & 75.5 & & & & & & & & & \\
\hline DPLiavid Densily bom/b3 & 93.83 & & & & & & & & & \\
\hline Eloviont & & 4 & 6 & 8 & 9.875 & & & & & \\
\hline Whesured DPFa्sid & 0.139 & 0.558 & 0.81 & 7.7461 & 7.91 & & & & & \\
\hline Zro Correctionsold & 0.01 & -0.000 & $-0.24 \pi$ & 0.091 & C.OWL & & & & & \\
\hline Cortectud DP Pad & 0.118 & 0.507 & 1.051 & 1.350 & 1.808 & & & & & \\
\hline ADt DI DP & 223 & 3.84 & 5.38 & 7.28 & 8.6 & & & & & \\
\hline Measured TPPgg & 178.6 & & & & $17 \pi .4$ & & & & & \\
\hline \multicolumn{11}{|l|}{ Meaceured Overall. } \\
\hline & & & & & & & & & & \\
\hline Computad DPA & 2.18 & 4.22. & 6.19 & 8.14 & 9.87 & & & & & \\
\hline Fraton-h & 0.17 & 0.41 & $0 . \pi$ & 1.05 & 1.42 & & & & & \\
\hline Acodertiont & 0.12 & 0.25 & 0.3 & 0.12 & 0.49 & & & & & \\
\hline Elovationt & 1.9 & 3.57 & 5.15 & 6.67 & 8.08 & & & & I & \\
\hline & & & & & & & & & & \\
\hline
\end{tabular}




\begin{tabular}{|c|c|c|c|c|c|c|c|c|c|c|}
\hline Runt & 838 & & & & 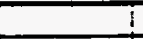 & ! & $\mathbf{i}$ & $i$ & $\dot{0}$ & $i$ \\
\hline Test Fuid & TCAF ? & & $i$ & 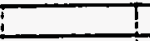 & 1 & $T$ & i & $\bar{\vdots}$ & 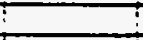 & \\
\hline Date: & 1 अप4831 & I & $!$ & i & 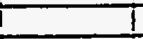 & 1 & $\div$ & $\mathbf{T}$ & $T$ & $T$ \\
\hline Time: & $18: 20$ & 1 & 1 & I & 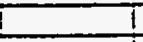 & 1 & 1 & $i$ & $!$ & $i$ \\
\hline Not & Anatricad d & Hisused & 1 & I & 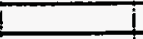 & 1 & 1 & 1 & I & 1 \\
\hline Flow Arantite & 0.003361 & 1 & $!$ & 1 & 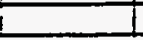 & i & 1 & 1 & t & \\
\hline Hen fow Aros 2 & $0.1 \% 5 \%$ & 1 & i & 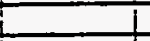 & 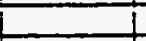 & & 1 & $i$ & 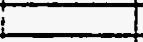 & \\
\hline Sx.Ptassure pig & 1801 & & & & & 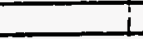 & 1 & + & + & \\
\hline Sat Temperature F(inlel) & 175.4 & & & & & & & & & \\
\hline La. Density dbm/13 & 76.96 & 76.96 & 76.78 & 76.5 & 76.55 & 76.52 & 76.36 & 76.43 & 76.45 & 76.31 \\
\hline Latent Hoar Btubm & 31.35 & 31.3 & 31.2 & अ.97 & 31.01 & 30.99 & 30.861 & 30.82 & 30.93 & 30.82 \\
\hline Spectio Her Bunbm/ & 0.311 & 0.3711 & 0.312 & $0.3 \mid$ & 0.313 & $0.3\}$ & 0.3141 & $0.374 \mid$ & 0.3 | & 0.374 \\
\hline La. Viscosingtsmithr & 0.352 & 0.3521 & 0.359 & 0.3551 & 0.3561 & 0.3561 & 0.3511 & 0.351 & 0.355 & 0.353 \\
\hline 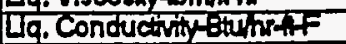 & 0.02021 & 0.0282 & 0.0281 & 0.02501 & 0.0250 & 0.0259 & 0.02581 & 0.0059 & 0.0059 & 0.0038 \\
\hline Uq. Prande No. & 4.31 & 4.3 & 4.3 & 4.3 & 4.3 & 4.3 & 4.3 & 4.3 & 4.3 & 4.3 \\
\hline Intolfow Rade-GPM & 4.6 & 1 & 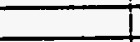 & 1 & & 1 & & & 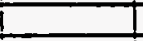 & \\
\hline Outlet Lig. Fow Rate-CPM & 0.9 & $T$ & & T & & 1 & 1 & 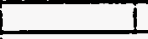 & & \\
\hline Outel Vep. Fow Rate-CPM & 2.31 & $i$ & & 1 & 1 & 1 & & & $t$ & \\
\hline Intal Temperateref & 172.6 & & & & & 1 & & $i$ & & \\
\hline Outlat Lia. Tomperanef & 174.4 & & & & & & + & 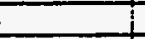 & & \\
\hline Outlet Vap. Tempermuref- & 1725 & & & & & & & & & \\
\hline Weler Fow Rate-GPM & & & & & & & & & & \\
\hline Wear inlot remp.f & 105.4 & & & & & & & & & \\
\hline Weter ounot Temp.f & 165.81 & & & & & & & & & \\
\hline Int Glbmhrite & 857071 & & Temp & T68.5ाL & Densty & 78.07 & & & & \\
\hline Subcooling $=$ & 28 & & & & & & & & & \\
\hline Fow Obsenvion & & & & & & & & & & \\
\hline & ZOFET & ZONED & ZONES 1 & ZON: 2 & ZOWIES & ZOFEO I & ZONE] I & LONEB I & COÁE & $205=10$ \\
\hline 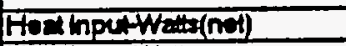 & 575.7 & 578.7 & 5782 & 580.11 & 590.8 & 585.6 & 5782 & 589.61 & 575.8 & 575.7 \\
\hline Hect LosewW" & 102 & 24 & 3.2 & 3.2 & 32 & 24 & 1.6 & 24 & 32 & 17 \\
\hline Hen Flowgurmine & $897 \%$ & 807 & 8क्ष & 1005 & 10211 & 10161 & $9 \% 8$ & 10116 & 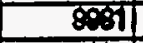 & $\$ 20$ \\
\hline Wall Temp. $13 \mathrm{~F}$ & 1812 & 1825 & 181.7 & 1हा & 183 & 182 & 184.3 & 183.1 & 188 & 1806 \\
\hline Waltemp.s4F & 787.8 & 181.1 & 1828 & 1825 & 983.4 & 1826 & 184.8 & 183.9 & 1838 & 1836 \\
\hline Wall tomp. 5 - & 1821: & 80.8 & 183.8 & 1853 & 1828 & 1828 & 183.8 & 1828 & 1828 & 180 \\
\hline Wall Tomp. $1{ }^{-}$ & TFE. & 181.8 & 1802 & 181.8 & 182 & 183.8 & 184.8 & 183.4 & 181.8 & 178.8 \\
\hline Wall remp. ${ }^{24}=$ & |8्2ठ01 & 181.8 & 1812 & 781.8 & 18द्या & 183.8 & T84.3 & 184.11 & 1828 & 1802 \\
\hline Instuem Tomp, $=$ & 773.6 & 173.5 & 174.8 & T75.5 & 775.3 & 775.4 & 176.J1 & 175.81 & 775.7 & 778,3 \\
\hline Ave. Wall Temp.t & 181.8 & T81.8 & 18T5| & Tह2ता & T828 & क्ष्टा & 184.2 & 783.51 & T82.1 & 780.6 \\
\hline AYg. Wall. & 7.8 & 7.81 & 6.7 & 6.11 & 6.8 & 6.8 & 7.6 & 7.2 & 6.6 & 3.8 \\
\hline Aomaining Subooolf & 0.6 & & & & & & & & & \\
\hline Exauty & -0.01 & 0.021 & 0.0 .1 & 0.08 & 0.08 & 0.11 & 0.13 & 0.15 & 0.17 & 0.19 \\
\hline 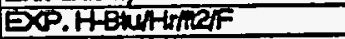 & T255 & 1273.8 & 1485.5 & 16129 & 1503,4 & 1483.8 & 1305.8 & 14042 & 1523.1 & 25327 \\
\hline COMPUIEDH & & & & & & & & & & \\
\hline Jillowk & 1743.4 & 1140.1 & Tos5.4 & 1081.6 & 10725 & 10408 & 1008. & 10056 & T012.1 & 7003.7 \\
\hline Sindy1(Cudo tube) & 178 & 1788.4 & T7is.2 & FFS.6 & 1808.7 & 18012 & 1758.8 & $17 / 6.8$ & 1785.8 & 172 \\
\hline Sady & T5428 & $75 \times 1.8$ & 15022 & 1530 & 1541.7 & 151 & 1545.7 & 1552 & 1515.61 & 158.6 \\
\hline (ALK) & 154889 & 154160 & 151853 & T5007B। & 74623 & $\overline{42878}$ & 74020 & 136372 & 13233 & 12558 \\
\hline (F) & 1.005 & $1.17 n$ & 1.475 & 1.6851 & 1.82 & 1.957 & 20ता & राष्टा & 238 & 231 \\
\hline$(\mathrm{FWO})$ & 255.7 & 299.61 & 370.1 & 425.8 & 4852 & 499.9 & 5891 & 557.8 & 383.6 & 603.3 \\
\hline$($ F.80L) & 17621 & 7705.61 & 170855 & 7785.81 & T758:.4 & 7791.81 & 1750.8 & 1758.3 & 774581 & 7748 \\
\hline & & & & & & & & & & \\
\hline & $\frac{120132}{789}$ & $20 i=4$ & $2017=0$ & $240 \sqrt{2}=8$ & 201010 & & & & & \\
\hline 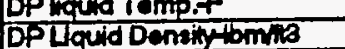 & $\begin{array}{r}78.8 \\
83.2 \\
\end{array}$ & & 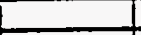 & & & & & & & \\
\hline 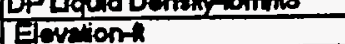 & 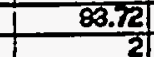 & & & & & & & & & \\
\hline Nereved DPFad & \begin{tabular}{r|}
2 \\
0.183 \\
\end{tabular} & & हा & 8 & 9.876 & & & & & \\
\hline & $\frac{0.183}{0.021}$ & 0.87 & T.071 & 1.78 & 2.25 & & & & & \\
\hline 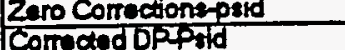 & $\frac{0.021}{0.1621}$ & -0.009 & -0.231 & 0.08 & 0.0 & & & & & \\
\hline & $\frac{0.162}{3.75}$ & 0.67 & 1.288 & $1.5 \mathrm{~s}$ & 2\%, & & & & & \\
\hline 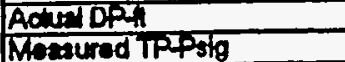 & 2.75 & 3.63 & 4.81 & 6.75 & 7.931 & & & & & \\
\hline 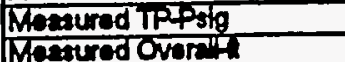 & 180 & & & & 778.8 & & & & & \\
\hline Wesuredoverlit & 203 & & & & & & & & & \\
\hline & & & & & & & & & & \\
\hline Computed DPh & 21 & 3.82 & 5.68 & 7.6 & 8.80 & & & & & \\
\hline Ficlonm & 0.12 & $\mathbf{0 . 8 5}$ & 0.62 & 0.83 & 1.38 & & & & & \\
\hline Acollerion & 0.08 & 0.18 & 0.28 & 0.38 & 0.39 & & & & & \\
\hline Exvent & 1.89 & 3.4 & 4.75 & 6.01 & 7.12 & & & & & \\
\hline
\end{tabular}




\begin{tabular}{|c|c|c|c|c|c|c|c|c|c|c|}
\hline Run" & $1 \quad 8391$ & 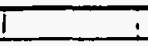 & 1 & $\perp$ & & 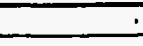 & I & $\therefore$ & $\therefore$ & \\
\hline Tostfuid & CAF8 & & & . & $:$ & . & & & & \\
\hline Date: & $9 / 2493$ & & & & & & & & & \\
\hline Time: & $8: 20$ & & & & & & & & & \\
\hline Note & Analyicalc & Ct is used: & 1 & & & & & & & \\
\hline Flow Area-42 & 0.00336 & 1 & T! & ! & & & & i & I & ! \\
\hline Hout flow Aroath & $0.19 \% 5$ & T & $T$ & $T$ & í & İ & & & & T \\
\hline Sat. Pressuropsig & 180.21 & T & $T$ & ? & i & $T$ & & & & $T$ \\
\hline Sal. Tempercture f(inlet) & 775.51 & 1 & I & 1 & $\mathrm{i}$ & i & $\mathrm{I}$ & 1 & I & I \\
\hline Liq. Densitytemn113 & 76.911 & 76.941 & 76.64 & 76.431 & 76.481 & 76.481 & 76.431 & 76.481 & 76.48 & 76.24 \\
\hline Latent HoditBtubm & 31.22 & 31.34 & 31.09 & 30.821 & 30.85 & 30.851 & 30.921 & 30.951 & 30.05 & $30 . \overline{6}$ \\
\hline Spocitio Hotbulbmif & 0.317 & 0.3111 & 0.318 & 0.3141 & 0.313 & 0.3131 & 0.314 & 0.3131 & 0.313 & 0.316 \\
\hline Lig. Visoosithlomithr & 0.351 & 0.362 & 0.357 & 0.354 & 0.3561 & 0.3551 & 0.354 & 0.3551 & 0.356 & 0.352 \\
\hline Lia. Conductivatursedf & 0.0252 & 0.0225 & 0.026 & 0.0259 & 0.0255 & 0.0259 & 0.0259 & 0.0259 & 0.0259 & 0.0258 \\
\hline Lig. PrandtiNo. & 4.3 & 4.3 & $4.3 !$ & 4.3 & 4.3 & 4.3 & 4.3 & 4.3 & 4.3 & 4.3 \\
\hline Inlet Flow Rato-GPM & 3.7 & & & & & & & & & \\
\hline Outlet Liq. Fow Ralo-GPM & 0.8 & & & & & & & & & \\
\hline Outlet Vao. Fow Rate-GPM & 21 & & & & & & & & & \\
\hline Inlol Tomperaturef & 172.8 & & & & & & & & & \\
\hline Outlet Liq. Temperaturef & 7751 & T & I & ! & & & & ! & ! & \\
\hline Oudet Vap. Temporaturef & 1721 & T & $\bar{i}$ & $\overline{\mathrm{i}}$ & T & I & 1 & T & T & \\
\hline WeWr Fow REW-GPM & $T$ & T & 1 & 1 & I & 1 & 1 & $T$ & $T$ & \\
\hline Wrer hilet Temp.f & 103.5 & 1 & I & 1 & 1 & 1 & 1 & 1 & 1 & \\
\hline Wewp outhot Temp.F & 164.7 & 1 & 1 & 1 & 1 & : & 1 & 1 & 1 & \\
\hline Inda Glbm/hr/R2 & 690639 & & & 167.81 & Density & 78.211 & & 1 & 1 & \\
\hline Subcoolingf & 2.7 & & ITemp & 1 & 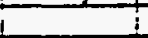 & $\longrightarrow$ & + & & + & \\
\hline \multirow{2}{*}{ Flow Observation } & & & & & & & & & & \multirow[b]{2}{*}{ ZONE10 } \\
\hline & ZONET & ZONE? & ZONES & ZONE4 & ZONES & ZONE6 & ZONE7 & ZONEB & ZONE9 & \\
\hline Head Input-Walts(net) & 567.7 & 573.5 & 579.6 & 5828 & 587.3 & 587 & 575 & 574 & 579.4 & 578.6 \\
\hline Hact Loss-Wadts & 10.1 & 2.31 & 3.21 & 3.2 & 3.2 & 2.4 & 1.6 & 2.4 & 3.1 & 16.8 \\
\hline Hoat Flox-Btuthrie & 9847 & 994ा & 10047 & 101021 & 101801 & 101751 & 9967 & 99501 & 1004 & 10000 \\
\hline Wal Temp. BF & 781.31 & 18281 & T81.91 & 18231 & 183.41 & 18251 & 183.81 & 18281 & 1824 & 780.8 \\
\hline WEITOMP. \$4F & 182.11 & 181.41 & 18201 & 1828 & 183.8 & 182.8 & 183.9 & 183.81 & 183.6 & 183.6 \\
\hline Wä Temo. 15F & 182.2 & 180.61 & 183.5 & 18281 & 183 & 1831 & 183.81 & 182.51 & 182 & 180.4 \\
\hline WE'Temp. \&1F & 172.8 & 181.81 & 180.4 & 181.71 & 1822 & 183.8 & 183.8 & 1891 & 181.4 & 179 \\
\hline Wall Temp. ILF & 1825 & 182 & 181.51 & 18211 & 1828 & 188.8 & 183.8 & 183.61 & 181.8 & 180.2 \\
\hline Instroan Temp.F & 173.7 & 173.6 & 974.0 & 175.8 & 175.6 & 175.6 & 175.8 & 176.61 & 176.6 & 176.6 \\
\hline Avg. Wall Tomp ff & 182 & 181.9 & 181.7 & 1824 & 1828 & 1831 & 183.7 & 183.1 & 1822 & 180.8 \\
\hline Avg. Wall DT.F & 7.9 & 7.9 & 6.3 & 6.2 & 6.8 & 7 & 7.5 & 7 & 6.2 & 3.7 \\
\hline Remaining Subcooff & 0 & & & & & & & & & \\
\hline Exit Cuality & & 0.03 & 0.05 & 0.081 & 0.111 & 0.14 & 0.16 & 0.19 & 0.22 & 0.25 \\
\hline EXP.HEtuHriar & 1248.8 & 7266.3 & 1589.5 & 1637.8 & 1504.8 & 1450.9 & 1330.8 & 1419.8 & 1628.6 & 269.8 \\
\hline COMPVTEDH & 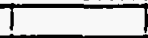 & & & & & & & & & \\
\hline Jallowk & Tभवत.Aा & $173 \pi$ & T220.41 & T1Tद2. & 1083.81 & 1082.61 & 7057.8 & T035.Tा & 1002 & 1000.8 \\
\hline Sady1(0udo tube) & 1754.1 & 17626 & 1784.81 & 1798.81 & 1804.61 & 1805.61 & 1750.4 & 1758.3 & 1787.6 & 1775.8 \\
\hline $\operatorname{sendr} 2$ & 1527 & 1538.61 & 1514.8 & 1535.4 & 1539.4 & 1541.61 & 1534.4 & 1532.81 & 1551.1 & 1559.8 \\
\hline$(R \cup 0)$ & 125019 & 1232301 & 121272 & 118778 & 115031 & 1114801 & 108185 & 904481 & 101008 & 98990 \\
\hline (F) & 1.005 & 1.322 & 1.601 & 1.790 & 1.969 & 2.116 & 2241 & 2.3641 & 2.478 & 2.568 \\
\hline (1-2) & 215.2 & 283.1 & 34.8 & 388.0 & 4282 & 4548 & 4825 & 508.11 & 531.7 & 651.9 \\
\hline (HBOI) & 1753 & 1700 & 1780.3 & 17928 & 1796.8 & $17 \% 59$ & 1748.8 & 1744.11 & 17515 & 17578 \\
\hline & & & & & & & & & & \\
\hline & ZONEZ & ZONE4 & ZONE6 & ZONEB & ZONE 10 & & & & & \\
\hline DP hquid Temp.F & 77.8 & & & & & & & & & \\
\hline DP Lquid Densitylomilis & 98.51 & & & & & & & & & \\
\hline Eevation-t & 2 & 4 & 6 & 8 & $9.8 \%$ & & & & & \\
\hline Moasured DPFsid & 0.218 & 0.783 & T.250 & 2.003 & $2 \approx$ & & & & & \\
\hline Zero Corrections-psid & 0.027 & -0.0091 & -0.241 & 0.09 & T.042 & & & & & \\
\hline Comected DPPald & 0.197 & 0.7921 & 1.5 & 1.913 & 2588 & & & & & \\
\hline Acter OPA & 2.08 & $9.4 \pi$ & 4.58 & 8.2 & 7.22 & & & & I & \\
\hline Mremoured TPPsig & 1802 & & & $\overrightarrow{1}$ & 178.4 & & & & & \\
\hline Maenured Overelli & 9.51 & & & & t & & & & i & \\
\hline & & & & & & & & & & \\
\hline Computad DR & 2 & 3.68 & 523 & 6.74 & 8.14 & & & & & \\
\hline Friction-t & 0.09 & 0.25 & 0.47 & 0.74 & 1.03 & & & & & \\
\hline Acosterdiont & 0.08 & 0.15 & 0.2 & 0.25 & 0.37 & & & & & \\
\hline Elovation-ft & 1.82 & 3.28 & 7.56 & 5.75 & 6.8 & & & & & \\
\hline & & & & & & & & & & \\
\hline
\end{tabular}




\begin{tabular}{|c|c|c|c|c|c|c|c|c|c|c|}
\hline Runy & 8401 & \pm & $\therefore$ & 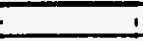 & - & $\dot{-}$ & - & - & 1 & \\
\hline Tostfluid & CAF 8 & $\therefore$ & $\therefore$ & 1 & 1 & 1 & 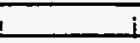 & $i$ & & \\
\hline Dalo: & $9 / 2493$ & & & & 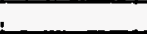 &. & . & & & \\
\hline Time: & $8: 20$ & & & & & & & & & \\
\hline Noto & Anabytical d & th is used & & & & & & & & \\
\hline Flow Avas+12 & $\mid 0.00336$ & & T & $T$ & ; & ; & ? & & & \\
\hline Hoal Fow Areatte & 0.19695 & & 1 & I & í & it & 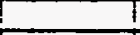 & & & \\
\hline Sat. Pressure Psig & 178.7 & T & T & 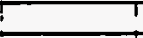 & $T$ & T & r & $r$ & & \\
\hline Sat. Tomporeure $f(n+0)$ & 174.8 & & 1 & i & $T$ & ! & 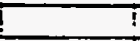 & 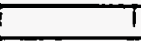 & & \\
\hline LG.Donsm $+b m / 13$ & 77.12 & 76.991 & 76.781 & 76.571 & 76.84 & 76.61 & 76.57 & 76.611 & 76.011 & 76.00 \\
\hline Letent Heriftulom & 31.49 & 31.84 & 31.21 & 31.031 & 31.001 & 31.091 & 31.081 & 31.07! & 31.07 & $\mathbf{3 0 . 6}$ \\
\hline Specitio thatenthms & 0.31 & 0.3171 & 0.3121 & 0.3131 & 0.3131 & 0.3181 & 0.3181 & 0.3131 & 0.318 & 0.315 \\
\hline Lig. Viecosihylom/hrs & $0.36 \pi$ & 0.352 & 0.3601 & 0.3661 & 0.357 & 0.357 & 0.366 & 0.357 & 0.357 & 0.30 \\
\hline Lig. Conductivity thesh tf & 0.02281 & 0.0251 & 0.03611 & 0.0001 & 0.0051 & 0.028 & 0.0251 & 0.025 & 0.02 & 0.0256 \\
\hline Liq. PrandtI No. & 4.3 & 4.31 & 4.31 & 4.31 & 4.3 & 4.3 & 4.3 & 4.3 & 4.3 & 4.3 \\
\hline Inlat Flow Rate-GPM & 2.8 & & & & & & & $\longrightarrow$ & & \\
\hline Outel Lia. Fow Rato-GPM & $0.7 !$ & & & & & & 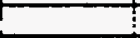 & & & \\
\hline Outlet Vap. Flow Rale-GPM & 1.91 & & & & i & $i$ & $i$ & & & \\
\hline Inlot Tomperauref & 172 & & & $T$ & $T$ & $T$ & & & & \\
\hline Outhof Lig. Tomperaturer & 774.61 & & & & $\mathrm{~T}$ & $\mathrm{~T}$ & $\Gamma$ & I & & \\
\hline Outol Vap. Tomperturof & 171.7 & & $T$ & T & ! & $!$ & $\Gamma$ & T! & & \\
\hline WCM FOW Aat-GPM & $T$ & I & T & 1 & 1 & $i$ & $T$ & 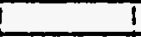 & & \\
\hline Wererinlot Temp, $f$ & 108.4 & & I & 1 & 1 & 1 & $L$ & & & \\
\hline Weter ortel Temp.F & 163.5 & 1 & 1 & 1 & i & & 1 & & & \\
\hline Intat G-bm/hrint2 & 5255165 & & Temp & 165.01 & Doneity & 78.64 & & 1 & & \\
\hline Subcooling f & 2.9 & & & i & 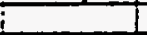 & 3 & L & 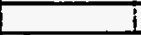 & & \\
\hline \multirow[t]{2}{*}{ Fow Observation } & & & & & & & & & & \\
\hline & ZONE1 & ZONE2 & ZONE3 & ZONE4 & ZONES & ZONE6 & ZONE & ZONE8 & ZOWE9 & ZOKEIO \\
\hline Hoad input-Watts(ned) & 572.3 & 574.4 & 576.7 & 580.9 & 576 & 576.1 & 575.7 & 575.3 & 576.4 & 576.5 \\
\hline Hesl Loss-Wets & 101 & 2.3 & 3.2 & 3.2 & 3.2 & 2.4 & 1.6 & 24 & 3.1 & 16.8 \\
\hline Hon Flusturtire & G9201 & 9857 & 9997 & $700 \% 1$ & 995 & 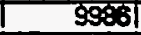 & 997 & 9972 & 9982 & 938 \\
\hline We TTOmo. BF & T80.5 & 18150 & 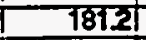 & 781.6ा & 7825 & 781.8 & 783.3 & गध्टां & 187.8 & 180.8 \\
\hline WaT Tomp. IfF & 1812 & 180.7 & 182.1 & 18221 & 183.11 & 182या & 783.5. & 183.71 & 7828 & 183 \\
\hline WeT Tomp. ISF & 1812 & 179.8 & 1828 & 18221 & 18221 & 18241 & 18291 & 1821 & 181.5 & 178.8 \\
\hline WCI TEmp. IIF & 172 & 187 & 179.7 & 181.2 & 181.6 & 182.91 & 189.4 & 18261 & 180.0 & 178.0 \\
\hline Wal Temp. ILF & 181.0 & 181.1 & 180.6 & 181.6 & 181.8 & 18.11 & 188.4 & 189.11 & 181.4 & 179.8 \\
\hline Introam Tomo.f & 172.81 & 173.6 & 174.3 & 176.21 & 174.0 & 174.9 & 176.21 & 1751 & 170 & 177.4 \\
\hline Avg.Wall Tomp.F & 1812 & 181.1 & 1800 & 981.8 & 182.11 & 1824 & 183.3 & 1826 & 181.7 & 180.3 \\
\hline Avo. Wall DT.F & 8 & 7 & 6.1 & 6.1 & 6.8 & 7 & 7.6 & 7.11 & 62 & 24 \\
\hline \multicolumn{11}{|l|}{ Remaining Subcooff } \\
\hline Exot Quality & 0.01 & 0.04 & 0.08 & 0.11 & 0.15 & 0.19 & 0.22 & 0.26 & 0.29 & 0.33 \\
\hline EXP.HBtwhinge & 1247.6 & 1417.3 & $\sqrt{50}$ & 1638.7 & 1474 & 1426.8 & 1304.7 & 14027 & 160.2 & 4081.6 \\
\hline \multicolumn{11}{|l|}{ COIMPOTEDH } \\
\hline Jalouk & 1785 & T758.6] & 7138.8 & 17दम5. & 7117.3 & 710.8 & 1000.71 & T076.81 & 7087.9 & T07. \\
\hline Fradvi(Cudotibo) & 77522 & 17832 & 173.9 & 178.1 & 1780.8 & 17815 & 1784.7 & 7783.81 & 17881 & 1776.2 \\
\hline $\operatorname{sind} / 2$ & 1531.6 & 1540.7 & 1549.7 & 1520.6 & 1511.9 & 1513.8 & 15202 & 1518.41 & 15ट्राड़ा & 1568.7 \\
\hline (R.LO) & 8404 & 92725 & 89820 & 87212 & 8610 & 80071 & 788001 & 732491 & 697:01 & 67814 \\
\hline (F) & 1.146 & 1.450 & 1.754 & 1.872 & 21ब्थ & 2.385 & 2468 & 2.6061 & 27811 & 281 \\
\hline (H) & 186.0 & 250.6 & 3023 & 304 & 37 & 401.2 & 428.1 & 440.61 & 471.3 & 486.0 \\
\hline \multirow[t]{3}{*}{ (FoU) } & 7751.4 & 17615 & 170.8 & 173 & 174.9 & 174.7 & 176.5 & 1773.8 & 1775 & 1784 \\
\hline & & & & & & & & 1 & & \\
\hline & ZONE2 & ZONEA & ZONE 6 & ZONEB & ZONE10 & & & & & \\
\hline \multicolumn{11}{|l|}{ DP hquid Tomp.f } \\
\hline \multicolumn{11}{|l|}{ 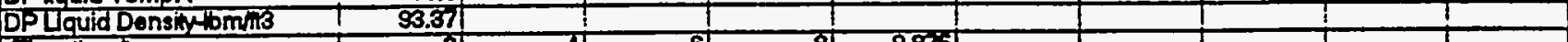 } \\
\hline Eeviliont & 2। & 4 & 61 & का & 9.875 & & & & & \\
\hline Theavured Dop काd & 0.205 & 0.980 & 7.505 & 2.3531 & 3.051 & & & & & \\
\hline Zero Corrections-pedd & 0.0.21 & -0.000 & -0.2 .11 & 0.091 & 0.021 & & & & & \\
\hline Comend Dpropd & 0.234 & 0.988 & 1.740 & 23.3 & 3.008 & & & & & \\
\hline Aotedoph & 2.07 & 3.18 & 4.00 & 5.57 & 8.48 & & & & 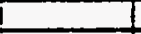 & \\
\hline Mearured TPPaig & 178.7 & & & & 178 & & & & 1 & \\
\hline \multicolumn{11}{|l|}{ Meapured Overall } \\
\hline & & & & & & & & & & \\
\hline Computed DF! & 7.81 & 3.28 & 4.6] & 5.88 & 7.04 & & & & & \\
\hline Ficton-t & 0.07 & 0.18 & 0.38 & 0.57 & 0.8 & & & & & \\
\hline Aocaleration- & 0.081 & 0.1 & 0.14 & 0.78 & 0.23 & & & & & \\
\hline Elovation-l & 1.91 & 2.98 & $4.7 \pi$ & 5.18 & 6.02 & & & & & \\
\hline & & & & & T & & & & $i$ & \\
\hline
\end{tabular}




\begin{tabular}{|c|c|c|c|c|c|c|c|c|c|c|}
\hline Runt & $\therefore \quad 8411$ & 1 & 1 & 1 & 1 & - & - & & & , \\
\hline Test Fluid & . CAF8 & & 1 & & 1 & & & & & \\
\hline Date: & . \$24 & & & & & & & & & \\
\hline Time: & $8: 20$ & & & & & & & & & \\
\hline Noto & Analytical c & dt is used & $\vdots$ & & & & & & & \\
\hline Flow Aresth2 & 0.003361 & & & & & $:$ & & & & \\
\hline Head Fow Area-li & 0.1959 & & & & & 7 & T & & & $T$ \\
\hline Sal. Pressuro Psig & 179.81 & & & $T$ & & 7 & & & & $T$ \\
\hline Sat. Temporature f(inlet) & 175.3 & $\pi$ & $\mathrm{T}$ & T & T! & $T$ & $\Gamma$ & $T$ & $T$ & $T$ \\
\hline Ua. Denstymins & 76.84 & 76.57 & 76.591 & 76.451 & 76.521 & 76.451 & 76.31 & 76.361 & 76.30 & 75.89 \\
\hline Later Hexterubm & 81.281 & 31.08 & 31.051 & 30.831 & 30.99 & 30.991 & 30.82 & $\mathbf{3 0 . 8 0 1}$ & $\$ 9.60$ & $\$ 0.47$ \\
\hline 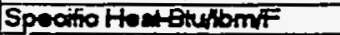 & 0.312 & 0.313 & 0.3131 & 0.3131 & 0.3131 & 0.313 & 0.314 & 0.3741 & 0.374 & 0.316 \\
\hline Lig. Visoositylbmilth & 0.36 & 0.356 & 0.357 & $0.365 i$ & 0.356 & 0.356 & 0.353 & 0.3541 & 0.354 & 0.37 \\
\hline Liq. Conductive uhr-tF & $1 \quad 0.02611$ & 0.0261 & 0.026 & 0.02591 & 0.02591 & 0.02591 & 0.0258 & 0.02581 & 0.0258 & 0.0255 \\
\hline Lig. Prand'No. & 4.3 & 4.3 & 4.3 & $4.3 i$ & 4.3 & 4.31 & $4 . \overline{3}$ & 4.3 & 4.3 & 4.3 \\
\hline Inlet Fow Rato-GPM & 1.9 & & & & & & & & & \\
\hline Outhet Liq. Flow Rato-GPM & 0.41 & & & & & & & & & \\
\hline Outiat Vap. Flow Rato-GPM & 1.11 & & & & & & & & & \\
\hline Inlot Temperaturef & 173.1 & & & & & & & & & \\
\hline Outat Lia. Temperaturef & 175.1 & & & & & & & & & $\mathrm{~T}$ \\
\hline Ouflet Vap. Temperaturef & 173.11 & & & T & $T$ & $\mathrm{~T}$ & & T & & \\
\hline WEP FOW PAT GPM & 1 & I & i & I & I & I & & T & i & \\
\hline Wabor hilet Temp.F & 104.71 & I & $i$ & i & $\frac{1}{1}$ & it & & $i$ & $i$ & \\
\hline Weter ould Temp.f & 159.4 & T & & $i$ & 1 & 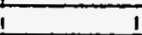 & & 1 & 1 & \\
\hline Infat G fb minerit2 & 357141 & & Temp & 165.31 & IDonaty & $78.7 \pi$ & & 1 & 1 & \\
\hline Subcoolingf & 22 & 1 & & 1 & 1 & i i & & 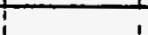 & 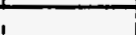 & \\
\hline \multicolumn{11}{|l|}{ Fow Observation } \\
\hline & ZONET & ZONE2 & ZONE3 & ZONEA & ZONE5 & ZONE6 & ZONE7 & ZONE8 & ZONE9 & ZONETO \\
\hline Hod lnput-Walts(nof) & 573.8 & 575.7 & 573.2 & 571.3 & 571.8 & 574.3 & 573.4 & 573.6 & 571.6 & 573.1 \\
\hline Hoal Loss-Wadts & 10.11 & 2.4 & 3.2 & 3.2 & 3.21 & 2.41 & 1.6 & 2.4 & 3.1 & 16.7 \\
\hline Heaflux-Btuptrine & 9948 & 99791 & 99881 & 90031 & 9912 & 995 & 99401 & 9941 & 9908 & 9934 \\
\hline Wal Tomp. 134 & 1811 & $182 \pi$ & 787.81 & $182 \pi$ & 183 & 18231 & 883.81 & 78251 & 1824 & 780.5 \\
\hline Wa Tomp. $14 \mathrm{~F}$ & 181.8 & 187 & 18261 & $78 ् 24 !$ & T83.81 & 182.7 & 784.21 & 183.51 & कि्3.1 & 1829 \\
\hline Wal Tomp. 15 F & 181.8 & 180.51 & 183.6 & 182.81 & 18281 & 182.81 & 183.01 & 182.01 & 182.1 & 180.1 \\
\hline WEV Temp. Iff & 173.1 & 181.51 & 180.1 & 1811 & 181.81 & 189.21 & 183.71 & 189.11 & 181.2 & 178.6 \\
\hline Well Tomp. I2F & 182.8 & 181.5 & 181.1 & 181.61 & 181.8 & 1892 & 184.11 & 1841 & 162 & 180 \\
\hline Infreen Temp.f & 174 & 176.21 & 175.1 & 175.7 & 176.4 & 176.71 & 176.3 & 176.11 & 176.1 & 178.1 \\
\hline Avg. Wall Tomp.F & 181.8 & 181.5 & 181.3 & 182 & 1823 & 182.8 & 183.8 & 183.1 & 1822 & 180.4 \\
\hline Avg. Wall DT.F & 7.3 & 5.9 & 5.8 & 5.9 & 6.5 & 6.6 & 7.1 & 6.6 & 5.6 & 1.9 \\
\hline \multicolumn{11}{|l|}{ Remaining Subcoolf } \\
\hline Exot Quality & 0.031 & 0.08 & 0.13 & 0.19 & 0.24 & 0.29 & 0.35 & 0.4 & 0.45 & 0.5 \\
\hline EXP.HBtuHnCe & 1362.4 & 1698.9 & 17202 & 1684.9 & 15362 & 1508.3 & 1401.7 & 1508.61 & 1765.5 & 5368.9 \\
\hline COMFUEDH & & & & & & & & & & \\
\hline Jalouk & रिशा। & Tद97.81 & 1769.41 & T133.8T & T738.4ी & 7750.31 & Tन20.6 & Tाद्षा & Tाइ5.8| & 7123.8 \\
\hline Szendy (Cudo ubo) & 1765.2 & 1779.4 & 175.51 & 178.8 & ITt7.4 & 1784.31 & 1789.2 & 1788.31 & 7788.21 & 7774.4 \\
\hline $\operatorname{sand} 2$ & 1562 & 1510.71 & 1507.31 & 1516.3 & 1512.9 & 7521.81 & 1532.11 & 1530.11 & 152921 & 1560.6 \\
\hline (PUO) & 636521 & 618611 & 586691 & 552281 & 5182 & 488171 & 45082 & 415191 & 38009 & 35182 \\
\hline (F) & 1.348 & 1.7221 & 204 & 22891 & 2505 & 26811 & 2868 & 3.0171 & 3.167 & 3.257 \\
\hline (रण्यत) & 169.8 & 218.3 & 259 & 20.4 & 317.7 & 341.4 & $3 \pi 24$ & 383 & 402.1 & 416.3 \\
\hline \multirow[t]{3}{*}{ (HOL) } & 1764.6 & 1778.3 & 1773.6 & 1776.3 & 174 & 17802 & 17842 & 178241 & 1779.3 & 1765.7 \\
\hline & & & & & & & & & & \\
\hline & ZONE2 & ZONE4 & ZONE6 & ZONE8 & ZONETO & & & & & \\
\hline DP Fquid Tomp.F & 79 & 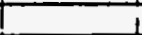 & & & & & & & & \\
\hline DPLiquid Densintbmit3 & 93.34 & & & & & & & & & \\
\hline Elovitiont & 2 & & & छा & 9.875 & & & $T$ & & \\
\hline Whesuredoppasd & 0.29 & 7.9541 & T.865 & 27911 & 3.638 & & & $T$ & & \\
\hline Zoro Comedions-orid & 0.021 & -0.0081 & -0.241 & 0.09 & C.042 & $T$ & & $T$ & & \\
\hline Corrocied opprid & 0.2001 & 1.163 & 2.100 & 2707 & 3.591 & T & & $T$ & & \\
\hline Aoten DPA & 1.95 & $27 \pi$ & 3.38 & 4.7 & 5.39 & i & & $i$ & & \\
\hline Meacurod TfFig & 179.81 & & & 1 & 178.2 & & & $\bar{t}$ & & \\
\hline \multirow[t]{2}{*}{ Heatured Overdih } & 7.141 & & & $i$ & & & & 1 & & \\
\hline & & & & & & & & 1 & & \\
\hline Computed DPA & 1.56 & 277 & 3.84 & 4.821 & 5.7 & & & i & & \\
\hline Fidion-A & 0.05 & 0.13 & 0.24 & 0.39 & 0.54 & & & & & \\
\hline Acoderational & 0.04 & 0.07 & 0.09 & 0.13 & 0.16 & & & $\vdots$ & & \\
\hline Elovation-t & 1.48 & 2571 & 3.5 & 4.31 & इा & & & & & \\
\hline & 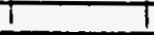 & & & & & $T$ & & & & \\
\hline
\end{tabular}




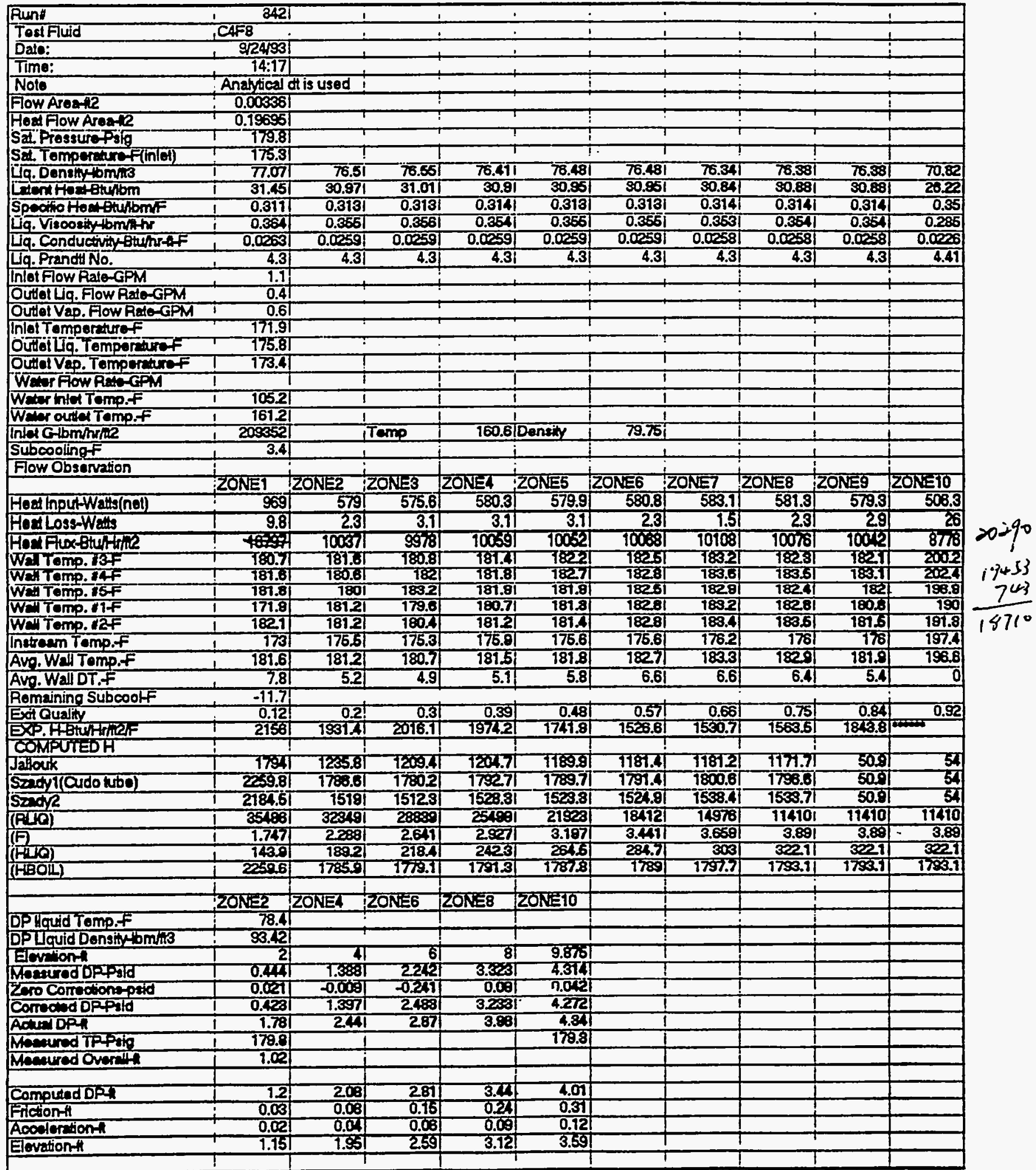




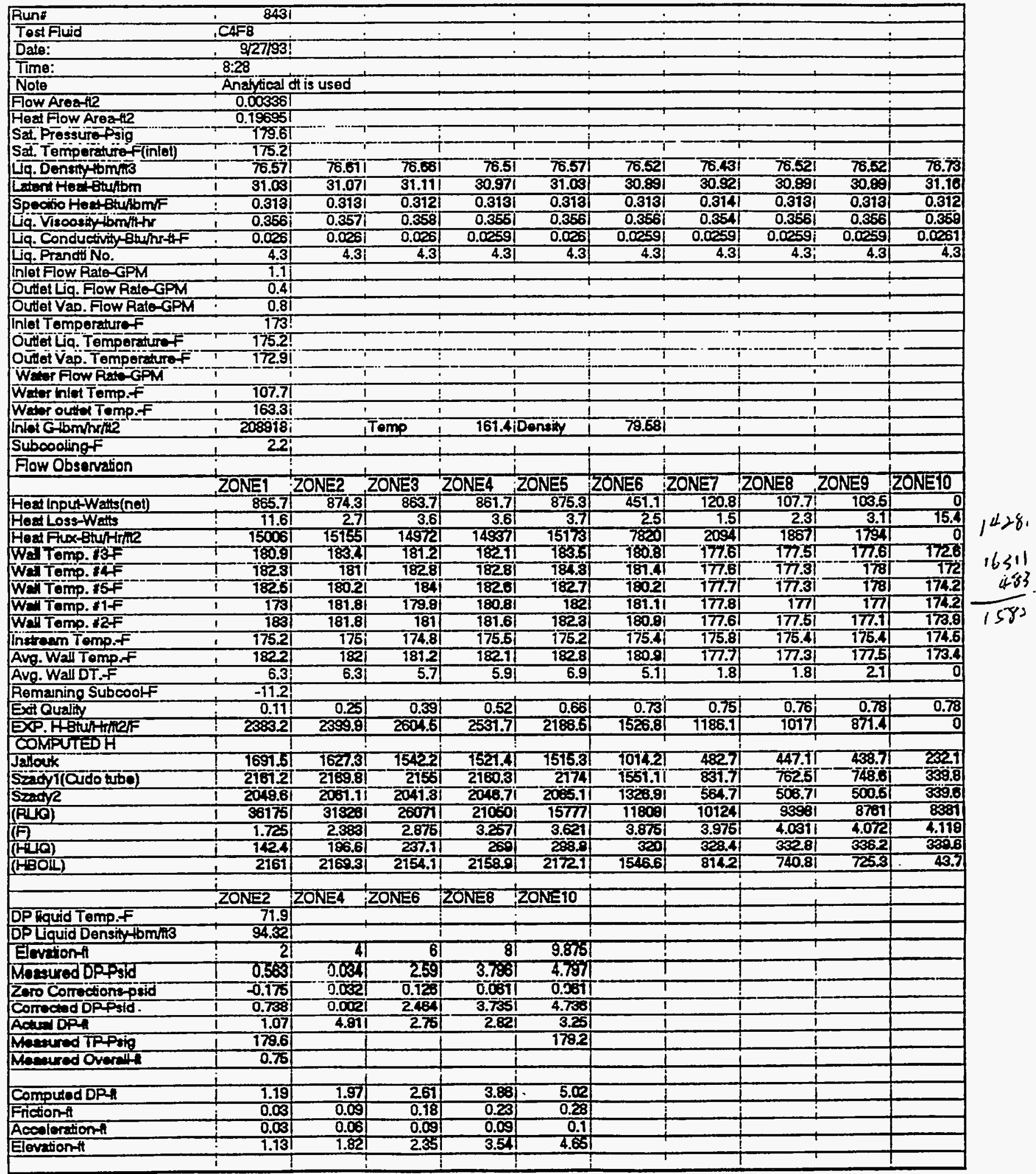




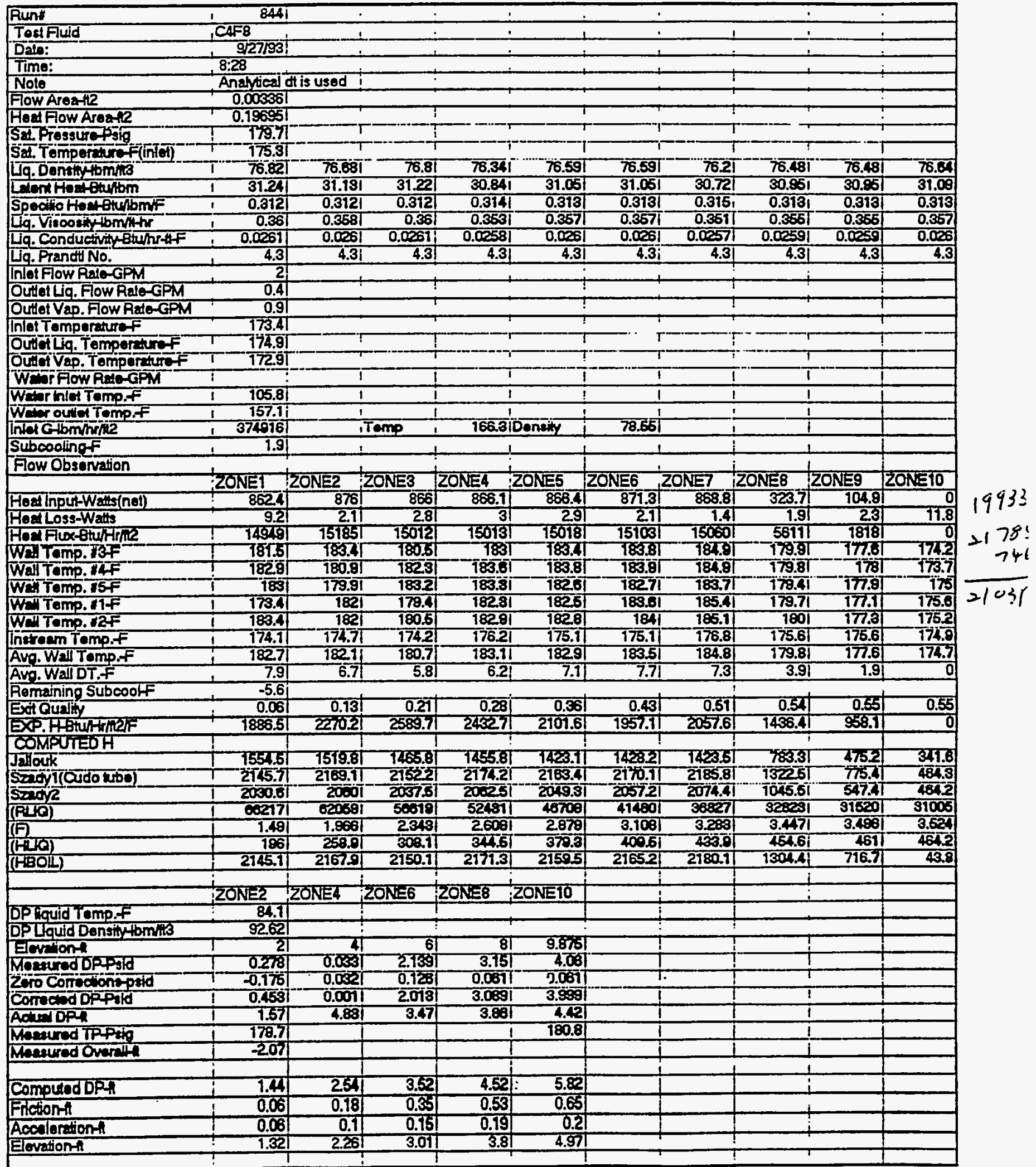




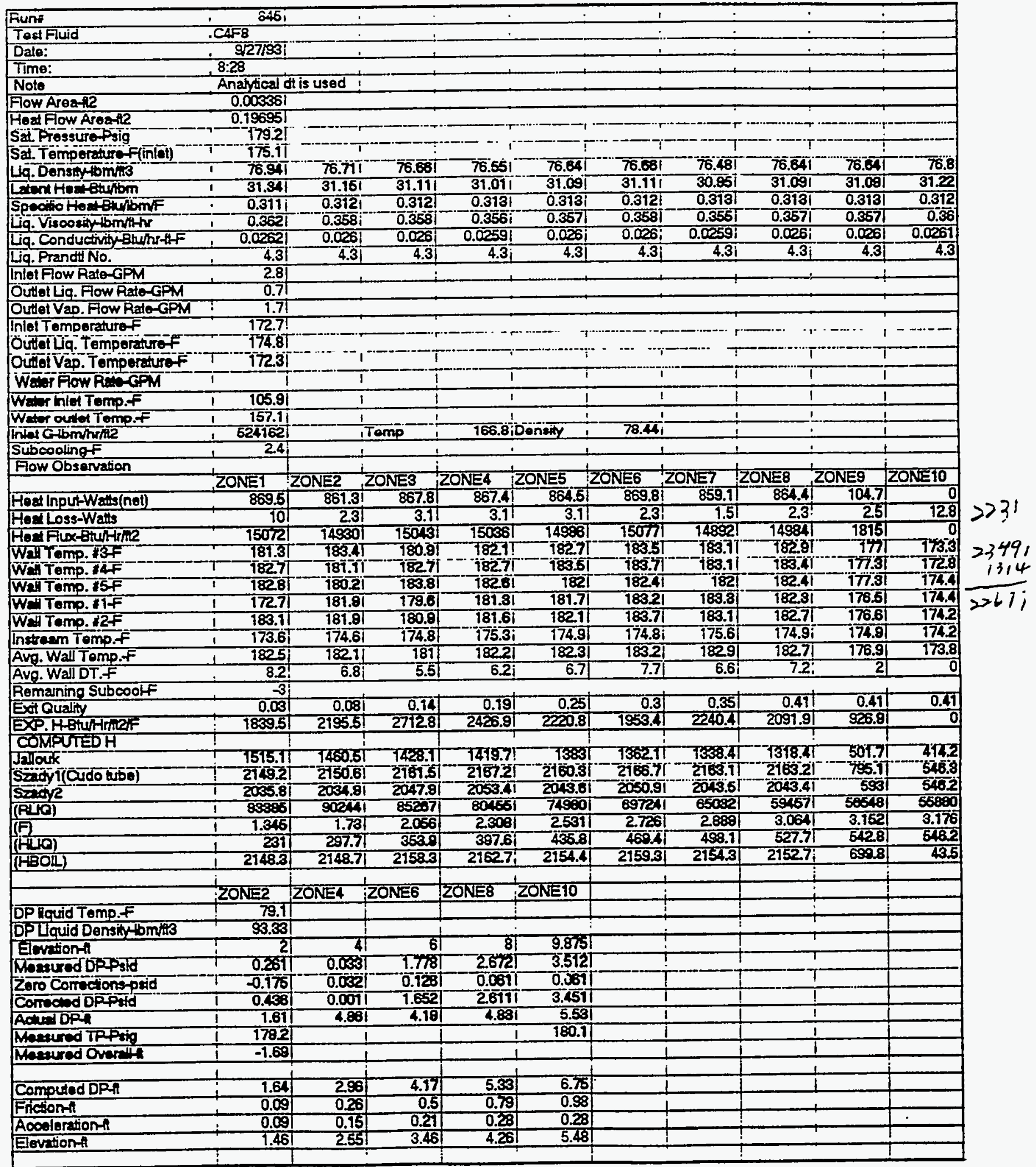




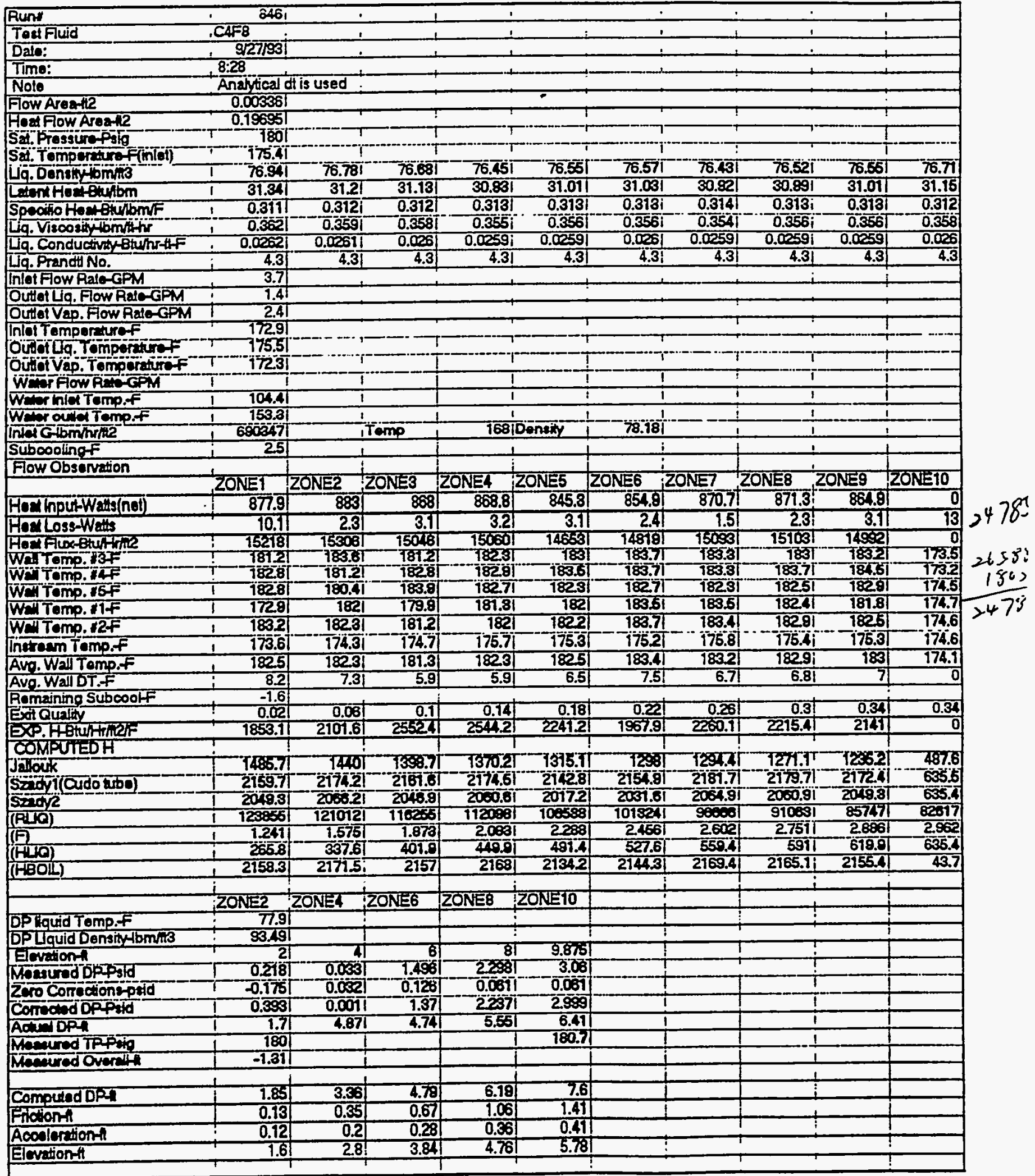




\begin{tabular}{|c|c|c|c|c|c|c|c|c|c|c|}
\hline Run" & 8471 & & & & & & & & & \\
\hline Tolffuid & CAF8 & & & & & & & & & \\
\hline Dats: & 929931 & & & & & & & & & \\
\hline Time: & $9: 42$ & & & & & & & & & \\
\hline Note & Analytical d & His used ! & & & & & & & & \\
\hline Flow Areattle & $0.00336 \mathrm{i}$ & & & & & & & & & \\
\hline Heat Fow Area-12 & 0.1955 & & t & & & & T & & & $i$ \\
\hline Sat Prossure Psig & 181.4 & & & & & T & i & $i$ & & 5 \\
\hline Sat. Temperaturo finlet) & 176 & $?$ & : & & & 1 & 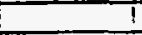 & ! & & \\
\hline Lq. Density tbmms & 76.75 & 76.81 & 76.68 & $76.41 !$ & 76.51 & 76.521 & 76.241 & 76.411 & $76.4 \pi$ & 76.36 \\
\hline Laterk Hext-Bulbm & 31.18 & 31.221 & 31.73 & 30.91 & 30.97 & 30.991 & 30.78 & 30.91 & 30.9 & 30.88 \\
\hline Specifo Heat Bumbm/F & 0.312 & 0.312 & 0.312 & 0.3141 & 0.313 & 0.313 & 0.3151 & 0.3141 & 0.379 & 0.314 \\
\hline Liq. Visoositylbminthr & 0.359 & 0.36 & 0.3581 & 0.354 & 0.355 & 0.3561 & 0.362 & 0.354 & 0.354 & 0.354 \\
\hline Liq. Conductivity BturnstfF & 0.0261 & 0.0267 & 0.026 & 0.0259 & 0.0259 & 0.0259 & 0.02581 & 0.02591 & 0.0259 & 0.0258 \\
\hline Lia. Prandi No. & 4.31 & 4.3 & 4.3 & 4.3 & 4.3 & 4.3 & 4.3 & 4.3 & 4.3 & 4.3 \\
\hline Inlet Fow Rate-GPM & 4.6 & 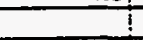 & 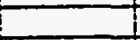 & & & & & & & \\
\hline Outlat Lig. Fow Rate-GPM & 9.41 & i & I & & & \pm & I & I & & \\
\hline Outlet Vap. Fow Rade-GPM & टरां & $T$ & 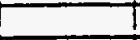 & $i$ & T & T & T & & & \\
\hline Inlot Temperature of & 173.5 & $i$ & $T$ & $T$ & 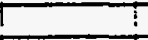 & $!$ & 1 & i & 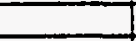 & \\
\hline Outlet Liq. Temperaturef & 774.81 & T & $\mathrm{T}$ & $T$ & $T$ & $T$ & t & & & \\
\hline Outlet Vap. Tomperature & 172.9 & $T$ & $\Gamma$ & 1 & 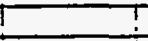 & T & I & I & {[} & \\
\hline Water Fow Rate-GPM & $i$ & 1 & 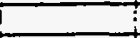 & I & i & I & I & $i$ & & \\
\hline Water hlot Tomp.f & 100.1 & $i$ & 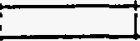 & T & I & $i$ & 1 & I & 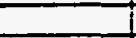 & \\
\hline Water outhet Temp.f & 149.81 & 1 & 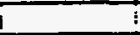 & 1 & $i$ & 1 & 1 & 1 & L & \\
\hline Intet G fbominrin 2 & 856628 & & Temp & 169.11 & Denaily & 77.84 & $I$ & 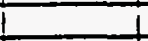 & L & \\
\hline Subooalingf & 2.5 & & & & & & I & i & & \\
\hline \multicolumn{10}{|l|}{ Flow Observation } & \\
\hline & ZONE1 & ZONE2 & ZONE3 & ZONEQ & ZONES T & RONE6 & ZONE7 & ZONE8 & ZONE9 & ZONE10 \\
\hline Heat Inpul-Wadte(net) & 856.6 & $\frac{258.2}{85}$ & 8511 & 857.5 & 855.5 & 860.3 & 856.4 & 847.6 & 85 & \multirow{2}{*}{\begin{tabular}{|r|}
851.8 \\
16.8 \\
\end{tabular}} \\
\hline Hoal Loss-Watts & 10.2 & 2.4 & 3.2 & 3.3 & 3.3 & 2.5 & 1.6 & \multicolumn{2}{|c|}{$\frac{4.1 .01}{2.41} \quad \frac{\cos }{3}$} & \\
\hline Hoa:flux-Eunthin & 1486 & 14876 & 14925 & 14864 & 14850 & 14973 & 14816 & 146931 & 14776 & 14765 \\
\hline Wall Temp. I3F & 1824 & $\mid 84.81$ & 1828 & 183.91 & 1852 & 186.3 & 186.21 & 183.91 & 1823 & 787.9 \\
\hline Wal Tomp $14 F$ & 183.7 & 7826 & 184.8 & 184.5 & 985.7 & 188.61 & 188.2 & 185.6 & 18.1. & 788.7 \\
\hline Wall Tomp. $15 F$ & 183.3 & $181.3 !$ & 184.0 & 184.6 & 189.3 & 185.81 & 185.7 & 183.8 & 183 & 180.6 \\
\hline Wall Tomp. IIF & 173.6 | & 188.31 & 181.8 & 1828 & 189.8 & 185.81 & 187 & 184.51 & 187.8 & 179.4 \\
\hline WeilTomp. ILF & 184.1 & 183.81 & 1828 & 183.5 & 183.8 & 186,8 & 187.1 & 185.51 & 183.1 & 181.2 \\
\hline Instream Tomp.f & 174.4 & 174.2 & 174.7 & 976.8 & 175.5 & 176.4 & 176.8 & 176.0 & 175.0 & 178.7 \\
\hline Avg. Wall Temp.F & 183.4 & 183.5 & 1828 & 184 & 184.4 & 186.1 & 186.4 & 184.6 & 1820 & 181.8 \\
\hline Avg. WallDT.F & 8.3 & 8.6 & 7.4 & 7.4 & 8.3 & 10.1 & 9.2 & 8.1 & 6.3 & 5.1 \\
\hline Remaining Subcooff & -0.8 & & & & & & & & & \\
\hline Ext Cuality & 0.01 & 0.041 & 0.07 & 0.11 & 0.14 & 0.17 & 0.2 & 0.24 & 0.27 & 0.3 \\
\hline EXP.HBturntrieF & 1788.1 & 1724.3 & 2023.6 & 2014.6 & 1796.5 & 1483.7 & 1619 & 1819.3 & 2341.4 & 2922.3 \\
\hline COMPUTEDH & & & & & & & & 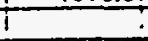 & & \\
\hline Jallouk & 7441.11 & Tక্36.81 & T350.81 & 7318.6 & 12827 & T250.41 & Tिडा & 7201.51 & 1188.4 & 7173.1 \\
\hline Szady (Cudo utbe) & 21427 & 2148.7 & 2154.1 & राह4.2 & 2159.2 & 2165.7 & 2175.4 & 2159.21 & 2167.1 & 2170.2 \\
\hline sendye & 2024.8 & 2024.8 & 2055.4 & 204.6 & 2005.8 & 2018 & 2051.6 & 2028.01 & 2057 & 1984.8 \\
\hline (PDa) & 1552285 & 151857 & 14748 & 149879 & 138183 & 1320888 & 129119 & 123120 & 117947 & 112978 \\
\hline$(F)$ & 1.156 & 1.46 & 9.78 & 1.805 & 2.111 & 2.268 & 2.391 & 2.527 & 2645 & 2752 \\
\hline$(H \cup O)$ & 294.3 & 369.1 & 440.7 & 493.2 & 638.5 & 578.1 & 611.2 & 645ा & 675.2 & 700 \\
\hline (HBOIL) & 21408 & 2140 & 21479 & 2155.6 & 2148 & 2151.9 & 21592 & 2939.8 & 2145.1 & 21453 \\
\hline & & & & & & & & & & \\
\hline & ZONE2 & ZONE & ZONE6 & ZONEB & ZONETO & & & & & \\
\hline DP hquid Temp.f & 79.3 & & & & & & & & & \\
\hline DPLiquid Density bm/13 & 93.3 & & & & & & & & & \\
\hline Elovation & 21 & 4 & 6 & 8 & 9.875 & & & & & \\
\hline Whasured DPFsid & 0.274 & 0.877 & T.302 & 2.058 & टह्या & & & & & \\
\hline Zro Correctons-psid & 0.007 & -0.0501 & -0.184 & 0.02 & 0.0281 & & & & & \\
\hline Cormond Dp pstd & 0.273 & 0.800 & 1.484 & 2.048 & 2.590 & & & & & \\
\hline Actual DPh & 1.83 & 3.18 & 4.53 & 5.91 & 7.17 & & & & & \\
\hline Meaqured TPPsig & 181.4 & & & & 180.7 & & & & & \\
\hline Masured Overellth & 1.32 & & & & & & & & & \\
\hline & & & & & & & & & & \\
\hline Computed DPA & 2 & 3.77 & 5.3. & 6.98 & 8.5 & & & & & \\
\hline Frictiont & 0.18 & 0.45 & 0.83 & 1.39 & 1.83 & & & & & \\
\hline Acouteretiont & 0.15 & 0.26 & 0.36 & 0.16 & 0.56 & & & & & \\
\hline Elvetiont & 1.69 & 3 & 4.16 & 5.2 & 6.12 & & & & & \\
\hline & & & & & & & & & & \\
\hline
\end{tabular}




\begin{tabular}{|c|c|c|c|c|c|c|c|c|c|c|}
\hline Aunt & 8481 & - & : & : & & & & : & & i \\
\hline Tostfluid & CAF8 & $\dot{-}$ & $\dot{-}$ & & & & & & & 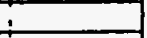 \\
\hline Date: & i 92993 & & $!$ & $!$ & & & & & & 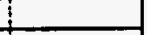 \\
\hline Time: & $9: 42$ & & & & & & & & & i \\
\hline Note & IAnalyical & th is used & ! & 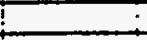 & & & $i$ & & & \\
\hline Fow Areath & 0.003561 & & & & & & & & & \\
\hline Hod Fow AYOAfL2 & 0.19695 & ! & ! & I & I & I & & $i$ & & \\
\hline Sat. Prossure trig & 180.6 & 7 & 1 & $\mathrm{i}$ & $!$ & 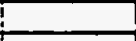 & & & & \\
\hline Sal. Tomperarefindet) & 175.7 & & & & i & & & & & \\
\hline La. Denstintomit3 & 76.66 & 76.82 & 76.821 & 76.431 & 76.011 & 76.67 & 76.22 & 76.48 & 76.52 & 76.5 \\
\hline Letent Houptenbm & 31.17 & 37.24 & 31.24 & 30.921 & 31.07 & 31.07 & 30.74 & 30.95 & 30.89 & 30.97 \\
\hline Specitio Hod othlom, & 0.312 & 0.312 & 0.312 & 0.3141 & 0.313 & 0.313 & 0.315 & 0.313 & 0.313 & 0.313 \\
\hline Lig. Visoosity tbmilitx & 0.358 & 0.361 & 0.36 & 0.354 & 0.357 & 0.357 & 0.352 & 0.356 & 0.356 & 0.355 \\
\hline 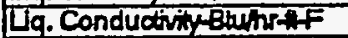 & 0.006 & 0.0261 & 0.0251 & 0.0259 & 0.026 & 0.026 & 0.0257 & 0.0259 & 0.0259 & 0.0259 \\
\hline Liq. Prandt No. & 4.3 & 4.3 & 4.3 & 4.3 & 4.3 & 4.3 & $4 . \overline{3}$ & 4.3 & 4.3 & 4.3 \\
\hline Infet Fow Rate-GPM & 5.5 & & & & & & & & & \\
\hline Outet Lig. Flow Pato-GPM & 7.4 & & & & & & & & & \\
\hline Outlet Vap. Flow Rate-GPM & 2.51 & 7 & & & & & & & & \\
\hline Inlat Tamperetureff & 174.2 & & & & & & & & & \\
\hline Outel Lg. Temperauref & 174.11 & & & & t & i & & & & \\
\hline Outat Vap. Tomperdiref & 172.5 & & & & & & & & & \\
\hline Wa Fow RAT-GPM & & & & & 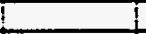 & & & & & \\
\hline Weter hlet Temp.f & 98.3 & 1 & 1 & 1 & 1 & $i$ & i & & & \\
\hline West outet Temp.f & 148 & & & & 1 & 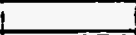 & & 1 & & \\
\hline 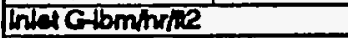 & 1022456 & & Temp & 169.3 & Donsiy & 7.9 & & & & \\
\hline Suboodingf & 1.5 & & & & & i & & & & \\
\hline Fow Observation & & & & & & & & & & \\
\hline & ZONEI & ZONE2 & ZONE3 & ZONE4 & ZONE5 & ZONE6 & ZONE & ZONEB & ZONE9 & LONE10 \\
\hline Hoal Input-Watts(net) & 867.7 & 862 & 859.1 & 858.8 & 875.4 & 858.4 & 865.8 & 31853.3 & 857.6 & 855 \\
\hline Heat Loss-Wous & 10.1 & 2.3 & 3.1 & 3.3 & 3.2 & 24 & 1.6 & 2.4 & 3 & 16.5 \\
\hline Hogl Flox-bourthrie & 150दा & 1492 & 14892 & 148521 & 15974 & 15018 & 14999 & 14565 & 74868 & 14827 \\
\hline Wall Temp. & 7821 & โ84.8 & 1828 & 183.8 & 184.81 & 785.7 & 185.4 & 183.9 & 182 & 381.5 \\
\hline Wa' Temp.14F & 185.6 & 182 & 183.8 & 184.6 & 185.8 & 185.8 & 188.4 & 185.8 & 783.8 & 785.8 \\
\hline Wa Tomp. 157 & 1832 & 181 & 184.4 & 1845 & 183.8 & 1852 & 185.8 & 183.7 & 1827 & 180 \\
\hline Well Temp.11F & 174.2 & 183 & 180.6 & 180.1 & 183.6 & 185.8 & 787.4 & 184.6 & 181.6 & 779.1 \\
\hline Wall Temp. \&2F & 184.1 & 1828 & 182 & 183.8 & 188.7 & 185.7 & 187.4 & 185.6 & 1820 & 1812 \\
\hline Instroem Tomp.f & 174.8 & 174.1 & 174.1 & 175.8 & 175 & 175 & 176.7 & 175.6 & 175.4 & 1755 \\
\hline Avg. Wall Tomp.F & 183.8 & 183.1 & 1822 & 184 & 184.11 & 185.5 & 186.7 & 184.6 & 1826 & 181.5 \\
\hline Avo. Wall DT.F & 7.8 & 8.3 & 7.4 & 7.5 & 8.4 & 9.8 & 9.3 & 8.3 & 6.5 & 5.4 \\
\hline Romaining Subcoolf & -1.3 & & & & & & & & & \\
\hline Ext Quality & 0.01 & 0.04 & 0.07 & 0.1 & 0.12 & 0.15 & 0.18 & 0.21 & 0.23 & 0.26 \\
\hline EXP. HQtur-HERE & 1925.7 & 1789.5 & 2011.8 & 19727 & 1800.4 & 15202 & 16125 & 1797.8 & 22112 & 2759.8 \\
\hline COMPUIEDH & & & & & & & & & & \\
\hline Jallock & 7418.31 & 7341.7 & $\{200\}$ & 1264.7 & 1233.31 & T2012.8 & Tद⿱ & 1762.3 & 1737.5 & TIT7.6 \\
\hline sendil(cudo wa) & 2767.4 & 2148.7 & 2Т47.4 & 218.7 & 2180.8 & 2172.8 & 2191 & 2178.4 & 2T728 & 2773.2 \\
\hline $\operatorname{san} 2$ & 2047.7 & 2020.0 & 2024.8 & 20122 & 20026 & 2018 & 20.7 .8 & 2051 & 1987.7 & 1988.4 \\
\hline (A山a) & 185740 & 180783 & 175698 & $1 \% 202$ & 16884 & 181011 & 158887 & 152048 & 1460.2 & 141627 \\
\hline$(F)$ & t.21 & 1.470 & 1.76 & 1.886 & 2.048 & 2188 & 229 & 242 & 2581 & 2.0 \\
\hline (1) & 356.7 & 434.1 & 5032 & 556 & 602 & 648 & 676.8 & 7126 & 74.5 & 77.7 \\
\hline$(1+804)$ & 21532 & 21428 & 213911 & 21521 & 21656 & 21535 & 21623 & 2153.7 & 21434 & 2140 \\
\hline & & & & & & & & & & \\
\hline & $20 N=2$ & ZOKEA & ZONEG & ZONE8 & ZONETO & & & & & \\
\hline DP hquid Tomp.F & 84.8 & & & & & & & & & \\
\hline DPUlovid Densih 4 bma & 9261 & & & & & & & & & \\
\hline Elovetion- & 2 & 4 & 61 & 81 & 9.875 & & & & & \\
\hline Mheutred DPpid & 0.251 & $0.7 \%$ & 3.18 & 1.7841 & 223 & & & & & \\
\hline Zero Correctons-psid & 0.001 & -0.058 & $-0.18 \pi$ & 0.02 & 0.068 & & & & & \\
\hline Comecod DPpald & 0.258 & 0.798 & $1.29 \pi$ & 1.774 & ह205 & & & & & \\
\hline Actual DPh & 1.94 & 3.34 & 4.82 & 6.34 & 7.75 & & & & & \\
\hline Mosured TPFig & 180.6 & & & & 180.8 & & & & & \\
\hline Mesesured Overallth & 0.66 & & & & & & & & & \\
\hline & & & & & & & & & & \\
\hline CompledDPA & 2.12 & 4 & 5.85 & 7.7 & 9.48 & & & & & \\
\hline Fichont & 0.21 & 0.59 & 1.08 & 1.62 & 221 & & & & & \\
\hline Acoderctionth & 0.2 & 0.35 & 0.47 & 0.58 & 0.7 & & & & & \\
\hline Eevent & 1.67 & 3.08 & 4.3. & 5.5 & 6.54 & & & & & \\
\hline & & & & & & & & & & \\
\hline
\end{tabular}




\begin{tabular}{|c|c|c|c|c|c|c|c|c|c|c|}
\hline Run: & $1 \quad 8491$ & 5 & 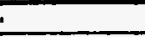 & & & $:$ & : & : & & $i$ \\
\hline Tostfuid & . $4 \mathrm{FB}$ & . & 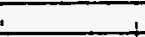 & & & . & $:$ & 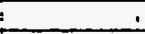 & 1 & \\
\hline Dals: & 1.9293 & $\vdots$ & 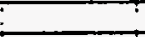 & 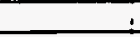 & & 1 & 1 & 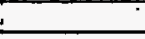 & & $\vdots$ \\
\hline Time: & $9: 42$ & & 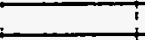 & & $\vdots$ & & & & & $!$ \\
\hline Note & - Analytical d & di is used & 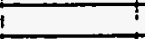 & & $i$ & : & $\dot{\square}$ & 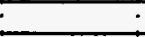 & 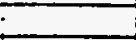 & 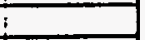 \\
\hline Flow Area-12 & 0.003561 & & & & & & & & & \\
\hline Hoat Aow Areati2 & 0.1955 & $T$ & $?$ & & $T$ & & 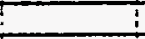 & 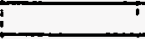 & 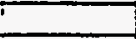 & \\
\hline Saf. Pressure Psig & 179.81 & I & 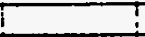 & 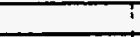 & 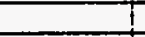 & i & $i$ & 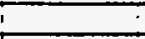 & 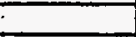 & \\
\hline Sat. Temperature F(inet) & 175.31 & 1 & 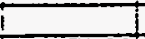 & ! & & & 1 & ! & 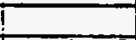 & \\
\hline Liq. Density fbm/13 & $\pi$ & 76.961 & 76.84 & 76.041 & 76.75 & 76.88 & 76.451 & 76.551 & 76.57 & 76.00 \\
\hline Latan Hoal-Bhuhbm & 31.39 & 31.351 & 31.281 & 31.091 & 31.18 & 31.13 & 30.981 & 31.011 & 31.03 & 31.11 \\
\hline Specitio Hoat-Bhubmir & 0.311 & 0.3711 & 0.312 & 0.373 & 0.312 & 0.312 & 0.313 & 0.313 & 0.313 & 0.312 \\
\hline Lig. Viscosity tbmithr & 0.368 & 0.352 & 0.36 & 0.357 & 0.359 & 0.358 & 0.355 & 0.3561 & 0.356 & 0.368 \\
\hline Lig. Conductivive Bunrtif & 0.0252 & 0.002 & 0.0261 & 0.026 & 0.0261 & 0.0201 & 0.02591 & 0.0259 & 0.065 & 0.065 \\
\hline Lig. PrandtiNo. & 4.3 & 4.3 & 4.3 & 4.3 & 4.3 & 4.3 & 4.3 & 4.3 & 4.3 & 4.3 \\
\hline Inlet Fow Rate-GPM & 5.5 & & & & & & & & 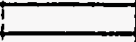 & \\
\hline Outal Lig. Fow Rade-GPM & 1.1 & & & 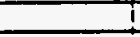 & i & 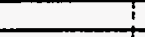 & 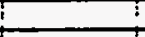 & 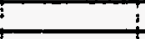 & 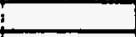 & \\
\hline Outlot Vap. Flow Rate-GPM & $2.6 !$ & & $!$ & 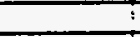 & & & 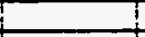 & $t$ & & \\
\hline Inlet Temperaturef & 172.4 & & $!$ & ! & 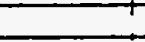 & & t & 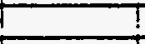 & $!$ & \\
\hline Outol Lq. Temperature & T74.11 & & + & ! & 1 & $T$ & $T$ & 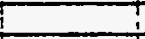 & 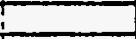 & \\
\hline Outuol Vap. Temperaturef & 172.4 & & 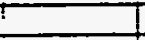 & T & 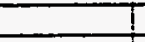 & & & T! & ! & \\
\hline Waer Fow Pade-GPM & 1 & $\vdots$ & 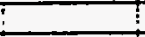 & 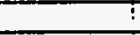 & $T$ & & $!$ & 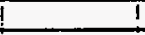 & $!$ & \\
\hline Water inlot Temp.f & 101.4 & & i & 1 & $!$ & 1 & 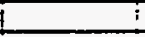 & 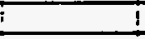 & 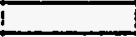 & \\
\hline Water outlet Tomp.f & 147.2 & & 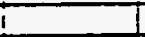 & 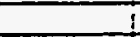 & 1 & 1 & 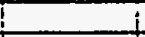 & $i$ & 1 & \\
\hline Intel Gtominine & 102323 & & Temp & 168 & Density & $77 . \infty 1$ & & & & \\
\hline Subcooling $F$ & 2.9 & & & & & & & & $i$ & i \\
\hline \multicolumn{11}{|l|}{ Flow Obsenvation } \\
\hline & ZONE & ZONE2 & ZONE3 & ZONE4 & ZONE5 & ZONE6 I & ZONE7 & ZONEB & ZONES & ZONEIO \\
\hline Head input+Watts(nel) & 1149.8 & 1153.8 & 1147.8 & 1158.8 & 11527 & 1150.2 & 481 & 1025 & 96.8 & 99.8 \\
\hline Hoad Loss-Walls & 10.61 & 251 & 3.4 & 3.41 & 3.3 & 2.5 & 1.4 & 21 & 2.6 & 14.1 \\
\hline Heat Flo $B$ turfine & 19sen & 20000 & 1938 & 20054 & 19981 & 2001 & 8338 & $77 \pi$ & $16 \%$ & 1732 \\
\hline Wai Temp. HF & 1823 & 186.9 & 18.61 & 184.11 & 184.1 & 185 & 179.8 & 176.7 & 776.5 & 1752. \\
\hline Wall Temp. \$4F & 184.8 & 1827 & 185.7 & 184.9 & 184.8 & โ84.71 & 179.71 & 178.7 & 17 & 175.7 \\
\hline Wall Temp. $15 \mathrm{~F}$ & 183.7 & 187.51 & 185.7 & 184.8 & 1829 & 183.8 & 179.31 & 178.7 & 177.1 & 175.7 \\
\hline WEITamp. IfF & 172. & 183.8 & 181.7 & 1827 & 184 & 185.51 & 180.1 & 178.8 & 1782 & 175.3 \\
\hline Wall Tomp.t2F & 184.6 & 183.8 & 183.8 & 188.7 & 184.4 & 186.4 & 179.9 & 176.9 & 176.8 & 175.4 \\
\hline Indroem Temp.f & 173.3 & 173.6 & 174 & 174.8 & 174.4 & 174.7 & 175.7 & 175.3 & 1762 & 174.8 \\
\hline Avo. Wall Tomp.F & 183.7 & 184.8 & 183.6 & 1842 & 984. & 184.8 & 179.8 & 176.7 & 176.6 & 175.5 \\
\hline Avg. Wall DT.F & 9.51 & 9.9 & 8.7 & 8.3 & 8.7 & 9.1 & 3.7 & 1.3 & 1.3 & 0.6 \\
\hline \multicolumn{11}{|l|}{ Remaining SubcooHF } \\
\hline Exit Quafiky & 0.011 & 0.01 & 0.08 & 0.12 & 0.15 & 0.19 & 0.21 & 0.21 & 0.21 & 0.22 \\
\hline EXP.HBtuHrine & 2091.7 & 2015.9 & 2233.81 & 24085 & 228.4 & 2191.8 & 2203.7 & 1326.2 & 1248.8 & 2978 \\
\hline OOMPUED & 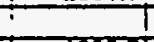 & & & & & & & & & \\
\hline Jallouk & 168321 & 1574.11 & 1502.4 & T45281 & 1407.71 & 1359.4 & 835.31 & 605.11 & 607.5 & 608.9 \\
\hline Seadyi(Sido tubo) & 2464.8 & 2473.4 & 2475 & 24875 & 2488 & 245:3.1 & 1628.4 & 874.41 & 8516 & 8727 \\
\hline sodr & 2384.6 & 2405.8 & 2405 & 24821 & 2477.9 & 2487.51 & 1369.2 & 737.81 & 739.8 & 74.9 \\
\hline$(R \operatorname{QD})$ & 183924 & 1800031 & 174604 & 168887 & 161218 & 154778 & 151270 & 148970 & 148285 & T4riog \\
\hline$(\mathbf{F})$ & 1.152 & 1.468 & 1.771 & 1.831 & 2.185 & 237 & 2.4R & 2484 & 2.497 & 2574 \\
\hline (HUQ) & $\mathbf{3 7 . 7}$ & 430.7 & 518.0 & 5Es.4 & 647.8 & 50.11 & 719.2 & 730.01 & 734.8 & 739.8 \\
\hline \multirow[t]{3}{*}{ (FOL) } & 24527 & 2450 & 2467.3 & 24827 & 2474.7 & 24808 & 15802 & 6528 & E296 & 627 \\
\hline & & & 1 & & & & & & & \\
\hline & ZONE2 & ZONE4 & ZONES & $20 N^{5}=8$ & ZONETO & & & & & \\
\hline \multicolumn{11}{|l|}{ DP Fquid Temp. $F$} \\
\hline \multicolumn{11}{|l|}{ DPLlquid Density 1 bmis } \\
\hline \multicolumn{11}{|l|}{ Eevation- } \\
\hline MesauradDPPsid & 0.25 & 0.76 & 1.168 & 1.535 & 2.518 & & & & & \\
\hline Zoro Com ctions-psid & 0.001 & -0.068 & -0.184 & 0.02 & 0.068 & & & & & \\
\hline Comecied DPPstd & 0.251 & 0.801 & 1.352 & 1.915 & 2485 & & & & & \\
\hline Achod DPA & 1.98 & 3.37 & 4.81 & 6.15 & 7.37 & & & & & \\
\hline Masarud TPPsig & 179.8 & & & & .79 .61 & & & & & \\
\hline \multicolumn{11}{|l|}{ Meavered Overnilt } \\
\hline & & & & & & & & & & \\
\hline Computad DR & 214 & 4.01 & 5.85 & 7.77 & 9.551 & & & & & \\
\hline Ficton-a & 0.2 & 0.87 & 1दा & 1.63 & 200 & & & & & \\
\hline Acoolerion-1 & 0.28 & 0.4 & 0.68 & 0.6 & 0.61 & & & & & \\
\hline Enration-f & 1.68 & 2.97 & 4.03 & 5.51 & 6.81 & & & & & \\
\hline & & & & & & & & & & \\
\hline
\end{tabular}




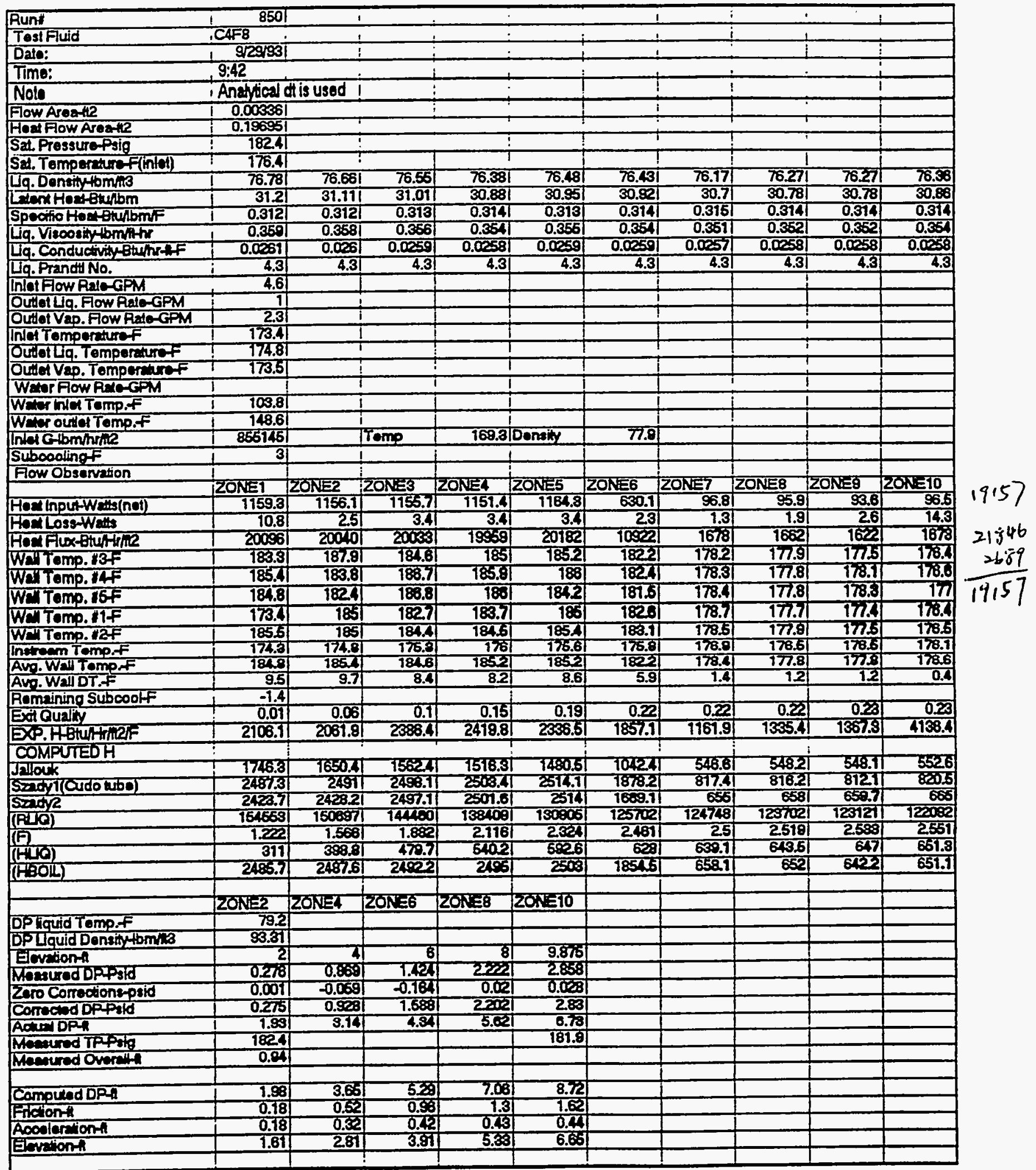




\begin{tabular}{|c|c|c|c|c|c|c|c|c|c|c|c|}
\hline Run: & 8511 & $\therefore$ & & & - & - & $\therefore$ & 2 & & & \\
\hline Tost Fuid & . $44-8$ & \pm & & & & & & & & 1 & \\
\hline Date: & $9 / 291931$ & & - & & & & & 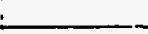 & & & \\
\hline Time: & $9: 42$ & & & & & & & & & & \\
\hline Note & Analytical o & th is used! & & & & & & & & & \\
\hline Flow Areatle & 0.003361 & & & & : & 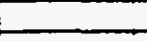 & & & & $!$ & \\
\hline Heat Fow Aroatt2 & 0.19595 & & & & & & & & & & \\
\hline Sat. Pressuro Psig & 181.7 & & & & & & & 1 & & & \\
\hline Sal Temperature finlet) & 176.1 & & ! & & 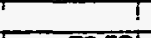 & 1 & 1 & 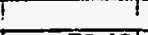 & & & \\
\hline Ua. Density lbmins & 76.841 & 76.641 & 76.591 & 76.431 & 76.521 & 76.5 & 76.34 & 76.431 & 76.521 & 76.36 & \\
\hline Ladont Hoar-Builbm & 31.28 & 31.091 & 31.05 & 30.921 & $30.99 !$ & $30.97 !$ & 30.841 & 30.921 & 30.991 & 30.80 & \\
\hline Spocite HostotulbmfF & 0.312 & 0.3131 & 0.313 & 0.3141 & 0.3131 & 0.3131 & 0.3141 & 0.314 & 0.313 & 0.314 & \\
\hline Lig. Visoosity bomphr & 0.361 & 0.357 & 0.357 & 0.3561 & 0.366 & 0.365 & 0.3531 & 0.364 & 0.3561 & 0.364 & \\
\hline Liq. Conductivity hrsf & 0.02611 & 0.0261 & 0.0261 & 0.02591 & 0.0259 & 0.02591 & 0.02581 & 0.02501 & 0.0259 & 0.0258 & \\
\hline Liq. Prandel No. & 4.3 & 4.31 & 4.3 & 4.31 & 4.3 & $4.3 !$ & 4.31 & 4.3 & 4.3 & 4.3 & \\
\hline Inlei Fow Rato-GPM & 3.7 & & & & & & & & & & \\
\hline Oútlet Liq. Fow Rate-GPM & 1) & & & & & 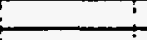 & $!$ & 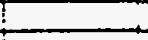 & & 7 & \\
\hline Outlet Vap. Flow Rate-GPM & 1.91 & & I & $\therefore$ & 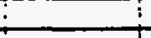 & 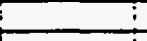 & i & I & & i & \\
\hline Inlet Temporaturef & 173.31 & & & & 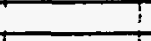 & 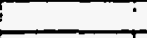 & & I & & & \\
\hline Outel Lia. Temperature f & 774.7 & & & & & & & & & & \\
\hline Outel Vap. Temperaturef & 172.51 & & 5 & & 1 & I & $!$ & ! & & & \\
\hline Water Fow Roto-GPM & 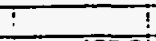 & $\div$ & 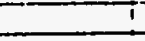 & 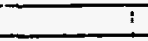 & $\vdots$ & 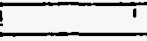 & $i$ & $i$ & & & \\
\hline Water hnlot Tomp.f & 103.81 & $\stackrel{\square}{!}$ & 1 & 1 & $i$ & I & $!$ & \pm & & & \\
\hline Water outiot Temp.F & 151.8 & i & & & 1 & 1 & $\vdots$ & I & & & \\
\hline Intal Glbmhrne & 689769 & & Temp & 768.310 & Density & 78.12 & & 1 & & & \\
\hline SubcoolingF & 2.8 & 1 & & & 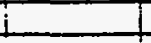 & $i$ & 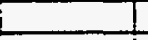 & 1 & & & \\
\hline Fow Observation & & & & & & i & L & & & & \\
\hline & ZONE1 & ZONE2 ; & ZONE3 & ZONE4 i2 & ZONES & ZONE6 & ZONE7 IS & ZONE8 & ZONES & ZONE10 & \\
\hline Hoad input-Watts(net) & 1158.1 & 1162.9 & 1159.2 & 1158.6 & 1159.2 & 644.8 & 98.2 & 97.2 & 94.2 & 97.5 & $1: 8$ \\
\hline Hoed Loss-Watts & 10.3 & 2.41 & 3.3 & 3.31 & 3.3 & 22 & 1.2 & 1.81 & 2.4 & 13.8 & \\
\hline Hod FLx-BuHHriL2 & 20075 & 20158 & 20094 & 200841 & 20094 & 11179 & 1702 & 16851 & $16 \mathbb{B}^{\prime}$ & 1690 & 215,7 \\
\hline Wall Temp. H3 & 183.1 & 187.5 & 184.2 & 184.8 & 185 & 182 & 177.4 & 177.21 & 176.7 & 778.4 & 26 \\
\hline Wal Temp. $14 F$ & 185.11 & 183.5 & 185.3 & 185.8 & 185.81 & 18241 & 777.71 & 177.21 & 177.3 & 176.8 & \\
\hline W21 Temp. $15 F$ & 184.6 & 18221 & 188.5 & 1801 & 184 & 181.4 & 178 & 177.21 & 777.7 & 17 & 178 \\
\hline WEV Tomp. IIF & 173.3 & 184.7 & 182.8 & 189.01 & 184.8 & 18251 & 178.2 & 177 & 170.6 & 170.4 & \\
\hline Weld Temp. ILF & 1852 & 184.7 & 184.1 & 184.51 & 185.1 & 18281 & 178 & 177.31 & 176.7 & 176.6 & \\
\hline Inetream Temp.f & 174 & 174.0 & 175.1 & 176.8 & 176.4 & 175.6 & 1762 & 176.8 & 175.4 & 176.1 & \\
\hline Avg. Wall Tomp.F & 184.5 & 185.11 & 182 & 185.1 & 184.9 & 182, & 177.9 & 177.2 & 177 & 176.8 & \\
\hline Avg. Wall DT.F & 9.6 & 9.3 & 8.2 & 8.41 & 8.6 & 6.11 & 1.61 & 1.3 & 1.5. & 0.4 & \\
\hline Remaining Subcooff & -2.7 & & & & & & & & & & \\
\hline Ext Quality & 0.03 & 0.081 & 0.14 & 0.19 & 0.25 & 0.28 & 0.28 & 0.29 & 0.29 & 0.3 & \\
\hline EXP.HBtuHrA2/F & 20927 & 2170.11 & 2445.4 & 2400.2 & 2337.9 & 1841.7 & 1075.3 & 1292.2 & 1069.8 & 3989.7 & \\
\hline COMPUTEDH & & & & & & & & & & & \\
\hline Jallouk & 7759.7 & 7705.61 & 7631.6 & 7569.61 & 1520.11 & 90751 & 520.51 & 521.4 & 520,5 & 524 & \\
\hline Srady (audo wbo) & 248221 & 2499.11 & 2498.6 & 2507.61 & 2504.61 & 1891.41 & 796.31 & 794.11 & 786.7 & 798.2 & \\
\hline $\operatorname{send} 2$ & $2417 . \pi$ & 2438.81 & 2438 & 2509.21 & 2508.6 & 16981 & 671.9 & 513.91 & 614.8 & 618.1 & \\
\hline (R्य) & 125358 & 1194281 & 112700 & 1084351 & 99082 & $\$ 37031$ & 92047 & 911201 & 90210 & 90005 & \\
\hline$(\mathbf{F})$ & 1.32 & 1.708 & 2.045 & 2.2091 & 2.526 & 2.678 & 2.728 & 2751 & 2.77 & 2.775 & \\
\hline (1) & 2827 & 366.4 & 438.8 & 49 & 54221 & 575.21 & 586.5 & 590.71 & 594.0 & 596.5 & \\
\hline$(\mathrm{HBOIL})$ & 2481 & 2496.4 & 2494.1 & 25012 & 2496.1 & 18735.5 & 671.8 & 665.5 & 651.9 & 668.6 & \\
\hline & & & & & & & & & & & \\
\hline & ZONE2 & ZONE4 & ZONE6 & ZONEB & ZONE 10 & & & & & & \\
\hline DP liquid Temp.f & 78.8 & & & & & & & & & & \\
\hline DP Lquid Densitymitis & 93.37 & & & & & & & & & & \\
\hline Everation-h & 2 & 4 & 6 & 81 & 9.875 & & & & & & \\
\hline MhasuredDPPsid & 0.287 & T.0181 & 1.6881 & 2.557 & 3.212 & & & & & & \\
\hline Zero Corrections-psid & -0.024 & -0.0811 & -0.250 & 0.0731 & 0.097 & & & & & & \\
\hline Comected DPfyid & 0.371 & 1.1 & 1.989 & 2.489 & 3.175 & & & & & & \\
\hline Actud DPA & 1.88 & 2.82 & 3.8 & 5.11 & 8.09 & & & & & & \\
\hline Menetred TPYPig & 181.7 & & & & 181.1 & & & & & & \\
\hline Mentured Overellith & 1.13 & & & & & & & & & & \\
\hline & & & & & & & & & & & \\
\hline Computad DRA & 1.78 & 3.28 & 4.67) & 6.321 & 7.88 & & & & & & \\
\hline Frictionft & 0.15 & 0.42 & 0.79 & 1.05 & 1.3 & & & & & & \\
\hline Accoleration h & 0.15 & 0.25 & 0.34 & 0.35 & 0.35 & & & & & & \\
\hline Elevation-a & 1.49 & 2.58 & 3.50 & 4.821 & 6.2. & & & & & & \\
\hline & & & & & & & & & & & \\
\hline
\end{tabular}




\begin{tabular}{|c|c|c|c|c|c|c|c|c|c|c|c|}
\hline Runt & 8521 & & ' & 1 & ; & & 1 & - & &. & \\
\hline Tost Fuid & CAF8 & & . & 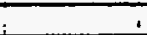 & : & & . & . & & . & \\
\hline Date: & $9 / 30 / 331$ & & & & & &. & & & & \\
\hline Time: & $10: 01$ & & & & & & & & & & \\
\hline Note & Anatyical d & ot is used & $i$ & & & & & & & & \\
\hline Flow Areati2 & $0.00336 !$ & & & $\vdots$ & & & & & & : & \\
\hline Hoat Fow Areath & 0.1955 & & - & & $\vdots$ & ; & & & & it & \\
\hline Šd. PTessure Psig & 178.8 & & ? & t: & $T$ & ! & $t$ & & & T! & \\
\hline Sad. Tomperauref(inlet) & 774.91 & & $T$ & & $T$ & 1 & $T$ & & & & \\
\hline [a.Denstingomins & 76.87 & 76.08 & 76.82 & 76.291 & 76.671 & 76.31 & 76.221 & 76.571 & 76.67 & 76.30 & \\
\hline 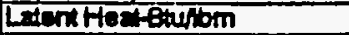 & 31.281 & 81.181 & 37.24 & 30.81 & 31.07 & 37.051 & $\$ 0.74$ & 31.081 & 31.07 & 30.80 & \\
\hline 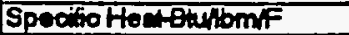 & $0.31 \pi$ & 0.3121 & 0.312 & 0.3141 & 0.318 & 0.3131 & 0.3151 & 0.3131 & 0.319 & 0.314 & \\
\hline Lig. Viecosity bomilitr & 0.3611 & 0.358 & 0.38 & 0.3631 & 0.857 & 0.3571 & 0.362 & 0.3661 & 0.357 & 0.354 & \\
\hline Lig. Conductiviturfiff & 0.02511 & 0.005 & 0.0251 & 0.0258 & 0.008 & 0.0261 & 0.0257 & 0.0261 & 0.0261 & 0.0258 & \\
\hline Lig. Prandd No. & 4.31 & 4.31 & 4.3 & 4.3 & 4.3 & 4.31 & 4.31 & 4.31 & 4.31 & 4.3 & \\
\hline Indofflow Patc-GPM & 28 & & & & & & & 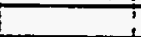 & & & \\
\hline Outal Lig. Fow Rato-GPM & 0.5 & & 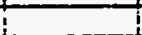 & & & & & 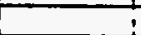 & & & \\
\hline Outhat Vap. Flow Rate-GPM & 1.71 & & & i & 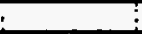 & & $T$ & & & & \\
\hline Inlel Temporaturef & 173.4 & & & $\frac{?}{4}$ & & & T & & & $t$ & \\
\hline OutelLiq. Temporaturef & 174.71 & & : & $T$ & $\mathrm{~T}$ & & $T$ & & & & \\
\hline Outer Vap. Temperaturef & 1722 & & $\mathrm{~T}$ & & 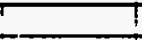 & & T & & & & \\
\hline WAOP FIOW REAT-GPM & 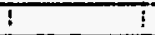 & & ! & $i$ & i & & $T$ & T & & & \\
\hline Water hetomo,f & 100.81 & & $!$ & 1 & I & I & $I$ & $\overline{5}$ & & i & \\
\hline Wespr outul Tomp.f & 157.71 & 1 & i & 1 & 1 & & 1 & 1 & & 1 & \\
\hline Intel Gibmhwithe & 524882 & & itemp & 168.8 & Density & 78.65 & $T$ & 1 & & & \\
\hline Subcoaling $F$ & 1.5 & 1 & 1 & 1 & 1 & 1 & 1 & 1 & & & \\
\hline Flow Obsenvion & & & 1 & & & & & & & & \\
\hline & ZONEI & ZONE2 & ZONES & ZONE4 I & ZONES & ZONE 6 & ZONET & ZONE8 & ZONE9 & ZONE10 & \\
\hline Hod Input-Wats(net) & 1154 & 1754 & 1754 & 1154 & 1154 & 784.6 & 184 & 11.1 & 10 & 5.2 & $1-8$ \\
\hline Heal Loss-Wats & 111 & 2.5 & 3.4 & 3.6 & 3.5 & 2.31 & 1.41 & 2 & 2.6ा & $\overline{15.7}$ & \\
\hline Henflux-BtuHurite & 20004 & 20001 & 20001 & 20004 & 20004 & 136011 & उ1S| & 19ी & 17 & 90 & 2374 \\
\hline Wal Tomp. B广 & 783.5 & 187.11 & 183.31 & 185.91 & T85.11 & 18टया & 779.9 & 777.51 & 778.0 & 778.2 & \\
\hline Wartomp. Ift & 185.2 & 183.11 & 185.2) & 1885. & 188.11 & 1828 & 779.7 & T7T:! & T78.8 & 178.6 & $52-3$ \\
\hline WEIT Tomp. 155 & 184.7 & 18151 & 1856 & 180.41 & 184.81 & 182.11 & 179.8 & 177.41 & 17.11 & 778.2 & \\
\hline WeI Tomp. Iff & 179.4 & 184.6 & 781.8 & 185.11 & 184.8 & 182.61 & 187 & 177.81 & 170.21 & 178.0 & 21551 \\
\hline WCHTOMP. ILF & 185.5 & 184.81 & 183.1 & 185.71 & 185.8 & 18201 & 180.5 & 177.81 & 176.8 & 178.4 & \\
\hline Inetroem Temp,ff & 173.0 & 174.71 & 774.11 & 178.41 & 176 & 175.1 & 176.7 & 17621 & 175 & 176.1 & \\
\hline Ang. Wall Teme.f & 182.7 & 184.8 & 183.2 & 188 & 185.9 & 1825 & 180.1 & 17751 & 176.6 & 178.4 & \\
\hline Avg. Wall DT.F & 9.9 & 9.2 & 8.2 & 8.7 & 9.3 & 6.81 & 32 & $2.3 !$ & 1.8 & 2.3 & \\
\hline Femaining Subcooff & -5.7 & & & & & & & & & & \\
\hline Ext Qualiny & 0.06 & 0.13 & 0.2 & 0.27 & 0.34 & 0.39 & 0.4 & 0.4 & 0.45 & 0.41 & \\
\hline \multirow{2}{*}{\multicolumn{11}{|c|}{$\begin{array}{l}\text { EXP.HBtuHrnefF } \\
\text { COMPUTEDH }\end{array}$}} & \\
\hline & & & & & & & & & & & \\
\hline Jallouk & T794.91 & 1745.T1 & 16721 & 7650.81 & 108 & 101 & 106.4 & 1081 & 708 & 106.2 & \\
\hline Szadyi(Cudo ubs) & 2778.4 & 2488.7 & 2460.8 & 2503.81 & 103 & 7081 & 708.41 & 1081 & 108 & 708.2. & \\
\hline $\operatorname{szady} 2$ & 2400.0 & 24229 & 2474.3 & 25121 & 100 & 1081 & 100.4 & 1001 & 1001 & 1062 & \\
\hline (RUA) & 925:-1 & 87072 & 7977 & 74481 & 74189 & 74487 & 7489 & 74484 & 74481 & 7448 & \\
\hline (F) & 1.498 & 1.5651 & 2.317 & 2.572 & $25 \not 2$ & 25721 & 2.572 & 2.572 & 2.572 & 2572 & \\
\hline (सUO) & 256.0 & 3360 & 398.0 & 7481 & 44.81 & 44.61 & 444.6 & 444.61 & 44.8 & 94.6 & \\
\hline \multirow[t]{3}{*}{ (1BOLIL) } & 2475.5 & 24847 & 2478.8 & $250 \times 2$ & 25072 & 2504.2 & 25012 & 25012 & 250121 & 350k2 & \\
\hline & & & & & & & & & & & \\
\hline & ZONE2 & ZONE & ZONE6 & ZONES & ZOREETO & & & & & & \\
\hline DP lquid Tomp.f & 75.8 & & 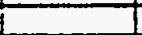 & & & & & & & & \\
\hline DPL'quid Density & 93.81 & & & & & & & & & & \\
\hline Elovation & 2 & का & 6) & 81 & 9.8751 & & & & & & \\
\hline Kosured DP4ard & 0.3007 & 7.398 & $7.3 \% 8$ & 2.505 & 3.791 & & & & & & \\
\hline Zaro Contactions-peid & -0.024 & -0.0811 & 0.208 & 0.0731 & 0.097 & & & & & & \\
\hline Combiad DPfird & 0.351 & 1.27 & 2300 & 2802 & 3.021 & & & & & & \\
\hline Acluadoph & 1.89 & 251 & 3.1 & 4.451 & 5.31 & & & I & & & \\
\hline Mresured tPpipg & 178.8 & & & 1 & 178.4 & & & 1 & & & \\
\hline \multirow[t]{2}{*}{ Mesured Overlll } & 0.76 & & & & & & & 1 & & & \\
\hline & & & & & & & & & & & \\
\hline Computed DPa & 1.55 & 279 & 3.97 & 5.28 & 6.9 & & & & & & \\
\hline Fitotion & 0.111 & 0.3 & 0.61 & 0.81 & 0.99 & & & & & & \\
\hline Acopleration-1 & 0.11 & 0.10 & 0.27 & 0.21 & 0.28 & & & & & & \\
\hline Elevetionh & 1.32 & ह2ा & 3.08 & 2.4. & 5.62 & & & & & & \\
\hline & & & & & & & & & & & \\
\hline
\end{tabular}




\begin{tabular}{|c|c|c|c|c|c|c|c|c|c|c|}
\hline Runt: & $853 i$ & & & & & 5 & & & 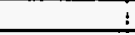 & $\leq$ \\
\hline Test Fluid & CAF 8 & & & & & & & & & 5 \\
\hline Date: & $9 \sqrt{3} 493$ & & & & & & & & & \\
\hline Time: & $10: 01$ & & & & & & & & & \\
\hline Note & Anayticalo & Htis used! & & & & & & & & \\
\hline Fow Asea-il2 & 0.003361 & & & & & & & & & \\
\hline Head Flow Area-f2 & 0.196951 & & & & & & & & & \\
\hline Sat. Pressure Prig & 179.21 & & & & & & & & & \\
\hline Sat. Temperature f(iniot) & 175.1 & & & & & & & & & \\
\hline Uq. Densitytominz & 76.871 & 76.571 & 76.75 & 76.31 & 76.521 & 76.551 & 76.171 & 76.431 & 76.45 & 76.31 \\
\hline Gent Hoaf-Btuhbm & 31.281 & 31.081 & 31.181 & 30.821 & 30.991 & 31.011 & 30.7 & $30.92 !$ & 30.93 & 30.82 \\
\hline Spectic Heat-Btuhbmif & 0.3111 & 0.3131 & 0.312 & 0.3741 & 0.3131 & 0.3131 & 0.315 & 0.3141 & 0.313 & 0.314 \\
\hline Liq. Viscosty-lom/ith & 0.3611 & 0.3661 & 0.359 & 0.3531 & 0.3667 & 0.3561 & 0.3511 & 0.3541 & 0.3651 & 0.353 \\
\hline Liq. Conductivitetuhrfif & 0.02611 & $0.026 i$ & 0.02611 & 0.02581 & 0.02591 & 0.02591 & 0.0257 & 0.0259 & 0.0250 & 0.0258 \\
\hline Liq. Prand No. & 4.3 & 4.31 & 4.31 & 4.3 & 4.3 & 4.31 & 4.31 & 4.3 & 4.3 & 4.3 \\
\hline Inlat flow Ralo-GPM & 2 & & & & & 1 & & 1 & & \\
\hline Outlel Lia. Fow Ralo-GPM & 0.41 & & & & & & & & & \\
\hline Outtel Vap. Flow Rato-GPM & 0.91 & & & & & & ; & & & \\
\hline Iniet Temperaturef & 173.1 & & & & & & & & & \\
\hline Outlel Liq. Temperaturef & $1 \% 5.9$ & & & & & & & & & \\
\hline Outfet vap.Temperaturef & 1731 & & & & & - & & & & 1 \\
\hline Wator Fow Rato-GPM & & & & & & & & & & \\
\hline Water hlat Temp.F & 101.41 & 1 & $!$ & 1 & \pm & 1 & $\therefore$ & 1 & & 1 \\
\hline Wator oudet Temp.F & 158.91 & $!$ & 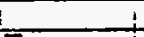 & 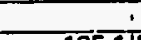 & 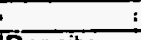 & 1 & 4 & 1 & & \\
\hline Ind Glbminnac & 376141 & & Tomp & 165.11 & Densiky & 78.811 & I & i & & \\
\hline Subcooling ${ }^{f}$ & 2 & 1 & $\square$ & 1 & 1 & . & i & $\perp$ & & \\
\hline Fow Observation & & & & & & $\perp$ & & & & \\
\hline & ZONE1 & ZONE2 & ZONES & ZONE4 & ZONES & ZONE6 6 & ZONE7 & ZONE8 & ZONE9 & ZONE10 \\
\hline Hoeflnput-Watts(nel) & 11527 & 1153.4 & 1157.5 & 1157.5 & 1153 & 691.4 & 307.2 & 96.5 & 87.5 & 99.4 \\
\hline Hoak Loss-Watts & 11.5 & 2.7 & 3.6 & 3.7 & 3.7 & 2.5 & 1.5 & 2.2 & 2.9 & 16.5 \\
\hline Heof Fux-BtuntrfnR & 199811 & 199381 & 200651 & 20055 & 19987 & 11985 & 53251 & 16731 & $15+7]$ & 1723 \\
\hline Wall Temp. I3 & 189 & 186.91 & 983.11 & 184.71 & 188.11 & 182.81 & 780.31 & 777.91 & 177.2 & 777.6 \\
\hline Wal Temp. $14 F$ & 184.91 & 7831 & 185.51 & 785.4 & 188.2 & 18201 & 180.31 & 778.11 & 777.7 & 777.8 \\
\hline Wai Temp. $15 F$ & 184.6 & 181.71 & 185.91 & 185.51 & 184.0 & 182.11 & 179.91 & 178.4 & 177.8 & 177.8 \\
\hline Wat Temp. \#1F & 173.1 & 184.11 & 181.4 & 184.11 & 184.11 & 1881 & 181.6 & 177.91 & 170.8 & 178.9 \\
\hline WeH Tomp. $2 F$ & 185.8 & 1841 & 188 & 184.7 & 184.61 & 18281 & 181 & 178.11 & 177.1 & 178 \\
\hline in theam Tomp.F & 173.8 & 175.21 & 174.4 & 176.3 & 175.4 & 175.31 & 176.9 & 175.8 & 776.7 & 178.3 \\
\hline Avg. Wall Tomp.f & 184.5 & 184.5 & 183.3 & 184.9 & 185.4 & 1827 & 180.6 & 178.71 & 177.3 & 177.9 \\
\hline Avg. Wall DT.F & 9.6 & 8.4 & 7.9 & 7.7 & 9 & 6.91 & 3.5 & 22 & 9.6 & 1.5 \\
\hline Remaining Subcooff & & & & & & & & & & \\
\hline Exat Quality & 0.08 & 0.18 & 0.28 & 0.38 & 0.48 & 0.54 & 0.57 & 0.58 & 0.58 & 0.59 \\
\hline EXP. HHBtuHAPRER & 2071.2 & 2381.21 & 2526 & 2599.61 & 2209.2 & 1747.5 & 1530.5 & 758.81 & 9552 & 1132 \\
\hline COMPUTEOH & & & & & & & & & & \\
\hline Jallouk & 1859.7 & 1794.6 & 1758.7 & 1734.6 & 81.2 & 8121 & 81.6 & 81.3 & 81.3 & 81.4 \\
\hline Szady (Oudo tube) & 2474.8 & 2491.61 & 2483.8 & 2510.41 & 81.2 & 81.21 & 87.6 & 81.3 & 87.8 & 81.4 \\
\hline Szady2 & 2407.6 & 2429.41 & 2420.7 & 2453.6 & 812 & 81.21 & 81.6 & 81.31 & 87.8 & 81.4 \\
\hline (RLO) & 65501 & 600061 & 52788 & 467011 & $46 \% 01$ & 46701 & 46701 & 467011 & 46701 & 46701 \\
\hline$(F)$ & 1.601 & 2.1491 & 2.58 & 2.889 & 2.889 & 28891 & 2.889 & 2.8891 & 2.889 & 2.869 \\
\hline (HบO) & 211.1 & 283.91 & 340.4 & 3824 & 3824 & 3824 & 382.4 & 38241 & 3824 & 3824 \\
\hline (HBOIL) & 24742 & 24004 & 2484.8 & 2507.4 & 2507.4 & $2507 \Omega$ & 2507.4 & 2507.4 & 2507.4 & 25074 \\
\hline & & & & & & & & & & \\
\hline & ZONE2 & ZONE 4 & ZONE6 & ZONE8 & ZONE10 & & & & & \\
\hline DP Rquid Temp.F & 75.6 & & 1 & & & & & & & \\
\hline DP Liquid Density bm/it3 & 93.81 & & & & & & & & & \\
\hline Eevationt & 2 & 4 & & 8 & 9.875 & & & & & \\
\hline Moseured DPPsid & 0.527 & 1.4. & 2.323 & 3.3851 & 4.281 & & & & & \\
\hline Zero Corrections-psid & -0.024 & -0.087 & -0.206 & 0.073 & 0.097 & & & & & \\
\hline Corrected DPPsid & 0.551 & $1.50 \mathrm{e}$ & 2.619 & 3.312 & 4.184 & & & & & \\
\hline Activ DPa & 1.42 & 2.081 & 243 & 3.581 & 4.28 & & & & & \\
\hline Masured TPPsig & 179.2 & & 1 & & 178.8 & & & 1 & 1 & \\
\hline Mecaured Overalle & 0.57 & & $\perp$ & & & & & & & \\
\hline & & & & & & & & & & \\
\hline Computad DP-I & 1.37 & 24 & 3.32 & 4.55 & 5.84 & & & & & \\
\hline Frietion-fi & 0.07 & 0.2 & 0.42 & 0.57 & 0.7 & & & & & \\
\hline Accolertion-li & 0.07 & 0.13 & 0.2 & 0.21 & 0.22 & & & & & \\
\hline Elevation & 1.23 & 2.04 & 27 & 3.77 & 4.98 & & & & & \\
\hline & & & & & & & & & & \\
\hline
\end{tabular}




\begin{tabular}{|c|c|c|c|c|c|c|c|c|c|c|c|}
\hline Runt: & 8541 & & & $\cdot$ & & - & $\therefore$ & - & 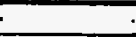 & & \\
\hline Tost Fluid &. C4F8 & & & $\therefore$ & 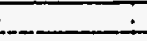 & . & & 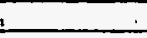 & & & \\
\hline Dato: & 930093 & & & & & & & & & & \\
\hline Time: & $10: 011$ & & & & & & & & & & \\
\hline Noto & Anatytical c & dt is used & & & & & & & & & \\
\hline Fow Avea-t2 & 0.003361 & & & & & & & & & I & \\
\hline Heat Flow Areartl2 & 0.19695 & & & & & & & & & & \\
\hline Saf. Prossure psig & 178.81 & & & & & & & & & & \\
\hline Sat Temperature Fintet) & 174.9 & & & & & & & & & & \\
\hline पq. Donstry bmin 3 & 76.551 & 76.571 & 76.081 & 76.471 & $7 \overline{6.55 T}$ & 76.521 & 76.221 & 76.411 & 76.47 & 76.30 & \\
\hline Laton Hoartunibm & 31.011 & 31.081 & 31.181 & 30.91 & 31.011 & 30.991 & 30.74 & 30.91 & 30.9 & 30.86 & \\
\hline Specitio Hout EtuntomiF & 0.313 & 0.3181 & 0.372 & 0.3141 & 0.3131 & 0.3131 & $0.315 !$ & 0.3141 & 0.314 & 0.314 & \\
\hline Liq. Viscosity & 0.356 & $0 . \overline{3561}$ & 0.358 & 0.354 & $0 . \overline{356}$ & 0.3561 & 0.3521 & 0.3541 & 0.354 & 0.354 & \\
\hline Lq. Conducivity Btuhr-uF & 0.0259 & 0.0251 & 0.0261 & 0.0259 & 0.0259 & 0.02551 & 0.0257 & 0.02591 & 0.0259 & 0.0258 & \\
\hline ig. Prandd No. & 4.3 & 4.31 & 4.3 & 4.3 & 4.31 & 4.31 & 4.31 & 4.31 & 4.3 & 4.3 & \\
\hline Inlotflow Rato-GPM & 1.1 & & & & & & & -1 & & & \\
\hline OutelLig.Flow Rato-GPM & 0.4 & & & & & & & & & & \\
\hline Outlet Vap. Flow Rate-GPIM & 0.7 & & : & & & 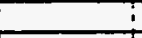 & & & & & \\
\hline Inlet Temperatur of & 172.5! & & & & & & ! & & & & \\
\hline Outlot Lig. TemperatureF & 175.5 & & & & $!$ & 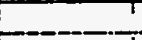 & & 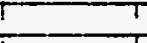 & & & \\
\hline Qutet Vap. Temperauref & 173.2 & T & $t$ & & & $i$ & & $T$ & & & \\
\hline Waler Flow Roto-GPM & & i & T & 1 & 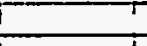 & 1 & & $i$ & 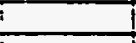 & & \\
\hline Wator inlot Tomp.f & 104.31 & 1 & I & \pm & \pm & $!$ & & I & I & & \\
\hline Whar outet Temp.F & 1631 & 1 & 1 & -1 & & 1 & & 1 & I & & \\
\hline indel $G-1 b m / n h / R 2$ & 208591 & & Temp & 1621 & Densiny & 79.461 & & 1 & I & & \\
\hline Suboodingf & 24 & & 1 & . & \pm & 1 & & 1 & 1 & & \\
\hline Flow Obsenvation & & & & & & & & & & & \\
\hline & ZONE1 & ZONE2 & ZONES & ZONE4 & ZONE5 & ZONE6 & ZONE7 & ZONE8 & ZONE9 & ZONEIO & \\
\hline Head InpLITWatts(nel) & 1168 & 1166.2 & 1163.4 & 1160.8 & 455.7 & 176.3 & 85.2 & 83.1 & 83.5 & 200.5 & $10<0$ \\
\hline Hoat Loss-Watts & 11.4 & 2.6 & 3.5 & 4.21 & 3.3 & 2.2 & 1.4 & 2.21 & 3 & 17.3 & 138 \\
\hline Hos Flox-sturfirite & 20247 & 20디 & 20167 & 201221 & 8593 & 3056 & 1477 & 1440 & 7447 & 3478 & \\
\hline Wartomp. 137 & 781.9 & 786.1 & 181.71 & 196.11 & 187.6 & 178.11 & 178.2 & 177.7 & 178.2 & 778.9 & \\
\hline Wa Tomp $4 \mathrm{~F}$ & 183.8 & 181.81 & 184 & TS5.91 & 987.8 & 178.91 & 178.2 & 177.7 & 78.5 & 779.4 & \\
\hline WAT TOmp. $15 \mathrm{~F}$ & 183.4 & 180.41 & 1842 & 183.81 & 180.7 & 177.7 & 178.11 & 177.61 & 178.5 & 178.7 & \\
\hline Wal Tomp. IIF & 172.5 & 182.81 & 180.4 & 18221 & 1811 & 178.81 & 178.9 & 177.7 & 177.8 & 178.3 & \\
\hline WCl'Tomp. IF & 184.41 & 183 & 181.8 & 1832 & 181.11 & 177.7 & 178.41 & 177.81 & 177.8 & 178.5 & \\
\hline Ingtream Temp.f & 175.3 & 175.21 & 174.7 & 176.01 & 176.8 & 175.41 & 176.71 & 175.0 & 975.0 & 178.1 & \\
\hline Avg. Wall Temp.F & 183.4 & 183.5 & 182 & 189.6 & 1812 & 178.3 & 178.41 & 177.7 & 178 & 178.8 & \\
\hline Avg. Wall DT.F & 7.2 & 7.31 & 6.4 & 128 & 5.5 & 27 & 1.6) & 1.81 & 2 & 25 & \\
\hline Remaining Subcooff & -15.8 & & & & & & & & & & \\
\hline Exid Quality & 0.16 & 0.34 & 0.52 & 0.7 & 0.78 & 0.81 & 0.83 & 0.84 & 0.85 & 0.88 & \\
\hline EXP.HBtuHAriter & 2827.7 & 2755.5 & 3156.7 & 1573.2 & 1573.3 & 1126.9 & 927 & 820.81 & 725.7 & 1388.6 & $\because 2 t$ \\
\hline COMPUTEDH & & & & & & & & & & & \\
\hline Jallouk & 2035.4 & 1902.1 & 1843.41 & 50.81 & 50.7 & 50.7 & 50.9 & 50.8 & 50.8 & 50.8 & 16122 \\
\hline Szadyi(Oudo tube) & 2507.8 & 25051 & 2408.2 & 50.8 & 50.7 & 50.71 & 50.9 & 50.8 & 50.8 & 50.8 & \\
\hline $\operatorname{sen} d y$ & 2450.8 & 2446.81 & 2456.4 & 50.81 & 50.7 & 50.7 & 50.8 & 50.81 & 50.8 & 50.8 & \\
\hline (RLA) & 35270 & 286891 & 21682 & 215921 & 21592 & 215321 & 21592 & 27592 & 21532 & 21382 & \\
\hline (F) & 1.87 & 2.6441 & 3.221 & 3.221 & उ.22i & 3.221 & 3.2211 & 3.221 & 3.221 & 3.221 & \\
\hline$(1-40)$ & 164.7 & 2181 & 265.8 & 265.31 & 265.3 & 265.81 & 265.31 & 266.31 & 265.8 & 265.8 & \\
\hline (1-BOIL) & 2507.7 & 2504.4 & 24552 & 2452 & 2455 & 24052 & 24652 & 2496.21 & $24 \% 52$ & 24052 & \\
\hline & & & & & & & & 1 & I & & \\
\hline & ZZONE2 & ZONEA & ZONE6 & ZONE 8 & ZONE 10 & & & 1 & \pm & & \\
\hline DP liquid Tomp.f & 75.5 & & & & & & & & & & \\
\hline DPLlquid Density 4 bm/th & 93.83 & & & & & & & & & & \\
\hline Elevation & & 4 & 6! & 81 & 9.875 & & & & & & \\
\hline Mestured DPFrid & 0.65 & 1.749 & 2.7881 & 3.978T & 4.981 & & & & & & \\
\hline Zoro Comectonsosid & -0.024 & -0.081 & $=0 . \overline{206]}$ & $\overline{0.0731}$ & 0.097 & & & & & & \\
\hline Conrectod DPPilo & 0.674 & 1.831 & 3.082 & 3.8701 & 4.8351 & & & & I & & \\
\hline Actual DPA & 1.19 & 1.461 & 1.581 & 2.521 & 2.931 & I & & 1 & I & & \\
\hline Measured TPFig & 178.8 & & & & 178.6 & $L$ & & I & I & & \\
\hline Mesuured Overall' & 0.38 & & & & 1 & $t$ & 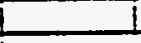 & 1 & 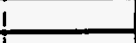 & & \\
\hline & $i$ & & & & & $i$ & $i$ & $\downarrow$ & I & & \\
\hline Computed DP & 1.1 & 1.79 & 251 & 3.74 & 4.65 & 1 & & & i & & \\
\hline Friaton-t & 0.04 & 0.11 & 0.19 & 0.24 & 0.3 & i & $t$ & & $\perp$ & & \\
\hline Accoleration- & 0.04 & 0.08 & 0.11 & 0.71 & 0.11 & & & & & & \\
\hline Elevation-ft & 1.02 & 1.59 & 221 & 3.39 & 4.23 & & & & & & \\
\hline & & & & & & & & & & & \\
\hline
\end{tabular}




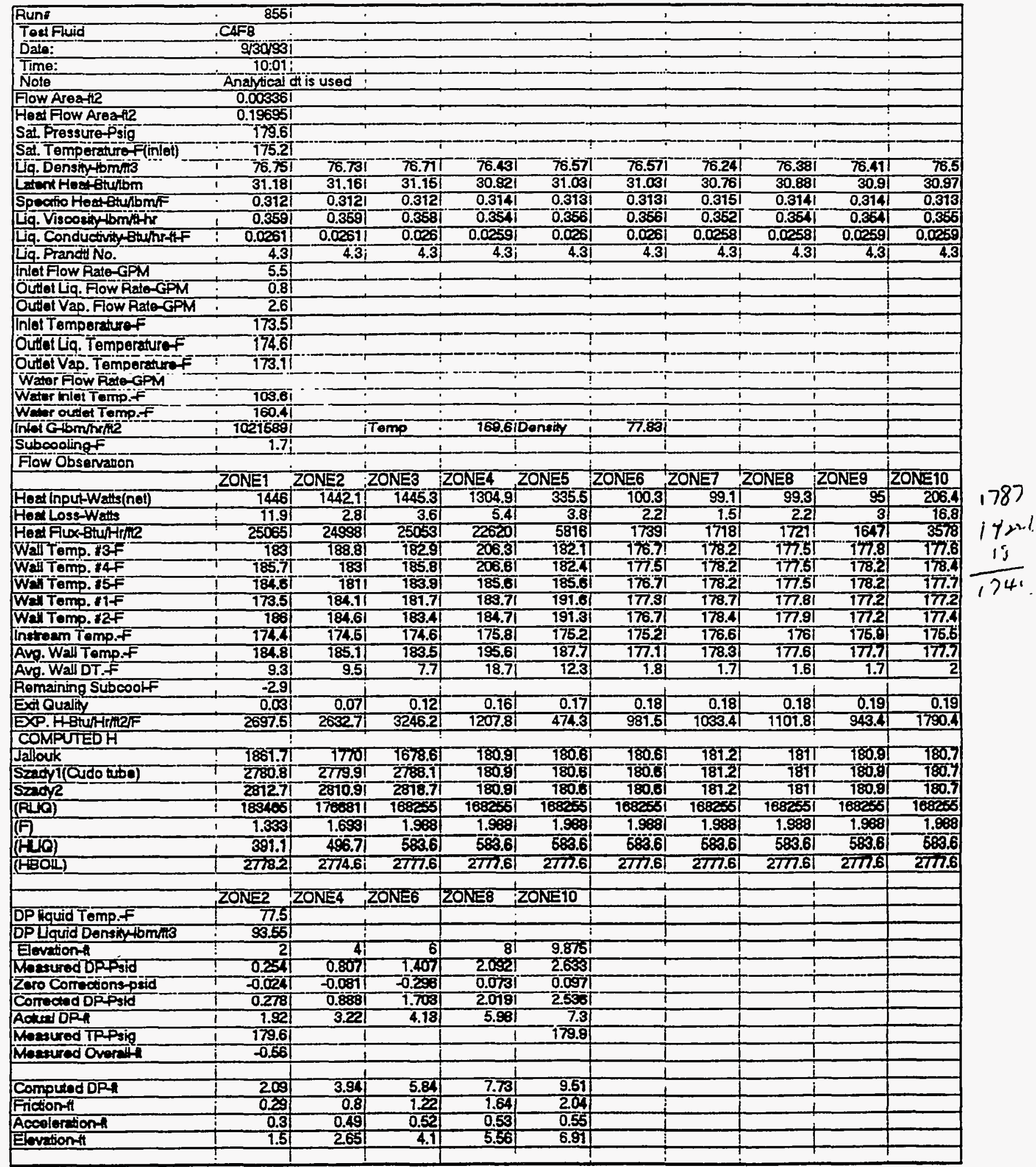




\begin{tabular}{|c|c|c|c|c|c|c|c|c|c|c|c|}
\hline Runt & 8561 & $\therefore$ & 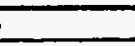 & \pm & $\therefore$ & $:$ & $\therefore$ & $\dot{-}$ & - & $\therefore$ & \\
\hline Test Fluid & CAF8 & 1 & 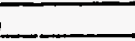 & & & & :- & & : & 1 & \\
\hline Date: & 9301931 & & & & & & & & & & \\
\hline Time: & $10: 011$ & & & & & & & & & & \\
\hline Note & Analyticald & dis used & & & & & & & & & \\
\hline Fow Area-t2 & $0.00336 !$ & & & & & & & & & & \\
\hline Hoal Fow Areatt2 & 0.19651 & & & & & & & & & & \\
\hline Sat. Prossure prig & -180 & & & $t$ & T & 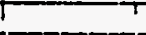 & & & & t & \\
\hline Sat. Temperaturo finiel) & 775.4 & & & $T$ & & & -1 & ?ריד & $i$ & $i$ & \\
\hline LG. Denstitomina & 76.781 & 76.731 & 76.68 & 76.411 & 76.51 & 76.521 & $76.17 !$ & 76.311 & 76.34 & 76.45 & \\
\hline Latent Heatsturibm & 31.21 & 31.161 & 31.11 & 30.91 & 30.97 & 30.991 & 30.7 & 30.821 & 30.841 & 30.93 & \\
\hline Specitio Heat BuntbmiF & 0.3121 & 0.3121 & 0.312 & 0.3141 & 0.313 & 0.3131 & 0.3151 & 0.3141 & 0.314 & 0.313 & \\
\hline Liq. Viscosity & 0.359 & 0.3591 & 0.358 & 0.354 & 0.356 & 0.3561 & 0.3571 & 0.3531 & 0.353 & 0.356 & \\
\hline Lq. Conductivity Btuhr-ffF & 0.03611 & 0.02611 & 0.026 & 0.0259 & 0.0250 & 0.02591 & 0.0257 & 0.02581 & 0.00258 & 0.0259 & \\
\hline íg. Prandt No. & $4.3 i$ & 4.3 & 4.3 & 4.3 & 4.3 & 4.31 & 4.3 & 4.31 & 4.3 & 4.3 & \\
\hline Inlet Fow Rato-GPM & 4.6 & & & & & & & & & & \\
\hline Outlet Lig. Flow Rato-GPM & 0.7 & & & i & & & i & is & 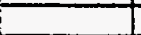 & & \\
\hline Outlet Vap. Flow Rato-GPM & $2.2 !$ & & & & & & & 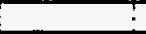 & & & \\
\hline Inlot Temperaturef & 173.5 & & & 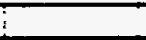 & & & & & & & \\
\hline Outot Liq. Temperature F & 774.91 & & & $\therefore$ & & & & T & 5 & & \\
\hline Oút Tap. Temperature $F$ & 173.5 & & & $T$ & & & I & $T$ & $\Gamma$ & & \\
\hline Warop Fow Rato-GPM & $-\frac{i}{i}$ & - & & $i$ & 7 & i & & $T$ & 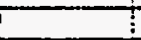 & $\vdots$ & \\
\hline Water inlot Tomp.f & 104.01 & $T$ & 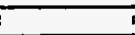 & $i$ & & $\mathrm{i}$ & T & $T$ & ! & 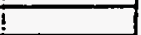 & \\
\hline Water ovilet Tomp.f & 1625 & $\overline{!}$ & 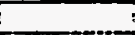 & $\vdots$ & & 1 & I & 1 & 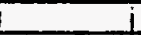 & i & \\
\hline Intol Gibminime & 8565281 & & Tomp & 169.11 & Density & 77.94 & $\bar{i}$ & 1 & $t$ & & \\
\hline Subcoolungf & $1.9 !$ & $?$ & 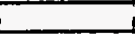 & 1 & & 1 & + & $i$ & 1 & 1 & \\
\hline Fow observation & & & & & & & & & & & \\
\hline & ZONET & ZONE2 & ZONE3 & ZONE 4 & ZONES & ZONEE & ZONE7 & ZONE8 & ZONE9 & ZONE10 & \\
\hline Hoad lnput-Watts(nol) & 1446.2 & 1445.4 & 1445 & 1253.8 & 326.8 & 96.1 & 94.9 & 95.4 & 94.4 & 198.8 & 348 \\
\hline HealLoss-Watts & 11.8 & 2.8 & 3.6 & 3.81 & 3.2 & 2.2 & 1.4 & 2.2 & 3 & 16.6 & \\
\hline Hos FLo BtuFr/Re & 25059 & 25055 & 25048 & 21734 & 565 & 1666 & 1बंडा & 1654 & 168 & 3446 & \\
\hline Wall temp. 13 & 18281 & 188.8 & 1829 & 185.7 & T79:2 & $17 \pi$ & 778.31 & 177.81 & 178 & 177.7 & $11=0$ \\
\hline Wat Tomp. & $185 . \pi$ & 183.17 & 788.1 & 786.51 & 179.6 & 177.8 & 178.31 & T7.4 & 178.5 & 18.3 & 1704 \\
\hline WEITOMp.15F & 184.6 & 181.4 & 183.8 & 185.11 & 1792 & $17 \pi$ & 178.31 & 177.71 & 178.6 & 777.7 & \\
\hline WEITOMp. $11 \mathrm{~F}$ & 173.6 & 184.61 & 181.8 & 183.8 & 179.91 & 177.5 & 178.71 & 177.81 & 177.4 & $7 \pi 2$ & 1734 \\
\hline Wall Temp. Ha & 185.9 & 184.51 & 183.4 & 184.81 & 179.8 & 171 & 178.6 & 178.11 & 177.5 & 177.4 & \\
\hline Intteen Tomp.f & 174.31 & 174.51 & 174.8 & $175 . \overline{81}$ & 175.5 & 175.81 & 176.9 & 176.31 & 178.2 & 175.7 & \\
\hline Avg. Wall Temp.F & 184.7 & 18521 & 183.6 & 185.31 & 179.6 & 177.31 & 178.4 & 177.8 & 178 & 177.7 & \\
\hline Avg. Wall DT $f$ & 9.3 & 9.61 & 7.6 & 8.41 & 3.9 & 1.8 & 7.5 & $1.4 !$ & 1.7 & 1.8 & \\
\hline Romaining Subcooff & 3.6 & & & & & & & & & & \\
\hline Ext Quality & 0.04 & 0.09 & 0.15 & 0.19 & 0.21 & 0.21 & 0.21 & 0.22 & 0.32 & 0.23 & \\
\hline DXP. HBturithar & 2697.9 & 2611.91 & 3277.3 & 2585.91 & 14642 & 900.61 & 11224 & 1144.2 & 95621 & 1910 & \\
\hline COMPUTEDH & & & & & & & & & & & \\
\hline Jallouk & 7940.81 & T\&3द.2! & T750.3] & T57! & 156.81 & T56.8 & 157.4 & 35721 & 157.1 & 156.9 & \\
\hline sect) (cudo hubब) & 2779.2I & 27823 & 2787.4 & $15 \pi$ & 158.81 & 756.81 & 157.41 & 157.21 & 157.ा & $\overline{156.8}$ & \\
\hline $\operatorname{sindy2}$ & 2810.7 & 2874.61 & 2820.8 & 157 & 150.8 & 156.81 & 157.4 & 157.21 & 157.11 & 156.9 & \\
\hline (स्मी) & 152934 & 146137 & 137927 & 137927 & 137927 & 137827 & 737927 & 137927 & 1379 & 187827 & \\
\hline (F) & 1.38 & 1.778 & 2.097 & 2.0971 & 2007 & 20971 & 2.097 & 2.097 & 2.097 & 2007 & \\
\hline (1) & 361.2 & 4627 & 53.4 & 534.41 & 534.4 & 534.4 & 534.4 & 634.41 & 634.4 & 534.4 & \\
\hline (HEOL) & 277.4 & 2778.3 & 27808 & 278081 & 2780.8 & 2780.81 & 2780.8 & 2780.8 & 27808 & 2780.8 & \\
\hline & & & & & & & & & & & \\
\hline & ZONE? & ZONE4 & ZONES & $\angle O K=B$ & ZONEIO & 1 & 1 & & & & \\
\hline DP kquid Tomp, F & i 78 & 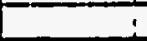 & & & & & & & & & \\
\hline DPLiquid Densinulbm/na & 93.68 & & & & & & & & & & \\
\hline Elovation-t & 2! & $4 !$ & & 81 & 9.875 & & & & & & \\
\hline 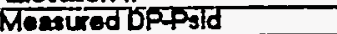 & 0.278 & 0.8291 & 1.588 & 23481 & 2964 & & & & & & \\
\hline Zero Corroctions-psid & -0.0241 & -0.0877 & -0.206 & 0.079 & 0.097 & & & & & & \\
\hline Comeciod DPPild & 0.302 & 0.91 & 1.884 & 2.275 & 2.837 & $!$ & 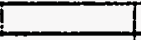 & & & & \\
\hline Actul DPA & 1.88 & 3.181 & 3.78 & 5.5 & 6.67 & 1 & $i$ & 1 & & & \\
\hline Mhesured IPPsig & 180 & 1 & 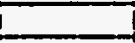 & 1 & 180.1 & 1 & 1 & 1 & 1 & & \\
\hline Meaured Overift & -0.10 & 1 & 1 & & & & & & & & \\
\hline & & & & & & & & & & & \\
\hline Computad DPA & 1.91 & 3.56 & 5.34 & 9.111 & 8.77 & & 1 & I & L & & \\
\hline Friction & 0.23 & 0.64 & 0.97 & 1.3 & 1.52 & & & & & & \\
\hline Accoleretion- & 0.24 & 0.39 & 0.42 & 0.43 & 0.44 & & & & & & \\
\hline Elevition-t & 1.44 & 2.52 & 3.95 & 5.38 & 6.7 & & & & & & \\
\hline
\end{tabular}




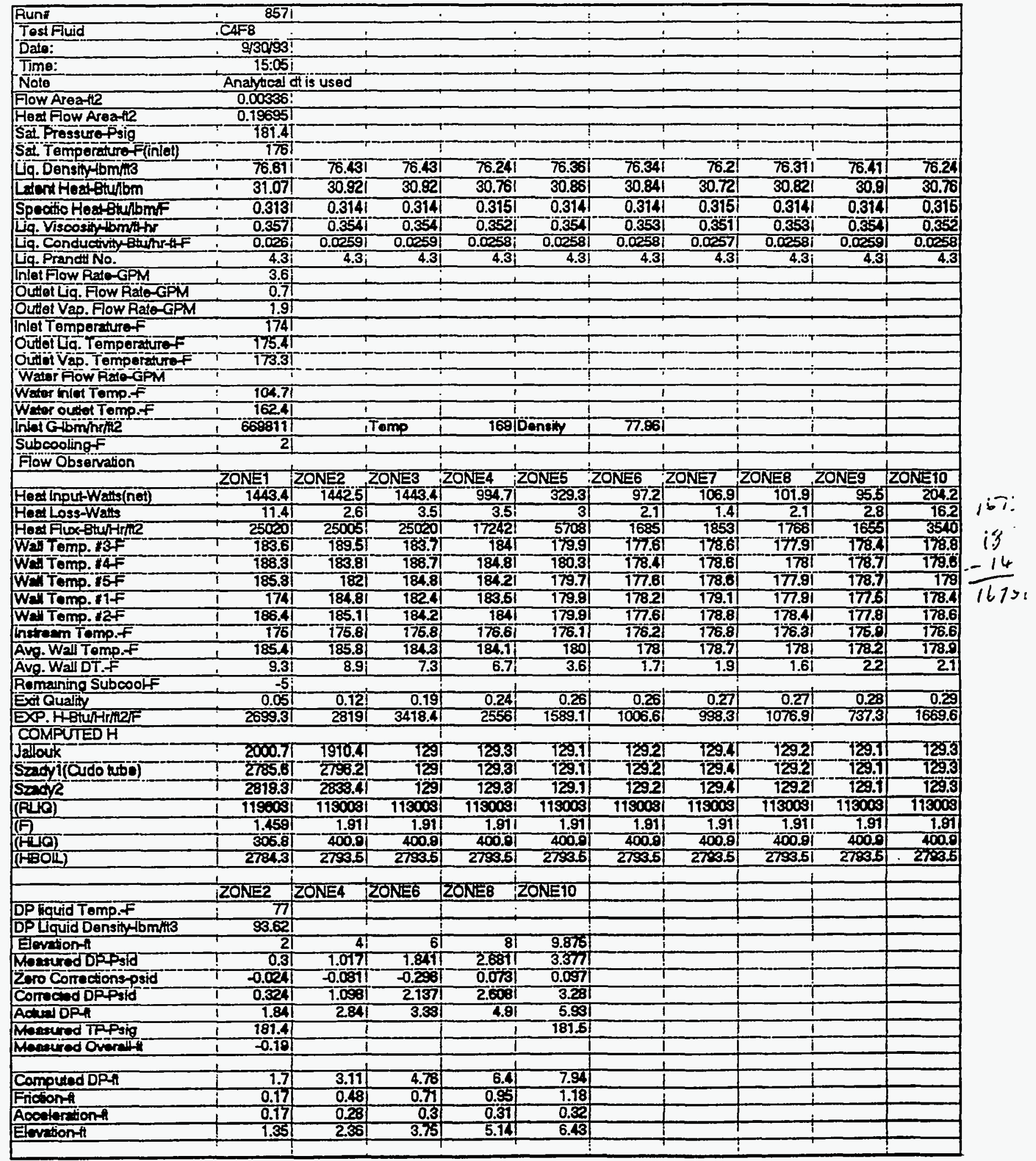




\begin{tabular}{|c|c|c|c|c|c|c|c|c|c|c|c|}
\hline Runy &.$\quad 8581$ & & . & . & 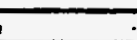 & • & ' & $\cdot$ & $\cdot$ & & \\
\hline Test Fluid & CAF8 & & 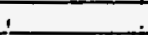 & . & & & & , & 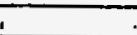 & & \\
\hline Dato: & 930993 & & & . & & & & & & & \\
\hline Time: & $15: 051$ & & & & & & & & & & \\
\hline Not & Analyicalo & ot is used: & 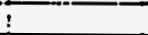 & & & & & & & & \\
\hline Fow Area-ti2 & $0.00336 !$ & & & & 7 & $i$ & & & & & \\
\hline Heat Flow Area-1L & $0.19 \% 51$ & & & & & & & & & & \\
\hline Sat. Pressuto Psig & 181.7 & & & T & 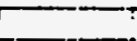 & & & & & & \\
\hline Sä. Tomperature f(inlet) & 776.11 & & $T$ & $i$ & $\mathrm{r}$ & !" & ! & $\underline{t}$ & & Ț & \\
\hline La. Densty & $76.57 T$ & $\overline{\bar{c}} . \overline{27}$ & 76.381 & $7 \overline{6.171}$ & 76.311 & 76.311 & 76.081 & 76.221 & 76.221 & 76.15 & \\
\hline Ladont Hedifuliom & 31.031 & 30.781 & 30.88 & 30.7 & 30.821 & 30.821 & 30.81 & 30.741 & 30.741 & 30.68 & \\
\hline Specino Hoctentbomp & 0.313 & 0.3141 & 0.314 & 0.3151 & 0.3141 & $0 . \overline{3141}$ & 0.3151 & 0.3151 & 0.315 & 0.316 & \\
\hline Liq. Visoosidibomition & 0.3661 & $0.352 i$ & 0.354 & 0.357 & 0.3531 & 0.3531 & 0.390 & 0.3521 & 0.3521 & 0.351 & \\
\hline Lig. Conductivat Btumraf & 0.026 & $0.0258 i$ & 0.02581 & 0.02571 & 0.02581 & $0.0258 i$ & 0.02551 & $0.0257 !$ & 0.02571 & 0.0257 & \\
\hline Liq. Prandilino. & 4.31 & $\overline{4.3 i}$ & 4.3 & 4.3 & 4.3 & $4 . \overline{3 i}$ & $4.3 i$ & 4.31 & 4.3 & 4.3 & \\
\hline Inlot Flow Rato-GPM & 2.9 & & & & & & & & & & \\
\hline Outlet Lig. Flow Ralo-GPM & 0.61 & & 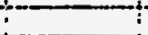 & & & & & & & & \\
\hline Outlet Vap. Fow Rato-GPM & $1.8 !$ & & & & & & & & & & \\
\hline Inlet Tomporaturof & 174.11 & & $T$ & $\mathrm{~T}$ & T! & ! & & $T$ & & $T$ & \\
\hline óutetlia. Tomperaturaf & 775.61 & & & i & i & & & $T$ & & $t$ & \\
\hline ÖUnot Vap. Tomperautrof & $173.8 T$ & & $\bar{i}$ & $\vec{i}$ & $T$ & $T$ & 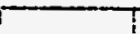 & $T$ & & 7 & \\
\hline Wator Flow RatoGPM & $i$ & i & i & $i$ & & & & $T$ & & if & \\
\hline Water inlet Tomp, f & 104.81 & & $t$ & T & i & 1 & 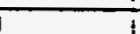 & $\mathrm{T}$ & & T & \\
\hline What outel Tempef & 16241 & & t & 7 & $\mathrm{t}$ & 1 & 1 & 1 & & T & \\
\hline Inilot G & 5403281 & & iTemp & 168.61 & Donsity & 78.071 & & $i$ & & & \\
\hline Subcooling & $2 !$ & & & & 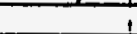 & 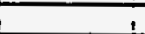 & 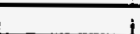 & 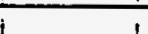 & & & \\
\hline \multirow[t]{2}{*}{ Fow Obsurvation } & $i$ & & & & & & & & & & \\
\hline & ZONET & ZONE2 & ZONEB & ZONEA & ZONES & ZONE6 & ZONE? & ZONE8 & ZONE9 & $Z O N=10$ & \\
\hline Hoed Input-Watts(net) & 1437.4 & 1438.7 & 1435.31 & $988.5 t^{\circ}$ & 326.90 & 96.8 & 105.91 & 101.11 & क5.11 & 209 & \\
\hline Hoat Loss-Watts & 17.5 & 2.7 & 3.5 & 3.51 & 3.1 & 211 & 1.4 & 2.11 & 2.8 & 16.5 & 700 \\
\hline Hog Flox-BtuHinRe & 24916 & 24904 & 24860 & 17135 & 5667 & 1678 & 1836 & 1753 & 168 & 3517 & \\
\hline Wa Tomp. 33 & 183.7 & 189.71 & 184 & 184 & 180.1 & 177.91 & 1792 & 178.61 & 179 & 779.3 & 18143 \\
\hline Wal Tomp $14 \mathrm{~F}$ & 786.61 & 783.91 & 188.9 & 185 & 180.4 & 978.7 & 179.21 & 178.8 & 179.1 & 178.8 & 1136 \\
\hline WAT Tomp.15F & 185.7 & 18221 & 185.11 & 784.81 & 779.91 & 177.91 & 179.3 & 178.51 & 179.11 & 179.4 & \\
\hline WEITTOMP. IIF & 174.11 & 185.21 & 182.6 & 183.8 & 180.1 & 178.41 & 179.6 & 178.81 & 178 & 178.9 & $176=9$ \\
\hline Wall Tomp. VAF & 186.61 & 185.41 & 184.6 & 184.41 & 180.11 & 177.8 & 179.81 & 178 & 178 & 179.1 & \\
\hline Inetraem Tomp.f & 176.2 & 176.6 & 176 & $176.0 \mathrm{i}$ & 176.3 & 176.3 & 177.4 & 178.7 & 176.7 & 17 & \\
\hline Avg. Wall Tomp, F & 185.7 & 186.11 & 1845 & 184भ & 180.11 & 1782 & 179.3 & 178.71 & 178.6 & 179.3 & \\
\hline Avg. Wal DT.F & 9.31 & 8.41 & 7.4 & 6.71 & 3.6 & 1.81 & 1.8 & 1.91 & 1.9 & 2.1 & \\
\hline Remaining SubcoofF & -6.6 & & & & & & & & & & \\
\hline Ext Quality & 0.071 & 0.151 & 0.24 & 0.3 & 0.32 & 0.33 & 0.33 & 0.34 & $0 . \mathbf{3 5}$ & 0.36 & \\
\hline EXP.HBturtinter & 2672.31 & 2956.2 & 3361.9 & $2576.4 !$ & 1587.8 & 907.4 & 999,3 & 9318 & 8837 & 16427 & \\
\hline \multicolumn{11}{|l|}{ COMMUTEDH } & \\
\hline Jejouk & 3087.51 & $7905.8 !$ & 708.71 & $709 !$ & 708.81 & 708.81 & T09.11 & T\$8.91 & 708.8 & Tि & \\
\hline Sżdy (Cudo ubo) & 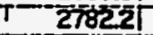 & 270.5 & 108.7 & 709 & 108.8 & 708.8 & 109.11 & $108.9 \mathrm{I}$ & 108.9 & 109 & \\
\hline Szadty & 2814.8 & 28381 & 108.7 & 1091 & 108.81 & 108.8 & 109.11 & 108.8 & 108.9 & 100 & \\
\hline (RLLO) & 95847 & 892081 & 892081 & 892681 & 89201 & 896081 & 892081 & 892031 & 89200 & 8920 & \\
\hline (F) & 1.538 & 2.041 & 2.0411 & 2.047 & 2.041 & 2.0411 & 2.0411 & 2.0411 & 2.031 & 2.041 & \\
\hline (अ) & -271.61 & 361.11 & 361.7 & 361.7 & 361.1 & 351.11 & 361.11 & 361.11 & 361.1 & 361.1 & \\
\hline \multirow[t]{3}{*}{ ( } & 2781.3 & 2797.5 & 27975. & 27975 & 27975 & 2797.51 & 2797.5 & 2797.5 & 2797.6 & 27975 & \\
\hline & & & & & & & & & & & \\
\hline & ZONE2 II & ZONEA & ZONEG & ZONE8 & ZONETO & & & 1 & & & \\
\hline DP lquid Temp.f & 77.6 & & & & & & & 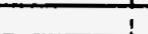 & & & \\
\hline DPTuquid Density tbmnin & \$9.5म & & $T$ & & & & & & & & \\
\hline Eivation-h & $2 !$ & 41 & 61 & 81 & 9.8751 & & & it & & & \\
\hline Wosured DP75s & 0.3041 & 1.1781 & 2075 & 2.9871 & 3.754 & & & T' & & & \\
\hline Zero Corrections-osid & 0.024 & 0.0811 & -0.206 & 0.073 & 0.097 & & & $t$ & & & \\
\hline Correctod opprd & 0.328 & 1.2591 & 2.371 & 2914 & 3.657 & t & & T & & & \\
\hline Adtad DPA & 1.84 & 2.581 & 2.891 & 4.821 & 5.21 & t & & $\frac{1}{1}$ & & & \\
\hline Moacured TPFoig & 181.7 & & 1 & 1 & 181.8 & I & & $i$ & & & \\
\hline \multirow[t]{2}{*}{ Mrenured overall } & -0.10 & & & T & & 1 & & $i$ & & & \\
\hline & & & & & & & & $I$ & & & \\
\hline Computad DP & 7.55 & 278 & 4रा & 5.75 & 7.2 & & & $\frac{1}{1}$ & & & \\
\hline Frition- & 0.13 & 0.37 & 0.58 & 0.77 & 0.94 & & & & & & \\
\hline Acostertion & 0.13 & 0.22 & 0.24 & 0.24 & 0.28 & & & & & & \\
\hline Elevation-t & 1.291 & 2.19 & 3.31 & 4.74 & 5.901 & & & & & & \\
\hline & & & & & & & & & & & \\
\hline
\end{tabular}




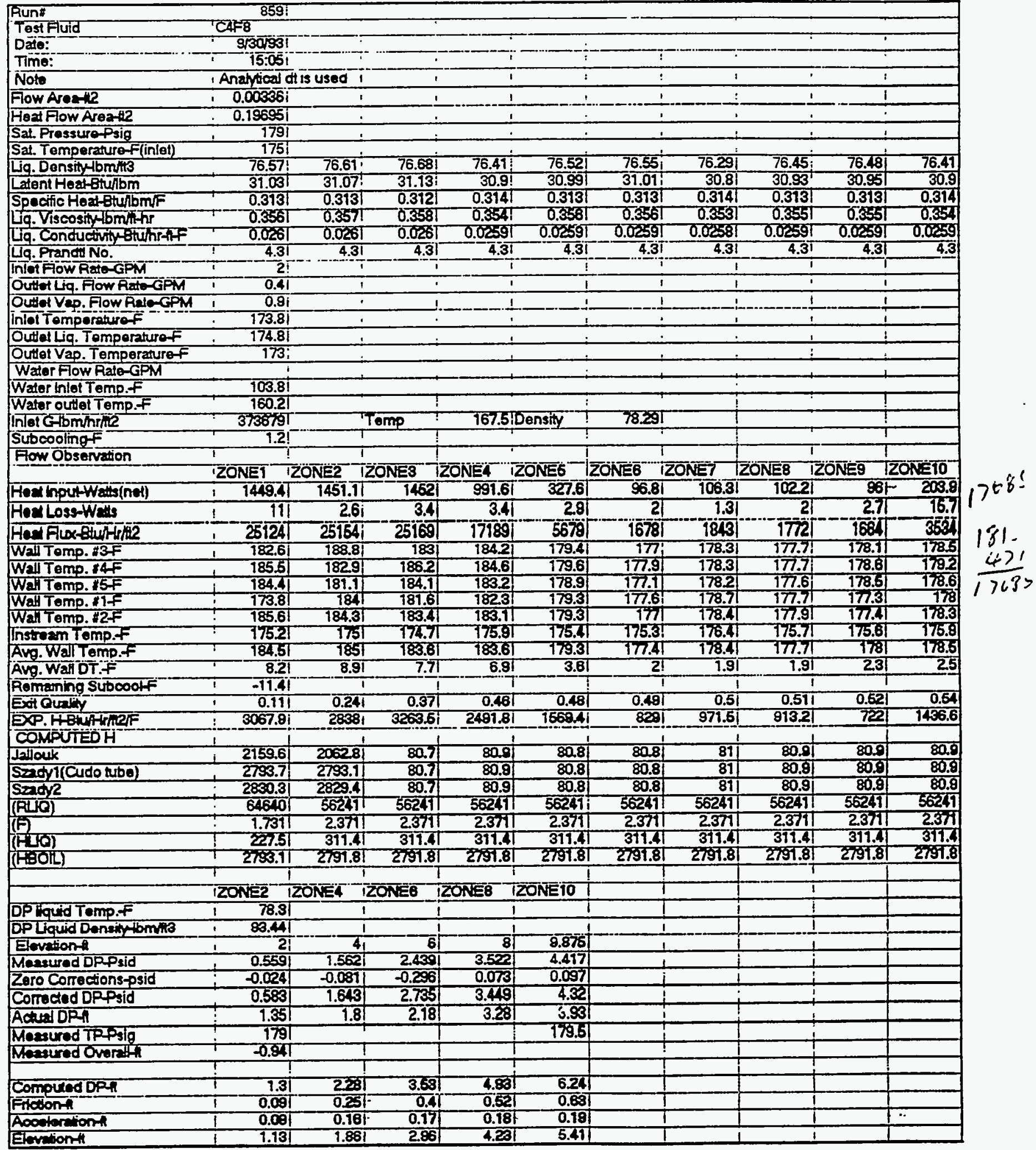




\begin{tabular}{|c|c|c|c|c|c|c|c|c|c|c|}
\hline Run: & 860 & & i & & & & & & & \\
\hline TOAFIU & CAF & & & & & & & & & \\
\hline Den: & $101 / 93$ & & & & & & & & & \\
\hline Time: & $11: 05$ & & & & & & & & & \\
\hline Not & Angtyod & ditused & & & & & & & & \\
\hline Flow ATeath & 0.00356 & & & & & 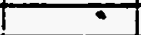 & & & & \\
\hline Hoct flow Aneate & 0.18635 & & & & & & & & & \\
\hline SElPFostiroph & 24.1 & & & & & & & & & \\
\hline Sel Tompermure f(nt) & 199 & & & & & & & & & \\
\hline 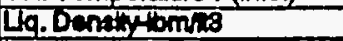 & 70.01 & 70.39 & 70.31 & 70.28 & 70.25 & 70.18 & 70.09 & 70.04 & 70.04 & \%o.31 \\
\hline 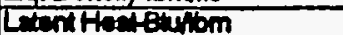 & 25.64 & 25.82 & $\mathbf{5 . 7 8}$ & 25.7n & 25.74 & ¿.6. & 25.58 & 25.58 & L556 & 25.00 \\
\hline Specinothenewhing & 0.357 & $0.35 \pi$ & 0.354 & 0.354 & 0.355 & 0.355 & 0.357 & 0.357 & 0.657 & 0.354 \\
\hline 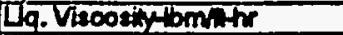 & 0.278 & 0.2791 & 0.278 & 0.27 & 0.278 & 0.278 & 0.276 & 0.27 & 0.2 .8 & 0.278 \\
\hline Lig. Conductivalurefref & 0.022 & 0.024 & 0.022 & 0.023 & 0.0023 & $0.00 \pi$ & 0.0222 & $0.0 \times 2$ & 0.0025 & 0.0024 \\
\hline Lq. PranddNo. & 4.3 & 4.42 & 4.42 & 4.42 & 4.2 & 4.48 & 4.43 & 4.43 & 4.48 & 4.12 \\
\hline Inith Fow PLCEPM & 5.8 & & & & & & & & & \\
\hline OT"LLIq. Fow Puta-GPM & 0,8 & & & & & & & & & \\
\hline OULAVE. Fow Rato-GPM & 3.5 & & & & & & & & & \\
\hline Inlet Temperder & 198.8 & & & & & & & & & \\
\hline 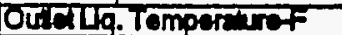 & T\$8.5 & & & & & & & & & \\
\hline OU'AVe. Tomperetirof & 797.4 & & & & & & & & & \\
\hline WEM FIOW RET-GPM & & & & & & & & & & \\
\hline Whatheterip.f & 1800 & & & & & & & & & \\
\hline Wher outel Temp.f & 185.8 & & & & & & & & & \\
\hline Intagominnite & 100460 & & tremp & 185.6 & IDentily & 77.2. & & & & \\
\hline Súbooolingf & $0 \hat{2}$ & & & & & & & & & \\
\hline Frow Obsantion & & & & & & & & & & \\
\hline & ZONET & ZONEZ2 & ZOKIES & ZOR:3 & ZONE5 & ZONEB & ZOKE & 20158 & ZORE9 & ZON=TO \\
\hline 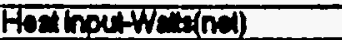 & 291.8 & 285.6 & 283.6 & 283.1 & 288.4 & 289.8 & 288 & 2824 & 2835 & 285 \\
\hline Han Loseway & 14.8 & 3.5 & 4.6 & 4.8 & 4.9 & 3.9 & 2.7 & 3.9 & 4.7 & है.1 \\
\hline 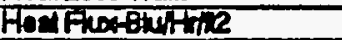 & 5019 & 756ा & 9916 & 28001 & 485 & 500 & 4087 & 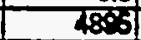 & क्वृत्रा & 7010 \\
\hline We TTamp. BJF & 2036 & Zृ2या & 20.8 & 3228 & एँ. & ב203 & \%3. & क्षियो| & 202 & 2007 \\
\hline WE TTOM. IfF & 203 . & Z026 & 201.0 & एटता & ๘ाइ & 20028 & य3। & 2028 & 200 & 202 \\
\hline We'tamp. I5F & 2030 & क्टा & 2028 & 201.8 & ॠ15 & 2018 & करत्म & 202द & प्र25) & 2017 \\
\hline WeTtomp.t1F & 188.8 & 2028 & 201.8 & Zण.8| & 201.7 & Zरा & 20 & 20028 & काठ & 200 \\
\hline We'Tomp. 124 & 303.7 & 2026 & 201. & 2018 & 201.7 & 2018 & क्ठ & 2008 & 201.8 & $\mathbf{2 0 0}$ \\
\hline nvieem Tomp.f & Z0.01 & 180 & 190.1 & 1922 & 1998 & 1896 & $\mathbf{2 0 0}$ & की & $\mathbf{2 0 0}$ & Tह०.T \\
\hline AVF. Wath Temp.f & 203.6 & 2026 & 201.6 & $202 \pi$ & 201.7 & दर्या & 2020 & 2006 & 2028 & 200 \\
\hline Avo. WCIDTFF & 3.3. & 3.3 & 23 & 27 & 2य & 26 & 27 & 2र्वा & 27 & 1.4 \\
\hline Romaing suboooff & -0.7 & & & & & & & & & \\
\hline Ext Quthy & 0.011 & 0.02 & 0.03 & 0.04 & 0.05 & 0.07 & 0.08 & 0.01 & 0.1 & 0.11 \\
\hline 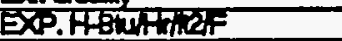 & 753.6 & 1488.4. & 21583 & 1816.4 & र2का. & 1897.11 & 18520 & 2दि8.2 & 268.7 & उस्नें8 \\
\hline CSHPUIEDH & & & & & & & & & & \\
\hline Jinouk & 83301 & 87530 & 8002 & 865.8 & 80835 & 817.8 & 810 & 80006 & 8000 & $7 \$ 5.7$ \\
\hline 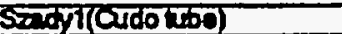 & Tद्धकी & T35:8 & 13978 & 7368 & 7403.8 & 7430 & Tरिबा & TEसमू| & TatoDS & 7200.7 \\
\hline 5od, 2 & 7134.6 & 1178.8 & 11126 & 1115.4 & TाE?.A & 11400 & Tासh & Tअव & THS2: & Tनिद्धा \\
\hline (स) & జाओ & 28165| & zars & 22003 & स्यूगत & 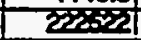 & ख्यस्दर्श & सृख्या & 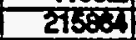 & संक्जि \\
\hline & 1.14 & 1.25 & 1.364 & 7.468 & 1.54 & 1.607 & T.Eक & T.72 & $1.7 i$ & 1.827 \\
\hline (F) & ஆाइ & 3527 & 45.1 & 45ा.ी & 778 & tor & कार्षा & 650 & 6758 & 681 \\
\hline (F.OU) & 1दादु) & 1362 & T386 & 73836| & ऊुड़ा & Tक्वJ & किण & 7381 & 1380: & 73750 \\
\hline & & & & & & & & & & \\
\hline & $201=2$ & ZONES & $20 N=6$ & $20 N=88$ & $\angle O N=50$ & & & & & \\
\hline DPToudtemp.f & $\pi$ & & & & & & & & & \\
\hline DPTlodd Denthis bm & उ3.6्ट & & & & & & & & & \\
\hline Eov'iont & & 4 & 6 & 8 & $9.8 \%$ & & & & & \\
\hline Nealred Dprand & o.37 & 0.681 & 1.18 & 1.570 & 1.850 & & & & & \\
\hline Zero Cortectome-pad & -0.05 ा। & -0.68 & 0320 & -0.063 & 0.02 & & & & & \\
\hline Comoud op and & 0.368 & 0.67 & 0.855 & 1.552 & 1.987 & & & & & \\
\hline Activioph & 1.81 & 3.85 & 8.08 & 7.47 & 9.18 & & & & & \\
\hline Weopred fipleng & 2मा. & & & & 2426 & & & & & \\
\hline memed over in & 3.3 & & & & & & & & & \\
\hline & & & & & & & & & & \\
\hline Compured Daxh & 216 & 7द्य & 63 & 8בा & 10.05 & & & & & \\
\hline Fichont & 0.18 & 0.4 & 0.6 & 0.8 & 1.14 & & & & & \\
\hline Rocetereiont & 0.1 & 0.15 & 0.10 & 03 & 0.20 & & & & & \\
\hline Encint & 7.87 & 3.63 & 5.30 & 7.60 & 8.65 & & & & & \\
\hline & & & & & & & & & & \\
\hline
\end{tabular}




\begin{tabular}{|c|c|c|c|c|c|c|c|c|c|c|}
\hline Runt & 8611 & & 1 & t & 1 & 1 & 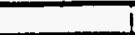 & $i$ & 1 & \\
\hline Tost Fuid & CAF $=8$ & & 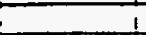 & 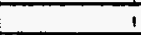 & 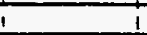 & $i$ & L & & I & \\
\hline Date: & $10 / 1 / 83$ & 1 & $L$ & & I & $\perp$ & & 1 & $\perp$ & \\
\hline Time: & $11: 05$ & & & & & + & & & & \\
\hline Note & Anatyoulc & dt is used & 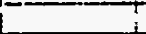 & & & 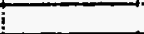 & & & & \\
\hline Fow Areat2 & 0.003361 & & 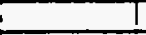 & & i & I & & $i$ & & \\
\hline Hod Fow Aresel2 & 0.1955 & & & & & I & & & & \\
\hline Set Pressure tig & 218.21 & & & & & i & & & & \\
\hline 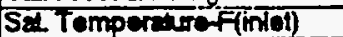 & 190! & & & & & & & & & \\
\hline La. Denthromins & 73.08 & 73.181 & 73.05 & 72.871 & 72.84 & 72.87 & 72.62 & 72.62 & 72.71 & 72.91 \\
\hline Lent Hod Bivibm & 28.08 & 28.16 & 28.1 & 27.84 & 27.82 & 27.94 & 27.74 & 27.74 & 27.81 & 28.08 \\
\hline Specito Hesterhtomf & 0.383 & 0.332 & 0.383 & 0.304 & 0.384 & 0.334 & 0.336 & 0.3361 & 0.385 & 0.384 \\
\hline Liq. Visoosing & 0.31 iी & 0.312 & 0.311 & 0.300 & 0.300 & 0.300 & 0.306 & 0.3061 & 0.307 & 0.31 \\
\hline 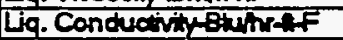 & 0.0238 & 0.02391 & 0.0239 & 0.0238 & 0.0237 & 0.0238 & 0.0236 & 0.0236 & 0.0237 & 0.0268 \\
\hline Liq. Prand No. & 4.34 & 4.34 & 4.34 & 4.35 & 4.35 & 4.35 & 4.35 & 4.35 & 4.35 & 4.34 \\
\hline Inlat Fow Rath-GPM & 5.7 & & & & & & & & & \\
\hline Outet Lq. Fow Rato-GP & $\pi$ & & & & & & & & & \\
\hline Oudot Vap. Fow Rato-CPM & (3) & & & & & & & & & \\
\hline Inlat Temperitrof & 188.6 & & & & & & & & & \\
\hline Ould Lig. Temperritrof & 189 & & & & & & & & & \\
\hline Oulat Veos. Temporetrof & 187.7 & & & & & & & & & \\
\hline WEOr flow PlW GPM & & & & & & & & & & \\
\hline Werer hatet Temp, ff & 130.5 & & & & & & & & & \\
\hline Werer outer Temp.F & 7828 & & & & & & & & & \\
\hline Flat Gomprine & 1012383 & & Temp & 184.1 & Densiy & 74.42 & & & & \\
\hline Subooding $F$ & 1.4 & & & & & & & & & \\
\hline Fow Observetion & & & & & & & & & & \\
\hline & ZONET & ZONE2 & ZON:33 & ZONE & ZONE: & ZONEE & ZONET & ZONEB & ZONE & $201=10$ \\
\hline Hod hortwethed & 2924 & 284 & 291 & 288.2 & 281.8 & 284.8 & 2865 & 283.8 & 281.7 & 289.6 \\
\hline Her'Lose-Wat & 11.7 & 2.7 & 3.5 & 3.6 & 3.5 & 2.5 & 1.7 & 2.6 & 3.4 & 98.5 \\
\hline 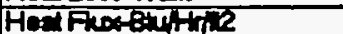 & 5039 & 4620 & 5041 & 4998 & 4885| & 4937 & 4568 & 4971 & 7838 & 5018 \\
\hline Wed Temp. 135 & 19.3. & 193.2 & क्टर2 & 393.5 & $35 \pi$ & 1S3.1 & $\$ 3.8$ & iş3.5| & क्ष.द. & 191.5 \\
\hline WET Tomp. I4 & 19.8. & 193.8 & 1920। & 193.71 & 783.11 & (94) & 193.9 & 793.7 & T94.1 & T92.7 \\
\hline WeT Temp. 85 F & 195.1 & 198.1 & 193,A & 1981 & 1928 & 1927 & 198.5 & 193.4 & 193.7 & 191.6 \\
\hline WET Tomp. DIF & 188.6 & 193.4 & 1922 & 19281 & 192.81 & 1985 & 198.7 & 193.31 & 1927 & 190.4 \\
\hline WCI Temo.124 & 194.8 & 199.6 & 182.8 & 199 & 1920 & 1927 & 193.7 & 193.7 & 198 & 150.0 \\
\hline Intrem Temp.f & 1895 & 189.7 & 189,4 & 190.1 & 180.2 & 180.11 & 191 & 191 & 180.7 & 189.7 \\
\hline Avg.WNITemp.f & 794.8 & 193 & T20 & 1982 & 1520 & 133.3 & 193.7 & 1936 & 193. & 191.4 \\
\hline Avp. Wal DT.f & 4.8 & 4.11 & 29 & 2.9 & 2.6 & 3 & 25 & 28 & 26 & 7.6 \\
\hline Remining Suboodf & 0.5 & & & & & & & & & \\
\hline Ext Cually & -0.01 & 0 & 0.01 & 0.02 & 0.03 & 0.04 & 0.05 & 0.01 & 0.09 & 0.03 \\
\hline Exp.मHuhne & 1005.8 & 1214.8 & 17412 & 1738.1 & 1970.3 & 1674.8 & 19742 & 2138.8 & 1956.6. & 3108.4 \\
\hline COM-UIEDH & & & & & & & & & & \\
\hline JE'ouk & 80.1 & 788.2 & 788.8 & 78.8 & 77056 & $772 \pi$ & 775.7 & 7702 & 783.3 & 77.6 \\
\hline 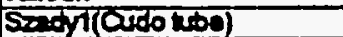 & T3878 & 1316.7 & T353.6 & 13512 & 7305.1 & सिख्डs.2 & 135 & 13005 & 1355 & 13778 \\
\hline $500+2$ & 1047.8 & 1026.7 & 10489 & 1050.8 & 1059.7 & 10621 & 10.22 & 1057.7 & $105 h 7$ & T6.8.8 \\
\hline (स्DO) & 218005 & ट12ब्ध & 210004 & 2100301 & 208316 & 205610 & 20.518 & 208601 & 20067 & 18028 \\
\hline$(F)$ & 1.004 & 1.009 & 1.25 & 1.349 & 1.44 & 1.555 & 1.503 & 1.658 & 9.78 & 7.782 \\
\hline (सU) & 307.61 & 3012 & 367.7 & 4058 & 434 & 4687 & 4602 & 492.8 & 617.9 & 655.6 \\
\hline (1.304 & 13327 & 1310.8 & 13213 & स्स्यरा & 13096 & 13140 & 1325 & 13150। & 13002 & उउसका \\
\hline & & & & & & & & & & \\
\hline & ZONE2 & ZONER & 2OR: $=6$ & $20 N=6$ & ZONE=10 & & & & & \\
\hline DProvid Tomoff & 79.7 & & & & & & & & & \\
\hline Dellavid Densixtomi3 & क्वयू & & & & & & & & & \\
\hline Exwiont & 2 & 4 & 6) & 8 & 9.870 & & & & & \\
\hline Mearuredidpepd & 0.27 & 0.537 & 3.067 & 1.38 & 3.757 & & & & & \\
\hline Zero Comectionsord & $-0.05 \pi$ & -0.089 & 0.238 & 0.008 & 0.002 & & & & & \\
\hline Correctod op pud & 0.83 & 0.570 & 0.83 & 7.388 & 1.726 & & & & & \\
\hline Acheloph & 1.8 & 3.57 & 6.01 & 7.46 & 8.17 & & & & & \\
\hline Meoured TPYh & ¿183 & & & & 216.8 & & & & & \\
\hline Meerured Over"li & 3.18 & & & & & & & & & \\
\hline & & & & & & & & & & \\
\hline Compered DP & 2.76 & 4.2. & 6.28 & 8.28 & 10.1 & & & & & \\
\hline Fifciont & 0.18 & 0.55 & 0.58 & 0.88 & 1.08 & & & & & \\
\hline Acoeleretion? & 0.04 & 0.18 & 0.18 & 0.2 & 0.27 & & & & & \\
\hline Eveliont & 1.97 & 3.78 & 5.52 & 7.21 & 8.75 & & & & & \\
\hline & & & & & & & & & & \\
\hline
\end{tabular}




\begin{tabular}{|c|c|c|c|c|c|c|c|c|c|c|}
\hline Runt & 8521 & & & $\mathrm{i}$ & & & & I & & \\
\hline Togfuid & CAF 8 & & & & & & & $i$ & & \\
\hline Date: & i $10 / 1 / 93$ & & & & & 1 & & & & \\
\hline Time: & $111: 05$ & & & & & & & & & \\
\hline Noto & Analytions & th is used & & & & & & $i$ & & \\
\hline Flow Arent? & 0.003361 & & & 1 & I & i & & 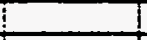 & & \\
\hline Hod Fow Aroant? & 0.13655 & & & & & & & 1 & & \\
\hline SE. Prossuretrig & 218.4 & & & & & & & $t$ & & \\
\hline SE Temper & 190.1 & & & & & & & & & \\
\hline La. Densty & 72.89 & 731 & 72.58 & 72.87 & 72.84 & 72.81 & 72.68 & 7262 & 7265 & 72.92 \\
\hline Len Heresuriom & 27.98 & 28.05 & 28.01 & 27.841 & 27.92 & 27.9 & 27.78 & 27.741 & 27.78 & 27.99 \\
\hline Specilothoneluphom, & 0.334 & 0.33 & 0.329 & 0.391 & 0.334 & 0.356 & 0.398 & 0.3561 & 0.383 & 0.394 \\
\hline Liq. Viscosithom/hr & 0.309 & 0.311 & 0.31 & 0.300 & 0.300 & 0.309 & 0.307 & 0.308 & 0.308 & 0.31 \\
\hline 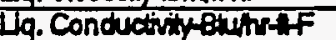 & 0.0238 & 0.0238 & 0.068 & 0.02381 & 0.0237 & 0.0237 & 0.026 & 0.00861 & 0.028 & 0.0288 \\
\hline Lg. Prende No. & 4.35 & 4.34 & 4.34 & 4.35 & 4.35 & 4.35 & 4.35 & 4.35 & 4.35 & 4.35 \\
\hline Inlet Fow Pate-CPM & 4.8 & & & & & & & & & \\
\hline OUACLA. Fow RTE-GPM & 7.1 & & & & & & & & & \\
\hline 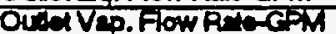 & 26 & & & & & & & & & \\
\hline Int tampercioref & 188.8 & & & & & & & & & \\
\hline 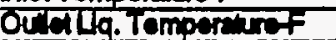 & 78.8 & & & & & & & & & \\
\hline GildVs. Tempereteref & 187.8 & & & & & & & & & \\
\hline WEM Flow PM-GPM & & & & & & & & & & \\
\hline Wererinket Temp,f & 180.0 & & & & & & & & & \\
\hline Wrier octil Temp.f & 189 & & & & & & & & & \\
\hline Ind Glbmmin? & 861000 & & Tomp & 984.81 & Denaity & 74.8 & & & & \\
\hline Suboodingt & 1.3 & & & & & & & & & \\
\hline Fow Obsenvion & & & & & & & & & & \\
\hline & ZONE1 & ZONEZ2 & ZONES & ZONE & ZONE 5 & ZONE6 & $\angle O N=7$ & LONE8 & ZORE & ZON=TO \\
\hline 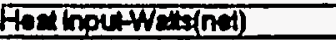 & 2028 & 284 & 267.5 & 283.8 & 281.6 & 284.8 & 288.7 & 283.4 & 278.3 & 260 \\
\hline Herlose-Wels & 11.6 & 2.6 & 3.5 & 3.5 & 3.4 & 25 & 1.6 & 26 & 3.4 & 18.3 \\
\hline 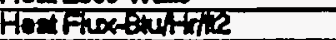 & 5077 & 492 & 4631 & प्बाना & 4887 & पिक्छ & 496 & 4918 & 48्21 & 4507 \\
\hline We Tomp. B⿸丆 & 194, & 33.6 & 3525 & 1983.3। & 1326 & 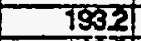 & 153.7 & 733.5 & TSS.4 & Tड़ी.6 \\
\hline WE" Tomp. OAF & 1946 & 198.6) & 1828 & 198.7 & 198 & 733.9. & 153.7 & 193.7 & $19 \sqrt{2}$ & 1925 \\
\hline WE Temp. $16 \mathrm{~F}$ & 150.8 & 198.4 & 198.6 & 1920 & 1827 & 1920 & 158.5 & 188.6 & 193.7 & 191.0 \\
\hline WDT Tomp. \#1F & 188.8 & 199.6 & 1920 & 1928 & 1927 & 199.51 & 198.7 & 198.81 & 1928 & 190.7 \\
\hline Wentomp. IRt & 156 & 198.8 & 18 & 1920 & 1527 & 19201 & 198.7 & 183.51 & 188 & 791.8 \\
\hline intreen Tomprf & 7801 & 189.8 & 18.8 & TS0.11 & 190.2 & 190.8 & 190.8 & 1811 & 1900 & 1860 \\
\hline Avo. Wall TomprF & 394.8 & 1936 & 1527 & 1932 & कृष्ठ & 1984 & 193.7 & 1935 & 193 & T91.5 \\
\hline Avo. Wall DT. & 4.6 & 3.8 & 2.7 & 2.9 & 24 & 20 & 2.6 & 2.3 & 2.3 & 1.4 \\
\hline Remuining Subcooff & 0.3 & & & & & & & & & \\
\hline Exalathy & o) & ठ.ण & 0.02 & 0.08 & 0.04 & 0.08 & 0.07 & 0.08 & 0.00 & 0.1 \\
\hline EXP. HE & T1a.8 & 123.7 & 17235 & Tद्या & 2078.8 & 1727.8 & 188.8 & 2158.5 & 2005.6 & 3137.8 \\
\hline ConifUIEDH & & & & & & & & & & \\
\hline Jelouk & 874.8 & 783.7 & 758.0 & 78.11 & 775.8 & 77.6 & 780.7 & 775.1 & 768.0 & 7385 \\
\hline 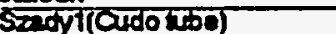 & ख्याइ & 1398.2 & 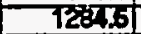 & Tद्यद.8 & T3er25 & T350.7 & T34086 & 7द्ध一:2 & $7 \overline{3058}$ & T278.4 \\
\hline $500 \sqrt{2}$ & 1050.2 & $10 \mathrm{C} .6$ & $\sec 8$ & 1050.0 & T0, & 10440 & 1050 & Tosi. & TOताS & $\$ 300$ \\
\hline (FUa) & 180050 & 1787 & 171000 & 175100 & 17340 & 17400| & TFË60 & 16850| & 10.55 का & TE्चव \\
\hline (F) & 1.004 & 1.12 & 1.301 & 1.418 & 1.517 & 1.002 & 1.678 & 1.741 & 1.808 & 1.872 \\
\hline (HD) & 2528 & 2020 & $310 x$ & 37.5 & 397.8 & 740.8 & ஆठ & 457.61 & 475: & 480.0 \\
\hline (स-304) & 1337.9 & 1314.4 & F⿻8.4 & उआद.5 & BTas & 1378.6 & 1325 & 1378.1 & 13016 & 1253 \\
\hline & & & & & & & & & & \\
\hline & ZONES & ZONEA & $\angle O K=6$ & $\angle O N=8$ & $\angle O N=10$ & & & & & \\
\hline Dplquid trmp.fF" & 802 & & & & & & & & & \\
\hline DPUlaud Dentity bmit & \$3.17 & & & & & & & & & \\
\hline Elovition & & & 6 & 8 & 9.875 & & & & & \\
\hline Mestred biplatd & Tyis & 0.600 & 1.17 & 1358 & 2071 & & & & & \\
\hline Zero Correctioneptid & -0.051 & -0.080 & 0.280 & -0.063 & $0.0<2$ & & & & & \\
\hline Comeand DP Pard & 0.680 & 0.046 & D.g. & 7.580 & 20,2 & & & & & \\
\hline 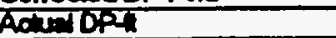 & 7.89 & 3.84 & 5.81 & 7.06 & 8.62 & & & & & \\
\hline Mhetured tiplatg & 218.4 & & & & टा6.8 & & & & & \\
\hline Masured overnili & 28 & & & & & & & & & \\
\hline & & & & & & & & & & \\
\hline Comperind DP & हागा & T.1 & 6.0. & 7.81 & 9.65) & & & & & \\
\hline Filctont & 0.12 & 027 & 0.45 & 0.6 & 0.8 & & & & & \\
\hline Aocaleretion & 0.05 & 0.11 & 0.16 & 0.18 & 0.दा & & & & & \\
\hline Elvetiont & 7.95 & 3.72 & 5.68 & 7.00 & 8.0 & & & & & \\
\hline & & & & & & & & & & \\
\hline
\end{tabular}




\begin{tabular}{|c|c|c|c|c|c|c|c|c|c|c|}
\hline \multirow{3}{*}{\begin{tabular}{|l|} 
Runt \\
Toeffid \\
Dato: \\
\end{tabular}} & \multicolumn{2}{|l|}{$1 \quad 8531$} & & & & & \multirow{2}{*}{$i$} & & & \\
\hline & CAF 8 & & & & & $I$ & & & & \\
\hline & $10 / 1 / 39$ & & & & & & & & & \\
\hline & $11: 05$ & & & & & & & & & \\
\hline Noto & Anatyica do & d is used & & $i$ & & & & & & \\
\hline Fow AYente & 0.00336 & & & & & & $i$ & & & \\
\hline Host Fow Arosed & 0.1965 & & & & & & & & & \\
\hline Sty Presturepsig & 217.7 & & & & & & & & & \\
\hline 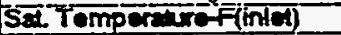 & 189.8 & & & & & & & & & \\
\hline Liq. Dengly bm/13 & 72.9 & 73.081 & 73.05 & 72.921 & 72.89 & 72.87 & 72.73 & 7276 & 72.76 & 72.69 \\
\hline 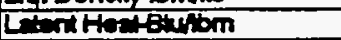 & 27.99 & 28.12 & 28.1 & 27.991 & 27.98 & 27.94 & 27.89 & 27.85 & 27.85 & 27.88 \\
\hline Specito Heatelubm/r & 0.334 & 0.333 & 0.333 & 0.34 & 0.334 & 0.334 & 0.335 & 0.335 & 0.396 & 0.334 \\
\hline Lig. Visoostyomithr & 0.31 & 0.311 & 0.311 & 0.31 & 0.300 & 0.309 & 0.307 & 0.300 & 0.308 & 0.300 \\
\hline Liq. Conducting hroff & 0.0238 & 0.0239 & 0.0239 & 0.0238 & 0.0238 & 0.0238 & 0.0237 & 0.0237 & 0.0237 & 0.038 \\
\hline Ĺtg. Prendel No. & 4.35 & 4.34 & 4.34 & 4.35 & 4.35 & 4.36 & 4.35 & 4.35 & 4.35 & 4.35 \\
\hline Inteffow RAt-GPM & 3.9 & & & & & & & & & \\
\hline 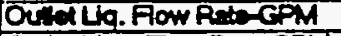 & & & & & & & & & & \\
\hline OUld VSP. Fow Rad GPM & 22 & & & & & & & & & \\
\hline Intel Tampercure of & 188.8 & & & & & & & & & \\
\hline Oofliti. Tomoordirof & 788.8 & & & & & & & & & \\
\hline 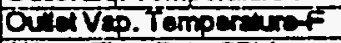 & 187.3 & & & & & & & & & \\
\hline Whor Fow P-CPM & & & & & & & & & & \\
\hline Wh tor hinet Tomp.f & 1820 & & & & & & & & & \\
\hline Werer orled Tomp.f & 183 & & & & & & & & & \\
\hline Lhiog Gbomhinh & 689146 & & TTomp & 185.6 & Donsity & 74.09 & & & & \\
\hline Swboodingf & 1 & & & & & & & & & \\
\hline Flow Obsenvetion & & & & & & & & & & \\
\hline & ZONE & ZONE2 & ZONE3 & ZONEA & ZORES & ZONE6 & IZONE7 & ZONEB & ZONEQ & $20 N=10$ \\
\hline 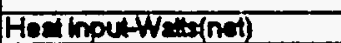 & 289.1 & 290.6 & 220.3 & 232 & 289.4 & 293.8 & 289.2 & 285.8 & 2200 & 269.8 \\
\hline Hod Lose-WE"s & 12.1 & 28 & 3.6 & 3.6 & 3.6 & 2.6 & 1.7 & 2.7 & 3.5 & 19.4 \\
\hline Had fox busthe & 5019 & 5037 & 3819 & 4023 & 5017 & 5008 & 5013 & 4568 & 3814 & 5000 \\
\hline WE Tomp. 13F & 794.4 & 333.3 & 791.9 & Tख्डा & 7925 & 13 & 193.8 & 193.4 & Tद्26] & 991.8 \\
\hline We Tomp. IIF & 1945 & 993.4 & 1922 & 783.2 & 1526 & 753.8 & 130.9 & 193.7 & 733,1 & 183 \\
\hline WN Tomp. ISF & 96.1 & 193.8 & 193.1 & 1925 & 1926 & 192.8 & 193.4 & 193.2 & 193.1 & 191.8 \\
\hline Well Tomp. StF & 188.8 & 193.5 & 192 & 1922 & 192.5 & 193.3 & 193.9 & 1920 & 1921 & 190.8 \\
\hline Wel Tamp. ILF & 1948 & 183.6 & 1824 & 7925 & 1928 & 1328 & 198.6 & 793.6 & 1928 & 191.6 \\
\hline Intresn Tomp.t & 189.0 & 189.3 & 189.4 & 189.8 & 190 & $150 . \pi$ & 150.6 & 150.5 & 190.6 & 180 \\
\hline Awo. Wall Tomp. F & 1847 & 193,4 & 1921 & 1927 & 152.7 & 1532 & 193.7 & 193.4 & 982, & 191.8 \\
\hline AUT.WADT.F & 4.6 & 3.9 & 2.8 & 2.6 & 24 & 28 & 2.9 & 2.6 & 2 & 1.8 \\
\hline Ramping Suboooff & 0.2 & & & & & & & & & \\
\hline Exa Cualy & & 0.02 & 0.03 & 0.04 & 0.08 & 0.07 & 0.09 & 0.1 & 0.11 & 0.18 \\
\hline 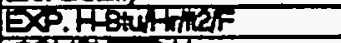 & 7069.8 & 12325 & 1483.1 & 7521.4 & 200.1 & 1753.8 & 1756.9 & 1850.1 & 1880.8 & 3252 \\
\hline CONOUIEDH & & & & & & & & & & \\
\hline Jsouk & 835 & 80096 & 685.8 & 705.6 & 795.0 & 789.8 & 791.8 & 782.3 & 6830 & 784.8 \\
\hline 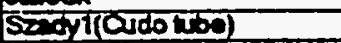 & 1337.4 & 7331.6 & 71180.8 & 7989.7 & 7365 & 1348 & 7362 & 135.1 & 17802 & 73द1.7 \\
\hline $500+2$ & $1005 \mathrm{a}$ & 1040.5 & 880.1 & 904 & 1047.8 & 100il & 705.3 & 1048.5 & 8808 & 1051.1 \\
\hline (Fua) & 125\%2 & 148287 & 141459 & 140440 & 138604 & 15न180 & 134656 & 182587 & 13083 & T2787 \\
\hline & 1.076 & 1.247 & 1.397 & 1.498 & 1.696 & 1.691 & 1.774 & 1.851 & 1.814 & 1.878 \\
\hline$\frac{10}{(1 \mathrm{H})}$ & 237.6 & 275.1 & 300.8 & 30.7 & 3526 & 378.0 & 3826 & 400.7 & सEST & 2075 \\
\hline$(\mathrm{H} 3 \mathrm{OH})$ & 1358.9 & 1327.7 & 7158.1 & 719.8 & 13232 & 1338.4 & 133009 & 13221 & 1187.8 & T3206 \\
\hline & & & & & & & & & & \\
\hline & ZONE? & ZONEA & $20 K=6$ & $2 O N=8$ & ZOEEIO & & & & & \\
\hline DP Iqud Tomp.F & 82.1 & & & & & & & & & \\
\hline 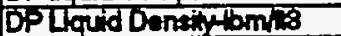 & प्र291 & & & & & & & & & \\
\hline Elowiont & & & & & 9.875 & & & & & \\
\hline Mampured DPfidd & 0.270 & 0.658 & T.282 & 1.730 & 234 & & & & & \\
\hline Zero Corrections-pend & $-0.06 \pi$ & -0.088 & 0.208 & -0.000 & 0.023 & & & & & \\
\hline Comodod of pidd & 0.358 & 0.697 & 7.004 & 1.762 & 232 & & & & & \\
\hline ACWWDP & 1.81 & 3.72 & 5.68 & 6.72 & 8.12 & & & & & \\
\hline Mecoured TPFक & 217.7 & & & & 216.8 & & & & & \\
\hline Memedred Overallh & 1.78 & & & & & & & & & \\
\hline & & & & & & & & & & \\
\hline Comoutad Das & 202 & 3.97 & 5.75 & 7.52 & 9.18 & & & & & \\
\hline Finctont & 0.09 & $\delta .2$ & 0.33 & 0.47 & 0.6 & & & & & \\
\hline Aocoteritiont & 0.05 & 0.08 & 0.11 & 0.14 & 0.16 & & & & & \\
\hline Everiont & 1.88 & 3.63 & 5.31 & 6.83 & 8.42 & & & & & \\
\hline & & & & & & & & & & \\
\hline
\end{tabular}




\begin{tabular}{|c|c|c|c|c|c|c|c|c|c|c|}
\hline Runt: & 864 & & & 1 & 1 & & & & & \\
\hline Texfind & CAF 8 & & & $\perp$ & & & & & & \\
\hline Dede: & 10133 & & & & & & & & & \\
\hline Time: & $11: 05$ & & & & & & & & & \\
\hline Now & Ansticald & dis used & & & & & & & & \\
\hline Flow Araste & 0.00036 & & & & & & & & & \\
\hline Hes Fow Areall? & 0.1865 & & & & & & & & & \\
\hline SEl Piosure prig & -218.4 & & & & & & & & & \\
\hline S.1 Temperenef(net) & 190.1 & & & & & & & & & \\
\hline Lq. Dentityomin & 72.87 & 72.97 & 72.89 & 7281 & 72.81 & 72.79 & 72.81 & 72.68 & 72.68 & 72.79 \\
\hline Lenthentem & 27.8त & 28.08 & 27.98 & 27.9 & 27.8 & 27.87 & 27.78 & 27.78 & 27.78 & 27.87 \\
\hline Specioflecterhomf & 0.34 & 0.384 & 0.384 & 0.351 & 0.35 & 0.355 & 0.383 & 0.386 & 0.38 & 0.385 \\
\hline Lig. Viscosh bom hr & 0.300 & 0.31 & 0.300 & 0.308 & 0.500 & 0.3081 & 0.307 & 0.3071 & 0.307 & 0.308 \\
\hline 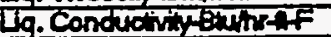 & $0.0 \mathrm{c} 3$ & 0.008 & 0.0288 & 0.0237 & 0.023 & 0.0237 & 0.028 & 0.068 & 0.028 & 0.028 \\
\hline Lq. PrendilNo. & 4.35 & 4.34 & 4.35 & 4.36 & 4.36 & 4.35 & 4.35 & 4.35 & 4.85 & 4.35 \\
\hline Int FoW REA-GPN & 3 & & & & & & & & & \\
\hline OU'NLIL. Fow RMGGP & 0.7 & & & & & & & & & \\
\hline OUNV Vo. Flow RE-GFM & 1.9 & & & & & & & & & \\
\hline Intat Temperenter & 188.8 & & & & & & & & & \\
\hline Orllig. Tomper trof & 189.5 & & & & & & & & & \\
\hline ot'a Vep. Tomper trof & 787.8 & & & & & & & & & \\
\hline WhM FOW AWOCPM & & & & & & & & & & \\
\hline Welorintertomp, & 181.7 & & & & & & & & & \\
\hline Wher oull Tompef & 183 & & & & & & & & & \\
\hline 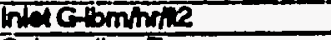 & E31\%31 & & Tenp & 184.8 & Denty & 74.3 & & & & \\
\hline Subooding & 1.2 & & & & & & & & & \\
\hline Fow Obsenvion & & & & & & & & & & \\
\hline & ZONET & ZONEZ2 & ZONES & $20 \mathrm{~K}=4$ & $2015=5$ & ZONE6 & ZONis & $20 \mathrm{ON}=8$ & $\angle O N=9$ & $x=10$ \\
\hline Hoal houtWarind) & 288.8 & 288.7 & 287.7 & 289.8 & 2882 & 288.7 & 287.7 & 288.8 & 2912 & 288.7 \\
\hline Hen Los WWE':s & 11.2 & 2.5 & 3.4 & 3.4 & 3.3 & 2.4 & 1.6 & 2.5 & 3.3 & 17.9 \\
\hline 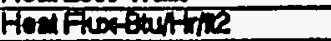 & 5005 & 5004 & 4587 & 503 & 4561 & 4970 & 4891 & 50003 & 3078 & 5007 \\
\hline We'Temp.13+ & 194, & 198.4. & iक्टा & 93.6 & 1920 & 193.6 & 193.0 & 193.8 & 193.6) & क्रा \\
\hline WCT Tomp. 14F & 194.6 & 133.5 & TS3.1 & 184 & Tळ3.8 & 195द्2 & Tar. & ह8., & 18ग0 & T88. \\
\hline WeT Tomp. 15 F & 1952 & 193.4 & 198.8 & TS20 & 193.1 & 198 & 198.6 & 198.6 & दा & TE28 \\
\hline We Tomp. IF & 188.9 & 199.0 & 1920 & 1827 & 1529 & 183.7 & 198.9 & 198.4 & 168 & 791.1 \\
\hline We'Tomp. V24 & 1940 & 193.6 & 183 & 183 & 188 & 1932 & 198.7 & $18 \pi$ & 18 & 797.0 \\
\hline hitrem tompat & $180 . \pi$ & 189.7 & 150 & T80.31 & 1808 & $180 \mathrm{~A}$ & 180.8 & 1800 & 7005 & 180.4 \\
\hline Av. We Tompa & 1950 & 7ख्या & 1920 & 1938 & 15.J & 1836 & अठ50 & 198 & Tक्यू & TR्रा \\
\hline Avo. We'DT. & 4.4. & 3.6 & 2.6 & 28 & 26 & 3 & 28 & 28 & 20 & 15 \\
\hline Remining suboootf & $=0.61$ & & & & & & & & & \\
\hline Exathy & 0.01 & 0.08 & 0.01 & 0.08 & 0.03 & 0.7 & 0.12 & 0.74 & 0.10 & 0.78 \\
\hline 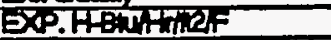 & T13.7 & 1350 & 1918.4 & 1817.6 & 19rरo & 16703 & 7730.4 & 18.6.0 & 1976 & 3555 \\
\hline CON:PUIEDH & & & & & & & & & & \\
\hline Je & 863.1 & 8748 & 818 & 81261 & 8028 & $80 \mathrm{iz}$ & 8000 & 798.11 & $80 i 2$ & $783 \pi$ \\
\hline Fodviा (cudotwo) & ß5. & 73821 & $738 \%$ & 7387.2 & 1330.7 & 13328 & 1389 & 13\%0.7 & 73783 & $18 \% 6$ \\
\hline sed 12 & TESB & $10: 28$ & 1037.8 & TOFA & ाष्का & Tok28 & 1080 & 10021 & 100 & TDE25 \\
\hline (FIO) & 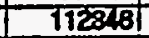 & 1104ता & 108002 & 10676 & Tons: & Tि्250 & 700658 & $\operatorname{ses} 16$ & 900 & 835 \\
\hline (F) & 1.112 & 1.307 & 1.49 & $1 . \approx 28$ & 1.741 & 1.846 & 1.834 & 2018 & 2003 & 2175 \\
\hline (मिक) & 1800.3 & ख्याब & 2578 & 327 & आद.7 & 38121 & 34.71 & उद3: & 3775 & अ.1 \\
\hline (F:O4) & 1300 & 13837 & 138 & Tर्325 & 1320 & 1325 & 1350.1 & स्ख़ा & 13318 & 7302 \\
\hline & & & & & & & & & & \\
\hline & 20152 & ZORES & $201=6$ & $201=8$ & $201=10$ & & & & & \\
\hline DPligud Temp.f & 83.1 & & & & & & & & & \\
\hline 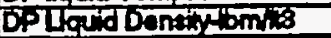 & कृता & & & & & & & & & \\
\hline Eventont & & 4 & 6 & 8 & 3.875 & & & & & \\
\hline Thaured bpapd & 0278 & $0 . \% 01$ & 1.35 & 200 & 260 & & & & & \\
\hline Zcro Corteciona ped & 0.051 & -0.0001 & 0.208 & 20.008 & 0.02 & & & & & \\
\hline Comond DP P Id & 0.3 & 0.774 & 1.120 & 2001 & 2581 & & & & & \\
\hline ACTW DPI & 7.8 & 3.57 & 5., & 6.28 & 7.47 & & & & & \\
\hline Whened tiplaing & 218.4 & & & & 277.9 & & & & & \\
\hline Whaned Overith & 0.0 & & & & & & & & & \\
\hline & & & & & & & & & & \\
\hline Compund bPI & 7.9n & 3.74 & 5.48 & 7.17 & 8.08 & & & & & \\
\hline Fricton- & 0.08 & 0.18 & 022 & 0.32 & 0.44 & & & & & \\
\hline Acontertiond & 0.04 & 0.08 & 0.08 & 0.11 & 0.12 & & & & & \\
\hline Exw'onh & I.85 & 3.65 & 5.17 & 6.70 & 8.01 & & & & & \\
\hline & & & & & & & & & & \\
\hline
\end{tabular}




\begin{tabular}{|c|c|c|c|c|c|c|c|c|c|c|}
\hline Runt & $1 \quad 855$ & 1 & & 1 & 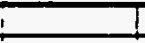 & & & i & 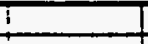 & \\
\hline Test Fuid & CAF8 & & & & i & & & & $i$ & \\
\hline Date: & $101 / 93$ & & 1 & & & & & & & \\
\hline Time: & $11: 05$ & & & & & & & & 1 & \\
\hline Nole & Analical d & ot is used & 1 & & & & ; & & & \\
\hline Flow Aread? & 0.003351 & & 1 & & & & & & & \\
\hline Hoes Fow Areal? & 0.1965 & & & & & & & & & \\
\hline Sa Prestur Pig & 218.4 & & & & & & & & & \\
\hline Sal Temperifref(inlet) & 190.1 & & & & & & & & & \\
\hline Lig. Dentitytoming & 72.81 & 72.84 & 72.87 & 7281 & 72.81 & 72.79 & 72.57 & 72.57 & 7267 & 72.78 \\
\hline Lterthostariom & 27.9 & 27.92 & 27.94 & 27.91 & 27.9 & 27.871 & 27.69 & 27.69 & 27.69 & 27.88 \\
\hline Spectio theserubmp & 0.335 & 0.341 & 0.334 & 0.3851 & 0.365 & 0.3351 & 0.356 & 0.386 & 0.356 & 0.395 \\
\hline Liq. visoosityminth & 0.308 & 0.3001 & 0.309 & 0.308 & 0.308 & 0.308 & 0.305 & 0.305 & 0.205 & 0.307 \\
\hline Lig. Conductivit Buhn- Ff & 0.0237 & 0.0237 & 0.0238 & 0.02371 & 0.0237 & 0.0237 & 0.0236 & 0.026 & 0.0038 & 0.0237 \\
\hline Liq. PrandtiNo. & 4.35 & 4.35 & 4.35 & 4.35 & 4.35 & 4.35 & 4.35 & 4.35 & 4.35 & 4.35 \\
\hline IndFow Rato-GPM & 21 & & & & & & & & & \\
\hline Oule Lig. Fow Pro-GPM & 0.4 & & & & & & & & & \\
\hline \multicolumn{11}{|l|}{ Oula Vso. Fow Rat-GPM } \\
\hline \multicolumn{11}{|l|}{ Inlatemperiuref } \\
\hline \multicolumn{11}{|l|}{ Orilig. Temperofyre } \\
\hline oretVep. Tomoer of & 188.1 & & & & & & & & & \\
\hline \multicolumn{11}{|l|}{ 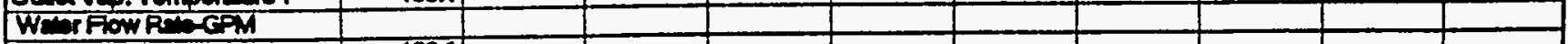 } \\
\hline \multicolumn{11}{|l|}{ Werer bied Temp.f } \\
\hline \multicolumn{11}{|l|}{ Whar octlel Temo.f } \\
\hline $\ln$ da Gbminhe & 374611 & & Temp & 1828 & Dentiy & 74.51 & & & & \\
\hline Subooding $f$ & 0.8 & & & & & & & & & \\
\hline \multicolumn{11}{|l|}{ Flow Obsenvition } \\
\hline & ZONE T & ZONEZ & $2 O N=3$ & 2OKIS & ZONES & ZON=6 & $20 N=7$ & ZONEE & ZONES & ZON=10 \\
\hline Hest houtwathet) & 2924 & 292 & 2922 & 2942 & 288.1 & 288.4 & 290.8 & 2925 & 2050 & 293 \\
\hline HontLosatwes & 11.7 & 2.7 & 3.5 & 3.6 & 3.5 & 2.6 & 1.7 & 2.6 & 3.4 & 78.9 \\
\hline 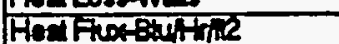 & 5059 & $50 \div 2$ & 5065 & 5100 & 4S9 & 400 & 5017 & $50 \%$ & 5720 & 5079 \\
\hline WeT Tomp. Ho & T\$, & $\{93.7$ & क्रद्ध & T\$3.8 & 1532 & \$क्. & Тभा. & 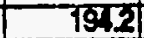 & |वा| & 1920. \\
\hline WEl Tomp. IIF & TSम5 & 193.7 & 153.2 & 197 & 198.8 & Tras & Tित्र & Tद्र.2 & To4.7 & 738.4 \\
\hline Wh Tremp. $55 \mathrm{~F}$ & 158.4 & 198.6 & 193.8 & 198 & 1932 & 193.11 & 793.8 & 199 & 194.I & 1926 \\
\hline WEN Tamp. BIF & 189.8 & 193.7 & 1928 & 193 & 193.11 & 198.7 & 194.8 & 193.7 & 193.1 & 191.4 \\
\hline WCITTemp. HAF & 196.1 & 198.0 & 1938 & 1932 & 1932. & 193.2 & 192: & 1942 & 198.5 & 19201 \\
\hline Ditroen Temp.f & 180.2 & 1802 & क्a.t & 180.91 & 180.3 & 1004 & 181.2 & 1812 & 1012 & 1806 \\
\hline Avo. WaltemptF & Tिर्व & 193.8 & 12 & Tक्86 & TE्द2 & 183.7 & Tg(2) & कूप & 7ख्या & 15 \\
\hline Au WEIDT.F & 4.3 & 3.3 & 2.7 & 29 & 27 & 3 & 28 & 26 & 21 & 1.8 \\
\hline Rem ing Suboooff & -1.6 & & & & & & & & & \\
\hline Exacharity & 0.02 & 0.05 & 0.08 & ठ.ग & 0.13 & 0.16 & 0.18 & 0.2 & 025 & 0.27 \\
\hline DX.HBuHRQR & 1173 & 1524 & 1878.7 & 1728.8 & $1887 \mathrm{~A}$ & 153.6 & 7818.4 & क्टाइ2 & 205.1 & 3163.7 \\
\hline \multicolumn{11}{|l|}{ 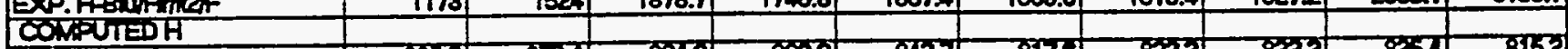 } \\
\hline Itakk & 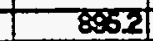 & 878.01 & 887.8 & 8803 & 84k7 & 817.61 & $8 \sin$ & $\operatorname{sen} 2$ & 8251 & 8752 \\
\hline 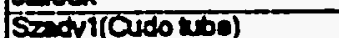 & 1370.7 & 13356 & T33.0 & 136 & 13520 & T3्3म4 & 7345.8 & न35021 & T358.8: & 1838 \\
\hline Fod & 1008.9 & 1008 & 100.8 & T10. & 1010.7 & 10 歹 & 1050 & 100.4 & $10 \%$ & 30.6 .1 \\
\hline (FUa) & 78750 & 76783 & 74401 & TSEII & 70100 & 8799 & 6004 & 63900 & 61600 & 68570 \\
\hline$(F)$ & 1.281 & 1.474 & 1.67 & 1.8281 & 1.958 & 2.0791 & 217 & 2.267 & 2.350 & 245 \\
\hline (1) & 167.8 & 2005 & 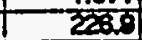 & 248 & 268 & 281.7 & 256.4 & 308.7 & उदासा & 338.6 \\
\hline \multirow{3}{*}{$(1+3011)$} & 1339.8 & 1338 & 13375 & उ3द2 & Tस्द्य.1 & 13602 & अदा.ग & 1344.81 & 13528 & उद. \\
\hline & & & & & & & & & & \\
\hline & ZOK:E2 & ZOKRET & $201=6$ & $20 N=8$ & $\angle 015=10$ & & & & & \\
\hline \multicolumn{11}{|l|}{ DP hqud Tomp.f } \\
\hline 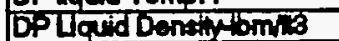 & क्टा4 & & & & & & & & & \\
\hline Exwiton & & & & & 9.875 & & & & & \\
\hline Kaecurnd DPapd & O.द्ध & 0.8801 & 7.568 & टअा & 3.084 & & & & & \\
\hline 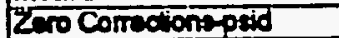 & 0.051 & 0.060 & 0.28 & -0.003 & 0.002 & & & & & \\
\hline Corrected Dph id & 0.3 & 0.803 & 1.30 & 237 & उ.णर्ण & & & & & \\
\hline Acter DPu & 1.88 & 3.91 & 5.02 & 5.58 & 8.68 & & & & & \\
\hline Menoured tpapho & दा8.4 & & & & 217.8 & & & & & \\
\hline Mecoured overalle & 1.10 & & & & & & & & & \\
\hline & & & & & & & & & & \\
\hline Compled DPA & 1.78 & 3.32 & 4.76 & 6.12 & 7.34 & & & & & \\
\hline Friciont & 0.04 & 0.09 & 0.18 & 0.24 & 0.32 & & & & & \\
\hline Aocaleriont & 0.03 & 0.04 & 0.05 & 0.07 & 0.08 & & & & & \\
\hline Erevitiont & 1.72 & 3.18 & 0.54 & 5.87 & 6.89 & & & & & \\
\hline & & & & & & & & & & \\
\hline
\end{tabular}




\begin{tabular}{|c|c|c|c|c|c|c|c|c|c|c|}
\hline Runt & 866 & & & & | & & & & i & \\
\hline Teeffind & CAFB & & 1 & & i & & & & & \\
\hline Dade: & $10 / 1 / 33$ & & 1 & & & & I & & & \\
\hline Time: & $15: 20$ & & & & & & & & $\frac{1}{1}$ & \\
\hline Not & Anatical & His used & & & & & & & & \\
\hline Fow Areakr & 0.00356 & & ț & - & & & & & & \\
\hline Hod flow Areat? & 0.19655 & & I & 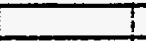 & & & & & & \\
\hline SDI Presture & 215.3 & & & & & & & & & \\
\hline SE Temper mofind) & 185 & & & & & & & & & \\
\hline Wh. Denthyomin & 73.21 & 73.05 & 73.18 & 73.711 & 73.08 & 73.00 & 7284 & T2. & 72.84 & $\overline{3}$ \\
\hline Lenthe exprom & 28.28 & 28.1 & 28.16 & 28.14 & 28.12 & 28.1 & 27.92 & 27.92 & 27.9 & 28.05 \\
\hline 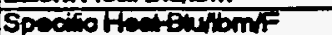 & 0.322 & 0.333 & 0.22 & 0.3881 & 0.388 & 0.32 & 0.384 & 0.324 & 0.334 & 0.388 \\
\hline Lig. Visooch thminh & 0.314 & 0.317 & 0.312 & 0.312 & 0.311 & 0.317 & 0.300 & 0.300 & 0.300 & 0.31 \\
\hline 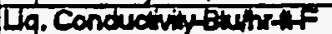 & 0.024 & $0.0 \times 3$ & 0.0230 & 0.0209 & 0.0230 & $0.0 \times 39$ & 0.030 & 0.0237 & 0.0037 & 0.005 \\
\hline Lig. PrandiNo. & 4.3 & 4.35 & 4.34 & 4.34 & 4.34 & 4.34 & 4.35 & 4.35 & 4.85 & 4.31 \\
\hline Int fow PMaGPM & 1.1 & & & & & & & & & \\
\hline OTllLG. Fow PS-GPM & 0.4 & & & & & & & & & \\
\hline 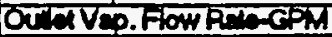 & 0.8 & & & & & & & & & \\
\hline Int temperenter & 787.8 & & & & & & & & & \\
\hline OLCLLg. Tomperetref & TE2 & & & & & & & & & \\
\hline ortuveo. femperilrof & 7872 & & & & & & & & & \\
\hline Wh FOW PDLAP & & & & & & & & & & \\
\hline Worhtatemp.t & 120.4 & & & & & & & & & \\
\hline Whereulet Tompif & 1822 & & & & & & & & & \\
\hline 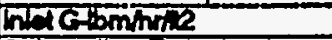 & 20002 & & TTenp: & 78.8 & Dening & 832 & & & & \\
\hline Subooalingt & 12 & & & & & & & & & \\
\hline Fow Observation & & & & & & & & & & \\
\hline & ZONE=1 & ZONEZ2 & ZONES & ZON:EX & ZONE6 & $\angle O N=6$ & ZON=7 & ZONiEB & $\angle O 1=9$ & $201=10$ \\
\hline 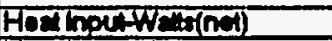 & 287.7 & 287.6 & 287.5 & 28.1 & 287.8 & 2858 & 287.8 & 2983 & 287.1 & 289.1 \\
\hline Herlosat Wets & 10.5 & 24 & 3.11 & 32 & 3.1 & 23 & 1.5 & 23 & 3.1 & 16.8 \\
\hline 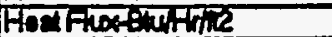 & 2687 & 458 & खिए। & 458 & $20 \%$ & 2803 & क्जा & 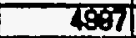 & 788 & TEX \\
\hline We Tromo. 135 & T93.1 & T925 & T9.01 & T827 & 7921 & 78:7 & 1883 & 183 & 18200 & TII.4 \\
\hline WhTomo.tif & 78356 & T9525 & 192 & 188 & 7924 & 7882 & 788.8 & 7882 & 7885 & 7825 \\
\hline WeTamo.16f & 194.1 & 1920 & 198 & Tद्यर्य & 191.8 & 1820 & 1927 & Tक्टी & \$क्य & TS1.0 \\
\hline Wentamo.tF & 787.6 & 1920 & 19T.5 & 182 & 1821 & 1820 & 188.2 & 18201 & 182 & TSo.1 \\
\hline WCITAmp. IAT & 7888 & 1920 & 182 & 15ट2] & 19टर & 1822 & 783.2 & 13 & क्यूत्या & 780.8 \\
\hline intreen fompof & 188.8 & 180.4 & द्या & 182 & 1893 & 160 & 1803 & 1802 & Fम2द & 78.6 \\
\hline AWo.WCTtempd & Tक्56 & 1920 & T्राइा & Tक्25 & 182 & Tिए: & 183.1 & कृख्य & 78्2: & TFS \\
\hline Avo. Wald DTf & 4.8 & 29 & 2द & 3.11 & 26 & 3.1 & 27 & 26 & 2दा & 7.5 \\
\hline Remening Suboodf & 3.2 & & & & & & & & & \\
\hline Extoumy & o.0. & 0.08 & 0.76 & 0.18 & 0.24 & 0.3 & 0.35 & 0.4 & 0.6 & $\mathbf{0 . 5 1}$ \\
\hline 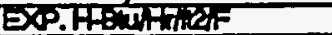 & T0ख्ड. & 1650.1 & 2035 & 7678.4 & 1918. & 15857 & $180^{2} x_{2}$ & 2003.7 & 30.58 & 378 \\
\hline COT:DTEOH & & & & & & & & & & \\
\hline INok & 3824 & 916.1 & 872 & 887.4 & 8805 & 870.7 & 8785 & 80.1 & 83 & 8056.0 \\
\hline 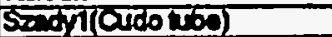 & 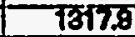 & 73235 & द्वस्2 & Fजद & 1320.6 & 13812 & T30.4 & छाइ5 & 13827 & 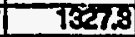 \\
\hline $5 \cdot d 2$ & Tớl: & $107 \pi$ & $10 \% 5$ & 10.57 .5 & $907 \pi .6$ & 10785 & 10348 & 1065.8 & 1083.0 & 10000 \\
\hline (PIO) & TOE & 3937 & 37001 & 365 & 32500 & 3002 & 28740 & 26501 & 2480 & टर्बा \\
\hline$(F)$ & 1.35 & 1.680 & 7.865 & 2.185 & 2.363 & 26 & 262 & 2.758 & 283 & 3.806 \\
\hline (सDO) & 1105 & 138.0 & |हत/ & 7716 & 1822 & 205.1 & 216.4 & 23.1 & 237.8 & 26.4 \\
\hline$\left(\mathrm{H} B \mathrm{O}^{1} \mathrm{~S}\right)$ & 1317.7 & 13228 & 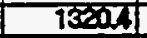 & 7313.3. & 13328 & 1318.5 & 1305 & |328.8 & 1द्ध一्टा.T & Tउस्टर \\
\hline & & & & & & & & & & \\
\hline & $20 N^{2} x_{2}$ & ZONET & $\angle O T=6$ & $\angle O N=8$ & $\angle O D=10$ & & & & & \\
\hline DPligud tomp. f $^{-}$ & 87 & & & & & & & & & \\
\hline DPILgudd Dentityomis & 82284 & & & & & & & & & \\
\hline Everiont & & & (8) & 81 & $9.8 \%$ & & & & & \\
\hline Mevered DPrord & 0.283 & 7.085 & 7.800 & 274 & 3.534 & & & & & \\
\hline 200 comeconped & 7.651 & -6.088 & o28a) & 200001 & 0.082 & & & & & \\
\hline ComeandDPapd & 0.87 & 1.07 & T.631 & हा: & 3.612 & & & & & \\
\hline Acterof & 9.85 & 25 & 2.,दा & 2.74 & 3.6 & & & & & \\
\hline Macoured tpropg & $\mathbf{2 1 5 . 8}$ & & & & दास: & & & & & \\
\hline Wecoured oventh & 1.38 & & & & & & & & & \\
\hline & & & & & & & & & & \\
\hline Competed DP: & 7.58 & 278 & 3.87 & 7.8. & 5.68 & & & & & \\
\hline Flach & 0.02 & 0.0 & o.01 & 0.12 & 0.16 & & & & & \\
\hline Acosterion- & ठ.ण & 0.02 & O.0.2) & 0.08 & 0.04 & & & & & \\
\hline Enliont & 9.53 & 2.78 & 3.71 & 4.01 & 5.46 & & & & & \\
\hline & & & & & & & & & & \\
\hline
\end{tabular}




\begin{tabular}{|c|c|c|c|c|c|c|c|c|c|c|}
\hline Runt & 867 & $i$ & & & 1 & 1 & & & I & \\
\hline Touffird & CAF 8 & & & & & & & & & \\
\hline Dato: & 10493 & & & & 1 & & & & & \\
\hline Timo: & $9: 30$ & & & & & & & & & \\
\hline Not & Andytioal c & dt is used & & & & & & & & \\
\hline How Araste & 0.00336 & & & & & & & & & \\
\hline How Row Arose? & 0.19696 & & & & & & & & & \\
\hline Sel Presuretsig & 200.4 & & & & & & & & & \\
\hline 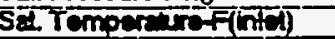 & 50.8 & & & & & & & & & \\
\hline La. Dentomis & 73.05 & 73.181 & 72.5 & 72.79 & 72.7] & 72.71 & 72.52 & 72.54 & $72.5 \times 1$ & 72.79 \\
\hline 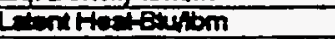 & 28.11 & 28.18 & 28.01 & 27.87 & 27.81 & 27.831 & 27.65 & 27.07 & 27.67 & 27.87 \\
\hline Specilio the elutiomif & 0.338 & 0.332 & 0.34 & 0.336 & 0.335 & 0.3361 & 0.337 & 0.337 & 0.337 & 0.395 \\
\hline ig. Visoos thom Wr & 0.311 & 0.312 & 0.31 & 0.308 & 0.307 & 0.3071 & 0.305 & 0.305 & 0.006 & 0.300 \\
\hline Lig. Conductivity ofurt of & 0.0020 & 0.0230 & 0.0238 & $0.023 \pi$ & 0.0257 & 0.02371 & 0.0236 & 0.0236 & 0.0260 & 0.0287 \\
\hline Lg. Prond No. & 4.34 & 4.34 & 4.34 & 4.35 & 4.35 & 4.35 & 4.36 & 4.35 & 4.36 & 4.30 \\
\hline Initit Flow Rut-GPM & 5.7 & & & & & & & & & \\
\hline OulaLLG. Fow RWA-GPM & 0.8 & & & & & & & & & \\
\hline 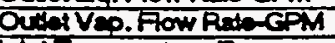 & 26 & & & & & & & & & \\
\hline 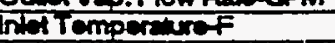 & 788.5 & & & & & & & & & \\
\hline 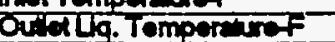 & Tि.8. & & & & & & & & & \\
\hline ovelvep. Temper hrof & 188.4 & & & & & & & & & \\
\hline Wear Fow PSW-GPM & & & & & & & & & & \\
\hline Whar hitel Temp.f & 120.1 & & & & & & & & & \\
\hline Wher ould Temp.f & 175.8 & & & & & & & & & \\
\hline tribet Goomphnine & 1017356 & & Temp & 184.4 & Density & 74.35 & & & & \\
\hline Subooolingf & 23 & & & & & & & & & \\
\hline Flow Obsenvetion & & & & & & & & & & \\
\hline & ZORET & ZONE2 & ZONEB & ZONE4 & 2ONES & ZOKNE6 & ZOK:=? & ZONE8 & ZONiÉ & ZONE $=10$ \\
\hline 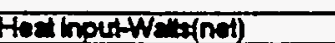 & 590 & $582 \mathrm{~d}$ & 5713 & 584.8 & 579.1 & 575.7 & 578.7 & 578.3 & 578.5 & 575.1 \\
\hline Hen Lors-WCS & 12.5 & 2.9 & 3.9 & & 3.9 & 2.9 & 1.9 & 2.9 & 3.7 & 80.8 \\
\hline Hed Fiox-Buthe & 70 & 10098 & 10005 & 1013 & 10038 & 997 & 10031 & 9990 & 10000 & 965 \\
\hline WaTtemp. 134 & 785.6 & 198.9 & 955.7 & 788.6 & 198.6 & 197.7 & 797.5 & 155.8 & 7964) & $19 \pi .8$ \\
\hline WETTOTD. \$4 & 158.8 & 798.3 & 798.7 & 187 & 1978 & 198.2 & 197.7 & 136.6 & 7965 & 797.9 \\
\hline Wa Temp. $16 F$ & (198.8) & 1980 & 197.6 & 190.80 & 190.5 & 197.4 & 197.5 & 195.6 & 195.7 & 194.8 \\
\hline WDT Temp. IIF & 788.5 & 195.8 & 196.6 & 195.7 & 198.2 & 197.7 & 198.11 & 190.8 & gित्य & 78्य \\
\hline WCI Tomp. \#24 & 186.7 & 195.0 & 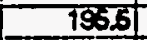 & 186.1 & 198.8 & 197.6 & 198.4 & 197.8 & कि:2 & $18 \pi, 4$ \\
\hline intreen Temp.F & 160.4 & 189.1 & 189.8 & 19001 & 100.7 & 190.6 & 181.4 & 191.8 & 101.3 & 180.4 \\
\hline Aup. Wel Temp.F & 7562. & 198. & 185.6| & 196.6 & 198.6 & T97.8 & 197.8 & 198.8 & 1956ी & 1840 \\
\hline AYP. WCIDT.F & 6.4 & 6.5 & 5.4 & 5.7 & 5.4 & 6.7 & 6 & 4.5 & 3.7 & 3.9 \\
\hline Romping Subooolf & 0.5 & & & & & & & & & \\
\hline Ex chony & -0.01 & 0.07 & 0.04 & 0.08 & 0.08 & 0.11 & 0.12 & 0.14 & 0.70 & 0.18 \\
\hline 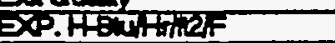 & 18007.4 & 1548.7 & 18द2 & 7781.8 & 1855.6 & 7469.7 & 1675.6 & 2198.8 & $250, \bar{B}$ & $\frac{1500}{2500}$ \\
\hline CONipUIEDH & & & & & & & & & & \\
\hline Inok & 324. & 7200.8 & 7157.8 & Tास.8 & 1705.5 & 709.8) & 7089.8 & 1077.7 & T0\%2 & 10052 \\
\hline 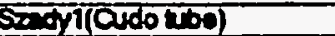 & 1898 & 1884 & 1883.9 & 1960\%7 & 18938 & 18986 & 1907.6 & 1904.1 & 1800.11 & $9600, \pi]$ \\
\hline sodve & 17/4.2) & 1723,8 & 1720 & 17495 & 174h.4 & 1737.6 & 1735.5 & 1750.8 & 1700 & $17 B A$ \\
\hline (RD) & 212820 & 211112 & 200228 & 20505 & 201202 & 19508 & 199648 & 188000 & $18+5506$ & TFम" \\
\hline & 1.004 & 1.12 & 1.404 & 1.567 & 1.683 & 1.8711 & 1.904 & 1.935 & 2070 & 2100 \\
\hline सिखा & उ이리 & 32.7 & 辰. & 777.81 & 510.9 & 544.8 & 574.1 & 5015 & 6207 & 6615 \\
\hline (18024) & 1896 & 18602 & 1872.8 & 1894 & 18862 & 1878.3 & 1890.1 & 1888.8 & 18838 & 7870.4 \\
\hline & & & & & & & & & & \\
\hline & ZON=32 & $20 N=4$ & $201=6$ & $201=8$ & ZONETO & & & & & \\
\hline DPloged Tomp.f & 7... & & & & & & & & & \\
\hline DPLquid Dentin & 9358 & & & & & & & & & \\
\hline Evevion & & & & & 9.875 & & & & & \\
\hline Whewrod DAphd & 0.288 & 0.63 & 7.ाओ & 1.648 & 2.171 & & & & & \\
\hline 20ro Corrochom pad & -0.00 & -0.063 & 0.161 & 0.008 & 0.084 & & & & & \\
\hline Cormechd DP Pid & $0.38 \pi$ & 0.672 & 0.97 & 1.616 & 2.07 & & & & & \\
\hline Actur DPh & 1.8 & 3.81 & 5.70 & 7.08 & 8.68 & & & & & \\
\hline homped thath & 3004 & & & & 218.6 & & & & & \\
\hline Menored Ovareth & 3.56 & & & & & & & & & \\
\hline & & & & & & & & & & \\
\hline Computed DFa & दार्ठा & 2.17 & 6.05 & 7.89 & 9.8 & & & & & \\
\hline filctiont & 0.17 & 0.0 & 0.75 & 1.13 & 1.54 & & & & & \\
\hline Aocoteriont & 0.1 & o.स & 0.20 & 0.83 & 0.48 & & & & & \\
\hline Exutiont & 1.52 & 3.53 & 5.01 & 6.30 & 7.63 & & & & & \\
\hline & & & & & & & & & & \\
\hline
\end{tabular}




\begin{tabular}{|c|c|c|c|c|c|c|c|c|c|c|}
\hline Run: & 8681 & 1 & & : & & & 1 & & 1 & \\
\hline Tost Find & ic4न्8 & $\mathbf{i}$ & & i & & & & & $!$ & \\
\hline Deta: & $10 / 493$ & 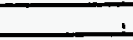 & & & & & & & - & \\
\hline Time: & $9: 30$ & & & & & & 1 & & & \\
\hline Now & Anatiol & Is used 1 & & & & & & & & \\
\hline Fow Arean? & 0.003361 & & & & & & & & & \\
\hline Hoc Fow Areare & 0.156951 & ! & & & & & & & & \\
\hline Sत् Proserur pig & 220.11 & & & & & & & & & \\
\hline SL tomoer & 790.4 & & & & & & & & & \\
\hline Lg. Dentantoms 3 & 73.06 & 73.18 & 73.05 & 72.71 & 72.70 & 727 & 7269 & 7254 & 7257 & 72.70 \\
\hline Lind Hanghiom & 28.1 & 28.21 & 28.1 & 27.85 & 27.85 & 27.87 & 27.02 & 27.67 & 27.61 & 27.65 \\
\hline 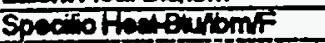 & 0.32 & 0.322 & 0.339 & 0.355 & 0.356 & 0.3501 & 0.337 & 0.387 & 0.356 & 0.385 \\
\hline Uf. Virootingomith & 0.317 & 0.318 & 0.317 & 0.308 & 0.300 & 0.008 & 0.304 & 0.306 & 0.306 & 0.308 \\
\hline Lig. Conductity ethr-fF & 0.02 & 0.020 & 0.028 & 0.0287 & 0.0237 & 0.0 .51 & 0.0255 & 0.028 & $0.0 \times 61$ & 0.0237 \\
\hline La. Prendino. & 4.34 & 4.3 & 4.34 & 4.36 & 4.35 & 4.35 & 4.38 & 4.35 & 4.36 & 4.35 \\
\hline Intat flow Puta-CDM & 4.8 & & & & & & & & & \\
\hline OUNLq. Fow P WE & 0.8 & & & & & & & & & \\
\hline 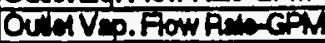 & 2.2 & & & & & & & & & \\
\hline Intet temperntefref & 188.8 & & & & & & & & & \\
\hline oralla. Temparime & 789.6 & & & & & & & & & \\
\hline outivep. Temoermef & 188.8 & & & & & & & & & \\
\hline Wer Fow PWW-GPM & & & & 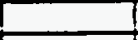 & & & & & & \\
\hline Wom linel Tomp ff & T18.8 & & & 1 & & & & & & \\
\hline Werrocilet Temp.f & 175.1 & & & & & & & & & \\
\hline hro Gommith & 853 & & Temp & 184.11 & Dandiy & 74.421 & & & & \\
\hline SuboodinfF & 24 & & & & & & & & & \\
\hline Flow Observetion & & & & & & & & & & \\
\hline & $201=1$ & 20Nen & $2 O N=8$ & ZON:4 & $20 N=5$ & $201=6$ & ZOHiei & $20 N=8$ & $2 \mathrm{OH}=9$ & $\angle O A=10$ \\
\hline Henthoutweral) & 5724 & 5826 & 575.1 & 580.1 & 5772 & 53.4 & 578.7 & 576.4 & 577.1 & 5724 \\
\hline Heallosatwe: & T3.1 & 3ी & 4.1 & 42 & 4.2 & 3.2 & 2 & 3) & 3.9 & 22 \\
\hline 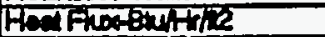 & sei2) & Toकी & 960 & 70056 & 10000 & छाס & 100्डा & 982 & 1000 & 9322 \\
\hline We Tamp. Ba & 183 & 7986 & T\$5.7 & [9332] & 158.8 & 188 & 797.6 & T\$5:| & 735.01 & T95.0 \\
\hline We Tomp. If & T88.4 & 156.7 & 135.6 & 796.8 & 187.8 & 1588 & 197.8 & 158.4 & 783.7 & 78 \\
\hline Weitomp. 15 F & 190.5 & 180 & 187.4 & 196.7 & 156.8 & 197.4 & 197.4 & 135.6 & 15501 & 78.8 \\
\hline Whitomp. 11 t & 188.5 & 190 & $15 \pi .6$ & 195.7 & 180 & 197.61 & 198.7 & 180.2 & ISETh & Tि्दूर \\
\hline WCThomp.124 & 1872 & 186 & 1956 & 198 & 196.1 & 197.61 & 188.8 & 197.11 & 1953 & कहूर \\
\hline Inmentemp & 168.4 & 188.0 & 189.4 & 180.5 & 180.5 & 1901 & 191.6 & 181.8 & TO12 & 1806 \\
\hline ANo.We' Tompa & 196.6 & कर.ग & 755.6) & IGर्म & 15SD| & 1978 & 1978 & 1582 & क5 & Tर्म.6 \\
\hline AVT Wa DT.F & 6.8 & 6.7 & 5.7 & 5.4 & 5.5 & 7 & 5.9 & 4.5 & 3.8 & 3.6 \\
\hline Remining subooff & 0.4 & & & & & & & & & \\
\hline Exaduly & 0 & 0.02 & 0.04 & 0.07 & 0.00 & 0.12 & 0.16 & 0.5 & 0.10 & 022 \\
\hline 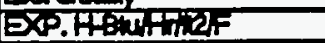 & 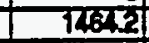 & 75035 & 1738.0 & 1838.7 & 18द्ध3: & 7क्या: & 7707.1 & स्2:01 & 2578.8 & 2747.8 \\
\hline CoI'OUIEOH & & & & & & & & & & \\
\hline JElk & 7207.8 & Tर्षsen & T18250 & गसद2 & 7147.0 & T1EST| & 77102 & T05974 & 105.0 & 10702 \\
\hline 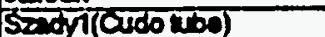 & 1808 & 1835 & 9875.7 & 185.6 & 1802 & 1885.5 & 180.4 & 1908 & 1906] & 18806 \\
\hline $5=\operatorname{dr} 2$ & 1703 & 770.8 & I715. & 1741.7 & 7737.1 & 17ठ0. & 175 & 1746.4. & 176.7 & F\$.4. \\
\hline (PLO) & Theres & T76378| & 173898 & 170801 & 16808 & 161861 & 168387 & 16\%5 & 1250.2 & 761078 \\
\hline$(F)$ & 1.004 & 1.24 & 1.474 & 1.61 & 1.78 & $1.50 \mathrm{a}$ & 201 & 2.101 & 2.18 & 238 \\
\hline$(\mathrm{H} D \overline{1})$ & -2027 & 3178 & 5at & 750 & 467.7 & 769 & 5256 & E528 & $6 \overline{8.1}$ & 609 \\
\hline$(\mathrm{FinO})$ & 18578 & 1878.8 & 18702 & 18823 & $1882 \mathrm{~d}$ & 1874. & 18025 & 98621 & 18850 & 18306 \\
\hline & & & & & & & & & & \\
\hline & $\angle O N=2$ & ZOMET & $20 \mathrm{O}=5$ & $20 x=8$ & $\angle O N=10$ & & & & & \\
\hline DPlgud Temp.t & 78 & & & & & & & & & \\
\hline DPLiqud Dentix omis & 83.18 & & & & & & & & & \\
\hline Eveliont & & 4 & & 8 & 9.875 & & & & & \\
\hline 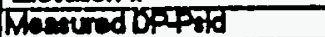 & 0.280 & 0.78 & 7238 & 7.81 & 2खा & & & & & \\
\hline Lero comechonopd & 7.046 & -0.088 & $0.16 \pi$ & 0.008 & $0.6 \pi$ & & & & & \\
\hline Comecied oppid & 0.08 & 0.81 & 7.087 & 9.807 & 2370 & & & & & \\
\hline ACh & 1.8 & 3.62 & 5.50 & 630 & 7.88 & & & & & \\
\hline Wheored TPF & खराग & & & & 278, & & & & & \\
\hline Wheoured overell & 3.20 & & & & & & & & & \\
\hline & & & & & & & & & & \\
\hline Computed Derl & हा? & 3.80 & 5.7. & 7.45 & 8.88 & & & & & \\
\hline Flotonn & 0.13 & ర.3\$ & 0.50 & 0.9 & 722 & & & & & \\
\hline Aocelentiont & 0.00 & 0.17 & 0.20 & 0.20 & 0.50 & & & & & \\
\hline Exwiont & 7.88 & 3.65 & 4.88 & $6: 20$ & 7.61 & & & & & \\
\hline & & & & & & & & & & \\
\hline
\end{tabular}




\begin{tabular}{|c|c|c|c|c|c|c|c|c|c|c|}
\hline Runt & 869 & & 1 & $:$ & $:$ & 1 & 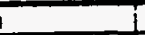 & i & & \\
\hline Tosf Fuid & CAF 8 & & & & 1 & 7 & 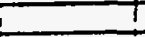 & I & & \\
\hline Date: & 104993 & & & & & & & 1 & & \\
\hline Time: & $9: 30$ & & & & & & & & & \\
\hline Noto & Anaviodo & सis used I & & & & & & & & \\
\hline Flow AroaR2 & 0.00356 & & & & & & & & & \\
\hline Host Fow Arase & 0.1क्S5 & & & & & & & & & \\
\hline Sat piesureporg & टरव.ा & & & & & & & & & \\
\hline 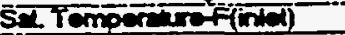 & 190.3 & & & & & & & & & \\
\hline Liq. Denengermis & 73.05 & 73.18 & 7297 & 72811 & 72.87 & 72.84 & 72.731 & 72.7 & 72.76 & 72.87 \\
\hline 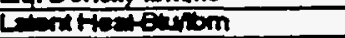 & 28.7 & 28.101 & 28.08 & 27.91 & 27.91 & 27.92 & 27.801 & 27.65 & 27.85 & 27.9 \\
\hline 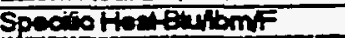 & 0.383 & 0.352 & 0.334 & 0.365 & 0.324 & 0.339 & 0.3551 & 0.3251 & 0.325 & 0.336 \\
\hline Iig. Visoosincibmintr & 0.311 & 0.3121 & 0.37 & 0.308 & 0.300 & 0.309 & 0.3031 & 0.300 & 0.303 & 0.300 \\
\hline Lig. Conductive Eurreff & 0.0230 & 0.0201 & 0.0288 & 0.0237 & 0.0088 & 0.0237 & 0.0237 & 0.0037 & $0.0 \times 3$ & 0.0080 \\
\hline Lig. Prendell No. & 4.3 & 4.34 & 4.34 & 4.35 & 4.35 & 4.35 & 4.35 & 4.351 & 4.85 & 4.35 \\
\hline Infet fow R-CPM & 3.9 & & & & & & & & & \\
\hline OL L Lig. Fow Rato-GPM & 0.7 & & & & & & & & & \\
\hline OCATVSOFOW RE-GPM & 1.8 & & & & & & & & & \\
\hline Ind tamoeriteref & T88.4 & & & & & & & & & \\
\hline 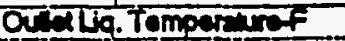 & 188.4. & & & & & & & & & \\
\hline 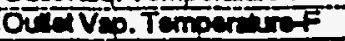 & 187.4 & & & & & & & & & \\
\hline Wabrflow RU-CPM & & & & & & & & & & \\
\hline Werer hitat Temp.f & 118.4 & & & & & & & & & \\
\hline Wer octed Tempof & 168.7 & I & & & & & & & & \\
\hline Intaraminn? & 60258 & & Tamp & 184.7 & Deniy & 74.42 & & & & \\
\hline Subooolinef & 1.9 & & & & & & & & & \\
\hline Flow Obrenvion & & & & & & & & & & \\
\hline & ZONET & 20152 & $2 O N=3$ & ZONE & ZONES & ZONiE6 & ZON:=4 & ZOR: 8 & ZONES & $201=10$ \\
\hline Heathoutwernot) & $5 \% .7$ & 578.5 & $5 \% 3$ & 580.5 & 578.8 & 58.6 & 577.3 & $5 \pi$ & 581.8 & 588.7 \\
\hline Hentosowas & 13.8 & 3.2 & 4.3 & 4.5 & 4.6 & 3.6 & 231 & 3.3 & 4.1 & 23.7 \\
\hline 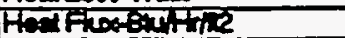 & 1006 & 10028 & 10000 & 1008 & 10050 & 10008 & 70007 & 70002 & $300 \%$ & 10170 \\
\hline Whemp. J84 & 195.7 & T\%8.8 & 155.5 & T56.1 & 7965 & 797.7 & 798.8 & T55. & 7870 & T8I.8 \\
\hline WeTtomo.14F & 198.8 & 195.8 & 198.4 & 7980 & 197.1 & Ts83 & Ts8.0 & 158.7 & 180 & 787.0 \\
\hline WD tomp. $15 f$ & 158.5 & 194.8 & 197.8 & 156.6 & 198.8 & 197.8 & 158.7 & 197.9 & 195 & 198.8 \\
\hline We Tomp. f1F & 188.6 & 155.7 & 134दी & 1956 & 196.8 & 1975ा & T97.5 & 195.4 & 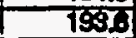 & 7922 \\
\hline WNT Tomp. IRF & 186.9 & 155.7 & 18:2 & 185: & 196.9 & 19751 & 197.7 & 1886 & 184A & 187 \\
\hline horem tomp.f & $780 \times 1$ & 180.11 & 186.5 & 180.3 & 180.J1 & 1802 & 180.8 & 1806 & 1000 & 1802 \\
\hline Avg. We tempots & $\$ 56 . \lambda$ & 15.3 & 1558 & 1963 & 1968 & 197.7 & 197.1 & 1955 & 18: & 79:56 \\
\hline AYP.WEDT.F & 6.5 & 62 & 52 & 5.4 & 5.7 & 7 & 6.1 & 4.8 & 3.7 & 3.8 \\
\hline Remening seboost- & -0.6 & & & & & & & & & \\
\hline Ex' Cut" & 0.07 & 0.04 & 0.07 & 0.1 & 0.13 & 0.76 & 0.19 & 0.32 & 0.3 & 0328 \\
\hline 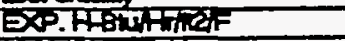 & 75365 & 16182 & क्साइ & 1858.5 & 7753.0 & T4:3.11 & IEखि & टाह9.6 & 270 & क्यद2 \\
\hline CoNOUIEH & & & & & & & & & & \\
\hline Jatouk & 12001 & T246.67 & Tद्ध8.ा & 72008 & 7778.8 & T16201 & 71508 & T13ä8 & T15005 & T100.0 \\
\hline Sodvi(cedorbo) & 18812 & 187 & 18814 & 18836 & 18686 & 789050 & 1896.6 & 183 & 19000 & 7802 \\
\hline Sect & 1727 & T76.6 & 17212 & 1730 & T738.6| & $7 \longdiv { 3 7 . 8 1 }$ & $17 \mathrm{Sa} .1$ & 7737.61 & 7726,1 & 17020 \\
\hline (FU) & T45112 & 141834 & isenet & 18901 & 190106 & 125771 & 121600 & $17 \% 001$ & TFEFA & 10780 \\
\hline$(F$ & $1.1 \%$ & 1.32 & 1.677 & 1.789 & $1.83 \pi$ & 2004 & 2.175 & 2खा & 2378 & 2478 \\
\hline (स) & 2का & उ063 & 356 & 37. & फूरो & 760 तो & 48त्रो & 603.7 & 6324 & $6 \times 62$ \\
\hline$(1+30)$ & 1870.7 & 787as & Tosan & 18876 & 18828 & 1881.01 & 7883.1 & 18005 & 18872 & $18 \times 2$ \\
\hline & & & & & & & & & & \\
\hline & $201=2$ & ZONET & ZON=6 & $\angle O A=88$ & $\angle O K=10$ & & & & & \\
\hline DPtigud Tomof & 77.5 & & & & & & & & & \\
\hline 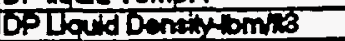 & 836 & & & & & & & & & \\
\hline Exwiont & & & & & 9.870 & & & & & \\
\hline Mavered DPPad & 03 & $0.88 \pi$ & 1.85 & 258 & 2808 & & & & & \\
\hline zero Correctone peld & 0.069 & -0.038 & 0.10才 & 0.008 & 0.08 & & & & & \\
\hline Coroctod Dapud & 0.830 & 0.021 & 7.274 & 2.1\% & $2 \pi 3$ & & & & & \\
\hline Acter Don & 1.0 & 3.81 & 5.10 & $5 . \%$ & 7.21 & & & & & \\
\hline Wheoured tfPth & 218.1 & & & & 217.0 & & & & & \\
\hline Mecuredoventll & 237 & & & & & & & & & \\
\hline & & & & & & & & & & \\
\hline Compitad DP & 7.56 & 3.621 & 5.17 & 6.68 & 8.68 & & & & & \\
\hline Frolont & 0.17 & क्टा & 0.46 & 0.74 & 1.02 & & & & & \\
\hline Acodienciont & 0.08 & 0.14 & 0.18 & 0.30 & 0.8 & & & & & \\
\hline Envinont & 1.83 & उसा & 45 & 5.60 & 8.72 & & & & & \\
\hline & & & & & & & & & & \\
\hline
\end{tabular}




\begin{tabular}{|c|c|c|c|c|c|c|c|c|c|c|}
\hline Runt & 8701 & $\Gamma$ & 1 & 1 & 1 & & 1 & 1 & i & \\
\hline Tonflud & CAFB & & & i & & & i & & I & \\
\hline Dato: & 10443 & & & & & & & $i$ & i & \\
\hline Timo: & $9: 30$ & & & & & & & & I & \\
\hline Nois & Anatical & At is used & I & & & & 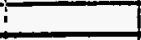 & & & \\
\hline Flow AToste & 0.00356 & 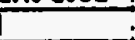 & 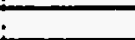 & & & & & & 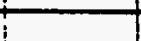 & \\
\hline Han Flow Arest? & 0.18051 & 7 & 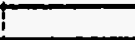 & & & & & & & \\
\hline Sal Proseurequg & 21821 & & & & & & & & & \\
\hline Sritempenter finte) & 189.3 & & & & & & & & & \\
\hline Wa. Dentinytom, 3 & 73.29 & 73.201 & 73.18 & 73.031 & 73.05 & 73.08 & 72.82 & 72.9 & $728 \mathrm{~g}$ & 7.63 \\
\hline Lent He-tempom & 28.8 & 28.81 & 28.21 & 28.081 & 28.1 & 28.12 & 27.90 & 27.89 & 27.59 & 28.00 \\
\hline 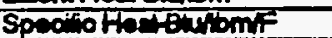 & 0.391 & 0.331 & 0.382 & 0.3831 & 0.353 & 0.383 & 0.334 & 0.384 & 0.3 & 0.358 \\
\hline Lia. Viroosin iom in & $0.3 \overline{14}$ & 0.374 & 0.378 & 0.317 & 0.317 & 0.397 & 0.31 & 0.31 & 0.3 & 0.311 \\
\hline 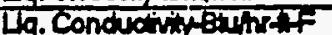 & ठ.0या & 0.024 & $0 . \bar{E}$ & 0.0238 & 0.0020 & 0.0000 & 0.068 & 0.068 & $0.0 \times 3$ & 0.028 \\
\hline Lg. Prandéno. & 4.34 & 4.34 & 4.34 & 4.34 & 4.34 & 4.34 & 4.35 & 4.55 & 4.55 & 4.37 \\
\hline 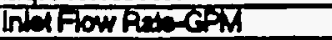 & रों & & & & & & & & & \\
\hline OT LLG. FHOW RAL-GFM & 0.6 & & & & & & & & & \\
\hline 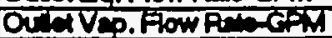 & $1.9 !$ & & & & & & & & & \\
\hline Int Tomperenter & 787.4 & & & & & & & & & \\
\hline ortilla. Tompereyre & T88.01 & & & & & & & & & \\
\hline of FVo Temoen & 188.8 & & & & & & & & & \\
\hline WEM FOW P GPM & & & & & & & & & & \\
\hline Wher hiet Temp.F & 118.2 & & & & & & & & & \\
\hline Wen outertemp.f & 169,4 & & & & & & & & & \\
\hline 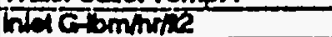 & 618 & & Treme & 1823 & ibenaiy & 74.0 & & & & \\
\hline Suboodingt & 1.9 & & & & & & & & & \\
\hline Flow Obsenvion & & & & & & & & & & \\
\hline & ZONiET & $\angle O N=32$ & $20 \sqrt{2}=3$ & ZONEA & ZONES & $20 \pi=6$ & $2 O N=7$ & ZONEB & ZOTEO & CON=10 \\
\hline Hocthortwanged & 5728 & 578.7 & 581.6 & 5826 & 57.7 & 579.7 & 578 & $5 \% 5.8$ & $583 \mathrm{~A}$ & 500.7 \\
\hline HaALoseWE'B & 128 & 3 & 4 & 4.1 & 4.1 & 3.1 & दे & 28 & 3.8 & 27.5 \\
\hline Hon Fior Busthin & sos & 999 & 90000 & 7001 & To56 & 1006 & \$35 & S672 & Tर्णाड8 & 1000 \\
\hline Watromp.t广 & \{द्8. & 7852 & Tदूर० & T5\$8| & 7950 & - दूर & T98.4. & T9म5 & 780 & T98.0 \\
\hline Writomp.ift & 785.4 & 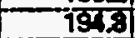 & 1565 & 155.8 & 1833 & 1875 & 183.4 & 1953 & 7850 & T91.1 \\
\hline We'tomp. 86 & 155.6 & 1द्या & 1900 & TS5I & 185.7 & 180.7 & 1902 & 194.8 & Tर्जा & 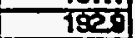 \\
\hline WE'TCTP. IT & 187.,. & 192.0 & 1932 & 19क्रा & 155.1 & 197 & 190.0 & 194.7 & TSस्ये & 78.8 \\
\hline We Temp. da & 1858 & 19RD| & 194.8 & 1552 & 195. & 1830 & 786.9 & 1558 & कित्य & 1832 \\
\hline netrem Tomp.F & 1886 & 1835 & 7830 & 18651 & 1820 & 188 & 1800 & 1800 & 76 & 7685 \\
\hline Avo.Waltempd & TS5.4 & 194.7 & ग्रा4 & 1551 & 7856 & 797.7 & 186.6 & Isतo & क्रूर्य & 1936 \\
\hline AGo.WCTDT.F & 6.5 & 5.7 & 6 & 5.4 & 5.7 & 7.3 & 62 & 4.5 & 8.8 & 3.7 \\
\hline Rem ining Subooff & -1.6 & & & & & & & & & \\
\hline Extautity & 0.0 & 0.03 & ర.t & 0.री & 0.18 & 0,2 & $0 \$$ & 0,8 & 0.87 & 0,38 \\
\hline DXP.ABV, & 1560.11 & 1746.7 & 79532 & 185.0 & 1782 & 73718 & ह5: & राक्या & $2 \times 5: 12$ & 278.7 \\
\hline COW:XUIEDH & & & & & & & & & & \\
\hline Jecok & $72: 0.8$ & $|\tan 2|$ & T2E.T & र्यमा & Tरeor & 72036 & Tद\&.7 & Th76.0 & T175.7 & Tri \\
\hline 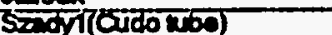 & दिज.ा & 7858 & T8:6it & 188 & 1688 & 18827 & 788250 & T8828 & T68.22 & $180 \times 3$ \\
\hline $50 x \sqrt{2}$ & 10972 & TFon & 178 & 17द्धर्ग & 1728.8 & 7727 & T7क. & Trचah & 77392 & T7F.TS \\
\hline (FIO) & 10709 & 1085 & 90000 & sesmi & 8Tts] & 87201 & 83507 & 789.21 & 74510 & 65120 \\
\hline$(F)$ & 1म्यों & 1.516 & T.7r & 1.888 & 2.18 & $22 \pi$ & 2000 & 2518 & $2 \mathbf{8}$ & $27 \pi$ \\
\hline (1) & 2768 & 2557 & उ10: & औरता & 378 & 400 & स्टूती & 73.1 & 7628 & 2824 \\
\hline$\left(\mathrm{H}: \mathrm{X}^{\prime}\right)$ & 78052 & Tद्ध一ा & $187 / 3$ & Tहरता & 18721 & 1876 & 18758 & T874.I & 18.5 & T\&्या \\
\hline & & & & & & & & & & \\
\hline & $201 .=2$ & ZONES & $20 N=6$ & $2011=8$ & $\angle O T^{\prime}=10$ & & & & & \\
\hline DPlgud Tomp.f- & 78.8 & & & & & & & & & \\
\hline 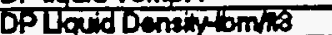 & $\$ 3.55$ & & & & & & & & & \\
\hline Exwiont & & ता & & 8 & 9.870 & & & & & \\
\hline 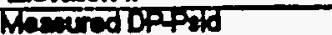 & 0301 & i & 7.85 & 2द7 & 3.188 & & & & & \\
\hline 20ro Correcton odd & 20.049 & -0.68 & 0.187 & 0.001 & 0.687 & & & & & \\
\hline Comeondophed & ס.j. & 1.08 & 1.46 & 2407 & 3.140 & & & & & \\
\hline Actuld DPA & 1.80 & 3.01 & 4.8 & 6.83 & 6.45 & & & & & \\
\hline Wemedtph & संख्यो & & & & 2140 & & & & & \\
\hline Mactered overils & 256 & & & & & & & & & \\
\hline & & & & & & & & & & \\
\hline Comoundor & क.8ा & 330 & 4.65 & -5.8: & 7.05 & & & & & \\
\hline Fictont & 0.007 & 0.10 & 0.35 & 0.58 & 0.74 & & & & & \\
\hline Acontering & 6.06 & 0.1 & 0.78 & 0.17 & OLI & & & & & \\
\hline Elymion & 1.68 & 3 & 2.18 & 5.2 & 6.1 & & & & & \\
\hline & & & & & & & & & & \\
\hline
\end{tabular}




\begin{tabular}{|c|c|c|c|c|c|c|c|c|c|c|}
\hline Aunt & 8711 & 1 & & & & & & & & \\
\hline Teg Fivid & CAF & & & & & & & & & \\
\hline Date: & 104493 & 1 & & & & & & & & \\
\hline Trmo: & $9: 30$ & & & & & & & & & \\
\hline Note & Andytion o & is used & & & & & & $!$ & & \\
\hline Fow Areale & 0.003561 & & & & & & & & & \\
\hline Host Fow Arear? & 0.195851 & & & & & & & & & \\
\hline SI Prostur pide & 217.8 & & & & & & & & & \\
\hline Sel fomperimotinied & 189.9 & & & & & & & & & \\
\hline Lig. Dentitiom 18 & 7328 & 72.971 & 73 & 72.89 & 72.82 & 72871 & 72.9 & 72.84 & 7283 & 7284 \\
\hline 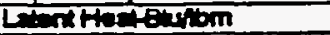 & 28.8 & 28.08 & 28.05 & 27.88 & 27.99 & 27.281 & 27.87 & 27.921 & 27.92 & 27.82 \\
\hline Speciso therewiomf & 0.3311 & 0.384 & 0.338 & 0.384 & 0.354 & 0.331 & 0.335 & 0.331 & 0.323 & 0.334 \\
\hline Lip. Vinoostom & 0.374 & 0.37 & 0.37 & 0.3001 & 0.31 & 0.3001 & 0.308 & 0.300 & 0.300 & 0.000 \\
\hline Lig. Conducting Bupreff & 0.024 & 0.028 & $0.0 \times 38$ & 0.0288 & 0.028 & 0.028 & 0.0237 & 0.0237 & 0.037 & $0.0 \times 3$ \\
\hline Uo. Prendino. & 4.34 & 4.34 & 4.34 & 4.35 & 4.35 & 4.35 & 4.35 & 4.35 & 4.35 & 4.56 \\
\hline 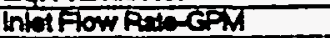 & 2 & & & & & & & & & \\
\hline 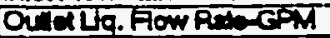 & 0.4 & & & & & & & & & \\
\hline 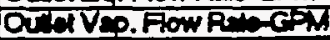 & II & & & & & & & & & \\
\hline thit Tempercters & 187.6 & & & & & & & & & \\
\hline ordule Tomper iref & 389.7 & & & & & & & & & \\
\hline ortep. Temperentof & 1873 & & & & & & & & & \\
\hline 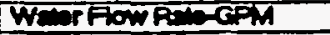 & & & & & & & & & & \\
\hline Whar thed Terme.f & 1192 & & & & & & & & & \\
\hline Wereoulat Tomp.F & 168 & & & & & & & & & \\
\hline FinG & 350250 & & Tenp & 180.61 & Dentity & 75.3 & & & & \\
\hline Sebooding F & 2.3 & & & & & & & & & \\
\hline Fow Observetion & & & & & & & & & & \\
\hline & ZONET & ZONE2 & 2OK:-3 & ZONEA & ZONi=5 & $20 \mathrm{ON}=6$ & 2ONE7 & ZONEE & $20 N=\theta$ & $2010=10$ \\
\hline 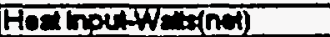 & 5692 & 580.8 & 581.8 & 583 & 580.6 & 581.1 & 578.9 & 577.1 & $58 \times 2$ & 360.7 \\
\hline Hod Losentwe & 13.3 & 3.1 & 4.2 & 4.4 & 4.4 & 3.4 & 2.1 & 3.1 & - & 23 \\
\hline Had FWE & 9881 & T00381 & 10087 & 1010 & 70089 & 1003 & 10035 & 10014 & TOT27) & 10067 \\
\hline We Tomp. Ha $^{3}$ & 185.1 & $\mid\{95.8 \mid$ & T9.8 & 9558 & 7952 & 197.8 & 198.7 & TS & \{874] & T9.8 \\
\hline WCTEmp.77F & T\$. & 19म. & 190 & 1962 & 198.6 & 188 & 158.8 & 196.6 & 7850 & 787.8 \\
\hline Wh Tamp. $15 \mathrm{~F}$ & 198 & 194.17 & 187 & 198.4 & 196 & 197 & 198.8 & 194.8 & 1955 & 183.6 \\
\hline WeTomp.t1F & 187.8 & 196.1 & 1339 & 1940 & 1856 & 1873 & 1972 & 1962 & 1926 & $3 \%$ \\
\hline Writamp.12f & 186.1 & 185.1] & 194.7 & 185. & 1956 & $187 . \pi$ & 1978 & 186.81 & 1826 & 1988 \\
\hline houm temp.f & 1885 & 189.7 & 186 & 180 & 1836 & 190.11 & 190.4 & 1502 & TEDद्या & 1653 \\
\hline 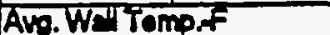 & 195.7 & 35521 & 19र० & 195.8 & 1950 & 197.4 & 196.0 & 135.8 & I9\$0 & Tor? \\
\hline AYOWEDT F & 6.7 & 5.1 & 2.8 & 5.4 & 5.5 & 6.8 & 6 & 4.7 & 30 & 35 \\
\hline Paminim subcooff & -26 & & & & & & & & & \\
\hline ExTCunty & 0.03 & 0.00 & 0.15 & 0.31 & 0.30 & 0.32 & 0.38 & 0.4 & 0.6) & 0.58 \\
\hline 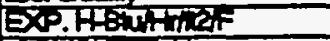 & 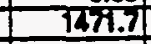 & 15958 & 210 & 18826 & 1815 & 747 & 767.7 & 2753.4 & उद्ध一ा & 2837.8 \\
\hline Co'M-OIIEDH & & & & & & & & & & \\
\hline Jieck & T3E3.4 & 1373.7 & 72812 & T2/2: & 1202 & T201.1 & 12520 & $723 / 5$ & 7250 & T2eibs \\
\hline 5. & 185201 & 1885.6 & 188.58 & 18827 & 18362 & 189.8 & 183030 & 18877 & $78 \% 35$ & $76: 27$ \\
\hline $\operatorname{sed} \sqrt{2}$ & 1690.7 & 172 & 1700.6 & 1787.8 & 1782 & 1750.89 & 1737.1 & 17322 & 174 & 1731.6 \\
\hline (RO) & 3750 & 713051 & 6.514 & 60031 & 58104 & 53731 & 49162 & 448801 & 20702 & 3530 \\
\hline (F) & 1.301 & 1.660 & 1.867 & 230 & 2.30 & 25561 & 2.707 & 2856 & 28 & 3.121 \\
\hline (स्प) & -151 & 217.7 & 2572 & 28756 & 318ב & 356 & 365.7 & 375 & 30.1 & 7702 \\
\hline (F:ODQ & 78515 & 18843 & 1882 & 8506 & 18358 & 1837.8 & 7883.7 & 78825 & 18:23: & 78835 \\
\hline & & & & & & & & & & \\
\hline & $\angle O N=32$ & $\angle O N=4$ & $2011=6$ & $120 N^{2} 38$ & $201 x=10$ & & & & & \\
\hline DPligud tomp.f & 802 & & & & & & & & & \\
\hline 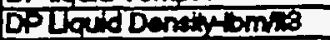 & \$3.17 & & & & & & & & & \\
\hline Exveriont & & 4 & 8 & 8 & 9.875 & & & & & \\
\hline Howred DFPhd & 0.37 & 7.130 & 1.88 & 2720 & 3.65 & & & & & \\
\hline zoro Combotion poid & 0.0 & 20.088 & 0.101 & 0.003 & 0.081 & & & & & \\
\hline Comond op pad & 0.419 & 1.171 & 1.78a & 272 & 3.601 & & & & & \\
\hline Acterd DP & 7.78 & 2.78 & 2.35 & 4.801 & $5.6 \pi$ & & & & & \\
\hline Wheved ippeng & 217.8 & & & & 216.8 & & & & & \\
\hline Mecoured oversh & 1.89 & & & & & & & & & \\
\hline & & & & & & & & & & \\
\hline Computed Der & T.64 & 2.98 & 4.01 & 5.12 & 6.08 & & & & & \\
\hline Fichan & 0.04 & 0.12 & 0.22 & 0.34 & 0.48 & & & & & \\
\hline Acocteriont & 0.08 & 0.03 & 0.00 & 0.12 & 0.16 & & & & & \\
\hline trentong & 7.56 & 2.8 & 3.77 & T.68 & 5.4. & & & & & \\
\hline & & & & & & & & & & \\
\hline
\end{tabular}




\begin{tabular}{|c|c|c|c|c|c|c|c|c|c|c|}
\hline Rent & 8721 & $i$ & & 1 & & 1 & & 1 & 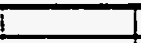 & \\
\hline Testfurd & CAFB & $i$ & & 1 & I & i & t & 1 & 1 & \\
\hline Date: & $10 \overline{4}$ & $!$ & & & & i & 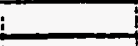 & $\downarrow$ & 1 & \\
\hline Tims: & $13: 12$ & & & & & 1 & $L$ & 1 & 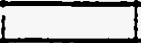 & \\
\hline Nob & Anationd d & is used & & & & & 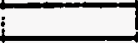 & 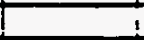 & 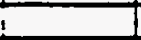 & \\
\hline Fiow Area-12 & 0.003561 & & & & & & & i & 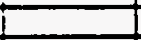 & \\
\hline Hort Fow Aron? & 0.1955 & & & & & & & & & \\
\hline Sn prosere phg & 2183 & & & & & & & & & \\
\hline S工 Tomper & 180 & & & & & & & & & \\
\hline Lg. Denoinyomis & 73.08 & 72.89 & 72.55 & 72.811 & 72.84 & 727 & 72.82 & 72.85 & 7268 & 727 \\
\hline 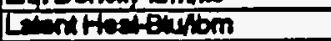 & 28.12 & 27.98 & 28.01 & 27.8 & 27.21 & 27.87 & 27.74 & 27.78 & 27.78 & 27.83 \\
\hline Spectoherighromr & 0.388 & 0.381 & 0.334 & 0.385 & 0.384 & 0.355 & 0.300 & 0.305 & 0.350 & 0.350 \\
\hline Ca. Viroostyismir & 0.31 & 0.500 & 0.31 & $-\overline{308}$ & 0.300 & 0.308 & 0.50 & 0.306 & 0.307 & 0.807 \\
\hline 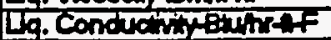 & 0.0 & $0.0 \times 3$ & 0.038 & 0.023 & 0.0007 & 0.0237 & 0.025 & $0.0 \mathrm{ES}$ & 0.008 & 0.025 \\
\hline LLG. Prend" To. & 4.34 & 4.35 & 4.34 & 4.35 & 4.35 & 4.35 & 4.35 & 4.5 & 4.3 & 4.35 \\
\hline Inforfow $R$ WE & होi & & & & & & & & & \\
\hline 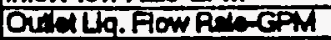 & 0.2 & & & & & & & & & \\
\hline 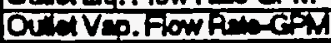 & T.1. & & & & & & & & & \\
\hline 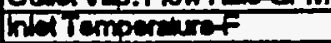 & 188.8 & & & & & & & & & \\
\hline 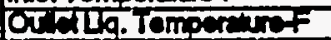 & T90.1 & & & & & & & & & \\
\hline 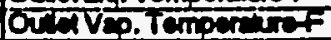 & 887.8 & & & & & & & & & \\
\hline WMAFOW RMGAM & & & & & & & & & & \\
\hline Werertint Tomp of & T18.4 & & & & & & & & & \\
\hline Wer oul Tomp & 160.0 & & & & & & & & & \\
\hline Intabomme & उुद्या & & Tanp & 787.4 & Dening & 75.00 & & & & \\
\hline Subooolingt & 1.7 & & & & & & & & & \\
\hline Fhow Obsem ion & & & & & & & & & & \\
\hline & ONET & $20 \mathrm{O}=2$ & $\angle O R=3$ & ZON=6 & ZOR=5 & $\angle O R=6$ & ZON: $=7$ & $\angle O K=B$ & ZONE9 & $201=10$ \\
\hline Hodhortwsingl) & 5552 & 56.0 & 5723 & 5724 & 5675 & 567.1 & 5555 & 563.5 & 574.8 & $5 \% .4$ \\
\hline HenLosutwas & 13.6 & 32 & 4.8 & 4.5 & 4.5 & 3.5 & 23 & 3.8 & 4.1 & 23.0 \\
\hline 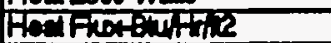 & \$स्या & 8्यात & क्वां & Sos & \$8) & 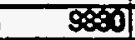 & $\mathbf{8 0 0 8}$ & 978 & 8. & 868 \\
\hline We tromo.B & T\$5.11 & 18B & TS52 & कि.ा & 188.6 & 187.4 & 197.5 & 155.8 & 18 & T84.8 \\
\hline WET Temp. ItA & 785.8 & T35. & TS32 & TS8.4 & T871 & 188 & 197.1 & 195.9 & TE.2 & T87.7 \\
\hline WeTtomp.t5F & 198.1 & 194.8 & 1972 & 198.7 & TS3.8 & 197.1 & 198.0 & 18 & दक्ष & 184 \\
\hline WeTanp.AT & 188.8 & 195.8 & 199.1 & 195.8 & 195.7 & 1972 & 187.7 & 185.7 & 1800 & क्रिय \\
\hline WE Tamp. IIL' & 1862 & 155.8 & 1946 & 1958 & 1956 & 197.4 & 187.7 & 188.8 & TिD: & Tहदर \\
\hline Intreem temp.t & 1800 & 180 & T20: & 1002 & 18ूर्य & $180 \mathrm{al}$ & 10I & 100 & Tor & 1000 \\
\hline Avo. Wertompot & 7850 & TSEd & T5.5 & कि. & Tब53 & Fम, & 757.8 & is.7 & W6 & 396 \\
\hline Avo.WaDT & 6.1 & 2.9 & 4.8 & 5.4 & 5.5 & 6.6. & 5.8 & 2.4 & 3.7 & 3.6 \\
\hline Remining suboootf & -28 & & & & & & & & & \\
\hline Extouty & 0.08 & 0.00 & 0.76 & oर्द & 0.5 & ठ.3 & 0.58 & 0.42 & 0.47 & 0.39 \\
\hline 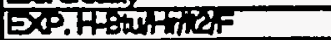 & 7563.8 & 1585.7 & 2050 & 1815X & 178.8 & 1483 & $15 \% .7$ & स्ट्य.71 & 25750 & 27072 \\
\hline COT-UVIEDH & & & & & & & & & & \\
\hline Fouk & Tर्ण.1. & T2008 & 120 & T25 & 1301 & 1250| & Tziera & 12175 & Te्राD & Tais \\
\hline 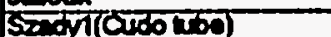 & $18 \times 3.2$ & 1887,2 & 78725 & 1878.8 & 18:06 & 18त2 & 7874.7 & 1870.8 & 1860 & 78802 \\
\hline $\operatorname{sed} \sqrt{2}$ & 16775 & 1710.0 & 7765 & 17236 & 178.5 & T715.9 & 17258 & IT7.S & 778.7 & $7 \% 5.7$ \\
\hline$\left(R^{2}\right)$ & $77 / 69$ & 74891 & 70 & 6025 & 61816 & 67586 & 55514 & CSON & 4007 & $\$ 0005$ \\
\hline (F) & T.ख्या & 1.687 & 1.96 & टाह5 & 2053 & 2518 & 2.632 & 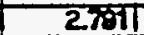 & 203 & 3.038 \\
\hline (1) & 778.7 & $32 \pi$ & 258 & 36. & 300 & 3427 & उE्टर2 & 381.11 & ऊल्या & 756.0 \\
\hline$\left(\mathrm{H} 3 \mathrm{CH}^{4}\right)$ & $18 \times 5$ & Tछू: & 187i & 18758 & T86an & 18572 & 78752 & 18355 & 188 & 1872 \\
\hline & & & & & & & & & & \\
\hline & $201=3$ & $20 N=4$ & $\angle 0 \%=6$ & $201=8$ & $201=10$ & & & & & \\
\hline DPPI Gud Temp.f & 805 & & & & & & & & & \\
\hline 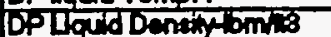 & 83.18 & & & & & & & & & \\
\hline Eeverd & & & 6 & & 9.875 & & & & & \\
\hline Mheorred Deyphd & ठ.3ठ & 7.74 & 7.897 & 2801 & 3.68 & & & & & \\
\hline Zero Cormeoton pard & -0.06 & 20.08 & $0.16 \pi$ & 0.008 & $0.0 \mathrm{sn}$ & & & & & \\
\hline Correctsdopend & 0.805 & 1.982 & 7.700 & 2.801 & 3.500 & & & & & \\
\hline Acter Dow & T.8त & 278 & 4.21 & 4.60 & 6.02 & & & & & \\
\hline 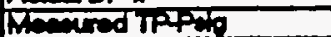 & पद्टर & & & & Eंग.8 & & & & & \\
\hline Mneured dven & 1.78 & & & & & & & & & \\
\hline & & & & & & & & & & - \\
\hline Compundory & T.Eय & 285 & 2.12 & 5.10 & 6.18 & & & & & \\
\hline Ficiont & 0.05 & 0.18 & 0.28 & 0.33 & 0.5 & & & & & \\
\hline Aoodentont & 0.04 & 0.08 & 0.00 & 0.12 & 0.15 & & & & & \\
\hline Exhriont & 1.56 & $\sum 70$ & 3.5 & 4.त & 5.47 & & & & & \\
\hline & & & & & & & & & & \\
\hline
\end{tabular}




\begin{tabular}{|c|c|c|c|c|c|c|c|c|c|c|}
\hline Runt & $1 \quad 8731$ & 1 & 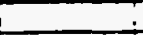 & 1 & 1 & & $\therefore$ & & & \\
\hline Test Fuid & CAF & $\div$ & & & i & & & & & \\
\hline Dete: & $i \quad 10493$ & & & & & & & & & \\
\hline Trme: & $13: 12$ & & & & 1 & 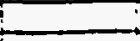 & & & & \\
\hline Note & Andy youd & is used I & 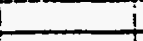 & & & & & & & \\
\hline Flow Araste & 0.005361 & 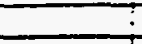 & & & $t$ & & & & & \\
\hline Heal Fow Areat & 0.19555 & & & & & & & & & \\
\hline Sa Presture oigh & $2\} 6.51$ & & & & & & & & & \\
\hline Sel Temperitrof $($ intel $)$ & 189.4 & & & & & & & & & \\
\hline Lig. Denting & 73.05 & 731 & 73.08 & 7282 & 72.85 & 72.55 & 727 & 7279 & 7278 & 72.97 \\
\hline Lenthenteribm & 28.1 & 28.05 & 28.12 & 27.891 & 28.01 & 28.01 & 27.85 & 27.871 & 27.87 & 28.08 \\
\hline Specito theneribmf & 0.383 & 0.383 & 0.388 & 0.321 & 0.334 & 0.34 & 0.355 & 0.386 & 0.386 & 0.324 \\
\hline Lig. Vifoosin tbm hh & 0.317 & 0.37 & 0.311 & 0.311 & -0.31 & 0.31 & 0.308 & 0.3081 & 0.209 & 0.81 \\
\hline Lig. Conductivin Surreff & 0.003 & 0.0238 & 0.0209 & 0.038 & 0.008 & 0.0288 & 0.0237 & $0.003 \pi$ & $0.02 \pi$ & 0.0020 \\
\hline Lig. Prend No. & 4.34 & 4.34 & 4.34 & 4.35 & 4.34 & 4.35 & 4.35 & 4.35 & 4.36 & 4.3 \\
\hline Inth fow Re-GeM & 1.2 & & & & & & & & & \\
\hline 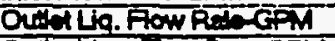 & 0.4 & & & & & & & & & \\
\hline OuAVED. Fow RN-GPM & 0.8 & & & & & & & & & \\
\hline hex Tempermer & 188.81 & & & & & & & & & \\
\hline OTHLG. Tompar trof & 789.81 & & & & & & & & & \\
\hline ortives. Temper ins & 187.9 & & & & & & & & & \\
\hline Werfow RM GPM & & & & & & & & & & \\
\hline Were trid Temo,f & 117..4 & & & & & & & & & \\
\hline Wher orier Temp.f & 17 & & & & & & & & & \\
\hline InTGomnNe & दार्टा & & Temp & 176.71 & Densiy & 76.20 & & & & \\
\hline Subooding & 1.7 & & & & & & & & & \\
\hline Fow Obsenvion & & & & & & & & & & \\
\hline 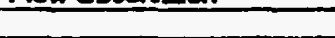 & ZONET & $20 \mathrm{~K}=2$ & $20 N=3$ & $201=4$ & ZON=5 & $\angle O N=6$ & ZONE & $20 K=8$ & ZONES & $20 n=10$ \\
\hline Heilhoutwated & 5727 & 577.6 & 574.6 & 578.4 & 573.8 & 574.4 & 578.3 & 574 & 237.8 & 153.6 \\
\hline Heat Lox-Wa: & 74.8 & 3.41 & 4.6 & & 5.1 & 4.1 & 29 & 4.5 & 6.8 & $\mathbf{2 8 . 8}$ \\
\hline Hod FW Buptis & Sक्षा & ToOt2 & 960 & 1005 & \$95 & S50 & T00्य & 950 & सिष्ठ & 203 \\
\hline Whiomp. 13F & Tुग & 755.11 & TS3. & 1938 & 756.7 & 155.4 & 155 & 2040 & 2001 & 189.7 \\
\hline We Tomp. 14 & T9.7. & किता & is5.1 & 1952 & 756.6 & 190.0 & 180 & 304.8 & काम & 797.7 \\
\hline We Tomo. $16 \mathrm{~F}$ & 195.1 & 193.4. & TSB,A & 195 & 1948 & 1945 & 15S2 & दि। & 200.7 & 1852 \\
\hline WeTtomp. I1F & 1888 & 194.8 & 1820 & 184 & 194.0 & 1955 & 155.7 & 194.81 & 198.1 & Tद्य.: \\
\hline We romp.tzt & 185.8 & 19स5 & 183.8 & 1956 & 196.7 & 1954 & 1960 & 1952 & 1832 & TE45 \\
\hline Intreen tomph & 7891 & 186.6 & 189 & 1809 & 780.01 & 18000 & 100.5 & $100 \mathrm{~A}$ & 150 & 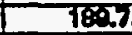 \\
\hline AWo. WN TompA & Tिका & क्रिजा & रद्ध5: & 1950 & 1020 & 755\$ & 185.7 & 183 & 150 & गूल \\
\hline AY.WEIDTF & 4.0 & 4.4 & 4.2. & 4.4. & 4.7 & 5.1 & 2.8 & 8.3 & 88 & 2.8 \\
\hline Remining Suboodf & -6.0 & & & & & & & & & \\
\hline Exherly & 0.08 & 0.18 & 0.21 & 0.37 & 0.48 & 0.68 & 0.68 & 0.75 & 0.7 & 0.82 \\
\hline EXo.PHE, & 2015:2 & स्2512 & 2050 & 2008 & हाँेत्ये & 19618 & 201511 & TI87.8 & 34 & 6780 \\
\hline COW:UTEDH & & & & & & & & & & \\
\hline Jotk & 730.11 & T\$5T.7 & Tद्याओ & 7310.51 & 720.6 & 72839 & 72835 & 3.7 & 37.7. & E5.5. \\
\hline 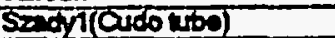 & 1800 & 187.1 & 1877. & 1889.7 & 7875.8 & 18753 & 1835 & 5.7. & 8.7 & 35. \\
\hline $\operatorname{sen} x$ & Tारण & 1721.7 & 1711.9 & 1727.8 & 178.3 & 1718.4 & 1737 & 36.7 & 55.7 & 84.0 \\
\hline (स्व) & क्जान & 40082 & 3535ा & 31840 & 28910 & 22518 & 18232 & 18282 & Tिद्रता & $18 \times 25$ \\
\hline (F) & 1.538 & 2.013 & 2368 & $2 \approx 2$ & 287 & 3.085 & 3288 & 3.23 & 3.208 & 3258 \\
\hline (1) & 155.8 & 1712 & 2078 & ट्राइ & 353 & 2726 & 2000 & 28001 & 303 & 2603 \\
\hline$(130 \mathrm{~d})$ & TE88.8 & 7878.8 & $78 \% 5$ & 18925 & 1873 & 1874.8 & 18815 & 8875 & 1887.6 & 8887.0 \\
\hline & & & & & & & & & & \\
\hline & $20 \mathrm{~N}=2$ & $20 N=7$ & $201=6$ & $20 N=8$ & $201=10$ & & & & & \\
\hline DP lequd temp.f & 828 & & & & & & & & & \\
\hline 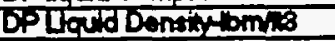 & E288 & & & & & & & & & \\
\hline Exvition & दा & (4) & (B) & 8 & 9.875 & & & & & \\
\hline Whated DFend & 0.5 & 1.388 & 2518 & 3.46 & 4.88 & & & & & \\
\hline zerocomectomperd & 20.00 & $=0.083$ & क.ाजा & 0.008 & $0.08 \pi$ & & & & & \\
\hline Cortecud Daphd & 0.560 & 1.45 & 2167 & 3.18 & 4.815 & & & & & \\
\hline Act DPU & 1.46 & 238 & 3.5. & $\$ 89$ & 9.9 & & & & & \\
\hline Whenred TfPLig & टंख्ड & & & & 2ट15.8 & & & & & \\
\hline Neceured over it & -1.38 & & & & & & & & & \\
\hline & & & & & & & & & & \\
\hline Computed DPa & T.ख্জ| & दूव & 32.1 & 3.83 & 7.75 & & & & & \\
\hline Fichont & 0.02 & 0.007 & 0.18 & 02 & 0.5 & & & & & \\
\hline Aooterrion & 0.02 & 0.01 & 0.08 & 0.08 & 0.00 & & & & & \\
\hline Eloritont & 7.35 & 23 & 3.06 & 3.68 & द.दा & & & & & \\
\hline & & & & & & & & & & \\
\hline
\end{tabular}




\begin{tabular}{|c|c|c|c|c|c|c|c|c|c|c|}
\hline Runs & 8721 & & $!$ & - & - & I & 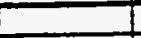 & & & \\
\hline Toqf Fuid & CAF & & & & & $i$ & 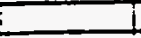 & & & \\
\hline Date: & 101533 & & & & & 1 & 1 & & & \\
\hline Tome: & $8: 34 \quad 1$ & & & & & 1 & $L$ & & & \\
\hline Note & Anstioge & it is used & & & & & 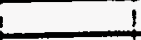 & & & \\
\hline Flow Arane & 0.00586 & & & & & & & & & \\
\hline Had Fow Areare & 0.1955 & & & & & & & & & \\
\hline Sel piesturepal & 279.4 & & & & & & & & & \\
\hline Sel Tempere & โ80.6 & & & & & & & & & \\
\hline Lل. Dentatom, & 73.08 & उ.छा & 7328 & 72.87 & 73 & 73.031 & 72.68 & 727 & Fe्ना & 726 \\
\hline Lenthateriom & 28.t2 & 28.81 & 28.8 & 27.84 & 28.051 & 28.00 & 27.78 & 27.87 & 27D & 27.5 \\
\hline 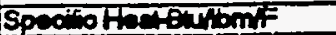 & 0.328 & 0.331 & 0.331 & 0.384 & 0.38 & 0.3981 & 0.360 & 0.355 & 0.355 & 0.32 \\
\hline [0. Visoovitiom/Th & 0.311 & 0.314 & 0.314 & 0.3001 & 0.37 & 0.371 & $\overline{0.307}$ & 0.300 & $0.30 \mathrm{3}$ & 0.300 \\
\hline 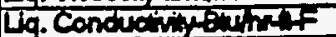 & $0.0 \times 6$ & 0.03 & 0.00 & 0.03 & 0.0283 & 0.028 & $0.0 \times 5$ & $0.0 \times 3$ & $0.08 \pi$ & 0.028 \\
\hline Lig. Prund" No. & 4.34 & 4.34 & 4.30 & 4.35 & 4.34 & 4.3 & 4.35 & A.SS & 4.8 & 4.55 \\
\hline Ind flow PW-CPM & 5.7 & & & & & & & & & \\
\hline OClNLL. Fow R-GPM & 0.8 & & & & & & & & & \\
\hline OUAV VE. HOW R'A-CPM & 2.5 & & & & & & & & & \\
\hline Inticemper & 788 & & & & & & & & & \\
\hline 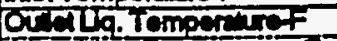 & 780 & & & & & & & & & \\
\hline or Vep.Tamoner & 187.0 & & & & & & & & & \\
\hline Wer Flow RINGAM & & & & & & & & & & \\
\hline 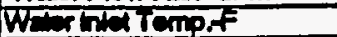 & 105.0 & & 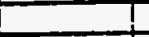 & & & & & & & \\
\hline Werroult temper & 1618 & 1 & 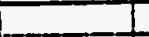 & & & & & & & \\
\hline Irichmine & गठठका & & temp & 183.71 & IDentity & 74.53 & & & & \\
\hline Suboodingt & 25 & & & & & & & & & \\
\hline Flow Observison & & & & & & & & & & \\
\hline & ZORES & ZONEZ & ZONIES & ZOKE & $2012=5$ & $2 \mathrm{ON}=6$ & $20 N=7$ & 2ON=8 & 2010 & $201=10$ \\
\hline Hed inpotwathon) & 873.6 & 861.6 & 856 & 858 & 8525 & 850.7 & 858.8 & 868.1 & 8827 & 851.1 \\
\hline Hed Lostwe's & 127 & & 4) & 4 & 3.8 & 28 & 1.9 & 3) & 3.0 & 27.5 \\
\hline Hen Finterthine & 75ाराप & 76855 & Tद\&8 & $148 \%$ & Th778 & 747L & 7487 & 75018 & 7Rogn & Toret \\
\hline We Tremp.i3F- & T:5.5 & \$\$.ा & TS5:2 & 7835 & TS5.7 & 197] & 798.1 & 787.5 & 78..ा & 1852 \\
\hline Wrtomp.84t & 788.6 & 195.7 & 79836 & 797.81 & 79.50 & 1972 & 7981 & T88.3 & 780 & 799.8 \\
\hline WrTtomp. 16F & Tि1.5 & 155.0 & 153.5 & 157.01 & 195 & 197. & 153.0 & 1562. & 783.3 & 785.5 \\
\hline WE Tomp. If & 1609 & 150.81 & दिला & 1856 & 156्य & 150.61 & 786.5 & 1805 & 7560 & 1820 \\
\hline WeTtamp.tat & 1800 & 186.81 & 7556 & 18331 & 18..1 & 188.31 & 188.8 & 197.6 & 786 & TधमA \\
\hline Irean tempdF & 185 & 18835 & 18350 & 780.ी & 1830 & 1805 & 7856 & I80, & क्षs & 180 \\
\hline Akp. Wa'tempd & 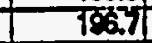 & Tक6. & T5्5) & 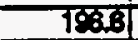 & $1 क ् 6$ & $\mid 87$ & 158. & 197.7 & सू० & 780 \\
\hline ALP.WCOTA & 6.7 & 7.4 & 8.8 & 5.8 & 5.8 & 6.8 & 6.8 & 6.6 & 5.0 & 4.6 \\
\hline Reminin suboodt & -0.2 & & & & & & & & & \\
\hline Exh outhy & & 0.08 & 0.0 .0 & 0.6 & 0.12 & 0.76 & 0.10 & 03 & 025 & 628 \\
\hline ExP.मEM & 2in.o & कामी & 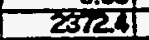 & Z565. & 250.08 & 2773.8 & दह5डा & C25388 & 2530 & 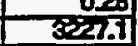 \\
\hline CoT:OUIEDH & & & & & & & & & & \\
\hline Jouk & 15658 & T68,01 & TAFाद. & 78 sint & Т358: & Tिस:01 & 73058 & Tered & 78030 & 1837 \\
\hline 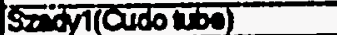 & 2008 & 20.JP & २०णन & 230.10 & \%8.6 & Zस्टा & 23051 & क्या. & $76: 50$ & TE.7 \\
\hline $\operatorname{sed} 2$ & 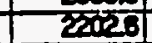 & होत. & 2100 & 278.8 & ¿द्ध一 & 22ख. & 2द्याट्2 & 2085 & 1850 & 783.7 \\
\hline (R्य) & 212007 & 207501 & अणत्र & 187884 & 180037 & 18538 & 783080 & I721621 & TFizis: & trदि \\
\hline$(F)$ & 1.068 & 1.334 & 7.598 & 1.787 & 1.82 & टक्णा & 2.758 & $22 \pi$ & 22 & $22 i$ \\
\hline (F) & 396 & 700.11 & 47.7 & 5321 & 577d & 617.5 & 6508 & 6830 & 6850 & 6835 \\
\hline$(1 \mathrm{~B} 30 \mathrm{~T})$ & 2028 & Z3505 & 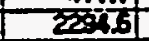 & अं7द्या & क्ष.7.7 & 2207.8 & 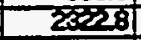 & Z2ता & zist & 2821 \\
\hline & & & & & & & & & & \\
\hline & $201 n=2$ & ZON=6 & $20 N=6$ & $201=38$ & $201=10$ & & & & & \\
\hline DPTgud temp.t & 79.1 & & & & & & & & & \\
\hline 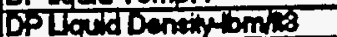 & 93.83 & & & & & & & & & \\
\hline Ehertion & 2 & 4 & 8 & 81 & 9.87 .5 & & & & & \\
\hline Wherred DPupard & 02001 & 0.7501 & $T .18 \pi$ & T.83 & 250 & & & & & \\
\hline $200 \mathrm{Con}$ cione odd & -0.008 & -0.002 & 0.02 & ठ.ण्टा & 0.05 & & & & & \\
\hline Corrend DP 4 यd & 0.865 & 0.84 & 1.171 & 1.816 & टर्डs & & & & & \\
\hline Aon Dor & 9.88 & 3.6ी। & 6.80 & 6.01 & 8.00 & & & & & \\
\hline 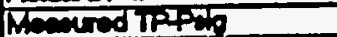 & ¿ंब्रा & & & & सब & & & & & \\
\hline Weenred ovellit & 0.78 & & & & & & & & & \\
\hline & & & & & & & & & & \\
\hline Compurad Das & 2.7 & 2.08 & 5.0 & 7.7 & छ. प. & & & & & \\
\hline Ficion- & 0.10 & 0.0 .01 & o.9त & 7.45 & हो & & & & & \\
\hline Aockerrion-1 & 0.16 & 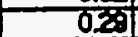 & 0.3 & 0.46 & 0.8 & & & & & \\
\hline Enctonth & 1.82 & 3.21 & 4.6] & 5.70 & 6.8) & & & & & \\
\hline & & & & & & & & & & \\
\hline
\end{tabular}




\begin{tabular}{|c|c|c|c|c|c|c|c|c|c|c|}
\hline Runs & 8751 & & & & & & & & & \\
\hline Toet Fuid & C4F 8 & 1 & & & 1. & $\perp$ & & & & \\
\hline Deto: & 1 10/533 & 1 & & & i & 1 & & & & \\
\hline Time: & 12.051 & & & & & & & & & \\
\hline Not & Anatyiced: & is used & & & & & & & & \\
\hline Fow AYaAt2 & 0.00386 & & & & & & & & & \\
\hline Hey fow Areat? & 0.18565 & & & & & & & & & \\
\hline Sil prosureptig & 219 & & & & & & & & & \\
\hline 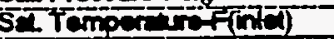 & 190.8 & & & & & & & & & \\
\hline L. bentinyomis & 7297 & 73.081 & 73.08 & 72.70 & 72.87 & 72.321 & 7234 & 728 & $\frac{72.2}{301}$ & 7270 \\
\hline Whithesemom & 28.08 & 28.12 & 28.12 & 27.80 & 27.84 & 27.801 & 27.67 & $27 . \pi$ & 27.76 & 27.06 \\
\hline 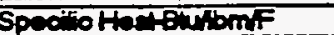 & 0.39 & 0.3389 & 0.333 & 0.396 & 0.334 & 0.334 & 0.357 & 0.356 & 0.326 & 0.356 \\
\hline ig. Viecoth fom & 0.37 & 0.391 & 0.317 & 0.308 & 0.300 & 0.31 & 0.305 & 0.306 & 0.306 & 0.308 \\
\hline Lo. Conducting the & 0.0238 & 0.0250 & 0.0200 & 0.02371 & 0.0238 & 0.0238 & $0.0 \mathrm{c}$ & 0.0025 & 0.023 & 0.0567 \\
\hline La. Prendino. & 4.34 & 4.34 & 4.34 & 4.35 & 4.35 & 4.35 & 4.35 & 4.35 & 4.36 & 4.35 \\
\hline ntoflow R-GPM & 4.8 & & & & & & & & & \\
\hline 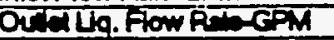 & 0.8 & & & & & & & & & \\
\hline OCI VED. Fow RM-GPM & 2.1 & & & & & & & & & \\
\hline 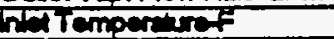 & 788.7 & & & & & & & & & \\
\hline 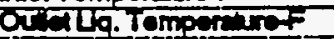 & 789.6 & & & & & & & & & \\
\hline ocelve. Tomperetrof & 188.2 & & & & & & & & & \\
\hline 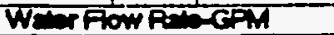 & & & & & & & & & & \\
\hline Werthla Tompof & 100.9 & & & & & & & & & \\
\hline Whar ach Tomp.F & 171.1 & & & & & & & & & \\
\hline 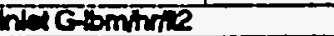 & 851378 & & Tamo & $184.5 \pi$ & iDantiny & 74.32 & & & & \\
\hline Subooolingt & 1.6 & & & & & & & & & \\
\hline Fow Obsewrion & & & & & & & & & & \\
\hline & $20 \mathrm{Ni}=\mathrm{T}$ & $2 O N=2$ & ONES & ONiE4 & ZONE5 & $\angle O N=6$ & 2ONE & LONE8 & $\angle O N=9$ & $20 N=10$ \\
\hline 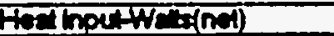 & 861 & 8622 & 865.5 & 864.4 & 860.7 & 859.8 & 861.8 & 8572 & 435.6 & 102.1 \\
\hline Heelostwe: & $1 \hat{2}$ & 2.8 & 3.7 & 3.8 & 3.7 & 2.7 & 1.8 & 2.8 & 3.4 & 77.4 \\
\hline Hod Fin & 1485 & 74918 & 75000 & 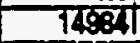 & 1450 & 72800 & Tदक5 & 15082 & 7501 & $17 \%$ \\
\hline We Tomp. 164 & $1 \%$ & 998.7 & 750.0 & 796.7 & 796.8 & 797.7 & 798.0 & T97.5 & किस & $180, \mathrm{~A}$ \\
\hline WE TOMp. If & 997.2 & 796.2 & 797.6 & Fot.o & 997.1 & 1978 & 198.8 & 798.2 & 7857 & 7908 \\
\hline Wล Temp. 85 & 187.7 & 195.8 & 198.8 & 197.8 & 190.8 & 797. & 798.8 & 188 & 180.1 & Tनु० \\
\hline WE Tomp.tif & 188.7 & 19000 & 194.8 & 195.8 & 196.7 & 190.9 & 190 & 1972 & 1890 & 18005 \\
\hline We'tomp.taf & 187.4 & 187 & 180.0 & 198.8 & 198.6 & 197.1 & 190.11 & 798.2 & 1942 & 18006 \\
\hline hatem Temp.F & $189 . \pi$ & 189.2 & 1603 & 1906 & 180.1 & 1856 & 791.8 & 781. & की & โक्ण0 \\
\hline Av. Wh Tamof & 997. & 7972 & 196.1 & 196.0 & $196 \pi$ & 79756 & 19809 & 1975 & 1940 & 1804 \\
\hline AL. WEDT .F & 6.7 & 7.2 & 6.1 & 5.7 & 5.7 & 6.9 & 7 & 6 & 32 & 0.1 \\
\hline Rem Ining Scboooff & -1.5 & & & & & & & & & \\
\hline Edachy & 0.02 & 0.05 & 0.00 & 0.18 & 0.18 & 0.2 & 0.24 & 0.28 & 0.80 & 0.8 \\
\hline 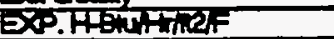 & एवin & 2068.7 & 2460.7 & $28(18)$ & 28403 & 2167.7 & 2दि.8 & 2465 & 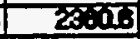 & \\
\hline CONDUEDH & & & & & & & & & & \\
\hline Jinotk & 15268: & 74880 & 7650 & 7457.6 & 73978 & B367.8 & 7358 & 7828 & 1623 & 162210 \\
\hline Sadvil(व, & zoti & 2316.9 & 2028 & 2335 & 236.1 & Z्सा.T & 2336.2 & 1620 & 182. & 16220 \\
\hline $50 \mathrm{~d}+2$ & 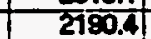 & 2188.4 & $21 \overline{8}$ & 2214.4 & 2028 & 2190.5 & 228.0 & 1620 & 1620 & 1620 \\
\hline (स्म) & 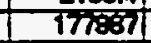 & 172418 & 165848 & 181284 & 153632 & 147041 & 145518 & 715558 & 743518 & 1425T: \\
\hline (F) & 1232 & 1.5 & 1.741 & 1.818 & 2.075 & 23:4 & 232 & 232 & 28 & 232 \\
\hline (स०का & 319.7 & अवा & 7656 & 5023 & $543 \pi$ & 5505 & $\overline{6003}$ & 6003 & 600.5 & 6003 \\
\hline (स:504) & ख्या7. & 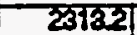 & 2016.6 & 238.7 & zका & 2315 & $203(4)$ & 2334.4 & $23 x$ & 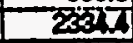 \\
\hline & & & & & & & & & & \\
\hline & $20 N=2$ & ZONET & $\angle O N=8$ & 2010.88 & $20 k=10^{\circ}$ & & & & & \\
\hline DP liqud Tomp.f & 83.4 & & & & & & & & & \\
\hline DP Laud Denotymis & 8272 & & & & & & & & & \\
\hline Eovition- & & & & & 9.875 & & & & & \\
\hline Wenund DP 4 da & O38 & 0.882 & 7.37 & 2000 & 2783 & & & & & \\
\hline Zoro contocions pesd & -0.058 & $=0.032$ & 0.02 & 0.0ट्य & 0.034 & & & & & \\
\hline Compond Dp pod & 0.388 & 0.8001 & 1.357 & 2078 & 2720 & & & & & \\
\hline Actudora & 1.88 & 3.88 & 4.5 & 8.06 & 7.18 & & & & & \\
\hline Weored tip & सार्ण & & & & 218.8 & & & & & \\
\hline Whoured owedilt & -0.50 & & & & & & & & & \\
\hline & & & & & & & & & & \\
\hline Compued be & 201 & 3.50 & 5.41 & 7.04 & 8.72 & & & & & \\
\hline Frotom & 0.17 & 0.4 & 0.70 & 1 & 1.52 & & & & & \\
\hline Acoderciont & 0.16 & 0.24 & 0.33 & 0.42 & 0.46 & & & & & \\
\hline Exrionth & 7.68 & 3.06 & 430 & 5.5 & 6.78 & & & & & \\
\hline & & & & & & & & & & \\
\hline
\end{tabular}




\begin{tabular}{|c|c|c|c|c|c|c|c|c|c|c|}
\hline Runt & 878 & I & & $i$ & & & $I$ & & 1 & \\
\hline TOA Find & CAFB & & & & & & & & & \\
\hline Data: & 1बए5क्डा & & & & & & 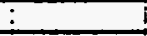 & & 1 & \\
\hline Timo: & $12: 051$ & & & & & & & & & \\
\hline Now & Analyied d & dis used & & & & & & & & \\
\hline Flow ARCAR & 0.0058 & & & & & & & & & \\
\hline Hed Fow A Seat? & 0.19606 & & & & & & & & & \\
\hline SI Promureptig & 2021 & & & & & & & & & \\
\hline SL tomporne & 190.71 & & & & & & & & & \\
\hline Lq. bentomis & 73.05 & 78.111 & $\frac{73.111}{28.21}$ & 7268 & 7281 & $\frac{72 \pi}{2781}$ & 7262 & $\frac{72.73}{27891}$ & 72.70 & 72.68 \\
\hline Limingerstom & 28.1 & 28.141 & $28.14 \mid$ & 27.78 & $\frac{27.9}{0351}$ & & & & 27.80 & 27.78 \\
\hline 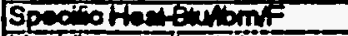 & 0.33 & 0.3831 & 0.33 & 0.386 & 0.335 & 0.325 & 0.396 & 0.355 & 0.356 & 0.356 \\
\hline 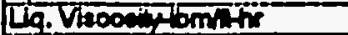 & 0.379 & 0.3121 & 0.312 & 0.507 & 0.308 & 0.307 & 0.308 & 0.307 & 0.506 & 0.307 \\
\hline 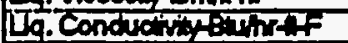 & 0.000 & 0.0030 & 0.0000 & 0.020 & $0.0 \mathrm{Bi}$ & 0.087 & 0.0206 & 0.0027 & 0.0057 & $0.0 \times 6$ \\
\hline LG. Prend" No. & 0.0 .34 & 4.34 & 4.34 & 4.35 & 4.35 & 4.36 & 4.35 & 4.35 & 4.30 & 4.35 \\
\hline 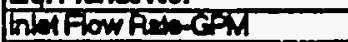 & 3.9 & & & & & & & & & \\
\hline OCALG. Fow ROAPM & 0.8 & & & & & & & & & \\
\hline Ou & 1.9 & & & & & & & & & \\
\hline Inth tamperitrof & 788.6 & & & & & & & & & \\
\hline 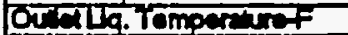 & isen & & & & & & & & & \\
\hline 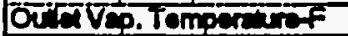 & 7885 & & & & & & & & & \\
\hline 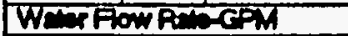 & & & & & & & & & & \\
\hline Wower line temp,ff & 111.8 & & & & & & & & & \\
\hline Wex owel Tomp f & 172.5 & & & & & & & & & \\
\hline Tha Glbmhath? & 650 & & tremp & 787.6 & Deneity & 74.32 & & & & \\
\hline Suboocingt & 2री & & & & & & & & & \\
\hline Fow Obeanstion & & & & & & & & & & \\
\hline & SNET & STE? & $\angle O N=8$ & $20 N=2$ & $\angle O R=5$ & $Z O N=6$ & $201=7$ & $\angle O N=8$ & $\angle O N=9$ & $20=10$ \\
\hline 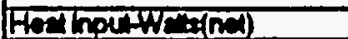 & 868.4 & 870.8 & 868.5 & 858.8 & 860.0 & 8827 & 887.8 & 57.5 & 88.8 & 97.2 \\
\hline Henlose-WE & 128 & & 4 & 2.1 & 4 & & 1.8 & 2.8 & 3.4 & 18.9 \\
\hline Hod fionturtis & $750 \overline{8}$ & 75065 & 75080 & 7600 & 14976 & 148050 | & 74850 & sर्जो & $770 \mathrm{~m}$ & 7680 \\
\hline W: Tomo. IF & $18 \%$ & 798.8 & 195.7 & 397.8 & 79800 & 788, & 797.5 & 180 & दिर्ड़ी & 9905 \\
\hline Writomp. IL & T87.t & 1983 & 7978 & 797.9 & 7975 & 798.8 & 797.7 & 796.4 & T912 & T80.7 \\
\hline We tomp. 167 & TुB.t & 753.11 & 1980.0 & 997.8 & 197 & 198 & 1982 & 185.8 & 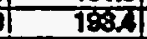 & 781. \\
\hline We'tomp. Af & 188.6 & 193.01 & 199.7 & 190.0 & 156.5 & 198.11 & 798.7 & 19.11 & द्वात्मा & 7500 \\
\hline Wr'Tamp. VIA & 187.8 & 186.01 & 18500 & 7973 & 1972 & 187.9 & 188.7 & 78n, & Iकाना & 780. \\
\hline nemom tampt & 760. & 189.2 & 1802 & 780.8 & 790.8 & 980.7 & 767 & 1506 & 180.5 & 780.6 \\
\hline ALP.WeT Temort & 187.5ी & 1972 & 750 & 1974 & 197.1 & 1922 & 1582 & 106 & कारी & 30.8 \\
\hline ALO. WADOT.F & 7 & 7.3 & 6 & 5.9 & 6.1 & 6.8 & 6.5 & 3.8 & 1.8 & \\
\hline Reoming Scboodf & -1.7 & & & & & & & & & \\
\hline Exh chathy & 0.02 & 0.07 & 0.11 & 0.16 & 0.2 & 0.35 & 0.20 & 0.32 & 0.38 & $\mathbf{0 . 3 8}$ \\
\hline 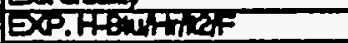 & टाओम & 30150 & 2460 & \$5T4.8. & 34856 & टाष्ञस & एवस्टर्या & 25322 & 1501.7 & \\
\hline CONiPUIEDH & & & & & & & & & & \\
\hline$J=100 x$ & 75975) & $75 \% 8.8$ & रदिध5| & 748.8 & 74832 & 7458] & दिाठण & Toख़ी & 5780 & 518.8 \\
\hline 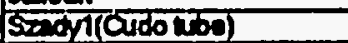 & zक्ष. & 3650 & 2325 & 208.7 & 2383.8 & 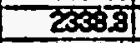 & $23 \pi$ & 188 & 835 & 897. \\
\hline $\operatorname{sen} 1 / 2$ & 21828 & 2187 & 219.5 & $22 \pi 16$ & 20006 & 20819 & 205.8 & 77305 & 0.1 & $\$ .7$ \\
\hline (सLO) & Tिकास & 738746 & 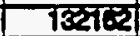 & 12713 & 12062 & 11बसाठ5 & 107800 & 10185 & क्जाराया & 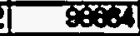 \\
\hline & 12रो & 1.53 की & 1.85 & 2.077 & 2.195 & 2342 & 2478 & 2508 & 2.68 & 2.64 \\
\hline (1) & 27रमी & 3422 & 20030 & 47.7 & 756 & 512.7 & Eम66 & 67.7 & Egan & 6827 \\
\hline$\left.(A: O)^{2}\right)$ & 232006 & 20200 & $3 \mathbf{3 1 7 4}$ & 20252 & द्सक्र & 205 & एका & 787201 & 732 & 7278 \\
\hline & & & & & & & & & & \\
\hline & $20 \%=2$ & $\angle O N=4$ & $20 \%=6$ & $20 N=8$ & $20 \pi=10$ & & & & & \\
\hline DPPloud tomp.f & 828 & & & & & & & & & \\
\hline 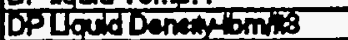 & 82.87 & & & & & & & & & \\
\hline Everiont & & & & & 9.875 & & & & & \\
\hline 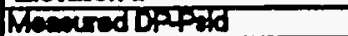 & 0.307 & $0 . \%$ & 1.5त & $2383 \pi$ & 3.1 & & & & & \\
\hline 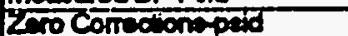 & -0.068 & -0.0 .2 & 0.02 & 0.02 & 0.002 & & & & & \\
\hline 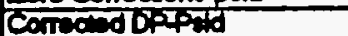 & 0.805 & 0.00 & 7.351 & 2508 & 3.000 & & & & & \\
\hline Actw Don & 7.68 & 3.14 & 2506 & 6.59 & 8,5, & & & & & \\
\hline 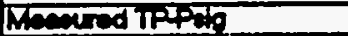 & .2002 & & & & 30,7 & & & & & \\
\hline Menred overalla & -0.00 & & & & & & & & & \\
\hline & & & & & & & & & & \\
\hline Compind Dax & 1.80 & 3.40 & (2.5 & 6.37 & 7.2 & & & & & \\
\hline Frotont & 0.12 & 0.35 & 0.6 & 0.5 & 7.16 & & & & & \\
\hline Acocheriont & 0.17 & 0.18 & 0.25 & 0.32 & 0.82 & & & & & \\
\hline Enclont & 7.68 & 280 & 2.08 & 5.32 & 8.46 & & & & & \\
\hline & & & & & & & & & & \\
\hline
\end{tabular}




\begin{tabular}{|c|c|c|c|c|c|c|c|c|c|c|}
\hline Runt & 877 & 1 & & & 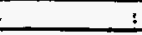 & 1 & 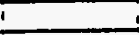 & 1 & 1 & \\
\hline Tost Fuid & CAf 8 & 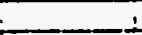 & & - & i. & & j. & i & I & \\
\hline Data: & 10593 & & $\vdots$ & $\vdots$ & $i$ & $i$ & $i$ & i & $\perp$ & \\
\hline Time: & $14: 361$ & & & & & & & 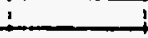 & & \\
\hline Nob & Andricado & Af is used & & & & & & & & \\
\hline Flow Arest? & 0.00336 & & & & & & & & & \\
\hline Host Fow Areat? & 0.19351 & & & & & & & & & \\
\hline 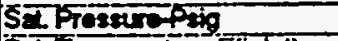 & -201 & & & & & & & & & \\
\hline 5. Tomper & 190.7 & & & & & & & & & \\
\hline Lig. Dentiblomin & 7297 & 72.95 & 73.05 & 72.681 & 7289 & $72 \pi$ & 72.62 & 72.68 & 72.73 & 72.57 \\
\hline Lind thentembin & 28.08 & 28.01 & 28.1 & 27.781 & 27.82 & 27.811 & 27.74 & 27.78 & 27.69 & 27.09 \\
\hline 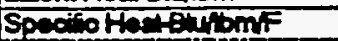 & 0.334 & 0.381 & 0.333 & 0.365 & 0.389 & 0.3551 & 0.386 & 0.366 & 0.35 & 0.396 \\
\hline iq. Visootingming & 0.31 & $0.3 \pi$ & 0.317 & 0.307 & 0.300 & 0.307 & 0.305 & 0.307 & 0.307 & 0.306 \\
\hline 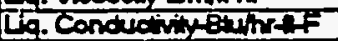 & $0.0 \times 3$ & 0.0038 & 0.000 & $0.0 \times 68$ & 0.0231 & 0.023 & 0.0286 & 0.0236 & 0.003 & 0.0203 \\
\hline Llq. Prandt No. & 4.34 & 4.34 & 4.34 & 4.35 & 4.35 & 4.35 & 4.35 & 4.35 & 2.55 & 4.35 \\
\hline Inte Fow Rate-GAM & 3 & & & & & & & & & \\
\hline Ould Lig. Fow RathaM & 0.6 & & & & & & & & & \\
\hline Dutu Vso. Fow Rele-GPM & 1.8 & & & & & & & & & \\
\hline That tomoer irs of & 188.7 & & & & & & & & & \\
\hline 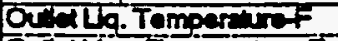 & 1928 & & & & & & & & & \\
\hline Oratvo. Tomperser & 188.5 & & & & & & & & & \\
\hline WMA Fiow Ran-GPM & & & & & & & & & & \\
\hline Whar hint Temp.F & 119.8 & & & & & & & & & \\
\hline Wer oult Temp.f & 173.5 & & & & & & & & & \\
\hline IntGommé & 531750 & & Tamp & 184.7] & Deniny & 34.21 & & & & \\
\hline Suboooling & 2 & & & & & -1 & $L$ & & & \\
\hline Fow Obsenvion & & & & & & & & & & \\
\hline & ZONE=? & ZONEZ & $20 \mathrm{KN}=8$ & 20104 & ZONES & $\angle O N=6$ & ZON $=7$ & ZONIE8 & ZONES $=9$ & $\angle O N=10$ \\
\hline 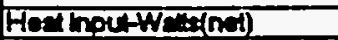 & 855.7 & $8 \% 5.4$ & 869.1 & 868.7 & 887.7 & 858.7 & 542.8 & 200.6 & 81.0 & 89.8 \\
\hline HeALoseWE"t: & 127 & & 4 & 4.1 & 4 & 28 & 1.8 & 25 & 3.4 & 78.8 \\
\hline Hen Fixb'sthis & 75006 & 15001 & $750 \mathrm{0}$ & 75038 & 1505 & 15021 & क्साता & 347 & 7दा4 & 7658 \\
\hline WeTromp.135 & 153.1. & 198.7 & 198 & 197.5 & 797.1 & 19.11 & 155.5 & 19टरा & T8T.4 & T90.7 \\
\hline WE Temo.rat & 197.1 & 198.2 & 797.5 & 198.7 & 197.7 & 198.2 & 195.8 & 15250 & 797.81 & 180.8 \\
\hline WT Themp. 16F & 198.1 & 198.8 & 139 & 198.8 & 197.11 & 1972 & 1888 & 198.7 & 1865 & T822 \\
\hline WETramp. IIF & 188.7 & 1989 & 194.7 & 198.7 & 198,6 & 188 & 185.8 & 1925 & 7812 & 180.7 \\
\hline Wet Tomp. Hat & 197.8 & 158.9 & 198.1 & 197. & 187.8 & 188 & 18.1 & 189 & 193 & 980.0 \\
\hline Intrem temp.f & 18.7 & 189.8 & -1892 & 1838 & 78023 & 180.7 & 101 & 1808 & 1000 & 192 \\
\hline Awo. Whitrompt & Tड़/2 & 1972] & 156.1 & 197.7 & 1972 & 198.T & 1550 & 1920 & 793 & 791 \\
\hline AV. WeIDT.F & 6.8 & 6.7 & 6 & 62 & 6.8 & 6.7 & 45. & 1.9 & 12 & 0 \\
\hline Romining suboooff & 3 & & & & & & & & & \\
\hline Excowity & 0.04 & 0.08 & 0.15 & o.zi & 6.2.7 & 0.38 & 0.37 & 0.3 & 0.801 & 0.39 \\
\hline 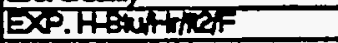 & द्वाठ & ट210 & 2518.8 & 2710.8 & 2383.8 & 2321 & 2103.1 & 178.8 & Tex] & (2) \\
\hline COM:PUIEDH & & & & & & & & & & \\
\hline Ja ôk & 7ET.6.6 & 1587. & 15518 & 75356 & 150.6 & 150.71 & 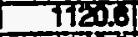 & E21 & 7652.1 & 475.0 \\
\hline 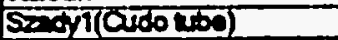 & 2324. & 200.8 & 230.4 & 237.1 & 238 & 234.81 & 18102 & 17a & 730.01 & 780.0 \\
\hline sodr & 2180.8 & 2183.81 & 218.2 & 22289 & खृाइा & 2200.51 & 16828 & 800 & 3512 & 67.8 \\
\hline (FDO) & T10162 & 1040. & 97817 & 82074 & 8532 & 790001 & 73810 & Tôs: & ESor & est. \\
\hline (F) & 1.385 & 1.685 & 1.802 & 2200 & 2400 & 257 & 2.63 & 2750 & 2.78 & 23 \\
\hline (स) & 260.8 & 3043 & 3572 & 37.1 & 7256 & 7501 & 4840 & 46.7 & 5017 & 6027 \\
\hline$\left(\mathrm{H}^{3} 3 \mathrm{C}^{4}\right)$ & 2325 & स्या & 20256 & 23105 & 23.1.1 & 23557 & 1830 & 170.7 & 6742 & 77.1. \\
\hline & & & & & & & & & & \\
\hline & $201=3$ & $201=4$ & $20 \times=6$ & $\angle O N=8$ & $\angle O N=10$ & & & & & \\
\hline DPRoud temp.f & 83.5 & & & & & & & & & \\
\hline 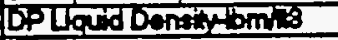 & S27 & & & & & & & & & \\
\hline ENention & & 4 & 6 & & $9.8 \%$ & & & & & \\
\hline Mh:ared DPप & 0302 & 1.101 & 1.8.01 & 2750 & 3585 & & & & & \\
\hline 2 ro com chompind & $\$ 0.068$ & 0.002 & 0.02 & $0.0=1$ & 0.034 & & & & & \\
\hline 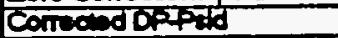 & 0.35 & 1.1003 & 1.85ा & 2क्3 & 3.5008 & & & & & \\
\hline 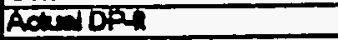 & 7.88 & टखा & 4.01 & 4.5 & 5.68 & & & & & \\
\hline Wheored TPA & 20 & & & & 3014 & & & & & \\
\hline Masered overith & -0.78 & & & & & & & & & \\
\hline & & & & & & & & & & \\
\hline Comprad Dax & $9 . \pi$ & 3.11 & 4.371 & 5.70 & 7.24 & & & & & \\
\hline Frotion- & 0.06 & 0.2या & 0.45 & 0.84 & 0.51 & & & & & \\
\hline Acooterriont & 0.08 & 0.13 & 0.10 & 0.22 & 0.23 & & & & & \\
\hline Exwiont & 154 & 278 & 3.78 & 4.88 & 6.23 & & & & & \\
\hline & & & & & & & & & & \\
\hline
\end{tabular}




\begin{tabular}{|c|c|c|c|c|c|c|c|c|c|c|c|}
\hline Runt & $1 \quad 878$ & T & 5 & \pm & $\therefore$ & I & i & & I & & \\
\hline Tort Find & CAFB & & & & 1 & & 1 & & I & & \\
\hline Dada: & $10 \sqrt{533}$ & & & & $i$ & & & & 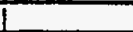 & & \\
\hline Time: & $14: 36$ & & & & & & & & & & \\
\hline Not & Anstios & dis used & & & & & & & & & \\
\hline Fow Areate & 0.0056 & & & & & & & & & & \\
\hline Hod Fiow Areare & 0.1855 & & & & & & & & & & \\
\hline SElProters & 257.7 & & & & & & & & & & \\
\hline SIT Tomper & 783.8 & & & & & & & & & & \\
\hline La. Dentintom 8 & 73.24 & 73.08 & 73.08 & त2क्या & 7297 & 72.89 & 72.78 & 7278 & $72 . \pi$ & 7281 & \\
\hline 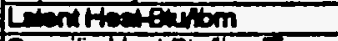 & 28.20 & 28.08 & 28.12 & 27.89 & 28.08 & 27.91 & 27.8 & 27.81 & 27.80 & 27.8 & \\
\hline Spectiothenteribmis & 0.352 & 0.338 & 0.363 & 0.3841 & 0.324 & 0.334 & 0.365 & 0.335 & 0.805 & 0.385 & \\
\hline Ug. Visooentomint & 0.318 & 0.317 & 0.311 & 0.31 & 0.31 & 0.300 & 0.307 & 0.303 & 0.00 & 0.008 & \\
\hline 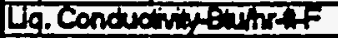 & 0.024 & 0.038 & $0.0 \mathrm{x}$ & 0.028 & 0.0008 & 0.008 & 0.0801 & 0.0831 & 0.0081 & O.0E3] & \\
\hline Lg. PrendiNo. & 4.3.3 & 4.3 & 4.34 & 4.551 & 4.34 & 4.55 & 4.35 & 4.35 & 4.35 & 4.85 & \\
\hline In & 2.7 & & & & & & & & & & \\
\hline 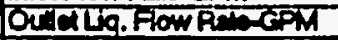 & 0.4 & & & & & & & & & & \\
\hline 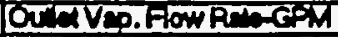 & 1 & & & & & & & & & & \\
\hline 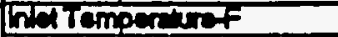 & 187.7 & & & & & & & & & & \\
\hline 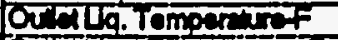 & Tश2 & & & & & & & & & & \\
\hline 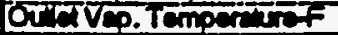 & 788.2 & & & & & & & & & & \\
\hline 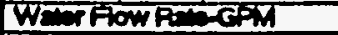 & & & & & & & & & & & \\
\hline Wem trat Temp.f & 118.8 & & & & & & & & & & \\
\hline Werowntempt & 7728 & & & & & & & & & & \\
\hline ntatammitic & 372851 & & temp & 18251 & IDenain & 74.57 & & & & & \\
\hline Subooctinge & 2.1 & & & & & & & & & & \\
\hline Fow Observion & & & & & & & & & & & \\
\hline & ZONEEI & ZON:E2 & $20 N=3$ & 2OnEL & 12ON=5 & $20 N=6$ & $20 \mathrm{~N}=7$ & $\angle O N=6$ & $\angle O N=8$ & $20 N=10$ & \\
\hline Feal houtwand & 851.5 & 867.6 & 8848 & 8882 & 8ह51 & 870 & 30.ग & 83.8 & 78.0 & 90.1 & \\
\hline Hen Losentwes & 128 & & 4 & 4.11 & 4 & का & 1.8 & टह & 3.4 & 19 & \\
\hline Font Fion binthic & 74700 & 75021 & 7483 & $752.50 \mid$ & 7690 & 15031 & 6001 & $7 \mathbf{6 3 8}$ & TE:3 & 7682 & $1-9 x=$ \\
\hline WE Tamp. B & 785.8 & 198.1 & 795.1 & 78621 & TSo.J & 197.8 & नक्यद्य & Fंत्रा & क्ष: & 80.0 & $-1.5=$ \\
\hline WE Tomp. IfF & 183.5 & 195.7 & T97.4 & 181 & 190.5 & 138 & igrot & 191.4 & वि13 & 180 & , \\
\hline WE T/cmp. 165 & 187 & 195.0 & 1584 & 187 & 180.1 & $1975 \mid$ & T9x. & 1920 & TSEs & ग912 & 4,0 \\
\hline Wertrong. $11 F$ & 187.7 & 150.51 & 194.4 & 155.6 & 1855 & 187.1 & 1983 & 18ह & क्य, & 189. & 1968 \\
\hline Wiltemp.tit & 1830 & 198, & 18] & 198.1 & TE3.1 & 197.2 & 19:0 & स्ज.ग & क्ष.I & 768 & 377 \\
\hline hatemtampa & 78.7 & 7896 & 168.8 & 788.3 & 1627 & 180 & 1908 & 1805 & 1505: & 1503 & 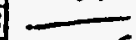 \\
\hline AW. We Tompf & Tक्6म & 1560| & 155: & 196्रा & 7532 & 7575| & 7801 & Tिका & Tजू] & TSE-1 & 18205 \\
\hline Avo.WNDT. & 7 & 6.4. & 5.8 & 5.8 & 5.8 & 6.0 & 3.6 & f.11 & 1 & 0 & \\
\hline Remining seboodt & -4.8 & & & & & & & & & & \\
\hline Exacher & 0.06 & 0.74 & 023 & 0.31 & 0.80 & 0.77 & O.5T & Dbic & $\mathbf{0 . 3 3}$ & 0.5 & \\
\hline EXP.HA:MThM= & ह173.य & 2035) & 25125 & 2550 & 250.18 & ETtel & Tद्या| & 7577.7 & 13702 & 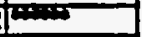 & \\
\hline COMPUIEDH & & & & & & & & & & & \\
\hline Jeork & Th6.21 & 7601.8 & $160 x+$ & T5:38, & 7550 & 878 & 8250 & 84.01 & 897. & 824] & \\
\hline 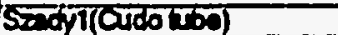 & 22355 & 252.0 & खर्डाष्ट & 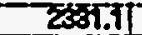 & 268.8 & 89.8 & 845 & हात्रा & $87 \pi$ & 84.4 & \\
\hline $5 \operatorname{sod} 2$ & 275.7 & ह180.7 & 218. & 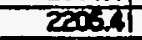 & 2दि & कर्या & 840 & 84.4 & 8रा & हम, & \\
\hline (F०) & 70118 & 712501 & 84000 & 58डाठ & 5157 & 5167 & 5ाइत्) & 57678 & 61670 & काजित् & \\
\hline$(F)$ & 1.450 & 1.872 & रखण & 247 & 2.70 & 2.70 & 2.700 & 270 & 270 & 2700 & \\
\hline$(1+0)$ & 105 & ऊक्रा1 & 3000 & 3836 & 355 & 3670 & 357.0 & 387.0 & 3873 & 3575 & \\
\hline (H:304) & 2363 & 23201 & 23786 & Zeas & $2 \operatorname{sen}$ & 23018 & 250 & 2058 & $2 \cos$ & 2000 & \\
\hline & & & & & & & & & & & \\
\hline & $20 \times 152$ & ZONEA & $\angle O B=6$ & ZON=8 & $\angle O M T=10$ & & & & & & \\
\hline DPloged remp.f & \begin{tabular}{|l|}
87.8 \\
\end{tabular} & & & & & & & & & & \\
\hline 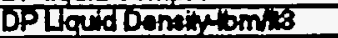 & कू० & & & & & & & & & & \\
\hline Eovitiont & 2 & का & 6 & 81 & 8.875 & & & & & & \\
\hline Thetred Dephod & 0385 & 7.278 & टाया & 3.1801 & 7.0002 & & & & & & \\
\hline ¿ro Cormecionopind & $=0.068$ & -0.002 & 0.02 & o.ce्या & 0.084 & & & & & & \\
\hline Comandoptid & 0.318 & 1.885 & 212 & 3.174 & 3.8 .8 & & & & & & \\
\hline Actud & 1.87 & 231 & 3.47 & 4.051 & 7.5 & & & & & & \\
\hline Wheoured tiptiph & 217.7 & & & & 217.7 & & & & & & \\
\hline Mecoured overaith & of & & & & & & & & & & \\
\hline & & & & & & & & & & & \\
\hline Comped ber & 1.53 & $2 \pi$ & 3.7. & 4.891 & 6.8 & & & & & & \\
\hline Fricton- & $0.0 \%$ & 0.18 & 0.3 & o.t. & 0.52 & & & & & & \\
\hline Aoontriont & 0.00 & 0.00 & 0.14 & 0.15 & 0.16 & & & & & & \\
\hline envionn & 1.6 & 216 & 3द्धा & 7.4. & 5.68 & & & & & & \\
\hline & & & & & & & & & & & \\
\hline
\end{tabular}




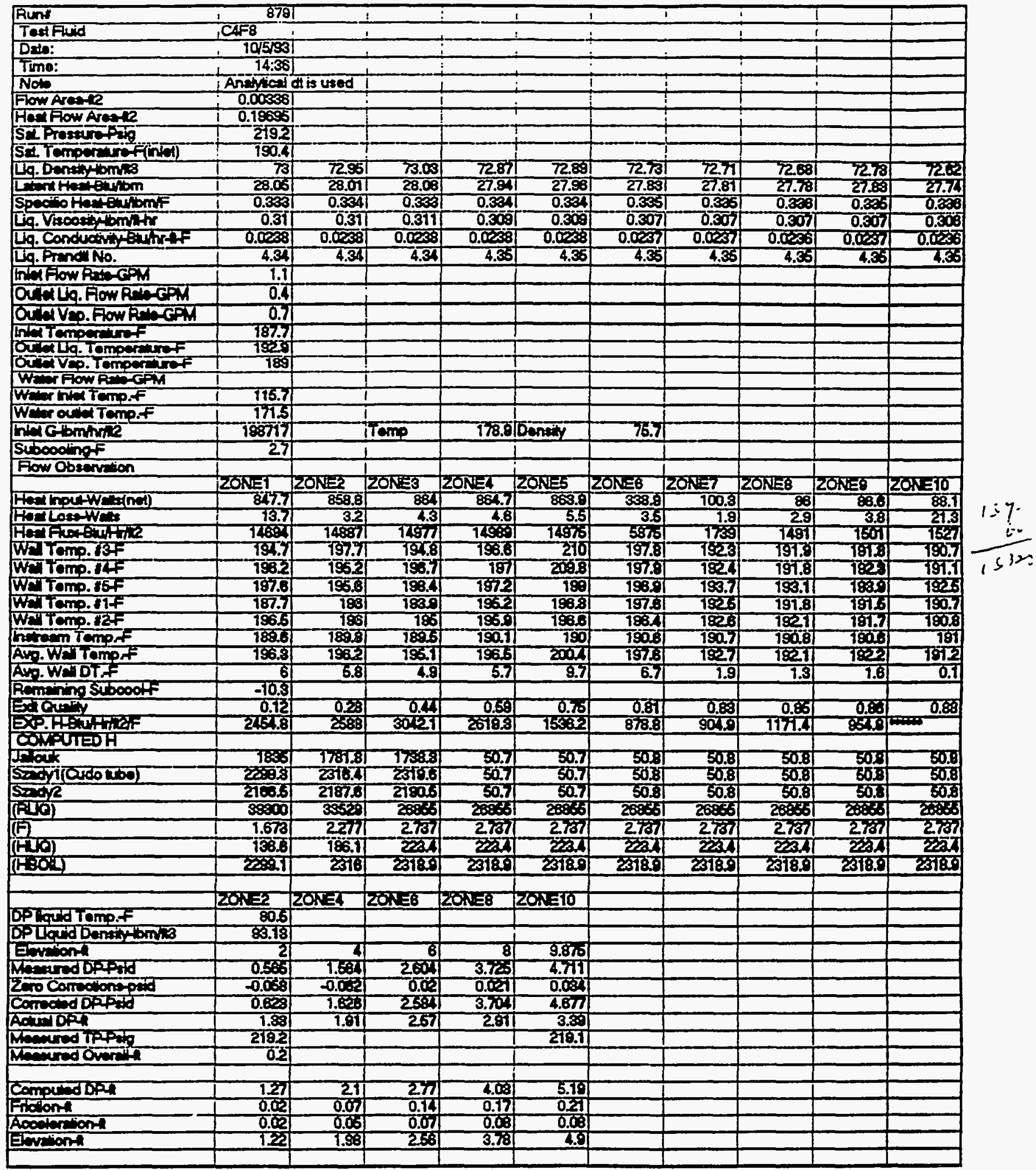




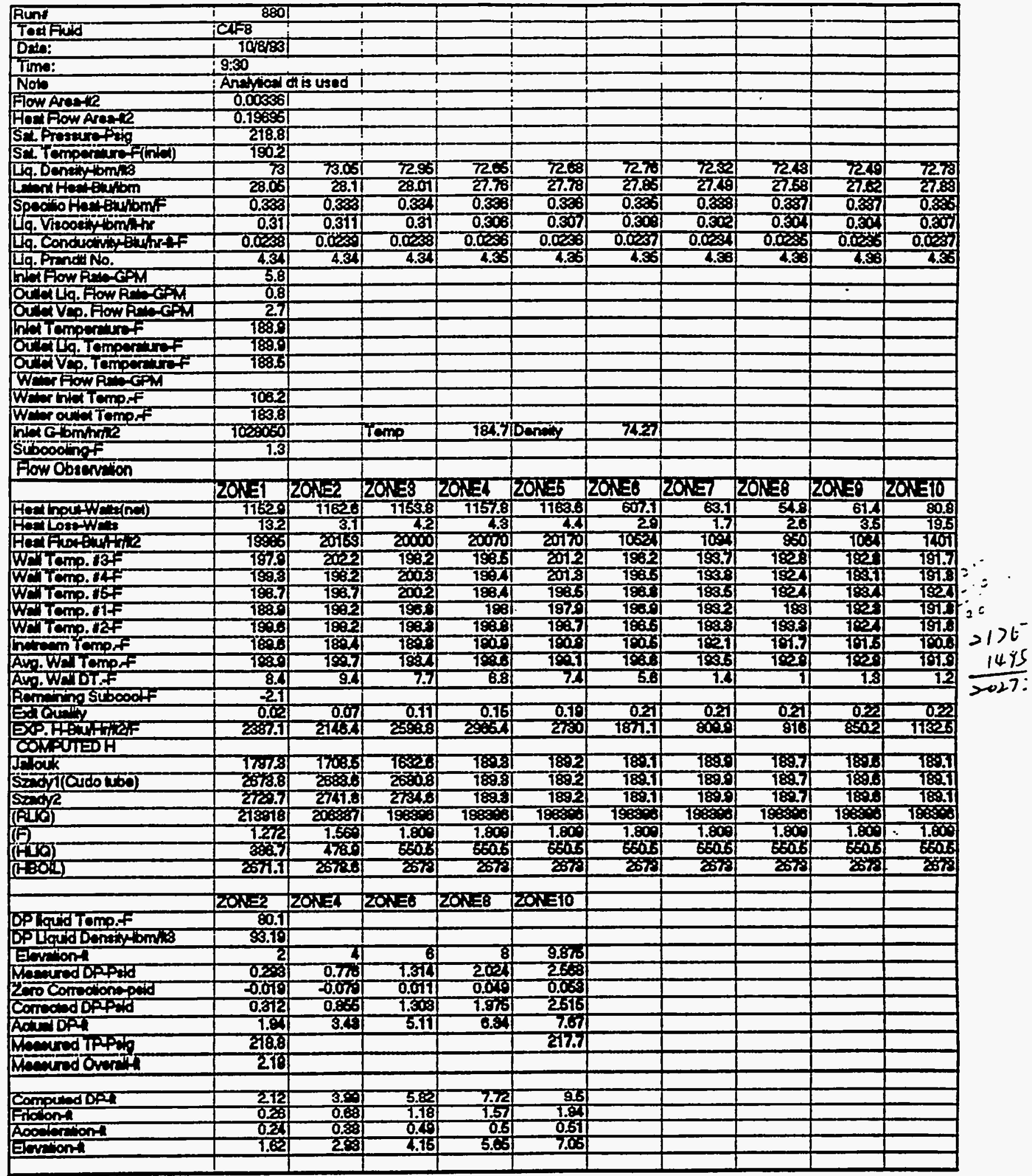




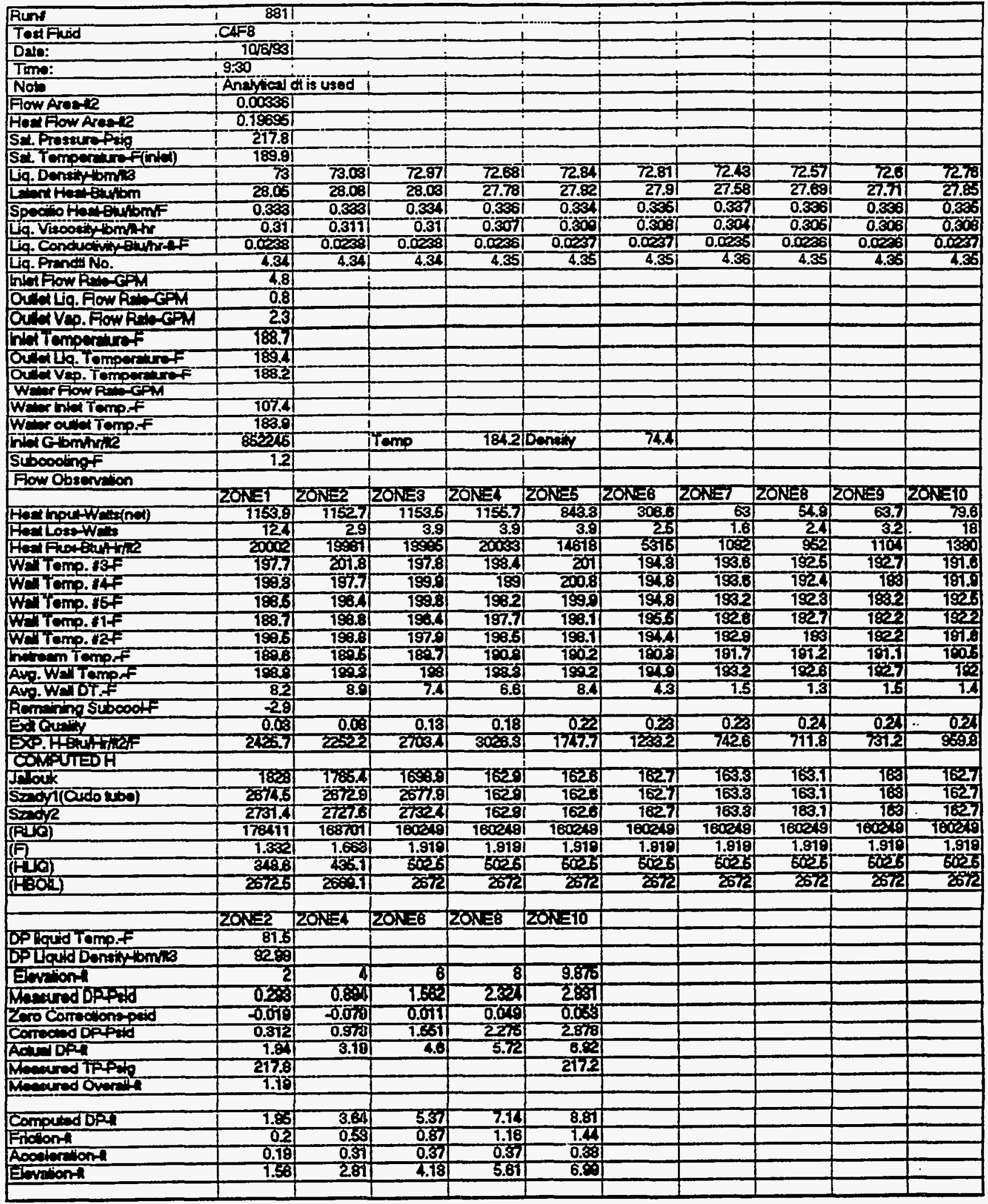




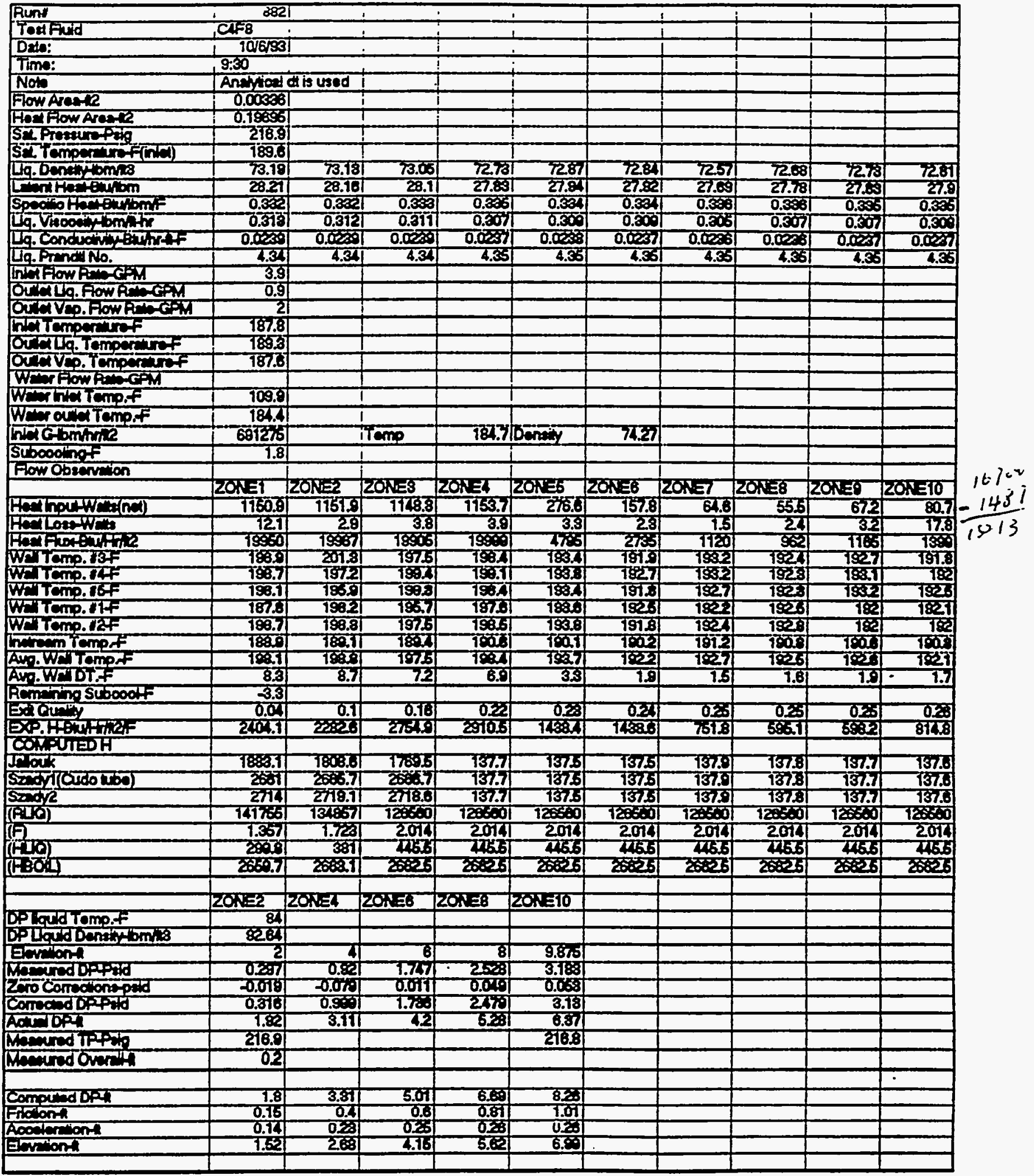




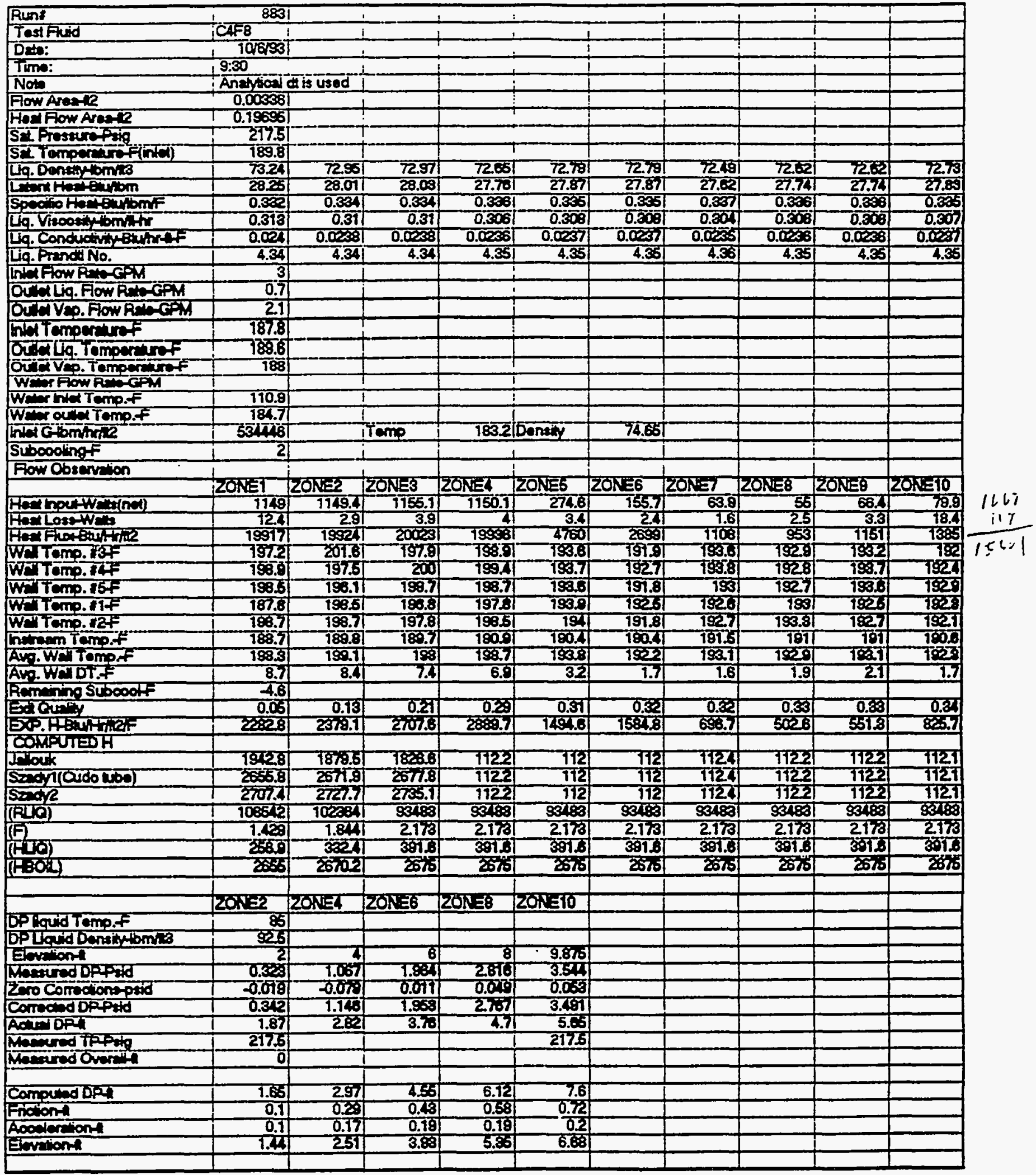




\begin{tabular}{|c|c|c|c|c|c|c|c|c|c|c|c|}
\hline Run & $1 \quad-894$ & 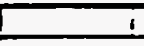 & 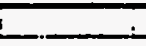 & & - & 1 & - & $\div$ & 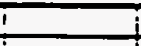 & & \\
\hline Teभf Find & CAF & $\div$ & 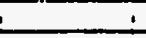 & - & 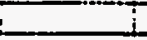 & $i$ & 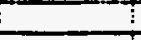 & 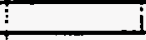 & & & \\
\hline Dese: & 1 10633 & 1 & 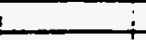 & & & - & & 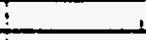 & & & \\
\hline Time: & $12: 30$ & & & & & & & $i$ & 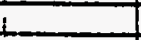 & & \\
\hline Not & Anghion: & dis used & & & & & & I & & & \\
\hline flow Areare & 0.00386 & & & & & & & I & & & \\
\hline HoI Fow Ares? & 0.1855 & & & & & & & I. & & & \\
\hline SE Presure? & 216.6 & & & & & & & & & & \\
\hline 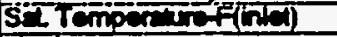 & T89.5! & & & & & & & & & & \\
\hline La.benn & 7320 & 72.89 & 7257 & 7287 & 7282 & 72.87 & 72.57 & 727i & 7278 & 72.70 & \\
\hline Lenthecteriom & 28.SI & 27.821 & 28.03 & 27.81 & 27.99 & 27.84 & 27.69 & 27.87 & 27.88 & 27.85 & \\
\hline 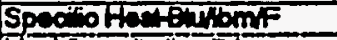 & 0.331 & 0.331 & 0.324 & 0.356 & 0.39 & 0.3811 & 0.363 & 0.380 & 0.28 & 0.35 & \\
\hline La. Visoosilinith & 0.314 & $0 . \overline{3001}$ & 0.31 & $0 . \overline{c 03}$ & 0.31 & 0.300 & 0.500 & 0.207 & 0.507 & 0.503 & \\
\hline 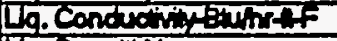 & क्या & 0.0231 & 0.003 & 0.035 & 0.008 & 0.0230 & 0.0201 & 0.0034 & 0.005 & $0.0 \times 5$ & \\
\hline Lig. PrendiNo. & 4.34 & 4.55 & 4.34 & 4.35 & 4.35 & 4.5 & 4.35 & 4.35 & 4.85 & 4.35 & \\
\hline hel Fow Ponecin & 2.1 & & & & & & & & & & \\
\hline OCALA. FOW RDECPM & 0.4 & & & & & & & & & & \\
\hline 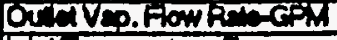 & 7.11 & & & & & & & & & & \\
\hline Intremperntur & 787.6 & & & & & & & & & & \\
\hline 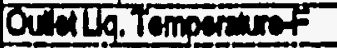 & 18915] & & & & & & & & & & \\
\hline 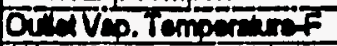 & $\overline{188}$ & & & & & & & & & & \\
\hline 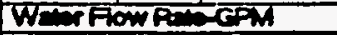 & & & & & & & & & & & \\
\hline Werrinket TempdF & 110.1 & & & & & & & & & & \\
\hline 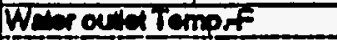 & $184 \times 3$ & & & & & & & & & & \\
\hline Interominos & उ7क: & & tenp & 181.6. & Denaty & 75.07 & & & & & \\
\hline Suboodingt & 1.0 & & & & & & & & & & \\
\hline Fow Obsenvion & & & & & & & & & & & \\
\hline & $201=1$ & $2 O H N_{2}$ & 20153 & $20 N=4$ & $201+5$ & $20 \times 1=6$ & Zoner & $20 \mathrm{l}=$ & $\angle O N=0$ & $20 \mathrm{~N}=10$ & \\
\hline Hellopt Weinal & 17632 & 11622 & TIES & T158.8 & 274 & 154.4 & E.J. & 55.4 & 66.8 & 80.7 & 16.61 \\
\hline 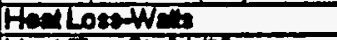 & 125 & 3 & 4 & 5 & 3.5 & 2.4 & 1.8 & 2.6 & 3.3 & 78.4 & .174 \\
\hline 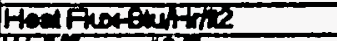 & TSOा & 1997 & क्रिश्रा & 2078 & 4750 & $26 \%$ & Tाङ & 50 & 1738 & 1300 & \\
\hline We Tomp. 13 & 79801 & खणाडा & 7975 & 21द.ण & 735? & 19Tडा & T98.71 & T31 & 18201 & T95.0 & 15977 \\
\hline WrTamp.14F & 198.6 & 1978 & 200 & 275.2 & 1583 & 1827 & 1935: & 19:0 & 1982] & T81.7 & \\
\hline Wh Tamp. 767 & 1882 & 195.91 & 189.0 & 2005 & 1382 & 191.7 & 133 & 15्रण & 1835 & 18023 & \\
\hline We'Tomp. ItF & 187.6 & isal & $1 S 3$ & 197.11 & 138.5 & 1928 & T925) & 198.11 & Ts2a & Tित. & \\
\hline Wh Temp.12t & 188.6 & 1988 & 197.7 & 197ग & 1989 & 191.5 & 192रा & 153.5 & 1820 & 1953 & \\
\hline Iniren tampa & 1885 & T803 & 188.7 & 1850 & 1801 & 190.11 & 18132 & 180.7 & To5 & 1650 & \\
\hline AW.WAT Tempof & क्जा & 19: & 1975| & 2030 & क्रा5 & 1522) & 793 & क्या। & 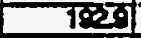 & 153.7 & \\
\hline AN.WETDT.F & 8.7 & 7.7 & 72 & 15.7 & 3.3 & 1.91 & 1.8 & 23 & 28 & T3 & \\
\hline Remoning scboodt & -7.5 & & & & & & & & & & \\
\hline Extoung & 0.0 & 0.2 & 0.31 & 0.42 & 0.46 & 0.48 & 0.47 & 0.67 & 0.68 & 0.0 & \\
\hline EXP.HFErThe & 3052 & 258.8 & टrreo & 1द्राइ & 7ख्या & 138त्A & 6320 & दाता & 5ा0. & 4098 & \\
\hline COMPIIEOH & & & & & & & & & & & \\
\hline Jeak & क्र०ता & 1585.I & 84.41 & 84,61 & 845 & 845 & 84.8 & 8.7 & 870) & 84.0 & \\
\hline 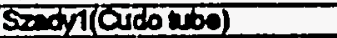 & 265.7 & 26808 & 84,4 & 84.01 & 845 & 845ा & 84.8 & 84.7 & 82.01 & 89.6 & \\
\hline $\operatorname{sen} \sqrt{2}$ & 2770.7 & 273.0 & 84, & 84.0 & 845 & 8450 & 84.8 & 84.7 & 840. & 87.0 & \\
\hline (स) & 76000 & 683231 & 68320 & 68820 & 68808 & 688501 & 6850: & 088201 & 6830 & 6830 & \\
\hline (F) & 5.568 & 20.51 & 2031 & 2081 & 208 & 2001 & 2.08 & 2081 & 2007 & 200 & \\
\hline (FD) & ZIES & खाइ & ऊदाइ & खाया & ऊसा & खराइ & 3ख12 & 2812 & खरा & 2812 & \\
\hline$\left(E=0^{2}\right)$ & 25012 & 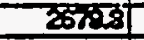 & 35 & 2013 & 37s: & 20728 & 2073 & 257.3 & $25 \times 1$ & कstis & \\
\hline & & & & & & & & & & & \\
\hline & $201 \times 2$ & $200^{2}=5$ & $\angle 0^{\prime}=6$ & $201=8$ & Zonsio & & & & & & \\
\hline DPlogud temp.t & 856 & & & & & & & & & & \\
\hline DPLoudd Deneingmis & क्यार्य & & & & & & & & & & \\
\hline Exwiont & 2 & & & & 9.875 & & & & & & \\
\hline Tremured op 4 ded & 0.305 & 7.383 & 2801 & 3228 & 7.065 & & & & & & \\
\hline 2600 correcionsped & 0.010 & $-0.0 \%$ & 0.07 & 6.069 & 0.038 & & & & & & \\
\hline Comodoplond & 0.474 & 7.488 & हसा & 3.19 & A.083 & & & & & & \\
\hline Aculari & 1.72 & 218 & 3.12 & 3.89 & 4.5 .1 & & & & & & \\
\hline 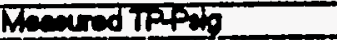 & टा6. & & & & 217 & & & & & & \\
\hline Trenured diverlin & -0.70 & & & & & & & & & & \\
\hline & & & & & & & & & & & \\
\hline Compered dox & 1.46 & 2301 & 3.801 & 5.8. & 6.64 & & & & & & \\
\hline Finconth & 0.07 & 0.10 & 0.81 & 0.4 & 3.60 & & & & & & \\
\hline Aconter on & 0.08 & 0.12 & 0.18 & 0.19 & 0.74 & & & & & & \\
\hline Exrions & 1.321 & 2क्ष & 3.दा & 4.76 & 6.01 & & & & & & \\
\hline & & & & & & & & & & & \\
\hline
\end{tabular}




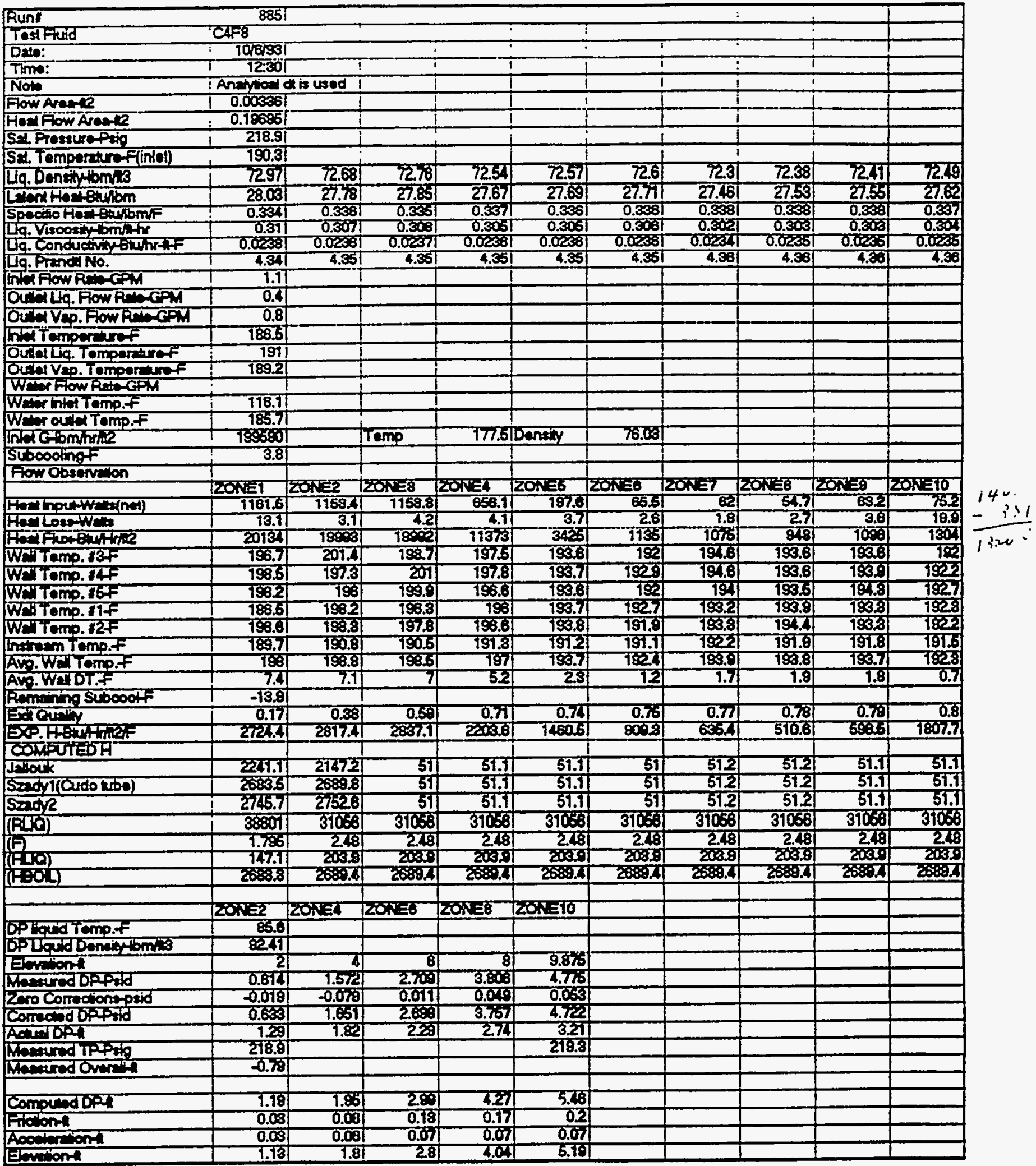




\begin{tabular}{|c|c|c|c|c|c|c|c|c|c|c|}
\hline nun: & 3861 & & i & & & - & & $\therefore$ & & \\
\hline $7+\ldots=$ & $.04 / 8$ & & & & & & & & & \\
\hline nsts: & - 10/7/93! & & & & & $\vdots$ & & & & \\
\hline Time: & $8: 43$ & & & & & & & & & \\
\hline Nots & - Anstriced & dis used & 1 & & & & & & & \\
\hline Flow AYoatR & 0.00356 & & & & & & & & & \\
\hline Hod Flow Areat2 & 0.196965 & & $\mathrm{t}$ & & & & 1 & i & & \\
\hline SElptessurepig & $! \quad 179.41$ & & $!$ & i & & 1 & $!$ & i & & \\
\hline Se. Temperturef(inla) & 175.2 & & I & T & & 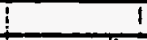 & 1 & 1 & & t \\
\hline Lig. Lensitoming & 78.781 & 76.84 & 15.181 & 5.51 & 10.5 & 10.21 & n.X81 & 10.401 & n.0.1 & 10.11 \\
\hline Lexpriecionsiom & 31.21 & 31.26 & 31.21 & $3 \overline{0.971}$ & Si.011 & 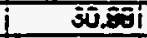 & $\overline{\mathbf{3 0} .001}$ & \$i.jol & $\dot{s i v j}$ & $\$ 1.10$ \\
\hline Fpiofio How-6tubm/F & 0.372 & 0.612 & 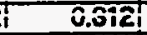 & o.jial & 0.575 & 0.5101 & $0.5 i 91$ & $0.313 i$ & 0.35 & 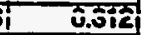 \\
\hline Liq. Viscositytomith & 0.359 & $c .3$ & र.ख़ी & G.ख्रE & C.5E & ה.खू| & 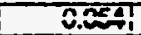 & C.NFE! & כ.xsi & 2.00 \\
\hline 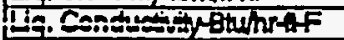 & $0.0261 !$ & ก.9251 & n.0251! & n.mag! & n.m59 & 2.2050! & n.mx: & 3.0230 & n.xeso & n.ms \\
\hline Lig. Prandillo. & 4.3 & 4.3 & 4.3 & 4.3 & 4.8 & 4.31 & 4.31 & $4.8 !$ & 43 & 4 \\
\hline Inlat Flow Axis-GPM & 5.51 & & 1 & i & & & $?$ & & & \\
\hline Outhet Lin How Rato-GPM & 11 & & $t$ & 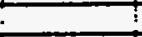 & & & & $\therefore$ & & T \\
\hline OUEA VeD. Fow RER-GPM & 2.81 & & ? & 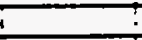 & & & $\bar{z}$ & 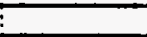 & & T! \\
\hline Intet Tomperefuref & 173.61 & & & & & & & & & \\
\hline Oulut Li Tomoer Trof & $174.2 T$ & & 1 & ! & 1 & $!$ & $i$ & ! & & 1 \\
\hline 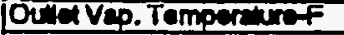 & 172.51 & & T & ! & t & ! & & $i$ & & \\
\hline 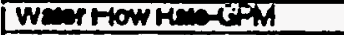 & 1 & & $i$ & I & I & 1 & 1 & 1 & & $t$ \\
\hline Wom inta Tomo.f & 125.11 & & I & 1 & 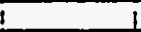 & 1 & I & $i$ & & 1 \\
\hline 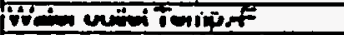 & 167.87 & & 1 & 1 & & 1 & 1 & $:$ & & 1 \\
\hline Whachorintion & 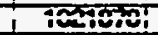 & & Tamp & 155! & Dansini & 7.50 & & I & & \\
\hline Subcoctingf & $1.8 !$ & & & & & & & & & \\
\hline \multicolumn{11}{|l|}{ Fow Observation } \\
\hline & ZONET & ZONE2 & :ZONEZ & LONIET & $\angle O N=5$ & TZONEB & KKNET & 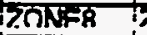 & $\boldsymbol{L} \mathbf{N}=\mathbf{A}$ & bxג-का \\
\hline Heal hputwatined) & 289.11 & 288.6 & 28851 & 288 & 2924 & 292 & 281 & 289.5 & 289.7 & 2885 \\
\hline Fon Losg-Well: & 10.4 & 24 & 3.31 & 3.31 & 3.21 & दो & 7.6 & 231 & 3.11 & 162 \\
\hline Ifo- Fion-whthe - & 50iा & 5003 & 5001 & 45681 & 506 & $\begin{array}{l}3050 \\
18081\end{array}$ & केंगा & 5018 & 5स्वर्या & 5007 \\
\hline WE Tamp. IfF & 180.81 & $\frac{180}{180.1}$ & $\frac{180.11}{18001}$ & $\frac{180.61}{1811}$ & $\frac{180.91}{1811}$ & 181.61 & 180.9 & 179.01 & 180.81 & 978.1 \\
\hline WE TOMp. 15F & 180.7 & Tि.8 & 787.7 & 180.7 & 18000 & 180.71 & 781 & Tत्यद्या & 10.01 & $1 / 8.4$ \\
\hline Wintamp. itf & 173.61 & 1803 & 179.31 & iकृसी & iक्रिम & iक्षित & iôद厶 & 1701 & itant & TRिA \\
\hline 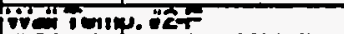 & उक्ण & 163.8 & 779.81 & i20.41 & 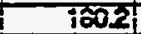 & i50.J1 & Tिक्सी & 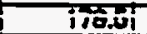 & 7rost & iि0.6 \\
\hline frisentamp & 174.3 & 174 & 174.3 & 975.51 & 775.3 & 37.4 & 78.1 & T.7E.7! & 755 & I7.S. \\
\hline A:F, Wall Temp.t & 1805 & 1802 & 180 & 180.6 & 1805 & 991 & 191 & 179.9 & ?:34 & ?? \\
\hline A.p. Wath DT.F & 5.9 & 5.9 & 5.4 & 4.91 & 5 & 5.4 & 4.7 & 3.4 & 3.7 & 22 \\
\hline Remining Subcooff & 0.6 & & & & & & & & & \\
\hline Extoristy & -0.011 & 0 & 0.011 & 0.ర्टा & 0.031 & 0.04 & $0 . \infty 1$ & 0.06 & 0.071 & 0.08 \\
\hline Exp.HBtwhther & 8481 & 840.9 & \$2या & 1011.61 & T014.5 & क्रा & T0द्य. II & 7487.7 & 135251 & 279.1 \\
\hline COWWUIEDH & & & & & & & & & & \\
\hline Jeloik - & $746, \mathrm{Al}$ & 743.7 & 735.7 & 73281 & 738.21 & 737.11 & 732.01 & 73421 & 7ant & 731 \\
\hline Fendy (Qudo tube) & 1235.2 & 12325 & 1250.9 & 12का & T2554 & +-125751 & -12528 & 1257.8 & 1250.11 & 12585 \\
\hline seagrt & प्रक्यम & Y48 & S28 & 957.4 & 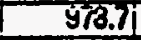 & Y77.11 & $\mathbf{5 7 2 8}$ & प्रत्ग & IU18.81 & 1044.8 \\
\hline (म्य) & 180012 & 165508 & Fors & 18स्म & 1625761 & iói281i & i 600080 & ifoces & 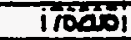 & itatu \\
\hline (in) & 1.004 & 1.004 & i. & 1.301 & 1.401 & 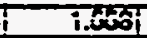 & i. & 1.8011 & $i ., 131$ & 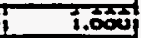 \\
\hline 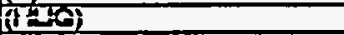 & 3्रू. & 34.6 & 3568 & 舟事 & काज्ञ & 장요 & सक्सी & $\approx$ & & \\
\hline \multirow[t]{3}{*}{ 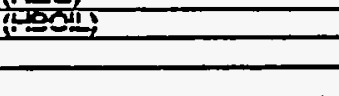 } & 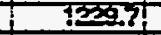 & .2258 & 1235 & 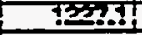 & 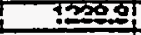 & 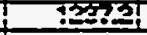 & F现! & 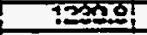 & भ⿸厂二 & द्यदू \\
\hline & & & & & & & & & & \\
\hline & ZONES I & ZONEST & ZONEE & ZOKLE8 & 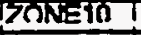 & & & & & \\
\hline DP loud temo.f & 80.41 & & & & & T & 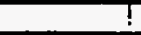 & & & i \\
\hline 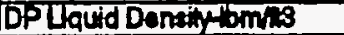 & 93.15 & & & & & & & & & ! \\
\hline Evevilion & 2 & & & 8 & $9.8 \%$ & & & & & \\
\hline Meacured DPPsid & 0.4551 & 0.525 & 0.7521 & 1.369 & $1.628 \mathrm{i}$ & & & & & \\
\hline Zero Cortiochonapad & 0.763 & -0.04 & -0.129 & 0.171 & 0.121 & & & & & \\
\hline Correand op pod & 0.677 & 0.565 & 0.5011 & 1.169 & 1.507 & $I$ & & $1=-1$ & & I \\
\hline Fou Do्य & 13.31 & 3.61 & 501 & 7.52 & 9.761 & I & 1 & 1 & & 1 \\
\hline 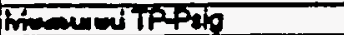 & 770.4 & & & & 7.0.3. & & & 1 & & \\
\hline Meaured Ovarlith & 1.5 & & & & & & + & $i$ & & \\
\hline \multirow{2}{*}{\multicolumn{11}{|c|}{ Computed DP }} \\
\hline & & & & & & & & & & \\
\hline Frictions & $0.15 t$ & 0.35 & 0.581 & D.8nt & शारा & & & & & \\
\hline Acoelarctiont & 0.04 & 0.15 & 0 आा & ర.द्ठा & o.3ता & & i & & & \\
\hline Elevetiont & 1.96 & 3.74 & 5.43 & 7.07 & 8.561 & & & & & \\
\hline & & & & & & & & 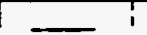 & & \\
\hline
\end{tabular}




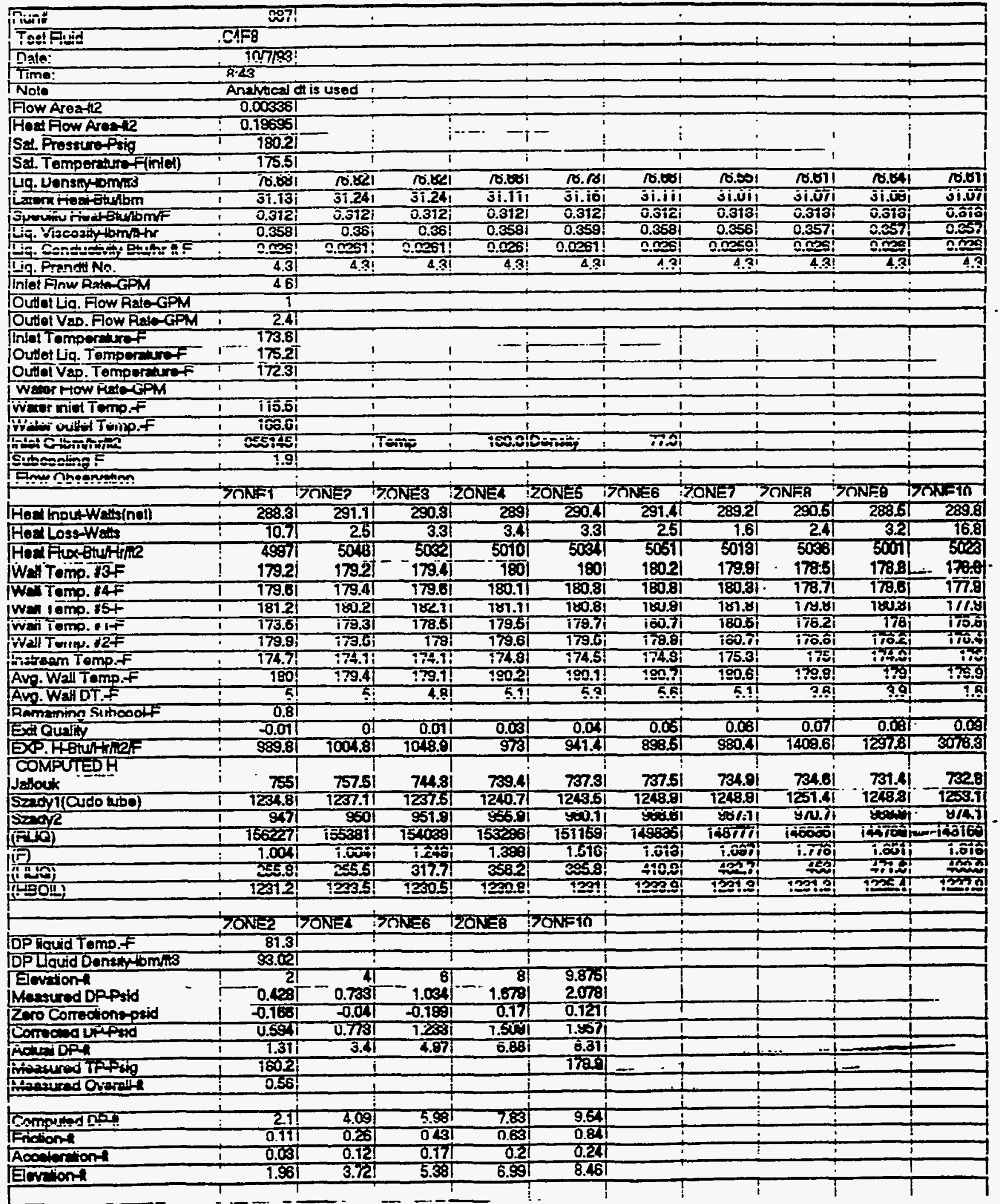




\begin{tabular}{|c|c|c|c|c|c|c|c|c|c|c|c|}
\hline Thint & & का: & & & & - & - & & - & & \\
\hline 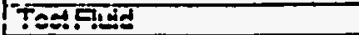 & & $24=0$ & - & & & & & & & & \\
\hline nete: & & 1n?7\%?! & & & & & & & & & \\
\hline Time: & & $8: 43$ & & & & & & & & & \\
\hline Note & & Analutical of & is used & & & & & & & & \\
\hline Fow Arast2 & & 0.003361 & & & & & & & & & \\
\hline Houf Flow Areat? & & 0.196951 & & & & & ; & 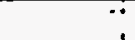 & , & & \\
\hline ISat. Pressure-Psig & ! & $\frac{178.41}{178.81}$ & $!$ & - & 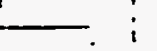 & & 1 & ( & & & $\vdots$ \\
\hline 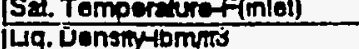 & 1 & $\frac{174.81}{75.821}$ & 77.081 & ro. & 16.731 & 6.81 & r.781 & no.n1 & No.ष्टा & r.8.1 & 10.68 \\
\hline Leren fioustubm & - & $31.24 i$ & 31.411 & $3 i .341$ & 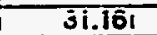 & $31.22 i$ & $\overline{3 i .2 i}$ & $31 . i 5 i$ & si.çal & Ji. $24 i$ & si.ioj \\
\hline Srevilio Hostbterbm/F & - & 0.3121 & $0.311 i$ & 0.5i11 & $\overline{0.5 i c i}$ & $\overline{0.512 i}$ & $0.37 \overline{k i}$ & 6.51दi & 5.5ici & 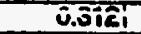 & 0.5iz \\
\hline Lig. Viseositylominht & 1 & $0.36 i$ & 0.331 & 0.202 & C.ర్జ & C.LE! & C.ञ5E & O.జ्WE, & O.Ni & 0.51 & . \\
\hline 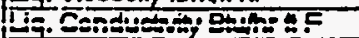 & . & n.mx?! & a.max! & 9.2xi! & $2.2 x \leq 1$ & ..xx?! &..$x x ! !$ & nm! & n.m:! & a.xa! & n.ms \\
\hline Lif. Prangen Na. & & 4.31 & 4.3, & $4.3 !$ & $4.3 !$ & 4.31 & $4.3 !$ & 43 & $4.3 !$ & $43 !$ & 4 \\
\hline Inlel Fow Rato-GPM & & $3.7 !$ & & & & & & & & & \\
\hline Outel Lio. Fow Rato-GPM & & 0.94 & & & & & & & & & \\
\hline Oưfolvap.Flow Rato-GPM & ? & $2 i$ & & & & & & & & & \\
\hline $\begin{array}{l}\text { Inlot Temporaturef } \\
\text { Outet Lia. Temporziñof }\end{array}$ & $i$ & $\begin{aligned} 1731 \\
174.11\end{aligned}$ & -. & & i --- & & $-\cdots$ & $\vdots$ & $i$ & & \\
\hline Outel Vap. Temporencef & $\mathrm{I}$ & 177.31 & $T$ & & $:$ & i & I & 1 & ! & $T$ & 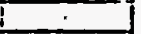 \\
\hline WCO FOW Hat GPM & 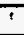 & & & $\dot{5}$ & $\bar{\vdots}$ & 3 & $i$ & -1 & $i$ & & 1 \\
\hline vivater hiot Tomo,F & 1 & $113.1 i$ & 1 & 1 & 1 & 1 & L. & 1 & 1 & & 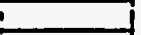 \\
\hline Fiven urivel Temp.f & 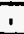 & i0̄.31 & 1 & $\dot{i}$ & 1 & $\therefore$ & $\cdot$ & i & 1 & & 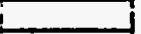 \\
\hline 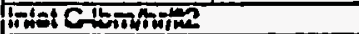 & 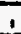 & SOASE! & & $T=\sin$ & 10.710 & insing & 7...1! & 1 & 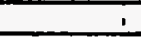 & & $i$ \\
\hline Subsestung 5 & & 9.8 & 1 & & & $\perp$ & 1 & + & 1 & & \\
\hline Tmu hospuntion & & & & & & & & & & & \\
\hline & & $5 \pi+2 ?$ & กरन 3 & उतNF- & NFR & $2 \pi=5$ & 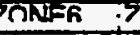 & त्राजन & Nine & INFa & \\
\hline Hod hoLt-Wetteinol) & & 29.1 & 291.1 & 279 & खर्टा & 28231 & $28 \pi$ & 289.7 & 290.81 & 28801 & खा.ा \\
\hline Hod Loss-Wats & - & 11.11 & 2.61 & 3.4 & 3.51 & 3.41 & 2.51 & 1.61 & 241 & 321 & 17.8 \\
\hline Heal Fux-8turthile & : & 5ा15ा & 50461 & 48581 & 502 & 4898 & 49751 & 5 प्टा & 50खा & 50081 & 5016] \\
\hline Wall Temp. "3F & i & 179.41 & 17921 & 1791 & 179.81 & 179.81 & 1801 & 179.51 & 1781 & 178.11 & 178.0 \\
\hline Wall Tomp. Iff & $I$ & 179.6 & 179.11 & 179.61 & 179.81 & 180.11 & 180.71 & $1 \overline{9.81}$ & 178.11 & $178.8 !$ & 178 \\
\hline Wan lomp. $15 F$ & $\cdot$ & 1811 & $1(9.5)$ & 181.31 & 100.61 & 180.41 & 180.01 & 160.81 & $1 / \mathrm{d.91}$ & $1 \mathrm{P.8L}$ & $-\ldots-1+A+B$ \\
\hline Wall Tomp. If & 1 & 1731 & i79.11 & i76.2. & $i 79.51$ & $\overline{179.41}$ & $10021-$ & 10us| & 171.01 & iti.4. & -170 \\
\hline WDl Temp. d2F & - & i7s.8. & $i 73.31$ & i75.6i & 179.5 & 79.3: & 179.71 & 180.8 & ito.li & iT. & ino. \\
\hline $6000001-01000 .-7$ & & 174.11 & $173.2 i$ & $7.50 .5 !$ & .7 .21 & .79 .21 & .74 .31 & :74.5! & $i 74.1 i$ & 179.1! & .79 .7 \\
\hline 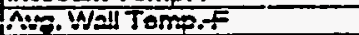 & $t$ & $180 !$ & 179.2 & 7.98 & 180 & $179.8 !$ & 190.1! & $92 !$ & $170.9:$ & .7251 & 17 \\
\hline AWg. Wall DT.F & & 5.5 ! & 5.7 & 5 & $5.2 !$ & 5.4 & $5.8 !$ & 53 & 3.91 & 3.9 & $2 ?$ \\
\hline Remaning Suboootf & & 0.4 & & & & & & & & & \\
\hline Ext Cuality & & 01 & 0.07 & 0.021 & 0.09 & 0.051 & 0.051 & 0.081 & 0.09 & 0.711 & 0.12 \\
\hline EXP. HBtuHMR2R & & 908.4 & 878 & 968 & 909.4 & 900.81 & 854 & 947.6 & स्ट्टि & 1293.5 & 2435.11 \\
\hline COMPUTEDH & & & & & & & & & & & \\
\hline Jatouk & ! & $\pi 7.51$ & 750.51 & 731.51 & 7481 & 730.41 & 734.91 & 737.81 & 735.91 & 732.11 & 735.8 \\
\hline Szadyi(Cudo ubb) & t & 124.61 & 1235.41 & 8209.61 & 1243.7 & 122281 & 1234.81 & 124281 & 1243.81 & 1240.71 & 1250 \\
\hline 5zaoye & I & 501.41 & $\sin 6.61$ & Y.221 & 508.21 & 307.11 & Solv1 & 504.81 & 587.41 & Youj. & 5ogs \\
\hline |Fिं & I & 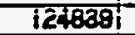 & 1234161 & I2i88i & 1216001 & IISAFi & iitrot & 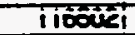 & 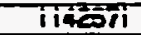 & iī̄ & iifite \\
\hline in & T & 1.0041 & 1.1441 & 1.357 & 1.5तI & i. .531 & $i .8001$ & i..201 & i.STA & 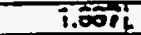 & - C.W \\
\hline (स०थ) & 1 & $214.5 !$ & $2+3.91$ & 291.7 & 3231 & 348.7! & 27.0. & wh: & क्जय & 201 & 표. \\
\hline (19901 & 1 & 12125 & $12 \times 32$ & 13039 & Tzs.1! & 1213.1 & 132 & 330! & $2200 ?$ & .253! & 193 \\
\hline & & & & & & & & & & & \\
\hline & & CONE2 IL & ZONER & ZONEE & HE8 & SNETO & $i$ & & & & \\
\hline DP liquid tomo.f & & 86.61 & & & & & $i$ & & & & \\
\hline 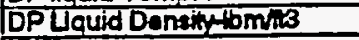 & & 92दा & & & & & & & & & \\
\hline Evation-l & & & & & & 9.875 & ! & & & & \\
\hline Mosured DPPsld & $\mid$ & $0.405 i$ & 0.7481 & 1.009 & $1.772 !$ & 2.2271 & & $!$ & & & \\
\hline Zero Combctionsered & i & $-0.16 \overline{1}$ & $=0.041$ & -0.189 & 0.171 & $0.12 \pi$ & & $!$ & 1 & & \\
\hline Comocho uphad & 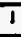 & 0.571 & 0.7801 & 132 & 1.0021 & L.1481 & 1 & 1 & $I$ & & I \\
\hline 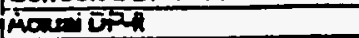 & 8 & 1.331 & 5.34 & $4 . \bar{\pi}$ & 6.621 & $7.35 i$ & 1 & 1 & I & & $i$ \\
\hline minowitis & 1 & 7.6 .4 & 1 & & 1 & Trost & 1 & 1 & $i$ & & \\
\hline Mesesured Ojaralls & $\perp$ & $=207 !$ & $i$ & 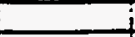 & i & & $i$ & $i$ & & & \\
\hline & & & & & & & & & & & \\
\hline Compesed OP- & & 203 & $3.9 !$ & 5. & 7.43 & on?! & & & & & \\
\hline Fristiont & & 0.08 & 0.191 & तख्या & nदा & 0.61 & & & & & \\
\hline Acosterition: & $:$ & 0.051 & 0.65 & 0.131 & $0.167-$ & 0.107 & + & & & & \\
\hline Eleveriont & & 1.911 & 3.611 & 5.2द & 6.81 & 8.20 & & & & & \\
\hline & & & & & & & & & & & \\
\hline
\end{tabular}




\begin{tabular}{|c|c|c|c|c|c|c|c|c|c|c|}
\hline Tintis & उद्व! & & & - & & 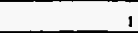 & & . & & . \\
\hline Tag कhind & .159 & & & & & & & & & \\
\hline nate: & 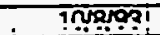 & & & & & & & & & \\
\hline Timn & 8.35 & & & & & & & & & \\
\hline Note & Analupard & it is used & & & & & & & & \\
\hline Flow AYoat2 & 0.003361 & & & & & & & & & \\
\hline Hock Row Aroad? & 0.1935 & & & & & & & & & \\
\hline Sat. Pressuro-Psig & 179.81 & $!$ & 1 & 1 & 1 & & $\ldots+n=-1$ & 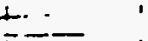 & & ! \\
\hline Sal Temperaire fintel) & 175.31 & $T$ & $T$ & & 1 & -3 & & & $\therefore \quad i$ & \\
\hline 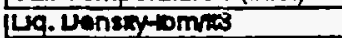 & 76.841 & 15.981 & 76.821 & 50.751 & 10.81 & ro.pl & 20.701 & 0.781 & $\sqrt{6 . \overline{81}}$ & 76.71 \\
\hline 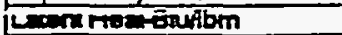 & 31.261 & $31.35 i$ & $5 i .24$ & $3 i .181$ & $5 i .221$ & Si.iô & $\overline{3 i .1 \overline{6}}$ & $3 i .21$ & 3i.21 & ș.ítoj \\
\hline 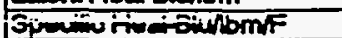 & $0.512 !$ & $5.377 i$ & 0.312 & $0.312 \mathrm{i}$ & U.जici & U.. $\bar{i} 2 \bar{i}$ & 0.3121 & $0.512 i$ & O.STA| & 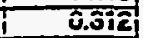 \\
\hline b. 'Piscosth-1bm/liht & $0 . x_{1}$ & 0.3621 & $0.35 !$ & - .2599 & $0.36 !$ & $0.05 \mathrm{SI}_{1}$ & 3..56! & 0.351 & C..SEI & 0.50 \\
\hline 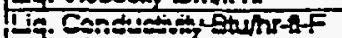 & 0.2xs!! & $3.0 x 29 !$ & $2.0259 !$ & n.xx?! & 2.xx!! & c.nesi & 2.조! & n.xa!! & a.ma!! & ה.m \\
\hline Lim Drand NA. & 4.31 & $4.3 !$ & $4.3 !$ & 4.3 & $4.3 !$ & $4.3 !$ & 4.31 & $4.3 !$ & $4.3 !$ & $1 . ?$ \\
\hline Intet Fow Rato-GPM & $2.9 !$ & & & & & & & & & \\
\hline Oudel Lia. Fow Ralo-GPM & 0.7 ! & & & & & & & & & \\
\hline Outlet Vad. Flow Rate-GPM & 2.31 & & & & & & & & & \\
\hline Inlet Tomperaturef & 1731 & & & & 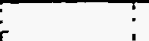 & 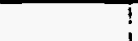 & & 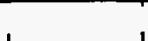 & & 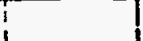 \\
\hline Oullot Liq. Tomperaturof & 174.71 & & -1 & - & $!$ & $\frac{1}{1}$ & & 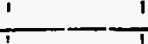 & & $!$ \\
\hline jouter vap. Temperaurof & $17 \overline{81}$ & $T$ & ! & $T$ & T & $T$ & & 7 & & \\
\hline Wear How RowolisM & 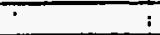 & ' & 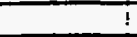 & 1 & 1 & T & & $i$ & 1 & 1 \\
\hline Fiver inial Tomp. & i ij.zi & 1 & i & 1 & $:$ & 1 & & $:$ & \pm & \pm \\
\hline ivider unthit Temo.f & ín7.8; & I & 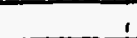 & 1 & 1 & 1 & & 1 & $=-1$ & \\
\hline 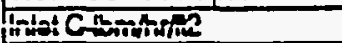 & $53526 !$ & & Tomp & 1032 & Jonsity & $7 . x_{1}^{1}$ & & 1 & & 1 \\
\hline Subcedinge & $2.3 !$ & & & & & +1 & & ב. & & \\
\hline Fow Rhompans? & & & & & & & & & & \\
\hline & ZONET & ZONE? & TONFE & TOKNE & ZONES T2 & ZONE6 & ZONET: & TZONE8 & รतNE-9 I & CIN=To \\
\hline 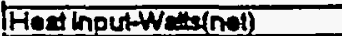 & 287 & 289.81 & 288.81 & 290.8 & 2882 & 289.4 & 290.2 & 288.6 & 253 & 289.8 \\
\hline HeatLoss-Was & 11.81 & 2.81 & 3.7 & 3.8 & 3.81 & 2.8 & 1.81 & 2.71 & 3.6 & 99.7 \\
\hline Hosfinexthithes & 4975 & 5001 & 50031 & 500321 & 4061 & का818 & 50001 & 50081 & 5ाबा & 5020 \\
\hline JWail Tomp. ISF & 178 & 178.41 & 178.81 & 179.51 & 1801 & 18021 & 179.9 & 178.61 & 179.81 & 177.5 \\
\hline Wal Tomp. BAF & 178.61 & 178.6 & 179.61 & 179.01 & 180.81 & 180.91 & 780.41 & 179.11 & 1786 & 179 \\
\hline Wal lomp. $15 \mathrm{~F}$ & 160.21 & $7 \% .5$ & 181.91 & 181.11 & 1871 & 187.81 & 781.71 & 160.01 & 180.61 & 18.01 \\
\hline iwem Tomp. 1F & $i 73 i$ & i70.5i & $i \pi .8 i$ & i79.11 & i7S.d & $i 80.7$ & 100.01 & 178.11 & Tfo.6 & 170.2 \\
\hline 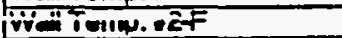 & $i 73.11$ & $i 78.51$ & 778.51 & 178.31 & 179.31 & $100 i$ & 100.01 & i76.81 & 780.01 & inte \\
\hline$f= \pm \pi n T=m p . F$ & $174 !$ & $173.5 !$ & $\$ 74.1 !$ & 177.41 & 17421 & 374.41 & .74 .5 & $.74 .0_{1}$ & 72.01 & 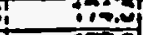 \\
\hline A. Y Y III Temp.F & $179 !$ & 178.51 & 178.5 & $179.9 !$ & !ga! & ?929! & ?ma! & 179.41 & 17921 & 779 \\
\hline QY WallOT:E & 1?! & $4.8 !$ & 4 & 53 & $5.5 !$ & $52 !$ & $50 !$ & 49 & 4.9 & दू \\
\hline & तरों & & & & & & & & & \\
\hline Fxicushy & - क्ता & 0.01 & 0.061 & 0.06 & 0.051 & तळा & 0.11 & 0121 & $n+21$ & ก) \\
\hline 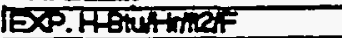 & $\mid 002$ & 105381 & 718.61 & 968.21 & SEaZ1 & 798.41 & 856.81 & 1018.71 & 7105.1 & 3851.6 \\
\hline COKDUTEOH & & & & & & & & & & \\
\hline jJallouk & $\pi 7.71$ & 775.21 & 7602 & 758.8 & 7481 & $787.9 !$ & 745.81 & $740.1 !$ & 74751 & 73224 \\
\hline (Szadpi (audo tbo) & 1200.41 & 1209.81 & 123211 & 1237.81 & 1239.11 & 12201 & 124071 & 1237.11 & $1251.9 !$ & 1243.11 \\
\hline 50092 & प्र8/,A1 & 547.81 & Stanal & 5011 & 540.1 & पcosel & 500.11 & 50001 & Yoen & sour \\
\hline unul| & 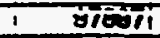 & Frioii & somini & 80001 & 500001 & Yilas! & oroen & 8 810991 & $\infty$ & oanul \\
\hline$|\overline{1}|$ & i.5\%4 & i.iô & 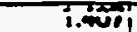 & istoi & $1.1 \alpha_{1}$ & i.jogi & 1.0901 & c.रिका & Eion & $2 \leq 10$ \\
\hline (it) & 7.5 .7 & 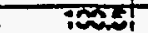 & a4.21 & 2,4 & क? & जू: & $245 i$ & NDE & tim & జूs \\
\hline 然 & :2xi! & 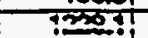 & 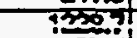 & 4 sol & t质? & $12 ? 3.1$ & 1203 & 9209 & $: 34 n 5$ & \\
\hline & & & & & & & & & & \\
\hline & पE2 & 7CX:-2 & Tतs & $20 N=8$ & D.CXNEI0 & & & & & \\
\hline DP houid Tamo.f & 78.4 & & $-\infty=-7$ & & & & & 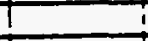 & & \\
\hline DPLiquid Densitutomin & \$3.4. & & & & & & & & & \\
\hline fenvionth & $2 !$ & 4 & 6ा & & 9.8751 & & & T & & \\
\hline Meseeured DPPsid & 0.3871 & 0.8471 & 2211। & $2.002 i$ & 2.뻬 & & & & & $!$ \\
\hline Zero Combetionspsid & 0.1571 & -0.0021 & 0.9681 & 0.1691 & 0.111 & & & $!$ & & \\
\hline Comóa d L & 0.21 & 0.86001. & 1.201 & 7.8781 & 2071 & 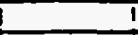 & & 1 & & \\
\hline Manion & 2 & 5.38 & क.5. & 6.16 & 7.81 & & & $i$ & & \\
\hline 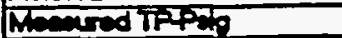 & 779.8 & & & & TिE5 & & & & & \\
\hline Moceured Overelli & 24 & & & & & & & & & \\
\hline & & & & & & & & & & \\
\hline Computad DR & 1.38 & 3.77 & 5.78 & 7.74 & 8.68 & & & & & \\
\hline Fridion & 0.051 & 0.731 & 0.221 & 0.321 & 0.44 & & & & & \\
\hline Acoslaration-t & ก०8 & 007 & 0.091 & 0.11 & 0.131 & & & & & \\
\hline Elevation & 191 & 3.581 & 5.17 & 6.7 & 8.091 & & & & & \\
\hline & & & & & & & & & & \\
\hline
\end{tabular}




\begin{tabular}{|c|c|c|c|c|c|c|c|c|c|c|}
\hline Runt & 8901 & 1 & & - & & 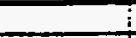 & & $!$ & $!$ & \\
\hline Toxt Find & C4F & $i$ & & & & I & & & 1 & \\
\hline Dath: & 10283 & & & & & & & & & \\
\hline Time: & $8: 35$ & & & & & & & & & \\
\hline Not & Antyicalo & th is used & & & & & & & & \\
\hline Fow Arest? & 0.003361 & +2 & I & & & & & i & i & \\
\hline Heal Fow Arost? & 0.1558 & & & & & ! & & & & \\
\hline SElpresure phig & $-18 \pi$ & & & & I & $T$ & & & & \\
\hline Su Tempar ure finte) & 175.8 & & & & -1 & & & & & \\
\hline Lag. Deneinytomis & 76.84 & 76.781 & 76.73 & 76.591 & 76.001 & 76.591 & 76.59 & 76.71 & $76 . \pi$ & 76.55 \\
\hline Demithensuriom & 3128 & 31.21 & 31.18 & 31.051 & 31.17 & 37.05 & 31.051 & 31.15 & 31.15 & 31.01 \\
\hline 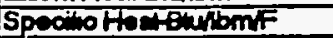 & 0.312 & 0.3121 & 0.312 & 0.3181 & 0.312 & 0.3781 & 0.3181 & 0.312 & 0.312 & 0.318 \\
\hline Lig. Virooshylominhr & 0.36 & 0.350 & 0.359 & 0.3571 & 0.358 & 0.357 & 0.357 & 0.358 & 0.358 & 0.366 \\
\hline 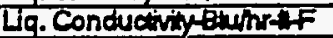 & 0.0851 & 0.0651 & 0.02011 & 0.0061 & $0.0 \infty 6$ & 0.061 & 0.026 & 0.025 & 0.028 & 0.0250 \\
\hline UL.Prand" No. & 4.3 & 4.3 & 4.3 & 4.3 & 4.3 & 4.31 & 4.3 & 4.31 & 4.3 & 4.3 \\
\hline IntA FIOW RXE-GPM & 2 & 1 & & & & & & & & \\
\hline 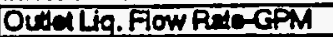 & 0,4 & 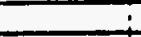 & & & & & & & & \\
\hline OuनT Vep. How RaA-GPM & 1.5 & & & & & ! & & & & \\
\hline Intat tomocremer & 1729 & & & & & & & & & \\
\hline 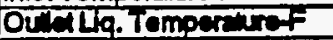 & 375.7 & & & & & & & & & \\
\hline Orita Vep. Tomperthref & 172.4 & & & & & & & & & \\
\hline WEM FOW PM CPM & 1 & 1 & 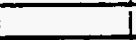 & L & & & 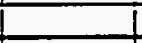 & & & \\
\hline Wortintel Temp.F & 117 & 1 & 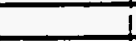 & 1 & 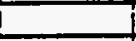 & & & & & \\
\hline Whar ould Tomp.F & 168.7 & 1 & & 1 & 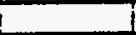 & 1 & & & & \\
\hline Inded Gibmment2 & 372610 & & Temp & 168.6 & Denthy & 78.07 & & & & \\
\hline Subooding & 29 & 1 & & 1 & & i & & & 1 & \\
\hline Fow Obsenvion & & & & & & & & & & \\
\hline & ZON $=1$ & ZONEES & $2 O N=3$ & ZONET & $2 O N=5$ & $20 N=6$ & $201=7$ & ZOKiS8 & ZONES & $\angle 0 N=10$ \\
\hline 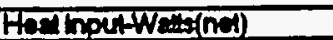 & 287.7 & 200.7 & 203 & 294.7 & 291.5 & 2928 & 291.4 & 2921 & 2900 & 267.8 \\
\hline Hentoss-We"s & Tद्2 & 2.8 & 3.8 & 3.8 & 3.8 & 28 & 1.81 & 2.8 & 3.6 & 19.8 \\
\hline 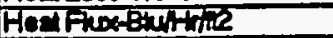 & 2580 & 5068 & 5065 & 51081 & 5053 & 5007 & 50511 & $50 \% 1$ & 5031 & 4642 \\
\hline We Tremp. 13 $=$ & 778.5 & 178.0 & 779.2 & 180.11 & 780.7 & 1800 & 180.81 & 778.01 & 778.6 & 777.8 \\
\hline WeT Tomp. If & T78.8 & $\mid 781$ & $1 \% 9.7$ & 180.81 & 1808 & 181.6 & 180.81 & 779.6 & 178. & 178 \\
\hline WhI Tomp.15F & 180.8 & 180 & 1822 & 181.61 & 181.3 & 181.7 & 181.9 & 180.71 & 180.7 & 179 \\
\hline WeI Tomp. IfF & 172.8 & 178.11 & 1782 & 179.7 & 179.8 & 18121 & 180.01 & 179.61 & 1782 & 178.6 \\
\hline Wall Tomp. IZF & 179.5 & 179.11 & 178.8 & 179.8 & 179.6 & 180.7 & 180.0 & 180.41 & 178.6 & 1772 \\
\hline Instrean Tomp.f & 174 & 174.8 & 174.5 & 175.11 & 174.8 & 175.11 & 176.1 & 174.6 & 174.6 & 175.3 \\
\hline Avo. Wall TompF & 179.5 & 77 & 16 & 180.4 & 180.4 & 181.4 & 180.9 & 179.8 & 17.5 & $\sqrt{753}$ \\
\hline Avo.WaHDT & 5.2 & 4.5 & 42 & 5.1 & 5.4 & 6 & 5.6 & 5 & 2.3 & 24 \\
\hline Aemining Subcooff & 0.4 & & & & & & & & & \\
\hline Esat curly & 0 & 0.02 & 0.05 & 0.07 & 0.1 & 0.12 & 0.751 & 0.18 & 0.2 & 0.2 \\
\hline 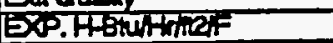 & \$5त् & Tt25 & Tा8्र० & 10028 & 940.7 & 845 & 908.01 & 1015.6 & 1168.1 & 19:21 \\
\hline COMPUIEOH & & & & & & & & & & \\
\hline Jitork & 8002 & 79921 & $78 \pi .1$ & 7828 & $7 r 0.8$ & 7882 & $7 \approx 201$ & 759.8 & 753.1 & 78.2 \\
\hline इradvi(Cudotibo) & स्यरत. & 12650 & सिम्8 & 1250.1 & 12दूर & 1246.1 & 124.7 & 1244.8 & 12द1.1 & T198.8 \\
\hline $\sec d x$ & 8382 & 9472 & 9527 & 9620 & 954.8 & 9589 & 5578 & 958.8 & 952 & 8035 \\
\hline (ADO) & 67848 & 67241 & 65687 & बद्या & ब्ञन8 & 60789 & 58960 & 5697 & 35352 & కख़心 \\
\hline 17 & 1.004 & 1.26 & 1.548 & 7.748 & 1.818 & 2057 & 2184 & 23081 & 2614 & 2502 \\
\hline (1) & 131, & 1636 & 2027 & 3822 & उका. & 2506 & 286.4 & 30211 & 3762 & 3द्यर1 \\
\hline (FBOA & 12050 & 1230| & 120 & 12455 & 12836 & 1241.8 & 12308 & 12388 & 1232 & 71865 \\
\hline & & & & & & & & & & \\
\hline & $\angle O N=2$ & ZONRZ & ZOANE & ZON:S8 & $2017=70$ & & & & & \\
\hline DP houid tomp.t & 80 & & & & & & & & & \\
\hline 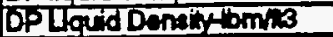 & $\$ 32$ & & & & & & & & & \\
\hline Eeviont & 2 & & 6 & 81 & $9.8 \%$ & & & & & \\
\hline Weatred opyond & 0.373 & 0.888 & 2383 & 2831 & 298 & & & & & \\
\hline Zero Correctiona-peid & 0.157 & -0.002 & 0.85 & 0.161 & 0.17 & & & & & \\
\hline Cormecud Depeld & 0.210 & 0.860 & 7.44 & 2.788 & 2.870 & & & & & \\
\hline Actud DPA & 200 & 3.11 & 4.0 & 5.02 & 8.61 & & & & & \\
\hline 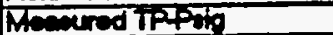 & 187 & & & & 179.0 & & & & & \\
\hline Weseured Overell & 207 & & & & & & & & & \\
\hline & & & & & & & & & & \\
\hline Compered DP & 7.89 & 3.4.81 & 4.82 & 6.13 & 7.31 & & & & & \\
\hline Frictionn & 0.03 & 0.08 & 0.15 & 0.23 & 0.32 & & & & & \\
\hline Aocalerriont & 0.02 & 0.04 & 0.05 & 0.07 & 0.08 & & & & & \\
\hline Everion & 1.84 & उ.3ा & 4.62 & 5.83 & 6.9 & & & & & \\
\hline & & & & & & & & & & \\
\hline
\end{tabular}




\begin{tabular}{|c|c|c|c|c|c|c|c|c|c|c|}
\hline Runt & 8971 & & & $\cdot$ & - & & : & & & \\
\hline Test Fuid & CAF & & & & : & & & & & \\
\hline Date: & 10893 & & & & & & & & & \\
\hline Time: & 8.35 & & & & & & & & & \\
\hline Noto & Analytical & dt is used & & & & & & & & \\
\hline Flow Areatt? & 0.003561 & & & & & & & & & \\
\hline Hod fow Aros-42 & 0.19655 & $\mathbf{i}$ & $\mathrm{i}$ & $!$ & 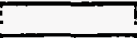 & & T & & & \\
\hline Sad Pressure Pig & $181.5 T$ & T & i & & 7 & $T$ & ? & & & \\
\hline Sal Temperatur of(intol) & 176 & & -1 & & & & & & & \\
\hline Liq. Denaty tomis & 76.681 & 76.571 & $76.68 !$ & 76.521 & 76.571 & $76 . \overline{81}$ & 76.47 & 76.51 & 76.52 & 76.41 \\
\hline Ltant Hea - Qwithm & 31.111 & 31.031 & 31.11 & $30.89 i$ & 31.03 & 30.851 & 30.9 & 30.87 & 30.80 & 30.8 \\
\hline Spectio Hestoultomit & 0.312 & 0.3131 & 0.3121 & 0.3131 & 0.313 & 0.3131 & 0.314 & 0.373 & 0.313 & 0.314 \\
\hline Liq. Visoositymhr & 0.358 & 0.3561 & 0.3581 & 0.356 & 0.356 & 0.355 & 0.3541 & 0.3561 & 0.356 & 0.354 \\
\hline Lig. Conductitubers fof & 0.026 & 0.0251 & $0.026 !$ & 0.02591 & 0.026 & 0.02591 & 0.0259 & 0.0259 & 0.0250 & 0.0250 \\
\hline Liq. Prandid No. & 4.31 & 4.31 & 4.31 & 4.31 & 4.3 & 4.3: & 4.31 & 4.3 & 4.3 & 4.3 \\
\hline Inlet Flow Rate-GPM & 1.11 & & & & & & & & & \\
\hline Outlot Lig. Fow Rata-GPM & 0.41 & & & & 7 & i & : & & & \\
\hline Outet Vao. Flow Rate-GPM & 0.81 & $T$ & ; & & & & & i & & \\
\hline Inlet Temperesurof & 173.91 & & $\overline{:}$ & ? & 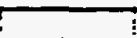 & T & ! & & & \\
\hline Outlot Lig. Tomporiaref & 176.41 & T & $T$ & T & $i$ & T & ! & & & \\
\hline Owet Veo. Temperefor of & 173.41 & $T$ & T & 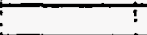 & $\bar{i}$ & T & $T$ & & & \\
\hline Wald Fow Pato-GPM & $\mathrm{i}$ & $T$ & T & i & I & $\bar{i}$ & 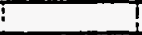 & $T$ & & \\
\hline Weter indat Temp.F & 118.5 & T & i & & I & T & i & T & & \\
\hline Wely outen Tomp.f & $1 \approx .6$ & 1 & 1 & 1 & 1 & 1 & i & $\bar{i}$ & & \\
\hline Intal Glbmhin & $205 \% 21$ & & ITemp & 166.81 & Denaiy & 78.41 & & & & \\
\hline Suboodingf & 2.1 & + & $i$ & +1 & & +1 & 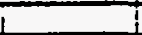 & & & \\
\hline Flow Obsenvation & & & & & & & & & & \\
\hline & ZOKE & ZONES & ZONE3 & ZONEA : & ZONES & ZOKiE6 : & ZONE? & ZONE8 & ZONES & $201=50$ \\
\hline Heat houtWa:-(net) & 288.3 & 282.2 & 2872 & 287.6 & 288.1 & 289.81 & Z8ा & 287.2 & 289.1 & 2036 \\
\hline Her Loss-Wall & 12.11 & 281 & 3.81 & $3.9 !$ & 3.8 & 2.81 & 7.81 & 2.81 & 3.6 & 20 \\
\hline Heaffox-Bulth/R2 & 4997 & 45281 & 49781 & 46851 & 49gत & 50201 & 4EZ31 & 4978 & कणाI & G94 \\
\hline Waltemo. औ' & 178.81 & 179.11 & 979.41 & 180.11 & 1805 & 18081 & 180.7 & 179.81 & T78. & 78 \\
\hline WET TEmp. RLF & 179.11 & 179.21 & 779.81 & 180.21 & 780.8 & 187.61 & 181.2 & 180 & 178.8 & 778.8 \\
\hline Wal Tomp. $55 F$ & 1811 & 1801 & 1824 & 181.41 & 187.71 & 181.61 & 182.2 & 181.71 & 180.7 & 178 \\
\hline WEH TOMP. IIF & 173.8 & 979.21 & 178.4 & 179.51 & 179.8 & 181.81 & 181 & 180.11 & 178.7 & 176.8 \\
\hline WEIT Tamo. REF & 179.9 & 179.41 & 178.8 & 179.8 & 179.6 & 180.7 & $181.2 \mid$ & 180.81 & 778.0 & 777.6 \\
\hline Instream Temp.ff & 174.81 & 175.21 & 174.8 & 175.41 & 175.2 & 775.61 & 175.9 & 175.51 & 775.4 & 175.8 \\
\hline Avg. Wall Temp.F & 179.7 & 1792 & 479.11 & 180,31 & 180.3 & 181.41 & 181.3 & 180.3 & 779.4 & โ78. \\
\hline Avo. Wall DT.F & 4.7 & 3.8 & 4.1 & 4.7 & 4.9 & 5.5 & 5.1 & 4.51 & 3.8 & 2 \\
\hline Remaning Suboooff & -24 & & & & & & & & & \\
\hline Exd Qualy & 0.02 & 0.07 & 0.121 & 0.161 & 0.21 & 0.251 & 0.31 & 0.31 & 0.30 & 0.45 \\
\hline ExP.HBhuthiner & 105.5 & 1295.6 & 1214.21 & $10 \approx 8.5 \mid$ & 1024.8 & 909.61 & 95821 & 1097.81 & 136.] & 256.8 \\
\hline COMPUTEDH & & & 1 & & & & ! & & & \\
\hline Jallouk & 85. & 8ख्य.ता & 785.51 & 79271 & 787.4 & 78751 & $7 \pi 21$ & 775.21 & 775.2 & 7678 \\
\hline 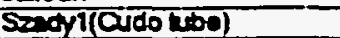 & 7253.8 & T27.11 & 1231.4 & 1258 & 1238.1 & 124241 & 1232 & $\mid 2238.8$ & 12105 & Tद्वा.1 \\
\hline $5 \operatorname{sed} 2$ & 94.6 & $\$ 65.7$ & $941.8 !$ & $9 \overline{86.51}$ & 948.8 & 95851 & 94281 & 947.8 & 9322 & 9प्र \\
\hline (RบB) & उ7is & 36017 & 34178 & 32088 & 30051 & 292201 & 275031 & $25 \%$ & 2397 & द्रान \\
\hline (F) & 1.302 & 1.6501 & 1.986 & 2.187 & 2.38 & 25001 & 2718 & 2.870 & 3.016 & 3.187 \\
\hline (1+40) & 1063 & 134.91 & 159.5 & 178.61 & 186 & 20031 & 2321 & 234.61 & 248.1 & 2565 \\
\hline (स्य) & $1230 \pi$ & 12256 & $120 \overline{0}$ & 122181 & 12315 & 12404 & 120.6 & T2335 & 72372 & 722 \\
\hline & & & & & & & & & & \\
\hline & ZONE2 & ZONEA & ZONE6 & ZONES & ZOKETO & & & & & \\
\hline DP gquid Tomp.f & 812 & & & $i$ & $\mathrm{i}$ & & & & & \\
\hline 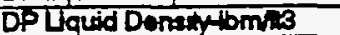 & \$3.03ा & & : & $:$ & 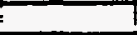 & I & & & & \\
\hline Elevetion & & 4ा & 6 & 8 & 9.8751 & & & & & \\
\hline Meatured DPPad & 0.37 & 1.0831 & 2.73 & 287 & 3.224 & & & & & \\
\hline Zero Corredionspesd & 0.167 & -0.002 & 0.058 & 0.169 & 0.11 & & & & & \\
\hline Corroctod DP pad & 0.182 & 9.688 & 1.780 & 2.रण & 3.514 & & & & & \\
\hline Act-1DP & 2.07 & 2.8 & 3.86 & 4.65 & 5.4 & & & & & \\
\hline Whatered TPPag & 187.5 & & & & 7607 & & & & & \\
\hline Meavered Overalli & 1.51 & & & & & & & & & \\
\hline & & & & & & & & & & \\
\hline Comouted oph & 7.55 & 275 & 3.78 & 4.74 & 5.56 & & & & & \\
\hline Friction- & 0.02 & 0.09 & 0.08 & 0.13 & 0.18 & & & & & \\
\hline Aocolereiont & 0.01 & 0.02 & 0.03 & 0.04 & 0.05 & & & & & \\
\hline Elevetionth & 1.531 & 2ख & 3.60 & 4.57 & 5.32 & & t & & & \\
\hline & & & & & & & & & & \\
\hline
\end{tabular}




\begin{tabular}{|c|c|c|c|c|c|c|c|c|c|c|}
\hline Run: & 8श्रा & 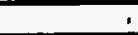 & i. & & & , & & 1 & & \\
\hline Tost Fluid &.$C 4 F$ & . & 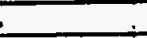 & & & & & & & \\
\hline Dats: & 108931 & & & & & & & & & \\
\hline Time: & $8: 35$ & & & & & & & & & \\
\hline Note & Anabytical o & th is used ! & $!$ & & 1 & & & & & \\
\hline Flow Aroarte & 0.003361 & -1 & $i$ & & $i$ & & & & & \\
\hline Horlfow Aroat? & 0.19695 & & & & & & & & & \\
\hline S:Ptessure pig & 1822 & T: & ! & T! & & & & & & \\
\hline Sal. Tamperaure F(inlai) & 176.3 & 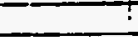 & ? & & & & & & & \\
\hline Ug. Dantioytomina & 76.041 & 76.781 & 76.731 & 76.481 & 76.5 & 76.451 & 76.31 & 76.431 & 76.48 & 76.38 \\
\hline Lenthentexplom & 31.00 & 31.21 & 31.181 & 30.951 & 31.01 & 30.81 & 30.82 & 30.21 & 30ल2 & 30.88 \\
\hline Specirotherembemf & 0.318 & 0.3121 & 0.312 & 0.3131 & 0.313 & 0.3131 & 0.314 & 0.3141 & 0.314 & 0.314 \\
\hline Lig. Viscositubmithx & 0.357 & 0.3591 & 0.359 & 0.3551 & $0.356 !$ & 0.3551 & 0.353 & 0.354 & 0.354 & 0.354 \\
\hline Lq. Conductivity eumstiF & 0.0261 & 0.02511 & 0.02611 & 0.0259 & 0.0259 & 0.02591 & 0.0258 & 0.0209 & 0.0259 & 0.0258 \\
\hline Lg. Prande No. & $4.3 !$ & $4.3 i$ & 4.3 & 4.31 & 4.3 & 4.3 & 4.3 & 4.31 & 4.3 & 4.3 \\
\hline Intot Fow Rac-GPM & 4.61 & & & & & 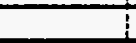 & & & & \\
\hline OulitLig, Fow RatoGPM & $0 . \overline{91}$ & & & : & & & & & & \\
\hline Outet Vap. Fow Rate-GPM & 231 & & & & & & & & & \\
\hline Indet Tompormuref & 173.8 & & $i$ & & & & & & & \\
\hline outella. Tomper Tref & T75.81 & & I & & & & & & & \\
\hline ótive. Tompertiref & 773.5 & & I & & & & & & & \\
\hline WEST FIOW REA-GPM & -1 & 1 & 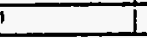 & & & & & & & \\
\hline Wher hilat Tomp.ff & 109.81 & & $I$ & $i$ & & & & & & \\
\hline Whar octet Temp.f & 170.4 & & 1 & 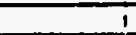 & & & & & & \\
\hline net G lomhwh & 852477 & & iTemp & 170.4I & Dansity & 77.65 & & & & \\
\hline Subcoolingf & 25 & & - & + & & & & & & \\
\hline Fow Obsenvation & & & & & & & & & & \\
\hline & ZOKEST & $20 N=32$ & ZONES & ZONEK T & $20 N=5$ & ZOKE & ZONEI & ZONES & ZONEO & $\angle O N=T 0$ \\
\hline 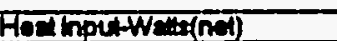 & 403.5 & 40.26 & 408.8 & 4028 & 408.6 & 4052 & 400.7 & 403.1 & 705 & 400.1 \\
\hline Heallose-WC: & 221 & 5.31 & 721 & 8.9 & 9.81 & 9.7 & 7.7 & 8.5 & 8.5 & 45.7 \\
\hline Hearfioxewhathe & क9शी & 69ां। & 70521 & छाया & \$35! & $7 \overline{0 B}$ & Eखा & क\$8] & 7028 & 68 \\
\hline We rromp. Ha & 180 & Tहण1 & T80.46 & 181 & दिन्ड & 7810 & 787. & 780.7 & Fon & T79.1 \\
\hline Wh Tomp. ILF & 180.6 & 180.11 & 1812 & 181य & 181.8 & 1826 & 18ट्रा & 181.8 & Tळ0 & 18 \\
\hline WaTTomp. $16 f$ & 1825 & 180.8 & 183.6 & 1825 & 1822 & 1828 & 183.8 & 1821 & 181.0 & 179.8 \\
\hline WE'Tomp. ITF & 173.8 & 180.4 & 179.2 & 180.8 & 180.6 & $182 \pi$ & 18261 & 181.21 & 1850 & 177.5 \\
\hline WETTOMP. Hस & 1812 & 18051 & 1801 & 180.6 & 1805 & 181.8 & 1827 & 1822 & 180 & 778.4 \\
\hline natram Tomp.F & 174.9 & 174.3 & 174.51 & 175.61 & 175.3 & 175.7 & 176.3 & 175.8 & 175.8 & 176 \\
\hline Avo. Wall Tomp.f & 181.1 & 180.4 & 1802 & 181.3 & 181.8 & 1824 & 1826 & 1815 & 1802 & 1792 \\
\hline AYo. Wall DT.F & 5.9 & 5.81 & 5.4 & 5.3 & 5.7 & 6.3 & 6 & 5.4 & 4.8 & 2.8 \\
\hline Remaining subcooff & & & & & & & & & & \\
\hline Ex'Qusty & -0.01 & 0.01 & $0 . \overline{2}$ & 0.04 & 0.5 & 0.07 & $0 . \mathbf{6}$ & 0.1 & O.tता & 0.78 \\
\hline EXP.ABurnear & 1183.8 & 1206.4 & 1310.4 & 13012 & 1236.8 & 11112 & TIET3 & 12378 & TE:3.] & टरS: \\
\hline COMPUIEOH & & & & & & & & & & \\
\hline Ja'ouk & 919.6। & 915.ता & 90201 & 838.81 & 8ु5ा & 876.11 & $\mathbf{8 8 5 3 5}$ & छहरा & 85.11 & 851.7 \\
\hline 50dvil(ado wo) & 7661.8 & 14501 & $14 \% 3.0$ & 1457.81 & T45:.0 & 14781 & T474.2 & 747850 & T4:ast & T470.4 \\
\hline $\operatorname{send} 2$ & 1262 & 220.7 & 1202 & 12द्या & 124 & 121.I & 12475 & 1247.0 & 1250 & 1246.8 \\
\hline (PLA) & 160002 & 15517 & $158 \% 00$ & 15377 & 14897 & 14trint & 74600 & 1429 & 140.5 & Fo\% \\
\hline$(5)$ & 1.000 & 1.004 & 1.32 & 1.401 & $1 . \infty 2$ & 1.746 & 1.815 & 1.891 & 2ब्रा & 2.105 \\
\hline (1) & 2553 & 2हा & 3354 & उ.8. & 476 & 446ा & 450. & 48 & 5158 & 550 \\
\hline (स-30I) & 145929 & 14532 & 1465 & $1452 \times 1$ & 14535 & 14656 & 145931 & 7457.8 & 14502 & 74515 \\
\hline & & & & & & & & & & \\
\hline & $20 \mathrm{ON}=2$ & ZONE4 & 1205: & ZORES & $\angle O K=T 0$ & & & & & \\
\hline DP houid Temp.f & 85.8 & & 1 & & & & & & & \\
\hline DPLLquid Densiy tomins & क्टाना & & & & & & & & & \\
\hline Elevitiona & 2 & का & 6ा & & 9.8751 & & & & & \\
\hline Measured DP phla & 0.391 & 0.74 & T.978 & 1.73 & 2175 & & & & & \\
\hline Zero Comectionsepd & 0.157 & -0.002 & 0.936 & 0.169 & 0.13 & & & & & \\
\hline cormed Dppild & ozin & 0.76 & 1.002 & 1.585 & 2005 & & & & & \\
\hline Actud & $T .86$ & 3.60 & 5.38 & 6.78 & 8.06 & & & & & \\
\hline Mescured Tfphth & 18्23 & & & & $182 \pi$ & & & & & $\because$ \\
\hline Meneured overall & 0.18 & & & & & & & & & \\
\hline & & & & & & & & & & \\
\hline Competad DQA & दर्श & $4.06 \mid$ & 5.छा & $7 . \pi 1$ & 9.48 & & & & & \\
\hline Edctions" & 0.11 & 027 & 0.47 & 0.9 & 0.93 & & & & & \\
\hline Acoling & 0.05 & ठ.दी & कर्य & 0 क्षो & 0301 & & & & & \\
\hline 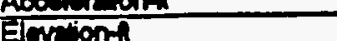 & 1.3 & 3.50 & 527 & 6.63 & $8 . \overline{3}$ & & & & & \\
\hline & & & & & & & & & & \\
\hline
\end{tabular}




\begin{tabular}{|c|c|c|c|c|c|c|c|c|c|c|}
\hline Runt & . 8931 & & & $\therefore$ & 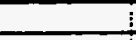 & $\vdots$ & 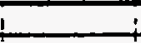 & 1 & 1 & \\
\hline TestFluid & CAF & $\therefore$ & & $\perp$ & & i & $i$ & $i$ & $i$ & \\
\hline Date: & 10893 & $\overline{-}$ & & & & $i$ & 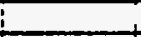 & & & \\
\hline Timo: & $15: 411$ & & & & & & i & & & \\
\hline Nole & Analytical d & dt is used I & & & & 1 & 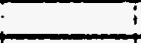 & & & \\
\hline Flow Aresti2 & 0.003561 & & $\therefore$ & & & & & & & \\
\hline Hod Fow Area-12 & 0.19695 & & & & & & & & & \\
\hline Sal Prossuro prig & 181.5 & T & $T$ & + & & $!$ & I & & & \\
\hline SE. Tomporaturef(inlat) & 176 & -1 & $5:$ & & & 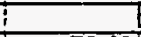 & $\square$ & & & \\
\hline Lig. Densty tominis & 76.81 & 76.971 & 76.84 & 76.611 & 76.64 & 76.48 & 76.48 & 76.561 & 76.57 & 76.43 \\
\hline Letanx Hox-Bustom & 31221 & 31.321 & 31.261 & 31.07 & 31.09 & 30.85 & 30.95 & 31.01 & 31.03 & 30.92 \\
\hline Specifo Heal-BuhbmiF & 0.312 & 0.3711 & 0.312 & 0.318 & 0.313 & 0.313 & 0.313 & 0.313 & 0.318 & 0.314 \\
\hline Liq. Viscosingminhr & 0.36 & 0.3511 & 0.361 & 0.357 & 0.357 & 0.355 & 0.355 & 0.356 & 0.356 & 0.354 \\
\hline 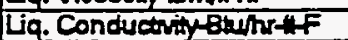 & 0.02611 & 0.02521 & 0.02611 & 0.0261 & 0.026 & 0.0259 & 0.0259 & 0.0259 & 0.008 & 0.0259 \\
\hline Lia. Prend No. & 4.31 & 4.31 & 4.31 & 4.31 & 4.3 & 4.3 & 4.3 & 4.31 & 4.3 & 4.3 \\
\hline Inlot Fow Rato-GPM & 4.6 & & & & & & & & & \\
\hline Outot Lig. Fow PatG-GPM & 1.11 & $\div$ & & & & & & & & \\
\hline Outot Vap. Fow Rato-GPM & $2.5 !$ & & 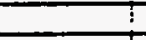 & & i & & i & & & \\
\hline Inlat Tomper auref & 173.51 & $\cdot$ & 1 & & & 7 & & & & \\
\hline Ou Cl Lia. Tempormar & 78.61 & T! & & I & & & & & & \\
\hline OUtat Vad. Tomporturaf & 1729 & T! & & $T$ & & & & & & \\
\hline WDOPFOW RAO-GPM & 1 & T & I & T & & & & T & & \\
\hline Werer Inter Temp.f & 128.61 & $!$ & $!$ & 1 & 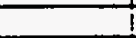 & 7 & I & 1 & 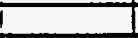 & \\
\hline We ouldel Temp.f & 169.6 & $T$ & 1 & 1 & & & & I & I & \\
\hline Indel Glomperit2 & 852477 & & Tamp & 170.41 & Density & 77.66 & & 1 & 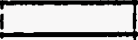 & \\
\hline Subcoolingf & 25 & $\perp$ & & 1 & 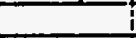 & & & I & I & \\
\hline Fow Obsention & & & & & & & & & & \\
\hline & ZONEI & ZONE2 & ZONE3 & ZONE4 T & ZONE5 & ZONE6 & ZONE & ZONE8 & ZONE9 & $\angle O N=10$ \\
\hline Hor holtwathot) & 199.7 & 199.8 & 204.1 & 199.4 & 218.8 & 215 & 201.11 & 198.4 & 201.4 & 198.6 \\
\hline HeCLoso-Was & 18 & 4.31 & 5.81 & 6.81 & 7.2 & 8.6 & 4.91 & 5.91 & 6.3 & 34.7 \\
\hline Hes flox bustride & 34621 & 3455 & 3538 & उदहB। & 3783 & $372 \pi$ & 3488 & $3+38$ & 341 & 3408 \\
\hline WeTtemp. & 778.5 & 178.81 & 178.6 & 179.11 & 179.4 & 179.5 & 1792 & 17821 & 177.9 & 171.5 \\
\hline We Tomp t4F & 178.51 & $178 . \pi$ & 178.8 & 178 & 179.5 & 180.11 & 179.8 & 178.61 & 178.6 & 778 \\
\hline Wall Tomo. $16 F$ & 180.81 & 179.6 & 181.4 & 18021 & 180.2 & 180.2 & 180.8 & 179.8 & 179.7 & $17 \overline{8.3}$ \\
\hline Wall Tomp. $1 F$ & 173.5 & 178.7 & 177.8 & 178.81 & 178 & 180.2 & 179.7 & 178.7 & 177.6 & 178.3 \\
\hline WCll Tomo. L2F & 179 & 178.81 & 178.4 & 178.81 & 178.7 & 179.4 & 179.8 & 179.31 & 177.8 & 176.7 \\
\hline Instream Temp.f & 174.2 & 173.7 & 174 & 175 & 174.9 & 175.6 & 175.61 & 175.31 & 175.2. & 176.8 \\
\hline Av. Wall Temp.f & 1792 & 178.6 & 178.4 & T79.3 & 179.4 & 180 & 179.8 & 178.9 & 178.2 & 77.3 \\
\hline Avp Wat DT F & 4.8 & 4.81 & 4.3 & 4.1 & 4.3 & 42 & 4.7 & 3.51 & 29 & 1.3 \\
\hline Remaning Subcooff & 1.8 & & & & & & & & & \\
\hline Ext Quatisy & -0.02 & -0.07 & of & 0.01 & 0.01 & 0.02 & 0.08 & 0.04 & 0.05 & 0.00 \\
\hline EXP. HBturthal= & च23 & 724.4 & 8205 & 83921 & 888.4 & 880.7 & 858.1 & 9927 & 11881 & 5it.1 \\
\hline COMPUTE H & & & & & & & & & & \\
\hline Jalouk & 609.4 & 60I.4T & 6IE.II & काI & ह4.1 & 68.1 & 50.1 & 617.61 & E्या & 60.1 \\
\hline Sendy I(Odo tho) & 1009.7 & 1028.97 & 1009.4 & T034 & 10828 & 1079.4 & T077.7 & 1041.41 & 1069 & 108.1 \\
\hline seade & 738.7 & 733.61 & 748.5 & 741.81 & 7926 & 7905 & 761.1 & 758.01 & 760.1 & 787.8 \\
\hline (RUO) & 155088 & 154887 & 15478 & 1559141 & 154586 & 154205 & 152950 & 1518291 & 140000 & 740001 \\
\hline (F) & 1.009 & 1.0061 & 1.009 & 1.0021 & 1.250 & 1.375 & 1.46 & 1.54 & 1.607 & 1.680 \\
\hline (मि) & 2549 & 254.71 & 254.8 & 277.6 & 3200 & 340.9 & 3725 & 391.5ा & 408.6 & 462 \\
\hline$(13014)$ & 1024,41 & 10208 & 1032 & 10273 & 10734 & 1057 & 1037.8 & 100261 & 10280 & T0125 \\
\hline & & & & & & & & & & \\
\hline & ZONEZ & ZONE 4 & $20 K=6$ & ZONBS8 & ZONE & & & & & \\
\hline DP lquid Tamp.f & (85.4. & & & & 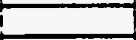 & & & & & \\
\hline DPLguid Densidom/13 & 9244 & & & 1 & 6 & & & & & \\
\hline Eeveriont & 21 & 4 & 6 & हा & 9.875 & & & & & \\
\hline Meatured De्phad & 0.321 & 0.491 & 1.7001 & 1.34 & 9.658 & & & & & \\
\hline Zoro Correcliona-pesid & 0.157 & -0.002 & 0.858 & 0.169 & 0.71 & & & & & \\
\hline Compoted Dppide & 0.255 & $0.4 \% 81$ & 0.75 & 1.17 & 1.558 & & & & & \\
\hline Achel DPA & 1.98 & 3.921 & 5.84 & 7.47 & 9.01 & & 1 & & & \\
\hline Mracured TPQh & 181.5 & 1 & 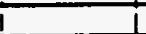 & 1 & 181.4 & & & & & \\
\hline Mocoured overallh & 0.10 & $i$ & 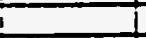 & $i$ & $i$ & & & & & \\
\hline & 1 & & & & & & & & & \\
\hline Compund DRA & 2.1 & 4.2 & 6.17 & 8.07 & 9.82 & & & & & \\
\hline Friction- & 0.1 & 0.2 & 0.36 & 0.531 & 0.7 & & & & & \\
\hline Acoderctiond & of & 0.06 & ס.Tा & 0.44 & 0.17 & & & & & \\
\hline Everiont & 2) & 3.98 & $5 . \pi$ & 7.4 & 8.55 & & & & & \\
\hline & & & & & & & & & & \\
\hline
\end{tabular}




\begin{tabular}{|c|c|c|c|c|c|c|c|c|c|c|}
\hline \multicolumn{11}{|l|}{ Run! } \\
\hline Tosf Fuid & $\frac{\text { CAF } 8}{1011931}$ & & & & & & & & & \\
\hline Timo: & 8.52 & & & & & & & & & \\
\hline Noto & Anaysticalc & dis used & & & & & i & & & \\
\hline Fow Areste & 0.00336 & & & & & & & & & \\
\hline Hod Fow Areat? & 0.18696 & 1 & $i$ & & & & & & & \\
\hline 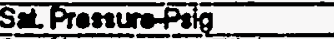 & 902 & & & & & & & & & \\
\hline Su. Tomper wrot finde) & 129.8 & & & & & & & & & \\
\hline Lig. Dentitytomia & 86.62 & 86.89 & 86.65 & 86.16 & 86251 & 86.21 & 86.25 & 86.451 & 86.67 & 86.21 \\
\hline 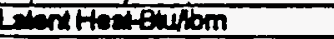 & 39.18 & 39.321 & 39.28 & 38.881 & 38.96 & 38.9n & 38.96 & 39.181 & 39.14 & 38.98 \\
\hline Speatio Hederelomf & 0.278 & $0.27 \pi$ & $0.2 \pi$ & 0.279 & 0.278 & 0.2781 & 0.278 & 0.2781 & 0.278 & 0.278 \\
\hline Liq. Vizoosityllom/htr & 0.571 & 0.576 & 0.575 & 0.559 & 0.552 & 0.5611 & 0.52 & 0.5681 & 0.59 & 0.66 \\
\hline Lig. Conductivity theth & 0.0326 & 0.0327 & 0.0323 & 0.0323 & 0.0324 & 0.0324 & 0.0224 & 0.0520 & 0.0038 & $0.0 \times 24$ \\
\hline Lig. PrandéNo. & 4.86 & 4.881 & 4.87 & 4.811 & 4.821 & 4.82 & 4.821 & 4.85 & 4.85 & 4.83 \\
\hline Intof flow RAG-GPM & 4.9 & & & & & & & & & \\
\hline 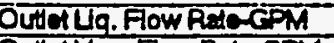 & 1.2 & & & & & & & & & \\
\hline OuthIVAp. Fow Rat-GPM & 2.6 & & & & & & & & & \\
\hline nde tompormeref & 124 & & & & & & & & & \\
\hline 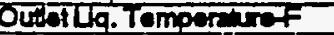 & 125. & & & & & & & & & \\
\hline Outavep. Tompowetrof & 121.11 & T & & & & & & & & \\
\hline WEM FIOW PEO-GM & & & & & & & & & & \\
\hline Westortal Tompoff & 85.8 & & & & & & & & & \\
\hline Wer oclded Tomp.f & 119.5 & & $i$ & 1 & & & & & & \\
\hline nha Glbmhnthe & 1000110 & & iTamp & 118.4 & Dentily & 87201 & & & & \\
\hline Suboodlingf & 5.9 & & & & & & & & & \\
\hline Fow Obsenvition & & & & & & & & & & \\
\hline & ZONET & ZONE2 & ZONE3 & ZONEA & ZONi=5 & ZON $=6$ & ZONi=7 & ZONE $=8$ & $20 N=8$ & $O N=10^{\circ}$ \\
\hline Hod hoptwethr(nel) & 201 & 290.11 & \begin{tabular}{|l|}
287.5 \\
\end{tabular} & 292 & 287 & 288.8 & 291.8 & 2886 & $\mathbf{2 5 8 6}$ & \\
\hline 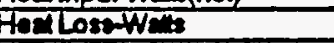 & 6.4 & 1.5 & 1.9 & 23 & & 1.4 & & 1.3 & 1.8 & 9.8 \\
\hline 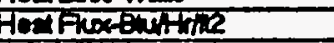 & $50 \mathrm{mh}$ & 5000 & 497 & 5062 & 2675 & 498 & 50389 & 5008 & 7850 & 5010 \\
\hline Wel Tomp. $13 F$ & 133.5 & 133.4 & 1325 & 135 & 134.6 & 133.7 & 134.91 & 131.2 & 1808 & 7505 \\
\hline Wू Tomp.14 & 133.8 & 139 & 1320 & 135 & 7322 & 134.11 & 134.8 & 137.7 & 130.9 & 1322 \\
\hline Wal Tomo. $15 f$ & 135.7 & 134 & 735.8! & 138.41 & 134.8 & 134.8 & 135.7 & 133.61 & 1325 & 131.8 \\
\hline W=1 Tomo. $11 \mathrm{~F}$ & 124 & 133.4 & 131.8 & 135.5 & 133.8 & 134 & 135.8 & 13281 & 128.8 & 130.6 \\
\hline WNI Tomo. 124 & 134 & 133.41 & 131.8 & 135 & 733.6 & 133.6. & 735.7 & 13271 & 1802 & 130.0 \\
\hline netrean Temo,f & 123.0 & 722.01 & 123.7 & 126.11 & 725.6 & 125.7 & 125.5 & 124.31 & 122 & 125.4 \\
\hline avg. Wall Tomp.F & 134,3 & 133.9 & 1321 & 135.5 & 734.11 & 1342 & 134.9 & 13231 & 130.7) & 1372 \\
\hline Av. Wall DT. 7 & 10.1 & 10.2 & 8.8 & 9.11 & 8.4 & 8.2 & 9.2 & 7.8 & 6.8 & 5.6 \\
\hline Remaining Suboostf & 4.8 & & & & & & & & & \\
\hline Exat Cuality & -0.03 & -0.03 & -0.02 & -0.011 & of & 0 & 0.07 & 0.02 & 0.02 & 0.08 \\
\hline SXP.1म & 498.8 & 49483 & 565.6 & 3535 A & हली & 60.6.6. & 516.1 & ब236 & 72.4 & 920 \\
\hline COMAPUIEDH & & & & & & & & & & \\
\hline solk & $\frac{568.9}{8055}$ & $\frac{56.11}{89.8}$ & $\frac{524}{804}$ & $\frac{58.71}{9.2221}$ & $\frac{571.8}{910.2}$ & 5720 & $\begin{array}{l}578.8 \\
90222\end{array}$ & $\begin{array}{l}577.31 \\
513.11\end{array}$ & $\frac{5827}{912 \pi}$ & 509 \\
\hline 579 & $\frac{80.0}{6838}$ & $\frac{8917.81}{658.61}$ & 658.1 & $\frac{9.2 .1}{678.41}$ & $\frac{610.4}{650.8}$ & 6518 & $\frac{\theta<2}{68}$ & $\frac{580}{250}$ & 57ad & $6882^{2}$ \\
\hline (स्वa) & 116974 & 115860 & 116002 & 119660 & 118770 & T1900/ & 118000 & 11586001 & 114500 & $=715800$ \\
\hline (F) & 1.006 & 1.006 & 1.000 & 1.008 & 1.000 & 1.008 & 1.206 & 1.458 & 1.560 &.$\quad 1.60$ \\
\hline (1) & 367.4 & 268.8 & 2600 & 268.8 & $258 \mathrm{an}$ & 268.6 & 3428 & 387 & 7238 & 7621 \\
\hline (FBOL) & 897.6 & 8893 & 80 & 9146 & 9028 & 9038 & 900.1 & 8802 & 8000 & 895 \\
\hline & & & & & & & & & & \\
\hline & ZON: & ZONREA & $20 k=6$ & $201=8$ & $20 N=10$ & & & & & \\
\hline DPlavid Tamp. & 78 & & & & & & & & & \\
\hline DPUquid Denaty bom/18 & 83.78 & & & & & & & & & \\
\hline Exvetionth & & 4ा & & 81 & 9.875 & & & & & \\
\hline 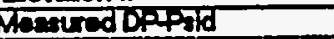 & 0.25 & 0.3601 & 7.355 & $0.98 \pi$ & 1.207 & & & & & \\
\hline Eero Comectonepsid & 0.163 & 0.0011 & 0.985 & 0.1821 & 0.00 & & & & & \\
\hline 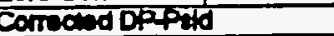 & 0.128 & 0.8051 & 0.47 & 0.8251 & 1.147 & & & & & \\
\hline ACt1D DP & 1.90 & 3.841 & 5.87 & 7.321 & 8.82 & & & & & \\
\hline 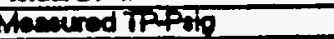 & 602 & & & & 87.1 & & & & & \\
\hline hemured overith & 5.17 & & & & & & & & & \\
\hline & द12 & 435 & & 8 多 & 10,77 & & & & & \\
\hline 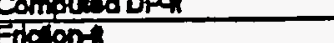 & $\frac{2.12}{0.12}$ & 0.201 & (0.00 & 0.59 & 0.83 & & & & & \\
\hline oelertion & & of & 0.00 & ర.2. & 0.28 & & & & & \\
\hline Elvetiont & & दो & 5.33 & 7.59 & 9.06 & & & & & \\
\hline & & & & & & & & & & \\
\hline
\end{tabular}




\begin{tabular}{|c|c|c|c|c|c|c|c|c|c|c|}
\hline Runt & 8961 & 1 & 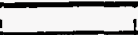 & $i$ & & 1 & & & 1 & \\
\hline Touf Fuid & $.24=8$ & & & & & 1 & & & & \\
\hline Defe: & 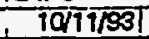 & i & & & & 1 & & 1 & I & \\
\hline Time: & $15: 20$ & & & & & $t$ & & & $i$ & \\
\hline Noto & Ancyion o & His used! & & & & 4 & & & & \\
\hline Fow Arest? & 0.003561 & & & & & & & & & \\
\hline Hes flow Areat & 0.15055 & & & & & & & & & \\
\hline Sz Prestur Psig & 86.4 & & & & & & & & & \\
\hline SITempormentintel) & $\sqrt{27.4}$ & & & & & & & & & \\
\hline Le. Dentity tom/3 & 86.64 & $86 . \overline{721}$ & 88.64 & 86.871 & 86.421 & 86.51 & 86.41 & 86.591 & 88.64 & 86.6 \\
\hline Len Her Bxutbm & 39.28 & 39.34 & 39.28 & 39.06 & 39.1 & 39.171 & 39.091 & 39.241 & 3928 & 39.20 \\
\hline Speoiso toefourbomf & 0.27 & 0.27 & 0.27 & 0.2781 & 0.278 & 0.2781 & 0.278 & 0.27 & 0.277 & 0.277 \\
\hline Lig. viscosithm & 0.574 & 0.677 & 0.574 & 0.5661 & 0.6671 & 0.571 & 0.567 & $-0 . \overline{6} 73$ & -0.576 & 0.57 \\
\hline La. Canductive insth & 0.0327 & 0.0327 & 0.0527 & $0.0325 i$ & 0.0325 & 0.00861 & 0.05251 & 0.03271 & 0.00301 & 0.0327 \\
\hline Lia. PrandiNo. & 4.87 & 4.88 & 4.87 & 4.84 & 4.84 & 4.851 & 4.84 & 4.87 & 4.87 & 4.87 \\
\hline Inlat flow Rade-GPM & 4.8 & & & & & i & & +3 & & \\
\hline Outat Liq. Fow Rato-GPM & 1.11 & & & & I & & & & & \\
\hline Outlet Vap. Flow Reto-GPM & 2.61 & & & & i & & & & & \\
\hline Inla Tompersturef & T291 & & & & & & & & & \\
\hline 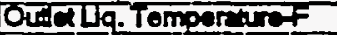 & 723.1 & & & & & & & & & \\
\hline Ovid Vy.Temper & 719.4 & & & & & & & & & \\
\hline Wear Fow RMACPM & & I & T & & & 1 & & & & \\
\hline Water thet Temo.f & 751 & 1 & $T$ & 1 & & I & & & & \\
\hline Werer ould Temp.f & 118 & 1 & $\bar{I}$ & 1 & & & & & & \\
\hline Inde Glominti2 & 1000521 & & Temp & T18.81t & Dentity & 87.34 & & & & \\
\hline Subcoolingf & 4.5 & & & 1 & & 1 & & 1 & 1 & \\
\hline Fow Observation & & & & & & & & & & \\
\hline & $20 N=1$ & ZONE2 & ZORES & ZOKEA is & ZONES & ZONEG & ZONET & ZONEB & $\angle O N=9$ & $\angle O N=10$ \\
\hline 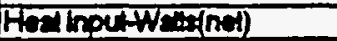 & 2385 & 291.0 & 290.8 & 289.6 & 2927 & 282 & 283.8 & 291.4 & 2क्या & 2928 \\
\hline Had LosedWa'ts & 6.3 & 1.5 & 1.9 & 2.11 & 2 & 1.4 & $\pi$ & 1.3 & 1.11 & 9.5 \\
\hline Fedfor buithin & 5001 & 50001 & 5052 & 50201 & 5074 & 5018 & कावां & 5051 & 50601 & 5087 \\
\hline Weltomp. HF & 13: & 1528] & 132 & उआ & 133.4 & Tद्यर्म & $\mid \overrightarrow{3}$ & 330.0 & Tद.8. & 7290 \\
\hline WET Tomp. 14 & Tर्डर्ट & 1328 & 13218 & 134 & 133.5 & 133.T & 1328 & 731.31 & 130.8 & 731.2 \\
\hline WE Tomp. R5f & 1325 & 131.7 & 133.1 & 134.61 & 1326) & 132 की & 1325 & 131 & 150 & 120.1 \\
\hline WedTomp. A1F & 122.8 & 13251 & 131 & 134 & 132.6 & 1324 & 134.11 & 131.0 & 1292 & 209.0 \\
\hline Wल11 Tomp. .2F & 1327 & 132.61 & 131.5 & 133.9 & 1323 & 1324 & 133.7 & 131.9 & 1298 & 120 \\
\hline Lnctream Temp. $F$ & 123.2 & $122 \pi$ & 1232 & 124.8 & 124.5 & 124 & 124.6 & 123.5 & 1232 & 123.4 \\
\hline Avo. Wall tomp. 7 & 1324 & 1328 & 131.8 & 1342 & 1327 & 1328 & 133.2 & 937.3 & 720 & 120 \\
\hline Ave. WatDT.F & 9 & 9.6 & 8.3 & 9.11 & 8 & 8.3 & 8.4 & 7.6 & 6,4 & 6.3 \\
\hline Rematning Suboootf & 3.4 & & & & & & & & & \\
\hline Ext Cually & -0.0 & -0.02 & -0.01 & of & 0.01 & 0.01 & 0.62 & 0.03 & 0.0 .9 & 0.0 \\
\hline EX.H8tw & 555.7 & $5 \overline{5.41}$ & 6028 & 550.8 & 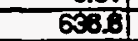 & 6005 & 583,4 & 668.0 & F5: & 870.6 \\
\hline COKPUIEUH & & & & & & & & & & \\
\hline Jalok & 56321 & 753.01 & 585.8 & 570.81 & $5 \% 1$ & 57.7I & 578.8 & 587] & 590,6 & 5835 \\
\hline sodyi(cudolub) & 898.6 & 8931 & 890 & 900.81 & 914.11 & 900.61 & 9082 & 977 & क्राब & 63050 \\
\hline $5 x+\sqrt{2}$ & 655.8 & 65751 & 658.5 & 6500 & 6578 & -658.7 & 67 & 670.8 & 6834 & 675.7 \\
\hline (नी०) & 113928 & 1133861 & 11308 & 1156961 & 115093 & 116379 & 11350 & 111437 & 11005 & $10 \times 00$ \\
\hline (F) & 1.006 & 1.0081 & 1.000 & 9.008 & 1.158 & 1.378 & 1.522 & 1.65 & 1.782 & 1.801 \\
\hline (सिका & $2 \overline{8}$ & 2525 & 2028 & 263.81 & 300 & 35211 & 396.9 & 431.8 & 46032 & 7838 \\
\hline 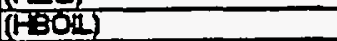 & 88.1 & 8035 & 891.9 & 5018 & 9020 & 8002 & 898 & 89 & 8810 & 8787 \\
\hline & & & & & & & & & & \\
\hline & $20 N=2$ & ZONET & $20 \pi=6$ & ZONEB & $201=10$ & & & & & \\
\hline DP squid Temp. & 71.8 & & & & & & & & & \\
\hline DPUlquid benaty tom/3 & 94.82 & & & & & & & & & \\
\hline Elovion & & 4 & & & 9.875) & & & & & \\
\hline Whatred DPphad & 0.388 & 0.451 & T.1.58 & 9.184 & 1.480 & & & & & \\
\hline Cero Comectionsprid & 0.153 & $0.03 \pi$ & $0.8 \mathrm{s5}$ & 0.702 & 0.031 & & & & & \\
\hline 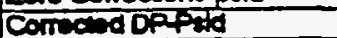 & 0.150 & 0.363 & 0.628 & 1.002 & 1.35 & & & & & \\
\hline Actur Dan & 1.96 & 3.77 & 5.60 & $7.0 \mathrm{~d}$ & 8.47 & & & & & \\
\hline Mavered tpeph & 864 & & & 1 & 835 & & & & & \\
\hline Maetured overith & 7.82 & & & & & & & & & \\
\hline & & & & & & & & & & \\
\hline Compered DR & 212 & द.या & 6.3 & क्दा & 9.98 & & & & & \\
\hline Fintont & 0.12 & 0.24 & 0.42 & 0.86 & 0.94 & & & & & \\
\hline Acoederention- & o & D & 0.17 & o.2. & o.3. & & & & & \\
\hline Exitiont & द & (4) & $5 . \pi$ & 729 & 8.7ा & & & & & \\
\hline & & & & & & & & & & \\
\hline
\end{tabular}




\begin{tabular}{|c|c|c|c|c|c|c|c|c|c|c|}
\hline Runn! & $1 \quad 896$ & 1 & & $\dot{-}$ & & $\vdots$ & & 1 & & \\
\hline Tastfud & CAFB & 1 & i & 1 & & $\perp$ & & & & \\
\hline Date: & 1Q12123 & & $i$ & & & & & 1 & & \\
\hline Time: & $8: 40$ & & & & & & & $t$ & & \\
\hline Now & Anthicald & If used: & & & & + & & & & \\
\hline Fow Areste & 0.00356 & & & \pm & & 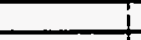 & & & & \\
\hline Hed flow Aroate & 0.1865 & & & & 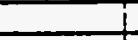 & & & & & \\
\hline 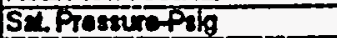 & 87.51 & & & & & & & & & \\
\hline 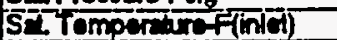 & 128.11 & & & & & 20 & & & & \\
\hline La.Denatylomis & 88.49 & 88.01 & 86.85 & 86.151 & 88.18 & 88.221 & 86.181 & 88.81 & 88.4 & 88.4 \\
\hline 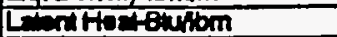 & 39.16 & 39.251 & 39.21 & 38.881 & 38.911 & 38.94 & 38.811 & 39.071 & 39.00 & 39.00 \\
\hline Specino HostimbinfF & 0.278 & $0.27 \pi$ & 0.27 & 0.2791 & 0.278 & 0.278 & 0.278 & 0.278 & 0.278 & 0.278 \\
\hline Liq. Visoostrtominh & 0.57 & 0.5781 & 0.572 & $0.55 \%$ & 0.66 & 0.5611 & 0.66 & 0.565 & 0.657 & 0.667 \\
\hline 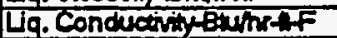 & 0.03261 & 0.0327 & 0.03261 & 0.03231 & 0.0024 & 0.0324 & 0.0324 & 0.0385 & 0.0825 & 0.0325 \\
\hline Lig. Prendt No. & 4.85 & 4.871 & 4.86 & 4.811 & 4.821 & 4.821 & 4.82 & 4.81 & 4.84 & 4.84 \\
\hline Inla Fow RMagdM & 4.91 & & & & & & & 1 & & \\
\hline OLACILG. Flow RA-GPM & 11 & & & & & & & & & \\
\hline OulloIVap. Flow RaA-GPM & 261 & & & & 1 & $\therefore$ & & 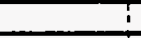 & & \\
\hline Inta Temperituref & 12द.5 & $T$ & i & i & $T$ & 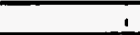 & & & & \\
\hline 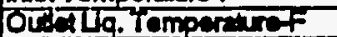 & 725.61 & $T$ & i & & $!$ & ? & & & & \\
\hline Oude vep. Tomper urof & 120.9 & $\because$ & 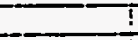 & & $!$ & 7 & & & & \\
\hline WEM Flow PAL-GPM & & 1 & & 1 & I & I & & & & \\
\hline Werer inter Teinp.f & 83.7 & 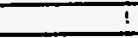 & 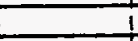 & 1 & & 1 & 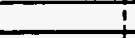 & & & \\
\hline Wer ould Temp.f & 119.3 & 1 & 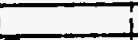 & $\frac{1}{1}$ & & 1 & & & & \\
\hline lntabmhnte & 1018072 & & Tamp & 120.11L & Dansiny & 87.151 & & & & \\
\hline Subooolingt & 3.6 & 1 & L & 1 & & 1 & & & & \\
\hline \multirow[t]{2}{*}{ Fow Observation } & & & & & & 1 & & & & \\
\hline & 2ON=1 & ZONEZ2 & ZONE3 & ZOR=4 & ZONE5 & ZORE6 & ZONE? & ZONE8 & ZON:EO & $20 N=10$ \\
\hline 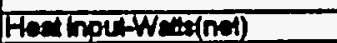 & 27.6 & 278.8 & 2853 & 288.7 & 209.7 & 209.6 & खा & 2827 & 268 & 26.1 \\
\hline 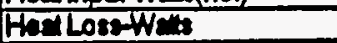 & 10.3 & 2.3 & उ। & 3.21 & 3.11 & 221 & 1.5 & 2. & 27 & 15.4 \\
\hline 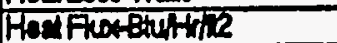 & $47|3|$ & 4781 & क्जा & 5001 & stes & 50001 & 5180 & \$SOO| & 460 & 7685 \\
\hline We romo. Baf & 7368 & T35. & T35. & $|35.1|$ & 13:.1 & T32 & 7382 & |स्32 & T883 & 1822 \\
\hline Wer Tamo. .4 & 1385 & 134.0 & 735.51 & 138या & 138.1 & 135.8 & 138.1 & 133.8 & T33. & 735.8 \\
\hline WE TOMP. 165 & 137.8 & 134.11 & 13821 & 130.61 & 135.8 & 135.31 & 135.31 & 133.81 & 1320 & 137.7 \\
\hline WE TOMD.DIF & 124.51 & 135.21 & 133.8 & 138.81 & 135.4 & 135.7 & 137.8 & 134.21 & 1310 & 931.0 \\
\hline WEll Tomp. .24 & 137.51 & 13521 & 134.4 & 13621 & 135.8 & 135.61 & 136.0 & 134.61 & 1स्32द & 732 \\
\hline Intraen Tomp.f & 124.1 & 123,1 & 123.7 & 128.11 & 125.0 & 125.71 & 125.8 & 124.71 & 124.6 & 124.8 \\
\hline Avo.Wall Tomp.f & 137 & 13521 & 134.7 & $138 \mathrm{Al}$ & 1355 & 1355 & 136.4 & 133.81 & 1322 & 1324 \\
\hline Avo.WNDTF & 127 & 11.61 & 10.8 & 101 & 9.4 & 9.6 & 102 & 8.9 & 7.8 & 7.5 \\
\hline \multicolumn{11}{|l|}{ Reming Suboooff } \\
\hline Exaloulty & -0.0 .2 & $=0.01$ & of & o. & $0 . \overline{01}$ & 0.02 & 0.03 & 0.08 & 0.03 & 0.06 \\
\hline DXP. HETHARE= & 373.1 & 475.11 & 458.8 & सहैं। & इ3का & 532.41 & 503.91 & 5507 & 587.8 & 617.0 \\
\hline \multicolumn{11}{|l|}{ 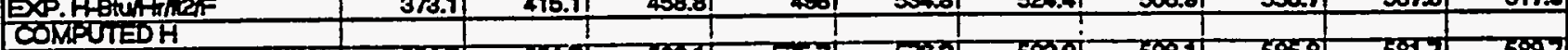 } \\
\hline Jisouk & 356.7 & 355.7 & $55 \pi 1$ & 575.71 & 5762 & 5828 & 358.1 & 585.81 & 581.7 & 5827 \\
\hline Szodvi (Cudo tubl) & $8 \% .4$ & 87.9 & 895.11 & जाI & द्याओ & 9248 & 94.7 & 916.11 & 85xi & SOL \\
\hline sond? & $\approx 28.7$ & हया & 653.7 & 85.81 & 888.11 & 68.7 & 705.6 & $6 \% 5$ & 603 & 67.2 \\
\hline (RLb) & $11 \% 0 \mathrm{~m}$ & $116 \approx 081$ & 11653 & 1198021 & 118288 & 117601 & 118487 & $11 \not 280$ & T1335 & T12010 \\
\hline (F) & 1.008 & 1.0081 & 1.006 & 1.0061 & 1.209 & $1.4 \approx 51$ & 1.596 & $1 . \pi 1$ & 1.803 & 1.80 \\
\hline (F) & 357.81 & 268.81 & 267 & 26861 & 36,4 & 3901 & $25 \pi$ & 45521 & 4807 & 5030 \\
\hline (HBO4) & $8 \pi .1$ & $87 \pi .6$ & 887.11 & क्रया & 9058 & 9005 & 914 & 875.81 & 8475 & 82,4 \\
\hline & & & & & & & & & & \\
\hline & ZONEE2 & ZONE & ZONE6 & ZOTRE8 & $2 O N=10$ & & & & & \\
\hline \multicolumn{11}{|l|}{ DP Tquid Tamp.F } \\
\hline \multirow{2}{*}{\multicolumn{11}{|c|}{ DPLquid Dantitemin }} \\
\hline \multicolumn{4}{|l|}{ Event } & & & & & & & \\
\hline \multicolumn{11}{|l|}{ Whendoplopd } \\
\hline \multicolumn{11}{|l|}{ 2erocometions ptid } \\
\hline \multicolumn{11}{|l|}{ Comendopy } \\
\hline Actud DPR & 2.3 & 4.181 & 8.18 & 7.881 & 9.64 & & & & & \\
\hline Whemed if & 875 & & T & & 855 & & & & & \\
\hline Menoured Overelin & 267 & & I & & & & & & & \\
\hline & & & & & & & & & & \\
\hline Compund DPA & हार & 4.251 & 625 & 8.15 & 9.9i1 & & & & & \\
\hline Fichont & 0.12 & 0.26 & 0.47 & 0.73 & 1.02 & & & & & \\
\hline Acotertiond & 0 & 0.07 & 0.2 & 03 & D.34 & & & & & \\
\hline Etrentiont & द) & उ.क्2 & 5.58 & 7.74 & 8.55 & & & & & \\
\hline & & & & & & & & & & \\
\hline
\end{tabular}




\begin{tabular}{|c|c|c|c|c|c|c|c|c|c|c|}
\hline Run: & i 897 & $\dot{-}$ & - & 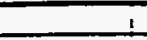 & & - & & 1 & & \\
\hline Test Flid & CAF 8 & - & & & & $i$ & 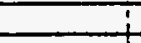 & 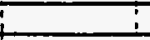 & & \\
\hline Dato: & 10/2य3 & & & & & $\perp$ & & $i$ & & \\
\hline Time: & $8: 40$ & & & & & & & $i$ & & \\
\hline Nois & Anatytical of & is used I & & & & & & & & 1 \\
\hline Fow Areale & 0.003361 & & & & 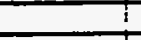 & & & & & \\
\hline Hon Fow Arese & 0.19695 & & & & 7 & 7 & & 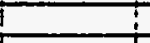 & & \\
\hline SEl pipsoure pig & $85.7 T$ & & & & & $T$ & & & & \\
\hline S. temperaturef(inlet) & 127.61 & ! & & & & & & & & \\
\hline La. Densty & 86.521 & 86.041 & 88.6 & 88.171 & 8624 & 85.31 & 86.281 & 86.521 & 86.57 & 88.42 \\
\hline 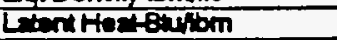 & $39.18 !$ & 39.281 & 39.28 & 38.9 & 38.85| & 39.011 & 39.99 & 39.18 & 3921 & 39.1 \\
\hline 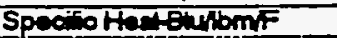 & 0.278 & $0.27 \pi$ & 0.271 & 0.2781 & 0.278 & 0.278 & 0.278 & 0.2781 & $0.27 \pi$ & 0278 \\
\hline Lig. Visoostibminh & $0.57 \pi$ & 0.574 & 0.574 & 0.569 & 0.5511 & 0.564 & 0.563 & 0.5711 & 0.57 & 0.567 \\
\hline Lig. Conductivit Buntif & 0.03261 & 0.0527 & 0.0027 & 0.0324 & 0.0324 & 0.0325 & 0.00321 & 0.03261 & 0.003 & 0.0325 \\
\hline Lig. Prendéno. & 4.86 & 4.87 & 4.87 & 4.891 & 4.821 & 4.83 & 4.831 & 4.861 & 4.86 & 4.84 \\
\hline Inlat Pow Rato-GPM & 4.11 & & & & & & & ; & & \\
\hline Oellat Lia. Fow Rato-GPM & $1.1 T$ & & & & & & & 1 & & \\
\hline Oulet Vsp. Fow Rato-GPiM & 24 & & & & & $\therefore$ & & 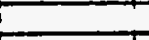 & & \\
\hline Inifl Temowerturef & 12421 & $i$ & 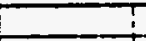 & $!$ & & & & & & \\
\hline 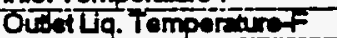 & T25.11 & $?$ & & & & & & & & \\
\hline Ootal Vap. Tamperacer & 119.7 & $T$ & 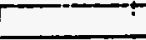 & & & 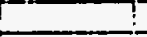 & & $T$ & & \\
\hline Whar Fow Rate-GP & i & $!$ & 1 & $!$ & 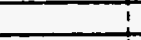 & $!$ & & 1 & & \\
\hline Wewer ind Temp.f & 85.1 & 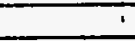 & 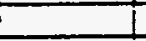 & 1 & & 1 & 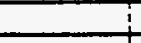 & 1 & & \\
\hline Werer out Temp.f & 119.3 & $\bar{i}$ & 1 & -1 & & 1 & 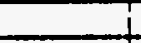 & 1 & & \\
\hline Intel Gomhrike & 855108 & & itamp & 118.41 & Deneity & 87.43 & & $i$ & & \\
\hline Subcoobingf & 3.4 & 1 & & & i & 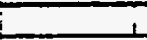 & & 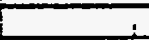 & & \\
\hline \multicolumn{11}{|l|}{ Frow Observetion } \\
\hline & ¿ZONE1 T2 & ZONE2 & ZONES & ZONE4 - & ZONE5 I & ZONEE 1 & $201=7$ & ZONEB I2 & ZONEQ & $20 N=10$ \\
\hline 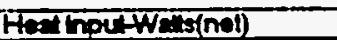 & 290.75 & 29: & 290.21 & 297.1 & 268 & 287.9 & 290.3 & 290.3 & 291 & 2695 \\
\hline Hert Los:WE:B & 17.4 & 2.5 & 3.4 & $3.6 !$ & 3.4 & 2.5 & 7.6 & 2.4 & 3.11 & 17.6 \\
\hline 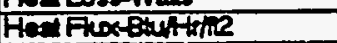 & इ0ख्डा & $50 \% 2$ & $50<01$ & 50461 & 48581 & 459 & $50<21$ & $50<21$ & 5001 & 5018 \\
\hline 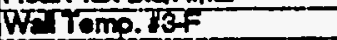 & 135.8 & अख़ा & 132 & 735.81 & 735.6 & 735.7 & 7352 & T3221 & 7332 & 132 \\
\hline WE Tomp. IAF & 735.3 & 133.91 & 134.8 & 735.81 & 135.4 & 735.31 & 135.21 & 1328 & 1327 & 7333.6 \\
\hline WE Tamp. 155 & 136.8 & 133.31 & 135.8 & 1381 & 134.5 & 134.81 & 134.4 & 1324 & 131.8 & 131.0 \\
\hline We Tomp. If & 1242 & 134.91 & 1327 & 1381 & 134.9 & 135.11 & 138.4 & 133.4 & 131 & 131.7 \\
\hline Well Tomp. & 137.71 & 134.31 & 133.6 & 135.8 & 134.7 & 135.11 & 1361 & 133.8 & 131.8 & 132 \\
\hline Instram Temp.F & 123.9 & 12321 & $\overline{123.21}$ & 126 & 125.61 & 12521 & 125.31 & 123.91 & 123.6 & 122,6 \\
\hline Avo. Wall Tomp.F & 166.3 & 13421 & 133.8 & 135.9 & 1349 & 135 & 135.6 & 1331 & 131.8 & 1322 \\
\hline AY W WallDT T & 12.2 & 10.81 & 10.3 & 9.7 & 9.11 & 9.5 & 9.9 & 8.8 & 8 & 7.6 \\
\hline \multicolumn{11}{|l|}{ Remoining Subcooff } \\
\hline Exhouly & 0.02 & -0.0 & & 0.01 & $0 . \mathbb{2}$ & 0.031 & 0.04 & 0.05 & 0.08 & 0.00 \\
\hline Ex.HE & 474 & 459.0 & 4878 & इद्रो.7 & 547.7 & 5226 & 507.7 & 550.7 & 6327 & 678.8 \\
\hline \multicolumn{11}{|l|}{ COMTUTED } \\
\hline Ifork & 5531 & 561.ग्र & $550 . \pi$ & 574.11 & 57.7 & 5712 & 58501 & $588.9 T$ & 5878 & 5958 \\
\hline 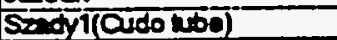 & 803.6 & 900.8 & 898 & 921.81 & 914.7 & 977.8 & 924.6 & 918.61 & Se00 & geid \\
\hline sadye & 6881 & 660.31 & 658.1 & 688.91 & 681.1 & 68721 & 687.8 & 680.61 & 682 & 678.8 \\
\hline (P्यक) & क्col & 974061 & 97405 & \$9391 & 98007 & 9sesn & Scon & ssen & $92 \%$ & gecira) \\
\hline (F) & 1.006 & 1.0061 & 1.006 & 1.304 & 1.482 & 1.62 & 1.765 & 1.887 & 1.898 & 2002 \\
\hline (स्ठO) & खर्या & 231.81 & 23ाइी & 30231 & 346.7 & 379.61 & 403.8 & 435.4 & 4560 & 787.2 \\
\hline$(\mathrm{H} 30 \mathrm{C})$ & 89.6 & 895.4 & 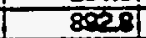 & 9100 & 8973 & 8sil & 8972 & 8847 & 8्वा & 825 \\
\hline & & & & & & & & & & \\
\hline & ZOTis? & ZONET & ZONiE6 & LOT:B & $\angle O N E=$ & & & & & \\
\hline \multicolumn{11}{|l|}{ DP toud Tomo.f } \\
\hline DP Latad Densinytiom/3 & S3.02 & & & & & & & & & \\
\hline Everion- & 2) & 4ा & 6 & 81 & 9.8751 & & & & & \\
\hline Whearedopende & ठद्यों & 0.4351 & 7.602 & उ.ख्डा & 7.5881 & & & & & \\
\hline Zero Comectionsosd & 0.158 & 0.04 & 0.80 & $0.1 \$ 8$ & 0.108 & & & & & \\
\hline Corrected Dapadd & 0.178 & 0.305 & 0.700 & 1.108 & 1.485 & & & & & \\
\hline Acted DP- & 1.88 & 3.65 & 5.3 & 6.781 & 8.15 & & & & & \\
\hline Memped Tfph & 86.7 & & & & E5.7 & & & & & \\
\hline \multirow[t]{2}{*}{ Neverred overalla } & 1.67 & & & 1 & $i$ & & & & & \\
\hline & & & & & & & & & & \\
\hline Comprtad DPa & 2.09 & 4.69 & 5.95 & $7.72 !$ & 9.38: & & 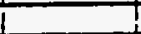 & & & \\
\hline Fictonth & 0.09 & 0 ख्या & 0.4 & $0 . \approx 2$ & 0.87 & & & & & \\
\hline Aooderiond & of & 0.11 & 0.10 & 0.231 & 0.29 & & & & & \\
\hline Elviciont & 2) & 3.7 & 5.35 & 6.801 & 8.21 & & & & & \\
\hline & & & & & & & & & & \\
\hline
\end{tabular}




\begin{tabular}{|c|c|c|c|c|c|c|c|c|c|c|}
\hline Aunt & 8381 & & & - & & & & & & \\
\hline Taff Fud & . 4 AF & - & & $\therefore$ & & & & & - & \\
\hline Date: & i 101293 & & & & & & & & & \\
\hline Time: & $8: 40$ & & & & & & & & & \\
\hline Nots & Añayicals & this used & & & & & & & & \\
\hline Fow Aras? & 0.00356 & & I & I & & & & & & \\
\hline Hed Fow Arond? & 0.19696 & 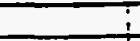 & & & & & & & & \\
\hline Selpiesuresig & 86.8 & & & ! & & & & & & \\
\hline Set fomperetrof(nied) & 127.6 & 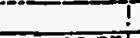 & & $?$ & & & & & & \\
\hline Wq. Donsty tomin 3 & 86.56 & 86.051 & 88.57 & 88.11 & 86.201 & 86.29 & 88.181 & 8.4 & 86.,1n & 86.87 \\
\hline 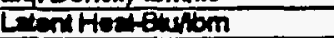 & 39.21 & 39.291 & 3922 & 38.84 & 38.851 & 38.991 & 38.97 & 39.09 & 39.71 & 3.00 \\
\hline SpecisotherewiomiF & 0.27 & 0.277 & 0.27 & 0.2791 & 0.278 & 0.278 & 0.278 & 0.278 & 0.278 & 0,278 \\
\hline is viscostrubomintr & 0.572 & 0.575 & 0.572 & 0.5571 & 0.561 & 0.563 & 0.56 & 0.567 & 0.558 & 0.568 \\
\hline 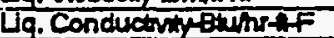 & 0.0326 & 0.0327 & 0.0326 & 0.03231 & 0.0324 & 0.0324 & 0.0324 & 0.0351 & 0.0585 & 0.085 \\
\hline Lq. PrandidNo. & 4.85 & 4.871 & 4.86 & 4.811 & 4.82 & 4.831 & 4.82 & 4.841 & 4.86 & 4.84 \\
\hline Inlet How Put-GPM & 3.3 & & & 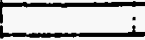 & 1 & i & & 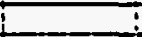 & 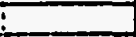 & \\
\hline Outellig. Fow Pata-GM & 0.7 & & & i & & it & & & 1 & \\
\hline OUth Vep. Flow Rato-GPM & 2.11 & & & & & & & & 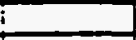 & \\
\hline Intomperanef & 12421 & & & & & & & & & \\
\hline OUALG. Tompermef & 1252 & ! & & & & & & & & \\
\hline OuClvep. Temperiefef & 120.6 & ! & & 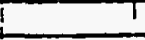 & 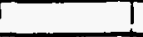 & & & & & \\
\hline Way Fow Rate-GPM & T & 1 & & 1 & 1 & 1 & 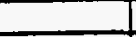 & & & \\
\hline Wher Inter Tomp.f & 85.8 & & & 1 & $E$ & $T$ & & & & \\
\hline Worr oclut Temp.f & 119.8 & & & I & & & & & & \\
\hline instabmhnt & 68949 & & Temp & $118.4 \pi$ & Deneiy & 87.461 & & & & \\
\hline Subcoolingt & 3.4 & 1 & & 1 & & & & & 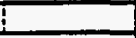 & \\
\hline Fow Obenvention & & & & & & & & & & \\
\hline & ZONE 1 & ZONE2 & $20 \mathrm{~N}=3$ & ZONE4 & ZON:55 & $20 N=6$ & ZOKE & ZONE 8 & $\angle O N=9$ & $\angle O N=10$ \\
\hline 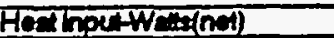 & 1291.4 & 2922 & 291 & 200.1 & 287.1 & 288.5 & 250.8 & 291.3 & 1200 & - 2898 \\
\hline HenLose-We: & 11.2 & 25 & 3.31 & 3.51 & 3.4 & 2.1 & 1.6 & 24 & 3.1 & 17.4 \\
\hline Het finatewhin? & 50511 & 50501 & 5074 & 5069 & प्वnा & 500ा & 50ता & 5001 & 5021 & 5015 \\
\hline We romp. & 7352 & खआया & 1372 & 935.61 & 135.7 & 734.01 & -1361 & Tस्3.II & I3:20 & 7324 \\
\hline WETTEP. IA & 935.4 & 733.9 & 134.7 & 135.7 & 1358 & 135.81 & 135.8) & 130.6 & 133.2 & 133.8 \\
\hline WEITOMP. 15 F & 136.3 & 133.21 & 135.6 & 1381 & 1345 & 134.61 & 135 & 133.Tा & 1320 & 737.8 \\
\hline WDT Tomp, ह1F & 124.2 & 134.8 & 132.8 & 1381 & 134.8 & 134.9 & 138.8 & 134.11 & 131.6 & 131.8 \\
\hline WतIT"mp:12F & 137.7 & 134दा & 133.6 & 135.61 & 134.6 & 134.81 & 136.71 & 134.7 & 182 & 1322 \\
\hline Instrementempt & 123.7 & 123.11 & 123.6 & 126.41 & 125.6 & 125.3 & 125.91 & 127.61 & 124.4 & 124.8 \\
\hline Avp.Wal Tomp.F & 1362 & 1342 & 133.8 & 135.8 & 134.8 & 1349 & 13.9 & 133.7 & 1325 & 1324 \\
\hline AVO. WNAT DT & 122 & 10.9 & 10 & 92 & 9 & 9.3 & 10 & 8.8 & 7.8 & 7.4 \\
\hline Ramining Suboooff & 1.9 & & & & & & & & & \\
\hline Exaloualy & -0.01 & of & 0.01 & 0.02 & 0.03 & 0.04 & 0.05 & 0.081 & 0.01 & 0.00 \\
\hline Exp.FBur & 413.8 & 465.8 & 505.8 & 56.7 & 5545) & 538,2 & 5055 & 557.9 & EकाB & 60.1 \\
\hline CONRUIED & & & & & & & & & & \\
\hline Jibuk & 574.7 & 573.11 & 5526 & 57421 & 569.71 & 57281 & 5802 & 58051 & 5821 & 568 \\
\hline Szodyl(cudowtod & 9022 & 916.41 & soe & 92 & 914 & 916.81 & 9253 & 920.1 & 91838 & 9250 \\
\hline Sedt? & 6628 & Eab & 681.7 & 68201 & 658.8 & 6896 & 674.6 & 685.11 & 6825 & Eस: \\
\hline (P्थि) & $787 \%$ & 78301 & 78456) & 79000 & द्वाष & कार्या & 780.82 & 746001 & 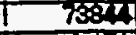 & 73207 \\
\hline$(F)$ & 1.006 & 1.0001 & 1.191 & 1.4681 & 1.696 & 1.700 & 1.89 & 2.0571 & 275 & $22 \pi$ \\
\hline (F) & 185.1 & 18र्ण. & 23 & 284. & 3207 & उ5a21 & 3752 & 70051 & 423 & 406 \\
\hline$\left(\mathrm{H}: \mathrm{O}^{2}\right)$ & 8092 & 9725 & 860 & 9120 & s007 & 89 & 505 & 68्रा & 82 & 827 \\
\hline & & & & & & & & & & \\
\hline & $20 N=2$ & ZONEC & $20 \times=6$ & $201 \times=8$ & $201=50$ & & & & & \\
\hline DPlgud Tomp.f & 18 & & & . & 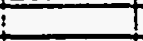 & & & & & \\
\hline 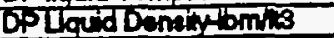 & 928 & & & & & & & & & \\
\hline Exw'tont & & का & & 8 & 9.875 & & & & & \\
\hline Mimeredoppid & 0.285 & 0.5161 & T.77 & 1.571 & 1.308 & & & & & \\
\hline Zero Correcionopida & 0.158 & $0 . \overline{0}$ & 0.88 & 0.768 & 0.100 & & & & & \\
\hline Cortsond DFpid & 0.120 & 0.475 & 0.889 & 1.3081 & 1.825 & & & & & \\
\hline AOT & 1.94 & 3.61 & 4.58 & 6.381 & 7.58 & & & & & \\
\hline Wemed Traph & 86.8 & & & & 8.7 & & & & & \\
\hline Menered Oventil & 1.83 & & & & & & & & & \\
\hline & & & & & & & & & & \\
\hline Computed oph & 2.08 & उ.S3! & 5.65 & 7.331 & 8.891 & & & & & \\
\hline Ficion- & 0.06 & 0.16 & 0.3 & 0.481 & 0.57 & & & & & \\
\hline Aocentiont & 0 & 0.1 & 0.15 & 0.19 & 0.2 & & & & i. & \\
\hline Eviont & 2 & 3.67 & 5.21 & 6.651 & 7.991 & & & & & \\
\hline & & & & & & & & & & \\
\hline
\end{tabular}




\begin{tabular}{|c|c|c|c|c|c|c|c|c|c|c|}
\hline Runt & 8991 & & o & & & & $\therefore$ & - & 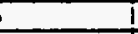 & I \\
\hline Teat Fuid & CAF8 & $\therefore$ & & & & 1 & & $\dot{-}$ & . & \\
\hline Dato: & . $1072 / 293$ & & & & $!$ & $\dot{-}$ & & & . & 1 \\
\hline Time: & $8: 40$ & & & & & & : & 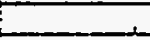 & & \\
\hline Noto & Analytica of & tis used 1 & & & & & & & & \\
\hline Flow Areat? & 0.003561 & & i & & & & $\dot{-}$ & is & & \\
\hline Heat Flow Arest? & 0.19551 & & & & & 7 & $t$ & 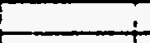 & & \\
\hline Sa Presture paig & $85.3 T$ & & & & 7 & $?$ & t & $\frac{1}{i}$ & $t^{\prime}$ & \\
\hline Se्d Temperaturof(iniel) & 127.31 & & & $\pi_{10}$ & & & $\frac{1}{1}$ & & & \\
\hline Liq.Dentinyomin & 88.391 & 86.421 & 86.391 & 85.11 & 86.24 & 86.291 & 86.271 & 86.471 & 88.49 & 86.42 \\
\hline Ltent Hod Bturbm & 39.091 & 39.11 & 39.07 & 38.841 & 38.55 & 38.991 & 38.981 & 39.141 & 39.16 & 39.1 \\
\hline Speciofterenubmf & 0.278 & 0.2781 & 0.278 & 0.2781 & 0.2781 & 0.2781 & 0.2781 & 0.2781 & 0.278 & 0.278 \\
\hline Lig. Visoosit Fom mi tr & $0.565 i$ & 0.567 & $0.566 i$ & 0.5571 & 0.5611 & $0.563 i$ & 0.5521 & 0.5691 & 0.67 & 0.657 \\
\hline Lia. Condueting suhaff & 0.03251 & $0.0325:$ & $0.0325 !$ & 0.03231 & 0.0524 & $0.0324 i$ & 0.03241 & 0.03281 & 0.0326 & 0.0325 \\
\hline Lia. Prandt No. & 4.831 & $4.84 i$ & 4.84 & 4.811 & $4.82]$ & 4.831 & 4.831 & 4.851 & 4.85 & 4.84 \\
\hline Intot fow Pata-GPM & $2.5 !$ & & & & & & & & & \\
\hline OWd Lig. Fow Rato GPM & 0.4 & & & & & & & & & \\
\hline Ou'st Ves. Fow Rata-GPM & 2.31 & & í & & T & & & 1 & 1 & \\
\hline Inlal Temperature f & 124.81 & & T & & & 1 & 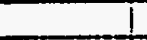 & $i$ & 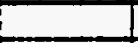 & \\
\hline Ortet La. Tomperturef & 125.7 & & & $T$ & T & & & $T$ & & \\
\hline Outa vop. Tomperaturef & 120.4 & & T & & !e & & & & & \\
\hline Wetor flow Pato-GPM & 1 & 1 & I & 1 & I & & & $T$ & I & \\
\hline Werer thlex Temp.f & 84.51 & I & i & 1 & i & I & & I & I & \\
\hline Weror out Temp.F & 118.7 & i & I & +1 & & 1 & & 1 & 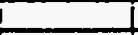 & \\
\hline Indel Gibmhri, & 62201 & & Tomp & 777.21 & Dansiy & 87.52 & & I & E & \\
\hline Suboooling $F$ & 2.5 & 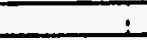 & 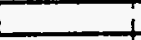 & & & & & $i$ & $i$ & \\
\hline Fow Obsenvetion & & & & & & & & & & \\
\hline & ZONEI & ZONE2 2 & ZONE3 & ZONEA i & ZONES & ZONE6 II & ZONE? Z & $2 O N=8 \quad 12$ & $\angle O N=9$ & $\angle O N=10$ \\
\hline 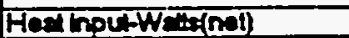 & 289.31 & 29021 & 277.5 & 290.8 & 28.01 & $288 . \overline{8}$ & 289.8 & 291 & 288.8 & 203 \\
\hline HoatLoss Wast & 10.7 & 2.4 & 3.21 & 3.4 & 3.21 & 2.3 & 1.51 & 22 & 2.8 & 16.0 \\
\hline Heat Fux-thithe & 50151 & 50501 & 48121 & 50321 & 4973 & 50008 & 5023 & 50741 & 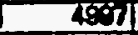 & 5070 \\
\hline WA Tomp. 13F & T3द.5ा & T3उ. & $\overline{1} \mathbf{3} . \pi$ & 135 & 135 & 1342 & 134.8 & $13 \div 91$ & 1396 & 13.7 \\
\hline Wa Tomp. $14 \mathrm{~F}$ & 134.8 & 133.8 & 134.2 & 135 & 734.9 & $1 \overline{34.71}$ & 134.0 & 13261 & $132 \pi$ & 733.4 \\
\hline WNTOmo. $15 F$ & 135.4 & 13291 & 135 & 13.5 .5 & 133.9 & 134.11 & 1341 & 13221 & 137.4 & 130.8 \\
\hline WEITomp. \#1F & 124.8 & 133.71 & 132.11 & 135.11 & 133.8 & 134.61 & 135.81 & 13321 & 1502 & 1372 \\
\hline Wellomp. ILF & 136.61 & 133.71 & 1327 & 1351 & 139.5 & 134.31 & 135.51 & 133.51 & 130.7 & 131.8 \\
\hline Thatsem Tomp.f & 125 & $1245 i$ & 124.71 & 726.41 & 125.61 & $1 \overline{25} .31$ & 125.47 & 12421 & 125.1 & 124.6 \\
\hline Avg. Wall Tomp.F & 135.31 & 133.71 & 13321 & 13521 & 134.11 & 134.4 & 135 & 1327 & 1312 & 131.8 \\
\hline Avo. Wal DT. & 10.1 & 8.9 & 8.3 & 8.5 & 8.2 & 8.8 & 9.4 & 8.3 & 6.8 & \\
\hline Remaning Subooolf & 0.51 & & & & & & & & & \\
\hline Exdourly & of & 0.071 & $0 . \overline{C E}$ & 0.04 & 0.05 & 0.07 & 0.081 & $0 . \pi$ & 0.17 & 0.18 \\
\hline 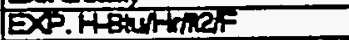 & 497.81 & 56381 & 5827 & 550.51 & EOR,B & 5671 & 533.71 & 611.3 & T2i & $722 \pi$ \\
\hline COMRUTEDH & $!$ & & & & & & & & & \\
\hline Jeouk & 589.41 & 577.71 & 558.21 & $5 \% .11$ & 568.7 & 570.11 & 573.21 & 57261 & 5720 & 36001 \\
\hline 5radyloudo (w) & $907 . \pi$ & 905.71 & 8886 & 92181 & 9i2 & 915ा & 978.81 & 914.41 & 9112] & 928.1 \\
\hline sedr & 667.81 & 68.71 & 84721 & 6.1.41 & 655.5 & 80.11 & 806.01 & 668.91 & 67IA & 687 \\
\hline (Pण्व) & 605601 & 600111 & 59008 & 5907 & 58078 & 57088 & 56217 & 547001 & 597801 & SBt:3 \\
\hline (F) & 1.006 & 1.214 & 7.534 & 1.7491 & 1.927 & 2.007 & 230 & 2.372 & 249 & 2.600 \\
\hline (10) & 157 & 189.31 & 233 & 2728 & उ012। & 32501 & 342 & 3606 & 368 & 70350 \\
\hline$(1+304)$ & 9055 & 9020 & 8828 & 913.71 & 901.4 & solा & sots & 893.8 & 8872 & 851 \\
\hline & & & & & & & & & & \\
\hline & iZONEZ & ZONEA & $201=6$ & ZONEB & IZONE:T0 & & & & $L$ & \\
\hline DP hquid fomp.fF & 829 & & 1 & & & & & & & \\
\hline DP Liquid Denty & \$27 & & & & & & & & & \\
\hline Eleviont & 2 & & & 81 & 9.875 & & & & & \\
\hline 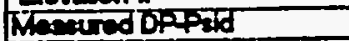 & 0.32 & 0.7281 & 2.74 & 1.98 & -2504 & & & & & \\
\hline Zero Corrodionsosid & 0.158 & 0.04 & 0.808 & 0.168 & 0.100 & & & & & \\
\hline Comected DP phd & $0.18 \pi$ & 0.6801 & 7328 & 1.848 & 2401 & & & & & \\
\hline Ach Dopa & 1.87 & 3.16 & 4.53 & 5.52 & 6.8 & & & & & \\
\hline Mantred Tiphap & 86.8 & & & & 856 & & & & & \\
\hline Maned overill & 7.33 & & & & & & & & & \\
\hline & & & & & & & & & & \\
\hline Comperted DPR & 7.93 & 3.51 & 52 & 6.76 & 8.21 & & & & & \\
\hline Frictont & 0.05 & 0.13 & 0.24 & 0.37 & 0.52 & & & & & \\
\hline Aconierion- & 0.04 & 0.08 & 0.17 & 0.14 & 0.17 & & & & & \\
\hline Ervent & 7.84 & 3.39 & 4.85 & 6.25 & 7.52 & & & & & \\
\hline & & & & & & & & & & \\
\hline
\end{tabular}




\begin{tabular}{|c|c|c|c|c|c|c|c|c|c|c|}
\hline Rent & 9001 & 1 & & & & 1 & 1 & i & $i$ & \\
\hline Tatfind & . C4F & & & - & & & & & I & \\
\hline Dala: & . 101293 & & & & & & $i$ & & & \\
\hline Time: & $.8: 10$ & & & & & & i & 1 & 1 & \\
\hline Noto & Ansyical & dis used & T & $i$ & & & & & $i$ & \\
\hline FTOW ATOLAR & 0.00536 & & I & T & & & & & & \\
\hline Hon Fow ArosR & 0.15695 & & & $?$ & & & 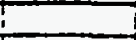 & & & \\
\hline Set. Prossurepig & 87 & 1 & & 1 & & $T$ & $T$ & & T & \\
\hline Set tomporicref(n) & 727.8 & & 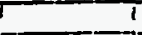 & $T$ & & & I & & & \\
\hline Lq.Densinyomis & 86.81 & 86.221 & 86.251 & 85.831 & 86.11 & 86.171 & 86.08 & 865! & 88.3 & 86.24 \\
\hline Lent Hor Butwom & 39.011 & 38.941 & 38.86 & 38.751 & 38.84 & 38.91 & 38.83 & 38.801 & 39.01 & $\mathbf{3 8 . 5 5}$ \\
\hline Specino Hedenulomir & 0.278 & 0.2781 & 0.278 & 0.279 & 0.279 & 0.2781 & 0.279 & 0.278 & $0 . \overline{2} 8$ & 0.278 \\
\hline [ig. Viscosh & 0.564 & 0.5611 & 0.5201 & 0.5531 & 0.557 & 0.5591 & 0.557 & 0.562 & 0.584 & 0.561 \\
\hline Liq. Conducivit Btumfaf & 0.0325 & 0.0524 & 0.0324 & 0.03221 & 0.0323 & 0.05241 & 0.0323 & 0.0324 & 0.0025 & 0.0324 \\
\hline LGg. Prande No. & 4.83 & 4.821 & $4.82 !$ & $4.79 !$ & 4.811 & 4.811 & 4.8 & 4.82 & 4.88 & 4.82 \\
\hline Inlet flow Pua-GPM & 1.71 & & & & & & & & & \\
\hline 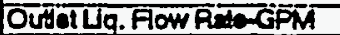 & 0.31 & $\therefore$ & + & 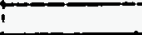 & & & & & & \\
\hline OWATVM. Fow RAT-GPM & $1.7 !$ & & $\div$ & 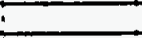 & & & & & & \\
\hline Inket Tompereteref & 725.21 & & $\stackrel{1}{!}$ & 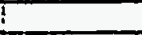 & & & & & I & \\
\hline 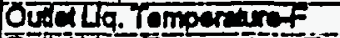 & 126.31 & & & & & & & & & \\
\hline Dinave Tomper mer & $12 i .81$ & & $\overline{!}$ & ! & & & & & & \\
\hline 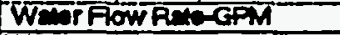 & -1 & $\overline{1}$ & 1 & $I$ & & $i$ & & & $T$ & \\
\hline Wator hilel Tomp.f & 88.2. & 1 & $i$ & $i$ & & i & & & $T$ & \\
\hline 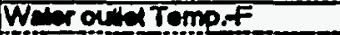 & 1206 & 1 & & 1 & & & & & I & \\
\hline 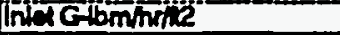 & 353656 & & Temp & 177.71 & Density & 87. ही & & & & \\
\hline Subooding f & 26 & 1 & & $i$ & & & & & & \\
\hline \multirow{2}{*}{ Fow Obsenvition } & & & & & & & & & & \\
\hline & RONS1 & ZONES & ZOKE3 & ZONE & $20 N=5$ & $20 N=6$ & ZONE? & ZONE $=8$ & 12ON:=8 & $\angle O O N=10$ \\
\hline Hod houtwerest) & 209.7 & 297.4 & 296.81 & 2945 & 20 & 294.4 & 2000 & 2005 & $\cos$ & 357.1 \\
\hline Hoel Lose-We: & 10.8 & 241 & 3.2 & 3.3 & 3.2 & 231 & 1.8 & 2.31 & 20 & 16.7 \\
\hline 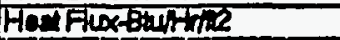 & 502 & 50511 & 5145 & 5105 & 5131 & 5ाष्ठा & 5613 & $50 \mathrm{~s} 8$ & 606 & 7800 \\
\hline Wistamp. 13 & 13.7 & और & १33.71 & $13.1 T$ & 735.3 & उद्य & 735.7 & T32? & अओ & k्टा \\
\hline WET Tomp.14F & 734.8 & 133.4. & 1342 & 1352 & 135.1 & 135.11 & 135.8 & 138 & 1325. & T35.6 \\
\hline WET Tomp. 15F & 135.4 & 132.81 & 135.4 & 135.51 & 134.3 & 134.31 & 134.7 & 132.7 & 131.8 & 131.4 \\
\hline WEl Tomp.01F & 125.2. & 133.81 & 1324 & 135.11 & 130.8 & 134.7 & 136.8 & 134.11 & 1306 & 131.4 \\
\hline WET Tomp.12F & 135.8 & 133.81 & 1328 & 735.11 & 133.7 & 134.6 & 136.2 & 134.51 & 1513 & 731.8 \\
\hline hitrem Tomo.f & 1255 & 125.7 & 1255 & 127.11 & 126.4 & 1261 & 1265 & 12551 & 1352 & 125.6 \\
\hline Avo, Wall Temp.t & 1352 & 133.8 & 133.3 & 1352 & 134.3 & 134.7 & 135.7 & 133.4 & 737.7 & T32 \\
\hline AVT.WalloT. & 9.8 & 7.8 & 7.5 & 7.9 & 7.6 & 8.4 & 9 & 7.7 & 63 & 6.8 \\
\hline Remaining sebooof & -0.4 & & & & & & & & & \\
\hline Elat Cuadis & 0 & 0.02 & 0.06 & 0.01 & 0.06 & 0.17 & 0.13 & 0.15 & 0.78 & 0.19 \\
\hline 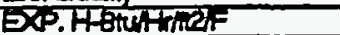 & 512.51 & 645.8 & Exटा & 646.71 & 673.5 & 6018) & 559.6 & 656.8 & 800.31 & 740.7 \\
\hline \multicolumn{11}{|l|}{ COMPUIEDH } \\
\hline Jelok & $59 \pi$ & 59001 & 5861 & $57 \cdot \pi$ & 58001 & 5807 & 5824 & $5 \pi 51$ & 5R. & 3512 \\
\hline 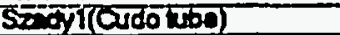 & 60.41 & 975.41 & 9302 & 937.7 & $\$ 50.11$ & 98558T & 922:81 & 978.51 & 97881 & 885.6 \\
\hline $5 \sqrt{2} \sqrt{2}$ & 6022 & $674.7 \pi$ & 682 & 6881 & 680.4 & 68.7 & E.an & 55251 & $6 \pi$ & 650 \\
\hline (थण) & 91207 & 40001 & उ969ा & 38084 & 384 & 37619 & 36008 & 354601 & 3467: & 39708 \\
\hline (F) & 1.12 & 1.2551 & 1.7891 & 20321 & 2ह5ा & 2401 & 2.67 & 27791 & 2क्षा & 3.068 \\
\hline (HDO) & 1285 & $16 \overline{6} . \overline{6}$ & 2055 & 30.11 & 2589 & 28001 & 3003 & 318.91 & 3231 & 351. \\
\hline \multirow[t]{3}{*}{ (स3OII) } & sord & 913.51 & 9192 & 925.7 & şand & 917.71 & 914.2 & 90551 & 80 & 8857 \\
\hline & & & & & & & & & & \\
\hline & 120NE2 & ZONET & 20106 & ZONE & $201=10$ & & & & & \\
\hline \multicolumn{11}{|l|}{ DP havid tomo,f } \\
\hline \multicolumn{11}{|l|}{ 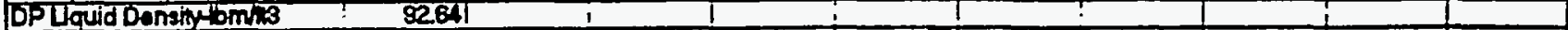 } \\
\hline Eovtiont & 21 & का & 6ा & 81 & 9.875 & & & & & \\
\hline Reseuredopsidd & 0.331 & 0.971 & 2401 & 23:01 & 3.017 & & & & & \\
\hline ¿eno correctiong pad & 0.168 & 0.01 & 0.80 & 0.763 & 0.160 & & & & & \\
\hline Comeoned DP Pyd & 0.187 & 0.874 & 1.509 & 2258 & हक्षा & & & & & \\
\hline Roteidoph & 1.89 & 284 & $3 . \infty$ & 4.881 & 5.74 & & & & & \\
\hline 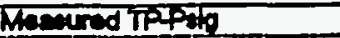 & 87 & & & & 862 & & & & & \\
\hline \multicolumn{11}{|l|}{ Mesured Overill } \\
\hline \multirow{2}{*}{\multicolumn{11}{|c|}{ Computed DPh }} \\
\hline & & & & & & & & & & \\
\hline Fiction- & 0.09 & 0.1 & 0.18 & 0.31 & 0.42 & & & & & \\
\hline Aocoler iont & 0.03 & 0.05 & 0.07 & $0.09 !$ & 0.71 & & & & & \\
\hline Everion & 1.55 & 2.88 & 3.99 & 4.801 & 6.03 & & & & & \\
\hline & & & & & & & & & & \\
\hline
\end{tabular}




\begin{tabular}{|c|c|c|c|c|c|c|c|c|c|c|}
\hline Runt & 9011 & & & & & & & & & \\
\hline Testifund & CAF 8 & & & & & & & & & \\
\hline Dato: & 1012391 & & & & & & & 1 & & \\
\hline Time: & $-15: 131$ & & 三 & 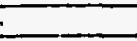 & & T & $i$ & $t$ & & \\
\hline Not & 'Anaryeal & dis used I & $\div$ & 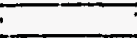 & 1 & $\overline{!}$ & 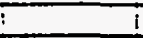 & $i$ & i & \\
\hline Fow Araxte & 10.005661 & 1 & 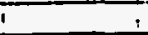 & $i$ & - & 1 & 1 & 1 & i & \\
\hline Host Fow Aroent2 & 0.196851 & 7 & \pm & 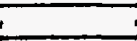 & - & $!$ & $!$ & 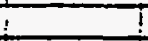 & 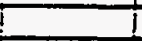 & \\
\hline S.l Proseurrofig & 1861 & 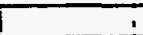 & $\bar{i}$ & 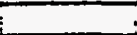 & . & 1 & 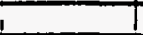 & $\overline{1}$ & L & \\
\hline Sat Temperatrefo(inlet) & 127.1 & & 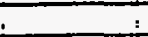 & 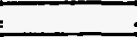 & i & $\mathrm{i}$ & 1 & +1 & 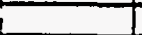 & \\
\hline Lig. Dansity tom/ras & 86.34 & 86.241 & 86.34 & 86.05 & 86.17 & 86.24 & 86.13 & 88.31 & 86.32 & 86.25 \\
\hline Ltan Heathenthm & 39.031 & 38.95 & 39.031 & 38.8 & 38.9 & 38.951 & 38.87 & 39.011 & 39.02 & 38.86 \\
\hline Specifie Hoatetunbmif & 0.278 & $0.278 !$ & 0.278 & 0.279 & 0.278 & 0.2781 & 0.279 & 0.2781 & 0.2781 & 0.28 \\
\hline Liq. Viscositytominth & 0.5851 & $0.561 !$ & 0.5651 & 0.556 & 0.5591 & 0.5611 & 0.5581 & 0.5641 & 0.5671 & 0.552 \\
\hline Lig. Conductivy otuhrstf & 0.005 & 0.0324 & 0.00525 & 0.0523 & 0.0024 & 0.0524 & 0.0023 & $0.0056 \mathrm{~T}$ & 0.03651 & $0.0<24$ \\
\hline Liq. Prand No. & 4.831 & 4.821 & 4.831 & $-\overline{4} . \overline{8}$ & 4.811 & 4.821 & 4.811 & 4.831 & 4.831 & 4.82 \\
\hline InIfH Fow Rato-GPM & 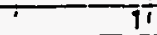 & i & $i$ & & ! ! & ! & & T & 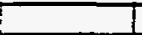 & \\
\hline Outat Lia. Fow Rato-GPM & 0.41 & & & & 7 & 1 & & + & t & \\
\hline Outot Vap. Frow Ruto-GPM & $0.9 i$ & 1 & $\cdot$ & 5 & & 1 & 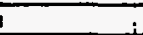 & 3 & $i$ & \\
\hline Intel Tempormuref & 124.71 & $:$ & + & $i$ & 1 & 1 & $i$ & 1 & 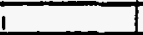 & \\
\hline Outalia. Tompereturef & 126.41 & $t$ & . & . & 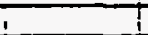 & 7 & 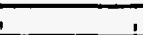 & & - & \\
\hline Outat Vep. Tomparzteref & 921.81 & & & & & & & & & \\
\hline Wator Fow Pate-GPM & & & & & & & & & & \\
\hline Water hilal Tomp.f & 85.91 & & 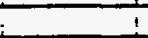 & 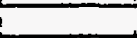 & & & & 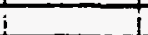 & 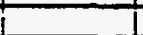 & \\
\hline Wator outel Tomp.f & 120.61 & & & & & $i$ & & & & \\
\hline Inlat Glommonic & 209945 & & Tomp. & 175 & Toensity & 87.97 & & & & \\
\hline Suboooling & 2.4 & & -2 & 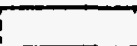 & & 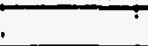 & & $!$ & & \\
\hline Fow observen & & & 1 & 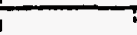 & & i & & T & ? & \\
\hline & 'ZONET & $Z O N=2 \quad 1$ & $\angle O N=\overline{3}-12$ & ZONE & ZONES & 'ZONE 6 'T & ZONET & ZONEB & ZONES & $\angle O N=10$ \\
\hline Heal hortwers(not) & 291.71 & 293.21 & 289.8 & 2952 & 298.21 & 295.41 & 291.81 & 2911 & 295.8 & 288 \\
\hline Hod Lose-W: & 10.6 & 2.31 & 3.11 & 3.31 & 3.2 & 241 & 1.61 & 2.31 & 29 & 17.1 \\
\hline Hedfolowhthith & 5056, & 50821 & 5035 & 5117 & 573 & 51211 & 50000 & 5041 & 5110 & 4900 \\
\hline WNil Temp. 13+ & 133.7 & 1323 & 132iा & 133.81 & 93421 & 13301 & 1345 & 131.9 & 131 & Tु. \\
\hline Wall Tomp. If & 133.9 & 131.71 & 1326 & 133.9 & 134 & 134.4 & 134,8 & 1324 & 131.0 & 1320 \\
\hline Wat Tomp. $15 \mathrm{~F}$ & 134.6 & 1312 & 134.4! & 136.4 & 133.11 & 133.6 & 133.8 & 132 & का & 130.0 \\
\hline Wa Tomo. $81 F$ & 124.7 & 1324 & 130.7 & 133.9 & 1331 & $133.6 !$ & 135.61 & 133.1 & 150.7 & 131 \\
\hline Wh Tอmo. 27 & 1351 & 1326 & 1312 & 133.8 & 132.81 & 133.7 & 735.81 & 133.7 & 135.5 & कु. \\
\hline Instrom Tomp.F & 125ा & 125.61 & 125 & 126.7 & 1201 & 125.61 & 126.21 & 125.21 & 125.1 & T25.6 \\
\hline Avo Wa Temo. & 134.81 & 1323 & 131.7 & 134 & 1332 & 133.81 & 134.8 & 1328 & 130.8 & 737.5 \\
\hline AYo. Wallot.F & 9.11 & 6.41 & 6.4 & 7.11 & 7 & 8 & 8.4 & 7.2 & 5.6 & 5.8 \\
\hline Rombing swoootf & -2.71 & & t & & $T$ & & & & & \\
\hline Exacualy & 0.021 & 0.001 & 0.00 & 0.131 & 0.10 & 0.21 & 0.321 & $0.2 \pi$ & 0.31 & .3 .30 \\
\hline 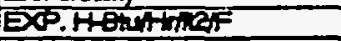 & 557.4 & 791.81 & 7824 & 728.9 & 7342 & 638.61 & 600.11 & 707.41 & $\operatorname{sen} 2$ & 558.8 \\
\hline COARUTEDH & $i$ & & -1 & & & & & & & \\
\hline Inlouk & ह5०र्वा & 5 क्त.ा & 588.1 & 56 & $589 . \overline{3}$ & 583.31 & 573.41 & $5 \% 21$ & 675.21 & 6722 \\
\hline Sradvil(Oedo tube) & 910.3 & 917.1 & $\operatorname{sos} 2$ & 9288 & उद्वा & 9235 & क्टा8 & 91421 & Saso & 9726 \\
\hline $\operatorname{sen} 2$ & 6712 & 67 & 668.2 & 688.7 & छा & 600.4 & 678.8 & 671.4 & 67.7 & 65.6 \\
\hline (स्ति) & 24000 & 23550 & 225361 & 201 & 2058 & 1997 & 1918ी & 18728 & TFen & 16400 \\
\hline 10 & 7.367 & 1.816 & 2.215ा & 2.574 & रक्षो & 3.068 & 3.25 & 3.488 & 3.681 & 3.80 \\
\hline (स्प) & 1028 & 135.7 & 165.6 & 189.8 & 210.81 & $2 \circlearrowright 9.6$ & 246 & 2223 & $27 \pi .1$ & 2000 \\
\hline$\left(\mathrm{H} \otimes \mathrm{OL}_{\mathrm{L}}\right)$ & 900.8 & 916.11 & Sob.31 & Se8.1 & 9र2या & 917.8 & 315.0 & $\$ 08.81$ & 912.4 & 903.0 \\
\hline & & & 1 & 1 & & ! & & & & \\
\hline & T2ONE2 & ZON=4 & ZONEB & ZONEB & TZONETO & & & & & \\
\hline DPT Ruld tomp.f & 184.6 & T & 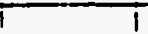 & 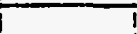 & 1 & $T$ & $\Gamma$ & & & \\
\hline D'Plged Dansithm/3 & 9267 & 1 & $T$ & $\Gamma$ & $t$ & $i$ & $i$ & & & \\
\hline Eloviont & 2 & $4 i$ & 61 & 8 & 9.875 & T & 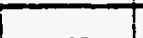 & & T & \\
\hline Mentered DFpaid & 0.358 & 7.7911 & 2873 & 2553) & 3.705 & & & & & \\
\hline Zero Comections-peid & 0.168 & 0.04 & 0.8981 & 0.143 & 0.1031 & $i$ & 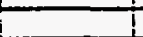 & & & \\
\hline Correated DP Pid & ठदा & 1.151 & 1.97 & 281 & 3.602 & 1 & 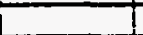 & & & \\
\hline Actul oph & 1.8 & 2.37 & 3.14 & 3.89 & 2581 & t & 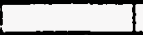 & & & \\
\hline Wheneured TPFPig & 88 & $i$ & 4 & & 85.61 & & & & & \\
\hline Thensured Ovorelita & 0.671 & & & & & T & & I & & \\
\hline & & T & & & i & & & & & \\
\hline Computed DPen & T.यां & 2.481 & 3.41 & 730 & 5.णा & & & & & \\
\hline Frtolont & 0.02 & 0.081 & 0.121 & 0.18 & 0.281 & $T$ & $T$ & & & \\
\hline Acoster ond & 0.021 & 0.081 & 0.04 & 0.061 & 0.07 & I & I & & & \\
\hline Etriont & 1.61 & 2दा & उ.21 & 4.01 & 7.681 & & r & & & \\
\hline & $\mathrm{i}$ & 1 & 1 & 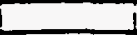 & $i$ & 1 & $I$ & I & & \\
\hline & & & & & & $i$ & 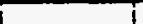 & & & \\
\hline
\end{tabular}




\begin{tabular}{|c|c|c|c|c|c|c|c|c|c|c|}
\hline Runt & 9021 & $\dot{-}$ & & & & $\therefore$ & 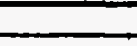 & $\dot{-}$ & - & \\
\hline Test Fuid & .CAF8 & & & & & & & $\div$ & - & \\
\hline Dato: & . 10193931 & & & & & \pm & & & & \\
\hline Tima: & $8: 30$ & & & & & & & & & \\
\hline Nots & Analycica ed & tis used & & & & & & & & \\
\hline FlOW AOERT & 0.003651 & & & & & & & & & \\
\hline Has Fow Ares 2 & 0.1955 & & & & & $?$ & I & ! & & \\
\hline 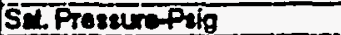 & 87.61 & & & & & ! & T & t & t & \\
\hline 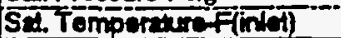 & 928.2 & & 1 & 1 & I & $-T$ & 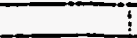 & $T$ & I & \\
\hline Lig. Density & 86.32 & 86.81 & 86.351 & 881 & 86.18 & 86.241 & 86.13 & 88.851 & 8.4. & 86.8 \\
\hline Ltent Her Exhbm & 39.02 & 39.031 & 39.05 & 38.761 & 38.87 & 38.551 & 38.87 & 39.051 & 39.00 & 39.01 \\
\hline Speciso Hoestuphomf & 0.278 & 0.2781 & 0.278 & 0.279 & 0.279 & 0.278 & 0.279 & 0.2781 & 0.278 & 0.278 \\
\hline 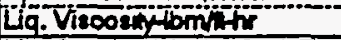 & 0.564 & 0.5651 & 0.565 & 0.5641 & $0.558 T$ & 0.5617 & 0.568 & 0.5651 & 0.567 & 0.584 \\
\hline Liq. Conductivity BurffF & 0.0325 & 0.03251 & 0.0025 & 0.00221 & 0.03201 & 0.0034 & 0.0323 & 0.0085 & 0.0585 & 0.005 \\
\hline La. Prandty No. & 4.831 & 4.831 & 4.89 & 4.79 & 4.81 & 4.821 & 4.81 & 4.841 & 4.84 & 4.88 \\
\hline Interfow Rate-CPM & 4.91 & & & & 7 & & & & & \\
\hline Outot Lia. Fow RA-GPM & 0.71 & & & & & & & 1 & & \\
\hline Outhol Vep. Fow Rex-GPM & 2.61 & & & & i & & & $\div$ & $\vdots-$ & \\
\hline Inlet Tomperearef & 125 & - & & - & $i$ & 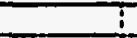 & & & & \\
\hline ou & T25.4 & & & 1 & I & & & $t$ & 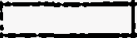 & \\
\hline 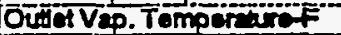 & 120.95 & & & & $i$ & & & $I$ & & \\
\hline Wha Fow RLA-GPM & 1 & $T$ & 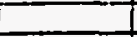 & $!$ & 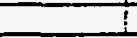 & I & & 1 & i & \\
\hline Wer hitat Temp.f & 75.9 & $!$ & 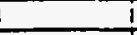 & 1 & 1 & 1 & & I & I & \\
\hline Worer oul Tomp.f & 108.8 & 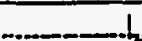 & 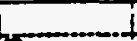 & 1 & $\frac{1}{4}$ & 1 & & 1 & 1 & \\
\hline Inta clomhine & 1902038 & & Temp & 119.11 & Densiny & 87.81 & & I & I & \\
\hline Subooding f & $32 !$ & 1 & 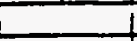 & 1 & 1 & $i$ & 1 & 1 & 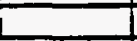 & \\
\hline Flow Obsenvition & & & & & & & & & & \\
\hline & ZONE 2 & ZONES : & ZONE3 & ZONEA & $Z O N=5$ & ZONES I2 & ZONET & ZZNE8 & $\angle O K=9$ & $201=10$ \\
\hline Heathorhwstat) & i $5 \% 0.81$ & 576.5 & $5 \pi . t$ & 579 & 575.8 & 581.81 & 574.81 & 57.4 & 5802 & 579.0 \\
\hline HorLLose-Wa"t & 9.8 & 22 & 3 & 3.11 & 31 & 2.2 & 1.51 & 2.1 & होग & 15.4. \\
\hline Hod FWo Exurwe & 985 & S98 & $1000 \pi$ & 10051 & 997 & 1008 & SGa1 & छजा4 & TOSOI & 1007 \\
\hline WE'Temp. $13+$ & $=737$ & 137.61 & 138.61 & $137.7 !$ & $7385 !$ & 138.81 & 139.1 & 1359 & 1351 & 735.4 \\
\hline Wettemp.t4F & 737.8 & T38.1 & 737.81 & 138.8 & 138.31 & 138.7 & 139 & 138.81 & 138.7 & 139 \\
\hline WeTtamp.16F & 138.2 & 135.21 & 138.51 & 138.81 & 138.7 & 137.81 & 137.9 & 135.91 & T36.8 & 334.2 \\
\hline WeIT Tomp. $11 F$ & 1251 & $138.7 \pi$ & 13,8 & 137.71 & 137 & 137.81 & 140 & 138.71 & 134 & 134 \\
\hline We'Tomp. 24 & 1391 & 136.71 & 135.7 & 137.7 & 136.91 & 138.81 & 139.8 & 137.6 & 134.6 & 334.8 \\
\hline Intream Temp.f & 126.71 & $125 i$ & $i 24.01$ & 127 & 126.21 & 125.67 & 126.2 & $124.0 i$ & 124.6 & 125 \\
\hline Aug. Wall Tamp.F & 138 & $136.8 ;$ & 1362 & $138.1 i$ & 1372 & 1382 & 132. & 136.61 & 135 & 135.5 \\
\hline Avo. Well DT 7 & 125 & $11.3 !$ & 10.8 & 10.71 & 10.6 & 12.1 & 125 & 11.21 & 9.9. & 9.8 \\
\hline Aomaining Subcooff & T.11 & & & & & & & & & \\
\hline Ext Qualis & -0.07 & 0.01 & 0.02 & 0.04 & 0.051 & 0.07 & 0.081 & 0.11 & 0.17 & 0.12 \\
\hline Exp.FBuntmen= & 796.61 & 88201 & 926.4 & 9405! & 943.61 & 8336 & F5.21 & 883.7 & 7013.8 & 10021 \\
\hline COMPUTEDH & & & & & & & & & & \\
\hline Jeloek & Eख3.T & 8424 & 8056 & 800781 & 8xi & 785,1 & 73321 & $7 \% 3$ & 7925 & 7820 \\
\hline 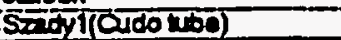 & 128 & 12898 & 1293.9 & 13205 & $7 \overline{3} \overline{3.06}$ & 7318.81 & 13द्यरत & 1315.31 & 73228 & 735 \\
\hline $\sec \sqrt{2}$ & 10533 & 906321 & 10505 & 1088.7 & 107321 & 106061 & T00.7. & 1051 & 10702 & 1097.7 \\
\hline (ADB) & 1188800 & $1182 \pi$ & 118500 & 117391 & T14821 & 11खाया & 11083 & $107 / 8$ & 1058 & $1045 \mathrm{M}$ \\
\hline (F) & 1.006 & 1.008 & 1.46 & 1.656 & 1.68 & 2.001 & 220 & 234 & 275 & 250 \\
\hline (f) & 368.3 & 26821 & 306.7 & 45381 & $505 \pi$ & 56.7 & 58.7 & Eका & 620.1 & 68 \\
\hline$(\mathrm{B} B \mathrm{OL})$ & 12821 & 1285,1 & 1282 & 13028 & 12383 & 128621 & 128: & 126581 & 1255 & 12525 \\
\hline & & & & & & & & & & \\
\hline & ZOKE2 & ZONES & ZONE 6 & ZONi=8 & ZON=10 & & & & & \\
\hline DP higud tomp.fF & 77.7 & & & & & & & & & \\
\hline DPP Lquid Denaiy & \$3.52 & & & & & & & & & \\
\hline Evertion & & & & & 9.8751 & & & & & \\
\hline Mha:tred Dpuprd & 0.308 & 0.5831 & 7.68 & 7.5071 & 1.898 & & & & & \\
\hline Zcro Corrocionspeid & 0.172 & $0.0 \mathrm{TI}$ & 0.879 & 0.टा & 0.116 & & & & & \\
\hline Correand op pud & 0.134 & 0.5221 & 0.818 & 1.29 & 1.697 & & & & & \\
\hline Acted DPa & 1.94 & 3.401 & 5.14 & 8.511 & 7.87 & & & & & \\
\hline Wheotrod TPph & 87.8 & & $L$ & & 848 & & & & & \\
\hline Mocured Overill & 4.67 & & & 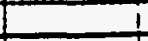 & & & & & & \\
\hline & i & & & & & & & & & \\
\hline Complued DF & 214 & 4.16 & 6.09 & 8.041 & 9.91 & & & & & \\
\hline Frotont & 0.15 & 0.4 iा & 0.7 & 1.21 & 1.7 & & & & & \\
\hline Aocolermionth & 0.12 & 0.3 & 0.42 & 0.53 & 0.64 & & & & & \\
\hline Ereveionth & 1.871 & 3.44 & 4.9 & 5.371 & 7.581 & & & & & \\
\hline & 10 & & & $i$ & & & & & & \\
\hline
\end{tabular}




\begin{tabular}{|c|c|c|c|c|c|c|c|c|c|c|}
\hline Runt & 9031 & & & & & $\therefore$ & & & & \\
\hline Tost fuid & $. C 4=8$ & $\therefore$ & & - & & & $\vdots$ & & : & \\
\hline Date: & $1013 / 93$ & & & & & & & & & \\
\hline Time: & $8: 30$ & & & & & & & & & \\
\hline Note & Analytical o & dit is usod & & & & & & & & \\
\hline Fow Areath & 0.003361 & & & & & & & & & \\
\hline Hed Fow Arast2 & 0.1965 & & & & I & & & & & \\
\hline S1. Pressure psig & 8721 & & & & & & & & & \\
\hline Sal Tomperature finiel) & 127.9 & & & & & & & & & \\
\hline Liq. Denthy tomir & 86.31 & 86.31 & $86.3 !$ & 86.051 & 86.221 & $867 i$ & 86.21 & 86.371 & 88.4.2 & 86.32 \\
\hline Letont Hoatom & 39.031 & 39.011 & $39.01 i$ & 38.81 & 38.941 & 38.981 & 38.92 & 39.081 & 39.1 & 39.02 \\
\hline Spocito Hedeupomf & 0.2781 & $0.278 i$ & $0 . \overline{2} 7 \overline{8}$, & 0.279 & 0.278 & $0.27 \overline{8} i$ & $0 . \overline{27} \overline{8} 1$ & $0.278 i$ & 0.278 & 0.278 \\
\hline Lig. Visoosintbm/hir & $0.665 i$ & 3.566 & 3.5641 & $0.5660_{1}$ & $0.56 \pi$ & 0.6521 & $0.56 i$ & $0.565 i$ & 0.5671 & 0.664 \\
\hline Liq. Conductin Binh & 0.0325 & $0.0025 i$ & 0.03251 & $0.0523 ;$ & 0.03241 & $0.0324 i$ & 0.0324 & 0.03251 & 0.00551 & 0.0325 \\
\hline Liq. Prende No. & 4.831 & $4.83 i$ & $4.83 !$ & 4.8 & $4.82 !$ & 4.831 & 4.827 & 4.84 & 4.84 & 4.83 \\
\hline Inlat Fow Rare-GPM & 4.11 & & & & & & $\ldots$ & & & \\
\hline Outallig. Fow RAOGFM & 0.71 & & & & & & 4 & & & \\
\hline Outet Vop. Flow Rato-GPM & $2.2 !$ & & & & & & & & & \\
\hline Intet Temperater & 124,3 & & & & & & & & & \\
\hline Outot Liq. Temperinge & 123.3 & & & & & $T$ & & & & \\
\hline Ouir Vap. Tompereturef & 121 & & & 1 & & 1 & & & & \\
\hline WaW Fow Rato GPM & & 1 & & 1 & $!$ & T & i & & & \\
\hline Werthet Temp.F & 78.81 & 1 & & $\mathbf{i}$ & I & 1 & & $!$ & 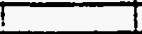 & \\
\hline Wex owe Temp.F & 108.6 & $i$ & & -1 & I & 1 & & I & I & \\
\hline 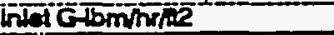 & $i \quad 85452$ & & Tome & 71976 & Densiny & 87.321 & & & & \\
\hline Subcooling & 3.61 & $\check{-}$ & -2 & & & 1 & & $I$ & $L$ & \\
\hline Fow Obsonvation & & & & & & & & & & \\
\hline & ZONEI & ZONE2 & $20 \mathrm{NE} 3$ & ZONE 4 & ZONE5 T & ZONEE 18 & ZONE & ZONEB & ZONESG & $201=10$ \\
\hline Hed houtwater(nol) & 5722 & 5773 & 57.5 & 578.8 & 574.3 & 570.9 & 57.2 & 575.31 & 580 & 5816 \\
\hline HeatLoss-Wa & 81 & 1.8 & 2.4 & 2.51 & 25 & 1.8 & 1.2 & 1.8 & 22 & 12.7 \\
\hline Hed flox-Buthtrine & 9918 & 10005 & क्णां & 10001 & 9551 & 98981 & 9953 & 997 & I003 & 1000 \\
\hline Waltomp. 137 & 738.8 & 137.4 & 736.8 & 137.51 & 138 & 137.7 & 138.8 & 135.81 & 1353 & 135 \\
\hline Wal Tomo.ouF & 137.21 & 135.4 & I3it. & 137.81 & 737.81 & 138.8 & 138.8 & 936.4 & 138 & 138.8 \\
\hline Wallomo. 15F & 137.71 & 134.71 & 138.81 & 138.21 & 936.91 & 137.61 & 137.7 & 135.41 & 134.6 & 133.9 \\
\hline Wall Tomp. I1F & 124.81 & 138.81 & 134.01 & 130.71 & 136.01 & 137.41 & 139.2 & 138.61 & 139.7 & 153 \\
\hline Wall Temp. I2F & 138.11 & 136.8 & 135.6 & 137.11 & 136,4 & 137.81 & 138.3 & 137.61 & 1345 & 13.8 \\
\hline ingtreem Tomo. & 125 & 12521 & 125.2 & 126.71 & 125.71 & 125.41 & 125.8 & 124.81 & 1245 & 125.1 \\
\hline Ang. Wall Tomp.F & 137.3 & 136.41 & 136 & 137.6 & 136.8 & 137.81 & 138.7 & 136,4 & 1320 & 135 \\
\hline AV WE WI DT.F & I1.9 & 10.7 & 10.3 & 10.41 & 20.7 & 11.91 & 125 & 11.1 & 9.9 & 9.4 \\
\hline Rempining Subootf & 1.2 & & & & & & & & & \\
\hline Ext Quelty & -0.01 & 0.01 & 0.03 & 0.04 & 0.08 & 0.08 & -0.1 & 0.17 & 0.18 & 0.16 \\
\hline 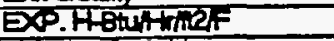 & 835.11 & 965.81 & 969.81 & 964.71 & 334.71 & 831.41 & 798.2 & 897.7 & 1019.I & 1087.8 \\
\hline COMPUEDH & & & & & & & & & & \\
\hline Ja & $8 x$ & 856.81 & 81021 & 8T7.6! & $850 \mathrm{AI}$ & $\$ 3.81$ & 7925 & 788.11 & 7891 & 783.1 \\
\hline Sadvi(cudotso) & 1283.7 & 12916 & 12956 & T374.81 & 13038 & 1330.81 & 13121 & 1307.81 & 1374.9 & 138568 \\
\hline $5 \operatorname{sen} 2$ & 1054 & 108211 & $10526 !$ & 1070.51 & $10 \overline{5.81}$ & 100981 & 105281 & 1051.4 & 1050 & 1072 \\
\hline (R्a) & 988851 & 991181 & 978741 & 970081 & 9489 & 92018 & 90680 & 889821 & 86816 & 8509 \\
\hline (F) & 1.008 & 1.0081 & $1.53 \pi$ & 1.7911 & 2005 & 2.1881 & 2.346 & 2504 & 2646 & 27 \\
\hline (स्य) & 2326 & 24727 & 3557 & 41551 & 46481 & 506.21 & 543.1 & 578.81 & 613.J & 6265 \\
\hline$(1+80)$ & 12812 & 12835 & 1286.61 & 13009 & 1283.8 & 1274.81 & 1280.3 & 1268.9 & 12835 & 1273.9 \\
\hline & & & & & & & & & & \\
\hline & ZONEZ & ZONET & ZONEE & ZONEE & ZONETO & & & & & \\
\hline DP hquid Temo.F & $\pi .9$ & & & & & & & & & \\
\hline 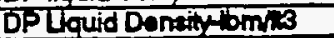 & 93.48 & & & & & & & & & \\
\hline Elovitiont & & का & 6ा & 81 & 9.875 & & & & & \\
\hline Whaved DPAnd & 0.332 & 0.701 & T.912] & T.86T & 2359 & & & & & \\
\hline zoro Controctonold & 0.172 & 0.0?Tा & 0.841 & 0.21 & 0.776 & & & & & \\
\hline Cormad Daphd & 0.18 & 0.0381 & 1.07 & 1.05 & 2.158 & & & & & \\
\hline Ach Dol & 1.8 & 3.21 & 4.7 & 5.91 & 7.11 & & & & & \\
\hline 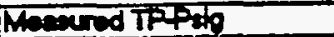 & 872 & & I & 1 & 84.81 & & & & & \\
\hline Macured Overall & 4.84 & & & i & $i$ & & & & & \\
\hline & $i$ & & & & & & & & & \\
\hline Computed DPa & 207 & 3.551 & 5.76 & 7.581 & 9.321 & & & & & \\
\hline Frictort & 0.11 & 0.32 & 0.6 & 0.98 & 1.36 & & & & 1 & \\
\hline Acosteriont & 0.1 & 0.24 & 0.33 & 0.42 & $0.5 \pi$ & & & & & \\
\hline Eloretont & 1.85 & 3.39 & 4.82 & 6.2 & 7.451 & & & & & \\
\hline & & & & & & & & & & \\
\hline
\end{tabular}




\begin{tabular}{|c|c|c|c|c|c|c|c|c|c|c|}
\hline Runt & 9041 & & & & 1 & & & & I & \\
\hline TOStFud & CAF : & & & & & & & & $\cdot$ & \\
\hline Das: & 101383 & & & & & & & & & \\
\hline Tims: & $12: 10$ & & & & & & & & & \\
\hline Now & Anaticid & His used & & & & & & & & \\
\hline Fow Areatid? & 0.003361 & & & & & & & & & \\
\hline Hed Fow Aroal? & D.t8ख5| & & & & & & & & & \\
\hline Sal Preseurephig & 87 & & & & & & & & & \\
\hline St Tomperitroffintet) & 127.8 & & & & & & & & & \\
\hline Lig. Densithtomin & 86.42 & 85.25 & 88.27 & 88.11 & 86.2 & 863 & 88.21 & 86.34 & 8855 & 86.80 \\
\hline Lenthemember & 3.1 & 38.88 & 38.88 & 38.81 & ऊ.8.2 & 38.99 & अ8.85| & 39.01 & 30.06 & 39.03 \\
\hline Specino How exulomp & $0 . \overline{27}$ & 0.278 & 0.278 & 0.27 & $0 . \mathbf{2 7 8}$ & 0.278 & 0.278 & 0.278 & 0.278 & 0.278 \\
\hline Liq. Viscositytom/ hr & 0.557 & 0.562 & 0.562 & 0.557 & 0.58 & 0.583 & 0.581 & $0.5 \%$ & 0.560 & 0.565 \\
\hline 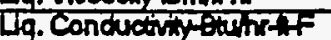 & 0.0385 & 0.0524 & $0.0 \mathrm{~s}$ & 0.03201 & 0.002 & 0.0324 & 0.0324 & 0.0585 & 0.0525 & 0.0525 \\
\hline Liq. PrendiNo. & 4.84 & 4.821 & 4.8 & 4.81 & 4.82 & 4.83 & 4.82 & 4.83 & 4.84 & 4.80 \\
\hline Intet Fow PWe-GPM & 3.3 & & & & & & & & & \\
\hline OU.TLLG. Fow ALW-GPM & 0.7 & & & & & & & & & \\
\hline OuHAVD. Fow RNE-CPM & 1.8 & & & & & & & & & \\
\hline Inta Tompermuref & 123.0 & & & & & & & & & \\
\hline Ou'tulq. Tamparmefe & 12301 & & & & & & & & & \\
\hline ovelvep Tempernere & 121.4 & & & & & & & & & \\
\hline Wen flow Rate-GPM & & & & & & & & & & \\
\hline Wererhlet Temp.t & $\mathbf{7 7 . 7}$ & & & & & & & & & \\
\hline Were oule Tomp.t & क्षी & & & & & & & & & \\
\hline n'telomproc & 687001 & & Temp & 118.11 & Densiy & 87.31 & & & & \\
\hline Subooongt & 3.8 & & & & & & & & & \\
\hline Flow Obsenviten & & & & & & & & & & \\
\hline & $200 N_{1}=1$ & $20 N_{i=2}$ & $201=3$ & $201=4$ & $20 \pi=65$ & $\angle O N=6$ & $20 \mathrm{~K}=1$ & $\angle O O^{1}=8$ & $201=9$ & $2019=10$ \\
\hline 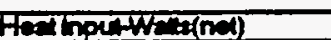 & 5775 & 579 & 5778 & strf & 577.8 & 577.8 & 57.2 & 57.1 & $5 \pi$ & 57 \\
\hline Hoelost-Was & 7.1 & 1.6 & 2द & यदो & 2,2 & 1.7 & 1.1 & 1.6 & 2) & 712 \\
\hline 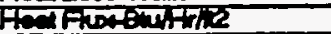 & क्णना & 10006 & 70018 & किस्रो & 700त्ड & Tक्णिं। & 70005 & T000ग & 70002 & 1000 \\
\hline WeTtemp. Bद & 7350 & 138.8 & 136.2. & 136.6 & 137.7 & 737.0 & 138 & 1356 & T352 & उद्य.: \\
\hline We'tremp.84F & 138.4. & $\mid E \mathbf{S}$ & अ3.4. & 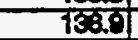 & 137.8 & 138 & Tऊ.ग & गह3.ग & 15 & Fo3, \\
\hline WeTtemo.16 & 136.0 & Tु, स्र & 738.4 & T37.5 & 736.4 & 1375 & T37.11 & 155.7 & अण & I3́5: \\
\hline WतIt Tamp. & 120.8 & 135.7 & 134.8 & T358 & 138 & 137.र्म & 138.6 & 136.4 & 133,1 & 1327 \\
\hline WeTremo.tat & Tु3.J & 135.8 & 135.8 & 188 & 136 & 137.0 & 138.6 & 137.4 & |अरा & 13.1. \\
\hline Instrem temp.f & T21.5 & द25.6 & 125. & T28.4 & 125.8 & 125.3. & 125.6 & 725 & T22.0 & 120 \\
\hline Avo. Waltomp.f & 3 & 135.8 & 135.8 & 138.7 & 13.6 & 137.8 & 138.1 & 138,8 & 33., & J34.7 \\
\hline AWO.WEDT.F & 71.5 & 9.8 & 9.8 & 9.8 & 10.8 & iर & Iटा & 10.0 & 8.4 & 9.2 \\
\hline Aloming Subooff & 0.8 & & & & & & & & & \\
\hline Ext कuthy & 0.01 & O.de & 0.07 & 0.08 & 0.03 & O.I & 0.181 & 0.16 & 0.17 & 0.10 \\
\hline 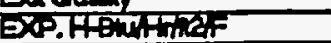 & 8380 & Tora.1 & 1005.0 & Tन्द्य & 97es & ह्धाता & 81.0 & 818. & 10051 & 10832 \\
\hline COT-PUTEDH & & & & & & & & & & \\
\hline Intak & 8क्रा & 8558 & 817.1 & 8127 & 8051 & 8013 & 800 & & Fan & 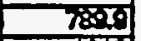 \\
\hline 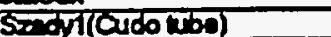 & भद्या & स大5.8 & स250| & FB. & किस56 & T30.5. & 13001 & 130332 & 73036 & 1318 \\
\hline $50 \mathrm{~d} 2$ & 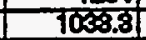 & TEO & 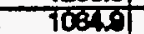 & Tणादय & 10080 & 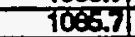 & 1069 & 10475 & TDETA & 1057.1 \\
\hline$(R+a)$ & 79276 & TS3it & 77783 & $767 / 2$ & 74574 & 72160 & \%क्ष & E87\% & 605 & 6030 \\
\hline & 5.000 & T.208 & 9.678 & 1.352 & 218 & टर्3 & 2.563 & $2 \%$ & 2ळa & 3.03 \\
\hline$\frac{1}{12}$ & 1858 & 245.6 & उ20.1 & अ.4 & 452 & द्वा5 & (528) & 53211 & 6822 & 68088 \\
\hline$(\mathrm{A}: 0 \mathrm{O})$ & 72825 & 12828 & F289.7 & T297.3 & 72006 & 72837 & F28: & T270.11 & TL78 & T272 \\
\hline & & & & & & & & & & \\
\hline & $20 \%=3$ & $20 \mathrm{~N}=4$ & ZONES & $28 \times 1=88$ & $201=10$ & & & & & \\
\hline DPlgudtamp.t & 77.8 & & & & & & & & & \\
\hline DPLoud Denta tomis & 83.40 & & & & & & & & & \\
\hline 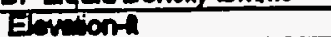 & 2) & 4 & 6 & 8 & 9.870 & & & & & \\
\hline Thenered DPFadd & 0.3\%) & 0.837 & 2375 & 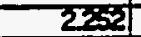 & 2732 & & & & & \\
\hline zero Combotions-pedd & 0.172 & 0.031 & 0.8यो & ठदी & ס.7न6 & & & & & \\
\hline Comectad Dophd & 0.18 & 0.820 & 1.30 & रवर्यो & 2.676 & & & & & \\
\hline Actoloph & 1.85 & 285 & 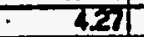 & 5.26 & 6.88 & & & & & \\
\hline Whecured tiptha & 87 & & & & $8 \pi 2$ & & & & & \\
\hline Themed over & 2.67 & & & & & & & & & \\
\hline & & & & & & & & & & \\
\hline Comperad DPI & 1.80 & 3.06 & 5.08 & 6.6. & 7.8 & & & & & \\
\hline Fiforl & 0.08 & 0201 & 0.07 & ठ.दूरा & 7.35 & & & & & \\
\hline Accortention & 0.00 & 0.18 & 0ही & ठ.3. & 0.4 & & & & & \\
\hline arutent & 9.82 & 3.00 & द.र्दा & हुओ & 6.12 & & & & & \\
\hline
\end{tabular}




\begin{tabular}{|c|c|c|c|c|c|c|c|c|c|c|}
\hline Runt & 9051 & & & & & & & & & \\
\hline Test Fuid & CAF8 & & & & & 1 & & & & \\
\hline Data: & $.1013 \times 3$ & & & & & & & & & - \\
\hline Tims: & $12: 10 !$ & & $\therefore$ & 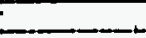 & & $i$ & & & & $i$ \\
\hline Note & Analytical of & th is used & 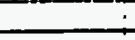 & & & & & & & 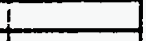 \\
\hline Flow Areath & 0.005361 & & & & & $\div$ & $i$ & & - & \\
\hline Hed Flow Area-12 & 0.1595 & & 1 & 1 & $i$ & the & ! & & $\vdots$ & \\
\hline Sat Pressuropig & $87.1 \Gamma$ & & $i$ & $i$ & & & & & + & \\
\hline Sn Tomporaturef (nlet) & 127.8 & & & $\bar{\sigma}=\overline{0}$ & & -1 & $\begin{aligned} 1 \\
-5=1\end{aligned}$ & & $T$ & \\
\hline U9. Dentity tom/n & -86.8 & 86.17 & 86.22 & 86.071 & 86.18 & 86.221 & 86.17 & 85.291 & 88.3 & 8632 \\
\hline Latent Hoenfulbm & 3.011 & 38.91 & 38.841 & 38.871 & 38.87 & 38.84 & 38.9 & 38.891 & 39.01 & 38.85 \\
\hline Specifo Her ewibmi & 0.278 & $0 . \overline{2} \overline{81}$ & 0.2781 & 0.2791 & 0.27 & 0.278 & 0.278 & 0.278 & 0.278 & 0.278 \\
\hline Lig. Visooshibmhhr & 0.564 & 0.5591 & 0.5611 & $0.556 i$ & 0.5581 & $0.561 i$ & $0.5591^{\circ}$ & 0.5631 & 0.564 & 0.681 \\
\hline Lig. Conductintyomptif & 0.0525 & 0.03241 & 0.0324 & 0.0523. & 0.03231 & 0.0324 & 0.0324 & 0.0324 & 0.035 & 0.0324 \\
\hline Lig. Prandt No. & 4.831 & $4.8 \%$ & 4.82 & $4.8 i$ & 4.811 & 4.821 & 4.811 & 4.831 & 4.83 & 4.82 \\
\hline Inlel Fow Rate-GPM & 2.5 & & & & & & : & & & \\
\hline Outel Lia. Fow Rato-GPM & 0.75 & & & & & & i & & & \\
\hline Outel Vap. Flow Rate-GPM & 1.7 & & & & & & $\therefore$ & & & \\
\hline Inlet Tomperediref & 724.5 & & $T$ & $T$ & 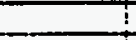 & & & & & \\
\hline Oufalig. Tompar & $125.1 T$ & & 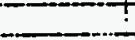 & & $i$ & & & & & \\
\hline Ouer vep. Temper uref & 127.91 & & $\therefore$ & $\vdots$ & & & & & 1 & \\
\hline Water Fow Rato-GPM & 1 & & 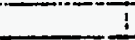 & 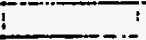 & $i$ & $\stackrel{!}{!}$ & 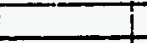 & & $i$ & \\
\hline Wear nlet Temp.f & 78.3 & $:$ & 1 & 5 & 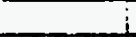 & 1 & 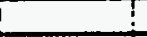 & & 1 & \\
\hline Wrin ould Tomp.f & 100.31 & 1 & 1 & 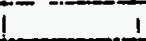 & 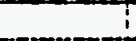 & 1 & 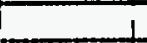 & $i$ & 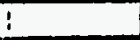 & \\
\hline In & 5211051 & & Tamo & 178.91 & Density & $87.34 i$ & & & 1 & \\
\hline Suboooling ff & 3.3 & & & & 工 & 1 & & & $i$ & \\
\hline Fow Obsenvition & & & & & & & & & & \\
\hline & ZONET & ZONE2 & ZONE3 & ZONE4 & ZONES & ZONE6 & ZONE? 2 & ZONEB & ZONES & 20 Ne10 \\
\hline Heat houtwallanit) & 574.1 & 572.3 & 574.7 & 577.9 & 575.81 & $5 \sqrt{2.6}$ & 574.4 & 5729 & $5 \% 0.6$ & 573.7 \\
\hline Hoet Loss-Wes & 6.7 & 1.51 & $2 \pi$ & 2.11 & 2.11 & 1.6! & 1.1 & 1.5 & 1.8 & 10.8 \\
\hline Heat Fiox Buthrie & 9921 & 99201 & 9021 & क्वादा & 9981 & 99281 & 9557 & 9351 & \$91 & sद्या \\
\hline WET TOM. 13F & T3535 & 736.7 & 135.9 & 736.7 & 138.1. & 738.11 & 73321 & T35.7! & 7351 & 735 \\
\hline We Tomp. $84 F^{-}$ & 13851 & 1351 & 137.21 & $137^{1}$ & 137.8 & 138.7 & 138.8 & 136.71 & 7355 & 138.7 \\
\hline WEUTOMD. 15F & 137 & 139.51 & 138.81 & 737.51 & 138.81 & 137.81 & 137.3 & 135.81 & 1396 & 133.8 \\
\hline Wed Tomp. Af & 124.51 & 135.61 & 134.11 & 135.91 & 138.2 & 137.71 & 138.5 & 136.4 & 133.7 & 1328 \\
\hline Wa Temp. d2F & 137.1 & 135.7 & 1352 & 136.11 & 135.8 & 137.81 & 138.7 & 137.4 & 1344 & 134.1 \\
\hline intrean Temp.f & 125.2 & 1261 & 125.7 & 126.61 & (262) & 125.71 & 126 & 玟. & 125.2 & 125.6 \\
\hline Ang. Wall Temp,F & 135.5 & 735.81 & 135.6 & 136.8 & 136.7 & 138.11 & 138.2 & 136.4 & 136.7 & 132.2 \\
\hline Avo. WaUDT F & 10.9 & 9.31 & 9.4 & 9.71 & 10.1 & 121 & T1.8 & 90.7 & 9.1 & 8.8 \\
\hline Rempining Suboooff & -0.7 & & & & & & & & & \\
\hline Ex' Qualy & of & 0.03 & 0.06 & 0.091 & 0.12 & 0.15 & 0.18 & 0.27 & 0.23 & 0.28 \\
\hline DX.HBuHrher & 915.11 & 1068.5 & 10542 & 101921 & 90.81 & 850.6 & 847.4 & 9324 & 70002 & $7 \operatorname{tach}$ \\
\hline COMPUIEDH & & & & & & & & & & \\
\hline Jak & 8882! & 85521 & 833.7 & 818.7 & 8122 & 803.61 & 80 茼 & 3978 & 351 & \\
\hline Sradi (ado twbe) & 1281 & 1233.91 & 1205.8 & 13029 & 1355.2 & 1298.9 & 1300 & 1300 & 1200 & 7800.2 \\
\hline $\operatorname{sen} 12$ & $1005 x \mid$ & 108421 & 9064.9 & 1069.7 & 1070.8 & 10524 & 1068.1 & 1050.4 & 103816 & 1069 \\
\hline (FUO) & 600356 & 59776 & 578811 & 560001 & 54628 & 528161 & डाना & 48983 & 2760 & 2500 \\
\hline$(F)$ & 1.164 & 1.5621 & 1.94 & 2232 & 2485 & 2.72 & 2.909 & 3.106 & 3.281 & 3.438 \\
\hline (Fin) & 181.8 & $242 \pi$ & 3001 & 348.61 & 3878 & 42201 & 453.81 & 483.7 & 571 & 6550 \\
\hline (FBOL) & 12858 & 1291.11 & 12828 & 12945 & 1233 & 12838 & 12875 & $127 / 2$ & T2721 & $127 / 5$ \\
\hline & & & & & & & & & & \\
\hline & RONis & ZONEA & ZONEG & ZONiE8 & ZONETO & & & & & \\
\hline DP havid temp.f & 761 & & & & & & & & & \\
\hline 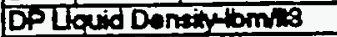 & 83.71 & & & & & & & & & \\
\hline Eoviond & & 4 & & 8 & 9.875 & & & & & \\
\hline Me:uredóspyed & 0.383 & 7.07 & 2477 & 2.8751 & 3.202 & & & & & \\
\hline Zero Contotion-ond. & 0.172 & 0.0 पा & 0.87 & 0.21 & 0.178 & & & & & \\
\hline 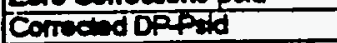 & 02181 & 1.080 & 1.800 & 2.456 & 3.068 & & & & & \\
\hline Acter DPA & 1.81 & 2831 & 3.84 & 4.68 & 5.68 & & I & & & \\
\hline Wemered iptip & 87.1 & & & +1 & 846. & & & & & \\
\hline Mecoured Overalla & 4.34 & & 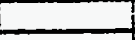 & 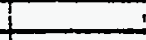 & 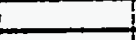 & & & & & \\
\hline & 1 & & & & & & $\perp$ & & & \\
\hline Computed DPA & 1.73 & 3.77 & 4.4.2. & 5.79 & 6.53 & & & & & \\
\hline Froton-1: & 0.07 & 032 & 0.44 & 0.73 & 1.06 & & & & & \\
\hline Acoutariond & 0.07 & 0.13 & 0.18 & 0.21 & 0.31 & & & & & \\
\hline Exwionth & 1.59 & 2.76 & 3.78 & 4.73 & 5.56 & & & & & \\
\hline & & & & & & & & & & \\
\hline
\end{tabular}




\begin{tabular}{|c|c|c|c|c|c|c|c|c|c|c|}
\hline Run: & 9061 & & & & & & & $\therefore$ & - & \\
\hline Test Fuid & CAF 8 & & & & & & & & 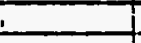 & \\
\hline Det: & 1013331 & & & & & & & & & \\
\hline Tims: & $12: 10 !$ & & & & & & & & & \\
\hline Now & Ânayticas d & is used & & & & & & & & \\
\hline Flow Arast'? & 0.003361 & & & & & & $\sum_{1}^{\prime}$ & 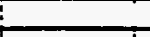 & & \\
\hline Hent Fow Aseal? & 0.19655 & & & & & & & & & \\
\hline Sip prossure Bitg & $84.8 T$ & & & & & & & & ! & \\
\hline SA Temperatefofiniet) & 126.31 & & & & & & & & & \\
\hline La. Dentinytomis & 86.521 & 86.39 & 86.45 & 86.321 & 86.37 & 86.4 & 86.42 & 86.48 & 86.63 & 85.46 \\
\hline Lenthostom & 39.181 & 39.071 & 39.18 & 39.021 & 39.08 & 39.091 & 39.11 & 3.151 & 39.18 & 39.18 \\
\hline 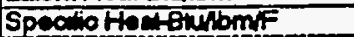 & 0.278 & 0.2781 & 0.278 & 0.278 & 0.278 & 0.2781 & 0.2781 & 0.278 & 0.278 & 0.278 \\
\hline Lja. Viroogny & 0.571 & 0.5651 & 0.568 & 0.5641 & 0.5661 & 0.5671 & 0.567 & 0.57 & 0.57 & 0.568 \\
\hline Lq. Conductivit Btumpraf & 0.03251 & 0.03251 & 0.0526 & 0.03251 & 0.03251 & $0.0525 i$ & 0.05251 & $0.08 \times 1$ & 0.0381 & 0.036 \\
\hline Lq. Prand No. & 4.861 & 4.841 & 4.85 & $4.83 !$ & 4.84 & 4.841 & $4.84 !$ & $4.85 !$ & 4.88 & 4.85 \\
\hline Inlot Flow Pate-GPM & $1.8 !$ & & & & & & & i & 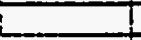 & \\
\hline Ō tot Lig. Fow Rato-GPM & 0.7 & & & & & & & & & \\
\hline Outer Vep. Flow Rive-GPM & 1.31 & & & & 1 & 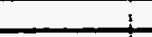 & & 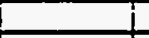 & & \\
\hline Inhat Temperminef & 131 & $?$ & ! & & & & & & & \\
\hline Oivalug. Tempararef & 123.8 & $i$ & 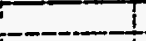 & & & & & & & \\
\hline OUta vap. Tomperatrof & 1211 & $T$ & $i$ & & 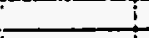 & $!$ & I & 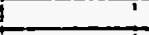 & & \\
\hline WES Flow RAOGPM & 1 & 1 & 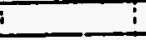 & 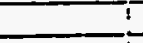 & 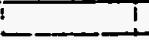 & 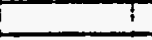 & & 1 & I & \\
\hline Waternkt Temp fF & 77.8 & 1 & $!$ & & 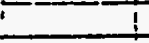 & I & & $I$ & I & \\
\hline Weroulet Temp.f & 109.7 & 1 & $i$ & $i$ & $\perp$ & 1 & & 1 & I. & \\
\hline lñablomin' & 3765501 & & Temp & Iftic & Densing & 87.661 & & I & i & \\
\hline Subooding f & 3.31 & & 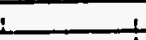 & & & & & 1 & 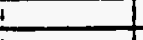 & \\
\hline Fow Obsenvition & & & & & & & & SONAS & i & \\
\hline & ZONET ; & ZONE? . & 20NS3 & Nيe日 4 & ZONE5 & ZONE6 iz & 2015 & ZONEB il & ZONES & $x=10$ \\
\hline Hos inplatwetelnel) & 573.2 & $5 \overline{2.31}$ & 576.3 & 573 & 574.6 & $5 \pi .8$ & 573.8 & 5724 & 5724 & 574.7 \\
\hline HonLosew & 6.7 & 1.5 & 2.11 & 211 & 2.1 & 1.61 & 1.11 & 1.51 & 1.9 & 10.8 \\
\hline Host Flox-Buth & 99861 & 99201 & 9950ा & 99331 & $95 \pi$ & \$9या & 9898 & 9321 & 9ses & 9562 \\
\hline Wet Tomp. $13 F$ & 133.61 & $134.7 !$ & 133.8 & $134.6 \mathrm{~T}$ & $135.1 T$ & $738 \pi$ & 738.11 & 138.81 & 13301 & T33.5 \\
\hline Wa Tomp. I4F & 134.61 & (335! & 135.1 & - & $135 . \pi$ & 738.61 & 136.6 & 135.11 & 134.a1 & 1372 \\
\hline WA Tomp.15F & $135 . \pi$ & $13241^{\circ}$ & 138.51 & 135.61 & 134.8 & 1361 & 135.4 & 134.11 & 1329 & 132.1 \\
\hline We: Tomp. & 123 & 133.7 & 1321 & 133.71 & $\overline{34} 2$ & 135.7 & 136.51 & 134.81 & 131.2 & 131.2 \\
\hline Wh Tomp. & 13521 & 133.81 & 133 & 134.11 & 1342 & 136.11 & 136.81 & 135.8 & 1326 & 1326 \\
\hline ineteen TompA & 123.8 & $.24 .7 i$ & 124.3 & 125.11 & 124.8 & $i 24.6 i$ & 124.5 & 124.11 & 123.0 & 124.3 \\
\hline Ang. Wall Tomp.t & 134.6 & 133.81 & $1335 ;$ & $134.7 i$ & 734.8 & 136,31 & 135.3 & 134.7 & 133.11 & 133.3 \\
\hline AVO.WEDT.F & 10.3 & 8.7 & 8.7 & 921 & $9 . \overline{6}$ & 11.2 & 11.3 & 10.2 & 8.7 & 8.6 \\
\hline Remining suboodf & -23 & & & & & & & & & \\
\hline Exhaual & 0.02 & 0.081 & 0.11 & 0.13 & 0.17 & ठ.दा & 0.5 & 0.29 & 0.331 & 0.37 \\
\hline 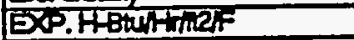 & 868.9 & 1146.71 & 1145.2. & 10824 & 103.8 & 885 & 877.I & 975.51 & 11384 & 11623 \\
\hline COMPUTIEDH & & & & & & & & & & \\
\hline Jefk & 889 & 860.8 & 840.1 & 8696 & 8115 & 8रा & 801.3 & 795.11 & 7382 & 7355 \\
\hline 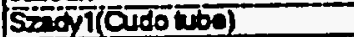 & TET29] & 7260.61 & $12 \overrightarrow{2} 2 \mid$ & 7387.71 & 1287.7 & 728256 & 1283.7 & 1283.61 & 12835 & 7297.8 \\
\hline $500 \sqrt{2}$ & - ब्रा & 1008.8 & 10519 & 10501 & 1054.8 & 1050.11 & 1051.1 & 1048.81 & 1045: & 10527 \\
\hline$(A \underline{O C})$ & मद्वाया & 41834 & 40058 & 38606 & 36800 & 350081 & 3350 & 31क्2 & 2903 & 2005 \\
\hline (F) & 1.358 & 9.8002 & $23 \pi 1$ & 25741 & 2800 & 3.1381 & 3.378 & 3.5021 & 3.874 & 2.008 \\
\hline (H) & 76.9 & 21621 & -3635 & 3051 & अयदो & 375.7 & 404.8 & $33 i .4$ & 756601 & 48.7 \\
\hline$(P: 30)$ & रिश्या & 1278.5 & $\sqrt{2782}$ & 1287.7 & 1दिया & 1273.71 & 12732 & 1257.11 & 138 & 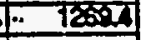 \\
\hline & & & & & & & & & & \\
\hline & ZONEE & ZONEA & ZONi=6 & $20 \mathrm{~N}=8$ & $120 K=10$ & & & & & \\
\hline DPrigud tome.tF & 75.9 & & 1 & & & & & & & \\
\hline DPLLquid Dentitionis & 93.77 & & $i$ & & & & & & & \\
\hline Evertion & & & & & 9.875 & & & & & \\
\hline Wheured DFphad & 0.496 & 1.23 & 278 & 3.035 & 3.81 & & & & & \\
\hline Zero Correctomptid & 0.172 & 0.047 & 0.851 & 0.21 & 0.178 & & & & & \\
\hline 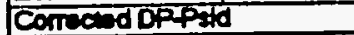 & 0.277 & 1357 & 1.947 & 28881 & 3.004 & & & & & \\
\hline Acter DP & 1.71 & 2251 & 3.20 & 3.681 & 4.58 & & I & & & \\
\hline Meaured Tripig & 848 & & 1 & & 824 & & I & & & \\
\hline Meneured Over & 4 & & 1 & & 1 & & 1 & & & \\
\hline & $\perp$ & & 1 & & & & 1 & & & \\
\hline Computad DPA & 1.52 & 268 & 3.7 & $4.73 \mathrm{i}$ & 5.68 & & & & 1 & \\
\hline Fictont & 0.01 & 0.17 & 0.35 & 0.58 & 0.84 & & & & & \\
\hline Aooderion & 0.05 & 0.01 & 0.13 & 0.181 & 023 & & & & & \\
\hline Eleverionth & 1..2दा & 2391 & 3.32 & 3.57 & 2.67 & & & & & \\
\hline & & & & & & & & & & \\
\hline
\end{tabular}




\begin{tabular}{|c|c|c|c|c|c|c|c|c|c|c|}
\hline Runt & $907 i$ & ' & & & $\therefore$ & - & & 1 & & \\
\hline Tosffuid & CAFB & & & & & & 1 & 1 & & \\
\hline Dets: & 1वाइय3। & & & & & & & & & \\
\hline Time: & $12: 10 !$ & & & & & & & & & \\
\hline Noto & Anayical d & is is used & . & & $\therefore$ & & & & & \\
\hline Fow Areath & 0.003351 & & : & & & & & & & \\
\hline Hoet Fow Aras 12 & 0.1955 & & $\therefore$ & & 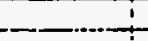 & & $\therefore$ & & & \\
\hline Sal Pressuro Psig & -85.61 & & 1 & & 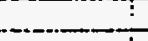 & & & & & \\
\hline S. Tomperatofinlol) & 126.81 & & & & & & & & & \\
\hline Lig.Dentiny & 86.241 & $86.29 !$ & 86.351 & 86.181 & 86.24 & $8629 !$ & 86.291 & 86.341 & 86.89 & 86.20 \\
\hline Lerd Has Bturibm & $38.951^{\circ}$ & $\overline{3} 8.991$ & $39.05 !$ & 38.911 & $38.95 i$ & 38.891 & 38.991 & 39.081 & 39.07 & 38.89 \\
\hline 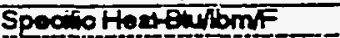 & 0.278 & $\bar{u} .278 \mathrm{i}$ & $0.278 i^{-}$ & $0.278 i$ & $0 . \overline{278}$ & 0.278 & $0.278 !$ & 0.278 & 0.278 & 0.278 \\
\hline Liq. Visoos fommin & $0.56 \mathrm{ii}$ & 0.5631 & $0.565 i$ & 0.561 & 0.567 & 0.5631 & $0.5631^{-1}$ & 0.5651 & 0.566 & 0.560 \\
\hline Lia. Conductivither & $0.0324:$ & 0.0324 & 0.0325 & 0.0324 & $0.0324:$ & $0.0324 ;$ & 0.0324 & 0.03251 & 0.0325 & 0.0324 \\
\hline Lig. Prand No. & $4.82 !$ & 4.83, & 4.841 & 4.82 & $4.82 i$ & 4.831 & $4 . \overline{83 !}$ & 4.891 & 4.84 & 4.83 \\
\hline Intet flow Rato-GPM & 1 & & & & & & & & & \\
\hline OWA Lia Fow Rate-GPM & $0.7 !$ & & & & & & $=$ & & & \\
\hline Outot Vap. Flow Rato-GPM & 0.61 & & & & & & i & 1 & & \\
\hline Intel Tomperaturef & 123.4 & & 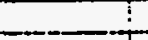 & & : & : & $\therefore$ & & & \\
\hline OW Liq Tempersuref & 124.8 & & ? & & ! & & & & & \\
\hline ÖUAT Vap.TomperareF & 1223 & & ! & & 1 & 1 & $!$ & & & \\
\hline WEAT Flow Rata-GPM & $i$ & & $i$ & & $i$ & 1 & 1 & & & \\
\hline Waler trilet Tamp. $F$ & 79.51 & 1 & & & 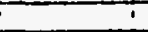 & $i$ & $t$ & 1 & & \\
\hline Wer oulet Temp.F & 109.6 & I & $\therefore$ & & 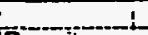 & $\ldots$ & I & ! & & \\
\hline Inde Gbmininc & 20067 & & Temp & 115.7 & Donsity & 87.86 & $I$ & i & & \\
\hline Subooding of & 3.4 & 7 & & 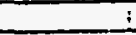 & $i$ & & & $\mathrm{~L}$ & & \\
\hline \multicolumn{11}{|l|}{ Fow Obsenvation } \\
\hline & ZONET : & ZONE2 & ZONE3 2 & ZONEL i & ZONE5 I2 & ZONE6 & ZONE7 : & ZONEB & $20 N=8$ & $20 N=10$ \\
\hline Hoat houtwesnot) & 572.61 & $5 \overline{5.8}$ & 579.3 & $5 \overline{8.11}$ & 57 & 5825 & 575.7 & -574 & 573.2 & 5726 \\
\hline Hentose-Was & & 1.6 ! & 2.11 & 221 & $22 !$ & 9.7 & 1.11 & 1.7 & 2 & 11.8 \\
\hline 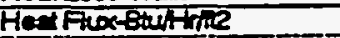 & 实安 & 99831 & 10042 & 100211 & 10002 & 10057 & 9969 & sos & 30000 & 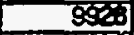 \\
\hline We Tomp. $13 f$ & T32\% & उखा & 1327 & T3द21 & $135 !$ & 138.81 & $135.8 T$ & 134 & 7330 & 130 \\
\hline Waltomp. I4F & 134 & $132 \overline{3}$ & $13 \pi .21$ & 134.81 & 135. 1 & 135.81 & 736.81 & 135.51 & $13 \pi 8$ & 137.4 \\
\hline WEITOmo. $85 F$ & 134.41 & 131.7 & 138 & $13 \overline{8} . \overline{1}$ & 134.21 & 135.4 & 135.11 & 134.41 & 135 & 1320 \\
\hline Wal Tomo. IIF & 123.41 & $132 \overline{81}$ & 131.5 & 133.11 & 133.51 & $135 . \overline{41}$ & 138.81 & 135 & 1321 & 131.6 \\
\hline Wall Tomp. $12 F$ & 134.91 & $132.8 i$ & 1322 & 133.71 & 139.6 & 1361 & 136.61 & 1361 & 1328 & 1320 \\
\hline intrem Tomo.t & 125.6 & 125.31 & 124.8 & $125.8_{1}$ & 125.6 & 125.31 & 125.31 & 125 & 124.7 & 125.3 \\
\hline Avo. Wall Tomp.f & 134 & $133 i$ & 1327 & 134.11 & 1342 & 1361 & 136.1 & 135 & 733.1 & 133.7 \\
\hline Avo. WaIDT.F & 81 & 7.2 & 7.3 & $7.8 i$ & 8.71 & 1021 & 10.3 & 9.6 & 8 & 8 \\
\hline Romaining Subooff & -6.51 & & & & & & & & & \\
\hline EATQuaty & 0.051 & 0.12 & $0.180^{-}$ & $0.26 !$ & 0.33 & 0.41 & 0.08 & 0.55 & 0.63 & 0.69 \\
\hline EXP.FBturingat = & 1248.31 & 1386.7: & $7376.3 !$ & 1289.3! & 1237.31 & 988.11 & $9 \times 2$ & 1039.71 & 1Z5T. & 1248.8 \\
\hline \multicolumn{11}{|l|}{ COMIPUTEDH } \\
\hline Jaber & Ђ7231 & 897.81 & -874.7 & $885.3 !$ & 85351 & 847 & 8374 & 86.8 & 823.1 & 8121 \\
\hline Solvil(ado ube) & 7288.81 & 3289.91 & 12003 & 1299.81 & 1296.iा & 13001 & 2928 & 7269.7 & 1201 & 12334 \\
\hline $5 \sin 2$ & 1005.61 & 7038.41 & $1008 . \overline{6 !}$ & $10 \overline{70.31}$ & $10559 !$ & 1069.51 & 1000.65 & 1050.4 & 1057.1 & 1038 \\
\hline (R्DO) & 238001 & 223601 & 20522 & 189601 & $17151 i$ & $15 \sqrt{561}$ & 13592 & 118191 & 1000 & 897 \\
\hline (F) & 1.2 & 2287 & 2.8651 & 3.3041 & 3.721 & 4.0651 & 4.3961 & 4.718 & 5.010 & 5.395 \\
\hline (स्य) & 721.81 & 7727 & 216.2 & 248.7 & 278.5 & 305.71 & 350.61 & 354.11 & 378. & 397 \\
\hline \multirow[t]{3}{*}{ (HBOA) } & 1288,4 & 1288.91 & 7208.31 & 12065 & 12918 & $129 \times 4$ & 128561 & 1280.71 & 12002 & 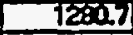 \\
\hline & & & & & & & & & & \\
\hline & ZONEE & ZONET & ZONEE & ZONEB & ZONETO & & & & & \\
\hline DP Fquid Temp.f & 75.91 & & & & & & $!$ & & & \\
\hline DPLlouid Denthym/33 & 33.77 & & & & & & & & & \\
\hline Eovitiond & & & & 81 & 9.875 & & & & & \\
\hline 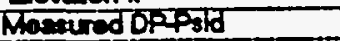 & 0.3501 & 7.595 & 3.7551 & 3.68 & 4.537 & & & & & \\
\hline Zero combotiong orid & 0.172 & $0 . \overline{0.71}$ & 0.84 & 0.21 & 0.118 & & & & & \\
\hline Correcud DP pid & 0.187 & 1.5581 & 2.318 & 3.470 & 4.91 & & & & & \\
\hline ACTDDPA & 1.88 & 1.781 & 260 & 289 & 3.34 & & & & & \\
\hline Whered ippla & 85.8 & 1 & & & 83 & & & & & \\
\hline Moatered Overlith & 3.67) & 1 & & & & & & & & \\
\hline & & & & & & & & & & \\
\hline Computed DPI & 1.15 & 1.321 & 2.61 & 3.22 & 3.781 & & & & & \\
\hline Fictornt & 0.03 & 0.71 & 0.2. & 0.35 & 0.51 & & & & & \\
\hline Aconier tion & 0.03 & 0.05 & 0.08 & 0.12 & 0.16 & & & & & \\
\hline Elovionth & 7.091 & 9.71 & 2.391 & 2.75 & 3.11 & & & & & \\
\hline
\end{tabular}




\begin{tabular}{|c|c|c|c|c|c|c|c|c|c|c|}
\hline Run: & So81 & 1 & & & & - & & 1 & I & \\
\hline Toeffuid & $\mathrm{CAF}_{\mathrm{B}}$ & . & & & 1 & & I & 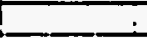 & 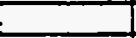 & \\
\hline Dalo: & $10=433$ & & & & I & & & 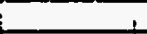 & & \\
\hline Timo: & $8: 21$ & & & & & & & & & \\
\hline Noto & Analyical of & Itis used : & & & $i$ & & & & & \\
\hline Flow Ares-12 & 0.003561 & & & & & & i & & & \\
\hline Hoel Fow Araste & 0.19655 & & & & i & & i & 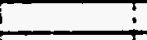 & & \\
\hline Sel Prossuro pig & 86.61 & & & & $T$ & T & $\mathbf{i}$ & & & \\
\hline 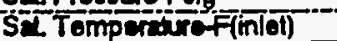 & 127.51 & & & & 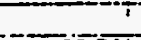 & & 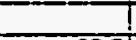 & -T & $T$ & \\
\hline Uq. Denaty tom & 86.371 & 86.27 & 88.31 & $86.17 i$ & 86.24 & 86.291 & 88.31 & 86.37 & 86.42 & 88.4 \\
\hline Ltent Hosteuthom & 39.081 & 38.881 & $39.01 !$ & 38.91 & 38.951 & 38.891 & 39.011 & 39.081 & 39.1 & 39.11 \\
\hline 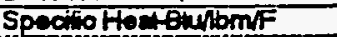 & 0.2781 & 0.2781 & 0.278 & $0.278 i^{\circ}$ & $0.278 !$ & 0.2781 & 0.278 & 0.278 & 0.378 & 0.278 \\
\hline Uiq. Visoot & 0.566 & 0.5621 & 0.564 & 0.5591 & 0.5611 & 0.5631 & 0.564 & 0.5651 & 0.567 & 0.568 \\
\hline Lig. Conductivity therrif & $0.0325 i$ & $0.0324 !$ & 0.0325 & $0.0324:$ & 0.03241 & 0.0324 & 0.0325 & 0.03251 & 0.038 & 0.0505 \\
\hline Liq. Prendt No. & 4.84 & 4.831 & 4.83 & $-4.81 i$ & 4.82 & 4.831 & 4.831 & 4.841 & 4.84 & 4.85 \\
\hline Inlot Fow Ars-GPM & $4.9 i$ & & & & & & & & & \\
\hline OUdel Uq. Fow Rato-GPM & $0.7 \pi$ & & $\cdot$ & & & & & & & \\
\hline Oulu Vep. Flow Rate-GPM & 2.51 & & $i$ & 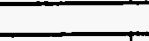 & & & 1 & & & \\
\hline Intal Tomperaturef & 123.9 & & & & & & 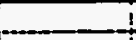 & & & \\
\hline OUTAĹt Temperturef & 123.61 & & & & !lt & & $i$ & & & \\
\hline OŨT Vap. Tomper trof & 121.11 & & & & & & & & & \\
\hline WLa Fow RALGPM & $-\frac{1}{1}$ & & 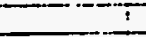 & 1 & & & & & & \\
\hline We tor hilet Tomp,f & 801 & $!$ & $i$ & 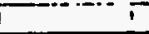 & $!$ & 1 & & 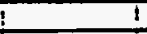 & & \\
\hline Werer ould Tomp.f & 1029 & 1 & 1 & 1 & $i$ & 1 & & 1 & & \\
\hline inda & 11018647 & & Tamp & $118.81 \overline{1}$ & Denty & 87.21 & & 1 & 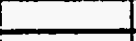 & \\
\hline Subooolingf & i.6 & $\perp$ & 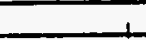 & 1 & $I$ & 1 & & 1 & 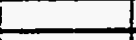 & \\
\hline Fow Obsenvation & & $i$ & $!$ & 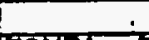 & $!$ & (19) & & i & & \\
\hline & ZONEI & $6 \sqrt{2}-i 2$ & ZONE3 T2 & 201542 & ZONE5 IS & ZONE6 & ZONEI & ZONE 8 & 2010 & $204=10$ \\
\hline Hodlnot Wartinet) & 8695 & 870.3 & 855.51 & 838.3 & 866.5 & 851.8 & 8628 & 864.7 & 8s52 & 8550 \\
\hline HenLoss-Wats & 7.51 & 1.7 & 2.1 & 241 & 251 & 7.9 & 1.2 & 1.8 & र्या & 11.8 \\
\hline Hon Fiofenthrie & 15072 & 1506801 & 150003 & 14835 & 950001 & 74940 & T45631 & 14969 & 160:8| & 750T0 \\
\hline We tomo.134 & 1365 & 139.8 & 33.1t & 136] & 7405 & दाना & 7100.8 & 137.31 & 7372 & 135,7 \\
\hline WaI Tomp.114 & 13. & 138.4 & 140 & 132.2 & 340.5 & 141.41 & 740.4 & 139.4 & 138.4 & 721.8 \\
\hline WET Tomp. 16 F & 13821 & 135.81 & 140.4 & 139.6 & 139 & 140.81 & 139.3. & 1381 & 1386 & 139 \\
\hline Well Tamo. dIf & 128.8 & 13721 & 135.8 & 137.81 & 138.21 & 140.51 & 141 & 138.4 & 135.0 & 1326 \\
\hline WEHTOmp. $12 f$ & 138.6 & 137.41 & 1372 & 137.8 & 138.41 & 141.31 & 141.7 & 139.7 & 136.7 & 135.1 \\
\hline Intream Tomp.f & 124.8 & 125.41 & 1252 & 位i & 125.61 & 125.31 & 125.27 & 124.81 & 1245 & 124.4 \\
\hline Avg. Wall Tomp.t & 137.8 & 137.7 & 137.8 & 738.81 & 139 & 140.9 & 140.4 & 138.61 & 1359 & 1350 \\
\hline Aur. Well DT 7 & 123 & 11.6 & 11.8 & 11.91 & 127 & 14.0 & 74.5 & 1321 & 11.8 & 10.8 \\
\hline Remaning Subooff & 0.5 & & & & & & & & & \\
\hline Exito outity & of & 0.02 & 0.04 & 0.05 & 0.09 & 0.11 & 0.13 & 0.15 & 0.17 & 0.2 \\
\hline 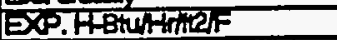 & सित्रा & 1304.1 & Tिका.1 & 13524 & 1178.4 & 999.4 & 1008.7 & 1138.8 & 1275.1 & 1387 \\
\hline COMPUTEDH & & & & & & & & & & \\
\hline Jeack & $10 \% 1$ & Tozien! & \$8तB| & 5501 & 97621 & 301 & 918.7 & 9031 & 80.11 & 8892 \\
\hline sandy ICdo ube) & 1550.21 & 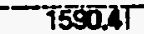 & 7587.4 & 1596.91 & 160121 & 1598.11 & $7 \bar{E}=28$ & 1004.71 & 7007.1 & 16123 \\
\hline $\operatorname{sed} 2$ & 13824 & 139211 & 13016 & 13735 & 13721 & 1374351 & 1378.7 & 1383.11 & 136.2 & Tदा28 \\
\hline (FWa) & 117896 & 1176881 & 114821 & 1130881 & 110000 & 107074 & 104538 & 101589 & $\mathbf{9 8 4 5}$ & $\$ 5 \%$ \\
\hline & 1.008 & 1.322 & 1.778 & 1.987 & 2218 & 2.477 & 2507 & $2.75 \pi$ & 298 & 3.078 \\
\hline (सUC) & 257.81 & 35231 & 457.67 & 53021 & $59 \overline{98}$ & Eम4. & 691.0 & -73637 & 783.2 & 8172 \\
\hline (F:O4) & 157821 & $158 \sqrt{61}$ & 1574.7 & $15 \% 21$ & 15330 & 1552 & 1559.1 & 15512] & 75130 & 15ख2 1 \\
\hline & & & & & & & 1 & & & \\
\hline & ZONES I & ZONEA & ZONEE & $201=8$ & ZONEETO & & $\perp$ & $\perp$ & & \\
\hline DP Iqud Tomp.F & 76.8 & & & & & & & & & \\
\hline 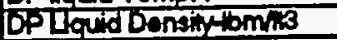 & 90.65 & & & & & & & & & \\
\hline Eov'tiont & & 41 & & $8 !$ & 9.875 & & & & & \\
\hline 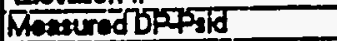 & 0.325 & $0.63 \pi$ & $7.75 \pi$ & $7.60 \mathrm{~m}$ & 7.88 & & & & & \\
\hline Zero Corractionspeid & 0.17 & 0.011 & 0.802 & 0.122 & 0.189 & & & & & \\
\hline Cormoled DPpsid & 0.148 & 0.620 & 0.809 & 1.461 & 1.72 & & & & & \\
\hline Actul DPa & 1.82 & 3.201 & a.s.5 & 6.251 & 7.85 & & & & & \\
\hline Fenured triphas & 86.6 & & 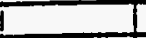 & 1 & 852 & & $I$ & & & \\
\hline Meceuredover & 41 & & & $i$ & 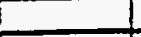 & & 1 & & & \\
\hline & & & & & & & & & & \\
\hline Computad DPA & 219 & 4.061 & 5.83 & 7.881 & 9.84 & & $!$ & & & \\
\hline Ficton- & 0.18 & 0.581 & 1.18 & 3.861 & 280 & & I & & & \\
\hline Arooferetion & 0.21 & o.4i & 0.57 & 0.73 & 0.9 & & 1 & & & \\
\hline Eleveliont & 1.81 & 3.07 & 4.17 & 5.18 & 6.07 & & & & & \\
\hline & & & & & & & & & & \\
\hline
\end{tabular}




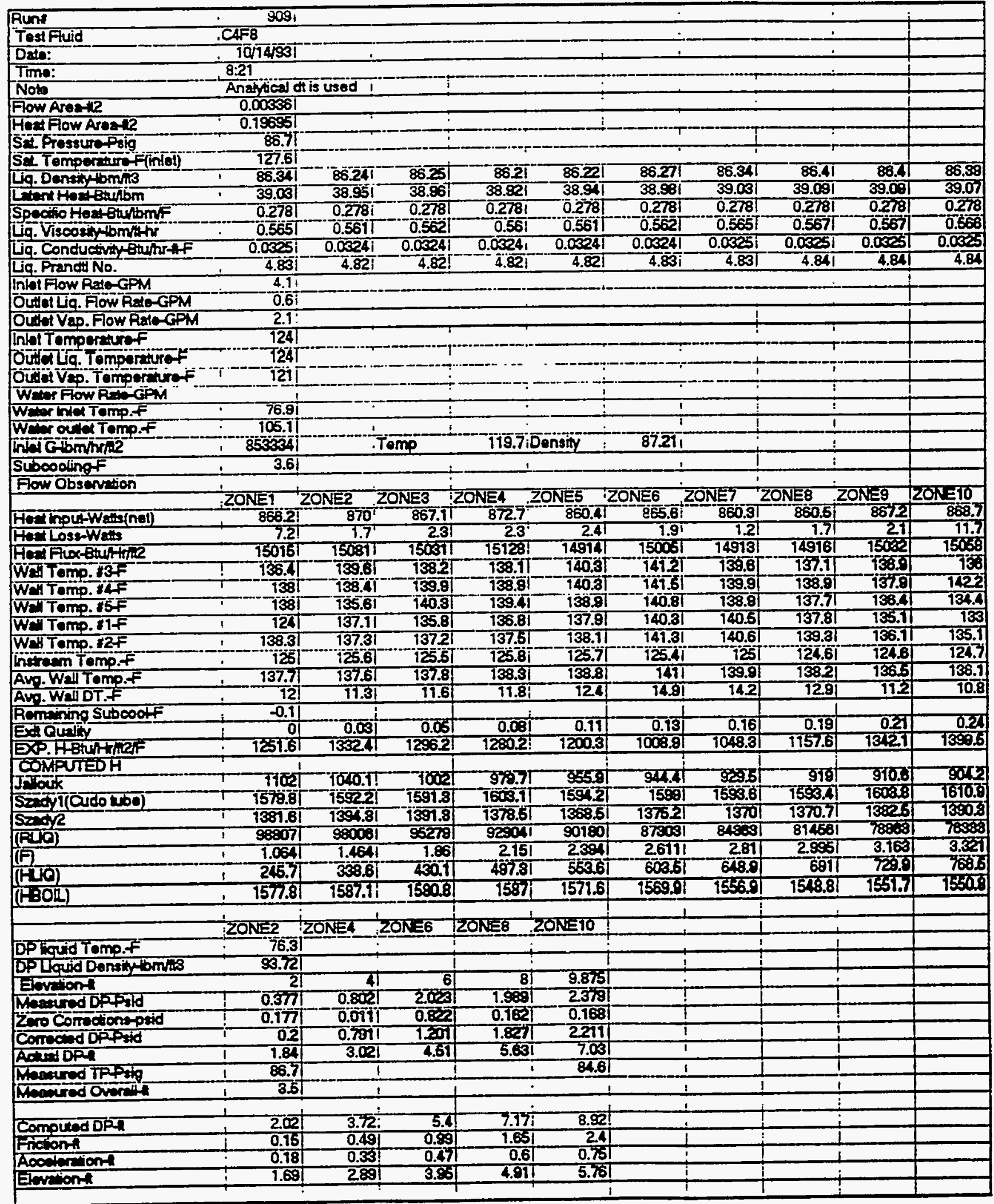




\begin{tabular}{|c|c|c|c|c|c|c|c|c|c|c|}
\hline Run & $\therefore \quad 310 i$ & & & & & & & . & . & i \\
\hline Tost Fluid & CAF8 & & & & & & & . & - & \\
\hline Date: & 101493 & & & & & & & t & & \\
\hline Time: & $8: 21$ & & & & & & & 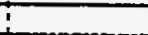 & & \\
\hline Note & Anatifical & of is used & & & & & & $\dot{i}$ & & \\
\hline Fow ANeste & 0.003561 & & & & & : & & & & \\
\hline Hext Fow Areat2 & 10.18651 & & & & & & & $\therefore$ & $\vdots$ & \\
\hline S21 Fioserrefig & 85.31 & & & & & T. & T & ! & & \\
\hline Sil tompor & 126.81 & & & & & $i$ & $i$ & i & & \\
\hline Liq. Dentinytbomis & 86.471 & 86.391 & $86.4 !$ & 86.321 & 86.351 & 86.891 & 86.451 & 88.64 & 88.54 & 88.52 \\
\hline 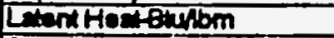 & 39.14 & 39.071 & $39.00 j$ & $3 \overline{9} .02 i$ & 39.051 & 39.071 & 39.13 & 39.181 & 39.10 & 39.78 \\
\hline Speciofter Qubmit & 0278 & 0.278 & 0.2781 & 0.2781 & 0.2781 & 0.2781 & 0.278 & $0.27 \%$ & 0.27n & 0.278 \\
\hline Lig. Viscostiominte & 0.5691 & 0.5661 & 0.567 & 0.5601 & 0.5651 & 0.5661 & 0.5681 & $0.5 \pi$ & $0.6 \%$ & -0.57 \\
\hline 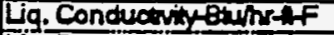 & 0.0325 & $0.0325 i$ & 0.0325 & 0.0325 & 0.03251 & 0.03251 & 0.03251 & 0.03251 & 0.058 & 0.023 \\
\hline Liq. PrandoNo. & 4.851 & 4.84 & 4.84 & 4.831 & 4.89 & 4.84 & 4.85 & 4.861 & $4.8 \overline{3}$ & 2.86 \\
\hline Inloffow fate-GPM & 3.3 & & & & & & & & & \\
\hline OU CUL. FOW RAOGPM & 0.5 & & & & & & & & & \\
\hline Outel Vap. Fow Parf-GPM & 1.9 & & & & & & & & & \\
\hline Intertompereturf & 923.11 & & & & & & & & & \\
\hline ouclitiq. Tompenter & 23.81 & & & & & & & & 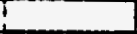 & \\
\hline outet Ve. Tompen uref & 120.71 & & & & & & & & & \\
\hline WhM Flow RaAg-GPM & 1 & 1 & T & $!$ & & 1 & I & & & \\
\hline Wer hintermp.t & 74.7 & & i & 1 & & & & I & & \\
\hline Wrer ovier Temp.F & 104 & $!$ & & 7 & & i & i & & & \\
\hline Intel Gibmhrne & 6883711 & & Temp & 718.610 & Density & 87.411 & & & & \\
\hline Suboodingf & 3.5 & $\dot{+}$ & & -1 & $=$ & 1 & $i$ & I & & \\
\hline Fow Obsenvion & & & & & & $i$ & & & & \\
\hline & ZZONET & ZONE & ZONES & ZONE 4 & ZONES & ZONE6 I & ZONE? & ZONES I & ZONES & $201=10$ \\
\hline Heit houtweret & 1 & $8726 !$ & 8725 & $-87 \pi .4$ & 875.51 & 873.61 & 873 & 870.7 & 878.8 & 8721 \\
\hline Hen Loss-Wes & 7 & 1.61 & 2.31 & $2.3 j$ & 2.31 & 1.8 & 1.1 & 7.7 & 2.1 & 71.5 \\
\hline 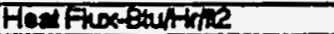 & T513:31 & $15126 !$ & 15T2ता & T5TBZI & T5176! & 15ाद्धा & 15T्टा & 750द्डा & $75 T 38$ & 15120 \\
\hline WelTomo. & 735.81 & 138.7 & 137.1 & 137.31 & 138.4 & 740.81 & 138.7 & 136.57 & 1832 & 135.8 \\
\hline WNTEMP.IAF & 137.71 & 135.51 & 738.81 & 137.81 & 139.61 & 940.51 & 1392 & $1382 !$ & 1378 & Tदा.4 \\
\hline Wall Tomo.157 & 137.2 & $135.7 \pi$ & 139.31 & $138 . \overline{1}$ & 1321 & 139.91 & 138.21 & 137.11 & 135.6 & 1356 \\
\hline WEl Temo.ntF & 123.1 & 136.31 & 134.71 & 135.91 & 137 & 139.51 & 1401 & 137.11 & 134,6 & 13्टा \\
\hline WEIT Tomp.12F & 137.3 & 136.31 & 1361 & $136.7 i$ & $137.3 i$ & 140.31 & 140.11 & 138.51 & 135.4 & 134.8 \\
\hline Inesem Temp.F & 124.2 & 124.35 & 124.6 & 125.11 & 124.91 & 124.7 & 124.31 & 123.81 & 1202 & 1230 \\
\hline AYp.Wall Tomp.F & 136.7 & $136.7 i$ & 136.7 & 137.4 & 138 & 140.11 & 1302 & 1375 & 1358 & 1353 \\
\hline Avo. WAII DT.F & 11.8 & $11.3 i$ & 11.4 & $11.6 i$ & 72.4 & 14.7 & 14.3 & 13 & (11.8) & 10.7 \\
\hline Remining Subooolf & -1.1 & & & & & & & & & \\
\hline Exalualy & 0.01 & 0.04 & 0.07 & 0.71 & 0.74 & 0.77 & 03 & 0.20 & 0221 & \\
\hline 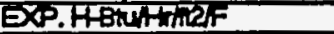 & 127 & 1353.6 & 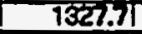 & 13सt.I & 12001 & $10526 !$ & 10522 & 11612 & 13\%, & 74036 \\
\hline CONFUTESH & & & & & & & & & & \\
\hline Jeck & 7033.91 & 3047.81 & 700881 & 3981 & -974.81 & 3581 & SसROI & -3<281 & 8030 & 80 \\
\hline Szovil(cudotwol & -157.351 & 1588.81 & 758.8 & 159.71 & 159.81 & $7565.7 \pi$ & 7597 & 1588.21 & T6马. I & 1653.8 \\
\hline Sadre & 187.7 & 13847 & 1384.8 & 1370.51 & 137251 & 1371.81 & 1368.7 & 1360.61 & $133 \mathrm{~B}$ & 73778 \\
\hline (Pय) & 78825 & 775881 & 74898 & 720191 & 698481 & 670681 & 64:17 & 61ร181 & 5872 & 56182 \\
\hline$(\mathbf{D}$ & 1.210 & 1.60 & 2056 & 23681 & 284 & 288 & 3.106 & 3.3181 & 3.500 & 3.83 \\
\hline (FLOB) & 286.6 & 318.6 & 3922 & 460.61 & 513.31 & 560.17 & 60.11 & 6421 & 680 & $3 \pi 4.0$ \\
\hline$(1+304)$ & 15746 & $15 \%$ & 1575.8 & 7581.81 & 1577.9 & 157.31 & 1561.6! & 15523 & 7551.7 & 15402 \\
\hline & & & & & & & & & & \\
\hline & $2 O N_{1}=2$ & ZON=4 & ZONE & ZONLE & ZONETO & & & & & \\
\hline DP Tqued tomp. $f$ & $\therefore \quad 772$ & & - & $\dot{-}$ & & & & & & \\
\hline DPLgud Density bonil 3 & 83.591 & & & & & & & & & \\
\hline Enimion & & 4 & & 81 & 9.8751 & & & & & \\
\hline Momired DFps]d & 0.199 & 0.89 & 2310 & 2388 & 2.89 & & & & & \\
\hline Ecro Correctionsped & 0.77 & 0.017 & 0.82 & 0.182 & 0.168 & & & & & \\
\hline Comeand DPritd & DI2 & 0.89 & 1.497 & $23 \times 1$ & 270 & & & & & . \\
\hline ACIDDP & $1.7 \pi$ & $2 \pi$ & 4 & 4.851 & 8.16 & & & & & \\
\hline Weaund if phe & 85.8 & 1 & I & $i$ & 833 & & & & & - \\
\hline Meenired Dver & 3.6 & I & I & 1 & & & & & & \\
\hline & & & & & & & & & & E \\
\hline Compered DPI & 1.781 & 3.231 & 4.67 & 6.171 & 7.67 & & & & & \\
\hline Fichont & 0.131 & 0.231 & 0.87 & 1.465. & 2.74 & $\perp$ & & & & 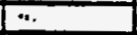 \\
\hline Acowerelion & 0.15 & 0.26 & 0.37 & 0.49 & 0.61 & & & & & \\
\hline Eluntiont & 9.511 & 25 & 3.4. & 4.22 & 4.8 & & & & & \\
\hline & & & & & & & & & & \\
\hline
\end{tabular}




\begin{tabular}{|c|c|c|c|c|c|c|c|c|c|c|}
\hline Runt & 9111 & & & & & & & & & \\
\hline Tost Find & CA & & & & 1 & & & & & \\
\hline Dado: & $.10 / 4831$ & & & & & 1 & 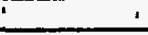 & 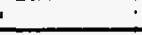 & & \\
\hline Trme: & $.8: 21$ & & & & $\therefore$ & $\therefore$ & $:$ & $\therefore$ & $\therefore$ & \\
\hline Not & Anatycal d & dtis used. & & & & 1 & 1 & $\therefore$ & 4 & \\
\hline Flow Area-? & 0.003351 & & & & & & & 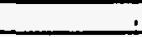 & \pm & \\
\hline Heat Fow Aroat? & 0.19655 & & & & & & & & & \\
\hline Sxt Pressuro prig & 84.21 & & & & & & & & & \\
\hline Sx. Temperature-finlef) & 125.91 & & & & & & & & & \\
\hline Lig. Denth tom & 86.52 & 86.49 & $86.52 !$ & 85.39 & 86.39 & 86.42 & 86.541 & 86.571 & 85.591 & 86.6 \\
\hline Lenthodentiom & 39.181 & $39.15^{r}$ & $39.78 !$ & $39 . \overline{0}$ & 39.07 & $39.1 !$ & 39.19 & $3922 !$ & 39.24 & 39.25 \\
\hline Specicherempmis & 0.2781 & 0.278 & 0.2781 & $0.278 !$ & 0.2781 & 0.278 & 0.277 & 0.277 & 0.277 & 0.27 \\
\hline Lig. Viscostintominhr & 0.5711 & $0.57 i$ & $0 . \overline{5} 711$ & 0.5651 & 0.5681 & 0.557 & 0.571 & 0.5721 & 0.578 & 0.573 \\
\hline 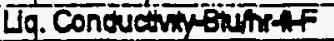 & 0.03281 & 0.03281 & 0.03881 & 0.03251 & 0.03251 & 0.03251 & 0.03281 & 0.03261 & 0.0531 & $0.0 \times 27$ \\
\hline Liq. Prand No. & 4.861 & 4.851 & 4.861 & 4.841 & 4.84 & 4.84 & 4.861 & 4.881 & 4.87 & 4.87 \\
\hline Inlet Fow PAT-GPM & 2.51 & $\ldots 1$ & -1 & 1 & +1 & $\mathrm{i}$ & i & & 1 & \\
\hline OWl Liq. Fow RWO-GPM & 0.31 & . & 1 & & & I & I & & - & \\
\hline Outat Vas. Flow Rado-GPM & 1.91 & & & & 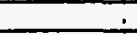 & $i$ & i & 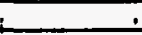 & - & \\
\hline Inift Temperaturof & 1231 & & & & $!$ & & t & i & i & \\
\hline Outet Lig. Temporatura F & 124.7 & & & & & & 1 & $i$ & $i$ & \\
\hline 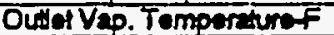 & 120.11 & & 1 & & $\mathrm{i}$ & I & 1 & $i$ & $i$ & \\
\hline Wator Flow Ralo-GPM & & & & & & 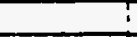 & 1 & & & \\
\hline Werthlet Temp.f & 72.3 & & & & & & & & & \\
\hline Weter ould Tomp.t & 1021 & & & & & & & & & \\
\hline Inist G-binmerie & 5200401 & & Temp & 116.81 & Densiny & $87.67 !$ & I & 1 & 1 & \\
\hline Subcoolingof & 291 & i & $\square$ & 1 & -2 & -1 & 1 & T & T & \\
\hline Flow Obsemvion & $!$ & I & 12 & I & 1 & I & 1 & T & $T$ & \\
\hline & ZONE1 is & ZONE2 & ZONE3 i & ZONEA 1 & ZONES it & ZONEE & ZONE7 is & ZONEB & ZONES & $20 \mathrm{~N}=10$ \\
\hline Heal houtwaratinet) & 870.81 & 865.4 & 870.51 & 874.11 & 875.4 & 871.1 & (8701 & -88.1 & 8653 & 8633 \\
\hline Hant Loss-Was & 7.41 & 7.7 & 24 & $24 !$ & 25 & 1.9 & $1.2 !$ & 1.81 & 22 & 125 \\
\hline Healflox-8huthrine & 15095 & $15001 i$ & 150001 & 15152: & 15174 & 15100 & 15031 & 75048 & 1459 & 15087 \\
\hline Wall Tomp. BF & 134.2 & 137.3! & 135.51 & $136.3 !$ & 138.71 & 139.6 & 137.9 & 135.7 & 135,1 & 134.8 \\
\hline Wall Tomp.14F & 136 & 134.11 & 137.41 & $137.1 !$ & 138.8 & 139.8 & 138.4 & 137.61 & 135.5 & 140.8 \\
\hline Weltomp. & 1361 & 133.51 & 138.1 & 137.5 & 137.4 & 138.9 & 137.4 & 136.2 & 134.6 & 1329 \\
\hline WeTtomo. Hif & $123 T$ & 134.9T & 133.37 & T35. & 136.61 & $738.6 !$ & 138.71 & Tß3.3. & T35.6 & 337.6 \\
\hline Weltomo. I2F & $\mid 381$ & 134.91 & 134.81 & 135.71 & 138.8 & 13951 & 13921 & 737.91 & 73.6 & 733.8 \\
\hline Instrom Temp.f & 123.8 & 124.71 & 123.9 & 124.7 & 124.7 & 124.61 & 123.8 & 123.6 & 12336 & 123.4 \\
\hline Avg. Wall Tomo.f & 135.61 & 135.31 & 136.21 & 136.61 & 137.4 & 13921 & 138.31 & 138.7 & 134.20 & 734.6 \\
\hline Av. Wa DT.F & 111 & 10.61 & 10.8 & 11.11 & 12 & $14 !$ & 13.8 & 125 & 10.8 & 10.7 \\
\hline Romaining Suboooff & 321 & & & & & & & & $i$ & \\
\hline Es Quali & 0.021 & $0.05 !$ & 0.11 & $0.15 i$ & $-03 i$ & 0.34 & 0.28 & 0.33 & 0.31 & 0.41 \\
\hline EXP.HBthinnaf & 137631 & 14257 : & 1421.1; & $1369.3 i$ & 12683 & 1075.3 & 1009.81 & 1207.7 & 1393 & 14056 \\
\hline COMPUTEDH & & & & & & & & & & \\
\hline Jalouk & 1103.9 & 10527 & 1027.8 & 1006.2 & 996.71 & 974.7 & $\$ 58.7$ & 947.8 & $\$ 36.8$ & 333.5 \\
\hline Sedvi(Cudotube) & $15 \% 0.5$ & 1569.1 & 15731 & 1587.7 & 1590.9 & 1588.0 & $15 \overline{80.11}$ & 1578.7 & 15775 & 75825 \\
\hline $5=1012$ & 137.A & 1359.11 & 1368.3 & 1353.31 & 1367.2 & 1353.2 & 13572 & 1356.11 & 1350 & 1351.7 \\
\hline (R्D句) & इSETII & 57469 & 547221 & 525601 & 49918 & 47200 & 44291 & 416321 & 3900 & 36700 \\
\hline (F) & 1.4171 & 1.911 & 2.3531 & 2.687 & 31 & $3.2 \%$ & 3.537 & 3.7731 & 3.838 & 4.203 \\
\hline (HL) & 218.7 & 297.81 & 368.4 & 420.71 & 4681 & 590.7 & 550.8 & 587.11 & E्21.8 & 653.8 \\
\hline$(130 \mathrm{OL})$ & 1569 & 1565.51 & 1568.4 & 1577.81 & 1577.3 & 1569.1 & 155761 & 15512 & 15T4.7 & 75म5.1 \\
\hline & 1 & 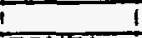 & & 1 & & & & & 1 & \\
\hline & सONE? & ZONES & ZONE 6 & ZON:SB & ZONEIO & & & & & \\
\hline DPtoid & 77.6 & $+\infty$ & & & -1 & & & & & \\
\hline 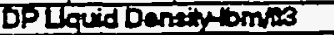 & 93.52 & & & & & & & & & \\
\hline Einviont & & 4 & & & 9.875 & & & & & \\
\hline Measured DPAPdd & 0.558 & 1.2. & 2653 & 2.848 & 3.488 & & & & I & \\
\hline Zero Cormectons-osid & $0.17 \pi$ & 0.017 & 0.82 & 0.162 & $0 . \overline{1681}$ & & & & & \\
\hline Corrected Dophd & 0.370 & 1.207 & 1.844 & 2.58ा & 3.25 & & & & & \\
\hline ACW:APA & 7.581 & 2.51 & 3.दा & 4.381 & 5.181 & & & & & \\
\hline Mo:tured TPFAtg & 84.21 & $\square$ & & & 8281 & & & & & \\
\hline Measured Overe"lith & 3.181 & $\underline{T}$ & & $i$ & & & & & & \\
\hline & 1 & i & & & & & & & $T$ & \\
\hline
\end{tabular}




\begin{tabular}{|c|c|c|c|c|c|c|c|c|c|c|}
\hline Runt & $912 !$ & & & & & & & & & \\
\hline Test filud & C्मि8 & & & & & & & & & \\
\hline Data: & 101493 & & $\therefore$ & - & $:$ & & & & & \\
\hline Tima: & 16.331 & & T & 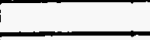 & $!$ & & & & & \\
\hline Nots & TAnayiedo & dis used! & $T$ & 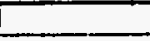 & $!$ & & : & $!$ & & \\
\hline Fow ATDall? & 0.003381 & 1 & $i$ & 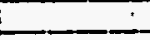 & $:$ & 1 & $i$ & \pm & & \\
\hline Hect Fow Arost? & $10.10055 i$ & 1 & $\therefore$ & & $\therefore$ & 1 & 1 & í & & \\
\hline Sel Proseuroping & 85.11 & : & $!$ & 1 & 1 & 1 & & 1 & I & \\
\hline Se Tempormuref(intel) & 126.51 & 1 & -1 & -1 & $i$ & 1 & 1 & -1 & I & \\
\hline LL. Donsityomin & 86.34 & 86.351 & $86 . \overline{37}$ & 8627 & 86.32 & 86.31 & 86.321 & 86.39 & 88.45 & 86.30 \\
\hline Leren Hent Bunbom & 39.031 & $39.05 !$ & 39.061 & 38.881 & 39.02 & 39.011 & 39.021 & 39.07 & 39.1 & 39.07 \\
\hline 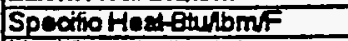 & 0.278 & 0.2781 & 0.278 & 0.2781 & 0.278 & 0.278 & 0.278 & 0.2781 & 0.27 & 0.278 \\
\hline Lla. Visoosity bmithit & 0.5651 & 0.565 & 0.568 & 0.562 & $0.584 !$ & 0.564 & 0.564 & 0.565 & 0.557 & 0.565 \\
\hline Ug. Conductivit Btentaf & 0.03251 & 0.03251 & 0.0355 & $0.0324^{1}$ & 0.05651 & 0.0325 & $0.03 \% 5$ & $0.00 \mathrm{E}$ & 0.0325 & 0.0325 \\
\hline Ua. Prand No. & 4.831 & 4.81 & 4.841 & 4.831 & 4.831 & $4.83 !$ & 4.831 & 4.84 & 4.8 . & 4.84 \\
\hline hablfow Rato-GPM & 7.7 & & ? & 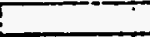 & 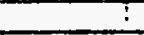 & & 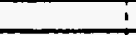 & & & \\
\hline 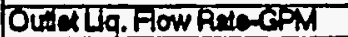 & 0.11 & & & & $i$ & & & $!$ & & \\
\hline 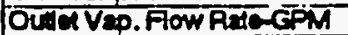 & 1.11 & & $\vdots$ & 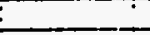 & $\therefore$ & & & 1 & I & \\
\hline Thiof temperiuref & 123.81 & & $\overline{1}$ & 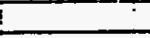 & $\therefore$ & & & & & \\
\hline Oc'letig. Tompermuref & 124.31 & 1 & $\div$ & i & 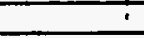 & $i$ & & 1 & I & \\
\hline Oetavep. Tomonter of & 121.81 & + & -1 & 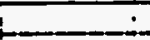 & $\therefore$ & $i$ & & I & I & \\
\hline Wen Fiow RaA GPM &. & $\div$ & 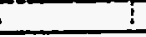 & 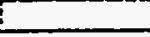 & $\therefore$ & 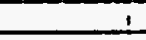 & $i$ & 1 & 1 & \\
\hline Weer hild tomp.f & $72.4 !$ & & & &. & 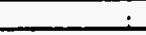 & & $\dot{5}$ & $\vdots$ & \\
\hline Werer outel Temp.F & 1061 & & & & & & & & & \\
\hline Intet Glom/hrin? & 3548791 & & Tamp & $918.1 ! \mathrm{C}$ & Denaty & $87.47 !$ & & $i$ & $\vdots$ & \\
\hline Stroooting ff & 2.7 & & 1 & 1 & i & i & & i & t & \\
\hline Fow Observition & & & $T$ & 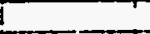 & 1 & & i & I & & \\
\hline & ZONE1 T & ZZONE2 T: & ZONES T & LONE4:2 & ZONES I2 & ZONE6- & ZONET & ZONES & ZONES & $201=10$ \\
\hline 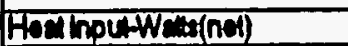 & $1 \quad 855.41$ & 868.5 & 870.61 & 871.61 & 875.8 & 85.11 & 8711 & 858.01 & 872 & 870.4 \\
\hline Fent Losentwa & 7.4 & 1.7 & 2.3 & 2.41 & 24 & 1.9 & 12 & 1.8 & 22 & 128 \\
\hline Henfingerme & 1500ा & T50001 & 5001 & 151091 & 15181 & 150.51 & 150:31 & 15057 & 15ा10 & 75068 \\
\hline WE" Temp. BF & 134.21 & 197.11 & 135.81 & 138 & 138.11 & 139.4 & 1388 & 138.8 & 1580 & 1350 \\
\hline Wh fomo.t4 & 1362 & 1341 & 137 . & 193.7 & 138,4 & 139.7 & 138.8 & 138.7 & 137.0 & 745.7 \\
\hline WNT Tomp. . & 1383 & 13321 & 138,4 & 137.31 & 7363 & 138.01 & 1382 & 737 & i35] & 7333 \\
\hline WeVtomp. M1F & 123.81 & 134.81 & 133.1 & 139.81 & 13501 & $138 . \pi$ & 1392 & 137.61 & $\sqrt{34.8}$ & 1320 \\
\hline Wall Tomp. Rf & 136.4 & 135.11 & 134.4 & 135.51 & 136.31 & 139.11 & 130.8 & 138.9 & 135.4 & 134.8 \\
\hline Instrean Tomp.f & 125 & 124,9 & 124.8 & 125.4. & 125.ा & 1251 & 125.1 & 124.7 & 122.6 & 124.7 \\
\hline Avg. Wait Temp.t & 135.81 & 135.31 & $135 . \pi$ & 136.2 & 136.91 & 139 & 139.1 & 137.8 & 735.8 & 135.8 \\
\hline AVo. WATOT.t & 10.11 & 9.7 & 9.6 & 10.11 & 11.1 & 13.11 & 13.3 & 12.4 & 10.7 & 10.4 \\
\hline Remining Swooctf & -6.21 & & & & & & & & & \\
\hline ExTounty & 0.0 .1 & 0.111 & 0.171 & 0.241 & 0.31 & 0.381 & 0.ख्ठा & 0.48 & 0.501 & 0.82 \\
\hline EXP. HEMLATIRA & 1485.7 & 1553.11 & 1577.31 & 1483.4 & 13722 & 1146.2. & 1133.7 & 121231 & 7618.7 & 7458.6 \\
\hline CONPUEDH & $T$ & 1 & 1 & & & 1 & & & & \\
\hline Jolok & 1138.9 & 1099.11 & $1052 \pi$ & 10181 & 1018.7 & 1007.81 & 1000.7 & ख्ञा & 978.01 & 972 \\
\hline Sendyi(audo rube) & 1678.4 & $15 \%$ & 158261 & 1591.81 & 158351 & 1580.11 & 159281 & 158721 & 1550 & 76320 \\
\hline $5 x+2$ & 730061 & T380.67 & $138 \pi$ & 130361 & 13955 & $138.6 i$ & 1357.7 & 13520 & - 36301 & 1350 \\
\hline (FDO) & 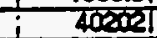 & 37961 & 35300 & 5268 & 30136 & 275301 & 24874 & ट्याह्ठा & 1S57t & 1602 \\
\hline & 1.604 & $224 \pi$ & 2763 & 3.177 & 3.55ा & 3.8811 & 4.10 & 4.488 & 4.750 & 5.021 \\
\hline$(\mathrm{F} D \mathrm{C})$ & 183.6 & 256.4 & 378.2. & उन्मा & 408.81 & प4R.6ा & 479.71 & 513.4 & 5452 & 575.1 \\
\hline$(\mathrm{P} B \mathrm{BO})$ & 7577.61 & 1576.7 & $15 \% .61$ & 7565.81 & 1584.71 & 1578.5 & 157.7 & 9560.1 & $7568 \pi$ & 1567.5 \\
\hline & & & & & & & & & & \\
\hline & 'ZONEZ? & 12OKEX & TEN=6 & ZONEB & ZONETO I & & & & & \\
\hline DPT ged tomp.t & 178.01 & & $!$ & $T$ & & & & & & \\
\hline 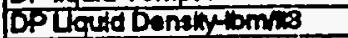 & 93.4 & & $T$ & $i$ & & & & & T & \\
\hline ETwions & 2 & 41 & 61 & 81 & 9.8751 & & & I & & \\
\hline Whoured Deppord & 0.6871 & 1.4651 & 3.081 & 3.5911 & 4.077 & $i$ & & $i$ & & \\
\hline 20ro Correctonspeid & 0.17 & 0.0211 & 0.821 & 0.162 & 0.1681 & 1 & & & & \\
\hline Correctod offidd & 0.791 & $1.44 \pi$ & 23031 & $3 . \overline{169}$ & $3 . \overline{0} 031$ & I & & & & \\
\hline Actue DPA & 9.35 & 1.2 & 281 & 3.37 & 4.17 & 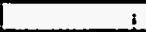 & & & & \\
\hline Whecured iflaphe & 85.1 & & & & 833 & & & & & \\
\hline Weatrod overdith & 3 & & 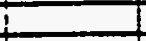 & & & & & & & \\
\hline & & & & & & & & & & \\
\hline Compred bph & 7.81 & दूरा & 3.โ2 & 4.031 & 4.911 & & & & & \\
\hline Fichon & 0.011 & 021 & 0.5 & 0.831 & T21 & & & & & \\
\hline Acostertionh! & $0 . \overline{0}$ & 0.131 & 0.21 & 0.29 & 0.41 & & & & & \\
\hline Eventons & 1.11 & 1.84 & 2.61 & 2शा & 3.3 & & & & & \\
\hline & & 1 & 1 & & & & & & & \\
\hline Comprodoris & 1.62 & टमा & 3.88 & 5.161 & 8.38 & I & & & & \\
\hline Ficiont & 0.171 & 0.301 & $0 . \pi 1$ & 7.781 & 7.83 & & & & & \\
\hline Aooster ion- & 0.971 & 0.21 & 0.291 & 0.391 & 0.511 & $\div$ & - & & & \\
\hline Elevation & 1.291 & $2.19 !$ & 2.531 & 3.591 & 4.141 & & & & & \\
\hline
\end{tabular}




\begin{tabular}{|c|c|c|c|c|c|c|c|c|c|}
\hline Run: & $913_{i}$ & & & & $\cdot$ & & $\therefore$ & - & $i$ \\
\hline Testfluid &.$C 488$ & & & & & $\therefore$ & $\therefore$ & & \\
\hline Date: & - 1014 & & & & . & $\therefore$ & $\therefore$ & 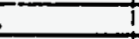 & \\
\hline Time: & $16: 35$ & & & & & & & & \\
\hline Note & Andyical d & th is used & & & & $\overrightarrow{1}$ & & & \\
\hline Fow Aront2 & 0.005361 & & & $\therefore$ & & 1 & & & \\
\hline Hent fow Area-l? & 0.19655 & & & $\therefore$ & & 1 & & & \\
\hline SaiPrestura $p_{s i g}$ & 851 & & & & & 7 & & & \\
\hline SEl Tempereturefin of) & 125.4 & & & & & 1 & & & \\
\hline La. Dentivitom/n3 & 86.251 & 85.341 & 86.321 & 86.321 & 86.31 & 86.35 & 86.351 & 86.851 & 86.37 \\
\hline Letent Hex-Burtom & 38.981 & $39.08 \mathrm{i}$ & 39.021 & $\overline{39.021}$ & 39.011 & 39.051 & 39.051 & 39.05 & 39.00 \\
\hline Specisotheretuhbmi & 0.2781 & $0.278 \bar{i}$ & 0.2781 & 0.278 & 0.2781 & 0.2781 & 0.278 & 0.278 & 0.278 \\
\hline Lig. Viscosity inth & 0.5521 & 0.5651 & 0.565 & 0.5541 & 0.554 & 0.5651 & 0.5651 & 0.565 & 0.568 \\
\hline Lig. Conductivin Bumr-hf & 0.05241 & 0.0325 & $0.0325 i$ & 0.0052 & $0.0325 i$ & 0.02551 & 0.05251 & 0.028 & 0.0325 \\
\hline Liq. Prandt No. & $4.82 !$ & 4.83 & $4.83 i$ & 4.831 & 4.831 & 4.841 & -4.841 & 4.84 & 4.84 \\
\hline Inlet Flow Rato-GPM & 1 & & & & & . & & & \\
\hline Outiof Liq. Fow Rate-GPM & $\overrightarrow{0}$ & & & & & & & & \\
\hline Outat Vap. Flow Aate-GPM & 0.6 & & & & & ! & & & \\
\hline Indot Tempereturef & 123.51 & & & 1 & & $i$ & & & \\
\hline OuAt Lq. Temperiture & 124.71 & & & 1 & & T & & & \\
\hline 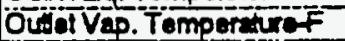 & 1228 & & & 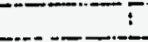 & & 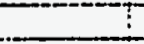 & 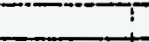 & & \\
\hline Wat Flow Rate-GPM & $\dot{\square}$ & 1 & & & $\div$ & $!$ & t & & \\
\hline Wour intet Temp.fF & 74.6 & $\therefore$ & $\div$ & - & $\because$ & 5 & $!$ & & \\
\hline Wetar outer Temp.F & 114.4 & & $-\ldots$ & $\therefore$ & : & 1 & 1 & & \\
\hline initeibminute & 2001 & Tomp & $116.4,0$ & Donsty & $87.75 i$ & 1 & $i$ & & \\
\hline Suboooling & 291 & & -4 & - & -1 & 3 & 1 & & \\
\hline Fow Obsenvation & & & & & . & & & & \\
\hline & ZONEI & ZONE2 ZONES & ZONE4 Z & ZONES :Z & ZONE6 & ZONE7 IZ & ZONES IZ & $\angle O K=9$ & $20 N=10$ \\
\hline Heat inpLIWEatinot) & 863.21 & $867.5 \quad 865.71$ & $867.3^{\circ}$ & 870.61 & $870.8 i$ & 869 & 864.11 & $\$ 5.6$ & 9 \\
\hline Heet Loss-Wals & 7.31 & 2.31 & 23 & 241 & 1.91 & 1.2 & $2 !$ & 1.8 & 9.3 \\
\hline Heat Fux-ountrile & 15050 & 15038 & 15024 & 15091 & 15098 & 15067 & 1497 & 1657 & 9710 \\
\hline Wh tamp. आ3=- & $\mid 3321$ & 136.31 & $135.1 !$ & 737.11 & 138.81 & 138.21 & 740.7 & 7282 & 728.1 \\
\hline Weif Temp. $2 \bar{F}$ & I35ा & 133.31 & 135.81 & 137.21 & 139.1 & 130.1 & 147.81 & 728.5 & 123 \\
\hline Wai Tomp. $5 F$ & 134.71 & 1324 & 135.71 & 135.81 & 137.91 & 137.4 & 137.31 & 1282 & 128.8 \\
\hline WaA Temp. IIF & 123.51 & 133.51 & 133.61 & 134.6 & 137.31 & 138.7 & 1381 & 127.4 & 127.8 \\
\hline WedTomp. $12 F$ & 135.7 & 133.81 & 13421 & 1351 & 138.31 & 1392 & 139 & 1275 & 128.4 \\
\hline Indream Tomp.f. & $125.5 !$ & 124.81 & 125.11 & 125.81 & 12521 & 124.91 & 126.81 & 1245 & $12 \overline{8}$ \\
\hline Avo. Wall Temp.f & 134.7 & 134.3 & 135.11 & 135.6 & 138.81 & 138.6 & 139.81 & 128 & T28.6 \\
\hline Avo.Wall DT. & 8.5 & 8.61 & 9.31 & 9.8 & 1261 & 1291 & 13.7 & 3 & 3.6 \\
\hline Remaining SubooolF & $-|23|$ & & & & & & & & \\
\hline Exaculy & 0.00 & 0.2 & 0.411 & 0.521 & 0.05 & 0.74 & 0.87 & 0.85 & 0.87 \\
\hline Exp.HBtuhth2 & 1776.8 & 17925 & 1621.7! & 1537.11 & 12182 & 11642 & 100.7 & 55.1 & 479 \\
\hline CIMPUTEDH & & & & & & & & & \\
\hline Jitok & T178. & 1129.1 & 1077.4 & 1031. & 105361 & 10403 & 105T. & 37.01 & 3828 \\
\hline Sadvi(audonbe) & 158.81 & 15805 & 15328 & 1583.7 & 1589.1 & 158.8 & $1581.7 \Gamma$ & 5829 & 5502 \\
\hline $5=012$ & 13801 & 13827 & 1394.1. & 13882 & 1350.81 & 1384.81 & 130081 & 4608 & 78.7 \\
\hline (ALQ) & ж18 & 208391 & 155331 & $\mid 3201$ & 10383 & 731 & 51211 & 3876 & 350 \\
\hline$(F)$ & 1.891 & 2.7711 & 4.0051 & 4.5 & 4.901 & 5.387 & 5.8181 & 6.010 & 6.1 \\
\hline$(15)$ & 1421 & 25.11 & 307 & 337.01 & 372 & 405 & 456.61 & 45.0 & \\
\hline$(1 \mathrm{BO}$ & 7586.41 & 1579.3. & 1579.3 & 1581.61 & T5823! & 15761 & 157051 & 450 & 478.7. \\
\hline & 1 & & & & & & 1 & & \\
\hline & $\mathrm{Ni}=2$ & ZONE4 ZZONE6 & ZONES & ZONE10 & & & & & \\
\hline DPPRusd Teme fF & 78.11 & -2 & & & & 1 & & & \\
\hline DPPUquid Densth tom/n & \$3.471 & $i$ & $\because$ & & & 1 & & & \\
\hline Evereliont & 2ा & 41 & 81 & 9.8751 & & & & & \\
\hline Nhécuredopsid & $0.8 \times 51$ & 1.7751 & 3.551 & 4.971 & & & & & \\
\hline Zero Cormetions-paid & 0.777 & 0.011 & $0.162 \mid$ & 0.168 & & & & & \\
\hline Comecied DPpsid & 0.65 & 7.78 & 3.7981 & 4.7431 & $T$ & & & & \\
\hline Actud DP & 1.07 & 1.391 & 2321 & 2.78 & 1 & & & & \\
\hline Whaured TPphiq & 85 & 1 & 1 & 832 & I & & 1 & & \\
\hline Mucured overall & 3 & 1 & 1 & 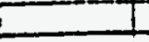 & 1 & & 1 & & \\
\hline & 1 & 1 & i & 1 & : & & 1 & 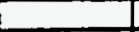 & \\
\hline Compued DPA & 0.99 & 1.57 & $282:$ & 3.94 &. & 1 & 1 & & \\
\hline Ficion-1 & 0.01 & 0.151 & 0.68 & 0.58 & 1 & & 1 & & \\
\hline Aconterion & 0.01 & 0.081 & 0.21 & $0 . \overline{23}$ & 1 & 1 & 1 & $L$ & \\
\hline Baviont & 0.911 & $1.4 \mathrm{MI}$ & 2.12! & 3.181 & & T & & & \\
\hline
\end{tabular}




\begin{tabular}{|c|c|c|c|c|c|c|c|c|c|c|}
\hline Runt & $914 !$ & & & & & & & & & \\
\hline Torf Fuid & C4F 8 & & & & & & & & & \\
\hline Delo: & 10/15931 & & & & & & & & & \\
\hline Time: & $8: 44$ & & & & & & & & & \\
\hline Not & Analyticalo & do is used & & & & & & & & \\
\hline Flow AYUAR & 0.003661 & & & & & & & $\vdots$ & & \\
\hline Heal Fow Arear? & 0.1855 & & 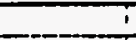 & 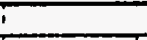 & & & & 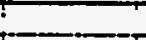 & & \\
\hline 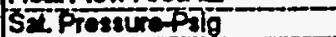 & -87.2 & & 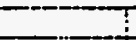 & 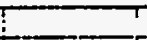 & & & & $t$ & & \\
\hline SLI Tomper trefindel) & i27.81 & & -1 & i & & & & 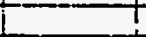 & $f$ & \\
\hline La. Denatytomns & 86.41 & $86 . \overline{251}$ & 86.21 & 86.121 & 86.17 & 86261 & 86.24 & 88.81 & 86.82 & 88.4 \\
\hline Lent Hen etwiom & 39.001 & 38.861 & 38.901 & 38.881 & 38.91 & $38 . \overline{951}$ & 38.85 & 39.011 & 3.02 & 39.00 \\
\hline 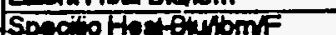 & 0.278 & 0.2781 & 02781 & 0.2791 & 0.278 & 0.2781 & 0.278 & 0.278 & 0.278 & 0.278 \\
\hline Wh. Viscosintom th & 0.567 & 0.5621 & 0.5611 & 0.558 & 0.559 & 0.5611 & 0.561 & 0.564 & 0.684 & 0.567 \\
\hline Lig. Conductivelenth & 0.0325 & $0.0324 i$ & 0.0324 & 0.05231 & 0.0324 & 0.00224 & 0.0524 & 0.00251 & 0.058 & 0.0325 \\
\hline Wg. Prand No. & 4.891 & 4.821 & 4.821 & 4.811 & 4.811 & 4.821 & 4.82 & 4.831 & 4.83 & 4.84 \\
\hline Inlat Fow Rate-GP & 4.81 & & & & & & & -1 & 1 & \\
\hline OUALLIG. FIOW RAR-GPM & 0.31 & & & & & & & $i$ & $i$ & \\
\hline OWA Vap. Flow REA-GPM & 2.41 & & $\mathbf{i}$ & $i$ & & & & & & \\
\hline Inlet tomperenter & 123.61 & & $\mathbf{T}$ & $i$ & & $!$ & $!$ & & & \\
\hline 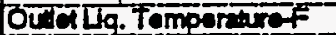 & 123.81 & & & & & & 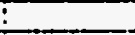 & & & \\
\hline oúda veo. Tomper & 121.5 & $i$ & it & 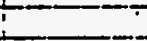 & & & : & & & \\
\hline WDA Flow A & $i$ & 1 & 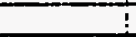 & ! & & & $!$ & t & † & \\
\hline Wertinte Temp.f & 73.01 & & & . & 1 & & 1 & 1 & I & \\
\hline Wer ouvertomeff & 100.7 & 5 & 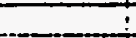 & -1 & $\ldots \ldots$ & & 1 & I & I & \\
\hline hlat o sominnt2 & 89769 & & Temp & $120 \pi$ & Deneidy & 87.767 & & 1 & $I$ & \\
\hline Suboodingt & 4.31 & & & 1 & & & 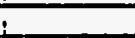 & I & I & \\
\hline Fow Obsenvion & $i$ & & & & & & & & & \\
\hline & ZZONET & ZONE=2 & ZONE3 & ZON=4 & ZONE5 & $\angle O K E=6$ & $2 O K=7$ & ZONES & ZONES & $20 N=10$ \\
\hline 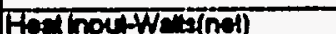 & 1149.8 & $1150.8 !$ & 1158.4 & 1153 & 1150.7 & 1750.6 & 1154.7 & 1148.5 & 1753 & 1155. \\
\hline HeVLose-Wens & 81 & $1.9 !$ & 26 & 2.61 & 27 & 2 & 1.3 & 1.9 & 23 & 13 \\
\hline How Fho buthres & 19क्या & 19960 & उ0015ा & 1998! & 99568 & 1क्या| & 20018 & 19800 & 15031 & 20003 \\
\hline We Trem. Bo & 737.81 & 1427 & 1601 & 7ato.1 & 7427 & 74201 & Tat2 & 138.11 & 138. & 7375 \\
\hline We Tomo. 4 & 1401 & 138.7 & 142ता & 140.91 & 19241 & 1427 & 741.7 & 747.81 & 733.7 & 746.8 \\
\hline WतTtomp. 54 & 739.91 & 138.91 & $142 \pi$ & 147.41 & 140.81 & 1424 & 740.5 & 130.21 & 137.5 & 135.8 \\
\hline Weltomo.11F & 123.61 & 139 & 137.4 & 138.71 & $140 !$ & 141.81 & 1424 & 13951 & 130.4 & 134 \\
\hline WelTomp. VIt & $140.3 i$ & 139.21 & 1391 & 139.4 & 140.3 & 143.4 & 142.7 & 1411 & 137.5 & 138.8 \\
\hline nom Temp. F & 124.61 & $125.5 i$ & 125.71 & 126.3 & -1261 & $125.6 \mathrm{i}$ & 125.6 & $125.2 i$ & 1321 & T21.6] \\
\hline Avo. Wall Tomp. ${ }^{\prime}$ & 1395 & 139.81 & 139.6 & $1403 i$ & $\$ 409$ & 1425 & 141.7 & 940 & 7372 & 138 \\
\hline AVo.WAIDT +5 & 14 & 13.3 & 13 & 13.11 & Ta & 15.91 & 15.2 & 13.9 & 11.8 & 125 \\
\hline Remaning Subooff & 0.1 & & & & & & & & & \\
\hline Exoun & of & 0.031 & 0.081 & 0.001 & 0.12 & 0.16 & 0.18 & $0.2 \pi$ & 035 & 021 \\
\hline 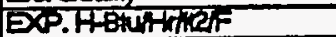 & T43.71 & 7494.61 & 1533.71 & $75 \times 01$ & 1426.7 & 1250.6 & 13172 & 14502 & 1325 & 16055 \\
\hline COIMUTEDH & & & & & & & & & & \\
\hline Jetk & Tस्बा & TZOTST & 7135.81 & T098.1! & 1087.7 & T064 & 1007.4 & 975.7 & \$33] & Sel. \\
\hline S.dyI(Odo tbo) & 1877.9 & 98521 & 1803 & 185281 & 1850 & 1848 & 1857.4 & 18524 & 1803 & रह्जा: \\
\hline $5=12$ & 1637.7 & 1678.81 & 1690.5 & 1701.81 & 1697 & 16888 & 1701.1 & 163261 & 1805 & 768712 \\
\hline (F) & 1150201 & 11म567 & $\pi 1274$ & 1088891 & 10पहBता & 100.51 & 9716 & 936291 & 89120 & 85800 \\
\hline (F) & 1.000 & 1.468 & 1.503 & टनाडा & 2481 & $2 \pi 7$ & 2.928 & 3.128 & 3.871 & 3.103 \\
\hline (FT) & $2 \approx 3.21$ & अवा & 4e्21 & 587.71 & 651.7 & -71321 & 767.7 & 810.4 & 8672 & हाठठ5 \\
\hline$(\mathrm{F} 304 \mathrm{~d})$ & 18153 & 183.81 & 18308 & 1830 & 18527 & 18135 & 18126 & |797.4 & 178 & 178.7 \\
\hline & & & & -1 & & & & & & \\
\hline & $\angle O K=2$ & ZONEX & ZONEE6 & ZONEB & $\angle O N=10$ & & & & & \\
\hline DPTqud Tomp.f & 78.5 & -2 & & $\rightarrow$ & $-1-$ & & & & & \\
\hline DPLlouid Dentitym/3 & उ3.4ग & & & & & & & & & \\
\hline Eviciont & & & 81 & 81 & $9.87 \%$ & & & & & \\
\hline 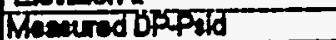 & ర.321 & 0.5031 & 7.680 & T.53ा & 1.878 & & & & & \\
\hline Zero Comectons peid & 0.168 & $-0.12 \pi$ & 0.681 & $02 \pi 1$ & 0.117 & & $T$ & & & \\
\hline Cormend Dophild & $0.18 \pi$ & 0.721 & 1.001 & $1.38 \mathrm{e}$ & 1.73 & & T & & & \\
\hline Acted Dat & 1.6 & 3.121 & 4.821 & 6.481 & 7.78 & & $T$ & & & $\therefore$ \\
\hline Wheored IP & 8721 & 1 & & i & 65.1 & & I & & & \\
\hline Manrodoverlin & 3.5 & 1 & & I & & & $t$ & & & \\
\hline & & 1 & & 1 & & & I & & & \\
\hline Compordoph & टाग & 4 & 5.5ा & 81 & 10.16 & & $\perp$ & & & \\
\hline ton & 0.18 & 0.7 & 1.48 & 2क6 & 3.65 & & I & & & \\
\hline Aoosteriont & 0.25 & 0.49 & 0.7 & 0.92 & 1.18 & & & & & \\
\hline Exming & 1.72 & ह.8ा & 3.74 & 4.ळा & 5.33 & & & & & \\
\hline
\end{tabular}




\begin{tabular}{|c|c|c|c|c|c|c|c|c|c|}
\hline Runt: & $915 i$ & & & & & & & & \\
\hline Toufluid & CAF8 & & & & & & & & 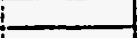 \\
\hline Date: & 1015931 & & & & & & & & \\
\hline Time: & $8: 44$ & & & & & & & & \\
\hline Note- - & Anaycica di & it is used & & & & & & & \\
\hline Flow Aroa-A2 & 0.003351 & & & & & & & & $!$ \\
\hline Hogt Fow Aveat2 & 0.1955 & & & & & & & & \\
\hline Sat Presstro ósig & 86.61 & & & & & & & & \\
\hline 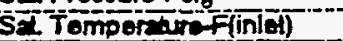 & 127.5 & 1 & & & & & & & \\
\hline La. Densty & 86.371 & -86.251 & 86.171 & $86.2 !$ & 86.27 & 86.31 & 86.351 & 86.42 & 86.42 \\
\hline Letord Heat Banthm & 39.061 & {$[-38.96 i$} & $=38.91^{\circ}$ & -38.92 & 38.981 & $39.01 i^{-}$ & 39.051 & 39.1 & 39.1 \\
\hline Spooito Hodentiom & 0.2781 & $\because .278 i$ & $-0.278_{1}^{-}$ & $3 \overline{2} 7 \overline{1}$ & 0.2781 & 0.278 & 0.2781 & 0.278 & 0.278 \\
\hline 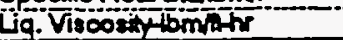 & $0.565 i$ & $0.562 \mathrm{i}$ & 0.559, & $0.56 i$ & $0.562 i$ & $0.5611^{-}$ & 0.5651 & 0.667 & 0.567 \\
\hline Liq. Conductivity $\mathrm{Btwhr}$ th & $0.0325:$ & 3.0324 & 0.0324 & 0.0324 & C.0324. & 0.03251 & 0.0325 & 0.00251 & 0.0325 \\
\hline Lig. Prand No. & $4.84 i$ & 4.821 & 4.811 & $-\overline{4.82 !}$ & 4.831 & 4.831 & 4.841 & 4.84 & 4.84 \\
\hline Inlat Fow Rato-GPM & $4.1 !$ & & & & & & & & \\
\hline OUACl LG. FOW RAT-GPM & $0.6+$ & & & & & & 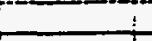 & & \\
\hline Outed Vep. Flow Rado-GPM & 2.11 & & & & & & & & \\
\hline Inlet Temperaturef & 123.9 & 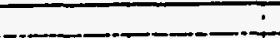 & & & & & & & \\
\hline ठwALig. Tomperature & 123.9 & & & & 5 & & & & \\
\hline Oula vap. Temperaturof & 1211 & $!$ & T & & 1 & I & T! & & \\
\hline WDI Flow RMO GPM & & I & T & & $!$ & $!$ & $T$ & & \\
\hline Weter nla Tamp.F & 74.9 & 1 & 1 & 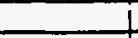 & $!$ & & $!$ & & \\
\hline Wor ould Tomeff & 99.6 & 1 & 1 & & 1 & & $T$ & & \\
\hline Indot G ibminhe & 864139 & Temp & 178.210 & Donaty & $87.8 T$ & & 1 & & \\
\hline Subooding f & $3.6 i$ & $\therefore$ & 1 & & 1 & & $i$ & 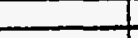 & \\
\hline Fow Obseavetion & & & & & & & $I$ & & \\
\hline & ZONE=1 T & ZONE2 :ZONES TL & ZONE 4 & ZONES & ZONE 6 12 & ZONET L & ZONEB $\mathrm{i}^{2}$ & ZONE3 & $20 x=10$ \\
\hline Hed houtwingl) & $11612 !$ & $1151.5 ! \quad 1158.4$ & -1152.2 & गामूा & 1150.8 & 1161 & 7159 & 1158.5 & 1160.8 \\
\hline Hen Loss-We's & 7.51 & 1.81 & $2.5 !$ & 2.5 & 1.91 & 1.21 & 1.8 & 2) & 125 \\
\hline 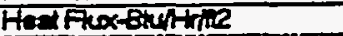 & ๘णछा & 19067 & 19931 & 198001 & 199181 & 201द्डा & 20091 & 20002 & 20118 \\
\hline Wal Temp. \$3F & 13721 & 142.15 & 739.61 & 141.6T & Tक्या & 140.81 & $138.5 T$ & 785.7 & 739.8 \\
\hline Wal Tomp. ILF & 139.5 & 937.4 & 740.31 & $\overline{147.61}$ & 1422! & $741 . \pi$ & $140.8 \Gamma$ & 138 & 145.7 \\
\hline Wal Tomp.15f & 130.3 & $738.3 i$ & $1412 !$ & 140.61 & 141.41 & 140.7 & 138.81 & 1352 & 134.5 \\
\hline WEH Temp. I $1 \mathrm{~F}$ & 123.9 & 138.31 & 138.21 & 139.31 & 140.81 & 141.81 & 139.11 & 134.11 & 133.8 \\
\hline Wall Tomo. Iaf & $139.41^{\circ}$ & 138.61 & 139.11 & $139.7 i$ & 142.41 & 1421 & 140.81 & 136.8 & 136.2 \\
\hline intrem tomp f & 124.81 & $125.5 i$ & 1261 & $125.8 i$ & $125 . \lambda_{1}$ & 1252 & 924.91 & 124.5 & 124.6 \\
\hline Ang. Wall Temp.F & 138.8 & 139.11 & 139.8 & 140.31 & $141.6 \mid$ & 14121 & 139.6 & 1353. & 137.8 \\
\hline Avg. Wall DT .F & 13.11 & $12 \pi$ & $129 i$ & 13.8 & 15.31 & 75.1 & 13.81 & 10.5 & 126 \\
\hline Remaining Suboootf & -1.41 & & & & & & & & \\
\hline Ed Qualis & 0.01 & 0.081 & $0.12 !$ & 0.15 & $0.18 !$ & 0.22 & 0.251 & 0.28 & 0.33 \\
\hline DX.HBtuHneF & 1531.8 & 1594.61 & 15.5 .61 & 1460.4 & 1301.8 & 1355.41 & 1456.7 & 1921.3 & 16132 \\
\hline COMPUTEOH & & & & & & & & & \\
\hline Jasouk & T257.3T & TM4TEI & $-1708 T$ & Tó & $70526 \pi$ & 1005.7 & 7004.7 & 978.1 & 958.8 \\
\hline 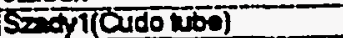 & 182.9 .31 & 1847.41 & 7846.51 & $78 \overline{40.81}$ & 1894.57 & 1857 & $18528 !$ & 1851.9 & 18586 \\
\hline $5 \operatorname{sog} 2$ & 16527 & 1679.61 & 1694.71 & 1686.71 & 168.61 & 1697.4 & 1691 & 16006 & 169.8 \\
\hline (PLD) & 982781 & \$712! & soīasi & 88767 & 82054 & 78907 & 752741 & 77500 & 6009 \\
\hline (F) & 1.247 & 2.103 & $2 \sqrt{231}$ & 27 & $255 i$ & 3.178 & 3.3991 & 3.586 & 3.783 \\
\hline (1) & 288.11 & $388 \pi$ & 567.41 & E253 & 68261 & $-73 \pi .01$ & 783.01 & 85021 & 8782 \\
\hline (1.30) & 1826.9 & 1827.11 & 1820.11 & 1816.4 & 181291 & 9814.6 & 1802.81 & $17 \%$ & $7 \% 5$ \\
\hline & & & & & & & $-i$ & & \\
\hline & ZONES & 2ONE6 & ZON: 8 & ZOKE $=10$ & & & & & \\
\hline DP hquid Tomp.f & 78.7 & & & & & & & & \\
\hline 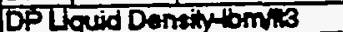 & 93.38 & & & & & & & & \\
\hline Exwliont & & $4 !$ & $8 !$ & 9.875 & & & & & \\
\hline Momed DFandid & 0.372 & 0.8081 & 1.99 & 2.45 & & & & & \\
\hline Znocomocions pesd & 0.158 & $=0.127$ & $0.27 \pi$ & 0.771 & & & & & \\
\hline Cormoled DPpild & 0.210 & 0.9551 & 1.779 & 2325 & & & & & \\
\hline ACW:AOR & 1.8 & 2.81 & 5.681 & 6.8 & & & & & \\
\hline Meapured TPQp & 86.8 & -1 & & 84.9 & & & & & \\
\hline M-evred Overill & 2831 & 1 & & $i$ & & & & & \\
\hline & & & & & & & & & \\
\hline Compund DPA & 1.92 & 3.51 & 7दा & 9.15 & & & & & \\
\hline Friction-1 & 0.2 & 0.61 & हटा & $3 \mathbf{8}$ & & & & & \\
\hline Acoler iond & 0.24 & 0.42 & 0.81 & 7.09 & & & & I & \\
\hline Eleveriont & 1.481 & 2.5Tा & 4.15 & 4.82 & & & & & \\
\hline
\end{tabular}




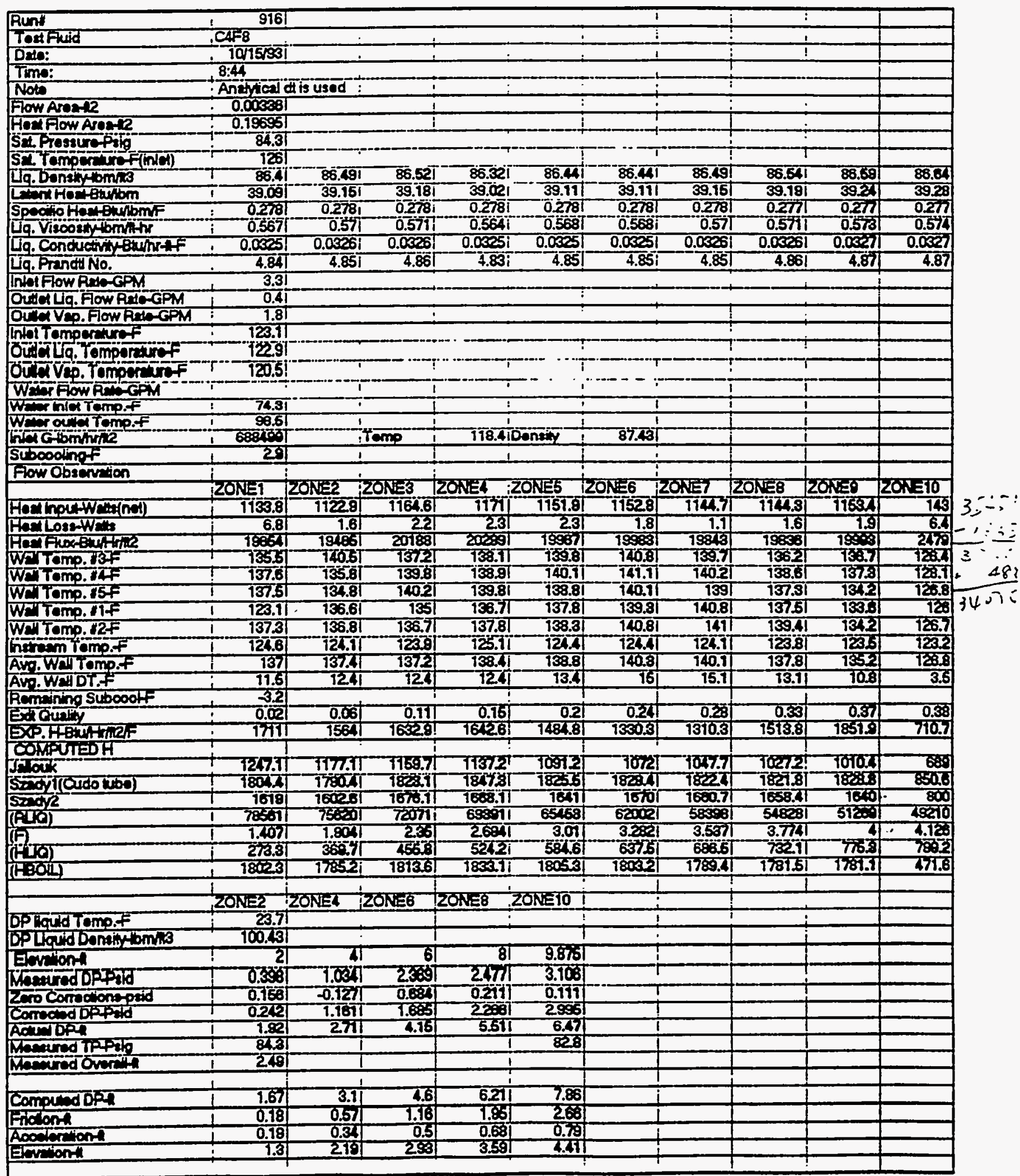




\begin{tabular}{|c|c|c|c|c|c|c|c|c|c|c|}
\hline Runt: & 9171 & & & & & & & ! & ! & \\
\hline Test Fuid & CAF 8 & & & & & & - & & & \\
\hline Data: & 101593 & & & & & & $\dot{\Sigma}$ & & & \\
\hline Time: & $8: 44$ & & & & & & \pm & & & \\
\hline Note & Analyocal d & th is used & & & & & & & & \\
\hline Flow Areatil2 & $0.00336 !$ & & & & & & & & & \\
\hline Hod Fow Aroante & 0.1995 & & & $i$ & i & & & & & \\
\hline Sat Pressure psig & $-341 !$ & & & & & & & & & \\
\hline SEl Tomperatur finlel) & 125.8 & & & & & & & & & \\
\hline Fo. Donsty tomins & 86.52: & 86.5511 & $86.39 i$ & 86.41 & 86.421 & 86.451 & 86.491 & 86.54 & 88.57 & \\
\hline 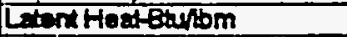 & $39.18 i$ & $\overline{39.14 !}$ & 39.071 & 39.001 & 39.11 & 39.131 & 39.15ा & 39.18 & 39.22 & \\
\hline Specitic Heat BuphomiF & 0.278 & 0.277 & 0.2781 & 0.278 & 0.2781 & 0.278 & 0.278 & 0.27 & 0.27 & \\
\hline Lia. Visoosin 13 minh & 0.571 & $0.569_{1}$ & $0.566 i$ & 0.567 & 0.5571 & 0.5581 & 0.57 & 0.571 & 0.572 & \\
\hline Lig. Conductivat BuhreffF & 0.03261 & 0.0326 & 0.0325 & 0.03251 & $0.0525 ;$ & 0.03261 & 0.03261 & 0.05251 & 0.036 & \\
\hline Lia.Prandeño. & 4.861 & $4.85 !$ & 4.34 & 4.841 & 4.841 & $4.85 !$ & 4.851 & 4.861 & 4.86 & \\
\hline nlot Fow Rate-GPM & 2.5 & & & & & & & & & \\
\hline Outer Liq. Fów Rato-GPM & 0.11 & & & & & & & & & \\
\hline Outlat Vap. Flow Rate-GPM & $1.8 !$ & & & $i$ & 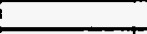 & & & & & \\
\hline Indal Temperguref & 122.81 & & & & & & & & & \\
\hline ơ & 723.3 & & & & & & 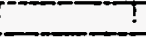 & & & \\
\hline OCol Vap. Temparairof & T2̈ii & & & T & & t & 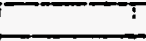 & & & \\
\hline We- Flow Rate-GPM & -7 & $i$ & & 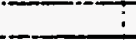 & 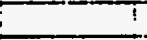 & $\bar{z}$ & 1 & & & \\
\hline Watandet Tomp.f & 74.9 & $\dot{i}$ & 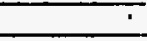 & $i$ & 1 & & i & & & \\
\hline Water outel Temp.F & 101.6 & $\underline{\square}$ & & $\therefore$ & 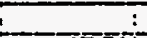 & & T & & & \\
\hline Indet Gibminne & 52076 & Temp & $\$ 17.810$ & Donsty & 87.571 & 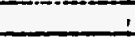 & 1 & & & \\
\hline SubooolingF & 31 & & & & & & $\perp$ & & & \\
\hline Flow Obsenvation & & & & & & & & & & \\
\hline & ZONET : & ZONE2 2ONE3 '2 & ZONE4 4 & ZONES: & ZONE6 & ZONE7 & ZONE8 & ZONE9 I & $20 N=10$ & \\
\hline Heathputwasinet) & 1151.7 & $1156.1 !-1159.31$ & $1159.3 !$ & 1154.81 & 1149.31 & 1755.91 & 9156.1 & 238.9 & -173.5 & \\
\hline Hea Loss-Wats & 7.41 & $1.7 !$ & 2.41 & 251 & $1.9 !$ & 1.31 & 1.8 & 1.5 & 7.8 & \\
\hline Hoal Fho-buthm & 13964 & 200101 & 200051 & 20018 & 19sen! & 20037 & 200101 & 414II & 3000 & $:$ \\
\hline We Temp $3 F$ & 134.71 & 139.8 & $137 !$ & $130.2]$ & 140.81 & 140.2 & 137.3 & 1285 & 1263.5 & - \\
\hline WE Tomp. I4F & $1372 !$ & 135.11 & $138 . \overline{2 !}$ & 139.61 & 140.8 & 140.6 & 139.2 & 129.11 & 127.9 & \\
\hline WA Tamp. $15 \mathrm{~F}$ & 136.97 & 13421 & 138.51 & 1381 & 139.7 & 139.2 & 137.7 & 128 & 128.8 & \\
\hline Wall Tomp. IIF & 122.8 & 136.11 & 1361 & 137 & 1391 & 140.9 & 1381 & 127.31 & 126.44 & \\
\hline Wall Tomp. ILF & 137.1 & 136.11 & 136.91 & 137.51 & 140.51 & 141.31 & 1401 & 127.6 & 126.7 & $\overline{: \sigma:}$ \\
\hline Intreen Temp. $F$ & 123.81 & 12421 & 124.71 & 124.61 & $1245 i$ & 124.3 & $124 . \pi$ & 123.8 & 123.6 & \\
\hline Avo. Wal Tomp. $f$ & 1365 & 136.8 & 137.41 & 138 & 140.11 & 140.4 & 1384 & 12.1 & 138 & \\
\hline Avg. WeilDT.F & 11.7 & $11.7 !$ & $11.8 !$ & 125 & 14.71 & 15.2 & 13.41 & 7.11 & 3.1 & \\
\hline Remaining Suboooff & -5.1 & & & & & & & & & \\
\hline Exa Cuay & 0.04 & $0.09 !$ & 0.211 & 0.27 & 0.32 & 0.38 & 0.44 & 0.15 & 0.46 & \\
\hline EAP.HBtuHRRF & 1710.3 & $1717.3 !$ & 1700.61 & 1558.8 & 1357.6! & 1315.2 & 1491.7 & 10062 & 958.6 & \\
\hline COMPUIEDH & & & & & & & & & & \\
\hline Jalouk & 7273.4 & 7186.11 & $7761.8 !$ & $-17 \pi$ & $T 1 \infty, 3 !$ & 108721 & 10721 & Exsor & 597.8 & \\
\hline Szady 1 (Cudo tha) & 1808.7 & 7817.61 & 1850.81 & 1827.8 & 182456 & 1820.51 & 1820.8 & 915Б & 8द्रा & \\
\hline sedp? & 1659.8 & 1647.91 & 1650.61 & 1845.2 & 1638.81 & 1615.4 & 164.81 & 7250 & 704.8 & \\
\hline (P्DO) & 58776 & 561471 & 49474 & 45582! & 421081 & 38988 & 354181 & उडst & 3250 & \\
\hline$(F)$ & 1.537 & 2.134 & 3.0681 & 3.391 & 3.7061 & 4.0011 & 4.2791 & 4.4. & 4.483 & \\
\hline$(\mathrm{FUn})$ & 239 & 3321 & 47821 & 527.91 & 57.11 & E23 & 665.61 & 65031 & 6895 & \\
\hline (12014) & 1807.3 & 1813.91 & 18201 & 18131 & 18051 & 1805.7 & 1799.9 & 75333 & 5530 & \\
\hline & & & & & $i$ & & 1 & & & \\
\hline & ZZONE2 & ZONE6 & ZONE8 - : & 2010 & & & & & & \\
\hline DF Equid Tomp.F & 80.6 & $i$ & 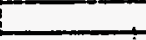 & & & & 4 & & & \\
\hline DPLFqud Densiy tom/13 & 93.12 & 1 & & & 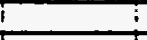 & & 1 & & & \\
\hline Elovition & & 6 & 81 & 9.875 & & & & & & \\
\hline Mhesured Dphid & 0.405 & 2709 & 2.081 & 3.682 & ! & & & & & \\
\hline Zero Corredion-paid & 0.1561 & -0.127 & 0.211 & 0.711 & $!$ & & & & & \\
\hline Cormeond oppitd & 0.209 & 1.371 & 2697 & $3.5 \pi 1$ & 1 & & & & & \\
\hline ACtw DPA & 1.74 & 2001 & 4.121 & 4.681 & I & & & & & \\
\hline Manered Tfysig & 84.11 & 1 & -1 & 828 & $!$ & & I & & & \\
\hline Manimed Overish & $216 i$ & 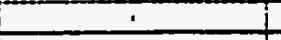 & 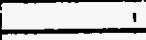 & & 1 & & 1 & & & \\
\hline & $\therefore$ & & & & & & & & & \\
\hline Comoutad DPA & 1.44 & 2541 & $5.18 i$ & 6.69 & & & & & & \\
\hline Frictiont & 0.16 & 0.44 & 1.51 & 1.89 & & & & & & \\
\hline Aoosterciont & $0 . \overline{14}$ & 0.25 & 0.55 & 0.58 & & & & & & \\
\hline Everiont & 1.17 & 1.95 & $3.12 !$ & 4.2.1 & & & & & & \\
\hline
\end{tabular}




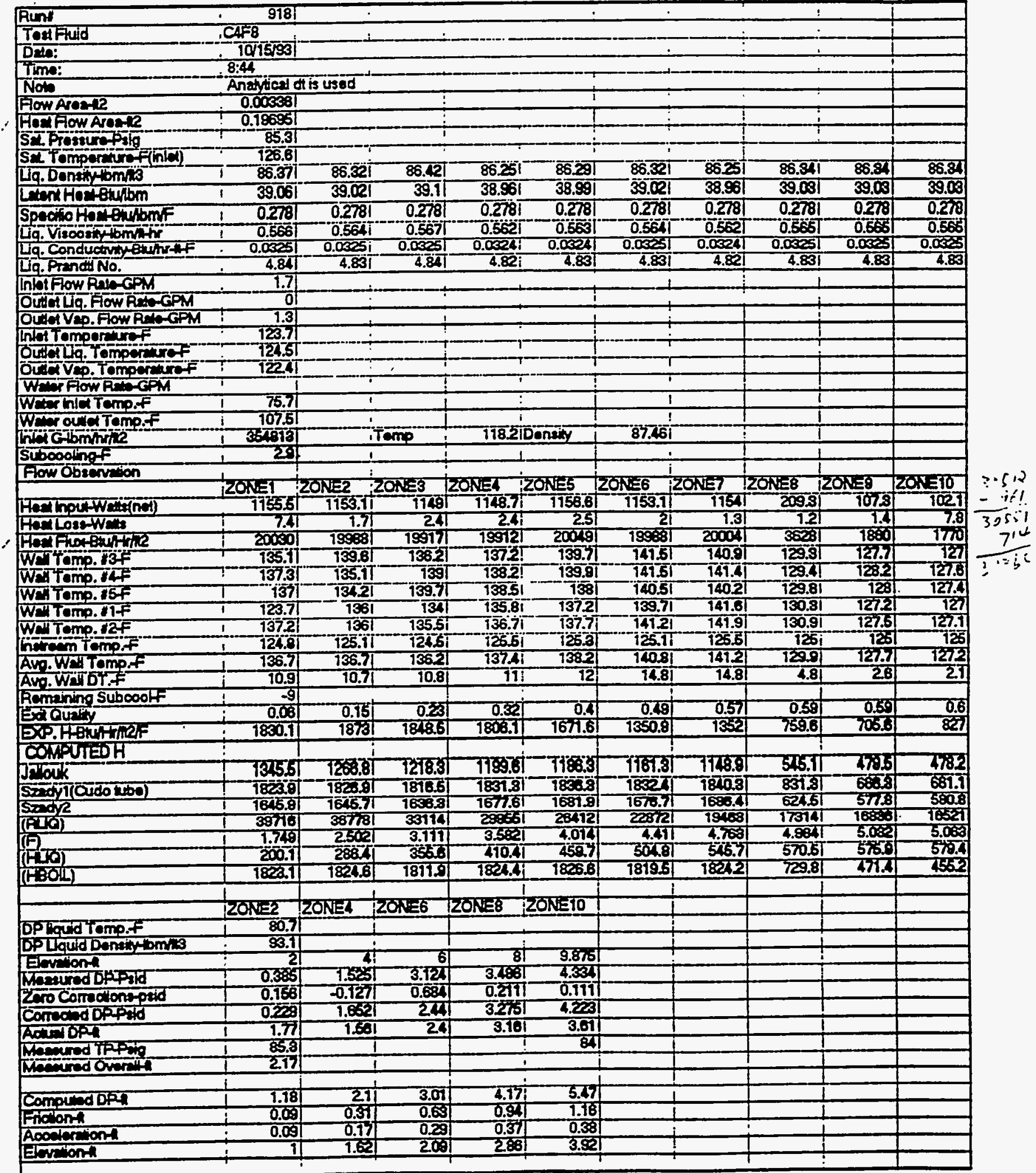




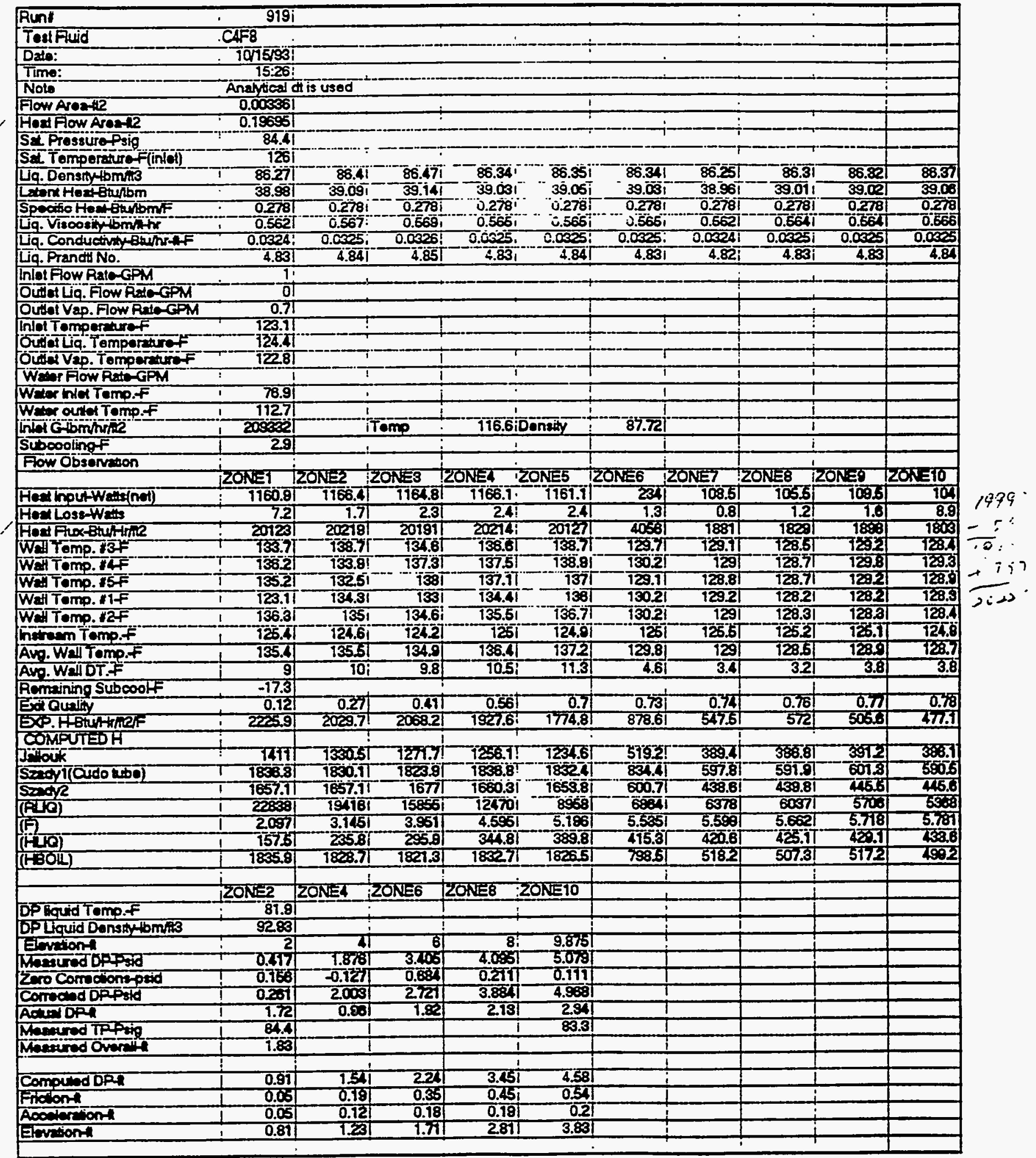




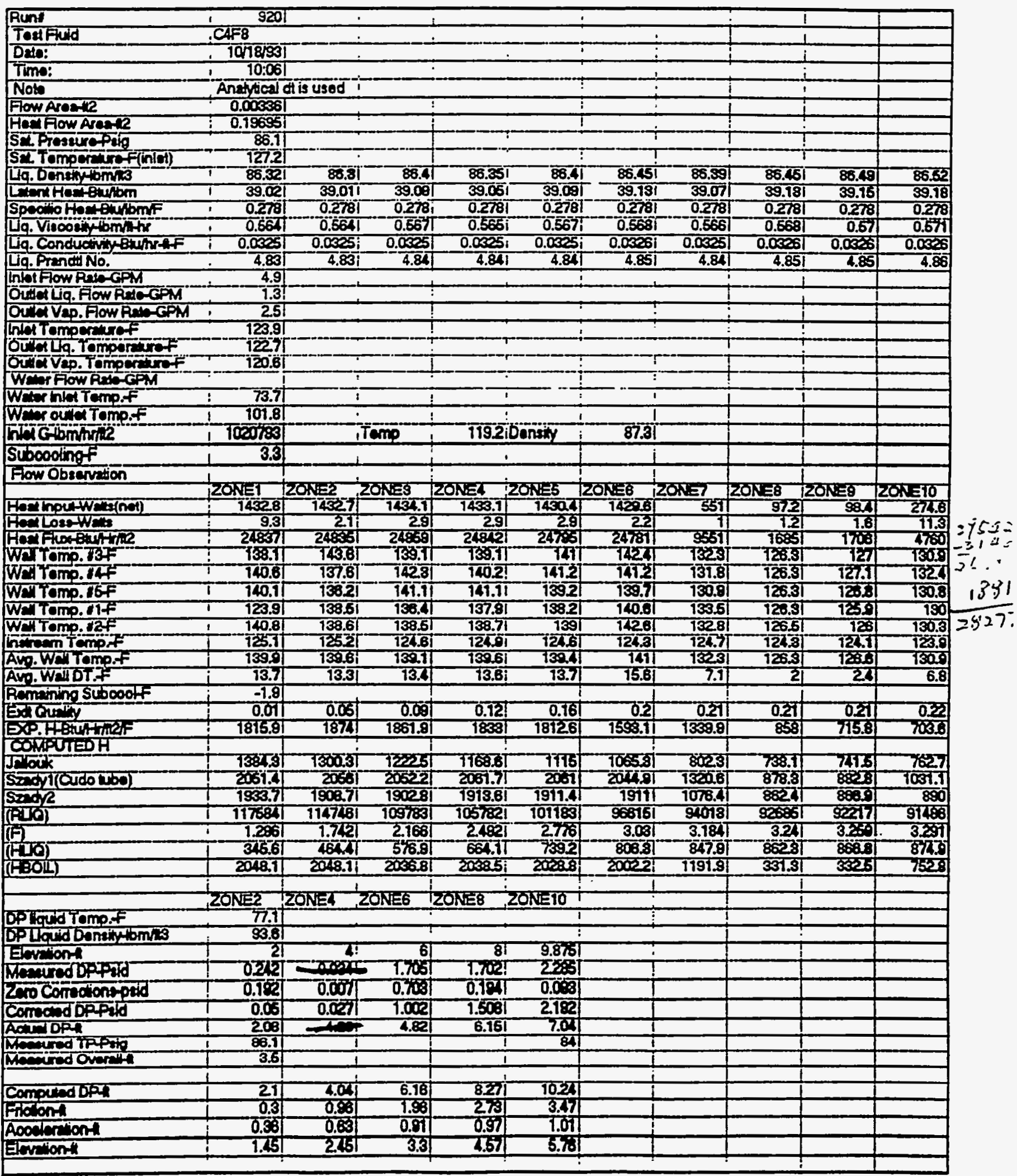




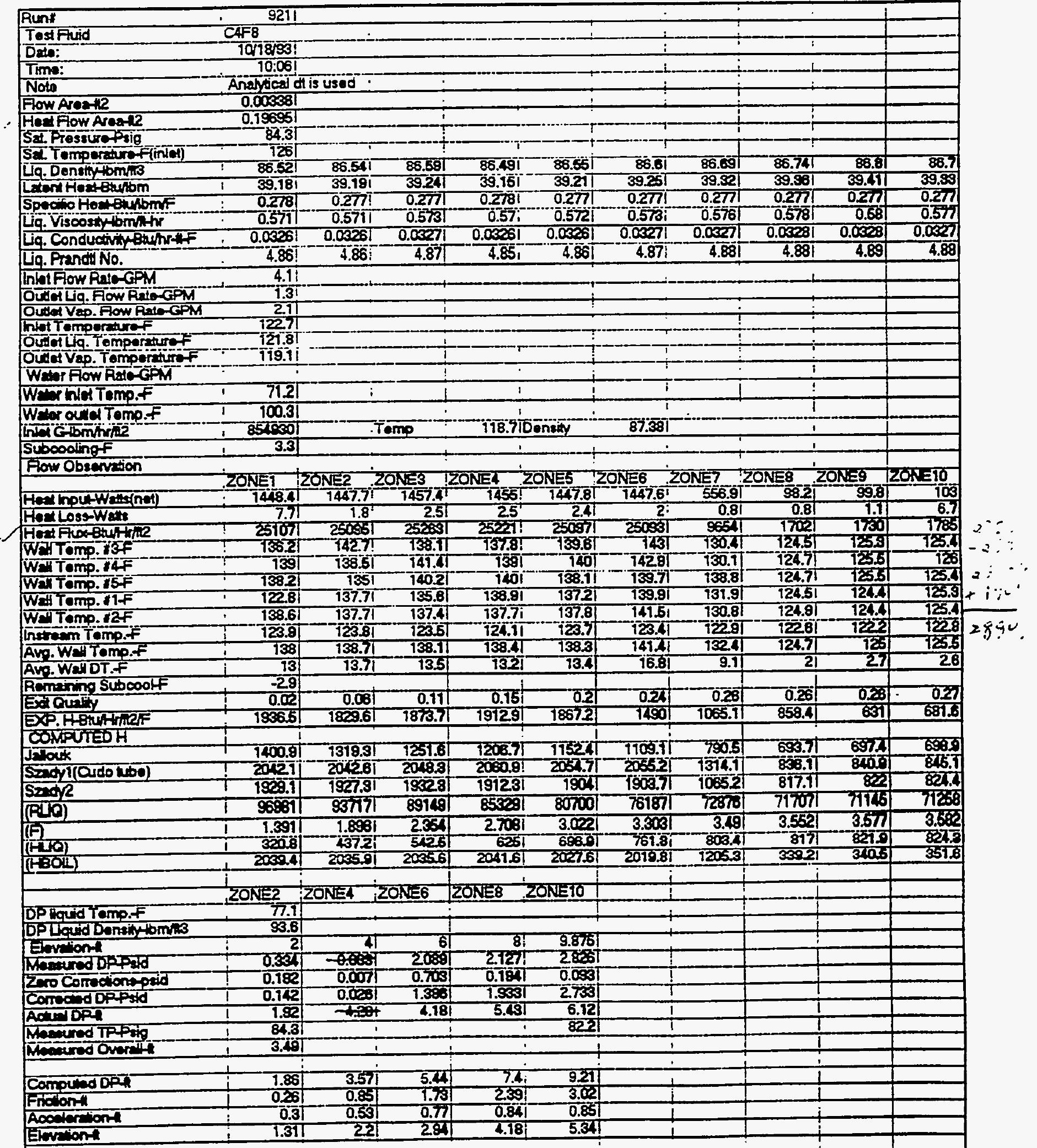




\begin{tabular}{|c|c|c|c|c|c|c|c|c|c|c|c|}
\hline Run: & $\therefore \quad 9221$ & & & - & 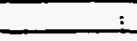 & $\therefore$ & - & & & & \\
\hline TerfFuid & . 4 AF 8 & & & & & & & $\therefore$ & & & \\
\hline Dथa: & 1 101893 & & & & & & $\dot{-}$ & - & & & \\
\hline fime: & $10: 061$ & & & & & & \pm & 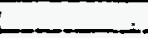 & & & \\
\hline Note & Analytical d & tis used & & & & & & & & & \\
\hline Flow Areante & 0.003501 & & & & & & 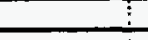 & 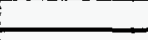 & & & \\
\hline Hex Fow Arapt2 & 0.19695 & & & & & 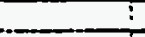 & 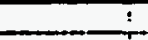 & 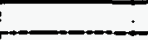 & & & \\
\hline SEl Pressure ping & 86 & & & & & ? & - & & & & \\
\hline 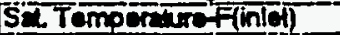 & 127.11 & & 1 & & & $?$ & & 1 & & & \\
\hline Lq.Densiny $46 \mathrm{~m} / 3$ & 86.45 & $86.371^{-1}$ & 86.41 & 86.321 & 86.37 & 86.41 & 88.41 & 86.491 & 86.521 & 86.47 & \\
\hline 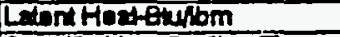 & 39.78 & 39.081 & 39.111 & 39.021 & 39.08 & 39.081 & 39.091 & 39.151 & 39.18 & 39.16 & \\
\hline Specilothengulome & 0270 & 0.278 & 0.278 & 0.2781 & 0.278 & 0.2781 & 0.278 & 0.281 & 0.27 & 0.278 & \\
\hline Lig. Viscosine & 0.5681 & 0.5661 & 0.5681 & 0.5541 & 0.5661 & 0.5671 & 0.557 & 0.571 & 0.571 & 0.569 & \\
\hline Lig. Conductinuetuhr-fF & 0.03261 & 0.05251 & 0.0325 & 0.03251 & 0.005251 & 0.00851 & 0.03251 & 0.0361 & 0.0365 & 0.0580 & \\
\hline Lig. Prendid No. & 4.85 & $4.84 i$ & 4.85 & 4.831 & 4.84 & 4.841 & 4.84 & 4.851 & 4.86 & 4.85 & \\
\hline Inlet Fow Raye-GPM & 3.3 & & & & & i & $\ldots$ & i & & & \\
\hline OLALIT. Fow ARTEAM & (1.2) & & & & & & & i & & & \\
\hline OLtA VAp. Flow RAG-CPM & 1.91 & & $i$ & & & & 1 & $i$ & 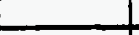 & & \\
\hline Inlat tomperaturef & 1228 & & & & & & 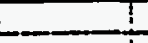 & 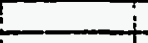 & & & \\
\hline Ou of Liq. Tomportere $F$ & 123.9 & & & & 1 & $T$ & 1 & 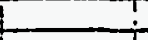 & & & \\
\hline 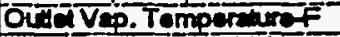 & 120.8 & ! & & & & I & i & 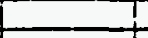 & & & \\
\hline We Fow RateGPM & -7 & $T$ & 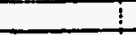 & $:$ & & T & $!$ & T & & & \\
\hline Wertrat Tamp.F & 70.11 & $!$ & 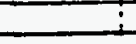 & $!$ & & I & 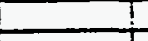 & $\frac{1}{1}$ & 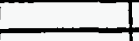 & & \\
\hline Weroutel Tomp.F & 104.4 & $\therefore$ & 1 & 1 & & 1 & T. & I & 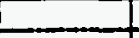 & & \\
\hline latadommine & 680.21 & & temp & 178.310 & Densiny & 87.441 & & 1 & 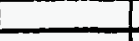 & & \\
\hline Suboooling & 45 & 1 & & 1 & & i & 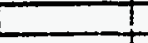 & $-i$ & 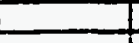 & & \\
\hline Fow Obsenvition & & & & & & & & & & & \\
\hline & ZONE $=1$ & ONEE 2 & 20 Nis 3 & SONiE4:2 & $20 N=5$ & ZON:56 it & 20Ni: 12 & LONes it & 20N=9 & $20 N=10$ & \\
\hline 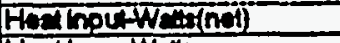 & 1449.6 & -1447.31 & 143.9 & 145.6 & 142291 & 14442 & 584.8 & 107.3 & 1023 & 104.8 & $1: \div$ \\
\hline HodLoss-Wels & 7.51 & 1.81 & 2.4 & 2.4 & 2.31 & 1.9 & 0.81 & 0.91 & 1.2 & 7.2 & \\
\hline Heat Floxbuhthe & 25128 & Z50681 & 25021 & $25069 !$ & 25012 & 25031 & 9700 & 17561 & $77(2)$ & 1817] & \\
\hline WATtomp. & 135. & $143 ., 1$ & 733 & 73835 & 738.71 & 1459 & 137.6 & 1258 & 1283 & 727 & $2:-\cdots$ \\
\hline WeTtomp. $14 \mathrm{~F}$ & 139.4 & 136.81 & 147.6 & 139.41 & 138.8 & 741.8 & 137.4 & 725.51 & 1273 & 127.6 & $17 \div 8$ \\
\hline WaI Tamp. $15 F$ & 138.7 & 135.71 & 140.9 & 140.21 & 138 & 138.31 & 130.7 & 12621 & स्टिग & 127.4 & \\
\hline WeU Tomp. IIf & 1228 & 138.11 & 135.91 & 137.1 & 137.2 & 139.61 & 131.8 & 126.21 & 128.1 & 128.8 & \\
\hline Wel Tamp.12F & 138.8 & 138.31 & 137.8 & 138.31 & 1382 & 141.31 & 131.61 & 126.31 & 128.1 & 127 & \\
\hline hot en temo.f & 12431 & 124.81 & 124.4 & $125.7 i$ & 124.81 & 124.81 & 724.61 & 124.17 & 1200 & 1243. & \\
\hline Av. Wall Tomp.t & 138.21 & $1392 i$ & 138.4 & 138.81 & 138.11 & 140.7 & 131.41 & 12631 & 725.7 & 1272 & \\
\hline Avo.Wall DT.7 & 12.9 & $132 !$ & 129 & 1251 & 121 & 14.9 & 6.4 & $2 \pi$ & 2. & 29 & \\
\hline Remainm Suboooff & -3.4 & & & & & & & & & & \\
\hline Exataly & 0.02 & 0.08 & 0.13 & 0.191 & 0.21 & 0.3 & 0.321 & 0.52 & 0.38 & 0.33 & \\
\hline 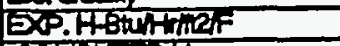 & 1940.5 & 1894.7 & 1965 & 20019 & $20 \approx 3.7$ & 16753 & 15503 & $8 \times 4$ & 65632 & 6312 & \\
\hline CoMPUIEOH & & & & & & & & & & & \\
\hline Iriak & 1245 & 13051 & 7259.81 & 7टर2गा & 1दाव.8 & 197251 & 7852 & Exiा & ख21 & ख3.8. & \\
\hline 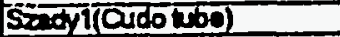 & 2008.7 & 2057.81 & 205151 & 2037.51 & 2038.6 & 206361 & 1320.7 & 77.6 & 881.7 & 68 & \\
\hline $\sin \sqrt{2}$ & 1358.61 & 196281 & T933.81 & 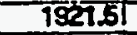 & 197. & 1918.7 & 10775 & 747 & 7512 & 735.8 & \\
\hline (P्यू) & 78287 & 75301 & 709761 & 87087 & Estos & 58001 & 5568 & 53791 & 53578 & ESE्दी & \\
\hline (i) & 1.दिम & 1.909 & 25001 & 20051 & 3257 & 3.571 & 3.772 & 3.8751 & $3.8 \pi$ & 3.894 & \\
\hline (सिख) & 26.6 & 368 & $4978 !$ & 5E5: & 635 & बघाT & 783.91 & 376.87 & 751.1 & 7842 & \\
\hline$(\mathrm{Fis01})$ & 2047.1 & $2052 \pi$ & दुरूख & 205331 & ट0146: & 200ा & 1200 & $37 \pi$ & $37 r .1$ & 3850 & \\
\hline & & & & & & & & 1 & & & \\
\hline & ZOKEE & ZONE4 & CONEE & 201,58 & ZONETO & & & & & & \\
\hline DPthird remp.f & 76.2 & & & & & & & & & & \\
\hline DPLIquid Domithem 13 & 93.73 & & & & & & & & & & \\
\hline Evintont & & 4 & 6) & 8ा & 9.875 & & & & & & \\
\hline 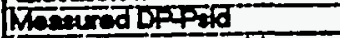 & 7.355t & 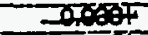 & 2467 & 281 & 3.36 & & & & & & \\
\hline Zero Combotiona ped & 0.182 & 0.007 & 0.708 & $0.78 \pi$ & 0.008 & & & & & & \\
\hline Comeciad DPS dd & 0.161 & 0.0201 & 1.7581 & 2403 & 3.2501 & & & & & & \\
\hline Actel DPA & 1.8 & thon & $\mathbf{3 . 6 8}$ & 4.001 & 5.28 & & I & & & & \\
\hline Whated trophe & 861 & 1 & & 1 & 83.8 & & 1 & & & & \\
\hline Mesoured oventil & 4 & 1 & & I & L & & & & & & \\
\hline & 1 & & & & & & & & & & \\
\hline Computed DP & 7.71 & 3.12 & 4.7. & 6.391 & 8.03 & & & & & & \\
\hline Fritiont & 0.18 & 0.05 & 1.361 & 7.97 & 246 & & $i$ & & $\perp$ & & \\
\hline Acoaler ionth & 0.22 & 0.4 & 0.67 & $0.6 \pi$ & 0.68 & & & & & & \\
\hline Elomiont & 1.261 & 2.07 & $2 \pi$ & 3.751 & 4.89 & & & & & & \\
\hline
\end{tabular}




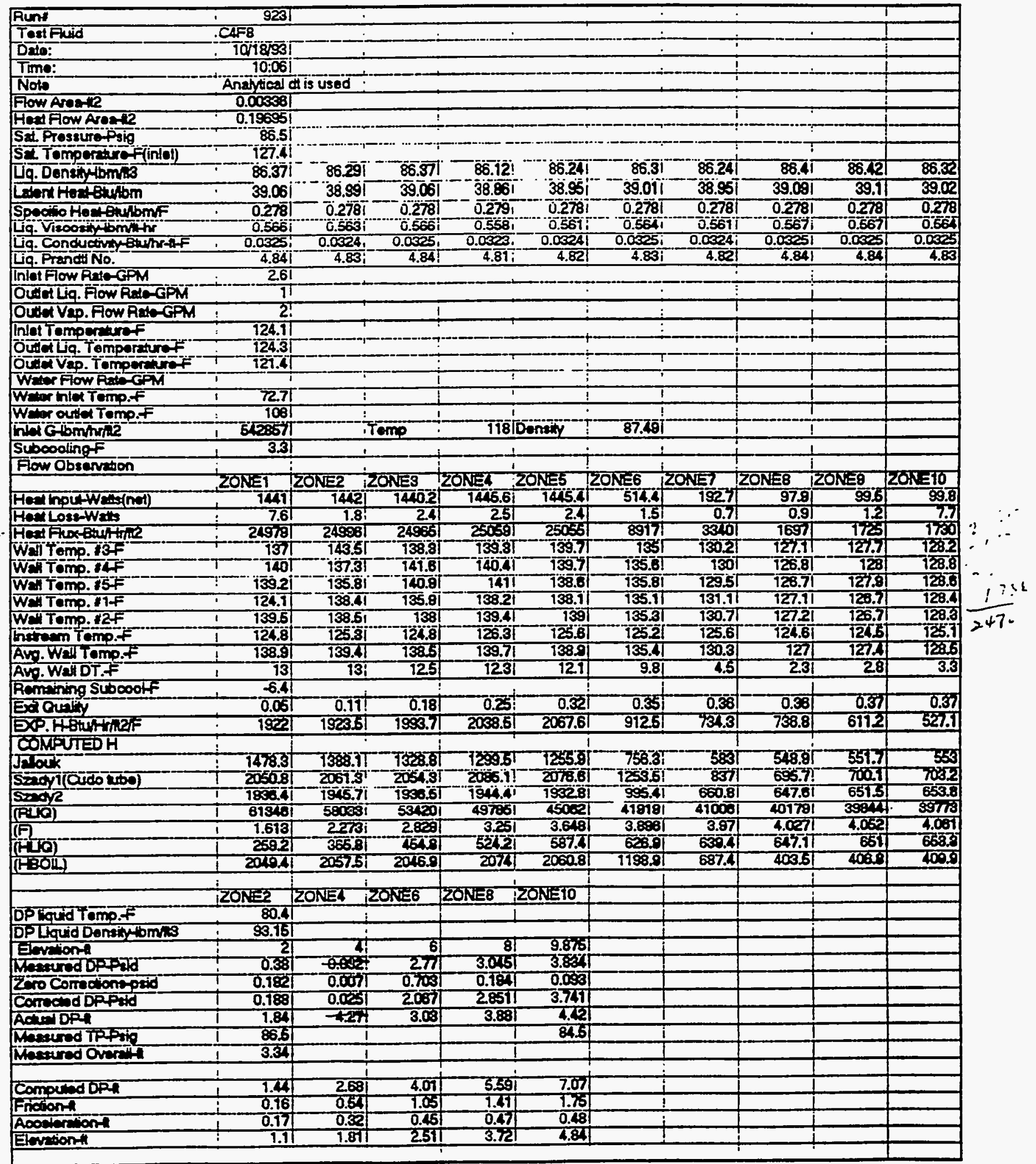




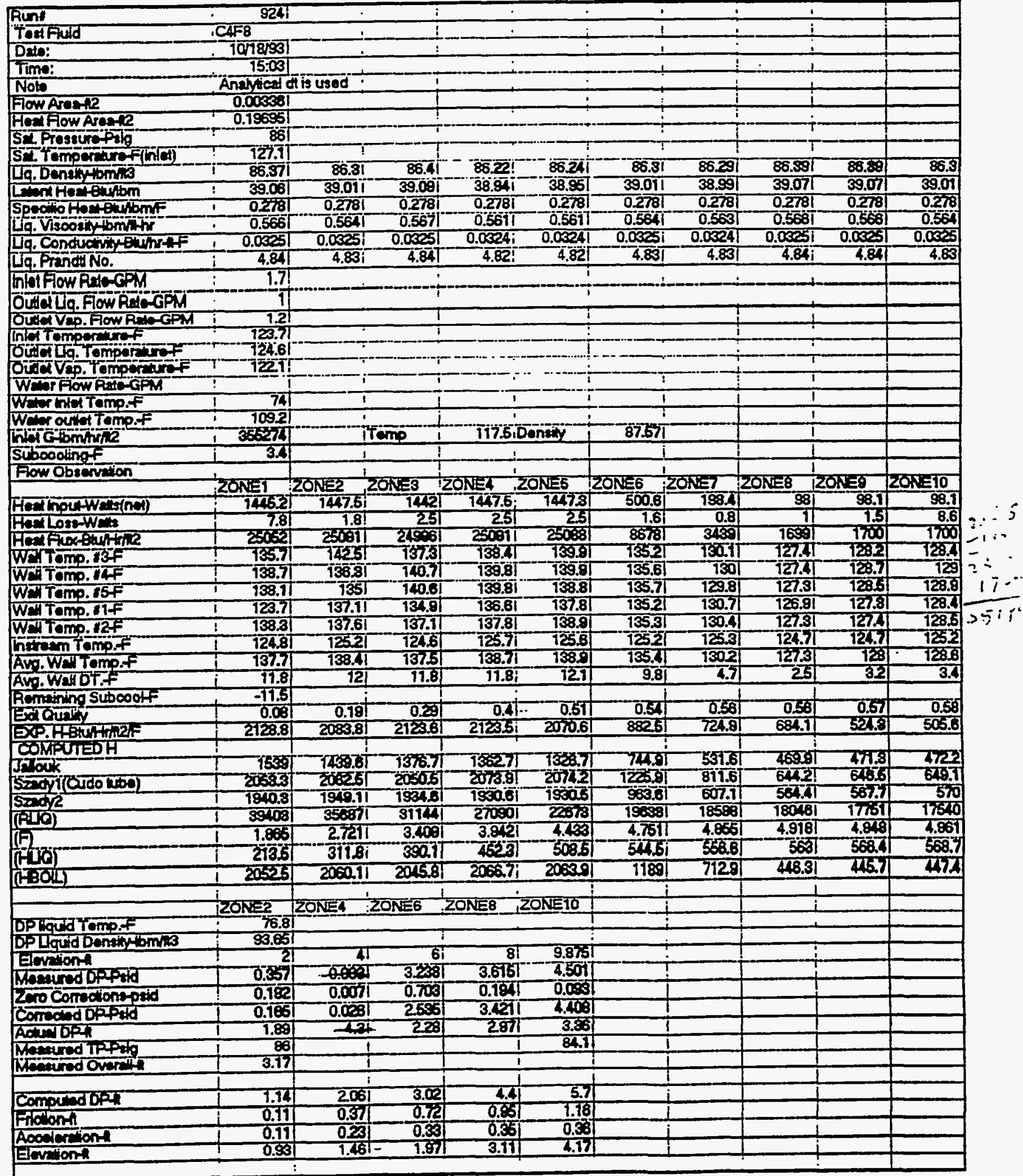




\begin{tabular}{|c|c|c|c|c|c|c|c|c|c|c|}
\hline Runt & 9251 & & & & & & & & & . \\
\hline Tostflind & CAF & & & & & & & & & \\
\hline Dato: & - $10018 \overline{8}$ & & & & & & & & & \\
\hline Time: & 15.031 & i & $\because$ & & & & 1 & $!$ & T & \\
\hline Now & - Antatyoed & dis used & I & - & & & $!$ & & $!$ & \\
\hline Fow Aren? & 10.00656 & & 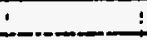 & $!$ & $=$ & $!$ & 1 & & 1 & \\
\hline Hon Flow AYost? & 0.19505 & & 4 & 1 & : & & $i$ & & i & \\
\hline SEl Pressuropyig & $85.7 !$ & & & - & - & $!$ & 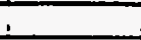 & & 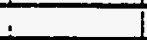 & \\
\hline Set Temporeteroffinlot) & 126.9 & & & & & & & & & \\
\hline Lq. Densiny 4 omm 3 & 86.2 & 86.34 & 86.41 & 86.2 & 86.25 & 86.29 & 86.24 & 86.32 & 86.34 & 86.24 \\
\hline Lotert Heat-Bhubm & 38.92 & 39.031 & 39.091 & 38.921 & 38.96 & 38.99 & 38.95 & 39.021 & 3.03 & 38.95 \\
\hline Spacifo Hosterhbmi & 0.281 & 0.278 & 0.278 & 0.2781 & 0.278 & 0.278 & 0.278 & 0.2781 & 0.278 & 0.278 \\
\hline Liq. Viscosing & 0.56 & 0.565 & 0.567 & 0.561 & $0.5 \overline{2}$ & 0.553 & 0.561 & 0.564 & 0.565 & 0.561 \\
\hline Ua. Conducivingethriff & 0.0324 & $0.0325 !$ & 0.03251 & 0.03241 & 0.0324 & 0.0324 & 0.0324 & 0.03251 & 0.0325 & 0.0524 \\
\hline Lig. Prandu No. & 4.821 & 4.831 & $-4.84 i$ & $4.82 i$ & 4.82 & 4.83 & 4.82 & 4.831 & 4.88 & 4.82 \\
\hline InhIFIOW RE-GPM & 11 & & : : & 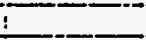 & & & & 1 & I & \\
\hline Orist Lig. Fow Rate-GPM & $\pi$ & $T$ & 3 & $i$ & $i$ & $i$ & & & $\bar{i}$ & \\
\hline Outel Veo.Fow RM-GPM & 0.6 & & 1 & 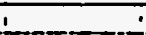 & $\therefore$ & & $\mathrm{i}$ & & $i$ & \\
\hline linter Temperewer & 123.11 & $\therefore$ & 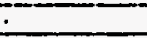 & & $\because$ & $\because$ & & & i & \\
\hline Outeflig. Temperaturof & 135 & 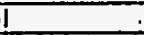 & $\dot{.}$ & . & + & $i$ & & & 1 & \\
\hline Outat Veo. Temporaturef & 1225 & & : & : & & & & & $i$ & \\
\hline Welp Fow Rate-GPM & & & & & & 1 & & & & \\
\hline Water inlet Temp.F & 7.5 & & . & . & & $\cdot$ & & & & \\
\hline Wert ould Temp.f & 113.8 & & & & & $i$ & & & & \\
\hline 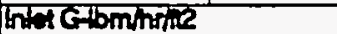 & $20052 \pi$ & & Tamp & 116.71 & Tbensity & 87.8 & & & & \\
\hline Swoooling & 3.81 & & $i$ & $i$ & & $!$ & & & & \\
\hline \multirow[t]{2}{*}{ Fow Observen } & T & & $\bar{\vdots}$ & $\bar{\vdots}$ & & & & & $T$ & \\
\hline & ZONEI & IZONE? & ZONE3 1 & LONEA : & ZONES & ZONE 6 & ZONET & ZONE8 & ZONES & $120 N=10$ \\
\hline Hod houtwatnet) & $1 \quad 1457.2$ & 1442.7 & 1448.81 & $1-\overline{1448.7 i}$ & 8972 & 273.1 & 198.81 & 96.81 & 11027 & 1015 \\
\hline HeCLose-Wats & 8.11 & 1.9 & 2.61 & 271 & 2.7 & 1.8 & 0.8 & 1.2 & 9.6 & 9.8 \\
\hline Hes Fior Buthite & 24913 & 25008 & $257 \pi 4$ & 25Tा21 & 15552 & 473 & 7713 & 16801 & 1780 & 753 \\
\hline Wall Tomp. 3 IF $^{\prime}$ & 135.11 & 147.8 & 136.21 & 138.5 & 144.5 & 134.8 & 128.6 & 128.1 & 1282 & 1282 \\
\hline WEH TOMO. RLF & 138 & $135.6 \mid$ & 13.7 & 139.61 & 143.5 & 135.4 & 128.4 & 128.11 & 128,4 & 128.7 \\
\hline WEIT TEmp. 155 & 136.11 & $133.6 !$ & $139.2 !$ & $139.1 i$ & 135.3 & 135.6 & 128 & 127.8 & 128.3 & 128.5 \\
\hline WEIT Temp.\$1F & 123.11 & 136 & 134.6 & 1361 & 735.5 & 137.4 & 128.8 & 127.8 & 127.4 & 1282 \\
\hline 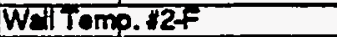 & 137.4 & 136.71 & 136.21 & 137.51 & 138 & 137.1 & 128.8 & 128.11 & दिवाठ & 1225 \\
\hline inctrom temp.f & |Z25.81 & 125 & 124.61 & 125.81 & 125.5 & 125.3 & 125.6 & T25. & 1201 & 125.6 \\
\hline Ang. Wahtomp.t & 138.11 & |3751 & 138.7 & T38.81 & 137.8 & 135.8 & 128.5 & Tद्व & 128 & 728.4 \\
\hline AY. WaOT. & 9.7 & 11.4 & 10.8 & 11.41 & 11.4 & 10.3 & 2.8 & 2.8 & 20 & 2.7 \\
\hline Romoning suboooff & -212 & & 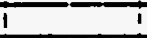 & & & & & & & \\
\hline Ext Cusaly & 0.15 & 0.881 & 0.511 & 0.691 & 0.8 & 0.84 & 0.85 & 0.881 & 0.81 & 0.89 \\
\hline EXP.HEWhThET & 256 & 2194.71 & 2336.6 & 2209.81 & 1367.8 & 460.2 & 671.1 & 5801 & 614 & 666.4 \\
\hline COAPUIEDH & $T$ & & & & & & & & & \\
\hline Leook & 7615.2. & 1510. & 1बaरा & 742851 & $\mathbf{4 5 2}$ & 453 & 45.21 & 45.8 & 7529 & 251 \\
\hline Sadvil(ado tubo) & 2003.8 & 20551 & 2053.8 & 2074.41 & 45.4 & 45.3 & 45.4 & 45.8 & 45.8) & 45.4. \\
\hline $\operatorname{send} 2$ & 1919.4 & 1941य & 1940.7 & 1935.5 & 45.4 & 45.3 & 45.4 & 45.3 & 45.8 & 75.4 \\
\hline (RDO) & 22002 & 18405 & 13968 & 9757 & 9751 & 9751 & 9751 & 9751 & 9751 & 9751 \\
\hline (F) & 2.252 & 3.4001 & 4.362 & 5.051 & 5.06 & 5.05 & 5.05 & 5.05 & 5.05 & 5.05 \\
\hline (स) & 167.9 & 2581 & उयदा & 379.81 & 379.9 & 379.9 & 379.9 & 379.91 & 379.0 & 379.9 \\
\hline (A०OL) & 20.35 .51 & 2853.81 & 2351.ाI & 2070.2 & 20702 & 2070.2 & 20703 & 2070.2 & 20702 & 20102 \\
\hline & +1 & & & 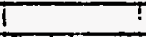 & & & & & & \\
\hline & TONES & ZONE4 & IZONEB & LZONEB & ZOONETO & & & & T & \\
\hline DPl kqud Temp.f & 178.2 & & 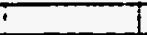 & & 1 & & & & 1 & \\
\hline DPLoud Dansth bom/3 & \$3.73 & 1 & I & 1 & 1 & & & & & \\
\hline Exvetiont & 2 & 4 & 6] & 81 & $9.8 \%$ & & $i$ & & & \\
\hline 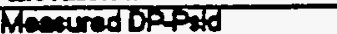 & 0.753 & 1.5801 & 3.883 & 4.351 & 5.357 & & & & & \\
\hline Zero Cometions-pid & 0.182 & $0.007 !$ & 0.703 & 0.189 & 0.053 & & & & & \\
\hline Corrocind ofy id & 0.567 & 1.946 & 3.161 & 4.168 & 5.224 & & & & & \\
\hline ACNDDR & 7.23 & $1 . T^{t}$ & 1.2दा & 1.75 & 2.07 & & & & & \\
\hline Mearured t7p & 85.7 & & & & 83.5 & & & & & \\
\hline Messured Overeh & $3.6 \pi$ & & & & & & & & & \\
\hline & & & & & & & & & & \\
\hline Compoxpla & 0.87 & 7.81 & टका & 3.381 & 4.4 & & & & & \\
\hline Frotont & 0.00 & 0.21 & 0.39 & 0.51 & 0.59 & It & & & & \\
\hline Acoderitiont & 0.08 & 0.181 & 0.32 & 0.29 & 023 & $T$ & & & I & \\
\hline Elovetonn & 0.75 & 1.11 & 1.61 & 2.561 & 3.66 & 1 & & & $i$ & \\
\hline
\end{tabular}




\begin{tabular}{|c|c|c|c|c|c|c|c|c|c|c|}
\hline Runi & 8261 & & & & & & & & & \\
\hline Tost Fuid & CAF10 & & & & & . & 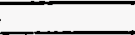 & & & \\
\hline Dalo: & $1110 \overline{93} \bar{i}$ & & & & & & & & & \\
\hline Time: & $11: 17$ & & & & & & & & & \\
\hline Note & Analyticald & ot is used: & & & & & & & & \\
\hline Flow Area-tL2 & $0.00336 i$ & & & & & & & & & \\
\hline Heal Fow Area-fl? & 0.196951 & & & & & & & & & \\
\hline Sat.PTessurePsig & 107.31 & & & & & & & & & \\
\hline Sat. Temperaturef(inlet) & 951.11 & & & & & & & & & T \\
\hline Liq. Density 4 bmitis & 81.981 & $82.13 !$ & 82.13 & 81.491 & 81.74 & 81.831 & 81.53 & 81.831 & 81.81 & 81.79 \\
\hline Lafont Hoetotuibm & 29.55 & 29.761 & 29.761 & 29.291 & 29.471 & 29.541 & 29.32 & 29.541 & 29.54 & 29.51 \\
\hline Spectho Hood etunbmif & 0.2851 & $0.285 i$ & 0.2851 & 0.2861 & 0.286 & 0.2861 & 0.2861 & 0.2861 & 0.2861 & 0.286 \\
\hline Lig. Viscositybm/lithr & 0.433 & $0.435 i$ & 0.4361 & 0.4231 & 0.428 & 0.431 & 0.4241 & 0.431 & 0.431 & 0.429 \\
\hline Liq. Conductivity BtuhrthF & 0.02511 & 0.02521 & 0.0252 & 0.02481 & 0.025 & 0.0251 & 0.02481 & $0.025 i$ & 0.025 & 0.025 \\
\hline Lig. PrandtiNo. & 4.92 & 4.92 & 4.92 & $4.89 i$ & $4.9 !$ & 4.911 & $4.89 j$ & 4.911 & 4.911 & 4.91 \\
\hline nlet Fiow Rate-GPM & $5.1 !$ & & & & & & & & & \\
\hline Outlot Liq. Flow Ralo-GPM & 1 & & & & & & & & & \\
\hline Outlot Vap. Flow Rate-GPM & 2.7 & & & & & & & & & \\
\hline Inlet Temperaturef & 149.11 & & & & & & & & & \\
\hline Outlet Liq. Temperaturef & T49.6 & & & & & & & & ? & \\
\hline Outlet Vap. Temperaturef & 145.8 & & & & & & & & & \\
\hline Water Flow Rato-GPM & & & & & & $i$ & & & & \\
\hline Water filot Temp.t & 91.41 & & & & $\bar{t}$ & + & & & $\div$ & \\
\hline Water outlet Temp.f & 113,41 & $!$ & T & & $=$ & $\div$ & & & $\cdot$ & $i$ \\
\hline Inet G-1bm/hr/it2 & 1002036 & & Tomp & $144.6 \mathrm{i}$ & iDensity & 82.331 & & & $!$ & $i$ \\
\hline Subcooling $f$ & 2 2! & $i$ & 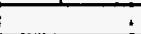 & & 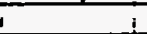 &. & 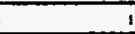 & & 1 & 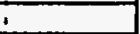 \\
\hline Fow Obsorvation & & & & & $\bar{i}$ & 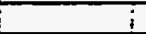 & & & & \\
\hline & ZONE1 & ZONE2 & LONE3 I & ZONEA : & ZONE5 & ZONE6 IE & ZONE? & ZONE8 & ZONE9 & ZONE10 \\
\hline Hoat Input-Walts(net) & 575.2 & 57 & 576 & 5727 & 57.5 & 575.3 & 576.4 & 576.9 & 575 & 577.7 \\
\hline HedLLoss-Watts & 8.71 & 1.91 & 2.61 & 2.81 & 2.7 & 2 & 1.4 & 21 & 251 & 14.3 \\
\hline Hoed Flox-BtuhHr/il2 & 9971 & $10002 !$ & 99851 & 9927 & 10011 & 9972 & 99921 & 10000 & 9967 & 10014 \\
\hline Wall Tomp. BF & 160.41 & 160.81 & 159.4 & 162 & $1 \sigma$ & 161.51 & 162.8 & 160.91 & 159.61 & 159.9 \\
\hline Wall Tomp. *4F & 160.8 & 159.21 & 160.3 & $162 \pi$ & 162.2 & 161.8 & 162.9 & 161.3 & 160.11 & 163.3 \\
\hline Wall Temp. $15 f$ & 761.31 & 158.51 & 161.4! & 162.51 & 160.8 & 161.31 & 162.1 & 160.11 & 159.1 & 158.5 \\
\hline Wall Temp $1=$ & 149.11 & 1601 & 157.8 & 169 & 160.9 & 161.31 & 1841 & 167.41 & 158.4 & 158.5 \\
\hline Wall Temp. MF & 162.31 & 159.91 & 158.7 & 161.51 & 160.91 & 161.3 & 163.71 & 162.21 & $159 !$ & 159.5 \\
\hline instream Temp.f & 149.7 & 148.91 & 148.9 & 162.31 & 151 & 160.6 & 152.71 & 150.61 & 160.61 & 160.7 \\
\hline Avg. Wall Tomp.f & 161.2 & 1601 & 159.11 & 161.81 & 161.21 & $161.5 i$ & 163.11 & 161.21 & 159.21 & 159.9 \\
\hline Avg. Wall DT. $f$ & 111 & 10.61 & 9.7 & 921 & 9.71 & 10.5 & 10.5 & 10.21 & 8.3 & 8.8 \\
\hline Romaining Subcooff & 0 & & & & 1 & 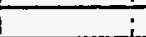 & & & & \\
\hline Exot Quality & of & 0.021 & 0.04 & 0.05 & 0.08 & 0.1 & $0.12 !$ & 0.14 & 0.16 & 0.18 \\
\hline EXP. HBtu/rin/2/F & 902.41 & 941.5 & 1029.5 & $1084.8:$ & 1027 & $947.6 i$ & 947.2 & 977.7 & 12024 & 1139.6 \\
\hline COMPUTEDH & & & & & & & & & & \\
\hline Jallouk & 949.41 & $905 . \pi 1$ & 868.61 & 8701 & 859.2 & 848.31 & 849.21 & 830.91 & 823.31 & 819.9 \\
\hline Szady (Cudo tube) & 1456.6 & 1454.6 & 7457.8 & 1488.2 & 1486.8 & 1484.31 & 1504.11 & 1495.8 & 1498.6 & 1508.4 \\
\hline Szadye & 1256.3 & 1251.5 & 1250.1 & 1275.91 & 1272.31 & 1241.61 & 12625 & 1256.2 & 1259.8 & 1270.7 \\
\hline (RLO) & 1514231 & 148908 & 145998 & 1471371 & 1425131 & 138865 & 137707 & 132840 & 129915 & 127018 \\
\hline (f) & 1.028 & 1.327 & 1.612 & 1.81 & 1.979 & 2.134 & 2356 & 23961 & 2.513 & $2 \sqrt{2}$ \\
\hline (म) & 291.7 & 375.41 & 456.11 & 511.31 & 561.1 & 604.81 & 640.5 & 679.11 & 71231 & 742.9 \\
\hline (HBOLL) & 1452.71 & 1446.31 & 144281 & $1467.8 i$ & 1459.7! & 1450.11 & 1464.4 & 1447.61 & 144201 & 1445.8 \\
\hline & $!$ & & & & $1 \quad 1$ & 1 & 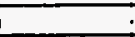 & & 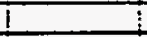 & \\
\hline & ZONE2 & ZONE4 & ZONE6 & ZONE8 & ZONE10 & & & & $i$ & \\
\hline DP liquid Temp.F & 81.3 & & & & & 1 & 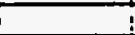 & ! & 1 & i \\
\hline DPliquid Density $-1 \mathrm{bm} / \mathrm{t} 3$ & 92.81 & & & & & & & & + & \\
\hline Elovation-t & $2 !$ & 41 & 61 & 81 & 9.875 & & & & 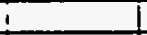 & \\
\hline Measured DPFsid & $-0.72 \pi$ & 0.571 & 0.881 & 1.3771 & 1.432 & & & & 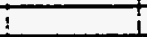 & \\
\hline Zero Contections-psid & 0.04 & -0.015 & -0.006 & -0.0361 & -0.021 & & & & i & \\
\hline Conected DPPsid & -0.181 & 0.5881 & 0.887 & 1.413 & 1.452 & & & & & \\
\hline Actual DPA & 255 & 3.511 & 5.251 & 6.591 & 8.65 & $T$ & $\Gamma$ & & I & \\
\hline Moasured TP poig & 107.81 & 1 & 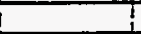 & & 0.3 & 1 & 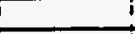 & & 1 & \\
\hline Mosalured Overdith & 188.38 & i & E & & & $!$ & 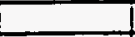 & & $I$ & \\
\hline & 21 & 1 & 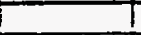 & & $I$ & 1 & & & i & \\
\hline Computad DPA & 217 & 4.131 & 6.061 & 7.971 & 9.79 & 1 & 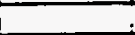 & & I & \\
\hline Friction-t & 0.16 & 0.47 & 0.731 & 1.1 & 1.511 & & & & $i$ & \\
\hline Acosleration- & 0.16 & 0.29 & 0.39 & 0.49 & 0.6 & & & & 1 & \\
\hline Elevation-t & 1.85 & 3.43 & 4.39 & 6.381 & 7.69 & & & & & \\
\hline
\end{tabular}




\begin{tabular}{|c|c|c|c|c|c|c|c|c|c|c|}
\hline Run: & 927. & & & & & & & & & \\
\hline Tostfluid & CAF10 & & & & & & & & & \\
\hline Date: & $11 / 1033$ & & & & & & & & & \\
\hline Time: & $11: 17 !$ & & & & & & $\vdots$ & & & \\
\hline Note & Analytical of & th is used & & & & & & & & \\
\hline Fow Area-t? & 0.003361 & & & & & & & & & \\
\hline Heal Flow Asea-1L2 & 0.1935 & & & & & & & & & \\
\hline Sal. Pressure-Pig & 105.81 & & & & & & & & & \\
\hline Sal. Tomperature-F(inlet) & 150.2 & & & & & & & & & \\
\hline Liq. Densitylbmn3 & 82.241 & $82.28 !$ & 82.131 & 81.78 & 81.89 & 821 & $81.72 !$ & 81.941 & 81.381 & 81.92 \\
\hline Latent Hoat Btulbm & $29.84 !$ & 29.881 & 29.76 & 29.51 & 29.581 & 29.651 & 29.461 & 29.621 & 29.65 & 29.61 \\
\hline Specific Heat Btulbmit & 0.2841 & 0.2841 & $0 . \overline{2851}$ & 0.2861 & 0.2851 & 0.2851 & 0.2861 & 0.2851 & 0.2851 & 0.285 \\
\hline Lig. Viscosity lbmithith & 0.4381 & 0.4391 & 0.4361 & 0.4291 & 0.4311 & 0.4331 & 0.4281 & $0.432 !$ & 0.4331 & 0.432 \\
\hline Liq. Conduavivity-8humpaff & 0.02531 & 0.02531 & 0.02521 & 0.0251 & 0.0251 & 0.02591 & 0.0240 & 0.02511 & 0.02511 & 0.0251 \\
\hline iq. Prandts No. & 4.931 & 4.931 & 4.921 & $4.9 i$ & 4.911 & 4.921 & 4.91 & $4.91:$ & 4.921 & 4.91 \\
\hline nlet Fow Rato-GPM & $4.3 !$ & & & & & & & & & \\
\hline Outiet Lig. Flow Ralo-GPM & 1 & & & & & & & & & \\
\hline Outlet Vap. Flow Rate-GPM & $2.4 !$ & & & & & & & & & \\
\hline Iniel Temperature of & 147.3 & & & & & & & & & \\
\hline Outlel Liq. Temperaturef & 149.5 & & & & & & & & & \\
\hline Outel Vap. Temperaturef & 145.1 & & & & & & & & & \\
\hline Water Fow Rato-GPM & & & & & & & & & & \\
\hline Water Inlel Temp.F & 90.81 & & & 5 & + & 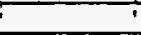 & $\therefore$ & & & \\
\hline Water outel Temp.f & 112.71 & & & $\cdot$ & & & i & & & \\
\hline Inlot Glbmhrnte & 8481671 & & Temp & 142.81 & Dengity & 82.651 & & & & \\
\hline Subcoolingf & $2.9 i$ & & & & & & 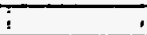 & & i & 1 \\
\hline Flow Observation & & & & & & $i$ & $i$ & & $i$ & \\
\hline & ZONEI : 2 & ZONE2 . & ZONE3 & ZONE4 : & ZONE5 IV & ZONE6 & ZONE7: & ZONE8 & ZONE9 T & ZONETO \\
\hline Heal Input-Watts(nef) & 576.5 & 578.8 & 576.8 & $573.5 i$ & 578.2 & 575.2 & 577.8 & 57.2 & 575.8 & 577.4 \\
\hline Heat Loss-Watts & $9:$ & 21 & 2.8 & 2.9 & 2.91 & 2.11 & 1.4 & $2.1 i$ & $2.6 !$ & 15.1 \\
\hline Hod $A \omega x$-Btu/han' & 9993 & 100331 & 9998 & 99411 & 10023 & $9971 !$ & 100161 & 90005 & 99811 & 10000 \\
\hline Wall Temp. \#3F & 159.1 & $160.1 i$ & 159 & 160.7 & 1612 & 1611 & 167.5 & 160.2 & 158.81 & 159.1 \\
\hline WaH Tomp. $4 \mathrm{~F}$ & 159.7 & 158.71 & I60.2! & $160.7 !$ & 161.5 & 161 & 169.8 & 160.9 & 159.6 & 162.3 \\
\hline Wall Temp. $85+$ & 160.11 & 157.81 & 161.2 & $161.2 !$ & 160.3 & 160.4 & 161.11 & T59.61 & 158.4 & 157.7 \\
\hline Wall Temp. 1F & 147.3 & 159.21 & 157.6 & 150.01 & 159.61 & 160.91 & 18261 & 760.51 & 157.8 & 157.6 \\
\hline Wall Temp. & 160.9 & $159.2 i$ & 158.2 & 160.11 & 159.9 & $161 !$ & 162.51 & $161.5 !$ & 158.4 & 158.6 \\
\hline Instream Temp.F & 148.31 & 148.11 & 148.81 & $150.8 \mathrm{i}$ & 750.21 & 148.61 & $151.1 !$ & 149.81 & 148.7 & 160 \\
\hline Avg. Wall Tomp.f & 1601 & 159.31 & 158.81 & 160.61 & 160.31 & 160.81 & 167.91 & 160.51 & 158.6 & 159.1 \\
\hline Ävg. WaliDT.F & $\pi 1.2$ & $10.7 !$ & 9.41 & $9.3 i$ & 9.71 & 10.81 & 10.3 & $10.2 !$ & 8.41 & 8.6 \\
\hline Remaining Subcoolf & 0.51 & & & & & & $\vdots$ & & & \\
\hline Exit Quality & $0 !$ & 0.021 & 0.04 & 0.06 & 0.091 & 0.111 & $0.13 i$ & $0.16 i$ & 0.18 & 0.21 \\
\hline 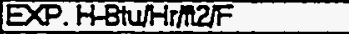 & 892.4 & $933.6 i$ & 1063.9 & 1038.9 & 1036.31 & 925.41 & 968 & $982.1 i$ & 1181.3 & $11 \approx 2.8$ \\
\hline COMPUIEDH & & & & & & & & & & \\
\hline Jallouk & क5ा.11 & 932.81 & 899.4 & $887.6 !$ & 869.3 & 856.91 & 858.91 & 847.71 & $84 \pi$ & 838.7 \\
\hline Szady1(Cudo tubo) & 1444.81 & $1447.4 !$ & 1455.6 & 1471.81 & 1475.7 & 1470.5 & 1400.5 & 1483.31 & 1484.1 & 1492.7 \\
\hline Szadid2 & 12461 & 1247.31 & 1251.2 & 1263.21 & 1265.21 & 1256.31 & 1247 & 1241.81 & 1243.8 & 1253.6 \\
\hline (RUQ) & 128705 & 1258011 & 123487 & 122473 & 118883 & 115274 & 113707 & 109585 & 106388 & 10355 \\
\hline$(F)$ & 1.0051 & 1.205 & 9.617 & 1.845 & 2.0431 & 2.219 & 2357 & 2.5091 & 2.642 & 2.76 \\
\hline (संणि) & 248.7 & 3131 & 400.31 & 457.71 & 506.61 & $549.8 !$ & 585 & 622 & 654.8 & 684.3 \\
\hline (HBOIL) & 14423 & 14425 & 1445.41 & 1456.91 & 145551 & 1444.5 & 1459.81 & 1445.9 & 9440.3 & 1443.2 \\
\hline & & 1 & 1 & & 1 & & $i$ & 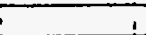 & & $T$ \\
\hline & ZONE2 . 2 & ZONE4 & ZONE6 & ZONE8 & ZONE10 I & & $i$ & & & \\
\hline DP liquid Temp.F & 761 & i & 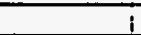 & $i$ & i & & & & & I \\
\hline DP Liquid Density-lbm/ti3 & 93.571 & & & & & & & & & \\
\hline Elevation & & 4 & $6 !$ & & 9.8751 & & & & & \\
\hline Measured DPPaid & -0.0591 & 0.7011 & 7.173 & $7 . \% 41$ & 7.8571 & & $T$ & & & \\
\hline Zero Correctons-psid & 0.041 & $-0.015 !$ & -0.0061 & -0.0361 & -0.021 & & & & & \\
\hline Cortected DPFild & -0.099 & 0.7161 & 1.119 & 1.741 & 1.877 & & T & & & \\
\hline Actual DPA- & 2.461 & 3.311 & 4.89 & 6.081 & 7.991 & & $T$ & $t$ & 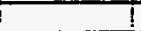 & \\
\hline Mossured TPPsig & 105.81 & 1 & & $i$ & 0.31 & & I & 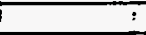 & & \\
\hline Measured Overall & 185.44 & $!$ & $T$ & & 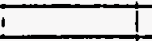 & & 1 & ! & & \\
\hline & 1 & & & & & & 1 & & & \\
\hline Computed DPAn & 2.08 & 3.811 & 5.451 & 7.061 & 8.59 & & 1 & & 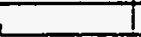 & \\
\hline Friction-th & 0.12 & 0.341 & 0.661 & 1.04 & 1.471 & & $i$ & & & \\
\hline Acceleration- & 0.111 & 0.22 & 0.311 & 0.39 & 0.48 & & $\frac{1}{1}$ & & & \\
\hline Elovation- & 1.85 & 3.251 & 4.48 & 5.631 & 6.ळा & & 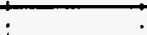 & & & \\
\hline & & & & & & & i & & & \\
\hline
\end{tabular}




\begin{tabular}{|c|c|c|c|c|c|c|c|c|c|c|}
\hline Runt & $928 i$ & & & & & & & & & \\
\hline Test Fuid & CAF10 & & & & & & & & & \\
\hline Dalo: & $11 / 10$ 931 & & & & & & & & & \\
\hline Time: & $11: 17 !$ & & & & & & & & $\therefore$ & - \\
\hline Nole & Analytical of & tis used & & & & & & & & \\
\hline Fow Area-ti2 & 0.003361 & & & & & & & & & \\
\hline Heal Fow Area-th & 0.196951 & & & & & & & & & \\
\hline Sat. Pressure Psig & $106 !$ & & & & & & & & & \\
\hline Sat. Temperature $F$ (inlel) & 150.3! & & & & & & & & & \\
\hline Lia. Density bmints & 82.371 & 82.241 & 82.021 & 81.741 & 81.851 & 89.561 & 81.761 & 81.911 & 87.981 & 81.83 \\
\hline Lotent Hoa tetuíbm & 29.891 & 29.841 & 29.68 & 29.471 & 29.551 & 29.681 & 29.491 & 29.591 & 29.05 & 29.54 \\
\hline Spocitic Hoot thulbm/F & 0.284 & 0.2841 & 0.2851 & 0.2861 & 0.2851 & $0.285 i$ & 0.2861 & 0.2851 & 0.2851 & 0.28 \\
\hline Liq. Viscositylbm/nthr & 0.439 & 0.4381 & 0.434 & 0.4281 & 0.43 & 0.4321 & 0.4281 & 0.4311 & 0.4391 & 0.43 \\
\hline Lig. Conductivity-8tumh-fif & 0.02531 & $0.0253 i$ & 0.02511 & 0.0251 & 0.0051 & 0.02511 & 0.0251 & 0.02511 & 0.02511 & 0.025 \\
\hline Sq. Prandt N̄o. & 4.931 & 4.931 & 4.921 & 4.91 & 4.911 & $4.99 i$ & $4.9 i$ & 4.911 & 4.921 & 4.91 \\
\hline Inlet Fow Rato-GPM & 3.5 & & & & & & & & $i$ & \\
\hline Outlet Lig. Fow Ralo-GPM & 1 & & & & & & & & & \\
\hline Outlet Vap. Flow Rato-GPM & 2 & & & & & & & & & \\
\hline Inlel Tomperaturof & $147.1 !$ & & & & & & & & & \\
\hline Outel Lig. Temperaturef & 150.11 & & & & & & & & 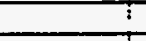 & \\
\hline Outlet Vap. Temperature of & 145.21 & & & & & & & & & \\
\hline Wador Fow Rato-GPM & 5 & & & & & & 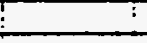 & & i & \\
\hline Wator hilet Temp.F & 921 & & & ' & - & & & $!$ & 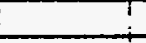 & \\
\hline Water out of Temp.F & 116.7 & · & & . & & & 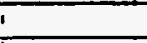 & & $i$ & 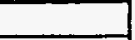 \\
\hline Inlot G-1bm/hr/h2 & 6910001 & & Tomp & 142.51 & Density & 82.731 & & & $i$ & 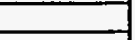 \\
\hline Subcooling & $3.2 !$ & $\div$ & & & $\therefore$ & & $:$ & - & $\therefore$ & 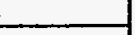 \\
\hline Flow Obsonvation & & & & & & & & 1 & 1 & \\
\hline & ZONE1 12 & ZONE2 : & ZONE3 & ZONE4 & ZONE5 12 & ZONE6 & ZONET I & ZONE8 & ZONE9 II & ZONE10 \\
\hline Heat Input-Walls(net) & 577 & 577 & 577 & 57 & $57 \pi$ & $57 i$ & 577 & $5 \pi$ & 577 & 577 \\
\hline Head Loss-Watts & 9.41 & 22 & 2.9 & 3 & $3 \mid$ & $22 !$ & 1.5 & $2.2 i$ & 2.71 & 15.9 \\
\hline Hoat Flox-EtuhHinte & 10002 & 10002 & 10002 & 10002 & 100021 & 10002 & 10002 & 100021 & 10002 & 10002 \\
\hline Wall Temp. Bł- & 158.91 & 160.11 & 759.2 & 160.51 & 161.51 & 167.3! & 161.5! & 1601 & 158.9 & 159.1 \\
\hline Wall Temp. 14F & 159.51 & 158.9 & 160.1 & 160.7 & 161.6 & 161.4 & 161.71 & 161.11 & 159.9 & 162.7 \\
\hline Wal Temp. $15-F$ & 159.91 & 1581 & 161.4 & 161 & 10.31 & 160.8 & 161.1 & 159.81 & 158.4 & 158.1 \\
\hline Wall Tomp. $11 F$ & 147.11 & 159.31 & 157.4 & 159.51 & 159.8 & 181.1 & 16241 & $1 \times 0.61$ & 157.8 & 157.6 \\
\hline Wall Tomp. 12f & 160.31 & 159.21 & 158.4 & 1601 & 159.91 & 161.2! & $1622 !$ & 161.71 & 158.4 & 158.8 \\
\hline In:truam Temp.f & 147.91 & $148.3 i$ & 149.6 & 1511 & 150.41 & 149.81 & $150.9 !$ & 150.11 & 149.7 & 150.5 \\
\hline Avg. Wall Tomp, $F$ & 159.71 & 159.41 & 158.8 & 160.41 & 160.41 & 161.21 & 161.81 & 160.61 & 158.7 & 159.3 \\
\hline Avg. WallDT.f & $11.3 !$ & 10.61 & 3.8 & 9: & $9.5 i$ & $10.9 !$ & 10.41 & $10.1 !$ & $8.5 i$ & 8.3 \\
\hline Romainino subcooff & 0.2 & & & & & & & & & \\
\hline Ext Quality & 0 & 0.03 & 0.05 & 0.08 & 0.111 & 0.141 & 0.17 & 0.2 & 0.28 & 0.26 \\
\hline EXP.HEturArne्यF & 885.3 & $941.5 i$ & 1133.6 & 1114.7 & 1047.5 & 917.8 & 959.11 & $991.5 !$ & 11728 & 1203.9 \\
\hline COMPUTEDH & & & & & & & & & & \\
\hline Jallouk & 970.7 & 949.3 & 921.8 & 970.7 & 8931 & $871.1 !$ & 83.81 & $850.6 !$ & 853.9 & \\
\hline Szadyl(Cudo tubo) & 1441.2 & 1446.2 & 1459.4 & 1475.41 & 1472.8 & 1470.3 & 1482.9 & 1479.11 & 1478.9 & 1489.1 \\
\hline Szadyz & 12127 & 1246.61 & 1256.9 & 1270.11 & 1264.9 & 1259.81 & $12 \% .11$ & $1235.1]$ & 1236.5 & 1247.6 \\
\hline (AUQ) & 102884 & 1020011 & $1000 \approx 3$ & 983181 & 948111 & 91333 & 891541 & 85521 & 82268 & 79800 \\
\hline (F) & 1.005 & 1.3591 & 1.725 & 1.975 & 2.194 & 2.3881 & 2544 & 27001 & 2.859 & 2.982 \\
\hline (HDO) & 211 & 285.41 & 362.8 & 416.11 & 461.91 & 50231 & 535.9 & 570 & 601.31 & 627.9 \\
\hline$(\mathrm{HBO} / \mathrm{L})$ & 1439.7 & 1442.51 & 1451.9 & 1464.31 & 1457.51 & 1450.51 & 1459.21 & 1450.3 & 1445 & 1450.9 \\
\hline & & & & 1 & & & $i$ & i & $i$ & \\
\hline & ZONE2 & ZONEA & ¿ZONE6 & ZONE8 & ZONE10 : & & $i$ & $\vdots$ & & \\
\hline DP liquid Temp.F & 75.5 & & & 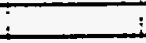 & 1 & 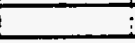 & : & & & \\
\hline DP Líquid Density lbmpit3 & $93.64 i$ & & & $\therefore$ & & & & & $\div$ & \\
\hline Elovation-h & & 4. & & $8:$ & 9.8751 & & & & & \\
\hline Mersured DPAsid & 0.0141 & 0.834 & 7.34 & 2.0021 & 2.252 & & & & & \\
\hline Zoro Cortectons-psid & 0.04 & -0.015 & -0.006 & -0.0361 & $-0.02 !$ & & $\div$ & & & \\
\hline Comected DPF sid & -0.054 & 0.849 & 7.346 & 2.0581 & 2.272 & & & & & \\
\hline Actual DPA & 2.38 & 3.08 & 4.5 & 5.53 & 7.31 & & & & I & i \\
\hline Mesured TP Psig & 1061 & & E & $i$ & 0.3 & & 1 & i & $\vdots$ & \\
\hline Mensured Overalth & 186 & & i & $i$ & : & 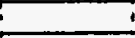 & $\therefore$ & $!$ & i & \\
\hline & 1 & & $i$ & 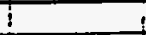 & & 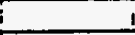 & 1 & i & 1 & \\
\hline Computed DPA & 1.96 & 3.56 & 5.06 & 6.52 & 7.9 & & $i$ & & 1 & \\
\hline Friction-th & 0.09 & 0.26 & 0.51 & 0.811 & 1.151 & & $i$ & & 1 & \\
\hline Acceloration-fl & 0.09 & 0.17 & 0.24 & 0.311 & 0.39 & & & 1 & & \\
\hline Elevation-H & 1.79 & 3.13 & 4.31 & 5.41 & $6.36 !$ & & & & $\vdots$ & \\
\hline & & & & & & & & & 1 & \\
\hline
\end{tabular}




\begin{tabular}{|c|c|c|c|c|c|c|c|c|c|c|}
\hline Runk & 929. & & & & & & & & & \\
\hline Test Fiud & C4F10 & & & & & & & & & \\
\hline Date: & $11 / 1093$ & & & & & & & & & \\
\hline Time: & 11:17: & & & & & & & & & \\
\hline Note & Analytical c & dt is used & & & & & & & & \\
\hline Flow Area-12 & 0.003361 & & & & & & & & & \\
\hline Heat Fow Area-fl2 & 0.19695 & & & & & & & & & \\
\hline Sal. Pressure Pig & 105.1 & & & & & & & & & \\
\hline Sat. Temperature F(iniel) & $150 . \overline{4}$ & & & & & & & & & \\
\hline La. Densty-1bmins & $82.13 !$ & $87.56 !$ & $81.94 !$ & $81.7 \overline{4}$ & $81.87 !$ & $81 . \overline{921}$ & 81.741 & 81.941 & 87.98 & 81.79 \\
\hline Latent Hoadentom & 29.781 & 29.631 & $29.62 !$ & $29.47 !$ & 29.571 & 29.611 & 29.471 & 29.62 & 29.65 & 29.51 \\
\hline Specitic Heatotunibmif & 0.2851 & 0.2851 & 0.2851 & $0.286 !$ & $0 . \overline{2851}$ & 0.2851 & 0.2861 & 0.285 & 0.285 & 0.286 \\
\hline Liq. Visoositylom/lithr & 0.4361 & $0 . \overline{432 !}$ & 0.4321 & $0.42 \overline{81}$ & 0.4311 & $0.432 i$ & 0.4281 & 0.4321 & 0.430 & 0.429 \\
\hline Liq. Conductivity-Btuhr-ffF & $0.0252 !$ & 0.0251 & 0.02511 & $0.025 i$ & 0.0251 & 0.02591 & 0.0251 & 0.02511 & 0.02511 & 0.025 \\
\hline Liq. Prandt Nó. & $4.92 i$ & $4.9 \overline{1}$ & 4.911 & $4.9:$ & $4.91 i$ & 4.911 & $4.9 !$ & 4.91. & 4.92 & 4.91 \\
\hline Inlet Fow Rate-GPM & 2.6 & & & & & & & & & \\
\hline Outlet Liq. Flow Rate-GPM & 0.7 & & & & & & & & & \\
\hline Outiet Vap. Fow Ralo-GPM & 1.8 & & & & & & & & & \\
\hline Inlet Temperature-F & 147.8 & & & & & & & & & \\
\hline Outtet Liq. Temperaturer & 150.4: & & & & & & & & & \\
\hline Outlet Vap. Temperaturef & $145.7^{T}$ & & & & & & & & & \\
\hline Water Flow Rato-GPM & & & & & & & & & & \\
\hline Water inlet Temp.F & 91.5 & & & & & & $i$ & & & $:$ \\
\hline Water outiol Temp.F & $113 . \overline{3}$ & & & & & & & & & \\
\hline Inlet Gibmihint2 & 5148301 & & Temp & $14 \overline{4 i}$ & Density & $82 . \overline{97} i$ & 7 & ? & 7 & $t$ \\
\hline Subcoolingf & $2.6:$ & & 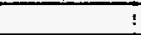 &. & 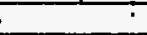 & & & & & T. \\
\hline Flow Observation & & & & : & & & $\vdots$ & & & $!$ \\
\hline & ZONE1: & ZONE2 & ZONE3 & ZONE4 & ZONE5 & ZONE6 & ZONE & ZONEB & ZONE9 : & IZONE10 \\
\hline Heat input-Watts(net) & 77.2 & 577.9 & 576.7 & 573.8 & 573.9 & 571.4 & 576 & 576.2 & 575.1 & $11 \quad 576.7$ \\
\hline Hoat Loss-Watts & 9.7 & 2.2 & 31 & 3.11 & 3.1 & $2.3 i$ & $1.5 i$ & 2.3 & 2.91 & 16.5 \\
\hline Hoat Flox-Bu/Hriti2 & 133681 & 10018 & 9997 & 99461 & 99481 & $9905 \mathrm{i}$ & 9985 & 99881 & 9969 & 9997 \\
\hline Wall Temp. अ & T58.6! & $159.9 !$ & 159.11 & 160.3 & t6ा1 & $161.3 !$ & 161.31 & 159.9 & 359.1 & 159.2 \\
\hline Wall Temp. X4F & 159.51 & 758.61 & 160.1 & 160.51 & 161.2 & 167.51 & 161.7 & 160.8 & 759.7 & 162.4 \\
\hline Wall Temp. $5 F$ & 159.91 & $157.9^{1}$ & 161.41 & $160.6 i$ & 1601 & 160.91 & 160.91 & 159.71 & 758.6 & 157.9 \\
\hline Wall Temp. 1F & 147.81 & 1591 & 157.4 & $159.2 !$ & 159.4 & 160.81 & 16211 & 180.51 & 157.5 & 157 \\
\hline Wall Temp. & 160.11 & $159.2 \mathrm{i}$ & 158.51 & 159.71 & 159.41 & 161.11 & 162.11 & 161.31 & 158.3 & 158.3 \\
\hline Instroam Tomp.F & $148.9 \mathrm{i}$ & $149.8 \mathrm{i}$ & $\overline{149.81}$ & ${ }^{15 T i}$ & $15 \overline{0.31}$ & 1501 & $-151 T$ & $749.9 i$ & 149.7 & 150.7 \\
\hline Avg. Wall Temp.F & $159.5 i$ & $159.2 i$ & 158.8 & 160.21 & 1601 & $161.2:$ & 161.61 & 160.41 & 158.61 & 159 \\
\hline Avvo Waäl DT.F & $10^{\circ}$ & 8.9 & 8.41 & 8.7 & 9.31 & 10.7 & 10.21 & $10.1 !$ & 8.5 & 7.8 \\
\hline Remaining subcoolf & $-2.8^{\prime}$ & & & & & & & & & \\
\hline Exat Quality & 0.031 & 0.061 & 0.11 & $0.14 i$ & 0.181 & $0.22 !$ & $0.26 \mathrm{i}$ & $0 . \overline{9}$ & 0.33 & 0.37 \\
\hline EXP.HBtuHr/h2/F & 13341 & $1122.8:$ & 1186.8 & 1143.2 & 1075.41 & $925.5 i$ & 981.91 & 9901 & 1174.3 & 1280.3 \\
\hline COMPUTEDH & & & & & & & & & & \\
\hline Jallouk & 1184 & 951.7! & 937.2 & 924.61 & 907.71 & 893.4 & 894.41 & $872.5 !$ & 866.4 & 868.4 \\
\hline Szzadyl(Cudo tube) & $167 \overline{61}$ & $1460.9 !$ & 14621 & 1470.2 & $1466.1 !$ & 146251 & $1479.1 !$ & 1472 & 1471.4 & 1484.4 \\
\hline seady2 & 1483.21 & 1261.11 & 12601 & 1265.41 & 1259.31 & 12531 & 1268.91 & 1259.81 & 1257 & 1240.3 \\
\hline (RUQ) & 76273 & 74327 & 71390 & 65047 & 65546 & 624961 & 60012 & $56463 !$ & 53378 & 50817 \\
\hline (F) & 1.3811 & $1.7 n$ & 2.1011 & 2.3511 & 2.5831 & 2.7881 & 2.957 & $3 . \overline{1461}$ & 3.312 & 3.446 \\
\hline (साLQ) & 229.3 & 25.41 & 349.3 & 391.41 & 429.61 & 463.61 & 492.31 & 523.11 & 550.6 & 573.6 \\
\hline (HBOIL) & 1672.11 & $1456.9 \mathrm{i}$ & 1455.4 & 1460.81 & 1453.71 & 1446.81 & 1460.61 & 1449.61 & 14452 & 1455.3 \\
\hline & & & & & & & : & & & \\
\hline & ZONE2 & ZONE4 & ZONE6 & ZONE8 & ZONE & & & & & \\
\hline DP liquid Temp.F & 75.81 & & & & & & & & & $i$ \\
\hline DP Liauid Densitylbm/t33 & 33.61 & & & & & & & & & \\
\hline Elevation-ft & & & $6 !$ & 8 & 9.875 & & & & & \\
\hline Measured DPPsid & 0.0481 & $1.075 !$ & 9.7731 & $2.507^{\top}$ & 2.841 & & & & & \\
\hline Zero Comections-psid & 0.041 & -0.015 & -0.0051 & -0.0361 & -0.021 & & & & & \\
\hline Corrected DPP sid & $0.008 !$ & 1.091 & 1.779 & 2.5431 & 2.861 & & & i & & \\
\hline Actual DPA & $2.27 !$ & 2.681 & 3.84 & 4.681 & 6.281 & & & $i$ & & 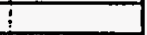 \\
\hline Measured TPPsig & 106.11 & ! & 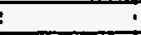 & 5 & 0.31 & 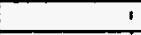 & ' & $\therefore$ & & \\
\hline Mensuted Overalta & 186.261 & $\therefore$ & 1 & $!$ & & i & $i$ & $i$ & 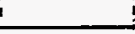 & $!$ \\
\hline & $\therefore$ & & & & & 1 & 1 & 1 & & \\
\hline Computed DPA & 9.67 & 2381 & 4.15 & 5.341 & 6.451 & & $\mathrm{i}$ & I & & \\
\hline Friction-t & 0.08 & 0.231 & 0.43 & 0.681 & 0.961 & & & & & \\
\hline Accoleration-ft & 0.08 & $0.73 i$ & 0.19 & 0.251 & 0.321 & & $\dot{5}$ & & & \\
\hline Elovation-H & 1.441 & 2.561 & 3.531 & $4.47 i$ & $5.17 i$ & & & & & \\
\hline
\end{tabular}




\begin{tabular}{|c|c|c|c|c|c|c|c|c|c|c|}
\hline Runt: & $930 i$ & & & & & & & & & \\
\hline Tost Fiuid & C4F10 & & & & & & & & & \\
\hline Date: & - i11/10i93i & & & & &. & & & & \\
\hline Time: & $15: 24 !$ & & & & & & & & & \\
\hline Nole & Anabrucal d & I! is used & & & & & & & & \\
\hline Flow Areatt2 & $0.00336 i$ & & & & & & & & & \\
\hline Heat Flow Area-li & 0.19695 & & & & & & & & & \\
\hline Sal. Prossure Psig & 106.5 & & & & & & & & & \\
\hline Sal. Temperaturof(inlel) & 150.6 & & & & & & & & & \\
\hline Ua. Density & 81.98 & 81.781 & $81.85 !$ & 81.611 & $81.74 !$ & $81.76 !$ & 81.621 & 81.781 & $81.79 !$ & 81.68 \\
\hline Latent Hoar-Btunbm & 29.651 & $29.5 !$ & 29.551 & 29.381 & 29.47 & 29.491 & 29.39 & 29.51 & 29.51 & 29.43 \\
\hline Specific Hear Qtulbm/= & 0.285 & 0.2861 & 0.2851 & 0.2861 & 0.286 & 0.2861 & 0.2861 & 0.2861 & 0.286 & 0.286 \\
\hline Lig. Visoosity $/ \mathrm{bm} / \mathrm{ihr}$ & 0.4331 & 0.4291 & 0.43 & 0.426 & 0.4281 & 0.4281 & 0.4261 & $0 . \overline{4291}$ & 0.4291 & 0.427 \\
\hline Lig. Conducturty-Btump fiff & $0.0251 !$ & 0.0251 & 0.0251 & 0.02491 & $0.025:$ & 0.0251 & 0.02491 & $0.025 i$ & 0.0251 & 0.0249 \\
\hline Liq. Prand No. & 4.921 & 4.9 & 4.911 & 4.91 & 4.91 & $4.9 !$ & 4.91 & 4.91 & 4.911 & 4.9 \\
\hline Inlet flow Rato-GPM & 1.8 & & & & & & & & & $:$ \\
\hline Outlet Liq. Flow Raio-GPM & $0.4 i$ & & & & & & & & & \\
\hline Outlet Vap. Flow Rate-GPM & $1.3^{\circ}$ & & & & & & & & & \\
\hline Inlet Temperature $F$ & $148.5 !$ & & & & & & & & & \\
\hline outtel Lia. Temperatureff & $151.4 !$ & & & & & & & & & \\
\hline Outtel Vap. TemperaturefF & $\overline{146.7 !}$ & & & & & & & & & \\
\hline WaterFow Rato-GPM & -1 & & & & & $T$ & & & & \\
\hline Water inlot Temp.F & 91.91 & & & & & & & & & \\
\hline Wator outtot Temp.F & 113.1 & & 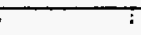 & 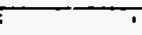 & & $\bar{r}$ & & & & T \\
\hline Intet Glb mihritz & 3569031 & & Temp & 140.61 & Density & 83.001 & 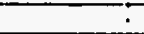 & $\cdot$ & & $t$ \\
\hline Subcoolingf & 2.11 & & & & & & & & & 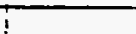 \\
\hline Flow observation & 1 & & & & & & & & & 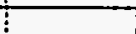 \\
\hline & ZONEI & ZONE2 . & ZONE3 : & ZONE4 & IZONE 5 I & IZONE6 & ZONE7 & ZONE8 12 & ZONE9 & ZONE10 \\
\hline Heal Input-Watts(nol) & 572.8 & 5772 & 574.91 & 571.5 & $573.5 i$ & 570.9 & $575.7 i$ & 574.4 & 572.8 & 5 \\
\hline HeadLoss-Watts & 10.21 & 2.4 & $3.2 !$ & 3.31 & 3.21 & $2.4 i$ & 1.6 & 2.41 & $3 !$ & 17.3 \\
\hline Hoal Flux-Btuhtrm? & 99201 & 10005 & 9966 & 90071 & 99411 & 98961 & 9979 & 9957 & 9280 & 9946 \\
\hline Wall Temp. "3F & 158.81 & $160.1 !$ & 158.81 & 160.5 & 161.41 & 161.81 & 761.71 & 160.4 & 159.51 & 159,3 \\
\hline Wall Tomp. "4F & 159.41 & 958.7 & 759.81 & 160.5 & 161.7 & बहा & 162 & 151.51 & 160.4 & 162.5 \\
\hline Wall Temp. 5 & 159.91 & 158 & 161.5 & 1611 & 160.41 & 161.31 & $161.5 !$ & $160.1 !$ & 159 & 1582 \\
\hline Wall Temp. MIF & 148.51 & 159 & 157.3 & 159.4 & 159.8 & 161.41 & 162.5 & 160.91 & 158.3 & 157.4 \\
\hline Wall Tomp. R2f & 160.21 & $159.2 !$ & 158.31 & 159.71 & 159.8 & 161.51 & 162.61 & 16्21 & 158.7 & 158.6 \\
\hline Instroem Temp.F & $1 \overline{49.71}$ & $15 \overline{0} . \overline{81}$ & 150.41 & 151.71 & 1511 & 150.81 & 151.61 & 150.81 & 150.7 & 151.3 \\
\hline Avg. Wall Tomp.f & 159.61 & 159.31 & 158.61 & 160.41 & 160.41 & 161.61 & 162.11 & 1611 & 15921 & 159.2 \\
\hline Avg. WalloT.f & $9.4 i$ & 8. & $7.7^{\circ}$ & 8.21 & 9 & 10.31 & 10 & 9.7 & 81 & 7.5 \\
\hline Romaining Subcooff & $3.6 i$ & & & & & & & & & \\
\hline Exit Quality & 0.031 & 0.001 & 0.15 & $0.2 !$ & 0.261 & $0.3 i j$ & 0.37 & $0.42 !$ & 0.48 & 0.53 \\
\hline EXP. HBtuHrH2/F & 1053.31 & 12511 & 1294.31 & $1207.8 i$ & 1107.6 & 95291 & 997 & $1023.3 i$ & 1236.3 & 1335 \\
\hline COMPUTEDH & & & & & & & & & & \\
\hline Jallouk & $1003.2 !$ & 985.2. & 950 & 95261 & $945.7 !$ & $931.2 !$ & 930.91 & $916.8 !$ & 908.1 & 907.1 \\
\hline Szadyl(Cudo tube) & 1451.7! & 1467.8! & 1462.51 & 9471.11 & 1468.61 & 7465.8! & 1479.4 & 1472.2! & 1471 & 1479.3 \\
\hline Szady2 & 1253.11 & 1269.2 & 1252.1 & 1208.41 & 1265 & 125021 & 1273.6 & 1264.51 & 1261.41 & 1268.4 \\
\hline (RLA) & $53011 !$ & 510541 & 47875 & 453691 & 420881 & 39037 & 36233 & 32964 & 299201 & 27048 \\
\hline (F) & 1.443 & 1.9221 & 2.328 & 2.6331 & 2.916 & 3.164 & 3.377 & 3.6041 & 3.806 & 3.982 \\
\hline (मயब & 178.91 & 238.7 & 288.8 & 327.21 & 362.1 & 392.81 & $-\overline{418.61}$ & 447.4 & 472.41 & 494.7 \\
\hline (HBOLL) & 1450.81 & $1465.7 i$ & 1458.71 & $1465.7 i$ & 1461.21 & 1456.41 & 1468.11 & 1458.31 & 1454.6 & 1460.6 \\
\hline & 1 & & $i$ & & & . & & . & & 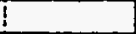 \\
\hline & :ZONE2 :2 & ZONE4 & ZONE 6 & ZONE 8 & ZONE10; & & & & & \\
\hline$\overline{\mathrm{DP}}$ liauid Temp.f & 76.51 & & - & & $=-\frac{1}{1}$ & & & & & \\
\hline DP Liquid Density & 93.5 & & & & & & & & & \\
\hline Elevation-t & & & $6 !$ & 8. & $9.875 !$ & & & & & \\
\hline Measured DP Psid & $0.083 !$ & 1.32ता & 2.0861 & 2.997 & 3.452 & & & & & \\
\hline Zero Corrections-psid & 0.041 & $-0.015 !$ & -0.0061 & -0.0351 & -0.02 & & & & & \\
\hline Comected DPFsid & $0.043 i$ & $1.336 !$ & $2.092 !$ & 3.0331 & $3.472 !$ & $!$ & & & & \\
\hline Actual DPA & 2.21 & $2.22 !$ & 3.18 & $3.8 \pi$ & $5.18 !$ & $T$ & & & & \\
\hline Moasured TPPsig & 106.51 & $\div$ & I & 1 & 0.31 & - & ¿ & 1 & & \\
\hline Measured Overalitt & 187.231 & 7 & 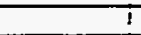 & $\bar{i}$ & $T$ & 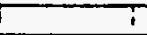 & : & $T$ & & $t$ \\
\hline & 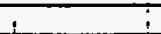 & & & & $!$ & $i$ & $T$ & $i$ & & 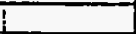 \\
\hline Computed DPA & $1.44 !$ & 2541 & 3.511 & 4.421 & 5.241 & . & $i$ & $\bar{i}$ & & 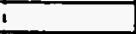 \\
\hline Frictionft & 0.05 & 0.15 & 0.281 & 0.451 & 0.641 & $!$ & 7 & 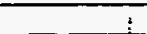 & & $i$ \\
\hline Accolerationtt & 0.051 & 0.081 & 0.12 & 0.171 & 0.231 & $=$ & $\bar{\vdots}$ & & & \\
\hline Elovation-t & 1.35 & 2.311 & 3.11 & 3.791 & 4.361 & & I & F. & & \\
\hline & & & & & & & $i$ & & & \\
\hline
\end{tabular}




\begin{tabular}{|c|c|c|c|c|c|c|c|c|c|c|}
\hline Runf & 331 & & & & & & & & & \\
\hline Test Flüid & CAF & & & & & & & & & \\
\hline Date: & $11710193^{\circ}$ & & & & & & & & & \\
\hline Time: & $15: 24$ & & & & & & & & & \\
\hline Noto & Anatyocato & tit is used & & & & . & & & & \\
\hline Flow Areatit & 0.00336 & & & & & & & & & \\
\hline Hox Flow Aveathl & 0.19695 & & & & & & & & & \\
\hline Sad. ProssuroPsig & 104.8 & & & & & & & & & \\
\hline Sat. Temperature-F(inlet) & 149.61 & & & & & & & & & \\
\hline Lig. Density-lbmitt3 & $82.05 !$ & 81.92 & $81 . \overline{981}$ & 81.76 & $81.85 !$ & 81.911 & 81.76: & $81.89:$ & 81.871 & 81.76 \\
\hline Latent Heat-Btulibm & 29.7 & 29.61 & 29.65 & 29.49 & $29.55 !$ & 29.59 & $29.49 !$ & 29.58 & 29.571 & 29.49 \\
\hline Specite Heatotunbm/t & $0.285 i$ & $0.285:$ & $0.285 i$ & $0.286:$ & 0.2851 & $0.285:$ & 0.286 & $0.285:$ & 0.2851 & 0.286 \\
\hline Lia. Viscosity-lbm/thr & $0.434 !$ & $0.432^{\prime}$ & 0.433 & $0.428:$ & 0.431 & $0.431^{\circ}$ & 0.428 & $0.431^{\circ}$ & $0.431 !$ & 0.428 \\
\hline Lia. Conductivity Btum & 0.0253 & 0.0259 & $0.0251^{1}$ & $0.025:$ & $0.025^{\circ}$ & 0.0251 & 0.025 & 0.025 & $0.025:$ & 0.025 \\
\hline Liq. Prandt No. & 4.92 & 4.91 & 4.92 & 4.91 & 4.91 ? & $4.91 !$ & $4.9 !$ & $4.91^{\circ}$ & 4.911 & 4.9 \\
\hline Inlet Flow Rate-GPM & $\frac{1}{1}$ & & & & & & & & & \\
\hline Outurtua. Fiow Rato-GPM & $0.4^{\circ}$ & & & & & & & & & \\
\hline Outlet Vao. Flow Rato-GPM & 0.8 & & & & & & & & & \\
\hline inlet Temperaturef & 147.1 & & & & & & & & & \\
\hline Outtot Liq. TamperaturoF & $150.9 !$ & & & & & . & & & & \\
\hline Outuet Vap. Temperaturef & 147.1 & & & & & & & & & \\
\hline Water Flow Rato-GPM & & & & & & & & & & \\
\hline Water Inlet Temp.f & 93.8 & & & & & & & & & \\
\hline Water outlet Temp.f & $120.1 !$ & & & & & & & & & \\
\hline Inlet G-bm/hr/th2 & $199999 !$ & & Temp & $136.7: \mathrm{L}$ & Density & $83.81 !$ & & & & \\
\hline Subcooling f & $2.5 i$ & & & & & & & & & \\
\hline Flow Observation & & & & & & & & & & \\
\hline & ZONET & ZONE2 & ZONE3 & ZONE4 & ZONE5 & ZONE6 & ZONE7 & Z̄ONE8 & ZONE9 & ZONE1O \\
\hline Hoat Inpurt-Watts(net) & 5741 & $577.6^{1}$ & 573.61 & $580.5 t$ & 5761 & $573.9 !$ & $575.7 !$ & $574.5 i$ & $5 \longdiv { 6 9 . 6 7 }$ & 243.9 \\
\hline Hoat Loss-Watts & 91.41 & 2.61 & 3.51 & $3.7 !$ & 3.61 & $2.7 i$ & 1.81 & 2.7 & 3.51 & 18.6 \\
\hline Hoad Flux-BuU/Hrit2 & 99501 & 100121 & 9943 & $100 \approx 31$ & 93851 & 99481 & 99791 & 99591 & 98741 & 4228 \\
\hline Wall Tomp. $3 F$ & 1571 & $158.3 !$ & 156.81 & $158.7 i$ & 159.41 & 160.31 & 160.5 & $159.7 i$ & 159.51 & 156.3 \\
\hline Wall Temp. $4 \mathrm{~F}$ & 1581 & 156.91 & 1581 & 1591 & 159.61 & 160.61 & 160.81 & $160.7 !$ & 160.21 & 157.4 \\
\hline Wall Temp. $\$ 5 F$ & 158.51 & 156 & 159.5 & $158.8_{i}$ & $158.5 i$ & 159.6 & $159.8 ;$ & 159.11 & 158.11 & $155 . \overline{9}$ \\
\hline Wall Temp. \#1F & $147.1 !$ & 157.3 & 155.4 & $157.5:$ & 158.1 & 159.81 & 160.71 & 160.2 & $157.2 !$ & 155.4 \\
\hline Wall Temp. "2f & 158.8 & $157.4 !$ & $156.4 \mathrm{i}$ & $158 !$ & $158.1 !$ & $159.8 !$ & 160.81 & $160.9^{3}$ & 1581 & 155.7 \\
\hline Instream Temp.f & 149.31 & $150^{\circ}$ & $149.7 !$ & $150.9 !$ & $150.4 \Gamma$ & $150.1 !$ & $150.9 i$ & $150.2:$ & 150.31 & 150.9 \\
\hline Avg. Wall Temo.F & $158.1 !$ & 157.5 & $156.7^{1}$ & 158.5 & $158.6^{\prime}$ & 160.1 & 160.5 & 160.1 & 158.61 & 156.1 \\
\hline Avg.Wali DT $\bar{F}$ & 8.3: & 7 & $6.5 !$ & $7.1^{\circ}$ & $7.7 !$ & $9.5 !$ & $9.2 !$ & 9.51 & $7.9 !$ & 5 \\
\hline Romaning Subcooff & $-7.7 t$ & & & & & & & & & \\
\hline Ext Quality - & 0.07 & 0.17 & $0.27 !$ & 0.371 & 0.471 & 0.571 & 0.671 & $0.77 !$ & 0.861 & 0.91 \\
\hline EXP.HEtuntrmerF & 1195.11 & $1425.7 i$ & 1529.5 & 1408.31 & 1292.71 & 104.41 & 1088.41 & 1051.61 & 1257.21 & 837.4 \\
\hline COMPUTED H & 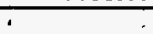 & & & $\because$ & : & 1 & & i & 1 & \\
\hline Jallouk & 1053.31 & 10181 & 997.51 & 9931 & 974.61 & 9527 & 963.91 & $9 \longdiv { 8 8 . 8 1 }$ & $77.8 !$ & 77.8 \\
\hline Szady I(Cudo tubo) & $1449.2 i$ & 1460.3 & 1453.31 & 1473.21 & $1463.8 i$ & $1459.3 i$ & 1469.6 & 1452.81 & 77.81 & 77.8 \\
\hline Szady2 & 1219.91 & 1262.9 & 1254.2 & 1275 & $1263.7 !$ & 1257.8 & $1267.5 i$ & $1259.4 !$ & 77.8 & 77.8 \\
\hline (RUQ) & 290081 & 26556 & 23510 & 20721 & $17620 !$ & 14576 & $11650 \mathrm{i}$ & 85901 & 8590 & 8590 \\
\hline (A) & 1.6331 & 2.37 ! & 2.9071 & $3.319 !$ & $3.707 !$ & 4.059 & 4.3631 & 4.697 & 4.6971 & 4.697 \\
\hline (संயठ) & $132 !$ & $184.9 !$ & 226.81 & 259.31 & 289.41 & $316.8 !$ & 340.81 & $3 \% 6.6 !$ & 366.61 & 366.6 \\
\hline (HBOIL) & $1448.8 !$ & T459.4 & $1451.4 !$ & 1470.5: & 14801 & 1454.3! & $1463.4 i$ & $1455.1^{\circ}$ & $7455.1 !$ & 1455.1 \\
\hline & ZONE2 : & ŻONE4 & ZONE6 & ZONE8 & ZONE10 & & & & & \\
\hline DP kquid Tomp.f & 76.41 & & & - & & & & & & \\
\hline DP Liquid Density $-1 \mathrm{bm} / \mathrm{t}$ 3 & 93.511 & $?$ & . & $\cdot$ & 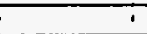 & $i$ & & & : & \\
\hline Elovaton-1 & 2. & 41 & $6 i$ & 81 & $9.875 i$ & T. & & 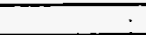 & & \\
\hline Measured DPFsid & 0.078 & $1.521 \mathrm{i}$ & 2.541 & 3.5391 & 4.1931 & . & & . & T & \\
\hline Zero Corrections-psid & $0 . \overline{041}$ & -0.0151 & -0.0061 & -0.0361 & -0.021 & 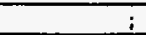 & & & & \\
\hline Contected DPPsid & 0.0381 & $1.636 \mathrm{i}$ & 2.5461 & 3.6351 & 4.213 & & & & & \\
\hline Actual DP-A & $2.22 !$ & $1.69 \mathrm{i}$ & 2.38 & $2.75 i$ & 3.871 & & & & 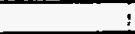 & \\
\hline Moesured TPPsig & 104.81 & . & . & 1 & 0.31 & 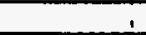 & 5 & & : & \\
\hline Measured Overallit & 184.061 & & & & & & & & & \\
\hline & & & & & & & & & & \\
\hline Computed DPA & 7.181 & $1.97 !$ & 2.ळ!! & 3.171 & 3.661 & & & & & \\
\hline Friction-t & 0.031 & 0.081 & 0.15 & 0.241 & 0.321 & i & & & & \\
\hline Accoloration-t & 0.021 & 0.051 & 0.081 & 0.121 & 0.181 & 1 & & & 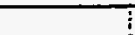 & \\
\hline Elovation-t & 7.131 & 1.851 & 2.391 & 2.891 & 3.181 & 1 & $\bar{l} 1$ & & 1 & \\
\hline
\end{tabular}




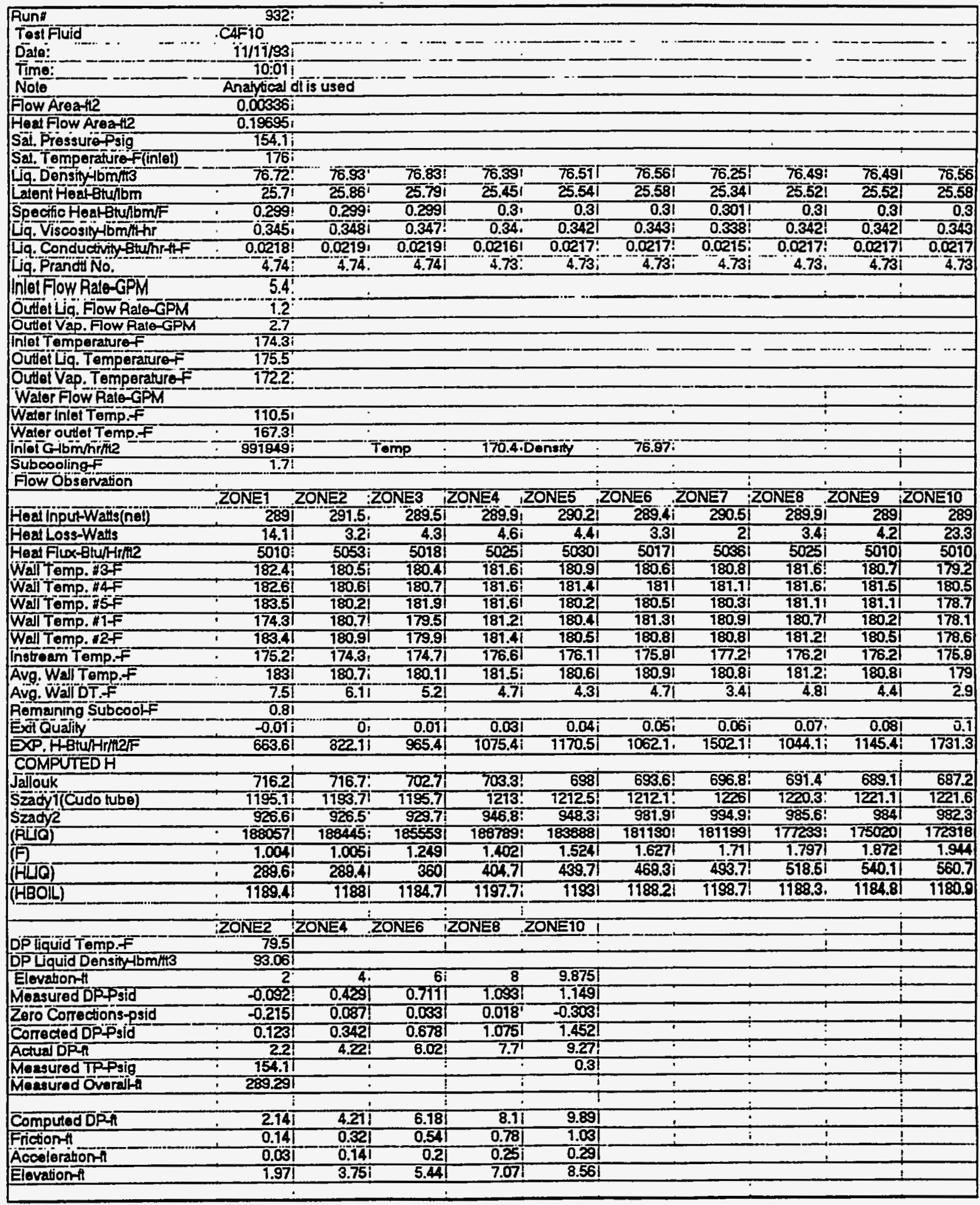




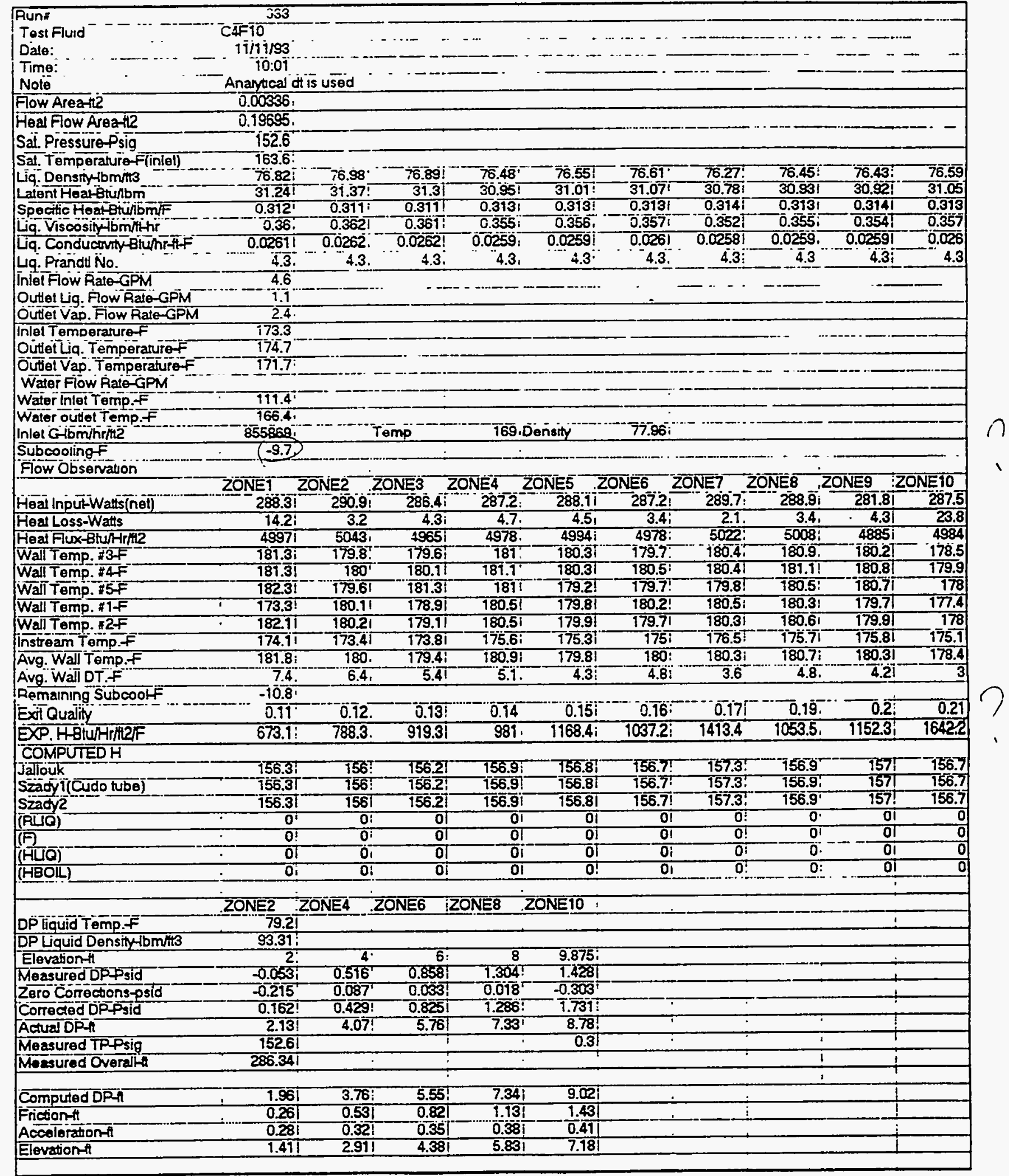




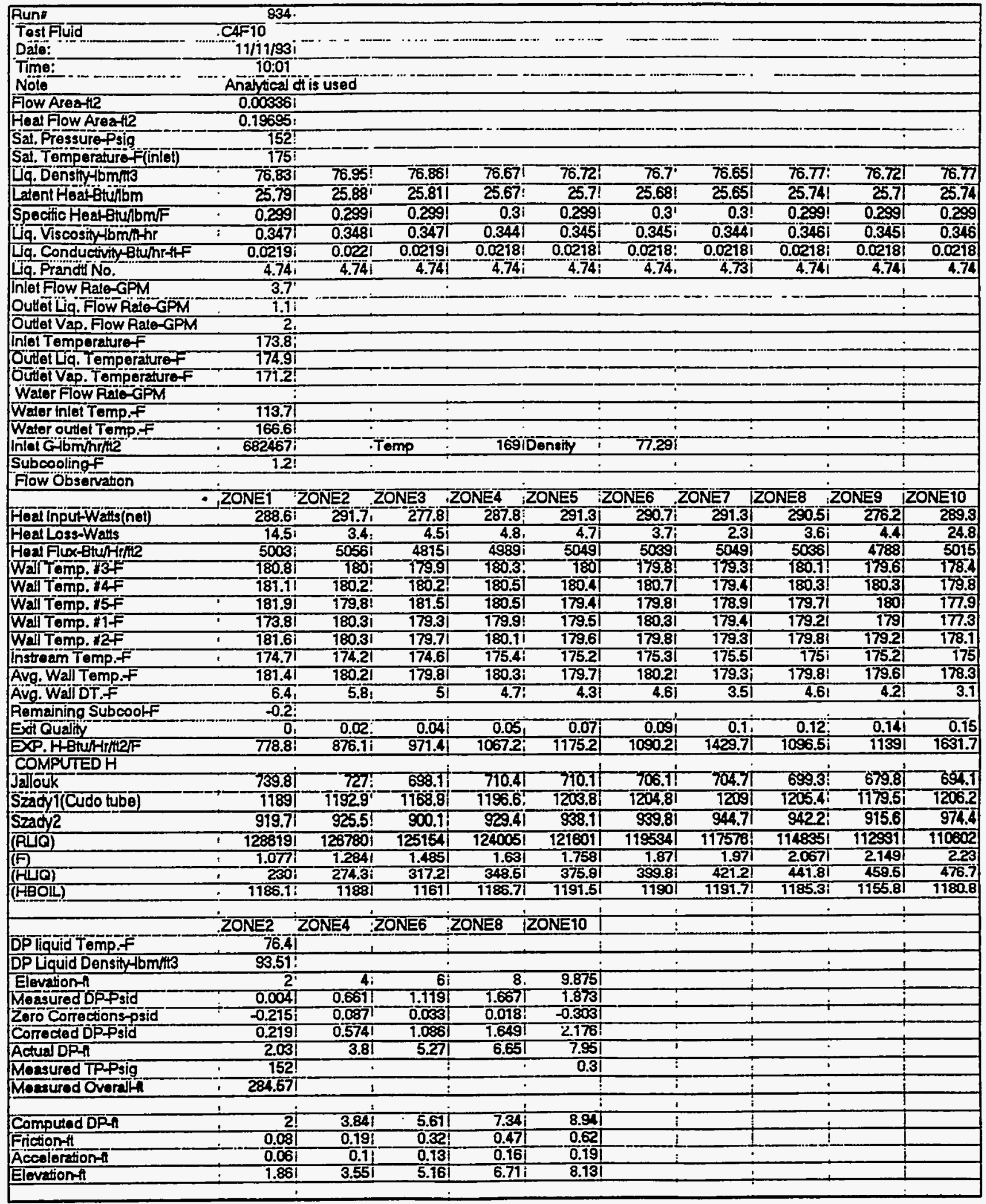




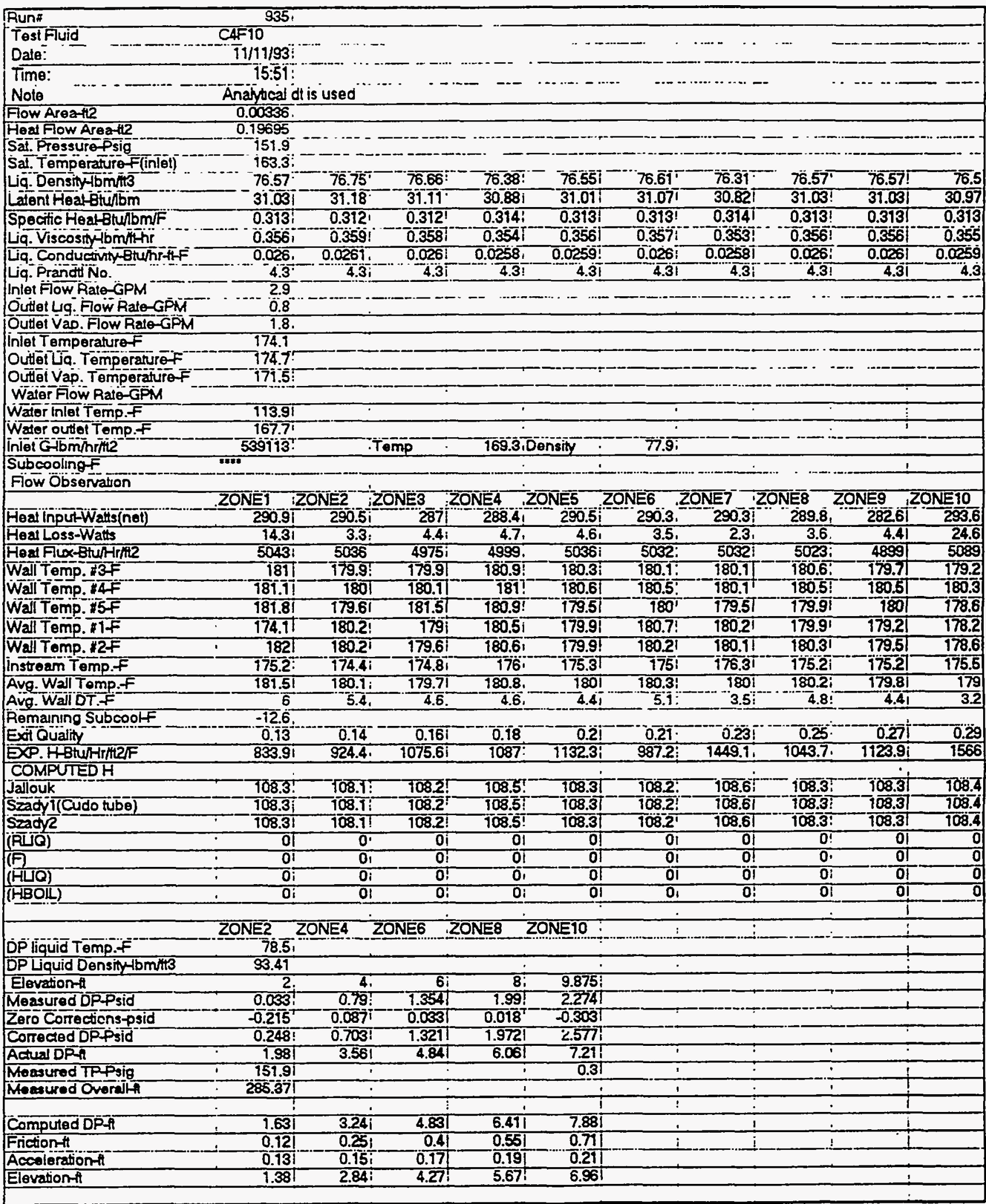




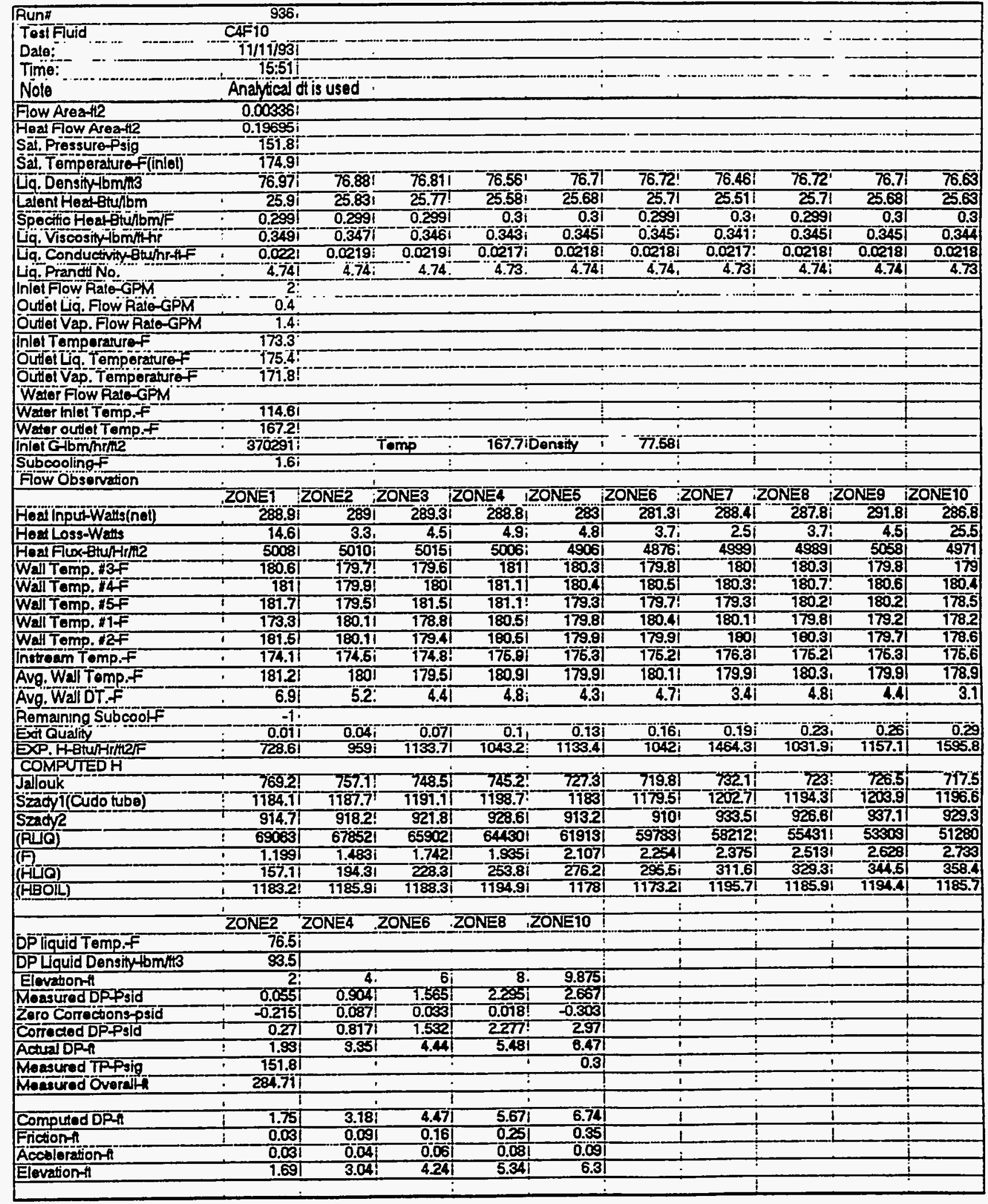




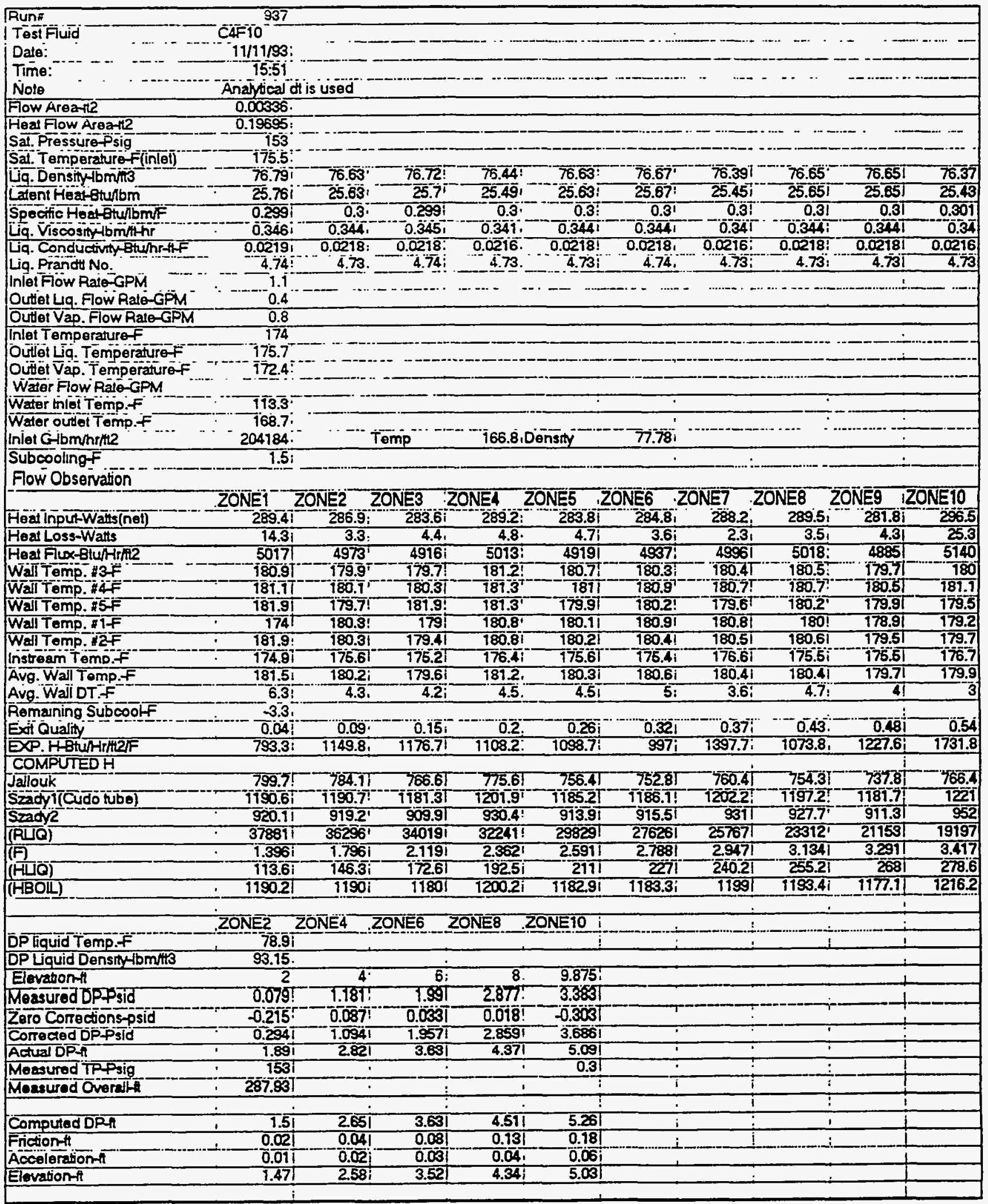




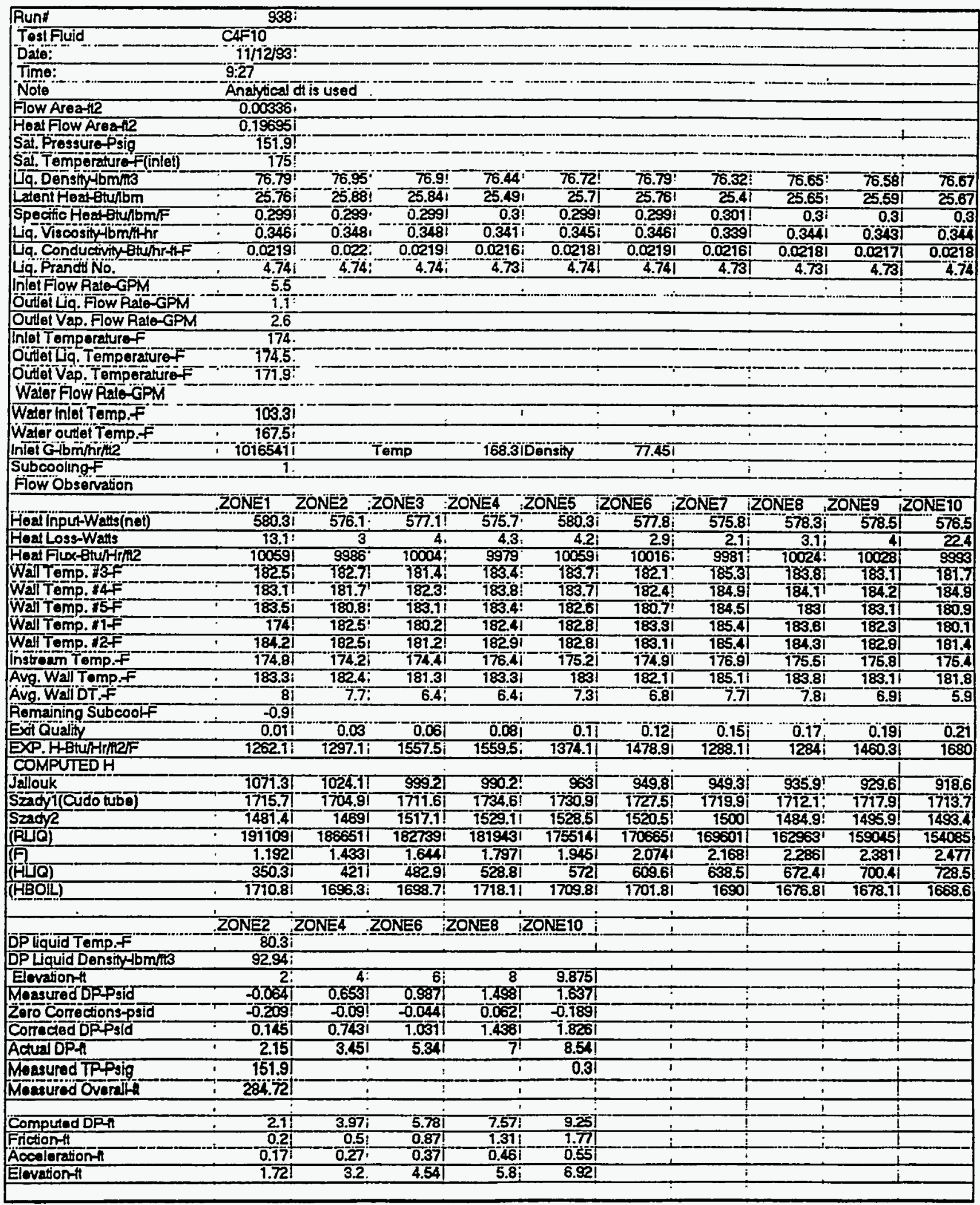




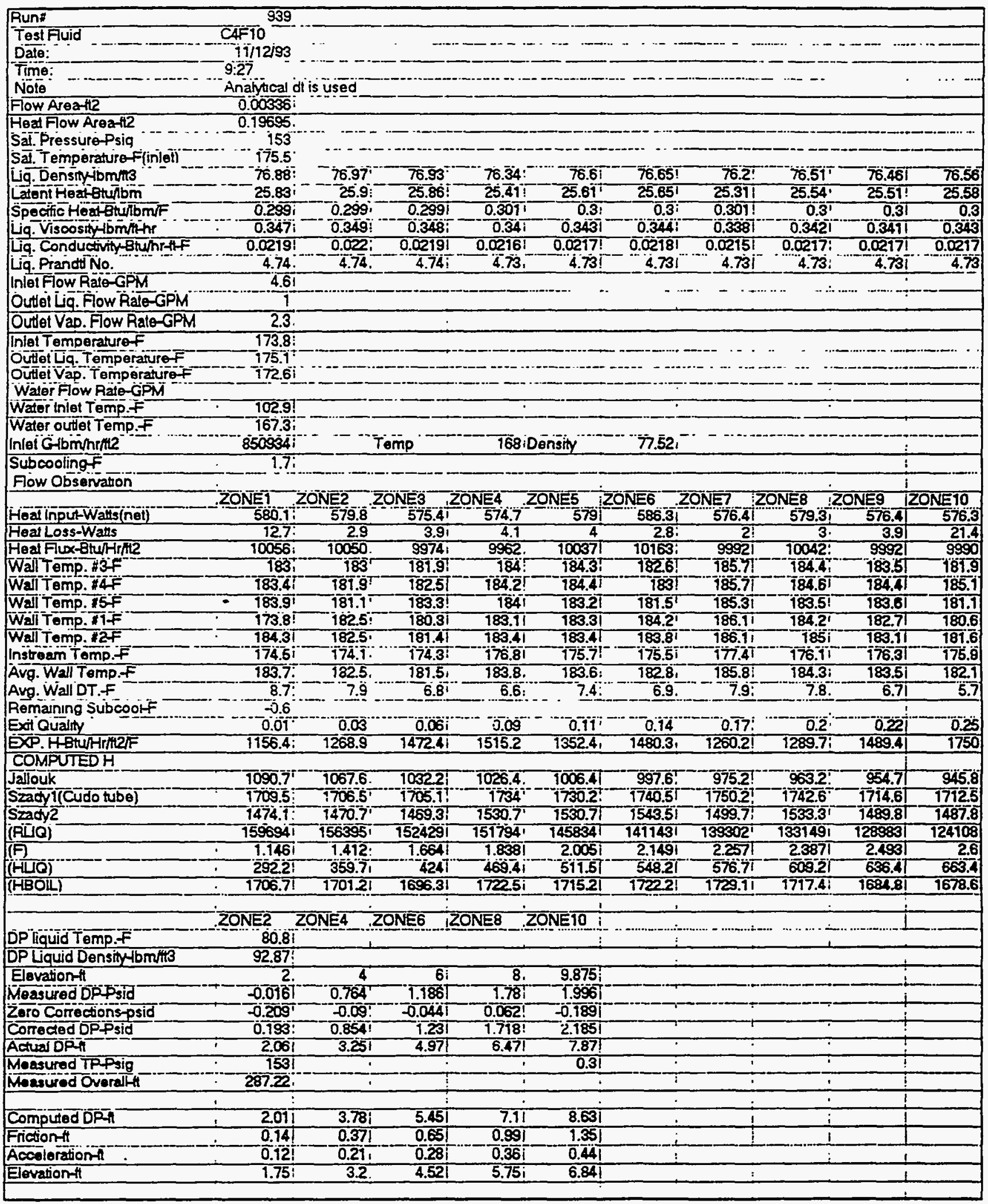




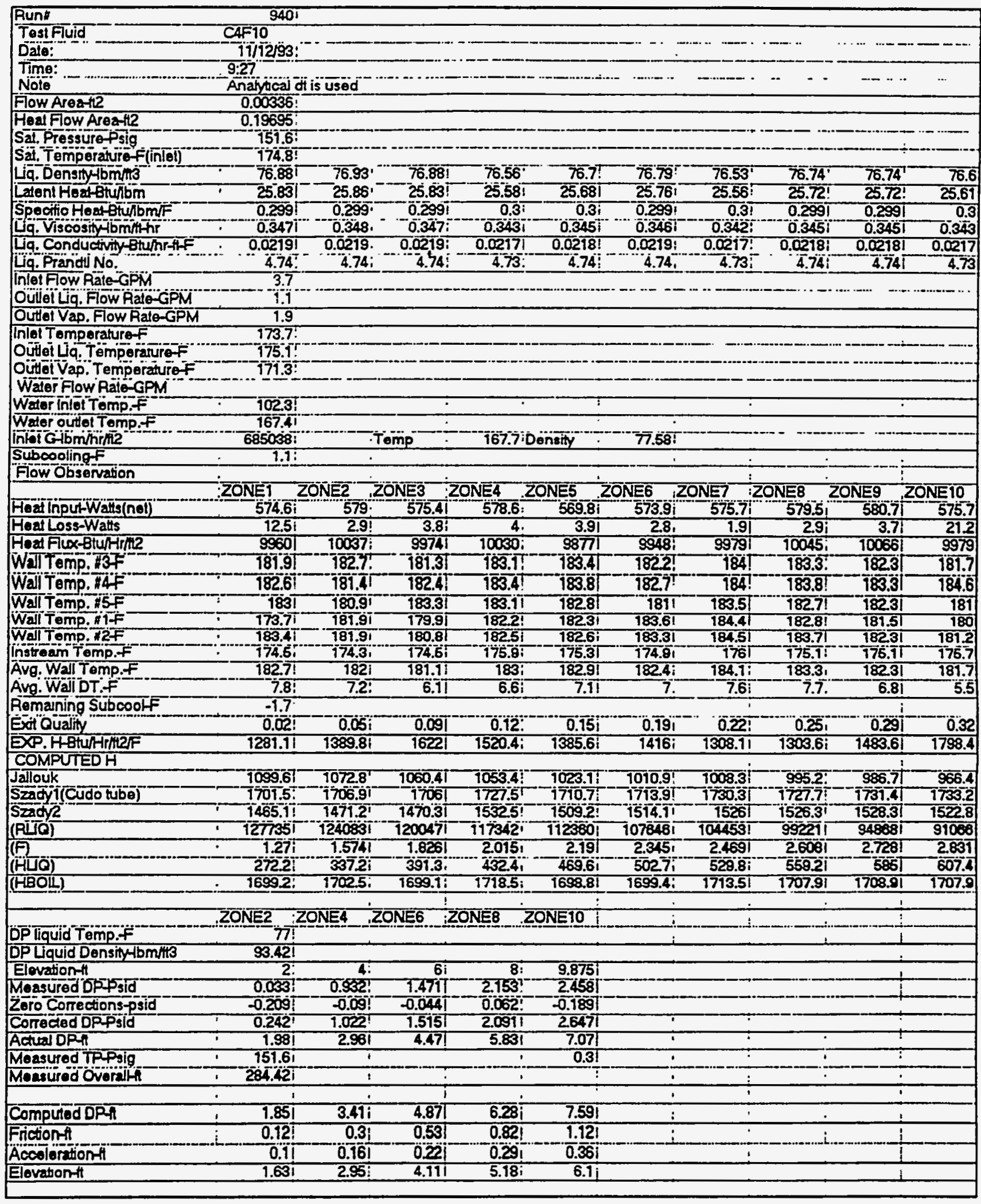




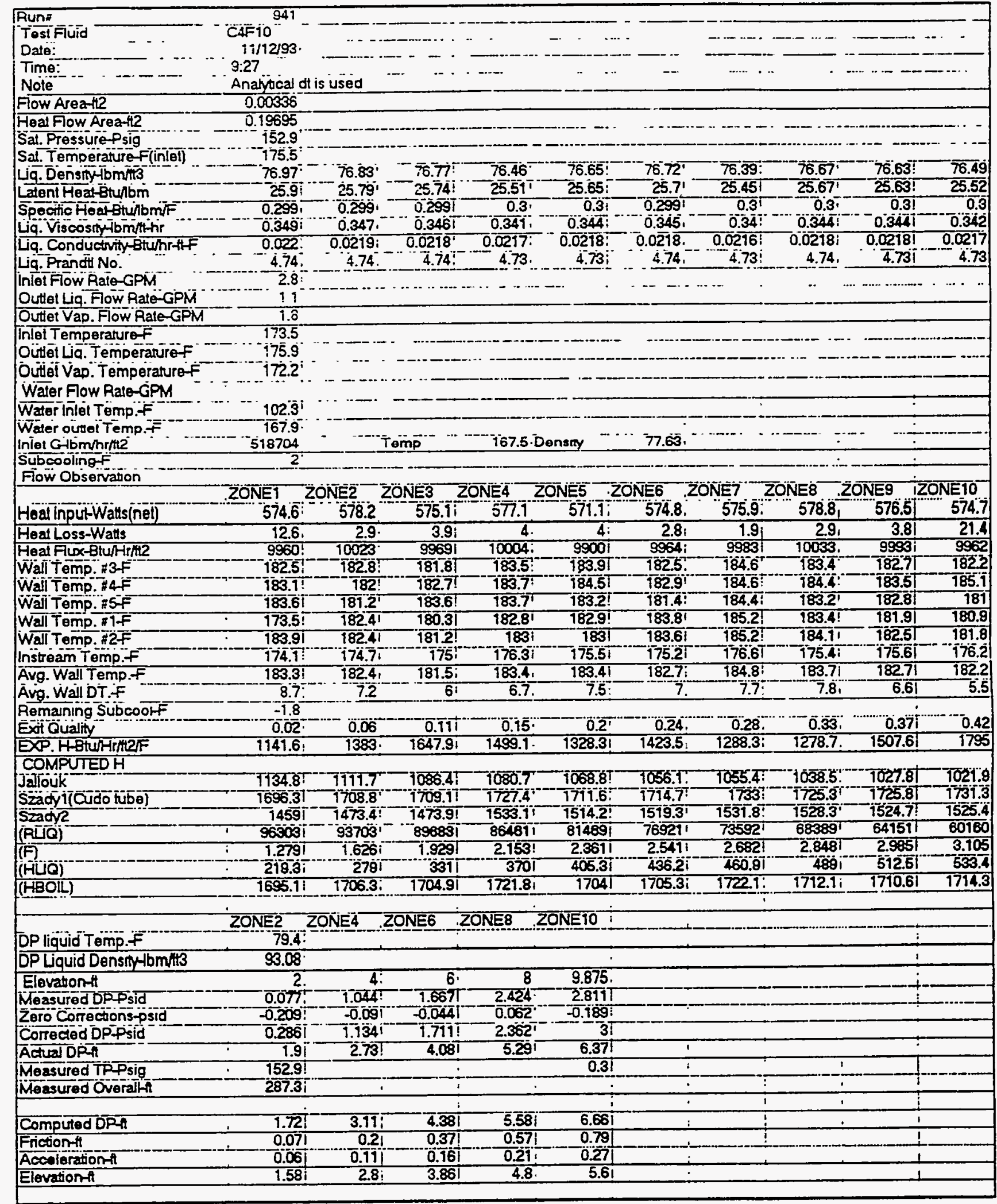




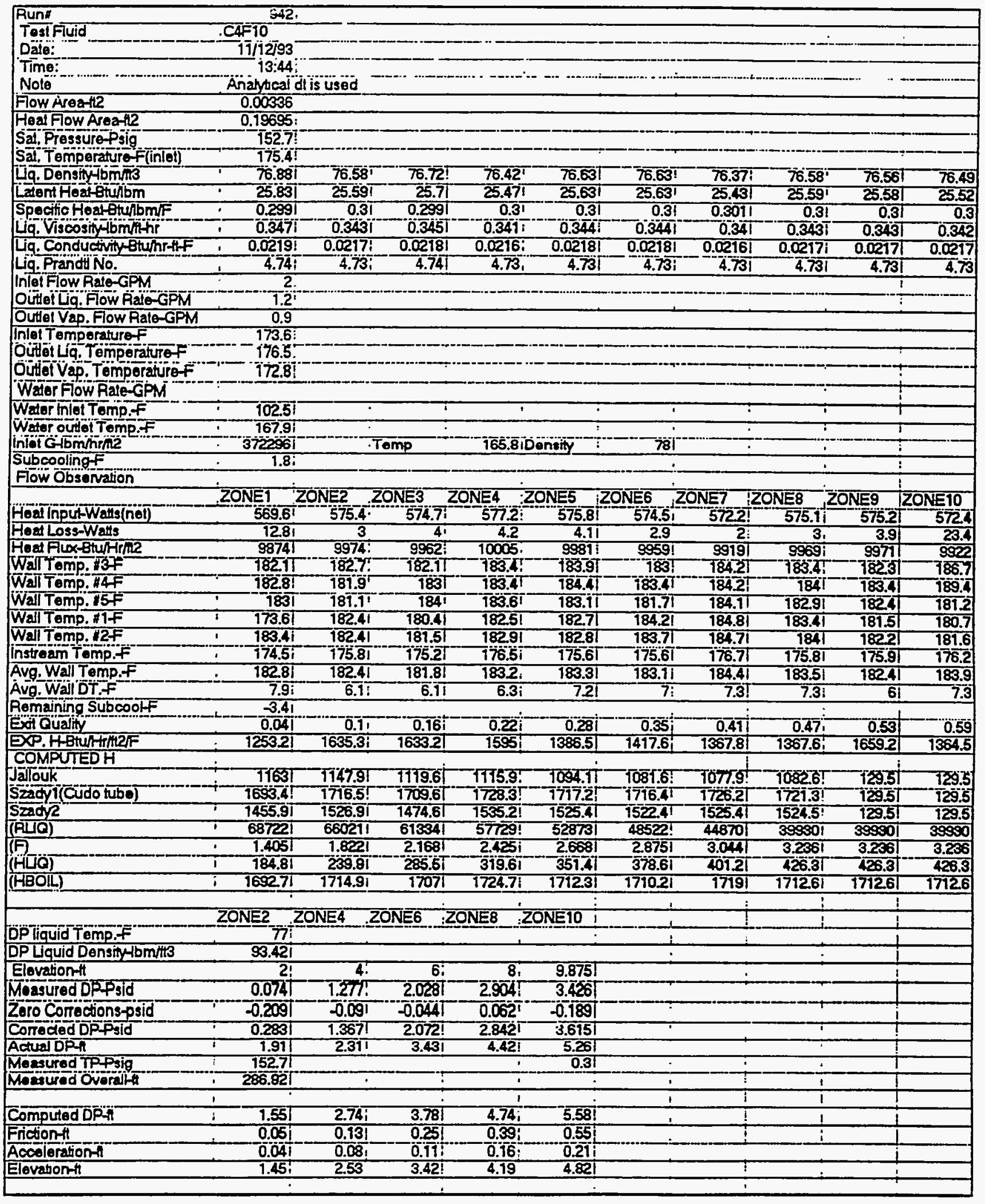




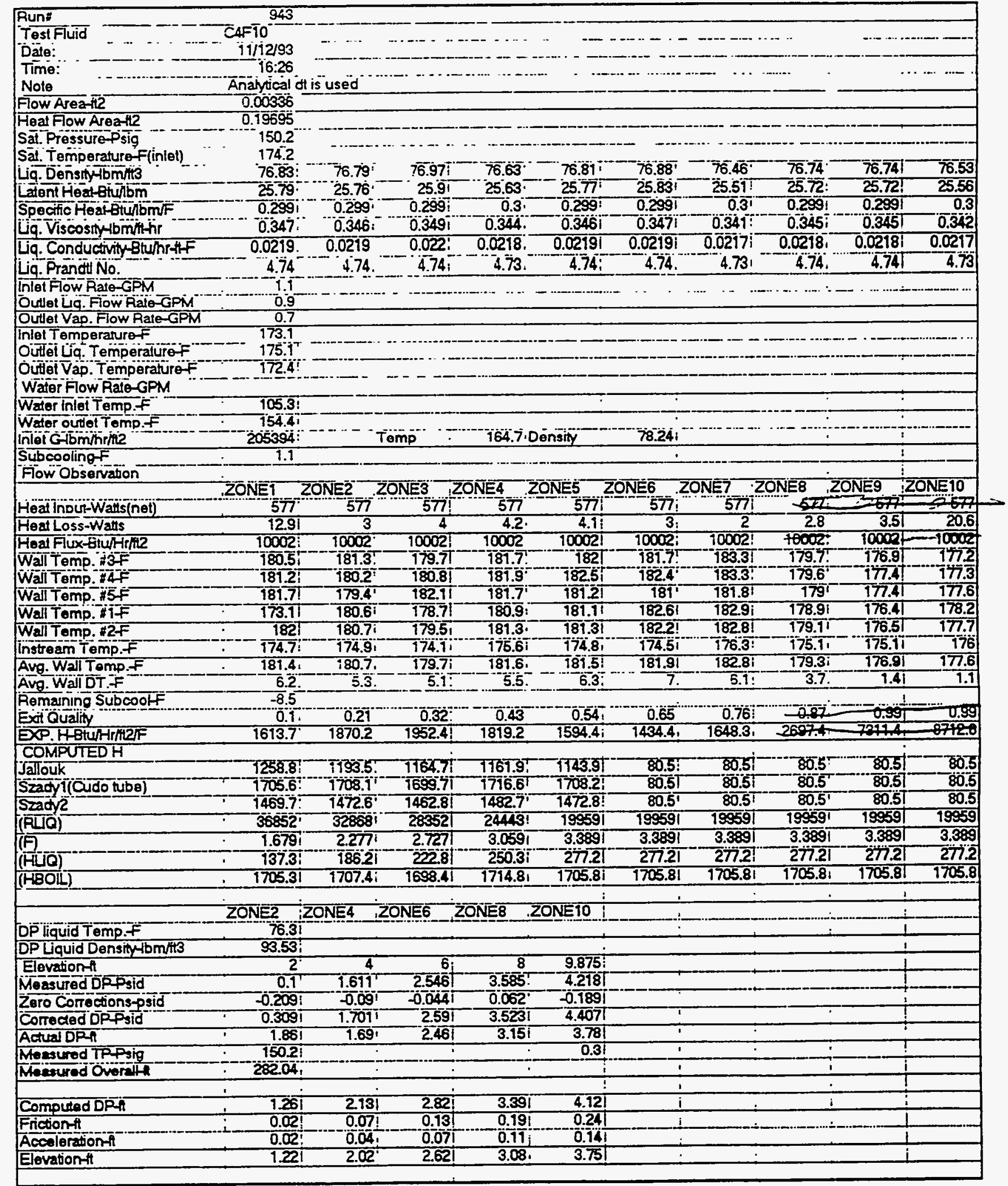




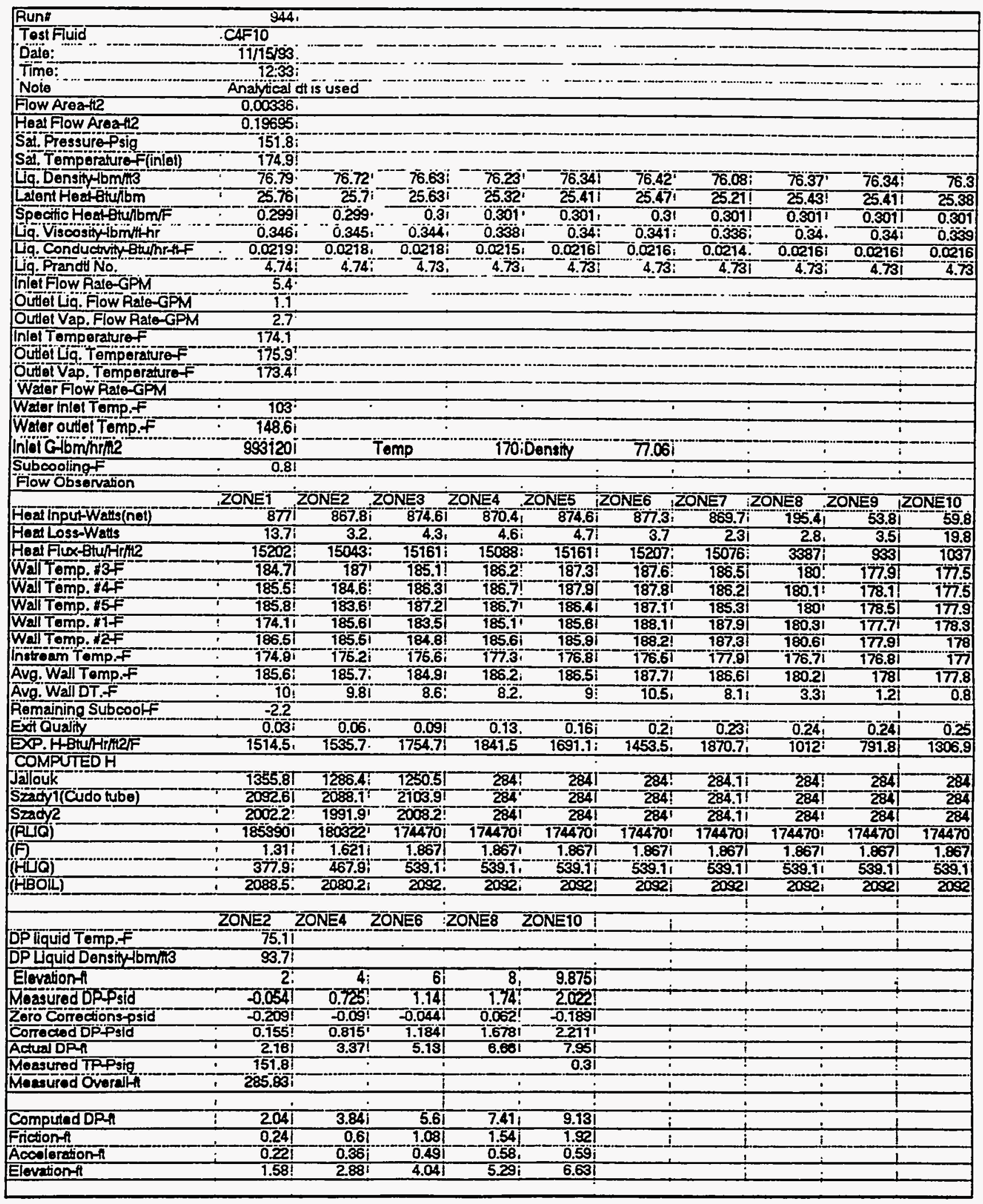




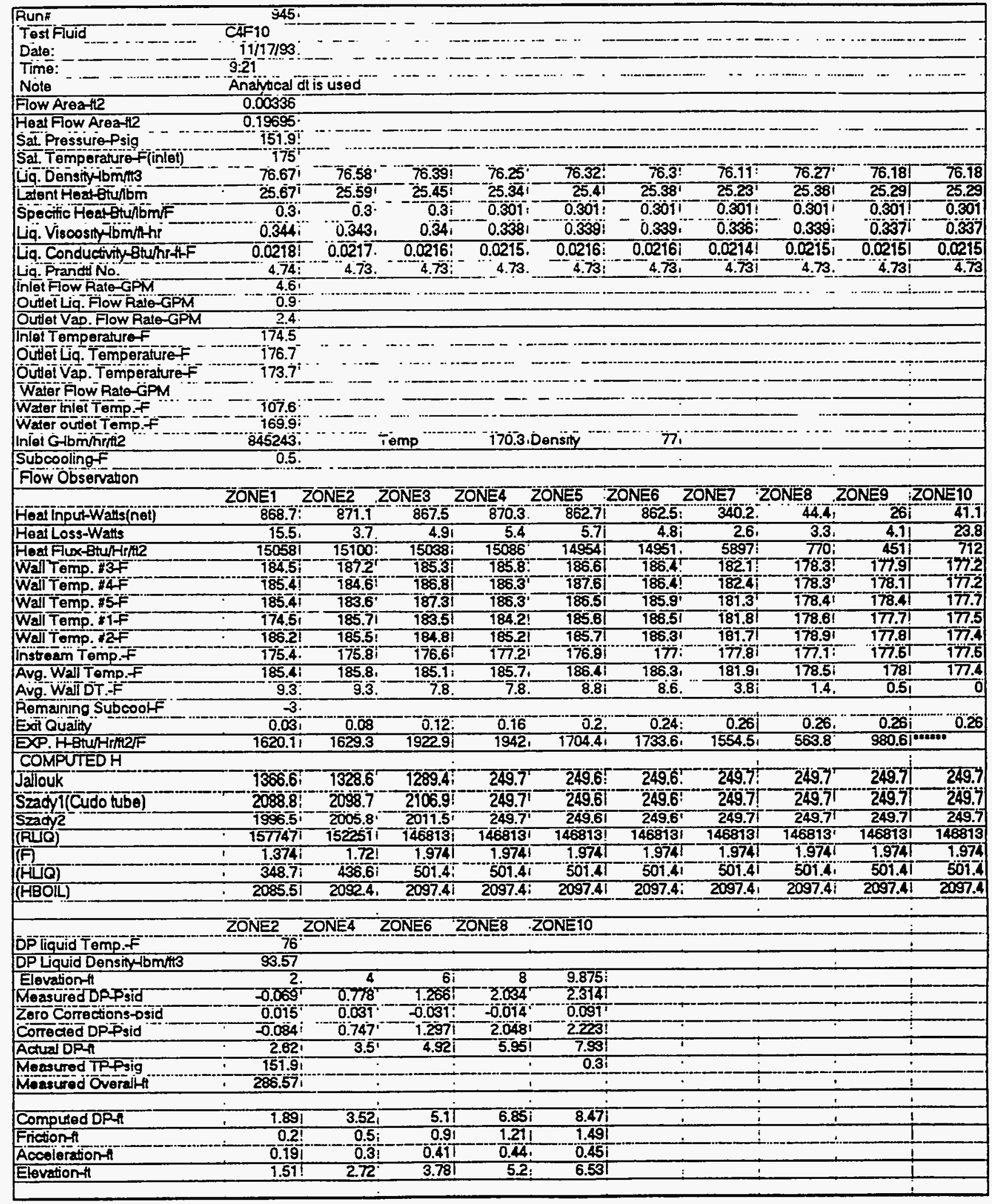




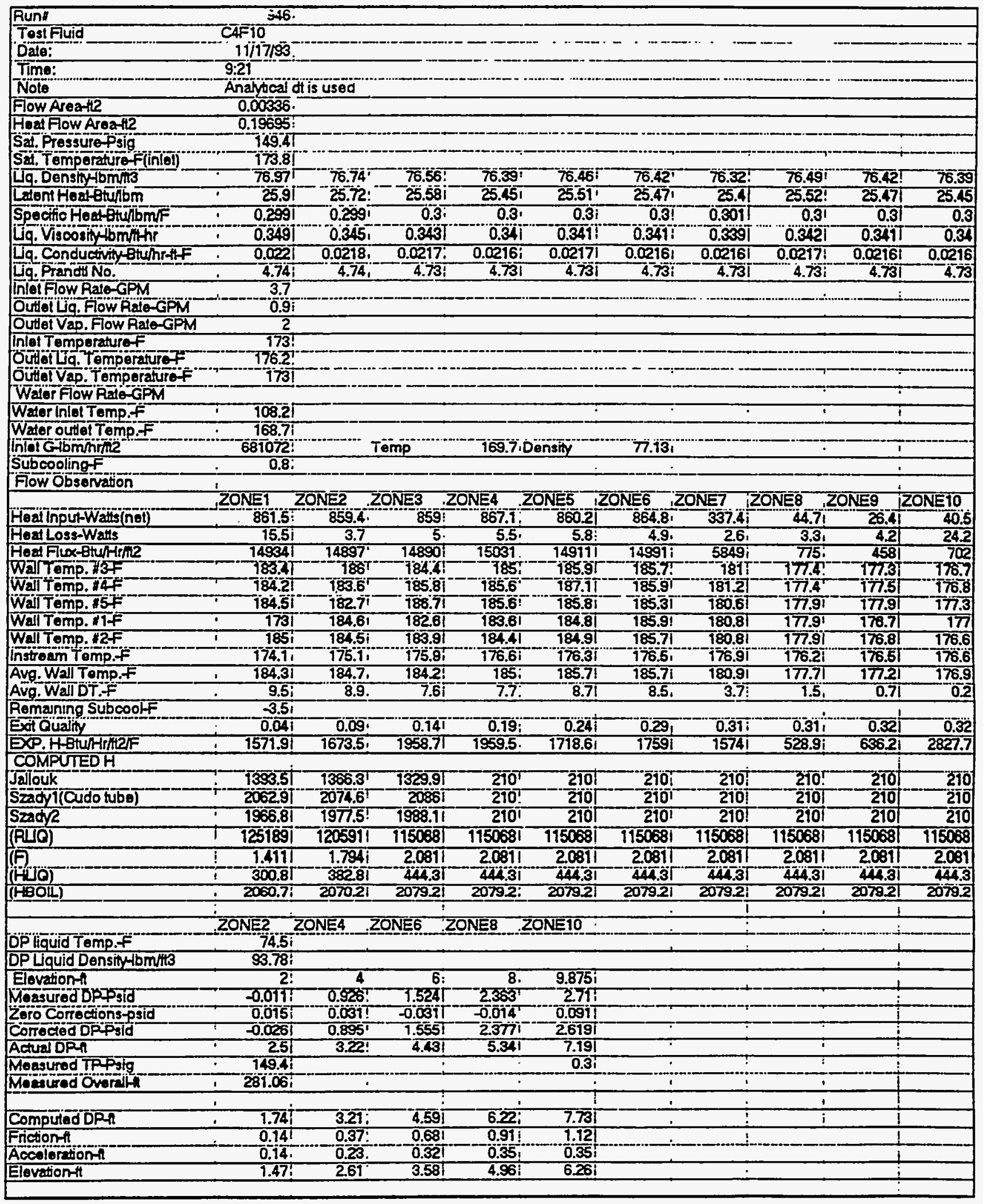




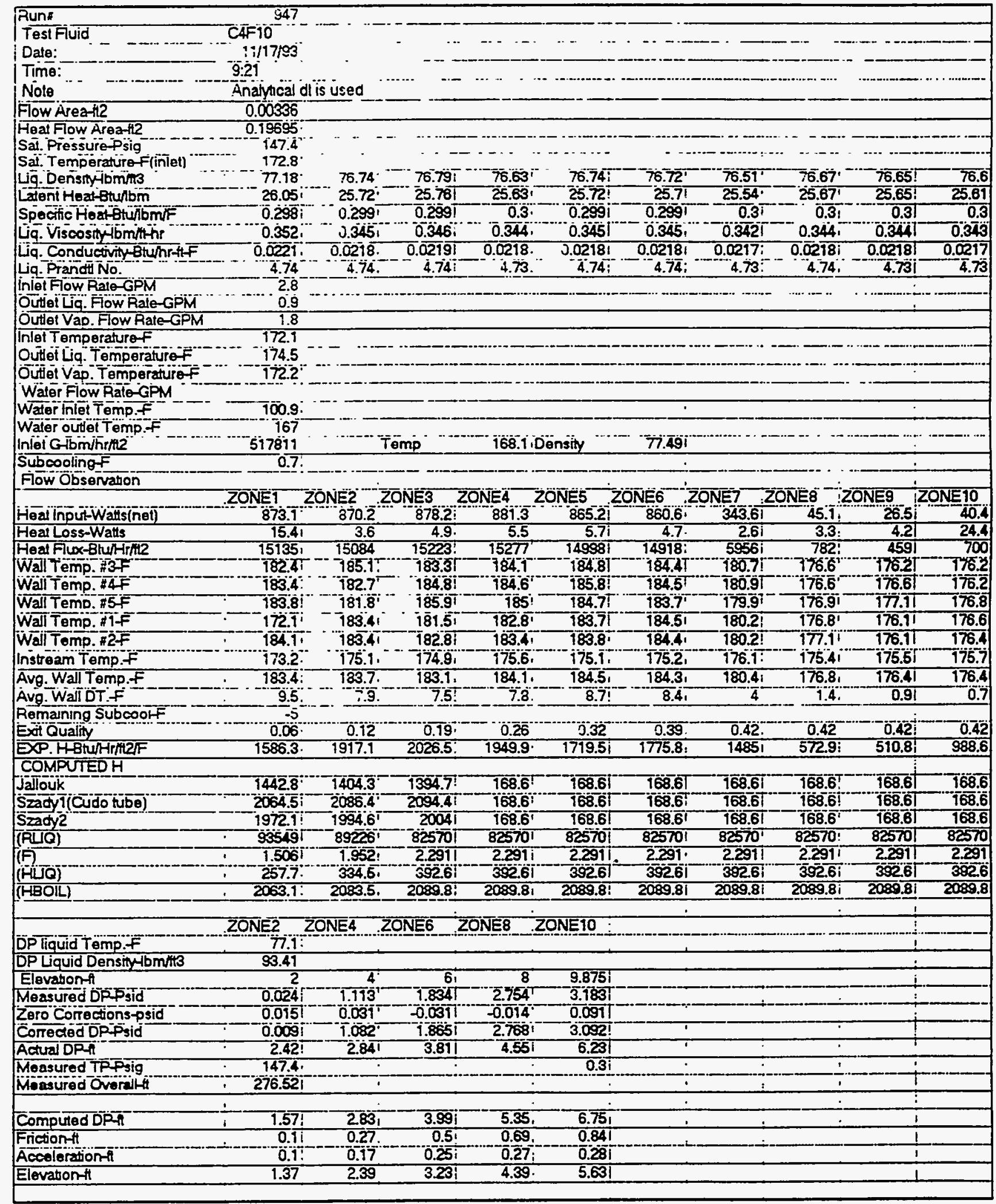




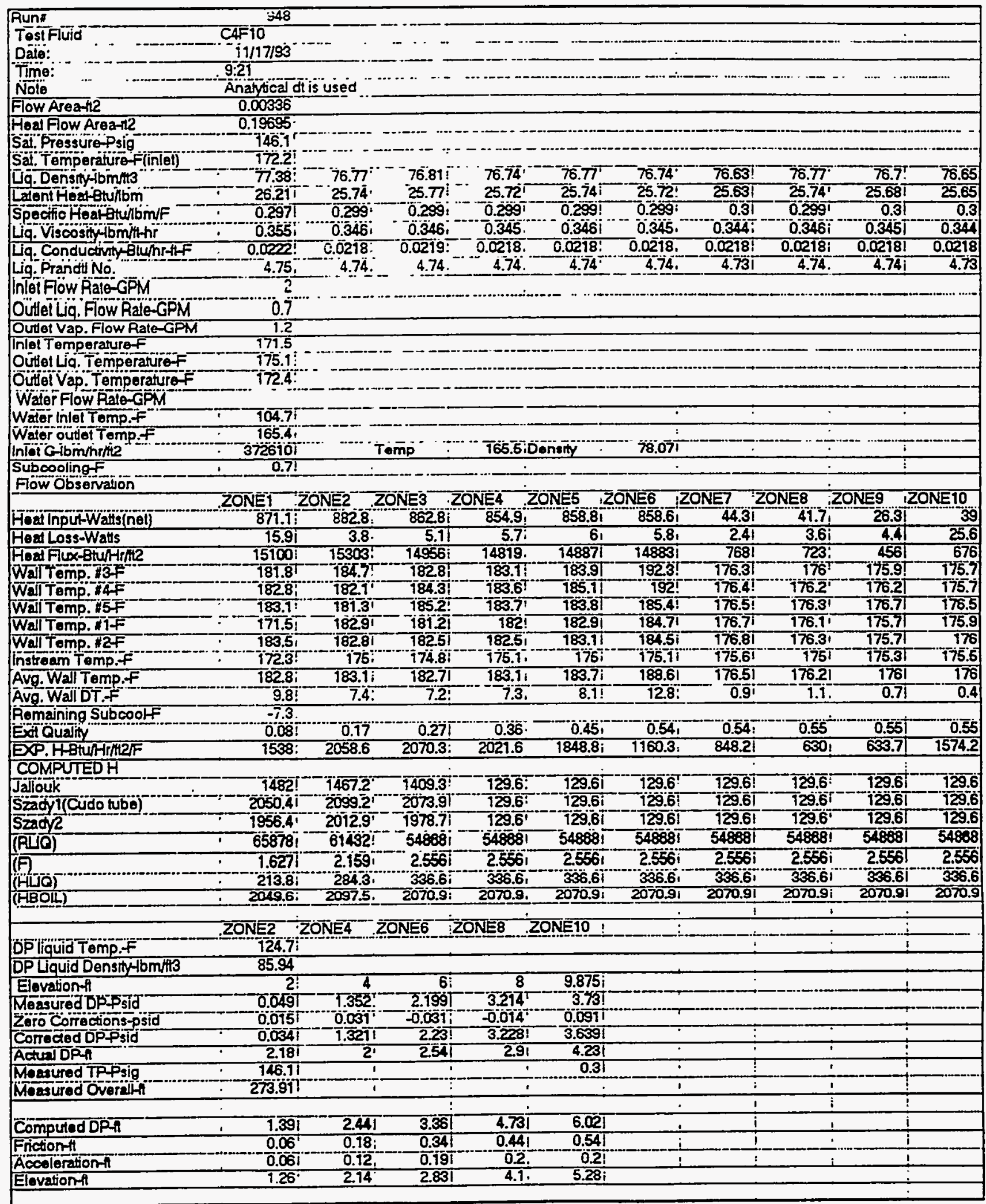




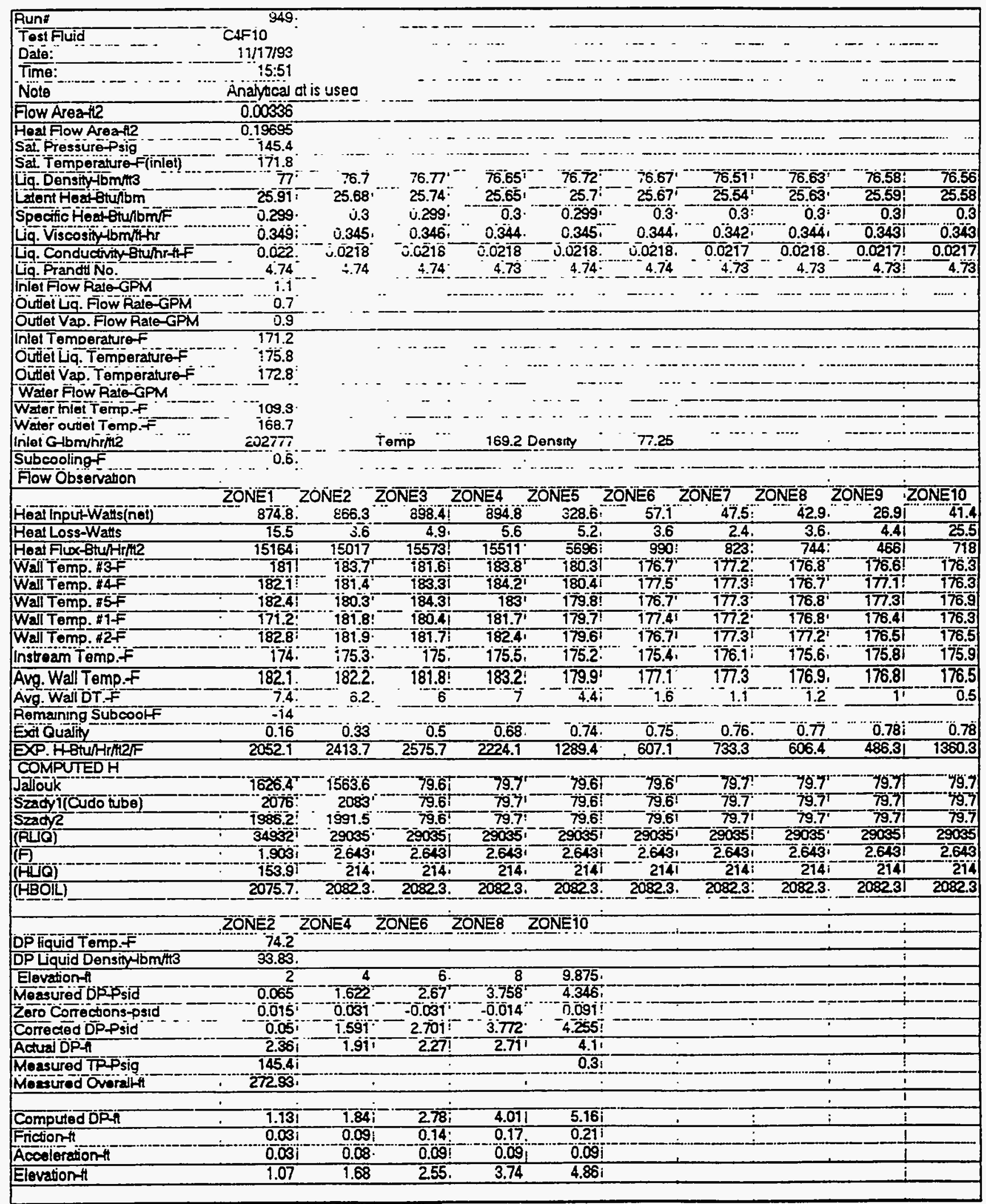




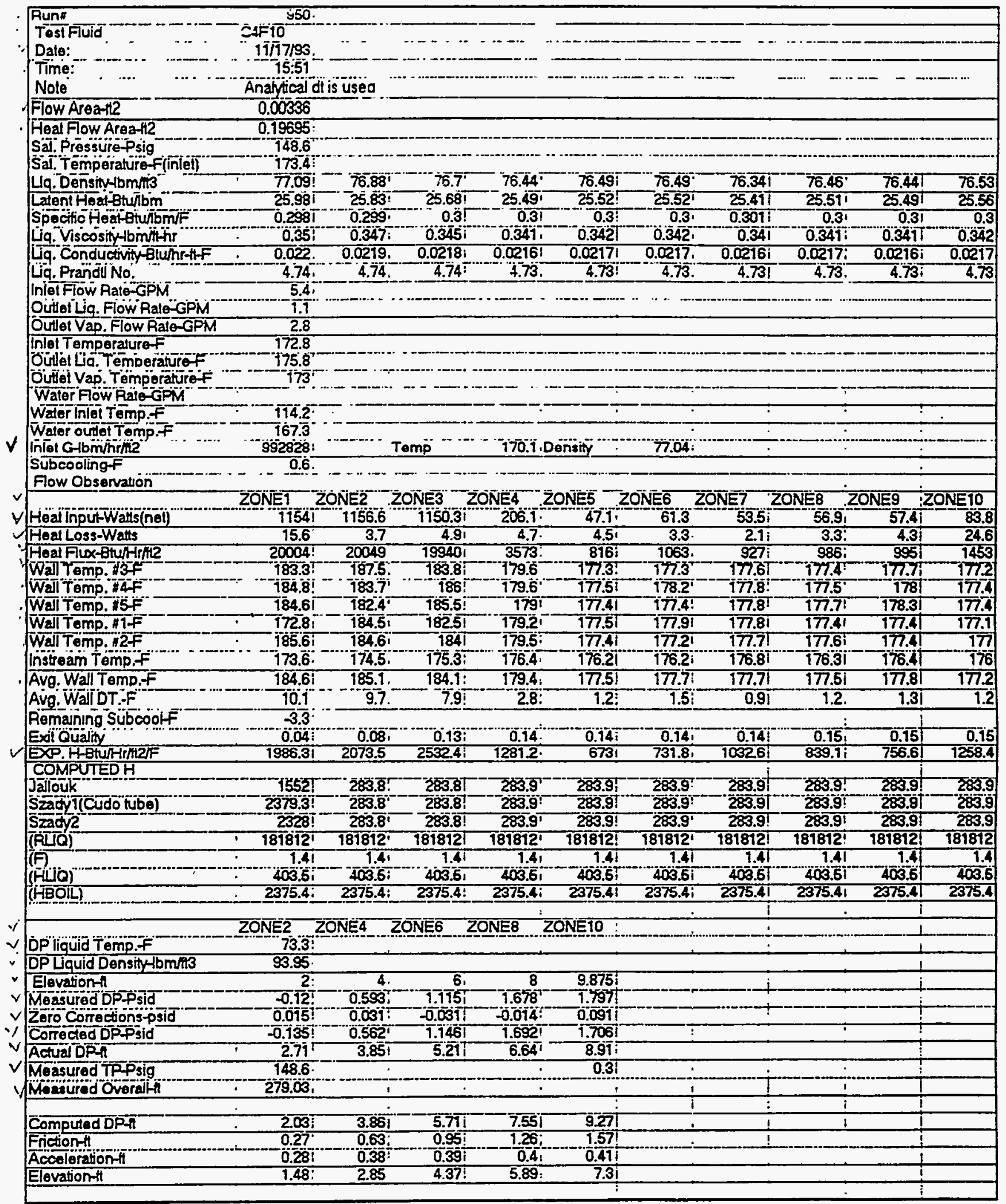




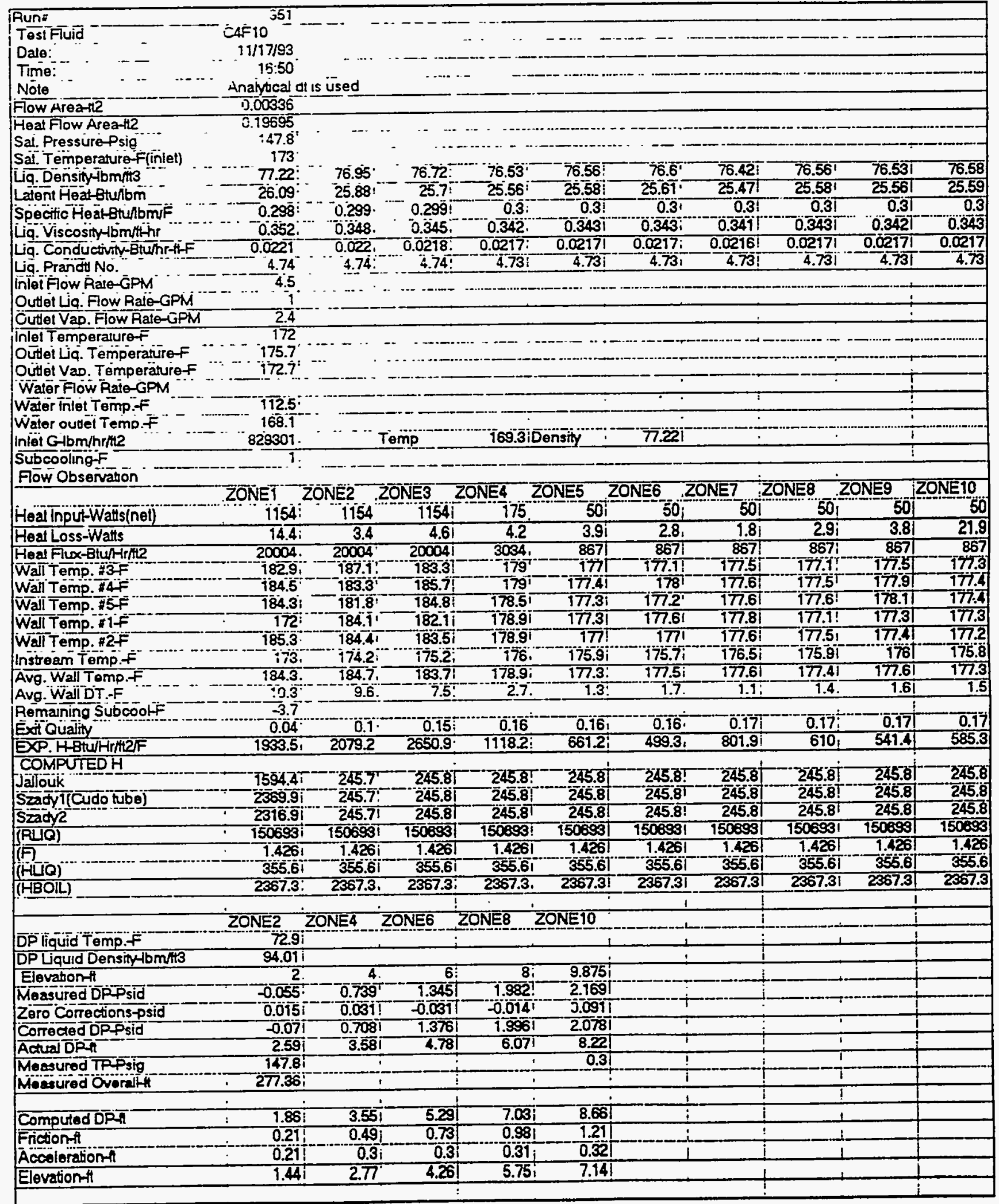




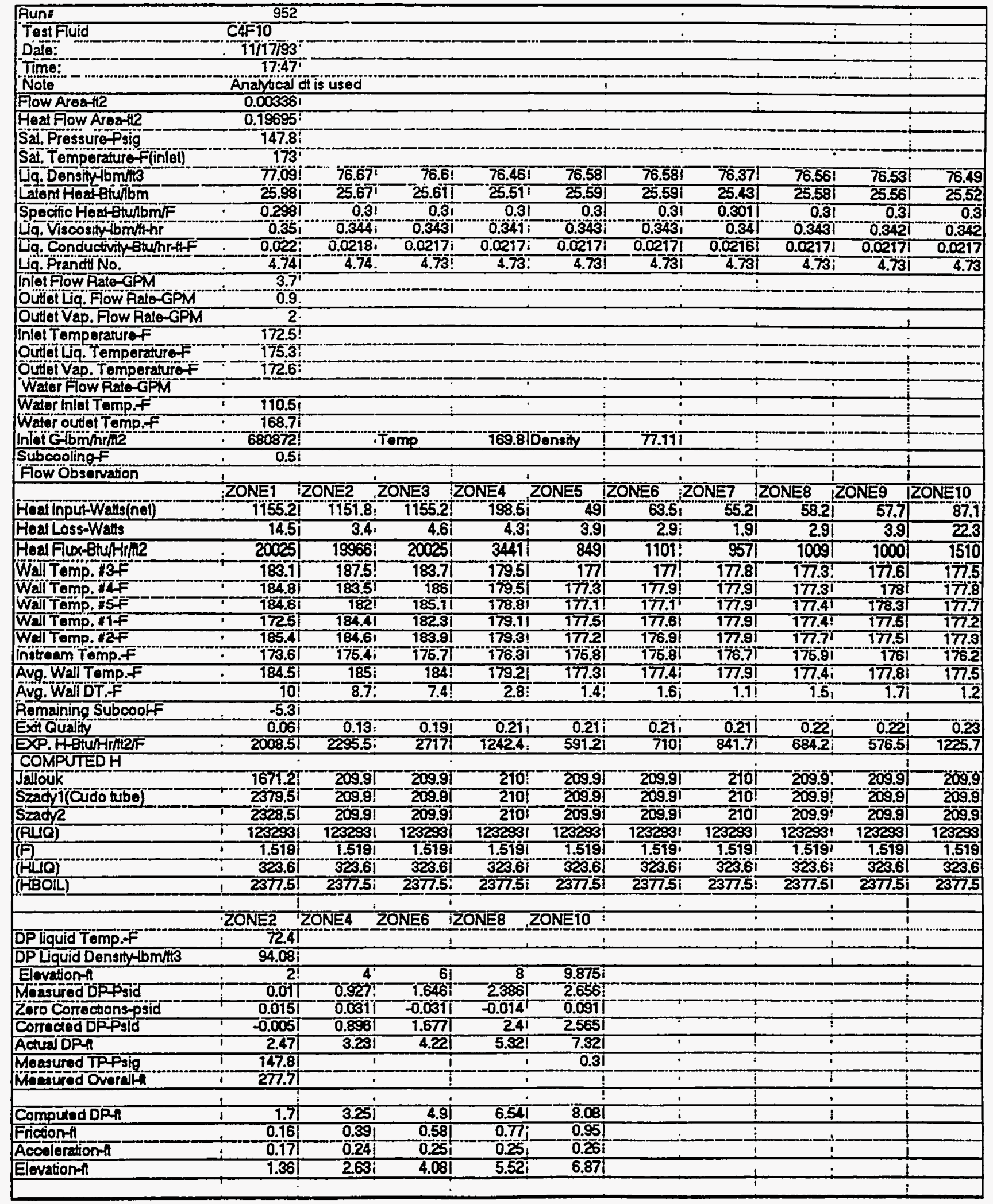




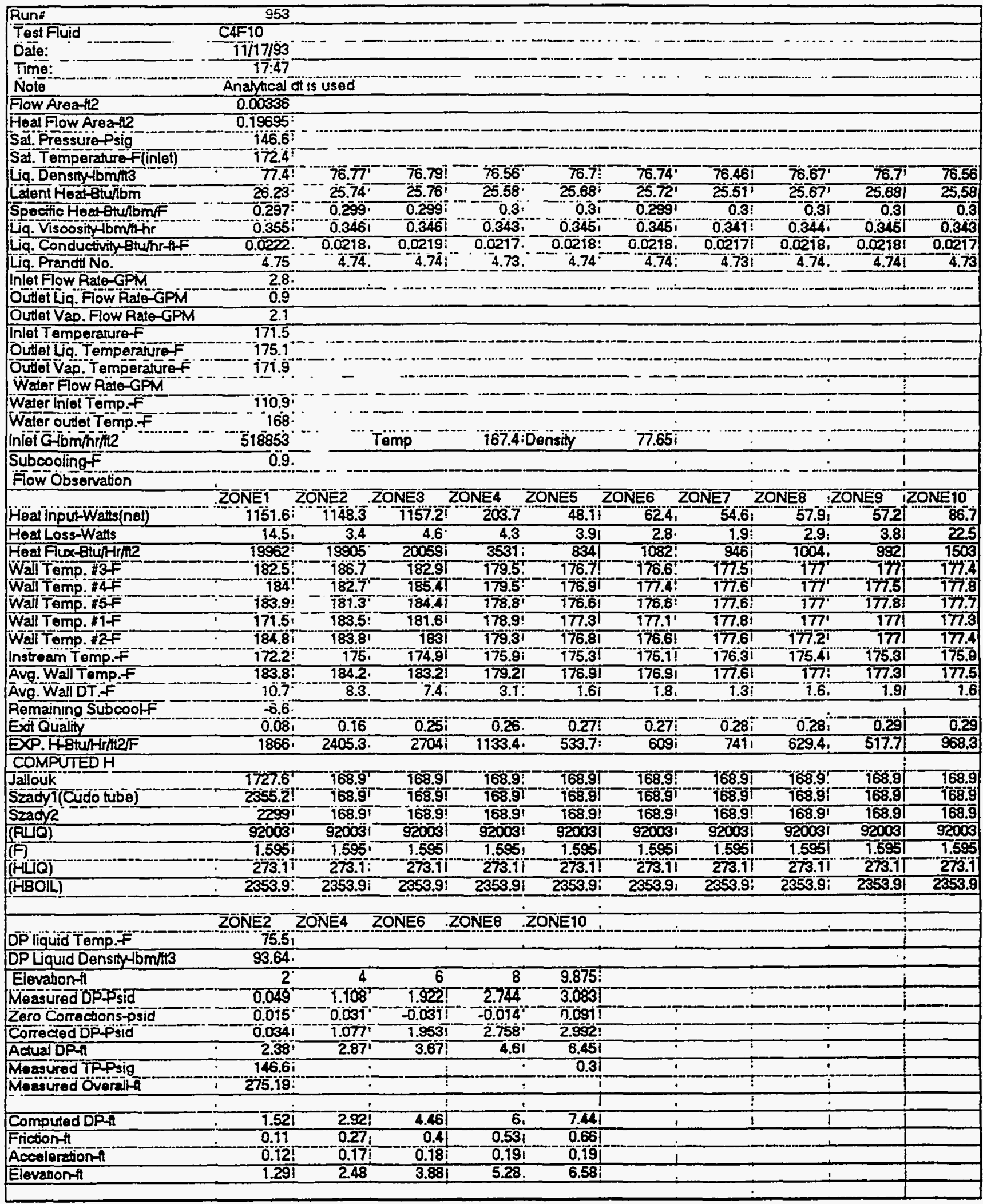




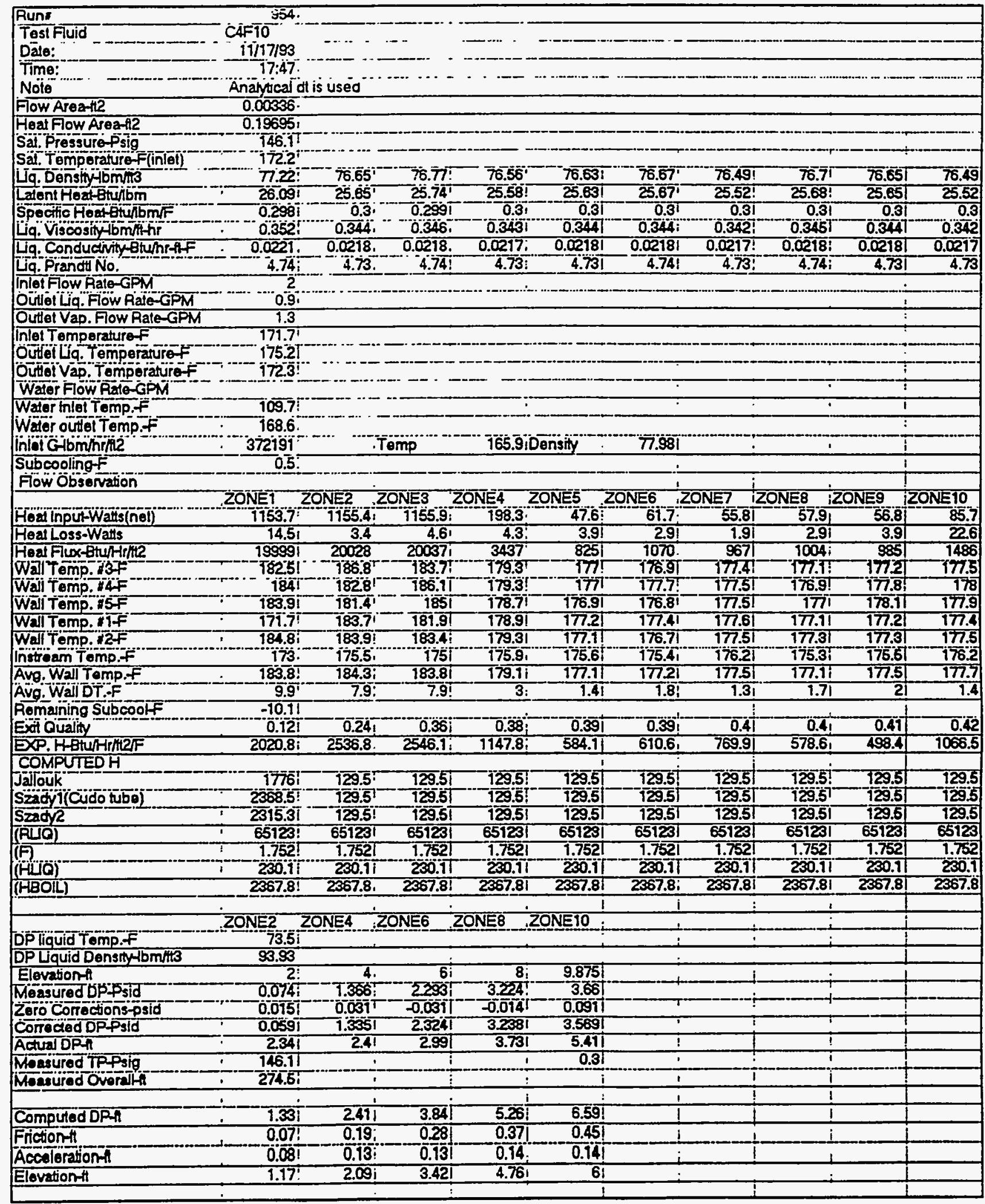




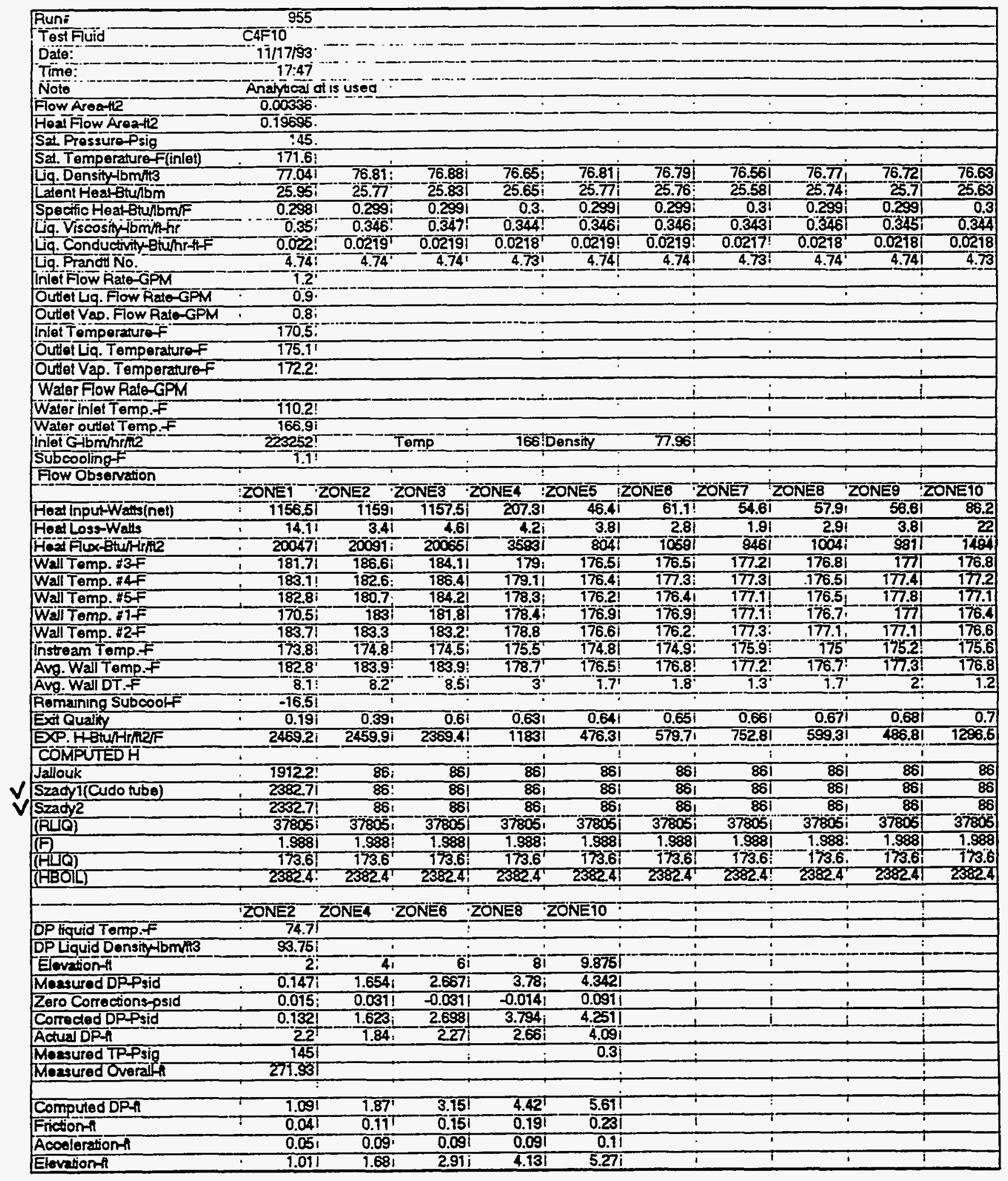




\begin{tabular}{|c|c|c|c|c|c|c|c|c|c|c|}
\hline Funt & 956 & & & & & & & & & \\
\hline Tostfiuld & 75\% CAFTC & $0-25 \% R 114$ & & & & & & & & \\
\hline Dade: & 12याB3 & & & & & & & & & \\
\hline Time: & 13:05 & & & & & & $L$ & & & \\
\hline Now & & & & & & & & & & \\
\hline Flow Areate? & 0.00336 & & & & & & & & & \\
\hline Hean Fow Areate & 0.19695 & & & & & & & & & \\
\hline Set. Prossupe Psig & 76.7 & & & & & & & & & \\
\hline Se. Temperature f(indel) & 730.5 & & & & & & & & & \\
\hline |ndat Fowreto-EPM & 4.8 & & & & & & & & & \\
\hline Intet Tomperaturef & 120.1 & & & & & & & & & \\
\hline InT Ghmomine & 987868 & & Tomp & 124.2 & Donsty & 88.24 & & & & \\
\hline Subooolingf & 1.4 & & & & & & & & & \\
\hline Fow Observilon & & & & & & & & & & \\
\hline & & & & & & & & & & \\
\hline & ZONE & $20 k=2$ & ZONE8 & 201: & ZONES & ZON:S6 & ZONE7 & ZONES & ZONE⿱一𫝀口 & $\angle O N=10$ \\
\hline Hadhoutwatar(ni) & 290.3 & 290.8 & 281.7 & 288.7 & 284.6 & 288.8 & 200.7 & 289.1 & 289.8 & 287.3 \\
\hline HeclLose-Wa's & 7.8 & 1.6 & 21 & 2.3 & 2.1 & 1.5 & 1.1 & 1.5 & 1.9 & 10.8 \\
\hline Hes: Floxturiffice & 5032 & 5041 & 4878 & 4970 & 4382 & 5008 & 5039 & 5017 & 5023 & 460 \\
\hline Wel Tomp. BF & 139.7 & 137.3 & 138.7 & 138.7 & 138.1 & 137 & 137.7 & 135 & 134.7 & 134.5 \\
\hline Wa tamp. 14 & 740 & 138.8 & 137.2 & 138.8 & 138 & 137.7 & 737.7 & 135.7 & 13.1 & 135.8 \\
\hline Wa Tomp. 165 & 140.8 & 736.81 & 137.9| & 138.5 & 737.4 & 136.8 & 137.3 & 135.1 & 134.6 & 1342 \\
\hline Wall Tomp. ITF & T29.11 & 137.51 & 135.7 & 138.8 & 137.3 & 137.8 & 738.6 & 735.6 & 134 & सुद. \\
\hline WET Tamp. ILF & 140.8 & 737.51 & T36.2 & Tگ্3.8 & 137.3 & 137.1 & 138.4 & 136.11 & T34.4 & 134.8 \\
\hline Instrasm Tomp.f & 129.4 & 128.8 & 128.3 & 130 & 1292 & 128.5 & 129 & 127.7 & 127.6 & 127.8 \\
\hline Avo. Wall Temp.f & 140.4 & 137.8 & 136.6 & 138.6 & 137.5 & 137.2 & 137.8 & 135.5 & 134.6 & 134.9 \\
\hline Avo.WalbT.F & 10.7 & 8.3 & 7.8 & 8.3 & 8.1 & 8.5 & 8.7 & 7.6 & 6.7 & 6.8 \\
\hline DPP.HBuntring & 469.3 & 609.4 & 614.5 & 598.7 & 610.6 & 591 & 578.4 & \$1.7 & 766.1 & 737.3 \\
\hline & & & & & & & & & & \\
\hline & ZONES & ZONET & $20 N=6$ & ZONEB & ZOWASTO & & & & & \\
\hline DPliquid Tomp.f & 75.3 & & & & & & & & & \\
\hline 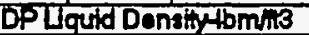 & 92.91 & & & & & & & & & \\
\hline Elovelionf & 2 & 4 & B] & 8 & 9.875 & & & & & \\
\hline Moaulred DP psid & -0.28 & 0.37 & 0.608 & 9.174 & 1.128 & & & & & \\
\hline Zoro Compations-psid & -0.318 & -0.008 & -0.04 & -0.005 & $=0.824$ & & & & & \\
\hline ComeandDPA & 0.038 & 0.378 & 0.648 & 1.169 & 7.45 & & & & & \\
\hline Actual DPh & 2.09 & 3.68 & 5.38 & 6.67 & 8.22 & & & & & \\
\hline & & & & & & & & & & \\
\hline
\end{tabular}




\begin{tabular}{|c|c|c|c|c|c|c|c|c|c|c|}
\hline Run: & $\$ 57$ & & I & J & i & i & & $\mid$ & & \\
\hline Tagt Fuid & $175 \%$ CAFIO & $0-25 \%$ R11 & & & 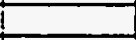 & & & & & \\
\hline Dats: & 127193 & & & & & & & & & \\
\hline Time: & $13: 05$ & & 1 & & i & & & & & \\
\hline Now & 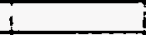 & & 1 & & 1 & & & & & \\
\hline Flow Area-d2 & 0.00336 & & & & & & & & & \\
\hline Heat flow Aroal? & 0.19695 & & & & & & & & & \\
\hline Sad. Pressuro Psig & 77.6 & & & & & & & & & \\
\hline Sal. Temperaturef f(inlal) & 137.1 & & & & & & & & & \\
\hline Intet Flowrate-GPM & 3.6 & & & & & & & & & \\
\hline indel Temperaturef & Tर्3,4. & & & & & & & & & \\
\hline intet Gibminite & 73566 & & Temp & 12.1 & Donsify & 86.11 & & & & \\
\hline Subcoolingf & 0.7 & & & & & & & & & \\
\hline Fow Observition & & & & & & & & & & \\
\hline & & & & & & & & & & \\
\hline & ZONEI & ZONE2 & ZONE8 & ZONE4 & ZONE6 & Zूण & ZONE? & LONE8 & $20 N=8$ & $\angle O N=10$ \\
\hline 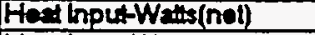 & 209.1 & 290.7 & 289.1 & 288.7 & 285.7 & 288.8 & 209.4 & 288.7 & 2001 & 286.7 \\
\hline HoanLoss-Was & 7.6 & 1.8 & टर & 23 & 22 & 9.8 & 1.7 & 1.5 & 1.8 & 71 \\
\hline Heal Flox Bturitrine & 5011 & 5030 & 5011 & 455 & 4952 & 5008 & 5017 & 497 & 3034 & 4970 \\
\hline Wall Temp. $3 F$ & 139.9 & 138.7 & 738.5 & 140.1 & 139.7 & 138.8 & 139.5 & 136.8 & 736.8 & 786.4 \\
\hline Wall Tomp. $14 \mathrm{~F}$ & 740.5 & 738.2 & 738.8 & 140.2 & 139.5 & 139.3 & 739.7 & 137.1 & T36.7 & 737.8 \\
\hline WAl Tomp. 15 F & 141 & 138 & 139.3 & 140.1 & 138.8 & 138.5 & 139.2 & 136.5 & 738.4 & 138.2 \\
\hline Wal Tomp. \#1F & 130.4 & 138.7 & 137.3 & 139.5 & 138.9 & 138.9 & 120.1 & 137.5 & 136.7 & 136.4 \\
\hline WaH Tomp. $12 f$ & 141.7 & 138.9 & 137.9 & 139.5 & 139 & 138.8 & 140.2 & 138 & 138.1 & 138.4 \\
\hline Instream Tomp.f & 131.2 & 130.8 & 1302 & 131.6 & 130.6 & 129.8 & 130.5 & 129.1 & 1292 & 129.8 \\
\hline Avg. Well Tamp.F & 140.6 & 138.6 & 138.2 & 140 & 139.1 & 138.8 & 139.7 & 137.1 & 1262 & 136.7 \\
\hline Avg. Wal DT.F & 9.2 & 7.8 & 7.7 & 8.8 & 82 & 8.7 & 8 & 7.8 & 6.8 & 6.6 \\
\hline 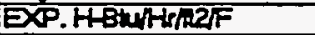 & 544.8 & 646.3 & 648.8 & 601.1 & $6 \infty 2$ & 575.7 & 556.6 & 635.9 & 738.8 & 760.4 \\
\hline & & & & & & & & & & \\
\hline & ZONE2 & ZONEL & $20 N=6$ & $20 N=8$ & $20 \mathrm{~N}=10$ & & & & & \\
\hline DP lquid Tomp.f & 72.3 & & & & & & & & & \\
\hline DP Liquid Density $1 \mathrm{bm} n \mathrm{nt} 3$ & 30.25 & & & & & & & & & \\
\hline Elevationst & & 4 & 6) & 8 & 9.875 & & & & & \\
\hline Mensured DFPhid & -0.181 & 0.02 & 1.018 & 1.67 & 9.804 & & & & & \\
\hline Zoro Corrections-psid & -0.316 & -0.0061 & -0.04 & -0.055 & 0.324 & & & & & \\
\hline Comeded DPP sid & 0.135 & 0.028 & 1.053 & 1.725 & 2.728 & & & & & \\
\hline Actud DPA & 1.94 & 3.28 & 4.74 & 5.78 & 7.14 & & & & & \\
\hline & & & & & & & & & & \\
\hline
\end{tabular}




\begin{tabular}{|c|c|c|c|c|c|c|c|c|c|c|}
\hline Runi & 958 & & $!$ & 1 & ! & & & 1 & 1 & \\
\hline Tasfiuld & $175 \%$ C4F 10 & $0-25 \%$ Rा1 & & & 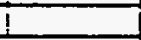 & & & & & \\
\hline Data: & 127130 & & I & $i$ & $\mathrm{i}$ & & & $T$ & & \\
\hline Time: & $13: 06$ & & 1 & & $\perp$ & - & I & & & \\
\hline Not & 1 & & 1 & 1 & 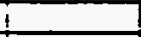 & & 1 & & & \\
\hline Flow Arant2 & 0.00336 & & & & & & & & & \\
\hline Hest Flow Areafe & 0.18695 & & & & & & & & & \\
\hline Set. Prossuto & 77.8 & & & 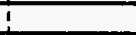 & I & & & & & \\
\hline Set. Tompertureffintel) & 131.3 & & & & & & & & & \\
\hline Indet Fowralo-GPM & 2.4 & & & & & & & & & \\
\hline intel temporater & 730.8 & & & & & & & & & \\
\hline Inlet Glbm/mine & $4 \operatorname{sos}_{2}{ }^{2}$ & & TTamp & 724.9 & TDensity & 86.18 & & & & \\
\hline Subcoolingf & 0.5 & & & & & & & & & \\
\hline Flow Obsenvion & & & & & & & & & & \\
\hline & & & & & & & & & & \\
\hline & ZOKE & 2OKE2 & $201 \pm 3$ & ZONE & ZO=5 & ZON & $20 N=7$ & 20125 & $20 N=0$ & ZONETO \\
\hline Healnpu-Weth(ned) & 283 & 2832 & 288.4 & 285.3 & 28.3 & 287.4 & 286.8 & 2312 & 2008 & 285.0 \\
\hline HanlooseWa: & 7.6 & 1.7 & 22 & 24 & 23 & 1.7 & 1.1 & 1.8 & 2 & 11.8 \\
\hline 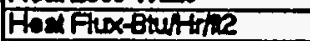 & 4961 & 4989 & $\overrightarrow{4990}$ & 4945 & 408 & 4982 & 48: & 4808 & 50ता & 4856 \\
\hline Wetromp. IJ & 139.8 & 138.8 & 139.1 & 140.3 & 140.5 & 169.7 & 1402 & 137.5 & 1372 & 137.2 \\
\hline WET Tamp. IfF & 140.1 & 738.6 & 139.8 & 140.8 & 740.3 & 760.8 & 740.8 & 738.1 & 737.6 & 1387 \\
\hline WE T Tamp. IFF & 140.8 & 1382 & 140 & 1405 & 139.4 & 139.4 & 139.8 & 137.5 & 137.2 & 137.1 \\
\hline Wal Tomp. $11 \mathrm{~F}$ & 1300.8 & 138.8 & 137.5 & 140 & 139.8 & 139.7 & 140.7 & 138.4 & 136.8 & 137 \\
\hline WL Tomp.12F & 141 & 139.1 & 938.1 & 740 & 139.8 & 139.8 & 140.8 & 139 & 135 & 737.2 \\
\hline Instresn Temp.f & 131.5 & 731.7 & 1302 & 137.7 & 130.8 & 130.5 & 131 & 129.6 & 120.7 & 130.3 \\
\hline Avo, Wal Tomp.f & 140.5 & 138.8 & Tङ.5. & 140.8 & 139.6 & 139.8 & 740.4 & 138.1 & 137.1 & 137.4 \\
\hline Avg. Well DT.F & 8.7 & 7.6 & 8.1 & 8.4 & 8.5 & 8 & 9.1 & 8.8 & 7.2 & 6.8 \\
\hline 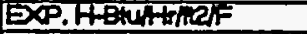 & 569.6 & 668.2 & 6182 & 6822 & 588.11 & E505 & 542 & 586.2 & 700.0 & 718.6 \\
\hline & TOKR=5 & उहागन & 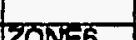 & कृVi=8 & द大 & & & & & \\
\hline DPFoud Tomp $f$ & LONee 39 & ZONES 4 & ZONE6 & ZONE8 & LONIED & & & & & \\
\hline DP Ulouid Donsity & 93.89 & & & & & & & & & \\
\hline Elevetions & $\frac{-2}{2}$ & 4 & & & & & & & & \\
\hline 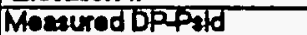 & $00 \frac{\pi}{81}$ & $0 \times 8$ & & 8 & 9.875 & & & & & \\
\hline & -0.070 & 0.878 & 1.422 & 2द्रा7 & 2.472 & & & & & \\
\hline 20ro Corrections-psid & -0.316 & -0.005 & -0.04 & -0.005 & -0.324 & & & & & \\
\hline Correctod DP Pigd & 0.2381 & 0.882 & 1.462 & $2.2 \%$ & 2.798 & & & & & \\
\hline Acued DPA & 1.77 & 2.88 & 4.08 & 4.88 & 6.08 & & & & & \\
\hline & & & & & & & & & & \\
\hline
\end{tabular}




\begin{tabular}{|c|c|c|c|c|c|c|c|c|c|c|}
\hline Runf & 59 & ! & $!$ & 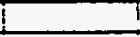 & I & $!$ & & & & \\
\hline Testfivid & $175 \%$ CAF10 & -25\%RT1 & & 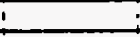 & 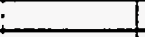 & & & & & \\
\hline Dadi: & 127193 & & & $i$ & 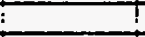 & $!$ & & & & \\
\hline Time: & $13: 05$ & & & & $i$ & $i$ & L & & & \\
\hline Now & 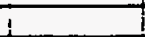 & 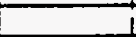 & 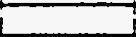 & 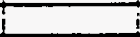 & 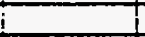 & & & & & \\
\hline Fow Areare & 0.00336 & & & & $i$ & & & & & \\
\hline Hen Fow Areade & 0.1955 & & & & $i$ & & & & & \\
\hline Sat Prossure Psig & 76.5 & & & 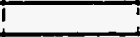 & & & & & & \\
\hline Sut. Temperature F(inloi) & 130.4 & & & I & & & & & & \\
\hline Intelfowrto-GPM & 4.7 & & & & i & & & & & \\
\hline intst temperauref- & 129 & & $!$ & & $i$ & 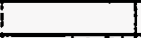 & & & & \\
\hline Inlat Gbintrine & 970854 & & Tomp & 1223! & Density & 88.58 & & & & \\
\hline Subcooling $F$ & 1.4 & & & & 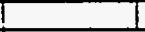 & & & & & \\
\hline Fow Observition & 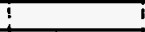 & 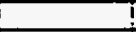 & i & 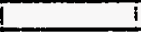 & $i$ & & & & & \\
\hline & $!$ & 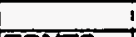 & 1 & 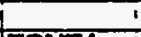 & 1 & 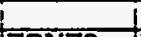 & & & & \\
\hline & ZONEI & ZONE2 & ZONE3 & ZONEA i & IZONES I & ZONE6 & ZONET & ZONER & ZONEO & ZONETO \\
\hline Hoal Inputwasinet) & +580.8 & 578.8 & 578.9 & 580.1 & 582 & 581.9 & 584.9 & 580.8 & $57 \overline{3.8}$ & 577.5 \\
\hline Heat Loss-Wast & 8.9 & 2 & 27 & 2.8 & 2.71 & 2 & 1.3 & 1.9 & 24 & 13.7 \\
\hline Hod Flox-Btwhthe & $100 \%$ & 10038 & 10085 & 10058 & 10089 & 10087 & 10189 & 70059 & 998 & T0011 \\
\hline Wat Tomp. $3 F$ & 140.5 & 740.8 & 139.7 & 141.8 & 141.8 & 7472 & 741.7 & 138.7 & 188 & 137.7 \\
\hline Wall Temp. " 4 F & 141.2 & 1392 & 140.7 & 142.1 & 141.9 & 141.8 & 141.7 & 139.4 & 138.8 & 145.6 \\
\hline WaT Tomp. $15 F^{2}$ & 141.7 & 138.3 & 147.7 & 1422 & 140.5 & 140.8 & 140.8 & 138.1 & 137.8 & 136,6 \\
\hline WeTTemp. IfF & 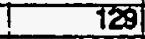 & 140.1 & 138 & 140.5 & 140.2) & 141.2 & 1422 & 33.4. & 138.7 & T38.6 \\
\hline We Tomp. 127 & 1424 & T40.1 & T38.8 & 740.91 & 740.61 & 747.5 & 7425 & 740.3 & 737.6 & 737.4 \\
\hline Instren Tomp.f & 129.5 & 128.7 & 128.1 & 129.9 & 128.8 & 128.3 & 128.8 & 127.4 & 127.4 & 127.5 \\
\hline Avq. Wall Tomp.f & 141.6 & 140.1 & 139.3 & 141.7 & 140.8 & 1412 & 141.8 & 139.2 & 137.7 & T37.8 \\
\hline Amg. Wald DT.f & 11.5 & 10.9 & 10.7 & 11.3 & 11.5 & 124 & 125 & 17.8 & 9.8 & 10 \\
\hline EX.HEMHARE & 878 & 918.7 & 883.8 & 802 & 873.9 & 810.6 & 808.7 & 8882 & 10112 & 1005 \\
\hline & & & & & & & & & & \\
\hline & ZONE2 & ZONE4 & ZONE6 & ZONE8 & ZONETO & & & & & \\
\hline DP lquid Tomp.f & 79.9 & & & & & & & & & \\
\hline DP Louid Density tomnt3 & 9288 & & & & & & & & & \\
\hline Elovion-l & 2 & 4 & 6 & 8 & 9.875 & & & & & \\
\hline Wesesured DPFsid & -0.237 & 0.518 & 0.884 & 1.413 & 1.413 & & & & & \\
\hline Zaro Corrections-psid & -0.316 & -0.006 & -0.04 & $-0.0 \leq 5$ & -0.324 & & & & & \\
\hline Corrected DFPsid & 0.039 & 0.524 & 0.927 & 7.468 & 1.737 & & & & & \\
\hline Actull DPA & 2.02 & 3.4 & 4.87 & 6.1 & 7.80 & & & & & \\
\hline & & & & & & $L$ & & & & \\
\hline
\end{tabular}




\begin{tabular}{|c|c|c|c|c|c|c|c|c|c|c|}
\hline Punt & 9501 & & & & & & & & & \\
\hline Test Fiud & $75 \%$ C4F10 & 0.25\% R11 & & I & & & & & & \\
\hline Dat: & 127193 & & i & 1 & & & & & & \\
\hline Time: & $13: 05$ & & & & & & & & & \\
\hline Now & & & & & & & & & & \\
\hline Flow Arealle & 0.00365 & & & & & & & & & \\
\hline Hed Flow ATende & 0.19895 & & & & & & & & & \\
\hline Sad. Pressuro Psig & 76.1 & & & & & & & & & \\
\hline St. Tomperaturef (intel) & 130.11 & & & & & & & & & \\
\hline Infotfowrate-GPM & 3.6 & & & & & & & & & \\
\hline Intel Tomperaturef & स्ञा & & & & & & & & & \\
\hline Intot Gibminitie & 743788 & & Tomp & 7821 & Donstisy & 88.57 & & & & \\
\hline Subootingt & 1.7 & & & & & & & & & \\
\hline Fow Obsenvion & & & & & & & & & & \\
\hline & & & & & & & & & & \\
\hline & ZONE & ZONEZ & IZONES & ZONEC & ZOWE5 & ZONE6 & ZONET & $201: 38$ & ZONE & 2ON:=T0 \\
\hline Hod houtWatulne) & 5792 & 576.5 & 579.8 & 579.4 & 5829 & 582 & 5823 & 577.8 & 578.7 & $5 \%$ \\
\hline Hod Loss-Walls & 7.9 & 1.7 & 2.3 & 25 & 24 & 1.8 & 13 & 1.7 & टंग & 125 \\
\hline 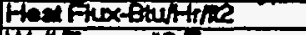 & $100 \% 0$ & 9933 & 1002 & 10024 & 10104 & 1008 & 10082 & 10018 & 9997 & 7006 \\
\hline WE Tomp. 134 & 710.8 & 140.8 & 139.8 & 141.8 & 142.1 & 741.5 & 74 & 138.8 & 738.4 & 738.8 \\
\hline WAITOMo."14F & 741.3 & 739.2 & 140.8 & 141.8 & 1422 & T62.1 & 1कर. & 1402 & 136.2 & 7420 \\
\hline Waltomp. $16 \mathrm{~F}$ & 741.8 & 138.6 & 141.7 & 142.1 & 141 & 141.8 & 141.8 & 138.8 & अ.री & 137.5 \\
\hline WhT Temp. RF & 1खा & 139.9 & 737.6 & 740.5 & 110.2 & 941.4 & T42. & $|36.8|$ & 1372 & 737.8 \\
\hline Waltamp. ILF & 742.Tा & 1401 & 738.5 & 740.8 & 140.8 & 7215 & 7428 & T40.81 & 138 & T38.6 \\
\hline Instrem Tomp.f & 129.5 & 128.7 & 128 & 128.8 & 128.8 & 128.3 & 128.6 & 1ट्टाइ & T27.4. & 128.2 \\
\hline Avg.Wall Tomp.f & 141.5 & 140 & 139.1 & 147.6 & 740.8 & 14t.6 & करस्टी & 139.7 & 1382 & 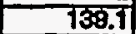 \\
\hline Av. Wall DT.F & 71.5 & 10.8 & 10.7 & 112 & 11.8 & 12.8 & 18.2. & 11.7 & 10.8 & 10.4 \\
\hline 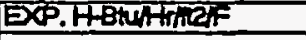 & 873.3 & 923.3 & 841 & 893.1 & 873.4 & 787 & 768.6 & 85.Tी & 857 & 8500 \\
\hline & & & & & & & & & & \\
\hline & $\angle O N=2$ & ZONEA & ZONE6 & ZONBEB & ZONESTO & & & & & \\
\hline DPFgud Tomp.F & 75.7 & & & & & & & & & \\
\hline DPLquid Donsingtomin & कृष्ठा & & & & & & & & & \\
\hline Eleventiont & 2) & 4 & 6 & 8 & 9.875 & & & & & \\
\hline Mosured DPFsid & -0.123 & 0.815 & T.3. & 7.800 & 2.137 & & & & & \\
\hline Zaro Correctionmpsid & -0.316 & -0.006 & -0.04 & -0.05 & -0.82 & & & & & \\
\hline Corrected DPphid & 0.163 & $0.8<1$ & 1.4 & 205 & 2461 & & & & & \\
\hline Ached DPA & 1.82 & 2.88 & 4.17 & 5.17 & 6.5 & & & & & \\
\hline & & & & & & & & & & \\
\hline
\end{tabular}




\begin{tabular}{|c|c|c|c|c|c|c|c|c|c|c|}
\hline Run: & 961 & & 1 & 1 & & & & & & \\
\hline Testfind & $75 \%$ CAF10 & $0-25 \%$ R114 & & i & & & & & & \\
\hline Date: & $127 / 3$ & 1 & 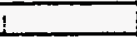 & 1 & & & & & & \\
\hline Time: & $17: 31$ & 1 & 1 & 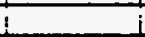 & & & & & & \\
\hline Nole & -0 & & & i & & & & & & \\
\hline Flow Areari? & 0.00365 & & & & & & & & & \\
\hline Hod flow Areste? & 0.19595 & & & & & & & & & \\
\hline Sal Pressuropsig & 76.9 & & & & & & & & & \\
\hline Sat. Tomperature-F(intel) & 130.6 & & 1 & & & & & & & \\
\hline Inlat Fowrate-GPM & 2.4 & & ; & & & & & & & \\
\hline inlat Temperaturef & 1292 & & & & & & & & & \\
\hline inhel Glbmhrile & 498116 & & TTomp & 121.8 & Donsily & 88.82 & & & & \\
\hline Suboooting $F$ & 1.4 & & 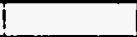 & & & & & & & \\
\hline Fiow Observation & & & & & & & & & & \\
\hline & 1 & & & & & & & & & \\
\hline & ZONET & ZONEZ & ZONEB & LONE4 & 20156 & ZONE6 & $20 K=7$ & ZONEB & ZONES & ZOWE \\
\hline Had input-Watis(nel) & 574.4 & 573.1 & 5705 & 567.5 & 5672 & 568 & 57.7 & 570.8 & 557.8 & 566.4 \\
\hline Hod Loss-Watis & 9.4 & 22 & 2.9 & 3 & 3 & 22 & 1.5 & 2.1 & 27 & 15.8 \\
\hline Hedflux-BurHine & 9957 & 9934 & 9889 & 9837 & 9832 & 986 & 9910 & 9888 & 894 & 9818 \\
\hline Wa Tomp. BF & 140 & 141.2 & 140.1 & 141.9 & 142.8 & 142.8 & 142.8 & 140.2 & 139.7 & 140 \\
\hline Wat Temp. N4F & 141 & 139.8 & 141.3 & T42.11 & 142.8 & 143.2 & T43.3. & 141.4 & 140.5 & TaB.6.5 \\
\hline WatTemp. 15F & 141.5 & 139.1 & 1424 & 14261 & 141.7 & 1423 & 1422 & 140 & 139.4 & 138.8 \\
\hline Wed Temo. flf & 129.2 & 140.51 & Tß3.3 & 140.7 & 140.8 & 742.8 & 145.5 & 741.11 & T38.8 & T38.4 \\
\hline WC Temp. ILF & 741.7 & 740.51 & 135.4 & 14.T.ग & 140.8 & 1423 & 143.8 & 142.2 & 130 & Tऊ.3 \\
\hline Instrem Tomp.f & 1302 & 1301 & 129.4 & 130.8 & 130 & 129.8 & 1302 & 128.8 & 728 & 129.8 \\
\hline Avp. Wall Tamp.f & 141.11 & 140.61 & 139.8 & 141.8 & 141.6 & 142.8 & 143.1 & 141] & 139.4 & 140 \\
\hline Avg. WaADT.F & 10.4 & 101 & 8.8 & 10.6 & 11.1 & 12.8 & 125 & 11.7 & 9.8 & 9.8 \\
\hline EX.HBuAlnatr & 857.4 & 993.8 & 996.1 & 989.8 & 888.3 & 7842 & 793.3 & 8426 & 990.8 & 1006.8 \\
\hline & ZONE2 & ZONE4 & 120NE6 & $20 N=8$ & ZONETO & & & & & \\
\hline DP Rquid Temp.F & 727 & & & & & & & & & \\
\hline DPU quid Density & 93.21 & & & 3 & & & & & & \\
\hline Envation-1 & 2 & 4 & 6 & 8 & $9.8 \% 5$ & & & & & \\
\hline Mescured DPFid & 0.003 & 1.008 & 1.763 & 2.61 & 2.874 & & & & & \\
\hline Zero Comoctonspsid & 0.316 & -0.008 & -0.04 & 0.055 & -0.324 & & & & & \\
\hline Corrected DPPsid & 0.319 & 1.105 & 1.783 & 2.685 & 3.188 & & & & & \\
\hline Aohted DPR & 1.62 & 2.47 & 3.48 & 4.18 & 5.31 & & & & & \\
\hline & & & & & & & & & & \\
\hline
\end{tabular}




\begin{tabular}{|c|c|c|c|c|c|c|c|c|c|c|}
\hline Aun: & $9 \approx 2$ & & 1 & & 1 & i & T & & & \\
\hline TaभFud & $75 \%$ CAF10 & 0-Z5\% & & & 1 & & 1 & & & \\
\hline Den: & 1 12/8/831 & & 1 & 1 & 1 & & 1 & & & \\
\hline Time: & $10: 00$ & & & & 1 & & i & & & \\
\hline Now & & & $i$ & & + & & i & & & \\
\hline Flow ATOLAR & 0.00536 & & & & & & & & & \\
\hline Heal fow Aseart? & 0.19095 & & & & & & & & & \\
\hline Sat. Prossure Pslg & 78.8 & & & & & & & & & \\
\hline Sed. Temperaturo finlet) & 1321 & & & & & & & & & \\
\hline Ind Frowne-GPM & 4.81 & & & & & & & & & \\
\hline Inter Tomperaturef & 129.51 & & & & & & & & & \\
\hline Inla Glom/hine & 988052 & & Tomp & 124.7 & Dansiy & 88.28 & & & & \\
\hline Subooding ff & 25 & & & & & & & & & \\
\hline Fow Observation & & & & & & & & & & \\
\hline & & & i & & & & & & & \\
\hline & ZONEA & ZONE? & ZONE & ZONE & ZONES & $\angle O N=6$ & ZONE7 & $\angle C A=8$ & $201=0$ & $20 \pi=10$ \\
\hline Hed lnpulW Whatned & 870.9 & 872 & 865.6 & 867.7 & 853.1 & 884.8 & 8627 & 857.8 & 8दरा & 8696 \\
\hline HarlossWa & 7.8 & 1.7 & 23 & 25 & 25 & 1.9 & 1.2 & 7.7 & दो & 11.8 \\
\hline Host fox Bulthil & 15098 & 15118 & 15000 & 15001 & 14931 & 1485 & 7485 & 160\% & T5TE: & 750072 \\
\hline WE Tomp. BF & 143.1 & 145.8 & 1426 & 745.6 & 146.7 & 146.8 & 188.1 & 1428 & 140.0 & 140.8 \\
\hline WE Tomp. IIF & 144 & 1424 & 144.4 & 748 & 148.6 & 146.6 & 148.2 & 14.2 & Tिद्र & 167.8 \\
\hline We'Tomp. 15F & 14.4 & 141.4 & 145.1 & 1781 & 14.7. & 145.6 & 745.6 & Tस्टि & 740.7 & $\$ 38.8$ \\
\hline WETTOM. IIF & 120.5 & 143.6 & 140.8 & 14.3. & 144.8 & 148 & 147.6 & 743.6 & 740.1 & 138.8 \\
\hline Wa Tomp. liz & 745.4. & 143.3 & 141.8 & 74.6 & 74.8 & 746.61 & 747.8 & 74.8 & रका & 710.4 \\
\hline Instresm Tomp,f & 130.2 & 130.1 & 129.7 & 7321 & 130.8 & 130.11 & 130.8 & 129.2 & 12.8.8 & 120.4 \\
\hline Avo. Well Tomp, f & 144.2 & 143.6 & 1426 & 145.6 & 146.8 & 14621 & 148.8 & T4.3.6. & दाता & सदा.2 \\
\hline Avo.WNDT.F & 13.3 & 12.8 & 12.1 & 128 & 18.8 & 15.4 & 15.2 & 13.7 & 11 & 112 \\
\hline EXP.HEWhrme & 1731.4 & 1977.1 & 12402 & 1174.7 & 10832 & 971.8 & 800.2 & 11012 & 1370.8 & 1350.7 \\
\hline & TOONE? & CORET & thases & का & & & & & & \\
\hline DP Wquid Temp,f & $\frac{\angle O N=2}{818}$ & ZONE4 & $20 N=6$ & ZONE8 & ZONE10 & & & & & \\
\hline DPLquid Density & $\frac{81.8}{92.18}$ & & & & & & & & & \\
\hline Erevionth & & & & & & & & & & \\
\hline Masurred DPAnid & $\frac{21}{-03281}$ & 4 & 6 & 8 & 9.8\% & & & & & \\
\hline Zero Comectionzerid & $\frac{-0.2281}{-0.2521}$ & 0.483 & 0.879 & 1.324 & 1.321 & & & & & \\
\hline Cormectopsid & $=0.2521$ & -0.083 & -0.037 & -0.051 & -0.25 & & & & & \\
\hline 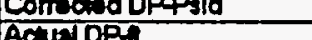 & $\frac{0.0251}{2.11}$ & 0.5161 & 0.916 & 1.375 & 1.347 & & & & & \\
\hline ACU1 DPA & $2 \pi$ & 3.41 & 4.88 & 6.25 & 7.8 & & & & & \\
\hline & & & & & & & & & & \\
\hline
\end{tabular}




\begin{tabular}{|c|c|c|c|c|c|c|c|c|c|c|}
\hline Runf & 9631 & 1 & i & & & & & & & \\
\hline Teqf Fuid & $175 \%$ C4F10 & -25\% RII & & & & & & & & \\
\hline Detw: & $1 \quad 12 / 8 / 93 \mid$ & 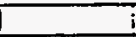 & $i$ & & & & & & L & \\
\hline Time: & $10: 00$ & I & 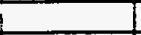 & & & & & & $t$ & \\
\hline Noto & 1 & & 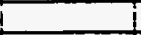 & & & & & & & \\
\hline Fow Areat 12 & 0.00386 & & & & & & & & & \\
\hline Heat Flow Areati2 & 0.19095 & & & & & & & & & \\
\hline Sa. Pressurofsig & 77.6 & 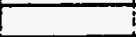 & & & & & & & & \\
\hline Sat. Tomperature F(inlet) & 131.11 & & & & & & & & & \\
\hline Inielfowme-GPM & 3.61 & & & & i & & & & & \\
\hline indel Temperaturef & 128.9 & 7 & 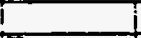 & & I & & & & & \\
\hline Intol Glbmmine & 742953 & & ITomp & 1227 & Donsity & 86.48 & & & & \\
\hline Subooolingf & 221 & & & & & & & & & \\
\hline Fow Obsenvion & $\mathrm{I}$ & I & 1 & & $!$ & & 1 & & & \\
\hline & $i$ & 1 & $i$ & & 1 & & & & i & \\
\hline & ZONE1 & ZONE2 & ZONEO & ZONEA & IZONEE & ZONE 6 & $20 \mathrm{~K}=7$ & ZONEB & ZONEO & $20 N=10$ \\
\hline 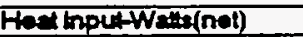 & 1851.3 & 864.5 & 860 & 853.2 & 8682 & 857.8 & 861.8 & 858.1 & 8627 & 8849 \\
\hline Hes Loss-Wats - & 8.1 & 1.9 & 26 & 2.7 & 27 & 2 & 1.3 & 1.9 & 23 & 13.3 \\
\hline Hext Fiox buthine & 14930 & 14986 & 74908 & 1493 & 14878 & 14881 & 14850 & 148\% & 14584 & 14682 \\
\hline Wa Tomp. \#FF & 141.5 & 144.3 & 1425 & 143.9 & 145.7 & 145.5 & 144.9 & 142.8 & 140.1 & 140.8 \\
\hline Wall Temp. I4F & 142.4 & 141.3 & 144.3 & 14.5. & 145.8 & 145.8 & 145.5 & 146.8 & 74.8 & 7472 \\
\hline WEH TOmp. $15 F$ & 1431 & 140.5 & 145.11 & 144.8 & 144.1 & 145 & 74.5.5 & 142 & 1405 & 33.8 \\
\hline Wal Tomp. IIF & 128.91 & 142.1 & 140.1 & 142.8 & 143.6 & 745.11 & 146.8 & 143.2 & 139.8 & 738.6 \\
\hline Wal Tomp. $12 F$ & 143.3 & 142.1 & 141.8 & 143.3 & 143.6 & 745.5 & 146.6 & 144.5 & 740.7 & 739.8 \\
\hline Intream Tomp.F & 120.7 & 129.8 & 129.6 & 130.8 & 130 & 129.4 & 130.1 & 128.8 & 128.7 & 129.3 \\
\hline Avg. Wall Tomp.f & 1426 & 142.5 & 142.1 & 144.1 & 144.8 & 145.4 & 145.5 & 143.2 & 140.6 & 14t.1 \\
\hline AM. WAHDT.F & 122 & 11.9 & 12 & 12.8 & 13.8 & 15.8 & 14.8 & 13.7 & 11.3 & 11.1 \\
\hline DP. HBuHtrier & 126.3 & 12522 & 1267.4 & 1180 & 1005.6 & 971.1 & 10112 & 1085.1 & 1327.8 & 9340,4 \\
\hline & & & & & & & & & & \\
\hline & ZONES & ZONET & ZONEE & ZONE8 & ZOKE $=10$ & & & & & \\
\hline DP Tquid Tomp.F & 76.2 & & & & & & & & & \\
\hline 6PUquid Donsity bom/13 & 92.81 & & & & & & & & & \\
\hline Elovetiont & 2 & 4 & 8 & 8 & 9.875 & & & & & \\
\hline Mesaured DPPsid & -0.006 & 0.83 & 1.389 & 2.035 & 2.232 & & & & & \\
\hline Zaro Corrections-psid & -0.2521 & -0.058 & -0.037 & -0.051 & -0.325 & & & & & \\
\hline Comeded DPP sid & 0.183 & 0.883 & 9.488 & 2.088 & 2.557 & & & & & \\
\hline Actud DPA & 1.84 & 2.88 & 4.06 & 5.11 & 6.84 & & & & & \\
\hline & & & & & & & & & & \\
\hline
\end{tabular}




\begin{tabular}{|c|c|c|c|c|c|c|c|c|c|c|}
\hline Rimn: & 964 & & i & & & & & & & \\
\hline Ta्मfind & $175 \%$ CAF10 & $-25 \%$ म114 & & & & & & & & \\
\hline Den: & $12 / 8 / 831$ & & I & & & & & & & \\
\hline Tims: & $10: 001$ & & 1 & & & & & & & \\
\hline Nots & & & & & & & & & & \\
\hline For Areate & 0.00356 & & & & & & & & & \\
\hline Hed flow ATeat2 & 0.1835 & & & & & & & & & \\
\hline Sel. Prossure Asto & 78 & & & & & & & & & \\
\hline Set Tompenturof(intol) & 737.4 & & i & & & & & & & \\
\hline InICT FowT-GPM & 2.41 & & & & & & & & & \\
\hline indet Tomperduref & $\{29.4$ & & & & & & & & & \\
\hline In'Gomntue & 755602 & & Tamp & 727 & Density & 86.48 & & & & \\
\hline Suboooting f & 2 & & & & & & & & & \\
\hline Fow Obscrvaton & t! & & & & & & & & & \\
\hline & 1 & & & & & & & & & \\
\hline & ZZONE1 & ZONE2 & ZONE3 & ZONEA I & ZONEE & $20 \mathrm{~K}=6$ & 2OK=1 & ZONE 8 & ZOK: & $201=10$ \\
\hline Hadinputwaterel) & 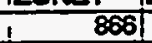 & 866 & 868 & $8 \times 5$ & 856 & 888 & $8 \times 8$ & 888 & 8 & 868 \\
\hline HeallosesWa & 8 & 1.8 & 2.5 & 27 & 27 & 2. & 7.4 & 2 & 23 & 14 \\
\hline 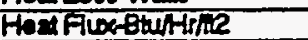 & 15012 & 15012 & Tड्राट & 15012 & 15012 & T5012 & 16012 & 15012 & 76012 & 75012 \\
\hline Wallomp. B & 141.4 & 144.1 & 142 & 144 & 145.8 & 145.8 & 148.1 & 143.6 & Tदा.S & 142.3 \\
\hline Wed Tomp. Iff & 142.81 & 141.3 & 743.8 & 74.4. & 145.8 & T45.8 & 148.71 & 145.1 & 763.8 & 168.8 \\
\hline WalTomp. $16+$ & 143.11 & 140.4 & 146 & T4.71 & 14 & 14.8 & 145.6 & 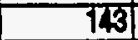 & Th.8 & T\$O. \\
\hline Writomp.l1F & 129.4 & 142 & T39.8 & 143.6 & 143.4 & 161 & T47.81 & 144.4 & 7506 & 136.8 \\
\hline WaTtomo. "Iz & 143.41 & 141.9 & 141.3 & 143.7 & 143.6 & 145.4 & 147.5 & 145.8 & TK1.6 & Tदा. \\
\hline Infrom Tomp.F & 1301 & 130.4 & 128.7 & 131.3 & 730.6 & 130.1 & अव & 729.8 & 20.7 & 130.4 \\
\hline Avo. Wdil Tomp.F & 1426 & 1428 & 141.8 & TAR.2. & T44.1 & 146.4 & 148.6 & 14.8 & 741.6 & TW24 \\
\hline Avg. Wall DT.F & 17.8 & 112 & 11.4 & 122 & 12.8 & 14.6 & 14.8 & 14 & 71.2 & 11.8 \\
\hline EXP.HBWWIRnRF & 1256.6 & 133.8 & 13172 & 120.8 & 1188.5 & 1006.7 & 1016.9 & 1069.1 & 1357.7 & 7350.6 \\
\hline & & & & & & & & & & \\
\hline & ZONEE & ZONES & $2 O N=6$ & $20 \pi=38$ & ZONETO & & & & & \\
\hline DP lquid Tomp.f & 24.1 & & & & & & & & & \\
\hline 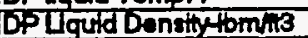 & 97.45 & & & & & & & & & \\
\hline Ererelont & 2) & 4 & 6 & & 9.875 & & & & & \\
\hline Mosuced brapsid & 0.049 & 7.1811 & T.8535 & 2.78 & 3.09 & & & & & \\
\hline Zero Cormections peld & -0.2521 & -0.0831 & -0.087 & -0.051 & -0.325 & & & & & \\
\hline Cormotophyd & 0.301 & T.244 & 7.87 & 2785 & 3.416 & & & & & \\
\hline AChis DP: & 1.75 & 2.69 & 3.48 & 4.38 & 5.44 & & & & & \\
\hline & & & & & & & & & & \\
\hline
\end{tabular}




\begin{tabular}{|c|c|c|c|c|c|c|c|c|c|c|}
\hline Runt & 965 & ; & $i$ & 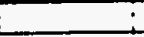 & 1 & $i$ & 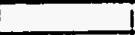 & & & \\
\hline Tost Fuid & $.75 \%$ CAF 10 & $-25 \%$ R114 & 1 & 1 & i & & & & & \\
\hline Dalo: & 12/8/33! & $!$ & $\therefore$ & i & 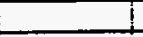 & & & & & \\
\hline Timo: & $10: 00_{1}$ & 1 & ; & i. & 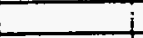 & & & & & \\
\hline Note & 1 & 1 & & 1 & 2 & & & & & \\
\hline Fow Ares-12 & 0.00366 & & & & & & & & & \\
\hline Hest Fow Areant? & 0.1905 & & & & & & & & & \\
\hline Set. Prossure Psiq & 109.1 & & & & & & & & & \\
\hline Sit. Temporatureff(inlot) & 150.3 & I & & & & & & & & \\
\hline Inlet flowra-GPM & 5 & & & & & & & & & \\
\hline Intol Tomperaturef & 748.9 & i & & & & & & & & \\
\hline Inlet Gbmhrne & 985669 & & Temp & 745.71 & Dansity & 82681 & & & & \\
\hline Subcoollingf & 1.4 & & & & & & & & & \\
\hline Fow Obsurvation & 1 & & & & & & & & & \\
\hline & & $i$ & 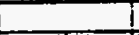 & ल & i & & & & & \\
\hline & ZONE & ZONE2 it & ZONEB 3 & 20NE4 & ZOFiEs & ZONiE6 & ZONE7 & ZONEB & ZONEO & ZONETO \\
\hline Heat lnpentwatts(nel) & 1860.3 & 870.6 & 888.5 & 868.8 & 871.5 & 869.8 & 871.1 & 860.8 & 874.7 & 867.6 \\
\hline Heat Lose-Watis & 10 & 2.3 & 3.1 & 3.2 & 3.2 & 2.5 & 1.6 & 2.3 & 20 & 16.4 \\
\hline Hes Flox-BtuHahe & 14913 & 15091 & $15000 \mid$ & 15025 & 15107 & 15079 & 15100 & 14918 & 15182 & 16089 \\
\hline Wail Tomp. 13F & 161.7 & 164.1 & 152 & 163.4 & 165.1 & 165.7 & 165.3 & 1 1ळ.8 & 181 & 160.7 \\
\hline Wal Tomp. W4F & 16.6 & 167.4 & 10.6 & 163.8 & 165.6 & 165.81 & 165.5 & 163.8 & 162.6 & 167.1 \\
\hline Walt Tomp. $15+$ & 162.81 & 10.1 & 164.4 & 164.8 & |ळ3.8 & 165.2 & 164.8 & 16्2ा & 160.8 & 758.4 \\
\hline WaI Tomp. IIF & 148.91 & 162.1 & 159.8 & $1 \approx 2.61$ & 168 & $1 \times 5.5$ & 168.7 & 163.2 & 160 & 768.7 \\
\hline Wad Tomp. ILF & 164.51 & $1 \sigma 21$ & 161.2 & 162.81 & 163.1 & 16.8 & 168.8 & 164.4 & 160.8 & 160.7 \\
\hline Instroen Temp.f & 149.51 & 149.7 & 149.7 & 151.11 & 150.3 & 149.9 & $15 t .2$ & 149.7 & 149.6 & 750.8 \\
\hline Avo. Wah Temp.F & 1629 & 1624 & 161.7 & 163.6 & 163.9 & $165.5 T$ & 165.8 & 163.2 & 161 & 161.3 \\
\hline Avg. Wat DT.F & 12.81 & 121 & 11.3 & 91.7 & 12.8 & 14.9 & 13.8 & 12.9 & 10.8 & 10 \\
\hline EP.HBWh-1h2/F & 1169.5 & 1255.71 & 13526 & 127,2 & 1169.51 & 1010.8 & 1086.5 & 1159.6 & 1360.4 & 1497.8 \\
\hline & & & & & & & & & & \\
\hline & ZONE2 & ZONE4 & ZONE6 & ZONE & $20 N=10$ & & & & & \\
\hline DP liguid Tomp.F & $1 \quad 81.5$ & & & & & & & & & \\
\hline DPLiquid Density 1 bm & 922 & & & & & & & & & \\
\hline Ejerationft & 2 & 4 & 6 & 8 & 9.875 & & & & & \\
\hline Massurad DPPSid & -0.185 & 0.524 & 0.88 & 1.468 & 1.589 & & & & & \\
\hline Zoro Corrections-psid & -0.2521 & -0.033 & -0.037 & -0.051 & -0.2251 & & & & & \\
\hline Correctad DPY sid & 0.067 & 0.557 & 0.917 & $7.517 \pi$ & 1.912 & & & & & \\
\hline Actual DPh & 212 & 3.49 & 5.1 & 6.29 & 7.09 & & & & & \\
\hline & & & & & & & & & & \\
\hline
\end{tabular}




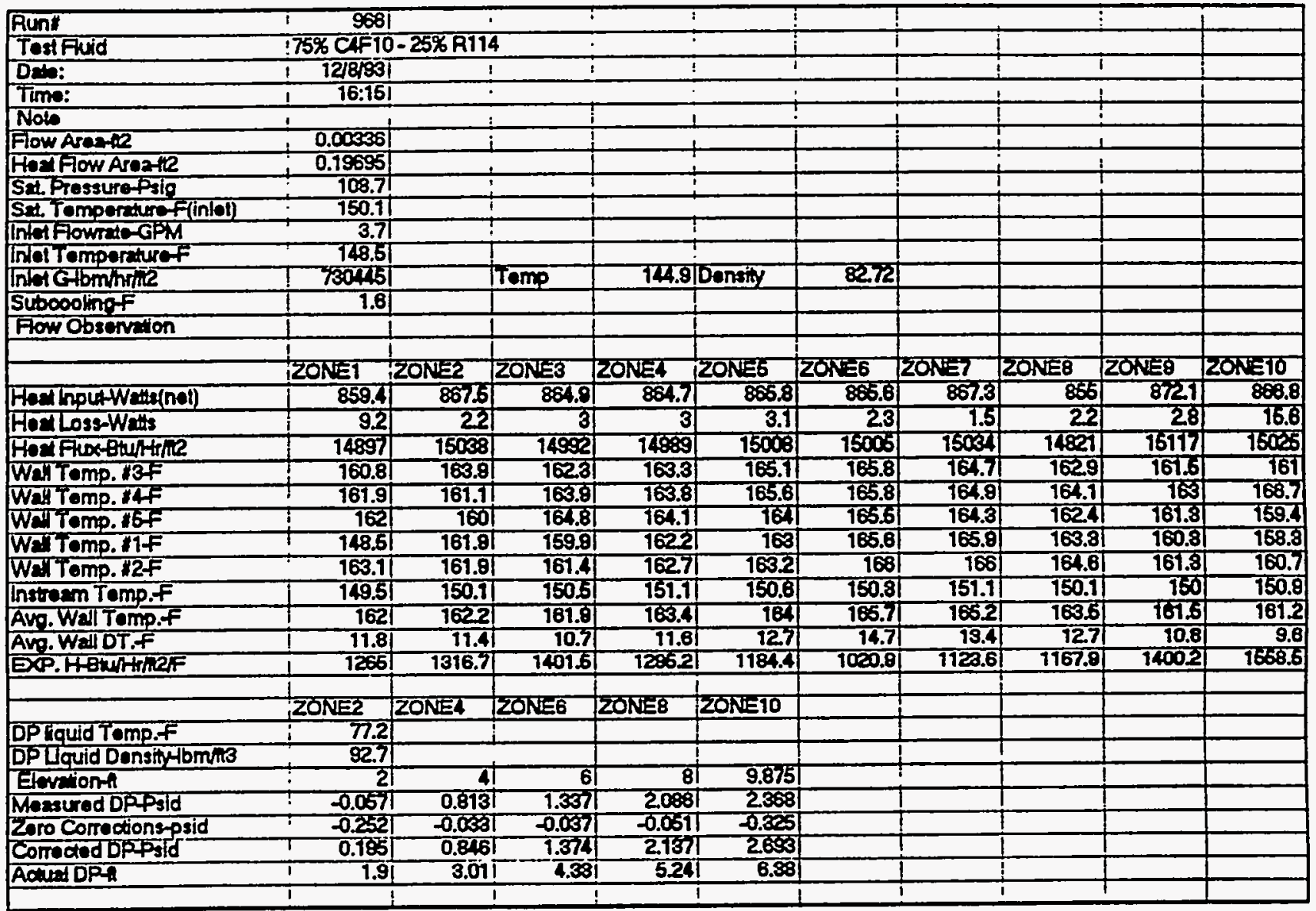




\begin{tabular}{|c|c|c|c|c|c|c|c|c|c|c|}
\hline Runt: & 967 & & & & . & & & $T$ & & \\
\hline Torf Fluid & $175 \%$ C4F 10 & $0-25 \% R 114$ & 4 & 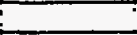 & I & I & & $T$ & T & \\
\hline Date: & $12 / 8 / 931$ & & $\div$ & 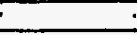 & $\therefore$ & & $i$ & 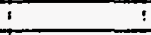 & 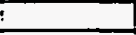 & \\
\hline Time: & $16: 15$ & & & & i & & 1 & 1 & 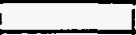 & \\
\hline Noto & $i$ & 1 & 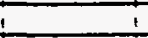 & 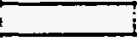 & 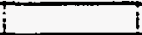 & & & & & \\
\hline Flow Area-d2 & 0.00336 & & İ & 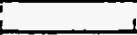 & 1 & & & & & \\
\hline Hox Fiow Areati2 & 0.19695 & & & & & & & & 1 & \\
\hline Sed. Pressurefsig & 107.3 & & & & & & & & & \\
\hline Sat. Temporature f(inlet) & 149.3 & & $\bar{\vdots}$ & 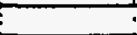 & $\cdot$ & i & & & & \\
\hline Indet flowrato-GPM & 2.51 & & i & 5 & i & & & & & \\
\hline indel temperaturef & 148.71 & & $\bar{\vdots}$ & $\vdots$ & $T$ & & & & & \\
\hline Intet Glbmhrnit & 4951461 & & Temp & 143.4 & Toensidy & 8299 & & & & \\
\hline Subcoolingf & 1.21 & & T-1 & -7 & 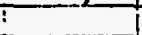 & 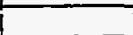 & i & & & \\
\hline \multirow[t]{3}{*}{ Fow Obsenvation } & 1 & $!$ & I & I & 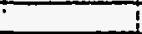 & & 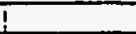 & I & I & \\
\hline & 1 & & 1 & + & & & 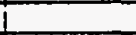 & & & \\
\hline & ZONE1 & ZONE? & ZONE3 & LONE4 & ZONE5 & ZONE & ZONET & ZONE8 & ZONEO & ZONE 10 \\
\hline Heal hpt W (net) & i 853.31 & 870.1 & 884 & 862.1 & 868.9 & 82.9 & 868.7 & 869.11 & $862 \pi$ & 57.8 \\
\hline Hod Loss-Watts & 10.6 & $25 !$ & 3.4 & 3.5 & 3.5 & 2.6 & 1.7 & 26 & 3.8 & 14.7 \\
\hline Haad Fux-BhuHrhle & 14965 & 15083 & 1497 & 1494 & 15027 & 14958 & 15024 & 15055 & 1494 & 1002 \\
\hline Wal Tomp. WF & 159.5 & 162.9 & 160.8 & 161.8 & 163.7 & 184.8 & 164 & 1627 & 180.4 & 151.8 \\
\hline Wall Tomp. "4F & 160.8 & $1 \approx 0$ & 1624 & 1622 & 164.1 & 164.8 & 964.5 & 763.7 & 180 & 152.4 \\
\hline Wal Tomp. $15-F$ & 160.81 & 158.9 & 164.ा & $1 \approx 2.7$ & $1 \approx 25 \mid$ & 163.6 & $1 \approx 5.4$ & 161.9 & 160.6 & 1527 \\
\hline Wa Tomp. IIF & 148.11 & 160.8 & 158.7 & 160.8 & 161.5 & $1 \approx 3.8$ & 164.9 & 163.2 & 159.4 & 156.8 \\
\hline Wal Tomp. B2F & T61.6 & 160.4 & TE.11 & 161.3 & 161.71 & 164.7 & 165 & T64.3 & 760.4 & 756.4 \\
\hline Instream Tomp.f & 148.9 & 150.71 & 149.7 & 150.7 & 150.1 & 148.8 & 750.8 & 749.8 & 749.8 & 150.6 \\
\hline Avg. Wall Tomp.f & 160.7 & 1811 & 160.5 & 181.9 & 162.6 & 1642 & 169.4 & 163.2 & 168.1 & 153.8 \\
\hline Ang. Wai DT.f & 11.1 & 10.2 & 10.11 & 10.5 & 11.7 & 13.7 & 129 & 12.7 & 17.8 & 9.8 \\
\hline \multirow[t]{3}{*}{ 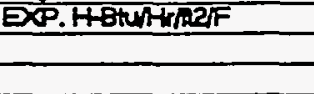 } & 1348.9 & 1479.61 & 14785 & 1630.7 & 1287.6 & 1091.8 & 1166.8 & 1188.21 & 836.6 & 304.1 \\
\hline & & & & & & & & & & \\
\hline & ZONE2 & ZONE 4 & ZONE & $20 \mathrm{~N}=8$ & ZONIETO & & & & & \\
\hline DP hquid Tomp.F & 7.1 & & & & & & & & & \\
\hline DPLiguid Densitylbm/13 & 92.71 & & & & & & & & & \\
\hline Elovetiont & 2 & 41 & 61 & 8 & 9.875 & & & & & \\
\hline Measured DPPsid & 0.0039 & 1.154 & 1.848 & 2774 & 3.201 & & & & & \\
\hline Earo Corrections-psid & -0.2521 & -0.0391 & -0.037 & -0.051 & -0.355 & & & & & \\
\hline Corrected DPF sid & 0.2911 & 1.187 & 1.885 & $28 \leq 5$ & 3.589 & & & & & \\
\hline \multirow[t]{2}{*}{ Actud DPA } & 1.731 & 2.41 & 3.48 & 4.04 & 4.8 & & & & & \\
\hline & 1 & +1 & -1 & 1 & & & & & 1 & \\
\hline
\end{tabular}




\begin{tabular}{|c|c|c|c|c|c|c|c|c|c|c|}
\hline Run: & 9681 & & $i$ & i & i & $i$ & 1 & & 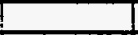 & \\
\hline Tert Fluld & $75 \%$ CAF10 & J.25\%R114 & & 1 & 1 & & i & & & \\
\hline Dade: & 1 129981 & 1 & 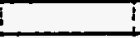 & 1 & I & & & 1 & 1 & \\
\hline Timo: & $12: 16$ & $!$ & 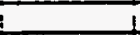 & 1 & 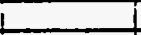 & & & & i & \\
\hline Nob & & & & & 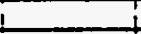 & & & & & \\
\hline Fow ATOA-12 & 0.00336 & & & & & & & & & \\
\hline Hed Flow Areant? & 0.1959 & & & & & & & & & \\
\hline Su. Prossure Psig & 106.2 & & & & & & & & & \\
\hline Su. Tomporaturo f $($ inlet $)$ & 148.7 & & & & & & & & & \\
\hline IndfFowrate-GPM & 4.9 & $\bar{i}$ & & & & & & & & \\
\hline Intet tomperaturef & 14798 & $?$ & & & & & & & & \\
\hline Intot Gibmhrine & 97अाओ। & & Tromp & Tक्छा & Densily & 83.08 & & & & \\
\hline Suboooling & 0.9 & & & & & & & & & \\
\hline Fow Obsorvation & ! & i & $\perp$ & 1 & 1 & & & & & \\
\hline & 1 & & & & 1 & & & & & \\
\hline & ZON=1 & ZONEZ I & ZONE3 & ZONE4 & ZONES & ZONiE6 & ZONE & ZONE8 i & ZONE9 & $20 N=10$ \\
\hline Hod input-Walts(nel) & 5832 & 579.7 & 5792 & 576.6 & 579.1 & 578.1 & 580.8 & 580.1 & 575.2 & 581 \\
\hline Healoss-Walls & 10 & 2.3 & 3.1 & 3.2 & 32 & 23 & 1.4 & 2.1 & 28 & 15.8 \\
\hline 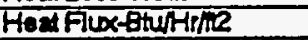 & 10109 & 10049 & 10040 & 9995 & 10038 & 10021 & 10058 & 10066 & 997 & 1007 \\
\hline WaA Tomp. H3F & 159 & 160.1 & 159.1 & 160.3 & 161.2 & 181 & 158.1 & 757.8 & 157.6 & 157.1 \\
\hline Waf Temp. IfF & 159.51 & 158.41 & 160.2 & 160.7 & 161.4 & 161.11 & 157.91 & 157.8 & 758.7 & 160.9 \\
\hline Wall Tomp. $15+$ & 159.8 & 157.81 & 161.4 & 160.7 & 160.3 & 160.5 & 158.4 & 157.3 & 167.6 & 156.1 \\
\hline Wh Temp. ITF & 147.8! & 159.21 & 157.51 & 759.31 & 759.61 & 161.21 & T61.7 & 157.41 & 755.6 & 155.2 \\
\hline Wall Tomp. $2 F^{-}$ & 160.9 & 159.11 & 158.51 & T59.81 & 159.8 & 161.1 & 161.3 & 157.9 & 156.11 & 156.6 \\
\hline Instruen Tomp.f & 148.8 & 148.7 & 1492 & 150.31 & 149.8 & 149.8 & 150.6 & 149.5 & 149.5 & 749.5 \\
\hline Ang. Wall Tomp.F & 159.8 & 15921 & 158.8 & 16.8 .8 & 160.3 & 181 & 159.4 & 157.7 & 157.1 & 1572 \\
\hline Av. WaHDT.F & 10.7 & 101 & 9.2 & 9.5 & 9.8 & 10.7 & 8.3 & 7.7 & 7.1 & 7.2 \\
\hline EXP.HEWWWMRE & gदा & 1000.3 & 1064.7 & 10523 & 1011.8 & $\$ 56.8$ & 1212.3 & 1305 & 139.8 & 739 \\
\hline & & & & & & & & & & \\
\hline & ZONE2 & ZONER & ZONE 6 & $201 \mathrm{~N}=8$ & ZONETO & & & & & \\
\hline DP lquid Tomp.f & 173.2 & & & & & & & & & \\
\hline DPLiquid Density tom/n3 & 93.15 & & & & & & & & & \\
\hline Elevion-fl & र & & 61 & 8 & 9.875 & & & & & \\
\hline Messured DPFsid & -0.234 & 0.4851 & 0.818 & 1.352 & 1.49 & & & & & \\
\hline Zoro Corrections-psid & -029 & -0.061 & $=0.03$ & -0.052 & -0.328 & & & & & \\
\hline Comodod DPPyid & 0.57 & 0.508 & 0.882 & 1.404 & 1.818 & & & & & \\
\hline Actud DPA & 2.14 & 3.61 & 53 & 6.5 & 7.8 & & & & & \\
\hline & & & & & & & & & & \\
\hline
\end{tabular}




\begin{tabular}{|c|c|c|c|c|c|c|c|c|c|c|}
\hline Run: & 99 & & & & & & $!$ & & & \\
\hline Test Fluid & $75 \%$ C4F 10 & $0-25 \%$ R114 & & & 1 & & $\bar{\vdots}$ & & & \\
\hline Date: & 122983 & $\longrightarrow$ & : & 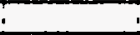 & 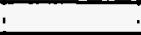 & & $!$ & & i & \\
\hline Timo: & $12: 16$ & 7 & 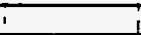 & 7 & & & $i$ & & $i$ & \\
\hline Nob & + & & 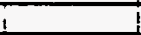 & ? & & & & & & \\
\hline Fow Area-12 & 0.00036 & & & & & & & & & \\
\hline Hent Fow Areaft' & 0.1969 & & & & & & & & & \\
\hline Sat. Pressuro-Psig & 908.11 & & & & & & & & 1 & \\
\hline Sat. Temperature-F(inlet) & 149.7 & T & i & & & & t & & + & \\
\hline Inlatflowrato-GPM & 3.7 & & T & T & & & & & & \\
\hline intel Temperaturef & 149.7 & & & 7 & & & & & & \\
\hline Inlot GHbmhrint & 731554 & & Tomp & 744.2 & TDensity & 82.85 & & & & \\
\hline Subcooling $f$ & 0 & & 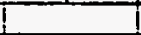 & 21 & 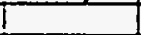 & & & & & \\
\hline Fow Observation & & i & i & 1 & & & i & & & \\
\hline & & & I & 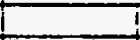 & & & & & & \\
\hline & ZZONET & ZONE2 & ZONE3 & ZONE4 & ZZONES & ZONE6 & ZONE7 & ZONEB & RONES & ZON:ETO \\
\hline Heal Input-Watts(nol) & 581.4 & 577.6 & 5727 & $5 \%$ & 578.4 & 578.8 & $5 \% .8$ & 577.4 & 559.1 & 574 \\
\hline HedLoss-Watts & 10.2 & 2.3 & 3.2 & 3.2 & 3.2 & 24 & 1.5 & 221 & 2.8 & 16.4 \\
\hline Head FLx-BhuHr/nL & 10078 & 10012 & 9927 & 98871 & 10044 & 10033 & 10050 & 10000 & 9885 & 9350 \\
\hline Wall Temp. \#3F & 160.1 & 161.2 & 160.5 & 161.4 & 162.6 & 162.1 & 159.2 & 159.2 & 158.8 & 158.6 \\
\hline Wal Temp. $4 F$ & 160.7 & 159.9 & 161.6 & 161.7: & 162.9 & 1 1ळ.2.3! & 159 & 159.4 & 159.8 & 162.3 \\
\hline Wall Temp. I5F & 160.8 & 159.2 & 162.7 & 161.7 & 767.6! & 161.81 & 159.9 & 159 & 758.8 & 157.7 \\
\hline Well Tomp. If & 149.7 & 160.11 & 158.6 & 160.7 & 160.7 & 16251 & 162.7 & 158.71 & 156.0 & 157.1 \\
\hline Wallomp. \#2F & 162 & 160.11 & 159.6 & 1611 & 161.2! & 1525! & 152.5 & 159.11 & 157.4 & 758.2 \\
\hline instroan Tomp.f & 150.5 & 150.71 & 151 & 151.4 & 151.1 & 150.91 & $151.8 !$ & 150.7 & 150.6 & 151 \\
\hline Avg. Wall Tomp.f & 160.9 & 100.81 & 160.1 & 161.41 & 161.8 & 1622 & 160.71 & 159.11 & 158.4 & 758.8 \\
\hline Avg. Wat DT.F & 9.9 & 9.21 & 8.8 & 9.5 & 10 & 10.81 & 8.4 & 7.9 & 7.3 & 7.3 \\
\hline DXP.HEturtrim & 1013.4 & 1091.61 & $1150.8 \mathrm{i}$ & 1037 & 999.7 & 9251 & 1195.7 & 126261 & 1345.1 & 1357.4 \\
\hline & 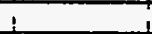 & 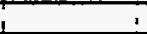 & & & & & $!$ & & 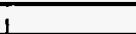 & \\
\hline & ZONE2 & ZONE4 & ZONE6 & ZONEB & ZONE10 & & 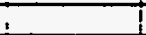 & & & \\
\hline DP Fquid Tomp.f & 71.4 & & & & -1 & & 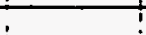 & & & \\
\hline DPL'quid Densitylbm/13 & 93.35 & & & & & & & & & \\
\hline Elovetiont & 2 & 41 & 6) & 8 & 9.8751 & & & & & \\
\hline Mescurad DPफid & -0.116 & $0 . \pi \pi$ & 1.217 & 1.8891 & 2.1531 & & $T$ & & & \\
\hline Zero Corrections-psid & -0.291 & -0.0431 & -0.043 & -0.0521 & -0.328 & & & & & \\
\hline Corrected DPpsid & 0.175 & 0.731 & 1.28 & 1.9411 & 2.481 & & $T$ & & & \\
\hline Actual DPR & 1.95 & 32 & 4.57 & 5.84 & 6.81 & & $\mathrm{~T}$ & & & \\
\hline & 1 & & & 1 & & & $i$ & & & \\
\hline
\end{tabular}




\begin{tabular}{|c|c|c|c|c|c|c|c|c|c|c|}
\hline Runt & ! 9701 & : & 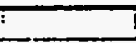 & $I$ & $i$ & $!$ & $:$ & $!$ & T & \\
\hline Tast fivid & $.75 \%$ C4F 10 & -25\%R114 & & & & 1 & $!$ & & t & \\
\hline Dan: & $.12 / 9331$ & & 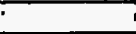 & i & $!$ & I & $!$ & $!$ & ! & \\
\hline Timo: & $12: 16 i$ & 1 & 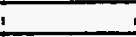 & $i$ & $i$ & 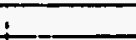 & $i$ & - & $:$ & \\
\hline Now & 1 & & & 7 & 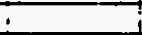 & i & 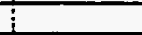 & & $!$ & \\
\hline Fow Araste & 0.00336 & & + & & 1 & & & & & \\
\hline Head flow Asoand: & 0.19595 & & & & & & & & & \\
\hline S2. Pressuro Prig & 107.3 & i & i & & & 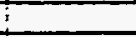 & I & & & \\
\hline Sat. Temperature f(inled) & 149.3 & ; & 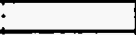 & & & $t$ & $i$ & & & \\
\hline Intelfowratc-GPM & 2.51 & t & $t$ & & 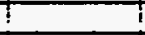 & $t$ & & & & \\
\hline intertomporturef & 148.5 & T! & 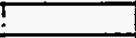 & & I & & & & & \\
\hline IntalGbmhrne & 496524 & & Tomp & 142.1 & Donsity & 832 & & & & \\
\hline Subooolingf & 0.8 & & & 1 & 1 & & & & & \\
\hline \multirow[t]{3}{*}{ Fow Obsorvation } & it & & I & 7 & T & & & & & \\
\hline & 1 & & & & 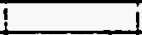 & 1 & & & & \\
\hline & ZONEI & ZONES T & ZONE3 & ZONE & ZONES & ZOW 6 & 12ONET & $20 N=8$ & ZON:39 & ZONE10 \\
\hline Healnpetwatu(not) & 585.7 & 581.8 & 573.7 & 57.4 & 580.8 & 580.1 & 584 & 587.7 & 670.5 & 674 \\
\hline HenLose-Wetts & 10.3 & 24 & 3.2 & 3.3 & 3.3 & 2.5 & 15 & 23 & 20 & T7.T \\
\hline Hest Flox-guifinth & 1074 & 70082 & 9945 & 9005 & $1008 \pi$ & 10058 & 10123 & 10083 & 9882 & 980 \\
\hline Wa'Tomp. BF & 159.6 & 160.8 & 1602 & 161 & 1623 & 1623 & 159 & 159.1 & 150 & 160 \\
\hline Wal Tomp.14F & 16021 & 159.2 & 161.8 & 961.1 & 162.4 & 1524 & 159 & 159.4 & 160 & 102.7 \\
\hline Wal Temp. $15+$ & 160.3 & 158.8 & TE24 & 161.31 & 161.2 & 961.8 & 159.8 & 150 & ibo & 158 \\
\hline WeTtomp.11F & 148.6 & 159.81 & 7682 & 159.8 & 160.4 & 162.1 & 1626 & 158.7 & 158.8 & 157.1 \\
\hline WCTomp. IL & 161.51 & 759.81 & 159.3 & $160.6]$ & 160.5 & 162.1 & 162.6 & 150 & 157.5 & 758.8 \\
\hline Instresm Tomp.F & 149.8 & 150.8 & 150.6 & 150.81 & 150.8 & 150.6 & 151.4 & 150.8 & 1505 & 1512 \\
\hline Avo. Well Tomp.f & 160.4 & 159.8 & 169.8 & 160.8 & 761.1 & 162.2 & 160.6 & 159 & 768.6 & 159.1 \\
\hline Avg. WadDT.F & 10.8 & 8.8 & 8.7 & 9.5 & 9.9 & 11.1 & 8.7 & 8 & 7.5 & 7.4 \\
\hline \multirow[t]{3}{*}{ EP.HEWHKIRT } & 968.1 & 1166.3 & 1143 & 1046.1 & 1010.7 & 906.3 & $11 \approx .3$ & 12620 & 7316.8 & 1339.1 \\
\hline & & & & & & & & & & \\
\hline & ZONE2 & ZONE4 & ZONEE & ZONEB & ZONE10 & & I & & & \\
\hline DPGquid Tamp.F & 70.4 & & & 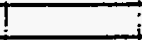 & & & 1 & & & \\
\hline DPLuquid Density 4 bmit3 & 93.46 & & - & $T$ & & & $\vdots$ & $!$ & & \\
\hline Eleventiont & 2 & 4 & $\overline{6}$ & 8 & 9.875 & & i & $i$ & & \\
\hline Mesaured DPFis & -0.015 & 0.9791 & 1.639 & 2466 & 2.871 & & $\therefore$ & 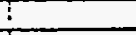 & & \\
\hline Lero Corrections-psid & -0.291 & -0.0431 & $=0.048$ & -0.052 & -0.3281 & & $T$ & I & & \\
\hline Comeded DPpid & 0.278 & $1.02 \pi$ & 9.682 & 2.518 & 3.199 & & & & & \\
\hline \multirow[t]{2}{*}{ Aculd DP } & 1.77 & 272 & 3.83 & 4.631 & 5.51 & & & & & \\
\hline & I & 1 & $i$ & 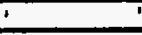 & & $i$ & 1 & $i$ & & \\
\hline
\end{tabular}




\begin{tabular}{|c|c|c|c|c|c|c|c|c|c|c|}
\hline Run" & 971 & & & & $i$ & 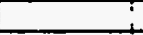 & & & & \\
\hline Testfluid & $175 \%$ C4F 10 & $-25 \%$ Rा14 & & & 1 & $i$ & $!$ & 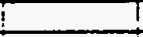 & I & \\
\hline Dato: & 1 121933। & & $!$ & i & 1 & $?$ & 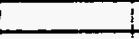 & & I & \\
\hline Time: & $12: 16 i$ & $!$ & $\therefore$ & 1 & 1 & $\vec{i}$ & 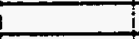 & & 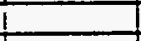 & \\
\hline Nole & $!$ & ? & & 1 & 1 & 1 & 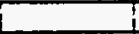 & & & \\
\hline Fow Arente & $0.00335 !$ & $i$ & & $\dot{.}$ & $i$ & 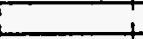 & & & & \\
\hline Heat Fow Areante & $0.19595 !$ & & & & I & 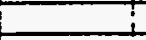 & & & & \\
\hline Sod. Pressure Psig & 106.51 & & & & $T$ & 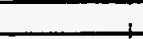 & & & & \\
\hline Sal. Temperature F(inlel) & 148.9 & & 1 & 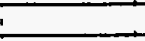 & 1 & - & I & i & & \\
\hline Inteffowrate-GPM & $5 i$ & & $\therefore$ & $\therefore$ & ! & 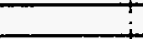 & & & & \\
\hline indel Temperaturef & 148.5 & i & $i$ & 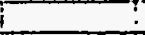 & & & & & & \\
\hline Intel Gibminric & S884441 & & Temp & 145.2I & Density & 82.671 & & & & \\
\hline Subcoolinge & $0.41^{\circ}$ & 7 & - & 5 & 1 & ! & & & & \\
\hline Flow Obsonviton & 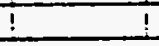 & 1 & 1 & 1 & 1 & 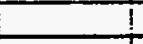 & & & & \\
\hline & 1 & : & $\vdots$ & 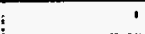 & $!$ & 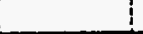 & i & 1 & 1 & \\
\hline & ZONEI IS & ZONE? : & ZONEB is & ZONEA & IZONES IS & $20{ }^{5}=6$ & ZONE? & ZONEB & ZONEO & $201=10$ \\
\hline Heat inpertwatis(nel) & 284.1 & 2842 & 286.4 & 2002 & 288.8 & $\mathbf{2 0 0}$ & 288.0 & 287.8 & 29.1. & 239.5 \\
\hline Heat Loss-Walls & $9.2 !$ & 2.1 & 27 & 28 & 27 & 2) & 1.3 & 1.8 & 2.4 & 13.8 \\
\hline Heat Fix -Bushthe & 4925 & 4926 & $4 \overline{55}$ & 5050 & 5008 & 5027 & 5008 & 4939 & 5018 & 5001 \\
\hline WaH Temp. \#3F & 158.11 & 157.1 & 156.61 & 157.4 & 157.8 & 157.1 & 155.8 & 154.8 & 154.8 & 1642 \\
\hline WaH Temp. :4F & 158.31 & 157 & 156.9 & 157.4 & 157.7 & 157.8 & 155.1 & 154.8 & 165.8 & 15.7. \\
\hline Well Tomp. I5F & 158.81 & 156.7 & 158.21 & 157.4 & 157.11 & 157.11 & 150.7 & 154.8 & 154.8 & 15..1 \\
\hline Wal Temp. If & 148.51 & 1572 & 155.61 & 156.9 & 156.7 & 157.5 & 157.5 & 15.5.6 & 763.4 & 153,8 \\
\hline Wal Tomp. $2 f$ & 158.71 & 15721 & 156.3 & 157.2! & 158.7 & 157.1 & 157.5 & 154.8 & 163.7 & 154.2 \\
\hline Instrosn Temp.fF & 149.31 & 148.91 & 149.21 & 150.3 & 150.1 & 149.71 & 150.51 & 149.2 & 749.1 & 148.6 \\
\hline Ang. Well Temp.F & 158.5 & 157.1 & 156.4 & 157.8 & 157.1 & 167.4 & 158.21 & 154.7 & 165.4 & 164.4 \\
\hline Avg. Wa!d DT.f & 91 & 81 & 6.8 & 6.7 & 6.7 & 7.4 & 5.5 & 5.8 & 6 & 4.8 \\
\hline EXP. HEtuhrner & $550.1 i$ & 615.61 & 716.8 & 7455 & 744.5 & 675 & 911.6 & 945.8 & 10028 & 1053.3 \\
\hline & & & & & & & & & & \\
\hline & ZONEE : & ZONE4 & ZZONES & ZONE8 & ZONETO & & & & & \\
\hline DP gquid Temp.f & 71.11 & & 1 & & & & & & & \\
\hline DPLiquid Dansity $1 \mathrm{bm} / \mathrm{i} 3$ & \$3.38 & & & & i. & & & & & \\
\hline Elovion & $2 i$ & $4 i$ & 6 & 8 & 9.875 & & & & & \\
\hline Mesured DPpsid & -0.2351 & 0.312 & 0.517 & 0.551 & 1.052 & & & & & \\
\hline Zero Corrections-osid & $\$ .3911$ & -0.0 .31 & -0.063 & -0.0521 & -0.328 & & & & & \\
\hline Cortectad DFPsid & 0.055 & 0.3051 & 0.56 & 1.0031 & 1.38 & & & & & \\
\hline Actual DP & 2161 & 3.9 & 5.8 & 7.20 & 8.79 & & & & & \\
\hline & 1 & 1 & 1 & 1 & I & & & & & \\
\hline
\end{tabular}




\begin{tabular}{|c|c|c|c|c|c|c|c|c|c|c|}
\hline Run! & : 9721 & & 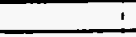 & 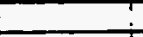 & & 1 & & 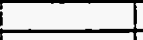 & & \\
\hline Test Fuid & $: 75 \%$ C4F 10 & $-25 \% \mathrm{A114}$ & $i$ & 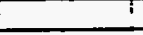 & & & & & & \\
\hline Dats: & $1 \quad 12993$ & ! & $\vdots$ & 1 & 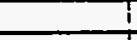 & 1 & & & 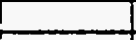 & \\
\hline Time: & $12: 16 !$ & 1 & + & $i$ & 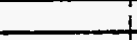 & & & & L & \\
\hline Note & ? & $\perp$ & - & 1 & 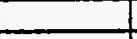 & & & & & \\
\hline Fow Areare? & +0.00336 & 1 & & 1 & & & & & & \\
\hline Hest Fow Arestie & 0.1959 & & + & 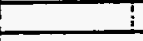 & & & & & & \\
\hline St, Prossure Psig & $107.8 !$ & & i & 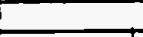 & & & & & & \\
\hline Sat. Temperaure ffinlot) & 149.6 & & 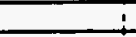 & 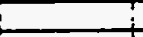 & & + & & & & \\
\hline Intet Fowride-GPM & 3.71 & & $!$ & 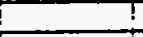 & & & & & & \\
\hline Intat remperituref & 149.51 & & $T$ & ! & 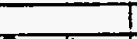 & & & & & \\
\hline Intal Gimminie & 7313951 & & Temp & T4.31 & Densidy & 82.831 & & & & \\
\hline Swocooling & $0.7 !$ & & I & 1 & & & & & & \\
\hline Fow Obsenvaon & : & 1 & $!$ & 1 & 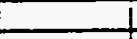 & i & & & & \\
\hline & 1 & $i$ & 1 & 1 & 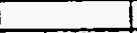 & 1 & 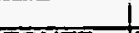 & 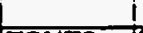 & & \\
\hline & ZZONE1 & ZONE2 I & $20 N=3$ & RONE4 I & ZONES & $20 N=6$ & ZONEE? I & ZONEB I & ZONEO & 201E \\
\hline Hanlnpu-Watls(not) & 12895 & 287.8 & 200.8 & 295.21 & 205.8 & 298 & 293.8 & 2928 & 205.7 & 292.7 \\
\hline Hed LoseWalls & 8.71 & 2 & $2 B$ & 27 & 26 & 1.9 & 12 & 1.8 & 23 & 13.4 \\
\hline Heat finoturhine & 5018 & 49891 & 5043 & 5117 & 5129 & 5166 & 5035 & 5057 & 5128 & 5074 \\
\hline WaH Tomp. KF & 158.5 & 157.7 & 157.21 & $158.4 !$ & 158.6 & 158 & 156.4 & 155.7 & 155.8 & 155.6 \\
\hline Wall Tomp. N4F & 158.5 & 157.4 & 157.51 & 158.31 & 158.8 & 158.41 & 156.31 & 155.81 & 1561 & 157 \\
\hline Wal tomp. $15 \%$ & 159.1 & 1572! & 158.6 & 158.3 & 157.9 & 157.81 & 156.9 & 155.41 & 155.41 & 155.1 \\
\hline Waltomp. ITF & $\{49.51$ & 157.7 & 156.11 & 158.31 & 157.4 & 158.11 & 159 & 155.4 & 15.11 & 165.3 \\
\hline Wultomp. $12 F$ & 159.8 & 157.81 & $156 . \overline{81}$ & 158.81 & 157.4 & 1581 & 158.8 & 155.81 & 154.4 & 155.8 \\
\hline Instrean Temp. F & 1501 & 14951 & 149.81 & 151.11 & 150.4 & 150.11 & 151.31 & 149.8 & 149.8 & 1502 \\
\hline Ave. Wall Temp.f & 1691 & 157.7 & 156.8 & 158.8 & 157.8 & 158.11 & 157.51 & 155.6 & 75 & 155.8 \\
\hline Avo. WanDT.F & 8.71 & 7.81 & 6.8 & 7 & 72 & 7.7 & 5.8 & 5.5 & 5.2 & 5.3 \\
\hline EP.HBtuHnLaf & 573.6 & 620.51 & 739.11 & 731.71 & 713.1 & 657.31 & 856.3 & 9227 & \$9.1 & $\$ 51.8$ \\
\hline & 1 & & & & & 1 & & & & \\
\hline & ZONE2 & ZONE4 & 201: & ZONE8 & ZONETO & & & & & \\
\hline DPliquid Temp.F & 76 & & & & & & & & & \\
\hline DPUquid Densitylbmn3 & 92.84 & & & & & & & & & \\
\hline Evation-t & 2 & 4 & 61 & $8 !$ & 9.875 & & & & & \\
\hline Neasured DPFsid & -0.168 & 0.5 & 0.855 & 1.4141 & 1.612 & & & & & \\
\hline zoro Correctonsupsid & 20.291 & $=0.068$ & -0.013 & -0.0521 & 0.3 .01 & & & & & \\
\hline Corrected DPpsid & 0.128 & $0.5 \%$ & 0.8881 & 1.4681 & 1.97 & & & & & \\
\hline ActulDPa & 2.08 & 3.54 & 5.16 & 6.42 & 7.69 & & & & & \\
\hline & i & I & 1 & 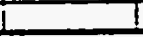 & & & & & & \\
\hline
\end{tabular}




\begin{tabular}{|c|c|c|c|c|c|c|c|c|c|}
\hline Runt & 9731 & & & & & & : & & \\
\hline Test Fuid & $75 \%$ CAF 10 & $-25 \%$ R114 & & 1 & 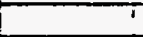 & 1 & & 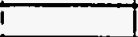 & \\
\hline Date: & 12ब331 & 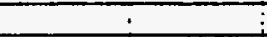 & & 1 & $i$ & $i$ & : & $i$ & \\
\hline Tume: & $12: 16$ & 1 & & $\dot{-}$ & 1 & 1 & $i$ & 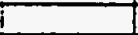 & \\
\hline Nots- & 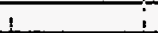 & i & & 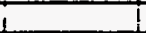 & & 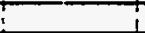 & 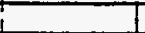 & & \\
\hline Fow Area-ile & 0.00335 & + & & 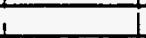 & & 1 & & & \\
\hline Head Fow Area-i2 & 0.19695 & $\frac{1}{1}$ & & 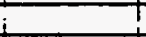 & 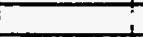 & & & & \\
\hline Sad. Prossuropsip & 107.8 & 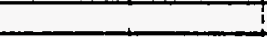 & - & 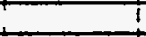 & & & : & & \\
\hline Sat. Temperaturef finlef) & 149.61 & & & T & 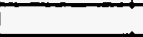 & & & & \\
\hline Inlet Flowrato-GPM & 2.51 & & & T & & & & & \\
\hline intal remperature & 148.71 & & & $!$ & & & & & \\
\hline Intat Glom/nine & प्35181 & romp & 7423 & Density & 83.21 & & & & \\
\hline Subcoolingf & 0.9 & ( & 1 & 1 & & $\Gamma$ & & & \\
\hline Frow Obsenvation & 1 & 1 & I & I & $\vdots$ & 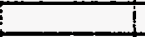 & I & 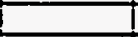 & \\
\hline & 7 & 1 & 1 & 1 & & I & & 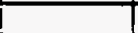 & \\
\hline & :ZONE1 IZ & ZONE2 IZONE3 & ZONEA & ZONES & ZOKE6 & ZONET & ZONEB I & ZONAE & $201 \mathrm{a}=10$ \\
\hline Heat inputWatis(net) & $1 \quad 288.4$ & \begin{tabular}{|l|l|}
287.4 & 291 \\
\end{tabular} & 205.4 & 293.3 & 295.8 & 203 & 291.71 & 205.0 & 291.8 \\
\hline Heal Loss-Watts & 8.8 & 27 & 27 & 27 & 2 & 12 & 1.8 & 24 & 13.6 \\
\hline Heat Fwo-gtuftifle & 4989 & 4882 & 5121 & 5084 & 5119 & 5079 & 5056 & 5120 & 5058 \\
\hline WaH Temp. BF & 158.3 & 157.7 & 158.8! & 158.8 & 158.5 & 156.6 & 156.2 & 158.1 & 155.8 \\
\hline Wall Tomp. t4F & 158.41 & 157.51 & 158.81 & 159.11 & 159 & 156.51 & 156.31 & 156.71 & 157.4 \\
\hline WaH Tamp. $5 F$ & 15921 & 157.2! & 158.81 & 158.31 & 158.11 & 157.21 & 156.11 & 158.8 & 15.8 \\
\hline WaH Tamp. If & 148.7 & 157.71 & $158.2 !$ & 157.6 & 158.51 & 159.2 & 155.8 & 154.8 & 155.4 \\
\hline Waltomp. & 159.61 & 157.71 & $158.2 !$ & 157.61 & $158.5 i$ & 159.11 & 156.31 & 155.1 & 155.9 \\
\hline Instren Tomp.fF & $749 . \overline{31}$ & 149.31 & $1 \overline{51.31}$ & 150.81 & $150.5 !$ & 151.51 & 150.5 & 150.8 & 150.8 \\
\hline Av. Wai Temp.F & 158.81 & 157.71 & 158.71 & $1682 i$ & 158.51 & 157.7 & 15621 & 155.8 & 165 \\
\hline Avo. Wall DT.F & 9.31 & 8.11 & 7.11 & 7.11 & 7.81 & 6 & 5.4 & 6.8 & 5 \\
\hline 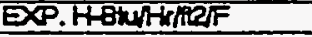 & 534.71 & 613.21 & 719.31 & 7141 & $656.8 i$ & 847.8 & $\$ 30.9$ & 973.6 & 1000.8 \\
\hline & 1 & 1 & & $\cdot$ & & 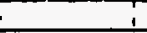 & 1 & 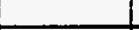 & \\
\hline & ZONEZ : & ZZONES & ZONE8 & .ZONEIO & & $L$ & 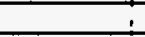 & 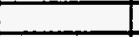 & \\
\hline DP liquid Temp.f & 74.8 & (15) & -2 & & - & 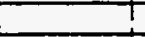 & 1 & 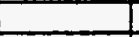 & \\
\hline DP Liquid Density 4 bm/13 & $92.99 !$ & 1 & 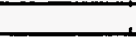 & & & & $t$ & 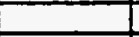 & \\
\hline Elencionth & दो & $4 !$ & $8 !$ & 9.8751 & & & & & \\
\hline Measured DP paid & -0.118 & 0.0551 & 1.8281 & 2.144 & i & 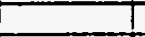 & & & \\
\hline Zero Comectonsesid & 0.2911 & 0.0 .01 & -0.0521 & -0.3281 & 1 & 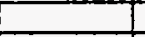 & & & \\
\hline Comedad DPFsid & 0.1731 & 0.6981 & 1.88 & 2.4721 & & & & & \\
\hline Actud DPA & 1.94 & 3.23 & 5.691 & 6.75 & $T$ & 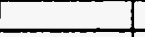 & & & \\
\hline & 1 & 1 & 1 & 1 & 1 & 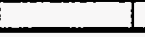 & & & \\
\hline
\end{tabular}




\begin{tabular}{|c|c|c|c|c|c|c|c|c|c|c|}
\hline Run! & $i \quad 974$ & $1-1$ & & & $\vdots$ & 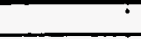 & T & i & 1 & \\
\hline Test Fuid & $175 \%$ CAF10 & $-25 \%$ R114 & & & $i$ & 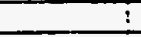 & $\because$ & 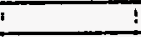 & $!$ & \\
\hline Dens: & $12 / 17 / 331$ & 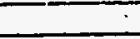 & & & $:$ & 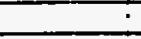 & I & i & $!$ & \\
\hline Tum: & $1 \quad 10: 29 !$ & 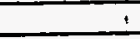 & ; & 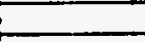 & & 1 & 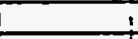 & $:$ & $!$ & \\
\hline Note & 1 & 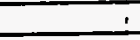 & 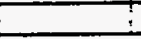 & $\therefore$ & 1 & $i$ & 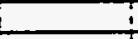 & 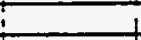 & 1 & \\
\hline Fow Areane & $\therefore 0.003361$ & - & 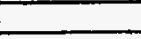 & - & $!$ & 1 & $L$ & & & \\
\hline Hed Fow Areate & 0.19095 & & & $\therefore$ & 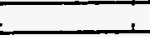 & & & 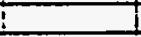 & & \\
\hline Se. Ptessuta Prif & 149.81 & & i & i & & & & $i$ & & \\
\hline SA, Tamperature F(iniet) & 169 & & 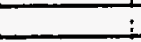 & 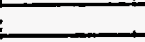 & $i$ & + & 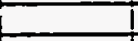 & I & 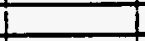 & \\
\hline Inlot FownteG GM & 5.3 & & & & $T$ & 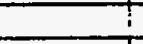 & 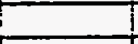 & & & \\
\hline infot tompersuref & 170.11 & & & & 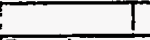 & & & & & \\
\hline InntGibminfe & 9717 & & Tomp & 165.4II & Density & 78.84 & & & & \\
\hline Suboooling & -1.11 & 1 & & $!$ & 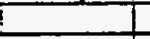 & & & & & \\
\hline Fow Observion & 1 & 1 & 1 & 1 & 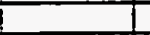 & & & & & \\
\hline & 1 & 1 & 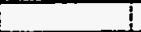 & i & 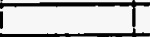 & & & & & \\
\hline & ZZONE1 T & ZONER I & ZONEB i & ZONE & \begin{tabular}{l|l} 
ZONE5 & 2 \\
\end{tabular} & 2ONE6 IS & ZONE & ZONE8 & ZONES & 2ORETO \\
\hline HedlnputWatent) & 868 & 865 & 855 & 868 & 868 & 863 & 858 & 868 & 886 & 868 \\
\hline HeallosstWats & 132 & $3.1 i$ & 4.1 & 4.41 & 4.4 & 3.3 & 24 & 3.2 & 3.4. & 19 \\
\hline Hodfux-Btunfrice & 15012 & 15012 & 15012 & 15012 & 15012 & 15012 & 15012 & 15012 & 15012 & 15012 \\
\hline Wathomp. & 182.7 & 184.5 & 1823 & 184.5 & 185.4 & 183.7 & 188.2 & 185.1 & 1822 & 175.6 \\
\hline WeVt Temo. $14 \mathrm{~F}$ & 180.7 & 181.9 & 183.6 & 185 & 185.7 & 183.8 & 1882. & 185.9 & 183.4 & 775.5 \\
\hline WadTemp. IfF & 183.8 & 180.5 & 184.5 & 185.1 & 184.5 & 18.8 & 1872 & 184.1 & 1828 & 775.4 \\
\hline WalTemp. IIF & 170.1 & 183.31 & 180.21 & $183.7 !$ & 183.5 & 185.81 & 188.4 & 185.8 & 1815 & 176.1 \\
\hline Wal Tomp. Iaf & 185 & T83.2! & 181.6 & 184.21 & 184 & 18621 & 188.81 & 186.8 & 1828 & 775.9 \\
\hline Introem Tomp.f & 170.61 & 170.41 & 171.41 & 174 & 173.1 & 1729 & 175.21 & 173.3 & 17.4 & 173.9 \\
\hline Avo. Wall Tomp.f & 183.8 & 183.21 & 181.91 & 184.81 & 184.4 & 1845 & 188.1 & 185.6 & 1826 & 175.7 \\
\hline AWo, Wall DT. & 12.51 & 12.11 & 9.8 & 9.91 & 10.8 & 10.9 & 122 & 11.8 & 8.5 & 1.1 \\
\hline QX.HBWHAREF & $1188.8 \mathrm{i}$ & $1235.9 i$ & 1528.4 & $1516.8 i$ & 7410 & 1377.6 & 1226.3 & 1298.4 & 174.1 & pones \\
\hline & 1 & & & & 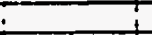 & & & & & \\
\hline & ZONE2 & ZONE4 & ZONE6 & ZONES & ZONETO & & & & & \\
\hline DP hquid Temp.f & 73.4 & & & 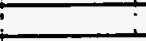 & & & & & & \\
\hline DPLguid Densivelomns & \$3.13! & & & 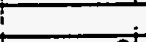 & & & & & & \\
\hline Elevationt & $2 !$ & $4 !$ & 61 & $8 !$ & 9.8751 & & & I & $\mathbf{i}$ & \\
\hline Messured DPFsid & -0.1791 & $0.521 !$ & 0.858 & 1.4821 & 9.711 & & & & & \\
\hline Zero Correctons-psid & -0.354 & -0.0451 & -0.0531 & $-0.02 \pi$ & -0.3611 & & & & & \\
\hline Comected DFPlid & 0.1751 & $0.568 !$ & 0.96 & 1.503 & 2.0721 & & & & & \\
\hline Aciul DPA & 2.041 & 3.69 & 5.411 & $6.7 \pi$ & 7.881 & & $T$ & & & \\
\hline & I & 1 & 1 & 1 & ! & 1 & 1 & 1 & 1 & \\
\hline
\end{tabular}




\begin{tabular}{|c|c|c|c|c|c|c|c|c|c|c|}
\hline Run: & $975 !$ & & & & & & & & & \\
\hline Tost Fuid & $75 \%$ C4F 10 & $-25 \% \overline{R 114}$ & & & & 1 & 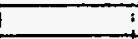 & 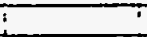 & T & \\
\hline Dato: & $1217 / 331$ & & . & $\div$ & i & 1 & 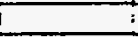 & 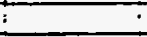 & : & \\
\hline Time: & $1 \quad 14: 13 i$ & & 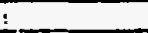 & $\dot{ }$ & $\div$ & 1 & 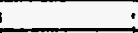 & $:$ & $\vdots$ & \\
\hline Note & 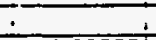 & & $\dot{5}$ & $\therefore$ & $!$ & $i$ & $i$ & 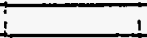 & 1 & \\
\hline Fow Areand & 0.005351 & & $\therefore$ & $\dot{-}$ & $i$ & 1 & $i$ & $i$ & 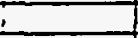 & \\
\hline Head flow Area-ile & 0.19055 & & 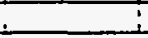 & 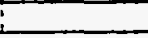 & & + & 1 & $i$ & $:$ & \\
\hline Sal. Pressure Psig & 144.8 & & & & & & & 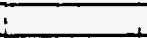 & & \\
\hline Sad. Temperture-F(inlet) & 1671 & & & & & & & t & $i$ & \\
\hline Inlet Flowrate-GPM & $3.9 i$ & & & & & & & & & \\
\hline inlot Tempercurefi & 158.2 & & 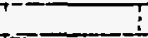 & $!$ & & ? & & + & 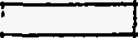 & \\
\hline Inist Gommsme & 7385521 & & TTemp & $152.8 !$ & Density & 79.351 & & $!$ & 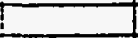 & \\
\hline Subcoolingf & -121 & & 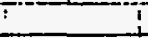 & 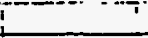 & & -7 & & $\bar{i}$ & 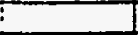 & \\
\hline Fow Observanon & i & & $i$ & 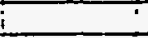 & 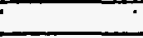 & 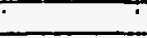 & $\vdots$ & 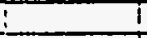 & 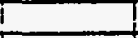 & \\
\hline & i & 1 & 1 & 1 & 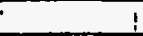 & 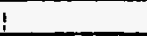 & & 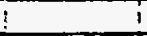 & 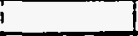 & \\
\hline & ZONEI: & ZONE2 & ZONE3 & ZONEA & IZONE5 & ZONE 6 & ZONET & ZONE 8 & ZONEO & ZOHEDTO \\
\hline Hext InpestWatts(net) & .870 .5 & 875.5 & $i \quad 871.11$ & 833.91 & 83.6 & 863.4 & 871.6 & 871 & 199.3 & 942 \\
\hline Heat Loss-Watts & 13.21 & 3.1 & 4.1 & 4.4 & 4.41 & $3.3 i$ & 2.4 & 32 & 3.1 & 19 \\
\hline 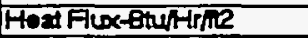 & 15090 & 15176 & 15100 & $14975 !$ & 149701 & 14956 & 15709 & 15098 & 365 & 1633 \\
\hline WaH Tomp. BF & $181.1 !$ & 192.9 & 180.9 & 182.4 & 183.81 & 182.4 & 18521 & 181.3 & 174.8 & 178.9 \\
\hline WaHTemp. \$4F & 181.9 & 180.6 & 182.31 & 182.81 & 184.4! & 182.5! & 185.8 & 183 & 175.5 & 174 \\
\hline Wall Tomp. $15 \mathrm{~F}$ & 182.21 & 979.6 & 183.11 & 183 & 182.91 & 182.91 & 184.6 & 181.51 & T75.1 & 173.9 \\
\hline WaH Temp. If & 168.2 & 181.7 & 178.81 & $182 !$ & 181.71 & 184.31 & 188.8 & 182रा & 174.2 & 1752 \\
\hline Wall Temp. & 183.41 & 181.5 & 180.21 & 182.11 & 18231 & 184.61 & 188 & 183.41 & $T 74.3$ & T74.8 \\
\hline Instroam Temp.F & 168.51 & 769.7 & 170.4 & 172.11 & 177.31 & 172 & 172.6 & 177.3 & 177.8 & 772.3 \\
\hline Arq. Wall Temp.f & 18221 & $181 . \overline{7}$ & 180.6 & 18261 & 182.81 & 1831 & 185.5 & 182.3 & 174,8 & 774.8 \\
\hline Avo. Wall DT.f & 13 & 11.91 & 9.51 & 9.81 & 10.8 & 11.11 & 12.2 & 10.3 & 3.8 & 1.8 \\
\hline EOP.HBWhinger & 1163.6 & 1276.5 & 1594.8 & 1528.4 & 1383.1 & 13426 & 1238.7 & 1468.2 & 10882 & 830 \\
\hline & 1 & & 1 & & & & & & & \\
\hline & 1ZONE2 & ZONEQ & ¿ZONE6 & ZONE8 & ZONEID & & & & & \\
\hline DP liquid Temp.fF & $i \quad 73.4$ & & & $!$ & 1 & 1 & & & & \\
\hline DP Liquid Densitylomin & \$3.13 & & & & & 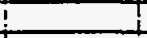 & & & & \\
\hline Elovetion- & $2 !$ & 4 & $6 !$ & 81 & 9.8751 & & & & & \\
\hline Messured DPPsid & -0.0991 & 0.739 & 1.25 & 1.9731 & 2.348 & & $T$ & & & \\
\hline Zero Corrections-psid & $=0.3541$ & $-0 . \overline{0} 4 \overline{5} \mid$ & -0.053 & -0.024 & -0.3511 & & 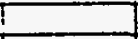 & & & \\
\hline Correded DPFid & 0.255 & 0.784 & 9.3031 & 1.997 & 2707 & & $T$ & & & \\
\hline Achul DPA & 1.881 & 327 & 4.68 & 5.761 & 6.681 & & 1 & & & \\
\hline & 1 & $L$ & 1 & 1 & 1 & $i$ & $i$ & 1 & i & \\
\hline
\end{tabular}




\begin{tabular}{|c|c|c|c|c|c|c|c|c|c|c|}
\hline Run: & $9 \quad 976$ & & 1 & 1 & 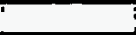 & T & 1 & 1 & 1 & \\
\hline Tost Fivd & $75 \%$ C4F10 & $0-25 \%$ ค114 & 4 & $i$ & i & 1 & I & $!$ & & \\
\hline Dais: & $: 1217 / 381$ & 1 & - & 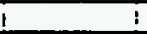 & 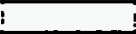 & $\overrightarrow{1}$ & i & $i$ & & \\
\hline Timo: & $14: 13$ & 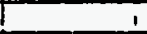 & 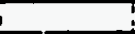 & $\mathrm{i}$ & i & i & I & & & \\
\hline Now & 1 & 1 & 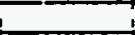 & + & 1 & & $i$ & & & \\
\hline Flow Aros-12 & 0.003361 & $i$ & & 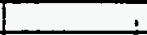 & - & & & & & \\
\hline Hos folow Aroalle & 0.19595 & & 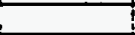 & & & & & & & \\
\hline Sel. Pressure +rig & $142.1 !$ & & $i$ & & & & & & & \\
\hline Sal. Tomportumef(inlel) & 765.91 & & 1 & & -1 & & & & & \\
\hline indelflowrate-GPM & 2.61 & & & & & T! & 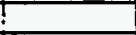 & & & \\
\hline Intel tomperauter & 165.61 & & 7 & $T$ & $T$ & T! & t & & & \\
\hline Intat Gomhine & 497031 & & Tomp & $758.9 !$ & Donsty & 80.711 & & & & \\
\hline Subooding & -0.71 & T & 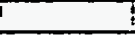 & 1 & 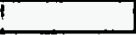 & 1 & I & & & \\
\hline Fow Obsonvation & 1 & $!$ & 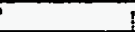 & $i$ & 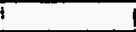 & T & I & & & \\
\hline & 1 & & & $t$ & $i$ & $i$ & i & & & \\
\hline & IZONE1 & ZONE2 is & ZONE3 & ZONEA 1 & ZONE5 & ZONE6 & IZONET & TZONEB & ZONNES & $201=10$ \\
\hline Heal Input-Watts(net) & 857.6 & 864.51 & 863.1 & $850 i$ & 8712 & 8527 & 857.8 & 148.7 & 945 & 872 \\
\hline HenLose-Wates. & 13.1 & $3.1 i$ & 4.1 & $4.4 !$ & 4.4 & 32 & 2.3 & 26 & 3.2 & 19 \\
\hline 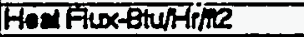 & 14858 & $14986 !$ & 14981 & 14909 & 15102 & 14854 & 14659 & 2578 & 1638 & 1512 \\
\hline WhI Tomp. "BF & 179.1 & $181.4 !$ & 179.6 & 181.21 & 182.6 & 180.8 & 183.5 & 174.11 & T724 & 772.6 \\
\hline Wat Tomp. 14f & 180.11 & $179 i$ & 781.11 & 787.61 & 183 & 180.9 & 183.7 & 173.9 & 172.7 & 172.9 \\
\hline Wall Tomp. $15+$ & 180.21 & $17 \pi .7$ & 181.8 & $181.8 !$ & 181.4 & 180.9 & 182.9 & 173.8 & 9725 & 172.8 \\
\hline Waltomp. Ift & $166.6 !$ & 780.1 & 77.5 & 180.17 & 180.7 & 18251 & 184 & 174.91 & T71.9 & 173.2 \\
\hline Whitomp. "t2F & 180.91 & $179.9 !$ & 178.9 & 180.7 & 181.17 & 182.7 & 18.1 & 175.2. & 171.8 & 173.1 \\
\hline Instresm Tomp.f & 167.81 & 169.71 & 169.5 & $17 !$ & -17 & 169.8 & 171.5 & 17.1 & 169.8 & 17.3 \\
\hline Av. Wall Tomp.f & 180.11 & 180.11 & 179.3 & 181.21 & 181.6 & 181.8 & 183.6 & 174.41 & 172.8 & 172.8 \\
\hline AMo. Wal DT.F & 11.8 & 9.71 & 9.1 & 9.51 & 10.8 & 10.8 & 11.5 & 42 & 2.8 & 1.8 \\
\hline 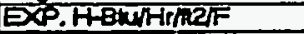 & $1259.5 i$ & 1541.31 & 1644.3 & 15581 & 1389.6 & 1384.8 & 1296.6 & 618.1 & 710.4 & 974.1 \\
\hline & & & & 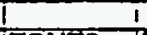 & 1 & & & & & \\
\hline & ZZONE2: & ZONE4 & ZONE 6 & ZONE 8 & ZONE10 & & & & & \\
\hline DP liquid Temp.f & 73.5 & 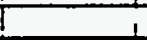 & & & & 1 & 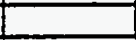 & & & \\
\hline DPLiquid Density Homit3 & 93.12 & & & & & 1 & 1 & & & \\
\hline Elevetiond & 2ा & 41 & 6 & 81 & 9.876 & & & & & \\
\hline Mhenured DPFsid & 0.0051 & 1.0781 & 1.781 & 2.72 & 3.171 & & & & & \\
\hline Zoro Correctionsepsid & $=0.354$ & $-0.045 !$ & -0.053 & -0.024 & -0.361 & & & & & \\
\hline Comedod DP sid & 0.359 & 1.1231 & 1.897 & 2.728 & 3.552 & & & & & \\
\hline Actial DPA & 1.88 & 2.631 & 3.87 & 4.4 & 5.13 & & & & & \\
\hline & 1 & 1 & $I$ & 1 & & & & & & \\
\hline
\end{tabular}




\begin{tabular}{|c|c|c|c|c|c|c|c|c|c|c|}
\hline Run: & 97 & & & & & & & & & \\
\hline TeAf Fisd & $175 \%$ CAF10 & $5-25 \%$ म 114 & & 1 & $T$ & $i$ & 1 & 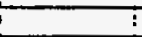 & $\vdots$ & \\
\hline Dafe: & $12 / 20193$ & 1 & 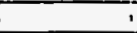 & i & 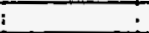 & I & 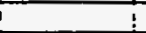 & 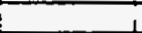 & 1 & 1 \\
\hline Time: & $.9: 25$ & 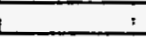 & 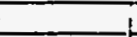 & & \pm & $\cdot$ & 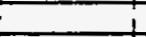 & 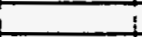 & 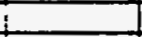 & \\
\hline Note & 1 & 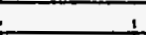 & & 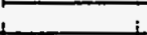 & ; & 1 & 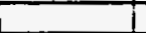 & i & i & \\
\hline Flow Areate & $0.00036 !$ & & & & 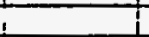 & & & & $i$ & \\
\hline Hoad Aow Arostil & 0.195951 & & & 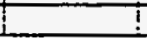 & 1 & $t$ & & & & \\
\hline Sad. Prossuropsio & $142 . \overline{81}$ & & & & 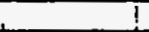 & 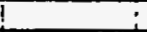 & i & 1 & 1 & \\
\hline St. Tomperaturof(inlet) & 166.21 & & & 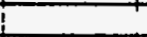 & $i$ & 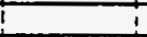 & $T$ & 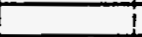 & 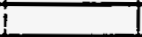 & \\
\hline Inlet Fownd-GPM & 5.31 & & & & ? & 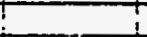 & T & & & \\
\hline intal Tomperature & 169 & & & & $!$ & & & & & \\
\hline Intatgiomnine & 10049311 & & Tomp & 162.31 & Donsing & 79.45 & & & & \\
\hline Suboooting & -28 & I & Tran & $\bar{i}$ & $i$ & 1 & 1 & 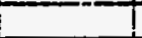 & & \\
\hline Fow Obsenvation & 1 & $i$ & 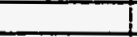 & $i$ & 1 & 1 & 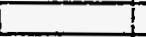 & & & \\
\hline & . & & & $i$ & $i$ & 1 & 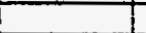 & & 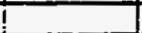 & \\
\hline & IZONET & ZONEZ & ZONEB I & ZONEA i & ZONE5 & RONES IS & ZONEA I & ZONEB & ZONEA & ZONE10 \\
\hline Heat inputWathol) & $i \quad 5823$ & 577,3 & 57821 & 580.1 & 580.4 & 5812 & 578.6 & 576.3 & 575.0 & 678.4 \\
\hline Host Loss-Wats & 9.41 & $21 i$ & 27 & 3 & 2.8 & 1.9 & 9.4 & 1.9 & 25 & 14.8. \\
\hline Heat Fux-BunHrine & $10094 !$ & 10007 & 10023 & 10056 & T005T & 10075 & 10047 & 9990 & 983 & 9910 \\
\hline Wall Temp. $3 F$ & 1801 & 179.4 & 177.9 & 180.9 & 179.3 & 177.8 & 179.91 & 178.1 & $17 \pi 2$ & 177.8 \\
\hline Woll Temp. "4F & 180.31 & 178.3! & 178.7 & 181.1! & 179.71 & 178.2! & 179.9 & 177.9 & 178.1 & 180.8 \\
\hline Wali Temp. 55 . & 180.71 & 977 & 779.8! & 180.7 & 179.11 & 177.41 & 179.1 & 177.11 & 176.6 & 976.3 \\
\hline Waf Tomp. 1F & $169 !$ & i79.2! & 176.61 & 181.31 & 179.4 & $1782 !$ & 181.11 & 7781 & 7\%5.81 & 176.8 \\
\hline Wall Temp. $+2 F$ & $|\overline{82}|$ & $178.9 !$ & 17\%. & "181.2! & 779.51 & 177.81 & 180.9 & 1781 & 175.8 & 777.5 \\
\hline Instram Tomp. $f$ & 169.11 & $167.8 !$ & $168.7 !$ & 177.71 & 170.31 & 170.1 & 1724 & 170.11 & 170 & $7 \pi .2$ \\
\hline Avo. Wal Tomp.f & 180.81 & 1791 & 177.61 & 1811 & 179.4 & 177.91 & 18021 & 177.8 & 176.8 & 177.8 \\
\hline Avg. Wall DT.f & 1121 & 10.7 & 8.1 & 8.81 & 8.71 & 7.31 & 7.31 & 7.31 & 6.1 & 6.2 \\
\hline ExP.HBhuHritar & $901.7 i$ & 935.41 & 9104.81 & 1136.81 & $1160.4 i$ & 1371.71 & 1371.41 & $1374.4 i$ & 16312 & 1595.2 \\
\hline & 1 & 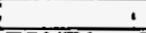 & 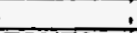 & & & $\therefore$ & 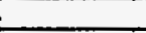 & & $i$ & \\
\hline & ZONE2 & ZONE 4 & ZONES & ZONE8 & ZONE10: & - & 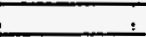 & & 1 & \\
\hline DP liquid Temp.f & $\quad 77.5$ & & & & & & & & & \\
\hline DP Liquid Density fomits & $92.66^{\circ}$ & & & & & & & & $i$ & \\
\hline Elevationd & 21 & $4 !$ & 6! & 8 & 9.875 & & & & $:$ & \\
\hline Measured DPpsid & -0.18 & 0.4691 & 0.7471 & 1.269 & 1.394 & & & & & \\
\hline Zoro Corrections-psid & -0.2961 & -0.0251 & -0.001 & -0.081 & -0.343 & & & & & \\
\hline Compoded DPPsid & 0.1161 & $0 . \overline{8} 31$ & 0.8111 & $9 . \overline{31}$ & 1.737 & & & & & \\
\hline Actuid DPA & 2.12 & 3.77 & 5.53 & 6.97 & 8.37 & & & & & \\
\hline & $t$ & 1 & 1 & i & 1 & i & & & & \\
\hline
\end{tabular}




\begin{tabular}{|c|c|c|c|c|c|c|c|c|c|c|}
\hline Runt & 9781 & & & & & & & T & $i$ & \\
\hline Togt Fuid & $175 \%$ C4F 10 & $3.25 \%$ मा1 & 4 & & 1 & $\vdots$ & 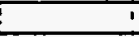 & 5 & 1 & $\perp$ \\
\hline Das: & 112120183 & 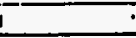 & & & 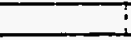 & $!$ & 5 & 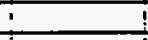 & $!$ & \\
\hline Timo: & $.9: 25$ & . & - & & $\dot{s}$ & & & $i$ & i & \\
\hline Noth &. & $=$ & 2 & - & 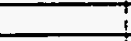 & $\therefore$ & :- & i & $!$ & \\
\hline Fow Aros-de & $0.00355 !$ & 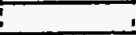 & 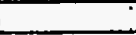 & $\therefore$ & & 1 & 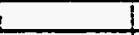 & & & \\
\hline Heat Fow Areade & $0.19695^{\prime}$ & & & & & & & & & \\
\hline Su. Pressurepsig & 141.5 & & & & $\therefore$ & & & & & \\
\hline Sxt. Tomperatureffinlol) & $165.6 !$ & & : & $\therefore$ & 7 & i & & & & \\
\hline IndFowrá-GPM & $4 !$ & & 7 & & & & & & & \\
\hline intel Tompersuref & 769.4 & & T & ! & & & & & & \\
\hline IntalGbibminte & 7595611 & & Tomp. & 161.71 & Dansity & 79.57 & & & & \\
\hline Suboooling of & -3.81 & I & - & +1 & & & & & & \\
\hline Fow Observation & $!$ & 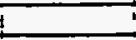 & 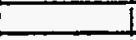 & 1 & & & & & & \\
\hline & 1 & 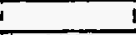 & 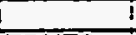 & T & & I & 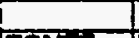 & & & \\
\hline & ZONEI & ZONE? & 20NE3 & ZONE4 I8 & ZONE5 & ZONE6 & 2ON: & LON:E8 & ZONE=O & $201=10$ \\
\hline HealnpentWatil(nel) & 1677.1 & $5 \% 82$ & 5812 & 5762 & 5815 & 5832 & 585.3 & 5802 & 680 & 581.6 \\
\hline Hoal Loss-Watts & 102 & 2.3 & 3 & 32 & 3.7 & 21 & 1.5 & 2.1 & 2.7 & 16.3 \\
\hline 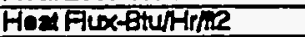 & 10004 & 10023 & 10075 & 9988 & 10080 & 1010 & 10146 & 10057 & 10004 & 1008 \\
\hline WaH Tomp. \#3F & 179.6 & 749.3 & 178.5 & 179.9 & 179.1 & 178 & 178.8 & 777.5 & 77 & 777.8 \\
\hline Wal Tomp. "If F & 180 & 178.1 & 179.4 & 1802 & 179.6 & 178.4 & 178.6 & 177.4 & 778 & 180.8 \\
\hline Wal Tomp. $15 F$ & 180.51 & 977.2 & 180.4 & 180.2 & 779.2 & 177.5 & 778.4 & 176.8 & 178.3 & 776.3 \\
\hline Wal Tomp. "lf & 169.4 & 779.1 & 776.8 & 779.71 & 178.9 & 178.4 & 180.5 & 17731 & 174.8 & 776.7 \\
\hline Walt Tomp. 27 & 181.51 & 778.8 & (in.71 & 979.7 & 179.21 & $\mid 78$ & 180 & 177.2 & 775.3 & 777.5 \\
\hline Instrem Tomp.f & 169.51 & $16 \overline{8} 8.7$ & 189.51 & 171.31 & 1702 & 170.11 & 171.3 & 16.6 & 169.8 & 770.8 \\
\hline AVo. Wat Tomp.f & 180.41 & 178.8 & 178.1 & 1801 & 179.21 & 178.11 & 179.8 & 177.2 & 778.8 & 777.9 \\
\hline AYg. Wall DT F & 10.41 & 9.7 & 8.1 & $8.2 !$ & 8.6 & 7.51 & 7.5 & 7.2 & 6.2 & 8.6 \\
\hline EP.HETUHR2R & 857.5 & 10036.3 & 1237 & 1210.9 & 1178.3 & 1344.7 & 1352.5 & 1403.6 & 160.8 & 15.6 .6 \\
\hline & + & & & 1 & & -1 & L & & & \\
\hline & ZONE2 : & ZONE4 & ZONE6 & ZONE & ZONE10 & $i$ & & & & \\
\hline DP hquid Temp.f & $\quad 7.91$ & & & & & $\dot{\vdots}$ & & & & \\
\hline DPLguid Density $4 \mathrm{bm} / \mathrm{n}$ & 92.62 & & & i & & & & 1 & & \\
\hline Elovationt & & 4 & 61 & $8 !$ & 9.875 & & & i & i & \\
\hline Messurad DPPsid & -0.089 & 0.7 & 1.152 & $1.818:$ & 2.061 & & & & & \\
\hline Zoro Corrections-psid & -0.296 & -0.025 & -0.084 & 0.0311 & -0.36 & & & & I & \\
\hline Correded DPFsld & 0.2071 & 0.725 & 7.2161 & 1.849 & 2.404 & & & & & \\
\hline Actua DPA & 1.95 & 3.3 & 4.78 & 5.97 & 7.14 & & & & $T$ & \\
\hline & $i$ & & i & & & i & & $t$ & $t$ & \\
\hline
\end{tabular}




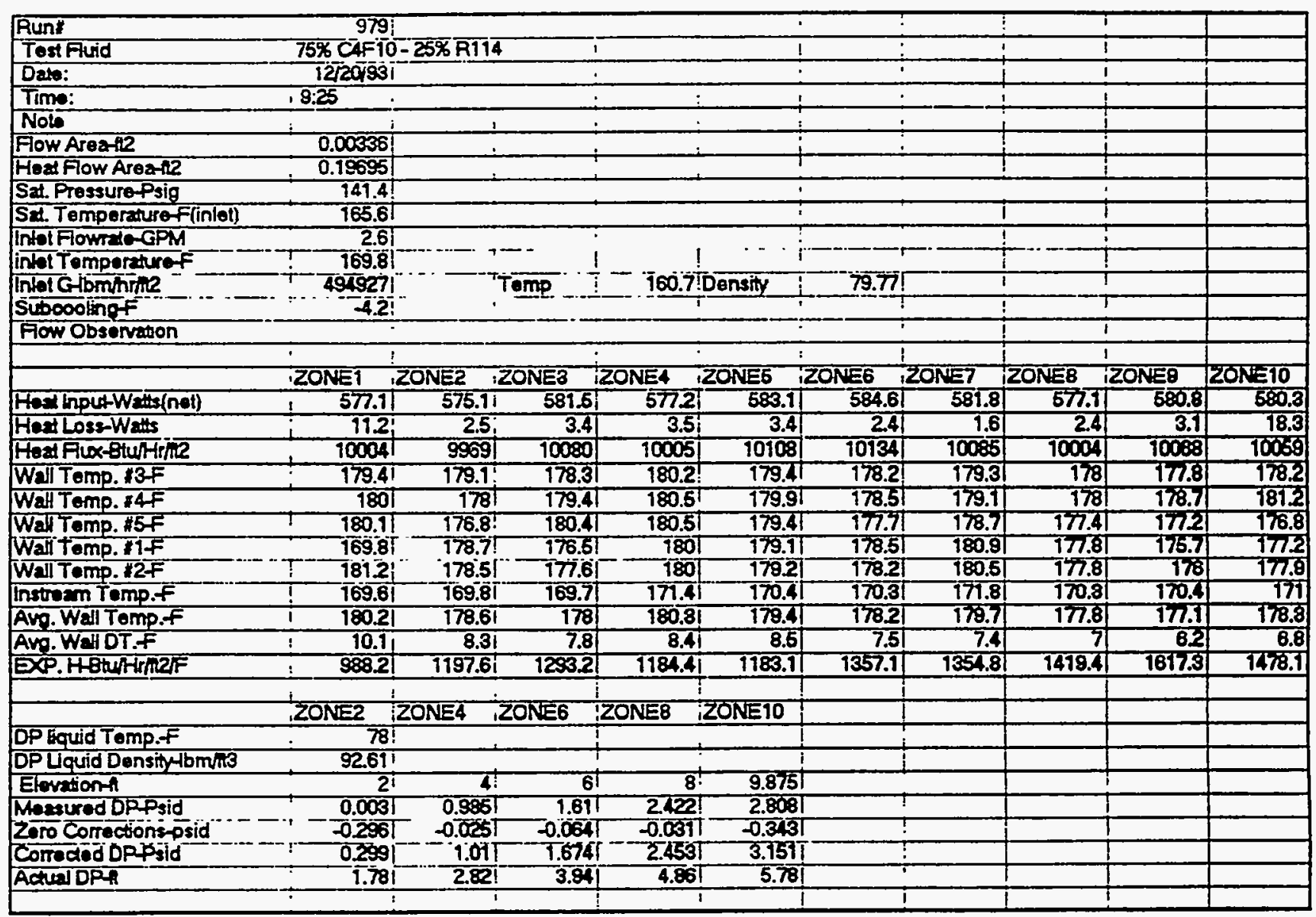




\begin{tabular}{|c|c|c|c|c|c|c|c|c|c|}
\hline Run: & 9801 & & & & & & 1 & i & \\
\hline Tarffivd & $175 \%$ C $4=10$ & $-25 \% R 114$ & $\cdot$ & & $!$ & 1 & 1 & i & \\
\hline Dals: & 1220183 & ' & I & - & ! & 1 & $!$ & $!$ & \\
\hline Time: & $9: 25$ & $:$ & $i$ & $i$ & $i$ & 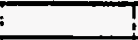 & & $i$ & \\
\hline Nate & & 1 & $!$ & $!$ & $i$ & 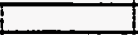 & & & \\
\hline Flow A Arease? & $0.00336 !$ & & & 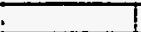 & & & & & \\
\hline Hes Flow Aroath2 & 0.19595 & & & & 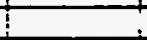 & & & & \\
\hline Set. Pressure Prig & 137.41 & & & & 1 & & & & \\
\hline Su. Temperaruf(inlol) & 163.8 & & • & & ? & : & & i & \\
\hline Intel Flowre-GPM & 2.61 & & 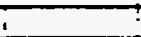 & & T & t: & & & \\
\hline Intof Tomporaturaf & 169.81 & $\div-$ & ? & & 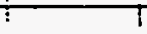 & $\Gamma$ & & & \\
\hline Inte Glom mhe & 7650061 & Tremp & 160.81 & IDengly & 79.751 & & & & \\
\hline Subooolingt & -61 & & 1 & 1 & 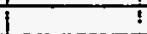 & 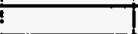 & & & \\
\hline Fow Obsenvion & \pm & & $i$ & 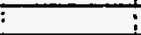 & 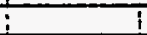 & i & i & $!$ & \\
\hline & $T$ & 1 & 1 & I & 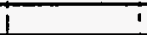 & 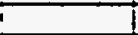 & & 1 & \\
\hline & RONEI & IZONE3 & ZONE4 & ZONES & iZONE6 & ZONE7 & ZONE $=8$ & TZONEO & ZONE \\
\hline Hosilnp(atWatts(nal) & 25321 & $230.5 \mathrm{i} \quad 290.3$ & 280 & 290.7 & 290.5 & 294.3 & 2885 & 27. & 2821 \\
\hline HexL Loss-Watts & $10.5 !$ & $23 i$ & 3.3 & 3.11 & 2.11 & 1.5 & 2.1 & 2.8 & 16.6 \\
\hline Hod Flox-tuntshe & $5082 !$ & $5018 !$ & 4854 & 5030 & 50061 & 5101 & 5001 & 782 & 4890 \\
\hline Wall Temp. \#3F & $176 !$ & 174.5 & 975.7 & 174.51 & 1731 & 174.41 & 173.1 & T72.6 & 973.3 \\
\hline Wall Tomp. naf & $176.1^{\circ}$ & $174.3 !$ & $175.7^{1}$ & 1751 & 173.6 & 174.21 & 172.8 & 173.2 & 174.6 \\
\hline Wall Tomp. $15+$ & $176.6 !$ & $173.7^{1}$ & 175.6 & $174.6 i$ & $172.8^{1}$ & 173.91 & 172.51 & 1725 & 172.6 \\
\hline Wat Tomp. & 169.81 & $174.9 T$ & $17 \overline{6}$ & 174.71 & $173.4 !$ & 175.71 & 1731 & 971.3 & 173.2 \\
\hline WaTTamp. ILF - & 176.91 & $174.8 \Gamma$ & 175.7 & $174 . \overline{81}$ & 172.91 & 775.31 & 173.11 & 171.6 & 173.4 \\
\hline Instresn Tomp. $F$ & $169.7 i$ & $1682 !$ & $16 \overline{21}$ & 168.11 & 167.91 & 969.5 & 167.7 & 167.8 & 168.8 \\
\hline Avo. Wall Tomp.F & 176.4 & 174.61 & 175.8 & 174.71 & 173.21 & 174.7 & 172.9 & 1722 & 178.4 \\
\hline Avo. Wall DT .f & $6.5 i$ & $6.2 i$ & 6.31 & 6.4 & 6.11 & 5 & 5) & 42 & \\
\hline EP.HBturimar & 785.51 & $809.6 i$ & 765.7 & 787.7 & \$27 & 1006.6| & 1005.4 & 11472 & 1171.6 \\
\hline & & & & & & & & & \\
\hline & ZONE2 & ZONE6 & ZONEB & ZONETO & & & & & \\
\hline DP hquid Temp. $f$ & $\pi 7.8$ & & & & & & & & \\
\hline DPLlouid Density $4 \mathrm{bm} / 13$ & 92.631 & & & & & & & & \\
\hline Evertion- & & 4 & 8 & 9.875 & & & & & \\
\hline Messured DPPsid & -0.0261 & 0.71 & 2.058 & 2.37 & & & $\mathbf{i}$ & T & \\
\hline zono Correctónsosid & -0.2061 & 0.0851 & 0.081 & 0.36 & & & & & \\
\hline Correchdopasid & 0.27 & 0.75 & 2.1 & 2.7रा & & & & & \\
\hline Actul DPA & 1.841 & 3.23 & 5.5 & 6.57 & & & & & \\
\hline & & & & & & & & & \\
\hline
\end{tabular}




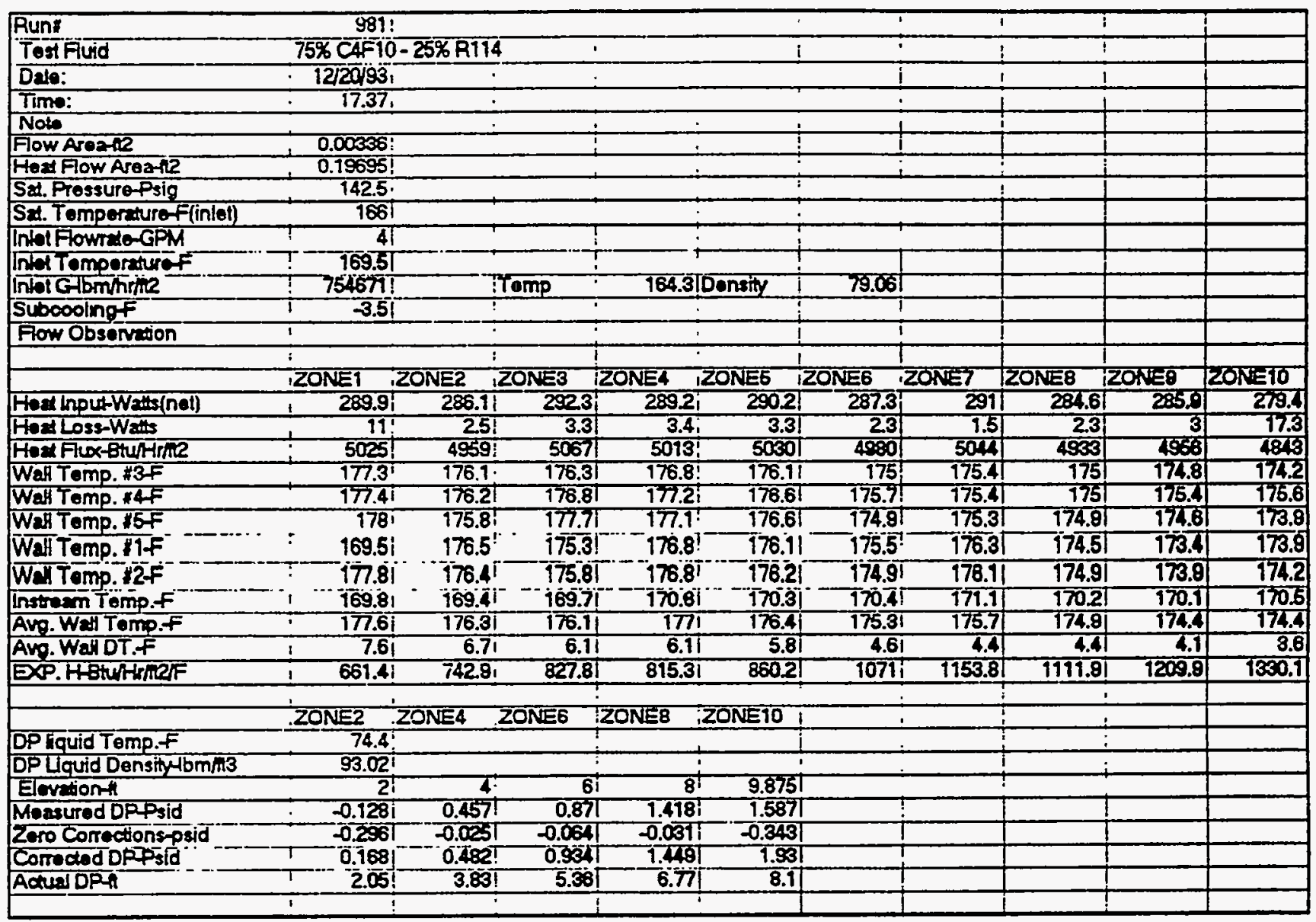




\begin{tabular}{|c|c|c|c|c|c|c|c|}
\hline Runt & & & & & & & \\
\hline $75 \%$ CAF 10 & $-25 \% R 114$ & & & & & & \\
\hline 12201931 & & & & i & 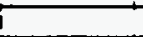 & & \\
\hline Time: & & 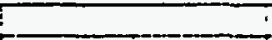 & & & & & \\
\hline Now & +2 & 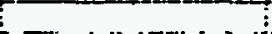 & & & & ! & \\
\hline 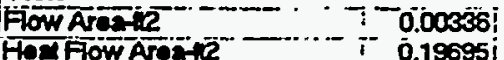 & ל-- & & $\ddot{-}$ & & & & \\
\hline 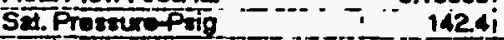 & & $T^{-}$ & 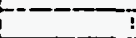 & 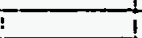 & & $i$ & \\
\hline SL. Tomperemuref(intel) & 7 & 7 & & 1 & & 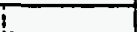 & \\
\hline IndFowre-CFM & $\because$ & $:$ & & & & I & \\
\hline indol Temperaturef & & & & & & & \\
\hline 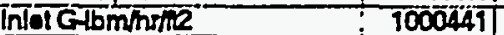 & Tamp & 164.11Density & 79.11 & & & & \\
\hline Subcooling & & & & & & & \\
\hline Fow Obsenvetion & & $\div$ & & 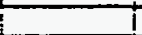 & & & \\
\hline & & & & & & & \\
\hline TZONEI & ZONE2 :ZONES & ZONEA ZONE5 & ZONE6 & ZZONET & ZONE8 & ZONLS & 2ONE \\
\hline Heal inputw ats(nal) & 291.61 2का & 294.612823 & 287.3 & 26.8 & 252या & 288.6 & -288.1 \\
\hline HenLose Wres & 2.4 & 3.21 & 2.2 & 1.5 & 22 & 28 & 18.2 \\
\hline 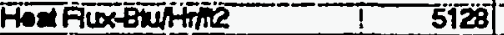 & 5055 & 51071 & 40001 & 5147 & 5068 & 2968 & क्षे \\
\hline Wall Tomp. 13F & $176.1 i$ & 176.81 & 174.81 & 175.6 & 175.1 & 174.7 & 173.8 \\
\hline Wall Tomp. I4F & 176.11 & 176.81 & 175.4 & 175.6 & 175.2 & 175.4 & 775.1 \\
\hline Wall Tomp. $15 f^{\prime}$ & 175.6 & 176.81 & 174.6 & 175.5 & $\overline{174.9 T}$ & 174.7 & 173.3 \\
\hline Wal Tomp. HIF & 176.4 & 176.7, & 175.3 & 176.5 & 174.81 & 773.6 & 173.3 \\
\hline W. Tomp. $2 F$ & 176.41 & 176.7 & $\$ 74.81$ & 1762 & 175.11 & 173.8 & 173.6 \\
\hline Instrasm Temp.F & 169.8 & 170.8 & 170.2 & 171.4 & 170.3 & 170.3 & 170.1 \\
\hline Avp.Wall Tomp.f & 176.91 & 176.81 & 175 & 175.9 & 175 & 174.6 & 173.8 \\
\hline Avg. Wall DT F & 6.21 & $5.7^{1}$ & 4.6 & 4.2 & 4.51 & 3.8 & 3.6 \\
\hline EXP.HBtuHAnRE & 812.4 & 877.11 & 10827 & E21.7 & 1128.6 & 2032 & Tद2:. \\
\hline & & & & & & & \\
\hline & ZONEA TZONEE & ZONES- ZONETO & & & & & \\
\hline БP hqud Tomp,F & $-1 ;=$ & & i & & & & \\
\hline DP Liquid Densin bom/13 & $T$ & $i$ & & & & i & \\
\hline Exvitiont & $\overline{41}_{1}$ & 9.875 & & & & & \\
\hline Massured DPPaid & $0.321 i$ & 1.1021 & 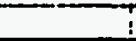 & & & & \\
\hline Zaro Connections-psid & $-0.025 ;$ & $-0.031 i$ & & & & & \\
\hline Corrected DPP sid & $0.345 !$ & $1.133 !$ & & & & & \\
\hline Actual DPA & 5.74 & 7.33 & & & & & \\
\hline
\end{tabular}




\begin{tabular}{|c|c|c|c|c|c|c|c|c|c|c|}
\hline Run & 9831 & 1 & 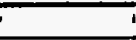 & 1 & 1 & $i$ & i & 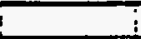 & $\vdots$ & \\
\hline Test Fluid & EOक्सेटAF10 & $3.50 \%$ R114 & & $i$ & 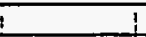 & 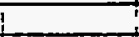 & 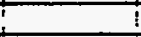 & 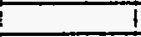 & 1 & \\
\hline Date: & $\therefore \sqrt{271931}$ & i & 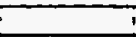 & 1 & $i$ & 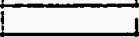 & 1 & 1 & $t$ & \\
\hline Time: & $12: 24$ & 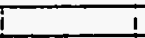 & 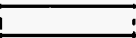 & $\therefore$ & 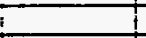 & 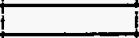 & 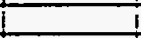 & $i$ & $i$ & \\
\hline Note & $:$ & 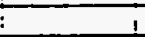 & 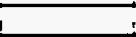 & $\therefore$ & 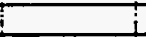 & 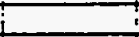 & 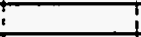 & 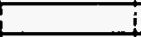 & 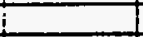 & \\
\hline Fow Areate & 0.00368 & . & 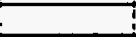 & $i$ & 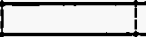 & 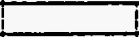 & $i$ & & 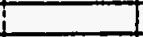 & \\
\hline Heat Fow Areatil & 0.19595 & & & $T$ & 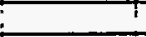 & $T$ & $?$ & & & \\
\hline Sat. Pressure-Psig & 75.31 & & & t & 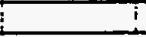 & $i$ & 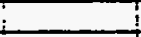 & 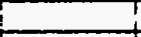 & & \\
\hline Sat. Temperature finlet) & 131.71 & I & 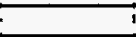 & 1 & 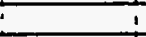 & 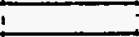 & 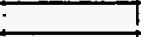 & & & \\
\hline Inlotfiowra-GPM & 4.91 & & & 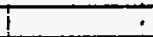 & - & 1 & 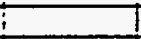 & & & \\
\hline intot Temperature fF & 129.6 & $i$ & $i$ & & i & i & 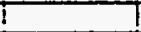 & & & \\
\hline Inlet Glbm/hrithe & 10058471 & & Temp & 124.2II & IDensity & 86.02 & & $T$ & 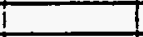 & \\
\hline Subcoolingf & $2.1 !$ & 1 & (1) & 1 & 1 & 1 & 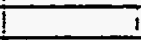 & 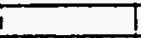 & $T$ & \\
\hline \multirow[t]{3}{*}{ Fow Observaton } & & $i$ & 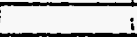 & $i$ & $!$ & $i$ & i & L & t & \\
\hline & $i$ & 1 & 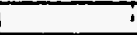 & 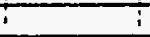 & $i$ & 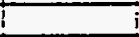 & $\bar{i}$ & & 1 & \\
\hline & .ZONE1 IS & ZONEZ & ZONE3 & ZONE4 & ZONES & ZONE6 & ZZONE? & ZONES I & ZONEQ & ZONETO \\
\hline Hoat Input-Watts(net) & 869.91 & $870 . \overline{3 i}$ & 872.9 & 868 & 873.9 & 878.6 & 867.4 & 8762 & 873.8 & 875.3 \\
\hline Heaf Loss-Watts & $8 !$ & $1.8 !$ & 24 & $2.6 i$ & 26 & 2 & 1.3 & 1.7 & 2.J & 11.8 \\
\hline Heat Flox-BtuhH/lic & 15079 & 15086 & 15131 & 15046 & 15148 & 15230 & 15036 & 15188) & 15147 & 15178 \\
\hline Wall Temp. \#3F & 1451 & 146.8 & 144.6 & 147.3 & 148.8 & 149.1 & 148.9 & 144.1 & 142 & 141.6 \\
\hline Wall Temp. F4F & 145.91 & 143.7 & 146.5 & 147.6 & 148.7 & 149.1 & 148.9 & 145.2 & 144.1 & 147.8 \\
\hline WETTOmp. I5F & 146.1 & 142.2 & 7472 & 7482 & 147.7 & 148.8 & 147.8 & 143.8 & 141.01 & 740 \\
\hline Wall Tomp. $1 F$ & 129.6 & 145 & 1दा & 146.4 & 1463 & 148.8 & 750.9 & 145.6 & 740.8 & 739.6 \\
\hline Wal Tomp. taf & 747.7 & 74.8 & T43.8 & 746.8 & 747 & T49.8 & 750.6 & 746.8 & 742 & 741.6 \\
\hline Instresm Temp.f & 130 & 130.1 & 129.8 & 131.5 & 130.4 & 129.8 & 131 & 129.1 & 1द8.8 & $\lceil\overline{9.7}$ \\
\hline Avg. Wall Tomp.f & 140.2 & 145 & 1446 & 767.4 & 147.4 & 148 & 149.4 & 746 & Th2य & T421 \\
\hline Avg. WaH DT.F & 16.6 & 14.2 & 74 & 152 & 16.8 & 18.5 & 17.8 & 15.2 & 126 & 91.7 \\
\hline \multirow[t]{3}{*}{ Exp.HBWhima' } & 973.8 & 10503 & 1079.6 & छ्डat & 9285. & 828.8 & 848.8 & 893.8 & T202B & E352 \\
\hline & & & & & & & & & & \\
\hline & $201:=2$ & ZOFVE & $207=6$ & ZONEB & ZONE10 & & & & & \\
\hline DP squid Tomp.f & 84.4 & & & & & & & & & \\
\hline DP Lquid Densithimik & 91.18 & & & & & & & & & \\
\hline Elevition-1 & 2 & 4 & 6 & 8 & 9.875 & & & & & \\
\hline Whasured DPpsid & 2.25 & 0.41 & 0.674 & T.T3 & 1.152 & & & & & \\
\hline 20ro Correctons-psid & -0.283 & -0.008 & $=0.030$ & $-0.0<2$ & 70.235 & & & & & \\
\hline Correctod Dperto & 0.001 & 0.435 & 0.73 & T.201 & 1.487 & & & & & \\
\hline Actul DPA & 2.71 & 3.51 & 5.17 & 6.46 & 7.98 & & & & & \\
\hline & & & & & & & & & & \\
\hline
\end{tabular}




\begin{tabular}{|c|c|c|c|c|c|c|c|c|c|c|}
\hline Runf & 984 & & & & & & & & & \\
\hline Tagfiudd & $50 \%$ CAFIO & $-60 \%$ R1T & & & & & & & & \\
\hline Dot: & 1227198 & & & & & & & & & \\
\hline Timo: & $13: 01$ & & & & & & & & & \\
\hline Now & & & & & & & & & & \\
\hline Flow Arade & 0.00038 & & & & & & & & & \\
\hline Hout Fow AToath & 0.18696 & & & & & & & & & \\
\hline Sed. Pressure Peig & 74.8 & & & & & & & & & \\
\hline Set. Tomperaurof(inted) & 131.3 & & & & & & & & & \\
\hline Intelflownde-GPM & 3.7 & & & & & & & & & \\
\hline Intel temporesuret & 720.5 & & & & & & & & & \\
\hline 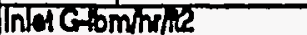 & 7650001 & & Tomp & T24.8] & Denthy & 85.88 & & & & \\
\hline Subooolngt & 1.8 & & & & & & & & & \\
\hline Flow Obsenviton & & & & & & & & & & \\
\hline & & & $20 \mathrm{x}=3$ & $70 \times=4$ & $207 j=5$ & $2015=6$ & $2019 \Rightarrow$ & $20 N \overline{8} 8$ & 20NE6 & $807=50$ \\
\hline Festhpu-Wattal) & $\frac{\text { ZONEE1 }}{868.9}$ & $\frac{20 \text { NE2 }}{888.2}$ & $\frac{\operatorname{Lin}}{859.8}$ & $\frac{24}{8713}$ & 873 & $\frac{106}{868.1}$ & 869 & 867.8 & 858.8 & $\frac{201 x=10}{859,8}$ \\
\hline HonLoss-Wats & 7.9 & 1.8 & 25 & 27 & 27 & 2.1 & 7.3 & 1.8 & 22 & $\sqrt{2.3}$ \\
\hline 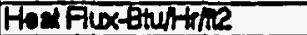 & 16062 & 15050 & 15069 & 1510द्ध & 15762 & 15018 & 76008 & 76078 & 15051 & 16007 \\
\hline Wal Temp. 13 F & 143.8 & 746.8 & 145.4 & 747.3 & 149.1 & 749.3 & 148.4 & 144.8 & 143.1 & 142.4 \\
\hline Wa Tomp.14F & 745.1 & 143.6 & 147.2 & 147.8 & 149.8 & 149.5 & 148.8 & 1482 & 145.8 & 148.8 \\
\hline Wall Tomp. "I5F & 145.4 & 1425 & 148.1 & 748.3 & 147.8 & 148.8 & 147.8 & 144.6 & Tदटर & 140.8 \\
\hline Wall Temp. IF & 729.5 & 34.7 & 743 & 146 & 766.6 & 748.8 & 750.2 & T46.1 & T42 & 739.9 \\
\hline Wa:T Tomp. ILF & 746.2 & 74.4. & 744.3 & 746.8 & 747 & 749.6 & 75032 & 747.4 & 743.7 & T42.2 \\
\hline Introsm Tomp.F & 730.4 & 130.8 & 130.4 & 131.6 & ज可 & 730.0 & T3T.2 & 129.8 & 123.8 & 1302 \\
\hline Avo.Wall Temp.F & 145.2 & 744.8 & 145 & 147.8 & 747.7 & 1492 & Tदि.1 & 745.8 & 143.8 & 7दि. \\
\hline Avg.WallDT.F & T4.1 & 18.8 & 13.9 & 75 & 18 & 17.9 & 77.2 & 75.8 & 12.7 & 17.8 \\
\hline EXP.HBWHWR2F & 10705 & 1132 & $108 \pi .6$ & 1005.6 & 846,8 & 890.1 & 874.8 & 980.8 & 11852 & 12628 \\
\hline & & & & & & & & & & \\
\hline & $\angle O O N=2$ & ZONEA & $\angle O N E B$ & ZONEB & ZONETO & & & & & \\
\hline DP hquid Tomp.f & 78.6 & & & & & & & & & \\
\hline DPLiquid Donsity 4 bm/13 & कृ.02 & & & & & & & & & \\
\hline Elevation-h & & का & 6 & (8) & 9.875 & & & & & \\
\hline Mesaured DPprid & -0.138 & 0.77 & T.24 & 1.969 & 2.001 & & & & & \\
\hline Zoro Corrections-psid & -0.203 & -0.020 & -0.08 & -0.00 & -0.335 & & & & & \\
\hline Comectod DPsid & 0.155 & 0.8 & 1.280 & 1.987 & 2.48 & & & & & \\
\hline Actual DP-n & 1.88 & ट्रा & 4.27 & 5.25 & 6.51 & & & & & \\
\hline & & & & & & & & & & \\
\hline
\end{tabular}




\begin{tabular}{|c|c|c|c|c|c|c|c|c|c|c|}
\hline Run: & 985 & & & & & & & & & \\
\hline Togffuld & $150 \%$ CAF 10 & -50\%RT14 & & & & & & & & \\
\hline Deds: & $12 / 27183$ & & & & & & & & & \\
\hline Time: & 13.01 & & & & & & & & & \\
\hline Now & 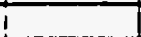 & & & & & & & & & \\
\hline Flow Aroade & 0.00365 & & & & & & & & & \\
\hline Hed Fow Areath & 0.18696 & & & & & & & & & \\
\hline Sat: Prossure Psip & 72.1 & & & & & & & & & \\
\hline Set. Tomperaturo f(inlet) & 129.4 & & & & & & & & & \\
\hline Indot Fowreto-GPM & 2.4 & & & & & & & & & \\
\hline Indot Tomperctires & 13.1 & & & & & & & & & \\
\hline Intat Glbmhrite & 495145 & & Tramp & 12 दू. & Density & 86.15 & & & & \\
\hline Subooolingf & 0.3 & & & & & & & & & \\
\hline Fow Obsonvation & & & & & & & & & & \\
\hline & ZONEA & ZON=? & ZOK: $=3$ & 2ON: $=4$ & ZON:35 & $20 x=6$ & हका $=7$ & ZONE & 2ONE⿱一𫝀口 & $20 x=10$ \\
\hline Heal input-Waltinel) & 858.7 & 883.8 & 868 & $8 \% 2$ & 860.7 & 857.6 & 857.8 & 869.3 & 870.8 & 869.4 \\
\hline HonLLose-Wasts & 9.3 & 2.1 & 2.8 & 3.2 & 3 & 23 & 1.6 & 22 & $2 B$ & 36.5 \\
\hline Host Flox-buHrine & 14972 & 15051 & 14960 & 14888 & 7480 & 14888 & 15063 & 15069 & 15083 & $160 \%$ \\
\hline WaI Tomp. BF & 143.9 & 146.1 & 143.7 & 147.3 & 148.1 & 147.6 & 149.4 & 145.1 & 143.1 & 143.8 \\
\hline Wal Tomp. I4F & 145 & 142.8 & 145.6 & 147.5 & 147.8 & 147.8 & 749.8 & 145.8 & 146.2 & 150.2 \\
\hline Wa Tomp. $15 F$ & 145.1 & 141.2 & 76.8 & 147.8 & 945.8 & 148.8 & 148.7 & 144.1 & 942.8 & 141.8 \\
\hline Wall Tomp. ItF & 729.1 & 14.1.1 & 747.4 & 146.5 & 146 & 147 & $15 \pi$ & 746.9 & T42.1 & 747.6 \\
\hline Wal Tomp. IIf & 7462 & T43.7 & T42.7 & 748.0 & 746.1 & T47.4 & 751.1 & 148 & 143.2 & 743.7 \\
\hline Intream Temp.f & 720.4 & 130.1 & 122. & 1322 & 130.6 & 130 & 131.8 & 129.5 & 129.7 & 131.1 \\
\hline Avo. WaH Temp.F & 146.1 & 1442 & 143.4 & 747.8 & 146.4 & 747.8 & 769.9 & 148 & T143.3 & 744.2 \\
\hline AYo.WalDT.F & 15 & 15.4 & 13.6 & 14.4 & 162 & 16.6 & 77.4 & 15.8 & 12.9 & 12.5 \\
\hline 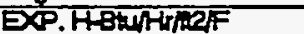 & 8890 & 1121.6 & 1110,3 & T0692 & 884.7 & $89 \pi$ & 864.5 & 953.8 & 1169.6 & $\sqrt{2000.6}$ \\
\hline & & & & & & & & & & \\
\hline & ZONE2 & ZONE4 & Z2ONi=6 & ZONES & 2ONETO & & & & & \\
\hline DP Lquid Tomp.f & 70.9 & & & & & & & & & \\
\hline DP Liquid Densityming & 82.61 & & & & & & & & & \\
\hline Elovetion & 2 & 4 & 6 & 8 & 9.875 & & & & & \\
\hline Moesured DPFild & 0.024 & 1.148 & 1.836 & 2.698 & 2.982 & & & & & \\
\hline Zne Comections-osid & -023 & $-0,088$ & -0.039 & -0.082 & 0.35 & & & & & \\
\hline CorrededDPPsid & 0.317 & 1.171 & 1.878 & 273 & 3.377 & & & & & \\
\hline Acrual DRn & 1.61 & 2.30 & 3.3 & 4.02 & 5.05 & & & & & \\
\hline & & & & & & & & & & \\
\hline
\end{tabular}




\begin{tabular}{|c|c|c|c|c|c|c|c|c|c|c|}
\hline Run & 988 & & & & & & & & & \\
\hline Tost fivid & $50 \%$ CAF 10 & $-50 \%$ RाTh & & & & & & & & \\
\hline Dats: & 122083 & & & & & & & & & \\
\hline Time: & $10: 40$ & & & & & & & & & \\
\hline Nat & & & & & & & & & & \\
\hline Fow Arasde & 0.00536 & & & & & & & & & \\
\hline Hon Fow Arose? & 0.18686 & & & & & & & & & \\
\hline Sel Prossure Pig & 75.8 & & & & & & & & & \\
\hline Su. Tomperaturef(intet) & ॠू. & & & & & & & & & \\
\hline Intet Fowme-GPM & 4.9 & & & & & & & & & \\
\hline indet tempercicure & 130.5 & & & & & & & & & \\
\hline Incr Glbmhntile & 1006058 & & temp & 1228 & Dansiny & 8821 & & & & \\
\hline Suboooing & 1.6 & & & & & & & & & \\
\hline Fow Obsenvion & & & & & & & & & & \\
\hline & ZOाE & $\angle O N=2$ & $\angle O N=3$ & $20 \pi=1$ & $\angle O N=6$ & $20 N=6$ & $201: 3$ & ZONE8 & $\angle O A=3$ & $20 N=10$ \\
\hline 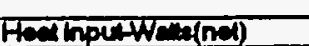 & $\frac{67.3}{67}$ & 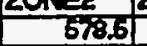 & $\frac{60}{672.8}$ & 6720 & 578.8 & 6750 & 680.8 & 6754 & 65at & 678.3 \\
\hline HenLoso-WC:s & 8.5 & 1.8 & $2 \pi$ & 27 & 25 & 1.9 & 1.4 & 1.8 & 22 & 13.1 \\
\hline Hesl fux sulfithe & Iod: & TOE & 886 & 899 & 996 & 988 & 1008 & 997 & 70068 & 10085 \\
\hline Wa Tomp. P\$ & 1452 & 14.8 & 1428 & 748 & 145.4. & 145.8 & 148.3 & 143.8 & 9422 & 7428 \\
\hline 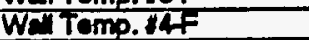 & 145.8 & 143 & 143.0 & 146.8 & 145.6 & 148 & 148.2. & 14.5 & 14.8 & $\sqrt{18.2}$ \\
\hline WeTtomp. & 148.8 & 747.8 & 144.7 & 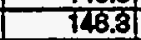 & 144.7 & T45.2 & 747.1 & 143.8 & 741.2 & 741.3 \\
\hline Waltomp. If & 130.5 & 744.8 & 741.8 & 746.6 & Th.4 & 145 & 150 & 745.5 & 741 & 741.8 \\
\hline WeT Tomp. ILt & 78 & कामी & 742.8 & 146.8 & 74.5 & 1452 & 349.7 & 146 & 76. & 742.6 \\
\hline Instrem Tomp. $f$ & 130.8 & 730.4 & 120.8 & 13.1 & 135.6 & आ & 1328 & 1302 & 150.1 & 735.3 \\
\hline Avo. Wel Tomp.f & 146.2 & T4.5 & 7द्2.8 & T46.8 & T4R: & 745.5 & 748.7 & 14.6 & TELT & 1428 \\
\hline AWo. WEll'DT.F & 15.4 & 13.2 & 12.6 & 12.7 & 127 & 14 & 15.4 & 14 & $T 1.6$ & 112 \\
\hline 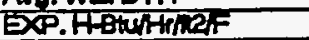 & 650.11 & 759.0 & 785.6 & 780.8 & 784 & 710.6 & 653.5 & 74 & 874.0 & 687.1 \\
\hline & & & & & & & & & & \\
\hline & ZON:S & ZONEA & $\angle O K=6$ & 201:58 & ZONETO & & & & & \\
\hline DPT tqud tomp.f & 76.7 & & & & & & & & & \\
\hline DP Lquid Densitutom/n3 & क्रा & & & & & & & & & \\
\hline Elentiont & 2) & 4 & 6 & 8 & 9.875 & & & & & \\
\hline Mesured Dppidd & 0.182 & 0.418 & 0.750 & 9.57 & 1.161 & & & & & \\
\hline 20 ro Corractions-psid & -0.218 & ठ.014 & 0.006 & 0.009 & -0.881 & & & & & \\
\hline 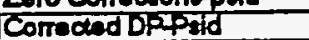 & 0.008 & 0.205 & 0.678 & 7.16ा & 7.482 & & & & & \\
\hline ActuidDPA & 20 & 3.59 & 5.27 & 8.8 & 8.08 & & & & & \\
\hline & & & & & & & & & & \\
\hline
\end{tabular}




\begin{tabular}{|c|c|c|c|c|c|c|c|c|c|c|}
\hline Runt & 387 & & & & & 7 & I & ! & i & \\
\hline Test Fuld & $50 \%$ CAF 10 & $0.50 \%$ RTT & & & & $T$ & & $T$ & & \\
\hline Dats: & $12 / 2898$ & & & & & & & t & & \\
\hline Timo: & $10: 40$ & & & & & & & $T$ & & \\
\hline \multicolumn{11}{|l|}{ Noto } \\
\hline Flow Aros-de & 0.0036 & & & & & & & & & \\
\hline Hod fow Arost? & 0.1855 & & & & & & & & & \\
\hline Sad. Pressurepsig & 75.1 & & & & & & & & & \\
\hline Sut Tompereture fintet) & 131.6 & & & & & & & & & \\
\hline \multicolumn{11}{|l|}{ Int Fowmito-GPM } \\
\hline \multicolumn{11}{|l|}{ indat Tempersturef } \\
\hline Inta Glommente & 761785 & & Temp & 7229 & Density & 65.2ता & & & & \\
\hline Subcoolingt & 25 & & & & & & & & & \\
\hline \multicolumn{11}{|l|}{ Fow Obsarvion } \\
\hline & & & 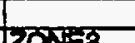 & 70125 & Z्रOIE5 & दठर:66 & ZONE? & 5 करण & 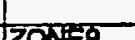 & \\
\hline Had houtWalu(nel) & $\frac{20 N=1}{580.0}$ & $\frac{2010 .}{580.11}$ & $\frac{601}{67.0}$ & $\frac{20 N=4}{578.4}$ & \begin{tabular}{|l|l|}
58120 \\
5812
\end{tabular} & $\frac{1200}{580.8}$ & 20.10 & 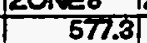 & 587.1 & $\frac{\angle U N=10}{585}$ \\
\hline Hed Loss-Whits & 7.8 & 1.7 & 2.3 & 2.5 & 2.4 & 1.8 & 1.3 & 1.7 & 2.1 & र25 \\
\hline Heal Flex-Btu/Hrile & 100 ग & 10068 & 70018 & 10028 & 10075 & 10008 & 10108 & 10007 & 1017 & 10दा \\
\hline Wa Tamp. *3F & काषा & 14.6 .6 & 143.1 & 745.5 & T45.6 & 146 & 747.3 & 143.7 & 142.8 & 145 \\
\hline WE Tomo. I4 & 144.4 & 142.6 & 14.8 & 945.8 & 145.8 & 748.5 & 147.3 & 144.9 & 143.8 & 146.5 \\
\hline We' Tomp. I5 & 715.3 & 141.8 & 145.8 & 745.9 & 145.1 & 145.8 & 148.5 & 143.6 & 1421 & 741.7 \\
\hline Wal Temp. IIt & 129.1 & 143.8 & 74.8 & T45.6 & 144.8 & 745.4 & 749.1 & 745 & 141.8 & 741.8 \\
\hline Wal Tomp.i2f & 746.8 & 143.4. & 742.8 & 745.4 & T4.5 & 145.8 & 748.7 & 745.7 & 7427 & 72.8 \\
\hline Instrem Tomp.f & 129.4 & 730.8 & 730.3 & अ्ट.4 & 131.3 & 130.8 & 132 & 13 & 130 & 731.7 \\
\hline Avg. Wai Tomp.f & 745.1 & 148.5 & 116 & 745.7 & 725 & 145.8 & 147.8 & 144.8 & 742.6 & 140.1 \\
\hline Avg. WalDT. $F$ & 152 & 128 & 122 & 12.8 & 13.2 & 14.8 & 75.8 & 14.1 & 12.1 & 11.0 \\
\hline \multirow[t]{3}{*}{ EXP.HBhirtrialis } & E5T.8 & 787.4 & 897.8 & 7835 & 753.51 & 691 & $\$ \$ \$ 0.5$ & 709.3 & 841.1 & 876.6 \\
\hline & & & & & & & & & & \\
\hline & ZONE2 & ZONE4 & ZONE6 & $\angle O N=38$ & ZONETO & & & & & \\
\hline Dp havid temp.f & 72.3 & & & & & & & & & \\
\hline DP Lqud Donstom/ns & \$247 & & & & & & & & & \\
\hline Elovetiont & 2) & 4 & 6 & 8 & 9.875 & & & & & \\
\hline Mozsured DRP pid & -0.088 & 0.673 & 1.17 & 1.76 & 3.89 & & & & & \\
\hline Zaro Correctons-osid & -0.278 & 0.014 & 0.058 & 0.000 & -0.33 & & & & & \\
\hline CorrededDPY rid & 0.13 & 0.658 & 7.774 & 7.751 & टथा & & & & & \\
\hline Actua DPA & 9.83 & 3.18 & 4.57 & 5.03 & 6.88 & & & & & \\
\hline & & & & & & & & & & \\
\hline
\end{tabular}




\begin{tabular}{|c|c|c|c|c|c|c|c|c|c|c|}
\hline Runt & 9881 & & & & & & & & & \\
\hline Tarf Fud & $50 \%$ CF 10 & -50\% RIT & & & I & & & & & \\
\hline Den: & 122083 & & i & i & 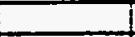 & & & & & \\
\hline Time: & $10: 40$ & & & & & & & & & \\
\hline Nots & & & & & & & & & & \\
\hline Frow Areal2 & 0.00356 & & & & & & & & & \\
\hline Hest flow AMente & 0.18050 & & & & & & & & & \\
\hline Set Pressure phio & 74.4 & & & & & & & & & \\
\hline Sel Tompor wre flintel) & 131 & & & & & & & & & \\
\hline Ind Fowrato-GP & 2दा & & & & & & & & & \\
\hline Intel Tomperituref & 728.8 & & & & & & & & & \\
\hline Intat Gomphilie & $2950 \% 3$ & & Tomp & 1202 & Donaty & 86.59 & & & & \\
\hline Stooooding f & 2.1 & & & & & & & & & \\
\hline Fow Obtervelion & & & & & & & & & & \\
\hline & & & & & & & & & & \\
\hline & 201:1 & ZONi32 & $20 N=3$ & $20 N=4$ & $201=6$ & $\angle O N=6$ & ZON=7 & $\angle O N=8$ & $\angle O N=\theta$ & $\angle O N=10$ \\
\hline 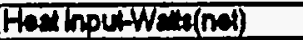 & 5812 & 580.7 & 578.7 & 578.8 & 580 & 57.4 & 5828 & 677.4 & 676.8 & 584 \\
\hline Fan Lose-We'ts & 8.1 & 1.7 & 2.3 & 2.7 & 25. & 1.8 & 1.4 & 1.8 & 23 & 13.6 \\
\hline 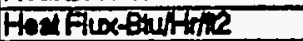 & $100 \%$ & 1063 & 10051 & 10030 & 1005 & 10014 & 10068 & 1000 & (986) & 10123 \\
\hline WAl Tomp. 13F & 73.7 & 14 & 1424 & 145.8 & 145.8 & 145.7) & 148 & 143.8 & 1425 & 14 \\
\hline Wall TOmp. ILF & 744.4 & 142.1 & 143.6 & 146.2 & 145.7 & 145.8 & 148.7 & 74.8 & 743.5 & 747.4 \\
\hline Wal Tomp. 16 & 145 & 140.9 & 74.6 & 1462 & 744.3 & 145.1 & 147 & 143.1 & 141.7 & Tद2. \\
\hline Wal Tomp. IIF & 728.8 & 743.8 & 740.8 & 746 & TaR.4 & 745.1 & 749.8 & 745.5 & दाता & 1427 \\
\hline War Tomp. ILF & 148 & 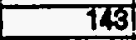 & 747.8 & 745.7 & 14.4 & 146.8 & 749.4 & 1462 & 1क्र.1 & 143.5 \\
\hline Instroem tomp.f & 128 & अ.1 & 129.7 & 1326 & 130.9 & 130.5 & बक्र2: & భ2.7. & 1द्2.6 & 731.5 \\
\hline Avo. WA Tomp.t" & 14.8 & 143.1 & 7422 & 146.1 & 144.7 & 145.5 & 148.5 & 144.6 & Ta22] & 14 \\
\hline AW.WEIDT. & 16.8 & 125 & 12 & 18 & 13.8 & 14.5 & 16.8 & 14.6 & 12.1 & 12.1 \\
\hline 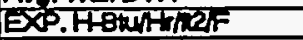 & 657.6 & 8024 & 8531 & 770.2 & 753.8 & 628 & 609 & 690.8 & 825.8 & 837.8 \\
\hline & & & & & & & & & & \\
\hline & ZONE2 & ZONEA & ZOKEEB & ZONEB & ZONETO & & & & & \\
\hline DPPloqud Temp.f & 78.1 & & & & & & & & & \\
\hline DPLiquid Density - tom/3 & 9200 & & & & & & & & & \\
\hline Elevetion & 2 & 4 & & 8 & 9.876 & & & & & \\
\hline Meatured DPFidd & 0.017 & 0.556 & $1 . \overline{672}$ & 2425 & 2.704 & & & & & \\
\hline Zoro Comactons-psid & -0.218 & 0.074 & 0.068 & 0.008 & -0.35 & & & & & \\
\hline CoTrectedDPF Id & 0.235 & 0.982 & T.81B & 2.476 & 3.035 & & & & & \\
\hline Actula DPA & 1.74 & 2.62 & 3.69 & 4.49 & 5.45 & & & & & \\
\hline & & & & & & & & & & \\
\hline
\end{tabular}




\begin{tabular}{|c|c|c|c|c|c|c|c|c|c|c|}
\hline Runt & 9891 & & & & & & & & & \\
\hline Test Fuid & $50 \% \operatorname{cis} 39$ & $5.50 \times 9114$ & & & & & & & & \\
\hline Dais: & 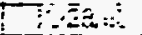 & & & & 1 & & & & & \\
\hline Time: & $-74.07 \%$ & & & & & & & & & \\
\hline Now & & & & & & & & & & \\
\hline Fow Arealle & 0.00538 & & & & & & & & & \\
\hline Healfow Arearte & 0.19695 & & & & & & & & & \\
\hline Sat. Prassure Prig & 75.8 & & & & & & & & & \\
\hline Sat. Tompercture f(inlel) & 132.1 & & & & & & & & & \\
\hline Intel fowrde-GPM & 4.9 & & & & & & & & & \\
\hline intef Temperaturet & 130.5 & & & & & & & & & \\
\hline Intelgbmintire & 1008133 & & Temp & 122.5 & Density & 83.20 & & & & \\
\hline Suboodingf & 1.6 & & & & & & & & & \\
\hline Fow Observation & & & & & & & & & & \\
\hline & ZOONET & 2ONEद & ZONESOS & ZONiE4 & ZONES & $\angle O K=6$ & ZONE=7 & ZONEB & ZONES & $20 N=10$ \\
\hline Heal nputWas(not) & 288.8 & 285. & 285 & 287.6 & 28.1 & 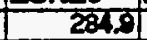 & 293.0 & 290.8 & 287.7 & 291.0 \\
\hline Hea Loss-Wats & 8.2 & 9.7 & 23 & 2.6 & 2.8 & 1.7 & 1.2 & 1.6 & 2 & 12.3 \\
\hline 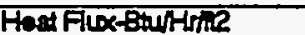 & 5008 & 486 & 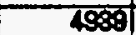 & 4805 & 4951 & 483 & 5035 & 5041 & 4887 & 5000 \\
\hline Wal Temp. KFF & 144.4 & 14.2.4 & 141.7 & 14.6 & 143.4 & 7R1.8 & 144.8 & 141.5 & 140.8 & 140.7 \\
\hline Wall Tomp. IfF & 144.7 & 142 & 147.8 & 144.6 & 142.8 & 142.2 & 144.2 & 1412 & 741 & 142.3 \\
\hline Wad Tomp. 15F & 145.4 & 140.8 & 742. & 744.6 & 142.4 & 142 & 943.1 & 140.4 & 1402 & 140.4 \\
\hline Wall Temp. $11 F$ & 130.5 & 1426 & 140.7 & 146 & 142.7 & 142.7 & 145.1 & 142.1 & 139 & 141.2 \\
\hline Wall Tamp. ILF & 145 & 142.7 & 141.2 & T4.8 & 142.6 & 142.4 & 144.8 & 142.3 & 736.8 & 7दा.3 \\
\hline Instroam Tomp.F & 130.6 & 129.3 & 129.8 & 1326 & 131.5 & 137 & 132.3 & 129.8 & 720.8 & 131.1 \\
\hline Avo. Wall Tomp.f & 14.8 & Tदिया & 141.4 & 14.7 & 1427 & 142.2. & 144.3 & 141.5 & 140 & 7द12 \\
\hline AnO.WAHT.F & 74.1 & 12.8 & 17.8 & 11.8 & 10.8 & 11 & 11.8 & 11.6 & 9.9 & 9.9 \\
\hline EXP.HBWHAnef & 353.8 & 384.1 & 417.8 & 421.6 & 460.7 & 450.8 & 4329 & 439.4 & 502 & 513.6 \\
\hline & & & & & & & & & & \\
\hline & ZONE2 & ZONER & ZONE6 & ZONEB & ZONETO & & & & & \\
\hline DP rquid Tomp.f & 75.5 & & & & & & & & & \\
\hline DPLiguid Densintom/3 & S2.13 & & & & & & & & & \\
\hline Elevelionf & & 4 & 6 & 8 & 9.875 & & & & & \\
\hline Measured DPP sid & -0.18 & 0.274 & 0.451 & 0.848 & 0.834 & & & & & \\
\hline Zoro Correctons-pesid & 2.218 & 0.074 & 0.056 & 0.008 & -0.331 & & & & & \\
\hline Corroded DPFid & 0.028 & 0.801 & 0.35 & 0.834 & 1.165 & & & & & \\
\hline Actual DPA & 200 & 3.84 & 5.75 & 7.16 & 8.6 & & & & & \\
\hline & & & & & & & & & & \\
\hline
\end{tabular}




\begin{tabular}{|c|c|c|c|c|c|c|c|c|c|c|}
\hline Runt & $\begin{array}{r}990 \\
\text { Fag }\end{array}$ & करण & & i & & & & & & \\
\hline Tanfluid & & - $60 \%$ RIIL & & & & & & & & \\
\hline Data: & $1228 / 93$ & & & & & & & & & \\
\hline Time: & 14.07 & & & & & & & & & \\
\hline Now & & & & & & & & & & \\
\hline Flow Areat? & 0.00358 & & & & & & & & & \\
\hline Fenflow Areate & 0.18536 & & & & & & & & & \\
\hline Set. Ptessureplio & 75.8 & & & & & & & & & \\
\hline Set. Tempernture fe(intat) & 132.1 & & & & & & & & & \\
\hline InA Fowr2L-GPM & 3.6 & & & & & & & & & \\
\hline Finet Tomperaturef & 331.1 & & & & & & & & & \\
\hline nat Gbmhitie & 747482 & & Temp & 1223 & Donsty & 88.87 & & & & \\
\hline Subcoollingf & Tा & & & & & & & & & \\
\hline Fow Obsenvelon & & & & & & & & & & \\
\hline & $2 O N=1$ & 2OKE:C & ZOKES & $20 N=4$ & ZONES & $201=6$ & 20kE=? & $20 N=8$ & ZONESO & $201=10$ \\
\hline Heal houtwated & 2028 & 2808 & 2888 & 200.1 & 2850 & 287.5 & 2क्वा & 293.7 & 289.8 & 200.7 \\
\hline Healloss-Wed & 7.4 & 1.6 & 2। & 2.5 & 2.2 & 1.6 & 1.11 & 1.5 & 1.8 & 11.4 \\
\hline Hed Flux-Buinthe & 5075 & 502 & 4983 & 5000 & 4868 & 2884 & 5077 & 5091 & 6008 & 5060 \\
\hline Wel Tomp. 13+ & 144 & 14द्टा & 141.8 & 165.4 & 141 & 142.3 & 145.8 & 141.9 & 1411 & 142 \\
\hline Wall Tomp. ILF & 144.9 & 1423 & 142 & 145.3 & 143.7 & 142.8 & 145.8 & 142 & 141.3 & 143.2 \\
\hline Waltomp. 15F & 145.2 & 141.9 & 1425 & Ta5.6 & 1428 & 142.6 & 14.5. & 14ा & 160.7 & 141 \\
\hline Wall Tomp. ItF & 131.1 & 142.9 & 140.8 & 146.8 & 143.6 & 143.1 & 147.8 & 143 & 140.1 & 142.5 \\
\hline Wall Tomp.12t & 146.4 & 143 & 1दा & 146.8 & 143.11 & 142.8 & 146.7 & 143.2 & 1402 & -1623 \\
\hline Instroen Tomp.t & T30.8 & 120.2 & 120.1 & 133.2 & 131.6 & 130.8 & 13.1 & 130.1 & 120.8 & 131.6 \\
\hline Aug. Wall Tomp.f & 144.8 & T428 & 141.8 & 745.6 & 743.8 & 1427 & 746.8 & 1622 & 140.7 & 142.2 \\
\hline Ang. Wal DT. & 19.9 & 19.8 & (2) & 12.2. & 97.5 & 11.7 & 12.6 & 11.9 & 10.5 & 10.4 \\
\hline 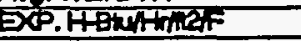 & 265.8 & 378.8 & 414.4 & 412.8 & 4828 & 420 & 402.6 & T28.2 & A75.2. & 488.8 \\
\hline & & & & & & & & & & \\
\hline & ZONE2 & ZONEA & ZONE & ZONIES & 20NE10 & & & & & \\
\hline DP toquid Temp.f & 77.2 & & & & & & & & & \\
\hline DPLquid Dons $4 \mathrm{bm} / \mathrm{Br}$ & 97.85 & & & & & & & & & \\
\hline Elevation-a & & 7) & & 8 & 9.875 & & & & & \\
\hline Mes:ured DPFild & -0.145 & 0.458 & 0.756 & 7.278 & 1.37 & & & & & \\
\hline 20 cortoctone-perd & -0.278 & 0.04 & 0.058 & 0.009 & -0.381 & & & & & \\
\hline Correded DPFild & 0.078 & 0.421 & 0.703 & 1.267 & $1 . \pi 1$ & & & & & \\
\hline Actur DPA & 201 & 3.58 & 5.22 & 6.41 & 7.87 & & & & & \\
\hline & & & & & & & & & & \\
\hline
\end{tabular}




\begin{tabular}{|c|c|c|c|c|c|c|c|c|c|c|}
\hline Runt & $991 !$ & $T$ & T & & & & & & 1 & \\
\hline Tost Fuld & $50 \%$ C4Fin & $7.50 \%$ Rा14 & & $T$ & & & & & T & \\
\hline Dat: & 12 2893 & & 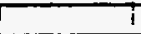 & $i$ & & & & & & \\
\hline Time: & 14.07 & & & & & & & & 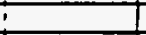 & \\
\hline \multicolumn{11}{|l|}{ Now } \\
\hline Fow Aresed & 0.00035 & & & & & & & & & \\
\hline Hodf Fow Areata2 & 0.1958 & & & & & & & & & \\
\hline Sut Pressura Paig & 76.7 & & & & & & & & & \\
\hline Sed. Temperature F(inled) & 132.7 & & & & & & & & & \\
\hline Intelfowrate-GPM & 2.4. & & & & & & & & & \\
\hline \multicolumn{11}{|l|}{ intat Temperaturet } \\
\hline Intot G $b$ mhrine & LS630 & & Tomp & Tदा & Density & 86.48 & & & & \\
\hline Subooolingf & 1.31 & & & & & & & & & \\
\hline \multicolumn{11}{|l|}{ Fow Observetion } \\
\hline & & & & & & & & & & \\
\hline & ZONET & ZONES & ZONES8 & $20 N=4$ & ZONES & ZONEB & ZONES & ZONE8 & ZONEO & ZONETO \\
\hline Hod inputwe"tnel) & 233.4 & 200 & 288.8 & 290.7 & 288.1 & 287.9 & 2332 & 294.1 & 2392 & 290.8 \\
\hline HedLoss-Was & 7.8 & 9.6 & 2.1 & 25 & 22 & 1.6 & 1.2 & 1.6 & 1.9 & 91.8 \\
\hline 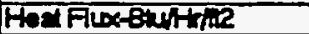 & 5008 & 5027 & 4963 & 50391 & 4959 & 4591 & 5082 & 5098 & 5018 & 5043 \\
\hline Wall Tomp. 13F & 144.2 & 143 & 1424 & 146.1 & 144.8 & 143 & 148.3 & 1423 & 141.3) & 1427 \\
\hline Wal Temo. \$4F & 144.5 & 142.6 & 142.7 & 145.9 & 144.5 & 143.7 & 146.3 & 142.5 & 741.9 & 144.2 \\
\hline Wal Temo. $75+$ & 145.4 & 141.6 & 143.3 & 146.2 & 143.3 & 143.1 & 145 & 141.8 & 120.8 & 141.8 \\
\hline Wall Temp. If & $131.4 \mid$ & T43.4 & दका & 146.81 & 143.8 & 143.8 & 147.8 & 143.9 & 7क0. & 142.8 \\
\hline Wa Tomp. T2F & T46.91 & 143.3 & 74t.6 & 746.8 & 743.6 & 143.5 & 747.3 & 144 & 740.3 & T42.7 \\
\hline Instren Temp,f & 131.4 & 130.8 & 130.8 & 1342 & 1322 & 137.8 & 133.1 & 130.8 & 130.8 & 1328 \\
\hline Avg. Wal Temp. F & 145.81 & |43.11 & 141.8 & 146.8 & 143.8 & 748.4 & 148.5 & 1428 & 140.8 & 7268 \\
\hline Ang. Wed DT.F & 18.8 & 125 & 10.8 & 11.8 & 11.4 & 11.5 & 12.81 & 11.9 & 9.र्व & 9.7 \\
\hline \multirow[t]{3}{*}{ EXP.HBthwn?F } & 378.4 & 400.61 & 469.5 & 45.8 & 453.8 & 438 & 408 & 739.6 & 603.8 & 618.1 \\
\hline & & & & & & & & & & \\
\hline & ZONE2 & ZONER & ZONE6 & ZONEB & $201=10$ & & & & & \\
\hline DP Fquid Tomp.f & 77.5 & & & & & & & & & \\
\hline DPLiquid Density bminz & 91.92 & & & & & & & & & \\
\hline \multicolumn{11}{|l|}{ Elovetion? } \\
\hline Wesured DP4sid & -0.083 & 0.652 & 1.1 बा & 1.84 & 2.085 & & & & & \\
\hline Zero Corrections-psid & उ.द्रा & 0.074 & 0.058 & 0.009 & -0.3 & & & & & \\
\hline Comected DPPSid & 0.135 & 0.618 & 1.108 & $1.8 \%$ & 2.386 & & & & & \\
\hline Actul DP & 1.9 & 3.17 & 4.63 & 5.46 & 6.51 & & & & & \\
\hline & & & & & & & & & & \\
\hline
\end{tabular}




\begin{tabular}{|c|c|c|c|c|c|c|c|c|c|c|}
\hline Run! & 992 & & 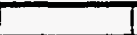 & & & & & & & \\
\hline Tert Fuld & $50 \%$ CAF10 & D-50\%RाT & & & & & & & & \\
\hline Den: & 12299 & & & & & & & & & \\
\hline Time: & 8.61 & & & & & & & & & \\
\hline Now & & & & & & & & & & \\
\hline Fow Areathe & 0.00336 & & & & & & & & & \\
\hline Herl Fow Arean? & 0.19035 & & & & & & & & & \\
\hline Sat. Proszurofig & 105 & & & & & & & & & \\
\hline Sal. Tempermuref(int) & 150 & & & & & & & & & \\
\hline Indelfowrete-GeM & 5 & & & & & & & & & \\
\hline Intalomperdinef & 149.6 & & & & & & & & & \\
\hline InTGGomhin & 3078 & & Tomp & 7243.6 & Denciy & 83.031 & & & & \\
\hline Subooolingt & 0.4 & & & & & & & & & \\
\hline Fow observation & & & & & & & & & & \\
\hline & & & & & & & & & & \\
\hline & ZONiET & $201=2$ & ZOWE3 & ZONiz4 & ZON=5 & $20 N=6$ & LONE & $20 \mathrm{~N}=8$ & T2ON=9 & $201=10$ \\
\hline 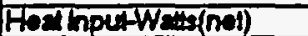 & 859.1 & 859.8 & 867.6 & 881.7 & 875.7 & 875.21 & 859.8 & 8605 & 884.4 & $8 \pi$ \\
\hline HenLose-Wes & 9.8 & 2.1 & 2.8 & 3 & 3 & 23 & 1.5 & 7.91 & 27 & 75.9 \\
\hline 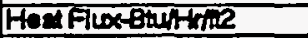 & 14892 & 1507 & 15038 & 14827 & 15180 & 1517ता & 14804 & 14918 & 1498 & 15098 \\
\hline WE Tamp. \# & 165.1 & 157 & 164.6 & 167.5 & 168.7 & 169.4 & 167.8 & 163.7 & 165.4 & 168 \\
\hline WaH Temp. IfF & 166.2 & 163.9 & 1662 & 167.8 & 16 & 169.3 & 167.4 & 163.8 & 168.5 & 171.8 \\
\hline WEA TOMP. IEF & 966.4 & 162 & 167.1 & 1682 & 167.3 & 169.11 & 168.7 & 1623 & 165.2 & 163.8 \\
\hline Wall Tomp. ITF & 149.6 & Tए5 & TE्2.1 & 7672 & 168.8 & 169.11 & 772.6 & 7628 & Tะ3.6 & 76.8 \\
\hline Wal Tamp. IRF & 768.7 & 764.8 & 163.6 & 767.2 & 188.8 & 169.61 & 7724 & 163 & $78 \pi, 4$ & 165.4 \\
\hline Instrom Tomp.f & 749.7 & 749.4 & 750 & 153.2 & 151.8 & 151.8 & 153.7 & 751.3 & 750.0 & $\sqrt{522}$ \\
\hline AYq. WaH Tamp.f & 168.6 & 96.2 & 1ब्र.T & 187.7 & 767.3 & T6Sב & 169.8 & 163.1 & 165 & 160.2 \\
\hline Avg. WaH DT of & 18.2 & 15.1 & 13.4 & 13.8 & 14.7 & 172 & 15.4 & 11.1 & 13.4 & 13.8 \\
\hline EXP.HBWHTREF & 817.7 & 998.8 & 1118.6 & 1081.8 & 10्31.7 & 800 & 868.1 & 1338,8] & 1114.8 & 1188.7 \\
\hline & & & & & & & & & & \\
\hline & ZONES & ZONES4 & ZONE6 & ZONEB & ZONETO & & & & & \\
\hline DP hquid Tomp.f & 78.7 & & & & & & & & & \\
\hline DPL Luid Donsityomin & 91.68 & & & & & & & & & \\
\hline Elevention & & 4 & 6 & 8 & 9.875 & & & & & \\
\hline Measured DPSPad & 0.184 & 0.475 & 0.830 & 7.319 & 1.361 & & & & & \\
\hline Zero Cortections-psid & -0.28 & -0.077 & ర.018 & 0.072 & -0.338 & & & & & \\
\hline Corrected DP4 sid & 0.058 & 0.492 & 0.82 & 7.301 & 1.57 & & & & & \\
\hline Actud DPA & 2.11 & 3.56 & 5.2 & 6.57 & 7.98 & & & & & \\
\hline & & & & & & & & & & \\
\hline
\end{tabular}




\begin{tabular}{|c|c|c|c|c|c|c|c|c|c|c|}
\hline Run: & 993 & & & & & & & & T & \\
\hline Test Fund & $50 \%$ CF10 & $5-50 \%$ A114 & & & & & & & & \\
\hline Date: & $12 / 2893$ & & & & & & & & & \\
\hline Time: & $12: 38$ & & & & & & & & & \\
\hline Now & & & & & & & & & & \\
\hline Fow Areare & 0.00356 & & & & & & & & & \\
\hline Heal Fow Arest? & 0.1959 & & 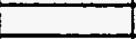 & & & & & & & \\
\hline Sed. Prossure Psig & 104.4 & & & & & & & & & \\
\hline Sat. Temperaturef(indot) & 149.7 & & & & & & & & & \\
\hline Intet Fownde-GSM & 3.8 & & & & & & & & & \\
\hline Intal Tomperativet & 149.3 & & & & & & & & & \\
\hline Ind Glom/nrine & 75365 & & Tomp & 143.1 & Densily & 83.11 & & & & \\
\hline Subooolingf & 0.4 & & & & & & & & & \\
\hline Fan Observation & & & & & & & & & & \\
\hline & $20 N=1$ & $20{ }^{N}=2$ & ZONEB & LONE & LZONiES & $20 N=6$ & $20 \mathrm{~T}=7$ & ZONEB & KONE8 & $20 N^{\prime}=10$ \\
\hline Hoat inputWatinget) & 884.7 & 875.11 & 867.1 & 881 & 874.5 & 875.4 & 868.9 & 865.5 & 868.1 & 870.8 \\
\hline Host Lose-Wels & का & 2 & 26 & 3 & 2.9 & 22 & 3.51 & 1.8 & 25 & 15.7 \\
\hline Hant flux-8turtshe & 1480 & 15169 & 15031 & 14505 & 15169 & 15174 & 149751 & 15003 & 14881 & 1508 \\
\hline Wet Tamp. 35 & 165 & 168.7 & 164.1 & 167.6 & 168.7 & 169.4 & 167.8 & 163.5 & 164.5 & 168.3 \\
\hline Well Temp. 145 & 166.1 & 163.7 & 165 & 168 & 169.1 & 1692 & 1672 & 163.6 & 165.6 & 172 \\
\hline Well Temp. 15 F & 166.2 & 161.6 & 168.6 & TE8.1 & 166.9 & 168.6 & 168.8 & 161.8 & 164.1 & 184.1 \\
\hline Wall Temp. IfF & 749.3 & 1651 & T61.8 & T67.5 & T65.5 & 160 & T72.8 & 162.9 & T62.8 & T64.2 \\
\hline Wail Tomp. Baf & 768.2 & 764.61 & TE3.3. & 167 & T68.5 & T69.8 & 1725 & TE29 & 763.6 & T65.8 \\
\hline Instreem Temp.f & 149.5 & 149.8 & 150.1 & 153.1 & 151.4 & 151 & 153,3 & I50.7 & 1506 & 162.3 \\
\hline Avo. Wall Temp.t- & 168.4 & 185 & 183.8 & 767.8 & 167.3 & 169.1 & 169.8 & 163 & 164.1 & 160.4 \\
\hline Avo. Wel DT.F & 16.2 & TAS & 18 & 14 & 15.2 & 17.4 & 15.8 & 97.8 & 129 & 19.5 \\
\hline DAP. HBuntrnat & 925.4! & 1045.1 & 1168.8 & 1084.1 & 998.6 & $87 \overline{3.8}$ & 948.4 & 1295.4 & 7167.6 & Tसर.8 \\
\hline & & & & & & & & & & \\
\hline & ZONE2 & ZONES & $207 \mathrm{~N}=6$ & 20158 & $201: 50$ & & & & & \\
\hline DPP iguid Tomp.f & 81.3 & & & & & & & & & \\
\hline DPUquid Densily ymith & 91.51 & & & & & & & & & \\
\hline Elevationnt & 2 & 4 & 61 & 8 & 9.875 & & & & & \\
\hline Measured DPFisid & $-0.07 \pi$ & 0.78 & 13 & 1.883 & $2.07 / 1$ & & & & & \\
\hline Eero Corractionsepsid & 0.223 & -0.017 & 0.018 & 0.012 & $\$ .388$ & & & & & \\
\hline Corrected DPFAd & 0.162 & 0.745 & 1241 & 1.874 & 2.413 & & & & & \\
\hline Actuld DPR & 1.94 & 3.1Tा & 4.46 & 5.56 & 6.69 & & & & & \\
\hline & & & & & & & & & & \\
\hline
\end{tabular}




\begin{tabular}{|c|c|c|c|c|c|c|c|c|c|c|}
\hline \multicolumn{11}{|l|}{ Run: } \\
\hline Torf Fud & T50\% CAF 10 & D-50\% मा1 & & & I & & & & & \\
\hline Das: & 1122998 & & & & $i$ & & & & T & \\
\hline Time: & 12.38 & & & & & & & & & \\
\hline \multicolumn{11}{|l|}{ Now } \\
\hline Fow ATeanL & 0.00336 & & & & & & & & & \\
\hline Hod Flow Areat2 & 0.18695 & & & & & & & & & \\
\hline Sed Prossurefrig & 103.8 & & 1 & & & & & & & \\
\hline Sed. Temperaturef(inot) & 149.4 & & & & & & & & & \\
\hline Intetflowreto-GPM & 2.5 & & & & & & & & & \\
\hline \multicolumn{11}{|l|}{ intel Tomperation } \\
\hline Intet Gibm/nine & 497891 & & fomp & 140.9 & Donsity & 89.48 & & & & \\
\hline Subooolingf & 2 & & & & & & & & & \\
\hline \multicolumn{11}{|l|}{ Flow Observelion } \\
\hline & CON=1 & ZONE? & $201: 53$ & $20 \mathrm{~N}=4$ & $20 \mathrm{~N}=6$ & $20 \mathrm{R}=6$ & टका=? & $20:=8$ & $20 \mathrm{~N}=9$ & $\angle O N=10$ \\
\hline Hadlnpentwatnel) & 8673 & 868.7 & 865.7 & 853.7 & 8556 & 8840 & 884 & $883 x$ & 8050 & 881.0 \\
\hline HeNLose-WM & 8.2 & 2.1 & 28 & 3.1 & 3 & 23 & 1.5 & 2 & टा & 18.6 \\
\hline Hod Floxthulthin & 14982 & 15058 & 15008 & 1497 & 76000 & 14882 & Tद्बका & 14868 & 15035 & 1490 \\
\hline Waltomp. \%F & 184 & 168.1 & 163.4 & 168.8 & 157.8 & 167.9 & 1682 & 163.8 & 164.1 & 166.5 \\
\hline Wall Tomp. I4F & 765.1 & 162.9 & 165.1 & 167.4 & 168.1 & 168 & 167.5 & 163.8 & 165.4. & 172.3 \\
\hline Wal Tomp. 157 & 165.4 & 160.8 & 166.2 & 167.8 & 168.2 & 167.3 & 16.3 & 162.3 & 163.6 & 164.1 \\
\hline Wal Temp. 11F & 747.4 & 164 & 780.7 & 16.8 & 125.8 & 167.6 & 172.2 & 1528 & 162.4 & 164.3 \\
\hline Wall Tomp. IIF & TE.7 & i6A & T624 & 168.8 & 165.8 & 767.8 & 17T.9 & 162.9 & 16 & 185.7 \\
\hline Instrosm Tomp.f & 147.7 & 149.8 & 148.6 & 1520 & 151.1 & 150.6 & 1532 & 150.4 & 150.8 & 152.3 \\
\hline AVO.WCl Tomp.t. & 165.8 & 164.8 & 1628 & 187.8 & 168.6 & 167.7 & 169.6 & I632) & 163.7 & 160.6 \\
\hline Avg. Wa'DTAF & 18.8 & 13.7 & 12.6 & 13.7 & 14.7 & 18.4 & 15.7 & 12. & 127 & 13.6 \\
\hline \multirow[t]{3}{*}{ 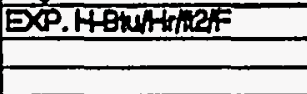 } & 885.3 & 1101.6 & 1188.0 & 700.8 & 1000.0 & छाटर्ण & 561.8 & T268.6 & Tा8्व & 10032 \\
\hline & & & & & & & & & & \\
\hline & ZONE2 & ZONE4 & $20 K=6$ & ZONE8 & ZOKETO & & & & & \\
\hline DP tigud tamp.f & 822 & & & & & & & & & \\
\hline DPLquid Densin tom/3 & 91.411 & & & & & & & & & \\
\hline Eleviont & 2 & 7 & 6) & 8 & 9.875 & & & & & \\
\hline Mesured DPFald & 0.018 & 1.077 & 1.73 & 2491 & 2.869 & & & & & \\
\hline Zero Comectonsporid & -0.23 & -0.017 & 0.018 & 0.012 & 0.356 & & & & & \\
\hline Correctsd DPS Id & 0.211 & 7.03 & T.72 & 2478 & 3.205 & & & & & \\
\hline ACUMDPA & 1.71 & 2.6 & 3.6 & 4.48 & 5.3 & & & & & \\
\hline & & & & & & & & & I & \\
\hline
\end{tabular}




\begin{tabular}{|c|c|c|c|c|c|c|c|c|c|c|}
\hline Runt & का & & i & ! & ! & & & & T & \\
\hline Tosf Fudd & $150 \%$ C4F 10 & -50\%RT16 & & i & 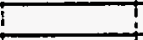 & & & & 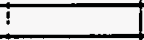 & \\
\hline Dats: & 1222998 & & i & & ! & & i & & I & \\
\hline Time: & $12: 38$ & & $!$ & & 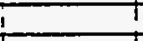 & & & & $I$ & \\
\hline Now & & & & & I & & & I & $I$ & \\
\hline Flow Areas 2 & 0.00336 & & & & & & & & & \\
\hline Heal Fiow Ares-12 & 0.19596 & & & & & & & & & \\
\hline Set Pressure Psig & 104.8 & & & & & & & & & \\
\hline St. Temperature f (inlot) & 149.8 & & 1 & & & & & & & \\
\hline Intel Fowrato-GPM & 5.11 & & & & & & & & & \\
\hline inhol Tomperaturef & 149.3 & & & & & & & & & \\
\hline Intel Glbmhink & 7007982 & & TTemp & 744.9 & Donstity & 828 & & & & \\
\hline Subooolingf & 0.6 & & & & & & & & & \\
\hline Flow Obsorvation & & & & & & & & & & \\
\hline & & & & & & & & & & \\
\hline & ZONE1 & LONEE & LONE'B & शिONE & $20 \mathrm{~N}=5$ & $\frac{2 O N=6}{5 \times 2}$ & $\frac{12 O N=7}{67 \pi}$ & $\frac{\text { ZONEB }}{667}$ & ZONE9 & $20 N=10$ \\
\hline 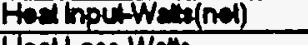 & 675.8 & 577.5 & 567.5 & $\frac{568.3}{36}$ & \begin{tabular}{|r|}
574.8 \\
227 \\
\end{tabular} & \begin{tabular}{|r|}
570.2 \\
1.7
\end{tabular} & $\frac{011}{12}$ & 1.7 & 22 & $\begin{array}{r}068.0 \\
3.7\end{array}$ \\
\hline HeclLose-Wa"s & 8.6 & 1.9 & 2.4 & 26 & $\frac{2.41}{\cos 2 !}$ & $\frac{1.1}{20}$ & कoges & g.17 & क्वरा & क्5.1 \\
\hline Had flox-bultine & 9881 & 9907 & 9837 & 8851 & $\frac{9984}{1608}$ & $\frac{9884}{1594}$ & $\frac{8608}{161.2}$ & 159.9 & 153.6 & $\frac{8001}{1600.6}$ \\
\hline Wall Temp. HF & 760.9 & 1623 & 160.2 & 162 & $\begin{array}{r}160.8 \\
161\end{array}$ & $\begin{array}{l}159.4 \\
155.7\end{array}$ & 180.9 & 159.8 & 161 & 164.1 \\
\hline Wat Tomp. $14 \mathrm{~F}$ & 167.4 & 160.8 & 161.3 & 1627 & $\begin{array}{r}161 \\
159.8\end{array}$ & 159.4 & T59.8 & 158.8 & 758.4 & 150 \\
\hline Wall Temp. $15 F$ & 162.3 & 159 & 160.2 & 161.2. & $\frac{159.8}{150}$ & 100.7 & 122 & 159 & 1576 & 109 \\
\hline Wal Temp. $11 F$ & 749.3 & 160.3 & 158 & 167.21 & 160 & & जहा? & 5503 & 158 & 159 \\
\hline W2H Tomp. T2F & 165.81 & 760.8 & 758.8 & 161.4 & 160 & 160.4 & 1612 & 159.3 & 158 & 759.8 \\
\hline Instream Temp.F & 149.4 & 149.7 & 149.5 & 157.8 & 1512 & 150.9 & 1524 & 750.5 & 150.8 & 151.4 \\
\hline Ang. Wefl Tomp.F & 1628 & 181.1 & 759.6 & 761.8 & 160.2 & 159.8 & 181 & 158.4 & 158.0 & 760.5 \\
\hline Avg. WallDT.f & 13 & 11.8 & 9.8 & 8.5 & 8.6 & 8.5 & 8.2 & 8.5 & 8.2 & 8.7 \\
\hline DXP.HBuHrner & 788.4 & 857.6 & 10्टाठा & 1033.2 & 11ब्2 & 1169.3 & दि1. & 1164.8 & 1216.7 & 17382 \\
\hline & & & & & & & & & & \\
\hline & ZONE2 & ZONEA & $20 N=6$ & ZONE & ZONE 10 & & & & & \\
\hline DP Fquid Tomp.F & 75.8 & & & & & & & & & \\
\hline DPUquid Density & 92.1 & & & & & & & & & \\
\hline Elevitionth & & का & 6 & 8 & 9.876 & & & & & \\
\hline Massured DPphid & -0.193 & 0.399 & 0.677 & 1.106 & 3.166 & & & & & \\
\hline Zero Corrections-psid & -0.223 & -0.017 & 0.019 & 0.012 & 3.356 & & & & & \\
\hline Comeded DPP sid & 0.03 & 0.418 & 0.658 & T.0035 & 7.502 & & & & & \\
\hline Actual DPA & 2.17 & 3.72 & 5.58 & 7 & 8.37 & & & & & \\
\hline & & & & & & & & & & \\
\hline
\end{tabular}




\begin{tabular}{|c|c|c|c|c|c|c|c|c|c|c|}
\hline Runt & 996 & 7 & & & & & & 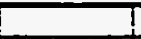 & & \\
\hline TerfFud & $150 \%$ CAF 10 & $-50 \% R 114$ & 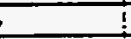 & 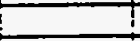 & & & & & & \\
\hline Dan: & 1 12:993| & 1 & & & & & & & & \\
\hline Timo: & $12: 381$ & $i$ & & & & & & & & \\
\hline Now & 1 & & & & & & & & & \\
\hline Fow Arente & 0.00386 & & & & & & & & & \\
\hline Hon Fow Arest? & 0.1955 & & & & & & & & & \\
\hline Se. Pressurepsio & 104.1 & & & & & & & & & \\
\hline 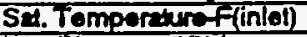 & 149.8 & & & & & & & & & \\
\hline Inlel Fowre-GPM & 3.8 & & & & & & & & & \\
\hline Intatemperetere & 749.1 & & & & & & & & & \\
\hline Inter Glommine & 753702 & & Temp & Ta.3. & Densty & 83.03 & & & & \\
\hline Subooolingf & 0.5 & & & & & & & & & \\
\hline Flow Obsenvelion & & & & & & & & & & \\
\hline & 12ONES & $20 N i=2$ & 201:3 & 2015 & $201=6$ & ZOT: & LONiel & $\angle O N=8$ & $\angle O N=0$ & $20 h^{\prime}=10$ \\
\hline 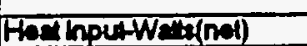 & 5712 & 568. & 568 & $5 \% 3.8$ & 53.4 & 558.7 & 557.8 & 585.8 & 6830 & 563 \\
\hline HorlLoss-Walls & 8.7 & 1.9 & 25 & 2र्ग & 2.5 & 1.8 & 12 & 1.8 & 24 & 14.3 \\
\hline Hed Fux-Buthifie & sol| & 986 & 9768 & 9773 & \$10 & 9858 & 984 & 8803 & 8gat & $97 / 5$ \\
\hline Waliomp, $13 \mathrm{~F}$ & 160.6 & 1621 & 160.5 & 1622 & 161 & 159.7 & 161.5 & 160.3 & 160.8 & 1612 \\
\hline WNTtomp. $4 \mathrm{~F}$ & 161.1 & 160.8 & 161.1 & 1626 & 161.2 & 159.8 & 161.1 & 1602 & 161.8 & 16.8 \\
\hline Wall Tomp. I5 & 162. & 153! & 1605 & 161.3 & 160.8 & 159.7 & 160.1 & 159.8 & 159.1 & 156.8 \\
\hline Wal Tomp. It & 149.11 & 160 & 758.1 & \$E.8: & 160 & 160.9 & 162.1 & 150.8 & 168 & 169.6 \\
\hline Wallomp. If & 165.8 & 160.7 & 159 & 161.1 & TE्. & 160.8 & 167.5 & 159.5 & 158.4. & 160.3 \\
\hline Instrem Tomp.f & 149.6 & 149.8 & 1505 & 1523 & 1512 & 161 & 1523 & 150.8 & 150.6 & 161.8 \\
\hline Avo. Went Tomp.t & 1624 & 160.9 & 159.7 & 161.7 & 1655 & बढ्र. & T61.3 & 169.7 & 1585 & 161.1. \\
\hline Avo.Wali DT.F & 12.3 & 11.1 & 8.7 & g) & 8.8 & 8.8 & 8.5 & 8.6 & 8.4 & 8.8 \\
\hline 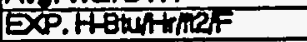 & 8032 & 883.1 & 1717.4 & To\$7.8 & 1128.2 & 1745.1 & 1156.1 & 1767 & 11682 & गाI \\
\hline & & & & & & & & & & \\
\hline & ZONE2 & ZONE4 & $20 \mathrm{~N}=6$ & $20, \sqrt{1}=8$ & 20K:=10 & & & & & \\
\hline DPliguid temp.f & 74.5 & & & & & & & & & \\
\hline DPUquid Dempityom/3 & 923 & & & & & & & & & \\
\hline Everion- & & 4 & 6 & 8 & 9.875 & & & & & \\
\hline Mesured DPFsid & -0.005 & 0.616 & I.tटी| & 1.70 & 1.916 & & & & & \\
\hline Zero Correctome-perd & $=023$ & $=0.017$ & 0.098 & 0.012 & $\$ .388$ & & & & & \\
\hline Correotsd DPF & 0.128 & 0.63 & 1.TCe & 1.697 & टंडा & & & & & \\
\hline Aclud DPR & 2) & 3.29 & 4.75 & 5.84 & 7.07 & & & & & \\
\hline & & & & & & & & & & \\
\hline
\end{tabular}




\begin{tabular}{|c|c|c|c|c|c|c|c|c|c|c|}
\hline Run* & 997 & & i & & & & & & 1 & \\
\hline Test fuvid & I50\% CAFTO & $0-50 \%$ RाT & & 7 & i & & & & $T$ & \\
\hline Date: & 122093 & & $T$ & & T & & & & T & \\
\hline Time: & $14: 46$ & & ! & & T & : & & & T & \\
\hline Now & & & & T & 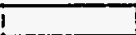 & 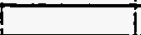 & & & I & \\
\hline Flow Areste & 0.00336 & & & & & & & & i & \\
\hline Heat flow Areatic & 0.19695 & & & & 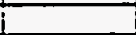 & & & & 1 & \\
\hline Sat. Pressure-Psig & 103.4 & & & & & & & & $i$ & \\
\hline Sat Temperaturef (inlet) & 140.2 & & & & 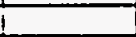 & & & & & \\
\hline Indet Fowrate-GPM & 2.5 & & & & & & & & & \\
\hline indel tomperaturef & 748.3 & & & & & & & & & \\
\hline Intol Glbmhrite & 497551 & & Tomp & 740.8 & Joensity & 83.46 & & & & \\
\hline Suboootingf & 0.9 & & & & & & & & & \\
\hline Fow Observation & & & & & & & & & & \\
\hline & ZONET & ZONE2 & ZONEB & ZONE4 & ZONE6 & ZONE6 & ZONES & ZONEB & TZONES & $20 N=10$ \\
\hline Heal trout-Weitu(not) & 570.4 & 557 & 565 & $5 \$ 5.7$ & $5 \% 5.5$ & 5682 & 5653 & 5526 & 57.5 & 5682 \\
\hline Hed Lose-Wats & 8.4 & 7.8 & 2.4 & 2.7 & 2.5 & 1.7 & 1.21 & 1.8 & 2.3 & 144 \\
\hline Hod Flux-Buithin? & 9888 & 9809 & 9794 & $\$ 908$ & 9689 & 9816 & 9797 & 9751 & 9907 & 9815 \\
\hline WaH TOmp. IF & 160.7 & 15 & 159.8 & 103 & 161.3 & 159,8 & 1522 & 960.5 & 1602 & 187.9 \\
\hline Well Tomp. $14 \mathrm{~F}$ & 1612 & 150.5 & 160.7 & 163.3 & 761.4 & 159.4 & 167.8 & 160.2 & 161.6 & 165.6 \\
\hline Wal Tomp. $15 \mathrm{~F}$ & T61.9 & 158.7 & 159.6 & 167.8 & 100.4 & 159.3 & 160.5 & 159.2 & 159.1 & 160.4 \\
\hline Wal Tomp. IIf & 948.8 & 159.8 & 157.7 & 161.7 & 160.5 & 160.7 & 153.1 & 159.7 & 158 & 160.5 \\
\hline Wall Tomp. $2 f$ & 165.4 & 160.5 & 158.2 & 167.8 & 160.8 & 160.5 & 15.2.2 & 159.8 & 758.8 & 161 \\
\hline Introam Tomp.fF & 148.5 & 149.7 & 150 & 152.3 & 1512 & 150.8 & 1527 & 150.4 & 150.8 & 152.1 \\
\hline Avg. Wall Tomp.f & 162.3 & 150.7 & 159.1 & 1525 & 100.7 & 159.7 & बढ्वा & 159.8 & 769.6 & 161.8 \\
\hline AMg. WaldT.F & 13.4 & 10.8 & 8.7 & 9.7 & 9.1 & 8.8 & 8.8 & 9 & 8.8 & 8.8 \\
\hline 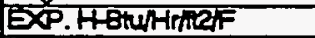 & 740.6 & 928.9 & 1131.3 & 10102 & 1089.4 & 1137.1 & 1111.2 & 1078.7 & 11318 & 10513 \\
\hline & & & & & & & & & & \\
\hline & ZONEE & ZONEC & ZONE6 & ZONE8 & ZONE10 & & & & & \\
\hline DP Gequd Tomp.f & 782 & & & & & & & & & \\
\hline DPUlquid Density & 91.25 & & & & & & & & & \\
\hline Elovation & & 4 & 6 & $\overline{8}$ & 9.875 & & & & & \\
\hline Measured DPPsid & -0.015 & 0.887 & 7.520 & टZ5 & 2.506 & & & & & \\
\hline Zoro Corrections-osid & -0.203 & -0.077 & 0.00 & 0.012 & -0.306 & & & & & \\
\hline Corected DPFasd & 0.208 & 0.904 & 1.507 & 2.238 & $2 \% 52$ & & & & & \\
\hline ACHADDP- & 1.84 & 2.84 & 4 & 4.84 & 5.87 & & & & & \\
\hline & & & & & & & & & & \\
\hline
\end{tabular}




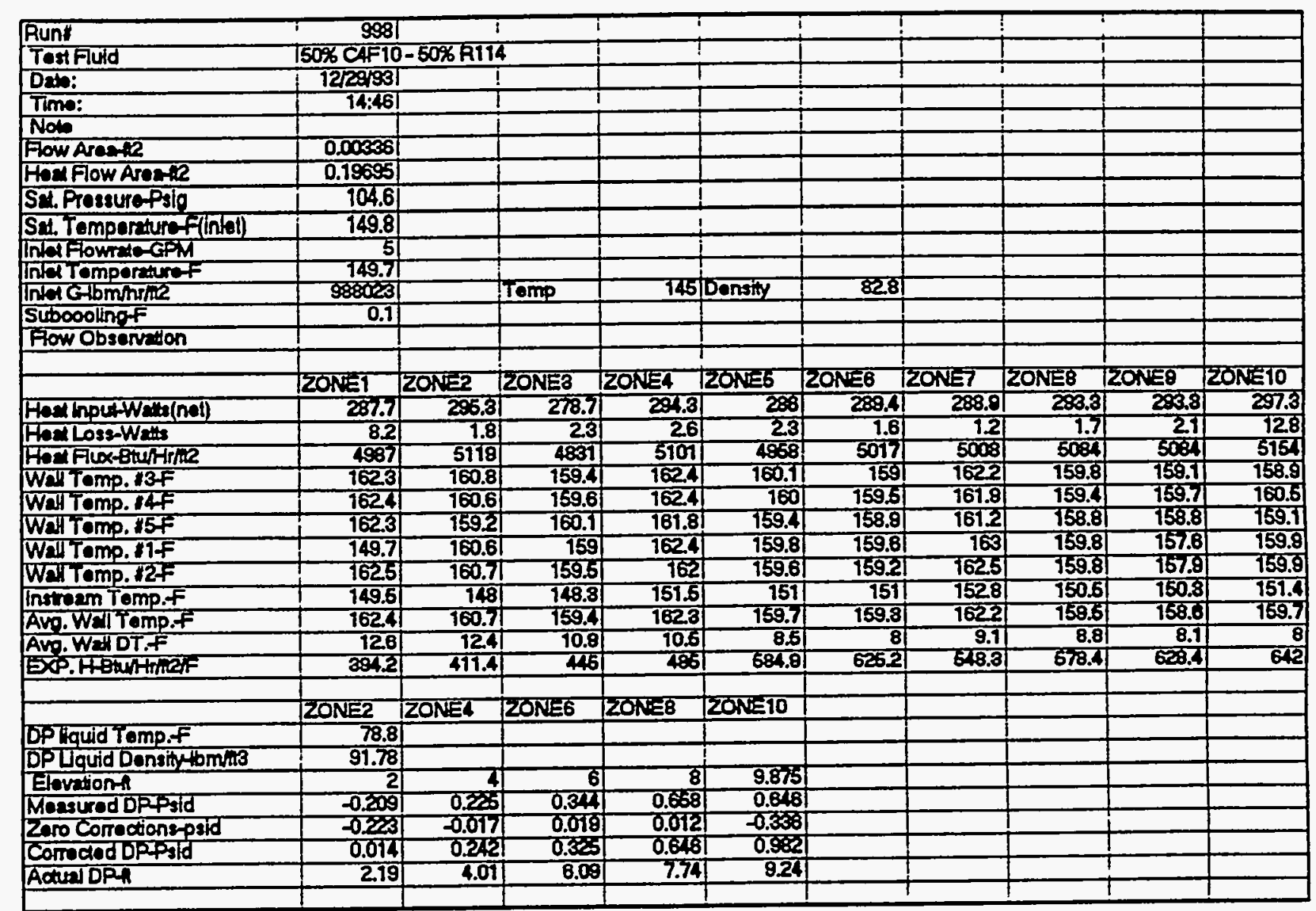




\begin{tabular}{|c|c|c|c|c|c|c|c|c|c|c|}
\hline Runt & ' 999 & & & T & ! & & & & & \\
\hline Torf Fuid & $150 \%$ CAFTO & J.50\%RाT & & t & t & 1 & & & & \\
\hline Dats: & 1 1229393 & ! & $T$ & i & $i$ & i & & & $T$ & \\
\hline Time: & $14: 46$ & 7 & T & 7 & 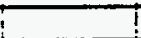 & ! & & T & T! & \\
\hline Nab & & & 1 & 1 & 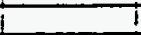 & i & & & $T$ & \\
\hline Flow Areare & 0.00336 & & i & & 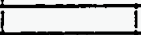 & I & & & & \\
\hline Hexfow Areate & 0.19695 & 1 & $\frac{1}{1}$ & & 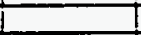 & & & & & \\
\hline Sat. Pressure P sip & 101.2 & & 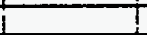 & & & & & & & \\
\hline Sat. Tomperaturof(inled) & 148 & 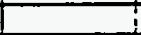 & 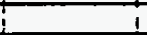 & + & 1 & $t$ & & $i$ & & \\
\hline Intel Fowrate-GPM & 3.8 & i & 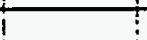 & & & & & & & \\
\hline intol Temperaturef & 747.9 & & & i & & & & & & \\
\hline Intot Glbm/hriliz & 7535201 & & TTamp & Tक्3.2. & Denety & 83.1 & & & & \\
\hline Subooolingf & 0.1 & & & & & & & & & \\
\hline Fow Observation & & & & & & T & & & & \\
\hline & T & & $\mathrm{i}$ & & & $T$ & & & & \\
\hline & TZONEI & दिONES & IZONE3 & ZONEA is & ZONES & TZONE6 & ZONET & ZONE8 & ZZONEO & ZON: $=10$ \\
\hline Herelnoutwatsinet) & 2872 & 2952 & खां & 205.4 & 288.5 & का & 287.8 & 2921 & 258 & 290.7 \\
\hline HodLoss-Was & 8.4 & 1.8 & 2.4 & 2.6 & 2.31 & 1.7 & 9.2 & 1.7 & 23 & 13 \\
\hline Hed Fix Btut? & 4978 & 5117 & 504 & 5721 & 4968 & 5020 & 4969 & 5053 & $51 \overline{3}$ & 5030 \\
\hline Wall Temp. I3F & 160.3 & 159.3 & 158.1 & 160.8 & 158.8 & 157.7 & 160.7 & 758.1 & 157.6 & 757.4 \\
\hline We' Temp. 14F & 160.5 & 158.9 & 158.4 & 160.8 & 158.6 & 158.3 & 160.6 & 157.8 & 158.t & 169.2 \\
\hline Wall Tomp. 15 & 161 & 157.6 & 158.9 & 160.4 & 157.7 & 757.6 & 159.7 & 157.3 & 157.4 & 157.7 \\
\hline Wall Temp. If & 747.8 & 150 & 157.7 & TED.5ा & 758.4 & 158.3 & $16 t .1$ & 758.2 & 756.8 & 157.9 \\
\hline Wall Tomp. \#LF & 967.8 & 159.2. & 158.2 & $\mid 60.51$ & 158.2 & 757.7 & 150.8 & 158.3 & T58.6 & T58.1 \\
\hline Instroam Tomp.f $f$ & 748.7 & 147.7 & 147.2 & 750.4 & 149.8 & 749.8 & 150.8 & 148.7 & 148.5 & 150 \\
\hline Avg. Wall Temp. F & 160.8 & 159.9 & 158.t & 160.7 & 158.2. & 158 & 160.6 & 157.9 & 1572 & 758.1 \\
\hline Ang. Wat DT.f & 12.8 & 11.8 & 10.7 & 101 & 8.4 & 8.4 & 9.8 & 9 & 8.4 & 7.8 \\
\hline ED. Hewerther & 305.8 & 234.8 & 2727 & ह11.1 & 6012 & 698.8 & 6221 & 561.8 & 608.0 & 873.4 \\
\hline & & & & & & & & & & \\
\hline & ZONE2 & ZONE 4 & ZONEE & ZONVE & ZONETO & & & & & \\
\hline DP Fouid Temp.F & 75 & & 1 & & & & & & & \\
\hline DP Lquid Densith $1 \mathrm{bm} / \mathrm{B}$ & 9219 & & & & & & & & & \\
\hline Elovetion- & 2 & 4 & 6 & 8 & 9.875 & & & & & \\
\hline Nessured DPpasid & -0.137 & $0.474 \mid$ & 0.727 & 1.2त & 1.349 & & & & & \\
\hline Zero Corrections-psid & -0.23 & -0.017 & 0.018 & 0.012 & -0.385 & & & & & \\
\hline Corredodopsid & 0.088 & 0.431 & 0.708 & T.20 & $1.6 \% 5$ & & & & & \\
\hline Actual DPA & 2.07 & 3.69 & 5.43 & 6.78 & 8.04 & & & & & \\
\hline & & & & & & & & & & \\
\hline
\end{tabular}




\begin{tabular}{|c|c|c|c|c|c|c|c|c|c|c|}
\hline Runt & 1000 & & & & & & & & 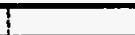 & \\
\hline Tast Fiuld & 150\% CAFIO & $0-50 \%$ मा1 & & & & & & & & \\
\hline Dats: & 122993 & & & & & & & & & \\
\hline Timo: & $14: 46$ & & $i$ & T & I & & & & & \\
\hline Now & $i$ & & & & & & & & & \\
\hline Fow Arestie & 0.00358 & & & & & & & & & \\
\hline Haxt Flow Areate & 0.19695 & & & & & & & & & \\
\hline Set. Prossuro prio & 104 & & & & & & & & & \\
\hline Sat. Temperature F(inlel) & 149.5 & & 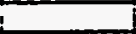 & & & & & & & \\
\hline Intetflowralo-GPM & 2.5 & & & & & & & & & \\
\hline Intet Tomporaturet & 748.8 & & & & & & & & & \\
\hline Intot Glbmhrite & 785756 & & Tromp & 743.2 & Densiny & 83.1 & & & & \\
\hline Subooolingt & 0.7 & & & & & & & & & \\
\hline Flow Observition & & & & & & & & & & \\
\hline & & & & & & & & & & \\
\hline & TZOK:E & ZONES & $20 N=3$ & ZONE & ZOT:E5 & $20 N=6$ & ZONE? & $20 \mathrm{~K}=8$ & TZONES & $20{ }^{N}=10$ \\
\hline 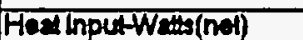 & 283.9 & 221 & 284.8 & 209.9 & 288.1 & 395 & 28.8 & 28921 & 1206 & खर्ड \\
\hline HodLoss-Wath & 8.3 & 1.8 & 23 & 28 & 24 & 1.7 & 93 & 1.7 & 22 & 13 \\
\hline Hoal FlDeBtuHrthe & 4921 & 5003 & 4830 & 5025 & C968 & 50ुठां & 7838 & 5018 & 5057 & 5032 \\
\hline Wall Temp. 13F & 161.8 & I61 & 150.8 & 162.8 & 161.2 & 160.8 & 16 & 160.2 & 7595 & 159.6 \\
\hline Wall Tomp. & 162 & 160.5 & 160.1 & 162.7 & $16 \pi$ & 160.7 & 128 & 159.8 & TEO & 1612 \\
\hline Wall Tomp. $16+$ & 16्2.6 & 159.51 & 160.7 & 1625] & $160 . \pi$ & 159.9 & 162 & 155.4| & 159.3 & 159.5 \\
\hline Wal Tomp, PIF & 748.8 & 160.7 & T59.1 & TE28 & T60.7 & 160.6 & 703.8 & T60.7 & 758.8 & T59.7 \\
\hline WalTomp. ILF & $1 \approx 3.7$ & 160.81 & 759.6 & FE. & TE0.5 & 760.2 & T\%3.5 & 100.7 & 7585 & 160 \\
\hline Inftrasm Tomp.F & 149 & 147.8 & 749.4. & 1527 & 151.4 & T51.1 & 152.8 & 150.7 & 950.0 & 152 \\
\hline Avg. Watl Tomp.F & 162.6 & 160.8 & 159.7 & 1627 & 100.0 & 100.4 & 163 & 160.2 & 159.1 & 100 \\
\hline Avg. Wall DT If & 13.9 & 12.7 & 10 & 8.8 & g) & gi & 9.8 & 9.8 & 8.8 & 7.8 \\
\hline EXP.HBWhHrnak & 369.8 & 397.2 & 2825 & हा29 & 85.1. & 56, & 4978 & Eम1.81 & 6015 & 647.4 \\
\hline & & & & & & & & & & \\
\hline & ZONE? & ZONEA & ZONE6 & ZONEB & ZONETO & & & & & \\
\hline DP Squid Tomp.f & 76.4 & & & & & & & & & \\
\hline БPUgudd Densiytbm/13 & क2.04 & & & & & & & & & \\
\hline Elovetion- & & दा & $\overline{\mathbf{B}}$ & 81 & 9.875 & & & & & \\
\hline Mossured DFFagld & -0.056 & 0.54 & 0.993 & T.611 & 1.858 & & & & & \\
\hline Zoro Correationz-psid & -0.223 & -0.017 & o.06 & 0.012 & -0.83 & & & & & \\
\hline Comected DPPAld & 0.128 & 0.557 & 0.974 & 1.539 & टाद्धा & & & & & \\
\hline Actul Dan & 1.98 & 3.47 & 4.88 & 8.00 & 7.14 & & & & & \\
\hline & & & & & & & & & & \\
\hline
\end{tabular}




\begin{tabular}{|c|c|c|c|c|c|c|c|c|c|c|}
\hline Runf & $\begin{array}{r}1001 \\
1509 c^{4}=10\end{array}$ & & & & ! & & & & & \\
\hline Tost Find & $150 \%$ CAF 10 & $0.50 \%$ RTI4 & & & & & & & & \\
\hline Dats: & $12 / 3 \% 93$ & & $i$ & & $!$ & & & & $t$ & \\
\hline Timo: & $13: 18$ & & $i$ & & I & & & & i & \\
\hline \multicolumn{11}{|l|}{ Now } \\
\hline Flow Arante & 0.00365 & & & & & & & & & \\
\hline Heal flow Aread & 0.19505 & & & & & & & & & \\
\hline Sef Prossure-Psig & 136.8 & & & & & & & & & \\
\hline Sod. Temperature $F$ (intal) & 165.2 & & . & & & & & & & \\
\hline \multicolumn{11}{|l|}{ Intat Fowrete-GPM } \\
\hline infot Tempersturet & IE़ & & & & & & & & & \\
\hline Intot Glbmhing & 1000121 & & Tomp & 1627 & DDansiy & 79.78 & & & & \\
\hline Subcoolingf & 3.8 & & & & & & & & & \\
\hline \multicolumn{11}{|l|}{ Fow Observation } \\
\hline & & & & & & & & & & \\
\hline & ZONET & ZONEE & ZONE8 & $20 N^{2}=4$ & TZONE5 & ZONE6 & RONE7 & ZONE8 & ZONES & 2ON=10 \\
\hline Hoal Input-Watth(not) & 855.7 & 8727 & 874.4 & 878 & 875.4 & 87.8 & 878.7 & 8528 & 875.5 & 873.4 \\
\hline Hed Loss-Wats & 10.9 & 2दा & 3.3 & 3.4 & 3.4 & 2.8 & 1.8 & 23 & 3 & 17.1 \\
\hline Hod Flox-BuHAr/R2 & 15006 & 151281 & 15167 & 15185 & 15174 & 15218 & 15232 & 14956 & 15183 & 15140 \\
\hline Wat Temp. BH & 182.9 & 183.8 & 182.5 & 184 & 185.7 & 188.5 & 185 & 182.3 & 180.6 & 180.7 \\
\hline Wall Tomp. Iff & 183.6 & 180.9 & 184 & 184.3 & 185.8 & 188.7 & 183.8 & 182.3 & 9823 & 188.5 \\
\hline We Tomp. $15+$ & 183.3 & 179.6 & 185.1 & 184.8 & 184.3 & 186.1 & 182.7 & 181.3 & 180.7 & 978.3 \\
\hline Wah Tomp. AT & 169 & 182.5 & 180.4 & T82.7 & 182.9 & T85.4 & 187.4 & 787.2 & $7 \% 9.1$ & 778.3 \\
\hline Wat Tomp. \$2F & T\&8 & 182.1 & 181.5 & 783.8 & 783.5 & 188.61 & 787.1 & 182.7 & 779.7 & 180.1 \\
\hline Introm Tomp.f & 768.7 & 168.8 & 19.4. & 17.3 & 170.6 & 170.8 & 171.8 & 170.5 & 170.7 & 970.7 \\
\hline Avg. Wal Tomp.F & 184 & 1828 & 1821 & 183.8 & 1842 & 188.4 & 185.2 & 181.8 & 1805 & 780.8 \\
\hline Avg. Wad DT.t & 14.8 & 18 & 12 & 11.8 & 12.8 & 14.9 & 12.7 & 10.7 & 8.1 & 9.4 \\
\hline \multirow[t]{3}{*}{ DXP.HBthiknlar } & 1029.8 & 116 & 1281.5 & T214.6 & 7770.6 & 1018.8 & 11982 & 1384.6 & 1673.8 & 1611.3 \\
\hline & & & & & & & & & & \\
\hline & ZONE2 & ZONE & $2015=6$ & ZONEB & ZONETO & & & & & \\
\hline DP Fquid Temp.fF & 76.8 & & & & & & & & & \\
\hline DP Liquid Donsity bmin & 91.88 & & & & & & & & & \\
\hline Elevation-l & & दा & $\overline{8}$ & 8 & 9.875 & & & & & \\
\hline Mestured DPFsid & -0.76 & 0.51 & 0.88 & 1.30 & 1.517 & & & & & \\
\hline Zero Corrections-psid & -0.002 & -0.0 & -0.018 & 0.008 & -0.363 & & & & & \\
\hline Corrocted DPPsid & 0.153 & 0.53 & 0.500 & $1.8 \pi$ & 1.85 & & & & & \\
\hline Acrual DPA & 2.08 & 3.68 & 5.28 & 8.74 & 8.05 & & & & & \\
\hline & & & & & & & & & & \\
\hline
\end{tabular}




\begin{tabular}{|c|c|c|c|c|c|c|c|c|c|c|}
\hline Run: & 10021 & & & & I & & & & & \\
\hline T०A FWd & $150 \%$ CAF10 & $0-50 \% R T 1$ & & & & 1 & & & & \\
\hline Deat: & 1223098 & 1 & I & & I & & & & & \\
\hline Tims: & $13: 18$ & 1 & 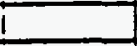 & & & & & & & \\
\hline \multicolumn{11}{|l|}{ Now } \\
\hline Flow Areane & 0.00336 & & & & & & & & & \\
\hline Had Fow ATEAR2 & 0.18695 & & & & & & & & & \\
\hline Set. Pressure Psig & 138.6 & & & 1 & 1 & & & & & \\
\hline Set. Temoerature f(inlal) & 165.1 & & & & & & & & & \\
\hline Tnket Fownd-GPM & 3.9 & & & & & & & & & \\
\hline inlat tomporthef & 169.1 & & & & & & & & & \\
\hline Indet G-lbm/hrine & 743223 & & Tomp & 1623 & Density & 79.88 & & & & \\
\hline Suboooling $F$ & -4 & & & & & & & & & \\
\hline \multirow{2}{*}{\multicolumn{11}{|c|}{ Fow Obsenvetion }} \\
\hline & & & & & & & & & & \\
\hline & ZOENET & ZONE2 & $\angle O C N=8$ & 20N: $=4$ & ZONE6 & ZOK:E6 & CONE7 & $\angle O K=8$ & ZONEO & $\angle O B=10$ \\
\hline Heal lnpulwathel & 8685 & 8672 & 874.6 & 876.7 & 8727 & $874 A$ & 859.6 & 86 & 876 & 871.8 \\
\hline HarLore-Wait: & 11 & 2.4 & 3.3 & 3.5 & 3.5 & 27 & 1.7 & 24 & 3 & 17.8 \\
\hline Hox Flux Btunhsher & 15007 & 1503 & 15159 & 15187 & 75728 & 15157| & 15072 & Tद्वर्ट & T5ा68 & 15112 \\
\hline Wall Tomp. BF & 1828 & 183.9 & 1825 & 184.1 & 188 & 187 & 1852 & 1824 & 181 & 781.6 \\
\hline Wall Tomp.14t & 183.6 & 181.2 & 184.8 & 184.8 & 188.4 & 1872 & 184 & 182.6 & 1827 & 187.3 \\
\hline Wal TOMp. ISF & 183.5 & 777.6 & 185.1 & 184.7 & 184.6 & 188.4 & 183.1 & 181.6 & 180.8 & 179.1 \\
\hline Wall Tomp ATt & ख़ी & T8ट.8 & 380.4 & T83.4. & 783.1 & 186.7 & 188 & TBT2 & i79.4 & 779.4 \\
\hline Wal Tomp. 12A & 785.0 & 7823 & 787.8 & 383.8 & 183.7 & 187 & 187.5 & 7825 & $180 . \pi$ & 181 \\
\hline Instroem Tomp.f & 168.8 & 169.2 & 169.8 & 177.4 & $1 \% 0.6$ & 170.3 & 177.8 & 170.5 & 970.8 & $1 \pi 1.1$ \\
\hline AV. Wall Tomp.F & 184 & 18261 & 1822 & 184.2 & 182.5 & 188.8 & 185.8 & 182 & 180.8 & 181.7 \\
\hline \multirow{4}{*}{ 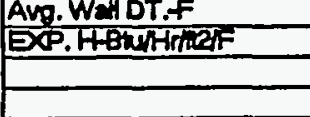 } & 14.4 & 127 & 11.8 & 121 & 132 & 15.8 & 18.1 & 10.8 & 0.5 & 9.8 \\
\hline & 1043.9 & 1188.4 & 1205.1 & 1254.6 & 7748 & SEB.8. & T1524 & 1375.8 & 1684. & 1620.0 \\
\hline & & & & & & & & & & \\
\hline & ZOKE? & ZONEA & $\angle O N=6$ & ZONE 38 & ZONEETO & & & & & \\
\hline DP Squid Tomp.f & 76 & & & & & & & & & \\
\hline \multicolumn{11}{|l|}{ DPLiquid Density } \\
\hline Elovition- & हो & 4 & 6 & 8 & 8.875 & & & & & \\
\hline Mhesurad DPFald & -0.038 & 0.774 & 1.351 & 1.85 & 2.272 & & & & & \\
\hline Zero Corrections-psid & -0.312 & -0.021 & 5.016 & 0.006 & 5.388 & & & & & \\
\hline Comeded DPFid & ठ.201 & 0.79 & 1.353 & 1.979 & 2.65 & & & & & \\
\hline ACUialDPA & 1.83 & 3.18 & 4.48 & 5.68 & 6.69 & & & & & \\
\hline & & & & & & & & & & \\
\hline
\end{tabular}




\begin{tabular}{|c|c|c|c|c|c|c|c|c|c|c|}
\hline Run\# & 1003 & & & & I & 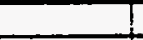 & & : & 1 & \\
\hline Test Fuid & $150 \%$ CAF10 & $.50 \%$ R114 & & & i & & 7 & 5 & $\vdots$ & \\
\hline Data: & $12 / 30939$ & & I & & T & ti & í & i & i & \\
\hline Time: & $13: 18$ & & $i$ & & & & & I & & \\
\hline \multicolumn{11}{|l|}{ Not } \\
\hline Flow Arestle & 0.00336 & & & & & & & & & \\
\hline Hoat flow Aroake & 0.19695 & & 1 & & & & L & & I & \\
\hline Sat. Pressure Psig & 135.5 & & & & & & $t$ & I & 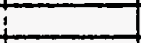 & \\
\hline Sat. Temperaturof finlel) & 164.6 & & & & & & 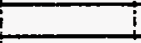 & 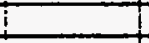 & & \\
\hline Indet Fowrato-GPM & 2.6 & & & & & & 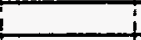 & & & \\
\hline \multicolumn{11}{|l|}{ inlat Tempereturef } \\
\hline Inlet Glbm/nrme & 469189 & & Tomp & 158.811 & Donsity & 80.45 & & & & \\
\hline Subcoolingf & 3.6 & & & & & & & & & \\
\hline \multicolumn{11}{|l|}{ Fow Obsenvation } \\
\hline & $T$ & & 1 & & & & & & & \\
\hline & ZONEA & ZONES & ZONE3 & 2OW: & ZONES & ZONEE 1 & ZONET & LONE8 & ZONEO & $2 O N=10$ \\
\hline Heal hot Westal) & I870.7 & 8672 & 8732 & 871.4 & 876.8 & 881.9 & 8721 & 8528 & 8722 & 615 \\
\hline HealLoss-Watt & 10.81 & 2.41 & 3.3 & 3.4 & 3.4 & 261 & 1.6 & 24 & 3 & 18.8 \\
\hline Hoad FLx-BtuhHiriR & 15030 & 15032 & 15133 & 15105 & 15200 & 15287 & 15117 & 14958 & 15118 & 10881 \\
\hline Wall Tomp. "अF & 181.8 & 183.2 & 181.8 & 183.8 & 185.4 & 185.8 & 184.8 & 1824 & 1812 & 179.8 \\
\hline Wall Temp. 14F & 182.7 & 180.4 & 183.5 & 184.1 & 185.4 & 1862. & 183.7 & 1822 & 1829 & 783.3 \\
\hline Wal Tomp. " 5 F & 1826 & 178.8 & 184.6 & 184.2 & 183.7 & 185.3 & 982.4 & 180.9 & 180.8 & 178.2 \\
\hline Wal Tomp. IF & 168.21 & 182.11 & 179.6 & 1826 & 1828 & 185.7 & 186.7 & 181.11 & 179.3 & 778.2 \\
\hline Wall Temp. IRF & 18.,2 & 181.61 & 780.9 & 183.3 & 183.4 & 185.8 & 188.6 & 182.5 & 160 & 178.4 \\
\hline Instrean Tomp.F & 168.8 & 169.5 & 169.6 & 171 & 170 & $1 \% .1$ & 171.4 & 170.1 & 170.8 & $17 \pi$ \\
\hline Avo. Wall Tamp.F & 1828 & 181.8 & 181.5 & 183.7 & 183.8 & 185.81 & 184.8 & $181.8 !$ & 180.8 & 179.8 \\
\hline Avg. Wall DT.F & 13.8 & 17.6 & 17.2) & 12 & 13.2 & & 12.8 & 111 & 9.8 & 8.3 \\
\hline \multirow{3}{*}{ EXP.HBturhrnerF } & 1090.3 & 1290.8 & 135.8 & 1259.6 & 1154.8 & 10032 & 1185 & 135421 & 1533.8 & ॠ81.6 \\
\hline & & & & & & & & & & \\
\hline & ZONE2 & ZONEG & ZONE 6 & ZONE 8 & ZONETO & & & & & \\
\hline DP rquid Tomp.f & $\pi$ & & & & & & & & & \\
\hline DPLiquid Donsitylbmit3 & 91.87 & & & & & & & & & \\
\hline Elevationt & 2 & 4 & 6 & 8 & 9.8751 & & & & I & \\
\hline Measured DRPsid & 0.041 & 1.088 & 1.827 & 2.63 & 3.085 & & & & & \\
\hline Zoro Correctionsepsid & -0.302 & -0.02 & -0.016 & 0.006 & -0.338 & & & & & \\
\hline Corrected DPAsid & 0.343 & 1.108 & 1.86 & 2.624 & 3.448 & & & & & \\
\hline ACtual DPA & 1.67 & 2.59 & 3.58 & 4.45 & 5.17 & & & & & \\
\hline & & & & & & & & & & \\
\hline
\end{tabular}




\begin{tabular}{|c|c|c|c|c|c|c|c|c|c|c|}
\hline Runt: & 1004 & & & & & & & & & \\
\hline Tost Fuld & $50 \%$ CAFTO & $0-50 \%$ RT14 & & & 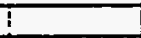 & & & & $T$ & \\
\hline Daw: & 1233093 & 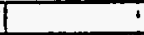 & 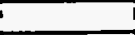 & 1 & $i$ & & & & i & \\
\hline Time: & $15: 04$ & 1 & & & & & & $!$ & $i$ & \\
\hline Now & & $i$ & & & & & & & i & \\
\hline Flow Aras-12 & 0.00336 & I & & & & & & & & \\
\hline Hon flow Arest? & 0.19685 & 1 & & & & & & & & \\
\hline Sed. Prossuraprip & 137.6 & & & & & & & & & \\
\hline Sat. Temperiture f (intef) & 165.5 & & & & & & & & & \\
\hline Inlat Flowrato-GPM & 5.3 & & & & & & & & & \\
\hline inct Tomperaturof & 168.7 & & & & & & & & & \\
\hline Intet Glbminilie & 1007518 & & Tomp & 103.5 & Donstity & 79.64 & & & & \\
\hline Suboooling $f$ & 3.2 & & & & & & & & & \\
\hline Fow Obsenviton & & & & & & & & & & \\
\hline & & & & & & & & & & \\
\hline & ZONET & ZONE2 & LONE 8 & LONEL & $20 N=6$ & $20 N=6$ & ZONE & TZONE 8 & ZZONEO & $\angle O N=10$ \\
\hline 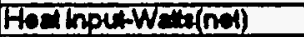 & 575.8 & 5824 & 587.6 & 583.2 & 586.4 & 584.1 & 584.8 & 578.8 & \begin{tabular}{l|l|}
3 & 685.8 \\
\end{tabular} & 584.4 \\
\hline HoxLoss-Welt & 10.3 & 23 & 3.1 & 3.3 & 3.2 & 24 & 1.6 & 23 & 29 & 16.1 \\
\hline Hod Flox bluthite & 9981 & 1008 & 10186 & 10108 & 10165 & 10125 & 10128 & 10050 & 10148 & 10130 \\
\hline Wal Tomp. 13F & 182 & 1822 & 181.9 & 182.8 & 183.6 & 183.7 & 184.8 & 1825 & 1802 & 179.7 \\
\hline Wall Temp.nfF & 782.5 & 180.8 & 182.7 & 183.2 & 183.7 & 184 & 184.7 & 182.8 & 187.6 & 182.9 \\
\hline Wall Tomo. I6F & 183 & 178.5 & 183.5 & 183.4 & 182.6 & 183.7 & 183.7 & 181.6 & 180.1 & 178.6 \\
\hline Waltomp.11F & 768.7 & 181.8 & 779.9 & 1829 & 781.8 & 784.2 & 785.9 & 1828 & 778.6 & 178.6 \\
\hline Wal Tamp. ThF & 7द्य.4 & 181.8 & 180.8 & 182.6 & 181.9 & 184.1 & 785.8 & 183.6 & 180 & 779.8 \\
\hline Instroen Tomp.F & 168.4 & 167.81 & 168.8 & $17 \pi$ & 170.5 & 170.7 & 172 & 170.5 & 170.7 & 170.8 \\
\hline Avg. Wall Tomp.f & 183 & 181.7 & 181.3 & 183.1 & 1825 & 183.8 & 185 & 182.6 & 180.8 & 179.9 \\
\hline Ang. Wall DT.F & 14.1 & 13.4 & 128 & 11.8 & 11.5 & 12.7 & 12.5 & 11.6 & 9.1 & 8.7 \\
\hline ER.HBWuHnar & 708.7 & 752.31 & 8305 & 868.3 & 880.8 & 794.6 & 808.8 & 868 & 17123 & 1169.5 \\
\hline & & & & & & & & & & \\
\hline & LONE2 & ZONE4 & ZONEE & ZONE8 & ZOWE & & & & & \\
\hline DP havid Temp.F & 8.7 & 1 & 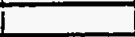 & & & & & & & \\
\hline DPLquid Densitybm/k3 & 91.79 & & & & & & & & & \\
\hline Eloration-h & & या & & 8 & 9.875 & & & & 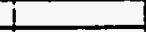 & \\
\hline MossuredDPFsid & -0.203 & 0.421 & 0.722 & 1.207 & 7.202 & & & & & \\
\hline Zero corrections-pqd & 20.3020 & -0.021 & -0.016 & 0.000 & -0.303 & & & & & \\
\hline Comeded DPS Sd & 0.000 & 0.451 & 0.738 & T.दण & 1.585 & & & & & \\
\hline ActualDPA & 2.13 & 3.81 & 5.58 & 7.05 & 8.5 & & & & & \\
\hline & & & & & & & & & & \\
\hline
\end{tabular}




\begin{tabular}{|c|c|c|c|c|c|c|c|c|c|c|}
\hline Run: & 1005 & & & & ; & & $T$ & $i$ & $T$ & \\
\hline Test Fivid & $50 \%$ CAFT & $0.50 \% R 11$ & & I & $T$ & & T & & ! & \\
\hline Dat: & $12 / 3193$ & & 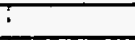 & T & $!$ & & $i$ & & i & \\
\hline Time: & 15:04 & & ; & $!$ & $i$ & & 1 & T & 7 & \\
\hline Now & & $T$ & & T & $\mathrm{T}$ & & i & & & \\
\hline Flow Areatal2 & 0.00336 & & I & & $I$ & & & & & \\
\hline Hoct flow Areate & 0.19695 & & 1 & & $\frac{1}{1}$ & & & & & \\
\hline Set. Pressuropsig & 136.7 & & I & & & & & & & \\
\hline Sat. Temperature f (inlol) & 165.1 & & $\therefore$ & & 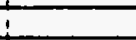 & & $i$ & I & & \\
\hline Intelflowruto-GPM & 3.9 & & T & & & & & & & \\
\hline intel Tomperaturef & 16 & & & & & & & & & \\
\hline Intot Gbmintive & 742862 & & Temp & 162.5 & TDonsty & 79.82 & & & & \\
\hline Subcooling $F$ & -3.9 & & & & & & & & & \\
\hline \multirow[t]{3}{*}{ Flow Obsenvation } & T & & & & & & & & & \\
\hline & $i$ & & $T$ & & $T$ & & t & & & \\
\hline & IZONET & KZONEZ & ZONE & ZONEA & TZONES & ZOOEE & RONE? & ZONEB & TZONES & ZOKE:TO \\
\hline Heat input-Wate(not) & $1 \quad 5727$ & 15724 & 578.1 & 579.4 & 575.8 & 57.4 & 574.6 & i) 574.2 & 574.3 & 677.8 \\
\hline Heal Loss-Wads & 10.5 & 2.3 & 3.2 & 3.3 & 3.3 & 2.5 & 1.6 & 2.4 & ट. & 16.4 \\
\hline Hod Flex-BtuAfinR & 9807 & 992 & 10021 & 1004 & 9981 & 9905 & 9960 & 953 & 9856 & 10018 \\
\hline Wall Temp. IF & 182.2 & 1821 & 181.8 & 183.5 & 184 & 184 & 185 & 182.4 & 180.1 & 178.8 \\
\hline Wall Temp. \$4F & 1827 & 180.8 & 1824 & 183.6 & 184.1 & 184.5 & 185 & 182.6 & 181.3 & 183 \\
\hline Wall Tomp. $15 F$ & 183 & 179.3 & 183.6 & 183.7 & 182.8 & 184.1 & 183.8 & 1815 & 180 & 178.6 \\
\hline Wal Tomp. If & 16 & 181.8 & 179.8 & 183 & 1823 & 184.8 & 185.7 & 1832 & 178.3 & 178.2 \\
\hline Wall Tomp. $12 F$ & 184.7 & 181.8 & 180.8 & 183 & 182.3 & 184.5 & 185.8 & 183.8 & 779.7 & 179.3 \\
\hline Instreen Tomp.f & 169 & 168.1 & 169.6 & 171.8 & 170.8 & 170.7 & 171.8 & 170.4 & 170.8 & 171 \\
\hline Avo. Wall Tomp.f & 1832. & 181.7 & $\overline{181.2}$ & 183.5 & 182.8 & 184.81 & 185.1 & 1827 & 180.1 & 178.8 \\
\hline Ang. Wat DT.F & 13.7 & 18.11 & 91.1 & 11.2 & 11.8 & 13.2 & 12.8 & 11.8 & 8 & 8.3 \\
\hline \multirow[t]{3}{*}{ EXP.HBth/trine } & 724.6 & 757.31 & 890 & 897.1 & 858.7 & 763.1 & 77.6 & 838.5 & 11024 & 1200 \\
\hline & & & & & & & & & & \\
\hline & ZONE2 & TZOKEA & ZONEG & ZONEB & ZONETO & & & & & \\
\hline DP rquid Tomp.f & 77.3 & & & & & & $t$ & & & \\
\hline DP Liquid Density $1 \mathrm{bm} / \mathrm{m}$ & 91.94 & & & & & & & & & \\
\hline Elovation & & 4 & 6 & 8 & 9.875 & & & & & \\
\hline Measured DF Fsid & -0.115 & 0.641 & 1.121 & 1.756 & 1.934 & & & & & \\
\hline Zero Corrections-psid & -0.300 & -0.02 & -0.016 & 0.006 & -0.333 & & & & & \\
\hline Corrected DPYsid & 0.187 & 0.651 & 1.137 & 1.753 & 2.287 & & & & & \\
\hline Actud DPA & 1.97 & 3.42 & 4.88 & 6.06 & 7.28 & & & & & \\
\hline & & & & & & & & & & \\
\hline
\end{tabular}




\begin{tabular}{|c|c|c|c|c|c|c|c|c|c|c|}
\hline Runit & 1006 & ! & 1 & & & & & $!$ & $!$ & \\
\hline Test Fuid & $150 \%$ C4F10 & $-50 \%$ A116 & & & & & I & $t$ & T & \\
\hline Dato: & 1 12/30/931 & 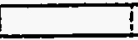 & & 1 & I & 1 & I & i & I & \\
\hline Timo: & $15: 041$ & 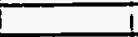 & & & & 7 & 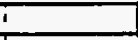 & $I$ & i & \\
\hline Now & $i$ & & & & & 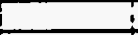 & $\div$ & 1 & & \\
\hline FOW ANOAR & 0.003361 & & & & & & I & & & \\
\hline Hod Fow Areat? & 0.1955 & & & & & & & & & \\
\hline St. Pressuro Paip & 136.4 & & & & & & & & & \\
\hline Sa. Tomperature f(indet) & 165 & & & & & & & & & \\
\hline Intet Fowrate-GPM & 26 & & & & & & & & & \\
\hline intel Temporaturef & 167.1 & & & & & & & & & \\
\hline Int Glomhine & 497129 & & Tomp & 760.8 & Dansiny & 80.12 & & & & \\
\hline Subcooling ff & -2.7 & & & & & & & & & \\
\hline Fow Observaton & & & & & & & & & & \\
\hline & ZOK:=T & ZिONES & ZOKE: & ZOTRE & ZONEБ & ZONE6 & ROKET & T2ONES & ZZONEO & 2ONET0 \\
\hline Hexhoutwalned) & 6762 & 578.4 & 5780 & 577.4 & 576.8 & 578.9 & 578.0 & 5795 & 677.7 & 677.1 \\
\hline HerLoss-Wats & 10.4 & 2.3 & 3.2 & 3.3 & 3,3 & 25 & 7.8 & 24 & 3 & 16.8 \\
\hline Hod Fiox-eturhime & 998 & 9910 & 10000 & 10000 & 9983 & 10003 & 10052 & 10045 & 1004 & 10004 \\
\hline WAll Tomp. *अ & 781.8 & 181,5 & 181.5 & 183 & 183.7 & 183.8 & 184.7 & 182.3 & 180.8 & 180.2 \\
\hline Wal Tomp. 44 & 182.3 & 180.6 & 1822 & 183.1 & 783.8 & 184.8 & 184.7 & 183 & 181.7 & 183.4 \\
\hline Wad Tomp. "VFF & 1824 & 179.3 & 183.7 & 183.3 & 182.8 & 183.8 & 183.6 & 181.8 & 180.6 & 179 \\
\hline Walt Tomp. $11 F$ & 167.1 & 181.3 & 179.4 & 182.7 & 1821 & 184.2 & 185.6 & 183 & 179.6 & 179 \\
\hline Wallomp. I2f & 184.1 & 181.2 & 180.2 & 182.7 & 182. & 184.31 & 185.5 & 183.7 & 180.1 & 179.9 \\
\hline Inftrean Tomp.f & 168.8 & 168 & 170.1 & 171.4 & 770.8 & 170.7 & 771.7 & 170.5 & 170.8 & 171.1 \\
\hline AVo. Wal Temp. $F$ & 1827 & 181.1 & 180.8 & 183 & 1827 & 184.11 & 184.8 & 182.7 & 1806 & 180.8 \\
\hline Avg. Wald DT.F & 15.8 & 12.7 & 10.8 & 11.2 & 11.8 & 12.91 & 12.7 & 11.8 & 9.1 & 8.7 \\
\hline EXP.HBtuHnRER & 6520 & 784.1 & 073.4 & 886.8 & 858.4 & 776.1 & 798.7 & 8653.8 & 1004.7 & 11435 \\
\hline & & & & & & & & & & \\
\hline & ZONE2 & ZONET & $20 k=6$ & ZONE8 & $\angle O N=10$ & & & & & \\
\hline DP hquid tomp,f" & 78.5 & & & & & & & & & \\
\hline DPUludd Donsitybm/13 & 91.81 & & & & & & & & & \\
\hline Elowiont & & 4 & 6 & 8 & 9.875 & & & & & \\
\hline Messured DPAsid & -0.054 & 0.827 & 1.458 & 227 & 2.534 & & & & & \\
\hline Zoro Corrections-psid & -0.302 & -0.021 & -0.018 & 0.008 & -0.323 & & & & & \\
\hline Comeded DPPad & 0.248 & 0.8411 & 7.474 & हस्ता & 2.857 & & & & & \\
\hline ACINDPA & 1.85 & 3.07 & 4.23 & 5.18 & 6.18 & & & & & \\
\hline & & & & & & & & & & \\
\hline
\end{tabular}




\begin{tabular}{|c|c|c|c|c|c|c|c|c|c|c|}
\hline Runt & 1007 & & & & & & & 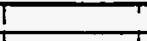 & & \\
\hline Test Fivid & $150 \%$ CAF 10 & $0-50 \%$ R114 & & & $\mathrm{i}$ & i & & & $T$ & \\
\hline Dat: & $12 / 31 / 83$ & & $T$ & & $!$ & i & I & & $T$ & \\
\hline Time: & $.8: 24$ & 1 & 1 & & - & i & I & & ? & \\
\hline Now & 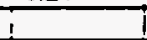 & i & : & & & $i$ & & & $i$ & \\
\hline Fow Arasti2 & 0.00336 & & 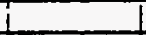 & & & L & & & 1 & \\
\hline Hodflow Arose & 0.19695 & & 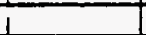 & & 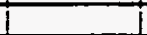 & 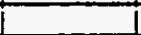 & I & & I & \\
\hline Sat.Pressuro ofsig & 136.4 & & 1 & & : & & & & 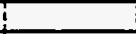 & \\
\hline Sel. Temperatur of (inlol) & 165 & & 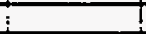 & & & & & i & 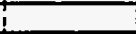 & \\
\hline Inlat Frowrato-GPM & 5.3 & & & & & & & 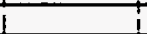 & & \\
\hline intel Tomperaturetf & 169 & & & & & & & & & \\
\hline Inlat Glbminrine & 1008896 & & tremp & 162.81 & IDensity & $79.7 n$ & & & & \\
\hline Subcoolingf & -4 & & & & & & & & & \\
\hline Fow Obsenvation & $i$ & & & & & & & & & \\
\hline & 1 & & 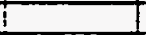 & & $i$ & & & & & \\
\hline & ZONE1 & LONE2 & ZZONE8 & ZONE4 & TZONES & ZONE6 & RONET & ZONEB & ZONEO & ZONETO \\
\hline Hod lnput-Wats(nel) & 2928 & 20.6 & 285.4 & 205.5 & 293.11 & 2862. & 289.2 & 286.6 & 288.8 & 28.7 \\
\hline Hoad Loss-Watts & 10 & 2.21 & 2.8 & 3.11 & 3 & 2 & 1.41 & 2 & 25 & 15.4 \\
\hline Hoa fuxbturhrm & 5075 & 5037 & 4947 & 512द्या & 50811 & 4981 & 5013 & 4958 & 4918 & 5178 \\
\hline WaH Tomp. 137 & 179.7 & 777.6 & 176.8 & 179.5 & 178.7 & 175.6 & 17.6 & 175.6 & 175 & 176.6 \\
\hline Wall Temp. \#4F & 179.5 & 177.6 & 177 & 179.5 & 178.9 & 176.2 & 177.4 & 175.5 & 775.6 & 178 \\
\hline Waff Tomp. $\$ 5 F$ & 180.11 & 776.5 & 178 & 179.3 & 178.2 & 178 & 978.8 & 175.51 & 175.1 & 175.8 \\
\hline Wal Temp. \#TF & 169 & 178 & 175. & 180.3 & i77.9. & 178 & 179.2 & T76.21 & 774.4. & 176.6 \\
\hline Wail Temp. IRF & 180.8 & 778.T & $|75.3|$ & 779.7 & 178 & 177.5 & 778.5 & 776.4 & T74.4 & 778.3 \\
\hline Instrom Tomp.f & 168.7 & 167 & 168.8 & 170.4 & 169.9 & 169.8 & 172.8 & 970.4 & $7 \% 02$ & 1n \\
\hline Avg. Wan Tamp.f & 180 & 177.8 & 178.5 & 179.7 & 178.8 & 178.6 & 177.9 & 175.8 & 774.8 & 176.0 \\
\hline Ang. WatDT.F & 11 & 10.8 & 9.4 & 8 & 8.1 & 8.4 & 5 & 5.21 & 4.6 & 5.4 \\
\hline 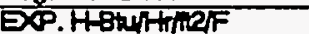 & 459.51 & 475.3 & 5द्धा.6 & 658 & इ5.7 & 772.11 & क्षा & 962.6 & 1063.7 & 861.3 \\
\hline & & & & & & & & & & \\
\hline & ZONE2 & ZONET & $\angle O N=6$ & ZONE8 & ZONETO & & & & & \\
\hline DP Equid Tamp.f & 727 & & & & 1 & & & & & \\
\hline DP Uquid Density 4 bmit3 3 & 92.42 & & & & & & & & & \\
\hline Erovetion-k & 2 & 4 & 6! & 8 & 9.875 & & & & & \\
\hline Messured DPpid & -0.207 & 0.205 & 0.436 & 0.746 & 0.719 & & & & & \\
\hline Zero Comections-psid & -0.305 & 0.02 & -0.009 & 0.0081 & -0.35 & & & & & \\
\hline Comedod DP sid & 0.086 & 0.185 & 0.446 & 0.74 & 7.056 & & & & & \\
\hline Achued DP/ & 2.14 & 4.3 & 6.16 & 7.87 & 9.54 & & & & & \\
\hline & & & & & & & & & & \\
\hline
\end{tabular}




\begin{tabular}{|c|c|c|c|c|c|c|c|c|c|c|}
\hline Run: & 1008 & & & & & & & & T & \\
\hline Tagffiuld & $150 \%<4=10$ & D-50\%RT1 & & & $\vdots$ & & & & t & \\
\hline Dati: & 1 12/31/801 & $T-1$ & i & & $i$ & & I & & $T$ & \\
\hline Tima: & $19: 24$ & i & i & & $i$ & & & & & \\
\hline Now & & I & & & 1 & & & & & \\
\hline Flow Areate? & 0.00355 & & & & & & & & & \\
\hline Heal Flow Arose? & 0.19695 & & & & 1 & & & & & \\
\hline Sut. Prossurefsig & 137.1 & & & & i & & & & & \\
\hline Set. Tompercure flintel) & 165.3 & & & & 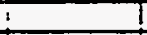 & & & & & \\
\hline Indetflowrate-GPM & 3.91 & & & & & & & & & \\
\hline intel Tomperaturef & 769.91 & & & & & & & & & \\
\hline Intot Glomhrice & 747400 & & Temp & 163.4 & Donsty & 79.68 & & & & \\
\hline Subooolingf & -4.6 & & & & +2 & & & & & \\
\hline Fow Obsenviton & & & & & & & & & & \\
\hline & & & & & & & & & & \\
\hline & ZONET & ZONE2 & ZONES3 & ZONEA & ZZONES & $20 N=8$ & ZZONES & ZONE8 & ZZONEO & $\angle O N=10$ \\
\hline Hod houtWathel) & 294.9 & 291.1 & 283.4 & 291.3 & 291 & 283.8 & 2008 & 287.4 & 286.0 & 297 \\
\hline Hod Loss-Weds & 9.9 & 2.1 & 27 & 3.1 & 29 & 2 & 1.3 & 1.9 & 25 & 14.9 \\
\hline Had Flux-biunthe & 5912 & 5048 & 4918 & 5101 & 5049 & काना & 5035 & 4982 & 4968 & 5148 \\
\hline Wall Tomp. H $3 F$ & 180.8 & 178.3 & 177.8 & 180.7 & 179.7 & 1765 & 178.3 & 176.2 & 175.3 & T77.4 \\
\hline Wall Tomo. Ift & 180.8 & 178.8 & 177.7 & 180.8 & 179.6 & 176.8 & 178.2 & 175.8 & 776.1 & 78.7 \\
\hline Wail Tomp. 157 & 181.2 & 177.1 & 178.7 & 180.4 & 178.8 & 177 & 177.4 & 176 & T75.6. & 776.1 \\
\hline Wall Tomp. ItF & 76.9 & 778.7 & 176.6 & 18.4. & 179.2 & T78.6 & 779.8 & $\mid 77$ & 374.7 & 176.6 \\
\hline Wartomp. PLF & 78्2.1 & 778.8 & 177 & 780.8 & 1792 & 778.2 & 179.1 & 177 & $\mid 75$ & 776.8 \\
\hline Instruem Temp.f & 170 & 168.8 & 168.8 & 172.11 & 170.9 & 170.7 & 173 & 170.6 & 170.5 & 171.6 \\
\hline Avg. Wall Tomp.F & 181.2 & 178.51 & 177.2 & 180.8 & 179.2 & 172 & 178.0 & 178.4 & 175.8 & 17.1 \\
\hline Avg. Wall DT.f & 11 & 101 & 8.7 & 8.4 & 8.1 & 6.8 & 5.3 & 5.7 & 4.8 & 5.9 \\
\hline EXP.HBHWRTRE & क्5: & 504.8 & 564.5 & 604.11 & 20 & 778.1 & 848.7 & 874.8 & 1078.4 & 873.7 \\
\hline & & & & & & & & & & \\
\hline & ZONE2 & ZONEA & ZONEE & 201:38 & T2ONE=10 & & & & & \\
\hline Dp froud Tomp.F & 78.1 & & & & & & & & & \\
\hline DPUquid Density tomnio & 9207 & & & & & & & & & \\
\hline Eovetion & & 4 & $\mathbf{6}$ & 8 & 9.875 & & & & & \\
\hline Masulred DPpyid & -0.744 & 0.458 & 0.739 & 1.207 & 1.365 & & & & & \\
\hline Zero Comections-prid & -0.306 & 0.02 & -0.000 & $-0.0<1$ & -0.356 & & & & & \\
\hline Comecod DPF' & 0.181 & 0.46 & 0.748 & 1.265 & 1.681 & & & & & \\
\hline Actual DPAn & 2.02 & 3.80 & 5.58 & 7.01 & 8.41 & & & & & \\
\hline & & & & & & & & & & \\
\hline
\end{tabular}




\begin{tabular}{|c|c|c|c|c|c|c|c|c|c|c|}
\hline Runit & 1000 & & $j$ & & & $\perp$ & & & & \\
\hline Test fuid & $50 \%$ C4F 10 & $0.50 \% R 114$ & & & & $\frac{1}{1}$ & & & & \\
\hline Date: & $+12 / 31 / 931$ & & 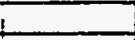 & & & & & & & \\
\hline Time: & $12: 19$ & & & & & & & & & \\
\hline \multicolumn{11}{|l|}{ Nolo } \\
\hline Fow Aroatil & 0.00336 & & & & & & & & & \\
\hline Hax Fow Aros R? & 0.1859 & & & & & & & & & \\
\hline Sat, Prossure Prig & 137.3 & I & I & & & & & & & \\
\hline Sed Tomperature f(inled) & 165.4 & 1 & $i$ & & & & & & & \\
\hline indet Fowrete-GPM & 26 & 1 & 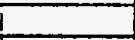 & & & & & & & \\
\hline intet Temperaturof & 169.7 & & & 1 & & & & & & \\
\hline Inlot Glbmhrn2 & 496910 & & Tamp & 16111 & Density & 80.09 & & & & \\
\hline Suboooling $F$ & -4.31 & & $L$ & -1 & & & & & & \\
\hline \multicolumn{11}{|l|}{ Fow Obsenvation } \\
\hline & & & & & & & & & & \\
\hline & ZONEI I & ZONE2 & ZONE3 & ZONEA & ZONES & ZONEE & ZONE? & ZONE8 & ZONES & ZONE=TO \\
\hline Hod inputWas:(nol) & 203.4 & ख9.3 & 288.1 & 2921 & 2927 & 285.7 & 200.8 & 28.1 & 286.7 & 291 \\
\hline HodLoss-Wadts & 9.9 & 2.1 & 2.7 & 3.1 & 2.9 & 2 & 1.3 & 1.9 & 2.6 & 15.1 \\
\hline Hanflaxtumitile & 5080 & 5048 & 4560 & 50081 & 5074 & 4552 & 5041 & 4994 & $45 \%$ & som \\
\hline Wall Temp. PaF & 180.8 & 178.41 & 172 & 181 & 179.8 & 178.51 & 178.6 & 178.4 & 175.0 & 177.5 \\
\hline Wall Tomp. \#4F & 180.7 & 178.11 & 177.7 & 180.91 & 179.9 & 177 & 178.5 & 175.0 & 176.9 & 178.0 \\
\hline WATTOMg $16 \mathrm{~F}$ & 181.3 & 176.01 & 178.7 & 180.5 & 178 & 171 & 177.6] & 176.1 & 775.7 & 178.4 \\
\hline Wal Tomp. IF & 168.7 & 178.81 & 176.2 & 181.5 & 179.5 & 179 & 180.2 & 177.31 & 174.9 & 97 \\
\hline Wall Tomp. $12 f$ & 182.6 & 178.8 & 176.6 & 181.1 & 179.4 & 178.5 & 979.5 & 177.3 & 175.2. & 177.1 \\
\hline Instrean Temp.F & 170 & 168.31 & 169.8 & 173 & 171 & 170.5 & 173 & 170.5 & 170.5 & 171.9 \\
\hline Avg. Wall Tomp.F & 181.4 & 178.5 & 176.9 & 181 & 179.5 & 177.4 & 178.9 & 176.6 & 775.5 & 177.4 \\
\hline Avg. Wall DT.F & 11.1 & 101 & 7.4 & 7.7 & 8.2 & 6.7 & 5.6 & 5.9 & 4.8 & 5.3 \\
\hline \multirow[t]{3}{*}{ DP. HBturtrine } & 457.4 & 505.11 & 670.1 & E53.71 & 617.2 & 74.6 & 895 & 850.1 & T0ख्रe & 900.4 \\
\hline & & & & & & & & & & \\
\hline & ZONE2 T2 & ZONE4 & ZONEG & ZONE8 & $20 N=10$ & & & & & \\
\hline DP liqudd Tomp. $F$ & $\begin{array}{l}78.81 \\
\end{array}$ & & & & & & & & & \\
\hline DP Liquid Densin $16 m / 18$ & 81.8 & & & & & & & & & \\
\hline Enertiont & 2 & 4 & 6 & 8 & 9.875 & & & & & \\
\hline Measured DPPsid & -0.094 & 0.621 & 1.069 & 1.684 & 1.33 & & & & & \\
\hline Zero Comections-psid & -0.305 & 0.02 & -0.009 & -0.028 & -0.356 & & & & & \\
\hline Correctod DPPsid & 0.211 & 0.001 & 1.078 & 1.712 & 2.257 & & & & & \\
\hline Acual DPA & 1.91 & 3.5 & 4.84 & 6.00 & 724 & & & & 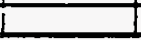 & \\
\hline
\end{tabular}




\begin{tabular}{|c|c|c|c|c|c|c|c|c|c|c|}
\hline Run: & $\quad 1010$ & & 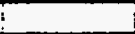 & $i$ & & I & 1 & 1 & & \\
\hline Tast Fuid & $125 \%$ C4F10 & $3-75 \% R 114$ & & & & & $T$ & t & & \\
\hline Date: & $: \quad 1 / 11 / 94$ & & & 1 & 1 & $\mathbf{i}$ & & & $=$ & \\
\hline Timo: & $.8: 29$ & & & 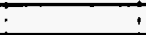 & t. & 1 & 1 & 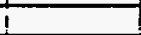 & & \\
\hline Now & 1 & 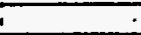 & 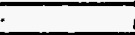 & 1 & $!$ & & $I$ & 1 & $\mathrm{i}$ & \\
\hline Fiow ATE-A2 & 0.00365 & & & 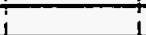 & 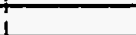 & & & & & \\
\hline Hos fow Aros-12 & 0.18695 & & & & & & & & & \\
\hline Sat. Prossurapsio & 127.5 & & & & i & & & & & \\
\hline Sut. Tempereture f(inlol) & 165.6 & & & ! & & & & & & \\
\hline InLA Fowrin-GPIM & 5.2 & & & T & $t$ & & & & & \\
\hline intet tomperaturef & 168.31 & & & & & & & & & \\
\hline Int Glbmhthic & $98 \% 0 \% 9$ & & ITomp & TE231 & Donsity & 8027 & & & & \\
\hline Subcoolingf & -2.71 & & & . & & & i & & & \\
\hline Fow Obsonvion & 1 & I & 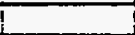 & & & & & & 1 & \\
\hline & 1 & 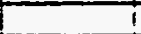 & $!$ & & 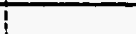 & & & & & \\
\hline & ZONEI & ZONE2 & ZONE3 & ZONE4 & IZONES & ZZONE6 & RONET & ZONEB & ZOKEO & ZONETO \\
\hline HexlnoctWatalnet) & 873,3 & 865.8 & 85.5 & 855 & 8526 & 870.8 & 8726 & 8591 & 8680 & 868.5 \\
\hline HostLoss-Wets & 1211 & 28 & 3.8 & 3.9 & 3.8 & 2.9 & 1.9 & 2.8 & 3.6 & 12.8 \\
\hline Hoxt Flox-Buhtrhe & 15138 & 15008 & 14968 & 14994 & 14953 & 15091 & 15128 & 15065 & 1500 ? & 15005 \\
\hline Wal Tomp. \#F & 183.3 & 985.4 & 184.1 & 185.4 & 186.5 & 187.4 & 188.2 & 184.8 & 183.3 & Tह22 \\
\hline Walt Tomp. Iff & 184.7 & 9828 & 185.8 & 186 & 988.8 & 187.6 & 188.2 & 188 & 184.8 & 188.8 \\
\hline Wal Temp. 154 & 184.3 & 181.1 & 186.8 & T86.4 & 185.1 & 188.7 & 187.2 & 184 & 983.2 & 180.6 \\
\hline Wall Tomp.tif & 168.3 & 184.11 & 181.6 & 184.3 & 183.8 & 1872 & 189.3 & 185.6 & 182.4 & 979.8 \\
\hline Wal Tomp. If & 986.3 & 183.71 & 183.1 & 184.91 & 184.3 & 187.3 & 189.4 & 186.8 & 183.1 & 1821 \\
\hline Instroam Tomp.f & 168.81 & 169.11 & 169.4 & 171 & 170 & 1701 & 171.7 & 170.4 & 170.4 & 170.7 \\
\hline AVo. Wal Tomp.F & 184.5 & 184 & 183.7 & 785.61 & 185 & 1872 & 188.5 & 185.4 & 183.4 & 1826 \\
\hline AY. WallDT, $F$ & 15 & 142 & 13.8 & 13.8 & 14.8 & 16.5 & 16.1 & 14.4 & 12.8 & 17.3 \\
\hline EXP. HEtuthmer & 1008.1 & 1056.8 & 11027 & 10828 & 108.8 & 9122 & 90.8 & 10402 & 1220.6 & 1337.1 \\
\hline & & & & & & & & & & \\
\hline & ZONE2 & ZONEA & ZONE 6 & ZONEB & ZONETO & & & & & \\
\hline DPliquid Temp.f & 81.7 & & & & & & & & & \\
\hline DPLiguid Density 4 bmin3 & 90.79 & & & & & & & & & \\
\hline Elovetionth & 2 & दा & 6 & 8 & 9.875 & & & & & \\
\hline Measured DPPsid & -0.185 & 0.523 & 0.903 & 1.438 & 1.45 & & & & & \\
\hline Zoro Corrections-peid & -0.317 & 0.101 & -0.078 & 0.049 & -0.350 & & & & & \\
\hline Correated DP sid & 0.132 & 0.42 & 0.81 & 1.389 & 1.816 & & & & & \\
\hline Achual DPA & 2.03 & 3.77 & 5.00 & $6.56 \mid$ & 7.91 & & & & & \\
\hline & & & & & & & & & & \\
\hline
\end{tabular}




\begin{tabular}{|c|c|c|c|c|c|c|c|c|c|c|}
\hline Run: & 10171 & & & & 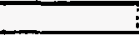 & 1 & & 7 & $T$ & \\
\hline Test fluid & $.25 \%$ C4F10 & $-75 \% R 114$ & 4 & 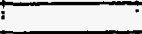 & 1 & T & & 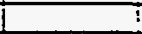 & 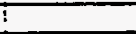 & \\
\hline Dafo: & $1 / 11 / 94$ & & & & 1 & 1 & & 1 & $\mathrm{i}$ & \\
\hline Timo: & $.8: 28$ & & i & $\cdot$ & $\cdot$ & $\mathrm{i}$ & & i & i & \\
\hline Note & 1 & & 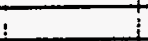 & 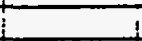 & 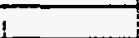 & & & 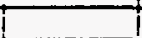 & & \\
\hline Flow Areaft2 & 0.003361 & & & & 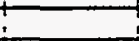 & & & & & \\
\hline Fea flow Area-fle & $0.19 \% 5$ & & & & & & & & & \\
\hline Sat. Pressuropsig & 127.91 & & & & & & & & & \\
\hline Sat. Temperaturo of (inlot) & 165.81 & & & & & & & & & \\
\hline Inlet Fowrate-GPM & $3.9 !$ & & T & $T$ & + & 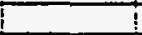 & & 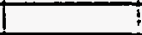 & 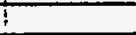 & \\
\hline inlot Temperaturef & 168.8 & & & 7 & ? & $i$ & & & $\mathrm{~T}$ & \\
\hline Intat Glbmhrile & 747575 & & Themp & TE्2ा & TDensity & 80.32 & & & $i$ & \\
\hline Subcoolingf & $-3 \mid$ & & $i$ & & & & & & & \\
\hline \multirow[t]{3}{*}{ Flow Observation } & $i$ & & i & 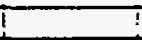 & $!$ & & & & & \\
\hline & $!$ & & 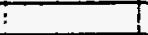 & $i$ & $i$ & & & $t$ & $t$ & \\
\hline & ZONE1 & ZONE2 & ZZONE3 T: & ZZONEA & ZONES & ZONE6 & ZONET & ZONE8 & ZONEO & LONETO \\
\hline Heal hout-Watts(net) & 87.41 & 869.5 & 868.2 & 83.2 & 859.3 & 874.4 & 876.1 & 869.8 & 868.4 & 872.1 \\
\hline Heat Loss-Watts & 11.4 & 2.6 & 3.5 & 3.8 & 3.7 & 2.8 & 1.9 & 2.7 & 3.4 & 19.2 \\
\hline Hoat Flux-Btuithinl & 15200 & 15072 & 15015 & 150671 & 15069 & 15157 & 15187 & 15077 & $150 \%$ & 15122 \\
\hline Wall Temp. \#F & 184 & 185.9 & 184.8 & 186.8 & 187.9 & 188.5 & 189 & 185.6 & 184.8 & 189.8 \\
\hline Wall Tomp $4 \mathrm{~F}$ & 184.8 & 188.4 & 188.2 & 187.5 & 188.2 & 188.5 & 189 & 187 & 185.6 & 190.1 \\
\hline Wall Temp. "5F & 185.21 & 181.51 & 187.3 & T87.81 & 188.3 & 188 & 188 & 184.8 & 184.1 & 189.8 \\
\hline Wall Temp. $\$ 7$ & 168.81 & 184.71 & 1821 & 185.8 & 185.81 & 188.4 & 190.4 & 186.41 & $\{83.2$ & 781.5 \\
\hline WaH Tomp. $2 F$ & 1871 & 984.31 & 183.5 & 986.4 & 785.71 & 188.5 & 190.31 & 187.7 & 184.1 & 783.7 \\
\hline Instrean Temp.f & 969.21 & $169.5 i$ & 169.8 & $172.2:$ & 170.8 & 170.61 & 172.31 & 170.81 & 170.8 & 171.7 \\
\hline Avg. Wall Temp.F & 185.31 & 184.61 & 184.11 & 187 & 188.4 & 188.41 & 189.3 & 980.31 & 184.3 & 184.2 \\
\hline Avg. Wall DT.F & 15.41 & 14.4 & 13.8 & 14.11 & 14.8 & 17.11 & 18.4 & 14.81 & 12.8 & 11.8 \\
\hline \multirow[t]{3}{*}{ EXP.HEhHning } & 8801 & 1047.1 & 1104.81 & 1055.81 & 1011.7 & 88821 & 828.6 & 1017.5 & 1170.8 & T28.1 \\
\hline & 1 & & & & & & & & & \\
\hline & .ZONE2 & ZONE4 & ZZNE 6 & ZONE8 & ZZONETO & & & & & \\
\hline \multicolumn{11}{|l|}{ DP liquid Temp.f } \\
\hline \multicolumn{11}{|c|}{ 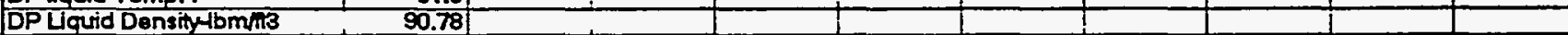 } \\
\hline Elevation-1 & & 4 & 6 & 8 & 9.875 & & & & & \\
\hline Messured DPPsid & -0.081 & 0.776 & 1.3141 & 1.987 & 2.151 & & & & & \\
\hline Zoro Corrections-psid & -0.317 & $0.101 !$ & 0.078 & ర.0.61 & -0.356 & & & & & \\
\hline Comectad DPPsid & 0.237 & 0.675 & 1.392 & 1.968 & 2.517 & & & & & \\
\hline \multirow[t]{2}{*}{ Actual DPA } & 9.84 & 3.37 & 428 & 5.57 & 6.65 & & & & & \\
\hline & $t$ & & & & & & & & & \\
\hline
\end{tabular}




\begin{tabular}{|c|c|c|c|c|c|c|c|c|c|c|}
\hline Runt & 10121 & & & & & 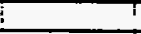 & & i & $?$ & \\
\hline Test Fuld & $25 \%$ CAF 10 & $-75 \%$ R114 & & $!$ & & - & & $t$ & i & 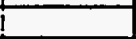 \\
\hline Date: & $: 1 / 11 / 94$ & & & & 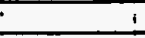 & 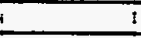 & 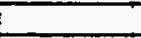 & & $i$ & L \\
\hline Time: & $8: 29$ & ; & $:$ & $:$ & 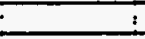 & 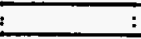 & 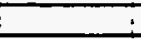 & 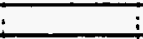 & $\vdots$ & 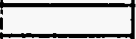 \\
\hline Note & $:$ & 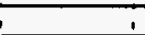 & 1 & 1 & 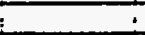 & 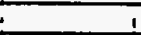 & 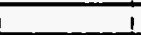 & 1 & 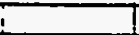 & 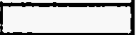 \\
\hline Fow Arow & 0.003561 & F & 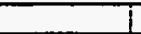 & & - & 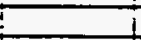 & 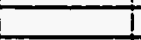 & & & \\
\hline Heat Flow Aroatic & 0.19655 & 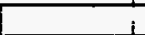 & 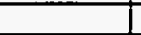 & & $!$ & 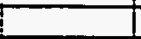 & & & & \\
\hline Set. Pressure Psig & 127.41 & & & & 1 & 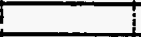 & & & & \\
\hline Sat. Temperature-finlel) & 165.51 & & & & 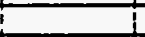 & 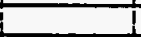 & & & & \\
\hline Inlet Fowrato-GPM & 2.61 & $!$ & 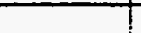 & & i & 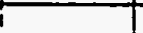 & & & & \\
\hline indet Tomporture F & 168.21 & $i$ & 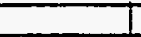 & & 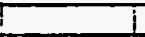 & & & & & \\
\hline Intot Glbmhrne & 500205 & & Temp & 1601 & IDensity & 80.631 & & & & \\
\hline Subcoolingf & -2.7 & & & & & & & & & \\
\hline Fow Observation & 1 & & & & 1 & 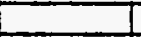 & & & & \\
\hline & 1 & & & & & & & & & \\
\hline & ZONEI & ZONE2 IS & ZONE3 & ZONE4 & ZONES & ZONE6 & ZONE & ZONEB & $\angle C O N=9$ & ZON:S10 \\
\hline Hed lnpet-Watts(nel) & 853.7 & 853.8 & $8 \$ 0.6$ & 865.4 & 874.6 & 865.5 & 8527 & 861.7 & 867.8 & 868.3 \\
\hline Fed Lose-Watls & 127 & 291 & 4.1 & 4.2 & 4.21 & 3.31 & 23 & उ.2। & 3.9 & 224 \\
\hline Hox Flex-Btu/fir/le & 14972 & 14975 & 14918 & 15001 & 15161 & 15003 & 14854 & 14937 & 1498्रा & 15051 \\
\hline Wall Temp, $3 F$ & 183 & 185.3 & 184.3 & 185,8 & 187.3 & 188.1 & 188.9 & 186.1 & 185 & 184 \\
\hline Wall Temo. I4F & 184.1 & 182.7 & 188 & 188.8 & 187.4 & 1882 & 188.9 & 187.1 & 1882 & 1903 \\
\hline Wall Tamp. $15+$ & 184.41 & 181.3 & 187.5 & 186.8 & 185.8 & 187.3 & 187.91 & 185.31 & 184.5 & 1823 \\
\hline Wail Temp. HF & 138.2 & 1841 & $\mid 81.8$ & T84.81 & 184.71 & $\mid 87.61$ & T89.81 & $\mid 86.81$ & 1835 & 787.6 \\
\hline Walt Temp.tiz & 185.51 & 783.61 & 183.4 & 185.31 & 1851 & T87.9T & 789.9 & T88.2 & 18.,.4 & T83.8 \\
\hline Instosm Tomp.F & 168.91 & 169.7 & 170 & 171.21 & 170.5 & 170.5 & 172.4 & 171.1 & 1712 & 172 \\
\hline Ave.Wall Tomp.f & 184.31 & 183.81 & 183.9 & 185.81 & 185.7 & 187.81 & 189.1 & 188.71 & 184.7 & 184.4 \\
\hline Avg. We्l DT.f & 14.7 & 13.5 & 132 & 14 & 34.6 & 16.8 & 16 & 14.9 & 12.8 & 11.7 \\
\hline DAP.HBWhimeh & 1020.3 & 1107.4 & 1130.1 & 1057.8 & 10427 & 90261 & 354.4. & 1000.8 & 1168.8 & 1283.5 \\
\hline & 1 & $=$ & & & 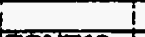 & & & & & \\
\hline & ZONE2 & ZONE4 & ZONE6 & ZONE8 & ZONE10 & & & & & \\
\hline DPliquid Temp.f & i $\quad 80.9$ & & & & 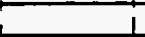 & 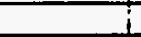 & & & & \\
\hline DPLIquid Density 1 bmuni3 & 90.87 & & & & & & & & & \\
\hline Elevation & 2) & 4 & 6 & 8 & 9.875 & & & & & \\
\hline Messured DPPsid & 0.026 & 1.1 & 1.811 & 2.6531 & 2809 & & & & & \\
\hline Zero Corrections-psid & -0.377 & 0.701! & $=0.078$ & 0.0 .61 & -0.3561 & & & & & \\
\hline Corrected DPFsid & 0.343 & 0.999 & 1.88 & 2.604 & 3.255 & & & & & \\
\hline Actuld DPA & 1.64 & 2.72 & 3.39 & 4.371 & 5.3 & & & & & \\
\hline & $i$ & $!$ & 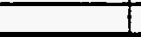 & 1 & 1 & & & & & \\
\hline
\end{tabular}




\begin{tabular}{|c|c|c|c|c|c|c|c|c|c|c|}
\hline Run: & 10131 & & & & 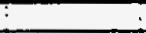 & i & $\cdot$ & & $!$ & \\
\hline Testfiuld & $25 \%$ CAF10 & $0-75 \%$ R114 & & & i & 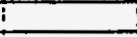 & $!$ & 1 & $i$ & \\
\hline Date: & $1 / 11 / 94 i$ & & & 1 & 1 & 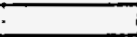 & $i$ & & 1 & \\
\hline Time: & $13: 281$ & & $\dot{-}$ & . & 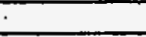 & & 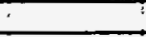 & & 1 & 1 \\
\hline Note & . & & $:$ & ? & & + & $:$ & & 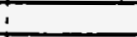 & \\
\hline Fow Areatt2 & 0.003361 & & 1 & 1 & & & 1 & & & \\
\hline Host Fow Aroa-12 & 0.195951 & & & $i$ & & $T$ & $i$ & 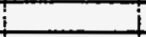 & 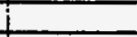 & \\
\hline Sut. Pressure Psig & 1281 & & & & & 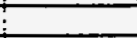 & & & & \\
\hline Sal. Temperature-f(inlot) & 165.81 & & & & & 1 & 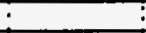 & 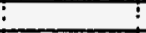 & 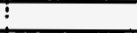 & \\
\hline Inlot Fowrato-GPM & 5.3 & & & 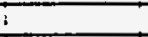 & & 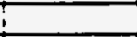 & & & T & \\
\hline inlet Temperature fF & 168.9 & & & 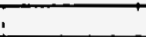 & & 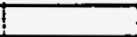 & $\therefore$ & & & \\
\hline Intat Gibm/hrint & 1012445 & & Themp & 16.71 & Density & 80.05 & & & & \\
\hline Subcooling & -3.11 & $\mathbf{t}$ & 1 & & 1 & - & & & & \\
\hline Fow Obsenvion & ; & $;$ & : & & $T$ & $T$ & 1 & & & \\
\hline & 1 & 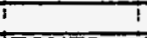 & 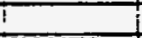 & & $\vdots$ & & 1 & & & \\
\hline & IZONET & ZONEZ & IZONE3 & ZONEA & ZONES & ZONE6 & ZONET & ZONE8 & ZONVE & $1201=10$ \\
\hline Hoal toput-Watts(nel) & \begin{tabular}{|l}
581.1 \\
\end{tabular} & 581.4 & 580.7 & 5827 & 578.3 & 588.8 & 583.8 & \begin{tabular}{|l|}
581.3 \\
\end{tabular} & 589.9 & 678.8 \\
\hline Hed Lose-Watis & 10.7 & 2.4 & 3.3 & 3.51 & 3.4 & 26 & 1.7 & 2.5 & 32 & 17.6 \\
\hline Hedflox-BuHAr/2 & 10073 & 10078 & 10065 & 10101 & 10024 & 10172 & 10120 & 10076 & 10087 & 998 \\
\hline Wal Tomp. "3F & 182.2 & 1826 & 183.1 & 184.2 & 184.7 & 184.9 & 1862 & 183.6 & 183.8 & 1812 \\
\hline Wal Temp. $4 \mathrm{~F}$ & 182.7 & 181.71 & 184.7 & 184.4 & 185.11 & 185.5 & 186.2 & 184.2 & 1842 & 183.8 \\
\hline Wal Temp. 15 F & 183.51 & 180.71 & 185.2 & 184.91 & 184.8 & 185.4 & 185,3 & 183,1 & 1829 & 180.8 \\
\hline Wall Tomp. AfF & T68.91 & 78241 & |85.6| & T83.3! & T82.9 & T85. & 187.1 & 784 & 781.8 & 780.3 \\
\hline Wall Tomp. ${ }^{2}=$ & 784.91 & 78241 & द81.7| & 183.71 & 1828 & 185.5 & 787.11 & T84.5T & 1822 & 781.3 \\
\hline Instruam Temp.f & 169.3 & 169.1 & 169.4 & 171.11 & 170.3 & 170.3 & $172 \pi$ & 170.8 & 170.7 & 171 \\
\hline Avg. Wadl Temp.F & T83.3. & 782.31 & 182.4 & 184.2 & 183.8 & 185.8 & 188.4 & 1803.81 & 1828 & 181.5 \\
\hline Avg. Wall DT.f & 13.6 & 12.7 & 125 & 12.61 & 13 & $\mathbf{1 4 . 5}$ & 13.81 & 12.8 & 11.7 & 10 \\
\hline \multirow[t]{3}{*}{ 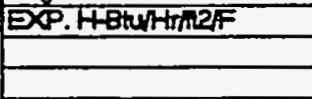 } & 74231 & 792.31 & 804 & 798.81 & 769.8 & 609.6 & 7321 & 798.2 & 8538 & 997 \\
\hline & 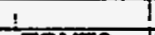 & & & & & & & & & \\
\hline & ZONE2 & ZONE4 & $\angle O N=6$ & ZONE8 & ZONETO & & & & & \\
\hline DP liquid Temp.f & 81.1 & & & & & & & & & \\
\hline DP Liquid Densitylbmit3 & 90.85 & & & & & & & & & \\
\hline Elevation-t & 21 & 4 & 6 & 8 & 9.8751 & & & & & \\
\hline Messured DPPsid & -0.231 & 0.344 & 0.7261 & 1.35 & 1.278 & & & & & \\
\hline Zero Correctons-psid & -0.305 & 0.1061 & -0.027 & 0.005 & $-0.3 \times 5$ & & & & & \\
\hline Corrected DPFsid & 0.075 & 0.238 & 0.763 & 1.255 & 1.613 & & & & & \\
\hline Actual DPA & 2.13 & 4.11 & 5.44 & 6.82 & 8.31 & & & & & \\
\hline & 1 & & & & & & & & I & \\
\hline
\end{tabular}




\begin{tabular}{|c|c|c|c|c|c|c|c|c|c|c|}
\hline Run: & 10741 & 1 & 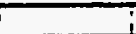 & i & $\cdot$ & & 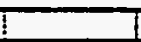 & 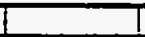 & & \\
\hline Testfluid & $125 \%$ C4=10 & $.75 \%$ R114 & 4 & 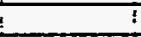 & 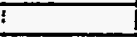 & 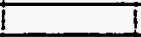 & 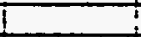 & 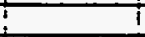 & 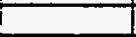 & \\
\hline Dade: & $1 / 11 / 941$ & & ! & 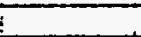 & & 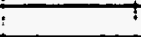 & + & 1 & 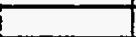 & \\
\hline Time: & $13: 32$ & & & 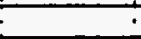 & $\cdot$ & $\vdots$ & I & i & 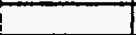 & \\
\hline Nob & . & $:$ & 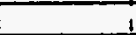 & 1 & $:$ & 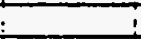 & 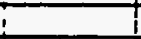 & + & 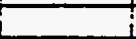 & \\
\hline Flow Aresti2 & +0.003361 & 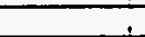 & 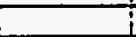 & 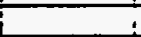 & 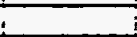 & $i$ & 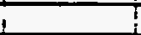 & 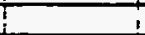 & 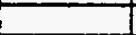 & \\
\hline Heat flow Areate & 0.19035 & & & 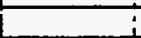 & 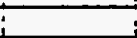 & 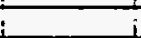 & $i$ & 1 & 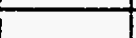 & \\
\hline St. PressurePsig & 126.61 & & 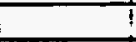 & 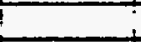 & & T & 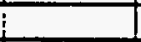 & & & \\
\hline Sat. Temperature of (inlet) & 165.11 & & 1 & 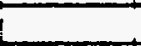 & & 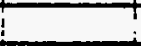 & & 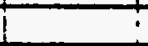 & 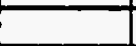 & \\
\hline Inlet Fowrade-GPM & 3.91 & 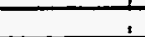 & i & i & & I & 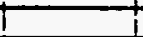 & 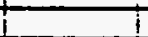 & 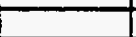 & \\
\hline intet Temperature & 169 & 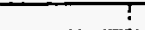 & 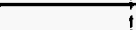 & i & 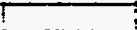 & 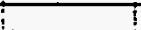 & 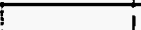 & 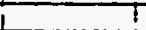 & 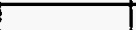 & \\
\hline Intet Glbmmine & 745451 & & Temp & 163.4 & Donsity & 80.001 & & & & \\
\hline Suboooling $F$ & -3.9 & t & s & $!$ & 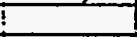 & & & $i$ & 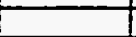 & \\
\hline Fow Observation & 1 & i & 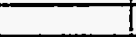 & & 1 & & & & 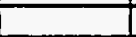 & \\
\hline & 1 & ! & 2 & & 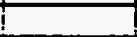 & & 1 & f & 2 & \\
\hline & ZONE1 & ZONE2 I2 & ZONE3 & ZONE4 & ZONE5 & ZONE6 & ZONE7 & ZONES T & ZONE9 & 20NE10 \\
\hline HesthputWettalnel) & $\quad 6762$ & 577.3 & 573.9 & 574 & 5726 & 578.7 & 578.7 & 574.7 & 674.8 & 574.6 \\
\hline Head Lose-Wathe & 10.8 & $2.4 !$ & 3.3 & 3.5 & 3.4 & 2.6 & 1.7 & 251 & 32 & 17.8 \\
\hline Head Fuxestu/Hi/le & 9988 & 10007 ! & 9948 & $9950 !$ & 9920 & 10031 & 10031 & 9952 & 994 & 9959 \\
\hline Wall Tomp. HF & 182.21 & 1823 & 182.6 & 184.3 & 184.9 & 184.8 & 185.8 & 183.1 & 182.7 & 181.6 \\
\hline Wall Temp. \$4F & 182.61 & $181.1 !$ & 183.61 & 184.8 & 185.2 & 18521 & 185.7 & 183.6 & 183.8 & 184.3 \\
\hline Wall Temp. 5 Fं & 183.71 & 180.4 & 184.7 & 185.8 & 184.1 & 185.1 & 184.9 & 1826 & 1828 & 181.2 \\
\hline Wat Temp. BtF & |б9| & दष्ट.2. & 180.1 & T\&3.61 & 183 & Tठ5.31 & 186.7 & 183.61 & 780.9 & T80.4 \\
\hline Wa Tomp. T2F & 184.71 & 18231 & 181.1 & $\mid 83.91$ & 1829 & 18521 & 186.7 & 1हद.2 & Tह1.4 & Tह1.5 \\
\hline Instroam Tomp.f & 169.5 & 168.9 & 169.4 & 1712 & 169.8 & 169.8 & 171.6 & 170.1 & To1 & Th2 \\
\hline Avg. Wall Temp.f & 183.3 & 182 & 181.8 & 184.6 & 183.8 & 185.1 & 1881 & 183.4 & Tह23 & 181.8 \\
\hline Avg. Wall DT ff & 13.3 & 12.6 & 12 & 128 & 13.6 & 14.8 & 13.9 & 128 & 11.7 & 10.1 \\
\hline EXP.HAW/H/n2/F & 7482 & 794.4 & 828 & 774.8 & 7324 & 677.8 & Tद1.81 & 774.1 & 848.4 & 881.1 \\
\hline & 1 & & & & 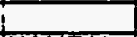 & & & & & \\
\hline & ZONE? & ZONE4 & ZONE6 & ZORE & ZONEIO & & & & & \\
\hline DP lquid Temp. $F$ & 81.4 & 1 & L & & & & & & & \\
\hline DPLIquid Denstitumbmis & 90.82 & 1 & 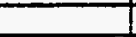 & & & & & & & \\
\hline Elevation & 2 & 4 & 6 & 8 & 9.875 & & & & & \\
\hline Measured DPPSid & -0.145 & 0.582 & 1.14 & 1.785 & 1.941 & & & & & \\
\hline Zero Conctions-psid & -0.306 & 0.106 & -0.021 & -0.0051 & -0.355 & & & & & \\
\hline Corroded DPPsid & 0.161 & 0.476 & 1.187 & 1.79 & 2.278 & & & & & \\
\hline Actual DPA & 1.98 & 3.681 & $4.7 \pi$ & 5.85 & 7.11 & & & & & \\
\hline & & & & & 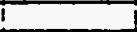 & & & & & \\
\hline
\end{tabular}




\begin{tabular}{|c|c|c|c|c|c|c|c|c|c|c|}
\hline Run: & $1015 !$ & & I & 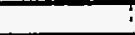 & & & ? & & 1 & \\
\hline Tost Fivid & $25 \%$ CAF 10 & $-75 \%$ R114 & & & $i$ & 7 & 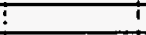 & & & \\
\hline Date: & $1 / 11 / 94$ & & & & i & 1 & $I$ & $\bar{T}$ & 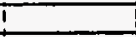 & \\
\hline Time: & $13: 32$ & & & $\therefore$ & i & $\because$ & 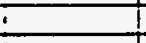 & & 1 & \\
\hline Now & 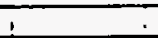 & & & 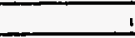 & 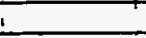 & & $i$ & & & \\
\hline Fow Arost2 & 0.003351 & & & i & i & 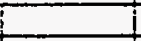 & & & & \\
\hline Hest Fow Area-de & 0.1955 & 1 & 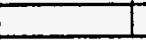 & $i$ & 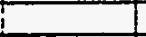 & & & & & \\
\hline Sat Prossure Psig & 127.4 & & & & & & 1 & & & \\
\hline Sat. Temperature-F(inlet) & 165.51 & & i & 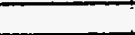 & 1 & ! & ! & & & \\
\hline Inlolflowrate-GPM & 2.61 & & $\mathrm{~T}$ & & & i & I & I & & \\
\hline indet Temperaturef & 168.4 & 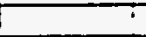 & T & & & & & & & \\
\hline Intat Gbmhrite & 4983441 & & Temp & 1ह2 & Donsity & 80.32 & & & & \\
\hline SubcoolingF & -2.91 & & & 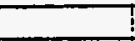 & 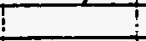 & & & $!$ & & \\
\hline \multirow{3}{*}{ Fow Observation } & $!$ & 1 & 1 & 7 & 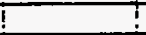 & T & T & I & & \\
\hline & 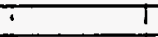 & T & $i$ & 7 & T & i & $i$ & $i$ & & \\
\hline & .2ONE1 is & ZONE2 I & ZONEB I & ZONE4 & IZONES & ZONE & ZZONE7 & ZONEB & ZONEO & ZONE10 \\
\hline Heat Inputwatte(not) & $5802 !$ & 581.11 & 572 & 576.8 & 576.3 & 578.6 & 578.6 & 577.7 & 678.8 & 677.1 \\
\hline Heal Loss-Watts & 10.8 & 2.4 & 3.4 & 3.5 & 3.5 & 2.6 & 1.7 & 2.5 & 3.2 & 18.2 \\
\hline Hod FLx BtuhHrit? & 10057 & 10073 & 10005 & 99981 & 9990! & 10030 & 10030 & 10014 & 1002 & 10004 \\
\hline Wall Tomp. $3 F$ & 182.2 & $182.6 !$ & 183.1 & 184.3 & 185 & 185.3 & 186.4 & 183.9 & 183.8 & 1822 \\
\hline Wall Tomp. 14F & $182.7 !$ & 781.61 & 184.11 & 184.7 & 185.5 & 185.8 & 186.5 & 184.5 & 184.4 & 185 \\
\hline Wat Tomp. \#5F & 183.6 & 180.71 & 185.6 & 185.4 & 184.61 & 185.7 & 185.81 & 183.5 & 183.1 & 181.4 \\
\hline Wat Tomp. I1F & 168.41 & 98231 & 180.5 & 183.7 & 183.3 & 185.61 & 187.2 & 184.51 & 181.7 & 780.8 \\
\hline Wall Tomp. ILF & $184.4 \Gamma$ & 182.31 & 181.61 & 183.9 & 183.11 & 185.5 & 187.31 & 185.21 & 1822 & 781.6 \\
\hline instroam Temp.f & 768.81 & $969.6 T$ & 170.11 & 171.4 & 170.51 & 170.6 & 172.2! & $17 \pi$ & 170.8 & 171.7 \\
\hline Avg. Wall Temp.F & $183.2 !$ & 18221 & 182.31 & 184.5 & 184.71 & 185.6 & 188.81 & 184.31 & 182.8 & 1822 \\
\hline Avg. Wed DT F & $14 \pi$ & 12.31 & $11.8 \mathrm{i}$ & -12.7 & 13.21 & 14.61 & 19 & 12.9 & 11.8 & 10 \\
\hline \multirow[t]{3}{*}{ EXP. HBtu/Hrhl2/F } & $719.9 i$ & 829.41 & 899.81 & 788.8 & 758.31 & 689.5 & 717.1 & 778.2 & 865.1 & 995.8 \\
\hline & & & & & 1 & & & & & \\
\hline & ZONE2 & ZONE4 & ZONE & ZONE8 & ZONE10 & & & & & \\
\hline DP liquid Temp.f & 181.9 & & & & & & & & & \\
\hline DPLiquid Density/bm/n & 90.77 & & & & & & & & & \\
\hline Elevation-t & 2! & 41 & 61 & 8 & 9.875 & & & & 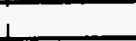 & \\
\hline Mossured DPPsid & -0.056 & 0.8221 & 1.529 & 2.317 & 2.606 & & & & & \\
\hline Zoro Corractions-psid & -0.3061 & 0.1061 & -0.0211 & -0.005 & -0.365 & & & & & \\
\hline Corrected DFf sid & 0.251 & 0.781 & 1.5561 & 2.322 & 2.941 & & & & & \\
\hline ACUal DPA & 1.811 & 3.241 & 3.99 & 4.88 & 5.89 & & & & & \\
\hline & & & & & & & & & & \\
\hline
\end{tabular}




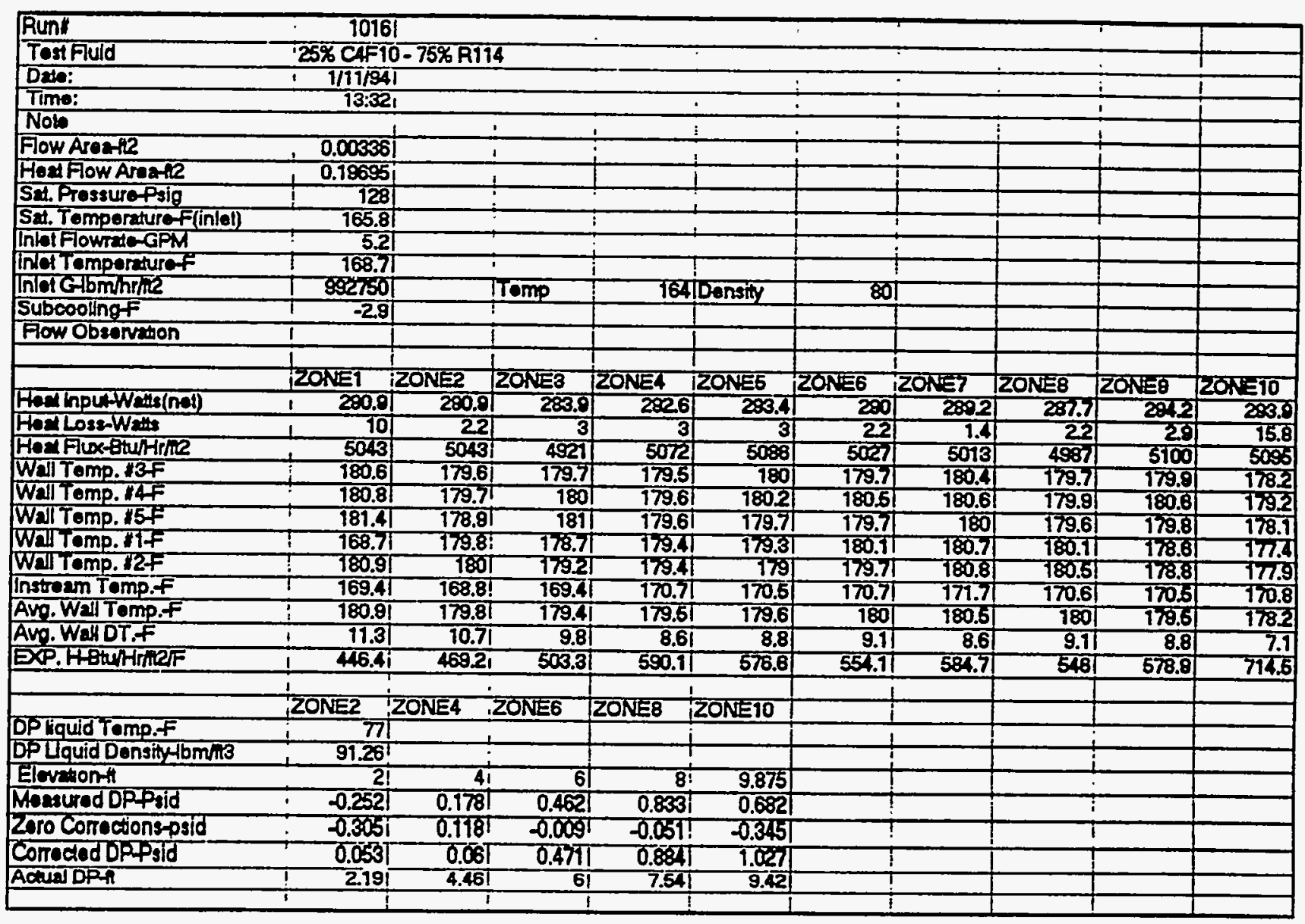




\begin{tabular}{|c|c|c|c|c|c|c|c|c|c|c|}
\hline Run: & 10171 & & & & - & • & 1 & 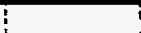 & $i$ & 1 \\
\hline Test Furid & $25 \%$ C4F10 & $3-75 \%$ Riti & & & $\cdot$ & $\cdot$ & & & 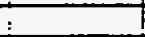 & t \\
\hline Date: & $1 / 11 / 94$ & & & & & & 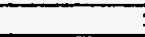 & : & $\div$ & 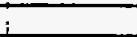 \\
\hline Time: & 16:55. & & $\therefore$ & & & & $\dot{0}$ & & & $i$ \\
\hline Noto & & & & & & & & & 1 & \\
\hline Fow Area-12 & 0.00336 & & . & & $:$ & $\vdots$ & 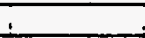 & & $i$ & \\
\hline Heaf Fow Area-t2 & $0.19595 !$ & & & & 3 & 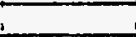 & . & & 1 & \\
\hline Sat. Pressure-Psig & 127.1 & & & & & & $\div$ & & 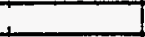 & \\
\hline Sat. Temperaturef(inlel) & $165.4 \mathrm{i}$ & & & & & & & & & ! \\
\hline Inlet Fowrate-GPM & 3.91 & & & & ! & 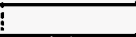 & & & ; & \\
\hline intot Temoeraturefe & 169.3 & & & & & & & & & \\
\hline Intot Gibminrine & 7451551 & & Tentp & $163.6 !$ & Tonsty & 80.05 & & & $:$ & \\
\hline Subcooling $f$ & -3.91 & & ! & & 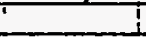 & 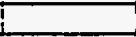 & & $\vdots$ & 1 & \\
\hline Flow Obsorvation & 1 & 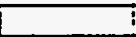 & $i$ & & 1 & 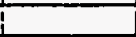 & 1 & & I & \\
\hline & I & 1 & 1 & & 1 & 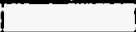 & 1 & & 1 & \\
\hline & ZONNEI is & ZONEE I & IZONEB & ZONEA : & ZZONE6 & ZONE6 & ZONE7 & ZONE8 & ZZONEO & $20 N=10$ \\
\hline Heat lnoutWather(nol) & 289.9 & 290.7 & $1 \quad 281.8$ & 288 & 288.8 & 288.1 & 1) 287.4 & 288.1 & 2802 & 2882 \\
\hline Heat Loss-Watts & 10 & 22 & 2.9 & 3.1 & 2.9 & 21 & 1.5 & 2.2 & 2.8 & 15.8 \\
\hline Heat Flux-BturHrne & 5005 & 5009 & 4885 & 4992 & 4997 & 499 & 4982 & 4959 & 5018 & 4986 \\
\hline Wall Temp. \#3F & 181.2 & 179.9 & 179.7 & 180.8 & 180.7 & 179.9 & 181.6 & 180.3 & 179.9 & 179.8 \\
\hline Wall Temp. \$4F & 181.4 & 179.9 & 179.9 & 180.8 & 180.6 & 180.4 & 181.7 & 180.2 & 180.6 & 180.2 \\
\hline Wall Temp. $5 F$ & 182.2 & 178.8 & T80.9 & 180.6 & 179.6 & 179.6 & 181 & 179.6 & 179.7 & T78.9 \\
\hline WaH Temp. \#1F & 169.3 & 180.2 & 97.7 & 180.6 & 180.2 & 180.3 & 182.1 & 180.9 & 178.8 & T78.5 \\
\hline Wall Tomp. $2 F$ & 1825 & 180.8 & 179.1 & 180.5 & 179.9 & 180 & 182.1 & 181.4 & 179 & 178 \\
\hline Instresm Tomp.f & $169.9 !$ & 168.8 & 16921 & 171.6 & .170 .41 & $1 \% 0.4$ & 171.9 & 170.4 & 170.4 & 77.4 \\
\hline Avg. Wall Temp.f & 181.81 & 180.1 & 179.4 & 180.7 & 180.1 & 180.1 & 181.7 & 180.5 & 779.8 & 178.2 \\
\hline Avg. WaHDT.f & 11.71 & 10.8 & 9.8 & 8.91 & 9.4 & 9.4 & 9.6 & 9.9 & 8 & 7.6 \\
\hline DCP.HOWhina & $428.6 i$ & 458.31 & 4921 & 55261 & 528.8 & 529.8 & 520.3 & 5032 & 5590 & 681.3 \\
\hline & i & & 1 & & 1 & & 1 & & & \\
\hline & ZZONE? & ZONE4 & ZOONE6 & ZONE8 & ZONEIO & & & & 1 & \\
\hline DP liquid Tomp.f & 80.9 & & & & & & & & & \\
\hline DP Lquid Densitylbm/13 & 90.87 & & 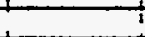 & & & & & & & \\
\hline Elevation- & 21 & 4 & 61 & 8 & 9.8751 & & 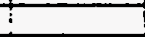 & & & \\
\hline Messured DPPsid & 0.189 & 0.374 & 0.7851 & 1.28 & 1.273 & & 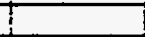 & & & \\
\hline Zero Conections-psid & -0.3051 & 0.118 & -0.009 & -0.0511 & -0.345 & & $T$ & & & \\
\hline CorrectedDPFid & 0.1161 & 0.258 & 0.794 & 1.3311 & 1.618 & & $t$ & & 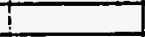 & \\
\hline Actual DPA & 2.051 & 4.081 & 5.381 & 6.691 & 8.3 & & 1 & & $T$ & \\
\hline & $T$ & 1 & $T$ & 1 & 1 & 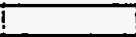 & $i$ & & $T$ & \\
\hline
\end{tabular}




\begin{tabular}{|c|c|c|c|c|c|c|c|c|c|c|c|}
\hline Run: & 1 & 1018 & 1 & & & $!$ & 1 & 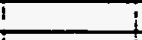 & $i$ & $\dot{s}$ & \\
\hline Tost Fluid & & $25 \%$ CAF10 & - 75\%Rा14 & 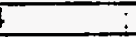 & 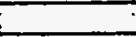 & 1 & 1 & 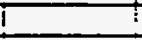 & 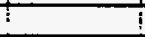 & 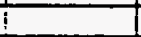 & \\
\hline Date: & 7 & $1 / 11 / 34 \mid$ & -1 & 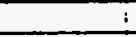 & 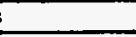 & ; & 1 & $I$ & & 1 & \\
\hline Time: & : & $16: 55 i$ & & & ; & $\therefore$ & $\cdot$ & $\cdot$ & $\dot{4}$ & $:$ & \\
\hline Note & 1 & & $\dot{2}$ & $\dot{1}$ & 1 & $\vdots$ & 1 & $!$ & 1 & $!$ & \\
\hline Fow Arasti2 & . & 0.003361 & $\therefore$ & i & 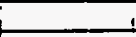 & 1 & & $\dot{i}$ & $\vdots$ & 1 & \\
\hline Hesf Fow Areate & i & 0.195951 & & 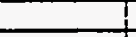 & 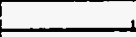 & + & i & ; & ; & $i$ & \\
\hline Sat. Pressure Psig & & 126.3 & & & & 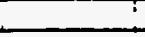 & & 1 & 1 & $i$ & \\
\hline Sat. Temperature-f(inlet) & & 165 & & 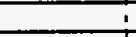 & 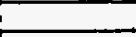 & & & & 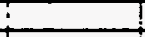 & & \\
\hline Inlot Fowrate-GPM & & 261 & & & & 1 & 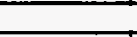 & & & & \\
\hline inst tremperaturef & & 167.9 & & $:$ & 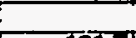 & $i$ & 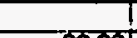 & & $i$ & 1 & \\
\hline Intet Glommine & T & 498371 & & Tomp & $161.7 !$ & Densty & 80.35 & & 1 & 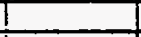 & \\
\hline Subooolingf & & -2.91 & & & & $\vdots$ & I & 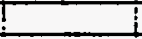 & $i$ & 1 & \\
\hline \multirow{3}{*}{ Fow Observation } & 1 & & & & 3 & $\vdots$ & 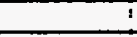 & 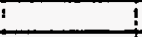 & $!$ & 1 & \\
\hline & 1 & & : & ; & 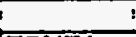 & 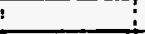 & 1 & 1 & $\mathbf{i}$ & i & \\
\hline & & ZONET : & ZONE2 12 & ZONEB & ZONEA: & ZONNES if & ZONE6 I & ZZONE7 i & IZONE8 & IZONES & ZONETO \\
\hline Heal inputWatts(net) & 1 & 287.6 & 288.3 & 2828 & 286.2 & 285.71 & 284.81 & 1285.11 & 283.8 & 287.4 & 287.2 \\
\hline Hoad Lose-Watts & $\dot{-}$ & $10.2 !$ & 2.3 & $3.1 !$ & $32:$ & $3.9 !$ & $2.3 !$ & 1.5 & 23 & 3 & 16.4 \\
\hline Hed Flox-Bu/Hr/ile & & $49855 !$ & 4997! & $4902 !$ & $4951 !$ & 4952! & 4937 & 49फा & 4919 & 498 & 4978 \\
\hline Wall Temp. HF & 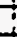 & 180.51 & 179.61 & 179.9 & 180.41 & $180.9 \mathrm{i}$ & 180.3 & 181.3 & 1802 & 180 & 178.9 \\
\hline WaH Temp. IAF & & 180.71 & 179.6 & 180.11 & 180.4 & $181.1 !$ & 181.1! & 181.31 & 180.31 & 180.6 & 779.9 \\
\hline Wall Temp. $15{ }^{2}$ & 7 & 181.6 & 178.71 & 181.11 & 180.4 & 180.31 & 180.4 & 180.9 & 179.9 & 179.7 & T78.5 \\
\hline Wall Tomp. HF & & 167.9 & 179.91 & 178.7 & 180 & 180 & 180.9 & 181.7 & 180.8 & 178.7 & 178 \\
\hline Woll Temp. "I2F & 1 & 182.2 & 180 & 179.1 & 180 & 179.81 & 180.61 & 181.7 & 181.2 & 179 & 178.4 \\
\hline Instroan Temp.f & $\dot{-}$ & $168 . \overline{6}$ & 168.31 & 169.71 & 170.9 & 170.2 & 170.21 & 171.2 & 170.1 & 170.1 & 17 \\
\hline Avg. Wall Tomp.f & 7 & 181.3 & 179.81 & 179.5 & 180.3 & 180.3 & 180.7 & 181.4 & 180.6 & 178.0 & 178.7 \\
\hline Avo. Wand DT.F & 1 & 124 & 1121 & 9.51 & 92 & 9.9 & 10.3 & 10 & 10.2 & 9.3 & 3.5 \\
\hline \multirow[t]{3}{*}{ 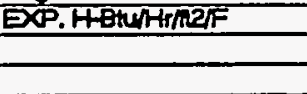 } & 1 & 4012 & 44.31 & 514.61 & ED0.7 & 501.5 & 481.61 & 486.4 & 484.8 & 687.1 & 6626 \\
\hline & & 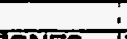 & & & & $i$ & & & & & \\
\hline & & ZONE2 & ZONE4 & ZONES & ZONES & ¿ZONE10 & & & & & \\
\hline \multicolumn{12}{|l|}{ DPliquid Temp.fF } \\
\hline \multicolumn{12}{|l|}{ DPUquid Densitubmit3 } \\
\hline Elovation & ' & 2 & $4 !$ & $6 !$ & 8 & 9.875 & & 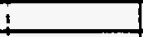 & & & \\
\hline \multicolumn{12}{|l|}{ Menured DPPsid } \\
\hline \multicolumn{12}{|l|}{ Zero Contections psid } \\
\hline Correctod DPPas & T & 0.172 & 0.4221 & 1.088 & 1.768 & 2.168 & & $T$ & & & \\
\hline \multirow[t]{2}{*}{ Actua DPA } & & 1.971 & 3.81 & 4.851 & 5.94 & 7.361 & & $i$ & & & \\
\hline & $i$ & ! & 1 & ! & $i$ & ! & 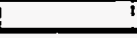 & 1 & & & \\
\hline
\end{tabular}




\begin{tabular}{|c|c|c|c|c|c|c|c|c|c|c|c|}
\hline Run: & & $1019 !$ & & & $\vdots$ & & 1 & 1 & T & 1 & \\
\hline Test Fluid & & $25 \%$ C4F 10 & $-75 \%$ R11 & & $\vdots$ & $:$ & 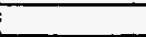 & & 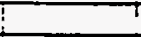 & & \\
\hline Date: & 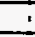 & $1 \quad 1 / 12 / 941$ & & & $\dot{-}$ & 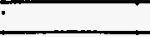 & $\vdots$ & 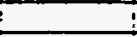 & 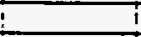 & $t$ & \\
\hline Timo: & $\therefore$ & $12.23 i$ & $i$ & : & & $:$ & 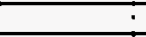 & 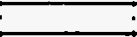 & i & $i$ & \\
\hline Noto & $\therefore$ & 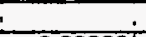 & $\dot{4}$ & 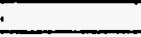 & & 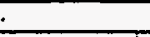 & 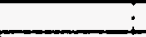 & 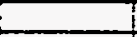 & & 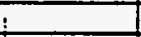 & \\
\hline Fow Areanti2 & & 0.003361 & & & ; & 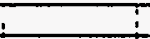 & $i$ & $i$ & & & \\
\hline Heat Flow Areati2 & & $0.19095:$ & & & & 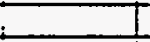 & & & & & \\
\hline Sat. Pressure-Psig & & $97.2 !$ & & & & & $i$ & 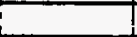 & & 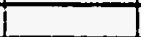 & \\
\hline Sat. Temperaturef(inlel) & & 1501 & & & & $\vdots$ & 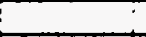 & & & & \\
\hline Inletflowrate-GPM & & $5 i$ & & & : & 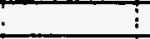 & 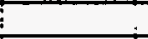 & & & T & \\
\hline intet Temperaturef & & 148.81 & & & $i$ & & & & & 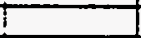 & \\
\hline Intat Glbmhrie & & $98861 \Gamma$ & & Tomp & 142.91 & Density & 83.21 & & & & \\
\hline Subcooling $f$ & & $1.2 i$ & & & $i$ & $:$ & 1 & 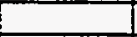 & & $T$ & \\
\hline Fow Observation & & & & & : & $i$ & i & 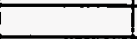 & & & \\
\hline & 5 & (2) & & 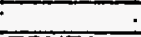 & 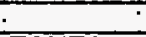 & 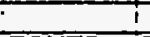 & 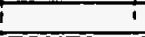 & 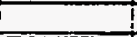 & & & \\
\hline & & ZONEE I & ZONE2 & ZONE3 1 & IZONE4: & ZZONES IZ & ZONEG & ZONE7 & ZONES I & IZONEO & ZOKETO \\
\hline Heat InputWatts(net) & 1 & 874 & 862.1 & 867.7 & 86751 & 1853.6 & 8611 & 868.3 & 865.1 & 859.4 & 8702 \\
\hline Hoat Loss-Watts & & $12.3 !$ & 2.8 & 3.9 & $4 i$ & 4 & $3 !$ & 2.11 & 2.9 & 3.6 & 21.4 \\
\hline Head Fix Btu/Hr/112 & & $15150 !$ & 14944. & 150411 & $15038 ;$ & 14970 & 149251 & 15051 & 14956 & $150 \%$ & 15084 \\
\hline Wall Temp. "3F & & 165.61 & 167.6 & 166.4 & 168 & 170.1 & 170.3 & 971.6 & 167.2 & 1662 & 166.8 \\
\hline Wall Temp. \#4F & & 166.51 & 164.7 & 168.3 & $168.6 !$ & 170.3 & 170.3 & 171.6 & 168.4 & 1672 & 172.6 \\
\hline Wall Tomp. $\$ 5 F$ & & 167.3i & 163.2 & 169.4 & 169.4: & 168.6 & 170.31 & 170.3 & 166.3 & 1652 & $1 ळ 5.1$ \\
\hline Wall Tomp. 115 & & 148.81 & 165.7 & 163.51 & 166.9 & 167.31 & 170.31 & 1729 & 168.4 & 164.4 & 16.3 \\
\hline Wall Temp: & & 169.11 & $165.6 !$ & 165.21 & 167.8 ! & 767.51 & $170 . \pi$ & 17281 & 169.6 & T\$5.8 & Tष्ठ.2 \\
\hline Instrem Temp.f & & $149 r$ & 149.8 & 150.7! & $151.6^{1}$ & $151 T$ & 150.61 & 15261 & 150.7 & 150.5 & 151.8 \\
\hline Avg. Wall Temp.f & i & $167 . \overline{1}$ & 165.81 & 165.81 & 16821 & 168.41 & 170.31 & $171.8 !$ & 1681 & 165.7 & 107 \\
\hline Avg. Wati DT.F & 1 & 17.41 & 15.41 & 15.11 & 15.91 & 16.71 & 191 & 18.6 & 16.61 & 14.6 & 14.5 \\
\hline EXP.HBtuhinfl2/F & $!$ & 868.71 & S68.8! & 888.11 & 943.11 & $893.8 !$ & $784.6 \mathrm{i}$ & 811 & 9032 & $10 \% 0.9$ & 1037.6 \\
\hline & & $i$ & & & 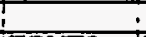 & 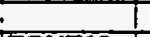 & 1 & & & & \\
\hline & & ZONE2 I: & ZONE4 & ZONES & ZONEB & ZONETO & & & & & \\
\hline DP liquid Temp.f & & 83.3 & & & $=$ & 2 & i & & & & \\
\hline DPLiquid Densitylbmit3 & & 90.621 & & & 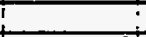 & & 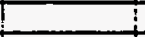 & & & & \\
\hline Elevation-f & & 21 & 41 & 6 & 81 & 9.875 & & & & & \\
\hline Measured DPPsid & & -0.2421 & 0.32 & 0.756 & 7.248 & 1.233 & ! & & & & \\
\hline Eoro Corrections-psid & & -0.296 & -0.0971 & -0.005 & 0.0381 & -0.168 & T & & & & \\
\hline Corrected DPpsid & & 0.0541 & $0.417 \pi$ & 0.777 & 1.2ा & 1.401 & & & & & \\
\hline Actual DP & ! & 2081 & $3 . \times 3 !$ & 5.2 & 6.621 & 8.33 & & & & & \\
\hline & $T$ & 1 & 1 & 1 & 1 & $i$ & i & & & & \\
\hline
\end{tabular}




\begin{tabular}{|c|c|c|c|c|c|c|c|c|c|c|}
\hline Run: & 10201 & & & & $\mathrm{i}$ & $i$ & & $i$ & & \\
\hline Test Fuid & क\% CAF & $5-75 \%$ RT14 & & 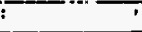 & ' & 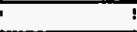 & $!$ & ! & 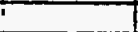 & \\
\hline Date: & - $1 / 12 / 941$ & & & & & & 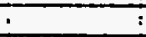 & 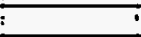 & 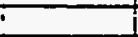 & \\
\hline Time: & $14: 27$, & & & $\cdot$ & $\dot{-}$ & $\vdots$ & $i$ & $i$ & $i$ & \\
\hline Nolo & $\cdot$ & . & . & & - & 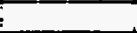 & ? & $?$ & 1 & \\
\hline Fow Aronit2 & $0.003636 !$ & & & & $i$ & 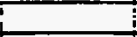 & $i$ & $i$ & 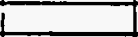 & \\
\hline Hoat Flow Area-12 & 0.19595 & & 1 & $t$ & 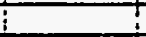 & $t$ & & 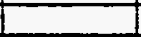 & & \\
\hline Sat. Prossure Psig & 96.31 & & i & 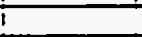 & i & 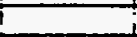 & & 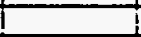 & & \\
\hline Sat. Temperature-F(inlet) & 149.51 & & & 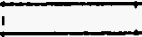 & i & 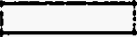 & & 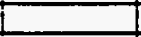 & & \\
\hline Inlel Flowrate-GPM & 3.81 & & & & 4 & & 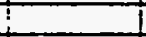 & 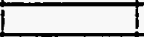 & & \\
\hline intel Temperature F & 148.41 & & & & & & $!$ & & & \\
\hline Intot Glbmhrine & 7557541 & & Temp & 1421 & Density & 83.34 & & & & \\
\hline SubcoollngfF & 1.11 & & & i & The & 1 & 1 & & & \\
\hline Fow Obsenvation & 1 & & 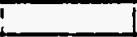 & & T & & 1 & & & \\
\hline & $!$ & 1 & 1 & & 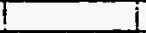 & & 1 & & & \\
\hline & RONEI I & ZONE2 : & ZONES & ZONE4 I & IZONES & ZONE6 I & IZONE? I & ZONE8 & ZONEO & ZOHE $=10$ \\
\hline Foad InputWatts(nat) & $1 \quad 865.7$ & 864.61 & 1851.6 & 881.4 & 8528 & 856.8 & 858.5 & 8529 & 8675 & 8615 \\
\hline Hoal Loss-Walts & $12 \pi$ & $27 !$ & 3.8 & 41 & 41 & 3 & 2 & 28 & 3.8 & 21.9 \\
\hline Heat Flox-Btuhirine & 15005 & $14987 !$ & 14935 & 14932 & 14956 & 15005 & 15055 & 14938 & 15038 & 1498 \\
\hline Wall Temp. BF & 165.2! & 167.5 & 166.31 & 168 & 170.3 & 170.5 & 571.1 & 1672 & 168.4 & 167.2 \\
\hline Wall Tomp. $44 F$ & 166.21 & 164.6 & 168.1 & $168.7 !$ & 170.3 & 170.6 & 171.1 & 168.1 & 167.3 & 1727 \\
\hline Wall Temp. 15 F & 166.51 & $1629:$ & 169.1 & 169 & 168.51 & 170.3 & 169.7 & 168 & 165.81 & 165.3 \\
\hline Wall Temp. HFF & 748.41 & 165.61 & 163.4 & 767 & 167.61 & 770.4 & $172 \pi$ & T68या & 765.5| & 164.4 \\
\hline Wal Tomp. izfF & 168.25 & 165.41 & 165 & 767.41 & 767.71 & 7\%.9. & 17R.9 & Tऊ্.6! & Tक5.4 & T65.4 \\
\hline Tnstrosm Temp.f & 148.81 & 149.8! & 150.31 & 151.71 & 150.81 & 150.6 & 152.11 & 150.3 & 1502 & 151.9 \\
\hline Avg, WaH Tomp.F & 168.51 & 165.81 & 165.7 & 168.21 & 168.51 & 170.5 & 171.2 & 187.81 & 185.8 & 167.2 \\
\hline Avg. Wall DT.F & 17 & 15.31 & 14.7 & 15.81 & 17 & 19.21 & 18.4 & 16.81 & 14.8 & 14.6 \\
\hline Exp. H-GWHAlizer & 880.31 & 978.71 & 1014.31 & 8451 & 87721 & 783.81 & 818.2 & 888 & 10002 & 1021.1 \\
\hline & 1 & & 1 & 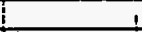 & 1 & 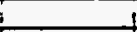 & 1 & & 1 & \\
\hline & ZONE2 & ZONE4 & ZONE6 & ZONEB & ZONE10 : & 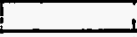 & 1 & 1 & L & \\
\hline DP hquid Temp.f & $80.2 !$ & & & 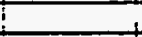 & 4 & & $i$ & 1 & L & \\
\hline DPLiquid Densitydbmin & 90.941 & & & 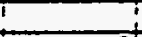 & $i$ & & & & & \\
\hline Elevation & 21 & 41 & 61 & 81 & 9.875 & & 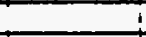 & & & \\
\hline Mosesured DPPsld & -0.1391 & $0.611:$ & 1.226 & 1.857 & 2.016 & & i & & & \\
\hline Zero Contectionspsid & -0.2931 & $-0.097^{\circ}$ & -0.005 & 0.0381 & $\$ 0.1681$ & & 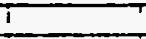 & & + & \\
\hline Conected Dppsid & 0.157 & 0.7081 & 1.231 & 1.829 & 2.1841 & & $!$ & & & \\
\hline Actul DPA & 1.911 & $3.14 !$ & $4 . \overline{2}$ & 5.57 & 7 & & $T$ & & & \\
\hline & 1 & 1 & $i$ & & 1 & 7 & $T$ & & & \\
\hline
\end{tabular}




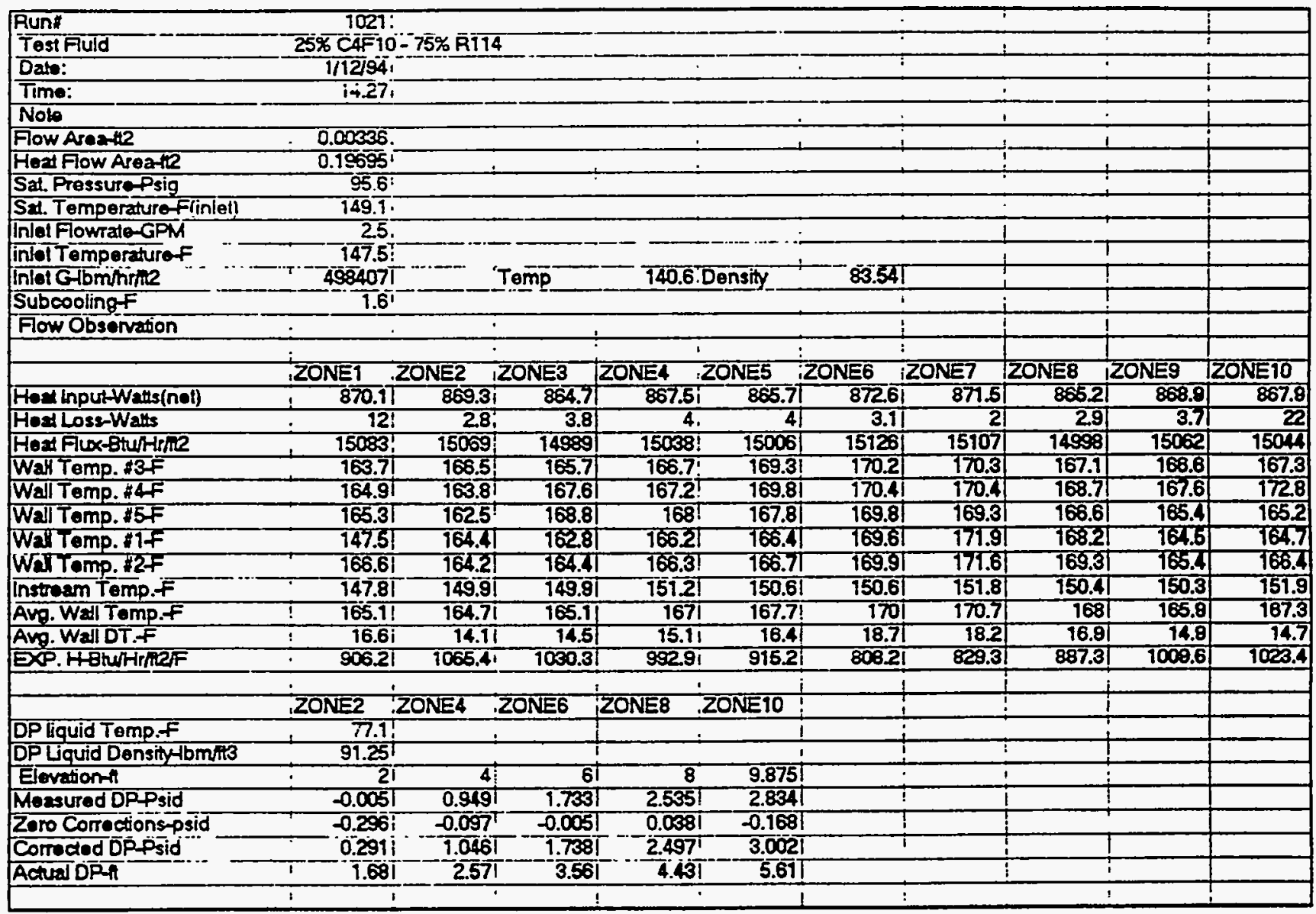




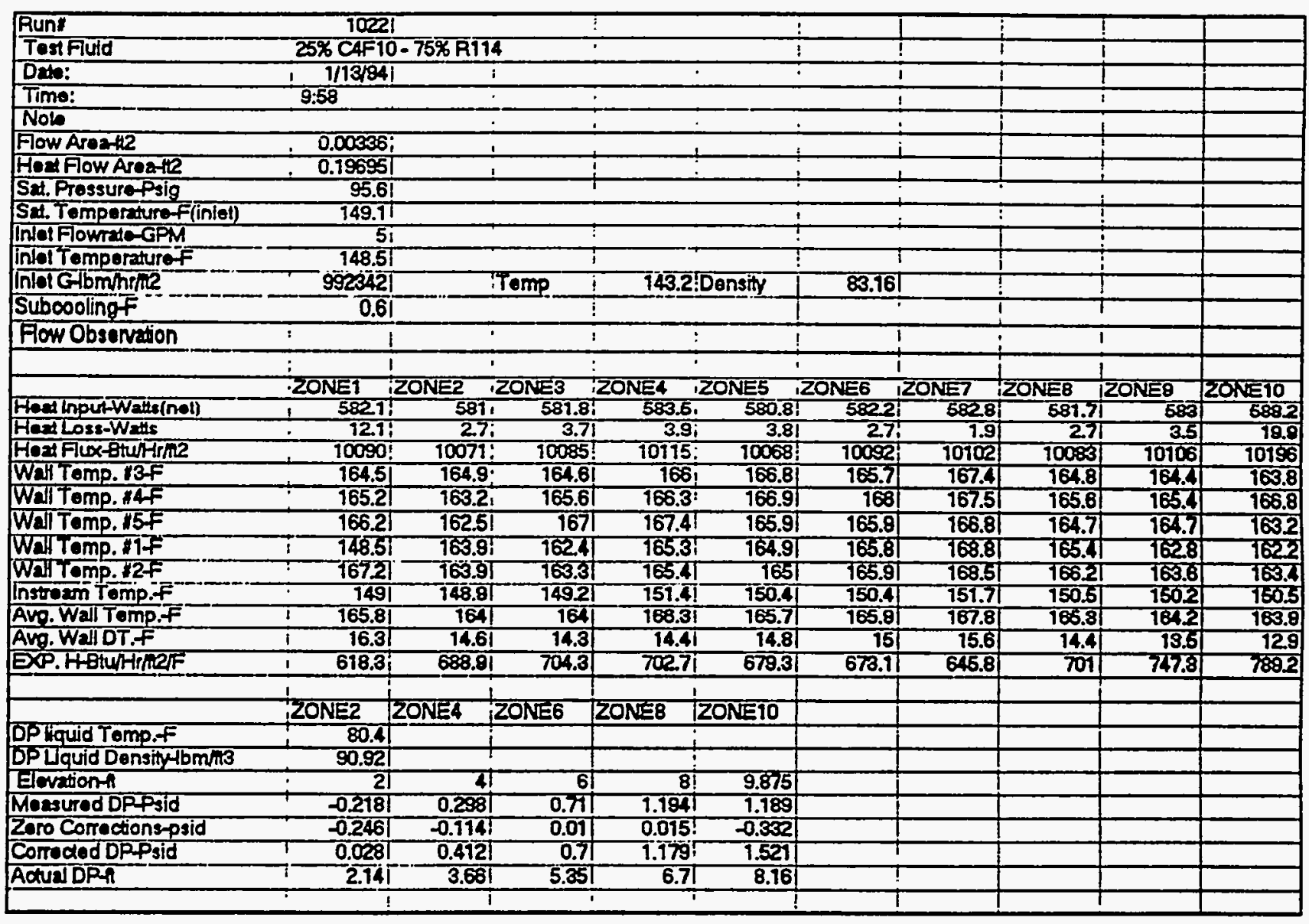




\begin{tabular}{|c|c|c|c|c|c|c|c|c|c|}
\hline Run: & $1023 !$ & & & & & - & 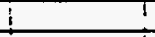 & ! & \\
\hline Test Fuid & $25 \%$ C4F $10-75 \%$ R 114 & & & 5 & & & $\therefore$ & & \\
\hline Dato: & $1 / 13 \overline{9} 4$ & & & $!$ & ; & i & $!$ & $!$ & \\
\hline Time: & $9: 58$ & & i & $:$ & ; & 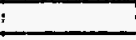 & 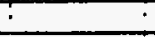 & : & \\
\hline Note & . & 1 & 1 & $:$ & 1 & 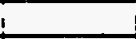 & $!$ & 1 & \\
\hline Fow Aroatt? & $0.00336 !$ & $\therefore$ & 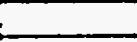 & 1 & 1 & 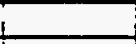 & 1 & 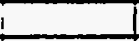 & \\
\hline Heat Flow Area-f2 & $0.19595 !$ & & & $i$ & $i$ & $i$ & & & \\
\hline Sat.Pressure-Psig & $95.6 !$ & $\vdots$ & $\vdots$ & 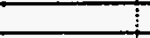 & 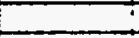 & 5 & 1 & 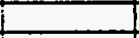 & \\
\hline Sat. Temperature F(inlet) & 149.11 & & & + & & & 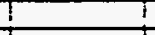 & 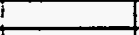 & \\
\hline Iniet Fowrate-GPM & 3.8 & & & $i$ & & & $!$ & & \\
\hline inlet Temperature- & $148.6 !$ & i & 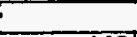 & $\div$ & 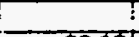 & 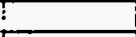 & i & & \\
\hline Indet Gibmhrne & 754431 & Temp & 143 & Density & 83.19 & & 1 & i & \\
\hline Subcooling $F$ & $0.5 !$ & & & I & ! & 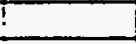 & $T$ & & \\
\hline Fow Observaton & & & - & $!$ & $\therefore$ & : & I & 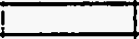 & \\
\hline & 1 & $:$ & 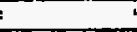 & $\mathbf{i}$ & 1 & 1 & $!$ & 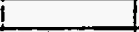 & \\
\hline & ZZONEI -ZONE2 & ZONEB I & ZONE4 & ZONES II & ZONE6 1 & IZONE7 & IZONEB I & ZONEO & ZONETO \\
\hline HealinputWats(nol) & . $583.3 \mathrm{i}-580.4$ & 578.81 & 5812 & 58121 & 582.8 & 583 & 31581.9 & $5 \% .8$ & 687.0 \\
\hline Hoad Loss-Wath & 2.2. & 3 & 3.2 & 3.21 & 2.3: & 9.5 & 22 & 2.8 & 16.5 \\
\hline Hear Flux-Bturifint & $10111 !$ & 10035 & 10075 & .0075 & 10104 & 10106 & 10087 & 10042 & 10191 \\
\hline Wall Temp. 3F & $164.4 i$ & 164.21 & 166.4 & 167.4 & 166 & 167.5 & 164.8 & 164.8 & 164.3 \\
\hline Wall Temp. "4F & $165.1 ! \quad 163$ & $165.3 i$ & 166.8 & 167.41 & 166.1 & 167.5 & $165.6:$ & 165.8 & 167.4 \\
\hline WaH Temp. HF & $166.2 \ldots 162.5$ & 167.11 & 167.8 & 166.31 & 166.6 & 166.9 & $164.7 !$ & 164.6 & 163.5 \\
\hline Wall Temp. If & $148.6:-163.8$ & 161.91 & 165.6 & 165.31 & 166.31 & 169 & 165.4 & 1628 & 162.7 \\
\hline Wall Temp. \#2F & 167.2 & 162.81 & 165.9 & 165.21 & 166.6 & 168.4 & 166.31 & 163.5 & 164 \\
\hline Înstroam Temp. & $149.1 !$ & 149.4 & 152 & 150.41 & 15021 & 151.8 & 150.41 & 150 & 1512 \\
\hline Avg. Wall Temp.F & 165.7 & 163.61 & 166.7 & $168.1 !$ & 166.31 & 167.9 & 165.41 & 184.1 & 164.4 \\
\hline Avg. Wall DT. $f$ & 1621 & 13.71 & 14.2 & 15.21 & 15.61 & 15.8 & 14.51 & 13.8 & 127 \\
\hline DX. HBWHrintr & $625.4 i$ & 73271 & 709.8 & 6631 & 6481 & 639.5 & 95.41 & 738 & 801.2 \\
\hline & inge & & & 1 & $!$ & $\vdots$ & 1 & 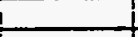 & \\
\hline & ZONE2 ,ZONE4 & ZONE6 & ZONE8 & ZONEIO & & & $\mathrm{i}$ & i & \\
\hline DP Gquid Temp.F & 81.31 & & & 1 & & & & & \\
\hline DP Liquid Density 4 bm/13 & $90.83 !$ & 1 & 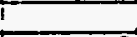 & & & & & & \\
\hline Elovation-h & $2 !$ & 61 & 8 & 9.875 & & & & & \\
\hline Mensured DPPsid & -0.1341 & 1.086 & 1.705 & 1.829 & & & & & \\
\hline Zero Corrections-psid & -0.2461 & 0.01 & 0.015 & -0.352 & & & & & \\
\hline Corrected DPPsid & 0.587 & 1.0761 & 1.69 & 2.161 & & & & & \\
\hline Actual DPA & 1.99 & 4.09 & 5.81 & 7.04 & & & & & \\
\hline & 1 & $\overrightarrow{1}$ & it & & & t & & & \\
\hline
\end{tabular}




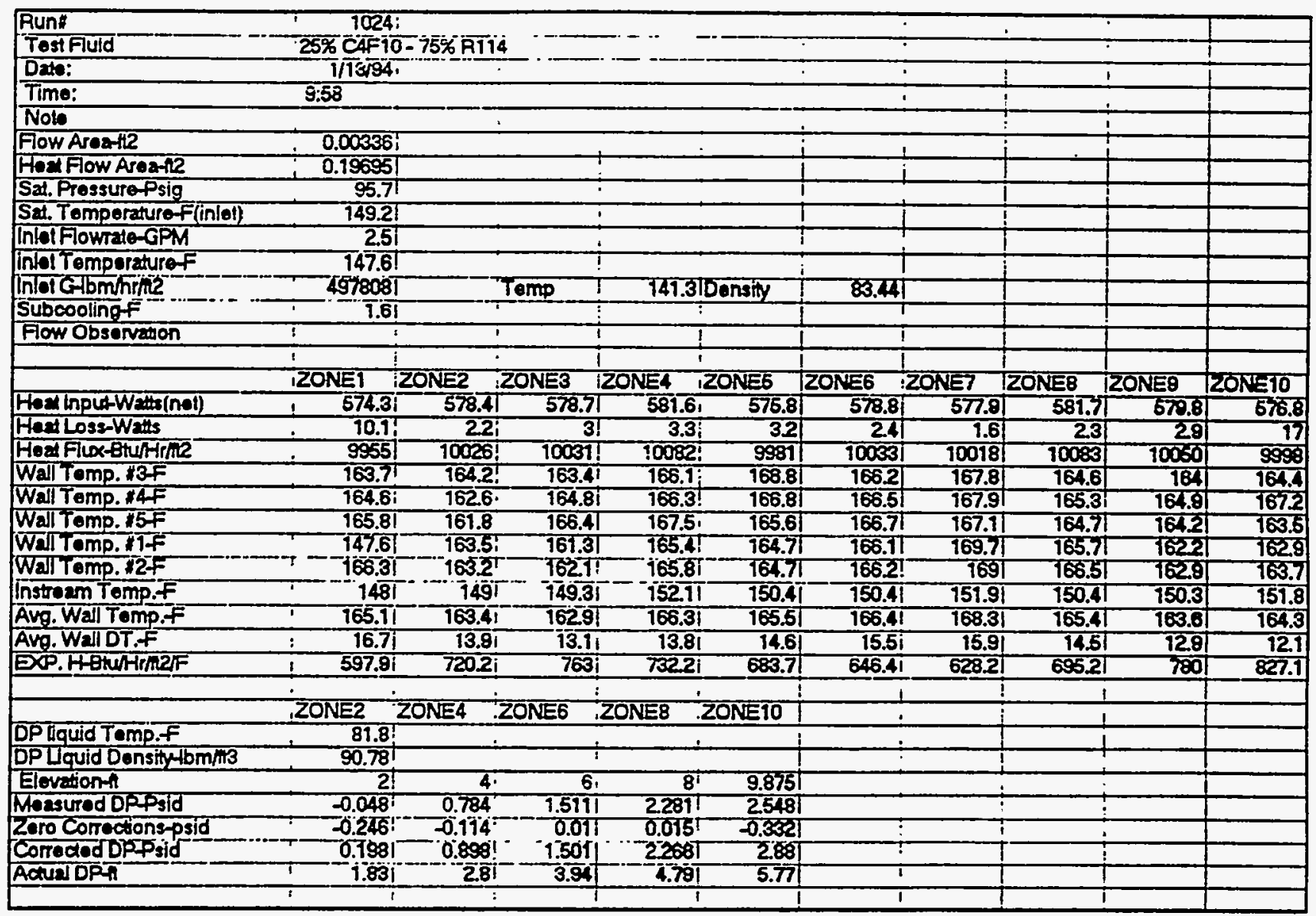




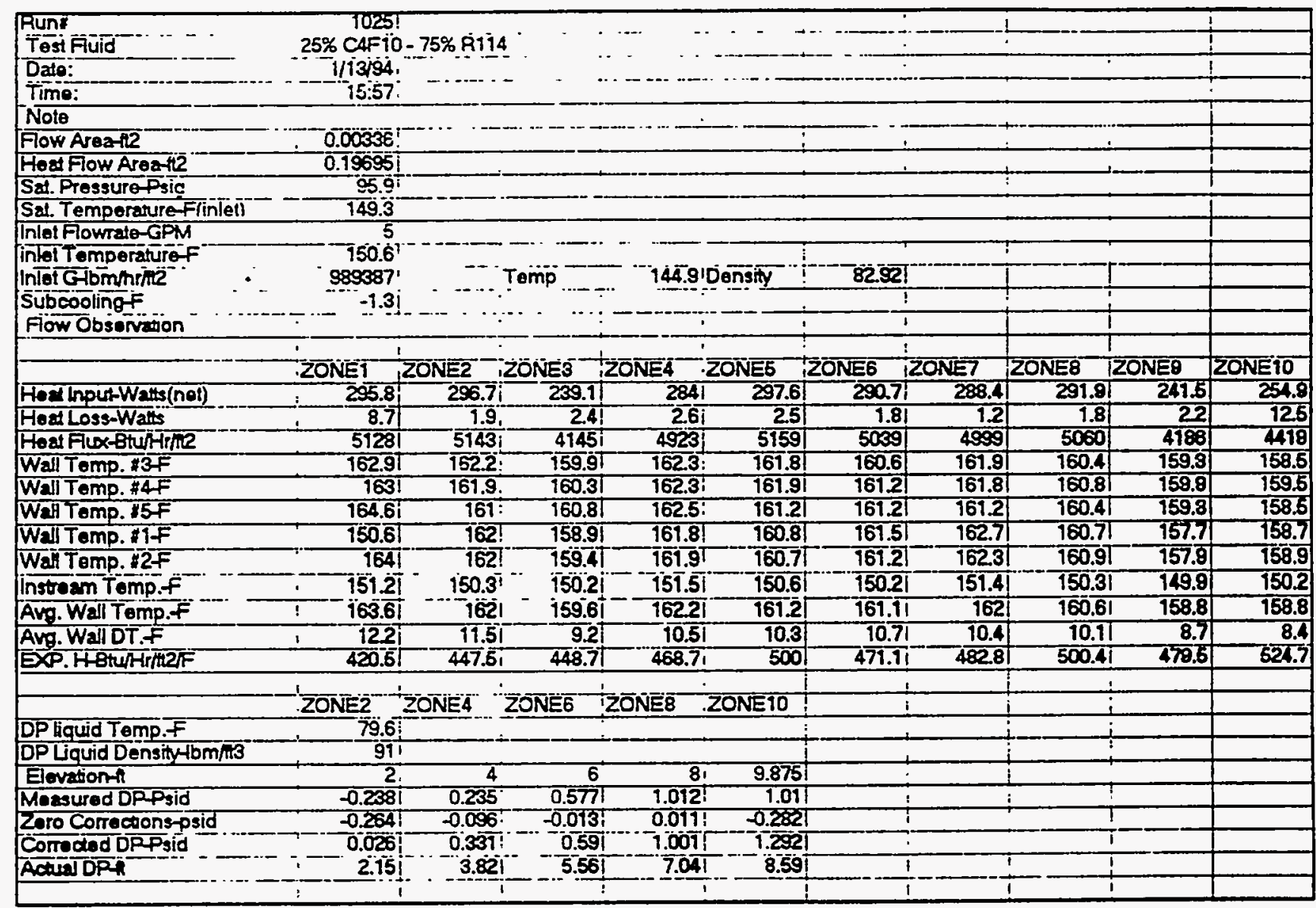




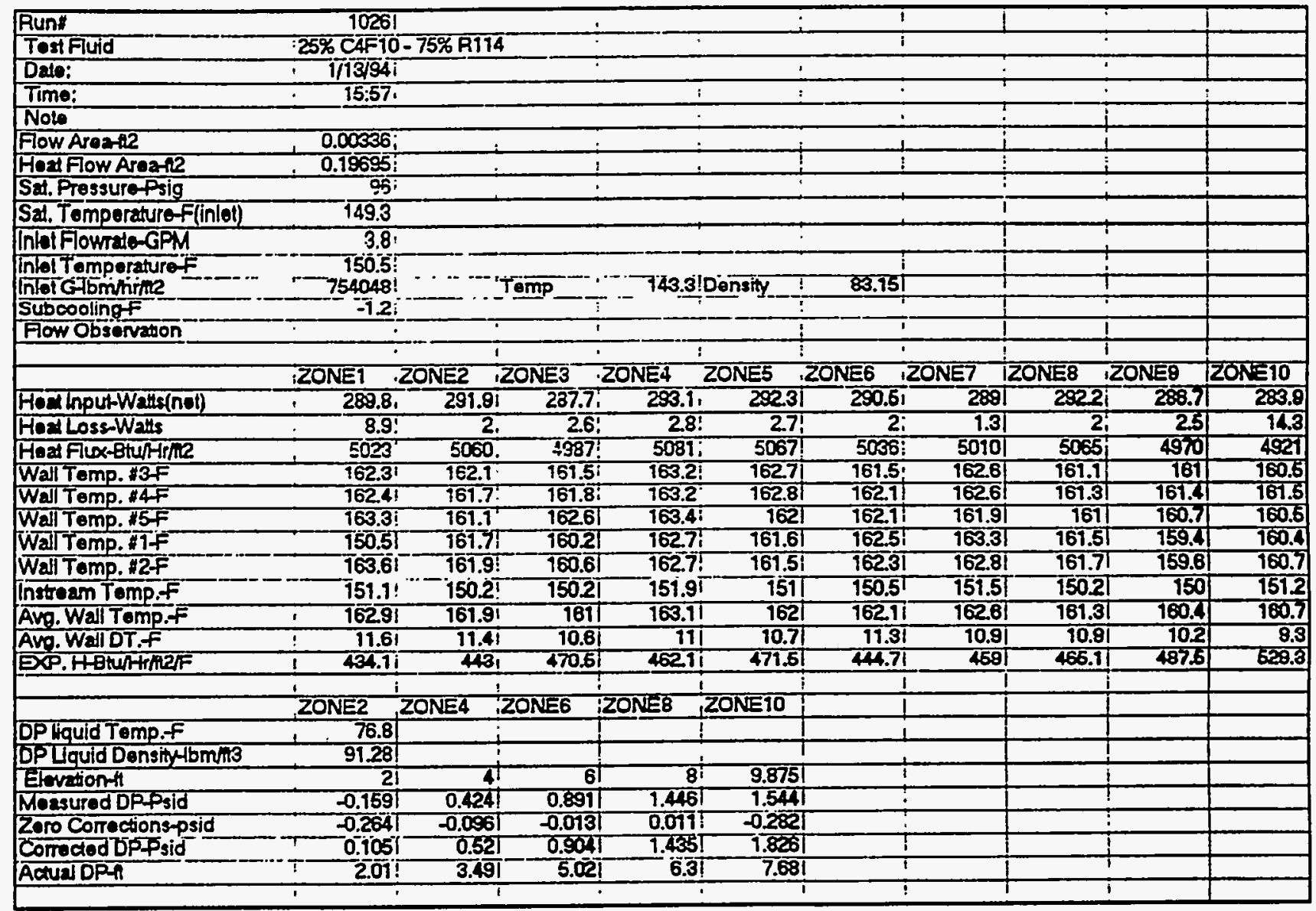




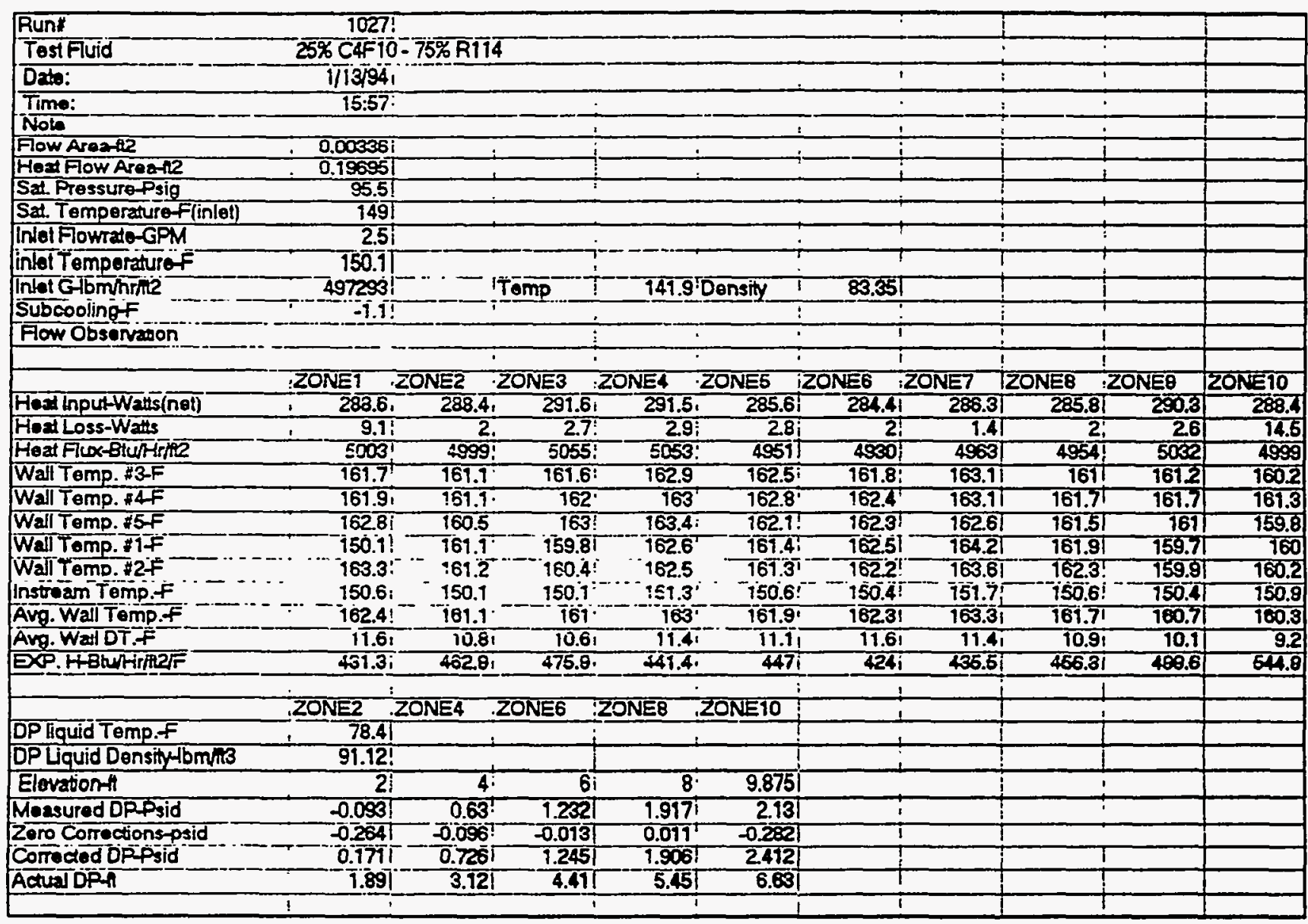




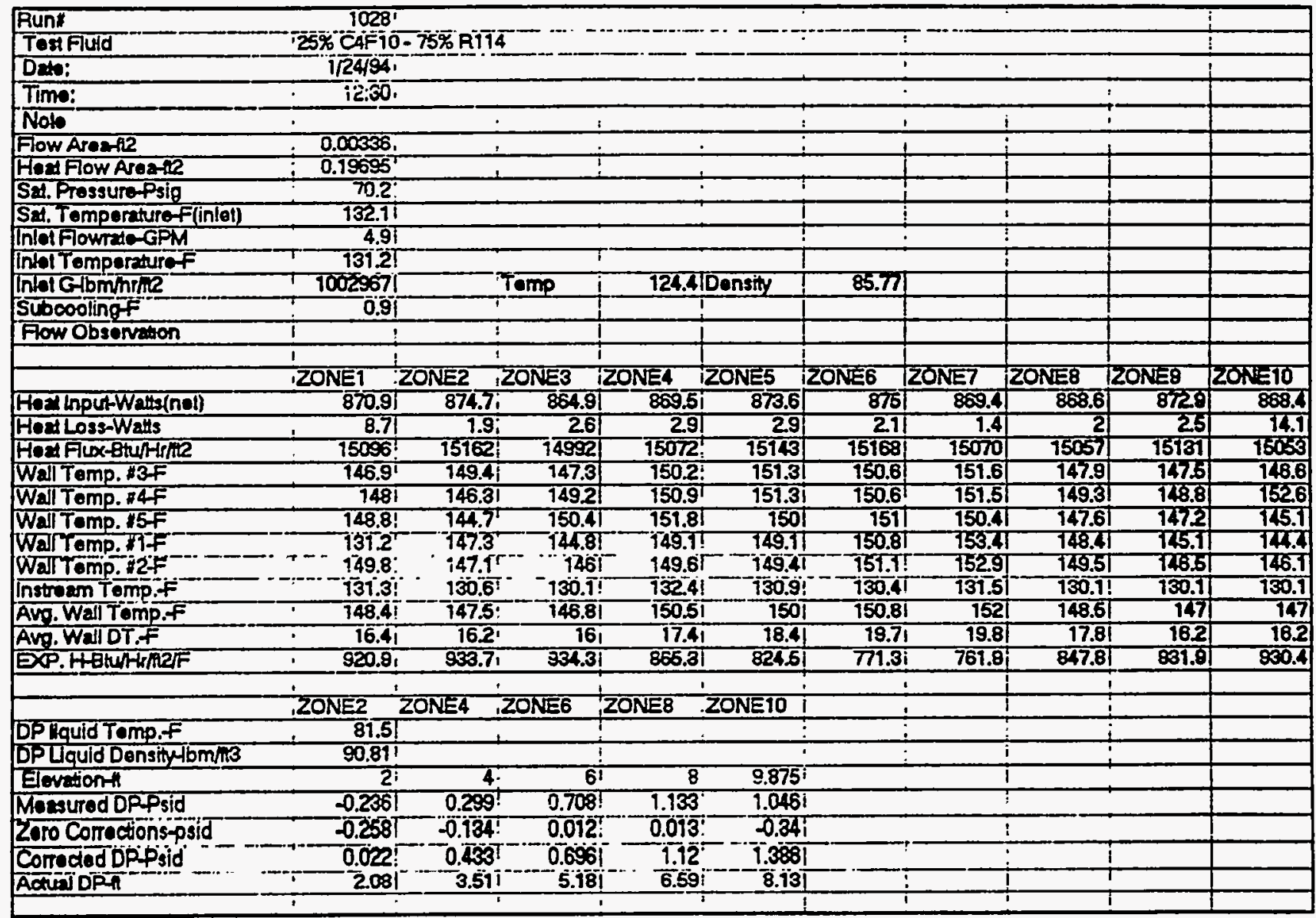




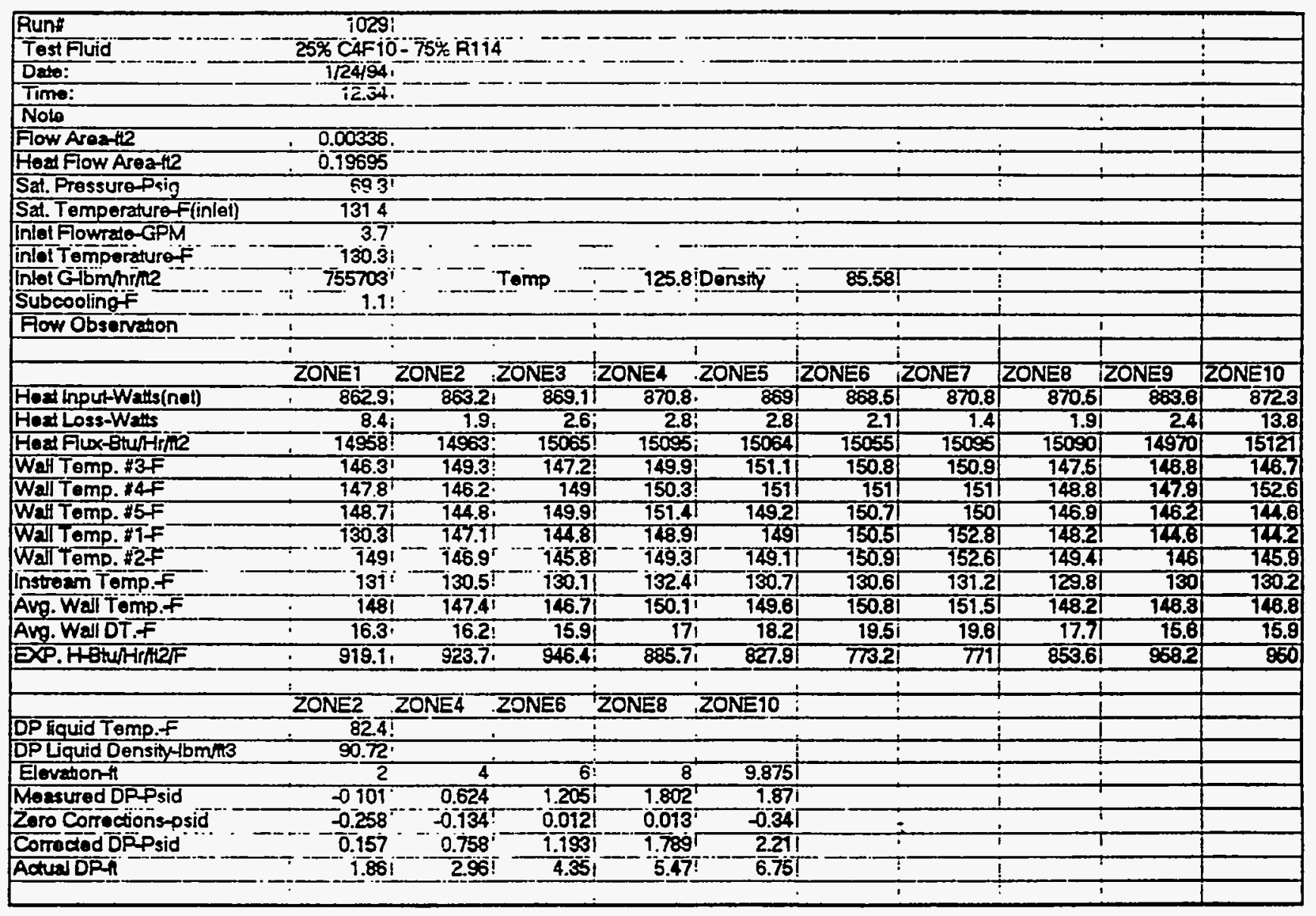




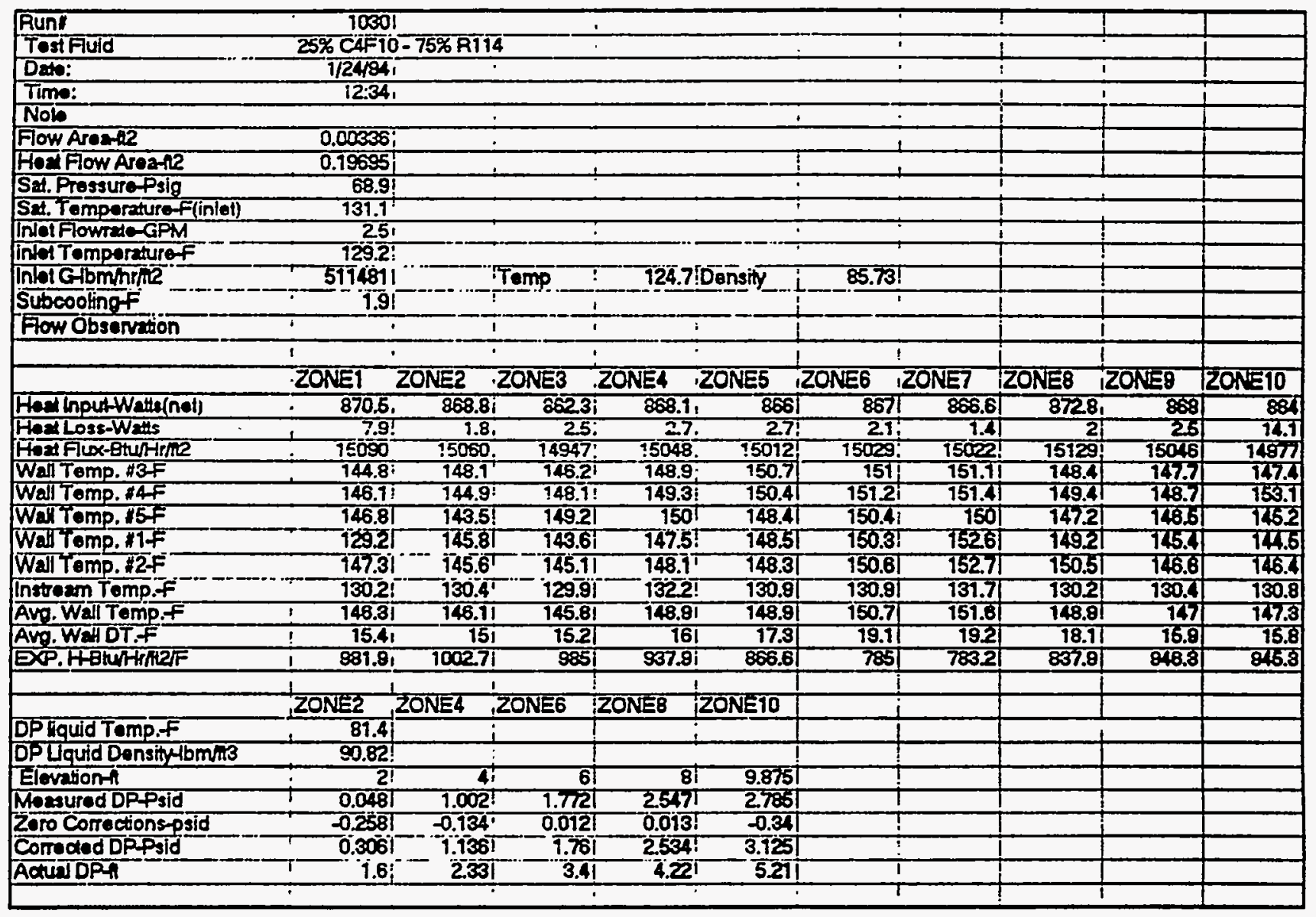




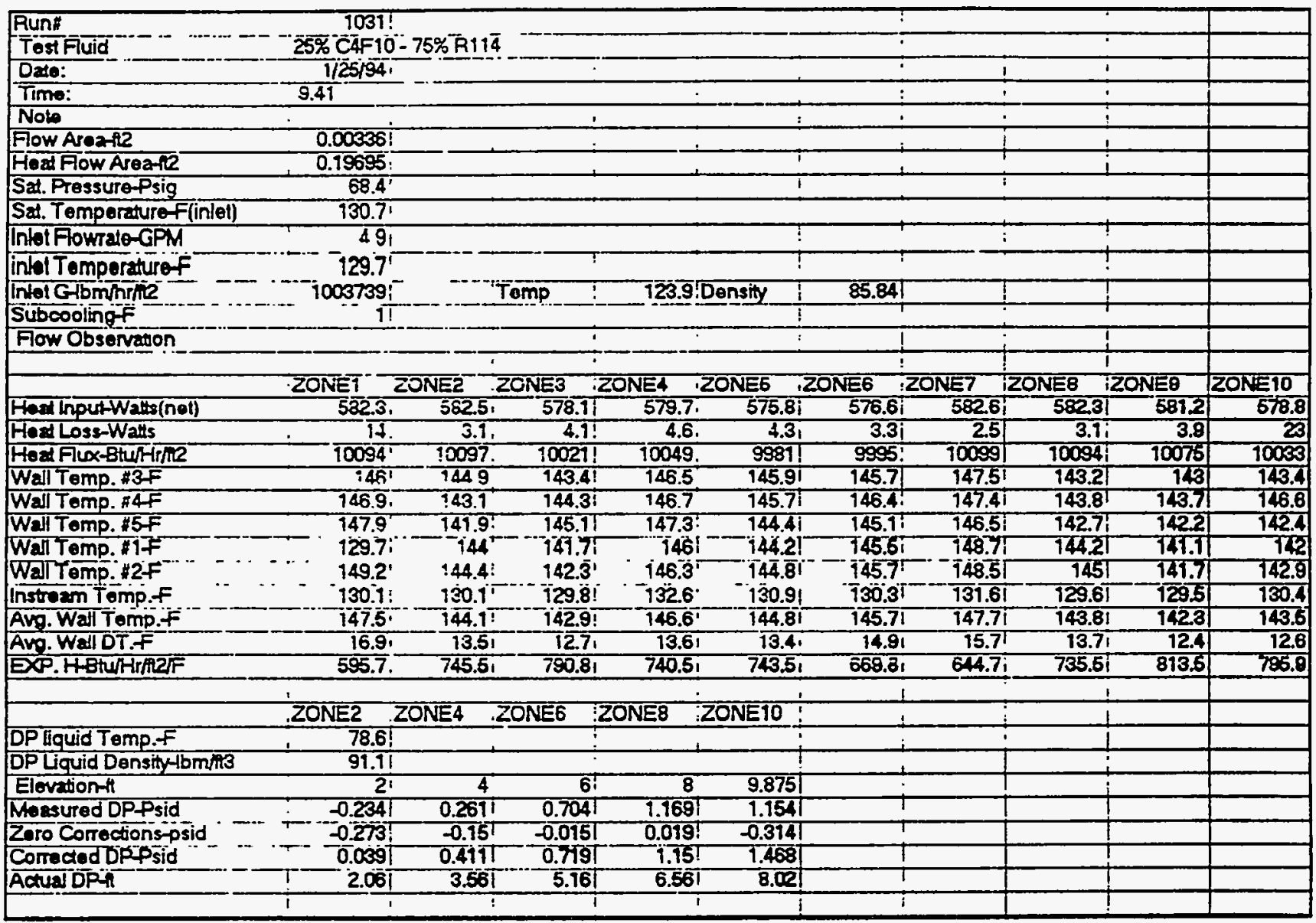




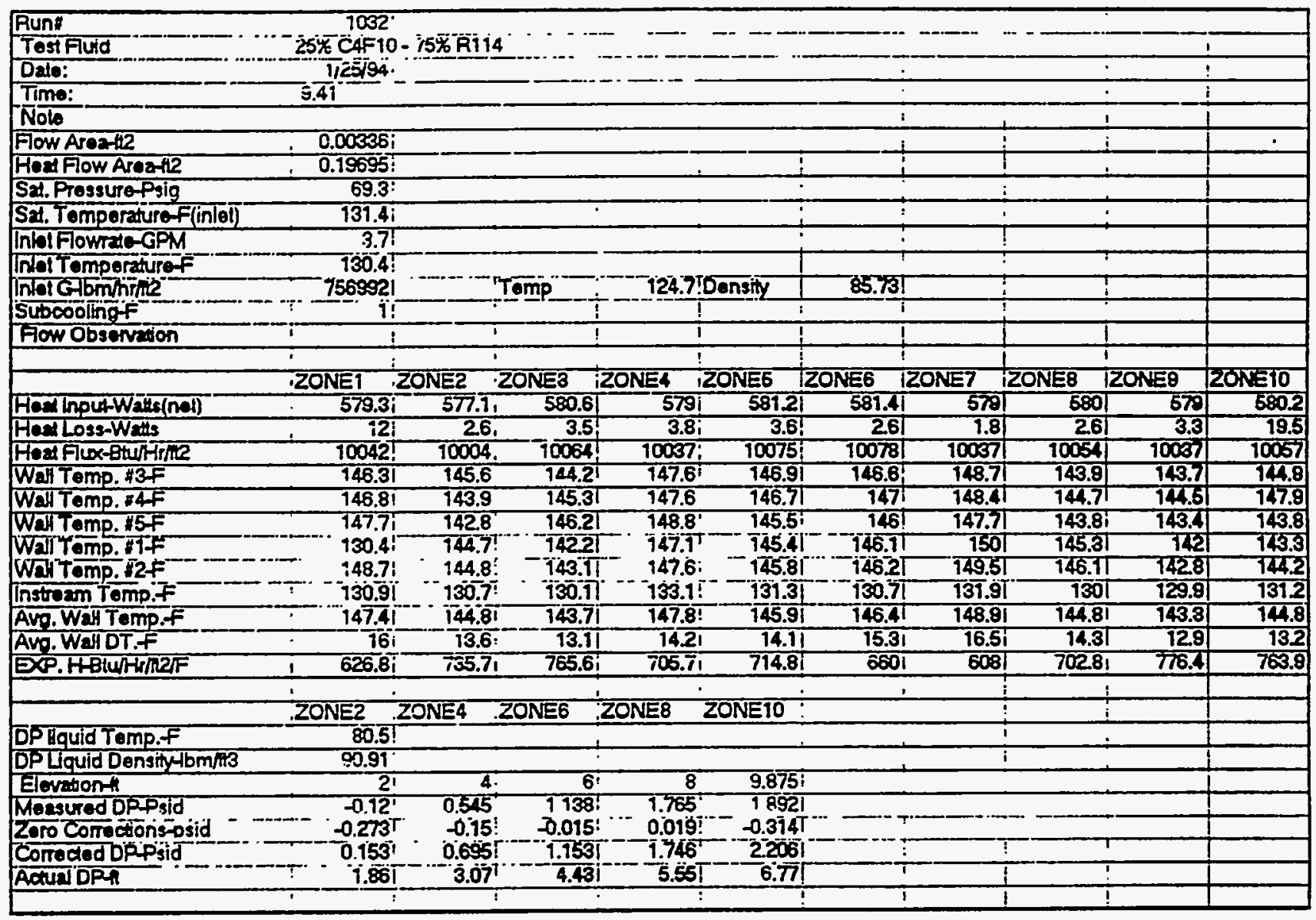




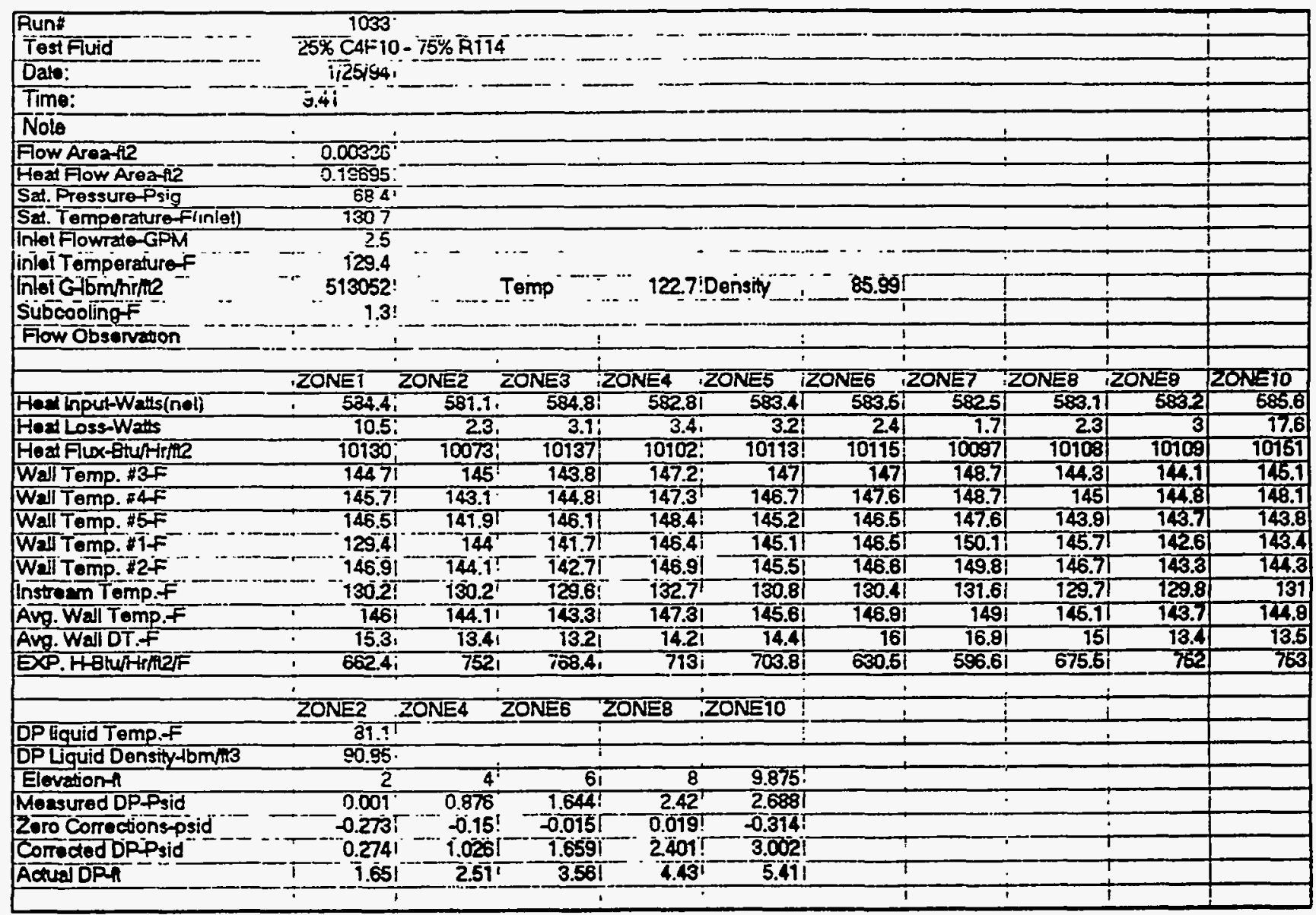




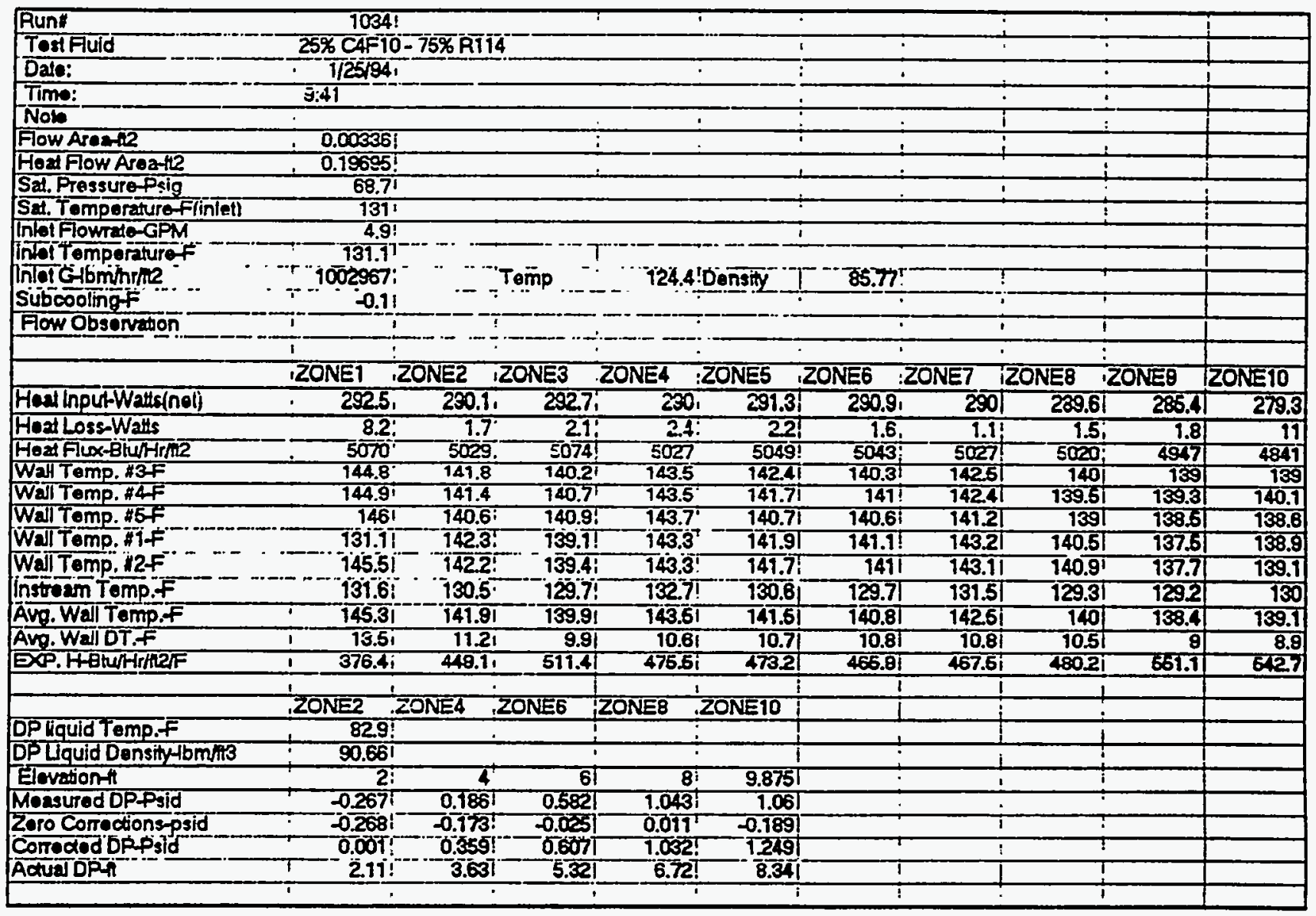




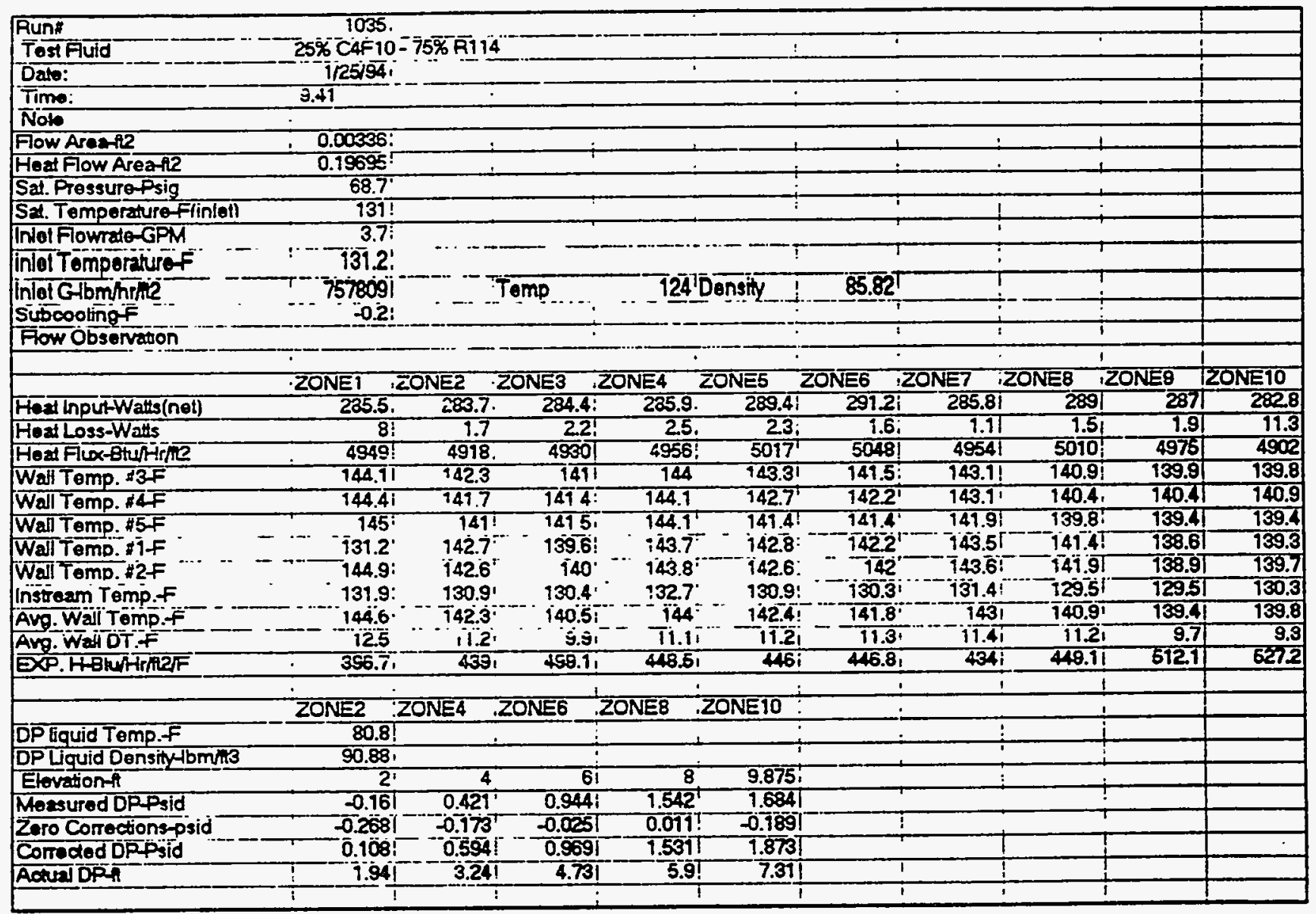




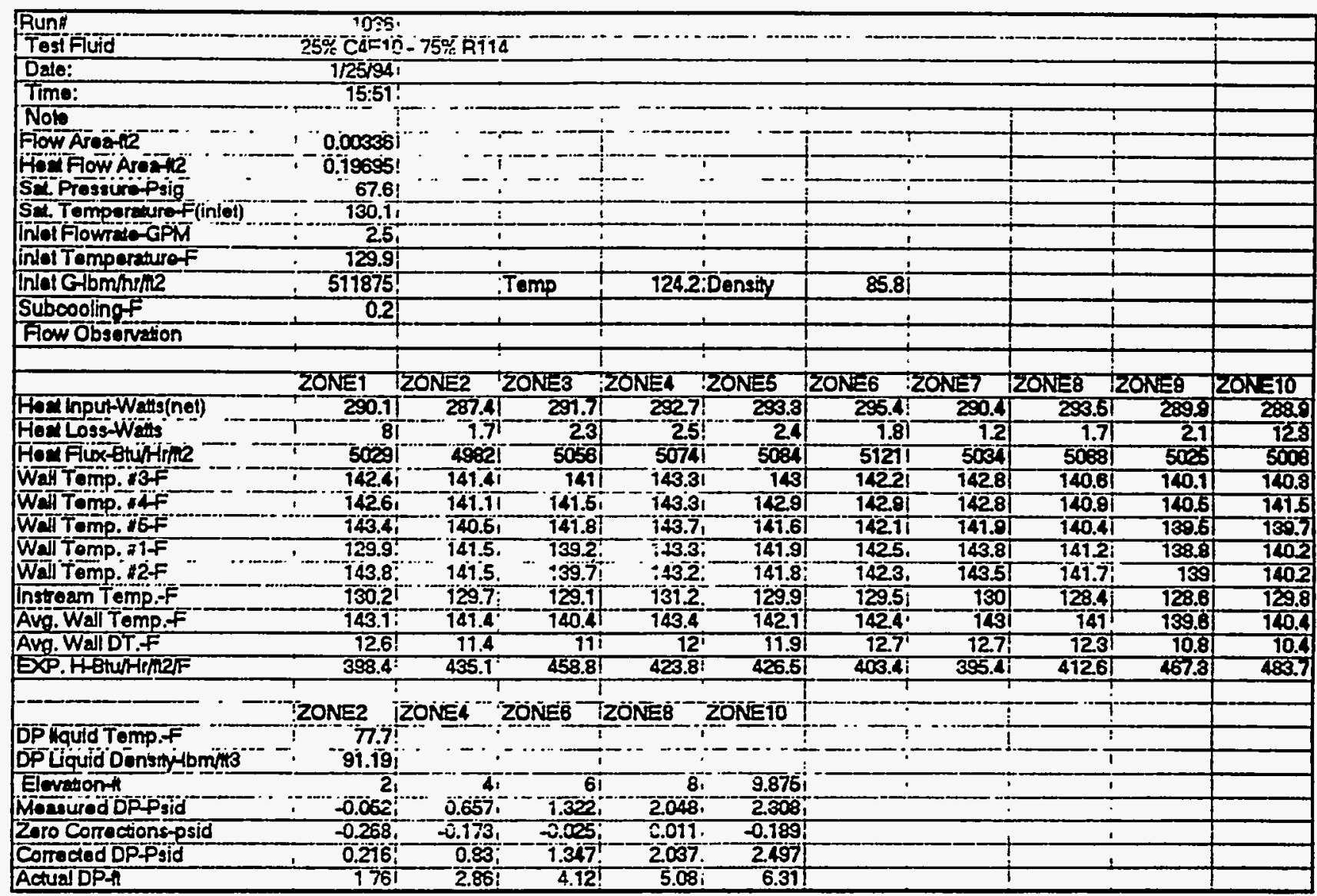




\section{DISTRIBUTION}

\section{Paducah Gaseous Diffusion Plant}

J. M. Fitzgerald

Library

\section{$\underline{\text { Portsmouth Gaseous Diffusion Plant }}$}

\section{G. Bobo}

Library

\section{Lockheed Martin Utility Services, Inc. - Oak Ridge}

S. H. Park

Library 